\title{
PROCEEDINGS OF THE
}

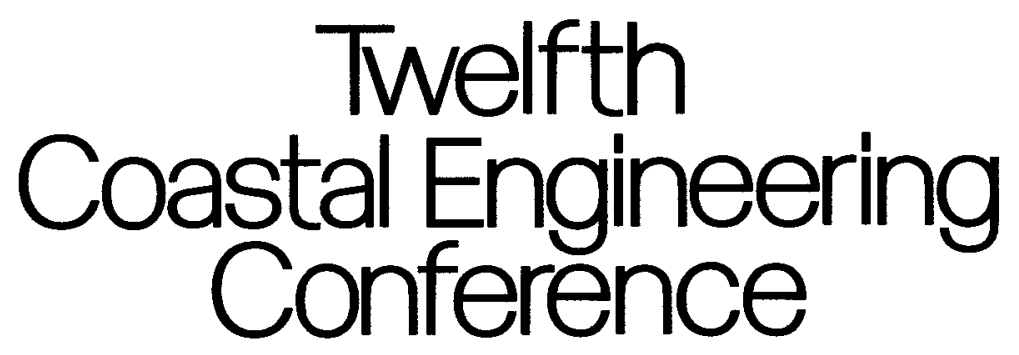

September 13-18, 1970 - Washıngton, D C.

\section{Volume I}

Sponsored by the

Coastal Engıneerıng Research Councıl

Technical Council on Ocean Engıneerıng

Waterways, Harbors and Coastal Engıneerıng Divisıon

National Capital Section

of ASCE

American Shore and Beach Preservation Association International Association for Hydraulic Research

Published by the American Society of Civil Engineers 345 East 47th Street, New York, N Y 10017 


\section{ACKNOWLEDGMENTS}

This Conference was sponsored jointly by the Coastal Engineering Research Councll of the American Society of Civil Engineers and the American Shore and Beach Preservation Association Cooperating organizations were

Waterways, Harbors, and Coastal Engineering Division, ASCE

National Capital Section, ASCE

Technical Council on Ocean Engineering, ASCE

International Association for Hydraulic Research 


\section{FOREWORD}

The Twelfth Conference on Coastal Engineering marks the twentieth year since the first conference was held at Long Beach, Callfornia, in October 1950 It appears appropriate at this time to present a brief history of the research counclls which have sponsored these conferences over the years

In March 1949 the late Professor Boris A Bakhmeteff of Columbia University was invited to the University of California, Berkeley, to present a special lecture entitled, The Engineer in the World of Today At that time Professor Boris A Bakhmeteff was charman of the Research Committees of both the American Society of Civil Engineers and The Engineering Foundation He observed in his discussions with Dean Morrough P O'Brien during his stay in Berkeley that there were numerous research councls in the country to sponsor and coordnate research in various fields, but none existed for studying waves and related problems Following this suggestion Dean O'Brien prepared a proposal for the formation of a Councll on Wave Research and submitted it to The Engineering Foundation through the Hydraulics Division of the American Society of Civil Engineers The A S C E Committee on Research approved the proposal and recommended to The Engineering Foundation that a grant be made to establish a Counc1l on Wave Research The Engineering Foundation Board at 1ts sem1annual meeting in June 1950 approved of the Councll with the grant becoming effective in October 1950 The Counc1l was thus formed with Dean O'Brien as Charrman and $\mathbf{J} \mathbf{W}$ Johnson as Secretary

In 1963 The Engineering Foundation revised its policy on the support of Research Councls With approval of The Engineering Foundation the Council on Wave Research proposed to the Board of Direction of the A S C E that the Councl be incorporated in the A S C E and be renamed the Coastal Engineering Research Counc1l The A S C E approved of the establishment of the Council in May 1964 and designated the Waterways, Harbors, and Coastal Engineering Division to serve as laison between the Council and the A S C E Research Committee Dean M P O'Brien remains as Charman of the Council with R L Wiegel as Vice-Chairman, and $\mathbf{J}$ W Johnson continuing as Secretary 
In October 1950 the University of California conducted an Institute on Coastal Engineering in Long Beach, Calıfornia This was conceived as a local meeting of engineers and scientists interested in shoreline problems The alm was to a1d engineers by summarizing the present state of the art and science related to the design and planning of coastal works rather than to present a series of original scientıfic contributions The program consisted of thirty five invited papers, copies of which were distributed to those in attendance This series of papers represented a rather thorough summary of coastal engineering as then practiced The quality and scope of the papers and the need for a comprehensive and modern treatment of the subject convinced the sponsors of the conference that publication in a single volume was desirable rather than piecemeal in the scientific and technical journals The newly-formed Council on Wave Research secured funds to underwrite the publication costs from the Engineering Foundation and the publication was entitled The Proceedings of the First Conference on Coastal Engineering

Because of the success of the Long Beach Conference, invitations to cosponsor additional conferences were extended to the Council by various societies, universities, etc, with the result that subsequent conferences were held in Houston, Texas (1951), Cambridge, Massachusetts (1952), Chicago, Illino1s (1953), Grenoble, France (1954), Gannesville, Flonda (1957), The Hague, Netherlands (1960), Mexico City, Mexico (1962), Lisbon, Portugal (1964), Tokyo, Japan (1966), London, England (1968), and Washington, D C (1970) In addition, conferences on Shıps and Waves (Hoboken, New Jersey, 1954), Coastal Eng1neering Instruments (Berkeley, Californı, 1955), and Civl Engineering in the Oceans I (San Francisco, Calıfornı, 1967) also were sponsored by the research councils

The proceedings of the various conferences were published by the Council on Wave Research with assistance from The Engineering Foundation unt1l the formation of the Coastal Engineering Research Counc1l in 1964, after which the proceedings have been published and distributed by the A S C E

In vew of the fact that the term coastal engineering appears to have been first used generally in connection with the Long Beach conference the following quotation from the preface of the proceedings of that conference is of interest

"A word about the term "Coastal Engineering" 1s perhaps in order here It is not a new or separate branch of engineering and there is no implication in tended that a new breed of engineer, and a new society, is in the making 
Coastal Engineering is primarily a branch of Civil Engineering which leans heavily on the sciences of oceanography, meteorology, fluid mechanics, electronics, structural mechanics, and others However, it is also true that the design of coastal works does involve many criteria which are foreign to other phases of civil engineering and the novices in this field should proceed with caution Along the coastlines of the world, numerous engineering works in various stages of disintegration testify to the futllity and wastefulness of disregarding the tremendous destructive forces of the sea Far worse than the destruction of insubstantial coastal works has been the damage to adjacent shorelines caused by structures planned in ignorance of, and occasionally in disregard of, the shoreline processes operative in the area"

It is believed that the proceedings resulting from the conferences held over that past twenty years have done much to improve the art and science related to the design and planning of coastal works throughout the world

J W Johnson, Secretary

Coastal Engineerıng Research Councll 


\section{CONTENTS}

ACKNOWLEDGMENTS

111

FOREWORD

V

VOLUME I

WAVE THEORY, MEASUREMENTS AND ANALYSES

Chapter 1

CANADIAN WAVE CLIMATE STUDY - THE FORMATIVE YEAR

Laurence Draper

Chapter 2

OCEAN WAVE RESEARCH IN SOUTHERN AFRICA

J A Zwamborn, C. van Schaik, and A Harper

Chapter 3

SWELL AND STORM CHARACTERISTICS FROM COASTAL WAVE RECORDS

Warren C Thompson

Chapter 4

MAXIMUM WAVE HEIGHT PROBABILITIES FOR A RANDOM

NUMBER OF RANDOM INTENSITY STORMS

L E Borgman

Chapter 5

SPECTRAL COMPUTATIONS ON PRESSURE WAVE GUAGE RECORDS

Manuel Mendes de Carvalho, Fernando Silveria Ramos,

and Carlos de Campos Moraes

Chapter 6

ANALYSIS OF WAVE RECORDS

D Lee Harris

Chapter 7

COMPARISON OF PRESSURE AND STAFF GAGE RECORDS

D Esteva and D Lee Harris

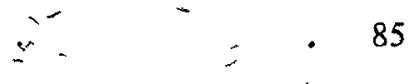




\section{CONTENTS}

Chapter 8

DIRECTIONAL SPECTRA FROM WAVE-GAGE ARRAYS

N N. Panıcker and L E. Borgman

Chapter 9

EQUILIBRIUM RANGE SPECTRA IN SHOALING WATER

Takeshı Ijıma, Takahıko Matsuo, and Kazutamı Koga

Chapter 10

WAVE INVESTIGATIONS IN SHALLOW WATER

Winfried Siefert .

Chapter 11

EXPERIMENTAL STUDIES ON THE GENERATION OF WAVES IN SHALLOW WATER

C T Kuo and Fredenck L W Tang

Chapter 12

WAVE FORECASTING FOR THE WEST COAST OF INDIA

J Dattatrı and P S. Renukaradhya

Chapter 13

COMPILATION OF OCEAN AND LAKE WAVE STATISTICS

John Simpson Hale

Chapter 14

MACRO-TURBULENCE FROM WIND WAVES

Chin Yuan Lee and Frank D Masch

Chapter 15

SHEAR STRESS OF SEA BREEZE ON A SWASH ZONE

Shih-Ang Hsu

Chapter 16

ENERGY LOSSES UNDER WAVE ACTION

P D Treloar and A Brebner

Chapter if

BOTIิดM BOUNDARY SHEAR STRESSES ON A MODEL BEACH

P G Telekipand $M$ W Anderson 


\section{CONTENTS}

Chapter 18

VARIATION OF LONGSHORE CURRENT ACROSS THE SURF ZONE

Edward B Thornton

Chapter 19

HORIZONTAL WATER PARTICLE VELOCITY OF FINITE AMPLITUDE WAVES

Yuıchı Iwagakı and Tetsuo Sakaı

Chapter 20

A SYSTEM FOR MEASURING ORBITAL VELOCITIES IN WAVES

M M Kolpak and P S Eagleson

Chapter 21

SHOALING OF FINITE-AMPLITUDE WAVES ON PLANE BEACHES

Robert K -C Chan and Robert L Street

Chapter 22

PERIODIC WAVES SHOALING IN WATERS OVER STEEPLY SHOALING BOTTOMS

Harihara Rama Ayyar

Chapter 23

BREAKING WAVE SETUP AND DECAY ON GENTLE SLOPES

Li San Hwang and David Divoky

Chapter 24

AIR ENTRAINMENT AND ENERGY DISSIPATION IN BREAKERS

Alfred Fuhrboter

Chapter 25

PROBABILITIES OF BREAKING WAVE CHARACTERISTICS

J Ian Collins

Chapter 26

CHARACTERISTICS OF WAVES BROKEN BY A LONGSHORE BAR

E Clark McNarr, Jr and Robert M Sorensen

Chapter 27

VELOCITY FIELDS IN THE BREAKER ZONE

M D Adeyemo 


\section{CONTENTS}

Chapter 28

DEVELOPMENT OF UNDULAR BORES IN ROUGH CHANNELS

$O$ Hawaleshka and S B Savage

Chapter 29

COMPUTER MODELLING OF DIFFRACTION OF WIND WAVES

Shou-Shan Fan and L E Borgman

473

Chapter 30

INTERACTION BETWEEN WAVES AND CURRENTS

Ivar G Jonnsson, Christian Skougaard, and

John D Wang

Chapter 31

EXPERIMENTS OF WAVE REFLEXION ON IMPERMEABLE SLOPES

Carlos de Campos Moraes

509

Chapter 32

LIMITING CONDITION FOR STANDING WAVE THEORIES BY PERTURBATION METHOD

Yoshito Tsuchiya and Masataka Yamaguch

Chapter 33

HIGHER ORDER THEORY FOR SYMMETRICAL GRAVITY WAVES

Peter L Monkmeyer

Chapter 34

ANALYTICAL APPROACH ON WAVE OVERTOPPING ON LEVEES

Hiroyosh1 Sh1-1ga1 and Tsug1o Kono

Chapter 35

IMPULSE WAVES GENERATED BY LANDSLIDES

J W Kamphuis and R J Bowerıng

Chapter 36

WAVES GENERATED BY A PISTON - TYPE WAVEMAKER

Ole Secher Madsen

Chapter 37

PREDICTION CURVES FOR WAVES NEAR THE SOURCE OF AN IMPULSE

Robert L Miller 


\section{CONTENTS}

Chapter 38

DIMENSIONAL ANALYSIS - SPURIOUS CORRELATION

M S Yalın and J W Kamphuis

VOLUME II

COASTAL SEDIMENT PROBLEMS

Chapter 39

COASTAL PROCESSES FROM SPACE PHOTOGRAPHY

Maynard M Nichols

Chapter 40

REACH OF WAVES TO THE BED OF THE CONTINENTAL SHELF

Richard Silvester and Geoffrey R Mogndge

Chapter 41

ISOPACHOUS MAPPING OF THE LOWER PATUXENT ESTUARY

SEDIMENTS BY CONTINUOUS SEISMIC PROFILING TECHNIQUES

Newell T Stiles and Donald R Wiesnet

Chapter 42

OBSERVATION OF SEDIMENT MOTION BY UNDERWATER-TELEVISION

Gunter Luck

Chapter 43

HISTORY OF THE DUTCH COAST IN THE LAST CENTURY

W T Bakker and D Sj Joustra

Chapter 44

STUDIES ON THE SHORE PROCESS AND WAVE FEATURES OF THE WESTERN COAST OF TAIWAN

Kenneth S T Chang and Frederick L W Chang

Chapter 45

STUDY OF MUDBANKS ALONG THE SOUTHWEST COAST OF INDIA

N S Monı

Chapter 46

TURBULENCE AND SEDIMENT CONCENTRATION DUE TO WAVES

Kıyoshı Horıkawa and Akıra Watanabe 


\section{CONTENTS}

Chapter 47

EFFECTS OF NONUNIFORM WAVE ENERGY IN THE LITTORAL ZONE

Victor Goldsmith and Joseph M Colonell

Chapter 48

LITTORAL TRANSPORT AND ENERGY RELATIONSHIP

L Bajorunas

Chapter 49

SYNOPTIC OBSERVATIONS OF SAND MOVEMENT

David B Duane

799

Chapter 50

UNDERWATER SURVEY SYSTEM FOR RADIONUCLIDE-TAGGED SEDIMENT TRACING

E $\mathrm{H}$ Acree, $\mathrm{H} \mathrm{R}$ Brashear, and $\mathrm{F} \mathrm{N}$ Case

Chapter 51

PROCESSING AND ANALYSIS OF RADIOISOTOPIC SAND

TRACER (RIST) STUDY DATA

H R Brashear, E H Acree, F N Case, P A Turner, and D B Duane

Chapter 52

CLASS OF PROBABILITY MODELS FOR LITTORAL DRAFT

William R James

Chapter 53

PROPERTIES OF LONGSHORE BARS IN THE GREAT LAKES

James H Saylor and Edward B Hands

Chapter 54

SAND TRANSPORT PHENOMENA ON COASTS WITH BARS

$M$ Dyhr-Nielsen and Torben Sorensen

Chapter 55

LABORATORY TESTS OF LONGSHORE TRANSPORT

John C Fairchild

867

Chapter 56

INFLUENCE OF GRAIN SIZE ON LITTORAL DRIFT

Jose Castanho 


\section{CONTENTS}

Chapter 57

COMPUTATION OF ALONGSHORE ENERGY AND LITTORAL

TRANSPORT

Theodor R Mogel and Robert L Street

Chapter 58

CRATER SINK SAND TRANSFER SYSTEM

Douglas L Inman and Roland W Harris

919

Chapter 59

USE OF VOLCANOES FOR DETERMINATION OF DIRECTION

OF LITTORAL DRIFT

Per Bruun and Gisli Viggosson

Chapter 60

OFFSHORE BEACH NOURISHMENT SCHEME

Robert A Dalrymple

Chapter 61

BEACH NOURISHMENT AT VIRGINIA BEACH, VIRGINIA

James W Bunch

Chapter 62

UNDERWATER MOUND FOR THE PROTECTION OF DURBAN'S

BEACHES

J S Zwamborn, G A W Fromme, and J B Fitzpatrick

975

Chapter 63

LABORATORY TESTS ON ARTIFICIAL SEAWEED

W A Price, $\mathrm{K}$ W Tomlinson, and D $\mathrm{H}$ Willis

995

Chapter 64

DYNAMICS OF A COAST WITH A GROYNE SYSTEM

W T Bakker, E H J Kleın Breteler, and A Roos

1001

Chapter 65

EXPERIMENTAL STUDY OF THE HYDRAULIC BEHAVIOUR OF INCLINED GROYNE SYSTEM

Jülıo Patrıarca Barcelō 


\section{CONTENTS}

Chapter 66

LAND RECLAMATION AND GROIN-BUILDING IN TIDAL FLATS

Heie Focken Erchinger

Chapter 67

EFFECT OF GROYNES ON ERODED BEACHES

W A Price and $\mathrm{K}$ W Tomlinson

1053

Chapter 68

CHARACTERISTICS OF SHINGLE BEACHES THE SOLUTION TO SOME PRACTICAL PROBLEMS

A M Muir Wood

1059

Chapter 69

COMPARISON OF FLUVIAL AND COASTAL SIMILITUDE

Bernard Le Méhauté

1077

Chapter 70

STUDY OF DURBAN HARBOR SILTING AND BEACH EROSION Jan Malan Jordaan, Jr

CHAPTER 71

ESTUARY INLET CHANNEL STABILIZATION STUDY USING A HYDRAULIC MODEL

Theodore $\mathrm{T}$ Lee

Chapter 72

MODEL TESTS AND STUDIES FOR PORT RASHID, DUBAI

Eric Loewy

Chapter 73

STUDY OF TOPOLOBAMPO'S BAY, MEXICO, ENTRANCE

Héctor López Gutiérrez and Danrel Cervantes Castro

Chapter 74

TIDAL INLET PROBLEMS ALONG THE NEW ENGLAND COAST

Oscar E Arpin

Chapter 75

OFFSET COASTAL INLETS

Miles O Hayes, Victor Goldsmith, and

Carl H Hobbs III 


\section{CONTENTS}

Chapter 76

FIELD STUDY OF TIDAL INLET, BIMINI, BAHAMAS

W Harrison, $R$ J Byrne, J D Boone, and

$\mathrm{R}$ W Moncure

Chapter 77

RECENT HISTORY OF EROSION AT CAROLINA BEACH, N C

Limberios Valhanos

Chapter 78

RIO GRANDE BAR THE CASE HISTORY OF A LAGOON

OUTLET INTO A TIDELESS SEA

V F Motta

1243

Chapter 79

SCOUR AROUND A CIRCULAR CYLINDER DUE TO WAVE MOTION

Donald R Wells and Robert M Sorensen

Chapter 80

COMPARISON OF MODEL AND BEACH SCOUR PATTERNS

John B Herbich

1281

Chapter 81

VARIATION OF TOPOGRAPHY OF SEA BED CAUSED BY THE CONSTRUCTION OF BREAKWATERS

Shoj1 Sato and Isao Irie

Chapter 82

EQUILIBRIUM PROFILES OF MODEL BEACHES

Irvathur Vasudeva Nayak

Chapter 83

STOCHASTIC ANALYSIS OF BEACH PROFILE DATA

Choule J Sonu and Myron H Young

Chapter 84

FIELD STUEDY OF SURATHKAL BEACH

P Suryaprakasa Rao and P Kassim

1365

Chapter 85

SEASONAL BOTTOM CHANGES, BOLINAS BAY, CALIFORNIA

J W Johnson 


\section{CONTENTS}

Chapter 86

SEDIMENT DISTRIBUTION AND ITS RELATIONS TO

CIRCULATION PATTERNS IN BOLINAS BAY, CALIFORNIA

$P$ Wilde and $T$ Yancey

Chapter 87

ON SUCCESSIVE SALTATION OF A SAND GRAIN BY WIND

Yoshito Tsuchiya

VOLUME III

COASTAL STRUCTURES AND RELATED PROBLEMS

Chapter 88

RESEARCHES ON SEA-WALLS

$S$ Naga1

1431

Chapter 89

PRESSURE OF WAVES AGAINST VERTICAL WALLS

M E Plakıda

Chapter 90

PLUNGING WAVE PRESSURES ON A SEMI-CYLINDRICAL TUBE

Yuan Jen and Pang-Mou Lin

Chapter 91

LOADINGS ON LARGE PIERS IN WAVES AND CURRENTS

Hsiang Wang

1491

Chapter 92

EXPERIEMENTS WITH SAND-FILLED FLEXIBLE TUBES

Per Roed Jakobsen and Arne Hasle Nielsen

Chapter 93

PRESSURE OF FLOATING ICE-FIELDS ON PILES

Joachım Schwarz

Chapter 94

BREAKWATER AND QUAY WALL BY HORIZONTAL PLATES

Takeshı Ijıma, Shıgeo Ozak1, Yasuhıko Eguch1, and Akıra Kobayashı 


\section{CONTENTS}

Chapter 95

INFLUENCE OF BREAKER TYPE OF RIPRAP STABILITY

John P Ahrens

Chapter 96

DAMAGE FUNCTIONS FOR A RUBBLE-MOUND BREAKWATER UNDER THE EFFECT OF SWELLS

Juan B Font

Chapter 97

HYDRAULIC RESISTANCE OF ARTIFICIAL CONCRETE BLOCKS

Nobuo Shuto and Hiroshi Hashimoto

Chapter 98

USE OF ASPHALT IN BREAKWATER CONSTRUCTION

$\mathrm{K}$ d'Angremond, J J Th Span, J Van der Weide, and $\mathrm{A} \mathbf{J}$ Woestenenk

1601

Chapter 99

HONOLULU REEF RUNWAY DIKE

Robert Q Palmer and James $\mathbf{R}$ Walker

Chapter 100

FLOATING BREAKWATER FOR RESERVOIR MIRINAS

Kenneth Chen and R L Wiegel

Chapter 101

FORCES ON A PONTOON IN THREE-DIMENSIONAL WAVES

J Eie, A Traetteberg, and A Torum

Chapter 102

RESONANCE OF MOORED OBJECTS IN WAVE TRAINS

A J Hermans and G F M Remery

Chapter 103

HYDRODYNAMIC FORCES OF TWIN-HULL VESSELS

Shen Wang

1701

Chapter 104

BEHAVIOR OF A SLENDER BODY IN SHALLOW-WATER WAVES

Hsiang Wang and Li-San Hwang 


\section{CONTENTS}

Chapter 105

OIL BOOMS IN TIDAL CURRENTS

Ralph H Cross and David P Hoult

PART 4

COASTAL, ESTUARINE, AND ENVIRONMENTAL PROBLEMS

Chapter 106

ENVIRONMENTAL PLANNING OF A BAY AND COASTAL LAGOON SYSTEM

Norman T Gilroy

Chapter 107

DETERIORATION AND RESTORATION OF COASTAI WETLANDS

Sherwood M Gagliano, Hyuck J Kwon, and

Johannes $L$ van Beek

Chapter 108

RESEARCH FOR THE COASTAL AREA OF THE DELTA REGION OF THE NETHERLANDS

J J Dronkers

Chapter 109

SEDIMENT POLLUTION IN COASTAL WATERS

$J$ van de Kreeke

Chapter 110

RECIRCULATION IN SHALLOW BAYS AND RIVERS

R K Price, $R$ A Dalrymple, and R G Dean

Chapter 111

NATURAL FLUSHING ABILITY IN TIDAL INLETS

I B Mota Oliveira

Chapter 112

MATHEMATICAL MODEL OF MIXING IN NEW HAVEN HARBOR

Michael D Disko, Robert A Norris, and

Francis C Lutz

Chapter 113

FLUSHING PATTERN OF NON-REACTIVE EFFLUENTS

Adel M Kamel 


\section{CONTENTS}

Chapter 114

THE MEASUREMENT OF TIDAL WATER TRANSPORT IN CHANNELS

R E Klein

Chapter 115

WATER CURRENT METER FOR MEAN FLOW MEASUREMENTS

David Smith and W Harrison

Chapter 116

TRAPPING OF OUTFALL CONSTITUENTS BEHIND SILLS

$\mathrm{T}$ Carstens and A Sybberg

Chapter 117

SUSPENDED LOAD CALCULATIONS IN A TIDAL ESTUARY

Brian A O'Connor

Chapter 118

PARTICLE VELOCITY MEASUREMENTS WITH A LASER DEVICE

C A Greated and N B Webber

1951

Chapter 119

DENSITY CURRENTS AND TURBULENT DIFFUSION IN LOCKS

A Roy Halliwell and Martın O'Dell

Chapter 120

MEASUREMENT OF DENSITY CURRENTS IN AN IDEALIZED MODEL

Timm Stuckrath

Chapter 121

COMPUTATION OF STORM SURGE

Richard Silvester

1995

Chapter 122

STATISTICAL PREDICTION OF HURRICANE STORM SURGE

C Y Yang, A M Paris1, and W S Gaither

Chapter 123

ANALYSIS OF HURRICANE TIDES AT PADRE ISLAND, TEXAS

Frank D Masch, Robert J Brandes, Floyd R Hill,

and William A White 


\section{CONTENTS}

Chapter 124

TURBULENCE IN HURRICANE-GENERATED COASTAL CURRENTS

Stephen P Murray

Chapter 125

JAMAICA BAY HURRICANE BARRIER

Frank L Panuzio

Chapter 126

MODEL STUDY OF THE TRANSFORMATION OF TSUNAMIS IN

URADO BAY

Shigehısa Nakamura, Yuichı Iwagak1, and

Yoshito Tsuchiya

2089

Chapter 127

TSUNAMIS SOME LABORATORY AND FIELD OBSERVATIONS

Fredric Raichlen

Chapter 128

HEAD LOSS AT TSUNAMI-BREAKWATER OPENING

Yoshryuk1 Ito

Chapter 129

TIDAL WAVES IN SCHEMATIC ESTUARIES

Hans Vollmers and Jürgen Sündermann

Chapter 130

DEFORMATION OF ROTATIONAL TIDAL CURRENTS IN SHALLOW COASTAL WATER

Harald Gohren

Chapter 131

RESONANCE IN HARBORS OF ARBITRARY SHAPE

J11n-Jen Lee and Frednc Raichlen

Chapter 132

SPECTRAL RESPONSE OF HARBOR RESONATOR CONFIGURATIONS

William James

Chapter 133

HARBOR STUDY FOR SAN NICOLAS BAY, PERU

James M Keith and Emmett J Murphy 


\section{CONTENTS}

Chapter 134

TIDAL MOTION IN BAYS

O H Shemdin and R M Forney

2225

Chapter 135

SURFACE OSCILLATIONS IN A TANK CAUSED BY A

SUBMERGED JET

Juan B Font, Omar Azpura, and Flora Aranguren

Chapter 136

EFFECT OF LONG PERIOD WAVES ON HYDROGRAPHIC SURVEYS

Orville T Magoon and Willıam O Sarhn

Chapter 137

SELECTION OF DISPOSAL AREAS FOR SPOIL FROM BALTIMORE

HARBOR

John F Hoffman

Chapter 138

WAVES GENERATED BY LARGE SHIPS AND SMALL BOATS

$M$ M Das and $\mathrm{J}$ W Johnson 



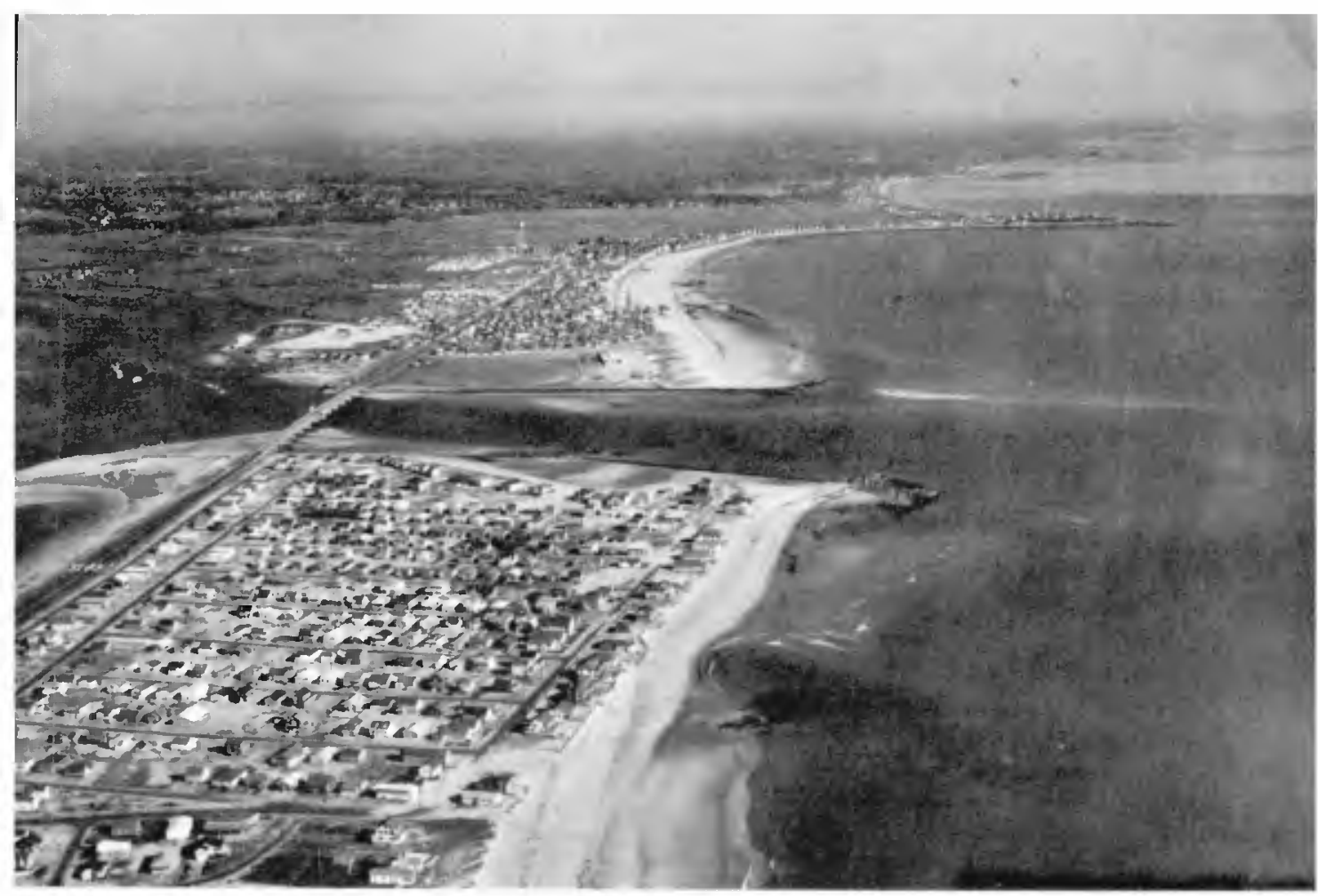

Hampton Harbor, New Hampshire

PART I. WAVE THEORY, MEASUREMENTS, AND ANALYSES

Webhannet River, Maine

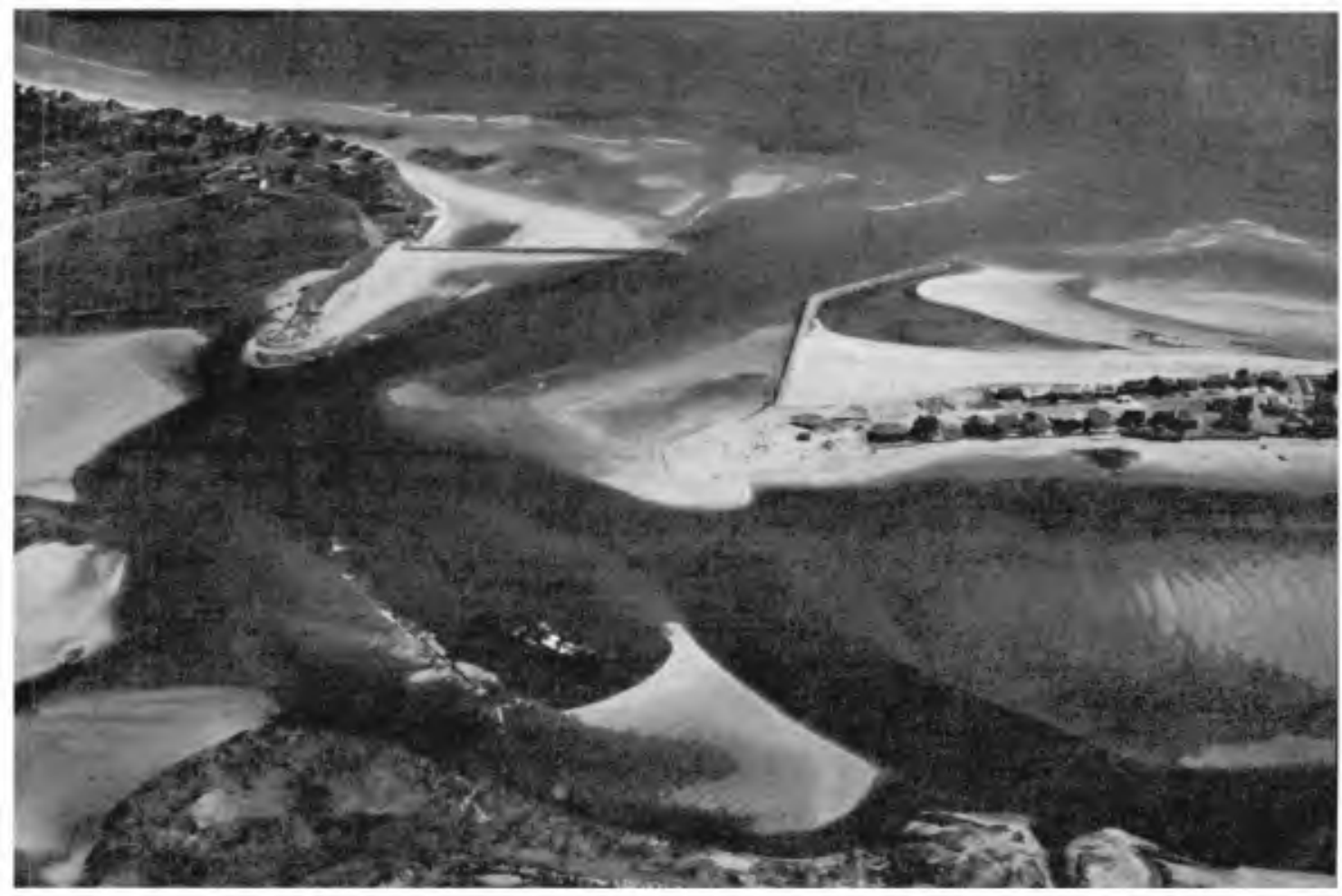





\title{
CHAPTER 1
}

\author{
THE CANADIAN WA VE CLIMATE STUDY - THE FORMATIVE YEAR
}

\author{
Laurence Draper
}

\author{
Nationa 1 Institute of Oceanography, Great Britain \\ Director, Canadian Wave C11mate Study, 1968-69 \\ Department of Public Works
}

\begin{abstract}
Th1s paper describes the need for, and the factors involved in, the establishment of a Wave Climate Study, 11lustrated with particular reference to the Canadian Wave Climate Study
\end{abstract}

\section{INTRODUCTION}

The shortage of sea wave information is world-wide, and that wh1ch does exist has usually been obtained for specific purposes and is of value only in a very limited area of the existing information, a large part in incomplete in comparison with the desirable minimum of a year's continuouslysampled conditions, and much of that which has been collected systematically has been analyzed in ways which are difficult to interpret Canadian waters were no better, and no worse, documented than most others, but the Canadian Department of Dublic horks, probably the major potential user of wave data, became concerned at the lack of information The engineering design division had the foresight to see that techniques had advanced sufficiently to justify the setting up of an organization to devote 1 tself solely to the collection of wave data to meet future needs Because of the size of the country, the project envisaged was probably larger than any similar project which had previous1y been undertaken anywhere else in the world Treasury approval was obtained for an exploratory phase, w1 th a nominal budget of $\$ 183,000$, and the author was privileged in being asked by the Chief Engineer of the Design Directorate, $M r$ G Millar, to direct the study in 1 ts formative year from October, 1968

\section{GENERAL JUSTIFICATION FOR A WAVE CLIMATE STUDY}

The first part of any such study is largely an administrative exercise A1though the need to spend money is more than obvious to anyone who has ever had to dream up design wave characteristics, it has to be justified in some detail to the controller of the purse strings Part of the justification 


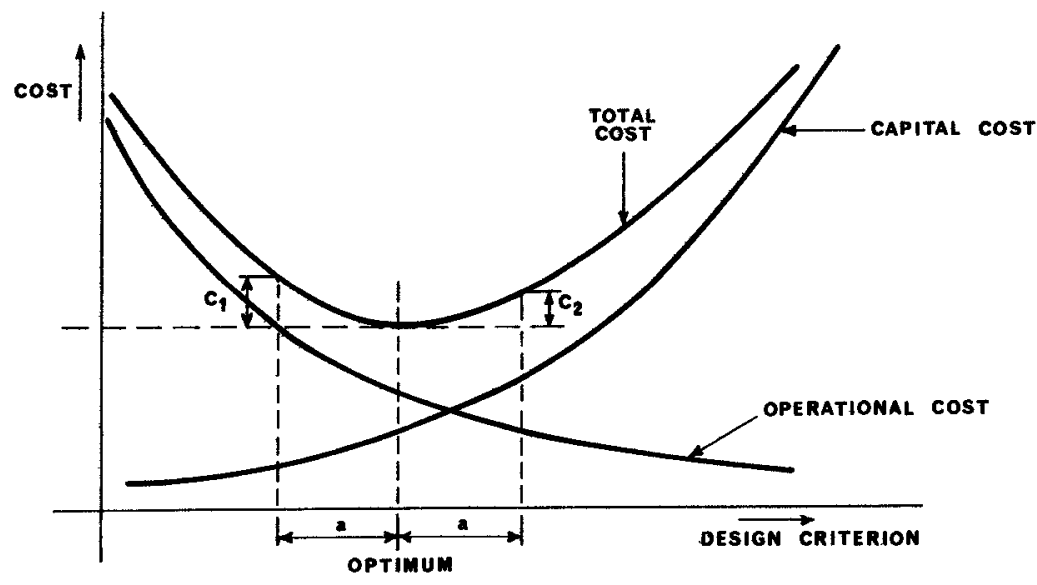

Figure 1 Schematic Relationship between Cost and Design Criteria

is not difficult to find, wherever structures have been built in exposed locations there must have been fallures which have been expensive to remedy, and $1 \mathrm{t}$ is $11 \mathrm{kely}$ that $1 \mathrm{t}$ would be easy to find major repair bills totalling far in excess of the 11 kely cost of any wave climate study It is just concelvable that no major repair works have been necessary, in which case this is again a forceful argument in the minds of the Treasury officials as $1 \mathrm{t}$ is most likely that the structures have been overdesigned, and that the initial capital outlay has, been excessive. Figure 1, based on a diagram in a paper by $J$. Ploeg, suggests that there is a fairly sharp optimum cost of design for coastal structures, so that without a fairly good estimate of wave cond1tions, which are major parameters, 1t would be nearly impossible to come close to the optimum, and the cost would necessarily increase As a consequence of this, $1 \mathrm{t}$ is reasonable to infer that the structure which is ultimately the cheapest w11 require occasional expenditure on maintenance and repair of storm damage Wave conditions are only one, albe1t major, of a number of parameters affecting the ultimate cost of a structure The cost of fallure is very much more than the actual rebuilding cost, for example, if a breakwater fails, in addition to the basic repair cost the re $w_{111}$ be the cost of overloading alternative ports and transport systems, and also the social costs, the latter may well have been completely neglected in many assessments and include the disruption of normal patterns of $11 \mathrm{fe}$ and true unemployment costs (not just those of unemployment benefit), and possibly the costs of loss of $11 \mathrm{fe}$ 
It cannot be pretended that good wave information alone w11l enable a designer to define the optimum precisely, but 1t should appreciably reduce deviations from the optima and make important savings in expenditure

\section{STANDARDS FOR DESIGN}

In the development of standards and criteria for the design of marine structures it is necessary to have reliable information, in some deta11, on wave conditions which do, and are 11able to, occur The only way to ensure that criteria for waves are realistic is to study them, instrumentally and theoretically, in all geographical areas

Uses for wave information are legion Apart from the obvious ones of design parameters for specific projects, and design standards in general, the results of a comprehensive wave climate study can be used for the assessment of utilization of all kinds of surface vehicles, ships, hydrofoils and hovercraft, drilling, pile-driving and pipe-laying vessels, and for many other c1vil, as well as military, purposes

\section{PERSONNEL AND ORGANIZATION}

The minımum personnel requirements of such a study, apart from a study Director, are for one or more Civil Engineers with marine experience, and preferably with a mathematical apt1tude, a Hydrodynamicist with oceanographic experience to be responsible for data collection, for each field team a competent electronic engineer able to plan his own programme and work on his own, often in remote situations, and a technician able to work with him and on small boats Diving experience in the field team would also be a useful attribute

The field team should preferably be supervised by the oceanographer, and the analysis of results would be undertaken jointly by him and the civil engineers, who would then be responsible for treatment of data and its interpretation for engineering purposes Apart from technical prowess, enthusiasm for and appreciation of the value of the whole project are essential in every member of the whole study The field team must have the ability to meet and win the confidence of all persons whose cooperation is required for the success of an installation

The engineers in the project must also be involved in the field work to help them appreciate the problems and difficulties involved, but their main task will be in the interpretation and application of data to real engineering problems They will find themselves called upon to advise and pronounce on al1 manner of problems connected w1th waves The study Director will probably have some difficulty in keeping a balance such that the group continues its research, and also helps those needing advice and often more substantial assistance, especially as they may well be the people on whom his field staff rely rather heavily for support in installing, running and maintaining equipment 
The study must maintain close links with two groups who are genera11y regarded as distinct entities - at one end 15 the construction organization which may not be research oriented, and at the other end is the oceanographic research organization which might not be too interested in detalled practical applications of 1 ts work The study should, if at all possible, house all its staff in close proximity to each other within one building, and also have its own laboratory and at least some workshop facilities. Only in this way can full discussion, essential in such a project, take place and all members of the staff be able to appreciate the problems and needs of the other parts of the project.

TASKS OF THE STUDY

\section{Existing Data}

One of the first tasks w11 be the tracking down and collection of 211 documents purporting to give wave information The data in each one w111 have to be assessed, and 11sted according to 1 ts geographical location, so that when future needs arise 1 t can be retrieved quickiy. It is quite probable that very 11 ttle data achieving the required standards w111 be found

\section{Known future needs}

Many projects can be foreseen in genera1, if not in detal1, several years in advance, so that a picture can be bui1t up showing where requirements for wave information are 11 kely to be concentrated This information can of ten be obtained from area Public Works Authorities, Port and Harbour Authorities and major resource exploiters such as 011 and mineral companies

\section{DEVELOPMENT OF A PLAN OF OPERATION}

When all the existing data has been collected and opinions on future needs have been assembled (previous attempts at wave recording are indications that there may be more interest in those areas), $1 \mathrm{t}$ will be possible to propose areas in which general coverage $s$ tations should be placed. Usually these should be in open waters, placed so that each station samples wave conditions which are 11 kely to be significantiy different from those at any adjacent station. Possible specific locations can usualiy be determined from hydrographic charts of the area, in conjunction with land maps to give an indication of shore conditions, accessibility and density of habitation. The locations must be as exposed as possible and not suffer significantly from refractive effects, be shielded by reefs or sand bars, or be sheitered by long stretches of shallow water If an underwater cable is to be used, a 'safe' landing zone must be available fa1rly convenientiy, tidal currents should be as small as possible. Possible locations for housing the recording equipment, such as lighthouses or other officially-owned buildings on a coast, may be found from charts and land maps, the authorities concerned are of ten only too pleased to he1p by giving introductory letters for project personne 1 to present to 11ghthouse staff and others, asking them to give assistance during site surveys. 
When specific locations have been decided $1 \mathrm{t}$ is then necessary to make site surveys. The actual visit to each site at which a cable may be brought ashore entalis inspection on foot, preferably at low tide, to determine an actual route for the cable. The route must be as protected as possible to avoid sharp rocks and areas where boulders are $11 \mathrm{ab} 1 \mathrm{e}$ to be moved by high waves A major hazard for surface instruments is that they may be in collision with shipping, be stolen, or even used as targets for shooting practice, submerged instruments are less vulnerable, but can easily be trawled up, or the cable may be wrecked by a dragging anchor, in either case al1 local marine authorities and opinions must be sounded to determine the $11 \mathrm{k} 11$ hood of misadventure These w111 usually range from black despair to high opt1mism, the real answer usually lies between the two, and a crystal bal1 may well be needed before the final decision is taken

Loss of information can, and w111, occur through many different causes, in fact, wave recording is a highly hazardous operation, at least from the point of view of the resulting data In planning the Canadian Study, it is intended to leave each recorder in operation for two years so that gaps in the first year's records can be patched with data from the second In the relatively rare cases when the first year's records have insignificant gaps, the instrument can be w1 thdrawn and moved elsewhere In 1ce-free waters a year means twelve calender months, but where the water is frozen over for part of the time, the "year" is taken to be the mean duration of time when substantial 1ce-cover 1 s absent

\section{ASSESSMENT OF INSTRUMENTS}

There is no universal wave recorder, but for most studies it may wel1 be possible to standardize on one sub-surface pressure recorder, one surface buoy and one through-surface recorder from the technical points of view of expertise in servicing and costs of spares, the virtues of having as few different types of instrument as possible are self evident. In the Canadian Study, 1t was 1 nitially intended to standardize on three types

(1) N.I O Shipborne wave recorder This can only be used for routine data collection where a vesse 1 is stationary at one place for long periods - for example, on Light Vessels or Weather Sh1ps, or where a vessel often revisits a station or small area for routine sampling purposes In our case the two Canadian Ocean Weather Sh1ps which operate on station $P$ in the North Pacific were each already fitted with a Shipborne wave recorder The only remaining light Vesse1 in Canadian waters, the LURCHER, was scrapped as an economy measure less than a month before the equipment was due to be installed One instrument was installed on the PORTE DAUPHINE, a Great Lakes Institute vesse1, the one destined for LURCHER should be installed on a vessel belonging to the Canadian Centre for Inland Waters 
(2) Pressure recorder If at all possible the recorder should be capable of being monitored, so that a fault can be recognised at a very early stage To achieve this it is necessary to use an underwater cable, but cables suffer bad1y from the vagaries of the sea, and may be damaged in a variety of ways which can reduce the insulation for long-term installations it is essential that any damage which 1mpairs the signal should be recognised at once In a recorder which transmits back a voltage or a current, diminution of the insulation can result in an attenuated signal reaching the shore unit, and the presence of the trouble may not be recognised for a long time Even when it is known that the record is in error, it may not be possible to determine with any certainty the time of onset of the trouble, and many perfectly good records may have to be scrapped Possibly the most elegant alternative is the use of the frequency modulation system in which a pressure signal is converted in the transducer into a frequency signa1, so that a frequency is transmitted up the cable. As long as the frequency can be discriminiated from background noise, regardless of the state of the cable, a shore unit will record the correct pressure Fallure, when 1 t comes, w111 be catastrophic, and w111 be 1mmediately recognisable

At the time when the Study was looking into the problem, on1y two types of $F M$ wave recorders were commercially avallable After testing one of each $1 \mathrm{t}$ was decided to standardize on the instrument designed and built by the National Research Council in Ottawa

(3) Surface buoy These instruments are intended for use where the water is deep and a pressure un1t cannot be used, or where the distance to shore makes the cable cost prohibitive They transmit by radio to a shore station, which may be twenty or thirty miles away One of each of two different types were tested, and $1 \mathrm{t}$ was decided to standardize on the Waverider buoy bu1t by Datawe11 of Ho11and

Through-surface recorder In the first stages where only general area data was sought, there was no need for a through-surface recorder (they generally need to be fixed to an existing structure)

\section{DATA HANDLING}

In the Canadian Study 1 t was intended $1 n_{1}$ tially to record by pen on paper charts, there were several reasons for this The assessment of instruments was not due to be completed unt11 the autumn of 1969 , and until decisions had been made it was not possible to decide the type of digital equipment needed to match the outputs In remote outstations the use of sophisticated equipment, especially if it is untried, can waste a great deal of time Even if all the equipment had been well proven and was 11 kely to give no trouble, the shortage of skilled technical staff would have reduced the number of 1nstallations which could be made in a given time. The philosophy at present is that if good chart records can be obtanned they can be analyzed cheaply at headquarters by people other than technicians 



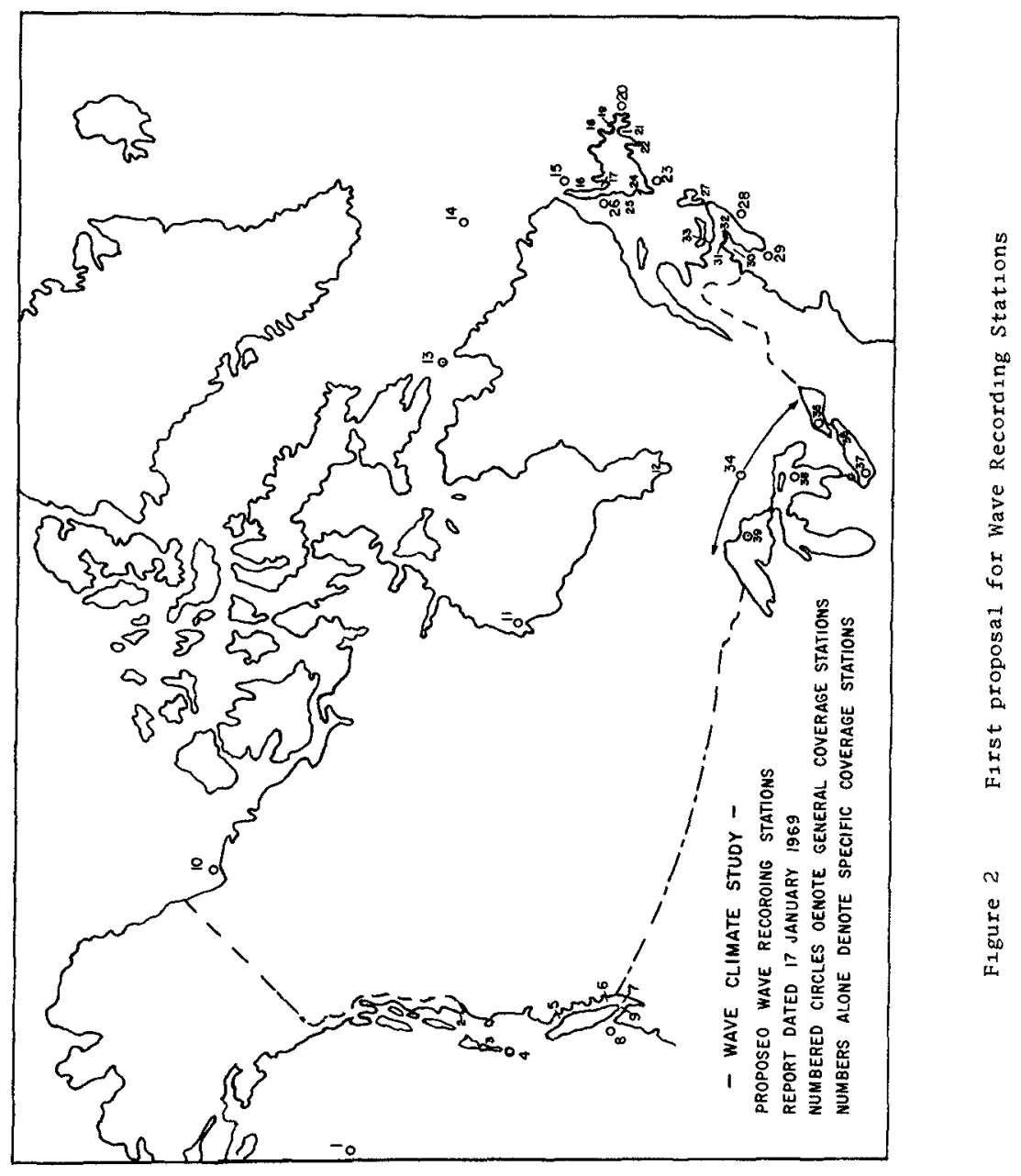


One technique which needs to be 1nvestigated is the transmission of data over existing telephone 1ines back to headquarters This would reduce the amount of data logging equipment needed, and have the great advantages that only one data logger would be required and could therefore be of high quality, and also that servicing staff would be easily available It would have the disadvantage that spurious signals might be injected into the records by parts of the system out of our control and that recording might not always be possible at the required time due to conjested lines

The intention is to process the data, whether on charts or tape, following the recommendations given by the author at the 1966 conference

\section{WIND DATA}

A very important source of information from which wave data can be calculated is wind data Measured or even observed wave data is sparse, but measured wind data is much more common and in many p1aces is avallable for ha1f a century or more For calculations of extreme wave conditions the wind data may not be in the most useful form, the information needed is the most probable value of the highest mean wind, over durations up to 24 hours, 11kely to be exceeded once in some long period of time such as 20 , 50 or even 100 years If the basic measurements of mean hourly wind speed have been consistent $1 y$ made for at least ten years, an extrapolation can be made from them. The author $1 \mathrm{~s}$ at present aware of only two countries where wind data 15 available in the required form, these being the Un1ted K1ngdom ${ }^{3}$ and Canada ${ }^{4}$, where the data has recent1y been obtalned by this Study If this mean-hourly data 1 s avallable, a further requirement for wave forecasting is information on the ratios of the mean wind speed over periods of from six to twenty four hours to the highest mean hourly speed in each storm For Canada this was done for the 20 most severe storms at 21 typical stations, the mean speed over 24 hours was between $60 \%$ and $80 \%$ of the greatest mean hourly value, and the mean speed over $s 1 x$ hours was between $80 \%$ and $90 \%$ of the greatest mean hourly value For the U.K some ratios have been calculated by Shellard (private communication) and the percentages were a 11ttle higher

\section{REFRACTION STUDIES}

Any study group established to provide wave information w111 rapidly find itself being called upon to produce refraction diagrams Several methods of doing this have been published, and one must be chosen and adapted to suit the computational fac1lities avallable, 1deally the output should be drawn by a plotter In the Canadian Study, the method devised by the U S Coasta1 Engineering Research Center was adapted 5 , with the output presented on a Benson Lehner plotter

\section{THE PROPOSALS AND ESTIMATED COSTS FOR THE CANADIAN STUDY}

To achieve reasonable coverage of Canadian waters, 39 stations were designated ( $F_{1 g}$ 2), 9 being on the Pacific coast, 5 in northern waters, 19 on the Atlantic coast and 6 on the Great Lakes Details of the specific proposals are not given here, as they are of local value only The estinated total cost is just over one million dollars, spread over five years 
The staff requirements are for five persons in the first year building up to ten in the fourth year, the total number of man-years amounting to 40 At the end of the five years the study ought to achieve reasonable coverage of Canadian wave conditions, with data processed and presented in a standardized manner

\section{CONCLUSIONS}

The needs for wave information are many, but the cost of a major comprehensive study is more than justified by the probable economies in construction and repair costs alone

In the running of a wave c1imate study, a $10 t$ w111 depend on the recruitment of staff with not on1y the appropriate ab111ties but with the sort of pioneer enthusiasm which is necessary in a sma11 new project, in which there are few precedents and where improvisation is needed in isolated locations Such a group must not only collect and analyse data, but 1 t must be capable of acting as a consultant to interpret its findings and advise those who need the data and who w111, in a11 probability, f1ock to 1 t in increasing numbers Indeed, maybe 1 ts major prob1em will be that of finding a way to $11 \mathrm{mit}$ the $t$ ime spent in solving particular localized problems, as this can easily interfere with the task of making and interpreting field measurements - the first priorities of the study At this stage it is hard to estimate what w111 happen after five years, but the author's feelings are that the group w11 be so involved in many projects that 1 ts continuation w11 be essential to the proper design of marine works in Canada, and that its usefulness in many other spheres as well will ensure its survival both as a data collecting and an engineering consultant agency

\section{ACKNOWLEDGEMENTS}

The author wishes to thank all those people, too numerous to mention, w1thout whose guidance, enthusiasm, friendship and hard work this project would never have become viable He also wishes to express his appreciation to the Director of the National Institute of Oceanography, Dr G $E$ Deacon, $C B E, F R S$ for his permission to undertake the task, and to his col1eagues at Worm1ey who held the fort so admirably during his year's absence 
REFERFNCFS

1

PLOEG, J

DRA PER, L

3 SHELIARD, HC

4 DRAPER, I and WIJ, $\mathrm{H} \mathrm{J}$

$5 \mathrm{WU}, \mathrm{H} \mathrm{J}$
1968 A General Discussion on the Selection of a Design Wave Second Marine Engineering Seminar Department of Public Works, Ottawa, Canada

1966 The anrlysis and presentation of wave data - a plea for uniformity Proc 10th Conf Coastal Engg 1, 1-11

1965 Extreme wind speeds over the United Kingdom for periods ending 1963 Climatological Memo No 50, Meteorological office

$196^{\circ}$ Extreme mean wind speeds over Canada Marine Fing ineering Report No 14 , Department of Public Works, Ottawa, ranada

1969 The application of a numerical method and computer program in a water-wave refraction study Marine Eng ineering Report No 12, Department of Public Works, Ottawa, Canada 



\title{
CHAPTER 2
}

\section{OCEAN WAVE RESEARCH IN SOUTHERN AFRICA}

\author{
by \\ J A Zwamborn ${ }^{*}$, C van Schalk*, A Harper ${ }^{*}$
}

\begin{abstract}
Wave recording on a national basis in South Africa commenced in 1967, when an "Ocean Wave Research" project was initiated, almed at obtaining reliable data on sea conditions around the shorelines of the Republic and South West Africa and to improve on wave prediction techniques

Various instruments are being used in this programe Some of the problems encountered in practice with these instruments and the agreement between the results obtained using various wave recorders simultaneously at the same station are discussed An indication of the volume of wave data so far obtained and the methods of analysing them are included
\end{abstract}

\section{INTRODUCTION}

The realisation that accurate data on the wave conditions along the country's entire coastline and also over the continental shelf were indispensable for further coastal development and nearshore operations, has become evident in South Africa several years ago Although wave records had been collected on an ad hoc basis in certain localities for many years, 1t was not unt11 February, 1967 that the "Ocean Wave Research" project was initiated This project was instigated by the South African National Committee for Oceanographic Research and is supported by various authorities and private bodies and a1ms at collecting wave data on a national and systematic basis The work is being carried out by a research team from the Hydraulics Research Unit of the CSIR's National Mechanıcal Englneering Research Institute

Xead, Senior Technical officer and Research Assistant respectively
of the Hydraulics Research Unıt of the CSIR's National Mechanical
Engıneering Research Institute, Stellenbosch, Republic of South Africa 


\section{OBJECTIVES AND PROGRAMME OF THE OCEAN WAVE RESEARCH PROJECT}

The main objectives of the Ocean Wave Research project are to record and analyse wave conditions along the $4000 \mathrm{~km}$ of coastline of South Africa and South West Africa, to correlate wave and wind data and to assist the South African Weather Bureau with wave forecasting

Wave recording stations are being operated or are being installed about $300 \mathrm{~km}$ apart, along the entire coast1ine Moreover, records are supplemented by deep sea data obtained from five research vessels operating in the country's coastal waters (see Figure 1)

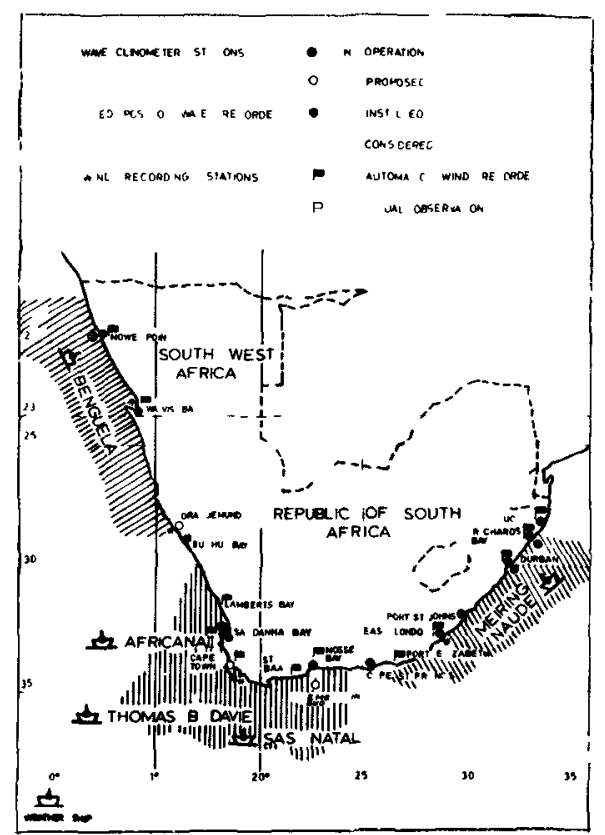

FIGURE 1 LOCATION OF WAVE RECORDING STATIONS

Most of the nearshore recording stations are in relatively shallow water of 10 to $30 \mathrm{~m}$ depth and the records obtained are only of local application Using we11-known refraction techniques, however, a11 the individual records are converted into deep sea values which not only apply to the recording area itself but they apply to much longer stretches of coastlines initially estimated to be as long as $300 \mathrm{~km}$ Avallable data have confirmed the reliability of this estimate but 
extensive correlation studies between the data from adjoining stations are being continued to establish the optimum spacing of recording stations

The full programme w111 run unt11 1975 at the earliest It $1 \mathrm{~s}$ possible that recordings at only a few key stations w111 continue on a permanent basis after this data

\section{MEASURING INSTRUMENTS}

When data from instruments are not ava1lable, v1sual wave records co1lected elther from merchant ships or from 11ghthouses have to be used The Ocean Wave Research project, however, a1ms at using only data obtained from wave recording instruments and relying as 1ittle as possible on visual observations Instruments using optical, accelerometer, acoustic and pressure measuring systems are being used to obtain the wave recordings

Wave clinometer

The wave clinometer is an optical instrument developed in South Africa by the CSIR primarily for measuring wave direction As can be seen in the photograph in Figure 2, it consists of a specially adapted telescope which 1 s mounted on a stand and in such a way that 1 ts axis can be inclined at one of three fixed angles, namely $3^{\circ}, 5^{\circ}$ or $7 \frac{1}{2}^{\circ}$ to the horizontal The instrument is installed on shore at a fixed compass direction and the angle of inclination of the telescope fixed at one of these angles so that it can be focussed on the observation point at sea To measure wave direction, a horizontal cross hair in the telescope is aligned with the wave crests by turning the telescope about its axis and the angle is recorded on a graduated disc mounted on the teles cope

Wave helghts and wave perıods are measured by observing the movements of an anchored floating buoy using the graduations provided in the telescope between which the image of the buoy moves The wave helghts obtained agree closely with the so-called significant wave height, Hs By measuring the time for 20 waves to pass the buoy, the average wave period can be determined This 1 found to represent both the significant wave period $\mathrm{T}_{\mathrm{S}}$ and the zero crossing wave period 
$T_{z}$ closely. The buoys used are usually inflatable polyform spheres $0.5 \mathrm{~m}$ in diameter. Unfortunately, they are easily lost, due to such reasons as theft, running over by ships and interference by fish. Moreover, they do not usually last longer than about six months due to wear and tear. Since they are not costly, they are normally replaced at four monthly intervals.

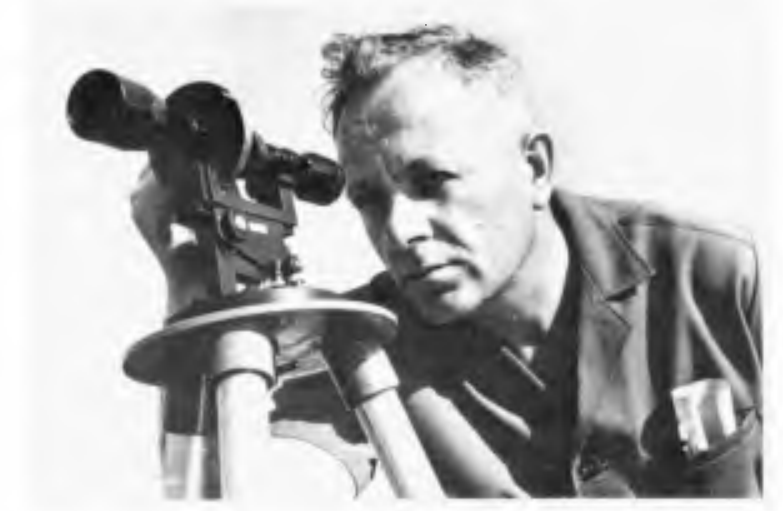

FIGURE 2: WAVE OBSERVATION WITH WAVE CLINOMETER

Greater accuracy is achieved when the telescope is inclined in the $5^{\circ}$ or $7 \frac{1}{2}^{\circ}$ positions, thus the instrument should preferably be sited on a high vantage point. The water depth at the observation point should not be less than about $12 \mathrm{~m}$, which means that the slope of the sea bottom should preferably be steep.

At present a total of eleven clinometers are installed around the coast, most of them in lighthouses (see Figure 1). The recordings are hand-punched on computer cards to facilitate subsequent analyses. Recordings can only be made during the hours of daylight and are normally made at 5.00, 10.00 and 15.00 hours G.M.T.

\section{Shipborne recorder}

The well-known NIO shipborme wave recorder is an accelerometer type instrument and consists of two identical units fitted under the waterline on each side of the ship's hu11. Each unit contains an accelerometer and a static water pressure transducer, connected to a recorder unit normally installed in the ship's wheelhouse ${ }^{1}$. The total vertical displacements of the ship are obtained by a double integration 
(electronically) of the output of the accelerometers and averaging the two results True wave height record is obtained after a correction 1s made for the ship's movements relative to the water surface by using the outputs of the pressure transducers Wave directions are estimated by the crew using the ship's compass

NIO recorders are installed on all five research vessels which operate in the areas shown hatched in Figure 1 Records of $15 \mathrm{~min}$ duration are made every six hours at $600,1200,1800$ and 2400 hours G M T dally The records are in graphical form and adjustments have to be made to the wave periods to eliminate the influence of the ship's speed In the past the graphic records were converted into digital form and punched on tape with a D-Mac pencil follower but arrangements have been made to install paper tape punch units in 1ine with the graphical recorders to facilitate computer analyses of the data

The NIO recorders give reliable results but should be calibrated at regular intervals and should be operated preferably by the ship's officers, or the scientist on board the research vessels

\section{Boersma recorder}

The Boersma wave herght meter is also an accelerometer type instrument It is normally installed on a float and connected to an integrating and recording unit on board ship In the early stages of the project the float, which $1 \mathrm{~s} 1 \mathrm{~m}$ square, was launched from the weather ship (see Figure 1) but due to frequent cable breakage and instability of the float in heavy seas, it was later decided to install the accelerometer in the cabin of the $51 \mathrm{~m}$ long weather ship Records are obtained daily for $15 \mathrm{~min}$ durations at $600,1200,1800$ and 2400 hours GM T The records are in graphical form but a paper tape punch unit will be installed shortly

\section{Wave rider system}

The Datowe II wave mder system is an accelerometer type instrument and consists of a telemetering buoy of spherical shape, anchored at sea and connected to a shore based recelving and punching unit The buoy contains an accelerometer and transmitting unit, whereas the shore units contain the recelver, programmer, pen-recorder and punch-unit Three buoys can be used with one recelver unit and they can be anchored 
up to $50 \mathrm{~km}$ from the shore station

A Datawell wave rider system 1s installed at Mosse 1 Bay (see Figure 1) where wave height records of $30 \mathrm{~min}$ duration are obtained automatically at $600,1200,1800$ and 2400 hours G M daily The records can be obtained either in graphical and/or punched form

The shore unit of the wave rider system must be installed at a "civilised" place, since regular attendance is required for proper functioning The Mossel Bay unit is installed at the radio station and has generally worked satisfactorily The buoy is anchored some $35 \mathrm{~km}$ from the shore $1 \mathrm{n} 100 \mathrm{~m}$ deep water, which $1 \mathrm{~s}$ close to the shipping lanes The buoys have proved to be the weakest $11 \mathrm{nk}$ in the system, since over a period of nine months, one buoy was lost and a second buoy was salvaged twice after it was presumed to have gone adrift It is difficult to decide whether a reward should be offered for the retrieval of a lost buoy, since this may encourage the removal of the buoy on purpose'

\section{Normal echo sounder}

Normal echo sounders, which are acoustic type instruments installed on small boats, e $g$ skiboats, are often used to obtain synoptic wave records to measure the effects of wave refraction and diffraction Provided the boat $1 \mathrm{~s}$ small and wave periods are relatively long, e $g$ as in the case with swell, accurate wave records can be obtained with these instruments

\section{INES wave recorder}

The INverted Echo Sounder or INES wave recorder is an acoustic type instrument developed by the Hydraulics Research Unit of the CSIR It is a self-contained wave recorder which consists of a normal echo sounder housed in a waterproof canister placed in a supporting frame on the sea bottom The transducer is fitted in the 11d of the canister and the acoustic waves are reflected from the water surface, thus giving a continuous wave record (see Figure 3)

INES recorders are operating at three stations at present and another four stations will be operative shortly (see Figure 1) The instrument is left unattended on the sea bottom for up to five weeks, glving $15 \mathrm{~min}$ duration records at $600,1200,1800$ and 2400 hours 
G.M.T. daily. These records are in graphical form and are converted into digital form by a D-Mac pencil follower.

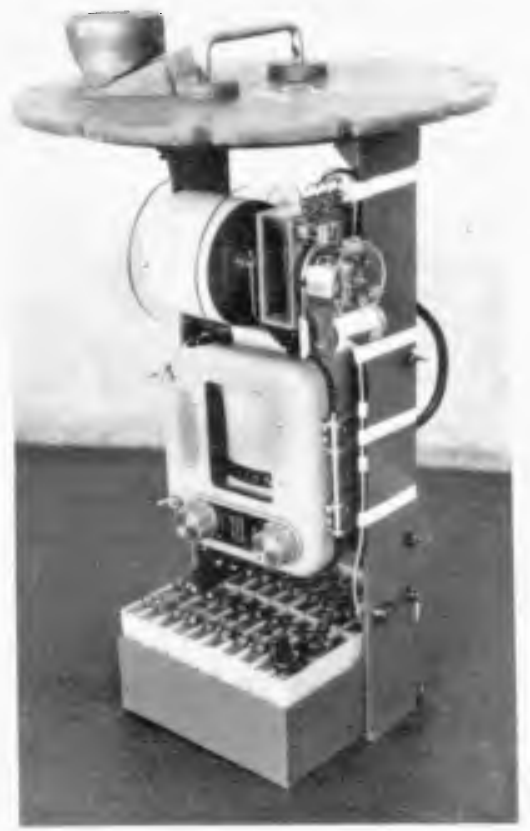

FIGURE 3: INES WAVE RECORDER

The INES recorders can be placed in any depth of water up to $36 \mathrm{~m}$ deep. Marker buoys indicate the position of the instrument, which are normally placed by divers to ensure that the tripod stand, which support the instrument, is in an upright position. They are serviced at monthly intervals normally by divers (see Figure 4) when the canister containing the instrument is released from the stand and brought to the surface. After servicing, it is taken down again. When operating in deep water, a spare serviced instrument is usually taken down by the diver on his first dive to replace the instrument which requires servicing. With this procedure, only one dive per instrument is required.

Good results have been obtained with these recorders but many problems have had to be overcome, e.g. leakage of the canister, breaking of the stylus and failure of the marker buoy mooring system. 


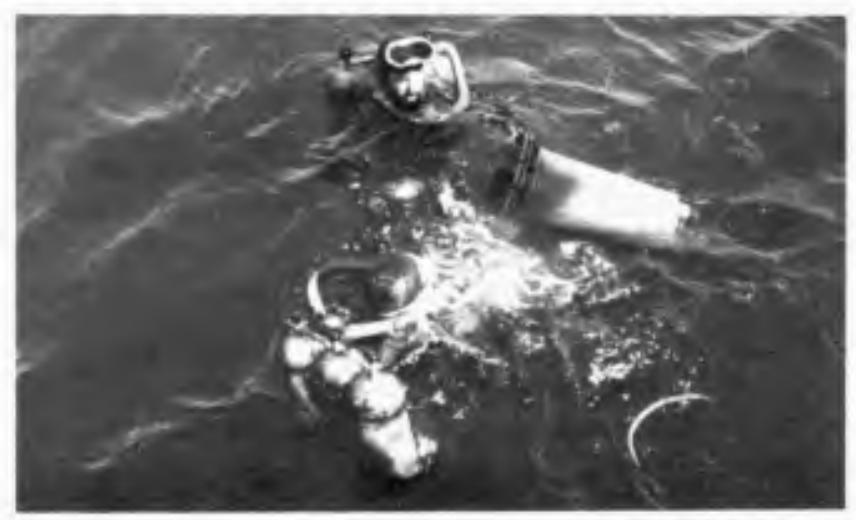

FIGURE 4: PLACING OF INES WAVE RECORDER BY DIVERS

\section{Ospos recorder}

Ospos recorders are also being used. They are of the pressure type and are self-contained and can be left unattended for about four weeks. They are placed either on the sea bottom or suspended from an anchor on the sea bottom to "float" at a known distance, preferably 1 m below the lowest expected wave trough below the water surface. In deep water, therefore, there is considerable movement of the suspended instrument with wave action and this affects the results adversely. Moreover, since the depth of the transducer must be accurately known, the conversion from pressure changes to wave heights is dependent on the depth of submergence.

The instrument records pressure variations for durations of $15 \mathrm{~min}$ four times per day, usually at $6.00,12.00,18.00$ and 24.00 hours G.M.T. The conversion from pressures to wave heights is very laborious and inaccurate and this recorder is used only if no other instruments are available.

\section{CORRELATIONS BETWEEN VARIOUS INSTRUMENTS}

Since the statistical analyses are based upon records obtained from different instruments, it is essential to check that their outputs are in good agreement ${ }^{2}$. 
Comparis on of the wave directions read from a wave clinometer (1nclined at $5^{\circ}$ ) with those obtained from aerial photographs specially made for this purpose showed a maximum deviation of $10^{\circ}$ and an average deviation of $5^{\circ}$

A special correlation test was arranged at a point due west of Robben Island (Cape Town) in a water depth of $274 \mathrm{~m}$ on 2nd october, 1969 from 1500 to 1600 hours S A S T Simultaneous recordings were made using the following instruments

(a) Boersma wave height meter (using the float)

(b) INES wave recorder

(c) Ospos wave recorder

(d) NIO wave recorder on research vessel Africana II (NIO A)

(e) NIO wave recorder on research vessel Benguela (NIO B)

A comparison of wave height and wave period distributions using a $30 \mathrm{~min}$ period of the records, are given in Figures 5 and 6

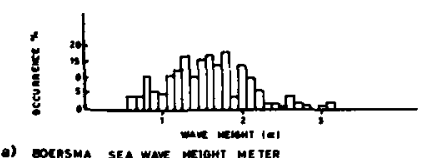

a) Bochsma SEA waV weloht METER

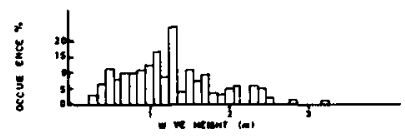

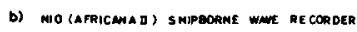

:

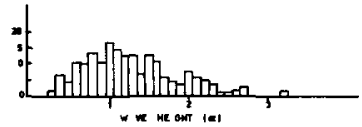

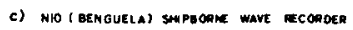

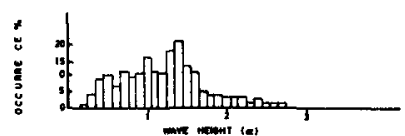

d) WES WAVE REC OMOER

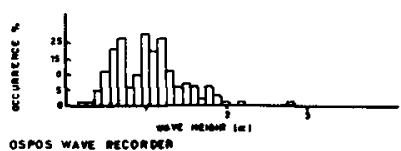

a) asmos wave ieconoun

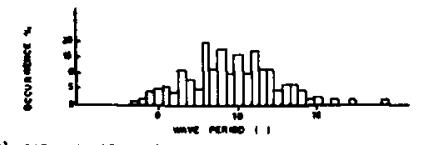

- Dotinsma sea mave Peniod secono

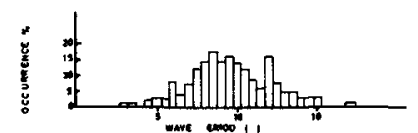

b) NIO (AFRICANA I) SHAPBCANE mUE RECORDER

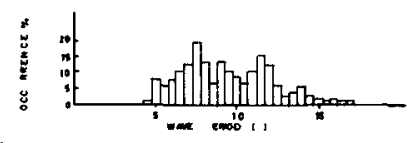

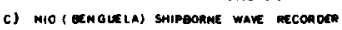

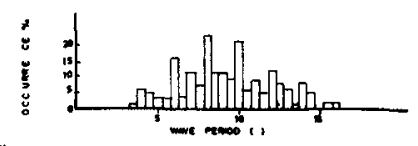

d) wes wave reconoen

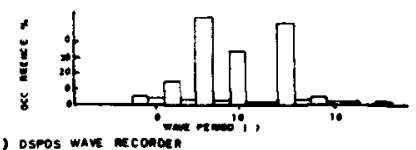

FIGURE 5 WAVE HEIGHT DISTRIBUTIONS FIGURE 6 WAVE PERIOD DISTRIBUTIONS 
The results show that the records of NIO recorders, installed on two different ships, are almost identical The Boersma wave buoy seems to over-estimate the wave heights, especially for the lower wave group, whereas the Ospos generally gives wave helghts which are too low The INES records generally agree well with the NIO records, except for the maximum wave height Wave perıods agree we11 with each other except for the ospos records

In Figure 7 the smoothed energy density for a constant time interval of 18 seconds is plotted versus the frequency as obtained from the results of recordings with the INES wave recorder and NIO wave recorders on two different ships It will be noted from this plot that the frequencies for maximum spectral density lie close to each other, although there are noticeable differences in the values for maximum spectral density, with the INES wave recorder giving the lowest values

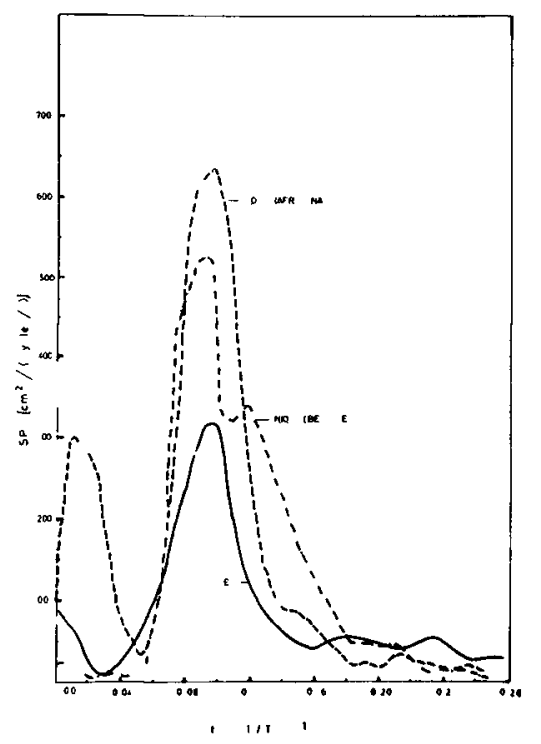

FIGURE 7 WAVE ENERGY SPECTRUM 


\section{AVAILABLE DATA}

Data on ocean wave characteristics for the Ocean Wave Research project have been collected using the above-mentioned wave recording instruments since February, 1967 although wave data for certain areas have been collected since 1961 The number of recording stations has increased with the passage of time and the present situation is as shown in Figure 1

The following instruments are in use

11 Wave clinometers

5 NIO shipborne wave recorders

2 INES wave recorders

1 Wave rider system

1 Boersma wave height meter

1 Ospos wave recorder

The amount of data aval lable from each instrument in its particular area or position is shown in Table 1 and Figure $8^{4,5,6,7}$

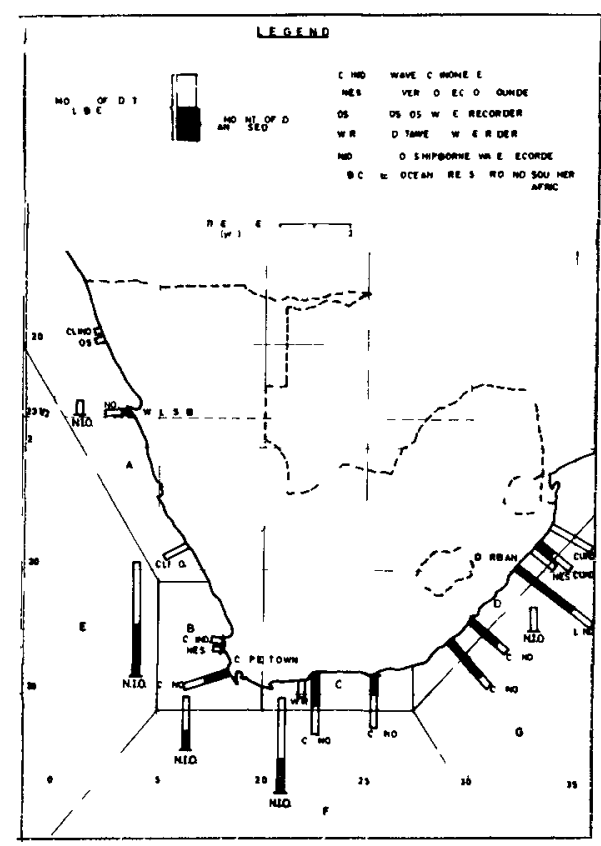

FIGURE 8 AVAILABLE WAVE DATA, MAY, 1970 


\section{WAVE ANALYSIS}

The distance of approximately $300 \mathrm{~km}$ between wave recording stations, was assumed to be adequate to give a good overall coverage of the actual sea conditions along the coast of Southern Africa Since, however, wave data are obtalned from both shallow and deep water, they have to be converted to deep sea values to obtain a basis for comparison This conversion is made using conventional refraction theories The method used for the construction of the wave refraction diagrams by computer, is that described by Wilson of the U S Army Coastal Engineering Research Center ${ }^{8,9}$ The correction of all wave directions to deep sea values is made by use of graphs on which deep sea wave direction versus nearshore direction at the recording point (found from the above refraction diagrams) are plotted

The method used for the conversion of nearshore wave heights to deep sea values, is that developed by Dorrestein ${ }^{10}$ whereby the wave direction graphs referred to above are used to obtain the height correction factors This method was found to be reliable and much quicker than the conventional method using pairs of orthogonals In certain cases a correction for shoaling water also has to be made ${ }^{11}$ Wave clinometer records

Wave parameters obtained from the clinometer records are as follows

(a) significant wave height $\left(\mathrm{H}_{\mathrm{s}}\right)$

(b) significant wave period $\left(\mathrm{T}_{\mathrm{s}}\right)$ or zero crossing wave period $\left(\mathrm{T}_{\mathrm{z}}\right)$

(c) wave direction

The clinometer records are converted to data applicable to deep sea by using the above-mentioned methods for the correction of the wave heights and directions This entalls the production by computer of refraction diagrams for each recording station using the most recent bathymetric chart The wave clinometer records are obtained directly on hand-punched computer cards to facilitate the analysis procedure The actual deep sea corrections, statistical analyses and tabulation of the data are now a11 done by computer

\section{Continuous wave records}

The wave records obtained from the INES, wave rider, NIO and Ospos 
instruments a11 lend themselves to the same type of analysis, although the records of the Ospos, which gives pressure fluctuations, first have to be converted to water level fluctuations A computer programme is avallable to obtain the following wave characteristics from these records

(a) cumulative wave height $(\mathrm{H})^{x}$ distribution

(b) cumulative zero crossing wave period $\left(\mathrm{T}_{2}\right)^{\mathrm{x}}$ distribution

(c) cumulative crest perıod $\left(\mathrm{T}_{\mathrm{c}}\right)$ distribution

(d) characteristic wave heights $\mathrm{H}_{50}, \mathrm{H}_{13}$, or $\mathrm{H}_{\mathrm{s}}, \mathrm{H}_{1}$ and $\mathrm{H}_{\max }$

(e) characteristic zero crossing wave periods $\left(\mathrm{T}_{\mathrm{z}}\right)_{50},\left(\mathrm{~T}_{\mathrm{z}}\right)_{13}$ or $\mathrm{T}_{\mathrm{s}}$ and $\left(\mathrm{T}_{\mathrm{z}}\right)_{1}$

(f) characteristic crest period ( $\left.\mathrm{T}_{\mathrm{c}}\right)_{50}$

(g) the period of the maximum wave in the sample ( $\mathrm{T}_{\mathrm{Hmax}}$ )

To reduce the cost of analysis by computer a simplified programme will be prepared to give the following information

(a) wave helghts $\mathrm{H}_{90}, \mathrm{H}_{50}, \mathrm{H}_{13}, \mathrm{H}_{10}, \mathrm{H}_{1}$ and $\mathrm{H}_{\max }$

(b) zero crossing wave periods $\left.\left(\mathrm{T}_{\mathrm{z}}\right)_{50},\left(\mathrm{~T}_{\mathrm{z}}\right)_{13}, \mathrm{~T}_{\mathrm{z}}\right)_{10}$ and $\left(\mathrm{T}_{\mathrm{z}}\right)_{1}$

(c) wave period of the maximum wave height viz $\mathrm{T}_{\mathrm{Hmax}}$

(d) wave crest period $\left(\mathrm{T}_{\mathrm{c}}\right)_{50}$

The wave rider system already produces a punch tape output, but the records from the other instruments are in graphical form and must be converted to punch tape on the D-Mac pencil follower before computer analysis is possible

Statistical analysis and presentation of data

The deep sea wave characteristıcs are analysed to yield seasonal and yearly directional distributions An example of the distribution of significant wave helghts is shown in Figure 9 Standard forms of presentation are also used for cumulative distributions and exceedance curves The standards used and methods of presentation are shown in Figure 10

\section{COMPARISON OF WAVE DATA}

Cumulative distributions of wave direction, height and period for the various recording stations are plotted in Figures 11, 12 and 13

* For definition of terms, see Appendix 

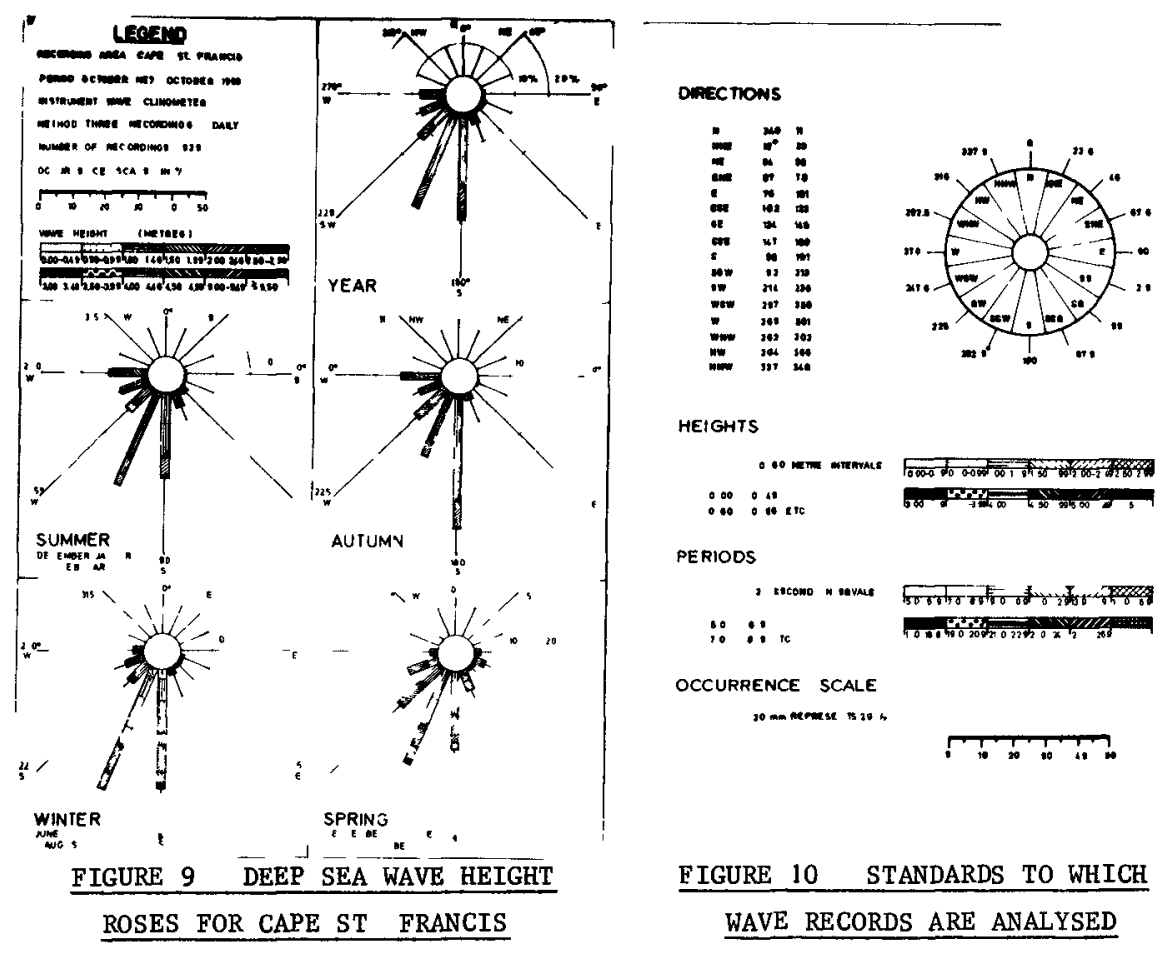

HE:GHTS

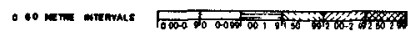

$$
\begin{aligned}
& 000: 01 \text { ar }
\end{aligned}
$$

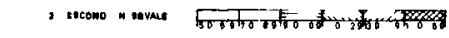

$$
\begin{aligned}
& \because \because: \text { rc }
\end{aligned}
$$

OCCURRENCE SCALE

30 momeremse is an $n$

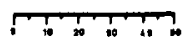

E IGURE 10 STANDARDS TO WHICH

WAVE RECORDS ARE ANALYSED

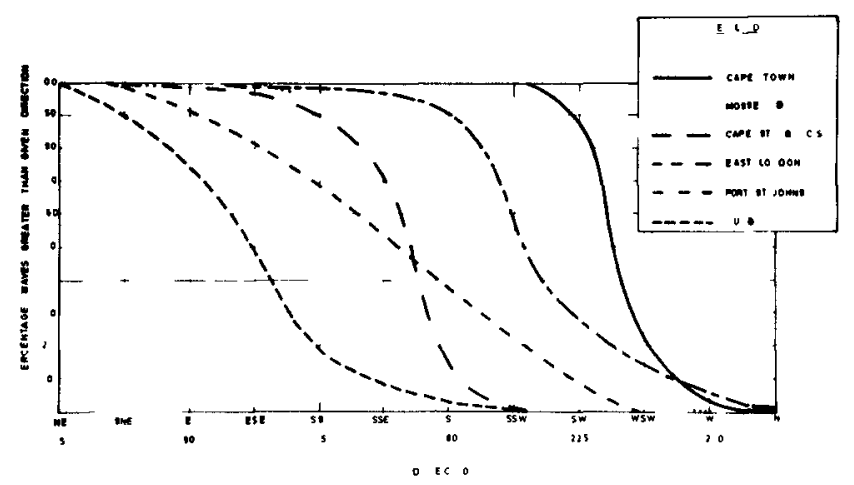

FIGURE 11 COMPARISONS OF WAVE DIRECTIONS CAPE TOWN TO DURBAN 


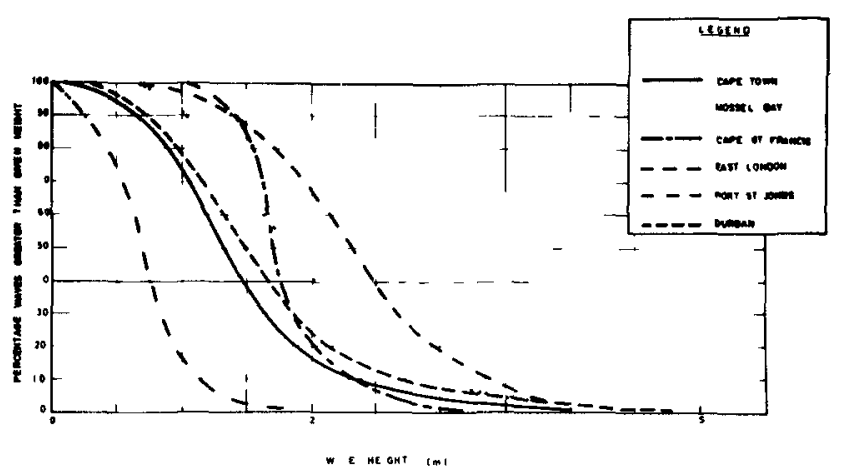

FIGURE 12 COMPARISONS OF WAVE HEIGHTS CAPE TOWN TO DURBAN

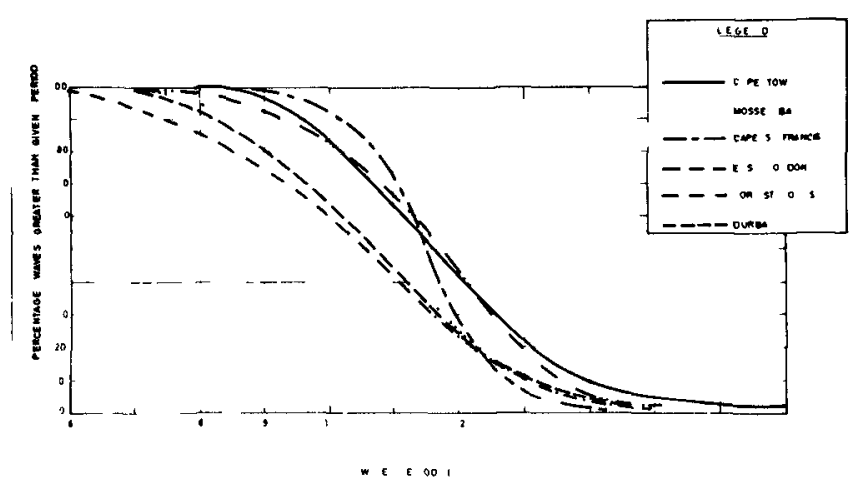

FIGURE 13 COMPARISONS OF WAVE PERIODS CAPE TOWN TO DURBAN

These figures show that there is an overall swing in the directions of wave approach from SW towards SE around the coast from Cape Town to Durban There is no obvious trend in the variation of wave helght along the coast, except that at East London and Port St Johns the waves are significantly higher and lower respectively than at the other stations The wave period distributions indicate that there are two distinct areas, namely East London, Mossel Bay and Durban with an average median wave period of $107 \mathrm{~s}$ and Port St Johns, Cape St Francis and Cape Town with an average median wave period of $116 \mathrm{~s}$ From these data the distance between stations of $300 \mathrm{~km}$ so far 
appears to be satisfactory More data will have to be obtained from some of the stations, however, before final conclusions on the sultabi11ty of this distance can be drawn

\section{CONCLUSIONS}

Since the commencement of the Ocean Wave Research project in 1967, data on wave conditions along the east and south coasts of Southern Africa have been systematically compiled The first data on wave conditions along the west coast will become avallable early in 1971 Already the project has provided the basic design data for various harbour and other coastal works, as well as for the present oll prospecting operations on the continental shelf

\section{ACKNOWLEDGEMENTS}

The energy spectra included in this paper were determined by $\mathrm{J}$ Forrer and $L$ Langenegger Their assistance is acknowledged with thanks

\section{REFERENCES}

1 TUCKER, M J T A shipborne wave recorder Proc of the Ist Conference on Coastal Engineering Instruments Council of Wave Research The Engineering Foundation, 1956

2 HARPER, A Correlations between various ocean wave recording instruments Oceanography in South Africa Symposium, Durban, 1970

3 BLACKMAN, R B , TUKEY, J W The measurement of power spectra Dover Publications, Inc, New York

4 Ocean Wave Research Report No 1 Wave and wind conditions for the Natal and Western Cape coastal areas CSIR Report MEG 665, Pretor1a, Apr11, 1968

5 Ocean Wave Research Report No 2 Handleiding vir golfvoorspe1lings aan die Suld-Afrikaanse kus WNNR Vers lag MEG 736, Pretorıa, Januarıe 1969

6 Ocean Wave Research Report No 3 Collection and analysis of ocean wave data South African practice CSIR Report MEG 741, Pretorı, January, 1969

7 Ocean Wave Research Report No 4 Wave and wind conditions for the Cape St Francis and Mosse1 Bay coastal areas CSIR Report MEG 804, Stellenbosch, 1969

8 WILSON, W S Development of a method for numerical calculation of wave refraction CERC, Tech Memo No 6, Washington, 1964 
9 WILSON, W S A method for calculating and plotting surface wave rays CERC, Tech Memo No 17, Washington, 1966

10 DORRESTEIN, $R$ Simplified method of determining refraction coefficients Jour Geophys Res Vo1 65, 1960

11 GROEN, $P$ Zeegolven Konink11jk Nederlands Meteorologish Instituut No 111 'S Gravenhage, 1949

\section{APPENDIX}

\section{GLOSSARY OF TERMS}

$\mathrm{H}$ - wave helght the vertical distance between a wave crest and the preceding wave trough

$\mathrm{T}_{z} \quad$ - the average wave period obtained by dividing the duration of the entire wave record (in secs) by half the number of times the actual water profile crosses the mean water level

$\mathrm{T}_{\mathrm{c}} \quad$ - the mean crest period obtained by dividing the duration of the entire record (in secs) by the number of crests in the record

$\mathrm{H}_{\mathrm{S}} \quad$ - the average height of the highest one third of the waves occurring in a specified recording period

$T_{s} \quad-$ the average period of the highest one third of the waves occurring in a specified recording perıod

$\mathrm{H}_{\max } \quad-$ the greatest vertical helght from crest to preceding trough in the record

$\mathrm{T}_{\text {Hmax }} \quad$ the period of $\mathrm{H}_{\max }$

$\mathrm{H}_{50}-50$ per cent of the waves occurring in a specific recording period are higher than $\mathrm{H}_{50}$

$\mathrm{H}_{135}-135$ per cent of the waves are higher than $\mathrm{H}_{13}{ }_{5}\left(\mathrm{H}_{135}=\right.$ $\mathrm{H}_{\mathrm{S}}$ when distribution is Raleigh) 
30

COASTAL ENGINEERING
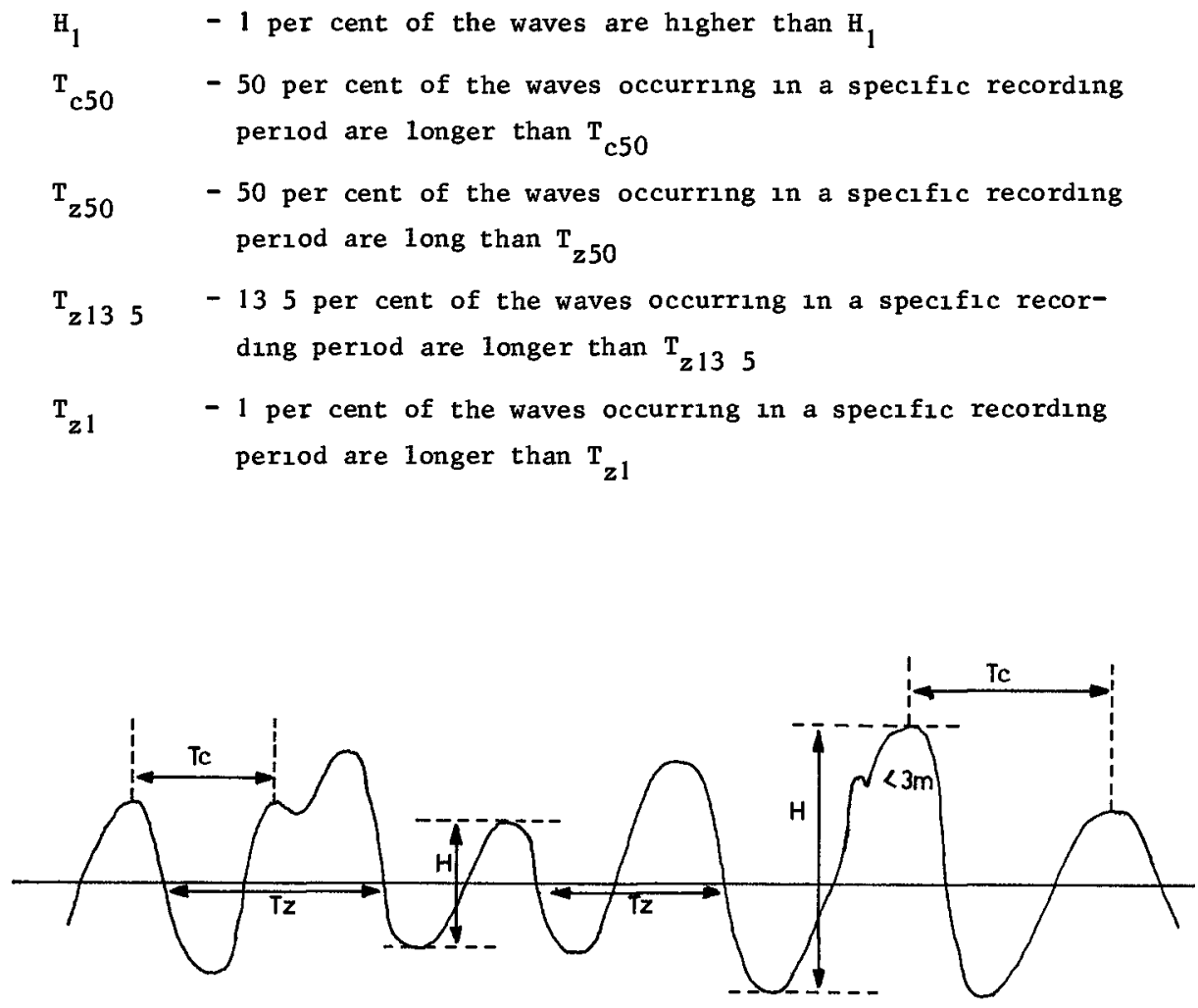


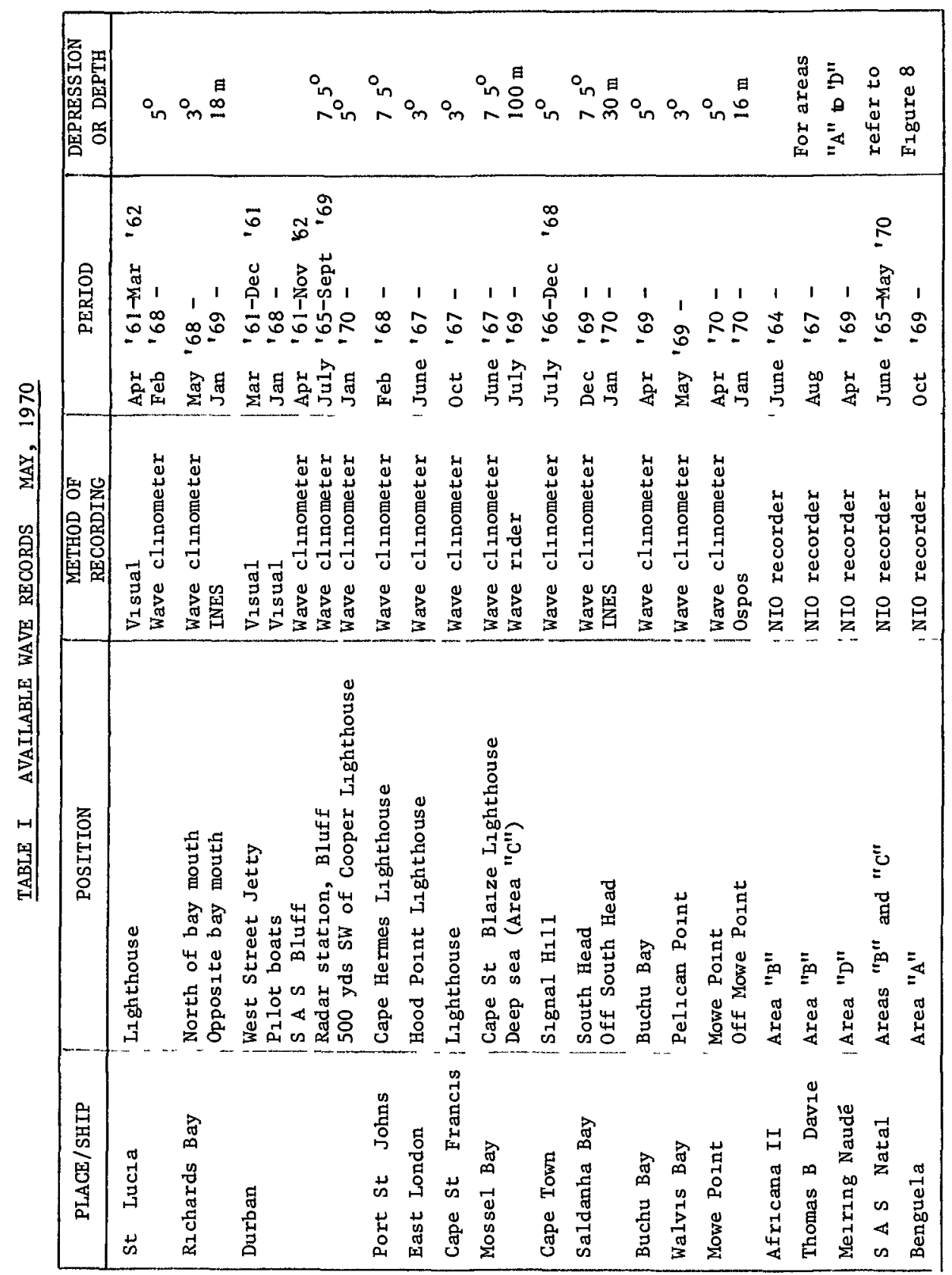





\title{
CHAPTER 3
}

\author{
SWELL AND STORM CHARACTERISTICS \\ FROM COASTAL WAVE RECORDS
}

Warren C Thompson, Professor of Oceanography

Nava1 Postgraduate Schoo1, Monterey, California

\section{ABSTRACT}

Manua 1 analysis of strip-chart records from a conventional wave sensor at Monterey, Californıa was found to yleld a linear frequency shift associated with each arriving swell train, from which the or igin time and travel distance of the swe 11 could be computed The use of surface weather maps allows identification of the source, and thereby yle lds an accurate determination of the deep-water arrival direction of the swe 11 for usc on the local coast The wave records are analyzed for the frequency of the individual waves composing wave groups, $\mathrm{f}_{\mathrm{g}}$, consistency of the results obtained Indicates that $f_{g}$ is equivalent to the frequency of maximum energy density $f_{\text {max }}$, obtained by spectral analysis Five swell sets studied were found to originate in North Pacific storms advancing toward Monterey The seas in the fetch were fully arisen at the time of computed swe 11 or $1 \mathrm{gin}$, and the surface to geostrophic wind ratio was 083 The dominant swell emerged from the fetch at a time when its group velocity equalled the velocity of the fetch towa rd Monterey

\section{INTRODUCTION}

Barber and Ursell in 1948 published non-directional power spectra of ocean waves recorded on the coast of Cornwall, and showed that the spectra 1 peak of swell arriving from a distant source shifts continuously toward higher frequencies, or short periods, with time This shift is the result of dispersal of the broad band of frequency components generated in a sea that takes place during the propagalion of the waves to a distant station, the energy in each component being transmitted at a velocity proportional to the frequency of the component, 1 e, at group velocity Barber and Ursell used the rate of increase in the frequency of the observed energy peaks to estimate the distance and time of swell origin, and identified swell sources at distances of 1200,2800 , and $6000 \mathrm{ml}$ les from the coastal wave station

Subsequently, Munk, et al (1963) and Snodgrass, et al (1966) conducted notable experiments in wave measurement and analysis in the Pacific basin In these studies, waves recorded dally and semi-dally at selected stations over several months were analyzed to determine the ir energy spectra, $E(f, t)$, as functions of frequency and $t$ ime The spectra were used to construct frequency-time $(f-t)$ graphs on which were drawn contours of energy density The resulting topography revealed pronounced linear ridges of high energy slanting across the grafh wuch represent the arrival of swell trains generated by individual storm located in the South Pacific and South Indian Oceans at distances up to about 10,000 nautical miles (antipodal circumference 
of the earth) The distance from the storm to the station, and the origin time of the swell, were derived from the slope of the ridge line and from Its intercept with the zero-frequency ordinate, respectively

As part of the study by Munk, Snodgrass, and colleagues, wave records from multiple-sensor arrays located on the continental shelf off San Clemente Island, Californid, and Honolulu, Hawalı, were analyzed for their direction spectra, $E(f, \theta, t)$, from which the directions to the swell sources, corrected for refraction, were determined Comparison of the origin of swell trains computed from the wave data with the location of storms on weather charts gave good agreement

The present paper introduces a non-spectral method of determinıng the frequency-time distribution of a swell train from coastal wave records The f-t data thus obtained may be used to derive the source distance and origin time of the swell in the manner referred to above This information, in turn, when used with synoptic weather charts permits identification of the storm, and provides a simple, accurate means of deriving the swe 11 arrival direction in deep water off the recording station The wave frequency is determaned manually from wave groups appearing in strip-chart records Evidence is offered to the effect that the frequency derived from wave groups, $f_{g}$, is equivalent to the frequency of maximum energy concentration, $f_{\text {max }}$, that is obtained from spectral analysis

This study also probed into the conditions prevaling in the storm, and provided information concerning the stage in the storm history, and the state of development of the sea in the fetch, when the observed swe 11 was produced In addition, information was obtained on the relationship of the surface wind to the geostrophic wind prevarling in the fetch at the time of computed swell origin by an indirect means in which the surface wind was inferred from the frequency peak of the recorded swell train

\section{SYNOPTIC WAVE TRAINS}

The wave records used in this study covered a period of 4-1/2 months in February-March 1967, November 1967, and March 1969, and were obtained using a conventional pressure sensor (Snodgrass Mark IX mode1) located in shallow water in southern Monterey Bay, Californıa (Figure 1) The records were analyzed for wave height and period by procedures described in the next section

Time plots of these parameters reveal an endless sequence of distinctive patterns representing the arrival of individual wave trains, each generated by a synoptic weather event occurring locally or somewhere in the Pacific ocean These synoptic wave trains sometimes arrive singly, and at other times two or more occur simultaneously Nearly al1 swel1 trains recorded by the Monterey gauge have been found to originate in the North Pacific Ocean Southern Hemisphere swe11, which are observed occasionally on the adjacent open coast, experience extreme refractive divergence in reaching the Monterey gauge and are seldom detected 
Because of the sheltered location of the wave gauge, most of the 4-1/2 month period was marked by the arrival of swell trains, but seas generated in several local storms, and occasional low wind waves, were also recorded In addition, there were several long quiet intervals of up to 12 days duration during which the wave height did not exceed 05 foot, and much of the time fell in the range from 01 to 03 foot

Swell trains from distant storms are typified in a time plot bv a narrow band of periods which decrease continuously with time The swell helght begins to increase about the time the first periods appear, reaches a peak, and eventually diminishes beyond detection This characteristic height-period pattern is 1 llustrated in Figure 3 , in which may be seen three swell trains which arrived successively at Monterey during otherwise quiet sea conditions

The initial period recorded among 13 swell trains studied ranged from 145 to 213 seconds, with the exception of the swe11 event of 22-28 March 1967 in which an initial period of 275 seconds was followed by a number of measurements above 20 seconds In most swe11 trains, the period distribution terminates at a fairly well-defined cutoff in the range of 10 to 14 seconds, which reflects tho submergence of the waning swell train below the rising energy leve 1 of is $w$ waves

During those extended intervals when the wave height was half a foot or under, the periods were broad band and random much of the time, possibly reflecting the arrival of diffusely scattered wave energy on several occasions, however, the period data clearly revealed the presence of a swe 11 train when no trace of it was visible in the wave-height plot The wave period is thus seen to be a sensitive indicator of swell even when the energy leve 1 is below that of the background

Waves from coastal storms, as seen in a time-series graph 11ke that of Figure 3 , are revealed by very short initial periods which increase with time and by a wide period scatter, characteristics which are representative of the broad spectrum in a sea Periods decrease following passage of the wave peak and display a more limited scatter, which is consistent with the dispersive nature of young swell trailing in after the storm Local seas, such as are caused by the commonly occurring sea breeze, produce a random scatter of periods ranging downward from about 6 seconds No further attention will be directed to local wind waves or young swell in this paper

\section{ANALYSIS OF WAVE RECORDS}

\section{Wave Period}

The wave data used in this study were recorded on Esterline-Angus strip-chart records, and were analyzed manually The recorder was programmed to write a 5-minute fast trace ( 3 inches per minute) once every hour and a slow trace (3 inches per hour) the remainder of the time, from which, respectively, wave periods and wave heights were obtained 
As may readily be seen in a wave record, ocean waves characteristically display at intervals discrete wave groups composed of a variable number of more or less periodic waves The average period of the individual waves contained in a wave group, $T_{g}$, is the period parameter used in this study $T_{g}$ may be determined from a wave record by measuring the time interval, $\Delta t$, for the passage of a selected number of waves, $n$, as 11 lustrated in the dlagram below The measurement is made between those equivalent parts of the wave form that the analyst judges to give the best measure in each case, e $g$, between selected crests, troughs, centers of wave mass, or centerline crossings

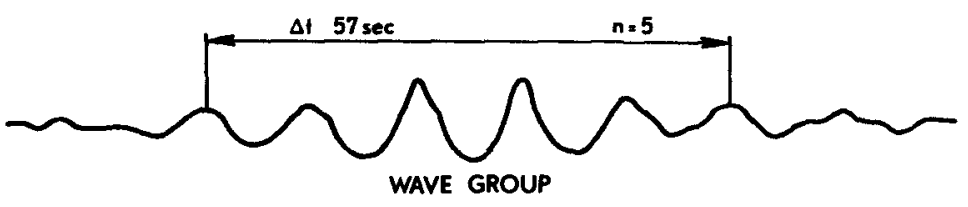

$T_{g} \frac{\Delta l}{n} 114 \mathrm{sec}$

At this point it is desirable to inquire into the physical significance of $\mathrm{T}_{\mathrm{g}}$ since $1 \mathrm{t}$ forms the basis of this study Within the conceptual framework of a sea surface composed of harmonic components of various period, extension of the rules for waveform interference stated by Manley (1945) to an ocean-wave spectrum leads to the conclusion that the apparent or mean period of the waves forming a group, or beat, $T_{g}$, is equal to the period of that component present having the greatest energy density In the case of swe11, the component of peak energy density can be considered to be the value $\mathrm{T}_{\max }$, or its reciprocal $\mathrm{f}_{\max }$, that would be obtalned from spectral analysis of the wave records As stated above, the frequency of the energy peak, $f_{\text {max }}$, has been used with good success for calculating the origin time and trave 1 distance of swell The wave records analyzed in this study have not been subjected to spectral analysis for direct proof of the equivalence of $\mathrm{T}_{\mathrm{g}}$ and $\mathrm{T}_{\max }$, however, the writer believes that the consistency of the results obtained using $\mathrm{T}_{g}$ in examination of the wave-generating conditions at the swe11 source, which are described below, can have been achieved on $1 y$ if this condition were met

With regard to practical considerations in the analysis of wave records, the wave-group method of determining the period has the important advantage over the conventional significant-period $\left(T_{s}\right)$ procedure of quick application It also yields a number of values of $\mathrm{T}_{\mathrm{g}}$ in $\mathrm{S}$ the 20-minute or so length of wave record needed to obtain $T_{S}$ In addition, two or three wave groups in 
superposition are frequently observed in the wave records, and the wave-group method has the further important advantage of providing the periods of each A time-series graph of wave data shows the simultaneously occurring periods to be associated with independent wave trains from different sources, and thus provides additional insight into the synoptic nature of the wave trains present For best results in the application of the wave-group method, the judgment of an analyst having a basic understanding of wave-interference phenomena is required The task of discriminating individual wave groups and measuring $\mathrm{T}_{\mathrm{g}}$ remains for the present beyond the capability of machine programming

In order to assure the quality of the period measurements in this study, care was taken to avold phase shifts and also the small secondary swell waves that occasionally appear in a wave group, and to account for occasional missing waves in a sequence Not infrequently during quiet intervals the waveform assumed a variety of highly irregular shapes In spite of their bumpy appearance these waves often occurred in a regular sequence, and the values of $\mathrm{T}_{\mathrm{g}}$ obtained were found to be consistent with the periods derived from sets of we11-defined waves

For the interval covered by this study, the value of $\mathrm{T}_{\mathrm{g}}$ derived from every swell group Identıfied in the wave records was measured and graphed Within the limits of Figure 3, for example, no data have been omitted, and all waves, whether sea or swe11, are represented The occurrence of obviously spurıous perıod values was found to be quite low

The period data reveal variable amounts of scatter when graphed as a time series, as may be seen in Figure 3 The scatter is attributed to the spectral bandwidth of the waves, and to subjectivity inherent in the selection and measurement of the wave groups In regard to the latter, the precision of period measurements is estimated to be about 02 second for a group consisting of 5 waves, and 01 second or less for groups of 10 or more waves An ldea of the overall precision can be obtained from the observation that out of 582 wave groups (of 4 or more waves per group) composing a randomly selected month of data, $62 \%$ contalned 4 to 6 waves, and $9 \%$ had more than 10 waves The number of waves in a group was found to be independent of the period

\section{Wave He1ght}

Wave heights, determined to 01 foot, were derived from one-hour and three-hour samples of the slow-trace portions of the strip-chart record using a shortcut method developed for the purpose The method involves estimating visually the crest amplitude, $A_{67 \%}$, such that one-third of the wave envelope exceeded this amplitude The trough amplitude was estimated in the same way The crest and trough amplitudes, which differ because of wave assymmetry in shallow water, were summed to give $\mathrm{H}_{67 \%}$, and then converted into the significant wave height, $H_{S}$, using the empirical relationshıp $\mathrm{H}_{\mathrm{S}}=135 \mathrm{H}_{67 \%}$ obtained from the statistical helght parameters given 
by Plerson, Neumann, and James (1955) The method requires a strıp-chart recording made at a speed such as to compress the waves time-wise but not beyond the point at which individual crests and troughs can be distinguished

This slow-trace method of height analysis is a simple operation which involves no counting of waves It has the important advantage of speed over other manual technıques, and can effect a major savings of time when a number of analyses are made The method can be applied much more quickly, for example, than the analysis method that is based on the identification and measuremont of a single wave in a fast-trace record having a height that represents some percentage level in a statistical height distribution Another advantage of the slow-trace method is that the helght variability associated with wave groups and energy bursts which frequently are present in the records is averaged out by treating a long record This results in a wave-height time series that displays relatively little scatter, as is illustrated in Figure 3 Intervals of unusually high or low wave groups having durations of 10 or 15 minutes are not uncommon, and bias the heights derived from the conventional 20-minute fast-trace Random comparisons showed the heights derived from the slow-trace method and the conventional zero-upcrossing method to agree within $20 \%$ in most instances

Wave-height information is not needed for the purpose of obtaining the swell origin distance and trave 1 time from a wave record, although it is useful, when examined in conjunction with the wave period, for deducing the synoptic characteristics of arriving wave trains in a time-series graph The relative wave height is needed, however, for identification of the period of the peak waves in a swell train, $T_{p}$ The latter is used for probing into the state of development of the sea that produced the swell and for estimating the surface wind at the time of swell origin, as discussed below

\section{Shallow-Water Effects}

It is possible, in theory, for the frequency of maximum energy density in swell to shift across the spectrum as the waves travel from deep water to the sensor because of shoaling transformations A shift of this sort would alter the source distance and origin time computed for the swe11 It is also possible for the largest waves to appear at the sensor either earlier or later than in deep water for the same reason Advance or retardation of the swe11 peak would, in turn, result in erroneous values deduced for the surface wind speed in the fetch, which is discussed later Accordingly, an analysis was made to determine the magnitude of the shift in the wave peak with both frequency and time, and these changes were found to be negligible

An idea of the magnitudes of the shallow-water coefficients at the sensor site, and their variation with frequency, may be gained from Figure 2 The coefficients, as given by linear wave theory, are defined as follows

$$
\begin{array}{ll}
\text { Shoaling coefficient } & \mathrm{K}_{\mathrm{s}}=\left(\mathrm{C}_{\mathrm{o}} / 2 \mathrm{~V}\right)^{1 / 2} \\
\text { Refraction coefficient } & \mathrm{K}_{\mathrm{r}}=\left(\mathrm{d}_{\mathrm{o}} / \mathrm{d}\right)^{1 / 2} \\
\text { Hydrodynamic coefficient } & \mathrm{K}_{\mathrm{h}}=(\cosh \mathrm{kh})^{-1}
\end{array}
$$


where $\mathrm{C}=$ phase (wave) velocity, $\mathrm{V}=$ group velocity, $\mathrm{d}=$ wave-orthogonal separation, $k=$ wave number $(2 \pi / I), L=$ wave length,$h=$ mean sensor depth (24 feet), and subscript o refers to deep water Energy losses dut to bottom friction were considered negligible because of the relatively narrow, deep shelf offshore Accordingly, the energy density at the sensor, $E(f)$, relative to that in deep water, $E_{0}(f)$, for a given frequency component in an arriving wave train is given by

$$
E(f)=\left(K_{s} K_{r} K_{h}\right)^{2} E_{o}(f)
$$

The curve of $\mathrm{K}_{\mathrm{h}}$ applies on ly to Swe 11 Train 1 of 21-23 February 1967 (Figure 3) which arrived from $311^{\circ}$ The variation of $\left(\mathrm{K}_{s} \mathrm{~K}_{\mathrm{r}} \mathrm{K}_{\mathrm{h}}\right)$ with frequency is seen to be small over the range of wave periods observed (20 to 12 seconds) Curves of $\left(\mathrm{K}_{s} \mathrm{~K}_{\mathrm{r}} \mathrm{K}_{\mathrm{h}}\right)$ for North Paciflc swell arriving from other directions also display relatively small variation, but differ somewhat in magnitude

\section{SWELL ORTGIN AND DIRECTION}

\section{Determination of Swe 11 Source}

Using spectral analysis of wave records, Munk, et al, (1963) observed that a swel1 train arriving from a distant storm is revealed as a prominent ridge line in the spectral density topography on a frequency-time ( $f-t$ ) graph, and noted that ridge lines are linear or very nearly so ( $\mathrm{df} / \mathrm{dt}=$ constant) A straight ridge line (f-t line), in the context of linear wave theory, implies a point source for the swell in space-time, and provides a simple means for determining the apparent source distance and origin time Thus, if the energy associated with each frequency component, $f$, generated in the sea is propagated at group velocity, $V_{f}=g / 4 \pi f$, over distance $D$ from the source to the wave gauge in travel time $\Delta t=t-t_{0}$, measured from the origin time $t_{0}$, then

$$
V_{f}=g / 4 \pi f=D /\left(t-t_{0}\right)
$$

The slope of the $f-t$ line is then $d f / d t=g / 4 \pi D$ Accordingly, the apparent distance to the swe 11 source is given by

$$
\mathrm{D}=\frac{1515}{\mathrm{df} / \mathrm{dt}} \text { (naut mi), }
$$

where $f$ is in cycles/second and $t$ is in hours The apparent origin time is given by the intercept of the $f-t$ line with zero frequency

In the present study, the values of $\mathrm{T}_{\mathrm{g}}$ derived from the Monterey wave records were converted to $f_{g}$, and $f-t$ graphs were constructed Examples of three swell trains (from Figure 3) are shown in Figure 4 in which the linear character of the $f_{g}$ distribution with time may be seen From the 4-1/2 months of analyzed wave data, 13 swell trains were selected for study Straight-1ine fits to each set of $f_{g}$ data ylelded source distances ranging from 1515 to 4270 naut $\mathrm{m} 1$ 
Wave records from a single gauge provide no directional information for use in locating the coordinates of a fetch that produced a given set of swe 11 In fact, directional information at the Monterey gauge site would have little value for this purpose because refraction caused by extreme sheltering results in a nearly unidirectional approach for all swe 11

The storm responsible for a recorded swe 11 train was identified, however, using the computed origin time and distance combined with six-hourly synoptic sea-level pressure analyses produced by the Fleet Numerical Weather Central, Monterey In a 11 swe 11 cases analyzed, an arc of radius D drawn from Monterey on a weather chart of time $t_{0}$ was found to intersect a storm system A11 of

the swel1 sets studied were found to originate in eastward-moving cyclonic storms in the North Pacific Ocean The absence of southerly waves among the swell sets selected is probably due to extreme energy reduction by refraction in passing around the Monterey Peninsula, since Southern Hemisphere swe 11 is recorded on the adjacent open coast

In view of the fact that a storm system produces swell for the duration of Its passage across the ocean, it appears that the point source computed from the wave records represents that moment and location in the storm history at which the maximum energy density in the sea directed toward Monterey was generated

\section{Deep-Water D1rection}

The deep-water wave direction, $\psi_{0}$, is a parameter required for nearly all coastal englneering applications involving waves in shallow-water Direct measurement of this quantity is difficult and expensive as conventionally done because 1 t requires synchronous operation of closely spaced multiple wave sensors and complex data-analysis procedures In addition, the sensor array is placed on the bottom in shallow water, and shoaling and refraction transformations must be applied to the data in reverse in order to obtain the deep-water direction spectrum if that is desired For these reasons directional wave recording remains for the present in the realm of research and is not performed on an operational basis

With regard to swell, the procedure described in the previous section, by which the source is ldentified using coastal wave data from a single sensor in connection with weather charts, permits construction on the appropriate weather chart of a great-circle trajectory from the swell source to the coastal station, and thereby provides an accurate measure of $\psi_{0}$ at the station as we11 as for other locations on the adjacent coast Noting that the energy in a sea is proportional to the fourth (or fifth) power of the wind speed, the swe 11 trajectory can logically be considered to originate at that point in the fetch where the wind velocity directed toward the coastal station is a maximum The deep-water swe11 directions derived in this manner for five selected storms producing swe 11 recorded at Monterey are listed in Table 1 , and can be seen in relation to the fetch trajectories of each storm in Figure 5 As implied above, the deep-water wave direction is also the fetch azimuth from Monterey

The beamwidth, or angular intercept, of the fetch as viewed from a coastal station, is determined by both the fetch width and distance, and varies during 
the $11 \mathrm{fe}$ of a storm For the purposes of this study the fetch width was defined as that swath of the wind field directed toward Monterey within which the winds can generate $70 \%$ or more of the wave energy generated by the peak winds in the fetch By this definition, the fetch beamwidth at Monterey, which is also the directional bandwidth of the arriving swe 11 , was $3^{\circ}$ to $4^{\circ}$ at the time of computed swe 11 or 1 gin for the five storms studied (Table 1) These values appear to be typical for most swell trains arriving at codsta 1 stations from distant sources Accordingly, for practical engineering purposes, swell arriving on the Pacific Coast can be considered unidirectional, and to originate at the source computed

In the directional recording of Southern Hemisphere swe 11 at San clemente Is land, Calıfornia, and Honolılu, Hawaı1, Munk, et al (1963) and Snodgrass, et al (1966) found the computed swell source to lie pretty consistently to the left of the fetch location obtained from weather charts The authors suggested that the deviations were the result of local refraction uncertainties and insufficient accuracy in the relative positioning of the sensors Deflection of the swel1 direction by ocean-current systems also appears to be a possibility For North Pacific swell arriving on the Pacific Coast, oceancurrent deflection might be expected to amount to several degrees from the great-circle swe 11 trajectory

Long-Range Triangulation

The results of this study indicate that the source of a swe11 train can be identified with certainty in most instances using the wave date from a single sensor coupled with weather charts, but occasionally more than one storm is present simultaneously at the computed origin distance This was the case with Storm 3 shown in Figure 5 (Braunstein, 1970)

It appears that positive identification can be obtained consistent ly and automatically by long-range triangulation using the swe 11 trave 1 distances derived from two sensors placed some miles apart along the coast The feasibility of this was tested using wave sensors at Monterey and Bolinas, California, separated by 90 naut $\mathrm{m} 1$, for the swel1 train of 18-20 March 1969 The two stations happened to be nearly in line with the swell direction in this case, and the computed origin times and distances yielded an almost identical conmon source

It is probable that location of swe 11 sources by long-range triangulation w111 not give direction resolution that is as good as can be obtained using weather charts, however, the precision of deep-water swe11 directions obtained by this means may prove to be fully adequate for practical engineering purposes

\section{GENERATING CONDITIONS IN THE STORM}

\section{Swe 11 Origin in Relation to Storm History}

With the objective of determining the relationship between the computed swe11 source and the history of development of the storm, five swel1 trains recorded at Monterey were selected and the storms that produced them were intensively studied by Braunstein (1970) and the author through the use of 
FNWC s1x-hourly synoptic weather charts and storms are presented in Table 1

Using objective methods devised for the purpose, Braunstein measured from successive weather maps the dimensions of the effective generating area and its azlmuth and distance from Monterey From this information, distancetime (d-t) graphs were prepared for the five storms throughout their development A d-t graph for the fetch area that produced the swe 11 train of 21-23 February 1967, shown in Figure 3, is presented in Figure 6 The point of maximum wind in the fetch is shown by the dashed 11ne, and the front and rear limits of the fetch are indicated by the solid lines the speed of advance of the fetch with respect to Monterey is given by the slope of the curves The wind area is observed to have travelled rapidly in its early stages but s lowed perceptıbly about $00 \mathrm{z}$ on 17 February

The movement of the wind field may also be seen in Figure 5, which shows the fetch tracks for the five storms The dots mark six-hourly positions of the point of maximum winds directed toward Monterey, and the small circle indicates the computed swel1 origin time The fetch trajectories labelled 1,2 , and 3 are associated with the storms that produced the three successive swel1 trains of Figures 3 and 4 The fetch tracks should not be confused with the trajectories of the storm centers, which are not shown

The computed swe11 source derlved from the Monterey wave records may be seen in Figure 6 to lie within the fetch observed from the weather maps Also plotted are the lowermost, uppermost, and peak period components identified in the swel1 The lines emanate from the swell source and arrive at the gauge $(\mathrm{D}=0$ naut $\mathrm{ml}$ ) at the times indicated The group velocity of each component is given by the slope of Its line It may thus be seen that the peak swe 11 component observed was generated when its group velocity coincided with the speed at whIch the fetch approached Monterey

The histories of all five storms studied were found to be simllar They were characterized during their early phase of development by a high fetch speed, followed by reduced speed as the storm matured The swe 11 source was located within the fetch envelopes in four cases, and near the fetch margin in the other The swell was found to emerge when the fetch velocity and the group velocity of the peak swe 11 component generated were approximately coincident It is significant that the peak geostrophic wind velocity in the $f_{1}$ ve storms occurred from 20 hours earlier to 33 hours later than the computed swell origin time, and that it exceeded the geostrophic wind observed at the time of computed swel1 origin by as much as 33 knots, thus indicating that the $t$ ime of swell or 1 gin is largely independent of the time of occurrence of the maximum wind speed in the type of synoptic situation dealt with, 1 e, swe 11 from an approaching storm The requirement for generating the maximum energy in the sea appears to be equivalence of the fetch velocity with the group velocity of the lowest frequency components with significant energy that can be generated by the surface wind present at the moment

\section{Fully Arisen Sea and the $V_{s} / \mathrm{V}$ Ratio}

The condition in which the fetch travels at the velocity of the lower frequency components generated permits those components to remain under the 
influence of the generating winds for a long effective duration over a long effective fetch, thus allowing maximum energy bulldup If it is assumed that North Pacific storms ordinarily permit the development of fully arisen seas directed toward Monterey, and if it is further assumed that the peak period generated is conserved during its propagation to Monterey, then the peak swell period observed, $T_{p}$, can be used to estimate the surface wind speed that generated the sea from sultable spectral models for the fully arisen sea Entering the Plerson-Moskowitz (1964) spectrum with the values of $T_{p}$ associated with the five selected storms yielded the surface winds listed in Tab le 1

Of particular interest in the forecasting of ocean waves from weather charts is the surface to geostrophic wind ratio Accordingly, the surface winds obtained in the manner described were used to calculate $\mathrm{V}_{\mathrm{s}} / \mathrm{V}_{\mathrm{g}}$ for each storm The geostrophic wınd used was that measured at the time of computed swell origin from the $12-\mathrm{mb}$ band in the strongest portion of the pressure gradient occurring along that storm radius lying normal to the surface wind direction toward Monterey The values obtained are listed in Table 1 and are illustrated 1n Figure 7

As may be seen in the figure, the surface to geostrophic wind ratio is in close agreement for all five storms Its mean value, 0 83, is in general accord with values quoted for the North Pacific, the North Atlantic, and the Greenland and Norweglan Seas (reviewed by Aagaard, 1969) The magnitude of the values obtained, along with their internal agreement, provide strong evidence to the effect that the seas producing the swell in all five storms were fully arisen or nearly so

In the case of a non-fully arisen sea, the peak swell period measured would yield a low value of $\mathrm{V}_{\mathrm{s}} / \mathrm{V}_{\mathrm{g}}$ that would lie on the left side of the curve in the figure The smaller value of $v_{s} / v_{g}$ for storm 4 (0 78) might indicate that the sea was not quite fully developed, or it might reflect greater aırmass stability typical of the fall of the year (November) The other four storms occurred in February and March when alrmass stability is normally less, and their average value is a little higher ( 085 )

The consuderation that perhaps most North Pacific storms may produce fully arisen seas is of potential importance with regard to forecasting swell on the west coast of North America

\section{APPLICATIONS AND FUTURE DEVELOPMENTS}

This study has demonstrated the fact that the source and deep-water direction of swell can be obtained by simple methods involving the manual analysis of coastal wave records from a conventional wave gauge and the use of ocean weather charts The procedures described are best sulted for use in economic situations and geographic regions where the availability of funds or technical expertise is limited, or computer facilities are not available, and a source of labor can be tapped 
Perhaps the most difficult problem in present-day swe 11 forecasting is making an accurate prediction of the time of arrival of the peak waves Based upon results obtained in this study, the author anticipates the development of a new method of swe 11 prediction in which forecast products will include the rate of change of swell frequency with time, and the arrival time and frequency of the peak waves

On coasts subject to costly shoreline damage by heavy swell, such as occasionally occurs on the California coast due to Southerly Swell, there is increasing need for an integrated program of forecasting and monitoring wave conditions The author foresees the development of a swe 11 warning system consisting of a network of coastal wave sensors from which recorded wave data $w_{1} 11$ be transmitted automatically to an analysis center for continuous machine processing storm systems will be Identified and tracked by long-range triangulation The deep-water swel1 characteristics along the coast w1 11 be obtained by forecasting and by swe 11 analysis for use in estimating the wave conditions expected on local beaches

\section{ACKNOWLEDGMENTS}

Th1s work was supported by the office of Naval Research Foundation Grant to the Naval Postgraduate School, and by the Fleet Numerical Weather Centra1, Monterey

\section{REFERENCES}

Aagaard, K, 1969 Relationsh1p between Geostrophic and Surface Winds at Weather Ship M J Geoph Res, 74, 13, 3440-3442

Barber, B F , and F Urse11, 1948 The Generation and Propagation of Ocean Waves and Swe11 Phl1 Trans Roy Soc, A, 240, 527-560

Braunstein, $W \mathfrak{J}, 1970$ Origin of Swe11 Recorded at Monterey, California M S Thesis, Naval Postgrad Sch, Monterey

Manley, R G , 1945 Waveform Analysis Wiley \& Sons, N Y , 275 pp

Munk, W H , G R Mlller, F E Snodgrass, and N F Barber, 1963

Directional Recording of Swell from Distant Storms Phil Trans Roy Soc, A, 255, 505-584

P1erson, W J, G Neumann, and R W James, 1955 Practical Methods for Forecasting Ocean Waves H 0 Pub 603,284 pp

Pierson, W J , and $\mathrm{L}$ Moskowitz, 1964 A Proposed Spectral Form for Fully Developed Wind Seas Based on the S1m1larity Theory of S A K1taigorodski1

$\mathrm{J}$ Geophys Res , 69, 24, 5181-5190 


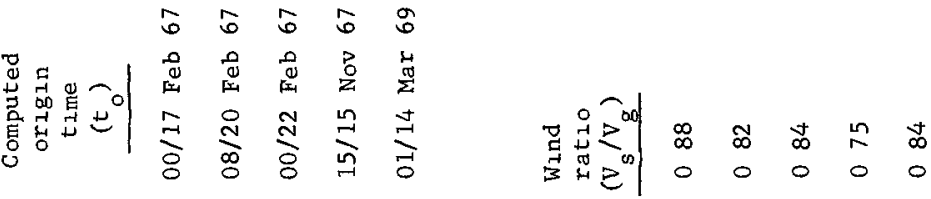

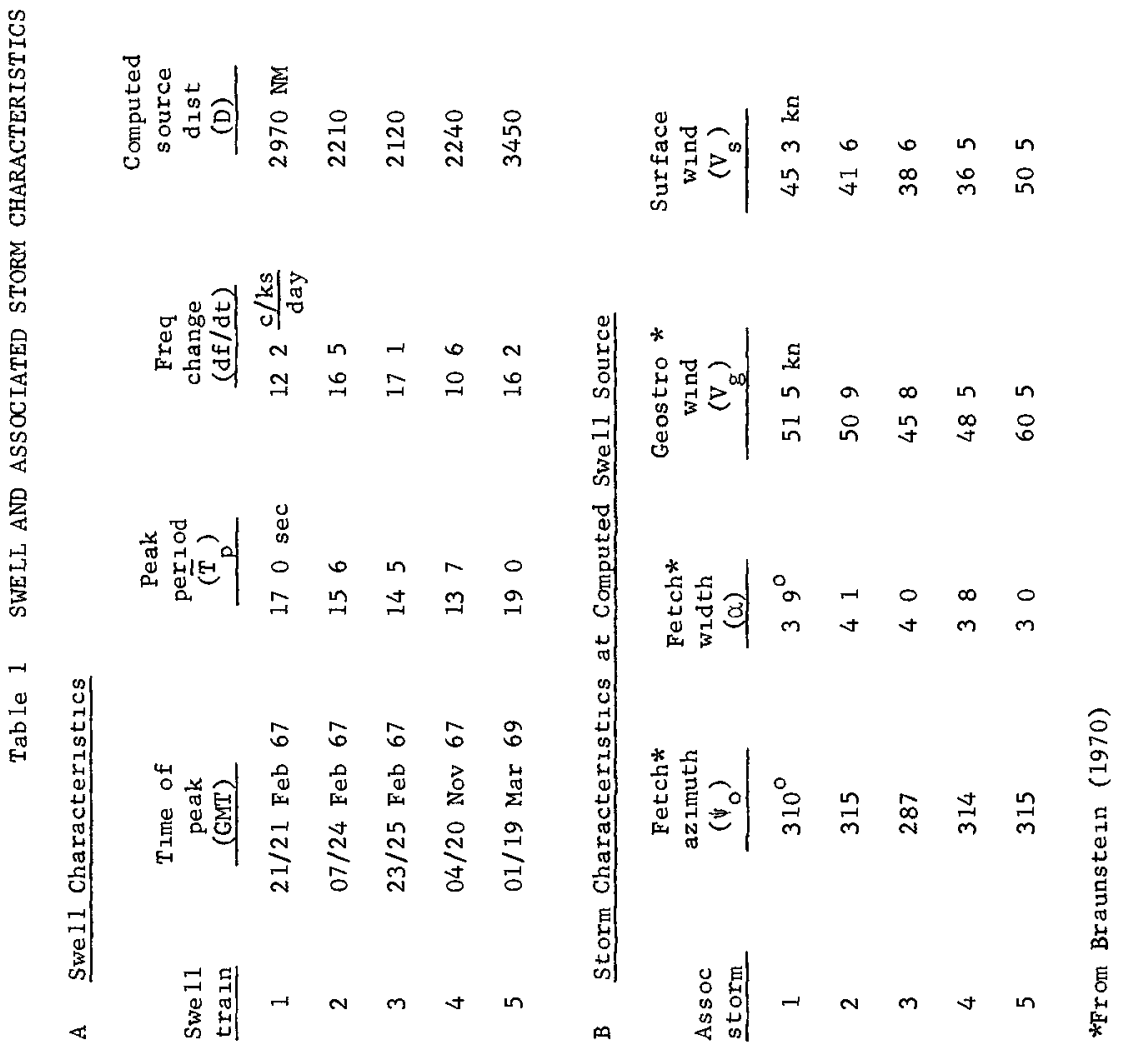




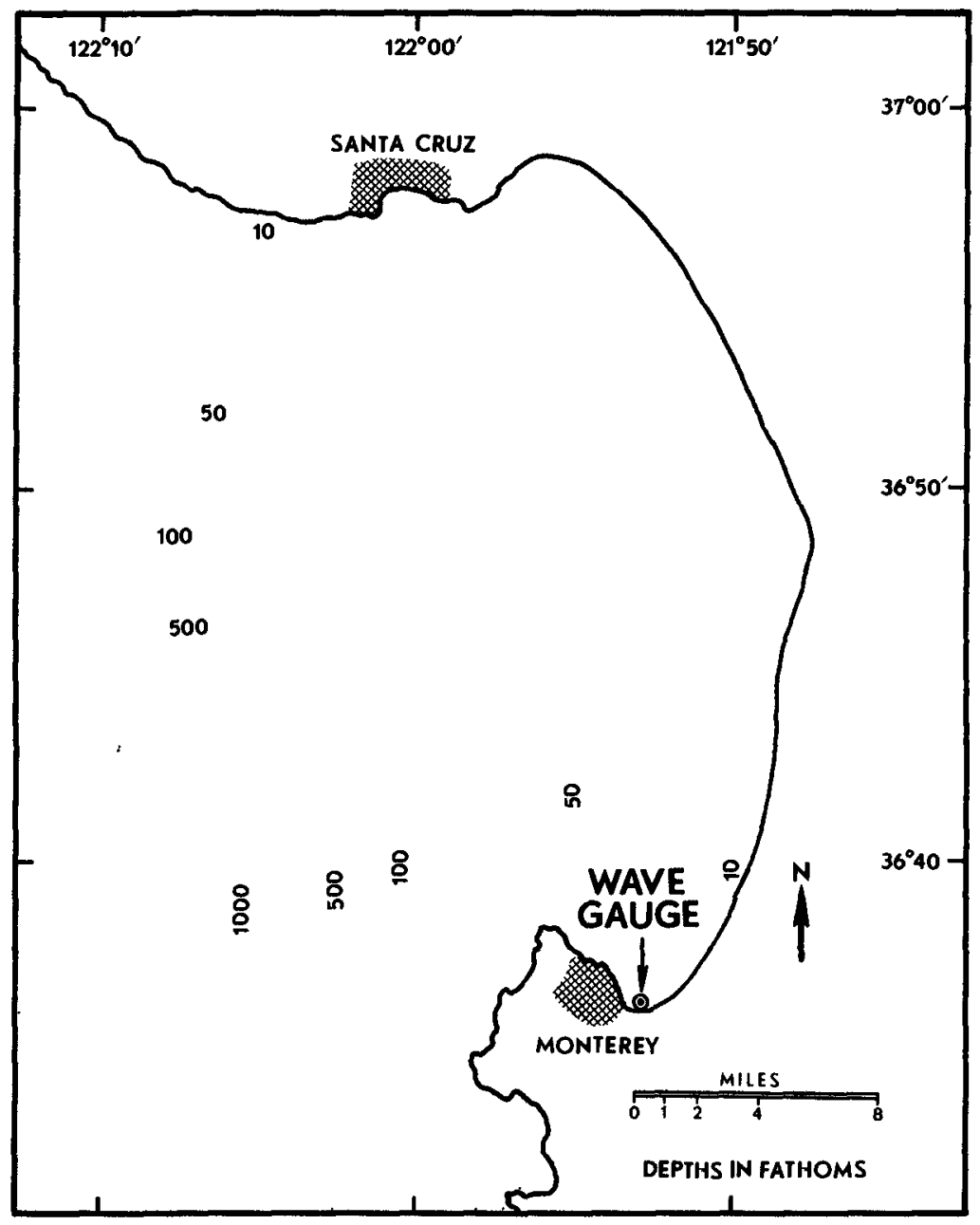

Figure I WAVE-GAUGE LOCATION IN MONTEREY BAY 


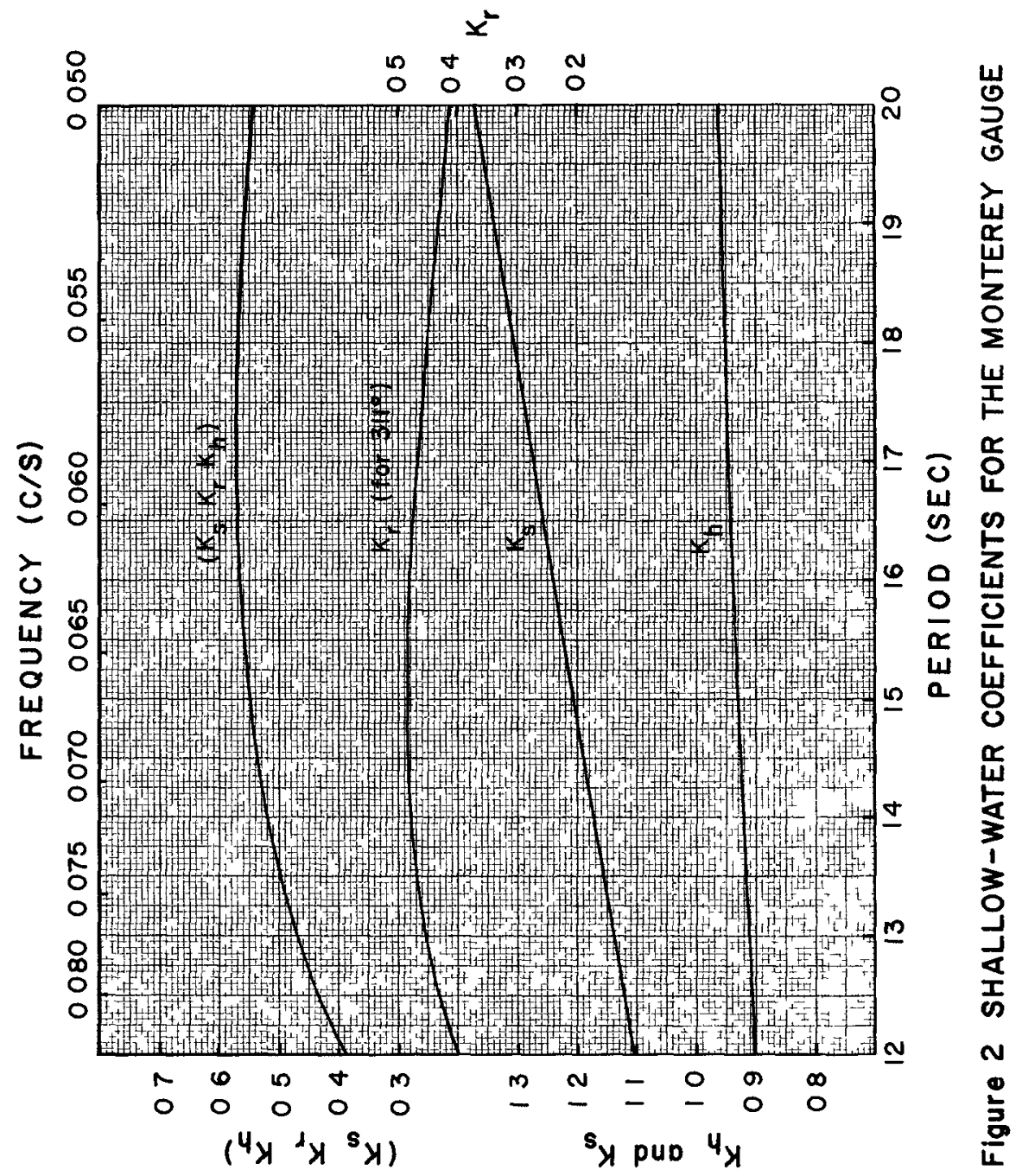




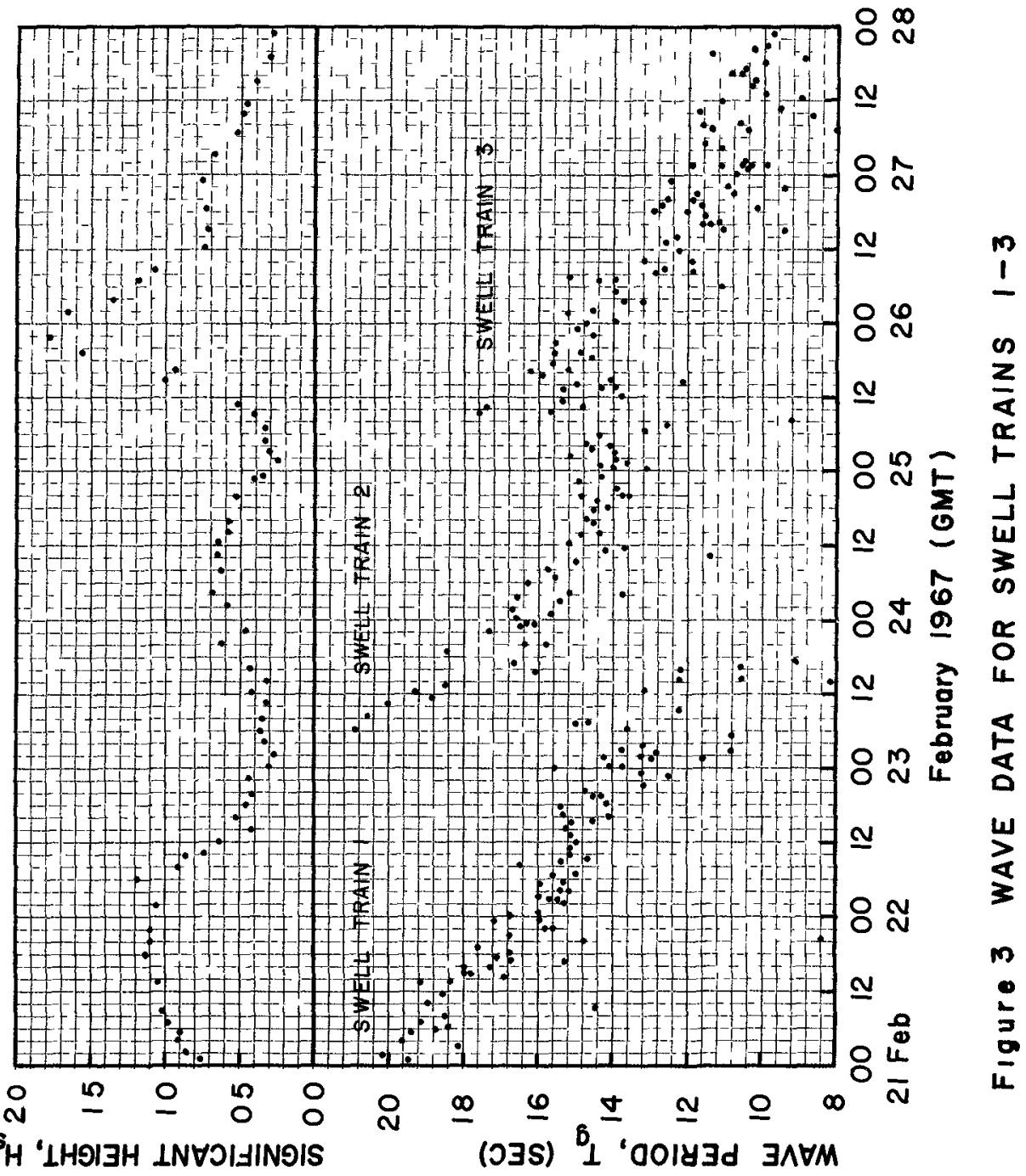




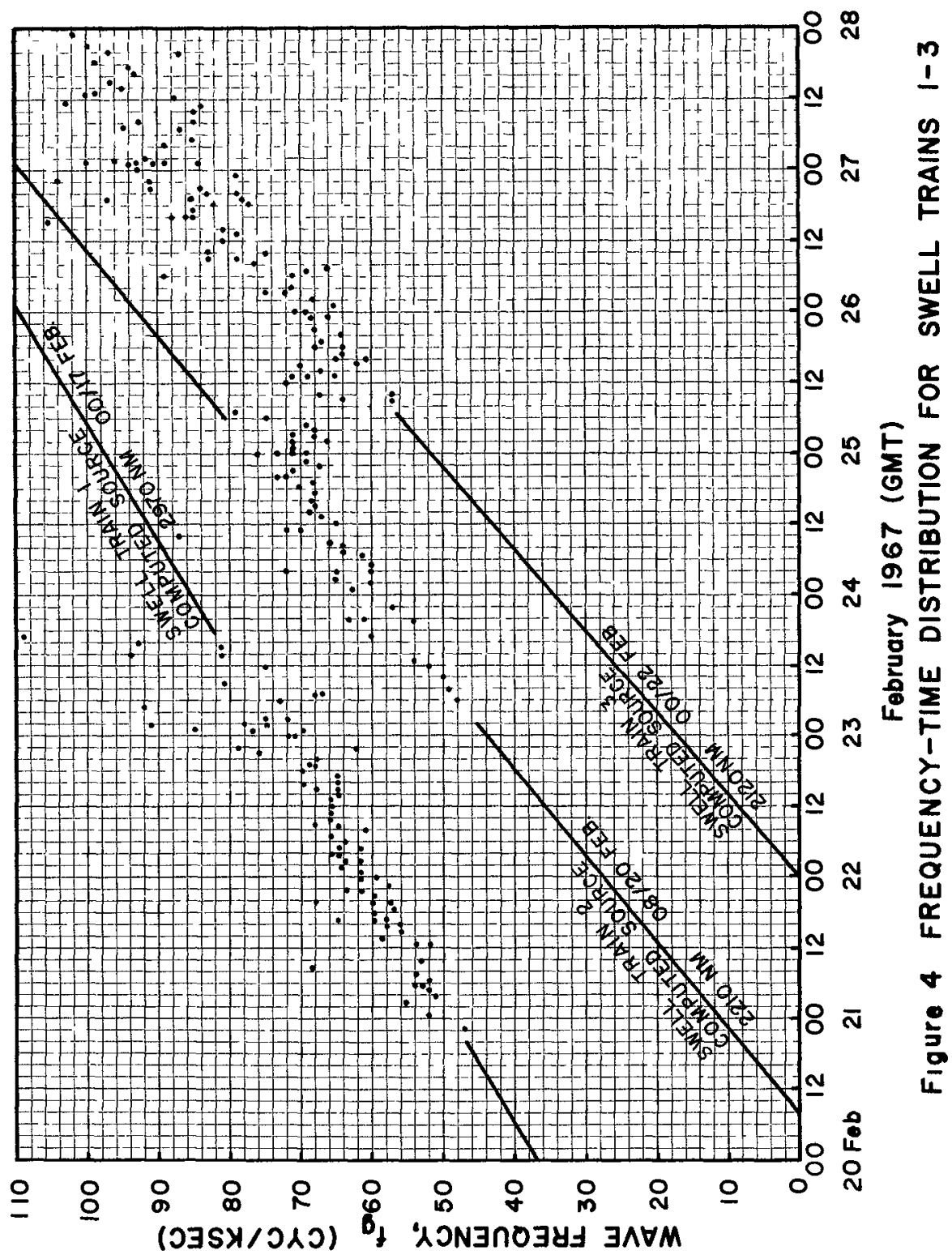




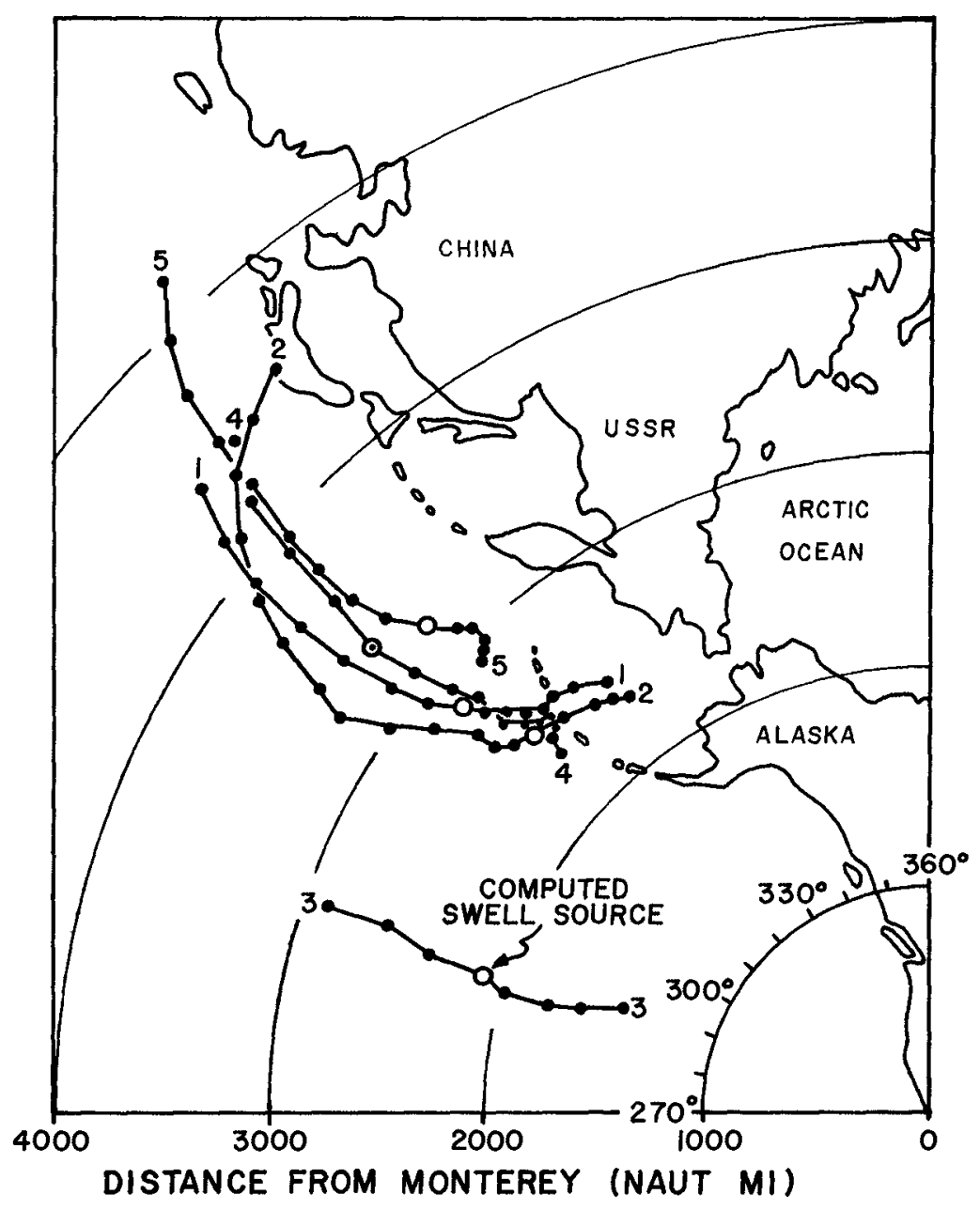

Figure 5 SIX-HOURLY FETCH LOCATIONS FOR STORMS 1-5 (after Braunstein, 1970) 


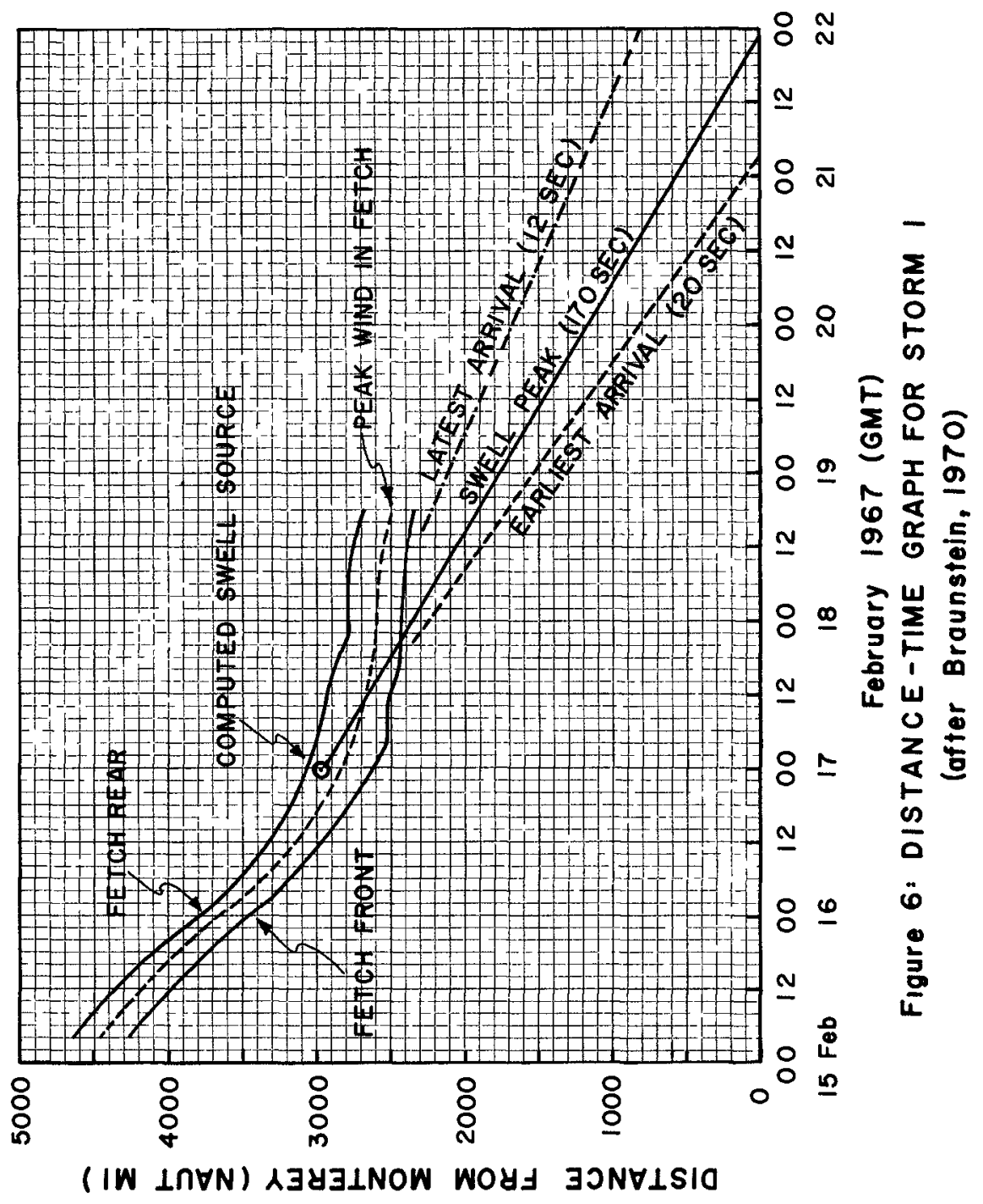




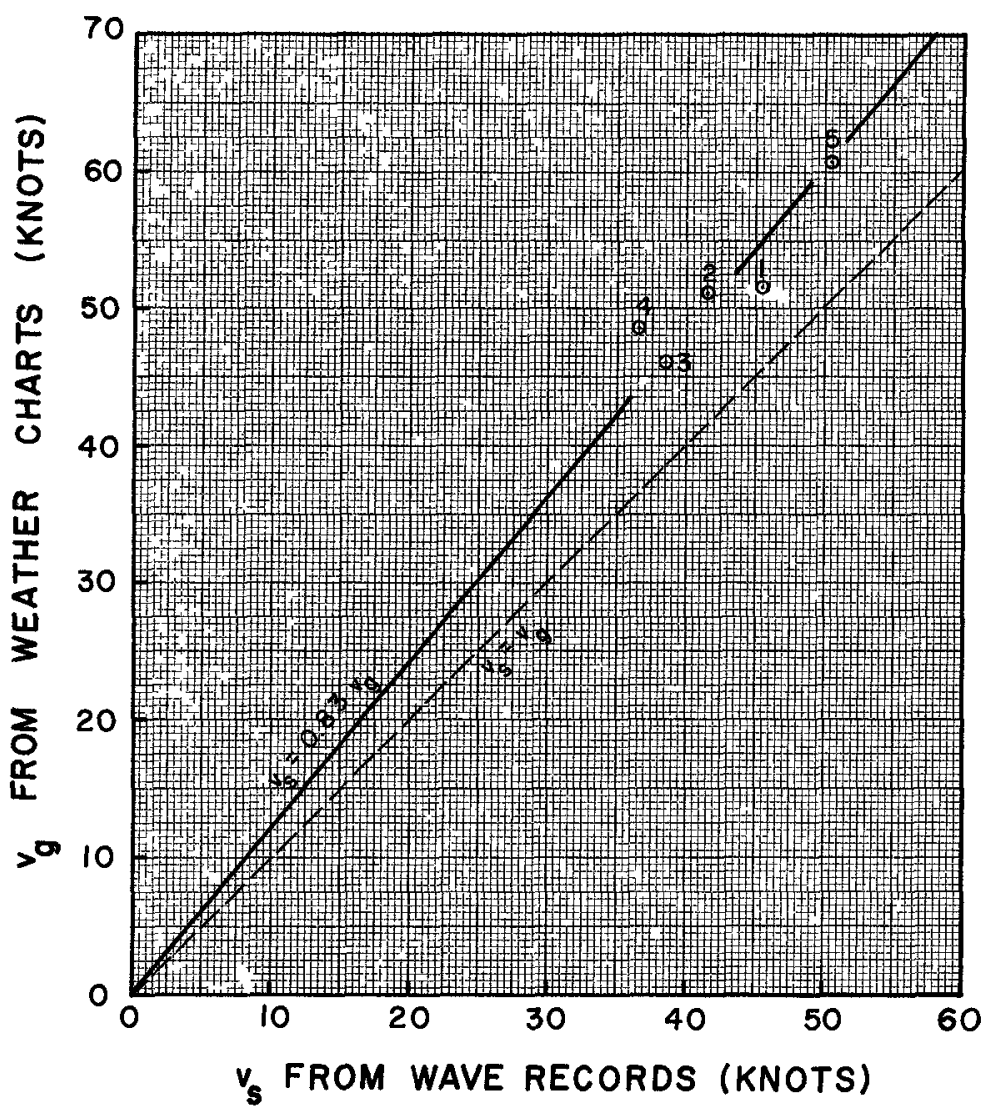

FIgure 7 RELATIONSHIP BETWEEN SURFACE AND GEOSTROPHIC WINDS AT COMPUTED SWELL SOURCE FOR STORMS 1-5 (after Braunsteln, 1970) 


\title{
CHAPTER 4
}

\section{MAXIMUM WAVE HEIGHT PROBABILITIES FOR}

\section{A RANDOM NUMBER OF RANDOM INTENSITY STORMS}

\author{
L E Borgman \\ Professor of Geology and Statıstics \\ Un Ivers I ty of Wyoming
}

\begin{abstract}
A very general model is presented for the probability distribution function for wave heights in storms with time-varying intensities Some of the possible choices for functions in the model are 1 isted and discussed Techniques for determining the "equivalent rectangular storm" corresponding to a given historically recorded storm are developed The final model formula expresses the probabilities for a random number of random length storms each with random intensities
\end{abstract}

\section{INTRODUCTION}

The probability 1 aw for the largest of $N$ independent, identically distributed random variables is covered quite well in statistical and scientific literature Gumbel (1954) provides an excellent survey of the main elements of the theory His book (Gumbel, 1958) gives a very complete bibl lography and many additional details

The application of these techniques to determine probabilities for the largest ocean wave heights in a sequence of $N$ identically distributed and Independent waves was developed by Longuet-Higgins (1952) What modIf Icatıons are necessary to yield maximum wave probabılıtıes for storms which vary in intensity with time? Furthermore, how would one obtain probabilities for the maximum wave in a random number of such timevaryıng storms? These questions will be considered in detall in the following

\section{PRELIMINARY ASSUMPTIONS}

The basic assumptions needed in the development are

(1) The probability distribution function

$$
F_{H}(h)=P[H \leq h]
$$

for wave helghts is known as a function of time-varying intensity parameters Here, and in later deviatıons, P[] will denote the probability of the event indicated within the square brackets The intensity parameters in $F_{H}(h)$ may be the root- 
mean square wave height, a, If the Rayleigh distribution is used

$$
F_{H}(h)=\left\{\begin{array}{ll}
1-e^{-h^{2} / a^{2}}, & \text { for } h \geq 0 \\
0, & \text { for } h<0
\end{array},\right.
$$

or the $r \mathrm{~ms}$ wave height, $a$, and the breaking wave height if the clipped Rayleigh distribution is used

$$
F_{H}(h)= \begin{cases}\frac{1-e^{-h^{2} / a^{2}}}{1-2^{-H_{b}^{2} / a^{2}}}, & \text { if } 0 \leq h \leq H_{b} \\ 0 & \text { otherwise }\end{cases}
$$

Another possibility is the Rice distribution outlined by Longuet-Higgins and Cartwright (1956) which depends on the $r \mathrm{~m} s$ wave helght and a parameter, $\varepsilon$, which is determined from the spectral density for the water level elevations

(2) It will also be assumed that each wave height is statistically independent of the helghts of 1 ts neighbors This assumption is largely one of convenience The theory is much harder without it However, it has been shown theoretically that the limiting distribution for the maximum of random variables which are what is called "m-dependent" of each other is the same as the limiting distribution for independent random variables (Watson, 1954) The term, m-dependent, used here means that random varlables in the sequence with more than $\mathrm{m}-1$ other random varıables between them are statistically independent of each other It seems reasonable to assume that a wave height is at most interdependent with the first several wave heights occurring before and after $1 t$ and essentially independent with waves further back into the past or forward into the future Hence $m$-dependence seems reasonable for wave heights

Since the limiting distribution is the same for independent as well as m-dependent random varıables, one can tentatıvely presume the independence assumptions for wave heights will not lead to badly incorrect conclusions it would appear that the independence assumpti on would lead to a conservative estimate of the maximum wave height probabılıtıes, in any case Longuet-Higgins and others have made this same assumption and it will be made here also

(3) It will also be presumed that there is a known, or estimated, function $T(t)$ such that for any small time interval dt, the number of waves in the interval is given by $d t / T(t)$ 


\section{A SINGLE TIME-VARYING STORM}

Consıder first $N$ identıcally-distributed, independent wave heights, each with probability distribution function, $F_{H}(h, a)$ Here a denotes the set of one or more intensity parameters which characterize the intensity of the sea conditions For this situation, let $\mathrm{H}_{1}, \mathrm{H}_{2}, \mathrm{H}_{3}$, , $H_{N}$ be the $\mathrm{N}$ waves The largest wave in the sequence will be less than or equal to $h, f$, and only $I f$, every one of the waves are less than or equal to $h$ Thus

$$
P[\max H \leq h]=P\left[H_{1} \leq h, H_{2} \leq h, H_{3} \leq h, \quad, H_{N} \leq h\right]
$$

But since the wave heights are assumed independent of each other,

$$
P[\max H \leq h]=P\left[H_{1} \leq h\right] P\left[H_{2} \leq h\right] P\left[H_{3} \leq h\right] \quad P\left[H_{N} \leq h\right]
$$

Finally since the $N$ waves are taken to have the same probabılıty distribution function

$$
P[\max H \leq h]=\left\{F_{H}(h, \underline{a})\right\}^{N}
$$

Suppose now that the time-varyıng storm can be subdivided into steps as shown in Table I below

TABLE I

FINITE STEP APPROXIMATION TO THE TIME-VARYING STORM

\begin{tabular}{l|cccc}
\hline $\begin{array}{c}\text { Time } \\
\text { Interval }\end{array}$ & $\begin{array}{c}\text { Number } \\
\text { of Waves }\end{array}$ & Intensity & $\begin{array}{c}\text { Interval } \\
\text { Width }\end{array}$ & $\begin{array}{c}\text { Period of } \\
\text { Waves }\end{array}$ \\
\hline $\mathrm{t}_{0}$ to $\mathrm{t}_{1}$ & $\mathrm{~N}_{1}$ & $\vec{a}_{1}$ & $\Delta \mathrm{t}_{1}$ & $\mathrm{~T}_{1}$ \\
$\mathrm{t}_{1}$ to $\mathrm{t}_{2}$ & $\mathrm{~N}_{2}$ & $\underline{a}_{2}$ & $\Delta \mathrm{t}_{2}$ & $\mathrm{~T}_{2}$ \\
$\mathrm{t}_{2}$ to $\mathrm{t}_{3}$ & $\mathrm{~N}_{3}$ & $\vec{a}_{3}$ & $\Delta \mathrm{t}_{3}$ & $\mathrm{~T}_{3}$ \\
$\mathrm{t}_{\mathrm{m}-1}$ to $\mathrm{t}_{\mathrm{m}}$ & $\mathrm{N}_{\mathrm{m}}$ & $\mathrm{a}_{\mathrm{m}}$ & $\Delta \mathrm{t}_{\mathrm{m}}$ & $\mathrm{T}_{\mathrm{m}}$ \\
\hline
\end{tabular}

The probabilities for the maximum wave in the entire storm will be the product of the probabilities for each of the steps

$$
\begin{aligned}
P[\max H \leq h] & =\prod_{J=1}^{m} P\left[\max H \leq h \text { in the } j^{\text {th }} \text { step }\right] \\
& =\prod_{J=1}^{m}\left\{F_{H}\left(h, \underline{a}_{j}\right)\right\}^{N_{J}}
\end{aligned}
$$

It is being assumed that the waves within a step change intensity sufficiently slowly so that, to a fair approxkmation, they may be taken 
as being identically distributed

It follows that the natural logarithm of $P[\max H \leq h]$ can be written

$\log P[\max H \leq h]=\sum_{J}^{m} N, \log F_{H}\left(h, \underline{a}_{J}\right)$

If

$$
N_{J}=\Delta t_{J} / T_{J}
$$

is substituted into eq (8), one gets

$$
\log P[\max H \leq h]=\sum_{j=1}^{m}\left(1 / T_{J}\right) \log F_{H}\left(h, a_{J}\right) \Delta t,
$$

Now let $m \rightarrow \infty$ and $\max \Delta t, \rightarrow 0$ By the usual definition of an integral,

$$
\log P[\max H \leq h]=\int_{t_{0}}^{t}[1 / T(t)] \log F_{H}(h, \underline{a}(t)) d t
$$

It is presumed that the integrand is continuous and uniformly bounded so that the stepwise expression in eq (10) becomes eq (11) in the limit

\section{A SERIES APPROXIMATION}

The distribution function $F_{H}(h, a(t))$ will be expanded in a power series about some convenient value $h_{0}$ Several possibilities for $h_{0}$ are the breakıng wave helght, $H_{b}$, (If the maximum wave is probably going to be close to breakıng), and the "expected" probable maximum, $V$, (which w1 11 be defined later) Thus, let

$$
\log F_{H}(h, \underline{a}(t))=b_{0}(t)+b_{1}(t)\left(h-h_{0}\right)+b_{2}(t)\left(h-h_{0}\right)^{2}+
$$

It is presumed that the distribution function is differentiable to the required order so that

$$
\begin{array}{ll}
b_{0}(t)=\log F_{H}\left(h_{0}, a(t)\right) & \\
7\left(1^{\prime}\right) b_{1}(t)=\frac{d}{d h} \log F_{H}(h, a(t)), & h=h_{0} \\
\left(2^{\prime}\right) b_{2}(t)=\frac{d^{2}}{d h^{2}} \log F_{H}(h, a(t)), & h=h_{0} \\
\text { etc } &
\end{array}
$$

If eq (12) is substituted into eq (11), one gets

$$
\log P[\max H \leq h]=B_{0}+B_{1}\left(h-h_{0}\right)+B_{2}\left(h-h_{0}\right)^{2}+
$$

with

$$
B_{k}=\int_{t_{0}}^{t_{m}}\left[b_{k}(t) / T(t)\right] d t, \quad k=0,1,2 \text {, }
$$


The evaluation of $\mathrm{B}_{0}, \mathrm{~B}_{1}, \mathrm{~B}_{2}$ to whatever number of terms is desıred gives a convenient representation of the probabılıty of maximum he ight as

$$
P[\max H \leq h]=\exp \left\{B_{0}+B_{1}\left(H-h_{0}\right)+B_{2}\left(h-h_{0}\right)^{2}+\right\}
$$

Presumably the first few terms would be sufficient for most situations since the higher order derivatives for most distribution functions become negligible as $h$ grows large

\section{THE COMBINATION OF SEVERAL STORMS}

Another advantage of the representaion in eq (14) is that it facilitates the determination of probabilities for the maximum for the combined wave helghts in several storms This is under the supposition that the $s$ ame $h_{0}$ has been used for all the storms

Let $P_{r}[\max H \leq h]$ denote the probability that the maximum wave in the $r^{\text {th }}$ storm is less than or equal to $h$ Suppose there are $R$ storms to be considered Then the probabilities for the maximum wave in the combined set of wave helghts would be

$$
\log P[\max H \leq h]=\stackrel{R}{\Sigma_{=}} \log P_{r}[\max H \leq h]
$$

The function of $h$ expressed by $P_{r}[\max H \leq h]$ can be evaluated from eq (11) for each storm Alternatively, let $B_{k r}$ be the $B_{k}$ value from eq (15) for the $r^{\text {th }}$ storm Then If the same $h_{0}$ was used for each storm, one may write

$$
\log P[\max H \leq h]=\sum_{r=1}^{R} B_{0 r}+\sum_{r=1}^{R} B_{1 r}\left(h-h_{0}\right)+\sum_{r=1}^{R} B_{2 r}\left(h-h_{0}\right)^{2}+
$$

That is, the $B_{k r}$ can be added storm by storm If $B_{k}$ is redefined as $\sum_{i=1}^{R} B_{k r}$ then eq (16) gives the distribution function for the maximum helght in the combined set of waves

The above development is appropriate for hindcasting the probabılitıes for maximum heights in storms whose fundamental time-varying intensities were measured or are known from other considerations What about probabilities for future periods of time, say the next hundred years? One could take the historical record as given by Wilson (1957) and determıne $B_{0}, B_{1}, B_{2}$ for each storm Then the probability density jointly for $\left(B_{0}, B_{1}, B_{2}\right)$ could be estimated from the data and used to make the extension to the future

This procedure appears to have grave disadvantages in that $\left(\mathrm{B}_{0}, \mathrm{~B}_{1}\right.$, 
$B_{2}$ ) are not "Intuitıve" quantitıes whose meanings are easy to interpret One runs the risk of makıng mistakes because the un reasonableness of values arising apparently from the data are not recognized $A$ more trustworthy procedure would appear to be to shift over to intuitively interpretable values

To fill this need, the concept of an "equivalent rectangular storm" will be introduced A rectangular storm is defined to be one in which the intensity, wave period, and distribution function for the height of a single wave remain constant during the duration of the storm The "equivalent rectangular storm" corresponding to a given historical storm will be that rectangular storm which leads to the same values of $B_{0}, B_{1}$, and $B_{2}$ as the historical storm The constants for the "equivalent rectangular storm'" will have intultıve meanıng in characterızıng the severity of the storm and in making predictions for the future

\section{PROBABILITIES FOR A RECTANGULAR STORM}

A special development will be made for the maximum wave height in a rectangular storm as related to the intensity parameters a and the number of waves, $N$ Let $w(h, a)$ be defined for $N$ independen $\bar{t}$, Identically distributed random wave heights, each with distribution function $F_{H}(h)$, as

$$
w(h, \underline{a})=N\left[1-F_{H}(h, \underline{a})\right]
$$

Then the distribution function for the maximum value may be written approximately (Cramer, 1946, p 286 , eq 2862 , Borgman, 1961, pp 3296 - 3297, see eq (6)) for large values of $N$ as

$$
P[\max H \leq h]=\{F(h, \underline{a})\}^{N}=\left(1-\frac{w(h, a)}{N}\right)^{N} \approx e^{-w(h, a)}
$$

Hence

$$
\log P[\max h \leq h] \approx N\left[1-F_{H}(h, a)\right]
$$

Gumbel (1954, p 13, eq 2 11) defines the "expected" largest value, $V$, of a variate to be the value $V$ which satisfies the equation

$$
w(V, \underline{a})=N\left[1-F_{H}(V, \underline{a})\right]=1
$$

This has a physical interpretation in that $I-F_{H}(V, a)$ is the probability, $\mathrm{P}[\mathrm{H}>\mathrm{V}]$ Multiplying this probability by $\mathrm{N}$ gives the expected number of times wave helghts will exceed $V$ in the $N$ occurrences Hence $V$ is that value such that on the average there will be exactly one exceedance in the $\mathrm{N}$ wave heights

From eq (22) 


$$
N=\left[1-F_{H}(v, \underline{a})\right]^{-1}
$$

This can be inserted into eq (21) to give the approximation

$$
\log P[\max H \leq h] \approx \frac{1-F_{H}(h, a)}{1-F_{H}(V, a)}
$$

Now suppose that, paralleling eq (12), one expands $F_{H}(h)$ in a power series about $h_{0}$

$$
F_{H}(h)=c_{0}+c_{1}\left(h-h_{0}\right)+c_{2}\left(h-h_{0}\right)+
$$

Then keepung only the terms to second order

with

$$
\begin{aligned}
\log P[\max H \leq h] & \approx \frac{1-C_{0}-C_{1}\left(h-h_{0}\right)-C_{2}\left(h-h_{0}\right)^{2}}{1-C_{0}-C_{1}\left(V-h_{0}\right)-C_{2}\left(V-h_{0}\right)^{2}} \\
& =B_{0}+B_{i}\left(h-h_{0}\right)+B_{2}^{\prime}\left(h-h_{0}\right)^{2}
\end{aligned}
$$

$$
\begin{aligned}
& B_{0}=\left(1-c_{0}\right) /\left[1-c_{0}-c_{1}\left(v-h_{0}\right)-c_{2}\left(v-h_{0}\right)^{2}\right] \\
& B_{1}^{s}=-c_{1} /\left[1-c_{0}-c_{1}\left(v-h_{0}\right)-c_{2}\left(V-h_{0}\right)^{2}\right] \\
& B_{2}^{\prime}=-c_{2} /\left[1-c_{0}-c_{1}\left(v-h_{0}\right)-c_{2}\left(v-h_{0}\right)^{2}\right]
\end{aligned}
$$

The value of $a, N$, and $V$ for the equivalent rectangular storm will be determined by equating $B_{0}^{\prime}, B_{1}^{*}$, and $B_{2}^{*}$ to the $B_{0}, B_{1}$, and $B_{2}$ respectively given by eq (15) for the historical storm Thus, to second order, the equivalent rectangular storm will be producing the same probabilities for maximum wave helghts as did the historical storm

The equations to be solved are

$$
\begin{aligned}
& D=1-c_{0}-c_{1}\left(v-h_{0}\right)-c_{2}\left(v-h_{0}\right)^{2} \\
& B_{0}=\left(1-c_{0}\right) / D \\
& B_{1}=-c_{1} / D \\
& B_{2}=-c_{2} / D
\end{aligned}
$$

Here, $h_{0}$ is regarded as a previously selected (and thus known) value to expand about Now the ratios

$$
\begin{aligned}
& R_{1}=\left(B_{1} / B_{0}\right)=-C_{1} /\left(1-C_{0}\right) \\
& R_{2}=\left(B_{2} / B_{0}\right)=-C_{2} /\left(1-C_{0}\right)
\end{aligned}
$$


can be computed from the values of $B_{0}, B_{1}$, and $B_{2}$ If $R_{1}$ and $R_{2}$ are substituted into the expression for $B_{0}$, one gets

$$
B_{0} R_{2}\left(V-h_{0}\right)^{2}+B_{0} R_{1}\left(V-h_{0}\right)+\left(B_{0}-1\right)=0
$$

Hence $V$ can be determıned from eq (30) as a quadratic solution

Now $F_{H}(h, a)$ is typically a monotone decreasing function of storm intensity for $f$ Ixed $h$ That is, a higher storm intensity normally means that there is a larger probability of exceeding the fixed $h$ value or a smaller probability of being less than or equal to that $h$ value But eq (23) states that

$$
F_{H}(V, a)=1-\frac{1}{N}
$$

Hence, the storm intensity can be determined from the value of $\mathrm{N}$ which is usually known, approximately at least, from other considerations If the intensity of a is a vector, reasonable interrelations between the components of a must be imposed

In summary the computational procedure for determınıng $V$ and $a$ for the rectangular storm is as follows

(1) Calculate $R_{1}$ and $R_{2}$ from eq (29)

(2) Determıne $V$ from eq (30)

(3) Compute a from eq (31) and the value of $\mathrm{N}$

\section{PROBABILITY GENERATING FUNCTIONS}

In developing the probabilities for the maximum height in a random number of random length and random intens Ity storms, it Will be natural to introduce various probability generating functions $A$ probability generating function for a random variable $N$ is defined to be the infinite series

$$
G_{N}(s)=n^{\infty} \underline{\underline{S}}_{0} P[N=n] s^{n}
$$

These functions have closed form for many probability laws (Borgman, $1961, \mathrm{p} 3305$, eq (21) - (27)) Two examples of particular usefulness are the probability generating functions for the Poisson and the negatıve binomial probabılity laws (W/llıamson and Bretherton, 1963, pp $9-10)$

$$
\begin{aligned}
\text { Poisson } \begin{aligned}
P[N=n] & =e^{-\lambda} \lambda^{n} / n^{\prime} \\
G_{N}(s) & =\exp [-\lambda(1-s)]
\end{aligned} \\
\text { Negative binomial } P[N=n]=\left(\begin{array}{c}
r+n-1 \\
n
\end{array}\right) p^{r} q^{n}
\end{aligned}
$$




$$
G_{N}(s)=p^{r}(1-q s)^{-r}, p+q=1
$$

The mean and variance of the Poisson is $\lambda$ The corresponding mean and variance of the negative binomial are respectively

$$
\begin{aligned}
& \text { mean }=r q / p \\
& \text { varlance }=r q / p^{2} \\
& \text { where } p+q=1
\end{aligned}
$$

The negative binomial parameters, $p, q$, and $r$, can be estımated from the mean $\bar{N}$ and variance $(N)=s^{2}$ by the method of moments as

$$
\begin{aligned}
& \hat{p}=\bar{N} / s^{2} \\
& \hat{q}=1-p \\
& \hat{r}=\bar{N} \hat{p} / \hat{q}
\end{aligned}
$$

\section{PROBABILITIES FOR A RANDOM LENGTH STORM}

Suppose a rectangular storm has a random length $\mathrm{N}$ and fixed intensity, a What is the probability law for the maximum wave height in the storm? Let $G_{N}(s)$ be the probability generating functions for $N$

By eq (2I), the approximate probability law for $\mathrm{H} \mathrm{given}$ a particular value of $N=n$ is

$$
P[\max H \leq h \mid N=n] \approx\left\{\exp \left[1-F_{H}(h, a)\right]\right\}^{n}
$$

Then for a random number of waves

$$
\begin{aligned}
P[\max H \leq h] & \approx \sum_{n=0}^{\infty} P[\max H \leq h \mid N=n] P[N=n] \\
& =\sum_{n=0}^{\infty} P[N=n]\left\{\exp \left[1-F_{H}(h, \underline{a})\right]\right\}^{n} \\
& =G_{N}\left(\exp \left[1-F_{H}(h, \underline{a})\right]\right)
\end{aligned}
$$

A comparison of eq (44) with eq (32) will justify substituting the exponential for the argument $s$ of the probability generating function

In practice one could use the guessed values of $\mathrm{N}$ and $\mathrm{s}^{2}$ together With the negative binomial probability law to determine the function $\mathrm{G}_{\mathrm{N}}(\mathrm{s})$ Alternatively another probability generating function could be us ed

PROBABILITIES FOR RANDOM LENGTH AND RANDOM INTENSITY STORMS

If a is also random, then eq (45) must be regarded as a probability given that intensity $=\underline{a} \quad$ Let 
$\underline{f_{1}}(\underline{a})=$ probabılıty densıty for $\underline{a}$

Then

$$
\begin{aligned}
P[\max H \leq h] & =\int_{-\infty}^{\infty} P[\max H \leq h \mid \underline{I}=\underline{a}] \underline{f_{\underline{1}}}(\underline{a}) d \underline{a} \\
& \approx \int_{-\infty}^{\infty} G_{N}\left(\exp \left[1-F_{H}(h, \underline{a}]\right) f_{\underline{1}}(a) d \underline{a}\right.
\end{aligned}
$$

\section{PROBABILITIES FOR A RANDOM NUMBER OF RANDOM LENGTH}

\section{AND RANDOM INTENSITY STORMS}

The final complication is to introduce a probability law for the number of storms, $K$, which may occur in the $t$ ime interval for which predictions are made Let $G_{K}(s)$ be the corresponding probability generating function By the identical same argument leading to eq (45),

$$
\begin{aligned}
P[\max H \leq h] & =k \sum_{k=0}^{\infty} P[\max H \leq h \mid K=k] P[K=k] \\
& =\sum_{k=0}^{\infty}\left\{\int_{-\infty}^{\infty} G_{N}\left(\exp \left[1-F_{H}(h, a)\right]\right) f_{1}(a) d a\right\} P[K=k]
\end{aligned}
$$

or

$$
P[\max H \leq h]=G_{K}\left(\int_{-\infty}^{\infty} G_{N}\left(\exp \left[1-F_{H}(h, \underline{a})\right]\right) f_{\underline{I}}(\underline{a}) d \underline{a}\right)
$$

The number of waves in a given storm may depend on a Hence the formula can be made a 11 ttle more general by introducing the conditional probability generating function for $\mathbf{N}$ given a This final version of the formula would be

$$
P[\max H-h]=G_{K}\left(\int_{-\infty}^{\infty} G_{N \mid \underline{a}}\left(\exp \left[1-F_{H}(h, \underline{a})\right]\right) f_{\underline{1}}(\underline{a}) d \underline{a}\right)
$$

\section{SOME FINAL COMMENTS}

(1) The application of the above formula will obviously require a digital computer and detalled analysis of the historical data for the particular location of interest

(2) The negative binomial appears to be the best cholce for the two probability generating functions al though, at least for Gulf of Mexico hurricanes, there is some basis for using the simpler Poisson probability generating function for $G_{K}(s)$ 
(3) The possible choices for $F_{H}(h, a)$ were discussed at the beginning of the paper Without more detalled information, the Rayleigh distribution appears to be as good a cholce as any (Goodnight and Russe11, 1963)

(4) The choice of $f_{1}($ a) would have to depend strongly on the analysis of historical data or on meteorological considerations Hence it is hard to make a guess as to a reasonable cholce However, a form of the gamma density would seem to be a good first guess

(5) In this whole discussion, the randomness of wave period has been ignored A more adequate model would certainly include this source of variation

(6) An alternative approach to the maximum wave height might be made through the statistical theory of maxima and minima of a random function Unfortunately, when such an approach 15 attempted, theoretical difficultires arıse very quickly information on wave crest elevation probabilities can be obtained, however, by the random function type of analysis

\section{ACKNOWLEDGMENT}

The research reported was supported in part by the Chevron 011 Field Research Company under a research gift to the University of Calıforn Ia, Berkeley, and in part, by the Coastal Engıneering Research Center, $U S$ Army Corps of Engineers under Contract DACW-72-69-C-000 The author gratefully acknowledges their financial assistance in the study

\section{REFERENCES}

Borgman, $L E$ (1961) The frequency distribution of near extremes, Journal of Geophysical Research, $\underset{\sim \sim}{66}$, pp 3295-3307

Cramer, Herald (1946) Mathematical Methods of Statistics, Princeton University Press, Princeton, New Jersey

Goodnight, $R$ C and Russell, $T L$ (1963) Investigation of the statistIcs of wave heights, Jour Waterways and Harbors DIV, ASCE, WW2, paper 3254 , pp 29-54

Gumbel, E J (1954) Statistical theory of extreme values and some practical applications, Applied Math Series 33, National Bureau of Standards, $U S$ Govt Printing Office, Washington, D C

Gumbel, E J (1958) Statıstıcs of Extremes, Columbıa Unıv Press,N Y 
Longuet-Higgins, $M S$ (1952) On the statistical distribution of the helghts of sea waves, Jour Marıne Res, 11, pp 245-266

Longuet-Higgins, $M S$, and Cartwright, D E (1956) The statistical distribution of the maxima of a random function, Proc Royal Soc, A, 237, pp 212-232

Watson, G S (1954) Extreme values in samples from m-dependent stationary stochastic processes, Ann Math Statıstıcs, 25, pp 798800 


\section{CHAPTER 5}

SPECTRAL COMPUTATIONS ON PRESSURE WAVE GAUGE RECORDS

by Manuel Mendes de CARVALHO*

Fernando Silveira RAMOS*

Carlos de Campos MORAES*

\section{ABSTRACT}

With a view to establish sea wave data processing procedures to be applied to records obtained at the Portuguese coast, a detaled study is made of different chol ces of certain parameters used in one-dimensional spectral analysis of a pressure wạ ve gauge record Statistics computed by the selected spectral procedure are then compared with results of a Tucker-Draper analysis of the same record Finally a hindcast of sea conditions for the date and place of the record is made by different methods and comparisons with previous results are presented

\section{1 - INTRODUCTION}

The study of the coastal sea wave regime in Portunal needs development both in programming an adequate deployment of sea wave gauges and in what concerns es tablishing the best data processing procedures The authors form a research team charged at present with the study of the instrumentation and use of an irregular wa ve flume at $L N E C$ it is known that for a perfect simulation of irregular sea waves a detalled knowledge is necessary of the wave regimes to be reproduced There are two phases to consider the first is a qualitative one in which methods, or sea wave data analysis and computation procedures are discussed and developed, the second is quantitative, results from the first being extensively applied, to try and acquire a more exact knowledge of the configuration of sea waves in the zone of interest.

The present paper relates to the above mentioned first phase it represents the study path followed by the authors Although most of the techniques presented are already known, it is thought that eventually some usefulness may be derived from reading it, particularly in pointing out some doubts and difficulties inherent in the me thods used The work being stıll restricted to the first phase, only one record was used This record was made by a pressure wave gauge

2 - CONDITIONS OF WAVE MEASURING, RECORDING AND ANAL YSIS

In the approaches to Leixões Harbour (In the northwestern coast of Portugal) a pressure wave gauge is installed on the bottom at a mean depth of $22 \mathrm{~m}$ ( $F$ ig 1) It is an autonomous St Chamond Granat pressure wave gauge, type LNH Its working schedule is as follows at both $0900 \mathrm{hrs}$ and $2100 \mathrm{hrs}$ a twenty-minute record ing period starts, when the surface waves exceed $4 \mathrm{~m}$ it automatically produces a twenty-minute record every two hours Normally a 250 milibar manometer is in use The present study is based on a twenty-minute record made at 347 GMT on Decem ber $18^{\text {th }}$, 1968 For the record digitalization a Boscar LNF 630 projector by Benson, France, was used This projector provides a twelvefold magnification of the 35 $\mathrm{mm}$ film from the wave gauge and can also handle 16 and $70 \mathrm{~mm}$ films The computa tions were made in the LNEC's computing centre in a NCR-Elliott 4130 computer with 24 k 24-bit words and 3 magnetic tape handlers

3 - SPECTRAL ANALYSIS

Estımates of the energy spectrum, $P(f)$, were obtained by the indirect method through the autocovariance function $c(\tau)$

$$
P(f)=\int_{-\infty}^{+\infty} c(\tau) e^{-12 \pi f \tau} d \tau=T F[c(\tau)]
$$

where TF means Fourier transform A measure of the mean energy of the waves

* - Trainee Research Officers, Laboratorio Nacional de Engenharia Civil (LNEC), Lisbon, Portugal 
during the record duration $T_{R}$ is given by

$$
c(0)=2 \int_{0}^{+\infty} P(f) d f
$$

31 - Lag and spectral windows

If $Z(t)$ is the water surface elevation above an arbitrary level as a function of time, $t$, and at a certain place, then the autocovariance function is the mean value of $z(t) z(t+\tau)$ along time

$$
c(\tau)=\lim _{T \rightarrow \infty} \frac{1}{T} \int_{-T / 2}^{T / 2} Z(t) Z(t+\tau) d t
$$

$c(\tau)$ expresses covariance between $Z(t)$ and $Z(t+\tau)$, that is, between water sur face elevations at any two instants separated by a time lag of $\tau$ secs In case there are no periodicities in $Z(t)$, then $c(\tau)$ tends to zero as $\tau$ tends to infinity if there is a periodicity in $Z(t)$, then an oscillation about zero, with the same period, appears in $c(\tau)$ In practice, the autocovariance function, $c(\tau)$, is computed as the mean value of $Z(t) Z(t+\tau)$ in the avalable recording interval, whose length or, rather, duration, is $T_{R} c(x)$ never really damps out to zero, not only because $T_{R}$ is finite but also owing to periodicities which always occur in records* This leads to the necessity of truncating** the autocovariance function, that is, to consider instead of c( $x$ ) the neh function $c(\tau) \quad D(\tau)$ where

$$
D(\tau)=\begin{array}{ll}
1 & ,|\tau| \leq T_{M} \\
0 & ,|\tau|>T_{M}
\end{array}
$$

$D(\tau)$ is the rectangular lag window and $T_{M}$ is the maximum lag of the autocovarlance function Instead of $D(\tau)$ and to get more stability in the spectrum estımates, orner functions or lag windows, $D_{1}(\tau)$ are used, which are also identically zero out side the interval $\left(-T_{M}, T_{M}\right)$ and take the value 1 for $\tau=0$ Using these lag windows, Which is unavoidable in practice, leads to the fact that, for every frequency, one gets a wheighted average of neighbouring values of the record spectrum The Wheighting function is $Q_{1}(f)=T F\left[D_{1}(\tau)\right]$, the so-called spectral window corresponding to the lag window $D_{1}(\tau)$ In this study the Parzen, Tukey, Hamming, Bartlett and rectangular lag windows were considered Their lag and spectral versions are presented in $F$ ig 3

It is known that to each window there corresponds a statistical estimator for the spectrum To chose one among these, various criteria have been proposed, most of them based in the minimization of the mean square error of the estimator or some function or functional of it (See, for instance, Jenkins and Watts [4]) According to this kind of criteria the rectangular window distinguishes itself by being considerably worse than the others mentioned, which in turn are similar to one another in performance In consequence the rectangular window should be avoided and the choice among the Parzen, Tukey, Hamming and Bartlett windows becomes of secondary importance, as compared, for example, with the choice of the maximum lag for $c(\tau)$, to be consiJered in 36 However, differences do exist and a choice had to be made

The fact that the side lobes of the Parzen spectral window are much smaller and that its small variance originates narrower confidence intervals for the spectrum estimates led to its selection For this window, the number of degrees of freedom of the spectrum estimates is $371 \mathrm{~T}_{R} / \mathrm{T}_{M}$ The spectral window bandwidth is

* - As shown later, (see $F$ Ig 2) the autocovariance function relative to the record considered is far from showing any tendency to become zero

** - Of course, if $c(\tau)$ equalled zero only outside a very large interval a truncation would be in order 


$$
b=\frac{1}{\int_{-\infty}^{+\infty} D^{2}(\tau) d \tau}=\frac{1}{\int_{-\infty}^{+\infty} Q^{2}(f) d f}=\frac{186}{T M}
$$

and it is approximately the frequency interval between two practically independent spectrum estimates It is seen that the smaller is $b$, the greater is the resolution of the estımates, $1 \mathrm{e}$, the smaller is the influence exerted on an estimate by estimates in neighbouring frequencies Applying the rectangular lag window is the same as simply truncating the autocovariance finction The snectrum shtaned is termed the raw spec trum The raw spectrum would be exactly equal to the true record spectrum if the autocovariance function were zero for $|\tau|$, TM, which never happens in sea wave and lysis Using any other of the mentioned windows produces smoothed estimates, that is, more stable estimates $F$ or a given window, if $T M$ is vamied then stability and resolution increase or decrease in opposite senses Fig 4 shows the raw spectrur computed for $T_{M}=80 \mathrm{~s}$ and its Parzen smoothed version

\section{2 - Prewhitening and prefiltering}

It is known that spectral windows delete accidental detals of the spectrum, that is, they smooth it in the process of smoothing, the spectrum estimate in a given fre quency is influenced by the estimates in neighbouring frequencies $A$ sharp peak is "spread" over its neighbourhood in a way similar to the spectral window configuration 5 This leads to the alteration of the true form of the spectrum To avoid this dif ficulty as much as possible, Blackman and Tukey [7] suggested a prewhitening, that is a preliminary digital filtering of the record aiming to reduce the imiportance of its spectrum peaks This is bringing the spectrum closer to that of a white noise

The suggested digital filtering is in general

$$
y_{t}=a x_{t}+b x_{t-1}
$$

$\left\{x_{t}\right\}$ being the input time series and $\left\{y_{t}\right\}$ the output time series The input and output spectra, $P_{x}(f)$ and $P_{y}(f)$ respectively, are then related by

$$
P_{y}(f)=\left(a^{2}+b^{2}+2 a b \cos 2 \pi f \Delta t\right) P_{x}(f)
$$

In this way the spectral window will be applied to a spectrum without sharp peaks and the obtained spectrum $P$ ( $f$ ) may then be corrected for prewhitening by (7)

Computations showed that, in the present case, estimates were practically the same with and without prewhitening This led to giving up the use of prewhitening As for other kinds of prefiltering, it was not deemed necessary to eliminate the spy rious energy of very low frequencies $A$ first difference filter was nevertheless con sidered, as is suggested by Jenkins [4], results showing that it was not adequate, as its influence reaches regions of too high frequencies

\section{3 - Confidence intervals}

Let $\bar{\Gamma}(f)$ be the smoothed spectrum estimate at frequency $f$, $P(f)$ the true value of the spectrum, $x^{2} v$ a random variable following the chi-square distribution with $v$ degrees of freedom and $b$ the spectral window bandwidth it can be proved that $2 T_{R} b \bar{P}(f) / P(f)$ is a random variable following the chi-square distribution with $2 T_{R} b$ degrees of freedom

$$
2 T_{R} b \frac{\bar{P}(f)}{P(f)}=x^{2} T_{R} b
$$

This permits confidence intervals to be constructed for the estimates Those intervals will be

$$
\left(\frac{v}{x_{v}(1-\alpha / 2)} \bar{P}(f), \frac{v}{x_{v}(\alpha / 2)} \bar{P}(f)\right)
$$


where $1-\alpha$ is the confidence level and $x_{v}(k)$ is a number such that

$$
P\left(X_{v}^{2} \leq x_{v}(k)\right)=k
$$
In $F I g$ 5, curves of variation with of $A(\nu)=\frac{v}{x_{v}(1-\alpha / 2)}$ and $B(\nu)=\frac{\nu}{x_{v}(\alpha / 2)}$
for $1-\alpha=80 \%$ are presented

This was the confidence level used in the computations made Most spectrum fi gures in this paper show a $80 \%$ confidence zone obtained in the manner described

\section{4 - Sampling interval}

From a continuous record a time series can be extracted using a certain sampling interval $\Delta t$ (digitalization) If $F_{D}$ is the frequency above which spectrum values are negligible one must have

$$
F_{D} \leq \frac{1}{2 \Delta t}
$$

to avoid the so called aliasing $F N_{N}=\frac{1}{2 \Delta t}$ is the Nyquist frequency Aliasing is a consequence of the digitalization of the ${ }^{2}{ }_{\text {recond }}$ In fact, the result of digital computations is not the true spectrum $P(f)$ but the aliased spectrum

$$
P_{a}(f)=\sum_{q=-\infty}^{+\infty} P\left(f-\frac{q}{\Delta t}\right)
$$

If condition (11) is met, however, then (12) gives $P(f)$ values between 0 and $F_{D}$ Having fixed $T_{M}=80 \mathrm{~s}$ (and so, $b=0023 \mathrm{cps}$ ), which is equlvalent to having fixed resolution and stability of the estimates, computations were carried out with $\Delta t=05,10,15,20,25$ and $30 \mathrm{~s}$, corresponding to Nyquist frequencies of 1 , 0 , $033,025,020$ and $016 \mathrm{cps} A s F_{D} \approx 025 \mathrm{cps}$ in the present case, alıasing should be negligible for $\Delta t=05,10,15$ and $20 \mathrm{~s}$ In fact, it was seen that estimates were practically the same $F$ or $\Delta t=25$ and $30 s$, that is, $F_{D}>F_{N}$, aliasing is already strongly apparent (FFig 6)

The conclusion is that inasmuch as there is no aliasing, the sampling interval may be as high as wished In these computations, $\Delta t=2 \mathrm{~s}$ could have been used

\section{5 - Cutoff frequency}

For reasons of economy, computation of spectrum values should not be carried much beyond $F$ Frequency $F$ up to which calculations are made will be called cutoff frequency ${ }^{D}$ One should then have $F_{C}<F_{C}<F_{N}$ The choice of $F C$ can have great influence in practical computation of the spectral moments

The spectral moment of order $n$ is defined as

$$
m_{n}=\int_{-\infty}^{+\infty} f^{n} P(f) d f
$$

For even values of $n$ and since $P(f)$ is an even function, we may write

$$
m_{n}=2 \int_{0}^{+\infty} f^{n} p(f) d f
$$

In practice, estımates of $m_{n}$ (even $n$ ) may be obtained from

$$
m_{n}=2 \Delta f \sum_{r=1}^{k}(r \Delta f)^{n} P(r \Delta f)
$$

With $k \Delta f=F_{C}$ and $f$ being the frequency interval between two adjacent estımates of $P(f)$ 
Theoretically we have $m_{0}=c(0)$ and so it is suggested that, in practice and with a view to calculating spectral moments, $F_{C}$ be chosen such that

$$
c(0)=2 \Delta f \sum_{r=1}^{k} \rho(r \Delta f)
$$

with $F F=k \Delta f$

Fig 7 shows the variation with $F_{C}$ of $m_{q}$ values obtained from (15) it is seen that $F_{C}$ should be chosen between $025^{C}$ and 03 cps approximately

36 - Maximum lag for the autocovariance

The choice of $T_{M}$ is very important, as on it are closely dependent the resolution and stability of estimates $A$ great $T_{M}$ produces high resolution estimates owing to the narrow bandwidth of the spectral Window, smoothing is however small and in consequence estimates present greater instability if $T_{M}$ is small then the inverse is true we get small resolution and great stability A compromise is therefore necessa ry between stability and resolution

Three criteria for the choice of $T_{M}$ were considered

a) The window closing technique

This technique is suggested by Jenkins (4) and consists in considering successi vely increasing $T_{M}$ values, or, equivalently, decreasing b values (window closing), whlch will have the effect that initially obscured spectrum detals will become more defined There are no rules to decide when certain detalls, as, for instance, a peak which is beginnıng to show, are real or due to instability Jenkins suggests that three spectra should be presented, computed for $T M$ values from the range where the initial form of the spectrum starts to change, that is, where after the initial convergence of shape a divergence begins to appear foIg 8 shows that the general spectrum configuration is kept untıl $T_{M}$ reaches about $70 \mathrm{~s}$ Afterwards, a swellıng begıns to appear around frequency $012 \mathrm{cps}$ and it becomes quite distinct when $T_{M} \approx 90 s T_{M}$ value of about $80 \mathrm{~s}$ seems therefore indicated

b) Using the rectangular lag window

If the autocovariance function $c(\tau)$ is zero for $|\tau|>\tau_{0}$, using the rectangular lag window with $T_{M}>\tau_{0}$ produces the true record spectrum 'If one uses $T_{M}<\tau_{0}$, negative values may appear for the spectrum, which is an indication that $T M$ is not sufficiently high (Barber $\{6]$ ) This could eventually serve as a criterion for the chol ce of $T_{M}$ Yet, in practice, with sea wave records, the autocovariance function never really comes to zero however great we may make $T$ T This is due to periodici ties which always turn up in natural records and the result is that negative values may always arise whatever the $T_{M}$ Fig 9 shows that, though $T_{M}$ was increased tenfold, negative estimates show no tendency to disappear On the other hand, when the autocovariance function looks like the one pictured in Fig 2 , where there are re gions of almost total damping, as in the neighbourhood of $\tau=44 \mathrm{~s}$, a rectangular lag window truncation in that region should produce a spectrum equal to one from a re cord for which $c(\tau)=0$ from that region on This spectrum should not exhibit nega tive values, that is really the case when, in the present computations, $T_{M}=445 s$ was used, as is seen in Fig 9 The conclusion to draw is that using the rectangular lag window is not a satisfactory way to decide on $T_{M}$

c) Variation of $E$ with $T_{M}$

One parameter to which the name spectrum width is generally given is

$$
\varepsilon=\sqrt{\frac{m_{0} m_{4}-m_{2}^{2}}{m_{0} m_{4}}}
$$

where $m_{n}$ is defined by (13) $\varepsilon$ values computed by (15) from spectra in which $T_{M}$ 
$\checkmark a t$ led from 22 s to $222 \mathrm{~s}$ and where $F$ was constantly equal to 03 cps are presented in table 1 and graphicaliy in Fig $C_{11}$ it is seen that for $T M>70 s$ the $\varepsilon$ value varies only very slightly This may mean that a sufficiently high value for $T M$ has been reached

Table 1

\begin{tabular}{|c|c|c|c|c|c|c|c|c|c|}
\hline \multicolumn{7}{|c|}{ VARIATION OF $T_{M}$} & \multicolumn{3}{|c|}{ 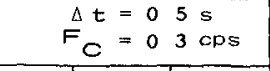 } \\
\hline$T_{M}$ & 22 & 445 & 50 & 60 & 70 & 80 & 89 & 100 & 2225 \\
\hline$\varepsilon$ & 061 & 056 & 055 & 055 & 054 & 054 & 054 & 054 & 053 \\
\hline$m_{0}$ & 050 & $0 \quad 51$ & 051 & $0 \quad 51$ & $0 \quad 51$ & 051 & $0 \quad 51$ & 051 & $\begin{array}{ll}0 & 51\end{array}$ \\
\hline$f_{\text {peak }}$ & 0085 & 0080 & 0080 & 0080 & $0 \quad 080$ & $0 \quad 080$ & $\begin{array}{ll}0 & 080\end{array}$ & 008 & 2 peaks \\
\hline$P\left(f_{\text {peak }}\right)$ & 317 & 455 & 479 & $5 \quad 15$ & $\begin{array}{ll}5 & 41 \\
\end{array}$ & $5 \quad 59$ & 569 & 577 & $\begin{array}{l}\text { smo- } \\
\text { othing }\end{array}$ \\
\hline
\end{tabular}

These 3 criteria considered, it was decided to use $80 \mathrm{~s}$ as an adequate value for $T_{M}$ ' which is about 6 to 7 times the period corresponding to the peak frequency of the spectrum

\section{7 - Record partition}

The record was partitioned in four 5-minute parts Table 2 and Fig 10 show results of computations made $m$ values increase clearly from the $1^{\text {st }}$ to the $2^{\text {nd }}$ part and from the $2^{\text {nd }}$ to the 3 rd part The $3^{\text {rd }}$ and $4^{\text {th }}$ part values are about the same Spectrum variations may have different reasons They may result from the fact that we are now dealing with four different samples and variation may be mere ly statistical in nature These variations should, perhaps, be considered large, which might mean that a five-minute period is too short for getting a representative re-

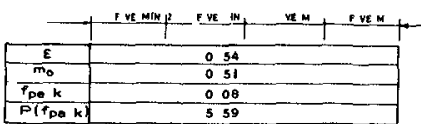

\begin{tabular}{|c|c|c|c|c|}
\hline$\varepsilon$ & 051 & 053 & 054 & $0 \quad 54$ \\
\hline$m_{0}$ & 039 & 045 & 057 & 060 \\
\hline$f_{\text {peak }}$ & 009 & 008 & 008 & 008 \\
\hline p(fpeak & 318 & 480 & 575 & 574 \\
\hline
\end{tabular}

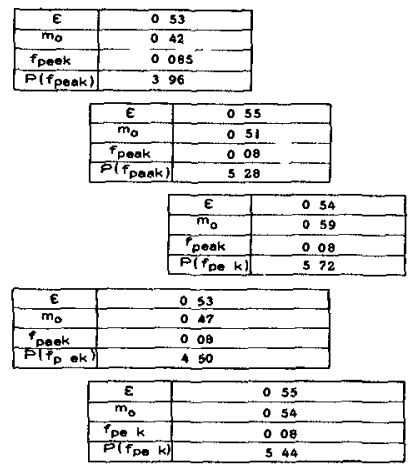
cord Since $m$ is a measure of the mean energy of sea waves, another reason for the variations may be that the record should not be considered completely stationary In future, when an extensi ve study is undertaken of records made at the same date as this one, the matter may be cları fied further

$38-\frac{\text { Variation of some statistics with } T_{M^{\prime}} \Delta t}{\text { and } F_{C}}$

Tables 1,3 and 4 show the variation of $\varepsilon$, $m_{0}, f_{\text {peak }}$ and $P\left(f_{\text {peak }}\right)$ with $T_{M}, \Delta t$ and $F_{C}$ res pectively Variation of $\varepsilon$ with the same parameters is also shown in Fig 11

As said in 35 variation of $\Delta t$ will not influen ce spectrum estimates, as long as $F_{D} \leq F_{N}$ This is illustrated in table 3

Convergence of $\varepsilon$ values when $T M$ increases is apparent from table 1 and $F i g ~ 11$, from $T=70 \mathrm{~s}$ onwards $f_{\text {peak }}$ is practically the same for all $T_{M}$ but $P\left(f_{\text {peak }}\right)$ increases with the closing of the spectral Window since the influence of neighbouring low values steadily decreases This increase, however, is small for $T_{M}>80 \mathrm{~s} \mathrm{~m}$ values vary only slightly with $T_{M}$ and $F C$ as seen in tables 1 and $4 \mathrm{~F}$ is clear that $F_{C}$ should 
exert no influence on $f_{\text {peak }}$ and $P\left(f_{\text {peak }}\right)$ Cholce of $F_{C}$ is nevertheless very important for the calculation of $\varepsilon$ as is seen in Table 4 and $F$ ig 11 , and should be made according to 35

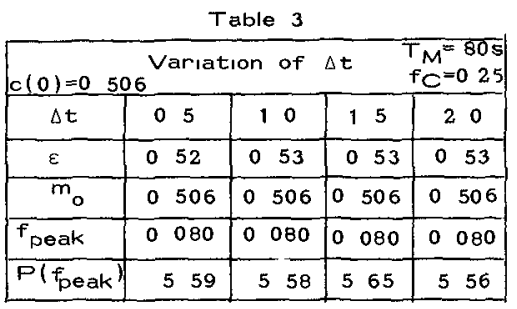

Table 4

\begin{tabular}{|c|c|c|c|c|c|c|c|c|c|c|}
\hline \multicolumn{2}{|c|}{$c(0)=0 \quad 506$} & \multicolumn{6}{|c|}{ Variation of $F_{C}$} & \multicolumn{3}{|c|}{$\begin{aligned} \Delta t & =05 \\
T_{M} & =805\end{aligned}$} \\
\hline${ }^{6} \mathrm{C}$ & $\begin{array}{ll}0 & 150\end{array}$ & 0 & 200 & 0 & 250 & 0 & 300 & & 650 & 1 \\
\hline$\varepsilon$ & 045 & 0 & 50 & & 052 & 0 & 52 & & 79 & 095 \\
\hline$m_{0}$ & $0 \quad 495$ & 0 & 505 & & 506 & 0 & 506 & 0 & 507 & 0508 \\
\hline \multicolumn{2}{|c|}{$f_{\text {peak }}$} & \multicolumn{6}{|c|}{008} & & & \\
\hline \multicolumn{2}{|c|}{$P\left(f_{\text {peak }}\right)$} & \multicolumn{6}{|c|}{559} & & & \\
\hline
\end{tabular}

39 - Conclusions

The considerations made from 31 to 38 led to the adoption of the following characteristics for the final computation of the bottom spectrum

- No prefiltering

- Window Parzen

- Maximum lag $80 \mathrm{~s}$

- Cutoff frequency $03 \mathrm{cps}$

- Sampling interval $05 \mathrm{~s}$ (although this could have been wider the corresponding computations were already avallable)

- 80\% confidence intervals

The number of degrees of freedom was 55 and the bandwidth 0023 cps The re sulting values for $\varepsilon, m_{0}, f_{\text {peak }}$ and $P\left(f_{\text {peak }}\right)$ are presented on table 1 Value comput ed for c(0) was 05056

This final bottom spectrum is shown in Fig 12

Table 5

4 - WAVE STATISTICS AND WAVE HEIGHT DISTRIBUTION

41 - Computations of wave statistics by the Tucker-Draper method

The Tucker-Draper method $[8],[9]$, was used to compute the sta tistics of Table 5 By this method, the fundamental values read from the record are $T_{Z}$ (mean zero up crossing perıod) $H_{1}$ (sum of the high est crest with the lowest trough), $\mathrm{H}_{2}$ (sum of the second highest crest $w$ th the second lowest trough) To obtain surface values $H$ from bottom values $H$ the classical Hydrodynamics Formula was used

where d $($ depth $)=233 \mathrm{~m}$

$$
H_{1}=H \operatorname{ch} \frac{2 \pi d}{L}
$$

$L($ wave length $)=\sqrt{\text { gd }}\left(1-\frac{\pi d}{468 T_{Z}^{2}}\right) T_{Z}^{*}$

$g$ being the acceleration of gravity

Computations were based on the digitalızed record using a sampling interval $\Delta t=05 \mathrm{~s}$

42 - Wave height distribution compared with the Rayleigh oistribution

Wave height in the present paper is the distance between the le-

* - This formula is derived from $c=\sqrt{\frac{g L}{2 \pi}}$ th $\frac{2 \pi}{L}$ by a series expan-

sion of the th and substituting $L$ by its approximate value

$L=\sqrt{g d} T_{Z}$

\begin{tabular}{|c|c|}
\hline$T_{z}$ & 108 \\
\hline$E$ & $0 \quad 58$ \\
\hline$H_{1}$ & 45 \\
\hline $\mathrm{H}_{2}$ & 35 \\
\hline$H^{\prime}$, & 71 \\
\hline$H^{\prime}{ }_{2}$ & 55 \\
\hline$\left(H_{s}\right)_{1}$ & 28 \\
\hline$\left(H_{s}\right)_{2}$ & 24 \\
\hline$\left(H^{\prime}{ }_{s}\right)_{1}$ & 44 \\
\hline$\left(H^{\prime}{ }_{s}\right)_{2}$ & 38 \\
\hline$\left(m_{0}\right)_{1}$ & 048 \\
\hline$\left(m_{0}\right)_{2}$ & 035 \\
\hline
\end{tabular}


vels of a crest and the preceding trough For the record considered, Fig 13 shows that the wave distribution is different from the Rayleigh distribution, which was tn be expected sınce $\varepsilon \approx 051$, a value greater than the lımit (0 4) below which, accord ing to Cartwright and Longuet-Higgins $[10]$, wave heights follow reasonably well that distribution

\section{5 - COMPAFISON BETWEEN WAVE STATISTIOS OBTAINED FROM THE PRESSURE RECORD BY SPECTRAL ANALYSIS AND THE TUCKER- -DRAPER METHOD}

In Table 6 wave statistics obtained from the pressure record by spectral analysis and the Tucker-Draper method are compared

Both for $m$ and $H_{s}$ a pair of values is presented corres ponding to calculations masde from $\mathrm{H}_{1}$ e $\mathrm{H}_{2}$

6 - CORRECTION TO SUIRFACE OF PFESSURE RECORD STATISTICS

Though the alm of the nork undertaken is mainly the pro cessing of digital data extracted from wave records, which makes unimportant the nature (surface or bottom) of the record used, a comparison of results arising from a weather hindcast with those from spectral analysis and the Tucker-Draper me thod is possible only if a correction to surface is applied to the latter Formula (18) was used with and without the instrument

Table 6

\begin{tabular}{|c|c|c|}
\hline & $S P$ & $T-D$ \\
\hline$\varepsilon$ & 054 & 058 \\
\hline \multirow{2}{*}{$m_{0}$} & \multirow{2}{*}{$0 \quad 51$} & 048 \\
\hline & & 035 \\
\hline \multirow{2}{*}{$H_{s}$} & \multirow{2}{*}{284} & 28 \\
\hline & & 24 \\
\hline
\end{tabular}
factor 125 which is recommended by the makers of the LNH type pressure wave gauge as adequate for the correction to sur face of the significant wave height The same formula, without the instrument fac tor, and the linearity hypothesis for spectrum decomposition as a sum of infinitesimal sinusoids leads to the following relation between surface and bottom spectra, $P_{S}(f)$ and $P_{d}(f)$ respectively

$$
P_{s}(f)=P_{d}(f) \mathrm{ch}^{2} \frac{2 \pi d}{L(f)}
$$

where $L(f)$ is the wave length corresponding to frequency $f$

It should be noted that (1) the instrument factor is very important and an in vestigation should be made to determine if the recomriended value of 125 is adequa te for sea wave regime at the Portuguese Coast, (2) formula (20) is based on an unverified hypothesis, namely, on linearity of the spectrum decomposition as a sum of Infinitesimal sinusoids in Fig 14 results of the surface correction for the spec trum are shown Some pertinent remarks are the following

- The peak of the surface spectrum occurs at frequency $0085 \mathrm{cps}$, which is very close to the one of the bottom spectrum

- The existence of a second peak at 011 cps may or not have a physical significance (as, for instance, a local storm superimposed on the pre-existent one) as pointed out in 36 a)

- Up from $f=013 \mathrm{cps}, P(f)$ values increase without any physical sjgnjficance Owing to the great depth $(233 \mathrm{~m})$ of the recording wave gauge, ch $\frac{2 \pi}{\mathrm{d}} \mathrm{va}-$ lues increase rapidly to absurd results from that frequency on

7 - WAVE HINDCAST FOR DECEMEER $18^{\text {th }}, 1968$

\section{1 - Analysis of the synoptic charts}

Examining the synoptic charts for some days prior to December $18^{\text {th }}$, 1968 , it is apparent that the pressure field has taken a little varying shape in the last three days ( FIg 15), the isobarics followlng approximately a northwesterly direction, and that Leixões is included in the generating area over which this three-day storm actuated As will be seen later the sea should be considered as fully arisen 


\section{2 - Approximate determination of the fetch to be considered}

In Fig 16 , the maximum fetch relating to the meteorological situations of the storm days $\left(16^{\text {th }}, 17^{\text {th }}\right.$ and $\left.18^{\text {th }}\right)$ is delimited by a dashed line After a mean wind speed during the storm is fixed, it can be seen that any of the indicated fetches is greater than the minımum fetch corresponding to a fully arisen sea

73 - Wave refraction up to the wave gauge site

Recorded values at Leixốes at a depth of $233 \mathrm{~m}$ cannot be transformed in off shore values without some criticism

As examination of $\mathrm{Fig} 17^{*}$ reveals, waves following a course close to NW have a refraction coefficient given by $\mathrm{He} / \mathrm{Ho}$ (where He is wave height at the harbour entrance and Ho the corresponding off-shore value) which is independent of period (the latter varying between 10 and $17 \mathrm{~s}$ ) and has a nearly constant value of 07 ap proximately This is the adopted value for a correction of statistics for off-shore conditions, according to a rough, yet seemingly adequate, procedure for the present case Concerning the course followed by waves the following remarks should be noted It was not possible to secure radar photographs made at Leixões and so there is no direct information on which course to consider However, the shape of the isobarics shows that that course should be close to NW On the other hand, for a better accuracy of the $\mathrm{He}_{\mathrm{e}} \mathrm{H}_{0}$ coefficient, a refraction plan including the wave gauge site should be avallable, indeed, elements provided in $[2]$ concern only the harbour entrance Therefore the adopted 07 value should not be accepted unreservedly

74 - Hindcast

741 - Observations from North Atlantic Weather Ships

For a wave hindcast for the day the pressure record was made, knowledge of wind, sea and swell data provided by the North Atlantic Weather Ships is necessary According to their position and keeping in view the NW direction of the storm, the most important ships are, by increasing order of distance to Leixões $K$ shıp $(730$ $\mathrm{km}), \mathcal{s h i p}(1500 \mathrm{~km})$ and $C \operatorname{sh} 1 \mathrm{p}(2400 \mathrm{~km})$

$K$ ship is the only one included in the zone common to the three fetches indicated in Fig 16 and, even so, she is outside the minimum fetch corresponding to a fully arisen 30-knot wind sea, as will be seen later Tnis is then the ship from which the most important information stems, as regards waves at Lelxões (Fig 18 )

We may roughly guess at the day and the period of time in which waves recorded at Leixôes were passing by each one of the three ships (Table 7) Once more we are simplyfying things by assuming that the propagation speed is $V_{T}=g T / 4 \pi$ and considering the period range to be 8 to $16 \mathrm{~s}$

Table 7

\begin{tabular}{|c|c|c|c|c|c|c|}
\hline \multirow{2}{*}{$\frac{2}{\vec{t}}$} & \multirow{2}{*}{$\left.\mid \begin{array}{lll}4 & x \\
0 & 0 & 0 \\
0 & 0 & 0\end{array}\right]$} & \multirow{2}{*}{$\begin{array}{c}T \\
(s)\end{array}$} & \multirow{2}{*}{$\begin{array}{l}V_{T} \\
(m / s)\end{array}$} & \multirow{2}{*}{ 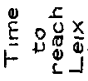 } & \multicolumn{2}{|c|}{$\begin{array}{l}\text { Ship passed } \\
\text { by }\end{array}$} \\
\hline & & & & & day & $\mathrm{hr}$ \\
\hline \multirow[b]{2}{*}{$k$} & \multirow{2}{*}{730} & 8 & 62 & 33 & 16 & 19 \\
\hline & & 16 & 125 & 16 & 17 & 12 \\
\hline & \multirow{2}{*}{1500} & 8 & $\begin{array}{ll}6 & 2\end{array}$ & 67 & 15 & 9 \\
\hline & & 16 & 125 & 33 & 16 & 19 \\
\hline \multirow{2}{*}{$c$} & 00 & 8 & $\begin{array}{ll}6 & 2\end{array}$ & 108 & 13 & 16 \\
\hline & & & 12 & 54 & 15 & 22 \\
\hline
\end{tabular}

Table 8

\begin{tabular}{|c|c|c|c|c|c|}
\hline \multirow{2}{*}{$\frac{a}{5}$} & \multicolumn{3}{|c|}{ Swell } & \multicolumn{2}{|c|}{ Sea } \\
\hline & Dir & $H(m)$ & $T(s)$ & $H(m)$ & $T(s)$ \\
\hline$K$ & $\mid \begin{array}{c}W \\
\text { iconst }\end{array}$ & $\left(\begin{array}{c}6 \\
\text { (const) }\end{array}\right.$ & $\begin{array}{c}12 \\
\text { (const) }\end{array}$ & $\left.\begin{array}{cc}9 & 5 \\
(8-10\end{array}\right)$ & $\begin{array}{c}10 \\
\text { (const) }\end{array}$ \\
\hline J & $\begin{array}{c}276 \\
270-282)\end{array}$ & $\begin{array}{cc}7 & 5 \\
(7-8) \\
\end{array}$ & $\begin{array}{c}12 \\
\text { (const) }\end{array}$ & $\begin{array}{cc}65 \\
(6-7) \\
\end{array}$ & $\begin{array}{c}85 \\
(8-9)\end{array}$ \\
\hline$c$ & - & - & - & $\begin{array}{c}5 \\
(4-6)\end{array}$ & $\left(7^{8}-9\right)$ \\
\hline
\end{tabular}

Table 8 is a résumé of sea and swell conditions recorded by each one of the three ships in such a way that waves could have arrived at Leixões during the twenty-minute recording period at 3 a $\mathrm{m}$ on December $18^{\text {th }}$, 1970

* - Reproduced from Fig 5 in $|2|$ 
Another important problem in the application of the two most common forecast Ing methods (SMB and PNJ) concerns the choice of a mean wind speed likely to have occurred during the storm Fig 16 shows that the mean values of wind speed recorded at $K$ ship on the two days before Dec $18^{\text {th }}$ are

$$
\begin{array}{lll}
\text { Dec } 16^{\text {th }} & U=42 \text { knots } & \text { (a) } \\
\text { Dec } 17^{\text {th }} & U=32 \text { knots } & \text { (b) }
\end{array}
$$

Assuming that the sea is fully arısen (Leixöes being included in the generatıng area) it will be interesting to know land-recorded values at the closest weather station to Leixões (Pedras Rubras)

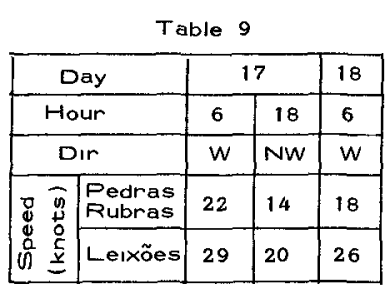

Mean value at Leıxões (sea) $U=25$ knots (c) With $U$ values indicated in (a), (b) and $(\mathrm{c})$ a mean value should now be adopted if a Neumann spec trum is chosen corresponding to $U=30$ knots according approximately with the pressure spectrum (Fig 14) and assuming a fully arisen sea, the following minı mum values are obtained for duration and fetch $t=$ $=23 \mathrm{~h}, F=518 \mathrm{~km}$ Hence, in fixing U, only Lei xões and $K^{\mathfrak{T}}$ ship should be considered ( $K$ ship is even beyond $F$ in case $U=30$ knots)

In short with mean $U$ values of 25 knots (at Leixões) and 32 knots (durıng the previous 24 hours) and now reasoning backwards, the choice of $u=30$

knots seems to be adequate as the mean prevaling wind speed which brought about the fully arisen sea relative to the Dec $18^{\text {th }}$ storm

742 - Forecasts of the $S M N^{*}$ $17^{\text {th }}$ and $18^{\text {th }}$ was "strong northwesterly waves" which corresponds to wave heights between 25 and $55 \mathrm{~m}$

743 - The Sverdrup-Munk-Bretschneider Method (S M B )

$F$ or $U=30$ knots and assuming a 800 nautical mile fetch corresponding approxima tely to Dec $18^{\text {th }}$, we get $t=50 \mathrm{~h}, H_{\mathrm{s}}=609 \mathrm{~m}, \mathrm{~T}_{\mathrm{s}}=14 \mathrm{~s}$

There is small accuracy in the choice of fetch, but observing Fig 1-7 on page 19 in [3] one can see that wave characteristics vary only slightly between 800 and 1000 miles The mean wind speed of 30 knots can be assumed constant during two days which corresponds approximately to $t=50 \mathrm{~h}$

Wave forecast for the wave gauge zone, takıng into account a He/Ho value of 07 is then $\mathrm{H}_{\mathrm{s}}=43 \mathrm{~m}$

744 - Pierson-Neumann-James method ( $P N J$ )

As mentioned above a 30 -knot Neumann spectrum waschosen according approxımately with the pressure spectrum (Fig 14)

The corresponding hindcast statistics have the following off-shore values

$$
\begin{array}{llll}
H_{s} & =66 \mathrm{~m} & H_{\text {av }} & =3 \mathrm{~m} \\
H_{1 / 10}=83 & T_{\text {av }} & =85 \mathrm{~s} \\
& f_{\text {peak }} & =008 \mathrm{cps}
\end{array}
$$

Taking into account the correction for the wave gauge zone we get

$$
H_{\text {av }}=21 \mathrm{~m} \quad \mathrm{H}_{\mathrm{s}}=44 \mathrm{~m} \quad H_{1 / 10}=57 \mathrm{~m}
$$

* - Serviço Meteorológıco Nacional (Portuguese Weather Service) 
8 - COMPARISON OF COMPUTED AND HINDCAST SURFACE SIGNIFICANT WAVE HEIGHT VALUES

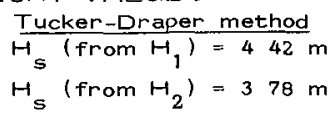

Taking the arithmetic mean and correcting to surface with ch $\frac{2 \pi d}{b}$ only, we get $H_{s}=41 \mathrm{~m}$ If the instrument factor 125 recommended by Chatou is used, then $H_{s}=5$ i $\mathrm{m}$

PN」method $H_{s}=44 \mathrm{~m}$

$S M B$ method $H_{s}=43 \mathrm{~m}$

$\subseteq M N$ forecast for Dec $18^{\text {th }}$ Wave helghts between 25 and $55 \mathrm{~m}$

These values are close to one another and so it seems reasonable to conclude that at Leıxões, on December $18^{\text {th }}$, 1968, the surface sıgnificant wave helght was 4 to $5 \mathrm{~m}$

9 FINAL SUMMARY OF THE MORE NOTEWORTHY ASPECTS OF THIS PAPER

Partial detaled conclusions were drawn in preceding chapters of seemingly more salient aspects A summary of important points is intended

- The sampling interval may be as high as 2 s if spectral analysis only is

- If a smaller interval is used, which means a higher Nyquist frequency $(F N)$, then a convenient cutoff frequency $(F$, ) should be chosen, possibly according to coincidence of $\mathrm{m}$ and $c(0)$

- A good estimate of the spectrum width depends on a well balanced choice of some parameters, especially on the cutoff frequency

- A good choice of maximum lag for the autocovariance function is of great importance to spectral analysis

- Results from record partition seem to indicate that a more careful study must be made of the necessary record duration

- Some aspects of wave data acquisition which are well-known yet should be kept in mind concern - correction to surface of bottom spectra (lack of linearity) and of bottom statistics (adequate instrument factor)

- great influence of depth on wave attenuation

waves

- faulty characteristics of pressure records for spectral analysis of sea

- For hindcast and related meteorological problems, the usual difficulties arise in fetch determination (to the resolution of this problem, directional spectra may provide a useful contribution) it is also important to make a realistic criticism to sea wave data secured from meteorological services, indeed, it is frequent to detect absurd correlations between wave heigths and periods and sometimes falla cious distinctions are made between sea and swell

As final conclusion we would like to stress the importance of a close collaboration between different techniques used in Maritime Hydraulics This collaboration is escential for an intimate connexion in the development of both the physical (meteorolvgy, oceanography, fluid mechanics' and the mathematical approaches 


\section{ACKNOWLEDGEMENTS}

The authors are grateful to the Instituto Hidrografico for the facilities granted concerning reading and digitizıng wave records They are also in debt to Dr Anthím:o de Azevedo from the Serviço Meteorologico Nacional for the meteorological elements provided and their interpretation

\section{REFERENCES}

[1] - Bonnefille, Germain, Lepetit - Statıstıques des houles naturelles mesures par le houlographe autonome type LNH, Houlle Blanche no 8, 1967

[2] - Rels de Carvalho, J $J$ e $D$ Vera-Cruz - Regıme de ondulação no Porto de Lelxões, Estudo realizado para a Sacor, LNEC, Julho de 1964

[3] - Shore Protection, Planning and Design, Technical Memorandum no 4, CERC (formerly Beach Erosion Board), 3rd Edition, June 1966

[4] - Jenkins, $G M$ and $D G$ Watts - Spectral Analysis and its applications, Holden-Day, San Francisco, 1969

[5] - Barber, NF - Experimental Correlograms and Fourier Transforms, Pergamon Press, 1961

[6] - Barber, NF - A plea for the rectangular lag window, Ocean Wave Spectra, Prentice-Hall, Englewood Cliffs, 1963

[7] - Blackman, $R B$ and $J W$ Tukey - The Measurement of Power Spectra from the Point of View of Communications Engineering, The Bell System Technical Journal, January and March, 1958

[8] - Tucker, $M \cup$ - Analysis of Records of Sea Waves, Proceedings of the Ins titution of Civil Engineers, October 1963

[9] - Draper, L M - The Analysis and Presentation of Wave Data - A plea for Uniformity, Froceedings of the $x$ th Conference on Coastal Engineering, Tokyo, 1966

[10] - Cartwright, $D E$ and $M S$ Longuet-Higgins - The Statistical Distribution of the Maxima of a Random Function, Proceedings of the Royal Society, $A$, 237,1956 


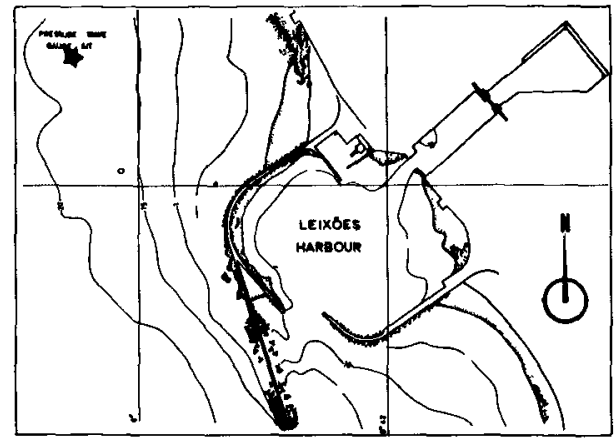

Fig 1 - Wave gauge site

Fig 4 - Raw and smoothed spectrum

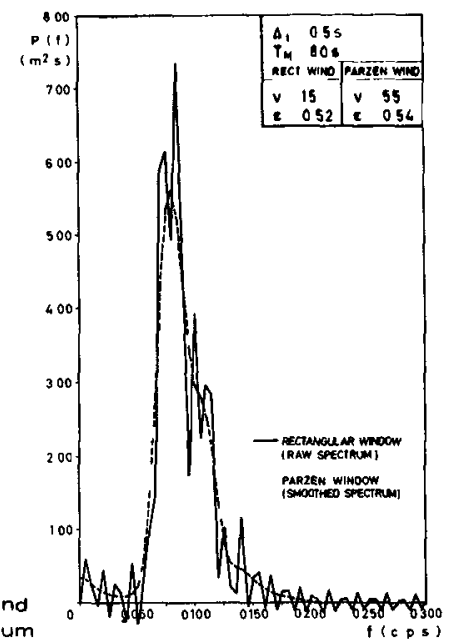

Fig 2 - Autocovariance function
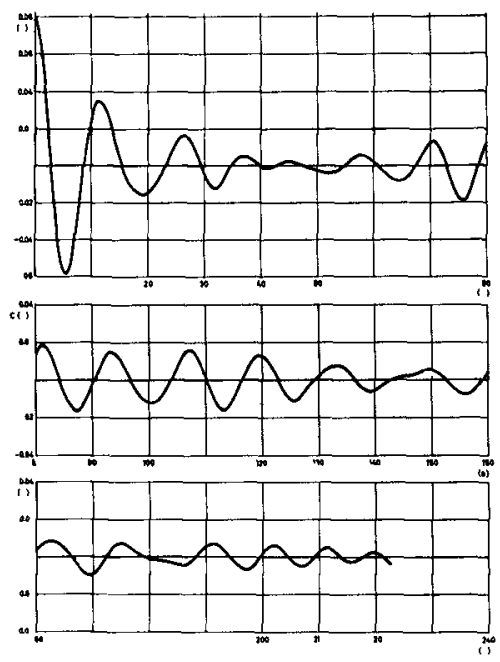

Fig 3 - Windows considered
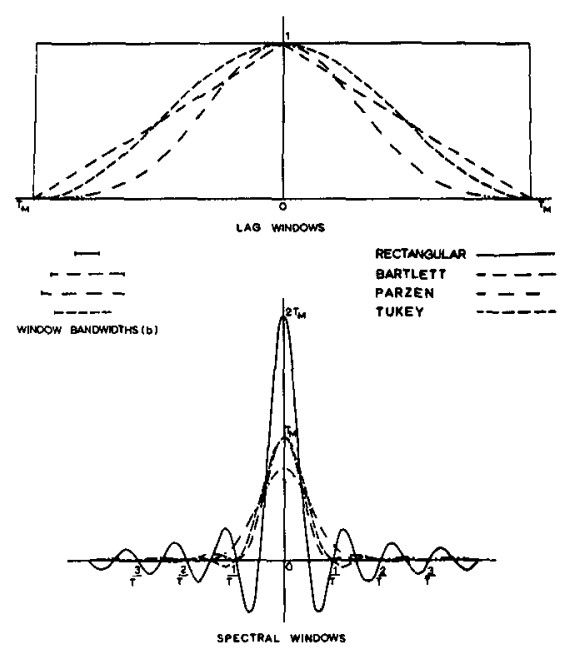

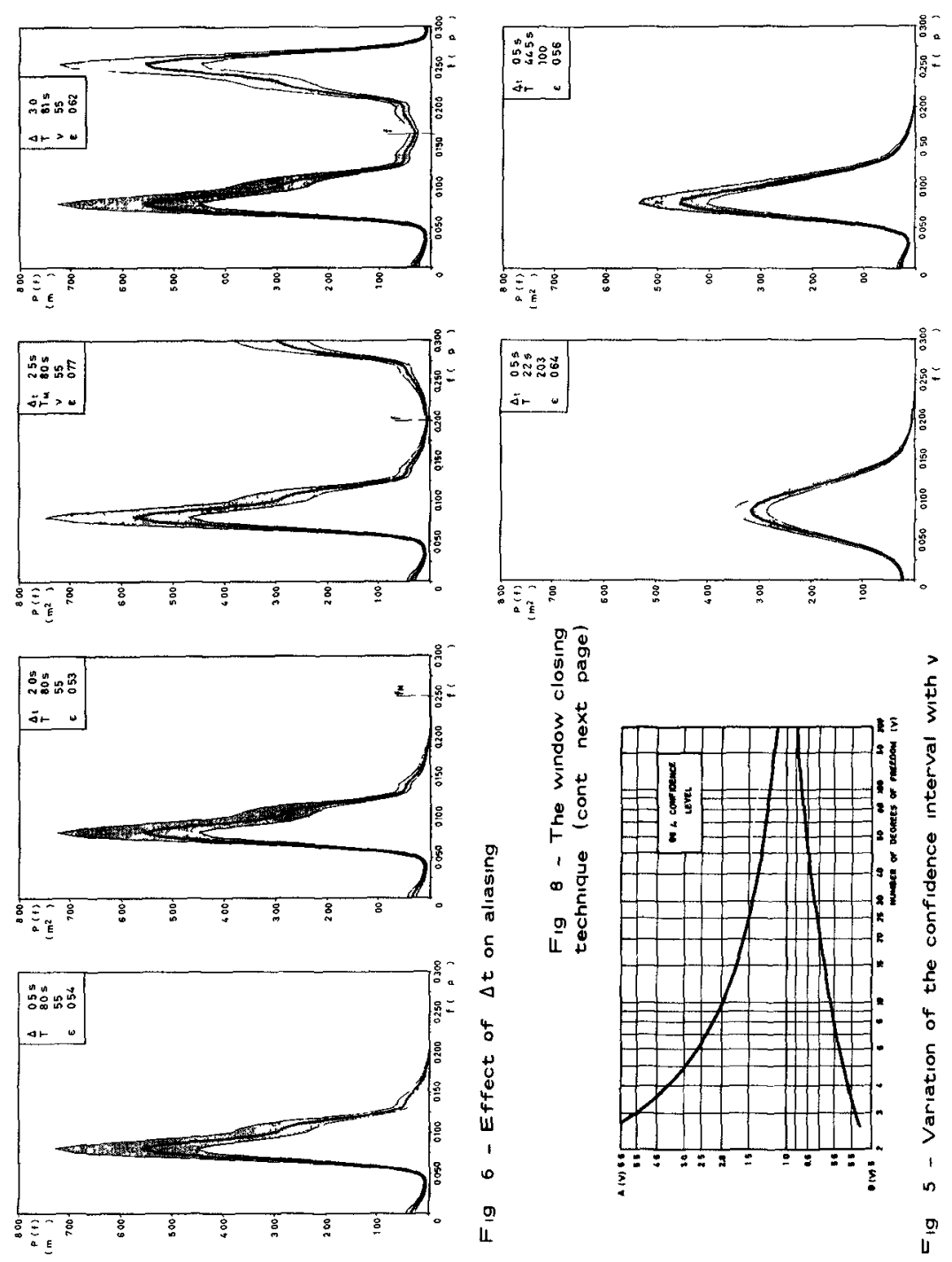

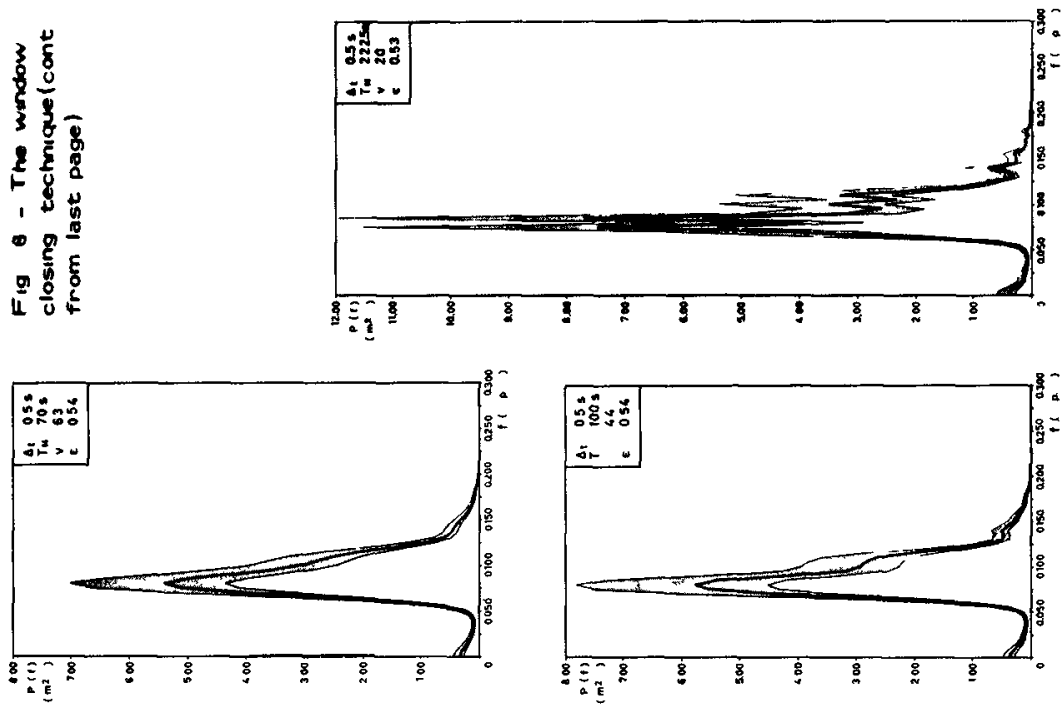

(4)
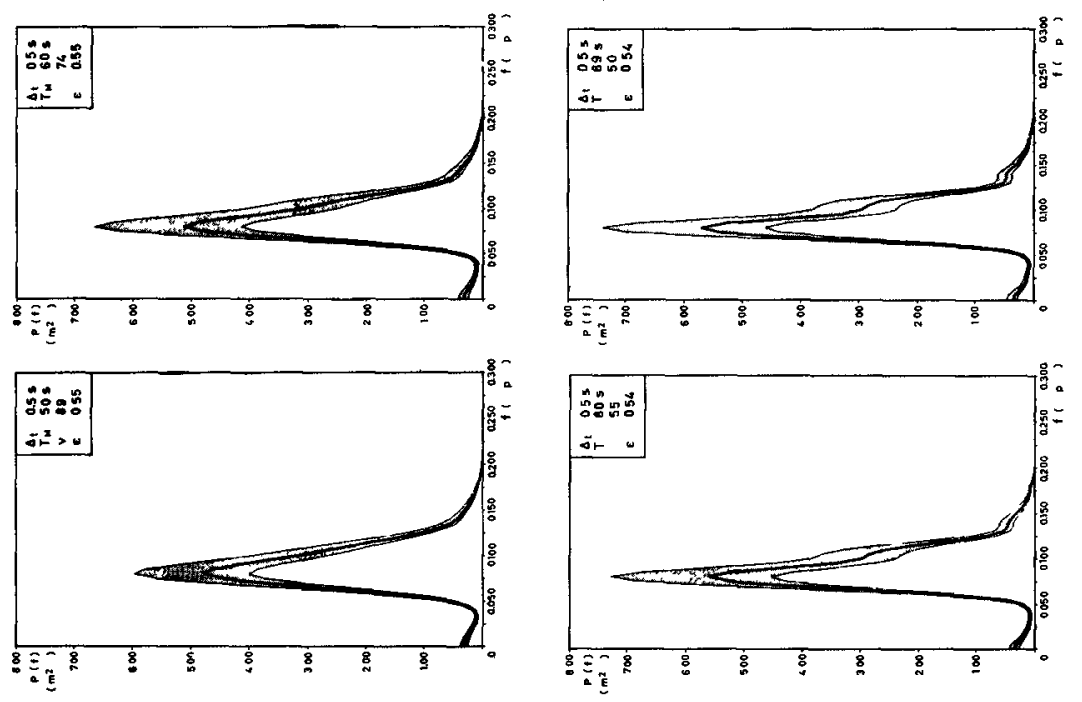

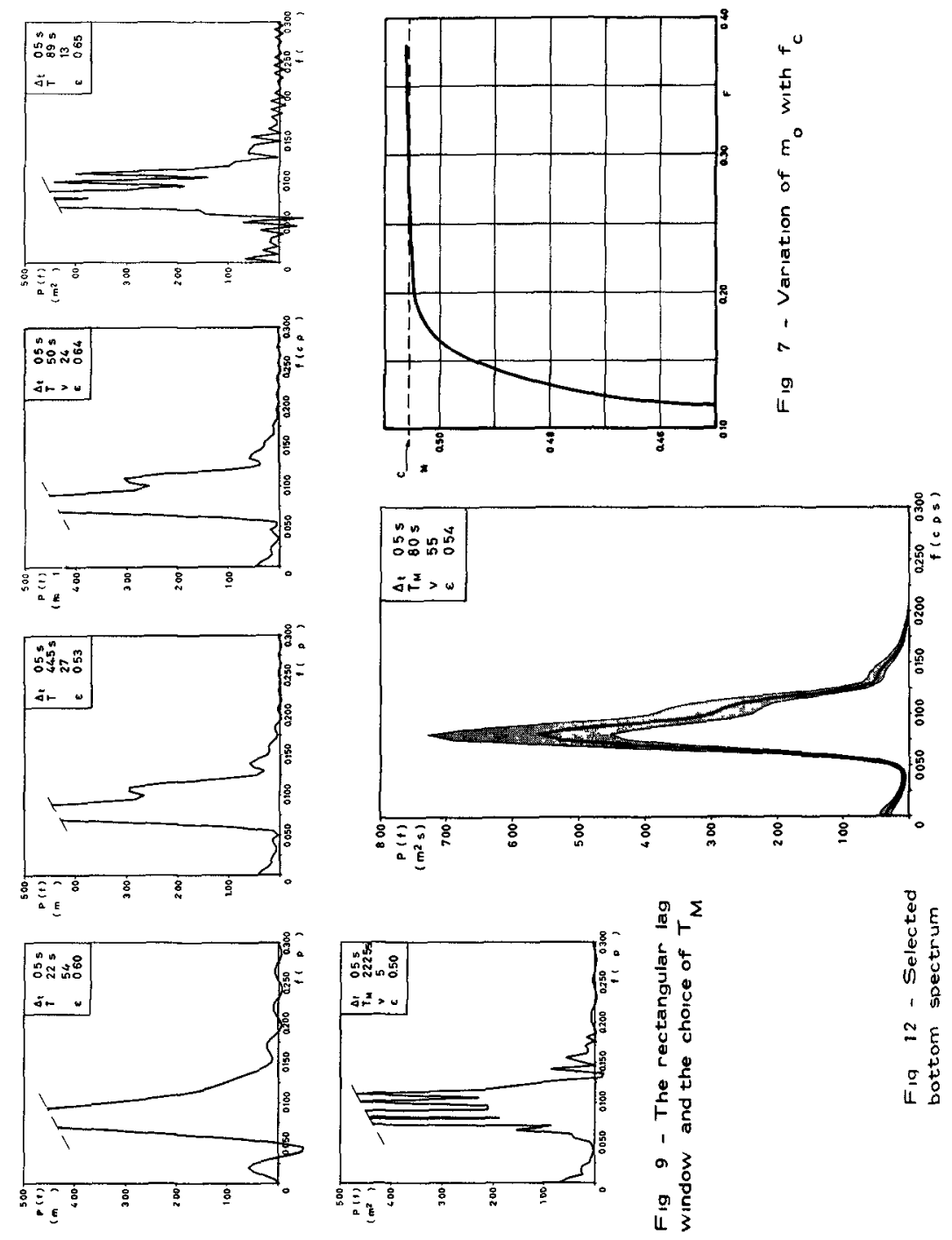

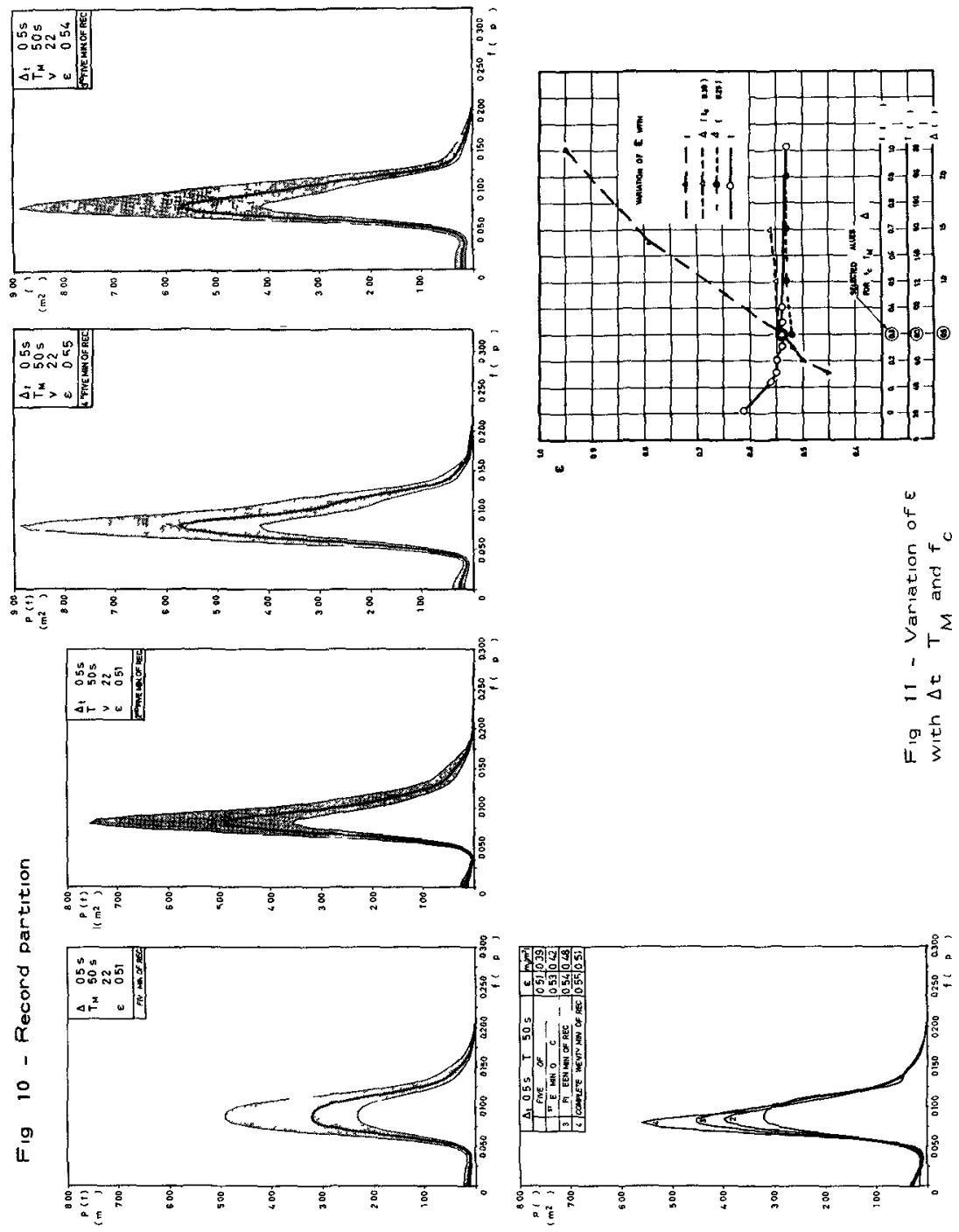

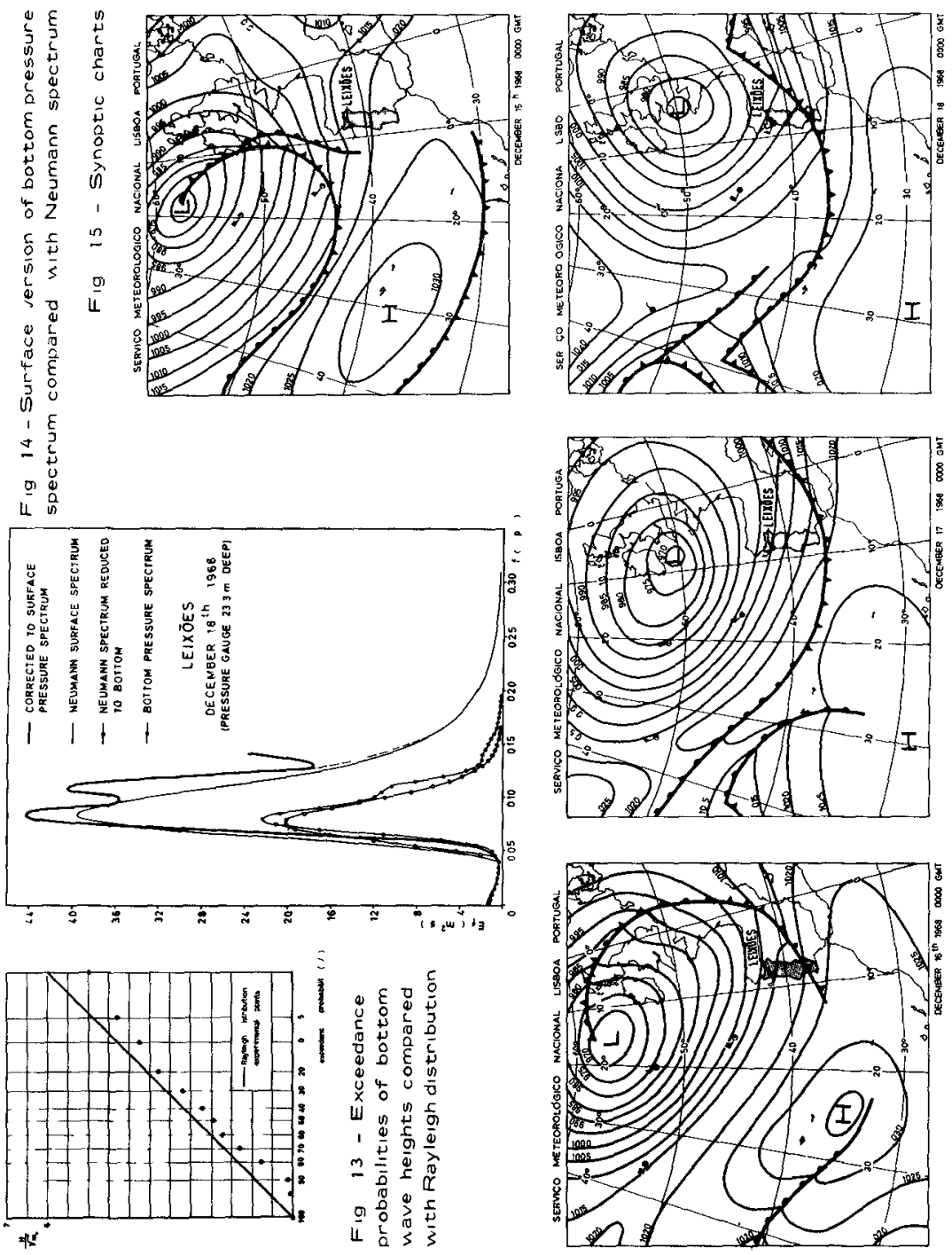
Fig 16 - Fetch delimitation
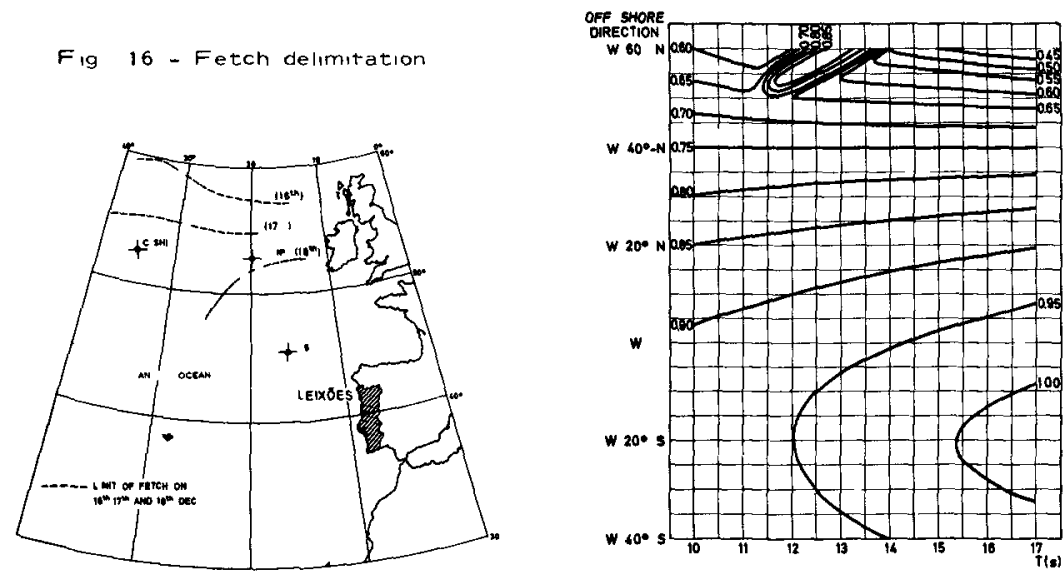

Fig 17 - Refraction coefficients at

Leixóes harbour entrince

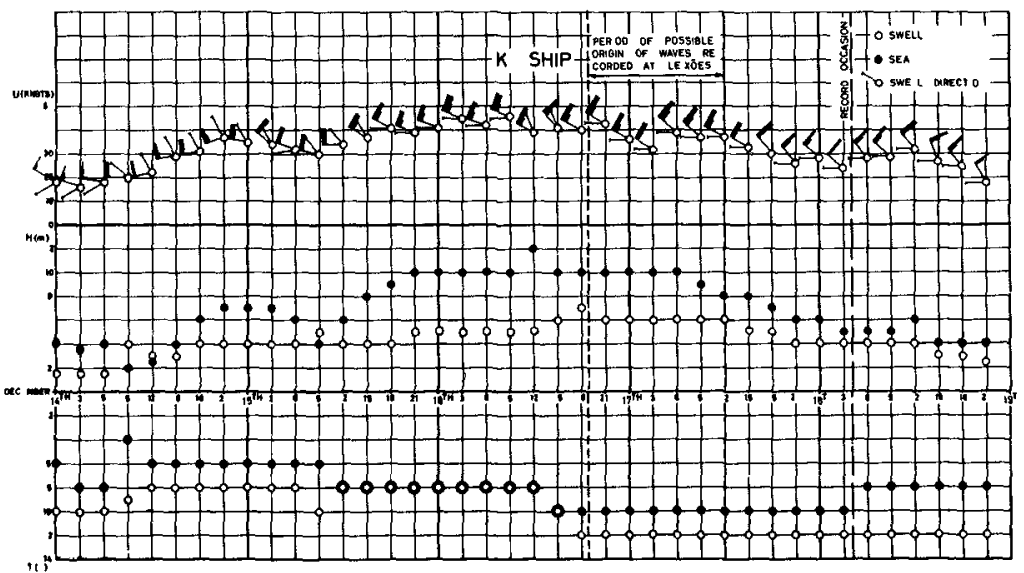

Fig 18 - Ouservations at North

Atlantic $K$ weather shis 



\section{CHAPTER 6}

\section{THE ANALYSIS OF WAVE RECORDS}

by

D Lee Harris

Research Division, U S Army Coastal Eng1neering Research Center Washington, D C

\section{ABSTRACT}

Data obtalned from two surface profile wave gages and two pressure wave gages at the Steel Pler in Atlantıc City, New Jersey, are used to check the consistency of the analysis variables obtained from a given set of records by several commonly used analysis procedures

All estimates of the characteristic helght tested are found to be correlated better than 86 The estimates of characteristic period are not so satisfactory and in some cases are below 25 Consideration of several proposed definitions of the characteristic period indicates that they are based more on convenience in data processing than on application of the derived data Consideration of the use of wave data in engineering design shows that no one definition of the period can be satisfactory for all applications The best definition of the characteristic wave period for a given engineering problem can be specified only when the dynamic aspects of the problem have been identified

\section{INTRODUCTION}

The concept of a "significant wave height" and a "significant wave period" which can be used to characterize a wave field is appealingly simple It suggests a simple transition from the experimental results in a laboratory wave tank and the theoretical results obtained with monochromatic wave theory to the phenomena that occur in the real ocean

Th1s concept was first introduced when sallors were asked to report the helght and the perlod of " the larger, well formed waves, and omit entirely the low and poorly formed waves " as part of the synoptic weather reports from ships Comparison of early wave gage records, with visual observations, led to the opinion that the wave helght "H "given by v1sual observers was the average helght of the one-third highest Individual waves, "H $1 / 3$ " Figure 1 , taken from Ross (1966) and based on an earlier figure by Cartwright (1962) provides some perspective on the reliability of this approximation Figure 1 is based on 905 pairs of visual and instrument observations from a weather ship equipped w1th a shipboard wave recorder For the data included in this figure $\mathrm{H}_{1 / 3}=$ $11 \mathrm{H}_{\mathrm{v}}$ Comparisons of shipboard observations by two or more observers are given by Hogben and Lumb $(1964,1967)$ and 11kew1se show considerable scatter 


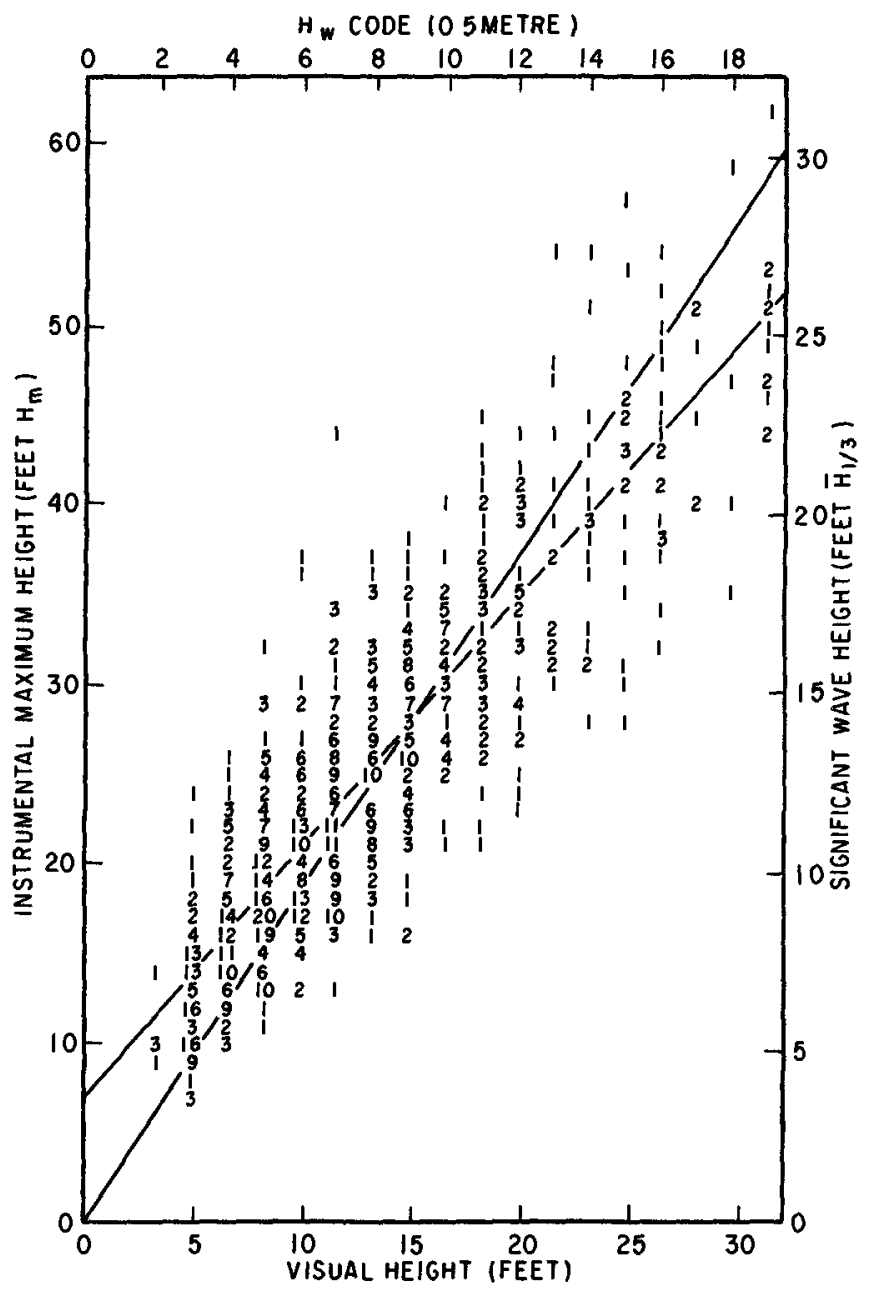

Figure I Comparison of instrument and visually observed heights (after Ross)

For the data in the above figure $\mathrm{H}_{1 / 3}=11 \mathrm{H}_{v}$ 
Many wave records have been analyzed by listing the he1ghts and periods of individual waves for the determination of $\mathrm{H}_{1 / 3}$ and the corresponding period $\mathrm{T}_{1 / 3}$ This process is rather tedious and not truly objective, for there is no purely objective way of making a clear distinction of which perturbations on the record are waves that should be counted and which are ripples that should be omitted

This problem is 11lustrated by F1gure 2, where simultaneous records from four wave gages, located within a 12-foot circle on the Steel Pier in Atlantic City, are shown Note that some of the waves appearing on the surface gage records do not appear on the pressure records, and that almost any procedure for determining which waves should be counted would accept some waves on one surface record that would be rejected on the other

\section{OTHER DEFINITIONS OF WAVE PERIOD}

To obtain a more objective measure of the wave period, various writers (Draper, 1966, Tucker, 1961) have suggested using the average period of all zero up-crossings as the characteristic period Both of these depend somewhat on the resolution of the wave recording system and Tucker's depends on a practical method for determining the zero line Draper suggests that it can be estimated by eye Thus two different estimates may lead to two different values if interest is centered on the wind-generated waves, it would be more appropriate to consider crossing of a trend line The difference would not be important with large waves and small tides, but it could be significant with large tide ranges and small waves This latter combination can be important in sedimentation problems

Since 1965, 1t has been customary at CERC to identify the most prominent period in a 7 -minute wave record as the significant wave period

To clarlfy the meaning of wave period estimates, it is useful to note that according to the linear theory for monochromatic progressive waves,

$$
\begin{aligned}
h(t) & =\bar{h}+A \cos (k x-\sigma t-\phi) \\
w & =\sigma A \frac{\sinh k(z+D)}{\sinh k D} \sin (k x-\sigma t-\phi) \\
u & =\sigma A \frac{\cosh k(z+D)}{\sinh k D} \cos (k x-\sigma t-\phi) \\
p & =\rho A C^{2} k \frac{\cosh k(z+D)}{\sinh k D} \cos (k x-\sigma t-\phi) \\
k^{2} C^{2} & =\sigma^{2}=g k \tanh k D=\left(4 \pi^{2}\right) / T^{2}
\end{aligned}
$$

where $w, u$ are the vertical and horizontal components of velocity, $p$ the 


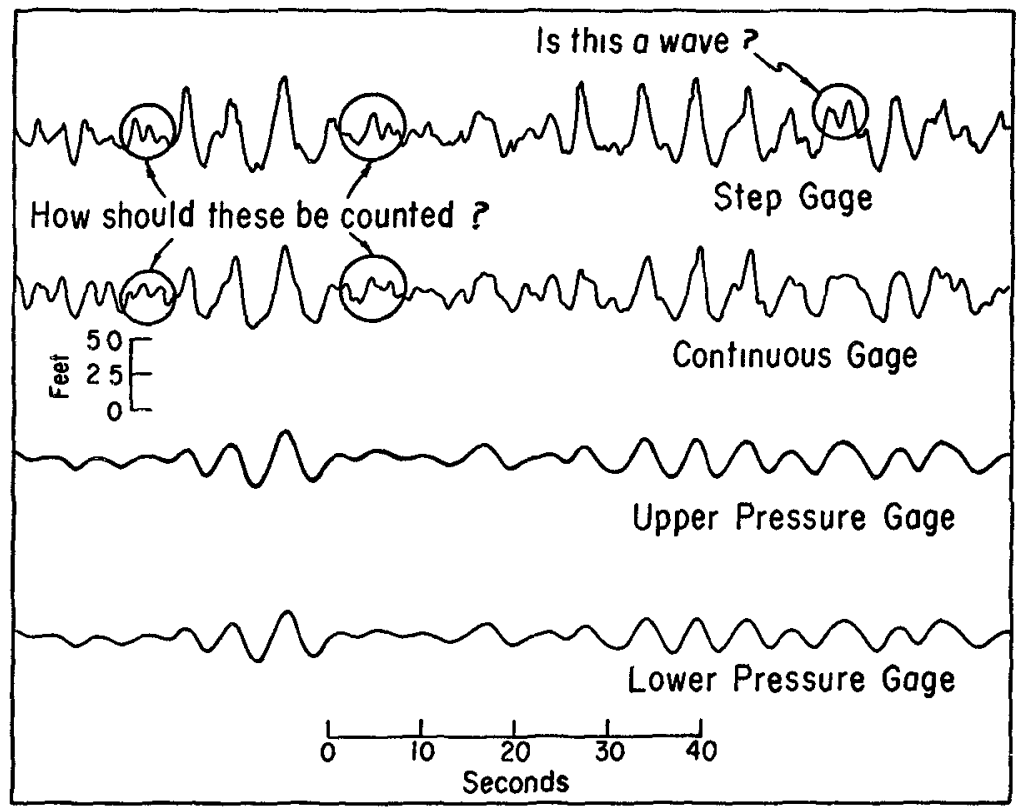

Figure 2 A sample of the simultaneous record from four wave gages at the Steel Pier in Atlantic City, $N J$, illustrating the difficulty in deciding which perturbations are to be considered as waves in determining $\mathrm{H}_{1 / 3}$ Records (a) and (b) are for pressure gages, (c) and (d) are pressure gages with (d) about 6 feet below (e) 
pressure, $k$ the wave number, $\sigma$ the frequency, $\phi$ the phase at $k x=\sigma t, C$ the phase speed, $T$ the period, $\rho$ the density and $g$ the acceleration of gravity The origin of $z$ is taken at the water surface and the upward direction is taken as positive Since, for any given value of $D$, there is a one-to-one relationshıp between perıod, frequency, wave number, wave length and phase velocity, functions which loglcally depend on any one of these parameters may be expressed unambiguously as functions of the period

In the more general case when several wave trains are present, it is more useful to consider

$$
h(t)=\vec{h}+\sum_{m=1} A_{m} \cos \left(k_{m} x-\sigma_{m} t-\phi_{m}\right)
$$

Similar expressions may be readily constructed for $u, w$, and $p$ in general $k_{m}$ and $x$ should be regarded as vectors with $k_{m} x$ as the scalar product of the vector wave number, $k_{m}$ and the position vector $x$ This detail is not important here, however, for if the gage position is fixed, $\mathrm{k}_{\mathrm{m}} \mathrm{X}$ is constant for each $\mathrm{m}$, and may be absorbed into the phase angle $\phi_{\mathrm{m}}$ This procedure will be followed in the remainder of the paper If the sequence of $\sigma_{m}$, important in a given application is known in advance, as in the case of astronomical tides, the most expedient analysis procedure for the calculation of the amplitudes $A$ would be a least squares evaluation of the coefficient $s, a_{m}, b_{m}$, in the expression

$$
\begin{aligned}
h(t)=\bar{h}+ & \sum_{m=1}\left[a_{m} \cos \sigma_{m} t+b_{m} \sin \sigma_{m} t\right] \\
A_{m}^{2} & =a_{m}^{2}+b_{m}^{2} \\
\phi_{m} & =\tan ^{-1} b_{m} / a_{m}
\end{aligned}
$$

If the sequence of $\sigma_{m}$ is not known in advance, and this is the usual case, the amount of arithmetic involved in the solution can be greatly reduced by choosing $\sigma_{m}=2 m \pi / T_{a}$, where $T_{a}$ is the period of record selected for analysis

It can be shown by Parseval's relation that the sum of all the $A_{m}^{2}$ is equal to the variance of $h(t)$ It can also be shown that the variance of $h(t)$ is proportional to the average potential energy of the wave (Kinsman, 1965, p 145ff) According to Taylor (1937), this is the idea Rayleigh had in mind when he first introduced the concept of an energy spectrum

If one value of $A_{m}^{2}$ say $A_{s}^{2}$ is much larger than all of the others, it seems natural to select the corresponding period, $T=2 \pi \mathrm{s} / \mathrm{T}$ a as the significant period If there are many $A_{m}^{2} ; s$ with nearly the same magnitude it may be desirable to regard the true energy as a continuous function of 
the frequency and the computed $A_{m}^{2}$ as estimates of this function, integrated over a small frequency interval centered on $\sigma_{m}$ When this procedure is followed, it seems natural to define the significant period as the period corresponding to the peak energy density per unit frequency if the wave energy is known, or estimated, as a continuous function of the frequency, a simple transformation of variables provides the energy as a function of the wave period Thus, the significant wave period could be defined as the period of maximum energy density per unit period

Short definitions of six proposed measures of the significant wave period are listed below

1 The average perıod of the one-third highest waves, $T_{1 / 3}$

2 The period most prominent in the record, $\mathrm{T}_{\mathrm{CERC}}$

3 The average period of all waves, $\mathrm{T}_{\mathrm{a} 11}$

4 The average period of a11 waves that cross the mean water level, $\mathrm{T}_{\text {ZUC }}$

5 The period of maximum energy density, $\mathrm{T}_{\mathrm{PM}}$

6 The period corresponding to frequency of maximum energy density, $\mathrm{T}_{\mathrm{FM}}$

The last of these seems most surtable for a study of wave dynamics, and is suggested as a standard The first two definitions have been proposed for convenience in collecting data, and the third and fourth to make the determinations more objective and reproducible

\section{OTHER DEFINITIONS OF WAVE HE1GHT}

Since the energy of a simple wave 1 s proportional to the square of the wave height, it is natural to define a measure of the wave height in terms of the square root of the average energy Th1s estimate, called the root-mean-square wave height by Tucker (1961) is equivalent to the standard deviation of the wave record and 15 defined by

$$
\mathrm{H}_{\mathrm{RMS}}=\left[\frac{1}{\mathrm{~N}} \sum_{\mathrm{n}=1}[\mathrm{~h}(\mathrm{n} \Delta \mathrm{t})-\overline{\mathrm{h}}]^{2}\right]^{1 / 2}
$$

where $h(n \Delta t)$ is the water surface elevation at time $t=n \Delta t$, and $\bar{h}$ is the mean water level for the analysis interval Thus $\mathrm{H}_{\mathrm{RMS}}$, unl1ke $\mathrm{H}_{1 / 3}$, has a clear physical definition and can be easily determined by either digital or analog computers Both theoretical and empirical evidence suggest that the average value of the ratio $\mathrm{H}_{1 / 3} / \mathrm{H}_{\mathrm{RMS}}$ is about 4 The actual value obtained from a given observation depends on the full wave spectrum 
Since 1965, it has been customary at CERC to estimate the significant wave height as the $N^{\prime}$ th highest wave in a 7 -minute wave record where $\mathrm{N}$ is a function of the selected period and is approximately $1 / 6$ of 420 seconds divided by the "significant period" This procedure can be performed very rapidly by making use of suitable transparent nomograms, but the determination of the "most prominent period in the record" is too subjective to be readily programed for a computer

Draper (1966), making use of some work by Tucker (1961), proposed a more objective system which can be really programed The standard deviation of the wave record called $H_{\text {RMS }}$ by Tucker is estimated from the highest and lowest water elevations in the record, and the signif 1 cant wave height is estimated as the product of the sum of the highest and lowest departures of the surface elevations from its mean position and a factor, which depends on the period of zero up-crossings

\section{A COMPARISON OF THE PARAMETERS AS EVALUATED BY SEVERAL DEFINITIONS}

Since 1966, CERC has been making a digital record from the step resistance wave gage at the Steel P1er in Atlantic City, New Jersey A computer program has been developed for calculating each of the measures of wave height and period discussed above with the exception of $\mathrm{T}_{\mathrm{CERC}}$ and $\mathrm{H}_{\mathrm{CERC}}$ which must be obtained manually Th1s program has been used to analyze the records from November and December of 1966

It has been found, for the records analyzed, that all of the measures for wave helght are highly correlated The correlation matrices for the principal measures are shown in Tables I and II Consequently, It appears to make relatively little difference how a record is analyzed to obtain wave height, since very nearly the same answer is obtained for any method The Fast Fourier Transform algorithm of Cooley and Tukey (1965), often called the "FFT" was used to analyze records 1024 seconds (17 minutes, 4 seconds) long for the computation of energy spectra This permits a detailed definition of the spectrum with a frequency resolution slightly better than $10^{-3}$ Hertz

Spectra with resolution per unit frequency similar to that obtained with the auto-correlation technique (B1ackman and Tukey, 1958), were obtained by averaging across frequency bands of constant width Spectra in terms of energy density per unit perıod were computed by averaging across frequency bands of varıable width

The correlation matrices for the various measures of the wave period are given in Tables III and IV, and a comparison of a few of the individual estimates in Table $V$ It can be seen that two estimating procedures which may agree to within 1 second in some cases, may differ by as much as 10 seconds in other cases The correlation appears to be better for the higher waves This is shown in Figure 3, in which the ratio of $T_{F M}$ to $T_{C E R C}$ is shown as a function of $H_{R M S} H_{R M S}$ is a measure of wave height defined on page 6 


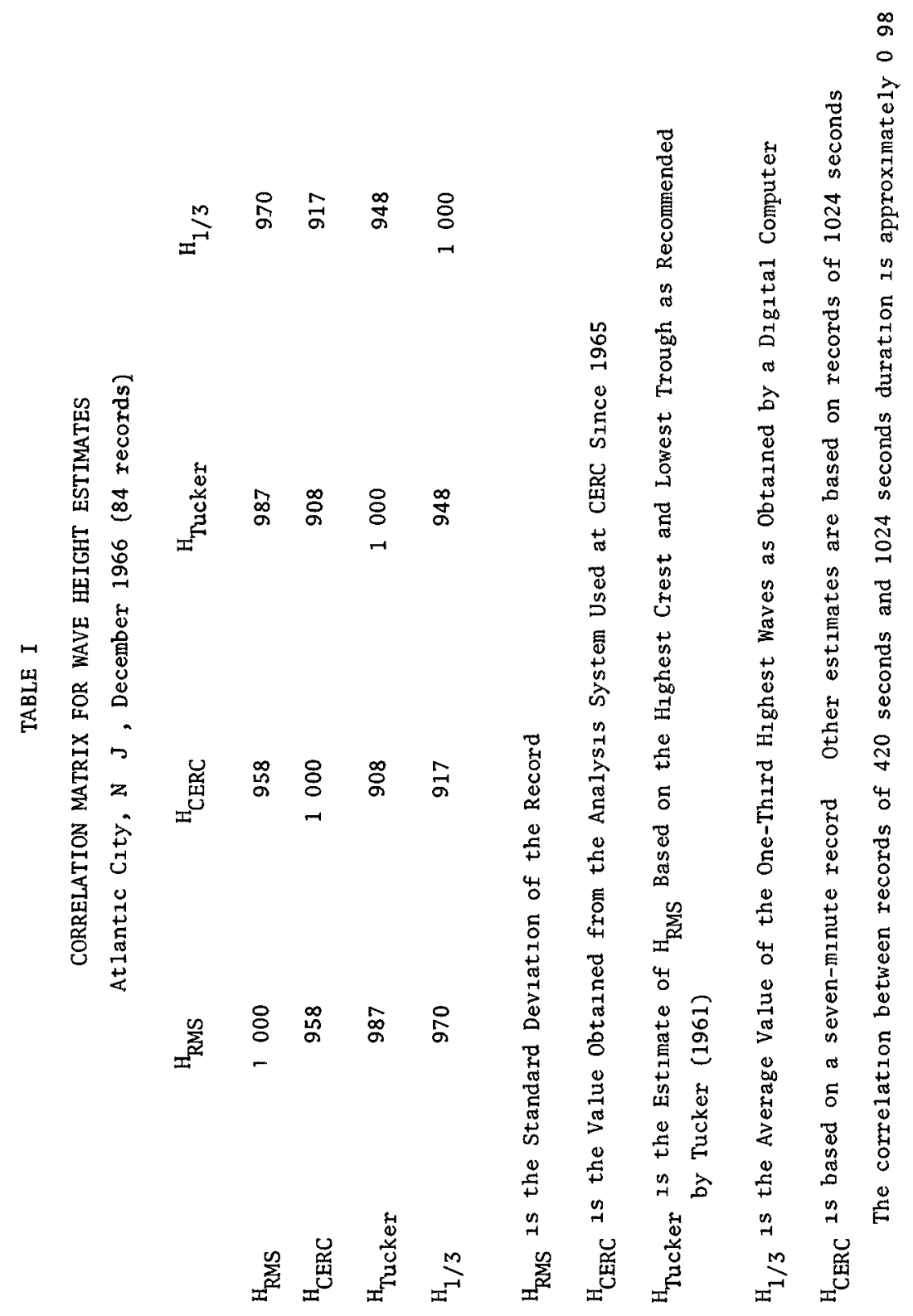


疍

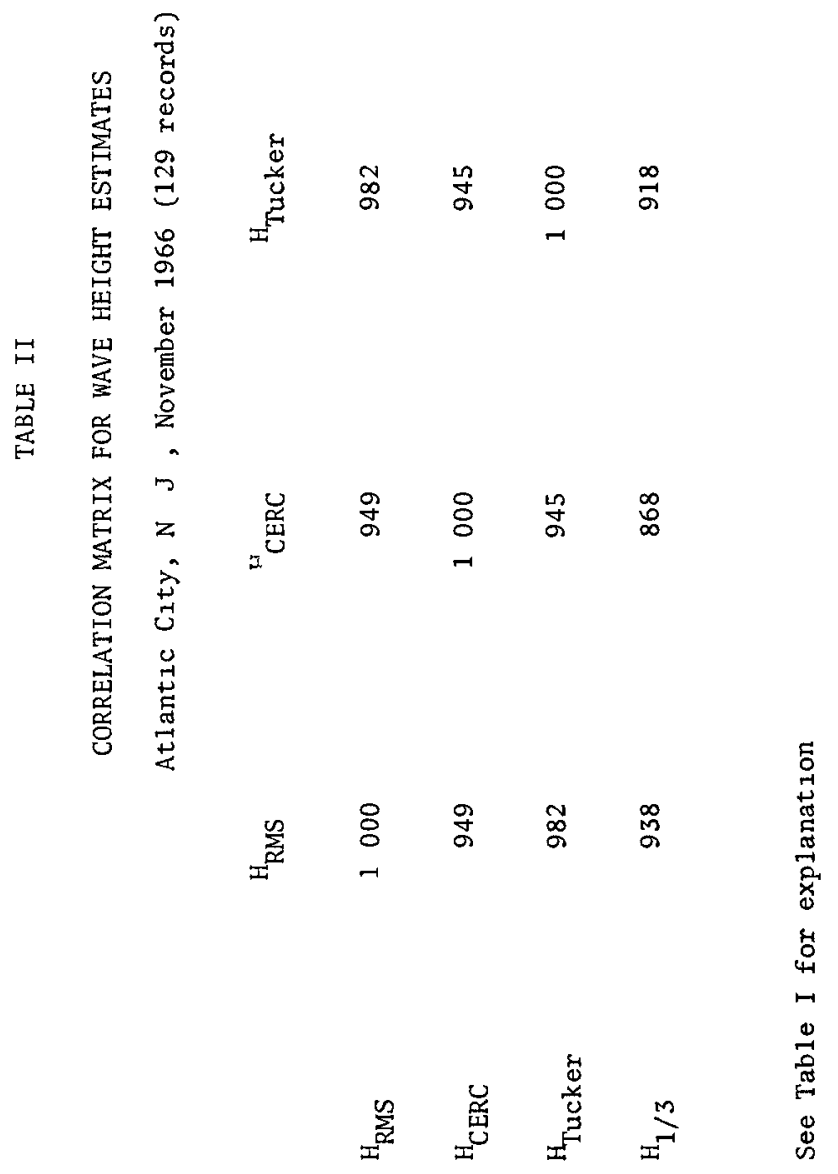




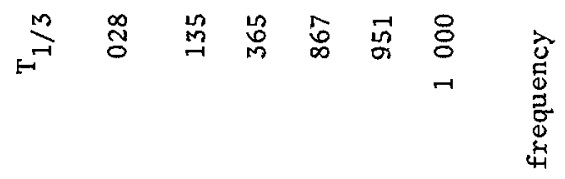

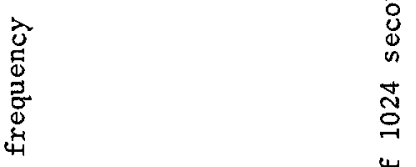

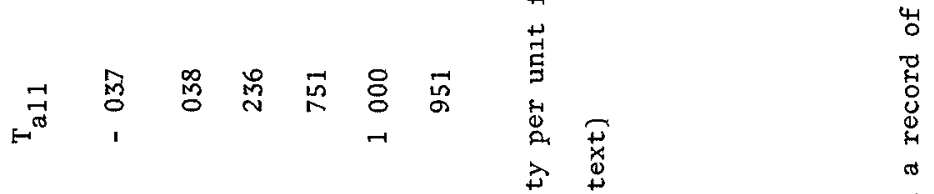

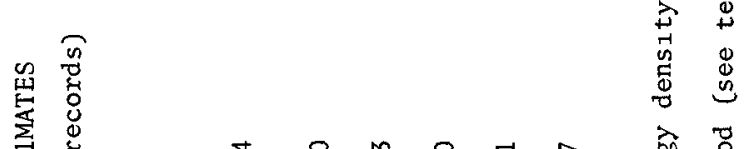

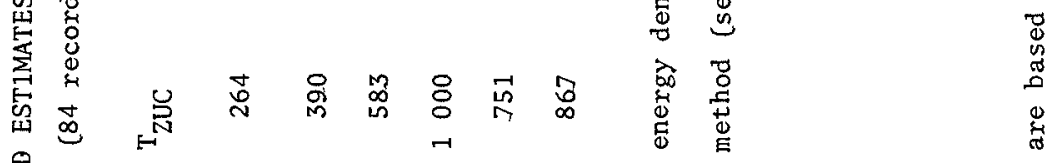

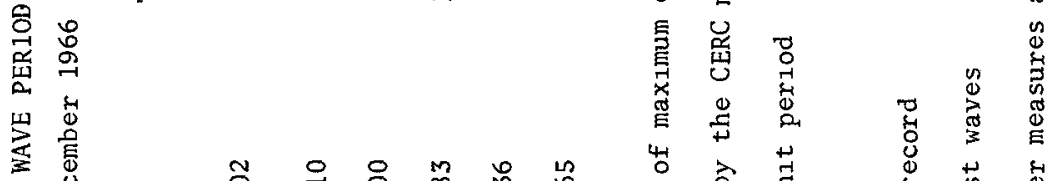

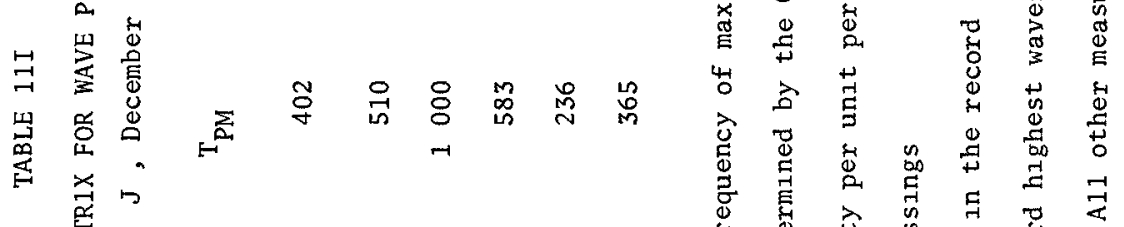

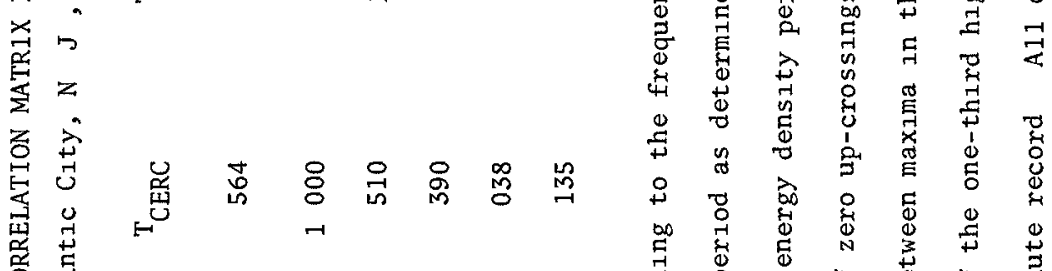

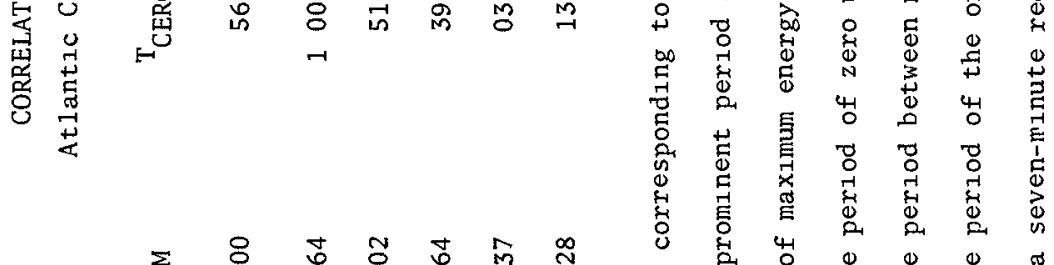

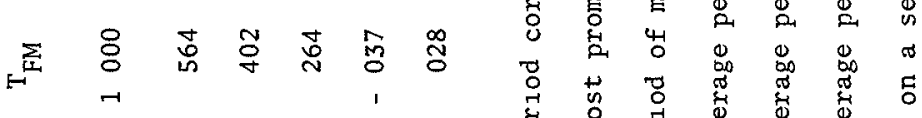

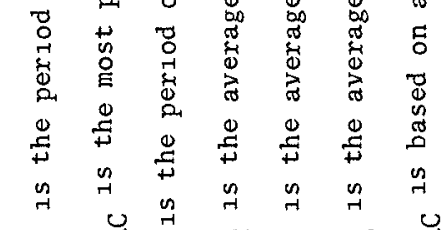

$$
\begin{aligned}
& \text { 蛋曷 }
\end{aligned}
$$




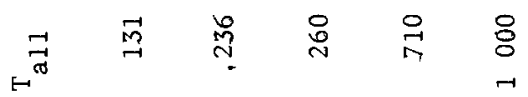

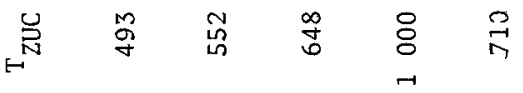

$$
\begin{aligned}
& \text { II } \\
& \text { 兽总 号 }
\end{aligned}
$$

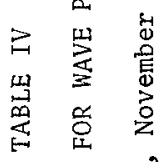

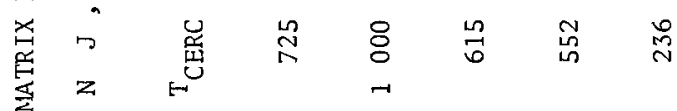

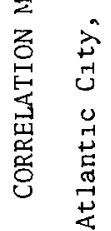

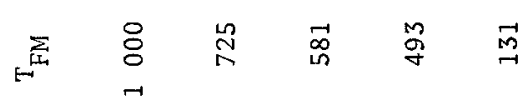

$$
\begin{aligned}
& \text { 5. } \\
& \text { 营 } \\
& \text { 定 }
\end{aligned}
$$

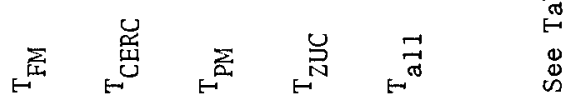




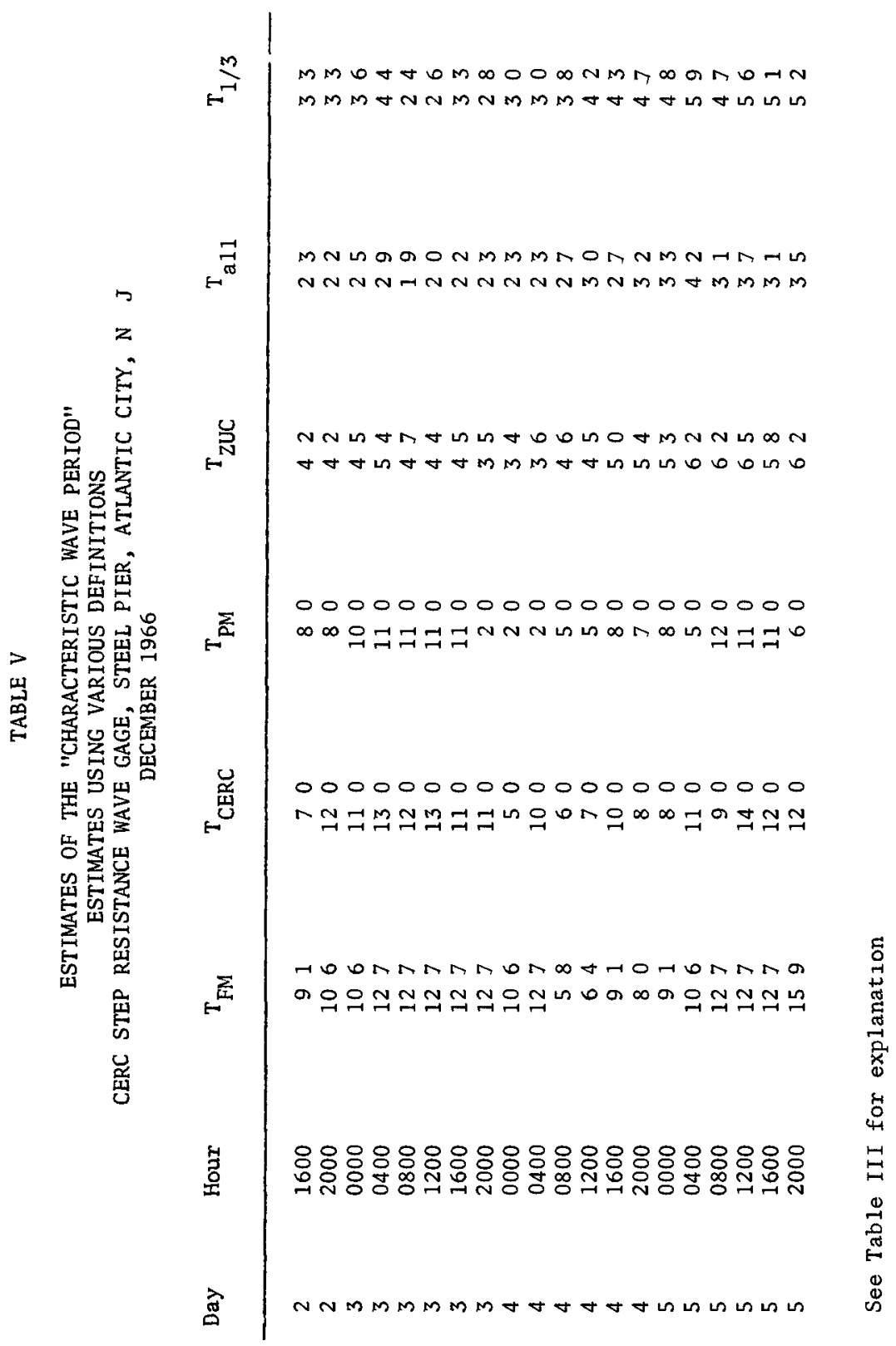




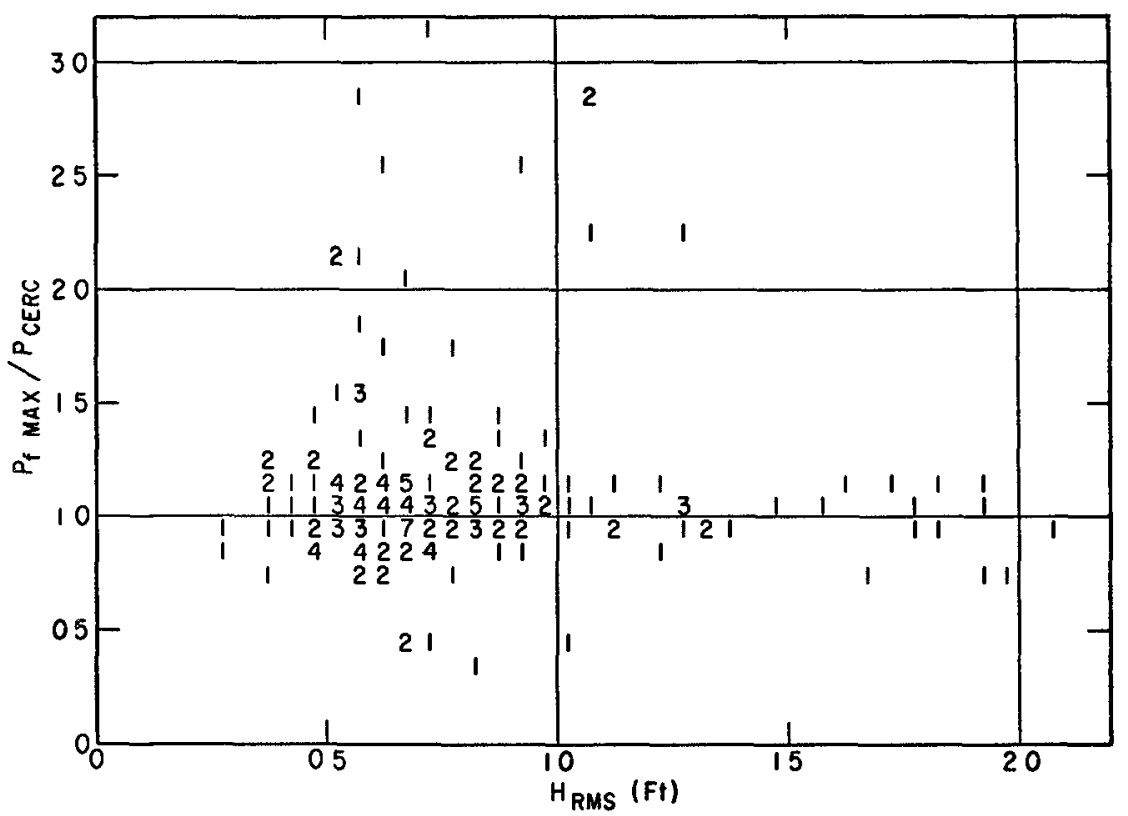

Figure 3 The ratio of $\mathrm{T}_{\mathrm{f}} \max$ to $\mathrm{T}_{\mathrm{CERC}}$ as a function of $\mathrm{H}_{\mathrm{RMS}}$ The numbers represent the number of cases in which the observation fell in $01 \times 005$ foot square 
THE INTERPRETATION OF A CHARACTERISTIC WAVE PERIOD

For linear wave theory there is a one-to-one correspondence between period, frequency, wave length, wave number and phase velocity Because of this, experimental results obtained with monochromatic waves are often tabulated or graphed as a function of period A careful analysis, however, may show that one of the other variables is more fundamental to the problem Thus in coastal engineering design, the wave period may be used to specify either the characteristic time scale or the characteristic length scale of some process due to waves A little reflection on the physical processes involved will show that no one variable can provide the best estimate of the time and space scales for all processes due to a given sea state This can be seen most clearly by considering a Fourier Transform of the wave record form

The energy spectrum for the wave height can be expressed in the

$$
E_{h}\left(\sigma_{m}\right) d \sigma=A_{m}^{2}
$$

The corresponding expressions for the spectra of the velocity components are obtained from (2) and (3) in the form

$$
\begin{aligned}
& E_{w}\left(\sigma_{m}\right) d \sigma=A_{m}^{2} \sigma_{m}^{2}\left[\frac{\cosh k(z+D)}{\sinh k D}\right]^{2} \\
& E_{u}\left(\sigma_{m}\right) d \sigma=A_{m}^{2} \sigma_{m}^{2}\left[\frac{\cosh k(z+D)}{\sinh k D}\right]^{2}
\end{aligned}
$$

The term $\sigma_{m}^{2}$ produces an amplification of the height spectrum with increasing frequency Thus if the height spectrum is flat or contains two or more nodes of nearly the same value, the peak of the velocity spectrum, on the surface, is likely to occur at a higher frequency than the peak of the helght spectrum The terms in square brackets decrease with depth and decrease more rapidly with increasing frequency Thus the peak of the velocity spectrum will have a tendency to shift toward Jower frequencies with increasing depth

$$
\begin{aligned}
& \text { Equation (5) can be used to eliminate } c^{2} \text { from (4) to obtain } \\
& \qquad p=\rho g A \frac{\cosh k(z+D)}{\cosh k D} \cos (\sigma t-\phi)
\end{aligned}
$$

The horizontal displacement, $x$, may be obtained from (3) by integration with respect to time to obtain

$$
x=A \frac{\cosh k(z+D)}{\cosh k D} \sin (\sigma t-\phi)
$$

The corresponding spectra are 


$$
\begin{aligned}
& E_{p}(\sigma) d \sigma=\rho^{2} g^{2} A^{2}\left[\frac{\cosh k(z+D)}{\cosh k D}\right]^{2} \\
& E_{X}(\sigma) d \sigma=A^{2}\left[\frac{\cosh k(z+D)}{\cosh k D}\right]^{2}
\end{aligned}
$$

At the surface the peak of these spectra must agree with the peak of the helght spectra, but the high-frequency components are attenuated w1th depth more than the low frequency components Thus with relatively flat or bimodal spectra, there is a tendency for the peak of the spectrum to shift toward low frequencies

Other transformations, which will produce other changes in the spectra, and in the period which seems to be most 1 mportant will be appropriate to some engineering problems

It seems that no one definition of the "significant period" for a wave field in which waves of several frequencies are present, can provide the best value for use in all engineering calculation

\section{SUMMARY AND CONCLUSIONS}

The wave-height estimates obtained from any particular wave gage by any of the analysis procedures tested are consistent, in the sense that the ranking of estimates obtained by one procedure will be nearly the same as that obtained by any other analysis procedure tested But a scale correction may be necessary to obtain the best fit between data analyzed by two different procedures

The period data obtained by different analysis procedures are not consistent and the estimates of a characteristic wave period have i1ttle value unless the procedure used in obtaining the period estimate is known Comparisons between wave periods may be more satisfactory when the data are stratified in some way which makes the data sample more homogenous Restricting attention to waves more than 3 feet high is one such stratification which improves the consistency of the estimates Other forms of stratification may also be useful

It appears that the best procedure for engineering design will be to disregard the tabulated periods, and to consider every period that might reasonably occur, along with the given height estimate, to determine the critical conditions or the design wave

The best choice for a design wave for a particular environment depends on the problem to be considered No single value can be sufficient for all problems 


\section{REFERENCES}

Blackman, R B and Tukey, J W, The Measurement of Power Spectra, American Telephone and Telegraph Co, 1958, reprinted by Dover Publications, New York

Cartwright, D E, "A Comparison of 1nstrumental and Visually Estimated Wave Heights and Periods Recorded on Ocean Weather Sh1ps", National Institute of Oceanography, October 1962

Cooley, J W and Tukey, J W, "The Fast Fourler Transform", IEEE Spectrum, Vo1 4, p 63-70, 1967

Draper, L "The Analysis and Presentation of Wave Data - A Plea for Uniformity", Proceedings of Tenth Conference on Coastal Engineernng, ASCE, New York (1967)

Esteva, D and Harris, D L, "Analysis of Pressure Wave Records and Surface Wave Records", Proceedings of Twelfth Conference, ASCE, Wa shingt on (1970)

Hogben, $\mathrm{N}$ and Lumb, $\mathrm{F} \mathrm{E}$ with Appendix by Cartwright, $\mathrm{D} \mathrm{E}$ The Exesentation of Wave Data from Voluntary Observing Shrps, National Physical Laboratory, Ship Division, London, 1964

Hogben, $\mathrm{N}$ and Lumb, F E, Ocean Wave Statrstrcs, National Physical Laboratory, Ministry of Technology, London, 1967

Kinsman, B , Wrnd Waves, Prentice-Ha11, Inc, Englewood Cliffs, N J (1965) P 676

Ross, D B, "Recent Developments in Remote Sensing of Deep Ocean Waves", The New Thrust Seoward, Transactions of the Third Annual MTS

Conference, the Marine Technological Society, Washington, $D$ C (1967), p 371-393

Taylor, G 1 , "The Spectrum of Turbulence", Proceedings, Royal Society, A164, $p$ 476-490, 1938 Reprinted in Friedlander, $S K$ and Topper, L Turbulence, Classze Papers on Statzstrcal Theory, Interscience, New York, 1961

Tucker, M J , "Simple Measurement of Wave Records", Proceedings of the Conference on Wave Recording for Crvil Engineers, N I O (1961) Also in Dock \& Harbor Authority, Vo1 42, p 231 


\section{CHAPTER 7}

COMPARISON OF PRESSURE AND STAFF WAVE GAGE RECORDS

by

D Esteva and D Lee Harris

Research Division, U S Army Coastal Englneering Research Center Washington, D C

\section{ABSTRACT}

Simultaneous records from two pressure gages located at different depths, a step-resistance relay gage, and a continuous-wire staff gage have been collected at Atlantic City, $\mathrm{N} J$

Spectra and cross-spectra are computed using the Fast Fourier Transform Algorithm (FFT) method proposed by Cooley and Tukey Individual harmonics of the pressure energy spectra are compensated for pressure at tenuation according to classical theory Results indicate better agreement is obtained between the wave helght and the spectra computed from the compensated pressure gages and those computed from the continuous-wire staff gage than between the two surface gages

Values of coherences are near 98 in the energy-containing part of the spectrum, and are always larger for the pressure-continuous wire staff cases than for the two surface gages whlch are displaced from each other only 12 feet in the horizontal

\section{INTRODUCTION}

Two basıcally different types of wave gages are widely used by coastal engineers One, called a surface-profile gage, produces a record which is considered to represent the actual elevation of the water surface at a point for each instant of time The other, called a pressure gage, produces a continuous record of the pressure at some fixed position beneath the surface The amplitude of the pressure pulses generated by waves is at tenuated with depth, and short waves are attenuated more than long waves

To compensate for this attenuation, a theoretical correction is commonly applied to the record from a pressure gage Several recent comparisons of the records from surface-profile gages with compensated records from pressure gages have shown systematic differences (Hom-ma, Horıkawa and Komor I (1967)) In general, the differences have been attrubuted to inadequacy of the compensation formula

The Coastal Engineerıng Research Center (CERC) has establıshed a facility at the Steel Pier in Atlantic City, New Jersey, for obtaining simultaneous records from several wave gages This installation is being used to compare surface-profile gages of varıous desıgns, and to obtain more information about the performance of pressure gages 
A comparison of the records obtalned from two pressure transducers, a step resistance relay gage described by Williams (1970), and a continuous wire gage is presented in this paper

\section{THE INSTALIATION}

The step-resistance wave gage has been installed for many years on one of the pilings supporting the Steel Pier at Atlantic City The other three gages were installed on a 4-inch outsfde diameter, heavy-duty steam pipe jettied into the sand bottom and secured to the Pler deck about 12 feet to the northeast of the step resistance gage The lower pressure transducer is Immediately above the bottom of the continuous wire gage and the upper pressure transducer about 55 feet above the first All gages are on the seaward end of the pier, about half a mrle from the mean water line

The mean depth at the gage site was determined as 155 feet MLW by lead line soundings a few days before and after the experiments Extensive surveys a few months earlier and later snowed that the gage site was near the center of a shallow depression The depth within 200 feet of the instruments varied from 110 to $166 \mathrm{feet}$, with an average value near 140 feet MLW A sketch of the installation is shown in Figure 1

The signal from each sensor is obtained in the form of a DC voltage All signals are transmitted to the CERC laboratory in Washington by telephone line

The transmission was accomplished by using channels two through five of the IRIG multiplex channels as described in TELEMETRY STANDARDS, June 1962, Document 106-60, and in many other publications on telemetry The transmission coefficient for the system is near unity for all frequencies less than 6 hertz In the laboratory, the signals are separated and converted back to DC voltages A digital voltmeter is used to measure the signal and the voltage is recorded on computer compatible magnetic tape at a rate of four samples per second from each gage

\section{THE ANALYSIS PROCEDURE}

The Fast Fourzer Transform Algorithm (FFT) suggested by Cooley and Tukey (1966) was used to analyze observations 1024 seconds ( 17 minutes and 4 seconds) long This procedure gives 1024 harmonics with periods of 1 second or longer The inftial record, expressed as a departure from the mean, was multiplied by a cosine Bell Taper function as suggested by Bingham, Godfrey and Tukey (1967) prior to the analysis in order to decrease the leakage of energy fetween spectral lines That is to say, the FFT was applied to the series

$$
\tilde{Y}(n \Delta t)=\frac{1}{2}(Y(n \Delta t)-\bar{Y})\left(1-\cos \frac{2 \pi n \Delta t}{T}\right)
$$


Linear monochromatic wave theory was used to obtaln the compensation function needed to compute the amplitude of the surface disturbance from the observed pressure disturbance for each harmonic according to the equation

$$
C(m)=\frac{\cosh (k(m) H)}{\cosh (k(m) G)}
$$

where $C(m)$ is the compensation function for the $m^{\prime}$ th harmonic, $k(m)$ is the wave number of the $m^{\prime}$ th harmonic, $G$ is the height of the pressure transducer above the bottom and $\mathrm{H}$ is the mean thlckness of the water column above the bottom during the observation The wave number is given by the implicit equation

$$
(2 \mathrm{~m} \pi \mathrm{T})^{2}=\mathrm{gk}(\mathrm{m}) \tanh \mathrm{k}(\mathrm{m}) \mathrm{H}
$$

where $T$ is the lenoth of the observation (1024 seconds in this study) and $g$ is the acceleration of gravity Thus the compensation factor appropriate to each specific frequency is applied to that harmonic

The Fourler Transforms were used to compute energy spectra for each gage record, the compensated record from the pressure transducers, and the cross-spectra between the records from the continuous wire gage and each of the other gages

The detalled spectra obtained in this way contain more than 1000 individual spectral lines The results are easler to grasp if some of this detall is suppressed, so the individual spectral values have been grouped into bands of 17 lines each

\section{RESULTS}

Eıghty-three observations, taken 2 hours apart durıng December 19-26, 1969 were analyzed A sample of the resulting spectra, as obtained directly from the records of the four gages and from the compensated pressure records is shown in Figure 2 The spectrum from the continuous-wire record has been superimposed on all others The data in this Figure are normalized with respect to the frequency band with period between 3 and 1969 seconds The short-period cutoff was ımposed because the spectrum of the pressure record at higher frequencies has little correlation with the surface spectrum The long-perıod cutoff was imposed because the step-resistance gage shows an excessive amount of energy at longer periods for some of the observations The spectra computed from the compensated pressure records agree very well with that from the continuous-wire record within this perıod band

Figures 3 and 4 show a comparison of the wave helghts as estimated from the contınuous-wire gage and from the pressure gages compensated fur hydrodynamic attenuation as described above The root mean square wave helght, which is equal to the standard deviation of the wave record, is used as a measure of the wave helght because unlike the "significant wave helght" it is clearly and objectively defined Figure 5 shows the same comparison for 
the records obtained from the step resistance relay gage A tendency for the step resistance gage to record higher waves than the other gages is apparent This tendency has also been noted by Hom-ma, Horıkawa and Komorı These authors attributed this effect to wave runup on the gage or its support It has been determined that wave runup has affected the records from the step resistance wave gage at Atlantic City Th1s step gage is no longer being used

Different mountıng arrangements have been used at some other locations It seems likely that the installation at Atlantic City leads to larger runup than that experienced at some other installations The possibility that the differences between the records of the step gage and the continuously variable gages is due to the digltal nature of the record from the step gage was investigated by truncating the resolution of the continuous wire gage to correspond with that of the step gage The results of the analysis of the truncated record did not differ significantly from those of the analysis of the original record

It should be noted that the agreement between the compensated pressure records and the continuous-wire record is better than that between the two surface-profile records The continuous-wire gage has been used as the standard in this comparison partly because of this better agreement and partly because wave runup is known to affect the accuracy of the step resistance gage

The average factor needed to convert the wave helghts as determued from the upper pressure transducer compensated by indıvidual lines to those determined from the continuous-w1 re gage was found to be 98 with a correlation coefficient of 999 For the lower pressure transducer this factor becomes 104 , w1th a correlation coefficient of 999 Even when the entire spectrum is compensated by the factor computed for the frequency of maximum energy density the factor is 108 with a correlation of 997 The agreement reported here is much better than most of those cited in the review paper by Grace (1970) The 1mproved agreement is believed to result partly from the use of a more satisfactory surface gage system, partly because the FFT procedure permits a more precise determination of the frequencies of maximum interest than the procedures used by earlier investigations, and partly because the correction was applied to the individual harmonics in the spectrum

5 ACCURACY OF THE COMPENSATION FACTOR AS A FUNCTION OF FREQUENCY

Hom-ma, et al, have studied the function $n(f)$ defined by the relation

$$
E(f)_{s f x}=n(f) E(f)_{c p}
$$

where $f$ is the frequency, and the subscripts $s$ fr and $c p$ refer to the surface wave record and the compensated pressure record The function $n(f)$ has been computed from our data for all bands containing as much as 5 percent of the total energy in a glven spectrum The function $n(f)$ based on all records from the upper pressure transducer is shown in Figure 6 The mean value of $n(f)$ is plotted as a circle and the standard deviation is shown by a vertical line A similar plot based only on those observations in which $\mathrm{H}_{\mathrm{RMS}}$ exceeded 1 foot is given in 
Figure 7 The two values for which no standard deviations are shown consisted of single observations Similar results, but with a little more scatter, were derlved for the lower pressure transducer, as shown in Figures 8 and 9

It is noted that both the deviation of the mean value of $n(f)$ from its theoretical value of unity and the scatter of the individual values is greater for low waves than high waves, and greater for the lower pressure transducer than for the upper one From these results we are led to belleve that the deviation results more from the presence of pressure impulses caused by factors other than surface gravity waves than from nonlinear effects due to the finite amplitude of the wave This is especially likely at high frequencies where the large value of $c(m)$ would greatly amplify small impulses In general, we feel that values of $c^{2}(\mathrm{~m})$ greater than 25 should not be used at this installation

\section{CROSS SPECTRUM RESULTS}

The cross spectra calculations for band widths of 0017 Hertz showed a coherence of 95 between the continuous-wire gage and the pressure gages in most bands containing more than 5 percent of the total energy in the spectrum The coherence between the two surface gages was slightly lower but still above 90

Computations of the phase lags between the continuous-wire and the pressure gages showed that the phase of the wave advances slightly with increasing depth This effect tends to increase wath frequency A phase shift of this kind has been predicted by Battjes (1968) and Mel and Chu (1970) 


\section{REFERENCES}

Bingham, C , Godfrey, M D and Tukey, J W, 1967, "Modern Technıques of Power Spectrum Estimation", IEE Trans Audio Electro-acoustics, Vo1 AU15, No 2, pp 56-66

Battjes, J A, "Refraction of Water Waves", Journal of the Waterways and Harbors Div, Proceedings of the American Society of Civil Englneers, Vol 94, 1968, pp 437-450, No WW4

Cooley, J W and Tukey, J W "The Fast Fourier Transform", IEEE Spectrum, Vo1 4, p 63-70, 1967

Draper, L, "Attenuation of Sea Waves w1th Depth", La Houllle Blanche, Vo1 12, No 6, 1957, pp 926-931

Gerhardt, J R, Jehn, $K$ H and Katz, I , "A Comparison of StepPressure - and Continuous-Wire-Gauge Wave Recordings in the Golden Gate Channel", Transactions of the Amerıcan Geophysical Unıon, Vo1 36, No 2, 1955, pp 235-250

Glukhovskı, B K, "Study of Wave Attenuation with Depth on the Basis of Correlation Analysis", Meteorologlya 1 Gidrologiya, No 11, 1961, pp 22-30, Electrucıte de France, Centre de Recherches et d'Essais de Chatou, Traduction 1343

Grace, Robert A, "How to Measure Waves", Ocean Industry, Vo1 5, No 2, 1970 , pp 65-69

Harrıs, D L , "The Analysıs of Wave Records", Coastal Engıneering, Proceedings of Twelfth Conference, 1970

Hom-ma, M Horıkawa, K, and Komorx, S, "Response Characterıstics of Underwater Wave Gage", Proceedings of the 10 th Conference on Coasta1 Engineerıng, Tokyo 1966", pp 99-114

Me1, C C and Chu, Vıncent, "Slowly Varyıng Stokes Waves", Journal of Fluld Mechanics (1970)

Wr1liams, Leo C , Technical Memorandum No 30, Dec 1969, U S Army Corps of Engıneers, Coastal Englneerıng Research Center, 1969 
LEGEND TO FIGLPES

Fig 1 Sketch of installation (not to scale)

Fig 2 Computed frequency energy spectra from continuous wave gage superımposed on computed spectra from a) compensated upper pressure gage, b) uncompensated upper pressure gage, c) compensated lower pressure gage, d) uncompensated lower pressure gage, e) step resistance relay gage

F1g 3 Comparison of RIS heights, compensated upper pressure gage vs continuous wire gage

Fig 4 Comparıson of RMS helghts, compensated lower pressure gage vs continuous wire gage

F1g 5 Comparison of RMS heights, step resistance relay gage vs continuous wire gage

Fig 6 The function, $n(f)$ for the upper pressure gage for all samples

Fig 7 The function, $n(f)$ for the upper pressure gage for high wave samples

Fig 8 The function, $n(f)$ for the lower pressure gage for all samples

Fig 9 The function, $n(f)$ for the lower pressure gage for high wave samples 


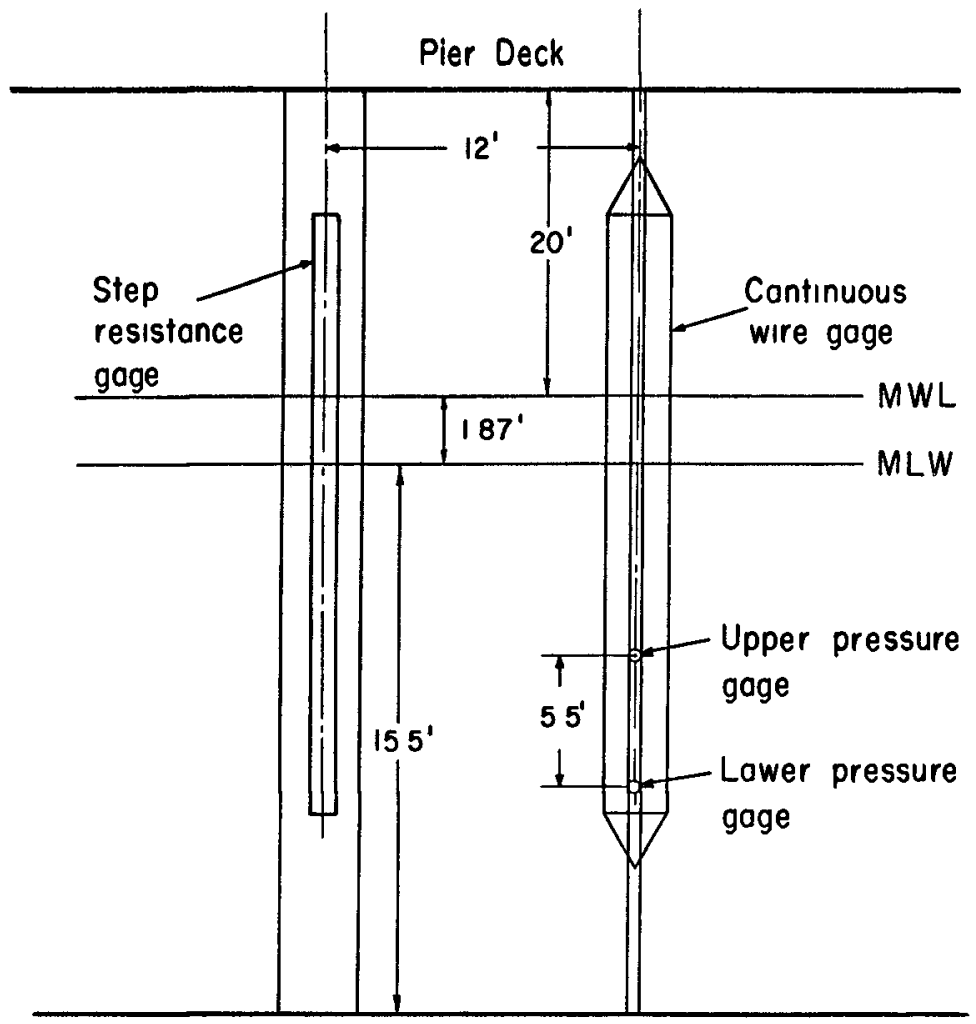

Fig 1 Sketch of Installation (not to scale) 


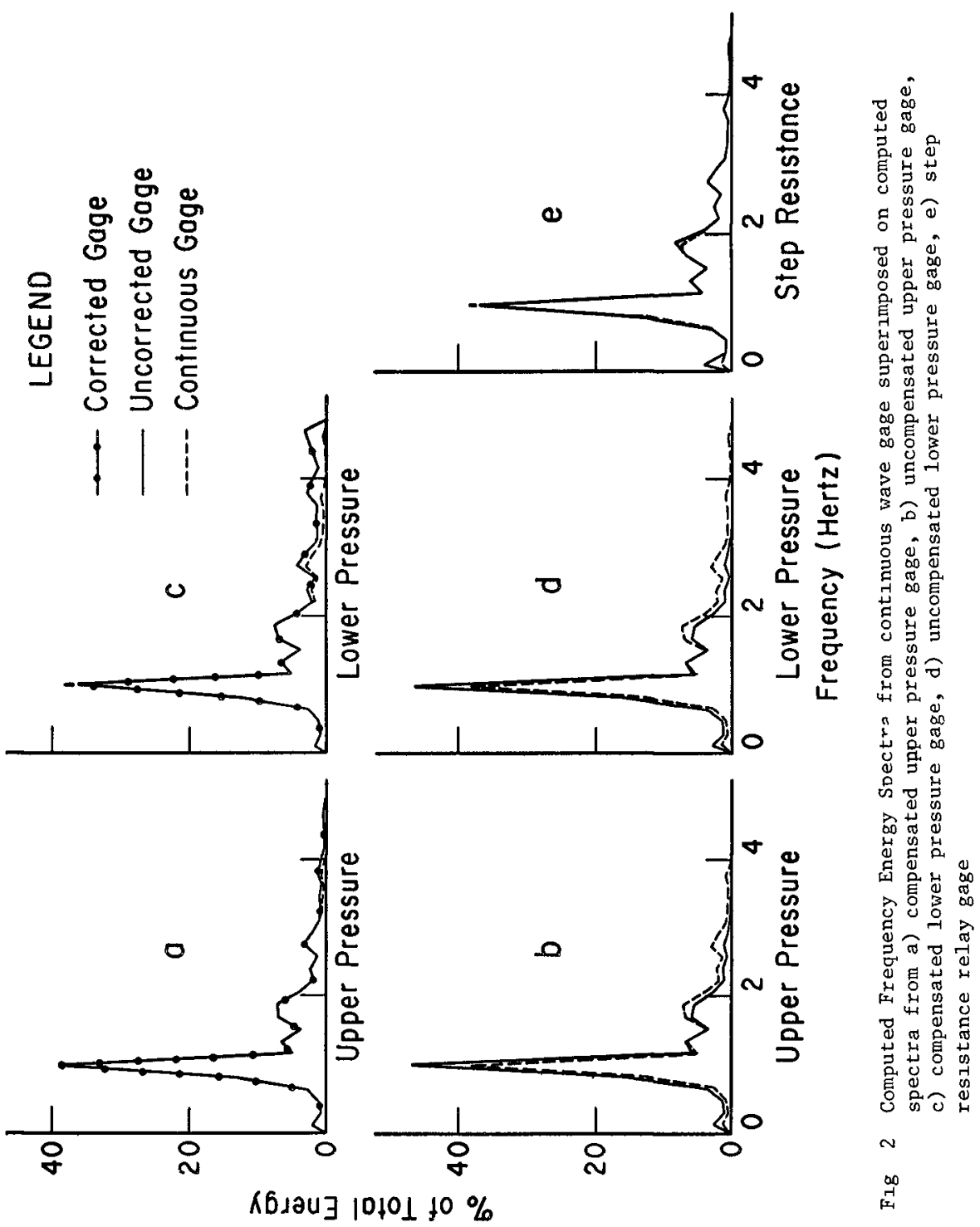




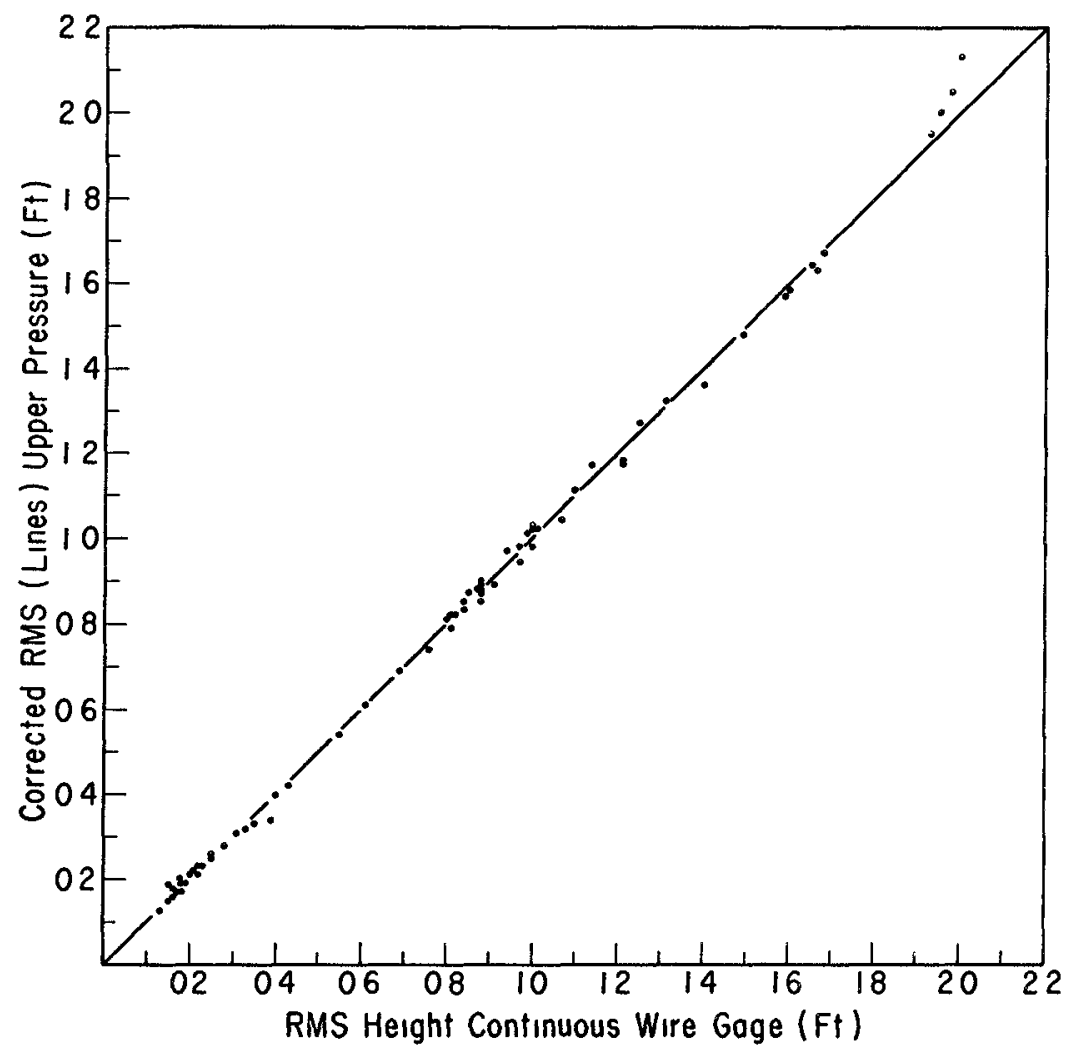

Fig 3 Comparison of RMS heignts, compensated upper pressure gage vs continuous wire gage 


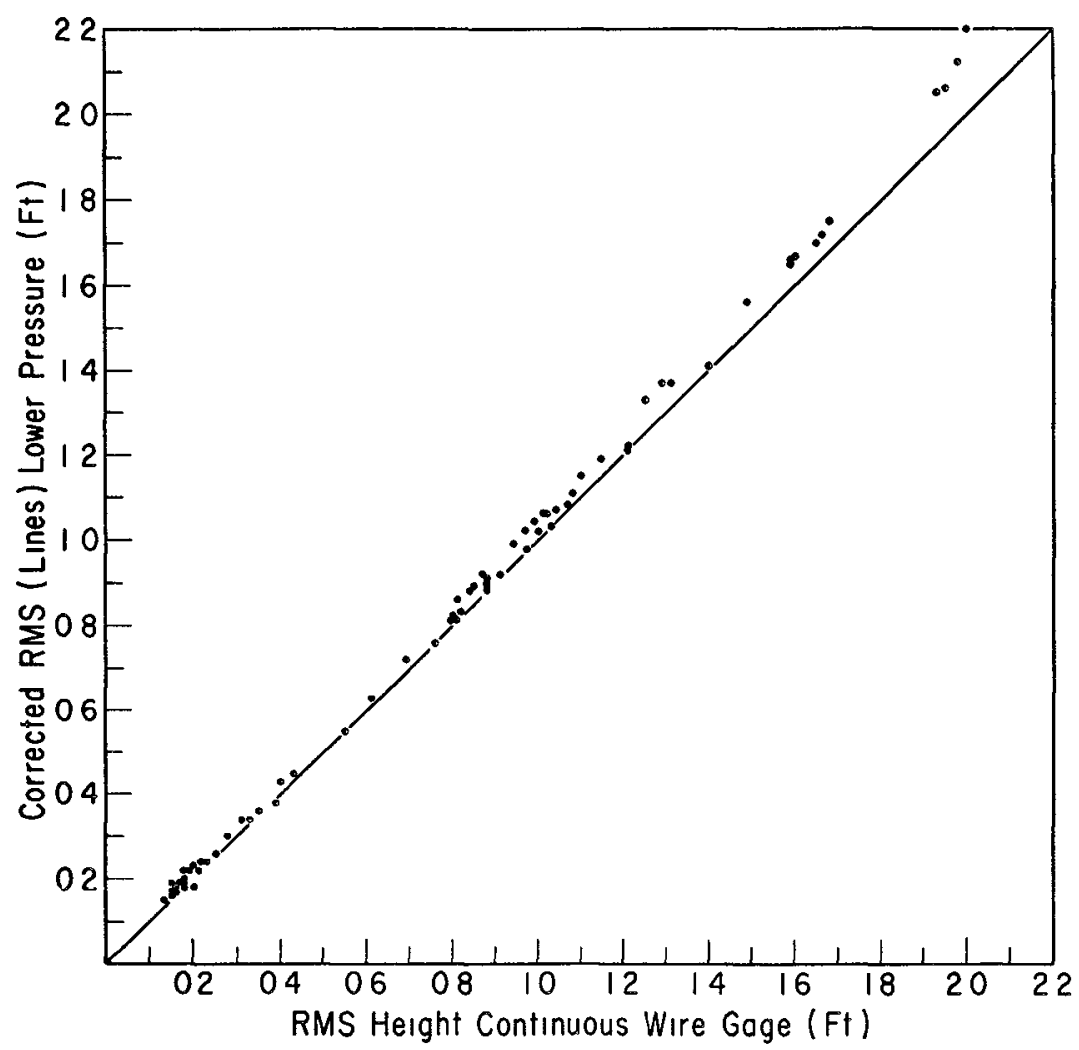

Fig 4 Comparison of RMS heights, compensated lower pressure gage vs contınuous wire gage 


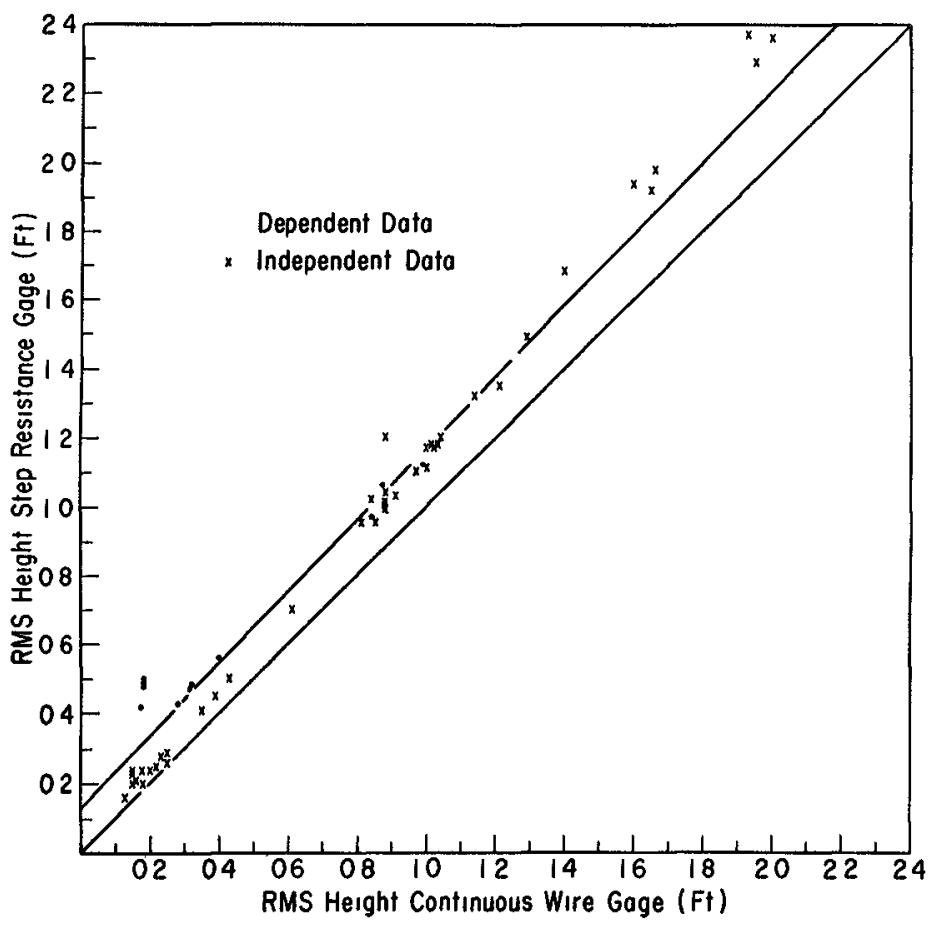

F1g 5 Comparison of RMS helghts, step resistance relay gage vs continuous ware gage 


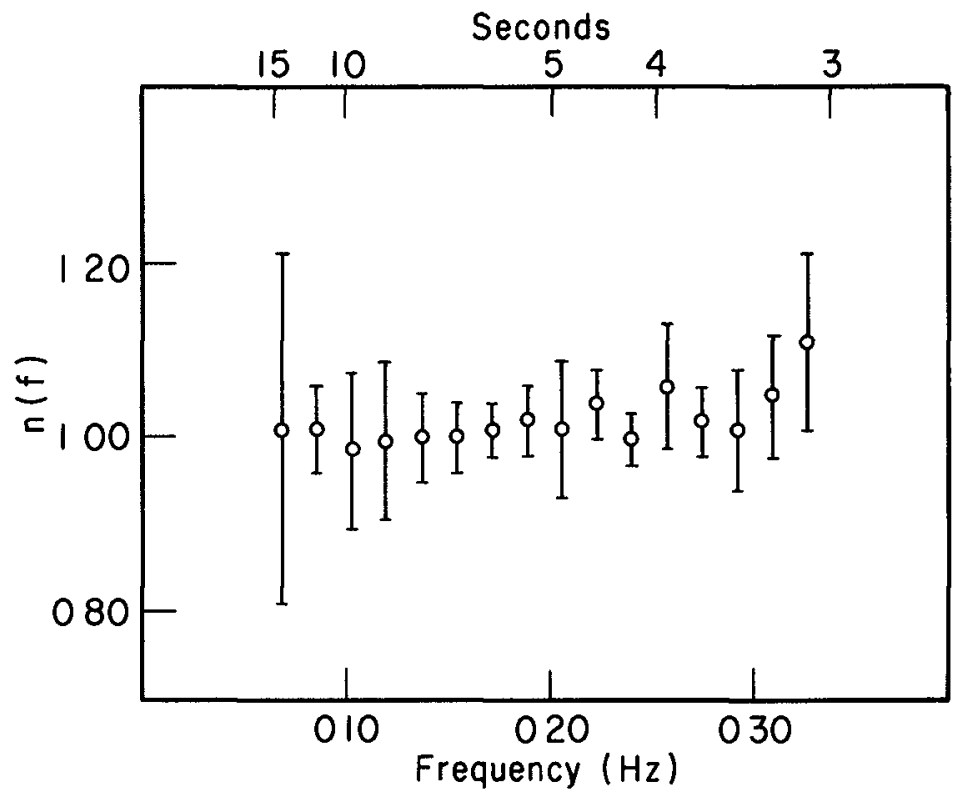

Fig 6 The function, $n(f)$ for the upper pressure gage for all samples 


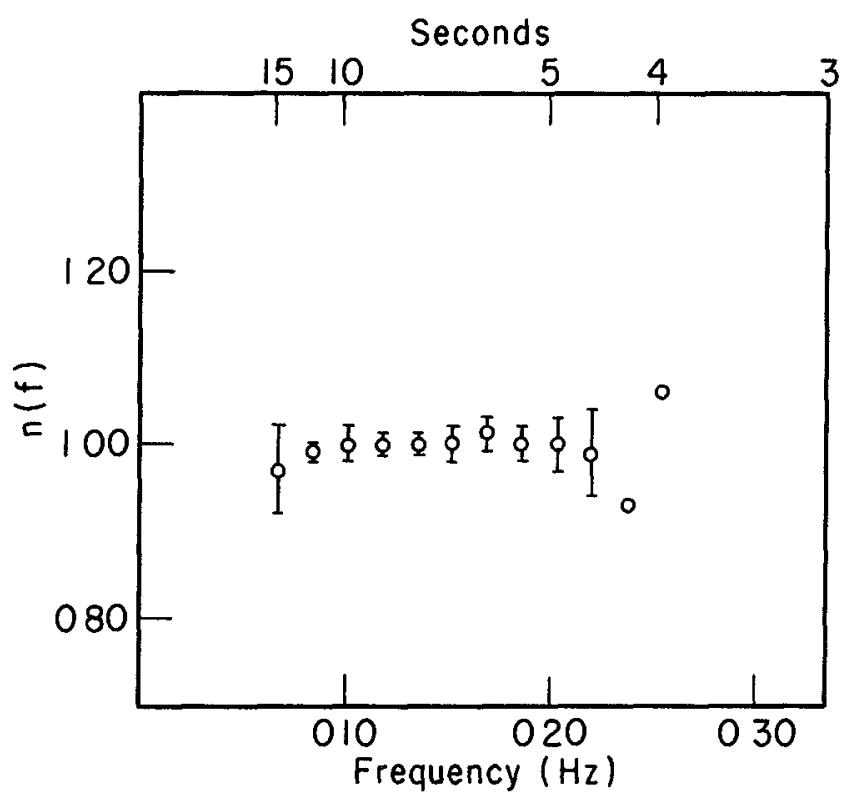

Fig 7 The function, $n(f)$ for the upper pressure gage for high wave samples 


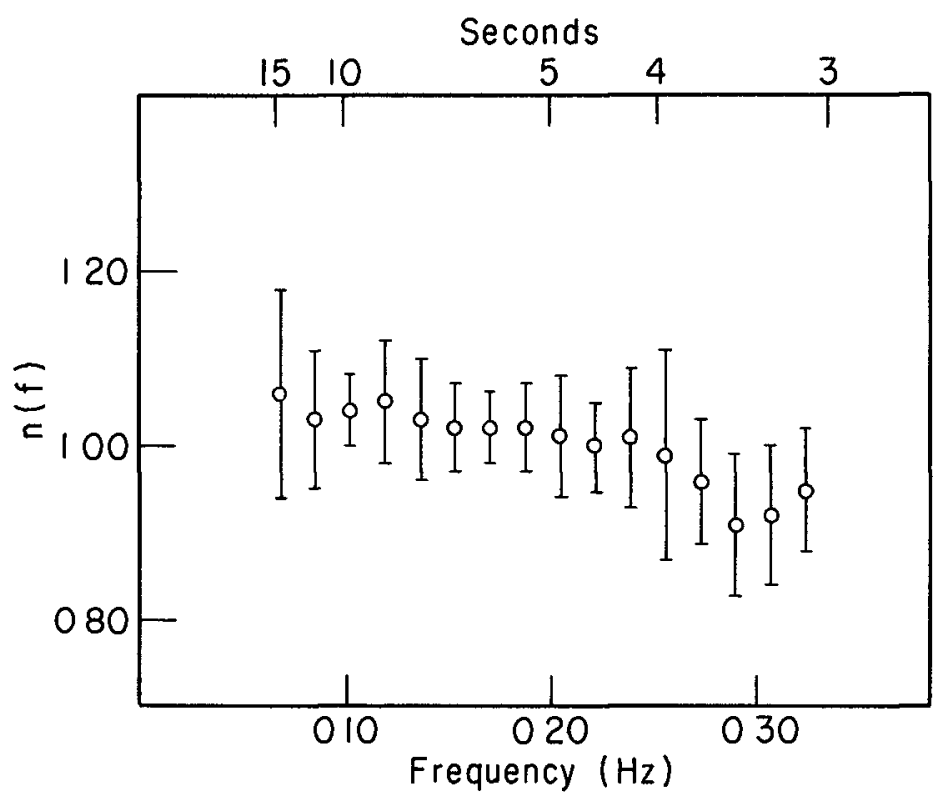

Fig 8 The function, $n(f)$ for the lower pressure gage for all samples 


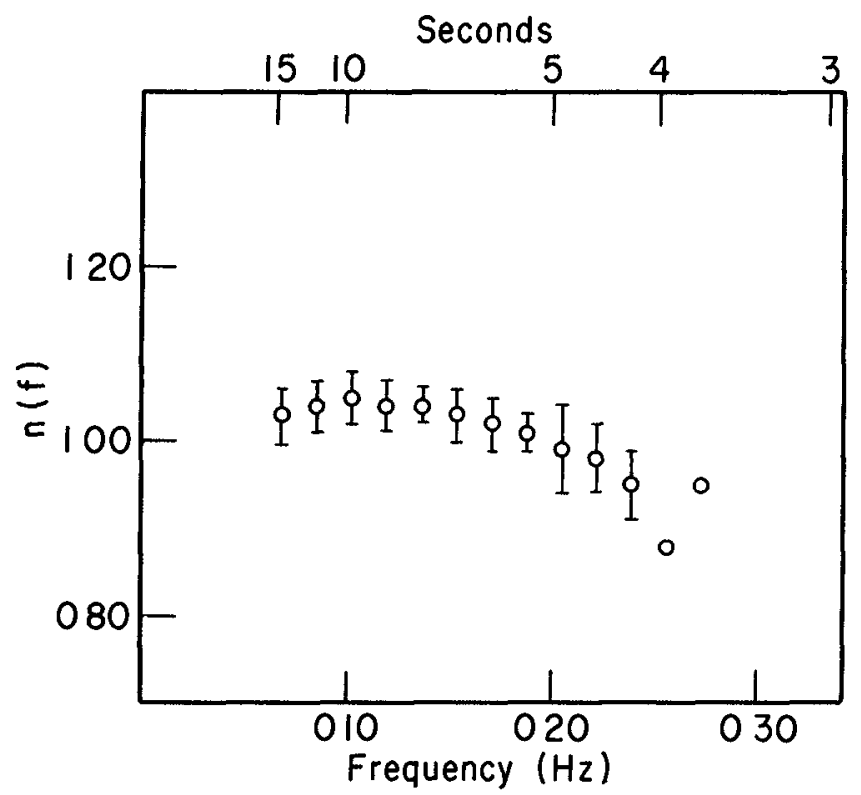

Fig 9 The function, $n(f)$ for the lower pressure gage for high wave samples 


\title{
CHAPTER 8
}

\section{DIRECTIONAL SPECTRA FROM WAVE-GAGE ARRAYS}

by

\author{
N N Panicker \\ Graduate Student, Dept of Civil Engineerıng \\ Unıversity of California, Berkeley, Calıf \\ and \\ L E Borgman \\ Professor of Geology and Statistics \\ University of Wyoming, Laramie, Wyoming
}

\section{SYNOPS IS}

The ocean surface may be considered to be composed of many waves traveling at different directions with different frequencies A graphical plot showing the allocation of wave energy to the different component frequencies and directions is the directional spectrum Directional spectrum has many applications in Coastal Engineering Herein an analytical procedure is developed to obtain the directional spectrum from records of an array of wave gages The two methods developed are the "locked phase method" and the "random phase method The locked phase method can be used to obtain the distribution of both phase as well as energy of the waves with respect to frequency and direction and is a deterministic approach The random phase analysis, on the other hand, is more suitable for wind waves in the ocean and yields just the distribution of energy alone as in most other procedures of spectrum analysis The procedures programmed for computers are checked using simulated data and laboratory data Wave records of the Pacific Ocean obtained off Point Mugu, Calıfornia, on a 5-gage array were analyzed using the method developed and examples of the directional spectra obtained are presented

\section{INTRODUCTION}

When confronted with a design or operation in the ocean environment, an engineer invariably needs to know how high the waves are and from which direction they are coming Had ocean waves been single sinusoids, one could immediately obtain the height and direction of the waves But waves in the ocean do not look like sinusoids At best 
the ocean surface may be thought of being the result of adding together very many sinusoids of various frequencies traveling in vax lous directions Therefore one has to specify the particular wave for which the height and direction are desired As frequency, $i e$, inverse of the period of the component wave, is the least easily changeable wave parameter, the component wave may be specified by its frequency So, the problem is to obtain the wave amplitude $a_{1}$ and direction $\theta_{1}$ for the various component frequencies, $f$ But to consider the ocean surface to be composed of a finite number of sinusoids is a poor approximation A better approximation would be obtained if one lets the number of component simple harmonic waves to approach infinity and the frequency interval, $\Delta f$, between them to approach zero The individual wave amplitudes, $a_{1}$, In this case must approach zero in ordex for the overall wave heights to maintain a finite, mean square value However, in this limiting process the quantity $a_{i}^{2} / 2 \Delta f$ remains finite and therefore $1 t$ can be plotted as a continuous function of frequency If the quantity $\mathrm{a}_{1}^{2} / 2 \Delta \mathrm{f}$ is plotted against frequency, the area under the curve within the frequency band of $\Delta f$ is $a_{1}^{2} / 2$, but this is proportional to the wave energy contributed by that frequency band because the energy of a simple harmonic wave of amplitude $a_{1}$ in a medium of unit weight $\gamma$ is $\mathrm{Ya}_{1}^{2} / 2$ per unit width of crest, see, for example, Wiegel (44) In the same manner the number of component directions may be assumed to approach infinity to yield a continuous function of direction and the area undel that cuive for any angular width will be proportional to the energy of the waves traveling in those directions for a particular frequency Alternately, the above two plots can be combined into a twodimensional plot which will show the distribution of energy with respect to frequency and direction This is the Directional Spectrum

\section{USES OF DIRECTIONAL SPECTRA}

Directional spectrum shows the distribution of wave energy against frequencies and directions Therefore, it specifies the wave climate more completely than any other way, ideally when only some particular information like the predominant wave perıod and helght alone are needed, that can be obtained from the directional spectrum $R M S$ wave height, for example, is the volume under the directional spectrum Scott (37) and Neumann and Plerson (34) provide equations for the significant wave helght from $R M S$ wave height assuming different distributions for the frequency spectrum As Wiegel (46) pointed out, there are still situations where the significant wave concept is useful to the design engineer, but it might be possible to obtain that information from the directional spectrum Nevertheless, there are many engineering problems where directional spectra are the necessary input data for the correct design or prediction For example, for the study of the diffraction of wind waves directional spectrum 1 s necessary and, in fact, Wiegel and Mobarek (45) and Fan (19) successfully used it for the study of diffraction of laboratory wind waves Refraction of ocean waves is another problem where the directional spectrum is needed and Karlsson (24) used it for practical cases In the design of offshore towers and piles directional spectrum is useful for the analysis of vibration and three-dimensional analysis 
of structures with torsional loads Malhotra and Penzien (29) have succeeded in developing the procedure for analyzing tower structures using the spectrum and indicated the need for the directional spectrum For structures subjected to random forces, in general, a design by the method of simulation on a computer is highly suited and directional spectra are the sultable input data Borgman (11) showed how this can be done for offshore pile structures by linearizing wave forces Directional spectrum is necessary for the prediction of the response of ships and floating drilling vessels to sea conditions as the spectra of their motions can be obtained from the directional spectra of the sea It is, in fact, used by naval architects (see for example, Abkowitz, Vassilopoulos and Sellars (1)) Even for the design of an ocean outfall sewer, directional spectrum may be useful because, as Wiegel (43) pointed out, mixing and wave spectra are related Once a single wave model for longshore transport is established, directional spectrum may prove to be specifically cut out for the study of littoral transport because it provides at once the three crucial parameters of the problem, viz the wave energy, frequency and direction Another major use for directional spectrum is in wave forecasting and hindcasting In hindcasting the location of the origin of storms and the path of swell can be deduced from the variation of the non-stationary directional spectra, see Munk et al (32)

When directional spectra become available for desired locations, several other uses also may be found for them The problem at present is its non-avallability for almost any place Here again, barring the huge expenses involved in the collection of necessary data, the main hurdle is the lack of a valid, dependable computational procedure to obtain directional spectra in a routine manner

\section{REVIEW OF METHODS USED}

The most direct way of obtaining the directional spectrum is to obtain the sea surface elevations over an area by stereophotographs and to analyze these data to get the directional spectra This was done in the Atlantic Ocean by $W J$ Plerson and his group, see cote et al (17), and in a much more modest scale by $I_{j}$ Ima et al (23) From the story of the Stereo Wave Observation project [Cote et al (17)] one realizes how arduous and expensive this method is Kinsman (25) therefore doubts whether it will ever become habit forming Another successful method applicable for deep ocean is to take records of the elevation and tilt of a free floating buoy as used by Longuet-Higgins, Cartwright and Smith (27) From the water surface elevation and the slopes in two coordinate directions they computed the first five coefficients ( 1 e two harmonics) of the Fourier series expansion of the directional spectrum To remove the appearance of negative energy they used a smoothing function $\omega_{2}=\frac{8}{3} \cos ^{4} \frac{\varphi}{2}$ The net result of having a directional spectrum represented only up to two harmonics and then smoothing it is to get a very broad angular width for the spectrum If more accuracy is needed, records of the water surface curvatures are to be obtained Ewing (18) also obtained a sequence of ten records 
of the directional spectra from the motions of a floating buoy located in the North Atlantic

The use of an array of wave reoorders to measure wave parameters and the computation of directional spectrum from it was tried by several workers Barber (3) was probably the first to suggest it The wave-gage array could be one-dimensional (line array) or twodimensional If one can make sure that no wave comes from one of the sides of a line array, a line array can be used to obtain directional spectrum Barber and Doyle (4), described a procedure to get the directions of swells by using just two gages stevens (39) described the procedure to obtain directional spectrum from a line array and used it for an array in Buzzards Bay Macovsky and Mechlin (28) described a possible method of using a line array of inverted acoustic fathometers mounted on the deck of a submarine Two-dimensional arrays, in general, would be more appropriate for a general situation Barber (6) discussed a general theory of gage arrays and suggested ways to compare the directional resolving power of different arrays Mobarek (31) found that the discrete energy method was the most successful of the methods for the estimation of directional spectra for 1 aboratory wind waves Fan (19) used a 4-gage array in the form of a star and compared the Fourier transform method and Least Square Method by simulation technique to obtain the directional spectra He found that the Fourier Transform Method gave better results for higher frequency components and the Least Square Fitting Method gave better results for lower frequency components Two-dimensional arrays have also been used in the ocean Munk, Miller, snodgrass and Barber (32) used three bottom pressure gages forming an equilateral triangle with sides about $900 \mathrm{ft}$ in $330 \mathrm{ft}$ of water and obtained the direction of long period swell from the data Bennett, Pittman and Austin (7) and Bennett (8) described a 6-gage array in the form of a Pentagon with one gage at the center, which was used off Panama City, Florida in the Gulf of Mexico at depths of $63 \mathrm{ft}$ and $104 \mathrm{ft}$ Bennett (8) essentially used the procedure of Munk et al (32) by fitting a single wave of a particular frequency to the cross-spectrum equations This may, perhaps, be sufficient to obtain the direction of $1 \mathrm{ong}$ period swell But as Tukey (42) pointed out, in analyzing or thinking about a computational process involving several layers of approximations, or the propagation of sampling fluctuations through several layers of tranformations, step-by-step analysis is not $11 \mathrm{kely}$ to be enough and an analysis of the overall process is needed

A few other methods have also been reported Nagata (33) measured orbital motions with electromagnetic current meters and used it to obtain the directional spectra Ford, Timme and Trampus (20) used a triset sensor made up of three vertical surface-penetrating wave staffs located at the corners of a right triangle of side about $5 \mathrm{ft}$ to obtain the three outputs, viz two components of wave slope and the average wave amplitude at the sensor From these they calculated the directional spectrum accurate up to 2 harmonics just in the same way as Longuet-Higgins et al (27) did Simpson (38) had a similar arrangement but the probes at the apices of the right triangle 
measured orbital velocities instead, and the side of the triangle was about $3 \mathrm{ft}$ By this arrangement he could obtain the first 4 harmonics of the directional spectra Suzuki (40) proposed another method of determining the directional spectra of sea waves using a wave gage and a wave direction meter which can record $X$ and $Y$ component of wave force acting on a bottom mounted sphere

There may, perhaps, be many more ingenious ways of obtaining the directional spectra But it seems to the authors that wave gage arrays might be the most convenient arrangement for collecting data in a routine way to determine the directional spectra Hence a general theory for the determination of directional spectra from records of wave gage arrays and a computer program for it were developed $A$ brief description of the theory and the results obtained are described bel ow The equations for use when the wave gages measure surface elevations are presented here The detailed development of the general case $w_{1} 11$ be given in a separate report

\section{A THEORY FOR GAGE ARRAYS}

\section{The Modes of Analysis}

Two modes of analysis are developed - the locked phase method and the random phase method The locked phase mode of analysis is essentially a deterministic approach where the phases of the component waves are assumed to be fixed Hence the analysis provides both the distribution of energy with frequency and direction as well as the distribution of phase angle with frequency and direction In the random phase method the phases of component waves are considered to be random and independent of each other, hence they average out in the analysis The locked phase mode of analysis is appropriate to situations where phase is locked to particular values, such as in a wave tank with flapper The random phase mode of analysis, on the other hand, is applicable to situations where phase changes randomly with time as in narrow band surf or wind waves

\section{Locked Phase Mode of Analysis}

The wave surface elevation, $q$, at a given instant of time $t$, is considered to be the result of superposition of a large number of simple harmonic waves each with its own frequency and direction Let the amplitudes of the component waves be $a_{0}, a_{1}, a_{2}, a_{m}, a_{M}$ and frequencies $f_{0}, f_{1}, f_{2}, f_{m}, f_{M}$ and let them propagate in all directions between $-\pi$ and $\pi$ Let $\varphi$ be the phase and $\theta$ the direction of wave Let the coordinates of gage $y$ in an array be $x_{y}$ and $y_{y}$ and let the wave number be $k=2 \pi$ wave length Then the water surface elevation at gage $j$ at time $t$ can be written as

$$
q_{y}(t)=\sum_{m=0}^{M} \int_{-\pi T}^{\pi} a_{m}(\theta) \cos \left[k x_{j} \cos \theta+k y_{j} \sin \theta-2 \pi f t+\varphi_{m}(\theta)\right]
$$


The subsequent development will make use of the Fast FourierTransform technique [see Cooley \& Tukey (16), Cochran et al (15), Bergland (9) or Bingham, Godfrey and Tukey (10)] Let the length in time of the water surface elevation record be $T$ and let the discrete time interval of recording be $\Delta t$ Let $T$ and $\Delta t$ be such that $N=\frac{T}{\Delta t}$ be a power of 2 One gets the complex amplitude spectrum $A_{m}$ by taking the FFT of the surface elevation record $q_{n}$

$$
A_{m}=\Delta t \sum_{n=0}^{N-1} q_{n} e^{-12 \pi m n / N}
$$

Let the directional distribution be represented as a finite Fourier series in complex form as below

$$
\begin{aligned}
a_{m}(\theta) e^{1 \varphi_{m}(\theta)} & =F(\theta) \\
& =\frac{a_{0}+1 a_{o}^{\prime}}{2}+\sum_{n=1}^{N}\left[\left(a_{n}+1 a_{n}^{\prime}\right) \cos n \theta+\left(b_{n}+i b_{n}^{\prime}\right) \sin n \theta\right]
\end{aligned}
$$

To determine the directional spectrum one therefore has to evaluate the coefficients $a_{0}, a_{0}^{\prime}, a_{1}, a_{1}^{\prime}, b_{1}, b_{1}^{\prime}$ etc After going through some mathematical manipulations, one can come up with the following two equations for the real and imaginary parts of the FFT coefficients $A_{m}$ of the surface elevation record for each gage

$$
\begin{aligned}
& R\left(2 A_{m}^{(J)} / \pi T\right)=a_{0} A_{0_{J}}^{*}-\left(a_{1}^{\prime} A_{1 J}^{*}+b_{1}^{\prime} B_{1 J}^{*}\right)-\left(a_{2} A_{2 J}^{*}+b_{2} B_{2 J}^{*}\right) \\
& +\left(a_{3}^{\prime} A_{3 j}^{*}+b_{3}^{\prime} B_{3 j}^{*}\right)+\left(a_{4} A_{4 j}^{*}+b_{4} B_{4 j}^{*}\right)- \\
& \operatorname{lm}\left(2 A_{m}^{(\jmath)} / \pi T\right)=a_{0}^{\prime} A_{0 J}^{*}+\left(a_{1} A_{1 J}^{*}+b_{1} B_{1 J}^{*}\right)-\left(a_{2}^{\prime} A_{2 j}^{*}+b_{2}^{\prime} B_{2 j}^{*}\right) \\
& -\left(a_{3} A_{3 \jmath}^{*}+b_{3} B_{3 \jmath}^{*}\right)+\left(a_{4}^{\prime} B_{4 j}^{*}+b_{4}^{\prime} B_{4 j}^{*}\right)+
\end{aligned}
$$

In these equations,

$$
A_{n j}^{*}=2 \cos n B J_{n}(k D)
$$

and

$$
B_{n J}^{*}=2 \sin n \beta J_{n}(k D)
$$

whe re

$$
\begin{aligned}
A= & \text { angle of gage } \mathrm{J} \text { from origin } \\
J_{\mathrm{n}}(\mathrm{kD})= & \text { Bessel function of order } \mathrm{n} \text { with argument } \\
& \mathrm{kD} \text { in which } \mathrm{k}=\text { wave number and } \mathrm{D}=\text { distance } \\
& \text { of the gage from the origin }
\end{aligned}
$$

Once the coefficients $a_{n}, a_{n}^{\prime}, b_{n}$ and $b_{n}^{\prime}$ are evaluated from the above equations up to the number of harmonics feasible with the number of gages, one may obtain the energy and phase by the following relationships 


$$
\begin{aligned}
& a_{m}^{2}(\theta)=|F(\theta)|^{2} \\
& \varphi_{m}(\theta)=\arg [F(\theta)]
\end{aligned}
$$

Random Phase Analys 1s

Let $p(f, \theta)$ be the directional spectral density function valid for $f>0$ and $-\pi \leq \theta<\pi$ Then it can be shown, after Pierson and Marks (35), that the water surface elevation $q(x, y, t)$ at gage $J$ at $t i m e t$ can be symbolically written as

$q(x, y, t)=2 \int_{0}^{\infty} \int_{-\pi}^{\pi} \sqrt{p(f, \theta) d f d \theta} \cos (k x \cos \theta+k y \sin \theta-2 \pi f t+\varphi)$

$q(x, y, t)$ here ends up with a Gaussian probability density for any fixed $x, y$ and $t$, because of the normal convergence criterion [Brown (14), Loeve (26), Takano (41)] The cross covariance between water surface elevations at two gages therefore turns out to be independent of phase The directional spectrum may be represented as a finite Fouriex series of the form

$$
p(f, \theta)=\frac{a_{0}}{2}+\sum_{n=1}^{N}\left(a_{n} \cos n \theta+b_{n} \sin n \theta\right)
$$

Here the Fourler series coefficients $a_{n}$ and $b_{n}$ are to be evaluated In terms of the co- and quad- spectrum for each pair of gages the following two equations can be written down

$$
\begin{aligned}
& \text { Co-spectrum }{ }_{J} l=\pi\left[a_{0} A_{0 J}^{*}-\left(a_{2} A_{2 J}^{*}+b_{2} B_{2 J}^{*}\right)+\left(a_{4} A_{4 J}^{*}+b_{4} B_{4 J}^{*}\right)-\right. \\
& \text { Quad-spectrum }{ }_{J \ell}=\pi\left[\left(a_{1} A_{1 \jmath \ell}^{*}+b_{1} B_{1 \jmath \ell}^{*}\right)-\left(a_{3} A_{3 \jmath \ell}^{*}+b_{3} B_{3 \jmath}^{*}\right)+\left(a_{5} A_{5 j \ell}^{*}+b_{5} B_{5 j \ell}^{*}\right]\right.
\end{aligned}
$$

The co-spectrum and quad-spectrum can be calculated from the FFT coefficients on gage $J$ and gage $l$ The quantities $A_{n}^{*}$ and $B_{n}^{*}$ are as bel ow

$$
A_{n}^{*}=2 \cos n \beta J_{n}(k D)
$$

and

$$
\mathrm{B}_{\mathrm{n}}^{*}=2 \sin \mathrm{n} \beta \mathrm{J}_{\mathrm{n}}(\mathrm{kD})
$$

where $\quad \beta=$ angle between gage $J$ and gage $\ell$

$$
\begin{aligned}
& \mathrm{D}=\text { distance between the gages, } \\
& \mathrm{k}=\text { wave number }=2 \pi / \text { wave length and } \\
& J_{n}=\text { Bessel function of order } n
\end{aligned}
$$

The unknowns are the Fourıer series coefficients $a_{0}, a_{1}, b_{1}, a_{2}$, $b_{2}$ etc There are two equations for each parr of gages and two unknowns for every harmonic 
Least square analysis is used to make coefficient estimates In both procedures, a unidirectional wave train will produce analytical results spread over an angular band width, because only a finite number of Fourier coefficients can be estimated

\section{COMPUTATION OF DIRECTIONAL SPECTRA}

A very general computer program was developed to compute the directional spectrum, the details of which will be reported subsequently The analytical procedure and the scheme of computation was verified for their validity and workability by computation of known directional spectra using the scheme The directions obtained out of the computer checked very well with the known directions of the simulated wave as well as regular waves generated in the laboratory when the respective data were fed in However, there was considerable angular spread in the results obtained for directions These were due to leakage, finlte length of data and the truncation of Fourler series representing directional spectra It was also noticed that there was considerable negative energy showing up in the spectra As the negative energy caused by the presence of one wave may foul up with the positive contribution from another wave in the system, the presence of negative energy may affect appreciably the directional resolution Hence this had to be cured For this a non-negative smoothing function $w_{2}(\varphi)$ was applied where

$$
W_{2}(\varphi)=R_{N} \cos ^{2 N}\left(\frac{\varphi}{2}\right)
$$

in which $\mathrm{R}_{\mathrm{N}}$ is a coefficient to be obtained for each harmonic Borgman (12) has described the procedure to apply this smoothing The smoothing, however, broadens the angular spread of the directional spectrum and decreases the value of the spectral peak The problem of desmoothing the spectra seems to be very important and perhaps Medgyessey (30) may yield some clues

The scheme of computation developed was used to compare the directional resolving power of some two-dimensional arrays by simulating a single wave train and comparing the response to it from different gage arrays Figures 1 and 2 tabulate the relevant quantities for comparison The difference between the two tables is that the quantities in Table 1 are obtained without $W_{2}$ smoothing, whereas the quantities in Table 2 are smoothed A comparison shows that all the arrays considered give the direction correctly, but there is a difference in the angular spreads and the values of spectral peaks For a single wave train the spectrum should have been theoretically a Dirac delta function, 1 e a spike So, the narrower the angular spread and the higher the peak, the better the resolving power of the array By this token, out of the five gage arrays tested, the CERC array seems to be the best Figure 3 gives the plots of smoothed directional spectra obtained for different arrays for various input directions 


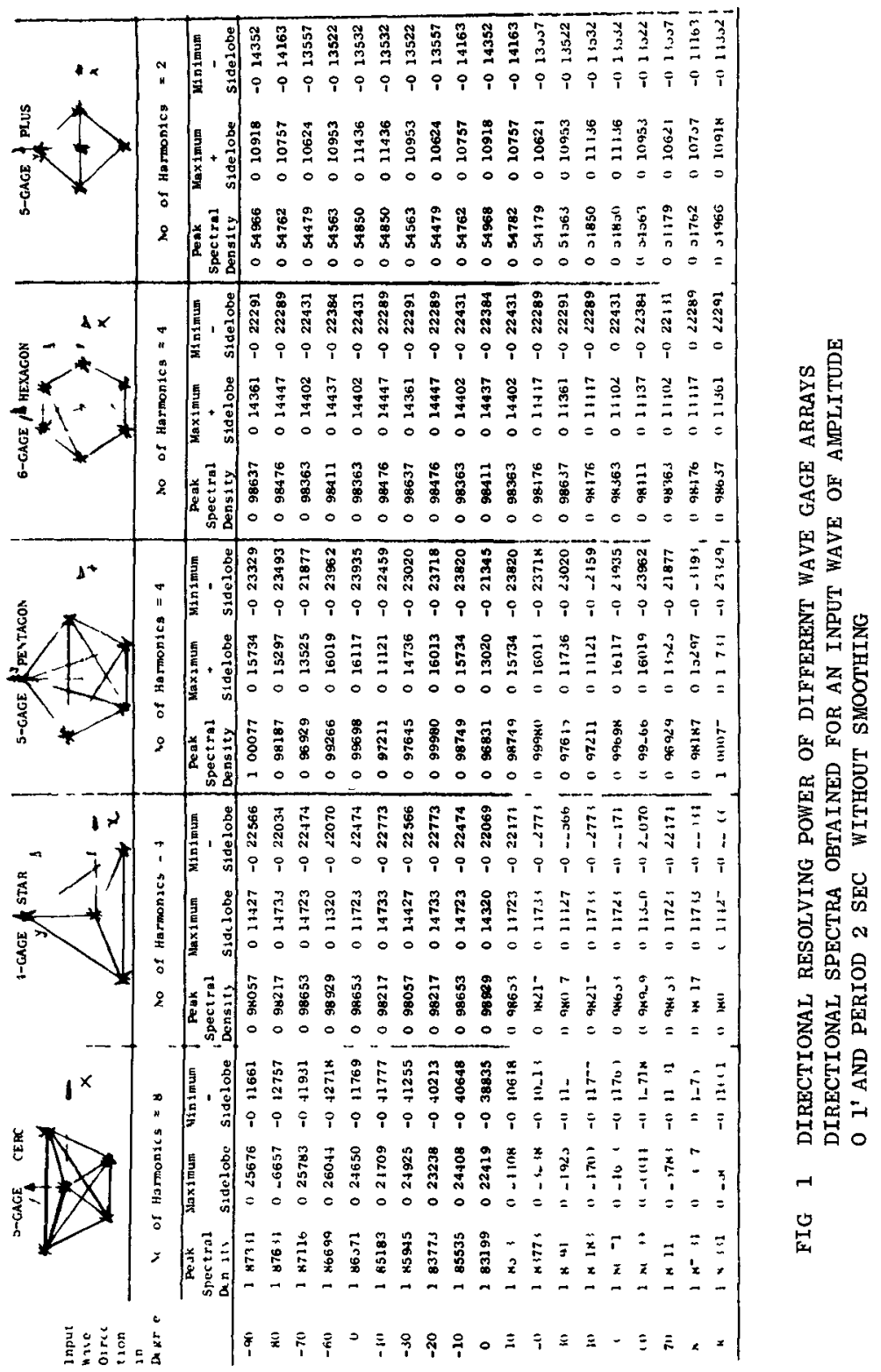




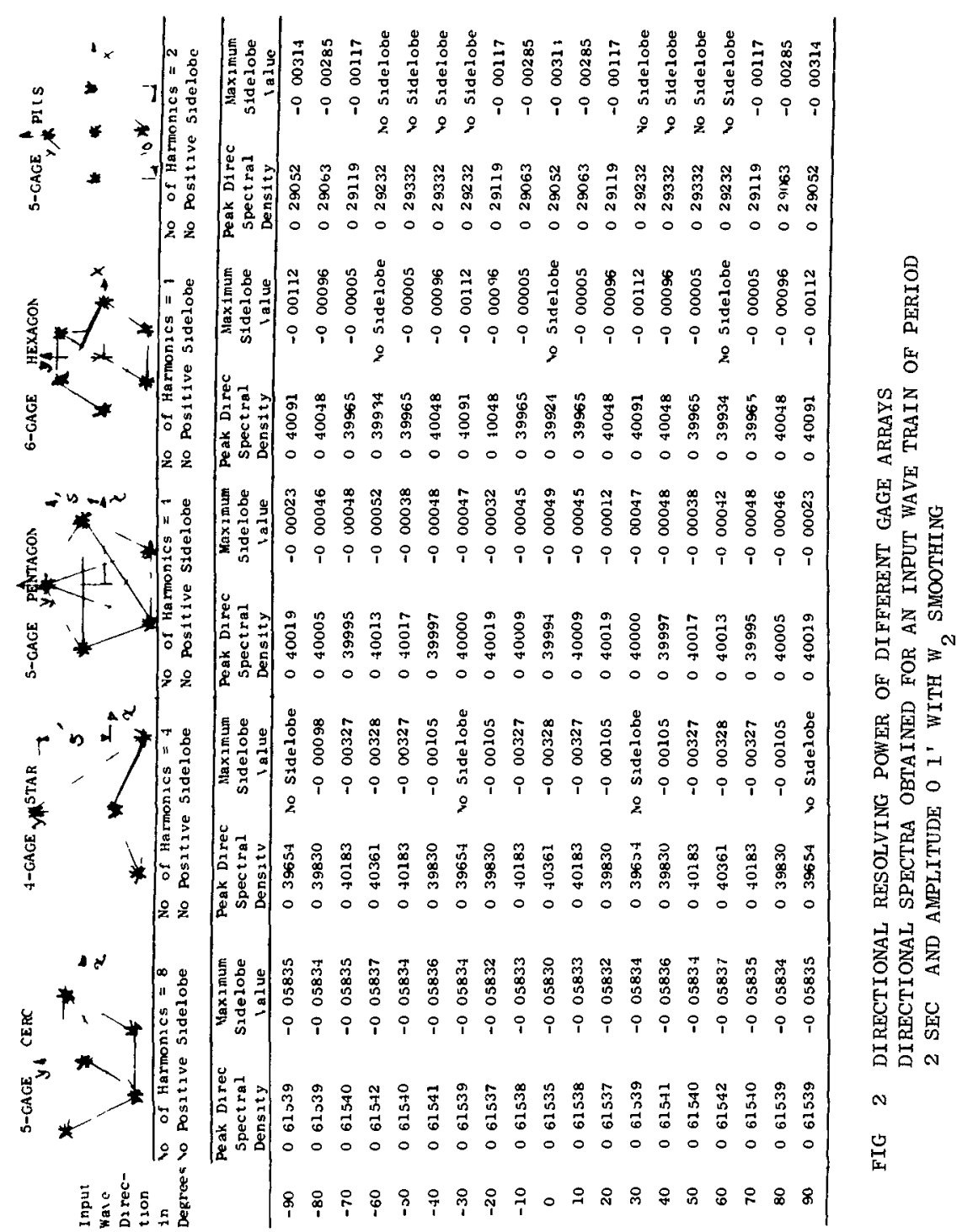




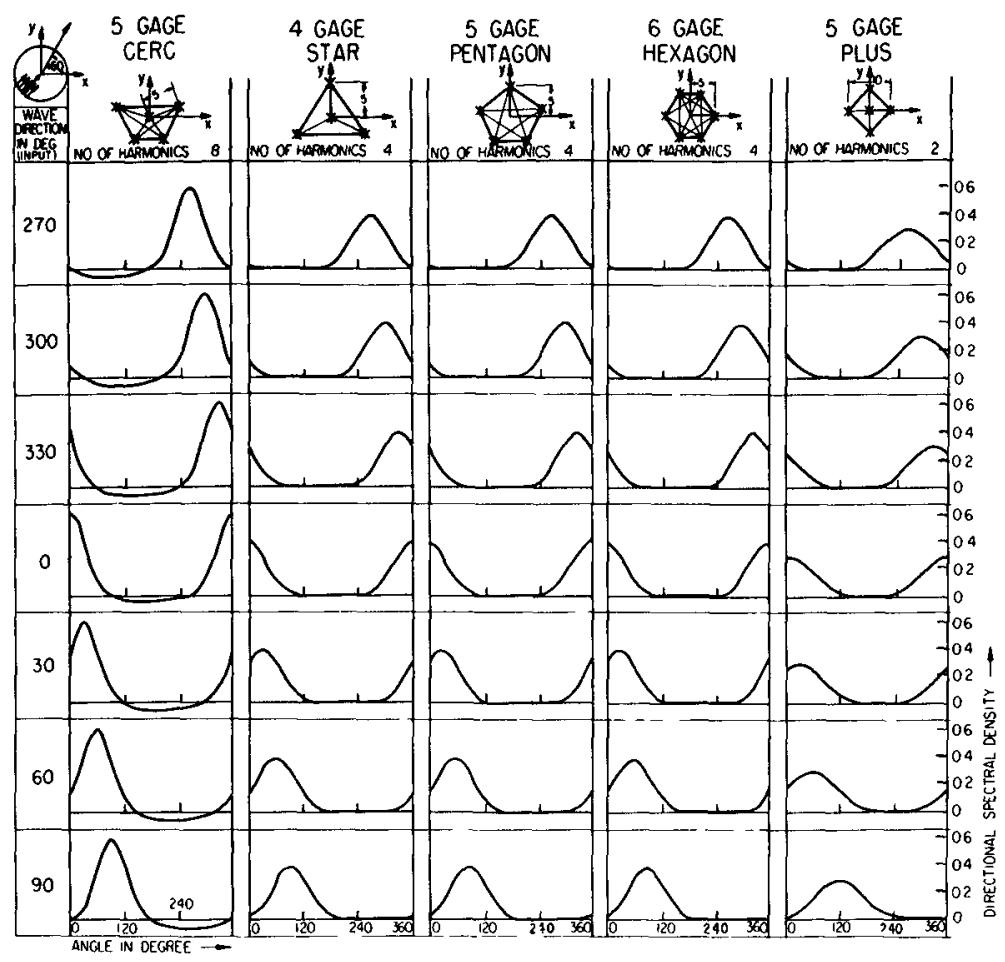

FIG 3 DIRECTIONAL RESOLVING POWER OF GAGE ARRAYS DIRECTIONAL SPECTRA OBTAINED ON DIFFERENT ARRAYS WITH W SMOOTHING FOR FREQUENCY $05 \mathrm{~Hz}$ FOR A SINGLE WAVE TRAIN ÖF PERIOD 2 SEC AND AMPLITUDE $011^{\prime}$ COMING FROM DIFFERENT DIRECTIONS 


\section{Analysis of Data From the Pacific Ocean}

Figure 4 gives the location map and Fig 5 the gage orientation for a 5-gage array put up by the Coastal Englneering Research Center in the Pacific Ocean off Point Mugu, California This array was specifically designed for the determination of directional spectrum of ocean waves of perlods between $7 \mathrm{sec}$ and $25 \mathrm{sec}$, see Borgman and Panicker (13) for the design The gages were subsurface pressure transducers placed 3 feet from the bottom at a water depth of 30 feet and 1600 feet away from the shore The wave data were analyzed for directional spectrum using the method developed Some examples of results obtained are given below

The cholce of a frequency band for averaging the spectra was made by studying Fig 6 which shows the effect of the use of different frequency bands for averaging When the band width is large, the confidence interval of the spectral estimate is close, but the spectrum obtalned is rather too smooth, see, for example, the spect rum obtained when averaging is made in blocks of 64 FFT coefficients, 1 e , in band width of $64 / 1024 \mathrm{~Hz}$ The spectrum is so smooth that it does not show the bimodality indicated by most other cases with block averaging in narrower frequency band width But when the block averaging is done in too narrow a frequency width, as in block averaging of 4 Fourier coefficients, the spectrum shows much erratic nature Tentatively, therefore, it was decided to average in blocks of 32 FFT coefficients and 16 FFT coefficients Figure 7 shows a comparison of the spectra obtalned at the different gages They compare well but there is some discrepancy at peak frequencies Figures 8 and 9 show typical directional spectra obtalned for the locality using the random phase method of analysis described The spectral densities shown are based on pressure in ft of water and not adjusted for surface elevations The dominant directions would not be affected, anyway Figure 8 shows a comparison of the directional spectra obtained at two different times Notice the arrival of the prominent swell in the morning of March 28 Figure 9 shows a contour plot of directional spectrum obtained with a block averaging of 16 FFT coefficients, or a frequency band width of $16 / 1024 \mathrm{~Hz}$ The typical bimodal spectrum of the Pacific coast can be seen with the ridges showing the strong sea and swell, both being shown to come generally from the West South West

\section{CONCLUSIONS}

The following conclusions may be drawn from the above discussions

1 Use of wave gage arrays seems to be well suited for the determination of directional spectra in a routine manner

2 The analytical procedure and the scheme of computation developed seem to work well for the different situations tested, viz numerically simulated data, laboratory data and ocean data 


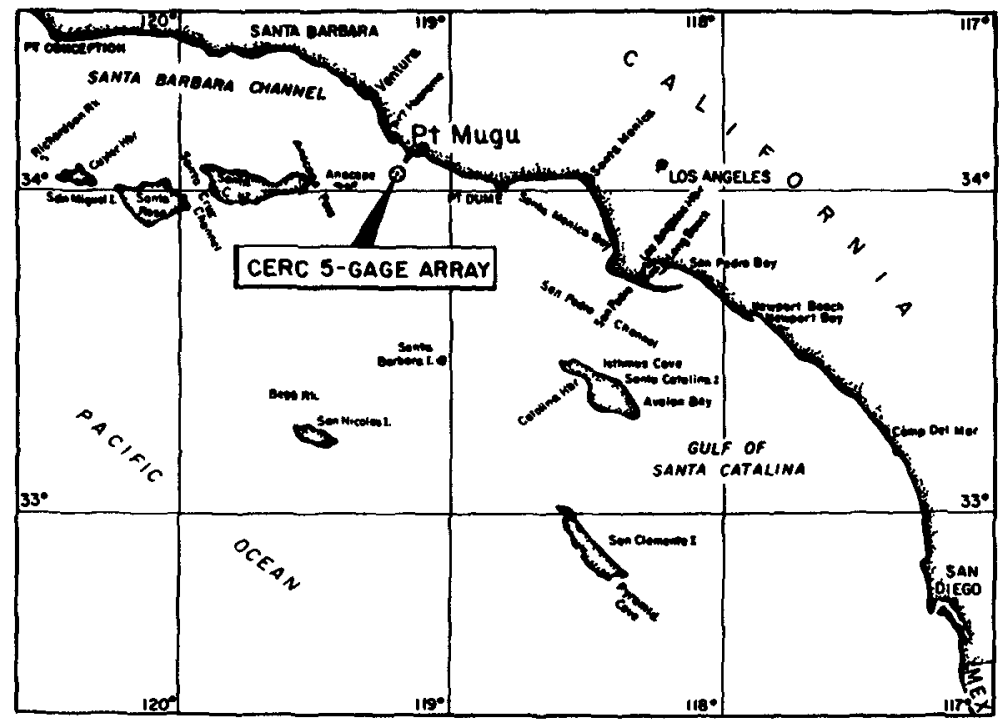

FIG 4 LOCATION MAP OF CERC 5-GAGE ARRAY OFF POINT MUGU,
CALIFORNIA

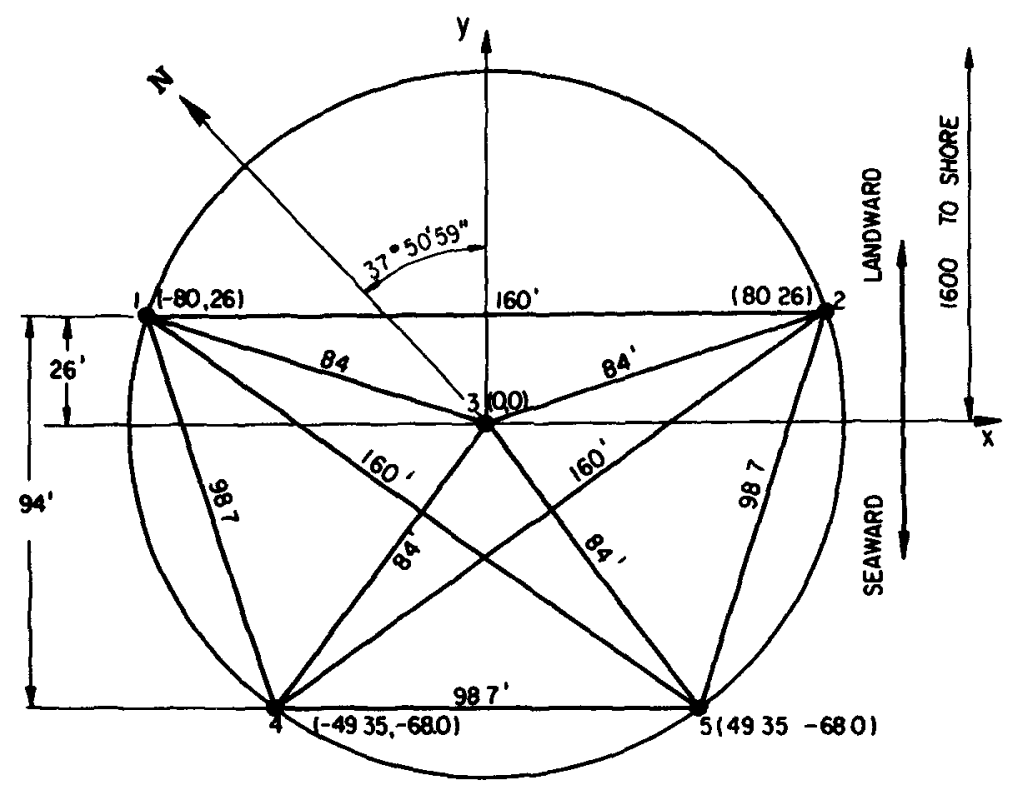

FIG 5 CERC ARRAY OF WAVE GAGES OFF POINT MUGU, CALIFORNIA 


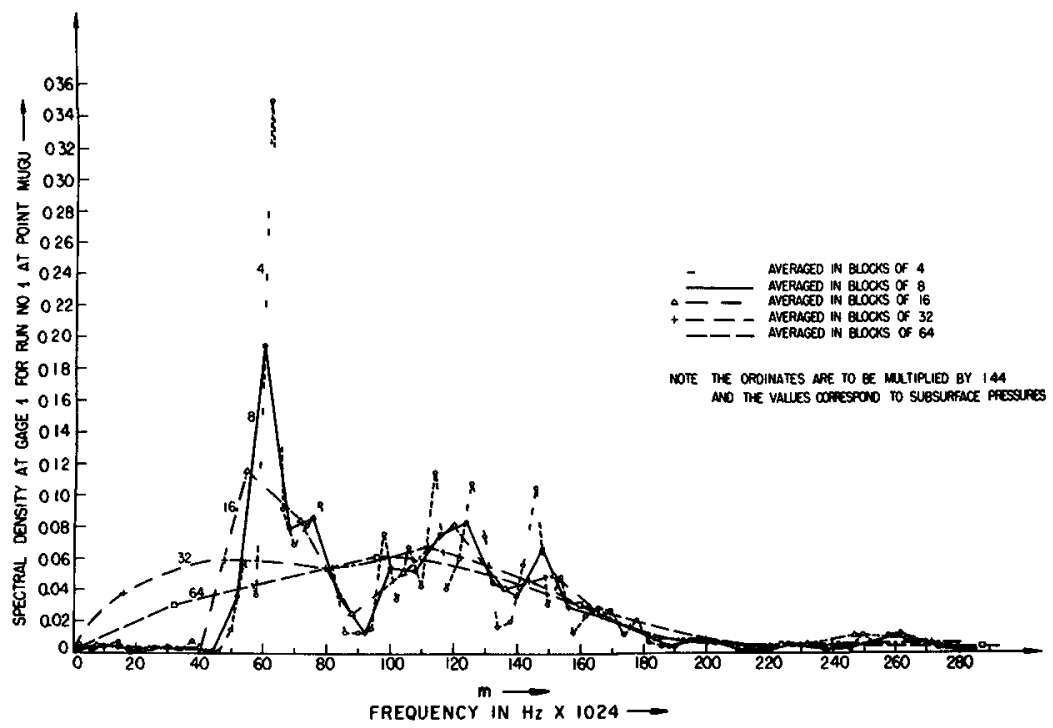

FIG 6 SPECTRA AT GAGE 1 OFF POINT MUGU FOR RUN NO 1 , AVERAGE IN BLOCKS $4,6,16,32$ and 64

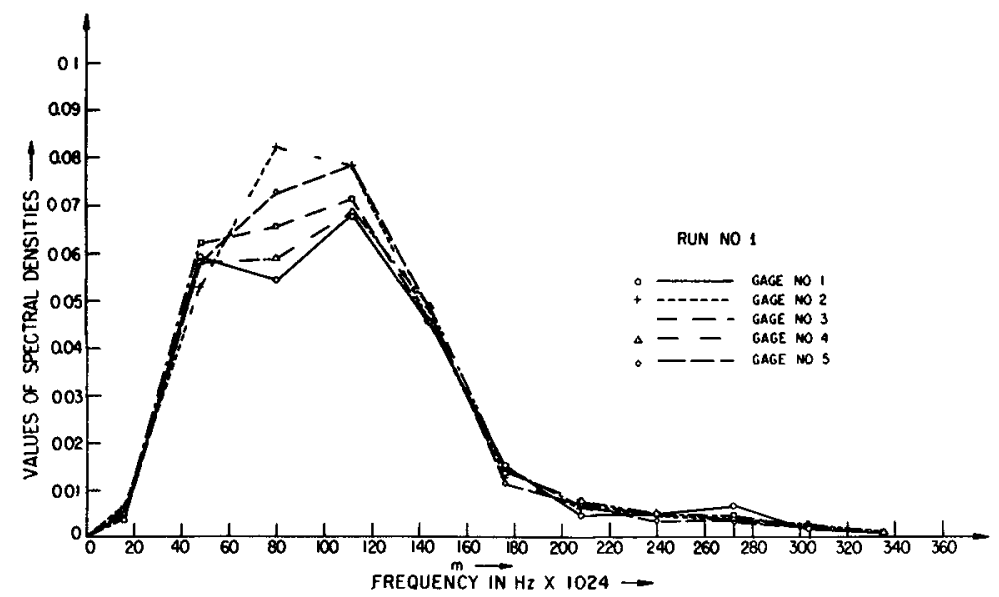

FIG 7 SUBSURFACE PRESSURE SPECTRA AT POINT MUGU AT GAGES $1,2,3,4$ AND 5 AVERAGED IN BLOCKS OF 32 


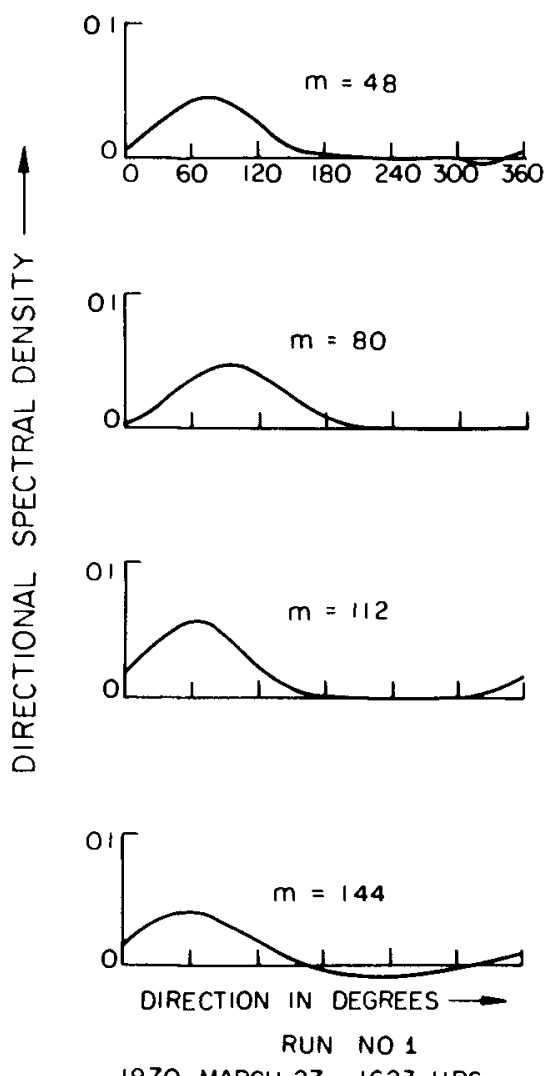

1970 MARCH $27 \quad 1623$ HRS
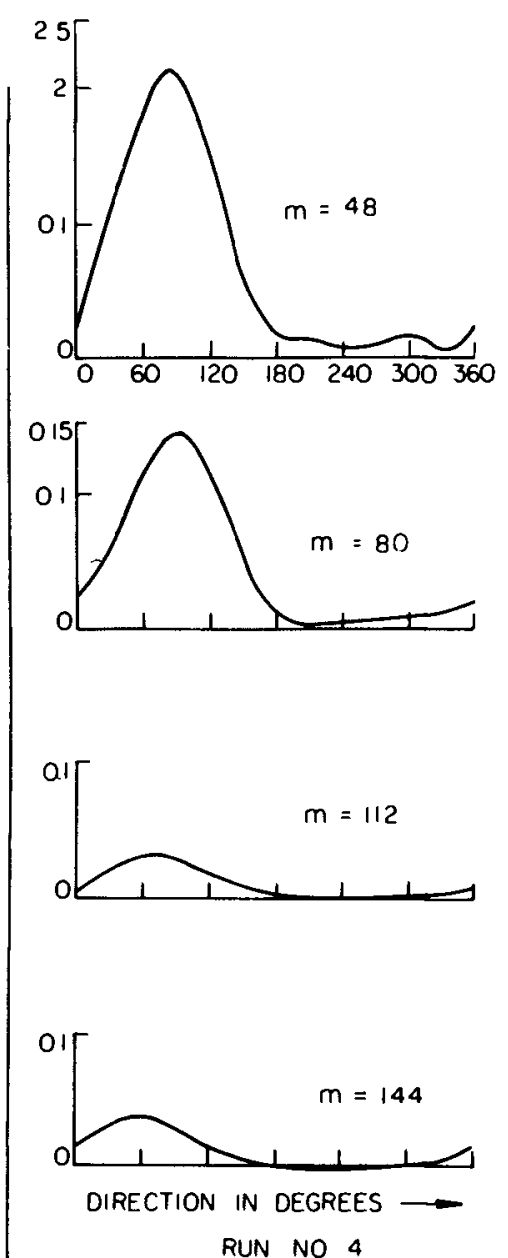
1970 MARCH $28 \quad 0239$ HRS

\footnotetext{
NOTE FREQUENCY $t=\frac{m}{1024}$ LENGTH OF BLOCKS IN AVERAGING SPECTRA $=32$ NO OF HARMONICS TO WHICH DIRECTIONAL SPECTRAL DENSITY FUNCTION IS REPRESENTED $=4$
}

FIG 8 DIRECTIONAL SPECTRA OBTAINED FROM CERC 5-GAGE ARRAY OFF PT MUGU, CALIFORNIA 


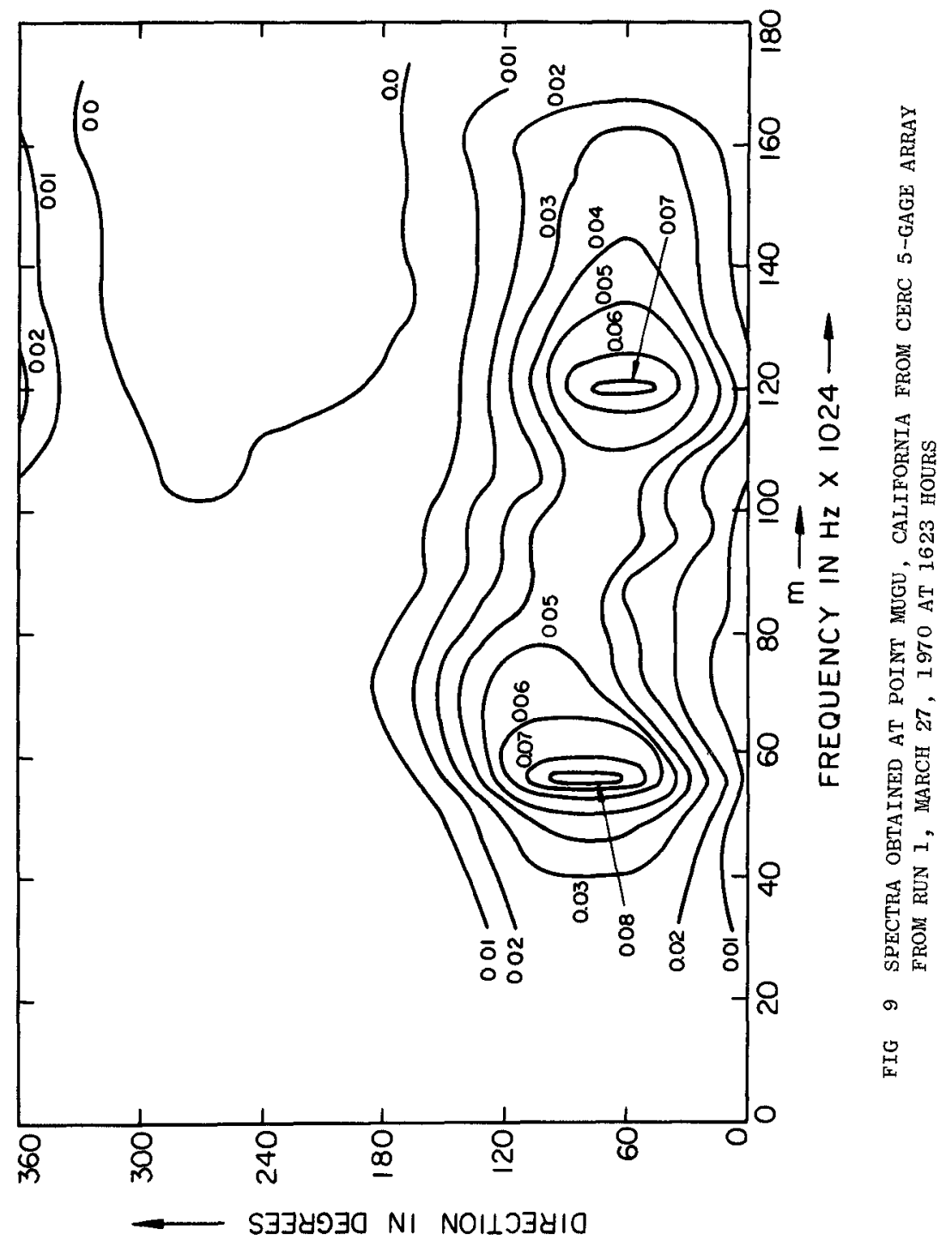


3 Much more research work is needed in almost all aspects of the problem

\section{ACKNOWLEDGMENT}

The work presented herein was partially supported by contract DACW 72-68-C-0016 with the Coastal Englneering Research Center, Corps of Engineers, U S Army The authors express their appreciation to fellow research workers and to the staff of the Coastal Englneering Research Center who were extremely helpful in every aspect of the work Professor R L Wiegel of the Unıversity of California, Berkeley, contributed greatly to the quality of the work by discussions, encouragement and other help which are highly appreciated The assistance of Mr Dennis Dufalla of the Computer Science Division of the University of California, Berkeley, is also gratefully acknowledged

\section{REFERENCES}

1 Abkow1tz, M A, Vassilopoulos, L A and sellers, F H, "Recent developments in seakeeping research and its application to design," Transactions, Society of Naval Architects and Marıne Engıneers, vol 74, pp 194-259, 1966

2 Abramowitz, $M$ and Stegun, I A, Handbook of Mathematical Functions, Dover Publications, Inc, New York, 1965

3 Barber, N F , "Finding the direction of travel of sea waves," Nature, vol 174, pp 1048-1050, Dec 4, 1954

4 Barber, N F and Doyle, D, "A method of recording the direction of travel of ocean swell," Deep-Sea Research, vol 3, pp 206213,1956

5 Barber, N F, "Some relations to be expected between the directional spectra of swell observed at different times and places on the ocean," New Zealand Journal of Science, vol 1, no 2, pp $330-341$, June, 1958

6 Barber, N F, "Directional resolving power of an array of wave detectors," Ocean Wave Spectra, Prentice-Hall Inc, Englewood Cliffs, $N$ J, pp 137-150, 1961

7 Bennett, $C M$, Pittman, $E$ P and Austın, G B, "Data processing system for multiple series analysis of ocean wave induced bottom pressure fluctuations," lst U S Navy Symposium on M1litary Oceanography, pp 379-415, June 1964 
Bennett, C $M$, "A directional analysis of sea waves from bottom pressure measurements," Transactions of the National Symposium on Ocean Sciences of the Atlantic Shel $\mathrm{f}$, Marine Technology Soc1ety, 1965

9 Bergland, G D, "A guided tour of the FFT," IEEE Spectrum, pp 41-52, July 1969

10 Bingham, C, Godfrey, $M$ D and Tukey, J W, "Modern technıques of power spectrum estimation," IEEE Transactions of Audio and electroacoustics, vol AU-15, No 2, pp 56-66, June 1967

11 Borgman, L E, "Ocean wave simulation for engineering design," Proceedings of the ASCE Conference on Civil Englneering in the Oceans, San Francisco, pp 31-74, Sept 1967

12 Borgman, L E , "Directional spectra models for design use," Technical Report HEL 1-12, Hydraulic Englneering Laboratory, University of California, Berkeley, June 1969

13 Borgman, L E and Panıcker, N N, "Desıgn Study for a Suggested Wave gage array off Point Mugu, Calıfornia," Technical Report HEL 1-14, Hydraul ic Engineering Laboratory, Unıversity of Calıfornıa, Berkeley, January 1970

Brown, $L J$, "Methods for the analysis of non-stationary time series with applications to oceanography," Technical Report, HEL 16-3, Hydraul 1c Engineering Laboratory, University of California, Berkeley, May, 1967

15 Cochran, $w \mathrm{~T}$, et al , "What is the FFr?" IEEE Transactions on Audıo and Electroacoustics, vol AU-15, no 2 , pp 45-55, June 1967

16 Cooley, $J W$ and Tukey, $J W$, "An algorithm for the machine calculation of complex Fourier sexies," Mathematics of computation, vol 19, pp 297-301, April 1965

Cote, $L \quad J$ et al , "The directional spectrum of a wind generated sea as determined from data obtalned by the Stereo Wave Observation Project," Meteorologıcal Papers, New York Unıverm sity, vol 2 , no 6 , June 1960

18 Ewıng, J A, "Some measurements of the directional wave spectrum," Journal of Marıne Research, vol 27, no 2, 1969

19 Fan, S S, "Diffraction of wind waves," Technıcal Report HEL 1-10, Hyd raulıc Engineering Laboratory, Unıversity of California, Berkeley, October 1968

20 Ford, $J$ R, Timme, $R$ C and Trampus, A, "A new method for obtaining the directional spectrum of ocean surface gravity waves,' presented at the Marine Technology Society 3 rd Annual National Conference, Surface Wave Measurements Session, June 1967 
21 Graybili, $F$, "An introduction to linear statistical models," McGraw-Hill Book Co, Inc, New York, 1961

Hasselman, $K$, Munk $W$ and MacDonald, $G$, "Bispectra of ocean waves," Ch 8 of Time Series Analysis edited by M Rosenblatt, John Wiley \& Sons, Inc, 1963

IJ1ma, T and Matsuo, T , "Study on waves in surf zone,"

Proceedings, 15th Conference on Coastal Engineering in Japan, 1968

Karlsson, T , "Refraction of continuous ocean wave spectra,"

Proceedings of the ASCE, Journal of the Waterways and Harbors Division, vol 95, no WW4, pp 437-448, Nov 1969

Kınsman, B , Wınd Waves, Prentıce-Ha11, Inc, Englewood Cliffs, N J, 1965

Loeve, M, Probability Theory (3rd edition), D van Nostrand Co , Inc, New York, 1963

Longuet-Higgins, M S, Cartwright, D E and Smith, N D, "Observations of the directional spectrum of sea waves using the motions of a floating buoy," Ocean Wave Spectra, PrenticeHall Inc, Englewood Cliffs, $\mathrm{N} \mathrm{J} \mathrm{,} \mathrm{pp} \mathrm{III-132,} \mathrm{1961}$

Macovsky, M L and Mechlin, G, "A proposed technique for obtaining directional wave spectra by an array of inverted fathometers," Ocean Wave Spectra, Prentice-Hall, Inc, Englewood Cliffs, N J, pp 235-242, 1961

Malhotra, A $\mathrm{K}$ and Penzien, $J$, "Stochastic analysis of offshore tower structures," Report No EERC 69-6, Earthquake Englneering Research Center, University of Calıfornia, Berkeley, May 1969

30 Medgyessy, $P$, Decomposition of superpositions of distribution functions, Publishing House of the Hungarian Academy of Sciences, Budapest, 1961

31 Mobarek, I E, "Directional spectra of laboratory wind waves," Proceedings of the ASCE, Waterways and Harbors Division, vol 91, no WW3, August 1966

Munk, $W$ H , Mller, $G R$, Snodgrass, $F$ E, and Barber, $N F$, "Directional recording of swells from distant storms," Royal Society of London, Philosophical Transartions, Series A, vol 255, pp 505-584, Ap ril 18, 1963

33 Nagata, $Y$, "The statistical properties of orbital wave motions and their application for the measurement of directional wave spectra," Journal of the Oceanographic Soclety of Japan, vol 19 , no $4, \overline{1964}$ 
34 Neumann, $G$ and Pierson, $W J$, "Known and unknown properties of the frequency spectrum of wind generated sea," Ocean Wave Spectra, Prentice-Ha1l Inc, Englewood Clıffs, N J, pp 9-25, 1961

35 Plerson, $W J$ and Marks, $W$, "The power spectrum analysis of ocean wave records," Transactions, American Geophysical Union, vol 33,1952

36 Plerson, $W \mathrm{~J}$, "Wind generated gravity waves," Advances in Geophysics, vol 2, Academic Press Inc, New York, pp $\overline{93-178}$, 1955

37 Scott, J R , "A sea spectrum for model tests on long-term ship prediction," Journal of Ship Research, Society of Naval Architects and Marine Englneers, vol 9, no 3, Dec 1965

38 Simpson, $J \mathrm{H}$, "Observations of the directional characteristics of sea waves," Geophysical Journal of the Royal Astronomical Society, vol 17, pp 93-120, January 1969

39 Stevens, R G, "On the measurement of the directional spectra of wind generated waves using a linear array of surface elevation detectors," Unpublished manuscript, Reference No 65-20, Technical Report of Woods Hole Oceanographic Institution, Woods Hole, Mass, April 1965

40 Suzukı, Y, "Determination of approximate directional spectra for coastal waves," Report of the Port and Harbor Research Institute, Japan, vol 8, no 4, pp 43-101, December 1969

41 Takano, $\mathrm{K}$, "On some limit theorems of probability distributions," Annals of the Institute of Statistical Mathematics, Tokyo, vol 6, pp 37-113, 1954

42 Tukey, J W , The sampling theory of power spectrum estimates," Symposium on Application of Autocorrelation Analysis to Physical Problems, Woods Hole, Mass, pp 47-67, June 1949

43 Wlegel, R $\mathrm{L}$, "Some englneering aspects of wave spectra," Ocean Wave Spectra, Prentice-Hall, Inc, Englewoods Cliffs, N J, pp 309-321, 1961

44 Wlegel, $R$ L , Oceanographical engineering, Prentice-Hall Inc, Englewood $\mathrm{Cl} 1 \mathrm{ffs}, \mathrm{N} \mathrm{J}, 1964$

45 Wregel, $R \quad L$ and Mobarek, I E , "Diffraction of wind generated water waves," Proceedings, Tenth Conference on Coastal

Englneerıng, vol I, p 185-197, 1966

46 Wlegel, $\mathrm{R} \mathrm{L}$, "Waves and their effects on pile-supported structures," Technical Report HEL 9-15, Hydraulic Engineering Laboratory, University of California, Berkeley, March 1969 


\title{
CHAPTER 9
}

\author{
Equzlıbrium Range Spectra in Shoaling Water \\ by \\ Takesh工 IJIma', Takahzxo Matsuo ${ }^{(2)}$ and Kazutam Koga $a^{(3)}$
}

\section{Abstract}

in shoalıng water on sloping beach, waves break by hydraulic instabinty due to the finiteness of water depth, so that frequency spectrd of waves in surf zone must have any limitirg form siml lar to the equalibrium spectrum given by Fhillips(1958) In this paper, authors have derived an equilibrium form of spectra for surf waves from the limiting wave condition at constant water depth by Mche(1944) and froin breaking wave experiments on sloped rottom by Iversen(I952) The results are compared with surf wave spectra obtained from field observations by means of stereo-type wave meter devised by the authors (1968).

By means of this spectrum and by deep water wave spectra for various wind conditions, significant wave heights and optimum periods of limiting waves in surf <one are calculated

\section{Introduction}

By dimensional considerations, Phillips(1958) has shown that in the frequency spectrum of deep water wind waves the energy spectral density $\Phi(\sigma)$ of saturated high frequency component is proportional to -5 powers of frequency as shown by the following equation

$$
\Phi(\sigma)=\beta g^{2} \sigma^{-5}
$$

where $g$ is gravity acceleration and $\beta$ is non-dimensional constant, for which Phıllips has given numerıcally 00117 from observed data and Plerson(1964) has obtained 000810 from observed spectra on the North Atlantic Ocean

Lq 1 is independent of wind conditions and Is anterpretted as represencing a limiting spectrum for wave breaking in deep water in the state of hydraulic instability

As for waves breasang in shoaling waler, the limating wave height is deternined by $n$ stabllity dependong on water depth $h$ and the simlar limiting spectrum should exist, whych, of course, is maepencent on wind conditions but depends or.ly on water depth and gravıty acceleration in the range of gravity waves

(1) Professor of Kyushu Unıversity, Fuluoka, Japan

(?) Lecturer, DoIta Technical Col lege, Doita, Japan

3) Ilaster Course Student, Kyushu Unıversıty 
Here, we assume stralght shore line with constant slope and waves incident normally, so that one-dumensional waves without refraction effect

\section{Derivation of equilibrium range spectrum}

From observed wave record of a trann of waves at a point of depth $h$ in surf zone, apparent indıvidual wave helght $\widetilde{H}$ and wave period $\widetilde{T}$ of all the waves are measured in spite of breaking and non-breaking and then wave length $\widetilde{L}$ of the wave at water depth $h$ are calculated by small amplitude wave relation Then, we obtain a scatter dzagram of $\widetilde{\mathrm{H}} / \widetilde{\mathrm{L}}$ related to $\mathrm{h} / \widetilde{\mathrm{L}}$ as shown in Fig $\mathrm{I}$, which was obtained from wave record of 17 munutes long at depth of 57 meters on the Coast of Myazakı (Pacıfıc Coast of Kyushu)

The envelope curve through the upper limit of scattered point in the figure is interpreted to show the Ilmuting wave condition for tre depth $h$

For a train of single sınusoldal waves, the limlting condition at constant depth for $h / L$ larger than about $I / 20$ is given by Mcche(1944) as follows

$$
H / L=\frac{1}{7} \tanh \frac{2 \pi h}{L}
$$

For deep water $(\mathrm{h} / \mathbf{L} \rightarrow \infty), \mathrm{H} / \mathrm{L}, \mathrm{s} \mathrm{l} / 7$ and for shallow water long waves $(h / L \rightarrow 0)$, eq 2 gives $H / h \approx 09$, whlch 1 s somewhat larger than 078 by solıtary wave theory For the bottom of constant slope, we have not yet theoretjcal reldtion but Iversen $\left(19^{\prime}, 2\right)$ has shown experımental relations of $\mathrm{H}_{b} / \mathrm{H}_{0}^{\prime}$ and hy/ $\mathrm{H}_{0}^{\prime}$ to $H_{0}^{\prime} / L_{0}$ for bottom slopes $\alpha=1 / 10,1 / 20,1 / 30$ and $1 / 50$, where $H_{0}^{\prime}$, Lo are deep water wave helght and length and $H_{b}, h_{b}$ are wave helght and water depth of breakers From these experimenual relations, an emp $\perp$ rical lelation of $\mathrm{H} / \mathrm{L}$ and $h /$ Lsimlar to eq 2 is ohtalned as follows

$$
H / L=\frac{1}{7} \tanh \left[f(\alpha) \frac{2 \pi h}{L}\right], f(\alpha)=\cosh 35 \alpha^{3 / 3}
$$

where $\alpha$ is bottom slope Above relatıons are shown $1 \mathrm{n}$ F'g 1 for $\alpha=0$ and $1 / 10$ Eq 3 may gıve somewhat larger values for $h / L<1 / 20$ for single sınusoldal waves but for actual randum waves the form of eq 3 may be assumed to represent approxımate relatıons of llmıtıng apparent wave helght $\widetilde{H}$ and length $\widetilde{L}$ derived from apparent wave perıod $\widetilde{T}$ for all $h / \widetilde{L}_{*}$ Accordıngly, we assume next relation for limeting apparent waves

$$
\frac{\widetilde{H}_{\max }}{L}=C_{1} \tanh \left[f(\alpha) \frac{2 \pi h}{\widetilde{L}}\right]
$$


Where $C_{1}$ Is assumed to be constant

As for actual waves with continuous spectrum, the limzting wave height $\widetilde{\mathrm{H}}_{\max }$ Is interpreted as the result that all the phases of component waves whose periods are within narrow range of perıod band between $\widetilde{T}-\Delta \widetilde{T} / 2$ and $\widetilde{T}+\Delta \widetilde{T} / 2$ centered at perzod $\widetilde{T}$ happened to colncide and thejr spectral wave helghts were summed up to attain the limiting helght $\widetilde{\mathrm{H}}_{\text {max }}$ And for sufficiently narrow width of $\Delta \widetilde{T}$, the component waves within the period band are consldered to have nearly equaI spectral wave helght $\mathrm{H}_{\mathbf{T}}$ proportional to the limlting wave helght $\widetilde{\mathrm{H}}_{\text {max }}$, that Is,

$$
H_{T} \propto \widetilde{H}_{\max }
$$

Therefore, from eq 4 we have

$$
H_{T}=\text { const } \tilde{L} \tanh \left[f(\alpha) \frac{2 \pi h}{\tau}\right]
$$

Assuming that the number of component waves wathin the period band IS $\mathbb{N}$ and they are mutually independent, the total energy density wathin the period band of $\tilde{T}-\Delta \tilde{T} / 2$ and $\tilde{T}+\Delta \widetilde{T} / 2$ is

$$
N \cdot \frac{\rho g}{8} \operatorname{conat} \tilde{L}^{2} \tanh ^{2}\left[f(\alpha) \frac{2 \pi h}{\tau}\right]
$$

Accordingly, the total frequency spectral energy density $\Phi(\sigma) \Delta a b e t w e e n$ $\alpha-\Delta \sigma / 2$ and $\sigma+\Delta \sigma / 2$ is given by the followng equation.

$$
\Phi(\sigma) \Delta \sigma=\operatorname{const} N \tilde{L}^{2} \cdot \tanh ^{2}\left[f(\alpha) \frac{2 \pi h}{\widetilde{L}}\right]
$$

The number of component waves $\mathbb{N}$ in above equation is considered to become large when the perıod band $\Delta \widetilde{q}$ becomes wade, but to become swall when the perıod $\widetilde{T}$ becomes large for limlted length of wave record Accordingly, we may assume the following relation

$$
\text { corrat } N=\operatorname{conat} \Delta \tilde{T} / \tilde{T}=\operatorname{conat} \Delta \sigma / \alpha,(\alpha=2 \pi / \tilde{T})
$$

Now, eq 7 is written as follows

$$
\begin{aligned}
\Phi(\sigma) \Delta \sigma & =\text { const } L^{2} \cdot \tanh ^{2}\left[f(\alpha) \frac{2 \pi h}{L}\right] \\
& =\operatorname{const}(2 \pi)^{2} \frac{h^{5 / 2}}{g^{1 / 2}} \frac{\tanh ^{2}\left[f(\alpha) \frac{2 \pi h}{L}\right]}{\left(\frac{2 \pi h}{L}\right)^{2}\left(\frac{\sigma^{2} h}{g}\right)^{1 / 2}} \Delta a \\
& =\operatorname{const}(2 \pi)^{2} \frac{h^{\frac{5}{2}}}{g^{1 / 2}} F\left(\alpha, \frac{\sigma^{2} h}{g}\right) \Delta a
\end{aligned}
$$


where

$$
F\left(\alpha, \frac{\sigma^{2} h}{g}\right)=\frac{\tanh ^{2}\left[f(\alpha) \frac{2 \pi h}{L}\right]}{\left(\frac{2 \pi h}{L}\right)^{2}\left(\frac{\sigma^{2} h}{g}\right)^{1 / 2}}
$$

In above equations, $2 \pi \mathrm{h} / \mathrm{L}$ is determined as a function of $\alpha^{2} \mathrm{~h} / \mathrm{g}$ by the following equation

$$
a^{2} h / g=\frac{2 \pi h}{L} \tanh \frac{2 \pi h}{L}
$$

In eq 10 , when $\sigma^{2} \mathrm{~h} / \mathrm{g}$ becomes large, $2 \pi \mathrm{h} / \mathrm{L}$ tends to $2 \pi \mathrm{h} / \mathrm{L}_{0}$ and $F$ tends to $\left(\sigma^{2} b / g\right)^{-5 / 2}$, and when $\sigma^{2} h / g$ becomes small, $F$ tends to $f^{2}(\alpha)\left(\sigma^{2} h / g\right)^{-1 / 2}$ Accordingly, from eq 9 it is seen that

$$
\begin{array}{ll}
\Phi(\sigma)=\operatorname{conat}(2 \pi)^{2} g^{2} \alpha^{-5} & \text { for deep water waves } \\
\Phi(\sigma)=\operatorname{cont} \cdot(2 \pi)^{2} f^{2}(\alpha) h^{2} \alpha^{-1} & \text { for shallow water waves }
\end{array}
$$

Comparing eq 12 wIth eq 1 ,

$$
\text { conat. }(2 \pi)^{2}=\beta
$$

Thus, eq 9 is determined as follows

$$
\Phi(\sigma)=\beta \frac{h^{5 / 2}}{g^{1 / 2}} F\left(\alpha, \frac{\sigma^{2} h}{g}\right)
$$

Above equation is considered to represent the equilibrum spectrum of surf waves

\section{Comparisons with observations}

The observation of surf waves is difficult because of various troubles in setting and malntanng wave meters The authors(1968) have devised a stereotype wave meter, whth whych field observations at Myyazakı Coast and Nata Coast of Kyushu were carried out Fig 2 is an example of observed frequency spectra at Nata Coast. F1g.3 and 4 are non-dimensional plot of frequency spectra at Myyazakı- and Nata Coast, respectively, in whlch the curves are equilibrium spectra by eq 14 for $\alpha=0$ and $1 / 10$ whth $\beta=000810$.

In Fig.3, measured spectral densities for large $\sigma^{2} \mathrm{~h} / g$ attain to the equillbrium values but for small $\sigma^{2} \mathrm{~h} / \mathrm{g}$ measured densities are lower than the latter 
This seems to be due to the fact that few waves are breakıng by the effect of fincte depth and only high frequency waves are saturated by local wind In Flg.4 measured energy densities almost attan to the equilibrium values, which mean that almost waves are breaknng by the effect of finlte depth

4 Estımation of limıting slgniflcant waves in surf zone

Assuming that eq 14 Is the liming spectral energy density at water depth $h$, the limsting spectrum for given wınd condltions at the depth may be estimated by the following considerations

In Fig 5, suppose that varıous spertra are drawn in linear scale and the curve $M N$ is equilibrium spectrum at given water depth When the spectrum of offsea wind waves Is given by the curve MWQ, the spectrum to be observed at the point of depth $\mathrm{h}$ should be gaven by the curve $M C Q$, which is the limiting spectrum for the given wind condition. When the spectrum of offsea swell 1 s given by the curve ASQ, the spectrum at depth $h$ should be the curve $A B C Q$, whlch is the llmitIng spectrum for glven swell. Flg 6 shows an example of offsea wind wave spectrum for wind speed $U=I 5 \mathrm{~m} / \mathrm{s}$, fetch length $F=400 \mathrm{~km}$, whlch Is proposed by the authors is shown in Appendıx, and equilıbrıum spectra at water depth $h=8$ meters for bottom slope $\alpha=0$ and $1 / 10$

F'Ig $7(a)(b)$ are significant wave helght and Its optimum perıod of limiting waves calculated by above-derıved limtıng spectmum at varıous water depth for fetch length $100 \mathrm{~km}$ and $400 \mathrm{~km}$ wIth wind speed $15 \mathrm{~m} / \mathrm{s}$ and $30 \mathrm{~m} / \mathrm{s}$

\section{Conclusions ana remarks}

Omiting the effect of wave refraction and directional distribution of wave spectmum, the equzlıbrum spectrum of surf waves at water depth $h$ Is represented by eq 14, by means of which and a proposed fetch- or duration spectrum of offsea wind waves the lizuting spectrum at any depth 1 s estimated as shown in Fig 6

Up to date, for deslgn purposes of surf zone structures the limating wave helght by such an equation 2 or 3 is frequently used as design wave helght But in some cases, it seems to be more reasonable to uee such a limiting spectrum as above The effect of directional distribution of wave spectrum should be considered in future 


\section{Appendix}

Derivation of Duration and Fetch Spectrum

We assume the general form of frequency spectrum as follows

$$
\Phi(\sigma)=\alpha_{*} g^{2} \sigma^{-5} \exp \left\{-\beta_{*}\left(\frac{g}{\sigma \sigma_{*}}\right)^{4}\right\}
$$

where $g$ is gravity acceleratı,n, $U_{*}$ is friction velocity of w॰nd and $\alpha_{*}$, $\beta$ * are non-dimensional constants

Above spectrum has the raximum energy density $\Phi\left(\sigma_{0}\right)$ at frequency $\sigma_{0}$ as follows

$$
\Phi\left(\sigma_{0}\right)=\alpha_{*} g^{2} e^{-\frac{5}{4}} \sigma_{0}^{-5} \quad \text { (A2) } \quad \frac{\sigma_{0} \pi_{*}}{g}=\left(\frac{4}{5} \beta_{*}\right)^{1 / 4}
$$

from walch we obtaun

$$
\beta_{*}=\frac{5}{4}\left(\frac{\sigma_{0} \Pi_{*}}{g}\right)^{4} \quad \text { (A4) } \quad \alpha_{*}=\frac{e^{5 / 4}}{g^{2}} \Phi\left(\sigma_{0}\right) \sigma_{*}^{5}
$$

The time developement of the maximum energy density of wind generated-wave spectrum is given by Phrllips(1966) as follows

$$
\Phi\left(\sigma_{0}\right)=A \frac{D_{*}^{4}}{g^{2}} \frac{\operatorname{senh}\left(\mu \sigma_{0} t\right)}{\mu \sigma_{0}}, A=240\left(\frac{\rho_{a}}{\rho_{\omega}}\right) \approx 3.46 \times 10^{-4}
$$

where $\rho_{a}$ and $\rho_{w}$ are densities of air and water and $\mu$ is coupling coefficient of aur-sea interaction

Substrtuting eq 44,45 and $A 6$ into eq AI, we obtain as duration spectrum

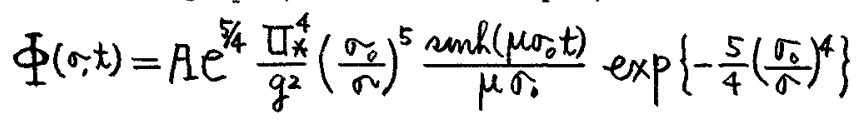

Meanwhlle, Plerson(1964) has proposed the following spectrum for fullydeveloped wind waves

$$
\Phi(\sigma)=\alpha g^{2} \sigma^{-5} \exp \left\{-\beta\left(\frac{g}{\sigma-\bar{U}}\right)^{4}\right\}
$$

where $\alpha=000810, \beta=074$ and $U$ is mean wind speed at 195 meters high above mean sea surface, which is related to $U_{*}$ and $U_{10}$ (w wrd speed at 10 meters above mean sea level) by the following empirical relationships

$$
\Pi=K \Pi_{*} \quad K=160+\frac{1}{\sqrt{C_{10}}}
$$




$$
\Pi_{10}=\frac{\Pi_{*}}{\sqrt{C_{10}}} \quad C_{10}=\left(080+0.114 \Pi_{10}\right) \times 10^{-3}
$$

For any given wınd speed, the maxımum energy densıty $\Phi\left(\sigma_{\boldsymbol{\theta}}\right)$ of duration spectrum should lie always on the saturated spectrum curve of that wind speed given by eq $A 8$, so that equating eq $A 6$ to eq $A 8$, we obtain next relation for $\sigma_{0}$ at any duration time $t$

$$
\frac{A}{\alpha} \pi_{*}^{4}\left(\frac{\sigma_{0}}{g}\right)^{4} \frac{\sinh \mu \sigma_{0} t}{\mu}=\exp \left\{-\beta\left(\frac{g}{K \pi_{*} \sigma_{0}}\right)^{4}\right\}
$$

Eq A7 together with eq All gives duration spectrum

Fetch length $x$ is related to duration time $t$ by the following equation

$$
x=\frac{1}{2} C_{0} t
$$

where $C_{0}$ Is the phase velocity of waves wh th frequency $\sigma_{0}$

Thus, fetch spectrum is obtained from duration spectrum as follows

$$
\Phi(\sigma, x)=A e^{5 / 4} \frac{U_{*}^{4}}{g^{2}}\left(\frac{\sigma_{0}}{\sigma}\right)^{5} \frac{\sinh \left(2 \mu \sigma_{0} x / c_{0}\right)}{\mu \sigma_{0}} \exp \left\{-\frac{5}{4}\left(\frac{\sigma_{0}}{\sigma}\right)^{4}\right\}
$$

By the relation $\sigma_{0}=g / C_{0}$, duration and fetch spectrum is written as

$$
\begin{aligned}
& \Phi(\sigma, t)=A e^{5 / 4} g^{2} \sigma^{-5} \frac{\operatorname{ainh}\left(\mu \frac{g t}{u_{*}} / c_{0}\right)}{\mu} \cdot \frac{1}{\left(C_{0} / u_{*}\right)^{4}} \exp \left\{-125 \frac{\left(g / u_{*}\right)^{4}}{\left(C_{0} / u_{*}\right)^{4}}\right\} \\
& \Phi(\sigma, x)=A e^{5 / 4} g^{2} a^{-5} \frac{\sinh \left(2 \mu \frac{g *}{u_{*}} /\left(\frac{c_{0}}{u_{*}}\right)^{2}\right)}{\mu} \frac{1}{\left(C_{0} / u_{*}\right)^{4}} \exp \left\{-1.25 \frac{\left(g \sigma / u_{*}\right)^{4}}{\left(C_{0} / u_{*}\right)^{4}}\right\}
\end{aligned}
$$

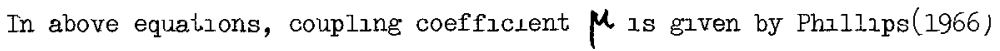
ac a function of $\mathrm{c} / \mathrm{U}_{*} \quad$ But when fetch spectrum is given by eq AI5, the significant wave neight $\mathrm{H}_{1 / 3}$ is shown by followng equation

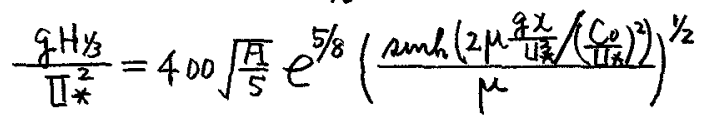

And also from observation data, wlgnuficant wave helght is empurically related to wand speed hy the following equation by WIlson(1965)

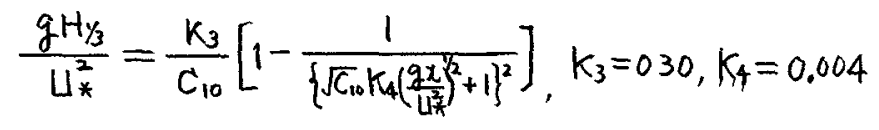


Equating above two equations, coupling coefficient $\mu$ is obtained for $\tau_{\odot} / U_{*}$ w1th parameter $\sigma_{*}$ as shown in Fig AI F1g 42 is an example of fetch spectrum References

Phillips, O M (1958) "The Equilibrium Range in the Spectrum of Wind-Generated Waves " J Fluzd Mechanzos, Vol 4

Mzche,A (1944) "Mouvements ondulatolres de la mer en profondeur constante ou decrolssante " Annales des ponts st chaussues, Vol 114

Iversen, H W (1952) " Laboratory Study of Breakers "Gravity waves Nat Bur Standards Cir No 521

Plerson, $W J$ and L Moskowntz(I964) "A Proposed Spectral Form for Fully Developed Wind Seas based on the Simllarity Theory of S A K talgorodskıl " $J$ Geoph Res VoI 69 , No 24

Ijlma, $I^{\text {r }}$ and T.Matsuo(1968) "Observatıon of Surf Waves by Stereo-Wave Meter " Coastal Enguneering in Japan, Vol 11

Phillips, o M (1966) "The Dynarazcs of the Upper Ocean " Cambridge Unzversity Press

Wilson, B W (1965) " Numerical Prediction of Ocean Waves in the North Atlanilc for December, 1959 " Deutsch Hydr Z $J_{g} 18$ Ht 13 

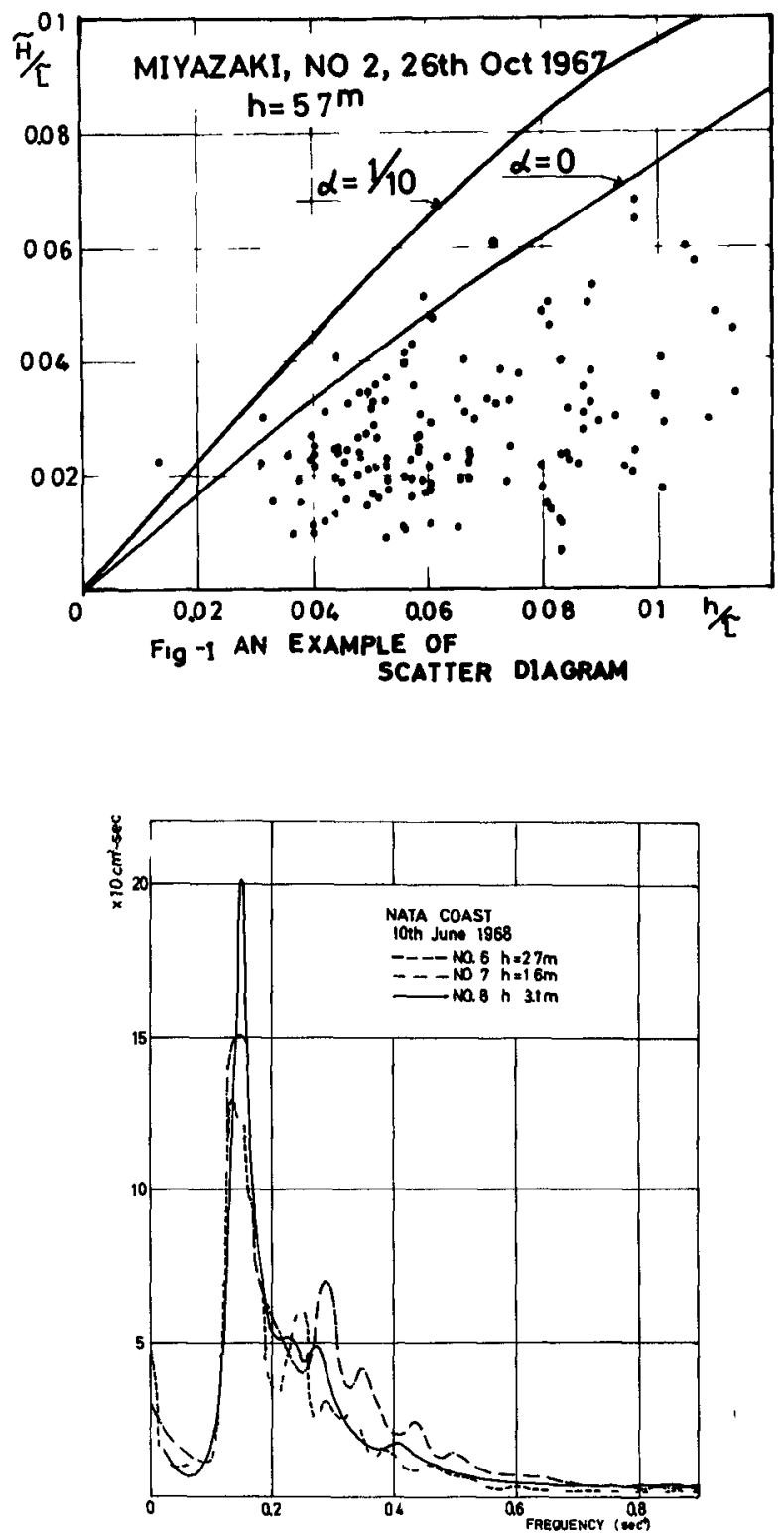

Fig 2 EXAMPLES OF FREQUENCY SPECTRA 

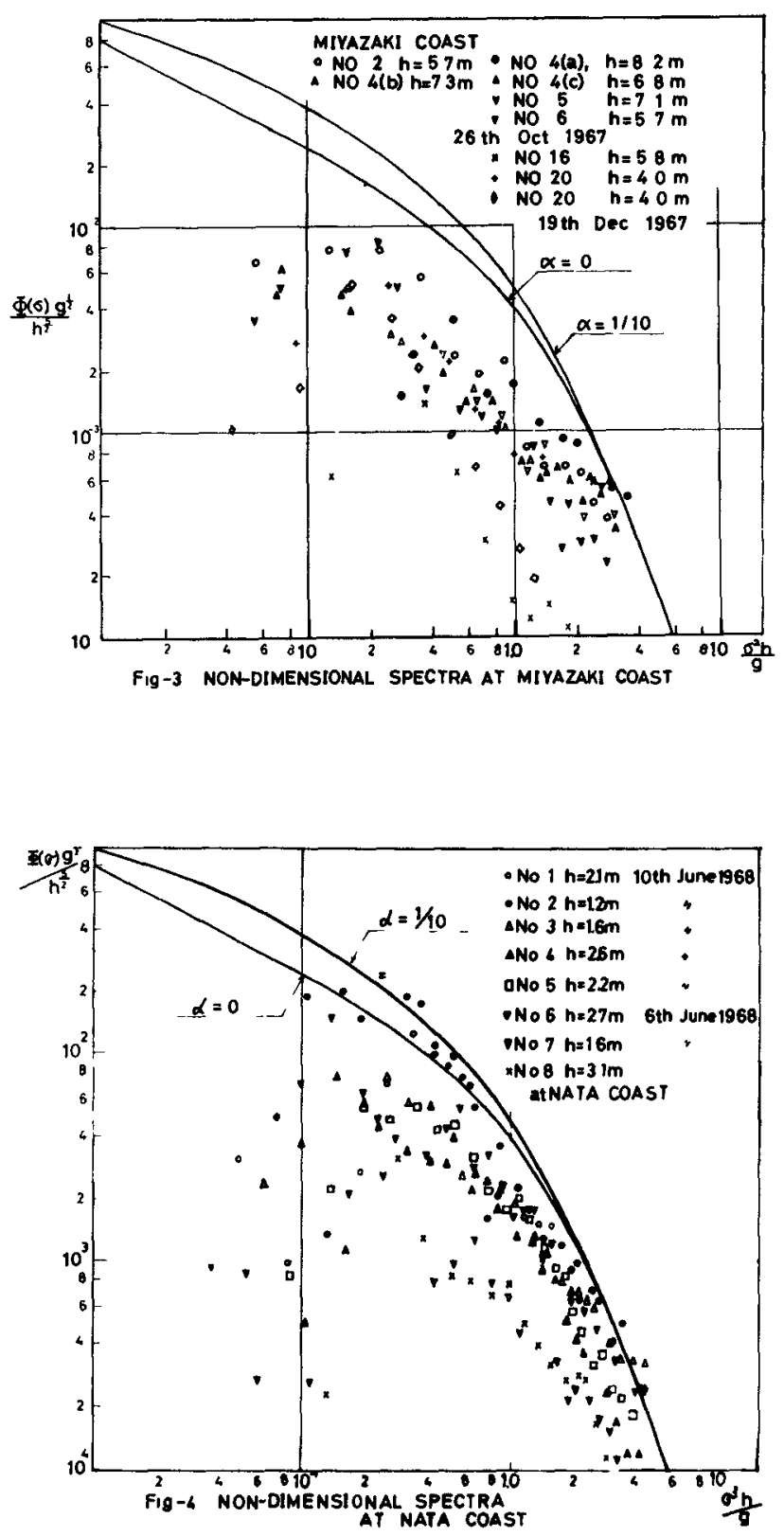


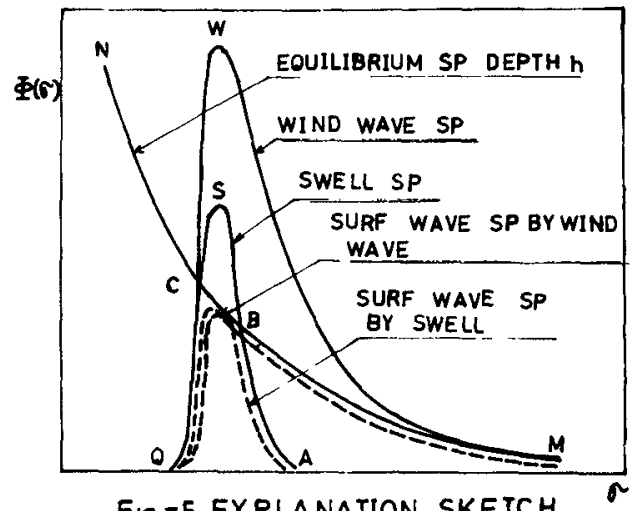

Fig -5 , EXPLANATION SKETCH

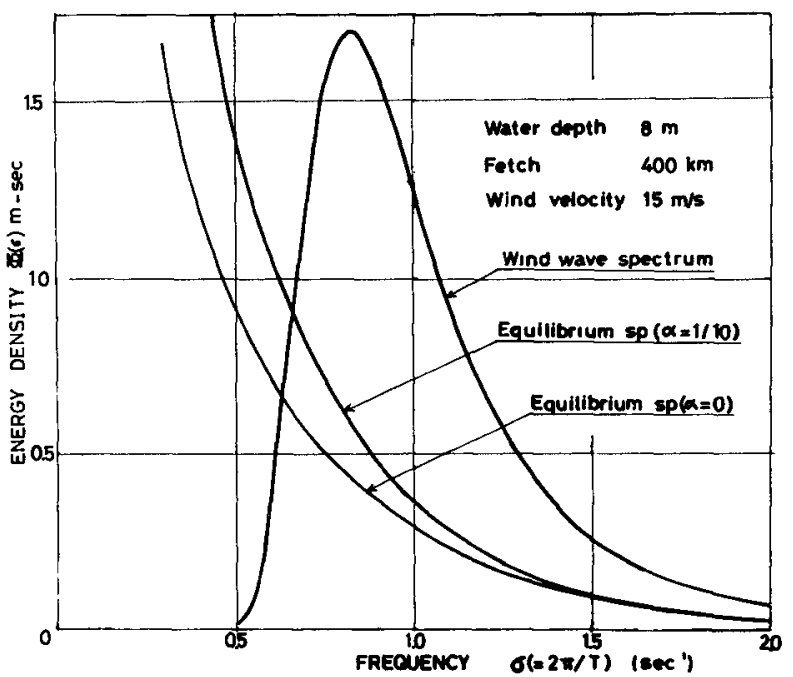

Fig -6 Limiting Spectra at Depth of 6 meurs tor an Off sea Wind Wave Spectrum 


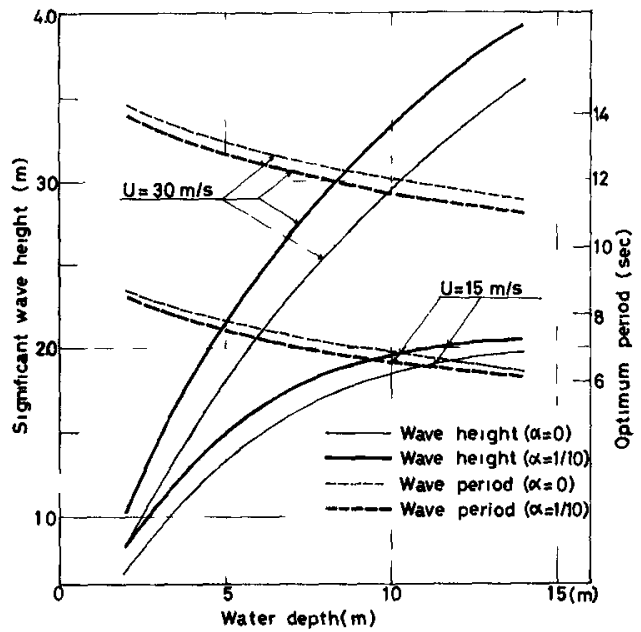

FIg-7(a) LIMITING SIGNIFICANT WAVES FOR FETCH LENGTH $100 \mathrm{KM}$

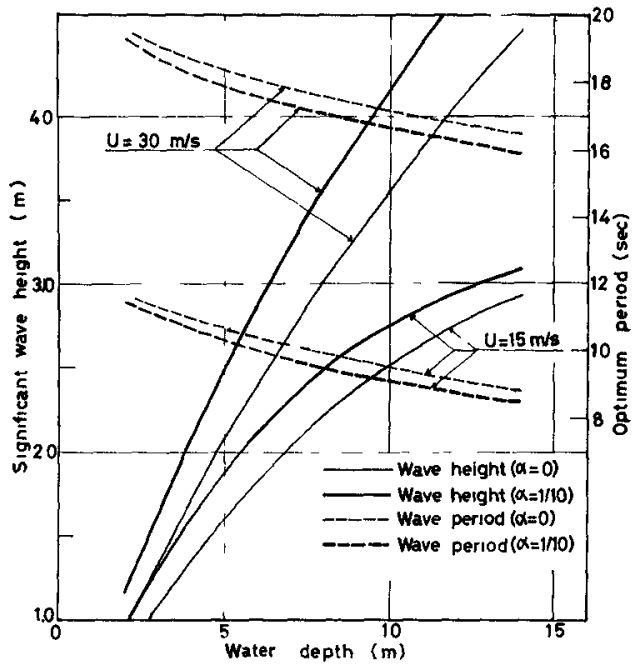

FIg - $($ b) LIMITING SIGNIFICANT WAVES FOR FETCH LENGTH $400 \mathrm{KM}$ 

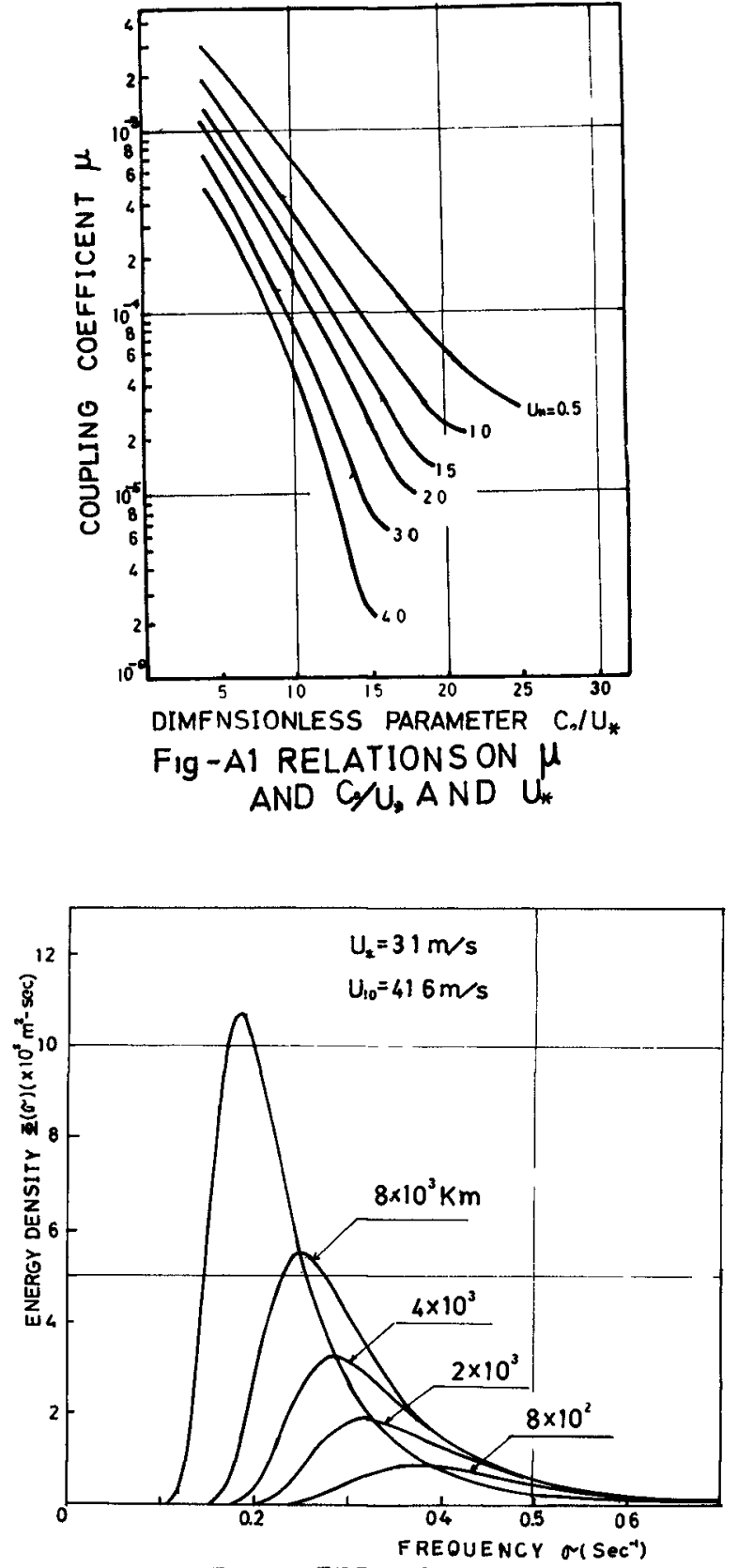

F1g -A2 FETCH SPECTRUM 

CHAPTER 10

WAVE INVESTIGATIONS IN SHALLOW WATER

Dr -Ing. Winfried Siefert

Strom- und Hafenbau Hamburg

Forschungsgruppe Neuwerk, Cuxhaven, Germany

Abstract

$==ニ===$

Examination of the significant heights of zero-crossing waves in the Elbe Estuary has ylelded two noteworthy results: 1 In the deeper water of the estuary, the value of the quotient relating the significant and the mean wave helghts is larger than on the bordering tidal flat. 2. The value of this function is dependent on the helght of the waves; on the tidal flat this dependency is considerably more sensative than in deeper water. With increasing wave helght the value of significant wave helght divided by mean helght becomes smaller

The propagation direction of waves moving onto the tidal flat is contingent upon the position of intertidal channels Such channels sharply reduce the possible propagation directions The waves nearly always move up-channel regardless of the wind direction

It is possible to derive special wave period and wave helght distributions representing the conditions in very shallow water 
I. Introduction

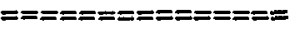

As part of a general research program investigating the hydrologlc, hydrodymamlc and morphologlc characteristIcs and changes in the Elbe Estuary, wave measurements are carried out (Iaucht, 1968, and Flg 1). Few recordings - at least in Germany - have been made of waves in the nearshore zone, consequently, no data was avallable for the Elbe Estuary at the beginning of this program Only a minimal amount of information on wave behavior in morphologically complex nearshore areas is found in the literature (Koele and de Bruyn, 1964; Wregel, 1964, US Army, 1966). It was therefore necessary to tallor an investigation program to fit these special conditions

Concurrent wave measurements have been made at 6 stations, and in 1970 an additional 7 stations on the tidal flat will be added. Of the present 6 stations, 3 are located on the edge of the Elbe channel in water depths from 5 to $10 \mathrm{~m}$, and 3 are located on the tidal flat in depths of less than $2 \mathrm{~m}$ (Fig. 2) Expanded records of 2 to 5 minute duration are recorded at preset intervals, following the Wemelsfelder principal. The results presented here are based primarily on the "Hundebalje" station located between the dune Island Scharhorn and the Island of Neuwerk on the "high tidal flat" bordering an intertıdal channel system At mean high tide the water reaches a depth of $15 \mathrm{~m}$ at this point.

II. Interpretation of Wave Records

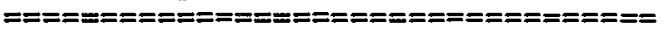

\section{A Slgnificant Wave Values}

Expanded records from the varıous stations give only wave helght and period and thereby only part of the signi- 
ficant wave characteristics which must also include wavelength, wave propagation direction and velocity the latter Items can be obtalned from radar and aerlal photographs, however, these techniques don't yield the helght and perlod (Slefert, 1969) An attempt is presently being made to combine aerial photo analysis with wave gauge records to bring together the helght, frequency and directıonal spectrums

Following currently accepted methods, the records were analyzed for the mean values $\overline{\mathrm{H}}$ and $\overline{\mathrm{T}}$ of the zerocrossing waves as well as $\mathrm{H}_{1 / 3}, \mathrm{TH}_{1 / 3}$, and $\mathrm{H}_{1 / 10}$. From these values, the ratıos $\mathrm{C}_{1 / 3}, \mathrm{C}_{1 / 10}$, etc were calculated

It is commonly believed that wave parameters such as the mean or significant wave height are of somewhat limsted use However, even in an area with extreme depth variations, these values still serve to give the engineer a relatively good picture of the waves Therefore, for preliminary work the calculation of spectrums can be omitted in favor of using the significant wave values For construction planning, characteristics such as mean, signlficant and maximum wave helghts and periods are more useful than spectrum analyses.

In the investigation area it is common to have several wave systems coming from different directions converging and overlapping one another. These systems can have quite different heights and periods depending on their origin and age A typical Gaussian distribution, as found in the theoretical treatment of Cartwright and Longuet-Higgins, or a Rayleigh distribution for a very narrow wave spectrum, are seldom present (Koele and de Bruyn, 1964). When several systems overlap at becomes impossible to measure a mean wave-length or height of each system The hlgh degree of scattering in the wave 
values precludes analytical treatment of data from this area until the mathematics of non-linear superposition is developed to a point where the spectrum can be calculated for shallow water (Wregel, 1969).

In splte of these restrictions, the measured wave heights yield some interesting facts regarding the changes that take place in waves as they move into shallow water

\section{B. Integration of the Dominant Wind Values}

The values $\bar{H}$ and $\bar{T}$ from the wave records were classified according to wind velocity and direction The wind parameters prevalling during the 6 to 12 hour period preceding the measurement were categorized into various wind sectors (Schrader, 1968) For wind directions from SW to $N$, the waves from the open sea are responsible for the waves in the channels and on the tidal flat. For these directions, the wind conditions prevaling outside the tidal flat area (Scharhornriff station, Fig. 2) must be considered. The division of the land wind directions ( $N$ to SW) is based solely upon the topography near the recording station In Fig 3, therefore, the wind sectors at the Scharhornriff station apply to the entire investigation area, whereas the sectors at the Hundebalje station apply only to records from this station.

Aerial photographic analysis of the wave-length changes as waves move into shallower water showed that classic wave theory could not be applied in this topographically complex region (Slefert, 1969). As a result, the attempt has been made to work with the changes in wave helght as the waves progress into shallower water. 
III Waves in Shallow Water

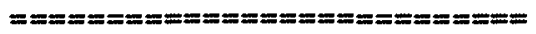

\section{A Comparison of Significant Wave Values in Deep and Shallow Water}

In order to achleve a reliable shallow water - deep water comparison, over 500 records from the 6 recording stations were analyzed for both mean and significant wave values using the zero-crossing method. This amounted to a comparison of the tidal flat readings (from the ScharhornWest, Scharhorn-Slid and Hundebalje stations) with the deeper water results (from the Scharhornriff, Scharhorn-Nord and Luchtergrund stations (see Fig. 2))

\begin{tabular}{c|c|c} 
Wave Parameter & Tidal Flat & 8 m Water Depth \\
\hline $\mathrm{C}_{\tau}=\frac{\mathrm{T}_{\mathrm{H}_{1 / 3}}}{\overline{\mathrm{T}}}$ & 1.11 & 1.30 \\
$\mathrm{C}_{1 / 3}=\frac{\mathrm{H}_{1 / 3^{*}}}{\overline{\mathrm{H}}}$ & 149 & 1.52 \\
$\mathrm{C}_{1 / 10}=\frac{\mathrm{H}_{1 / 10}}{\overline{\mathrm{H}}}$ & 1.76 & 1.90 \\
$\mathrm{H}_{1 / 10}$ & 1.18 & 124 \\
$\mathrm{H}_{1 / 3}$ & & 124
\end{tabular}

*) see Figure 4

All of the values show a tendency toward uniformity as the waves reach shallower water, $\mathrm{le}_{\mathrm{e}} \mathrm{H}_{1 / 10}$ and $\mathrm{H}_{1 / 3}$ become somewhat smaller in relation to the mean wave height. This is caused by a form of "sorting" in which the highest waves, which control the values of $\mathrm{H}_{1 / 10}$ and $\mathrm{H}_{1 / 3}$ in deeper water, are removed from the spectrum in shallower water. Therefore it is understandable that the ratio $C_{1 / 10}$ should become smaller faster than the ratio 
$\mathrm{C}_{1 / 3}$ The change in the wave spectrum is also clearly seen in the decreasing values of $C_{\tau}$ on the tidal flat

So one can not rely on having constant wave ratıos in the coastal area This applies to the wave helghts as well as the perlods. The value of $C_{\tau}=\frac{{ }_{\mathrm{H}_{1} / 3}}{\bar{T}}$ for the deeper Elbe Estuary, as well as off the Dutch coast (Svasek, 1969), is 130 , whereas on the tidal flat the value drops to 111 This lower figure corresponds well to the 1.10 calculated by Sibul (1955) for shallow water in a windwave channel (see Wlegel, 1964).

A further point should be mentioned which apparently is frequently overlooked in the evaluation of zero-crossing waves As Fig. 4 shows, using a deep water station (Scharhornriff) and a tidal flat station (Hundebalje) as examples, the ratio $\mathrm{C}_{1 / 3}=\frac{\mathrm{H}_{1 / 3}}{\mathrm{~F}}$ is dependent not only on location but also on the mean wave height $\mathrm{H}$. Higher waves produce a more uniform wave spectrum; on the tidal flat the uniformity is achleved more quickly than in deeper water.

Variances in the directional spectrum of the waves as they reach the tidal flat are shown in Fig. 5 where the maln wave systems seen in two serles of aerial photographs are presented (see also Slefert, 1969). In the inner portion of the Elbe Estuary, nearly all wave directions can be developed depending upon the wind However, this multiplicity of propagation directions is almost instantly reduced as the waves enter the tidal channels. The illustration shows propagation directions from WNW and $E$, a difference of $120^{\circ}$ in the deep Elbe channel This difference is reduced to $30^{\circ}$ in the Hundebalje channel. This would seem to indicate that waves always move up-channel on the tidal flat regardless of wind direction 


\section{B. Distributions of Wave Perlods and Wave Heights}

Everyone is aware of the large scattering in the relation of mean wave helghts and perlods, especially under the influence of complex topography and tidal conditions. Furtheron it is known that the heights and perlods of indlvidual waves seem to be nearly without any relation The only facts that become clear are that the highest wave in a spectrum does not belong to the longest perlod and that the longest perlod belongs to a wave of about mean helght. Therefore it is useful to split further investigations into analyzing separate perlod and helght distributions

Fig. 6 gives some characteristic period spectra in the form of cumulative distributions of the ratio of individual and mean periods in Gaussian paper. The comparison gives an interesting result: The distributions of Bretschnelder and Putz are not the same, but simllar, and both are derived for deep water waves. The spectra in the Elbe channel and the adjacent breaker zone ( 3 to $12 \mathrm{~m}$ ) as well as those on the tidal flats ( 1 to $3 \mathrm{~m}$ ) are different from those. The period spectrum of the waves obviously becomes wider with decreasing water depth. So It seems that the well-known theoretical Raylelgh-distribution is characteristic only for waves in very shallow water. The analytical variation of this distribution is possible by ralsing the factor "2" in the formula of the Rayleigh-distribution with increasing water depth.

Opposite developments can be noticed with the wave helghts To the usually used Raylelgh-distribution after Longuet-Higgins there belongs a value $C_{1 / 3}=1.60$. Many authors found that this value is not always constant (see Wlegel, 1964). Investigations in the Elbe estuary show that the mean value is less than 160 and that the quotient becomes smaller with increasing wave helght, but not at any polnt with the same rate (Flg. 4). The cumulative distributions of the wave helghts in Fig. 7 give characteristic 
curves for every $\mathrm{C}_{1 / 3}$. As $\mathrm{C}_{1 / 3}$ decreases with increasing mean wave height, the wave helght spectrum then must become narrower. This apparent relationship between the width of the spectrum and $\mathrm{C}_{1 / 3}$ can be expressed as a relation between the factor $\varphi$ in the formula of the wave helght distribution

$$
P\left(\frac{\mathrm{H}}{\overline{\mathrm{H}}}\right)=1-\mathrm{e}^{-\frac{\pi}{4}}\left(\frac{\mathrm{H}}{\overline{\mathrm{H}}}\right)^{\varphi}
$$

and $\mathrm{C}_{1 / 3}$ :

\begin{tabular}{c|c|c|c|c|c|c|c}
$\mathrm{C}_{1 / 3}$ & 1,35 & 1,40 & 1,45 & 1,50 & 1,55 & 1,60 & 1,65 \\
\hline$\varphi$ & 2,94 & 2,70 & 2,50 & 2,32 & 2,15 & 2,00 & 1,86
\end{tabular}

This relation is valid up to $998 \%$ and more. The following progress of the curves in Fig. 7 to a finite value indicates the influence of water depth, as there must be a maximum wave height even with the propability zero.

For a certain mean wave helght in a certain place on the tidal flat, one can get the value of $\mathrm{C}_{1 / 3}$ by Fig. 4, and further on Fig. 7 gives the complete wave height distribution in dependence on $\mathrm{C}_{1 / 3}$.

Summarizing the investigations it can be stated that the period spectrum of waves progressing into shallow water becomes wider while at the same time the height spectrum becomes narrower. This fact has to be considered during further investigations, but we hope to find some general characteristics that may be applied to sımilar areas elsewhere. 
C Wave Helghts on the Tidal Flat

Tidal conditions result in the wave height on a tidal flat belng controlled by the water level, le., the tidal phase In addition to the tidal fluctuations, the water level is strongly influenced by wind thereby yielding complex relationships:

a) Wave height as a function of wind and water level

b) Water level as a function of wind

Some considerations may illustrate this double dependency of wave helght on wind. When a constant water level is present (or in water deep enough to make such fluctuations insignificant) relatıonships can be established between wave helght and wind. The Hundebalje station serves as a good example to show the influence of the wind on the water level. In the German Bight, and particularly near the coast, strong SW to $N$ (sea) winds result in an accumulation of water and therefore increased mean tıdal levels; $N E$ to $S$ (land) winds drive the water away from the coast and result in lower water levels. Because wave helght is dependent on wind velocity and water level, higher waves can form on the tidal flat under sea wind conditions In contrast, land winds produce an increase in wave helght coupled with a lowering of the water level The wave helght can increase only as long as the first effect remalns larger than the second; further increases in wind velocity will result in a decrease in wave helght. It is therefore possible that hurricane force winds from some directions will produce no waves at all on the tidal flat if it falls dry.

This relatively simple hypothesis could be varified on the basis of one year's measurements at the Hundebalje station The events during land wind conditions could be particularly well defined during a long east wind period 
Figure 8 shows the mean wave height $\overline{\mathrm{H}}$ in relation to the wind velocity from various sectors for three water levels ( $\mathrm{HW}+1 \mathrm{~m}$, HW, $\mathrm{HW}-0.5 \mathrm{~m}$ ). As would be expected, the individual values are scattered. For clarity only the lines of highest $\overline{\mathrm{H}}$ are shown. It can be seen that during periods of land winds from sectors 1 to $3\left(346^{\circ}\right.$ to $210^{\circ}$ ) the waves never reach an $\bar{H}$ of over $37 \mathrm{~cm}$ on the tidal flat. For a ratio between significant and mean wave helght of $\frac{\mathrm{H}_{1 / 3}}{\overline{\mathrm{H}}}=1.46$ (F1g. 4) the significant wave height for this location during $N$ to $S W$ winds is

$$
\mathrm{H}_{1 / 3}=54 \mathrm{~cm} \text {. }
$$

From Figure 8 it can be seen that the highest waves do not develop when the water level (at a wind velocity of 19 to $22 \mathrm{~m} / \mathrm{sec}$ ) reaches the HW level $(1.9 \mathrm{~m})$, but rather a level some $20 \mathrm{~cm}$ lower. Thus, with a water depth of $1.7 \mathrm{~m}$, mean wave heights up to $37 \mathrm{~cm}$ can be expected.

The maximum possible wave height is also of partıcular interest. The maximum helght will develop under westerly winds when both wave helght and water depth are increasing. It is too early to set an absolute height limit based on only one year's measurements, particularly when these include relatively few storm-wind perıods. During $W$ to NNW wind (sector 6, Fig 3) with beaufort force 10 (about $27 \mathrm{~m} / \mathrm{sec}$ ) waves with a mean helght of $60 \mathrm{~cm}$ were recorded. The significant wave height was $85 \mathrm{~cm}$. The recording is being continued.

The dependency of wave propagation direction on water depth is shown in the fact that the highest waves on the tidal flat develop during $W$ to NNW wind (sector 6). During these times the Hundebalje recording station on the tidal flat lies directly in the wind shadow of the island of Scharhörn (F1g 3). The fetch of about $1.5 \mathrm{~km}$ over water depths from 0 to $19 \mathrm{~m}$ is not sufficient to generate mean wave heights of over $50 \mathrm{~cm}$. The relation- 
ship in Fig. 9 between wave height on the tidal flat (Hundebalje) and in about $8 \mathrm{~m}$ depth off the tidal flat (Lichtergrund) shows that the highest waves are recorded on the flat at the times when the hlghest waves off the flat are recorded (sector $6,268^{\circ}-354^{\circ}$ ).

It should again be stressed that the results obtained from the Hundebalje station apply only to its immediate area. The station was placed on a tidal channel in order to measure waves by their ingress on the tidal flat. On the higher parts of the flat away from channels, other conditions prevall which result in smaller waves. The wave spectrum on the tidal flat exhlbits extreme local varlations.

\section{List of Symbols}

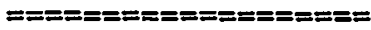

$\mathrm{C}_{1 / 3} \quad$ Ratio of Wave helghts $\frac{\mathrm{H}_{1 / 3}}{\overline{\mathrm{H}}}$
$\mathrm{C}_{1 / 10} \quad$ Ratio of Wave helghts $\frac{\mathrm{H}_{1 / 10}}{\overline{\mathrm{H}}}$
$\mathrm{C}_{\tau} \quad$ Ratio of Wave periods $\frac{\mathrm{T}_{1 / 3}}{\overline{\mathrm{T}}}$
$\mathrm{H} \quad$ Mean helght of the zero-crossing waves
$\overline{\mathrm{H}}_{\text {max }} \quad$ Maximum from number of mean wave helghts
$\mathrm{H}_{1 / 3} \quad \begin{aligned} & \text { Slgnificant wave helght }=\text { average helght of the } \\ & \text { hlghest one-thlrd of the zero-crossing waves }\end{aligned}$
$\mathrm{H}_{1 / 10} \quad \begin{aligned} & \text { Average helght of the highest one-tenth of the } \\ & \text { zero-crossing waves }\end{aligned}$
$\mathrm{HW} \quad \begin{aligned} & \text { Mean high tide } \\ & \mathrm{T}\end{aligned}$
$\mathrm{T}_{\mathrm{H}} \quad \begin{aligned} & \text { Mean perlod of the zero-crossing waves } \\ & \text { crossing waves (1.e. the slgniflcant waves) }\end{aligned}$


V References

$\mathrm{I=}=\mathrm{=}=\mathrm{=}=\mathrm{=}$

Koele, L.A. and de Bruyn, P.A.: Statıstical Distrıbution of Wave Heights in Correlation with Energy Spectrum and Water Depth. Proceedings, 9th Conference on Coastal Engineering, 1964

Laucht, H.: Ursachen und Ziele der Hamburger Küstenforschung an der Elbmïndung. Hamburger Klistenforschung, Heft 1, 1968

Schrader, J.P.: Kennzelchnende Seegangsgroßen für drel MeBpunkte in der Elbmundung. Hamburger Kuistenforschung, Heft 4, 1968

Slefert, W.: Seegangsbestımmung mit Radar und nach Luftbildern. Hamburger Küstenforschung, Heft 7, 1969

Svasek, J.N.: Statistical Evaluation of Wave Conditions in a Deltalc Area. Proceedings, Symposium Research on Wave Action, Delft, Holland, 1969

U.S. Army Coastal Englneerıng Research Center: Shore Protection, Planning and Design. Technical Report No. 4, 1966

Wiegel, R.L.: Oceanographical Engineering. Prentıce-Hall Inc., Englewood Cliffs, N.J. 1964

Wiegel, R.L.: Waves and their Effects on Pile-Supported Structures Proceedings, Symposium Research on Wave Action, Delft, Holland, 1969 
German Bight

Fig 1

with Investigation Area of the Forschungsgruppe Neuwerk

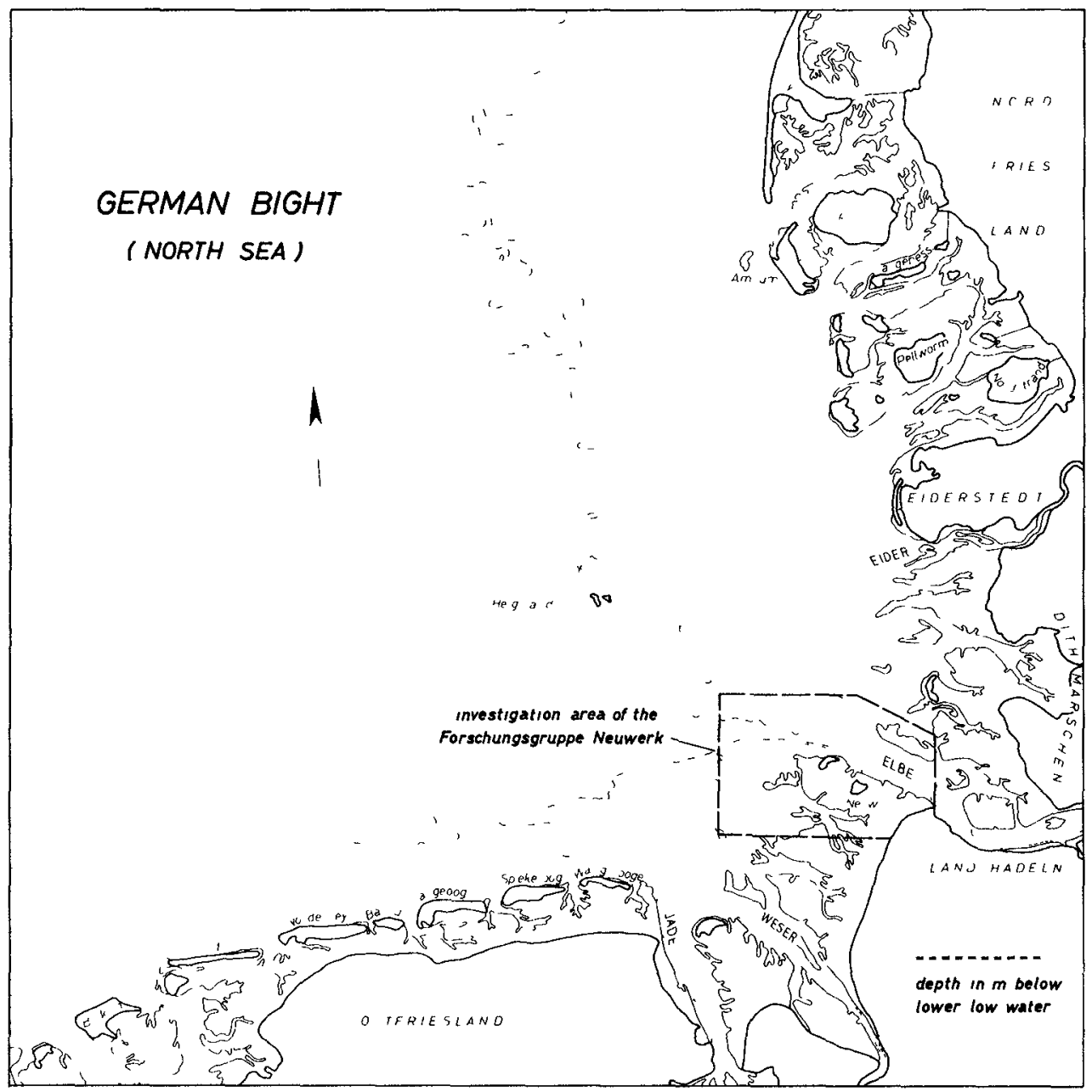




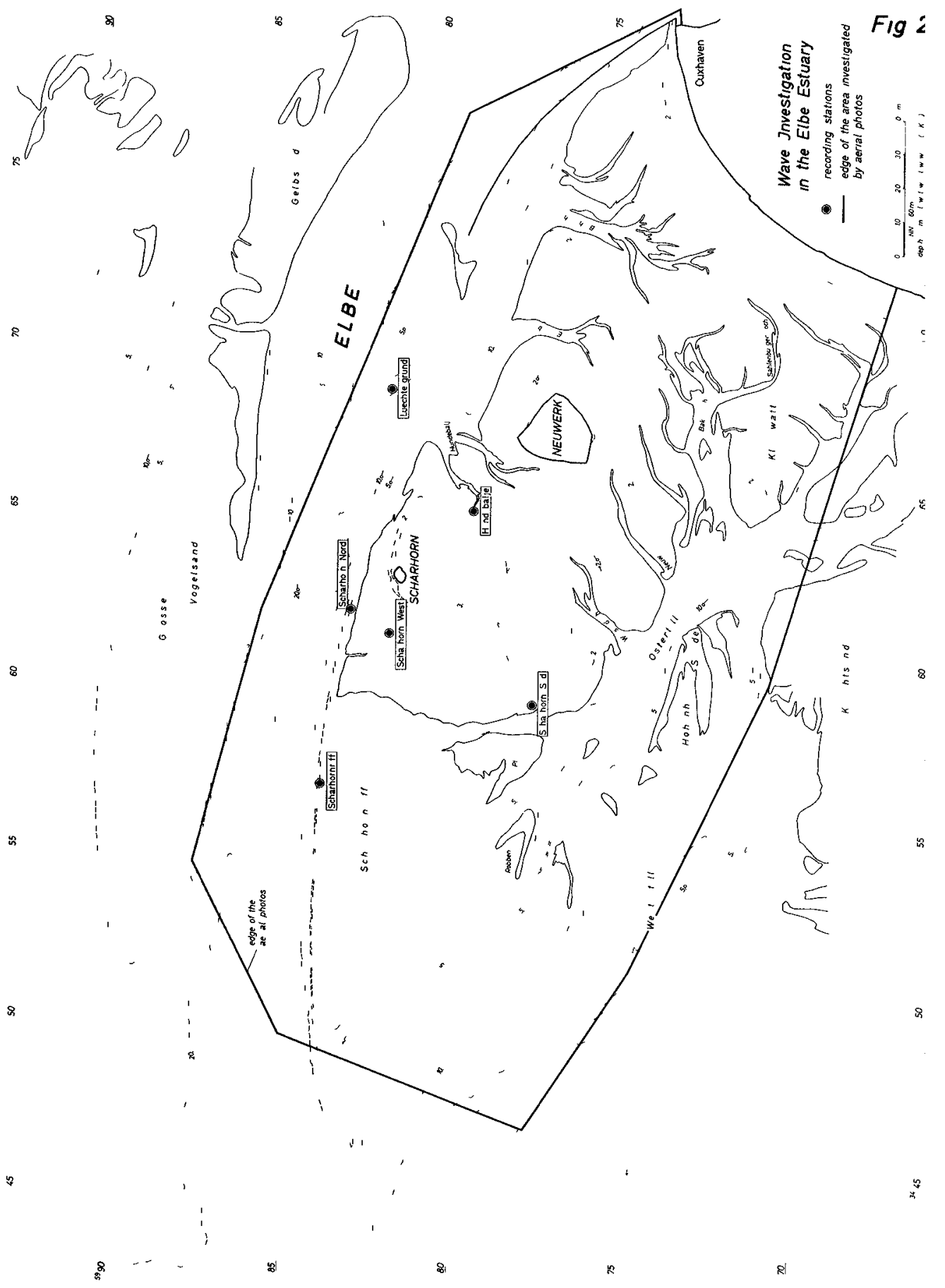




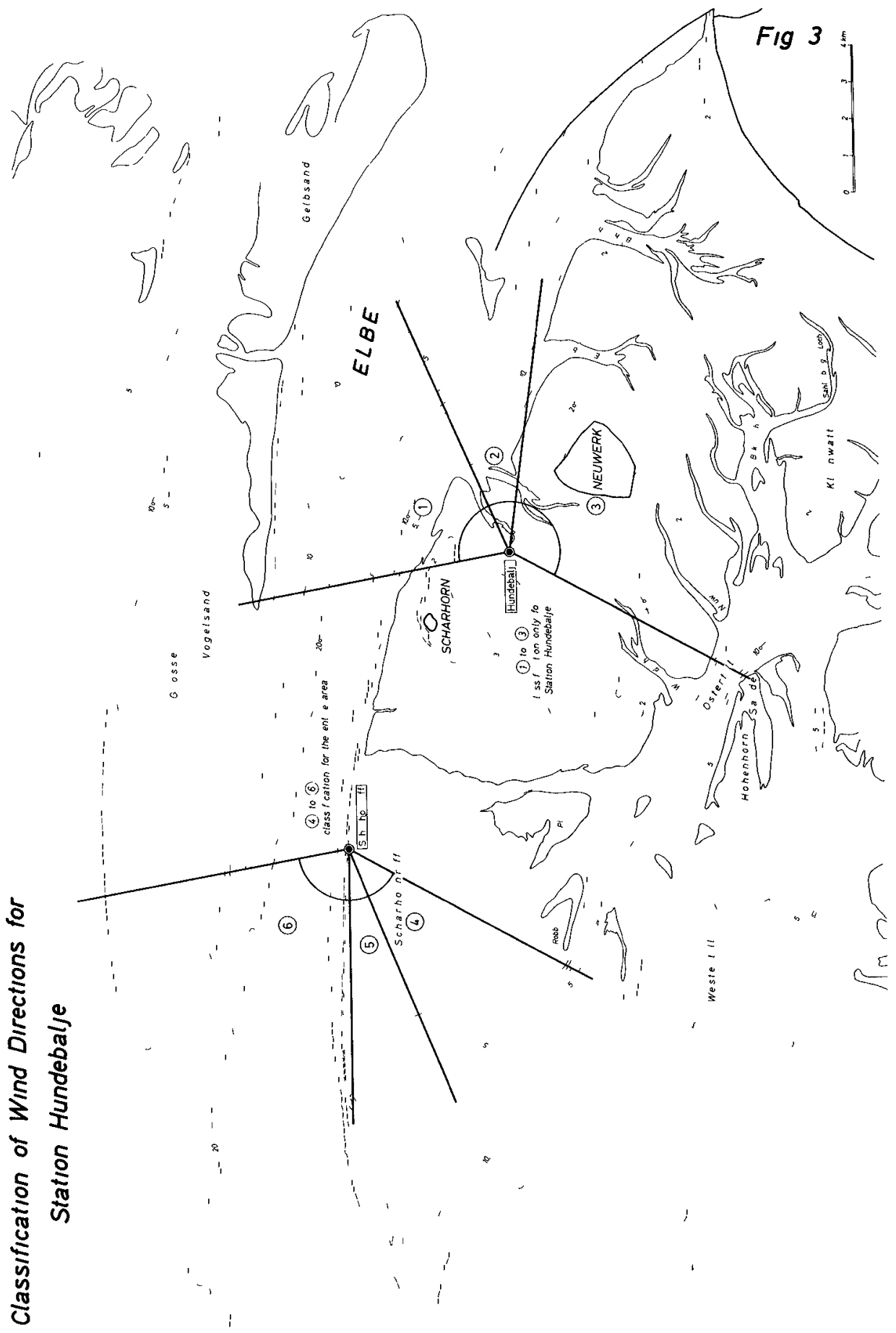


Quotient of Significant Wave Height $H_{1 / 3}$ and Mean Wave Height $\bar{H}$ versus $\bar{H}$
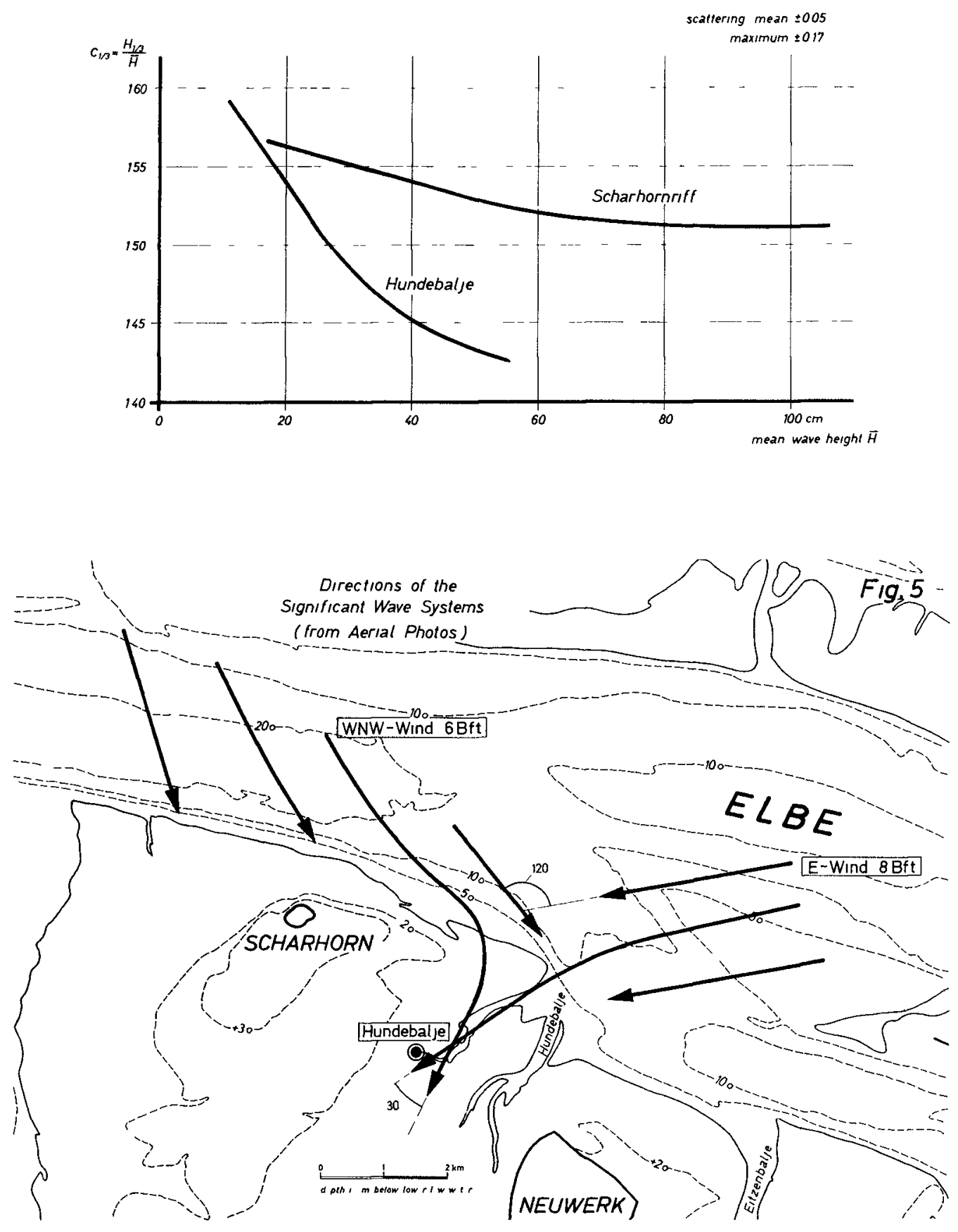
Fig 6

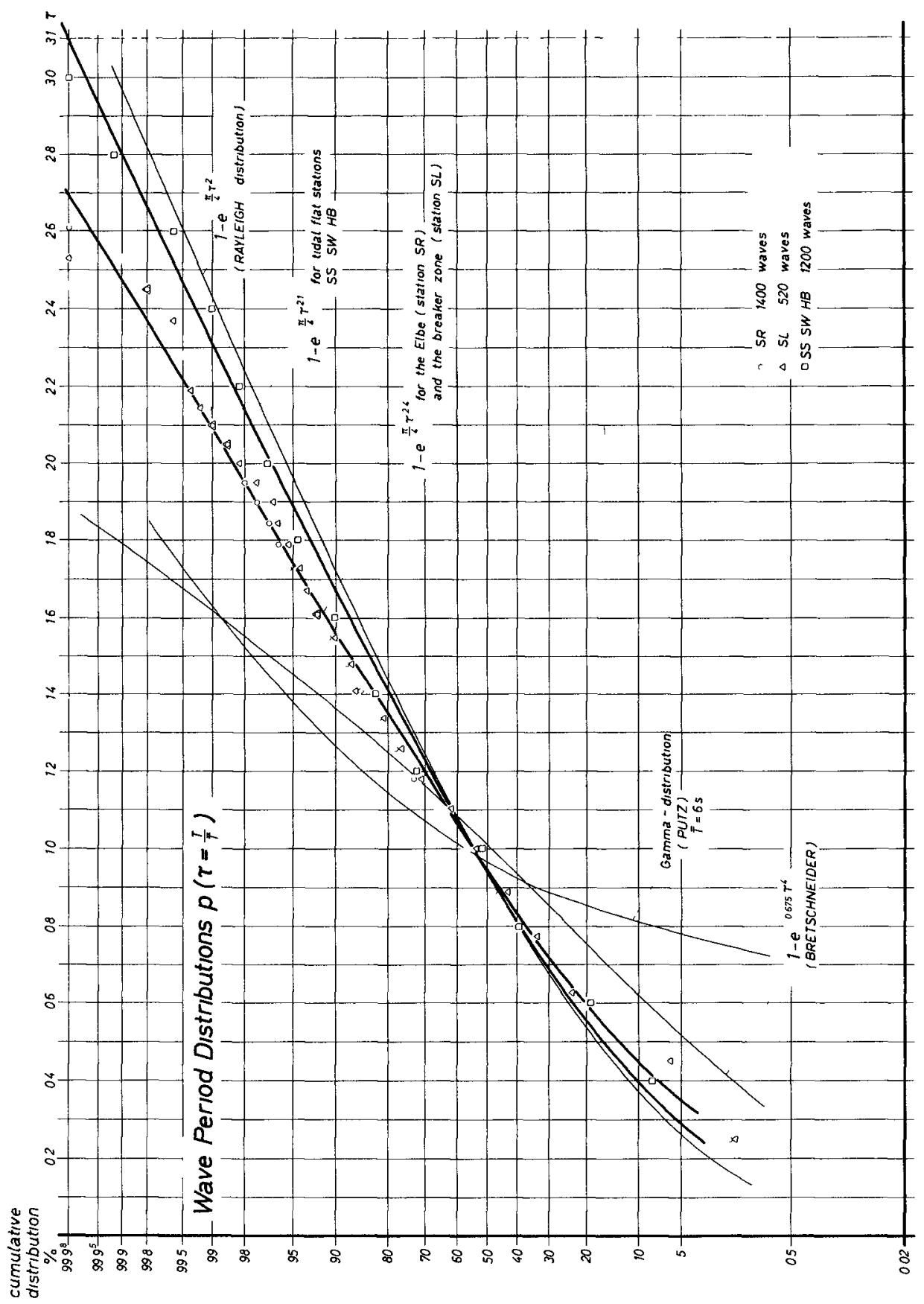


Fig 7

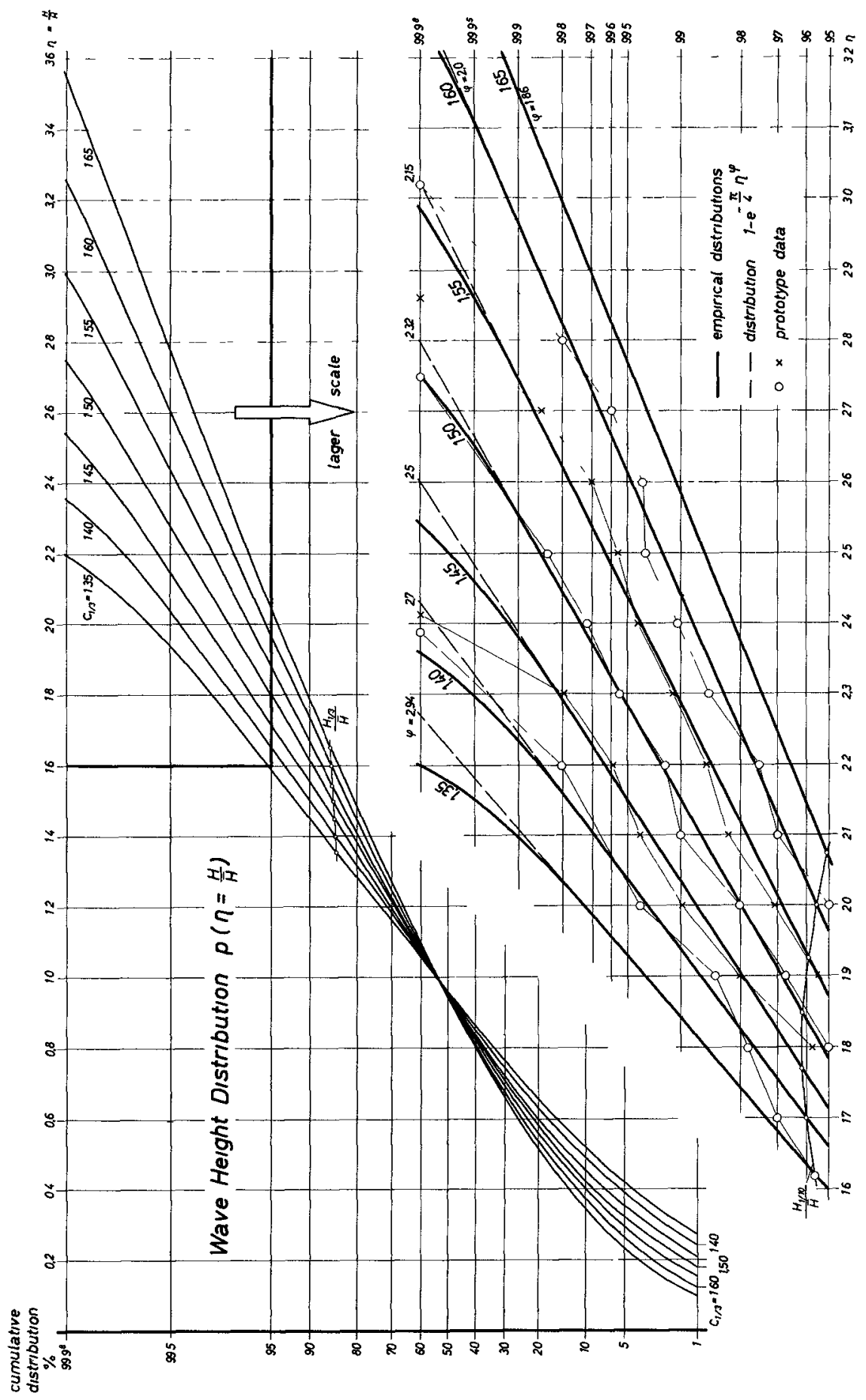


$F / g 8$

\section{Station Hundebalje \\ Relation between Wave Heights and Wind \\ (Depth of Water $19 \mathrm{~m}$ at HW)}
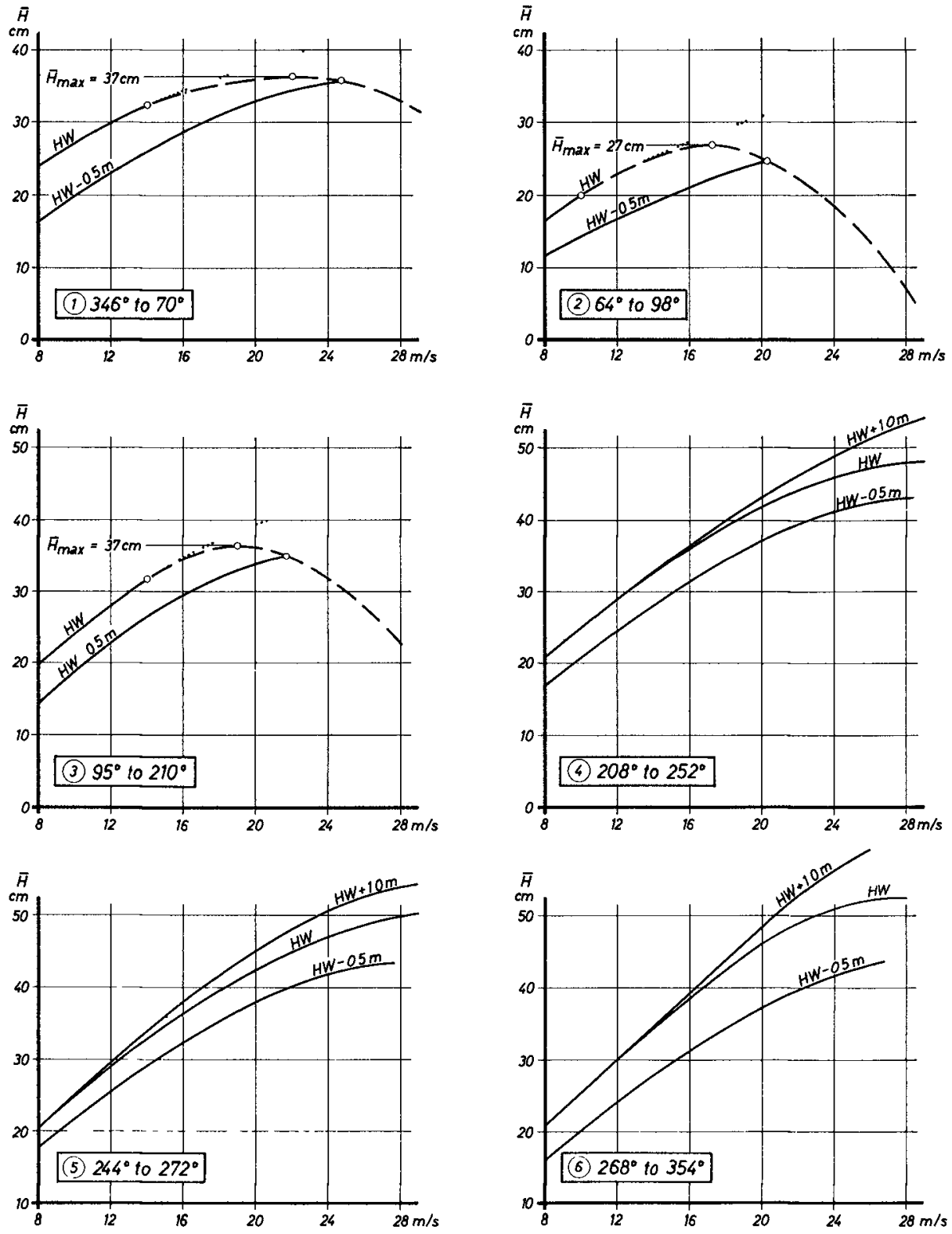


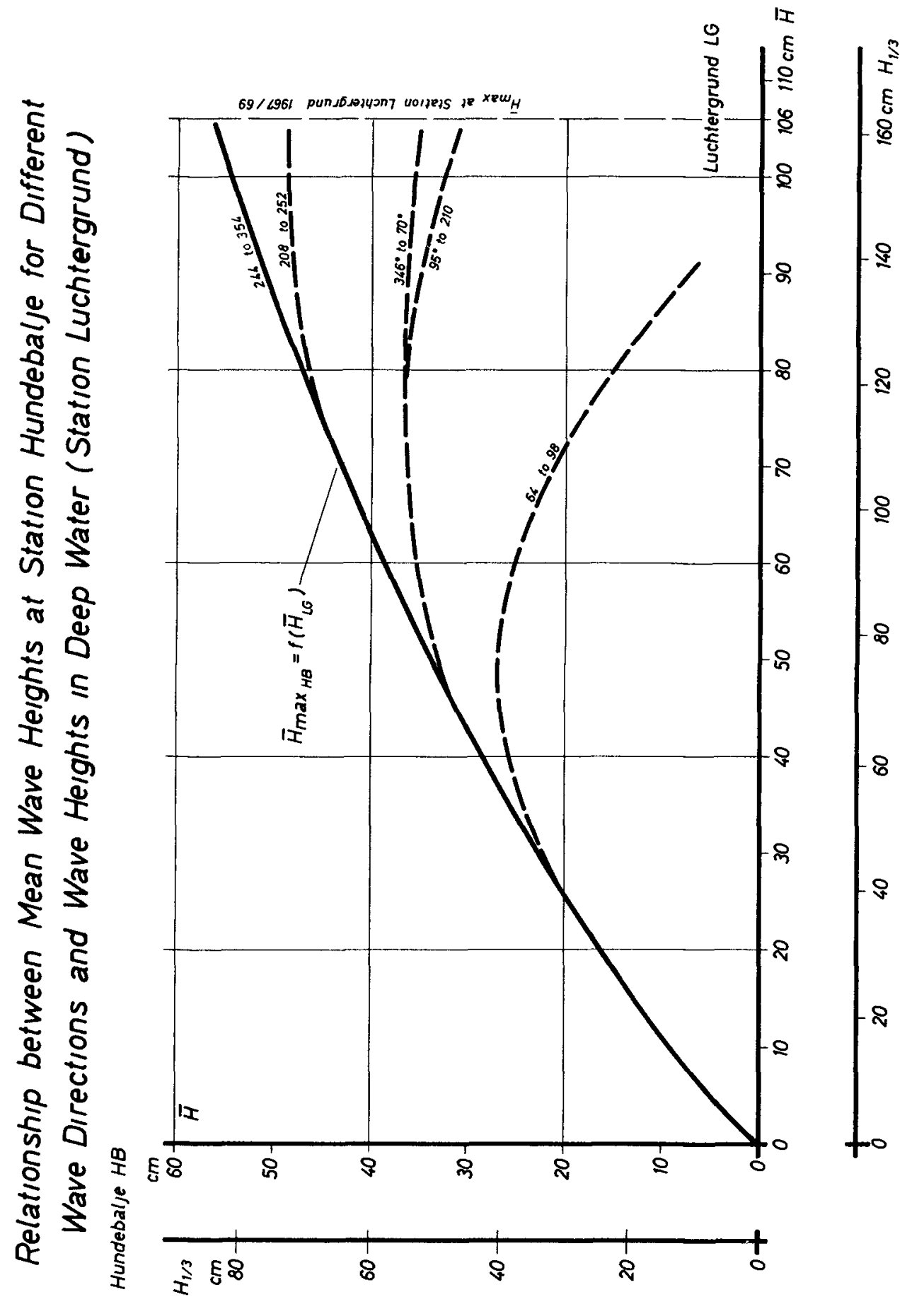




\section{German Bight}

Fig 1

with Investigation Area of the Forschungsgruppe Neuwerk

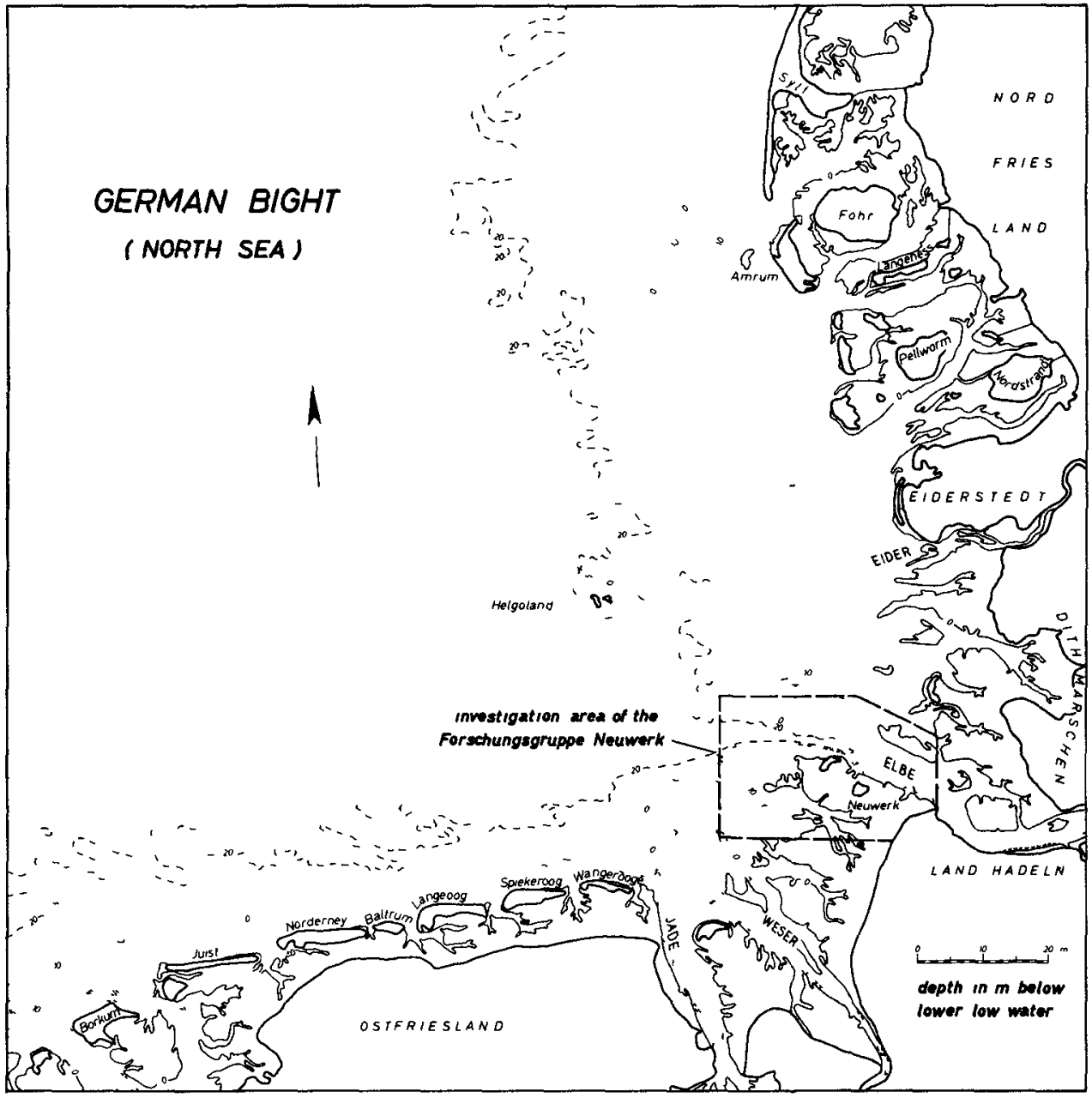




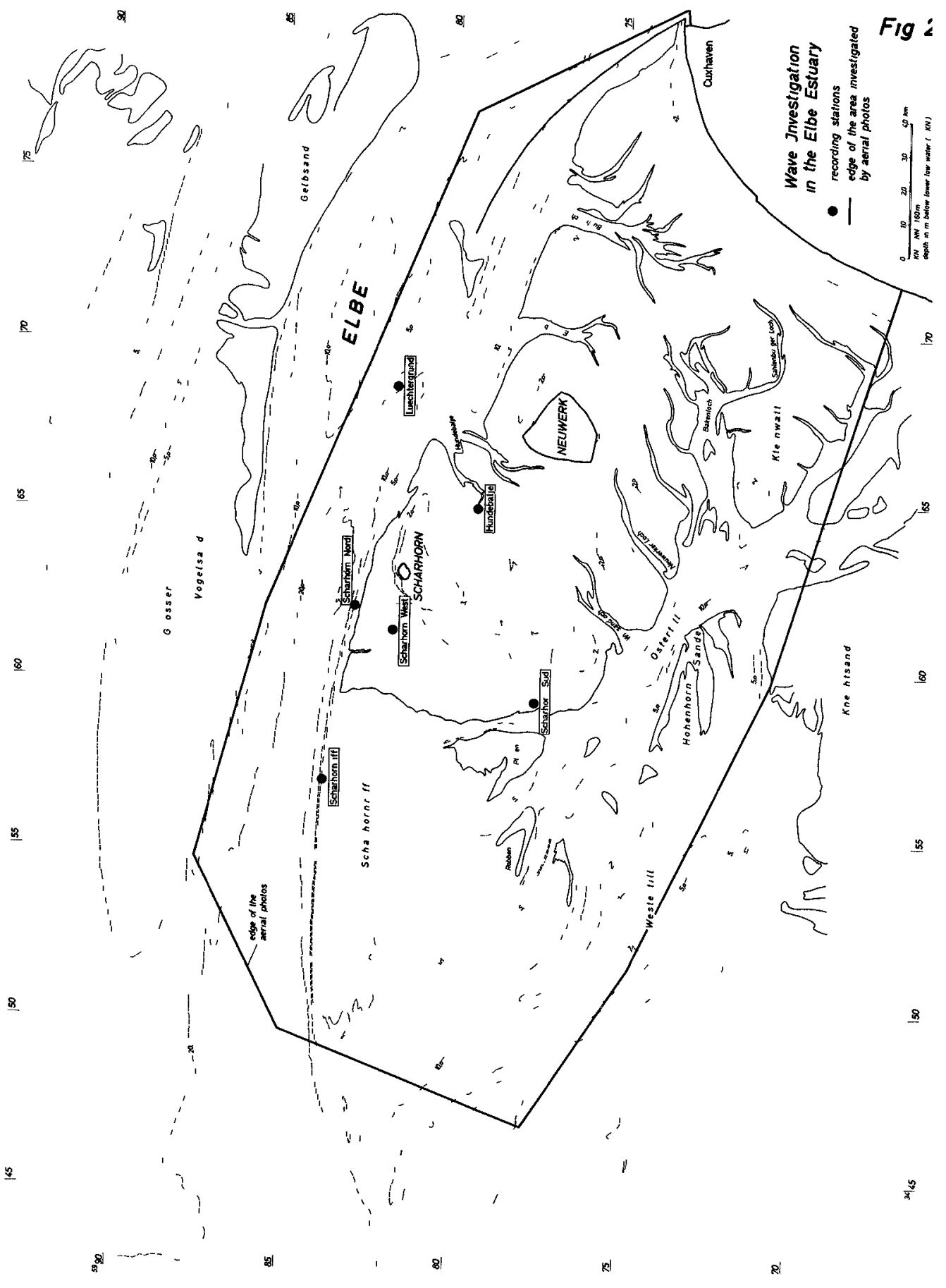




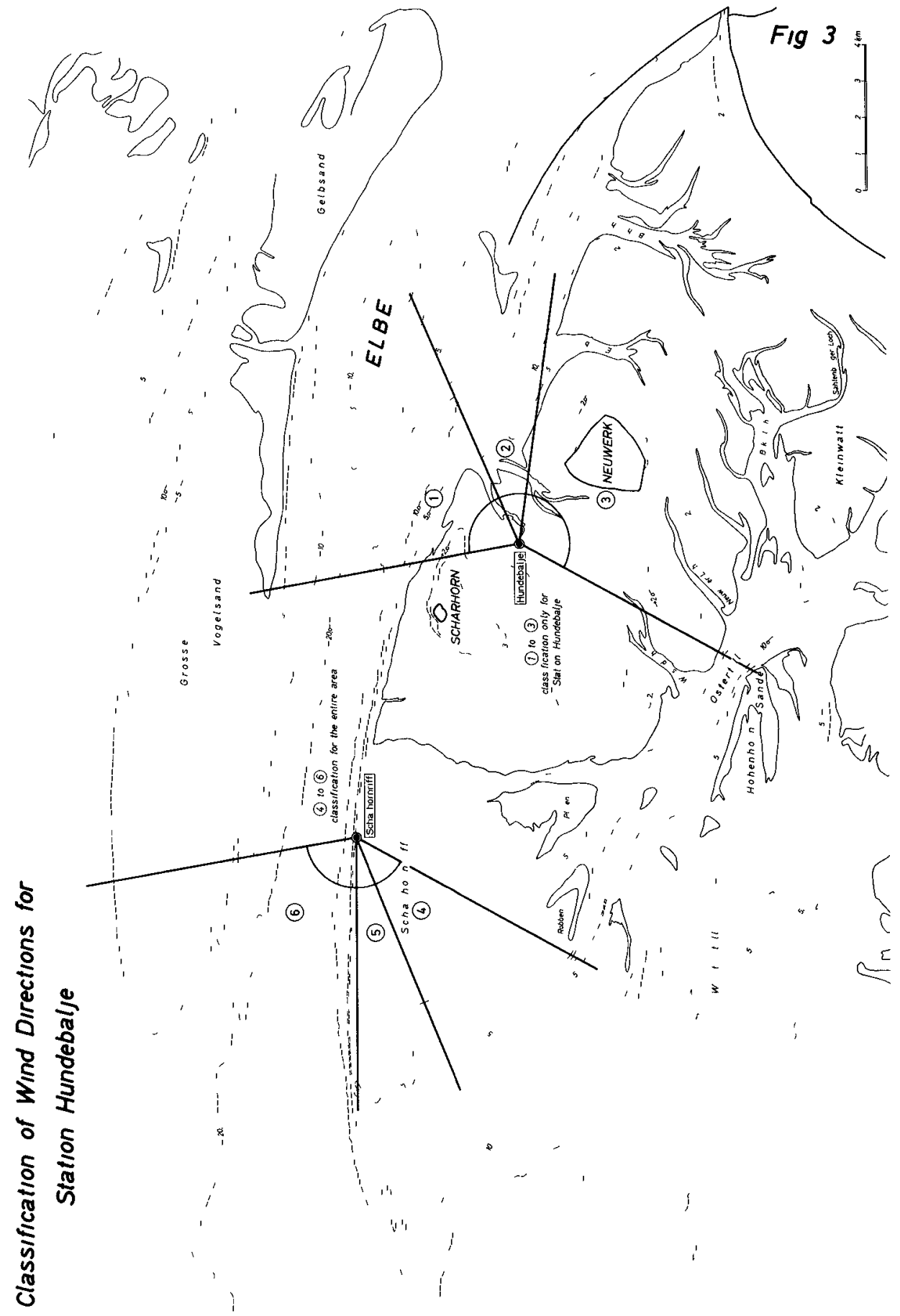


Quotient of Significant Wave Height $H_{1 / 3}$ and Mean Wave Height $\bar{H}$ versus $\bar{H}$
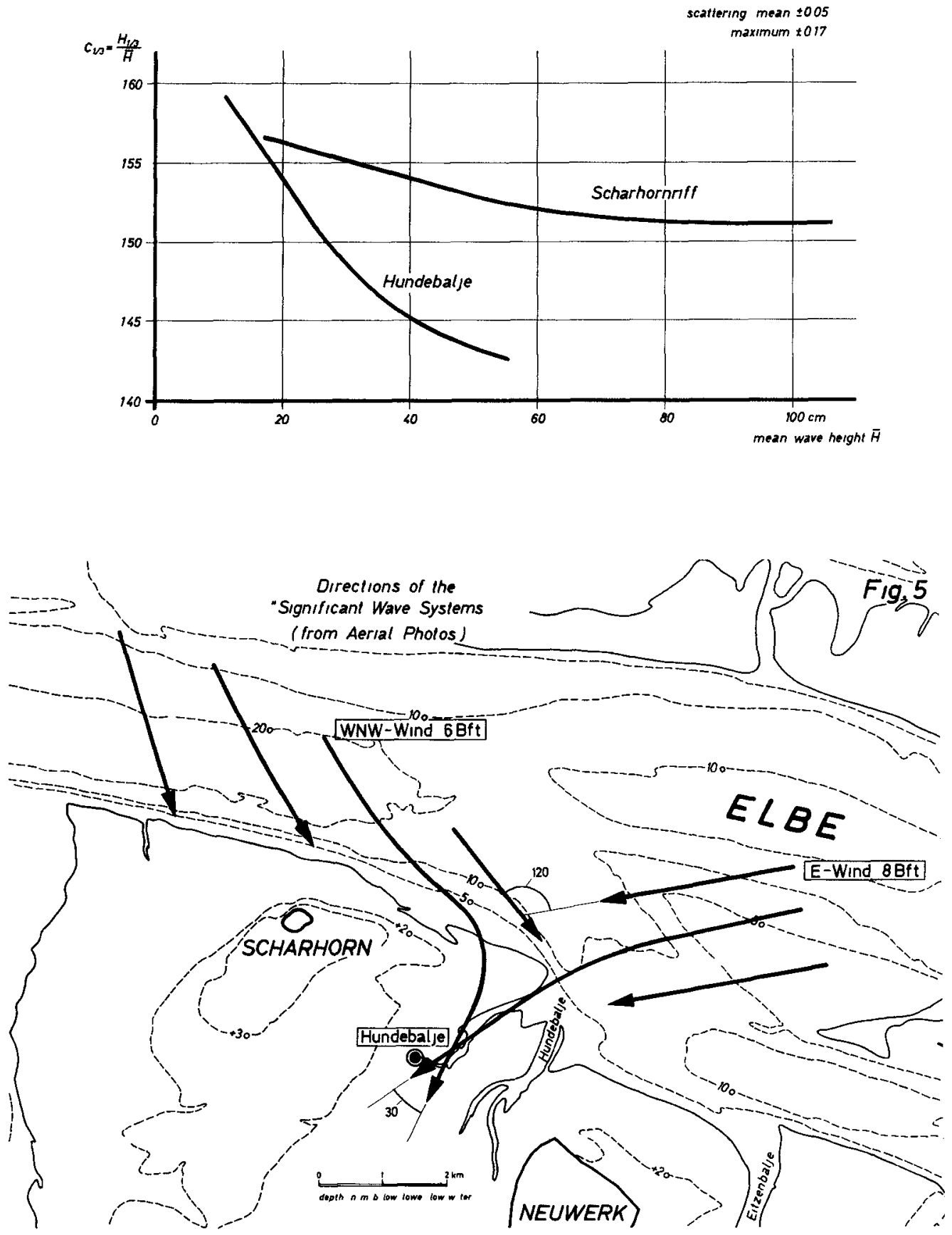
Fig 6

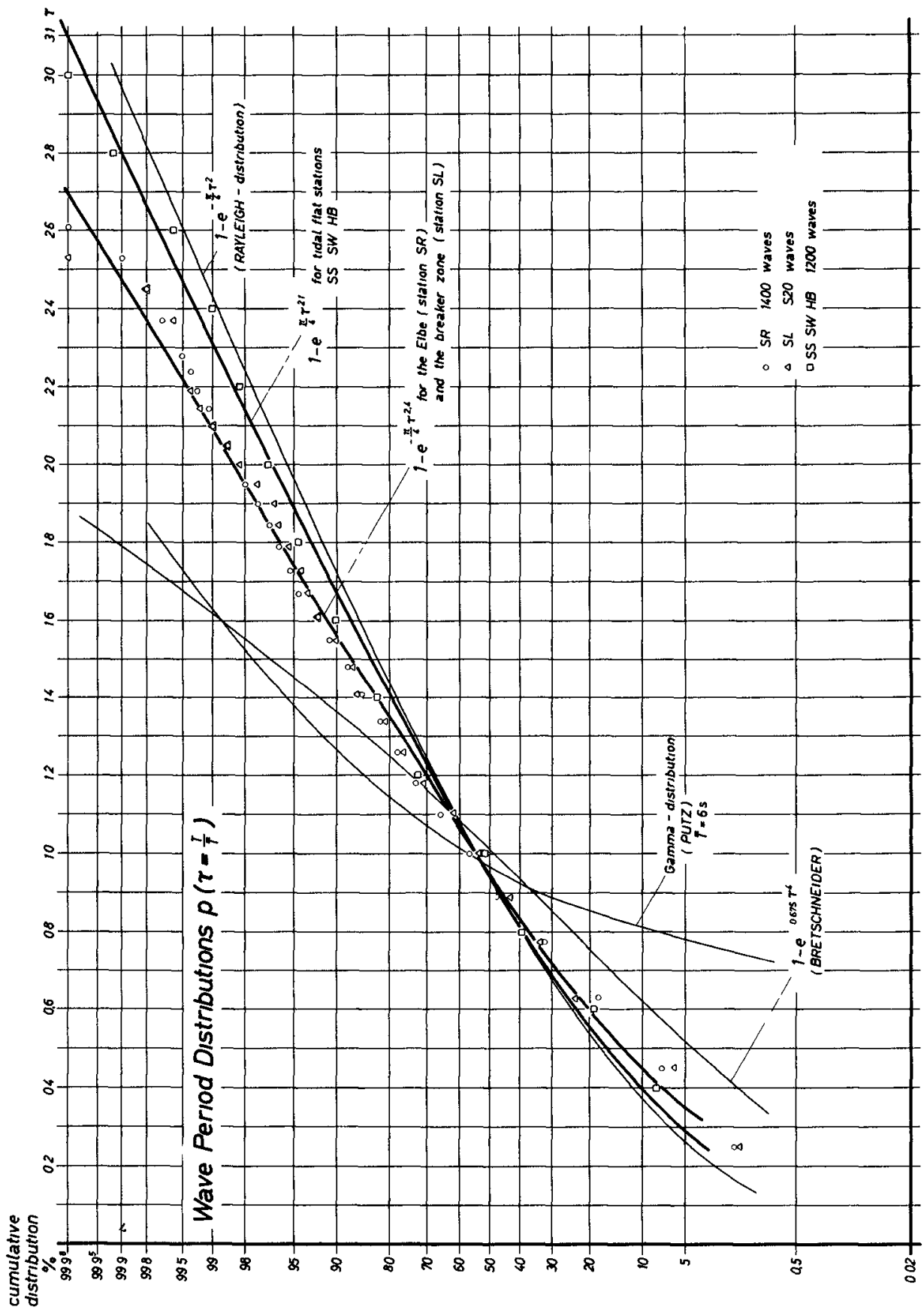


Fig 7

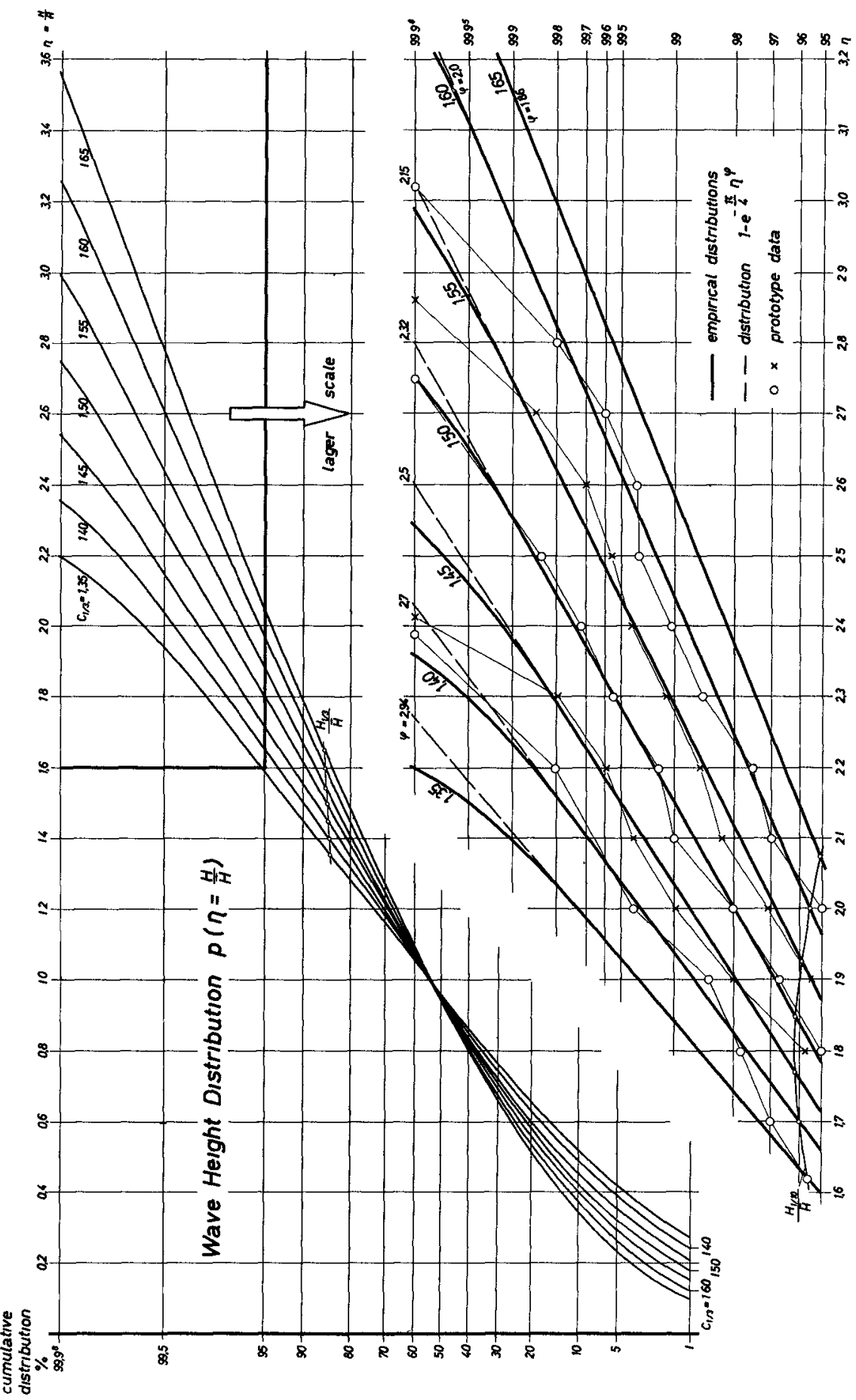


Station Hundebalje

Relation between Wave Heights and Wind (Depth of Water $19 \mathrm{~m}$ at HW)
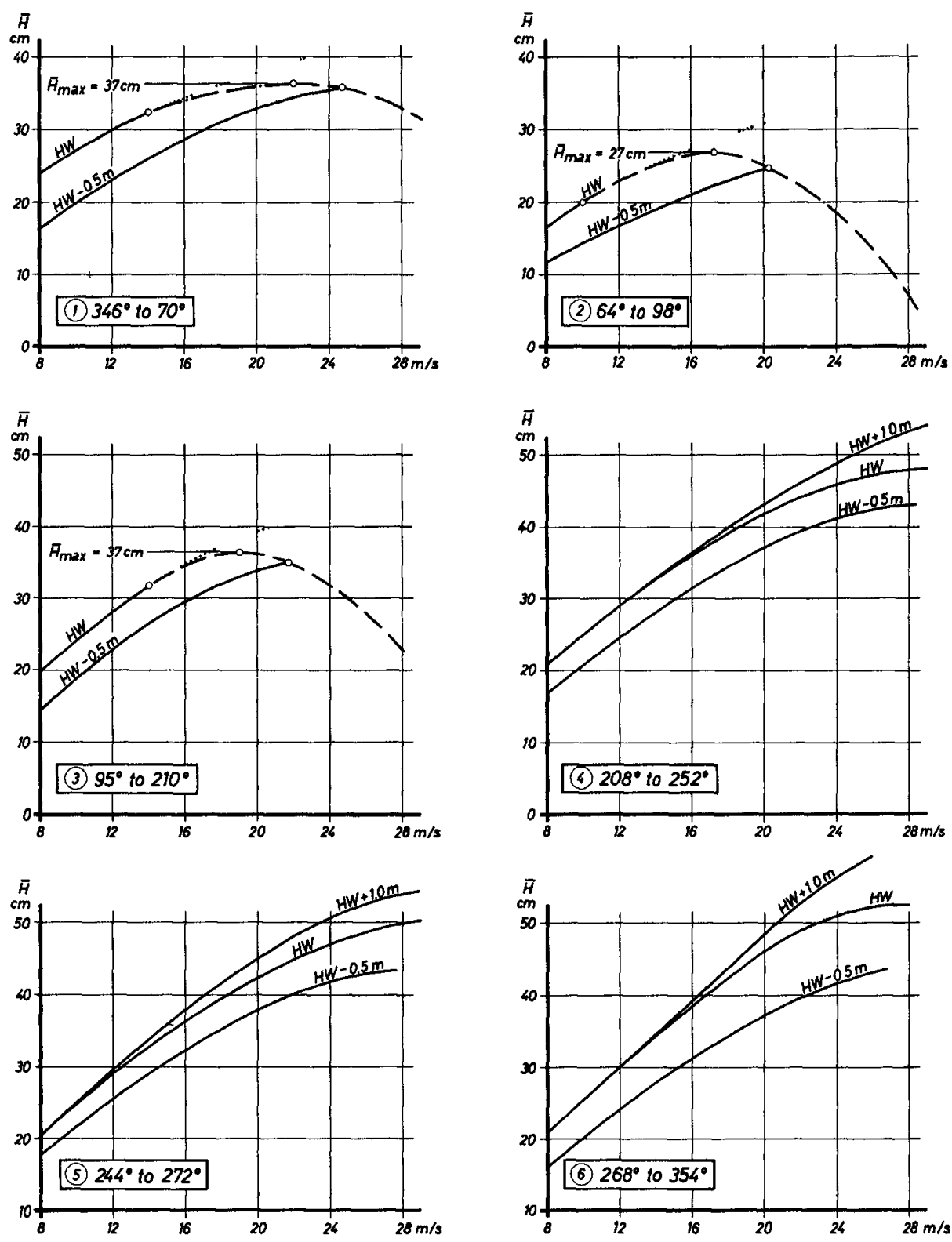


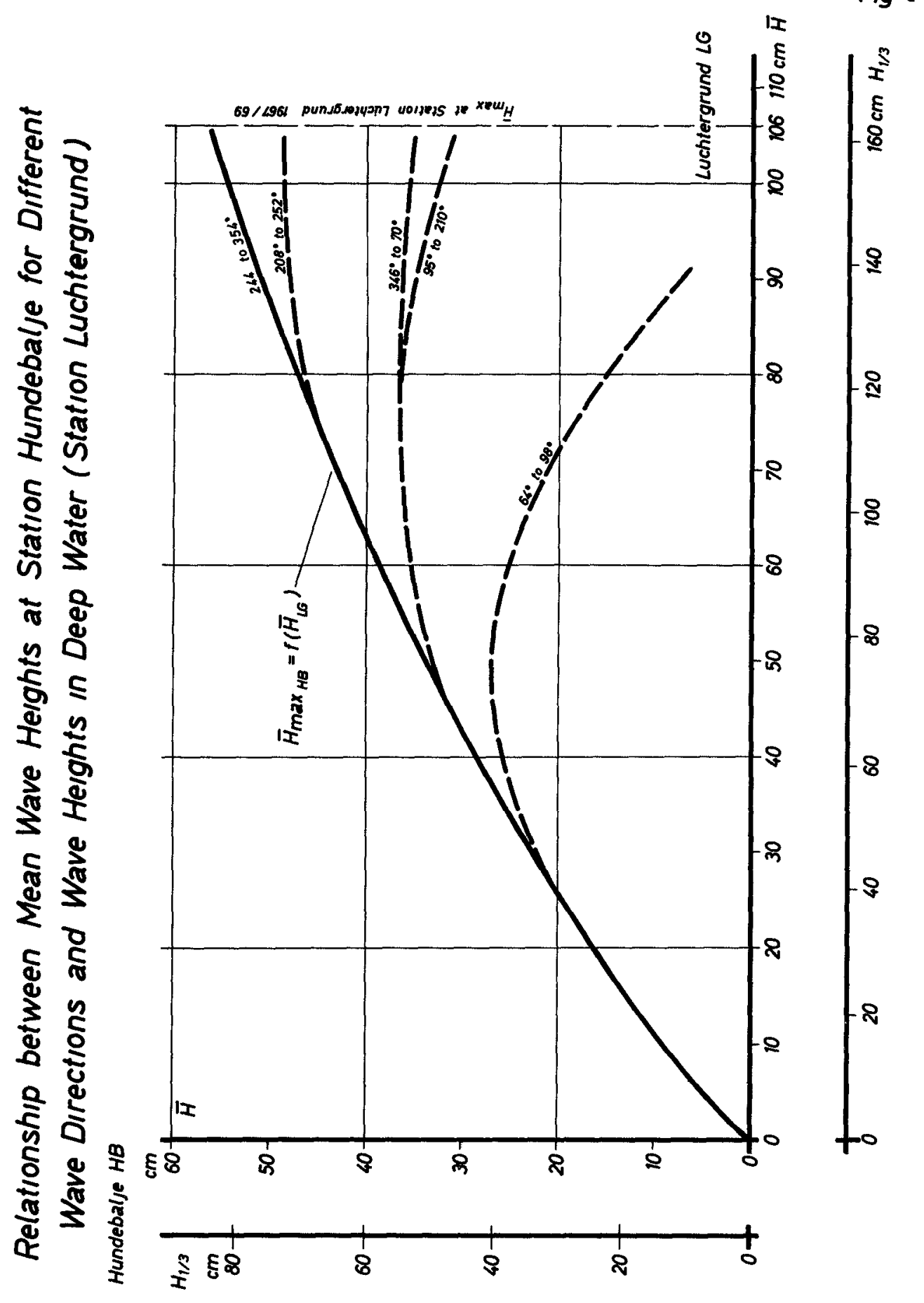




\title{
EXPERIMENTAL STUDIES ON THE GENERATION OF WAVES IN SHALLOW WATER
}

\author{
C. T. Kuo \\ Associate Professor \\ Frederick L. W. Tang, Dr. Eng. \\ Profeseor and Chairman \\ Hydraulic Engineering Department \\ Cheng Kung University \\ Tainan, City, Taiwan \\ Republic of China
}

\section{SYNOPSIS}

The seas in front of China coast such as Yellow Sea, East China sea and Taiwan straito are all located on continental shelf. In coneequence, waves approaching these coasts are generated in shallow water area in comparsion with wave length. The author has developed tangible calculation procedures for evaluating the wave features in such areas in stationary or moving fetches (1). However, the basical formulas of calculation are derived from experimental data of Bretechneider and Thijese. In order to investigate the generating process of shallow water wind waves and obtain more detailed informations for correcting calculation criteria, a series of experiments have been performed at a wind tunnel of 75 meters in length. Various investigations on the relationships between waves and wind as well as water depth ard to be submitted in this paper.

In addition, the situation of wind wave coexistance with regular wave is studied from experiments, because it resembles the aperposition of refracted and local wind waves on the western coast of Taiwan.

\section{SIGNIFICANT WAVE FEATURES}

From the experiments, the wave heights and periods increase with wind velocities and fetch lengths, however, they reach fully arion state more rapidly than deep water waves, only a fow minutes in the experiments. After fully arisen, a portion of high waves begin to break and reduce their heights slightly, however, the waves recover their heights after advancing a shovt distance and then they break partially, auch phenomena repeat again and again especially in the cases of the wind being strong. The ware periods remain increasing within the fetch length in these experiments, however, it can be expected that the wave periods and heights may become constant if the fetch length is long enough. 
The experimantal curves of $6 H_{1} / U^{2}$ versus $G F / U^{2}$ with perameter $\mathrm{Gd} / \mathrm{U}^{2}$ as shown in $\mathrm{Fig}_{\mathrm{g}} \mathrm{I}^{3}$ have the same tendency with the curves of Bretschneider (2), however, our data are larger than those of Johnson, Huft and Hawada, and oven larger than the values predicted from the fotch graph of SMB in the region of $\mathrm{gF}^{\mathrm{F}} / \mathrm{U}^{2}$ in Fig. 2 are located below breaking lint and Bretschneider's seady state of friction coefficients equal to 0.01 . In Fig. 3 the experimental result of the relationship between $\mathrm{gT}_{\frac{1}{3}} / \mathrm{V}$ versus $\mathrm{gF}^{\mathrm{F}} / \mathrm{U}^{2 /}$ is guite agreed with deep water waves.

Statistical characteristics of wave helghts and periods have also been calculated. As hown In Fig. 4 and 5 the probability distribution corresponds sufficiently to Gaussian's rather than Rayleigh's. The ratias between various $1 / n$th wave helghts are as follows.

$$
\begin{aligned}
& H_{y /} / H_{\text {ave }}=1.38 \\
& H_{y / d} / H_{1 / 3}=1.16 \\
& H_{\max } / H_{1 / 3}=1.39
\end{aligned} \quad\left\{\begin{array}{l}
1.60 \\
1.27 \\
1.64
\end{array}\right)
$$

Any of the numbers 18 smaller than the theoretical value of Longuet-Higgins (in parenthesis). The ratios of $1 / n$th wave helght to root-mean-square wave helght Hrms are as follows.

$$
\begin{aligned}
& \mathrm{H}_{\text {avd }} / \mathrm{H}_{\mathrm{rms}}=0.94 \\
& \mathrm{H}_{1 / 3} / \mathrm{H}_{\mathrm{rms}}=1.30 \\
& \mathrm{H}_{\mathrm{K}_{0}} / \mathrm{H}_{\mathrm{rms}}=1.55
\end{aligned}
$$

They ceen to be little concern with spectral widh paraneter.

On the whole, there 18 no substantial difference botween the statistioal properties of wind waves in shallow and deep water.

\section{SPECTRAL ANALYSIS}

The power epectra are calculated from experimental records by Blackman-Tukey' method. Sampling time interval it is $1 / 10-$ $1 / 15$ sec., total number of data $18 \mathrm{~N}=800$, maximua time lag a equals 40 , and folding frequency $\mathrm{fm}=5 \mathrm{cps}$ (or $0.375 \mathrm{cps}$ ). The degree of freedom calculated by Tukeys formula 1 s 40 , is consequence, confidence limit in $10 \%$ is $0.73-1.30$.

At early stage, the spectra grow continuousiy as the fetch length boconing longor tili saturation tate 1 seached. While the fetch lengths extend, the lower frequency parts of the epectral curves increase their densty, as Fig. $6,7,8$. 
The relationship between wave spectra and wind raloeity has the eame teldency of deep water waves. Namely in sane fetch length and water depth, the spectra grow with wind velocity being Increasing. As Fig. 9 o 11 , if the depth 1 . sma11, the epeotral denaity 1 omaller than that of deep water waves, as chown in Fig. 12, 13, 14, for oame witd velocitJ. The greater the wind velocity is the earlier the low frequency portion of epectrum developed, and the wider the frequency band becomes. In any case, the high frequency side of equilibrium range decade remarkably and can be represented by $f^{-n}$.

The oonnection between $T / 3$ and optimum period Top 18 to be $T y_{3}=1.23$ Top $1 n$ our experiments, and $T / / 3=1.23$ Tave $=1.23$ irme, accordingly Trms = Tave = Top.

Phillpo pointed out thy shape of spectral curves in high frequency aide should be:

$$
\Phi(f)=\beta E^{2} f^{-5}
$$

However, Hamada proved that $\bar{\Phi}(\mathrm{f})^{5}=\beta \mathrm{g}^{2} \mathrm{r}^{-n}$, and $\mathrm{n}$ w111 be larger than 5 in deep water wave spectra. Our experimento also reveal $n=7-10$ in shallow water waves.

The relationahip between $H$ and $E=2 \int \Phi(f)$ dr 1 e the same as deep water waves, namely $H / / 3=2.83 \sqrt{\mathrm{E}}$. As ohom in Fig. 15 .

\section{STUDIES ON THE COPERPOSITION OF REGULAR AND WIND WAVES}

According to the special topography of western coast of Taiwan, the beach is very flat and the waves approach from Taiwan Straite are breaking on offehore vare. The distanoe between bare and the wain coast or sea dike 18 otill a 1 ong as $5 \mathrm{~km}$. Loonl wind waves are overlapping on the waves after broken. To investigate such a phenomenon in order to offer design criteria for sea dikes, we generated regular waves by flap type wave generator and blow wind simultaneousiy in the same wind wave channel. The regular wave spectra are shown in Fig. 16 and the opectra of wind wave while regular wave are not to be existing are ahown in Fig. 17 . Fig. 18 shows the result spectra of overlapping. Apparently there are two kinde of wave existing independently. However, if the regular wave steopmeas is large such a phenomenon disappears as Fig. 19. The compriton of the energy of resultant wave energy and energy calculated by linear sumbation 1 s shown in Fig. 20. In case of broken waves superposed by wind waves as the case of western coast of Taiwan. Wave helght in front of sea dike can be approximately calculated by $\mathrm{H}^{2}=\mathrm{Hw}^{2}+\mathrm{H}_{\mathrm{R}}^{2}$. 


\section{CONCLUSTON}

From experimental data described above following conclusions can be made.

1. The generation procedure of shallow water waves is closed resembling to deep water waves, however, the duration for fully arisen is much shorter.

2. In the range of our experiments wave period seems not to be ignificantly influented by water depth. The wave height increases with fetch length increasing, however, they are smaller than deep water waves due to the influence of water depth.

3. If wave spectra of shallow wave area are available, the significant wave height can also be calculated by $\mathrm{H} \mathrm{Y}_{3}=2.83 \sqrt{\mathrm{E},}(\mathrm{E}=2 / \mathscr{f}(\mathrm{f}) \mathrm{df})$.

4. In the problem concerning superposition of two series of waves, if the oteepness is small, the resultant wave heights can be calculated by linear summation of their energies.

\section{REFERENCE}

1. Froderick L. W. Tang: Researches on Calculation of Waves on Long Shoaling Beaches. Jour. of Civil and Hydraulic Engineering, Taiwan Provincial Cheng Kung University. Feb. 1970.

2. Breschneider: Generation of Wind Waves Over a Shallow Bot tom. B. E. B. Tech. Memo No. 51 Oct. 1954. 


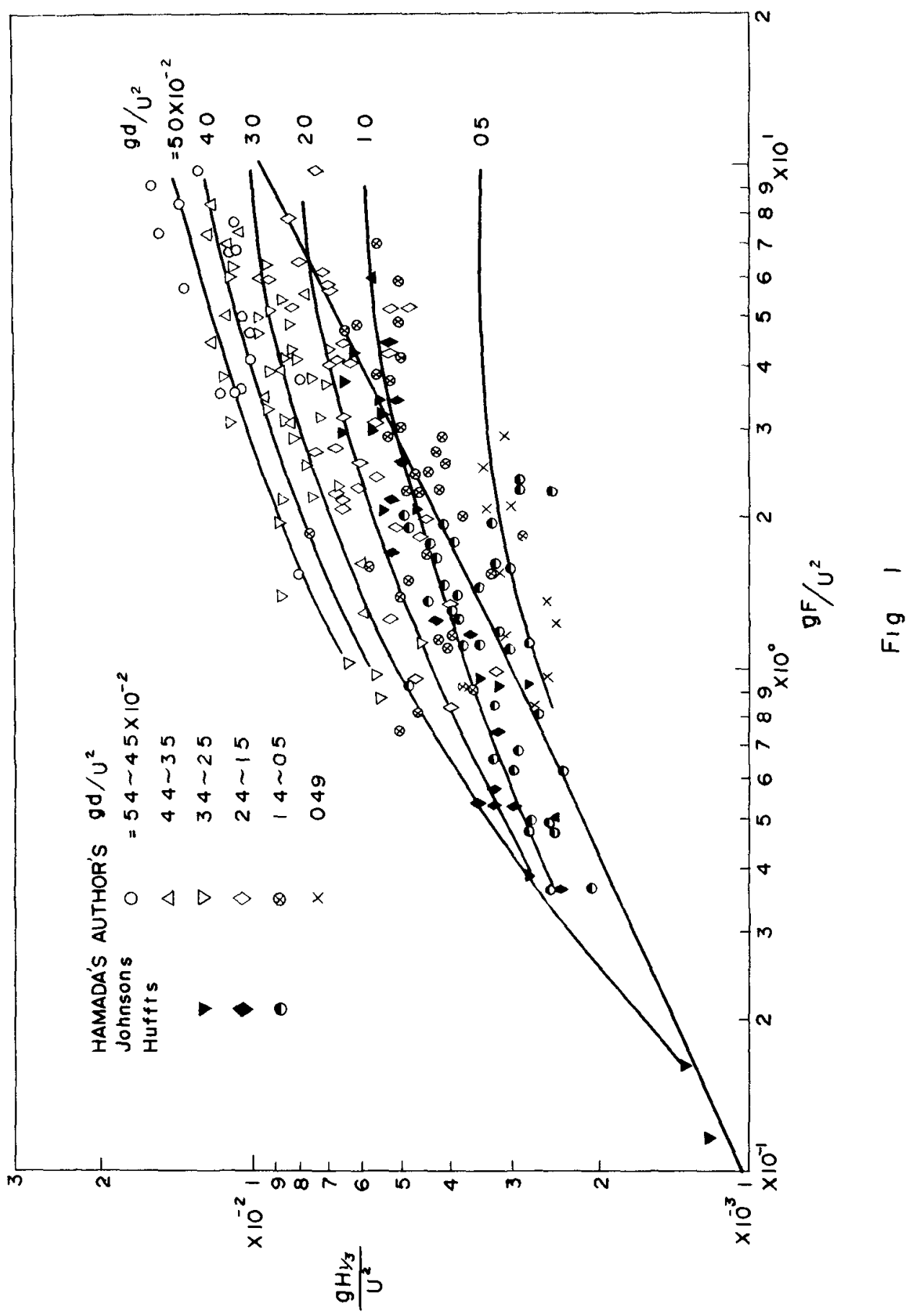




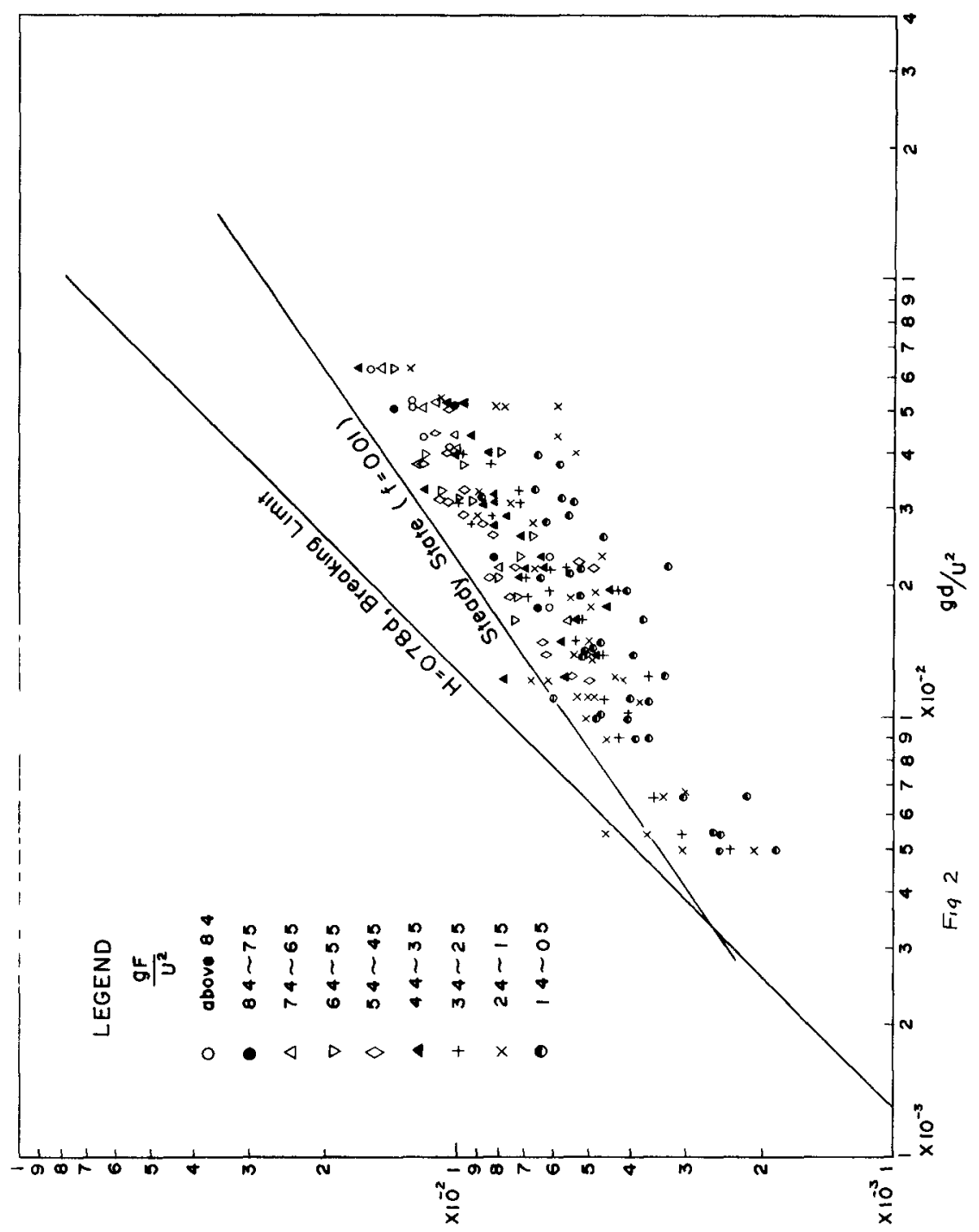

部 


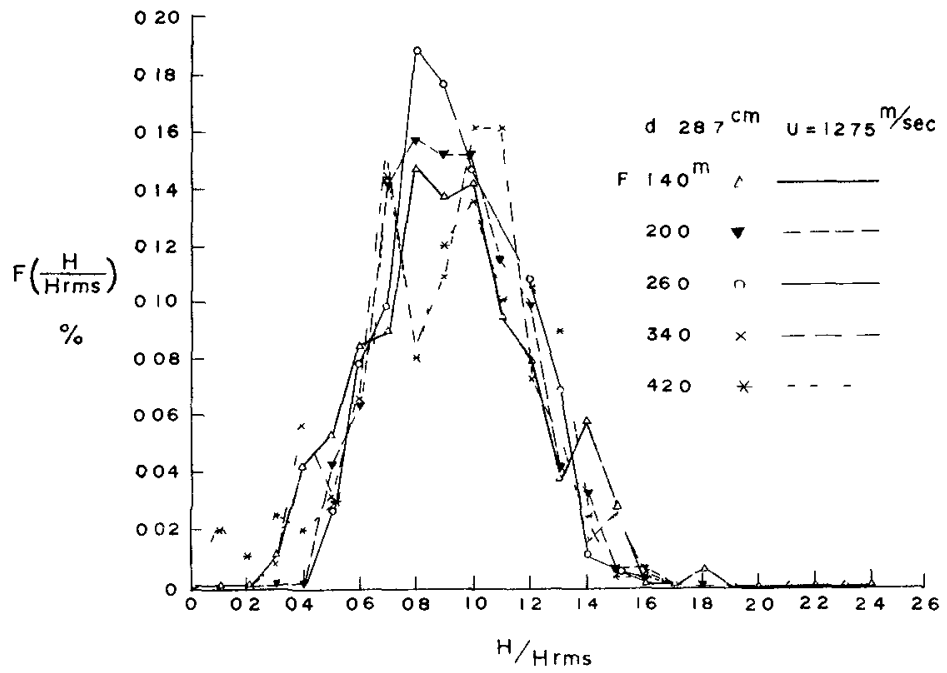

FIg 4

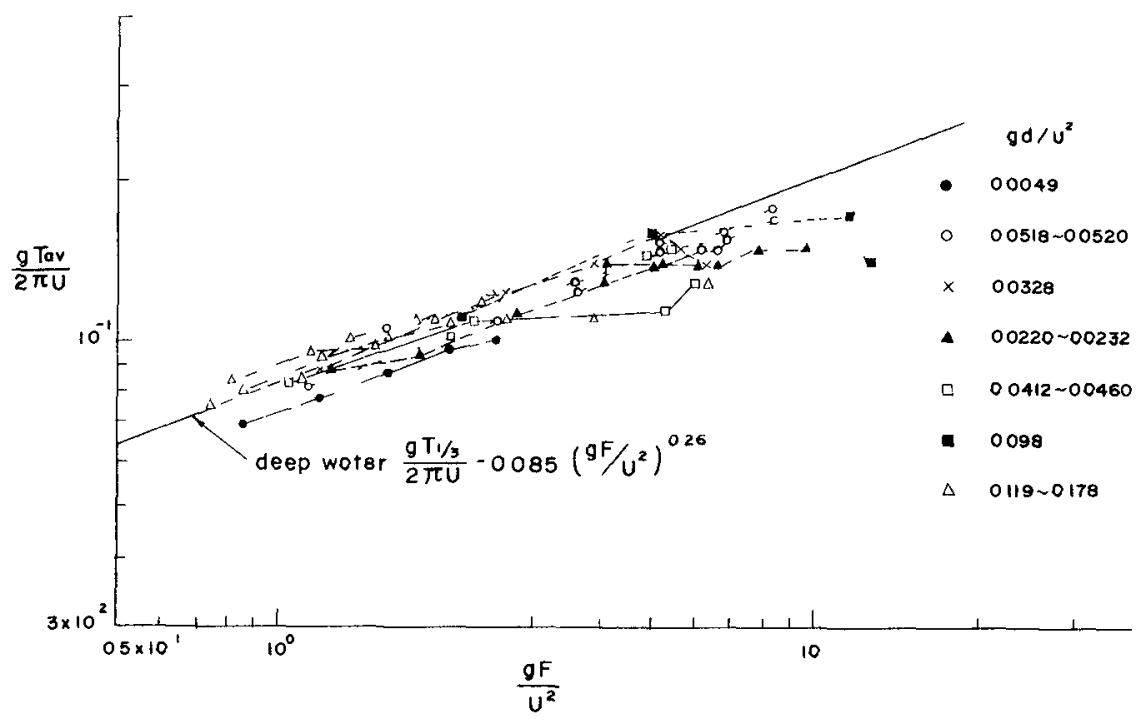




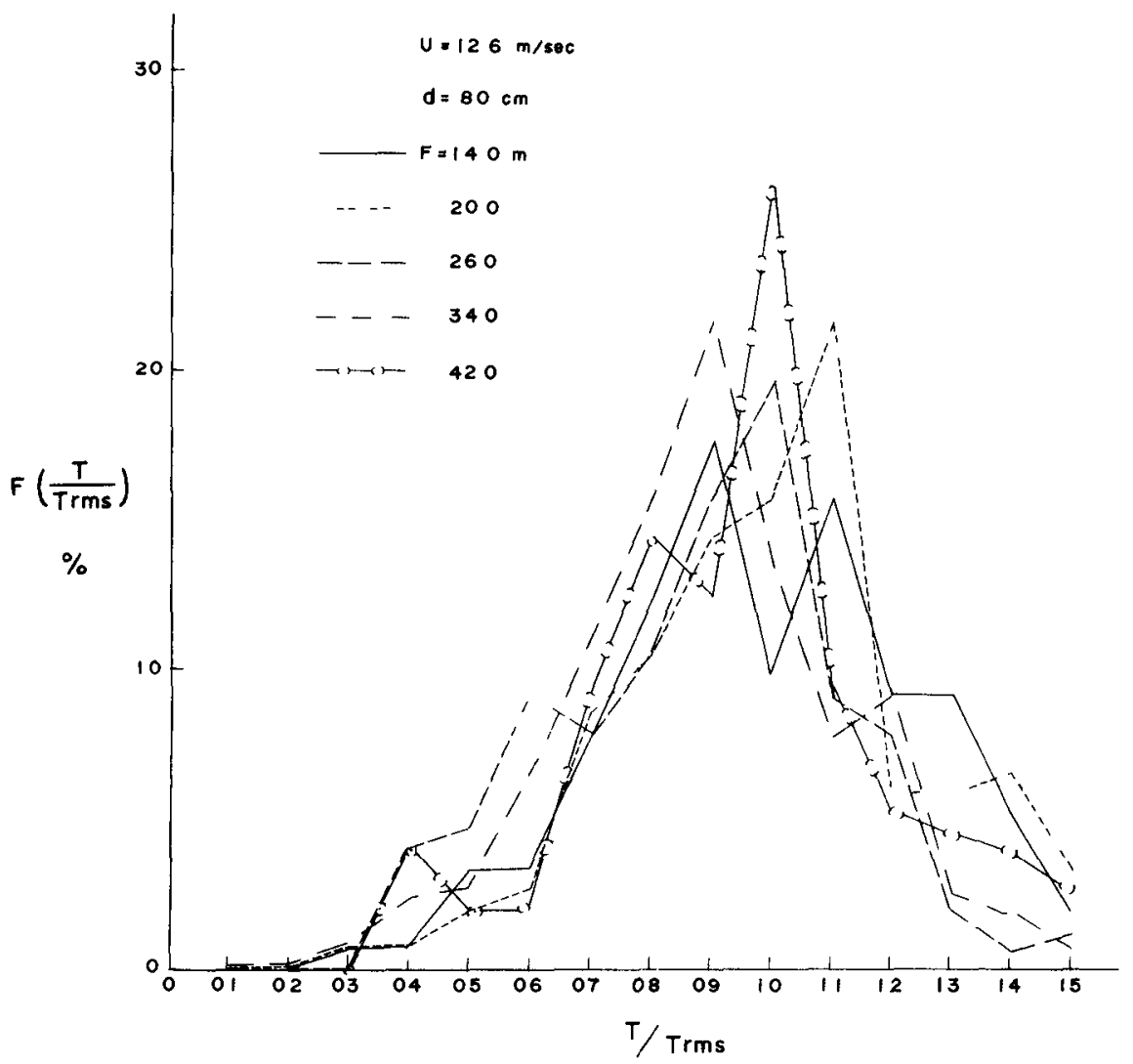

Fig 5 


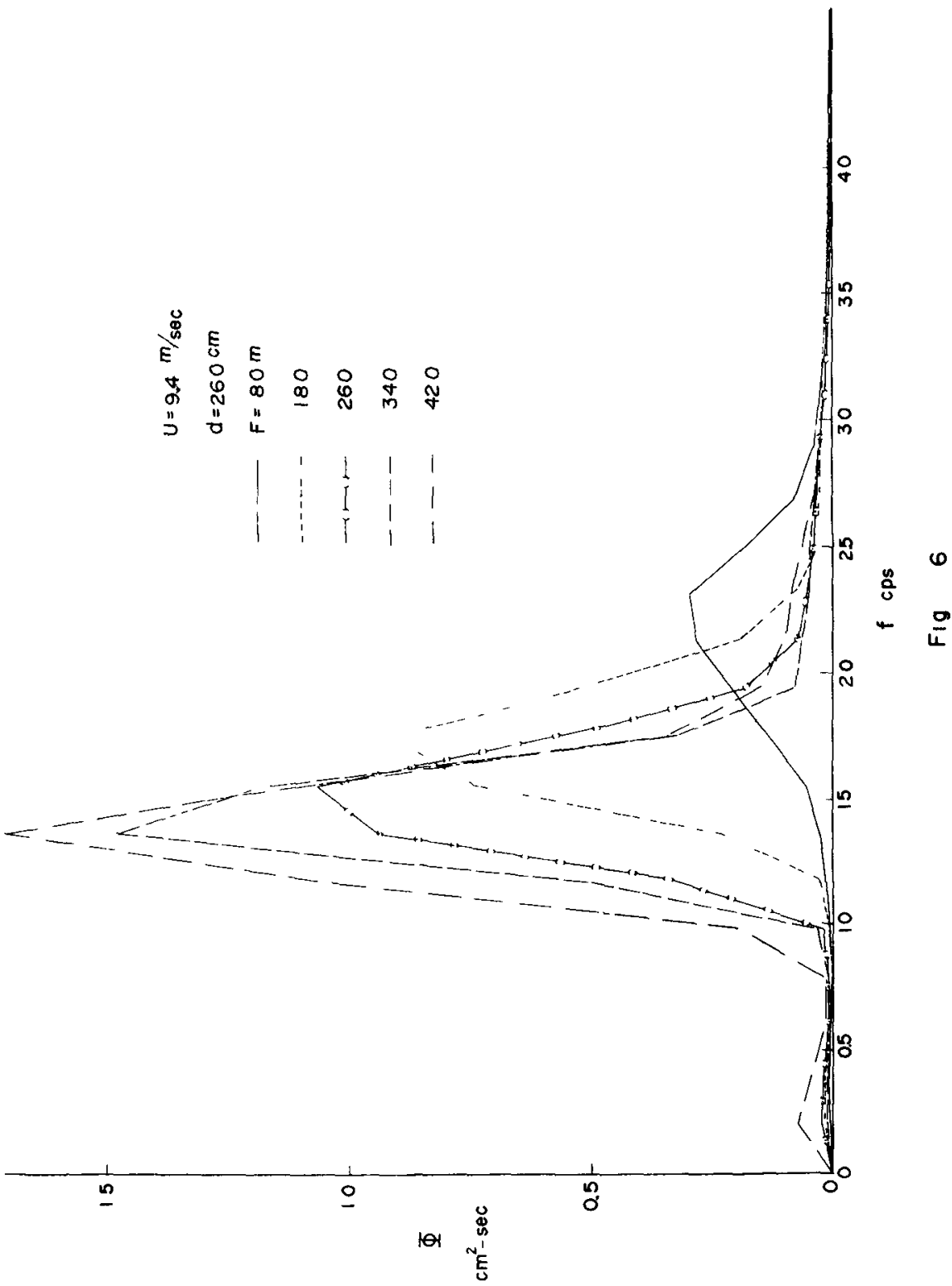




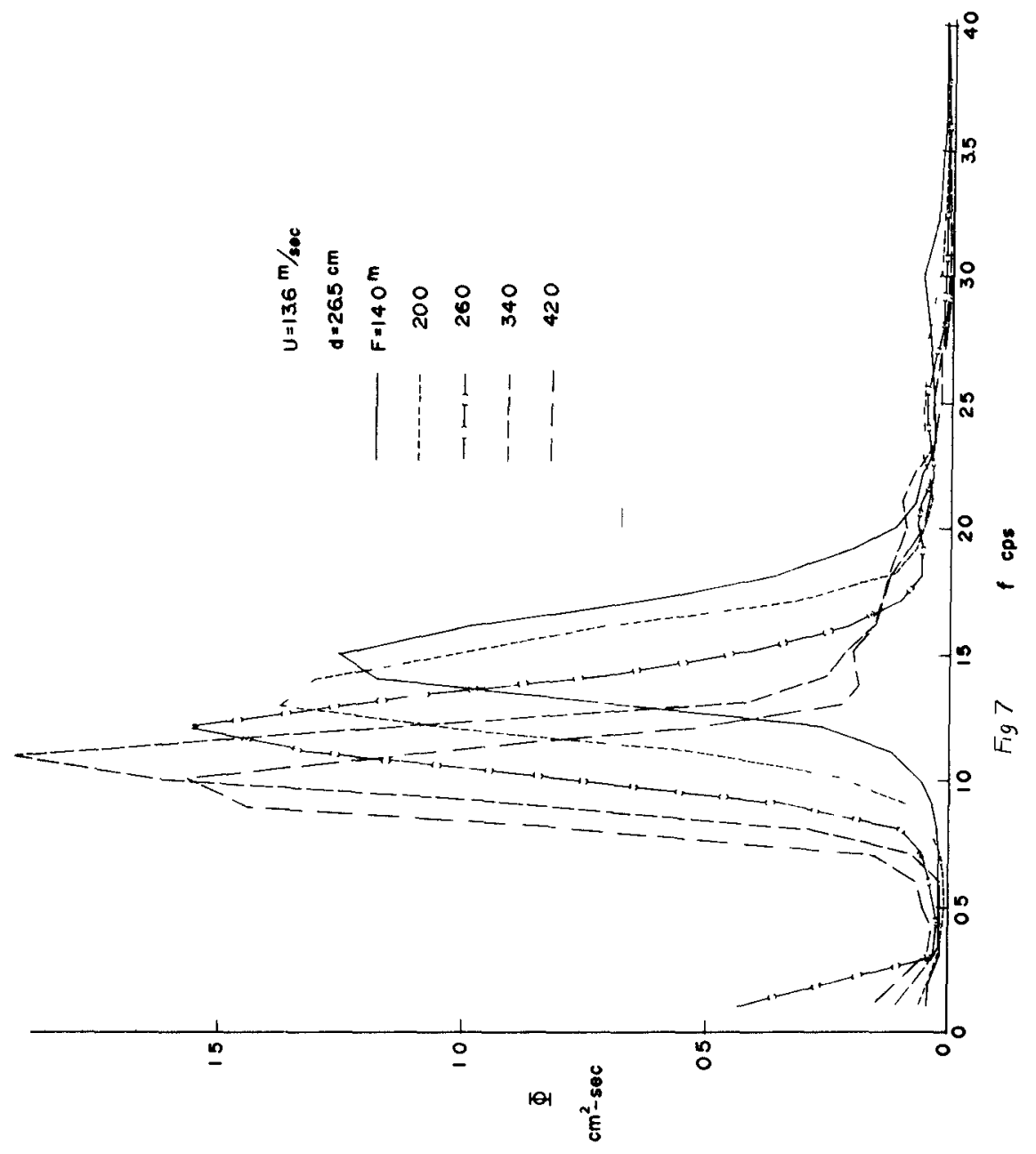




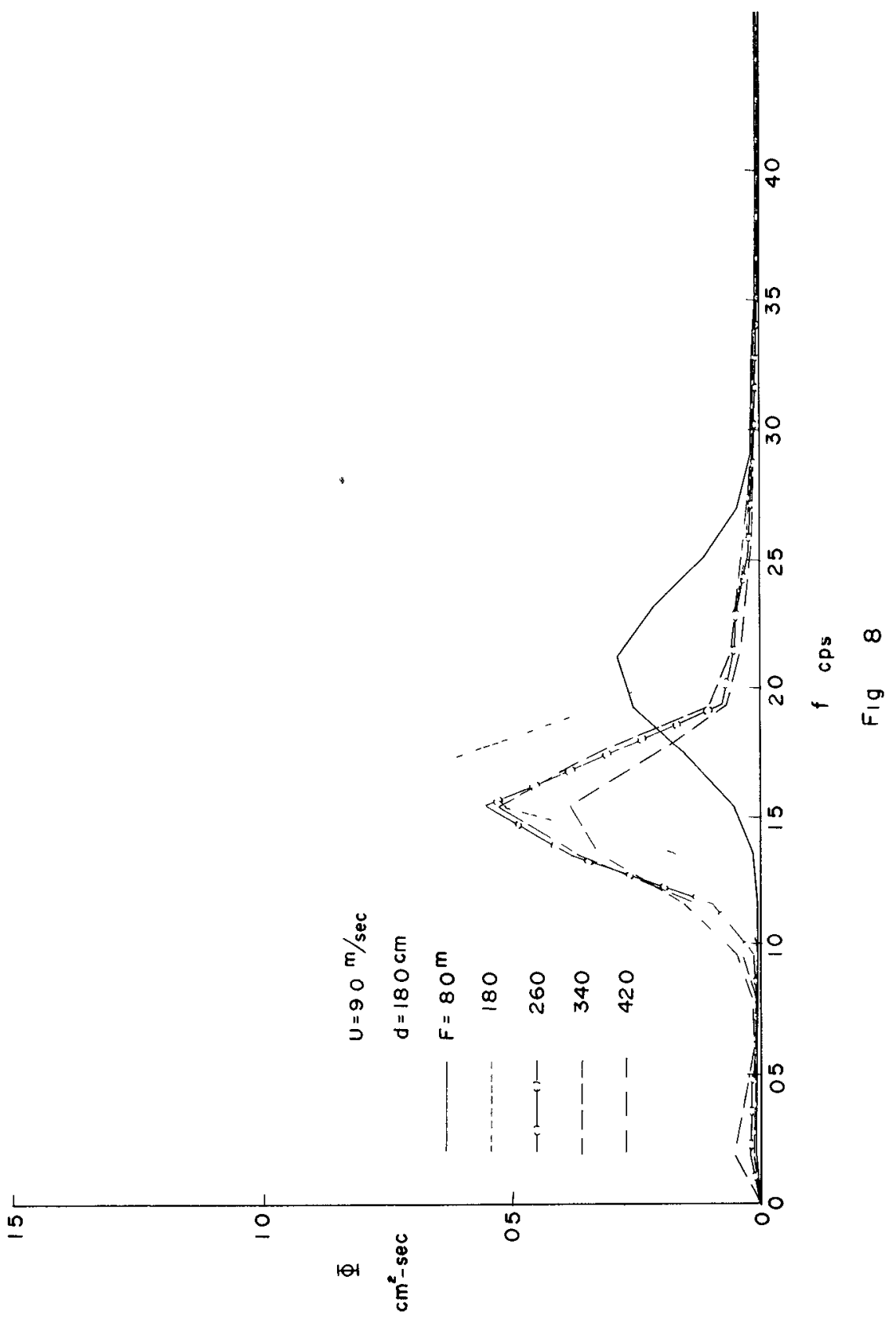




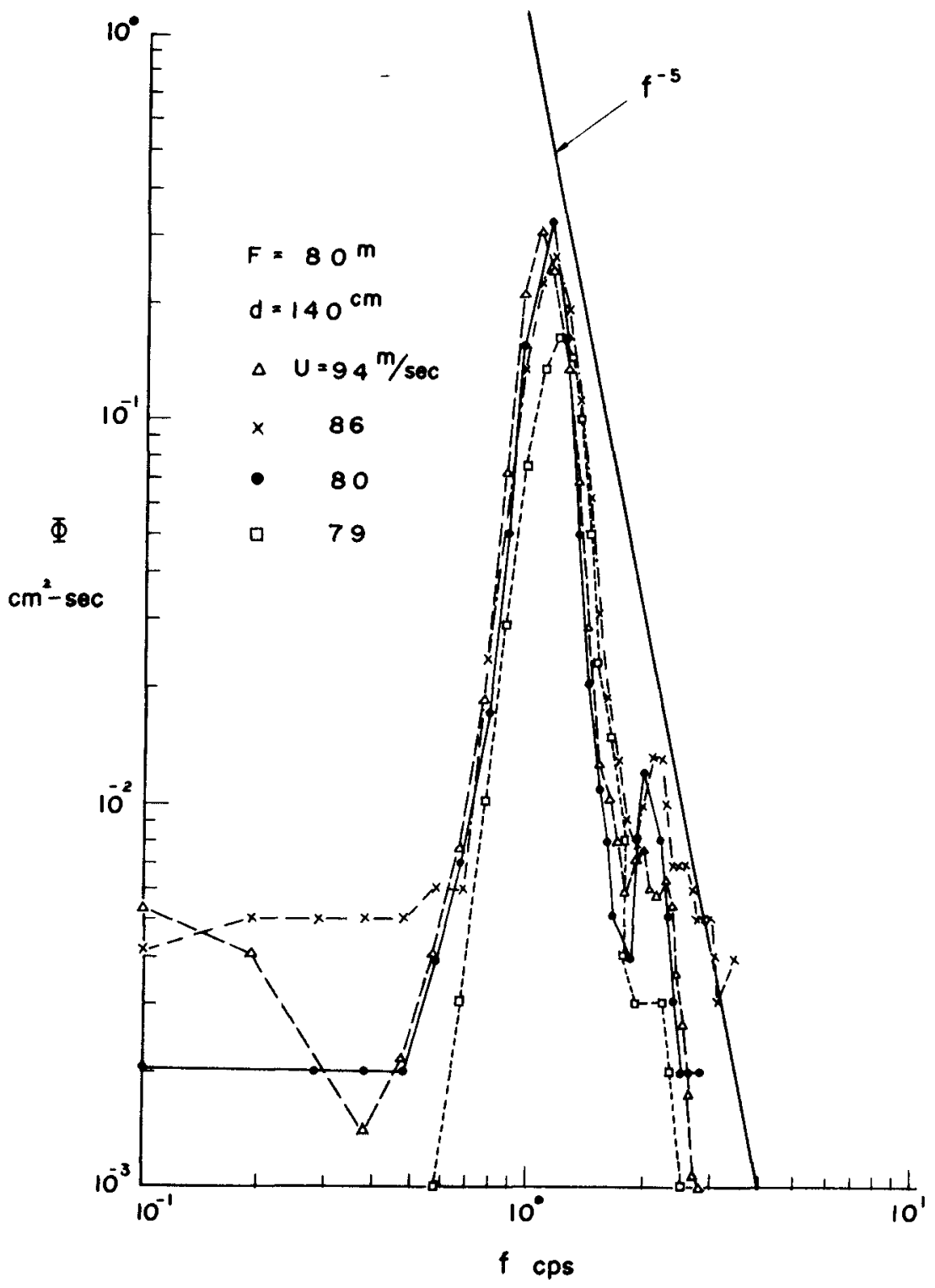

Fig 9 


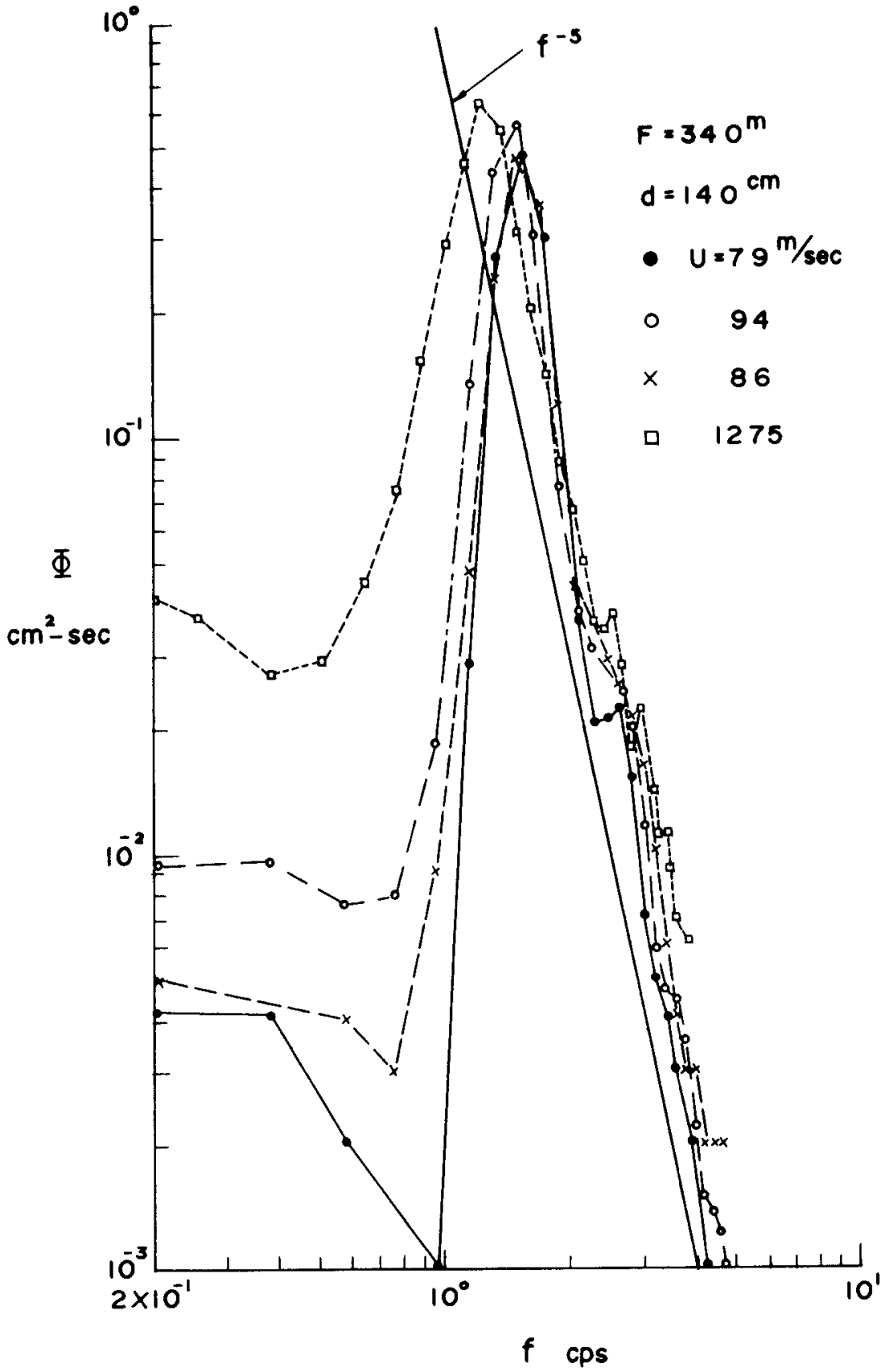

Fig 10 
192

COASTAL ENGINEERING
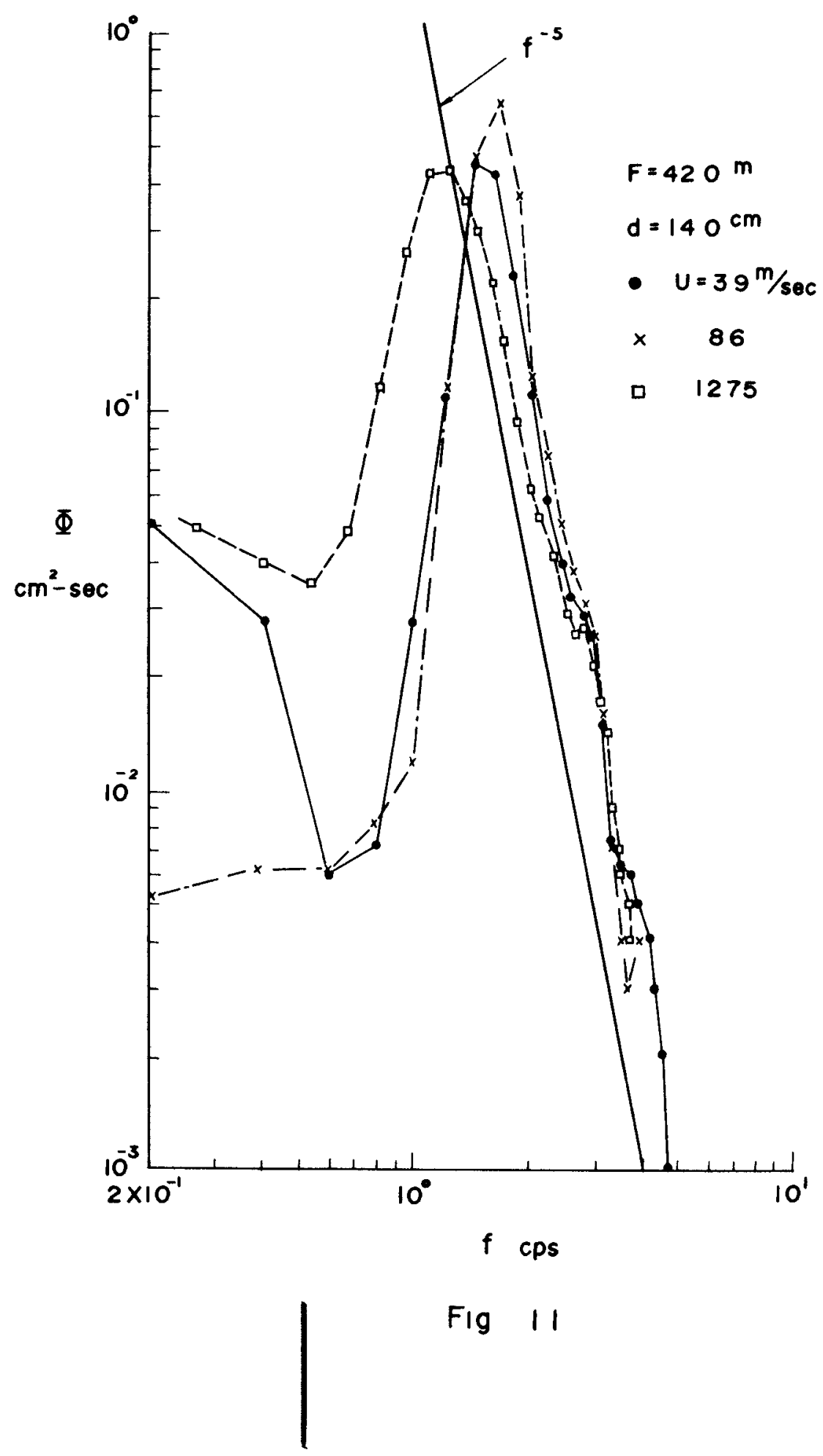

Fig 11 


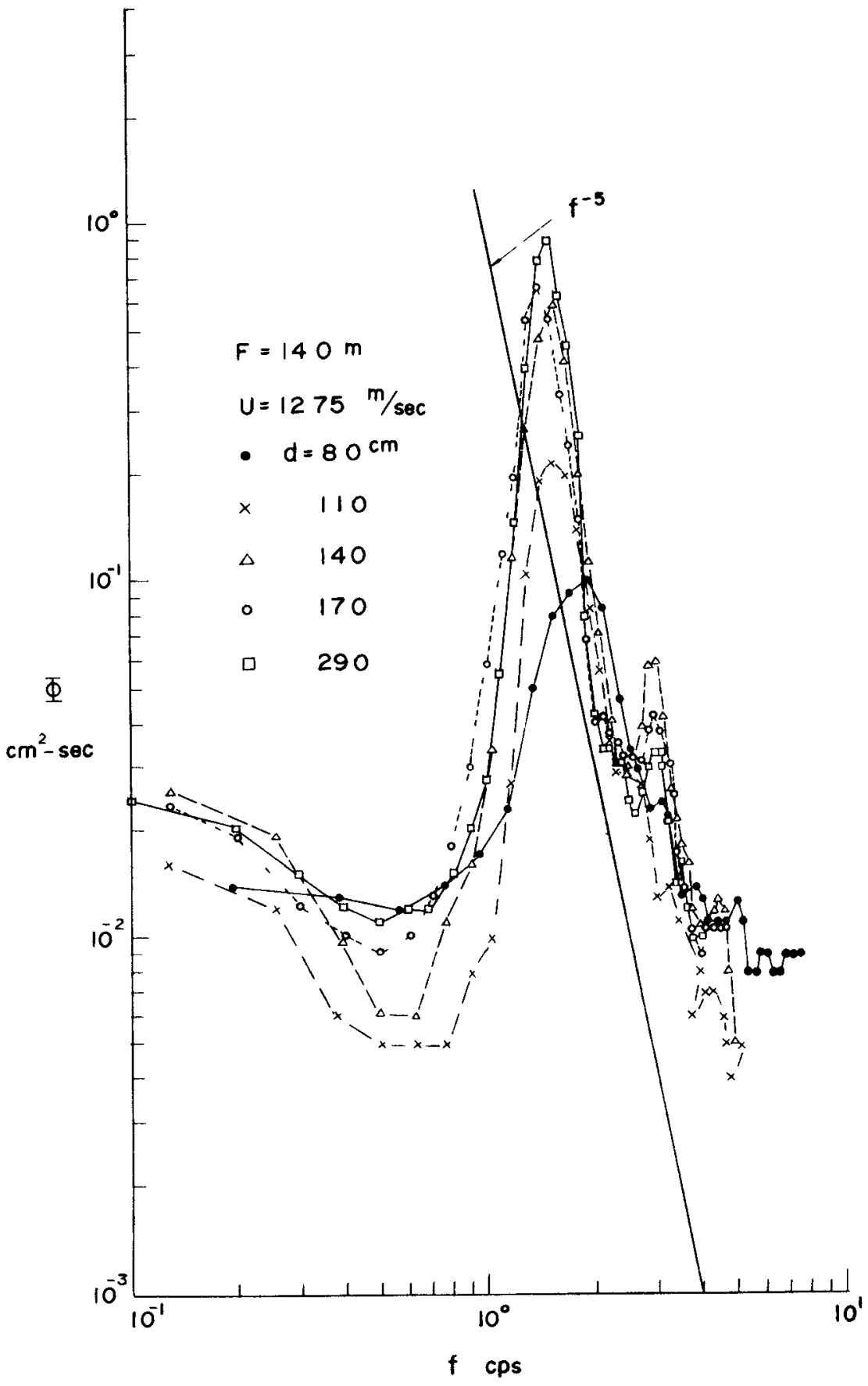

Fig 12 


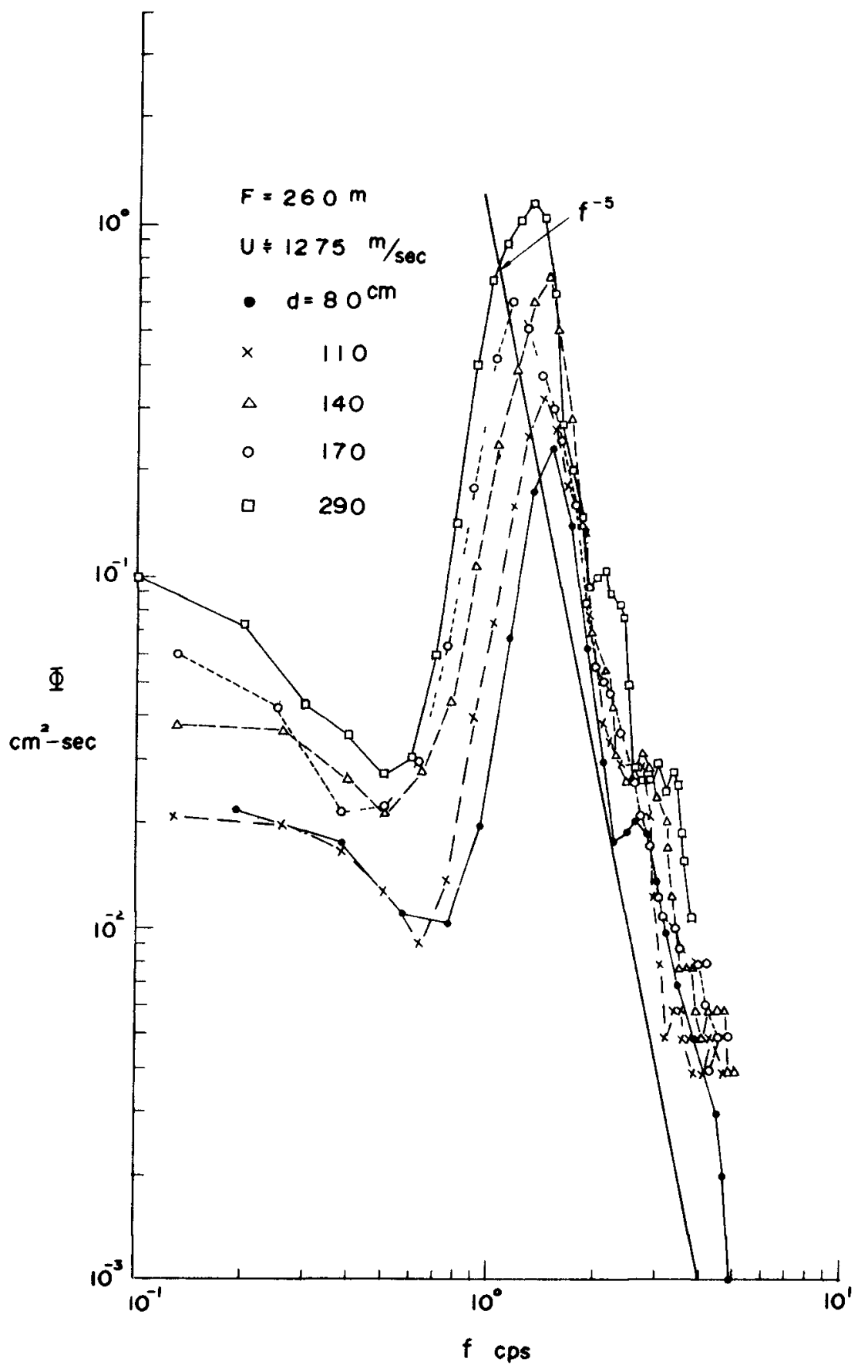

Fig 13 


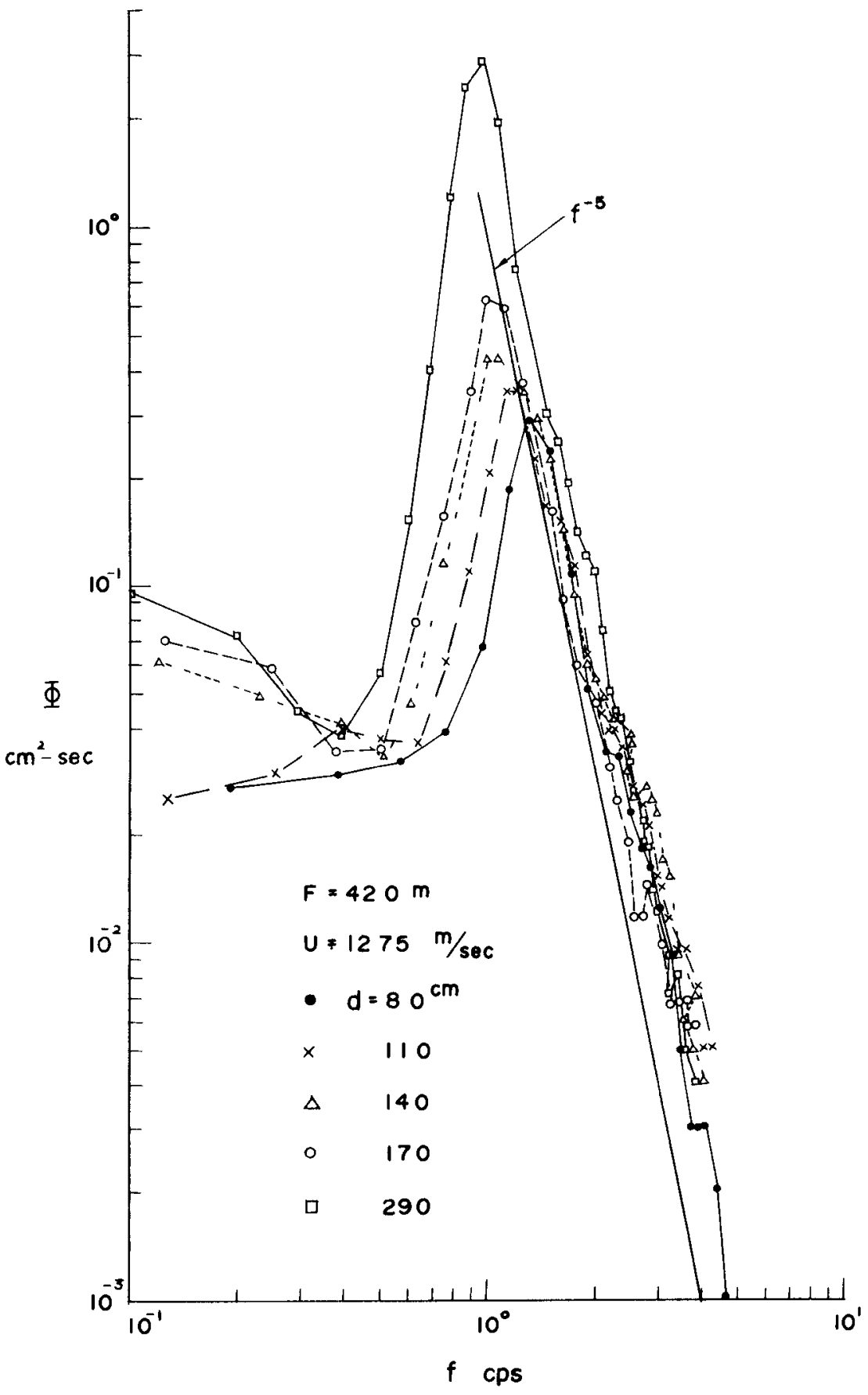

Fig 14 


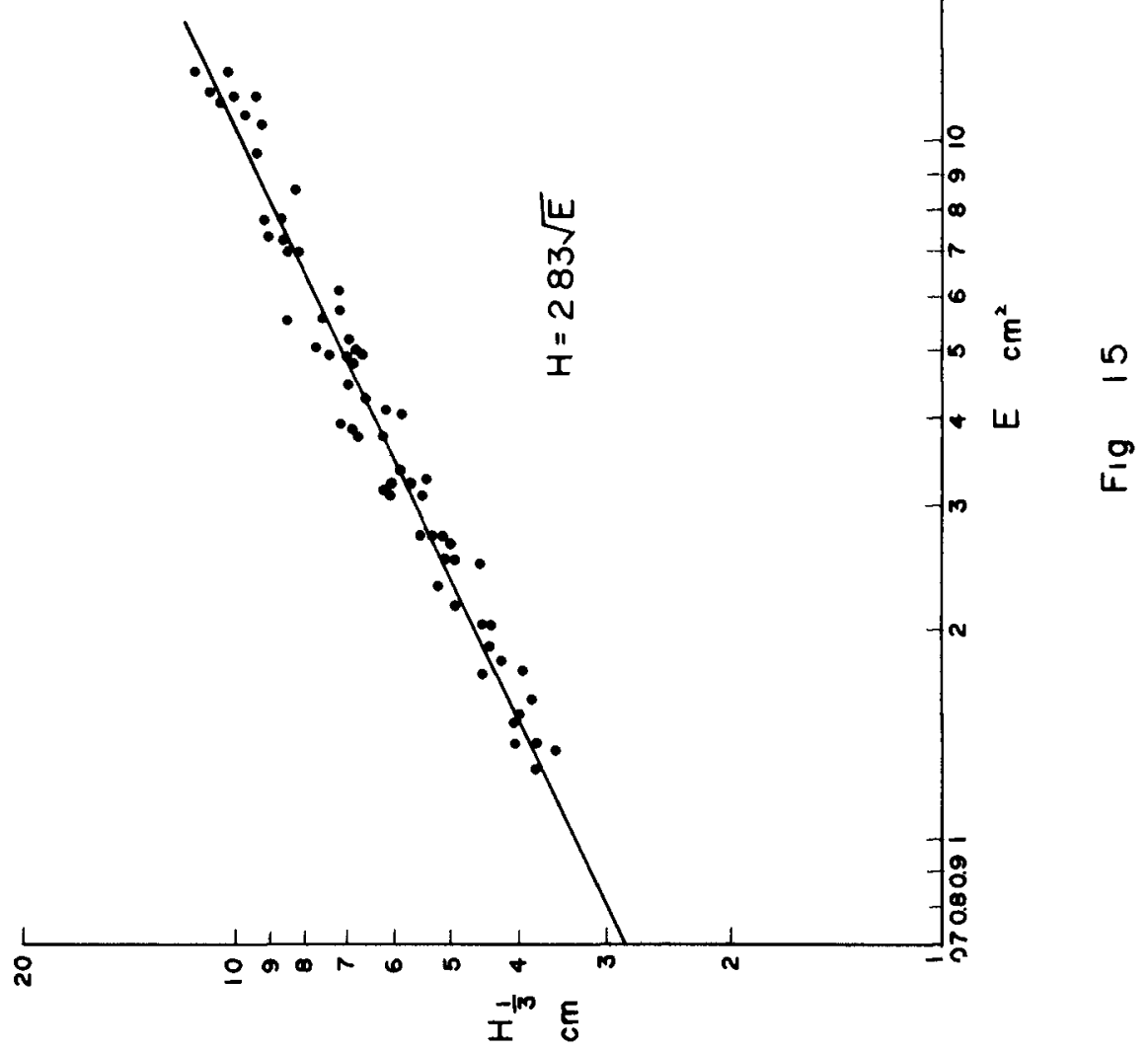


SHALLOW WATER

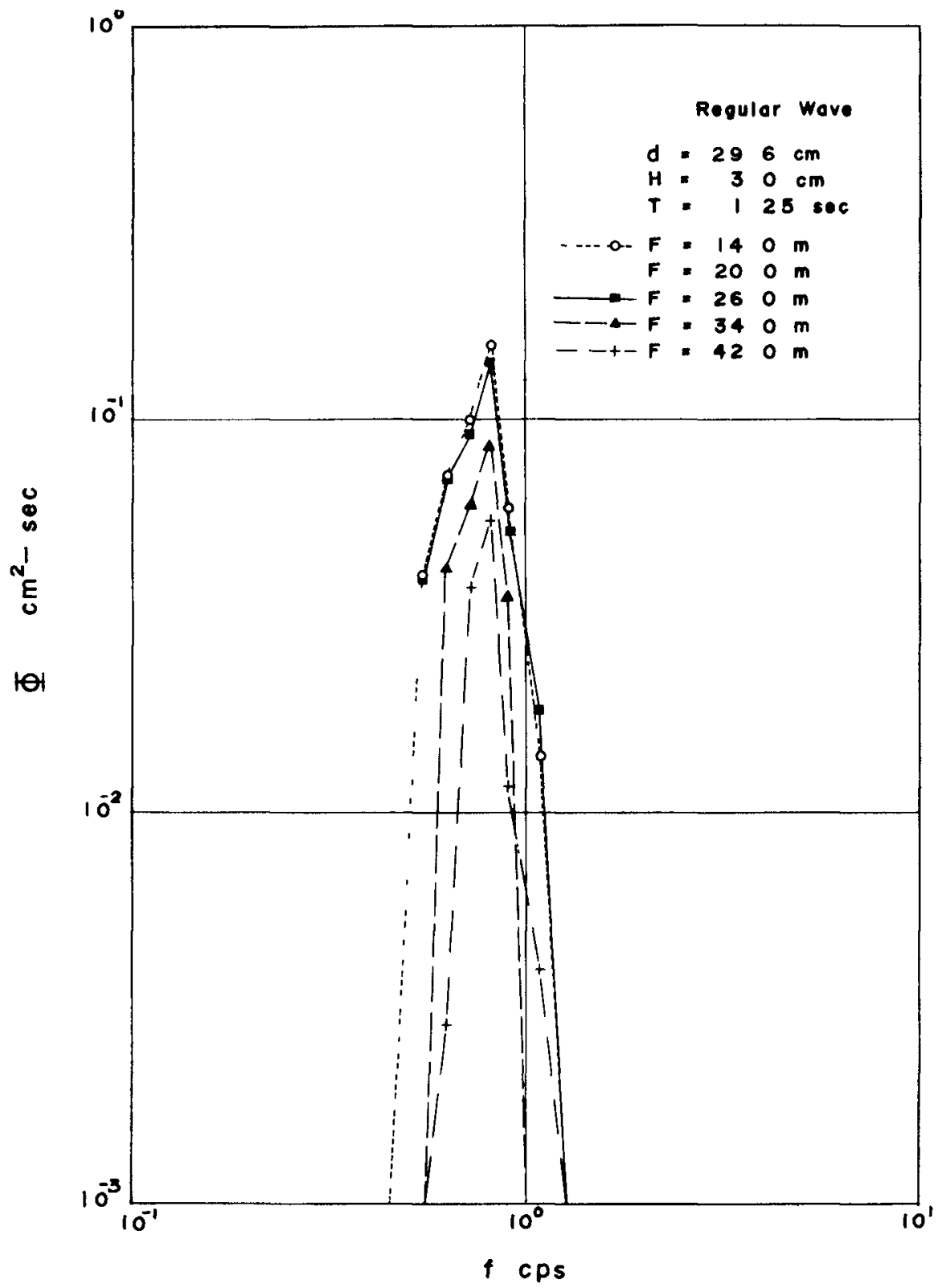

Fig 16 


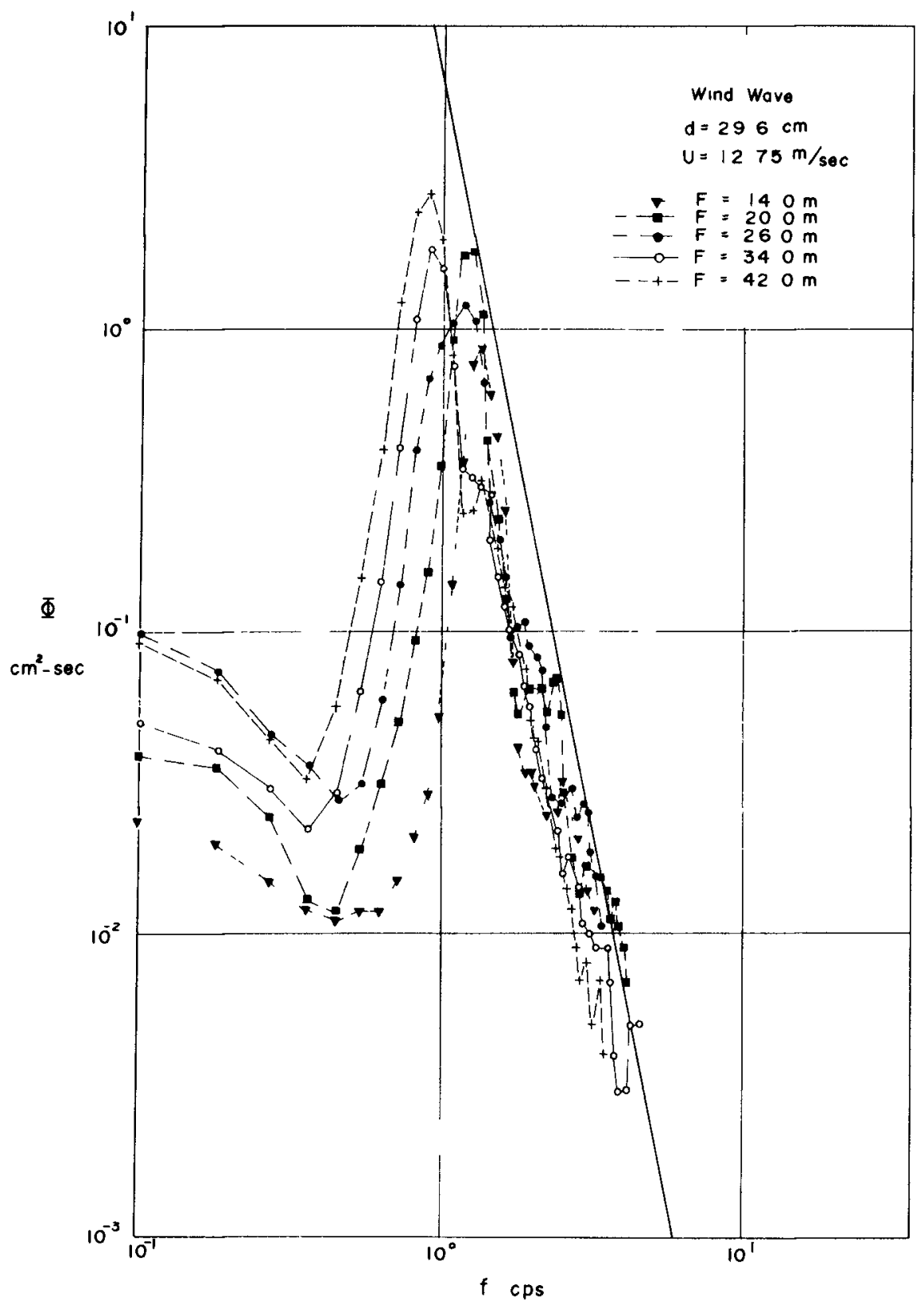




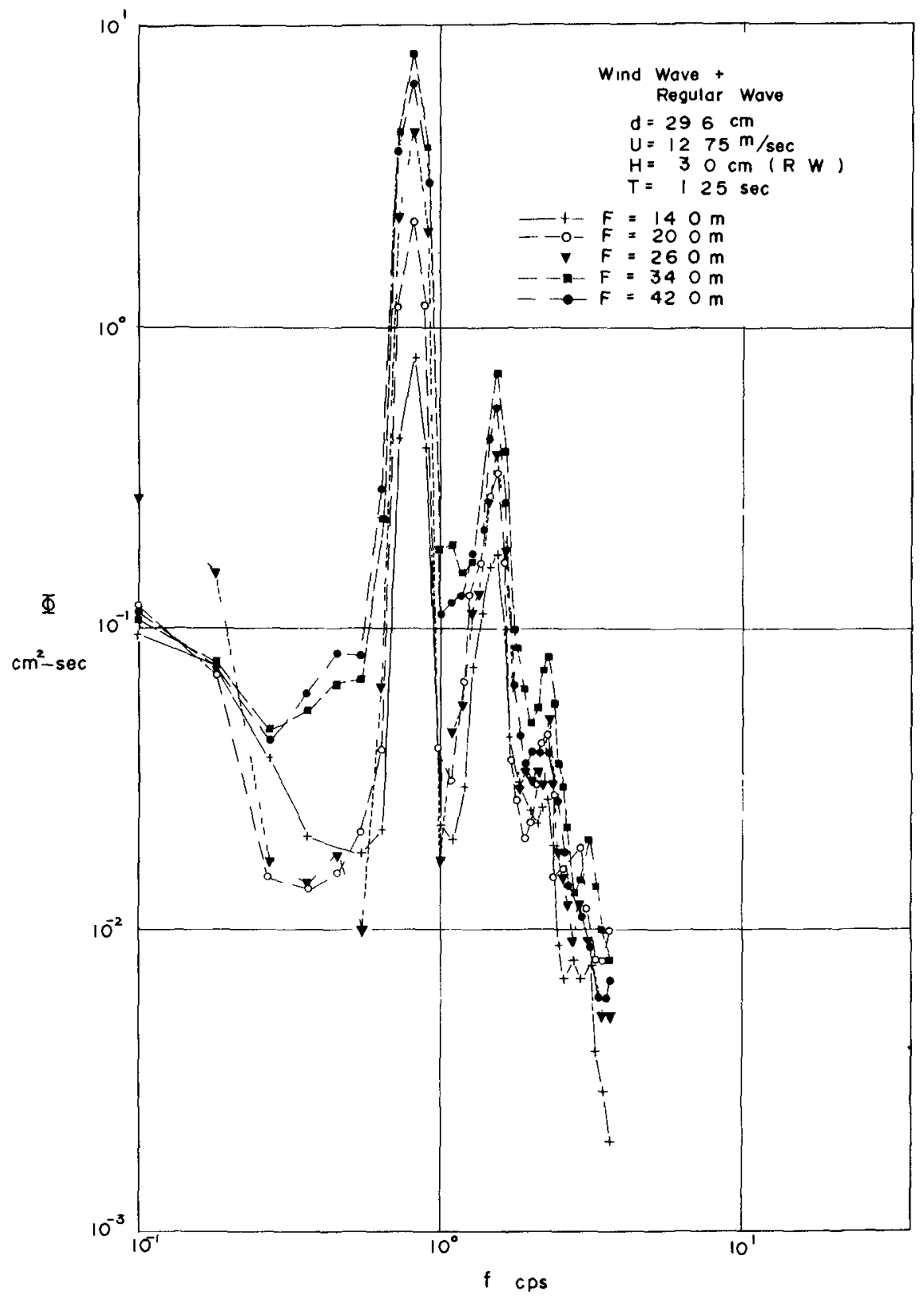

Fig 18 


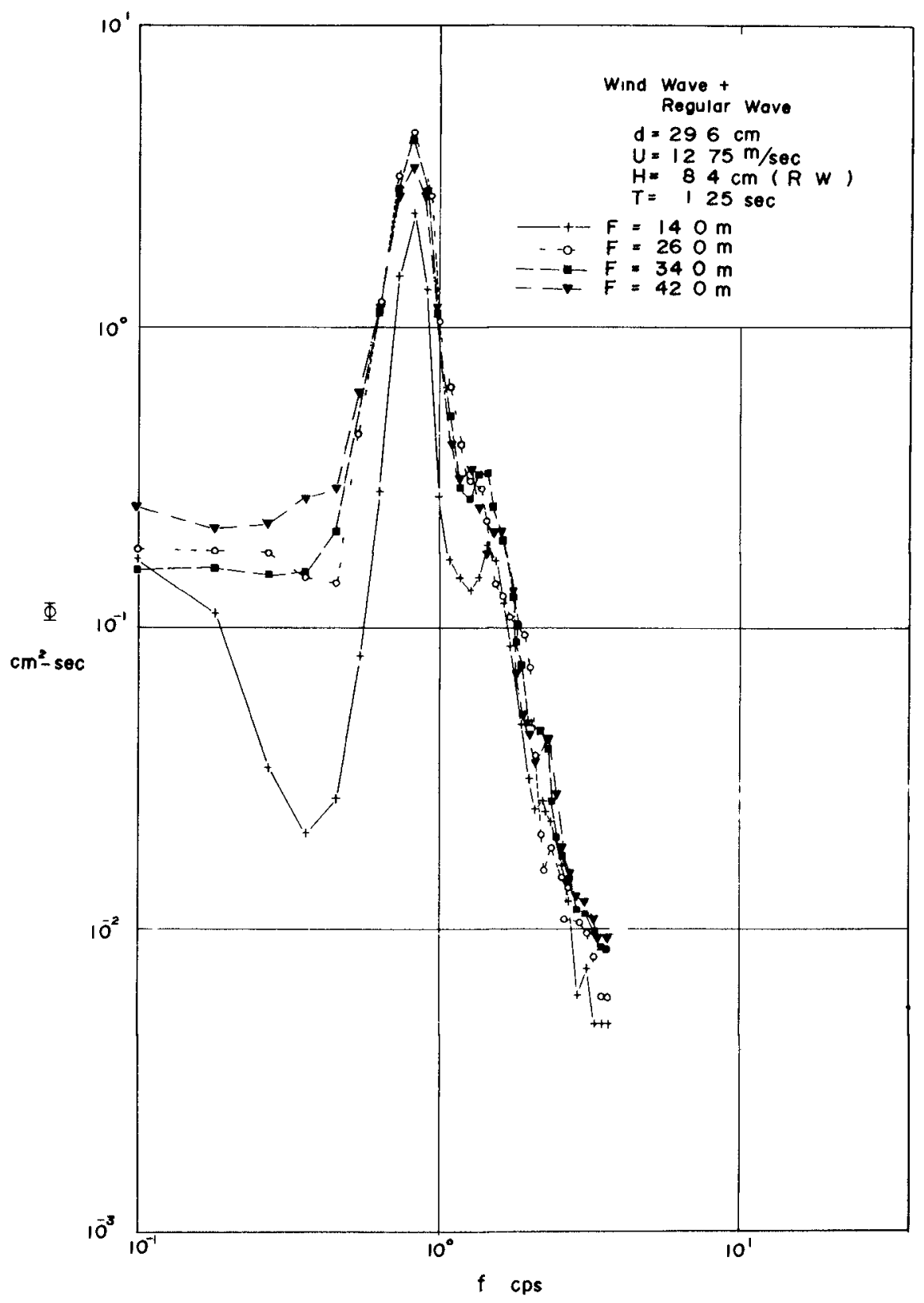

Fig 19 


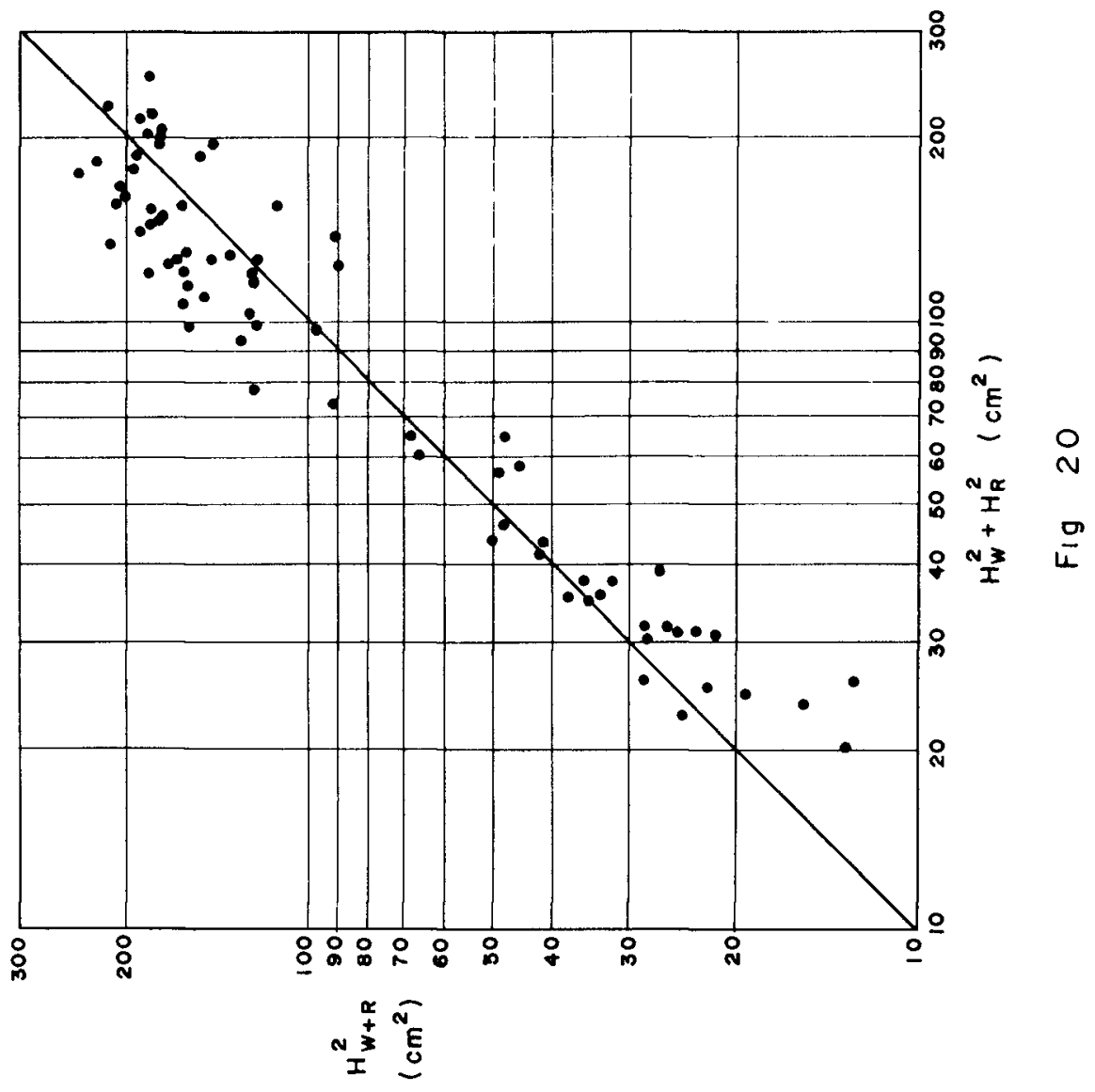





\title{
CHAPTER 12
}

WAVE FORECASTING FOR THE WEST COAST OF INDIA

$$
\text { J. DATTATRI * }
$$

AND

PS. RZNUR ARADHYA **

\begin{abstract}
ABSTRACI
The applicability of the general Wave Forecasting procedures like the SMB and the PNJ methods, to the Indian coasts is studied. The study consisted in analysing the synoptic oharts to obtain the necessary wind characteristics. The computed wand characteristics were used in the above Forecasting methods to yield significant wave heights These were compared with the wave characteristics as recorded by a sub-surface pressure type recorder after suitable modifications to account for the attenuation of wave pressure with depth.

The predicted wave heights compare well with the recorded wave heights and the SMB method predicts wave heights better for the case studied.
\end{abstract}

1. INTRODUCTION. Adequate foreknowledge of waves is essential for any coastal Engineering work. One of the ways of obtaining this necessary information about the wave characteristics is by installing wave recorders at surtable places along the coastline to obtain continuous reoords of waves. These records are statistically analysed to yield the required design wave height data. There are hardly any such recorders installed on the Indian coasts to the authors' knowledge. As such in the absence of any

* Department of Civil Engineering, Karnataka Regional Engineering Coll ege, Surathkal, India.

* Formerly Post-Graduate student, Marine structures Section, Karnataka Regional Engineering College, Surathkal, India. 
recorded wave data, in most cases the only alternative will be Wave Forecasting to get the required design wave height.

The two commonly used nethods of Wave Forecasting are the Sverdrup-Munk-Bretschncider method (hereafter referred as the SMB method) and the Pierson-Neumann-James method (PNJ method). These methods are based almost entirely on field data and as suoh can be used with confidence in areas from where the field data is taken. These nethods cannot be directly used for the Indian coasts without verifying thelr applicability.

The primary aim of the present paper is to study the general applicability of these Wave Forecasting methods to Ind Ian Coasts. For this purpose the South-West Monsoon period of 1968 was considered for the analysis, since it is during this season the stront monsoon winds generate the heavier seas. This work was possible thanks to the Wave Recorder installed near the Mangalore Harbour Project area situated $10 \mathrm{KM}$ to the south of the college.

2. ANALYSIS: The analysis consisted in Hindcasting the waves by the SMB and the PNJ methods for the Mangalore area on the West Coast of India from known meteorological conditions and comparing the Hindcast waves with the recorded waves.

The study conszsted of three stages, (1) analysis of weather maps (synoptic charts) to get the required meteorological data, (ii) wave Hindcasting (iii) analysis of wave records and comparison of recorded waves with Hindeast waves.

2.1 Meteorological Data:- The Indian Daily weather maps published twice a day by the Indian Meteorological Department, Poona, were used to obtain the required meteorological data (wind speed, direction, 
and duration, Fetch length and widh and Decay distance). These weather maps are prepared with very scanty data, particularly over the Arabian Sea and the Bay of Bengal, which render any data based on then very approximate. The present analysis is subject to that limitation.

2. (a) Wind characteristics:- The wind velocity over the generating area was determined by computing the Geostropic Wind velocity(5)* from the isobar spacings and applying the corrections for isobar curvature and the air-sea temperature differences as given in $T . R-4(6)$ to yield the surface wind speeds.

The wind direction was assumed to be parallel to isubars and the wind duration was fixed by a study of the changes in the successive synoptic charts.

Comparison with a few observed wind velocities from ships' reports showed that the computed wind velocities were not much differing from the observed velocities.

The use of the geostropic wind equation over the generating area for the Mangalore coast can be questioned. G.F. Taylor ( $\overline{\text { ) }}$ recommends that the geostropic wind equation should be used for latitudes above 200. The generating areas for the Mangalore coast generally $11 \mathrm{es}$ between $10^{\circ}$ and $18^{\circ}$ latitude $\mathrm{N}$. (Latltude of Mangalore is $\left.12^{\circ} 52^{\prime} \mathrm{N}\right)$ The equation the refore is likely to give inaccurate results with tendencies for the computed wind velocities being on the higher side. In the absence of any other method for finding the wind velocity, the Geostropic wind equation inspite of its limi tations was used.

* Nunerals in parenthesis refer to corresponding items in the list of references given at the end. 
2.1(b) FETCH:- The generating areas were demarcated on the synoptic charts according to procedures laid down in T.R-4(6). For most cases in the monsoon periods the isobars are straight and practically run Hast-West and the generating areas can be marked with a fair degree of accuracy The fetch length, wadth, decay distances and angles required for the PNJ method were di rectly scaled from the synoptic charts.

\subsection{WAVE HINDCASTING: As mentioned earlier the SMB and the PNJ} methods were used for wave hindcasting. The procedure for the SMB method was as that given in $T \cdot R-4(6)$ while that for the PNJ method was that given in Pierson et al (3).

The wave heights, periods and the time of arrival at the required hindcasting point were determined according to the standard procedures.

\subsection{WAVE CHARACTERSTICS - RECORDEDs The observed wave heights were} obtained from the analysis of the wave records, recorded by the wave recorder.

The Wave Recorder is of the frequency nodulated pressure type. The Recorder is kept at a depth of 6.2 metres from the M.W.I. and the water depth at the place is 7.5 metres.

The wave heights as recorded need to be modified to account for the attenuation of wave pressure with depth. From theoretical Wave Hyd rodynamics (7) the surface wave height $H$ is related to $H$ ' obtained from a pressure recorder by the following equation.

$$
H=H^{\prime} \frac{\operatorname{Cosh} 2 \pi d / L}{\operatorname{Cosh} 2 \pi d / L(1-3 / d)}
$$




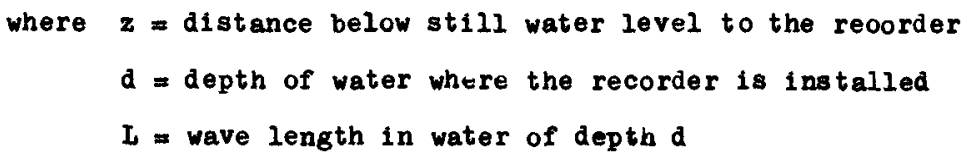

In the above attenuation factor the wave length $I$, must be known. $A$ close look at any wave record will show the wave heights and periods continuously changing. If the attenuation factor is to be applied for each individual wave helghts based on its period, with no computer facilities avallable, the work is very tedius and time consuming. As an approximation 1 t was decided to use the average period of the waves in the sample under consideration for computing the above at tenuation faotor.

To Investigate the probable error involved, some random samples of the wave records who selected, each of atleast 15 minutes duration. The significant wave height were calculated using attenuation factors based on the individual perlods of each wave in the 15 minute record as well as the average period. The results of this investigations is shown in Table 1.

This rand om survey as summarised in Table - 1, shows that the percentage error involved if the average period is used to be less than 6\% This is insignificant particularly in view of the findings that an additional factor together with the above attenuation factor may be required to get the correct surface wave heights from a subsurface recorder. As such the attenuation factor is based on the average period only.

The attenuation factor as given by Equation - 1 has been recognised to be inaccurate to correlate the underwater wave pressure with the surface wave heights. Based on simultaneous surface and sub-surface observations many investigators have proposed an additional 


\section{TABLE - 1}

\begin{tabular}{|c|c|c|c|c|}
\hline \multirow{2}{*}{$\begin{array}{l}\text { Sample } \\
\text { No. }\end{array}$} & \multicolumn{3}{|c|}{ Significant wave heights } & \multirow{2}{*}{$\begin{array}{l}\text { Percentage } \\
\text { error based on } \\
\text { individual } \\
\text { period } \\
\text { attenuation } \\
\text { factor }\end{array}$} \\
\hline & $\begin{array}{l}\text { Wi thout } \\
\text { attenuation } \\
\text { factor } \\
\text { Inches }\end{array}$ & $\begin{array}{l}\text { With } \\
\text { attenuation } \\
\text { factor based on } \\
\text { average period } \\
\text { inches }\end{array}$ & $\begin{array}{l}\text { With attenua- } \\
\text { tion factor } \\
\text { based on } \\
\text { individual } \\
\text { factor } \\
\text { inches }\end{array}$ & \\
\hline 1 & 24.40 & 29.00 & 30.00 & -3.3 \\
\hline 2 & 28.40 & 34.00 & 36.00 & -5.5 \\
\hline 3 & 2700 & 32.20 & 33.70 & -4.45 \\
\hline 4 & 43.60 & 53.00 & 53.20 & -0.38 \\
\hline 5 & 47.20 & 57.50 & 58.00 & -0.86 \\
\hline 6 & 44.76 & 55.60 & 59.00 & -5.75 \\
\hline 7 & 68.90 & 85.50 & 87.00 & -1.73 \\
\hline 8 & 64.00 & 78.50 & 81.20 & -3.20 \\
\hline 9 & 58.60 & 69.20 & 69.80 & -0.86 \\
\hline 10 & 50.89 & 63.50 & 63.10 & +0.63 \\
\hline 11 & 21.26 & 26.70 & 25.40 & +5.1 \\
\hline 12 & 18.60 & 22.70 & 23.80 & -4.60 \\
\hline 13 & 38.50 & 52.30 & 53.30 & -1.87 \\
\hline 14 & 2660 & 3480 & 37.00 & -5.80 \\
\hline 15 & 26.90 & 35.60 & 37.50 & -5.00 \\
\hline
\end{tabular}

factor to be used. As summarised by Masashi Homma etal (2) this factor designated $\mathrm{n}$ is normally taken as a constant for the analysis This modifies equation - 1 to equation $-1(a)$ as follows

$$
H=n H^{\prime} \frac{\operatorname{Cosh} 2 \pi d / L}{\operatorname{Cosh} 2 \pi d / L(1-z / d)} \quad \ldots \quad \ldots 1(a)
$$


Seiwell (2) recommends a value of 1.35 for $n$, in Japan (2) a value of 1.3 to 1.5 is used, while laboratories Natzonal DiHydraulique, France (2) recommends a value of 1.25 , on the other hand Draper and Glukhorskiy (2) have presented formulas giving $n$ as a function of relative water depth $d / L$. Cicslak and Kowalski (1) recommend a ralue of 1.25

In the absence of any definite criterza to $f i x$ this value of $n$, $n=1.25$ is used in the analysis

3. REDUCTION OF DEEP WATER WAVES TO SHALLOW WATMRS The waves as predicted by the Forecasting methods are deep water waves. Since the Wave Recorder is in shallow waters, the predicted deep water waves have to be reduced to shallow water waves to allow for refraction and shoaling. With the crests travelling parallel to coasts, the bottom contours also being practically parallel to the coast and the coast being a very flat one, the refraction ana shoaling coefficient are very nearly unity so that the waves as predicted are directly compared with the recorded waves.

4. RESULIS OF IHE ANALYSIS: Fig. 1 gives the significant wave heights as predicted by the SMB and the PNJ methods and the recorded significant wave heights over the active monsoon months of June and July. It is observed that the predicted wave heights are consistently smaller than the recorad heights for smaller wind velocities (upto 15 Knots). For wind velocities greater than 15 knots there is a strong tendency for the predicted wave heights to be more than the recorded heights. Between the two methods, the SMB method appears to be closer to the recorded wave heights than the INJ method. 
Fig. 2 and Fig.3 show the comparison between the predicted wave heights and the recorded wave heights for the two methods. No doubt there is scatter since we are dealing with data which are subject to errors but the reasonable agreement cannot escape the attention.

The other results of the study reported elsewhere (4) can be summarised as;

(1) the generating areas for the waves that reach the Mangalore area on the West Coast of Ind 20,11 e between $10^{\circ}$ and $18^{\circ}$ latitude North and $64^{\circ}$ and $74^{\circ} \mathrm{E}$ longitudes

(2) the fetch lengths in the monsoon period 11 es between 200 to $600 \mathrm{NH}$

(3) the maximum calculated wind velocity is about 40 Kno ts while generally it is around 25 knots

(4) the general direction of wave travel is between $\mathrm{S} 35^{\circ} \mathrm{W}$ and $N 65^{\circ} \mathrm{W}$ since the range of wind direction is found to be between these limits. The predominant di rection is from west.

5. CONCLUSIONS-- The planning, design and construction of any Coastal Engineering structure requires knowledge of wave characteristics. At present on the Indian Coast in most places the required design wave height is obtained from Wave Forecasting.

In this study undertaken at the Coastal ingineering Section of the Karnataka Keglonal Engineering College, Surathkal, India the primary aim was to investigate the applicablilty of the general wave Forecasting methods like the PHJ and the SMB methods to Indian Coasts.

The present analysis indicates that the predicted wave heights compare well with the recorded wave heights and between the two 
methods used, the SMB method predicts wave heights which are nearer to recorded wave helghts.

6 ACKNOWLEDGEMENTS Wuch of the work reported herein was undertaken by the Junior author (PSR)(4) in partial fulfilment of the requi rements for the degree of Master of Technology (Marine Structures) of the University of Mysore, Mysore.

Acknowledgement is gratefully made to Prof. Ev. Nielsen formerly UNLSCO Professor in Coastal kngineering in the College, who initiated Coastal Engineering studies in the College and was in overall charge of the project. The authors thank the authorities of the Mangalore Harbour Project for kund permission to use the wave records. The paper is published with the pernission of the College authoritzes.

\section{References}

1. Cicslak A and Kowalski T., "Wave pressure attenuation" XXII International Navigation Congress Section II, Subject 5, Paris 1969.

2. Masashi Himma, Riyoshi Horikawa and Shuzo Komori, "Response Characterstics of underwater wave gauge", Coastal Engineering in Japan Votg, 1966

3. Pierson W.J., Neumann G. and James R.W., "Practical methods for observing and Forecasting ocean waves", U.S. Navy Hydrographic office, Pub.No.603, 1955.

4. Renukaradhya P.S., "Wave Forecasting and Hindcasting for the West Coast of India", thesis submitted to the University of Mysore in partial fulfilment of the requi rements for the Master of Technology in Marine Structures.

5. Taylor G.F., "Elementary Meteorology", Prentice Hall, Inc., Eaglewood chiffs, N.J. 1954. 
6. U.S. Army Coastal Engineering Research Center, "Shore Protection, Planning and Design", Technical Keport No.4, 1966.

7. Wiegel R. L., "Oceanographical Engineering", Prentice-Hall, Inc., Eaglewood cliffs, N.J., 1964. 


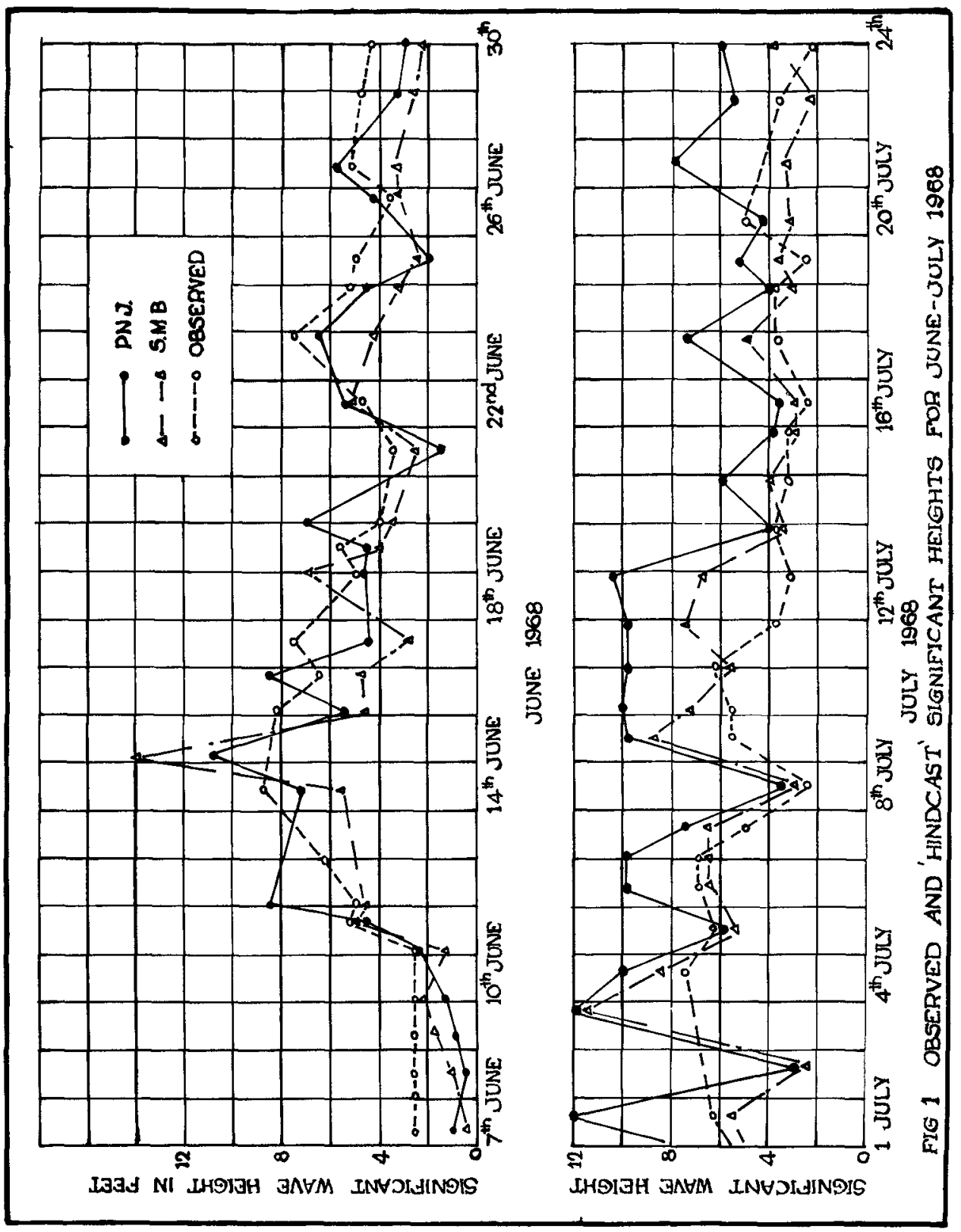




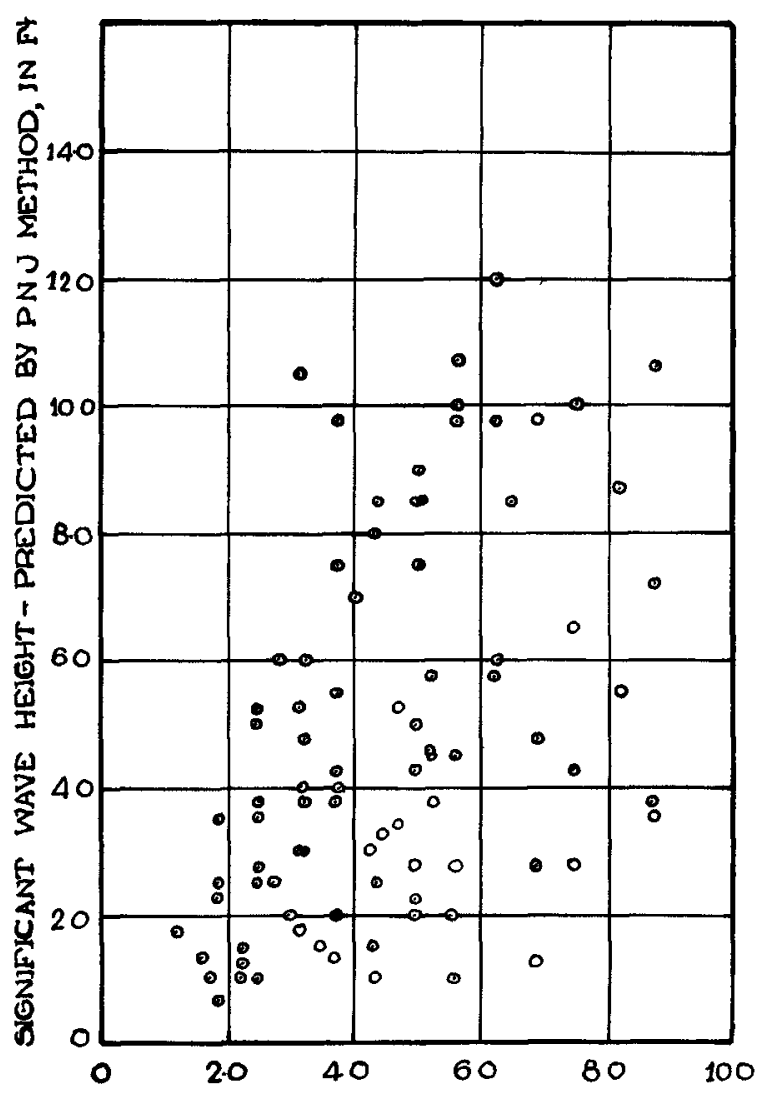

SIGNIFICANT WAVE HEIGHT - RECORDED, IN FH

FIG 2 SIGNIFICANT WAVE HEIGHTS PAEDICTED [DN J METHOD] VERSUS RECORDED 


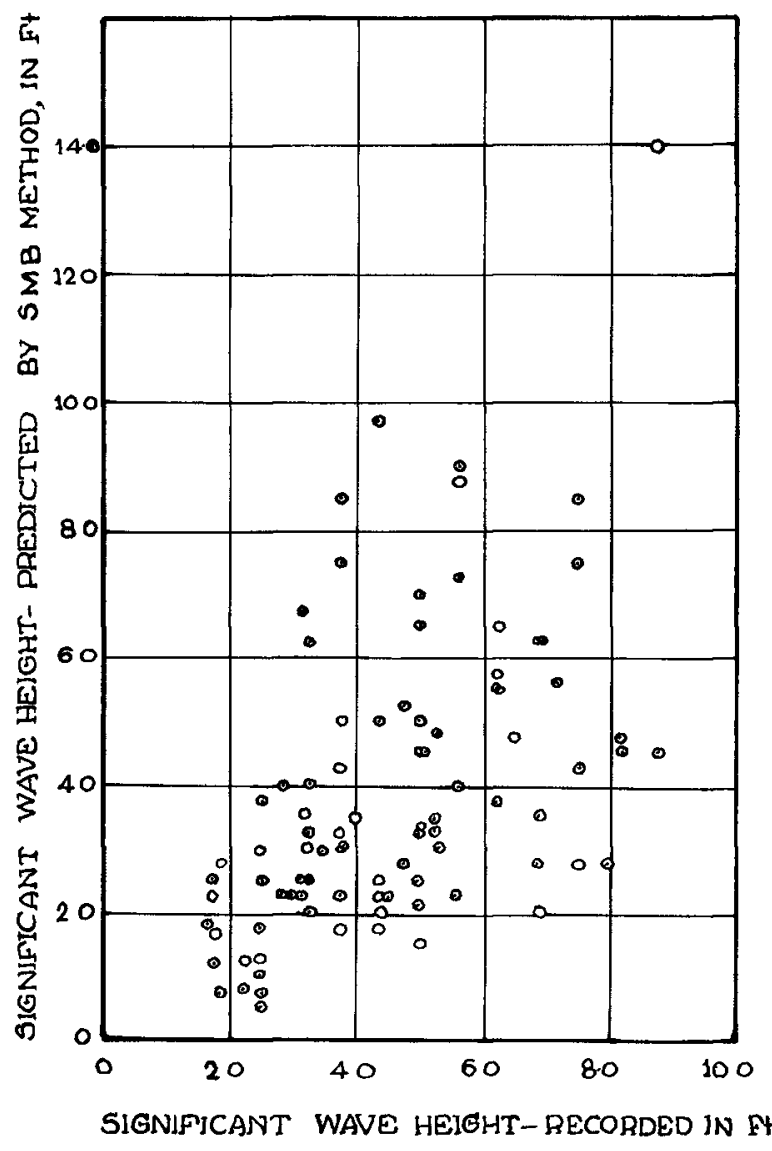

FIG 3 SIGNIFICANT WAVE HEIGHTS PREDICTED [SM B METHOD] VERSUS RECORDED 



\section{CHAPTER 13}

COMPILATION OF OCEAN AND

LAKE WAVE STATISTICS

John Simpson Hale

Head, Coastal Engineerıng Group

Los Angeles County

Los Angeles, California

RESUME AND COMMENTS

The wave program described in this paper is one intended to provide wave statistics by having our Los Angeles County IIfe guards observe the wave heights, directions and periods.

Mark-sense cards and high speed datd processing equipment and computers are used to handle the volumes of statistics.

While some scientists are planning very accurate, costly solutions to the problems of wave statistics, agencies are reluctant to venture into an expensive, elaborate wave survelliance program requiring a large quantity of personnel.

I feel that neither the accuracy of present duy design or construction methods warrant "split hair" accurucy in wave survelllance.

The method described in this paper will provide a practical solution if the statistics are handled as follow

1. Wave statistics are gathered continuously over many years of time.

2. A standard is set to check the observed values.

3. Large rare damaging storm conditions will be developed by any reasonable means avillable until enough years of observation have passed to provide adequate statistics. 
TEXT

With good wave statistics, we can provide a design that will prevent the insipient faliure that occurs, occasionally, with various coastal structures such as coastal homes, hotels, jetties, groin systems with sand fills, breakwaters, bulkhead walls, etc.

This wave compllation program coupled with surveys showing shoreline movement is all that is needed to design coastal structures for todays coast line profiles as well as for predicted coast Ine profiles that might exist 20 years from now.

This paper will be confined to the problems of wave statistics.

Wave statistics, used in my work, must include wave heights, periods and directions and be complled in a way that gives the results of dally observation, individual storm energies, resulting azımuths, resulting energies, root mean square wave heights and average periods.

Our wave observation program is planned to be a continuous program for years to come and will include observations in areas of special interest along miles of Los Angeles County Coast Line.

Hindcast wave statistics have been complied on the deep water side of the island maze that surrounds our county, but the effects of island sheltering and wave refraction have to be calculated to make these wave statistics usable as design waves along the shores of the continent. This is a laborious method of compling design wave values even with the use of present day computers. The method doesn't have the accuracy or the usefulness that the wave complation system discussed in this abstract will Illustrate.

After investigating deep water buoy type wave gauges, shallow water pressure gauges, and many other systems, we decided to tabulate wave statistics by visual observations from chosen points along our shoreline.

The chosen points were shoreline areas of future design, areas where man-power is aval lable and areas involving unusual shoreline culture and contours. There were 
points where much of the tabulated data could be converted, by applying wave refraction calculations, to deep water waves. The deep water waves could then be used to tabulate wave statistics in coast line areas, where wave observations were not recorded. Thls was to endble us to provide wave statistics for larger codst line areas.

A gredt volume of work is eliminated in data retrieval and tabulation by the use of mark sense cards and the I. B. M. 1620 and 360 systems. Our process w11l supply the wave statistics needed by the County of Los Angeles at littie additional cost and may be tric lype of program that many coastal engineering groups can implement.

The procedure enables us to use County lifegunrd personnel without interruption of their dally duties. The lifeguards will mark the wave obscrvations on mark-sense cards which are edsily edited and processed with our I. B. M. 1620 computer. Neither personnel time to edit, nor the cost of machine time is a large quantity. The direction of the waves is observed with a vane compass, the periods are timed with the second hand on a wrist watch or stop watch and the average wave heights will be obtalned by conparing wave heights to swimmers, rocks, surfboard riders, etc. Observations are made twice a day with edch observation being over a period of five minutes or more The wave conditions are averaged during each observation and the resulting values marked on the specially prepared marksense cards.

This statistical sample is quite small but is often enlarged considerably by the fact that the lifeguards observe the waves for a number of minutes prior to the above five minute period.

While the most accurate immediate use of these statistics wlll be for beach improvement projects, observations over many years will provide enough statistics to allow us to make accurate calculdtions for rock construction and other types of coastal structures.

The mid range wave conditions, in our County, are those most lmportant in shaping the bedches because of the large energy provided as the results of their frequency of occurrence.

Just one year's observation will result in average statistical waves close to the correct values for the entire family of wind formed waves.

Years of tabulating the above statistics would enable us to do the following

1. In the design of a barrier groin sytem, we can determine from the tabulated resultant wave direction 
the plan view alignment of the shoreline and the realignment with changing storm waves.

2. By defraction calculatıons, we can determine the wave heights inside a Harbor.

3. He can determine the helght of grouns from wave statistics. Uprush resulting from the comriled statistics can be added to the tidal averages to determine how high a groin has to be to provide for sand over topping.

5. Knowing the beach profile movement, we can use the wave statistics to determine the helght of bulldings on the shoreline, the type of foundations to be used, the helght of the bulkhead walls and revetment structures.

6. From the wave statistics we can determine the size rock for revetments, breakwaters and jetties and the dynamic and static forces that constal structures must resist. The designs of these and many other types of coastal structures are dependent on the Coastal Englneer's having these basic wave statistics.

After recording the name of the beach, the position on the beach and the date, the lifeguards record the average breaking wave heights, directions and periods. These tabulations are made twice everyday during the year.

The following list shows symbols and a resume of the basic formulas used by our I. B. M. computers to complle these wave statistics.

\section{List of Symbols}

$H_{b}=$ Breaking height of wave observed by ilfeguards

$\mathrm{d}^{\mathrm{b}}=$ Breaking depth of wave obs 2 rved by lifeguards

$n$ = Value used in computation of shoaling co-efficient

$\mathbf{R}=p 1=3.1416$

L = Shallow water length of wave

$L_{0}=$ Deep water length of wave

$H_{0}=$ Helght of deep water wave

$T=$ Feriod of wave observed by infeguards

$c$ = Inshore velocity of wave

$C_{0}=$ Deep water wave velocity

$\mathrm{g}=$ Gravity

En = Energy in milions of foot lbs/foot of beach

$N=$ Number of observations

$W$ = Weight of water 
Computations

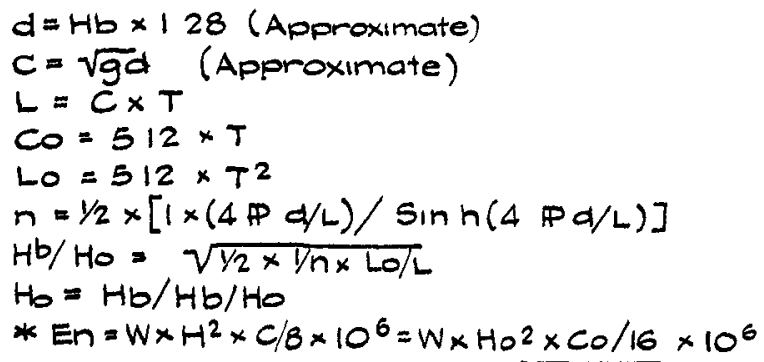

Root Mean Square Wave Helght $=\sqrt{\mathrm{LHb}^{2 / \mathrm{N}}}$

Arlthmatic Average For $T=\leqslant E n \times 16 /\left(W \times \leqslant H^{2} \times 512\right)$

* Greater accuracy was obtalned by converting $\mathrm{Hb}$ to Ho and calculating En by the use of the following

$E_{n}=W \times \mathrm{Ho}^{2} \times \mathrm{Co} / 16 \times 106$

All energy values are in millions of foot pounds.

Most of these listed formulas are commonly used in the Coastal Englneering field, but the solutions used for resulting azlmuths, energles and average perlods are special innovations Resulting energy is calculated by making vectors from each observation The vectors are formed by wave direction and calculated energy the machine mathematically joins the vector ends and calculates the resultant direction and energy The formula for the average period calculation is shown in the preceeding tabulations

The program tabulates each dally observation and will calculate the resulting azlmuths, energy, root mean square wave height, and average period for any time duration the computer operator desires

once the data has been edited and sorted for the I B M 1620, it takes only 7 minutes to tabulate an entire years observations for one point on the county coast line

This type of tabulation will very quickly give the design engineer wave statistics that he can convert to any type of design use Accuracy is well within the limits needed The data processing program for the I B M 1620 and 360 systems is avarlable in the office of the Los Angeles County Englneer, where it is filed as "Library Program No TG016" 


\section{BIBLIUG RAPHY}

1. SHORE PRUTECTION, PLAN ING AND DESIGN (Technical Report No.4--third edition, 1966) published by The Department of The Army, Corps of Engineers, Codstal Engineering Research Center.

2. COMPILATIUN OF OCEAN AND LAKE WAVE STATISTICS by John $S$. Hale. I B. M. 1620 Data Processing System, Library Program No. TGul6 in the Department of County Engineer office, Los Angles County 1969.

3. HANDBO $\mathrm{K}$ OF HYDRAULICS, fifth edition, by Horace K1ng, 1963 .

* 4. wave statistics fur stVen deEF water stations along THE CALIFORNIA COAST prepared by the National harine Consultants: 1960 .

*5 A STATISTIC LL SURVEY OF OCEAN WAVF Cr SOUTHIRN CALIFORNIA WATERS prepared by the Marine Advisors, 1.961

*6. A STATISTICAL STUDY OF WAVE CONDITIONS AT FIVE O EN SEA LOCALITIES ALONG THE CALIFORNIA COAST prepared by The University of California, Scripps Institution of Oceanography, 1947 .

7. BEACHES AND CUÁSTS by Cuchala1ne A. M. K1ng, 1960

8. TH MOVEMENT OF BE $\mathrm{TCF}$ SAND by James C. Ingle, 1966.

*Thanks to the Department of The Arny, Los Angeles District, Corps of Engineers for Loan of This Material. 


\title{
CHAPTER 14
}

\author{
MACRO-TURBULENCE FROM WIND WAVES
}

Chın-Yuan Lee ${ }^{1}$ and Frank D Masch $^{2}$

\author{
ABSTRAC'T
}

Laboratory studies in a wind wave flume were carried out to investigate the macro-scale turbulence associated with wind waves and white cap conditions Velocity fluctuations in water were measured with a hot film anemometer and parametric correlations between wind waves and turbulence characteristics were established Measured data were recorded in analog form, digitized and stored on magnetic tape Auto-covariance functions and power spectral density functions were then obtained for all sample records

Results showed that the depth of the penetration of the macro-scale turbulence increased rapidly with wind speed but the rate of penetration diminished at the higher wind speeds This rate of macro-turbulence penetration was found to vary inversely with wave height and wave steepness Most turbulent fluctuations having frequencies equal to or higher than the frequency of the ambient surface waves were confined to the zone of macro-turbulence penetration although some disturbances such as vortex rings and other turbulence associated with white cap wave conditions occasionally penetrated to greater depths It was found that the energy dissipation increased with wave height and that almost all wave energy dissipation was concentrated near the water surface

\section{INT RODUCTION}

Turbulence has been analyzed and described variously in terms of instantaneous velocity fluctuations, correlation functions, power spectral density functions eddy viscosity mixing length and turbulent shear stress Yet, much of the experimental work on fluid turbulence has been on the relatively special cases of steady flow or plane couette flow Most of this experimentation has been largely conducted in wind tunnels, pipes and conduits Very little experimentation has been on the "basics" of the phenomenon of macro-turbulence created by wind induced waves

The objectives of this investigation are (1) To obtain some insight into the phenomenon of macro-turbulence in large natural bodies of water due to wind induced waves and (2) To establish empirical parametric correlations between the major interrelated elements of the macro-turbulence phenomenon Underlying this investigation is the requirement to seek feasible practical solutions to the subsidiary objectives of pollution control based on the efficient use of the natural mixing and dispersion processes in natural bodies of water

1 Assistant Professor of Civil Engineering Tri-State College, Angola, Indiana

2 Special Consultant for Fiscalın, Zurich, Switzerland and Austin (on leave from The University of Texas at Austin) 
Energy from wind is imparted to water through shear stress at the interface, the "sheltering effect" on the lee side of individual waves, or the action of turbulent pressure fluctuations of the arr stream Some of the energy is absorbed in the form of a thin surface layer of flow called wind drift Some energy is dissipated through viscosity in orbital motions of water particles Additional dissipation occurs when surface drift interacts with orbital motions and wave breaking As the wind grows stronger and the waves approach fully developed conditions, energy dissupation continues to increase until an equilibrum state is reached with respect to energy input from the wind It appears that the increased energy dissipation results mostly from intensified interaction between the wind, wind drift, wave breaking, and orbital motion of water particles Since these are maxnly surface phenomena, it can be inferred that the macro-scale turbulence induced by wind waves is highly anisotropic Thus, auto-correlation and power spectrum functions appear to be the most viable means of analysis Furthermore since the diffusive action of turbulence is believed to be determined mainly by the larger eddies, primary emphasis is placed on the lower frequency portion of the power spectrum

Systematic measurements in a wind wave flume gave sample records of surface waves and velocity fluctuations for various combinations of water depths and wind speeds Assuming random Gaussian processes, each sample record was regarded as representative of the entire ensemble Comparisons of auto-correlation functions of surface waves and those of velocity fluctuations permitted evaluation of an arbitrarily defined depth of penetration of macro-turbulence Relative intensity of turbulence at different depths from the surface can also be inferred from power spectrum functions This information permits a more realistic assessment of the effects of wind and waves on the mixing and dispersion processes in natural bodies of water

\section{EXPERIMENTAL APPARATUS AND TEST PROCEDURES}

\section{Experimental Apparatus}

All experiments were carried out in a $70 \mathrm{ft}$ wind wave flume at The University of Texas at Austin This flume is $11 \mathrm{ft}$ wide $2-1 / 2 \mathrm{ft}$ deep and is equipped with a 4500 cfm blower The blower is attached so that ax 1 is drawn in at the upwind end of the closed flume through a vaned intake and then discharged through the blower to the atmosphere at the downwind end of the flume The wind velocity was controlled by a vane installed at the throat section in the flume near the blower This arrangement had the advantages that wind speed inside the flume could be effectively reduced without causing large pressure drops in the flume, and by leaving the entrance unobstructed the streamlined guide vane produced relatively uniform inflow of the wind at all wind speeds Wind velocities up to 40 fps were obtainable in the flume and were measured wath a standard Prandtl-type pitot tube connected to a Uehling Type-B inclined draft gage Wave absorbers were installed at the end of the flume to help dampen out oscillations and reduce reflections

Continuous records of the heights and periods of the wind generated waves were measured with parallel wire wave gages inserted through the top cover of the flume The outputs from the wave gages were recorded on a two channel oscillograph equipped with carrier preamplifiers 
To measure velocity fluctuations in fluids, many approaches have been tried Macovsky [1] found that impurities in water prevented stable operation of hot-wire anemometers Beginning in 1949 studies were undertaken at M1T to develop an instrument for turbulence measurements in water which would be free from the troubles encountered by hot-wires This effort resulted in an impact tube-pressure cell combination, Ippen, et al [2] which measured the fluctuation in dynamic head at the tip of the impact tube by means of the change in electrical capacitance between a diaphragm and a fixed electrode However any device based on this principle has relatively low natural frequency, picks up static pressure fluctuations as well as the dynamic head associated with the velocity fluctuations and is suited best for measurements of low intensity turbulence Lumley [3] reported the use of a hot-thermistor probe for measuring turbulence The main disadvantage of this device is the difficulty in attaining high frequency response

The measurements involved in this study were made with the Heat Flux System Model 1010 constant temperature hot-film anemometer This instrument consists basically of a power supply, wheatstone bridge, feedback amplifier, and readout meter The bridge has two fixed resistance legs, a cylindrical sensor, and a three stage resistance decade The frequency response reported by the manufacturer ranges from $0 \mathrm{cps}$ to $1,000 \mathrm{cps}$

The cylindrical hot-film sensor consists of a pyrex glass rod coated with a thin platinum $f_{1} l m$ and sputtered with a thin layer of quartz The platinum and quartz coatings are each approximately $10^{-5}$ inches in thickness The quartz coating reduces bubble formation and electrical shunts across the sensor This helps to eliminate the requirement for pure water and greatly reduces the effect of contamination of the surface The sensor used in this study was 0002 inches in diameter and 0040 inches long

In order to obtain a good calibration, an effort was made to as sure the kinematical resemblance between the conditions of operation and calibration Since a fixed sensor inserted into a water body under wave action experiences a periodic flow that changes direction continually each cycle a special calibration unit was built This unit consisted of a half-horsepower electrical motor and a Vickers transmission and gear combination that supported an aluminum rod which held the sensor holder in a vertical position whle undergoing an "irrotational rotation" This resulted in a periodic circular motion of the sensor relative to the still water The amplitude of the circular motion could be adjusted by varying the position of the supporting points Prior to a series of measurements analog wave records were obtained and samples of wave heights were chosen at random intervals and plotted against the corresponding wave periods After about 30 waves had been obtained and plotted in this way a distance equal to the model wave amplitudes was taken as the distance from the axis of the wheel to the supporting points of the aluminum rod This corresponded to the amplitude of the circular motion for the sensor During calibration, the rotating speed of the wheel was first maintained constant long enough for the angular speed to be measured and for the output from the hot-film anemometer to be observed and recorded As some effect of the supporting rod vibration was present during calibration, the average value of the output was assumed to represent the true sensor velocity A second speed was then maintained and recorded as before Repetition of this process before each series of velocity fluctuation measurements enabled the determination of the mean velocity experienced by the sensor under various wave actions 
The sensor stand was placed 60 feet from the entrance section of the flume This location provided a long fetch yet remained some distance from the downwind end of the flume During actual operations two external sources contributed to the vibration of the sensor, and gave signals irrelevant to the velocity fluctuations The first was the vibration of the flume due to the blower and motor The second was the vibration of the support rod for the sensor unt caused by the drag of the wind and waves in the flume The following measures were taken to reduce the vibration of the sensor First, the sensor stand was bullt of steel channels solidly welded together and was used to support the sensor independently of the flume Secondly, the support rod was firmly attached to the sensor stand inserted through a hole in the top cover of the flume, and fastened with a very thin wire at the lower end near the sensor to dampen out vibrations Finally the throat section connecting the flume and the blower was cut and re-sealed by masking tape to practically eliminate the transmission of vabration from the blower to the flume

Water in the flume was kept clean by regular replacement Before any measurements were taken with the hot-film sensor, new water was allowed to stand in the flume at least 24 hours to reduce the arr content of the water and thus, the chance of bubble formation at the surface of the sensor which tended to create hot spots on the sensor surface and cause "erosion" of the quartz coating If the quartz coating is deteriorating the cold resistance of the sensor drifts and no meaningful measurements are possible Under such conditions the sensor soon fails completely

\section{Test Procedures}

All experiments associated with the present study were designed in such a way that the characteristics and relative intensities of wind wave turbulence at various distances below the still water surface could be compared with one another under statistically constant wave conditions ln order to attain this objective, the water depth in the wave flume was first set at a predetermined level, a constant wind speed was maintained and the sensor was inserted into the water to measure the velocity fluctuations at a fixed point below the actual water surface, and a sample record was measured The elevation of the sensor was then changed successively until sample records were obtained for a number of points unformly spaced over the depth Usually the spacing was taken to be 01 foot and the uppermost point was about 005 to 01 foot below the still water level, depending upon the wave conditions This constituted one series of sample records from which information could be derived and the lower limit of penetration of the macroscale turbulence associated with the wind waves estimated The wind speed was then varied and the experiment repeated Finally, the water depth was also varied to provide new wave conditions under different wind speeds

Theoretically, sample record should be as long in real time as possible but there a re several limitations to this requirement To avoid the appearance of a squeezed or even blurred trace and to minimize human error in the digitizing process widely spaced records are desirable These considerations favor a higher chart speed which means a shorter sample record in real time for a constant length of strip chart Since the dimensions of the digitizing machine (the Pencil Follower) limited the effective chart length to about three feet $(90 \mathrm{~cm})$ a chart speed of $20 \mathrm{~mm} / \mathrm{sec}$ was selected for all measurements This chart speed and resulting chart length represented an actual operation time of 45 seconds and covered from 90 to 150 waves When digitized, from 1800 to 3000 discrete points were obtained to describe the original sample record 
ANALYSIS OF RESULTS

\section{Basic Data}

Measurements of surface waves and velocity fluctuations we re made at water depths of 07,10 and $12 \mathrm{ft}$ respectively Three different wind speeds were applied successively at each water depth resulting in three series of measurements reflecting the variations of wate $r$ particle velocity fluctuations at different depths A total of 96 sample records were obtained Wind velocity profiles at the center of the flume and contour lines of wind velocity in a cross-section were also obtained Generally speaking, the wind velocity distributions in the wave flume we re relatively uniform and the maximum cross-sectional velocity ranged from 13 to $40 \mathrm{fps}$ with the corresponding Reynolds number varying from $244 \times 10^{4}$ to $751 \times 10^{4}$

\section{Mathematical Considerations}

It is desirable at this point to make a summary analysis of the variables and the relations involved in the application of the stochastic process model approach adopted for this study There are many useful expositions and interpretations of the mathematical rationale underlying such methods in standard texts on stochastic methods, noise theory and random process analysis $[4,5,6]$

Suppose a stationary random process $y(t)$ has been given in the form of a time history record The mean value $\mu$ is

$$
\mu=\lim _{\Gamma \rightarrow \infty} \frac{1}{T} \int_{0}^{T} y(t) d t
$$

where $T$ is the length of the record The mean square value 2 is

$$
\psi^{2}=\lim _{\mathrm{T} \rightarrow \infty} \frac{1}{\mathrm{~T}} \int_{0}^{\mathrm{T}} \mathrm{y} 2(\mathrm{t}) \mathrm{dt}
$$

and the positive square root of this value is called the root mean square or rms value The variance, $\sigma^{2}$, is

$$
\sigma^{2}=\lim _{T \rightarrow \infty} \int_{0}^{T} \quad[y(t)-\mu]^{2} d t
$$

The positive square root of the variance is called the standard deviation By expanding Eq (3), it can be shown that

$$
\mu^{2}=\sigma^{2}+\psi^{2}
$$


The auto-covariance function $C(\mathcal{I})$ is defined as

$$
C(T)=\lim _{T \rightarrow \infty} \frac{1}{T} \int_{-T / 2}^{T / 2} y(t) \quad y(t+\tau) d t
$$

when the mean value of $\mu$ of the process is assumed to be zero when $\mu \neq 0$

$$
C(\tau)=\lim _{T \rightarrow \infty} \frac{1}{T} \int_{-T / 2}^{T / 2}[y(t)-\mu][y(t+\tau)-\mu] d t
$$

In Eqs (5) and (6) $\mathcal{J}$ represents a time lag $C(\mathcal{J})$ is also frequently called the "autocorrelation function" although this term should be applied to the normalized ratio $\mathrm{C}(\mathfrak{I}) / \mathrm{C}(0)$

The power spectral density function $P(f)$ may be defined as

$$
P(f)=\int_{-\infty}^{\infty} C(\mathcal{J}) \quad e^{-12 \pi f \tau} d \tau
$$

where $f$ is the frequency of the fluctuation of the process at a certain point in the frequency domain and $1=\sqrt{-1}$ it can be seen that $P(f)$ and $C(\mathcal{T})$ are Fourier transforms of each other From the stationary hypothesis the auto-covariance function is an even function of $\mathcal{T}$, and from Eqs (5) and (6), it can be seen that $C(\mathcal{T})$ is a real function Thus,

$$
\begin{aligned}
C(\mathcal{T}) & =\int_{-\infty}^{\infty} P(f) e^{12 \pi f \mathcal{T}} d f \\
& =\int_{-\infty}^{\infty} P(f) \cos 2 \pi f \mathcal{T} d f+1 \quad P(f) \sin 2 \pi f \mathcal{T} d f \\
& =\int_{-\infty}^{\infty} P(f) \cos 2 \pi f \mathcal{T} f=\text { real function }
\end{aligned}
$$

By inverse transformation or a similar argument

$$
P(f)=\int_{-\infty}^{\infty} C(\tau) \cos 2 \pi f \tau d \tau
$$

Since $C(\mathcal{T})$ is an even function $P(f)$ should also be even The above relations for the real valued two-sided power spectral density function $P(f)$ may be simplified to

$$
P(f)=2 \int_{0}^{\infty} C(\mathcal{T}) \cos 2 \pi f \widetilde{J} \mathcal{J}
$$

The physically realizable one-sided power spectral density function $V(f)$ is defined by

$$
V(f)=2 P(f)=4 \int_{0}^{\infty} C(\widetilde{\xi}) \cos 2 \pi f \widetilde{S} \tau
$$


For practical applications, the record lengths are limited and the y values involved in the actual calculations a re uniformly distributed along the time axis Discrete forms of the above equations for numerical calculations, are given as follows

$$
\mu=\bar{y}=\frac{1}{N} \sum_{I=1}^{N} \quad y(1)
$$

where $N 18$ the total number of data points The auto-covariance $C_{M}{ }^{1 s}$ given by

$$
C_{M}=\frac{1}{N-M+1} \sum_{1=1}^{N} \sum^{-M}+1 \text { (I) } y(I+M-1)
$$

where $1=1,2,3 \quad \mathrm{~N}$ and $M=1,2,3 \quad \mathrm{Ml}$ In this study $\mathrm{Ml}$ is assumed to be $\mathrm{N} / 10$ The lag time $\mathcal{I}=(\mathrm{M}-1) \Delta \mathrm{t}$ for a data sampling interval $\Delta t$

The power spectral density function $V(f)$ is calculated from

$$
\begin{aligned}
& \mathrm{V}_{\mathrm{J}}=\text { one-sided power spectral density function } \\
& =2 \Delta t\left\{C_{1}+2 \sum_{K=2}^{M I-1} C_{k} \cos [(K-1)\right. \\
& \left.(J-1) \pi /(M I-1)]+C_{M I} \quad \cos [(J-1) \pi]\right\}
\end{aligned}
$$

where $J=1,2,3 \quad$ Ml This is an estimate of the power spectrum associated with the discrete covariance function defined by Eq (13) rather than the continuous function of $\mathrm{Eq}$ (16) For this discrete cosine transformation the spectral estimates at any frequency are affected by the energy in neighboring frequencies it is apparent, therefore, that a smoothing operation is desirable ln this study, the Hanning procedure, [7], was performed on the above raw spectrum $V_{J}$, to obtain the so-called refined spectrum $U$

$$
\begin{aligned}
& \mathrm{U}_{1}=05 \mathrm{v}_{1}+05 \mathrm{v}_{2} \\
& \mathrm{U}_{\mathrm{MI}}=05 \mathrm{v}_{\mathrm{Ml}}-1+05 \mathrm{v}_{\mathrm{MI}} \\
& \mathrm{U}_{1}=\mathrm{v}_{1}+05\left(\mathrm{v}_{1-1}+\mathrm{v}_{\mathrm{I}+\mathrm{l}}\right)
\end{aligned}
$$

where $1+2 \quad 3 \quad 4 \quad(M 1-1)$ Note that in order to facilitate computer programming, the notation is such that $U_{1}$ stands for $C(0) U_{z}$ for $C(\Delta t), U_{1}$ for $C[(1-1)$ t $]$, etc Eqs (12), (13) (14) and (15) form the basis for the numerical evaluation of the autocovariance and power spectral density function

\section{Digitizing and Computing Procedures}

To digitize the sample records each paper chart was placed on a $51^{\prime \prime} \times 54$ " table with a magnetic field below the surface The curve on the chart was traced with a special "penc1l" which responded to the magnetic field and identified its position in terms of $x-$ and $y$-values in arbitrary but predetermined units These digitized values as well as calıbration and identification data were stored on a magnetic tape through an incremental 
tape recorder By means of a high speed digital computer, the raw data were decoded and transformed into grad point data uniformly spaced on the time axis using a straightline fit Wave characteristzcs auto-covariance and power spectral functions were calculated and then tabulated and plotted on microfilm

\section{Interpretation of Results}

A random process may be classified as waded-banded if it consists of fluctuations of various frequencies narrow banded if the range of frequencies is limited The auto-covariance function of a wide-band random process diminishes rapidly as the lag time is increased for a nar row-band random process the diminishing rate is slow [I] Inspection of the auto-covariance plots for each test series (Fig 1 is illustrative of a typical test series) revealed that the surface wave fluctuations appeared as narrow-band random processes and velocity fluctuations appeared as wide-band random processes near the water surface and narrow-banded at greater depths Thus the spatial pattern of transition of the velocity records from one rype of fluctuation to the other gives an estimate of the depth of penetration of wind-wave induced macro-turbulence

For each power spectral plot of surface wave record (Fig 2 is typical) there is only one major peak which always centers around the frequency corresponding to the mean wave period which, for this study, varied from about 038 to 063 seconds For velocity fluctuations measured near the water surface, there is also a major peak centering a round the frequency of the mean surface wave, but the smaller peaks at higher frequencies are relatively significant However for velocity fluctuations measured at a considerable distance below the surface, the trend is again toward the dominance of a major peak centering around the frequency of the mean surface wave

The power spectral density function for random data describes the general frequency composition of the data in terms of the spectral density of ats mean square value Integration of the power spectral density function between two frequency values represents the amount contributed to the mean square value by the power spectrum within these limits Comparison of all the power spectra for a given series of experiments $(1$, for a given combination of water depth and wind speed) permitted the determination of the common lower and upper frequency $11 \mathrm{mits}$ for the first major peak at the mean surface wave frequency lntegration of the power spectrum between these two limits was designated by $A_{1}$ Beyond the upper lamit of $A_{1}$ the power spectrum decreased in magnitude rather rapidly until a point was reached beyond whxch 1 t began to flatten out The change in the slope of the power spectrum was generally somewhere around $8 \mathrm{cps}$ A common frequency value of this turning point for each series of experiments was also determined from the power spectrum lntegration of the power spectrum between the upper limit of $A_{1}$ and this point was designated as $A_{2}$ it was assumed that $A_{1}$ essentially represents the contribution from wave motion to the mean square value whyle $A_{2}$ represents the contributions from macro-scale turbulent motions Although turbulent motions of larger scale comparable to wave motions supposedly were compounded into $A_{1}$, the effect was assumed insignificant and the ratio of $A_{1}$ to $A_{2}$ was regarded as an indicator of the relative strengths between wave motion and wind wave induced tu rbulence In other words, a small $A_{1} / A_{2}$ ratio implies a high turbulent intensity due to wind waves 
Results

Relying mainly on the spatial variation of auto-covariance the depth of penetration of macro-turbulence was estimated for each combination of wind speed and water depth These data are plotted in Figs 3 through 6 Figure 3 shows that the depth of penetration increases rapidly with maximum wind speed in the flume when the wind speed is relatively low, but the rate of increase diminishes rapidly at higher wind speeds The maximum wind speed was taken from wind velocity profiles at 03 to 04 feet above the still water level In Fig 4, it is seen that when the wave height is small, the depth of penetration increases rapidly with wave height but as the wave height is increased, resistance to increase in depth of penetration becomes stronger In Fig 5 , the ratio, $\mathrm{H}_{1 / 10} / \mathrm{T}^{2} 1 / 10^{\text {, }}$ which is proportional to wave steepness using the one-tenth wave height and the corresponding wave period is plotted against depth of penetration At small steepnesses the penetration increases rapidly, but for the steeper waves, there appears to be a lower limit to the penetration Since the wave speed $c$ is proportional to the wave period $T$, $\mathrm{U}^{2} / \mathrm{T}_{1 / 10}$ is related to the wave age $\mathrm{c} / \mathrm{U}$, where $\mathrm{U}$ is the wind speed Using the same maximum wind speed as in Fig 3, Fig 6 indicates that the depth of penetration correlates quite well with wave age

Values of $\mathrm{A}_{1} / \mathrm{A}_{2}$ also were plotted against water depths Figure 7 is typical of these plots It is clear that the relative turbulent intensity is much higher near the water surface than below This tendency is much more evident with lower wind speed than with high wind speed On the other hand the overall relative intensity of turbulence is much higher for high wind speed (CS loV0l series) where the return flow is stronger than for low wand speed (e g CS 10V04)

For the surface waves, a small $A_{1} / A_{2}$ ratio means widening of the frequency band or a confused sea surface condition It was interesting to note that the ratio $A_{1} / A_{2}$ correlated quite well with the wave steepness in a way which suggests that the white cap wave condition and consequently the turbulence level in a wind wave system grow in accordance with the characteristic wave steepness

\section{SUMMARY AND CONCLUSIONS}

It is well known that larger eddies generate smaller eddies through inertial interaction thereby transferring energy to the smaller eddies which consume most of the kinetic energy of turbulence through viscous dissipation However Von Karman and Lin [7] assumed that the eddy diffusivity might be regarded as a parameter determining the character of the turbulence in the lower-wave number range In other words the diffusive action of turbulence is determined mainly by the larger eddies Hence, it can be expected that once the behavior of the macro-turbulence is understood, the relative intensity and spatial distribution of the smaller energy-dissipating eddies could be inferred This point is reinforced by the tendency for confinement of macro-turbulence to a surface layer a few wave heights in thickness and the existence of high relative turbulent intensity near the water surface This confirms the finding of Stewart and Grant [8] who based on a study of energy-dissipating eddies concluded that almost all wave dissipation is concentrated near the water surface and decreases rapidly with depth from the surface Thus rapid and effective mixing near the water surface can be expected in a body of water subject to wind waves 
While direct application of the results of this laboratory study to field situations has to be confirmed by further investigation, the results derived here disclose, qualitatively, the general tendencies under comparable field situations The main conclusions from this study may be summarized as follows (1) The depth of the macro-scale turbulence penetration due to the wind induced waves increases rapidiy with maximum wind speed but the rate of increase diminishes at higher wind speeds There 15 a limiting depth of macroturbulence penetration which is not exceeded even if the wind velocity is further ancreased (2) The rate of increase in the depth of penetration of macro-turbulence varies inversely with wave height, wave length and wave steepness and (3) Most turbulent fluctuations having frequencies higher than the mean frequency of ambient surface waves are confined to the zone of macro-turbulence penetration This suggests that wind wave induced turbulence is a phenomenon confined essentrally to the surface layer with thickness of a few wave heights However, certain vortex rings or some disturbances associated with white cap wave conditions occasionally penetrate to greater depths, but the elements of the vortex spirals deteriorate into an admixture of random, small scale eddies

\section{ACKNOWLEDGMENT}

Th1s study was supported in part by the Federal Water Quality Administration under Grant 5 RO1 WP00705

\section{REFERENCES}

[1] Macovsky, M S , "The Measurement of Turbulence in Water", Rep No 670, Dand Taylor Model Basin, Washington, D C, 1948

[2] 1ppen, A $T$, Tankin, $R \mathrm{~S}$, and Raichlen, $F$, "Turbulence Measurements in Free Surface Flow with an Impact Tube-Pressure Cell Combination", Tech Rep No 20, Hydro Lab, MIT, Cambridge, Mass 1955

[3] Lumley, J L , "The Constant Temperature Hot-Thermistor Anemometer", Symposium on Measurement in Unsteady Flow ASME, Hydraulics Divsion Conf Worcester, Mass 1962

[4] Bendat Julius S, and Piersoll, Allen, G, Measurement and Analysis of Random Data, John Wrley and Sons Inc 1966

[5] Lee, Y W, Statistical Theory of Communication, John Wiley and Sons, Inc, 1963

[6] Blackman $R$ B , and Tukey, $J$ The Measurement of Power Spectra from the Point of View of Communications Engineering, Dover Publications, inc, 1958

[7] Karman Th von, and Lin, C C , Advances in Applied Mechanics, 2, 1, 1951

[8] Stewart, $\mathrm{R} \mathrm{W}$, and Grant, H L , "Determination of the Rate of Dissipation of Turbulent Energy Near the Sea Surface in the Presence of Waves", Journa1 of Geophysical Research, Vol 67, No 8, July, 1962 


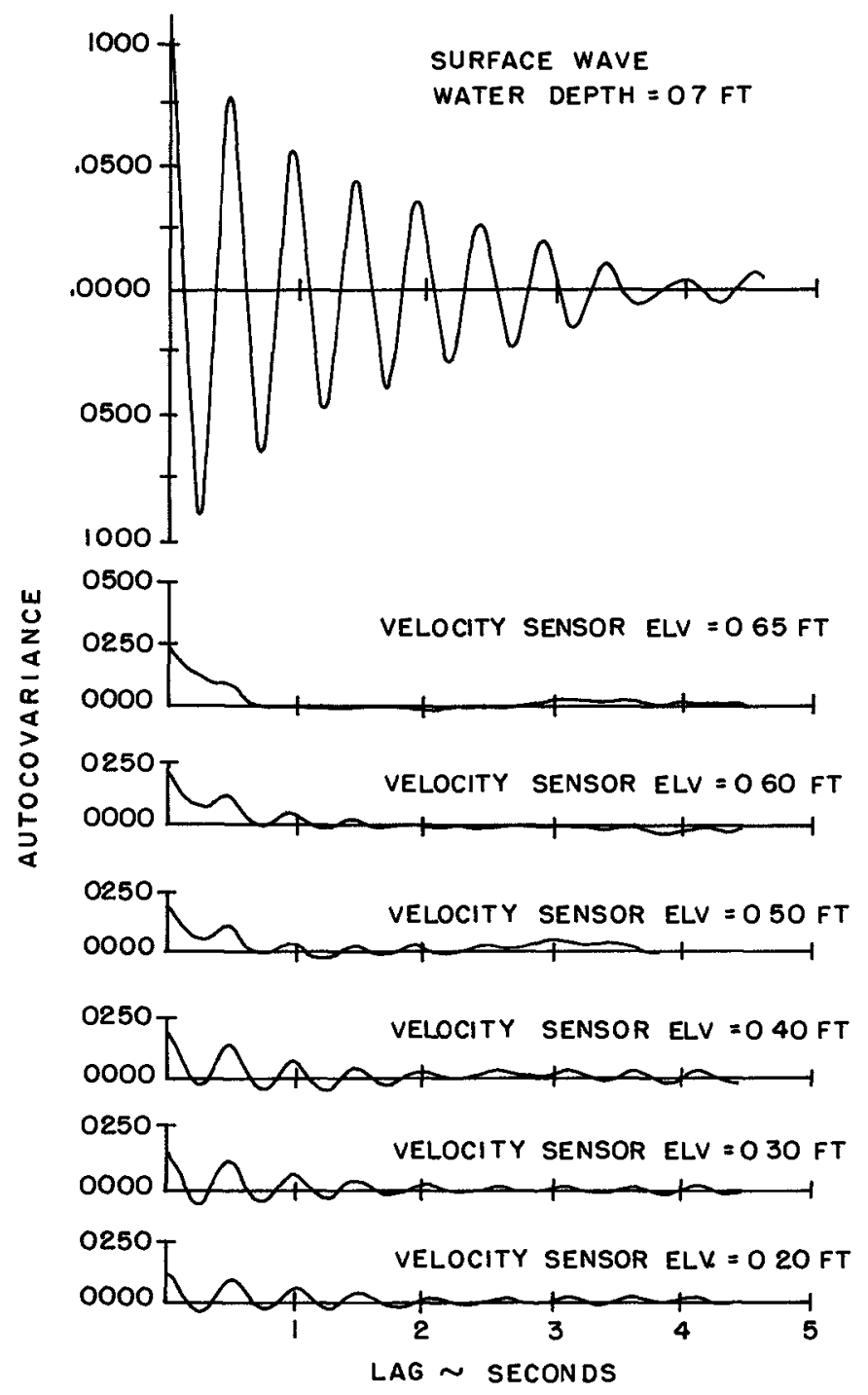

FIG I AUTOCOVARIANCE VS LAG TIME 
234

COASTAL ENGINEERING

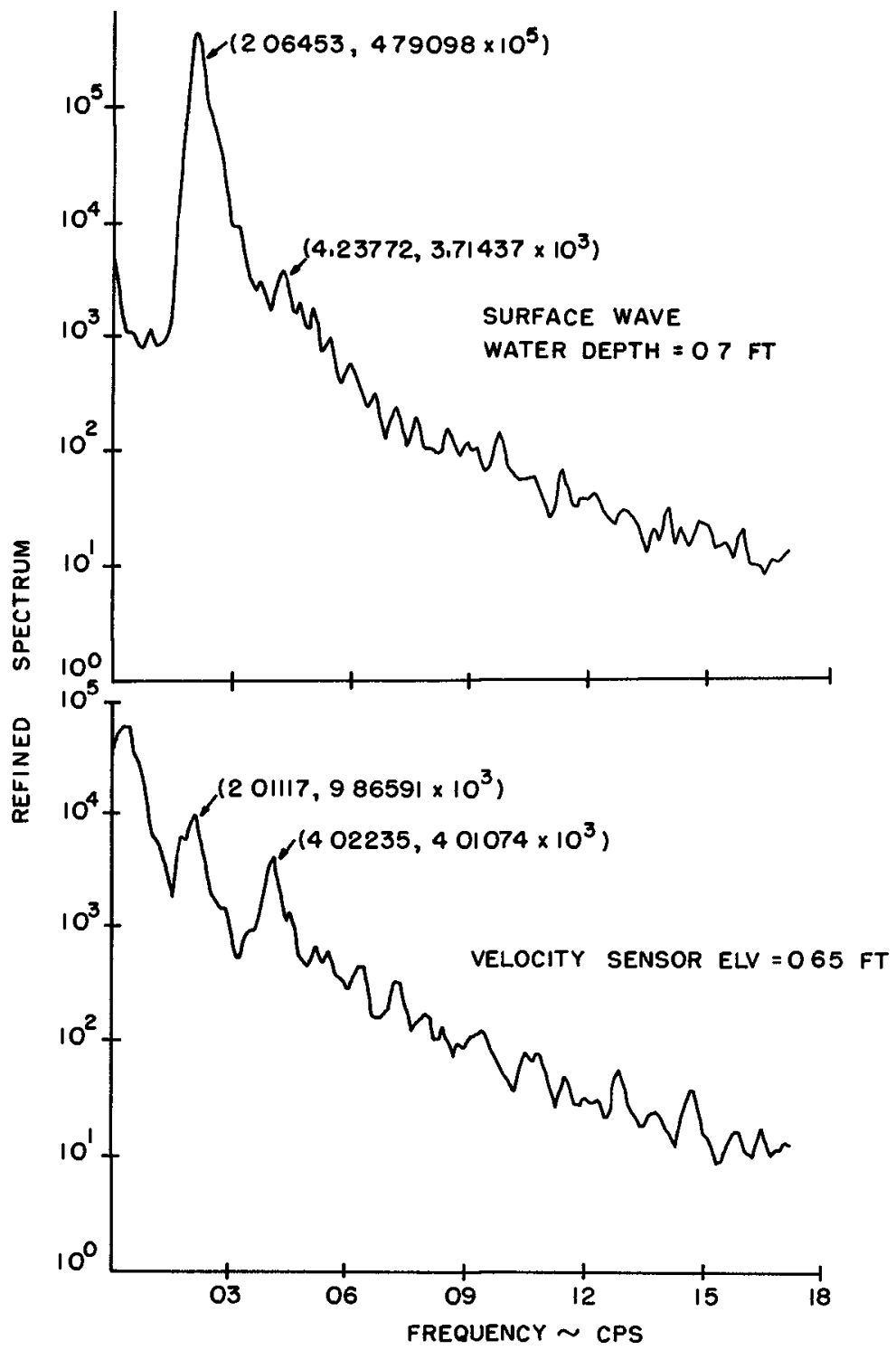

FIG RA POWER SPECTRAL DENSITY VS FREQUENCY 


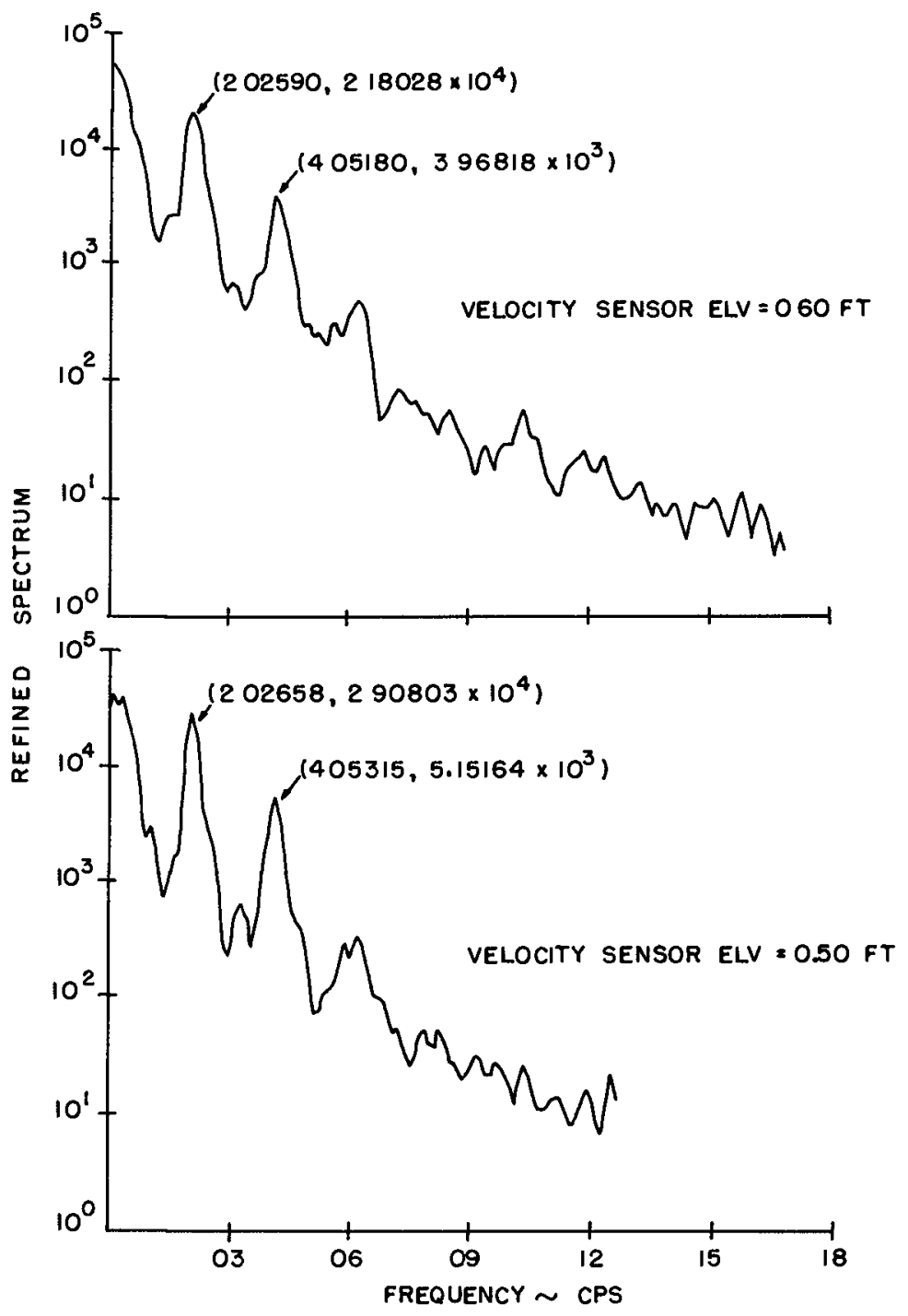

FIG 2B POWER SPECTRAL DENSITY VS FREQUENCY 


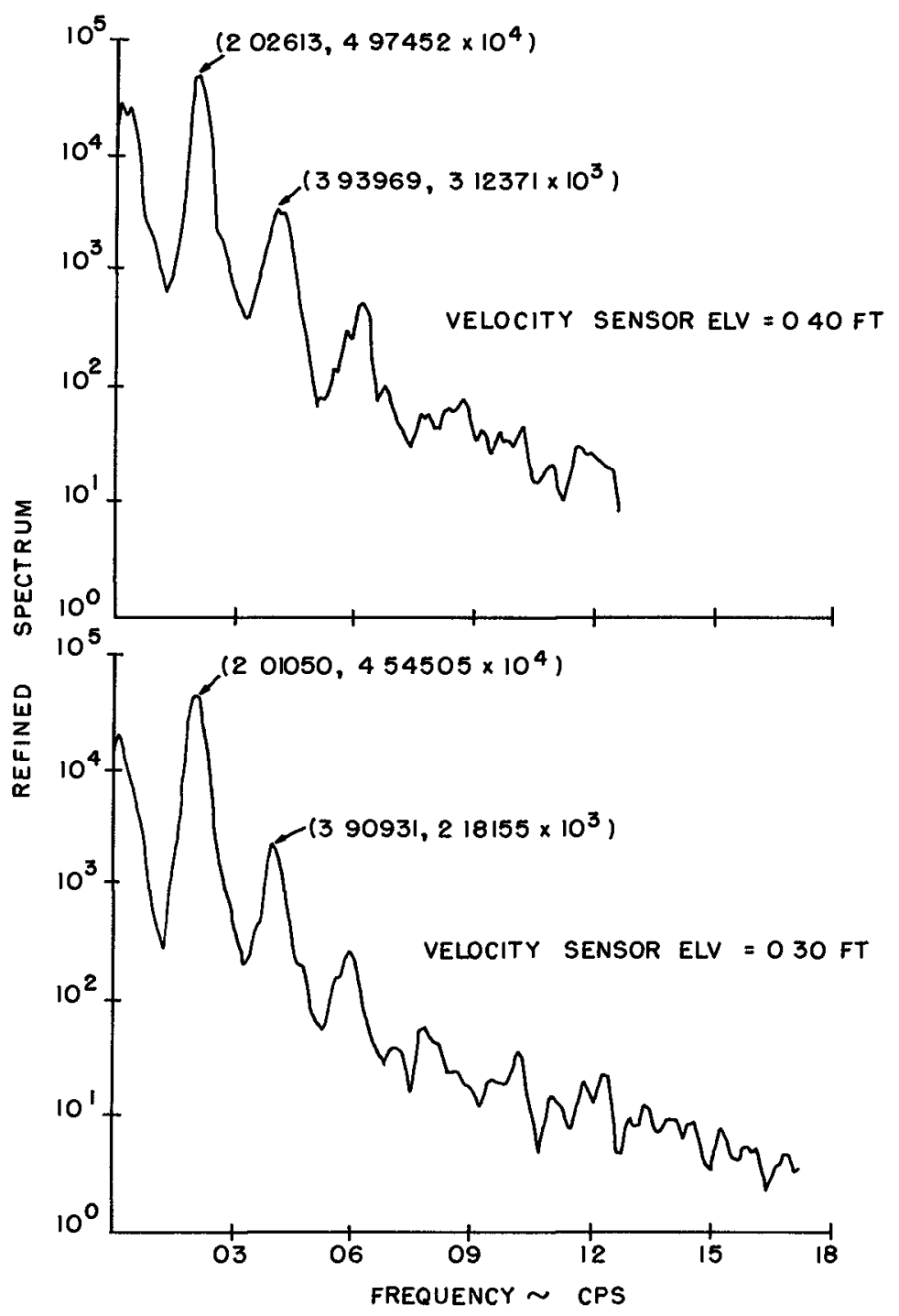

FIG $2 \mathrm{C}$ POWER SPECTRAL DENSITY VS FREQUENCY 


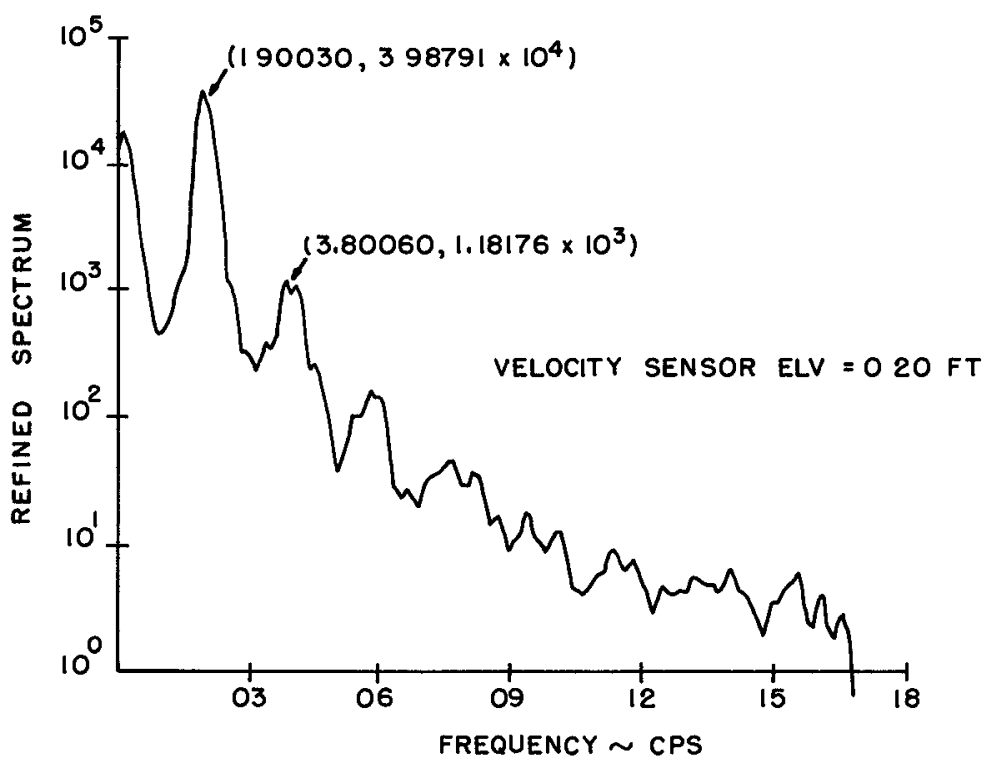

FIG 20 POWER SPECTRAL DENSITY VS FREQUENCY 


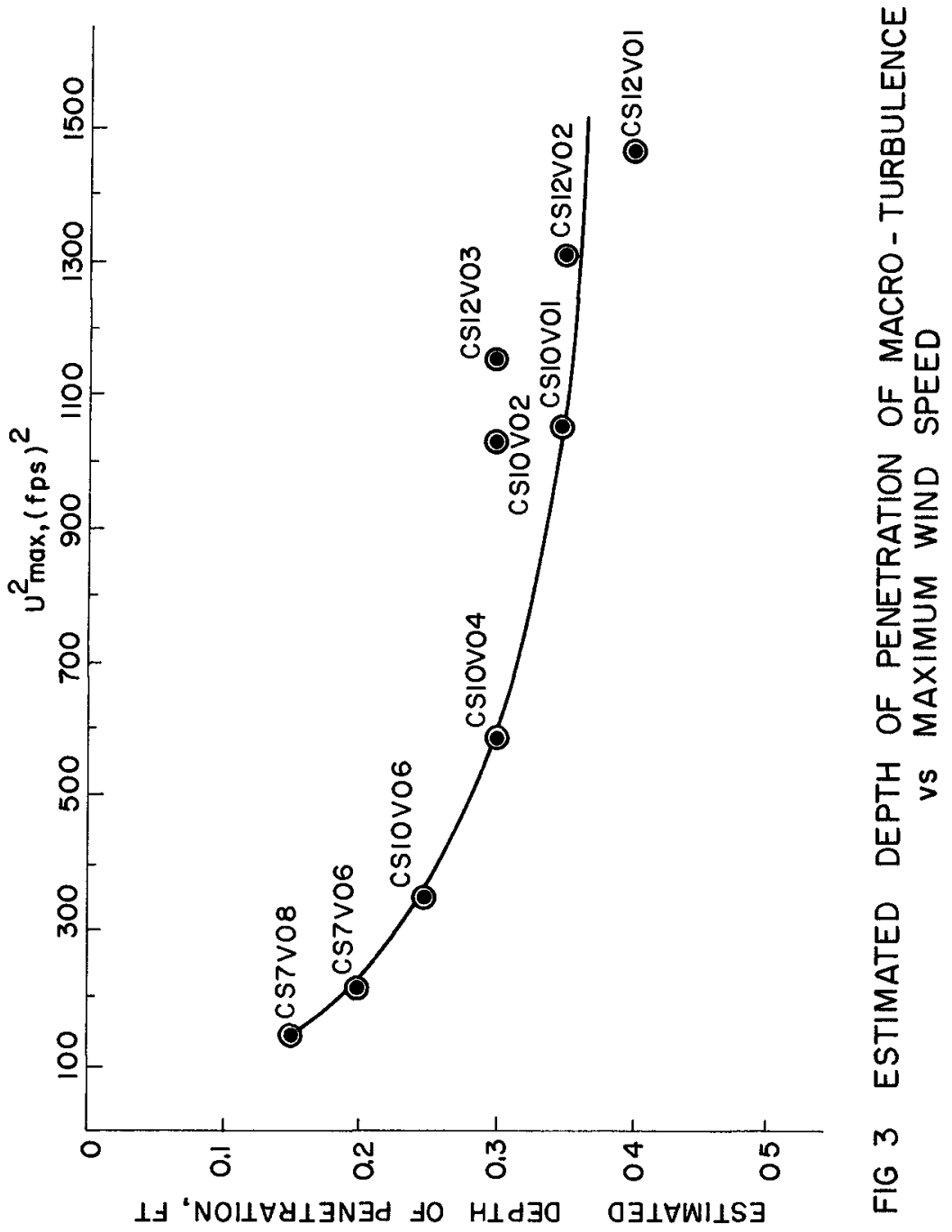




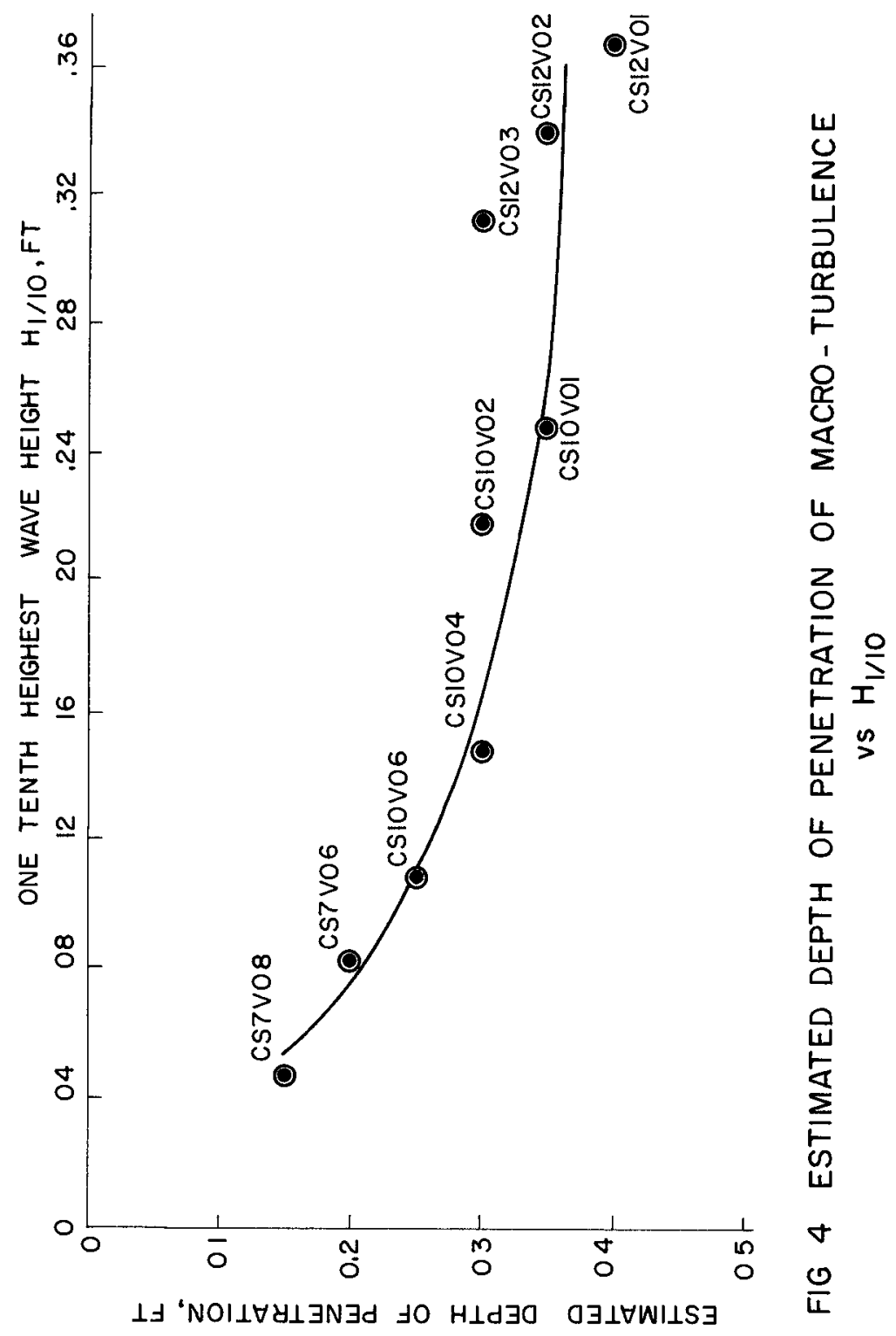




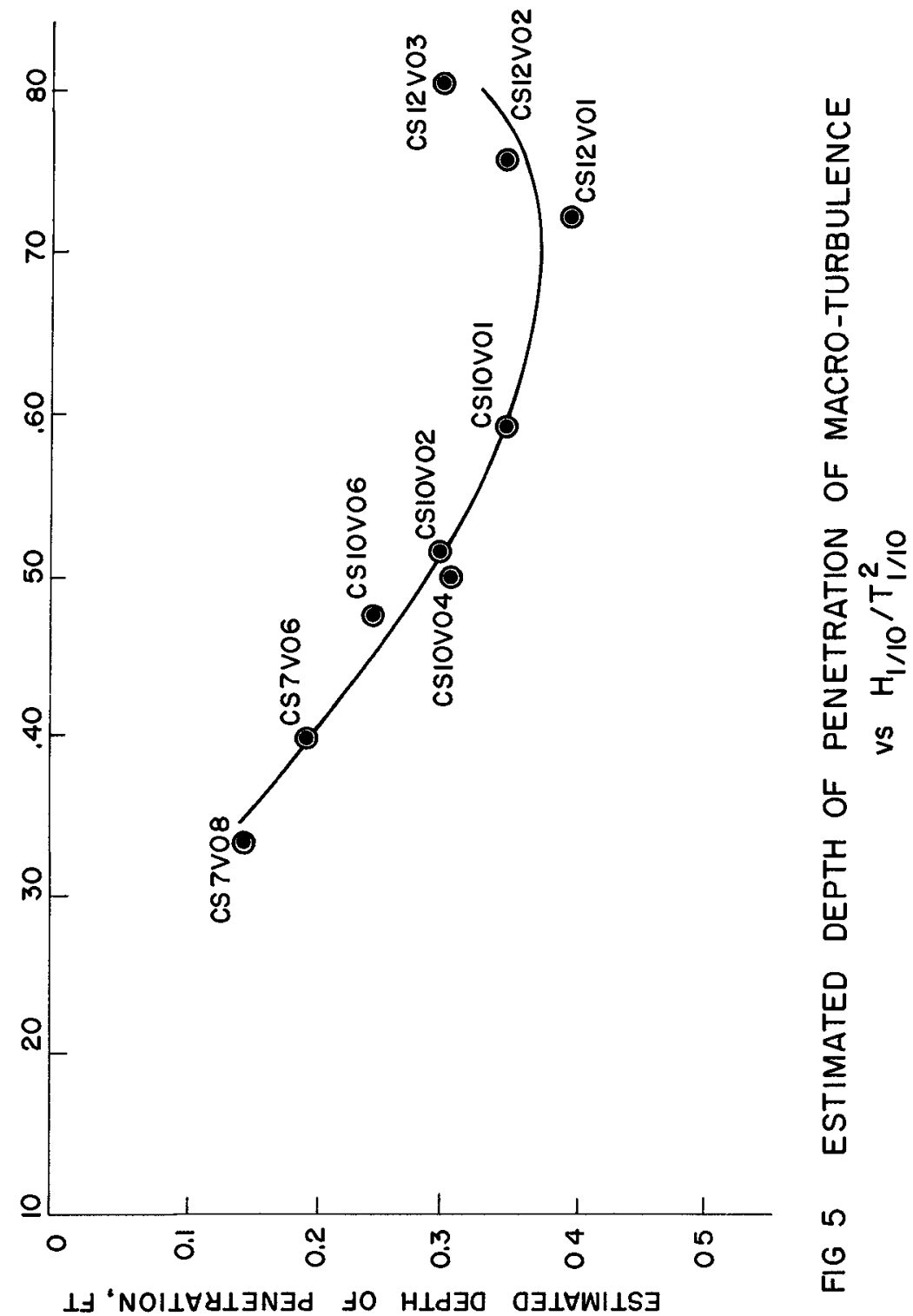




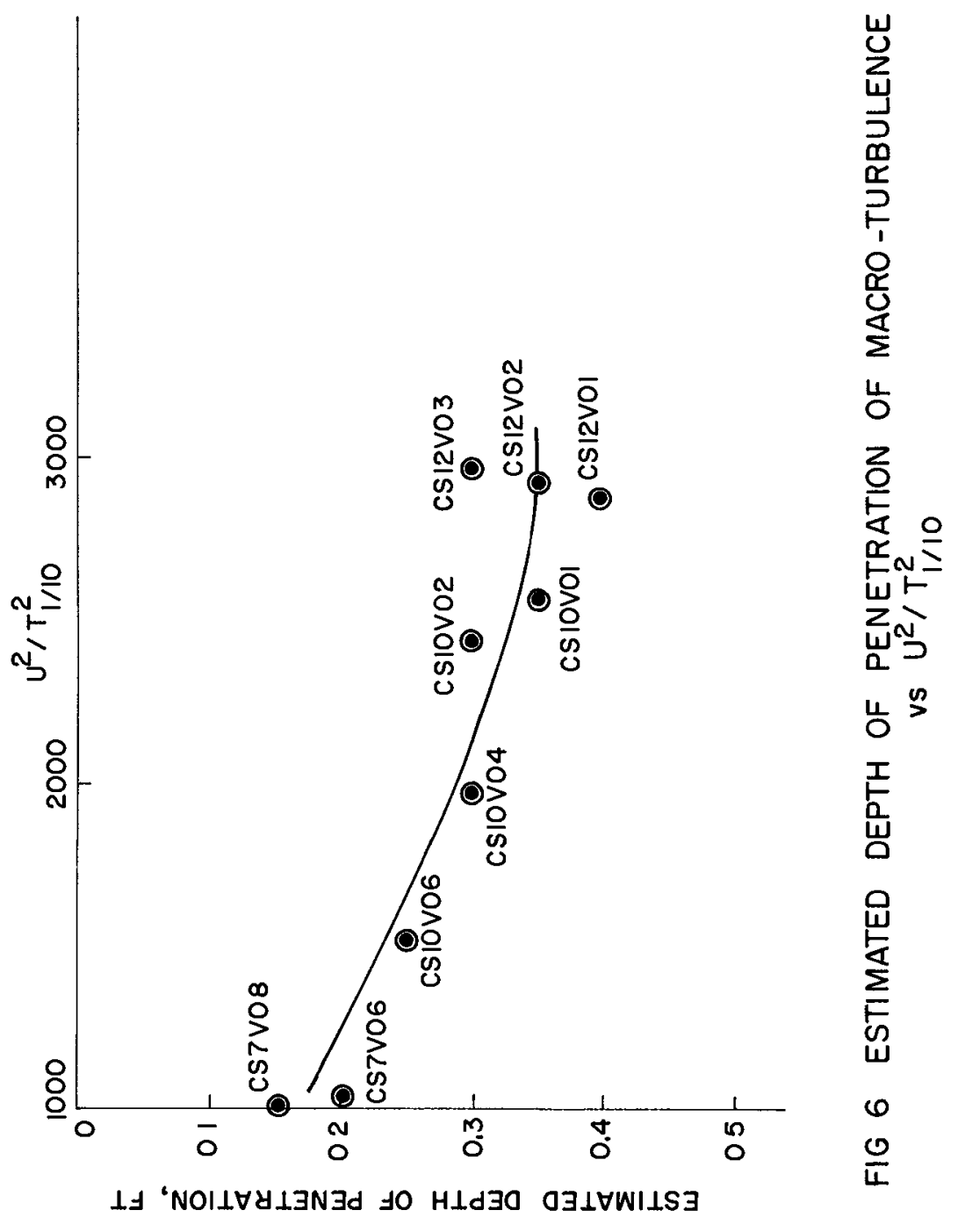




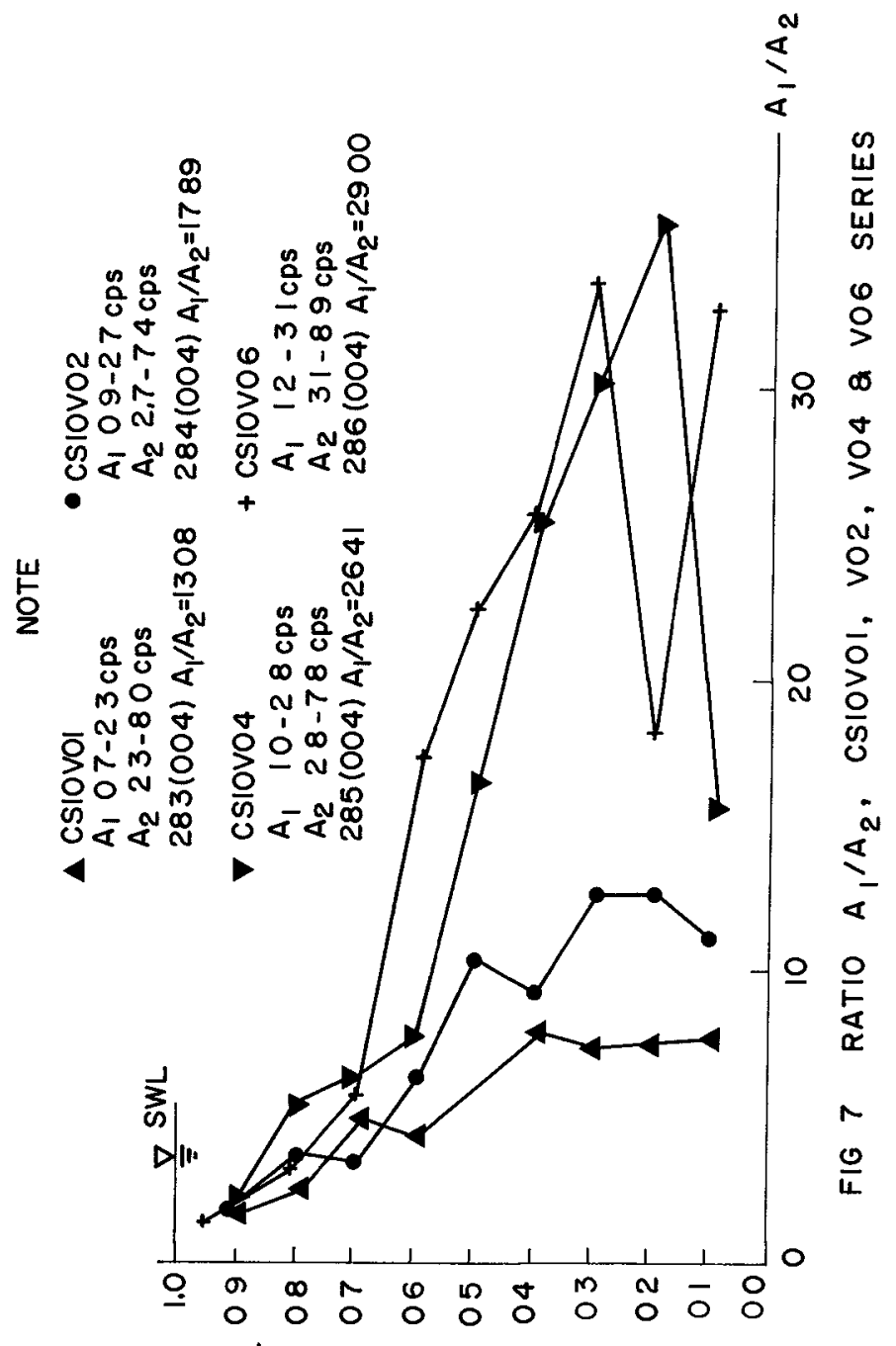

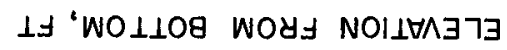




\title{
CHAPTER 15
}

THE SHEAR STRESS OF SEA BREEZE ON A SWASH ZONE

By Shih-Ang Hsu

Coastal Studies Institute

Loulsiana State University

Baton Rouge, Loursiana 70803

\begin{abstract}
Measurements of shear stress under the effect of a sea breeze were made by simultaneous wind and temperature profiles over a shore near Fort Walton Beach, Florida It was found that the sea breeze in the surface boundary layer is in the atmospheric free-convection regime The measured shear stress coefficient is in conformity with that obtained by other investigators by the sea surface tilt method under the unstable condition For coastal applications, the result is found to be more reliable than those assumed coefficients obtained under neutral stabllity for this localized coastal wind system
\end{abstract}

\section{INTRODUCTION}

In coastal areas, especially on tropical, subtropical, and marine desert coasts, and on the shores of relatively large lakes, we can observe in the course of a day the reversal of onshore and offshore winds, called sea breeze and land breeze, respectively Perhaps the most lucid symopsis of the main features of these local wind systems has been presented by Defant (1951) Baralt and Brown (1965) have complled an excellent annotated bibliography on this subject It should be noted that whereas a hurricane or storm may cause extensive damage to coastal structures, the sea breeze is a persistent phenomenon and may, in the long run, be more important For instance, loss of beach sand Into the backshore area as a result of sea breeze is an urgent engineerıng problem, examples are found especially in coastal Peru, Lzbya, Florida, and the Cape Hatteras National Seashore

The mesoscale structure as a function of space and time and particularly the hypothesis of land- and sea-breeze systems which are governed by the circulation theorem have,been observed and verified on the Texas coast by Hsu (1970) Some of the microstructure of the frontal characteristics and the diurnal clockwise rotation of the system with time owing to the Corlolis effect have been observed and presented elsewhere (Hsu, 1969a), this paper will study the surface shearing stress aspect of the sea breeze 
The sea breeze is effectıve in generating waves and currents (Murray, personal communication), which in turn transport sediments in the littoral environment (Sonu, personal communication) Also, eolian sand transport (Sonu, personal communication) and air pollution (Neiburger, 1969) in the coastal zone are closely controlled by this localized wind system Since in the nearshore areas that are frequently affected by diurnal local wind systems the lapse rate is rarely adiabatic and buoyancy forces must be considered, the wind shear stress coefficients obtained under the near-neutral condition (see, e $g$, Wu, 1969) cannot be used under these local wind conditions, as previously pointed out by Hsu ( $1969 \mathrm{~b}$ ) It is hoped that this study will fulfill the need for coefficient values under the sea breeze situation

\section{THEORETICAL CONSIDERATION}

The following derivation for the sea breeze regime in the surface boundary layer is due to McPherson (1968) and is an expanded version of that presented by Estoque (1961)

It is presumed that in the layer immediately above the surface the viscous forces acting on a fluid element are much larger than the inertial forces, thus the horizontal momentum equations and the thermodynamic equation for the layer become

$$
\frac{\partial}{\partial z}\left(K \frac{\partial u}{\partial z}\right)=0
$$

and

$$
\frac{\partial}{\partial z}\left(K \frac{\partial \theta}{\partial z}\right)=0
$$

where $\mathrm{K}$ is an eddy exchange coefficient, assumed to be the same for both heat and momentum transfer, $u$ is the magnitude of the horizontal wind, and $\theta$ is the potential temperature If $K$ is known as a function of height $\mathrm{z}$, the equations can be integrated to obtain $u$ and $\theta$ in the boundary layer

Under conditions of free convection-that 1 , when the buoyant forces dominate the mechanical forces--the expression of $\mathrm{K}$ used is

$$
K=\lambda z^{2}\left(\frac{g}{\theta}\left|\frac{\partial \theta}{\partial z}\right|\right)^{1 / 2}, R_{I}<-003
$$

as discussed by Priestley (1959) Here $\lambda$ is an empirical constant, $g$ Is the acceleration of gravity, and $\bar{\theta}$ is a layer-mean potential temperature 
In the layer in which the Richardson number $\left(R_{1}\right)$ is evaluated Eqs (1) and (2) may be written as

$$
\mathrm{K} \frac{\partial \mathrm{u}}{\partial \mathrm{z}}=\mathrm{U}_{*}^{2}
$$

and

$$
\mathrm{K} \frac{\partial \theta}{\partial \mathrm{Z}}=\theta_{*} \mathrm{U}_{*}
$$

where $U_{*}, \theta_{*}$ are termed the "friction velocity" and "friction temperature" respectively From Eqs (4) and (5) we obtain

$$
\frac{\partial u}{\partial Z}=\frac{U_{*}}{\theta_{*}} \frac{\partial \theta}{\partial Z}
$$

and

$$
\frac{\partial \theta}{\partial \mathrm{Z}}=\frac{\theta_{*}}{\mathrm{u}_{*}} \frac{\partial \mathrm{u}}{\partial \mathrm{Z}}
$$

For the free-convection regime, we use Eqs (3) through (7) and obtain

$$
\frac{\partial u}{\partial z}=\left[\frac{U_{*}^{5} \bar{\theta}}{\lambda^{2} g\left|\theta_{*}\right|}\right]^{1 / 3} z^{-4 / 3}
$$

and

$$
\frac{\partial \theta}{\partial Z}=\left[\frac{U_{*}^{2} \theta_{*}^{2} \bar{\theta}}{\lambda^{2} g}\right]^{1 / 3} z^{-4 / 3}
$$

Eqs ( 8 ) and (9) can be integrated to give

$$
\begin{aligned}
& \mathrm{U}=-3\left[\frac{\mathrm{U}_{*}^{5} \bar{\theta}}{\lambda^{2} \mathrm{~g}\left|\theta_{*}\right|}\right]^{1 / 3} \mathrm{z}^{-1 / 3}+\text { Constant } \\
& \theta=-3\left[\frac{\mathrm{U}_{*}^{2} \theta_{*}^{2} \bar{\theta}}{\lambda^{2} \mathrm{~g}}\right]^{1 / 3} \mathrm{z}^{-1 / 3}+\text { Constant }
\end{aligned}
$$


The expressions for turbulent shear stress $(\tau)$ and surface shear stress $\left(\tau_{0}\right)$ are

$$
\tau=\rho U_{*}^{2}
$$

and

$$
\tau_{0}=\rho C_{z} U^{2}
$$

where $\rho$ is the density of air and $\mathbf{C}_{z}$ is a dimensionless "shear stress" or "drag" coefficient for the height $z$

Combining Eqs (12) and (13) gives

$$
C_{z}=\left(\frac{U_{*}}{U_{z}}\right)^{2}
$$

Thus, from simultaneous temperature- and wind-profile measurements in the atmospheric boundary layer and from Eqs (8) through (14), the values of $\mathrm{C}_{z}$ and $\tau_{0}$ are obtained

\section{FIELD EXPERIMENT AND DATA ANALYSIS}

\section{Field Site and Experrment}

The experiment site (see Figs $I$ and 2) was located on the Gulf Coast near Fort Walton Beach, Florida The site $\left(86^{\circ} 43^{\prime} \mathrm{W}, 30^{\circ} 24^{\prime} \mathrm{N}\right)$ has an approximate east-west shoreline orientation It has been used to study the local wind system (Hsu, 1969a) The experiment related to the present study was designed to measure the temperature and wind profiles In the surface boundary layer as shown in Eqs (8) through (11) and was performed during the month of May 1970

\section{Instrumentation}

The main instrument used for this study was a Thornthwalte Wind Profile Register System (Mode1 106) with 6-unit, 3-cup fast response mounted $20,40,80,160,240$, and $320 \mathrm{~cm}$ above the beach surface (F1.g 1) The anemometers have a distance constant of better than 1 meter and are rugged enough to withstand limited exposure to a marine atmosphere (Seesholtz, 1968) Note that the system is portable so that during the experiment it is quickly and easily moved to a desired location

Temperatures were measured at 170,360 , and $550 \mathrm{~cm}$ above a grass- 


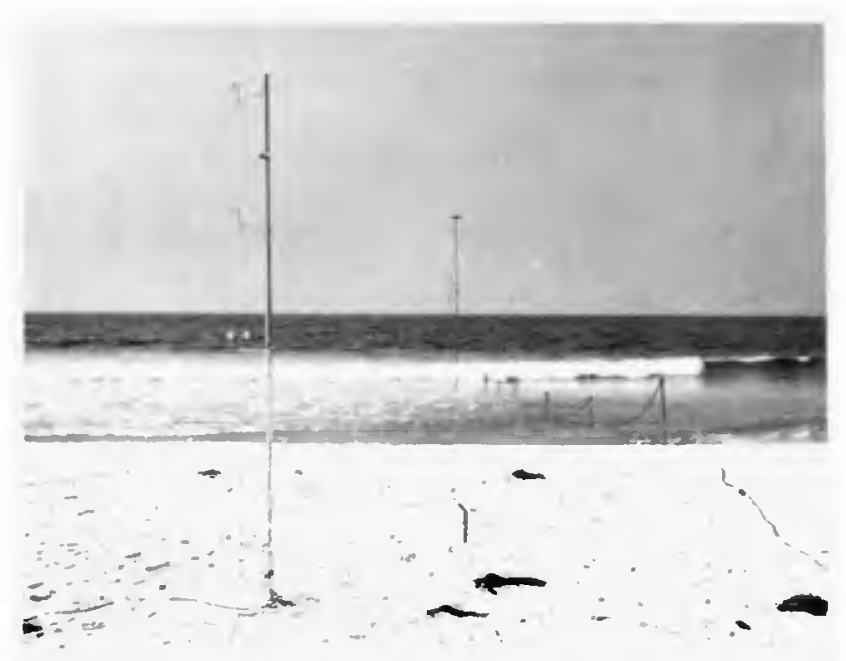

Fig. 1. Cup anemometer array located in the swash on the coast of the Gulf of Mexico near Fort Walton Beach, Florida. Hot-wire anemometer, also part of the instrumentation, is shown in the background.

free berm surface by three identical recording hygrometry systems (Taylor Instrument Company, Series $76 \mathrm{~J}$, having readings within \pm 1 percent of any given chart range). The sensors were mounted on a 10-meter meteorological tower. An all-purpose wind-recording system (Science Associates, Inc., No. 162) at the 10-meter level above the surface made wind speed and direction measurements as the reference level. For detailed information about these instruments and their installation, relevant manuals should be consulted.

\section{Data Reduction}

Wind velocities measured by the Thornthwaite Wind Profile Register System were recorded by a Thornthwatte's Digital Printout Recorder (system mode1 706), which uses the well-known Polaroid reproduction process. A system of mirrors and mechanical level movements moves the image of a row of counters by small increments along the length of the film card. During the experiment an exposure of the register image is made on command from a timer after each movement of the image. A 15-minute time interval was used for the present study. The readings of the counters are then entered on the film card at the start of the period and again at the end. The difference is obtained and the corresponding wind speed in centimeters per second is determined from the appropriate conversion table supplied by the manufacturer. 


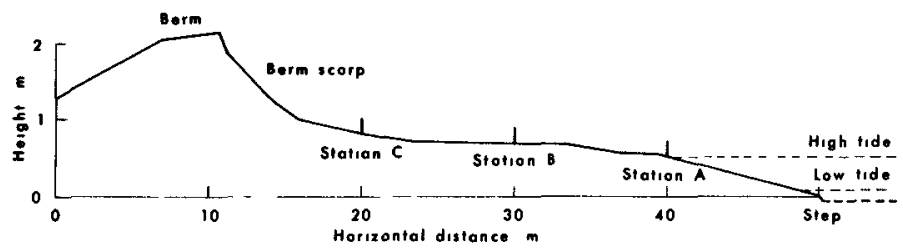

Fig 2 Beach profile on the coast of the Gulf of Mexico near Fort Walton Beach, Florida, during the experimental perıod, between May 20 and May 26, 1970

\section{Method of Data Analysis}

Our Immediate concern is to examine the validity of Eq (10) under sea breeze conditions First, by integrating Eq $(10)$ between any two heights $a$ and $b$, we have

$$
\mathrm{U}_{\mathrm{b}}-\mathrm{U}_{\mathrm{a}}=3\left[\frac{\mathrm{U}_{*}^{5} \bar{\theta}}{\lambda^{2} \mathrm{~g}\left|\theta_{*}\right|}\right]^{1 / 3}\left(\mathrm{a}^{-1 / 3}-\mathrm{b}^{-1 / 3}\right)
$$

This may be written

$$
y=a_{0}+a_{1} x
$$

where

$$
\begin{aligned}
& y=U_{b}-U_{a} \\
& a_{0}=0 \\
& a_{1}=3\left[\frac{U_{*}^{5} \bar{\theta}}{\lambda^{2} g\left|\theta_{*}\right|}\right]^{1 / 3} \\
& x=a^{-1 / 3}-b^{-1 / 3}
\end{aligned}
$$

We plot the observations for each run in the form $y$ against $x$, plotting on point for each avallable pair of heights $\mathrm{Eq} \mathrm{(15a)} \mathrm{shows} \mathrm{that,} \mathrm{if}$ the data do follow the free-convection form, the points lie on a straight line This method is similar to that used by Webb (1970) Eqs (15a) and (15b) have also been obtalned by the least-squares technique Table 1 gives as an example the least-squares values of each correlation 


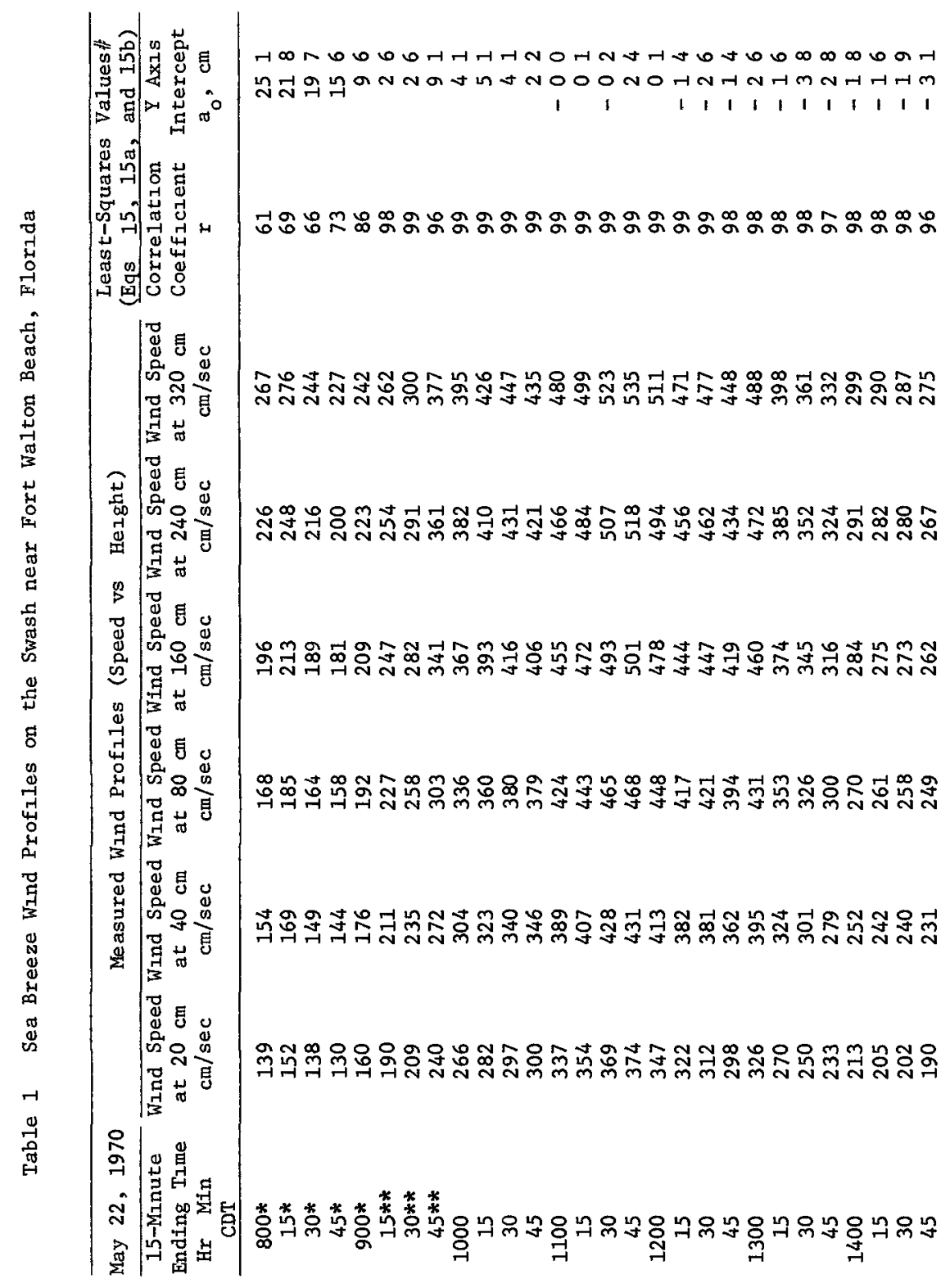




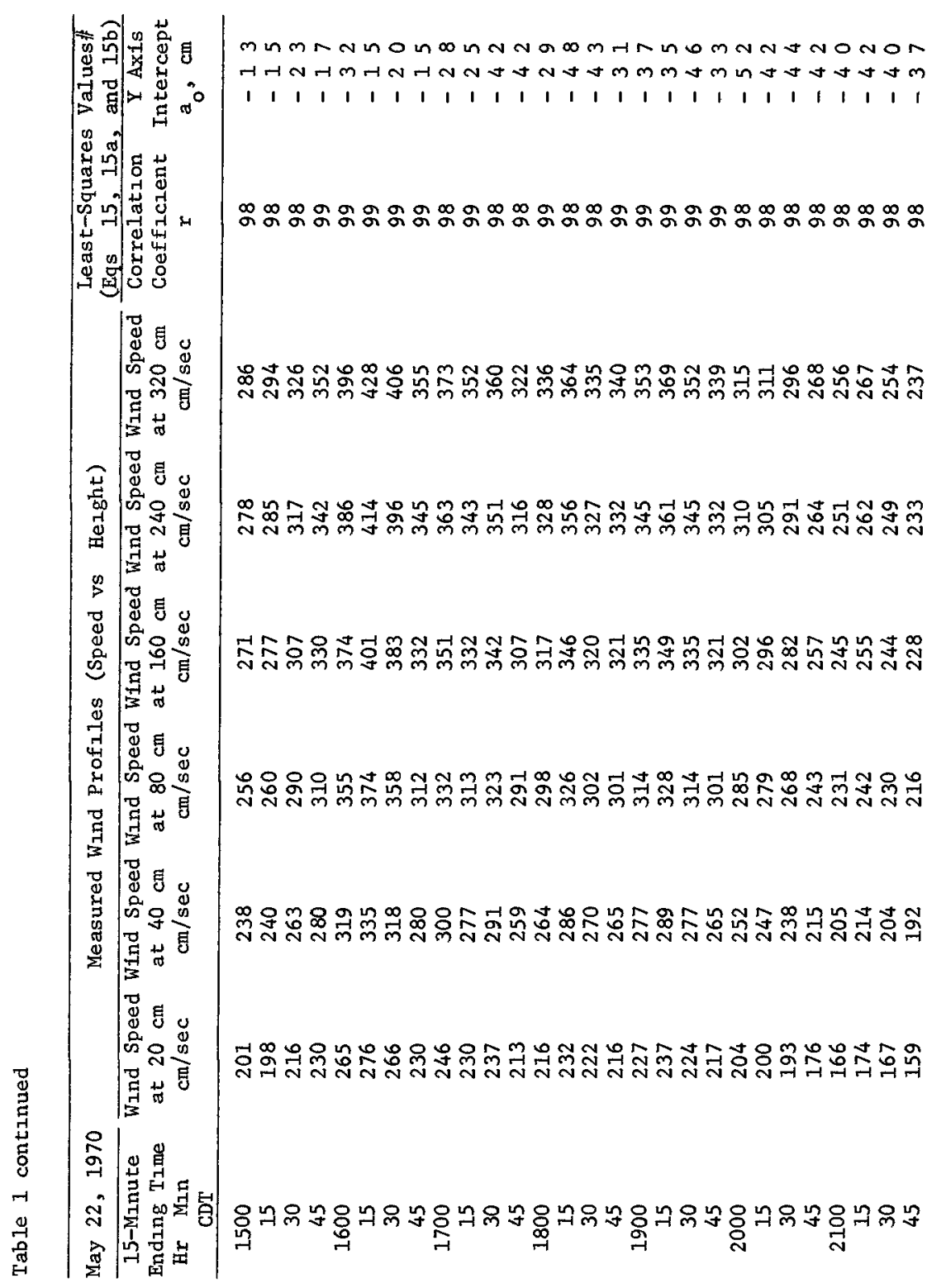




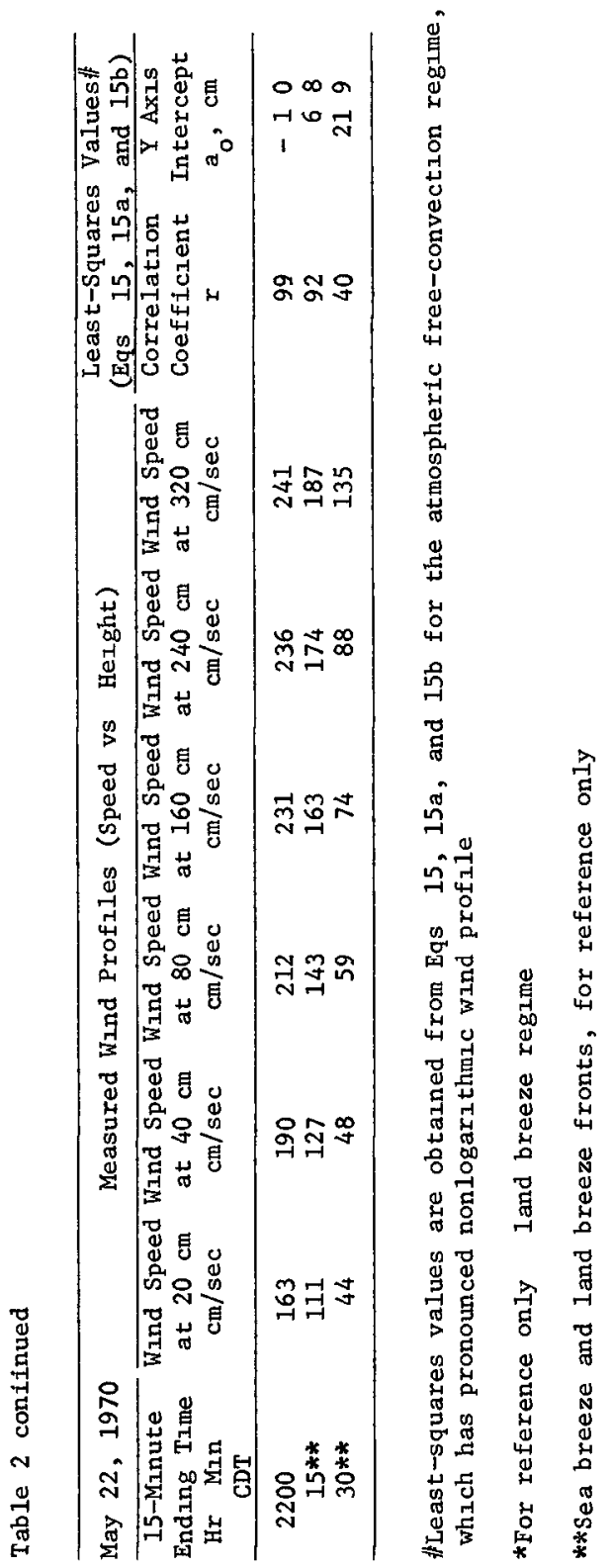


coefficient $(r)$, as well as the y-axis intercept $\left(a_{0}\right)$ It can be seen from this table that, in the whole sea breeze range from 1000 to 2200 $\mathrm{CDT}, \mathrm{r}>96$ and $-52 \mathrm{~cm}<\mathrm{a}_{0}<52 \mathrm{~cm}$, which $1 \mathrm{~s}$ within the experimenta1 error In other words, these observations verify that the sea breeze is in the atmospheric free-convection regime and that its wind profile in the surface boundary layer can be represented by Eq

Sımılar analyses were made for temperature profıles under sea breeze conditions whenever wind profiles were measured Thus, the $U_{*}$ and $\theta_{*}$ values are obtained from these observations, as mentioned previously Note that, on the basis of the findings by Priestly (1959), Deardorff and Willis (1967), and Dyer (1967), the value of $\lambda$ was chosen to equal unity and was used in the present analysis

\section{SURFACE SHEAR STRESS OF THE SEA BREEZE}

Since the main purpose of this paper is to provide the shear stress coefficient $\left(C_{z}\right)$ under the sea breeze condition, and since usually $Z=10$ meters is taken as reference leve1, Figure 3 shows the required results It can be noted Immediately from this figure that the $c_{10} \times 10^{3}$ value for a given wind speed is greater than that under neutral and stable conditions (e $g$, Roll, 1965) This is not surprising Inasmuch as the sea breeze itself is set up by the differential heating between land and water (Hsu, 1970) Thus the buoyancy forces must play the dominant role, as demonstrated in $\mathrm{Tab} l e \mathrm{I}$

The result is consistent with the findings by $J$ and $M$ Darbyshire (1955), who showed that atmospheric stability has a very marked effect on the tilt of the water surface in response to the wind In measuring the surface slope of a lake under different thermal conditions, they obtained stress coefficients that, for a given wind speed, were twice as great in unstable cases as in stable ones According to Roll (1965), the Darbyshires' result is also in conformity with the findings of several authors in different regions of the world (see references given by Ro11, 1965)

Since the shear stress coefficient may also be affected by the fetch of the wind ( $\mathrm{e}, \mathrm{J}$ and $\mathrm{M}$ Darbyshire, 1955, and Ro11, 1965), the annotated bibliography complled by Baralt and Brown (1965) for the sea breeze structure in various parts of the world and a summary of local winds by Defant (1951) may be consulted Furthermore, since the land and sea breeze systems, which are governed by the circulation theorem, have been verified by Hsu (1970), the fetch may be estimated from wind observations (see Eq $8 \mathrm{In} \mathrm{Hsu,} \mathrm{1970),} \mathrm{provided} \mathrm{that} \mathrm{the} \mathrm{value}$ of the coefficient of friction can be estimated on the basis of accurate geomorphological survey of the coastal area in question (see also the discussions by Haurwitz, 1947) An example of the mesoscale structure, including the fetch study of the sea breeze, is given by Hsu (1970)

As for coastal applications, Murray (personal communication) found that the computed shear stress value based on Figure 3 for a given wind speed fits his wind-induced current prediction and observation under the 


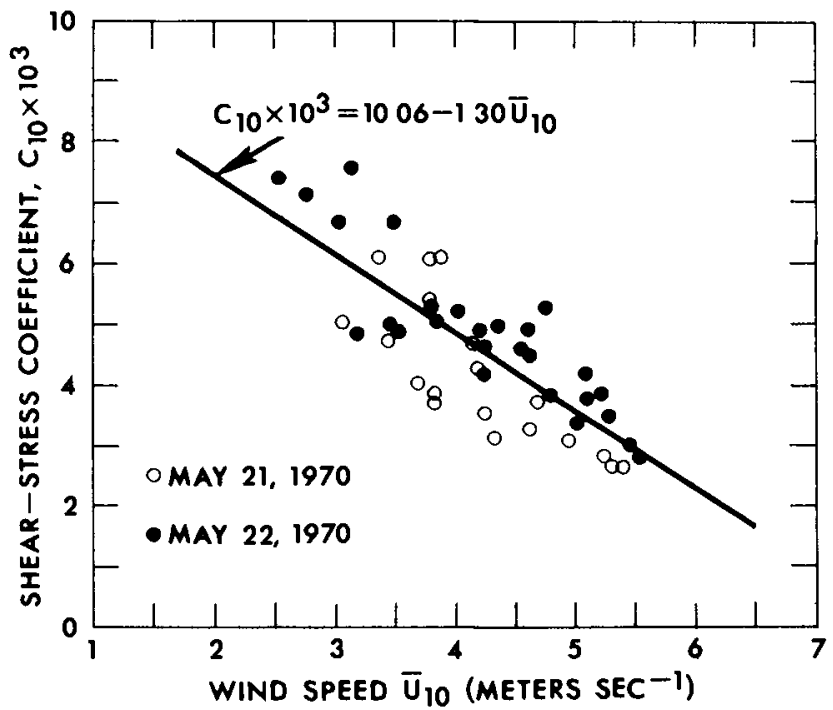

F1g 3 Measured shear stress coefficients under the sea breeze condition Each point is calculated from a 15-minute time average from both alr temperature and velocity profiles Correlation coefficient of the least-squares fit for the stralght line is 080

sea breeze condition more rellably than that based on the neutral stability coefficient (e $\mathrm{g}$, Wu, 1969)

\section{CONCLUDING REMARKS}

While this study is intended to provide the shear stress coefficient under the sea breeze condition, caution should be exercised in applying the result, which may not be applicable to other coastal winds, such as the land breeze, coastal mountain and valley winds, and other synoptic and subsynoptic wind systems It is suggested that before this result can be applied some knowledge of sea breeze meteorology may be needed In this connection, papers by, among others, Defant (1951), Baralt and Brown (1965), and Hsu (1969a and $b$ and 1970) may be consulted

\section{ACKNOWLEDGMENTS}

This study was supported by the Geography Programs, Office of Naval Research, through the Coastal Studies Institute, Loulsiana State University, under Contract N00014-69-A-0211-0003, NR 388002 Appreciation is expressed to personnel of Eglin Alr Force Base, particularly to Marshall Cartledge, for permission to occupy the site during the experiment Thanks also go to Norwood Rector and Stanley Sandifer, who helped perform the experiment 


\section{REFERENCES}

baralt, $G$ L , and $R$ A Brown, 1965, The land and sea breezes an annotated bibliography Final Report on Mesometeorological Field Studies, Dept of Geophysical Sciences, Univ Chıcago, $61 \mathrm{pp}$

Darbyshire, $J$, and $M$ Darbyshire, 1955, Determination of wind stress on the surface of Longh Neagh by measurement of tilt Quart $J$ Roy Meteorol Soc, $81333-339$

Deardorff, $J$ W, and $G$ E Willis, 1967, The free-convection temperature profile Quart J Roy Meteorol Soc, 93 166-175

Defant, F , 1951, Local winds Compendium of Meteorology, Am Meteorol Soc, pp 655-672

Dyer, A J, 1967, The turbulent transport of heat and water vapour in an unstable atmosphere Quart $J$ Roy Meteorol Soc, 93 501-508

Estoque, M A, 1961, A theoretical investigation of the sea breeze Quart $\mathrm{J}$ Roy Meteorol Soc, 87 136-146

Haurwitz, B , 1947, Comments on the sea-breeze circulation J Meteorol, 4 1-8

Hsu, S -A, 1969a, Land- and sea-breeze fronts near $50 \mathrm{~cm}$ on the Gulf coast Bull An Meteorol Soc, 50 880-882 , 1969b, Comments on paper by $\mathrm{J}$ Wu, 'Wlnd stress and surface roughness at alr-sea Interface ' $J$ Geophys Res , 745562 1970, Coastal air-circulation system observations and empirical mode1 Monthly Weather Rev, 98 487-509

McPherson, R D, 1968, A three-dimension numerlcal study of the Texas coast sea breeze Technlcal Report No 15, Atmospherlc Sclence Group, College of Englneerıng, Unıv Texas, Austın, 252 pp

Neiburger, M , 1969, The role of meteorology in the study and control of air pollution Bull Am Meteorol Soc, 50 957-965

Priestley, C H B, 1959, Turbulent transfer in the lower atmosphere Chlcago (Univ Chlcago Press), $130 \mathrm{pp}$

Ro11, H U , 1965, Physics of the marine atmosphere New York (Academlc Press) 
Seesholtz, J R, 1968, A field Investıgation of air flow immediately above ocean surface waves Mass Inst Technol Dept Meteorology Technical Report, $138 \mathrm{pp}$

Webb, E K, 1970, Profile relationships the $10 \mathrm{~g}-1$ inear range, and extension to strong stability Quart $J$ Roy Meteorol Soc, 96 $67-90$

Wu, J, 1969, Wind stress and surface roughness at a1r-sea interface $\mathrm{J}$ Geophys Res, 74 444-455 


\title{
CHAPTER 16
}

ENERGY LOSSES UNDER WAVE ACTION

by

\author{
P D Treloar, \\ Research Associate, \\ Department of Civil Engineering, \\ Queen's University at Kingston
}

and

\author{
A Brebner, \\ Professor, \\ Department of Civil Engineering, \\ Queen's University at Kingston
}

\begin{abstract}
Wave-height attemuation measurements were made in two Identical flumes of $d_{1} f f e r e n t$ widths and the results used to separate bottom energy losses from sidewall energy losses These energy losses, in the form of rates of energy dissipation, were then compared with their theoretical values as calculated by solving the linearized Prandt1 boundary layer equations and evaluating the Rayleigh dissipation function Using these results, an adjusted formula for the wave-height attenuation modulus was determined

\section{INTRODUCTION}

Up to the present time no direct measurements of the sidewall and bottom rates of energy dissipation in laminar boundary layers, produced by progressive, oscillatory gravity waves in a wave flume, have been made A comparison between these experimental values and their related theoretical values would be valuable as the results could be used to produce an adjusted formula for the wave-height attemuation modulus $\alpha$, which is defined by the equation
\end{abstract}




$$
\frac{H}{H_{0}}=e^{-\alpha x}
$$

where $H_{0}$ is the wave-height at position $x=0$ and $H$ is the subsequent wave-height at positior $x$, in the direction of wave propagation Eagleson (1) and Iwagakı and Tsuchiya (2) measured the 5ottom shear forces produced by a wave by measuring the shear on a plate fitted flush with the flume bottom Their results led to values of $\alpha_{b}$, (where $\alpha_{b}$ is the attenuation modulus which results from the bottom boundary layer), which did not agree with the theoretical values of $\alpha_{b}$, the formula for which is derived by the authors mentioned In model harbour tests it is often necessary to have an accurate estimate of this attemuation modulus in order that the prototype wave-heights may be interpreted from the model measurements This is because harbour models are constructed according to the Froude modelling law whereas the model wave amplitude attenuation is normally a viscous phenomenon

\section{GENERAL APPROACH}

In order to separate bottom and sidewall friction effects it is necessary to have two flumes which are identical except for their widths Since the space rate of change of average wave power per unit plan area, $\overline{\partial P} / \partial \mathrm{x}$, is equivalent to the sum of the average time rates of energy dissipation per unit plan area on the bottom and sidewa11s, $d \bar{E}_{\mathrm{b}} / \mathrm{dt}$ and $\mathrm{dE}_{\mathrm{W}} / \mathrm{dt}$ respectively, the following simultaneous equations can be written

$$
\begin{aligned}
& B_{1} \partial \bar{P}_{1} / \partial x=-\left(B_{1} d \bar{E}_{b} / d t+2 h d \bar{E}_{w} / d t\right) \\
& B_{2} \partial \bar{P}_{2} / \partial x=-\left(B_{2} d \bar{E}_{b} / d t+2 h d \bar{E}_{w} / d t\right)
\end{aligned}
$$

where $B$ is flume wldth, $h$ is still water depth and the subscripts 1 and 2 refer to the two separate flumes If the quantities $\partial \overline{\mathrm{P}}_{1} / \partial x$ and $\partial \bar{P}_{2} / \partial x$ are known, then the equations can be solved for $d E_{b} / d t$ and $\mathrm{dE}_{\mathrm{W}} / \mathrm{dt}$ These equations must be solved for the case when the wave parameters, wave period $\mathrm{T}$, wave height $\mathrm{H}$ and still water depth $h$, are the same in both flumes because then the values of $\mathrm{d}_{\mathrm{W}} / \mathrm{dt}$ and $\mathrm{d}_{\mathrm{b}} / \mathrm{dt}$ are the same in the two flumes Thus it becomes necessary to determine experimental values for $\partial \mathrm{P} / \partial \mathrm{x}$ in the two flumes Now since $\bar{P}$, from first order wave theory, can be written as

$$
\bar{P}=\gamma H^{2} C_{g} / 8 \quad-\ldots-\cdots-3
$$

where $\gamma$ is the specific welght of the fluid and $C_{g}$ is the wave group velocity, it is possible to write $\partial \bar{P} / \partial x$ as

$$
\partial \bar{P} / \partial x=\gamma H / 4 d H / d x C_{g} \quad \ldots \quad-\ldots-4
$$


for constant $\mathrm{T}$ and $\mathrm{h}$ First order waye theory was used because the horizontal water particle yelocities are described best, in this case, by this theory, (Le Mehauté et al (3)), and because the first order approximation to the solution of the waye equation satisfies the boundary conditions at the free surface as well as other theories, (Dean (4)) It now becomes obyious that it is necessary to determine wave height versus distance attenuation curves for waves with the same periods and depths in the two flumes Equation 4 can then be evaluated and equations 2 solved

\section{EXPERTMENTAL TECHNIQUE}

The Coasta1 Engineering Laboratory of Queen's University at Kingston has two smilar flumes of width $2 \mathrm{ft}$ and $3 \mathrm{ft}$ and length $150 \mathrm{ft}$ They are constructed from concrete and the inside walls were cement plaster which has been sanded smooth and painted However, in order to obtain sufficient difference between attemuation rates in the two flumes, an alumimm sheet wall was constructed in the $2 \mathrm{ft}$ flume to make a temporary flume 6 inches wide The flumes had identical motors and sumllar flap-type paddles

The wave-heights in the 6 inch flume were measured using a carbon-strip probe and those in the $3 \mathrm{ft}$ flume using a capacitance plate probe Both of these instruments have simllar accuracy, $( \pm 0002 \mathrm{ft})$ in comparison with a hook gauge Risaltex "horse-ha1r" mats were used to make beaches which absorbed the energy of the incident waves The reflection coefficient was always less than $5 \%$

The useful measuring length for each flume was about $25 \mathrm{ft}$ out of a total length of some $150 \mathrm{ft}$ Th1s distance was not long enough to produce a sufficient number of wave-height versus distance data points For this reason the flume was "lengthened" using the following known method which is described by Battjes (5) The wave-helghts were measured at two stations $25 \mathrm{ft}$ apart, wave-height at $\mathrm{x}=0 \mathrm{ft}$ and wave-helght at $\mathrm{x}=25 \mathrm{ft}$ The wave paddle was then adjusted so that the wave-he1ght measured at $x=25 \mathrm{ft}$ was now produced at $x=0 \mathrm{ft}$ in the flume The wave-height produced at $\mathrm{x}=25 \mathrm{ft}$ is now the wave-helght which would have been produced at $\mathrm{x}=50 \mathrm{ft}$ if the flume had been long enough By repeating this procedure the flumes were "lengthened" considerably In fact the paddle was adjusted so that the wave-height measured at $x=0 \mathrm{ft}$, for each "lengthening" increment, was within $\pm 03 \mathrm{~mm}$ of the required wave-height The difference in wave-height decrements for waves of initial height differing by this small quantity would be very small Each measurement was performed three tumes, the wave-height decrement being taken as the average of these values

The wave-heights at stations were measured by moving the measuring device over a distance of one half wavelength on both sides of the station, thus measuring two maxima and two minuma of the 
inc1dent-reflected wave interference profile Then, assuming linear attenuation over a short distance, and assuming first order wave motion, the wave-height at a station can be shown to be equal to

$$
H=1 / 8(A+3 B+3 C+D)
$$

where, in this case, $\mathrm{A}, \mathrm{B}, \mathrm{C}$ and $\mathrm{D}$ are the consecutive values of the maxima and minma taken from the recorder profile

\section{DIMENSIONAL ANALYSIS OF ENERGY DISSIPATION}

The rate of energy dissipation in the laminar bottom boundary layer can be written in the functional form

$$
d E_{b} / d t=f\left(a_{\delta}, T, \mu, \rho\right)
$$

where $\mu$ is fluid viscosity, $\rho$ is the fluid density and $a_{\delta}$ is the length of the bottom fluid particle motion Using dimensional analysis this equation becomes

$$
d \bar{E}_{b} / d t=\rho a_{\delta}^{3} / T^{3} \cdot \phi\left(a_{\delta}^{2} / T \nu\right) \quad-\quad-5
$$

where $\nu$ is kinematic viscosity It is well known that for laminar flow conditions a viscous force is proportional to the first power of a velocity, $1 \mathrm{e}$

\section{Force $\propto$ velocity}

Now the rate at which work is done, or energy dissipated, is given by the relationship

Rate of work = Force $\mathrm{x}$ average velocıty

1 e Rate of work $\alpha$ (velocity) $^{2}$

or in this case

$$
d \bar{E}_{b} / d t \propto u_{0}^{2}
$$

is taken, where $u_{0}$ is the horizontal velocity of fluid particles at the upper limit of the boundary layer Thus equation 5 can be written in the form

$$
d E_{b} / d t=\rho\left(a_{\delta} / T\right)^{3} \cdot \phi\left(\left(a_{\delta} / T\right)^{2} \cdot T / v\right)
$$


which, upon putting $u_{0}=$ constant $x a_{\delta} / T$

$$
d \bar{E}_{b} / d t=\rho u_{0}^{3} \phi_{1}\left(u_{0}^{2} T / r\right) \quad-\quad-\quad-\quad 7
$$

In order that equation 6 can be satisfied, $\phi$ must be of the form

$$
\phi=\text { constant }\left(\mu_{0}^{2} T / \nu\right)^{-1 / 2}--
$$
the following is obtained

$$
\begin{aligned}
& \mathrm{dE}_{\mathrm{b}} / \mathrm{dt} \text { experimental }=\text { const } \mathrm{dE}_{\mathrm{b}} / \mathrm{dt} \text { theoretical } \\
& =\mathrm{D} \mathrm{dE}_{\mathrm{b}} / \mathrm{dt} \text { theoretical }
\end{aligned}
$$

and in a similar way the sidewall rate of energy dissipation functional relationship produces

$$
\begin{aligned}
& d E_{\mathrm{w}} / \mathrm{dt} \text { experimental }=\text { konst } \mathrm{dE}_{\mathrm{w}} / \mathrm{dt} \text { theoretical } \\
& =\mathrm{F} \mathrm{dE}_{\mathrm{w}} / \mathrm{dt} \text { theoret } 1 \mathrm{cal}
\end{aligned}
$$

The subscript "theoretica1" indicates the theoretical equation for the rate of energy dissipation $\mathrm{D}$ and $\mathrm{F}$ are constants

From the above analysis it is obvious that, once the rates of attenuation curves for wave-height have been determined in the two flumes for waves with the same period and water depth, the constants $\mathrm{D}$ and $\mathrm{F}$ can be determined One experiment only is required However nine experiments were performed in order to reduce the effect of experimenta1 error The periods used were 091,108 and 121 seconds The water depths were $41,56,79$ and 100 inches The selection of these values ensured that the wavelength to depth ratios would be in the range $0<L / h<\bar{c} 10$, the lower limit being for deep water waves and the upper limit being the conmonly accepted 1 mit for cnoldal waves Maximm wave steepness was about 005 To ensure that the boundary layers remained laminar, the criterion for laminar oscillatory boundary layers under waves derived by Collins (6) was used, this being that

$$
R_{E}=\frac{\mu_{0} \delta}{\nu}<160
$$


where $\delta$ is a boundary layer thickness parameter defined as

$$
\delta=(\nu T / \pi)^{1 / 2}
$$

In addition, the water surface was kept clean and the condition of an inmobile surface never occurred Energy dissipation as a result of surface films was not considered to be of importance

\section{RESULTS AND CONCLUSIONS}

The experments were performed as described and the results tabulated The next step was to fit a suitable equation to the experimental results so that equation 4 could be evaluated. This was done by assuming exponential attemuation and linearizing equation 1

$$
1 \text { e } \ln H=\ln _{0}-\alpha x
$$

Using the least squares technique and a welghting function of $\mathrm{H}^{2}$, the values of $\alpha$ and $H_{\text {}}$ were determined Table 1 shows the experimental values of $\alpha$ together with their related theoretical values The data was found to fit this form very well The fit was tested using the parameter

$$
R^{2}=1-\frac{\sum\left(H-H_{l}\right)^{2}}{\sum\left(H_{l}-\bar{H}\right)^{2}}
$$

where

$$
\bar{H}=\frac{1}{n} \sum_{l=1}^{n} H_{c}
$$

$\mathrm{n}$ is the number of data points obtained for a particular attenuation curve and $H_{12}$ s the wave-height at position $x_{1}$ along a flume The values of $\mathrm{k}^{2}$ were better than 0995 in all cases

Grosch and Lukasık (7) determıned an attemuation equation for finite amplitude waves This equation was also tried and the results of aggression analysis showed that their equation fitted the data almost as well as the exponential equation However, the latter, because of its easier mathematical form, was used for evaluating equation 4 
With experimental yalues of $\alpha$ and $\mathrm{H}_{0}$ determined, the values of rates of energy dissipation on the bottom and sidewails can be separated For constant yalues of $T$ and $h$, the constants $D$ and $F$ w1 11 not yary with change in $\mathrm{H}$ as both the theoretical and experimental rates of energy dissipation are then functions of $\mathrm{H}^{2}$ only Tables 2 and 3 show theoretical and experimental rates of energy dissipation, calculated for an $H$ value of $20 \mathrm{~mm}$, for the bottom and sidewalis respectively, together with the values of D and $F$ The values of $D$ and $F$ are

$$
\begin{aligned}
& D=148 \pm 015 \\
& F=094 \pm 009
\end{aligned}
$$

at the $95 \%$ confidence lumits These values show that theory considerably underestimates the rate of energy dissipation on the flume bottom, whereas for the sidewalls, theory is very close to experiment The fact that first order theory and experuments agree quite well for energy dissipation on the side walls, whereas this is not the case for bottom losses, cannot be satisfactorily explained It is not thought that energy dissipation at the fluid surface as a result of surface films is the cause for the approx $50 \%$ difference since the experimental surface was certainly not immobile by any stretch of the imagination

Using a method sumllar to that demonstrated by Eagleson (1), an equation for the adjusted attenuation modulus for bottom and sidewalls was determined

$$
\alpha_{b+w}=\frac{k}{B}\left(\frac{T V}{\pi}\right)^{1 / 2}\left(\frac{148 B k+094 \sinh 2 k h}{2 k h+\sinh 2 k h}\right)-\ldots
$$

where $\mathrm{k}=2 \pi / \mathrm{L}$ and $\mathrm{L}$ is wayelength Th1s adjusted modulus would, for the case of a wide, shallow flume, be considerably larger than the well-known theoretical value 


\section{TABLE 1}

TABLE OF THEORETICAL AND EXPERIMENTAL ATTENUAT ION MODULI

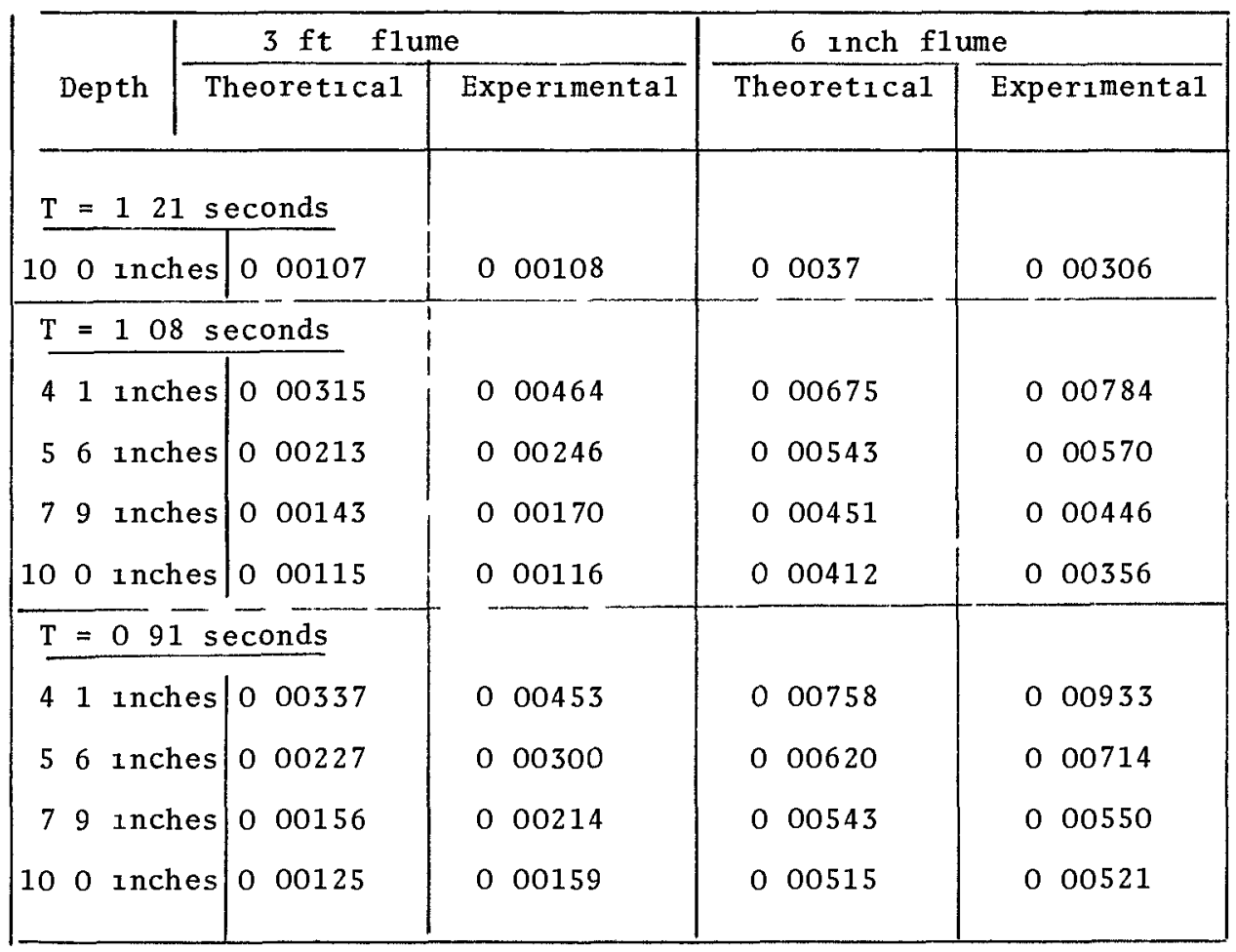


TABLE 2

TABLE OF THEORETICAL AND EXPERIMENTAL VALUES

$$
\text { of } d \bar{E}_{b} / d t
$$

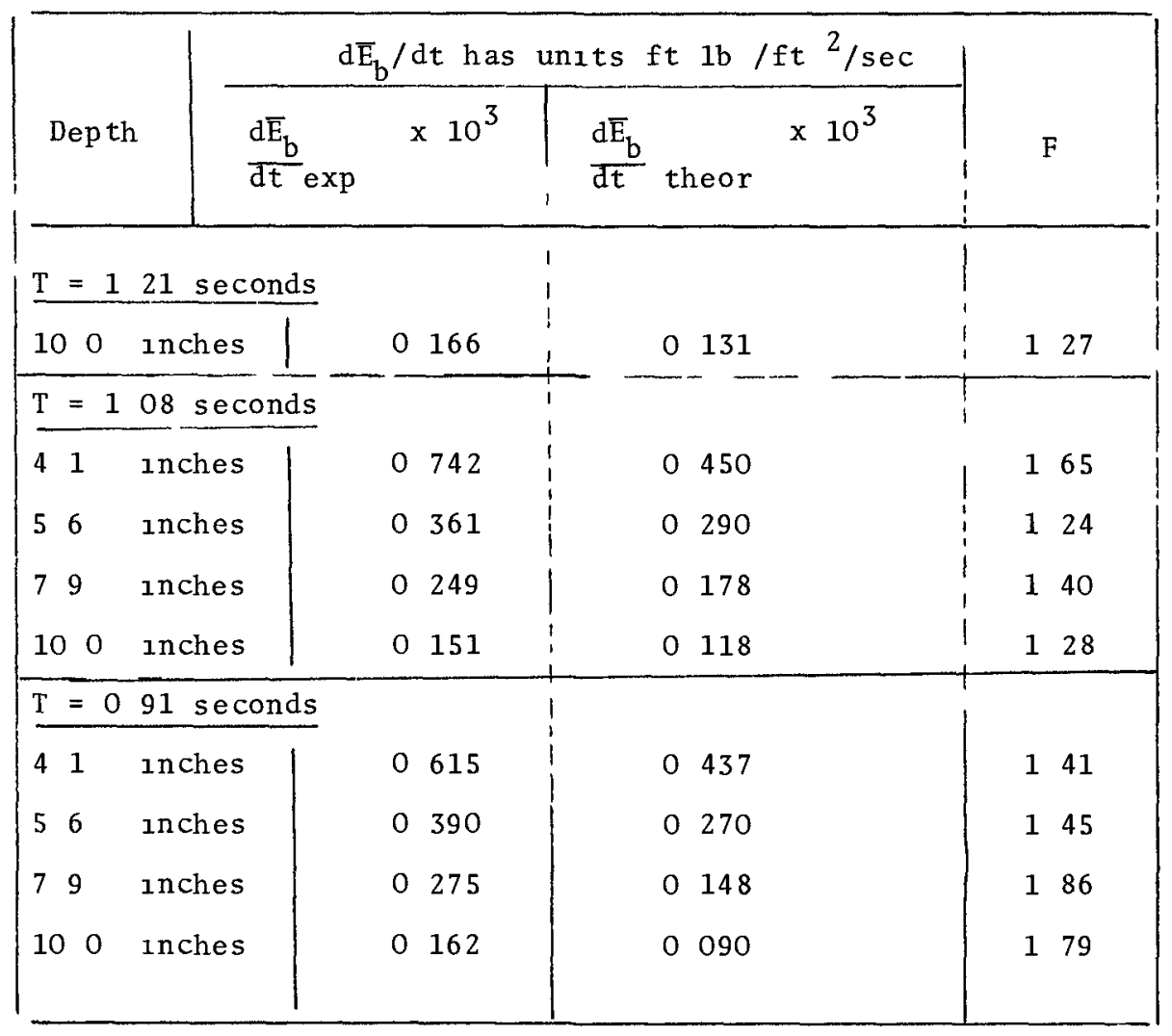


TABLE 3

TABLE OF THEORETICAL AND EXPERIMENTAL VALUES of $d \bar{E}_{\mathrm{w}} / \mathrm{d} \mathbf{t}$

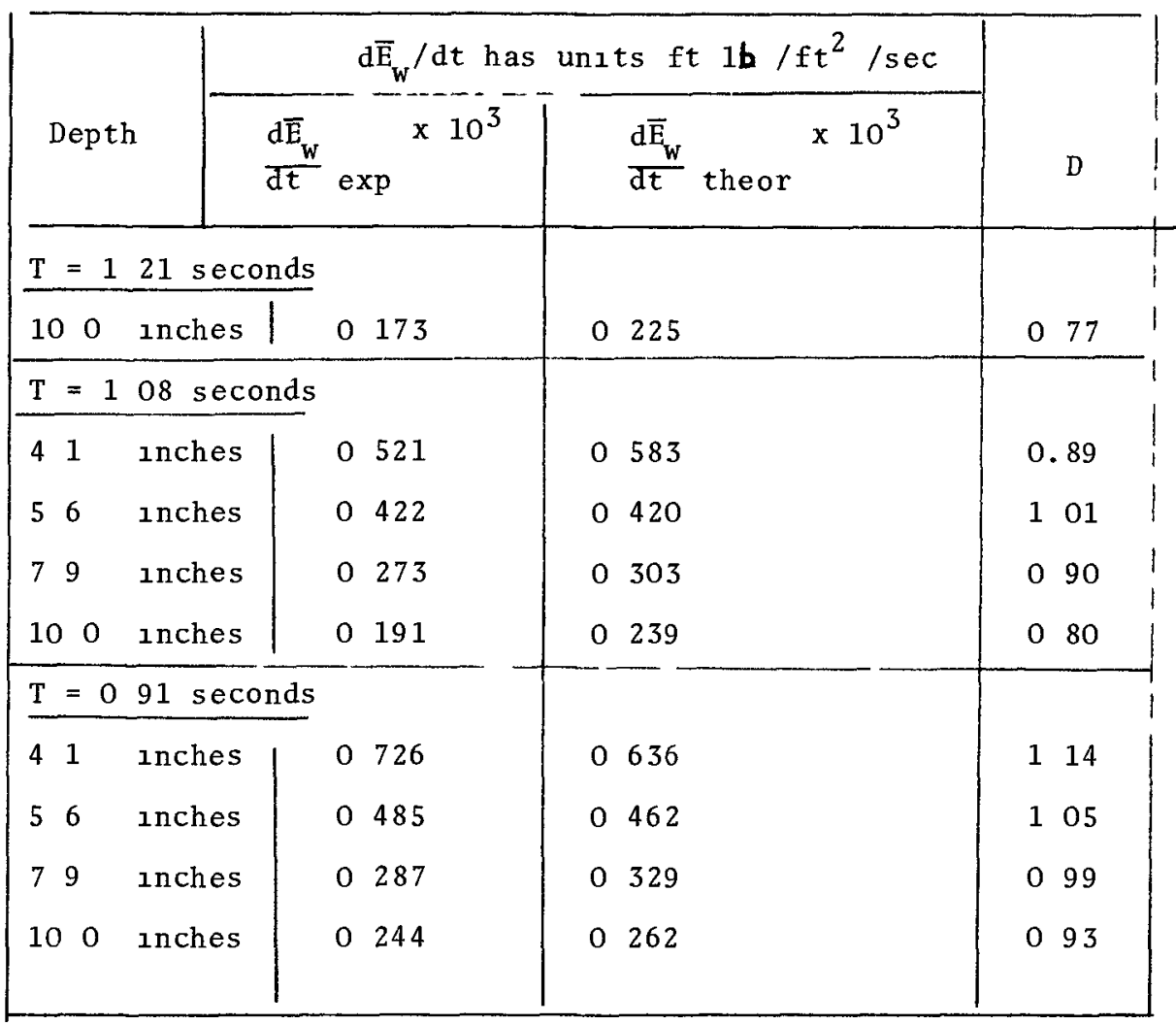




\section{REFERENCES}

Eagleson, P S (1962), "Laminar Damping of Oscillatory Waves", Proc A S C E, Vol 88, pp 155-181, Hy 3

Yu1ch1 Iwagak1 and Yosh1to Tsuch1ya, (1966), "Laminar Damping of Oscil1atory Waves Due to Bottom Friction", Coasta1 Englneering Conference, Tokyo, 1966 , pp $149-174$

Le Mehauté, B , Divorky, D and Lin, A, (1968), "Shallow Water Waves A comparis on of Theories and Experiments", Coasta1 Engineering Conference, London, 1968

Dean, R G (1965), "Stream Function Wave Theory, Validity and Application", Coastal Engineering Specialty Conference, Santa Barbara, 1965

Battjes, J S (1965), "Wave Attenuation In A Channe1 W1 th Roughened Sides", Coastal Engineering Specialty Conference, Santa Barbara, 1965

Collins, J I (1963), "Inception of Turbulence At the Bed Under Perlodic Gravity Waves", Jour Geophs Res, Vo1 68, pp 6007-6014

Grosch, C E and Lukasık, S J (1963), "Discussion of Laminar Damping of Oscillatory Waves", Proc A S C E, Vo1 89, No Hy1, pp 232-239 



\title{
CHAPTER 17
}

\author{
BOTTOM BOUNDARY SHEAR STRESSES ON A MODEL BEACH
}

\author{
by \\ P G Telekı \\ Department of Geology, Loulsiana State Unıversity, Baton Rouge, and \\ U S Army Coastal Engineering Research Center, Washington, D C
}

and

$M W$ Anderson, A M , ASCE

Department of Structures, Materials and Fluıds Unıversity of South Florıda, Tampa

\section{SYNOPSIS}

The maximum amplitude of shear stress in the bottom boundary layer of water waves was evaluated with a Preston probe inclined on a 1125 slope beach Near bottom velocity profiles were obtained in laminar and developing turbulent flow conditions from which the experimental boundary layer thicknesses were evaluated Agreement between experimental bottom velocities and those calculated from Alry theory deteriorate with decreasIng depth on the beach resulting in lower shear stress values than predicted by linear theory The measured boundary layer thickness on the slope exceeds the predicted for horizontal bottom, increasing shoreward to some critical depth outside the breaker zone from where it decreases shoreward The influence of roughness on the shear stress distribution is considerable in the "offshore" reglon, but becomes negliglble near the breaker zone on a smooth bottom the coefflclent of friction agrees with Kajıura's expression

\section{INTRODUCTION}

In order to quantıtatively evaluate nearshore sedıment transport, It must be recognized that substantially more is required to be known about oscillatory boundary layers, the magnitude of energy dissipation due to bottom friction and fluld turbulence and about the effect of permeability in various sediments, in other words about the physical phenomena near the fluid-solid interface our present knowledge on the flow of fluld near the bottom boundary is negligible because analytical solutions of mass transport exist only for laminar flow and for horızontal impermeable boundary Nature prescribes, in contrast, sloping beaches, 1oose boundarıes and waves undergoing transformation and breaking Furthermore, at this time we cannot yet solve the equations for turbulent boundary layer in unidirectional steady flow, let alone when waves are present One of the early efforts of investigating the nature of the oscillating laminar 
boundary layer was Eagleson's (1959) Solutions for the horizontal boundary were given by Longuet-Higgins (1958), Grosch (1962) and Iwagakı, et al (1967) Turbulent boundary layers were investigated by Jonsson (1963) and Horlkawa and Watanabe (1968) The authors of the latter reference employed KajIura's (1968) theory for turbulent boundary layers with some success Direct measurement of boundary shear stress and evaluation of the friction coefficient have been made by Eagleson (1959) and lwagakı et al (1965) The indirect method of velocity measurements has been applied by Jonsson (1963), Horıkawa and Watanabe (1968) and Sleath (1968) Sleath's results are particularly interesting because he used a permeable bottom boundary One of his conclusions was that increase in the permeability of a sandy bottom brings ab out an increase in the near bottom mass transport velocity Experıments by Horıkawa and Watanabe, employing the hydrogen bubble technique, hold out promise toward understanding not only the measurement of instantaneous velocities and boundary layer thickness but a very critical aspect of boundary layer research, namely phase differences between the velocities within and outside of the boundary layer and between the local velocities and the boundary shear stress

The use of the Preston probe for evaluating boundary shear stress from measurements of dynamlc pressure is well known from literature for unidirectional turbulent flows 1 ts theoretical development is due to Preston (1954) who used the probe on smooth boundaries Evidence for its applicability to rough boundarles was presented by Hwang and Laursen (1963) and Ghosh and Roy (1970) Nece and Smith (1970) used an enlarged verston of the probe on loose boundary of a tidal estuary with partial success The Preston probe, as presented in this report, has not been applied to oscillating flows nor to a sloping bottom in presence of waves, pressure gradients will necessarlly be present in the direction of flow Hsu (1955) and Patel (1965) have glven proof of the probe's use in pressure gradients In addition, Hsu (1955) extended its use to laminax flow

The alm of this paper is to report on some aspects of the oscillating flow near the bottom, in particular about resistance on a sloping beach and its manifestation in velocity and shear stress distributions near a solid boundary The theoretical developments of Kajlura (1968) for the oscillating boundary layer were followed Velocity and shear stress measurements were obtalned for various wave conditions, a fixed slope, smooth and a rough boundary in a wavetank, using the indirect method of a Preston probe

\section{THEORETICAL CONSIDERATIONS}

For the case of unsteady flow of a viscous, incompressible flund flow the Navier-Stokes equations of motion, two-dimensional case, is given as

$$
\rho\left(\frac{\partial u}{\partial t}+u \frac{\partial u}{\partial x}+v \frac{\partial u}{\partial z}\right)=-\frac{\partial p}{\partial x}+\mu \frac{\partial^{2} u}{\partial z^{2}}
$$


where the $x$-axis is positive in the direction of wave propagation, $z$ is the vertical coordinate measured positive upward, $\rho$ is the fluld density, $\mu$ is the viscosity, $p$ is pressure and $u, v$ are the local velocity terms in the horizontal and vertical directions respectively, defined

$$
u=-\frac{\partial \phi}{\partial x}
$$

and

$$
v=-\frac{\partial \phi}{\partial z}
$$

so that the condition of continuity is met by

$$
\frac{\partial^{2} \phi}{\partial x^{2}}+\frac{\partial^{2} \phi}{\partial z^{2}}=0
$$

given $\phi$ as the velocity potential Defining $d$ as the local water depth positive upward from the free surface, $U$ as the free stream velocity, and $\delta$ the boundary layer, the boundary conditions are
$z=-d, u=0, v=0$
at the bottom
$z=\delta, u=U(x, t)$
at the outer edge of the boundary layer
$z \rightarrow \infty, u=U(x, t)$
at the free surface

Introducing harmonic velocity components

$$
\begin{aligned}
& U(x, t)=\bar{U}(x)+U^{\prime}(x, t) \\
& U^{\prime}(x, t)=U(x) e^{i \sigma t}, \sigma=2 \pi / T \\
& \bar{U}^{\prime}(x, t)=0 \\
& u(x, y, t)=\bar{u}(x, y)+u^{\prime}(x, y, t) \\
& v(x, y, t)=\bar{v}(x, y)+v^{\prime}(x, y, t) \\
& p(x, t)=\bar{p}(x)+p^{\prime}(x, t)
\end{aligned}
$$

we integrate Eq 11 over one wave period and obtaın

$$
\rho\left(\frac{\partial \bar{u}}{\partial t}+\vec{u} \frac{\partial \bar{u}}{\partial x}+\overline{u^{\prime} \frac{\partial u}{\partial x}}+\bar{v} \frac{\partial \bar{u}}{\partial z}+\overline{v^{\prime} \frac{\partial u}{\partial z}}\right)+\frac{\partial \bar{p}}{\partial x}-v \frac{\partial^{2} \bar{u}}{\partial z^{2}}=0
$$

for the flow in the boundary layer Using the same procedure, the averaged expression for the flow outside the boundary layer will be

$$
\rho\left(\frac{\partial \bar{U}}{\partial t}+\bar{U} \frac{\partial \bar{U}}{\partial x}+\overline{U^{\prime} \frac{\partial \bar{U}^{\prime}}{\partial x}}+\bar{v} \frac{\partial \bar{U}}{\partial z}+\overline{v^{\prime} \frac{\partial \bar{U}^{\prime}}{\partial z}}\right)+\frac{\partial \bar{p}}{\partial x}-v \frac{\partial^{2} \bar{U}}{\partial z^{2}}=0
$$


Because its effect is negligible we can omit the viscous term in Eq 19 and extract the pressure term

$$
-\frac{\partial \bar{p}}{\partial x}=\rho\left(\frac{\partial \bar{U}}{\partial t}+\bar{U} \frac{\partial \bar{U}}{\partial x}+\overline{U \cdot \frac{\partial U^{\top}}{\partial x}}\right)
$$

having also neglected the terms contalning $v$, assuming that the vertical velocity 1a small Substituting Eq 110 into Eq 18 , gives

$$
\frac{\partial \bar{u}}{\partial t}+\bar{u} \frac{\partial \bar{u}}{\partial x}+\overline{u^{\prime} \frac{\partial u}{\partial x}}-\left[\frac{\partial \bar{U}}{\partial t}+\bar{U} \frac{\partial \bar{U}}{\partial x}+\overline{U^{\prime} \frac{\partial U}{\partial x}}\right]=\mu \frac{\partial^{2} u}{\partial z^{2}}
$$

whlch describes the total velocity distribution

Based on the parameter $\delta / L \ll 1$ (where $L$ is the wavelength), we make the assumption that the contributions represented by the nonlinear convectıve terms (except turbulence) are numerically not significant Therefore we rearrange $\mathrm{Eq} 111$ to show the defect velocity relationship

$$
\frac{\partial}{\partial t}(\bar{u}-\bar{U})=\frac{\partial}{\partial z}\left(\frac{\tau}{\rho}\right)
$$

where

$$
\begin{gathered}
\tau=\mu \frac{\partial u}{\partial z} \\
\tau=K_{L} \frac{\partial u}{\partial z}
\end{gathered}
$$

are the laminar and turbulent horızontal shear stress relationships respectively, and the dynamic viscosity $\mu=\rho \nu$ Eq 112 is the expression for osclllatory mean motion in the boundary layer based on potential theory According to Schlichting (1960), the validity of $\mathrm{Eq} 112$ can be established if the laminar boundary layer thickness

$$
\delta_{L}=(2 v / \sigma)^{\frac{1}{2}}
$$

\section{The Laminar Case}

Recalling that $u$ is the horizontal velocity in the boundary layer and $U$ just outside the layer, so that

$$
\begin{aligned}
& u(x, y, t)=U(x, t)=a / d \quad C \sin (k x-\sigma t) \\
& \lim _{z \rightarrow \delta}
\end{aligned}
$$

where $C$ is the wave celerity, "a" the wave amplitude and $k=2 \pi / L$ is the wave number Grosch (1962) has shown that for a/d « 1 or a/d near $(k x-\sigma t)=0$ the linearized theory in the laminar case provides an adequate description of the flow because the sum of all the terms $O(a / d)$ in the nonlinear solution for the bottom shear stress equal the linear solution and are hence negligible The solution becomes analogous to the Blasius series for steady flows 
The steady state solution of the velocity in the boundary layer can be written as

$$
u_{0}=\mathrm{U}\left[\sin (k x-\sigma t)-e^{-z / \delta_{L}} \sin \left(k x-\sigma t-z / \delta_{L}\right)\right]
$$

where the subscript "o" refers to boundary condutions, $\delta_{L}$ is defined in $\mathrm{Eq} 115$ and

$$
\mathrm{U}=\frac{\pi \mathrm{H}}{\mathrm{T}} \sinh ^{-1} \mathrm{kd}
$$

Is the velocity immedately outside the boundary layer

Iwagakl, et al (1967) based on Grosch's solution obtalned the approximate expression for the bottom shear stress

$$
\tau_{0} /\left(\rho \mathrm{u}_{0}{ }^{2}\right)=\mathbb{R}^{-\frac{1}{2}} \sin (\mathrm{kx}-\sigma \mathrm{t}-\pi / 4)
$$

where the local Reynolds number is

$$
\mathbb{R}=\frac{{ }_{u_{0}}{ }^{2} \mathrm{~T}}{2 \pi \nu}
$$

and the phase difference between $U$ and $\tau_{0}$ is $\pi / 4$

The maximum shear stress was glven by Iwagakl, et al (1967) as

$$
\begin{aligned}
\frac{\tau_{0 \max }}{\rho g H} & =\frac{\sqrt{2 v}}{g \sinh k d}\left(\frac{\pi}{T}\right)^{2 / 2} \\
\tau_{0 \max } & =\hat{\rho U}\left(\frac{2 \nu \pi}{T}\right)^{1 / 2}
\end{aligned}
$$

whlch shows that the amplitude ^, of the local boundary shear stress is a function of the local free stream velocity amplitude (therefore the water particle excursion distance) and a boundary layer thickness The wave helght $H=\frac{1}{2} a$ is applicable to sinusoldal wave profiles

In the lamnar case we can describe the flow regime with the appropriate Reynolds number, which should contain the parameters for the boundary layer thickness, local velocity and viscosity Hence, re-arranging Eq 25 , we get

and

$$
\begin{aligned}
& \mathbb{R}^{-\frac{1}{2}}=\frac{1}{U}\left(\frac{2 \nu \pi}{T}\right)^{\frac{1}{2}}=\frac{U}{U} \quad \frac{\sqrt{2}}{\delta_{L}} \\
& \mathbb{R}^{\frac{1}{2}}=\frac{U \delta_{L}^{\prime}}{V}=\mathbb{R}_{S}
\end{aligned}
$$

where $\delta_{I}^{\prime}=(\nu / \sigma)^{\frac{1}{2}}=\delta_{I} / \sqrt{2}$ for smooth bottom The Reynolds number in $\mathrm{Eq} 28$ is Identical with Kajlura's (1968) and that of Horlkawa and Watanabe (1968) 
For laminar flow we now nominally define the wave friction coefficient to be of the form

$$
c_{f} \hat{U U}=n\left(\tau_{0} / \rho\right)
$$

where $n$ must be determined from experıments For the case of smooth bottom laminar flow Kajiura (1968) approximates the amplitude of the friction coefficient by

$$
\hat{C}_{f}=1 / \mathbb{R}_{S} \text { for } \mathbb{R}<200
$$

for the case of the smooth boundary Introducing now $z_{0}$, as the characteristic roughness length, so that when

$$
D=30 z_{0}
$$

we have the equıvalent Nikuradse's roughness expressed, we follow Kajlura's notation and write the Reynolds number for the rough boundary as

$$
\mathbb{R}_{R}=\frac{\hat{U D}}{v}
$$

and the friction coefficlent can be expressed as

$$
\hat{\mathrm{C}}_{\mathrm{f}}=170\left(\frac{\hat{\mathrm{v}}}{\sigma \mathrm{z}_{\mathrm{o}}}\right)^{-2 / 3}
$$

\section{The Turbulent Case}

From experıments of steady turbulent flow (e $g$, see clauser, 1954) we know that the turbulent boundary layer structure is threefold, consisting of the inner or laminar sublayer at the wall, the outer or defect layer near 1 ts outer edge, the two connected by the overlap layer A simılar breakdown for oscillatory layers was suggested by Jonsson (1966)

The general form of the turbulent velocity profile can be written as

$$
\frac{u}{u *}=A \log \left(\frac{z u^{*}}{v}\right)+C
$$

whlch in the presence of roughness on the wall is modified to read

$$
\frac{\mathrm{u}}{\mathrm{u}^{*}}=\mathrm{A} \log \left(\frac{\mathrm{zu} *}{v}\right)+\mathrm{C}-\frac{\Delta \mathrm{u}}{\mathrm{u}^{*}}
$$

where $\Delta u / u^{*}$ represents the vertıcal shift of the logarıthmıc profile caused by the roughness elements, $\mathrm{A}$ and $\mathrm{C}$ are constants to be apprassed experimentally and

$$
(u *)^{2}=\tau / \rho
$$

Similarly

$$
\left(u_{0}^{*}\right)^{2}=\left(\tau_{0} / \rho\right) \quad z=0
$$

is the shear velocity 
and the shear stress is given by

$$
\tau=\mathrm{K}_{\mathbf{z}} \partial \mathrm{u} / \partial \mathbf{z}
$$

$\mathrm{K}_{\mathrm{z}}$ is Kajlura's (1968) eddy viscosity ( $1 \mathrm{e}$, the Boussinesq effective vis cosity) of the form

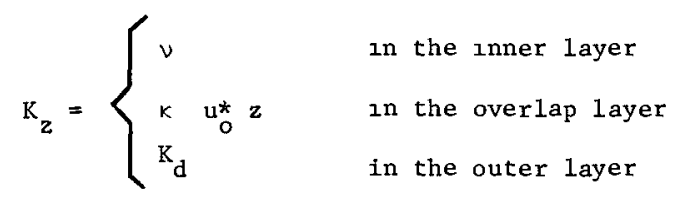

where $\mathrm{K}_{\mathrm{d}}=\mathrm{K} \hat{\mathrm{U}} \delta_{\mathrm{L}}^{\prime}, \mathrm{K}=02$ is a universal constant, $\delta_{\mathrm{L}}^{\prime}$ is defined in $\mathrm{Eq}$ 115 and $k=04$ is Kármán's unlversal constant Eq 36 is in relation to a smooth boundary For the rough case the overlap expression of Eq 36 applies for $z_{0}<z<\delta$, except when $\left(\delta_{L}^{\prime} / D\right)<1$ in which case

$$
\mathrm{k}_{\mathrm{z}}=553 \mathrm{ku}_{\mathrm{o}}^{*} \mathrm{z}_{\mathrm{o}}
$$

for the laminar sublayer Kajıura's eddy viscosity assumption is based on analogy to steady state flow It presents the possibility, however, of being an improved estimate because it takes the structure of the layer into consideration But as Clauser (1956) pointed out for steady flow, "the turbulent eddies introduce shearing stresses for which no reliable method of calculation exists", and this phenomenon should only be more complex in time perıodic flows

Even small changes in bottom roughness will drastically alter the profile of the turbulent boundary layer, which makes the vertical distribution of $\mathrm{K}_{\mathrm{z}}$ difficult to establish Liu (1967, as quoted by Kline, 1969, vo1 I, p 529) showed that changing $z_{0}$ can result in a change of $\mathrm{K}_{\mathbf{z}}$ by a factor of four in the defect layer Indeed, Horikawa and Watanabe (1968) showed that $K_{z}$ attenuates with respect to $z$ on both smooth and rippled boundarles, therefore further research is needed before accepting the formulations given $1 \mathrm{n} \mathrm{Eq} 36,37$, and 38

The friction coefficient for the smooth bottom in turbulent flow is given by Kajiura as

$$
\hat{c}_{f}=\left(\mathbb{R}_{S} m_{L}\right)^{-2} \text { for } \mathbb{R}_{S}>200
$$

with

$$
\mathrm{m}=\frac{1}{2} \sqrt{\frac{\mathrm{K}}{\mathrm{N}}}, \quad \mathrm{N}=\text { Const } \simeq 12 \quad \text { assumed }
$$




$$
\begin{aligned}
& y_{L}=\frac{2 D_{L}}{\sqrt{k N} \delta_{L}} \\
& D_{L}=N \nu / \hat{u}^{*}
\end{aligned}
$$

where $y_{I}$ is the distance between the bottom and the lower limit of the overlap layer, we obtann the the ald of Eq 28 the approximate expression

$$
\hat{\mathrm{C}}_{\mathrm{f}}=\left(\frac{\hat{\mathrm{a}} \mathrm{t}}{\hat{\mathrm{U}}}\right)^{2}
$$

For rough boundaries Kajlura gives

$$
\hat{\mathrm{c}}_{\mathrm{f}}=\left(\frac{60 \mathrm{z}_{\mathrm{o}}^{\sigma}}{\mathrm{kOy_{ \textrm {R } }}}\right)
$$

where $y_{R}$ is the upper limit of the laminar sublayer

$$
\text { For } \hat{\mathrm{U}} / \sigma \mathrm{z}_{\mathrm{o}}<1000 \mathrm{Eq} 314 \text { can be approximated by Eq } 213
$$

\section{The Preston Probe}

Preston (1954) assumed that in turbulent flow on a smooth boundary a region must exist close to the wall in which the "law of the wall" of the form

$$
\frac{\mathrm{U}}{\mathrm{u}^{*}}=\mathrm{f}_{1}\left(\frac{\mathrm{zu^{* }}}{\mathrm{v}}\right)
$$

applies, so that the local shear stress at the boundary $\tau_{0}$, can be related to the velocity distribution by medsuring the differential pressure $\Delta p$, with a modified Pitot tube in contact with the wall The inter-relations are

$$
\frac{\Delta \mathrm{p}}{\tau_{0}}=f_{2}\left(\frac{\mathrm{u} * \mathrm{~d}}{\nu}\right)
$$

and

$$
\frac{\tau_{0}}{\rho v^{2}}=f_{3}\left(\frac{\Delta \mathrm{pd}^{2}}{v^{2} \rho}\right)
$$

where $f_{2}, f_{3}$ denote functional dependence, and $d$ is the outside diameter of the probe The logarithmlc expression obtalned by Preston (1954)

$$
\log _{10} \frac{\tau_{0} \mathrm{~d}^{2}}{4 \rho v^{2}}=\overline{2} 604+7 / 8 \log _{10} \frac{\Delta \mathrm{pd}^{2}}{4 \rho v^{2}}
$$

was modified by Hsu (1955) for the Jaminar sublayer (hence for laminar 
boundary layers) to read

$$
y *=\frac{1}{2} \log _{10}\left(\frac{8}{4+t^{2}}\right)+\frac{1}{2} x *
$$

and for the turbulent portion

$$
\mathrm{y}^{*}=\log _{10} \mathrm{k}+\frac{7}{8} \mathrm{x}^{*}
$$

Where $y^{*}=\log _{10}\left(\tau_{o d} / 4 \rho \nu^{2}\right)$ and $x^{*}=\log _{10}\left(\Delta \rho d^{2} / 4 \rho \nu^{2}\right), t=$ the rat1o of inner to outer diameter of the stagnation tube and should be in the vicinity of $06, k=I(t)$ and $I(t)$ is given by Hsu in tabulated form

Preston also advanced the hypothesis that the relationships presented In Eqs 42 and 43 are 1 ndependent of the $x$-wise pressure gradient in the turbulent boundary layer Patel (1965) indicated, however, that for severe favorable and adverse pressure gradients the Preston probe overestimates skin friction The analytical solution given by Yalin and Russe11 (1966)

$$
\tau_{0}=\rho\left(\alpha U^{2}+\beta g S \delta\right)
$$

given $g$ as the gravitational acceleration and $\alpha, \beta$ as empirical constants, takes into consideration the instantaneous position of the free surface $S$, thus the pressure gradient It is clear therefore, that to avold the influence of vertical accelerations on the local static pressure, as well as the influence of wave set-down near the breaker zone, 1nitial evaluation of flow parameters with the Preston probe must be restricted to $S=0$, $1 e$, to the wave crest and wave trough, or approximately $(\mathrm{kx}-\mathrm{t})=0$

\section{EXPERIMENTAL APPARATUS AND PROCEDURE}

Experıments were carried out in a fixed level, concrete floor, concrete and plexiglas-wall wavetank designed by the authors and constructed in 1968-69 at Loulsiana State University Dimensions of the channel were $65 \times 3 \times 3$ feet and it is shown in Figure 1 A fixed angle beach was constructed at the downstream end of the tank w1th a slope of 1125 , covered with aluminum sheet to provide a smooth surface For part of the tests sand of uniform size was attached to the beach face in thickness of one grain dimension $\left(z_{0}=00123 \mathrm{ft}\right)$

Resistance type wave gauges and the Preston probe were suspended from a forward-reverse gear, varıable speed carriage and positioned with the ald of point gauges A Sanborn Model 150 oscillograph served as the excitation source and recording unit for the wave and pressure recording Waves with fixed periods of $T=10,15,20$ seconds were generated with a bottom-hinged paddle-type wavemaker The surface configuration of these waves were quite as ymetric for low frequencies and high amplitudes, consequently they were damped using various combinations of baffles following recommendations of Keulegan (1968) An undamped short perıod wave train is shown in Figure 1 
Figure 1. Experimental set-up

showing the wavetank, instrument carriage with Preston probe, and pressure transducer. Beach is nearest observer.

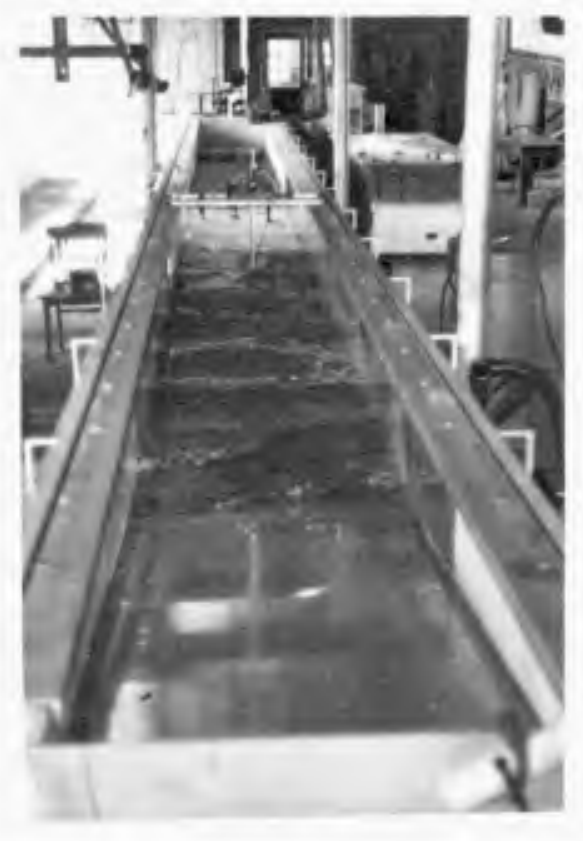

Since the Preston probe was to be used in wave boundary layers, the calibration procedure had to conform to the oscillatory motions experienced. For this purpose a variable stroke, variable frequency calibrating apparatus was built whose real time response was recorded by a linearsyn differential transformer. This enabled calculation of both the pressurevelocity relationship for the probe and the associated time lag. Differential pressures were sensed by a Pace 90B pressure transducer.

For the given wave periods the wavemaker stroke was changed to provide two wave amplitudes, noted as "large" and "small" in the graphs.

Water depth was fixed at 2.0 feet in the horizontal floor portion of the tank. Waves were measured in this part of the channel as well as adjacent to the Preston probe, aligned with the sloping bottom (Figure 2). The definition sketch for the probe is presented in Figure 3. Measurements of the differential pressure were made under the wave crests and troughs only by orienting the probe upslope and downslope, keeping other conditions the same. Adjustments for the phase difference between the surface, the free stream velocity and the velocity in the boundary layer were recorded. Measurements were carried out first on a smooth, then on a roughened bottom keeping other conditions the same. Water temperature was regularly recorded during the experiments. 


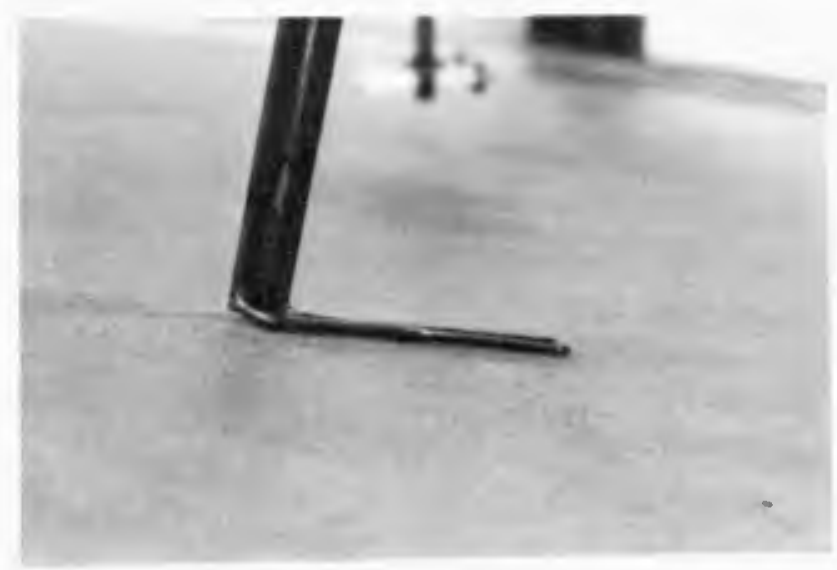

Figure 2. Preston probe al1gned with rough boundary. Static probe is in front.

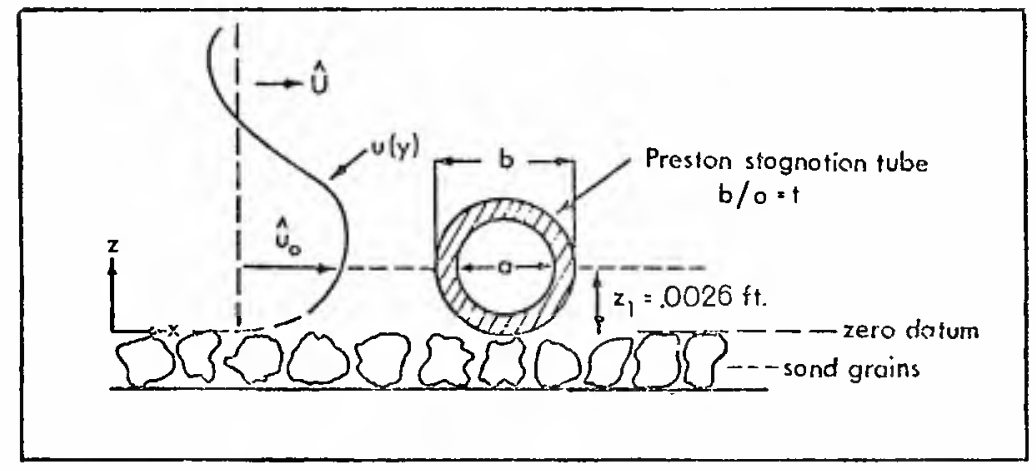

Figure 3. Preston probe resting on rough boundary, definition sketch

\section{EXPERIMENTAL RESULTS}

Rigorous evaluation of the velocity distributions on a slope, relative to waves undergoing transformation and without specific knowledge about boundary layer structure, growth and separation during a wave cycle will have to awalt further experimentation and a solution to the nonlinear phenomena experlenced. Results presented on the velocity profiles on a sloping bottom are therefore exploratory only. In Figures 4, 5, 6, a set of profiles are illustrated from one of the experiments conducted on a smooth bottom with "small" amplitude waves. 

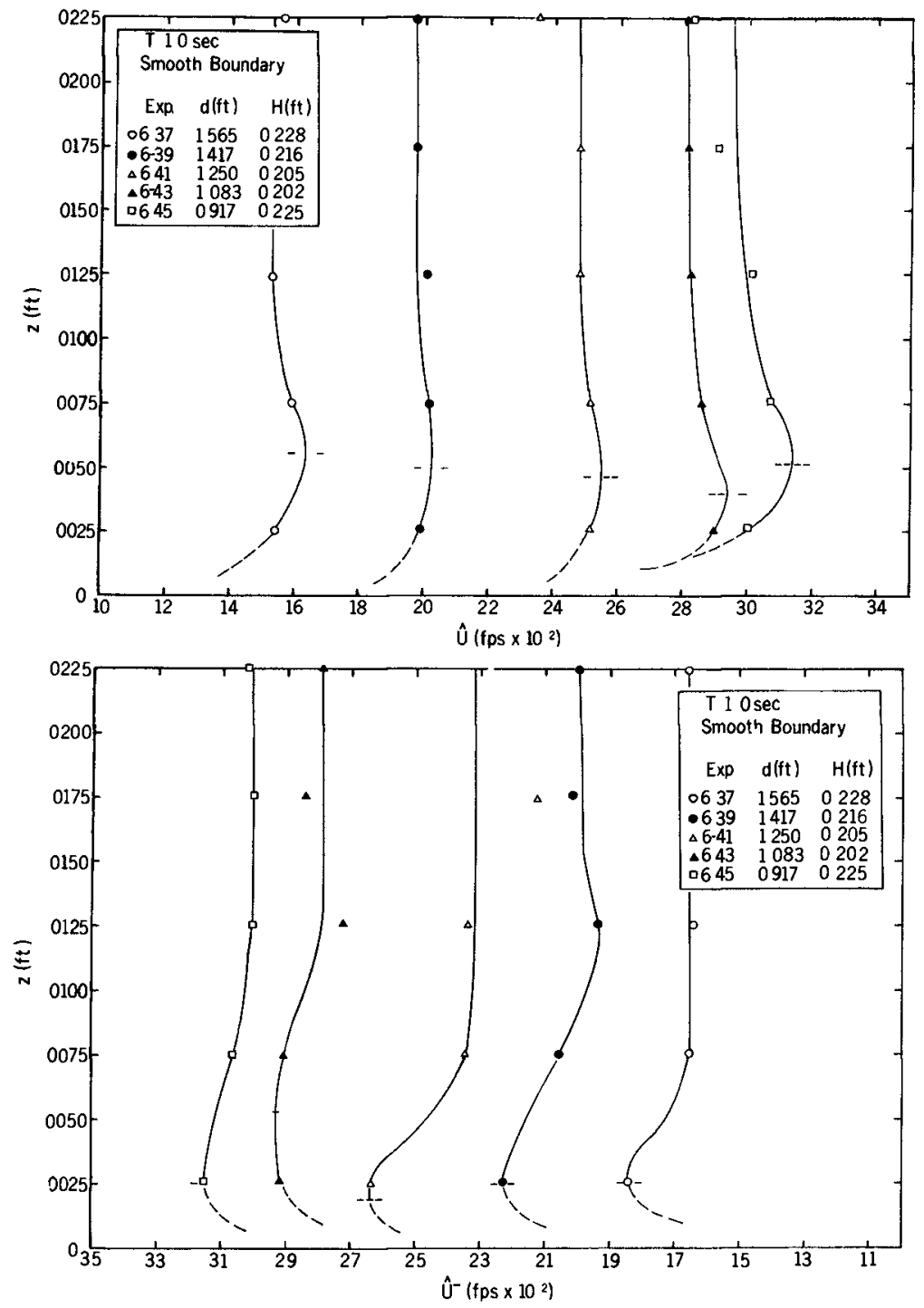

Figure 4 Experimental velocity profiles for $\mathrm{T}=10$ second on smooth boundary Upper graph shows measurements under wave crests, lower graph, wave troughs 

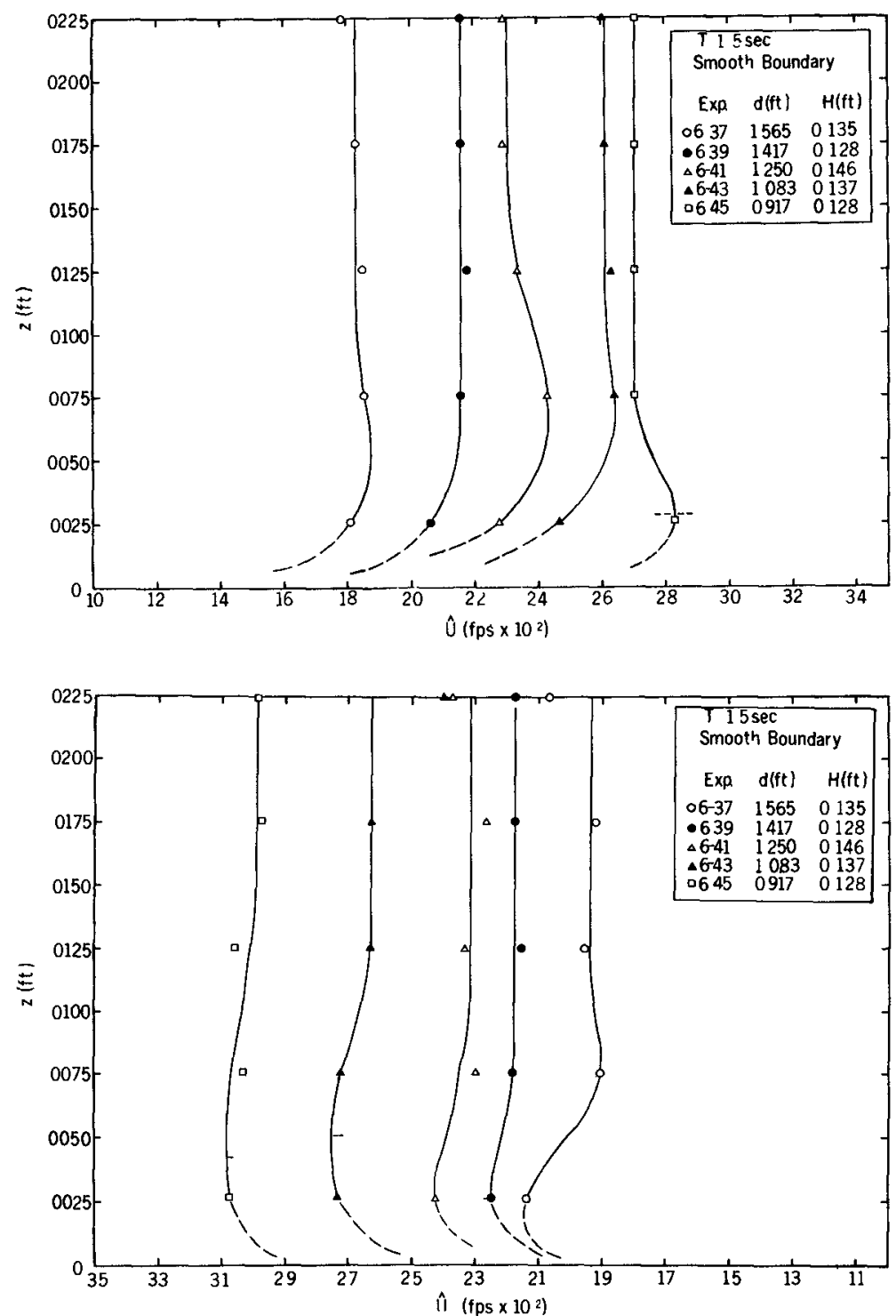

Figure 5 Experimental velocity profiles for $T=15$ seconds on smooth boundary Upper graph refers to wave crests, lower refers to wave troughs 

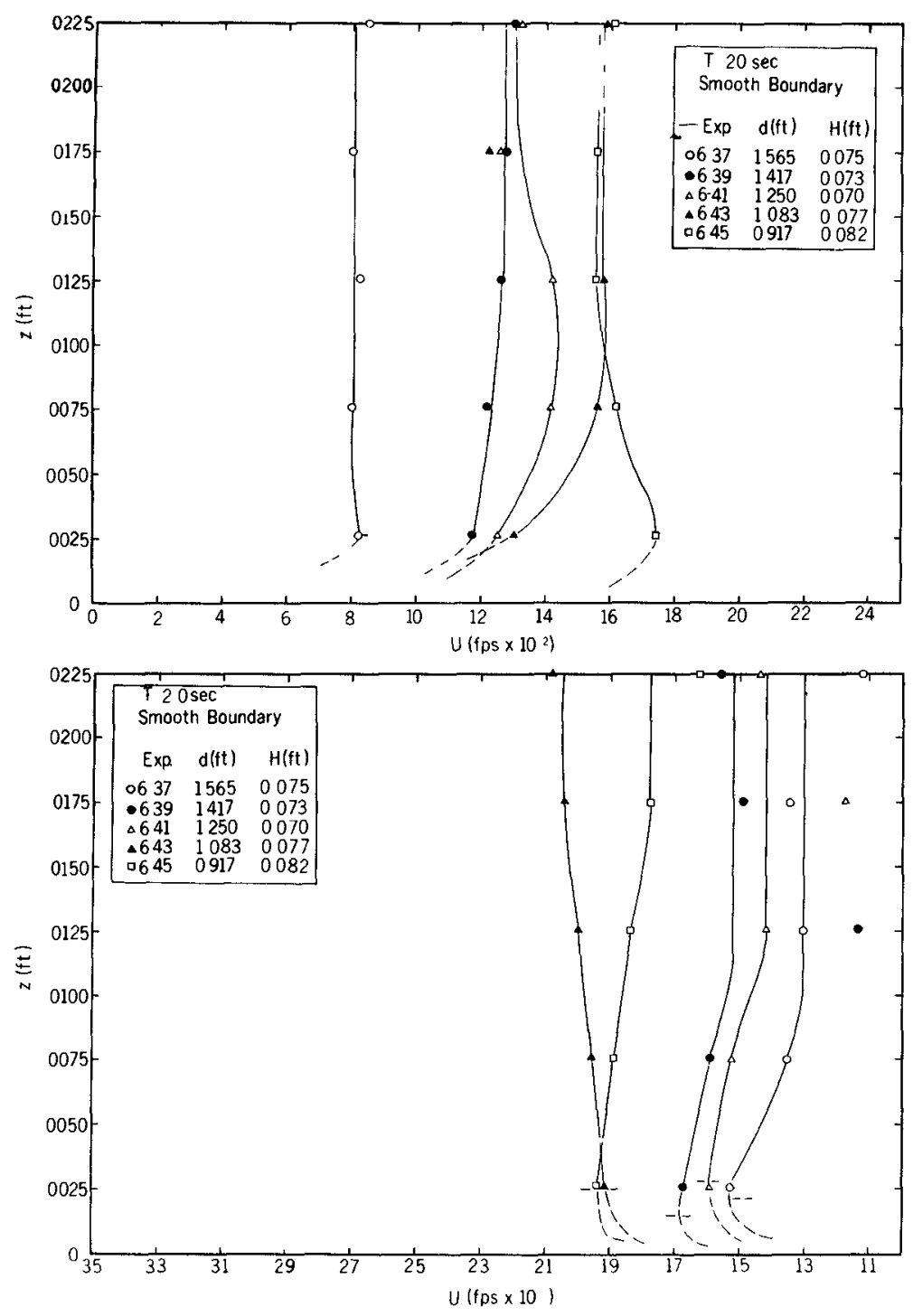

Figure 6 Experimental velocity profiles for $T=20$ seconds on smooth boundary Upper graph measurements with respect to wave crests Lower graph wave troughs 


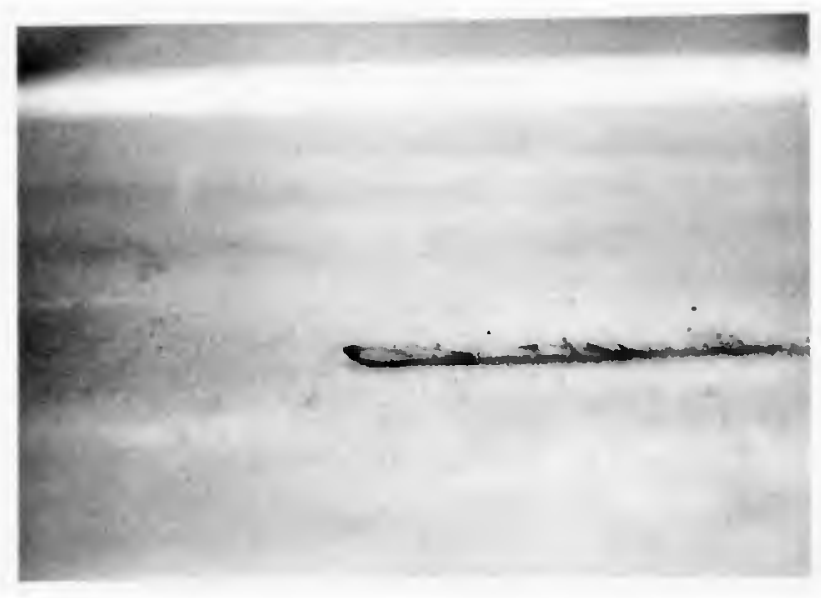

Figure 7. Boundary layer obtained with injected dye on a rough boundary.

Visual confirmation of the presence of a bounaary layer is shown in Figure 7, showing displacements of a dye streak upslope, indicative of incremental mass transport with successive wave cycles. The dashed lines of Figures 4 - 6 refer to the elevation of the maximum velocity in the boundary layer $a_{0}$ max following the procedure of Eagleson, Dean and Peralta (1958). Experimental values of $\hat{0}$, were found to agree very well for "deep" water on the slope, but the agreement declined for decreasing water depth and the linear model was found to overestimate the measured values. It should be noted the maximum reflection $\left(\mathrm{H}_{r} / \mathrm{H}_{f}\right)$ was less than $10 \%$ for any one test. Grosch's (1962) criterion for neglecting nonlinear terms in the $\mathrm{N}-\mathrm{S}$ equation was $\mathrm{a} / \mathrm{d}<.01$; it was exceeded in all cases. The effect of the convective velocity terms should be additive under the crest and subtractive under the wave trough with respect to linear values (Eq. 2.3).

Experimental data on boundary layer thicknesses are presented in Table 1 for various combinations of wave amplitude, water depth and wave period. Values of $\hat{\delta}$ exceed the theoretical $\delta_{\text {I }}$ (Eq.1.15), by factors of 3 to 8 . Boundary layer thicknesses were found to be greater under wave crests than under wave troughs, this is no doubt influenced by vertical accelerations prior to attainment of $\hat{U}_{\max }$ under the crest. Growth of the layer is also affected by the contribution of upslope mass transport which allows a longer excursion distance for its development. This phenomenon occurs on both smooth and rough boundaries. The effect of roughness is to increase $\hat{\delta}$. The contribution is more under wave crests and this is to be expected because the rate of boundary layer growth accelerates with increasing roughness element size. Wave amplitude influence on $\hat{\delta}$ was difficult to discern, more tests are needed to evaluate this relationship. In some cases, boundary layers were observed to grow to some maximum value offshore of the breaker zone from which point $\hat{\delta}$ diminished shoreward, the reversal usually taking place at $d \simeq 1.0$ foot. 
Table 1 EXPERIMENTAL BOUNDARY LAYER THICKNESSES ( In $10^{-3}$ feet)

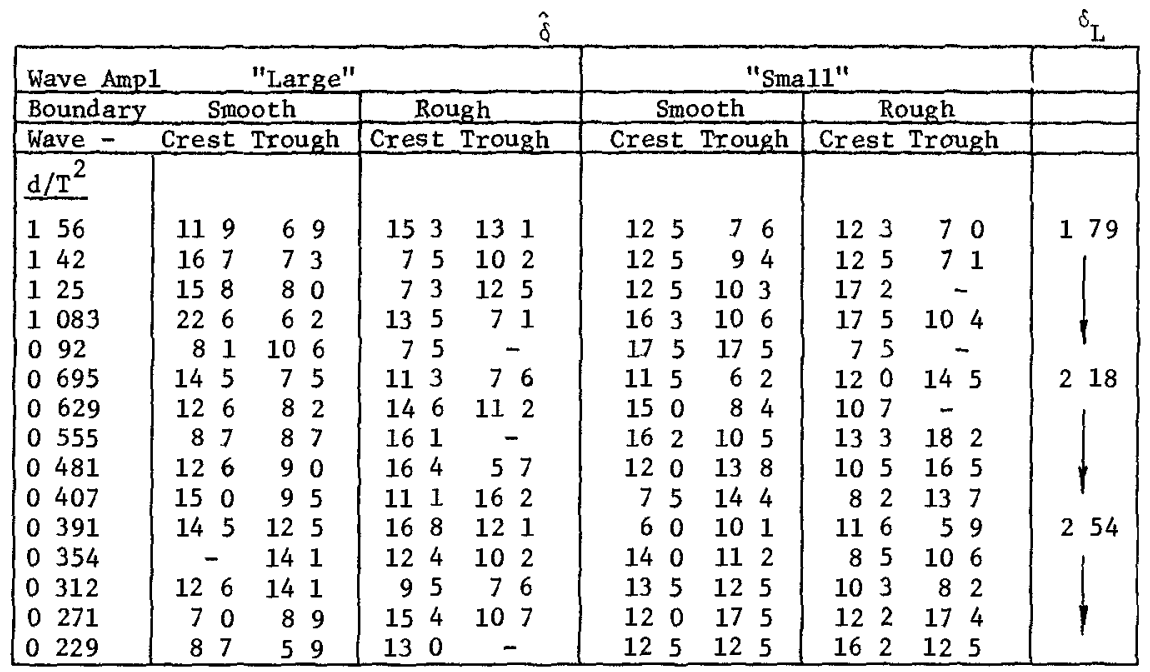

The classification of boundary conditions in terms of the prevalent flow regime, 1 e to establish where lamınar gives way to turbulent flow is a difficult task Kajıura specified the transition region as

$$
\begin{aligned}
25 & <\mathbb{R}_{\mathrm{S}}<650 \text { for smooth bottom } \\
\text { and } \quad 100 & <\mathbb{R}_{\mathrm{R}}<1000 \text { for rough bottom }
\end{aligned}
$$

Collins' (1963) critıcal Reynolds number of 113 (by transformation) is in the range for IR $\mathrm{I}_{\mathrm{S}}$ Both ranges are wide and until a universal velocity distribution for osclllatory boundary layers is established, we do not know when to assume inception of turbulence or when full turbulence appears Considerable data fell into the transition ranges specified above The critical Reynolds numbers of $\mathbb{R}_{\mathrm{S}}=250$ for the smooth bottom and $\mathbb{R}_{\mathbb{R}}=500$ for the rough boundary are proposed

Evaluation of boundary shear was based on Eqs 45 and 46 , using the given critical Reynolds numbers Maximum amplitudes of the shear stress were corrected for phase lag The distribution of $\tau_{0}$ max upslope 15 shown in Figure 8 corresponding to wave crests and in Figure 9 for wave troughs

Although the rate of 1ncrease varies depending on wave period and amp11tude, the trends are linear The dependence on wave amplitude is clearly discernible, higher shear stresses are associated with "large" amplitude waves In "deep" water inxtial values of $\tau_{0}$ max are smaller undex wave troughs than under wave crests Convergence was noted, however, for both cases of boundary conditions on nearing the breaker zone, indicating also that the effect of roughness used becomes negligible for very shallow water 

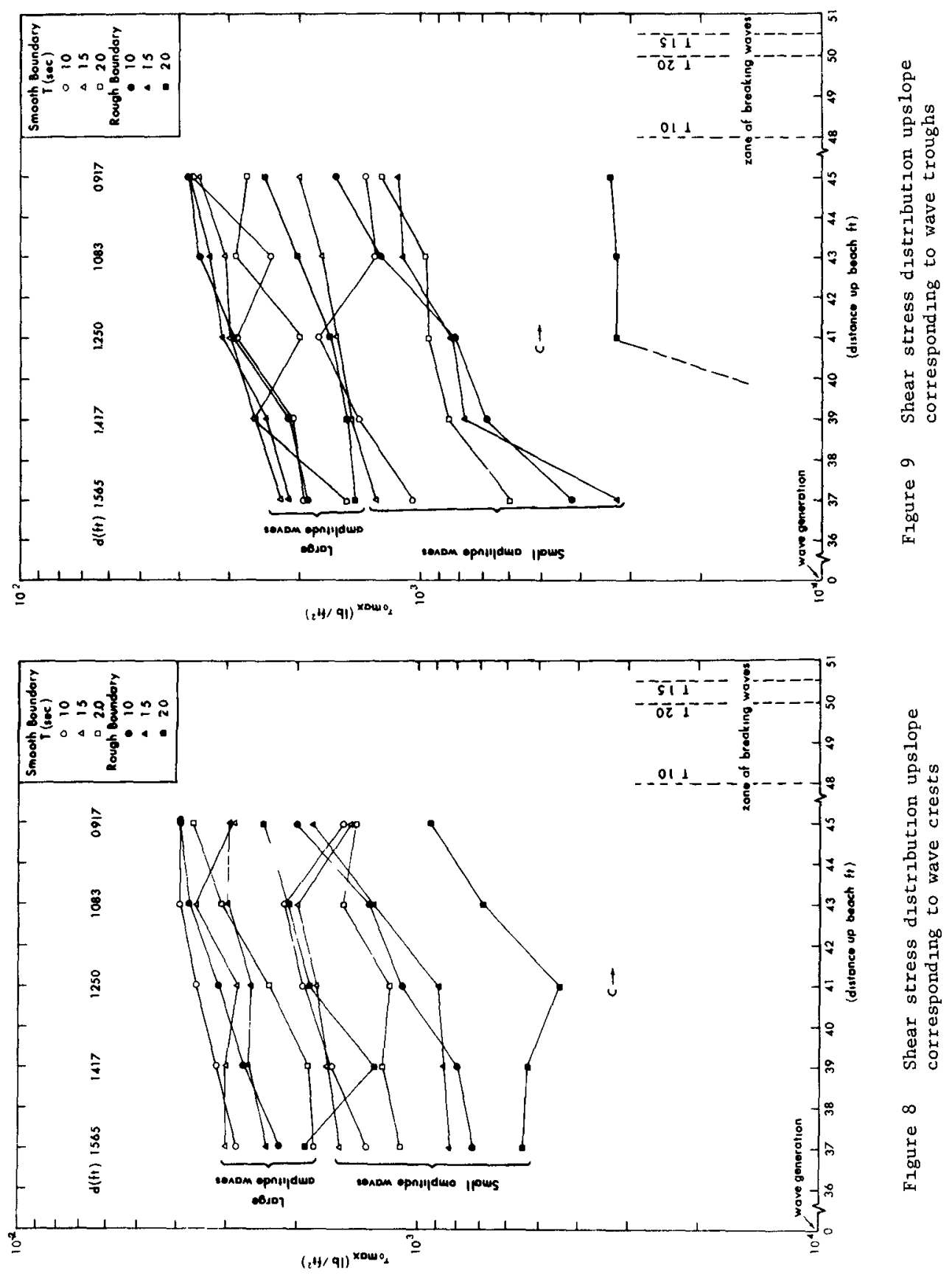


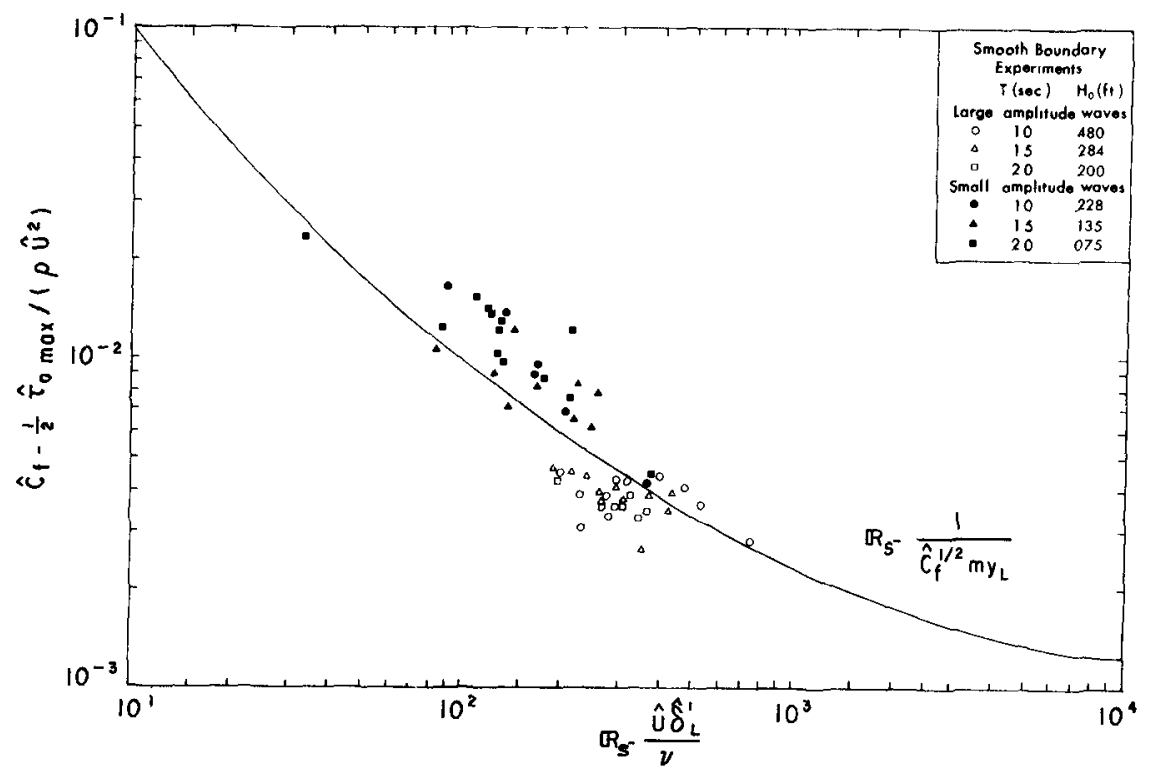

Figure 10 Relation between the coefficient of friction $\hat{C}_{f}$ and the Reynolds number $\mathbb{R}_{S}$ for smooth boundary

The relation between the shear stress and the corresponding velocity is dependent upon the coefficient of friction, whose value is a function of boundary conditions and the local flow regime For the smooth bottom Figure 10 is presented where agreement between Kajlura's curve and the computed $\hat{C}_{f}$ Is very good for $n=\frac{1}{2}$ ThIs is in contrast with $n=2$ for the rather widely used $\vec{C}_{f}$ ( e $g$, see Eagleson, 1959) It is shown that the friction coefficient increases for decreasing wave amplitude

In presence of boundary roughness, Eq 213 ss applicable when $0 / \sigma z_{0}<1000$, and this condition is validated for the test cases In Figure 11 the linear trends show that the friction coefficient increases for increasing wave frequency, decreasing Reynolds numbers and decreasing wave amplitude 


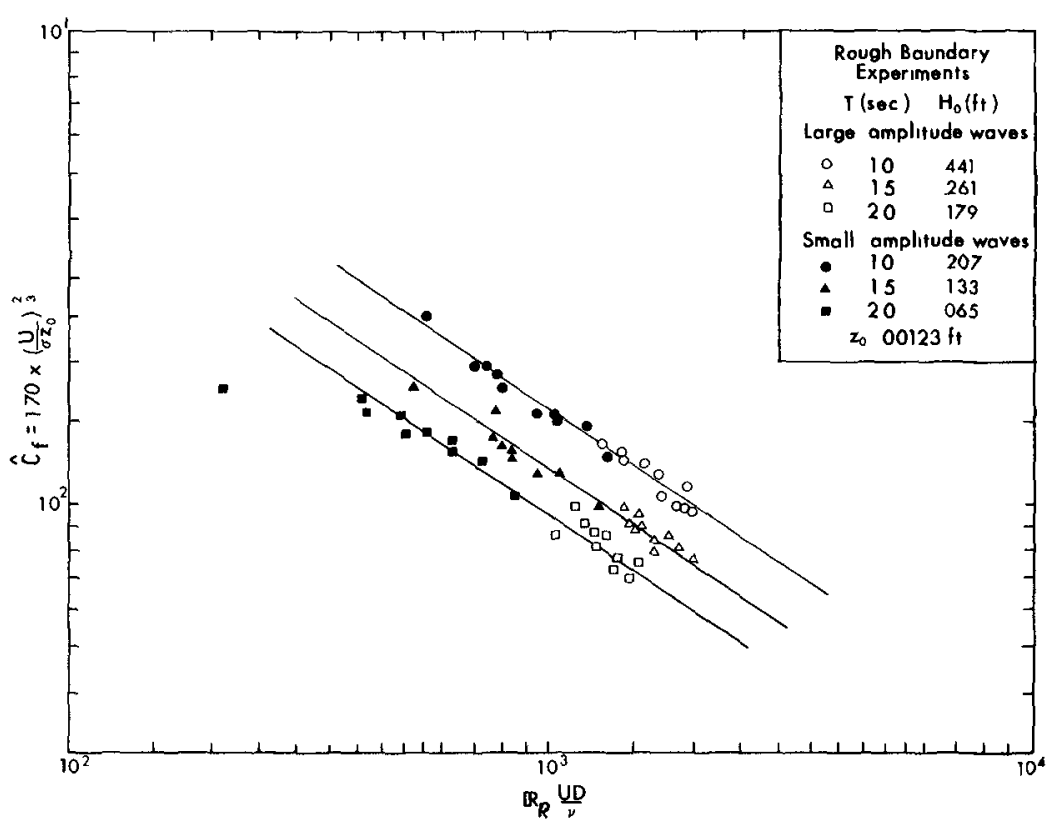

Figure 11 Relation between the coefficient of friction $\hat{C}_{f}$ and the Reynolds number $\mathbb{R}_{R}$ for rough boundary

\section{CONCLUSIONS}

Laboratory investigations of boundary layer thickness, near bottom velocity profiles and bottom friction were carried out on a slope with the ald of a Preston probe in hopes of inftiating greater understanding about littoral sediment transport The use of Preston probe is limited under oscillating flow conditions although its simplicity of construction and applicability to both laminar and turbulent flows is appealing Wave boundary layers are complex phenomena in time and space and their analysis on a sloping bottom presents considerable difficulties The thickness of fully developed layers greatly exceeds the theoretical, and this is probably the result of non-negligible vertical accelerations and the presence of mass transport The nonlinear effects could not be appraised for the velocity distribution and the agreement between the theoretical and experimental reference velocity only occurs for relatively deep water The shear stress distribution appears to have a linear relation to decreasing water depth, with higher amplitudes as wave helght is increased 
Clauser, F, 1954, Turbulent boundary layers in adverse pressure gradients, $J$ Aero Sci, 21 91-108

Clauser, F, 1956, The turbulent boundary layer, Adv in Appl Mech, 4 1-51, Academic Press, New York

Collins, J I , 1963, Inception of turbulence at the bed under periodic gravity waves, J Geophys Res 68(21) 6007-6014

Eagleson, P S, 1959, The damping of oscillatory waves by laminar boundary layers, Mass Inst Tech, Hydrodyn Lab Tech Rpt 32, $37 \mathrm{p}$

Eagleson, $P$ S, Dean, $R$ G and Peralta, L A, 1958, The mechanics of the motion of discrete spherical bottom sediment particles due to shoaling waves, U S Army Corps of Engrs, Beach Erosion Board Tech Memo 104, $41 \mathrm{p}$

Ghosh, S N and Roy, N, 1970, Boundary shear in open channel flow, Proc ASCE, J Hydr DIV , 96 (HY4) 967-994

Grosch, C E, 1962, Laminar boundary layer under a wave, Phys of Fluids $5 \quad 1163-1167$

Horikawa, $\mathrm{K}$ and Watanabe, A, 1968, Laboratory study on oscillatory boundary layer flow, Coastal Engineering in Japan 11 13-38

Hsu, E Y, 1955, The measurement of local turbulent skin friction by means of surface Pitot tubes, David Taylor Model Basin Rpt 957

Hwang, L S and Laursen, S M, 1963, Shear measurement technique for rough surfaces, Proc ASCE, J Hydr Dıv 89(HY2) 19-37

Iwagakı, Y , Tsuchiya, $\mathrm{Y}$ and Chen, $\mathrm{H}, 1967$, On the mechanısm of lamınar damping of oscillatory waves due to bottom friction, Bull Dis Prev Research Instıtute, Kyoto Unıversity, 16(3), 49-75

Iwagak1, Y, Tsuchıya, $\mathrm{Y}$ and Saka1, M, 1965, Basıc studies on the wave damping due to bottom friction, Coastal Engrg in Japan, 8 37-49

Jonsson, I G , 1963, Measurements in the turbulent wave boundary 1ayer, Proc IAHR Conf, London, 85-92

Jonsson, I G, 1966, On the exIstence of universal velocity distrubutions In an oscillatory turbulent boundary layer, Coastal Eng Lab Tech University, Denmark, Basıc Res Prog Rpt 12 2-9

Kajiura, K, 1968, A model of the bottom boundary layer in water waves, Bul1 Earthquake Res Inst, Tokyo Unıversity 46 75-123

Keulegan, $G \mathrm{H}, 1968$, Wave damping effects of screens, $U \mathrm{~S}$ Army Corps of Engıneers Waterways Exp Sta Rpt 2-12, 62 p

K1ıne, S J, Morkovin, M V , Sovran, G and Cockrel1, D J , 1969, Computation of turbulent boundary layers - 1968, Proc AFOSR-IFPStanford Conf, vol I, p 529

Longuet-Higgins, M 'S, 1958, The mechanics of the boundary layer near the bottom in a progressive wave, Proc 6th Conf Coastal Englneerıng, Galnesville, Fla, Ch 10 184-193

Nece, $R$ E and Smzth, J D, 1970, Boundary shear stress in rivers and estuaries, Proc ASCE, J Waterways \& Harbors Dıv 96(WW2) 335-358

Patel, V C , 1965, Calibration of the Preston tube and 11mitations on its pressure gradients, J Fluid Mech 23(1) 185-208

Preston, J H, 1954, The determination of turbulent skin friction by means of PItot tubes, J Royal Aero Soc 58 109-121

Schlichting, H, 1960, Boundary layer theory, McGraw-Hil1, N Y $647 \mathrm{p}$

Sleath, J F A, 1968, The effect of waves on the pressure in a bed of sand in a water channel and on the velocity distribution above it, unpub Ph D Thesis, St John's College, Cambridge, $141 \mathrm{p}$

Yalın, M S and Russell, R C H 1966, Shear stresses due to long waves, J Hydr Res , 4(2) 55-98 


\title{
CHAPTER 18
}

\author{
VARIATION OF LONGSHORE CURRENT \\ ACROSS THE SURF ZONE
}

\author{
Edward B Thornton \\ Assistant Professor of Oceanography \\ U S Naval Postgraduate School \\ Monterey, California
}

\section{ABSTRACT}

The wave-induced longshore current variation across the surf zone is described for a simplified model The basic assumptions are that the conditions are steady, the bottom contours are stralght and parallel but allow for an arbitrary bottom profile, the waves are adequately described by linear theory, and that spilling breakers exist across the surf zone Conservation equations of mass, momentum, and energy, separated into the steady and unsteady components, are used to describe second order-wave-induced phenomena of shoaling waves approaching at an angle to the beach An expression for the longshore current is developed, based on the alongshore component of excess momentum flux due to the presence of unsteady wave motion Wave set-down and set-up have been included in the formulation Emphasis in the analysis is placed on formulating usable predictive equations for engineering practice Comparison with experimental results from the laboratory and field show that if the assumed conditions are approximately fulfilled, the predicted results compare quite favorably

\section{INTRODUCTION}

A knowledge of the variation and extent across the surf zone of the longshore current is important in design considerations of structures placed in the littoral area This kind of information is particularly important for groins or similar structures designed to impede sand movement since the longshore current is a primary mechanism for sand transport On the other hand, it is often desirable to have natural bypassing about jetties constructed for navigational purposes at inlets and harbors The distribution of effluent, introduced onto beaches and into the littoral zone, is also influenced by the currents in the surf zone There is a very real need for a more complete understanding of the littoral zone so that further improvements and preservation of our beaches can be based on more rational and concrete approaches

The study of the area in and about the surf zone presents a difflcult problem due to Its very complex nature A proper treatment of the surf zone must consider a three-dimensional problem of unsteady fluid motion and is further complicated by moving interfaces at the upper and lower boundarles, that 
is, at the water surface and sediment bottom The hydrodynamics of the littoral zone can be characterized by two idealized systems of elther a longshore current or rip current system which can occur for seemingly simllar conditions Thus, it is necessary to make defincte and simplifying assumptions in order to make the problem tenable to a theoretical approach

This analysis considers the steady-state distrubution of quantities on a line normal to the shoreline A schematic of the surf zone area is shown in Figure 1 The analysis is restricted to the case of an arbitrary bottom profile with straight and parallel contours in the y-direction (parallel to the beach) The $\mathrm{x}$-direction is perpendicular to the beach

\section{CONSERVATION EQUATIONS}

A convenient starting point for this analysis is a statement of the general conservation equations of mass, momentum, and energy fluxes applicable to unsteady flow The analysis is not concerned with the internal flow structure of the fluid, hence, the derivation can be simplified by integrating the conservation equations over depth Conservation equations which have already been developed by Phillips [I] are used and are presented below

The conservation equations are applied to wave motion, but they are equaliy applicable to general turbulent motion The unsteady velocity field of the wave motion can be expressed in the same manner as in the treatment of turbulent motion as the sum of lts mean and fluctuating parts

$$
\vec{u}=\left(U_{I}(x, y, t)+u_{I}^{\prime}(x, y, z, t), w(x, y, z, t)\right) \quad I=1,2
$$

where $(1,2)$ refer to the horlzontal coordinates $(x, y)$, respectively, and $z$ is the vertical coordinate The tensor notation ls used only for horizontal components of water particle motion The mean current is assumed uniform over depth for simplicity The pressure term can be stated similarly These expressions can be substituted into the mass, momentum, and energy equations, and the mean and fluctuating contribution Identified

The conservation equations are averaged over depth and time (one can consider averaging over a few wave periods) For the case of waves superposed on a mean current, all the wave motion is identified with the fluctuating quantity which, when integrated over the total depth, can contain a mean contribution due to the waves The time averaging of the equations for a general development, belng over a short interval compared to the total time, does not preclude long term unsteadiness in the mean motion

\section{A Conservation of Mass}

The general conservation of total mass per unct area can be expressed 


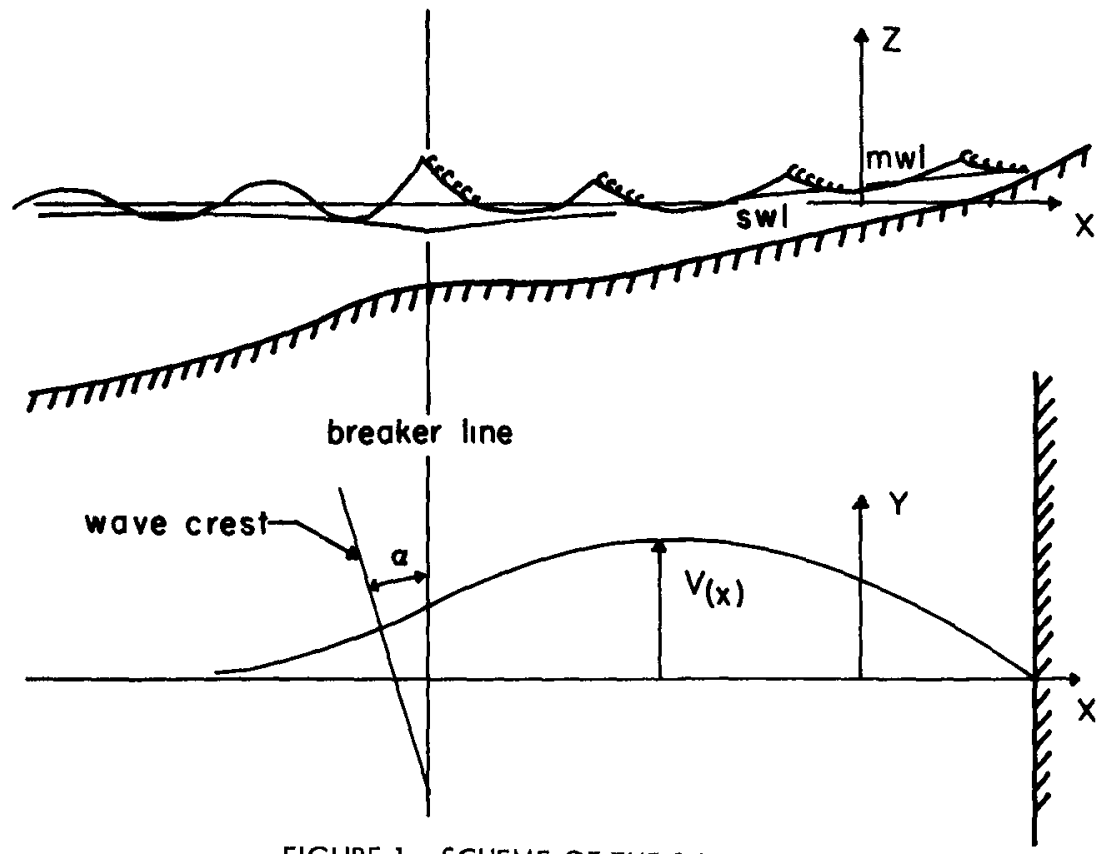

FIGURE 1 SCHEME OF THE SURF ZONE 


$$
\frac{\partial}{\partial t} \rho \mathrm{D}+\frac{\partial}{\partial \mathrm{x}_{1}} \tilde{\mathrm{M}}_{1}=0 \quad \mathrm{z}=1,2
$$

$D$ is the total averaged depth of water which can include a mean elevation $\bar{n}$, above (or below) the still water depth $h$, so that

$$
D(x, y, t)=(\bar{n}+h)
$$

The overbar shall be used to signify time averages The total mass flux $\tilde{M}_{1}$ can be partitioned into its mean and fluctuatIng components

$$
\tilde{M}_{1}=\bar{M}_{1}+M_{1}
$$

\section{$B$ Conservation of Momentum}

The equation defining the conservation of horizontal momentum is derived by integrating the momentum equation over depth and averaging in time The balance of total momentum per unit area can be expressed

$$
\frac{\partial}{\partial t} \tilde{M}_{1}+\frac{\partial}{\partial x_{J}}\left(\tilde{U}_{I} \tilde{M}_{J}+S_{I J}\right)=T_{1}+R_{1}
$$

Here $\tilde{M}_{1}$ denotes the total horizontal momentum per unit area Hence, the first term on the left represents the rate of change of the total mean momentum per unit area which includes both the current momentum and wave momentum

$\tilde{U}_{1}$ is the total mean transport velocity The second term on the left of Equation expresses the momentum flux of a steady stream together with an excess momentum flux term $S_{1 J}$ arising from the superposed unsteady motion, where

$$
S_{I J}=\int_{-h}^{n}\left(\rho u_{I}^{\prime} u_{J}^{\prime}+p \delta_{I J}\right) d z-\frac{1}{2} \rho g D^{2} \delta_{I J}-\frac{M_{I} M_{J}}{\rho D}
$$

and $\delta_{1 j}$ is the Kronecker delta The unsteady terms in the integral contain contribution from the mean motion, the last two terms represent the hydrostatic pressure and mean momentum flux contained in the integral term and 1 s subtracted out so that the term represents only the excess momentum flux due to the unsteady motion The last term is generally of higher order, but very near and inside the surf zone the term is of second order and consistent with this analysis, however, its maximum contribution is only four percent and this term will be neglected The term $T_{1}$ is given by

$$
\mathrm{T}_{1}=-\rho g(\bar{\eta}+h) \frac{\partial \bar{\eta}}{\partial \mathrm{x}_{1}}
$$

and represents the net horizontal force per unct area due to the slope of the free water surface 
$R_{f}$ Is the time mean averaged shear stress which must be incluad in any realistic treatment of the surf zone where dissipative effects occur The integrated form of the resistance term is given by

$$
R_{i}=\overline{\int_{-h}^{n} \frac{\partial \tau}{\partial x_{j}} d z}+\overline{\tau_{n i}}-\overline{\tau_{h i}} \quad 1, j=1,2
$$

where $\tau_{j z}$ Includes the comblned lateral shear stresses of waves and currents and the last terms are the surface and bottom shear stress respectively

In the development of the conservation equations, no restrictions are placed on the wave slopes or amplitudes Also, no restrictions are placed on the fluctuating motion so that the equations are equally applicable to wave or turbulent motion

\section{DESCRIPTION OF THE WAVE FIELD}

\section{A Waves outside the Surf Zone}

It is known that, due to the fluctuating water particle motion of the waves, there is a momentum flux component If the waves have a direction component parallel to shore, a longshore current can be generated due to changes in the longshore momentum flux component of the shoaling waves In waves, the momentum flux is the sum of the pressure and the product of two velocities It can be shown that the average momentum flux is nonlinear in wave height In order to speclfy the excess momentum flux of the waves, it therefore becomes necessary to consider nonlinear, or higher order, effects of the wave motion

The development presented here retalns terms to the second order in amplitude (first order in energy and momentum) and neglect all higher order terms The wave solution is substituted directly into the conservation equations providing a means for describing the wave-induced mean motions In making this substitution and dropping all terms of orders higher than the second, only knowledge of the first order (linear) wave water particle velocities and surface elevation is necessary This is because, in expanding and then averaging over the period, the terms involving higher order quantities in velocity and surface elevation go to zero The pressure must be known to the second order in wave height, however, the average second order pressure component can be determined from the furst order water particle velocities and surface elevation terms Thus, only the linear wave solution is required

B Waves Inside the Surf Zone

Inside the surf zone, energy is dissipated due to the generation of turbulence in wave breaking, bottom friction, 
percolation, and viscosity The waves in the surf zone constitute a non-conservative system in which the use of potential flow theory is no longer valid In fact, there is no analytical description avallable for the waves in the surf zone Hence, one is required to make rather gross assumptions and then to test these assumptions experimentally The linear wave theory wlll be retained as the input to the conservation equations, but with modification to the wave amplitude and speed The wave helght inslde the surf zone is controlled by the depth and is of the same order of magnitude Thus, even the second order theory in wave height is a rather poor assumption, but seems to agree surprisingly well with measurements of some phenomena.

Spilling breakers lend themselves to a physical treatment since the potential energy and momentum flux of the waves inside the surf zone can be expressed approximately in analytical form If the beach slope is very gentle, the spliling breakers lose energy gradually, and the height of the breaking waves approximately follows the breaking index curve The helght of the wave, $\mathrm{H}$, is then a function of the total depth, D, as given by

$$
H=K D
$$

where the breaking index, $\kappa=078$, as predlcted by the solitary wave theory

It is further assumed that the kinetic and potential energy are equally partitioned so that the total wave energy can be described in terms of the wave helght which is a function of the depth

$$
E=\frac{1}{8} \rho g H^{2}=\frac{1}{8} \rho g \kappa^{2} D^{2}
$$

This is a non-conservative statement of the energy distribution within the surf zone

The waves inslde the surf zone are assumed to retain their simple harmonic character so that the wave profile and water particle velocity are described by

$$
\begin{aligned}
& n=\frac{H}{2} \cos \left(k_{1} x_{1}-\sigma t\right) \\
& \vec{u}=\frac{H}{2} \frac{g}{c} \frac{k_{1}}{k} \cos \left(k_{1} x_{1}-\sigma t\right)
\end{aligned}
$$

where $k$ is the wave number, $\sigma$ the wave frequency and $c$ the wave speed The expression for the horizontal water particle velocity is based on the Alry wave theory and has been simplifled for shallow water 
In very shallow water, the waves are non-dispersive with the wave speed being only a function of the depth It has been found experimentally that a reasonable approximation to the wave speed in the surf zone is that predicted by the solitary wave theory

$$
c=\sqrt{g(H+D)}=\sqrt{g(I+K) D}
$$

Since the bottom contours are parallel, Snell's law can be used to account for the changes in wave direction due to refraction The wave angles are referred to the breaker wave angle which is the commonly measured angle in the study of the surf zone Hence,

$$
\sin \alpha=\frac{c}{c_{b}} \sin \alpha_{b}
$$

where the subscript "b" refers to the breaker line Refraction due to shear flow is neglected and can be shown to be of minor importance

The excess momentum flux tensor can be determined by substituting the wave expressions into Equation (6) In general terms of energy, group velocity, $c$, and wave speed, $c$, an expression applicable to both inside and outside the surf zone is given by

$$
S_{i j}=\left|\begin{array}{ll}
E \frac{c}{c} \cos ^{2} \alpha+\frac{E}{2}\left(\frac{2 c g}{c}-1\right) & \frac{E}{2} \frac{c g}{c} \sin 2 \alpha \\
\frac{E}{2} \frac{c}{c} \sin 2 \alpha & E \frac{c g}{c} \sin ^{2} \alpha+\frac{E}{2}\left(2 \frac{c}{c}-1\right)
\end{array}\right|(15)
$$

The effects of turbulence and surface tension have not been included

\section{LONGSHORE CURRENT FORMULATION}

The wave field has been completely specifled These results may now be substituted into the general conservation equations to describe wave-induced phenomena inside and outside the surf zone

Recalling the analysis is restricted to the case of an arbitrary bottom profile with straight and parallel contours in the $y$-direction The water depth is then a function of the $x$-direction only Since the distribution of mean properties of the wave field is a function of the depth, this eliminates any $\mathrm{y}$-dependence An exception to this was found by Bowen [2] Using the fact that incident waves can excite transversal waves, commonly called edge waves, he showed that if these waves are standing waves, or only slowly progressive, gradients in the mean water surface can be developed in the longshore direction which in turn can result in 
circulation cells Thus, a more exact formulation has to assume suitable spatial averaging in the longshore direction so as to preclude the effects of any transversal waves

It is assumed that wave reflection is negligible This assumption is justifiable outside the surf zone for gently sloping bottoms The present analysis is most valid for spilling breakers which implies a gently sloping bottom The wave reflection is least for this type of breaker condition and is assumed negligible inside the surf zone as well neglected

Shear stresses at the surface due to the wind are

The problem can be conveniently discussed by considering separately the areas outside and inside the surf zone A determination of the distribution of mass transport and energy of the waves is first necessary in order to solve for the wave-induced currents

\section{A Mass Transport Velocity}

Due to the absence of any $\mathrm{y}$-dependence, the mass conservation Equation (2) reduces to

$$
\frac{\partial \tilde{M}_{x}}{\partial x}=0
$$

Integration gives

$$
\tilde{\mathrm{M}}_{\mathrm{X}}=\text { constant }=0
$$

which must be equal to zero since the beach forms a boundary in the $\mathrm{x}$-direction This then says

$$
U_{X}=-\frac{M}{\rho D} \cos \alpha
$$

which states that there is a mean reverse current balancing the mass transport onshore due to the wave motion This must be true everywhere, both inside and outside the surf zone, to ensure that there is no accumulation of mass or growth of currents in the $\mathrm{y}$-direction in order to maintain steady-state conditions in accordance with the original assumptions

B Changes in the Mean Water Level

The changes in the mean water level must be determined inside the surf zone in order to specify the variation of wave energy since the waves are assumed proportional to the total depth of water The changes in the mean water level can be determined from the x-momentum equation given by

$$
\frac{\partial}{\partial x} S_{X X}=T_{X}=-\rho g D \frac{\partial \bar{\eta}}{\partial x}
$$


where shear stresses are neglected and time dependence, $y$-gradients and net mass flux in the $x$-dlrection are all zero This equation states that there is a change in the mean water level to balance the excess momentum flux of the waves Lonquet-Higgins and Steward [3] have solved Equation (19) for the case of wave approaching to an arbitrary plane bottom using linear wave theory Outside the surf zone they found

$$
\bar{\eta}=-\frac{H^{2}}{8} \frac{k}{\sinh 2 k h}
$$

where the negative sign indicates a set-down which is a function of the local conditions only

Inside the surf zone the changes in mean water level are again determined from Equation (19) It is assumed that the excess momentum flux tensor inside the surf zone can be expressed in terms of the energy and wave speed in the same form as in shallow water This assumption implies that even under the breaking waves, water particle motion retalns much of Its organized character as described by linear wave theory

The excess momentum flux decreases inside the surf zone due to the decreasing wave helght as energy is dissipated This results in a wave set-up inside the surf zone given by

$$
\vec{n}=K\left(h_{b}-h\right)+\vec{n}_{b}
$$

where the set-down at the breaker line $\vec{\eta}_{b}$ can be determined from Equation (20) and

$$
K=\frac{1}{1+\frac{8}{3 k^{2}}}
$$

Bowen et al [4] conducted laboratory studies verifying the theory predicting the changes in mean sea level for waves normally incident to the shoreline The effect of waves approaching at an angle is neglected in the above formula but was shown by Thornton [5] to result in a maximum change in the total depth of less than 2 per cent

\section{Distribution of Currents Outside the Surf Zone}

There Is a component of excess momentum flux directed parallel to the shore due to the oblique wave approach The question of whether a current can be generated is investigated by considering the general y-momentum equation Applying the previous assumptions, Equation (5) can be written

$$
\frac{\partial S_{x y}}{\partial x}=R_{y}
$$


where the time dependent term is zero, the gradients in the $\mathrm{y}$-direction are zero and the conservation of mass equation showed that $\tilde{M}_{X}=0$ If It Is assumed that outside the surf zone that energy is conserved, that is, no dissipation of energy, then the stress term will be zero Therefore, the change of momentum flux due to the waves and mean motion in the $\mathrm{y}$-direction is zero--there is no driving force for generating a current outside the surf zone The only waveinduced current far outside the surf zone is then due to the mass transport velocity which is weak

With the mean water profile and energy distribution specified, the variation of the longshore current across the surf zone can be determined The y-momentum equation ins the surf zone can be written the same as that outslde the surf zone Inside the surf zone energy is dissipated due to turbulence and bottom friction and the stress term is important In order to solve for the longshore current inside the surf zone, an appropriate description of the resistance term composed of both bottom and internal shear stresses is required

\section{Bottom Shear Stress}

It is desired to determine the combined bottom shear stress due to waves and currents It Is assumed that the total instantaneous bed shear stress for combined waves and currents is related to the velocity by

$$
\vec{\tau}_{h}=\rho \frac{f}{2} \vec{v}|\vec{v}|
$$

where $\vec{V}$ is the resultant instantaneous velocity vector of the combined wave and current motion, and $f$ is the friction factor Since the problem has been formulated as a combination of wave and current motion, it proves convenient to resolve the shear stresses into components in the direction of the wave and current components Resolving the component shear stresses in the direction of the velocity vectors results in the shear stress and velocity vectors being conformal Hence, the shear stress component for the wave motion can be written

$$
\vec{\tau}_{h w}=\vec{\tau}_{h} \frac{\vec{u}_{w}}{\vec{v}}=\rho \frac{f}{2}|\vec{v}| \vec{u}_{w}
$$

where $\vec{u}_{W}$ is the instantaneous velocity of the wave motion measured just above the frictional boundary layer near the bottom and $V$ Is the mean motion which was assumed uniform over the depth and in the longshore direction only

Similarly, for the shear stress in the direction of the mean current

$$
\vec{\tau}_{h y}=\vec{\tau}_{h} \underset{\vec{V}}{\frac{V}{\vec{V}}}=\rho \frac{f}{2}|\vec{v}| V
$$


If friction factors for the wave and current motion are now defined,

$$
f_{y}=f\left|\frac{\vec{v}}{v}\right| \quad f_{w}=f \frac{|\vec{v}|}{\left|\vec{u}_{w}\right|}
$$

This results in the shear stress components given in a form consistent with experimental results

$$
\begin{aligned}
\vec{\tau}_{h w} & =\rho \frac{f_{W}}{2} \vec{u}_{w}\left|\vec{u}_{W}\right| \\
\tau_{h y} & =\rho \frac{f_{y}}{2} V^{2}
\end{aligned}
$$

The shear stress for waves, as defined in Equation (28) is in the same form as given by Jonsson [6] for which information of the friction factor was found experimentally $\mathrm{He}$ found, further, that the friction factor was practically constant over an osclilation period The constancy of the friction factor for particular flow conditions is an important result which allows for a better analytical determination of the combined shear stress due to waves and currents Using the avallable data from several sources, he found that the friction factor for wave motion alone for rough turbulent boundary layers (as usually found in nature) could tentatively be represented by

$$
\frac{1}{4 \sqrt{f_{W}}}+\log \frac{1}{4 \sqrt{f_{W}}}=-008+\log \frac{\xi_{h}}{r}
$$

where $r$ is a measure of roughness, and $\xi_{h}$ is the maximum water particle excursion amplitude of the fluid motion at the bottom as predicted by linear wave theory

$$
\xi_{\mathrm{h}}=\frac{\mathrm{H}}{2} \frac{1}{\mathrm{slnh} \mathrm{kh}}
$$

Equation (30) is based on the roughness parameter $r$ being a measure of the ripple helght The wave friction factor is seen to be a function of the wave characteristics This is because, for granular beds consisting of a partucular grain size, the ripples adjust their dimensions according to the wave motion, and it is the ripple geometry that determines the effective roughness The difflculty in using the quadratic shear stress formula is in stipulating the friction factors The wave friction factor is seen to be a function of the wave properties for a deformable bed, that is, the fluid motion, whereas, the friction factor for steady currents for rough turbulent boundary layers is only a function of the system geometry It would seem reasonable to expect that for weak currents, as compared to the water particle motion of the waves, that the wave dynamics would dominate the hydrodynamical system For thls reason, it is desirable to use the combined bottom shear stress in terms of the wave 
friction factor alone, even though it is less well defined than the friction factor for steady currents

For the problem at hand, the bottom shear stress directed parallel to shore can be written in terms of the wave friction factor by combining Equations (27) and (28) which results in the form

$$
\tau_{h y}=\frac{\rho f_{y}}{2} v^{2}=\rho \frac{f_{w}}{2}\left|\overline{u_{w h}}\right| V=\rho f_{w} \frac{H}{2 \pi} \frac{g}{c} V
$$

where shallow water waves are assumed

\section{E Lateral Shear Stress}

It is necessary to include the lateral shear stress in the formulation If the lateral shear stress is not included, a velocity discontinuıty at the breaker line is predicted due to the abrupt change in momentum flux here The lateral shear stress effectively couples the adjacent elemental water columns together resulting in a diffusion of momentum in a direction perpendicular to shore The lateral diffusion of momentum flux seaward across the breaker line results in the momentum flux inside the surf zone driving the currents outside, the lateral diffusion of momentum shoreward results in the maximum velocity displaced shoreward

Considerable success has been achieved using Prandtl's mixing length hypothesis for speciflc problems This concept wlll be utilized to relate the internal shear stresses to the mean flow The expression for the internal shear stress can be given in terms of the mean turbulent Reynolds stress which is compared to a "Boussinesq" approach

$$
\tau_{x y}=-\rho \overline{u^{\prime} v^{\prime}}=\rho \varepsilon_{v} \frac{\partial V}{\partial x}
$$

where $\varepsilon_{\mathrm{v}}$ Is the kinematic eddy viscosity

The variation of the turbulent velocity component in the $y-d i r e c t i o n$ is given in accordance with Prandtl's hypothesis by

$$
v^{\prime}=\ell \cdot \frac{\partial V}{\partial x}
$$

where $\ell$ ' denotes a mixıng length which can fluctuate with time From Equation (32), the kinematic eddy viscosity can then be written

$$
\varepsilon_{\mathrm{v}}=-\left|\overline{\mathrm{u}^{\prime} \mathrm{\ell}}\right|
$$

For the case of superposed waves and currents, It is natural to consider the length over which momentum is transferred as equivalent to the water particle excursion due to the wave motion and the velocity fluctuation $u^{\prime}$ equal to that of the water particles in the waves 
Because $U^{\prime}$ and $\ell^{\prime}$ are in quadrature, the absolute value of the product is necessary in order to obtain a non-zero value The mixing length $\ell^{\prime}$ can be interpreted as a measure of the turbulent scale and $u^{\prime}$ as a measure of turbulent intensity This interpretation is not unreasonable physically Examination of actual energy spectra of turbulence occurring in the surf zone shows that most of the fluctuating energy is associated with the waves

The kinematic eddy viscosity can be evaluated by recalling Equations (12) and ( $30 \mathrm{a})$ It will be assumed for simplicity that shallow water wave conditions apply, so that

$$
\varepsilon_{v}=\frac{H^{2}}{8 \pi^{2}} \frac{g T}{h} \cos ^{2} \alpha
$$

Evaluating the internal shear stress term by substituting Equation (32) gives

$$
\int_{-h}^{n} \frac{\partial \tau x y}{\partial x} d z=\int_{-h}^{n} \rho \frac{\partial}{\partial x}\left(\varepsilon_{v} \frac{\partial V}{\partial x}\right) d z=\rho D \frac{\partial}{\partial x}\left(\varepsilon_{v} \frac{\partial V}{\partial x}\right)
$$

where $\varepsilon_{\mathrm{V}}$ and $\mathrm{V}$ are independent of $\mathrm{z}$

The total resistance term, including internal and bottom shear stresses, is then given by

$$
R_{y}=\rho D \frac{\partial}{\partial x}\left(\varepsilon_{v} \frac{d V}{d x}\right)-\rho \frac{f_{w}}{2 \pi} \frac{g}{c} H V
$$

F Distribution of Currents Inside the Surf Zone

The variation of the longshore current inside the surf zone can be solved by equating the changes in excess wave momentum to the resistance forces Substıtuting for $S_{x y}$
from Equation (15) gives

$$
\frac{\partial}{\partial x}[E \sin \alpha \cos \alpha]=R_{y}=\rho D \frac{\partial}{\partial x}\left(\varepsilon_{v} \frac{d V}{d x}\right)-\rho \frac{f_{W}}{2 \pi} \frac{g}{c} H V
$$

The helght, celerity, refracted angle of the waves, and, hence, changes in momentum flux inside the surf zone can be expressed in terms of the local depth of water Substituting Equations (10), (14), and (35) into Equation (38) gives

$$
A D^{3 / 2}\left(1-07 \frac{D}{D_{b}} \sin ^{2} \alpha_{b}\right) \frac{\partial D}{\partial x}=\rho D \frac{\partial}{\partial x}\left(\varepsilon_{v} \frac{\partial V}{\partial x}\right)-\frac{\rho K}{2 \pi} f_{w} \sqrt{\frac{\rho D}{(1+K)}} V
$$

where

$$
A=\frac{5}{16} \rho g \kappa^{2} \frac{\sin \alpha_{b}}{\sqrt{D_{b}}}
$$


Th1s is a general equation expressing the changes in momentum flux across the surf zone in terms of the total local depth of water Included in the formulation is the set-up of water and the effects of wave refraction inside the surf zone This equation is subject to the restriction that the waves be described as spilling breakers, and, hence, the depth continuously decreases shoreward from the breaker line Thus, the momentum flux decreases monitonically inside the surf zone since both the energy and wave angle decrease with decreasing depth

This development is simllar to that by Bowen [7] in an investigation limited to a plane beach in which he assumes linear bottom friction and constant kinematic eddy viscosity in order to obtain an analytical solution

The inclusion of the bottom friction requires Equation (39) to be solved numerically Also a general method of solution is sought for comparison to the arbitrary field conditions Boundary conditions imposed on the problem inside the surf zone are for $D=0, V=0$ corresponding to conditions at the intersection of the water line and the beach, and for $D=D_{b}, V=V_{b}$ corresponding to conditions at the breaker line

A similar solution can be sought outside the surf zone where it is now assumed that the driving force for the currents outside the surf zone is zero--the changes in the momentum flux directed parallel to shore are zero The $y$-momentum Equation (38) reduces to

$$
D \frac{\partial}{\partial x}\left(\varepsilon_{v} \frac{\partial V}{\partial x}\right)-\frac{f_{w}}{2 \pi} \frac{g}{c} H V=0
$$

where now the force driving the currents is due to lateral momentum flux resulting from a coupling of the adjacent vertical faces of the differential water column across the breaker line This is to say that currents outside the surf zone are being driven by the longshore currents inside the surf zone due to coupling across the breaker line

The boundary conditions imposed on the formulation outstde the surf zone are that the velocity approaches zero far away from the breaker line $(D \rightarrow-\infty)$ and that the velocities and velocity gradients inside and outside the surf zone match at the breaker line

\section{COMPARISON OF THEORY AND EXPERIMENT}

The laboratory results of Galvin and Eagleson [8] are used to test the predictive equations The only parameter that is necessary to be chosen is the roughness in order to utilize the predictive equations A value of $r=00033$ feet ( $1 \mathrm{~mm})$ was chosen for the concrete beach which is a reasonable value The kinematic eddy viscosity is completely specified by the kinematics of the flow fleld The resulting 
velocity distribution is shown in Figure 2 The distributions of the kinematic eddy viscosity and friction factor are also shown in Figure 2 The kinematic eddy viscosity is a maximum at the point of breaking, where the maximum momentum exchange would be expected to take place, and decreases to zero at the shoreline The friction factor varies slowly except near the beach where $f_{\mathrm{w}}$ increases very rapidly and approaches infinity at the intersection of the beach and the still water line

\begin{abstract}
Other cases and a more complete text Is given by Thomton [5] In general, surprisingly good correlation is found considering that the only parameter chosen is the roughness in order to completely specify the longshore velocity distribution
\end{abstract}

The same equations are applied to the fleld data taken by Ingle [9] The velocity distribution is shown in Figure 3 The bottom profile is shown in the same figure Information concerning the bathymetry outside the surf zone is lacking so a bottom slope of 001 Is assumed The kinematic eddy viscosity and friction factor distributions are also shown and are similar to those found for the laboratory beaches The friction factors for the laboratory and field are of the same order of magnitude, ownng to the fact that the friction factor is not only dependent on the roughness but also the wave characteristics The kinematic eddy viscosity for field conditions is several orders of magnitude greater than the value found for laboratory conditions owing to the greater turbulent scales

\title{
CONCLUSIONS
}

It was shown that the component of excess momentum flux due to the presence of the unsteady wave motion (sometimes called a "radiation stress") directed parallel to shore can generate longshore currents Changes in the excess momentum flux, as the waves shoal, must be balanced by a resistance force in order to maintain the assumed steady-state conditions The component of excess momentum flux perpendicular to the beach is responsible for wave set-down outside the surf zone and wave set-up inslde the surf zone These changes in the mean water level were included in the longshore current formulations

Logical means of introducing the friction factor associated with the bottom shear stress term was presented--the friction factor being related to the wave and bottom roughness characteristics A mlxing length hypothesis and the kinematics of the wave motion were combined in order to define the internal shear stresses Comparison of experimental results from the laboratory and field with the derived theory shows that the predicted results compare favorably if the assumed conditions are approximately fulfilled 


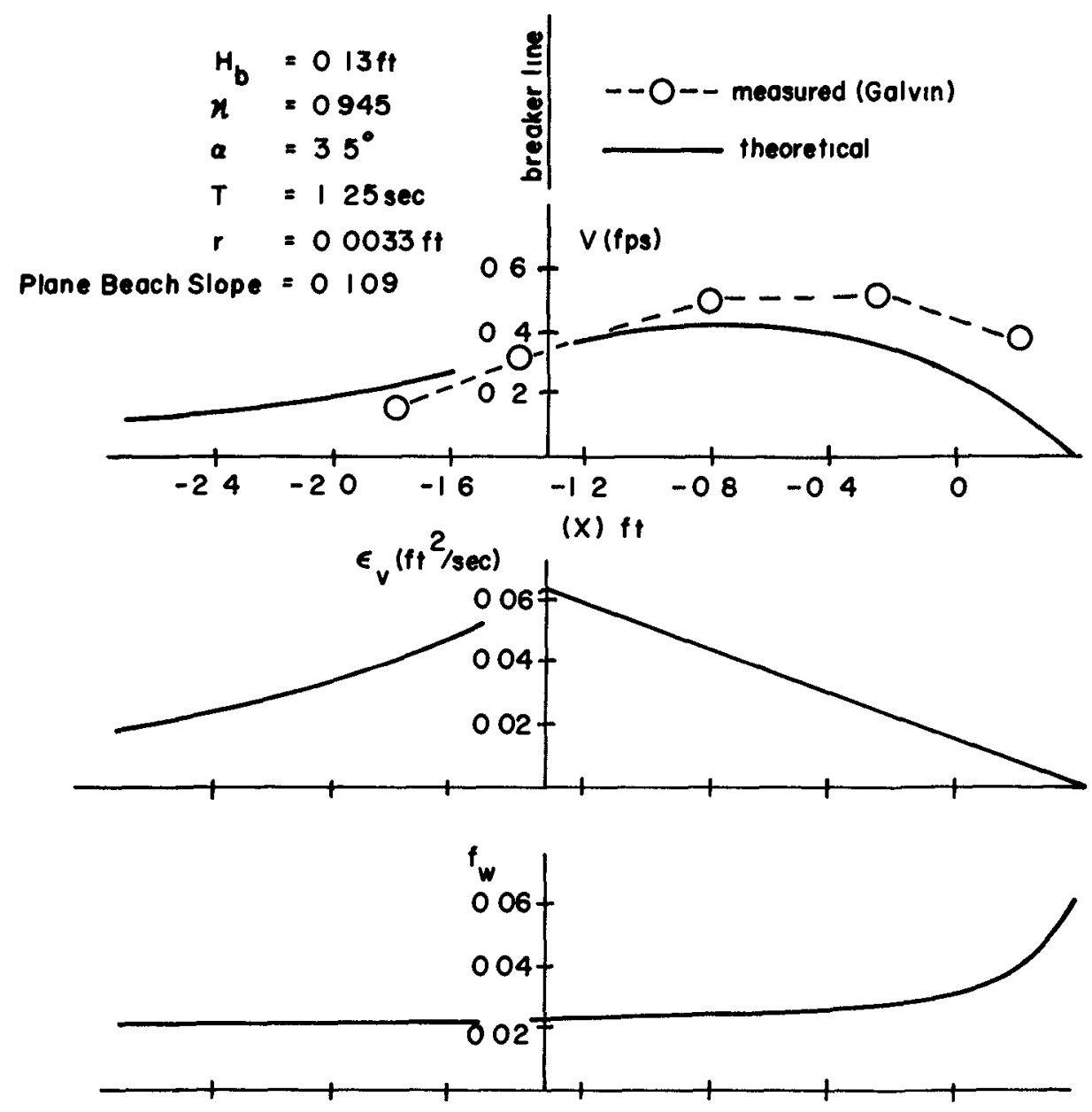

FIGURE 2 VELOCITY DISTRIBUTION ACROSS

THE SURF ZONE FOR LABORATORY BEACH 

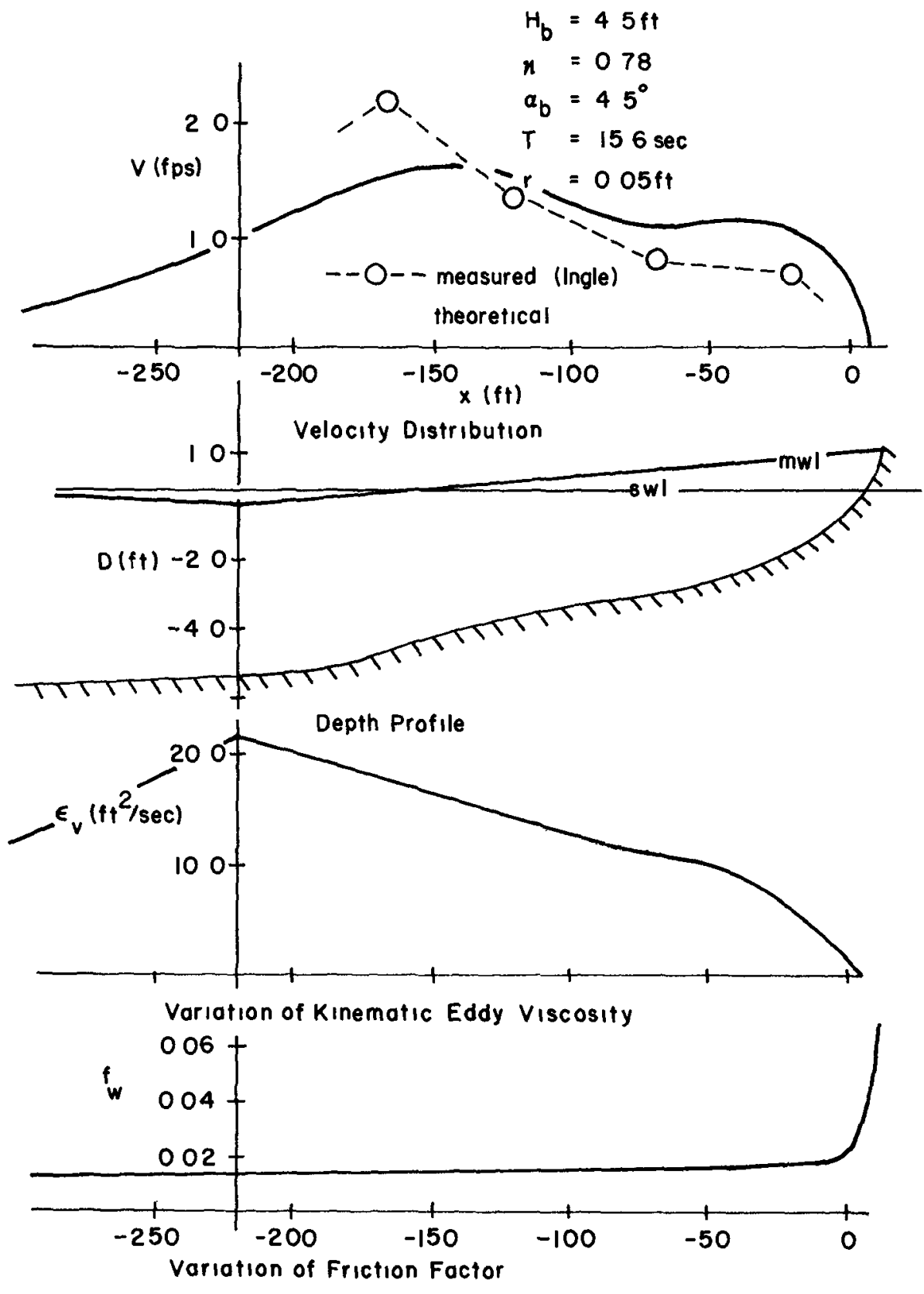

FIGURE 3 VELOCITY DISTRIBUTION ACROSS SURF ZONE, TRANCAS BEACH, CALIF ORNIA 
REFERENCES

1 Phillips, 011 , The Dynamics of the Upper Ocean, Unzversity Press, Cambridge, 22-70, 1966

2 Bowen, A J, Rip Currents, Doctoral Thesis, Scripps Institution of Oceanography, 1967

3 Longuet-Higgins, $M S$ and Stewart $R W$, A Note on Wave Set-up, J of Marine Res , 21 4-10, 1963

4 Bowen, $A \mathrm{~J}$, Inman, $\mathrm{D} \mathrm{L}$ and Simmons, $\mathrm{V} P$, Wave 'Set-down' and 'Set-up', J of Geophys Research, 73, 8 2559-2577, 1968

5 Thornton, E B , Longshore Current and Sediment Transport, Dept of Coastal and Oceanographic Engineering Tech Rpt No 5, University of Florida, 1969

6 Jonsson, I $G$, Wave Boundary Layers and Friction Factors, Proc Tenth Conf Coastal Eng, ASCE, 127-148, 1966

7 Bowen, A $\mathrm{J}$, The Generation of Longshore Currents on a Plane Beach, J of Marine Research, 27, 2 206-215, 1969

8 Galvin, $C \mathrm{~J}$ and Eagleson, $\mathrm{P} S \mathrm{~S}$, Experımental Study of Longshore Currents on a Plane Beach, Coastal Eng Res Center Tech Memo No 10, U S Army Corps of Engrs, Washington, D C, 1965

9 Ingle, $\mathrm{J} 0, \mathrm{Jr}$, The Movement of Beach Sand, Elsevier Publishing Co, Amsterdam, 1966 


\title{
CHAPTER 19
}

\section{HORIZONTAL WATER PARTICLE VELOCITY}

OF FINITE AMPLITUDE WAVES

\author{
Yuıchı Iwagakı \\ Professor \\ and \\ Tetsuo Sakal \\ Postgraduate \\ Department of Clvil Englneering \\ Kyoto Unzversity \\ Kyoto, Japan
}

\section{ABSTRACT}

This paper firstly describes two methods to measure vertical distribution and time variation of horizontal water particle velocity induced by surface waves in a wave tank These two methods consust of tracing hydrogen bubbles and using hot fılm anemometers, respectıvely

Secondly, the experimental results by the two methods are presented with the theoretical curves derıved from the small amplitude wave theory, Stokes wave theory of $3 \mathrm{rd}$ order, and the hyperbollc wave theory as an approxumate expression of the cnoldal wave theory

Finally, based on the comparison of the experimental data with the theoretical curves, the applicability of the finite amplitude wave theories, whlch has been studied for the wave profile, wave velocity, wave length and wave crest helght, is discussed from view point of the water particle veloclty

\section{INTRODUCTION}

The water particle veloclty induced by surface waves is one of the most important factors to solve the wave breaklng mechanlsm, wave forces acting on submerged structures, mechanism of suspension and diffusion of materials by waves, and so on However, there have been very little experimental data $1,2,3$, 4 , because of difficulty of the measurement Among the laboratory experiments of the water partıcle velocity, the data taken by Le Méhaute and others ${ }^{4}$ usıng tracers of neutrally buoyant particles show by the comparison with the predicted values from the varıous small amplitude and finlte amplitude wave theorles that no theory is uniformly valid This conclusion makes us confused in applying finlte amplitude wave theorles to practical problems It is necessary, therefore, to check the validity of the conclusion 
Recently, a new method to measure the water particle velocity was proposed, whlch is by using hydrogen bubbles as tracers, that is, tracing motions of hydrogen bubbles generated in water by electrolysis every very short period, to measure the water particle velocity induced by waves 5,6 This method is a very useful tool to measure vertical distriburions of the water particle velocity at a particular phase of waves on the other hand, in order to measure time variation of the water particle velocity, the hydrogen bubbles method is not so useful, but the use of a hot film anemometer is sultable, whlch was primarıly developed for turbulence measurements in water ${ }^{7}$ These two methods were applied in this study to measure the water particle velocity induced by waves in a wave tank As mentioned above, vertical distribution of the horizontal water particle velocity under the wave crest was measured by the method of tracing hydrogen bubbles, and time variation durıng one wave perıod at a definite helght from the tank bottom was measured by a hot film anemometer

This paper presents the experimental results of the horizontal water particle velocity obtalned by these two methods, and comparison with theoretical values calculated from the small amplitude wave theory, Stokes wave theory of $3 r d$ order $^{8}$, and the hyperbolic wave theory ${ }^{9}$ as an approximate expression of the cnoldal wave theory of 2nd approximation 10 Based on the comparison between the theories and the experiments, the applicability of the finite amplitude wave theories for the water particle velocity is discussed

\section{EXPERTMENTAL APPARATUS AND PROCEDURE}

\section{EXPERIMENTAL APPARATUS}

The wave tank at Department of Clvil Englneering, Kyoto University whIch has a wave generator of plston type was used in the experiments

Method of tracing hydrogen bubbles The experimental apparatus to measure the water particle velocity by this method is shown in Fis 1 A platınum ware of $005 \mathrm{~mm}$ in diameter was used as a negative pole to generate hydrogen bubbles in water One end of the wire was attached to the tank bottom and stretched vertically, and the other end was connected with the pointgauge set a few centimeters high above the wave crest helght Four pleces of copper plates $(15 \mathrm{~cm} \times 37 \mathrm{~cm})$, whlch were put on both slde wall glasses of the wave tank arround the negative pole, were used as positive terminals one wave gauge was mounted side by side with the negative pole in the transverse direction of the wave tank, whlle the other wave gauge was mounted as far as about $2 \mathrm{~m}$ from $1 t$ Two wave gauges were connected to the recorder The equipment to load pulse voltages on the terminal can supply output voltages of $400 \mathrm{~V}$, with the pulse perıod of $4 \approx 700 \mathrm{~ms}$ and the pulse wldth of $04 \approx 70 \mathrm{~ms}$ Photographs of hydrogen bubbles generated along the platinum wire and moving with flow were taken with a camera through the side wall glass

Method, of using hot film anemometer Two hot film anemometers of type $55 \mathrm{D} 05$ and thelr probes of type 55085 made by DISA were used The anemometer amplifies electrically heat convected from the probe which is one of bridge resistances The probe, as shown in Fig 2, consists of a thin metal film as electrically heated resistance and its support This anemometer is of 


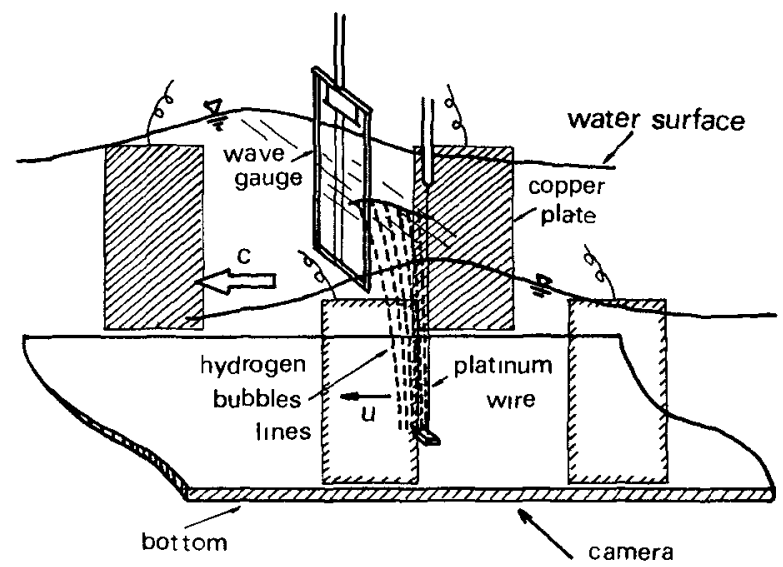

Fig 1 Schematic figure for measurement of water particle velocity by method of tracing hydrogen bubbles

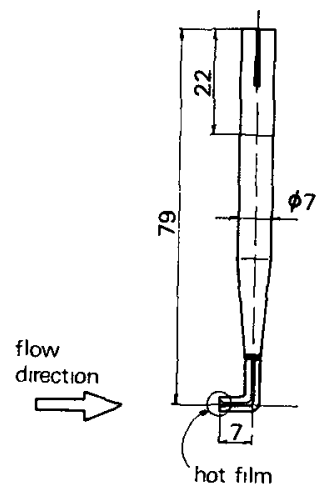

dimensions in mm

Fig 2 Probe of hot film anemometer 
constant-temperature type desıgned for measuring energy required to keep always probe resistance(therefore probe temperature) constant(operating resistance)

When fluld flows as shown in Fig 2, the probe can detect its current velocity, but not its opposite current velocity Therefore, if the probe is mounted contronting waves as shown in Fig $3(1)$, the record of the anemometer is reliable when the water level is higher than the still water level(full line), but not reliable when the water level is lower than the still water level and the flow direction becomes opposite In addition, the probe can not detect opposite change of the flow direction on the other hand, if the probe is mounted following waves as shown in Fig 3(2), the record is reliable even when water level is lower than the still water level(full line), but the other part of the record is not reliable

In the experiments, two probes and one wave gauge were mounted side by side in the transverse direction Especially, two probes of the hot film were set at the same helght, confronting each other(see Fig 4) The other wave gauge was mounted as far as about $2 \mathrm{~m}$ from the probes All of two hot film anemometers and two wave gauges were connected to the recorder

\section{EXPERTMENTAL PROCEDURE}

Method of tracing hydrogen bubbles Among the waves generated by the wave generator, one wave which became fully stable and was not influenced by the reflected waves from another end of the tank was selected Just before the crest of this wave passed over the negative pole, pulse voltage of a proper period $\Delta t$ began to be loaded Lines of hydrogen bubbles generated along the platinum wire are transported by the flow in the direction of wave propagation under the wave crest The photograph in this state was taken by a $35 \mathrm{~mm}$ camera At the same time, the records of waves at the wire position and as far as about $2 \mathrm{~m}$ from the wire were taken When pulse voltage is loaded on the terminal, the wave gauge can detect it and the disturbance appears in the record Therefore, the time $t_{0}$ when the first pulse was loaded can be detected ( If the time when the crest of a selected wave passes over the wire is put as $\left.\mathrm{t}=0, \mathrm{t}_{0}<0\right)$

The $m$ th and $(m+l)$ th hydrogen bubble lines were selected on the fllm, so that the arithmetic average $t_{0}+\{m-(1 / 2)\} \Delta t$ of $t_{0}+(m-1) \Delta t$ and $t_{0}+m \Delta t$ becomes the smallest(see Photo 1 ) Reading the distance between these $m$ th and $(m+1)$ th lines and dividing lt by $\Delta t$, the horlzontal water particle velocity $u$ at the phase of $t / T=\left[t_{0}+\{m-(I / 2)\} \Delta t\right] / T$ was obtalned approximately ( $T$ the wave period) This process was repeated at different helghts above the tank bottom, and the vertical distribution of the horizontal water particle velocity was obtained

Test conditions are shown in Table 1 , Where $h$ is the water depth, $H$ the wave helght, and $p$ the pulse width The opening and shutter speed of the camera were always 14 and $1 / 125 \mathrm{sec}$

Method of using hot film anemometer After the value of operating resistance of two hot film anemometers was set to be $100 \sim 115$ time of cold resistance value, the calibration curves of two anemometers were determined by 
(1)
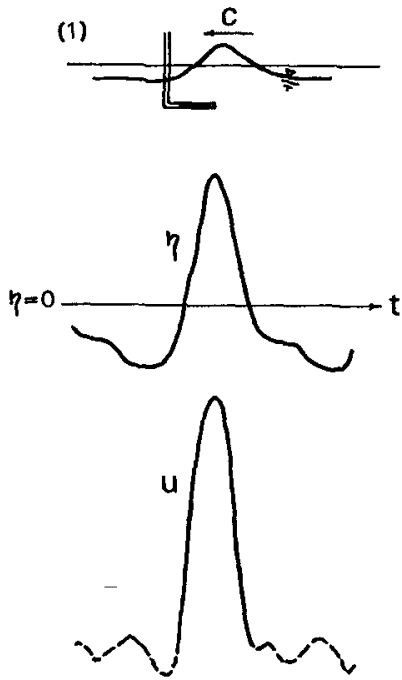
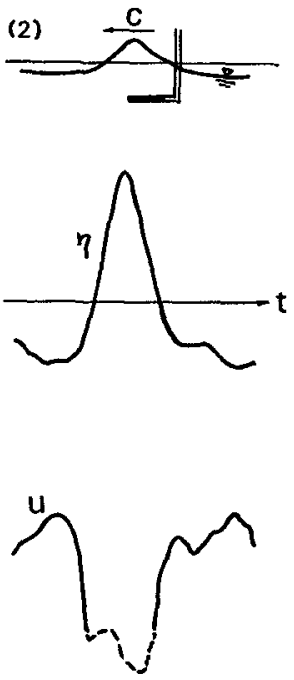

$\mathrm{U}=0$
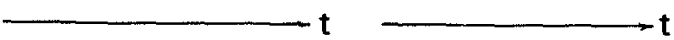

Fig 3 Relation between direction of probe and record of hot film anemometer

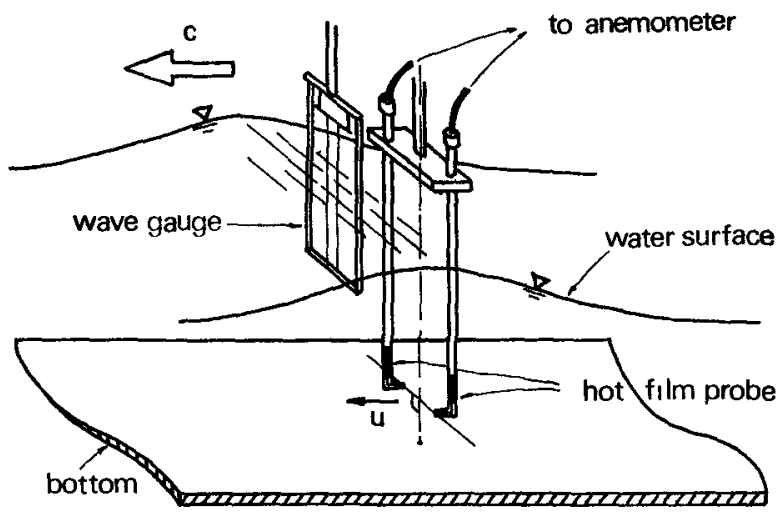

Fig 4 Schematic figure for measurement of water particle velocity by hot flim anemometer 


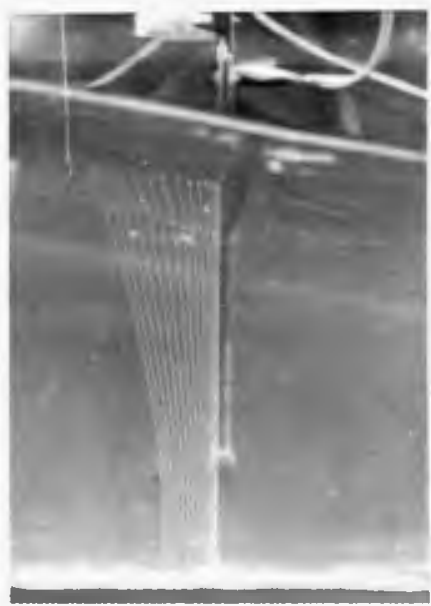

Photo 1 Hydrogen bubble tracer

Table 1 Conditions of experiments by method of tracing hydrogen bubbles

\begin{tabular}{c|c|c|c|c|c|c}
\hline $\mathrm{h}(\mathrm{cm})$ & $\mathrm{T}(\mathrm{sec})$ & $\mathrm{H}(\mathrm{cm})$ & $\Delta \mathrm{t}(\mathrm{msec})$ & $\mathrm{p}(\mathrm{msec})$ & $\mathrm{T} \sqrt{\mathrm{g} / \mathrm{h}}$ & $\mathrm{H} / \mathrm{h}$ \\
\hline 30.5 & 0.93 & 9.08 & 40 & 20 & 5.3 & 0.298 \\
20.9 & 0.96 & 7.22 & 20 & 10 & 6.6 & 0.345 \\
16.0 & 0.94 & 5.22 & 20 & 10 & 7.4 & 0.326 \\
13.0 & 0.95 & 3.98 & 20 & 10 & 8.3 & 0.306 \\
16.0 & 1.10 & 4.91 & 20 & 10 & 8.6 & 0.307 \\
13.0 & 1.10 & 4.13 & 20 & 10 & 9.6 & 0.318 \\
29.8 & 1.74 & 7.12 & 50 & 20 & 10.0 & 0.239 \\
21.0 & 1.74 & 6.47 & 30 & 15 & 11.9 & 0.308 \\
21.0 & 1.76 & 6.44 & 20 & 5 & 12.0 & 0.306 \\
21.0 & 1.89 & 6.24 & 20 & 10 & 12.9 & 0.297 \\
16.0 & 1.73 & 5.18 & 20 & 5 & 13.6 & 0.324 \\
13.0 & 1.93 & 3.66 & 20 & 7 & 16.8 & 0.282 \\
\hline
\end{tabular}


moving two probes with the carrier The records of two confronting anemometers were read out using two calibration curves The full lines in Fig $3(1)$ and (2) were selected, whlch are the part of the record of the anemometer confronting waves and that of the other anemometer following waves These two curves were drawn up slde down each other on the same flgure, matching the phases of the waves, as shown In Fig 5 In general, as mentioned above, two records do not represent zero values but overlap where the real values of the velocity are zero In order to obtain the time variation of the horizontal water particle velocity during one wave perıod, the records were modlfied assuming that the phase of $u=0$ was located where the absolute value of the upper record was equal to that of lower one, as shown in Fig 5

In the experiments, the water depth $h$ was constant, and the wave period $T$ and the wave helght $H$ were variable Test condıtions are shown in Table 2 , where $\mathrm{z}$ is the ordinate taken upwards from the still water level, and $\mathrm{z}_{\mathrm{p}}$ is the depth of the probe, so that $z_{p}+h\left(z_{p}<0\right)$ means the helght of the probe above the tank bottom As shown in Table 2, the value of $\left(z_{p}+h\right) / h$ was always 005

\section{EXPERIMENTAL RESULTS}

F1g 6(1) (12) show the experimental results of the horlzontal water particle velocity at the phase of the wave crest measured by the method of tracing hydrogen bubbles under the conditions of Table $1 \mathrm{u} / \sqrt{\mathrm{gh}}$ as dimensionless expression of $u(g$ the gravity accerelation) is taken as an abscissa, and $(\mathrm{z}+\mathrm{h}) / \mathrm{h}$ as dimensionless expression of the helght from the bottom taken as an ordinate, whth parameters of $\mathrm{T} \sqrt{\mathrm{g} / \mathrm{h}}, \mathrm{H} / \mathrm{h}$ and the wave phase $t / T$ In this figure, the experimental data are denoted by circles, and, for the same values of $T \sqrt{g / h}, H / h$ and $t / T$, the vertical distributions based on the small amplitude wave theory, Stokes wave theory of 3 rd order ${ }^{8}$ and the

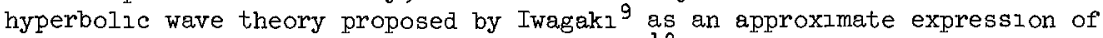
the cnoldal wave theory of 2 d approximation 10 are shown for comparison with full, broken and chaln lines, respectively

The thick full lines in Fig $7(1) \sim(12)$ show the profiles of the waves measured simultaneouly with the vertical distribution of the velocity As in Fig 6, the wave proflles based on the three theorles are also shown for comparison in the figure

The thick full lines in Flg $8(1) \sim(10)$ show the continuous records of time variation of the horizontal water particle velocity during one wave period at $\left(z_{p}+h\right) / h=005$ measured by the hot film anemometers under the condition of Table 2 and the wave profiles recorded simultaneouly other three thin lines show the theoretical curves as in Fig 7

\section{DISCUSSION}

The error of the velocity measured by the method of tracing hydrogen bubbles, which results malnly from the error of reading films and the error of pulse period, wlll be approximately 3\% Also, the error of the velocity measured by the hot film anemometer occurs in the stage of determining the calibration curve, which will be approximately $3 \%$ too 


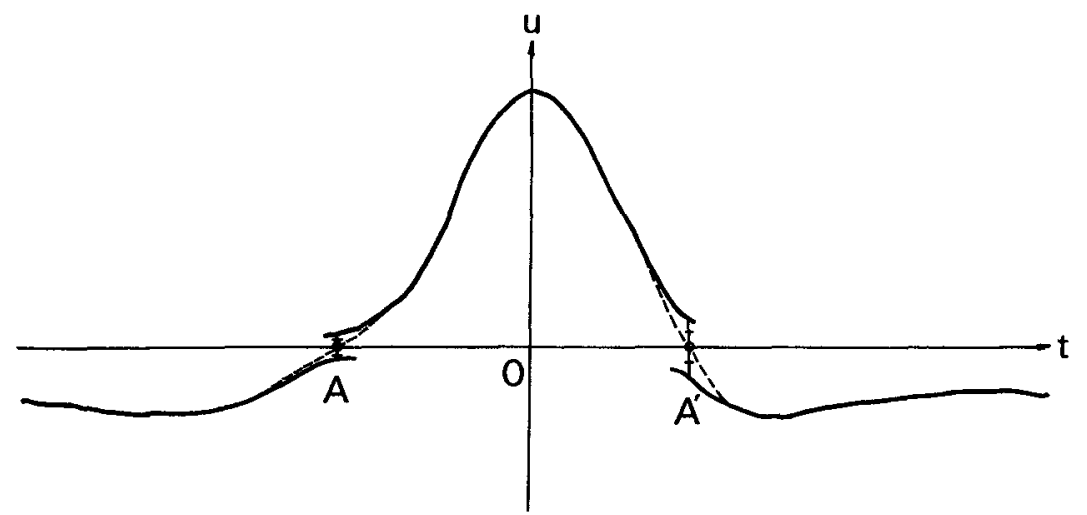

Fig 5 Schematic diagram for modification of record of water particle velocity by hot film anemometer

Table 2 Conditions of experiments by using hot film anemometer

\begin{tabular}{c|c|c|c|c|c|c}
\hline $\mathrm{h}(\mathrm{cm})$ & $\mathrm{T}(\mathrm{sec})$ & $\mathrm{H}(\mathrm{cm})$ & $\mathrm{z}_{\mathrm{p}}+\mathrm{h}(\mathrm{cm})$ & $\mathrm{T} \sqrt{\mathrm{g} / \mathrm{h}}$ & $\mathrm{H} / \mathrm{h}$ & $\left(\mathrm{z}_{\mathrm{p}}+\mathrm{h}\right) / \mathrm{h}$ \\
\hline 160 & 103 & 672 & 080 & 81 & 0420 & 005 \\
160 & 130 & 686 & 080 & 102 & 0429 & 005 \\
160 & 158 & 604 & 080 & 124 & 0378 & 005 \\
160 & 183 & 721 & 080 & 143 & 0448 & 005 \\
160 & 208 & 705 & 080 & 163 & 0441 & 005 \\
160 & 239 & 489 & 080 & 187 & 0306 & 005 \\
160 & 263 & 506 & 080 & 206 & 0316 & 005 \\
160 & 284 & 413 & 080 & 222 & 0258 & 005 \\
160 & 307 & 411 & 080 & 240 & 0257 & 005 \\
160 & 340 & 366 & 080 & 266 & 0229 & 005 \\
\hline
\end{tabular}




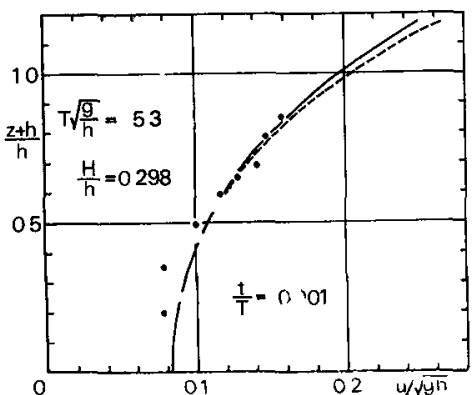

(1)

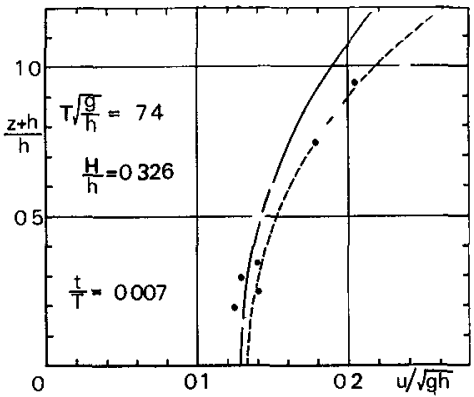

(3)

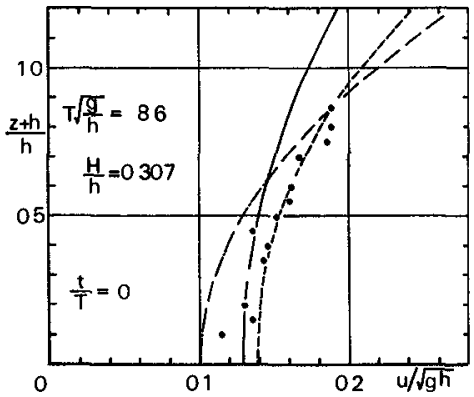

(5)

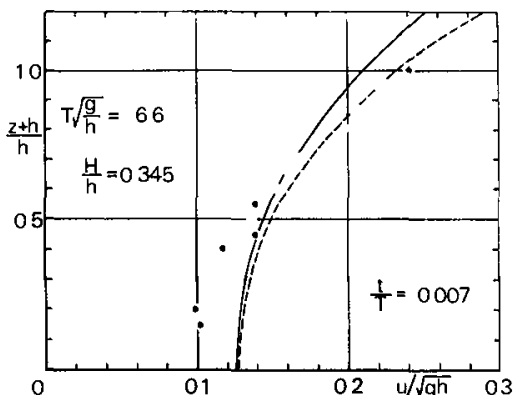

(2)

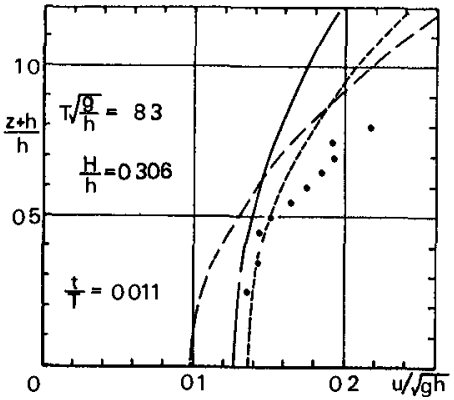

(4)

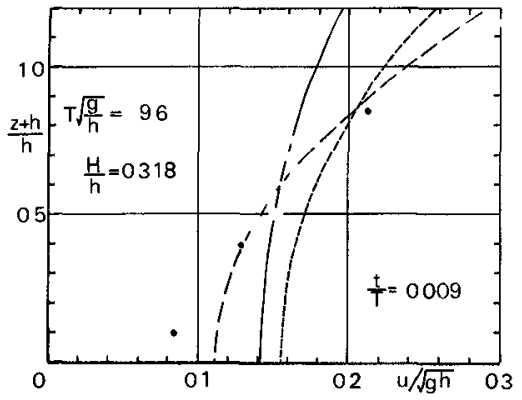

(6)

Fig 6(1) (6) Vertical distribution of horizontal water particle velocity at phase of wave crest (full line small amplitude waves, broken line Stokes waves, chain line hyperbolıc waves) 


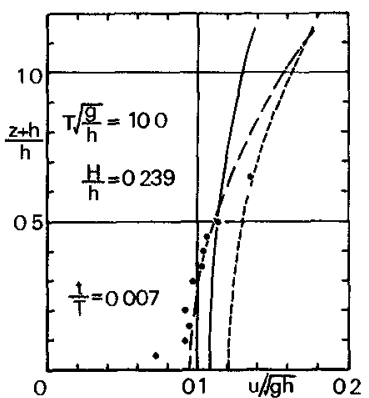

(7)

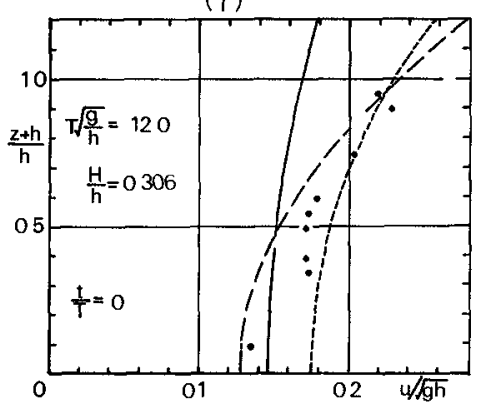

(9)

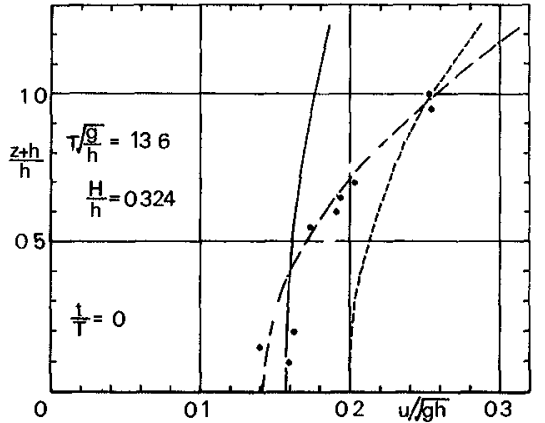

(11)

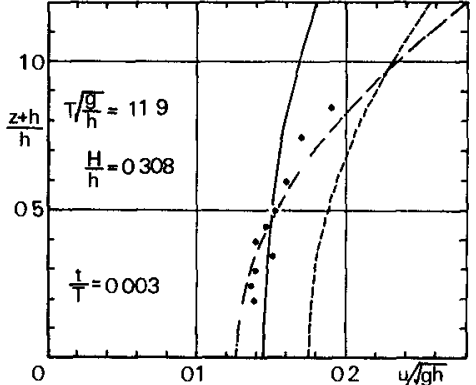

(8)

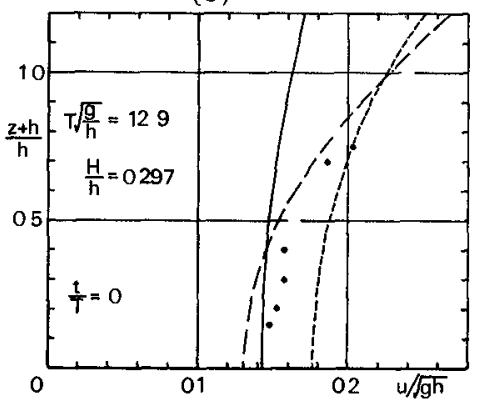

(10)

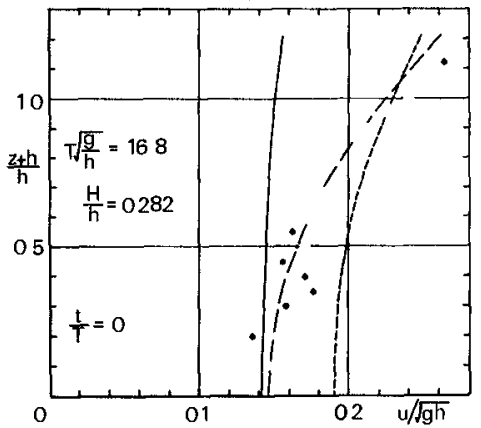

(12)

F18 6(7) (12) Vertical distribution of horizontal water particle velocity at phase of wave crest(full line small amplitude waves, broken line Stokes waves, chain line hyperbolle waves) 


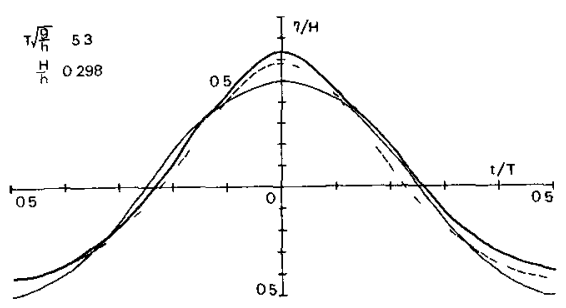

(1)

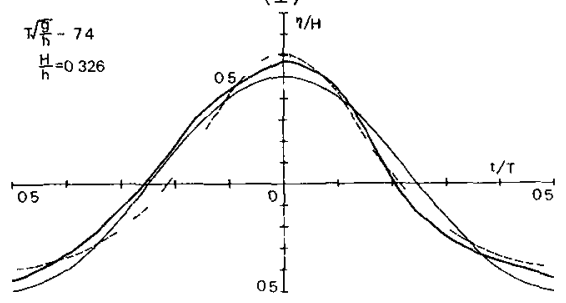

(3)

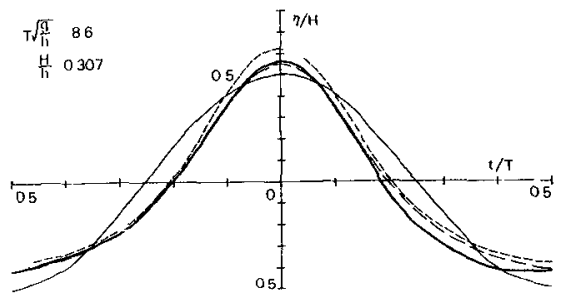

(5)

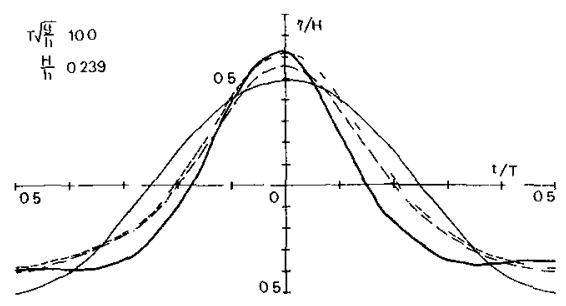

(7)

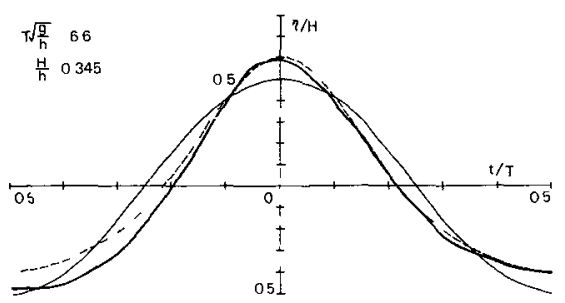

(2)

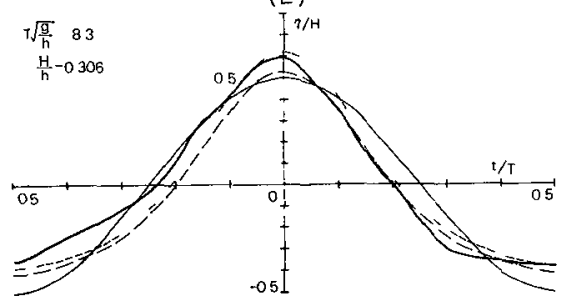

(4)

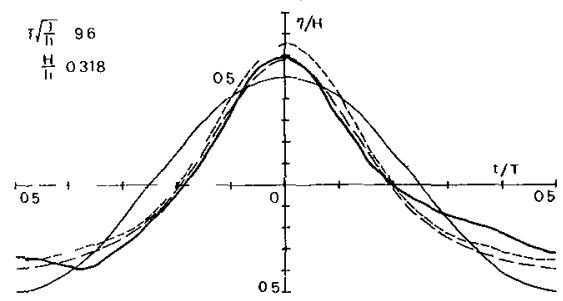

(6)

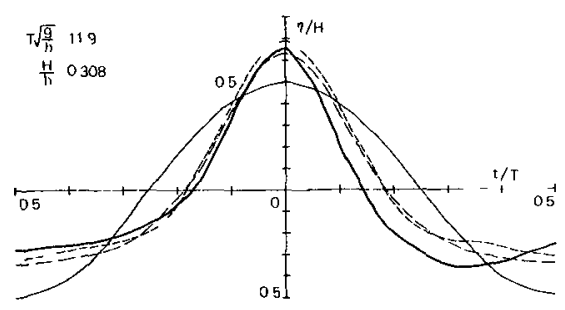

(8)

Fig $7(1)$ (8) Time varlation of water surface corresponding to Fig 6(thick full line experiment, thin full line small amplitude waves, thin broken line Stokes waves, thin chain line hyperbolic waves) 

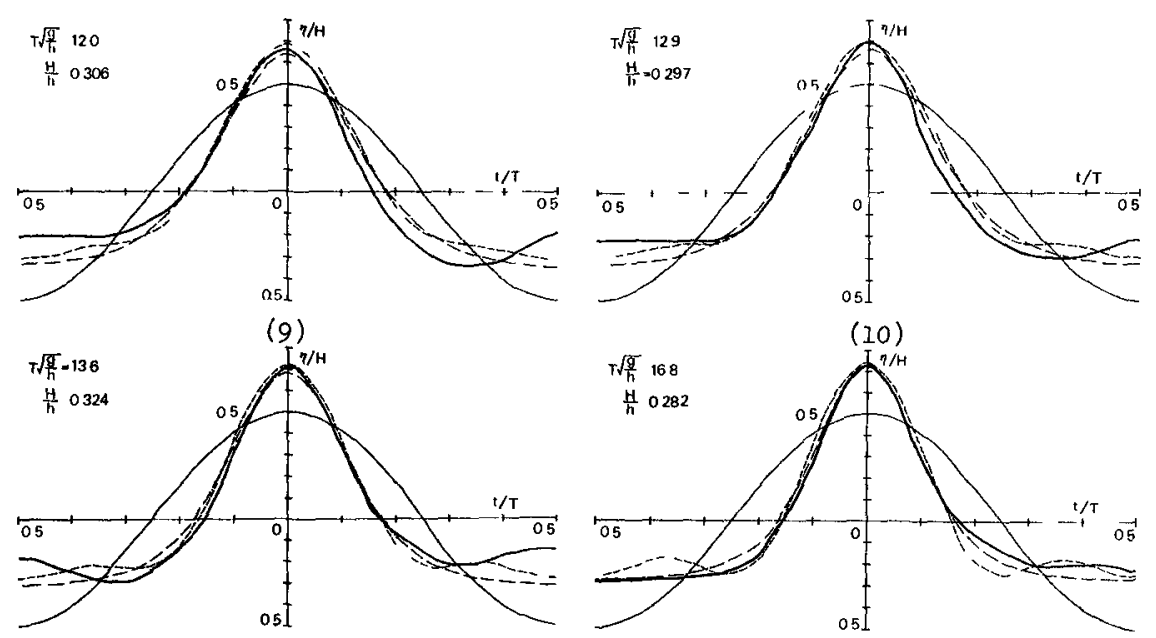

(11)

(12)

Fig $7(9) \sim(12)$ Time variation of water surface corresponding to Fig 6(thick full line experiment, thin full line small amplitude waves, thin broken line Stokes waves, thin chain line hyperbolıc waves)

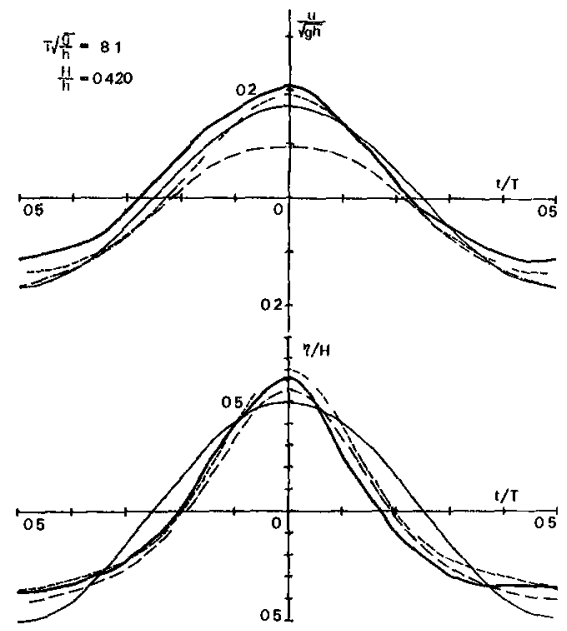

(1)

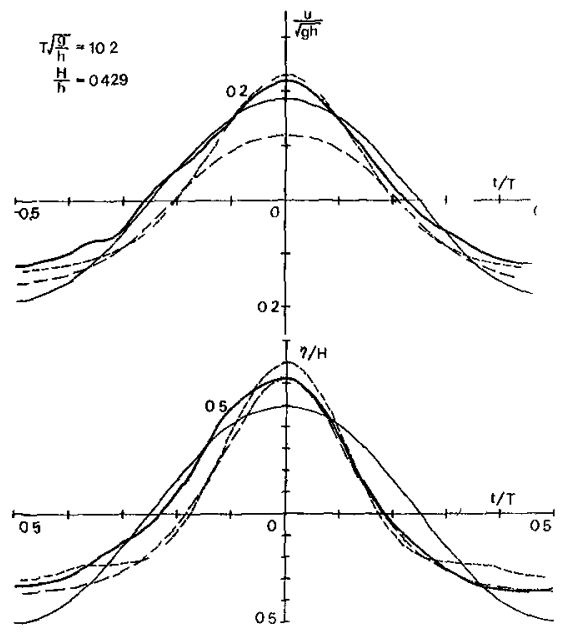

(2)

FIg 8(1),(2) Time variation of horizontal water particle velocity and corresponding water surface(thick full line experiment, thin full line small amplitude waves, thin broken line Stokes waves, thin chain line hyperbolic waves) 


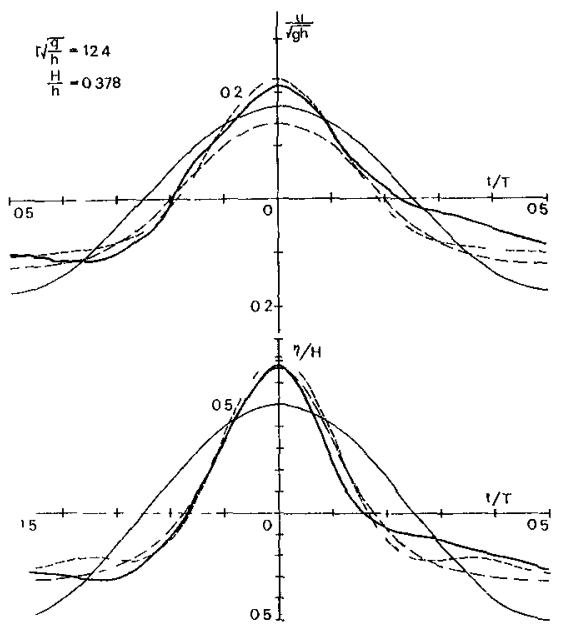

(3)

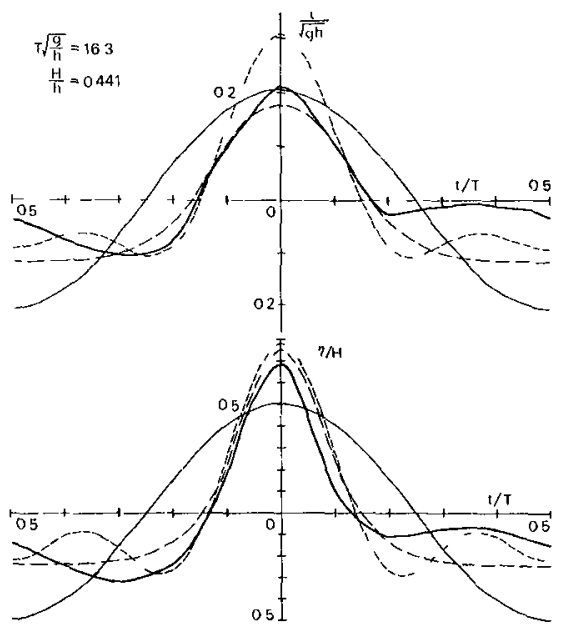

(5)

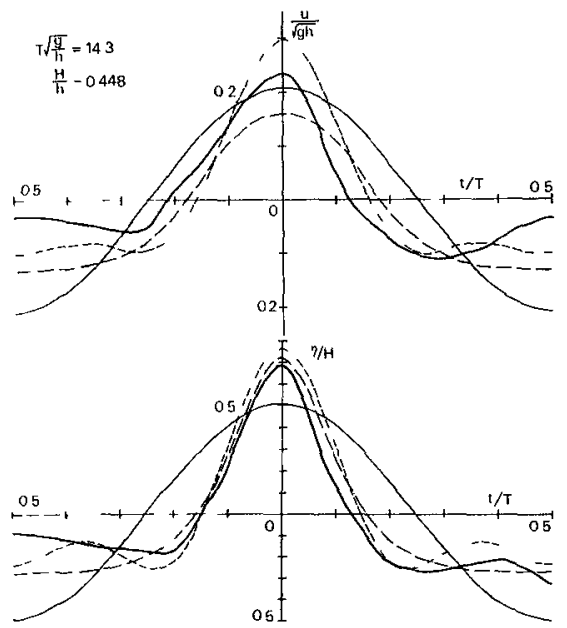

(4)

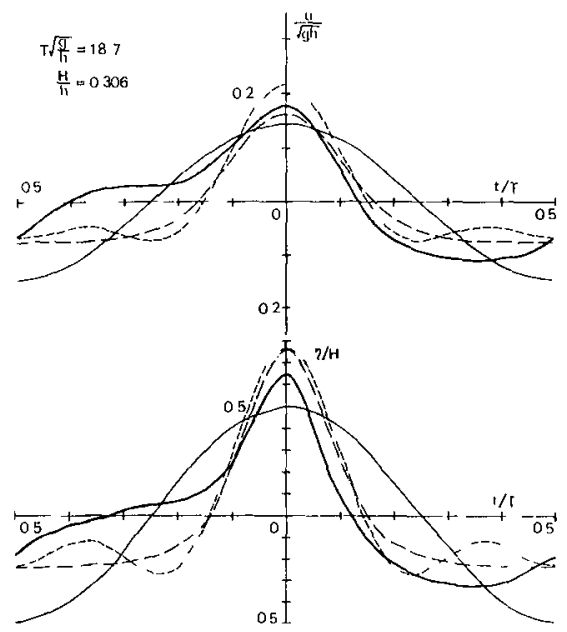

(6)

Fug 8(3)u(6) Time variation of horizontal water particle velocity and corresponding water surface(thick full line experiment, thin full line small amplitude waves, thin broken line Stokes waves, thin chain line hyperbolic waves) 


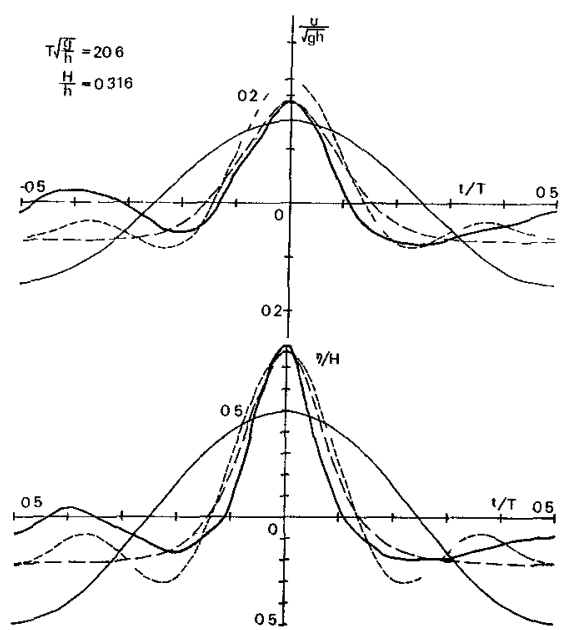

(7)

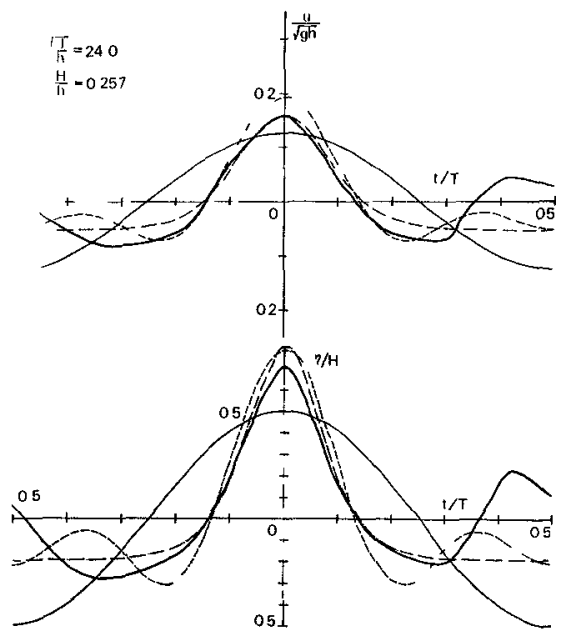

(9)

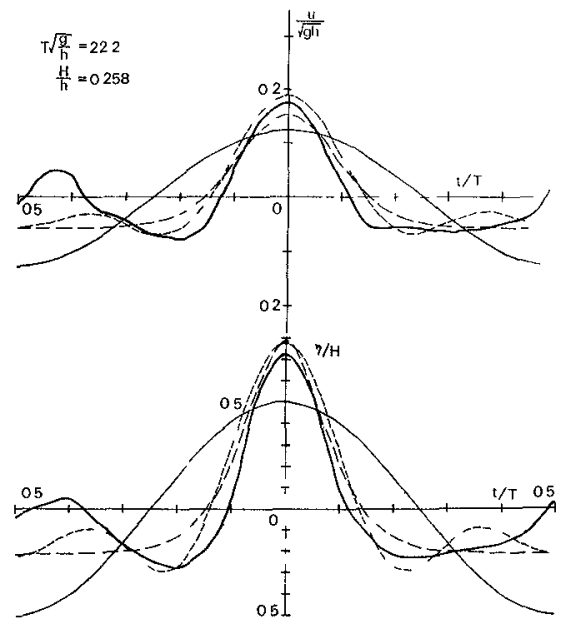

(8)

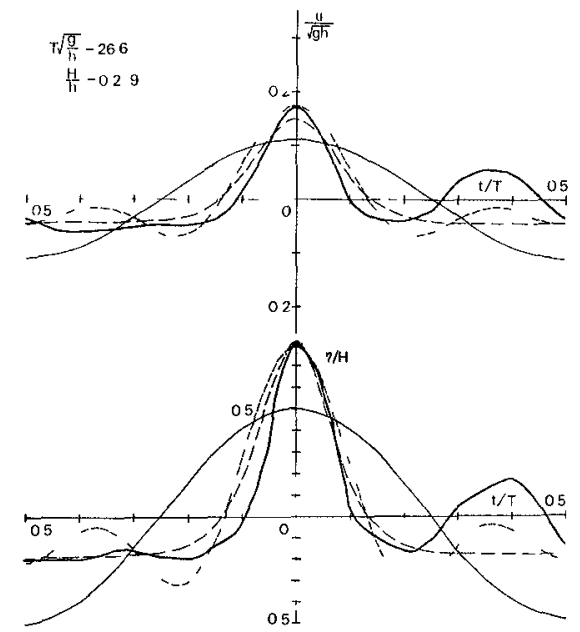

(10)

F1g 8(7) (10) Time varıation of horizontal water particle velocity and corresponding water surface(thick full line experiment thin full line small amplitude waves, thin broken line Stokes waves, thin chain line hyperbolic waves) 
In F.g 6 , the ralues of wave phase $t / T$ are not always zero, but the absolute values excst between 0 and 001 , whjch means that the data taken are regarded as at the phase of the wave crest Generally speaking, among the theoretical curves of vertical distributions of the horlzontal velocity, those of the small amplitude wave theory are steepest and the curves of the hyperbollc wave theory are most gentle The value of the velocity based on the small amplitude wave theory is smaller all over the depth than that of Stokes wave theory, and the dufference between them becomes largest at the water surface on the other hand, the value based on the hyperbolic wave theory, in most cases, Is smaller than that of the small amplitude wave theory near the bottom, but larger than that of Stokes wave theory near the water surface

The experimental data were obtalned generally at every helght of $5 \%$ of the water depth from the bottom However, in the region where $(\mathrm{z}+\mathrm{h}) / \mathrm{h}<0 \mathrm{l}$, the velocity field induced by waves was disturbed by the obstacle of $5 \mathrm{~mm} \mathrm{~h}$ gh to attach the platinum wire to the bottom, and in the region where $(z+h) / h>10$ the velocity could not be measured because of insufficlent generation of bubbles In the case when $\mathrm{T} \sqrt{\mathrm{g} / \mathrm{h}}=53$, the experumental values have the same trend as two theoretical distributions based on the small amplitude wave and Stokes wave theories In the cases when $\mathrm{T} \sqrt{\mathrm{g} / \mathrm{h}}=66$ and 74 , the experimental values agree falrly with those of Stokes waves In the case when $\mathrm{T} \sqrt{\mathrm{g} / \mathrm{h}} \geqslant 8 \quad 3$, the vertical distributions of the velocity based on the hyperbolic wave theory are also presented In cases when $T \sqrt{g / h}>86$, the experjmental data are plotted between the theoretical curves of Stokes and hyperbolic waves In general, when $\mathrm{T} \sqrt{\mathrm{g} / \mathrm{h}} \geq 10$, the vertucal distribution of the velocity under the wave crest can be explained well by the hyperbolıc wave theory as an approximate expression of the cnoldal wave theory rather than stokes wave theory

Fig $7(1) \sim(12)$ show the comparisons between theories and experiments of the wave profile corresponding to each case of Flg 6 as mentioned above It is evident that the experimental results of the wave profile are close to those of two finite amplitude wave theorles rather than that of the small amplitude wave theory However, it $1 . s$ very difficult to decıde which finıte amplitude wave theory is fit to the experiment on the wave profile from the figures In other words, the difference between two finlte amplitude wave theories is not clear in the case of the wave proflle, but very clear for the vertical distribution of the velocity It should be noticed that this fact is very signiflcant when the wave force acting on submerged structures is estimated by the theory Especially remarkable differences of the velocity between stokes wave and hyperbolıc wave theories appear near the bottom and above the still water level

The probes of the hot fllm anemometer were mounted as high as $5 \%$ of the water depth above the bottom rather than near the water surface, because of avolding risk of damage by exposing the probe in alr Tendencles of three theoretical curves of time variation of the velocity shown in FiB 8 are that the theoretical curves of Stokes and hyperbolic waves are sharper near the phase of the wave crest and flatter near the phase of the wave trough than that of the small amplitude wave theory, in the same manner as in the wave profile However, in the cases when $\mathrm{T} \sqrt{\mathrm{g} / \mathrm{h}}=81$ and 102 , the velocity of hyperbolic waves at the trough is greater than that at the crest This trend, Jhlch is of the cnoldal wave theory ltself, occurs near the bottom only when 
$\mathrm{T} \sqrt{\mathrm{g} / \mathrm{h}}<120$ and the value of $\mathrm{H} / \mathrm{h}$ is large Under the wave crest, the theoretical value of $\mathrm{u} / \sqrt{\mathrm{gh}}$ of Stokes waves ls largest and that of hyperbolic waves is smallest near the bottom when $\mathrm{T} \sqrt{\mathrm{g} / \mathrm{h}} \leqslant 163$, and the value of hyperbolic waves shifts in the middle of small amplitude waves and Stokes waves when $\mathrm{T} \sqrt{\mathrm{g} / \mathrm{h}} \geqq 187$

The experimental results of time variation of the horizontal velocity during one wave period show that, in general, the wave crest is sharper and the wave trough is flatter than those of small amplitude waves, in the same manner as the theoretical curves of finlte amplitude waves It is seemed that the experimental curves near the crest are close to the theoretical values of Stokes waves when $\mathrm{T} \sqrt{\mathrm{g} / \mathrm{h}} \leqq 124$ and to those of hyperbolıc waves when $\mathrm{T} \sqrt{\mathrm{g} / \mathrm{h}} \geqq$ 163 , although secondary waves appear at the wave trough In the vertical distributions of the velocity shown in Fig 6, as mentioned above, the experimental values agree falrly with the hyperbolıc wave theory when $\mathrm{T} \sqrt{\mathrm{g} / \mathrm{h}} \geqq$ 10 However, in the experimental results of time variation of the velocity, the experimental values agree roughly with the theoretical curves of Stokes waves at least when $T \sqrt{\mathrm{g} / \mathrm{h}} \leqq 124$ One of reasons of the discrepancy may be the difference of the values of $H / h$ as shown in Table 1 and 2 , that $1 \mathrm{~s}$, when $\mathrm{T} \sqrt{\mathrm{g} / \mathrm{h}}$ $\leqq 163$ In Flg 8 , values of $\mathrm{H} / \mathrm{h}$ are greater than 04 In most cases, and considerably large compared with those in Fig 6

In F1g 8, measured wave profiles are close to theoretıcal profiles of finite amplitude waves rather than those of small amplitude waves However, differences between measured profiles and computed profiles based on two finjte amplitude wave theories are not so clear as in the horizontal velocity

In all cases of the experiments by the hot $f_{1} l m$ anemometer, Reynolds numbers defined as

$$
\mathrm{N}_{\mathrm{R}}=\sqrt{\pi / \nu T} \mathrm{H} / \sinh (2 \pi \mathrm{h} / \mathrm{L})
$$

were calculated ( $\nu$ the kinematic viscosity) The maximum value among them is smaller than the critical Reynolds number $\mathrm{N}_{R}=160$ for transition from laminar to turbulent boundary layer by waves ${ }^{1}$ Also, it was conflirmed that the calculated thickness of the laminar boundary layer in this case, which is assumed to be the helght from the bottom where the velocity becomes 99\% of the velocity at the outside of the boundary layer, was $48 \mathrm{~mm}$ at maximum and smaller than the helght of the probes from the bottom, $8 \mathrm{~mm}$

\section{CONCLUSION}

The authors described two methods to measure the hor Izontal water particle velocity induced by waves in the wave tank, the method of tracing hydrogen bubbles and the method of using a hot fllm anemometer Experimental results of the vertical distribution of the velocity under the wave crest measured by the former method and time variation of the velocity during one wave period at a definite height from the bottom by the latter method were presented Discuccions were made compared with the theoretical values based on the small amplitude wave theory, Stokes wave theory of 3 rd order and the hyperbolic wave theory as an approximate expression of the cnoldal wave theory of and approximation 
After all, the following conclusions were obtalned The reglon, where the hyperbolic wave theory should be applied to the horizontal water particle velocity rather than Stokes wave theory, is $\mathrm{T} \sqrt{\mathrm{g} / \mathrm{h}} \geqq 10$, which was found for 1 ts vertical distribution under the wave crest, and $T \sqrt{g / h} \geqq 14$ for Its time varlation during one wave perıod. It should be noted that, in computing the wave force acting on submerged structures, Stokes wave theory may give too small values of the horlzontal water particle velocity at the water surface in the reglon $\mathrm{T} \sqrt{\mathrm{g} / \mathrm{h}} \geqq 10$

\section{ACKNOWLEDGEMENT}

Part of this investigation was accomplished with the support of the Science Research Fund of the Ministry of Education, for which the authors express their appreciation Thanks are due to $\mathrm{Mr} \mathrm{T}$ Kawashima for his help during this investigation

\section{REFERENCES}

1) Morison, $J$ R and $R$ C Crooke The Mechanies of Deep Water, Shallow Water, and Breakıng Waves, $U$ S Army, Coros of Engıneers, B E B, Tech Memo No 40, March, 1953, pp 1-14

2) Ellıott, John G Interim Report, Hydrodynamics Laboratory, Calif Inst of Tech, Contract Noy-12561, U S Navy, Bureau Yards and Docks, July, 1953

3) Goda, Y Wave Forces on a Vertıcal Circular Cylınder, Experiments and a Proposed Method of Wave Force Computation, Port and Harbour Technical Research Institute, Minıstry of Transportation, Japan, Repert No 8, 1964

4) Le Méhauté, B , D Divoky and A Lin Shallow Water Waves A Comparıson of Theories and Experiments, Proc 1lth Conference on Coastal Englneering, 1968, pp 86-107

5) Schraub, F A, S J Klıne, J Henry, $P$ W Runstadler, Jr and A Llttell Use of Hydrogen Bubbles for Quantitatıve Determenation of Time-Dependent Velocity Flelds in Low-Speed Water Flows, Trans ASME, Jour of Basic Englneerıng, Vol 87, 1965, pp 429-444

6) Horlkawa, IF and A Watanabe Laboratory Study on Oscillatory Boundary Layer Flow, Proc llth Conference on Coastal Englneering, 1968, pp 467486

7) Richardson, $E V$ and $R$ S McQuivey Measurement of Turbulence in Water, Jour of the Hydraulies Division, ASCE, Vol 94, No HY2, Proc Paper 5855, March, 1968, pp 411-430

8) Skjelbrela, I Gravity Waves, Stokes' Third Order Approximation, Table of Function, Counc1l on Wave Research, The Englneerıng Foundation, 1959, pp $1-337$

9) Iwagakı, Y Hyperbolıc Waves and Thelr Shoaling, Proc 1lth Conference on Coastal Englneerıng, 1968, pp 124-144

10) Laltone, E V The Second Approximation to Cnoldal and Solıtary Waves, Jour of Fluld Mechanics, Vol 9, 1961, pp 430-444

11)Collins, J I Inception of Turbulence at the Bed under Periodic Gravity Waves, Jour of Geophysical Research, Vol 68, No 21, 1963, pp 6007-6014 



\section{CHAPTER 20}

\section{A SYSTEM FOR MEASURTING ORBITAL VELOCITIES IN WAVES}

by M. M. Kolpak ${ }^{\dagger}$ and $P$ S Eagleson *

Abstract

A single-ended, cylindrical hot-film sensor and a directionvane transducer are studied as instruments for measuring flow flelds in laboratory waves Errors in the hot-film measurements are discussed in terms of sensor voltage and water temperature drufts, and directional sensitivity The response of the direction-vane transducer is discussed in terms of the parameter $b / r$, which is the ratio of the vane chord length to the radius of curvature of the measured orbital flow

The instruments are tested in a laboratory wave system, using stationary and traversing measuring techniques The velocity measurements so obtained are compared to those obtained by a photographic technique, to determine instrument error The flow speed comparisons indicate that the maximum hot-film error in flow speed measurements is between \pm 5 and \pm 10 lnches/sec for the range 1 to 11 inches $/ \mathrm{sec}$ tested The flow direction comparisons indicate that the direction vane response is subject to errors larger than $5^{\circ}$ for $\mathrm{b} / \mathrm{r}>01$

The study was carried out under the sponsorship of the Coastal Englneering Research Center at the Massachusetts Institute of Technology and Is reported in full detall in the M.I T. Hydrodynamics Laboratory Report No 118

\section{Introduction}

This is a study of the utility of a hot-film sensor and a direction-vane transducer in the laboratory measurement of velocity flelds in water waves It was motivated by a need for orbital data which is easier to obtain than by photographic techniques The photographic techniques, in which neutrally buoyant particles are suspended in the wave flow and photographed, have the advantage that they produce relatively accurate data, but the disadvantage that the reduction of such data to flow speed and direction is so time consuming and tedious that the quantity of such data reduced to date is rather limited

† Sr Research Engineer, Pan American Petroleum Corporetion

* Head, Dept of Clvıl Engıneerıng, Massachusetts Institute of Technology 
As an alternative to this method of measurement, a study was made of the use of the hot-film and direction-vane, which have electrical. outputs that can be digltized and subsequently processed by computer to reduce the task of data reduction

For flow speed measurements the $6 \mathrm{mll}$ diameter, single ended, cylindrical hot-film sensor shown in Fig (1) was studied The sensor consists of an electrically heated metal film which conducts heat to the surrounding flow in proportion to the flow speed When positioned so that its cylindrical axis is perpendicular to the plane of flow in a two dimensional wave flow field, the sensor can measure flow speed regardiess of flow direction

For measurements of flow direction, the direction-vane transducer shown in Fig (1) was studied It consists of a neutraliy buoyant vane $125^{\prime \prime}$ long, $03^{\prime \prime}$ wide and 004" thick, attached to a rotateable shaft Fluid drag tends to align the vane with the direction of flow By monitoring the angulax orientation of the shaft with a sensing device in the transducer housing, it is possible to obtain measurements of flow dixection in a two dimensional wave flow fleld

The two instruments are mounted back to back on a strut and submerged to any location where simultaneous measurements of flow speed and direction are desired

\section{The Hot-Fin Sensor}

Experience with the hot-film sensox in this study showed that

1) The water in which measurements are made must be flitered to prevent output signal drift due to accumulation of dirt particles around the sensor

2) The watex must be deaerated to below about $16 \mathrm{ppm}$ at $70^{\circ} \mathrm{F}$ dissolved gas content to prevent formation of gas bubbles on the sensor surface and the accompanying signal drift

3) The overheat ratio, whlch is a measure of the degree to which the sensor is heated above the water temperature, must be less than about 115 to further prevent formation of gas bubbles on the sensor surface

4) The presence of the sensor in the orbital flow field of wave motion can result in velocity and thermal disturbances in the flow which tend to remain in the vicinity of the sensor and interfere with measurement These disturbances are lilustrated schematically in Fig (2) where the wake emanating from the sensor is shown at four different times during the passage of a wave past a stationary sensor since the motion is orbital, the wake is orbital. And since the water in the wake is warmer than the surrounding water the walke tends to rise and have a spiral shape Whenever a wave trough is passing the sensor, the sensor is crossed by previously formed loops of Its own wake Since the sensor is sensitive not only to flow speed but to water temperature, wake crossings therefore result in the aberrations of output signal schematized in Fig (2e) and (2f). 


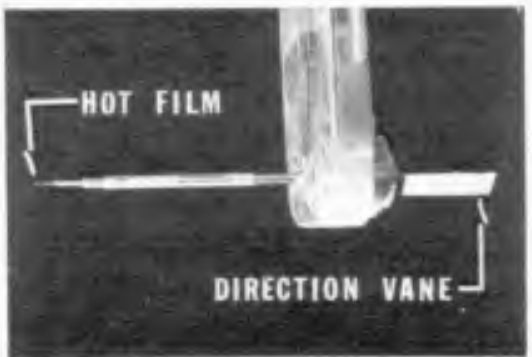

FIGURE (1) Hot-film Sensor and Direction-Vane Transducer mounted on Strut. 


\section{HEATED - WAKE EFFECTS}

WAKE-TRACE CONFIGURATION UNDER PASSING WAVE

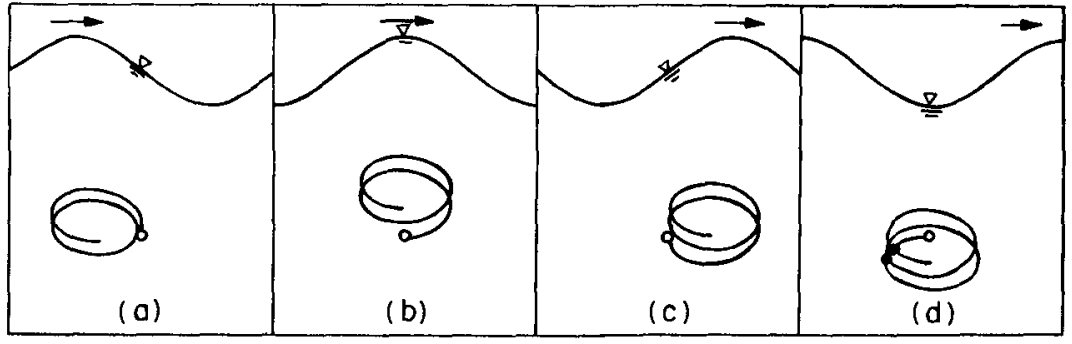

- STATIONARY SENSOR

- WAKE CROSSINGS

CONDITIONS FOR WAKE CROSSINGS

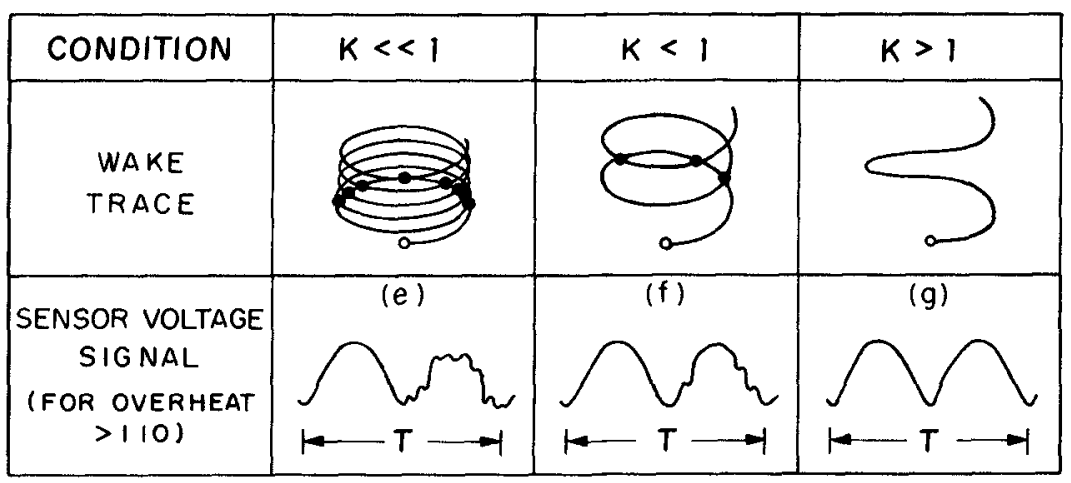

$$
K=\frac{W_{S}}{(2 A / T)}
$$

$W_{s}=$ VERTICAL VELOCITY OF BOUYANT WAKE

$2 A=$ MINOR AXIS OF PARTICLE ORBITS

$T=$ WAVE PERIOD 
Through experiments It was found that signal disturbances due to heat wake crosings are reduced to tolerably low levels when the overheat ratio is kept between 102 and 1 12 At the lower figure, however, the hot-film becomes relatively more sensitive to long term varıations in water temperature than to velocity so that it is desirable to operate at around 110

We may obtain estimates of the sensitivities of the hot-film by use of Kıngs law type emplrical heat transfer equations for example, at 110 overheat ratıo a $1 \%$ drift in the sensor's output voltage corresponds to a $5 \%$ velocity error Also, a $1^{\circ} \mathrm{F}$ change in water at 110 overheat ratıo results in a $3 \%$ velocity error unless corrections for temperature are made

5) The hot-film sensors tested in this study have an undesirable sensitivity to flow direction, in spite of efforts by the manufacturer to avold it A typical calibration for directional sensitivity is lllustrated in Flg (3) whlch shows the result that the normalized sensor output voltage varies with flow direction when the direction of a constant speed flow is varied but kept perpendicular to the sensor axis at all times The points are representative of a range of constant flow speeds and overheat ratios and tend to fall on the same curve whlch is very nearly sinusoldal Even though the maximum variation is only about $\pm 3 \%$ In voltage, this however translates to a $\$ 15 \%$ veloclty error unless corrected for Such corrections were possible in the present study since the calibration points collapsed to a single sinusoldal curve, but even so, flow direction had to be known before the corrections could be made Hopefully such sensitivity can be reduced as the art of sensor fabrication Improves

\section{The Direction-Vane Transducer}

Experience with the direction vane in shallow water waves has shown that 1) The vane can properly respond to changes in flow direction, but only near the free surface where the orbits are more nearly clrcular than near the bottom boundary of the flow where they are hlghly eccentric ellipses lvear the bottom the vane tends to lag the flow direction The problem of predicting the vane's response error due to considerations of inertia and friction of the moving parts is complicated by the difficulty of obtaining a solution of the vane's dynamic equation of motion, which is highly non-linear Furthermore, the forcing function in the equation, flucd drag on the vane, is not known for the general unsteady case, including the effects of virtual mass and separation Although some approximations might be made about the drag force and numerical methods might be employed to solve the equations, no solutions were obtalned in this study Rather, it was decided to carry out some quantitative experiments first to establish criterıa for the vane's response

2) Apart from the dynamics, the fact that the vane is $r i g+d$ whereas the flow is curvilinear means that the vane will generally lag the flow by an amount which $I$ s a function of $b / r$, where $b$ is the chord length of the vane and $r$ is the radius of curvature of the flow past the vane This is lllustrated, Fig (4) where, schematically, we have, inltially, several partieles on cither side of the vane identified by 
DIRECTIONAL SENSITIVITY OF HOT-FILM SENSOR
$V^{\prime}=$ OIRECTIONAL SENSITIVITY FACTOR $=V_{\theta} / V_{A}$
$V_{\theta}=$ SENSOR VOLTAGE FOR FLOW DIRECTION $\theta$
$V_{A}=$ AVERAGE VOLTAGE OVER $360^{\circ}$ RANGE

- experimental points for all combinations, of OVERHEAT $=110,107,105$

VELOCITY $=35,774,1212,1626 \mathrm{IN} / \mathrm{SEC}$

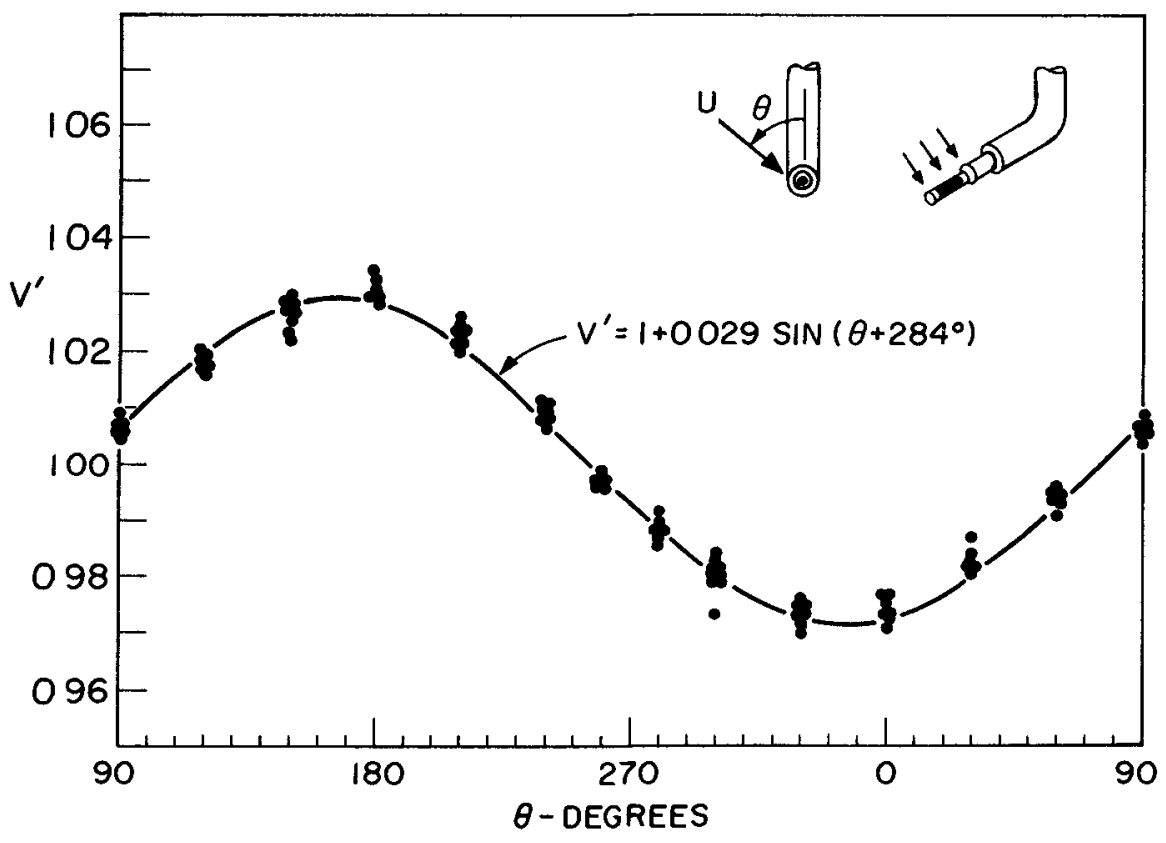

FIGURE (3) 
KINEMATIC INCOMPATIBILITY

$$
r<b
$$

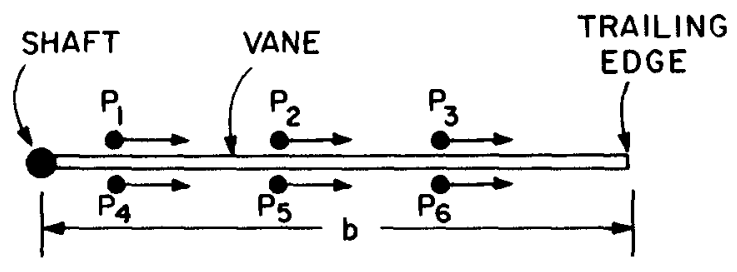

FIGURE (4a)

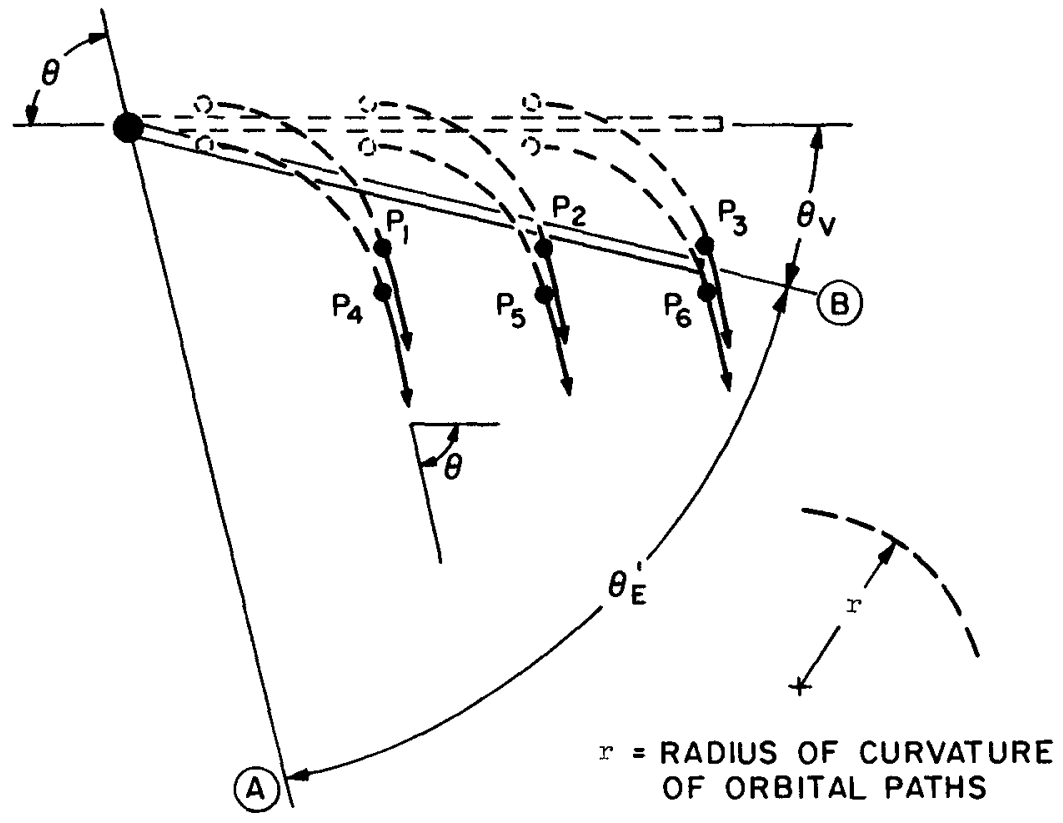

FIGURE (4b) 
small circles, Fig (4a) For the sale of illustration, let the vane at this time be aligned with the direction of the flow It is seen that if the particles undergo portions of their undisturbed orbital motion, they move to the new locations shown in Fig (4b), but the rigld vane cannot possibly respond by pivoting about a fixed shaft and yet remain between the particles without deforming In fact, the vane will more likely point in the $\boldsymbol{\theta}_{\mathbf{v}}$ direction rather than in the actual direction $\boldsymbol{\theta}$ of the flow This lilustration is for the case of $b / r>1$, but it can be extended for the case $b / r<1$, and we realıze that this purely kinematic consideration shows that the vane will in general lag the flow An approximate calculation of the resulting directional error, $\theta_{E}$, yields

$$
\theta_{E}=\operatorname{Sin}^{-1}[b / 2 r]
$$

which gives $\theta_{E} \leq 5^{\circ}$ for $b / r \leq 01$

To obtain experimental verification of this and some other quantitative aspects of the hot-film's response, measurements of the same flow field were made by the instruments and by a photographic technique and the results were compared to estimate instrument error

\section{Measuring Techniques}

The instruments were utilized in a number of ways

1) The "stationary" technique - here the instruments are submerged to a given depth and kept stationary for several wave periods' duration This is the simplest measuring technique, however, the response of the vane is rather limıted by the small orbital radius of curvature near the wave tank bottom

2) The "transversing" technıques - these are represented in Fig (5) for the case of towng the instruments vertically upwards during measurement Refering to Fig ( $5 a$ ), as the instruments are towed upwards at uniform speed we obtain data along a straight line depth-time characteristic A-B If the flow is periodic, repeating Itself exactly, every wave period, then portion C-B may be shıfted by one wave period to $D-E$, so that the data can be viewed within the time domain of a single perıodic wave Thua, by making several traverses, a.ll at different beginning phase times, it is possible to fill the y-t plane with data Later, by subtracting the towing velocity rector from the relative flow vector measured, we obtain a mapping of the flow fleld along a vertical section through the fllow The advantage of such a technique is that the radius of curvature and the flow speed of the relative flow past the vane are both larger than for the stationary technique and the vane response improves However, such improvements in response occur only during times when the vertical component of the flow Is opposite to the direction of towing Thus to obtain data of superior relative accuracy a set of upward traverses is needed for $T / 4 \leqslant t \leqslant 3 T / 4$ and a set of downward traverses for $0 \leq t \leq T / 4$ and $3 T / 4 \leq t \leq T$, where $T$ is the wave period and $t$ is time

3) The "dual tow" technique - where it is possible to obtaln measurements of flow direction using only the hot-film sensor and not the direction vane transducer The procedure is to first fill the 


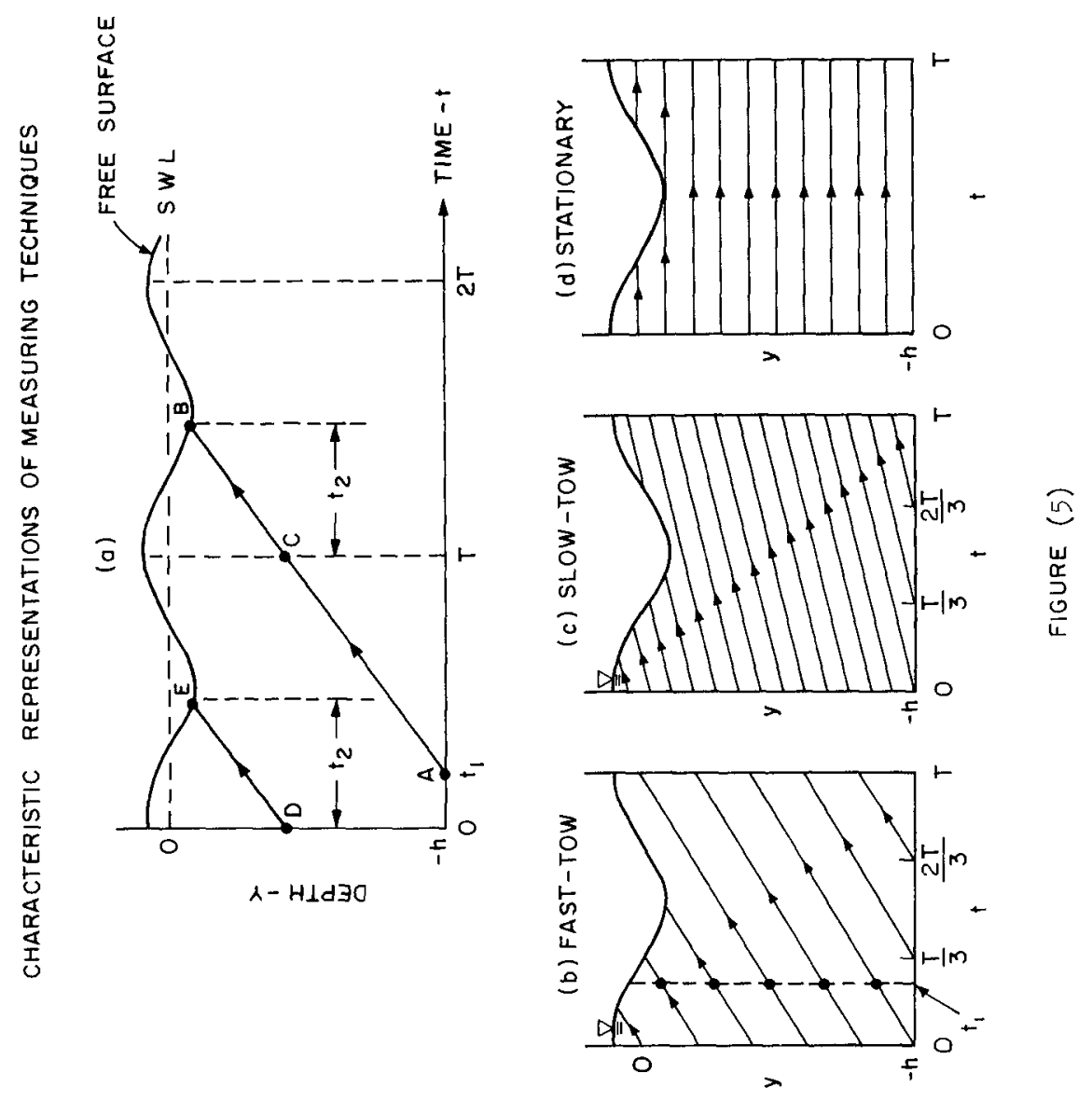


depth-time plane with measured relatıve flow speed data, $R_{1}(y, t)$, having made traverses at towlng speed $S_{1}$ Then the measurements are repeated but at a different towing speed, $S_{2}$, glving relative flow speed data, $\mathrm{R}_{2}(\mathrm{y}, \mathrm{t})$ The result is that at every point in the $\mathrm{y}-\mathrm{t}$ plane, we now have the four knowns, $R_{1}, S_{1}, R_{2}$, $S_{2}$, from whlch it is possible to solve for the two unknowns, $U$, the locel flow speed, and $\theta$, the local flow direction With Fig (6) representing the vector diagram of the variables the Cosine Iraw for triangle $A B C$ is,

$$
R_{1}^{2}=S_{1}^{2}+U^{2}-2 S_{1} U \operatorname{Cos}(\pi-\theta)
$$

and for triangle ABD,

$$
R_{2}^{2}=S_{2}^{2}+U^{2}-2 S_{2} U \operatorname{Cos}(\pi-\theta)
$$

Subtracting and rearranging, we obtain

$$
U=\left[\frac{\left(S_{2}^{2}-R_{2}^{2}\right) S_{1}-\left(S_{1}^{2}-R_{1}^{2}\right) S_{2}}{S_{2}-S_{1}}\right]^{1 / 2}
$$

from which $U$ can be obtalned since $S_{1}, S_{2}, R_{1}$ and $R_{2}$ are known Equation (3) may then be used to solve for

Since Equation (4) yields only the principal values of , additional information about whether the vertical component of $U$ is up or down, is required to resolve the ambiguity In practice, the position of the free surface may be used as an indicator

\section{Experimental Results}

Typical experimental results for the stationary technıque are shown in Fig (7), where the differences between hot-film measurements and photographic technique measurements are plotted against time for various depth locations, $y / h$, where $y$ is the depth at measurement and $h$ Is the total depth The hot-film error for this typlcal case is seen to be generally less than $1 \mathrm{ln} / \mathrm{sec}$ for the range of flow speeds, 1 to 11 in/sec tested These results are for plane progressive waves $43^{\prime \prime}$ high, $103 \mathrm{ft}$ long and having a 1474 sec period

Similar comparisons of flow direction data for the stationary technique are shown In Flg (8) It ls seen that near the free surface, $\mathrm{y} / \mathrm{h}=0138$, the direction-vane error is generally less than $10^{\circ}$ for this case, whereas near the bottom, serious errors occur

Fig (9) shows the decrease in direction errors achieved by utilizing the traversing technique

Fig (10) shows the directional errors for the case of the dualtow technique Errors generally less than $10^{\circ}$ were obtained even for locations we 11 below the water surface 
VECTOR DIAGRAM FOR THE DUAL-TOW TECHNIQUE

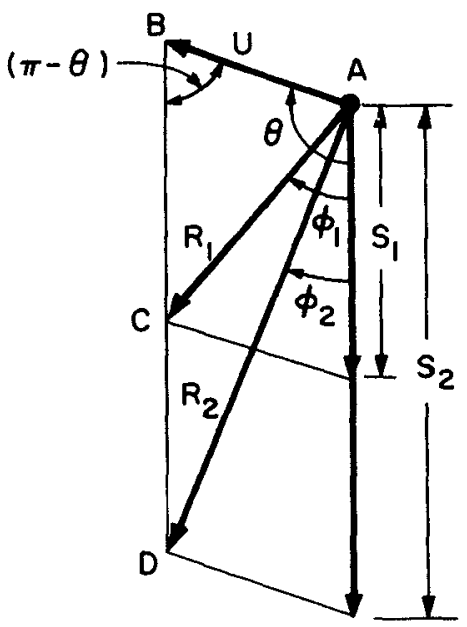

$s_{2} \neq s_{1}$

FIGURE (6) 


$$
\begin{gathered}
\text { VELOCITY ERROR } \\
\text { FOR } \\
\text { STATIONARY TECHNIQUE, STA - I5 } \\
U_{E}=U_{\text {HOT-FILM }}-U_{\text {PHOTOGRAPHIC }}
\end{gathered}
$$

\begin{tabular}{|c|c|c|c|c|c|c|}
\hline CURVE & (1) & (2) & (3) & (4) & (5) & (6) \\
\hline$-y / h$ & 138 & 354 & 465 & 589 & 708 & 830 \\
\hline
\end{tabular}

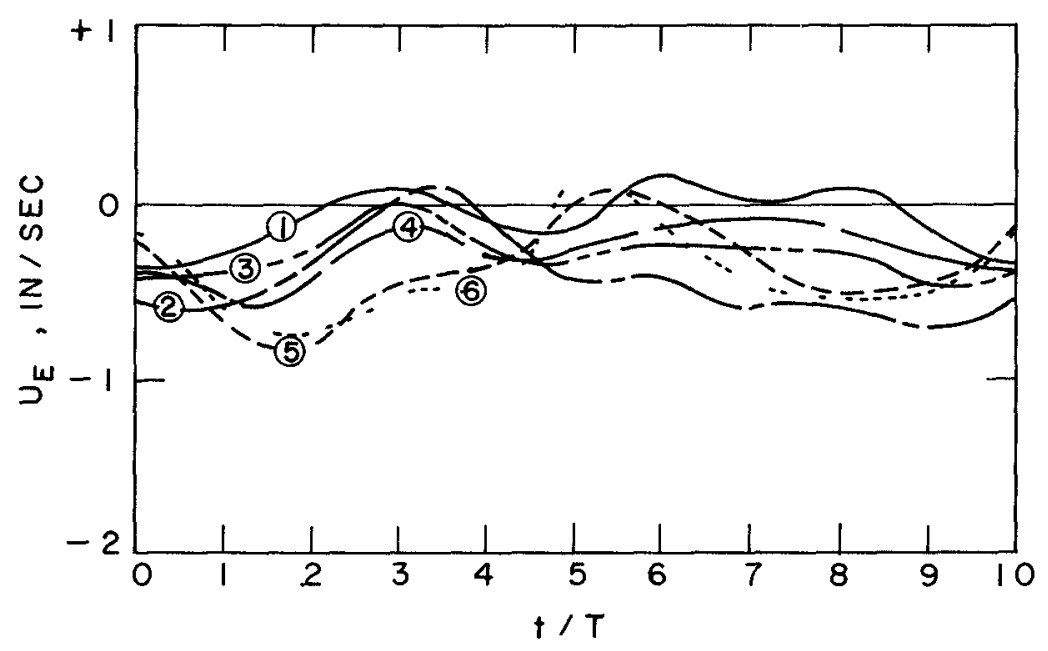

FIGURE (7) 


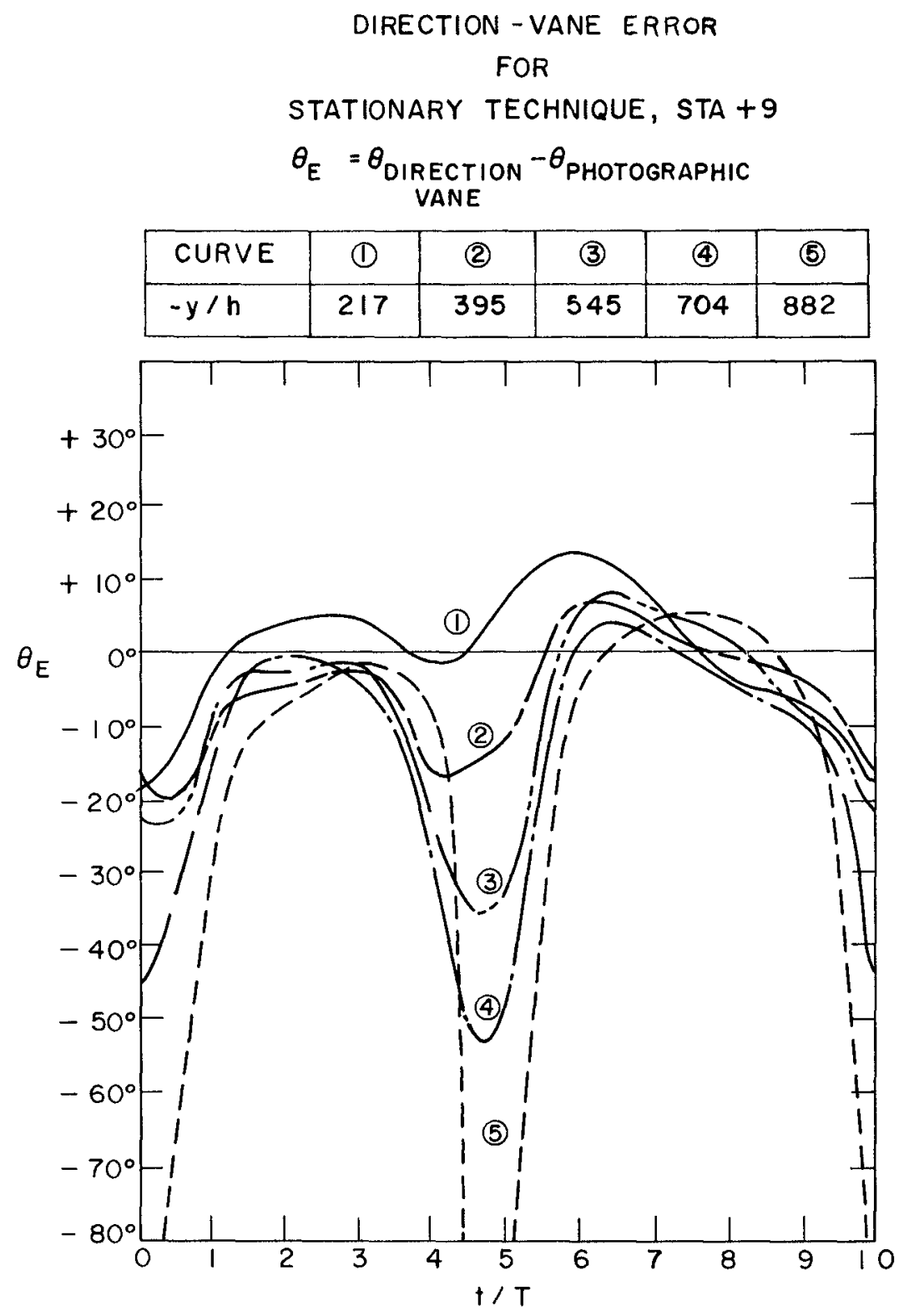

FIGURE (8) 
DIRECTIONAL ERROR

FOR

FAST - TOWS UPWARDS, STA-15

TOWING SPEED $=102 \mathrm{IN} / \mathrm{SEC}$

$\theta_{E}=\theta_{\text {FAST-TOW }}-\theta_{\text {PHOTOGRAPHIC }}$

\begin{tabular}{|c|c|c|c|c|c|}
\hline CURVE & (1) & (2) & (3) & (4) & (5) \\
\hline$-y / h$ & 138 & 354 & 465 & 589 & 708 \\
\hline
\end{tabular}

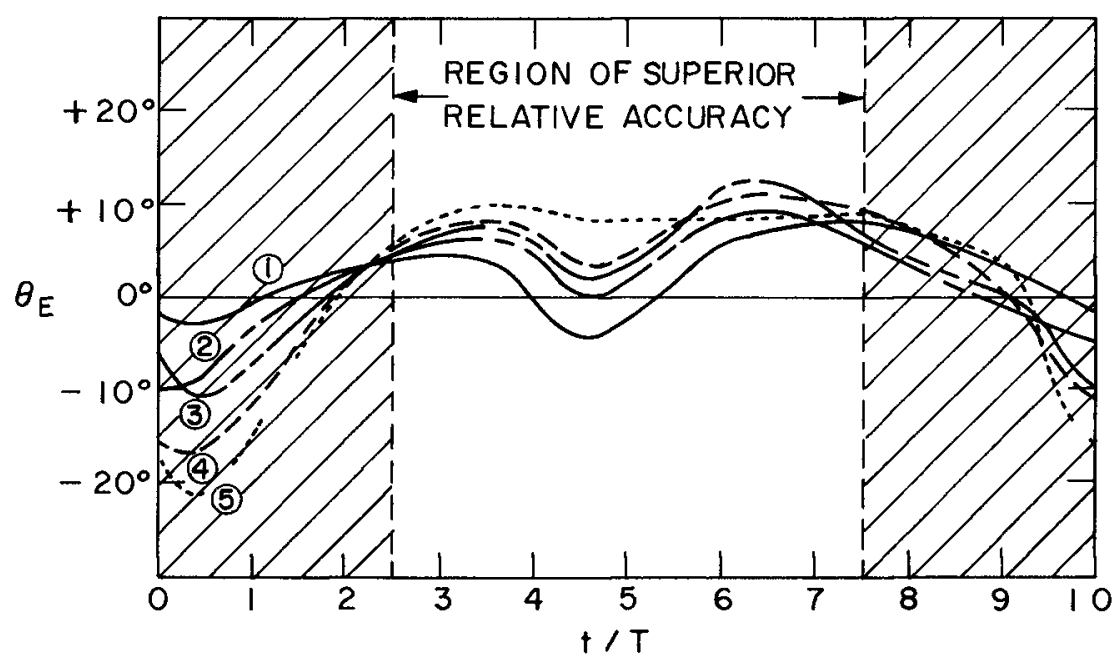

FIGURE (9) 
DIRECTIONAL ERROR

FOR

DUAL -TOW, STA - 15

TOWING SPEEDS $=0,252$ IN/SEC

$\theta_{\mathrm{E}}=\theta_{\text {DUAL-TOW }}-\theta_{\text {PHOTOGRAPHIC }}$

\begin{tabular}{|c|c|c|c|c|c|}
\hline CURVE & (1) & (2) & (3) & (4) & (5) \\
\hline$-y / h$ & 138 & 354 & 465 & 589 & 708 \\
\hline
\end{tabular}

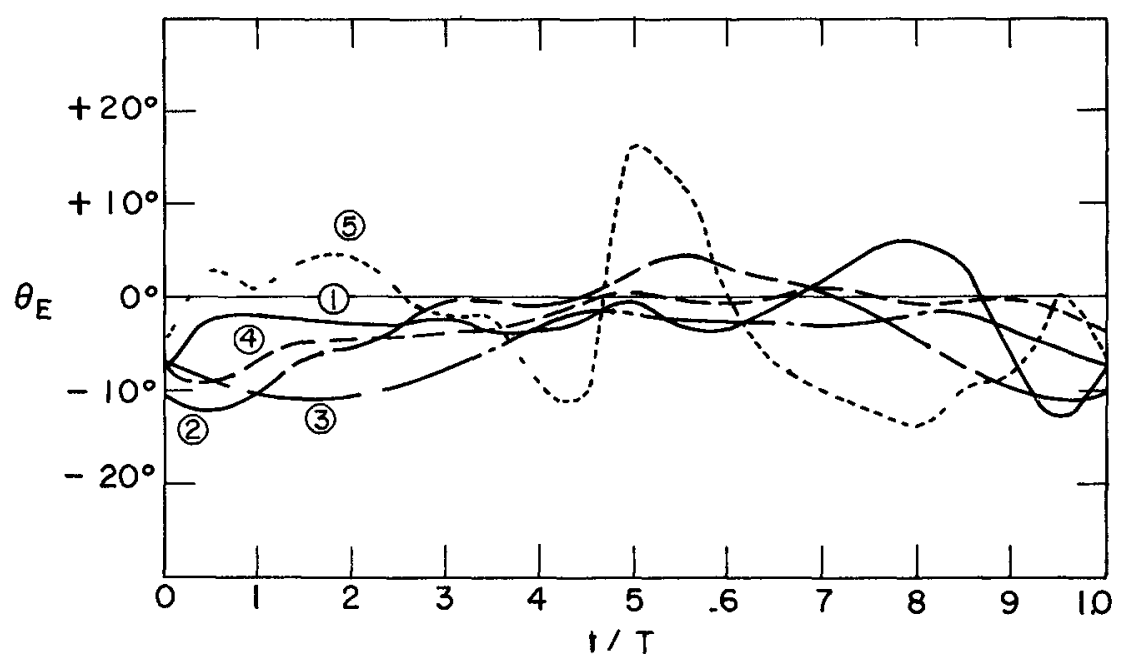

FIGURE (10) 


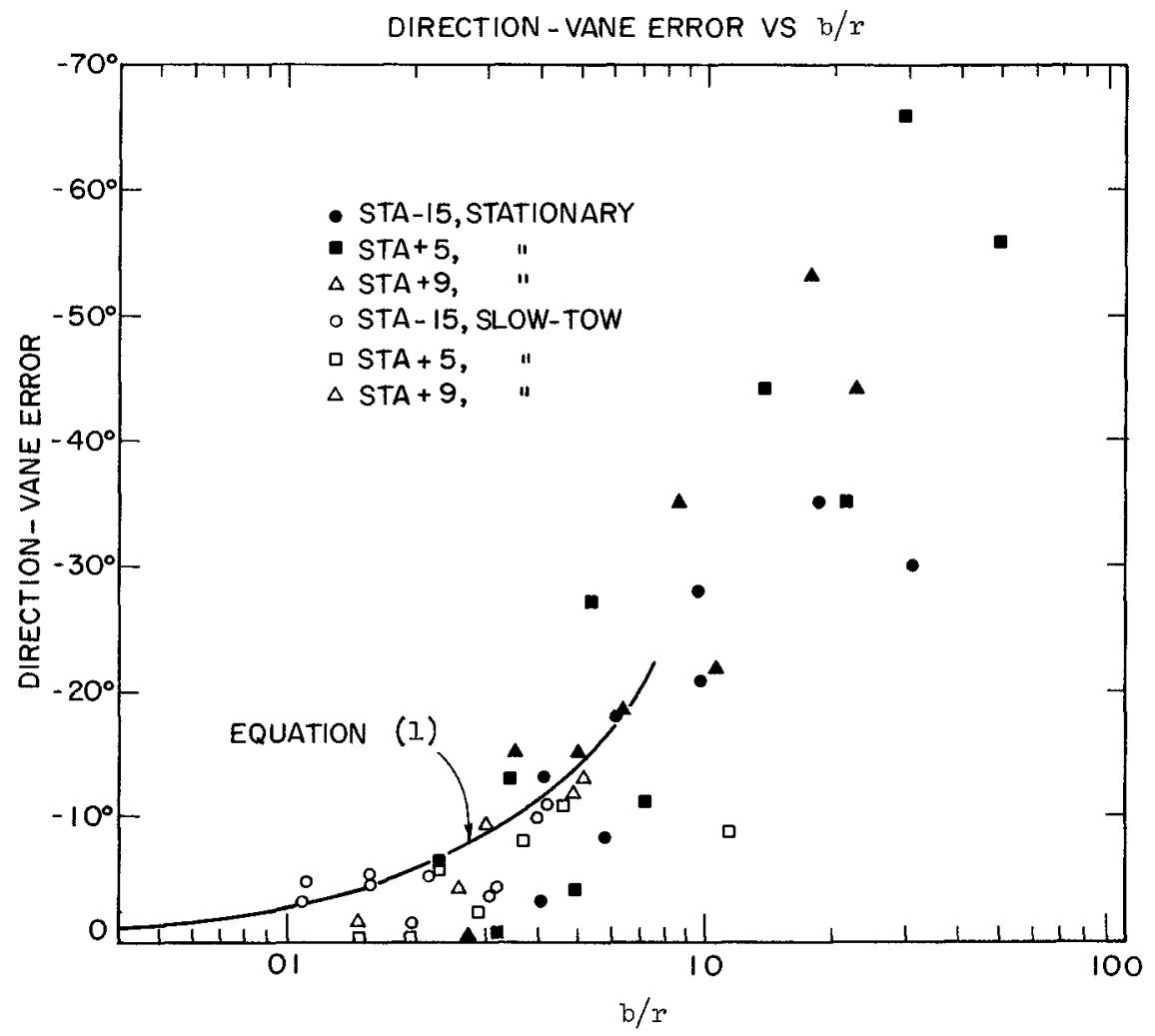

FIGURE (1I) 
Flg (11) shows experimental values for the vane plotted against the parameter $b / r$ The approximate relation for error given by Equation (1) appears to define the upper envelope of the points corresponding to small b/r falrly well The data thus confirm that $\theta_{E} \leqslant 5^{\circ}$ for $\mathrm{b} / \mathrm{r} \leqslant 01$

\section{Conclusions}

1) Since the problem of miniaturizing the physical dimensions of the vane sufficiently to make its chord length, b, an order of magnitude smaller than $r$, becomes prohıbitıve for the case of small laboratory waves, it appears that the direction-vane transducer is more sultable for larger scale wave flows, such as real ocean waves

2) The directional sensitivity of the hot-film sensor appears to limit its accuracy at present to $\pm 15 \%$ of the velocity measured unless corrections are made Apart from thjs, the sensor can measure flow speed to within at least $10 \%$ of the velocity

3) The dual-tow technique allows measurement of flow direction without the need for a separate direction measuring instrument It is not scale dependent and thus makes possıble measurements in small scale laboratory flow fields However, the flow fleld must be periodic for the technique to be valid 



\title{
CHAPTER 21
}

\section{SHOALING OF FINITE-AMPLITUDE WAVES ON PLANE BEACHES}

\author{
Robert K -C Chan ${ }^{1}$
Robert L Street, ${ }_{M}$ ASCE
}

ABSTRACT

This work focuses on the shoaling of large water waves w1th particular application to storm-generated waves and tsunamis The specific objective is the exact simulation on a digital computer of finlte-amplitude waves advancing on a beach of constant slope

The study is based on the simulation technique called SUMMAC (the Stanford-University-Modified Marker-And-Celi Method) The flow fleld is represented by a rectangular mesh of cells and by a line of hypothetical particles which defines the free surface Based on the Navier-Stokes equations, finıte-difference equations were derived so that the entire flow configuration could be advanced through a finite increment of time The pressure and velocity components are used directly as the dependent variables Through extensive analyses and numerical experiments, this scheme was found to be computationally stable if the cell size and the tume increment are properly selected

As a specific example, the dynamics of a solitary wave passing from a zone of constant depth onto a sloping beach were simulated Primary attention was focused on the details of the water particle motions and the changes in the amplitude and shape of the wave as it climbed the slope The computed results are compared with the experiments with good agreement

\section{INTRODUCTION}

The motion of water waves whose amplitudes are appreciable in relation to the water depth is nonlinear in nature (Stoker, 1957) Consequently the linearized theory (Lamb, 1945) does not provide adequate physical description of waves in the shallow-water zones Most of the existing nonlinear analytical theories deal with a steady-state solution (See, e $\mathrm{g}$, Laitone, 1960, Dean, 1965, Monkmeyer and Kutzback, 1965) However, in shallow water the primary interest is in the transient aspect of the wave processes It is quite difficult to treat timedependent problems of this kind without recourse to computational methods

At present several numerical methods are aval lable for computing waves in shallow water and nonlinear terms are included to some extent (See, Street, et al, 1970, for a detarled summary) These methods retain in their governing equations the terms representing the kinetic energy of the vertical motion to varyıng degrees of accuracy only

Department of Civil Engineering, Stanford University, Stanford, Calıfornıa 94305 
one space variable, namely $x$, is involved Vertical variation of the fluid variables has been eliminated by integration or a series expansion approach and use of boundary conditions These approximate theories generaliy produce good results for long waves of smali but finite amplitude-to-depth ratio However, more must be known about the internal distribution of pressure and velocity which must be incorporated in the governing equations of motion if one wishes to study waves of considerable amplitude

Chan and Street (1970a) proposed a computing technique for analyzing two-dimensional finlte-amplitude water waves under transient conditions The method, called SUMMAC, is a modified version of the Marker-And-Ce11 method (MAC) which was originally developed by Welch, et al (1966) The essence of the modifications consists of a rigorous application of the pressure boundary condition at the free surface and of an extrapolation of velocity components from the fluid interior so that inaccuracy in shifting the free surface is kept at a minimum

Thus, Chan and street (1970a) outlined the basic features of SUMMAC and established 1 t s viability as an engineering research tool The present work summarizes the earlier study, presents some essential new concepts and features, and finally, in giving a means of treating sloping beach problems, greatly broadens the realms of usage of MACtype programs

\section{THE SUMMAC METHOD}

The presently implemented SUMMAC is designed for simulating the unsteady motion of water waves in two space dimensions The fluid is regarded as 1 ncompressible and the effect of viscosity is considered to be negligible

To set up a computing network, the entire flow field is covered with a rectangular mesh of cells, each of dimensions $\delta x$ and $\delta y$ The center of each cell is numbered by the 1 ndices 1 and $J$, with 1 counting the columns in the $x$-direction and $j$ counting the rows in the $y$-direction of a fixed Cartesian coordinate system (Fig 1) The field-variable values describing the flow are directly associated with these cells (Welch, et a1, 1966) The fluld velocity components $u$ and $v$ and the pressure $\vec{p}$ are used as the dependent variables while the 1 ndependent variables are $x$ and $y$, the Cartesian space coordinates, and the time variable $t$

In addition, there is a line of marker particles whose sole purpose is to indicate where the free surface is located The marker-andce11 system provides an instantaneous representation of the flow field for any particular time during the evolution of the dynamics

By neglecting viscosity, we can reduce the Navier-Stokes equations for an incompressible fluid to

$$
\frac{\partial u}{\partial t}+u \frac{\partial u}{\partial x}+v \frac{\partial u}{\partial y}=-\frac{\partial p}{\partial x}+g_{x},
$$




$$
\frac{\partial v}{\partial t}+u \frac{\partial v}{\partial x}+v \frac{\partial v}{\partial y}=-\frac{\partial p}{\partial y}+g_{y}
$$

Here $g_{x}$ and $g_{y}$ are the components of gravity acceleration $A 11$ variables are dimensionless The continuity equation is

$$
\frac{\partial u}{\partial x}+\frac{\partial v}{\partial y}=0
$$

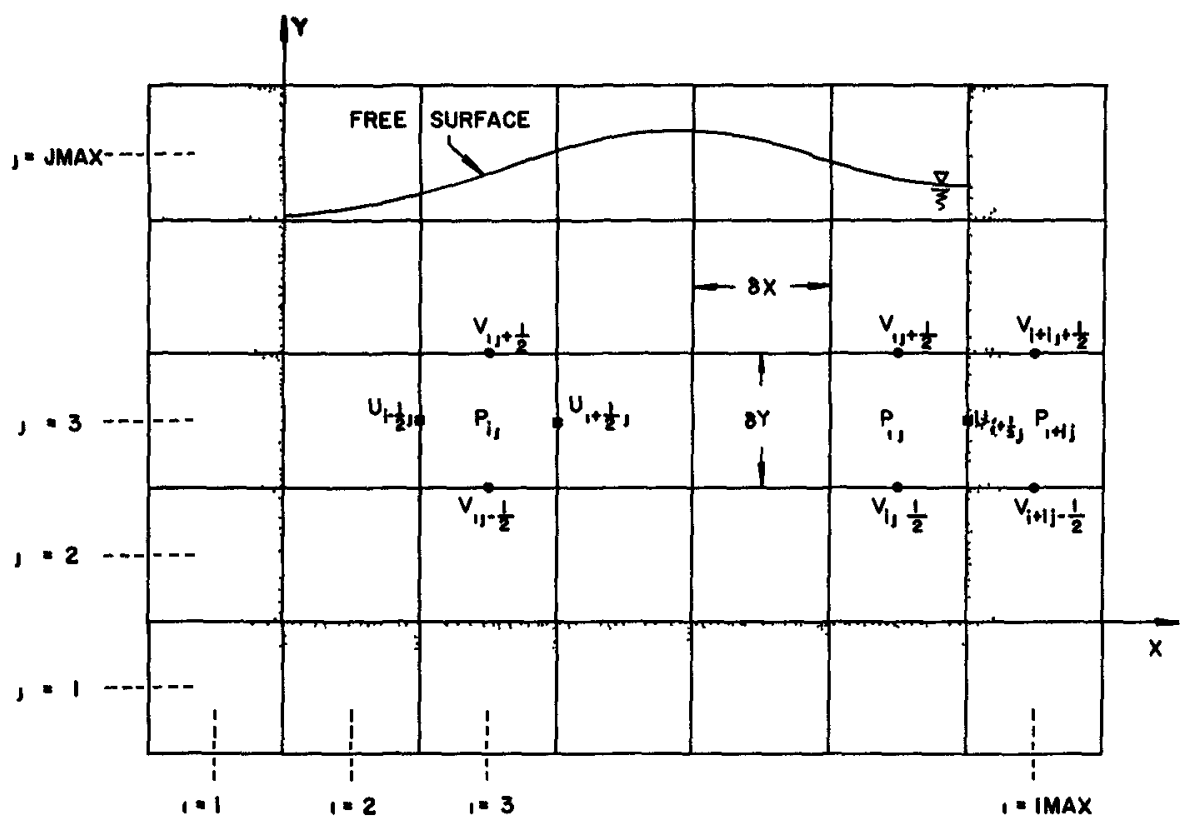

F1g 1 Cel1 Setup and Position of Variables

On the surface of a vertical impermeable wall, the boundary cond1tions are

$$
\mathrm{u}=0, \quad \frac{\partial \mathrm{v}}{\partial \mathrm{x}}=0 \text { and } \quad \frac{\partial \mathrm{p}}{\partial \mathrm{x}}=\mathrm{g}_{\mathrm{x}}
$$

Similar procedures can be followed to derive the boundary conditions for a horizontal solid boundary 


$$
v=0, \frac{\partial u}{\partial y}=0 \text { and } \quad \frac{\partial p}{\partial y}=g_{y}
$$

For incompressible fluids with very low viscosity, such as water, it is sufficlent ly accurate to use the single condition on the free surface

$$
p=p_{a}(x, t)
$$

where $\mathrm{pa}$ is the externally applied pressure at the free surface (Chan and street, 1970a)
A finlte-difference scheme can be derıved from Eqs

(3) for cel1 $(1,3)$ in Fig 1 as follows

$$
\begin{aligned}
& u_{1+\frac{1}{2} J}^{n+1}=u_{1+\frac{1}{2} J}^{*}+\delta t \cdot g_{x}+\frac{\delta t}{\delta x}\left(p_{1 J}-p_{1+1 j}\right), \\
& v_{1 j+\frac{1}{2}}^{n+1}=v_{1 j+\frac{1}{2}}^{*}+\delta t \cdot g_{y}+\frac{\delta t}{\delta y}\left(p_{1 j}-p_{1 j+1}\right), \\
& D_{1 j}^{n+1} \equiv \frac{u_{1+\frac{1}{2} 1}^{n+1}-u_{1-\frac{1}{2} 1}^{n+1}}{\delta x}+\frac{v_{11+\frac{1}{2}}^{n+1}-v_{11-\frac{1}{2}}^{n+1}}{\delta y}=0,
\end{aligned}
$$

where $D_{1}$ is the velocity divergence, $\mathrm{u}^{*}$ and $\mathrm{v}^{*}$ are contributlons to ${ }^{1} u^{n+1}$ and $v^{n+1}$, respectively, by pure convection Varıables with the superscript $n+1$ are related to the $n+1$ th time step while those lacking a superscript are evaluated, at the nth step If the original MAC scheme is used, $u^{*}$ and $v^{*}$ are evaluated by

$$
\begin{aligned}
& \left.u_{1+\frac{1}{2} J}^{*}=u_{1+\frac{1}{2} J}+\delta t \mid \frac{u_{i 1}^{2}-u_{1+1]}^{2}}{\delta x}+\frac{(u v)_{i+\frac{1}{2} j-\frac{1}{2}}-(u v)_{\left.2+\frac{1}{2}\right]+\frac{1}{2}}}{\delta y}\right] \text {, }
\end{aligned}
$$

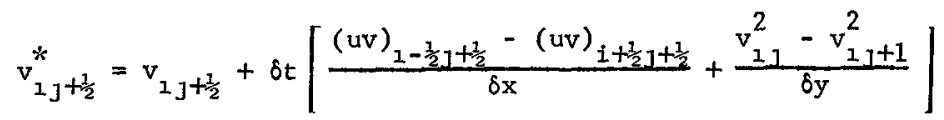
Substituting Eqs (7) and (8) into Eq (9) and requiring $\mathrm{D}_{1 \mathrm{j}}^{\mathrm{n}+1}=0$
lead to the pressure equation

$$
p_{1 \jmath}=\frac{1}{Z}\left(\frac{p_{1+1 \jmath}+p_{1-1 j}}{\delta x^{2}}+\frac{p_{1 \jmath+1}+p_{1 \jmath-1}}{\delta y^{2}}+R_{1 \jmath}\right),
$$


where

$$
\begin{aligned}
& z \equiv 2\left(\frac{1}{\delta x^{2}}+\frac{1}{\delta y^{2}}\right) \\
& R_{1]} \equiv-\frac{1}{\delta t}\left[\frac{u_{\left.1+\frac{1}{2}\right]}^{*}-u_{1-\frac{1}{2} 1}^{*}}{\delta x}+\frac{v_{11+\frac{1}{2}}^{*}-v_{11-\frac{1}{2}}^{*}}{\delta y}\right]
\end{aligned}
$$

Near the free surface "Irregular stars" (F1g 2) must be used to derive a special equation for pressure so that, in the discrete sense, the free surface condition $[\mathrm{Eq} \quad(6)]$ is applied at the exact location of the surface and not in a nearby cell center where $p$ is normally defined Let $\eta_{1}, \eta_{2}, \eta_{3}, \eta_{4}$ be the lengths of the four legs of the Irregular star' (Fig '2) and $\mathrm{p}_{1}, \mathrm{p}_{2}, \mathrm{p}_{3}, \mathrm{p}_{4}$ be the values of $p$ at the ends of these legs By expressing $\mathrm{p}_{1}, \mathrm{p}_{2}, \mathrm{p}_{3}, \mathrm{p}_{4}$ in terms of Taylor series expansions about the point $(1,1)$, Chan and Street (1970a) obtained

$$
\mathrm{p}_{1 \mathrm{~J}}=\frac{\eta_{1} \eta_{2} \eta_{3} \eta_{4}}{2\left(\eta_{2} \eta_{4}+\eta_{1} \eta_{3}\right)}\left[\frac{\eta_{3} \mathrm{p}_{1}+\eta_{1} \mathrm{p}_{3}}{\eta_{1} \eta_{3}\left(\frac{\eta_{1}+\eta_{3}}{2}\right)}+\frac{\eta_{4} \mathrm{p}_{2}+\eta_{2} \mathrm{p}_{4}}{\eta_{2} \eta_{4}\left(\frac{\eta_{2}+\eta_{4}}{2}\right)}+\mathrm{R}_{1 \mathrm{~J}}\right]
$$

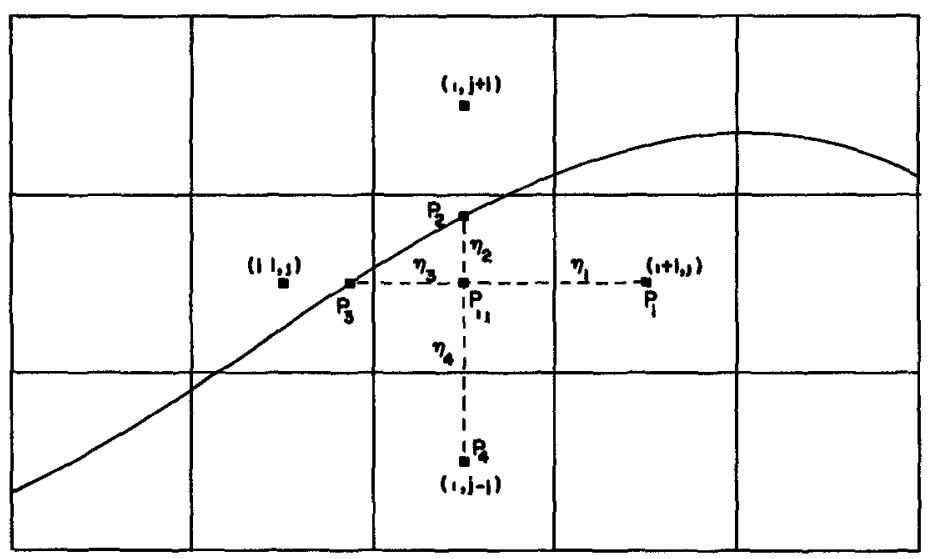

Fig 2 Irregular Star for P Calculations

A complete set of initial data -. the $u$ and $v$ fields and 
the position of a line of particles depicting the free surface -- are needed to start the computation The initial pressure $p$ need be known only approximately A hydrostatic distribution is adequate, because the $p$ field is obtainable if $u$ and $v$ are given Chan and Street (1970b) described a method of generating the flow fields of a solitary wave which may be used as the initial condition in simulating the shoaling process

The evolution of the fluid dynamacs is calculated in "cycles" or time steps At the start of each cycle the source term $R_{1}$ for each ce11 is evaluated by Eq (14) The pressure $p$ is computed only for those cells whose centers fall within the fluid region Equation (12) is used if the centers of the four nelghboring cells are all located on the fluid side of the free surface If any of these nelghboring centers lies beyond the free surface, then $\mathrm{Eq}$ (15) is used The Successive Over-Relaxation method is employed to solve for $p$, with Eqs (12) and (15) being the iteration formulas The iteration is terminated when the condition

$$
\left|p_{1 j}^{(m)}-p_{1 j}^{(m-1)}\right|<\varepsilon_{p}
$$

1s met for every cell, where (m) means the mth iteration and ${ }^{6} p$ is a predetermined small positive number

Now we can compute the new velocities using Eqs (7) and (8) Then, each free surface marker particle is advanced to 1 ts new position by

$$
\begin{aligned}
& x_{k}^{n+1}=x_{k}^{n}+u_{k}^{n+1} \delta t, \\
& y_{k}^{n+1}=y_{k}^{n}+v_{k}^{n+1} \delta t,
\end{aligned}
$$

where $x_{k}$ and $y_{k}$ refer to the position of the kth particle and the particle velocities $u_{k}$ and $v_{k}$ are interpolated from the velocity fields at the $n+1$ th time step Thus, a cycle is completed and the next one can be immediately started

\section{BOUNDARY CONDITIONS ON A SLOPE}

A set of boundary conditions can be rigorously obtalned for a plane beach of constant $s$ lope which coincldes with the diagonal of the cells (Fig 3) The maln consideration in deriving an equation for $p_{1}$ whlch lies on the beach face is the conservation of mass In Fig $3^{1}$ we require that the net flow into the triangular region equal zero at the $n+1$ th time step, viz, 


$$
u_{1-\frac{1}{2} J}^{n+1} \delta y-v_{1 j+\frac{1}{2}}^{n+1} \delta x=0
$$

By substituting Eqs (7) and (8) into Eq (19) we have

$$
p_{1 \jmath}=\frac{1}{z_{1}}\left(\frac{p_{1-1]}}{\delta x^{2}}+\frac{p_{1 \jmath+1}}{\delta y}+R_{1 \jmath}\right)
$$

where

$$
\begin{gathered}
\mathrm{z}_{1} \equiv \frac{1}{\delta \mathrm{x}^{2}}+\frac{1}{\delta \mathrm{y}^{2}} \\
\mathrm{R}_{1 \mathrm{~J}} \equiv \frac{1}{\delta \mathrm{t}}\left(\frac{\mathrm{u}_{1-\frac{1}{2} 1}^{*}}{\delta \mathrm{x}}-\frac{\mathrm{v}_{1 \mathrm{1}+\frac{1}{2}}^{*}}{\delta \mathrm{y}}\right)+\left(\frac{\mathrm{g}_{\mathrm{x}}}{\delta \mathrm{x}}-\frac{\mathrm{g}_{\mathrm{y}}}{\delta \mathrm{y}}\right)
\end{gathered}
$$

In the iterative solution of the $p$ field, Eq (20) is used at a beach cel1

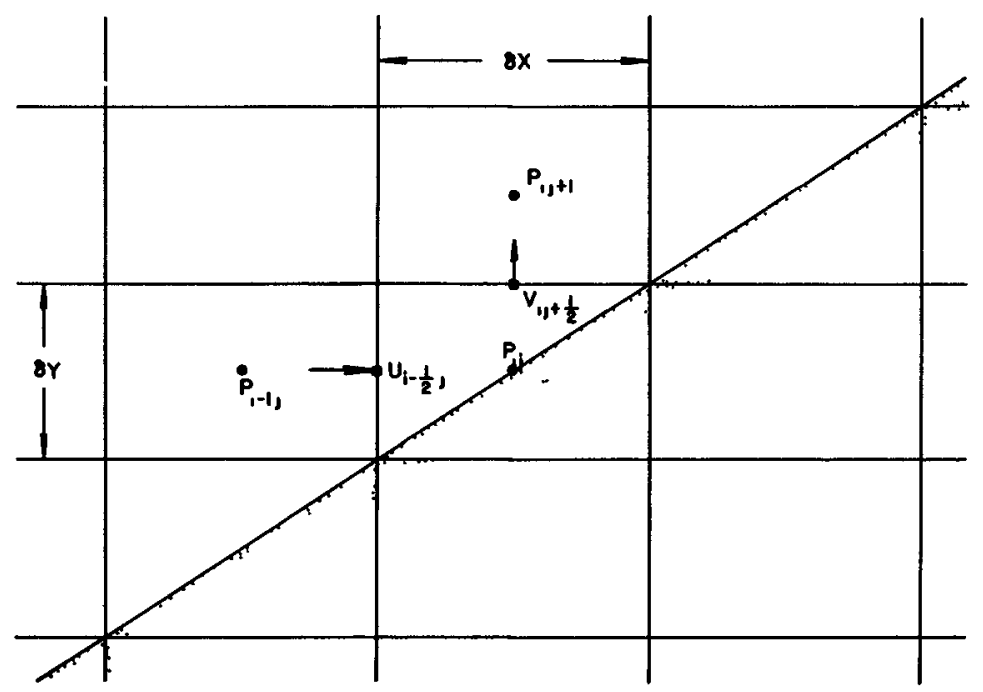

F1g 3 Definition sketch for Computing $p$ 
Now, consider the diamond-shaped control volume for computing the convective contribution $\mathrm{u}_{1+\frac{1}{2} \mathrm{~J}}^{*}$ (Fig 4) The total influx of the u-momentum is as follows

Through Face 1

$$
M_{1}=-\delta \times v_{1+1 \jmath+\frac{1}{2}}\left(\frac{u_{1+\frac{1}{2} \jmath}+u_{1+\frac{3}{2} \jmath+1}}{2}\right)
$$

Through Face 2

$$
\begin{aligned}
M_{2}= & \delta y\left(\frac{u_{1-\frac{1}{2} \sqrt{1}}+u_{1+\frac{1}{21}}}{2}\right)^{2} \\
& -\delta x\left(\frac{v_{11-\frac{1}{2}}+v_{11+\frac{1}{2}}}{2}\right)\left(\frac{u_{\left.1-\frac{1}{2}\right]}+u_{1+\frac{1}{2} 1}}{2}\right)
\end{aligned}
$$

Through Face 3

$$
M_{3}=\delta \times v_{1 j-\frac{1}{2}}\left(\frac{u_{1+\frac{1}{2} J}+u_{1-\frac{1}{2} J-1}}{2}\right)
$$

Through Face 4

$$
\begin{aligned}
M_{4}= & \delta x\left(\frac{v_{1+1]-\frac{1}{2}}+v_{1+1}+\frac{1}{2}}{2}\right)\left(\frac{u_{1+\frac{1}{2} 1}+u_{1+\frac{3}{2} 1}}{2}\right) \\
& -\delta y\left(\frac{u_{\left.1+\frac{1}{2}\right]}+u_{\left.1+\frac{3}{2}\right]}}{2}\right)^{2}
\end{aligned}
$$

The area of the control volume is $\delta \times \delta y$ Therefore, by relating the net inflow of the u-momentum to its rate of increase, we obtain

$$
\left(\frac{u_{1+\frac{2}{2} J}^{*}-u_{1+\frac{1}{2} J}}{\delta t}\right)(\delta \times \delta y)=M_{1}+M_{2}+M_{3}+M_{4}
$$

or, by rearranging,

$$
u_{1+\frac{1}{2} J}^{\prime}=u_{1+\frac{1}{2} J}+\frac{\delta t}{\delta \times \delta y}\left(M_{1}+M_{2}+M_{3}+M_{4}\right)
$$

The total $u_{1+\frac{1}{2}}^{n+1}$ is then

$$
u_{1+\frac{1}{2} J}^{n+1}=u_{1+\frac{1}{2} J}^{*}+\delta t g_{x}+\frac{\delta t}{\delta x}\left(p_{1 J}-p_{1+1 j}\right)
$$




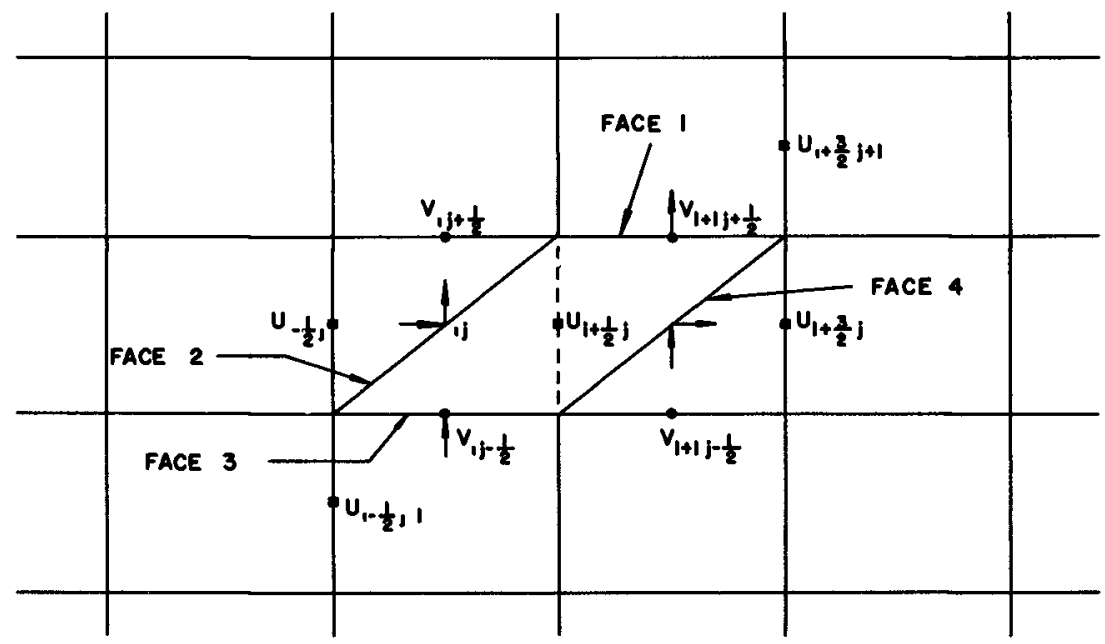

F1g 4 Definition Sketch for Computing $u$

If Face 4 is the beach face, then $M_{4}=0$ because there 1 s no flow across it Similarly, we use $M_{3}=\stackrel{4}{M}_{4}=0$ if both Face 3 and Face 4 are solid boundaries

To evaluate $v^{n+1}$ near the beach face, a different type of diamondshaped control volume 1 s used (F1g 5) Again, the concept of balancing momentum within the control volume is employed to compute the convective contribution $v_{1 j+\frac{1}{2}}^{*}$ The total influx of the $v$-momentum is as follows

Through Face 1

$$
M_{1}=-\delta y u_{1+\frac{1}{2} \jmath+1}\left(\frac{v_{1 j+\frac{1}{2}}+v_{1+1 j}+\frac{3}{2}}{2}\right)
$$

Through Face 2

$$
\begin{aligned}
M_{2}= & \delta y\left(\frac{u_{\left.1-\frac{1}{2}\right]+1}+u_{1+\frac{1}{2} 1+1}}{2}\right)\left(\frac{v_{1 j+\frac{1}{2}}+v_{1 j}+\frac{3}{2}}{2}\right) \\
& -\delta x\left(\frac{v_{1 j+\frac{1}{2}}+v_{1 j+\frac{3}{2}}}{2}\right)^{2}
\end{aligned}
$$


Through Face 3

$$
M_{3}=\delta y u_{1-\frac{1}{2} j}\left(\frac{v_{i j+\frac{1}{2}}+v_{1-1-\frac{1}{2}}}{2}\right)
$$

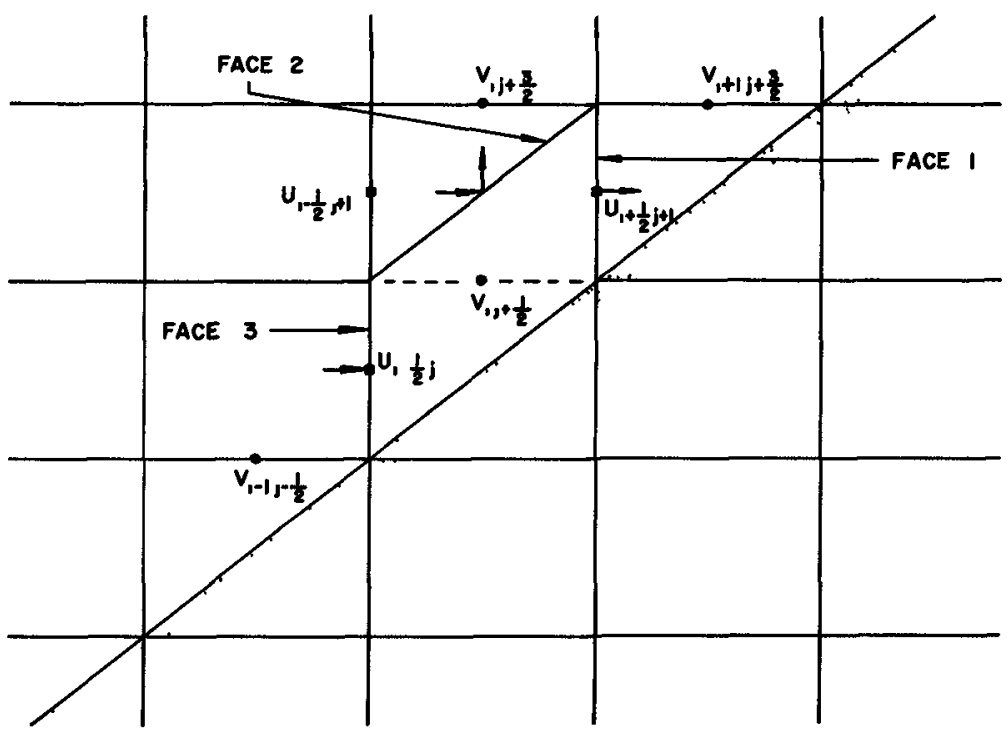

Fig 5 Definıtion Sketch for Computing v

Balancing the net inflow of the $v$-momentum with 1 ts rate of increase, we have

$$
\left(\frac{v_{1]+\frac{1}{2}}^{*}-v_{11+\frac{1}{2}}}{\delta t}\right)(\delta x \delta y)=M_{1}+M_{2}+M_{3}
$$

or

$$
v_{1 j+\frac{1}{2}}^{*}=v_{i j+\frac{1}{2}}+\frac{\delta t}{\delta \times \delta y}\left(M_{1}+M_{2}+M_{3}\right)
$$

The total $v_{1 j+\frac{1}{2}}^{n+1}$ is then

$$
v_{1 j+\frac{1}{2}}^{n+1}=v_{1 j+\frac{1}{2}}^{*}+\delta t g_{y}+\frac{\delta t}{\delta y}\left(p_{i j}-p_{1 j+1}\right)
$$




\section{APPLICATION TO THE SHOALING OF WAVES}

The initial position of the wave crest was chosen such that neither the left-hand wall nor the beach Influenced the wave at $t=0$ The beach slope used first was $S=005$ ( 1 on 20) Each cell had the dimensions $\delta x=050$ and $\delta y=0025$ The time increment was $\delta t=005$ The Initial wave height was $H_{0}=025$

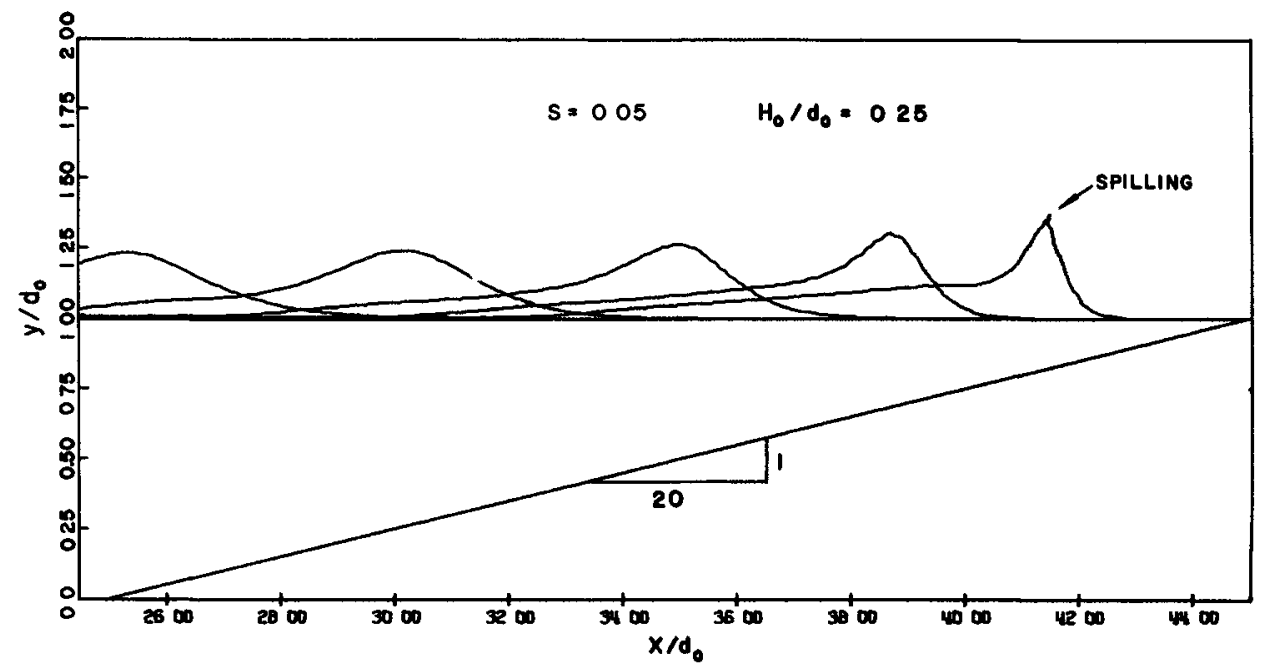

F1g 6 Shoaling on a 1/20 slope (Surface Profiles)

When the wave advances into the beach section where the water depth decreases, Its shape changes The wave profiles at various stages of shoaling are shown in Fig 6 It is seen that the wave shape gradually loses symmetry on the beach The wave has a steeper front than Its hind part which looks $1 \mathrm{l}$ ke a long tall At approximately $\mathrm{H} / \mathrm{d}=20$, where $H$ is the instantaneous wave height and $d$ is the local still water depth under the wave crest, the wave crest peaks up with a very slight tendency to curl forward This result is consistent with the observation of Ippen and Kulin (1955) that spil1ing rather than plunging occurs when a solitary wave of this magnitude breaks on the $1 / 20$ slope A11 these properties regarding the wave deformation also agree with the observations of Camfield and Street (1967)

The evolution of the fluid dynamics under the wave is best 111 ustrated by the contour plots of the $u$ and $v$ fields In Fig 7 (a) the time history of the distribution of $u$ is shown on the two outermost contour lines, $u=0015$ Then the value of $u$ is 
Increased by 0030 per line toward the wave crest Thus, $u=0375$ at the wave crest at $t=0$ When the wave is well up the beach $(t=2300), u=0675$ at $i$ ts crest In Fig $7(b)$ the motion of the $v$ field is shown To the left of the crest, the lowest contour line has $v=-0.015$, and $v$ decreases by 0030 per line toward the free surface To the right of the crest, the lowest contour line has $\mathrm{v}=+0015$, and $\mathrm{v}$ increases by 0030 per line toward the free surface The line $v=0$ lies between the lines $v= \pm 0015$ and is not shown
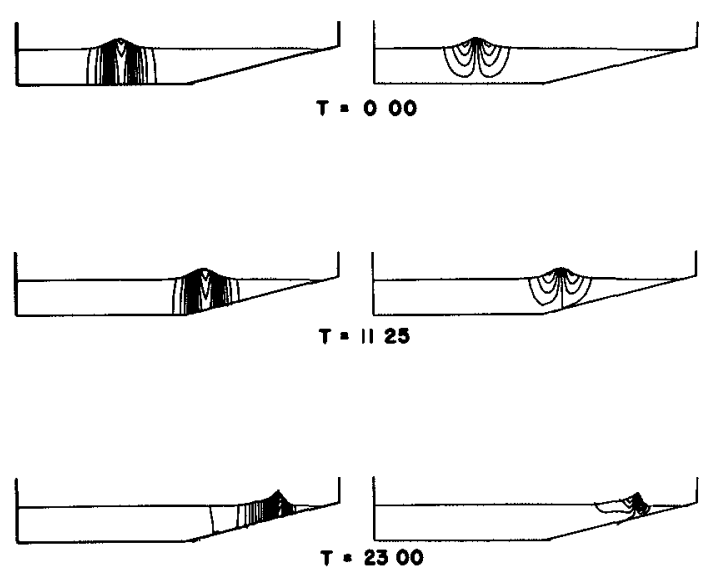
(a) $\mathrm{u}$
(b) $\mathrm{v}$

Fig 7 Shoaling on a $1 / 20$ Slope (Velocity Contours)

The contour lines in Fig 7 were computed by using a plotting program developed by Schreiber (1968) The facility used was an IBM 2250 graphic display device in which the computed contour lines are shown on a TV screen By directly photographing the surface of the screen, the contour plots were obtained Several motion pictures have also been made with this apparatus These graphical outputs prove to be valuable visual alds to an understanding of the physics in the waves

In Fig 8 the mass transport phenomenon in the mid-section of the beach is shown A vertical line of fluld particles are moved forward from their initial position $x=350$ by the passing wave The mass transport here is of the translation-type as opposed to the oscillationtype in the periodic waves Also, the wave induced motion on the beach is seen to be quite uniform throughout the water depth 


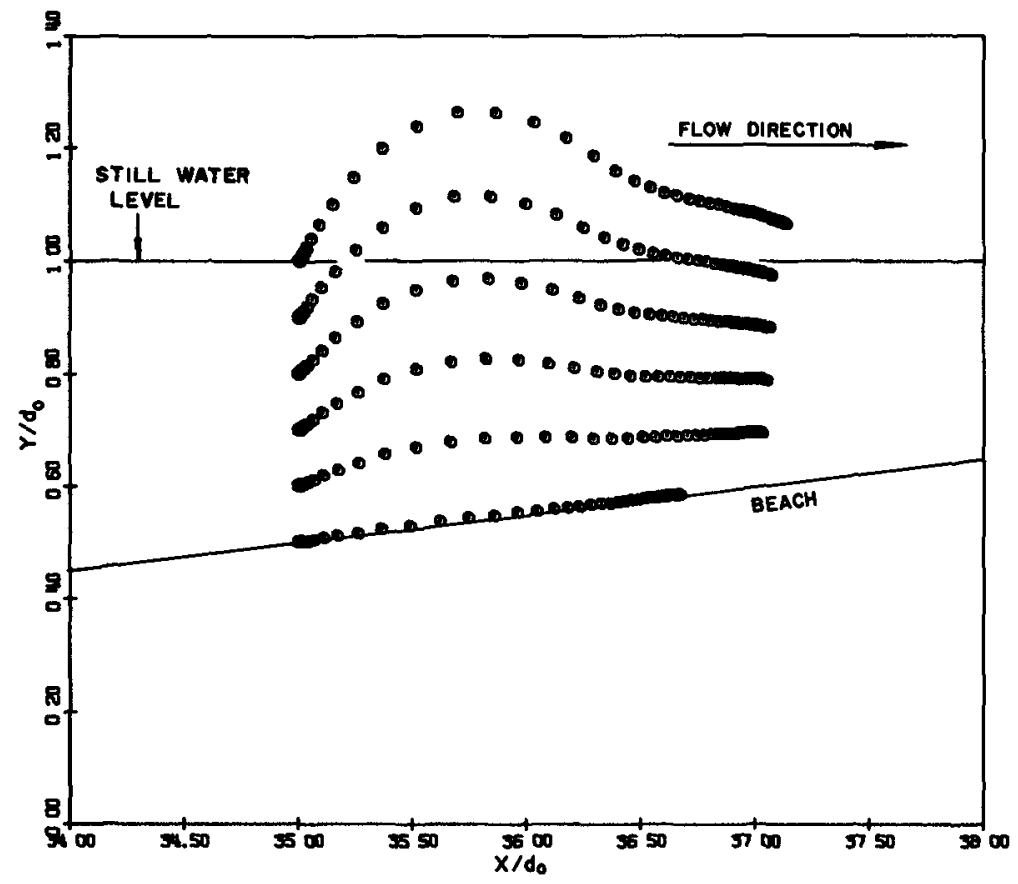

Fig 8 Shoaling on a $1 / 20$ slope (Particle Paths)

To study the growth of the wave helght $H$ as a function of the local water depth $\mathrm{d}$, computations were conducted covering a range of the initial helght-to-depth ratios $\left(\mathrm{H}_{0} / \mathrm{d}_{0}\right)$ The results are compared with the experiments of Ippen and Kulin (1955) in Fig 9 Ippen and Kulin did not Indicate the $H_{o} / d$ value associated with each data point, but $\mathrm{H}_{\mathrm{O}} / \mathrm{d}$ is an important parameter in predicting the wave growth The scatfering of the measured data cannot be reconciled without knowledge of this parameter However, the $\mathrm{H}_{\mathrm{o}} / \mathrm{d}$ ratio clearly has a profound effect on the wave's initial reaction to the slope Our results show that in the region $d / d>045$, a solitary wave with smaller $\mathrm{H}_{\mathrm{o}} / \mathrm{d}_{\mathrm{O}}$ has greater relative growth $\left(\mathrm{H} / \mathrm{H}_{\mathrm{o}}\right)$ The trend is reversed in the shallower region where $\mathrm{d} / \mathrm{d}_{\mathrm{o}}<045$ For $\mathrm{H}_{\mathrm{o}} / \mathrm{d}=010$ comparison was also made with the theories of Peregrine ( 1969 ), Madsen and Mel (1969) and the characteristics solution by Camfleld and Street (1967) (Fig 10) With the exception of the characteristics solution, these theories seem to agree quite we 11 


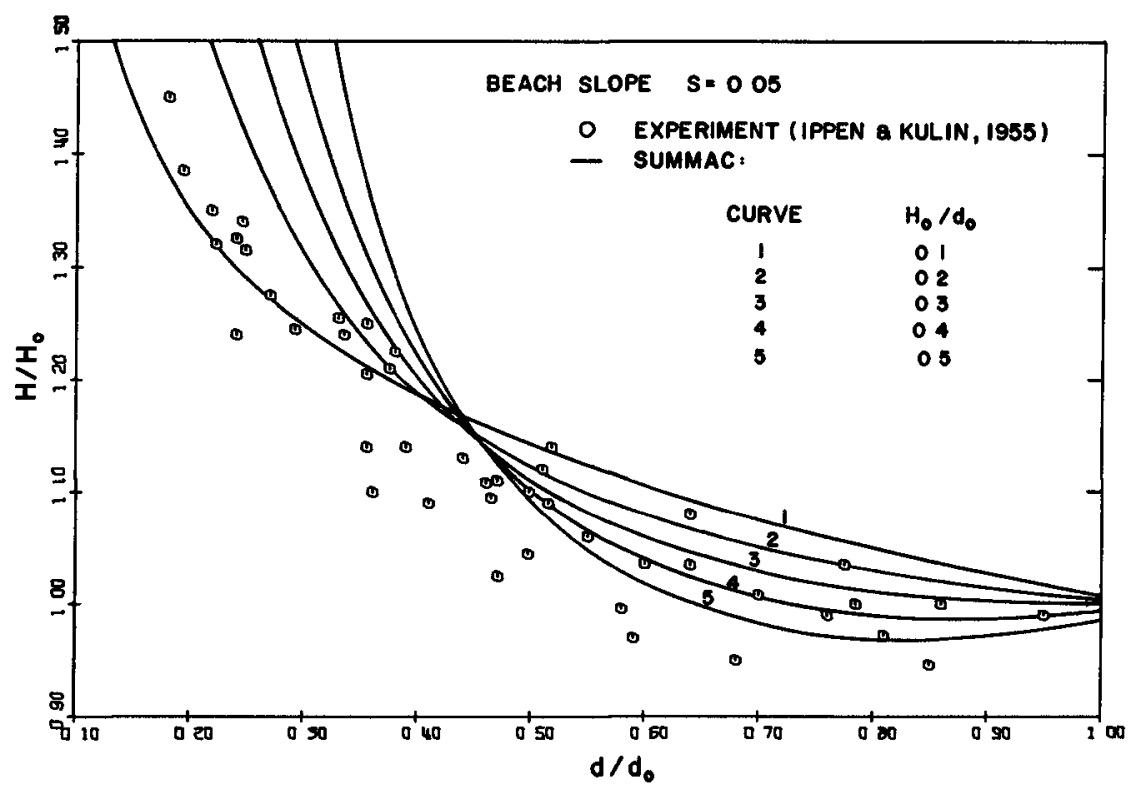

Fig 9 Growth of Wave Amplitude $(S=0$ 05)

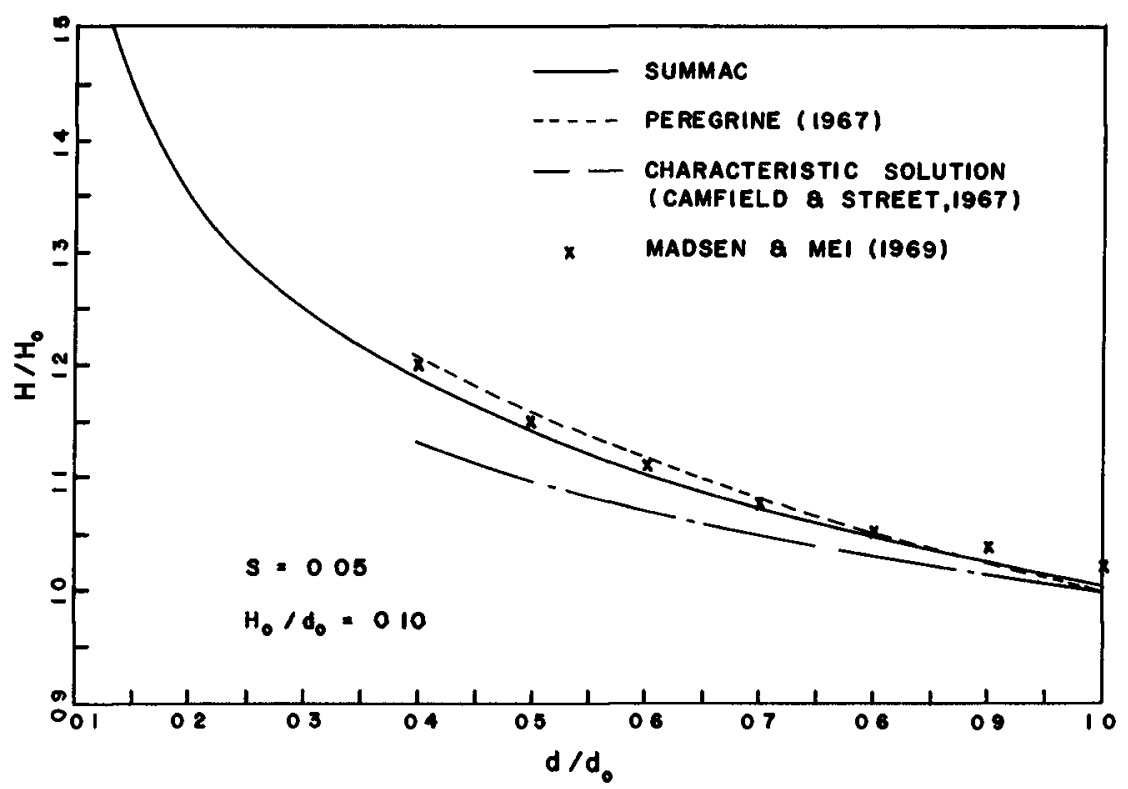

Fig 10 Comparıson of Wave Amplitude Growth with Other Theories $(S=0$ 05) 
Second, a wave w1 th $\mathrm{H}_{\mathrm{O}} / \mathrm{d}_{\mathrm{O}}=010$ was shoaled on a beach with $\mathrm{S}=0045$ In Fig 11 the local height-to-depth ratio $\mathrm{H} / \mathrm{d}$ is plotted against $x^{*} / d_{b}-1 / s$, where $x^{*}$ is the distance measured from the intersection of the still water level and the beach and $d_{b}(\approx 0085)$ is the breaking depth The solution agrees favorably with the measurement of Camfield and Street (1967)

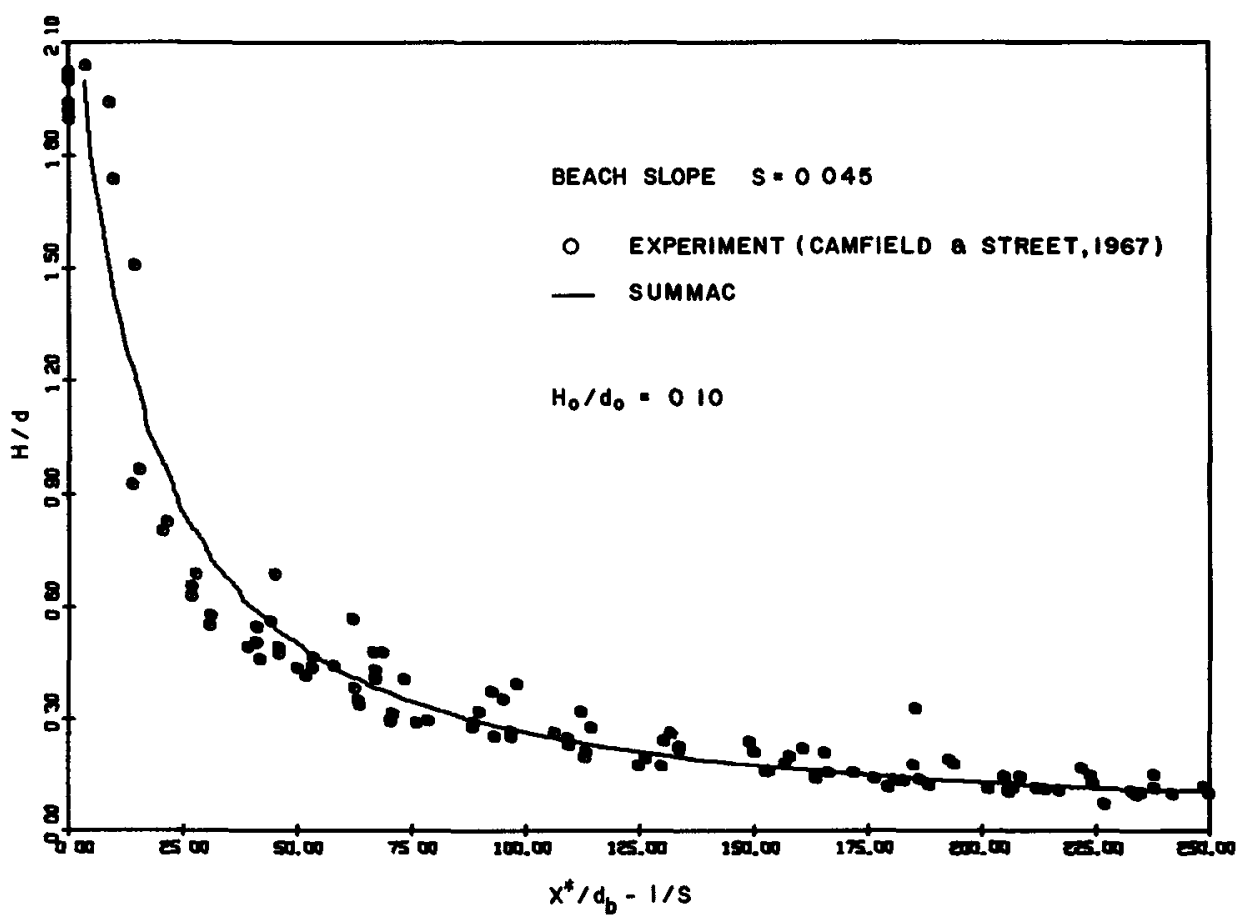

Fig 11 Growth of Wave Amplitude $(S=0$ 045)

We also simulated wave run-up on a $45^{\circ}$ slope which has direct application to the study of wave run-up on coastline structures such as rubble-mound breakwaters The cell size was $\delta x=\delta y=010$ The inltial wave helght was $H_{0} / d_{0}=048$ and a time increment $\delta t=005$ was used in computation The wave profiles at several stages of run-up are plotted in Fig 12 Quantitative comparison of these profiles with experiments was not made because of the difficulty in obtaining a computed profile which corresponds to a measured profile in time Nevertheless, the profiles closely resemble those observed by Street and camfield (1966) We find the calculated envelope of the wave crests slight ly higher than that of the experiment in the early stages of run-up, but the engineering interest is primarily in the prediction 
of the maximum height of run-up $\mathrm{R}_{\mathrm{o}} / \mathrm{d}_{\mathrm{o}}$ (F1g 12) The numerical model gave $R_{\mathrm{o}} / \mathrm{d}_{\mathrm{o}}=127$ wh1ch $1 \mathrm{~s}$ equal to the measured value (Street and Camfield, 1966)

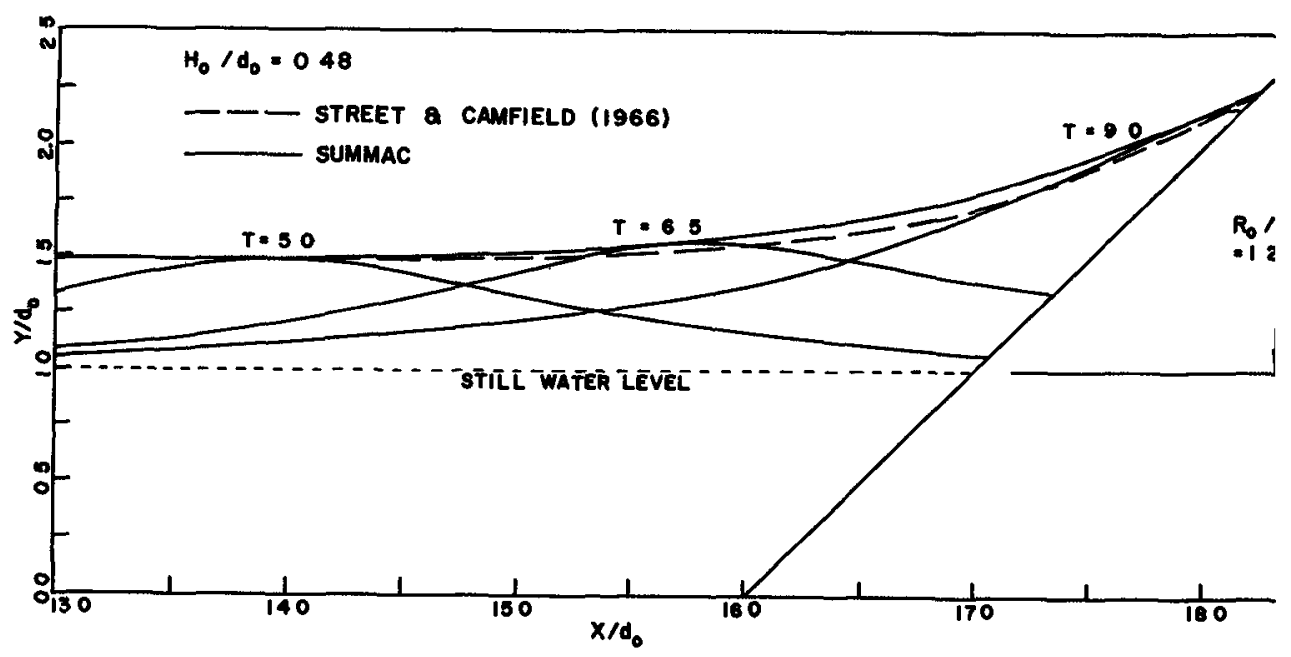

F1g 12 Run-up Profiles on a $45^{\circ}$ slope

\section{CONCLUS IONS}

The successful application of the SUMMAC technique to several physical problems indicates its usefulness as an engineering research tool for analyzing the dynamics of water waves in two space dimensions It is capable of providing accurate quantitative results as well as qualitative descriptions [e g, the prediction of wave run-up on a $45^{\circ} \mathrm{s}$ lope] In addition, rapid advance in the design of high-speed computing systems makes numerical modeling economically feasible

While it is possible to employ the SUMMAC technique to attack a wide variety of water wave problems, some limitations inherent in the method must be noted First, as a result of achieving a high degree of accuracy in applying the free surface pressure condition by using irregular stars, waves after breakıng cannot be simulated when breaking occurs, the computation must be terminated second, only non-turbulent flows are considered in our model Although laminar viscous damping has 11 ttle effect on large scale wave motions, energy dissipation due to the turbulence can be significant However, recent studies by Gawain and Pritchett (1970) and by Pritchett (1970) show that it is feasible to implement a phenomenological simulation of turbulence in the MAC framework 


\section{REFERENCES}

Camfleld, F E and Street, R L, Dept of Clvil Engrg Tech Rept No 81, Stanford University, Calif (1967)

Chan, R K -C and Street, R L, Jour Computational Physics, 6 , 1 (1970a)

Chan, $\mathbf{R}$ K -C and Street, $R$ L, Dept of Clvil Engrg Tech Rept No 135, Stanford University, Calif (1970b)

Dean, R G, Jour Geophysıca1 Research, 70, 18 (1965)

Gawain, $T H$ and Pritchett, $J$ W, Jour Computational Physics, $\underline{6}$, 1 (1970)

Ippen, $A$ T and Kulın, $G$, Dept of C1Vı 1 and Sanıtary Engrg Tech Rept No 15, Massachusetts Instrtute of Technology, Mass (1955)

Laltone, E V, Jour Fluid Mechanics, 9 (1960)

Lamb, Sir H, Hydrodynamics, Dover Publications (1945)

Madsen, $O S$ and Me1, C C , Jour Flu1d Mechan1cs, 39, 4 (1969)

Monkmeyer, P L and Kutzback, J E , Chapter 13, Coasta1 Englneerıng, Santa Barbara Specialty Conference, ASCE, New York (1965)

Peregrine, D H, Jour Flu1d Mechan1cs, 27, 4 (1967)

Pritchett, J W, Information Research Asso., Inc, IRA-TR-1-70 (1970)

Schreiber, D E , IBM Research Report, RJ499, IBM Research Laboratory, San Jose, Calif (1968)

Stoker, J J, Water Waves, Interscience Publishers, Inc, New York (1957)

Street, $R$ L and Camfield, F $E$, Dept of Civil Engrg Tech Note No $85(1)-66$, Stanford University, Calif (1966)

Street, $R$ L, Chan, $R$ K -C, and Fromm, J E, 8th Symposium on Nava1 Hydrodynamıcs, CIT, Pasadena, Ca1ıf (1970)

Welch, J E , Harlow, F H, Shannon, J P , and Daly, B J, Los Alamos Sclentific Lab Rept LA-3425 (1966) 



\section{CHAPTER 22}

\section{PERIOUIC WAVES SHOAIING IN WATERS OVER \\ STHEPLY SLOPING BOTTOMS}

by Dr.Harıhara Rama AYYAR

NSslstant Professor in Clvil Englneering

Indian Instz tute of Technology, ladras, India.

\section{Abstract}

The bottom bed slope pleys a vital role in the shoaling and breakjng of periodic waves. This study alms at the understanding of the effect of steep s] opes (range steeper than 1 10) on the breaking point and the breaker trajectory and the point of Impact. This range of slope is often met with in the near-shore structures. Analytical investigations and model studies are outlined. The influence of slope on each of the signiflcant breaker parameters is discussed.

\section{INRRODUCTIUII}

A train of periodic waves propogating on a sloping beach presents one of the most interesting and perhaps one of the most exciting phenomena in nature. Similar phenomend occur on the slopes of near-shore structures like sea-walls, preakers, dykes etc. A knowledge of the point of breaking and position of occurrence of maximum impact pressures due to breakers wlll be useful alds to the designer. It has been observed tnat beyond the point of breaklng $t i e$ particles rove in a confused manner. Vave theorles cannot be applied in this zone, at best till the breaking polnt only.

\section{2. "RRTODIC UAVES IMOPOGARING IN SHOALING WATERS}

It is know from IRY(1)'s theory of small amplitude waves, that the energy in a wave train is transported in the direction of wave propogation with the group velocity $\mathrm{C}_{\mathrm{G}}$. Considering a train of progressive waves advancing in water of variable deoth, whth change in depth being unform and small RAYLIIGH $(2)$ assumed that the mean rate of energy transport past all vertical sections is constant. runther it was assumed that since the change in depth is very graoual, the characteristics of the wave at any section are given by AIRY's equations. This leads to the relation

$$
\frac{H}{H_{0}}=\sqrt{\frac{2 \cosh ^{2} k h}{2 k \perp+\sinh 2 k h}}
$$


and

$$
\frac{H / I}{H_{0} / I_{0}}=\sqrt{\frac{2 \cosh { }^{2} k h}{2 k h+\sinh 2 k h}} \cdot \operatorname{Cotanh} k h
$$

where

$$
\begin{aligned}
& \text { Ho = Helght of the incident wave (deep water) } \\
& \mathrm{H}^{\mathrm{O}}=\text { Felgnt of wave at any given location } \\
& I_{0} \quad=\text { Length of Incident wave (deep water) } \\
& I^{\prime}=\text { Length of wave at any guven Jocatjon } \\
& \mathrm{h}=\text { Local depth of water bel ow SWL } \\
& \mathrm{k}=\text { Wave number }=2 \pi / \mathrm{L}
\end{aligned}
$$

Thus solution, however, does not predict breakjng, as It Is based on the IInear theory of AIRY.

STOKIR(3) analysed the breaking problem based on the classical non-linear wave theory and the method of characteristics. The solution is however not explicit and calls for zndividual treatment. Onjy solutions for a few special angles of bottom slope were obtazned. The solutions of STOKER do not preduct the breaking point correctly (LE MEEAUTE(4)) and also predicts a bore even when the wave travels on a horizontal bottom. Moreover the results of STOKER alvays predict a snj $11 \mathrm{ng}$ bre on mild slopes. On steep slopes nlunging breakers arc formed unvariably.

3. SOLUTION BASED ON CIASSIC IL NON-IINEAR SHALIOW WATER WAVE THEORY

The classical non-tinear shaljow water wave theory equations are

$$
\begin{aligned}
& \frac{\partial}{\partial x} u(\eta+h)=-\frac{\partial \eta}{\partial t} \\
& \frac{\partial u}{\partial t}+u \frac{\partial u}{\partial x}=-8 \frac{\partial \eta}{\partial x}
\end{aligned}
$$

where $u=$ particle velocity in the horizontal orrection ( 1 e. di rection of wave advance)

$x=$ horizontal distance from the oriman in the in the direction of wave advance.

$\eta=$ the vertzcal elevation of any vartzcle on the free surface above SWL

$\mathrm{h}=$ depth of water measured below SiL

$\mathrm{g}=$ acceleration due to gravity

$t=$ the time reckoned from the moment the wave enters the sloping bottom.

The origin Is taken at the intersection of SWL with the vertical at the foot of the slope (See Frg.1)

The equation can be expressed In terms of dımenstonless quantities

$$
x^{\prime}=\frac{x}{I} \text { where } I=n h
$$




$$
\begin{aligned}
\eta^{\prime} & =\frac{\eta}{h} \\
u^{\prime} & =\frac{u}{u_{0}} \quad \text { where } u_{0}=\sqrt{g h} \\
t^{\prime} & =\frac{t}{t_{0}} \quad \text { where } t_{0}=n \sqrt{\frac{h}{g}}
\end{aligned}
$$

The equations (3) and (4) wlll reduce to

$$
\begin{aligned}
\frac{\partial}{\partial x^{\prime}} u^{\prime}\left(1-x^{\prime}+\eta^{\prime}\right) & =-\frac{\partial \eta^{\prime}}{\partial t^{\prime}} \\
\frac{\partial u^{\prime}}{\partial t^{\prime}}+u^{\prime} \frac{\partial u^{\prime}}{\partial x^{\prime}} & =-\frac{\partial \eta^{\prime}}{\partial x^{\prime}}
\end{aligned}
$$

Along the characteristic directions

$$
\begin{aligned}
& \frac{d x^{\prime}}{d t^{\prime}}=u^{\prime}+C^{\prime} \\
& u^{\prime}+2 C^{\prime}+t^{\prime}=\text { Constant } \\
& \frac{d x^{\prime}}{d t^{\prime}}=u^{\prime}-C^{\prime} \\
& u^{\prime}-2 C^{\prime}+t^{\prime}=\text { Constant }
\end{aligned}
$$

It Is shown by GRLENSPAN( 8$)$ and KISHI( 6 ) that the inutial positive characteristic is given by

$$
x^{\prime}=t^{\prime}-\frac{1}{4} t^{\prime 2}
$$

In the $x-t$ plane. Ly changing over to a moving coordinate system, the slope of the wave front is obtalned as

$$
\frac{\partial \eta^{\prime}}{\partial x_{m}^{\prime}}=\left(1-\frac{1}{2} t^{\prime}\right) \frac{\partial u^{\prime}}{\partial x_{m}^{\prime}}
$$

By using the kinematic stablilty criterion that $u=c$

at the crest at the breaking opunt and using this relation in the integrated equetion (10)

$$
\eta^{\prime}=\left(1-\frac{1}{2} t^{\prime \prime}\right) c^{\prime}
$$

But the property of the inltial positive characteristic gives

$\begin{aligned} C^{\prime} & =1-\frac{1}{2} t^{\prime} \\ \text { Hence } \quad \eta^{\prime} & =\left(1-\frac{1}{2} t^{\prime}\right)^{2}\end{aligned}$

Surtable parameters are to be chosen to define the kinematics of the breaker. The following are chosen in this analysis. 
$\mathrm{h}_{1}=$ Denth at breaking point below SWL

$\mathrm{y}_{\perp}^{j}=$ Hezght of breaker crest above bed.

irom the reletzons (9) and (12) and the geometry of the breaker, the following final relations will result.

$$
\begin{aligned}
\frac{h_{B}}{h} & =\left(\frac{2 m}{1+2 m}\right)^{4 / 3} \\
\frac{y_{B}}{h} & =2\left(\frac{2 m}{1+2 m}\right)^{4 / 3} \\
\text { and } \quad \frac{y_{B}}{h_{B}} & =2 \\
\text { where } \quad m & =n \cdot \pi \cdot \frac{H}{L}
\end{aligned}
$$

inen friction and reflection effects are neglected, as was done in this analysis, one gets the relation (15) for the breaker geomeiry. This also confurms the thumb rule trat breakins occurs at about the point where the wave helght equals water depth. Ilowever the effects of reflection of wave energy are to be accounted for in the case of waves shoaling on steep slopes.

\section{IABORATORY STUDIES IN FRANZIUS-INSTITUT $A$ ND CONCLUSIONS}

Is a result of a large number of model tests conducted by the author in the iranzius Institute in the Technical Unıversity of Hannover, West Germany the following conclusions were arrived at, by which the determination of the characteristics of breakers is made possuble. Varıous bottom slopes (in the steep slope range) incident wave helght and incldent wave perlod were studied in a wave flume $45 \mathrm{mX} 0.5 \mathrm{mX}$ $0.9 \mathrm{mr}$ with a paddle type wave generator, with provisions for changing the period as well as the helgnt of the wave even during operation.

Instrumentatzon used consisted of a number of wave gauges of the parallel wire resustance type (gold-plated. to prevent any chemical action) of different lengths to sult the local depth of water. The wave orofjles were recorded by tnese wave gauges connectedtto a 3 channel electronic direct recording devices, which recorded the wave profile on a millimeter section paper run at the known sneed. To ensure perfect linear relationship between the water levil fluctuations and the records on paper special tyne of variable resustances vere introduced in the circult. This ensured linear relationship over a large range of wave helght. The visual determination of the point of breaking was almost zmpossible. Therefore the wove statistics was collected along the flume and also ahove tro slonin bottom. 
A method was developed to determine the bresknng nolnt from the wave stitistic thus collected, on the assumbtion that the trajectr $y$ of the breaker crest is a second degree curve. This follows from the fact that the particle vclocity at the breaker crest at the breakung pount is purely horizontal and eauel to $\mathrm{C}$, the phase velocity of the wave and in oddution the particles are subjected to a gravjtational acceleration $\mathrm{g}$. What this Is very much so Is demonstrated by the shane of the plunging breakers in nature. A computer nromramme was written using the method of least squares and solving the resulting simultaneous equatjons by the diagonal matrıx method (compact Gauss method). The comrutations were done in CDC-1604 digital computer. The breaking nount was stralghtaway determuned and then the breaker trajectory plotted. A back check was mada to verify the degree of the curve (FIg.2). The ngreement was culte good.

\section{THE RETARDATION OF THE BRDAKER}

From dumensional analysjs of the voriabjes involved, It was found that the factors infuencung the breaker characterustics are $\mathrm{H} / \mathrm{g} \mathrm{T}^{2}$ and $\mathrm{n}$ ( $\mathrm{T}$ is the period of the wave) which are the incldent wave steepness and the bottom slope function respectively. The variation of $\mathrm{Y}_{\mathrm{B}} / \mathrm{h}_{\mathrm{B}}$ w th $\mathrm{H} / \mathrm{g}$.T was studjed for all the slopes tested. The variation shorred the same trend for all the slopes. A relation was established os follows

$$
\frac{\mathrm{y}_{\mathrm{B}}}{\mathrm{h}_{\mathrm{B}}}=\log \left\{40 \cdot \mathrm{n}^{0.5} \cdot\left(\frac{\mathrm{g} \cdot \mathrm{HT}^{2}}{\mathrm{H}}\right)^{0.25}\right\}
$$

(Fug.3) Note that the curves show the varjation of $y_{B / h_{B}}$ rith bed slope as well os the vave steepness (compare theory $\mathrm{y}_{\mathrm{B}} / \mathrm{h}_{\mathrm{B}}=2$ )

The denth at the breakung nolnt wo 1.1 help us to fux the location of breakjng incebtion (FI. 4). For incldent waves of given denth and perrod, the breaking; deoth remalns almost constant for slopes flatter than 15. It was observed that for slones flatter than 15 , all the breaking action of the wave takes place shoreward of the breaking nount. For slopes steeder than 15 , the values of $h_{B}$ increases sharply due to the seaward retardation of the breaker.

6. THE INHLUEITCL OF A SLOPE ON DRLAIER

FIg. (5) shows the influence of the bottom slope on the breaking of a wave.

The reflection coeffacrent us nemlinibly smalj for 
a bed slope of 150 , Increases very slowly tIII a slope of 15 is reached and then increases sharply till the bed slope of 12 is reached and is then asymntotic till a factor of 10 is reached for vertical walls.

The breaking deptk $h_{B}$ Is almost constant till a bed slope of 15 is reached, since the reflection $7 \mathrm{~s}$ still very small of the order of $15 \%$. The small decreasing tendency seen in the breaking depth is indeed neglifible. For slopes steeper than 15 the breaking depth increases sharply showing that the breaker is retarded seawards. The breaker crest elevation $\mathrm{y}_{B}$ varies in a similar manner.

The variation of $y_{B} / h_{B}$ is of interest. The theoretical value of 2 is never reached. The forces of reflection are almost negligible and frictional losses are small compared to tne slopes 150 etc. For slopes flatter than 110 frictional losses increase. The effect of this is to reduce the potential energy growth and hence $\mathrm{y}_{\mathrm{B}} / \mathrm{h}_{\mathrm{B}}$ decreases. For $n=10$ to $n=5$, the breaking action occurs shoreward, the waves still overcome the forces of reflection. s slight decrease is registered. For $n<5, h_{3}$ increases sharply due to retardation of the breaker. $\mathrm{y}_{B}$ also Increases but at a slower rate. $\mathrm{y}_{\mathrm{B}} / \mathrm{h}_{\mathrm{B}}$ decreases. The lowest value of 1.10 is reached for breaking clapotis.

\section{THE POINT OF IMPACT}

The point of Impact of the breaker on the slope of the bottom boundary can be calrulated once $\mathrm{y}_{\mathrm{B}}$ and $\mathrm{h}$, are known. From the geometry of the slope (EUEHRBOETFR) (?)

$$
\Delta \mathrm{h}=-\mathrm{h}_{\mathrm{B}}+\mathrm{y}_{\mathrm{B}}\left\{1-\frac{1}{2 \mathrm{n}^{2}}\left(\sqrt{1+2 \mathrm{n}^{2}}-1\right)^{2}\right\}
$$

The determination of the point of impact was checked on the model. With pressure cells connected to electron'c direct recording devices (Honeywell ultraviolet light beam osclilograph), the impact pressure variation with respect to time is followed. Impact pressures were recorded at 6 points at $20 \mathrm{~cm}$ intervals along the slope simultaneously...The maximum pressure corresponds to the direct impact of the breaker on the slone which helps to locate the point of impact. Comparison of the calculated. values and measured values showed Jess than $8 \%$ error. The The deviation of the curve $\Delta \mathrm{h} / \mathrm{h}_{\mathrm{B}}$ for the slopes tested, from the curve computed from the I inear theory applicabie with reasonable accuracy for mild slopes is shown in Fig. (6).

\section{ACKNOWLEDGETLIVTS}

The work reported here is a nart of the doctoral work done by the author uncer the guzdnnce of I'rof.C.iouvé and 
Prof.W.Hensen in the Technical Unıversity of Hannover. The author expresses hls deep gratıtude to them and to Dr.Fuehrboeter for thelr help and guldance.

9.RETERENCES

1. AIRI, G.B.

2. RAYLEIGH, IORD

3. STOKER, J.J.

4. IE MEHAUTE, B.

5. GREEISPAN?H.P.

6. 站 ISHI, T.

7. FUEHRBOITTR, A.
On TIdes and Waves - Encyclopaedıa

Metrooolitana Vol 5, London, 1842. Hydrodynamıcal Notes Philosophical Magazıne, Vol 21,1911. The formation of Breakers and Bores Communications on Applied Mathematıcs, Vol 1, 1948.

A synthesis on Wave run-up Proceedings, American Soclety of Civil Engıneers, WW Dn., February 1968. On the breaking of water waves of fincte amplitude on a sloping beach - Journal of Fluld Wechanles, Vol 4, Part I, 1958. Transformatıon, Breakıng and run-up of a long wave of finite helght Proceedlngs 8th Conference on Coastal Inguneering, 1962 .

Der iruckschlag durch Brecher auf Delchbochungen - Mittellungen des Franzıus-Instituts, Heft 28, Ilannover, 1966. 


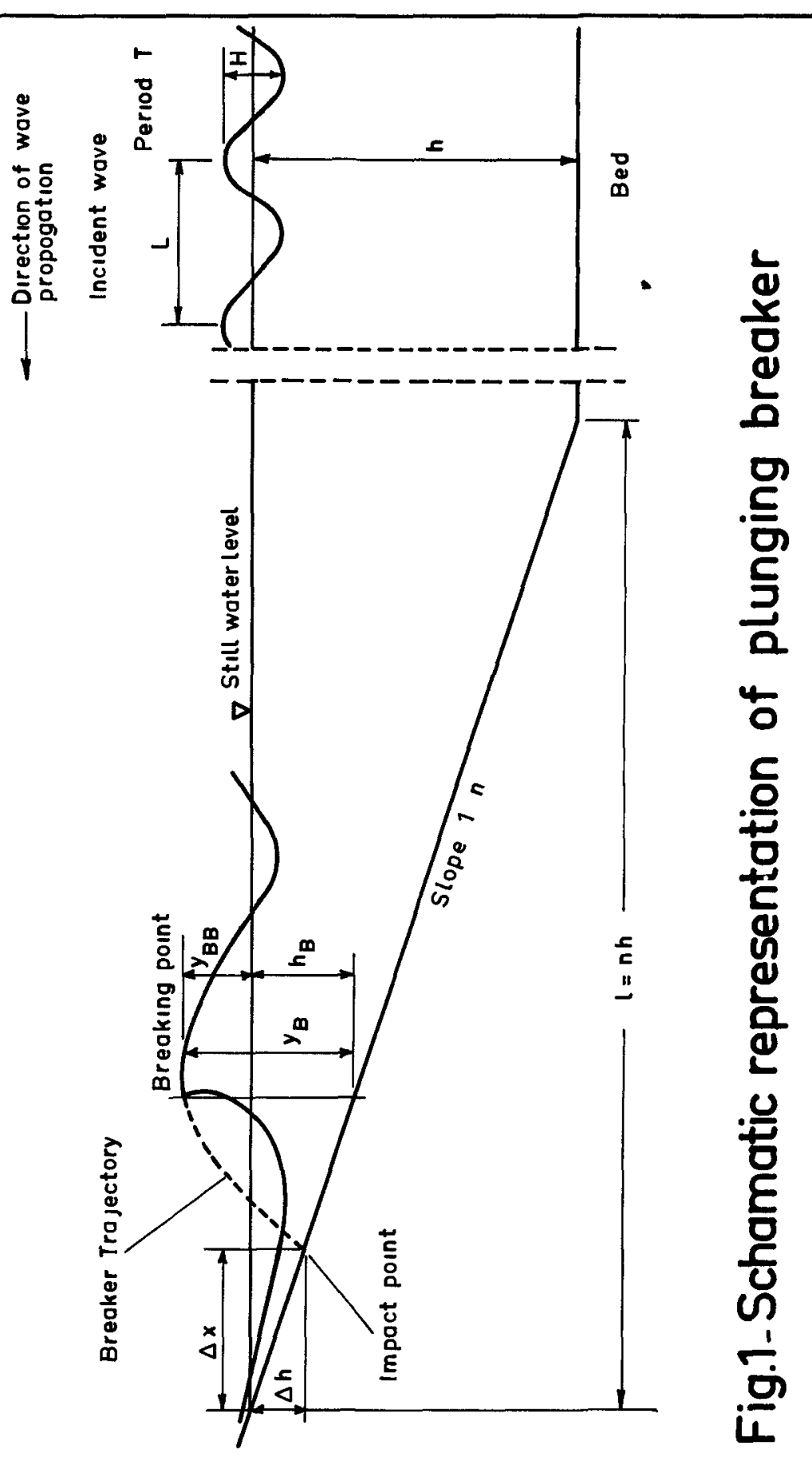




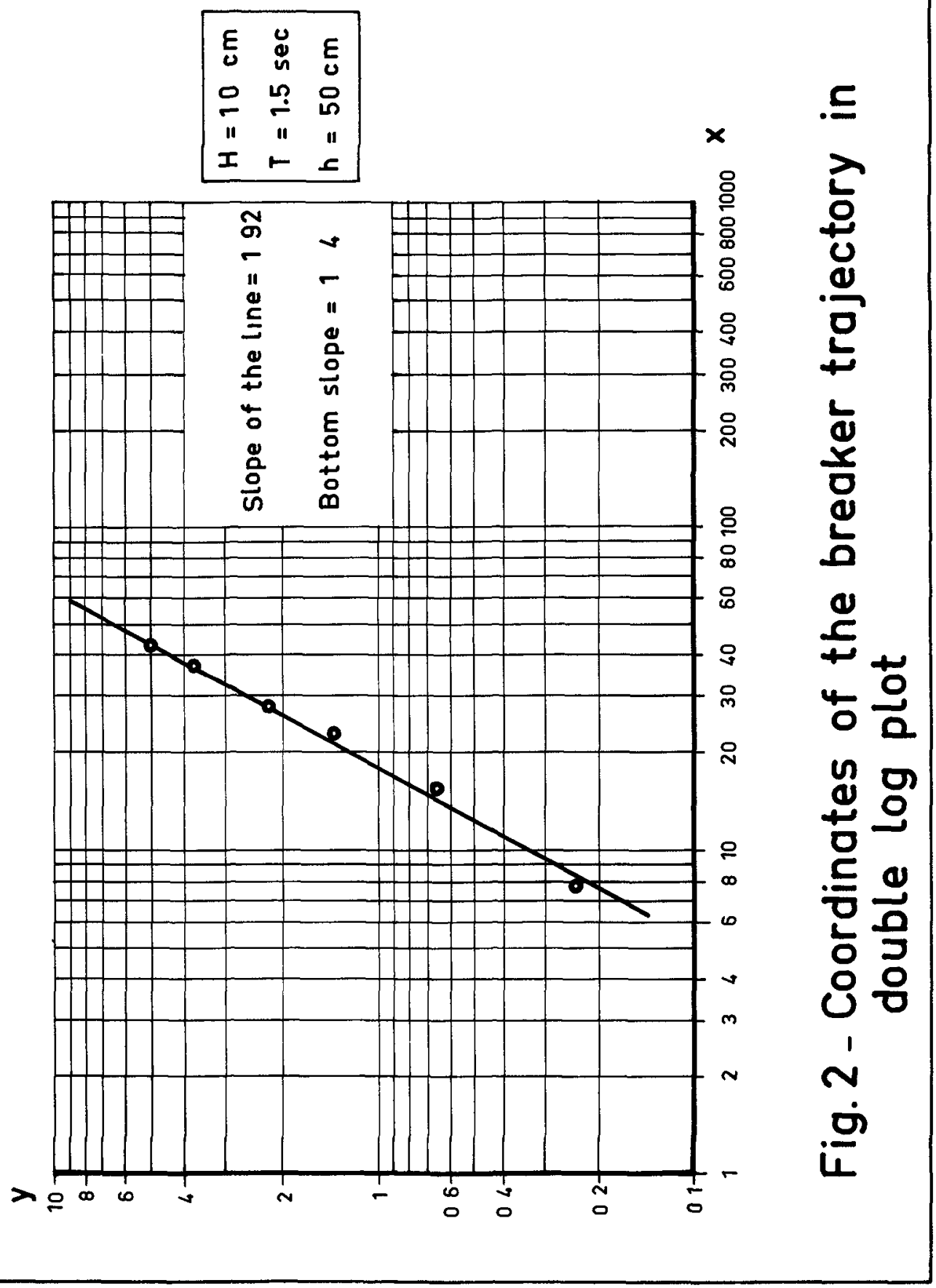




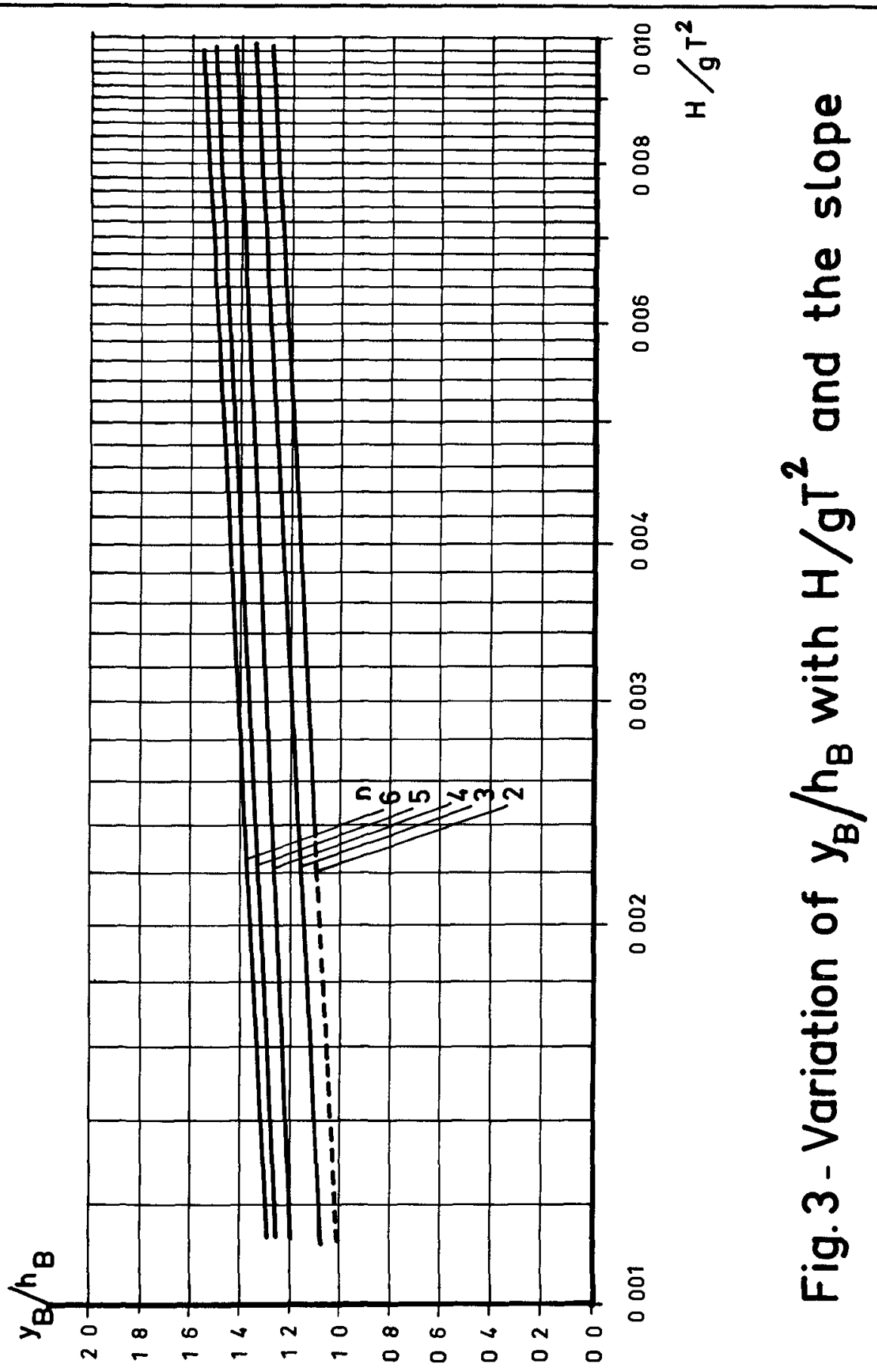




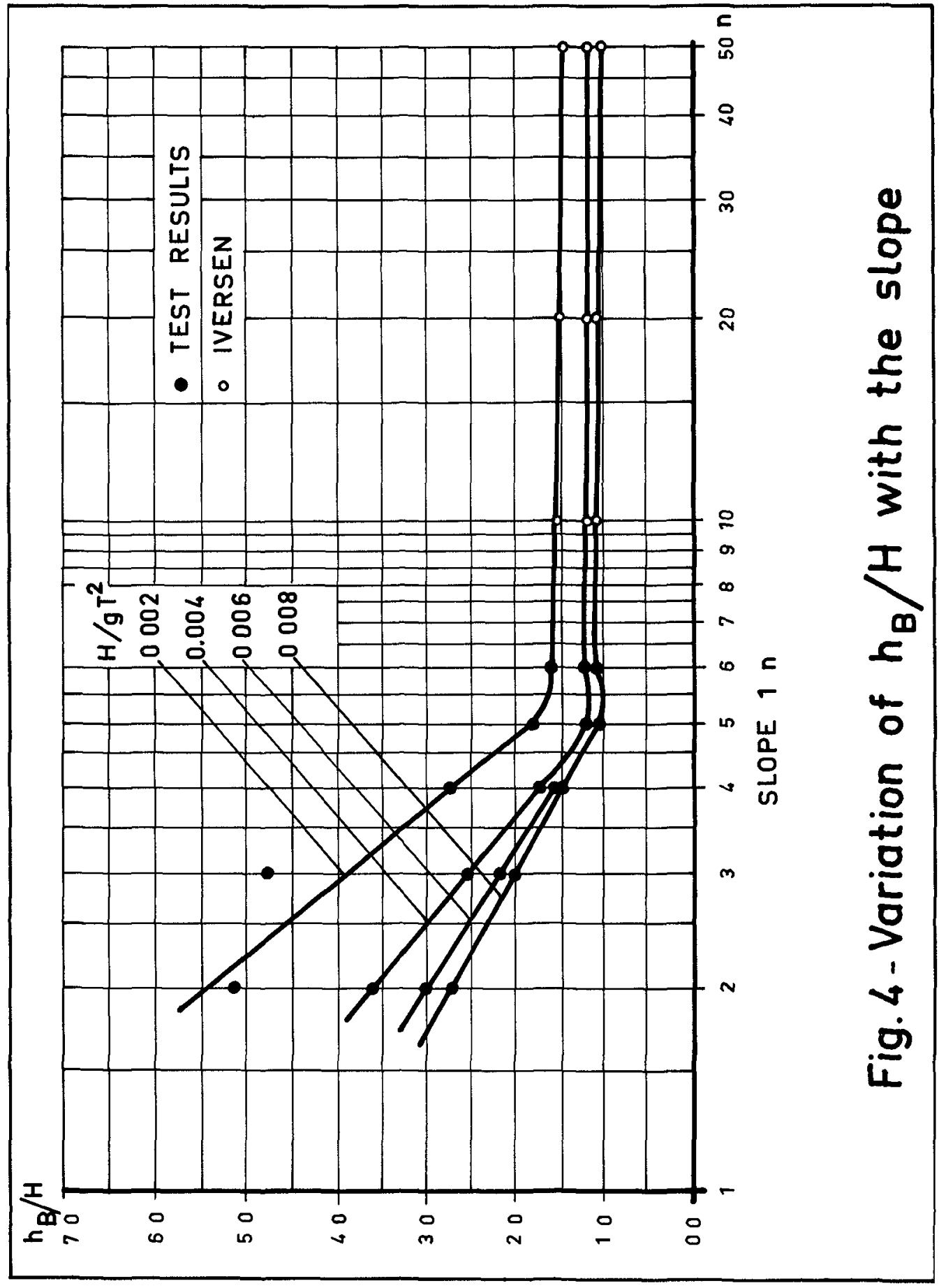




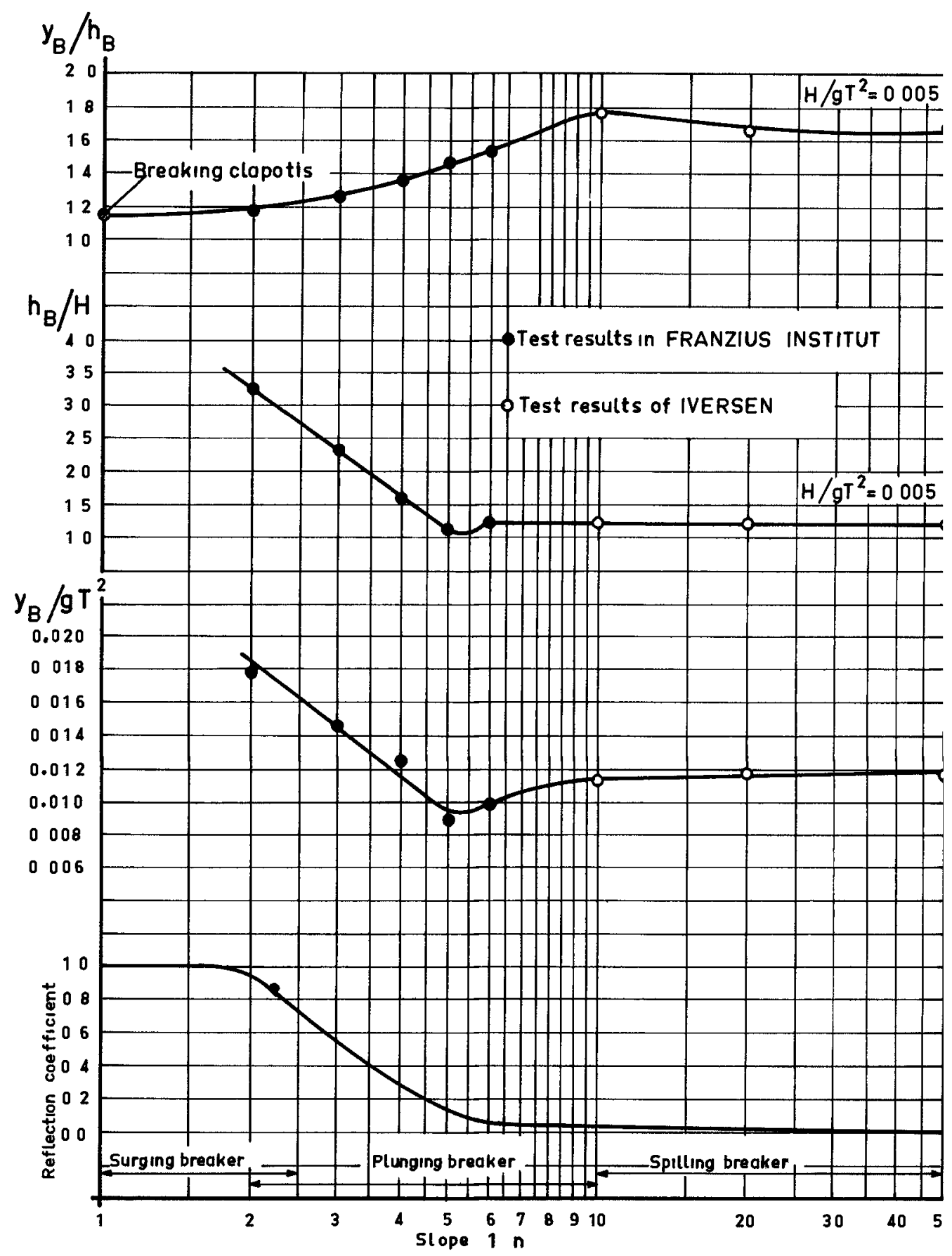

FIG. 5 - Influence of slope on breaking of waves 


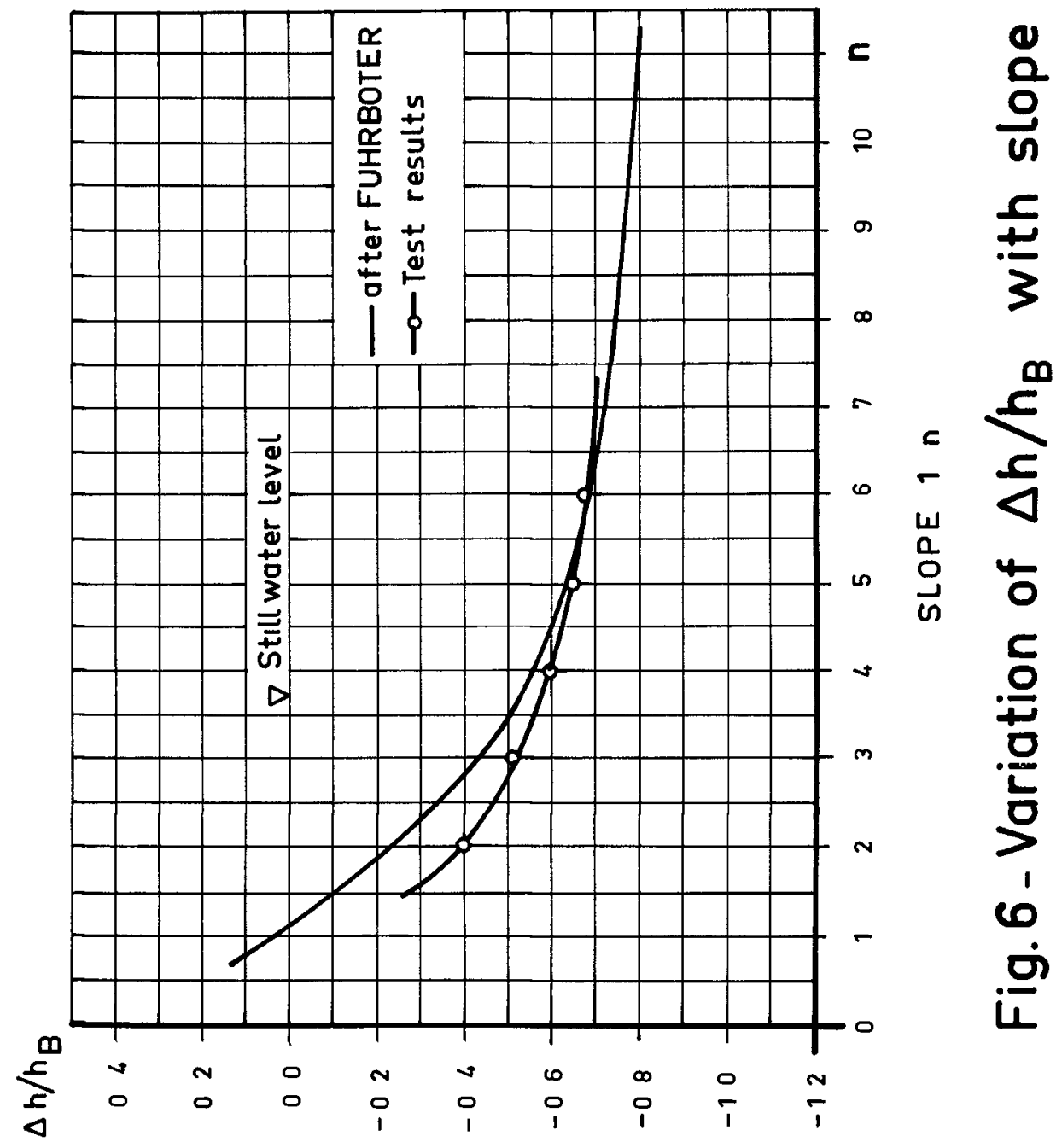




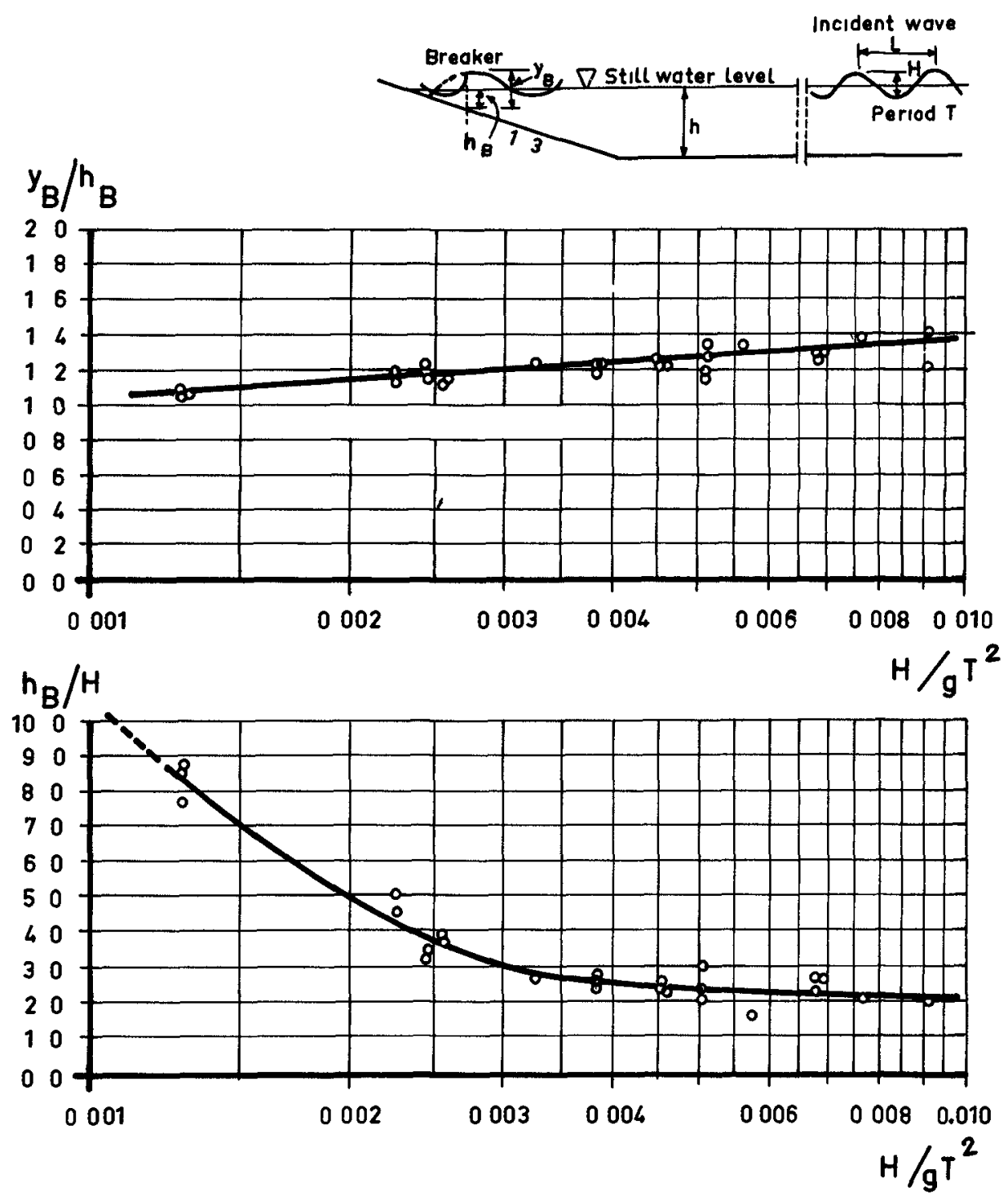

Fig. 7 -Test results for the slope 1:3 


\title{
CHAPTER 23
}

\section{BREAKING WAVE SETUP AND DECAY ON GENTLE SLOPES}

\author{
by \\ L1-San Hwang, Member \\ and \\ David Divoky, Visitor
}

\begin{abstract}
Waves of large amplitude on a gentle slope may form spilling breakers which propagate shoreward and are slowly transformed In addition, there occurs a modification of the mean water level termed wave setup An analytical description based upon consideration of momentum flux has been developed which predicts this wave setup and the decay history of breaking wave height The results have been compared with experiments and found quite satisfactory

The effect of wave setup on breaking wave transformation is particularly important near the shoreline, where setup dominates the vanishing mean depth
\end{abstract}

\section{INTR ODUCTION}

The transformation of a breakıng wave over a slope is a problem of obvious concern in the design and planning of coastal facilities A number of investigators have considered this topic, including Freeman and LeMéhauté (1964), Horıkawa and Kuo (1966), Le Méhauté (1962), Divoky, LeMéhauté, and Lin (1970), Nakamura, Sh1raısh1, and Sasakı (1966), and Street and Camfield (1966) The present contribution considers an essential aspect neglected in previous studies, the phenomenon of "wave set-up"

Set-up, while negligible seaward of the breaking point, becomes dominant with respect to still-water depth as the shore is approached It is apparent, then that any analysis of breaking wave height transformation should account for the set-up Experimental investigations of set-up have been made by Saville (1961) and by Bowen, Inman, and Simmons (1968) with the result that the maximum elevation may be a significant fraction $(\sim 50 \%)$ of the breaking wave height Additionally, measurements obtained during the Mono Lake explosion-wave tests indicated a set-up value equalling the maximum height of the superposed dispersive wave train (Van Dorn, et al, 1968) Hwang (1970) also investigated dispersive wave trains and found a fluctuating set-up of roughly half the peak wave height

Wave set-up has been investigated extensively in a series of papers by Longuet-H1ggins and Stewart $(1960,61,62,64)$ from an a nalytical approach The difficulty in applying their results to the

Tetra Tech, Inc, Pasadena, California 
surf zone, arises from the problem of finding an adequate description of the waves after breaking Bowen, Inman, and Simmons (1968) assumed that the wave height remains a constant fraction of mean water depth after breaking and found, from a momentum balance, a linear set-up variation

In this paper, a more detalled description of the wave transfor mation has been used so that height-decay and set-up are calculated simultaneously The approach roughly follows Divoky, LeMéhauté, and Lin (1970) with the wave decay computed from an energy-dissipation model The essential difference is that the set-up is included so that more realistic behavior is found near the shoreline, in particular, a significant surviving wave height is found at the still-water shoreline

\section{MODEL}

Two governing equations are adopted in the present model Firstly, a balance of forces across a fluld element (see, for example, LonguetHiggins and Stewart, 1964), as shown in Figure l, gives the momentum equation

$$
\frac{d M}{d x}+\rho g(h+g) \frac{d}{d x}=0
$$

where $M$ is the momentum flux, $h$ is the still water depth, and $\xi$ is the wave set-up The energy dissipation rate is assumed to be a fixed fraction, $B$, of that of a bore of the same height Then the standard formula (see, for example, Lamb, 1945) may be adopted--adjusted, of course, to conform to a moving jump, the result is

$$
\frac{d E}{d x}=B\left(\frac{d E}{d x}\right)_{B O R E}=B \frac{\rho g}{4} \frac{H^{3} D}{y_{t}\left(y_{t}+H\right)}
$$

In this expression the volume flux $Q$ has been taken to be $C D$ wheie $C$ is the wave celerity and $D$ is $(h+\xi), y_{t}$ is the depth below the trough (corresponding to the depth ahead of the jump) and $\left(y_{t}+H\right)$ corresponds to the depth behind the jump The assumption which has been made here is that $B$, which must account for all forms of energy dissipation, is constant within the breaking region

To implement this simplified model, a suitable wave description must be chosen in order to calculate $\mathrm{E}$ and $\mathrm{M}$ In this, we have followed the observation of LeMéhauté, Divoky, and Lin (1968), that the cnoldal wave theory of Keulegan and Patterson ( $1<40)$ appears to give the best description of periodic waves in moderately shallow water and that this description may be adopted for gently spilling breakers with moderate success, of course, any non-breaking wave theory wil be inadequate to describe violently plunging or surging conditions

The following equations of cnoldal wave theory have been used

$$
\eta=y_{s}-D=y_{t}-D+H c n^{2}\left[2 K(k) \frac{x}{L}\right]
$$




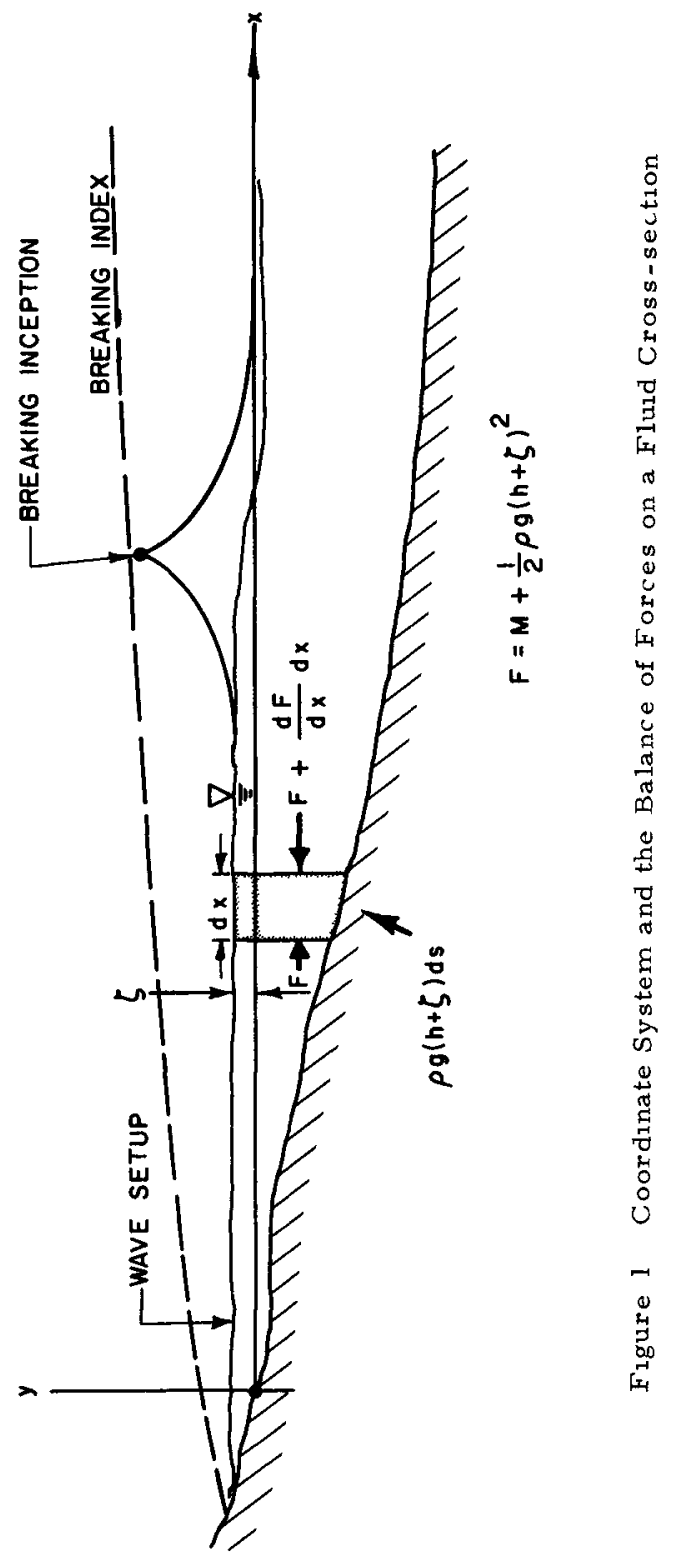




$$
\begin{aligned}
& L=\sqrt{\frac{16 D^{3}}{3 H}} k K(k) \\
& T \sqrt{\frac{g}{D}}=\sqrt{\frac{16 D}{3 H}}\left\{\frac{k K(k)}{1+\frac{H}{k^{2} D}\left[\frac{1}{2}-\frac{E(k)}{K(k)}\right]}\right\} \\
& y_{t}=D-H+\frac{16 D^{3}}{3 L^{2} H}\{K(k)[K(k)-E(k)]\}
\end{aligned}
$$

For given values of $H, D$, and $T$, Equation (5) is used in an iteration procedure to find the elliptic parameter $\mathrm{k}^{2}$, which then allows calculation of all other quantities In particula1, we have evaluated the energy as

$$
\begin{aligned}
E & =2\left(\text { POTENTIAL ENERGY) }=\rho g \int_{0}^{L} \eta^{2} \mathrm{dx}\right. \\
& =\rho g L\left\{-\left(\mathrm{y}_{\mathrm{t}}-\mathrm{D}\right)^{2}+\frac{\mathrm{H}^{2}}{3 \mathrm{k}^{2}}\left[1-\mathrm{k}^{2}+\left(2-4 \mathrm{k}^{2}\right)\left(\frac{\mathrm{y}_{\mathrm{t}}-\mathrm{D}}{\mathrm{H}}\right)\right]\right\}
\end{aligned}
$$

Calculation of the momentum flux from defining integrals poses a time-consuming and costly task even on a computer, since it involves repeated evaluation of the cnoidal wave properties We have made the greatly simplifying approximation that

$$
\mathrm{M} \approx \frac{3}{2} \frac{\mathrm{E}}{\mathrm{L}}
$$

a result essentially from Airy theory given by Longuet-Higgins and Stewart (1964) Following them, we consider this to be a not unreasonable assumption within the surf-zone, with the possible improvement that $E$ and $L$ are computed here from a more adequate wave theory

The solution of Equations (1) and (2) begins with specification of a set of initial values for height, depth, set-up, and period Equations (3) - (8) then give the wave properties at that point For an advance by an increment $\mathrm{dx}$ toward shore, Equation (2) supplies the corresponding energy loss dE An iteration procedure 1nvolving Equation (1) then gives new values of wave height and total mean depth consistent with the avallable energy

A word concerning calculation of the elliptic parameter is in order The cnoldal wave theory is of greatest interest when $k^{2}$ is extremely near unity A direct expansion of $E$ and $K$ in terms of $k^{2}$ is then limited by problems of round-off in $k^{2}$ This problem has been obviated by defining

$$
P=-\log _{10}\left(1-k^{2}\right)
$$


$E$ and $K$ are written as power series in $P$ rather than $k^{2}$, and all calculations involved in Eqs (3) - (8) similarly suppress $k^{2} \quad P$ is an easily manageable number and is roughly the length of the string of nines in the decimal form of $k^{2}$ In the present calculations $P$ has varied from less than unity to above fifty

\section{RESULTS}

A number of observations on wave transformation are summarized in Figure 2 The data of Horlkawa and Kuo (1966) for a slope of $1 / 65$ is shown in Figure 3 with the solid lines representing results of the present model with $B$ taken arbitrarily as 08 The general trend appears quite good, and a somewhat better fit might have been obtained with other values of $B$ The comparison indicates that the data scatter is accounted for by the variation in deep water steepness, so that the transformation is not solely a function of bottom slope

The dependence upon slope is indicated in Figure 4 Here, only slope is varied and the theoretical height transformation is shown It is noted that the present model is probably better for gentle slopes than steep slopes, since all calculations of wave properties are based upon the assumption that the water depth is constant and equal to the loca 1 value

Measurements of set-up are relatively few A comparison of the present model with observations of Saville (1961) is shown in Figures 5 and 6

The curves labelled "Calculated" represent the contributions to set-up in the breaking and non-breaking zones The latter has been computed from (Longuet-Higgins and Stewart, 1964)

$$
\xi=-\frac{1}{8} \frac{\mathrm{H}^{2} \mathrm{k}}{\sinh 2 \mathrm{kD}}
$$

where $k$ is the wave number The contribution due to breaking has been calculated by using observed values at the breaking point as initial values with $\xi$ taken as zero (the portion due to breaking) The figures show that the general trends and magnitudes are correct and that the observed set-up "patches together" the portions contributed before and after the breaking point

In all of the calculations shown so far $B$ has been taken arbitrarily as 0 , representing an energy dissipation rate $80 \%$ of that for a hydraulic jump of equal height The dependence of results on this parameter is indicated in Figure 7, showing set-up for the conditions of Figure 6 It is seen that $B=06$ would have shown better agreement with observation, but that the differences are relatively small

\section{DISCUSSION}

The model presented here appears, from limited comparison with data, to be capable of adequate prediction of set-up and height decay in the breaking zone Considerable improvement is possible, however 


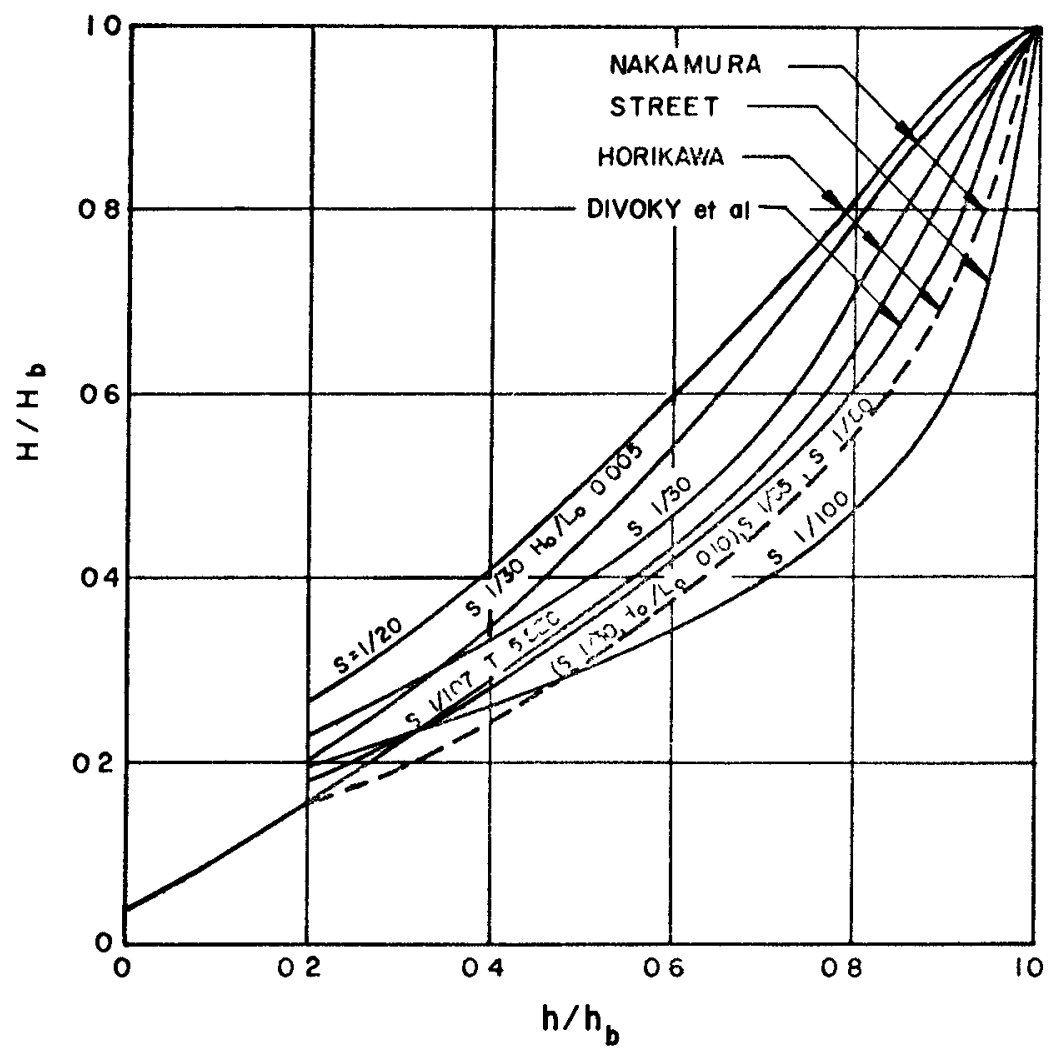

Figure 2 A Summary of Wave Transformation Data Shoreward of the Breaking Point 


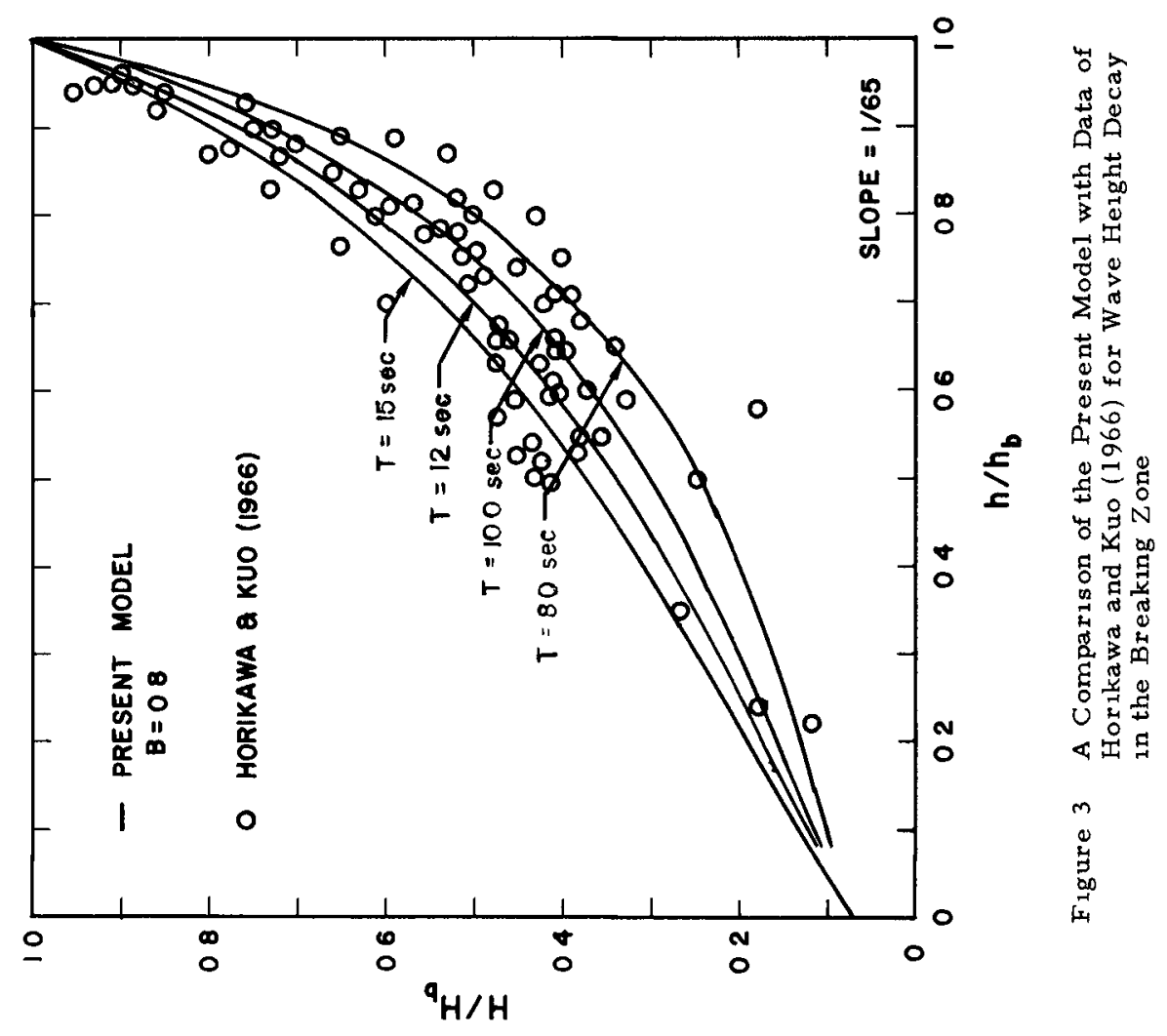




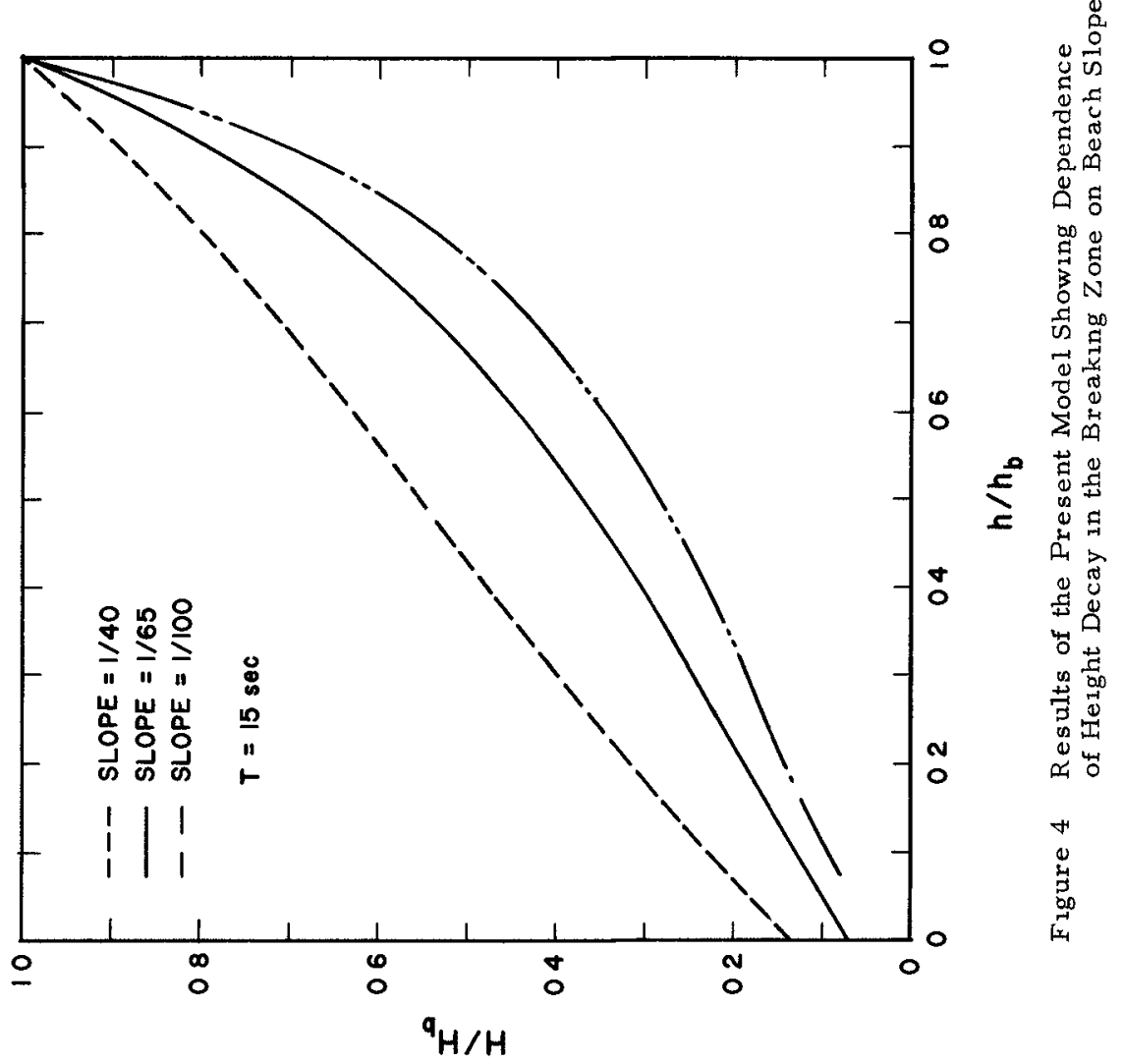




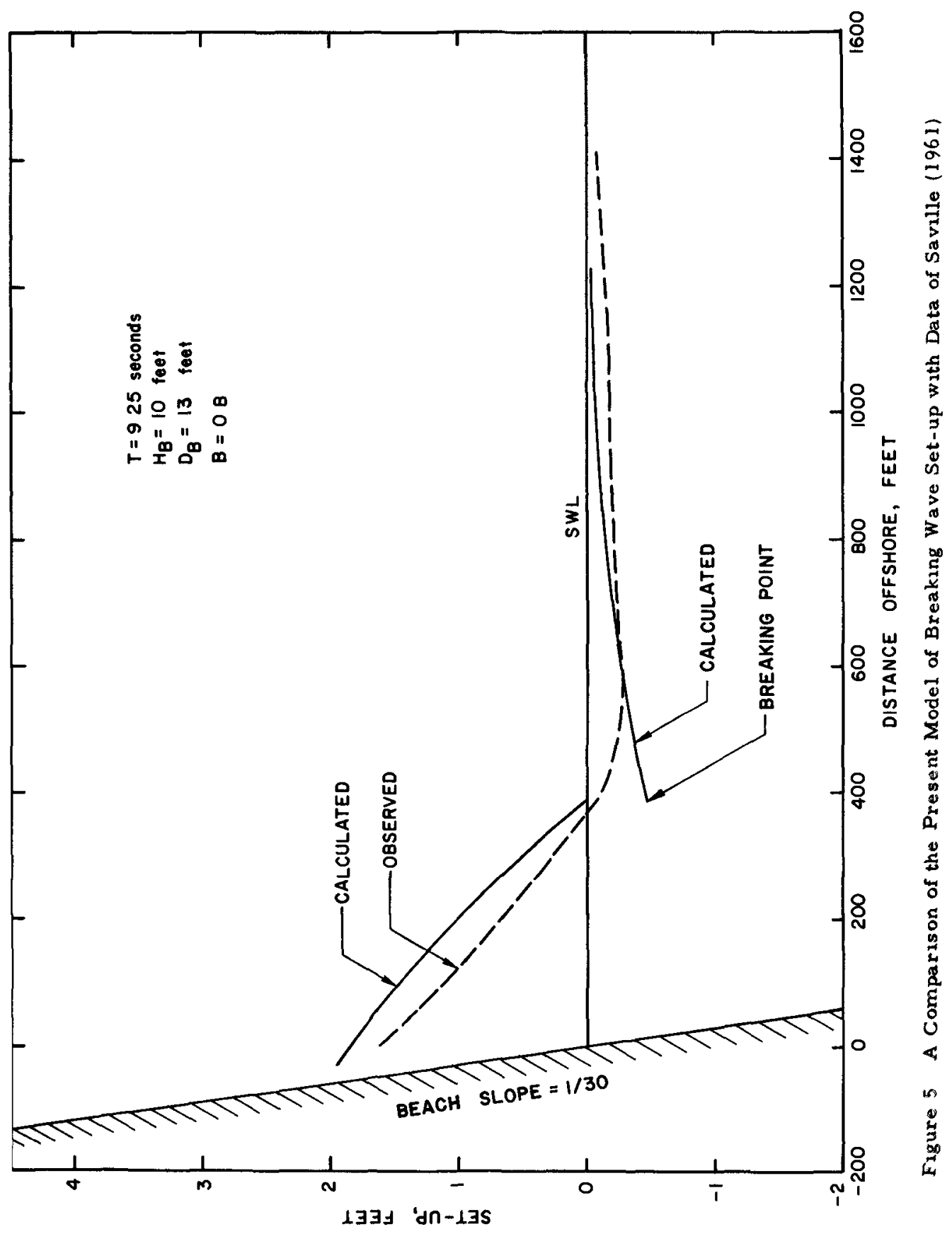




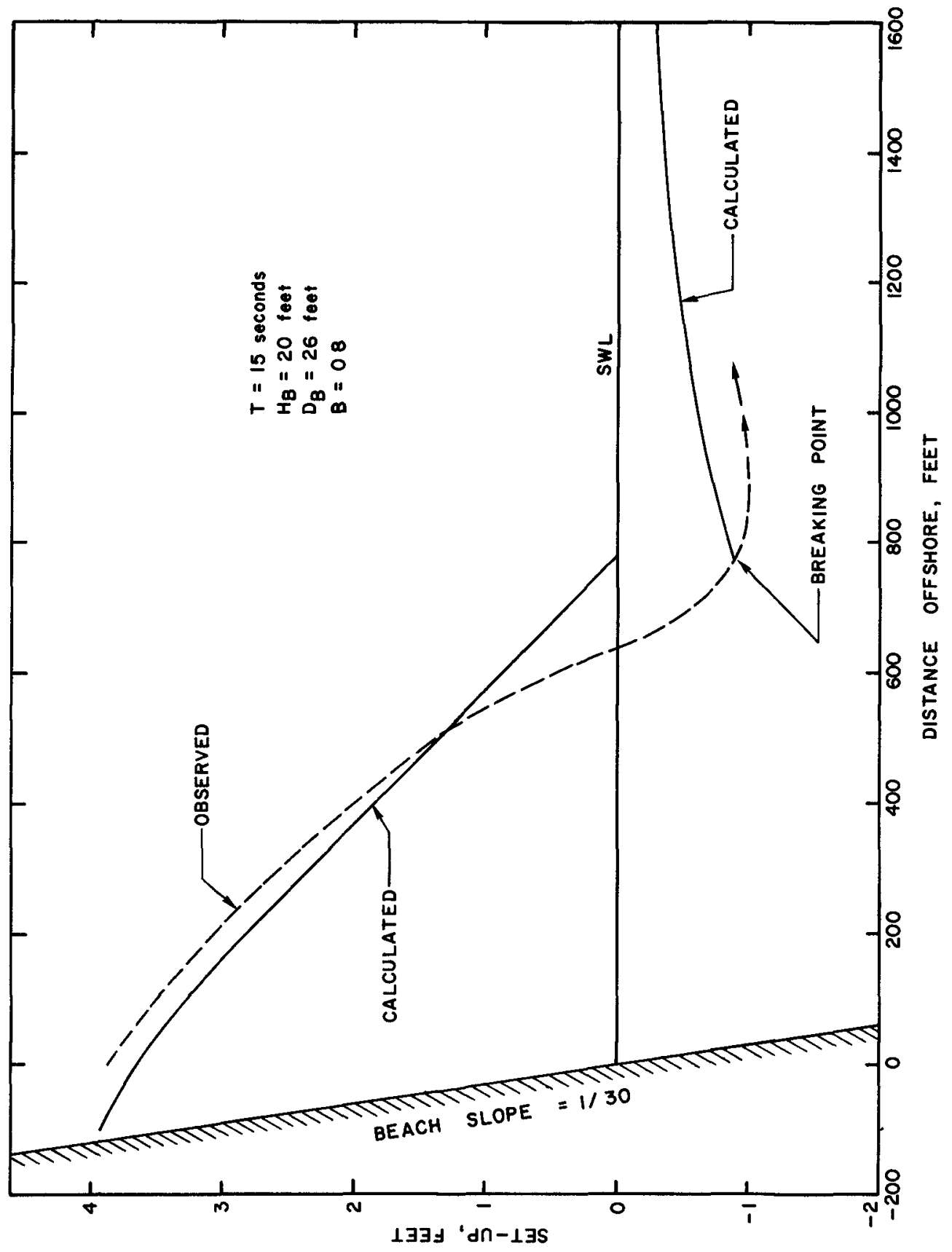




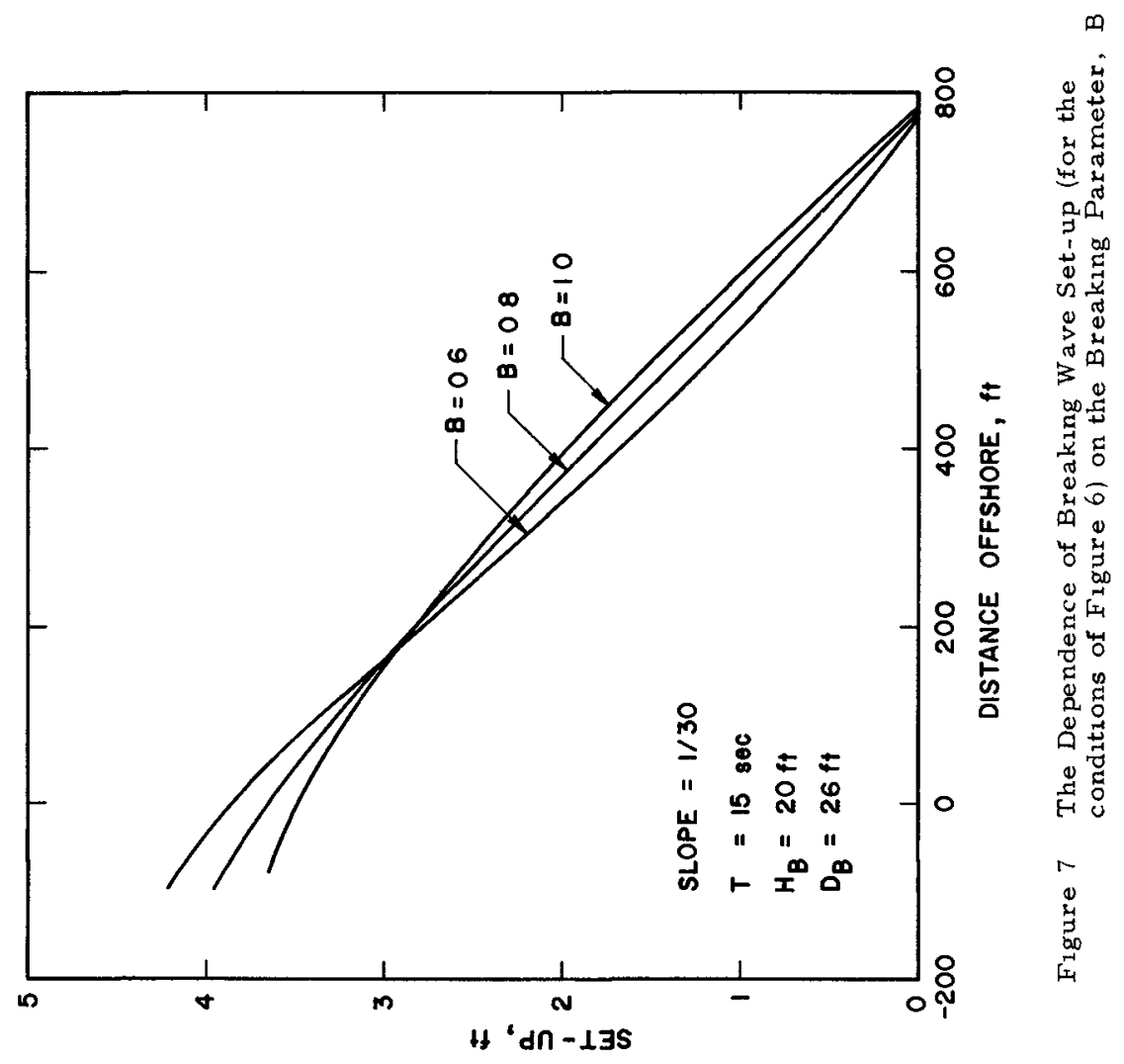


In particular, the approximation to the momentum flux given in Equation (8) is especially primitive as noted, $M$ can be computed directly from defining integrals, although this is difficult and inherently approximate for breaking waves if a non-breaking theory is adopted

The magnitude of the set-up at the shoreline is found, in accordance with the data of Saville (1961), to be quite large It is pointed out that the Saville data, given in field magnitudes, was actually obtained in very small scale tests Similarly, the data of Bowen, Inman, and Simmons (1968) was also taken at a small scale with periods on the order of one second and maximum set-up heights of a very few centimeters For this reason, comparis on with available data, including that of Saville, is somewhat problematic owing to the possibility of scale effects

An interesting phenomenon observed by Bowen, Inman, and Simmons was a strong tendency for the set-up profile to become tangent to the beach slope, their analysis, which assumed a breaking wave height equal to a constant fraction of the total water depth, also showed this feature The present model was unable to duplicate this, always showing a set-up profile convex-upward intersecting the beach, the data of Saville appears generally similar

\section{REFERENCES}

Bowen, A J , D L Inman, and V P Simmons (1968) "Wave 'Set-Down' and Set-Up, " Journal of Geophysical Research, Vol 73, No 8

Divoky, D J, B J LeMéhauté, and A Lin (1970) "Breaking Waves on Gentle Slopes, "Journal of Geophysical Research, Vol 75, No 9

Freeman, J C and B J LeMéhauté (1964) 'Wave Breakers on a Beach and Surge on a Dry Bed," ASCE, Journal of the Hydraulics Division, Vol 90, No HY2

Horikawa, K and C T Kuo (1966) "A Study on Wave Transformation Inside the Surf Zone, " Proceedings, Tenth Conf on Coastal Engineering, ASCE

Hwang, L1-San (1970) 'Wave Set-up of Nonperıodic Wave Train and Its Associated Shelf Oscillation," Journal of Geophysical Research, Vol 75, No 21

Keulegan, G and G W Patterson (1940) "Mathematical Theory of Irrotational Water Waves," J Res NBS, Vol 24, No 1

Longuet-Higgins, M S and R W Stewart (1960) "Changes in the Form of Short Gravity Waves on Long Waves and Tidal Streams," Journal of Fluid Mechanics, Vol 8

Longuet-Higgins, M S and R W Stewart (1961) "The Changes in Amplitude of Short Gravity Waves on Steady Non-Uniform Currents, " Journal of Fluid Mechanics, Vol 10

Longuet-Higgins, M S and R W Stewart (1962) "Radiation Stress and Mass Transport in Gravity Waves, with Application to 'Surf Beats'," Journa1 of Fluid Mechan1cs, Vol 13, Part 4 
Longuet-Higgins, $M S$ and $R$ W Stewart (1964) "Radiation Stresses in Water Waves, A Physical Discussion with Applications, " Deep-Sea Research, Vol 11

Nakamura, M, H Shirassh1, and $Y$ Sasak1 (1966) "Wave Decaying Due to Breakıng," Proc Tenth Conf on Coastal Engineering, ASCE

Saville, T (1961) "Experimental Determination of Wave Set-up," Proc 2nd Tech Conf on Hurricanes, Miami, Fla

Street, R L and F E Camfield (1966) "Observations and Experiments on Solitary Wave Deformation, "Proc , Tenth Conf on Coastal Englneering, ASCE

Van Dorn, W G, B LeMéhauté, and L1-San Hwang (1968) "Handbook of Explosion-Generated Water Waves Volume 1State of the Art," Tetra Tech Rept TC - 130, DASA - 1944 



\section{CHAPTER 24}

AIR ENTRAINMENT AND ENERGY DISSIPATION IN BREAKERS by Alfred FUHRBOTER ${ }^{1}$ )

\section{SUIMARY}

Even in shallow water, only a part of wave energy is lost by turbulent vascosity and bottom friction, most of wave energy transfer takes place in the narrow zone of surf at the shore.

Till to the point of breaking, the theoretical conception of an one-phase flow may be applied to the problem. From beginulng of breakıng, however, the effect of aeration can not be neglected.

From a simple physical consideration, the sudden reduction of wave helght and wave energy inslde the surf zone can be explauned by the entraunment of alr bubbles into the water. Except compression and surface tension effects, most of wave energy is stored at first by the static energy of the alr bubbles which are driven into the water.

Using ldeallzed assumptions for calculation (unlform concentration of alr bubbles a.s.o.), It can be shown that In a plunging breaker the wave energy is disslpated on a very short way (less than on wave length), for a spliling breaker however, this way is of the order of some wave lengths.

After formation of the alr-water-mixture, the energy of the alr bubbles is transformed by the microturbulence of the eddies in the turbulent wakes behlnd the uprising bubbles.

1) Unıversitatsdozent Dr.-Ing., FRANZIUS-INSTITUT, Technıcal Uncversity of Hannover, Germany 
So turbulence spectrum also ls connected whth the arr content of the water, this shall be of Importance for the sediment transport.

For model Investıgatıons of breakıng waves heavy scale effects are to be expected from the alr entranment.

\section{THEOHETICAL CONSIDERAIION}

Inslde the surf zone, wave helgth and energy are reduced suddenly by strong interactions, lt shall be demonstrated here that the major part of wave energy ls spent by surface interaction = alr entralnment. For this demonstration, only simple assumptions and linear wave theory are used, the results would be essentlally the same for hlgher order theories.

The total energy of a wave whth the helgth $H$ and the length I Is on the wadth $b$

$$
E=\frac{1}{8} \cdot Y \cdot b \cdot I \cdot H^{2}
$$

and a reduction of wave helght $\mathrm{dH}$ gives the reduction of wave energy dE

$$
d E=\frac{1}{4} \cdot Y \cdot b \cdot I \cdot H \cdot d H
$$

The wave length $L$ shall be stated to be constant during breakıng.

This loss of (statlc and dynamlc) energy must be transformed Into other sorts of energy, so in turbulent motion and at last into heat by fruction. But before the energy Is going into turbulence, there is a transfer mechanlsm by alr entrainment and foam production.

Fig. 1 shows a column of water with the helgth $\mathrm{y}$ and $\mathrm{a}$ volume of alr $\Delta y$, when mixed together in such a matter, that there is a nearly unlform distribution of alr bubbles in the water, the arr concentration $c$, related to the vo- 


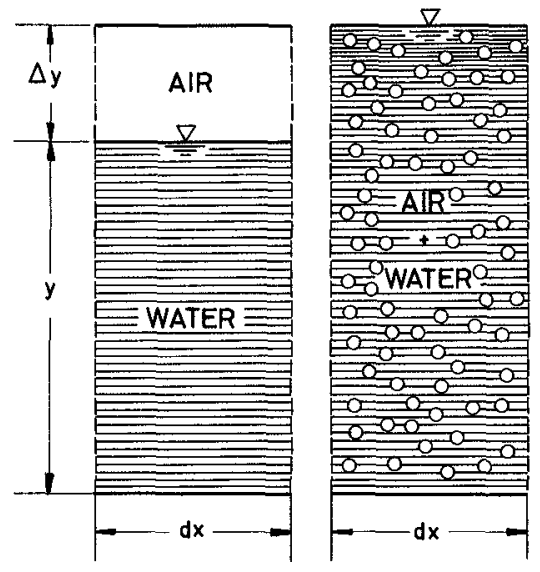

FIG. 1. - ENERGY CONSUII BY AERATION lumen wath the heaght $y+\Delta y$, $1 \varepsilon$

$c=\frac{\Delta y}{v+\Delta y} \ldots \ldots \ldots \ldots \ldots(3)$

Neglecting the density of alr, the static energy to bring a volume of alr into the cept\}. $\checkmark$ belor water surface is the same like to oring an equal volume of water into the helgnt d above surface. Hor the length $d x$, the wath $b$ and an (uniform) alr concentration c

therefore 1s (F1g. 1)

$$
\begin{aligned}
& d E_{\text {stat }}=\gamma \cdot b \cdot d x\left(c \cdot \frac{y^{2}}{2}+\frac{\Delta y^{2}}{2}(1-c)\right) \\
& \text { alr' In water" with } \\
& \text { water alr above } \\
& \text { level y }
\end{aligned}
$$

and with $\Delta y$ from Eq. 3

$$
d E_{\text {stat }}=r \cdot b \cdot d x \cdot \frac{y^{2}}{2} \cdot \frac{c}{1-c}
$$

This ls only the static energy which ls recessary to transfer the water column $y$ and the arr column $\Delta y$ into a foam column $y+\Delta y(F i g .1)$, additıonally, energy losses by effects of (adiabatıc) compression, surface tension and friction auring the movement of the bubbles occur, these effects, however, are of small order compared with the statıc energy rrom Eq. 5.

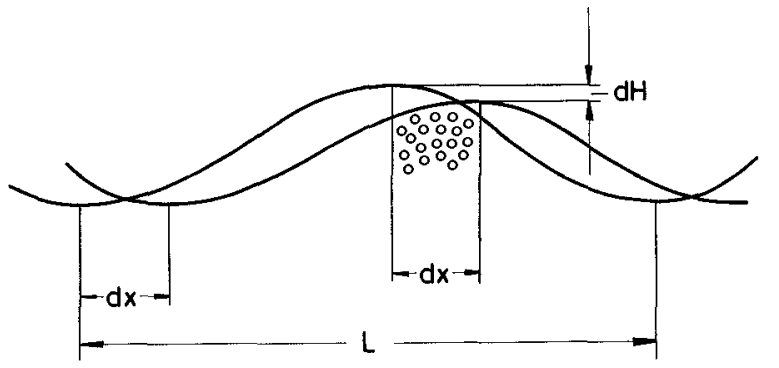

HIG. 2 .

- WAVE REIGHI KEDUC'LION 
Now the reduction of wave helght (Fig. 2) and the obligate loss of energy from Eq. 2 can be put together with Eq. 5 for the energy from aeration effect, with the assumption, that the energy amount $d E$ on the way $d x$ is spent completely for the aeration of a water column of the same length $d x$ and the helght $\mathrm{y}$

$$
\frac{d H}{d x}=-2 \cdot \frac{c}{1-c} \cdot \frac{y^{2}}{H L} \ldots \ldots \ldots \ldots \ldots . .
$$

Ihis is the differential equation for $H=H(x)$, the wave helght inslde the surf zone, for an evaluation, data are necessary for $c$ and 7 , if there is no uniform distribution of the alr bubbles, integrals have to be formed whych represent the static energy of the alr content.

But for a study only on the order of magnitude of aeration effect, for two special cases of breakers some rough assumptions can be made, so for the spllling breaker, that the depth of aeration is in a linear relation to the reduction of wave helght $\mathrm{H}(\mathrm{x})$

$$
\mathrm{y} \propto H(\mathrm{x}) \text { (spilling breaker) }
$$

Then an Integration of Eq. 6 gives with $H(x)=H_{b}$ for $x=0$ (point of breaking)

$$
H(x)=H_{b} \cdot e^{-2 \cdot \frac{c}{1-c} \cdot \frac{x}{3}}
$$

For a plunging breaker, In first approxımation the depth of aeration $y$ can be considered to be of same order of magnitude as the breaker height $\mathrm{H}_{\mathrm{b}}$ and to be constant during the process of breaking.

$$
\mathrm{y} \propto \mathrm{H}_{\mathrm{b}}=\text { const (plunglng breaker) }
$$

Here integration of Eq. 6 guves with $H(x)=H_{b}$ for $x=0$

$$
H(x)=H_{b} \sqrt{1-4 \cdot \frac{c}{1-c} \cdot \frac{x}{L}}
$$


On Fig. 3, the results of Iu. 7 and Eq. \& are presented for alr concentrations between $20 \%$ and $40 \%$ which may be probable for breaknng waves. It 1 s to be seen that for spliling breakers a way of some rave lengths is necessary for the recuction of inital wave helght $\mathrm{H}_{b}$, for the plunglng breaker, however, a part of one wave length Is enough for the total destruction of the wave, in agreement to observations in nature.

From wave theory as well as from the mechanlsm of the formation and the entralnment of the alr bubbles, very many questions are open for the problem. Here only should be pounted out that even a simple model demonstrates clear$1 y$ the importance of the aeration effect for the energy dissipation in surf zones, Eq. $?$ and Eq. 8 together with Fig. 3 show that the aeration effect for Itself is able to explain the high energy loss in breaking waves.
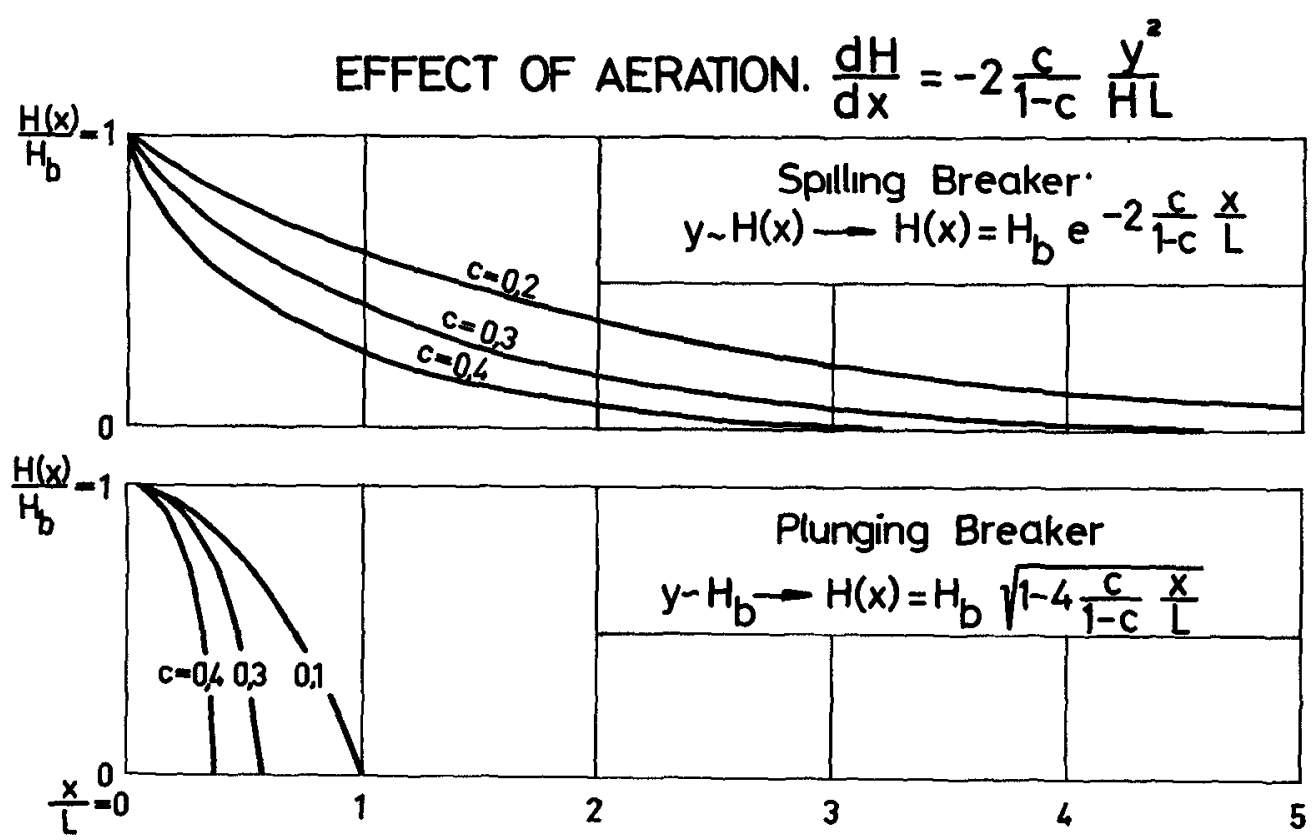

FIG. 3. - WAVE HEIGHT REDUCIITON FOR SFILLING AND ELUNGING BREAKERS 


\section{DISCUSSION AND CONCLUSIONS}

The static energy stored in the alr bubbles afterwards is transformed in turbulent mixing and eddy production curins the uprise of the bubbles to the surface. Wo the turbulence spectrum in the surf zone also is connected with aeration. The amount of alr entralnment, $1 . e$. the concentration and distribution of alr, is nearly unknorn, especlally for laree breakers, but lt seems to be sure that the alr entrainment $1 \mathrm{~s}$ not only a function of HROUDE's and liEYNCLIS' but strongly also from WEBLR's number. Surface tension and caplllary effects wil have influence on the procers of breaking more for small than for large waves, for wave helghts less than about one inch no areation takes place. The interaction between a gas and a fluld is a well krown but very complicated problem even for steaay conditiorsin Processing and Chemical Englneering, for breaking waves the insteady process makes the difficulties increase, this Is valid also for measurement of aly concentration end distribution.

Indirectly, the investigations of ShLALNEV and lorov (ref. 4) give some Ideas about the limits for scale models with breaking waves, they found for the specual case of wave forces on a concrete slope trat there are no scale effects for waves higher than $50 \mathrm{~cm}$, waves from s cm till $120 \mathrm{~cm}$ were used for the test series, the wave nelght of $120 \mathrm{~cm}$ beelng thought as the prototype. For wave hezthts less than $50 \mathrm{~cm}$, the forces scalea up after froudt's law became higher than measured with the prototype wave reight of $120 \mathrm{~cm}$, for waves $3 \mathrm{~cm}$ high more than two tumes. ${ }^{k}, 1 \mathrm{c}$ effect is explained by the authorsexvlicitely by the decreasing alr content. Similar considerations a out the influence of the aix content on imoact forces (chock forces) also are Elver in ref. 1 and $?$. stren the becinnine of oreakine as "vhıte copplng" at the 
wave crests is considered by HASSELMANn (ref. 3) to be a "strong Interaction" compared wIth the "weak Interaction" by bottom friction. Inside the surf zone, the water-alrinteraction must be expected much stronger tnan the waterDottom-interactions.

ThInklng the energy of an Incoming wave at the polnt of breaklng to be $100 \%$, It would be of highest interest to know how much percent of this energy ls spent by wateralr-interaction because only the difference can be effectIve for water-bottorn-lnteractıons, sedıment transport, wave set-up and rip currents, wave run-up a.s.o. Also of high Importance is the question how far scale moaels are able to reproduce these processes, when the aeration effect is reduced by suriace tension, the energy for the other effects mentioned above becomes hicher than In prototype. So all problems of litoral processes are Influenced by the aeration problem.

The production of aIr-water-mixtures by the different sizes and sorts of breakers therefore seems to be a first order problem for the physical process in surf zones. Measurements of alr concentrations and bubble distributions are necessary together with wave helght measurements in order to get a knowledge how much of the energy dissipation of a breaker is represented by the aeration effect given by E. 6 .

\section{REFERENCES}

1. FUURROTLR, A. Der Druckschlag durch Brecher auf Delchboschuncen, Mitteslungen des Franzıus-Instıtuts der Technıschen Universitat Hannover, Heft 28, 1966.

2. HUHRBOTER, A. Iaboratory Investzgatıon of Impact Forces, Haper No. 6, Symposzum Research on Wave Action, Delft, March 24-28, 1969. 
3. ILASSELMANN, $k$. Weak Interaction Theory of Ocean Waves, Bascc Developments in Fluzd Mechanics, Vol. 2, Academıc Press Inc., New York 1968.

4. SKLADNEV, M.F. and FOPOV, I.Ya.. Studzes of Wave Ioads on Concrete slope Protections of Earth Dams, Paper Nr. 7, Symposium on Wave Action, Delft March 24 - 28. 1969. 


\title{
CHAPTER 25
}

\author{
PROBABILITIES OF BREAKING WAVE CHARACTERISTICS \\ $J$ Ian Collins \\ Tetra Tech Inc \\ Pasadena California
}

\section{ABSTRACT}

Utılizıng the hydrodynamic relationships for shoaling and refraction of waves approaching a shoreline over parallel bottom contours a procedure is developed to transform an arbitrary probability density of wave characteristics in deep water into the corresponding breaking characteristics in shallow water A number of probability distributions for breaking wave characteristics are derived in terms of assumed deep water probability densities of wave heights wave lengths and angles of approach Some probability densities for wave heights at specific locations in the surf zone are computed for a Rayleigh distribution in deep water The probability computations are used to derive the expectation of energy flux and its distribution

\section{INTRODUCTION}

Many experimental investigations of breaking waves longshore currents and littoral drift have been made Sometimes the results have been expressed in terms of deep water wave characteristics and sometimes in terms of breaking wave characteristics making comparisons of varıous data a difficult task In some instances the wave characteristics at some intermediate water depth are given The laws of hydrodynamics can be used to relate deep and shallow water characteristics if bottom friction effects are neglected

Earlier theoretical work of LeMéhauté (1961) LeMéhauté and Webb (1964) and LeMehaute and Koh (1967) have proposed methods of computing shallow water wave characteristics in terms of deep water wave characteristics for periodic waves using first third and fifth order Stokian wave theories

It is well known that naturally occurring sea conditions can be characterized only in statistical terms Such terms include the spectrum the average wave height significant wave height mean wave period etc The sea state in deep water can be characterized by a probability distribution of wave heights wave lengths and angles of approach to the shore

The reason for the choice of characteristics in terms of probability distributions rather than the spectrum is purely for convenience in considering the behavor of waves in the surf zone as individual crests Relationships between spectra and probability of wave height and wave period have been demonstrated by Longuet-Higgins (1952 1957) Bretschnerder (1959) Collins (1967) and others

The usual method of treating a wave traveling in gradually varying water depths has been to use the wave theories for a horizontal bed and to account for the effects of bottom variation by considering energy conservation Biesel (1951) and many others and more recently Mer Tlapa and Eagleson (1968) have proposed theoretical approaches to wave propagation over variable depth The traditional method of treating waves over gradually varying bottom topography will be adopted It will also be assumed that the linear wave theory (first order) can be applied up to the point of breaking Non-linear effects (peak-up effect) which are important near breaking are, to some extent empirically taken into account in the breaking criteria 
In this paper an attempt has been made to apply the hydrodynamic theories of shoaling, refraction and wave breaking to statistical models of the sea Examples of computations are given for a plane beach Generally the wave characteristics in deep water are assumed and the corresponding characteristics in shallow water including the surf zone are computed

One-dimensional (wave height wave length or wave direction) and two-dim. ensional (wave height and wave length) probability distributions are given The general solution for a three-dimensional probability distribution is derived for two different wave breaking criteria

The results of the computations of wave probabilities in shallow water are used to compute expected values of energy flus in the surf zone as a function of depth of water A similar approach to determine longshore current dis tribution is proposed

GENERAL DISCUSSION

\section{Governing Fquations of Wave Transformation}

The dispersion relationship for waves is written

$$
L_{b} / L_{o}=\tanh 2 \pi d_{b} / L_{b}
$$

where the subscript b refers to conditions at breaking the subscript o refers to conditions in deep water (see figure l) $L$ is the wave length dis the water depth

Snell's law can be used

$$
L_{o} / L_{b}=\sin \alpha_{o} / \sin \alpha_{b}
$$

where $d$ is the angle of the wave crest with the shoreline

The choice of a suitable breaking criterion is required Experimental investigations on this phenomenon in two-dimensional wave tanks have been made notably by Iversen (1952) Hamada (1963) and Suquet (1950) The following criterion fits the data very closely (after LeMehauté and Koh 1967)

$$
\mathrm{H}_{\mathrm{b}} / \mathrm{H}_{\mathrm{o}}=076 \mathrm{~S}^{\mathrm{I} / 7}\left(\mathrm{H}_{\mathrm{o}} / \mathrm{L}_{\mathrm{o}}\right)^{-\frac{1}{4}}
$$

where $\mathrm{S}$ is the bottom slope and $\mathrm{H}$ is the wave height For waves breaking at an angle the bottom slope is actually $S \cos \alpha_{b}$ and $H_{o}$ should be replaced by $\mathrm{H}_{0} \cos ^{\frac{1}{2}} \alpha_{0}$ The breaking criterion becomes

$$
\mathrm{H}_{\mathrm{b}} / \mathrm{H}_{\mathrm{o}}=076 \mathrm{~S}^{1 / 7} \cos ^{1 / 7} \alpha_{b}\left(\mathrm{H}_{\mathrm{o}} / \mathrm{L}_{\mathrm{o}}\right)^{-\frac{1}{4}} \cos ^{3 / 8} \alpha_{\mathrm{o}}
$$

where $S_{1 s}$ the slope at breaking An alternate breaking criterion was also used

$$
H_{b} / d_{b}=072+56 \mathrm{~S}
$$

if bottom friction effects are neglected then the wave height change up to breaking can be computed from the conservation of energy as $\mathrm{H}_{\mathrm{b}}=\mathrm{K}_{\mathrm{r}} \mathrm{K}_{\mathrm{s}} \mathrm{H}_{\mathrm{O}}$ which over a beach with parallel bottom contours will be used in the form $s$ o
\[ H_{b} / H_{0}=\left\{\tanh k_{b} d_{b} l+2 k_{b} d_{b} / \sinh 2 k_{b} d_{b} \cos a_{b} / \cos x_{o}\right. \]

where

$$
k_{b}=2 \pi / L_{b}
$$

Equations 1,2 5 and 3 or 4 provide a system of equations such that a given deep water wave characterized by $H_{0} L_{0} \alpha_{0}$ yields a unique breaking wave 
BREAKING WAVE
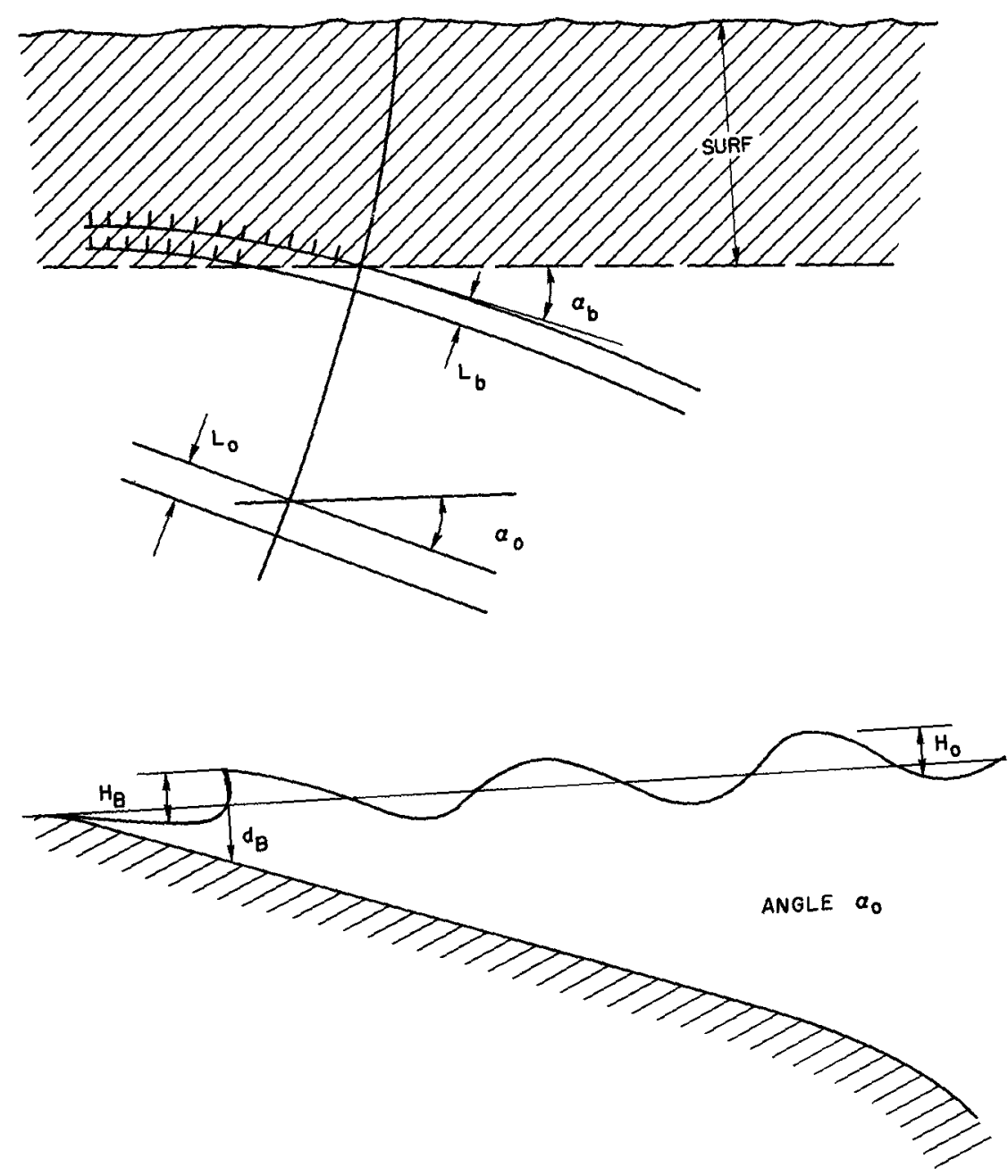

Illustration of Nomenclature Used

In Wave Transformation 
characterized by $H_{b} \quad L_{b} \quad \alpha$ In fact the solution of these equations for the breaking characteristics requires a step-by-step or iterative procedure since the dependent variables cannot be separated

Breaking Waves

The methods of the preceding section yield the information $H_{b} \quad L_{b} \quad \alpha_{b} \quad d_{b}$ for any specified deep water values $\mathrm{H}_{0} \mathrm{~L}_{\mathrm{o}} \alpha_{0}$ Another problem of interest can be stated given $H_{0} L_{o} \alpha_{o}$ - what is $H \quad L \quad \alpha$ at a specified location $d=d_{c}$ ? Three separate conditions must be recognized
(a) $d_{c}>d_{b}$
(b) $d_{c}<d_{b}$
(c) $d_{c}=d_{b}$

When $d_{c}$ is greater that $d_{b}$ Eqs 12 and 5 can be applied directly to compute $\mathrm{H}_{c} \mathrm{~L}_{\mathrm{c}} \alpha_{\mathrm{c}}$ The case $\mathrm{d}_{c}=\mathrm{d}_{\mathrm{b}}$ can also be included When $\mathrm{d}_{c}$ is less than $\mathrm{d}_{\mathrm{b}}$ the wave has broken farther offshore and some of its energy has been dissipated The decay of wave height after breaking has been studied by Horikawa and Kuo (1966) Street and Camfield (1966) Nakamura Shiraishi and Sasaki (1966) and Divoky LeMéhaute and Lin (1969) The experimental results are summarized in Figure 2 (after Divoky et al 1969) For purposes of the present study, the wave height at $d=d_{c}$ after breaking

$$
\mathrm{H}_{\mathbf{c}}=\mathrm{H}_{\mathrm{b}} \mathrm{d}_{\mathbf{c}} / \mathrm{d}_{\mathrm{b}}
$$

1 e a linear wave height decay from the breaking point to the shore

The angle and wave length of the broken wave at $d=d_{c}$ were computed from the refraction laws for long waves

$$
\begin{aligned}
& L_{c}=L_{b}\left(d_{c} / \alpha_{b}\right)^{\frac{1}{2}} \\
& \sin \alpha_{c}=\sin \alpha_{b}\left(d_{c} / d_{b}\right)^{\frac{1}{2}}
\end{aligned}
$$

Probability Distributions

Assuming that the statistical properties of the deep water wave characteristics are known the system of equations 2 through 5 will enable the determination of the statistical properties of shallow water waves up to breaking The problem can be stated given $p\left(H_{o} L_{o} \alpha_{o}\right)$ determine $p\left(H_{b} L_{b} a_{b} d_{b}\right)$ subject to the relationships of equations 1 through 5 At breaking only three conditions need to be specified (The fourth can be found in terms of the other three) The general solution is,

$$
p\left(H_{b}, L_{b} a_{b}\right)=p\left(H_{0} L_{o} a_{o}\right)|J|^{-1}
$$

where

$$
|J|=\left|\begin{array}{llll}
\partial H_{b} / \partial I_{o} & \partial H_{b} / \partial L_{o}, \partial H_{b} / \partial \alpha_{o} \\
\partial L_{b} / \partial H_{o} & \partial L_{b} / \partial L_{o} & \partial L_{b} / \partial \alpha_{o} \\
\partial \alpha_{b} / \partial H_{o} & \partial \alpha_{b} / \partial L_{o} & \partial \alpha_{b} / \partial \alpha_{o}
\end{array}\right|
$$

The partial derivities have to be determined from equations 2 through 5 Generally it is seen that if equations 2 through 5 are differentiated by each variable $\mathrm{H}_{0} L_{o} \alpha_{0}$ one at a time a system of 12 simultaneous equations are derived which have to be solved for the partial derivatives before substitution into 


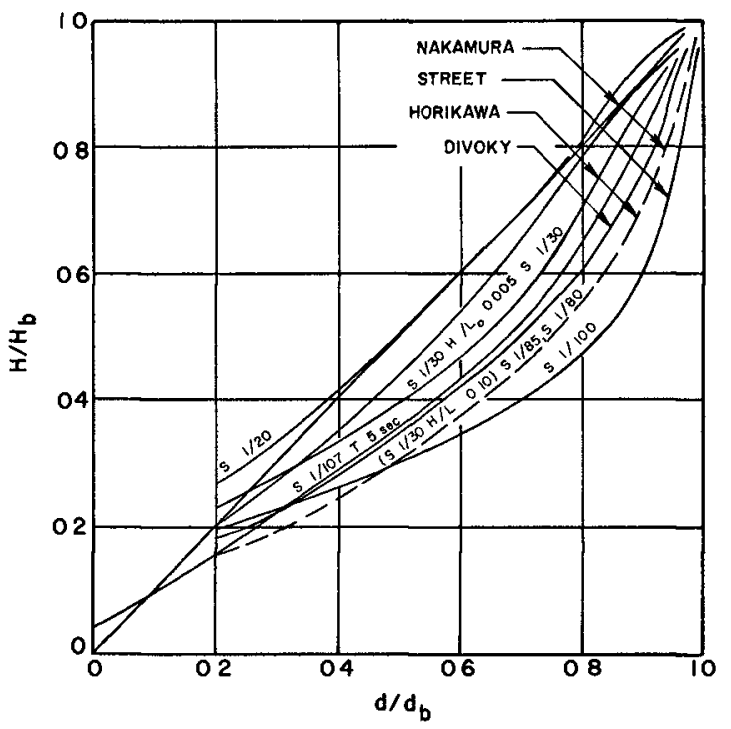

Figure 2 Summary of Experimental Data on Wave Height Decay After Breaking

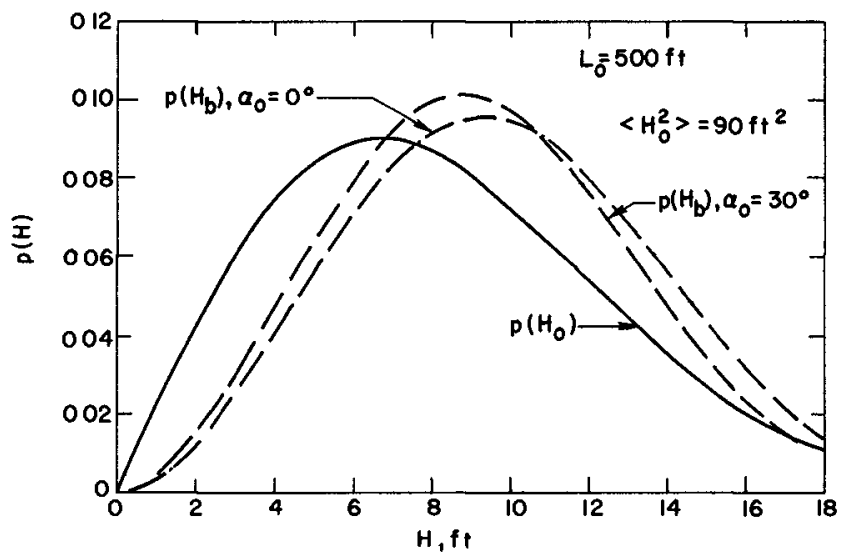

Figure 3 Probability Densities of Breaking Waves Height for Two Angles of Appraach in Deep Water 
equation 10 The entire procedure is straightforward (but tedious) unless $J$ contains zeros Special consideration must be given to regions where J changes sign

\section{SPECIFIC APPLICATIONS}

\section{One-Dimensional Probability Distribution}

Assuming that the wave characteristics are specified in deep water such that

$$
L_{0}=\text { constant } \quad \alpha_{0}=\text { constant }
$$

$\mathrm{H}_{\mathrm{o}}$ has the probability distribution

$$
\begin{aligned}
& \left.\left.\mathrm{p}\left(\mathrm{H}_{\mathrm{o}}\right)=2 \mathrm{H}_{\mathrm{o}} /<\mathrm{H}_{0}^{2}\right\rangle \exp \left[-\mathrm{H}_{0}^{2} /<\mathrm{H}_{0}^{2}\right\rangle\right] \\
& <>\text { denotes mean value }
\end{aligned}
$$

then

$$
\begin{aligned}
& p\left(\mathrm{H}_{b}\right)=p\left(H_{0}\right) \partial H_{o} / \partial H_{b} \\
& p\left(L_{b}\right)=p\left(H_{0}\right) \partial H_{o} / \partial L_{b} \\
& p\left(\alpha_{b}\right)=p\left(H_{0}\right) \partial H_{o} / \partial \alpha_{b}
\end{aligned}
$$

The values of the differentials can be determined analytically or they can be calculated numerically by finite difference approximations

Examples of some sample cases computed for one-dimensional deep water probabilities are given as Figures 3 through 5 The probabilities shown are computed for breaking characteristics at whatever location they occur Figure 3 illustrates the effect on the probability of breaking wave heights for different angles of approach in deep water This probability is not too sensitive to the angle of approach

Figure 4 indicates the effect of various deep water wave lengths on the probability distribution of breaking wave heights for a deep water angle of approach of $30^{\circ}$ As expected it is seen that increased deep water wave lengths lead to larger breaking wave heights The probability distributions are shifted toward higher values of waveheight Figure $b$ shows the effect of increased wave length in deep water on the angles of breaking waves for a Rayleigh-type deep water wave height distribution Once more as anticipated larger wave lengths tend to refract more and break more nearly parallel to the shore

Examples of computations for the probability densities of wave characteristics at a specific location are given as Figures 6 and 7 These two figures differ from figures 3 through 5 in that they present wave height probabilities at a fixed location Both broken and non-breaking waves are present All of the figures show a sharp rise in the probability density of wave heights at the breaking limit

\section{Two-Dimensional Probability Distributions}

The two-dimensional probability of breaking characteristics indicate the joint probability of breaking wave height and wave length Complete numerical computations have not been made since in this case the roots of the Jacobian must first be investigated One simplification which permitted numerical results to be obtained follows from the assumption that wave breaking occurs 


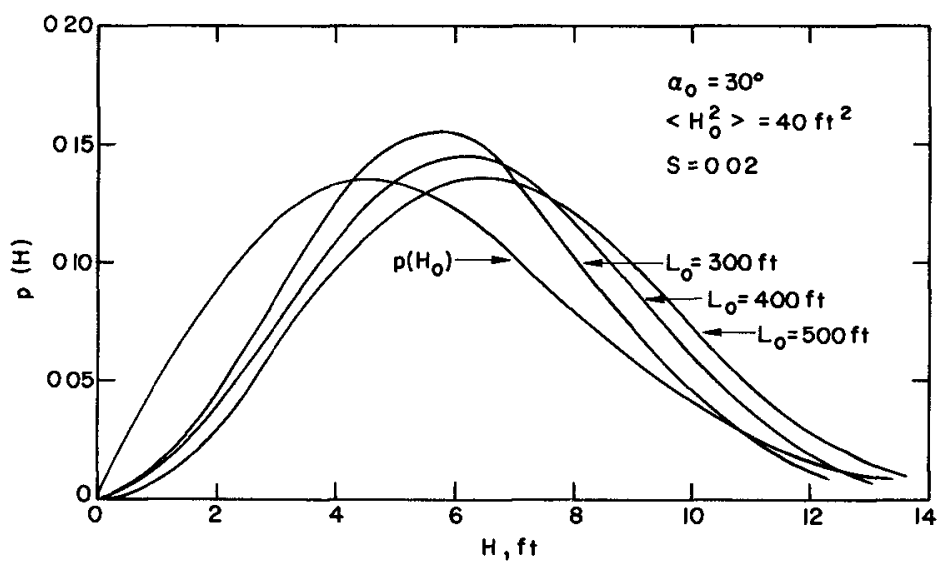

Figure 4 Probability Densities of Breaking Wave Heights for Varıous Deep Water Wave Lengths

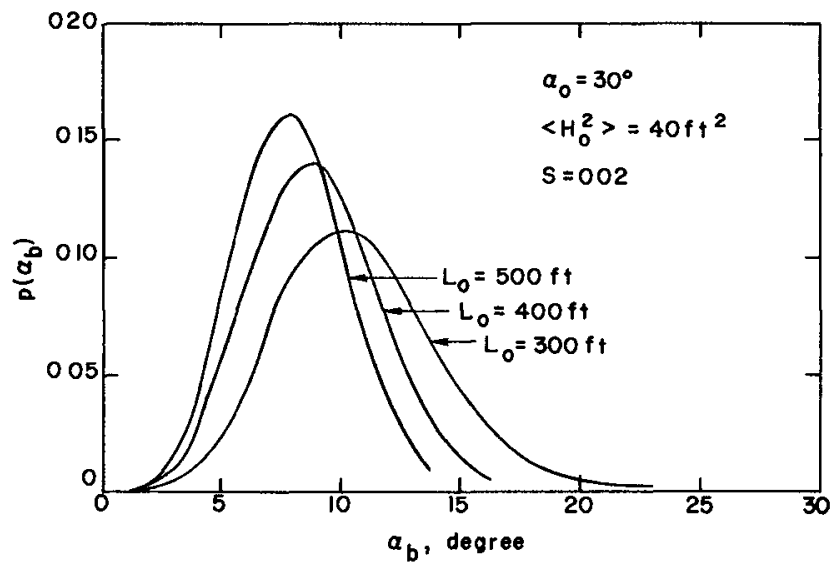

Figure 5 Probability Densities of Breaking Wave Angles for Varrous Deep Water Wave Lengths 


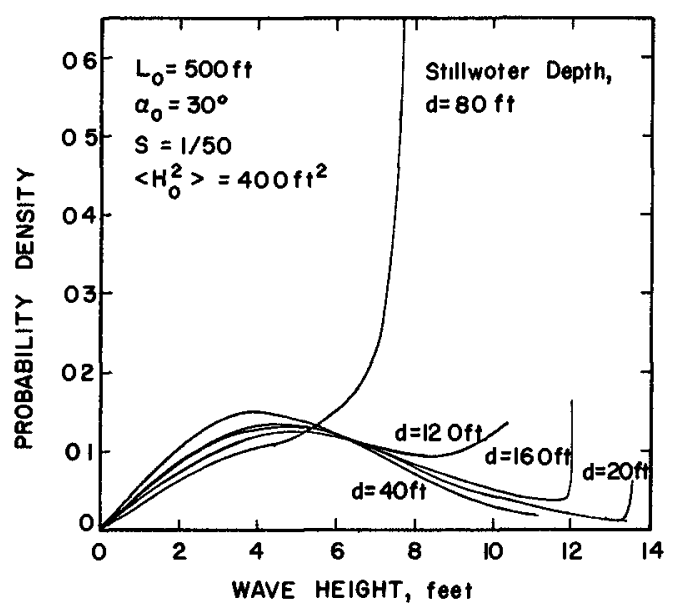

Figure 6 Probability Densities of Wave Heights at Specific Locations for $L_{0}=500 \mathrm{ft}$ $\alpha_{0}=30^{\circ} s=1 / 50$

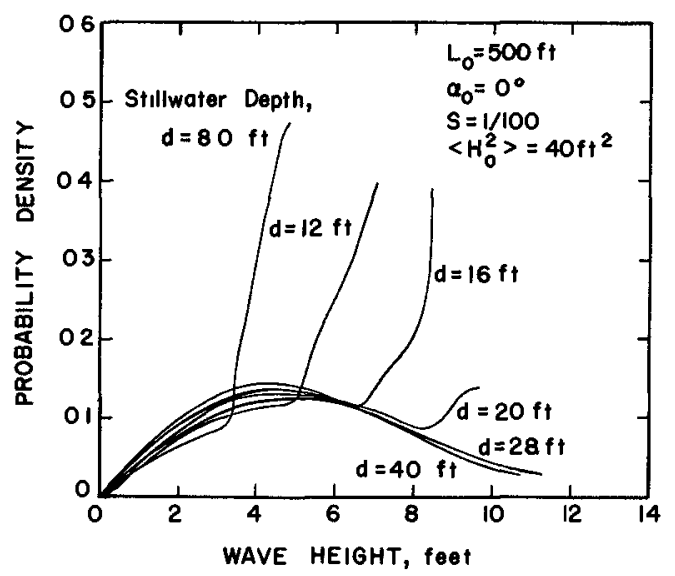

Figure 7 Probability Densilies of Wave Heights at Specific Locations for $L_{0}=500 \mathrm{ft} \quad \alpha_{0}=0^{\circ}$ $S=1 / 100$ 
in shallow water only, 1 e $d / L$ at breaking is small Then 1 follows that equation 5 becomes

$$
\mathrm{H}_{b} / \mathrm{H}_{0} \simeq\left[\begin{array}{ll}
\cos \alpha_{o} / \cos \alpha_{b} & 1 / 2 k_{d} q_{b}
\end{array}\right]^{\frac{1}{2}}
$$

equation 1 becomes

$$
\mathrm{L}_{b} / \mathrm{L}_{\mathrm{o}} \sim \mathrm{k}_{\mathrm{b}} \mathrm{d}_{\mathrm{b}}
$$

A comparison between equation 15 and equation 5 when $\alpha=0$ s shown as Figure 8 It is seen that the differences are very small when d/L is less than $0 \quad 05$

Substitution of equations 16 and 2 into equation 15 yields the interesting result

$$
\mathrm{H}_{b} / \mathrm{H}_{0}=05\left[\sin 2 \alpha_{o} / \sin 2 \alpha_{b}\right]^{\frac{1}{2}}
$$

which has the limit $\mathrm{H}_{b} / \mathrm{H}_{0}=\left[\mathrm{L}_{0} / \mathrm{L}_{b}\right]^{\frac{1}{2}}$ when $a_{o}=0$ as might be expected

The corresponding Jacobian is

$$
\begin{gathered}
J=\frac{\partial\left(\mathrm{H}_{o} L_{o}\right)}{\partial\left(\mathrm{H}_{b}\right.}=\left\{\frac{\left.L_{b}\right)}{L_{o}}-\frac{\cos \alpha_{b}}{\sin \alpha_{0}} \frac{\sin 2 \alpha_{o}}{\cos 2 \alpha_{b}}\right. \\
\left.-\frac{1}{6 A^{2}}\left(\frac{H_{o}}{L_{o}}\right)^{\frac{1}{2}}\right\} \frac{3 A}{4}\left(\frac{H_{o}}{L_{o}}\right)^{-\frac{1}{4}} \cos ^{3 / 8} \alpha_{o}
\end{gathered}
$$

where $A=076 \mathrm{~S}^{1 / 7}$

Figure 9 shows an assumed deep water probability distribution $p\left(H_{0} L_{o}\right)$ corresponding to a joint Rayleigh distribution with zero correlation between wave height and wave period (Bretschneider 1959) The corresponding breaking wave characteristics $p\left(H_{b} \quad L_{b}\right)=p\left(H_{0} L_{o}\right)|J|^{-1}$ are shown for $\alpha_{0}=0$ and $\alpha_{0}=30^{\circ}$ as Figures 10 and 11 for the breaking condition of equation 3 Figure 12 presents $\mathrm{p}\left(\mathrm{H}_{b} \mathrm{~L}_{b}\right)$ for the depth limited breaking criterion

It is seen from Figures 10 and 11 that the effect of the deep water angle of approach on breaking wave heights and lengths is relatively minor A comparison of Figure 10 with 12 shows that the breaking wave steepness is very sensitive to the choice of breaking criteria

Figure 13 compares the probability density of wave height determined from the one-dimensional computations and the marginal distribution for the twodimensional distributions using two breaking criteria 


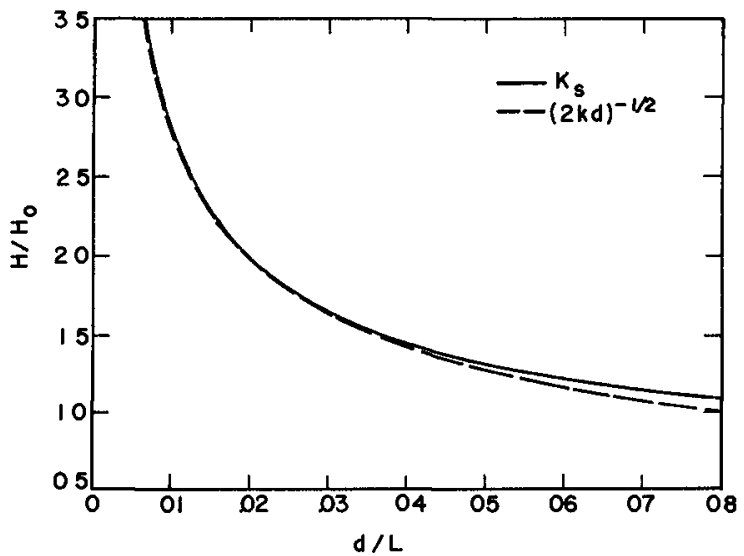

Figure 8 Comparison of Shoaling Coefficients Given by Equation 5 and Equation 17

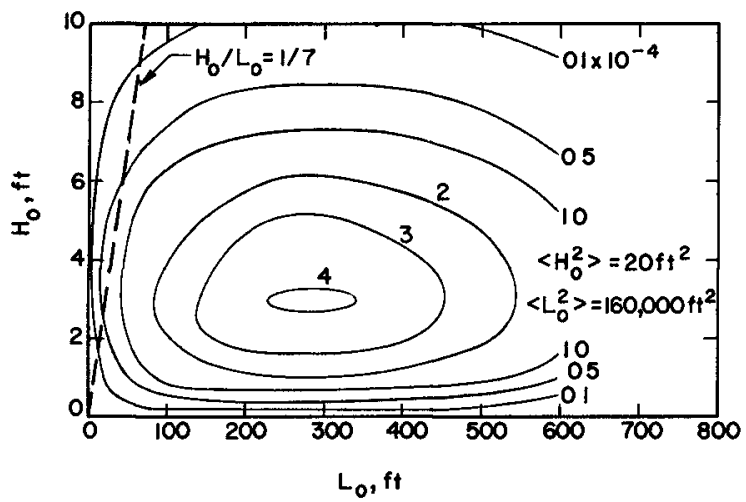

Figure 9 Two Dimension Probability Density of Deep Water Length and Wave Height for Joint Uncorrelated Raleigh Distribution 


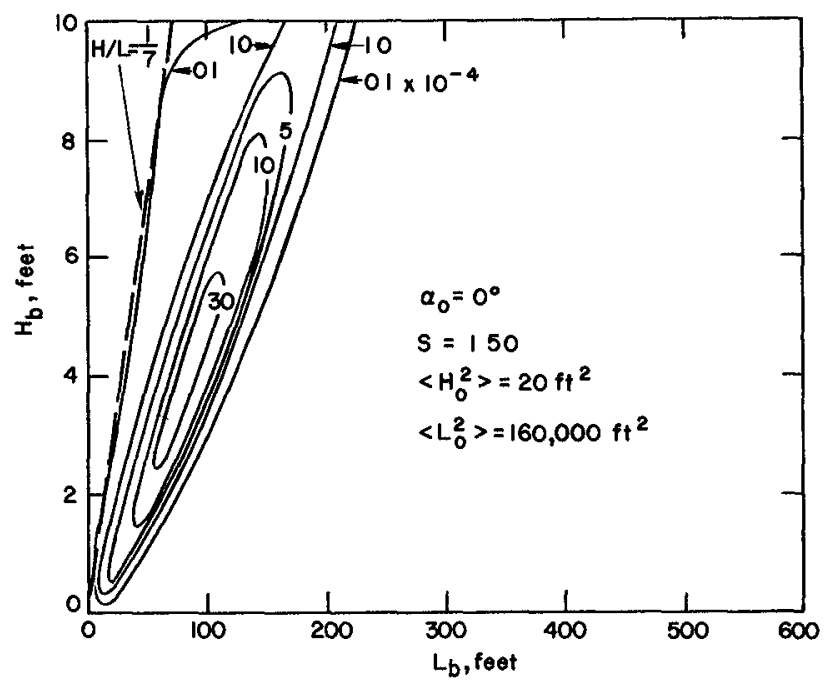

Figure 10 Two Dimensional Probability Density of Breaking Wave Characteristics for $\alpha_{0}=0$

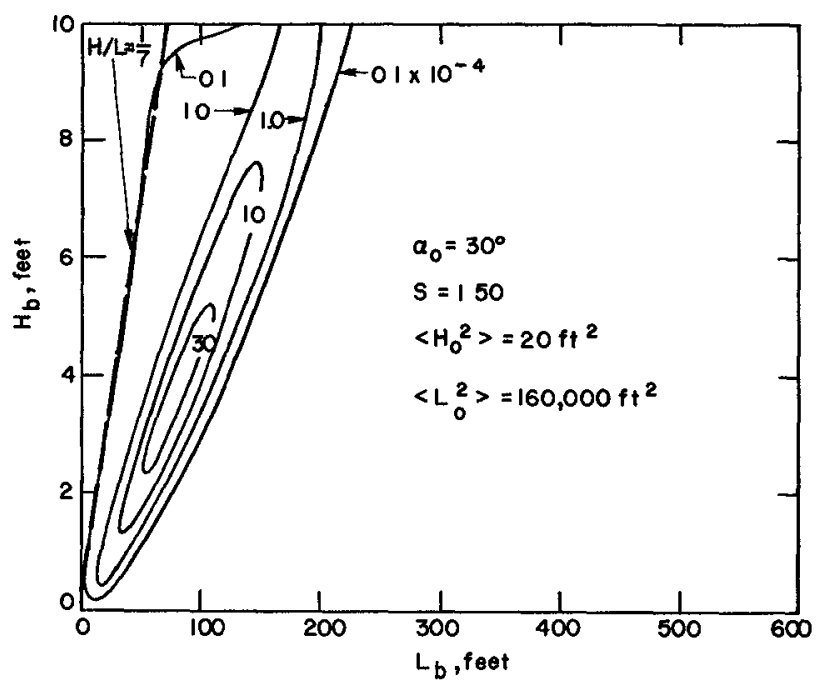

Figure 11 Two Dimensional Probability Density of

Breaking Wave Characteristics for $\alpha_{0}-30^{\circ}$ 


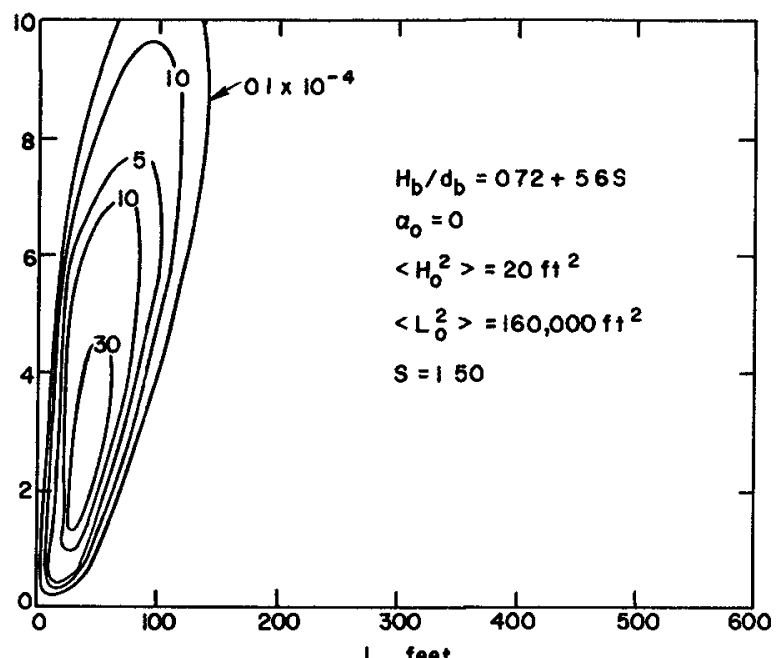

Figure 12 Two Dimensional Probability Density of Breaking Wave Characteristics for $\alpha=0$ and Depth Limited Breaking Criterion

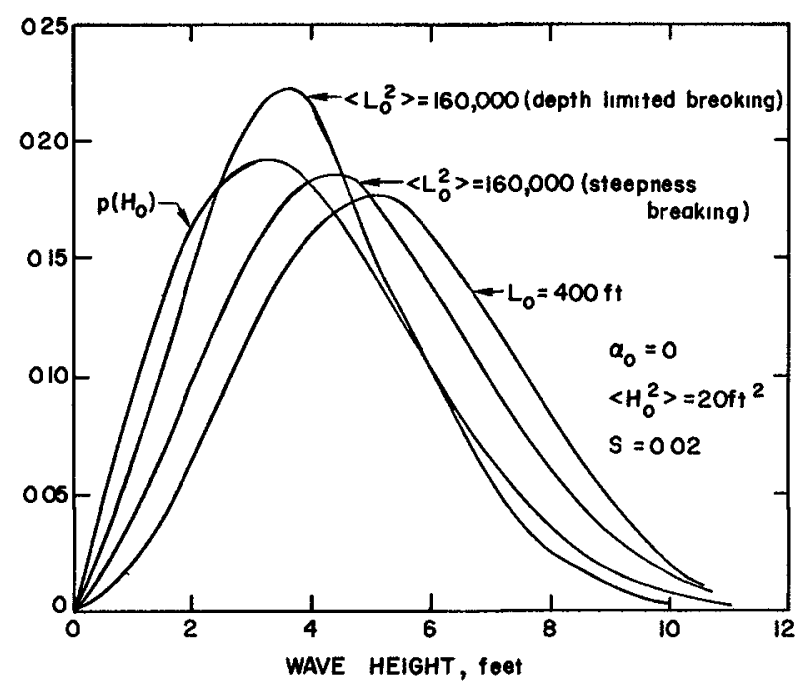

Figure 13 A Comparison of Marginal Distributions of Breaking Wave Heights from Two Dimensional Probability Densities with the Results for a One Dimensional Computation 
LONGSHORE CURRENT AND ENERGY FLUX FOR RANDOM WAVES IN THE SURF ZONE

The mean value of a function of $H \quad L$ and $\alpha 1 \mathrm{n}$ a random sea can be derived from the equation for uniform periodic waves following a well known theorem of probability given a function of $f(x, y)$ in which the probability distributions of $x$ and $y$ are known, the mean value of the function $f(x y)$ is given by

$$
\left\langle\mathrm{f}(\mathrm{x} y)=\iint \mathrm{f}(\mathrm{x} y) \mathrm{p}(\mathrm{x}, \mathrm{y}) \mathrm{dx} \mathrm{dy}\right.
$$

where $\mathrm{p}(\mathrm{x} y)$ is the joint probability density of $\mathrm{x}$ and $\mathrm{y}$ Hence a longshore current equation derived for uniform periodic wavesi e $V_{L}=V_{L}(H, L \alpha)$ can be applied to random waves if the random values of $H$ L and $\alpha$ are weighted correctly

The mean value of $V_{L}$ in a random sea is given by

$$
<\mathrm{V}_{L}>-\iint J \mathrm{~V}_{\mathrm{L}}(\mathrm{H}, \mathrm{L} \quad \alpha) \mathrm{p}(\mathrm{H} \quad \mathrm{L} \quad \alpha) \mathrm{dH} \mathrm{d} L \mathrm{~d} \alpha
$$

which can be considered as the result of superimposing all of the wave heights wave lengths and angles which are present and weighting the effect of each one by its probability of occurrence

A set of sample calculations for longshore and onshore energy flux has been worked out The mean longshore energy flux is given by

$$
\begin{aligned}
& \frac{1}{\rho g}<F> \\
& =\iiint \frac{1}{8} \sqrt{\frac{g}{2 \pi}} \frac{\mathrm{H}^{2} \mathrm{Ln}}{\sqrt{\mathrm{L}_{\mathrm{o}}}} \cos \alpha \mathrm{p}(\mathrm{H} \quad \mathrm{L} \alpha) \text { d H d L d } \alpha
\end{aligned}
$$

non-breaking

$$
+\iiint \frac{8}{3 \sqrt{3}} \sqrt{\frac{g}{2 \pi}}\left[\frac{(H, d)^{3}}{L_{o}}\right]^{\frac{1}{2}} \cos \alpha p(H \quad L, \alpha) d H d L d \alpha
$$

breaking

since

$$
\Gamma=\sqrt{\frac{2 \pi}{g}} \sqrt{L_{0}}
$$

Also by definition $p\left(\begin{array}{lll}H & L\end{array}\right) \mathrm{d} H \mathrm{H} L \mathrm{~d} \alpha \equiv \mathrm{p}\left(\mathrm{H}_{0}, \mathrm{~L}_{0} \alpha_{0}\right) \mathrm{dH} \mathrm{d} \mathrm{L}_{\mathrm{o}} \mathrm{d} \alpha$ o and the integrals can be approximated by summation

$$
\begin{aligned}
& \frac{1}{\rho \mathrm{g}}\langle\mathrm{F}\rangle \mathrm{L} \\
& =\frac{1}{8} \sqrt{\frac{\mathrm{g}}{2 \pi}} \sum\left\{\frac{\mathrm{H}^{2} \mathrm{~L}}{\sqrt{\mathrm{L}}} \cos \alpha \mathrm{p}\left(\mathrm{H}_{\mathrm{o}}, \mathrm{L}_{\mathrm{o}}, \alpha_{\mathrm{o}}\right) \Delta \mathrm{H}_{\mathrm{o}} \Delta \mathrm{L}_{\mathrm{o}} \wedge \alpha_{\mathrm{o}}\right\} \\
& \text { non-breaking } \\
& +\frac{8}{3} \sqrt{\frac{\mathrm{g}}{6 \pi}} \sum\left\{\left[\frac{(\mathrm{H} D)^{3}}{\mathrm{~L}_{\mathrm{o}}}\right]^{\frac{1}{2}} \cos \alpha \mathrm{p}\left(\mathrm{H}_{\mathrm{o}} \mathrm{L}_{\mathrm{o}}, \alpha_{\mathrm{o}}\right) \Delta \mathrm{H}_{\mathrm{o}} \wedge \mathrm{L}_{\mathrm{o}} \wedge \alpha_{\mathrm{o}}\right\} \\
& \text { breaking }
\end{aligned}
$$


The mean onshore energy flux is defined with sin $\alpha$ replacing cos a in equation 21 An example of the computations using equation 21 is given as Figure 14 Figure 14 illustrates the variation of onshore energy flux in the surf zone The variation of longshore energy flux is similar but absolute values of flux are smaller In this figure the deep water wave length and angle of approach were taken as constant and the deep water waves were characterized by a probability distribution of wave heights

\section{REFER ENCES}

Biesel F (1952) "Study of Wave Propagation in Water of Gradually Varying Depth" in Gravity Waves U $S$ Department of Commerce $N$ B S Circular 521

Bretschneider C L (1959) "Wave Varlability and Wave Spectra for Wind-Generated Gravity Waves" Technical Memorandum No 118 Beach Erosion Board August

Collins, J I (1967) "Wave Statistics from Hurricane Dora", Journal Waterways and Harbors Division A S C E May

Divoky D LeMéhauté and Lin A (1969) "Breaking Waves on Gentle Slopes", A S C E Annual Meeting New Orleans Lousiana February

Hamada T (1963) "Breakers and Beach Erosion" Port and Harbor Technical Research Institute Ministry of Transportation Japan

Horikawa K and Kuo, C T (1966) "A Study on Wave Transformation Inside Surf Zone" Proc l0th Conference Coastal Engineering A S C E Vol 1 Iverson H W (1952) "Laboratory Study of Breakers" in Gravity Waves U S Department of Commerce $N$ B S Circular 521

LeMehauté B (1961) "Theoretical Study of Wave Breaking at an Angle with a Shoreline" Journal of Geophysical Research February

LeMéhauté, B and Webb L (1964) "Periodic Giavity Waves Over a Gentle Slope at a Third Order of Approximatıon" Proc 9th Conference on Coastal Engineering, Lis bon

Miche R (1944) "Mouvemencs Ondulatorres de la Mer en Profondeur Constante ou Decroissante" Ann des Pouts et Chaus

LeMéhauté B and Koh, R C Y (1967) "On the Breaking of Waves Arriving at an Angle to the Shore" Journal of Hydraulic Research, Vol 5 No 1

Longuet-Higgins M S (1952) "On the Statistical Distribution of the Heights of Sea Waves", Journal of Marine Research, Vol 11 No 3

Longuet-Higgins M S (1957) "The Statistical Analysis of a Random Moving Surface" Phil Trans Royal Soc of London A-966

Me1, C C Tlapa G A and Eagleson, P S (1968) "An Asymptotic Theory for Water Waves on Beaches of Mild Slope", Journal of Geophysical Research Vol 73 No 14 July

Nakamura M , Shirouish1, H and Sasakı Y (1966) "Wave Decayıng Due to Breaking" Proc 10th Conference Coastal Engineering A S C E Vol I Street,R L and Camfueld, F E (1966) "Observations and Experiments on Solitary Wave Deformation", Proc l0th Conf Coastal Engineering A S E $E$ Vol 1

Suquet F (1959) "Experimental Study on the Breaking of Waves", La Hourlle Blanche No 3 May-June 


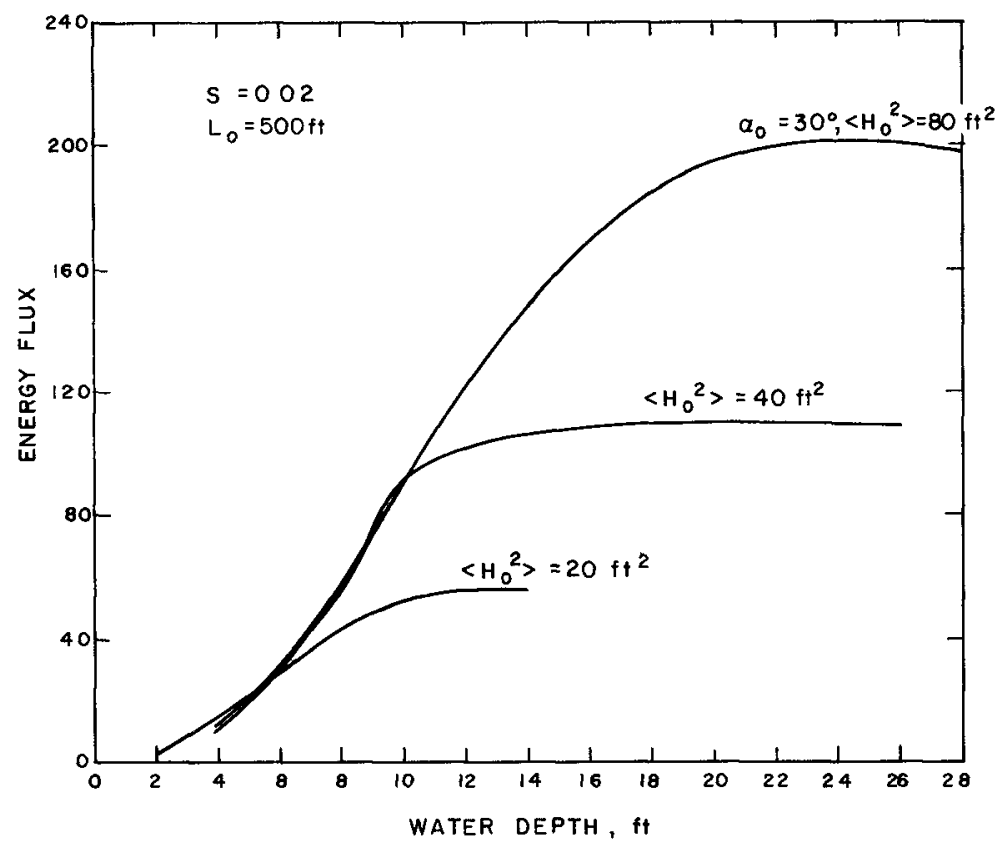

(a) Total Flux

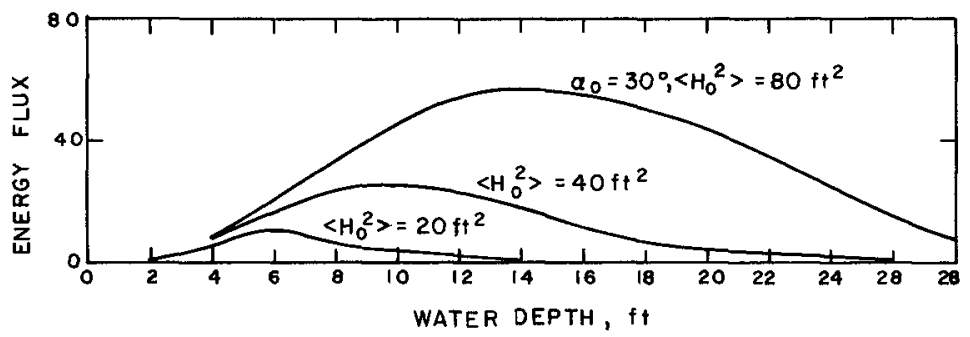

(b) Breakıng Flux

Figure 14 Onshore Energy Flux as a Function of Water Depth for Various Mean Square Wave Heights 


\section{ACKNOWLEDGMENT}

This work was originally performed for the Office of Naval Research Geography Branch under contract number N 00014-69-C-0107 


\title{
CHAPTER 26
}

CHARACTERISTICS OF WAVES BROKEN BY A LONGSHORE BAR

\author{
By \\ E Clark MCNair, Jr ${ }^{1}$ and Robert $M$ Sorensen ${ }^{2}$
}

ABSTRACT

A two-dimensional model submerged offshore bar was installed in a Texas A\&M Hydrodynamics Laboratory wave tank Monochromatic waves w7th a range of heights and periods were generated at this bar for three different depths of water over the bar For each wave, water surface time-histories were measured at points before and after the bar and spectral analyses of these measurements were performed

The analysis of each wave record yielded an equivalent wave height which is proportional to the square root of the wave energy per unit surface area The ratio of the reformed to incident equivalent wave helght is shown to relate to the ratio of incident wave height to water depth over the bar The predominant periods of the reformed waves are found to be the same as for the incident waves but the presence of energy at higher frequencies is also observed The cause of these higher frequency waves is discussed

\section{INTRODUCTION}

During parts of the year, generally the winter months, intense storms at sea generate waves that are quite steep These waves, upon reaching the shore, w1 11 erode material from the beaches and deposit this material some distance from the shoreline Continued deposition of material results in the formation of a lonqshore bar A typical bar formation will have a rather gently sloping face toward the sea, a rounded crest, and a steep slope downward into a trough in the lee of the crest These bar formations are generaliy of transient nature in shape and location

After a wave breaks over the bar and reforms, questions arise concerning the characteristics and energy of the resulting wave form, particularly if expensive structures are to be placed where the wave can act on them or $1 \mathrm{f}$ the shore is composed of material which can be carried away by the waves The object of the research described herein was to define some of the characteristics, particularly the heights and periods, of waves which have broken while passing a longshore bar and have reformed in the lee of the bar

1 Research Hydraulic Engineer, USAE Waterways Experiment Station, Vicksburg, Mississippi

2 Assoc Prof of $\mathrm{C} 1 \mathrm{v} 11$ Engineerıng, Texas A\&M University, Colleqe Station, Texas 


\section{REVIEW OF THE LITERATURE}

Invest 1 gat ions concerning breaking waves have been conducted by Ivers on (7), Nakamura ( 9 ), and others $(4,5)$ However, the beach slopes used in those studies were such that the water depths never increased shoreward of the breaking point of the waves The work of Ivers on showed that if the beach slope and the deepwater wave steepness are known, several characteristics of the breaking wave, including the height of the wave and the water depth at the breaking point, can be predicted

Investigations have also been performed $w 7$ th submerged rectangular breakwaters where waves approach the breakwater in fairly deep water, peak up and break over the relatively shallow structure, and then reform in the deeper water in the lee of the structure Tests conducted by Dick (1) with such breakwaters indicated that as a wave passed over the structure and broke, it was reduced to a fundamental wave whose period was the same as the 1 ncident wave but whose helaht was lower than the incident wave He also found lesser waves of higher frequency superimposed on the fundamental wave

Nakamura, Shiraish1, and Sasak1 (10) also conducted experiments with submerged breakwaters Their test results ind 1 cated alterations of wave length and period as the waves broke on the submerged dike for instance, their data showed that for a depth in the lee of the breakwater equal to 10 percent of the deepwater wave lenqth, the wave length in the lee of the breakwater was reduced to approximately 30 percent of the deepwater length and the wave period was reduced to approximately 60 percent of the deepwater peri od

Diephuis (2) has reported on a hydraul1c model study conducted at the Hydraulics Laboratory, Delft, Netherlands, to study the transmission of wave energy pas $t$ submerged bars Al though much of his data were taken $1 \mathrm{n}$ the range of wave lengths and herghts where surface tension and viscous effects play a large role in wave attenuation, his tests indicate that the wave heights in the lee of the bar where water depths are constant are some function of the deepwater wave steepness and that the heights of the reformed waves are more-or-less constant

\section{THE LABORATORY STUDY}

A laboratory experiment was devised to study the characteristics of gravity water waves after the waves had encountered a two-dimensional longshore bar and had broken while passing the bar A model simulating a longshore bar formation was designed and installed in a two-dimensional wave channel in the Hydromechanics Laboratory of Texas A\&M University Monochromatic, gravity water waves were generated toward this bar formation Most waves broke as they passed over the bar and then reformed as they entered the deeper water in the lee of the bar A $11 \mathrm{mit}$ on the waves studied was those waves that were on the verge of breaking but failed to do so These waves did however distort and develop shorter period components Re- 
cordings of the water-surface time-histories were obtained before and after the bar From the insight into the wave characteristics qained from these recordings, those characteristics which the wave possessed before reaching the bar were compared with the characteristics of the wave after it had reformed in the lee of the bar

The laboratory model simulating the bar formation was fabricated by fashioning wood $r i b s$ to a predetermined shape and affixing a stiff alumnum covering to the $r$ bs to form the bar surface The shape of the model, as shown in $\mathrm{F} i \mathrm{~g} \mathrm{1}$, was chosen arbitrarily, but is believed to have a counterpart in nature The helght of the model crest above the channel floor was $075 \mathrm{ft}$ and the total length of the model was $10 \mathrm{ft}$

The model was attached to the floor of the channel by drilling and tapping threaded ways into the floor and passing bolts through the model into the ways In this manner the model was securely fastened to the flume and showed $11 \mathrm{ttl}$ le tendency to shift under the oscillating forces created by the passing waves

The model was constructed with small tolerances between the aluminum covering and the walls of the channe 1 A caulking clay was forced into the spaces which did exist, to inhibit any leakage around or under the model

In nature, a longshore bar formation is a steeper discontinuity in an al ready sloping beach However, the sloping beach was not reproduced in this study The channel floor, at constant elevation, served as the ocean floor so that the effects of bottom slope were not studied

The wave channel in which this study was conducted is 2-ft wide by 3-ft deep by 120-ft long The walls of the channel are glass so that tests may be observed visually A pendulum-type wave generator was used to generate the monochromatic incident waves The displacement of the wave generator was determined by the eccentricity of the rod connecting the generator to a motor-driven flywheel The period of the generator was controlled by regulating the angular speed of the flywheel with the electric motor whose speed could be adjusted by the setting of a potentiometer A wi re-mesh wave filter was installed near the generator to attenuate the higher frequency nolse waves superimposed on the incident wave At the end of the flume, a permeable wave absorber $m i n$ mized reflection of the reformed waves back into the testing area

Water-surface time-histories were measured by a pair of capacitancetype wave probes The circuitry of this system includes a canacitance bridge of which the probe forms one leg Operation of the probe requires balancing the bridge by 1 mmersing the probe to its proper depth in the water and then balancing the bridge with an adjustable capacitor which forms another of the legs of the bridne With the circuit so balanced, any displacement of the water surface from the mean water leve 1 will cause an $1 \mathrm{mbal}$ ance of the circuit The magnitude and sense of the $1 \mathrm{mb}$ alance $1 \mathrm{~s}$ monitored and this signal is amplified The amplified signal is then recorded by a dual channel strip-chart recorder to yาeld a record of watersurface position versus time at each probe location 

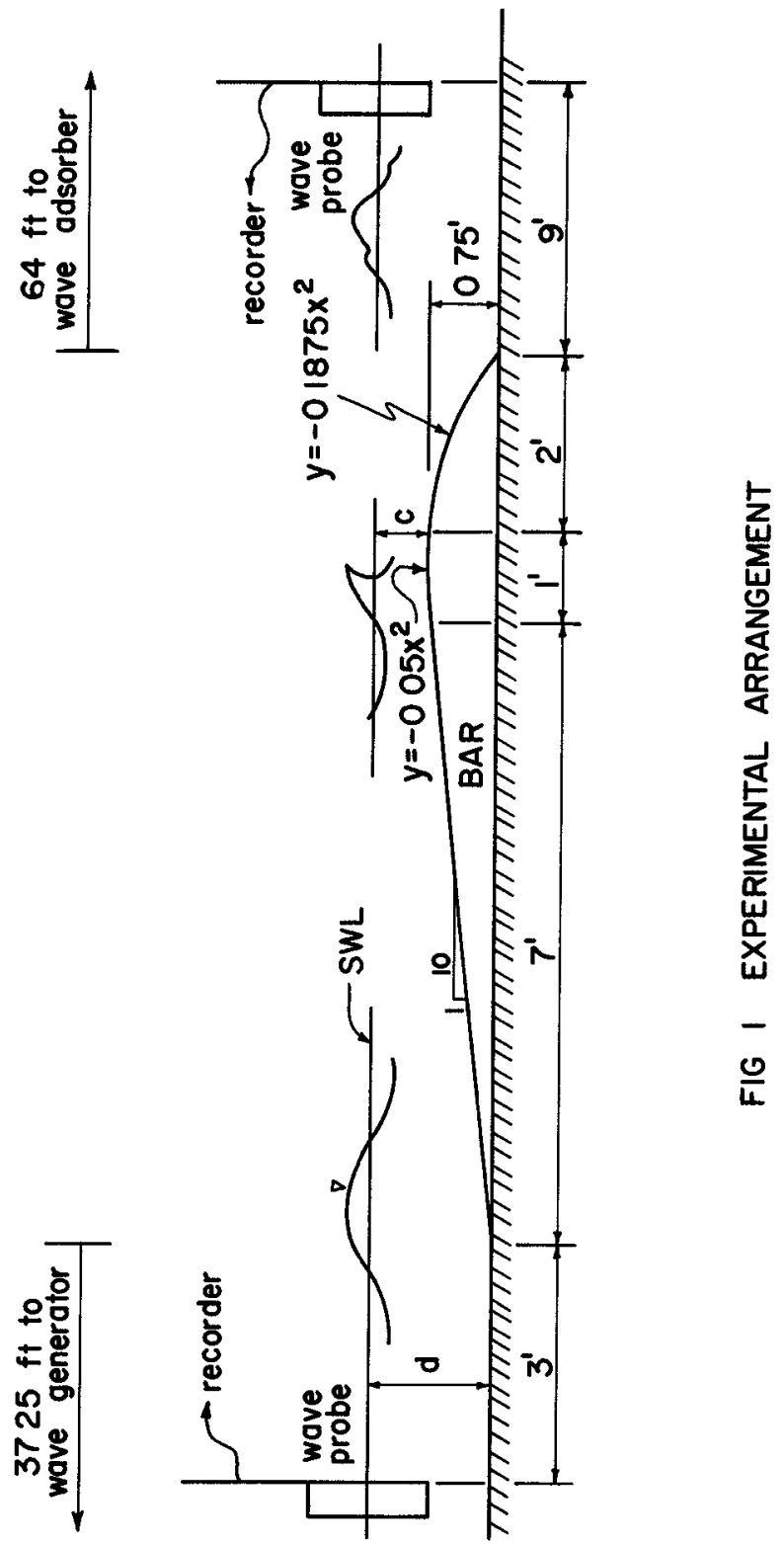
Several experimental runs $w$ th a range of wave characteristics were conducted for each of three water depths over the bar These depths over the bar crest, $C$, were $15 \mathrm{in}, 30 \mathrm{in}$, and 50 in giving total water depths, d, of $105 \mathrm{in}, 120 \mathrm{in}$, and $140 \mathrm{in}$

Two wave probes were installed in the channel at the locations shown in Fig 1 and calibrated before each set of test runs The calibration was checked quite often during the study

After each test run the wave channel was al lowed to sit for several minutes to allow any ripples on the water surface or any oscillation of the water in the channel to dissipate

$W_{1}$ th the water completely still, the wave probes and the recorder were switched on and the wave generator was started Records of approximately one minute in length of the water-surface time-histories at the two probe locations were taken The wave generator and recorder were then stopped, the water in the channel was allowed several minutes to still, and the test was repeated After this repeat test, the amplitude and/or period of the wave generated were changed and the entire procedure was repeated for the new wave train

In addition, a wave probe was attached to a movable carriage atop the wave channel and attempts were made to measure any reflected waves from the bar formati on by the envelope method (3) Although the procedure was repeated for waves of various steepnesses, in no instance were reflected waves detected

\section{EXPERIMENTAL RESULTS}

The laboratory data were obtained in the form of recorder traces of the water-surface time-histories for the two wave probe locations shown in Fiq 1 Samples of the recorder trace from each probe are shown in Fig 2

Wave heights were obtained from the wave records by measuring the vertical distance between the peaks and troughs of several successive waves and averaging these values This procedure was easily followed for the relatively uniform incident waves, but the irregular wave forms which developed in the lee of the bar presented some difficulty For those waves, the position of the crest and trough could not always be determned exactly, thus, there was some question concerning the helghts obtained Therefore, to provide a basis for comparison of test results as free as possible from subjectivity, a procedure was used in which an "equivalent" wave height was determned

In order to evaluate the equivalent wave height, it was necessary to assume that the recorder trace of the water-surface time-history is periodic and may be expressed as a Fourier series 


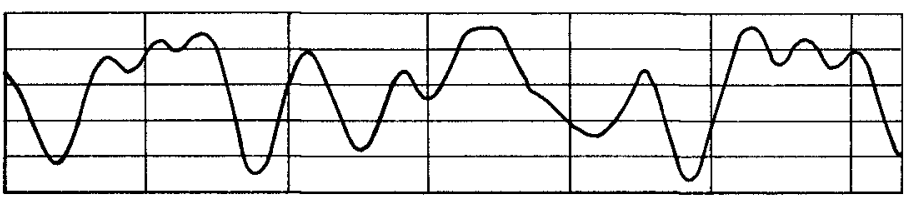

A Trace of the re-formed wave
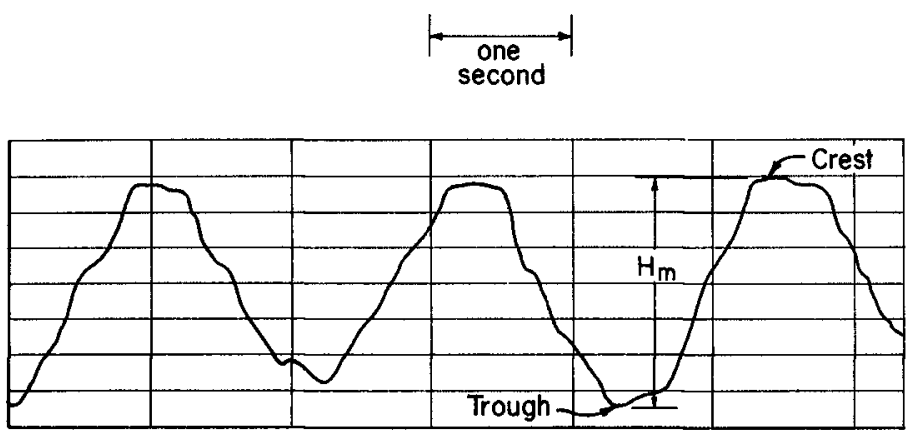

B Trace of the incident wave

FIG 2 A SAMPLE OF THE RECORDER TRACE 


$$
n(t)=\frac{a_{0}}{2}+\sum_{n=1}^{\infty}\left(a_{n} \cos n \sigma t+b_{n} \sin n \sigma t\right)
$$

Where $n(t)$ is the displacement of the water surface from mean water level at time $t, a_{0}$ is the mean displacement of the recorder trace from mean water level, $a_{n}$ and $b_{n}$ are Fourier coefficients, $n$ is an integer coefficient, and $\sigma$ is the angular frequency The Parseval theorem for periodic functions (6) gives

$$
\frac{\rho g}{\mathrm{~T}} \int_{0}^{\bar{T}} n(t)^{2} d t=\frac{\rho g}{8} \sum_{j=1}^{\infty} H_{j}^{2}
$$

where $\rho$ is the fluid density, a is the acceleration due to gravity, $\bar{T}$ is the $t i m e$ length of the wave record, $J$ is an integer, and $H_{3}$ is the height of each constituent wave comprising a complex wave form The right-hand side of Eq 2 may be recognized as the total energy per unit surface area in the wave form obtained by adding the energy of each constituent of the wave The incrdent equivalent herght, $H_{e q}$, may now be introduced and defined by

$$
\frac{1}{8} \rho g H_{e q}^{2}=\frac{\rho g}{T} \int_{0}^{\bar{T}} \eta(t)^{2} d t
$$

or

$$
H_{\text {eq }}=2 \sqrt{2}\left[\frac{1}{T} \int_{0}^{\bar{T}} n(t)^{2} d t\right]^{1 / 2}
$$

The values of $\eta(t)$ were measured for all tests at time increments of $005 \mathrm{sec}$ for record lengths, $\overline{\mathrm{T}}$, ranging from 20 to $25 \mathrm{sec}$ These values were punched onto cards and the equivalent wave helghts, as defined by Eq 3 , were evaluated numerically by a digital computer

Although the equivalent wave herghts need not have physical counterparts, they do provide an approximation of the measured wave helghts The plots in Fig 3 and 4 for $C=30$ in show this to be the case, particularly for the monochromatic incident waves The measured heights, $H_{m}$ and $h_{m}$, were obtained by averaging the helghts of several successive waves from the recorder traces $H_{m}$ is the measured height of the incident wave and $h_{m}$ is the measured height of the reformed wave heq is the equivalent height of the reformed wave, defined in the same manner as Heq Table 1 11sts pertinent data on the waves studied in these experiments 


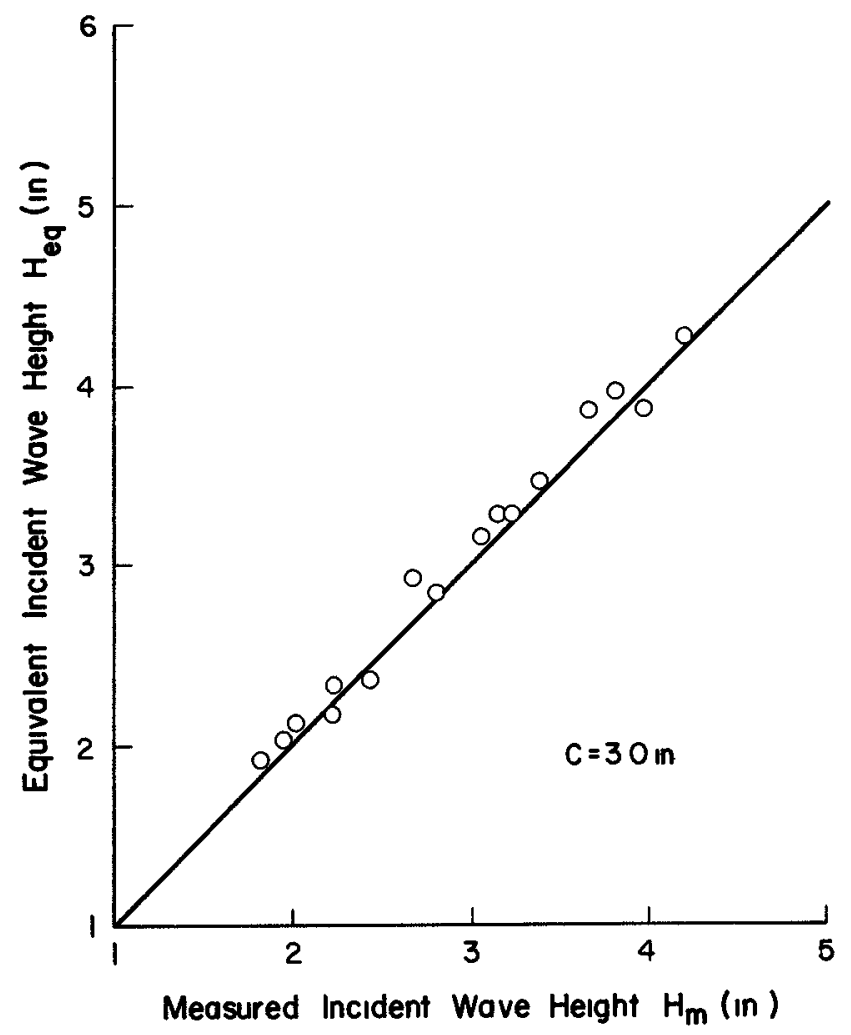

FIG 3-EQUIVALENT VERSUS MEASURED WAVE HEIGHT -INCIDENT WAVES 
LONGSHORE BAR

423

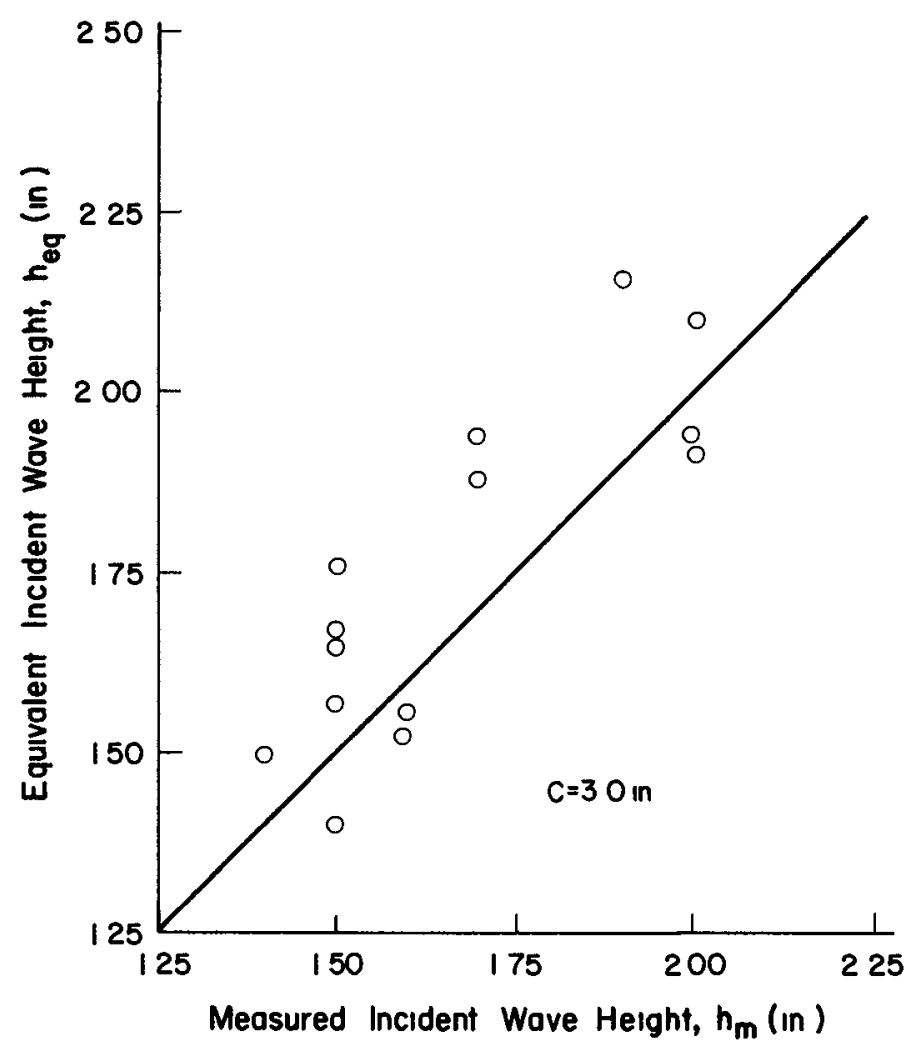

FIG 4-EQUIVALENT VERSUS MEASURED WAVE HEIGHT - REFORMED WAVES 
TABLE I - WAVE DATA

\begin{tabular}{|c|c|c|c|c|}
\hline $\begin{array}{l}\text { Run } \\
\text { Number }\end{array}$ & $\begin{array}{c}\text { Depth over Bar } \\
\text { C (nn) }\end{array}$ & $\begin{array}{l}\text { Wave Perrod } \\
\mathrm{T}(\mathrm{sec})\end{array}$ & $\begin{array}{l}\text { Heq } \\
\text { (in) }\end{array}$ & $\begin{array}{l}\text { heq } \\
\text { (in) }\end{array}$ \\
\hline 1 & 15 & 122 & 175 & 076 \\
\hline 2 & 15 & 100 & 190 & 064 \\
\hline 3 & 15 & 085 & 184 & 061 \\
\hline 4 & 15 & 073 & 162 & 051 \\
\hline 5 & 15 & 084 & 156 & 052 \\
\hline 6 & 15 & 122 & 154 & 053 \\
\hline 7 & 15 & 121 & 114 & 047 \\
\hline 8 & 15 & 092 & 133 & 052 \\
\hline 9 & 15 & 120 & 229 & 081 \\
\hline 10 & 15 & 169 & 204 & 063 \\
\hline 11 & 15 & 171 & 217 & 071 \\
\hline 12 & 15 & 121 & 252 & 078 \\
\hline 13 & 15 & 123 & 099 & 051 \\
\hline 14 & 30 & 224 & 304 & 193 \\
\hline 15 & 30 & 217 & 379 & 214 \\
\hline 16 & 30 & 219 & 203 & 149 \\
\hline 17 & 30 & 194 & 190 & 158 \\
\hline 18 & 30 & 193 & 268 & 190 \\
\hline 19 & 30 & 181 & 367 & 208 \\
\hline 20 & 30 & 157 & 236 & 140 \\
\hline 21 & 30 & 154 & 396 & 187 \\
\hline 22 & 30 & 158 & 314 & 157 \\
\hline 23 & 30 & 132 & 419 & 192 \\
\hline 24 & 30 & 133 & 338 & 176 \\
\hline 25 & 30 & 135 & 214 & 149 \\
\hline 26 & 30 & 110 & 202 & 140 \\
\hline 27 & 30 & 114 & 241 & 154 \\
\hline 28 & 30 & 110 & 320 & 167 \\
\hline 29 & 30 & 090 & 279 & 164 \\
\hline 30 & 50 & 126 & 345 & 265 \\
\hline 31 & 50 & 154 & 320 & 271 \\
\hline 32 & 50 & 198 & 521 & 278 \\
\hline 33 & 50 & 253 & 454 & 296 \\
\hline 34 & 50 & 192 & 434 & 242 \\
\hline 35 & 50 & 147 & 564 & 274 \\
\hline
\end{tabular}


Visual observations of the wave forms in the lee of the bar indicated that the water-surface history repeated itse $7 f$ in a time interval approximately equal to the period of the 1 ncident waves However, a more rigorous determination of the period of the waves in the lee of the bar may be made by conducting a spectral analysis of the wave form The assumption is made that the frequency at which the peak energy is located corresponds to the period of the wave The spectral analysis w1 11 show the frequency band in which the peak energy is located in both the incident wave and the reformed wave A comparison of the spectra of the 1 ncident wave and the spectra of the reformed wave will show if a shift in the location of the peak energy has occurred If a shift has occurred, then the period of the wave wil1 have changed during the breaking and reforming process

The spectral analysis may be carried out using the data which were gathered earlier for the determination of the equivalent wave heights $A$ method for determining the wave spectra is presented by Kinsman (8) and is outlined here with some silght changes in constants

With $N$ values of $n\left(t_{f}\right)$ spaced at time intervals $\Delta \tau$ and $w_{1}$ th $m$ as the $1 \mathrm{ag}$, the sample autocorrelation function, $r$, may be computed

$$
r(\nu \Delta \tau)=\frac{1}{N-v} \sum_{i=1}^{N-v} n\left(t_{1}\right) n\left(t_{1}+v \Delta \tau\right)
$$

where $v=0,1,2, \quad, m$ The $1 a g, m$, is some fraction of the length of the record, usually less than $01 \mathrm{~N}$

The Fourier transform of the autocorrelation function 15 the energy spectrum, which is required This transform may be effected by calculating the unsmootked estimates of the spectrum as follows

$$
\begin{aligned}
L_{\tau=0} & =\frac{1}{m}\left[\frac{1}{2}[r(0)+r(m \Delta \tau)]+\sum_{k=1}^{m-1} r(k \Delta \tau)\right] \\
L_{\tau \neq \nu} & =\frac{2}{m}\left[\frac{1}{2}\left[r(0)+(-1)^{\nu} r(m \Delta \tau)\right]+\sum_{k=1}^{m-1} r(k \Delta \tau) \cos \frac{\pi k v}{m}\right] \\
v=1,2, \quad, m-1, \text { and } & \\
L_{\tau=m} & =\frac{1}{m}\left[\frac{1}{2}\left[r(0)+(-1)^{m} r(m \Delta \tau)\right]+\sum_{k=1}^{m-1}(-1)^{k} r(k \Delta \tau)\right]
\end{aligned}
$$


where $L$ is the unsmoothed spectral estimates at $t i m e t=\tau$ The final smoothed estrmates of the spectrum, $\Phi$, are formed by the moving, weighted ave raqe

$$
\Phi(\nu \Delta \tau)=\sum_{i=0}^{m} a_{v, 1} L_{v}
$$

whe re $v=1,2, \quad, m-1$, and

$$
\begin{aligned}
& a_{v, v-1}=025 \\
& a_{v, v}=050 \\
& a_{v, v+1}=025 \\
& a_{v, 1}=0
\end{aligned}
$$

for $1 \neq v-1, v$, or $v+1$ For the end pornts,

$$
\left.\begin{array}{l}
\Phi(0)=\left(L_{0}+L_{1}\right) / 2 \\
\Phi(m)=\left(L_{m-1}+L_{m}\right) / 2
\end{array}\right\}
$$

Typical results of the spectral analysis are presented in Figs 5 through 10 These plots show that the peak energy of the incident wave and the peak energy of the reformed wave generally occur at the same frequency Thus, the indication is that the basic period of a wave does not change as the wave passes over the bar, breaks, and reforms in the lee of the bar

In all reformed wave records a second energy peak occurs at a higher frequency that is consistently twice the frequency of the 1 ncident wave In a few cases, the peak value of this higher frequency energy is equal to or slightly exceeds the reformed enerqy of the incident wave frequency (see Fig 8 for example) In most cases, the peak energy at this higher frequency 15 only 20 to 40 percent of the main peak of the reformed energy spectra

Also, in most of the reformed wave records analyzed, a very low level of energy with a frequency of approximately triple the incident wave frequency can be detected

The ratio of reformed to 1 ncident equivalent wave hejght, heq/Heq, has been plotted against the deep water wave steepness, Heq/ $\mathrm{T}^{2},(\mathrm{~F} 1 \mathrm{~g} 11$ ) and against the relative depth over the bar crest, C/T2, (Fig 12) As can be seen, no correlation is apparent in either of these figures 
Fig 13 shows heq/Heq plotted against the ratio of incident equivalent wave height to water depth over the bar crest, Heq/C The data for the $3 \mathrm{in}$ and $5 \mathrm{in}$ water depths plot together and produce a relatively well defined relationship between Heq/C and heq/Heq Some of the scatter may be caused by the fact that some waves break over the bar crest whlle others break ahead of the crest

The data for the 15 in depth fall below the other data, particularly for the low incident waves This is possibly due to scale effects in the breaking of these low amplitude waves

\section{CONCLUSTONS}

The objective of this investigation was to gain some understanding of the characteristics of monochromatic waves after they break over an offshore bar Generally, most of the energy in the reformed waves is at the same period as in the incident wave but a large portion of the energy shifts to higher frequencies The ratio of equivalent wave heights (square root of wave energy per unit surface area) before and after the bar depends primarily on the ratio of the incident wave height to water depth over the bar crest

Further tests are planned for $5 \mathrm{in}$ and greater (e $\mathrm{q} 7 \mathrm{in}$ and 9 in ) depths over the bar with waves that break and waves that do not break but are disturbed enough by the bar to cause the development of higher frequency components in the lee of the bar These tests should better define the relationship between heq/Heq and Heq/C They should also lead to a greater understanding of the reformed wave eneray spectrum Also, a closer look at the nature of the waves as they break as well as the point on the bar at which breaking occurs is needed

\section{ACKNOWLEDGEMENT}

The support of the $U S$ Army Corps of Englneers and the $U S$ Army Engineer Waterways Experiment Station, Vicksburg, Mississippi during the senior authors period of graduate study and research on the problem of breaking waves is gratefully acknowledged

\section{REFE RENCES}

1 Dick, T M, "On Solıd and Permeable Submerged Breakwaters", C E Research Reoort 59, Queen's University, Kingston, Ontar1o, Nov 1968, $144 \mathrm{p}$

2 Diephuis, J G H R, "Scale Effects Involving the Breaking of Waves", Proceedings, 6 th Conference on Coastal Engineering, Gaines ville, Palm Beach, and $M_{1}$ am1, Fla, 1957, Chapter 11 


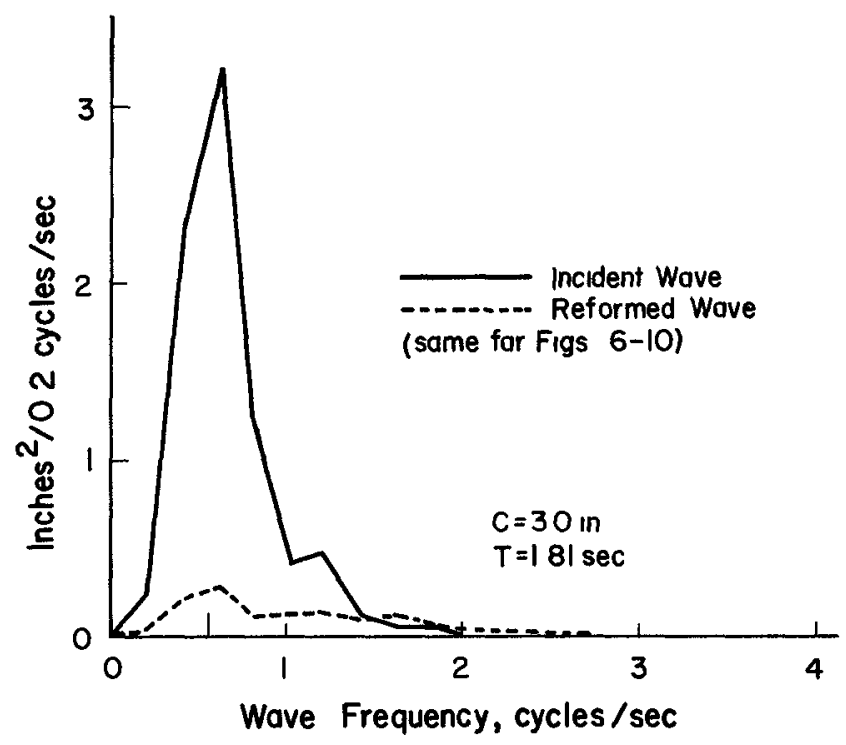

FIG 5 WAVE SPECTRA, RUN NO. 19

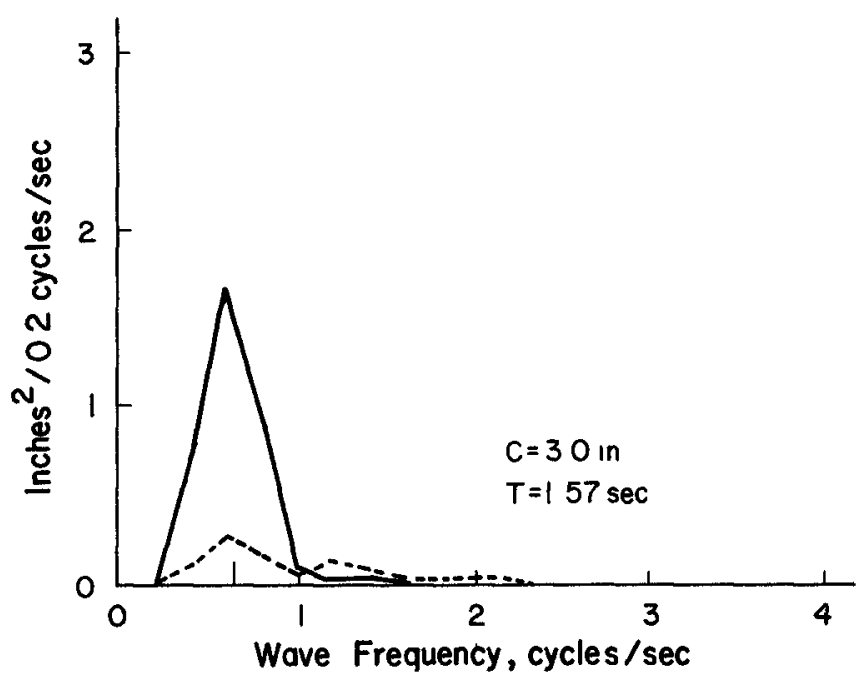

FIG 6 WAVE SPECTRA, RUN NO 20 


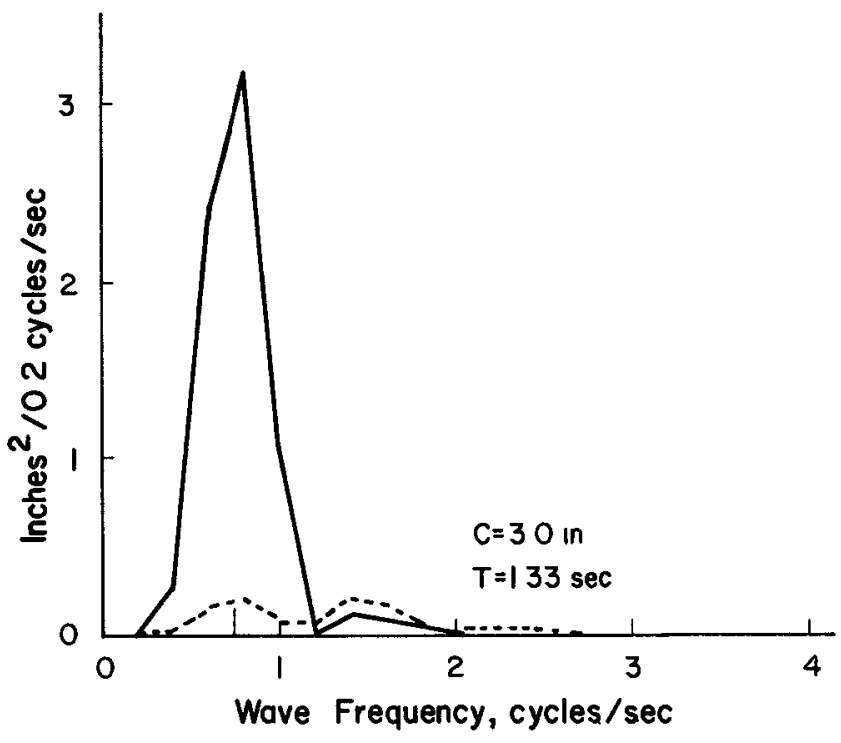

FIG 7 WAVE SPECTRA, RUN NO 24

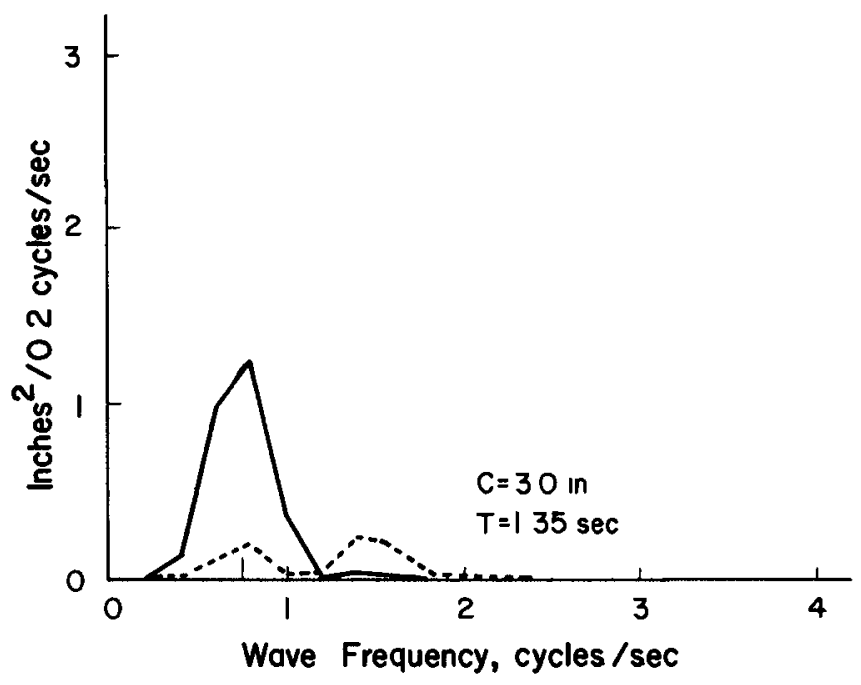

FIG 8 WAVE SPECTRA, RUN NO. 25 


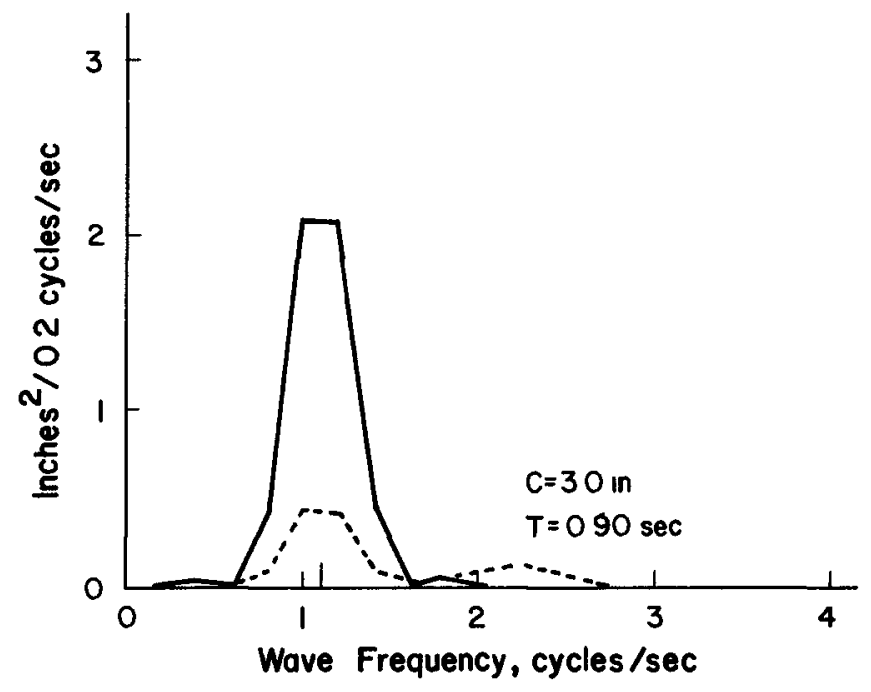

FIG 9 WAVE SPECTRA, RUN NO 29

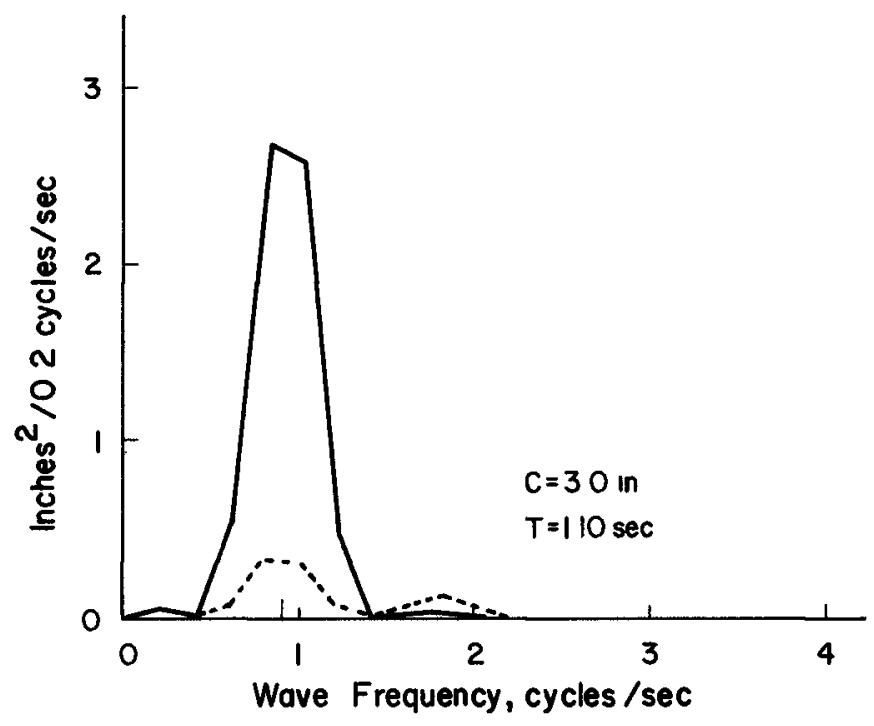

FIG 10 WAVE SPECTRA, RUN NO 28 


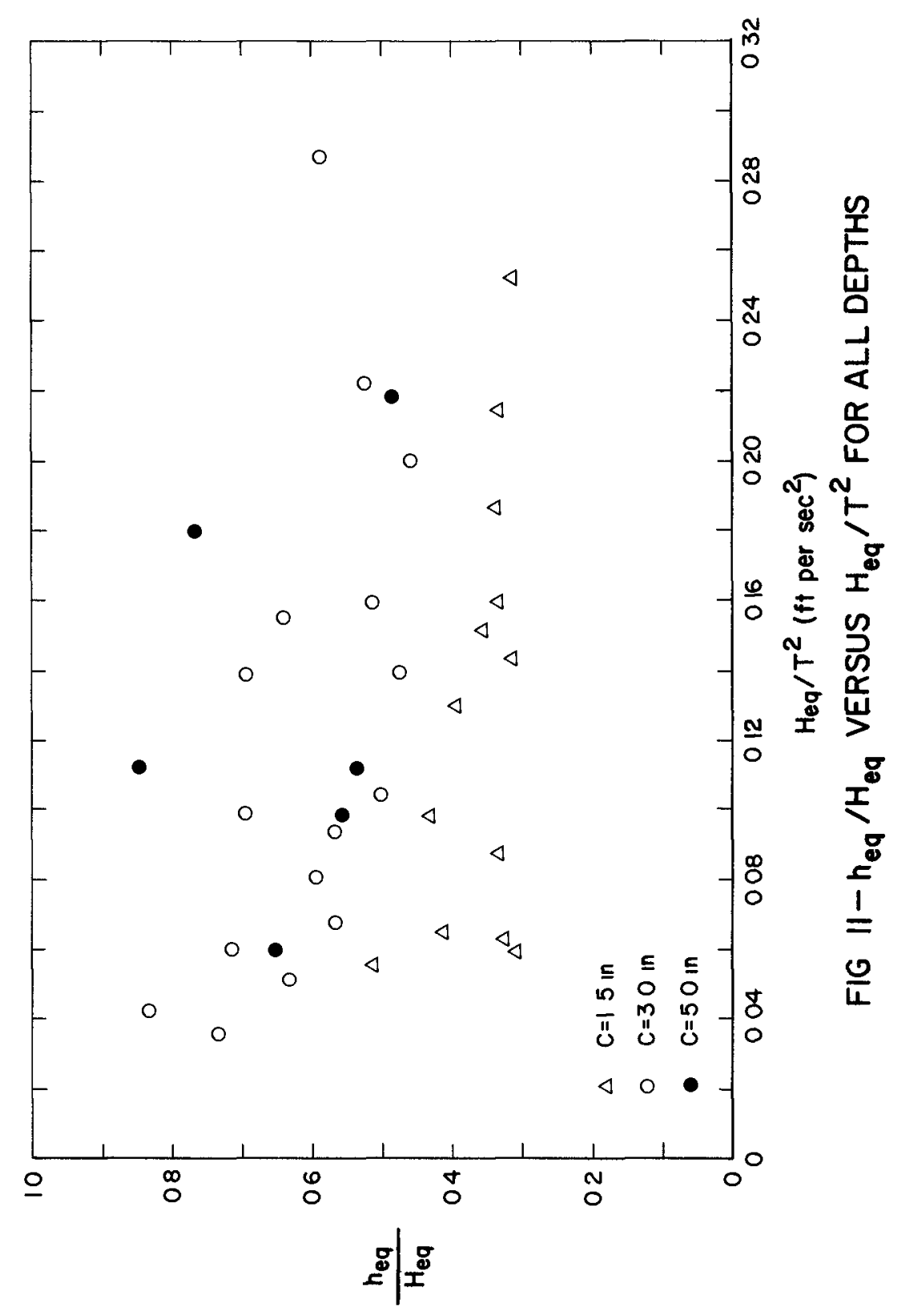




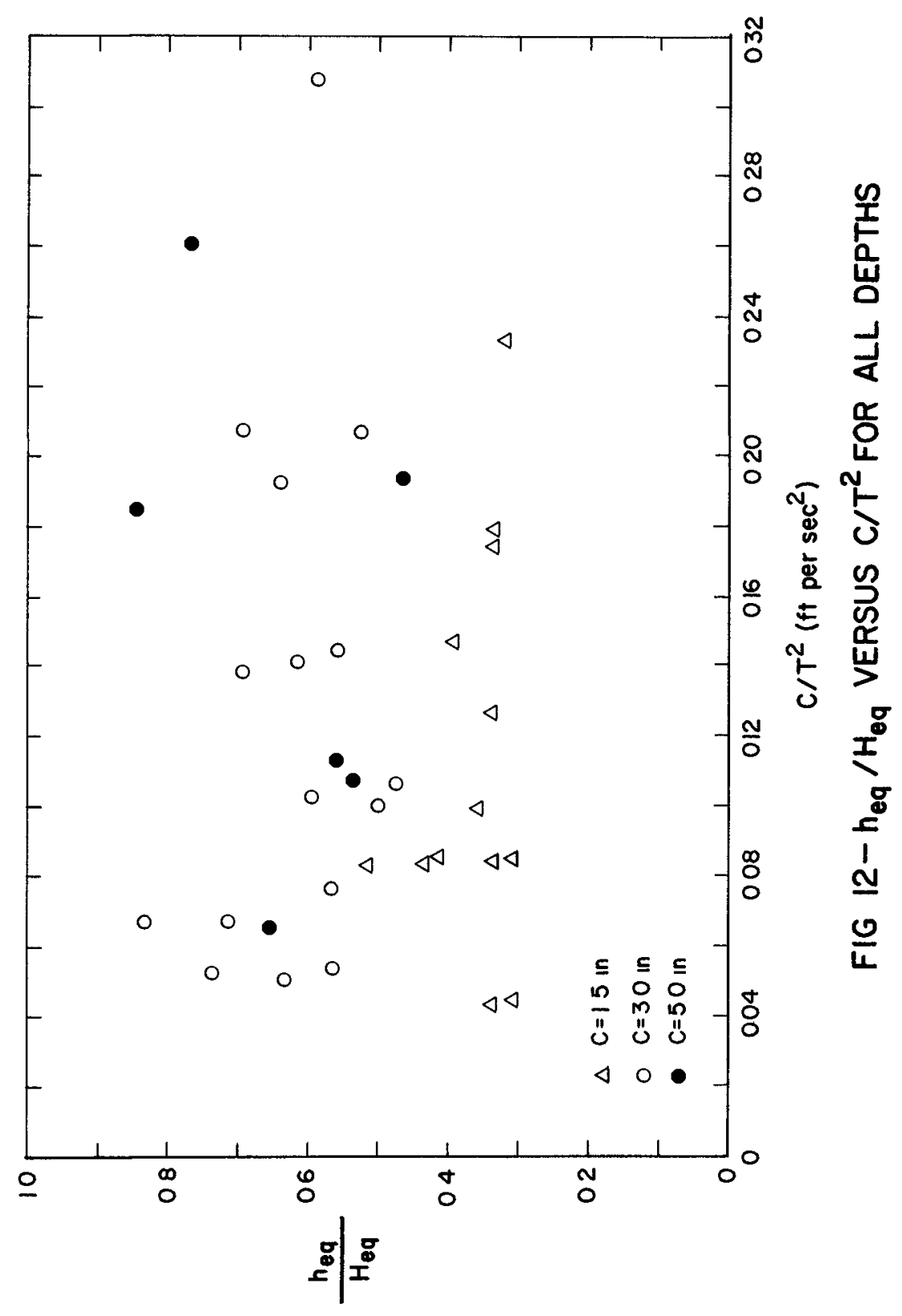




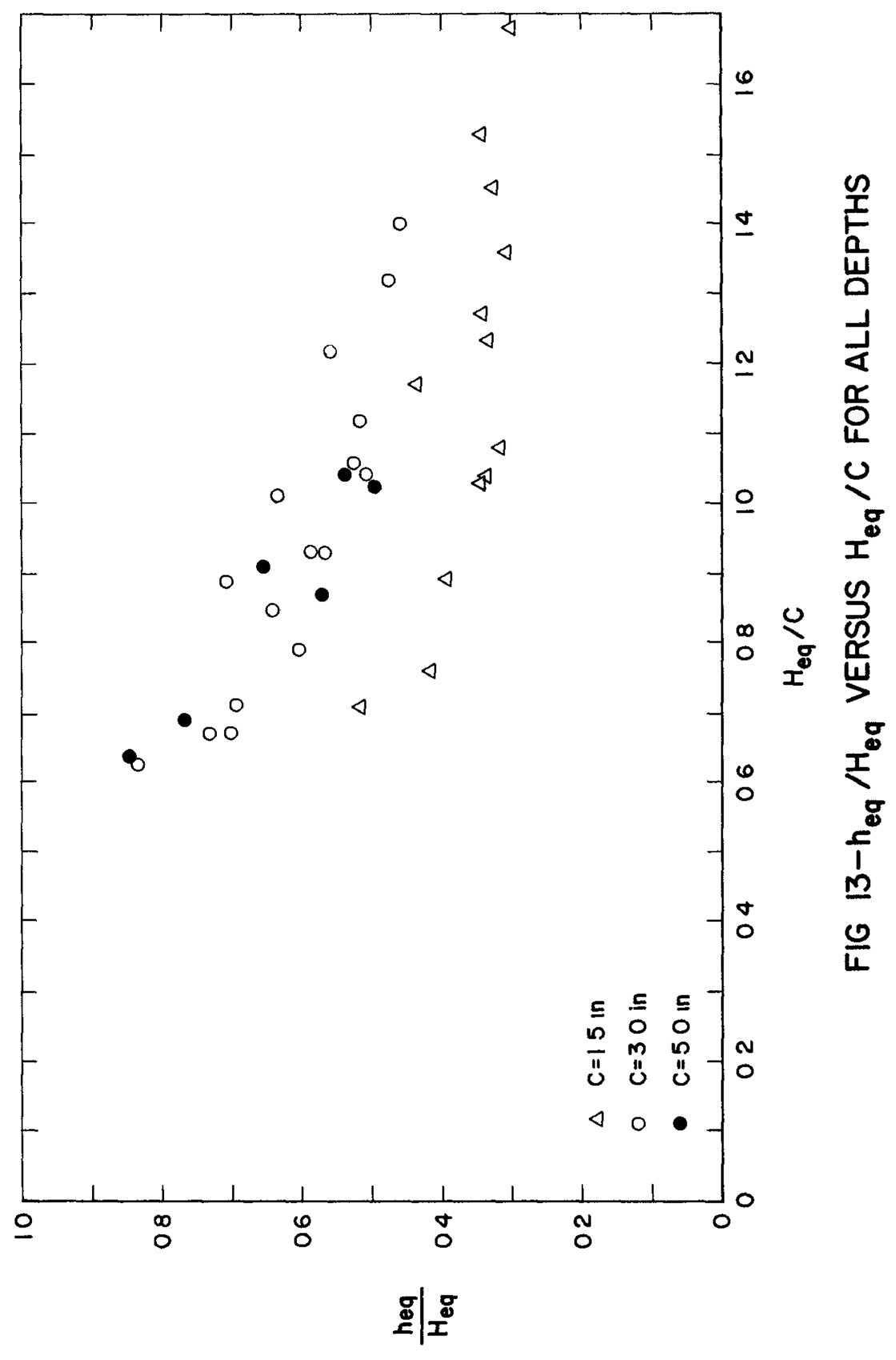


3 Herbich, J B , "Experimental Studies of Wave F1lters and Absorbers", Project Report No 44, Univers 1 ty of Minnesota, St Anthony Falls Hydraulic Laboratory, St Pau1, Minn, Jan, 1956

4 Hom-ma, M, and Horıkawa, K, "Wave Forces Against Sea Wa11", Proceedings, 9th Conference on Coastal Engineering, Lisbon, Portugai, 1954 , Chapter 31

5 Horikawa, K, and Juo, C, "A Study on Wave Transformation Inside Surf Zone", Proceedings, 10th Conference on Coastal Engineering, Vol 1, Tokyo, Japan, 1966, Chapter 15

6 Ippen, A T , ed, Estuary and Coastline Hydrodynamics, McGraw-H1 11 Book Co, New York, 1966

7 I verson, H W, "Laboratory Study of Breakers", National Bureau of Standards C7rcular 521, Gravity Waves Sympos 1 um, 1952

8 Kinsman, Blair, Wind Waves, Their Generati on and Propagation on the 0 cean Surface, Prentice-Hal1, Inc, Englewood Cliffs, N J, 1965, Chapter 9

9 Nakamura, M, Shiraıshı, H, and Sasakı, Y, "Wave Decayıng Due to Break ing", Proceedings, 10th Conference on Coastal Engineering, Vol 1, Tokyo, Japan, 1966, Chapter 16 


\section{CHAPTER 27}

\section{VELOCIIY FIFLDS IN HHE WAVE BREAKGR ZONE}

by

M. D. ADEYEMO, Ph.D. (Lond).

Faculty of Engineering, University of Lagos, Nigeria.

\section{SYNOPSIS}

The paper deals with the velocity fields in the neighbourhood of the breakers and the correlation between the wave asymetry and the velocity asymmetry.

The velocity measurements were made on two beach slopes 19 and 1.18. Earlier work (3) showed that the two slopes produced dufferent breaker types; the slope of 1:9 produced plunelng breakers and the slope of 118 spilling breakers.

The velocities (velocity - time history) of the water partucles were measured at a height of $5 \mathrm{~mm}$ above the bed using the hydrogen bubble method combined with cine photography.

Two types of horizontal velocity asymmetry were defined and investigated, namely

(1) horizontal velocity (Kagnitude) asymnetry

and (2) horizontal velocity (time) asymmetry.

It was found that there are both qualitative and quantitative correlation between the asymmetry of the wave, and the rsymmetry of the resulting velocity field. As a result of the correlation two elternative expressions are given for the horizontal velocity (magnitude) asymmetry.

\section{INTRODUCTION}

In fairly deep water, the water particle horizontal velocities at the bottom are approximately equal for the shoreward and seaward motions, but as the waves moves into progressively shallow water of about $\mathrm{d}<0.15$, the asymmetry of the wave gives rise to the velocity dufferential $I$ of the water particles in the beachward and seaward directions, the onshore velocity of the water particles being greater in magnitude and of shorter duration than the offshore velocity. This behaviour thus leads to definition of two types of horzontel velocity asymmetry, one besed on magnitude and the other on time. They are defined as follows.

Horlzontal velocity (magnitude) asymmetry =

Waximum homzontal shorepred velocity

Haximum horizontal seaward velocity 
Horizontal relocity (time) asymetry =

Time for the shoremard wotion

Time for the seaward motion

The study on wave asymetry where the effect of beach slope and shoaling on the magnitude of the wave asymmetry was examined had boen roported by the author(3). The present study is thus concentreted on the investigation of the relocity fields and the correlation of the velocity asyumetry and the wave asymmetry. The rave oonditifons used were the same as those used in the study on wave asymmetry $(3)$. The correlation of the ware asymetry and the asymmetry of the orbital velocity field will be discussed later in the paper. A revien of provious roxk on velocity studies of water partioles under the action of naves is giron below.

\section{Revier of Previous Work}

Iversen (1) examined the kinematios of the water movement in breakers. He used particles of a mixture of xylene, carbon tetraohloride and sine cedde for Plow visuallzation. The movement of the particles was rocorded on oine film, from which oach partiole velocity was obtained by noting the distance moved on the projected franos and the time interval of movement. The velooities obtained were plotted as rectors as shom in Fig. 1. Irersen, in addition, messured the backrash and orest velooities. He obtained the backwash velocities by averaging all particle velocities in the rogion of minimum depth in the backrash, and the crest velooities wero obtained from the gradient of the crest position - time history. Iversen found that the backmash velooity was higher on a stoep beach as compared to a flat beach. This was to be expeoted.

Hamade(5) studied the partiole velocity in ware motion in the region of the breakers. The veloolty of flon was measured by means of a ourrent meter which had several propellers driven by the action of water Plom. A direct elootric current in the circuit connected the current meter to an 0scillograph, with one element of the osoillograph corresponding to one of the propeliers. Hamada made oxbital velocity measurements $30 \mathrm{~m}$. from the bed. He noted the differential velocity in the shorenard and seaward diroctions. He found the ratio of backrand velocity to forwerd veloolty to vary between $1: 1.16$ to $1: 1.29$. Howover, his theorotical approach was to caloulate the orbital velooity by the Airy theory and to add an estimated "residual" velooity (oquivalent to the mass transport velooity). Since this nas always positive, the resulting values gave a differential between the forward and baokwand volocitios. His measurements are of intorest, but his theorotzoal evaluation negleots the existence of orbital velooity asymetry even in the absence of nass transport.

Inmen and Nasu(6) made a study of orbital velocity in shallow water at La Jolla California. The measurements nere made near the bottos and just seaward of the breaker zone in water depths ranging from 

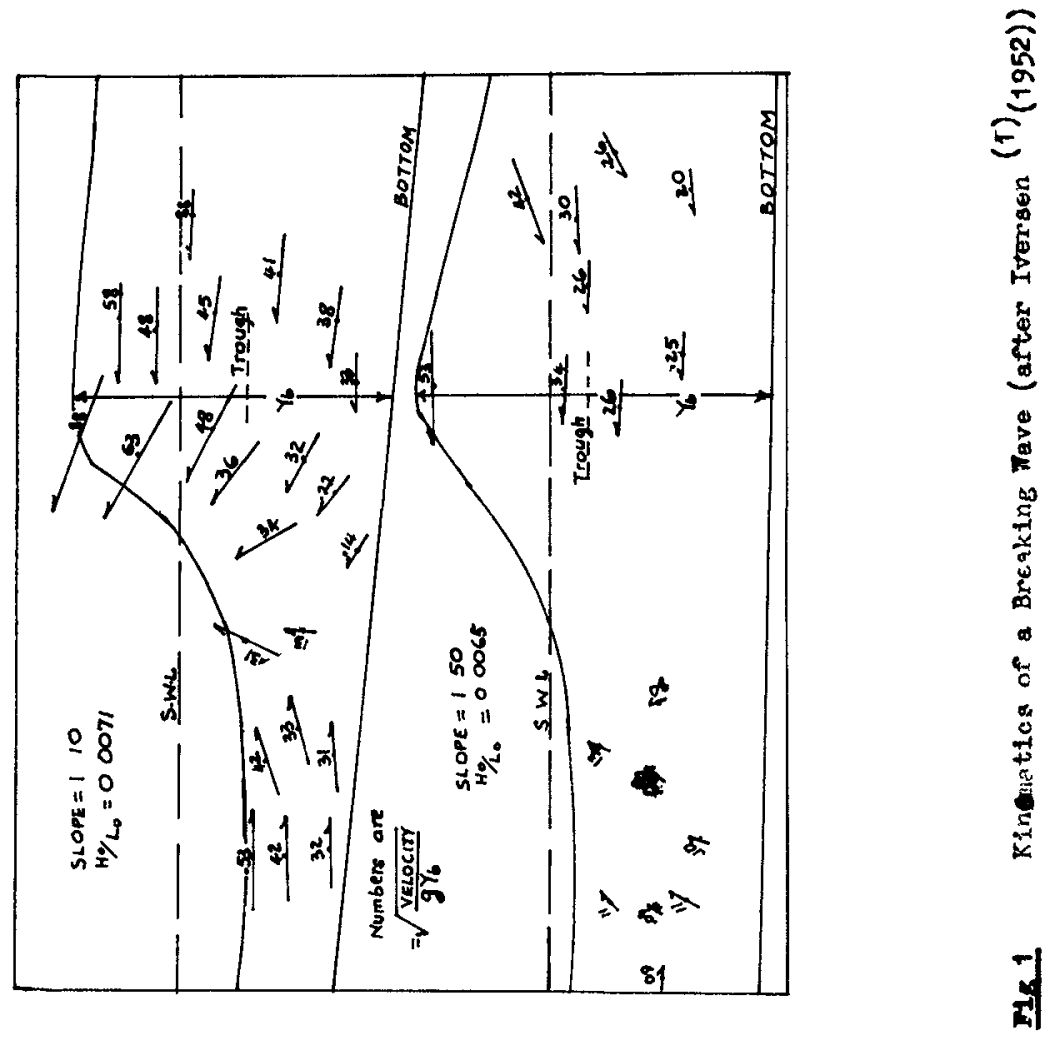
about five to fifteen foet and wave heights of up to seven and a half feet. The orbital ourrent ter consisted in the main of a cylindrioal rod fixed rigidly at one end like a cantilever, and the system was arranged such that the orbital velocity oould be interpreted from the bending of the rod oaused by the force exerted by the moving water. A pressure type wave meter was used with the ourrent moter, and both the wave meter and the ourrent meter were mounted on a tripod. The ourrent meter was mounted $0.82 \mathrm{ft}$. above the bottom. The bed had an average slope of about $\frac{1}{35}$ in the roglon where the orbital velocities were meacured. 35

Imman and Nasu oomented that the graph of the horizontal oomponent of orbital relooity as a funotion of time resenbled that of the mave pressure and they noted that the horizontal velooity seomed to be more dependent on the rate of ohange in level of the rater surface during the passage of the wave than on the sotugl, height of the wave. With reference to $\mathrm{Fig}_{1 \mathrm{~g}} 2$ after Inman and Nasu(6) they notod that in the figure, the fourth wave was almost as high as those proceding it, but showed a more gradual mise in level from the preoeding trough to the crest. The result was a very low crest velooity. In terms of the work reported in this paper, and in earlier papers by the author $(3,4)$ the statement of Inwen and Yasu oould be oondensed into the statement that for waves of the same height, greater veloolties are assooiated with Ereater wave slope asymutizy.

Inman and Nasu renaxiced that the meximum relooity under the rave orest was always onshore and that for almost all the waves analysed the nean onshore maximm velooity excoeded the off shore velooity. They ocmpared the maximu orbital velooities with Solitary and Airy-Stokes waves. They gave the sum of the orest and trough relocities along the botto al for both Alry and Stokes waves as:

$\omega=\mid$ Jorest $|+| v$ trough $\mid=\frac{2 \pi \|}{T} \frac{1}{\sinh \frac{2 \pi \mathrm{d}}{\mathrm{I}}}-$

For the Solitary wave, they gave the maximum oxbital velocity at the botton s0 as:

$$
\mathbf{s}=\frac{1}{2} \mathrm{NC}
$$

where $C=\sqrt{8(H+d)}$ is the veloolty of propagation of the wave orest. H is rave height and d water depth.

$N$ is dorined by the equations:

$N=\frac{3}{3} \sin ^{2}\left[u\left(1+\frac{2 \pi}{3 t}\right)\right]-$

and

$$
\frac{\mathrm{H}}{\mathrm{a}}=\frac{\mathrm{I}}{\mathrm{u}} \tan \frac{1}{2}\left[\mathrm{u}\left(1+\frac{\mathrm{H}}{\mathrm{d}}\right)\right]
$$




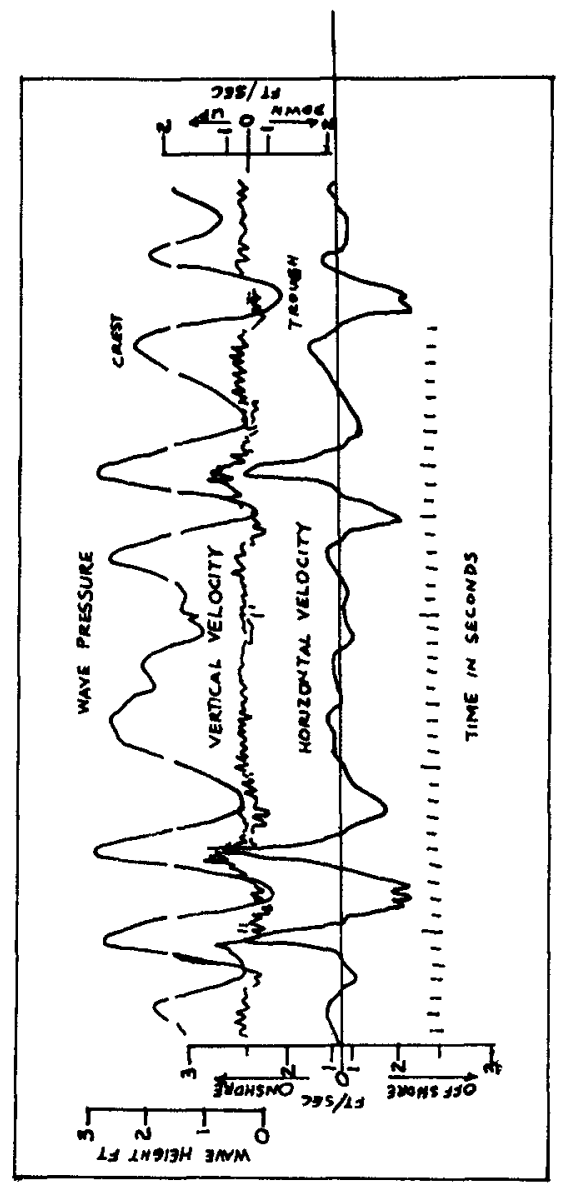

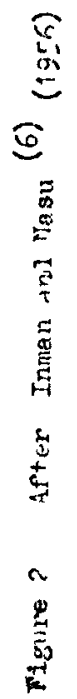


In man and Hasu(6) renarked that the measured velocities nere on the average in bettor agreement with the Solitary wave than with the Airy Stakes relations.

Ippen and $\operatorname{KuIn}(7)$ studied the internal relocities of the solitary wave at the break-point. They used droplets of a solution of xylene and n-butyl phthalate. The notion pioture camera they employed was operatod at 20 frases per second and they placed an auxiliary grid in the plane of the particles so thet on the photographs the grid lines could be projeoted down into the wave itseif. They conductsd their studies on two types of breakers; the plunging breaker on a slope of 0.065 and the spilling breaker on a slope of 0.023 . They compented that on the slope of 0.023 with a spilling breaker the maximum velooity was noarly equal to the crest celemity at the wave break point, and they noted that the maximun particle velooity seemed to oocur just slightly shoremand of the highest point of the orest.

some velocity atudies were also oarried out by Morison and Crooke(11) in a study of deep wator, shallow water and breaking waves. They used a nixture of carbon totrachloride, xylene and zinc oxide haring approximately the samo speciflo gravity as water. They found that the greatest horizontal perticle velooity ocourced when the wave was breaking, and they noted a relocity differential between the shoreward and seaward horizontal velooities. They concluded that the maxdmom horizontal partiole relocity at the orest of the wave might attain the ware celerity as the nave broke, but that the phonomenon was confined to a very narrow region of water at the grest of the wave. This was not borne out by uilier and Zeigler's $(8)$ observations.

\section{APPARATUS AND MBASURTHG TEGTNTOUES}

The teahnique used for the relocity reasurements in the studies presented in this paper is a method usually called the "hydrogen bubble mothod." Bssentialiy it oonsists of a fine wire which forms the negative electrode of a D.C. circuit, while two metal plates, positioned at each sido of the tank inth a reasonablo depth of the aetai inside the water, serve as the seoond electrode. Hydrogen bubbles are forned on the negative electrode. The arrangement was suoh that the support for the rire and metal plates servang as the positive electrode wexe integral, (see plate 1). Two advantages of suoh an errengement wero that, as measurements were made at different posztions along the beach, the apparatus could be noved intact. Secondly it was foumd that the quality of the bubbles was affeoted when the positive electrode was not olose enough to the wire, as the circuit then becane reak. The wire used in this work was 0.05 man dianeter platinum wire, with bress plates as the positive electrodes.

Cine photography was exployed and a suitable lighting arrangoment was therofore derised. The cawera used was a 16m Reflex Bolex at 64 frames per second. With a wave period of 0.8 seconds, this gave approximately 50 piotures per wave cycle. 


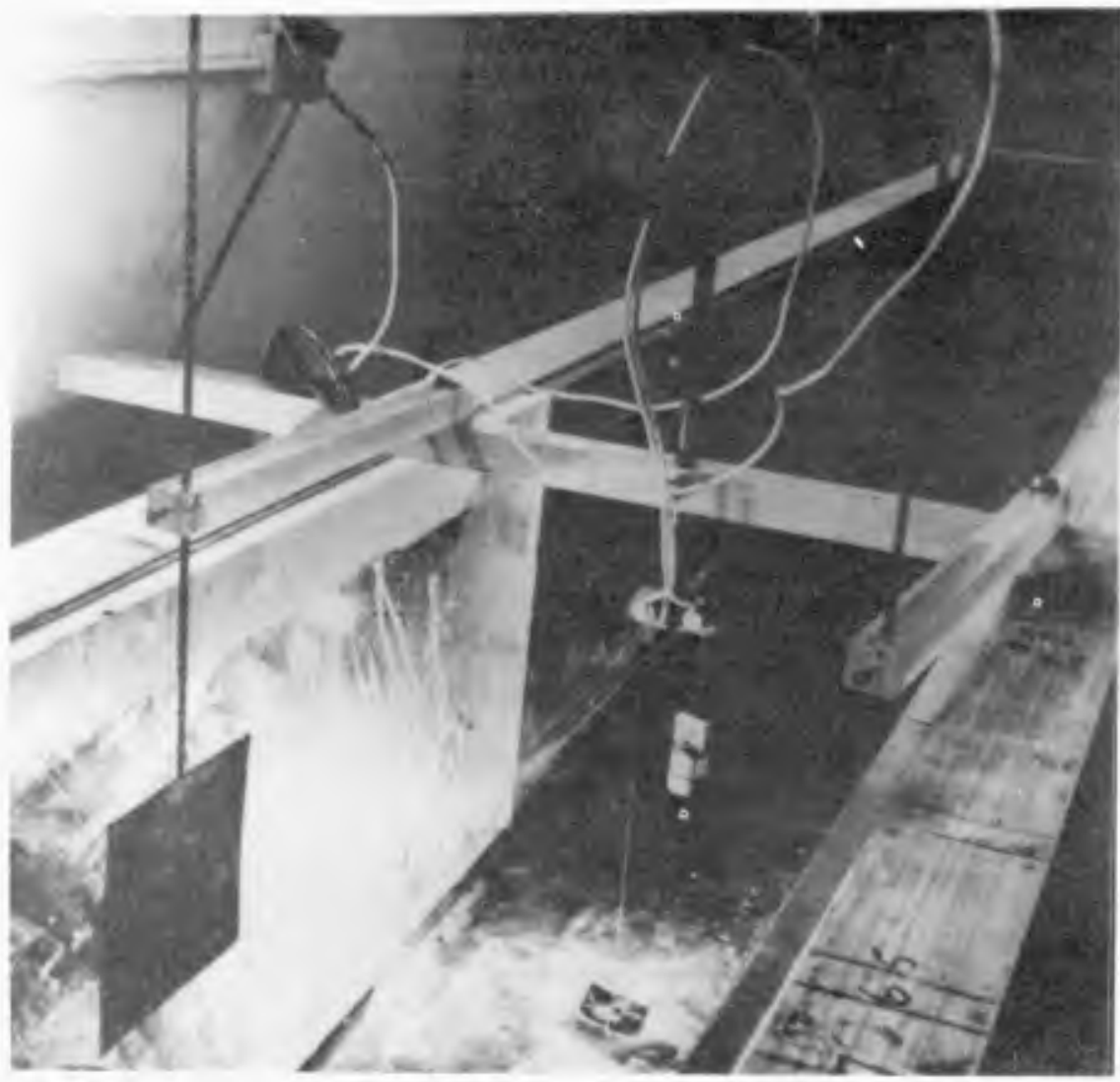

Plate I 
For the lighting arranganent, an Aldis Mutor 1000 Watt slide projector was used. The light source was located on the opposite side of the channel to the oamera, and orientated at on obilique angle to the channel. Slits wore used to prevent stray lights from soattoring in the wator.

In operation, one and of the wire was fixed to the beach with a tape, while the other end was passed over a support and was tensioned by a suitable reight. The relocities olose to the wire at the different positions were eraluated from the displaoement of the bubbles generated at different but knom times. The orbitel velocity measurenents wero made at a height of $5 \mathrm{~mm}$ above the bed, which was outaide the wave induced boundary later. These relocity measurenents were made at several positions along the beach on two beach slopes $1: 9$ and 1:18 in the regtion $2 / \mathrm{y}>0.15$ including the breakers. The two slopes were ohosen on the basis of previous experiments $(3,4)$ whioh indicated that they are respectively representative of oharracteristionlly stoep besohes with plunging breakers and flat bekohos with spilling breakers.

The ware profiles and henoe the different wave asymetmies for the differont positions along the two beach slopes were also obtained using the capacitanoe-wire probe together with a visicorder.

\section{EXPRETITHIAL DESULTS}

\section{Effeot of Shoeling on Orbitel Velooity Pield}

The velooity - tine history for a rull wave cyole at the different positions along the beach were obtained from the analyais of the cine files of hydrogen bubble blooks exitted from a vire positioned at these positions. The resulting ourves are shom superimposed in flgures 3 and 4. The two types of horlzontal velocity asymetry, one based on the magnitude of the shorvand-seanard velocities and referred to as the horizontal velooity (negnitude) asymetry and the other besed on the duration of the shorowari-seamand motion and rofermed to as the horizontal velooity (time) asymatry wero computed from the separate ourres of the velooity - time rariation for a wave cyole.

Figures 3 and 4 show how the orbitel velocity-time curte ohanges in form as the wave progresses inte shallower water. It can alsc be seen from the figures that the horizontal velocities inoreased in magnitude as the rere adranced into shallower water, and the greatest horisomtal relooities ocourred at the ware breaker position. It is interesting to note that the curres are closer together on the natter beach slope of 1:18 than on the slope of 1:9 but that on each beach slope, the velooity seems to have a constant value at all positions along the beach 0.4 seas 1.0 . half the wave period after the passage of the wave crest, although the constant velocity varies in magnitude Prom one slope to another. It is evident from figures 3 and 4 that for each position on the beach, the maximum horisontal shoreward velocity did not ocour direotly under the ware crest but at a time 0.09 seconds after the passage of the orest ( $T$ is rave period). 


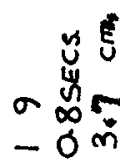

II II II

แ

$\alpha$

जे $\frac{w}{\alpha}$

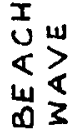

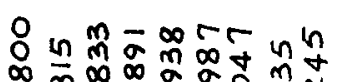

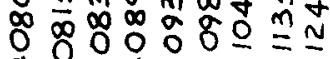
000000000 | " | || || " | " " 內 $==$ : $=$ = =

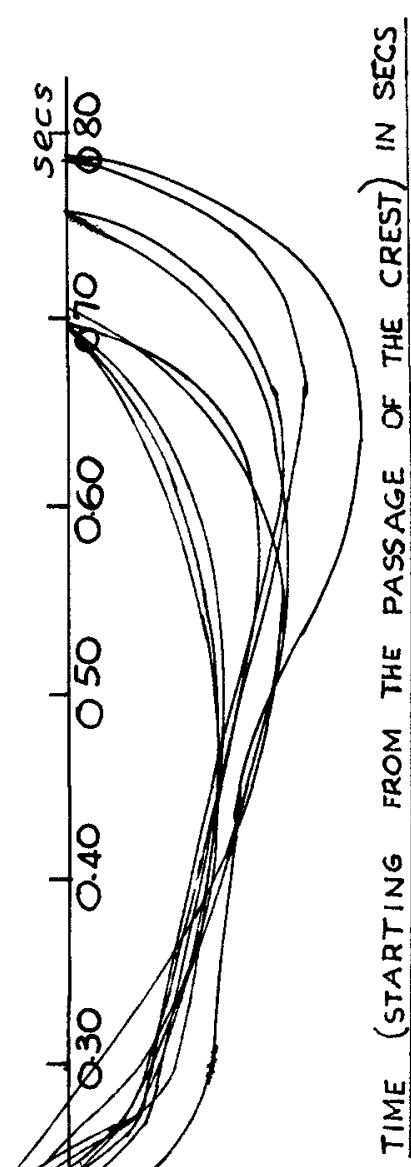

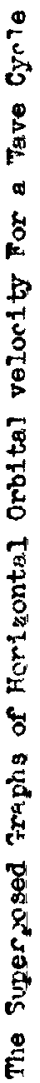

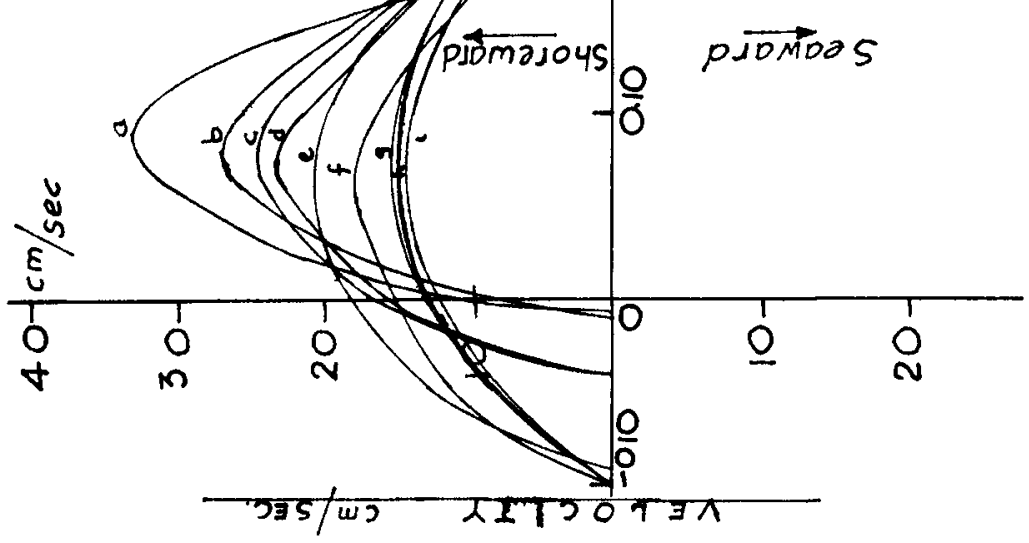

$\sum_{i=1}^{m}$ 

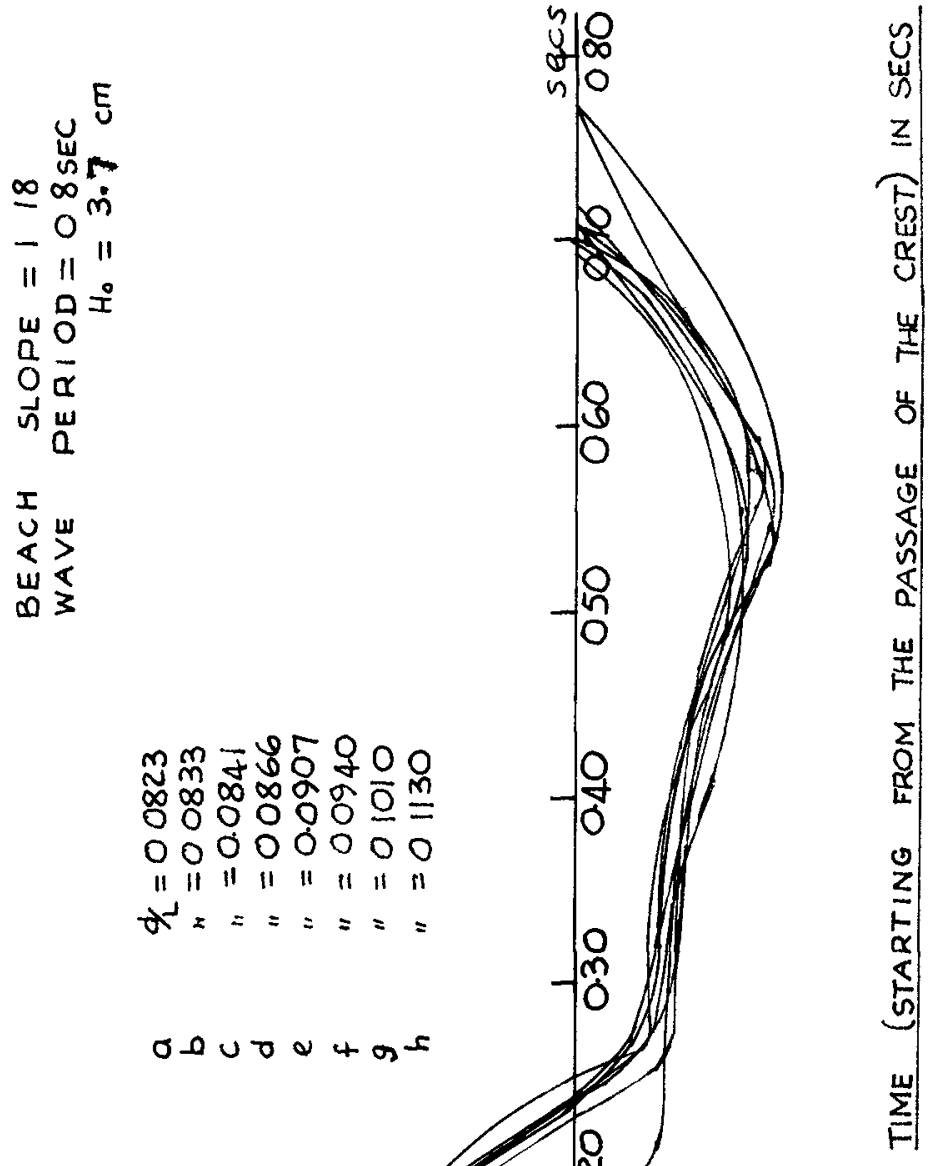

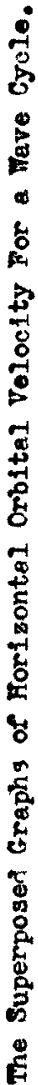

m

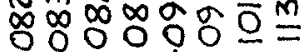
00000000 11 || || $|1||||| \mid$ $d x=====$

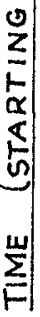
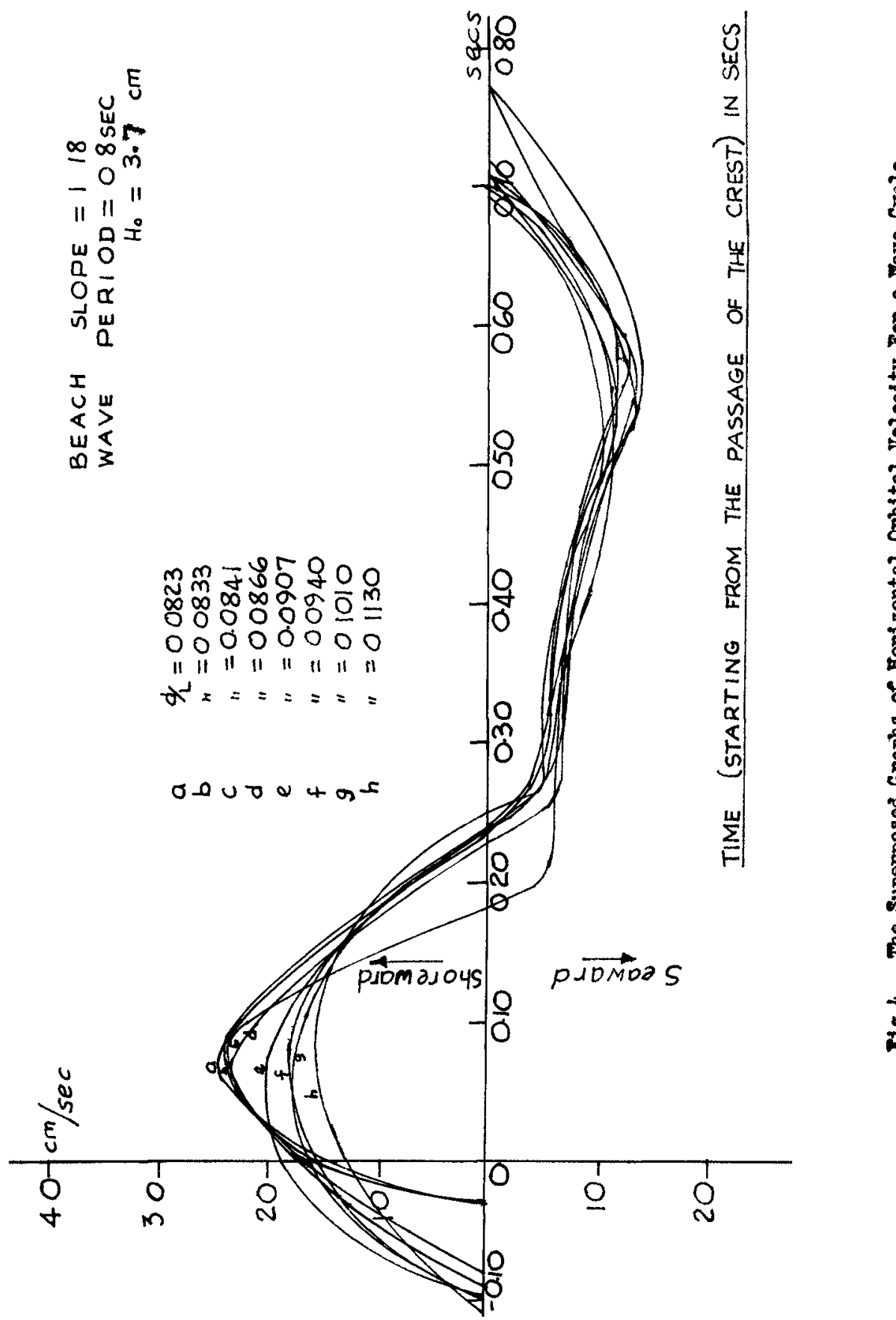

$\pm$

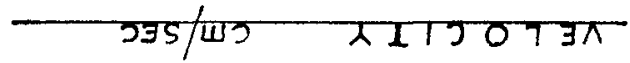


The graphs of horizontal velocity (magnitude) asymetry against d are shom in figure 5. It was found that for the two slopes of 1:9 and 1:18 the horizontal velooity (magnitude) asymetry inoreased as the wave advanced into shoaling water and in each oase was highest at the wave breaker position. At the $q$ value of 0.10 on the beach slope of 1:9 this asymetry wes found to be $1: 37$ whle on the beach slope of 1:18 it was found to be 1.585. At the wave break-point on the beach slope of 1.9 the value of this asymetry was found to be 1.63 while the oorrosponding value on the beaoh slope of $1: 18$ was 1.77 .

In teres of bsolute values of the horizontal velocities, the values on the steoper slope of 1:9 were found to be higher than those of on the flatter slope of $1: 18$, especially in the noighbourhood of the broekers, but the graph of the homzontal velooity (magnitude) asymetry against d (figuro 5) indicated that the asymetry values were higher on the liplatter slope. In other words larger beach material could be sot in motzon on the steep slope, but a greater shoreward - to - seaward differential rould act on material on the flattor slope.

The graphs of horizontal relooity (tiwe) asymetry against $\frac{d}{\mathrm{I}}$ are ahom in figure 6. These show inoreasing asywetry as the wave advances into shallower water. As noted in the introduction above, the horisontal velocity (time) asymetry was defined as the ratio of the the for the shoreward notion to the time for the seaward motion, thus a small muerical value compared with unity denotes inoreasing asymotry.

It was found that at the $/ /$ ralue of 0.10 the value of this asymetry on the slope of 1.9 wis 0.76 whilst on the beach slope of 1:18 the value was found to be 0.78 . At the wave breaker position on the beach slope of 1:9 the asymmetry became 0.37 whilst the oorrespanding value on the slope of $1: 18$ was 0.49 . Thus the shoreward motion takes a longer time on the flatter slope.

Fig. 7 illustretes the comparison between stokes theoretion wave proflle based on his thin approstmation, and typical profile obtained in the pxosent study. It om be seen that the Stokes Wave is less poaked then the experimental wave, and also, by virtue of its mathematical desoription, does not possess asymetry about the verticel axis.

Pigures 8 and 9 whioh compare the oxbital velooities based on Stokes theory with those obtrined experimentally, show that at a $\frac{d}{t}$ velue of 0.0833 , which is very olose to the breakers, the theory olearly doos not agreo with the easurements (seo sigure 9). Farthor eaward from the break-point, at a dalue of 0.1135 (figure 8), although the theoretios values wers still muah hi gher than the experimontal values, for anstanoe, by as muah as 73 of the experinontal value under the crest, and there was in addition a phase lag in the profile, nevertholoss the oamparison was better then in flgure 9. 


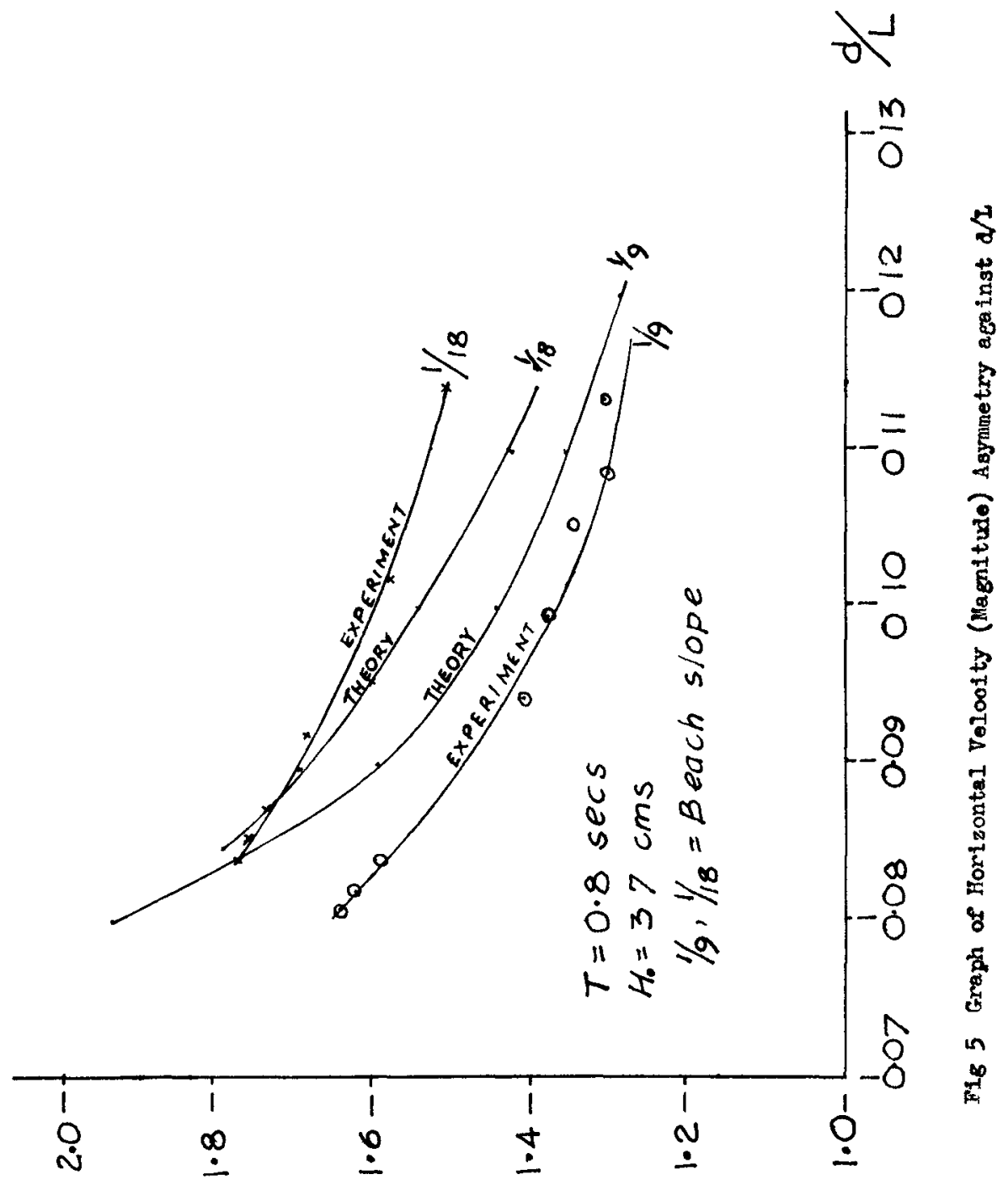

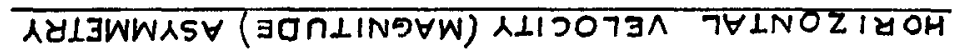




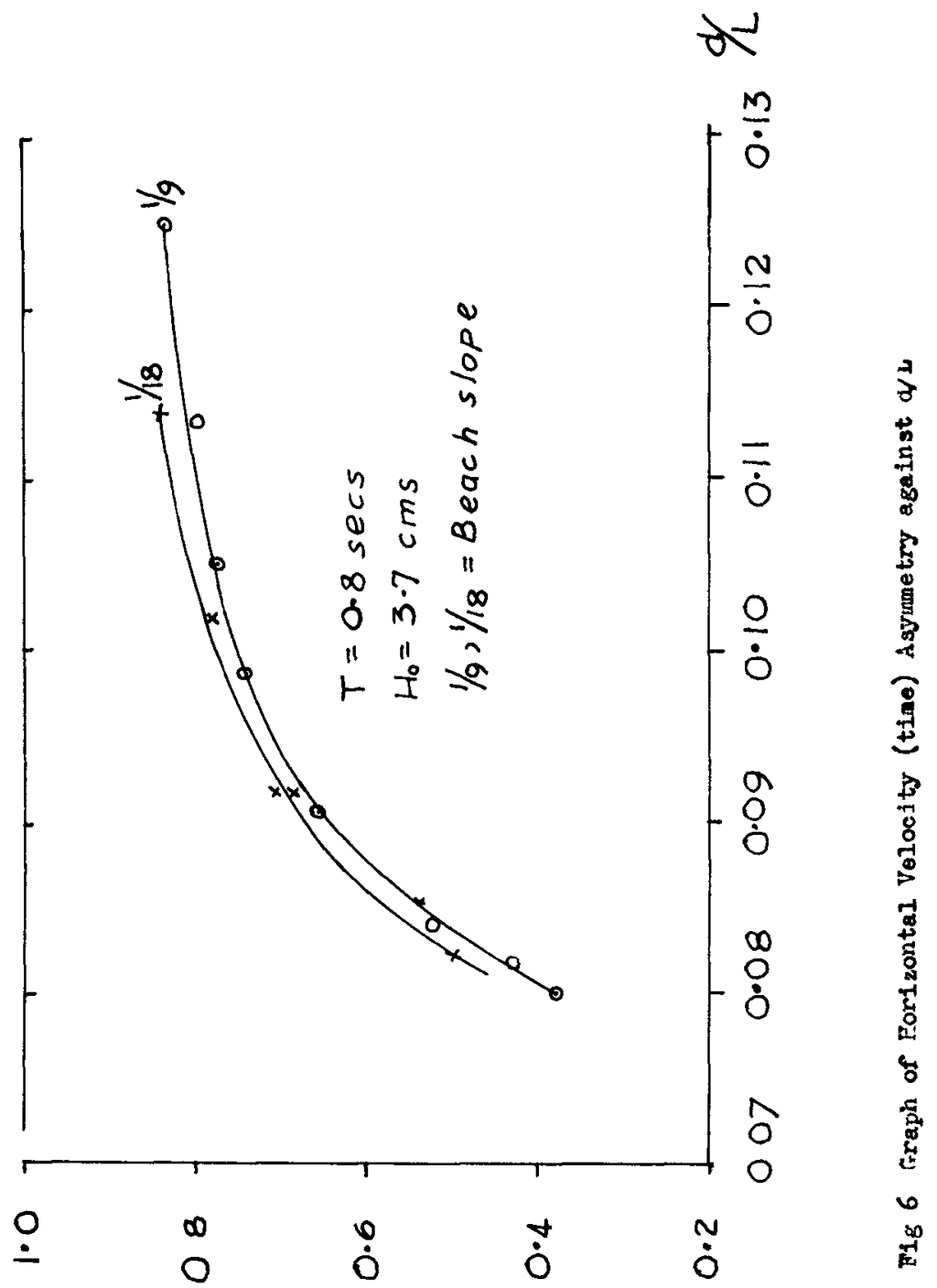

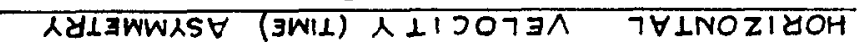



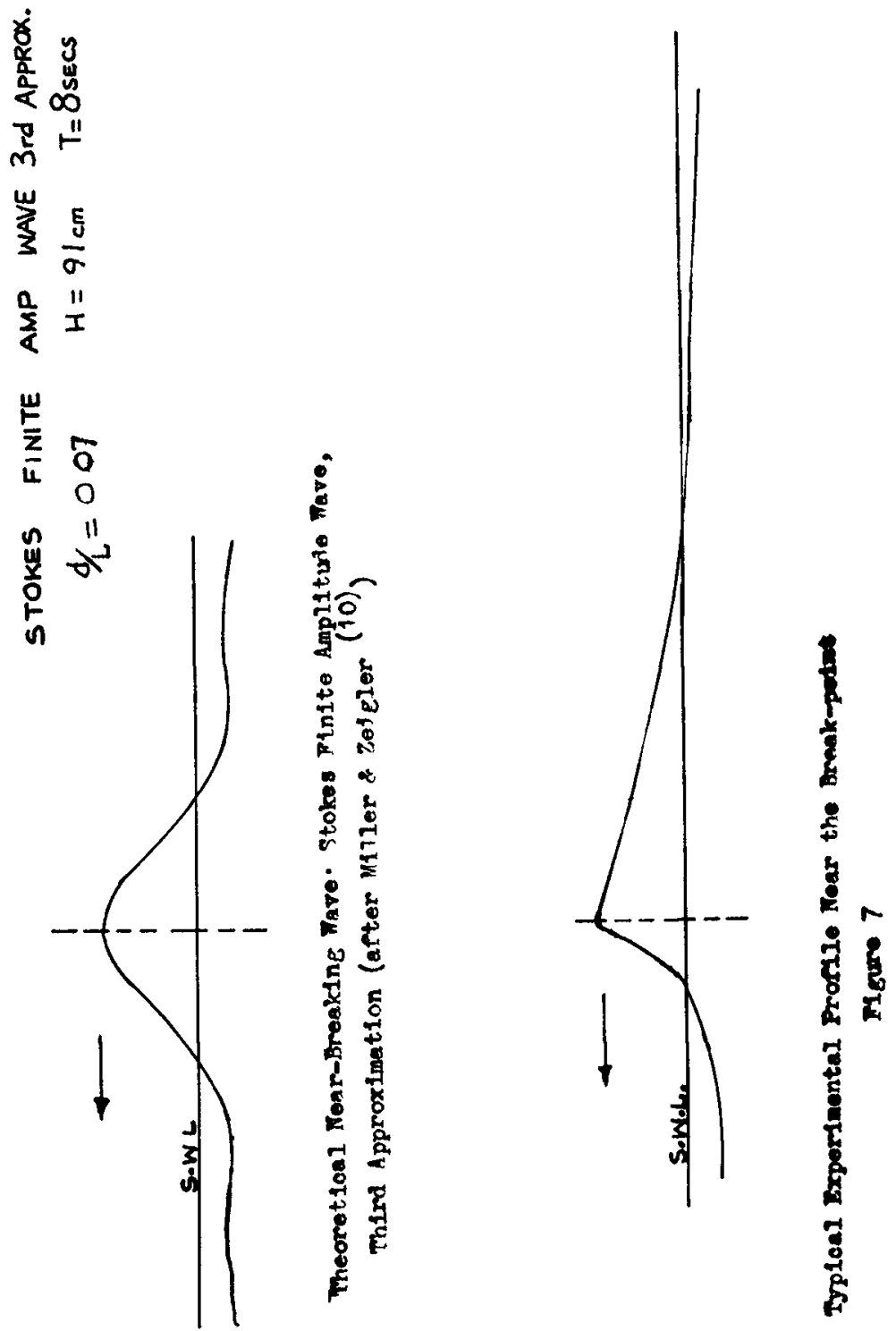
BREAKER ZONE

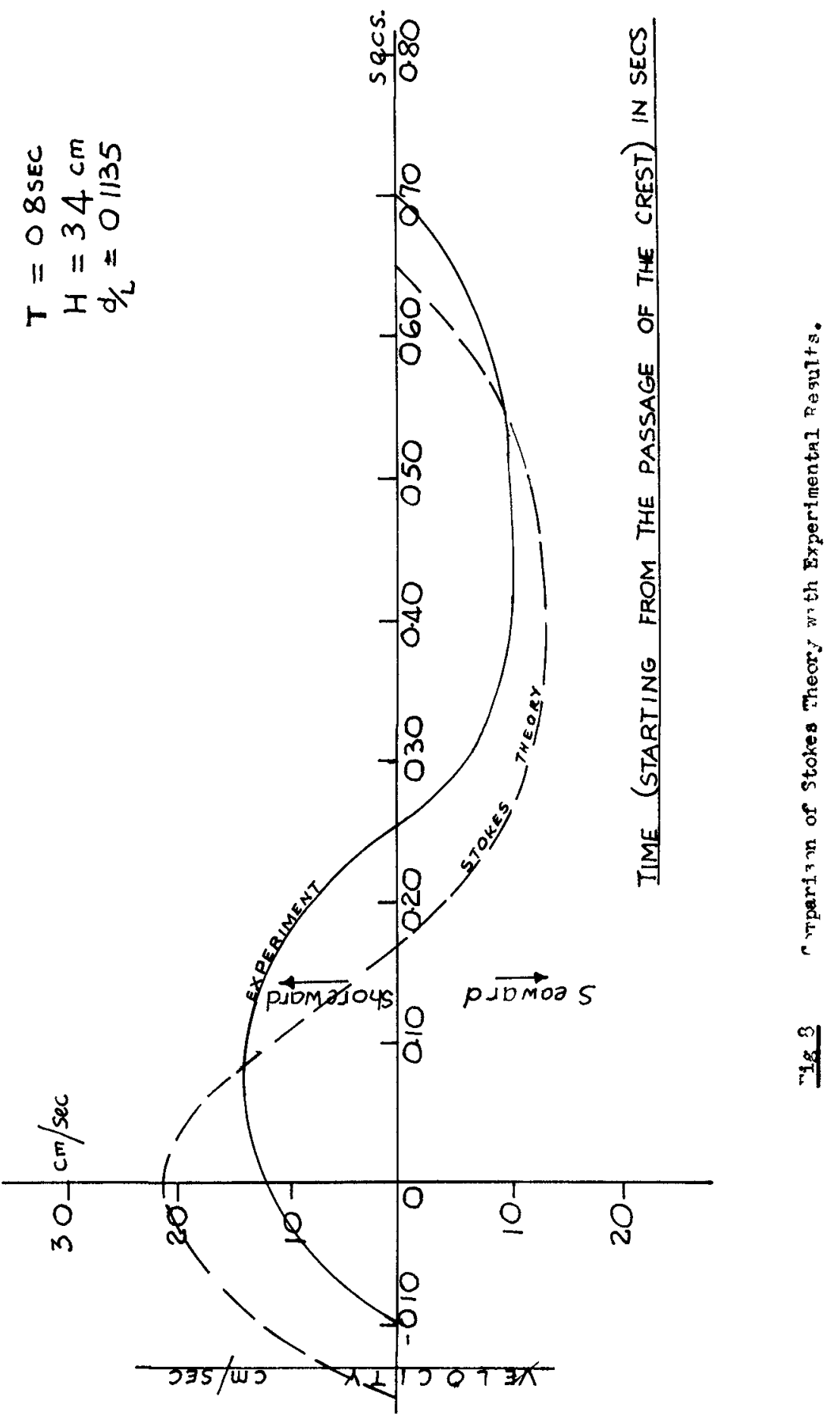




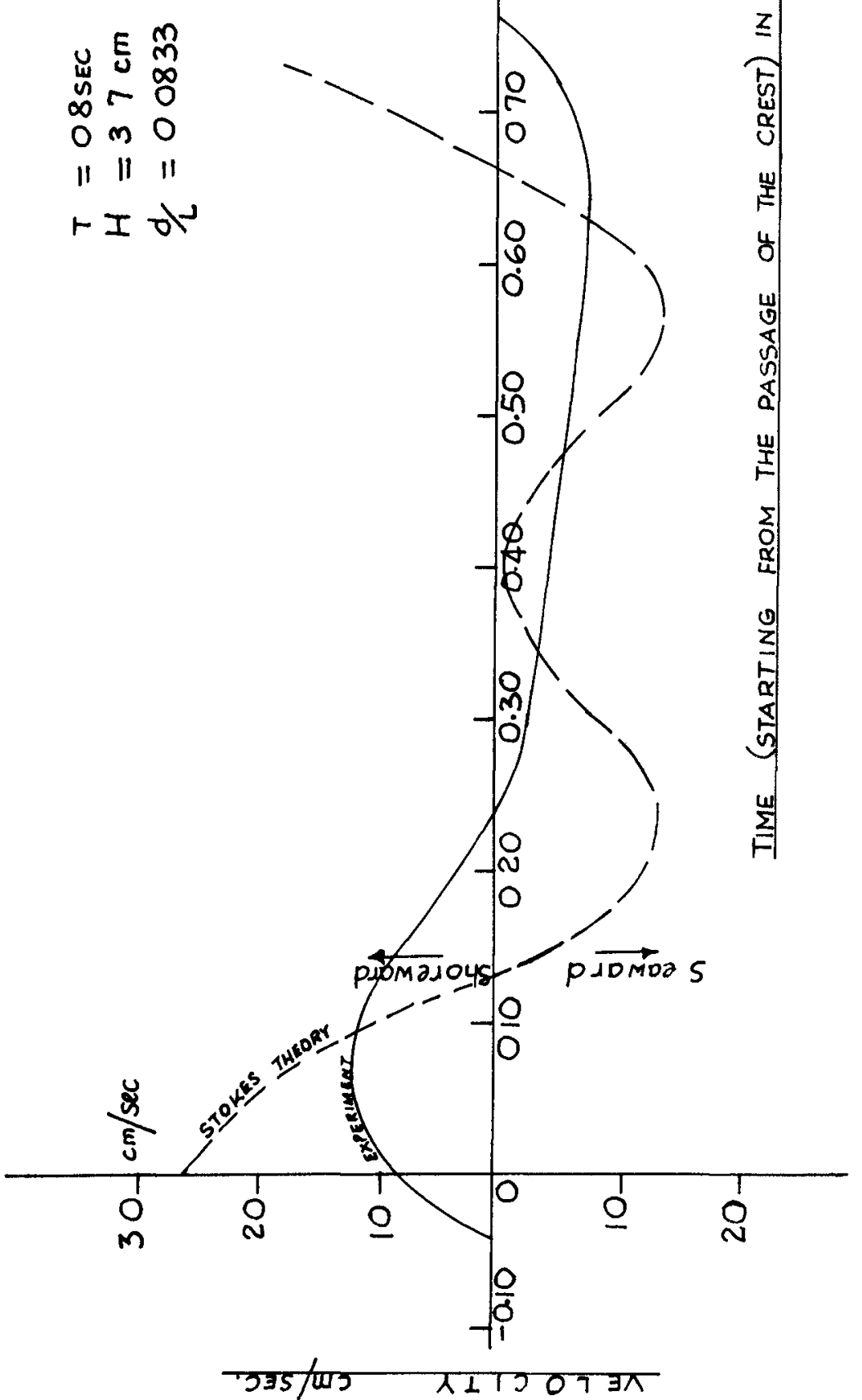

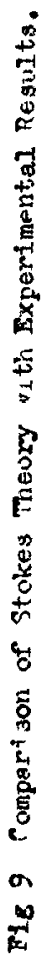


The horirontal component of water particle relooity at a point $(x, y)$ in water depth $a$ is giren to the second order of Stokes theory by the expression:

$$
\begin{aligned}
& 0=\frac{\pi H}{T} \frac{\cosh 2 \pi\left(\frac{y+d}{L}\right)}{\sinh \frac{2 \pi d}{L}} \cos 2 \pi\left(\frac{x}{L}-\frac{t}{T}\right) \quad+ \\
& \frac{3}{\left(\frac{\pi H}{T}\right)}\left(\frac{\pi H}{L}\right) \frac{\cosh 4 \pi\left(\frac{I+d}{L}\right)}{\sinh ^{4} \frac{2 \pi a}{I}} \cos 4 \pi\left(\frac{x}{I}-\frac{t}{T}\right)
\end{aligned}
$$

or in simplifiod form

$$
U=A \cos 2 \pi X+B 0004 \pi X
$$

An inspeotion of equation (5) shows that the velooity oomponents due to the firet term on the right hand side are positive under the ware orest and negative under the trough, nhilst the second ter is positive under both orest and trough, and gives maximum negative oomponents at $\frac{I}{4}$ and $\frac{2 \pi}{4}$ fron the orest. The not offeot is to increase the value of the magititude under the orest, and decrease it under the trough, thus producing asymetry in the magnitudes of the oxbital velooity. Fig. 10 ocmpares the ralues of the horizontal volooity (magnitude) asymetry given by the Stokes theory-equation(5) with the experimental rosults, and the theoretical values frox equation (10). It oan be ween that the divergenoe between the stokes prediotion and the experimental results was about $32 \%$ at a $\frac{a}{7}$ value of 0.11 , with the divergonce increasing shoreward but improring seaward.

For these reasons, one can conclude that Stokes theory is not direotly applioable to the study of relocities in the near breaker zone.

\section{Gorrelation Betwen Fare Asranetry and Velocitr Asymetrn}

An inportant aspect of the woxk oarried out by the author is the study of the oomelation botween the different types of wave asymetry and the correlation between these and the resulting relocity asymotry as the rave (pFogressed into shallon water. The first aspeot has been reported (3).

Figure 11 shows the graphs of both the horisontal relocity (eagnitude) asymetry and the horizontal velooity (time) asymetry plotted against the wave horizontal asymetry $H_{A}$ and $H_{A}{ }^{1}$ for the beach slope of 1:18. The graphs show that a oorrolation oxdsts between the velocity asymetry and the rave asjunetry. The eraph of horisontal velocity (megnituda) asymotry against wave horizontal asymotry $\mathrm{H}_{\mathbf{A}}$ indioatod that as the wave progressed into shallower water and the value of $\mathrm{H}_{\Lambda}$ docreased, indioating increased asymetry, 


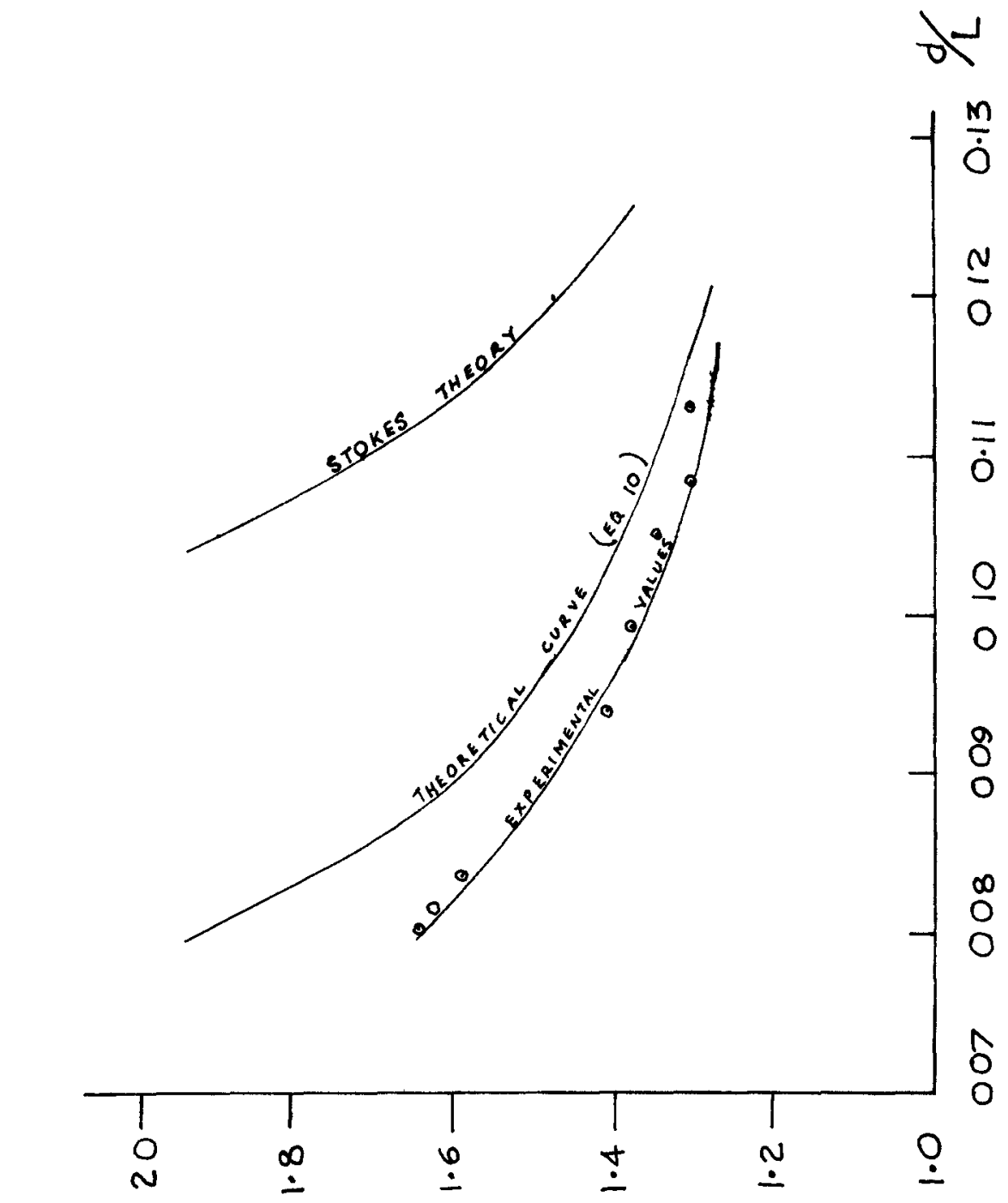

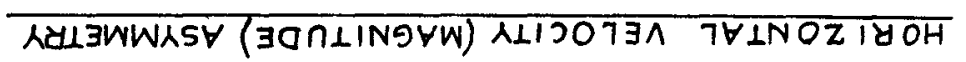

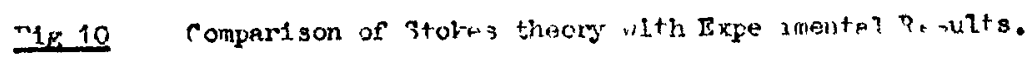




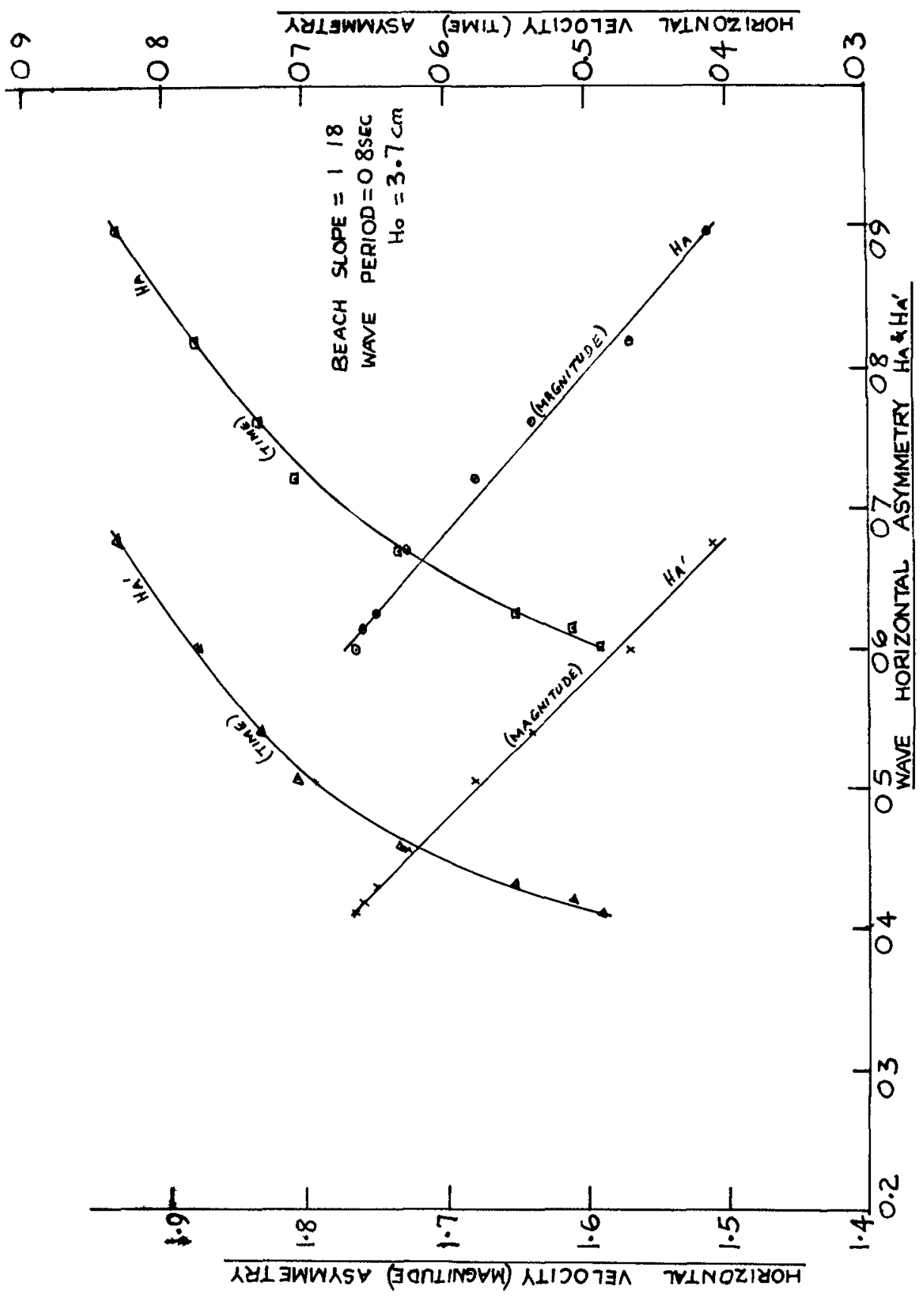

Fi gure 11 Graph of Velocity A aymetry ALaingt 7ave isymmetry. 


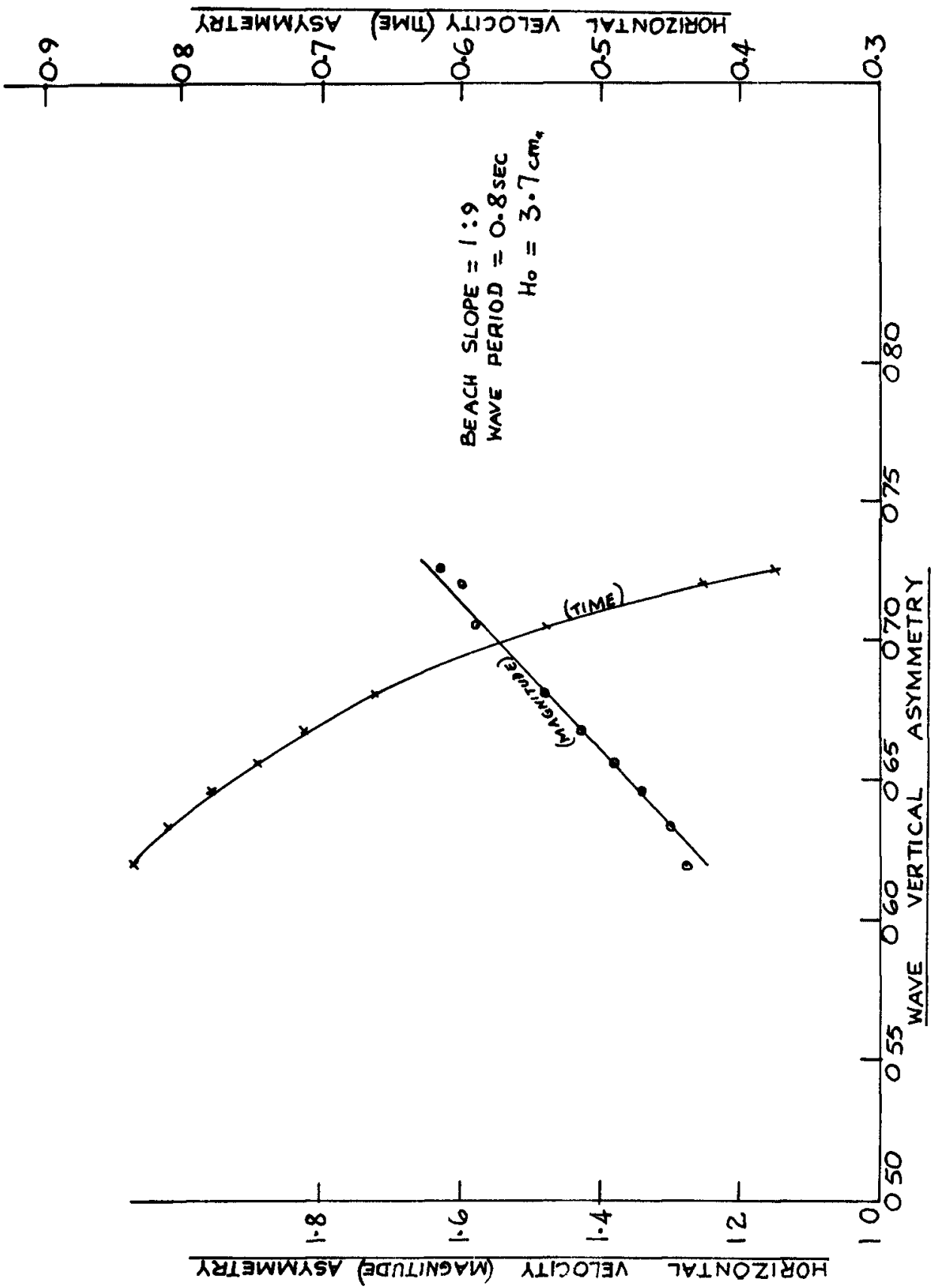

Fis 12 Graph of velocity Asymetry Against, Tave Asynmetry. 


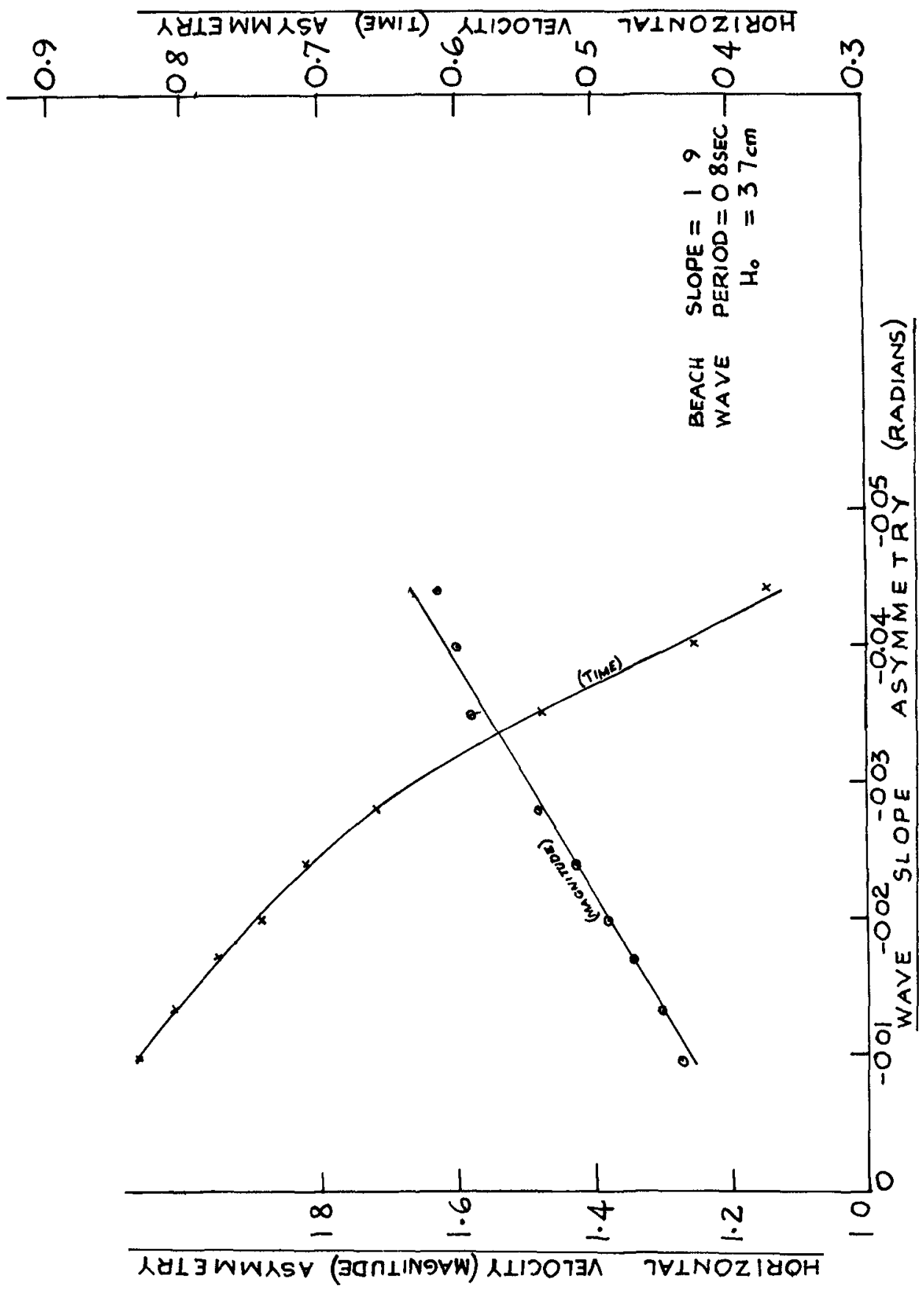

Fig 13 Frapl of velocity Asymmetry Agminst Fave Asymmetxy. 
the horisontal velocity (megnitude) asymetry also increased. For instance, when the value of the wave horizontal asymmetry $H_{\Lambda}$ is 0.80 the value of the horizontal velocity (magnitude) anymetry is 1.60, whereas when the value of $\mathrm{H}_{\mathrm{A}}$ becomes 0.70 the value of the horizontal velocity (nagnitude) asymetry increased to 1.685 very near the wave break-point, the ralue of $H_{\Lambda}$ becomes 0.60 the velue of the horizontal velooity (magnitude) asymetry is found to be 1.77. The greph of the horizontal volocity (magnitude) asymmetry against $B{ }^{1}$ followed a trond fairly similer to the graph of the horizontal velocity (magnitude) asymetry against $H_{A}$. The graph of the horizontal velooity (time) asymotry against $H_{\Lambda}$ and $H_{A^{1}}$ also show good correlations. The remaining graphs of the velocaty asymetry against the difforent types of wave asymetry all show reasonably good correlation botween the volocity asymotry and the ware asymanetry. (see figures 12 and 13). obtained:

As a result of the studies the following relationships wero

Let $A r=$ wave vertical asymetry = Vertical distance from orest to S.W.I. Total wave hoight.

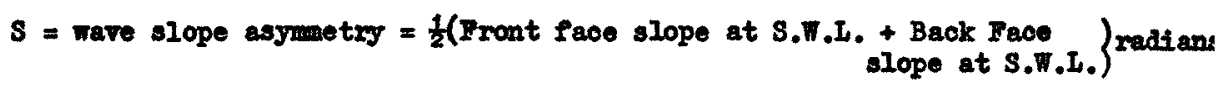

$$
\begin{aligned}
|s| & =\text { modulus of } s \\
\gamma & =\text { beach slope. } \\
V_{\text {FIM }} & =\text { Horizontal velocity (magnitude) asymmotry } \\
B_{A} \& H_{A} 1 & =\text { wave horizontal asymmetry }
\end{aligned}
$$

$H_{X}=$ Horizontel distance fron oreat to front face at S. I. I. Horizontal distance from crest to back face at S.W.I.

$\mathrm{H}_{\Lambda} 1=$ Horizontal distance from crest to preceding ware trough Horizontal atstence from crest to following ware trough

The ampirical rolationships botween $\nabla_{\mathrm{HW}}, s, \gamma, \Delta \mathrm{N}$ and $\mathrm{H}_{\mathcal{A}}$ aro:

$$
\begin{aligned}
& H_{\Lambda}=\frac{1.52}{e^{\gamma}}(1.18-\sinh A \tau) \\
& \nabla_{\text {HII }}=3.4 A T-0.78 \\
& A T=\frac{8.8}{e^{\gamma}} \tanh |s|+0.5
\end{aligned}
$$

From the Cnoidal wave theory as dereloped by Korterreg and De Vries(8), the wavn vertioel asymetry $A T$ is given by 


$$
A v=\frac{z_{0}-a}{H}=\frac{16 a^{3}}{3 L^{2} H}\left\{K_{(k)}\left[K_{(k)}-E_{(k)}\right]\right\}-
$$

where $J_{0}=$ distance fron the ooan botton to the wave crest

$$
\begin{aligned}
& \text { I = atill water depth } \\
& \text { H }=\text { wavo height (trough to crest) } \\
& \text { L }=\text { wave length }
\end{aligned}
$$

$K(k)=$ complete elliptio integral of the rixat kind.

$E(k)=$ complete ellaptio integral of the second bind.

$k=$ modulus of the elliptic integral

Froe oquations (9) and (7) wo have

$$
\nabla_{\mathrm{HCA}}=\left[\frac{18.1 \mathrm{~d}^{3}}{\mathrm{~L}^{2} \mathrm{H}}\left\{\mathrm{K}_{(\mathrm{k})}\left[\mathrm{K}_{(\mathrm{k})}-\mathrm{E}_{(\mathrm{k})}\right]\right\}-0.78\right]-
$$

substituting fox Av from eq. (8) into eq. (7) gives

$$
v_{\text {HIA }}=3.4\left\{\frac{8.8}{e^{\gamma}} \tanh |s|+0.271\right\}
$$

Also from the work of Biesel ${ }^{(2)}$, the wave slopo asymatxy $S$ is given by

$$
=m^{2}\left(\frac{H_{0}}{2}\right)^{2} \gamma\left\{\frac{3+\frac{\operatorname{ma}}{\tanh m d}-3 \text { ma } \tanh m d}{D^{2}(\sinh m \alpha)^{2} \tanh m d}\right\}-
$$

$$
\begin{aligned}
& \text { whor } D=1+\frac{\text { nd }}{\text { sinh } \cosh n d} \\
& \text { and } n=\frac{2 \pi}{I}
\end{aligned}
$$

substztuting s from oq. (12) into eq. (11) gires

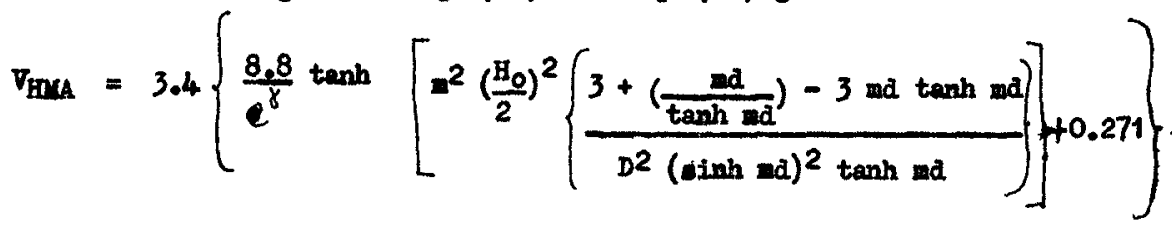


Thus equations (10) and (13) give expressions for $\nabla_{\text {HW }}$. Howerer, of the two, equation (10) gives closer values to the expermmontal results in this woxix, and is the theoretical curve shown on figure 5. Values of $K(k)$ and $B(k)$ are tabulatod in Kesoh and $\nabla 10 g 01(9)$ (1961).

\section{Coment}

Mgure 5 gives the impression that the theorotical prodiotions for horizontel velocity asymetry are aloser to the experimental values, for the steeper beach slope oategory. The correspondence is to rithis $5 \%$ in the region of $\Phi_{2}=0,10$ to 0.13 , and shoms a difference of about $8 \%$ rexy close to the breakexs where $\mathrm{a}$ is approxdmately 0.08 . In faot, sinilar percentages apply in the cast of the flatter beach slope, but the difference in the slope of the curves is more apparent and has the effoot of offsetting the correlation.

\section{Comolustons}

The results shew that the horizontal reloolties increase in shoaling water, and for both slopes of $1: 9$ and 1:18 the greatest horizontel relooities occurred at the wave broaker position. The curves of the velool ty-tive history flgures 3 and 4 show that the curves were cioser together on the slope of 1:18 than on the slope of 1:9. It was however found that the relooity seens to have a constant value on each beach slope at all positions along the beach hele the wave perfod after the passage of the wave orest; although the constant varies from slope to slope.

It was found that the meximin howlentel shoreward volocity did not ocour dirwotly mador the ware orest but at a tim $0.09 \mathrm{~T}$ soconds after the passege of the crest. In terms of the values of the horizontel relooities, the ralues on the steeper slope of $1: 9$ were higher than those on the flatter slope of 1:18 especially in the noighbourhood of the broakers, but it was found that the values of the horisontel velooity (nagnitudo) asymetry were higher on the Fatter slope. Nao the graph of the homeontal velooity (time) asymetry against di showed that the shorerard notion takes a longex tiwe on the flatter slope.

The author is of the vien that there mast be a correlation between the wave asymetry and the asymotry of the relooity field realting from the wave. The graphs of the differont types of wave asymetry defined by the author(3) against the veloci ty asymetry confirm that there are both qualitative and quantitative correlation between the wave asymetry and the velocity asymmetry. As a result of the correlation two alternative expressions aro given for the borisontal velocity (maenitude) asymetry, one based gn the Cnoldel wave theory and the other based on the work of Biesel $(2)$.

The paper also shows that Stokes theory is not directly applicablo to the study of the velooity patterns in the near breaker zone. 
In drawing conoluaions from the study of the velocity flelda associated with esymotrical waves on varylng beach slopes it is important to note that uflier and zelflor (10) observed three broad classes of wave asymetry on a beach of ans one slope. It is clear, as they sugsest, that in nature in addition to the inportance of beach slope, broaker shapes are affeoted by the interraction of different wave trains, and by the timing and nagnitude of the backwash. It seess probable that the observations of Inman and Iasu(6) that bicher orbital velooities are associated with wave shape asymetry wust be considered in relation to the faot that their measurenents were also nade in nature, and that more than one wave train is likely to be present.

The author considars that investigations of the velooity fields very close to the bed should erentually provide a quantztative basis for evaluating the acorvtion and dopletion of sediment under rave aotion. The megnitude and duration of the shoreward and seaward orbital veloofties are important in sediment movement under ware actions. The nagnitude of the madimun velooity is associated with initiating the motion and the asymetry of the velocity flolds deteraines the direotion and extent of the net movement. The author thus considers that the quantztative correlation of the wave charaoterstics with the asymetry of the orbital velooity flelds would most probably lead to the correlation of wave characteristica with sediment moroment.

\section{Acknowledgerente}

The author wishes to express his gratitude to Prof. A. H. Chilvor, the Chadwiok Professor of Civil and lunioipal Anginening, Oniversity Collogo, London, where this work was oarried out, and to Dr. P. H. Keap who supervised the work.

The author also Whahes to thank D. W. Vale, T. G. Gurman and Mick Gregory for their holp in the experimental aspecte of the rork.

\section{PESERTHIGSS}

(1) Irersen, H. W. - Vaves and breakers in shoaling water. Proc. Thind Conf. Coastal Inginoering 1952 Pp. 1-12.

(2) Bzesel, F. - Study of Wave Propagation in water of gracually verying dopth. Gravity waves circular No. 521 Nat. Bureau of Standards, Tashington D.C. 1951.

(3) Adeyemo, X. D. - Bffect of beach slope and shoaling on Wave Asy try. Proc. Eleventh Conf. Coastal Engineering, London. 1968. 
(4) Adeyomo, M.D. - Wave transformation in shalion water. La Houzlle Blanche No. 6. 1969.

(5) Hanada, T. - Breakers and beach erosions. Rept. Transportation Teoh. Res. Inst. Tokyo. Report No. 1. Deo., 1951.

(6) Inman, D. I. - Oxbital velocity assooiated with rave aotion and Nasu, N. near the breaker zone. Beach Erosion Board. Washington D.C. Teah. Werso No. 79, 1956.

(7) Ippen, A. T. - The shoeling and breaking of the solitary wave. and Kulin, G. - Proc. Fifth Conf. Coastal Ingineering. Sopt., 1954.

(8) Korteweg, D. J.- on the change of form of long waves adranoing and De Vries, $G$. in a rectengular canal, and on a new type of long stationary naves. Phil. Mag. 5th series 39, 1895 pp. $422-443$.

(9) Masch, F. D. - Cnozdal waves - Tables of Funotions Council on and Wiegel, R. L. Wave researoh. The Engineemng Foundation Rachmond, California. 1961. 129 pp.

(10) killer, R. I.

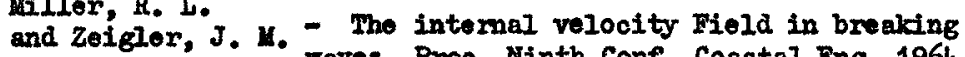

(11) Morison, J, R. and Crooke, R. C. - The mechanics of deep nater, shallo water broaking waves. Beach Erosion Board. Tech. Homo. No. 40. 1953. 


\title{
CHAPTER 28
}

\section{IHE DEVELOPMENT OF UNDULAR BORCS WITH FRICTION}

\author{
by
}

O Hawaleshka* and S B Savage**

\begin{abstract}
A theoretical and experimental study of the initial development of undular bores in two-dimensional, rectangular channels with and without boundary friction was performed Equations similar to those of Boussinesq, but including higher order and wall friction terms are presented and solved numerically by an implicit finite difference method A Pohlhausentype boundary layer monentum integral method is used to obtain the wall shear stress distribution under a developing long wave from the cons1deration of the boundary layer underneath it The solution is performed in a quasi-iterative manner proceedıng from the friction coefficient calculation for an initially assumed wave profile to the inclusion of this coefficient in the calculation of a new wave profile at an advanced time Comparisons of theoretical and experimental results are given For the initial development of tile undular bore with which the present work is concerned, the measurements are found to be in reasonable agreement with the theoretical predictions The effect of the wall shear stress manifests itself mainly in a slight reduction of the wave armlitudes
\end{abstract}

\section{INTRODUCTTONS}

Bores are transitions between two essentially uniform liquid flows A turbulent breaking zone is associated w1th "strong" bores, but if the depth change is relatively sma1l, the bores are termed weak and may consist of a train of undular waves following the head wave These waves are found to be closely approximated by the elliptic or cnoldal wave form of Korteweg and DeVries (1) This class of waves to which the solitary wave belongs as we11 is characterized by constancy of shape and a marked resistance to decay, in contrast to such waves as the non1inear shallow water waves of Aury that regardless of their initial smallness will eventually grow, steepen and break. Ursell (2) has

\footnotetext{
* Assistant Professor, Mechanica1 Engineering Department, Unıversity of Man1toba, Winnipeg, Manıtoba, Canada

**Associate Professor, Department of C1V11 Engineering and Applied I lechan1cs, McGi11 University, Montreal, Quebec, Canada
} 
distunguished various types of shallow water waves by the parameter $a \lambda^{2} / h^{3}$ where values of $a \lambda^{2} / h^{3} \gg 1, a \lambda^{t} / h^{3} \sim \alpha(1)$ and $a \lambda^{2} / h_{3}<<1$ correspond to the Alry theory, the nonlinear theory of cnoldal and solitary waves and the linearized theory respectively

Such bores may be created in many ways For example the opening of lock gates will form positive surges in the canal and negative ones within thelock A sudden stoppage of a turbine in a power station creates a positive surge in the supply channel and a negative one in the tall race channel Simllar bores are formed in tidal estuaries at rising tide

It is of interest to be able to predict the development of such bores and related waves, taking account of frictional effects Up till now, most investigations of undular bores were performed for condutions where these effects were neglected or were treated in an approximate manner by the use of, for example, some average value of a Chezy coefficlent (cf ex Benjamin \& Lighthil1 (3), Sandover \& Taylor (4), Sandover \& Zıenkıewıcz (5), Sturtevant (7), Peregrıne (6), Murota (8))

In this paper we treat the problem of an undular surge or an arbitrary long wave, considering the effect of boundary friction This shear effect is obtained from the computation of the development of the boundary layer underneath the advancing wave

\section{EXPERIMLNTAL SETUP AND PROCEDURE}

An alumnum channel with glass side walls was used during the tests, (fig 1) Its dimensions are 30 feet length, 12 inches wide and 18 inches deep, and was typically filled to a depth of four inches of water A close-fitting piston with a maximm stroke of three feet and with a continuously variable velocity was installed at one end of the channel and was actuated hydraulically It was found that with sultable combinations of piston stroke, speed and water depth a complete range of bores as well as a reasonable approximation to a solıtary wave could be produced Care was taken to provide seals around the wetted portion of the piston, effectively eliminating leaks

The surface profiles resulting from the motion of this piston were measured at several stations along the channel by streamlined, inmersed, variable-resistance probes and recorded together with the piston motion on a six channel recorder Electronlc filters were used to remove unwanted higher frequencies from the signals The wave-measuring equipment was bullt by Kempf-Remmers The accuracles obtained were essentially 1imited by the capillary effects of the water The probes themselves were of fiberglass construction in the shape of a symmetrical streamlined profile with conducting strips on each side of the probe's leadıng edge Production rum calibrations were done statically before each run, since a test dynamic calibration showed no appreciable difference

Some dye studues of the boundary layer under the advancıng wave were performed to record visually the bottom boundary layer development 
Photographic records of surface orofiles, in particular at the inltial stages of the plston motion were taken by both still and cune cameras

The test procedure involved the measurements of the wave profiles for various non-dimensional piston speeds $U_{p}$ from 01 to 05 and for still water depths ranglng from two to flve inches

THEORETICAL APPROACH

\section{$1 \quad$ Equations of Motion}

We make use of the equations derıved by $\mathrm{Su}$ and Gardner (9) for nonlinear dispersive shallow water waves These are in non-dimensional form

$$
\begin{aligned}
& \frac{\partial h}{\partial t}+\frac{\partial}{\partial x}(h \bar{u})=0 \\
& \frac{\partial(h \bar{u})}{\partial t}+\frac{\partial}{\partial x}\left[h \bar{u}^{2}+\frac{h^{2}}{2}-\frac{h^{3}}{3}\left(\bar{v}_{x t}+\bar{u} \bar{v}_{x x}-\bar{v}_{x}^{2}\right)\right] \\
& \text { where } \quad h=\frac{h^{*}}{h_{0}}, t=t^{*} \sqrt{\frac{g}{h_{0}}}, x=\frac{x^{*}}{h_{0}}, y=\frac{y^{*}}{h_{0}} \\
& \bar{u}=\frac{1}{h} \int_{0}^{h} \frac{u^{*}}{\sqrt{g h_{0}}} d y
\end{aligned}
$$

The starred variables are dimensional and $h$ is the local water depth, $t$ is time, $x$ and $y$ are the horizontal and vertical coordinates, $u$ is the horizontal component of velocity, $\bar{u}$ is the depth averaged velocity, $g$ is the gravitational acceleration and $h_{0}$ is the undisturbed reference depth, see fig 2

Equations (1) and (2) are similar to the Boussinesq equation but include the higher order terms

$$
\frac{\partial}{\partial x}\left[\frac { h ^ { 3 } } { 3 } \left\langle\bar{u} \vec{u}_{\psi x}-\bar{v}_{z}^{2}\right.\right.
$$

in equation (2) We can approximately account for the effects of wall friction by the addition of the term

$$
\text { Friction term }=-\frac{f h \bar{u}|\bar{u}|}{R}
$$

to the left hand side of equation (2), where $R$ Is the dumensionless hydraulic radius at any station,

$$
R=\frac{h b}{2 h+b}
$$

and $b$ is the dimensionless channel width $b / h_{0}$ 
2 Calculation of the friction coefficient from a Momentum Integra1 consideration of the boundary layer developed by a long wave

We now carry out an approximate momentum integral analysis in order to calculate the friction coefficient $f$, to be used for the friction term (3) which is added to equation (2) The notation in this section is somewhat different from the previous one and unless otherwise noted unstarred variables refer to physical quantities

Consıder the development of a lamınar two-dımensional boundary layer under a long wave advancing with speed $u_{F}$ into still water of depth $\mathrm{h}_{\mathrm{O}}$ In the boundary ldyer analysis the inviscid fluid velocity $\mathrm{u}_{1}$ is approximated as equal to the depth-averaged value obtained from the integration of the long wave equations Fig 3 shows the situation when brought to a quasi-steady state with respect to the wave front, and also defines the notation to be used In this quasi-steady frame the boundary layer equations are

$$
\begin{aligned}
& \frac{\partial u}{\partial x}+\frac{\partial v}{\partial y}=0 \\
& u \frac{\partial u}{\partial x}+v \frac{\partial u}{\partial y}+\frac{1}{\rho} \frac{\partial p}{\partial x}=-\frac{\partial^{2} u}{\partial y^{2}}
\end{aligned}
$$

where the symbols have the usual meanings Upon integration we obtain the common boundary layer momentum integral equation

$$
\frac{d \theta}{d x}+\left(2 \theta+\delta^{*}\right) \frac{1}{u_{e}} \frac{d u_{e}}{d x}=+\frac{\tau_{0}}{\rho U_{e}^{2}}
$$

where $\boldsymbol{\theta}$ and $\boldsymbol{S}^{*}$ are the momentum and displacement thicknesses respectively and the wall shear stress is defined by

$$
\frac{\tau_{0}}{\rho}=\left.\mu \frac{\partial u}{\partial y}\right|_{y=0}
$$

Equation (6) is solved by assuming a suitable form for the velocity profile in the boundary layer, a procedure first suggested by vo. i irs or Pohlhausen We take the fourth degree polynomia1

$$
\frac{u}{u_{e}}=f(\eta)=\frac{u_{w}}{u_{e}}-\left(\frac{u_{w}}{u_{e}}-1\right)\left(2 \eta-2 \eta^{3}+\eta^{4}\right) ; \eta=y / 5
$$

which satisfies the conditions of $f(\eta)=\frac{U_{w}}{V_{e}}$ at $\eta=0$, and $l$ for $\eta=1$ From this we can obtain $k_{1}=k_{1}(x)$ and $k_{2}=k_{2}(x)$ as defined

$$
\begin{aligned}
& \theta=k_{1} \delta \\
& \delta^{*}=k_{2} \delta
\end{aligned}
$$

We have as well

$$
\frac{d U_{e}}{d x}=-\frac{d u_{1}}{d x} \quad ; u_{e}=u_{w}-u_{1}
$$


$\frac{\tau_{0}}{\rho}=\left.\frac{\mathcal{s}}{\delta} \frac{t_{u}}{\partial \eta}\right|_{\eta=0}=-\frac{\mathcal{N}}{\delta}\left(2 u_{1}\right)$

$\frac{1}{2} \frac{d \theta^{2}}{d x^{2}}-\frac{1}{u_{e}} \frac{d u_{1}}{d x}\left(2+\frac{k_{2}}{k_{1}}\right) \theta^{2}=-2 \nu k_{1}\left(\frac{u_{1}}{u_{e}^{2}}\right)$

After definıng

$$
z=\theta^{2}
$$

and the Reynolds Number

$$
R_{a}=\frac{h_{0} \sqrt{g h_{0}}}{\mu}
$$

$$
\frac{1}{h_{0}} \frac{d z}{d x}-\frac{2}{u_{2}}\left(\frac{d u_{1}}{d x}\right)\left(2+\frac{k_{2}}{k_{1}}\right) \frac{z}{h_{0}}=-\frac{4 k_{1}}{R_{k}}\left(\frac{u_{1}}{u_{k}^{2}}\right)
$$

or, in non-dimensional form

$$
\begin{aligned}
& \frac{d \tilde{z}}{d \tilde{x}}-\frac{2}{\bar{u}_{e}} \frac{d \tilde{u}_{1}}{d \tilde{x}}\left(2+\frac{k_{2}}{k_{1}}\right) \tilde{z}=-\frac{4 k_{1}}{R_{e}} \frac{\tilde{u}_{1}}{\widetilde{u}_{e}^{2}} \\
& \text { where } \tilde{x}=x / h_{0}, \bar{z}=z / h_{0}^{2}, \tilde{u}_{1}=u_{1} / \sqrt{g h_{0}} \text { and } \tilde{u}_{e}=u_{e} / \sqrt{g h_{0}}
\end{aligned}
$$

Experımental conditions define $\mathrm{Re}$, and $\mathrm{k}_{1}$ and $\mathrm{k}_{2}$ are known from the assumed form of the boundary layer profife, equation (8) Equation (17) may be solved for $\bar{Z}$ and hence for $\boldsymbol{\theta}$ and $\mathcal{S}$ If we define the wall shear stress

$$
\tau_{0}=\frac{1}{2} \rho f u_{1}^{2}
$$

we may now calculate the coefficient of friction f from the equation

$$
f=-\frac{4 \mu}{5 u_{i}}
$$

The local value of $f$ is now used in the long wave equations (1)-(3) for the computation of the development of the wave profile

\section{Method of Solution}

Assuming a suitable initial profile for the long wave we may integrate equation (17) - using a fourth order Runge-Kutta numerical integration and thence determine all the parameters of the boundary layer developing under this surge In particular from equation (19) we obtain the friction coefficient distribution with $x$, corresnonding to this initial profile This friction coefficient is assumed to be constant 
over the wetted perimeter across any cross-section of the flow It is then fed into an implicit, finite central difference method of solution for equations (1)-(3), allowing the computation of a new wave profile at an incrementaliy advanced time This new wave profile is then used in equation (17) to calculate a friction coefficient distribution corresponding to 1 the solution proceeds in such a quasi-1terative fashion until the entire flow region of interest is covered

A square mesh spacing of $\boldsymbol{\Delta} x=\boldsymbol{\Delta} t=02$ was used in most computations The general finite differences solution followed the method outlined by Wachspress (10) The governing equations (1) - (3) are expressed as simultaneous, central (3-point) finite difference equations at each grid point at the desired time step and solved by a forward elimination, backward substitution technique (11ne inversion)

Special procedures are required near the starting and ending boundaries since full central differences cannot be evaluated there To reduce computation time, we specify initially a small portion of the $x$-axis $(t=0)$ and add new data points for the undisturbed liquid ahead of the advancing wave as it becomes necessary

\section{RESULTS}

Figure 4 shows the comparison of our theoretical results w1th and without the effect of friction We note that the main effect of the wall shear stress manifests itself mainly in the reduction of the wave peaks The troughs seem relatively unaffected This is in contrast to some experimental results given by Sandover and Zinkıewicz

(5) who note that the troughs become shallower as the friction increases

The value of the friction coefficient calculated in this manner is typically of the order of $30 \times 10^{-3}$ far from the head wave At the start of the wave the friction coefficient is very large but decreases rapidly w1th distance from the very beginning of the wave

Figures 5 and 6 give comparisons of experiments w1th theory w1th the friction taken into account The agreement is generally good in particular at the lower non-dimensional piston speed $u_{p}=01$ The first peak is well described but the theory indicates lower peaks for the trailing waves The troughs are well predicted The agreement becomes better with increasing time For $u_{p}=02$ the agreement is less good, the theory seeming to exaggerate the actual swings of the peaks and troughs The wavelength is well represented

The above remarks are confirmed by figures 7 and 8 , where we show the growth of the first peak and of the first wavelength from theory and experiment for cases with and without wall friction 


\section{CONCLUSIONS}

The method shown seems to reproduce the inltial development of the undular bore reasonably well It indicates the trends of behaviour regarding the influence of wall friction For the experimental conditions described herein the effect is fairly small

The analysis of the friction coefficient development is limited by the assumed form of the boundary layer velocity profile It is possible that velocity reversals may appear as the wave develops and undulations become larger These changes would invalidate the boundary layer approach described

The above method (without friction) has been extended and applied successfully to various problems where the depth is variable, in particular to the development of a solitary wave crossing a bar or a trench, the solitary wave climbing a beach and to the development of an undular bore moving up a beach These results will be given in a forthcoming paper

\section{BIBL1OGRAPHY}

1 Korteweg,D J \& deVries, G , 1895, Phi1 Mag (V), 39, 422-443

2 Urse11, F , 1953, The Long-Wave Paradox in the Theory of Gravity Waves, Proc Camb Phi1 Soc 39, 685-694

3 Benjamın, T B \& L1ghthı11, M J , 1954, On Cnolda1 Waves and Bores, Proc Roy Soc A, 224, 448-60

4 Sandover, J A \& Taylor,C , 1962, La Houllle Blanche, 17, 443-455

5 Sandover, J A \& Zınkıewıcz, O C , 1957, Experıments on surge waves, Water Power, 9, 418-424

6 Peregrane, D H ,1966, Calculation of the Development of the Undular Bore, J F1 Mech (25), 2, 321-330

7 Sturtevant,J , 1965, Implications of experiments on the weak undular bore, Phys of Fluids, vol 8,6

8 Muroka, A , 1966, Tenth Conf Coasta1 Engrg , 24, 382-395

$9 \mathrm{Su}, \mathrm{C} \mathrm{H} \&$ Gardner,C S , 1969, Derivation of the Korteweg-deVries Equation and Burger's Equation, J Math Phys ,10,3, 536-39

10 Wachspress,E , 1960, Numerical Solution of Boundary Value Problems, Mathematical Methods for Digital Computers, Ra1ston,A \& Wilf, H S ed, Wiley 
FIG I EXPERIMENTAL SETUP
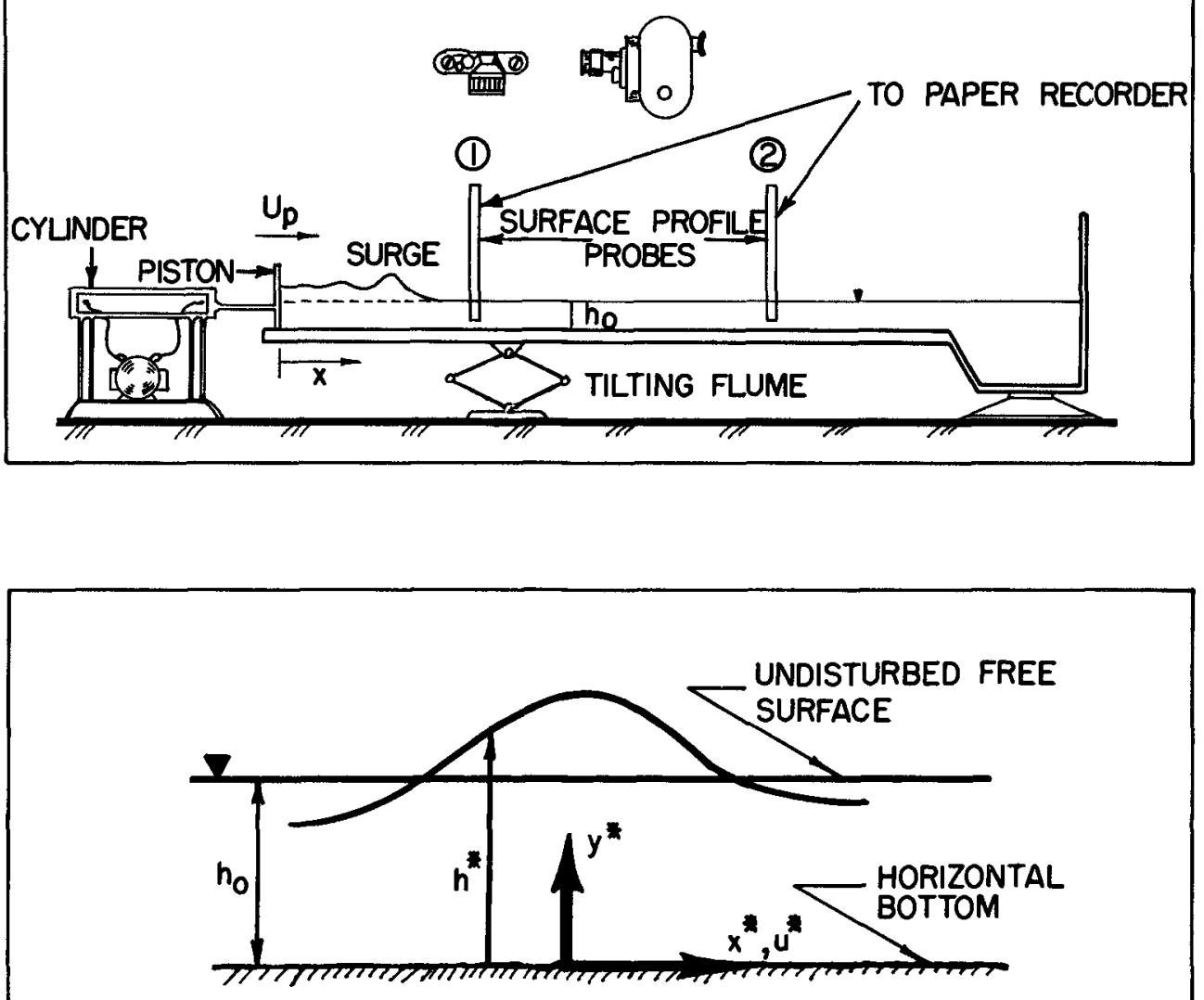

FIG 2 NOTATION 

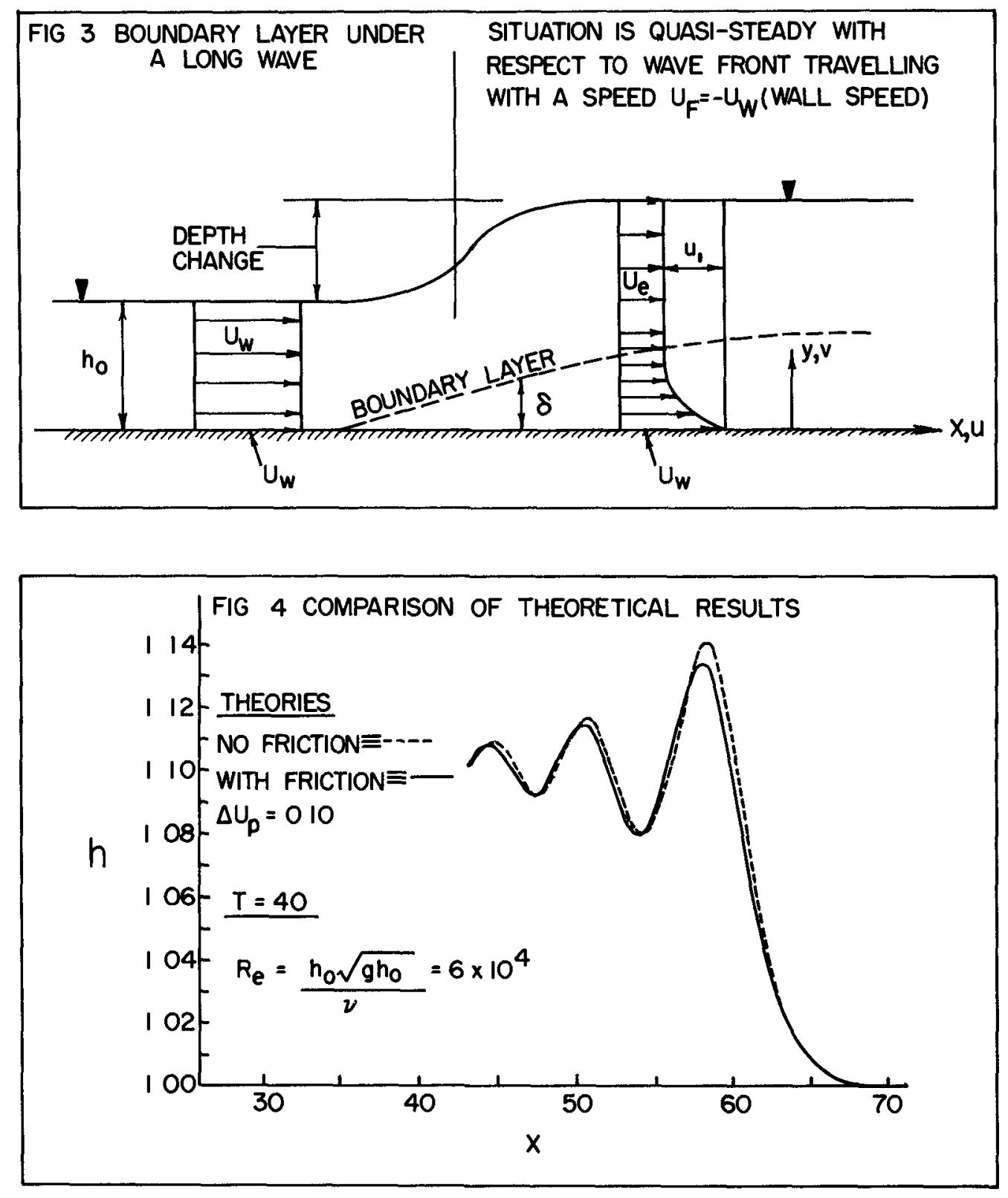
WAVE AMPLITUDES AT FIXED $X$

h

$$
U_{P}=01
$$

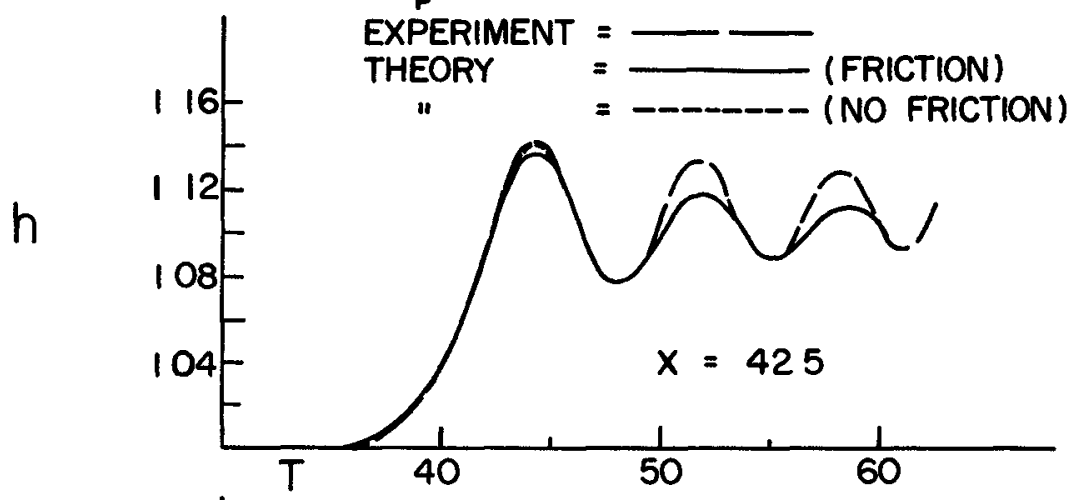

h

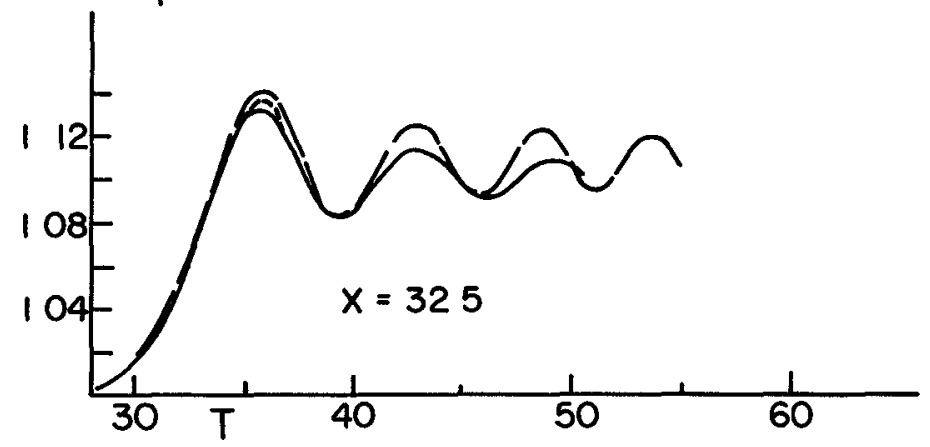

h

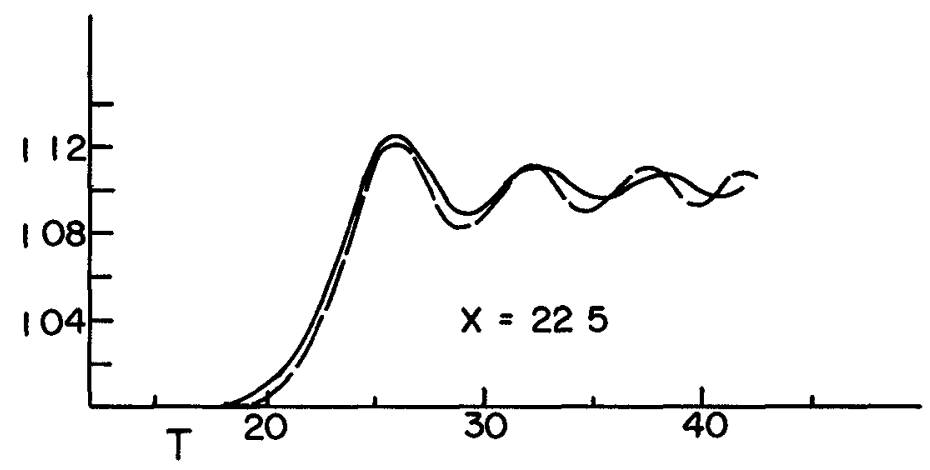

FIG 5 DEVELOPMENT OF AN UNDULAR BORE, $U_{p}=01$ 
WAVE AMPLITUDES AT FIXED $X$
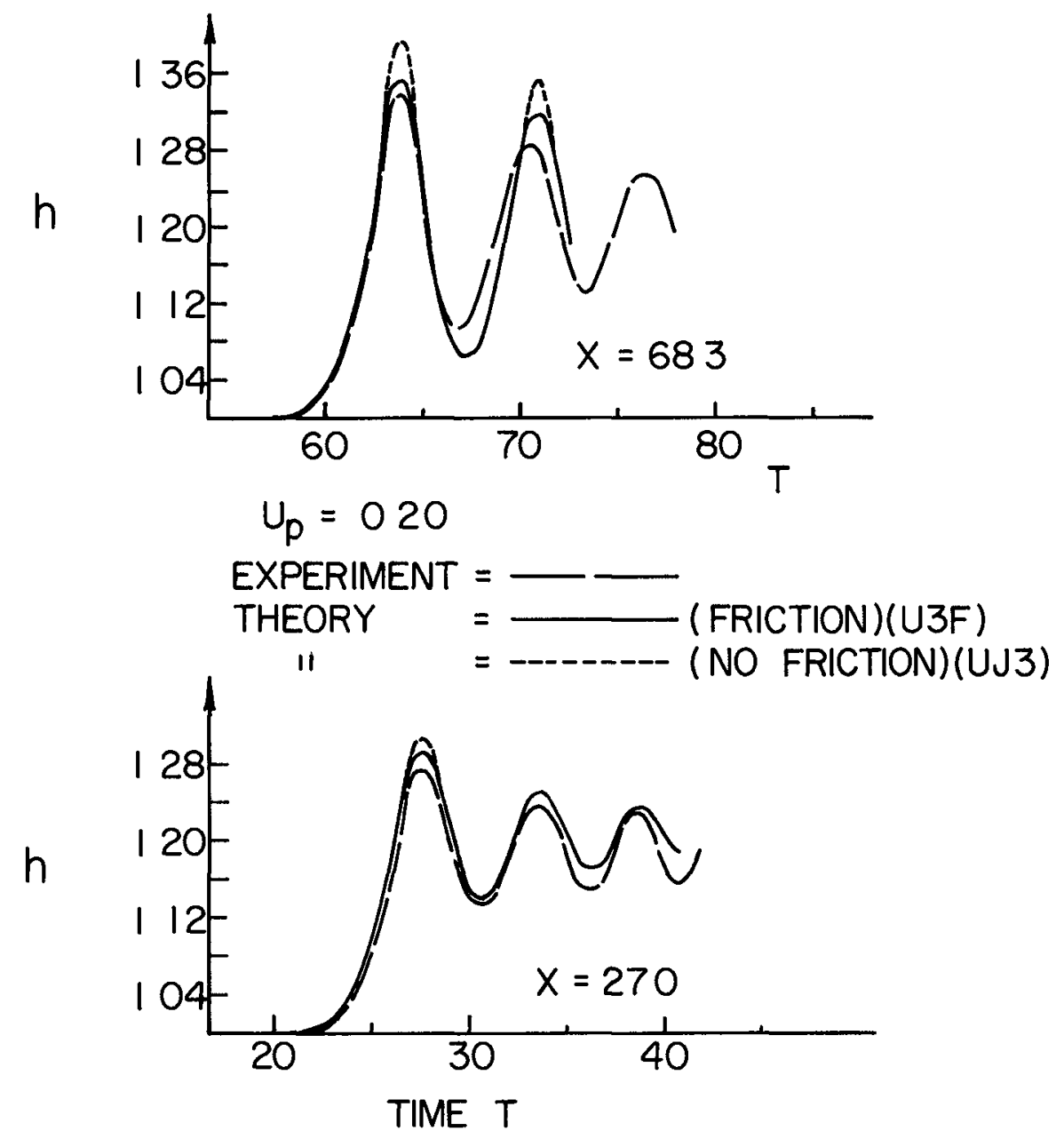

FIG 6 DEVELOPMENT OF AN UNDULAR BORE; $U_{p}=02$ 

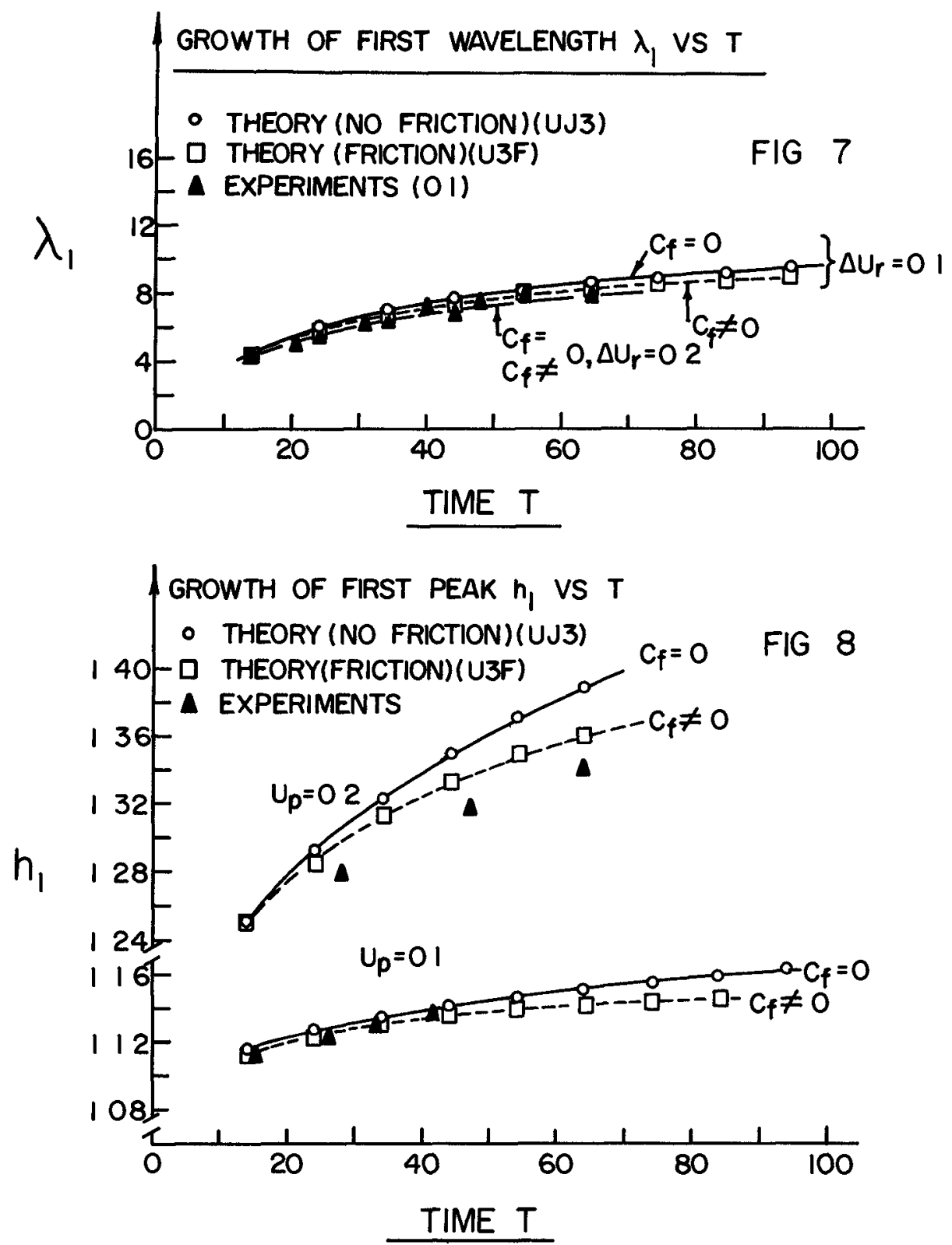


\section{CHAPTER 29}

\section{COMPUTER MODELLING OF DIFFRACTION OF WIND WAVES}

by

Shou-shan Fan ${ }^{1}$ and L E Borgman ${ }^{2}$

\section{ABSTRACT}

A digital computer model for diffraction of wind waves behind a breakwater is developed The model combines the hydrodynamic theories and the concept of directional spectra It 1 s designed so that it may be used not only for the study of the wind wave diffraction problem behind breakwaters but also for the investigation of experimental (or field) data analysis procedures of other kinds An extensive study of optimum data length, lag number and gage spacings in wave gage arrays is presented

\section{INTRODUCTION}

Unlıke monochromatıc waves, wind waves are quite complicated in nat ure Their helghts and lengths are Irregular The crest length along each wave crest is relatively short and their forms are not permanent Each portion of the water surface has a different shape The wave speed, frequency, and direction of advance vary from one wave to the next Because of the difficulties of an analytical treatment of such a complicated situation, an approach through simulation techniques offers many advantages [4]

The three types of simulation commonly used are laboratory (physical) electronic analog, and digital computer In the following, only the digital simulation procedures will be outlined

\section{SIMULATION OF WIND WAVES}

Nind waves can be simulated digitally in several ways, such as superposition and digital filter technıques [1] In the following development, only the filtering procedure will be employed

Let $y(t)$ be the wave surface elevations recorded by 4 gages in front of a brealwater Each of the $y(t)$ can be expressed by the summation of the products of $\mathrm{d}$ and $\mathrm{x}$ (see eq (1)) Here, $\mathrm{x}$ is a sequence of normal random deviates For computational convenience the random numbers have been selected so that they nave a zero mean and varıance of one

\footnotetext{
Former gradwate student, Unıversıty of Calıfornıa, Berkeley Oceanographer, Coastal Englneerıng Research Center, Washington, D C ${ }^{2}$ Former Associate Professor, Unıversıty of Calıfornia, Berkeley Professor, Unıversity of Wyoming, Laramie, Wyoming
} 
The " $\mathrm{d}$ " values are digıtal filter coefficıents and can be represented in terms of the Fourler coefficients, $A$ and $B$ obtained from the Fourler approximation of the system functions $K^{\mathrm{n}}(\mathrm{f})$ as shown by eqs (2)-(4)

The values of $K(f)$ are determined from the cross-spectral densities which are Fourler transform of the cross-covariance functions (eqs (5), (6))

If the probability distributions of the spectra are assumed to be circular normal (eq (10)), then the normalızed cross-spectral density functions can be expressed as the functions of Bessel functions (eqs (7)-(9))

In short, if the spectral forms of waves are assumed (such as that given by Bretschneider [5] and Plerson and Moskowitz [6] we can simulate the wind waves with digital computation

As already mentıoned previously these simulated random waves have very complicated natures and the conventional diffraction theories cannot be applied To deal with this kind of problem, we introduce the concept of directional spectrum

\section{CONCEPT OF DIRECTIONAI SPECTRUM}

Random waves can be thought as composing of infinite number of monochromatic component waves, each of which has a different frequency and phase and profagate along a different direction since it is known that, to a linear approximation, the conventional diffraction theories are applicable to these monochromatic waves, our problem has then become the question of "how to determine the directional spectrum?"

At the present time, there are several ways avallable for estimating the directional spectrum But, only one of those methods, [1,2] wil1 be discussed It considers the directional spectral density function as the product of $P S(f)$ and $D(\theta)$, where PS(f) is spectral density which varies solely with frequency and $D(\theta)$ is a function of direction and possible frequency (see eq (13))

There are several ways to estumate $\mathrm{D}(\theta)$ One of them is based on the assumption that $\mathrm{D}(\theta)$ is circular normal In that case, it can be approximated in terms of Bessel functions as shown in eq (14)

The spectral density function can be estumated by the conventional spectral analysis technique using elther the covariance function approach or the techniques arising from the fast Fourler Transform algorithm

\section{SIMULATION OF DIFFRACTION OF PLANE WAVES $[3,4]$}

Mathematically, the propagation of a plane wave is described by the boundary value problem with a second order partıal differential equation of elliptic type (eq (15)) and three boundary condltions (eqs 16,17,18) By applying the method of separation of varıables, the boundary value can be solved 
Due to the presence of a semi-infinite breakwater, located along the $\mathrm{X}$-axis with one tip at the origin and the other at $\mathrm{x}=+\infty$, an additional boundary condition of

$$
\frac{\partial \phi}{\partial y}=0
$$

should be introduced For the reason of generality and convenzence, a polar coordinate system is adopted here Accordingly, the amplitude for incident wave and diffracted wave can be determined

Since the diffraction coefficient, $k$ ' 1 s defined as the ratio of incident wave helght over the diffracted wave helght, It can be estimated by the modulus of $F(r, \theta)$ for the diffracted waves Their mathematical equations and solutions are given in the Appendix III

$\mathrm{V}$ THE APPLICATIONS OF SIMULATION TECHNIQUE

Simulation may be used to explain various features of data sampled from the field or to examine the consequences of selected theorles In addition, it can be used to determine (a) optimal data length (FIg 1) For this case, optional
length $=2048$
(b) optımal maximum number of lags (F1g 2) For this case, optional lag $=50$
(c) effects of smoothing on the spectral density estimates (FIg 3) The results have indicated that

(1) for shorter data length, there is a great difference between the outputs of unsmoothed and smoothed cases but the re seems to be no difference between hanning or Hamming smoothing

and that

(11) for very long record, there is no difference no matter whether they have been smoothed or not 
REFERENCES

Borgman, L E, "Ocean Wave Simulation For Engıneering Desıgn", Report HEL-9-13, Unıversity of Calıfornıa, Berkeley, October 1967

Borgman, L E, "Directional Spectra Models For Desıgn Use", Report HEL-1-12, Unıversity of Calıfornıa, Berkeley, 1969

Fan, Shou-shan, Cumming, J, Wlege1, $R$ L, "Computer Solution of Wave Diffraction by Semi-infinite Breakwater, Report HEL-1-7, University of Calıfornla, Berkeley, July 1967

Fan, Shou-shan, "Diffraction of Wind Waves", Report HEL-1-10, University of Calıfornıa, Berkelev, August 1968

Bretschneider, C L, "Wave Varıabılity and Wave Spectra For Wind Cenerated Gravıty Waves", BEB, TM-118, August 1959

Plerson, W J , Jr, \& Moskowltz, L , "A Proposed Form For Fully Developed Wind Seas Based On The Simılarıty Theory of S A Kıtalgovoskıl, Jour Geophy Res, Vol 69(1964), No 24 
APPENDIX I

Stmulation of Complicated Wind Wave Profiles

Let $y_{1}(t), y_{2}(t), \quad, y_{m}(t)$ be 11 - time serıes

$$
y(t)=\sum_{n=N}^{N} d_{n} x(t-n \Delta t)
$$

where $\mathrm{n}=0 \quad 1,2$

$$
\begin{aligned}
\Delta t & =\text { time nterval } \\
x & =\text { random deviates }
\end{aligned}
$$

and the digital filter coefficients

$$
\left.\begin{array}{l}
\mathrm{d}_{0}=A_{0} \\
d_{n}=A_{n}+B_{n} \\
d_{-n}=A_{n}-B_{n}
\end{array}\right\}
$$

where

$$
\begin{aligned}
& A_{n}=\frac{1}{F} \int_{0}^{F} R[\hat{K}(f)] \cos \left(\frac{n \pi f}{F}\right) d f \\
& B_{n}=\frac{1}{F} \int_{0}^{F} 1_{m}[\hat{K}(f)] \sin \left(\frac{n \pi f}{F}\right) d f \\
& F=\frac{1}{2 \Delta t}=\text { Nyquist frequency }
\end{aligned}
$$

and

$$
\begin{aligned}
\hat{\mathrm{K}}(\mathrm{f}) & =\mathrm{A}_{0}+\sum_{\mathrm{n}=1}^{N}\left\{\mathrm{~A}_{\mathrm{n}} \cos \left(\frac{\ln \pi \mathrm{f}}{\mathrm{F}}\right)-1 \mathrm{~B}_{\mathrm{n}} \sin \left(\frac{\mathrm{ln} \pi \mathrm{f}}{\mathrm{F}}\right)\right\} \\
\mathrm{f} & =\text { frequency, cps } \\
\mathrm{I} & =\sqrt{-1}
\end{aligned}
$$


The system function can be also written as

$$
\begin{aligned}
& k_{11}\left(f_{k}\right)=\left[s_{11}\left(f_{k}\right)\right] \\
& k_{m, 1}\left(f_{k}\right)=\left[s_{m, 1}\left(f_{k}\right)\right]^{\frac{1}{2}} \\
& k_{m, n}\left(f_{k}\right)=\left[s_{m,(m-1)}\left(f_{k}\right)-\sum_{j=1}^{n}\left[k_{m j}\left(f_{k}\right)\right]\left[k_{j n}\left(f_{k}\right)\right]\right\} / K_{n n}\left(f_{k}\right) \\
& k_{m, m}\left(f_{h}\right)=\left[s_{m m}\left(f_{k}\right)-\sum_{j=1}^{m-1}\left|k_{m j}\left(f_{k}\right)\right|^{2}\right]^{1}
\end{aligned}
$$

where

$$
\begin{aligned}
\mathrm{S}_{\mathrm{m} J}(f) & =\mathrm{CS}_{\mathrm{mJ}}(\mathrm{f})+1 \mathrm{QS}_{\mathrm{m} \mathrm{J}}(\mathrm{f}) \\
\mathrm{CS}_{\mathrm{m} J}(f) & =\text { c0-spectral densıty for gages } m \text { and } \mathrm{J} \\
\mathrm{QS}_{\mathrm{m} J}(\mathrm{f}) & =\text { quad-spectral densıty for gage } m \text { and } J
\end{aligned}
$$

Assuming the circular normal function (eq 10 below) for the angular distribution of energy at a given frequency, the normalized density between gages $m$ and $J$ can be expressed as

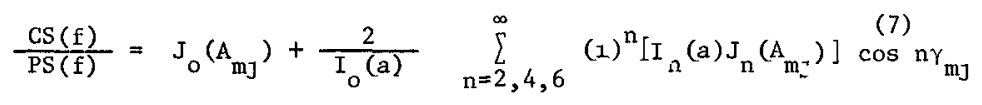

and the normalızed quad-(or quadrature-) spectral density between gages $\mathrm{m}$ and $\mathrm{J}$ is

$$
\frac{\mathrm{QS}(\mathrm{f})}{\operatorname{PS}(\mathrm{f})}=\frac{2}{I_{0}(a)} \sum_{n=1,3,5}^{\infty}(1)^{n}\left[I_{n}(a) J_{n}\left(A_{m j}\right)\right] \cos \left(n \gamma_{m j}\right)
$$

where

$$
\begin{aligned}
& I_{n}(a)=I_{-n}(a)=(1)^{-n} J_{n}(1 a) \\
& =\text { Modıfied Bessel Function of order } n \\
& a=a \text { circular normal parameter }=\begin{array}{l}
\text { measure of dispersion of the } \\
\text { cırcular normal }
\end{array} \\
& \alpha=\text { a circular normal parameter }=\begin{array}{l}
\text { modal direction of circular } \\
\text { normal energy distribut } 1 \text { on }
\end{array}
\end{aligned}
$$




$$
f(\xi, a)=\frac{e^{a \cos (\theta-\alpha)}}{2 \pi I_{0}(a)}
$$

$\theta=$ the angle between the positive $x$-axis and the direction of the wave propagation, measured counterclockwise

$\xi=$ the wave angle departure from the mean $=\theta-\alpha$

$A_{m j}=2 \pi D_{m j} / L$

$D_{m j}=d i s t a n c e$ between wave gages m and $\mathbf{J}$

$\mathrm{L}$ = wave length appropriate for the frequency, $f$

$\gamma_{m J}=$ the angle between direction of main energy and the line connected gage $m$ and gage $J$

$=\beta_{m, j}-\alpha$

$B_{m j}=$ the angle between the $x$-axis and the line connected gage $m$ and gage $J$

$1=\sqrt{-1}$ 
APPENDIX II

DIRECTIONAL SPECTRUM

1 Di rectiona1 spectral density function, $p(f, \theta)$

(a) It has the property of

$p(f, \theta)$ df $d \theta=\sum$ [Mean-Square Wave-Surface fluctuations] (df) $(d \theta)$

In other words,

$p(f, \theta) d f d \theta=$ varıance of sea surface fluctuations obtained by adding together on $1 y$ the waves with frequency and direction of travel in the (df,de) rectangle centcred (12) at $(f, \theta)$

(b) It gives the allocation of the total variance among the various frequencies and directions

(c) It can also be considered as an allocation of wave energy (since the wave energy per unit sea surface is proportional to the variance)

2 Estimation of directiona1 spectral density

$$
\begin{aligned}
p(f, \theta) & =\operatorname{PS}(f) D(\theta) \\
D_{f}(\theta) & =\frac{e^{a \operatorname{Cos}(\theta-\alpha)}}{2 \pi I_{0}(a)} \quad \text { (Circular Normal Function) } \\
& =\frac{1}{2 \pi}+\sum_{n=1}^{\infty} \frac{I_{n}(a)}{\pi I_{0}(a)} \operatorname{Cos}\left(n \theta-\frac{n \pi}{2}\right)
\end{aligned}
$$

( $\alpha$ and a may be functions of frequency in the gazeral case) 
APPENDIX III

Diffraction of Plane Wave

(A) BOUNDARY VALUE PROBLEM FOR PLANE WAVE PROPAGATION

$1 \quad$ Partial diff eq $\quad \frac{\partial^{2} \emptyset}{\partial x^{2}}+\frac{\partial^{2} \emptyset}{\partial y^{2}}+\frac{\partial^{2} \emptyset}{\partial z^{2}}=0$

2 Boundary Condztions

(1) At an Impervious and rigid bottom

$\frac{\partial \emptyset}{\partial z}_{z=d}=0$

whch $\mathrm{d}=$ stıII-water depth

(11) At the free surface

(a) Kınetıc Surface Boundary Condıtıon

$\frac{\partial \emptyset}{\partial z}=\frac{d}{d t}[n(x, t)]=\frac{\partial \eta}{d L}+\frac{\partial \eta}{\partial x}+\frac{\partial \eta}{\partial t}=\frac{\partial \eta}{\partial t}+u \frac{\partial \eta}{\partial t}$ at $z=n$ (non-1inear)

$\frac{\partial \varphi}{\partial z}=\frac{\partial \eta}{\partial t}$ at $z=0$ (1 Inear)

(b) Dynamic Surface Boundary Condition

$$
\begin{aligned}
& \frac{-\partial \emptyset}{\partial t}+\frac{1}{2}\left(u^{2}+v^{2}+w^{2}\right)+g n=0, \text { at } z=0 \text { (non-linear) } \\
& n=\frac{1}{g} \frac{\partial \emptyset}{\partial t} \text { at } z=0 \text { (11near) }
\end{aligned}
$$

3 Solutions

$$
\begin{aligned}
& \emptyset=F(x, y) z(z) T(t) \\
& \emptyset=A e^{-l k e t} \cosh [k(z+a)] F(x, y)
\end{aligned}
$$


For a plane wave travelling in the $y$-direction

$$
F(x, y)=e^{-1 k y}
$$

$$
\text { Sommerfeld's radiation condition } F=0\left(\frac{1}{r}\right)
$$

$$
r=\sqrt{x^{2}+y^{2}}
$$

$$
\begin{aligned}
\text { Wave amplitude, } & A & =\frac{A k c}{g} \cosh (k d) \\
\text { wave period, } & T & =\frac{2 \pi}{k c} \\
\text { wave velocity } & c & =\sqrt{\frac{g}{k} \tanh (k d)} \\
\text { wave length, } & L & =\frac{2 \pi}{k} \\
\text { wave number, } & \mathrm{k} & =\frac{2 \pi}{\mathrm{L}} \\
& & =\frac{A k c}{\mathrm{~g}} \cosh (k d) \sin [k(c t-y)]
\end{aligned}
$$

\section{B THE PROBLEM WITH THE PRESENCE OF A BREAKWATER}

\section{(semu-infinıte breakwater)}

1 an additional boundary condition

$$
\frac{\partial \emptyset}{\partial y}=0 \text { at } y=0, x \geq 0
$$

This 1mplies that

$$
\frac{\partial F}{\partial y}=0 \text { at } y=0, x \geq 0
$$


2 for incident waves

$$
F_{1}(r, \theta)=e^{-1 k r \cos \left(\theta-\theta_{0}\right)}
$$

3 the free wave surface can be given as

$$
=\frac{A l k c}{g}[\cosh (k d)] e^{l k c t} F(r, \theta)
$$

4 for dıffracted waves

$$
\begin{aligned}
& F_{d}(r, \theta)=f(\sigma) e^{-1 k r}\left[\cos \left(\theta-\theta_{0}\right)\right]+f(\sigma) e^{-1 k r}\left[\cos \left(\theta-\theta_{0}\right)\right] \\
& \sigma=2\left(\sqrt{\frac{k r}{\pi}}\right) \sin \frac{1}{2}\left(\theta-\theta_{0}\right) \\
& \sigma^{\prime}=-2\left(\sqrt{\frac{L r}{\pi}}\right) \sin \frac{1}{2}\left(\theta-\theta_{0}\right) \\
& f(\sigma)=\frac{1}{\sqrt{2}} e^{1 \pi / 4} \int_{-\infty}^{\sigma} e^{(1 \pi / 2) t^{2}} d t \\
& f\left(\sigma^{\prime}\right)=\frac{-1}{\sqrt{2}} e^{1 \pi / 4} \int_{-\infty}^{\sigma} e^{(1 \pi / 2) t^{2}} d t \\
& F_{d}(r, \theta)=\rho(r, \theta) e^{1 \xi(r, \theta)} \\
& a_{d}=\frac{A k c \rho}{g} \cosh (k d) \\
& D_{1} f f r a c t 1 o n \text { coefficient } \\
& k^{\prime}=\frac{2 a_{d}}{2 a_{1}}=\rho \\
& k^{\prime}=\left|F_{d}(r, \theta)\right|
\end{aligned}
$$




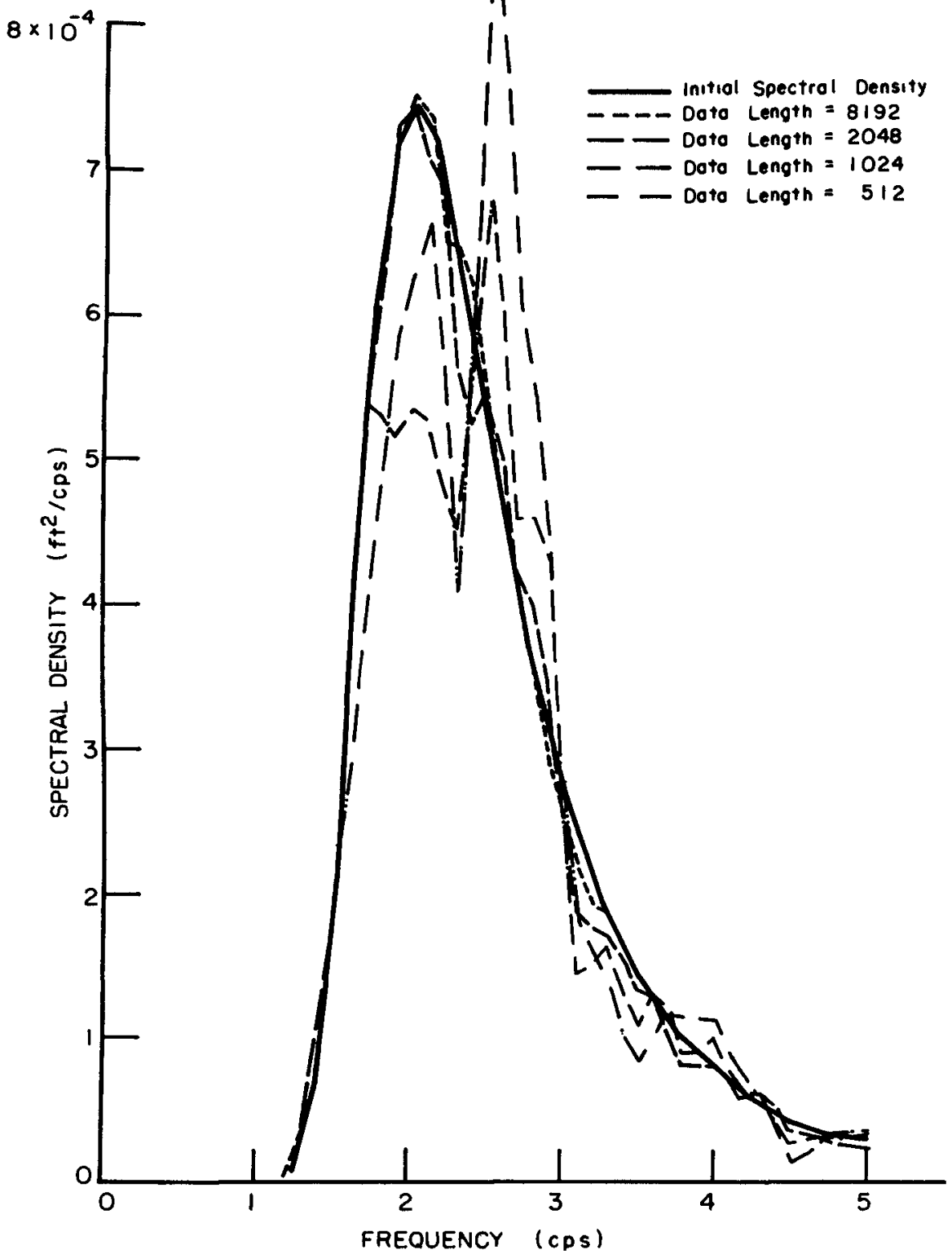

FIG I ESTIMATES OF SPECTRAL DENSITIES FOR VARIOUS LENGTH OF DATA 


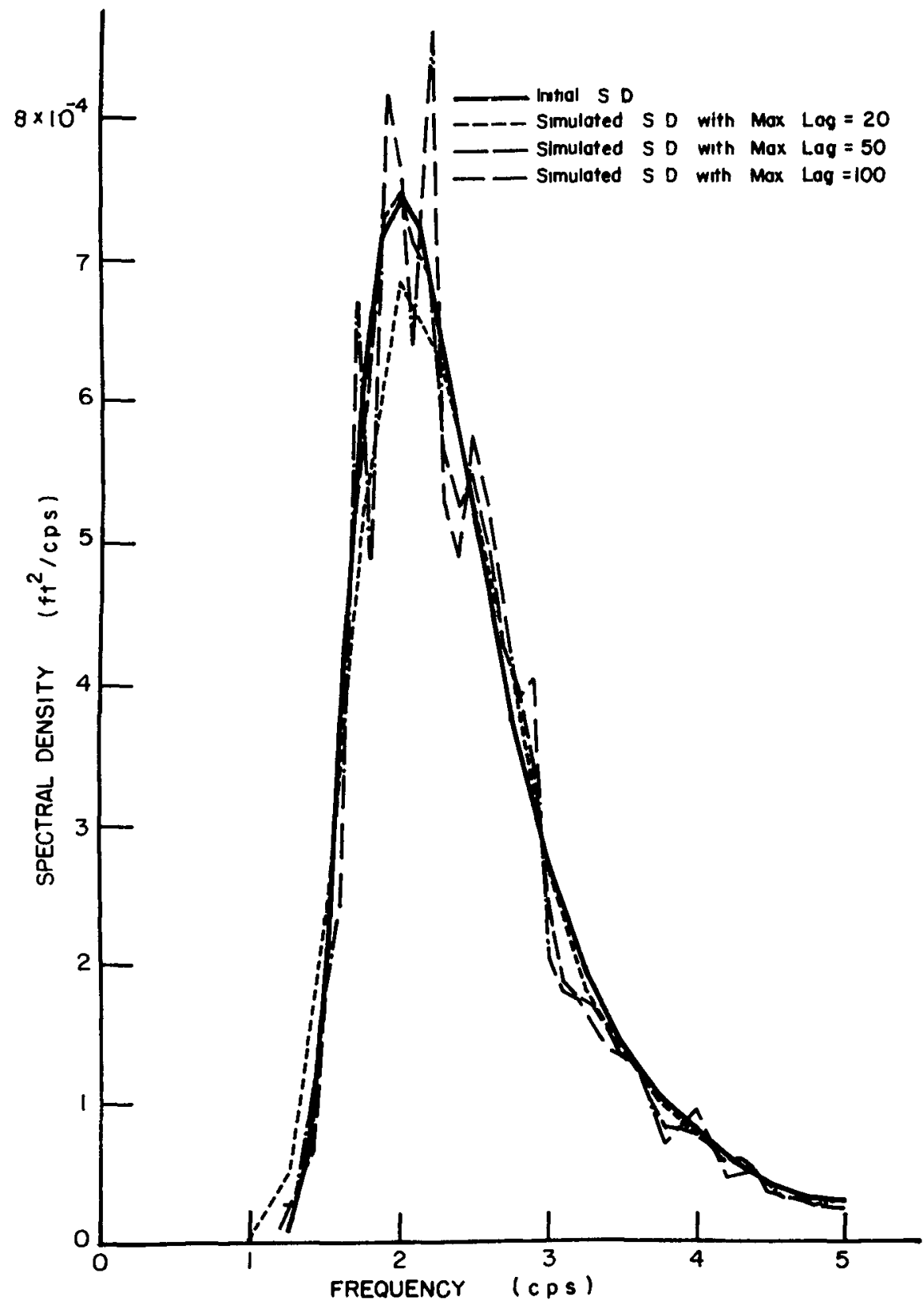

FIG 2 ESTIMATES OF SPECTRAL DENSITIES (2048 DATA LENGTH) FOR VARIOUS MAXIMUM LAGS ON THE COVARIANCE FUNCTIONS 


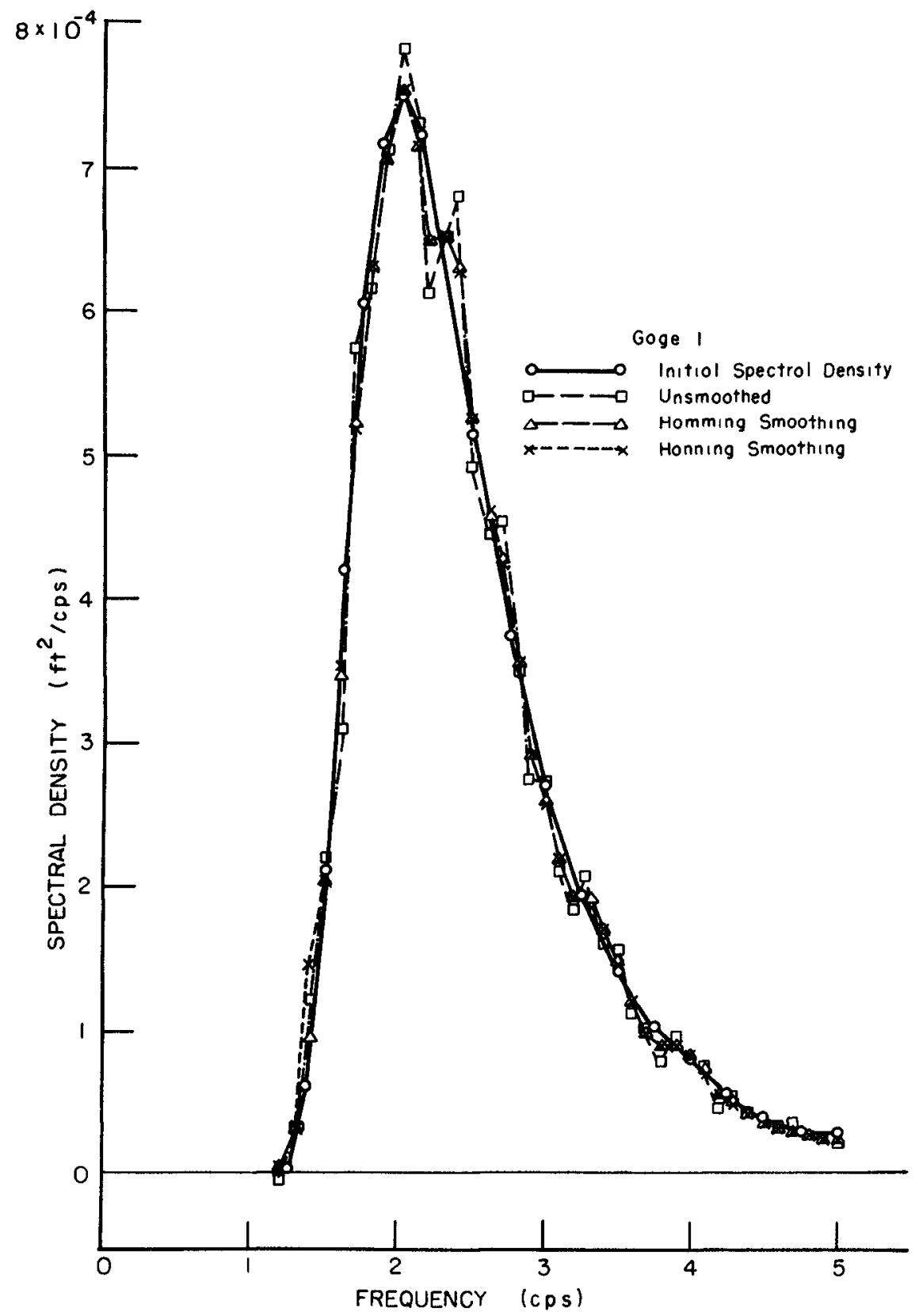

FIG 3 EFFECTS OF SMOOTHING ON THE SPECTRAL DENSITY ESTIMATES FOR DATA LENGTH 8192 AND MAXIMUM LAG 50 


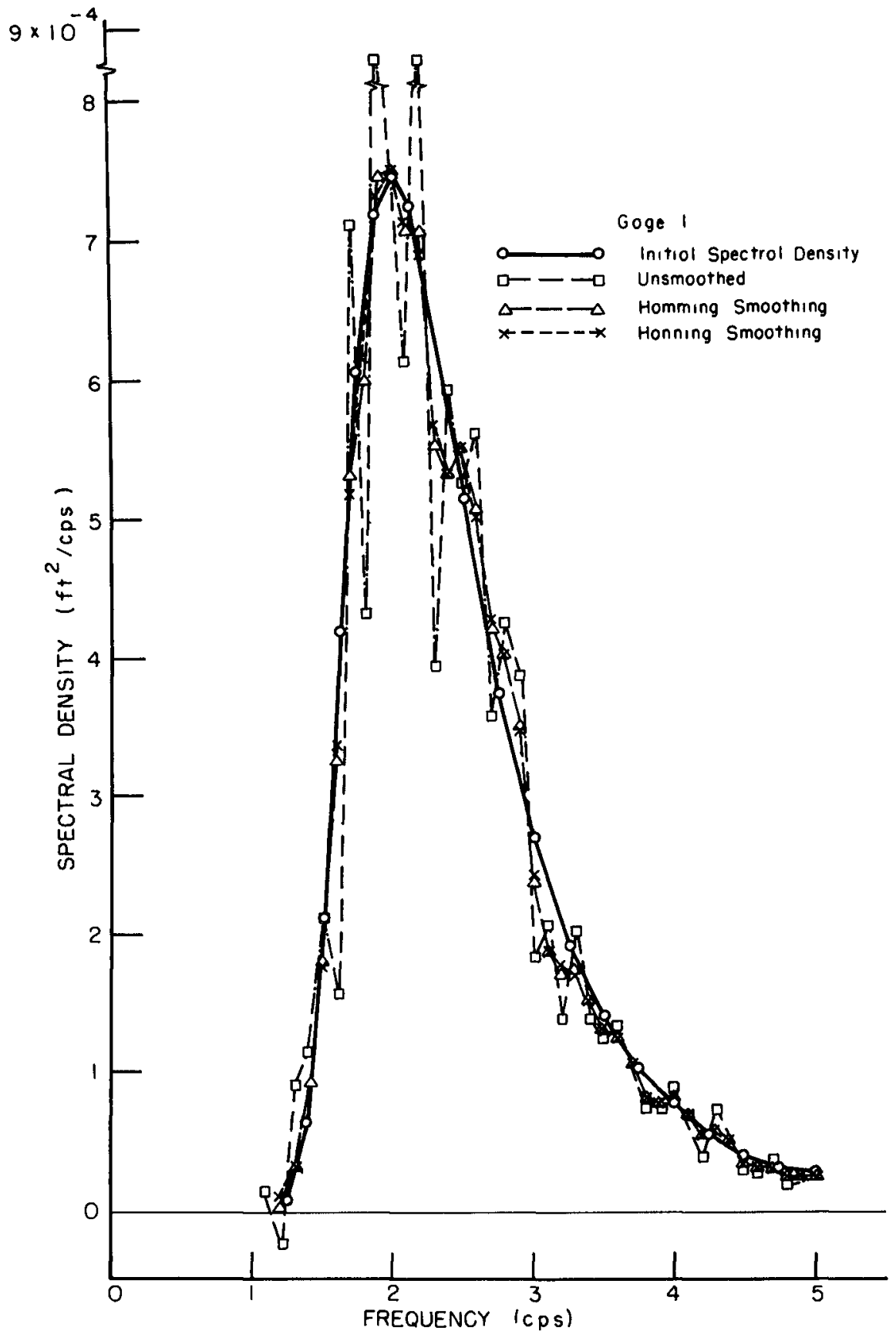

FIG 4 EFFECTS OF SMOOTHING ON THE SPECTRAL DENSITY ESTIMATES FOR DATA LENGTH 2048 AND MAXIMUM LAG 50 



\title{
CHAPTER 30
}

INTERACTION BETWEEN WAVES AND CURRENTS

\author{
Ivar $G$ Jonsson \\ Ph D, Coastal Englneerıng Laboratory \\ Christian Skougaard ${ }^{1}$ \\ M Sc , Coastal Englneerıng Laboratory
}

John D Wang

Graduate Student

Technıcal Unıversıty of Dermark

\section{ABSTRACT}

This paper presents some theoretical results of a general study of the interaction between surface gravity waves and a steady current Assuming irrotational flow and a second order Stokes wave motion, the main objects of the paper have been

a To present a simple graphical method for the computation of the wave length in a current field.

b To introduce the concept of the mean energy level for a periodic wave motion with a steady current superimposed.

c To utilize this for the calculation of the "current-wave set-down" for a two-dimensional motion with a constant discharge over a gently sloping bottom

d. To present a complete set of conservation equations for the case considered under point $c$

e To present graphs and tables for the variation in length and helght of wave for the case considered under point $c$

No experimental results are presented

\section{INTRODUCTION}

Aspects of non-linear interaction between gravity waves and a current motion have recelved increasing attention during the last lo years The works by Longuet-Higglns and Stewart [5], [6], and. Whitham [8] are already classics The mechanism is intimately connected with the so-called. radiation stress

However, there are stıll basıc features related to this problem that are not wadely known One of these $1 \mathrm{~s}$ the important concept of the mean energy level for a perıodlc, irrotational flow (For a pure wave motion the mean energy level was introduced by Lundgren [7]) One of the objects of the present study is to show how this concept can yleld the "currentwave set-down" in a simple way, and to demonstrate how it affects the conservation equations for a two-dimensional current-wave motion propa-

\footnotetext{
lPresent address Municipalıty of Roskilde, Denmark
} 
gating over a gently sloping bed (see Fig 5-A) The conservation equations for wave crests and energy are solved to yleld graphs and tables for the varlation in wave length and wave helght for this situation the wave length graphs and tables can be used, though, for any angle between wave front and current direction provided the current velocity is replaced by Its component in the direction of the wave orthogonal A graphical method for the determination of the wave length in a homogeneous current fleld will also be introduced This method permits a simple discussion of various domalns where different solutions are applicable

Energy losses are neglected in this paper The current velocity is assumed to be steady and constant over the water depth, and only surface waves are considered A second order Stokes expansion is used in the calculations

\section{NOTATION}

$\begin{array}{ll}C^{c} & (\mathrm{~m} / \mathrm{s}) \\ { }_{\mathrm{C}} & (\mathrm{m} / \mathrm{s}) \\ { }^{D} & (\mathrm{~m}) \\ \mathrm{E} & \left(\mathrm{Nm} / \mathrm{m}^{2}\right) \\ \bar{E}_{f} & (\mathrm{Nm} / \mathrm{m} / \mathrm{s}) \\ \mathrm{F}_{\mathrm{W}} & (\mathrm{N} / \mathrm{m}) \\ \mathrm{g} & \left(\mathrm{m} / \mathrm{s}^{2}\right) \\ \mathrm{H} & (\mathrm{m}) \\ \mathrm{h} & (\mathrm{m}) \\ \Delta \mathrm{h} & (\mathrm{m}) \\ \mathrm{k} & \left(\mathrm{m}^{-1}\right) \\ \mathrm{L} & (\mathrm{m})\end{array}$

MEL

MWL

$\begin{array}{ll}\mathrm{n} & (\mathrm{d} m \mathrm{~m} \text { less }) \\ \mathrm{p} & \left(\mathrm{N} / \mathrm{m}^{2}\right) \\ \mathrm{q} & \left(\mathrm{m}^{3} / \mathrm{m} / \mathrm{s}\right) \\ \mathrm{q}^{*} & (\mathrm{~d} \mathrm{~m} \text { less }) \\ \mathrm{s}_{\mathrm{v}} & (\mathrm{d} \mathrm{m} \text { less }) \\ \mathrm{T} & (\mathrm{s}) \\ \mathrm{t} & (\mathrm{s}) \\ \mathrm{U} & (\mathrm{m} / \mathrm{s}) \\ \mathrm{u} & (\mathrm{m} / \mathrm{s}) \\ \mathrm{w} & (\mathrm{m} / \mathrm{s}) \\ \mathrm{x} & (\mathrm{m})\end{array}$

Wave celerity

Wave group celerity

"Geometrical water depth" $(=\mathrm{h}+\Delta \mathrm{h}$, see Fig 4-A)

Mean specıfıc wave energy $\left(=1 / 8 \gamma \mathrm{H}^{2}\right)$

Mean energy flux per unlt wadth

Radiation stress

Acceleration due to gravity

Wave helght

"Physical water depth" (see Fig 4-A)

"Current-wave set-down" (see Fig 4-A)

Wave number $(=2 \pi / L)$

Wave length

Mean energy level (see Fig 4-A)

Mean water level (see Fig 4-A)

$c_{g r} / c_{r}$

Pressure

Discharge per unit width $(=\mathrm{h} \mathrm{U})$

Dimensionless discharge per unit width $\left(=\mathrm{q} /\left(\mathrm{c}_{0} \mathrm{~L}_{0}\right)\right)$

Slope of stralght line in Fig 3-B

Wave period

Tıme

Current velocity (positive in direction of c)

Horızontal particle velocity

Vertical particle velocity

Horızontal co-ordinate 


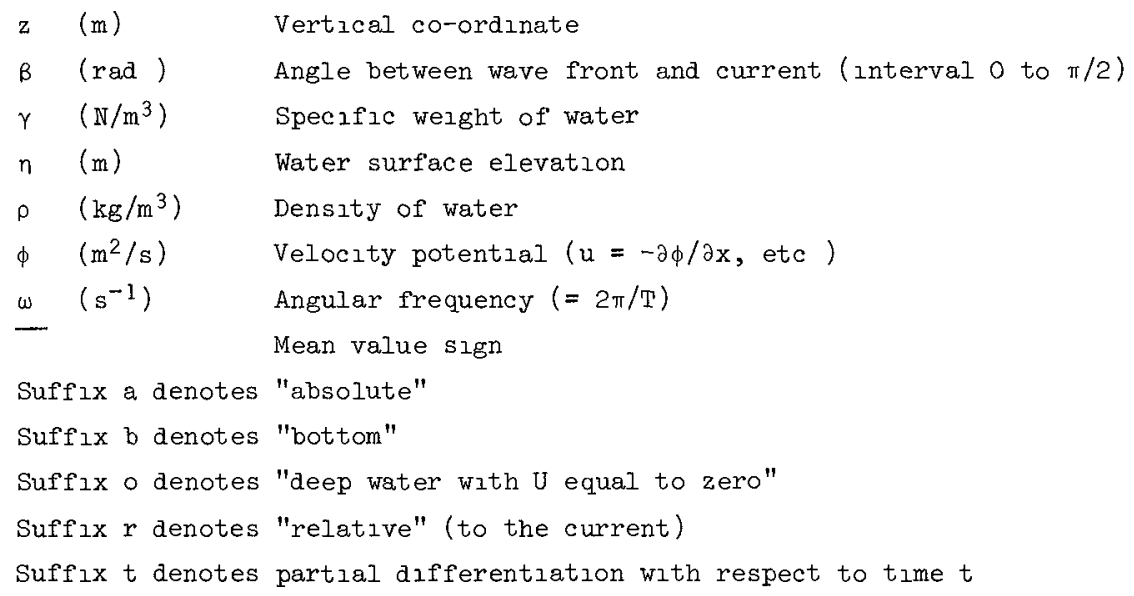

3 WAVES IN A HOMOGENEOUS CURRENT FIEID

Consider a region of constant water depth $h$, where the absolute wave period $T_{a}$ and the current velocity $U$ are given

WAVE LENGTHS

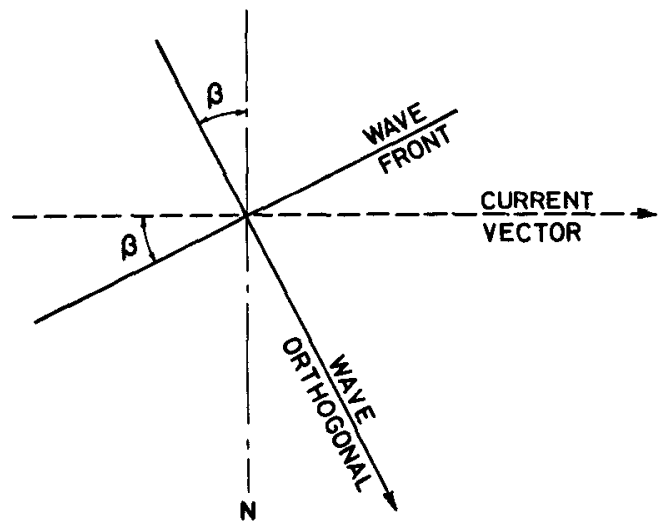

Fig 3-A Definition of the angle $B$

If $\beta$ is the angle between wave front and direction of current (Fig 3-A), the following four equations are avallable to determine the unknowns the absolute and relative wave celerities $c_{a}$ and $c_{r}$, the wave length $\mathrm{L}$, and the relative period $\mathrm{T}_{r}$

$$
\begin{array}{lll}
\mathrm{L}=\mathrm{c}_{\mathrm{a}} \mathrm{T}_{\mathrm{a}} & (3 \mathrm{l}) & \mathrm{I}=\mathrm{c}_{r} \mathrm{~T}_{r} \\
c_{\mathrm{a}}=c_{r}+\mathrm{U} \sin B & (33) & c_{r}=\sqrt{\frac{\mathrm{gL}}{2 \pi} \tanh \mathrm{kh}}
\end{array}
$$


where $k$ is the wave number The current velocity $U$ is considered positive to the same side of the normal $N$ as is indlcated by the positive direction of the wave orthogonal $\beta$ lies, by definition, in the interval

$0 \leq \beta \leq \pi / 2$ (3 1) to (34) now yield for determination of $\mathrm{L}$

$$
\left.\sqrt{\frac{h}{L} \tanh k h}=\sqrt{\frac{h}{L_{0}}\left[1-\frac{U \sin \beta T_{a}}{h}\right.} \frac{h}{L}\right]
$$

where $L_{0}$ Is defined as

$$
L_{0} \equiv \frac{g}{2 \pi} T_{a}^{2}
$$

$I$ e the deep water wave length wath $U$ equal to zero

(3 5) determines implicitly $h / L$ as a function of $h / L_{0}$ and

$U \sin \beta T_{a} / h$, and the equation can be solved by lteration (see chapter 6) It is not evident, however, whether a solution to (35) is unique - or whether It exists at all A graphical representation of (3 5) reveals this Writıng (3 5) as

$$
F(h / L)=G(h / L)
$$

It appears that graphlcally $h / L$ can be found as the value of the absclssa for the intersection of the $F$ - and G-curves, see Fig 3-B The F-curve is unique, and values can be extracted from a "conventional" wave table

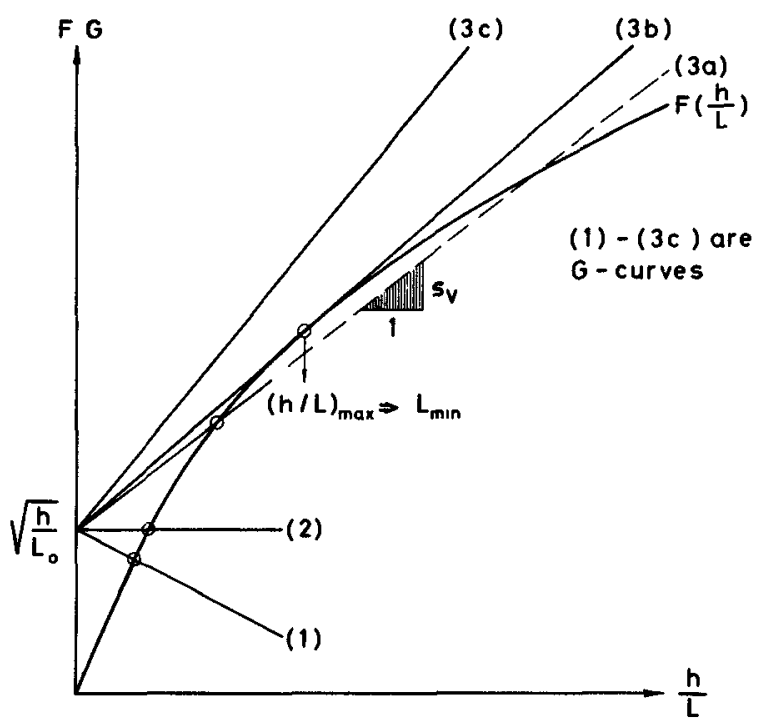

Fig 3-B Graphical determination of L (schematıcal) 
The G-curves are stralght lines through the point $\left(0, \sqrt{\mathrm{h} / \mathrm{L}_{\mathrm{O}}}\right)$, with a slope of 1 horizontal to $s_{\mathrm{v}}$ vertical, where

$$
s_{v}=-\sqrt{\frac{h}{L_{0}}} \frac{U \sin \beta T_{a}}{h}=-\sqrt{2 \pi} \frac{U \sin \beta}{\sqrt{B h}}=-\frac{U \sin \beta}{c_{0} \sqrt{h / L_{0}}}
$$

Here $c_{0}$ is defined as

$$
c_{0} \equiv \frac{g}{2 \pi} T_{a}
$$

$1 \mathrm{e}$ the deep water wave celerity whth $U$ equal to zero

Fig 3-B now enables us to discuss the solution(s) to (3 5) in detall ( $h, T$ and $B$ are assumed constant, so $s_{y}$ becomes directly proportional to - U) For $U$ positive we have case (1), and a unique solution exists for $L$ Th1s also applies to case (2), where U is zero For a negative current (case (3)), there are three possibilities In case (3a) there are theoretically two solutions for $L$ If $1 t$ is argued that the variation of $L$ must, for physical reasons, be continuous for $U \rightarrow 0$, only one solution is possible This means that the larger value for $\mathrm{L}$ should be chosen, as shown in the figure In case (3b) the G-curve is a tangent to the F-curve, and $U$ has attanned Its minimum value (always negatıve), as has the wave length, $L=L_{m i n}$ This case of "maximum counter-current" corresponds to

with

$$
\sqrt{\frac{h}{L} \tanh k h}=\frac{I}{1-n} \sqrt{\frac{h}{L_{o}}}
$$

$c_{\text {gr }}$ being the relative wave group celerity From (3 5) and (3 10) we then find that the absolute wave group celerity is zero for $L=L_{m i n}$

$$
c_{\text {ga }}=c_{\text {gr }}+U \sin \beta=0
$$

(It wlll be shown in chapter 5 that in the case of a two-dimensional wave motion $(\sin \beta=1)$ progressing against a current over a gently sloping bed, the wave height reaches infinlty at the water depth at which (3 12) is fulfilled) In case (3c) there is no solution for $L$ It is readily seen from Fig 3-B that - other things being equal - a positive current

"lengthens" the waves whereas a negative current "shortens" them

A full account of the graphical method, including its practical application, is found in [4] Solutions to (35) (with $\sin \beta=1$ ) are presented in chapter 6

For deep-water waves (for instance $h / L>05$ ) (35) can be solved explicitly

$$
L=\frac{1}{4} L_{0}\left[1+\sqrt{1+4 \frac{U \sin B}{c_{0}}}\right]^{2}
$$

MOMENTUM AND PRESSURE FORCES ("STRESSES")

In this paper wave helghts wlll be calculated only for two-dimenslonal flow Stresses and energy fluxes wlll therefore be calculated only 
for the special case $\underline{\beta}=\pi / 2$ Takıng mean values over perıod $T_{a}$, the following total force (normal "stress") over depth $h$ and per unit width is found in a section at right angles to the current vector (and so in this case also to the direction of wave propagation)

$$
\sigma=\frac{1}{2} \gamma h^{2}+\rho h U^{2}+F_{W}
$$

In this expression, $\mathrm{F}_{\mathrm{W}}$ is the radiation stress

$$
F_{W}=\frac{1}{16} \gamma H^{2}\left[1+2 \frac{2 k h}{\sinh 2 k h}\right]=\left(2 \frac{c_{g r}}{c_{r}}-\frac{1}{2}\right) E
$$

where $E$ Is the mean specific wave energy The first two terms in (3 14) are readily discernible

\section{ENERGY FLUX}

The mean energy flux over depth $h$ and per unlt wldth depends upon the zero level for potentıal energy, since a net current is present Taking the mean water level as level we find for $\underline{\beta}=\pi / 2$ in a section at right angles to the current vector

$$
\overline{\mathrm{E}}_{\mathrm{f}, \mathrm{MWL}}=\frac{1}{2} \rho \mathrm{h} \mathrm{U}^{3}+\left(\mathrm{U}+\mathrm{c}_{\mathrm{gr}}\right) \mathrm{E}+\mathrm{U} \mathrm{F}_{\mathrm{W}}
$$

This expression has already been given by Longuet-Higgins and Stewart [5]

The paradox arises that although (3 16) may be interpreted physicalIy, It is of no direct use for the calculation of the wave height variation on a non-uniform current (because of the variation in the mean water level) This will be elucldated in chapters 4 and 5

In these and the subsequent calculations the following restriction on a Stokes wave must be borne in mind For long waves the "Ursell parameter" $\left(\mathrm{HL}^{2} / \mathrm{h}^{3}\right)$ must not exceed a certain number

\section{THE MEAN ENERGY LEVEL}

As a computation of the wave helght variation requires a knowledge of the mean energy flux at any station corresponding to the same horizontal datum, it is imperative to determine the variation in the MWL over an "arbitrary" (here gently sloping) bottom In other words, the "set-down" of the MWL is wanted The discussion is confined to periodic, irrotational flows These two conditions are written in the frames in Fig 4-A (For simplicity we consider two-dimensional flow only)

In the figure we have a sloping, known bottom and a sloping, but unknown, MWL And we have a horizontal datum, $z=0$ The mean water level Is given by

$$
\bar{\eta}=z_{b}+h
$$

so the determination of the MWL calls in fact for a definition of the water depth $h$ In the present case, which is a second order theory in wave helght, but is also a "zero-order bottom-slope theory", it is natural to define the water depth from the mean bottom pressure 


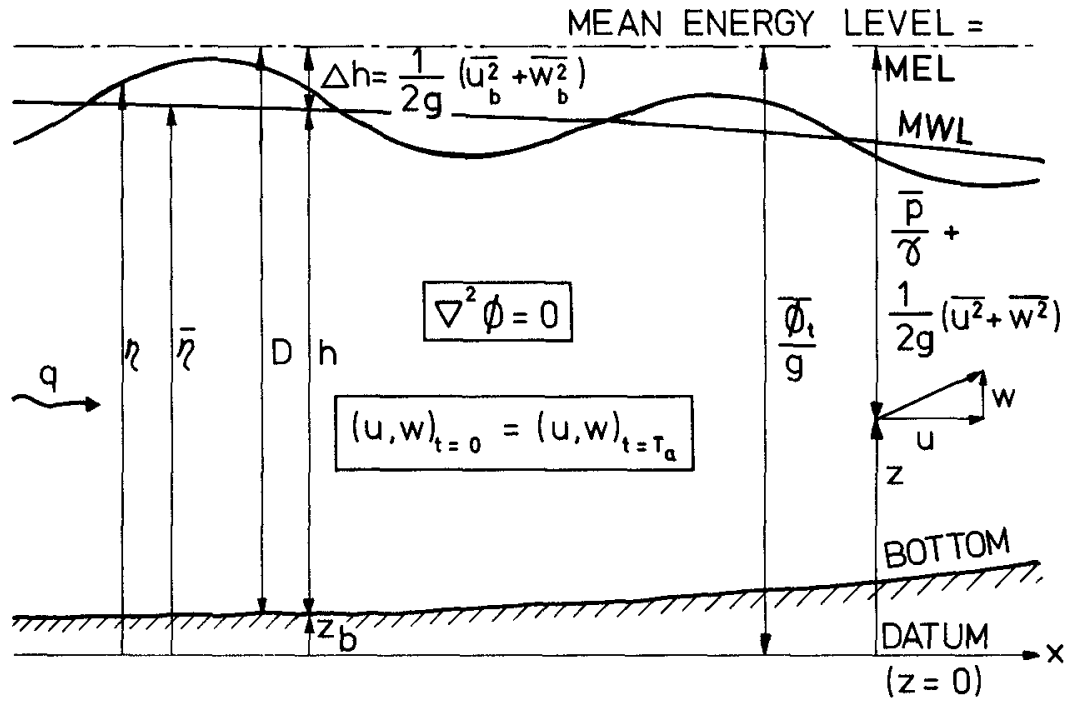

FIg 4-A Illustration of the "set-down" (schematical)

$$
\mathrm{h}=\frac{1}{\gamma} \overline{\mathrm{p}_{\mathrm{b}}}
$$

We stıll have not utilized the fact, that the flow is irrotational and perıodic The Bernoulli equation - presented with mean values over perıod $\mathrm{T}_{\mathrm{a}}$ - is therefore introduced

$$
\mathrm{z}+\frac{\overline{\mathrm{p}}}{\gamma}+\frac{1}{2 \mathrm{~g}}\left[\overline{\mathrm{u}^{2}}+\overrightarrow{\mathrm{w}^{2}}\right]=\frac{1}{\mathrm{~g}} \overline{\phi_{t}}=\text { const }
$$

( $\phi$ Is defined, so that $u_{1}=-\partial \phi / \partial x_{1}$ ) The fact that the right hand side Is independent of $x$ and $z$ is easıly ${ }^{1}$ seen from

$$
\frac{\partial}{\partial x_{I}}\left(\frac{\partial \phi}{\partial t}\right)=\overline{\frac{\partial}{\partial t}\left(\frac{\partial \phi}{\partial x_{1}}\right)}=0
$$

since the flow is periodic

This means that a constant horlzontal level is now found whlch is inherently connected with the flow Itself - and Independent of the arbitrary datum From the datum we can mark $\phi_{t} / g$ vertically, and we wlll ar$r$ rve at the same level for any $x$ This level wlll be called the mean energy level (MEL), since lt contains the three terms that are analogous to conventional steady hydraulies Combining $(41),\left(\begin{array}{l}4 \\ 4\end{array}\right)$ and (4 3) (at the bottom) we find the MWL

$$
\bar{n}+\frac{1}{2 g}\left(\overline{u_{b}^{2}}+\overline{w_{b}^{2}}\right)=\frac{1}{g} \overline{\phi_{t}}=\text { const }
$$

Since the constant in (45) as the distance from the datum up to the MEL, the set-down of the MWL - as defined by $\left(\begin{array}{ll}4 & 1\end{array}\right)$ and $\left(\begin{array}{ll}4 & 2\end{array}\right)$ - is 


$$
\Delta h \equiv D-h=\frac{1}{2 g}\left(\overline{u_{b}^{2}}+\overline{w_{b}^{2}}\right)
$$

(D could be termed the "geometrical depth", In contrast to $h$, which is a "physical depth") Assuming the discharge q to be finite anywhere, it appears from $(46)$ that the set-down $\Delta h$ is in fact the depression of the MWL at an arbitrary depth below the MWL at inflnite depth And this depression equals the mean velocity head at the bottom)

The actual analytical expression for the set-down in our two-dimensional combination of a steady current and a wave motion is found as follows It is assumed that locally, we can use the velocity potential corresponding to a horizontal bottom This Is - correct to second order with MWL as datum

$$
\begin{aligned}
\phi=-U x & +\frac{H c}{2} \frac{\cosh k(z+h)}{\sinh k h} \sin \left(\omega_{a} t-k x\right) \\
& +\frac{3}{32} \frac{c_{r}}{k}(k H)^{2} \quad \frac{\cosh 2 k(z+h)}{\sinh ^{4} k h} \sin 2\left(\omega_{a} t-k x\right) \\
& +\frac{g H^{2}}{8 h c_{r}} x+\left(\frac{1}{2} U^{2}+\frac{g H^{2}}{16 h} \frac{2 k h}{\sinh 2 k h}-\frac{g H^{2}}{8 h} \frac{U}{c_{r}}\right) t\left(\begin{array}{ll}
4 & 7
\end{array}\right)
\end{aligned}
$$

(4 7 ) assumes $\bar{n}=0$, so $\left(\begin{array}{ll}4 & 5\end{array}\right)-\left(\begin{array}{ll}4 & 7\end{array}\right)$ yıeld directly

$$
\Delta h=\frac{U^{2}}{2 g}+\frac{H^{2}}{16 h} \quad \frac{2 k h}{\sinh 2 k h}-\frac{H^{2}}{8 h} \frac{U}{c_{r}}
$$

for two-dimensional flow It wlll be observed in ( 48 ) that, in addition to the "current set-down" (first term) and the "wave set-down" (second term, see [7] and [1]), an interaction term appears (This last term was unfortunately missing from two previous publications from our laboratory Progress Report No 6 and a loose enclosure in Progress Repbrt No 7 This error was corrected in [3] p 20, where some other Corrigenda were also presented) Note that although (4 8) contains a negative term if U is positive, $\Delta \mathrm{h}$ can never become negatıve, according to (4 6)

It should be added that the set-down for a pure wave motion has been measured in a wave channel by Bowen et al [1] They found that the theory predicts the set-down outside the surf zone very well (They also found the set-up inside the surf zone)

\section{TWO-DIMENSIONAL WAVE TRANSFORMATION}

The two-dimenslonal current-wave system, whlch now wlll be consldered In some detall, is shown schematically in Fig 5-A The five main unknown quantities that we want to calculate are The wave length $L$, the current velocity $U$, the set-down $\Delta h$, the "physical water depth" $\mathrm{h}$ and the wave helght $H$ ( $c_{a}, c_{r}$ and $T_{r}$ can hereafter be found from (31)-(3 4), with $\sin \beta=1$ ) We assume that we know the absolute wave perlod $T_{a}$, the discharge $q$, the bottom topography $D=D(x)$, and the deep-water wave helght $\mathrm{H}_{\mathrm{O}}$ (where the current is zero) 


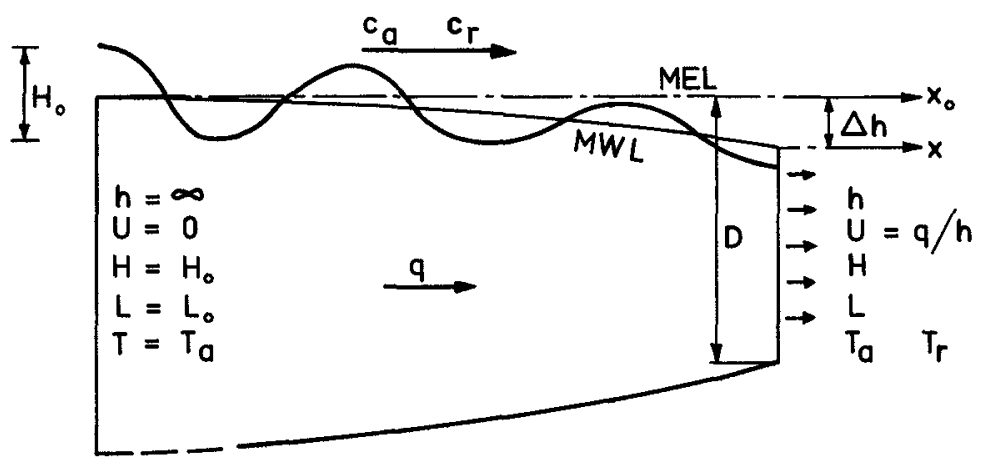

Fig 5-A Definition sketch for wave transformation

\section{CONSERVATION EQUATIONS}

To solve the problem, we must set up all the relevant conservation equations Only the energy concept presents us with some difficulty in this connection As pointed out in chapter 3, the expression for the mean energy flux with MWL as zero level cannot be used in a non-uniform flow since this level is constantly changing However, the existence of the MEL, which was found in the preceding chapter to be independent of the horlzontal coordinate(s), overcomes this difficulty

In accordance with Fig $5-A$ we find the following expression for the mean energy flux with MEL as reference

$$
\begin{aligned}
\overline{\mathrm{E}}_{f, M E L} & =\overline{\mathrm{E}}_{f, M W L}-(\gamma \mathrm{h} \Delta \mathrm{h}) \mathrm{U} \\
& =\left(1+\frac{\mathrm{U}}{\mathrm{c}_{r}}\right)\left(\mathrm{U}+\mathrm{c}_{g r}\right) \mathrm{E}
\end{aligned}
$$

using ( 3 16) and ( 4 8) (An erroneous expression for $\overline{\mathrm{E}}_{\mathrm{f}}$, MEL was pres In some earlier publications from this laboratory, see the comments to (4 8)) As, according to our assumptions, ( 5 2) must be constant, we can now write down the complete set of conservation equations

$$
\begin{aligned}
& \text { Wave crests } \omega_{a}=\omega_{r}+k U \\
& \text { Mass } \frac{d}{d x}(h U)=0 \\
& \text { Momentum } \frac{d F}{d x}+\frac{d}{d x}\left(\rho h U^{2}\right)-\gamma h \frac{d(\Delta h)}{d x}=0 \\
& \text { Energy } \frac{d}{d x}\left[\left(1+\frac{U}{c_{r}}\right)\left(U+c_{g r}\right)_{E}\right]=0 \\
& \text { Bottom topography } h+\Delta h=D
\end{aligned}
$$


(5 3) is the conventional way of expressing wave crest conservation In order to get to a practical formula, the expression for the relative wave celerity ( 34 ) must be used The mass and momentum equations have a direct physical interpretation and require no further comment $(-\mathrm{d}(\Delta \mathrm{h}) / \mathrm{dx}$ is the slope of the MWL) The energy equation is less obvious It should be noted, though, that (5 6) is a special case of Garrett's adiabatic invarlant expression, [2] It is directly seen from (5 6), that $\mathrm{H}$ reaches infinity at the water depth at which the absolute wave group celerity is zero It is interesting to recognize this "dynamical" limit as the "kınematical" limit (found in chapter 3), yielding the minimum wave length The last conservation equation may seem a trifle sophisticated, if not superfluous This is in a way true for a pure wave motion Our degree of approximation does not permit a discrimination between $D$ and $h$ for the determination of $\mathrm{L}, \mathrm{H}$ and $\Delta \mathrm{h}$ for this case However, when a current $1 \mathrm{~s}$ present, the first term in $(48)$ is by definition a zero-order term, and adjustment should be made for this, as described later 


\section{PRACTICAL EQUATIONS}

The conclusion of the foregolng discussion is that although the conservation equations arc fundamental, they are not applicable for direct calculations A new set of practical equations are therefore

$$
\begin{aligned}
& \sqrt{\frac{h}{L} \tanh k h}=\sqrt{\frac{h}{L_{0}}}\left[1-q^{*} \frac{h / L}{\left(h / L_{0}\right)^{2}}\right] \\
& h U=q \\
& \Delta h=\frac{U^{2}}{2 g}+\frac{H^{2}}{16 h} \frac{2 k h}{s i n h 2 k h}-\frac{H^{2}}{8 h} \frac{U}{c_{r}} \\
& \frac{H}{H_{O}}=\left[2 \frac{c_{r}}{c_{0}}\left(\frac{c_{g r}}{c_{r}}+\frac{q^{*}}{\left(c_{r} / c_{o}\right)\left(h / L_{o}\right)}\right)\left(1+\frac{q^{*}}{\left(c_{r} / c_{o}\right)\left(h / L_{o}\right)}\right)\right]^{-\frac{1}{2}} \\
& h+\Delta h=D
\end{aligned}
$$

wIth

$$
q^{*} \equiv \frac{q}{c_{0} L_{o}} \quad(513) \text { and } \quad \frac{c_{r}}{c_{o}}=\sqrt{\frac{h / L_{o}}{h / L} \tanh k h}
$$

(5 8) Is simply a rewriting of (3 5) with $\sin \beta=1$ (Now assumed to be valld for a gradually varying water depth) (59) and (5 11) are (5 4) and (56) integrated The momentum equation is replaced by the $\Delta$-expression (4 8) (which in fact can be deduced directly from the conservation equations)

The equations are solved as follows for glven values of $\mathrm{D}, \mathrm{q}, \mathrm{T}_{\mathrm{a}}$ and $\mathrm{H}_{\mathrm{O}}$ (not all combinations are admissable) Flrst $\mathrm{h}$ and $\mathrm{U}$ are found from ( 5 9) and $(512)$, whth $\Delta h=U^{2} / 2 g$ and subcritlcal flow assumed $L_{O}, c_{O}$ and $q^{*}$ are calculated from $(36),(39)$ and $(513)$ Next, L is found from (5 8), elther by iteration, or graphically, as explained in chapter 3 (wlth $\left.s_{v}=-q^{*} /\left(h / L_{0}\right)^{3 / 2}\right)$, or read from Fig 6-A, or interpolated in Table 6-a or 6-b $\mathrm{H}$ is calculated from $(511),(311)$ and $(514)$, or read from $F_{1 g}$ 6-B A new value of $\Delta h$ is then calculated from (5 10), (5 9) and ( 5 12) hereafter yleld the final value of $U$, whlch should be used to correct the first term in (5 10), to give the final value of $\Delta h$ (and $h$ ) In this way we should have taken account of all terms $O\left(\mathrm{H}^{2}\right)$ (The calculations can of course be repeated in an iterative manner to give "full numerical reciprocity" between $D$ and $h$ It should be borne in mind, though, that the increase in accuracy is formal) If $h$ were glven (measured), instead of $D$, the calculations and considerations are stralghtforward 


\section{TABLES AND GRAPHS FOR I AND $\mathrm{H}$}

\section{WAVE LENGTHS}

(5 8) was solved numerically by a Newton-Raphson iteration method on a digital computer The results are plotted in Fig 6-A ("constant discharge curves")

For $\mathrm{q}^{*}$ positive, the $\mathrm{h} / \mathrm{L}_{\mathrm{O}}$ - Ilmits have been so chosen that the Froude number is smaller than one, and $\mathrm{h} / \mathrm{L}$ goes up to about 05 Outside the latter limit, deep-water expressions wlll normally suffice Here (3 13) gives (wath $\sin \beta=1$ )

$$
\frac{L_{1}}{L_{0}}=\frac{1}{4}\left[1+\sqrt{1+4 \frac{q^{*}}{h / L_{0}}}\right]^{2}
$$

(The former limit is of course unrealistic Near such a high Froude number the curvature of the mean water surface becomes so large that the assumptions for our theory become invalid) The stralght line corresponding to $\mathrm{h} / \mathrm{L}$ equal to 005 indlcate that shallow-water condltions are not "typical" for the higher $\mathrm{q}^{*}$-values The shallow-water wave length is found from ( 58 )

$$
\frac{L}{L_{0}}=\sqrt{\frac{2 \pi h}{L_{0}}}+\frac{q^{*}}{h / L_{0}}
$$

of particular interest is the existence of maxima and minima for a number of positive $q^{*}$-values This is due to the fact that the steady increase in current velocity - as the depth decreases - tends to lengthen the waves, whlle the decrease in depth tends, in itself, to have the opposite effect The possibllity of horlzontal tangents can also be seen directly from (3 11) and the following expression, which is valid for constant values of $\mathrm{T}_{\mathrm{a}}$ and $\mathrm{q}$

$$
\frac{d L}{d h}=\frac{L}{2 h} \frac{\left(2 c g r-c_{r}\right)-2 U}{c_{g r}+U}
$$

For $\mathrm{q}^{*}$ negative, the upper limit for $\mathrm{h} / \mathrm{L}_{0}$ was chosen (arbitrarily) as 061 The lower limit was chosen so that the wave length $L$ comes as close to $I_{\text {min }}$ as the selected values of $h / L_{0}$ allow (see Table $6-b$ ) In the limit $\tilde{L}=L_{\mathrm{mln}}$ (see $(310)$ and the pertalning discussion) the numerical value of the Froude number wll be smaller than one, see [4] It is 1mmediately obvious that for $\mathrm{q}^{\mathrm{*}}$ smaller than - 005 (appr) deep-water conditions prevall, and $(61)$ can be used It will also be seen that in the case of a negative discharge we have the same trend as for no current I decreases monotonously with decreasing depth

In Table 6-a, numer lcal values of $L / L_{0}$ are presented for $\left|q^{*}\right| \leq 002$ (It should finally be mentioned that Fig $6-A$ and Tables $6-a$ and $6-\bar{b}$ can also be applied in the three-dimensional case, Fig 3-A Here we take as "current velocity" in (5 13) the component in the direction of the wave orthogonaI) 


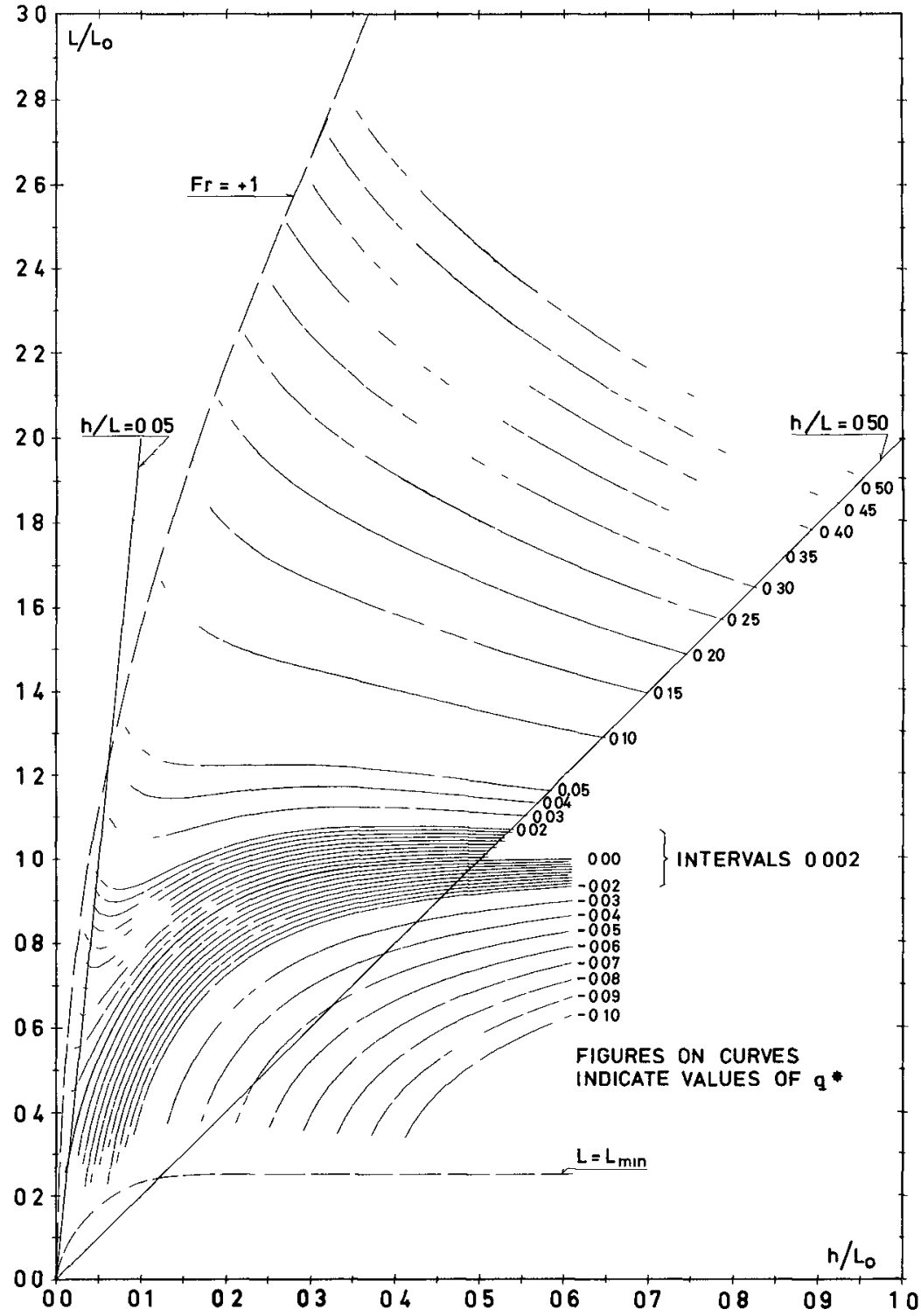

Fig 6-A "Constant discharge curves" for L/L。 


\begin{tabular}{|c|c|c|c|c|c|c|c|c|c|c|c|c|c|c|c|c|c|}
\hline$q^{\prime}$ & 0 & $+0002+$ & $+0004+$ & +0 & $006+$ & +0008 & +0 & 010 & +0 & $012+$ & +0 & 014 & +0 & $016+$ & +0018 & +0 & $0<0$ \\
\hline 000 & 0000 & & & & & & & & & & & & & & & & \\
\hline $\begin{array}{ll}0 & 01 \\
0 & 02 \\
0 & 03 \\
0 & 04\end{array}$ & $\begin{array}{ll}0 & 248 \\
0 & 347 \\
0 & 420 \\
0 & 480\end{array}$ & $\begin{array}{ll}0 & 450 \\
0 & 450 \\
0 & 491 \\
0 & 534\end{array}$ & $\begin{array}{ll}0 & 551 \\
0 & 560 \\
0 & 587\end{array}$ & & $\begin{array}{l}652 \\
628 \\
639\end{array}$ & $\begin{array}{ll}0 & 696 \\
0 & 691\end{array}$ & & $\begin{array}{l}763 \\
742\end{array}$ & $\begin{array}{l}0 \\
0\end{array}$ & $\begin{array}{l}830 \\
793\end{array}$ & 0 & 844 & 0 & 895 & o 946 & & 996 \\
\hline 005 & 0531 & o 575 & 0618 & 0 & 661 & 0703 & 0 & 745 & 0 & 786 & 0 & 828 & 0 & 869 & O 910 & 0 & 951 \\
\hline $\begin{array}{ll}0 & 06 \\
0 & 07 \\
0 & 08 \\
0 & 09\end{array}$ & $\begin{array}{ll}0 & 575 \\
0 & 614 \\
0 & 649 \\
0 & 681\end{array}$ & $\begin{array}{ll}0 & 613 \\
0 & 647 \\
0 & 679 \\
0 & 707\end{array}$ & $\begin{array}{ll}0 & 649 \\
0 & 679 \\
0 & 707 \\
0 & 734\end{array}$ & $\begin{array}{ll}0 & 6 \\
0 & 7 \\
0 & 7 \\
0 & 7\end{array}$ & $\begin{array}{l}686 \\
711 \\
736 \\
759\end{array}$ & $\begin{array}{ll}0 & 722 \\
0 & 743 \\
0 & 764 \\
0 & 785\end{array}$ & $\begin{array}{l}0 \\
0 \\
0 \\
0\end{array}$ & $\begin{array}{l}757 \\
774 \\
792 \\
810\end{array}$ & $\begin{array}{l}0 \\
0 \\
0 \\
0\end{array}$ & $\begin{array}{l}792 \\
804 \\
819 \\
835\end{array}$ & $\begin{array}{l}0 \\
0 \\
0 \\
0\end{array}$ & $\begin{array}{l}827 \\
835 \\
846 \\
860\end{array}$ & $\begin{array}{l}0 \\
0 \\
0 \\
0\end{array}$ & $\begin{array}{l}862 \\
865 \\
874 \\
884\end{array}$ & $\begin{array}{ll}0 & 897 \\
0 & 896 \\
0 & 900 \\
0 & 909\end{array}$ & & $\begin{array}{l}931 \\
9^{\circ} 6 \\
927 \\
9,3\end{array}$ \\
\hline 010 & 0709 & 0734 & 0758 & 07 & 782 & 0805 & 0 & 828 & 0 & 851 & 0 & 874 & 0 & 896 & 0919 & 0 & 941 \\
\hline $\begin{array}{ll}0 & 11 \\
0 & 12 \\
0 & 13 \\
0 & 14\end{array}$ & $\begin{array}{ll}0 & 735 \\
0 & 759 \\
0 & 780 \\
0 & 800\end{array}$ & $\begin{array}{ll}0 & 758 \\
0 & 780 \\
0 & 800 \\
0 & 819\end{array}$ & $\begin{array}{ll}0 & 780 \\
0 & 801 \\
0 & 820 \\
0 & 838\end{array}$ & $\begin{array}{ll}0 & 8 \\
0 & 8 \\
0 & 8 \\
0 & 8\end{array}$ & $\begin{array}{l}802 \\
822 \\
840 \\
856\end{array}$ & $\begin{array}{ll}0 & 824 \\
0 & 842 \\
0 & 859 \\
0 & 875\end{array}$ & & $\begin{array}{l}846 \\
862 \\
878 \\
893\end{array}$ & $\begin{array}{l}0 \\
0 \\
0 \\
0\end{array}$ & $\begin{array}{l}867 \\
882 \\
897 \\
910\end{array}$ & $\begin{array}{l}0 \\
0 \\
0 \\
0\end{array}$ & $\begin{array}{l}888 \\
902 \\
915 \\
928\end{array}$ & $\begin{array}{l}0 \\
0 \\
0 \\
0\end{array}$ & $\begin{array}{l}909 \\
921 \\
934 \\
946\end{array}$ & 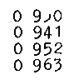 & $\begin{array}{ll}0 & 0 \\
0 & 0 \\
0 & 0 \\
0 & 9\end{array}$ & $\begin{array}{l}950 \\
960 \\
970 \\
980\end{array}$ \\
\hline $0 \begin{array}{ll}0 & 15\end{array}$ & 0 818 & 0836 & 0854 & 08 & 872 & 0889 & 0 & 906 & 0 & 923 & 0 & 940 & 0 & 957 & 0973 & 09 & 990 \\
\hline $\begin{array}{ll}0 & 16 \\
0 & 17 \\
0 & 18 \\
0 & 19\end{array}$ & $\begin{array}{ll}0 & 835 \\
0 & 850 \\
0 & 864 \\
0 & 877\end{array}$ & $\begin{array}{ll}0 & 852 \\
0 & 867 \\
0 & 880 \\
0 & 892\end{array}$ & $\begin{array}{ll}0 & 869 \\
0 & 883 \\
0 & 896 \\
0 & 907\end{array}$ & $\begin{array}{ll}0 & 8 \\
0 & 8 \\
0 & 9 \\
0 & 9\end{array}$ & $\begin{array}{l}886 \\
899 \\
911 \\
922\end{array}$ & $\begin{array}{ll}0 & 903 \\
0 & 915 \\
0 & 927 \\
0 & 937\end{array}$ & $\begin{array}{l}0 \\
0 \\
0 \\
0 \\
0\end{array}$ & $\begin{array}{l}919 \\
931 \\
942 \\
952\end{array}$ & $\begin{array}{l}0 \\
0 \\
0 \\
0\end{array}$ & $\begin{array}{l}935 \\
946 \\
957 \\
966\end{array}$ & $\begin{array}{l}0 \\
0 \\
0 \\
0\end{array}$ & $\begin{array}{l}951 \\
962 \\
972 \\
981\end{array}$ & $\begin{array}{l}0 \\
0 \\
0 \\
0\end{array}$ & $\begin{array}{l}967 \\
977 \\
986 \\
995\end{array}$ & $\begin{array}{ll}0 & 983 \\
0 & 992 \\
1 & 001 \\
1 & 009\end{array}$ & $\begin{array}{l}0 \\
18 \\
19 \\
1\end{array}$ & $\begin{array}{l}999 \\
007 \\
015 \\
03\end{array}$ \\
\hline 020 & 0888 & 0903 & 0918 & 09 & 933 & 0947 & 0 & 961 & 0 & 975 & 0 & 989 & 1 & 003 & 1016 & 10 & 0,0 \\
\hline $\begin{array}{ll}0 & 21 \\
0 & 22 \\
0 & 23 \\
0 & 24\end{array}$ & $\begin{array}{ll}0 & 899 \\
0 & 909 \\
0 & 918 \\
0 & 926\end{array}$ & $\begin{array}{ll}0 & 914 \\
0 & 923 \\
0 & 931 \\
0 & 939\end{array}$ & 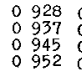 & $\begin{array}{ll}0 & 9 \\
0 & 9 \\
0 & 9 \\
0 & 9\end{array}$ & $\begin{array}{l}942 \\
950 \\
958 \\
965\end{array}$ & $\begin{array}{ll}0 & 956 \\
0 & 964 \\
0 & 971 \\
0 & 978\end{array}$ & $\begin{array}{l}0 \\
0 \\
0 \\
0 \\
0\end{array}$ & $\begin{array}{l}970 \\
977 \\
984 \\
991\end{array}$ & $\begin{array}{l}0 \\
0 \\
0 \\
1\end{array}$ & $\begin{array}{l}983 \\
990 \\
997 \\
003\end{array}$ & $\begin{array}{l}0 \\
1 \\
1 \\
1\end{array}$ & $\begin{array}{l}997 \\
004 \\
010 \\
016\end{array}$ & $\begin{array}{l}1 \\
1 \\
1 \\
1 \\
1\end{array}$ & $\begin{array}{l}010 \\
017 \\
023 \\
028\end{array}$ & $\begin{array}{ll}1 & 023 \\
1 & 029 \\
1 & 035 \\
1 & 040\end{array}$ & $\begin{array}{ll}1 & 0 \\
1 & 0 \\
1 & 0 \\
1 & 0\end{array}$ & $\begin{array}{l}03 t \\
04 \% \\
048 \\
052\end{array}$ \\
\hline 025 & 0933 & 0946 & 0959 & 09 & 972 & 0984 & 0 & 996 & 1 & 009 & 1 & 021 & 1 & 033 & 1045 & 10 & 057 \\
\hline $\begin{array}{ll}0 & 26 \\
0 & 27 \\
0 & 28 \\
0 & 29\end{array}$ & $\begin{array}{ll}0 & 940 \\
0 & 946 \\
0 & 952 \\
0 & 957\end{array}$ & $\begin{array}{l}0953 \\
0958 \\
0964 \\
0 \\
0\end{array}$ & $\begin{array}{ll}0 & 965 \\
0 & 971 \\
0 & 976 \\
0 & 980\end{array}$ & $\begin{array}{ll}0 & 9 \\
0 & 9 \\
0 & 9 \\
0 & 9\end{array}$ & $\begin{array}{l}977 \\
983 \\
987 \\
992\end{array}$ & $\begin{array}{ll}0 & 990 \\
0 & 995 \\
0 & 999 \\
1 & 003\end{array}$ & & $\begin{array}{l}002 \\
006 \\
011 \\
014\end{array}$ & $\begin{array}{l}1 \\
1 \\
1 \\
1\end{array}$ & $\begin{array}{l}014 \\
018 \\
022 \\
025\end{array}$ & $\begin{array}{l}1 \\
1 \\
1 \\
1\end{array}$ & $\begin{array}{l}0<5 \\
030 \\
033 \\
037\end{array}$ & $\begin{array}{l}1 \\
1 \\
1 \\
1\end{array}$ & $\begin{array}{l}037 \\
041 \\
045 \\
048\end{array}$ & $\begin{array}{ll}1 & 049 \\
1 & 053 \\
1 & 056 \\
1 & 059\end{array}$ & $\begin{array}{ll}1 & 0 \\
1 & 0 \\
1 & 0 \\
1 & c\end{array}$ & $\begin{array}{l}060 \\
064 \\
067 \\
069\end{array}$ \\
\hline 030 & 0961 & 0973 & 09840 & 09 & 995 & 1006 & 10 & 018 & 1 & 029 & 1 & 039 & 1 & 050 & 1061 & 10 & 072 \\
\hline $\begin{array}{ll}0 & 31 \\
0 & 32 \\
0 & 33 \\
0 & 34\end{array}$ & $\begin{array}{ll}0 & 965 \\
0 & 969 \\
0 & 972 \\
0 & 975\end{array}$ & $\begin{array}{ll}0 & 977 \\
0 & 980 \\
0 & 983 \\
0 & 986\end{array}$ & $\begin{array}{lll}0 & 988 & 0 \\
0 & 991 & 1 \\
0 & 994 & 1 \\
0 & 996\end{array}$ & $\begin{array}{ll}0 & 9 \\
1 & 0 \\
1 & 0 \\
1 & 0\end{array}$ & $\begin{array}{l}999 \\
002 \\
004 \\
007\end{array}$ & $\begin{array}{ll}1 & 010 \\
1 & 012 \\
1 & 015 \\
1 & 017\end{array}$ & & $\begin{array}{l}020 \\
023 \\
025 \\
027\end{array}$ & $\begin{array}{l}1 \\
1 \\
1 \\
1\end{array}$ & $\begin{array}{l}031 \\
034 \\
036 \\
037\end{array}$ & $\begin{array}{l}1 \\
1 \\
1 \\
1\end{array}$ & $\begin{array}{l}042 \\
044 \\
046 \\
047\end{array}$ & $\begin{array}{ll}1 & \\
1 & \\
1 & \\
1 & \end{array}$ & $\begin{array}{l}052 \\
054 \\
056 \\
057\end{array}$ & $\begin{array}{ll}1 & 063 \\
1 & 065 \\
1 & 066 \\
1 & 067\end{array}$ & & $\begin{array}{l}073 \\
075 \\
076 \\
077\end{array}$ \\
\hline 035 & 0978 & 0988 & 09991 & 10 & 009 & 1019 & 10 & 029 & 1 & 039 & 1 & 049 & 10 & 059 & 1068 & 10 & 078 \\
\hline $\begin{array}{ll}0 & 36 \\
0 & 37 \\
0 & 38 \\
0 & 39\end{array}$ & 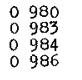 & 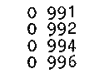 & $\begin{array}{ll}1 & 001 \\
1 & 002 \\
1 & 004 \\
1 & 005\end{array}$ & $\begin{array}{ll}1 & 0 \\
1 & 0 \\
1 & 0 \\
1 & 0\end{array}$ & $\begin{array}{l}011 \\
012 \\
014 \\
015\end{array}$ & $\begin{array}{ll}1 & 020 \\
1 & 022 \\
1 & 023 \\
1 & 024\end{array}$ & & $\begin{array}{l}030 \\
032 \\
033 \\
033\end{array}$ & $\begin{array}{l}1 \\
1 \\
1 \\
1 \\
1\end{array}$ & $\begin{array}{l}040 \\
041 \\
042 \\
043\end{array}$ & $\begin{array}{l}1 \\
1 \\
1 \\
1\end{array}$ & $\begin{array}{l}050 \\
051 \\
051 \\
052\end{array}$ & $\begin{array}{l}1 \\
1 \\
1 \\
1\end{array}$ & $\begin{array}{l}059 \\
060 \\
061 \\
061\end{array}$ & $\begin{array}{ll}1 & 069 \\
1 & 070 \\
1 & 070 \\
1 & 070\end{array}$ & & $\begin{array}{l}079 \\
079 \\
079 \\
079\end{array}$ \\
\hline 040 & 0988 & 0997 & 10061 & 10 & 016 & 1025 & 10 & 034 & 1 & 043 & 1 & 052 & 1 & 061 & 1070 & 10 & 079 \\
\hline $\begin{array}{ll}0 & 41 \\
0 & 42 \\
0 & 43 \\
0 & 44\end{array}$ & $\begin{array}{ll}0 & 989 \\
0 & 990 \\
0 & 997 \\
0 & 992\end{array}$ & $\begin{array}{ll}0 & 998 \\
0 & 999 \\
1 & 000 \\
1 & 001\end{array}$ & $\begin{array}{lll}1 & 007 & 1 \\
1 & 008 & 1 \\
1 & 009 & 1 \\
1 & 010 & 1\end{array}$ & $\begin{array}{ll}1 & 0 \\
1 & 0 \\
1 & 0 \\
1 & 0\end{array}$ & $\begin{array}{l}017 \\
017 \\
018 \\
018\end{array}$ & $\begin{array}{ll}1 & 026 \\
1 & 026 \\
1 & 027 \\
1 & 027\end{array}$ & & $\begin{array}{l}035 \\
035 \\
035 \\
035\end{array}$ & $\begin{array}{l}1 \\
1 \\
1 \\
1\end{array}$ & $\begin{array}{l}043 \\
044 \\
044 \\
044\end{array}$ & $\begin{array}{l}1 \\
1 \\
1 \\
1 \\
1\end{array}$ & $\begin{array}{l}052 \\
05 ? \\
052 \\
052\end{array}$ & $\begin{array}{ll}1 & \\
1 & \\
1 & \\
1 & \end{array}$ & $\begin{array}{l}061 \\
061 \\
061 \\
061\end{array}$ & $\begin{array}{ll}1 & 070 \\
1 & 070 \\
1 & 069 \\
1 & 069\end{array}$ & $\begin{array}{ll}1 & 0 \\
1 & 0 \\
1 & 0 \\
1 & 0\end{array}$ & $\begin{array}{l}079 \\
078 \\
078 \\
077\end{array}$ \\
\hline 045 & 0993 & 1002 & 10101 & 10 & 019 & 1027 & 10 & 036 & 10 & 044 & 1 & 052 & 10 & 060 & 1068 & 10 & 077 \\
\hline $\begin{array}{ll}0 & 46 \\
0 & 47 \\
0 & 48 \\
0 & 49\end{array}$ & 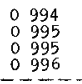 & $\begin{array}{ll}1 & 002 \\
1 & 003 \\
1 & 003 \\
1 & 004\end{array}$ & $\begin{array}{lll}1 & 011 & 1 \\
1 & 011 & 1 \\
1 & 011 & 1 \\
1 & 012 & 1\end{array}$ & $\begin{array}{ll}1 & 0 \\
1 & 0 \\
1 & 0 \\
1 & 0\end{array}$ & $\begin{array}{l}019 \\
019 \\
019 \\
020\end{array}$ & $\begin{array}{ll}1 & 027 \\
1 & 027 \\
1 & 027 \\
1 & 027\end{array}$ & & $\begin{array}{l}036 \\
035 \\
035 \\
035\end{array}$ & $\begin{array}{l}1 \\
1 \\
1 \\
1\end{array}$ & $\begin{array}{l}044 \\
043 \\
043 \\
043\end{array}$ & & $\begin{array}{l}05 \% \\
051 \\
051 \\
051\end{array}$ & $\begin{array}{ll}1 & 0 \\
1 & \\
1 & \\
1 & \end{array}$ & $\begin{array}{l}060 \\
059 \\
059 \\
058\end{array}$ & $\begin{array}{ll}1 & 008 \\
1 & 067 \\
1 & 067 \\
1 & 066\end{array}$ & $\begin{array}{ll}1 & 0 \\
1 & 0 \\
1 & 0 \\
1 & 0\end{array}$ & $\begin{array}{l}076 \\
075 \\
074 \\
074\end{array}$ \\
\hline 050 & 0996 & $\begin{array}{l}1004 \\
\end{array}$ & 10121 & 1 & 020 & 3027 & 10 & 035 & 1 & 043 & 1 & 050 & 1 & 058 & 1065 & 10 & 073 \\
\hline $\begin{array}{ll}0 & 51 \\
0 & 52 \\
0 & 53 \\
0 & 54\end{array}$ & $\begin{array}{ll}0 & 997 \\
0 & 997 \\
0 & 997 \\
0 & 998\end{array}$ & 1004 & 10124 & 4 & 0207 & & & & & & & $\begin{array}{l}050 \\
049\end{array}$ & $\begin{array}{r}1 \\
-\frac{1}{1} \frac{c}{c}\end{array}$ & $\begin{array}{l}057 \\
057 \\
0567\end{array}$ & $\begin{array}{ll}1 & 065 \\
1 & 064 \\
1 & 063 \\
1 & 062\end{array}$ & $\begin{array}{ll}1 & 0 \\
1 & 0 \\
1 & 0 \\
1 & 0\end{array}$ & $\begin{array}{l}072 \\
071 \\
070 \\
069\end{array}$ \\
\hline 055 & 0998 & & & & & & & & & & & & & & & & \\
\hline $\begin{array}{ll}0 & 56 \\
0 & 57 \\
0 & 58 \\
0 & 59\end{array}$ & $\begin{array}{ll}0 & 998 \\
0 & 998 \\
0 & 999 \\
0 & 999\end{array}$ & & & & & & & & & & & & & & & & \\
\hline 060 & 0999 & & & & & & & & & & & & & & & & \\
\hline NOTE & BELO & DOTT & TED & & & $E$ & NE & & 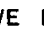 & & & & & & & & \\
\hline
\end{tabular}

Table 6-a Values of $\mathrm{L} / \mathrm{L}_{\circ}$ (posıtıve discharge) 


\begin{tabular}{|c|c|c|c|c|c|c|c|c|c|c|c|}
\hline$h / L_{0} q^{*}$ & 0 & -0002 & $-0 \quad 004$ & -0000 & -0008 & -0010 & -0012 & $-0 \quad 014$ & -0016 & $-0 \quad 018$ & $\begin{array}{lll}-0 & 020\end{array}$ \\
\hline 000 & 0000 & & & & & & & & & & \\
\hline $\begin{array}{ll}0 & 01 \\
0 & 02 \\
0 & 03 \\
0 & 04\end{array}$ & $\begin{array}{ll}0 & 248 \\
0 & 347 \\
0 & 420 \\
0 & 480\end{array}$ & 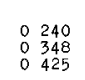 & $\begin{array}{ll}0 & 270 \\
0 & 367\end{array}$ & $\begin{array}{ll}0 & 169 \\
0 & 304\end{array}$ & 0227 & & & & & & \\
\hline 005 & O 531 & 0486 & 0439 & 0390 & o 336 & 0.272 & & & & & \\
\hline $\begin{array}{ll}0 & 06 \\
0 & 07 \\
0 & 08 \\
0 & 09\end{array}$ & $\begin{array}{ll}0 & 575 \\
0 & 614 \\
0 & 649 \\
0 & 681\end{array}$ & $\begin{array}{ll}0 & 537 \\
0 & 581 \\
0 & 619 \\
0 & 654\end{array}$ & $\begin{array}{ll}0 & 497 \\
0 & 546 \\
0 & 589 \\
0 & 626\end{array}$ & $\begin{array}{ll}0 & 456 \\
0 & 511 \\
0 & 557 \\
0 & 5997 \\
0\end{array}$ & $\begin{array}{ll}0 & 413 \\
0 & 474 \\
0 & 524 \\
0 & 568\end{array}$ & $\begin{array}{ll}0 & 365 \\
0 & 434 \\
0 & 490 \\
0 & 537\end{array}$ & $\begin{array}{ll}0 & 310 \\
0 & 391 \\
0 & 454 \\
0 & 505\end{array}$ & $\begin{array}{ll}0 & 223 \\
0 & 341 \\
0 & 414 \\
0 & 471\end{array}$ & $\begin{array}{ll}0 & 275 \\
0 & 369 \\
0 & 434\end{array}$ & $\begin{array}{ll}0 & 311 \\
0 & 39\end{array}$ & 0341 \\
\hline $\begin{array}{ll}0 & 10\end{array}$ & 0709 & 0684 & 0659 & 0633 & 0606 & 0578 & 0549 & 0519 & 0487 & 0452 & 0412 \\
\hline $\begin{array}{ll}0 & 11 \\
0 & 12 \\
0 & 13 \\
0 & 14\end{array}$ & $\begin{array}{ll}0 & 735 \\
0 & 759 \\
0 & 780 \\
0 & 800\end{array}$ & $\begin{array}{ll}0 & 712 \\
0 & 737 \\
0 & 760 \\
0 & 781\end{array}$ & $\begin{array}{ll}0 & 689 \\
0 & 715 \\
0 & 739 \\
0 & 761\end{array}$ & $\begin{array}{ll}0 & 664 \\
0 & 693 \\
0 & 718 \\
0 & 741\end{array}$ & $\begin{array}{ll}0 & 640 \\
0 & 670 \\
0 & 697 \\
0 & 721\end{array}$ & $\begin{array}{ll}0 & 614 \\
0 & 646 \\
0 & 674 \\
0 & 700\end{array}$ & $\begin{array}{ll}0 & 588 \\
0 & 622 \\
0 & 652 \\
0 & 679\end{array}$ & $\begin{array}{ll}0 & 560 \\
0 & 596 \\
0 & 628 \\
0 & 656\end{array}$ & $\begin{array}{ll}0 & 531 \\
0 & 570 \\
0 & 604 \\
0 & 634\end{array}$ & $\begin{array}{ll}0 & 500 \\
0 & 54 \\
0 & 578 \\
0 & 610\end{array}$ & $\begin{array}{ll}0 & 467 \\
0 & 512 \\
0 & 551 \\
0 & 585\end{array}$ \\
\hline 015 & 0818 & 0800 & 0781 & 0762 & 0743 & 0723 & 0703 & 0 682 & 0660 & 0638 & 0615 \\
\hline $\mid \begin{array}{ll}0 & 16 \\
0 & 17 \\
0 & 18 \\
0 & 19\end{array}$ & $\begin{array}{ll}0 & 835 \\
0 & 850 \\
0 & 864 \\
0 & 877\end{array}$ & $\begin{array}{ll}0 & 817 \\
0 & 833 \\
0 & 848 \\
0 & 861\end{array}$ & $\begin{array}{ll}0 & 800 \\
0 & 816 \\
0 & 831 \\
0 & 845\end{array}$ & $\begin{array}{ll}0 & 781 \\
0 & 799 \\
0 & 815 \\
0 & 829\end{array}$ & $\begin{array}{ll}0 & 763 \\
0 & 781 \\
0 & 798 \\
0 & 813\end{array}$ & $\begin{array}{ll}0 & 744 \\
0 & 763 \\
0 & 780 \\
0 & 796\end{array}$ & $\begin{array}{ll}0 & 725 \\
0 & 745 \\
0 & 765 \\
0 & 779\end{array}$ & $\begin{array}{l}0705 \\
0726 \\
0745 \\
0762 \\
0\end{array}$ & $\begin{array}{ll}0 & 685 \\
0 & 706 \\
0 & 726 \\
0 & 744\end{array}$ & $\begin{array}{ll}0 & 664 \\
0 & 687 \\
0 & 707 \\
0 & 726\end{array}$ & $\begin{array}{ll}0 & 642 \\
0 & 666 \\
0 & 688 \\
0 & 108\end{array}$ \\
\hline 020 & o 888 & 0873 & 0858 & 0843 & 0827 & 0811 & 0794 & o 778 & 0761 & 0743 & 0126 \\
\hline $\begin{array}{ll}0 & 21 \\
0 & 22 \\
0 & 23 \\
0 & 24\end{array}$ & 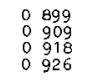 & $\begin{array}{ll}0 & 884 \\
0 & 895 \\
0 & 904 \\
0 & 912\end{array}$ & $\begin{array}{ll}0 & 870 \\
0 & 880 \\
0 & 890 \\
0 & 899\end{array}$ & $\begin{array}{ll}0 & 855 \\
0 & 866 \\
0 & 876 \\
0 & 885\end{array}$ & $\begin{array}{lll}0 & 839 \\
0 & 851 \\
0 & 862 \\
0 & 871 \\
0\end{array}$ & $\begin{array}{ll}0 & 824 \\
0 & 836 \\
0 & 847 \\
0 & 857\end{array}$ & 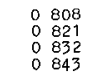 & $\begin{array}{ll}0 & 792 \\
0 & 805 \\
0 & 817 \\
0 & 828\end{array}$ & $\begin{array}{ll}0 & 776 \\
0 & 789 \\
0 & 802 \\
0 & 813\end{array}$ & $\begin{array}{ll}0 & 759 \\
0 & 773 \\
0 & 786 \\
0 & 798\end{array}$ & $\begin{array}{ll}0 & 742 \\
0 & 757 \\
0 & 770 \\
0 & 183\end{array}$ \\
\hline 025 & 0933 & 0920 & 0907 & 0894 & o 880 & 0866 & 0852 & 0838 & o 824 & 0809 & $0 \quad 194$ \\
\hline $\mid \begin{array}{ll}0 & 26 \\
0 & 27 \\
0 & 28 \\
0 & 29\end{array}$ & 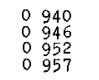 & $\begin{array}{ll}0 & 927 \\
0 & 934 \\
0 & 939 \\
0 & 945\end{array}$ & $\begin{array}{ll}0 & 914 \\
0 & 921 \\
0 & 927 \\
0 & 933\end{array}$ & $\begin{array}{ll}0 & 901 \\
0 & 908 \\
0 & 915 \\
0 & 921\end{array}$ & 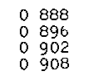 & $\begin{array}{ll}0 & 875 \\
0 & 882 \\
0 & 890 \\
0 & 896\end{array}$ & $\begin{array}{ll}0 & 867 \\
0 & 869 \\
0 & 877 \\
0 & 883\end{array}$ & $\begin{array}{lll}0 & 847 \\
0 & 856 \\
0 & 864 \\
0 & 871\end{array}$ & $\begin{array}{ll}0 & 833 \\
0 & 842 \\
0 & 850 \\
0 & 858\end{array}$ & $\begin{array}{ll}0 & 819 \\
0 & 829 \\
0 & 837 \\
0 & 845\end{array}$ & $\begin{array}{ll}0 & 805 \\
0 & 815 \\
0 & 823 \\
0 & 832\end{array}$ \\
\hline 030 & 0961 & 0950 & 0938 & 0926 & o 914 & 0902 & 0890 & 0877 & 0865 & 0852 & 0839 \\
\hline $\begin{array}{ll}0 & 31 \\
0 & 32 \\
0 & 33 \\
0 & 33 \\
0 & 34\end{array}$ & 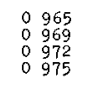 & 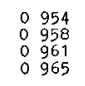 & $\begin{array}{ll}0 & 942 \\
0 & 947 \\
0 & 950 \\
0 & 954\end{array}$ & $\begin{array}{ll}0 & 931 \\
0 & 935 \\
0 & 939 \\
0 & 943\end{array}$ & $\begin{array}{ll}0 & 919 \\
0 & 924 \\
0 & 928 \\
0 & 932\end{array}$ & $\begin{array}{ll}0 & 907 \\
0 & 912 \\
0 & 917 \\
0 & 921\end{array}$ & $\begin{array}{ll}0 & 895 \\
0 & 901 \\
0 & 906 \\
0 & 910\end{array}$ & $\begin{array}{lll}0 & 883 \\
0 & 889 \\
0 & 894 \\
0 & 899\end{array}$ & $\begin{array}{lll}0 & 871 \\
0 & 877 \\
0 & 882 \\
0 & 887\end{array}$ & $\begin{array}{ll}0 & 859 \\
0 & 865 \\
0 & 871 \\
0 & 876\end{array}$ & $\begin{array}{ll}0 & 846 \\
0 & 853 \\
0 & 859 \\
0 & 864\end{array}$ \\
\hline 03, & 0978 & 0968 & 0957 & 0946 & o 936 & 0925 & 0914 & 0903 & 0 892 & 0881 & 0 869 \\
\hline $\begin{array}{lll}0 & 36 \\
0 & 36 \\
0 & 38 \\
0 & 38 \\
0 & 39\end{array}$ & 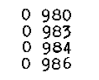 & 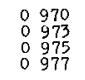 & $\begin{array}{ll}0 & 960 \\
0 & 962 \\
0 & 965 \\
0 & 967\end{array}$ & 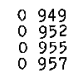 & $\begin{array}{l}0939 \\
0 \quad 942 \\
0 \quad 945 \\
0 \quad 947\end{array}$ & 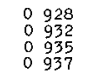 & $\begin{array}{ll}0 & 918 \\
0 & 921 \\
0 & 924 \\
0 & 927\end{array}$ & $\begin{array}{ll}0 & 907 \\
0 & 911 \\
0 & 914 \\
0 & 917\end{array}$ & 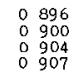 & $\begin{array}{ll}0 & 885 \\
0 & 889 \\
0 & 893 \\
0 & 897\end{array}$ & $\begin{array}{ll}0 & 874 \\
0 & 879 \\
0 & 883 \\
0 & 887\end{array}$ \\
\hline 040 & 0988 & 0978 & 0969 & 0959 & o 950 & 0940 & 0930 & 0920 & 0910 & 0900 & 0890 \\
\hline $\begin{array}{ll}0 & 41 \\
0 & 42 \\
0 & 43 \\
0 & 44\end{array}$ & 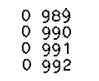 & 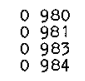 & $\begin{array}{ll}0 & 971 \\
0 & 972 \\
0 & 974 \\
0 & 975\end{array}$ & 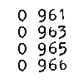 & $\begin{array}{ll}0 & 952 \\
0 & 954 \\
0 & 955 \\
0 & 957\end{array}$ & 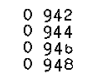 & $\begin{array}{ll}0 & 933 \\
0 & 935 \\
0 & 937 \\
0 & 939\end{array}$ & $\begin{array}{ll}0 & 923 \\
0 & 925 \\
0 & 928 \\
0 & 930\end{array}$ & $\begin{array}{ll}0 & 913 \\
0 & 916 \\
0 & 919 \\
0 & 921\end{array}$ & 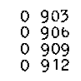 & $\begin{array}{ll}0 & 894 \\
0 & 897 \\
0 & 900 \\
0 & 902\end{array}$ \\
\hline 04, & o 993 & 0985 & 0976 & 0967 & o 959 & o 950 & 0941 & 0932 & 0 923 & 0914 & 0905 \\
\hline $\begin{array}{ll}0 & 46 \\
0 & 47 \\
0 & 48 \\
0 & 49\end{array}$ & 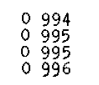 & $\begin{array}{l}0 \\
0 \\
0986 \\
0987 \\
0988\end{array}$ & $\begin{array}{r}0977 \\
0997 \\
0919 \\
0 \quad 998\end{array}$ & $\begin{array}{r}0969 \\
0970 \\
0971 \\
\\
\end{array}$ & $\begin{array}{r}0960 \\
0961 \\
-\frac{0}{0} 963 \\
964\end{array}$ & $\begin{array}{r}0951 \\
09 \\
0 \quad 953 \\
-0 \\
0954 \\
0956\end{array}$ & $\begin{array}{r}0943 \\
0 \\
0 \\
0 \\
0944 \\
0 \\
0947\end{array}$ & $\begin{array}{r}0934 \\
r_{0}^{0} 936 \\
0938 \\
0939\end{array}$ & $\begin{array}{r}0925 \\
-09927 \\
0929 \\
0931\end{array}$ & $\begin{array}{r}0916 \\
09919 \\
0921 \\
0923\end{array}$ & $\begin{array}{ll}0 & 908 \\
0 & 910 \\
0 & 912 \\
0 & 914\end{array}$ \\
\hline 050 & 0996 & 0989 & 0981 & 0973 & 0965 & o 957 & ० 949 & 0941 & 0933 & ○ 924 & 0916 \\
\hline $\begin{array}{ll}0 & 57 \\
0 & 52 \\
0 & 53 \\
0 & 54\end{array}$ & $\begin{array}{ll}0 & 997 \\
0 & 997 \\
0 & 997 \\
0 & 998\end{array}$ & $\begin{array}{ll}0 & 989 \\
0 & 990 \\
0 & 990 \\
0 & 990\end{array}$ & $\begin{array}{ll}0 & 981 \\
0 & 982 \\
0 & 983 \\
0 & 983\end{array}$ & 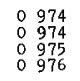 & 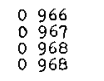 & 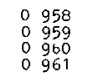 & 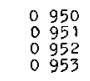 & $\begin{array}{l}0942 \\
09943 \\
0945 \\
0946\end{array}$ & 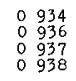 & 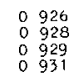 & $\begin{array}{ll}0 & 918 \\
0 & 920 \\
0 & 922 \\
0 & 923\end{array}$ \\
\hline 055 & 0998 & 0991 & 0984 & 0976 & 0969 & ० 962 & 0954 & 0947 & 0940 & 0932 & 0925 \\
\hline $\begin{array}{ll}0 & 56 \\
0 & 57 \\
0 & 58 \\
0 & 59\end{array}$ & 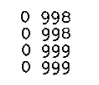 & $\begin{array}{ll}0 & 991 \\
0 & 992 \\
0 & 992 \\
0 & 992\end{array}$ & $\begin{array}{ll}0 & 984 \\
0 & 985 \\
0 & 985 \\
0 & 985\end{array}$ & 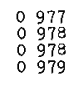 & $\begin{array}{l}0970 \\
09970 \\
0971 \\
0976\end{array}$ & 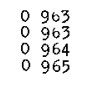 & 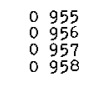 & $\begin{array}{l}0948 \\
0949 \\
0 \\
0950 \\
0951\end{array}$ & $\begin{array}{ll}0 & 941 \\
0 & 942 \\
0 & 943 \\
0 & 944\end{array}$ & 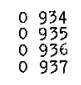 & $\begin{array}{ll}0 & 926 \\
0 & 928 \\
0 & 929 \\
0 & 930\end{array}$ \\
\hline 060 & o 999 & ० 992 & 0986 & 0979 & 0976 & 0966 & 0959 & 0952 & 0945 & 0938 & 0932 \\
\hline NOTE & BELOW & DOTTE & ED & $-\cos$ & URVE WE & HAVE & $h / L>0$ & & & & \\
\hline
\end{tabular}

Table 6-b Values of $L_{/} / L_{0}$ (negative discharge) 


\section{WAVE HEIGHTS}

The wave helghts - calculated from $(58)$ and ( 5 II) - are plotted in Fig 6-B Note how the "shape" of the curves corresponding to a constant positive discharge differs from that corresponding to $\mathrm{q}^{*}=0$ The outmost left part of these curves correspond to Froude numbers close to one (see also Fig 6-A, with comments) For negative discharges we end with inf1nite wave helghts in the limit $\mathrm{L}=\overline{\mathrm{L}} \mathrm{min}$

\section{NUMERTCAL EXAMPLES} racy

In the examples results are only given with normal slide-rule accu-

EX 7-a CALCULATtion of $\mathrm{L}, \mathrm{H}, \triangle \mathrm{h}, \mathrm{h}$ AND U FROM $\mathrm{w}_{\mathrm{a}}, \mathrm{q}, \mathrm{D}$ AND $\mathrm{H}_{0}$

Two-dimensional flow is assumed, and the following quantities are given

$\mathrm{T}_{\mathrm{a}}=80 \mathrm{~s}, \mathrm{q}=101 \mathrm{~m}^{2} / \mathrm{s}, \mathrm{D}=95 \mathrm{~m}, \mathrm{H}_{0}=16 \mathrm{~m}$ (corresponding to a deepwater steepness $\mathrm{S}_{\mathrm{O}}=16 \%$ )

In the first approximation, $H$ is put equal to zero in (5 10), so we find from ( 59 ) and ( 512$)$, by iteration

$\Delta \mathrm{h}_{1}=00583 \mathrm{~m}, \mathrm{~h}_{1}=94417 \approx 944 \mathrm{~m}, \mathrm{U}_{1}=107 \mathrm{~m} / \mathrm{s}$

From $(36),(39)$ and $(513) \mathrm{L}_{\circ}=15680^{2}=100 \mathrm{~m}$, $c_{0}=15680=125 \mathrm{~m} / \mathrm{s}, \mathrm{h} / \mathrm{L}_{0}=0.0944, q^{*}=000808$

By interpolation in Table 6-a we then find $L / L_{0}=0795 \Rightarrow L=795 \mathrm{~m}$

(without the current, the wave length would be $695 \mathrm{~m}$, for the same values of $D$ and $\mathrm{T}_{\mathrm{a}}$ )

$h / L=944 / 795=0119$, so we find from $(514)$ and $(311)$

$c_{r} / c_{0}=0709 \Rightarrow c_{r}=886 \mathrm{~m} / \mathrm{s}$ (w1thout the current the wave celerity

would be $869 \mathrm{~m} / \mathrm{s})$, and $\mathrm{c}_{\mathrm{gr}} / \mathrm{c}_{\mathrm{r}}=0853$

(5 11) then gives $H / H_{O}=0804 \Rightarrow H=129 \mathrm{~m}$ (w1thout the current the wave helght would be $150 \mathrm{~m}$, for the same value of $H_{\mathrm{O}}$ )

The new value of $\Delta \mathrm{h}$ from $(510) \quad \Delta \mathrm{h}_{2}=00583 \mathrm{~m}$

$+00078 \mathrm{~m}-00027 \mathrm{~m} \approx 00634 \mathrm{~m} \Rightarrow \mathrm{h}_{2} \approx 944 \mathrm{~m}=\mathrm{h}_{1}$ So w1thin slide-rule accuracy we find $\mathrm{U}=107 \mathrm{~m} / \mathrm{s}, \Delta \mathrm{h}=0.0634 \mathrm{~m}$ (w1thout the current the set-down would be $00094 \mathrm{~m}), \underline{\mathrm{h}}=94366 \mathrm{~m}(\approx 944 \mathrm{~m})$

Finally from $(31)$ and $(32) \quad c_{a}=795 / 80=993 \mathrm{~m} / \mathrm{s}$ and $\mathrm{m}_{\mathrm{r}}=$ $795 / 886=\underline{8.98 \mathrm{~s}}$

\section{EX 7-b CALCULATION OF WAVE HETGHT FROM BOTTOM PRESSURE CELL}

This example demonstrates how the wave helght $H$ can be determined for a two-dimensional flow, when the below mentioned parameters have been measured It also shows specifically the effect of a current 


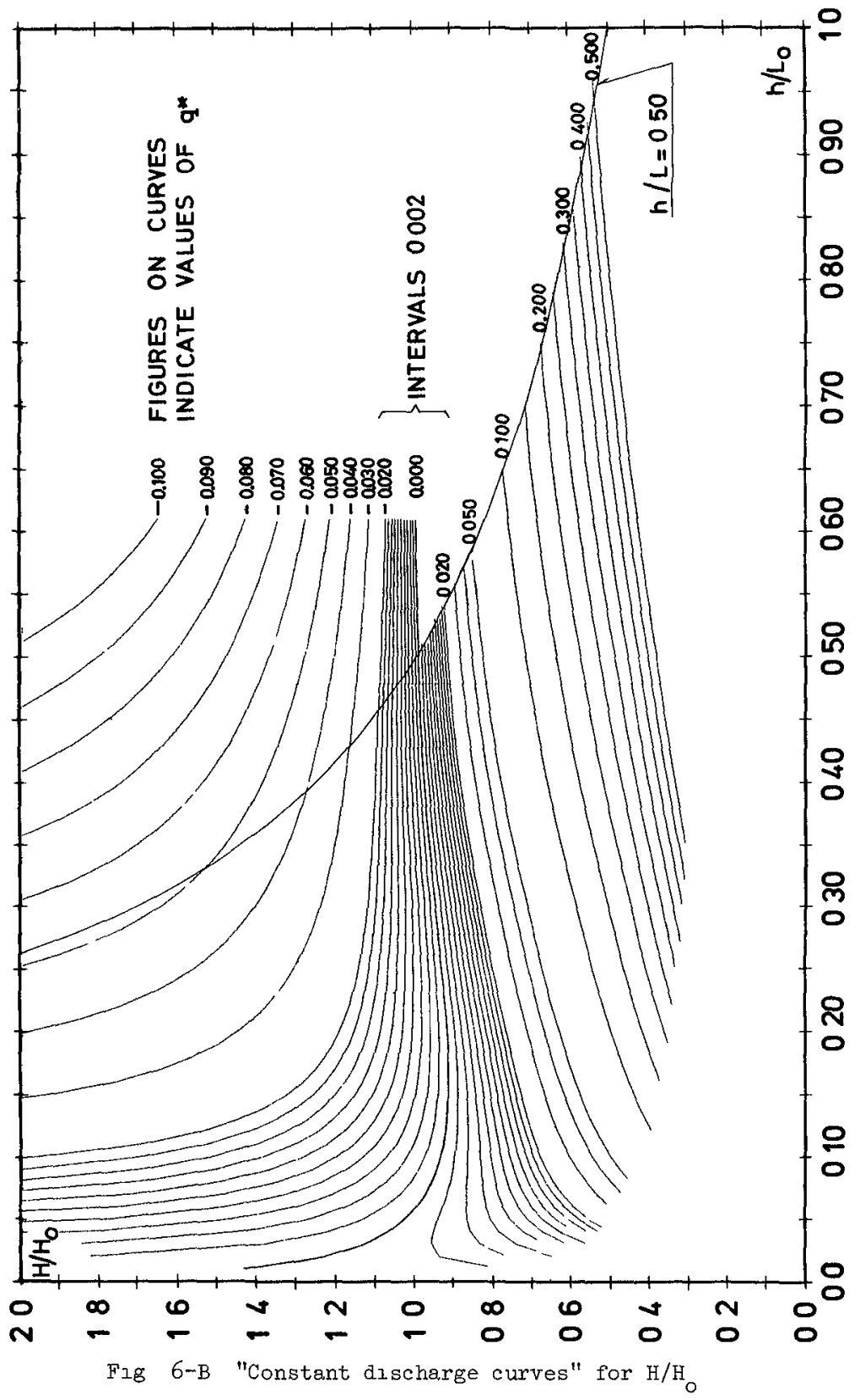


Measured parameters

$$
\begin{aligned}
\mathrm{h} & =850 \mathrm{~m} \\
\mathrm{U} & =-070 \mathrm{~m} / \mathrm{s} \quad \text { (1 e a counter-current) } \\
\mathrm{T}_{\mathrm{a}} & =60 \mathrm{~s}
\end{aligned}
$$

and $\max \Delta \mathrm{p}_{\mathrm{b}}=3400 \mathrm{~N} / \mathrm{m}^{2}$ ( $1 \mathrm{~N}=1 \mathrm{~kg} \mathrm{~m} / \mathrm{s}^{2}$ ), where $\Delta \mathrm{p}_{\mathrm{b}}$ Is the difference between actual pressure and hydrostatic pressure corresponding to MWL $(\equiv \gamma \mathrm{h}$ ) at the bottom, (here considered measured with a pressure cell at the bottom)

The calculations proceed thus

$$
\begin{aligned}
& \mathrm{L}_{0}=\frac{981}{2 \pi} \quad 60^{2}=562 \mathrm{~m} \\
& \mathrm{~h} / \mathrm{L}_{0}=850 / 562=0151 \\
& \mathrm{c}_{0}=\frac{981}{2 \pi} \quad 60=936 \mathrm{~m} / \mathrm{s} \\
& \mathrm{q}^{*}=850(-070) /\left(\begin{array}{lll}
9 & 36562)=-00113
\end{array}\right.
\end{aligned}
$$

Using linear interpolation in Table $6-\mathrm{b}$ we obtain

$$
L / L_{0}=0712
$$

hence

$$
\mathrm{h} / \mathrm{L} \equiv\left(\mathrm{h} / \mathrm{L}_{0}\right) /\left(\mathrm{L} / \mathrm{L}_{0}\right)=0151 / 0712=0212
$$

Using the first order expression only, we have for the wave amplitude

$$
\begin{aligned}
& \mathrm{H} / 2=\max \Delta \mathrm{p}_{\mathrm{b}} \quad \cosh \mathrm{kh} / \mathrm{\gamma} \\
& \cosh \mathrm{kh}=203
\end{aligned}
$$

and so the wave helght becomes

$$
\underline{H}=23400203 /(1000981)=141 \mathrm{~m}
$$

If the current were not noticed, then $I / I_{0}=0820$ from Table $6-a$ or $6-\mathrm{b}$ and $\mathrm{h} / \mathrm{L}=0151 / 0820=0184$

$$
\begin{aligned}
& \cosh \mathrm{kh}=175 \\
& \underline{\mathrm{H}}=23400175 /(1000981)=121 \mathrm{~m} \\
& \frac{\Delta \mathrm{H}}{\mathrm{H}}=\frac{141-121}{141} \quad 100 \%=14 \%
\end{aligned}
$$

1 e In this case, neglection of the current will give wave helght $14 \%$ too small (A positive current wlll have the opposite effect)

It is not difficult to find realistic sets of values for $h, U$ and $T_{a}$ that lead to hlgher values of $\Delta \mathrm{H} / \mathrm{H}$ (as defined above) Thls aspect shows the importance of considering the effect of a possible current The phe'nomenon may be one of the factors which can obscure the direct comparison in a coastal zone between wave helghts as measured at the water surface and as calculated from pressure cell recordings at the bottom 


\section{REFERENCES}

[1] Bowen, A J , Inman, D L , and Simmons, V P (1968) Wave "Set-Down" and Set-Up J Geophys Res 13, 2569-2577

[2] Garrett, C J R (1967) Discussion The Adıabatic Invariant for Wave Propagation in a Nonuniform Moving Medium Proc Roy Soc, A, 299, 26-27

[3] Jonsson, I G (ed ), (1969) Index to Reports 1-19 (1961-1969) Bas1c Res -Prog Rep 20 Coastal Engrg Lab and Hydraulic Lab, Techn Unıv Denmark

[4] Jonsson, I G (1970) Graphical Determination of the Wave Length in a Homogeneous Current Field Basic Res -Prog Rep 21, 19-26 Coastal Engrg Lab and Hydraulic Lab, Techn Unıv Denmark

[5] Longuet-Higgins, $M S$, and Stewart, $\mathrm{R} \mathrm{W}$ (1960) Changes in the Form of Short Gravity Waves on Long Waves and Tidal Currents $J$ Fluld Mech $8,565-583$

[6] Longuet-Hıggins, M S , and Stewart, R W (1961) The Changes in Amplitude of Short Gravity Waves on Steady Non-Unıform Currents J Flu1d Mech 10, 529-549

[7] Lundgren, H (1963) Wave Thrust [ $\equiv$ Radiation Stress] and Wave Energy Level Int Assoc Hydr Res, Proc 10th Congress 1, 147-151, London

[8] Whitham, G B (1962) Mass, Momentum and Energy Flux in Water Waves J Fluid Mech 12, 135-147 



\section{CHAPTER 31}

\section{EXPERIMENTS OF WAVE REFLEXION ON IMPERMEABLE SLOPES by Carlos de Campos MORAES*}

\section{ABSTRACT}

Results from experiments on the reflective power of smooth and rough impermea ble slopes are presented The importance of relative depth to the regular scatter dia gram of the function $R=R\left(\delta_{0}\right)$ and the need for an adequate computational wave theo ry through which no significant alterations are introduced in the determination of reflective power is pointed out Stokes 2nd order corrections are introduced These help find a superior value for the reflexion coefficient and destroy the mentioned regularity of the scatter diagram A regular scattering of experimental points, also of function $R=R\left(\delta_{0}\right)$, where, however, for constant steepness, reflexion decreases when relative depth increases, is found in rough slope tests in this case, the more inclined is the slope, the greater is the influence of roughness

\section{1 - INTRODUCTION}

Some of the approaches used for determining the reflexion coefficient of a given parameter are based on direct recording of incident and reflected wave trains (these are the "wave tall" and "subtraction" methods described in [1] by Goda and Abel, others on the recording of clapotis Calculation procedures using maxima and minima of these clapotis range from the simplest - immediate application of the small amplitude assumption through the well-known formula

$$
R=(\operatorname{Max}-\min ) /(\operatorname{Max}+\min )
$$

to more elaborate cases in which approximations of different orders are used Stokes II (as developed by Carry [2], Stokes III (as proposed by Goda and Abe [1]), cnoidal theory, etc More sophisticated methods exist as, for instance, Santon's and Marcou's method developed in Grenoble, which applies harmonic analysis to the clapotis profile as recorded at three points $[3],[4],[5]$

The present paper concerns results of tests where the reflective power of smo oth and rough impermeable slopes was studied

Use was made of the method of recording maxima and minima of clapotis The aim was above all to study the validity range of the linear theory small amplitude assumption and acquire an idea of errors introduced by its application

\section{2 - RANGES OF PARAMETER VARIATION IN TESTS}

Systematic tests were performed in a $20 \mathrm{~m}$ long and $080 \mathrm{~m}$ wide flume with a to tal depth of $055 \mathrm{~m}$ This flume is equipped with a monochromatic translation actuator Results of the tests are studied in the following

Absolute water depth (d) was kept constant and equal to $035 \mathrm{~m}$ since the slope con cerned a semt-indefinite plane Local or offshore relative depth, $d / L$ or $d / L o$, respecti vely varied then only owing to $L_{0}$ variation, that is, owing to $T$, which took values be tween 08 and $22 \mathrm{~s}$

In most cases concerning the presentation of results, periods 10,16 and $22 \mathrm{~s}$ were selected corresponding the following relative depths

\begin{tabular}{|c|c|c|}
\hline$T(s)$ & $d / L$ & $d / L o$ \\
\hline 10 & 025 & 022 \\
\hline 16 & 013 & 009 \\
\hline 22 & 009 & 005 \\
\hline
\end{tabular}
* - Trainee Research Officer, Laboratório Nacional de Engenharia Civil (LNEC), LiS 
Going along the decreasing order of relative depths, the first of those three ca ses presents waves satisfying Miche's condition for non-appearance of secondary crests (d/L $>0$ 15) These waves are best sulted for elementary computatıons, according to the linear theory smali ampiltude assumption The second case (which occupies an inter mediate position in the set of tests) includes waves corresponding to finite amplitude wave theories To them the different Stokes approximations may be applied, according to the rigour demanded The case of the ieast relative depth comprises waves calcula tion of which enters the domain of cnoidal wave theory"

As for steepness, its off-shore value $(80)$ varies approximateiy between 0 and 3\% These arethe most common limits for sea waves it was endeavoured to secure a great range of values corresponding to unbroken waves Aiso, tests were carried fur ther whenever breaking occured for increasing steepness However, beyond a certain point it was Impossible to get acceptabie clapotls from the point of view of reguiarity

The plane siope incilnation varied, for the set of tests, from the minımum $10 \%$ vaiue to vertical inclination

Roughness, which was introduced in the second part of the tests made, consisted of sand gived to the siope of course, the greater the intended roughness, the greater the used sand grain size The sift hole diameter $r$ was used to characterize absolute roughness This factor was made non-dimensionai by means of the wave iength The resulting parameter $\mathrm{r} / \mathrm{L}$, a measure of reiatıve roughness, took values between $13 \times 10^{-4}$ and $240 \times 10^{-4}$

\section{3 - TEST RESULTS}

\section{1 - Smooth siopes}

In Fig 1, a graphic diagram of $R=R\left(\delta_{0}\right)$, points corresponding to $10 \%, 15 \%, 20 \%$ and $30 \%$ slope tests are piotted

Fig 2 comprises four $R(80)$ plots corresponding to $40 \%, 50 \%, 100 \%$ and vertical siopes

in the first set of tests (FIg 1) points corresponding each one of the four in cinations cluster around similar curves in each inclination oniy an experimental scattering of points occurs, with no period separation

On the other hand, in the second set of tests a reguiar scattering of periods occurs For constant $\delta_{0}$ values a decrease of $\underline{R}$ is apparent when $\underline{T}$ increases $T h e$ same phenomenon is noted for run-up experiments (Fig 5)

\section{2 - Rough slopes}

In Fig 4 four diagrams relating to rough slope tests are seiected, absoiute rou ghness being in each case a constant

It is seen that in every case two type of point scattering are present, according to siope variation for $20 \%$ and $30 \%$ a requiar scattering of periods is observed, now, however, in the opposite sense for constant $\delta_{0}$ values, $\underline{R}$ and $\underline{I} v a i u e s$ increa se or decrease together For vertical inclination (and also for $1 \overline{00} \%$ inclinatıons, though no dlagrams are presented) again a regular scattering of periods is observed as in the case of smooth siopes

\section{4 - INTERPRETATION OF RESULTS}

\section{1 - Introduction}

With a view to simpilfying the language used, the following designations are adop

* - However, according to Uirseli's parameter

$$
u=\frac{H}{d}\left(\frac{L}{d}\right)^{2}
$$

cnoidai theory shouid be appired only for $u>100$ Taking into account the highest

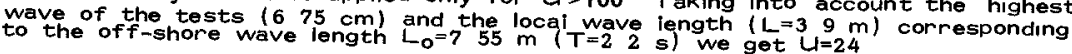


ted for characterization of the three mentioned scattering types

- experimental scattering

- smooth type regular scattering

- rough type regular scattering

An attempt shall be made to interpret occurrence of regular scattering in the high of an explanation relative to computations in a case and of physical order in another That is physical causes are perhaps related to energy dissipation phenomena which in turn are the reason for the decrease in the slope reflective power Changes undergo ne by the wave in its orbital velocity field, when it is propagating in decreasing relati ve depths, with an increasing turbulence and finally reaching breaking point or energy dissipation due to slope roughness are cases in which the physical factor is indisputable The decrease in reflective power may however be fictitious, that is, it may merely result from a inefficient computation method

\section{2 - Smooth slopes}

\section{1 - Influence of relative depth on reflexion computations}

It is known that the best suited wave theory for computations of the relevant pa rameters is determined by relative depth it should be noted, however, that the para meter $t / L$ (where $t$ is the horizontal distance measured from stıll water level to the slope base projection on the surface and $L$ is the local wave length) may be of great importance

\begin{tabular}{|c|c|c|c|c|c|c|c|c|c|c|c|c|c|}
\hline & & & & & & & & & $5 \mathrm{LOP}$ & & & & \\
\hline & & & & & $10 \%$ & $15 \%$ & $20 \%$ & $30 \%$ & $40 \%$ & $50 \%$ & $100 \%$ & $200 \%$ & vert \\
\hline & & & & & & & & & $t(\mathrm{~m})$ & & & & \\
\hline$d$ & $=035$ & $\mathrm{~m}$ & & & 350 & $\begin{array}{ll}2 & 33\end{array}$ & $\begin{array}{ll}1 & 75 \\
\end{array}$ & $\begin{array}{ll}117 \\
17\end{array}$ & 088 & $\begin{array}{ll}0 & 70\end{array}$ & $\begin{array}{ll}0 & 35\end{array}$ & $\begin{array}{ll}0 & 18\end{array}$ & 000 \\
\hline$T(s)$ & $L_{o}(m)$ & $L(\mathrm{~m})$ & $d / L_{0}$ & $d / L$ & & & & & $t / L$ & & & & \\
\hline 08 & 100 & $\begin{array}{ll}0 \quad 97\end{array}$ & 03506 & $\begin{array}{ll}0 & 3584\end{array}$ & 358 & $\begin{array}{ll}2 & 39\end{array}$ & $\begin{array}{|ll|}1 & 79 \\
\end{array}$ & $\begin{array}{ll}1 & 19 \\
\end{array}$ & 090 & $\begin{array}{ll}0 & 72\end{array}$ & $\begin{array}{ll}0 & 36\end{array}$ & $\begin{array}{ll}0 & 18 \\
\end{array}$ & 000 \\
\hline 10 & 156 & 142 & $0 \quad 2244$ & 02458 & 246 & $\begin{array}{ll}1 & 64\end{array}$ & $\mid \begin{array}{ll}1 & 23 \\
\end{array}$ & $\begin{array}{ll}0 & 82\end{array}$ & $\begin{array}{ll}0 & 61\end{array}$ & $\begin{array}{ll}0 & 49\end{array}$ & $\begin{array}{ll}0 & 25 \\
\end{array}$ & $\begin{array}{ll}0 & 12\end{array}$ & 000 \\
\hline 12 & 225 & 186 & $0 \quad 1558$ & $0 \quad 1881$ & 188 & 125 & $\begin{array}{ll}0 & 94\end{array}$ & $\begin{array}{ll}0 & 63 \\
\end{array}$ & $\begin{array}{ll}0 & 47\end{array}$ & $\begin{array}{ll}0 & 38\end{array}$ & $\begin{array}{ll}0 & 19\end{array}$ & $\begin{array}{ll}0 & 09\end{array}$ & $\begin{array}{ll}0 & 0\end{array}$ \\
\hline 14 & 306 & 228 & 01145 & $\begin{array}{lll}0 & 1535\end{array}$ & 154 & $\begin{array}{ll}1 & 02 \\
\end{array}$ & $\begin{array}{|ll|}0 & 77 \\
\end{array}$ & $\begin{array}{lll}0 & 51\end{array}$ & $0 \quad 38$ & $0 \begin{array}{ll}0 & 31\end{array}$ & $\begin{array}{ll}0 & 15\end{array}$ & $\begin{array}{lll}0 & 0 & 8\end{array}$ & 000 \\
\hline 16 & 399 & 269 & 00876 & $0 \quad 1301$ & 130 & $\begin{array}{lll}0 & 87\end{array}$ & $\begin{array}{ll}0 & 65\end{array}$ & $\begin{array}{ll}0 & 43 \\
\end{array}$ & 1033 & $\begin{array}{ll}0 & 26\end{array}$ & $\begin{array}{lll}0 & 1 & 3\end{array}$ & 1006 & 000 \\
\hline 18 & 505 & 309 & $\begin{array}{lll}0 & 0692\end{array}$ & $0 \quad 1132$ & 113 & $\begin{array}{ll}0 & 75\end{array}$ & $\begin{array}{ll}0 & 57\end{array}$ & 038 & $0 \quad 28$ & $\begin{array}{ll}0 & 23 \\
\end{array}$ & $\begin{array}{lll}0 & 1 & 1\end{array}$ & $\begin{array}{lll}0 & 06\end{array}$ & 1000 \\
\hline 20 & 624 & 349 & $\begin{array}{lll}0 & 0 & 561\end{array}$ & $0 \quad 1004$ & 100 & $\begin{array}{ll}0 & 67\end{array}$ & $0 \quad 50$ & $\begin{array}{ll}0 & 33\end{array}$ & $\begin{array}{ll}0 & 25\end{array}$ & $0 \quad 20$ & $\begin{array}{ll}0 & 10\end{array}$ & $\begin{array}{lll}0 & 0 & 5\end{array}$ & 1000 \\
\hline 22 & 755 & 388 & $0 \quad 0464$ & 00903 & 090 & $\begin{array}{ll}0 & 60 \\
\end{array}$ & $\begin{array}{ll}0 & 45\end{array}$ & $\begin{array}{ll}0 & 30\end{array}$ & $\begin{array}{ll}0 & 23 \\
\end{array}$ & $\begin{array}{lll}0 & 18 \\
\end{array}$ & 1009 & $\begin{array}{lll}0 & 0 & 4\end{array}$ & 000 \\
\hline
\end{tabular}

Schoemaker and Thissse have pointed out [6] that that parameter is the main cause of energy dissipation and indicated that for an almost total reflexion we must have $t / L \leqslant 025$, and for a very small reflexion then $t / L \geqslant 05$

In a small inclination slope (15\%, for Instance) two waves of different lengths, which propagate, before reaching it, in different relative depths, wil in the end have traveled over zones with same relative depth (of course one lagging behınd the other) Thus period does not exert a selective action This is the type of tests corresponding to "experimental scattering" which was found for $10 \%, 15 \%, 20 \%$ and $30 \%$ slopes

For strong inclinations (greater than 40\%), the fact that $t / L$ is small makes the two waves propagate in different relative depths when they bear on the slope Thus, a proper wave theory for computations is essential For the shorter wave a deep wa ter wave theory seems best suited while for the longer one a shallow water theory is indicated This seems to be the evident explanation for the occurence of the "smooth type regular scattering"

In fig 3 is shown the influence of inclination on $R$ for constant values of $T$ and 
cen be easily noted the different espect of the phenomenon in inciinations up to $40 \%$ and from this vaiue up to verticai in what concerns the shorter period ( 1 os) and the ionger ones ( $16 s$ and 2 2s)

422 - Miche's theory

The steepness maximum value, $\delta$ max, of a wave theoreticaily capable of totei re flexion on a siope which is at an angle $\alpha$ with the horizontai has been defined by Miche $[7]$ as

$$
\delta_{\max }=\sqrt{\frac{2 \alpha}{\pi}} \frac{\sin ^{2} \alpha}{\pi}
$$

the theoreticai reflexion coefficient being

$$
R^{\prime}=\frac{\delta_{\text {max }}}{\delta_{0}}
$$

for $\delta_{\text {mex }}<\delta_{0}$ If $\delta_{\text {max }} \geqslant \delta_{0}$ then $R '=1$

The ectuel refiexion coefficient is

$$
R=\rho R^{\prime}
$$

where $p$ is the so-calied slope intrinsic reflexlon coefficient

The diagram $R^{\prime}=R^{\prime}\left(\delta_{0}\right)$, Fig 6 , shows thet up to an $40 \%$ inciination and within the steepness range of the tests, $R$ ' vaiues present a first constant $100 \%$ "landing" The smailer the incilnation, the smalier is this ianding for slopes of inciination grea ter than $40 \%$ and about (but not quite) $3 \%$ steepness, theoreticai refiexion is aiways totai for steepness vaiues up to $3 \%$

Greater $\delta_{0}$ vaiues are more and more unlıkeiy in nature For instance, for a $100 \%$ slope it is $\delta$ max $=1126 \%$

This $40 \%$ inclination value (for results corresponding to the steepness range of the tests, as sald beforel separetes in fact the two domains of experimentei resuits experimentai scattering end reguiar scattering

423 - Stokes' 2nd order corrections

According to

$$
1 / \alpha=\frac{2 \pi}{\operatorname{th} \frac{2 \pi d}{L}}\left(1+\frac{3}{2 \operatorname{sh}^{2} \frac{2 \pi d}{L}}\right)
$$

$\alpha$ values were computed as e function of $d / L$

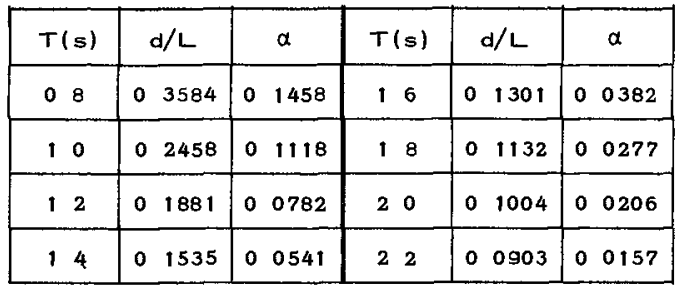

foilowing Carry's procedure [2] These d vaiues were used to compute

$$
D=\frac{M}{2 \alpha L}+\frac{m}{2 \alpha L}
$$

where $M$ and $m$ are maximum end minımum of the recorded ciapotis 
Fig 7 shows a diagram taken from [2] where the corrective value $\mu$ is determined which will allow the calculation of the new reflexion coefficient $\beta$ (vatue corrected according to Stokes' 2nd order theoryl

$$
\beta=\mu R
$$

where $R$ is computed from (1)

In Fig 2, the corrected experimental points (black simbols) and the uncorrected points (white simbols) are presented llnked by line segments which provide a measure

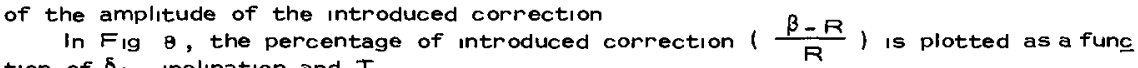
tion of $\delta_{0}$, inclination and $T$

The following points are noted

- Using a higher order wave theory will decrease the regular scattering of periods $\underline{R}$ being corrected for values close or equal to $100 \%$ Proximity to this value se ems to depend on the approximation order of the used wave theory

- Correction increases with period, 1 e, decreases with relative depth (see Fig $8)$, which agrees with what was sald above about the wave characteristics relating to depth

- Correction increases when either inclination or steepness increase

43 - Rough slopes

431 - influence of relative roughness reasons

In rough slopes, the regular scattering of periods is due, as sald above, to two

- "rough type regular scattering" for low inclinations $120 \%$ and $30 \%$ in the case of the tests performed) in which the greater the relative roughness ( 1 e the smaller is Lo relative to ) the greater the energy dissipation This explains that, for the same slope, longer period waves dissipate less energy

- "Smooth type regular scattering" for higher inclinations, in which, for the sa me absolute roughness, energy dissipation is smaller than in low inclination slopes The effect of the separation of the experimental points due to insufficient approximation of the used wave theory overrules the physical effect of energy dissipation through roughness

Fig 9 shows experimental results obtained for equal values of relative roughness. They confirm the given explanation for small inclinations, the "rough type regular scattering" disappears For vertical inclination the smooth type regular scattering re mains

2nd order corrections for rough slopes are not presented in this paper

\section{2 - Variation of the slope intrinsic reflexion coefficient}

According to formula $(4)$ and to Fig 10 diagrams, the intrinsic reflexion coefficient decreases with steepness until a minimum value is reached corresponding to $\delta_{0}=\delta_{\text {max }}$, afterwards it increases to values greater than $\delta_{0}$

When a slope's actual reflexion coefficient is estimated from the theoretical reflexion coefficient $R^{\prime}$ one should not take a constant $\rho$ value, taking the steepness in to account

\section{3 - Roughness influence on incllnation from the point of view of energy dissipation}

it is known that in a flume the maximum bottom orbital velocity for a wave whi ch is propagating with height $\underline{H}$ and length $L$ is glven by

$$
v_{\max }=\frac{H \pi}{\sin \alpha} \sqrt{\frac{g}{L}}
$$


and that this velocity value is concerned with energy dissipation by roughness

This $v_{\max }$ value increases very rapidly when inclination decreases, energy dissipa tion also increases as a consequence of roughness

Fig 11 shows, for a 30\% slope, the decrease of reflective power due to the roughness increase The same strong effect is not present for the case in which the Inclination is greater than $100 \%$

\section{REFEERENCES}

[1] - GODA, Yoshımı and ABE, Yoshıkı - Apparent Coefflcıent of Partial Reflectıon of Finite Amplitude Waves Report of the Port and Harbour Research Institute vol 7 No 3 September 1968

[2] - CARRY - Clapotıs partıel La Houlle Blanche No 4, Août - Septembre 1953 P $\quad 482-494$

[3] - SANTON, L , MARCOU, O - Enregistrement graphique du profil d'une houle produite en laboratoire Houlle Blanche № 3 de $1953 \mathrm{p}$ 411-417

[4] - SANTON, L - Enregistrement graphique du profil d'une houie de laboratoire, analyse harmonlque Proc 5th Conf Coastai Engıneerıng, Grenoble, France, September 1954 p 189-206

[5] - CASTRO, 0 - Etude du coefflcient de reflexion de la houle de laboratoire pour des talus plans, lisses et rugueux

[6] - SOHOEMAKER, H J P THIJSSE, TH - Investigations of the reflectlon of waves IAHR, 3th Meeting, Grenoble, September 1949

[7] - MICHE - Le pouvoir reflechissant des ouvrages maritımes exposés a 11 actlon de ia houle Annales des ponts et chaussées, année 121, No 3, 1951, p 285-319 


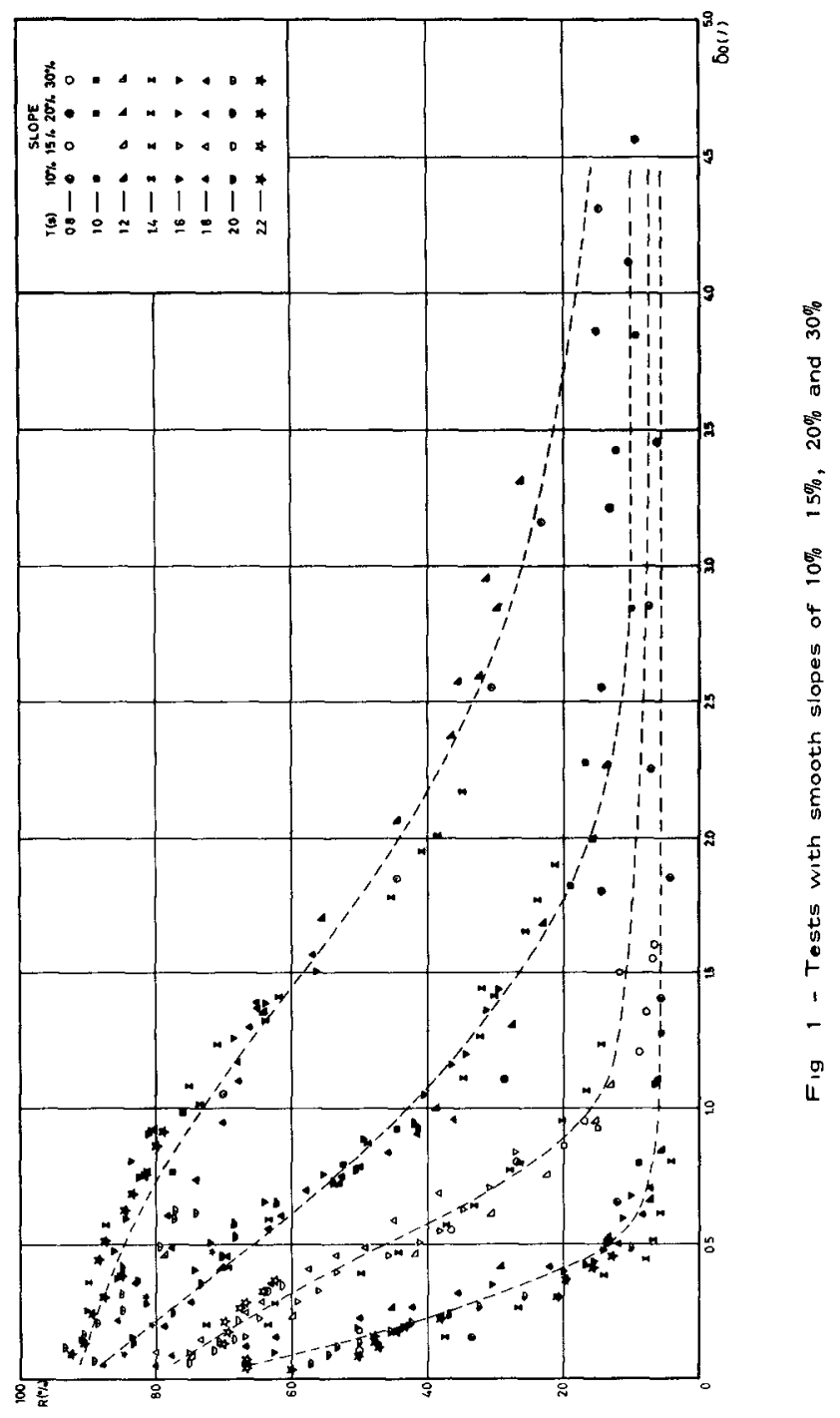



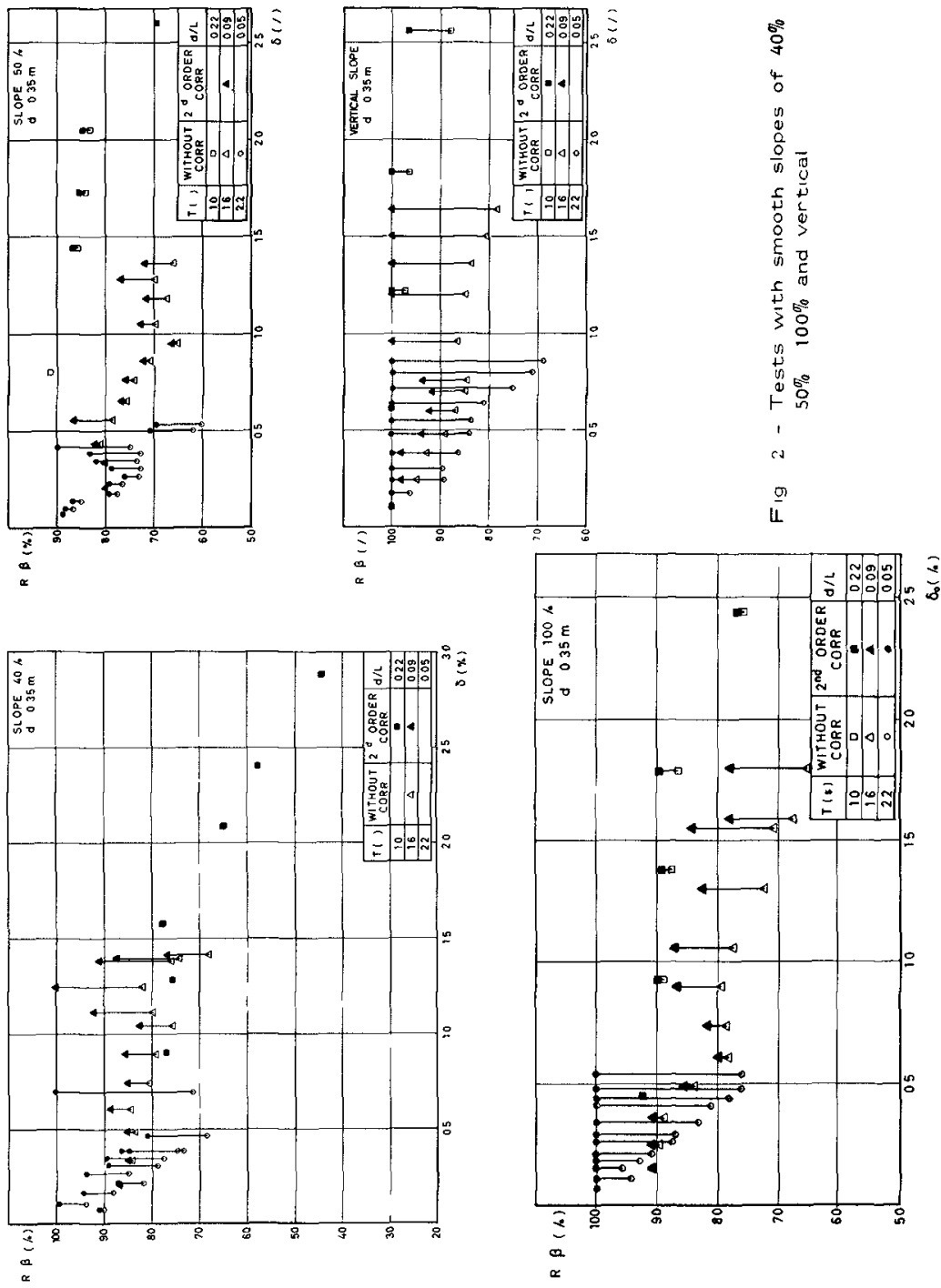

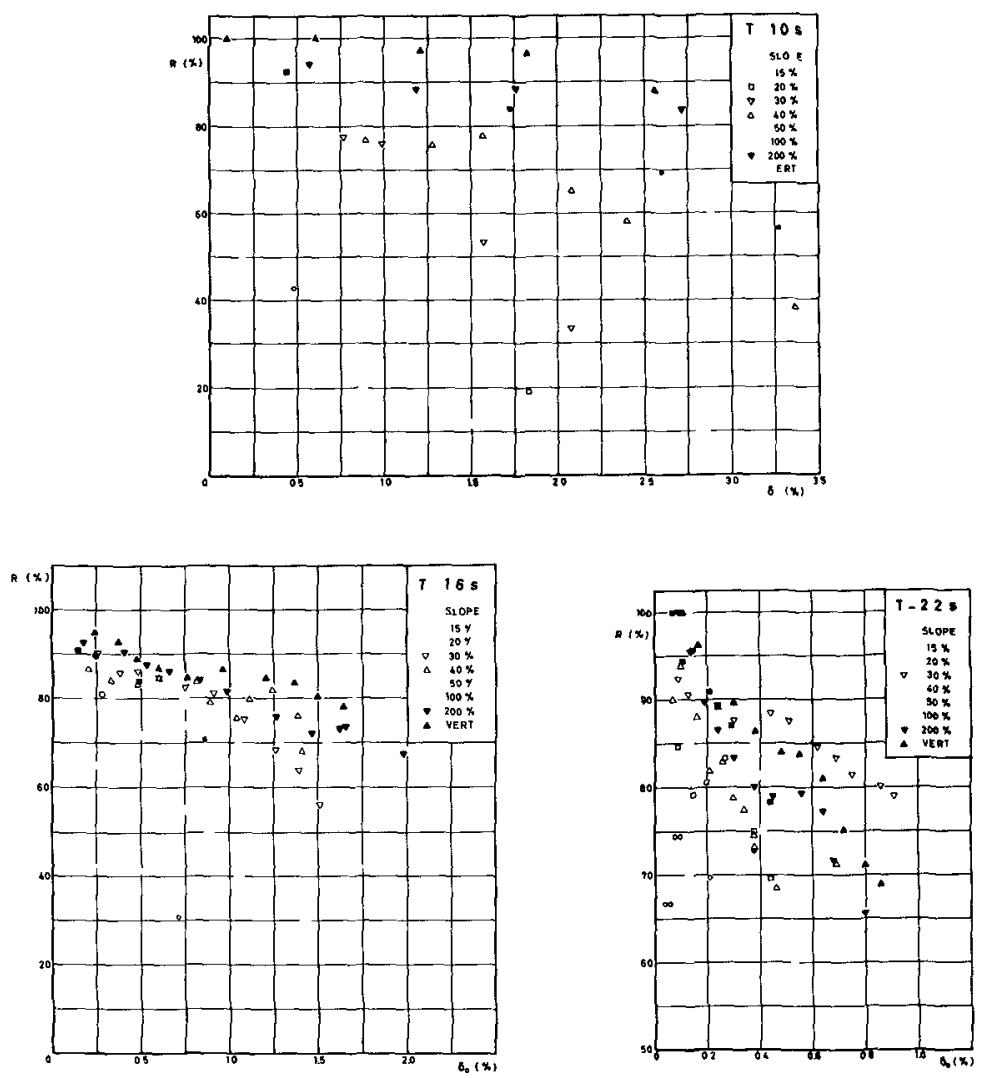

Fig 3 - Influence of inclination on $R$ 

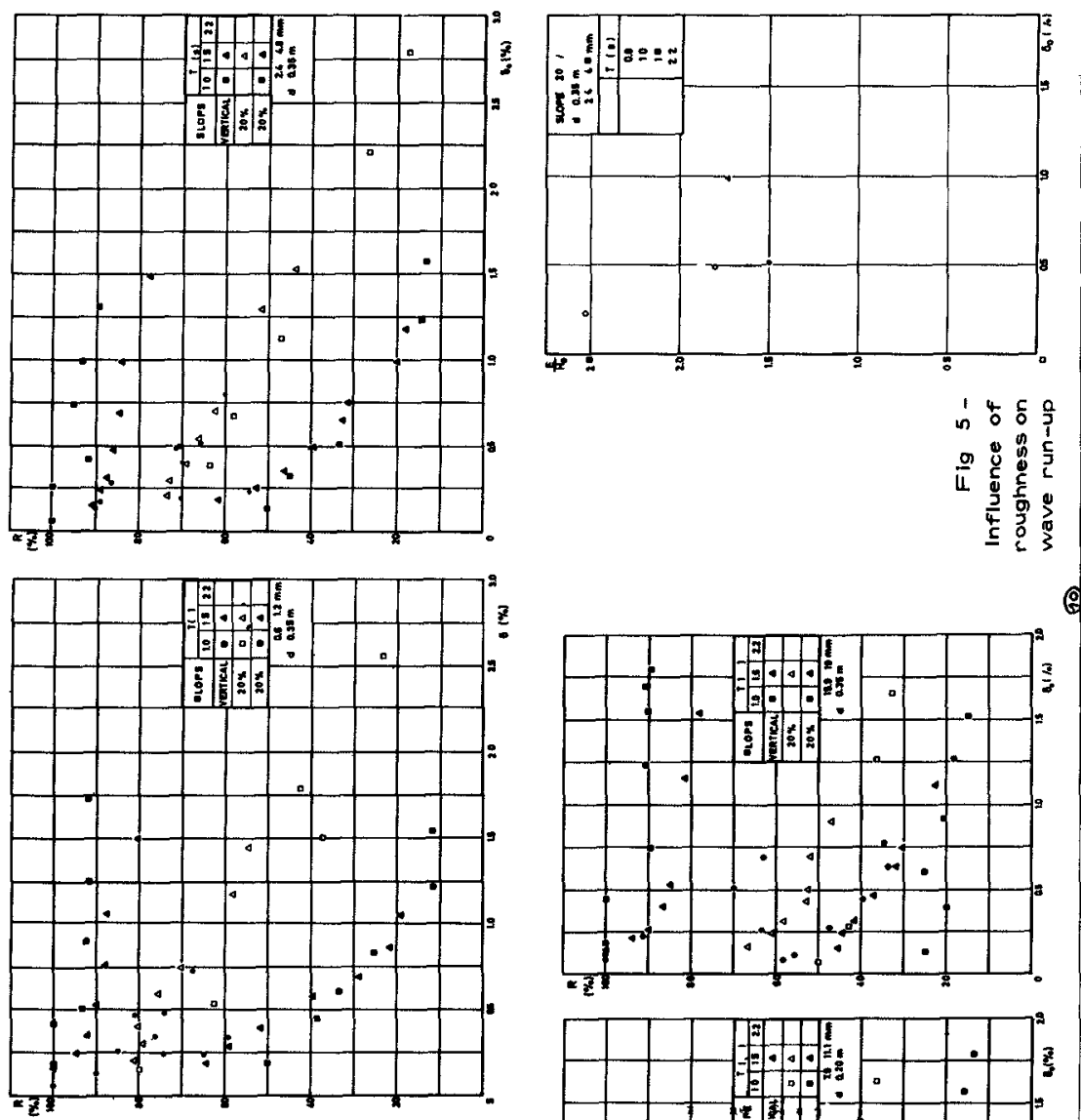

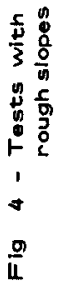

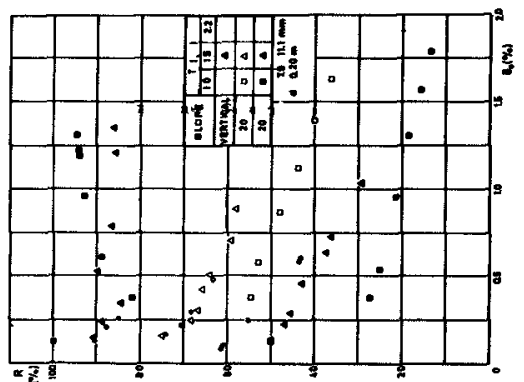




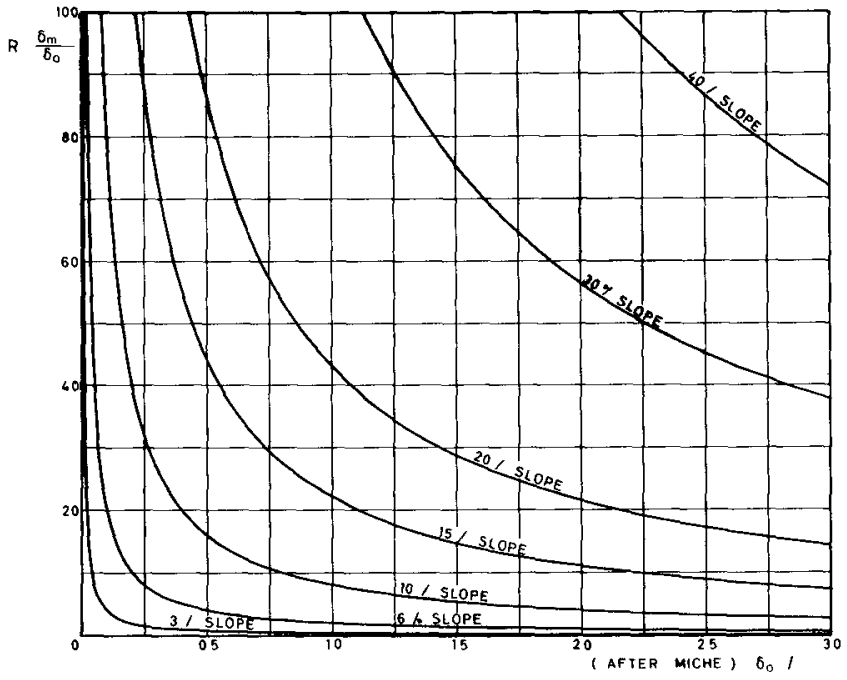

Fig 6 - Miche's theoretical reflexion coefficient

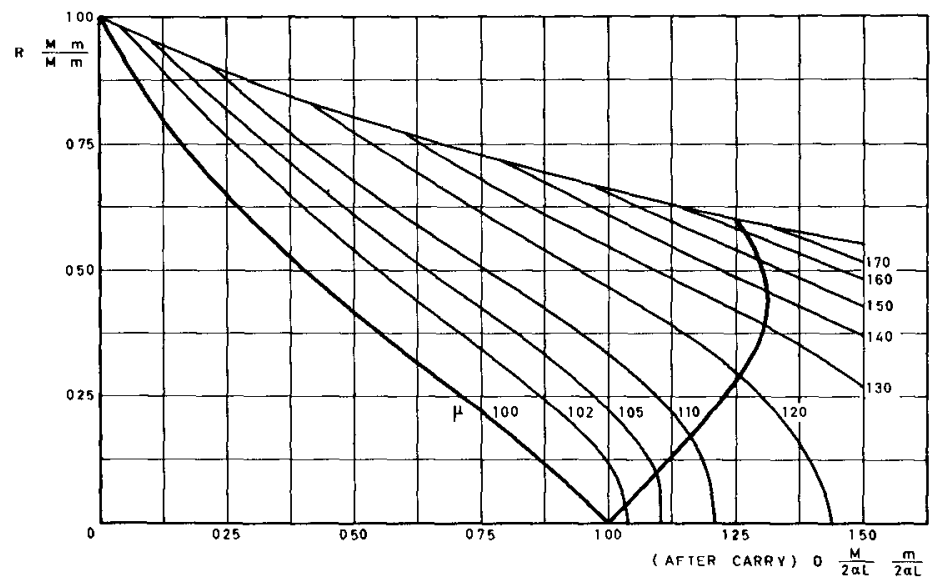

Fig 7 - Graph for the $2^{\text {nd }}$ order corrections 

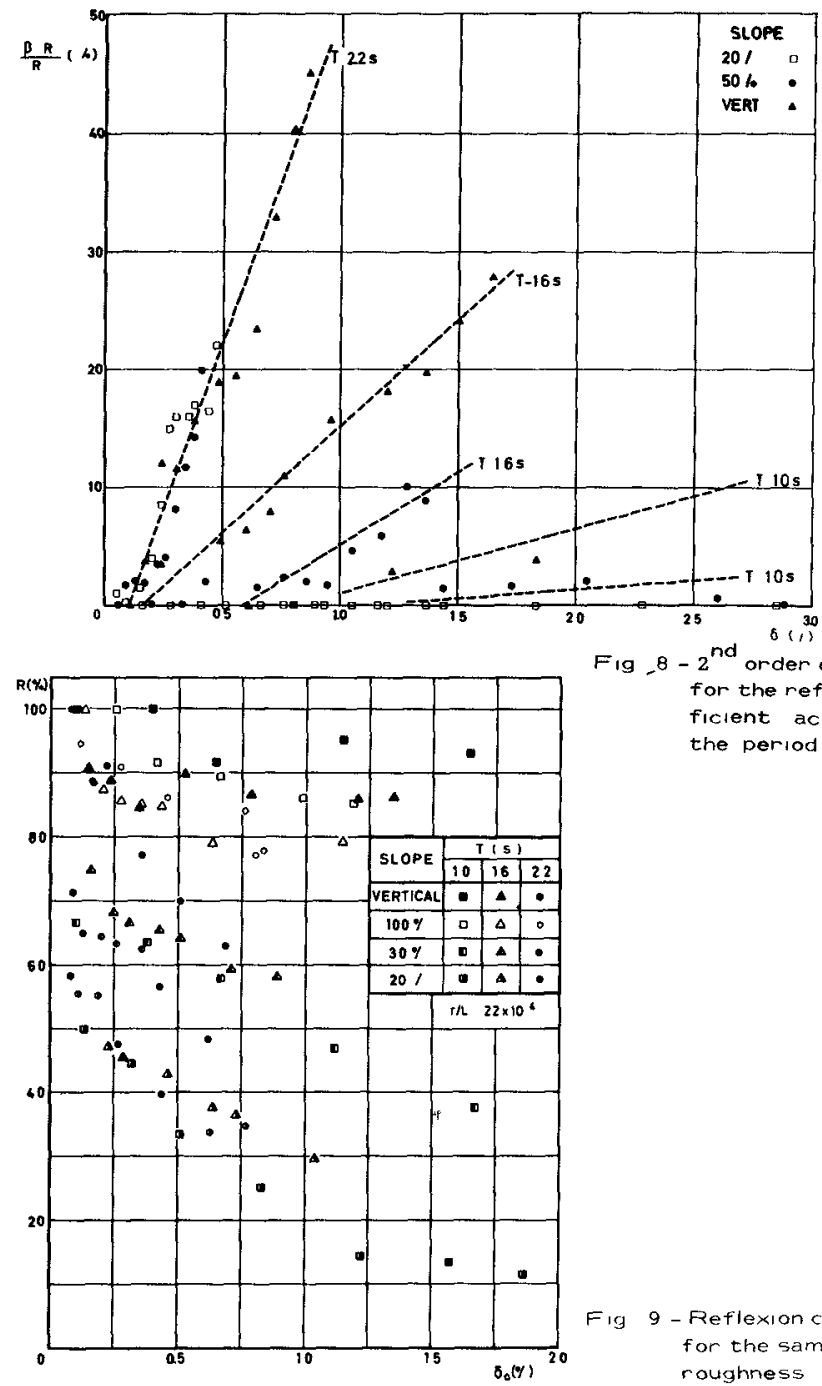

Fig $8-2^{\text {nd }}$ order corrections for the reflexion coef ficient according to the period and slope 

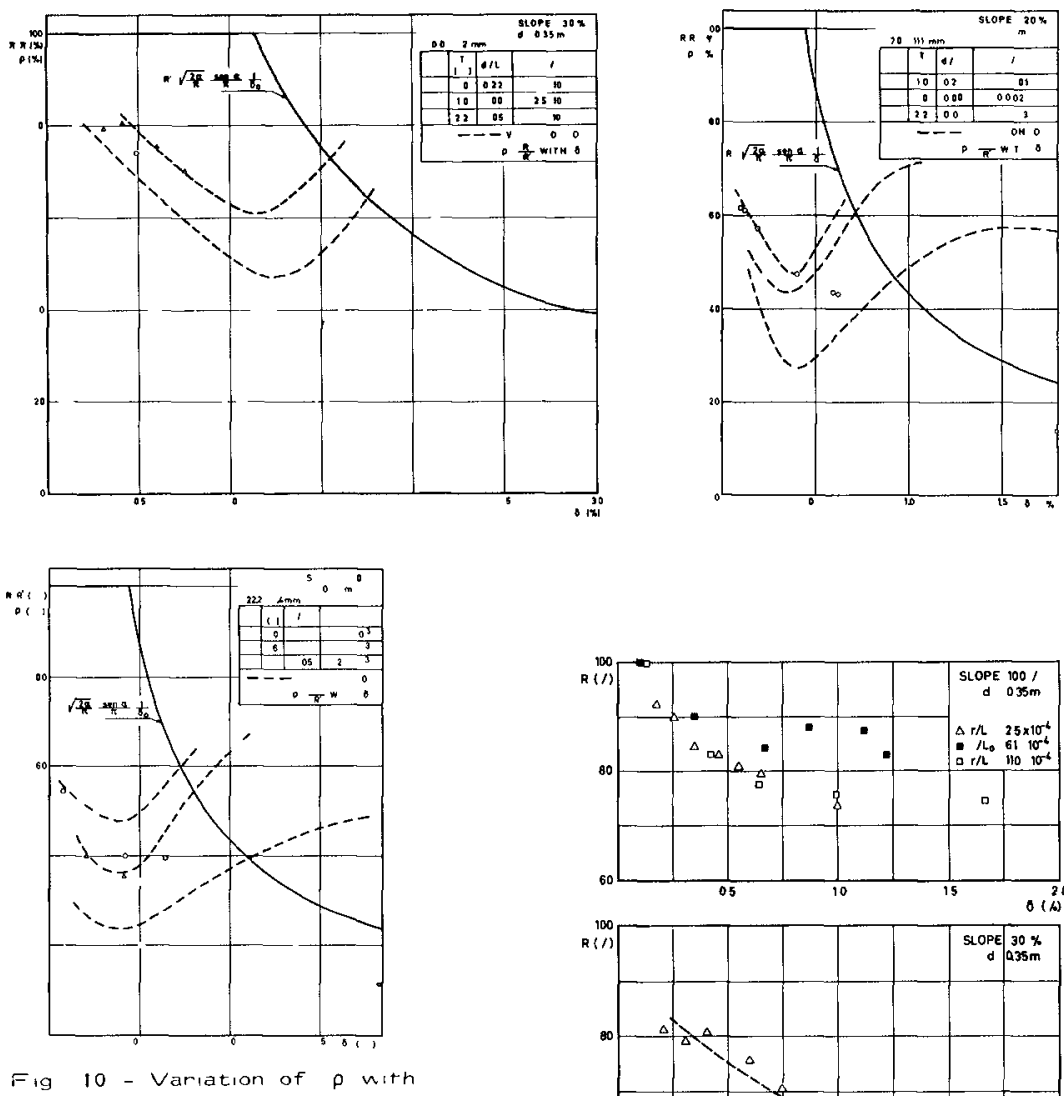

off-shore steepness

Fig 11 - Influence of roughness on
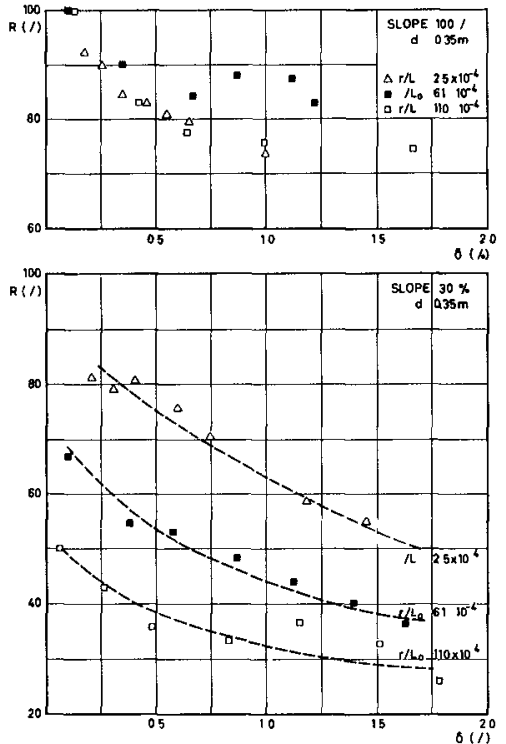

$R$ for different slopes 



\title{
CHAPTER 32
}

\section{LIMITING CONDITION FOR STANDING WAVE THEORIES}

BY PERTURBATION METHOD

\author{
Yoshı to Tsuchıya \\ Professor of Coastal Enganeering \\ and \\ Masataka Yamaguch \\ Research Assistant of Coastal Hydraulics \\ Disaster Prevention Research Institute \\ Kyoto Unı versity, Kyoto, Japan
}

\begin{abstract}
AESTRACT
The purpose of this paper is to make clear the validity and limsting condition for the application of the fimte amplitude standing wave theories by the perturbation method In a numerical example, the errors of each order solution of these theories for two non-linear free surface conditions are computed for varıous kinds of wave characterlstics and compared with each other

Some experıments on the wave pressure on a vertical wall by standing waves were carried out and a plot of the limiting condition for the application of these theories is proposed based on the comparison with theoretical curves

In addition, as an example of the application of these theories, the change of characteristics of wave pressure of standing waves accompanying the overtopping wave on a vertical wall is discussed
\end{abstract}

\section{INTRODUCTION}

Up to the present, the fincte amplitude standing wave theorles have been studied by many researchers such as Penny and Price, $\mathrm{K}_{\mathrm{s}}$ shı and etc In 1960, Tadjbaksh and Keller derıved the third order solution of finite aniplitude standing waves in shallow water by means of the perturbation method with two additional conditions Although the calculation for the higher approximation 1.s very complicated, this method has often been used to solve such non-linear differential equations, cecause approxımate solutions of arbitrary orders can be derived systematically Recently the fourth order solution of the finite amplıtude standing wave theorıes in shallow water was obtalned by Goda in 1966

The perturbation method is a formal one and no mathematical proof for the convergence of the solutions by this method is given Solutions by this method are only approximate ones consequently, the validity and limiting condition for the application of these theories to physical phenomena are sulject to question To clarify these problems, we have carried out some investigatıons by the numerical consideration of the valıdıty for two non-linear free surface boundary conditions using these theories and by the comparison between the theoretucal curves based on these theorles and experimental results for the wave pressure of standing waves 
Moreover, as an example of the application of these theorles, the change of the characteristics of the wave pressure of standing waves accompanying the overtopping wave is discussed in comparlson with the results of standing waves descrubed already

\section{NUMERICAL CONSIDERATION FOR THE BOUNDARY CONDITIONS AT FREE SURFACE}

\section{(1) Two Non-Linear Boundary Conditions at Free Surface}

Since these solutions are only approximate ones as mentioned above, the two non-linear boundary conditions at the unknown free surface, namely the kimematic condition prescribing the particle motion at the free surface and the dynamc condition describing the pressure at the free surface to be constant on the basls of Bernoulli's theorem are not satısfled rigorously by the se solutions and errors for these conditions always exist So, we investigate to what extent the perturbation solution of each order satisfies these conditions by computing numerically the errors of each order solution for these non-dimensionalized conditions for varıous kinds of wave characteristics expressed by dimensionless paraneters

The kinematic and dynamic conditions are given respectively as

$$
\begin{array}{ll}
v=\partial \eta / \partial t+u(\partial \eta / \partial x) & \text { at } \mathrm{y}=\eta \\
\partial \phi / \partial t+(1 / 2)\left(u^{2}+v^{2}\right)+g \eta=0 & \text { at } \mathrm{y}=\eta
\end{array}
$$

Let $\varepsilon_{1}$ and $\varepsilon_{2}$ be the errors of kinematic and dynamic conditions for the solutions These errors can be written in dimensionless form respectively as $\epsilon_{1}=[\sqrt{k / g}\{v-\partial \eta / \partial t-u(\partial \eta / \partial x)\} / \sqrt{g h}]_{\nu-\eta}$ $\varepsilon_{2}=\left[\left\{k \eta+(k / g)(\partial \phi / \partial t)+(k / 2 g)\left(u^{2}+v^{2}\right)\right] / k h\right]_{y-\eta}$

in which $x$ is the horizontal coordinate at the still water level, $y$ the vertical coordinate, directed upward positive, $t$ the time, $\phi$ the velocity potential, $u$ and $v$ the horızontal and vertical particle velocities respectively, $\eta$ the free surface elevation, $h$ the depth of water, $g$ the acceleration of gravity, $\mathrm{k}=2 \pi / \mathrm{L}$ and $\mathrm{L}$ the wave length

If these conditions are rigorously satisfied by the solutions, $\varepsilon_{1}$ and $\varepsilon_{z}$ would be identically zero However, as the solutions are approximate, the equality is not satisfied Accordingly, by substituting the se solutions into Eqs (3) and (4) and comparing the magnitude of the se errors wl th each of them, we can investigate which solution has the best fitness for these conditions

We introduce the followng criteria to evaluate the boundary condition errors

and

$$
\begin{aligned}
& \left(E_{1}\right)_{R}=\left(s_{1}\right)_{\max }-\left(\varepsilon_{1}\right)_{\min } \\
& \left(E_{2}\right)_{R}=\left(s_{2}\right)_{\max }-\left(s_{2}\right)_{\min }
\end{aligned}
$$

$$
\begin{aligned}
& \left(E_{8}\right)_{R}=\sqrt{\varepsilon_{1}^{2}} \sqrt{\left[\sum_{n=135}^{N}\left\{\left(\varepsilon_{1}\right)^{2}{ }_{n+1}+4\left(\varepsilon_{1}\right)^{2}+\left(\varepsilon_{1}\right)^{2}{ }_{n+1}\right\}\right] /\{3(M-1)\}} \\
& \left(E_{4}\right)_{R}=\sqrt{\varepsilon_{2}^{2}}=\sqrt{\left.\Gamma_{n=135}^{k-1}\left\{\left(\varepsilon_{2}\right)^{2}{ }_{n-1}+4\left(\varepsilon_{2}\right)^{2} n+\left(\varepsilon_{2}\right)^{2}{ }_{n+1}\right\}\right] /\{3(M-1)\}}
\end{aligned}
$$

which are according to Dean's criterla, where $\left(\varepsilon_{l}\right) \max$ and $\left(\varepsilon_{i}\right) \mathrm{mim}$ show the largest and smallest values of $\varepsilon_{1}$ and $\varepsilon_{2}$ calculated at each point respectively, $M$ is the number of calculation point and bar indicates the average, and the validity of the wave theorles can be investigated with the and of numerical calculations 


\section{(2) The Fitness for Boundary Condıtıons of the Theorles}

Fig 1 shows one of the time varlations of $\varepsilon_{1}$ and $\varepsilon_{2}$ at a vertical wall calculated for given values of $T \sqrt{g / h}$ and $h / H$ where $T$ is the wave perıod and $\mathrm{H}$ the amplitude of standing waves Notations 1, 2, 3 and 4 in Fig 1 indicate the first, the second, the third and the fourth order solutions respectively It 1 s found from the figure that the fourth order solution has the best fitness for these conditions of the four theoretical curves

The relation between $\left(\mathrm{E}_{1}\right)_{R}$ and $\left(\mathrm{E}_{2}\right)_{R}$ of each order solution at the wall and $\mathrm{T} \sqrt{\mathrm{g} / \mathrm{h}}$ in the case where the value of $\mathrm{h} / \mathrm{H}$ is constant, where black points in these figures designate the breakıng point of standing waves of each order solution calculated according to the criterıon derıved by Penny and Price are shown in Fig 2 It 1 s found from Fig 2 that the higher approximate solutions certainly have better fitness for these boundary conditions within a limited range of wave characteristics It is also found that the errors for these boundary conditions do not always decrease for the higher approximation, when the value of $\mathrm{T} \sqrt{\mathrm{g} / \mathrm{h}}$ becomes larger

Graphs expressing the areas where the boundary condition errors decrease for the higher approximation are given in Fig 3 according to each criterion Fig 3 shows that the perturcation solution of each order has a limited area of wave characteristics where the errors for the boundary conditions do not decrease by higher approximation, but there is a small difference between the areas for kınematıc and dynamic conditions

Fig 4 is the same as Fig 3 but is evaluated according to Dear's criterion, Eqs (7) and (8) for all phases of wave motion

\section{EXPERIMENT ON THE WAVE PRESSURE OF STANDING WAVES}

\section{(1) Experimental Equipment and Procedure}

The wave tank used in this experiment is $63 \mathrm{~m}$ long, $05 \mathrm{~m}$ wide and $065 \mathrm{~m}$ deep and belongs to the Ujigawa Hydraulıc Laboratory It has a piston type wave generator at the end and a calsson equipped with five pressure gages at about $395 \mathrm{~m}$ from the end In the experiment, the time variations in water level and wave pressure along the wall due to standing waves formed by wave reflection at the vertical wall were measured Since waves of sufficiently large helght which the value of $\mathrm{h} / \mathrm{H}$ Is smaller than 28 could not be generated in the case of the experiment whth uniform depth, a slope composed of $1 / 60$ ( $3 \mathrm{~m}$ long) and $1 / 400$ ( $20 \mathrm{~m}$ long) was constructed on the kottom of wave tank as shown in Fig 5, so that the waves of very large helght near wave breakıng were generated Experiments were carried out by changing the wave helght, whlle the wave perlod and the water depth were determined by keeplng the value of $\mathrm{T} / \mathrm{g} / \mathrm{h}$ constant The wave characteristics used in the experiment are tabulated in Table 1

\section{(2) Experimental Results and Considerations}

Fig 6 shows the comparison between the theoretical curves already discussed and the experimental results for the wave crest helght above the still water level $Z_{0} / \mathrm{H}$ The notations are the same as those in Fig 1 except for the notation SHUTO which indicates the second approximation to stationary long 
waves of fimte amplitude derived by Shuto as the interaction problem of cnoldal waves The theoretical curves agree comparatively well with the experimental results, but the theoretical curve of the second order approximate solution tends to deviate from the experimental results, as the value of $h / H$ becomes smaller and the value of $\mathrm{T} / \mathrm{g} / \mathrm{h}$ larger

Fig 7 is an example of the comparison between the theoretical curves and the experimental results for the wave pressure distribution along the wall at phase of wave crest, in whlch the theoretical wave pressure above the still water level is assumed to be triangular which connects the maximum point of elevation of water level wh a point of wave pressure at the still water level, where $p$ is the wave pressure and $\rho$ the density of flud The fourth order solution agrees well with the experimental values in the case of considerably large amplitude waves when the value of $\mathrm{T} \sqrt{\mathrm{g} / \mathrm{h}}$ is relatively small, but the other solutions glve excesslve values because of the insufficlency of approximation for calculation

Fig 8 shows the comparisons between the theoretical curves and the experimental results for wave pressure at a point on the wall at phase of wave crest, where the notation KISHI Is the second order approximate solution derlved by Kishl uslng the Penny and Price method When the value of $T / \sqrt{g / h}$ is relatively small as seen in Fig 8(a), the fourth order solution agrees well with the experimental values, but the lower order solutions deviate from the experimental results as the value of $\mathrm{h} / \mathrm{H}$ becomes small In the case where the value of $\mathrm{T} / \mathrm{g} / \mathrm{h}$ kecomes larger as shown $\mathrm{in} \mathrm{F}_{1 \mathrm{~g}} 8(\mathrm{e})$ and $(\mathrm{h})$, the fourth order solution also tends to deviate from the experimental results in the case of small $\mathrm{h} / \mathrm{H}$ The larger the value of $\mathrm{T} / \mathrm{g} / \mathrm{h}$ becomes, the larger the value of $\mathrm{h} / \mathrm{H}$, whlch the experimental values deviate from the theoretical curves corresponding to each order approximate solution except for the stationary long wave theory becomes The theoretical curve for the stationary long wave theory agrees relatively well with the experimental results for large $h / H$ as the value of $T \sqrt{g / h}$ becomes larger, but in the case of small $\mathrm{h} / \mathrm{H}$, it deviates rapıdly Thls may be due to 1 nsufficlency of approximation order for the calculation

Fig 9 indicates the comparison between the theoretical curves and the experimental results for the time varıations of water level and wave pressure on the wall It Is found that the experimental results approach the theoretical curves of the higher order approximate solutions in Fig 9(a), that they do not agree with the experimental results in Fig 9(b) due to the distortion of theoretical curves of the third and the fourth order solutions, and that the theoretical curve of stationary long wave agrees well with the experimental results only for the wave form

Using the results obtalned from the detalled comparison for the wave pressure of standing waves, a plot of the limıting conditions for the application of the theories is proposed in Fig 10 This figure shows that the limiting condition of the second order solution is confined to the area in which the value of $T \sqrt{g / h}$ is relatively small and the value of $h / H$ large Also thls shows that the area of poor correspondence between the theoretical results and the experimental ones exists, although the third and the fourth order solutions have relatively wide areas of applicability and that the area of correspondence for the stationary long wave theory is restricted to the area of relatively large value of $T \sqrt{g / h}$ 


\section{EXXERIMENT ON THE WAVE PRESSURE WITH WAVE OVERTOPPING}

\section{(1) Experimental Equpment and Procedure}

The experimental equipment used is same as in th previous section Experiments were carried out alternatively for the case of the wave pressure accompanying the overtopping wave and standing waves The characteristics of waves and the crest helght of wall are presented in Table 2

\section{(2) Experimental Results and Considerations}

The relation between the theoretical curves and the experimental results for wave crest helght above the still water level is shown in Fig 11, where the white circles indicate the case of standing waves, the other circles indicate the case of the wave pressure accompanying the overtopping wave and $\mathrm{Hc}$ is the crest helght of wall Experimental results for standing waves accompanying the overtopping wave agree relatively well with theoretical curves on the basis of the theories within a range of the experiment, although in the case of $\mathrm{T} / \mathrm{g} / \mathrm{h}=10$ they are a little less than the experimental values for the standing waves in spite of the scatter

Fig 12 shows the comparison between the theoretical curves of the fourth order solution calculated by taking into account the reduction of wave helght due to the wave overtopplng and the experimental results for the wave pressure distribution along the wall at phase of wave crest in the case of wave overtopping It is seen from Fig 12(a) that even if the wave helght in comparison with the crest hel ght of wall increases considerably, the change of the wave pressure due to the presence of wave overtopping may apparently disappear when the reduction of the wave height at the wall can be taken into account and that the theoretical curves agree well with the experimental results $F_{1 g} 12(b)$ describes the effect of the crest helght of the wall on the wave pressure distribution It is seen that the theoretical curve agrees well with the experimental results in spite of the difference in the crest height of wall

Fig 13 is the comparlson between the theoretical curves of wave pressure at a point on the wall at phase of wave crest and the experimental results for the overtopping wave The theoretical curves agree falrly well with the experimental results in spite of the existence of wave overtopping within a range of the limiting area for the application of the theories corresponding to each order solution found out in the previous section if the reduction of wave height at the wall can be taken into account However, when the wave helght increases considerably in comparison with the crest helght of wall and becomes near the breaking wave helght, the experimental results for the overtopping wave are a little less than those for standing waves This may be due to the change of the field of wave motion because of the increase of the rate of wave overtopping Accordingly, the value of $\mathrm{H} / \mathrm{Hc}$ is an important parameter which dominates the change of wave pressure caused by wave overtopping as well as the phenomenon of wave overtopping itself

F1g 14 shows the time variations of the water level and wave pressure, where the theoretical curve $1 \mathrm{~s}$ based on the fourth order solution The absolute values of experimental results for the overtopplng wave decrease in comparison with those of standing waves with the same wave characterlstics, whlle the values agree fairly well wh theoretical ones in both the case, if the reduction of wave helght 
Is taken into account But the second peak of double humped wave pressure record disappears and then an unsymmetric wave pressure record appears

The relation between the relative amplitude in the water level at the wall $\mathrm{H} / \mathrm{Hc}$ and the one for the overtopping wave $\mathrm{H} / \mathrm{Hc}$ is given in Fig 15 as evaluated by reducing of wave height due to the wave overtopping It is found that the rate of reduction of wave height is almost uniquely determined by the value of $\mathrm{H} / \mathrm{HC}$, regardless of the wave perrod

\section{CONCLUSIONS}

The main results of this investigation are summarized as follows

1 As a result of detarled numerical and experimental considerations for the fimte amplitude standing wave theorles, the limiting conditions for the application of these theories are presented and it is verified that the approximate solutions for various orders of the theories are valid within a certain range of $h / H$ and $T \sqrt{g / h}$ corresponding to each order of approximation

2 If the rate of reduction of wave helght at the wall caused by wave overtopplng can be taken into account, these theories are applicable for estimating the wave pressure of standing waves on a wall in the case where wave overtopplng exists within a range of the limiting area of applicability of the theorles except for relatively large values of $\mathrm{H} / \mathrm{Hc}$

\section{ACKNOWLEDGTMENT}

Part of this investigation was accomplished with the support of the Science Research Fund of the Ministry of Education, for whlch the authors express their appreclation Thanks are due to Dr Iwagak1, Professor, Department of Clvil Eng1neering Kyoto University, for his cordial encouragement during this investigation

\section{REFEFENCES}

Dean, R G (1967) Relative valıditıes of water wave theories Proc of Conf on Civil Eng in the Oceans, pp $1-30$

Goda, $Y$ and Kakızakı, S (1966) Studıes on standıng waves of finıte amplıtude and wave pressure Report of Port and Harbor Research Institute Mnnstry of Transport, Vol 5, No 10, pp 1-50 (1n Japanese)

Klshı, T (1957) Clapotis in shallow water Journal of Research of PWRI, Vol 2, Research Paper 5.

Penny, W G and Price, A T (1952) Finzte perıodic stationary gravity waves In a perfect liquid Phil Trans A, Vol 224, pp 254-284

Shuto, N (1968) Stationary long waves of finite amplitude Proc of the 15th Conf on Coastal Eng in Japan, pp 212-219 (in Japane se)

Tadjbaksh, I and Keller, J B (1960) Standing surface waves of finjte amplitude $J$ Flurd Mech, Vol 8, pp 442-451 


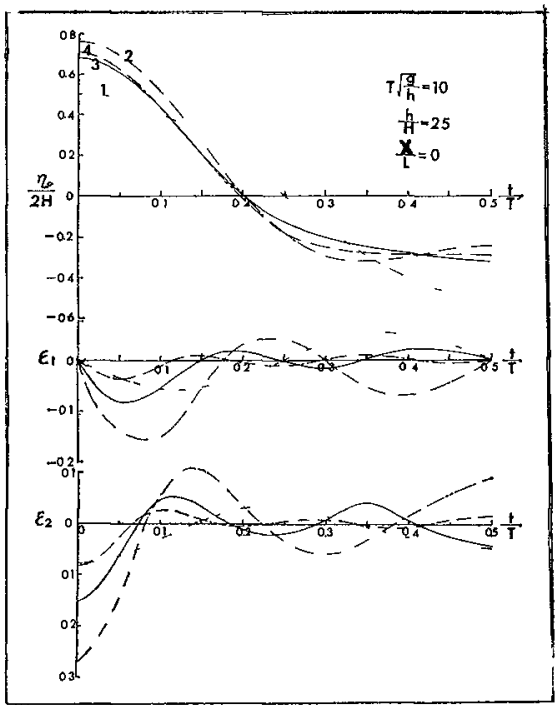

Fig 1 Time variations of $\varepsilon_{1}$ and $\varepsilon_{x}$ at vertical wall

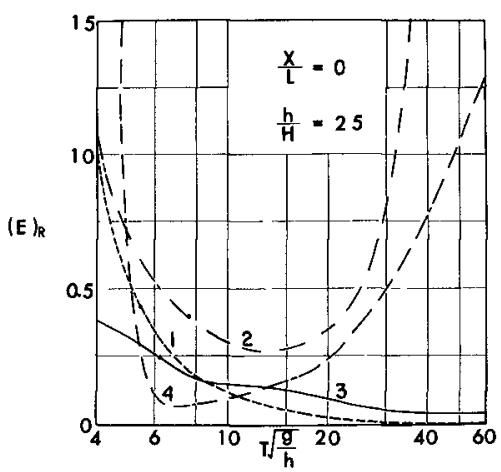

(a)

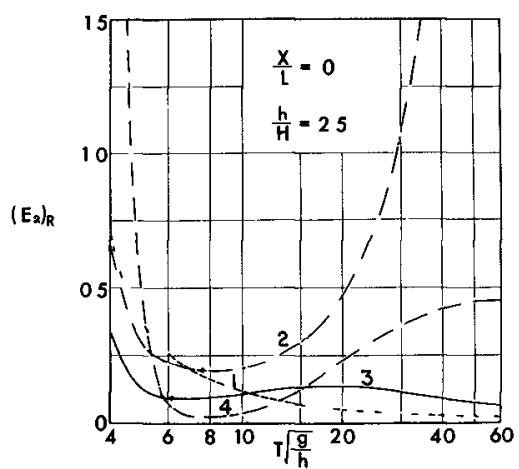

(b)

$F_{1 g} 2$ Relation between $\left(E_{1}\right)_{R}$ and $\left(E_{2}\right)_{R}$ of each order solution at wall and $\mathrm{T} \sqrt{\mathrm{g} / \mathrm{h}}$ 

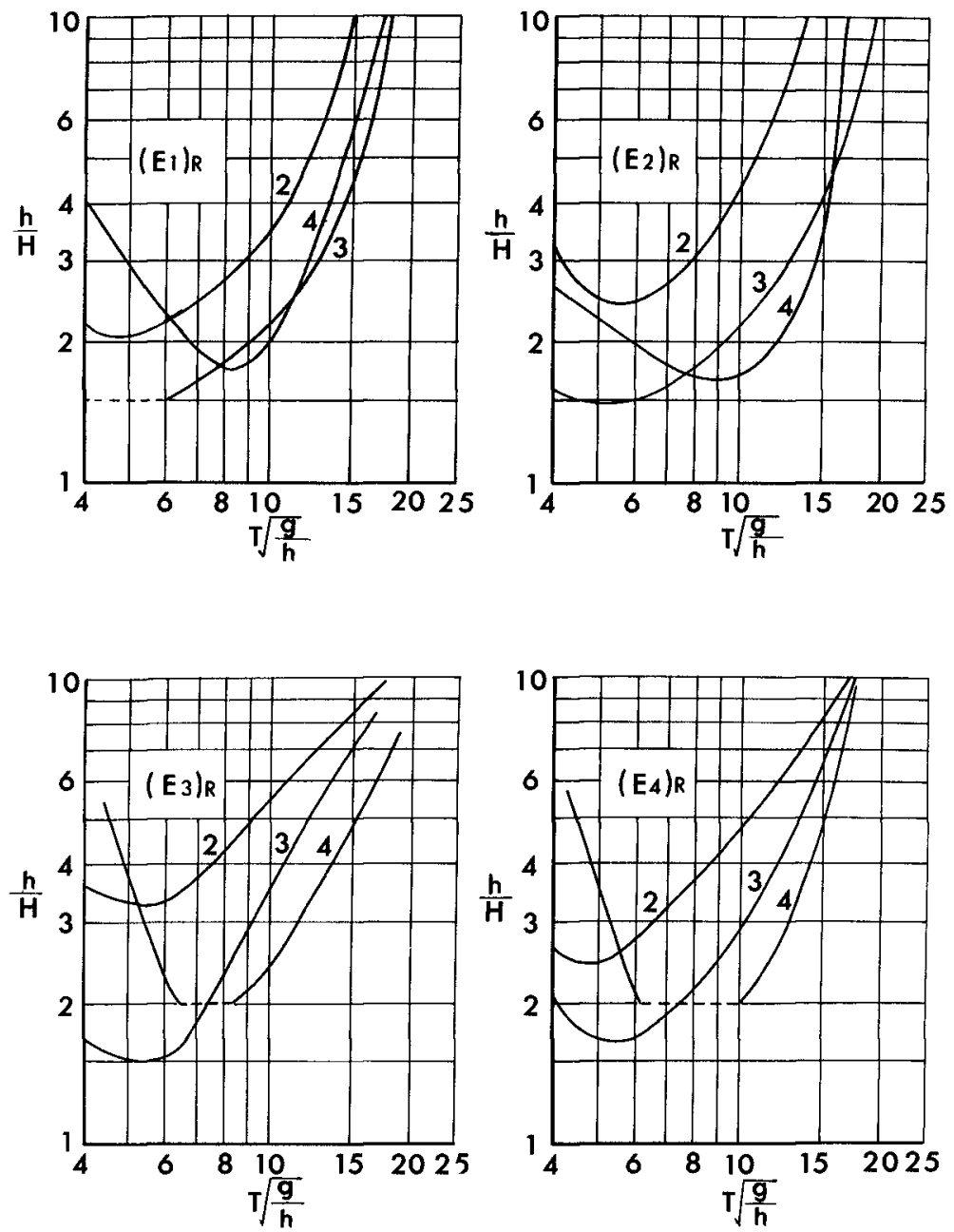

Fig 3 Decreasing areas, estimated approxımately, of free surface boundary condition errors for validity of finite amplitude standing wave theories by higher approximation 

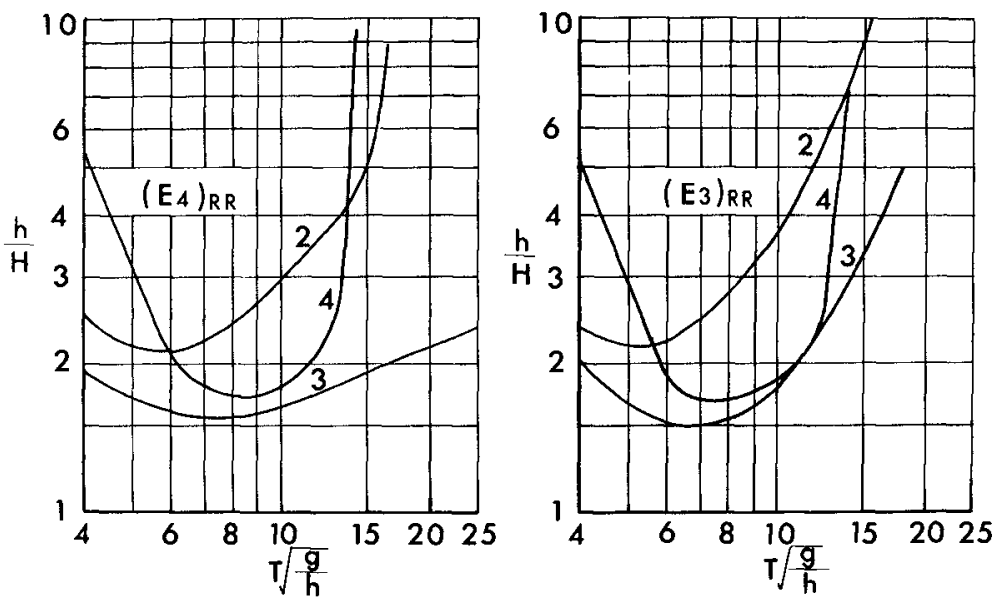

F1g 4 Decreasing areas, estzmated approximately, of free surface boundary condition errors for validıty of fincte amplitude standing wave theories by higher approximation 
Table 1 Wave characteristics used in the experiment on standing waves

\begin{tabular}{|c|c|c|c|}
\hline$T \sqrt{g / h}$ & $h(\mathrm{~cm})$ & $\mathrm{T}(\mathrm{sec})$ & $\mathrm{H}(\mathrm{cm})$ \\
\hline 6 & $\begin{array}{l}25.0 \\
200\end{array}$ & 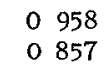 & $\begin{array}{rll}10 & 70 \sim 2 & 05 \\
6 & 72 \sim 3 & 18\end{array}$ \\
\hline 8 & $\begin{array}{ll}20 & 0 \\
25 & 0 \\
17 & 5\end{array}$ & $\begin{array}{ll}1 & 143 \\
1 & 278 \\
1 & 069\end{array}$ & $\begin{array}{rll}10 & 51 \sim 1 & 45 \\
7 & 11 \sim 2 & 97 \\
5 & 80 \sim 4 & 30\end{array}$ \\
\hline 10 & $\begin{array}{ll}20 & 0 \\
25 & 0 \\
17 & 5\end{array}$ & $\begin{array}{ll}1 & 427 \\
1 & 597 \\
1 & 336\end{array}$ & $\begin{array}{rll}10 & 00 \sim 1 & 42 \\
8 & 36 \sim 3 & 05 \\
4 & 97 \sim 3 & 00\end{array}$ \\
\hline 12 & $\begin{array}{ll}17 & 5 \\
20 & 0 \\
15 & 0\end{array}$ & $\begin{array}{ll}1 & 603 \\
1 & 714 \\
1 & 485\end{array}$ & $\begin{array}{lll}9 & 50 \sim 1 & 01 \\
5 & 93 \sim 2 & 98 \\
4 & 34 \sim 1 & 52\end{array}$ \\
\hline 14 & $\begin{array}{ll}15 & 0 \\
20 & 0\end{array}$ & $\begin{array}{ll}1 & 732 \\
2 & 000\end{array}$ & $\begin{array}{lll}9 & 21 \sim 1 & 23 \\
6 & 08 \sim 1 & 84\end{array}$ \\
\hline 16 & $\begin{array}{ll}15 & 0 \\
12 & 5\end{array}$ & $\begin{array}{ll}1 & 979 \\
1 & 807\end{array}$ & $\begin{array}{lll}9 & 20 \sim 1 & 01 \\
2 & 61 \sim 2 & 44\end{array}$ \\
\hline 18 & $\begin{array}{ll}12 & 5 \\
10 & 0\end{array}$ & $\begin{array}{ll}2 & 033 \\
1 & 818\end{array}$ & $\begin{array}{lll}6 & 78 \sim 1 & 03 \\
2 & 31 \sim 0 & 73\end{array}$ \\
\hline 20 & 100 & 2020 & $5 \quad 04 \sim 115$ \\
\hline
\end{tabular}

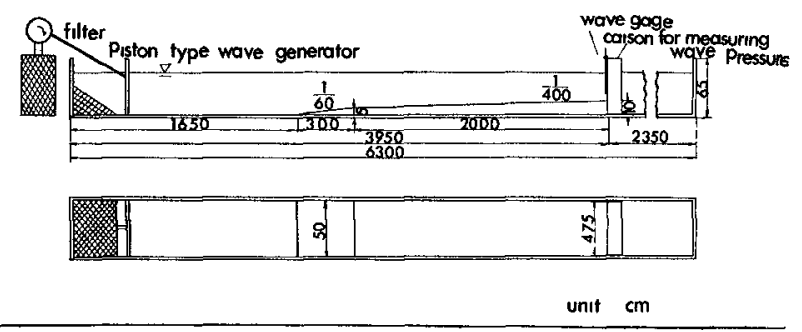

F1g 5 Schematic sketch of wave tank used 


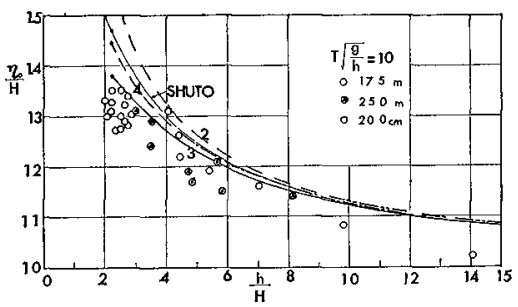

(a)

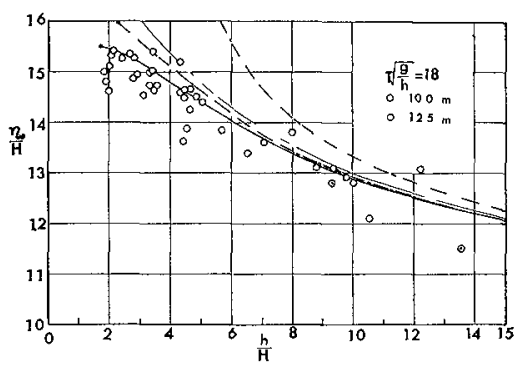

(c)

F1g 7 Comparison between theoretical curves and experimental results for wave pressure distribution along wall at phase of wave crest

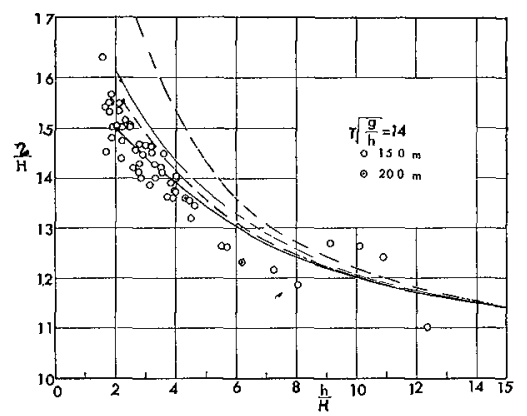

(b)

F1g 6 Comparıson between theore tical curves and experimental results for wave crest helght above still water level

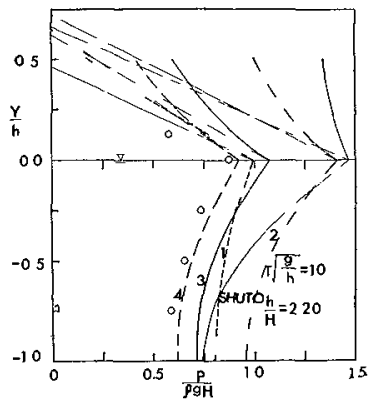




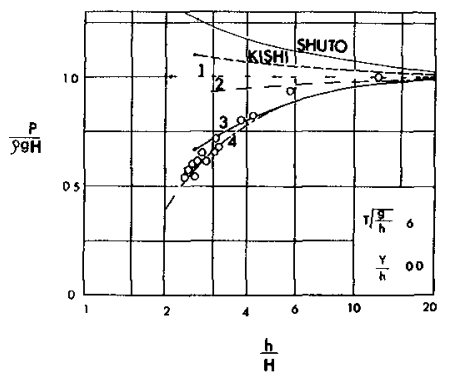

(a)

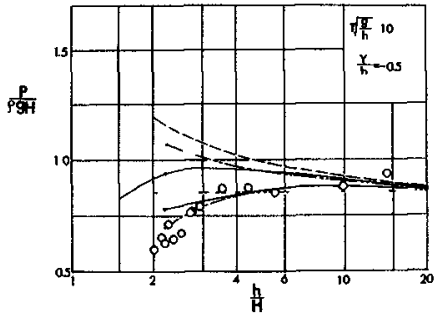

(c)

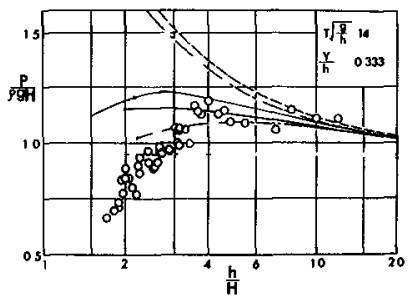

(e)

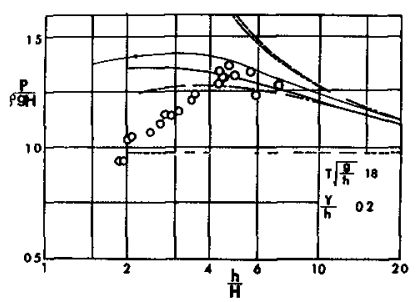

(g)

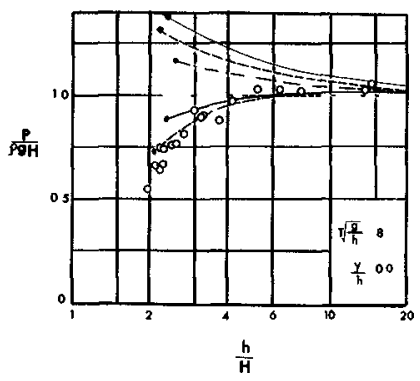

(b)

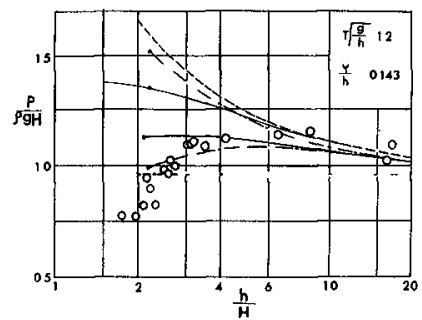

(d)

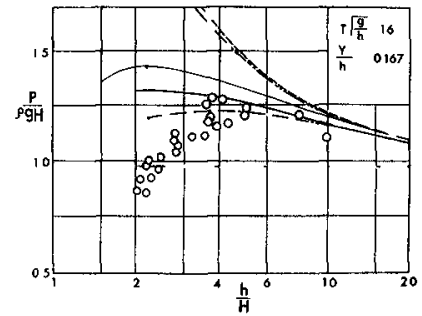

(f)

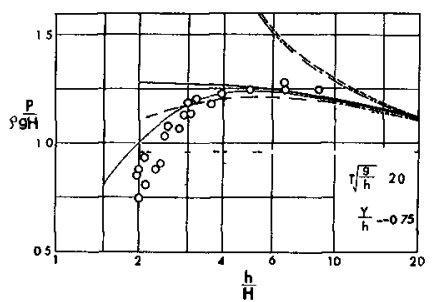

(h)

Fig 8 Comparıson between theoretıcal curves and experimental results for wave pressure at a point on wall at phase of wave crest 

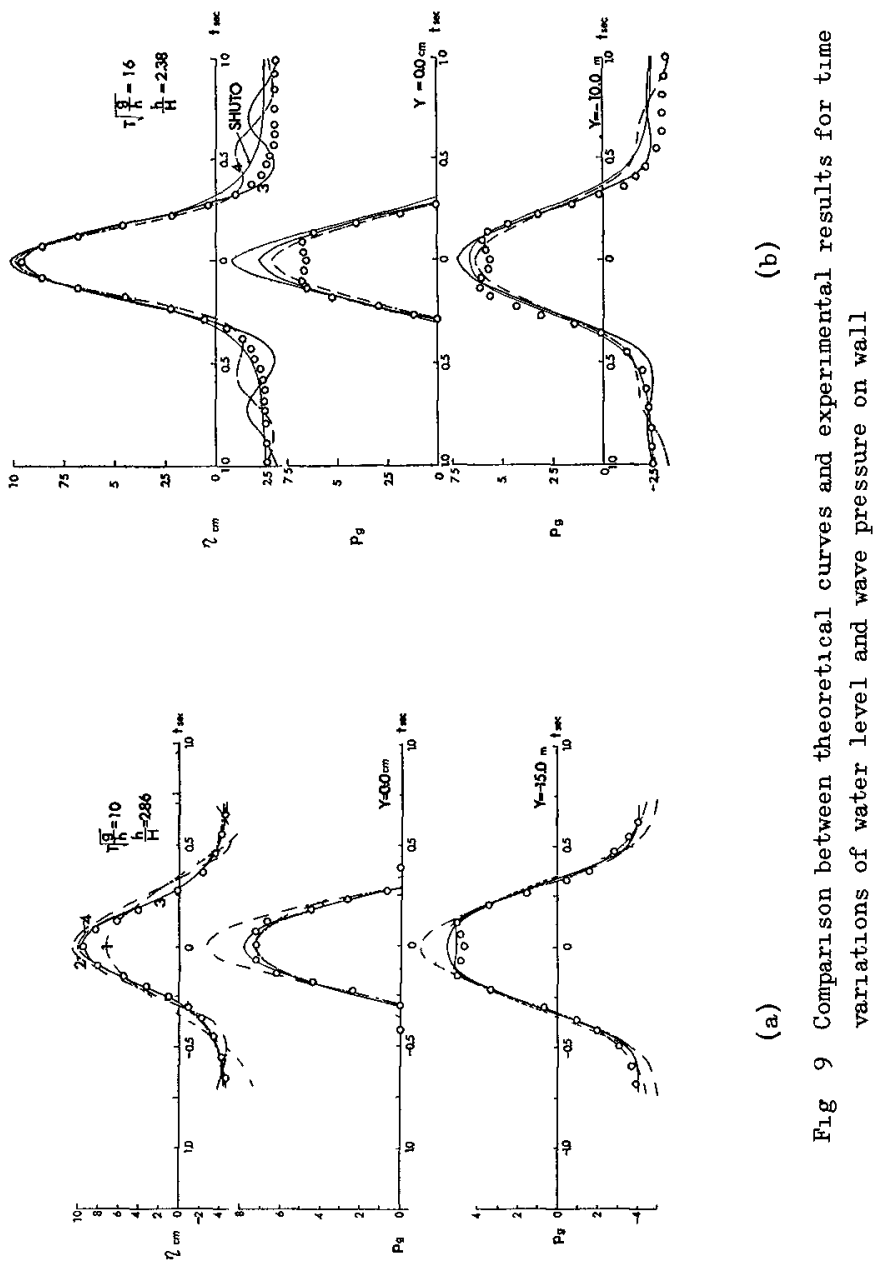

$\stackrel{9}{3}$

号哭

臣

$8 \$$

5 


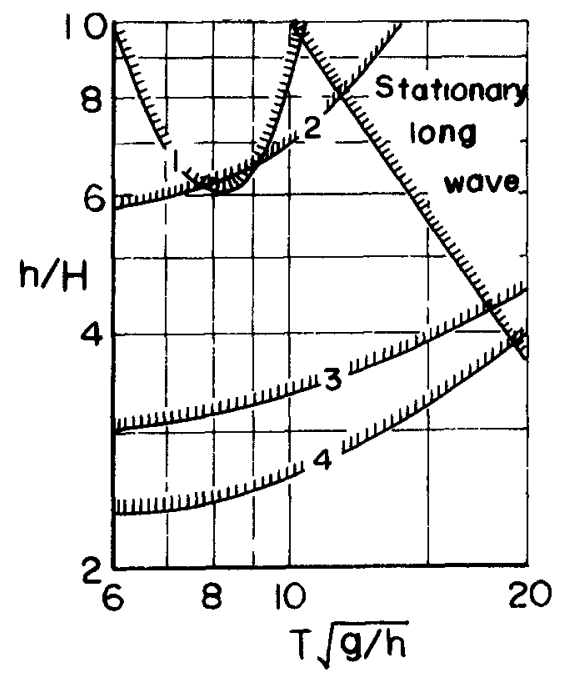

Fig 10 Lumiting conditions for application of fincte amplitude standing wave theories

Table 2 Wave characteristics used in the experiment on standing waves with wave overtopping.

\begin{tabular}{|c|c|c|c|c|}
\hline$T \sqrt{g / h}$ & $T($ sec $)$ & $h(\mathrm{~cm})$ & $\mathrm{Hc}(\mathrm{cm})$ & $\mathrm{H}(\mathrm{cm})$ \\
\hline 8 & 1143 & 200 & 50 & $1050 \sim 491$ \\
\hline 10 & $\begin{array}{ll}1 & 429 \\
1 & 336 \\
1 & 336 \\
1 & 336 \\
1 & 237\end{array}$ & $\begin{array}{ll}20 & 0 \\
17 & 5 \\
17 & 5 \\
17 & 5 \\
15 & 0\end{array}$ & $\begin{array}{ll}5 & 0 \\
2 & 5 \\
5 & 0 \\
7 & 5 \\
5 & 0\end{array}$ & $\begin{array}{rll}12 & 38 \sim 4 & 40 \\
9 & 87 \sim 2 & 89 \\
9 & 70 \sim 4 & 60 \\
8 & 53 \sim 5 & 26 \\
8 & 58 \sim 4 & 50\end{array}$ \\
\hline 12 & 1604 & 17.5 & 50 & $922 \sim 370$ \\
\hline 14 & I 732 & 150 & 50 & $958 \sim 40 I$ \\
\hline 16 & I 979 & 150 & 50 & $9.12 \sim 3.39$ \\
\hline 18 & 2033 & 125 & 50 & $828 \sim 304$ \\
\hline
\end{tabular}




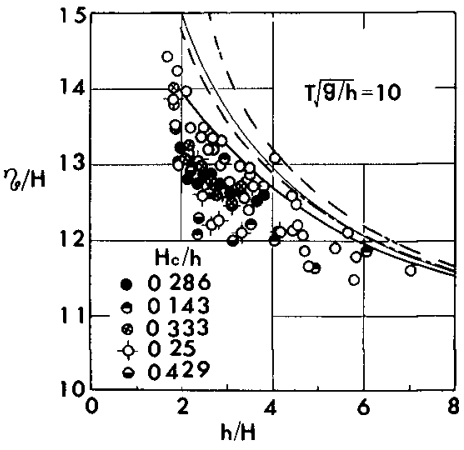

(a)

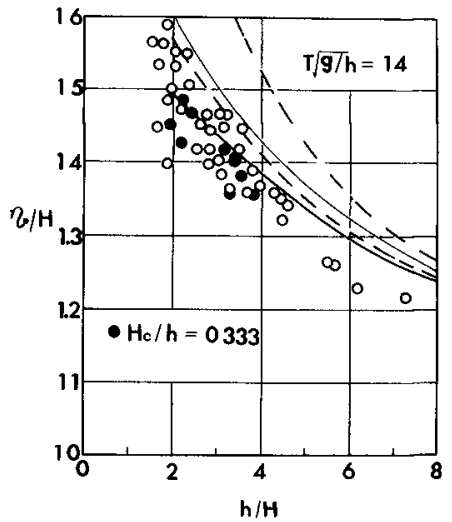

(c)

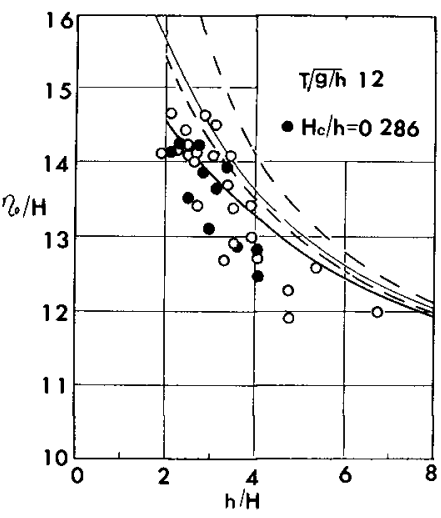

(b)

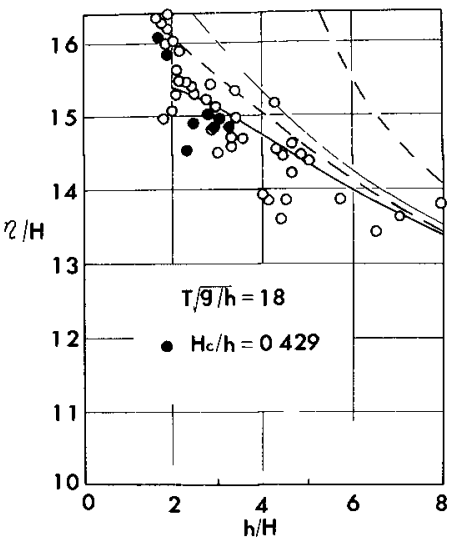

(d)

F1g 11 Comparison between theoretical curves and experimental results for wave crest helght above still water level in the case where wave overtopping exists 


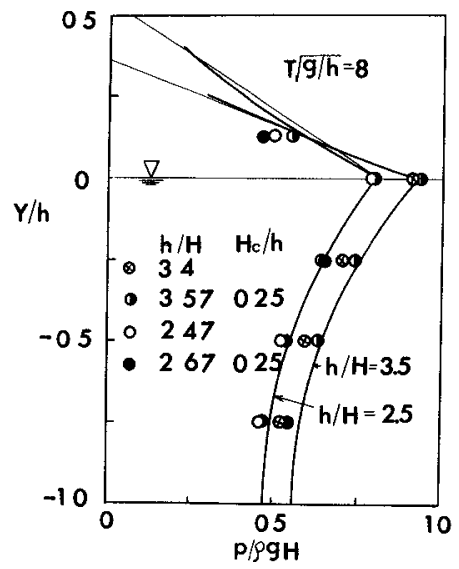

(a)

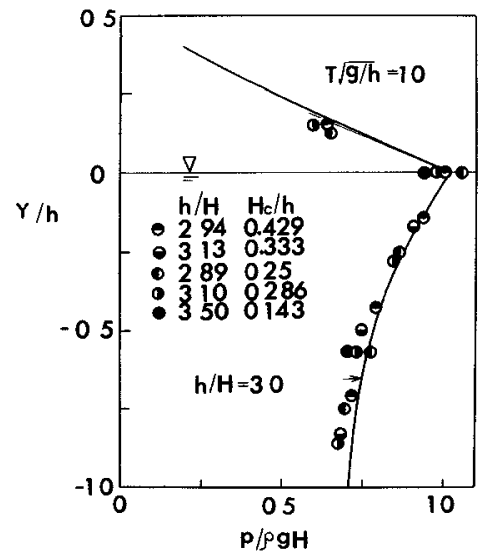

(b)

Fig 12 Comparison between theoretical curves and experimental results for wave pressure distribution along wall at phase of wave crest in the case where wave overtopping exists 


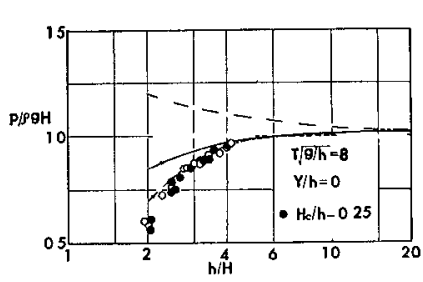

(a)

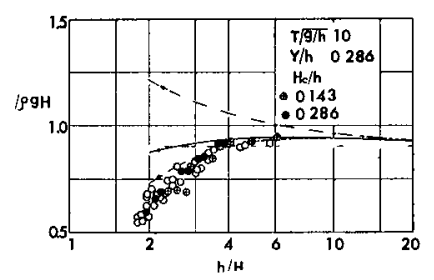

(c)

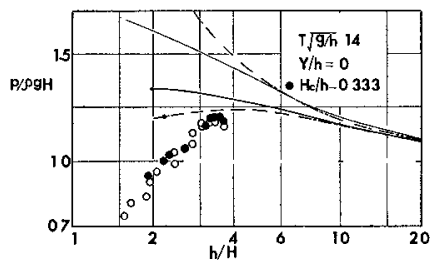

(e)

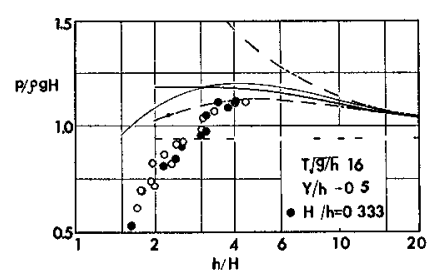

(g)

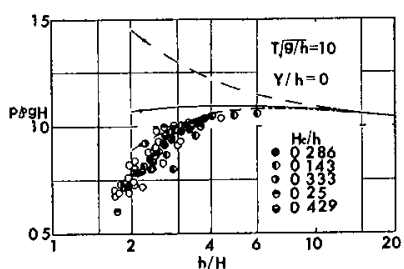

(b)

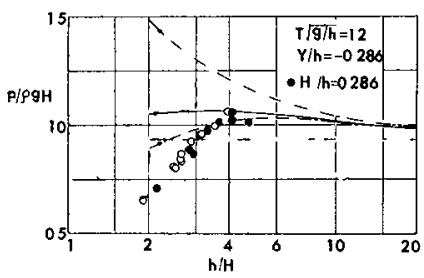

(d)

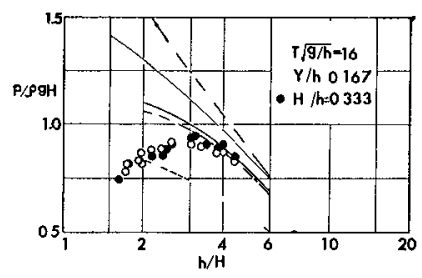

(f)

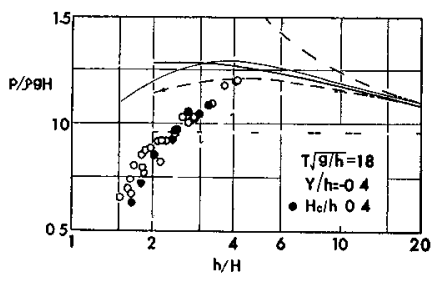

(h)

F1g 13 Comparison between theoretıcal curves of wave pressure at a point on wall at phase of wave crest and experimental results in the case where wave overtopping exists. 

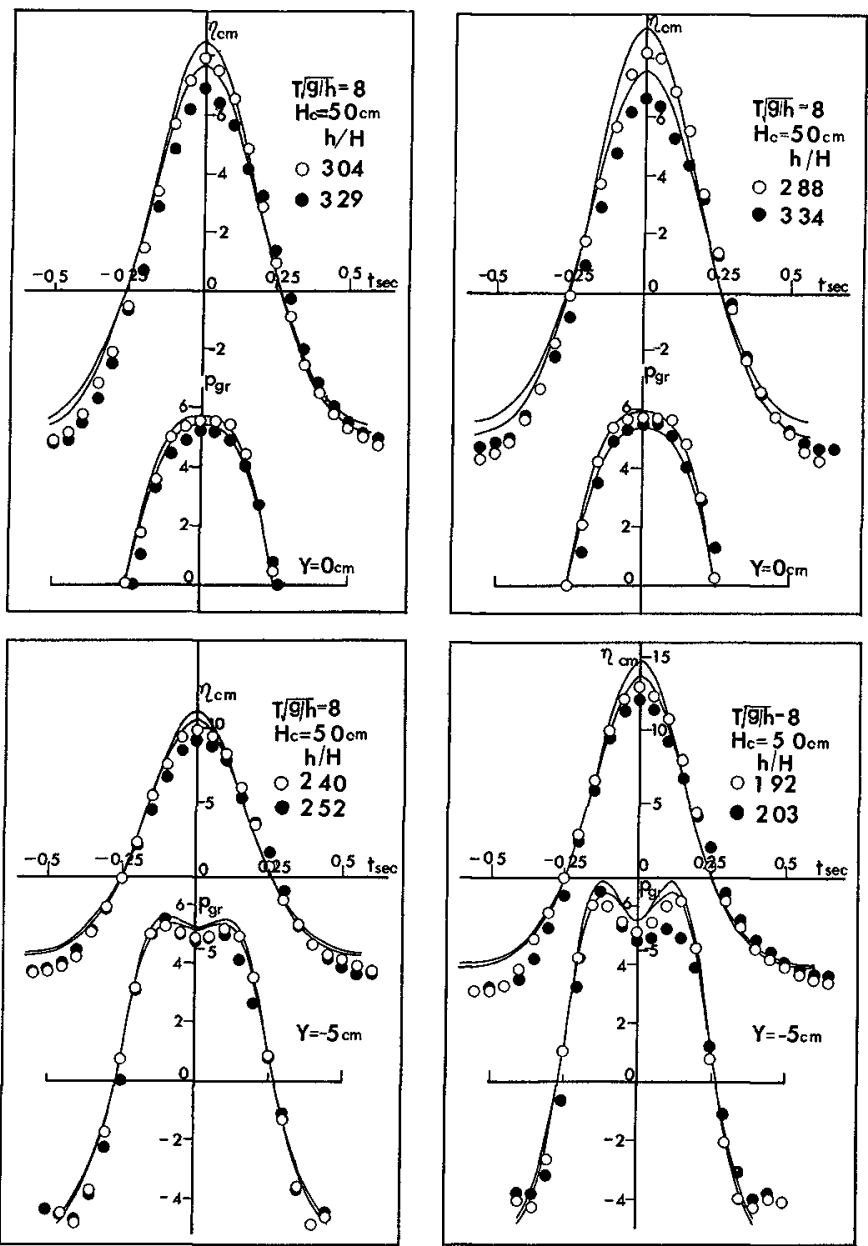

(a)

F1g 14 Comparison between theoretical curves and experimental results for time variations of water level and wave pressure on wall in the case where wave overtopping exists 


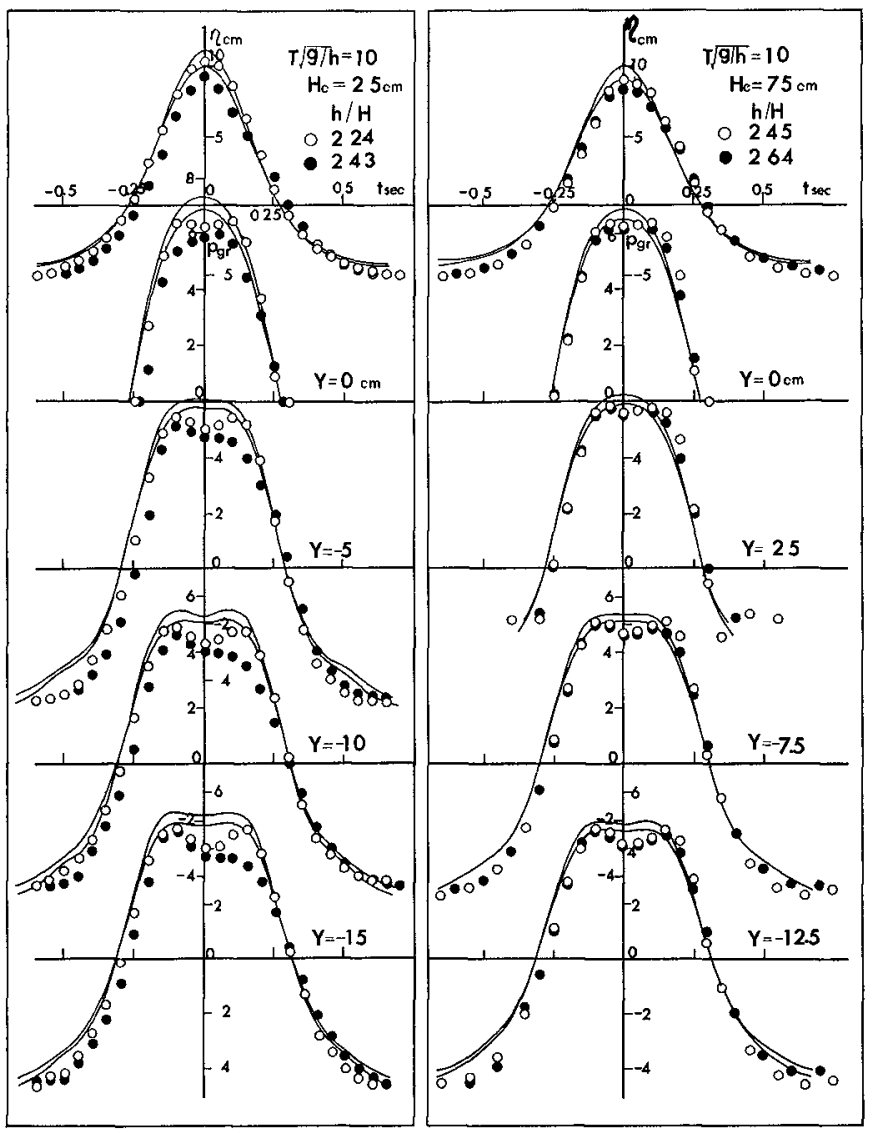

(b)

Fig 14 Comparison between theoretical curves and experımental results for time varıations of water level and wave pressure on wall in the case where wave overtopping exists. 


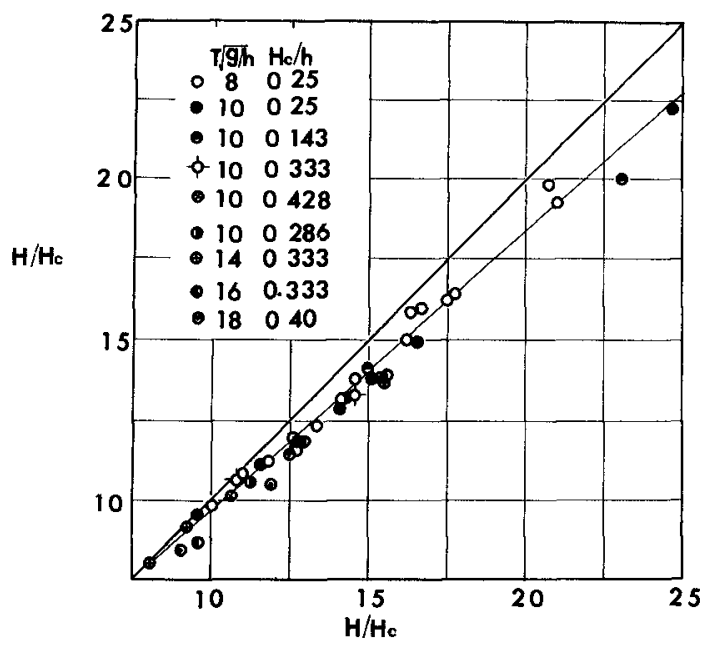

Fig 15 Relation between relative amplitude in water level at wall $\mathrm{H} / \mathrm{Hc}$ and one for overtopping wave $\mathrm{K} / \mathrm{Hc}$ 


\title{
CHAPTER 33
}

A HIGHER ORDER THEORY FOR SYMMETRICAL GRAVITY WAVES

\author{
by Peter L Monkmeyer \\ Professor of Civil Englneering \\ University of Wisconsin, Madison
}

\section{ABSTRACT}

A higher order theory ls presented for symmetrical, non-linear gravity waves As a consequence of the generality employed, the theory includes the full range of possible wave lengths, water depths and wave heights that may be encountered, and brings them into one unified formulation Thus, the theory encompasses both linear and non-linear waves, lncluding Alry waves, stokes waves, cnoldal waves and the solltary wave

Based on the work of Nekrasov, a complex potential in the form of an infinite series is developed to describe the flow fleld The potential satisfies the bottom (horizontal) condition as well as the kinematic surface condition exactly furthermore, the dynamic surface condition is satisfied by numerical calculation of the serles coefflclents whlch appear in the complex potential The calculation of these coefficients is accomplished by solving a set of non-linear algebralc equations, with the ald of a Newton-Raphson lteration procedure and matrix inversion

Coefflclents of the complex potential have been obtained for a flfth order analysis and preliminary results are presented ln tabular form A brief discussion of the characteristics of the waves, Including wave speed, wave shape and the helght of the highest possible wave follows

\section{INTRODUCTION}

Water waves and their characteristics have recelved a great deal of attention by mathematicians, geophysicists and englneers over the past century and a half in particular, numerous theorles have been developed to describe the characteristics of symmetrical, periodic, progressive waves Among the more classical papers are those by stokes (1847,1880), Raylelgh (1876), Boussinesq (1872), Korteweg and Devries (1895), Levi-civita (1925), and strulk (1926) Reviews of some of these works, as well as many more recent publications may be found in publications by stoker (1957), wiegel (1964) Kinsman (1965), Ippen (1966) and Neumann and Plerson (1966) No attempt will be made here to review the many recent contributions However, of particular anterest, especially for applications of the theory, are the works of Mash and wiegel (1961), Skjelbrela and Hendrıckson (1962), Laltone (1963) and Dean (1965)

The wealth of literature on the subject of periodic water waves reflects to some extent the lack of a unified approach An effort to resolve this problem was made by Nekrasov (1951), followed 
by Mllne-Thomson (1969) and Thomas (1968) Nekrasov first formulated the wave problem in general terms, and concluded his analysis with a non-linear integral equation

The present study reexamines the work of Nekrasov and his successors and presents it in a manner which should be more useful in practice More specifically, a method is developed to compute coefflclents which may be used to calculate the various characterlstics of the waves

Since the theory presented herein is general, it covers the entire range of possible wave lengths, water depths and wave helghts that may be encountered Thus it encompasses both linear and nonlinear waves including Alry waves, Stokes waves, cnoldal waves and the solıtary wave As a consequence it glves promise of simplifylng the cholce of the appropriate theory - a problem which curxently faces the practitioner

SOLUTION OF THE WAVE PROBLEM

\section{DEVELOPMENT OF THE THEORY}

The wave theory whlch will be developed herein applies to progressive, symmetrical, gravity waves moving over the free surface of an inviscid, incompressible liquid, in an oscillatory manner Furthermore the waves are two-dimensional and, except for the special case of Infinite depth, they move over a horizontal bottom No restriction 1 s placed on liquid depth, $D$, wave length, $L$, or wave helght, $H$ Hence the theory is comprehensive and includes the full range of constant-profile waves, from stokes waves to cnoldal waves and the solitary wave, as well as from small-amplitude waves to large-amplitude waves and the so-called "highest wave"

A train of oscillatory waves is moving from right to left over the surface of the liquid in question with wave speed, c By superlmposing a uniform flow from left to right of the same speed as that of the waves, the wave proflles are brought to rest The net effect is to provide a steady flow from left to right, bounded by the fixed profile formed at the free surface and the impervious boundary at the bottom The steady flow-fleld will be seen to be considerably more amenable to study than would be the unsteady, progressive-wave fleld

In Fig 1 the steady wave is depicted and the more important constants are defined For convenience in the development the coordinates are described in complex terms and the physical plane is the $z$-plane, where $z$ is the complex varlable and $x$ and $y$ are the real and imaginary axes respectively The y-axis is chosen to pass through the crest of the wave, $c$, in order to assure symmetry The free surface is defined by $y_{0}=y_{(}\left(x_{0}\right)$ and the still water level is located at $y=y$, a distance whlch remalns to be determined It should be noted shat the depth, d, usually defined as the distance from the still water level to the bottom will equal the sum of $y_{s}$ and $D$ 


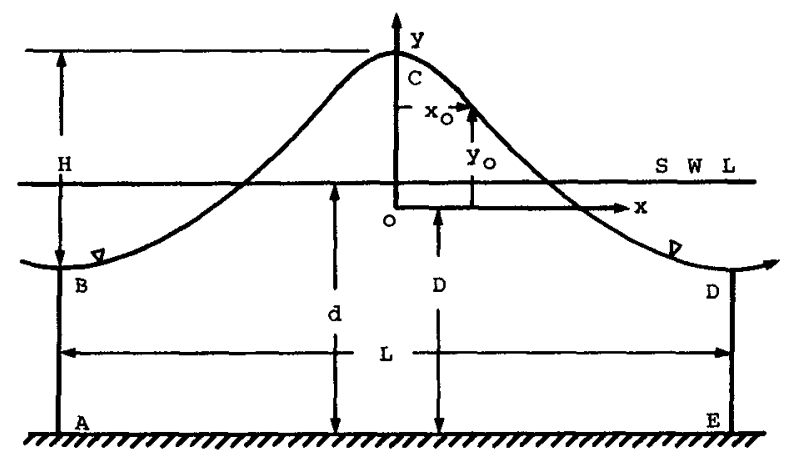

Fig 1 - The z-Plane

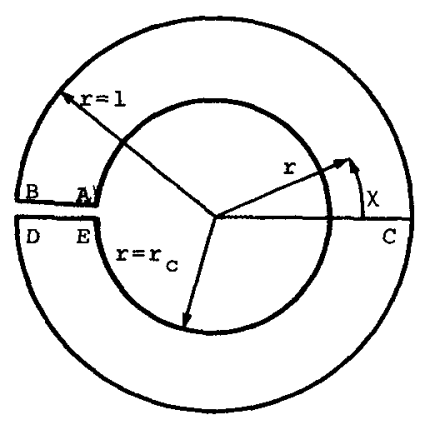

Fig 2 - The $\zeta$-Plane 
Since the flow is irrotational, an assumption which has been discussed by stokes (1847), the complex potential is given by

$$
W=\phi+1 \psi
$$

where $\phi$ is the potential function and $\psi$ is the stream function Furthermore, the complex potential is analytic and so the CauchyRlemann equations, which may be related to the velocity components, $u$ and $v$, are given by

$$
u=\frac{\partial \phi}{\partial x}=\frac{\partial \psi}{\partial y} \quad \text { and } \quad v=\frac{\partial \phi}{\partial y}=-\frac{\partial \psi}{\partial x}
$$

Substitution of these expressions into the continuity and irrotational conditions results in Laplace's equation for each function,

$$
\nabla^{2} \phi=0 \quad \nabla^{2} \psi=0
$$

respectively

At the free surface the kinematic boundary condition requires that the surface be a streamline for convenience, this bounding streamline is defined as

$$
\psi=0 \quad \text { at } y=y_{0}
$$

The dynamic boundary condition at the free surface is expressed by the Bernoull equation with pressure equal to zero,

$$
q_{0}^{2}+2 g y_{0}=k_{0} \quad \text { at } y=y_{0}
$$

where $q_{\text {is }}$ is the speed of a surface particle and $k_{0}$ is a constant

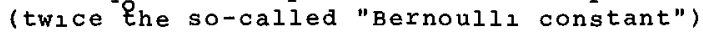

The lower boundary condition is kinematic and requires that the horizontal bottom be a streamline In order to reflect the volume rate of flow between the two bounding streaminnes,

$$
\psi=-C D \quad \text { at } y=-D
$$

where " $C D$ ' equals the two-dimensional flow rate observed in the $z-p l$ ane

Up to this point the relevant differential equations, together with the appropriate boundary condltions whlch describe the flow, have been presented The two fundamental problems which lmmediately present themselves are that the location of the free surface is unknown and the dynamic boundary condition is non-linear in order to deal with the first problem a conformal transformation will be employed The purpose of this transformation ls to redefine the problem in an auxiliary plane, the $\zeta$-plane, where the location of the free surface is known

The particular conformal transformation to be used, is an outgrowth of the work of Nekrasov (1951), as well as a subsequent 
analysis by Milne-Thomson (1969) and Thomas (1968) More specifically, it is an extension and generalization of the special deepwater case considered by Monkmeyer and Kutzbach (1965) The transformation is given as follows

$$
z=\frac{I L}{2 \pi}\left[\ln (\zeta)+\frac{\pi}{2 K} \sum_{\jmath=1}^{\infty} \frac{a}{\jmath} \exp \left[1 \operatorname{am}\left(-\frac{2 \jmath K}{\pi} \ln \zeta\right)\right]\right]
$$

where $K$ ls the complete elliptic integral of the first kind and the a 's are a set of real coefflclents whlch are as yet unknown Moreover, $\zeta$, which ls the complex variable describing the coordinates of the $\zeta$-plane is given in polar form by

$$
\zeta=r \exp (1 \chi)
$$

where $r$ is the radial coordinate and $\chi$ the angular coordinate of the -plane as shown in Fig 2 Finaliy, am() ls the amplitude of the elliptic integral of the first kind

One may verify, by application of the mapping function, Eq 7 , that the region bounded by ABCDE in the z-plane is a mapping of the equivalent region inside the unit circle in the $\zeta$-plane, subject only to the proper evaluation of the constant coefficients, a In fact it may be shown that the boundaries $A B$, DE and EA are mapped exactly from the $z-p l$ ane to the $\zeta$-plane, regardless of the values of the coefflcients, a The boundarles AB and DE transform exactly as a consequence of the perlodicity of the transformation The exact transformation of the lower boundary DE follows from the characteristics of the elliptic function, am(, The cholce of this particular conformal transformation was essentially dictated by the exact transformation of the bottom boundary As a by-product of the bottom transformation it is required that

$$
\frac{\mathrm{K}^{\prime}}{\mathrm{K}}=\frac{4 \mathrm{D}}{\mathrm{L}}
$$

where $K^{\prime}(m)=K(l-m)$, and $m$ is the parameter of the complete elliptic integral of the first kind This is a convenient formula since it permits a consideration of the entire range of waves for all wave lengths and depths In particular it facilitates inclusion of the two limiting cases, $L \rightarrow \infty$ and $D+\infty$, since $K+\infty$ in the first instance and $\mathrm{K}^{\prime} \rightarrow \infty$ in the second Eq 9 may therefore be used to convert $\mathrm{Eq} 7$ and many of the following equations, if the limiting case of the solitary wave is of interest or if cnoldal waves are to be expressed in terms of depth rather than wave length

The transformation can also be applied to the boundary conditions, Eqs 4,5 and 6 , to generate the equivalent conditions in the $\zeta-p l a n e$,

$$
\psi=0 \quad \text { at } r=1
$$


and

$$
q_{0}^{2}+2 g y_{0}=k_{0} \quad \text { at } r=1
$$

and

$$
\psi=-\mathrm{CD} \quad \text { at } r=r_{\mathrm{C}}
$$

where

$$
r_{c}=\exp \left(-\frac{2 \pi D}{L}\right)
$$

The validity of these representations of the boundary conditions is verified in Fig 2 Moreover it should be noted that Eqs 10 and 12 are precisely the boundary conditions for a portion of a clockwise irrotational vortex in the $\zeta$-plane, so that the complex potential for the flow in this plane may be written

$$
\mathrm{w}=\frac{1 \mathrm{CL}}{2 \pi} 1 \mathrm{n} \zeta
$$

By separating real and imaginary parts of this expression and rearranglng terms,

and

$$
\begin{gathered}
x=-\frac{2 \pi \phi}{c L} \\
r=\exp \left(\frac{2 \pi \psi}{C L}\right)
\end{gathered}
$$

It is therefore apparent that $x$ is a normalized form of the potential function and $r$ is the exponential of the normalized stream function

To this point the physical problem in the z-plane has been transformed to one in the $\zeta$-plane and two of the boundary conditions, Eqs 10 and 12 , have been satisfied by the complex potential, Eq 14 It therefore remains to satisfy the dynamic surface condition, Eq 11 , and this will be done by a proper cholce of the coefficients in the conformal transformation The remaining portion of this analysis is devoted to a method for calculating these coefficients so that the dynamic condition will be satisfied, approximately The degree of approximation will depend on the truncation of the infinite series which makes up the conformal transformation The greater the number of terms retalned, the more nearly the dynamic condition will be satisfied

Before proceeding to a calculation of the complex velocity, which will be needed in an examination of the Bernoulli condition on the free surface, it is necessary to substitute the complex potential in the $\zeta$-plane, Eq 14, into the conformal transformation, Eq 7 , in order to obtain the complex potential for the z-plane The result may be regarded as the general wave equation,

$$
z=\frac{w}{c}+\frac{1 L}{4 K} \sum_{J=1}^{\infty} \frac{a}{J} \exp \left[1 \text { am }\left(-\frac{4 J K}{c L} w\right)\right]
$$

For the limiting case of infinite depth, $K$ approaches $\pi / 2$ and am() approaches its argument Therefore Eq 17 reduces to the deep-water equation,

$$
z=\frac{w}{c}+\frac{2 L}{2 \pi} \sum_{J=1}^{\infty} \frac{a}{J} \exp \left[-1 \frac{2] \pi}{c L} w\right]
$$


on the other hand for the limiting case of lnfinlte wave length, lt should be noted that, after Eq 9 is introduced, $K$ approaches $\pi / 2$ and am () approaches gd(), the gudermannian Therefore, Eq 17 reduces to the solitary wave equation,

$$
z=\frac{w}{c}+\frac{12 D}{\pi} \sum_{j=1}^{\infty} \frac{a}{J} \exp \left[1 \operatorname{ga}\left(-\frac{J \pi}{2 c D} w\right)\right]
$$

Now along the free surface, $\psi=0$, where we wish to apply the dynamic boundary condition, Eq 17 becomes

$$
\mathrm{z}_{0}=\frac{\phi}{\mathrm{c}}+\frac{\mathrm{II}}{4 \mathrm{~K}} \sum_{\mathrm{J}=1}^{\infty} \frac{\mathrm{a} J}{\jmath} \exp \left[1 \text { am }\left(-\frac{4 \mathrm{~J}}{\mathrm{c} I} \phi\right)\right]
$$

or In terms of the dimensionless potential function, $x$,

$$
z_{0}=-\frac{L X}{2 \pi}+\frac{1 L}{4 K} \sum_{j=1}^{\infty} \frac{a}{J} \exp \left[1 \operatorname{am}\left(\frac{2 J K}{\pi} x\right)\right]
$$

Taking real and Imaginary parts of Eq 21 one obtalns

and

$$
x_{0}=-\frac{L X}{2 \pi}-\frac{I}{4 K} \sum_{J=1}^{\infty} \frac{a}{J} \sin \left(\frac{2 J K}{\pi} x\right)
$$

$$
y_{0}=\frac{I}{4 K} \sum_{\jmath=1}^{\infty} \frac{a}{\jmath} \operatorname{cn}\left(\frac{2 \jmath K}{\pi} x\right)
$$

wheresn(, and cn(, are Jacoblan elliptic functions These two equations are parametrically related through $x$ to define the shape of the free surface In order to make them somewhat more tractable, it is convenient to replace the elliptic functions by their expansions in sine and cosine series respectively (see Milne-Thomson (1950)) Eqs 22 and 23 therefore become

and

$$
x_{0}=-\frac{L}{4 K}\left(\frac{2 K X}{\pi}+\sum_{j=1}^{\infty} \frac{b}{J} \sin J x\right)
$$

$$
y_{0}=\frac{L}{4 K} \sum_{J=1}^{\infty} \frac{c}{J} \cos J x
$$

where

$$
b_{J}=\frac{2 \pi}{m^{1 / 2} k} \sum_{k=1}^{j} \frac{\frac{J}{k} a_{k} q^{J / 2 k}}{\left(1-q^{J / k}\right)}
$$

and

$$
c_{J}=\frac{2 \pi}{m^{1 / 2} K} \sum_{k=1}^{J} \frac{\frac{J}{k} a_{k} q^{J / 2 k}}{\left(1+q^{J / k}\right)}
$$

where

$$
k=3, \frac{2}{3}, \frac{3}{5}, 1
$$


and only integer values of $k$ are included in the summation, and where

$$
q=\exp \left(-\pi \frac{K^{\prime}}{K}\right)
$$

For the limiting case of deep water waves

$$
b_{J}=c_{j}=a_{J}
$$

whereas for the limiting case of the solitary wave

$$
b_{j}=\frac{4}{\pi} \sum_{k=1}^{J} a_{k}
$$

and

$$
\mathrm{Kc}_{\mathrm{J}}=\pi \sum_{\mathrm{k}=1}^{\mathrm{j}} \frac{\mathrm{J}}{\mathrm{k}} \mathrm{a}_{\mathrm{k}}
$$

with $k$ defined as for Eqs 26 and 27

In view of Eqs 24 and 25 it is now possible to reexpress the comples variable, $z$, that was given in Eq 21 , as follows

$$
\left.z_{0}=\frac{1 L}{2 \pi}\left[\ln \zeta_{0}+\frac{\pi}{2 K} \sum_{j=1}^{\infty}\left[\frac{c_{j}+b}{2} \zeta_{0}\right]+\frac{c_{j}-b}{2} \zeta_{0}-J\right]\right]
$$

where, on the free surface, Eq 8 reduces to

$$
\zeta_{0}=\exp (1 x)
$$

By differentiating Eq 32 , the complex operator

$$
\frac{d z_{0}}{d \zeta_{0}}=\frac{I I}{2 \pi} \frac{f\left(\zeta_{0}\right)}{\zeta_{0}}
$$

is obtained, where

and where

$$
\begin{aligned}
f\left(\zeta_{0}\right) & \left.=\frac{\pi}{2 k} \sum_{j=0}^{\infty}\left(\frac{\left.c_{j}^{+b}\right]}{2} \zeta_{0}\right]-\frac{c_{j}-b}{2} \zeta_{0}^{-j}\right) \\
& =R_{0} \exp \left(1 \theta_{0}\right), \text { say }
\end{aligned}
$$

$$
b_{0}=c_{0}=\frac{2 K}{\pi}
$$

Moreover, the modulus, $R_{0}$, of the function $f\left(\zeta_{0}\right)$ is given by

$$
R_{0}^{2}=\left[\frac{\pi}{2 k} \sum_{j=0}^{\infty} b_{J} \cos J X\right]^{2}+\left[\frac{\pi}{2 k} \sum_{J=0}^{\infty} c_{J} \sin J X\right]^{2}
$$

and the argument, $\theta_{0}$, of $f\left(\zeta_{0}\right)$ is given by 


$$
\theta_{0}=\cos ^{-1}\left[\frac{\frac{\pi}{2 k} \sum_{y=0}^{\infty} b_{j} \cos J x}{R_{0}}\right]
$$

The above relations may now be employed, together with Eq 14, to derlve an expression for the complex velocity, as follows

$$
u_{0}-1 v_{0}=\frac{d w_{0}}{d z_{0}}=\frac{d w_{0}}{d \zeta} \frac{d \zeta}{d z_{0}}=\frac{c}{f\left(\zeta \zeta_{0}\right)}=\frac{c}{R_{0}} \exp \left(-1 \theta_{0}\right)
$$

and therefore

$$
u_{0}=\frac{C}{R_{0}} \cos \theta_{0} \text { and } v_{0}=\frac{c}{R_{0}} \sin \theta_{0}
$$

and furthermore

$$
q_{0}^{2}=\frac{c^{2}}{R_{0}^{2}}
$$

The dynamic free surface condition, Eq 11, may now be written

$$
\frac{c^{2}}{R_{o}^{2}}+2 g y_{0}=k_{0}
$$

an expression which was apparently first derived by Nekrasov (1951) who proceeded to derlve a non-linear integral equation In addition, with the ald of the following dimensionless terms

$$
\begin{array}{ll}
x_{0}^{\prime} \equiv \frac{4 K}{L} x_{0}=\frac{K^{\prime}}{D} x_{0} & y_{0}^{\prime} \equiv \frac{4 K}{L} y_{0}=\frac{K^{\prime}}{D} y_{0} \\
c^{\prime} \equiv \frac{c}{\sqrt{\frac{g L}{4 K}}}=\frac{c}{\sqrt{\frac{g^{\prime}}{K^{\prime}}}} & \hat{K}_{0} \equiv \frac{K_{0}}{\frac{g L}{4 K}}=\frac{K_{0}}{\frac{g D}{K^{\prime}}}
\end{array}
$$

Eq 43 becomes

$$
c^{2}+2 y_{0}^{1} R_{0}^{2}=\hat{k}_{0} R_{0}^{2}
$$

where

$$
y_{0}^{\prime}=\sum_{j=1}^{\infty} \frac{c}{\jmath} \cos J x
$$

and, with the ald of trigonometric identities,

$$
R_{0}^{2}=\left(\frac{\pi}{2 k}\right)^{2}\left[D_{0}+2 \sum_{\jmath=1}^{\infty} D_{j} \cos J x\right]
$$

where

$$
D_{0}=b_{0}^{2}+\sum_{k=1}^{\infty} \frac{b_{k}^{2}+c_{k}^{2}}{2}
$$




$$
\begin{aligned}
& \mathrm{D}_{\mathrm{J}}=\mathrm{A}_{\mathrm{J}}+\mathrm{B}_{\mathrm{J}}+\mathrm{C}_{\mathrm{J}} \quad \mathrm{J}=1,2,3,4 \\
& A_{\jmath}=\sum_{k=j}^{\infty} \frac{b_{k-\jmath} b_{k}+c_{k-\jmath} c_{k}}{2} \quad j=1,2,3,4
\end{aligned}
$$

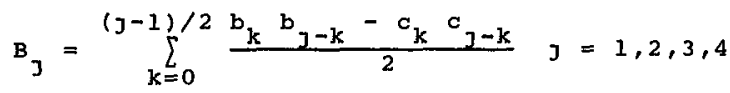

and

$$
\begin{aligned}
c_{J} & =\frac{1}{4}\left(b_{J / 2}^{2}-c_{j / 2}^{2}\right) & & \text { for } J=2,4,6,8 \\
& =0 & & \text { for } J=1,3,5,7
\end{aligned}
$$

By substituting Eqs 46 and 47 into $\mathrm{Eq} 45$, the problem of the general symmetrical wave of finite amplitude is reduced to one of finding the solution to the equation,

$$
\left.\left.c^{2}+2\left[\left(\sum_{j=1}^{\infty} \frac{c_{j}}{j} \cos \right] X\right)-\frac{\hat{K}_{o}}{2}\right]\left[\left(\frac{\pi}{2 K}\right)^{2}\left(D_{0}+2 \sum_{j=1}^{\infty}\left(A_{j}+B_{J}+C_{j}\right) \cos \right] X\right)\right]=0
$$

It is of interest to note that to this point no approximations have been made Therefore Eq 53 is an exact representation of the problem

In order to solve Eq 53 for a finite number of coefficients, it will be necessary to truncate the infinite trigonometric series which appear in the equation Therefore Eqs $46,47,48$ and 50 become

$$
\begin{aligned}
& \mathrm{y}_{0}^{\prime} \simeq \sum_{\mathrm{j}=1}^{\mathrm{n}} \frac{\mathrm{c} J}{\mathrm{~J}} \cos \mathrm{Jx} \\
& \left.R_{0}^{2}=\left(\frac{\pi}{2 K}\right)^{2}\left[D_{0}+2 \sum_{j=1}^{n} D_{j} \cos \right] x\right] \\
& D_{0} \simeq b_{0}^{2}+\sum_{k=1}^{n} \frac{b_{k}^{2}+c_{k}^{2}}{2} \\
& A_{J} \simeq \sum_{k=j}^{n} \frac{b_{k-\jmath} b_{k}+c_{k-\jmath} c_{k}}{2} \quad J=1,2,3,4 \quad n
\end{aligned}
$$
obtains

Furthermore, by combining and expanding these equations, one

$$
y_{0}^{\prime} R_{0}^{2} \simeq\left(\frac{\pi}{2 K}\right)^{2}\left[\sum_{k=1}^{n} \frac{c_{k}}{k} D_{k}+\sum_{j=1}^{n} \sum_{k=1}^{n} \frac{c_{k}}{k}\left(D_{k-j}+D_{k+j}\right) \cos j x\right]
$$

where absolute value signs are omltted on the subscripts of $D_{k-J}$, and furthermore, $D_{\ell}=0$ if $|\ell|>n$ since harmonics higher than the nth have been omitted, Eq 58 is not exact 
The expressions for $R^{2}$, Eq 55 , and $y^{\prime} R^{2}$, Eq 58 , may now be substituted into Eq $45^{\circ}$ By equating the coefficients of the harmonics, one obtains

$$
\begin{aligned}
& \mathrm{c}^{2}+2\left(\frac{\pi}{2 \mathrm{~K}}\right)^{2} \sum_{\mathrm{k}=1}^{\mathrm{n}} \frac{\mathrm{c}_{\mathrm{k}}}{\mathrm{k}} \mathrm{D}_{\mathrm{k}}=\hat{\mathrm{K}}_{\mathrm{o}}\left(\frac{\pi}{2 \mathrm{~K}}\right)^{2} \mathrm{D}_{\mathrm{o}}(0 \mathrm{th} \text { harmonic }) \\
& \begin{array}{l}
\sum_{k=1}^{n} \frac{C_{k}}{k}\left(D_{k-j}+D_{k+j}\right)=\hat{K}_{0} D_{J}(J \text { th harmonic, } J=1,2,3 \quad n) \quad(60) \\
\text { where absolute value signs are omitted on the subscripts of } D_{k-J} \\
\text { and furthermore } D_{\ell} \text { vanishes if }|\ell|>n
\end{array} \\
& \text { Since the unknown terms in Egs } 59 \text { and } 60 \text { are all functions of }
\end{aligned}
$$
using Eqs 23 and 9 ,

$$
\begin{aligned}
H=\left(y_{0}\right)_{x=0}+\left(-y_{0}\right)_{x=\pi}=\frac{L}{2 k} \sum_{j=1}^{n} \frac{a}{J} & =\frac{2 D}{k^{\prime}} \sum_{j=1}^{n} \frac{a}{J} \\
J & =1,3,5,7
\end{aligned}
$$

and in dimensionless form,

$$
H^{\prime}=2 \sum_{j=1}^{n} \frac{a_{j}}{J} \quad J=1,3,5,7
$$

Eqs 59,60 and 62 are therefore seen to constitute a set of $(n+2)$ equations in $(n+2)$ unknowns ( $c^{\prime}, \hat{K} \circ a_{1}, a_{2}, a_{3} a_{n}$ ) for any desired
value of the dimensionless wave helght, ${ }_{H}, 3$,

\section{COMPUTER SOLUTION}

In setting up the equations for computer solution, the coefficient, $\hat{k}$, is eliminated between the first of Eqs 60 ( $(j-1)$ and each succeeding equation $(j=2,3 \quad n)$, thereby reducing Eqs 60 to $(n-1)$ equations in $(n-1)$ unknowns $\left(a_{1}, a_{2}, a_{n-1}\right)$ for a fixed value of a After the unknown coefficients are assumed, the simultaneous solution of these $(n-1)$ non-linear equations is accomplished with the ald of a Newton-Raphson iteration By this technique the problem is reduced to one of obtaining the solution of a set of $(n-1)$ linear equations at each iteration The matrix is then inverted using triangular decomposition and a solution of the set of equations is obtained for corrections on the assumed values of $a_{1}, a_{2}$, $a_{n-2}$ and $a_{n-1}$ The entire procedure 1 s repeated until the corrections are simall enough to be neglected After the coefficients have been computed, Eqs 61,60 (first harmonic) and 59 are solved to yıeld $H^{\prime}, \hat{k}$ and $c^{\prime}$ respectively The entire procedure 1 s repeated lteratively until the coefflcients $\hat{K}$ and $c$ ' are evaluated for uniform-interval values of $\mathrm{H} / \mathrm{L}$, which are appropriate for tabular presentation 
Computations were made on the University of Wisconsin UNIVAC 1108 computer A fifth order solution was undertaken and some preliminary results are shown in Tables 1 and 2 In these tables the dimensionless wave helght, H/L, is calculated correct to \pm 000001 , while all other terms appearing in the tables were computed correct to at least the last place shown It should be noted that this precision is significant for the lower values of wave helght where the convergence of the a series is rapid and a fifth order solution is sufficient As the wave height increases, however, the truncation of the trigonometric series which replace the elliptic functions as well as the omission of higher harmonics in the development of $\left(Y_{0}^{\prime} R_{0}^{2}\right)$, result in a less accurate satisfaction of Eq 53 As a consequence the dynamic boundary condition, Eq 5, is only satisfied approximately By developing higher order solutions, greater than the fifth order solution considered here, the accuracy can be improved

\section{DISCUSSION}

No specific attempt will be made at this point to compare the new theory with those which exist The primary objective at the present is to develop the method of solution and to prepare sample tables of the coefficients

Nevertheless it is already possible to indicate some agreement with the existing theories In an earlier paper, Monkmeyer and Kutzbach (1965) compare the theory with that of stokes (1880) to reveal that the basic equation for deep water, Eq 18, is common to both theories stokes was, of course, limited in his ability to carry out computations to higher orders, and so restricted his attention to the well-known fifth-order theory The success of this theory in deep water has been the prime stimulus for using a fifth order approach in the present work Following stokes, Wilton (1914) developed a twelfth order solution for deep water waves and Monkmeyer and Kutzbach (1965) developed a fifteenth order solution These higher order computations resulted in little deviation from the fifth and third order theories, especially in the prediction of wave speed only in wave shape did the fifteenth order theory diverge from the lower order theories, as might be expected

Wave speed may be computed with the ald of Eq 59 However, since this equation demands considerable computation, a more convenient approach is to print out the wave speed together with the wave coefficients as shown in Tables 1 and 2 The wave speeds obtalned in Table 1 show excellent agreement with those of stokes' third and fifth order theorles for deep water waves (see Monkmeyer and Kutzbach, 1965)

In order to describe the wave shape or profile of a wave, one may choose to use the parametric set of equations,

$$
x_{0}=-\frac{L X}{2 \pi}-\frac{L}{4 K} \sum_{J=1}^{n} \frac{a}{J} \sin \left(\frac{2 \jmath K}{\pi} X\right)
$$


Table 1

FIFTH ORDER WAVE COEFFICIENTS

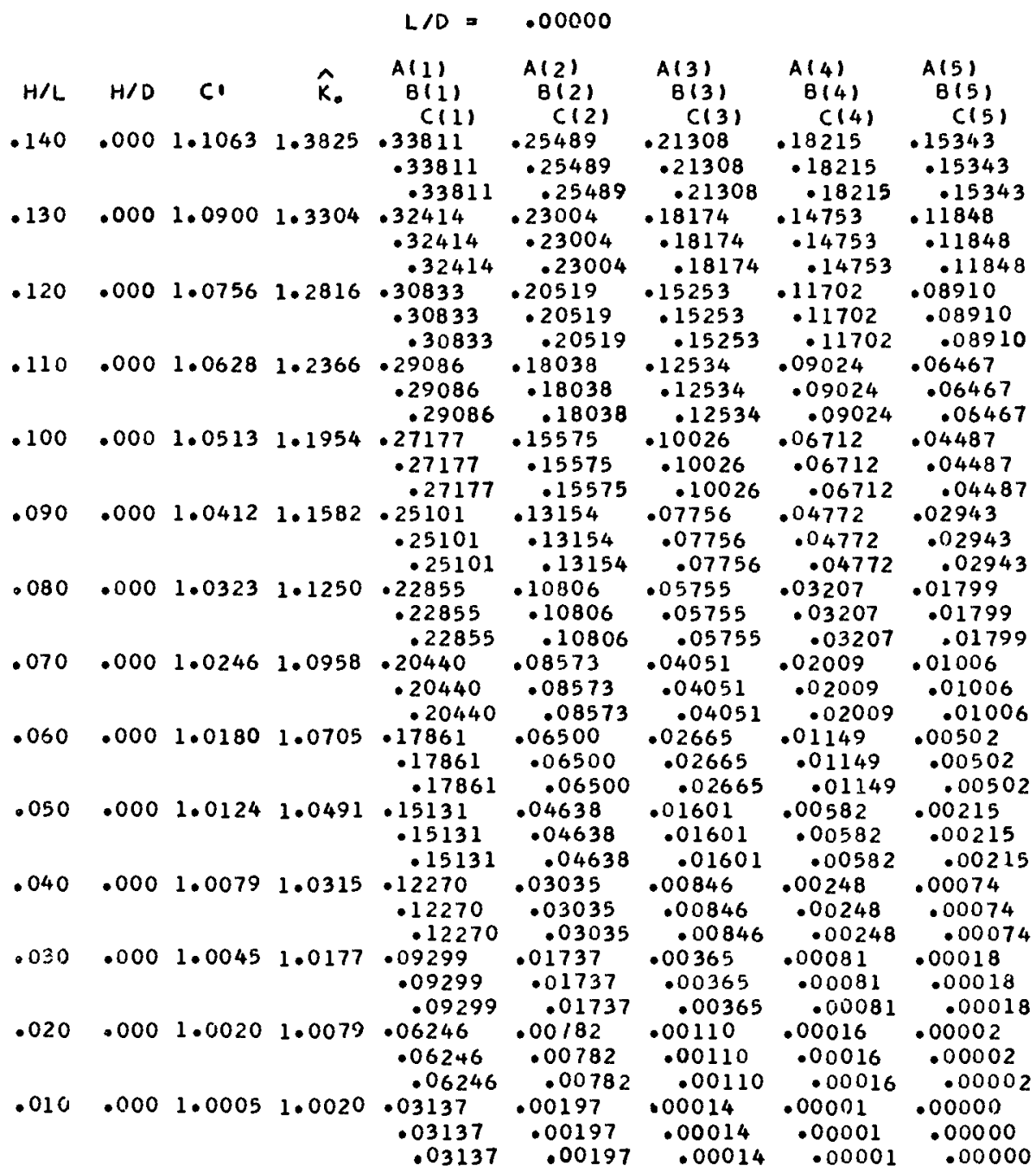

VALUES OF THE COMPLETE ELLIPTIC INTEGRAL OF THE FIRST KIND

$K=1.5707963 \quad K=I N F . \quad M=0.0000000 \quad K 1 / K=I N F$. 
Table 2

FIFTH ORDER WAVE COEFFICIENTS

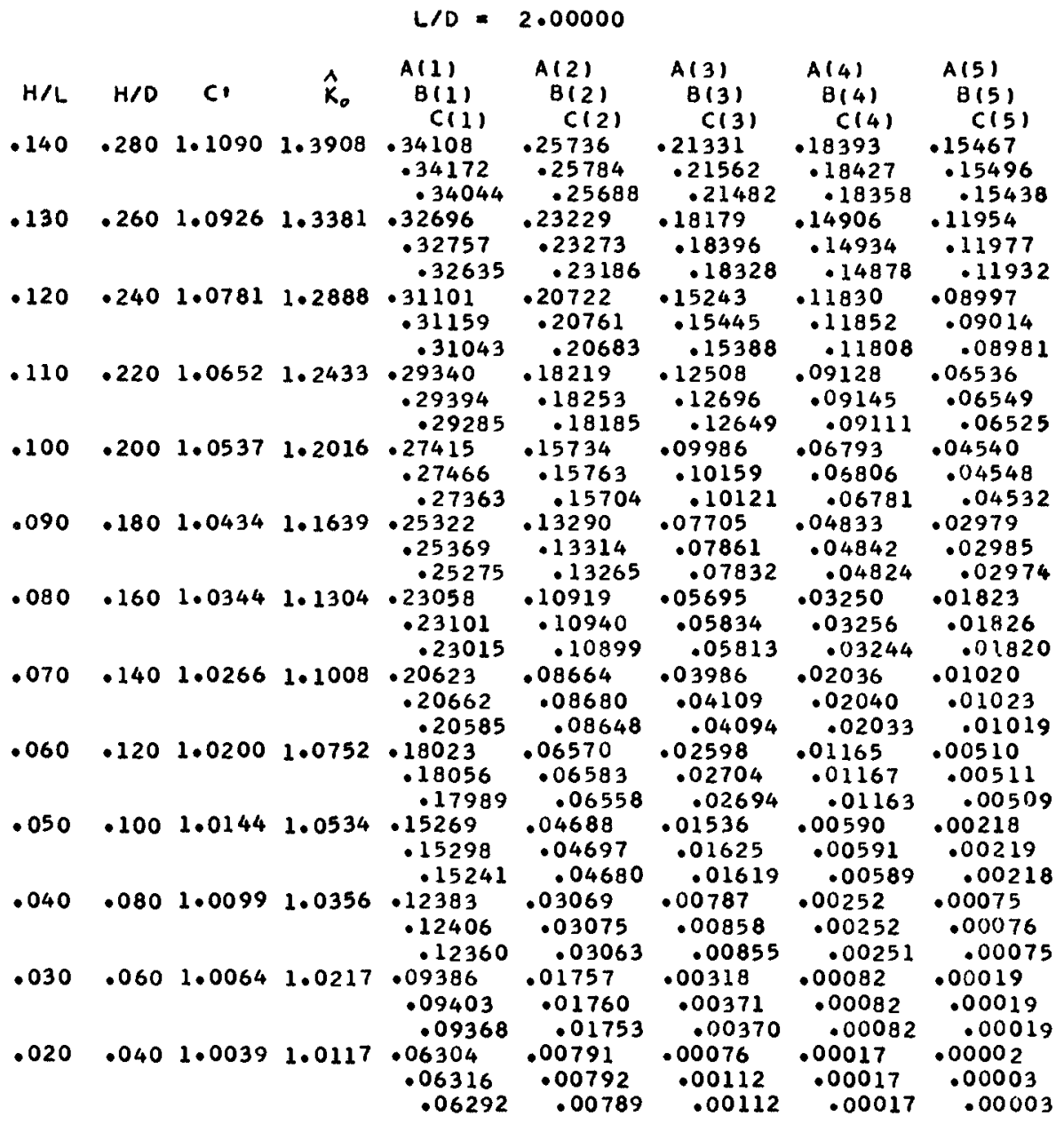

VALUES OF THE COMPLETE ELLIPTIC INTEGRAL OF THE FIRST KIND

$K=1.5825517 \mathrm{Kl}=3.1651034 \mathrm{M}=.0294372 \mathrm{~K} / \mathrm{K}=2.0000000$ 


$$
y_{0}=\frac{L}{4 k} \sum_{j=1}^{n} \frac{a_{J}}{\jmath} \operatorname{cn}\left(\frac{2 \jmath K}{\pi} x\right)
$$

or to avold the elliptic functions, but at the expense of some accuracy,

$$
\begin{aligned}
& x_{0}=-\frac{L x}{2 \pi}-\frac{L}{4 K} \sum_{j=1}^{n} \frac{b}{J} \sin J x \\
& y_{0}=\frac{L}{4 K} \sum_{j=1}^{n} \frac{c J}{J} \cos J x
\end{aligned}
$$

Numerlcal values relating $x_{0}$ and $y_{0}$ may be obtalned by substituting arbitrary values of the normalized potential function, $x$ This procedure is adequate for a graphical presentation of the wave shape stokes (1880) suggests that, with the ald of Lagrange's theorem (Whittaker and watson, 1963), the two equations may be reduced to one

It should be observed that the depth, D, differs from the depth to stillwater, d, by the elevation of the stillwater level, $y$ (see Fig 1) The computation of $y_{s}$, and therefore, d, may be accómplished by noting that the net area bounded by the free surface and the stillwater level vanishes Therefore

$$
0=\int_{0}^{L / 2}\left(y_{0}-y_{s}\right) d x_{0}
$$

By substituting the parametric profile expressions, Eqs 24 and 25 , the equation may be solved for the dimensionless stillwater elevation

$$
Y_{s}^{\prime}=\frac{\pi^{2}}{4 K} \sum_{k=1}^{n} \frac{b_{k} c_{k}}{k}
$$

Sample profiles for deep water waves are presented by Monkmeyer and Kutzbach

In order to study the characteristics of the highest possible wave it will be necessary to add one further restriction to those 1 mposed by Eqs 59, 60 and 61 This restriction, which was first suggested by Stokes, affects the Bernoulli equation which describes the dynamic upper boundary condition In effect stokes suggests that for a fluid particle on the surface to reach the highest possible point above the surface, the wave crest, it must give up all of its kinetic energy Hence at this point it has no velocity and the crest is a stagnation point The dynamic boundary condition, Eq 45, therefore reduces to

$$
2 y_{0}^{\prime}=\hat{\mathrm{K}}_{0} \quad \text { at } r=1, x=0
$$

or substituting for $y_{0}$ ' with the aid of Eq 23

$$
2 \sum_{k=1}^{n} \frac{a_{k}}{k}=\hat{k}_{0}
$$


Since this equation adds no new unknowns to those already appeaxlng $1 \mathrm{n}$ Eqs 59,60 and 62 its 1 nclusion results $1 \mathrm{n}$ a set of $(n+3)$ equations with ( $n+3)$ unknowns, $H$ ' no longer being axbitraxy but now considered as an unknown

For the deep water case using the fifteenth order theory Monkmeyer and Kutzbach show that

$$
(\mathrm{H} / \mathrm{L})_{\max }=01442
$$

Thls compares well with Michell's (1893) result of

$$
(H / L)_{\max }=0142
$$

and Havelock's (1919) conclusion that

$$
(\mathrm{H} / \mathrm{L})_{\max }=01418
$$

No precise computations of the highest wave have been made for the flnite waves considered herein However, Eq 57 has been used to determine whether or not the wave data obtalned in the preliminary computations includes waves that exceed the highest This check showed for example that for $L / D=60$, the maximum wave lies between $H / L=13$ and 14 , which is in good agreement with the breaking index curve of Reid and Bretschnelder (1953)

\section{CONCLUSION}

A higher order wave theory has been developed for the full range of waves from stokes waves to cnoldal waves to the solitary wave and from small amplitude waves to finite amplitude waves and the "highest wave' By means of a conformal transformation the problem is reduced to obtalning a solution to a non-linear set of equations Solutions of these equations using a high speed digltal machine have been obtained to fifth order for $L / D$ values of $00,20,40$ and 60 , and samples of this data are presented in tabular form

A consideration of some preliminary results as well as earlier results of the deep water case, suggest that the theory is in good agreement with existing theories Furthermore, it appears that this theory may provide a comprehensive practical means for wave analysis of the full-range of symmetrical waves from deep-water to shallow-water

\section{ACKNOWLEDGEMENTS}

The derivation of the basic theory was completed while the writex was a guest researcher at the River and Harbor Laboratory of the Technical University of Norway, Trondhelm, Norway in particulax the writer wishes to thank Director $H$ Berge and Dr $T$ Carstens for providing him with the opportunity to complete the theoretical analysis

Also, this research was supported in part by the National science Foundation and the state of Wisconsin under the university of Wisconsin sea Grant Program 
REFERENCES

Alry, $G$ B (1845) Tldes and waves Encyclop Metropol, London

Boussinesq, J (1872) Theorle des onde et de remous qui se propagent le long d'un canal rectangulalre horlzontal, en communiquant au liquide contenu dans ce canal des vitesses sensiblement parellies de la surface au fond, J Math Pures Appliquees, ser 2, vol 17 , pp 55-108

Dean, R G (1965) stream function wave theory validity and application Proceedings - A S C C Coastal Englneering Conference, Santa Barbara, 1965, pp 269-300

Havelock, $T H$ (1919) Perlodic irrotational waves of finite height Proc Roy Soc London, ser A, vol 95, pp 38-5l

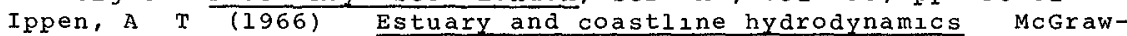
Hlll Book Co, New York

Kinsman, $B$ (1965) Wind waves Prentice-Hall, Inc, Englewood Cliffs, $N$ J

Korteweg, $D$ J and $G$ Devries (1895) on the change of form of long waves advancing in a rectangular canal and on a new type of long stationary waves Phl Mag, 5th ser, vol 39, pp 422-443

Ialtone, E V (1963) Higher approximation to nonlinear water waves and the limiting helghts of cnoldal, solitary and stokes' Waves Technical Memorandum No 133 , Beach Erosion Board, Corps of Engineers, U $S$ Army (now Coastal Engineering Research Center) Levi-Civita, $T$ (1925) Determination rigoureuse des ondes d'ampleur Elnie Math Annalen, vol 93, pp 264-314

Mash, F D and R L Wlegel (196i) Cnoldal waves-Tables of functions Council on Wave Research, The Englneering Foundation, University of California, Richmond, California

Michell, J $\mathrm{H}$ (1893) on the highest waves in water Phl Mag., ser 5 , vol 36 , pp $430-437$

Milne-Thomson, L M (1969) Theoretical hydrodynamics Fifth edition, Macmilian Co, New York, pp 428-435

Milne-Thomson, L M (1950) Jacobian elliptic function tables Dover Publications, Inc, $p$

Monkmeyer, $P$ L and $J$ E Kutzbach (1965) A higher order theory for deep water waves proceedings - A S E Coastal Engineering Conference, Santa Barbara

Nekrasov, A I (195I) The exact theory of steady waves on the surface of a heavy fluld Izdat Akad Nauk, SSSR, Moscow, Mathematical Reviews 15 (1965), 654 (translated by Math Res Ctr U S Army, Jniv of Wis)

Neumann, $G$ and $W$ J Plerson, Jr (1966) Principles of Physical oceanography Prentice-Hall, Inc, Englewood Cliffs, N J

Raylelgh, Lord, (1876) on waves Phl 1 Mag, ser 5, vol 1 , pp 257-279, also Scientific Papers, vol I, Cambridge University Press, 1899, pp 252-27I

Reid, $R$ and $C$ L Bretschnelder (1953) Surface waves and offshore structures, Texas A M Research Foundation, Technical Report, October

Skjelbreia, L and J A Hendrickson (1962) Fifth order gravity wave theory with table of functions National Englneering Science Co, Pasadena, Calif

stoker, J J (1957) Water waves Interscience Publishers, Inc, New York 
stokes, $G$ G (1847) on the theory of osclilatory waves Trans Camb Phl Soc, vol 8, p 441, also Mathematical and physical Papers, vol 1 , Cambridge Unıversity Press, 1880, pp 197-229

stokes, G G (1880) Supplement to a paper on the theory of oscillatory waves Mathematical and physical Papers, vol 1 , Cambridge Unıversity Press, pp 314-326

Strulk, D J (1926) Determination rigoureuse des ondes Irrotationnelles periodiques dans un canal a profondeur finie Mathematische Annalen, vol 95, pp 595-634

Thomas, J W (1968) Irrotational gravity waves of finite height a numerical study Mathematika, vol 15, part 2, Dec, pp $139-$ 148

Whittaker, $E$ T and watson, $G$ N (1963) A course of modern analysis 4 th edition, Cambridge University Press, Cambridge

wiegel, R L (1964) Oceanographical englneering Prentice-Hall, Inc, Englewood Cliffs, N J

wilton, $J$ R (1914) On deep watex waves Phil Mag, ser 6, vol 27 , pp $385-394$

\section{APPENDIX - NOTATION}

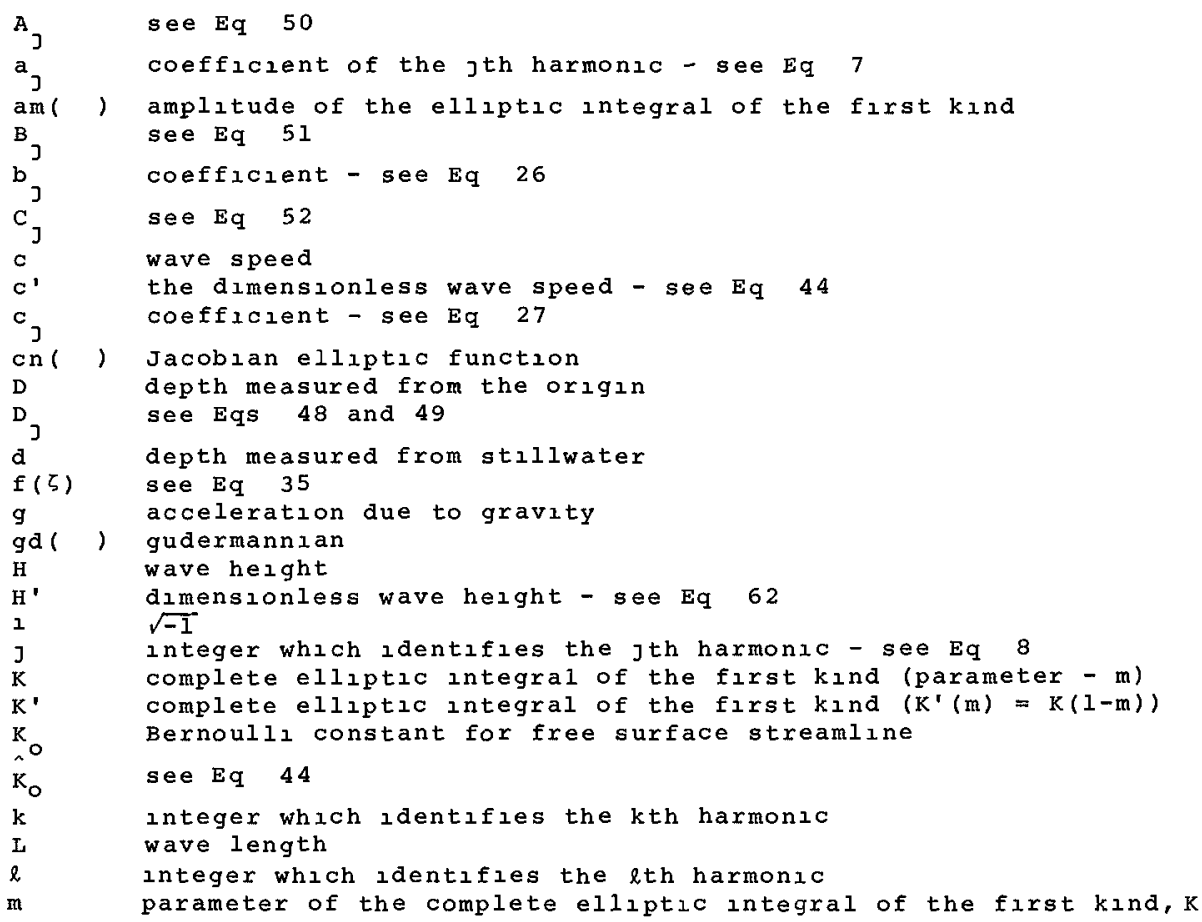


n

$q_{0}$

R。

$r$

$r_{c}$

sn

$u$

u

$v$

$v$

w

$\mathbf{x}$

$x$ '

$x$

$x_{0}^{\prime}$

Y

$y^{\prime}$

$y_{0}$

$y_{0}^{\prime}$

$y_{s}$

$\mathrm{y}_{\mathrm{s}}^{\prime}$

$z$

zo

$\zeta$

$\zeta_{0}$

$\theta$ 。

integer which ldentifies the highest harmonic and the order of the analysis

magnitude of the particle velocity at the free surface

modulus of $f\left(\zeta_{0}\right)-$ see $\mathrm{Eq} 38$, also $=\mathrm{c} / \mathrm{q}_{0}$

radial coordinate in the $\zeta$-plane

radius of AE in the $\zeta$-plane

Jacoblan elliptic function

$x$-component of the particle velocity

$x$-component of the particle velocity at the free surface

$y$-component of the particle velocity

$y$-component of the particle velocity at the free surface

complex potential $\equiv \phi+1 \psi$

horizontal coordinate in the z-plane

dimensionless horizontal coordinate

horizontal free surface coordinate in the $z$-plane

dimensionless horızontal free surface coordinate

vertical coordinate in the z-plane

dimensionless vertical coordinate

vertical free surface coorainate in the z-plane

dimensionless vertical free surface coordinate

still water elevation in the z-plane

dimensionless still water elevation

$\equiv \mathbf{x}+1 y$ and refers to the physical plane

complex variable at the free surface

$\equiv r \exp (1 x)$ and refers to the auxiliary plane

$=\exp (1 \chi)$, or $\zeta$ at the free surface

argument of $f(\zeta)$ - see Eq 39, also local slope angle of

the free suriace in the z-plane

$\equiv 31415927$

potential function

tangential coordinate in the $\zeta$-plane, also a normalized form of the potential function

stream function 



\section{CHAPTER 34}

ANALYTICAL APPROACH ON WAVE OVERTOPPING ON LEVEES H1royosh1 Sh1-1ga1, Dr Eng, M Eng, M of JSCE.* Tsug1o Kono, B Eng, $M$ of JSCE**

\section{Analytical Approach}

An analytical approach to evaluate the amount of overtopping for given conditions is studied in this paper Here, the wave overtopping is considered as a similar phenomenon to the flow over a welr changing the depth with respect to time

The theoret1cal approaches used by many scholars have been mostly based on the dimensional analysis In this paper, however, another kind of approach is tried, which is rather deterministic, in order to get a general vlew of wave overtopping mechanism

The well-known formula which expresses the discharge over a sharpedged weir is as follows

$$
q=\frac{2}{3} m \sqrt{2 g} y^{\frac{3}{2}}
$$

where $q$ is the discharge per unit width, in is the discharge coefficient and $y$ is the overflow depth It is usually admitted that Eq (1) is valid only for steady flow However, if we assume that $y$ does not change very rapldly with respect to tıme, Eq (1) may be used for the analysis of wave overtopping Writing that

$$
y=z(t)-z_{0}
$$

\footnotetext{
* Associate Professor, Aslan Institute of Technology, Bangkok, Tha1land

** Research Associate, Department of Givil Englneering, Tokyo Institute of Technology, Tokyo, Japan
} 
where $z(t)$ is the surface elevation of waves over the levee measured from SWL, and $z_{0}$ is the elevation of the top of the levee, we can obtain the quasi-steady equation for the overtopping discharge

$$
q(t)=\frac{2}{3} m \sqrt{2 g}\left(z(t)-z_{0}\right)^{\frac{3}{2}}
$$

Further, we write $z(t)$ as

$$
z(t)=\underset{m}{z} F(t)
$$

where $F(t)$ is a non-dimensional function of time which expresses the wave profile at the levee $\quad z_{m}$ is approximately equal to the wave run-up height but not the same one, because normally wave run-up height $R$ is measured without any overtopping If there is overtopping, the reflection rate must change and $R$ and $z_{\mathfrak{m}}$ cannot be of the same value Using Eq (2), Eq. (3) and Eq (4), we obtain.

$$
\begin{array}{ll}
q=\frac{2}{3} m \sqrt{2 g}\left(\mathrm{kH}_{0}\right)^{\frac{3}{2}}\left\{F(t)-\frac{z_{0}}{k_{0}}\right\}^{\frac{3}{2}} & \text { for } F(t) \geqq \frac{z_{0}}{z_{m}} \\
q=0 & \text { for } F(t)<\frac{z_{0}}{z_{m}}
\end{array}
$$

where $k$ is defined as:

$$
k=z_{m} / H_{0}
$$

If $m$ and $k$ are constant for a wave perıod, we integrate $q$ with respect to time

$$
\begin{aligned}
Q & =\int_{t_{1}}^{t_{2}} q d t \\
& =\frac{2}{3} m \sqrt{2 g}\left(k_{o}\right)^{\frac{3}{2}} \int_{t_{1}}^{t_{2}}\left\{F(t)-\frac{z_{o}}{k H_{o}}\right\}^{\frac{3}{2}} d t
\end{aligned}
$$


where $F(t)>\frac{z_{0}}{k H_{0}}$ for $t_{1}<t<t_{2} Q$ is the total discharge of overtopping for a period per unit width of the weir Eq (7) can be expressed as a non-dimensional form, which is

$$
\frac{Q}{\mathrm{TH}_{\mathrm{o}} \sqrt{2 \mathrm{gH}}}=\frac{2}{3} \frac{\mathrm{mk}^{\frac{3}{2}}}{\mathrm{~T}} \int_{\mathrm{t}_{1}}^{\mathrm{t}_{2}}\left\{\mathrm{~F}(\mathrm{t})-\frac{\mathrm{z}_{\mathrm{o}}}{\mathrm{kH}_{\mathrm{o}}}\right\}^{\frac{3}{2}} d t
$$

where $T$ is the wave period The left hand side is a kind of Froude Number Fig 1 and Fig 2 show the schematic diagrams for the definitions Eq (8) may be simplified if the wave profile can be approximated by triangular waves

$$
\frac{Q}{\mathrm{TH}_{\mathrm{o}} \sqrt{2 \mathrm{gH}_{\mathrm{o}}}}=\frac{2}{15} \mathrm{mk}^{\frac{3}{2}}\left(1-\frac{\mathrm{z}_{\mathrm{o}}}{\mathrm{kH}_{\mathrm{o}}}\right)^{\frac{5}{2}}
$$

For sinous waves we obtain Eq. (10)

$$
\frac{Q}{\mathrm{TH}_{\mathrm{o}} \sqrt{2 \mathrm{gH}_{\mathrm{o}}}}=\frac{4}{3} \mathrm{mk}^{\frac{3}{2}} \frac{1}{\mathrm{~T}} \int_{\mathrm{t}_{1}}^{\frac{\mathrm{T}}{4}}\left\{\sin \frac{2 \pi}{\mathrm{T}} t-\frac{\mathrm{z}_{\mathrm{o}}}{\mathrm{kH}_{\mathrm{o}}}\right\}^{\frac{3}{2}} \mathrm{dt}
$$

where $\quad t_{1}=\frac{T}{2 \pi} \sin ^{-1} \frac{\mathrm{z}_{\mathrm{o}}}{\mathrm{kH}_{\mathrm{o}}}$

Though Eq (9) looks very simple, it gives us fairly good results as is shown Fig 3, if we choose the value of $k$ carefully, while the value of $k$ is affected by so many factors Fig 4 shows the variation of $k$ with respect to $\mathrm{H}_{\mathrm{o}} / \mathrm{L}$ and $\alpha$, the slope of levees The value of $\mathrm{k}$ is much less than the value of $R / H_{o}$, which is for the case without overtopping, while both of them take the maximum value at $\tan \alpha=035$

Eq (9) may be arranged to ${ }_{A}^{\text {the }}$ conventional form obtained through dimensional analysis as follows 

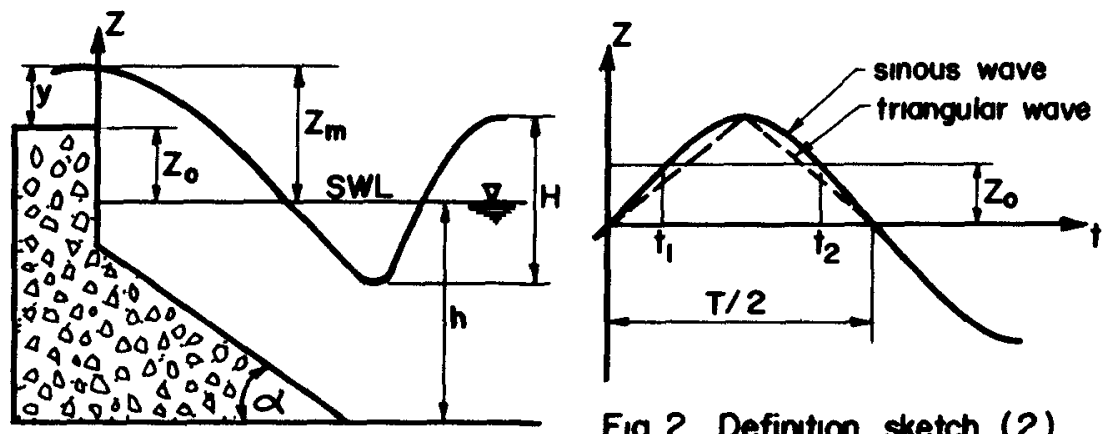

Fig 2 Definition sketch (2)

Fig I Definition sketch (1)

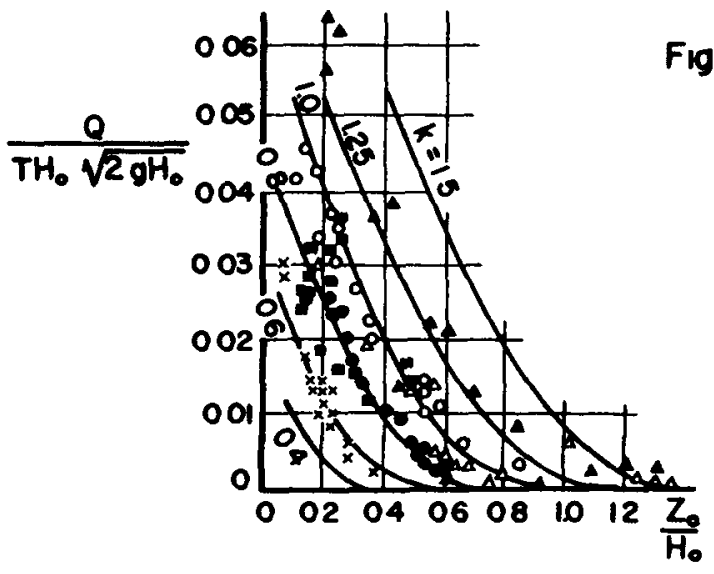

Fig $3 \mathrm{Eq}(9)$ and expermental data

\begin{tabular}{|c|c|c|c|}
\hline & $R U N$ & $\alpha, s$ & Note \\
\hline$x$ & 1 & $90^{\circ}$ & \\
\hline 0 & 2 & $30^{\circ}$ & \\
\hline$\bullet$ & 3 & $30^{\circ}, 90$ & \\
\hline$\Delta$ & 4 & $1 / 10,1 / 3$ & ., wind \\
\hline$\Delta$ & 5 & $V 10,1 / 6$ & $*$, wind \\
\hline- & 6 & $1 / 6$ & $*$ \\
\hline
\end{tabular}

- done by BE B

* done by WES

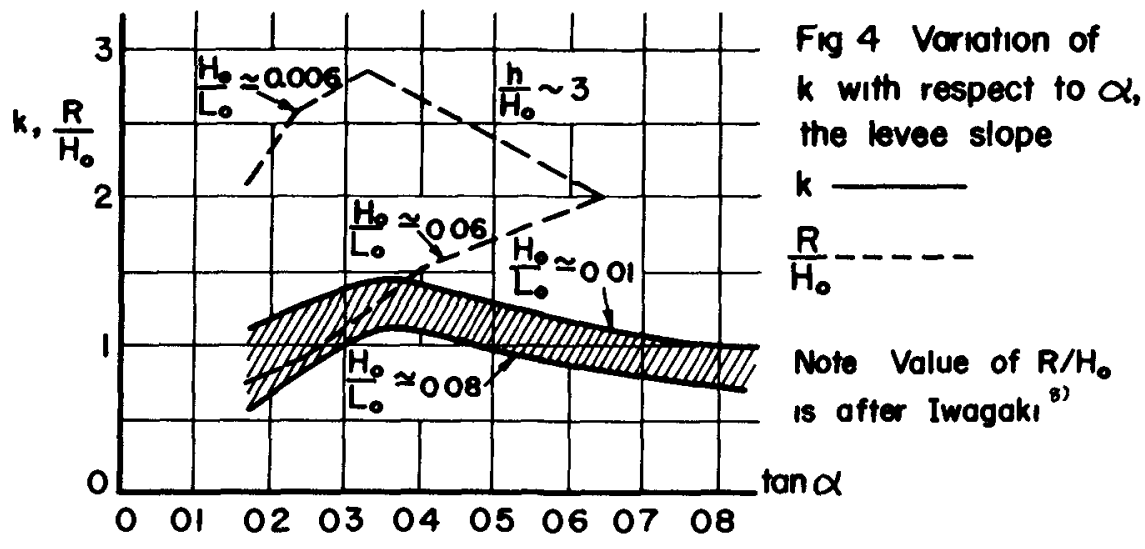




$$
\frac{2 \pi Q}{\mathrm{H}_{\mathrm{o}} \mathrm{L}_{\mathrm{o}}}=\frac{4 \pi}{15} \sqrt{2 \mathrm{gH}} \quad \frac{\mathrm{Tm}}{\mathrm{L}_{\mathrm{o}}}\left(1-\frac{\mathrm{z}_{\mathrm{o}}}{\mathrm{kH}_{\mathrm{o}}}\right)^{\frac{5}{2}}
$$

Normally, Eq (10) gives us the better results but Eq (9) also gives us a satisfactory result The effect of wind can also be expressed by the change of $k$, while the breakers' effect may not

\section{Computer Experiment}

For engineering purposes, what we need is the "prediction" Waveovertopping phenomenon is a combination of statistical and deterministic factors, since wave period and wave helght, for example, are statistical variables, while over-topping caused by a given wave can be a deterministic phenomenon In this section the authors treat each over-topping as a completely deterministic process for the given wave conditions, which may include some statistical characteristics The frequency response function being known, we can estimate the total amount of wave overtopping if all the informations of wave characteristics are available However, we face the following problem. Can we estumate the total amount of wave over-topping by the statistical parameters only ? Already this attempt has been done by Tsuruta and Goda by using authors' formula, Eq (9) They estimated the expected amount of overtopping for 1rregular waves by using $\mathrm{H}_{1 / 3}$ However, the authors would like to point out that the amount of over-topping depends upon the wave period as well as the wave height, and also that though Eq (9) 1 s dimensionless, it is not very convenient to use it directly as the response function, since it includes $H_{0}$ in both sides

Another important point which is related with the prediction problem is that the response function is highly non-linear Therefore the usual statistical values, $\mathrm{H}_{1 / 3}$ for example, may not be a suitable 
parameter at all In order to study this point, some numerical experiments were carried out at AIT in Bangkok

The following assumptions are made

1 For simplicity Equation (9) is used instead of sinuous wave formula in the modified form

2 Wave height $H_{0}$ and Wave period $T$ follow Rayleigh distribution

3 Cross correlation of $\mathrm{H}_{0}$ and $\mathrm{T}$ is very smal1

The modiflcation of $\mathrm{Eq}$ (9) $1 \mathrm{~s}$ as follows.

$$
\left.\begin{array}{rlrl}
\frac{Q}{\mathrm{z}_{0}^{2}} & =c^{\prime} \frac{(\zeta-1)^{5 / 2}}{\zeta} \tau & & (\zeta>1) \\
& =0 & & (\zeta \leqq 0)
\end{array}\right\}
$$

and

$$
\zeta=k \frac{H_{0}}{z_{0}}
$$

Eq (12) is convenient for prediction, since normally $z_{0}$ is given and the variable of left hand side is only $Q \quad H_{0} / \bar{H}_{0}$ and $T / \bar{T}$ may follow the following Rayleigh distributions

$$
\begin{aligned}
& \underset{\mathrm{p}\left(\frac{\mathrm{H}}{\mathrm{H}}\right)}{\overrightarrow{\mathrm{H}}_{\mathrm{O}}}=\frac{\pi}{2} \frac{\mathrm{H}_{\mathrm{O}}}{\overline{\mathrm{H}}_{\mathrm{O}}} \exp \left[-\frac{\pi}{4}\left(\frac{\mathrm{H}_{\mathrm{O}}}{\overline{\mathrm{H}}_{\mathrm{O}}}\right)^{2}\right] \\
& \mathrm{p}\left(\frac{\mathrm{T}}{\overline{\mathrm{T}}}\right)=27\left(\frac{\overline{\mathrm{T}})^{3}}{\mathrm{~T}} \exp \left[-0.675\left(\frac{\mathrm{T}}{\overline{\mathrm{T}}}\right)^{4}\right]\right.
\end{aligned}
$$

where $\quad p()$ means the probability density functions, and $\overline{\mathrm{T}}$ and $\overline{\mathrm{H}}_{\mathrm{o}}$ mean the ensemble averages 
Eq (16) and Eq (17) are only examples, since in fully developed H seas $p\left(\frac{o}{-}\right)$ is Gaussian Anyhow the numerical experiment is always possible if $\mathrm{p}\left(\frac{\mathrm{H}_{\mathrm{O}}}{\overline{\mathrm{H}}_{\mathrm{O}}}\right)^{\overline{\mathrm{H}}_{\mathrm{O}}}$ and $\mathrm{p}\left(\frac{\mathrm{T}}{\mathrm{T}}\right)$ are given

The procedure of numerical experiment is as follows

1 Prepare the necessary values for the numerical experiments They are $\mathrm{Z}_{\mathrm{o}}, \overline{\mathrm{T}}, \overline{\mathrm{H}}_{\mathrm{o}}$ and $\mathrm{k}$ If the levee slope is given, $\mathrm{k}$ may be estimated from Fig 4 m can be 06

2 Compute $\mathrm{T} / \sqrt{\mathrm{Z}_{\mathrm{o}} / \mathrm{g}}$ and $\mathrm{k} \overline{\mathrm{H}}_{\mathrm{o}} / \mathrm{z}_{\mathrm{o}}$

3 Generate $\mathrm{H}_{0} / \overline{\mathrm{H}}_{\mathrm{O}}$ and $\mathrm{T} / \overline{\mathrm{T}}$ whlch are statistically random but follow the given probability density Normally, if they are random, the cross correlation is rather small However, we should compute the cross correlation always

4 Compute the values of $T$ and $\zeta$, which are expressed by Eq (14) and $\mathrm{Eq}(15)$

5 Compute $Q / Z_{0}^{2}$ by the use of $\mathrm{Eq}$ (12)

6 Repeat the above procedure for a certain number of times, say one hundred

7 Compute the summation of $\mathrm{Q} / \mathrm{z}_{\mathrm{o}}^{2}$ Also compute $\mathrm{H}_{1 / 3}$ and $\mathrm{T}_{1 / 3}$ statistically

The above procedure is enough for design purposes, since we can estimate the total amount of overtopping Through this information, we can determine, for instance, the capacity of the pump to drain the water 
exerted by overtopping

The numerical experiments were carried out for the case that $\overline{\mathrm{H}}_{0}$, $\overline{\mathrm{T}}, \overrightarrow{\mathrm{T}} / \sqrt{\mathrm{Z}_{\mathrm{o}} / \mathrm{g}}$ and $\mathrm{k} \overline{\mathrm{H}}_{\mathrm{o}} / \mathrm{Z}_{\mathrm{o}}$ are all equal to unity the computer used for the experiment is IBM 1130 The results are shown in Table 1

Each run contalns one hundred of waves and $H_{01 / 3}, T_{1 / 3}, \bar{H}_{0}$ and $\bar{T}$ are statistically obtained For comparison, $\mathrm{H}_{1 / 3}$ and $\mathrm{T}_{1 / 3}$, are computed by Eq (18) and (19)

$$
\begin{aligned}
& \text { 'Computed' } \mathrm{H}_{\mathrm{o} 1 / 3}=160 \overline{\mathrm{H}}_{\mathrm{o}} \\
& \text { 'Computed' } \mathrm{T}_{1 / 3}=\overline{\mathrm{T}}
\end{aligned}
$$

For each wave the value of $Q / \mathrm{Z}_{0}^{2}$ is computed and its mean value is obtained through Eq (20)

$$
\frac{\sum_{i=1}^{100} \frac{\left(\frac{Q}{2}\right)}{z_{o}^{2}}}{100}=\bar{q}_{m}
$$

Agaln for comparison, $\bar{q}_{\text {computed }}$ is obtalned, which is defined by

$$
\mathrm{c}_{\mathrm{O}}^{\prime}\left(\frac{\mathrm{T}}{\overrightarrow{\mathrm{T}})_{\frac{1}{3}}} \frac{\left\{\left(\mathrm{H}_{\mathrm{o}} / \overrightarrow{\mathrm{H}}_{\mathrm{o}}\right)_{1 / 3}-1\right\}^{\frac{5}{2}}}{\left(\mathrm{H}_{\mathrm{o}} / \overrightarrow{\mathrm{H}}_{\mathrm{o}}\right)_{1 / 3}}=\overline{\mathrm{q}}_{\mathrm{c}}\right.
$$

From Table 1 the values of $\vec{q}_{c}$ are always greater than those of $\vec{q}_{m}$, which means $\vec{q}_{c}$ is in safety side for design purposes Among seven runs of experiment, which contain 700 waves, there is a case that $\bar{q}_{\mathrm{m}}=0945 \bar{q}_{\mathrm{c}}$ Thls fact shows us that for a quick estimation, the use of $\mathrm{H}_{\mathrm{ol} / 3}$ and $\mathrm{T}_{1 / 3}$ may be a good approximation However, the numerical experiments are more desirable for the actual design purposes 
3

\section{Conclusions}

As conclusions we may say the followings

1 Eq (9) gives us a falrly good prediction for the discharge of wave overtopping, though it contains two empirical factors

2 The above empirical factors, however, do not change vigorously at least for the existing experimental data

3 Theoret1cally speaking, the use of $\mathrm{H}_{\mathrm{ol} / 3}$ and $\mathrm{T}_{1 / 3}$ for the estimation of overtopping are doubtful

4 According to the limited number of computer experiments, the estimated values of over topping obtained by the use of $\mathrm{H}_{01 / 3}$ and $\mathrm{T}_{1 / 3}$ are always greater than the statistically obtained values

5 However, this formula may be useful by the use of $\mathrm{H}_{01 / 3}$ and $T_{1 / 3}$ in order to get the first approximation of wave overtopping caused by a set of irregular waves

6 For the design purposes the computer experiment is highly recomended since already we obtained the response function which is expressed by Eq (9) or Eq (12) 
Table 1 - Results of Numerical Experiment

\begin{tabular}{|c|c|c|c|c|c|c|c|}
\hline \multirow{2}{*}{ Run } & \multicolumn{2}{|c|}{$\mathrm{H}_{1 / 3}$} & \multicolumn{2}{c|}{$\mathrm{T}_{1 / 3}$} & \multicolumn{2}{c|}{$\overline{(1) /(2)}$} \\
\cline { 2 - 9 } & Measured & Computed & Measured & Computed & $\begin{array}{c}\text { (1) } \\
\text { Measured }\end{array}$ & $\begin{array}{c}\text { (2) } \\
\text { Computed }\end{array}$ & \\
\hline 1 & 135 & 145 & 110 & 112 & $383 \times 1 \overline{0}^{3}$ & $121 \times 10^{3}$ & 0317 \\
\hline 2 & 144 & 135 & 110 & 105 & 443 & 636 & 0695 \\
\hline 4 & 149 & 149 & 110 & 110 & 735 & 14.1 & 0521 \\
\hline 5 & 133 & 131 & 104 & 100 & 479 & 814 & 0590 \\
\hline 6 & 139 & 139 & 104 & 100 & 546 & 713 & 0765 \\
\hline 7 & 143 & 1.42 & 100 & 1.08 & 569 & 980 & 0581 \\
\hline
\end{tabular}

Note: All are non-dimensional variables

"Measured" means statistically obtalned values

"Computed" means the values obtained by mean values

(for example $H_{1 / 3}=16 \overline{\mathrm{H}}$ )

Each run includes 100 of waves 


\section{B1bliography}

1 Carrier, G F and Greenspan, H P Water waves of finıte amplitude on a sloping beach, Jour of $\mathrm{F} \mathrm{M}$, Vol 4, 1958

2 Gei, C Report on the model test of sea-dike cross-section of HsinChu T1dal Land, The Tainan Hyd, Lab Bulletın No 6, Ta1wan, Chına

3 K1kkawa, H, Sh1-1ga1, H and Kono, $\mathrm{T}$ On the experimental study of over-topping over the vertical wall, The Report of Annual Conf of JSCE, 1966 (In Japanese)

4 K1kkawa, H, Sh1-1ga1, H and Kono, T Fundamental Study of wave over-topping on levees, Coastal Englneerıng in Japan, Vol 11, 1968

5 Saville, Jr, $\mathrm{T}$ : Wave run-up on shore structures, Proc ASCE, Vo1 82, 1956

6 Sibul, $0 \mathrm{~J}$ and Tickner, E G Model study of over-topping of wind generated waves on levees with slopes of 13 and 6 , Beach Erosion Board Tech Memo No 80, 1956

7 Tsuruta, S and Goda, Y.. Expected discharge of 1rregular wave overtopping, Proc of Coastal Engineering, Vol II, 1968, London

8 Iwagaki, $\mathrm{Y}$, Shıma, $\mathrm{A}$ and Inoue, $\mathrm{M}$ Effects of wave height and sea water level on wave overtopping and wave run up, Coastal Englneering 1n Japan, Vo1 8, 1965 



\title{
CHAPTER 35
}

\section{IMPULSE WAVES GENERATED BY LANDSLIDES}

\author{
J W Kamphuis \\ Associate Professor of Clyll Englneering, \\ Queen's Unıyersity at Kingston, Canada \\ R J Bowering \\ EngIneer, Manitoba Water Resources, \\ Winnipeg, Canada
}

\section{ABSTRACT}

A study programme has been inltiated to investigate the Impulse waves generated by landslides orlginating entirely above the water surface It may be seen that the characteristics of this wave depend mainly on the slide volume and the Froude number of the slide upon impact with the water The resulting wave goes through a transition period For the highest wave (usually the first), the wave height becomes stable relatively quickly and decays exponentially during the period of transition, the wave period continues to increase for a long time, the velocity of propagation may be approximated very closely by solıtary wave theory

\section{IITTRODUCTION}

Impulse waves generated by landslides have long been a menace In certaln localities and the study of this phenomenon has been carrled out at an accelerated rate in the $1960^{\prime} \mathrm{s}$, perhaps as a result of disasters of major proportions such as at Valont (2) In coastal areas, these slides are also not uncommon and Miller (4) gives a number of examples

An attempt is made in this paper to determine the relation between the generated impulse waves and the varlous parameters of the slide and the recelving body of water Some studies of underwater slides and of slides under simplifled conditions have been performed, Wiegel (9), Prins $(5,6)$ and Wlegel et al (10), but little is known about slides orlginating above the water surface, sliding into the body of water at various angles

Theoretical work has also been done for simplified generating conditions e g Unokı and Nakano (7), Kranzer and Keller (3) and Ursell (8) In this paper no attempt has been made to express the results on a theoretical basis

The purpose of the present paper is to approach a highly complex, non-linear problem entirely experimentally, invoking as little simplification as possible The test series is yet incomplete and the analysis is part of a continuing program 


\section{EXXERTMENTAL TECHNIQUE}

Experıments were carrıed out in a $45 \mathrm{~m}$ long flume, $1 \mathrm{~m}$ wade, with water depths, d, ranging from 23 to $46 \mathrm{~cm}$ I The landslides were modelled using a tray ( $F_{1 g}$ l), loaded with a varying number of units of constant specific gravity and of zero porosity The tray was placed at varlous distances above the water to roll down a roller ramp In Fig 2 this ramp is shown with the tray in position at the top of the ramp The angle of the ramp with the water surface, $\theta$, was adjustable, as was the front slope of the tray, $\alpha$ The tray was released using a quick release mechanism and the velocity of impact with the water, $v$, was measured using a combination of stroboscope lllumination and prolonged photographic exposure of a series of lines on the top of the tray

The resulting waves were measured continuously at three locations, 335,945 and $171 \mathrm{~m}$ from the point of impact These locations are called $a, b$, and $c$ respectively

The record from the first wave probe (a) was also squared and integrated by analogue methods The wave energy was calculated, using a digital computer programme, by a combination of

$$
E_{0}=\rho g C_{a} \int n^{2} d t
$$

and

$$
E_{s}=\frac{8}{3} \mathrm{H}^{3 / 2} \mathrm{~d}^{3 / 2}
$$

where $E_{0}$ Is the energy contalned in a deep water wave using osclllatory wave theory, $\mathrm{E}_{\mathrm{S}}$ the energy by solitary wave theory, $\rho$ the water density, $g$ the acceleration of gravity, $C_{a}$ the phase speed of the wave at location $a, n$ the wave record, $t$ the $t$ ime and $H$ the maximum wave height The phase speed $C_{a}$, was measured using the elapsed travel time between probe a and an on-off probe, located at still water level, very close to probe a

\section{DIMENSIONAL ANALYSIS}

For the experimental study, any property, A, of the wave may be stated as a function of the following parameters

$$
A=f\left(l, w, h, v, \beta, \theta, p, \rho_{s}, \rho, \mu, B, d, x, t\right)
$$

where $l$, w and $h$ are the length, wadth and thlckness of the slide, $\beta$ is the angle between the front face of the slide and the horizontal $(\beta=\theta+\alpha), \rho_{s}$ is the density of the slide, $\mu$ is the dynamic viscosity of the water, $x$ is the distance from the point of Impact and $t$ is the elapsed time

1 A summary of the notation may be found at the end of the paper 


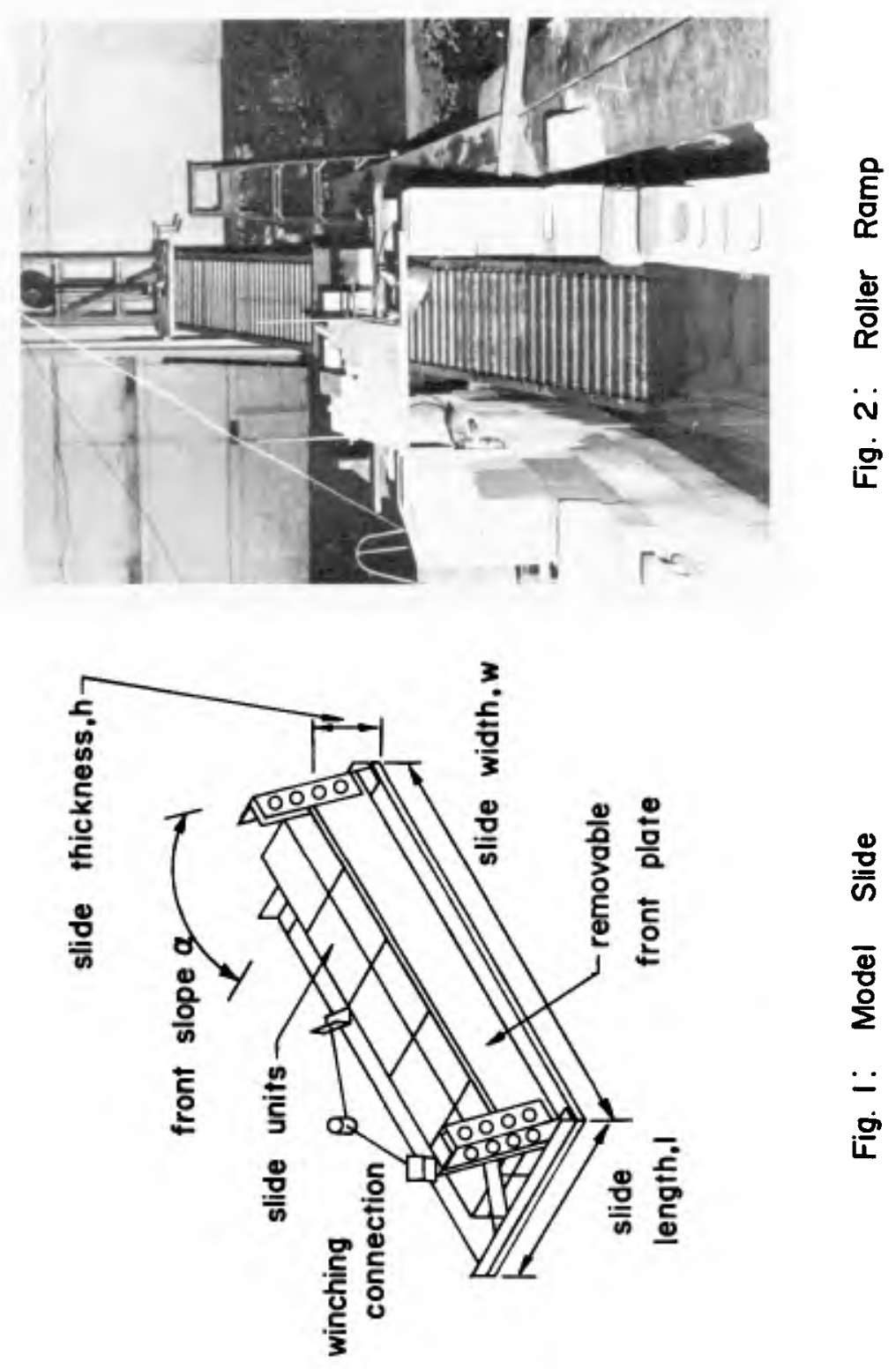


Eq 3 may be written in dimensionless form as

$$
\pi_{A}=\Phi_{A}\left(\frac{l}{d}, \frac{w}{d}, \frac{h}{d}, \frac{v}{\sqrt{g d}}, \beta, \theta, p, \frac{\rho s}{\rho}, \frac{\rho d \sqrt{g d}}{\mu}, \frac{x}{d}, t \sqrt{\frac{g}{d}}\right)
$$

Because the tests were performed in a two dimensional flume, the term w/d becomes irrelevant Early in the tests it became evident that the slide volume and the related kinetic energy of the slide upon impact with the water are very important terms and therefore $1 / d$ has been replaced by the two-dimensional slide volume per unit width expressed in dimensionless form as

$$
q=\frac{l}{d} \times \frac{h}{d}
$$

The kinetic energy upon impact can be obtained by the following combination

$$
k=\frac{1}{2}\left(\frac{s}{\rho}\right)\left(\frac{l}{d}\right)\left(\frac{h}{d}\right) \frac{v^{2}}{g^{d}}
$$

Because shear was negligible throughout, the Reynolds number can be neglected and $\mathrm{Eq} 4$ becomes

$$
\pi_{A}=\phi_{A}\left(q, \frac{h}{d}, \frac{V}{\sqrt{g d}}, \beta, \theta, p, \frac{\rho}{\rho}, \frac{x}{d}, t \sqrt{\frac{g}{d}}\right)
$$

where $\pi_{A}$ represents the dimensionless form of any dependent quantity A

Thus for maximum wave helght at any probe

$$
\frac{\mathrm{H}}{\mathrm{d}}=\phi_{\mathrm{H}}\left(q, \frac{\mathrm{h}}{\mathrm{d}}, \frac{\mathrm{v}}{\sqrt{\mathrm{gd}^{\mathrm{d}}}}, \beta, \theta, p, \frac{\rho}{\rho}, \frac{\mathrm{x}}{\mathrm{d}}\right)
$$

and for the wave energy at the first probe

$$
\frac{E}{\rho \mathrm{gd}^{3}}=\phi_{\mathrm{E}}\left(\mathrm{k}, \frac{\mathrm{h}}{\mathrm{d}}, \frac{\mathrm{V}}{\sqrt{\mathrm{gd}}}, \beta, \theta, \mathrm{p}, \frac{\rho \mathrm{s}}{\rho}\right)
$$

Here $\mathrm{q}$ has been replaced by $\mathrm{k}$, a more relevant parameter For the speed of propagation of the waves

$$
\frac{\mathrm{C}}{\sqrt{\mathrm{g}^{d}}}=\phi_{\mathrm{C}}\left(\mathrm{q}, \frac{\mathrm{h}}{\mathrm{d}}, \frac{\mathrm{v}}{\sqrt{\mathrm{gd}}}, \beta, \theta, \mathrm{p}, \frac{\rho \mathrm{s}}{\rho}, \frac{\mathrm{x}}{\mathrm{d}}, \mathrm{t} \sqrt{\frac{\mathrm{g}}{\mathrm{d}}}\right)
$$


and for the wave period

$$
T \sqrt{\frac{g}{d}}=\phi_{T}\left(q, \frac{h}{d}, \frac{V}{\sqrt{g d}}, \beta, \theta, p, \frac{\rho s}{\rho}, \frac{x}{d}, t \sqrt{\frac{g}{d}}\right)
$$

Throughout the tests, except for one 1solated test series, $p$ and $\rho_{s} / \rho$ were not varied $\left(p=0, \rho_{s} / p=27\right)$ Further work needs to be done to test the effect of these parameters The work also needs to be extended to higher values of slide volume, $q$ or kinetic energy, $k$

The test results therefore only apply to relatively small rock slides, originating above the water level

\section{TEST RESULTS}

The wave records obtanned were very much as expected At probe a, the wave train consisted of one large wave followed by a short train of smaller, tralling, osclllatory waves At probe $b$, the large leading wave had lengthened conslderably and decreased in helght It had passed some of its energy to the following wave train The train of oscillatory, following waves had also lengthened At probe $c$, the wave helght appeared to have reached a stable or almost stable value The wave train was now very long, the large wave itself having attained a wave length of the order of 20 to 30 times the depth of water

The first wave was always the highest in the train except for test series 52 where $\theta$ is $90^{\circ}$

Maximum Wave Helght The test results indlcate the dimensionless parameters in Eq 7 that exercise the greatest influence on the wave height are $q$, the volume per unit width of the slide and $\mathbb{F}=\mathrm{V} / \sqrt{\mathrm{gd}}$ the Froude number of the slide upon impact with the body of water The other parameters only introduce variations to the basic relationship All test results have been plotted against $q$

Typical test results are shown in Fig 3 and it may be seen that $\mathrm{H}_{\mathrm{c}} / \mathrm{d}$ varies directly with $\log \mathrm{q}$ This is not true for $\mathrm{H}_{\mathrm{a}} / \mathrm{d}$, the wave helght nearest the point of Impact From comparison of wave helghts at $a, b$ and $c$, It appears that $H_{c} / d$ is stable, $I$ e the wave helght does not decrease significantly beyond probe $c$ The subsequent figures focus on this stable wave helght and the other two measured wave helghts are related to it by a wave helght attenuation function discussed later in this section

The effect of the slide thickness on the stable wave helght is indicated in Fig 4 For thick slides, defined as $\mathrm{h} / \mathrm{d}>\frac{1}{2}$, the relation between $H_{c} / d$ and $q$ is relatively constant For thin slides, defined as $\mathrm{h} / \mathrm{d}<\frac{1}{2}$, a decrease in $\mathrm{h} / \mathrm{d}$ results in smaller wave helghts 

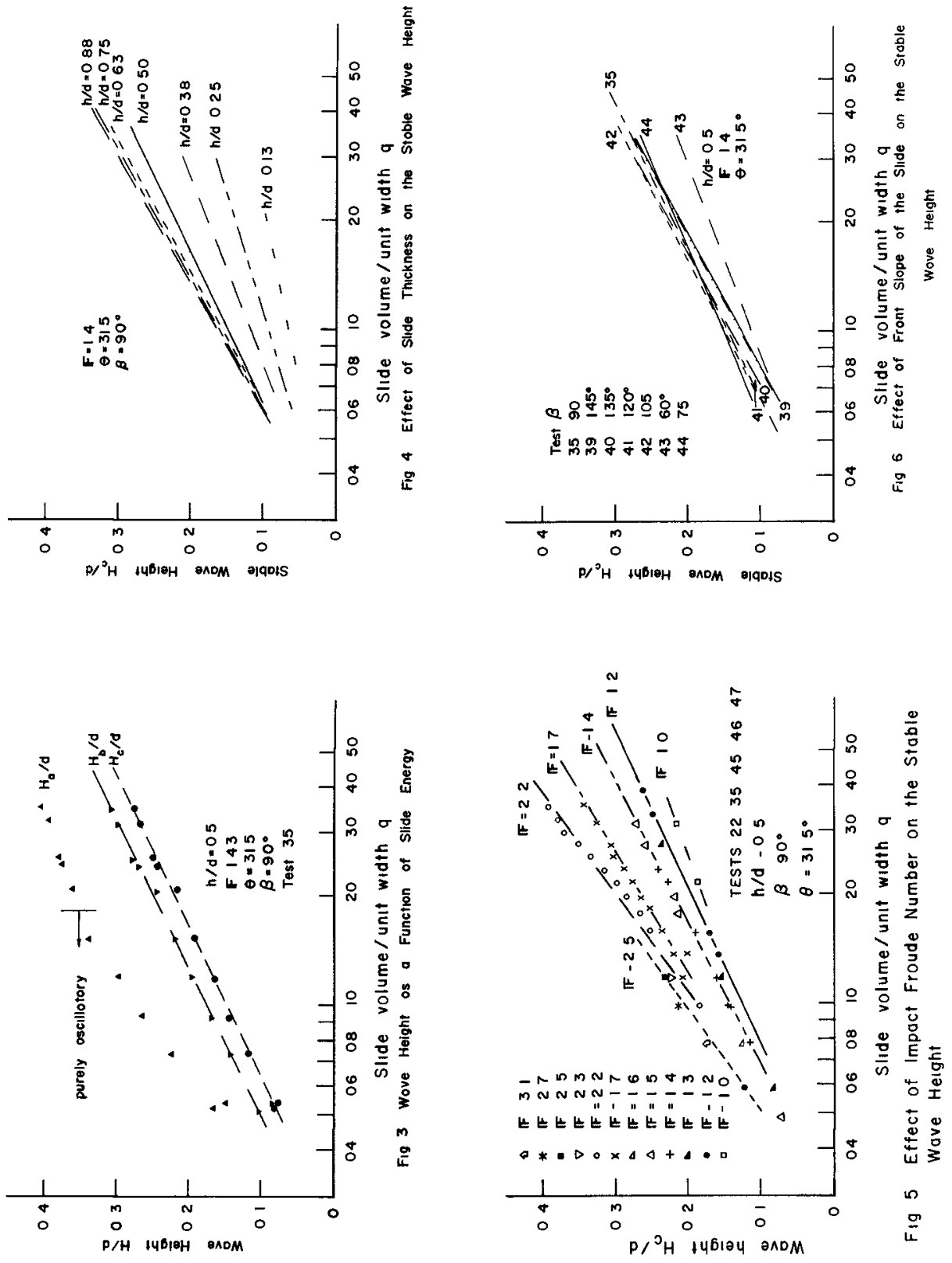

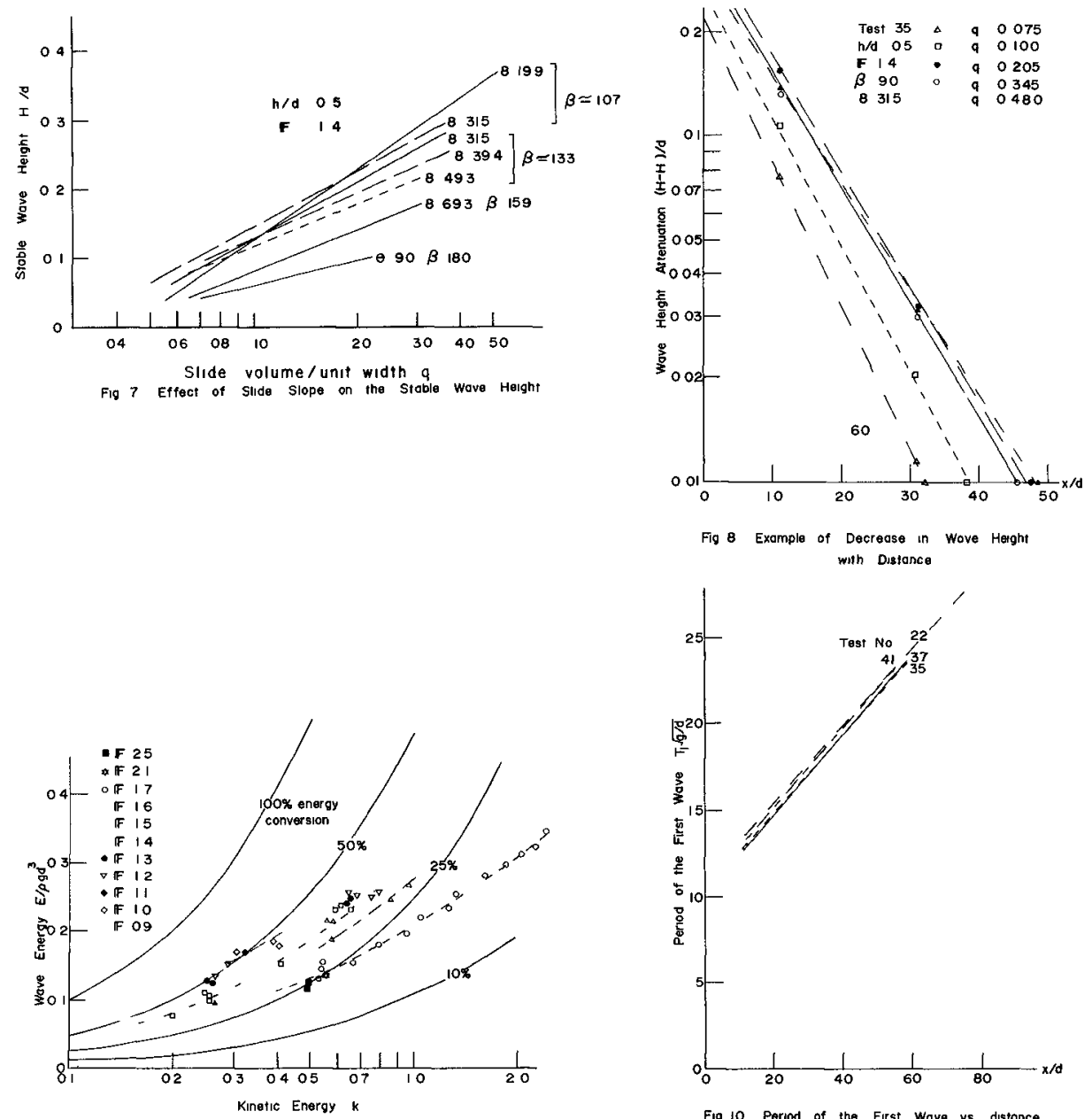

Fig 9 Effect of Impact Froude Number on Wove Energy 
For the range of Froude numbers tested ( $F 1 g$ 5) it may be seen that the stable wave helght increases significantly with the Froude number of the slide upon impact

An increase in the front slope of the slide, $\beta$, appears to Increase the slope of the relation between $\mathrm{H}_{c} / d$ and $\mathrm{q}$ For large slides the resulting wave helght increases with $\beta$, for small slides, it decreases with $\beta$ In addition, for $\beta<90^{\circ}$ there is a general reduction in agitation An example of the effect of $\beta$ on $H_{c} / d$ has been plotted in

As $\theta$ Increases, the generated wave 1 s decreased slightly except for small slides - Fig 7 The results of test series 51 and 52 have also been plotted - not to indrcate the effect of $\theta$ but to represent 8 possible overlap with the results of Prins $(5,6)$ and Wiegel $(9,10)$

In FIg 3 it may be seen that $\mathrm{H}_{a}>\mathrm{H}_{b}>\mathrm{H}_{\mathrm{c}}$ This can be attributed mainly to dispersion, 1 e the passing of energy from the highest wave to the tralling train and the lengthenang of the whole wave train Experimentally it was found that the decrease in wave helght is exponential and that the wave helght for most tests decreased little beyond probe $c$ An example is shown in Fig 8 The slope of the lines Is almost constant for all tests and the intercept varies as a function of $q$

Wave Energy The test results involving wave energy may be plotted similar to Figs 3 to 7 , but it is more convenient to plot them as a function of $\mathrm{k}$, the dimensionless kinetic energy As an example Fig 9 - the effect of Froude number has been plotted and on the same graph the lines of constant \% energy conversion have been shown

Velocity of Propagation The velocity of propagation, between probes $b$ and $c$, of the hlghest wave in the wave train was found to be within $1 \%$ of the following theoretical approximation for a solitary wave

$$
\frac{c}{\sqrt{g d}}=1+\frac{n_{\max }}{2 d}
$$

This corresponds to $\mathrm{Eq} 9$ because $n_{\max } / 2 \mathrm{~d}$ is simply another function of the right hand side of $\mathrm{Eq} 9$

Wave period $\mathrm{T}_{1} \sqrt{\mathrm{g} / \mathrm{d}}$, the dimensionless perıod of the furst wave in the wave train has been plotted agalnst distance along the flume in Fig 10 It may be seen that the wave period increases linearly with distance along the flume It was found that all the other parameters of $\mathrm{Eq} 10$ exercised a negliglble influence on the relation between $\mathrm{T}_{1} \sqrt{\mathrm{g} / \mathrm{d}}$ and $\mathrm{x} / \mathrm{d}$ 


\section{DISCUSSION}

The wave forms obtaıned from the tests varied from a pure osclllatory wave train to a wave approaching solıtary wave configuration, followed by an osclllatory wave train Comparison with Prins (5, Fig 10) would indicate his experience to be similar At no time during the tests were bores generated and therefore the results presented here can only be viewed as partial, also in this respect

Under these conditions the test results indicate that a stable wave helght is generally obtalned within 80 depths from the polnt of Impact

Maxımum Stable Wave Helght A definite relation exists between the stable wave helght and the volume of the slide This relation can be expressed as

$$
\frac{\mathrm{H}_{\mathrm{c}}}{\mathrm{d}}=\mathrm{C}_{1}+\mathrm{C}_{2} \log \mathrm{q}
$$

where both $\mathrm{C}_{1}$ and $\mathrm{C}_{2}$ are functions of the remaining dumensionless parameters of $\mathrm{Eq} 7$ But Fig 5 Indicates that the wave helght Is also highly dependent on Froude number Taking this into account, the relationship which approximates the test results most closely is

$$
\frac{H_{c}}{d}=F^{07}(031+020 \log q)
$$

This can be used as a good estimate of the stable wave helght for $005 \leq \mathrm{q} \leq 1 \mathrm{l}$, as long as the slide $\mathrm{ss}$ thlck, $1 \mathrm{e} \mathrm{h} / \mathrm{d} 2 \frac{1}{2}$, the front angle of the slide 1 s $90^{\circ}$ or greater and the angle of the slide plane 18 about $30^{\circ} \mathrm{Eq} 13$ can also be used as a conservative estimate for slides where $\beta<900$, or $\theta>30^{\circ}$ and an estimate of the bullt in factor of safety may be obtalned from the figures presented In this paper For the case $\theta<30^{\circ}$, Eq 13 could be unsafe

$\mathrm{Eq} 13$ is purely empirical Unokı and Nakano (7) conclude that theoretically $\eta \sim$ initial impulse since for each test serıes $V$ was kept almost constant, this reasoning should lead to $n \sim$ slide volume for each test series However, this appears not to be the case, indicating that the system is highly non-linear and that the theoretical approach of (7) overestimates the generated wave helght The theoretical approach of Kranzer and Keller (3) could perhaps be applied as an approxımation, if the "Inıtıal" water surface, immediately after impact of the slide, were known This is virtually impossible because of splash and because the slide takes a finite time to enter and travel through the body of water 
The changes in the relation of $\mathrm{H} / \mathrm{d}$ on $\mathrm{q}$ for the parameters treated in Figs 4 to 7 can be explained from the physical phenomenon It must be remembered that in all cases with the exception of Fig 5, the Froude number is constant, therefore slide volume is synonymous with kinetic energy on impact

Referring to Fig 4, as the slide becomes thinner, It imparts less energy to the water, dissipating more of the remalning energy by bottom impact

As the impact Froude number increases, Fig 5, the kinetic energy upon impact increases for the same slide volume This results in greater wave helghts as well as greater splash and bottom impact

Fig 6 indicates that a decrease in $\beta$ beyond $90^{\circ}$ causes the front of the slide to become more streamlined The slide then imparts less energy to the water

As $\theta$ decreases, the distance of travel of the slide through the water becomes greater and therefore it $1 \mathrm{~s}$ expected that a greater amount of the energy of the slide goes into wave generation Also, the generation mechanism changes to more of a pushing action

Wave Helght Attenuation Fig 8 is an example of the pattern of wave helght attenuation found generally Because the attenuation is represented by stralght lines of constant slope, the decrease in wave helghts can be expressed as

$$
\frac{H}{d}=\frac{H_{c}}{d}+C_{3} e^{-C_{4}\left(\frac{x}{d}\right)}
$$

where $\mathrm{C}_{3}$ is a function of the other parameters in $\mathrm{Eq} 7, \mathrm{C}_{4}$ appears to be constant at 008 For values of $01<q<10$, Eq 14 can be approxımated by

$$
\frac{H}{d}=\frac{H}{d}(\text { stable })+035 e^{-008\left(\frac{x}{d}\right)}
$$

Wave Energy For the range of values tested the wave energy is gener $\overline{a l l y}$ from $\frac{1}{4}$ to $\frac{1}{2}$ the kinetic energy of the slide upon impact For test 52 , the vertically falling slide only 10 to $20 \%$ of the kınetıc energy was converted into wave energy Thus the slide at an angle appears to be considerably more efficient than the vertical one The kinetic energy of the slide at the time of Impact is a rather difficult term to assess in the field An easier estimate is the potential energy of a slide and this figure has been used by Johnson and Bermel (1) and Wiegel (9) for laboratory generation of impact waves and by Miller (4) in assessing slides in the field The conversion from potential energy to kinetıc energy upon impact is entirely different 
In all three cases, however, and the complex mechanism in the field slides cannot be adequately sumulated by simplified laboratory models For that reason, kinetıc energy upon impact was used which leaves the transfer function of field potential energy to kinetic energy only to be determined, curcumventing an additional, similar function for each particular laboratory situation

Velocity of Propagation With wave lengths of the order of 20 to 30 times the depth, the waves are by definition shallow water waves and their characteristics can be approximated by the solitary wave theory, once the waves become of constant form Therefore the velocity of propagation should be approximated by the expression for the phase velocity of a solitary wave ( $\mathrm{Eq} \quad 11$ ) The close comparison between actual values and theoretical values was indeed surprising

Wave Period The various dimensionless parameters, other than the distance from the point of impact had very little influence on the wave perıod of the first wave and this wave perıod may be defined from plots simllar to Fig 10 as

$$
\mathrm{T}_{1} \sqrt{\frac{\mathrm{g}}{\mathrm{d}}}=11+0225\left(\frac{\mathrm{x}}{\mathrm{d}}\right)
$$

This indicates that, although wave helght and velocity of propagation of the first wave have reached a stable value at probe $c$, the wave period and wave length have not Therefore the first wave continues to stretch out, tending to become a true, solitary wave, of infinitely long period, with no or only slight decrease in wave helght The periods of the tralling waves exhibit the same tendency although their increase in period is not linear with $x / d$ through the range of $x / d$ tested

\section{PRELIMINARY COMPARISON WITH FIELD RESULTS}

Properly monltored field data are difficult to obtain because of the unexpected and disastrous nature of the slides In an attempt to see how relevant the model tests are and as an indication of the applicability of formulae such as Eq 13, the slides listed by Miller $(4, \mathrm{p} 66)$ were used as field examples

The model tests are two-dimensional and therefore can be expected to give a conservative indication for the three-dimensional field results

Most of the slides listed by Miller are rock slides and alı origlnated above the water surface The volume and width of the slide front are only mentioned simultaneously in three cases thus reducing the list to only three examples For one of these, the Shumabara Peninsula slide, the slide volume was greater than the range of $q$ tested For the other two, the Loen Lake slide of 1936 and the Tafjord slide of $1934 \mathrm{Eq} 13$ gives a reasonably close estimate wave helght

A great deal more work needs to be done in this area 


\section{CONCLUSIONS}

The impulsively generated waves resulting from the sliding of a rock mass into a body of water have been investıgated

A series of tests has been performed to determine the effect of varlous parameters describing the slide and the recelving body of water on the generated impulse waves In this test serles all slides were impervious and of rock density The volumes of the slides were kept relatively small and so the test results are only applicable to small and medium slzed slides of rock whlch orlginate above the water surface and slide into relatively large bodzes of water

For these conditions it was found that the wave helght stabilized very quickly and can be described as a function of the slide volume and Froude number on impact (Eq 13 ) All other parameters appear as relatively small modifications to this function The wave helght attenuation between a location close to the site of impact and the point where the wave helght has stabilized can be described by an exponential function ( $\mathrm{Eq}$ 15)

The wave energy varies for these tests from $\frac{2}{4}$ to $\frac{1}{2}$ of the input kinetic slide energy The velocity of propagation of the highest wave can be described adequately by solutary wave theory Its wave perlod increases linearly with distance for the distance range tested Some very preliminary work on field data indicates that $\mathrm{Eq} 13$ can be used to predict or hindcast field wave heights

\section{ACKINOWIEDGEMFNTS}

The authors are grateful to Dr A Brebner for inltiating this study and contributing during this program Also they are indebted to the National Research Council of Canada for financial assistance 
1 Johnson, $J \mathrm{~W}$, and Bermel, $\mathrm{K} \mathrm{J}$, "Impulse Waves in Shallow Water as Generated by Falling Welghts", Transactions American Geophysical Union, Vol 30, No 2, Apri1 1949, pp 223-230

2 Kıersch, George A, "Valont Reservolr Disaster", Clvil Englneerıng, March 1964, pp 32-39

3 Kranzer, Herbert C, and Keller, Joseph B, "Water Waves Produced by Explosions", Journal Applied Physics, Vol 30, No 3, March 1959, pp 398-407

4 Miller, Don J , "Giant Waves In Lıtuya Bay, Alaska", U S Geologıcal Survey Professional Paper 354-C, 1960

5 Prins, J E, "Water Waves Due to a Local Disturbance", Proceedıngs Slxth Conference Coastal Englneerıng, The Engineering Foundation Council on Wave Research, 1958 , pp 147-162

6 Prıns, J E, "Characteristıcs of Waves Generated by a Local Disturbance", Transactions American Geophysical Union, VoI 39, No 5, Oct 1958, pp 865-874

7 Unokı, S, and Nakano, M, "On the Cauchy-Polsson Waves Caused by the Eruption of a Submarıne Volcano", Oceonograph1c, May 4, pp 119-141

8 Urseil, F, "On the Waves Generated by a Local Surface Disturbance personal communication, mentioned in (10),

9 Wiegel, R L, "Laboratory Studies of Gravity Waves Generated by the Movement of a Submerged Body", Transactions American Geophysical Union, Vol 36 , No 5 , Det 1955, pp 759-774

10 Wregel, R L, Noda, E K, Kuba, E M, Gee, D M and Tornberg, G F "Water Waves Generated by Landslides in Reservolrs", Journal of the Waterways and Harbours Division, ASCE, Vol 96, No WW2, May 1970, pp 307-331 


\section{NOTATION}

$A=$ general dimensional expression for wave parameters,

$a=$ location of first wave measuring probe,

$b=$ location of second wave measuring probe,

$\mathrm{C}=$ velocity of propagation of the wave,

$c=$ location of third wave measuring probe,

$\mathrm{d}=$ depth of water,

$\mathrm{E}=$ wave energy,

$\mathrm{E}_{\mathrm{O}}=$ wave energy in deep water from oscullatory wave theory,

$\mathrm{F}_{\mathrm{S}}=$ wave energy by solitary wave theory,

$F=$ Froude number,

$f=$ dimensional function,

$\mathrm{g}=$ acceleration of gravity,

$\mathrm{H}=$ maxımum wave helght,

$\mathrm{h}=$ thickness of the slide,

$k=$ dimensionless kinetic energy of the slide,

1 = length of the slide,

$=$ porosity of the slide,

$=$ volume per unit width of the slide,

= wave perıod,

= time,

= velocity of impact of the slide,

$=$ width of the slide,

= distance from the point of impact of the slide,

$\alpha=$ angle between the bottom and the front face of the slide,

$\beta=$ angle between the front face of the slide and the horlzontal, $(=\alpha+\theta)$

$\eta$ = wave record,

$\theta=$ angle between the slip plane (roller ramp) and the water surface,

$\mu=$ dynamic viscosity of the water,

$\pi=$ general dimensionless expression for wave parameters,

$\rho=$ density of water,

$\rho_{\mathrm{s}}=$ density of the slide material,

$\Phi=$ dimensionless function,

$\phi=$ dimensionless function, 


\title{
CHAPTER 36
}

\author{
WAVES GENERATED BY A PISTON-TYPE WAVEMAKER
}

by

Ole Secher Madsen

Research Divisıon, $U$ S Army Coastal Engıneerıng Research Center Washington, $\mathrm{D} \quad \mathrm{C}$

\section{ABSTRACT}

When a wavemaker generates a finite number of waves, it has been found that one of the first and one of the last waves in such a burst is considerably larger than the average A mathematical model, based on the linearized governing equations, is used for the particular problem of the waves generated by a sinusoidally moving piston-type wavemaker starting from rest Theoretical results for the magnitude of the large wave relative to the average agree fairly well with experiments, however, the actual wave height is smaller in the experiments than predicted by theory It is shown, by extending the classical wavemaker theory to second order, that finite amplitude effects do not offer an explanation However, pistons rarely fit the tank dimensions exactly, and an approximate evaluation indicates that the discrepancy between predicted and observed wave helghts can be attributed to the effects of leakage around the piston

\section{INTRODUCTION}

One of the major problems encountered, when performing tests in a wave tank, is to account for the influence of reflected waves Within the framework of linear theory we can deal with this problem (see Urse11, et al, 1960), when the magnitude of the reflected wave 1s small compared with that of the incident wave However, in cases where the reflection from the far end of the tank is large, this is no longer possible To overcome this problem, some coastal engineering tests are performed using the "burst method", in which the wavemaker generates waves only so long as no significant reflection from the far end of the tank has yet reached the wavemaker After the wavemaker is stopped, time is allowed for the reflections to die out, before a new burst is generated This procedure essentially eliminates the influence of even large reflections, but as is often the case, eliminating one problem creates another

Figure 1 shows the surface profile recorded by a fixed gage 45 feet from a wavemaker, which generates a burst of 15 waves A prominent feature is evident One of the first and one of the last waves arriving at a particular station is considerably larger than the average The effects of these large waves on test results have been of concern to engineers at the Coastal Engineering Research Center (CERC) where e $\mathrm{g}$, rip-rap stability is determined from tests employing the burst method 


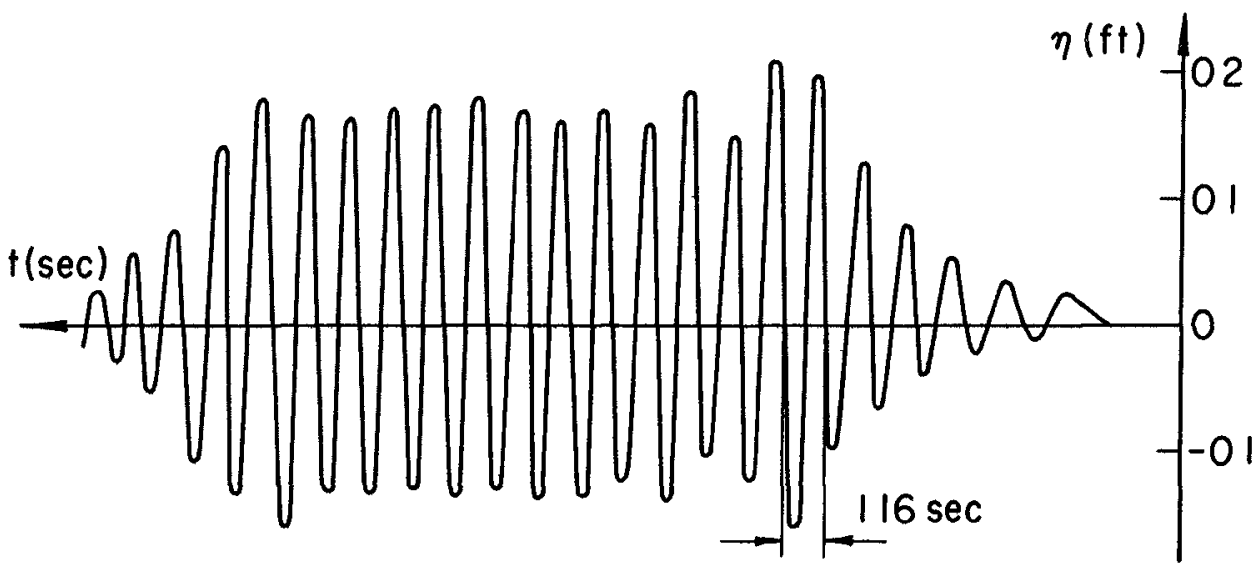

F1gure 1 Surface profile recorded 45 feet from a sinusoidal pistontype wavemaker starting from rest and generating 15 waves $(\mathrm{T}=116 \mathrm{sec}, \mathrm{h}=15 \mathrm{ft}$, Stroke of wavemaker $=033 \mathrm{ft}$

The study presented in the following was undertaken in an attempt to gain insight into the nature of these large waves and, if possible, to find a way to eliminate them

The large waves are clearly associated with the transient, $1 e$, the starting and stopping of the wavemaker, to which the classical wavemaker theory (Havelock, 1929, Biésel and Suquet, 1951, Ursell, et al, 1960) does not apply However, Kennard (1949) has solved the linearized governing equations based on the assumption of potential flow starting from rest, and we adopt his solution as the theoretical model for the particular problem of a sinusoidally moving piston-type wavemaker starting from rest (Section 2 1)

The question is How accurately will the linear theory predict the development of 1aboratory waves, which more often than not are quite nonlinear? From the theory of progressive waves we know that the second order nonlinear Stokes' wave sharpens the crest and flattens the trough when compared with the linear first order solution, but that the wave height remains unchanged Thus focusing on wave height, rather than amplitude, the linear solution is likely to cover at least slightly nonlinear waves Th1s is supported by the experimental confirmation of the classical wavemaker theory by Ursell, et al (1960) A much more serious limitation of the results from a linearized theory stems from the instability of sinusoidal waves, $1 \mathrm{e}$, for relatively short waves (depth/length $=\mathrm{h} / \mathrm{L}>0$ 216) the Benjamin-Feir side-band instability (Benjamin, 1967), and for moderately long waves $(\mathrm{h} / \mathrm{L}<009)$, the occurrence of secondary crests (Galvin, 1968, Madsen et al, 1970) 
With these limitations in mind, the theoretical results for wave heights are tested against experiments for three cases $(\mathrm{h} / \mathrm{L}=024$ 0197,0132 ) in Section 22 , and $1 t$ is found that the predicted and observed magnitudes of the large waves relative to the average agree reasonably well However, the theory overestimates the actual wave helght Th1s was also found by Ursell et al (1960) for waves of farrly large steepness and was attributed to possible nonlinear effects in Section 3 the classical (linear) wavemaker theory is advanced to second order, and it is found that finite amplitude effects cannot be considered responsible for the difference between observed and predicted wave herghts

An approximate evaluation of the amount of leakage through the gaps between the piston and the tank walls and the influence of this leakage on the height of the generated wave is performed in Section 4 it is found that the discrepancy between observed and predicted wave heights may be attributed to leakage around the piston, which establishes confidence in the wave helghts predicted by the linear theoretical model adopted

Section 5 discusses the possibility of utilizing the large effect of leakage on the helght of the generated waves to eliminate the large wayes in a burst

\section{LINEAR SOLUTION FOR A WAVEMAKER STARTING FROM REST}

\section{Theory}

Assuming irrotational motion the linearized equations governing the motion generated by a wavemaker (see Figure 2) are

$$
\begin{array}{ll}
\nabla^{2} \phi=\phi_{x x}+\phi_{y y}=0 & -h \leq y \leq 0 \\
\phi_{y}=0 & y=-h \\
n_{t}-\phi_{y}=0 & y=0 \\
\phi_{t}+g n=0 & y=0
\end{array}
$$

and at the wavemaker, which is characterized by its position, $\xi(y, t)$

$$
\phi_{x}=\xi_{t}(y, t)=u(y, t) \quad x=0
$$

where subscripts indicate partial differentiation and $g$ is the acceleration of gravity

Assuming the motion to start from rest, 


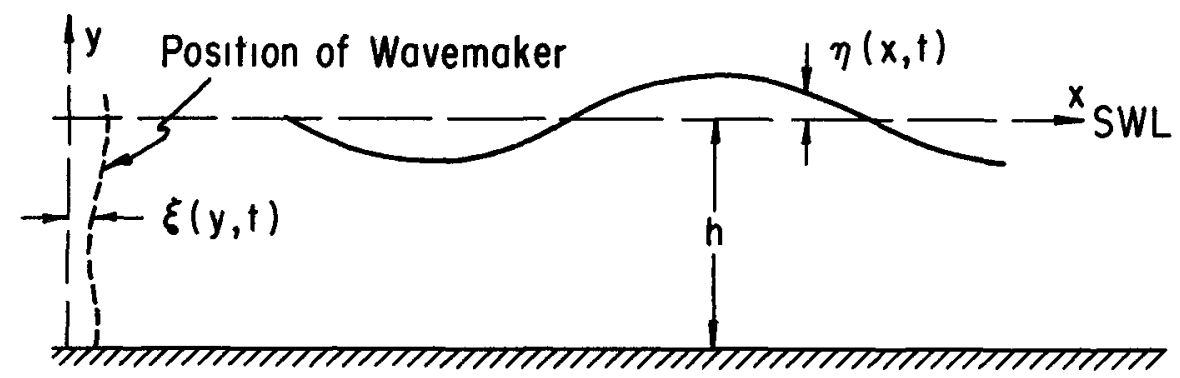

Figure 2 Definition of Symbols

$$
\phi(x, y, 0)=\phi_{t}(x, y, 0)=\xi(y, 0)=\xi_{t}(y, 0)=0
$$

the solution for the surface profile has been obtained by Kennard (1949)

$n(x, t)=-\frac{2}{\pi} \int_{0}^{\infty} d k \int_{0}^{t} d \tau \int_{0}^{-h} d y \quad \frac{\cos \sigma(t-\tau) \cos k x u(y, \tau) \cosh k(y+h)}{\cosh k h}$

where

$$
\sigma^{2}=g k \tanh k h
$$

$k$ being the wave number

For the particular case of a sinusoldal piston-type wavemaker, which runs for a length of time, $t^{\prime}$, we have

$$
u(y, t)=\left\{\begin{array}{lc}
0 & t \leq 0 \\
U \sin (\omega t+\delta) & 0<t \leq t^{\prime} \\
0 & t>t^{\prime}
\end{array}\right.
$$

and inserting this in (2 7), we can perform the integration with respect to $y$ and $\tau$ to obtain

$$
\begin{gathered}
n(x, t, \delta)=\frac{2}{\pi} \quad \frac{U}{\omega} \int_{0}^{\infty} d k \frac{\tanh k h}{k} \frac{\omega^{2}}{\omega^{2}-\sigma^{2}} \cos k x \\
{\left[(\cos \sigma t-\cos \omega t) \cos \delta+\left(\sin \omega t-\frac{\sigma}{\omega} \sin \sigma t\right) \sin \delta\right]}
\end{gathered}
$$

Realizing that

$$
\frac{u}{\omega}=\xi_{0}
$$


is the amplitude of the wavemaker motion and introducing the dimensionless variables (indicated by asterisks)

$$
\begin{aligned}
& \left(x^{*}, y^{*}\right)=h^{-1}(x, y) \\
& t^{*}=\sqrt{\frac{g}{h}} t \\
& k^{*}=k h
\end{aligned}
$$

the solution can be written

$$
\frac{\pi}{2} \frac{\eta(x, t, \delta)}{\xi_{0}}=I_{1} \cos \delta+I_{2} \sin \delta
$$

where

$$
I_{1}=\int_{0}^{\infty} d k^{*} \frac{\tanh k^{*}}{k^{*}} \frac{\omega^{*}}{\omega^{*^{2}}-k^{*} \tanh k^{*}}\left(\cos \sqrt{k^{*} \tanh k^{*}} t^{*}-\cos \omega^{*} t^{*}\right) \cos k^{*} x^{*}
$$

and

$I_{2}=\int_{0}^{\infty} d^{*} \frac{\tanh k^{*}}{k^{*}} \frac{\omega^{*}}{\omega^{*}-k^{*} \tanh k^{*}}\left(\sin \omega^{*} t^{*} \frac{\sqrt{k^{\star} \tanh k^{*}}}{\omega} \sin \sqrt{k^{*} \tanh k^{*}} t^{*}\right) \cos k^{*} x^{*}$

This solution as it stands is valid only so long as $t<t$ ' However, we may add the solution satisfying the boundary condition at $x=0$

$$
\phi_{X}^{\prime}=u^{\prime}(y, t)= \begin{cases}0 & t \leq t^{\prime} \\ -U \sin \left(\omega\left(t-t^{\prime}\right)+\delta^{\prime}\right) & t>t^{\prime}\end{cases}
$$

and due to the Iinearity of the governing equations the sum of these solutions will satisfy the boundary condition given by (29) provided

$$
\delta^{\prime}=\delta+\omega t^{\prime}-2 \mathrm{~m} \pi
$$

where $\mathrm{m}$ is an integer

Thus in short we may write the solution as

$$
n(x, t)=\left\{\begin{array}{lr}
n(x, t, \delta) & 0<t \leqq t^{\prime} \\
n(x, t, \delta)-n\left(x, t-t^{\prime}, \delta^{\prime}\right) \quad t>t^{\prime}
\end{array}\right.
$$

where the right-hand sides are calculated from (2 12)

The integrals $I_{1}$ and $I_{2}$ have removable singularities and are evaluated by numerical integration using the trapezoldal rule with a stepsize 
equa 1 to 005 the period of the integrand and the upper 11mit of integration equal to 16 The results were tested for accuracy by varying the stepsize and the upper 11mit of integration As a further check the numerical solution was found to approach the classical solution to the wavemaker problem (B1Esel and Suquet, 1951) as t became large

Taking $x=$ constant in $(212)$ we can compute the surface elevation at a particular station along the tank as a function of time, which corresponds to the surface profile recorded by a fixed gage if we define the wave helght as the difference in surface elevation between a trough and the preceding crest, we may describe the development taking place some distance from the wavemaker in terms of the sequence of wave heights as the waves arrive at this station For a station 30 times the depth from the wavemaker, the computed variation in wave helghts relative to the wave helght in the final periodic state, $\mathrm{H} / \mathrm{H}_{\mathrm{p}}$, is shown in Figure 3 for three depth-to-length ratios

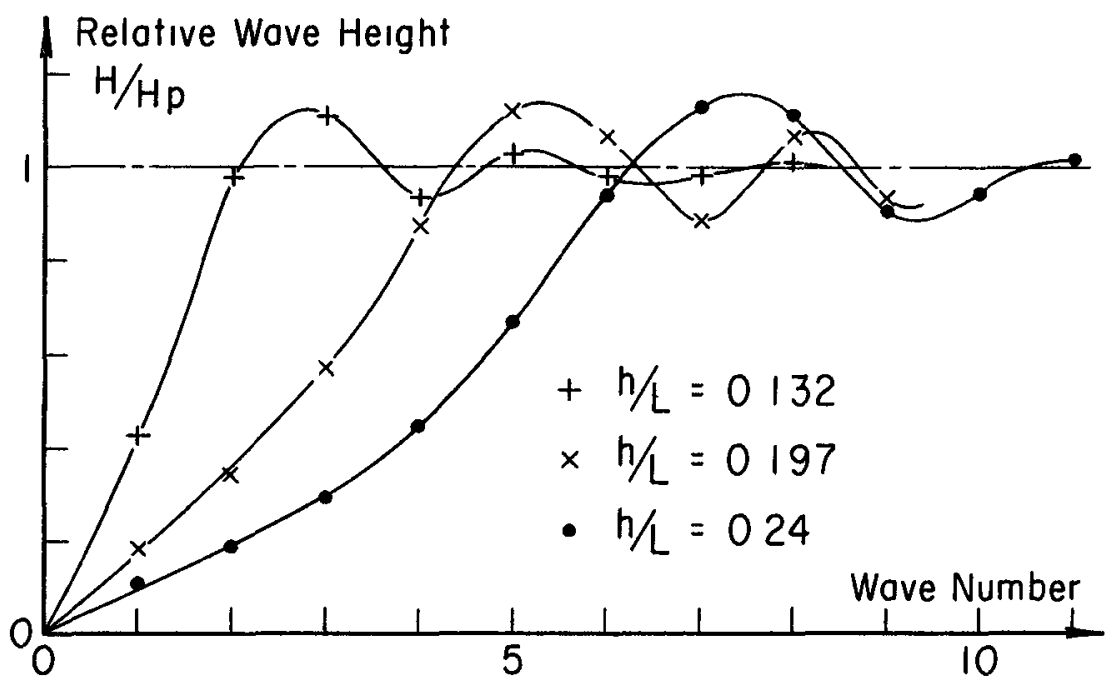

Figure 3 Computed variation in relative wave height of waves generated by a piston-type wavemaker starting from rest, as they reach a station $30 \mathrm{x}$ depth from the generator

1t shows that after the disturbance arrives, the wave helght increases, overshoots but finally attains the constant value corresponding to the final periodic state This type of behavior, which predicts a large first wave, is analogous to the response of a slightly damped mechanical system to an exciting force 


\section{Comparison with Experiments}

The large waves in a burst had previously been studied experimentally in CERC's 72-foot tank by John Ahrens, Hydraulic Engineer at CERC, and records of the surface profile obtained 16 and 45 feet from the wavemaker serve for a detalled comparison with the result computed from (2 12)

The wavemaker, piston type, is electronically controlled and was set to start from 1 ts mean position going backwards, 1 e, corresponding to $\delta=-\pi / 2$ in (2 9) The wayemaker was stopped manually, and if it was stopped off its mean position it would abruptly take this position Since it is very difficult to avoid this final impulse, which will influence the last part of the burst, the camparison 1 s only carried out for the first waves in a burst The depth, $h$, was 15 feet and for each period the experiment was repeated three times, giving practically identical results for the part of the burst used for the comparison

The experiments show second order effects (wave steepness $=H / L=$ $003-0$ 06) in that crest amplitudes are larger than trough amplitudes, however, as was indicated previously and in view of the results obtained in Section 3, this effect is essentially eliminated by comparing wave heights rather than amplitudes The comparison is presented in Table 1 as the variation in wave heights as the waves arrive at the particular stations, and it is obvious from the results that the actual wave heights differ considerably However, if we compare the variation in wave helghts relative to the wave height in the final periodic state, which for the experiments is taken as the average wave height in a burst excluding the large waves, the agreement between computed and experimental wave he1ghts in this sense seems good The larger discrepancy for the shortest wave, $\mathrm{h} / \mathrm{L}=024$, may be attributed to the Benjamin-Feir side-band instability

\section{APPROXIMATE SECOND ORDER WAVEMAKER THEORY}

The application of a linear theory as a predictor of the motion generated by a wavemaker starting from rest was found to be relatively successful The large difference between the actual computed and exper1mental wave heights noted in Table 1 is naturally of minor importance once a particular wavemaker has been calibrated However, if the observed discrepancy can be explained as other than the inadequacy of the linear solution, an understanding of the responsible mechanism will not only help us in designing more efficient wavemakers and maybe eliminate the need for calibrations, but it will give us confidence in the results obtained from the linearized governing equations, also for problems different from the one treated here We therefore proceed to investigate If the large difference in wave heights can be attributed to finite amplitude effects as Ursell et al (1960) suggested

Based on a Lagrangian formulation Fontanet (1961) derived the complete second order solution to the wavemaker problem His solution, however, is extremely difficult to evaluate, and for this reason we outline an approach using the more familiar Eulerian description Due to the nonlinear instabilities mentioned previously (Benjamin-Feir instability for short waves, secondary waves for long waves), we need 


\begin{tabular}{|c|c|c|c|c|c|c|c|c|c|}
\hline \multirow{3}{*}{$\begin{array}{l}\text { Wave } \\
\text { No }\end{array}$} & \multicolumn{2}{|c|}{16 feet from } & \multicolumn{2}{|c|}{ Wavemaker } & \multicolumn{4}{|c|}{45 feet from Wavemaker } & \\
\hline & \multicolumn{2}{|c|}{$\mathrm{H}$ feet } & \multicolumn{2}{|c|}{$\mathrm{H} / \mathrm{H}_{p}$} & \multicolumn{2}{|c|}{$\mathrm{H}$ feet } & \multicolumn{2}{|c|}{$\mathrm{H} / \mathrm{H}_{\mathrm{p}}$} & \\
\hline & Exp & Comp & Exp & Comp & Exp & Comp & Exp & Comp & \\
\hline 1 & 0305 & 0370 & 085 & 09 & 0155 & 0183 & 043 & 044 & \\
\hline 2 & 0368 & 0412 & 102 & 10 & 0305 & 0405 & 085 & 098 & $\stackrel{N}{n}$ \\
\hline 3 & $\underline{0} 387$ & $\underline{0 \quad 424}$ & 108 & 103 & $\underline{0} 387$ & $0 \quad 456$ & 108 & 111 & 0 \\
\hline 4 & 0345 & 0408 & 096 & 099 & 0354 & 0387 & 098 & 094 & 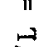 \\
\hline 5 & 0357 & 0418 & 099 & 102 & 0360 & 0424 & 100 & 103 & $\nexists$ \\
\hline 6 & 0362 & 0396 & 101 & 096 & 0361 & 0404 & 100 & 098 & \\
\hline 1 & 0142 & 0181 & 042 & 044 & 0061 & 0068 & $\begin{array}{ll}0 & 19\end{array}$ & 016 & \\
\hline 2 & 0331 & 0393 & 099 & 095 & 0112 & $\begin{array}{ll}0 & 138\end{array}$ & $\begin{array}{ll}0 & 34\end{array}$ & $\begin{array}{ll}0 & 34\end{array}$ & \\
\hline 3 & $\begin{array}{ll}0 \quad 352 \\
\end{array}$ & $0 \quad 457$ & 105 & 111 & 0194 & 0236 & 059 & 057 & $\hat{a}$ \\
\hline 4 & 0341 & 0384 & 102 & 093 & 0296 & 0363 & 091 & 088 & 0 \\
\hline 5 & 0334 & 0408 & 100 & 099 & $0 \quad 369$ & $0 \quad 468$ & 113 & 113 & 11 \\
\hline 6 & 0343 & 0412 & 102 & 100 & 0315 & 0442 & 096 & 107 & 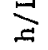 \\
\hline 7 & 0330 & $\begin{array}{lll}0 & 391\end{array}$ & 098 & 095 & 0318 & 0366 & 097 & 089 & \\
\hline 8 & 0333 & 0419 & 099 & 102 & $\begin{array}{ll}0 & 337\end{array}$ & 0444 & 103 & 108 & \\
\hline 9 & 0339 & 0416 & 101 & 101 & $\begin{array}{ll}0327\end{array}$ & 0384 & 100 & 093 & \\
\hline 1 & 0110 & $0 \quad 130$ & 030 & 028 & $0 \quad 045$ & $0 \quad 049$ & 015 & 011 & \\
\hline 2 & 0254 & 0287 & 071 & 062 & $\begin{array}{ll}0 & 068\end{array}$ & 0085 & 020 & 018 & \\
\hline 3 & 0420 & 0479 & 117 & 103 & $\begin{array}{lll}0 & 117\end{array}$ & 0136 & 035 & 029 & \\
\hline 4 & 0366 & $0 \quad 512$ & 102 & 110 & $\begin{array}{lll}0 & 170\end{array}$ & 0209 & 051 & 045 & \\
\hline 5 & 0369 & 0437 & 102 & 094 & 0275 & 0318 & 082 & 068 & $\stackrel{\sim}{\sim}$ \\
\hline 6 & 0368 & 0459 & 102 & 099 & $0 \quad 403$ & 0441 & 121 & 095 & 0 \\
\hline 7 & 0359 & 0465 & 099 & 100 & 0372 & $0 \quad 524$ & 112 & 113 & 11 \\
\hline 8 & 0364 & 0472 & 101 & 102 & 0300 & 0519 & 090 & 112 & $\bar{\epsilon}$ \\
\hline 9 & 0359 & 0466 & 099 & 100 & 0363 & 0427 & 109 & 092 & \\
\hline 10 & & & & & 0310 & 0446 & 093 & 096 & \\
\hline 11 & & & & & 0347 & 0474 & 104 & 102 & \\
\hline
\end{tabular}

Table 1 Comparıson Between Computed and Experımental Wave Heights (Depth $=15$ feet $)$

(Underlined values correspond to the largest wave) 
only consider intermediate wayes, $1 \mathrm{e}, 021 \leqq \mathrm{~h} / \mathrm{L} \leqq 009$ For large $t$ the solution to the first order (linear) equations gijen in Section 2 is the classical waye-maker theory (see e g, Bitsel and suquet, 1951) For a piston-type wavemaker,

$$
u(y, t)=\xi_{t}=U \sin \omega t, \xi=-\xi_{0} \cos \omega t
$$

and denoting the first order solution by a superscript, (1), the classical solution reads

$$
\begin{aligned}
\phi^{(1)=} & c_{0} \cosh k_{0}(y+h) \cos \left(k_{0} x-\omega t\right)- \\
& \sin \omega t \sum_{n=1} c_{n} \cos k_{n}(y+h) e^{-k_{n} x} \\
n^{(1)}= & n_{0} \sin \left(k_{0} x-\omega t\right)-\cos \omega t \sum_{n=1} n_{n} e^{-k_{n} x}
\end{aligned}
$$

and

$p^{+(1)}=p^{(1)}+\rho g y=$ pressure due to the wave $=$

$-\rho g\left[\eta_{0} \frac{\cosh k_{0}(y+h)}{\cosh k_{0} h} \sin \left(k_{0} x-\omega t\right)+\cos \omega t \sum_{n=1} n_{n} e^{-k_{n} x} \frac{\cos k_{n}(y+h)}{\cos k_{n} h}\right]$

where

$$
\begin{aligned}
& \mathrm{C}_{\mathrm{o}}=\frac{2 \mathrm{U} \sinh \mathrm{k}_{\mathrm{o}} \mathrm{h}}{\mathrm{k}_{\mathrm{o}} \mathrm{h}+\sinh \mathrm{k}_{\mathrm{h}} \cosh \mathrm{k}_{\mathrm{o}} \mathrm{h}} \frac{1}{\mathrm{k}_{\mathrm{o}}} \\
& g k_{0} \tanh k_{0} h=\omega^{2} \\
& C_{n}=\frac{2 U \sin k_{n} h}{k_{n} h+\sin k_{n} h \cos k_{n} h} \frac{1}{k_{n}} \quad(n \geqslant 1) \\
& g k_{n} \tan k_{n} h=-\omega^{2} \quad\left(n-\frac{1}{2}\right) \pi<k_{n} h<n \pi \\
& n_{0}=\frac{\tanh k_{0} h}{n_{1}} \xi_{0} \quad n_{1}=\frac{1}{2}\left[1+\frac{2 k_{0} h}{\sinh 2 k_{0} h}\right] \\
& n_{n}=\frac{2 \sin ^{2} k_{n} h}{k_{n} h+\sin k_{n} h \cos k_{n} h} \xi_{o}
\end{aligned}
$$

The first terms in (3 2), ( $\left.\begin{array}{ll}3 & 3\end{array}\right)$ and (3 4) represent a progressive wave, whereas the summations (inertia terms) express the correction necessary to account for the wavemaker motion not being exactly that 
of the particles in a progressiye wave with the given period and depth For long progressive waves, we know that the horizontal particle velocity is practically independent of $y$, and this suggests that the necessary correction, $1 \mathrm{e}$, the sum of the inertia terms, is small when a pistontype wavemaker generates long waves Simllarly, large corrections are needed when short waves are generated by a piston-type wavemaker This was clearly demonstrated in calculations by Blesel and Suquet (1951) We also note, ( 32 ) combined with $\left(\begin{array}{ll}3 & 6\end{array}\right)$, that the exponential behavior of the inertia terms means that their importance becomes negligible within a small distance, of the order $3 \mathrm{~h}$, from the wavemaker

In advancing the theory to second order we proceed as outlined by Stoker (1957) by assuming a perturbation solution and expanding the boundary conditions at the free surface and at the wavemaker around $y=0$ and $x=0$, respectively Denoting the second order solution by superscript (2) we have the governing equations,

$$
\begin{gathered}
\nabla_{\phi}^{2}(2)=\phi_{x x}^{(2)}+\phi_{y y}^{(2)}=0 \quad-h \leqq y \leqq 0 \\
\phi_{y}^{(2)}=0 \\
\phi_{y}^{(2)}+\frac{1}{g} \phi_{t t}^{(2)}=-\frac{1}{g}\left\{\left(\phi_{t y}^{(1)} \eta^{(1)}\right)_{t}+\phi_{x t}^{(1)} \phi_{x}^{(1)}+\phi_{y t}^{(1)} \phi_{y}^{(1)}\right\} \\
-\phi_{y y}^{(1)}{ }_{n}^{(1)}+\phi_{x}^{(1)} \eta_{x}^{(1)} \quad y=0
\end{gathered}
$$

where $\left(\begin{array}{ll}311 \\ 1\end{array}\right)$ is obtained by combining the dynamic and kinematic conditions to obtain a condition in $\phi^{(2)}$ only The second order profile, $\eta^{(2)}$, is given by

$$
n^{(2)}=-\frac{1}{g}\left\{\phi_{t}^{(2)}+\phi_{t y}^{(1)} \eta^{(1)}+\frac{1}{2}\left[\left(\phi_{x}^{(1)}\right)^{2}+\left(\phi_{y}^{(1)}\right)^{2}\right]\right\}_{y}=0
$$

and the boundary condition at the wavemaker

$$
\phi_{x}^{(2)}=-\phi_{x x}^{(1)} \xi \quad x=0
$$

where we have used that $\xi=O(n)$ except for very long waves (see $\left(\begin{array}{ll}3 & 7\end{array}\right)$ )

The troublesome part of the determination of $\phi^{(2)}$ from $(39),(310)$, (3 11) and (3 13) is the inhomogeneous equation (3 11) However, under the assumption of $t \rightarrow \infty, \phi^{(1)}$ and $\eta^{(1)}$ are as given by $\left(\begin{array}{ll}3 & 2\end{array}\right)$ and $\left(\begin{array}{ll}3 & 3\end{array}\right)$ and it can be shown that at $y=0$ the maximum value of the inertia terms is of the order $1 / 4$ of the term associated with the progressive wave so 
long as $\mathrm{h} / \mathrm{L}<1 / 4 \mathrm{It}$, therefore, seems to be a reasonable approximation to neglect the inertia terms when substituting $\Phi^{(1)}$ and $n^{(1)}$ into $(311)$ With this approximation the solution satisfying the equations (3 9) through ( 311$)$ is the well known Stokes' second order solution for progressive waves (see e $g$, Ippen, 1966)

$$
\phi_{P}^{(2)}=-\frac{3}{8} \omega \eta_{0}^{2} \frac{\cosh 2 k_{o}(y+h)}{\sinh ^{4} k_{0} h} \sin 2\left(k_{0} x-\omega t\right)
$$

and

$$
\eta_{P}^{(2)}=-\frac{1}{4} k_{0} \eta_{0}^{2} \frac{\cosh k_{o} h\left(\cosh 2 k_{0} h+2\right)}{\sinh ^{3} k_{0} h} \cos 2\left(k_{0} x-\omega t\right)
$$

However, we have not yet included the boundary condition $x=0$, (3 13), which reads

$$
\begin{aligned}
& \phi_{x}^{(2)}=-\phi_{x x}^{(1)} \xi(y, t)= \\
& {\left[C_{0} k_{0}^{2} \cosh k_{0}(y+h) \cos \omega t+\sin \omega t \sum_{n=1} C_{n} k_{n}^{2} \cos k_{n}(y+h)\right] \xi(y, t)}
\end{aligned}
$$

We have previously justified the neglect of the inertia terms in the boundary condition at $y=0$, however, this cannot be justified for all $y^{\prime} s$ at $x=0$ unless the wave length 1 s restricted further $\left(h / L<0\left(\begin{array}{ll}0 & 1\end{array}\right)\right)$ Introducing ( 31$)$ in ( 316 ) and using the relationship among $\eta_{o}$ and $C_{o}$ we obtain

$\phi_{x}^{(2)}=-\xi_{0}\left[\frac{g \eta_{0}}{\omega} k_{0}^{2} \frac{\cosh k_{o}(y+h)}{\cosh k_{o} h} \cos ^{2} \omega t+\sin \omega t \cos \omega t \sum_{n=1} C_{n} k_{n}^{2} \cos k_{n}(y+h)\right]$

Clearly the solution ( 314 ) does not satisfy this boundary condition Using $\cos ^{2} \omega t=\frac{1}{2}(\cos 2 \omega t+1)$ and taking only the periodic part of $\left(\begin{array}{ll}3 & 17\end{array}\right)$ we see that we have a residual periodic boundary condition at $x=0$

$$
\begin{aligned}
& \left.{ }_{\left(\phi_{R}\right.}^{(2)}\right)_{x}=\phi_{x}^{(2)}-\left(\phi_{P}^{(2)}\right)_{x}=-\frac{g n_{0}^{2} k_{0}^{2}}{2 \omega}\left\{\frac{n_{1} \cosh k_{o}(y+h)}{\sinh k_{o} h}-\right. \\
& \left.\frac{3}{2} \frac{\cosh 2 k_{0}(y+h)}{\sinh ^{3} k_{0} h \cosh k_{0} h}\right\} \cos 2 \omega t-\sin 2 \omega t \frac{\xi_{0}}{2} \\
& \sum_{n=1} C_{n} k_{n}^{2} \cos k_{n}(y+h)=U_{R}^{(2)}(y) \cos 2 \omega t+\sin 2 \omega t \sum_{n=1}\left(V_{R}^{(2)}(y)\right)_{n}
\end{aligned}
$$


Introduc1ng

$$
\phi_{\mathrm{R}}^{(2)}=\phi^{(2)}-\phi_{\mathrm{P}}^{(2)}
$$

in the governing equations, which are linear in $\phi(2)$ we see that $\phi_{\mathrm{R}}^{(2)}$ is the solution to $(318)$ and the homogeneous equations

$$
\begin{array}{ll}
\nabla^{2} \phi_{\mathrm{R}}^{(2)}=0 & -\mathrm{h} \leq y \leq 0 \\
\left(\phi_{\mathrm{R}}^{(2)}\right)_{y}=0 & y=-\mathrm{h}
\end{array}
$$

and

$$
\left(\phi_{R}^{(2)}\right)_{y}+\frac{1}{g}\left(\phi_{R}^{(2)}\right)_{t t}=0 \quad y=0
$$

$1 \mathrm{e}$, the same governing equations as those for the first order solution, except for the more complicated boundary condition (3 18) at $x=0$ The solution, however, can be found from the classical solution to the linear problem as a sum of $\phi_{R}{ }^{\prime} s$, which we may combine and the progressive part can be written as

$$
\phi_{\mathrm{R}, \text { Progressive }}^{(2)}=\mathrm{C}_{\mathrm{o}}^{(2)} \cosh \mathrm{k}_{\mathrm{o}}^{(2)}(\mathrm{y}+\mathrm{h}) \cos \left(\mathrm{k}_{\mathrm{o}}^{(2)} \mathrm{x}-2 \omega t+\psi\right)
$$

where $\mathrm{C}_{\mathrm{o}}^{(2)}$ and $\psi$ are found by combining the $\phi_{\mathrm{R}}{ }^{\prime} \mathrm{s}$ and

$$
4 \omega^{2}=g k_{o}^{(2)} \tanh k_{o}^{(2)} h
$$

We can therefore express the velocity potential far from the wavemaker to the second order

$$
\phi \simeq \phi_{\text {Progressive }}^{(1)}+\phi_{\mathrm{P}}^{(2)}+\phi_{\mathrm{R}}^{(2)} \text { Progressive }
$$

or in physical terms the periodic waves generated by a wavemaker can be expressed as

(1) A first harmonic linear wave of amplitude $\eta_{0}$

(2) A second harmonic coupled with the first harmonic to give the second order Stokes' wave corresponding to the Iinear solution

(3) A second harmonic free wave of small amplitude

This description agrees with that of Fontanet (1961), and when combined, 
we see that the surface elevation at a fixed value of $x$ can be expressed as

$$
\eta=\eta_{0}\left(\sin \omega t+\varepsilon_{1} \sin \left(2 \omega t+\psi_{1}\right)\right)
$$

where $\varepsilon_{1} \ll 1$ This type of surface elevation was shown by Ursell et al, (1960) to give a wave helght, $H=2 \eta_{0}\left(1+0\left(\varepsilon_{1}^{2}\right)\right)$ Thus for small $\varepsilon_{1}$, the wave helght of nonlinear waves, as recorded by a fixed gage, is practically the same as that predicted by a linear theory

This analysis was carried out assuming the final periodic state to be reached, and suggests that when results for perlodic waves obtalned from a linear theory are compared with experiments, a comparison of wave heights should essentially eliminate the influence of finite amplitude From this we conclude that the large difference between computed and experimental wave heights noted in Table 1 can hardly be attributed to nonlinear effects

4 THE INFLUENCE OF LEAKAGE ON THE HEIGHT OF THE GENERATED WAVES

The results of Ursell et at (1960) Indicated that the discrepancy between measured and predicted wave helghts increases from the order $3 \%$ to $10 \%$ with an increase in wave steepness from 003 to 0045 This does not agree with the experiments at CERC, which show a larger discrepancy (of the order 15\%) between theory and observation as well as the opposite trend, $1 \mathrm{e}$, decreasing discrepancy with increasing wave steepness In the experiments by Ursell et al the leakage around the wavemaker was reduced by a rubber foam lining between the piston and the wa11s and bottom of the tank, whereas no such provision was taken in the CERC experiments This suggests that leakage around the piston may have a large influence upon the helght of the generated wave A series of experiments performed at CERC (Tenney, 1969) serve as further evidence of the influence of leakage on the height of the generated waves Two holes were drilled through a piston, the area of the holes was approximately $029 \%$ of the wetted area of the piston It was found that the difference in wave height between the waves generated with these holes closed and open was of the order $28 \%-(16 \%-40 \%)$

\section{Waves Generated by an Oscillating Flow Through a Slot}

To evaluate the influence of leakage let us start by examining the waves generated by the oscillating flow through a slot which extends over the width of the generator

For a slot of helght $\Delta$ a distance $Y$ below the free surface, we have

$$
u(y, t)= \begin{cases}0 & y>-Y+\Delta \\ v_{0} \sin \omega t & -Y \leqq Y \leqq-Y+\Delta \\ 0 & y<-Y\end{cases}
$$


which, with the notation used in Section 3, gives the solution

$$
c_{0}=\frac{4}{2 k_{0} h+\sinh 2 k_{0} h} \frac{1}{k_{0}} v_{0}\left[\sinh k_{0}(-Y+\Delta+h)-\sinh k_{0}(-Y+h)\right]
$$

For small $\Delta$ we may write this as

$$
c_{0}=\frac{4 \sinh k_{o} h}{2 k_{o} h+\sinh 2 k_{o} h} \frac{1}{k_{0}} \quad\left\{v_{0} \frac{\Delta k_{o} \cosh k_{o}(-Y+h)}{\sinh k_{o} h}\right\}
$$

which written in this form by comparison w1th (3 5) clearly shows the generated progressive wave to be the same as that generated by a pistontype wave generator having a velocity given by

$$
U=U^{\prime}=v_{0} \frac{k_{0} \Delta \cosh k_{o}(-Y+h)}{\sinh k_{o} h}-h \leq y \leq 0
$$

In particular we see that for a gap between the wavemaker and the bottom of the tank, $Y=h$,

$$
U_{B}^{\prime}=v_{B} \quad \frac{k_{o} \Delta_{B}}{\sinh k_{0} h}=v_{B} \quad \frac{\Delta_{B}}{h} \quad \frac{k_{o} h}{\sinh k_{o} h}
$$

w1th indices introduced for clarity

Clearly, the influence of leakage between the sidewalls and the piston is not as easy to handle rigorously This leakage is probably one of the mechanisms responsible for the generation of transverse waves, however, if the width of the tank is small compared with the wave length of the generated waves, it seems physically reasonable that the waves generated by an oscillating flow, $v_{s}(y)$ sinwt, through a vertical slot of width $\Delta_{S}$ may be approximated as the waves generated by a piston-type wavemaker, having the prescribed motion

$$
u_{s}^{\prime}=v_{s} \frac{\Delta_{s}}{b}
$$

where $b$ is the width of the wave tank, and $v_{s}$ is the average of $v_{s}(y)$ over the depth

Thus in principle, if $v_{B}$ and $v_{S}$ are known, we can find, at least w1th some accuracy, the generated waves, as those generated by an ideal piston-type wavemaker having the prescribed motion

$$
u(y, t)=\left(U_{B}^{\prime}+U_{S}^{\prime}\right) \sin \omega t
$$

However, is the potential theory really appropriate? When the water is 
forced through the small gaps into the ambient fluid one might expect a considerable energy loss due to turbulence In a study of the forced heave motion of a rectangular cylinder of large draught, $1 \mathrm{e}$, sma11 distance between bottom and cylinder $\left(\Delta_{B} \approx 006 \mathrm{~h}\right)$, Svendsen (1968) found the radiated wave to be accurately predicted by potential theory Thus, an inviscid theory seems indeed to give reliable results, however, contrary to Svendsen's study, the major problem in our case is determining the velocities

\section{Determination of the Leakage Velocity}

In order to attack this problem we must first specify the conditions on both sides of the generator blade If we assume that the region behind the wavemaker is occupied by an absorber beach, which corresponds to the CERC 72-foot tank, a reasonable assumption is that waves are generated in both directions and that these waves are the same, but $180^{\circ}$ out of phase

With this assumption the pressure due to the sinusoldal motion (3 1 ), of the piston on the front side is given by (3 4 ) and the pressure due to the wave on the back of the piston is of equal magnitude but opposite sign If we restrict our analysis to moderately long wave $(h / L<1 / 4)$ the influence of the terms in (3 4) with exponential behavior in $x$, the inertia terms, is small compared with that of the term associated with the progressive wave Thus, we may approximate the pressure difference between the two sides of the piston, $\Delta p$, by

$$
\Delta \mathrm{p}=\mathrm{p}_{\text {front }}^{+}-\mathrm{p}_{\mathrm{back}}^{+} \simeq 2 \rho g \eta_{0} \frac{\cosh \mathrm{k}_{\mathrm{o}}(\mathrm{y}+\mathrm{h})}{\cosh \mathrm{k}_{\mathrm{o}} \mathrm{h}} \text { sinwt }
$$

where $\eta_{0}$ is given by ( 37 )

This pressure difference will produce a flow through the gaps between the piston and the sides and the bottom of the tank The velocity, $v$, of this flow may be estimated from Bernoul11's equation Neglecting friction and the unsteadiness of the motion we have

$$
v^{2} / 2 g=\Delta p / \rho g
$$

In particular we get for the gap at the bottom, $y=-h$, by introducing (4 8) and defining $v$ to be positive when directed towards the front of the piston, that

$$
v(y=-h)=-\operatorname{Sign}\{\sin \omega t\} 2 \sqrt{\frac{g \eta_{o}}{\cosh k_{o} h}} \sqrt{\sin \omega t}
$$

To comply with the type of boundary condition assumed in (4 1), we can expand the time dependence of $(410)$ in a Fourler Series This has been done by Keulegan (1967), and retalning only the term associated 
with sinwt, we get

$$
v(y=-h)=-222 \sqrt{\frac{g n_{0}}{\cosh k_{o} h}} \sin \omega t
$$

or with the notation used in Section 41 we have

$$
v_{B}=-222 \sqrt{\frac{g \eta_{0}}{\cosh k_{o} h}}
$$

To find the leakage velocity through the gaps along the sides of the piston, we proceed in a similar manner, and taking the average leakage velocity over the depth, $h$, as the mean of the velocity at $y=0$ and $y=h$ we get

$$
v_{s}=-111 \sqrt{g \eta_{0}}\left(1+\frac{1}{\sqrt{\cosh k_{o} h}}\right)
$$

\section{Decrease in Wave He1ght Due to Leakage Around the P1ston}

By substituting (4 12) and (4 13) into (4 5) and (4 6) respectively, we get from ( 4 7) that the leakage around the p1ston produces waves, whose characteristics are approximately those of the waves generated by an ideal piston-type wavemaker having the prescribed motion

$U^{\prime}=-\sqrt{g \eta_{o}}\left(222 \sqrt{\frac{1}{\cosh k_{o}^{h}}} \frac{\Delta_{B}}{h} \frac{k_{o} h}{\sinh k_{o} h}+111 \frac{\Delta_{S}}{b}\left(1+\sqrt{\frac{1}{\cosh k_{o} h}}\right)\right.$

Comparing (4 14) w1th (3 1) we see that the leakage around the p1ston w111 decrease the amplitude of the generated waves by an amount $\Delta n_{0}$, which may be found from

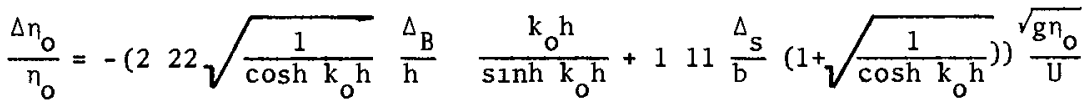

where $\eta_{0}$ is found from (3 7)

To test the validity of (4 15) some experiments were performed in the CERC 72-foot tank, which has a w1dth, $\mathrm{b}=15$ feet Measurements gave $\Delta_{B}=028$ inches and $\Delta_{S}=2 \times 01=02$ inches W1th water depth $h=15$ feet, the wave he1ght, $H_{\text {exp }}$, as recorded 16 feet from the generator serve for the comparison presented in Table 2 


\begin{tabular}{|c|c|c|c|c|c|}
\hline$h / L$ & $\begin{array}{c}\xi_{0} \\
\text { (feet) }\end{array}$ & $\begin{array}{c}\mathrm{H}_{\exp } \\
\text { (feet) }\end{array}$ & $\begin{array}{l}2 n_{0} \\
\text { (feet) }\end{array}$ & $\begin{array}{l}\text { Difference } \\
\text { Observed } \\
\%\end{array}$ & $\begin{array}{l}\text { Difference } \\
\text { from }\left(\begin{array}{ll}4 & 15\end{array}\right) \\
\%\end{array}$ \\
\hline \multirow{2}{*}{0132} & o 167 & $0 \quad 239$ & 0274 & 128 & 178 \\
\hline & 0251 & 0367 & 0411 & 107 & 146 \\
\hline \multirow{3}{*}{$\begin{array}{ll}0 & 197\end{array}$} & 0115 & 0228 & 0272 & 162 & 158 \\
\hline & 0173 & 0356 & 0410 & 132 & 129 \\
\hline & 0230 & O 477 & 0545 & 125 & 112 \\
\hline \multirow{3}{*}{0240} & 0125 & 0300 & 0352 & 148 & 127 \\
\hline & 0167 & 0390 & 0471 & 172 & 111 \\
\hline & 0196 & 0466 & 0553 & 153 & 102 \\
\hline
\end{tabular}

Table 2 Comparison of observed reduction in wave he1ght with that predicted from (4 15)

When considering the number of assumptions made in deriving (4 15), the agreement between predicted and observed reduction in wave height must be considered good It is therefore concluded that the main part of the discrepancy between observed and predicted wave heights noted in Table 1 can be attributed to the influence of leakage around the piston As a further check on the reasoning leading to (4 15), the reduction in wave height due to two holes in the piston, corresponding to the experiments by Tenney (1969) mentioned earlier, gave a predicted reduction of $21 \%$, wh1ch compares favorably w1th Tenney's $16-40 \%$

The neglect of friction and unsteadiness made in order to arrive at (4 9) was tested by mounting a fitted $3 / 4$ inch plywood board to the front of the piston blade This increased the thickness of the blade from $1 / 4$ inch to 1 inch, and since this had no significant effect on the height of the generated wave, this assumption seems justified

It 1 interesting to note that the area of the gaps around the piston is of the order $27 \%$ of the wetted area, and that leakage through these gaps reduces the wave helght by about 15\% Th1s large effect of leakage through a small area, which is even more pronounced in the experiments by Tenney, may explain the results of Ursell et al (1960), whose "seal" around the piston might not have been $100 \%$ effective for the large pressure difference between the two sides of the piston associated with the steepest waves When one considers the large gap, which may exist at the 
bottom in the case of a flap-type generator hinged at the bottom, the large effect of leakage through this gap may also explain why a group of Neyrpic engineers (1952) observed a discrepancy of $30 \%$ between theoret 1 m cal and measured wave helghts of waves generated by a flap-type wavemaker

\section{CONCLUSION}

In the preceding sections we have used a theoretical solution, based on the linearized governing equations, to predict the height of the waves generated by a piston-type wavemaker starting from rest By comparison with experiments it was found that the theoretical model predicted the relative wave helghts falrly accurately, and that the difference between predicted and observed wave heights could be attributed to the influence of leakage around the piston rather than to the inadequacy of the linear model Thus the material presented essentially serves to establish confidence in the theoretical model adopted

As stated in the lntroduction, the problem initiating this study was the large first and last waves in a burst (Figure 1) Since the large last wave can be eliminated by the use of a drop-gate or a similar structure, the preceding sections have concentrated on the development taking place as the wavemaker is started Computations show that the relative magnitude of the large first wave depends weakly on the position of the wavemaker, as it is started, and that this is smallest, when the wavemaker is started from 1ts extreme positions Th1s dependence 1s, however, Insignificant at stations far from the wavemaker, and consequently offers no solution to the problem of eliminating the large first wave

From the analogy between $F_{1}$ gure 3 and the response of a mechanical system to an exciting force, it is seen that the large first wave may be eliminated by starting the wavemaker slowly increasing the amplitude of its motion to the desired value If this is possible and how it is most efficiently achieved can be determined by means of our theoretical model Most wavemakers being of the type, which does not permit a change in stroke during operation, seems to render this solution impractical However, this is where it might be possible to utilize the surprisingly large effect of leakage on the helght of the generated waves By controlling $e \mathrm{~g}$ the area of the gap between the bottom and the piston, $1 \mathrm{t}$ would, in principle, be possible to change the height of the waves generated by a wavemaker having a constant stroke If this effect can be used to eliminate the large first wave remains to be seen However, this effect does seem to offer the possibility of generating amplitude modulated waves with a conventional wavemaker

\section{ACKNOWLEDGMENT}

The work described herein is a portion of the research program of the $U$ S Army Corps of Englneers and 1ts Coastal Engineering Research Center Permission to publish is appreciated The author wishes to thank Mr John Ahrens, Hydraulıc Eng1neer at CERC, for making his experiments avallable for comparison with the computed results 


\section{REFERENCES}

Benjamın, T B (1967), "1nstability of Perıodic Wave Trains in Nonlinear Dispersive Systems", Proceedings of the Royal Soclety, Series A, No 283

B1ésel, F and Suquet, F (1951), "Les Appare11s Générateurs de Houle en Laboratolre", La Houl11e Blanche, Vol 6, No 2, pp 147-165

Fontanet, P (1961), "Theorie de la Genération de 1a Houle Cylındrique par un Batteur Plan", La Houllle Blanche, No 1, pp 3-28

Galvin, C J , Jr (1968), "Shapes of Unbroken Perıodıc Gravity Water Waves" (Abstract), Transactions of the American Geophysical Union, Vol 49, No 1, p 206

Havelock, T H (1929), "Forced Surface-Waves on Water", Ph1losoph1ca1 Magazıne, Serıes F, Vol 8, pp 569-76

1ppen, A T (Edıtor) (1966), "Estuarıne and Coast1ıne Hydrodynamıcs", McGraw Hz11, New York

Kennard, E H (1949), "Generation of Surface Waves by a Moving Partition" Quarterly of Applied Mathematics, Vol 7, No 3, pp 303-312

Keulegan, G H (1961), "Tidal Flow in Entrances", Committee on Tidal Hydraulıcs, U S Army Corps of Engineers, Tech Bulletın No 19

Madsen, O S , Me1, C C and Savage, R P (1970), "The Evolution of Time-Perıodic Long Waves of Finite Amplitude", Journal of Fluid Mechanics, Vol 44, pp 195-208

Neyrp1c (1952), "Les Appareıls Générateurs de Houle en Laboratolre, La Houl11e Blanche, Vol 7 , No 6, pp 779-801

Stoker, J J (1957), "Water Waves", Interscience, New York

Svendsen, 1 A (1968), "On the Forces Induced on a Rectangular Cylinder by a Forced Heave Motion with Draught-Depth Ratio Close to Unity", Journal of Hydraulic Research No 4, pp 335-360

Tenney, L W (1969), "Wave Heıght Varıatıon Caused by Holes in the Wave Generator Blades", (Unpublıshed Report) Coastal Englneerıng Research Center

Urse11, F, Dean, R G, and Yu, Y S (1960), "Forced Small-Amplitude Water Waves A Comparison of Theory and Experiment", Journal of Flu1d Mechan1cs, Vol 7, Part 3, pp 33-52 



\title{
CHAPTER 37
}

\section{PREDICTION CURVES FOR WAVES NEAR THE SOURCE OF AN IMPULSE}

\author{
Robert L Miller \\ Department of the Geophysical Sciences \\ The University of Chicago
}

\section{The Problem}

The general problem of single-Impulse induced waves has been under experimental investigation by the author for several years The present paper is confined to a presentation of the wave modes which are to be expected for various permutations of displacement velocity and displacement length at the Impulse source other portions of the study will be published el sewhere

It is clear that a number of geometric arrangements in the laboratory may be made to generate impulse waves, for example, sudden upthrust or downdrop of a block on the tank bottom analagous to block faulting on the sea-floor in nature, or a hinged flap moving on the bottom analagous to the undulatory bottom motion which may occur during seismic disturbances on the sea floor However, it is my opınion that the Impulse generator which is most simple and most easily related to contemporary generalızed theory, is a single piston thrust at one end of the wave tank which pushes the fluid down channel (See Figure 1) As an additional simplification, the investigation is carried out for the flat bottom, fixed depth case, relegating the effect of varying depth to a later study

The parameters of the Impulse may thus be adequately described by the dimensionless variables $\ell / d$ (where $\ell$ is distance the pistion is displaced, and the constant, $d$, is the undistured water depth) and a piston Froude number $\bar{v} / \sqrt{g d}$ (where $\bar{v}$ is the piston speed and $\sqrt{g d}$ is the long wave velocity The range of dimensionless piston displacements and piston velocities were chosen to cover reasonably well (where information is available), the movements of natural impulse generators This includes coastal landslides enterıng a body of water, I ceberg calving, seismic movements of various kınds, and explosions None of the types of natural impulse generators referred to above are clearly modeled by a horizontal piston motion, although in many cases, the resultant of the natural motion may $f i t$ rather well, thus indicating the generality of this simple model

Permutations of $\ell / d$ and $\bar{V} / \sqrt{g d}$ (or $F_{\bar{V}}$ ) through the selected range yields a series of points on the $\ell / d, F_{\bar{y}}$ plane if a $\ell / d, F_{\bar{y}}$ plane is fixed at some arbitrary distance from the end of the piston motion, each point on the plane may be further described in terms of wave mode, some sort of wave speed, wave amplitude, etc If a single wave is tracked down channel, it will be indicated by a fixed point on the $\mathcal{l} / \mathrm{d}, \mathrm{F}_{\bar{y}}$ plane representing initial generating conditıons However, the nature of the wave mode, and the magnı tude of wave speed and amplıtude will change during the transformations which occur as the impulse wave progresses down channel Thus, although the position of the point on the $l / d, F_{\bar{v}}$ plane does not change for a series of distances from the piston, the value or nature of the parameters for that point, will change This yields a series of graphs consisting of $\ell / d, F_{\bar{V}}$ planes at various $f_{i x e d}$ 
distances from the piston, which indicate the changes in wave mode, Froude number and maximum amplitude (FIgures 5,6 and 7)

Since the Impulse waves are undergoing transformations and decay as they progress down channel, the question of a meaningful velocity must be considered In this study, two velocities are measured The first is a "phase velocity" A fixed point on the wave profile is tracked on multichannel oscillograph records it is assumed that the transformations take place slowly relative to the short distance between two recording stations, and that the error in this approximation is not large This method which yields an average velocity between points approximately 25 meters apart is in maximum error in the bore range near the piston where transformation is rapid and improves down channel Thus, 25 meters is regarded as a characteristic "transformation distance" for these wave channel experıments The Froude number representing this measurement of velocity is referred to as $F_{\bar{C}}$, and the results are shown in Figure 6

The second velocity in this study is the Boussinesq (1872) velocity of propagation of a volume element of the impulse wave We have succeeded in devising an electronic system which will measure this velocity but due to space limitations, the method and results must be published elsewhere

\section{Sensing and Recording System}

The surface time history of the Impulse waves is obtained through capacitance probe sensors at a series of stations along the channel (Figure 2, D) The wave sensor is basically an amplitude modulated square wave generator As the probe is immersed in the fluid, the capacıtance increases, resulting in a modulated envelope directly proportional to the instantaneous degree of Immersion for details see L F McGoldrick, 1969

The probe is constructed of \#17 gage hypodermic tubing with a polyethylene sleeve (O D $1194 \mathrm{~mm}$ ) forming the dialectrıc (FIgure 3a) Capacitive sensitivity is $20 \mathrm{pfd} / \mathrm{cm}$ giving a signal sensituity of $05 \mathrm{~V} / \mathrm{cm}$

The probe unit is driven by a $20 \mathrm{~V}$ D C power supply The wave information in D C volts from the probe actlvates an operational amplifier ( $F ı g$ 2,C and $F ı g$ 3) programmed as a subtractıng amplıfier, deleting the signal due to static water level (FIg 2,A), and also serving as the resultant output sensitivity control (FIg 2,E) This proved to be very useful when the experıments required an array of probes, and led to standardized calıbration for all probes The signal from the subtracting amplifier activates an oscillograph recorder for graphic analysıs of wave propertıes (Fıgure 4) 
The signal from the subtractıng amplifier also goes to a second operational amplifier programmed to interact with a capacitor to perform a graphic integration of the wave amplitude (Fıg 2,B and $F i g$ 3) The output from this unit activates an oscillograph recorder for graphic analysis of the wave volume (Figure 4) A ramp control is used to offset potential that may have accumulated in the integrator unit before the arrival of the wave

This brief summary given in this section will be considerably amplified in a forthcoming technical report, White and Miller, Tech Rept No 10, 1970

\section{Results}

Figure 5 shows the changes in wave mode as a function of distance from the impulse source Four initial wave modes are recognized These are, in order of increasing initial energy with the lowest first, "sinusoid", "solı tary", "undular bore" and fully developed bore (See Figure 4)

The following conclusions are drawn

1 The fully developed bore decays rapidly to the unbroken undular bore form (FIgs $5,6,7$ )

2 The lead wave of the undular form takes on the "sol I tary" mode leaving behind the rest of the "undular" wave in several cases, the second and even the third undulations in turn take on the "solı tary" mode (FIgs 5e,f)

3 The "sinusold" mode generated at short piston displacements transforms gradually as the trailing trough rises to the und Isturbed water level, and finally enters the "sol I tary" mode (FIgs $5 a-f$ )

The term "solı tary" is applied to the stable, symmetrical form which satisfies the following conditions

1) both leading and trailing portions asymptotıcally approach the static level

2) phase velocity agreed well wi th Laitones 1961 wave speed equation taken to the first three terms $\quad C=49 d\left(1+\frac{1}{2} \frac{H}{d}-\frac{3}{20}\left(\frac{H}{d}\right)^{2}+\right.$

3) amplitude as a function of volume, agrees well with the relationship given by Kuelegan and Patterson, $1940 \quad h_{1}=\frac{3 Q^{2}}{16 H^{3}}$ where $h_{1}$ is $\max$ amp, $Q$ is cross-sectional area, and $H$ is static water depth 
4 The Froude number ${ }^{2}$ gradient indicates no striking irregularıtıes, and Ind Icates that all wave modes are in the breakıng regime at approximately $F_{\bar{C}}=126 \quad$ (F/gure 6)

5 The experimentally establıshed line dividing the breakıng region from the unbroken region seems to be I ndependent of wave mode The breakıng wave region occupies about half of the $1 / d, F_{\bar{y}}$ plane very near the impulse source at $D / d=10$ and decreases steldily toward the upper $r$ ight corner at $D / d=188$ ( $F$,gure 5)

6 There appears to be no alternative to the conclusion that given a predictable distance down channel from the impulse source, all Impulse waves $w i l l$ assume the "most stable"l mode in agreement with the predicition of Boussinesq, 1872, I e the "solıtary" form has the minımum "moment of Instabılıty" and In agreement with the discussion in Keulegan and Patterson, 1940 p 87

7 The decay sequence for a fully developed bore at the impulse source is Bore $\longrightarrow$ undular bore $\longrightarrow$ lead undulation becomes solıtary, + trailıng undular bore consısting of remainıng undulations $\longrightarrow$ lead undulation sol itary + second undulation becomes solıtary $\longrightarrow$ in several runs three successive solitary forms have emerged from the preceeding undular form

\section{Discussion}

The graphs given in Figures 5,6 and 7 form a relatively complete set of prediction curves for waves created by a horizontal impulse, surface to bottom from these graphs, it is possible to predict the wave type, maxımum wave amplıtude, and maxımum "phase speed" for any I mpulse wave within the estımated natural impulse generating range These are open ended predictıons, however for example, the published $\not / d$ for "Shot Baker" at BIkı nı in the late 1940's is approximately 5 (Johnson and Bermel, 1949) The $F_{\bar{y}}$ value is undoubtedly very high but not avallable in the landslide case, I have estımated $\ell / d$ to be about 80 based on examination of landslide scars on coastal charts, if one takes avalanche figures for displacement velocity, the $F_{\bar{y}}$ values may easily exceed 400 Large scale seismic movements are particularly difficult to estımate, but for a submarıne overthrust, $l / d$ less than 10 and $F_{\bar{V}}$ less than 03 may be reasonable In general, natural impulses yielding $Y / d$ values in excess of 90 coupled $w i$ th $F_{\bar{V}}$ greater than 10

\footnotetext{
${ }^{2}$ Since this Froude number is based on an average phase velocity, it is insensitive to changes in velocity over the wave profile during transformatıons I have found that the Boussinesq velocity on the other hand does bring out these changes quite clearly
} 
should not change the pattern of the prediction graphs The wave mode will be elther an undular bore or fully developed bore, with the same transıtion and decay sequence found in this study, differing only in magnitude I feel extrapolation in this sense is justified The very low $\ell / d$ high $F_{\bar{y}}$ corner of the graphs is a different matter My present generating system will not produce impulses in this region it would appear that some other sort of Impulse generating mechanism should be devised for this region of the $\ell / d, F_{\bar{V}}$ plane

Finally, it seems suggestive that the linear theoretical models for Impulse waves of the type discussed in Lamb (1932, Art 238ff) are inadequate For example, Cauchy-Poisson wave trains are not generated by this type of impulse mechanısm in which the inıtial conditions include a velocity and surface elevation This is in clear contrast to the exper Iments of Prins 1958 where the I $\mathrm{nIt}$ ial condıtıons Include a surface elevation but no initial velocity it is interesting to note that Prins found wave modes similar to those described in this paper but also for the low energy impulse, obtaıned an oscillatory wave region of the Cauchy-Poisson type it is possible that a similar type of wave may exist in the low $\boldsymbol{l} / \mathbf{d}$ very high $F_{\bar{y}}$ region (Figure 5 ) which in my study was not investigated Further development of the more realistic nonli near models, Perigrine 1968,y is required to fit the "real" case 1969 ,

Acknowledgements are due to Robert $V$ White who is responsible for a major portion of the sensing and recording system, to $L F$ McGoldrick for advice and comment on almost all phases of the study, and to $J M$ Witting for several illuminating discussions This study was supported by the office of Naval Research Geography Branch, N0014-67-A-0285-0013 


\section{B,bl lography}

Boussinesq, J Theorie des ondes etc Liouville's Jour Vol 17, 1872

Johnson, $J W$ and $K J$ Bermel Impulsive waves in shallow water as generated by falling weights Trans A G U V 30, No 2, Apr I , 1949, pp 223-230

Kuelegan, G H and G W Patterson Mathematical theory of Irrotatıonal translation waves Jour of Res of Nat Bur Standards, Vol 24, R P 1272, 1940

Laıtone, E $V$ Higher approxımatıon to non-lınear water waves and the l Imıtıng helghts of Cnoldal, Solıtary and Stokes waves inst of Eng Res Tech Rept 89-6, Berkeley, Calıf, 1961

Lamb, H Hydrodynamıcs, 6th Edıtıon, Dover Press (Ist'Ed) 1932

MCGoldrick, L F A system for the generation and measurement of capillarygravity waves Office of Naval Research, Tech Rept No 3 , August 1969

Miller, $R \quad L$ and $R \quad V$ White A single impulse system for generating sol I tary, undulatıng surge and gravity shock waves in the laboratory Tech Rept No 5, Fluıd Dynamıcs Lab , Dept Geophys, Univ of Chicago, 1966

Perigrıne, D H Long waves in a channel of arbitrary cross-section Jour Fluid Mech $32,353-365,1968$

lbid, Solıtary waves in trapezoidal channels Jour Fluid Mech 35 , $1-6,1969$

Prıns, J E Characterıstıcs of waves generated by a local disturbance Trans A G U Oct 1958 pp $865-874$

White, $R \quad V$ and $R \quad L$ Miler An electrical system for sensing and analysıng wave propertıes Including Integration and different capabılıty Tech Rept No 10, Fluid Dynamıcs Lab, Dept Geophys Scl, Unıv of Chıcago (In press) 


\section{Figure Captions}

\section{FIGURE}

1 Schematic of wave generating and wave sensing system for details of the wave tank facılity see Miller \& White, 1966

2 Flow sheet for wave recording and integratıng systems

3 Detaıled schematıc of operatıonal amplıfiers, wave recordıng and integrating

3a Capacı tance probe unıt for sensing waves

4 The four possible wave modes due to a single hor izontal impulse The cross-sectional area of fluid displaced by the piston is matched against the cumulative curve (Integratıon over wave height) $B$ in the figure This produces a cut-off point (A) on the wave trace where the cross-sectional area under the wave trace is equivalent to the cross sectional area of the fluid volume at the impulse source due to the several damped back and for th oscillatıons which occur after the magnetıc brake has been applıed

5 The wave mode field as a function of impulse displacement $l / d$ and I, b, c, d,e, $f$ speed of displacement in Froude number form $F_{\bar{y}}$ or $(\bar{V} / \sqrt{g d})$ for $f i x e d$ distances from the impulse source $\ell$ is piston displacement d is undisturbed water depth $D$ is distance from impulse source and $\vec{V}=\ell / \mathrm{t}$ where $t$ is elapsed time during piston displacement Note that in $5 \mathrm{a}$ at $\mathrm{D} / \mathrm{d}=10$ closest to the source, there is no "sol I tary" wave region, whereas the "solıtary" region is significant $D / d=65$, and domınant at $D / d=150$

6 Froude number gradıent, $F_{\bar{c}}$, superımposed on the wave mode field $a, b, c$ at $D / d=10,65,150$ Note that the breakıng region retreats steadıly toward the upper right corner with increase in distance from impulse source, and that the breaker IIne always lies between $F_{\tilde{V}}=125$ and $13 C$

7 Maxımum wave amplıtude gradıent superımposed on wave mode field for $a, b, c \quad D / d=10,65,150 \quad$ Because of the relatively coarse contour interval, the abrupt drop in maxımum amplı tude from smooth to breakıng appears only in Fig $7 \mathrm{~b}$, at $\mathrm{D} / \mathrm{d}=65$ 


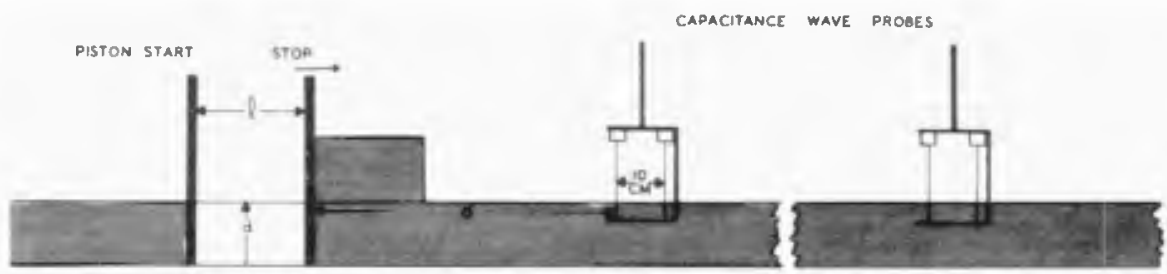

$\ell=$ HORIZ ONTAL. PISTON DISPLACEMENT

$d=$ STATIC WATER DEPTH

$0=$ DISTANCE FROM END OF PISTON

LOCATION

Fig. 1

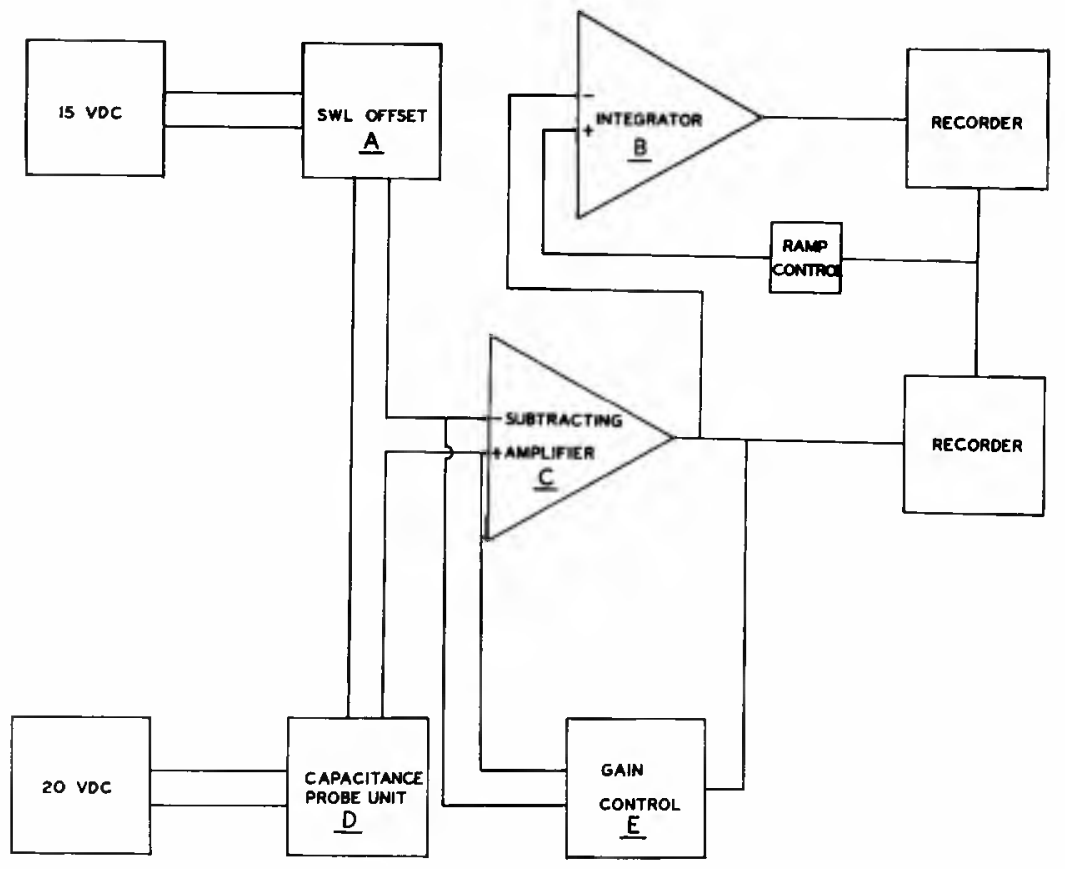

Fig. 2 

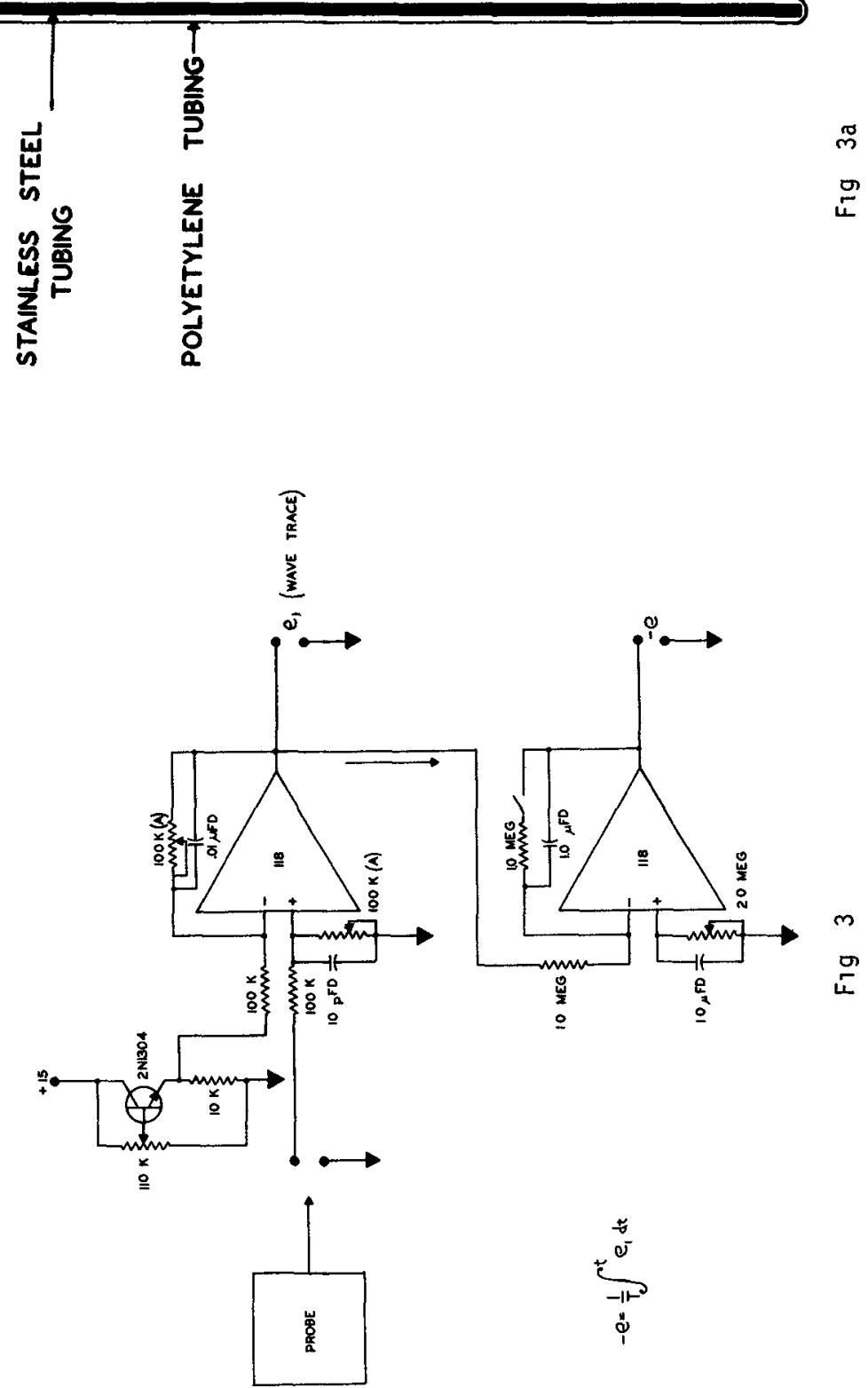
WAVE MODES

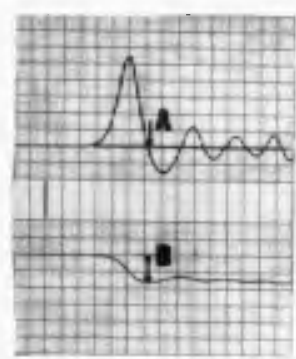

"SINUSOIDAL"

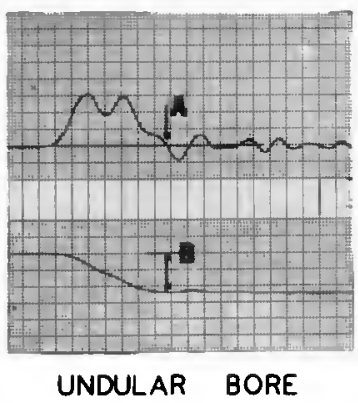

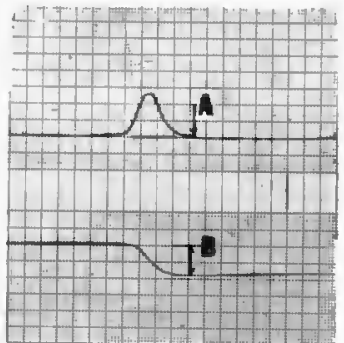

"SOLITARY"

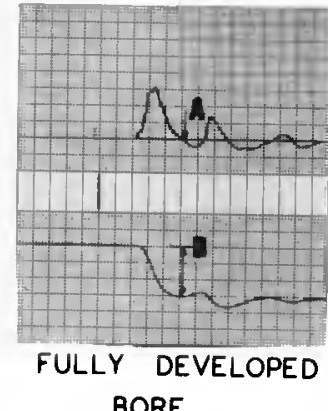

Fig. 4 


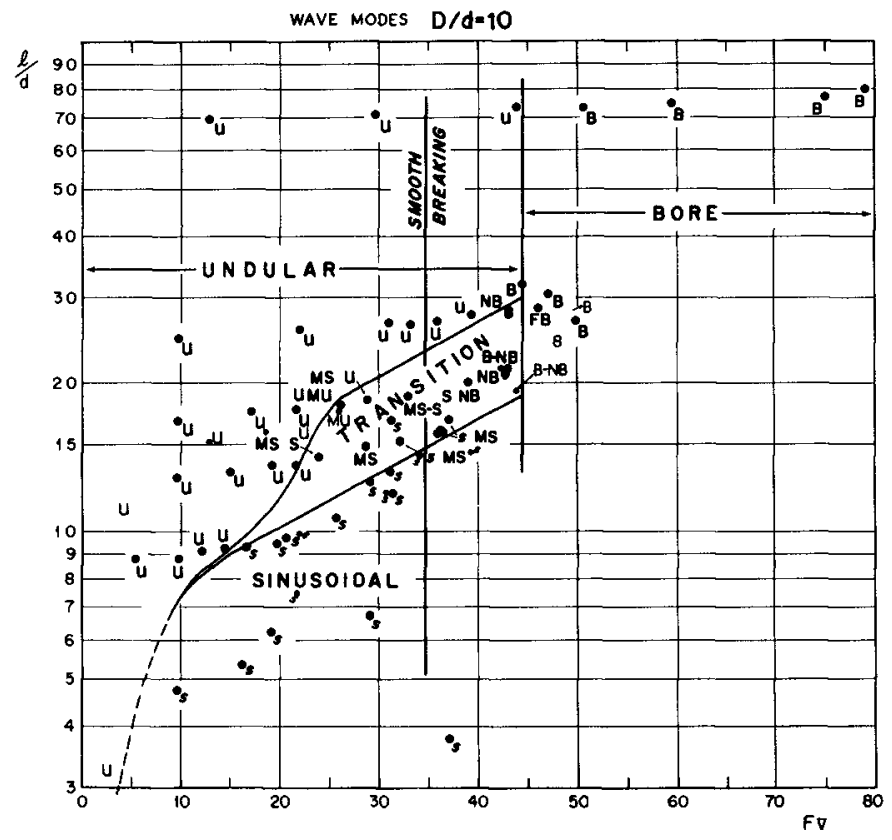

Fig $5 a$

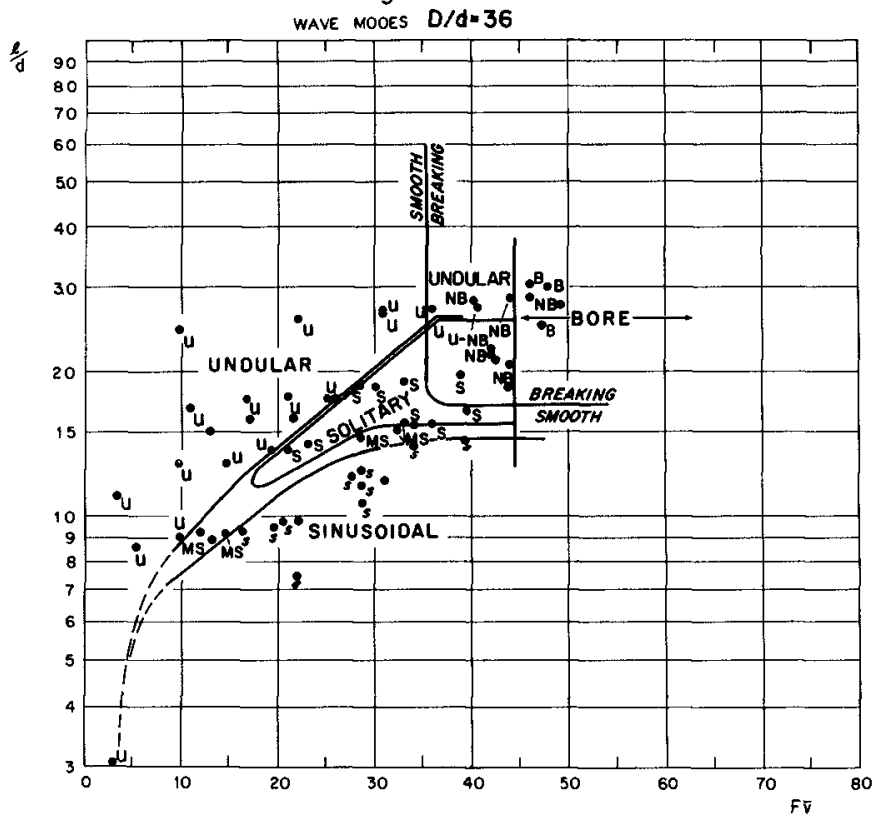

F1g 5b 


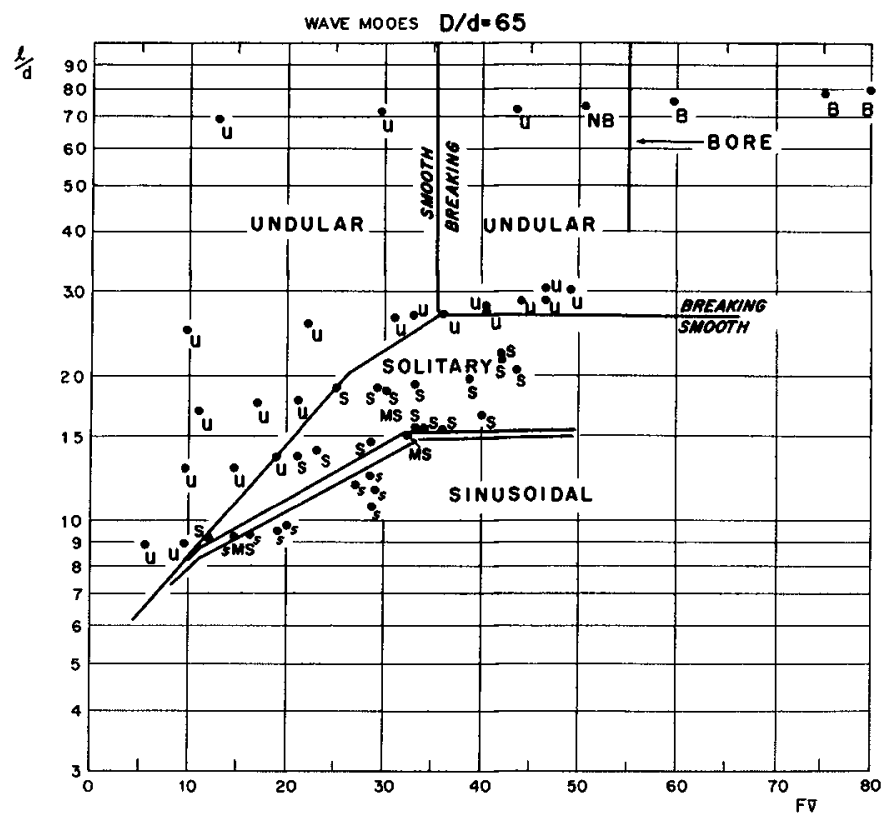

Fig $5 \mathrm{C}$

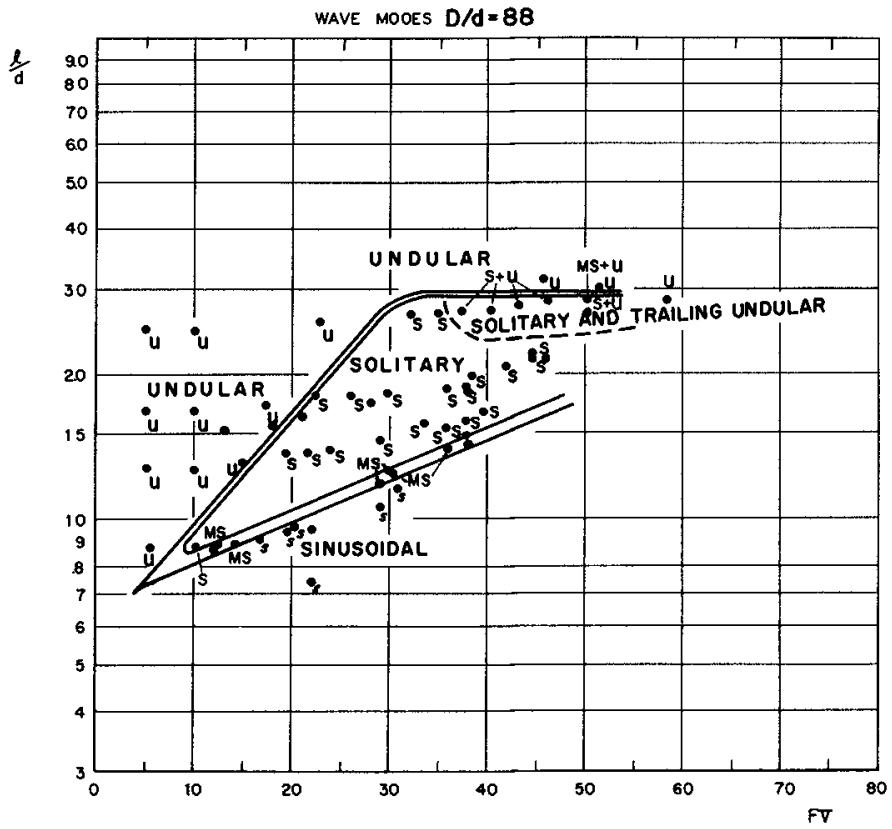

Fig 5d 


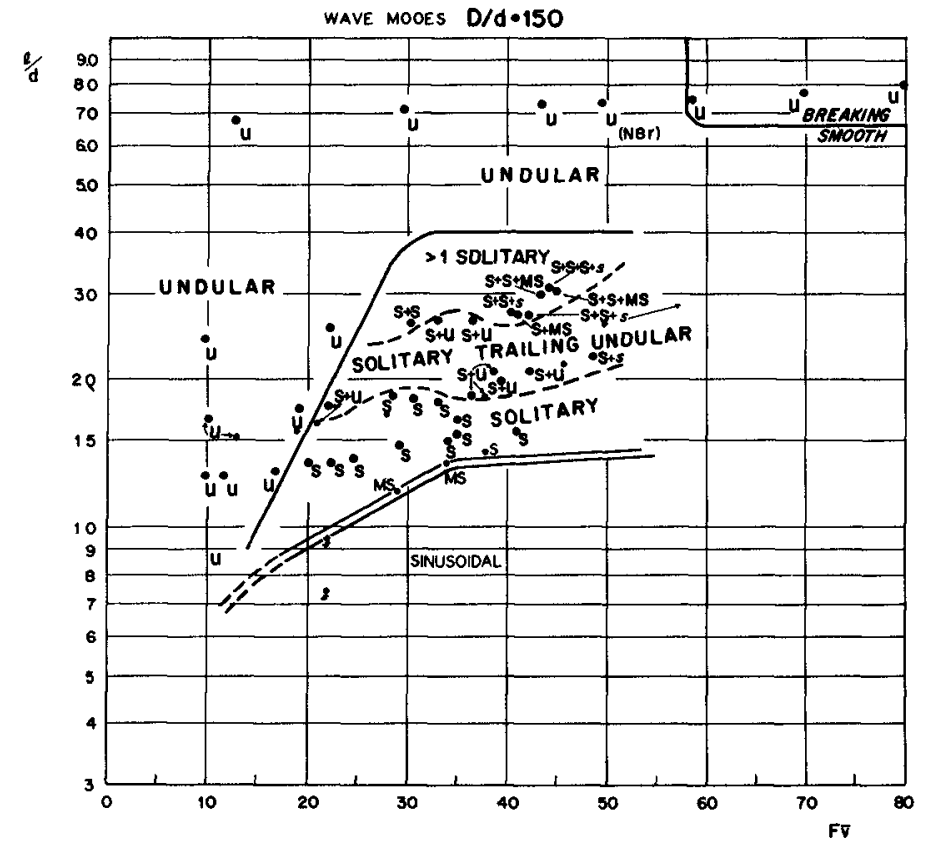

Fig $5 e$

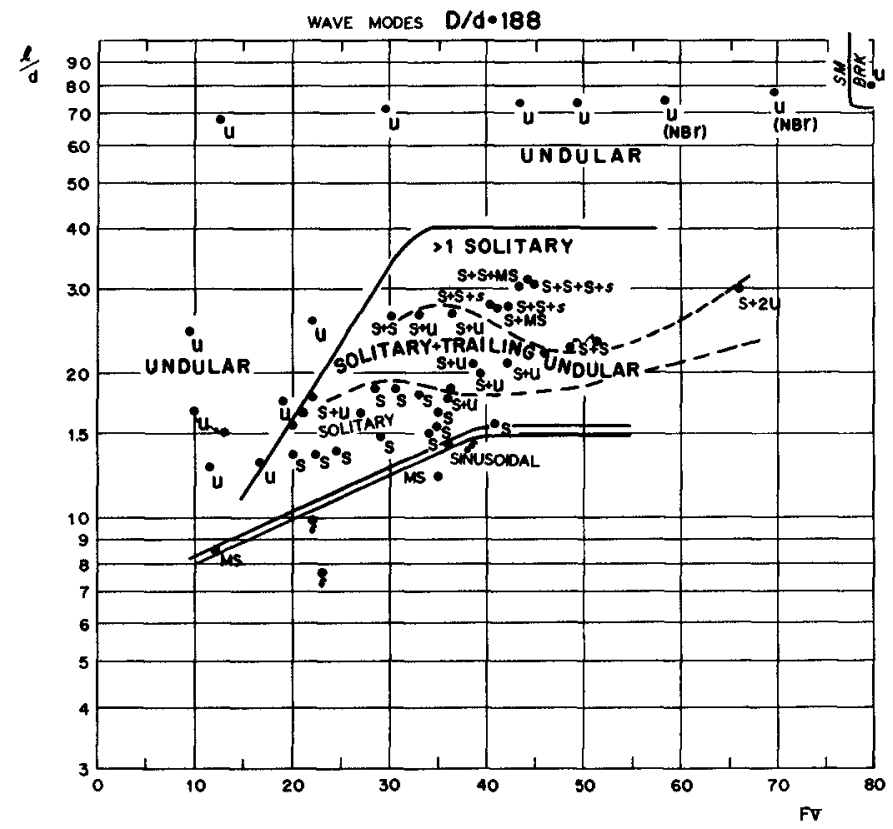

Fig $5 f$ 


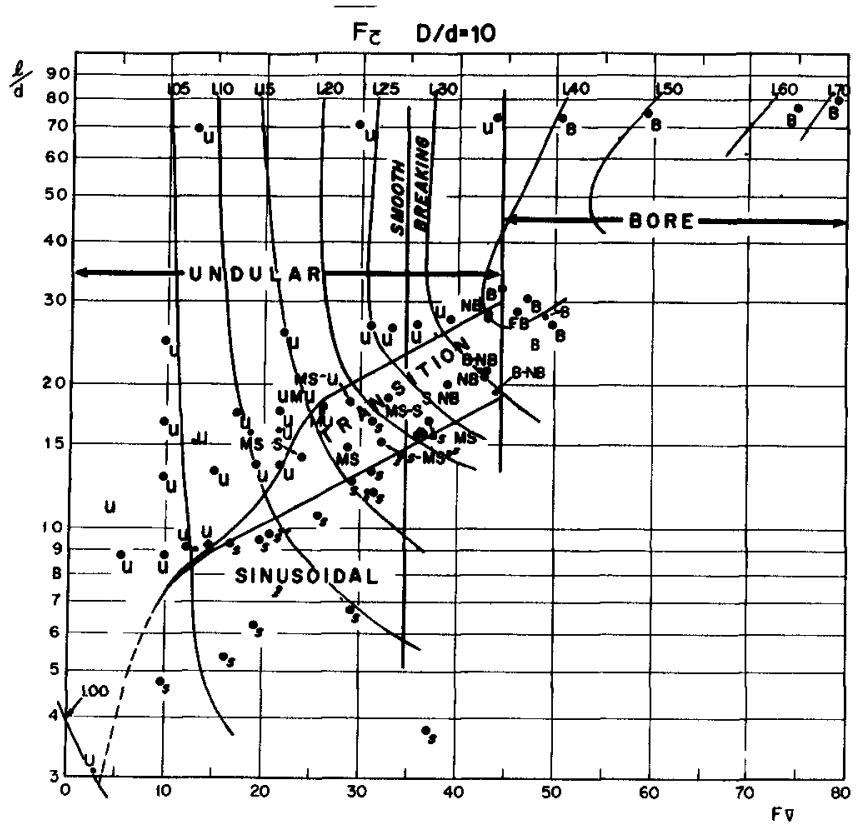

Fig 6a

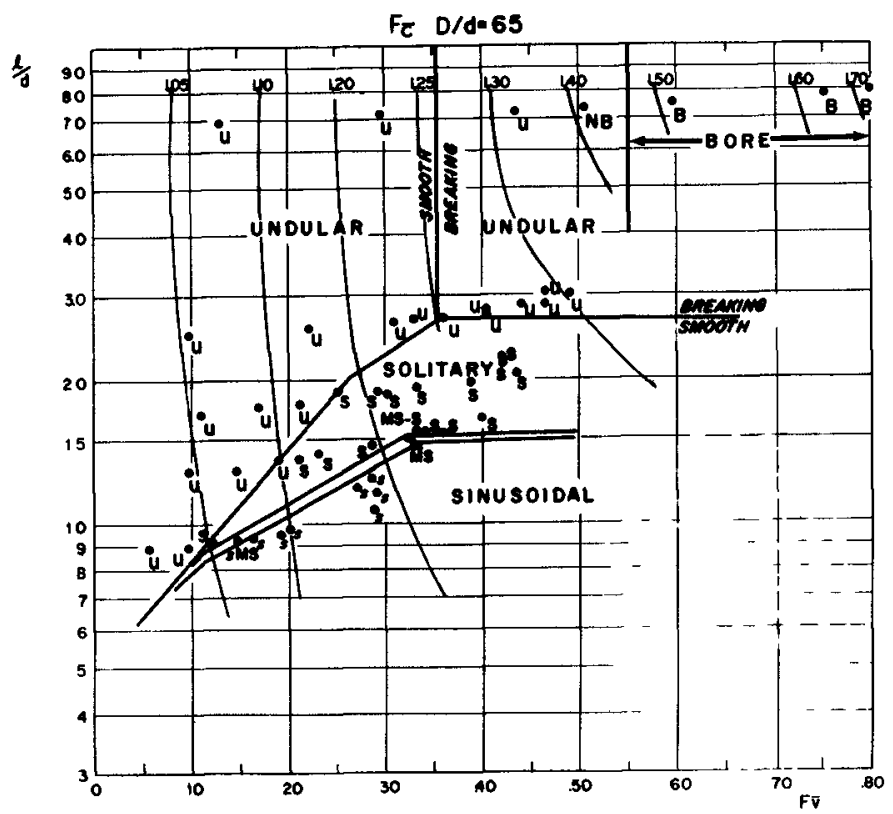

Fig $6 b$ 


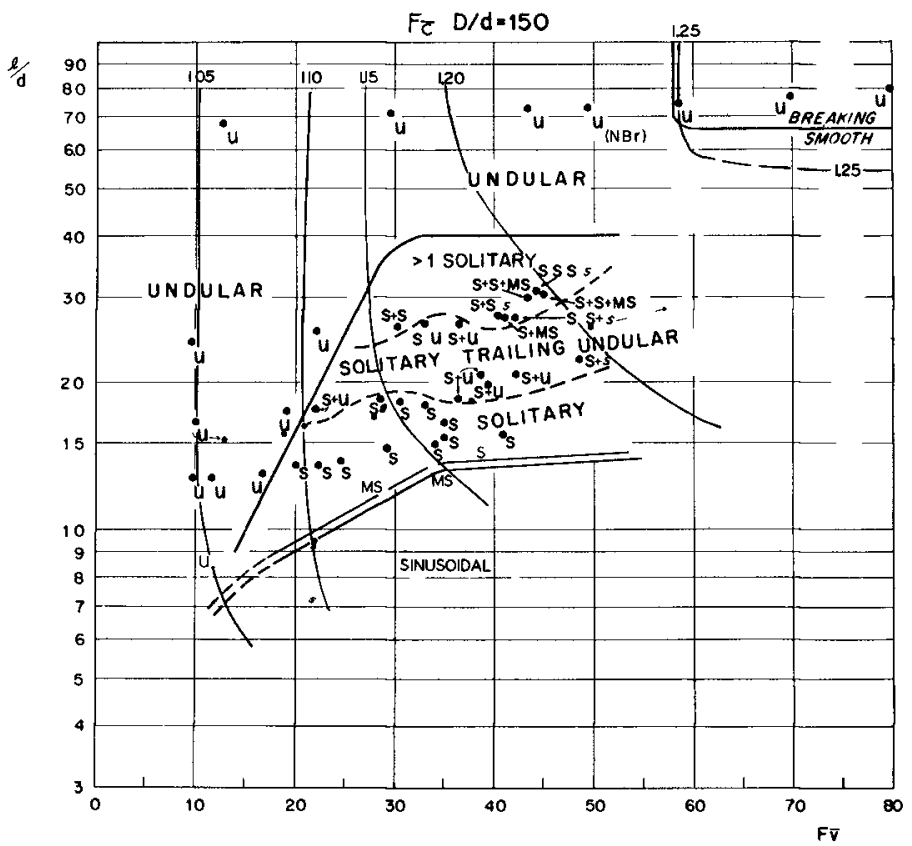

Fig 6c

$n d \mathrm{~d} \quad \mathrm{D} / \mathrm{d}=10$

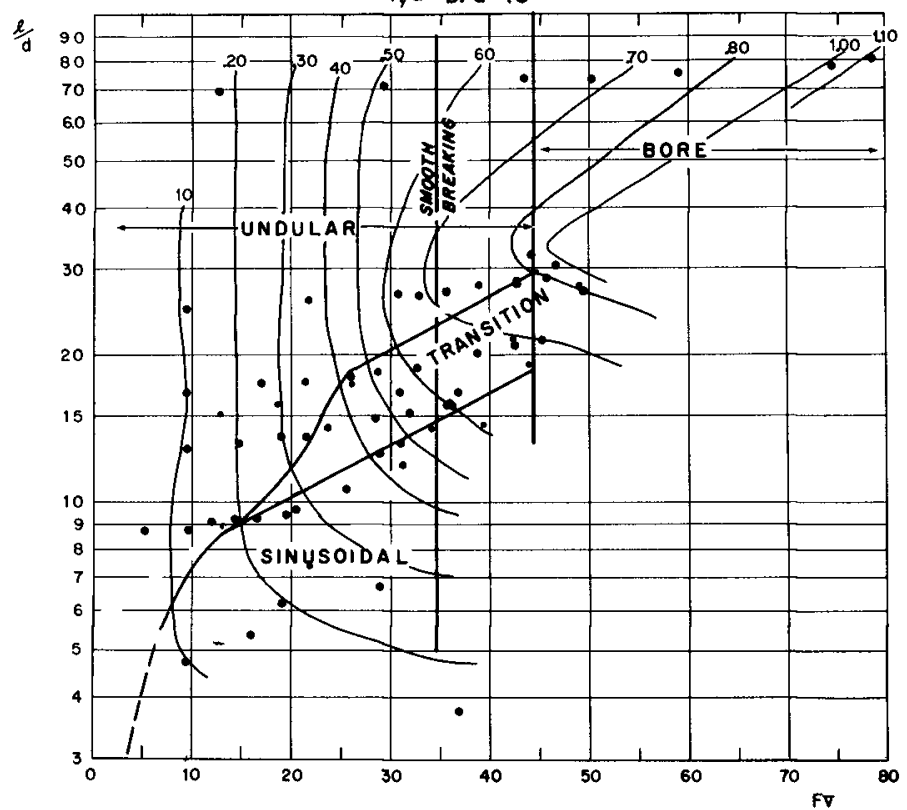

Fig $7 a$ 

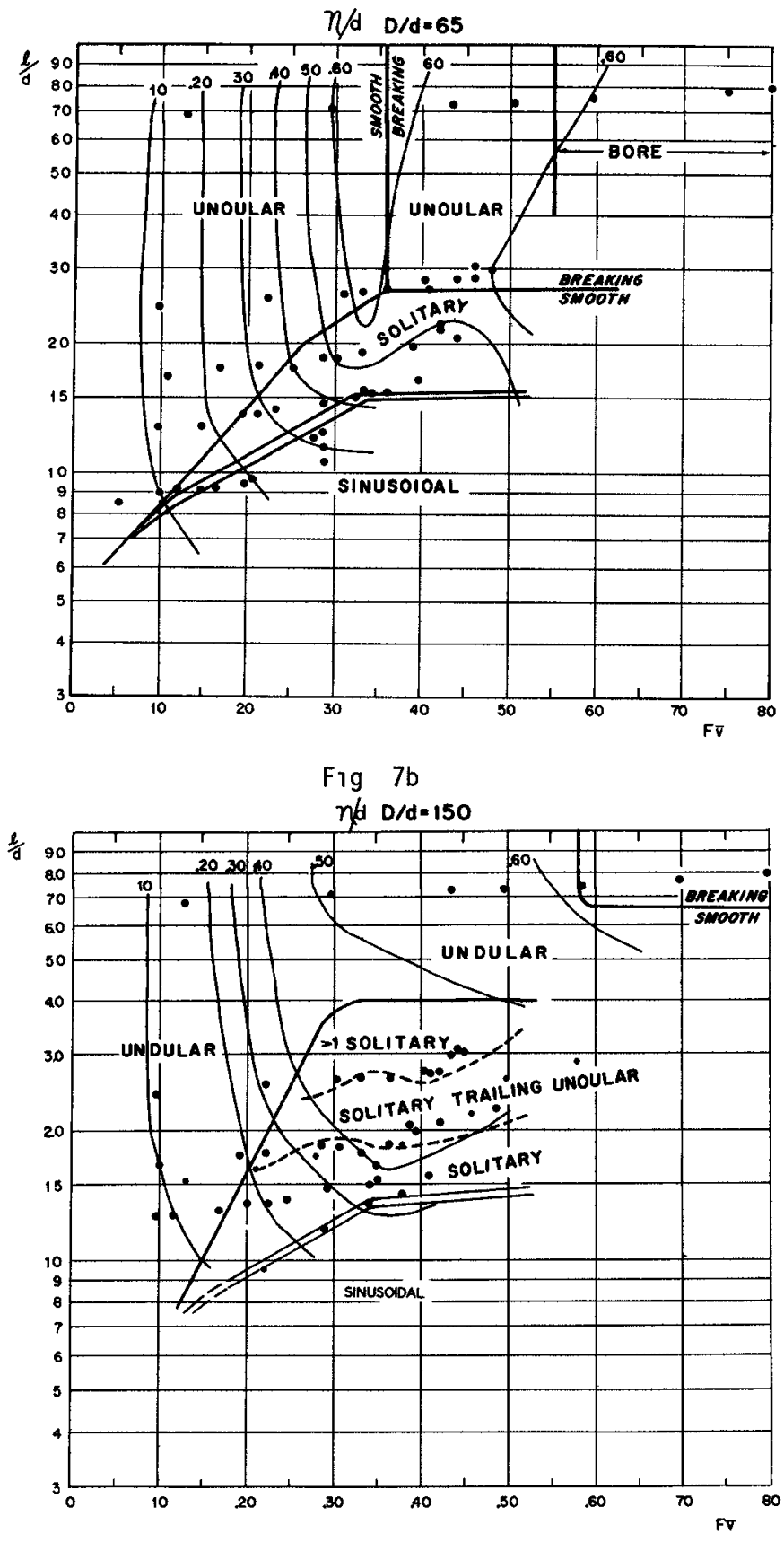

Fig 7c 


\section{CHAPTER 38}

\section{DIMENSIONAL ANALYSIS - SPURIOUS CORRELATION}

by

M S Yalin

Professor, Department of Civil Engıneerıng,

and

J W Kamphuis

Associate Professor, Department of Civil Engineerıng, Queen's Unıversity at Kingston, Canada

\section{ABSTRACT}

Dimensional analysis and errors leading to spurious correlation are presented using a number of practical examples

\section{INTRODUCTION}

The theory of dimensions has been subject of considerable controversy and only in the last few decades has it been agreed that dimensional methods can serve as a powerful tool in experimental investigations of physical phenomena Its effectiveness becomes especially noticeable when the number of factors involved is large and the theoretical knowledge is insufficient

However, 1lke any other mathematical tool, the theory of dimensions can supply correct and useful results only if it is properly applied One example of improper application is the introduction of an exaggerated and sometimes sumply of a nonexis tent correlation between the experimental results The purpose of the present paper 15 to analyse these kinds of spurious correlation

\section{OUTLINE OF GENERAL PRINCIPLES}

For detailed information on the principles of the theory of dimensions the reader is referred to Refs (1), (2), (3), here only those points will be mentioned which have a direct bearing on the present analysis

In genera1, a physical phenomenon corresponding to a specified geometry can be described by a number of independent quantities $a_{1}, a_{2} \quad a_{n}$ referred to as the characteristic parameters Accordingly any quantitative property $b$ of the phenomenon under consideration must be given by a functional relation such as

$$
b=f\left(a_{1}, a_{2}, \quad a_{n}\right)
$$

where the quantities $a_{1}$ and $b$ are usually dimensional Since all 
physical relations must be dimensionally homogeneous, using the $\pi$ theorem of the theory of dimensions, one can express the above relation in a dimensionless form as

$$
Y=\Phi\left(x_{1}, X_{2}, \quad X_{n-k}\right)
$$

Here, $k$ is the number of fundamental units, while $Y$ and $X_{j}$

$(\mathrm{j}=1,2, \quad n-k)$ are the following dimensionless power products

$$
\begin{aligned}
& Y=b{ }_{1=1}^{k} a_{1}^{\operatorname{II}_{1}} \\
& X=a_{J} \underset{1=1}{k} a_{1}^{\alpha_{1}}
\end{aligned}
$$

The dimensionless quantities $Y$ and $X_{j}$ can be interpreted as the dimensionless versions of $b$ and $a_{3}$ respectively The $k$ parameters $\mathrm{a}_{1}(1=1 \quad \mathrm{k})$ which are common $1 \mathrm{n}$ the expressions of $X_{j}$ and $Y$ are often called the basic quantities or the "repeaters"

Although elther system may be used in experimental work, Eq 2 has some definıte advantages over Eq 1 and these are described in standard works on the theory of dimensions, e $g$ Refs $(1,2,3)$ The most $1 \mathrm{~m}$ portant advantages are -
a) Eq 2 is independent of the system of units used,
b) the number of variables on the right hand side of the equation has been reduced by $k$,
c) the dimensionless variables $\left(X_{j}\right)$ are criterıa of simılarıty

VARIATION OF THE DIMENSIONLESS CHARACTERISTICS, SPURIOUS CORRELATION

In a correctly designed experimental investıgation, the variation of $Y$ is achieved by varying only one $X_{1}$ at a time In such cases, one deals with a set of functions of only one variable, as below

$$
Y_{j}=\Phi\left(\text { Const }_{1} \text {, const } t_{2}, \quad X_{J}, \quad \text {, const } t_{n}\right)=\phi_{J}\left(X_{J}\right)
$$

Accordingly in the following, the consideration of eq 2 will be replaced by its special case, Eq 5, while the subscript $\mathrm{J}$ will be omitted

Differentiating Eqs 3 and 4, one arrives at the following relations which represent the most general versions of variation of $Y$ and $X_{y}$

$$
\begin{aligned}
& d Y=b \underset{1=1}{\stackrel{k}{\underline{I}}} a_{1} \beta_{1}\left[\frac{d b}{b}+{ }_{1}^{k} \underline{\underline{E}}_{1} \beta_{1} \frac{d a_{1}}{a_{1}}\right] \\
& \frac{d Y}{Y}=d(\ln Y)=d(\ln b)+{ }_{1}^{k} \underline{\underline{E}}_{1} \beta_{1} d\left(\ln a_{1}\right) \\
& d X=a \underset{1=1}{k} a_{1} \alpha_{1}\left[\frac{d a}{a}+\sum_{1} \sum_{1} \alpha_{1} \frac{d a_{1}}{a_{1}}\right]
\end{aligned}
$$




$$
\frac{d X}{X}=d(\ln X)=d(\ln a)+\sum_{1=1}^{k} \alpha_{1} d\left(\ln a_{1}\right)
$$

Three typical methods of variation of $Y$ and $X_{j}$ are discussed below

Case I Variation of $Y$ and $X$ is achieved by varying $b$ and a only and by keeping the basic quantities $a_{1}$ constant Substituting $\mathrm{da}_{1}=0$ into Eqs 6 and 8, one arrives at

$$
\frac{d Y}{d X}=C \frac{d b}{d a}=C^{\prime} \frac{d b}{d X}
$$

where

$$
C=\frac{\substack{\Pi=1 \\ k \\ 1=1} a_{1}^{a_{1}}}{a_{1}} \quad \text { and } C^{\prime}=\prod_{1}^{k}=1 a^{\beta_{1}}
$$

Hence, the variation of $Y$ w1th $X$ is proportional to the variation of $b$ w1 th $a$ (and of $b$ w1th $X$ ) and therefore the dimensionless relationsh1p between $Y$ and $X$ is merely a scaled down version of the dimensional relationship between $b$ and $a$ (The scale of the ordinate is $\mathrm{C}$ times the scale of the abscissa)

For example, consider the decay of an impulsively generated wave with distance (4) The wave height $H$ (which should be 1 dentified with b) may be plotted against the distance, $s$ (to be identified with a) as in Fig la On the other hand, one can plot the dimensionless quantities $Y=\frac{H}{h}$ and $X=\frac{S}{5}$ against each other - F1g lb Since the common parameter $h$, the water depth (which is to be identified with one of $a_{1}$ ) has not been varied, Figs $1 \mathrm{a}$ and $1 \mathrm{~b}$ are merely scaled down versions of each other For the present example $\mathrm{C}=1$ and thus the curves in Figs $1 \mathrm{a}$ and $1 \mathrm{~b}$ are geometrically similar From Eqs 7 and 91 follows that

$$
\frac{d(\ln Y)}{d(\ln X)}=\frac{d(\ln b)}{d(\ln a)}=\frac{d(\ln b)}{d(\ln X)}
$$

is also valid, which implies that, in the case under consideration, the variation of $Y$ and $X$ in a logarithmic system is Identical to that of $b$ and $a$ (or of $b$ and $X$ )

Case II Variation of $Y$ and $X$ is achieved by keeping the parameter $\bar{a}$ constant and by varying $b$ and one or more of basic quantities $a_{1}$ Substituting da $=0$ into Eas 7 and 9 one obtains

$$
\frac{d(\ln Y)}{d(\ln X)}=\frac{d(\ln b)}{d(\ln X)}+m
$$



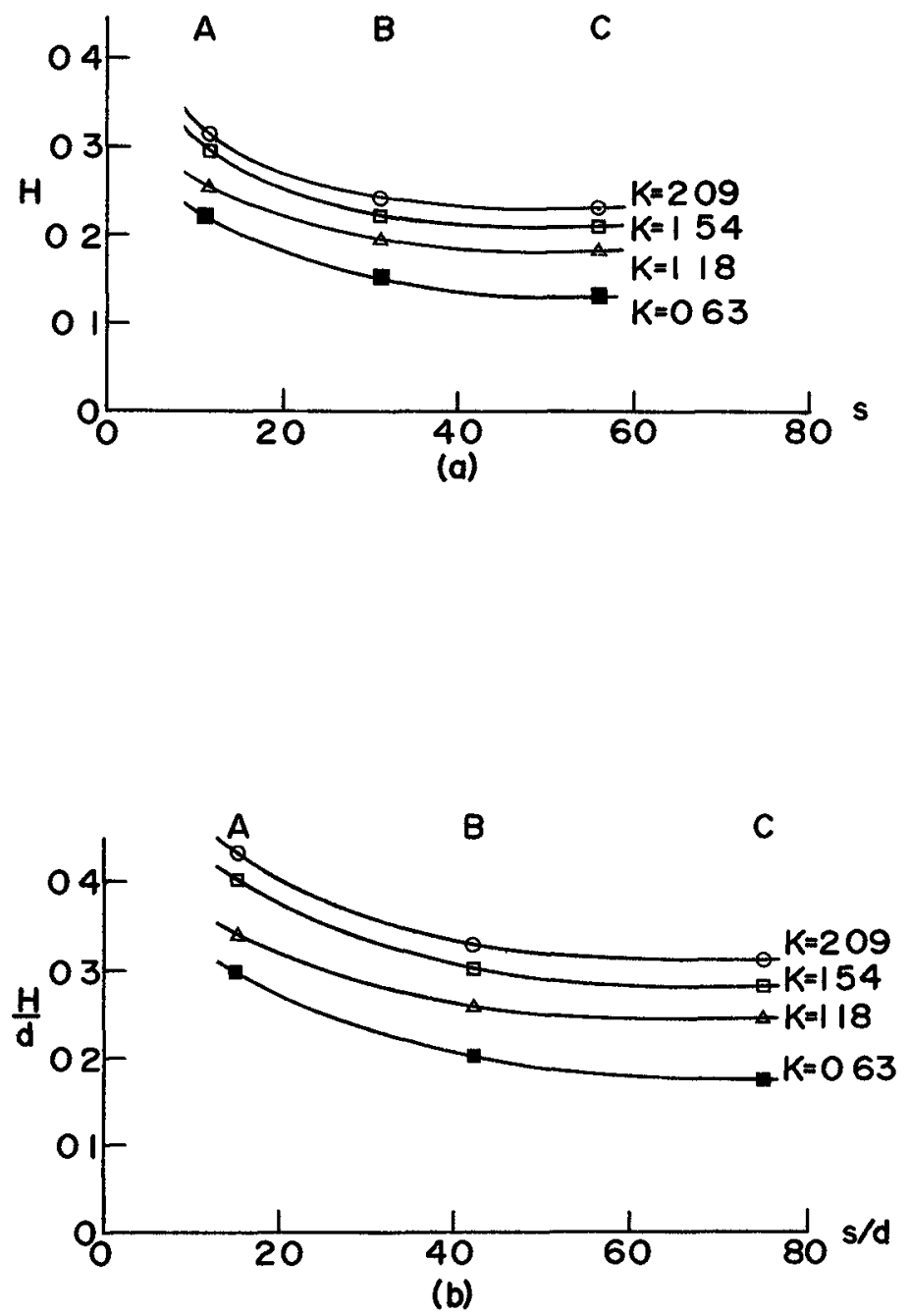

Fig I Dimensional and Dimensionless Plots 
where

$$
m=\frac{\sum_{1=1}^{k} \beta_{1} \frac{d a_{1}}{a_{1}}}{\sum_{1=1}^{k} \alpha_{1} \frac{d a_{1}}{a_{1}}}=\frac{\sum_{1=1}^{k} \beta_{1} d\left(\ln a_{1}\right)}{\sum_{1=1}^{k} \alpha_{1} d\left(\ln a_{1}\right)}
$$

If only one of the basic quantities, e $g a_{1}$ is varied, then the expression for $m$ reduces to

$$
\mathrm{m}=\frac{\beta_{1}}{\alpha_{1}}
$$

The present case is very common in practice

Consider, for instance, the famlliar plot of the drag force acting on a sphere in an infinite fluid flow (Fig 2) The dimensional relationship - analogous to $\mathrm{Eq} 1$ - $1 \mathrm{~S}$

$$
F=f(U, D, \rho, \mu)
$$

where $F$ is the drag force, $U$ the velocity of the undisturbed flow, $D$, the sphere diameter, $\rho$ the fluid density and $\mu$ the dynamic viscosity The quantities F, $\mu, U, D$ and $\rho$ must be identified with $b, a, a_{1}, a_{2}$ and $\mathrm{a}_{3}$ of $\mathrm{Eq} 1$ respectively

The dimensionless form - analogous to $\mathrm{Eq} 2$ - $1 \mathrm{~S}$

$$
Y=\frac{F}{U^{2} D^{2} \rho}=\phi(X)=\phi\left(\frac{U D_{p}}{\mu}\right)
$$

Note that Eq 16 contains four variables whereas Eq 17 contains only one, $1 \mathrm{e}$ the problem has been simplified considerably by introducing the dimensionless form' Let us now assume that the variation in flow is achieved by varying only one basic quantity (common parameter) $U$ The variation of $U$ alone will certainly induce the variation of the drag force $\Gamma$ and the experimental procedure carried out in this manner will form an example for the present case The experimental relation between $Y$ and $X$ shown in Fig 2 indicates that

$$
\frac{d(\ln Y)}{d(\ln X)}=-1
$$

1s valid On the other hand from the expression for $Y$ and $X$ (Eq 17) it follows that $\mathrm{m}=-2$ Accordingly, Eq 13 gives

$$
\frac{d(\ln b)}{d(\ln X)}=\frac{d(\ln Y)}{d(\ln X)}-m=1
$$


630

COASTAL ENGINEERING

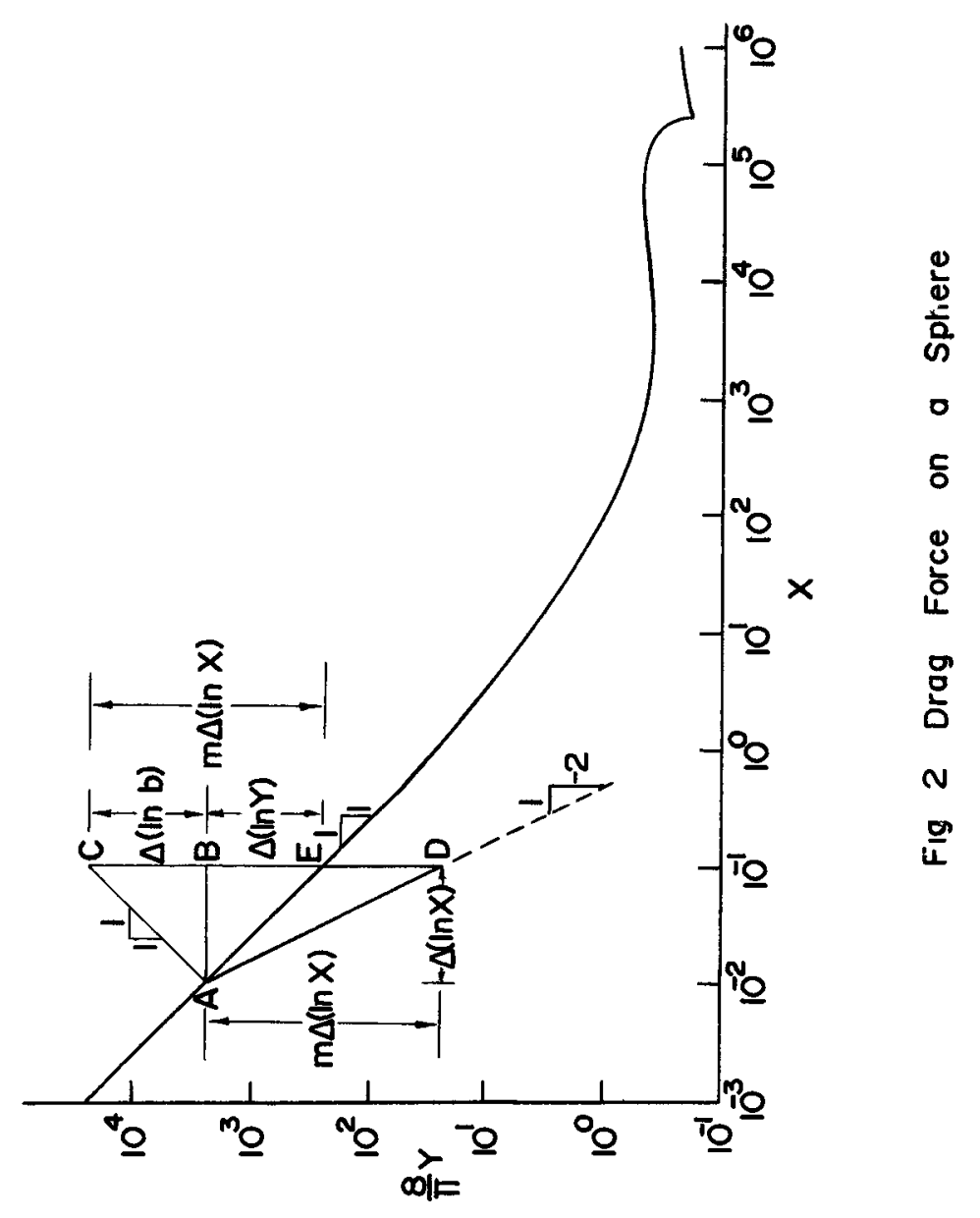


and in terms of finıte differences

$$
\Delta(\ln Y)=\Delta(\ln \mathrm{b})+\mathrm{m} \Delta(\ln X)
$$

In Fig 2 the increment $\Delta(\ln X)$ along the abscissa is shown by the horizontal distance $\overline{A B}$, the decrement $\Delta(\ln Y)$ along the ordinate being $\overline{\mathrm{BE}}$ In $\mathrm{F}_{1 \mathrm{~g}} 2$ the decrement $\overline{\mathrm{BE}}$ is shown as the algebra1c sum of the increment $\Delta(\mathrm{ln} b)=\overline{\mathrm{CB}}$ and the decrement $\mathrm{m} \Delta(\mathrm{ln} X)=\overline{\mathrm{BD}}=\overline{\mathrm{CE}}, 1 \mathrm{e}$ as implied by the equation above It follows, that the correlation between $Y$ and $X$, achieved by varying $a_{1}$ (and consequently $b$ ) is as legitimate as that achieved by varying a(and $b$ ) as in the previous case The difference is that in the case I the variation $\overline{A E}$ is achleved directly, whereas in the present case $1 \mathrm{t}$ yields itself as the "resultant" of the "component varıations" $\overline{\mathrm{AC}}$ and $\overline{\mathrm{AD}}$

Note, however, that the variation of $Y$ with $X$ can characterise the variation of $b$ with a only, as has been shown in the case I It cannot characterise the variation of $b$ with any of the common parameters $a_{1}$ observe from Fig 2 that the value of $Y$ decreases (along $\overline{\mathrm{AE}}$ w1th $\mathrm{X}$, whereas the value of $\mathrm{b}$ increases (along $\overline{\mathrm{AC}}$ ) w1th $\mathrm{a}_{1}=\mathrm{U}$

Case III Variation of $Y$ is achieved without varying $b$ Substituting $\overline{d(\ln b)}=0$ into Eqs 7 and 9 , one obtains

$$
\frac{d(\ln Y)}{d(\ln X)}=\frac{m}{1+\frac{d(\ln a)}{\sum_{1=1} \alpha_{1}} d\left(\ln a_{1}\right)}
$$

and if a is kept constant,

$$
\frac{d(\ln Y)}{d(\ln X)}=m
$$

$\mathrm{Fq} 19$ is simply Fq 13 w1thout the first term which reflects the influence of the variation of $b$, the dimensional term responsible for the existence of $Y$ Hence a correlation between $Y$ and $X$, obtained as described above is completely spurious Indeed the correlation implied by the present case can only be due to the variation of the common quantities $a_{1}$ If the parameters $a_{1}$ vary only, then the quantities $Y$ and $X$ also vary, and $Y$ can be plotted against $X$ in the form of a curve, even if $\mathrm{b}$ in actual fact does not depend on a at al1 WIth reference to Fig 2 , the curve of spurious correlation is the line $\overline{\mathrm{AD}}$ wh1ch implies the following case of $\mathrm{Eq} 19$

$$
\frac{\mathrm{d}(\ln Y)}{\mathrm{d}(\ln X)}=-2
$$

and which is due to the variation of the common quality $a_{1}=U$ alone Hence, even if $\mathrm{F}$ had not been a function of $\mu$ at a11, by applying the procedure described in the present case, one could still obtain a 
correlation between $Y$ and $X$, in the form of the line $\overline{A D}$, which would, of course, be completely spurious

Integrating $\mathrm{Eq} 19$, one obtains

$$
Y=C X^{m}
$$

which is a straight line in the logarithmic system of co-ordinates and where $C$ is given by

It follows that

$$
C=\frac{b}{a} \prod_{1=2}^{k} a_{1}\left(\beta-m \alpha_{1}\right)
$$

a) any correlation between $Y$ and $X$ obtained without varying $b$ is spurious (unless a special subset of Eq 16 is chosen, where $U, D, 0$ and $\mu$ are not independent, thus violating the basic assumptions),

b) in a $\log -\log$ system of co-ordinates, this spurıous correlation forms a stralght line,

c) the position and slope of the straight line of spurious correlation is, a priorı, predictable, it is determuned by Eqs 19 and 20

When analysing experimental plots, in case of doubt it is advisable to determune and plot the famly of stralght lines $S_{1}$ of spurious corre1ation (Fig 3) and to check the trend of the experimental points accordingly If the dimensional analysis has been performed 1mproperly and the original quantities are not truly independent, then the variation of $a_{1}$ brings about unexpected variation in $a$ or $b$ and spurious correlation will take place along a curve $\Gamma$ (F1g 3) This is also a point to be kept in mind

A specıal case occurs when experimentally

$$
Y=\phi(X)=C X^{m}
$$

and the lines of spurious correlation and genuine correlation coincide

Consıder, for example, the length $\Lambda=b$ of dunes formung on the bed of a two dimensional rough turbulent flow with a mobile bed According to (5), this length may be expressed by the following functional relation

$$
\Lambda=f\left(D, \rho, v_{*}, h\right)
$$

where $D=a_{1}$ is the grain size, $\rho=a_{2}$ is the flund density, $v_{*}=a_{3}$ is the shear velocity and $h=a$ is the flow depth

The relation above can be expressed in dimensionless form 


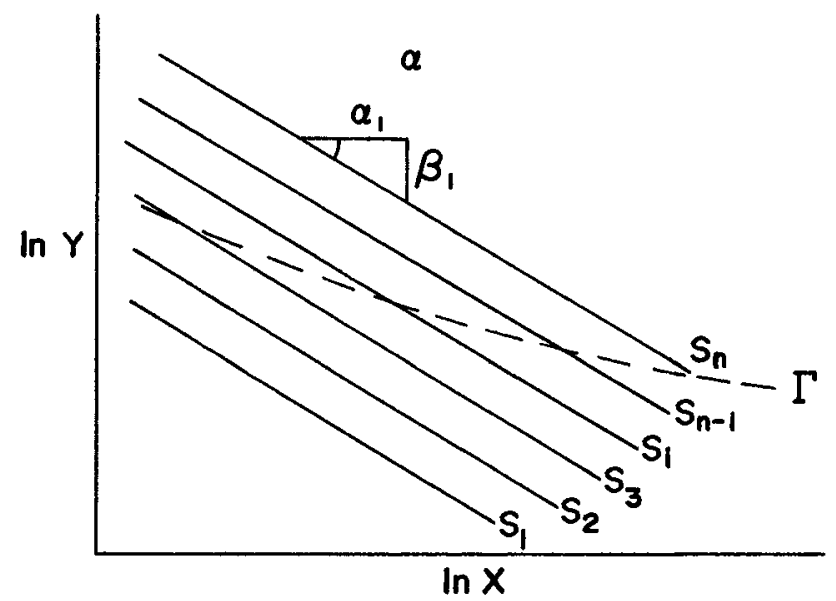

Fig 3 Lines of Spurious Correlation 
as follows

$$
\mathrm{Y}=\frac{\Lambda}{\mathrm{D}}=\phi(\mathrm{X})=\phi\left[\frac{\mathrm{h}}{\mathrm{D}}\right]
$$

If the grain size $D=a_{1}$ is varied only, then Eqs 20 and 21 give

$$
\mathrm{Y}=\mathrm{CX}
$$

which implies that the lines of spurious correlation in a $\log -\log$ system are straight lines with slope $m=1 \quad$ On the other hand, the experimental results (obtained from experiments where $\Lambda=b$ is varied over a large range) indicate that the genuine correlation between $\mathrm{Y}$ and $\mathrm{X}$ is given by the linear relation

$$
Y=2 \pi \quad X
$$

which also a straight line with the slope $\mathrm{m}=1$

The lines of spurious correlation and the real relationship can coincide as in example above only if the parameter $a_{1}$ is a

"spurious parameter" This can be deduced from Eqs 3, 4 and 20 and indeed, the relation above which can be expressed as

$$
\Lambda \simeq 2 \pi \mathrm{h}
$$

indicates (contrary to the theoretical expectation) that the dune length $\Lambda$ actually does not depend on the grain size $a_{1}=D$

\section{EXAGGERATION OF THE ACTUAL CORRELATION}

In practical applications, an existing correlation is often exaggerated by plotting conmon quantities along both co-ordinate axes (6) The explanation is given here in terms of a dimensionless system, however, the same argument is true for dimensional quantities

Consider the functional relation

$$
z=\psi(X)
$$

where $Z$ is the product

$$
\mathrm{Z}=\mathrm{Y} \mathrm{X}^{\mathrm{N}}
$$

and where $\mathrm{Y}$ implies

$$
Y=\phi(X)
$$

From Eq 28 one obtains

$$
d(\ln Z)=d(\ln Y)+N d(\ln X)
$$


and thus

$$
\frac{d(\ln Z)}{d(\ln X)}=\frac{d(\ln Y)}{d(\ln X)}+N
$$

This equation indicates how in a $\log -1 \log$ system of coordinates the rate of change with $X$ can be increased by plotting the product $Z=Y X N$ rather than sumply $Y$ versus $X$

For example if

$$
\frac{d(\ln Y)}{d(\ln X)}=\tan \theta=1
$$

then the plot $Y$ vs $X$ is a $45^{\circ}$ straight 11ne, as shown in Fig 4 a It is assumed that the experimental points forming this straight line are scattered in a "rıbbon" of the thickness $w_{a}$

If the product $Z=Y X^{N}$ is used as ordinate, and if for example the value of $\mathrm{N}$ is 2 , then from Eq 30

$$
\frac{d(\ln Z)}{d(\ln X)}=1+2=3
$$

is valid, which implies that the straight line becomes steeper by a factor 3, while the thickness of the scatter-ribbon decieases by a factor

$$
\frac{\mathrm{w}_{\mathrm{b}}}{\mathrm{w}_{\mathrm{a}}}=\sqrt{\frac{1+\mathrm{N}^{2} \tan ^{2} 0}{1+\tan ^{2} 0}}=\sqrt{\frac{5}{2}}=158
$$

(F1g 4b) Thus the relative thickness of the scatter ribbon, 1 e

$$
\frac{\text { thickness of scatter-ribbon }}{\text { length of the line }}
$$

1s reduced by factor

$$
\frac{1+N^{2} \tan ^{2} O}{1+\tan ^{2} O}=\frac{5}{2}=25
$$

One can say that the correlation can be umproved 25 times by plotting $Z=Y X^{2}$ rather than sımply $Y$ against $X$ Such an "Improvement" is nothing else but an optical illusion and is not legitimate, for the dimensionless version of the quantity $b$ under investigation, as supplied by the $\pi$ - theorem, is $Y$, not $Y X N$ These kinds of illegitimate plots are recognisable by the presence of the parameter a (which should be present only in the expression of $\mathrm{x}$ ) in the power product implying the ordinate

\section{SUMMARY}

The experimenter is free to choose between a dimensional representation, $\mathrm{Eq} 1$, or a dimensionless representation, $\mathrm{Eq} 2$ of the 

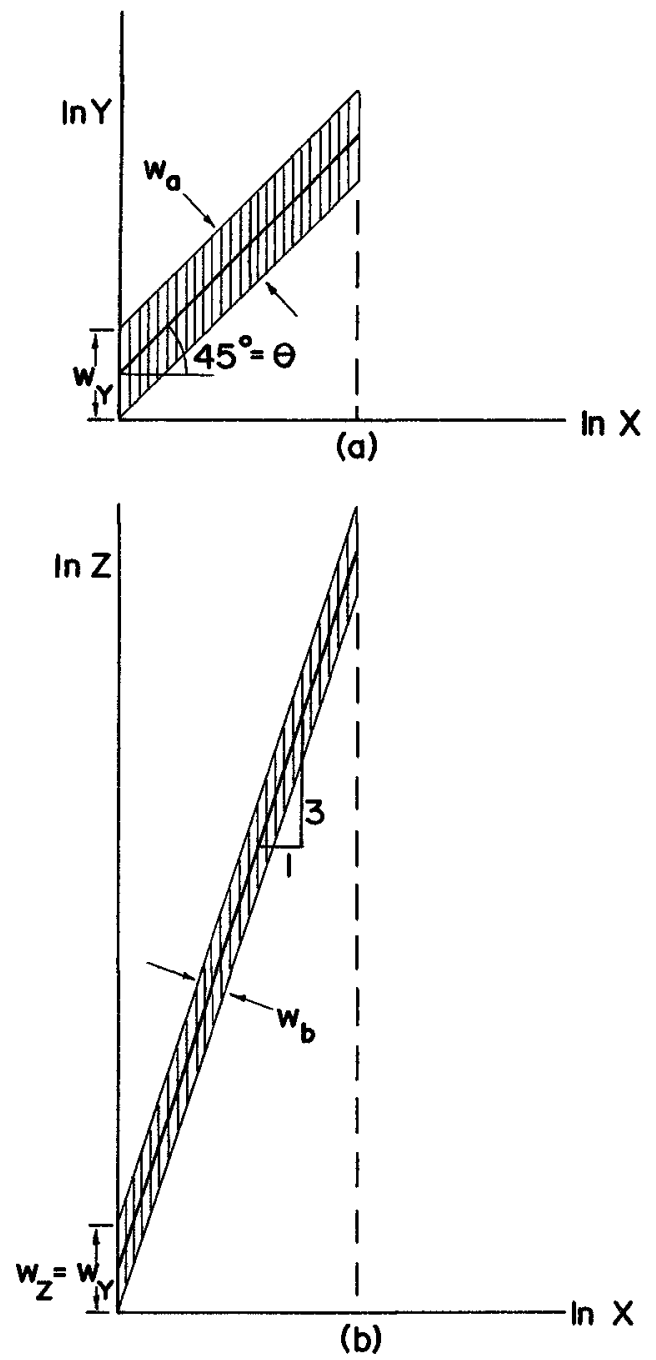

Fig 4 Exaggerated Correlation 
phenomenon under investigation. The latter is found to have certain obvious advantages Once a decision is made, a11 the subsequent analysis is expressed in terms of the system chosen For instance if the dimensionless system is chosen, then the results should not be interpreted in terms of the individual dimensional parameters that form the dimensionless variables since the real variables of the experiment are the dimensionless variables

The usual cause of spurious correlation is the appearance of common quantities along both co-ordinate axes This is true for both a dimensional and a dimensionless system This must not be confused with the appearance of common dimensional parameters along both co-ordinate axes when dimensionless variables are plotted against each other

In dddition, when considering dimensionless variables, care must be taken that the relationship between $Y$ and $X$ is not brought about only by variation of one or more of the repeating dimensional parameters common to both

\section{ACKNOWLEDGEMENTS}

The authors w1sh to thank Dr A Brebner who urged the writing of this paper

\section{APPENDIX 1 - REFERENCES}

1 Sedov, L I , Simllarıty and Dimensional Methods in Mechanics, Academic Press, New York, 1959

2 Langhaar, H L, Dymensional Analysis and Theory of Models, John W1 ley and Sons, New York, London, 1962

3 Birkhoff, G , Hydrodynamıcs, a Study in Logıc, Fact and Simı1ıtude, Princeton, Harvard University Press, 1950, Dover, 1955

4 Kamphuıs, J Wi11ıam and Bowerıng, Richard "Impulse Waves", 12 th Conference on Coastal Engineering, Washington, Sept 1970

5 Ya1ın, M S , "Geometrıc Propertıes of Sand Waves" Journal of the Hydraulics Division, ASCE, Vo1 90, No HY5, Sept 1964, pp 105-119

6 Benson, Manuel A, "Spurious Correlation in Hydraulics and Hydrology", Journal of the Hydraulics Division, ASCE, Vo1 91, No HY4, July 1965, pp 35-42 
APPENDIX II - NOTATION

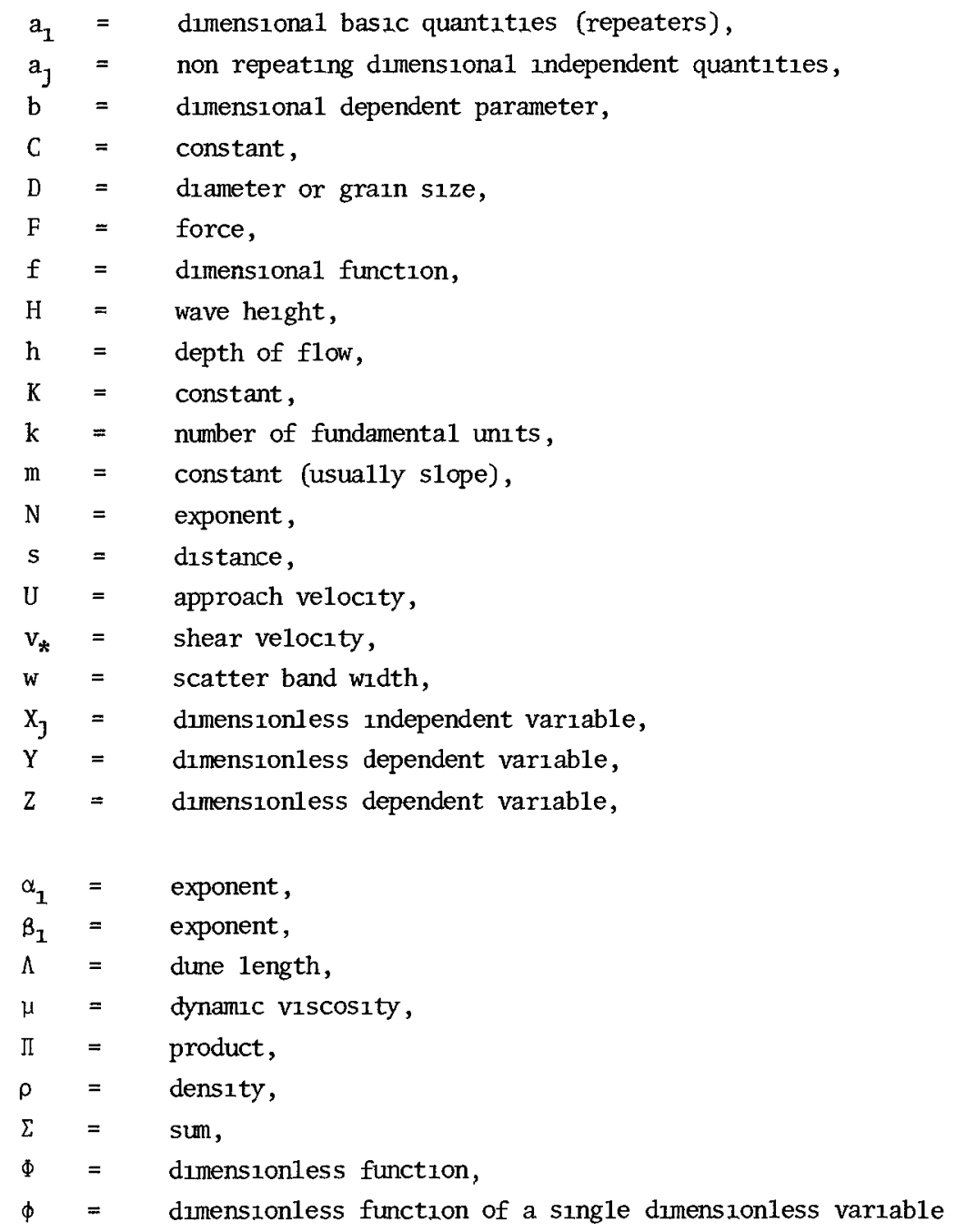




\section{CHAPTER 39}

COASTAL PROCESSES FROM SPACE PHOTOGRAPHY

By Maynard M Nichols

Virginia Institute of Marine Science

Gloucester Point, Virginia 23062, U S.A

\section{ABSTRACT}

Photographs taken from space provide a new source of data concerning coastal processes By utilizing the high vantage point and broad view orbiting space cameras can record little known large-scale processes at short time intervals Major outflow plumes, fresh-salt water "fronts", turbidity maxima and massive effluents are among the wide range of features displayed It is shown from selected examples how coastal processes can be evaluated from space photography and how the information may be of use for solving certain problems as a supplement to field and other remote sensing data By 1972 satellite photography w11l be avallable on a routine basis for many coasts of the world Englneers are urged to consider the potential for improving their information which space photography has to offer

\section{Introduction}

The "promise of space" has commanded a great deal of attention in recent years In particular, considerable credit has been given to the potential usefulness of space photography It has been asserted that space photography can record the location of fish-rich upwellings and mineral deposits, it can detect pollutants and diseased crops and inventory various natural recources, all on a global basis Though photography has contributed a great deal to our knowledge in many fields, study of coastal processes important to the coastal engineer, has been limited In recognition of this gap, this paper directs attention to space photography as a new source of information on aspects of coastal processes What coastal features can be recorded by space photography? How can processes be evaluated? And of what earthly good are space photographs to problems of coasta1 engineering?

Already more than one thousand photographs of coasts have been obtained from Gemini and Apollo flights which point up the possibilities of studying certain processes from space Recorded for the first time are major "fronts", massive effluents, and shelf-wide perturbations Dramatic as they are, these are only a prelude to photographs anticipated on a routine basis from the up-coming ERTS (Earth Resources Technology Sate1lites) program in 1972 
But before we can utilize space photography we need to consider the nature of coastal features as photographic subjects and the character of space photography itself

\section{Coastal Subjects}

In contrast to terrain features, coastal features vary widely from land to water, and with depth, from surface to subsurface Beaches, tidal flats, marshes, submerged bars, grass beds, bottom sediments, slicks, and plankton blooms are among the range of subjects Varying in geometric size from a few meters to tens of kilometers and with time, hourly, daily and month1y, they present a full spectrum of rapidly changing contrasts Best understood are those features of small geometric size and relative long time scale, least known are those of large-scale which occur at short time scales Because of the very wide range and transient nature of different coastal subjects, practical problems of recording photographic information are great Not only are reflecting qualities of the subject frequently masked by atmospheric phenomena but image contrast is often lost and illumination changed within the water Our understanding of these recording vagaries is $1 \mathrm{~mm}$ ted, and ground truth at the time of photography is seldom available It is small wonder why photography has not been extensively utilized for study of coastal processes as it has in other fields. Despite the fickleness of coastal subjects and their illumination, the orbital space camera has certain characteristics of advantage for study of coastal processes

\section{Character of Photography.}

From an altitude of over $200 \mathrm{~km}$, the space camera can record more than $40,000 \mathrm{sq} \mathrm{km}$ of coast, e $\mathrm{g}$ Figure 1 Its broad view and synoptic record make it possible to examine in toto large scale features of the water, wave patterns, and shoals, as well as marshland, coastal terrain and cultural features, all in a single photograph Even when only central parts of the phctograph are examined, geometric fidelity is good and lighting uniform over a relatively large area, greater than $8,100 \mathrm{sq} \mathrm{km}$. Such a quality is difficult to obtain from conventional low altitude aerial photography Because of the great distance above the optically turbulent atmosphere photographic "clarity" is good (Harvey and Myskowsk1, 1965), and color distortion small Whereas image scale is reduced to about $1 \cdot 1,000,000$ and effective resolution 11mited to 40-100 meters, detail "lost" by low resolution is more-or-less "integrated" into color or tone anomalies depicting major features Once in orbit the great strength of the satellite-borne camera is its ability to look at coastal phenomena at frequent intervals, once every 90 minutes to 17 days for a year or more

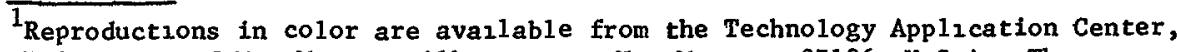
University of New Mexico, Albuquerque, New Mexico, 87106, U S A The illustrations used here do not fully portray the content of the original color photography
} 


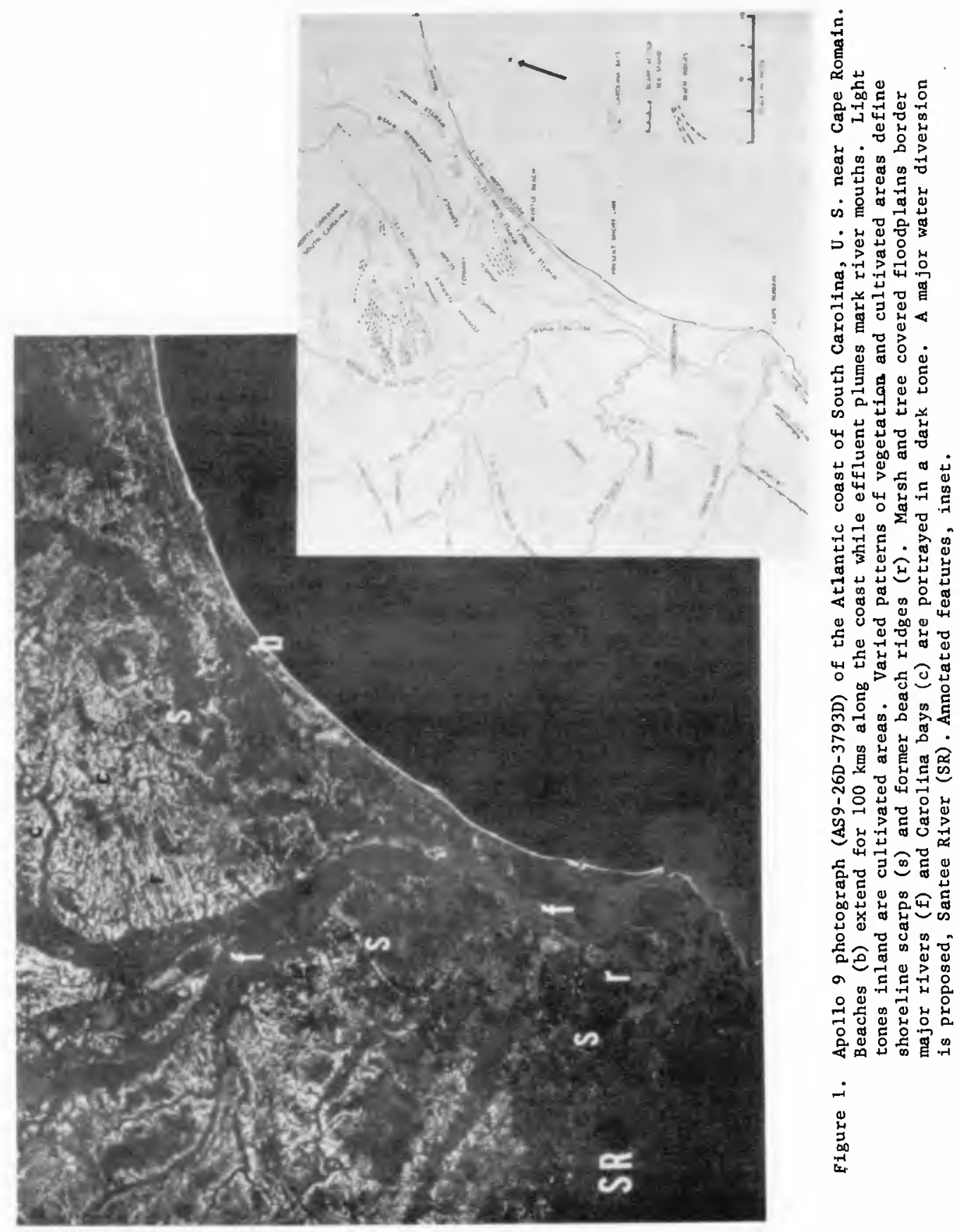


Waves

Because low resolution of the space camera "filters out" dimensional and directional detall, only long-period waves are recorded by photography They are observed mannly in glitter patterns around oceanic is lands and continental headlands When traced to storm-generating sources with the ald of meteorological satellite photography, long period wave data is of value for forecasting waves propagated into coastal areas Amenable to analysis are refraction patterns and landward changing wave dimensions that relate to decreasing water depth and the location of shoals, and in turn to wave-stirred sources of suspended materials or turbidity Procedural detalls for photographic analyses of glitter patterns are given by Cox and Munk (1954) and for spectral distributions of long perıod waves, by Llbby, et al (1969)

\section{Coastal Morphology}

Space photography is of value for portraying morphological features especially in regions where base maps are not avallable In remote areas like mangrove swamps and lce bound coasts they may be the chlef source of information on coastal configuration Where relief changes are small and difficult to observe on the ground, many coastal features often show up more clearly on photographs For example, linear features like ancient shoreline scarps (Figure 1) can be traced for more than $150 \mathrm{~km}$ despite their discontinuous nature with gaps of $20 \mathrm{~km}$, and subtle relief, less than $6 \mathrm{~m}$ The photographs revea1 coastwide trends more clearly than a good map other patterns often revealed clearly on photography include beach ridges and dunes, drainage systems, broad patterns of salt marsh, evaporite deposits, and limits of tidal flooding on flats spatial relations of these features can be further evaluated on a regional basis in a context of major sediment sources and dispersal routes

\section{Shoreline Changes}

In keeping with traditional use of aerial photography, space photographs can be used to measure changes in shoreline configuration and thus identify major sites of erosion or accretion With proper control, scaling and rectification (Colvocoresses, 1970) different positions of the shoreline can be established by comparing common image points taken from time to time according to practices for aerial photography (Moffitt, 1969, and Langfelder, 1968) old charts may be used to identify positions of former snorelines (F1g 2) which are comparable to those in space photographs if sufficient control is avallable However, relatively low ground resolution of present space photography limits application to shorelines having large changes (greater than about $300 \mathrm{~m}$ ) over extended periods such as deltalc coasts and storm-washed beaches Frequent coverage of shorelines by future satellites could record the opening or closing of tidal inlets in the aftermath of storms so that changes can be quickly assessed and corrective measures planned 

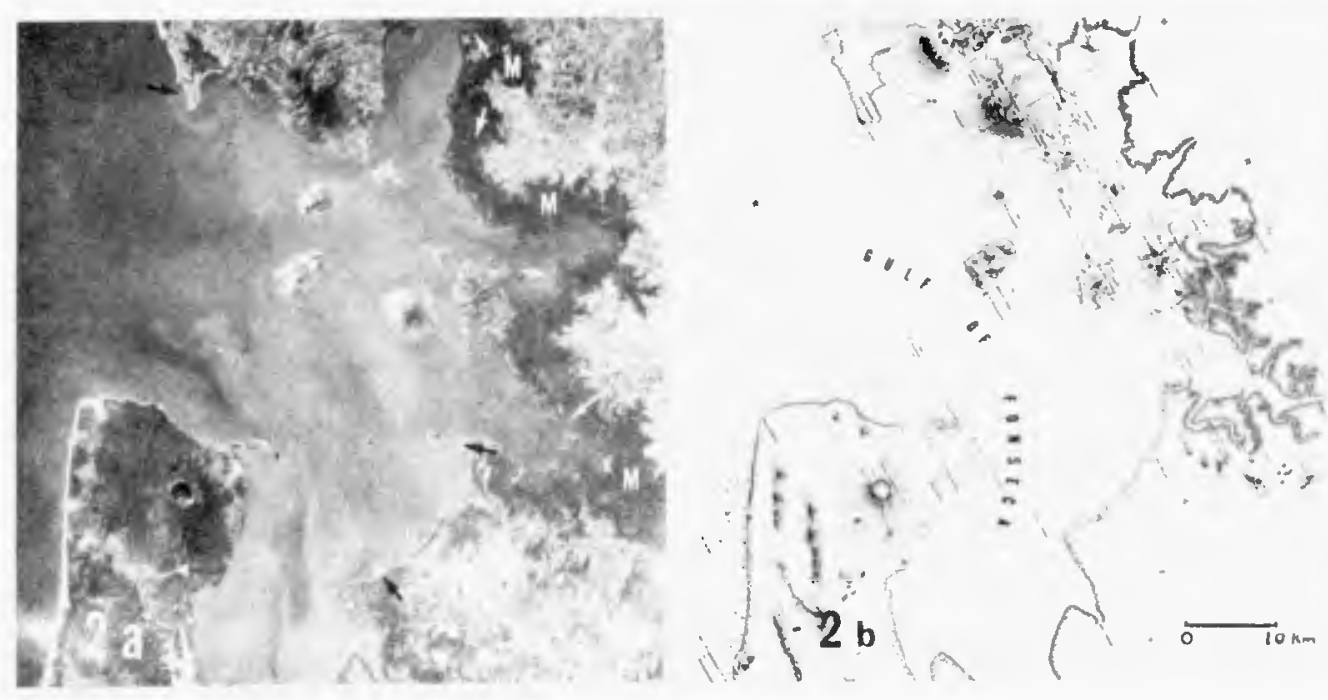

Figure 2A. Black and white reproduction of Apollo 9 color photograph (AS9-19-3019) over the Gulf of Fonseca, Pacific Coast of Honduras, March 9, 1969. Shore consists of mangroves (dark tone, M) as part of a landward extending zone $8 \mathrm{~km}$ wide. Locally barrier beaches (b) and spits (s) are visible. Light grey tone of the Gulf indicates turbid water supplied either by entering rivers or from wave-stirred tidal flats. Arrows directed landward indicate sites of major shoreline erosion, arrows seaward, sites of accretion.

Figure 2B. Corresponding hydrographic chart largely based on British Surveys of 1838 . Depths in fathoms.

\section{Coasta1 Drift:}

Color and tone anomalies that record the distribution of discolored water provide a visible tracer of drift along the coast. By sensing light scattered by suspended materials in near surface water, these anomalies are fashioned into distinct patterns which reflect the direction of flow. Most obvious are seaward expanding plumes representing suspended sediment discharged off mouths of bays and rivers. Often they are prolonged downstream as "streaks" from wave-stirred bank tops or shoals. Less obvious in relation to their source are great rip-like patterns extending seaward from shore in eddy configurations (Fig. 3A) or as narrow tongues (Fig. 3B). Where opposing flows occur, "fish-hook" patterns or asymetrical interfacial waves develop. Such indicators may be used together with other data to compile a coast-wide chart of drift for an area of interest. Similar indicators may be of use to evaluate the character and direction of flow. "ind structures like an interference eddy seaward of the jettiec :- 

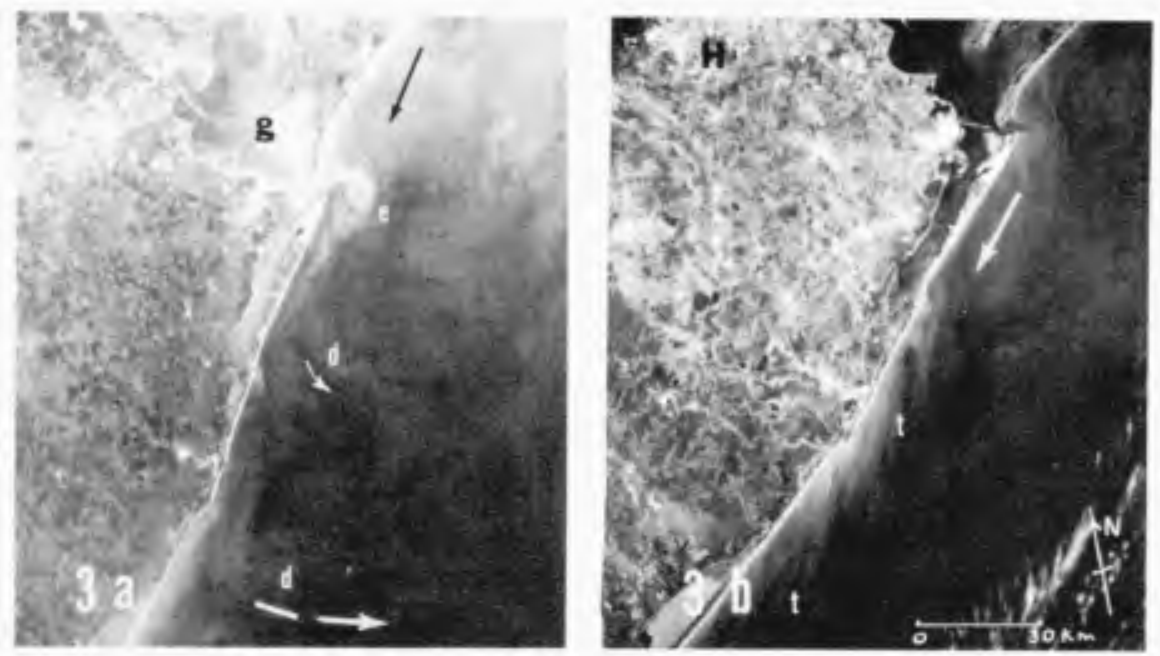

Figure 3A. Space photograph (S66-63064) taken from Gemini XII over the Texas Coast, U.S. Nov. 14, 1966. Eddy-like perturbations, (light grey tones) in the water mark offshore drift (d). In Galveston Bay $(g)$ light tone portrays sediment discharged from a dredging operation. Debris laden outflow forms an interference eddy (e) in the coastal drift (arrow).

Figure 3B. Space photograph (AS9-26A-3728A) of the same portion of the Texas Coast, near Houston (H) March 8, 1969. Narrow tongues extending seaward ( $t$ ) are in contrast to perturbations of figure $3 \mathrm{~A}$. Inferred coastal drift, arrow.

Galveston Bay (Fig. 3A). Within the Bay a light-colored streak marks turbid water eminating from a dredging operation. But, striking as the patterns are they are ephemeral and necessarily limited to suspended materials carried only in near surface water, often only a fraction of the total suspended load. Therefore, it seems essential to supplement the photographic record by extensive field measurements not only to gain a more complete "picture" of coast-wide drift but to "calibrate" the imagery as well.

\section{Offshore Drift:}

It was not until space photographs of the Gemini flights were available that the possibility of widespread dispersal of nearshore sediments was appreciated. First recognized by Stevenson (1967) in 
coastal water off Texas, turbid perturbations were traced as far as 150 $\mathrm{km}$ from shore. Just as rip currents relieve rising water levels in the surf zone these large scale features are believed to carry water offshore in response to a coast-wide build-up of sea level Later coverage of the same coast taken on Apollo 9 in 1969 portrays seaward extending tongues 22 $\mathrm{km}$ offshore A day later along the same coast (AS9-22-3464 and AS9-22-3465 not printed) after the wind had shifted from onshore to offshore with passage of a front, the tongues were largely replaced by dark masses of relatively "clear" water common to offshore areas Although the available examples are but a few snapshots of a whole "movie fi.lm" of rapidly changing drift patterns they do give an indication of widespread sediment dispersal. to be expected

In another example, figure 4, a large plume leads seaward from Cape Lookout, North Carolina seaward from the point, shoals are recorded as a light-toned shredded pattern representing large sand waves and these are interfingered with dark tongues representing tidal channels in deeper water Beyond the charted 11mit of the shoals, an indistinct plume extends $60 \mathrm{~km}$ offshore Its seaward edge is truncated by the Gulf stream and trails northward as a narrow tongue Concentrations of suspended material sampled in near-surface water close to the time of photography were relatively low, less than $2 \mathrm{mg} / \mathrm{L}$ and materials consisted of quartz sand (60\%) mixed with plankton and detritus Although the rate of drift is not recorded, the photograph shows where suspended sediment is going in relation to potential sources on the shoals or along the shore If this drift persists over the long-term, perhads it could be utilized as an avenue of transport to discharge pollulants across the shelf and into the ocean

Utilization of photography for measurement of current speed has been successfully accomplished using stero parallox techniques with aerial photography, Cameron (1952) and Waugh (1964) It seems feasible to use a similar approach with space photography if an economical means of "seeding" currents on a large scale can be developed

\section{Problems:}

In practice the user of space photography is faced with a number of problems which must be recognized The dynamic character of the subject creates a special problem for collecting ground truth In some areas it would seem that sampling of selected sites at the moment of photography would suffice for large areas but the wide range of environments, each with characteristics of 1 ts own, and the variety of unpredictable local conditions make ground truth data collection an enormous task

In addition to understanding the subject, use of the photography depends on an understanding of various colors, tones and density anomalies in the photograph Excellent progress has been made (Ross, 1969) in developing techniques which enhance images and extract spectral data to determine water depth and turbid patterns Yet, the three dimensional 
character of hydrographic imagery is incompletely understood; often turbid features cannot be differentiated from bottom features. It seems sensitometric techniques have exceeded our ability to reduce imagery data to meaningful and quantitative information.

At the present stage of progress space photography is no alternative for field sampling. Instead it can assist in planning field work, extend coverage and direct it to profitable sites. The need for rapid, synoptic and timely data on a regional basis demands that new dimensions in data collection and processing be added to improve our knowledge of coasta1 processes.

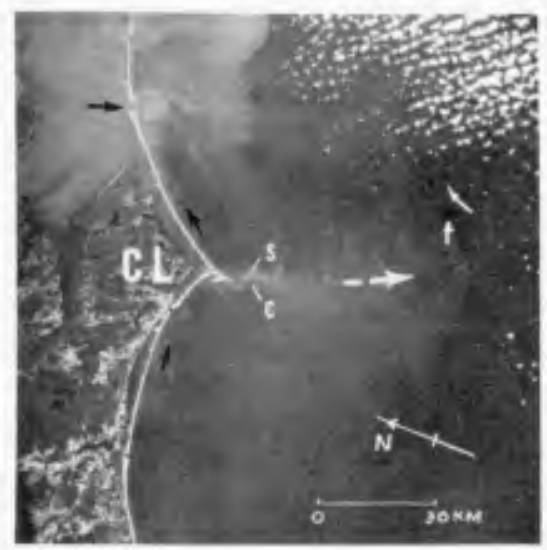

Figure 4. Black and white reproduction of color Apollo 9 photograph (AS9-20-3127) over Cape Lookout (CL), N.C. March 12, 1969. Seaward expanding plume (broken arrow) leads from shoals (s) dissected by tidal channels (c) Gulf Stream "front" (f), inferred coastal drift (arrow) nearshore. 


\section{References}

Cameron, H L , 1952 The measurement of water current velocities by parallax methods Photogrammétric Engineering, Vol 18, p 99-104

Colvocoresses, A P, 1970 ERTS-A satellite imagery Photogrammetric Eng1neering, June, p 555-560

Cox, C. and W Munk, 1954 Statıstıcs of the sea surface derived from sun glitter, effect of slicks Journal Marıne Research, Vol 2, P $\quad 2,22-226$

Harvey, D I and E P Myskowsk1, 1965 Physics of high altitude photography In Photographic considerations for aerospace, Hall, $\mathrm{H} \mathrm{J}$ and Howe11, H K, Eds, Lexington, Mass, Itek Corp, p $\quad 1-22$

Langfelder, J, Stafford, D and M Amein, 1968 A reconnalssance of coastal erosion in North Carolina School of Engineering, Dept of Civil Engineering, North Carolina State University, Raleigh, N C , contract report ERD-238, $127 \mathrm{p}$

Libby, L M , W F Libby, and S S Lawrence, 1969 Measurement of ocean waves in a satellite photography Rand Corp Miscell Docum AD696-492, p $1-9$

Moffitt, F H , 1969 History of shore growth from aerial photographs Shore and Beach, Vol 37, No 1, p 23-27

Ross, D S , 1969 Color enhancement for ocean photography In Oceans from Space, Badgley, P C, Mıloy, I and Childs, I, Eds, P 50-63

Stevenson, R E, 1967 View of the earth from space Journal of Geological Education, Vo1 16, no 3, p 83-90

Waugh, J E, 1964 Photogrammetric measurements of t1dal currents Journal of the Surveying and Mapping Division, Proc Am Soc Civil Engineers, paper no 3857, Su 1, p 17-29 


$$
\text { - }
$$




\section{CHAPTER 40}

Reach of Waves to the Bed of the Continental Shelf

RICHARD SILVESTER

Professor of Coastal Englneering

Aslan Institute of Technology, Bangkok, Thalland

(seconded from Dept of Civil Eng, University of Western Australia)

and

GEOFFREY R MOGRIDGE

Research Assistant

Queen's Unıversity, Ontario, Canada

(formerly Dept of Civil Eng, University of Western Australıa)

\section{ABSTRACT}

The physlography of Continental Shelves and their major composition of sediment indicate strongly their terrigenous origin and their smoothing by wave action This premise is supported by the geologic time over which waves have existed and the mass-transport velocity in these relatively shallow depths, particularly the net movement with in the wave boundary layer at the bed A given wave train arriving obliquely to the shore can transport material along the coast, both beyond the breaker line and within the surf zone It is shown that for equal over-all discharge in the two zones, the average sediment concentration offshore close to the bed need be reasonably small, indicating that transport near the beach could be a fraction of that from the breakers to the reach of the waves This latter 1 imit is shown to extend at least half way across the Shelf, with possibilities of greater reach when more realistic prototype conditions are introduced into experiments

\section{INTRODUCTION}

The margins of landmasses are made up mainly of mildly sloped under-water zones which are termed Continental Shelves The legally accepted 1 imit for these, where the slope increases substantially, has been taken as 100 fathoms ( 183 metres), although the accumulation of data over decades has shown (1) that the edge of this feature $1 \mathrm{~s}$ closer to 65 fathoms (120 metres)

Of the various classifications of Shelves the most important to the coastal englneer is the sediment accreted variety, which provides the widest marine margins avallgble and also make up the largest percentage area of all Shelves Hayes (2) reports that $80 \%$ of the inner Shelf is covered by gravel, coral, shell, sand or mud, the last two constituting around $70 \%$ Another important fact is that materlal is still being furnished to these zones by the rivers of the world $(3)$ 
Whilst the sediment is supplied at specific locations along the coastlines, it is spread relatively evenly along them by wave action and possibly currents Whilst geographers and geologists have stressed the role of tidal and other oceanic streams in this distribution process, it would seem that wave action could well be the predominant agent in this massive task The following observations would appear to support this thesis

(a) Where waves (partıcularly swell) arrive obliquely to a reasonab1y long length of coastine there is evidence of accretion at the downcoast area and a dearth of movable material in the upcoast reg ion The former occurs as an enlarged width of Continental Shelf and/or wide expanses of sedimentary plain such physlographic units $(4)$ are displayed on margins of enclosed seas as much as on boundaries of oceans where tidal currents are more pronounced

(b) There are indications that the net littoral drift in places is in the opposite direction to the strongest tidal streams For example on the Mozambique coast the predominant drift is northward whilst the strongest tides run southwards Th1s is understandable when it is realised that lengthy orbital water particle motions of tidal period will be circular and will suffer shearing stresses near the bed, and thus contain large scale vortices in both horizontal and vertical planes The net transverse movement over a tidal cycle, particularly near the bed may thus be negligible or random in any direction

(c) When a Continental Shelf consists of sediment over its complete w1dth it has a mean slope of $0002(1)$, being steeper near shore and milder at the edge The uniformity of this profile over long lengths of coastline, and from one landmass to another, does not seem to correlate with the vast differences in tidal range or tidal currents associated therewith As noted previously, totally enclosed seas, such as the Mediterranean, Baltic etc, should have Shelves only at river outlets if tidal action were the sole or major source of energy distribution

(d) The edge of the Shelf, demarcating the limit of sweeping action by waves, has already been noted as around 65 fathoms $(=390 \mathrm{ft}$ $=119$ metres) This is around the reach or 1 imlt of influence of 13 second waves It has been shown elsewhere (5) that waves of this period are predominant in the ocean wave spectra generated in the storm zones of the oceans Such waves traverse the oceans with very little loss of energy Hence most oceanic margins of the world recelve swell waves with perlods from 11 to 15 seconds The western margins of continents(6) in particular have swell incidence from the strong westerly gales in the $40^{\circ}$ to $60^{\circ}$ latitudes in both hemispheres In enclosed seas where the spectra of waves are 1 imited to the local storm centres, swell from major generating areas of the oceans being excluded, the shelf edge is not so deep In the Mediterranean it approximates 50 fathoms and in the Red Sea it is nearer to 30 fathoms, where sediment has accumulated

(e) A ublquitous feature of coastlines is the crenulate shaped bays formed by sedimentary sections between rocky headlands The sculpturing of this shape has been shown to be the work of waves arriving 
obliquely to the coast ${ }^{(7)}$ These bays are of vastly differing size, some encompassing many miles length of coast and some miles width of Shelf They indicate that waves can modify the sea bed to substantial depths and finally bring the bed and shoreline profiles into a compatible equilibrium with the direction of the most persistent swell waves in the adjacent ocean area The orientation of such bays has been used to determine the net sediment movement around the coastlines of the world $(4)$

(f) Finally, the oscillatory motion of the water particles at the bed produced by wave propagation involves a net advance each wave period which has a maximum value at the sea bed This is particularly so for small ratios of depth to wave length, and so is relevant to this discussion of predominant swell waves with periods from 11 to 15 seconds This net motion is termed mass transport and results from the viscous forces creating a boundary layer in the oscillatory motion of the water particles near the bed This boundary layer is in the order of 1 to 2 centimeters thick and yet the mass transport velocity is maximum within it Thus, if bed particles can be 1ifted temporarily from the floor during part of the wave cycle they can readily be carried forward in the net advance of the water itself As soon as the bed is so affected small ripples and then dunes form $(8)$, so creating a rough bed which changes the boundary conditions The ability of the waves to disturb bed particles is thus increased, so that sediment transport due to mass transport is enhanced The point of concern here is the comparison of this force with that of a tidal stream Whilst this latter may be strong at the surface, where it is most noticeable, it will be greatly reduced as the bed is approached, and be practically zero near the boundary layer previously mentioned Its transporting efficiency is further decreased by the vortices accompanylng $1 t$, as noted earlier

From the above discussion it is seen that waves appear to have exerted a great influence on the sediment existing on Continental Shelves The motions of particles are slow and are in fact zero for large periods of a year, when wave helghts or perlods are too small for adequate reach to the bed However, when $1 \mathrm{t}$ is realised that waves, both storm type and swe11, have been avallable on the oceans since their inception, there has been a surfelt of time for Shelves to be accumulated and even for vast expanses of landmasses to have been accreted

\section{LONGSHORE COMPONENT OF MASS TRANSPORT}

The mass transport velocity at the seabed has been derived theoretically for the case of waves of very small height, a smooth bed and laminar boundary layer( 9 ) An empirical relationship has also been found for the case of a rough bed and laminar or turbulent boundary layer (10) It is thus possible to compute this net advance in the direction of wave propagation at any point across the Continental Shelf when the wave characteristics are known

Assuming a wave arrives at the edge of a uniformly sloped Shelf at some given deepwater angle, it is possible to compute the shoaling and refraction coefficients for 1 t and find its angle to the shoreline during 
propagation towards the shore The variable wave height can be substituted into the bed mass-transport equation, assuming a roughness for the floor and the status of the boundary layer This latter is determined from a knowledge of the limiting wave height for a given wave train when the transition from laminar to turbulent boundary layer occurs The longshore component of the mass transport can be determined at each location to give its distribution across the Shelf

For smooth or rough bed and laminar boundary layer, we have

$$
\frac{\mathrm{U}_{\mathrm{L}} \mathrm{g} \mathrm{T}^{3}}{\mathrm{H}_{\mathrm{O}}^{2} \sin \alpha_{\mathrm{O}}}=\frac{\mathrm{k}}{\mathrm{A}}
$$

where $\mathrm{U}_{\mathrm{L}}=$ longshore component of mass-transport velocity at bed

$\mathrm{g}^{\mathrm{L}}=$ acceleration due to gravity

$\mathrm{T}=$ wave period in seconds

$\mathrm{H}_{\mathrm{O}}$ = deep-water wave helght

$\alpha_{0}^{0}=$ deep-water angle of crest to bed contour

$k^{0}=$ dimensionless factor dependent on

$$
\begin{aligned}
\mathrm{D} / \delta= & \mathrm{D}(\pi)^{\frac{1}{2}} / 46(\nu \mathrm{T})^{\frac{1}{2}} \\
\text { where } \mathrm{D}= & \text { dimension of bed roughness (grain diameter } \\
& \text { or ripple height) } \\
\delta= & \text { boundary layer thickness } \\
\nu= & \text { kinematic viscosity of seawater }
\end{aligned}
$$

Experimental values of $k$ derived by Brebner et al ${ }^{(10)}$ give values as In Figure 1

$$
\begin{aligned}
& A=\left(1-\sin ^{2} \alpha_{0} \tanh ^{2} 2 \pi d / L\right)^{\frac{1}{2}}\left[\tanh 2 \pi d / L\left(1+\frac{4 \pi d / L}{\sinh 4 \pi d / L}\right)\right] \sinh ^{2} 2 \pi d / L / \cos \alpha_{0} \\
& \text { where } \quad d=\text { water depth } \\
& \text { (The expression for } A \text { is graphed in Figure 2) }
\end{aligned}
$$

For the smooth and rough bed turbulent boundary layer condition, Brebner et a $1(10)$ found

$$
\frac{\mathrm{U}_{\mathrm{L}} \mathrm{g} \mathrm{T}^{26}}{\mathrm{H}_{\mathrm{O}}^{12} \sin \alpha_{0}}=\frac{289}{\mathrm{~A}^{06}} \text { for fps units }
$$

(Unlike Eq (1) this relationship is not dimensionally homogeneous, indicating the need for further experimental confirmation)

The transition from laminar to turbulent boundary layer as found by the same workers $(10)$ can be expressed as

$$
\frac{\mathrm{H}_{\mathrm{o}}^{2}}{\mathrm{~T}_{\nu}}=8150 \mathrm{~A}
$$

It will be seen in the subsequent calculations that turbulent boundary layer conditions exist for nearly all the waves and depths chosen 


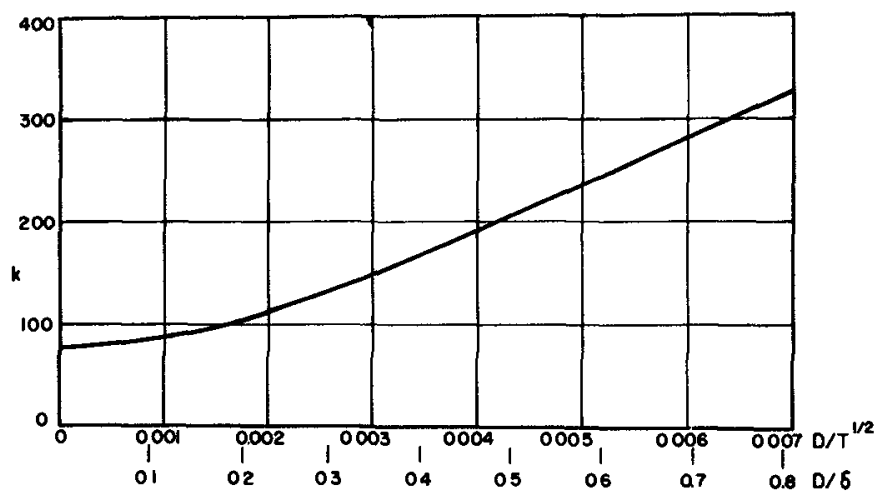

Fig 1 Values of $k$ in equation (1) from roughness helght in proportion to boundary layer thickness or $\mathrm{D} / \mathrm{T}^{\frac{1}{2}}$

\section{AVERAGE WAVE SPECTRA}

In order to determine some reasonable values of wave height and wave period to substitute in the above equations, the optimum storm. conditions of the oceans must be assessed As noted elsewhere (5), the 35 knot wind is the strongest that can normally generate a fully arisen sea Winds of higher velocity elther have limited duration or fetch (or both), so restricting the waves to $\mathrm{H}_{1} / 3=33 \mathrm{ft}$ and $\mathrm{T}_{\max }$ to 12 seconds The energy distribution curve(11) for this condition is depicted in Figure 3 where it is seen that the equivalent triangular distribution indicates upper and lower englneering limits of 194 and 42 seconds respectively Proportional energy values for wave bands centered on $8,10,12,14$ and 16 seconds result in wave train heights $\left(\mathrm{H}_{1 / 3}\right)$ of $32,49,53,42$ and 20 feet respectively, based upon proportional. areas under the curve

If these wave trains emerged from a fetch a long distance away they would arrive at the Continental Shelf separately The total energy avallable at any one time would depend upon the angular and radial dispersion from the fetch, but the resultant wave heights would be in proportion to those computed above for the discrete wave bands For the purpose of this comparison 1 t will be assumed that waves of all perlods are arriving from the one direction, with the crests angled at $50^{\circ}$ to the uniform and stralght underwater contours

In Figure 4 is depicted the Continental Shelf with uniform slope of 00021 Conditions at each $d / L_{0}$ value represent the mean for a certain width of Shelf, from which it can be seen that $\left(\Delta d / I_{0}\right)\left(b / I_{0}\right)=0002$

$$
\text { so that } \quad b=\left(\Delta d / I_{o}\right) 512 T^{2} / 0002
$$


At intermediate depths the differential $\Delta d / L_{o}$ is the difference of values midway between adjacent points, whilst at the extremeties of $\mathrm{d} / \mathrm{L}_{0}=001$ or 05 the outside half width is assumed the same as the inner half (e $\mathrm{g} \Delta \mathrm{d} / \mathrm{L}_{\mathrm{o}}=0$ 015-0 $005=0$ 01) The $\mathrm{b}$ values are listed in Table $\mathrm{I}$ The product $b U_{L}$ represents a mass transport discharge across the Shelf width $b$ per unit depth of liquid at the bed When multiplied by a thickness to which $U_{\mathrm{L}}$ is considered to apply ( $\frac{1}{2} "$ has been assumed), the discharge of water in this layer of liquid is obtalned The summation $\sum \mathrm{b} \mathrm{U}_{\mathrm{y}} / 24$ gives the sald discharge across the active width of the Shelf ( $\Sigma b$ ) for the wave train under consideration Although calculations have been carried out to $d / L_{0}=05$, the limit of disturbance of the respective components (derived later) is indicated by the double lines in Table I

\section{EQUAL SEDIMENT TRANSPORT IN SURF ZONE AND OFFSHORE}

For the wave conditions specified in Table I it is possible to compute the littoral drift in the surf zone by one of the many relationships avallable The one employed here is that presented by Castanho (12) which has been discussed elsewhere(13) The volume of sediment passing a plane normal to the beach per unit of time (e $\mathrm{g} \mathrm{ft}^{3} / \mathrm{sec}$ ) can be expressed as

$$
Q=\omega H_{o}^{2} L_{o} E_{r} \sin \alpha_{o} / 7 \Gamma Y_{s}
$$

where $Q=$ volume of sediment of specific weight $Y_{S}$ passing a plane normal to the beach per second $\left(\gamma_{s}=1101 \mathrm{~b} / \mathrm{ft}^{3}\right)$

$\omega=$ specific weight of seawater $\left(=641 \mathrm{~b} / \mathrm{ft}^{3}\right)$

$\mathrm{H}_{\mathrm{O}}=$ deep water wave height ( $\mathrm{ft}$ )

$\mathrm{L}_{\mathrm{o}}^{\mathrm{o}}=$ deep water wave length (ft)

$E_{r}=\frac{\text { energy dissipated }}{\text { longshore energy component }}$ (see Ref 13)

$\alpha_{\mathrm{b}}=$ angle of crest to beach at breaking ( $\mathrm{deg}$ )

$\alpha_{\mathrm{o}}=$ deep water approach angle (deg)

$\mathrm{T}=$ wave period (secs)

Values have been compuced in Table I for $\alpha_{0}=50^{\circ}$ and the respective wave heights and wave periods

Let it be assumed that an equal volume of material is passing the plane beyond the breaker line, out to the reach of each wave train Also let it be assumed that sediment particles are moving at the same net speed as the water near the bed ( 1 e in the $\frac{1}{2} "$ thick layer previously employed) Then the concentration of sediment by volume to accomplish this task can be calculated, as listed in Table I

It is seen that for the wave characteristics chosen the concentration necessary for equal transport offshore and in the surf zone are feasible Whilst the longshore velocity $U_{\mathrm{L}}$ is substantially reduced, the further from the beach and the longer the wave period, the widths of shelf over which these operate are substantially enlarged 


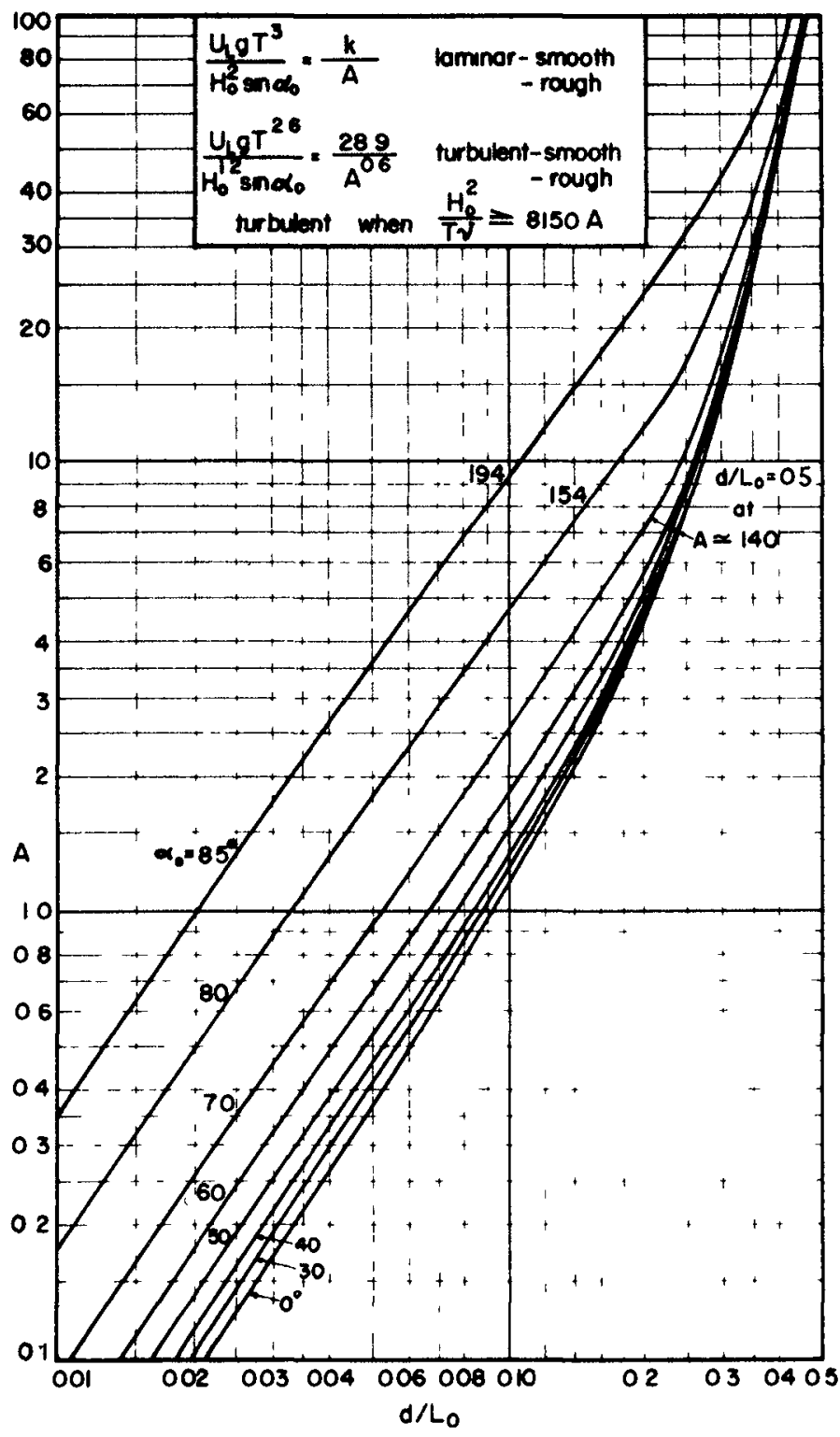

Fig 2 Factor A used in equations (1)(2) \& (3) 


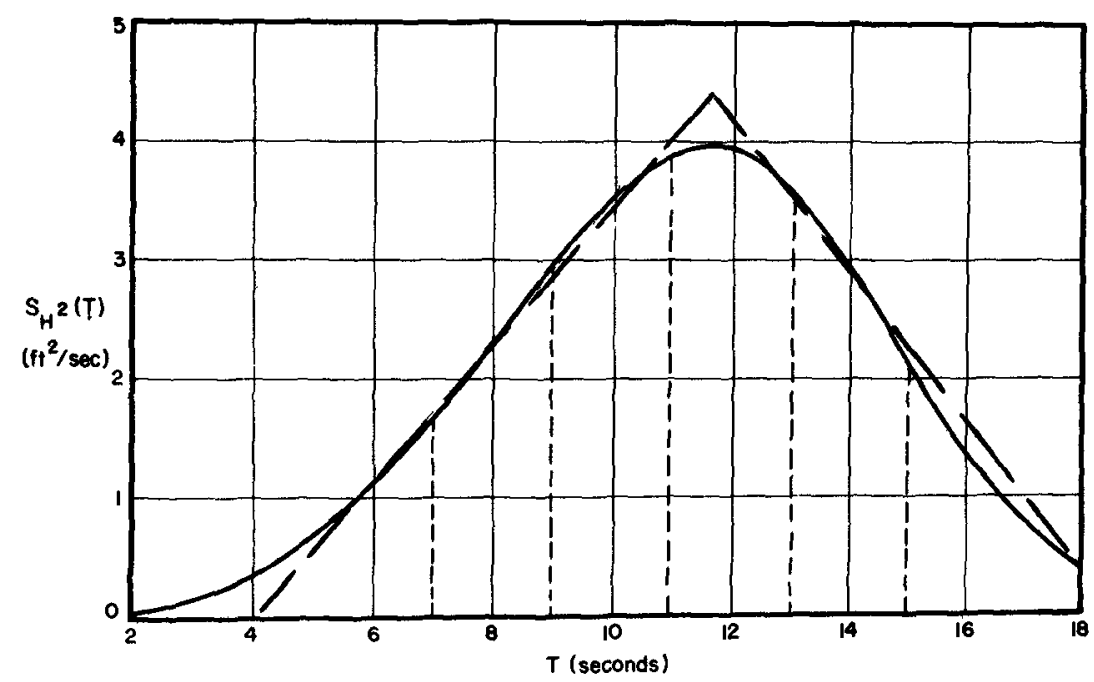

Fig 3 Energy distribution curve for a fully arısen sea generated by a 35 knot wind

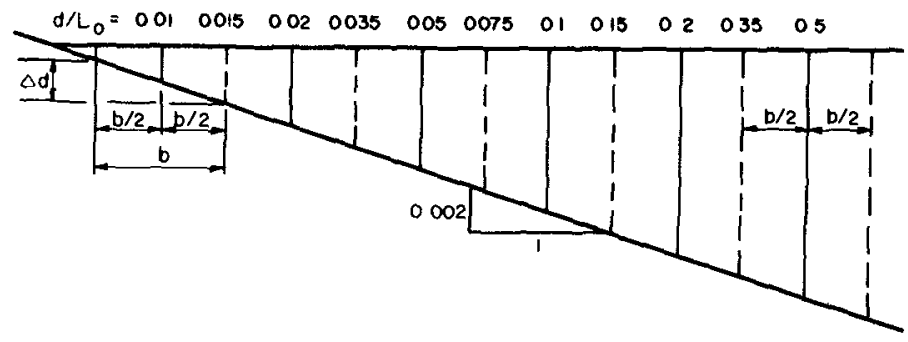

Fig 4 Assumed uniform Continental Shelf for purposes of calculations in Table I 
Table I Longshore mass transport in various wave trains across portion of the Continental shelf (See Figure 2)

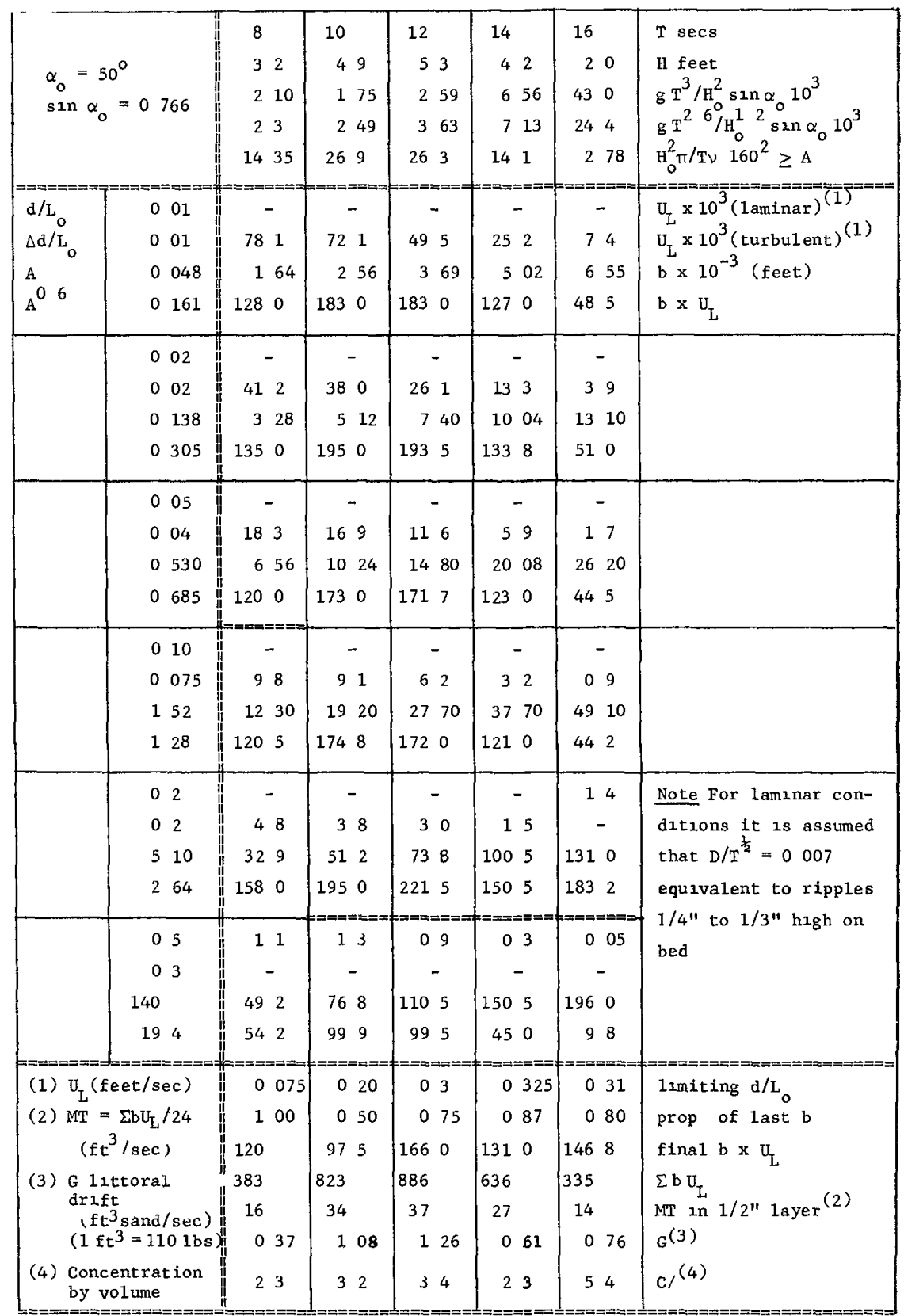




\section{INCIPIENT MOTION OF BED PARTICLES}

Before sediment particles can be carried forward and backward by the oscillatory motion of the water particles near the bed, they must be removed from the bed This topic needs much more active research, but results are available from a number of workers on the incipient motion of sand particles under wave action The differences and similarities of their results have been discussed elsewhere(8), but it can be concluded that, as the replication of bed conditions has tended towards the prototype scale of action, the greater has been found the reach of the waves The types of equipment employed in generating the necessary oscillatory water motion have been described elsewhere(14) A more recent $\mathrm{rig}$, used at the University of Western Australia(15), essentially oscillates a block of water with amplitudes and periods applicable at the sea bed Results from these tests are presented in Figures 5A \& B

The empirical formulae derived for incipient motion of sand particles on a flat bed can be put into similar dimensionless form as in equations (5) to (17)

The relationship by Abou seida ${ }^{(29)}$ cannot be written in such a form but a modification and iterative process carried out by Mogridge(25) permits it to be plotted as in Figure 5 The graph of Bonnefille and Pernecker (17) consisted of two curves which have been modified into one for the presentation in the figure The condition of the boundary layer has been indicated in the equations or been presumed (?)

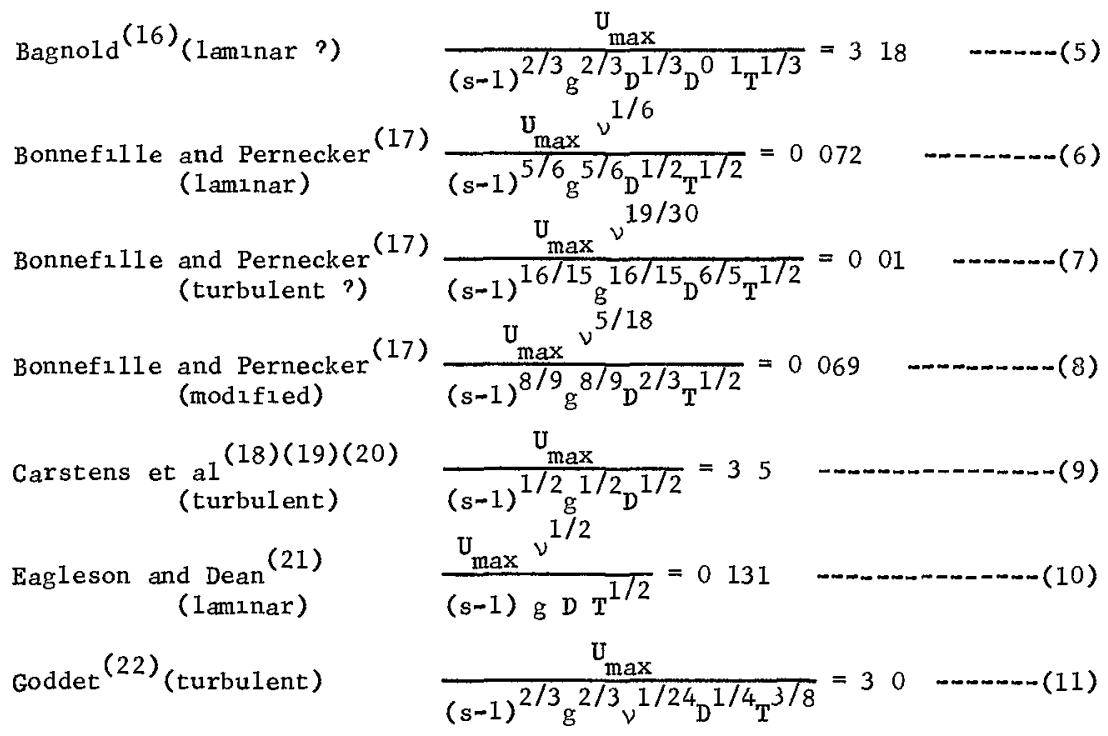




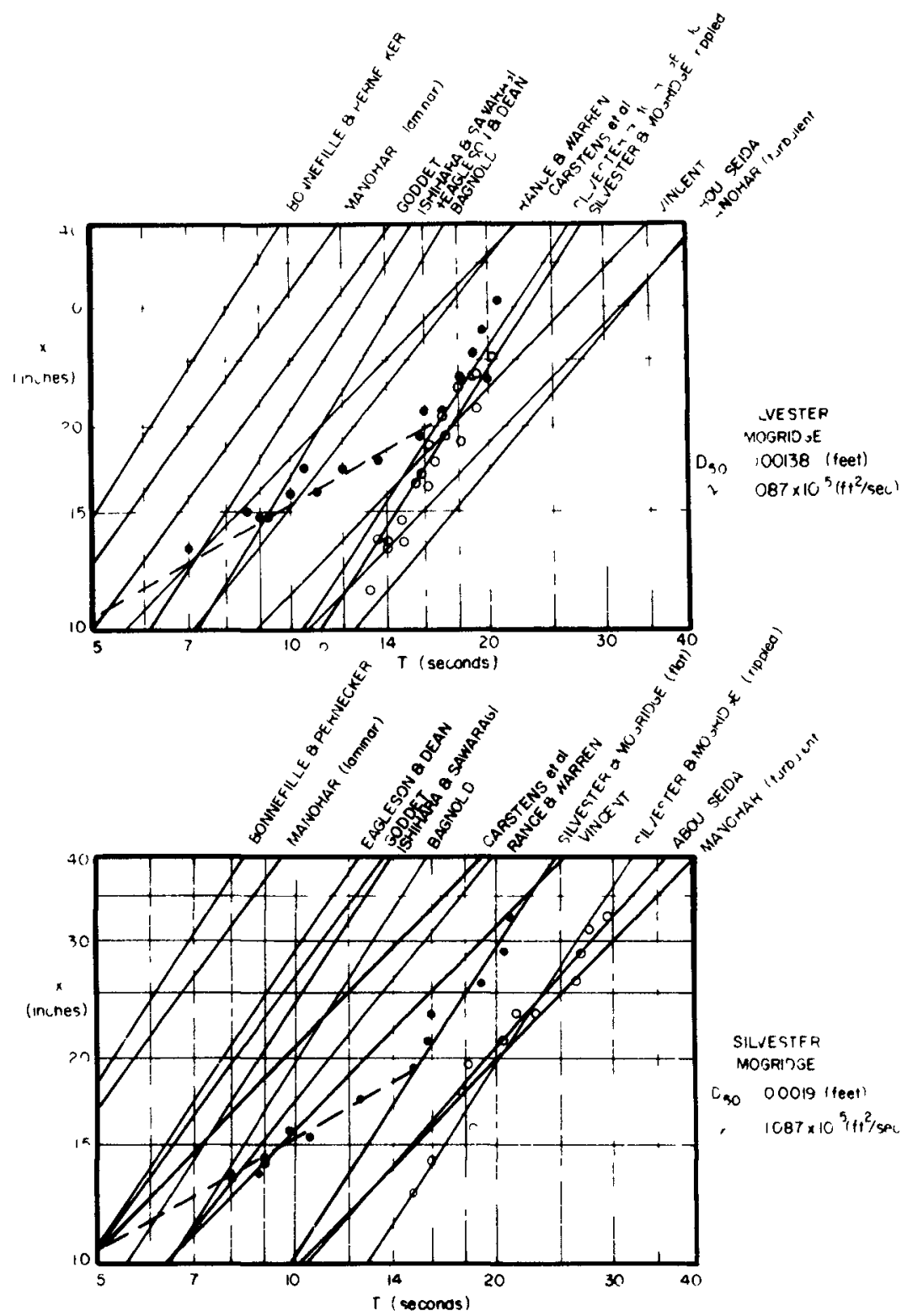

Fig 5 Wave perıods ( $T$ ) and amplitudes ( $\mathrm{x}$ ) at the sea floor to produce inciplent motion on a flat bed with sand particles of mean dia $A=000138 \mathrm{ft}(042 \mathrm{~mm})$ and $B=00019 \mathrm{ft}(058 \mathrm{~mm})$ 
Ishihara Sawarag 1 (23)

(turbulent)

Manohar ${ }^{(24)}$ (laminar)

Manohar ${ }^{(24)}$ (turbulent)

Silvester and Mogridge (flat bed)

(25)

Rance and Warren ${ }^{(26)}$ (sand) $\frac{U_{\max }}{(s-1)^{3 / 5} \mathrm{~g}^{3 / 5} \mathrm{D}^{2 / 5} \mathrm{~T}^{1 / 5}}=069$

$\frac{U_{\max }}{g^{3 / 4}(s-1)^{3 / 4} D^{1 / 4}}=0093$

$\frac{U_{\max } \nu^{1 / 3}}{(s-1)^{2 / 3} g^{2 / 3} \mathrm{D}^{2 / 3}}=0159$

$\frac{U_{\max }}{(s-1)^{04}{ }_{g}^{04}{ }_{D}^{02}{ }_{\nu}^{02}}=745$

$\frac{\mathrm{U}_{\max } \nu^{1 / 18}}{(\mathrm{~s}-1)^{7 / 9} \mathrm{~g}^{7 / 9} \mathrm{D}^{1 / 3_{\mathrm{T}}} \mathrm{T}^{1 / 2}}=0034$

Vincent ${ }^{(27)}$ (turbulent)
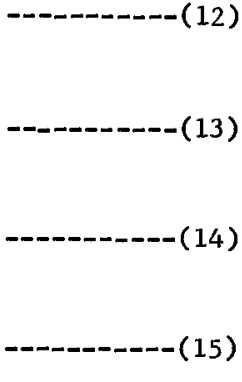

The $U_{\max } c$ an also be expressed in terms of amplitude of motion when the wave period is known or assumed, in which form these equations have been graphed in Figures $5 A \& B$, for $D_{50}=000138$ and 00019 ft respectively Also assumed in the figures is $\nu=1087 \times 10^{-5}\left(\mathrm{ft}^{2} / \mathrm{sec}\right), \mathrm{s}-1=16$ and $g=322 \mathrm{ft} / \mathrm{sec}^{2}$. It is seen immediately that these relationships are not compatible, probably through being extrapolated beyond the zones of verification

Also included in the figures are the results of the authors' tests, the black dots representing flat bed conditions and the open circles the rippled surface produced from prior oscillatory motion For the flat bed two relationships were found, one in which $x$ varied approximately as $\mathrm{T}^{1 / 2}$ for smaller $\mathrm{x}$ and $\mathrm{T}$ values, and the second in which $\mathrm{x}$ varied as $\mathrm{T}^{3 / 2}$ The transition from the one to the other occurred at $\mathrm{x}=205^{\prime \prime}$ and $T=17$ seconds in Figure $5 \mathrm{~A}$, and $\mathrm{x}=19^{\prime \prime}$ and $\mathrm{T}=15$ seconds in Figure 5B Sleath $(28)$, in his velocity measurements in the boundary layer at the bed of a wave tank, found a parameter $U_{\max } D_{50} / \nu$ which displayed the transition in the velocity profile from laminar to one still laminar but influenced by vortex formation around the grains of sand Appropriate substitutions from the above tests give values for incipient particle motion of 825 and 116 for the 000138 and $00019 \mathrm{ft}$ dlameter sands respectively

Sleath observed his critical condition to occur at a value approxlmating 50 He states "This may be compared with the value of $\mathrm{U}_{\max } \mathrm{D} / \nu=200$ obtalned from the formula proposed by Manohar (1955) (24) for the transition from laminar to turbulent conditions with sand of median diameter $=00445$ in It is probable that the phenomenon observer by Manohar and his colleagues was vortex formation rather than turbulence " 
Carstens et al ${ }^{(18)(19)(20)}$ from their tests with oscillating water in a condult obtained $u_{\max }$ values when certain changes in the bed were observed For the sand size $D=000097 \mathrm{ft}$ and $\nu=110 \times 10^{-6} \mathrm{ft}^{2} / \mathrm{sec}$ the following values of $U_{\max } D_{50} / v$ were obtained

$\begin{array}{lr}\text { boundary layer transit ion commences (sand bed) } & 563 \\ \text { boundary layer transit ion commences (smooth bed) } & 748 \\ \text { inclplent bed motion, bed undisturbed } & 713 \\ \text { spontaneous appearance of ripples } & 940 \\ \text { fully turbulent boundary layer (smooth bed) } & 119\end{array}$

From these values it seems quite probable that Manohar's observations were commencement of turbulence A significant observation of the above results is that incipient bed motion and even ripple formation occurred before full turbulence was experienced in the boundary layer It is at such a stage that mass transport will exert its influence on the bed particles As seen in Figures 5A\& $B$ the presence of ripples on the ocean floor will produce incipient motion at smaller amplitudes of the water particles for a given wave period, or at longer periods for a given amplitude However, the curves for this more realistic condition do not match the one equation, so the conservative flat bed relation of equation (15) has been put in terms which are graphed in Figure 6

With this diagram a wave of any specific period in a certain depth of water will have to have a minimum height in order to initiate particle movement on a flat bed The respective heights and periods for a fully arisen sea of a 35 knot wind previously derived are indicated in Figure 6 It 1 s seen that the 12 and 14 second waves can disturb sediment at over 200 feet depths This 18 half way across the Continental Shelf

It is believed that for similar wave conditions the ability of waves is greater than that indicated in Figure 6 due to the following prototype phenomena

1 Velocities of water particles near the ocean bed may be higher than those derived by first order theory

2 The interaction of wave trains of slightly differing period can generate greater instantaneous velocities than implied in the present analysis

3 Wave trains angled to each other produce vortices of large dimensions with associated turbulence which may disturb the bed more readily

4 In storms at sea, where fetches are changing location and orientation continually, waves may move in opposite directions so creating clapoti or partial clapoti These create h1gh water particle velocities near the bed They w11l also temporally bulld up furrows of material which w1ll be readily swept away when the standing wave has dispersed

5 Currents and internal waves associated with tidal action at the 


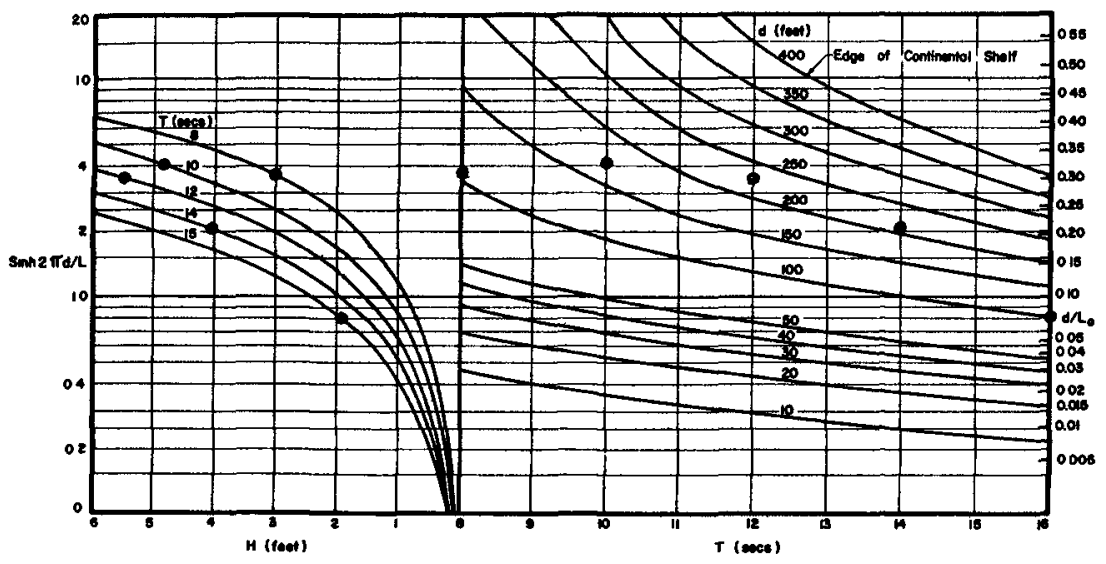

Fig 6 Wave conditions for inclpient motion on a flat bed for sand $\left(D_{50}=000065 \mathrm{ft}=02 \mathrm{~mm}\right)$

edge of the Shelf could assist the waves in disturbing the bed at these larger depths

6 Shell debris on the sea floor can inltlate movement of sand particles sooner than for rippled or smooth beds Carsten's tests(19) showed that,with small protruberances on the bed, motion is initiated at about half the water particle velocity of that for a smooth sand bed This implies in Figure 6 that the same reach can be effected with half the wave heights previously considered It can be shown by drawing curves in the left hand side of the figure with half the wave height for each period band, that for the original wave heights the reach is increased about $30 \%$ For example, the $55 \mathrm{ft}$ high 12 second wave whlch had a reach of $230 \mathrm{ft}$ for rippled bed conditions can influence a bed at $300 \mathrm{ft}$ depth which contalns she11-11ke debris on it

7 It is possible that fish 11 fe is concentrated heavily at the floor of the ocean Any motions by them on or near the bed will disturb sediment which the mass transport of the waves wl11 carry into and along the shore

These prototype conditions could increase the concentration of suspended sediment above those recorded in model studies They could also effect movement we11 above the boundary 1 ayer, on which the previous comparison was based 


\section{CONCLUSIONS}

1 Since the major proportions of Continental Shelves consist of sediment which has accreted to around the 400 feet depth, 1t would appear that wave action is the predominant spreading agent

2 The mass-transport due to wave action is at a maximum within the boundary layer at the bed where initial suspension of material occurs

3 Roughness of the sea floor due to the sediment particles or undulations formed thereon would appear to increase the mass-transport velocity and assist in the initial disturbance of the bed

4 For an oblique wave train, the mass-transport velocity at the bed, and its longshore component, can be computed at all depths across the Continental Shelf, to the limit where shoaling invalidates the theory employed

5 With certain sumplifying assumpt ions, it can be shown that for equal sediment transport within the surf zone and beyond the breaker line for a given wave traln, the volumetric concentration of sediment within the $1 / 2$ " bottom layer of water does not nave to exceed $5 \%$

6 Empirical relationships of wave and sediment characteristics for inclpient motion on a flat bed vary considerably, with those utilising prototype velocities and amplitudes in their experimental rigs showing a greater reach of wave action

7 Further tests are required to verify beyond all doubt that the average wave spectra to be expected over the continental Shelves can sweep sediment on them to their recognised outer limit These test should include normal marine debris on the bed, so that initiation of sediment motion and the frictional effects on the boundary layer can be studied

8 More analytıcal and experimental work needs to be carried out on mass-transport, both for simple wave trains and the more complex water and sediment systems 


\section{REFERENCES}

(1) Shepard F P Submarıne Geology (2nd Ed ) Harper Row, 1963

(2) Hayes M D "Relationship between coastal climate and bottom sediment type on the inner Continental Shelf" Marine Geo1 5, 1967, 111-132

(3) Stoddart D R "World erosion and sedimentation" Water, Earth and Man (Ed R J Chorley) Methuen (London), 1969

(4) Silvester $R$ "Sediment movement around the coastlines of the world" Proc Conf Inst Civil Engrs (London), 1962, 289-315

(5) Silvester $\mathrm{R}$ and $\mathrm{S}$ Vongvisessomjal "Computation of storm waves and swe11" Proc Inst C1vi1 Engrs (In Press)

(6) Davies J I "A morphogenic approach to world shorelines" Ann of Geomorph 으, 1964, 127-142

(7) Silvester R. "Stabilization of sedımentary coast1ınes" Nature (London) 188, 1960, 467-469

(8) Silvester R. "Sediment movement beyond the breaker zone" Trans Instn Engrs Aust CE12, 1970, 63-71

(9) Longuet-H1ggins M S "Mass transport in water waves" Phil Trans Roy Soc London, A245, 1953, 535-581

(10) Brebner A, J A Askew and S W Law "The effect of roughness on the mass-transport of progressive gravity waves" Proc 10th Conf Coastal Eng, Vo1 1, 1966, 175-184

(11) Silvester $R$ and $S$ Vongvisessomjal "Energy distribution curves of developing and fully arisen seas" $J$ Hyd Res (In Press)

(12) Castanho $\mathrm{J}$ "Breakıng waves and littora1 drıft" (in Portugese) Lab Nac de Eng Civil (Lisbon) Mem No 275, 1966

(13) Silvester $R$ "Beach profiles and littoral drift assessment" La Hour1le Blanche 24, 1969, 615-621

(14) Silvester $R$ "Modelling of sediment motion offshore" $J$ Hyd Res 8, $1970,227-259$

(15) Mogridge G $\mathrm{R}$ "Testing sediment movement due to wave action" Proc ASCE 96 (HY7), 1970, 1587-1604

(16) Bagnold R A "Sand movement by waves, some smal1 scale experiments with sand by very low density" Proc Inst Civil Engrs 27, 1947, 447-469

(17) Bonnefille $\mathrm{R}$ and $\mathrm{L}$ Pernecker "Debut d"Entrainment des Sediments per la Houle" Proc 11th Congress IAHR Vo1 V, 1965, 207-208 
(18) Carstens M R , F.M. Nel1son and H D Altınbılek "An analytıcal and experimental study of bed forms under water waves" Georgla Inst Tech Final Rep Proj A-798, 1967

(19) Carstens $M R$ and $F$ M Neilson "Evolution of a duned bed under oscillatory flow" J Geoph Res 72, 1967, 3053-3059

(20) Carstens M R ,F.M Neilson and H D Altınbilek "Bed forms generated in the laboratory under an oscillatory flow analytical and experimental study" Coastal Eng Res Centre, Tech Mem No 28, 1969

(21) Eagleson P S and R G Dean "Wave induced motion of bottom sediment particles" Trans ASCE 126 (Pt I), 1961, 1162-1189

(22) Goddet $\mathrm{J}$ "Etude du Début d'Entrainment des Materiaux Mobıles sous 1 'Action de la Houle" La Houllle Blanche 15, 1960, 122-135

(23) Ishıhara $T$ and $T$ Sawarag 1 "Fundamental studıes of sand drıfts" Coastal Eng in Japan $\underline{5}, 1962,59-65$

(24) Manohar $M$ IMechanics of bottom sediment movement due to wave action" Beach Erosion Board, TM 75, 1955

(25) Mogridge G R "Sedıment transport due to wave actıon"Thesis Unıversity of Western Australia in partial fulfilment of Master of Englneerıng Degree

(26) Rance P J and N.F Warren "The threshold of movement of coarse material in oscillatory flow" Proc 11th Conf Coastal Eng, Vo1 1, 1968, 487-491

(27) Vincent G E "Contribution to the study of sediment transport on a horizontal bed due to wave action", Proc 6th Conf Coastal Eng , 1958, 326-355

(28) Sleath J F A "Velocity measurements close to the bed in a wave tank" J Fluid Mech 42, 1970, 111-123 



\title{
CHAPTER 41
}

\section{ISOPACHOUS MAPPING OF THE LOWER PATUXENT ESTUARY SEDIMENTS BY CONTINUOUS SEISMIC PROFILING TECHNIQUES}

\author{
Newe11 $T$ Stiles and Donald $R$ Wresnet \\ $\mathrm{U} S$ Naval Oceanographic Office \\ Washington, D C 20390
}

\begin{abstract}
The thickness and extent of the sediment cover in the Patuxent Estuary has been determined using a high-frequency, high-spatıal resolution, shallow penetration, continuous seismic profiling system. From these data, an 1sopachous map was prepared The 1sopachous map provides the subbottom information required to determine optimum locations for placing test equipment on the river bottom

Mud filled depressions, acoustically transparent to $12-\mathrm{kHz}$ sound pulses, occur mainly to the north of Half Pone Point, and east of the present channel Based on the identification of first subbottom reflectors, these depressions are as much as 16 feet thick The dominance of the thicker deposits east of the channel and evidence of a submerged terrace indicates that either the channel has migrated to the west, or that the channel of the Patuxent River at this location was larger in the past and has subsequently filled in much of the material in the eastern edge Maximum penetration at the scarp of the submerged terrace was 36 feet beneath the water-sediment interface This study demonstrates the use of seismic profiling techniques for collecting and presenting data required for coastal englneering applications
\end{abstract}

\section{INTRODUCTION}

The Maryland State Road Commission is considering plans to build a bridge across the Patuxent River near Solomons Is land, Maryland (Figure 1) The proximity of the bridge to the existing naval test range located between Polnt Patience and Hooper Neck may require relocation of the test range A knowledge of the strength characteristics of the river bottom are critical for effective use of the test range Instrument packages may sink into soft muddy sediments and become impossible to locate or retrieve, other instruments could be damaged because of impact with hard sandy materials In order to delineate these two sediment materials, a seismic investigation was conducted from Town Point northward to Broomes Island (F1gure 2)

Th1s paper deals with the use of the 1sopachous map to present continuous seismic reflection data of the sediment cover in the Lower Patuxent Estuary An 1sopachous map shows the varying thickness of a designated stratigraphic unit by lines of equal thickness (1sopachous lines) The stratigraphic unit used in this study is the acoustically transparent sediment cover, which is composed of fine-grained silt- and clay-size sediments 


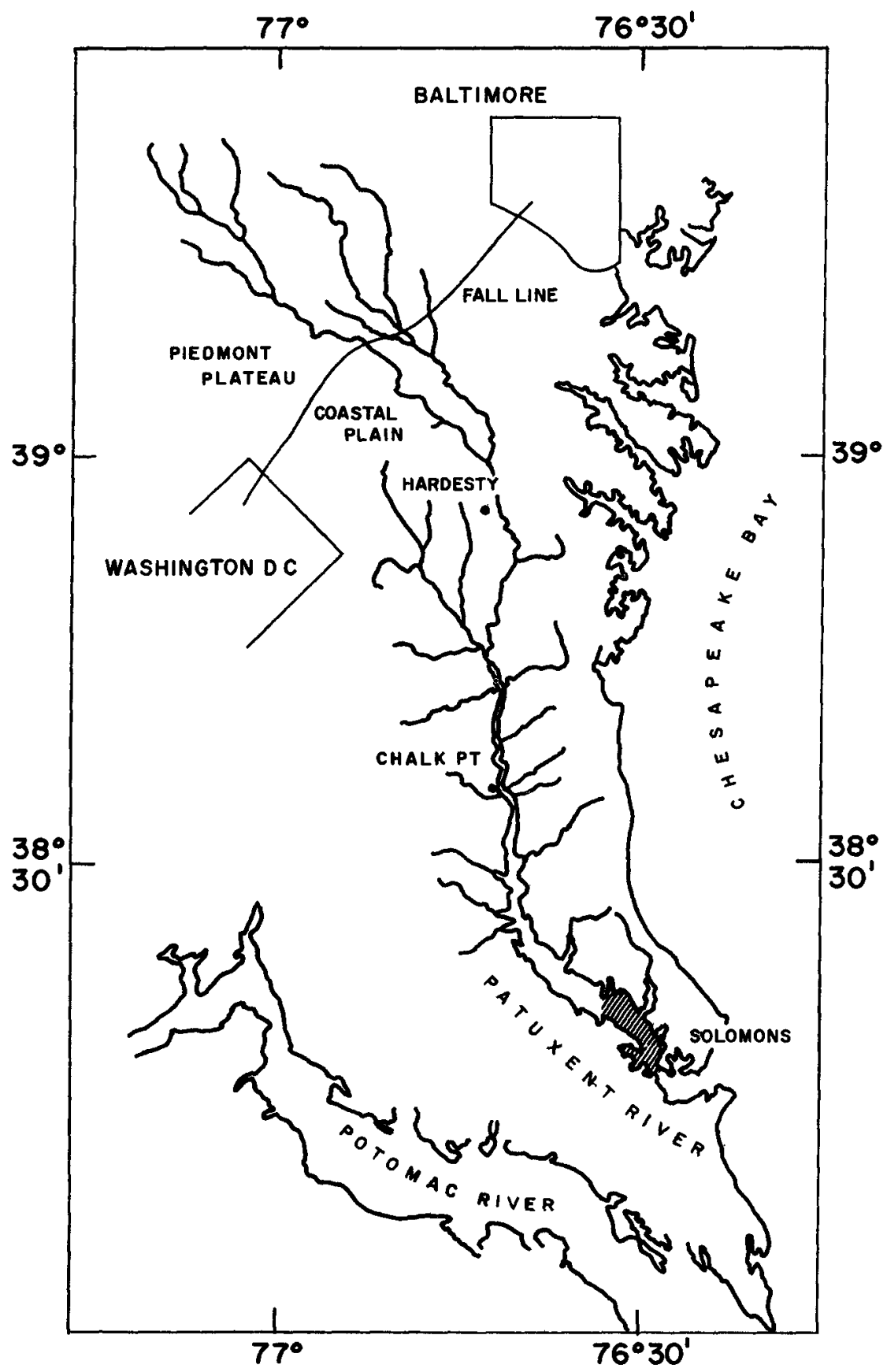

FIGURE 1 INDEX MAP SHOWING AREA OF STUDY 


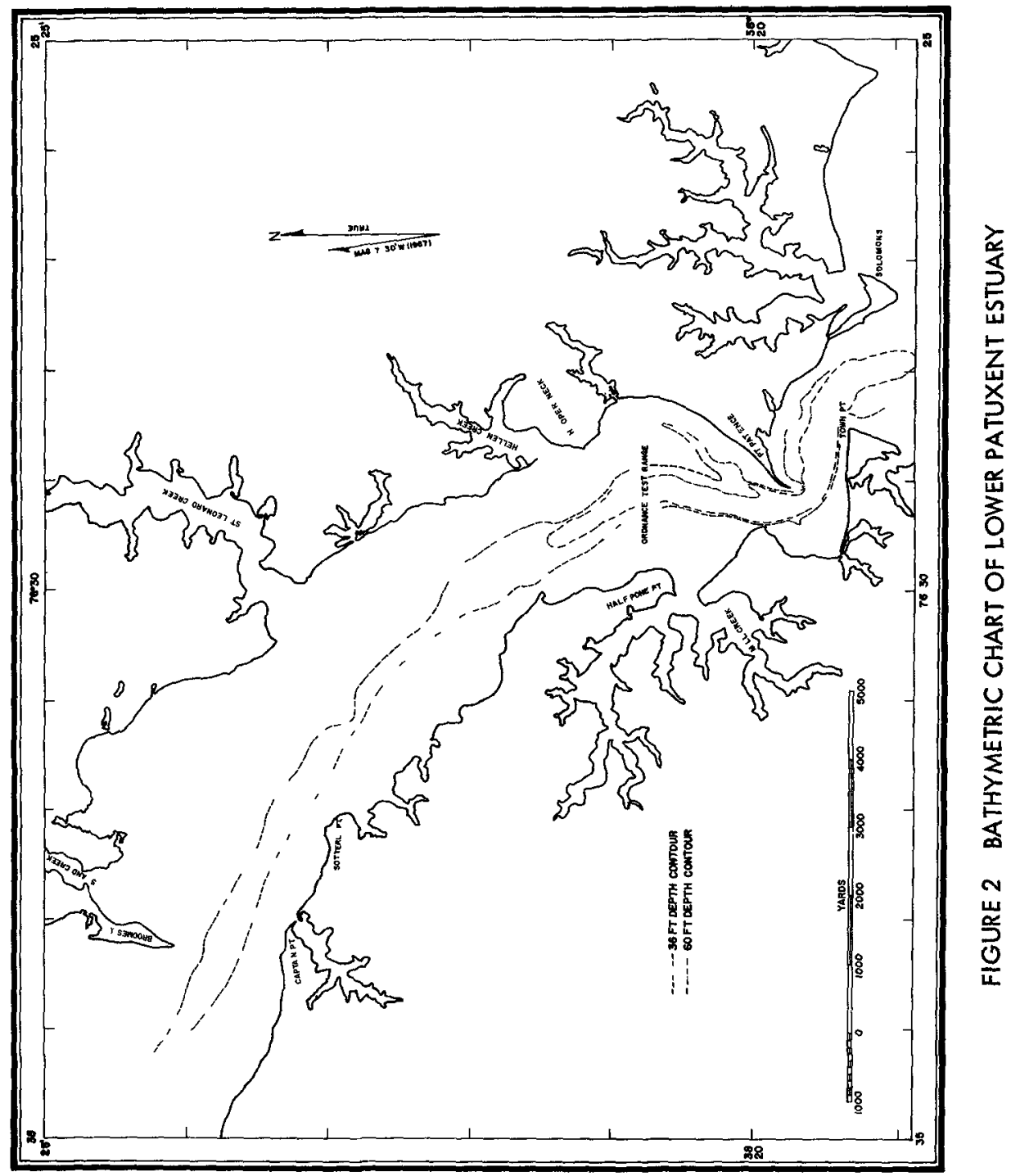


The Patuxent River drainage basin lies entirely within the state of Maryland and occupıes 930 square miles (Crooks, O'Bryan and others, 1967) The mouth of the patuxent Estuary is located where the river enters Chesapeake Bay The upper limit of the sea salt water ranges from 27 miles (Chalk Polnt) to 56 miles (Hardesty) above the mouth The portion of the waterway between the upper limit of tidal influence and the limit of sea salt intrusion is designated the tidal river (Pritchard, 1967, and Owen, 1969) Between the upper limit of the sea salt intrusion and the mouth of the river is the estuary The Patuxent has been termed moderately stratıfied by Pritchard (1967), and Owen states "The Patuxent Estuary has a normal two-layered flow associated with an intermittent three-layered flow "Chesapeake Bay itself is a moderately mixed estuary

Owing to the abundance of readily accessible erodible material in the Coastal Plain the sediment yield of the drainage basin is fairly high, 235 tons per square mile at Hardesty near the estuary head (Johns Hopkins University, 1966) Figure 3 shows the change in the lower estuary channel from 1859 to 1944 Although the location of this cross section is not shown, depth and width values correspond with the segment of the estuary between Helen Creek and Broomes Island (Figure 2)

\section{PROCEDURE}

Contınuous seismic profiles of the Patuxent River between Town Point and Broomes Island were recorded during the period 7 to 10 May 1968 Work was performed on a 45-foot utility boat provided by the U $S$ Nava1 Ordnance Laboratory Test Facility The transducer was rigidly mounted on the side of the boat three feet beneath the water surface A total of 19 tracks were run over a distance of 30 nautical miles (Figure 4) Boat speeds of about three to four knots were maintained for all traverses Positioning was determined from the two triangulation towers located at Point Patience

The seismic profiler, nicknamed a "Mud Penetrator" (Yules and Edgerton, 1964), consists of three main components (1) an acoustic transceiver and recorder (EGgG, Model 254 Seismic Recorder), (2) a transmitter/receiver transducer (EGGG, Model 228A Pinger Probe), and (3) a gasoline powered electric generator The system operates at an acoustic frequency of $12-\mathrm{kHz}$ which is sufficiently high so that only fine-grained sediments can be effectively penetrated

The technique for continuous seismic profiling of subsurface acoustic horizons uses a repetitive sound source which propagates acoustic wave fronts through the water and subsurface materials As these wave fronts encounter materials of contrasting acoustic impedance--normally manifestations of geologic stratification or boundaries--echoes are returned to the surface, sensed by the transducer, and then synthesized by the recorder to produce a real-time, continuous profile of the water column, 


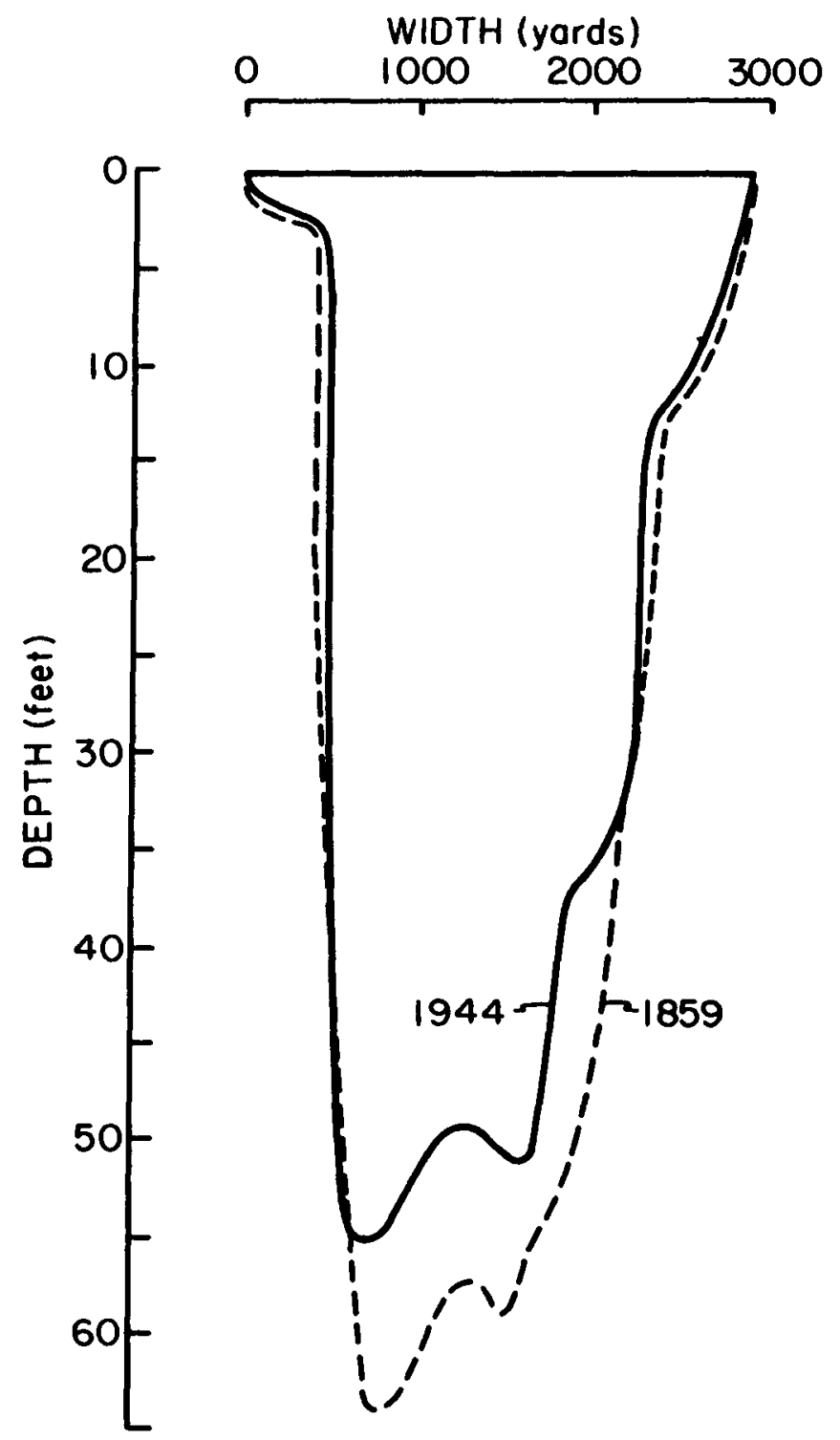

FIGURE 3. SEDIMENTATION IN THE PATUXENT ESTUARY. (AFTER JOHNS HOPKINS UNIVERSITY, 1966) 


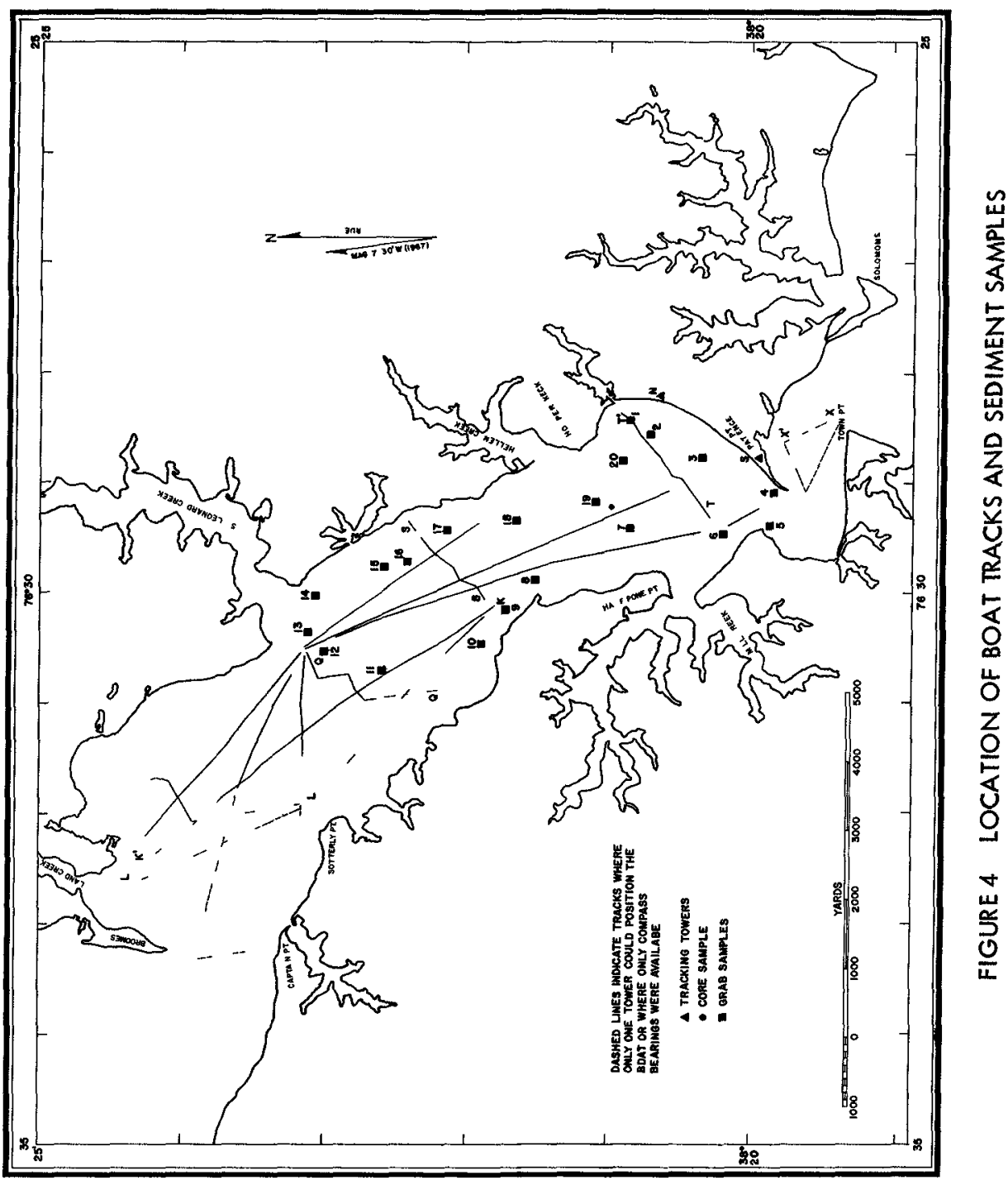


bottom, and subbottom The vertical scale is in time (m111iseconds) and the horizontal scale is a function of recorder-paper speed and ship speed The time values shown on the se1sm1c records are total travel times (two-way times) and must be halved for computing thickness Conversion of the time scale to thickness is accomplished by multiplying one-half the total travel time by a sound speed factor All depths in this report are based on a sound speed of 1500 meters per second in both the water and sediment. Th1s value corresponds closely w1th measured values on two core samples obtained near the mouth of the Patuxent Estuary (Stiles and Wiesnet, 1970).

Analysis of the seismic profiles in this investigation is based on the following premise. Observed subsurface geologic features on the selsm1c record are acceptable as proof of existence of subbottom structure However, the converse of this statement is not necessarily true The absence of subbottom structure on the seismic record does not always mean that a subbottom reflector does not exist The absence may indicate masking by a cover of sand, gravel, or other coarse-grained material For a given frequency, mud is normally much less attenuating than sand or coarser grain sediments In most cases where a mud cover over11es a coarser grain sediment mass, the impedance contrast will be sufficient to record the thickness of the mud Exceptions may exist where the thickness of the surface cover is too great for the acoustic energy of the particular se1sm1c system to penetrate and return to the sensor

Development of an isopachous map from selsmic profile records requires identification of a particular reflecting horizon which corresponds to a designated stratigraphic un1t, transferring the thickness values (e $\mathrm{g}$, in this paper, the distance between the first subbottom layer and the water-sediment interface) on to a track chart, and contouring these values

\section{DISCUSSION OF RESULTS}

Results of this investigation are presented in the form of annotated seismic profiles and an 1sopachous map Interpretation of the seismic records was based in part on previous work with this system 'Earlier studies by Breslau and Edgerton (1968) in the Gulf of La Spezia, and by Stiles and others (1969) in four Vietnamese rivers have shown that the acoustic energy of this system 1s not capable of penetrating coarse-grained sediments, such as sands or gravels, whereas, fine-grained materials, such as silts and clays, are transparent to the $12-\mathrm{kHz}$ sounds of the system After completion of the 1sopachous map, 20 sediment samples were collected Comparison between the sediment properties from these bottom samples which were collected simultaneously with seismic profiles corroborates the interpretation of seismic profiles in this investigation (Figure 5 and Table I)

The stratigraphic unit used in this study is the acoustically transparent sediment cover. This sediment cover is composed mainly of fine-grained silt- and clay-size sediments, 


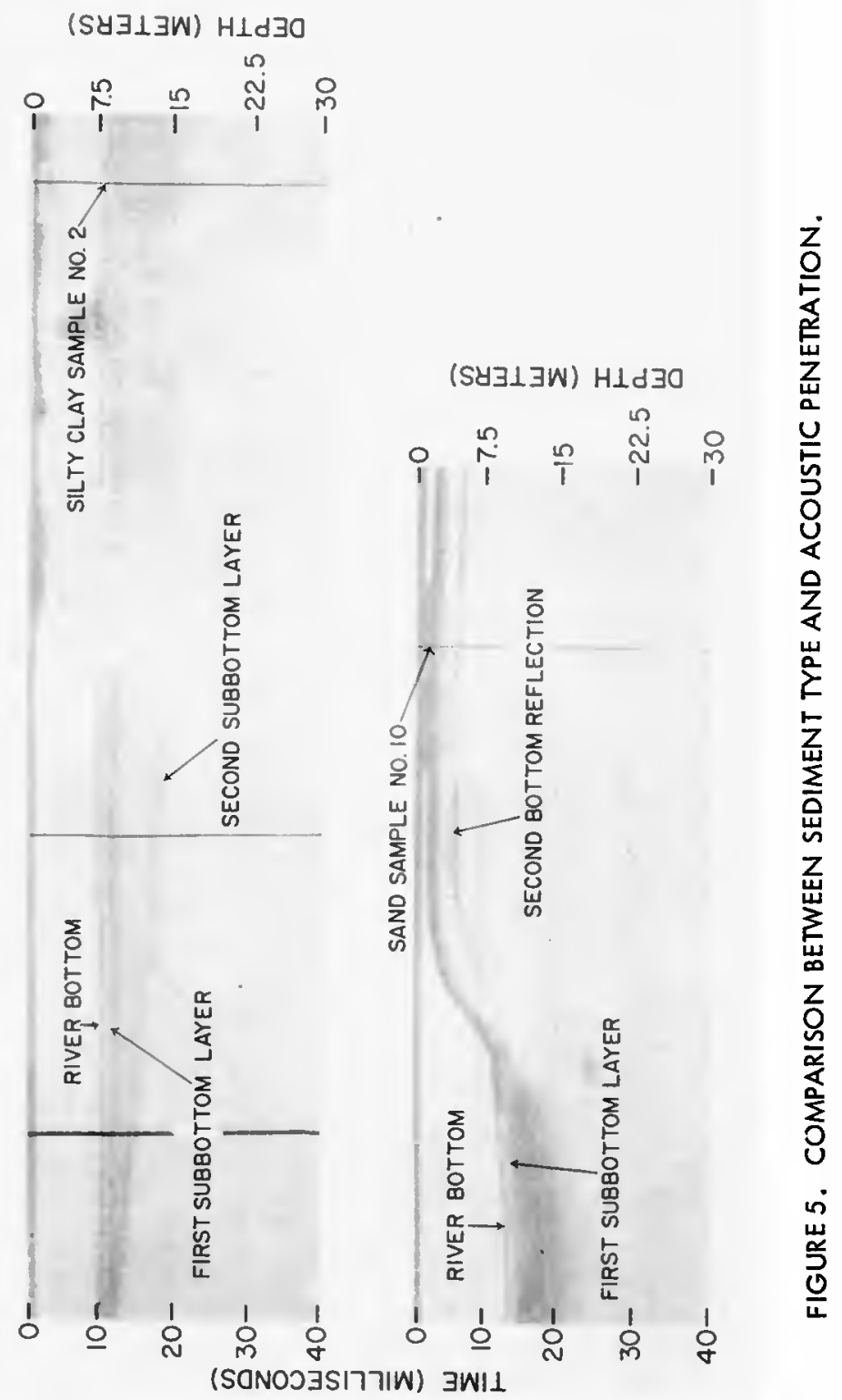




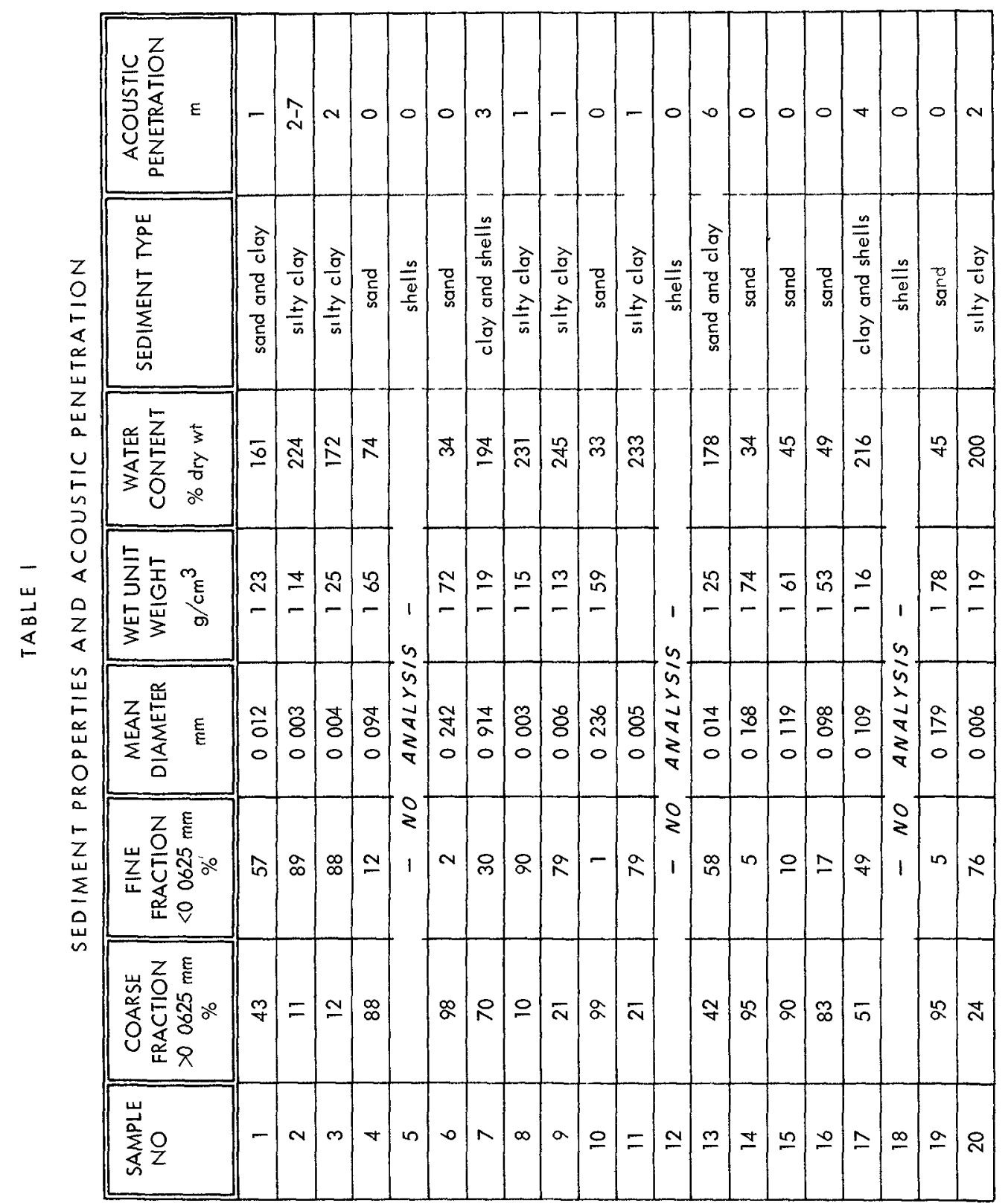


collectively referred to as mud The reflecting horizon used in all cases is the first subbottom layer Where penetration does not occur, the surface materials consist of coarse-grain sediments Both the opaque surface and subsurface reflectors are assumed to consist of similar sediments Selected longitudinal and transverse profiles are shown in F1gures 6 through 9 Location of these seismic profiles are shown in F1gure 4 The 1sopachous map is shown in Figure 10

Analysis of the seismic records shows numerous objects partially submerged on the river bottom The largest object is shown protruding from the bottom near the southern end of Profile L - L' (Figure 6), which is believed to be elther a part of the mooring buoy plotted on U S Coast and Geodetic Survey Chart No 553 or a sunken barge (James Green, Nava1 Ordnance Laboratory Test Fac1lity, Solomons, Maryland, oral communication) Green indicated that in addition to the barge there are many metallic objects scattered on the river bottom Some of these artifacts are probably the same objects observed on the seismic records (F1gures 7 and 9) In addition to the objects lying on the river bottom, many reflections presumably caused by fish were recorded in the water column (F1gure 9)

Subbottom layers are present in a majority of the seismic profiles north of Half Pone Point (F1gure 10) Depressions filled with mud are as much as 16 feet thick One of the clearest records of subbottom structure is shown on Profile $K$ - $K$ ' northwest of the "Black H111" structure (Figure 7) We assume that the subbottom reflecting layer is mainly composed of quartz sands or oyster shells (Table I) The same reflecting horlzon forms a terrace further northwest along Profile $K-K^{\prime}$ (F1gure 7) Similar terrace-shaped structures are present on the northeastern margin of Profiles $Q-Q^{\prime}$ and $S$ - $S^{\prime}$ (F1gure 8) A terrace consists of two parts, the inclined portion is called the scarp and the flat lying part is called the tread In this paper, the term terrace is used even if only the scarp portion of the terrace is observed on the seismic record Indications of terraces are seen on these bottom profiles and clearly show that they are composed of highly reflecting materials ( $1 \mathrm{e}$, sands or she11s) w1 th enough coherency to maintain slightly inclined slopes The vertical exaggeration between the profile record and the true slope angle should be realized The angle of the scarp shown in Profile Q - $Q^{\prime}$ is approximately 10 to 12 degrees $A$ submerged terrace $1 \mathrm{~s}$ visible on the northeastern edge of Profile $T-T^{\prime}$ (F1gure 9) The stairlike terrace is traced on the record as a second subbottom layer, and is clearly an abandoned feature not related to the present river channel Maximum penetration at the deepest portion of the lower scarp is approximately 36 feet beneath the water-sediment interface It is assumed that the first subbottom layer has a higher concentration of fine-grained sediments than those reflecting horizons previously discussed, and thus is partially transparent to the acoustic energy of the system

The feature labeled on Profile $K-K^{\prime}$ as a "Black $H_{1} 11$ " was viewed on several seismic records over this area The areal extent and shape 
ISOPACHOUS MAPPING

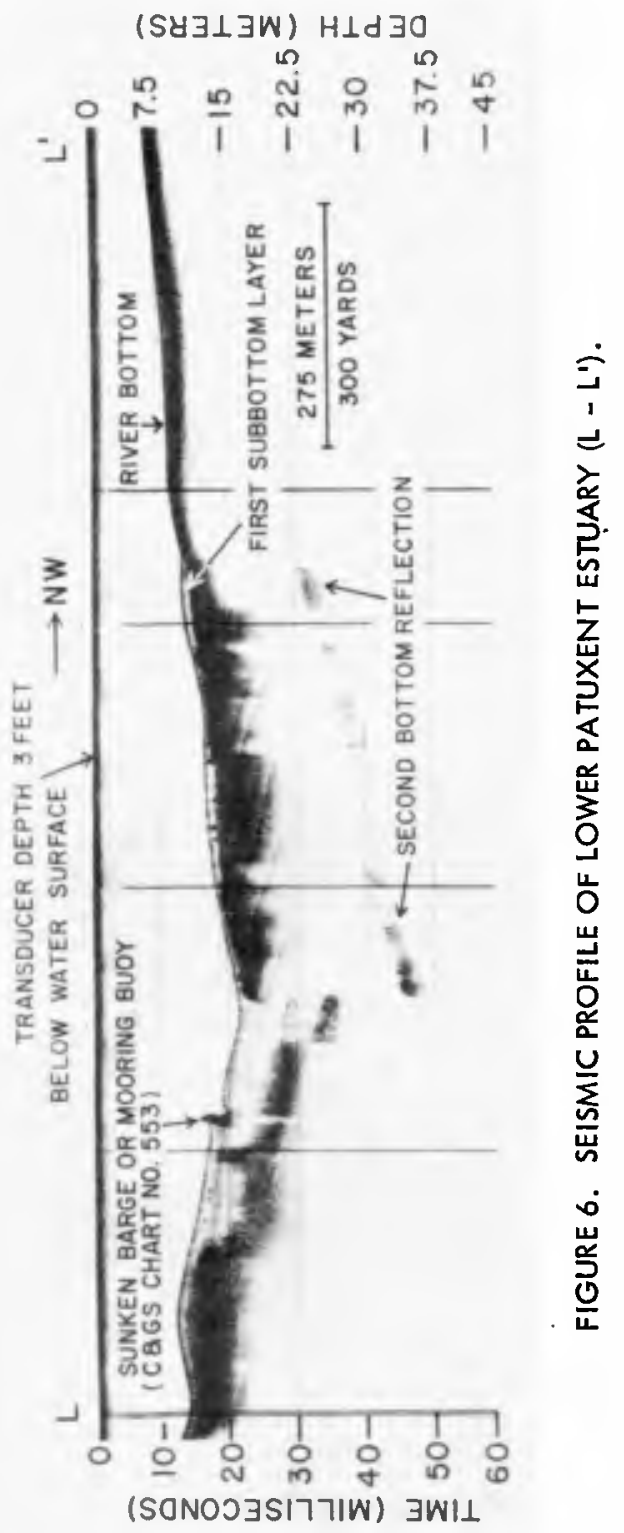




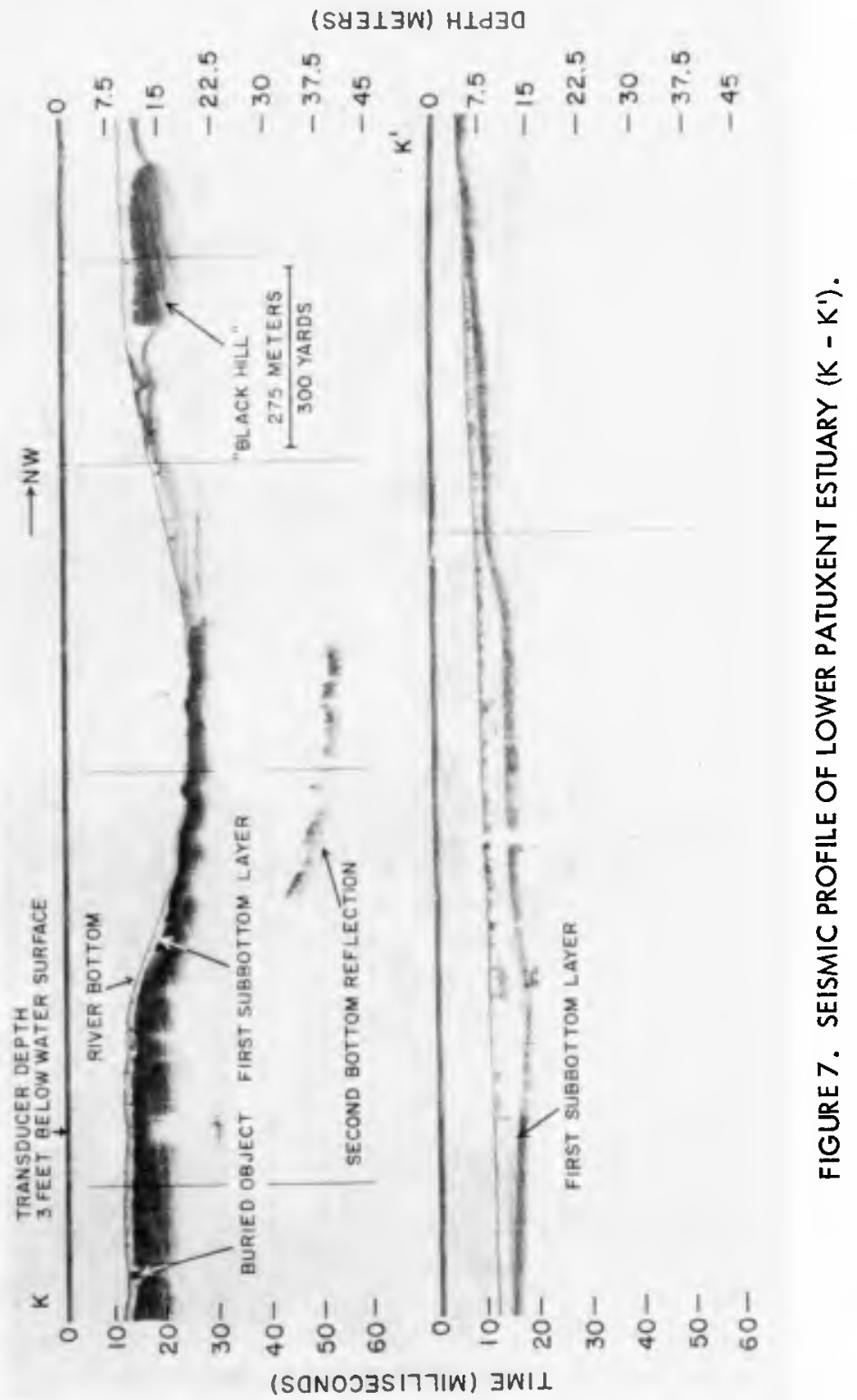




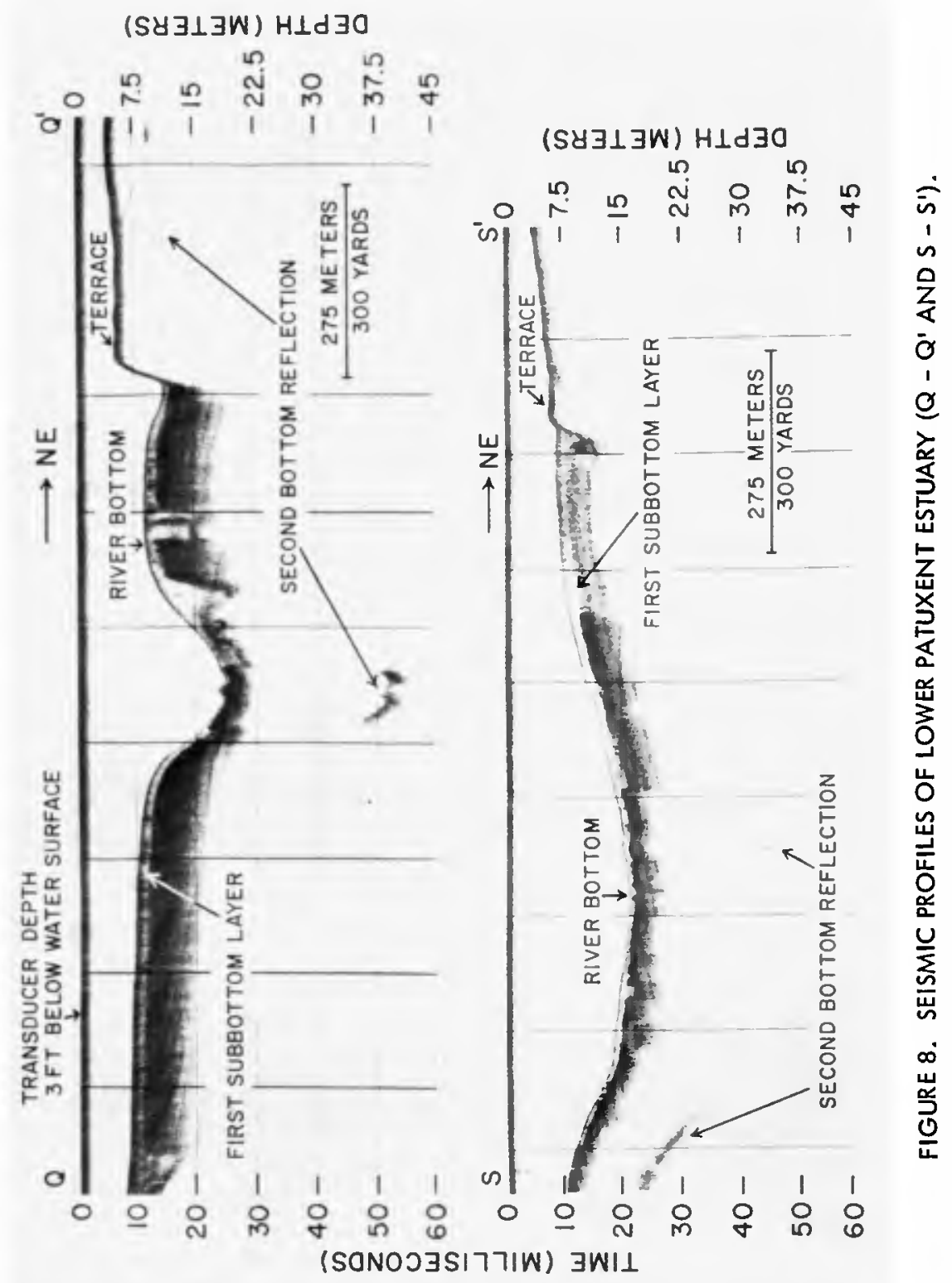




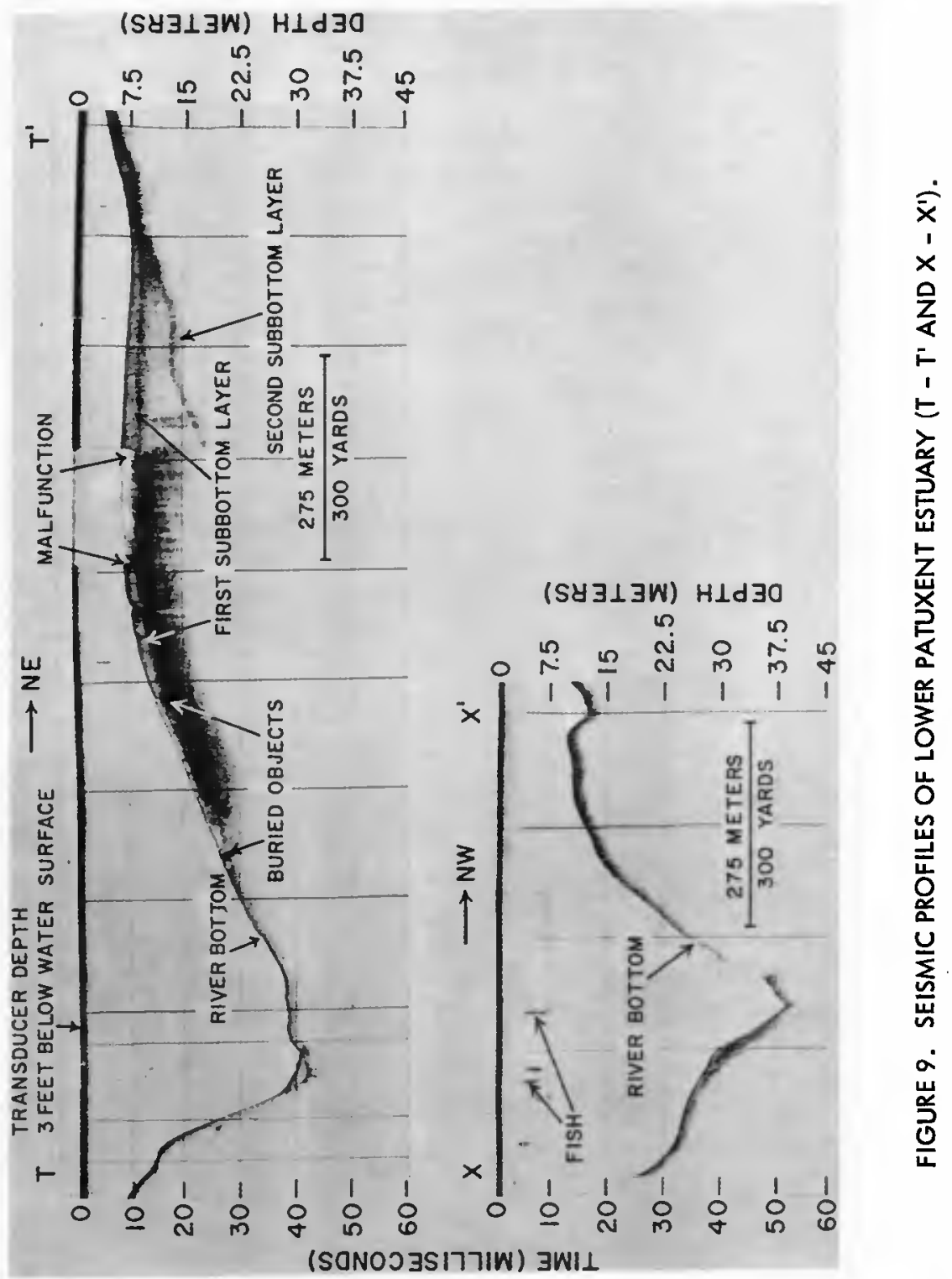




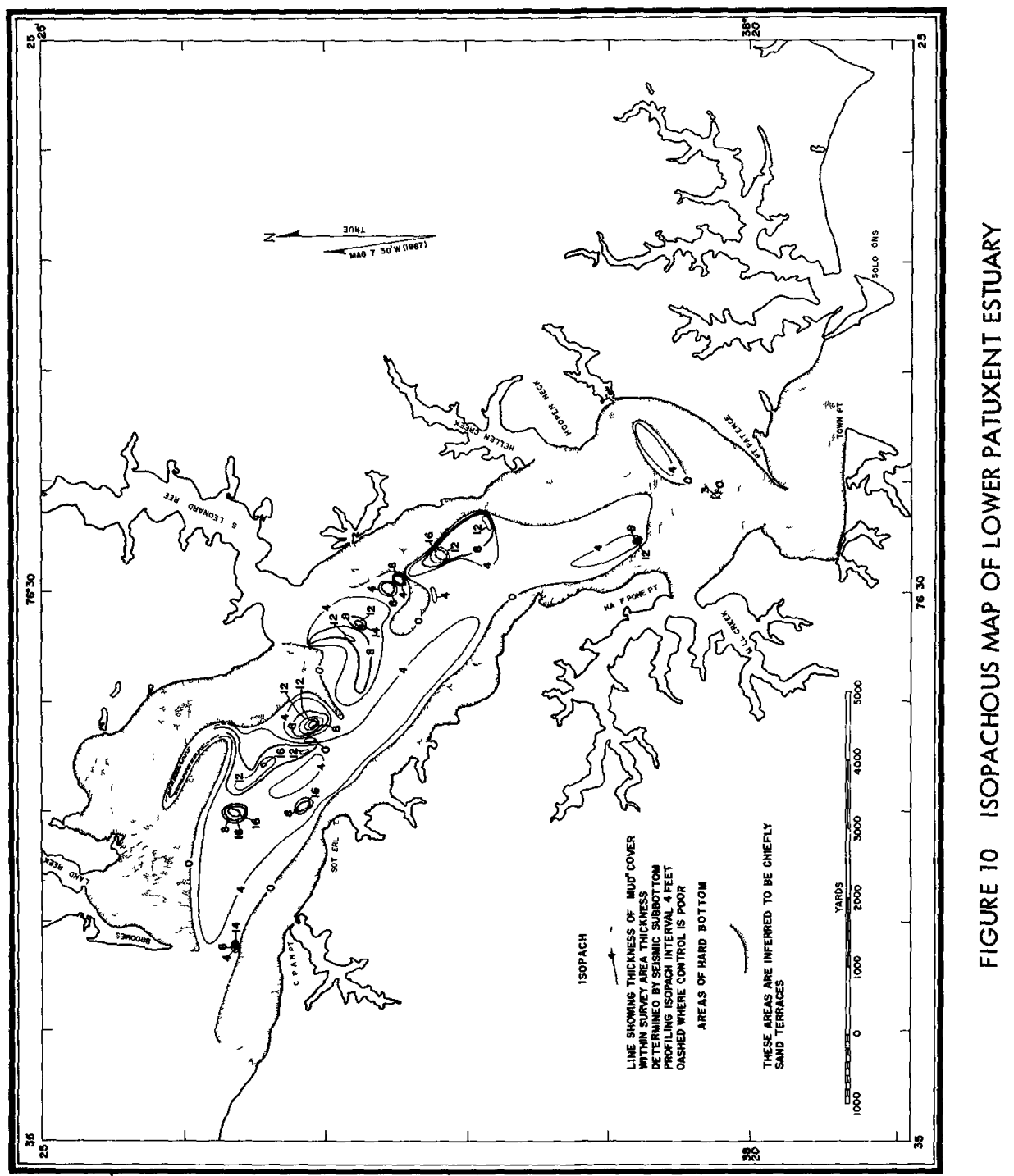


of this structure is shown on the 1sopachous map (F1gure 10) The structure is almost circular and lies directly northwest of the crescent-shaped mud hole at the mouth of St Leonard Creek The two almost c1rcular 16-foot mud holes between Sotterly Point and 1sland Creek also closely resemble in profile and plan view this "Black H111" structure Although the cause or nature of these structures is not known, it is believed that they may be oyster beds that have been covered during a period of prolonged sedimentation

\section{CONCLUDING REMARKS}

The work performed for this investigation demonstrates the stratigraphic variability of the surficial mud cover in the lower Patuxent River Estuary The thickness and extent of the mud cover is based on the occurrence of acoustic discontinuities seen on the seismic record These discontinuities occur only in areas where fine-grained materials, which are transparent to the $12 \mathrm{kHz}$ sound, overlie coarse-grained sediments Where penetration does not occur, the surface materials are coarse grained Both the opaque surface and subsurface reflectors are assumed to consist of similar materials

Depressions filled with mud occur mainly to the north of Half Pone Point (F1gure 10) The thickest mud layers lie to the east of the present channel These mud-filled depressions are as much as 16 feet thick The dominance of the deeper holes to the east plus the occurrence of a submerged terrace indicates that either the channel of the Patuxent River at this location was much larger in the past and has subsequently filled in much of the material on the eastern edge, or that the channel has migrated to the west

The presence of terraces on the edges of the channel was used as evidence for plotting the areas of hard bottom on the peripheral edges of the river (Figure 10) Although it was not possible to profile to the shoreline because the water was too shallow, it is safe to conclude that the materials which comprise the terraces extend also to the shoreline

The majority of the sediments below Half Pone Point allowed little acoustic penetration (FIgure 10) The presence of coarse-grained sediments is supported in part by divers from the Naval Ordnance Laboratory Test Facility These divers have indicated that the eliptical mud hole north of Point Patience, as shown in Figure 10, extends northward and is a part of the large deposit of mud shown in the center of the river channel These divers also have indicated that small mud holes $11 \mathrm{e}$ slightly north of Town Point

Recommendations for future study regarding the stratıgraphic variability of the surficial mud cover are directed toward two potentıal problem areas More work is needed in defining the sediment properties responsible for acoustic reflections in the study area Secondly, little is known of 
the riverine and estuarine dynamics causing the distribution of sediments Owen (1969) states that "a distinguishing feature of the Patuxent Estuary is 1ts intermittent transition from a two-1ayer flow system to a three-layer flow system, a transition which occurs most often in the month of April " On the basis of this concept of a twolayer flow system, 1 e, a net bayward (or seaward) flow of less saline (11ghter) water at the surface and a net landward flow of more saline (heavier) water near the bottom, Meade (1969) has presented evidence that bottom sediments in Atlantic Coastal plain estuaries are transported landward Some questions that remain to be answered are In the various flow layers is there some process going on differentiating sediment types? of the materials brought down by the river, how much and what type of material is trapped in the estuary? What is the spatial distribution and temporal variation of this flow system which may control the distribution of sediments?

Th1s study demonstrates the use of se1smic profiling techniques for collecting and presenting data required for coastal engineering

applications other possible coastal engineering applications for this type of work are (1) determining the thickness of a particular stratum for estimating dredging or excavation costs as well as for obtaining construction materials, (2) foundation studies, (3) harbor mapping, (4) slope stability studies, (5) spoil location, and (6) location of tunnels and pipelines

\section{ACKNOWLEDGEMENTS}

We would 11ke to express our special thanks to Dr Lloyd Breslau, former Head of the Coastal Oceanography Branch, $U S$ Naval Oceanographic Office, now with the $U S$ Coast Guard, whose guidance and valued advice during the field phase of this investigation are deeply appreciated His helpful comments and review of the manuscript are gratefully acknowledged

Appreciation 1s expressed to Dr Jerry Schube1 of the Chesapeake Bay institute, The Johns Hopkins University, Mr Leo Fisher of the $\mathrm{U} S \mathrm{~S}$ Naval Oceanographic Office, and $\mathrm{Mr}$ Ralph Hightower and $\mathrm{Mr}$ Arne Yensen of the $U S$ Naval Ordnance Laboratory for the1r suggestions and review of this manuscript 


\section{REFERENCES}

Breslau, L R , and Edgerton, $H$ E, 1968, The Sub-Bottom Structure of the Gulf of La Spezia SACLANT ASW RESEARCH CENTRE, Technica1 Report No $129,81 \mathrm{p}$

Crooks, J W , O'Bryan, D , and others, 1967, Water Resources of the Patuxent River Basin, Maryland U S Geological Survey Hydrologic lnvestigations Atlas $\mathrm{HA}-244,5$ sheets

Johns Hopkıns Unıversity, 1966, Report on the Patuxent River Basin, Maryland--Prepared by the members of the Water Management Seminar The Johns Hopkins Unıversity, Baltımore, Maryland, 228p

Meade, R H , 1969, Landward Transport of Bottom Sedıments in Estuaries of the Atlantic Coastal Plain Jour Sedimentary Petrology, Vol 39, No $1, p$ 222-234

Owen, W, 1969, A Study of the Physical Hydrography of the Patuxent River and its Estuary Chesapeake Bay Inst, The Johns Hopkins University Technica1 Report 53, 78p

Pritchard, D W, 1967, What is an Estuary Physical Viewpoint in Estuarıes, G $H$ Lauff (editor), AAAS Pub11cation No 83, p 3-5

Stıles, N T, Breslau, L R, and Beeston, M D, 1969, The Riverbed Roughness and Sub-Bottom Structure of the Main Shipping Channe1 to Sal Gon, RVN (Nga Bay, Long Tau, Nha Be, and Sai Gon Rivers) Proc, 12th Conf on the Naval Minefield, Nava1 Ordnance Laboratory Technical Report 69-69, Vo1 1, p 327-368

Stiles, N T, and Wiesnet, D R, 1970, Isopachous Mapping of the Lower Patuxent Estuary Sediments by Continuous Sersmic Profiling Techniques U S Naval Oceanographic Office Informal Report No $70-37,26 \mathrm{p}$

U S Coast and Geodetıc Survey, 1969, C \& GS Chart No 553, Chesapeake Bay, Patuxent River and Vicinıty, Maryland U S Dept of Commerce, Washington, D C , Scale 1 40,000, 8th Edition, Apri 1 14, 1969

Yules, J A, and Edgerton, H E , 1964, Bottom Sonar Search Technqques Undersea Technology, November, $\mathrm{p}$ 29-32 


\title{
CHAPTER 42
}

OBSERVATION OF SEDIMENTMOTION BY UNDERWATER-TELEVISION

\author{
Dipl Ing. Gunter Luck \\ Researchstation for Island- and \\ Coastprotection, Norderney, Germany
}

\begin{abstract}
For observation and interpretation of sedimentological movements an Underwater Television set-up is employed, which is discribed in the following paper the so long achıeved results and experiences are exemplified
\end{abstract}

THE TV SET-UP

Since springtıme 1968 an Underwater-Television set-up is employed for special sedimentological researches in the Julst-Norderney oceanlc region (East Frislan Islands), which is bestowed by the German Research Association (FIg 1). The TV set-up - in transportable execution - is constructed for use in free waters to a depth of $100 \mathrm{~m}$. The main equipments are as follows (Fig $2 a / b$ ).

1 TV camera in a cylindrical case of stalnless steel, resistant to pressure, with outflt for necessary correct contact when flxing the at times used slght- and lighting- 
supplements The TV camera is Iltted out with a multpole contact-plug for connection with the camera-cable and the wlreless control-transmission for focus. Dlameter $76 \mathrm{~mm}$; length $447,5 \mathrm{~mm}$; test pressure 48 atu, welght $6,7 \mathrm{~kg}$ in the alr and $4,5 \mathrm{~kg}$ in the water

1 Supplementary lens for free slght for general free water observations (opening angle $103^{\circ}$ in the alr and $72^{\circ}$ in the water).

2 Underwater widespread lights

1 Underwater photo camera with an underwater flashlight

1 Camera cable, length 100m, diameter $25 \mathrm{~mm}$

1 control unlt with electronlc regulator

I Distribution unit

1 Reproduction unit (screen diagonal. $36 \mathrm{~cm}$ )

I VIdeo tape recorder

Further there are providently kept ready accessorles and spare pleces such as tubes, incandescent-lamps, packlngs, servicebox and so on In anticipation lt was to be expected, that due to the small range of sight the set-up only can be used from an anchoring shlp Therefore the TV camera and the photo camera were mounted in a fourlegged frame (F1g 3), which can be placed on the sea floor by ald of a crane the reproductionunit, control-mechanisms, video tape recorder and so on were firmly installed on a shlp (Fig 4)

\section{PRIMARY INVESTIGATIONS AND EXPERIENCES}

In the first half-year of researches (summer 1968) above all the apparativ feasiblities of the TV set-up were tested, 
specially taking into consideration the particular conditions In the extreme turbid waters of the tidal flat In detall there was to find out

1 The most effectiveuse of the set-up (observations from anchoring or salling ship) Further the best distances from objectıv to sea bottom had to be found, simultaneously using the most favourable focal depth

2 Ascertainment of the most effectiv search light arrangement to recelve the most contrasting pictures as well as the best efficiency of light evolution.

3. Dependency of the TV work on meteorological conditions

4. Dependency of the observations on the different turbidness of the water

These investigations had the following results

1 In the turbid waters of the tidal flat the use of the TV set-up is only reasonable at continuous fine weather and during slack water (Fıg.5). As soon as the current exceeds the critical velocity of erosion or sedimentation, the rate of suspension increases so quickly, that it is no longer possible to gain an interpretable plcture (FIg.6).

2 After atmospheric disturbances and great water motion also during slack water the rate of turbidity in the tidal flat is for a longer time so intense (up to three and four days), that observations of the sea bottom are not possible 
Whereas after continuous fine weather lt might be possible to observe the sea bottom during the first and perhaps even the second turbulent tide. At wind forces of more than 6 Bft and at high current velocity connected with high waves an observation of sedimentary movements is absolutely ımpossible.

3. Even on the most favourable conditions the distance between the objective and the sea bottom must not exceed $35 \mathrm{~cm}$ (TV picture $40 \times 40 \mathrm{~cm}$ ).

4. The conditions improve obviously, when the set-up is employed in deeper waters abroad the tidal flats The visibility is much better and the times of observation can be extended (F1g.7). Nevertheless the range of turbidity is so extensive, that the distance objective - sea bottom only may amount to $55 \mathrm{~cm}$ (TV picture $60 \times 60 \mathrm{~cm}$ ).

5. These only small distances between objective and sea bottom, avallable due to the local boundary condltions, don't allow observations from the salling or drifting shlp. As for each observation the anchor has to be set, it is only possible to work statıonary and the researches take much time For this reason the $\mathrm{TV}$ method in the sediment research is severely limited.

SOME OBSERVATION RESULTS

In the research years 1969 and 1970 it was possible to perform almed sedimentological observations. Besıdes general observations of regional movements in the research area it was tried to gain 
knowledge of the sedimental process on the tidal flats and specially to find an optical definition of the critical velocity of erosion and sedimentation. Without entering into the detalls of the researches the maln results can be resumed as follows

I Only in exeptional cases the suspended matters and the sediments, transported on the bottom, are separated more or less exactly Consldering the hitherto existing observations a thus strict separation actually can ensue only within the compass of high current velocity and therefore strong sediment motion on the bottom ( $F 1 g$ ga/b) In regions with lower velocities, which nevertheless still are capable of transporting, a differentiation of the motion on the bottom and the transport of suspended matter is not possible Therefore the moved materials have to be consideres as one unz ty

2. As already mentioned the range of visibility very much depends on the meteorological conditions of the foretides. When the foretides were influenced by storm, the rate of turbidity is intensifled still for a longer time whereas as undisturbed progress of the foretides the rate of suspended matter for the present is stıll normal, even after commencing atmospheric disturbance and higher current velocity. Therefore at the same current velocity and otherwise similar conditions the concentration of the suspended matter differs very much

3 Of stIll more Importance to the suspended matter are the 
seasonable varıng blological condltions Thus the rate of suspended matter ls much higher on midsummer after full development of vegetation, than for example in winter or springtime After the long lasting quiet and warm summer 1969 in the whole oceanic region of Norderney the rate of suspended matter was much hlgner than in springtime 1970, whlch was preceded by an extreme long winter.

4 The physical process of suspension is essentially determined by the suspended matter's appearing not in equal dispersion but in flakes (FIg 9). These flakes are in nearly permanent hovering motion and only settle occasionally for short times durıng slack water At insıgnificant turbulence, when the preceding tides had been quiet and the current velocity is lower than some $15 \mathrm{~cm} / \mathrm{s}$, the flakes fall to the bottom in a sudden process As soon as the current velocity has risen to some $20 \mathrm{~cm} / \mathrm{s}$, the flakes are absorbed by the current.

At the microscopıcal and chemical analysis of these flakes one could discern particles of different sizes and orlgin, whlch adhere to a scarcely vislble organıc slıme, showlng a strong positive albumen- and occaslonally a cellulosereaction (Fig $10 \mathrm{a} / \mathrm{b}$ ). Here lt 1 s probably the question of reduction products of marine organisms This supposition 1 s conflrmed by the rich bacterlal trimming. These slimes, being scarcely perceptrble in the microscope, become well visible in the flash light beam and can be observed clearly In the television plcture as flakes In volume the slimes preponderate, whereas the quartz crystals and other mineral particles as well as diatom shells, chitin scraps, filaments 
etc prevall in welght.

Measuring and ascertaining the rates of suspended matters there was not pald enough attention to these flake structures untill now In the floating water these flakes and pure mineral particles are to be found close together During the sedimentation- and erosion-process this different physical deportment of flaky and pure mineral sediments causes different sedimentation- and erosion-phenomena, whlch however are temporary without transition

5 Seldom the transportation process near the bottom has a continuous run, but the sediment moves in intermittent veils Thls discontınulty also was to be observed at the progress of small ribs The motion of the sediment increases, when also shells are transported or animals move on the ground (FIg $11 \mathrm{a} / \mathrm{b}$ ) Thus a small $\mathrm{rlb}$ dlsappeared (length some $20 \mathrm{~cm}$, helght some $5 \mathrm{~cm}$ ), which first seamed to be rather stabel, whlle a crayfish was crossing it The whole sand supply at once turned to suspension

The discontinulty of the transport near the bottom is still additionally intensified, when the sediments come into the waveg' sphere of action. In this case the sediments move on paths, which relate to the direction of the current and waves When the waves work in the same direction as the current, the motion is rolling Do the directions mould an angle, this rolling progress passes over to a more saw-toothshaped path 
6. A series of researches was performed, especially tended to the optical ascertanment of the critical velocity of erosion and sedimentation In spite of a great expenditure however, so long It was not possible to undertake a singlevalued fixing of these critical velocities The process of sediment reception by the current proceeds most floating, locally however very much differing, wh thout a possiblilty of observing the beginning of this phenomenon The process of sedimentation is still more opaque, because only very $s \in I$ dom the whole sediment is deposited during slack water With diminishing velocity the process of erosion also does'nt turn slidingly to the process of sedimentation, as before the beginning of sedimentation there exists a higher range of velocity, which does'nt erose the material near the bottom any longer, but stlll transports more or less suspended matters.

\section{CONCLUSION}

The observations of sedimentological processes of motion with the underwater television set-up showed the limits of It's efficiency The extraordinary value of the so long achleved investigations is to be found especially in the fact, that the processes of motion were made visible and partly interpretable.

The sediment transport's predominant dependency on the meteorological conditions of the foretides and specially the development of the suspended matter as consequence to the brological phenomena was unobjectionably discerned and proved 
It was not possible to ascertain a connexion between the at times locally measured current-velocity, rate of suspended matters, grain distribution of the sediment near the bottom and so on. A separate treatment of the bottom-near transport of sediments and the suspended matters is only possible in those exceptional cases, in which high velocities put the whole bottom material in rolling, jumbing and at last pushing motion As a rule - in the tidal flats and oceanic regions whth low current velocity - the water contents flaky and pure sediments in proportionate distribution, whereat however at low turbulence the density of the suspended matter can increase with depth.

The in past times theoretical formed conceptions to ascertain sedimentological processes of motion, which predominantly depended on the current velocity and the critical velocity of erosion, are - appreciating the observation results, gained with the under water television - only little satisfilng. 


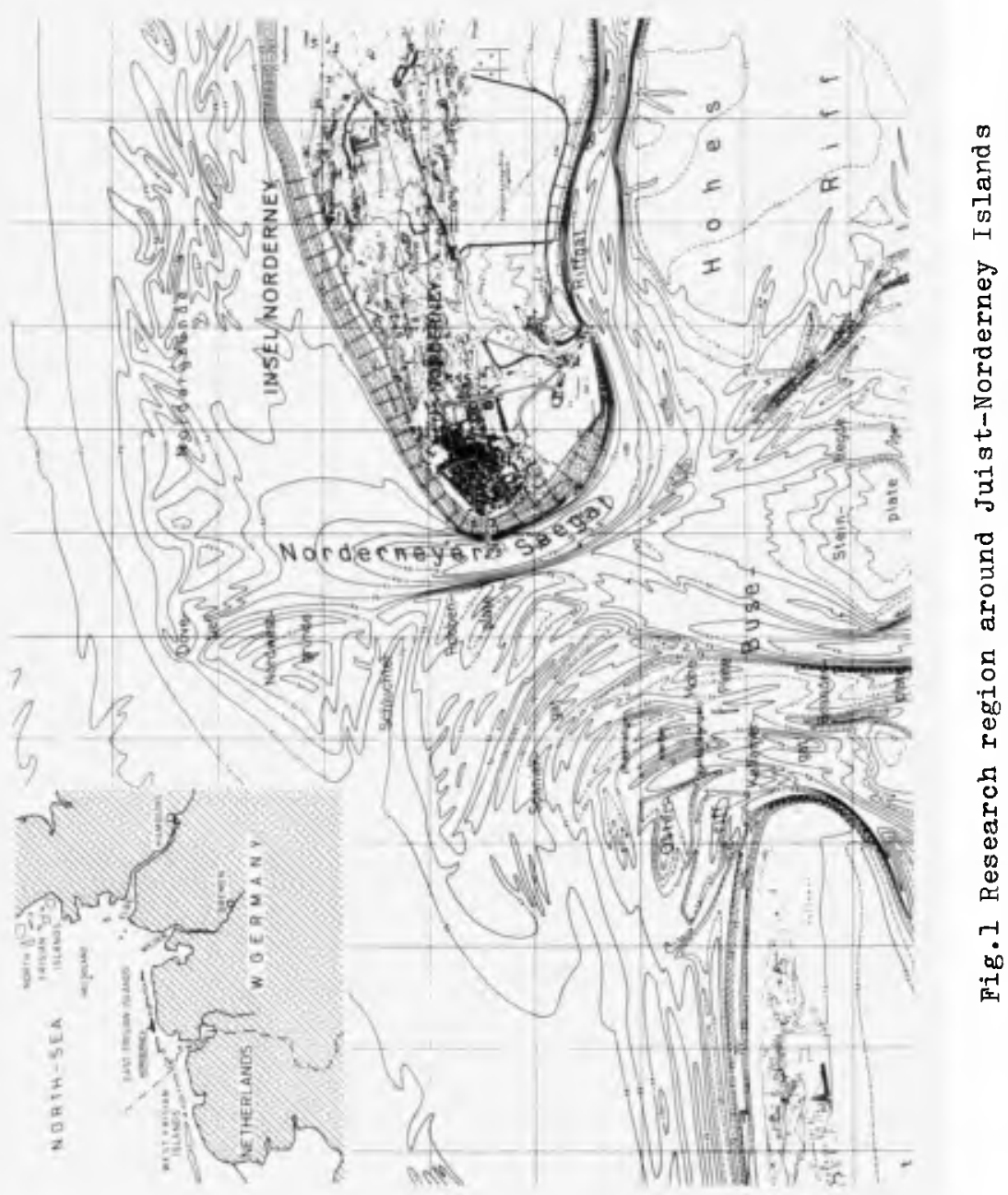




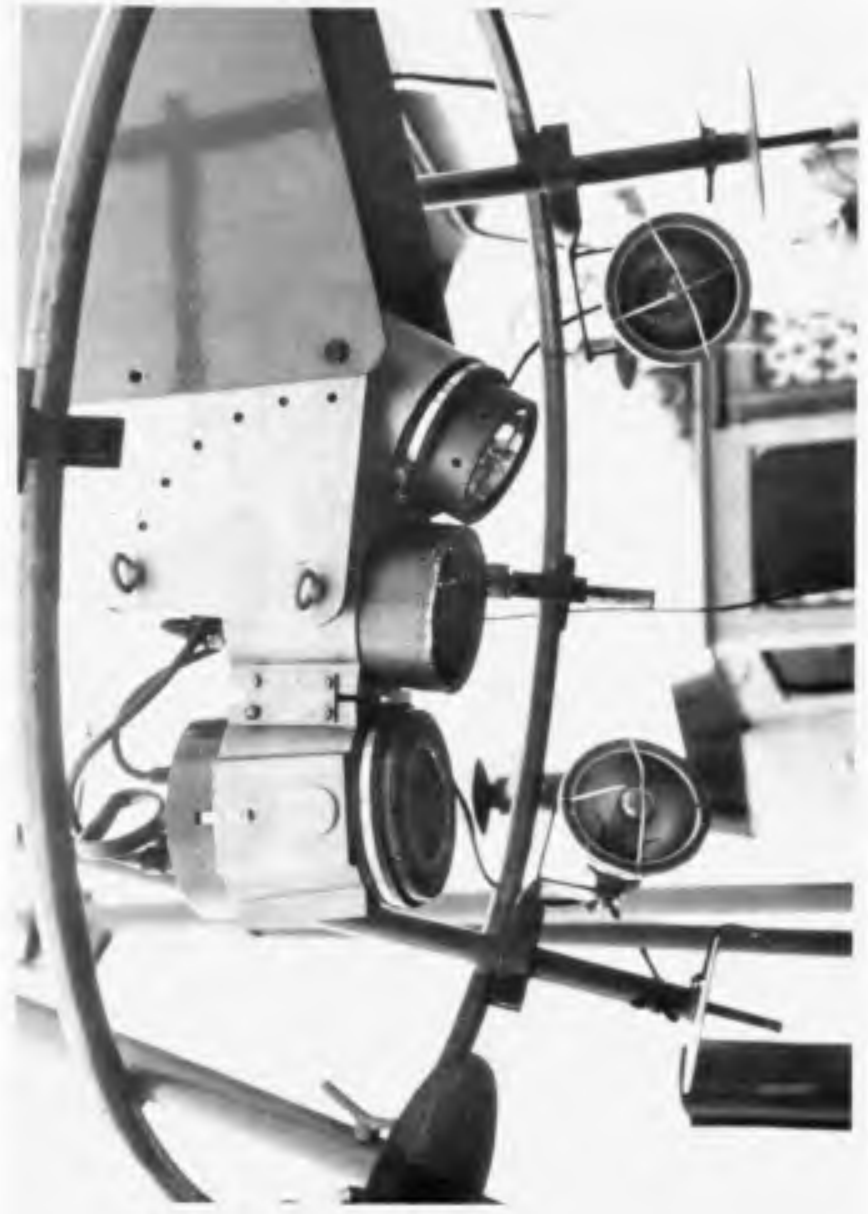

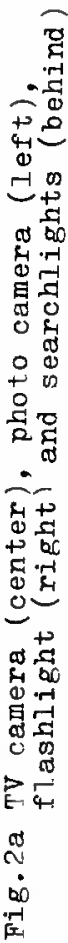




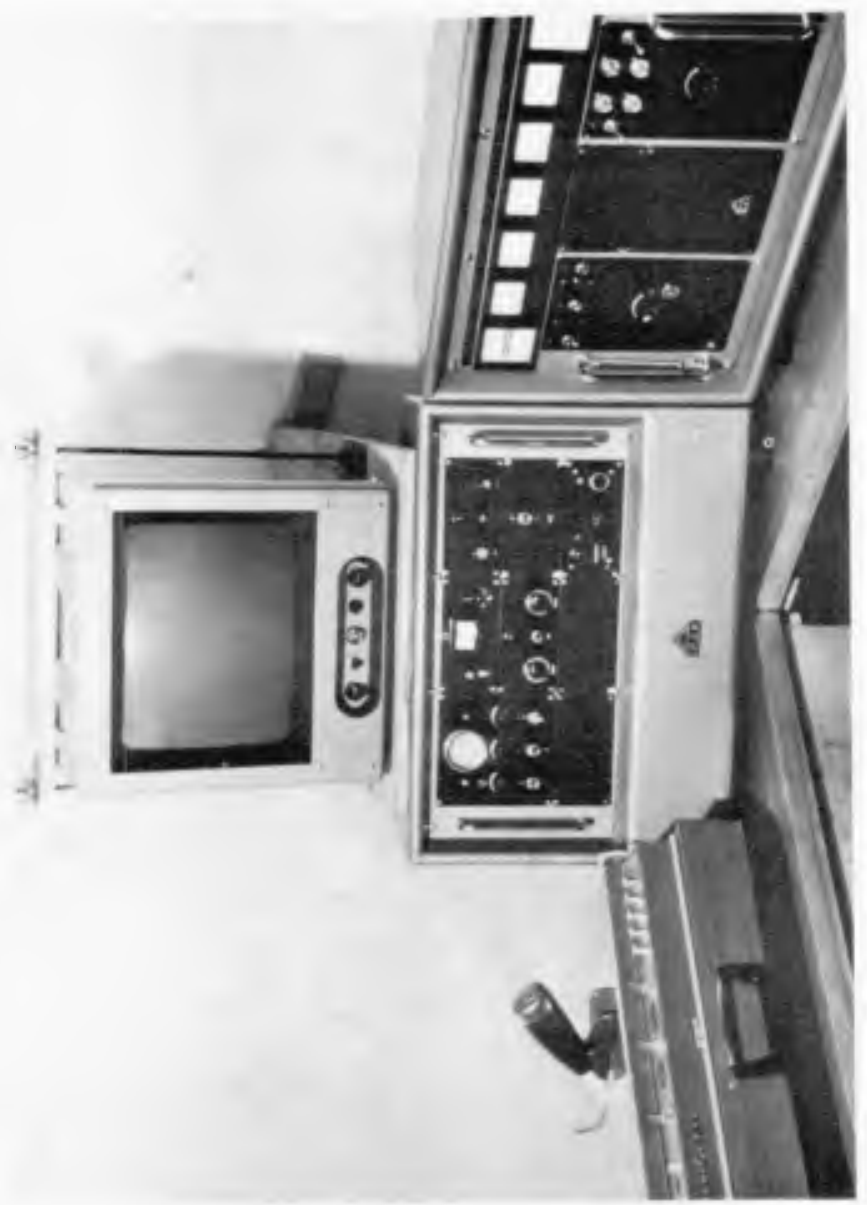

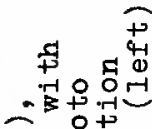
ant.c o

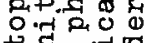
+ दु 4 응

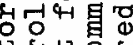
$+5+0$ द 5 \& 웅. 용 . 00. कि口 농엉 60 का 4 क्ष Tि 20 ra 명영 on + . . त 당 동옹 잉 $\rightarrow$ 死

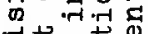
$>\rightarrow 000$ 0 द 4

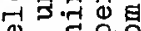
+ a 4.$0+5$ (1) 3 व 글 क्ष $0+0.1+$ . in 


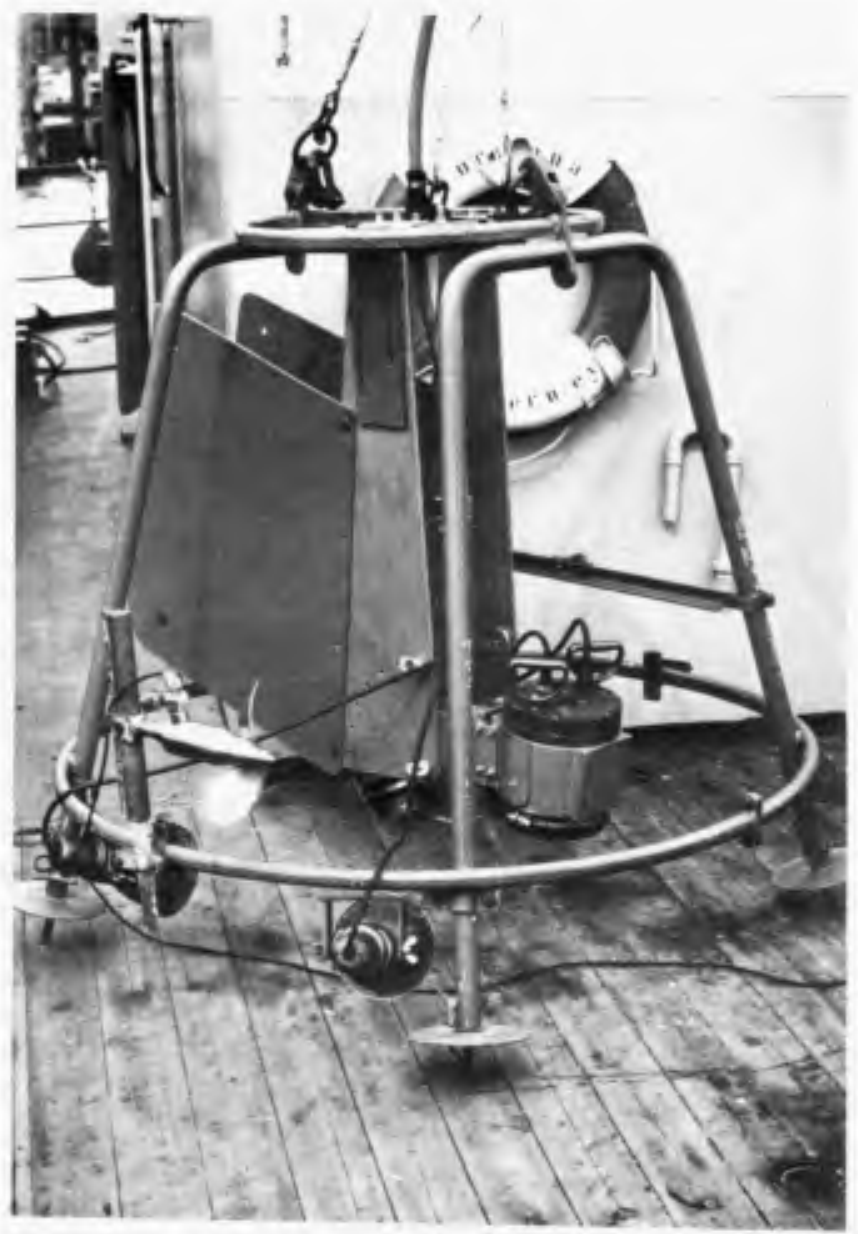

Fig. 3 Four-legged support frame on deck 


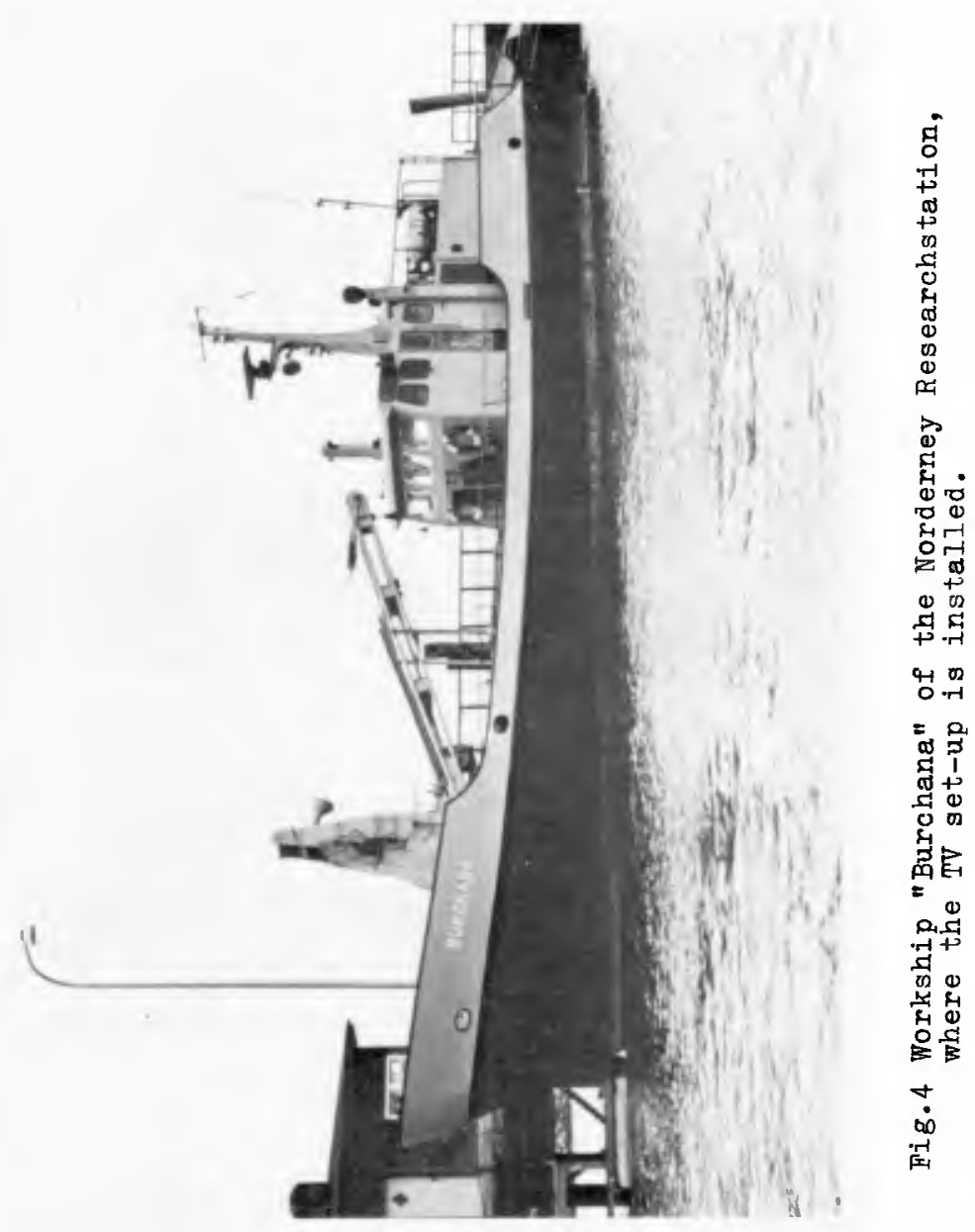




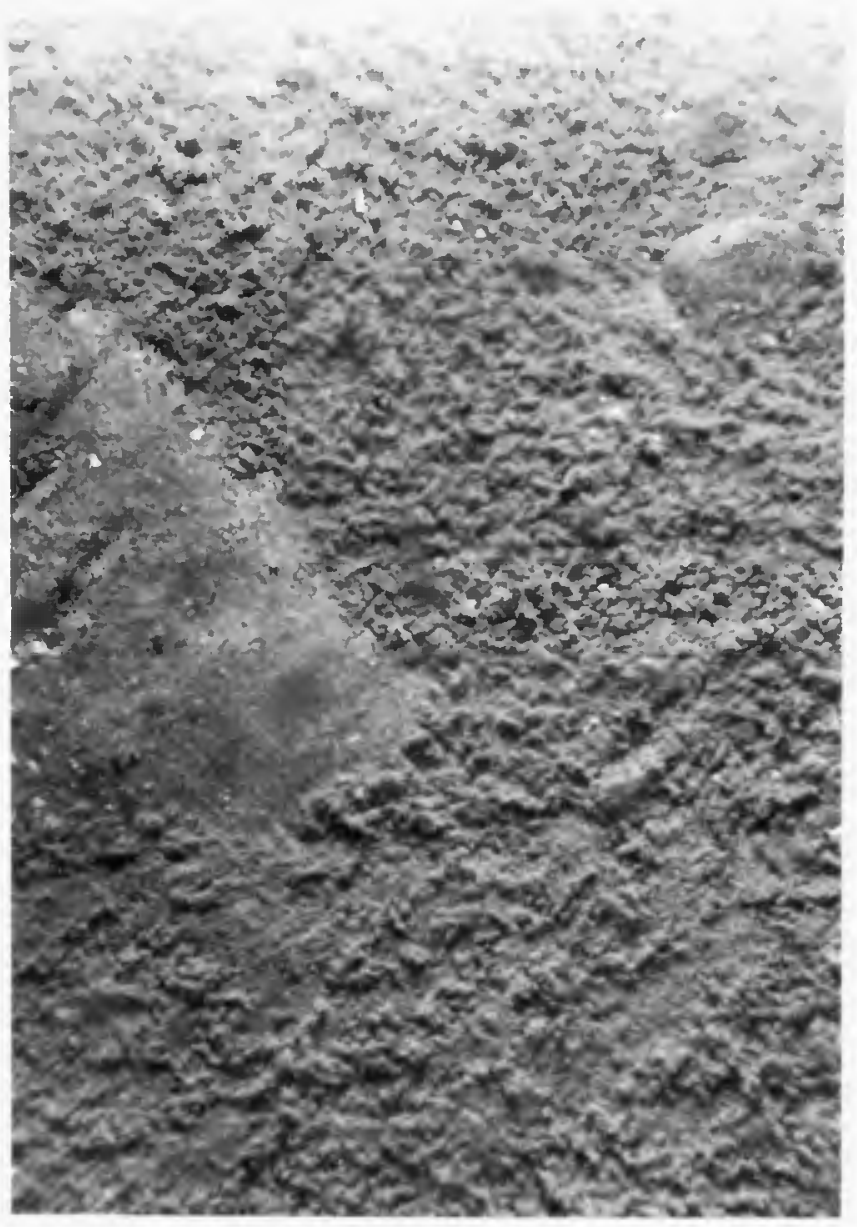

Fig. 5 Bottom in a narrow channel of the tidal flat during slack water. The ground is covered with flaky sediments. A small area of sand in the left-hand section of the photograph is still uncovered. 


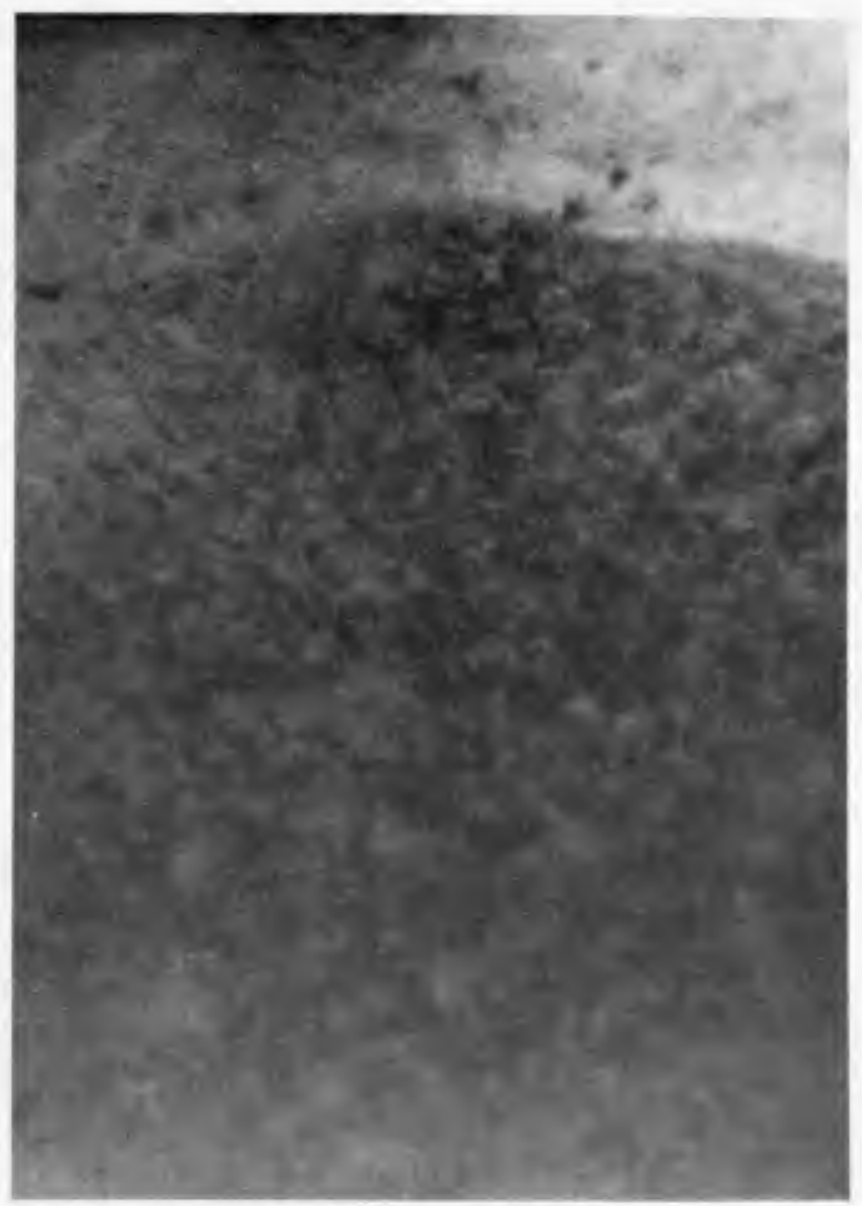

Fig. 6 At great water motion in the tidal flat it is impossible to gain an interpretable picture. 


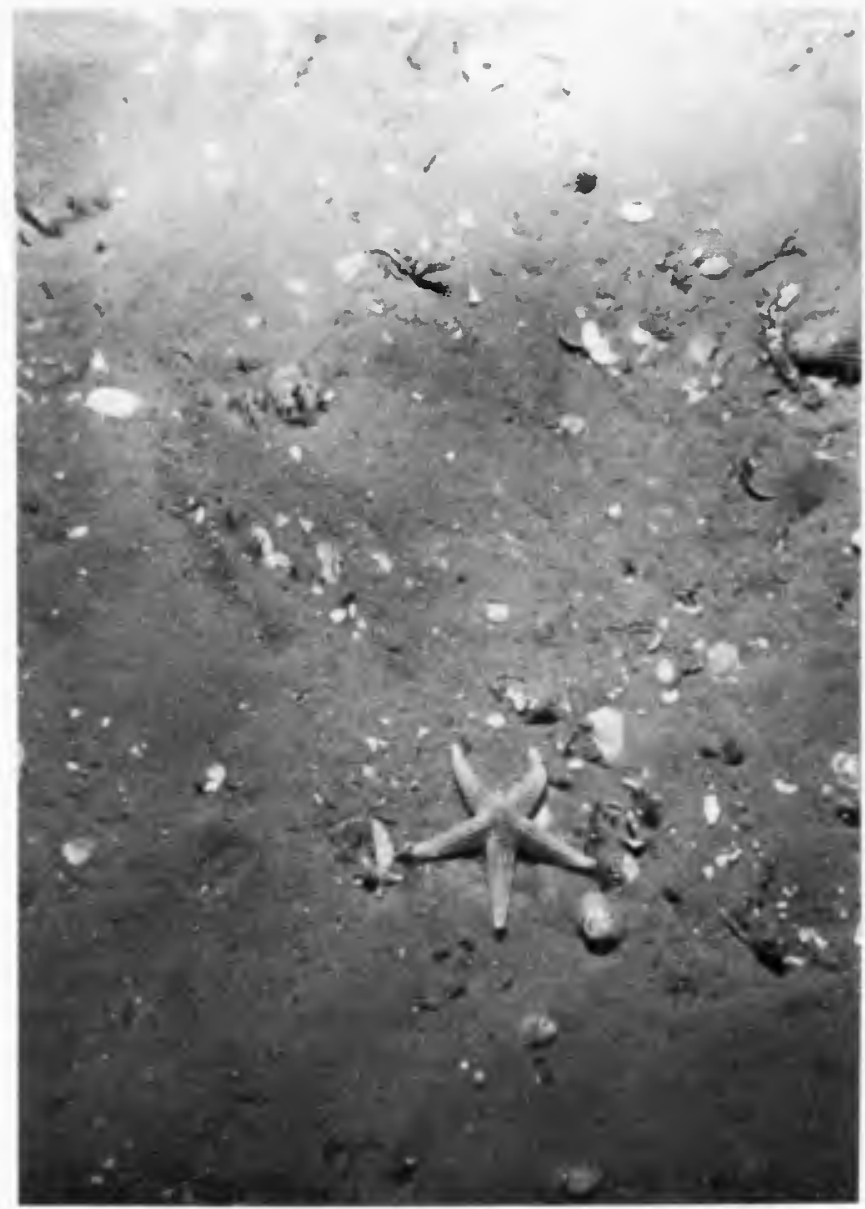

Fig. 7 Sea bottom in the north of Norderney island (water depth some $26 \mathrm{~m}$ ). The visibility is much better than in the tidal flat. 


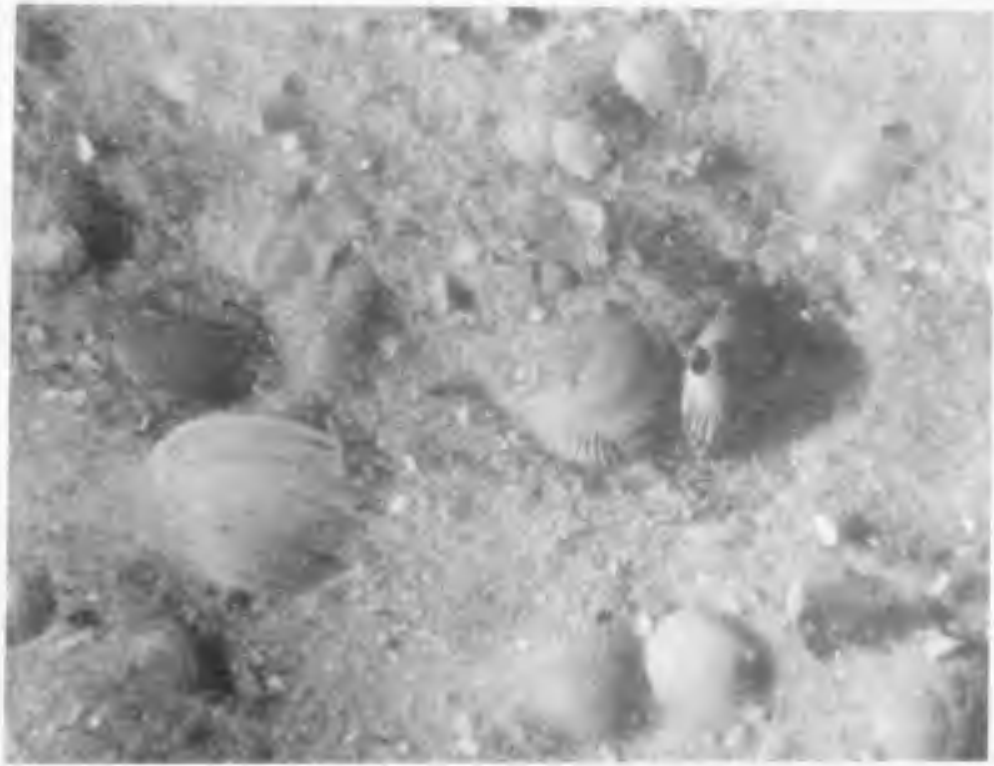

ถ્ํำ

न्न क्ष

도융

车实

금

คำ

더임

的

药

듬다

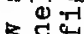

ह

단.

刃्व

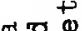

๙

A

$+$.

음

क्ष

需宁

E

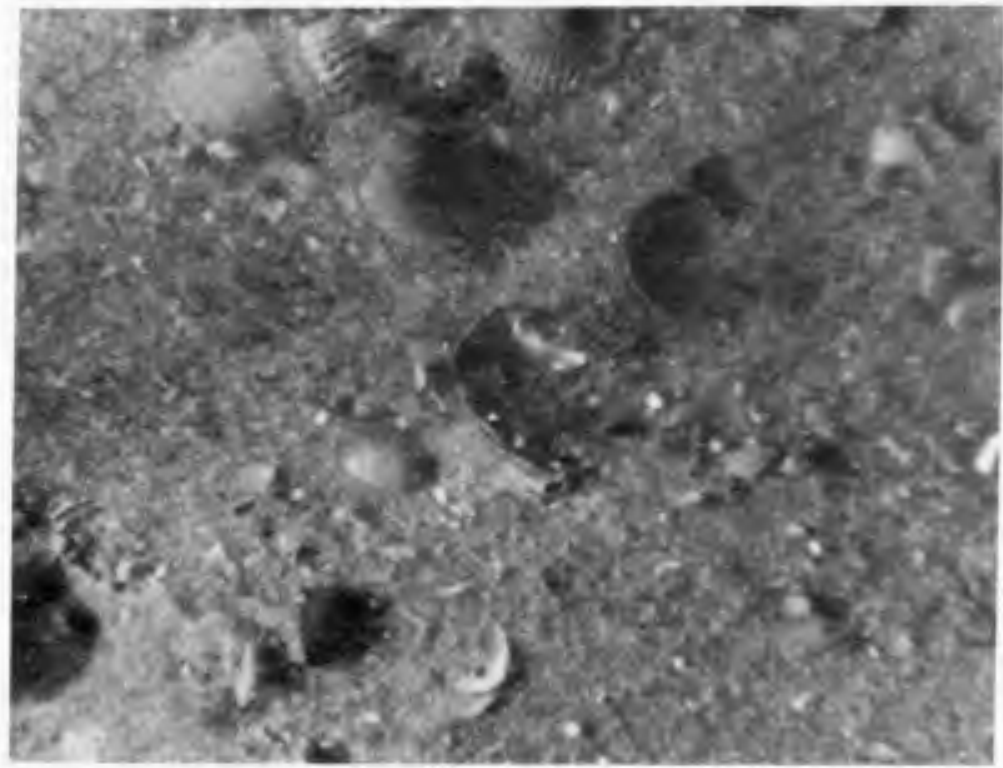

중

$\infty$

द家员

+ on

H-t

on

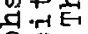

م.

$\$ 0$

4. तो bot

正

+क

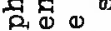

연

억겅응

एण

㚙 


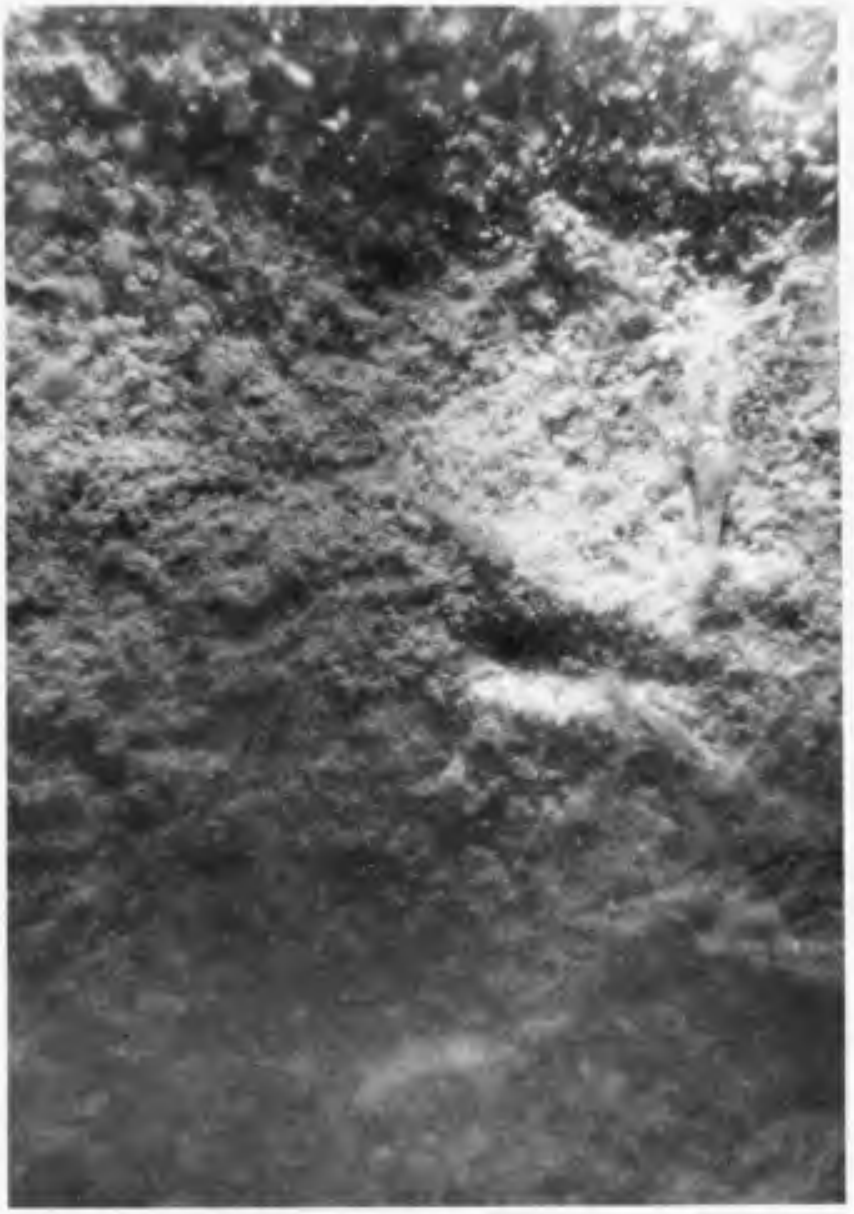

Fig.9 Flaky material covers the bottom for a short time during slack water. 


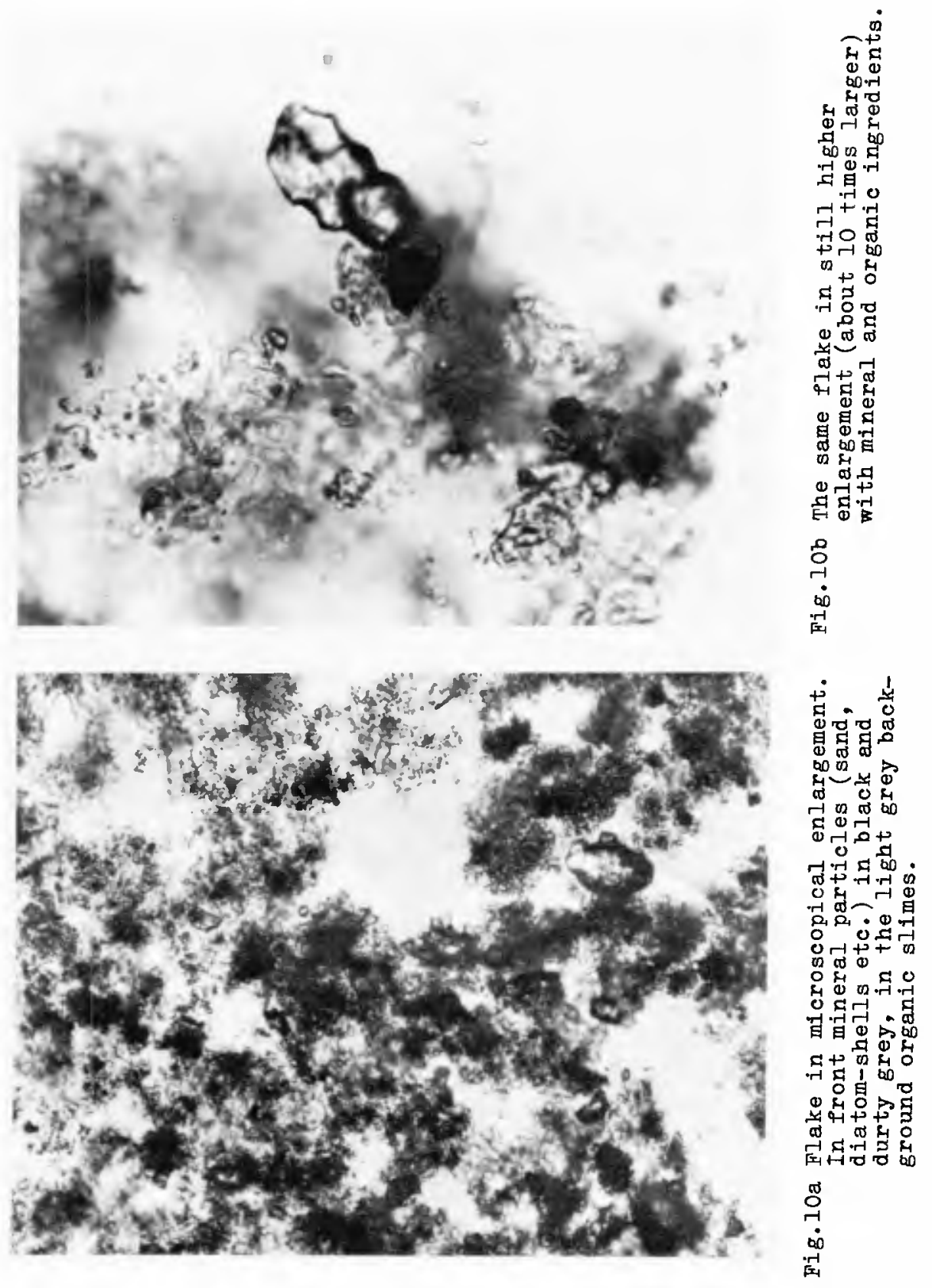




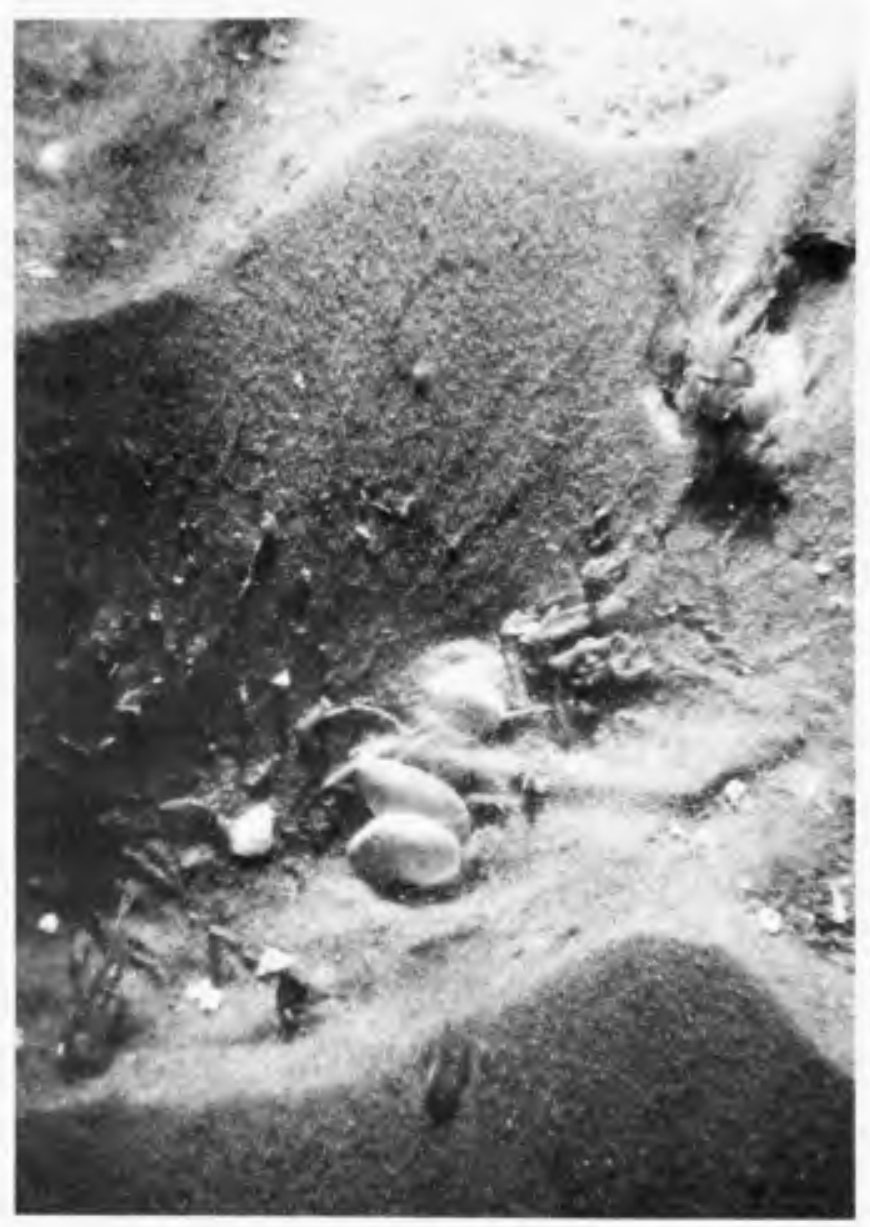

Fig.lla Ripplemark abroad the tidal flat in $18 \mathrm{~m}$ water depth, current velocity some $20 \mathrm{~cm} / \mathrm{s}$. 


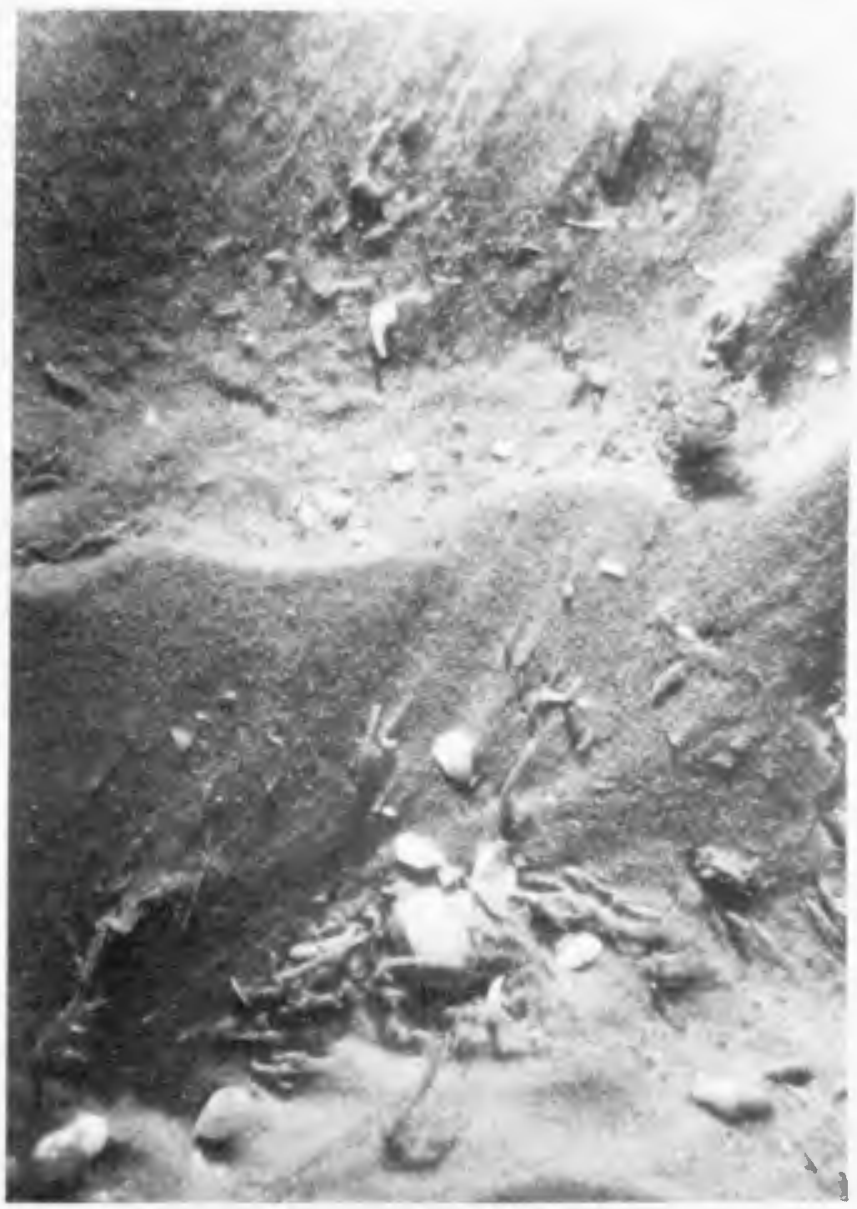

Fig.llb The same ripplemark one minute later. 


\section{CHAPTER 43}

THE HISTORY OF THE DUTCH COAST IN THE IAST CENTURY

$$
\text { by W.T. Bakker }{ }^{1)} \text { and D.SJ. Joustra }{ }^{2)}
$$

\section{ABSTRACT}

The purpose of this paper is

1. Publishing some avallable coastal measurements and computations of more than local importance

2 Investigation on the influence of groynes in practice.

3. Investigation on the motion of the gullies in the outer deltas.

The following conclusions are drawn

1. The gross littoral drift along the dutch coast is in the order of 1.5 to $2 \mathrm{mln} \mathrm{m}^{3} /$ year, (computed with the CERC-formula) the resulting net drift is mostly within the order of accuracy of the computation.

2. The erosion of the areas with groynes was much less than the erosion of the adjacent areas, partially this effect is due to lee-side scour but mainly to decreased erosion in the protected area.

3. The gullies in the outer deltas of the Wadden mainly turned clockwise, which is probably the direction of the resultant transport there.

\section{INTRODUCTION}

The dutch coast consists of three parts (fig. 1)

1. The Rhine-Schelde Delta

2. The uninterrupted coast of Holland

3. The Wadden area

It 1 s a sandy coast $\left(D_{m} \approx 200 \mu\right)$, the tidal difference varies between $4 \mathrm{~m}$ near the Belgium border, down to $1.5 \mathrm{~m}$ near Hook of Holland, to $1.3 \mathrm{~m}$ near den Helder and then up agann to $1.8 \mathrm{~m}$ near the German Island Borkum. The gully systems in the Rhine-Schelde delta and that of the inlets between the Wadden Islands in the North have quite a different shape, since the tidal basins are quite different. In the South the basins are rectangular with an open short side towards the sea and in the North they are rectangular with the long side parallel with the coast-iıne, this side is bordered by the Wadden Islands with narrow inlets in between. Therefore the Wadden cosst can be compared with lagoon coasts, as they occur in many parts of the world. The Wadden Sea is a tidal flat, which is submerged during every high tide. Most information wll be given about the Holland coast and the Wadden area, as DRONKERS [1] stresses the research in the Delta-area.

1) Senior Engineer Coastal Eng. Res. Dept., Directorate for Hydr. Res., Rijkswaterstaat

2) Lieutenant of Spec. Services 3rd Class Royal Dutch Navy Reserve 
The maln purpose of this paper 25 to supply data, which enables international comparison. This paper gives largely a summary of many reports of regional study-branches of Rijkswaterstaat, made over a period of about 50 years. From the former papers, covering the same subject, special attention is asked for WENTHOLT (1912)[2] who investigated especially the effect of groynes, van VEEN (1936) [3], who treated the origin of the Dutch coast and the shape of the gullies, van BENDEGOM [12], [22], [4], who investzgated the hydraulic laws for the motion of the gullies, van STRAATEN [5], who considered the directional effects of winds, waves and currents and concluded that the sand drift must be strong (west to east) along the northern barrier islands and small between Katwijk and Texel, EDELMAN and EGGINK [6] who drew morphological conclusions from the curvature of the coast. PER BRUUN and GERRITSEN [7] treated the cross section of gullies and the stability of coastal inlets. BIJKER and SVASEK [8] give a treatise about IJmulden harbour. The following paper gives more recent data than WENTHOLT, the conclusions of van VEEN, van STRAATEN, EDELMAN and EGGINK are reviewed in the light of modern theories about sand transport of CERC, BIJKER and SVASEK, making use of tidal computations.

\section{BEACH MEASUREMENTS}

Figure 2 and 3 show the erosion and accretion of the low-tide line, the high-tide line and the dune foot in periods of 10 year. First the 10-year average of each of these lines was determined, for instance 1856 - 1865, the distance between two successive 10-year arerages 18 plotted $1 \mathrm{n}$ f 18.2 and 3 . It $2 \mathrm{~s}$ indicated where groynes, seawalls or harbour moles are present and when they are constructed.

The area of the Holland coast (fig. 2) is highly influenced very much by the building of the harbour moles of Hook of Holland and IJmuiden in 1870. The low-tide line shows the influence of climatologic changes [5], overall much erosion between 1860 and 1880 and accretion between 1880 to 1900. These periodical changes are attenuated very much in the line of the dune foot and here one finds the general trend as indicated by EDELMAN and EGGINK [6] a general accretion of $\frac{1}{2} \mathrm{~m} /$ year, with erosion near Hook of Holland and den Helder ( $12 g .4)$. The Hondsbossche Seawall lies at the moment much further seaward than the adjacent dunes because of the erosion of the dunes.

The influence of the harbour moles (length $1400 \mathrm{~m}$ ) of IJmuiden built in 1870 is shown in detail in fig. 5. Fig. 2 shows, that the low-tide line and high-tide line obtain stabllity about 1900, but that the dune foot changes up to 1930. Fig. 5 glves an upper view on the 5-years average of the low-tide and high-tide line (seaward scale exagerated with respect to the longshore scale). The total gain was $9.10^{6} \mathrm{~m}^{3}$ in the North and $6.10^{3} \mathrm{~m}^{3}$ in the South [8]. In 1965 the harbour moles were enlarged to $3000 \mathrm{~m}$. Fig. 5 shows on the right hand side the change of the mean of the low-tide and high-tide line since 1965. Now the accretion on the South side is more than on the North side. Although a changing of the wave climate may have been of influence [5], [8], also the fact must be important, that at the moment an extensive area with only small 
currents prevents the entrainment of material from the surf zone around the head of the mole.

The changes of the Wadden Islands are shown in $f_{1 g} .3$. The changes at the ends are large compared to the changes in the middle of the islands, partly due to the changes in the gully-systems in the inlets, the silting up of a gully causes that a shoal grows together with the end of the island. After that, a sandwave along the coast is generated [9]. Obvious is the relatively large erosion on both ends of Texel. We shall return to this subject.

\section{GROYNES}

The Dutch groynes have a length of about $200 \mathrm{~m}$, the distances between the successive groynes can be found from fig. 6 to 8 (about $200 \mathrm{~m}$ ). In the consldered area they are broad-crested stone structures, lyıng at about mean sea-level. In order to investigate the behaviour of groynes in practice three areas were considered, on which groynes were constructed during the period of the measurements South-Holland $\mathrm{km} 97$ to $105(\mathrm{f} / \mathrm{g} .6)$, North-Holland $\mathrm{km} 8$ to 20 ( $f 1 g .7$, derlved from [11]) and Vlieland $\mathrm{km} 41$ to 52 ( $\mathrm{flg} .8$ ). Constructed is the 5-year average of the low-water line, (for instance 1858 to 1862 for 1860), the scale perpendicular to the reference-line is 10 times exagerated in fig. 8 and 20 times in fig. 6 and 7 with respect to the longshore direction. In each flgure two successive lines are plotted together, the black fields show the erosion in 10 years, the grey fields the accretion. The groynes built from 1853 - 1862 are plotted through the line of 1860, and so on. The hatched area gives the protected coastal area. Fig. 8 seems a striking proof of the benefit of groynes. The erosion near $\mathrm{km} 47$ to 51 in $1860 / 70$ can hardly be ascribed to the groynes $5 \mathrm{~km}$ away. The reduction of the erosion after the building of the groynes is quite clear. Of course, this does not mean that groynes are the most economic way of coastal protection.

The same kind of effect can be seen in fig. 6, 1860/70, although less convincingly. The influence of the lee-side scour plays here also a big role. A rough estimation of this lee-side scour (giving also a measure for the net littoral drift in this zone) about $100.000 \mathrm{~m}^{3} /$ year (erosion of about $2 \mathrm{~m} /$ year over $3 \mathrm{~km}$ ). Less clear still is fig. 7. The Northern part of this area is subject to the movement of the Schulpengat, the Southern branch of the inlet of Texel. The lee-side scour on the Northern side can be

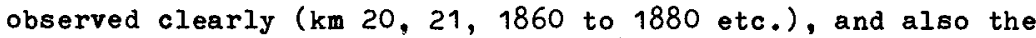
inverse the accretion near $\mathrm{km} \mathrm{13,14}$ between 1900 and 1910 on the luff-side.

Analysis of the effect of the groynes is very difficult. Comparison with unprotected parts of the beach has no sense, since groynes are constructed only on eroding beaches. A before- and after-comparison will be obscured by climatological changes (cf 128.3 , low-waterline)

We chose the areas of $\mathrm{f}_{18}, 6,7$ and 8 for comparison (all eroding beaches, gradually more and more protected with groynes) and computed for each area for each 10-year period the mean erosion/year of the protected part and of the unprotected part ( f $1 \mathrm{~g}, 9 \mathrm{a}, \mathrm{b}, \mathrm{c})$. Thus the erosion on the same area could be compared, when this 
area was protected (later on) or not (in the beginning)

In order to eliminate local influences all three areas were put together and again the mean erosion per year of the protected and of the unprotected area were computed (f18. 9d). An impression of the climatological changes gives fig. $9 e$, in which the mean regression and progression per 10-year period of the low-tide line of the uninterrupted coast of Holland is shown. Only the relative changes are of importance. In fig. $9 f$ finally the erosion of the protected part of $\mathrm{fig}$. $9 \mathrm{~d}$ is plotted aganst the erosion of the unprotected part, from which a considerable reduction of the erosion can be concluded. Although nearly all the considered unprotected areas were subject to lee-side scour, fig. 8 shows that the reduction is not mainly caused by that, but that the building of groynes reduced the erosion.

\section{SAND TRANSPORT BY WAVES AND TIDES}

Van STRAATEN [5] and EDELMAN and EGGINK [6] both give qualitative considerations about the sand transport by waves. Since their publications so many data have been collected, about the relation between the longshore component of the wave energy and the sand transport, that it is worthwhlle to apply such formulae to the dutch coast, in order to obtain more quantitative conclusions. However, these conclusions can be not better than the available data the visual wave observations on the dutch light-vessels. These measurements from 1949 to 1957 have been statistically treated by DORRESTEIN [10], glving for each wave condition, (characterized by height, period and direction of the waves) the probability of occurrence.

From [10], the longshore component of the wave energy flux $P_{1}$ has been computed, defined by

$$
\mathrm{P}_{1}=\frac{1}{8} \rho \mathrm{g} \mathrm{H}_{\mathrm{br}}{ }^{2} \mathrm{C}_{\mathrm{br}} \sin \varphi_{\mathrm{br}} \cos \varphi_{\mathrm{br}}
$$

in which $\rho g=$ specific weight water, $H=$ wave height, $C=$ wave celerity, $\varphi=$ angle of wave incidence and the index br refers to the breaker zone.

The exact way of computation is treated in [14]. The accuracy is very low, because it 15 impossible to get an accurate significant $H, C$ and $\varphi$ in the breaker zone from $v i s u a l$ wave data from a distance of $10 \mathrm{~km}$. It was necessary to make a lot of assumptions [14]. The results are given in the table above f18. 12 , and $f_{18}$. 10 shows a probability distribution.

It 1 s easy to compute from this the mean energy flux and the mean littoral drift $Q$ using the CERC-formula, which can be transferred $[14]$ in

$$
Q^{*}=2300 P_{1} \quad\left(Q^{*} \text { in } \mathrm{m}^{3} / \text { year, } P_{1} \text { in } W / m^{\prime}\right)
$$

However, the used data and formulae are not accurate enough to justify this computation, also because the probability distributlons are about symmetrical. Only along the Vlieland coast the resultant drift is significant (about $\frac{1}{2} \mathrm{mln} \mathrm{m}^{3}$ /year).

The gross littoral drift (sum of transport in both directions) is found to be of the order of 1.5 to $2 \mathrm{mln} \mathrm{m}^{3} / \mathrm{year}$ (thus about $1 \mathrm{mln}$ $\mathrm{m}^{3}$ in each direction). 
Assuming for instance no influence of tides, the distribution of the littoral drift over the surf zone has been computed by an adapted method proposed by SVASEK [8], the way of computation is pointed out in another paper of this conference [13]. The result for Scheveningen is shown in $f$ ig. 11 .

Considering which one is most important, the influence of waves or tides, in [15] and [16] the driving forces of waves and currents are investigated.

In order to get an impression of the influence of the tide, a tidal computation has been carried out with the numerical tidal. model of which $f_{18}$. 12 gives the scheme [19]. We assumed a gully system parallel and perpendicular to the coast on the ends the vertical tide was given, in the knots the vertical tide was computed and in the gullies the horizontal tide. The computerprogram was developed by BOOY according to the explicite leap-frog method, non-linear terms were considered, but Corlolis was neglected. In each gully at every time was computed $\sum_{*} B v h . \Delta t$, in which $B$ is the width of the gully, $v$ the current velocity, $h$ the water depth at that moment and $\Delta t$ the time step. From this, the resultant currents, Indicated in the upper figure of $\mathrm{flg} .12$ were found, about $3 \mathrm{~cm} / \mathrm{sec}$ in the shallow reglons and $6 \mathrm{~cm} / \mathrm{sec}$ in the deeper regions. A computation, taking the tidal currents into account, based on a simplified BIJKER-method (cf [8]) is in preparation.

\section{THE OUTER DELTAS}

The boundary conditions for the motion of the coast are glven by the inlets. Therefore it is important to consider the motion of the gullies. Fig. 13 and 14 give the motion of the gullies in the inlets of Texel and VIle respectively. The arrows give the motion of the gulizes since the last recording. What is known about these deltas?

The cross-section of the gullies reasonably fit in with the theory PER BRUUN and GERRITSEN [7]. As a variation HARING[20] found, that the quotient of the tidal volume (ebb $+f l o o d)$ and the total profile area of the gully was about $55 \mathrm{~cm} / \mathrm{sec}$, except for the inlet of Texel and the inlet of the VIle, where 1 t was $75 \mathrm{~cm} / \mathrm{sec}$ (cf DRONKERS [1]). This higher velocity might be some influence of littoral drift. Van VEEN [21] states, that the largest gullies are mainly orientated in the direction of the greatest water gradient, averaged per tide. If the tidal amplitude is everywhere the same (which is often not the case), this arection is perpendicular to the cotidal lines ( $f i g .17$ ) the gradient between $A$ and $B$ is much larger than between $A$ and $C$.

Two reasons can be given for erosion of the coast near these inlets As has been pointed out by van BENDEGOM [22], the submerging of the Wadden during flood tide takes place with larger velocities than the retreating of the water over the shoals during ebb-tide. Thus, the water looses here a part of Its sediment, which makes, that the Wadden shoals reach an equilibrium at about the mean water level. Now the relative sea-level rising in the Netherlands during the last 20 centuries was about $6 \mathrm{~cm} /$ century, which would result in a "sand hunger" of the Wadden shoals of about $1 \mathrm{mln} \mathrm{m} 3 /$ year (distributed over all inlets). 
However, this will mostly be confined to finer sediments ( $D_{m}$ about $100-150 \mu)$.

The second reason is that the water during the flood tide gets an acceleration, entering the inlet, but that during the ebb-tide it gets a retardation and this will give a jet-stream with vortexes on its side. Therefore in the gullies near the beaches, there is surplus of flood discharge and in the center gully a surplus of ebb discharge. The flood erodes the beaches and the ebb gives an outer delta, which can reach up to mean sealevel (Noorderhaaks in inlet of Texel. This delta gives a shelter against these waves, which would transport material away from the delta. As the waves come alternately from both directions, this process reinforces the erosion of the beaches near the inlets. Thus the erosion of Texel could be rather well explained [23]. After some time an equilibrium should be reached.

Two reasons can be given too for the motion of the gullies a meandering effect and a longshore sand drift. It will be clear, that the resulting sand drift perpendicular to the gully can not be derived from the velocity of the gully because of the meandering effect. The big sand transport in the gullies can be attributed to the high current velocities and this meandering.

The motion of the gullies and the effects of their orientation has been investigated ( 1 g. 16). The line in the middle of each of the bars gives the orientation of the gully in course of time. The width of the bar gives the development of representative cross section. As far as they were known, the time-integrated slope of the water-surface as a function of the orientation have been mentroned ( $\mathrm{fIg} .161)$ ). It gives no evidence about the van Veen-theory. In $\mathrm{flg}_{\mathrm{g}}$. 18, derived from fig. 16 , the turning of the gullies In the dutch Wadden delta is shown. Mainly they turn clockwise, although very slowly and there is a slight indication (correlation coeff..24), that the large gullies turn slower than the small ones.

\section{ACKNOWLEDGEMENT}

The authors gratefully acknowledge the ald of the Study Services Hoorn, IJmuiden and Delfzijl of Rijkswaterstaat in providing the data, published here.

\section{REFERENCES}

[1] dr. J.J. Dronkers, Research for the coastal area of the Delta region of the Netherlands, C.E.C., Washington 1970.

[2] 1r. L.R. Wentholt, Stranden en strandverdediging, Delft Doctor's Thesis 1912.

[3] 1r. J. van Veen, Onderzoekingen in de hoofden Delft, Doctor's Thesis 1936.

[4]. dr.1r. J. v. Veen, Eb- en vloedschaarsystemen in de Nederlandse getıjwateren. Tijdschr. Kon. Ned. Aardr. Gen. 67 pp. $45-65$.

\footnotetext{
1) These have been derıved from van Veen 21 and Ferguson 24
} 
[5]. dr. L.M.J.U. van Straaten, Directional effects of winds, waves and currents.

Geologie en Mijnbouw 23, 1961.

[6] $1 \mathrm{r}$. T. Edelman and drs. D.N. Eggink, Some characteristics of the dutch coast C.E.C. 1962.

17. Per Bruun and ir. F. Gerritsen, Stability of coastal inlets.

[8]. prof.dr.ir. E.W. Bijker and 1r. J.N. Svasek, Two methods for determination of morphological changes, induced by coastal structures. Int. Nav. Congr. 1969.

[9] 1r. W.T. Bakker, A mathematical theory about sandwaves. Shore and Beach, Oct. 1968.

[10] dr. R. Dorrestein, Wind and wave data of Netherlands lightvessels. Med. en Verh. K.N.M.I. no. 90.

[11] L. Knop

, Onderzoek Noordzeekust Petten-Huisduinen. Study Service Hoorn Rijkswaterstaat $\mathrm{nr} .584$.

[12] ir. L. van Bendegom, Beschouwingen over riviermorfologie. De Ingenieur 59, 24 jan. 1947.

[13. ir. W.T. Bakker, ir. E.H.J. Klein Breteler and A. Roos, The dynamics of a coast with a groyne system

04. ir. W.T. Bakker, Computation littoral drift with Svasekmethod. Rijkswaterstaat, Dept. for Coastal Res. Rep. W.W.K. 69-7.

[15. 1r. W.T. Bakker and H.J. Opdam, Influence of waves and tides on the littoral drift in the surf zone. Rijkswaterstaat, Dept. for Coastal Res. Rep.W.W.K. 70-8.

[1ם. H.J. Opdam

, A wave tide model for the dutch coast. M. Se. thesis, Delft 1970.

077. A.J. Bowen , The generation of longshore currents on a plane beach.

Journal of Marine Research 27 no. 2, May 1969.

[181. E.H.J. Klezn Breteler, Zandtransport bij Katwıjk. M Sc. thesis, Delft 1970.

G9l 2r. E.H.J. Klein Breteler, A tidal model for the coast of Hook of Holland - IJmuiden. Rijkswaterstaat, Dept. for Coastal Res Rep.W.W.K. 70-10.

[20] J. Harıng

, Oppervlakte van het dwarsprofiel van de Nederlandse zeegaten als functie van het getij-volume. Ri Jkswaterstaat, Delta Works, Rep. K 251

[21]. $1 \mathrm{r} \cdot \mathrm{J} \cdot \operatorname{van}$ Veen

, Zeegat van het Vlie (1934). Rijkswaterstaat, $D_{1} r$. Benedenrivieren

[22]. 1r. L. van Bendegom, Grondslagen der kustverdediging, Rijkswaterstaat.

[23] 1r. Th.J.C. W1 Jnant, Littoral drift near Texel. Rijkswaterstaat, Dept. for Coastal Res. 


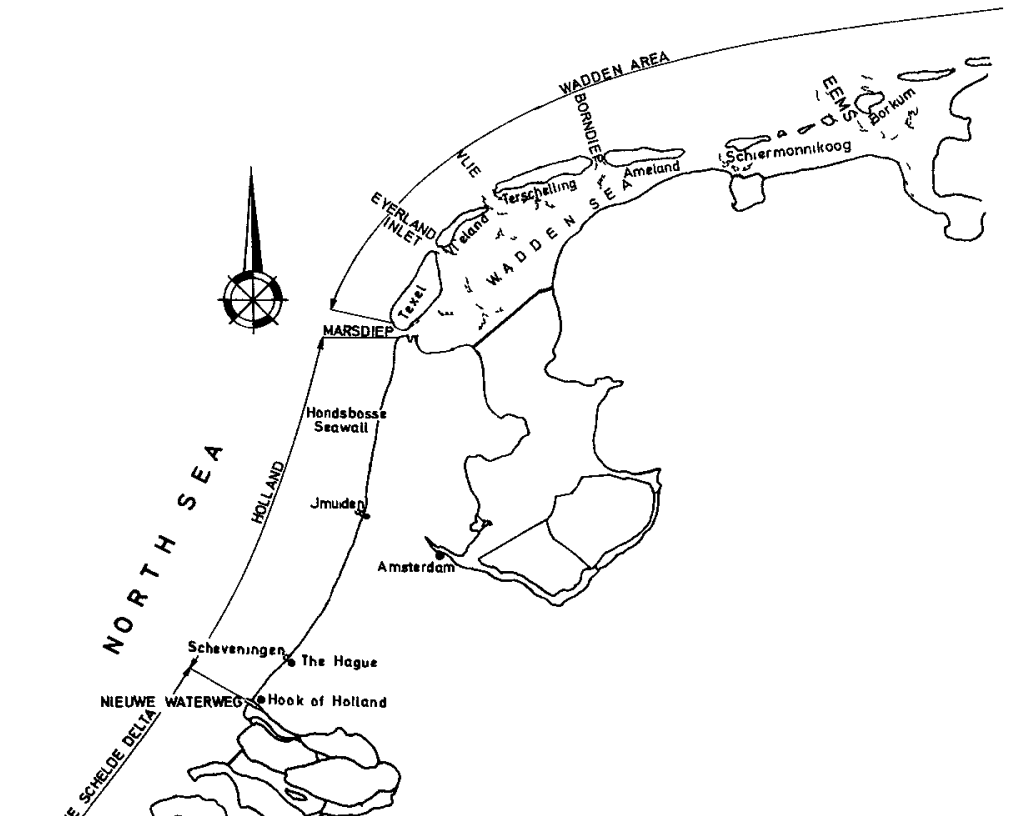

FIg1 THE DUTCH COAST

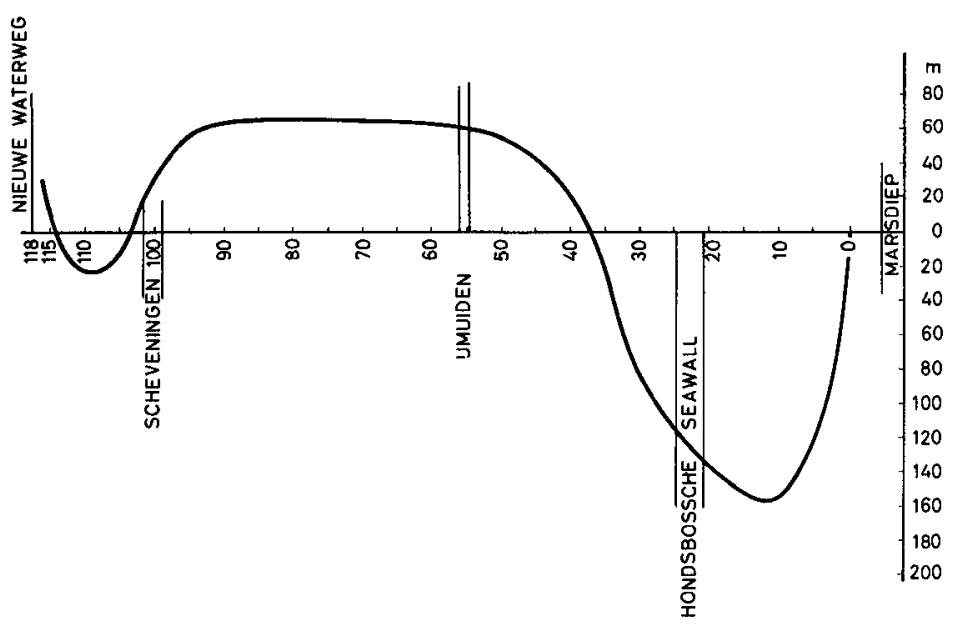

FIg 4 MEAN DUNE-FOOT MOVEMENTS FROM 1860 TILL 1960 BETWEEN NIEUWE WATERWEG AND MARSDIEP 
FOOT OF THE DUNE

HIGH WATER

LOW WATER

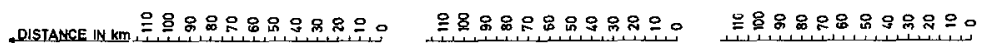

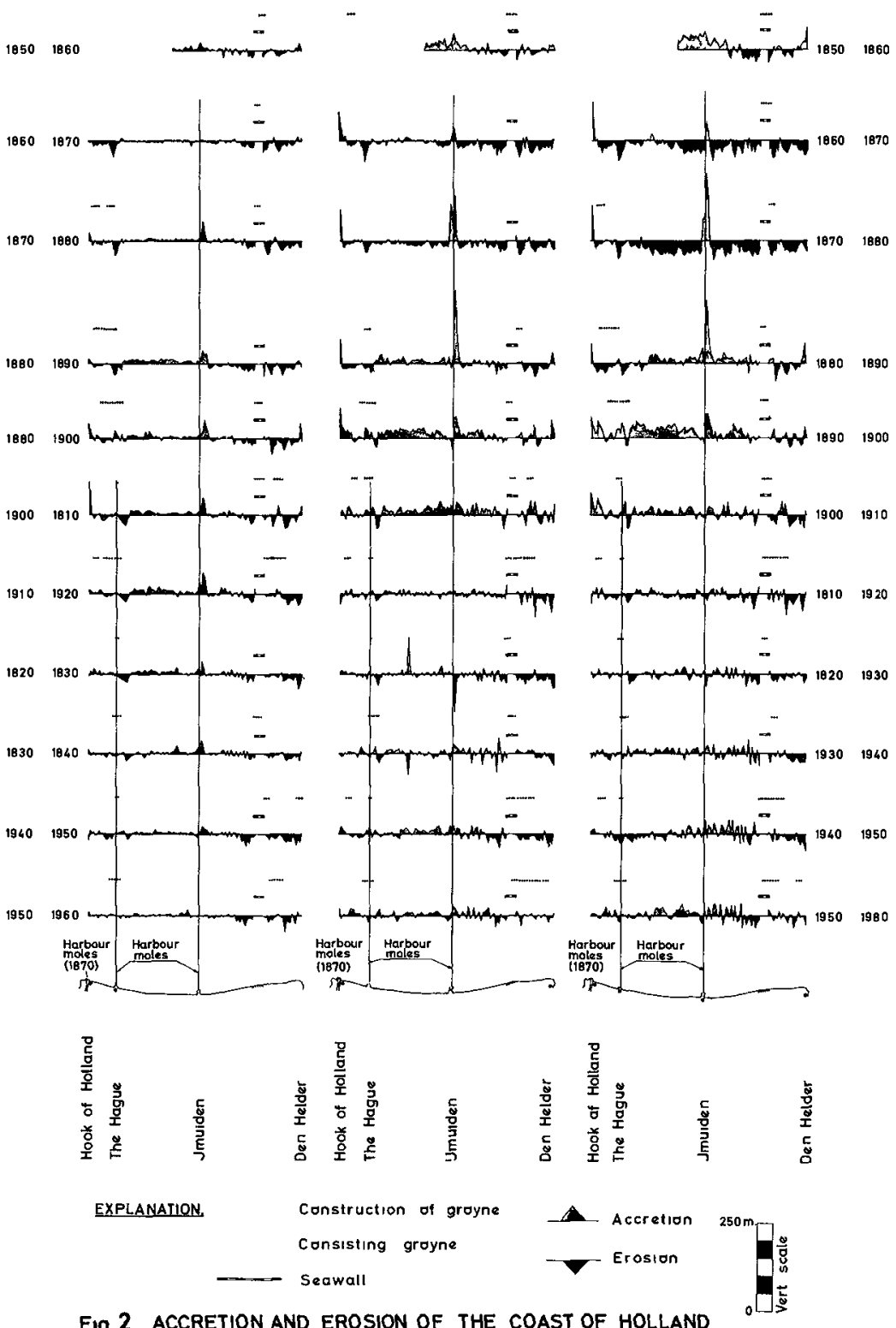

Fig 2 ACCRETION AND EROSION OF THE COAST OF HOLLAND 


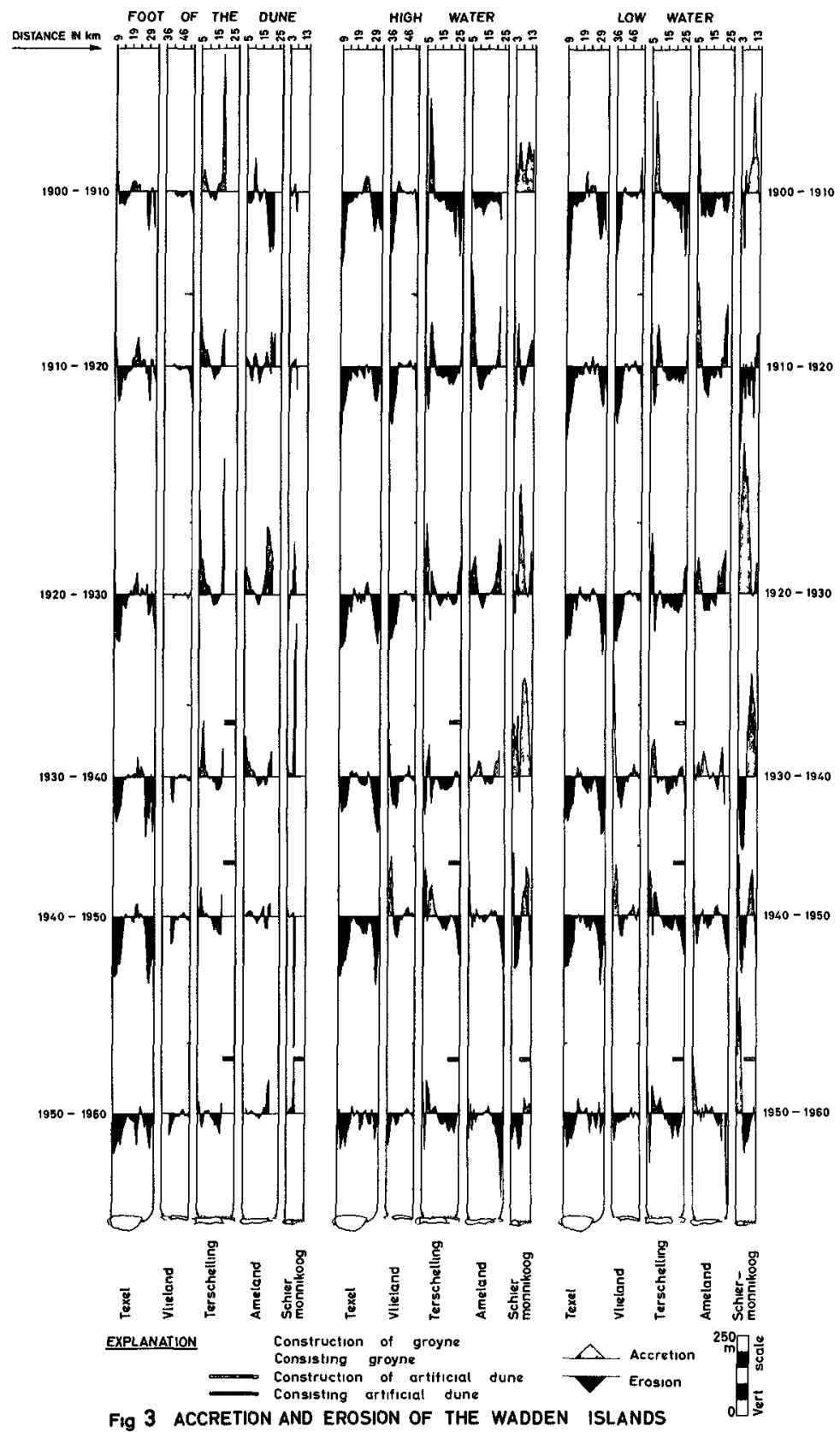




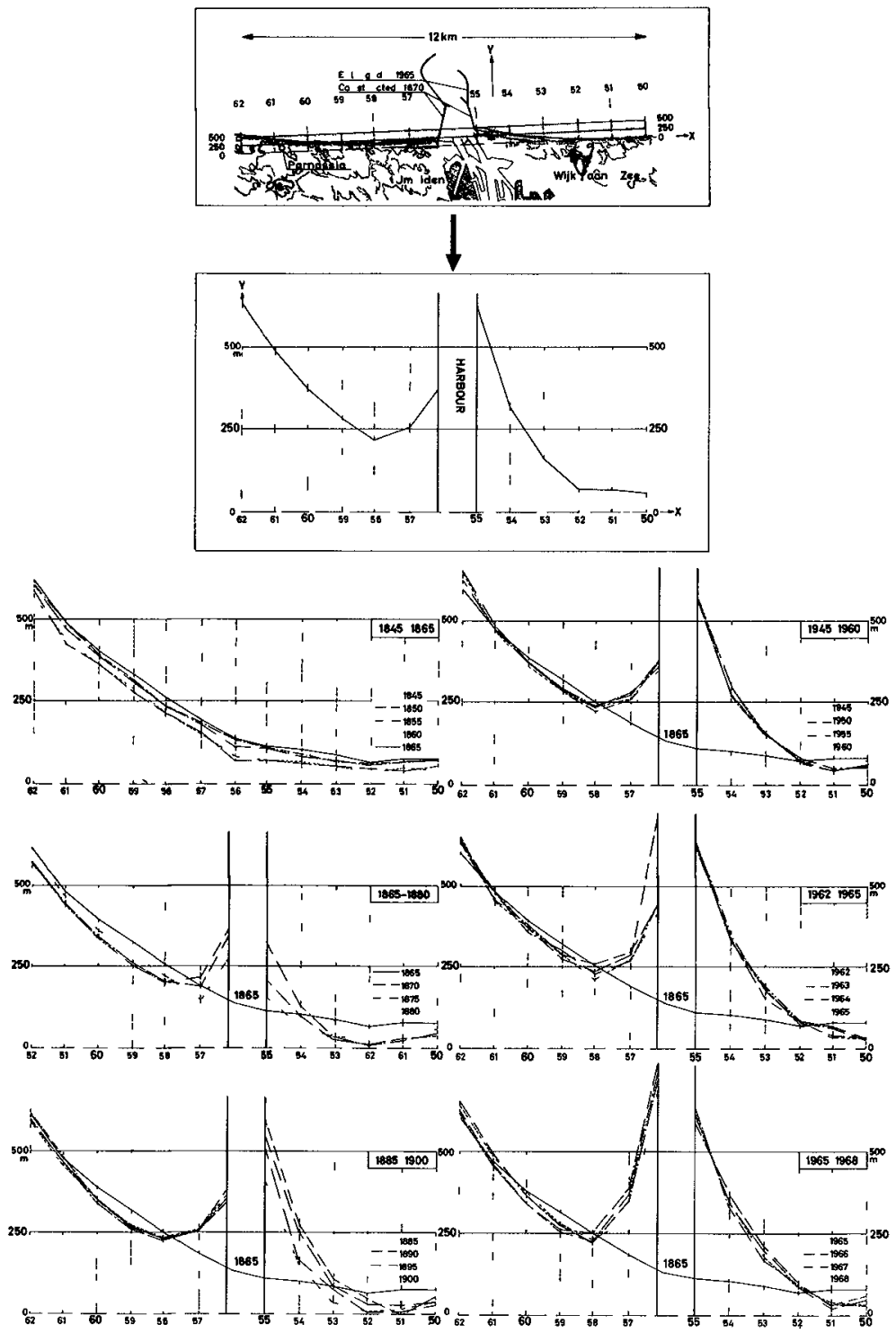

Fig 5 DEVELOPMENT OF the waterLine NEAR IJMUIDEN 


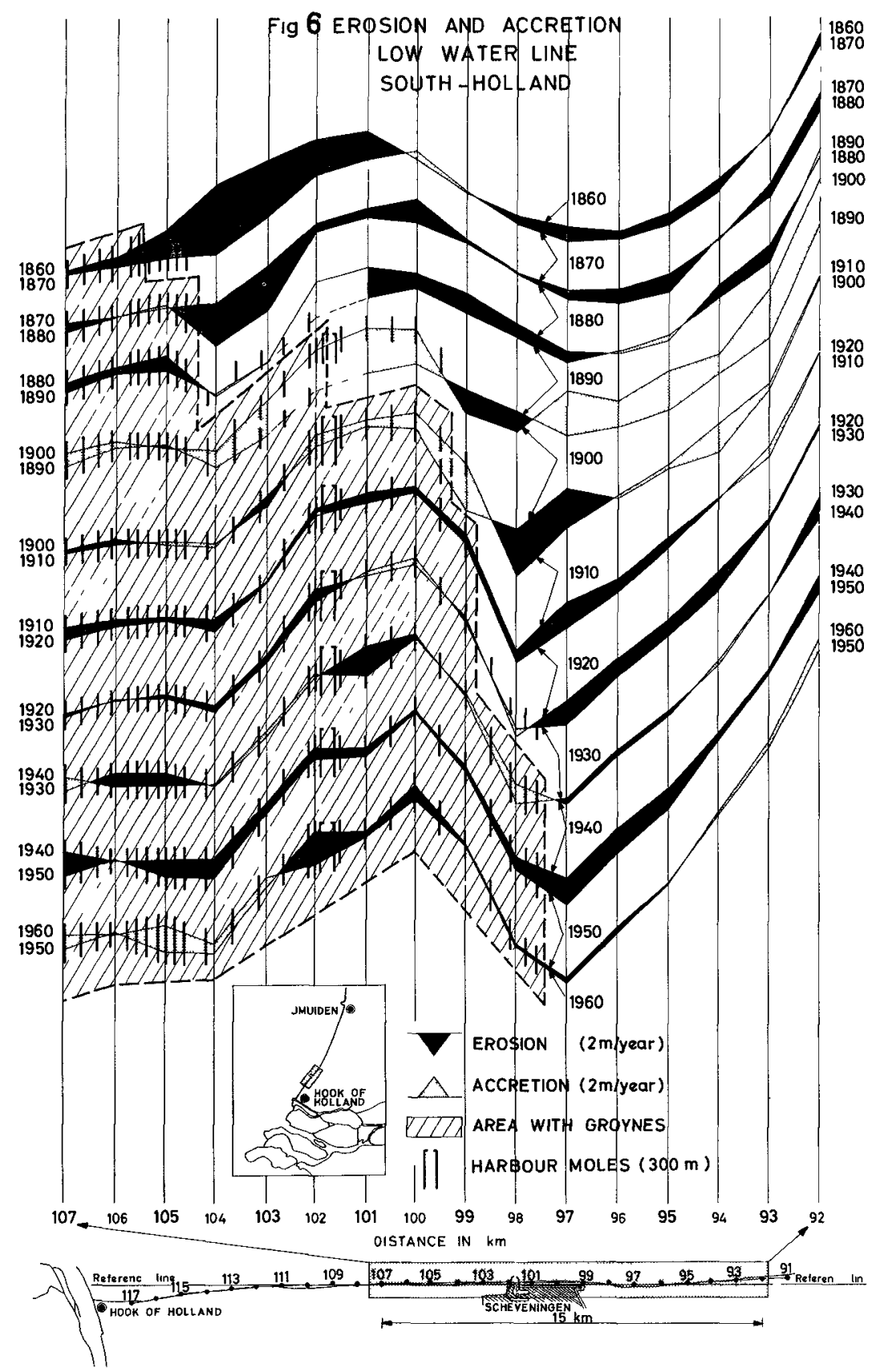




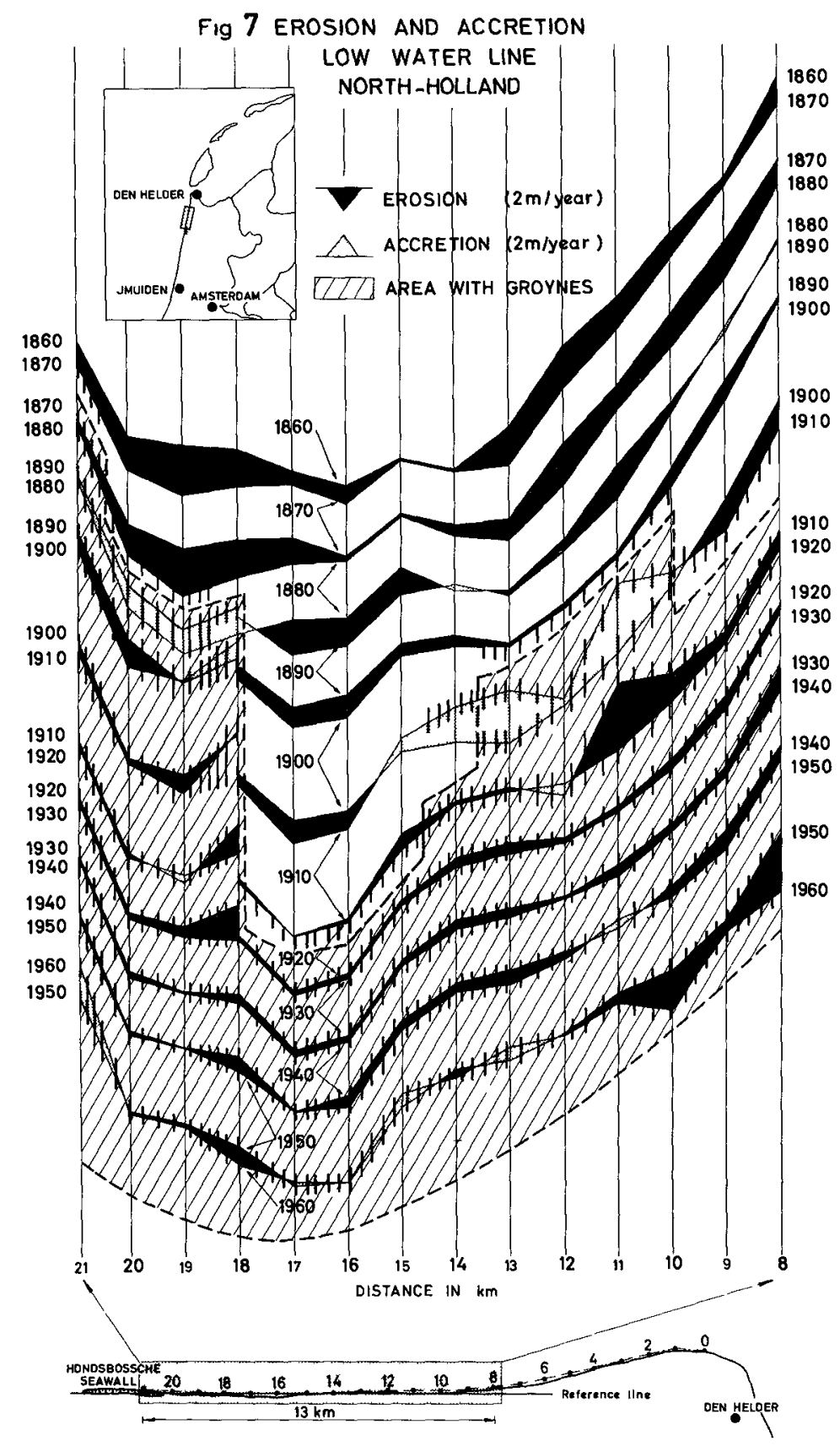


FIg 8 EROSION AND ACCRETION

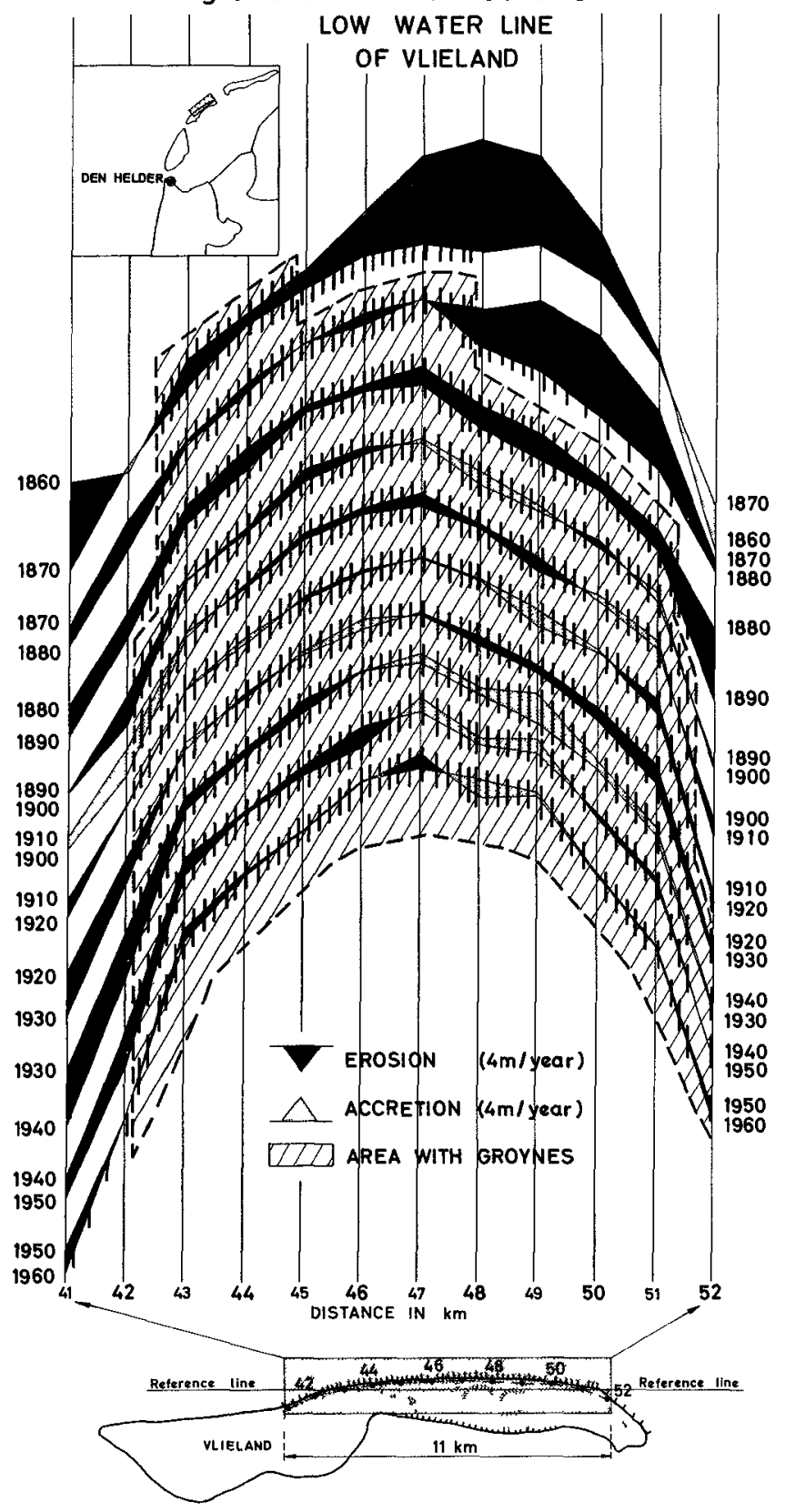



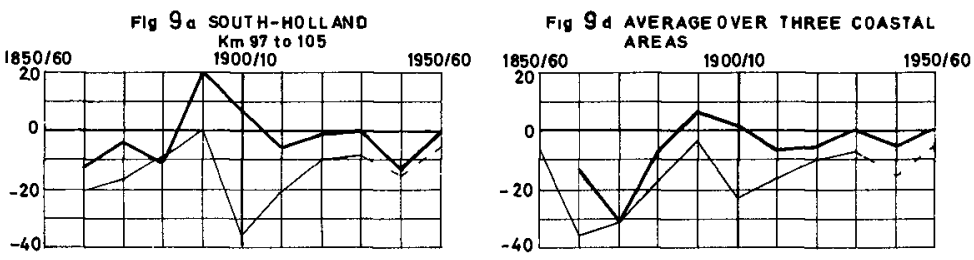

FIg 9b NORTH-HOLLAND

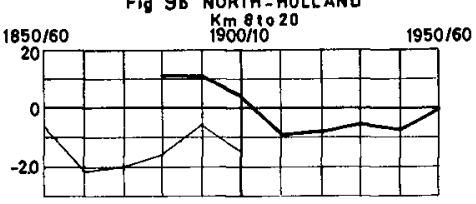

Fig 9 e AVERAGE OVER ALL UNINTERRUPTEO
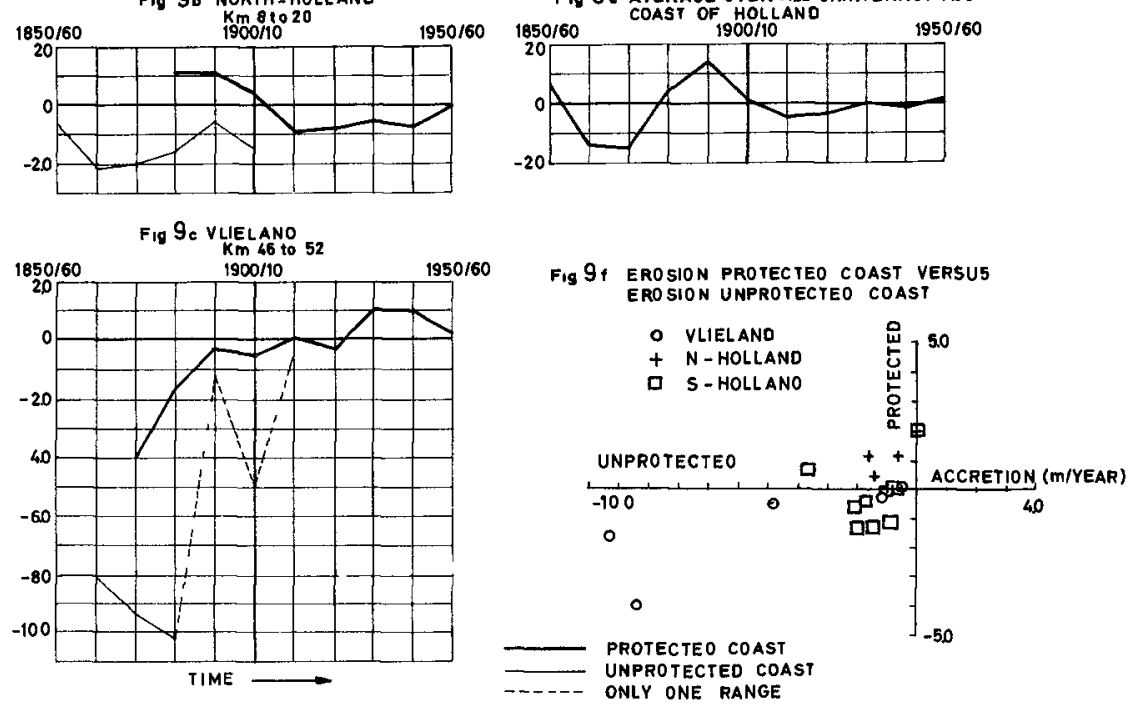

FIg 9 COMPARISON OF EROSION IN m/YEAR OF PROTECTED AND UNPROTECTED AREAS

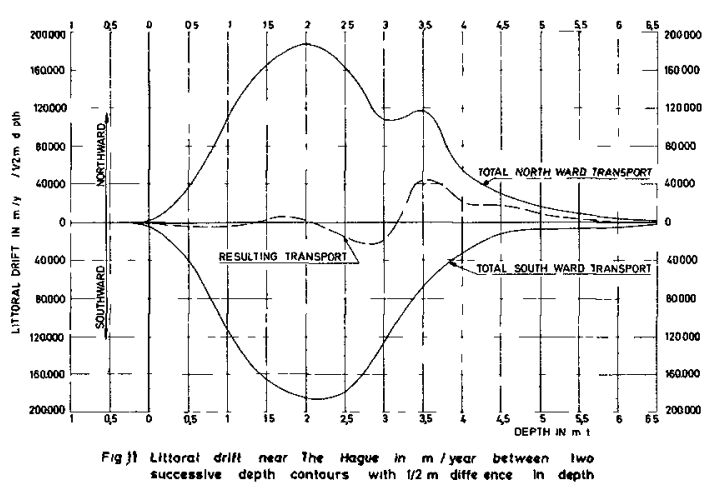




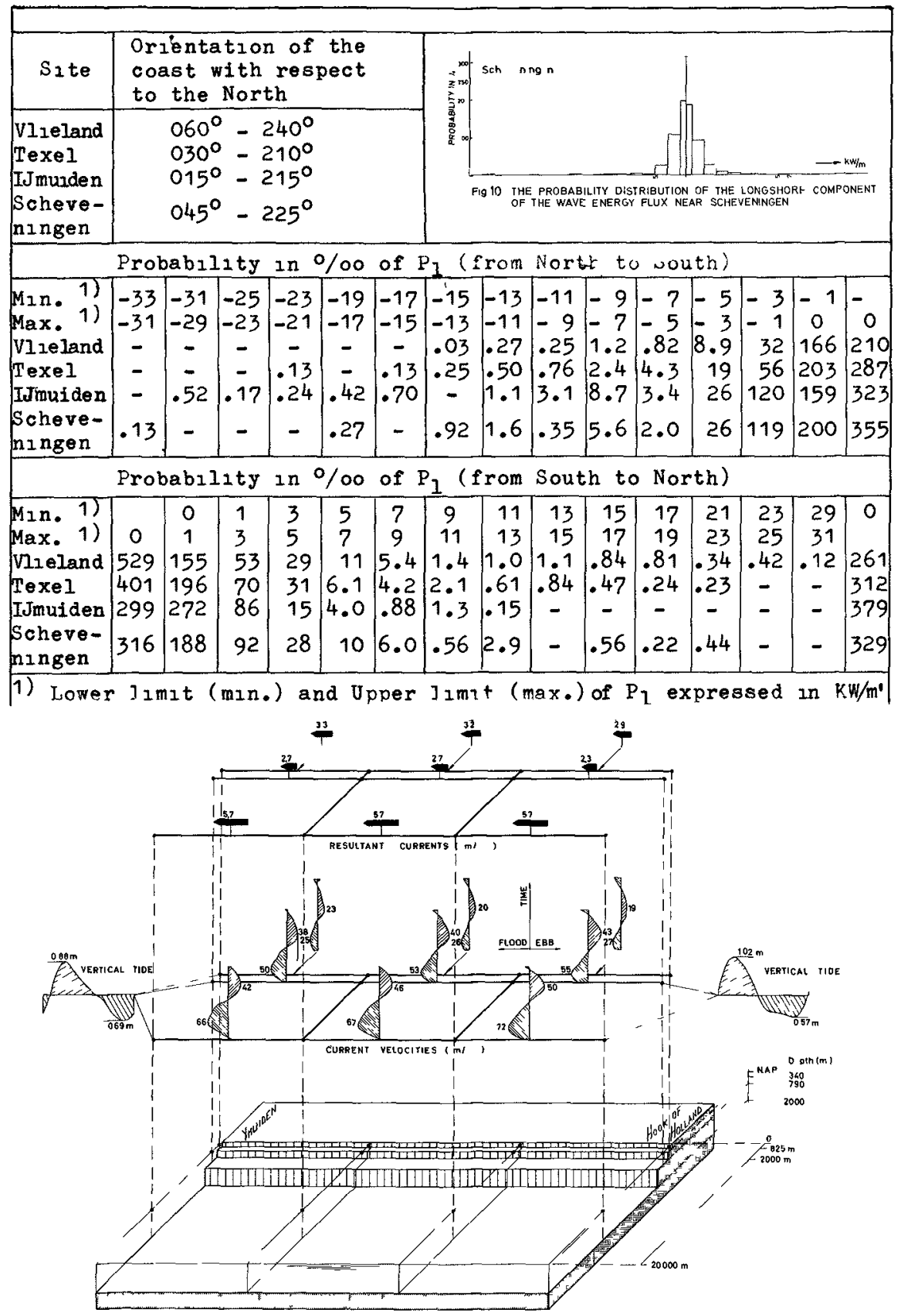

FIg 12 TIDAL MOOEL 







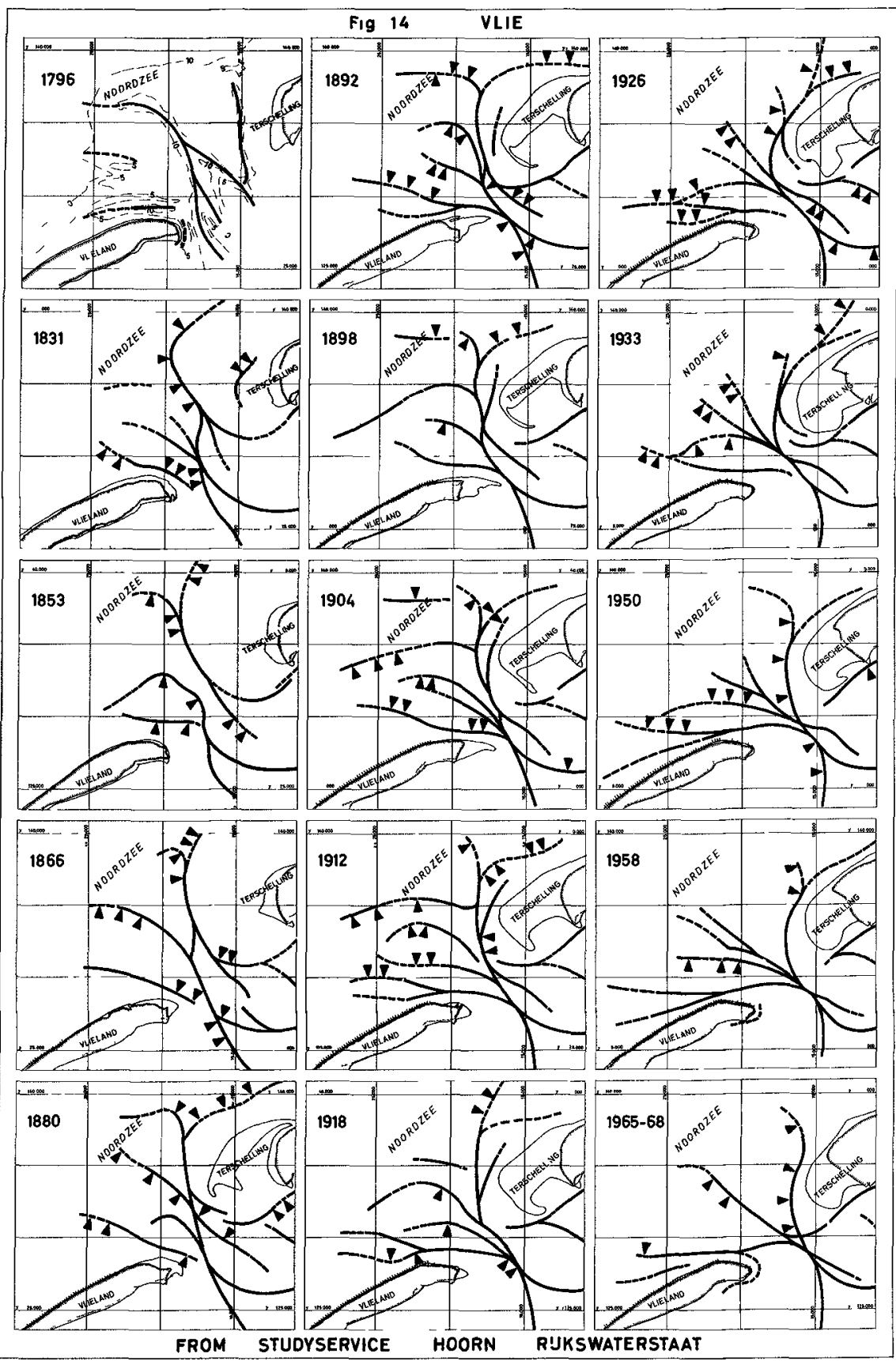



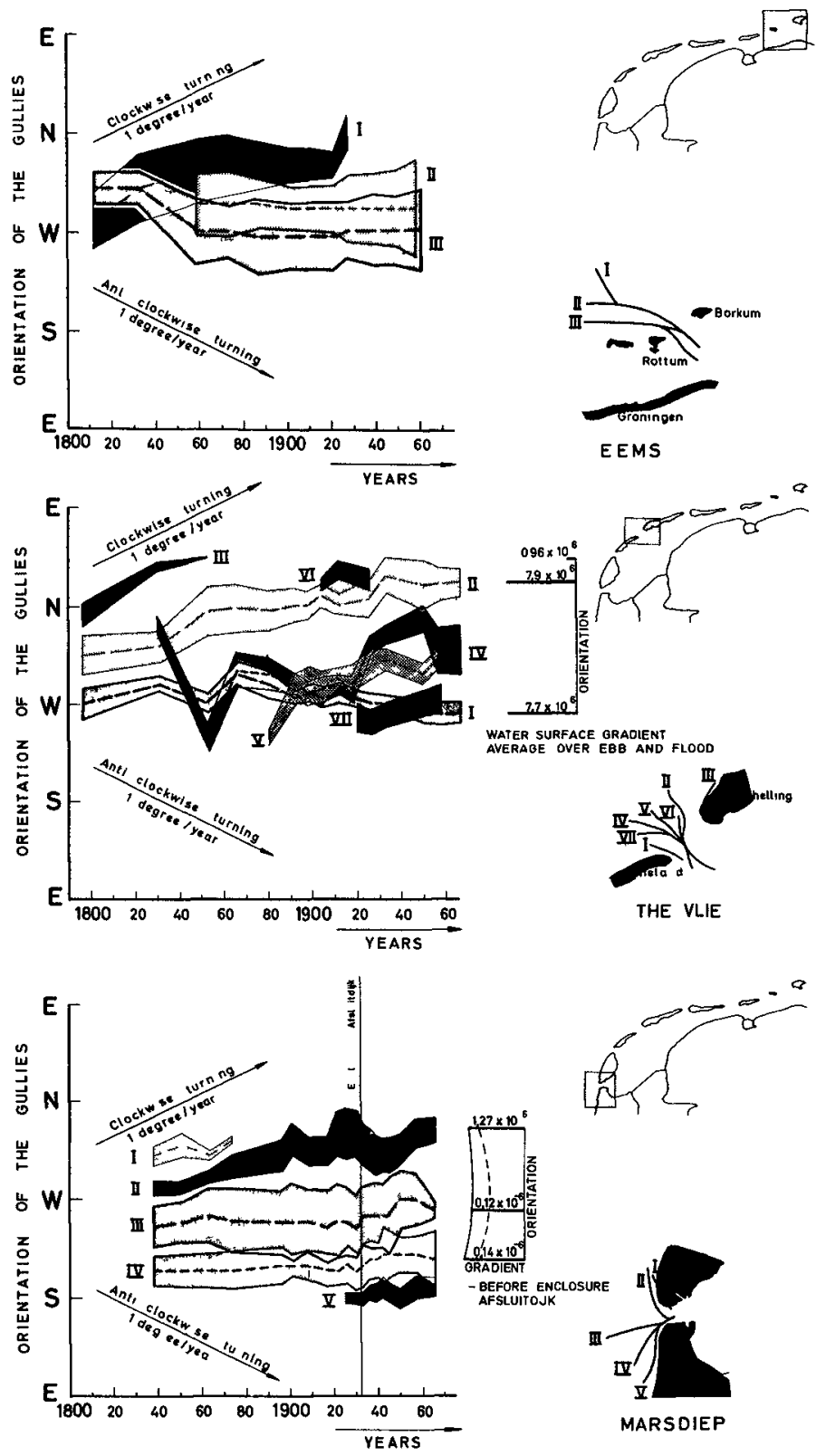

Fig 16 THE DIRECTION AND the WEt surface of the gulLies 


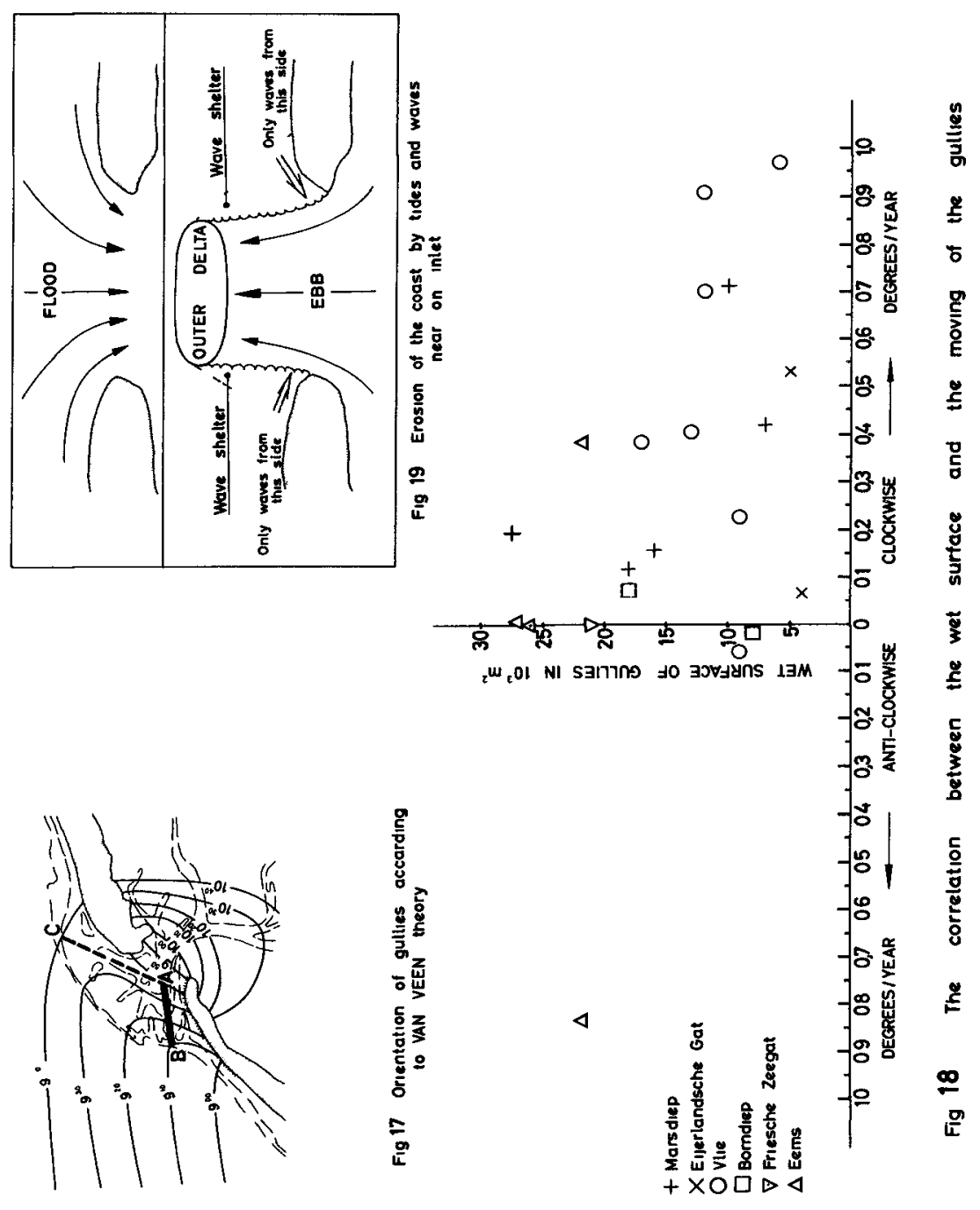




\title{
CHAPTER 44
}

\section{STUOIES ON THE SHORE PROCESS ANO WAVE FEATURES OF THE WESTERN COAST OF TAIWAN}

\author{
Kenneth S T Chang, Chief Engineer \\ Tarwan Land Resources Development Commission, R O C \\ Frederick L W Tang, Professor \\ Hydrau1ıc Engineerıng Department, Cheng Kung Unıversity, R $0 \mathrm{C}$
}

\section{SYNOPSIS}

The western coast of Taiwan has been accumulated to vast area of tidal flats by the sediment transported from numerous torential streams According to the aerial photographs, 53,800 hectares of tidal land are worth being reclaimed However, this coast suffers from waves generated by winter monsoons as well as waves and swells caused by typhoons in the summer Studies on the historical processes and future development of the shoreline are of great significance

The ancient topographical data in An-Ping and Tseng-Pa areas as well as recent coastal 11 ne variations of Tseng- $\mathrm{Pa}$ and Yulin are reported in this paper, also the general features of waves along this coast are described The future of the coast is predicted as a conclusion

\section{HISTORICAL OATA ON COASTAL CHANGE}

Taiwan is the eastern province of the Republic of China that is surrounded by the sea ( $F_{1} g$ 1) and has a total coastline of 1,566 k1lometers The type of the west coast 15 one of deposition, and there 15 a large area of tidal flats formed before long ago At the present there are 53,800 hectares tidal 1 and to be developed

According to historical data and results analyzed, this coast extended offshore since 1624 until 1961 as shown in Fig 2 and Table 1 Here An-Ping coast means the coast area nearby the city An-Ping, and Tseng-Pa coast means the coast area between rivers Tseng-wen and Pa-Chang

\section{WAVE CHARACTERISTICS ON THE COAST}

From September to March of last year, anticyclones of large intensity (some $1040 \mathrm{ml} 1 \mathrm{libars}$ ) occur continuously in the vicinity of Begal Lake and move due southeast Finally, they weaken and vanish in the Pacific Ocean 
While the anticyclone is moving, wind from NE or NNE direction blows over the East China Sea and Taiwan Strait wi th velocities of $10-20 \mathrm{~m} / \mathrm{sec}$ Due to the long duration of the wind, relatively large waves are generated According to measured data in the middle and south section of the coast, waves 2-3 meters high and 6-8 seconds in period exist on this coast most of the winter The steepness of these waves are large and the bed materials of the coast are as fine as $02 \mathrm{~mm}$ Apparently the sand of the coast is eroded by the waves and is moved in a southerly direction

In the summer months, winds from SW-S blow in the afternoon and the velocity rarely exceeds $10 \mathrm{~m} / \mathrm{sec}$ Waves generated by such wind are smal1, however, during these months typhoons frequently occur and cause considerable damage The waves caused by typhoons sometimes are 7 meters in height and 12 seconds in period These waves and swells also cause shoreline changes on the coast The coast is severely eroded and the sand drift moves offshore The coast does recover the sand when the winter monsoons begın

\section{RECENT SHORELINE PROCESSES OF TSENG-PA AND YULIN}

There are a series of offshore dunes along the Tainan coast After the Tseng-Wen polder was reclaimed, most of the dunes became more stable than before Only near the inlets between the dunes were changed seriously during the winter monsoons, thereby causing damage to the north dike of the polder Tseng-Wen Sometimes, owing to the fluctuation of climate and sea level during typhoons, the dunes are broken and new inlets are formed The tendency of shoreline processes in this area is one of erosion ( $F 1 g$ )

By means of hydrographic surveys and aerial photographs, we also provided valuable evidence of coastal and offshore changes in the Yulin area (Fig 4) Profiles in this area are shown in Fig 5 The shoreline process in this area is one of erosion

\section{CONCLUSIONS}

1 The western coast of Taiwan was the result of deposition by north to south drift during ancient times The sources of supply of sand drift was from high mountains and torential streams

2 According to the wave characteristics and bed material, this coast now suffers by erosion

3 In ancient times the sand supply exceeded that which was eroded The rivers have been regulated since the beginning of this century and also s011 conservation works have been constructed Thus the coast has begun to erode because of the sand supply which has been cons 1derably decreased 
4 Although the erosion is not serrous today, methods of shore protection must be undertaken The reclamation of tidal land is of great significance from the economical point of view

5 The shore process of this coast is very complicated and interesting, and detalled studies should be conducted 
SHORELINE PROCESSES OF AN-PING COAST

TABLE 1

\begin{tabular}{|c|c|c|c|c|c|}
\hline \multicolumn{2}{|c|}{$\begin{array}{l}\text { THE PEFIOD } \\
\text { OF } \\
\text { COASTAL } \\
\text { ACCRETION }\end{array}$} & PERIOD & $\begin{array}{l}\text { THE MAIN FORMATION AND } \\
\text { TYPES OF THE COAST }\end{array}$ & $\begin{array}{l}\text { THE UTILLZ- } \\
\text { TION OF } \infty \\
\text { AST AND } \\
\text { TIDAL LAND }\end{array}$ & $\begin{array}{l}\text { DATA } \\
\text { RESOURCES }\end{array}$ \\
\hline \multicolumn{2}{|l|}{$\frac{0}{x}$} & \multicolumn{3}{|c|}{ 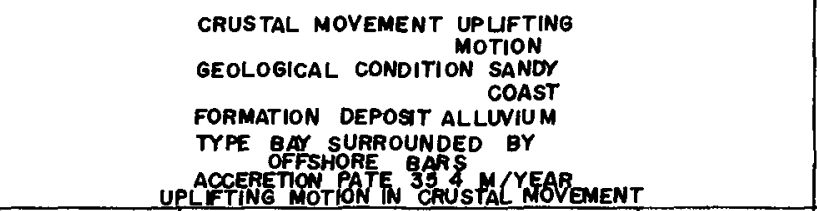 } & \multirow{2}{*}{$\begin{array}{l}\text { TAIWAN } \\
\text { HISTORY } \\
\text { TAWAN } \\
\text { HSIN HISTORY }\end{array}$} \\
\hline \multirow{3}{*}{ 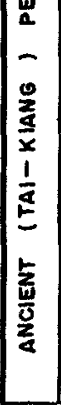 } & $\begin{array}{l}\text { FIRST } \\
\text { STAGE }\end{array}$ & $\begin{array}{l}\text { FROM } 1624 \\
\text { TO } \\
1662 \\
(38) \text { YEAR }\end{array}$ & $\begin{array}{l}\text { MANY OFF SHORE BARS FORMED } \\
\text { NATURAL HAREOUR EAY } \\
\text { AGE IN EARLY YOUNG STAGE }\end{array}$ & $\begin{array}{l}\text { VESSELS } \\
\text { ANCHORMGEO } \\
\text { W THE EAY } \\
\text { A SMALL } \\
\text { AMOUNT OF } \\
\text { SAY PANS }\end{array}$ & \\
\hline & $\begin{array}{l}\text { MDDLE } \\
\text { STAGE }\end{array}$ & $\begin{array}{l}\text { FROM } 1662 \\
\text { TO } \\
1722 \\
\text { (60 YEARS) }\end{array}$ & $\begin{array}{l}\text { SEDIMENTATION IN THE BAY BARS } \\
\text { INCREASED } \\
\text { AGE IN EARLY YOUNG STAGE }\end{array}$ & DITTO & $\begin{array}{c}\text { TAIWAN HSIN } \\
\text { HISTORY }\end{array}$ \\
\hline & $\begin{array}{l}\text { LAST } \\
\text { STAGE }\end{array}$ & $\begin{array}{l}\text { FROM } 1722 \\
\text { TO } \\
1822 \text { Y } \\
\text { (100 YEARS }\end{array}$ & $\begin{array}{l}\text { OFFSHORE BARS ENLARGED AND } \\
\text { CONNECTED WITH LANO FORNED } \\
\text { LAGOONS THE FOPM OF BAY VANISHED } \\
\text { AGE N YOUNG STAGE }\end{array}$ & $\begin{array}{l}\text { SALT PANS IN- } \\
\text { CREASED ANO } \\
\text { RECLAIMED } \\
\text { FISH PONOS }\end{array}$ & $\begin{array}{l}\text { KAOHSNNG } \\
\text { HAREOUR } \\
\text { HIS TORY }\end{array}$ \\
\hline \multicolumn{2}{|c|}{$\begin{array}{l}\text { RECENT } \\
\text { COASTAL } \\
\text { PERTOD }\end{array}$} & $\begin{array}{l}\text { FROM } \\
1822 \\
\text { TO } \\
1894 \\
(72 \text { YEARS })\end{array}$ & $\begin{array}{l}\text { CRUSTA MOVEMENT UPLIFTING MOTION } \\
\text { GEOLOGICAL CONDITION SANDY COAST } \\
\text { FORMATION DEPOSIT ALLUNIMM } \\
\text { TYPE LAGOONS SILTED UP LAND } \\
\text { OFFSHORE BARS BECAME NEW } \\
\text { COAST, PRODUCTED NEW } \\
\text { RIVERS AND NEW OFFSHORE } \\
\text { BARS FORMED AGE IN YOUNG } \\
\text { STAGE FATE } 279 \text { M/YEAR } \\
\text { ACCRETION RATE }\end{array}$ & $\begin{array}{l}\text { VESSELS } \\
\text { ANCHORACED } \\
\text { IN THE BAY } \\
\text { SALT PANS } \\
\text { AND FISH } \\
\text { PONOS INCR- } \\
\text { EASED AND } \\
\text { ALSO SETT- } \\
\text { LERS IN } \\
\text { CREASED }\end{array}$ & $\begin{array}{l}\text { KAOHSIUNG } \\
\text { HARBOUR } \\
\text { HISTORY }\end{array}$ \\
\hline \multicolumn{2}{|c|}{$\begin{array}{l}\text { NOWADAYS } \\
\text { PERIOD } \\
\text { (EARLY STAGE) }\end{array}$} & $\begin{array}{l}\text { FROM } \\
1894 \\
\text { TO } \\
1945 \\
\text { (5) YEARS) }\end{array}$ & $\begin{array}{l}\text { I AN-PING COAST BECAME } \\
\text { STABLE LAND } \\
2 \text { TSENG -PA COAST ( OLD SHAW - } \\
\text { LOONG COAST) NEW OFF-SHORE } \\
\text { BARS AND LAGOON FORMED } \\
\text { AGAIN AGE IN YOUNG STAGE } \\
\text { OTHER ITEMS SAME AS BEFOAE }\end{array}$ & $\begin{array}{l}\text { MODERN HAR- } \\
\text { BOUR WAS } \\
\text { CONSTRUCTED } \\
\text { MUCH MORE } \\
\text { SALT PANS } \\
\text { AND FISH PO- } \\
\text { NDS WERE } \\
\text { RECLAIMED }\end{array}$ & $\begin{array}{l}\text { HISTORY OF } \\
\text { TAIWAN ECO- } \\
\text { NOMICS } \\
\text { AND OLD } \\
\text { MAPS }\end{array}$ \\
\hline \multicolumn{2}{|c|}{$\begin{array}{l}\text { NOWADAYS } \\
\text { PERIOD } \\
\text { (NEW STAGE) }\end{array}$} & $\begin{array}{l}\text { FROM } \\
\text { 1945 } \\
\text { TO } \\
\text { (21 YEAFS) }\end{array}$ & $\begin{array}{l}\text { TYPE I AN-PING COAST ALREADY } \\
\text { FIXED OFFSHORE BAR AND } \\
\text { TIDAL LAND NO LONGER } \\
\text { EXISTING AGE NMATHE STMG } \\
2 \text { TSENO-PA CONST - OFFSHORE } \\
\text { BARS CONTINUOUSLY ACGRETEO } \\
\text { AND LAGO ON WHS FORMED }\end{array}$ & $\begin{array}{l}\text { TIDAL LAND } \\
\text { WAS DE- } \\
\text { VELOPED } \\
\text { WTH MODERN } \\
\text { TECHNICS }\end{array}$ & $\begin{array}{l}\text { HISTONY OF } \\
\text { TAIWAN ECO- } \\
\text { NOMICS ANO } \\
\text { OLD MAPS }\end{array}$ \\
\hline \multicolumn{3}{|c|}{$\begin{array}{l}\text { TOTAL } \\
\text { YEARS }\end{array}$} & $\begin{array}{l}\text { ACCRETION RATE } \\
\text { LAN-PING } 282 \text { M/YEAR } \\
\text { 2 TSENG-PA } 163 \mathrm{M} \text { YEAR }\end{array}$ & & \\
\hline
\end{tabular}


FIo

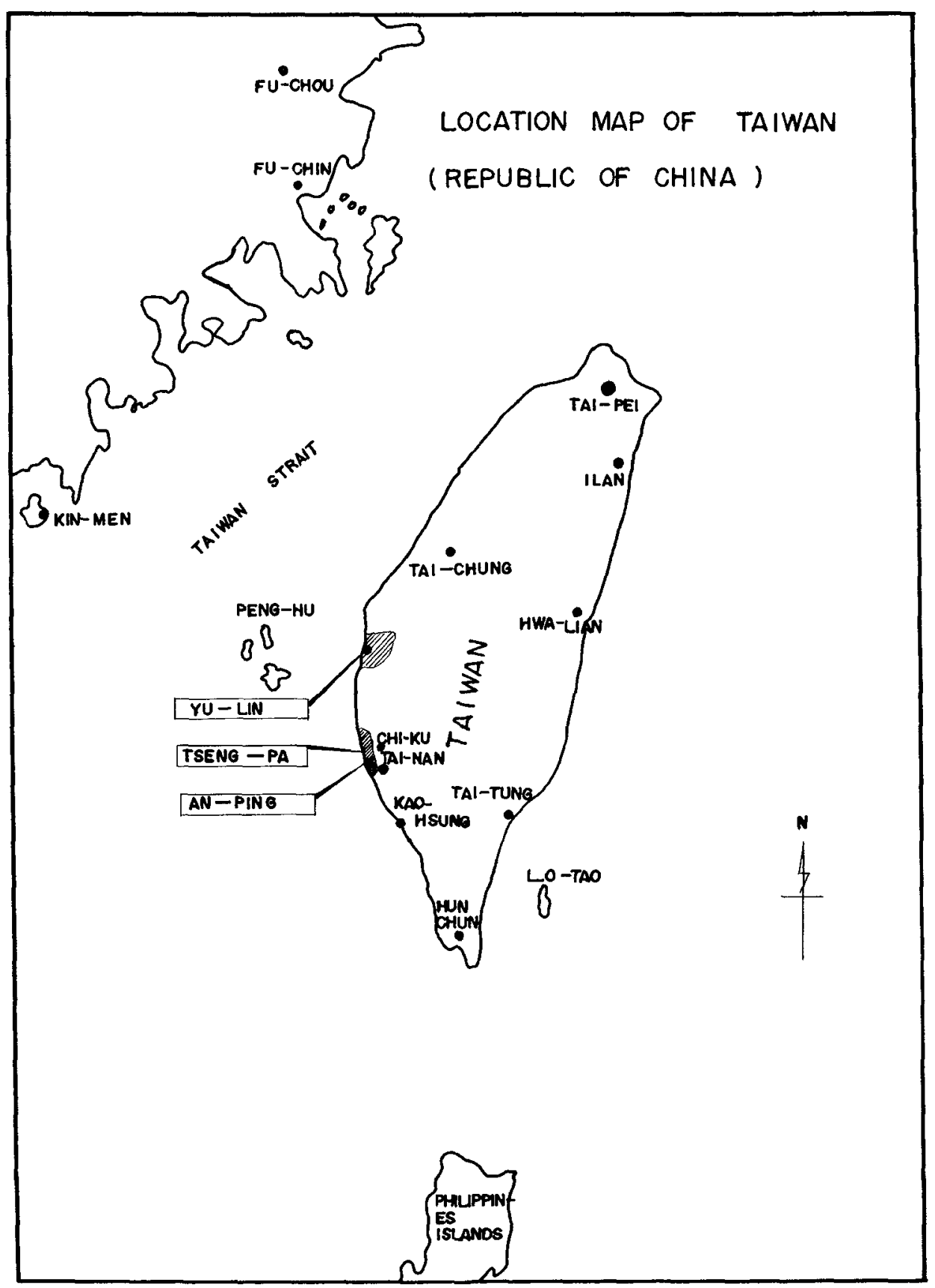




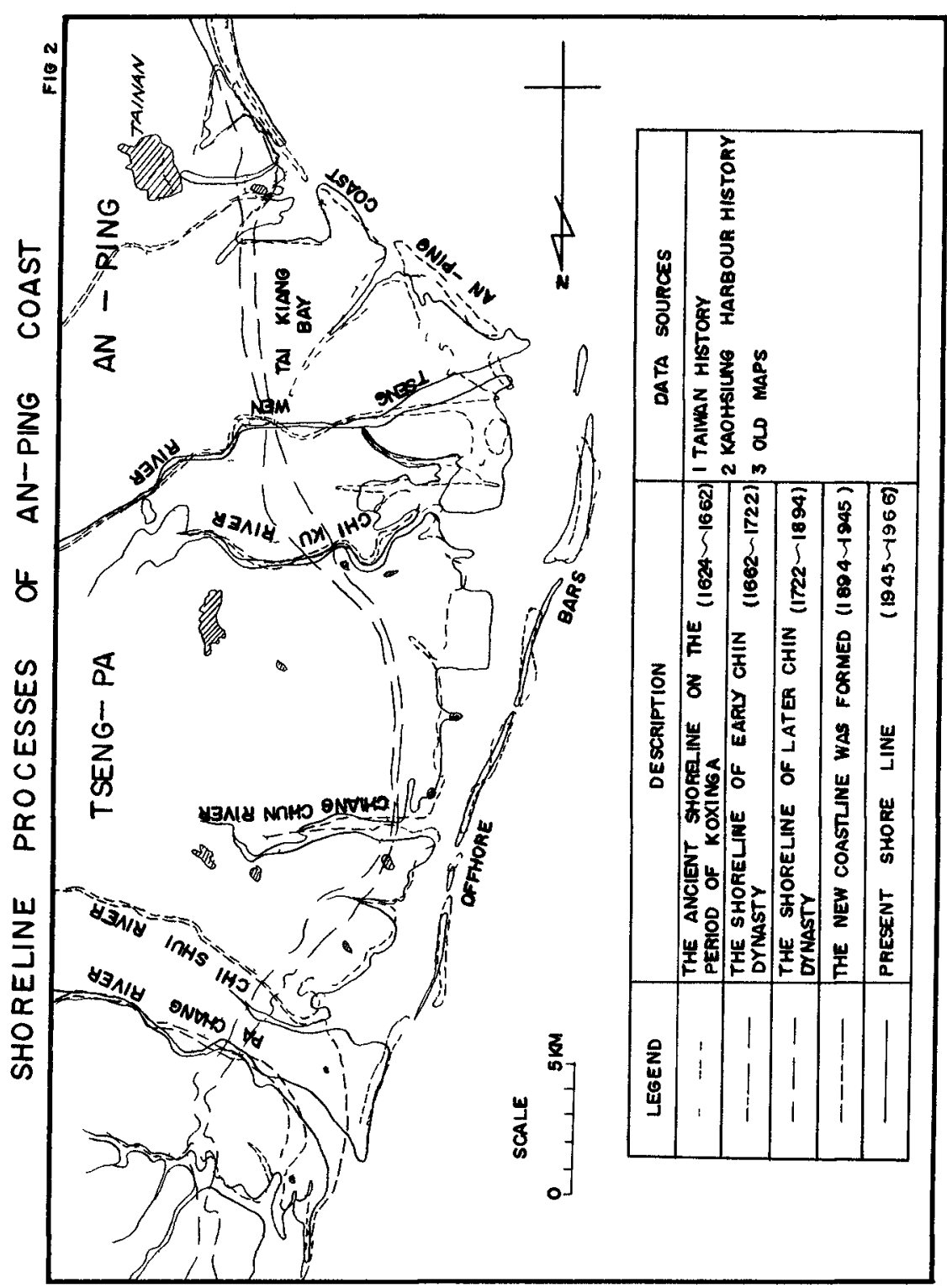






Fig 3 Map Showing the Varzation of Shoreline on Tseng-Wen River to Chıang-Chun River 


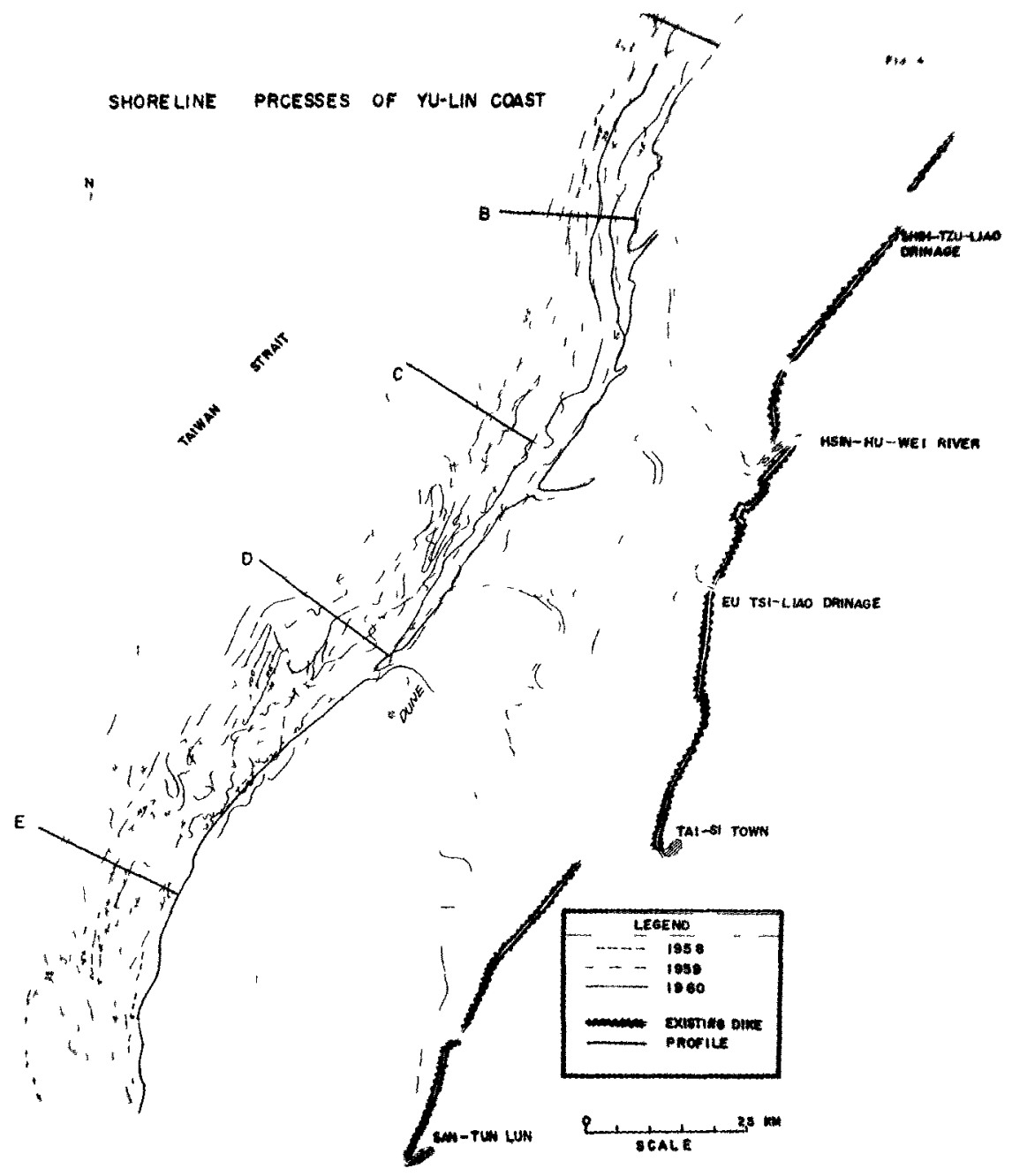

Fig 4 Shoreline Processes of Yu-Lin Coast 


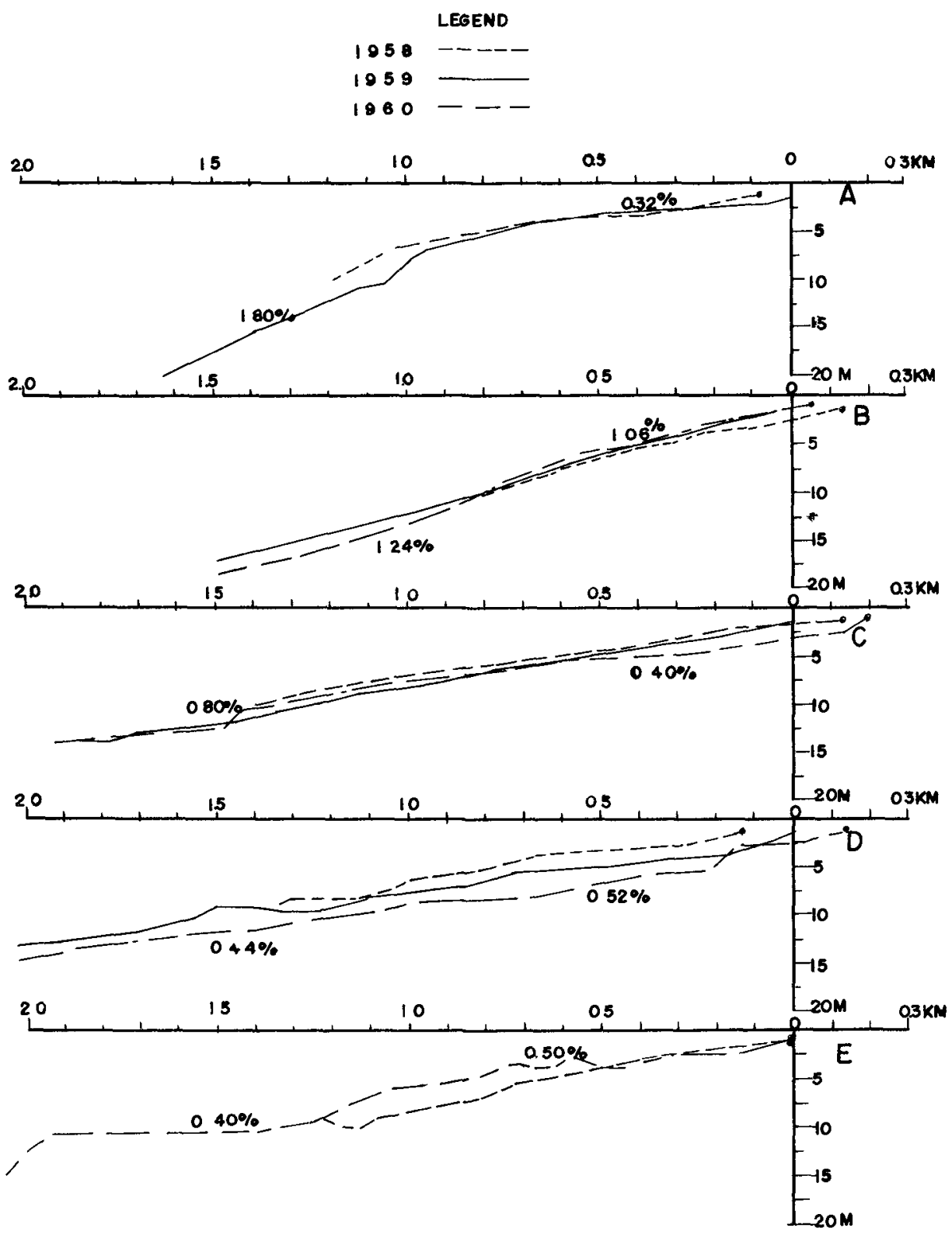





\section{CHAPTER 45}

STUDY OF MUDBANKS ALONG THE SOUTHUEST COAST OF INDIA

by N.S.MONI

\section{ABSTRACP}

The formation and behaviour of 'Mud Banks' is a phenomenon peculiar to the Southwest Coast of Indza. They are unique in their formation as well as functions. The paper deals with the history of mudbanks, their locations, causes of their formation, the nature of material and thezr sources. The bohaviour of the madbaiks and their influence on the stability of the cosst is also reported. The mudbanks act as storehouses of littoral material. ItIs also the initiator of erosion on its downdrift areas. Itis noted that the material stored in the mudbenks must be advantageously utIlised in stabilising the shores adjecent to It and for reclamation. Of extrome importance is the recognation that mudbanks are closely associated with the stability of the Southwest Coast of India and Is a factor to be reckoned in any protzeane of coastal development or protection inthis area.

\section{INTRODUCTION}

In the Southwest Coast of India, certain inshore areas have a special property of dampening wave actzon and producang regaons of calm water even during the rough monsoon sesson due to the disstpation of wave energy in the large quantity of colloldal suspension in the region. These regions are generally known as the 'Mud Banks''. They are unique in thenr formation as well as functions. The mudbanks form part of the sediment sctrvity along the coast. They directly influence the equilibrium conditions of shore in its vicinity. The mudbank regions are considered to be a 'boon' by the local populace as these aress which are calm during the monsoons abound in Prams, Sardines, Mackerals and Soles.

\section{LOCATION}

There are four well known mudbenks along the Southwest Coast of India- one near Cochin, one near Alleppey and two near Kozhlkode.

**

Deputy Director, Coastal Engineering Division,

Kerala Bnganeering Research Institute, Peechl, Kerala, INDIA

(now on deputation,as) Colombo Plan Participant (0.T.C.A)

Port and Harbour Research Institute, Hegase, Yokosuka, JAPAN 


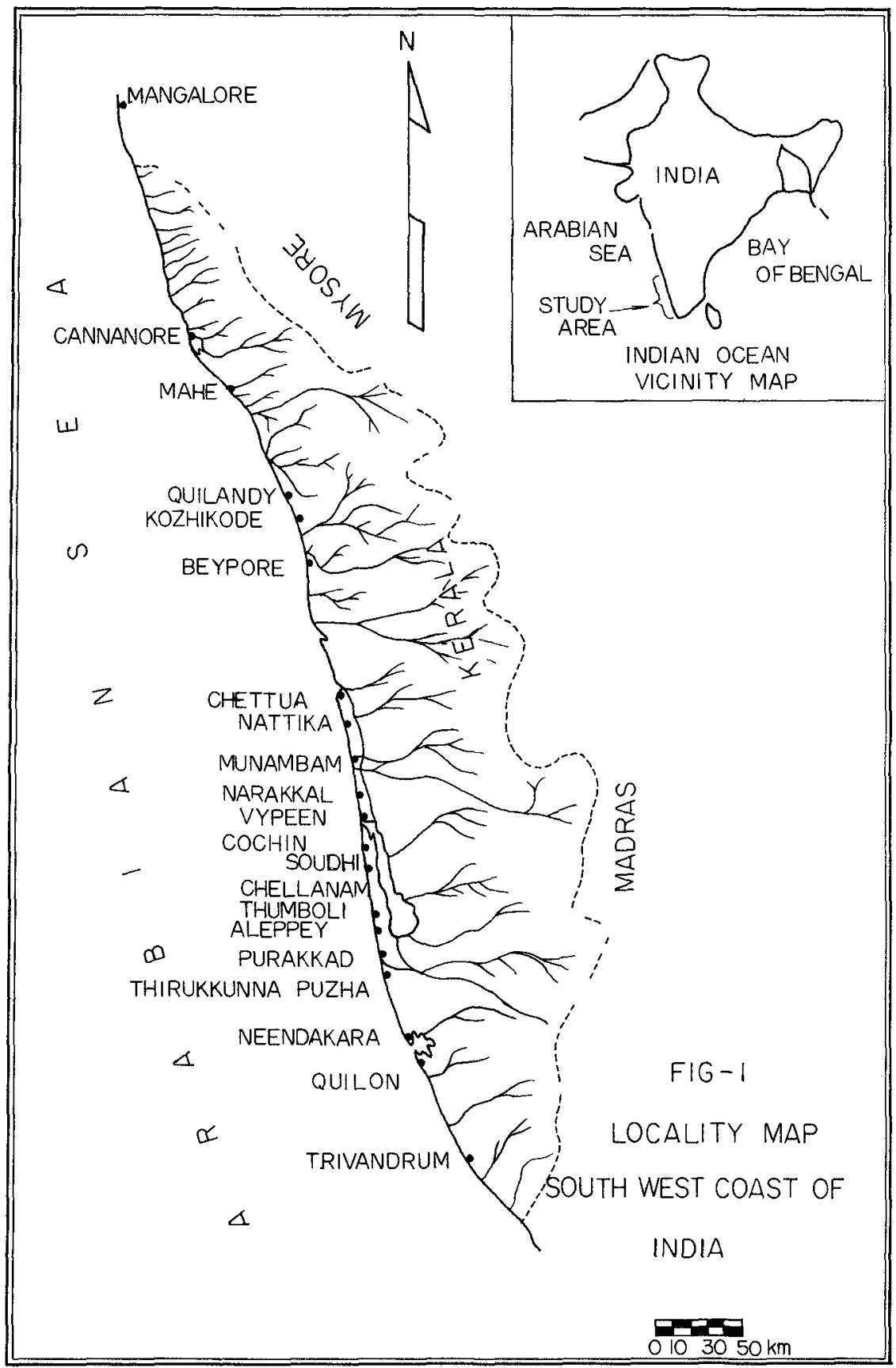


Historical records relating to the mudbanks at Cochin and at Alleppey are avalable from 1840. Of these four, three mudbanks are elther at or near the outlets of rivers and lagoons while the fourth one at Alleppey is not any where near an outlet. There are other mudbanks which are known to have existed during the past twenty years. They are located at.

Mahe (South of Mohe river outlet)

Beypore (South of Beypore river outlet)

Nattika (South of Chettua outlet)

Munambam (Soth of Percyar river outlet)

Soudhi (South of Cochin outlot)

Thumboli (North of Alleppey)

Thrikkunnapuzh (South of Thottapalli Artificial Cut )

The atudy area of $560 \mathrm{~km}$ and the location of the mudbanks are given in Figure 1

\section{HISTORY OF MUDBANKS}

The earliest known record of the expstence of mudbanks dates as far back as 1678, in Pankerton's 'Collections of royages and travels' given in the Admunatration Report of 1860 of Travancore (India). Dr.KIng, of the Geological Survey of India in his report 'Conszderations on the Smooth water anchorages or mudbanks of Narakkal (Cochin) and Alleppey' on Travencore Coast"(1881) has given an account of the migration and formation of the two mudbanks. According to his report, the range of magration of the Alleppey mudbank 18 trentyfour km between Alleppey and Purakkad and that of the Cochin mudbank 28 twenty $\mathrm{km}$ between Narakkal and Coch2n. an organised attempt to study the mudbanke was made by Sir R.C.Bristow, and these are detarled in h1s books 'History of Malabar Mudbanks', 1938 Vol.I and Vol.II. Th1s throws light on the origin, formation and other features of the mudbanics. The hastorical date of the cochan and Alleppey madbanks are giren in Figures $2 \mathrm{~A}$ and $2 B$.

\section{Cochin Mudbank}

The record of history of this mudbank 18 avallable from 1841. No perceptible change was reported between 1841 and 1861. From 1861 to 1881 it has moved southwards for $15 \mathrm{~km}$. In 1890 the mudbank was located north. Till 1924 the movement was gradual towards south. IH 1937, it crossed the Cochin approach channel and caused considersblo silting. It moved further south. Between 1950 and 1968 the mudbank was loceted both in the north and south of the Cochin outlet.

\section{Alleppey Mudbenk}

This mudbank was firat reported in 1678. Again it was located in the same region in 1725 . In 1827 it was reported $24 \mathrm{~km}$ south. In 1860 and in 1890 It was located near the Alleppey Plex (noxtherm limit). In 1896 it was $10 \mathrm{~km}$ south and in 1902, 25km south. In 1924 to 1928 It was located at the 1 lleppey Pier.Then onwards it was moring to the south.In 1937 it was 8km to the south of the Plex. From 1950 to 1968 It was located between 8 to $16 \mathrm{~km}$ south of the Alleppey Pler. 


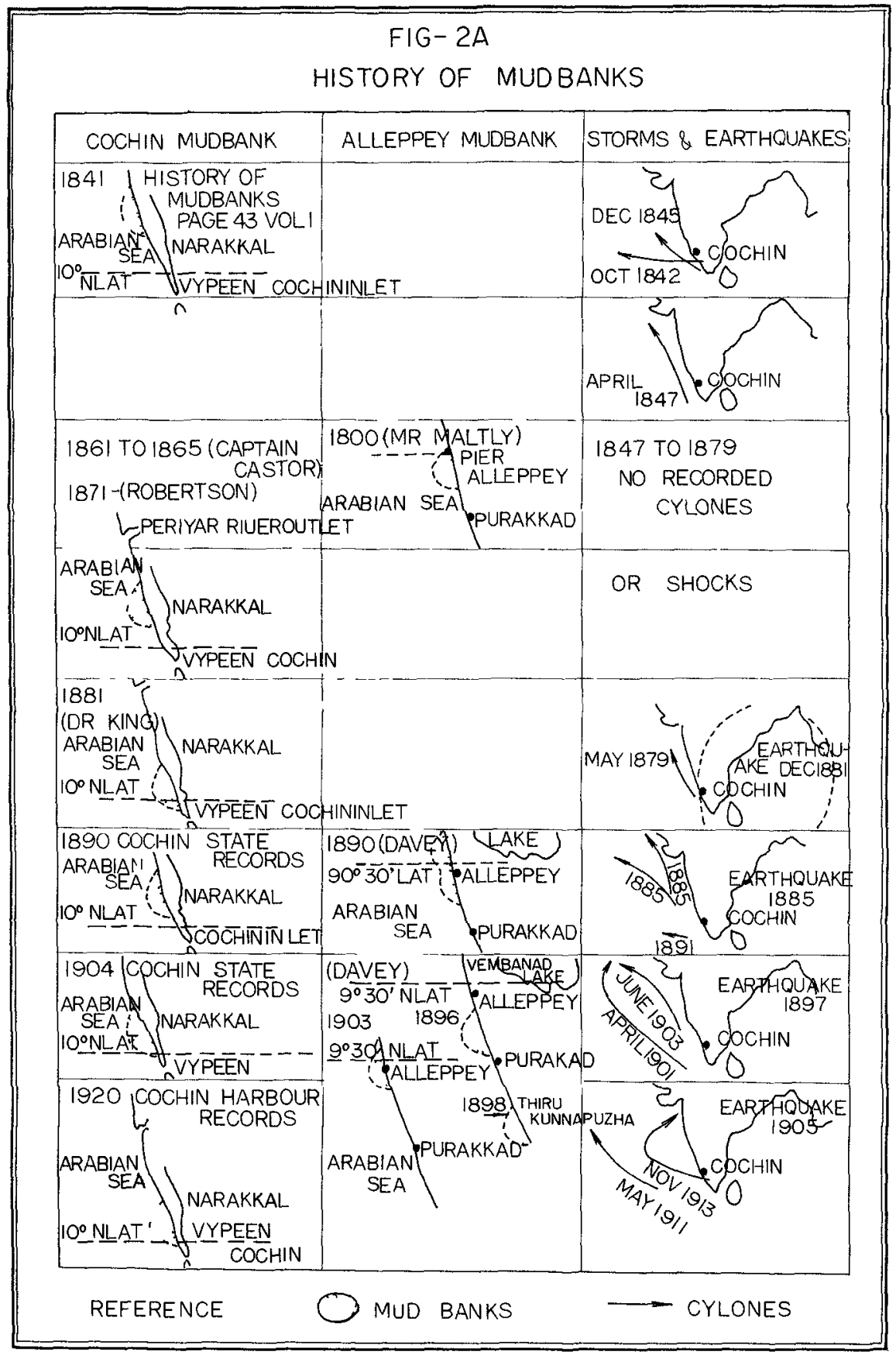




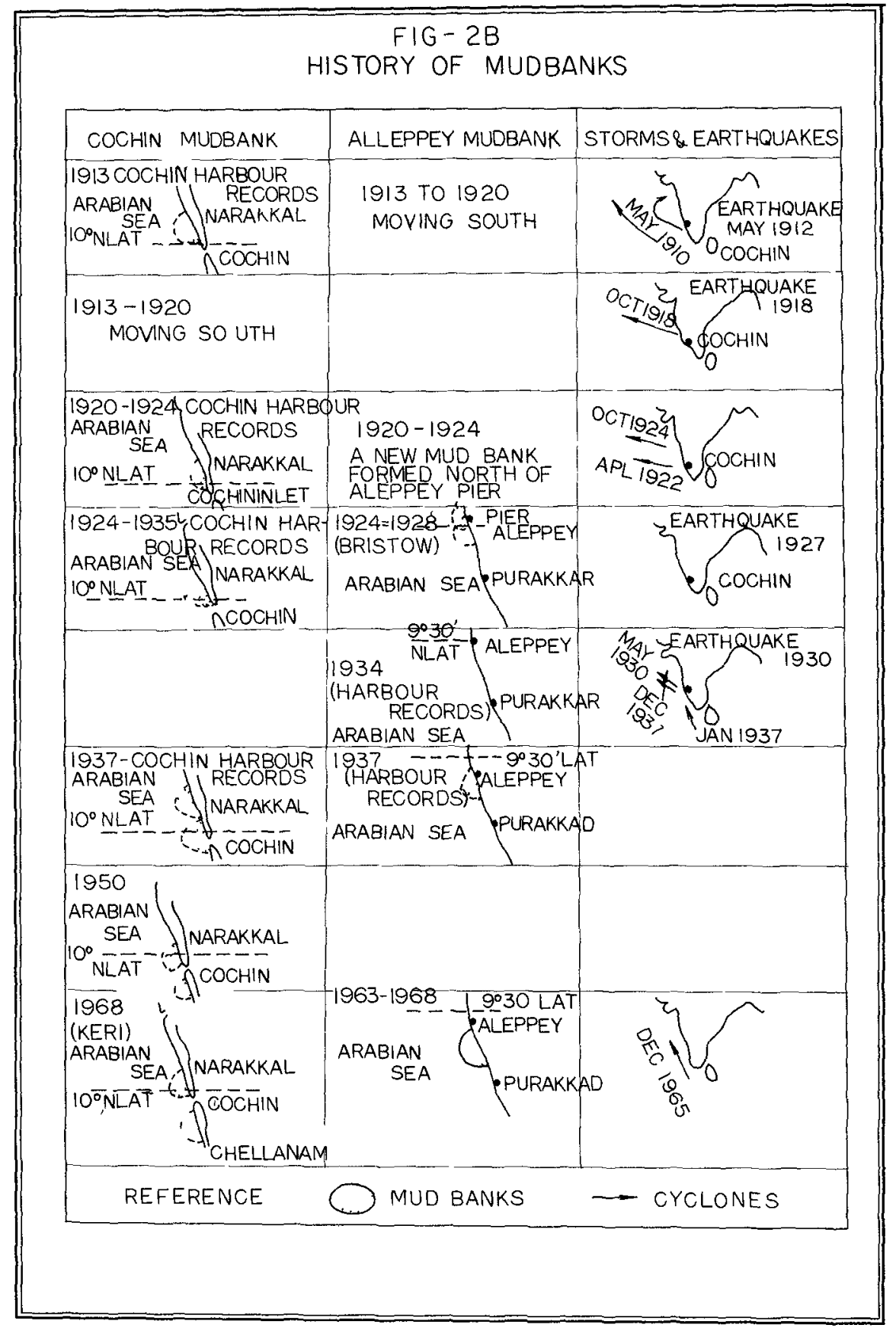




\section{PHYSICAL FACTORS AND LITTORAL MOVEMENTS}

The physical factors, shore effects and littoral drift have bearing on the mudbanks and and are detalled below.

Winds, Waves and Tides

The data was developed from the darly reather charts of the Indian Metereological Department. The predominant direction of the prevalling winds along the shore 18 from west and northwest during May to September(monsoon season). From the wave rose diagrams it is observed that during the monsoon season, the predomanant direction of waves 18 from west and those from northwestwest are contributory factors during July, August and September. The largest computed waves were between $5 \mathrm{~m}$ and $6 \mathrm{~m}$ in height The moan wave herght can be taken as $1.8 \mathrm{~m}$ with a period of 8 to 10 seconds. The tides are semidiurnal and vary with a mean range of $0.8 \mathrm{~m}$ at the south end to $1.8 m$ at the northern 1Imit. Storm tides occur all along the coast during the monsoon.

Offshore and Foreshore Zones

The continental shelf of thrs coast has a gradual slope upto 10 fathoms after which there 18 a steep fall. The distance of the 100 fathom line from the shore varies from $82 \mathrm{~km}$ at the north to $45 \mathrm{~km}$ at the south. In general the foreshore has a slope of 1 on 5 to 1 on 10 above LW with flatter underwater slopes. The foreshore slope in the mudbank regions are flatter compared to those on the adjacent sides. A typical example is given in Figure 3.

Field observations at selected reaches in the coast indicate that there are seasonal changes in the beaches. Erosion is experienced from April to September, after which the beach begins to accrete. The berm crest in certain cases fluctuates within wide limits - even upto $70 \mathrm{~m}$ in a season.

\section{Littorel Drift}

Littoral drift varies with the seasons of the year but as predomanantly from north to south. This predomznance is evident $\bar{A}_{\mathrm{m}}$ from the analysis of wave data, evidence from headlands and bays, migration of spits and inlets and observations of grouns and jetties.

\section{NATURE, FORHATION AND ORIGTN OF MUDBANKS}

Geologically the Southwest Coast of India ls of recent age, Its formation dating back to the early Tertiary period. Borings at Cochin show that there are deposits of alluvial material for $100 \mathrm{~m}$ to $125 \mathrm{~m}$ overlying rock. It is noticeable that the portion of the coast from Kozhikode to Trivandmu where the mudbanks are confined is coincident with the presence of alluvial belt backed by laterite deposits at no long distance from the cosst. The general distribution of sediments in the continental shelf of this coast indicate that 


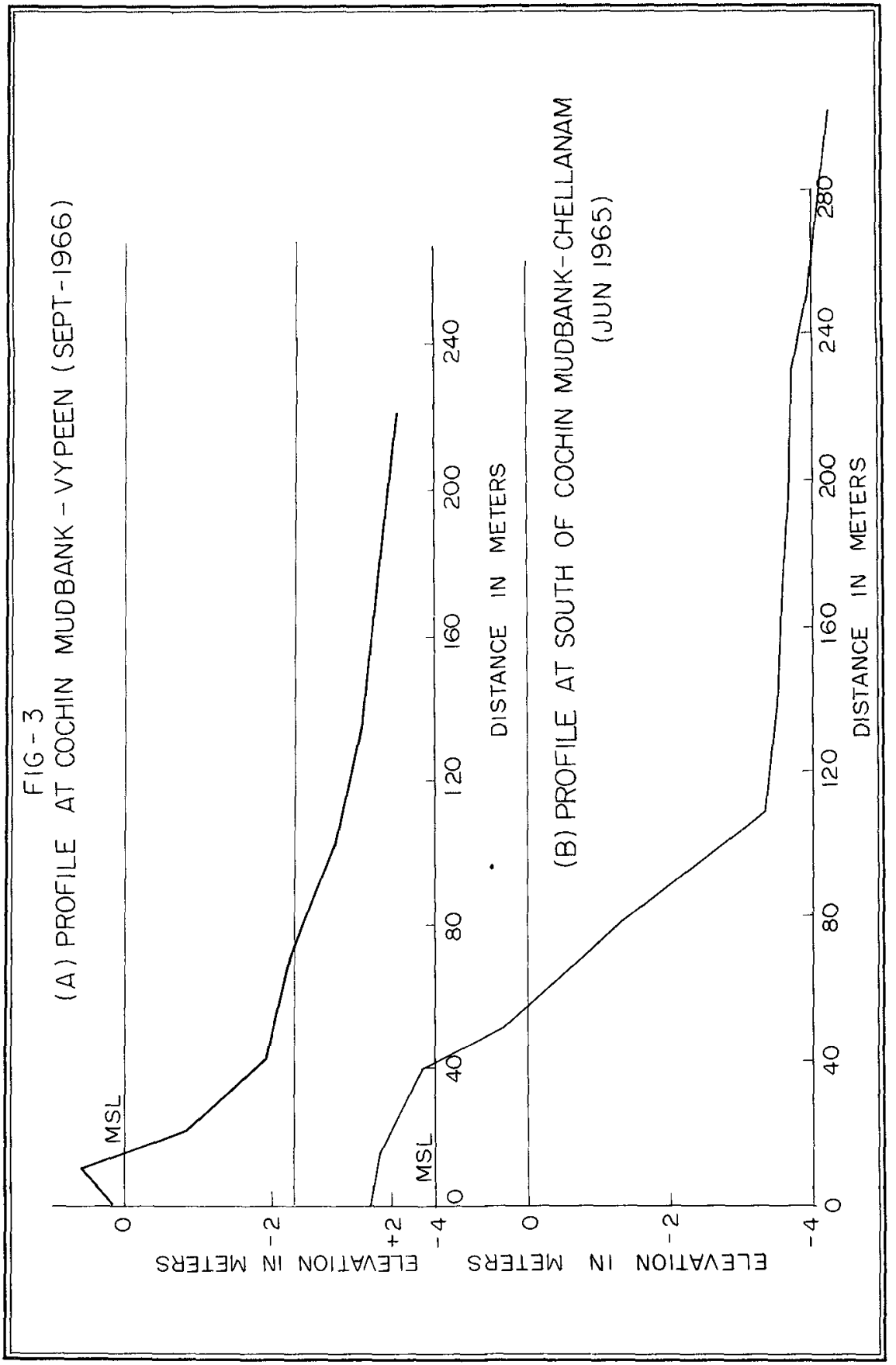


the inner shelf (upto 20 fathoms) consists of greenish black, poorly sorted clayeys and clayey silts and the outer shelf (20 fathoms) to 100 fathoms) consists of well sorted fine and medium sand with abundant shell fragments.

Analysis of samples from the seabed in the mudbank regzon reveal the followng. They are greenzsh black and deep slaty in colour and cohesive aind plastic to touch. Granulometric analysis: indicate that it consiats predomnantly of silt and clay fractions, with less than $5 \%$ of fine sand. (Figure 4) The heavy mineral fraction average $2 \%$. The camples taken at the top of the bed reveal that the top layer of the bottom mud is in a state of liquidity.

Samples from the sea bottom upto 10 m adjacent to the mudbanks and also from the inland backwaters were analysed and compared with the mudbank samples. The samples from the mudbanks and from the adjacent sea bed showed close resemblance and simllar characterestics. The granulometric analysis of samples taken from inland backwaters and mudbanks also showed similar charecterestics.

The mudbenks are situated near and not too distant from the river outlets except in the case of Alleppey mudbonk which 15 separated from the Vembanad backwater by a narrow strip of allurzal belt.

The analysis suggeats that the origan of the material in the mudbanks is from the deposits of laterzte and alluvial formation of the interzor brought by the rivers.

The madbanks are formediby a single or a combination of the undermentioned causes:

i) By the gradual depositzon of clay and silt brought by the rivers during the monsoon sesson

1i) By the throwing up of the already existing mud in the seabed by the waves $d$

izi)Their exists a vaterbearing strata along the coast.The appearence of the mudbanke 18 councident when the waterlevel in the backwaters adjacent to the coast 18 high. When the water level rises in the backwatwer during the monsoon, it is likely that silt and clay way be throw up through the waterbearing strata connecting the backwater and sea.

It Is Interesting to note that the effect of the mudbanks are felt about a week after the beginning of the monsoon. (Juscilvers and backwater systems discharge large quantities flood discharge and sediments into the sea through the numerous perennial and seasonal outlets and by seepage through barrier beaches separating the backwaters from the sea. Further the sea bed 18 agitated by the action of the waves and the material is thrown in suspension. The monsoon swells provide a continuous source of energy to maintain the colloidel suspension which alternately dampen the waves progressively and finally the sea becomes complotely calm within the area.

\section{BEHAVIOUR OF MUDBANKS}

Observation of the Cochin and Alleppey mudbanks Indicate that they change in shape and size with the sessons. The length along the coast varies from $6 \mathrm{~km}$ to $10 \mathrm{~km}$ and the midh upto $8 \mathrm{~km}$. By tracking the 


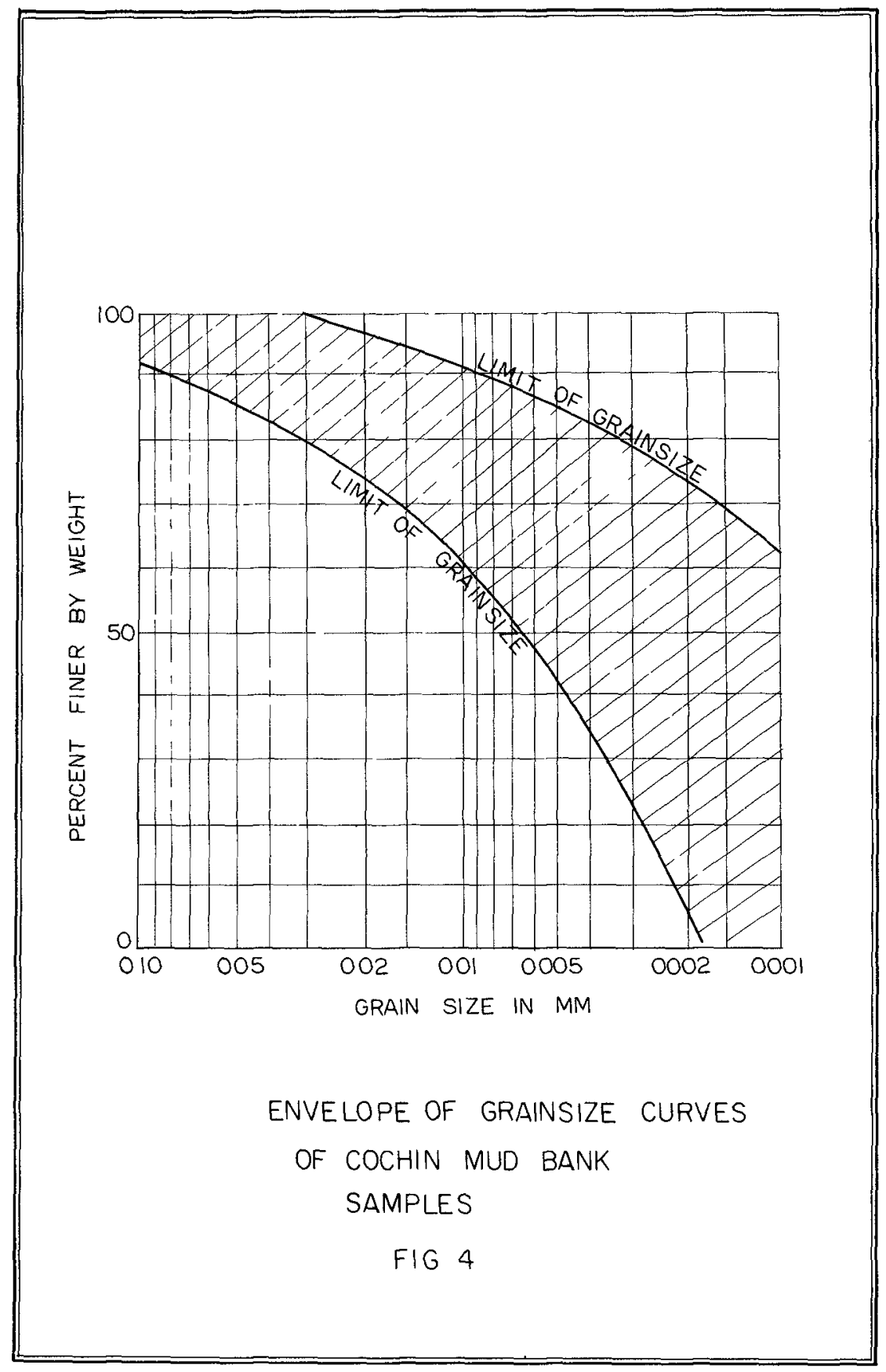


mudbanks it is noted that they migrate with the seasons, but ths predominant dirsction of movement is towards south (during June-July) These confirm the findings of ths earlier observations taken in 1937-1938. It 19 also noted that the migration of the mudbanks conform to a cyclic patterm similar to those of the uncontrolied inlets in this coast.

\section{MUDBANKS AND SHORE STABILITY}

The sffect of ths madbanks on ths squilibrium conditions of the coast adjacent to it was studied with particular reference to the Cochin and Allsppsy mabanks. The shors sectors located south of the two mudbanks are subjected to progressive shore recession. An sxamination of historical evidsncs and fisld observations indicats that ths mudbanks provide ths ksy to ths shore stability in thass two sactors.

Mudbanks affect the coastal procsssss in the following ways

1) Traps the littoral matsrial from ths updrift side and thereby prevsnt its downcoast movsment

11) Causes refraction of waves on its sides

11i)Causes accretion within the mudbank arsa

\section{Trapping of littoral material}

As the littoral material transported by ths alongshore movement reaches the mudbank, Its flrther movement 28 arrested as a rsault of ths dampsning of, the warss. Hence the in ths immsdiate downdrift is starved of littoral supply and the coast is eroded to maks up for ths daficisncy. Comparison aurvets prepared from authentic maps indicate that thare is consistent retrogression of shoreline, especially in the zones south of the Mlleppey and Cochin mudbanks. Ths shorslins had rsceded $360 \mathrm{~m}$ during the past 120 years south of ths Alleppsy mudbank and $600 \mathrm{~m}$ during the same period south of the Cochin madbenk (Isfisr Figure 5).

\section{Befraction of waves on the sidss of ths mudbank}

The mudbank act as a long, mds breakwatsr. Aa the waves approach the mudbank, they tsind to refract. The refracted waves turn towards the mudbank on 1 to leesids causing a reversal in ths gensral direction of ths drift from a nodal zone in the downcoast and causes movoment of material towards ths mudbank from its lesside. Observations confirm this phenomenon. Th1s aggravates the eroding tendency on the downcoast sids.

Accretion within ths mudbank

Within ths mudbank, ilttoral matsrial accumulates, thereby accreting the shore thin it. Abssncs of vaves preclude littoral movement through the mudbank and hence materials once trapped within it is prevsnted from moving out. The mudbank acts as a storehouse of littoral material. There is progressive growth of hsadland. The shorelins has advanced by $1250 \mathrm{~m}$ during the past 50 years in the Cochin zone and by 500m during ths past 15 years in the Alleppey zone. 


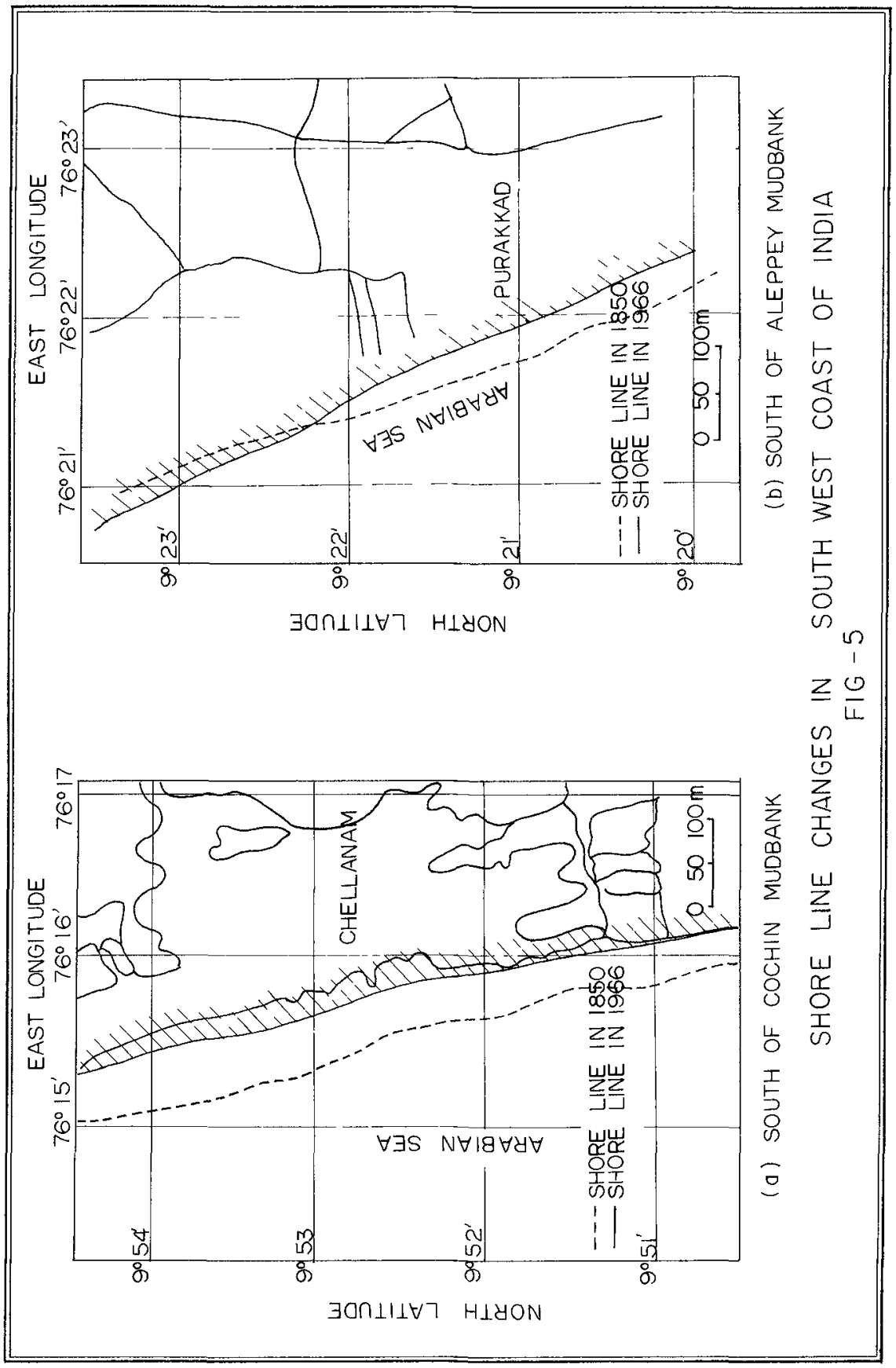




\section{BVALUATION}

Mudbanks have the speczal property of dampening wave action and producing areas of calm water even durzng the roughest monsoon Beason. They decisively influence the shore processes and are effoctirely disturbing the equilibrium conditione of the coast adjacent to them. They are acting as storehouses of littoral material. The material stored in the mudbanks must be advantageously utilised in stabilising the eroding shores on its downdrift side and for reclamation by a coordinated programme. Of extreme importance is the recognition that mudbanks are closely associated with the stability of the southwest coast of India and is a factor to be $\mathbf{x}$ studied further and reckoned $2 n$ any programme of coastal development or protection in this area.

\section{REFGRENCES}

1.Bristow,R.C., "The Hבstory of Malabar MudBank' 'Vol.I, Coch1n Port Trust., 1938 .

2.Bristor,R.C.,' 'The History of Malabar Mudbank' ', Vol.II, Coch2n Port Trust., 1938.

3.Joglekar,D.V.,Gole,C.V., Mulekar,S.N., 'Studies on siltation of Cochin Port' , Proceedings of XIX International Navigation Congress ., 1957.

4.Kerala Enganeer2ng Research Instztute, 'Annual Report' ',1964.

5.Damodaran, R., Hridayenadhan, C.,' 'Studzes of the MudBanks of the Kerala Coast" ', Symposzum on Coastal and Nearshore Oceanography, C.S.I.R., 1966 .

6.Monz,N.S.,Nambiar,K.K.K.," 'Study of seasonal variationsin the Beaches of the Kerala State', Irrigation and Power Journal, Vol.24 No.2,1966.

7.Richard T.Russel, 'Rzvers, Plazns, and Sea Cossts'', University of California Press, Berkeley.,1967.

8. Monz,N.S.,George, M.M.,Nambiar,K K.N.,' 'Study of Beach Profiles in \& near the Suff Zone of the Kerala Coast taken using Sled' ', Thirtyeighth Annual Research Session of C.B.I.P.,1968.

9. Kerala Enganeerang Research Instztute," Studies on Coastal Erosion" (Internal Report), 1969. 


\title{
CHAPTER 46
}

\section{TURBULENCE AND SEDIMENT CONCENTRATION} DUE TO WAVES

\author{
Kiyoshi Horıkawa \\ Professor \\ and \\ Akira Watanabe \\ Postgraduate
}

Department of Civil Engineering

University of Tokyo

Tokyo, Japan

\section{ABSTRACT}

A new electrolytic turbulenoe transducer has been developed in order to measure the turbulent velocity fluctuation superposed on the oscillatory flow velocity The aim of the present paper is firstly to describe the outline of this transducer and secondly to introduce some of the experimental results The main items of the results are, 1) the vertical distribution of turbulence intensity averaged over one wave cycle, where the turbulence is induced by ripples which appear on the movable bed of wave flume, and 2) the correlation between the turbulence intensity and the characteristics of sed iment particles at the same level such as their fall velocity and sediment concentration

\section{INTRODUCTION}

The authors have had a strong interest in the fundamental mechanism of suspended sediment due to wave action In the previous papers the authors have described their results related to the following subjects

1) The vertical distribution of suspended sediment concentration due to waves (Hom-ma and Horlkawa, 1962, Hom-ma, Hor Ikawa and Kajıma, 1965),

2) The general relationships to determine the size of sand ripples which appear on the movable bed owing to progressive waves (Hom-ma and Horikawa, 1962),

3) The critical water depth for the onset of bed material movement due to waves (Horlkawa and Watanabe, 1967), and

4) The measurement of velocity distribution in the vicinity of bottom boundary in the oscillatory flow field, from which the characteristics of shear stress and of eddy viscosity been evaluated (Horlkawa and Watanabe, 1968) 
All of these studies are successive approaches to clarify the basic mechanism of suspension phenomena in a wave field

The following equation is commonly used as the fundamental equation for determining the vertical distribution of suspended sediment concentration averaged over one wave cycle m

$$
\frac{d}{d z}\left(K_{z} \frac{d \bar{m}}{d z}\right)+w_{0} \frac{d \bar{m}}{d z}=0
$$

Where $w_{0}$ is the fall velocity of suspended sediment particle, $K_{z}$ is the vertical eddy diffusivity, and $z$ is the vertical axis taken upward from the bottom In order to solve the above equation the value of $K_{z}$ should be evaluated beforehand, hence in the previous papers the authors treated the above problem based on the assumption that $K_{z}$ could be replaced by the eddy viscosity $\epsilon_{m}$ This kind of treatment should be valuable from the englneering point of view, but may not be powerful to clarify the basic questionaries on the suspension phenomena, such as how to determine the concentration at a certain elevation near bottom which is used as a measure to define the vertical distribution of suspended sediment concentration

In the region where the sediment movement is active, the flow in the vicinity of sea bottom is, generally speaking, in the turbulent flow condition, and the vortices are generated behind sand ripples which are formed along the sea bottom These vortices induce the turbulence with higher frequencies in the oscillatory flow field These processes stated above must keep the motivative activities on suspension phenomena, hence it seems to be of essential importance to clarify the turbulence characteristics in an oscillatory flow field in order to understand the real state of questioned phenomena

\section{ELECTROLYTIC TURBULENCE TRANSDUCER (ELETT)}

In order to measure the mean velocity in an oscillatory flow field, Jonsson(1963) applied a miniature current meter, while Iwagaki(1969) applied a hot-film anemometer on the other hand the authors (1968) used the hydrogen bubble technique to measure the velocity in a thin boundary layer

With regard to the turbulence in an oscillatory flow no data is avallable to be used The main reasons why the measurement of turbulent velocity fluctuation in the vicinity of bottom boundary under the osciliatory flow is difficult are as follows

1) The direction and magnitude of the main flow in the oscillatory flow fleld vary in time differing from the states of steady flow field

2) The time variation of flow circumstance has been occurred in a rather short period such as one to several sec 
3) In the case of rippled rough bottom, a vortex with rela-

tively blg size(the diameter of vortex being one to several $\mathrm{cm}$ In laboratory) has been formed behind each ripple Under these complicated situations it is awfuliy difflcult to apply such a standardized measuring technique for obtaining each component of turbulent velocity fluctuation as a measurement of hot-film anemometer, the reliablilty of which has been verified in steady water flow Blnder(1967) reported the applicability of the electrokinetic turbulence meter, the principle of which is to measure the electric potential fiuctuations induced by the translation of electric double layers The disadvantage of this transducer is represented by the fact that the output is zero under the laminar flow condition That is to say, the output is affected by the flow characteristics even though the current velocity is the same

From these points of view the authors have been devoting their efforts to develop a new electrolytic turbulence transducer, herein after the authors will call it shortly as ELETT, and have a confidence in getting reliable data by using ELETT

Figure 1 shows the schematic diagram of the instrumentation system When the $D$ C voltage is charged between a cathode of platinum wire and an anode of carbon rod, both of which are immersed in water, some part of water nelghbouring those is electrolyzed to lons or gases The flow produces a variation in electric resistance between the electrodes Therefore the out-of-balance electric current in the Wheatstone's bridge is affected by the flow velocity at the cathode The actual length of sensing element $1 \mathrm{~s}$ about $1 \mathrm{~mm}$

In Figure 2 is shown the calibration curve between the velocity $V$ and the output of sensor $I$ Figure 3 shows the direction-sensitivity $M(\varphi)$ of the ELETT sensor, where $\varphi$ is the angle between the flow and a certain direction normal to the sensor

When a probe which consists of a palr of sensors as shown in Figure 1 is used, the ratio between the output from one sensor and that from the other depends on the flow direction

Table 1 is to summarize the processes how to determine the velocity component in a certain direction The output I is a function of the velocity $V$ and the direction angle $\varphi$, and is likely to be separated into two independent functions $S(V)$ and $M(\varphi)$ as shown in Equation(l) The calibration curves for $S(V)$ and $M(\varphi)$ are glven as shown in the previous Figures 2 and 3 By using Figure b in Table 1 , a newly defined function of $K(\varphi)$ can be calculated as shown in Figure $c$ The absolute value of $\mathrm{V}$, the velocity component $U$, the direction angle $\varphi$, and the outputs $I_{1}$ and $I_{2}$ are defined as given in the stated diagram Hence the outputs $I_{1}$ and $I_{2}$ are written as Equations (11) and (111) respectively, and the output ratio $I_{1} / I_{2}$ is expressed by the stated relation in Table 1 From Figure c the absolute value of direction angle $|\varphi|$ is deter- 


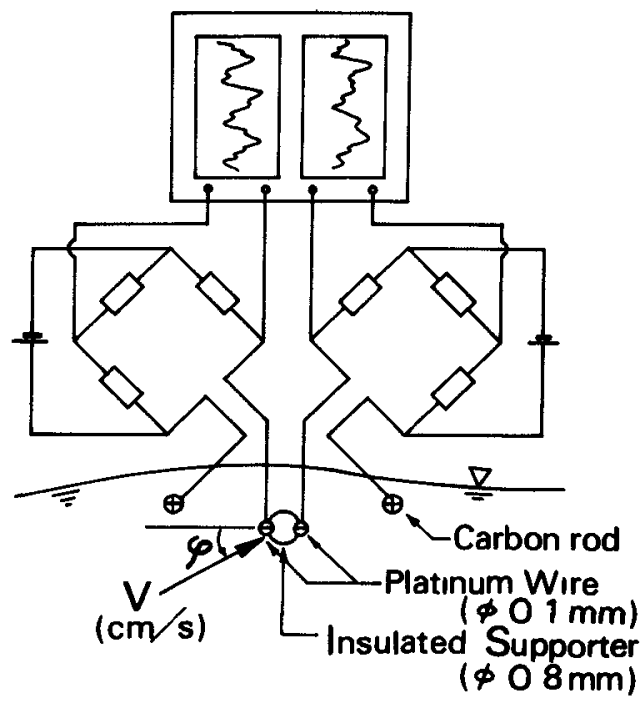

Fig. 1 Instrumentation system for velocity measurement.

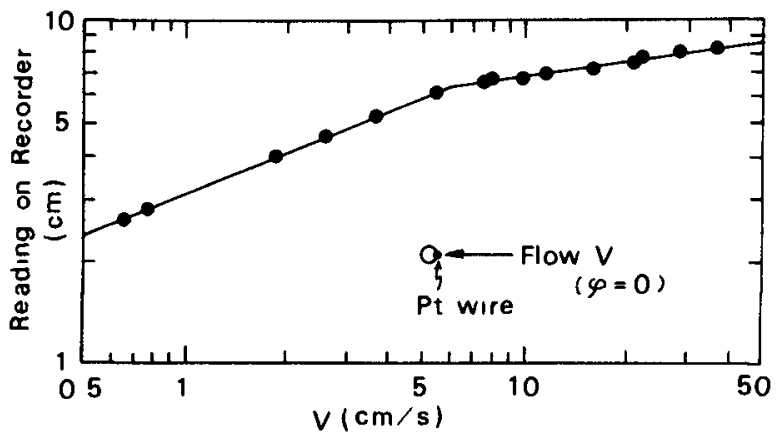

Fig 2. Calibration curve for ELETT 


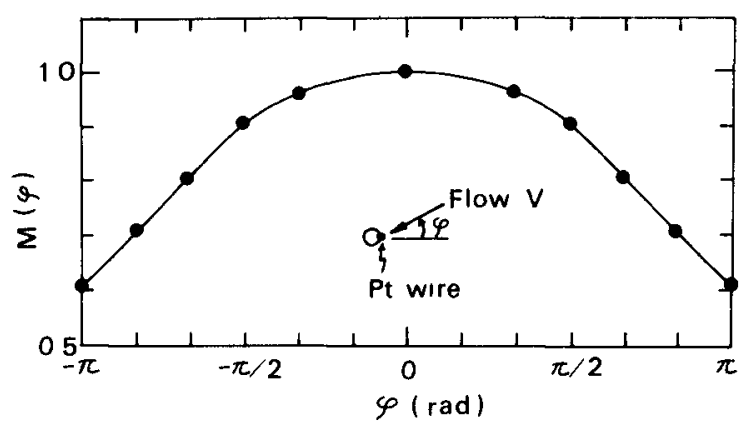

Fig 3 Direction-sensitivity of ELETT

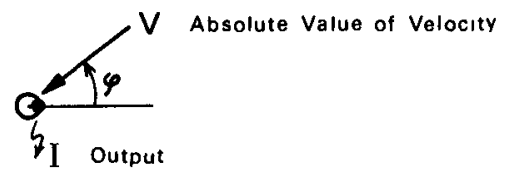

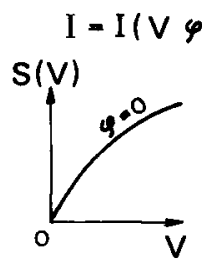

Fig a

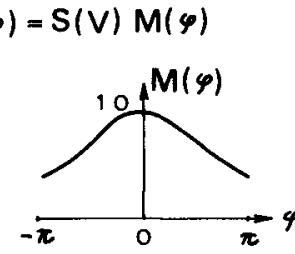

Fig b

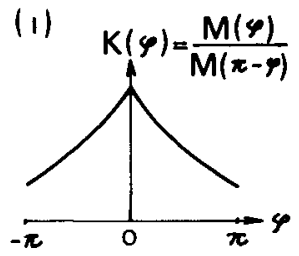

Fig C

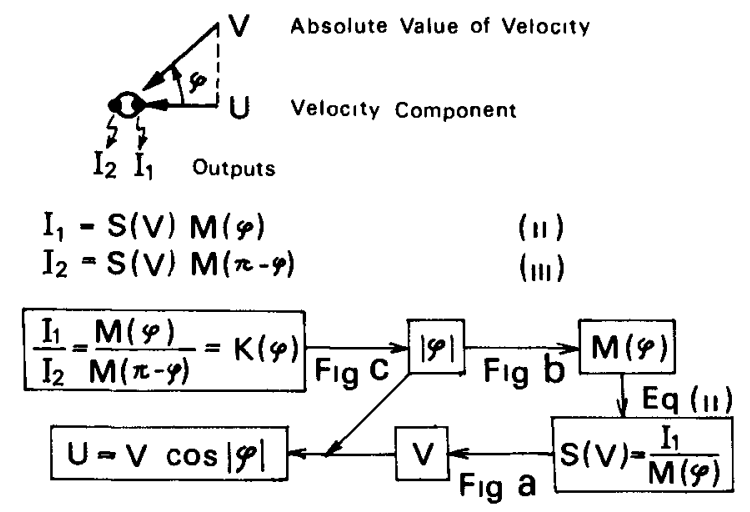

Table 1 Process diagram of velocity component reduction 
mined By using Figure $b$ we can get $M(\varphi)$, from which the value of $S(V)$ can be read by the help of Equation(11) Next the absolute value of $V$ can be obtained by using Figure $a$, and hence the value of $U$ is also calculated

\section{TURBULENCE CHARACTERISTICS MEASURED BY ELETT}

The laboratory measurements by ELETT was conducted in a wave flume under the following conditions the water depth was $30 \mathrm{~cm}$, the wave helght and perlod of fundamental mode were $7.93 \mathrm{~cm}$ and $135 \mathrm{sec}$ respectively, and the water temperature was $80^{\circ} \mathrm{C}$ The medium diameter and the sorting coefflclent of standard sand placed on the flume bed with the thlckness of $15 \mathrm{~cm}$ were $020 \mathrm{~mm}$, and 115 respectively The rise and pitch of ripples which appeared on the flume bottom were $095 \mathrm{~cm}$ and $57 \mathrm{~cm}$ respectively

Figure 4 indicates one example of the time history of the horlzontal velocity component whlch was measured by ELETT The thick line in this figure is for the measured horizontal velocity component $U$. Here lt is necessary to separate the turbulence fluctuation from the combined original record In the present case of laboratory experiment, it was found that the amplitude of the second harmonlc of surface wave profile was as small as $3 \%$ of the fundamental mode, through the harmonic analysis of the wave record Hence the authors defined temporarily the turbulence velocity fluctuations $u$ as the remainder after substracting the fundamental mode of the velocity $U_{1}$ from the corresponding original data of horlzontal velocity $U$ In Figure 4 the fundamental mode velocity was expressed by a thin line, whlle the turbulence velocity fluctuation defined above was expressed by a broken 1 ine The same procedure was applied to the vertical velocity component.

Figure 5 shows the amplitude ratio between the calculated value of the vertical velocity component on the basis of Alry's theory $W_{c a l}$ and the fundamental mode value of the measured vertical velocity component $W_{\text {meas. }}$. Thls diagram indicates that there exists a vertical motion in a considerably large scale even within a reglon near the bottom boundary for example $\hat{W}_{\text {meas }} \simeq 2 \mathrm{~cm} / \mathrm{sec}$ at $\mathrm{z} \simeq 05 \mathrm{~cm}$ According to the oscillatory turbulent boundary layer theory presented by KaJ1ura(1964, 1968), where the vertical component of velocity was neglected, the thickness of the turbulent boundary layer in the present case was estimated as about $35 \mathrm{~cm}$ Even if the above thickness is larger than the rise of ripple, the vertical velocity component seems not to be neglected owing to the fact that the stated value is comparable to the horlzontal one in the vicinity of ripples The authors believe that the Kajlura's theory on the oscillatory boundary layer flow should be modified with the consideration of the above stated fact

Here the turbulence intensity is defined as the root mean square of the turbulence fluctuation In Figure 6 


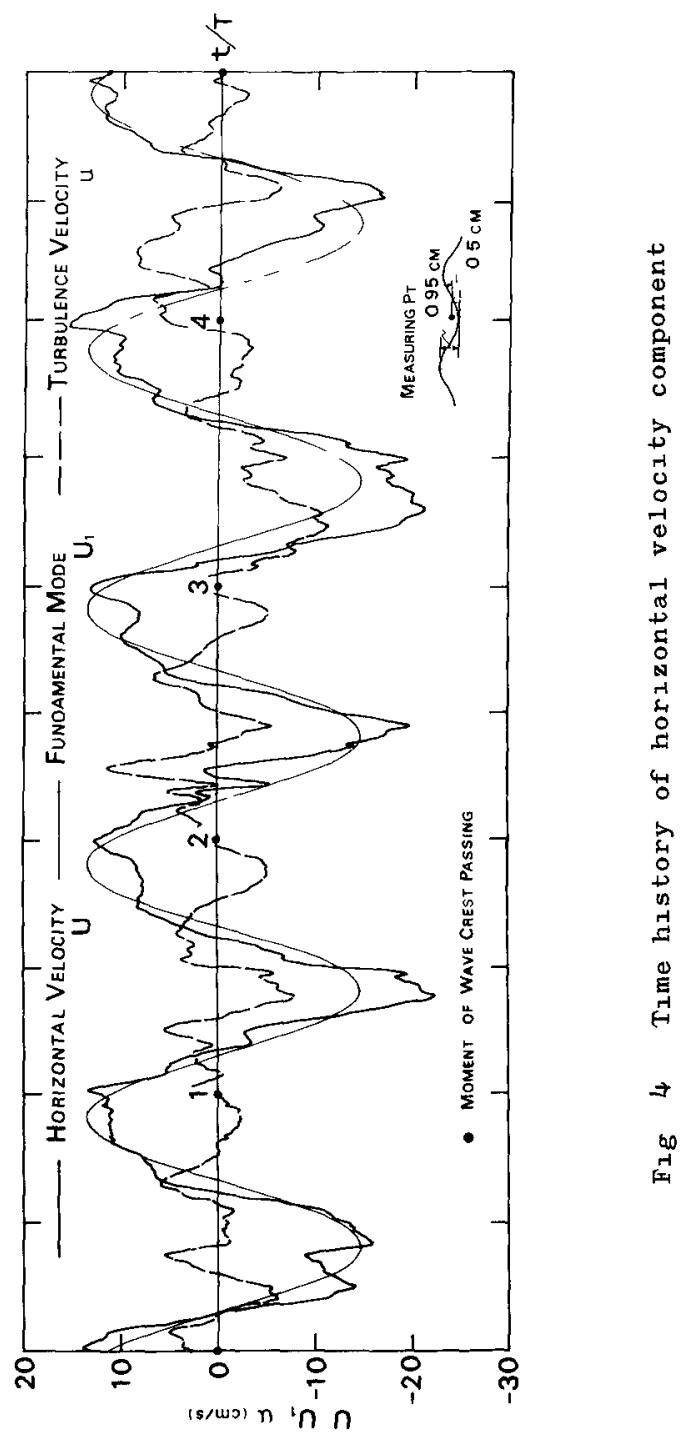




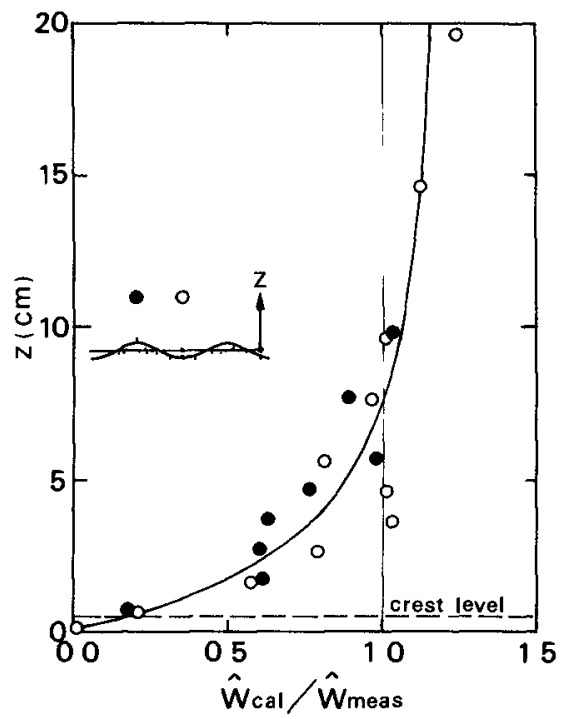

Fig 5 Amplitude ratio of $W_{\text {cal }}$ to $W_{\text {meas }}$

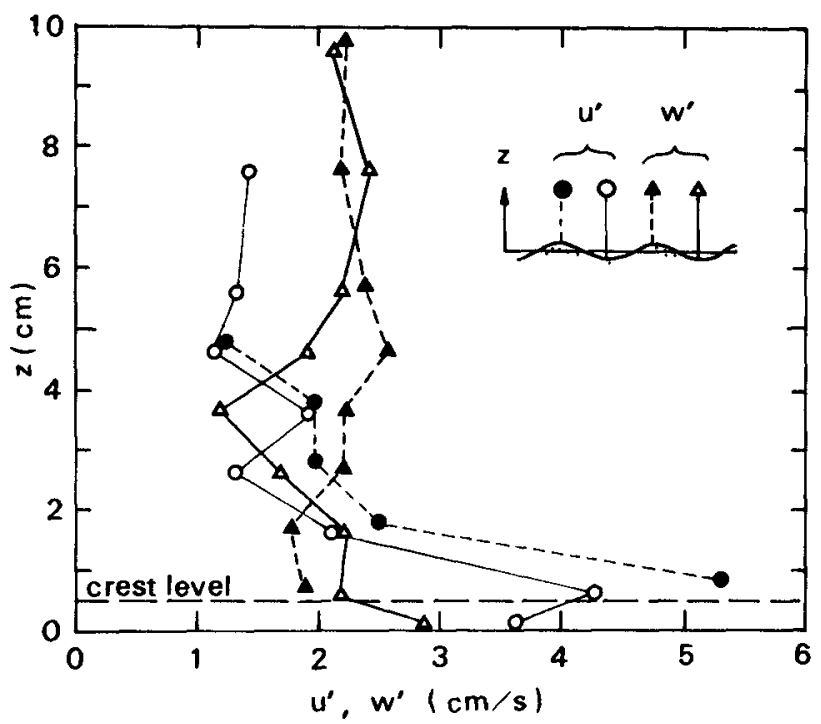

Fig 6 Vertical distributions of turbulence intensities, $u^{\prime}$ and $w^{\prime}$. 
are shown the vertical distributions of horizontal and verti cal components of turbulence intensity. This diagram indicates that the vertical intensity $w^{\prime \prime}=\sqrt{w^{2}}$ is nearly constant independently of the measuring position, while that the horlzontal intensity $u^{\prime}=\sqrt{\overline{u^{2}}}$ is very high near the bottom boundary. The value of $\left(u^{\prime z}+w^{\prime 2}\right)$, which ls related to the total energy of turbulence, is decreasing monotonously with the height above the bottom These are quite interesting and important facts to investigate the source mechanism of suspended materials from bottom

Figure 7 is for the normalized spectral energy density $F(n)$ of the horizontal component of turbulence fluctuation u given in Figure 4, where $n$ is a frequency. The range of the major energy content is situated at low frequencies less than 4 cps, and the so-called -7 power law seems to be applicable at higher frequencies larger than about $10 \mathrm{cps}$ From the latter fact the micro scale of turbulence in time is sald to be 0.1 sec This value seems to be pretty large compared with the result in an air flow, but is in falr agreement with the value obtained by Raichlen(1967), who measured the turbulence intensity of steady flow in an open channel by using a hotfilm anemometer. The spectrum computation was made under the condition of 1 ) the degrees of freedom being about 30 and 2) the lag window being the Hanning procedure.

The different expression of turbulence energy spectrum $n^{2} F(n)$, whlch is related to the rate of turbulence energy dissipation, is shown in Figure 8, from which two peaks are observed at $2 \sim 3$ cps and 7 cps Taking consideration of the osciliatory flow pattern in the vicinity of sand ripple and of the wave period of $135 \mathrm{sec}$, the authors believe that the former peak corresponds to the vortex or circulation formed behind a ripple, while that the latter peak corresponds to the turbulence itself induced by the vortex stated above This kind of approach must be quite valuable to find out 1) the essential reason why such a typical distribution curve as an $L$ shape is appeared in the vertical distribution of suspended sediment concentration, and 2 ) the critical elevation beyond which a certain sand particle can not exist as a suspended sediment.

\section{RELATIONSHIP BETWEEN SUSPENDED SEDIMENT CONCENTRATION AND TURBULENCE CHARACTERISTICS}

The photo-transistor type concentration meter was developed at the Coastal Englneering Laboratory, University of Tokyo(Hom-ma and Horlkawa(1963)) and has been Improved step by step during the last several years The circuit diagram of the present instrumentation system is shown in Figure 9 By using this instrument the time history of suspended sediment concentration was recorded under the same condition as the velocity measurement The above measurements were conducted along the vertical lines above both a crest and a trough 


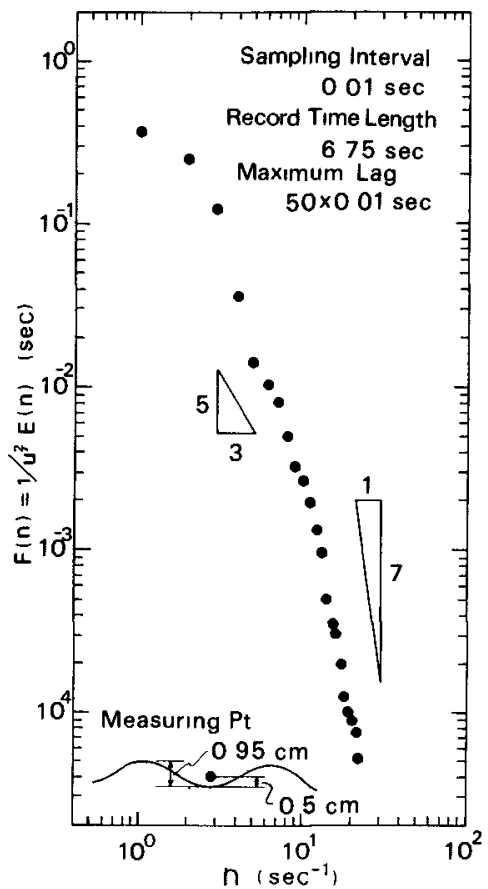

Fig 7 Spectral density distribution of horizontal turbulence component $(1), F(n)$

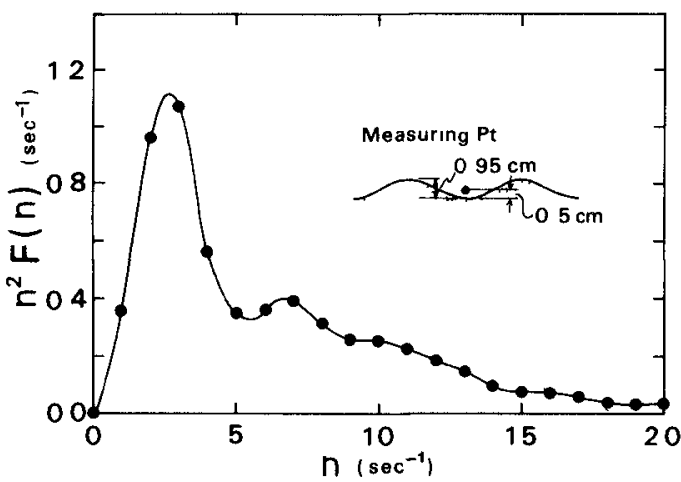

Fig 8 Spectral density distribution of horizontal turbulence component (2), $n^{2} F(n)$ 


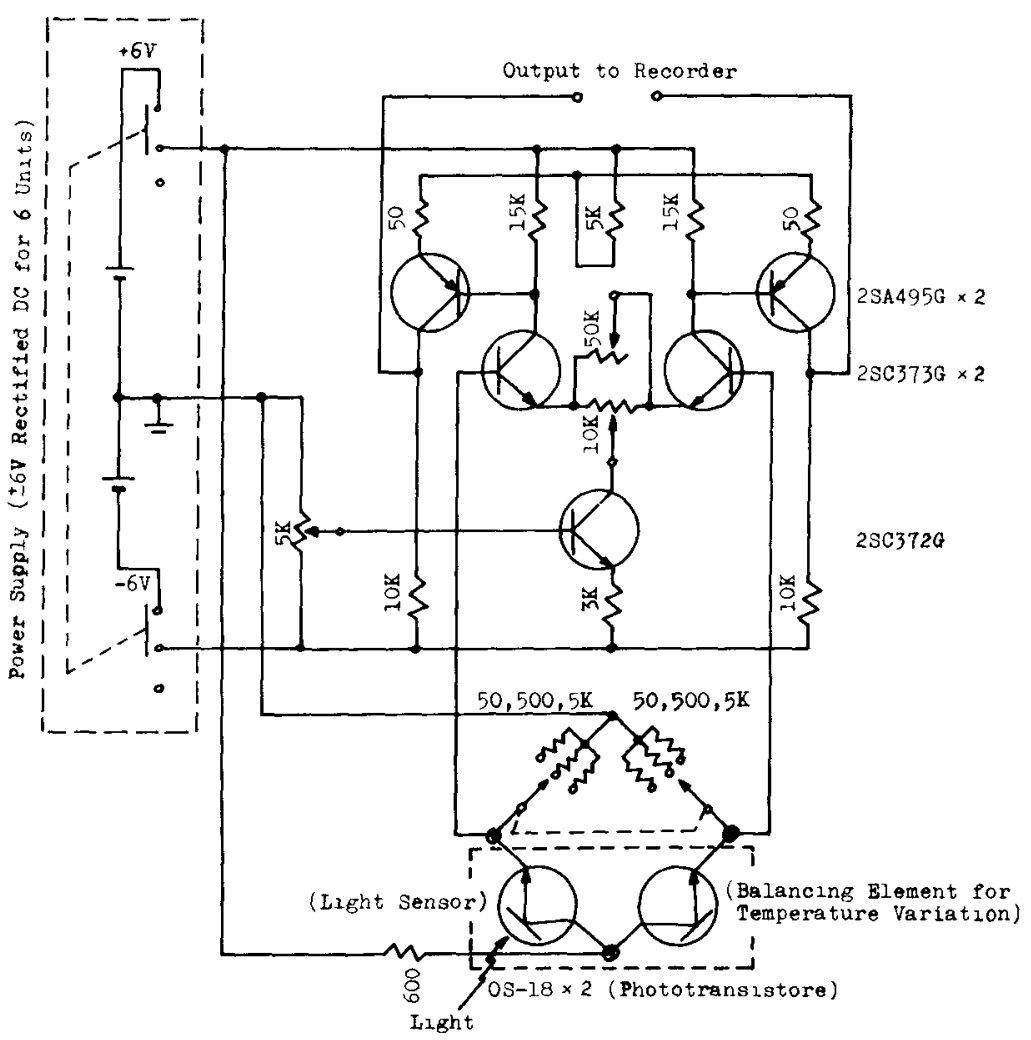

Fig 9 Circult diagram of photoelectric concentration meter unit 
of a certain sand ripple

From the recorded data were determined the time mean concentration $\bar{m}$ and the root mean sqare value of the fluctuation in sediment concentration $\sqrt{m^{2 /}}$, where m is its fluctuation determined as the difference between the lnstantaneous ralue of sediment concentration $m$ and the time mean concentration $\bar{m}$. In Figure 10 are shown the vertical distributions of $\overline{\mathrm{m}}, \sqrt{\mathrm{m}^{-\frac{2}{2}}}$ and $\sqrt{\mathrm{m}^{22}} / \overline{\mathrm{m}}$ The last $1 \mathrm{~s}$ the value of root mean square of m normalized by the local mean concentration From this diagram the following tendencies are observed

1) The vertical distribution of mean sediment concentration $\bar{m}$ has an L shape which has commonly been observed in field and in the previous experiments.

2) The vertical distribution of these values above the ripple crest are slightly different from, but are baslcally the same as those above the ripple trough.

3) The root mean square $\sqrt{m^{2 / 2}}$ has lts greatest value near the bed and decreases as the elevation increases

4) The distribution of the normalized value $\sqrt{\mathrm{m}^{\prime 2}} / m^{-1}$ roughly similar to that of the intensity of vertical turbulence component which is shown in Figure 6 .

Figure 11 is to show the comparison between the intensity of vertical turbulence component $w^{\prime}$ and the fall velocity $w_{0}$ of sediment particles sampled at corresponding elevations by using a syphon tube It should be mentioned that the magnitudes of $w^{\prime}$ and $w_{0}$ are comparable

As stated in INTRODUCTION of this paper Equation(1) is used as the fundamental equation to determine the vertical distribution of mean sediment concentration $\bar{m}$ By using the analized data of sediment concentration and of sediment fall velocity, the value of diffusion coefficient $K_{z}$ at each elevation can be evaluated on the basis of Equation(1)

On the other hand the following relationship can be taken as an analogue of the eddy viscosity $\epsilon_{m}$

$$
\epsilon_{m}=-\frac{\overline{\mathrm{UW}}}{\partial \bar{U} / \partial \mathrm{z}} \sim \frac{\overline{\ell(\partial \bar{U} / \partial \mathrm{z}) \mathrm{w}}}{\partial \overline{\mathrm{U}} / \partial \mathrm{z}} \sim \overline{\ell w} \sim \mathcal{J}_{E} \mathrm{w}^{\prime 2}
$$

where $u$ and $w$ are the turbulence fluctuation in horizontal and vertical directions respectively, $\bar{U}$ is the mean value of horizontal velocity component, z is taken positive upward, $w^{\prime}$ is the intensity of vertical turbulence fluctuation, $\ell$ is the mixing length, and $\mathcal{J}_{E}$ is the Eulerian integral time scale related to $w$ The value of $\mathcal{J}_{E}$ is calculated by the following equations

$$
\left.\begin{array}{l}
\mathcal{J}_{E}=\int_{0}^{\infty} R_{E}(\tau) d \tau \\
R_{E}(\tau)=\frac{\frac{w(t) w(t+\tau)}{w^{\prime 2}}}{\}}
\end{array}\right\}
$$




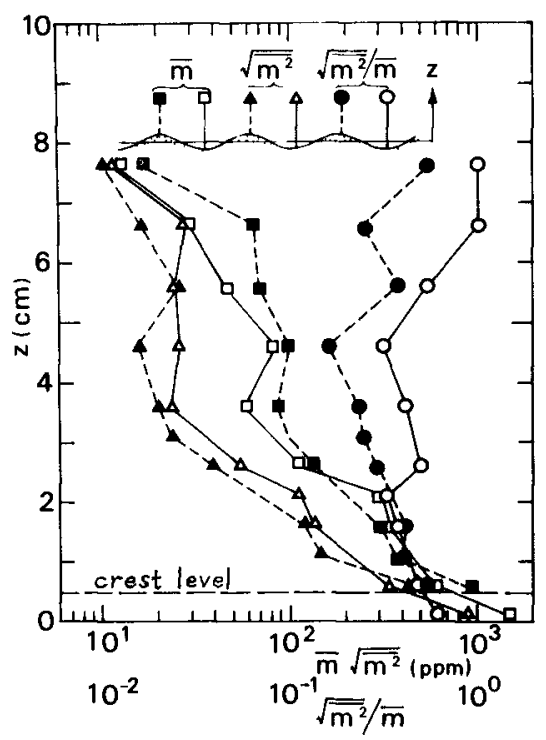

Fig 10 Vertical distributions of mean concentration $\bar{m}$, concentration fluctuation $\sqrt{\mathrm{m}^{12}}$ and $\sqrt{\mathrm{m}^{2 /}} / \overline{\mathrm{m}}$

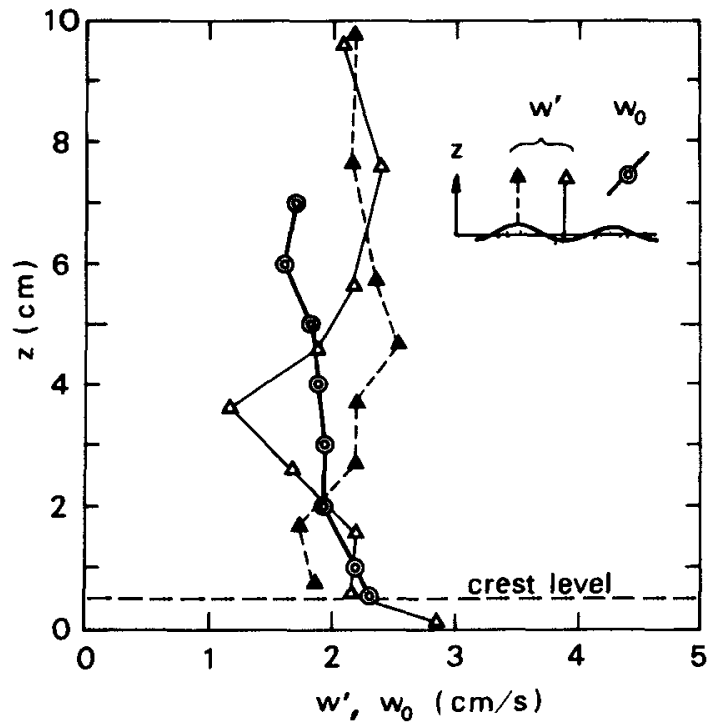

Fig 11 Comparison between vertical turbulence intensity $w^{\prime}$ and fall velocity $w_{0}$ 
where $R_{E}$ is a coefficient of Eulerian time correlation

In order to compare the vertical distribution pattern of $K_{z}$ with that of $\mathcal{J}_{E} w^{2}$, the values of both factors calculated through the stated methods were plotted in Figure 12 It is quite interesting that the distribution pattern of $\mathrm{K}_{z}$ is rather similar to that of $\mathcal{J}_{E} w^{\prime 2}$ There are still numerous uncertainties remained unsolved, hence it is certainly needed to accomplish further studies related to the following subjects, such as 1) to reevaluate the order of the term $\mathrm{d}\left(\frac{\mathrm{m} \cdot \mathrm{w}}{\mathrm{m}^{\prime}}\right) / \mathrm{dz}$ which was neglected in Equation (1), and 2) to find out the precise expression for $\epsilon_{m}$ The authors have the strong intention to improve the newly developed instrumentation system of ELETT in order to get more accurate data, and also to accumulate much more data with the alm of finding the generalized relationship between the diffusion coefficient $K_{z}$ and the eddy viscosity $\epsilon_{m}$

\section{CONCLUSIONS}

In order to clarify the mechanism of suspension phenomena due to progressive waves, it is of essentially importance to observe and to understand the turbulence fluctuation characteristics under the oscillatory flow condition on the basis of the above concept the authors developed a new anemometer named as ELETT(electrolytic turbulence transducer) and obtalned a certain amount of measuring data. This is at any rate the preliminary approach to attack the turbulence characteristics induced by oscillatory flows, hence there are many problems to be solved in future for example to establish the linearization of instrument output, to make clear the frequency characteristics of this instrument, and to find out more rational way of separating the turbulence fluctuation from the actual velocity record are the problems with which the authors have confronted at present.

The authors believe that the relationship among the turbulence intensity, the bottom roughness and the wave characteristics might be clarified in future owlng to the further accumulation of laboratory data The vertical distribution of turbulence intensity may be attacked theoretically on the basis of the turbulence energy equation The authors have an intention to proceed the present treatment to the following directions in order to understand more clearly the relationship between the suspended sediment concentration and

the turbulence characteristics These are

1) to reevaluate the effect of convective term on a suspended sediment concentration, and to reconsider the expression of eddy viscosity; these are to find out the rational expression for the diffusion coefficient and for the vertical distribution of suspended sediment concentration

2) to make clear the characteristic concentration at a certain elevation, which is used as a measure to determine the vertical distribution of suspended sediment concentration, the characteristic concentration stated above is 


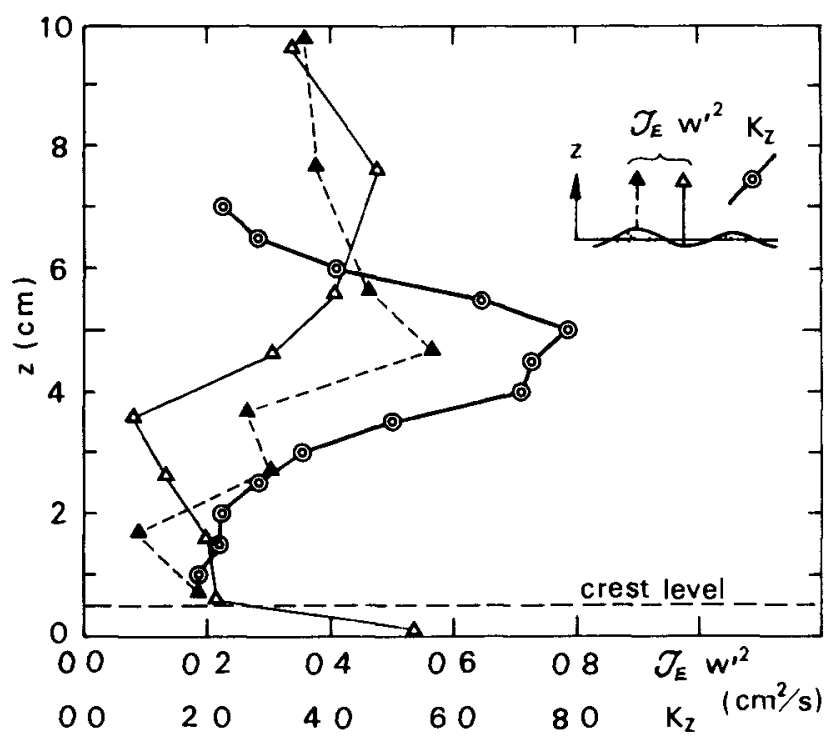

Fig 12 Comparison between analogue of eddy viscosity $\mathcal{J}_{E} w^{\prime 2}$ and eddy diffusivity $K_{z}$ 
expected to have a close relationship with the Reynolds stress in the vicinity of bottom The transducer reported here seems to be applicable to measure the Reynolds stress, therefore the pick-up and data processing system which are suitable for the present purpose should be developed in future.

\section{ACKNOWLEDGEMENT}

The work reported herein is a part of investigations carried out at the Coastal Engineering Laboratory, University of Tokyo, under the support of the Sclentific Research Fund of the Ministry of Education in Japan.

\section{REFERENCES}

Binder, G (1967) Potential fluctuations generated by turbulence on a small wire, Proc. 12th Congress, I $\quad \mathbf{A} \quad \mathrm{H} \quad \mathrm{R}$

Hom-ma, M. and K. Hor 1 kawa (1962) Suspended sediment due to wave action, Proc 8 th Conf on Coastal Engng

Hom-ma, $M$ and $K$ Horlkawa (1963) A laboratory study on suspended sediment due to wave action, Proc. 10th Congress, I A H R

Hom-ma, M, K Horıkawa and $\mathrm{R}$ Kajlma (1965) A study on suspended sediment due to wave action, Coastal Engng. in Japan, Vol. 8

Horıkawa, $K$ and $A$ Watanabe (1967) A study on sand movement due to wave action, Coastal Engng. In Japan, Vo1 10

Horlkawa, K. and A Watanabe (1968) Laboratory study on oscillatory boundary layer flow, Proc. 11 th Conf on Coastal Engng

Iwagakl, Y. and $T$ Sakal (1969) Experiments on water particle velocity of finite amplitude waves, Proc 16th Conf. on Coastal Engng in Japan (in Japanese)

Jonsson, I G (1963) Measurement in the turbulent wave boundary layer, Proc 10 th Congress, I A H R

Kajiura, $K$ (1964) On the bottom friction in an oscillatory currents, Bu11. Earthq Res Inst., Univ of Tokyo, Vol 42 .

Kajiura, K. (1968) A model of the bottom boundary layer in water waves, Bu11. Earthq Res. Inst., Univ of Tokyo, Vo1 46.

Ralchlen, F. (1967) Some turbulence measurements in water, Jour. Engng Mech Div, Proc. ASCE, Vol. 93, No EM 2. 


\section{CHAPTER 47}

EFFECTS OF NONUNIFORM WAVE ENERGY IN THE LITTORAL ZONE

$$
\begin{aligned}
& \text { Victor Goldsmith } \\
& \text { Joseph } M \text { and } \\
& \text { Colone } 11^{2}
\end{aligned}
$$

\section{Abstract}

$B$ i-weekly monitoring of four closely-spaced permanent beach profile stations located on the northeast end of Monomoy Island (Cape Cod) has revealed major variations in the amount of erosion and accretion occurring along this portion of the Massachusetts shoreline During the 27-month monitoring period a close relationship was observed between changes in the beach and offshore portions of the profiles Three distinct types of bars were noted

(1) Subtidal bars which are parallel to the shoreline and located one to two thousand feet off those portions of the shoreline undergoing relatively small amounts of beach erosion,

(2) Subtidal bars which are perpendicular to the shoreline and attached to areas of the shore undergoing large amounts of erosion, and

(3) Large intertidal bars which are oriented obliquely to the shore1 ine and associated with the formation of the ebb-tidal delta and the resulting wave refraction patterns

The large variations in erosion and accretion occurring along the beach at any one time are related to the nonuniform distribution of energy within the waves arriving at this section of the coastione This nonuniformity of wave energy is attributed to refraction of the waves around the irregular bathymetry offshore from Monomoy, and it appears to produce shoreline protuberances of sand which are flanked updrift and downdrift by erosional zones

Wave refraction calculations indicate zones of alternately converging and diverging orthogonals in the wave fronts impinging upon Monomoy Island, with a correlation observed between the zones of converging orthogonals, 1 e wave energy concentrations, and the areas of the beach presentiy undergoing the greatest amounts of erosion

\footnotetext{
Institute of Coastal Research, Department of Geology, University of Massachusetts, Amherst, Mass , 01002

${ }^{2}$ Associate Professor, Department of $C_{1} v_{1} 1$ Engineering, University of Massachusetts, Amherst, Mass , 01002
} 


\section{INTRODUCTION}

Rythmic beach topography and its relationship to adjacent intertidal and subtidal bathymetry has been discussed by several authors (Bruun, 1954, Robinson, 1960, Hom-ma and Sonu, 1963, Sonu et al, 1966, Bakker, 1968, Sonu, 1968, Dolan and Ferm, 1968, Van Beek, 1969, N1ederoda and Tanner, 1970) Attempts to relate nonuniform shorelıne changes to wave refraction over irregular offshore bathymetry has been $11 \mathrm{~m}$ ted to large-scale effects (Munk and Taylor, 1947, Shepard and Inman, 1950, Jordaan, 1964, Roberts, 1964) In this study the nonuniform wave energy distribution produced by wave refraction over uneven offshore bathymetry is related to the large variations in erosion and accretion occurring at closely-spaced intervals on Monomoy Island

Monomoy Island is located on the "elbow" of Cape Cod, Massachusetts Monomoy was formed in Holocene time as a sand spit in response to the longshore currents resulting from the dominant northeast and eastnortheast waves Impinging upon the outer beach of Cape Cod

Since June 1968, twelve beach profiles on Monomoy Is land and four profiles on Nauset Beach to the north have been monitored at b1-weekly intervals throughout the year Approximately twice per year, fathometer profiles have been run to extend these profiles seaward from the high tide line to a distance of one and one-half miles from shore Fathometer profiles parallel to the shore were also obtained The variations in erosion and accretion occurring at these closely-spaced profiles are quite significant This discussion is $11 \mathrm{mited}$ to the four northernmost profiles on Monomoy so that variations between these profiles, and their relationship to changes in the offshore region, may be examined in detarl ( F 19 1)

\section{BEACH PROFILES}

The northernmost profile, $M-1$, underwent 35 feet of erosion on the terrestrial portion of the profile in the first year of observations (June 1968 to June 1969) but was accretional during the second year (Fig 2) In the offshore area of the $M-1$ profile a series of intertidal swash bars had migrated to the northwest ( $1 \mathrm{e}$, onshore and into the page) during the period of these observations (Fig 2) This movement is a 
result of the refraction of northeast waves around the large ebb-tidal delta so that these waves actually approach from the southeast at the M-1 profile location One of the swash bars has become attached to the beach 300 feet north of the M-1 profile, causing a large accumulation of sand south of the bar at the M-1 profile ( $F 1 g$ 3)

The changes observed at the other three profiles suggest a slightly different interpretation but, nevertheless, 111 ustrate the close relationship between changes in the terrestrial and offshore portions of the profiles The second profile, M-6, underwent 30 feet of erosion the first year ( $F 1 g$ 4) However, the rate of erosion increased substantially during the second year such that by March 1970 the beach had retreated 135 feet at the M-6 location Since March 1970 this profile has undergone
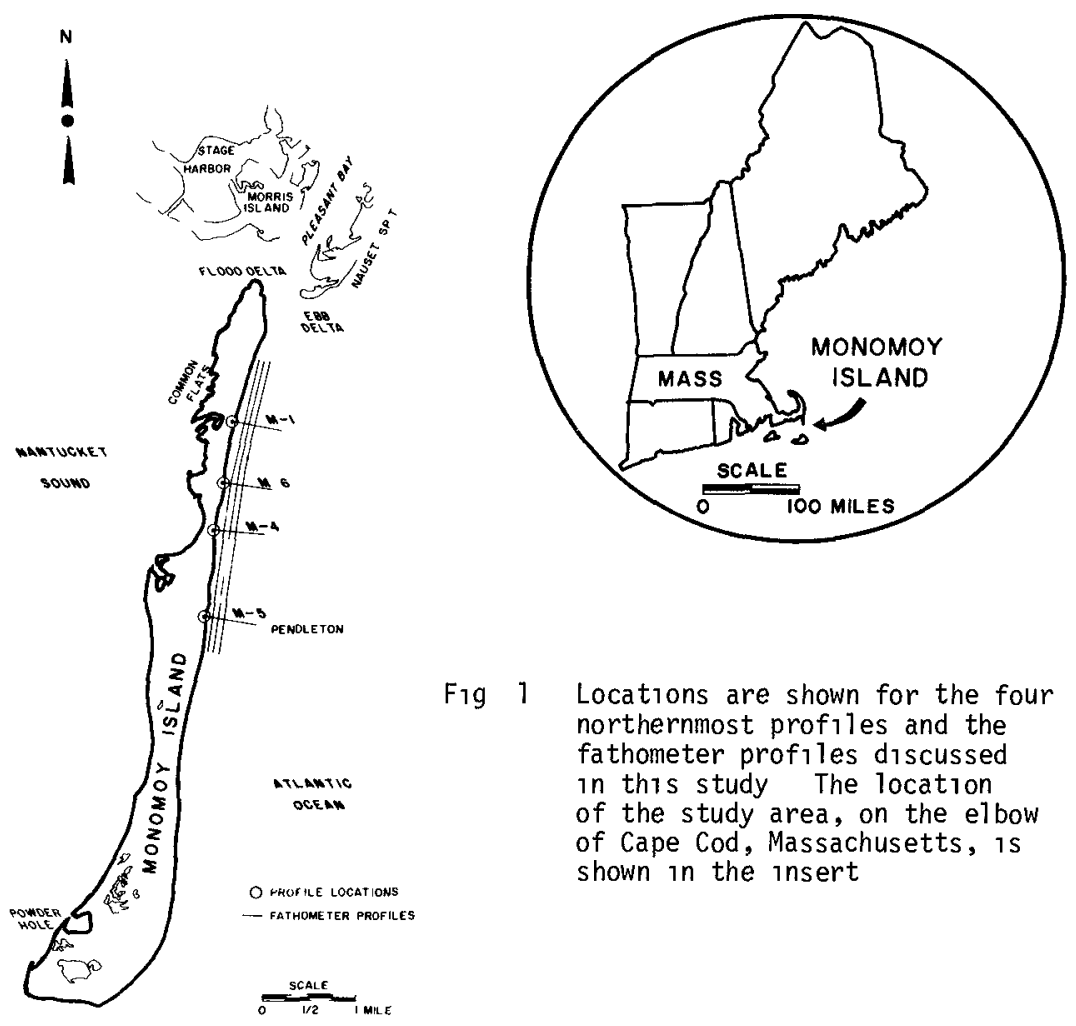

Fig 1 Locations are shown for the four northernmost profiles and the fathometer profiles discussed in this study The location of the study area, on the elbow of Cape Cod, Massachusetts, is shown in the insert 

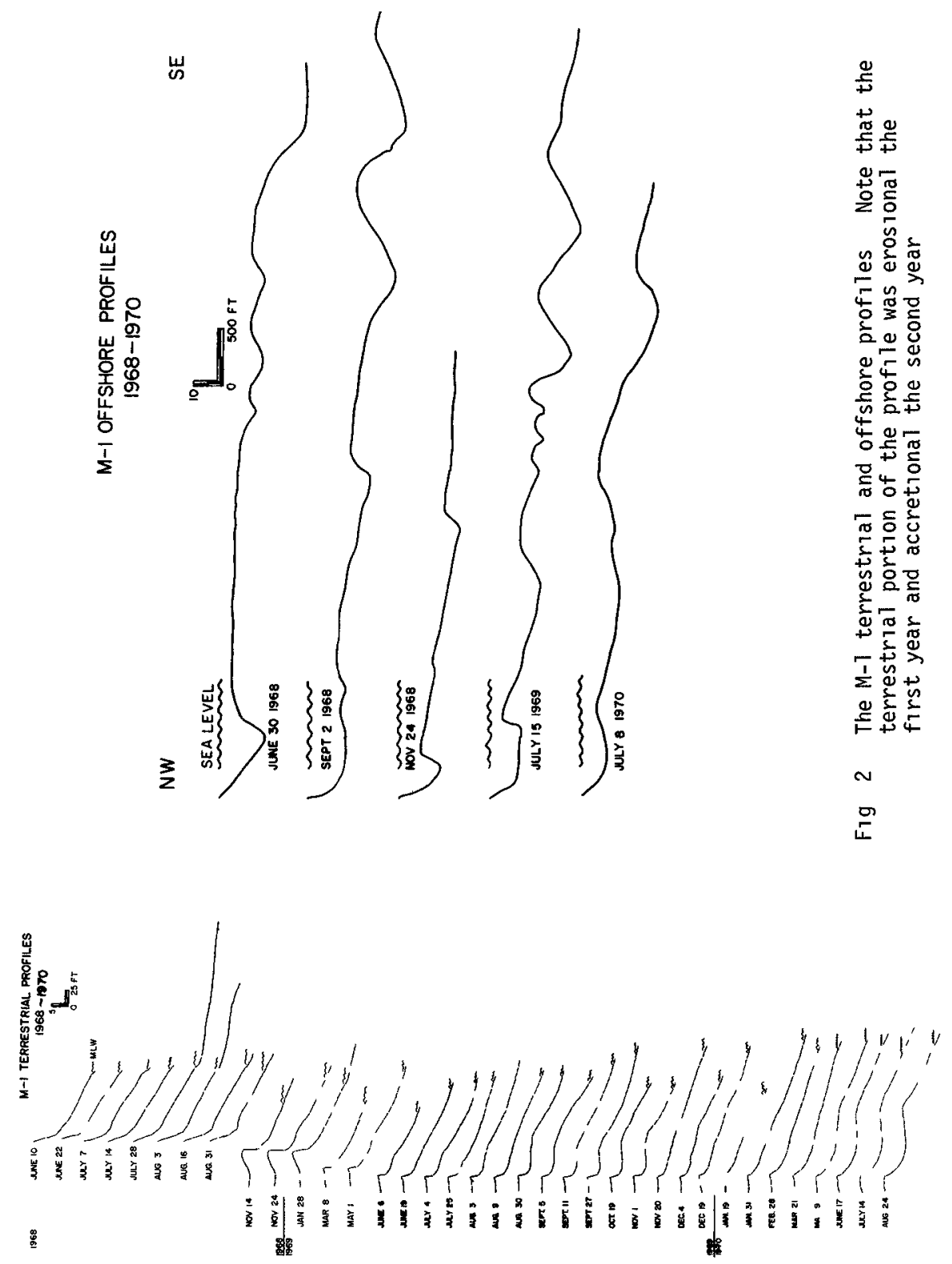

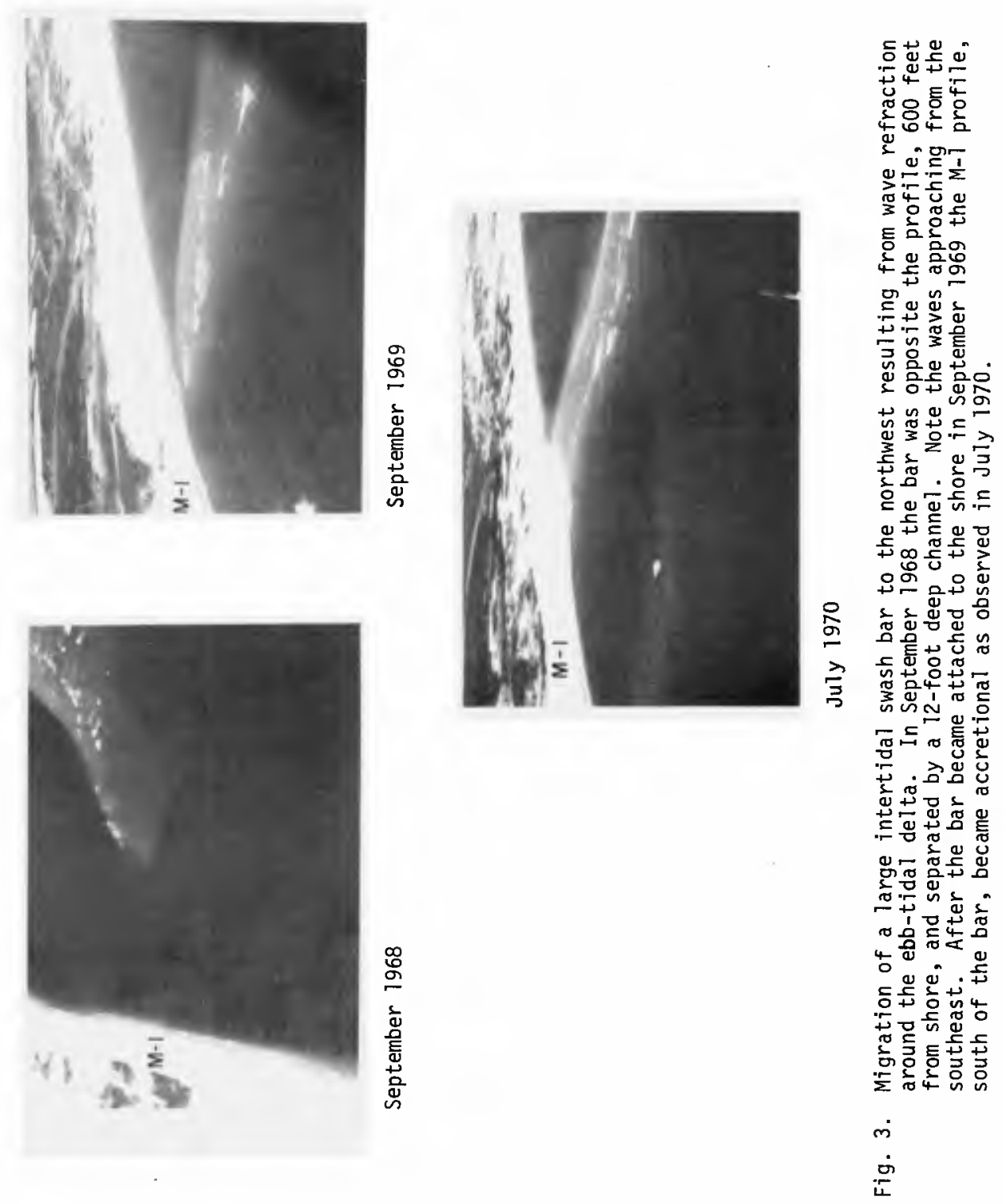


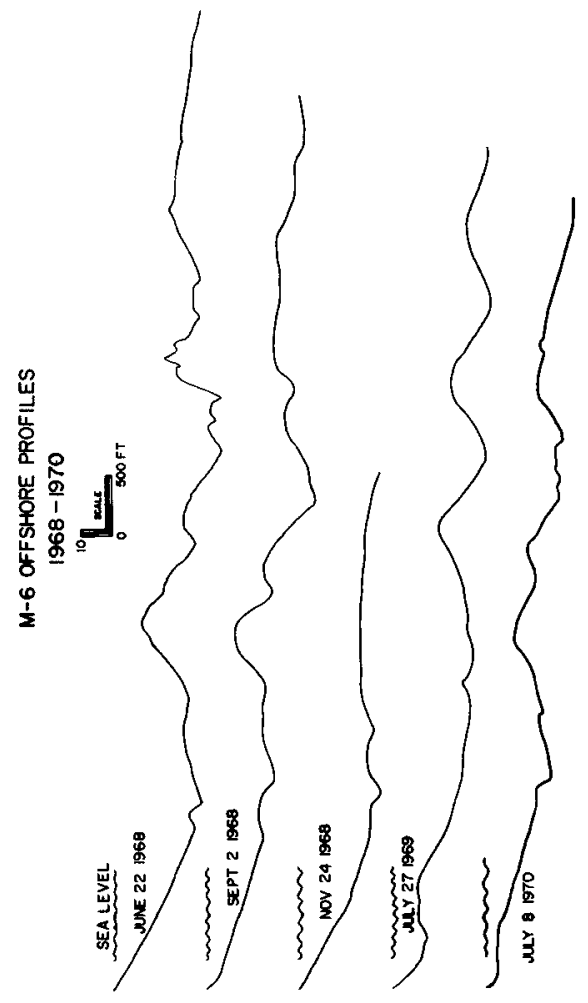

$\stackrel{2}{\frac{5}{4}}$

도용요

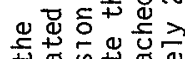

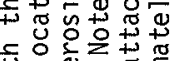

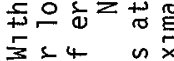

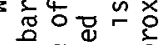
\%

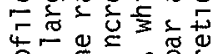

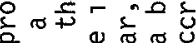
足

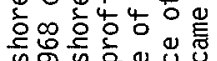

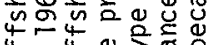

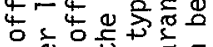

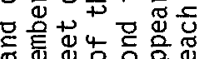

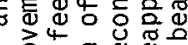

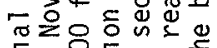

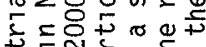

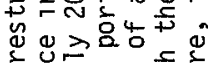

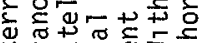

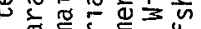
诸

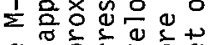

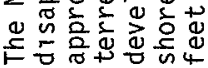

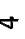

要

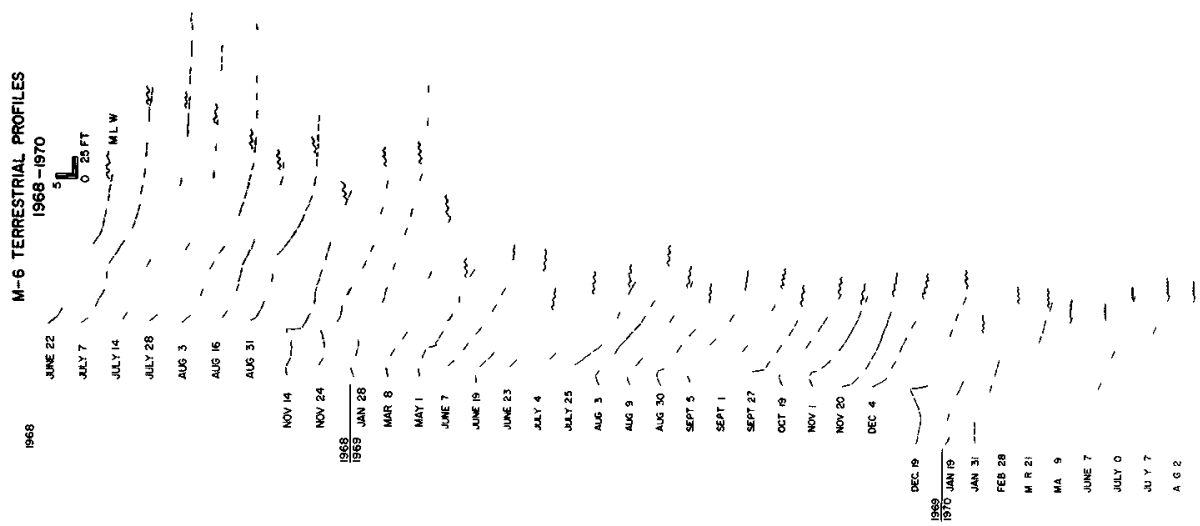


approximately 50 feet of accretion. In June 1968 a large bar with an elevation of twelve feet above the adjacent sea bed, was located approximately 2000 feet from shore and oriented parallel to the shore. By November 1968 this bar had disappeared from the profile and the terrestrial portion of the profile had become highly erosional. With the development of a new bar on the profile, as seen in the July 1970 fathometer profile, the rate of erosion decreased sharply and the profile became accretional. Associated with the severe erosion was the development of a shoreline protuberance of sand downdrift from the M- 6 profile (Fig. 5).

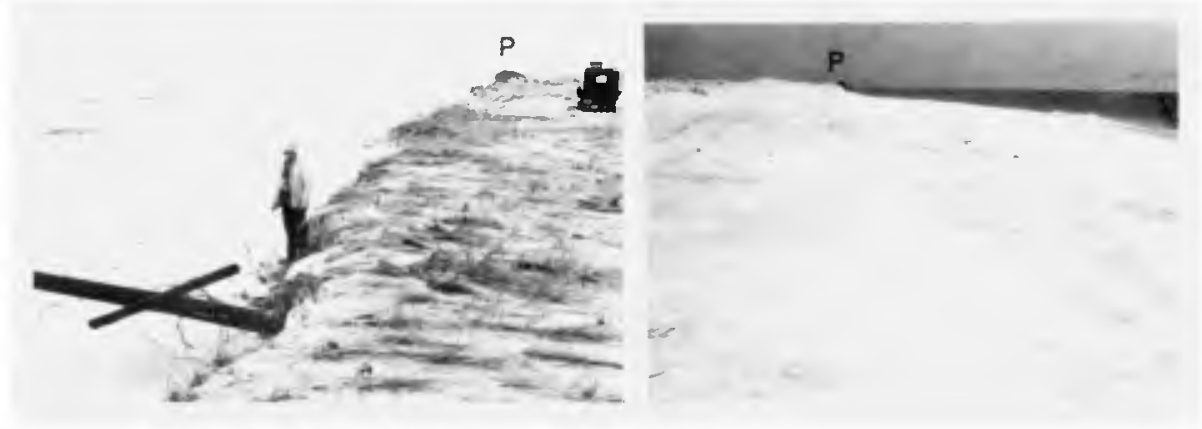

Fig. 5. Two views of the M-6 beach protuberance on December 19, 1969. The updrift erosional zone is shown on the left (looking south), and the accretional zone, downdrift from the view on the left, is shown on the right (looking north). Point $P$ is the same in each photograph.

The offshore extension of the protuberance, in the form of an attached subtidal bar oriented approximately perpendicular to the shoreline, is recorded in the July 1969 fathometer profile (Fig. 4).

The M-4 profile is located at the narrowest part of the island (600 feet wide at high tide), yet it underwent the least amount of change in both the terrestrial and offshore portions of the profile (Fig. 6).

The M-5 profile is located adjacent to a beach protuberance much like the one near the M-6 profile. Therefore, the M-5 profile is similar in behavior to the M-6 profile in that an offshore bar parallel to the shore, is present when the terrestrial portion is accretional and absent when the terrestrial portion is most erosional (Fig. 7). During the erosional 


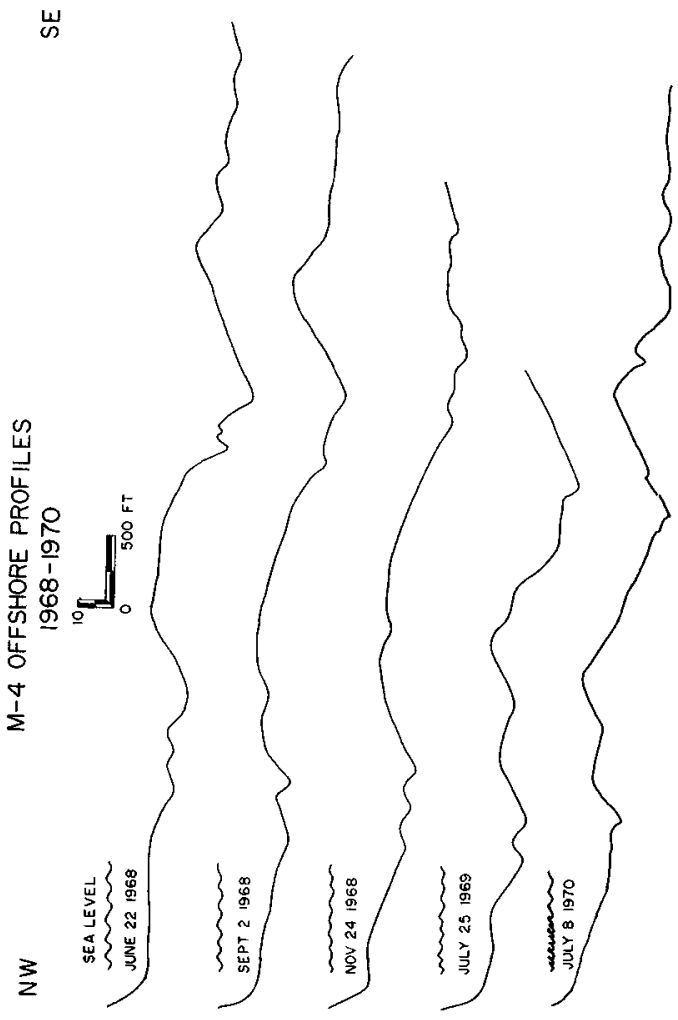

$\stackrel{+}{ \pm}$

雚萿

正

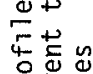

立蛋

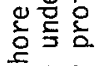

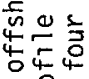

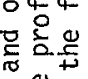

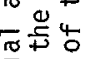
可它 논 0
+54
+5 +

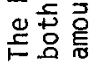

6

문

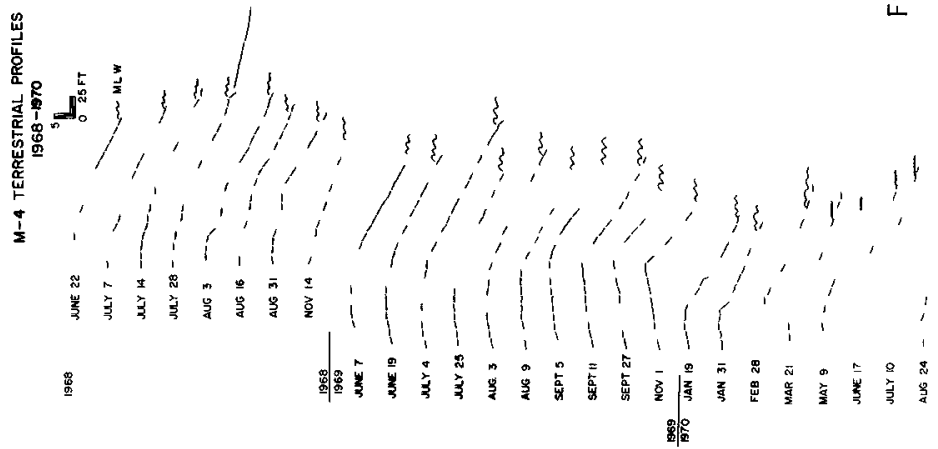



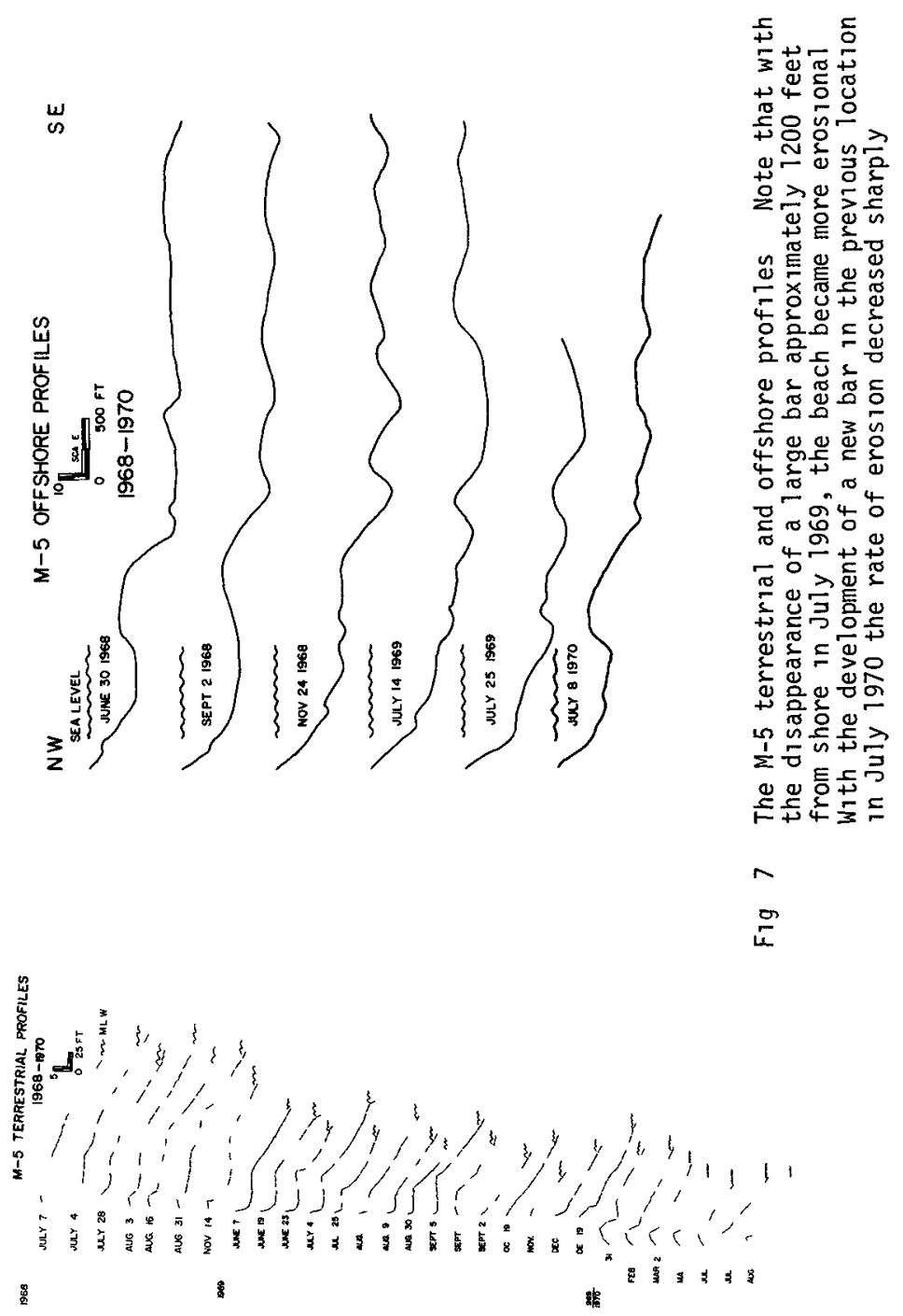
periods the second type of subtidal bar, which 15 perpendicular to the shoreline, began to form and 75 recorded on the July 25, 1969, fathometer profile The close relationship between erosional zones and the presence of subtidal bars perpendicular to the shoreline and attached to the shore 15 117ustrated in Figure 8

OFFSHORE PROFILES PARALLEL WITH SHORE

NORTH

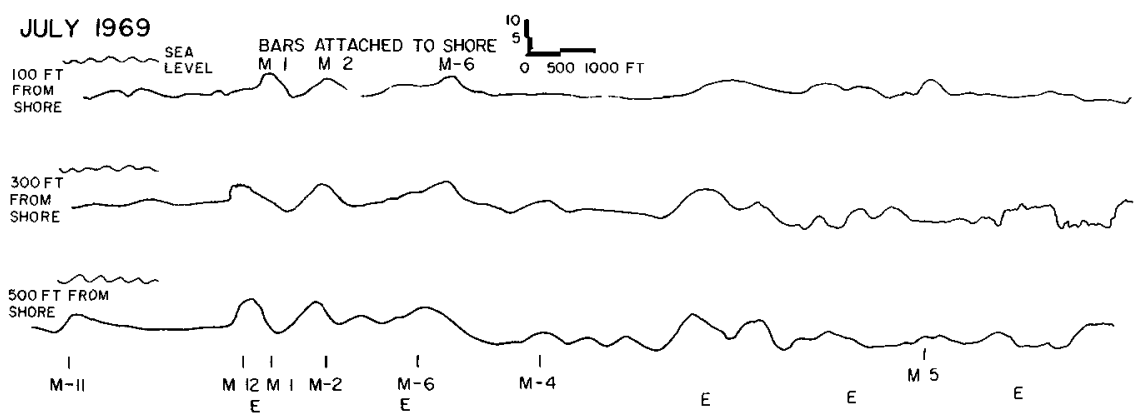

Fig 8 Fathometer profiles run parallel with, and 100, 300, and 500 feet from shore The areas of the Monomoy shoreline undergoing the severest amounts of erosion are indicated $w 1$ th the letter $E$ Note the correlation between the maximum erosion and the presence of nearshore subtidal bars which are perpendicular to the shoreline

A summary of the erosion or accretion occurring at these four closelyspaced profiles during the 27 months of observations is given in Figure 9 and Table 1 It is readily apparent that there is a large variation in the rate of beach retreat along this shoreline This suggests the hypothesis that there is a considerable spatial variation in the wave energy arriving at Monomoy Island and that the formation of the beach protuberances can be associated with this nonuniformity of wave energy in the littoral zone 


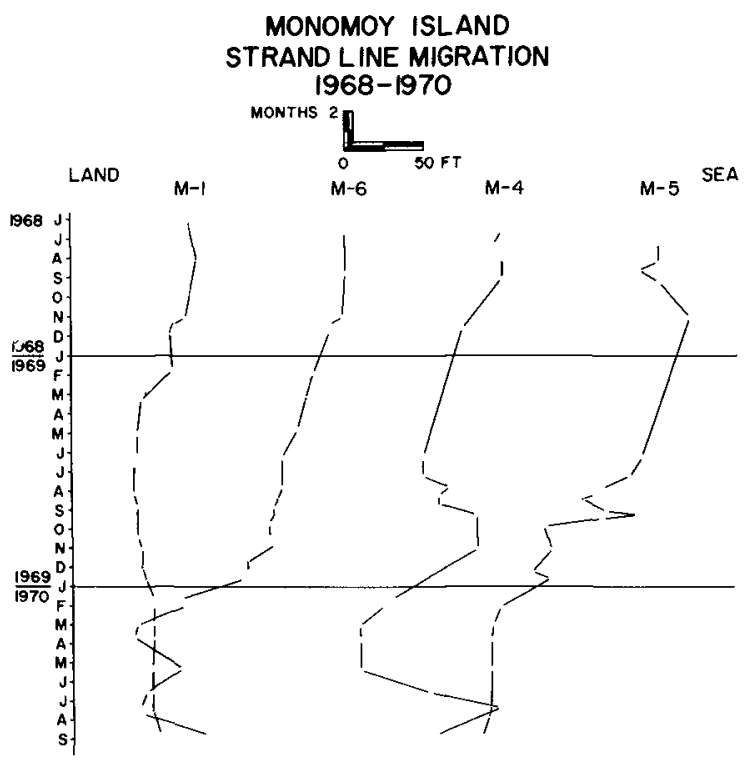

Fig 9 Rates of erosion and accretion at four permanent beach profiling stations located approximately one mile apart on Monomoy Is 1 and Leftward movement of the 11 ne under the profile number indicates erosion, while movement to the right indicates accretion

\begin{tabular}{|c|c|c|c|c|}
\hline & $M-1$ & $\underline{M-6}$ & $M-4$ & $M-5$ \\
\hline June 1968 - June 1969 & 35 & 30 & 35 & 0 Feet \\
\hline June 1969 - March 1970 & -10 & 105 & 40 & 75 \\
\hline March 1970 - August 1970 & $=7$ & -50 & $\underline{-48}$ & $\underline{6}$ \\
\hline TOTALS & 18 & 85 & 27 & 81 \\
\hline
\end{tabular}

Table 1 Summary of beach retreat at four profiles on Monomoy Island Negative amounts signify beach advance (accretion) 


\section{WAVE REFRACTION}

A mechanism by which zones of wave energy concentration can be produced is illustrated schematically in Figure 10. Waves passing
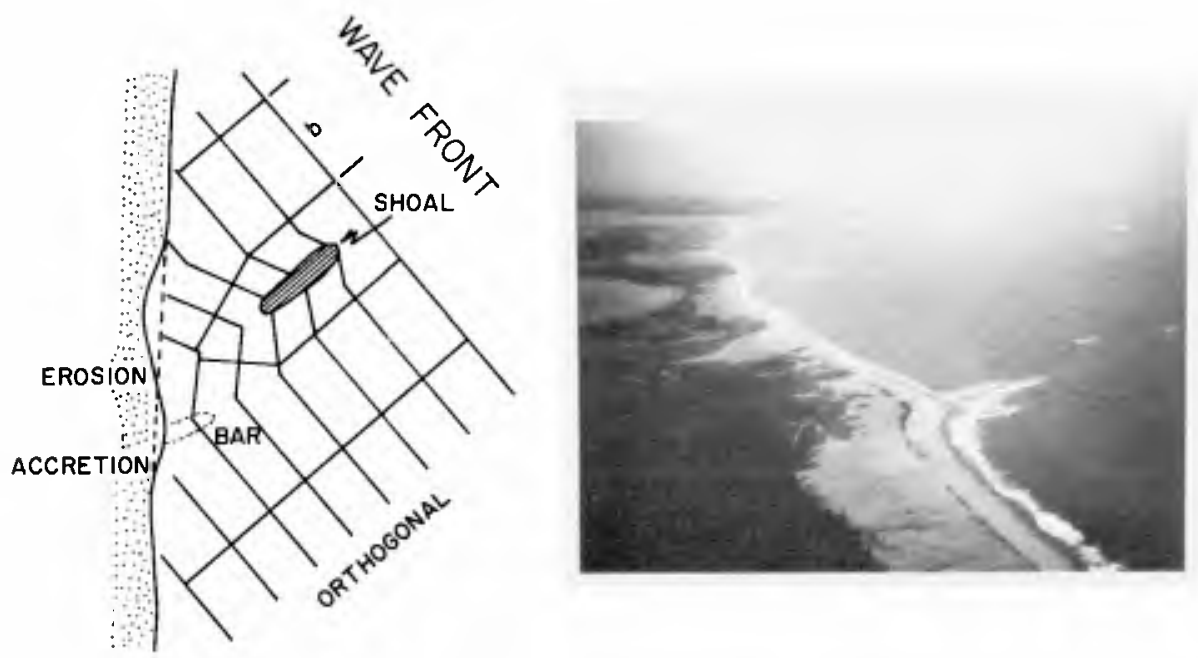

Fig. 10. A schematic diagram (left) illustrates the effects of wave refraction over irregular offshore bathymetry. This process results in differential erosion of the shoreline and causes segments of the shoreline to orient themselves perpendicularly to the dominant wave approach direction. Three beach protuberances are shown in the northward view of Monomoy Island (right).

over the highly irregular bathymetry offshore of Monomoy are refracted such that alternate zones of converging and diverging orthogonals are produced, indicating portions of the wavefront with increased or decreased wave energy concentration. When that portion of the wave front which contains the higher wave energy concentration impinges upon the shoreline, an increased rate of erosion can be expected to occur along that portion of the shoreline.

To test this hypothesis, a series of wave refraction computations were made with the aid of a digital computer ( $C D C 3600$ ), using the Stanford University Wave Refraction Program originally developed by Dobson (1967) and adapted by the authors for use at the University of Massachusetts Research Computing Center. The computational techniques employed by this program have been summarized by Mogel et al (1970). 
The bathymetric grid used in these computations is shown in Figure 11 The wave periods and approach directions chosen for the computations were based on the authors' personal observations of the dominant waves approaching Monomoy, and on deep water observations of the waves in the Marsden Square adjacent to Monomoy as compiled by the National Oceanographic Data Center The wave refraction diagrams resulting from the computations are illustrated in Figures 12 through 15 on the following pages 

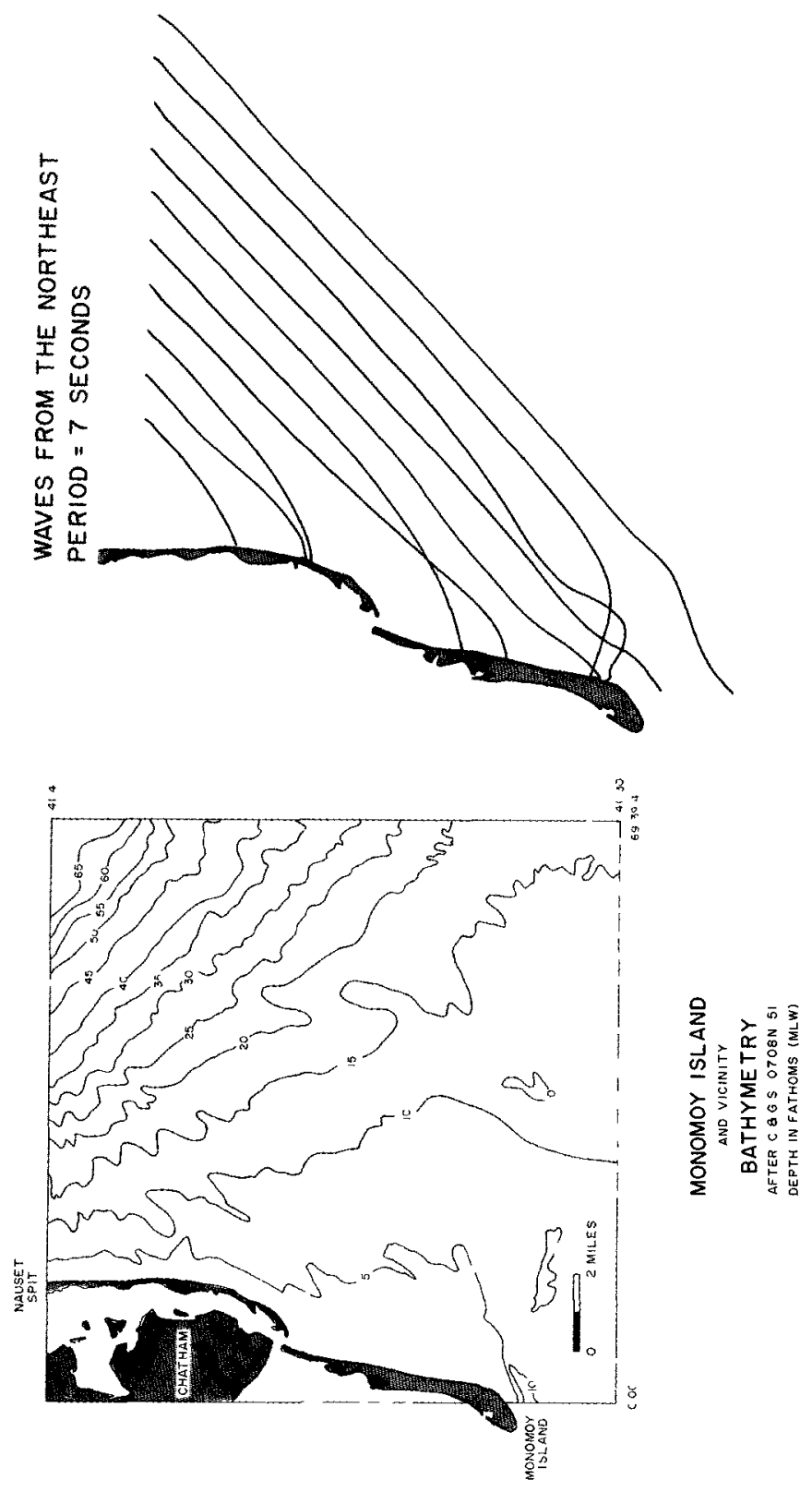

$\sim$

몬 


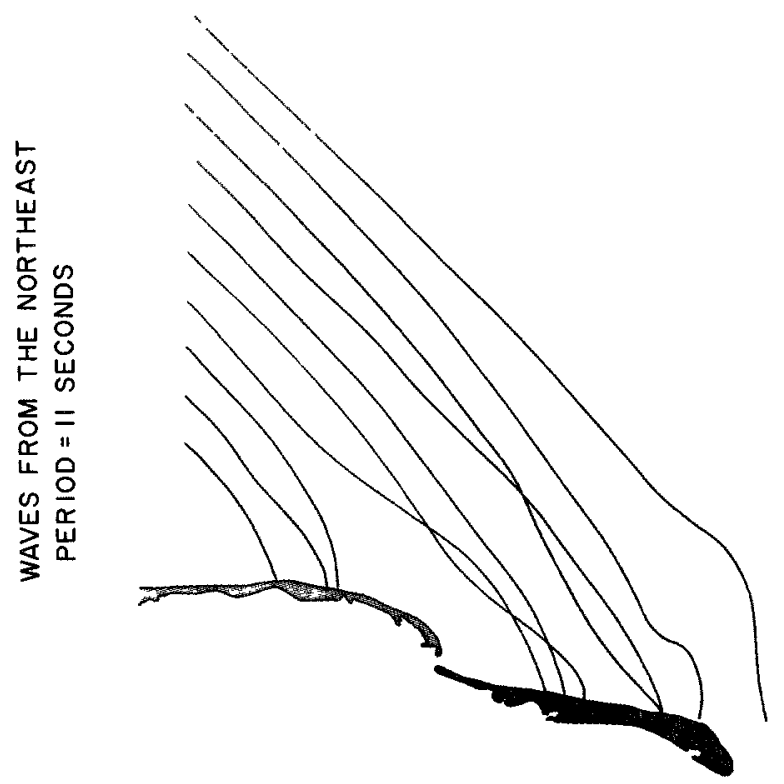

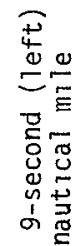

吾要

$\ddot{n}:$

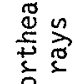

은

穵 동

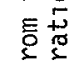

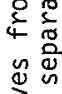

要两

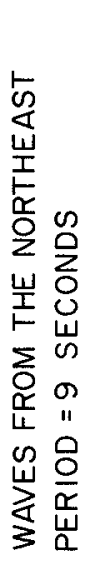

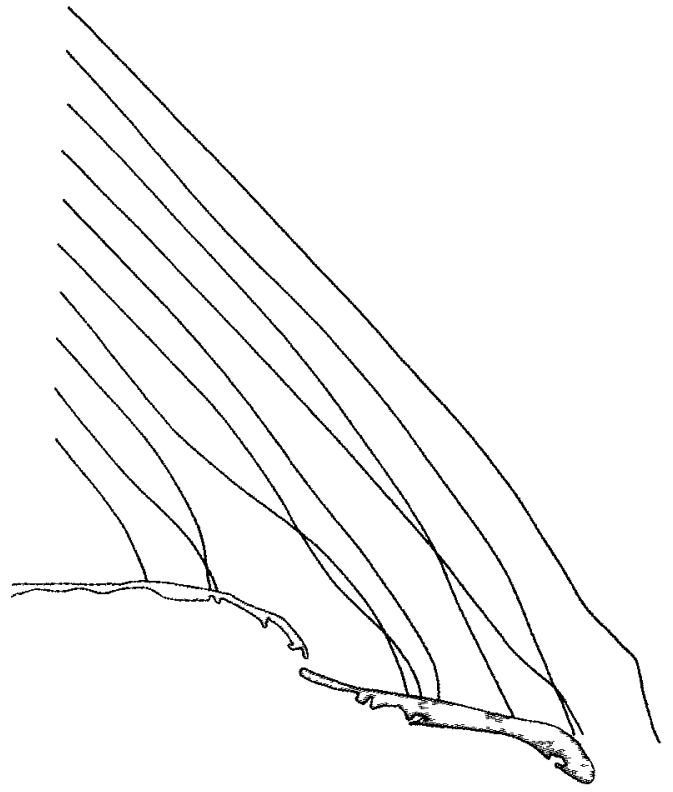

造变

究

혿

产这

"

ปั่

둥

ᄃㅇㅇ

苟占

4. 

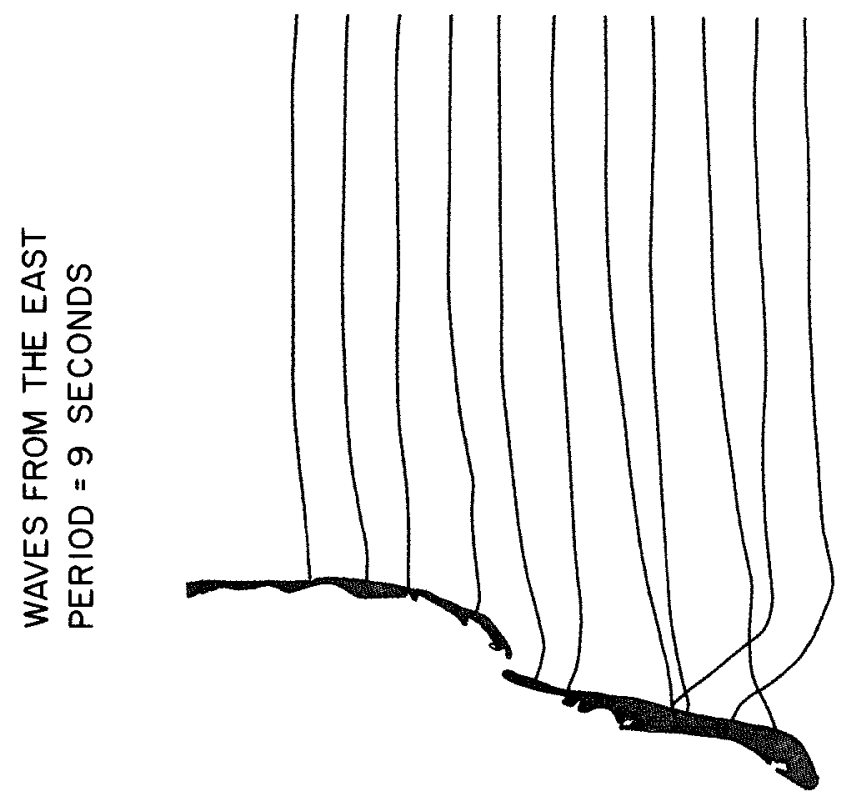

뀨

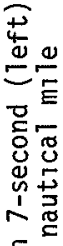

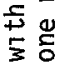

范告

\&

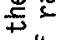

Eัㅇํㅇ

造 :

告范

पू

疍

5

焉

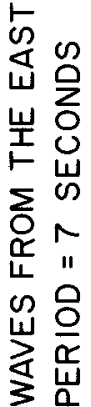

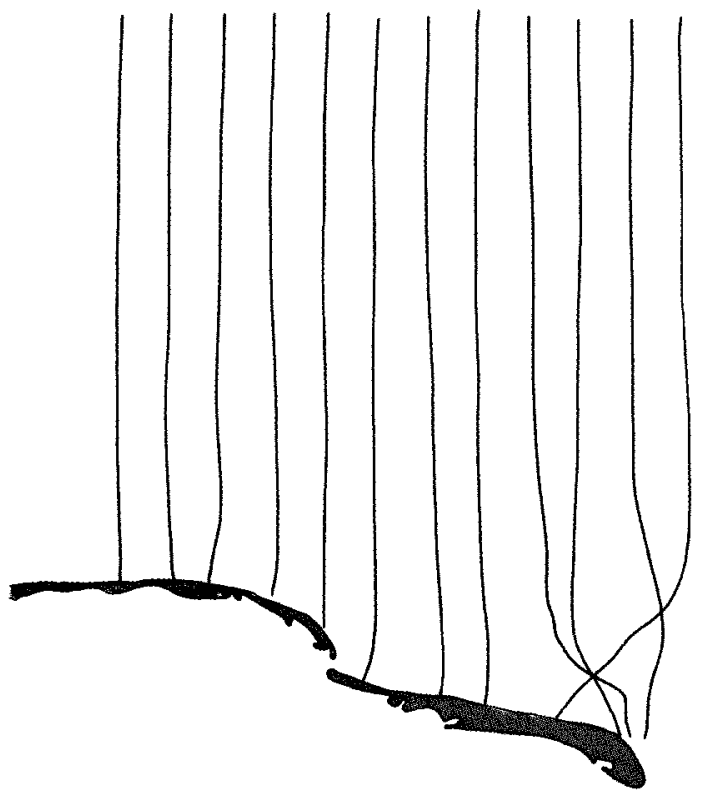

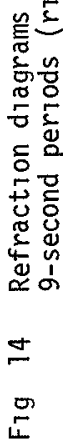


WAVES FROM THE EAST

PERIOD $=\|$ SECONDS

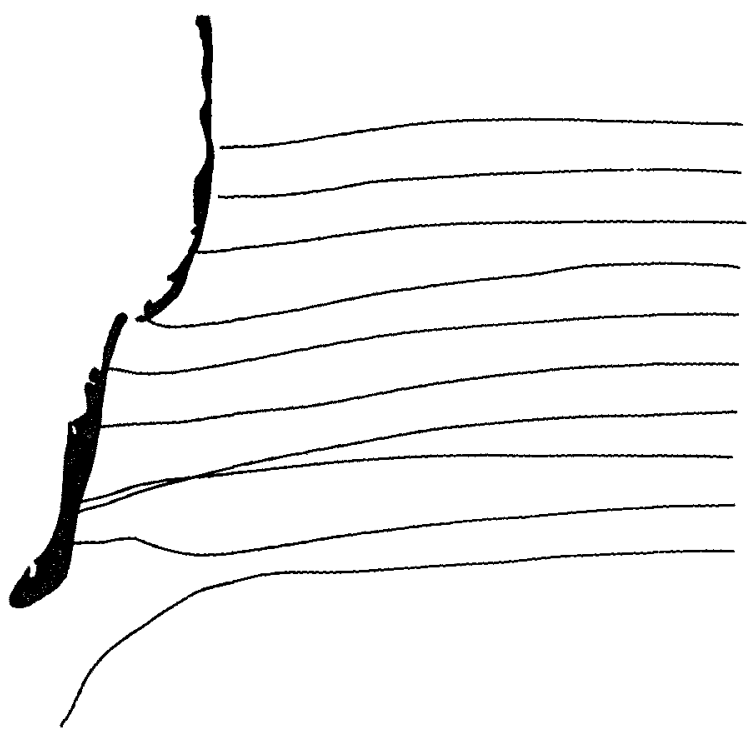

Fig 15 Refraction diagram showing rays for waves from the east with 11 -second period Initial separation of rays is one nautical mile

\section{CONCLUSIONS}

Wave refraction computations indicate that wave energy is distributed rather unevenly along closely-spaced intervals of the Monomoy Island shoreline Field data suggest a strong correlation between concentrations of wave energy and those portions of the shoreline which are undergoing increased rates of erosion producing shoreline protuberances of sand which are flanked updrift and downdrift by erosional zones This implies that areas of increased erosion can be predicted by wave refraction computations and that coastione changes resulting from variations in the offshore bathymetry may be similarly predicted 


\section{ACKNOWLEDGMENTS}

This work was supported by the Coastal Engineering Research Center, U S Army Corps of Engineers, Contract DACW 72-67-C-0004 Dr Miles 0 Hayes, supervisor of the project, provided much constructive discussion and criticism Thanks and appreciation are expressed to members of the UMass Coastal Research Group for their assistance Special acknowledgment goes to Yvonne Goldsmith, Frank Raffald1, Wayne Dowda11, John Gifford, Eugene Rhodes, Stewart Farre11, and Richard Callahan, who assisted in the field The University of Massachusetts Research Computing Center provided support through a grant to cover computer expenses The $U S$ Fish and $W_{1} l d l i f e$ Service granted permission to conduct the studies and provided storage facilities on the Monomoy Island National Wildi ife Refuge 


\section{REFERENCES}

Bakker, W T , 1968, A mathematical theory about sand waves and its application on the Outch Wadden Isle of VTieland Shore and Beach, october, p 5-14

Beek, J van, 1969, Systemat1c chanqes of rythmic beach topography (Abs) Geol Soc of Amer Ann Meeting, p 229

Bruun, Per, 1954, Migrating sand waves or sand humps, with special reference to investigations carried out on the Oanish North Sea Coast Proc Fifth Conf on Coastal Engrneering, p 269-295

Oobson, R S, 1967, Some applications of a digital computer to hydraulic engineering problems Technical Report No 80, Department of Civil Engineering, Stanford University, Stanford, California

Dolan, R and Ferm, J C, 1968, Crescentic landforms along the Atlantic coast of the U S Scrence $v$ 159, p 637-639

Hom-ma, M and Sonu, C, 1963, Rythmic pattern of longshore bars related to sediment characteristics Proc Eighth Conf on Coastal Engineering, p 248-278

Jordaan, J M, 1964, Effects of hydrographic changes due to nearshore dredger dumping on wave refraction and 11 ttoral sand balance Proc Ninth Conf on Coastal Engineering, p 310-322

Moge $1, T$ R, Street, R L, and Perry, B , 1970, Computation of alongshore energy and 11ttoral transport Proc Twelfth Conf on Coastal Engineering

Munk, $W$ and Taylor, M A, 1947, Refraction of ocean waves, a process linking underwater topography to beach erosion $J$ of Geol, $\checkmark 54, p \quad 1-26$

Niederoda, A W and Tanner, W F, 1970, Prel 1mınary study of transverse bars Marıne Geology, $v$ 9, $p$ 41-62

Roberts, J A, 1964, The reshaping of South Beach, Crescent City, Calif, after the tsunami of 27-28 March 1964 Paper presented before the Beach Erosion Control Conf, Cal if State Dept of Water Resources, Santa Monica, Calif , $18 \mathrm{p}$

Robinson, A H W , 1960, The hydrography of Start Bay and 1 ts relationship to beach changes at Hallsands Geogr J, v 127, p 63-77

Shepard, F P and Inman, 0 L, 1950, Nearshore circulation related to bottom topography and wave refraction Trans Am Geoph Un, $\checkmark \quad 31, p$ 196-212

Sonu, C J , McCloy, J M and McArthur, D S, 1966, Longshore currents and nearshore topographies Proc Tenth Conf on Coastal Engineering, p 525-549

Sonu, C J, 1968, Collective movement of sediment in the 11 ttoral environment Proc Eleventh Conf on Coastal Engineering, p 373-400 



\title{
CHAPTER 48
}

\section{LITTORAL TRANSPORT AND ENERGY RELATIONSHIPS}

\author{
L Bajorunas \\ National Oceanic and Atmospheric Administration \\ Detroit, Michigan 48226
}

The Inttoral transport rates in the Great Lakes were obtanned two distunctly different ways, long-term averages from dmft acoumlations and hourly averages were measured in the $S t$ clarr River which recenves sand from Lake Huron beaches Statistrcal analysis of both the recorded energy elements and measured sedument transport rates indrcates that a combrnation of energy elements and enviromental factors consisting of wave power and duration, current speed, and length of shoreline produces the best correlation whth the transport rate Dimensional analysis expands the process-response model by including sedzment-size and specific-werght parometers

\section{INTRODUCTION}

The St Clair River, which emerges from Lake Huron, carries 11ttle sediment during calm lake conditions, however, during storms, it transports significant amounts of sediment from Lake Huron beaches adjoining the river Extensive measurements were made during the spring and fall of 1965 to determine the amount and characteristics of the sediment transported both in suspension and as bedload by the St Clair River Simultaneous recordings were made of the energy elements in Lake Huron that could contribute to the pickup of sediment from the beaches and transportation downdrift into the St Clair River

\section{SEDIMENT TRANSPORT}

Measurement methods

The St Clair River heads at the southern end of Lake Huron (Fig where it passes through a restricted and narrow reach, "The St Clair Rapıds", with water velocities up to $2 \mathrm{~m} / \mathrm{s}$ and associated extreme turbulence Exposure to lake waves and very heavy vessel traffic prohibited the use of this reach and, therefore, a river cross-section further downstream was selected for measurement of sediment transport Insignificant deposition has been observed in the reach of the river between the lake and the metering section, which is at the shoal just downstream of the first river bend and some boat slips 


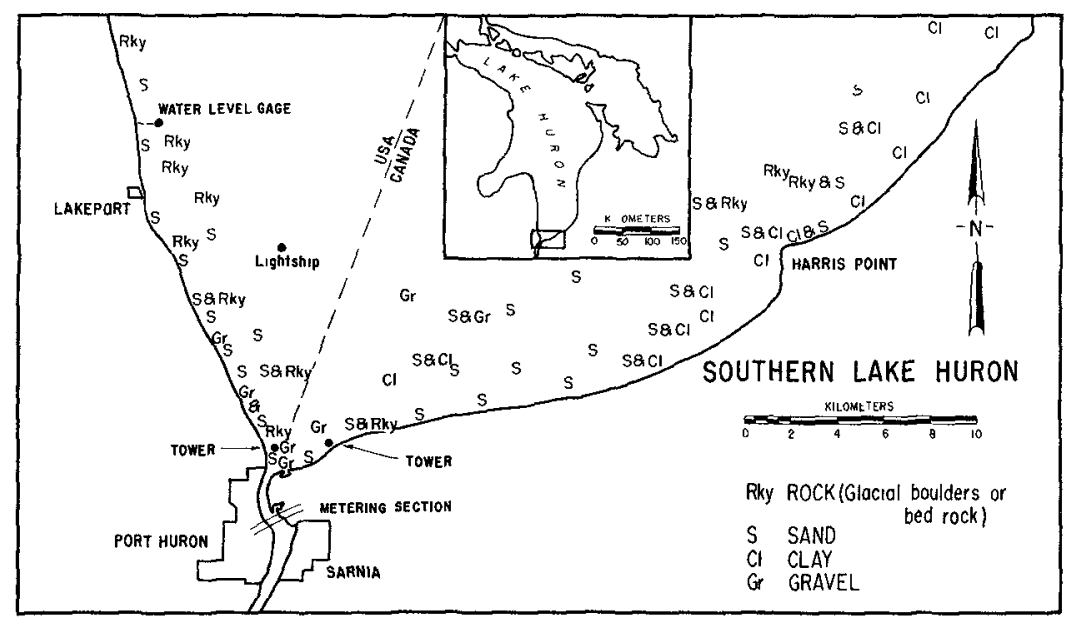

\section{Frg 1 Southern Lake Huron}

The river cross-section was divided into elght panels for the purpose of sediment measurement Water samples were taken and current velocities recorded at 01 depth intervals at the mid-vertical of each pane1 A second water sampler, simultaneously took samples at a constant 04 depth Each vertical was sampled twenty times, and the complete river crossing produced at least 160 samples $U$ S P-61 suspended sediment samplers and Price current meters were used in the data collection program The sand moving within five centimeters of the river bottom was trapped in an Arnhem bedload sampler The time required for sampling one panel, including the time for positioning of the survey boat, was about one hour it would have been possible to complete a river crossing in a day but weather and heavy vessel traffic caused unexpected delays and on only one occasion was a river crossing completed in a single day

\section{Transport rates}

The measured concentration of suspended sediment by weight ranged from 03 to $90 \mathrm{ppm}$, with an average of about $10 \mathrm{ppm}$ The sediment transport in suspension was determined using the standard method of the current velocity times the cross-sectional area times the suspended sediment concentration An average density of $1700 \mathrm{~kg} / \mathrm{m}^{3}$ was used to change the suspended sediment transport rate by weight into rate by volume Repeated sampling at the 04 depth shows that the concentration is subject to considerable variation with time in addition to the seemingly random variation some regular pulsation in sediment concentration was observed, which in no way can be related with the flow in the St Clair River 
The bedload was determined by extrapolating the measured rate in the sampler to represent the transport over the panel width Sediment movement along the river bed amounted to a fraction of one percent of the suspended sediment and was not considered further The measured rates of suspended and bedload sediment are given in Table 1 Table 1 also lists the sum of the measured values and the estimated total transport in the river In two cases the measurements were insufficient for a reasonable estimate Comparison of the measured portion of the transport with the estimated total gives some indication of the magnitude of errors

TABLE 1 SUSPENDED AND BEDLOAD SEDIMENT IN ST CLAIR RIVER

\begin{tabular}{llllllllll} 
Date & \multicolumn{4}{c}{ Rrver panezs } & & & $\begin{array}{l}\text { Sum Estrm } \\
\text { of total }\end{array}$ \\
1965 & 1 & 2 & 3 & 4 & 5 & 6 & 7 & 8 & meas trans
\end{tabular}

\begin{tabular}{|c|c|c|c|c|c|c|c|c|c|c|c|}
\hline 14 & May & 92 & 185 & 146 & 62 & 108 & $85^{*}$ & $62^{*}$ & $30 * 59$ & 3 & 77 \\
\hline 17 & $"$ & 41 & 66 & 53 & 73 & -- & -- & -- & 23 & 3 & 34 \\
\hline 19 & $"$ & 66 & 125 & 92 & 152 & 93 & 59 & 53 & $20 \quad 66$ & 0 & 66 \\
\hline 20 & $"$ & 64 & 87 & 109 & -- & - & -- & -- & -- & 0 & 48 \\
\hline 21 & $"$ & 27 & 75 & 133 & 129 & -- & -- & -- & 36 & 4 & 54 \\
\hline 3 & Jun & 89 & 139 & 102 & 92 & -- & -- & -- & 42 & 2 & 62 \\
\hline 4 & $"$ & -- & - & -- & -- & 68 & 77 & 53 & $\begin{array}{lll}19 & 21\end{array}$ & 7 & 68 \\
\hline 14 & $"$ & 226 & 356 & - & -- & - & -- & -- & 58 & 2 & 160 \\
\hline 15 & $"$ & $154^{*}$ & $264^{*}$ & 242 & 110 & 121 & 99 & 77 & 3368 & 2 & 110 \\
\hline 16 & $"$ & 104 & 162 & 164 & 107 & -- & -- & -- & 53 & 7 & 79 \\
\hline 3 & Nov & 32 & -- & -- & -- & -- & -- & -- & -- & 2 & - \\
\hline 4 & $"$ & 229 & 415 & 284 & 377 & -- & -- & -- & 130 & 5 & 190 \\
\hline 6 & $"$ & - & -- & -- & -- & 109 & 41 & 66 & $\begin{array}{lll}3 & 4 & 25\end{array}$ & 0 & 78 \\
\hline 9 & $"$ & 306 & 468 & 270 & 198 & 198 & 180 & $126 *$ & $54 * 162$ & 0 & 180 \\
\hline 10 & $"$ & -- & -- & -- & -- & -- & -- & 36 & 17 & 3 & -- \\
\hline 18 & $"$ & 405 & 640 & 464 & 486 & 375 & - & -- & 237 & 0 & 300 \\
\hline 19 & $"$ & - & - & - & - & -- & 95 & 89 & 4422 & 8 & 110 \\
\hline
\end{tabular}

$\begin{array}{rrrrrrrrrr}19 \mathrm{May} & 9 & 10 & 9 & 15 & 9 & 9 & 9 & 6 & 76 \\ 8 \mathrm{Nov} & 2 & 5 & 7 & 16 & 5 & 14 & 4 & 3 & 56 \\ \end{array}$

\section{Sedıment Characterıstics}

A detalled description of the sediments in St Clair River was published by Duane (1967) and, therefore, only a summary is given here The suspended sediment is mostly of the fine sand size, ranging from $011 \mathrm{~mm}$ to $017 \mathrm{~mm}$, with a few particles reaching $10 \mathrm{~mm}$ size Material is dominantly quartz although other minerals and organic matter are present The bedload sediment size ranges from $025 \mathrm{~mm}$ to $26 \mathrm{~mm}$ 
Samples from Lake Huron beaches show the same composition and grain-size characteristics as the suspended load and bedload of the St Clair River Areal extent of sand along lower Lake Huron is depicted in Fig 1 Mandelbaum (1966) reported that sediment in the St Clair River delta at the entrance to Lake St Clair contains some finer grain sizes than those measured in the river

\section{ENERGY ELEMENTS}

\section{Elements measured}

In addition to the primary variables, wind speed and direction, wave height and period, and current speed and direction, some elements probably only remotely related to the study were recorded, such as barometric pressure, and aIr and water temperature Lake level and river discharge data were aval lable from continuous data collection programs on the lakes Instrumentation was placed at five locations (see $F_{1 g}$ 1) on the Coast Guard Light Ship, on two specially erected towers, and on two submerged tripods Towers, as described by Duane and Saylor (1966) were placed near the east and west shores in $57 \mathrm{~m}$ and $66 \mathrm{~m}$ depths, respectively Submerged tripods were located between the shore and the towers in $15 \mathrm{~m}$ depth and supported current meters only Data pertinent to the sediment sampling periods are listed in Table 2

\section{TABLE 2 MEASURED ENERGY ELEMENTS}

\begin{tabular}{|c|c|c|c|c|c|c|c|c|c|c|}
\hline \multirow{4}{*}{\multicolumn{2}{|c|}{$\begin{array}{l}\text { Date } \\
1965\end{array}$}} & \multirow{4}{*}{$\begin{array}{l}\text { Lake } \\
\text { Zever } \\
m\end{array}$} & \multirow{3}{*}{\multicolumn{2}{|c|}{$\begin{array}{c}\text { Wrnd } \\
\text { Speed Persist } \\
\text { Drr }\end{array}$}} & \multicolumn{3}{|c|}{ East Shore } & \multicolumn{3}{|c|}{ West Shore } \\
\hline & & & & & Wave & Wave & Curps & Wave & Wave & Curs \\
\hline & & & & & Herght & Per & Speed I & Herght & Per & Speed \\
\hline & & & $\mathrm{m} / \mathrm{s}$ & $h r$ & $\mathrm{~cm}$ & $s$ & $\mathrm{~cm} / \mathrm{s}$ & con & $s$ & $\mathrm{~cm} / \mathrm{s}$ \\
\hline 14 & May & 17568 & $33 \mathrm{NE}$ & 4 & - & -- & -- & 6 & 30 & 16 \\
\hline 17 & & 17577 & $42 \mathrm{~W}$ & 10 & 18 & 33 & $10^{*}$ & 11 & 34 & 17 \\
\hline 19 & $n$ & 17576 & $40 \mathrm{~N}$ & 8 & 25 & 30 & 11 & 17 & 32 & 20 \\
\hline 20 & $n$ & 17577 & $43 \mathrm{~N}$ & 13 & - & -- & 9 & 12 & 31 & 18 \\
\hline 21 & $"$ & 17571 & $42 S E$ & 4 & - & -- & 9 & 6 & 34 & 17 \\
\hline 3 & Jun & 17580 & $55 \mathrm{~N}$ & 9 & 40 & 31 & 9 & 36 & 32 & 12 \\
\hline 4 & $"$ & 17577 & $39 \mathrm{~N}$ & 6 & 12 & 30 & 9 & -- & -- & 14 \\
\hline 14 & $"$ & 17584 & $76 \mathrm{~N}$ & 34 & 61 & 42 & $15^{*}$ & 37 & 36 & $20^{*}$ \\
\hline 15 & $"$ & $175 \quad 82$ & $88 \mathrm{~N}$ & 5 & 77 & 36 & 18 & 37 & 35 & 24 \\
\hline 16 & $"$ & 17583 & $75 \mathrm{~N}$ & 3 & 60 & 32 & $15^{*}$ & 29 & 32 & 15 \\
\hline 4 & Nov & $176 \quad 02$ & $56 \mathrm{~N}$ & 11 & 64 & 75 & 27 & 53 & 41 & $15^{*}$ \\
\hline 6 & $"$ & 17586 & $38 \mathrm{~S}$ & 25 & 8 & 37 & 3 & -- & -- & 21 \\
\hline 9 & $"$ & 17592 & $43 \mathrm{NW}$ & 22 & 41 & 52 & 40 & 46 & 45 & 10 \\
\hline 18 & $"$ & 17592 & $53 \mathrm{~W}$ & 42 & 31 & 62 & 110 & -- & -- & - \\
\hline 19 & $"$ & 17589 & $18 \mathrm{~W}$ & 15 & 29 & 54 & 10 & -- & -- & -- \\
\hline
\end{tabular}

Recorded current drrection on the Insted days was towards the St Claix River desprte the vamable wrnds

\section{*estrmated}


Overwater wind was recorded at the two towers and at the light Sh1p The wind over this rather small area was quite uniform The data listed were obtained from the tower near the west shore Wind persistence was defined as the period of time prior to sediment samp1ing having essentially the same wind speed and direction as that recorded during the sediment sampling period All higher wind speeds, lower speeds of less than one hour duration, and deviations of less than $45^{\circ}$ in direction were included in the determination of persistence

Waves were also recorded at the two towers and near the Light Shıp The wave gage near the Light Shıp was difficult to mantain due mainly to excessive cable length The data recorded at this location were insufficient for analysis Wave recordings taken at the two tower locations were more successful, but even in these locations some records were missing, as was the case on 18 November when the hlghest sediment movement was measured Heights listed in Table 2 are of significant waves, which are defined as four times the standard deviation of the wave gage record The linear average values read from wave spectra curves produced by the Coastal Engineering Research Center were multiplied by a factor of 27 to obtain significant waves The 27 factor was derived from data by Cole (1967) The wave periods are those at which maximum energy was produced Analyses of several 20 minute wave records taken at different tımes durıng the sediment sampling period were averaged to obtain the listed values No attempt was made to estimate the missing waves from wind records cole concluded on the basis of carefully recorded wind and wave data that existing wind and wave relatıonships were producing marginal results

Currents were recorded at four locations One current sensor was attached to each tower and the other two sensors were placed on the two tripods The propeller type current sensors were placed in a tube about $10 \mathrm{~cm}$ above the bottom These so-called ducted current meters were responsive to currents along the axis of the tube, which was oriented parallel to the shoreline The direction indicator was freely suspended and assumed the direction of dominant current Current speed and direction were both recorded, however, Table 2 lists only current speeds recorded on the tripods The currents at the times 1isted were always toward to the head of the river The meters frequently jammed during operation due to sand particles or weeds lodging between tube and propeller Some missing current data were estimated w1 th reasonable accuracy el ther from the records immediately preceding or following the missing records or from the currents recorded on the towers The estimated values are so indicated

Lake Huron levels and outflows are recorded by the $U S$ Lake Survey Levels are recorded by five water-level gages, w1 th those recorded at the Lakeport gage listed In addition to the 1ong-term gradual change of lake levels caused by the changing water volume there are frequently quite large local water level changes caused by wind and barometric pressure In contrast with the oceans, lunar tides in the Great Lakes are quite sma11, not exceeding $15 \mathrm{~cm}$ Durıng the sediment sampling periods, however, only insignificant short-term changes in lake level were recorded The flow in the St Clalr River has a small day-to- 
day varıation and was gradually 1 ncreasing from $4700 \mathrm{~m}^{3} / \mathrm{s}$ in May, to $4900 \mathrm{~m}^{3} / \mathrm{s}$ in June, and to $5200 \mathrm{~m}^{3} / \mathrm{s}$ in November

\section{ANALYS1S OF TRANSPORT FACTORS}

Littoral transport is affected by numerous factors basically related to the sediment supply and characteristics, and energy in its varlous forms A brief discussion of the more obvious factors and their numerical evaluation follows

\section{Sediment Characterıstics}

Recent investigations utılizing both models and the natural environment point to the important effect the sediment size distribution has on the movement rate in littoral environments However, no agreement exists on magnitude of that effect A comprehensive study of sediment movement in the ocean environment was made by lngle (1966) He found that sediment transport doubles with the change in sediment size from $014 \mathrm{~mm}$ to $020 \mathrm{~mm}$, and 1 ncreases by $20 \%$ for the change from $020 \mathrm{~mm}$ to $028 \mathrm{~mm}$ Model studies by Larras (1966) indıcate that the maximum transport takes place when the median diameter of the sediment is 09 $\mathrm{mm}$ Material finer or coarser than this moves at significantly lower rates For example, fine sand of the size on Lake Huron beaches moves at one third the rate of the $09 \mathrm{~mm}$ size sediment Contrary findings are reported by Iwagakı and Sawarag1 [Homma, (1966)] Their equation indicates that sediment movement decreases with increase of the square root of sediment diameter ln present study the dimensional analysis, discussed later, indicates that transport increases with the square root of sediment size This is in general agreement with findings by others for the fine to medium sand

Sediment must be freely avallable along the shoreline for equillbrium to exist between energy and the volume of sediment in movement In the study area, sand on the east shore extends northeastward for about $20 \mathrm{~km}$ and ends before Harris Point The areal extent of sand on the west shore cannot be clearly assessed, although it is estimated to be in the order of $20 \mathrm{~km}$ Sediment movement in this location is somewhat retarded by scattered rocks of glacial origin

\section{Effective Shoreline and Wave Duration}

Two limiting factors in sediment transport must be considered the length of unobstructed shoreline and the duration of wave action The wave duration is the limiting factor for very long shorelines, however, long shorelines without elther natural or man-made barriers to sediment movement rarely exist More frequently the length of unobstructed shorelines sets the limit to sediment transport Th1s phenomenon in nature, however, is rather complex due to the continuous variation of both the energy elements during a storm and the sediment characteristics along a shoreline Statistical methods must therefore be applied when considering observations in nature The effect of shoreline length was investigated by Bajorunas (1961) based on long-term drift accumulation on the updrift side of harbors or natural barriers Present 
investigations consider a variable duration of wave action over a given length of shoreline Persistence of wind speed and direction is used here as an index of the duration of waves

Effective Shoreline in the 1961 study varıed from 2 to $67 \mathrm{~km}$ with the wave duration undetermined Since sediment data was obtained from accumulations extending over long perıods, sometimes exceeding 90 years, it seems that any reasonable wave duration could be applicable and that the transport is, therefore, $11 \mathrm{mlted}$ by the effective shoreline rather than by wave duration It is difficult to isolate the effect of the shoreline in the empirical 1ittoral transport and energy equation derived in 1961 It was therefore necessary to replace that equation

$$
\mathrm{Q}=19 \mathrm{E}_{0} \sin \alpha_{0}\left(1-\mathrm{e}^{-0023 \mathrm{Scot} \alpha_{0}}\right)
$$

by the theoretically less desirable

$$
Q=45 \mathrm{E}_{\mathrm{o}} \sin 2 \alpha_{\mathrm{o}}\left(1-\mathrm{e}^{-004 \mathrm{~S}}\right)
$$

where $Q$ is the annual 11ttoral transport, cubic yards in equation (1) and $\mathrm{m}^{3}$ in (2)

$E_{O}$ is the annual deepwater wave energy, millions of foot-pounds in equation (1) and $10^{6}$ joules in (2)

$S$ is the effective length of shoreline, miles in (1) and $\mathrm{km}$ in (2)

$\alpha_{0}$ is the angle between the shoreline and the wave crest

Fig 2 contains the 1961 data which depicts transport per energy unit versus the effective shoreline

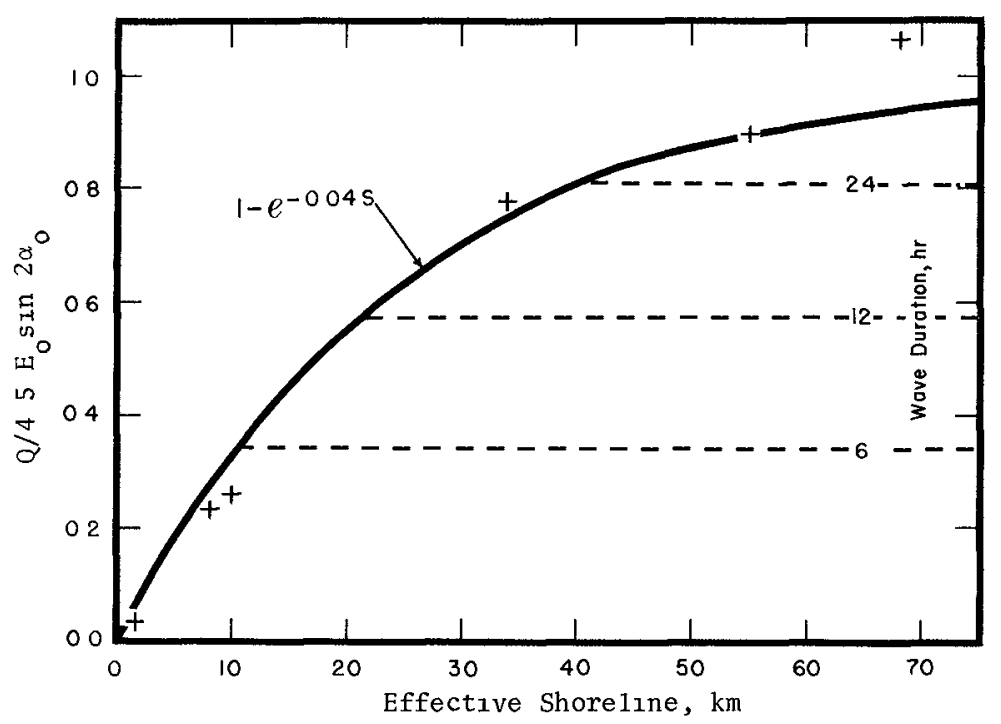

Fig 2 Effect of shoreline on Zittoral transport 
Wave Duration in the present study was based on recorded wind persistence and varied from 3 to 34 hours while the effective shoreline was about $20 \mathrm{~km}$ Data in Fig 3 indicate that the wave duration is a significant factor in sediment transport if less than about twelve hours The effective shoreline becomes the determining factor for longer periods and on these occasions the transport per energy unit remains more or less constant The effect of wave duration was expressed mathematically as (1 - e -0 07D) based on meager field data and on the assumption that 1 must have an exponential form similar to that derived for effective shoreline

The dependence of drift rates upon both the wave duration and effective shoreline requires selection and use of that factor which restricts the transport more

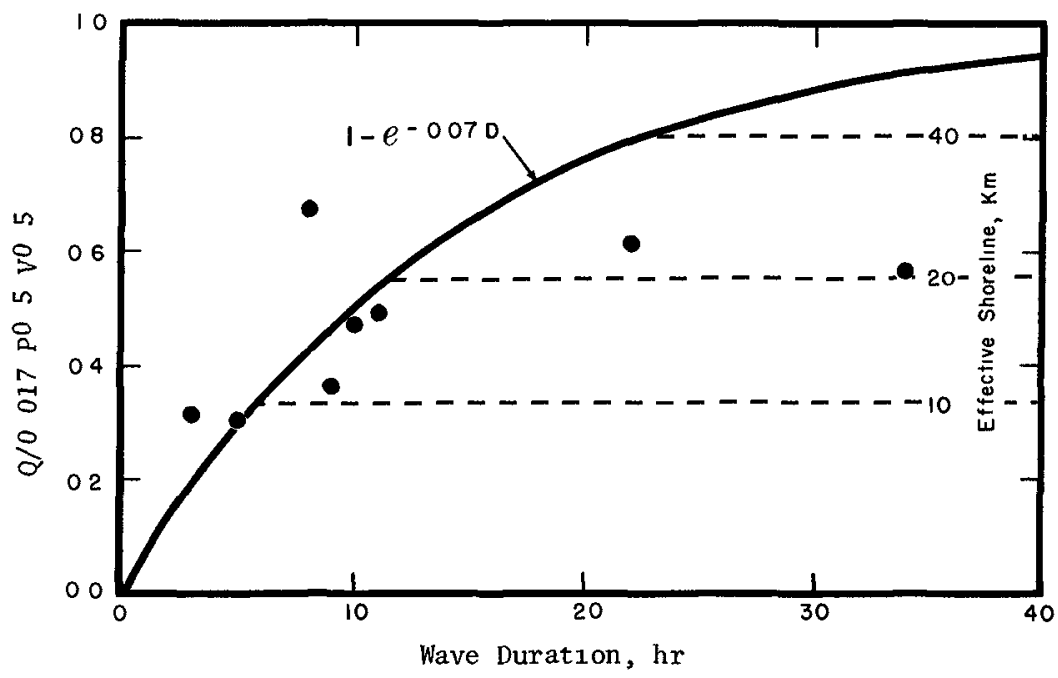

Fig 3 Effect of wave duration on inttoral transport

Energy Elements

Wave power, as used here, was derived from wave enexgy and wave frequency The wave energy per unit width of wave crest is expressed as

$$
E=0125 \gamma \mathrm{H}^{2} \mathrm{~L}
$$

where $\gamma$ is specific weight of water

$\mathrm{H}$ is wave height

$L$ is wave length

Considering the rather small waves in this particular case, the correction for wave shape was deleted and the wave length was expressed by $\mathrm{gT}^{2} / 2 \pi$, where $\mathrm{T}$ indicates wave period The energy transmitted to the 
shore by wave train in a $t$ me period $t$ is equal to $E_{t}=n E T^{-1} t$, where $\mathrm{n}$ is the ratio of wave group velocity to the phase velocity and is, In this case, approximately 05 The wave energy exerted over a time period $t$ is thus

$$
E_{t}=(32 \pi)^{-1} \gamma \mathrm{g} \mathrm{H}^{2} \mathrm{Tt}
$$

The above equation is correct for any unit system The equation can further be simplified to $E_{t}=960 \mathrm{H}^{2} \mathrm{Tt}$, if the international system (SI) is used where the wave energy is expressed in joules To conform with the sediment transport, $t=3600 \mathrm{~s}$ was used here Wave frequency, if considered as an independent factor, greatly improves the correlation between sediment transport and energy elements The combination of wave energy and wave frequency results in wave power $(P=E f)$, where the wave helght is the only significant element This would indicate that the wave helght and not the wave length or wave period has an effect on the transport rate

Currents It becomes obvious from the analysis of the data collected, that wave energy alone or in combination with the angle of wave approach is not sufficient to define the movement of material In this particular location, morever, with its prevalling winds from the west and currents from the east, the results would be erroneous When the waves and currents come from differing directions the sediment saltation is necessarily complex, however, net movement in the study area was always in the direction of the currents One can speculate then that waves lift the material and the currents transport it in the direction of flow Previous observations of shoaling in a harbor also indicate that sediment moves in the direction of currents [Bajorunas and Duane, (1967)]

Investigations of lake currents by Saylor (1966) indicate that currents generated by winds reach equilibrium with the wind at about 0033 of the wind speed This occurs in a rather short period of one to three hours Currents persist for days after wind cessation, and therefore have quite stable patterns in geographic locations where certain winds prevail, such as the Great Lakes wi th their predominantly westerly winds Currents in the open lake flow 10-15 degrees to the right of the wind direction with current speed and direction practically independent of surface wave activity Currents in nearshore locations are modified by shoreline configuration and, depending on that configuration, might flow against the wind Occurrences of this type were recorded frequently in the study area The data establishes a strong relationship between the volume of sediment being transported and the current speed There is no apparent upper IImI for this relationship

\section{RELAT IONSHIPS}

Logarithmic plotting (not shown) of the rate of sediment transport, adjusted for effective shoreline and wave duration, against a factor consisting of a combination of wave power and current speed indicates that transport is di rectly related to the square root of this factor and the relationship can be expressed as follows if $f(S, D)$ denotes the 
limiting effects either of the shoreline or the wave duration

$$
Q=039 \times(P \text { v })^{0} 5 \mathrm{f}(\mathrm{S}, \mathrm{D})
$$

where $x$ represents the unknown dimensions of the numerical factor Analysis indicates that sediment characteristics, expressed by sediment size $d$ and by specific weight of sediment in water $g(\rho-1)$, will satisfy the dimensional requirements Average values of the sediment in the study area, $d=014 \mathrm{~mm}$, and $\rho=24 \mathrm{~g} \mathrm{~cm}^{-3}$, allow us to determine the numerical value of $x$ Thus, the equation (6) becomes

$$
Q=39\left[\mathrm{P} \vee \mathrm{d}(\rho-1)^{-1} \mathrm{~g}^{-1}\right]^{0}{ }^{5} \mathrm{f}(\mathrm{S}, \mathrm{D})
$$

where $Q$ is 11ttoral transport, $\mathrm{m}^{3} / \mathrm{hr}$

- 3.9 is constant, dimensionless factor frequency

$\mathrm{P}$ is wave power, $\mathrm{J} / \mathrm{s}$, computed as hourly wave energy times wave

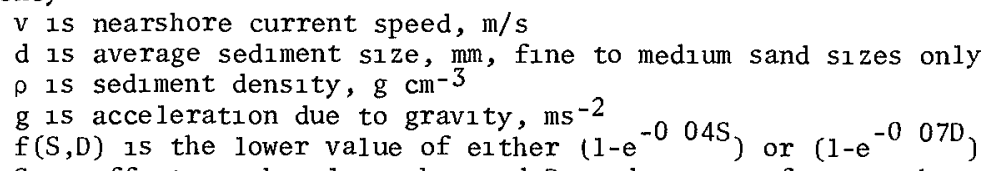
where $S$ is effective shoreline, $\mathrm{km}$, and $\mathrm{D}$ is duration of waves, $\mathrm{hr}$

Fig 4 provides a comparison of the measured transport with that computed by equations (2) and (7)

\section{SUMMARY}

The littoral transport rates in the Great Lakes were obtained two distinctly different ways The long-term averages were derived from drift accumulations over periods sometimes exceeding 90 years on the updrift side of harbors or natural barriers The hourly averages were directly measured in the St Clalr River into which sediment moves from Lake Huron beaches At the same time sediment characteristics were determined and recordings of the energy elements analyzed to relate them w1th the measured sediment transport rates

Analysis of data indicates that

1 Wave power and nearshore currents are the main elements in sediment movement On occasions when the current is flowing counter to the waves, sediment moves with the current One can speculate that waves are lifting material and the current is transporting it in the direction of flow Transport rate is directly related to the square root of wave power and current speed

2 Length of sediment-contributing shoreline, exposed to waves and currents, is a significant factor in determining the transport rate Analysis of data on shorelines varying from 2 to $67 \mathrm{~km} \mathrm{in}$ length indicates that transport grows exponentially with the shoreline length towards a steady-state value

3 History of acting elements, as expressed by wave duration, 


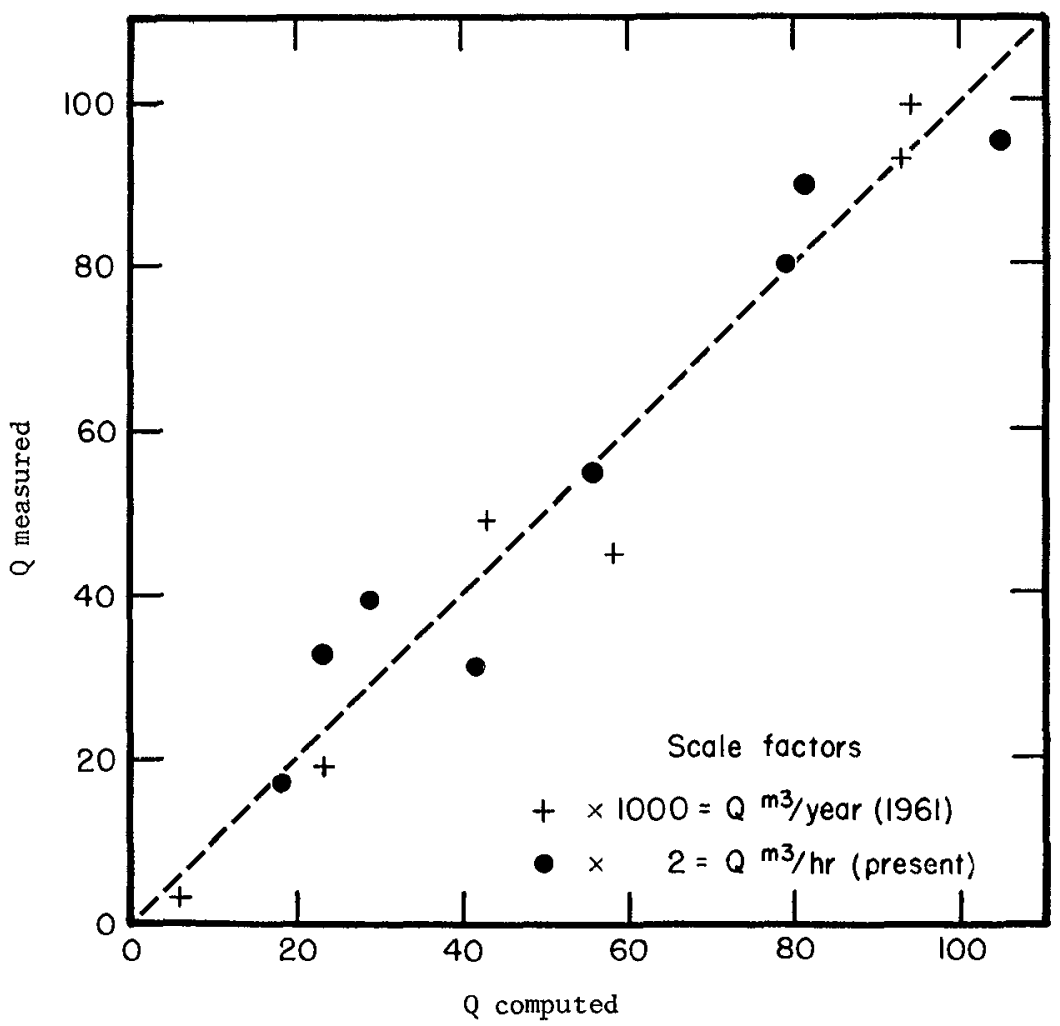

Fig 4 Compamson of measured littoral transport with computed

helps to explain the transport growth and is a limiting factor in cases of short wave duration on a long shoreline Wave duration, which varied from 3 to 34 hours, has a simllar effect to that of shoreline length, the effect increases exponentially with the increase in duration towards a steady-state value

4 Transport increases with the square root of sediment size This conclusion was reached indirectly by dimensional analysis and genera1ly agrees with observations in oceans and models for the fine and medium sand size

5 Equatıons utılizing wave energy, angle of wave approach, and shoreline length could provide reasonable estimate of sediment movement on an open shoreline along whlch the wind, waves, and current progress in the same direction They would fall, however, in the complex wave and current environment, in such environment the nearshore current speed and direction is an essential factor 


\section{ACKNOWLEDGEMENTS}

The field collection of data, laboratory procedures, and analysis were carried out in connection with the general research program of the Great Lakes Research Centex, U S Lake Survey, Army Corps of Eng1neers Permission granted by the Chief of Engineers to publish this information is appreciated Fieldwork was directed by $\mathrm{Dr} \mathrm{D}$ B Duane, presently with the U S Army Coastal Engineering Research Center Essential assistance was provided by $M r W$ P Ward in analysis of the sediment data and $M r \quad P \quad C$ Liu in wave energy calculations

\section{REFERENCES}

Bajorunas, L (1961) Littoral transport in the Great Lakes, Proc 7 th Coastal Eng Conf, Council on Wave Res, Unıv Calıf, Berkeley, 326-341

Bajorunas, L and Duane, D B (1967) offshore bars and harbor shoalIng, J Geophys, Res, 72, 6195-6205

Cole, A L (1967) An evaluation of wind analysis and wave hindcasting methods as applied to the Great Lakes, Proc Tenth Conf Great Lakes Res, Intern Asso for Great Lakes Res, Ann Arbor, Mich, 186-196

Duane, D (1967) Characterıstıcs of the sedıment load in the St Cla1r River, Proc Tenth Conf Great Lakes Res, Intern Asso for Great Lakes Res, Ann Arbor, M1ch, 115-132

Duane, D B , and Saylor, J H (1966) Portable Great Lakes research towers, Proc Ninth Conf Great Lakes Res, Intern Asso for Great Lakes Res, Ann Arbor, M1ch , 295-300

Homma, M (1966) Coastal sediment studies in Japan, Unıv of Tokyo, $11 \mathrm{p}$

Ingle, J C (1966) The movement of Beach Sand, Unıv of S Calif $221 \mathrm{p}$

Larras, J (1966) Cubes de sable charries par la houle parallelement a la cote, Anales des Ponts at Chaussees, Paris, 71-76

Mandelbaum, H (1966) Sedimentation in the St Claır River delta, Proc Ninth Conf Great Lakes Res, Unıv of Michıgan, Ann Arbor, 192-202

Saylor, J H (1966) Modification of nearshore currents by coastal structures, Misc paper, Lake Survey District, Corps of Engineers, Detrolt, Michigan, $14 \mathrm{p}$ 


\title{
CHAPTER 49
}

\section{SYNOPTIC OBSERVATIONS OF SAND MOVEMENT}

\author{
David B Duane \\ Chief, Geology Branch \\ U S Army Corps of Engineers \\ Coastal Engineering Research Center \\ Washıngton, D C
}

\section{ABSTRACT}

The U S Army Corps of Englneers' Coastal Engineering Research Center, in cooperation with the Atomic Energy Commission, inltiated a multi-agency program to create a viable radiolsotopic sand tracing (RIST) program other agency participants in this program have been the Los Angeles District, Corps of Englneers, U S A1r Force (F1rst Strategic Aerospace Division), U S Navy (Pacif1c Missile Range), U S Army Mobility Equipment Command, Nationa1 Aeronautics and Space Administration, and the State of California (Dept of Navigation and Ocean Development) CERC, together with the AEC's Oak Ridge National Laboratory has developed tagging procedures, hardware development, field surveys and data handling techniques that permit collection and analysis of over 12,000 bits of information per hour over a survey track of approximately 18,000 feet Data obtained with the RIST system can be considered as nearly symoptic observations of sediment transport in a single environmental zone or in adjacent beach, surf and offshore zones

Using sand tagged with lsotopes of gold, experiments have been carried out at several sites on the Califomia coast Surf, Point Conception area, Point Mugu, and Oceanside Data from the studies carried out in beach areas unmodified by littoral barriers indicate that under a given set of wave conditions the alongshore velocity of sediment transport differs from zone to zone such that transport seaward of peaking-breaking waves < transport on the beach face < transport in the plunge and surf zone Because of these differences, tracing surveys confined solely to the foreshore or offshore $z$ ones produce data only partially indicative of transport in the zone of immediate concern to coastal engineers

\section{INTRODUCTION}

Recognizing the engineering need to better understand coastal processes, the U S Army Corps of Englneers' Coastal Engineering Research Center, in cooperation with the Atomic Energy Commission, initiated a multi-agency program to create a viable radiosotopic sand tracing (RIST) program To date coopercting agencies have included the Los Angeles District, Corps of Engineers, U S Mobility Equipment Command, the U S A1r Force 1st Strateg1c Aerospace Division and Western Test Range, U S Navy Pacific Missile Range, the National Aeronautics and Space Administration, and the State of California Department of Navigation and Ocean Development In addition to the development of the techniques and technology necessary to trace nuclide labelled particles in the marine environment objectives can be summarized as understanding the mechanics 
of sediment movement, both entrainment and transport, patterns of movement, and the volume of sediment movement Relative to the application of these goals to englneering needs field experiments have been carried on straight coastal segments unaltered by englneering works as well as coastal segments effected by engineerıng works such as groins and harbor jettıes

Taggıng procedures, hardware development, field surveys and data handling techniques have been developed in cooperation with AEC's Oak Ridge National Laboratory that permit collection and analysis of over 12,000 bits of information per hour over survey track of approxımately 18,000 feet Detalls of mode 1 studies, tagging procedures, detection instrumentation, and data handling are discussed elsewhere in these proceedings (James, Acree et a1, and Brasheer et a1) However for clarity and perspective some statements about these 1 tems are necessary to this discussion Search for 1sotopes and tagging procedures sultable for sand tracing have emphasized two criteria, health physics and the engineering behavior of tagged sand (Duane and Judge, 1969, Acree et a1 1969) To date, 1sotopes of gold (198/199) have been used most extensively The gamma energy ( $04 \mathrm{Mev}$ ) is suitable as is the three day half life

Sand indigenuous to a study area is collected approximately 4 weeks before the scheduled field experiment and shipped to Oak Ridge National Laboratories where it is tagged and returned via common carrier to the field site Tagging is a surface tag but is done in such a manner as to simulate a mass tag (Stevens, et a1 1969) Tests were conducted at CERC to determine if the gold tagging process modified the characteristics of the untagged sand No detectable d1fferences in specific gravity occurred and there was no observable change in the shapes of grains overall hydraulic equivalence of the tagged sand to the natura1ly occurring sand was demonstrated by granulometric analyses conducted on the CERC Rapid Sediment Analyzer (a device used to determine the grain size distributional characteristics of sediment, especially grains in the size range from 62 to 2,000 microns, as they settle through a 1-meter column of water) Distance to be covered and areas involved in most field operations require a mobiledetector system with continuous transmission of data to an on-board data recording system The detector vehıcle developed is a ball like device which is towed behind an amphibious vehicle (F1g 1) Signal from the detectors is transmitted by cable to the recording system on-board the amphibious vehicle By means of a programmed interrogator other data pertinent to surveying is coordinated with the radiation data and read into the real time data display and on to punched paper tape

Surveying is accomplished at a speed of approximately three knots (5 $\mathrm{ft}$ per second) Data obtained with the RIST system can be considered as nearly synoptic observations of sediment transport in a single zone or on adjacent beach inshore and offshore zones

\section{FIELD EXPERIMENTS}

Field experiments involving tracing of sand simultaneously in one or more of the three environmental zones have been carrıed out at several California 
sites Surf, Pt Conception, Pt Mugu, and 0ceanside In each program at least one shore perpendicular line source was emplaced and followed for several days as the dispersal pattern continued to grow In some programs a point source was also utılized to more specifically identify the movement of sand from a particular location During most recent experiments conducted at Pt Mugu, as we11 as on the small beach in the harbor at oceanside, patterns of sedıment movement were observed that were similar to those observed during the initial experiments at Surf (Duane, 1970) These indicate that the observed patterns are real and operatıve on any beach, and indıcative of natural processes

At Pt Mugu the first line source (comprised of 30 packets each containing approximately $15 \mathrm{cc}$ of sand in water soluble material and placed 30 feet apart) was emplaced from approximately $-3 \mathrm{ft}$ MLLW to $-30 \mathrm{ft}$ MLLW Two wave sets were active a shorter period higher wave from the west was dominant The first RIST survey was made 5 hours after injection (F1g 2) Evident is a rapld dispersion in the swash zone, movement upcoast is greater in the breaker and surf zone than on the beach face At least one survey per day was made for the next 3 days as the dispersal pattern developed Swell during the 3 days was from the southwest $\mathrm{H}=3$ to $5 \mathrm{ft}, \mathrm{T}=12$ seconds, superposed was a wind chop directed from the northwest with an 8 second period Currents seaward of the breaker zone were north as were those measured in swash zone, although some reversals there were noted Typical of currents measured offshore are depicted in Figure 3 Seventy-two hours after injection a zone apparently vold of tagged material separated the offshore zone from the inshore $z$ one and the beach face This zone vold of labelled sand colncides with the approximate seaward limit of the zone of peaking and breaking waves

On 28 September oceanographic conditıons changed and a second line injection was made, approximately 1,000 feet downcoast This 11ne extended from approximately -1-foot MLLW to approximately $-18 \mathrm{ft}$ MLLW Patterns of sediment movement observed from this injection are unidirectional Swell during the next several days was from the northwest $H=3 \mathrm{ft} T=12 \mathrm{sec}$ Currents offohore and in the swash zone were downcoast (F1g 4) Zonal differential transport rate exists but is not of the same relative magnitude as that observed previously, movement of sand towards the beach face is also evident (FIg 5) The gap in radioactivity along the injection line and observed at tne first Pt Mugu drop coincident with the low tide breaker zone developed rapidly and seemed to grow in width To gather additional data on the movement of sand in and from the breaker zone during oceanographıc conditions then extant, a point source was emplaced there The dispersal pattern of tagged sand was monitored three times in 27 hours obvious movement is alongshore and to the beach and alongshore (F1g 6) Greatest alongshore rate is in the breaker zone and not the beach face No tagged sand was observed to move seaward through the surf and breaker zone

The oceanographıc mileaux adjacent to an engineerıng structure such as a breakwater is somewhat different than the mileaux adjacent an unmodified coastal segment This is amply demonstrated by the water depth in which sand was observed moving at Oceanside $-28 \mathrm{ft}$ MLLW (Fig 7) This depth is nearly twice that observed offshore at Pt Mugu, and under closely similar wave conditions At 0ceanside during the time of observations, no sediment was noted moving through the structure Clearly observed, however, was southward 
jetty and shore-paralle1 movement of nuclide labelled sand up to and slightly into the harbor entrance under a southerly directed current and wave regime (FIg 8) Several days later under northerly directed wave and current regime two point sources of labelled sand, were emplaced one in the harbor entrance at $-18 \mathrm{ft}$ MLLW and one of fshore at $-30 \mathrm{ft}$ MLLW Evident is a bifurcating transport system The sand placed in the harbor entrance moved into and down the dredged channels, the seaward point source moved northward para1le1 to the bottom contour and did not enter the harbor (Fig 9)

\section{SUMMARY}

Durıng perıods when only one wave train Impinges on the coastline unidirectional transport is evident In these instances, marked truncation of label led sand occurs updrift (within $10 \mathrm{ft}$ ) of the injection site Further these data indicate that under given wave conditions the alongshore velocity of sediment transport differs from the offshore zone through the inshore zone to the beach face such that transport seaward of peakıng - breaking waves < transport on the beach face < transport in the inshore (plunge and surf) zone A conceptual model for this process is Illustrated in Figure 10 Dimensions of these zones would change with tide and wave regime

Present technology and techniques make the RIST system a useful engineerIng and research tool However, while the identification of the zonal differentIal transport rate as well as the pattern of sediment movement is of some use to the practicing engineer, full practical value into design considerations will not be reached until the zonal volume rate of transport is obtained Types and dimensions of sedimentary structures observed offshore, in the surf zone, and on the beach are indicative of differing depths of active sand transport Isotope data and plugs of fluorescent and radioactive sand also indicate differing depths of sand movement in different environmental zones These differences, coupled with the differing lateral transport rates introduce complexities to realistic determination of volume sediment movement in the nearshore zone Simple diffusion models are unsuited and any studies confined to one environmenta zone cannot relate the true picture of sediment transport in the nearshore zones important to the coastal engineer Work withIn the RIST program is continuing toward a solution to the problem of volume rate of sand transport with indications of some success although it is premature to report that progress herein

\section{REFERENCES}

Acree, E H, Case, F N , Cutsha11, N H, and Brashear, H R, (1969), "RadioIsotopic Sand Tracer Study (RIST) Status Report for May 1966-Apri1 1968", ORNL-4341, Contract No W-7405-eng-26

Acree, E H, Brashear, H R , \& Case, F N (1970), "A System for Tracing Radionuclide-Tagged Sand", (this volume) 
Brashear, $H \mathrm{R}$, Acree, $\mathrm{E} H$, Case, $F \mathrm{~N}$, Turner, $\mathrm{P} A$, and Duane, $D \mathrm{~B}$ (1970), "Analysis of Radioisotopıc Sand Tracer Study Data" (this volume)

Duane, D B , (1970), "Tracıng Sand Movement in the Littoral Zone Progress in the Radiolsotoplc Sand Tracer (RIST) Study, July 1968-February 1969", U S Army Coastal Englneering Research Center, Miscellaneous Paper 4-70

Duane, D B, and Judge, C W, 1969, "Radıoısotopıc Sand Tracer Study, Point Conception, Calıfornia, Preliminary Report on Accomplishments July 1966June 1968", U S Army Coastal Englneering Research Center, Miscellaneous Paper 2-69

James, W R (1970), "A Class of Probabılıty Models for Luttora1 Drıft," (this volume) 


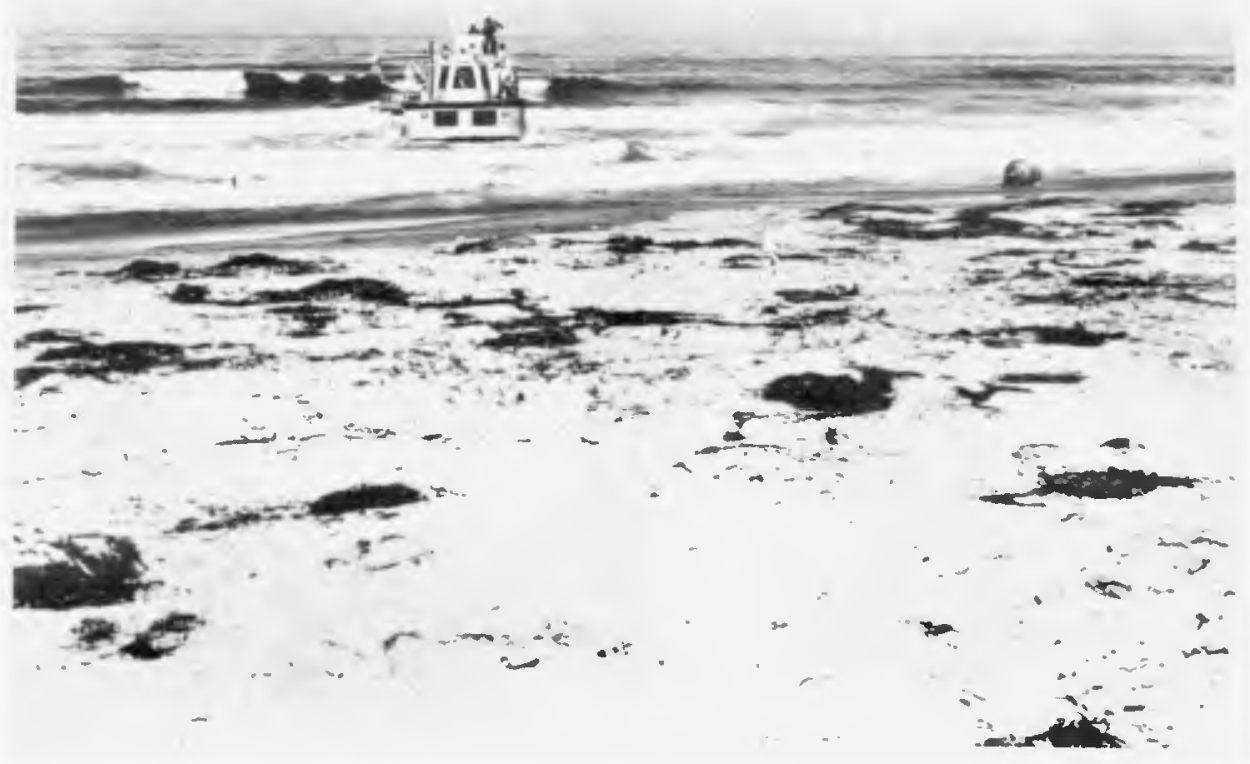

Amphibious LARC XV towing radiation detector vehicle entering surf. The ball-like device is connected to the LARC and the onboard data acquisition system by means of electromechanical cable.

FIGURE 1 


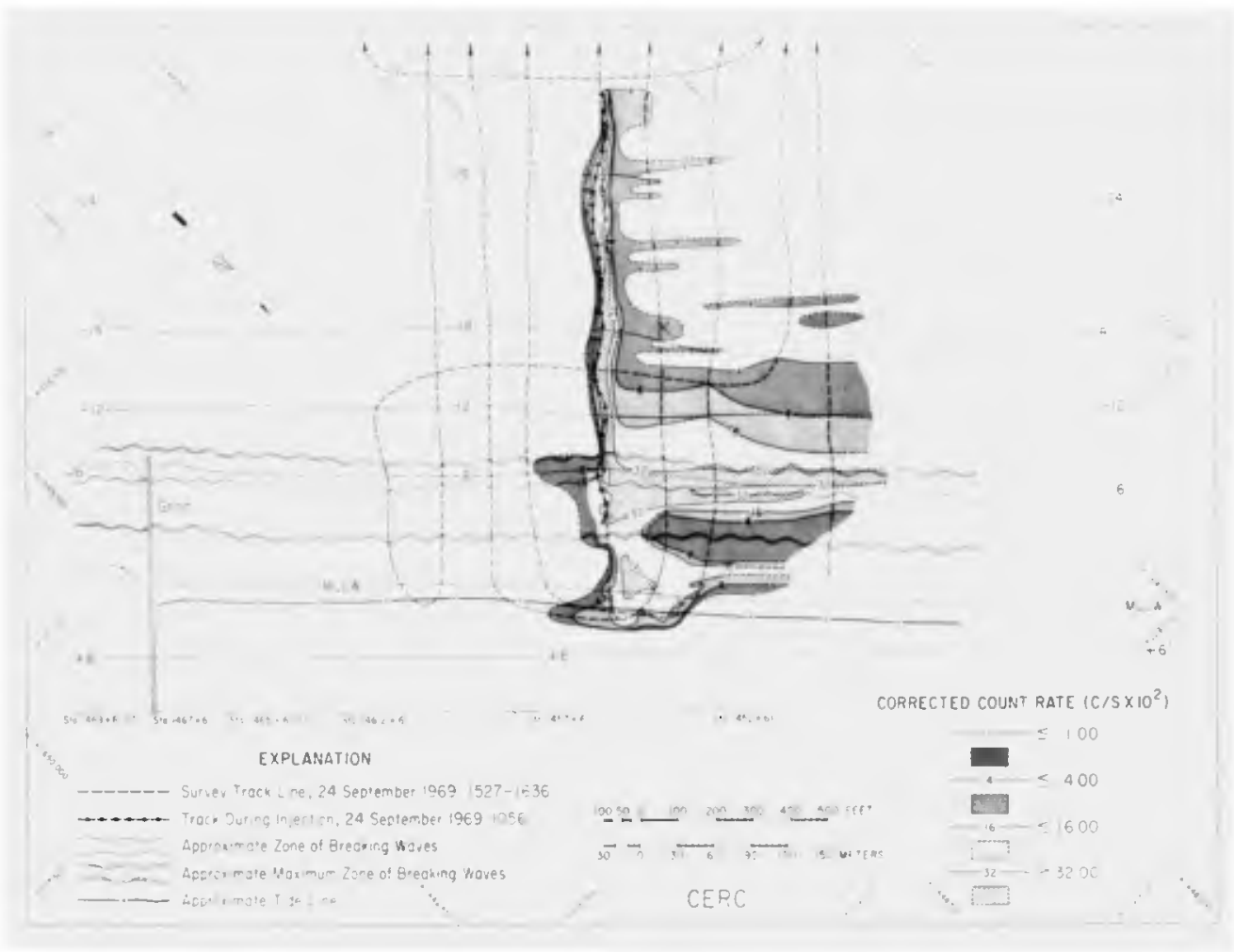

Dispersal pattern of gold-tagged sand observed approximately 5 hours after injection. Note that in this and all site figures, the groin had no panels emplaced and structurally was a pier. 


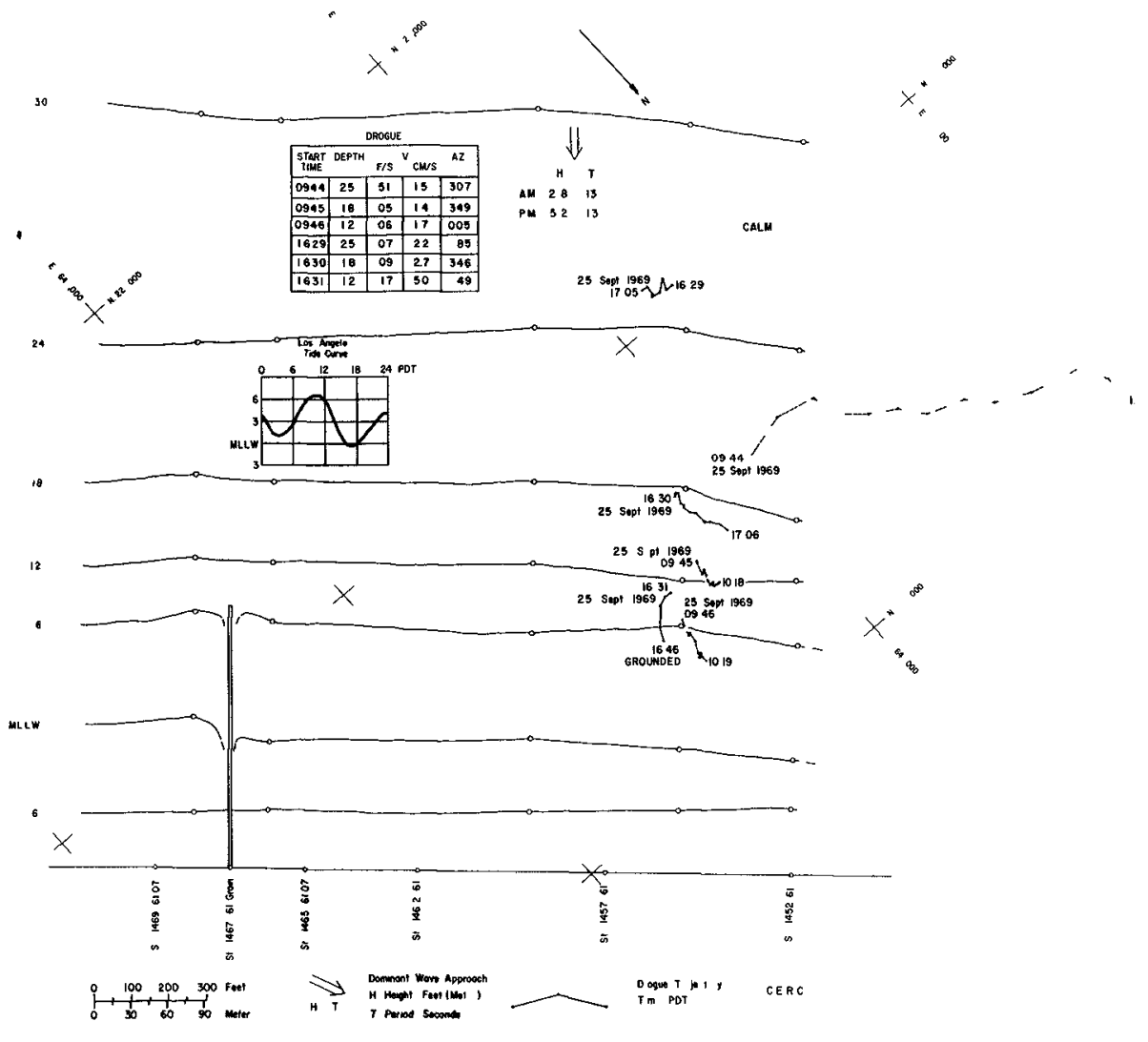

Current drogue patterns, velocitıes, and tide curve representatıve of condıtions during tests 24-27 September, 1970

FIGURE 3 


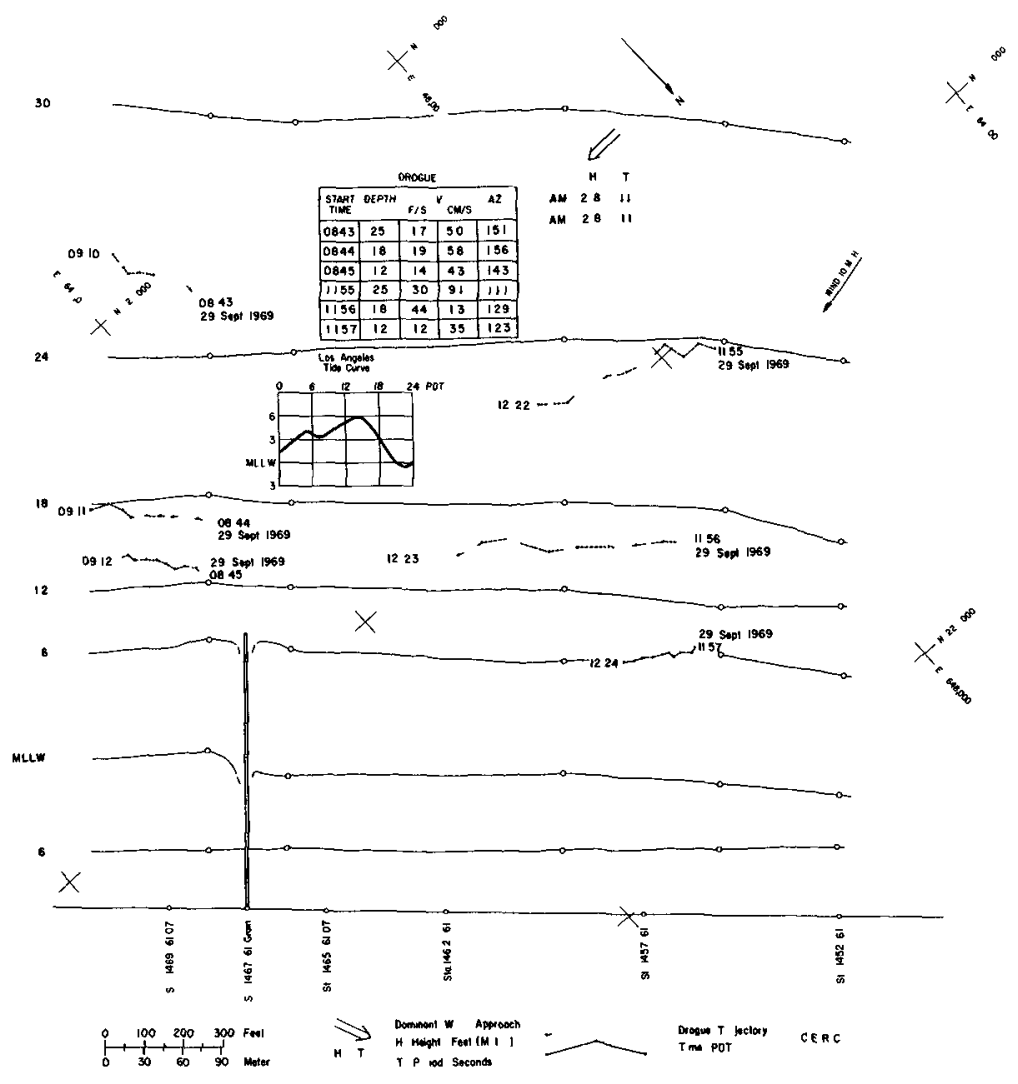

Current drogue patterns, velocities, and tide curve representative of cond1tions during tests 28 September-1 October, 1970

FIGURE 4 


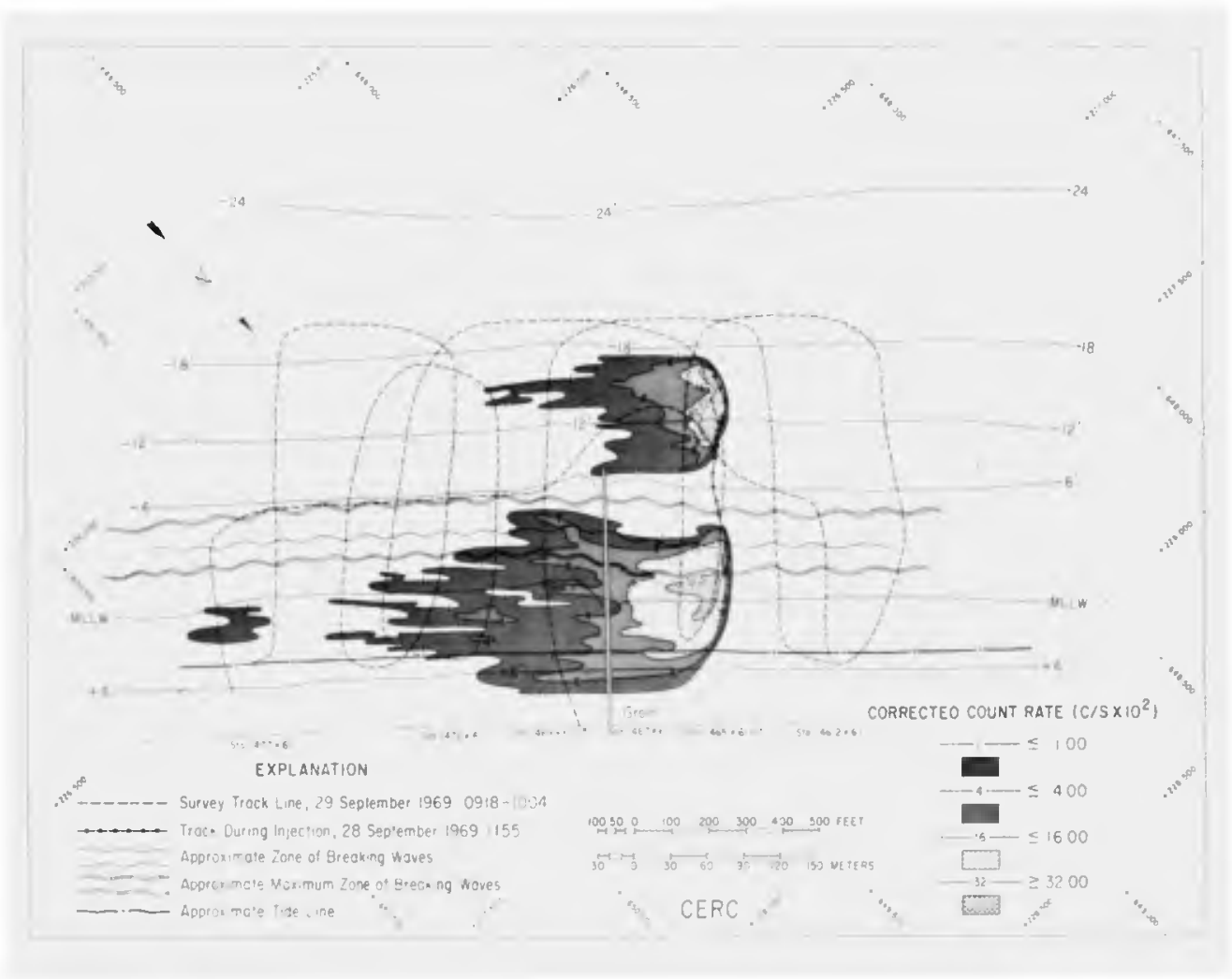

Dispersal pattern of gold-tagged sand observed approximately 23 hours after injection. Note patterns of sand moving through the pier-like structure. 


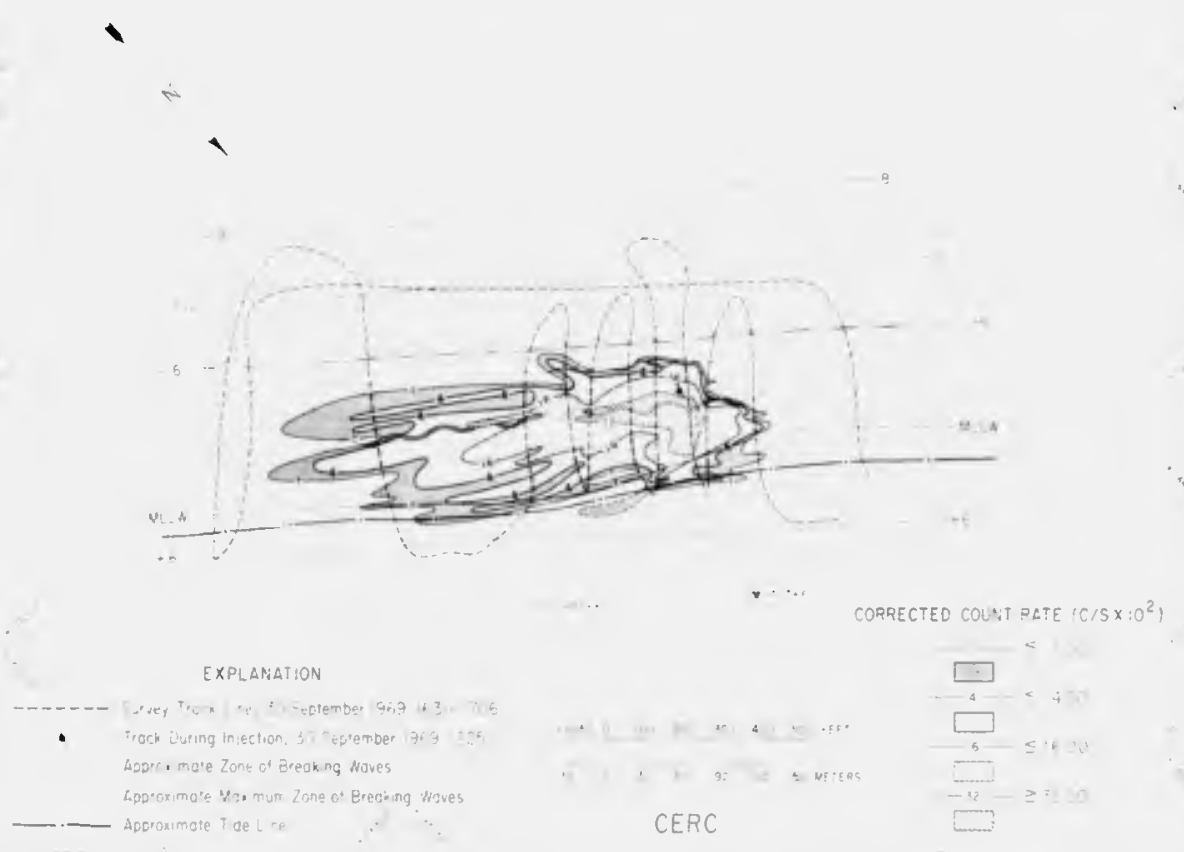

Dispersal pattern of gold tagged sand observed approximately 4 hours after emplacement as a point source in the surf zone.

FIGURE 6 


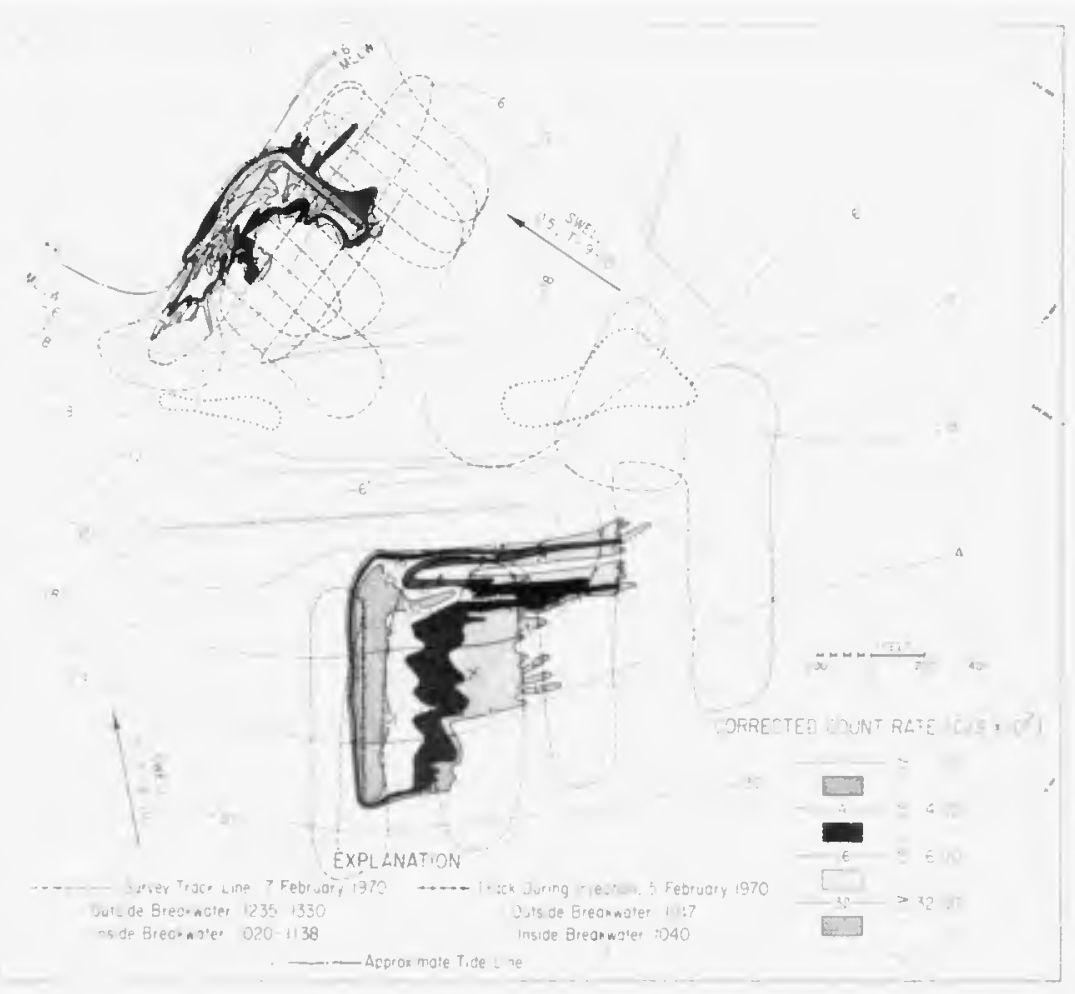

Dispersal patterns of gold-tagged sand placed outside and inside harbor approximately 48 hours after emplacement.

FIGURE 7 


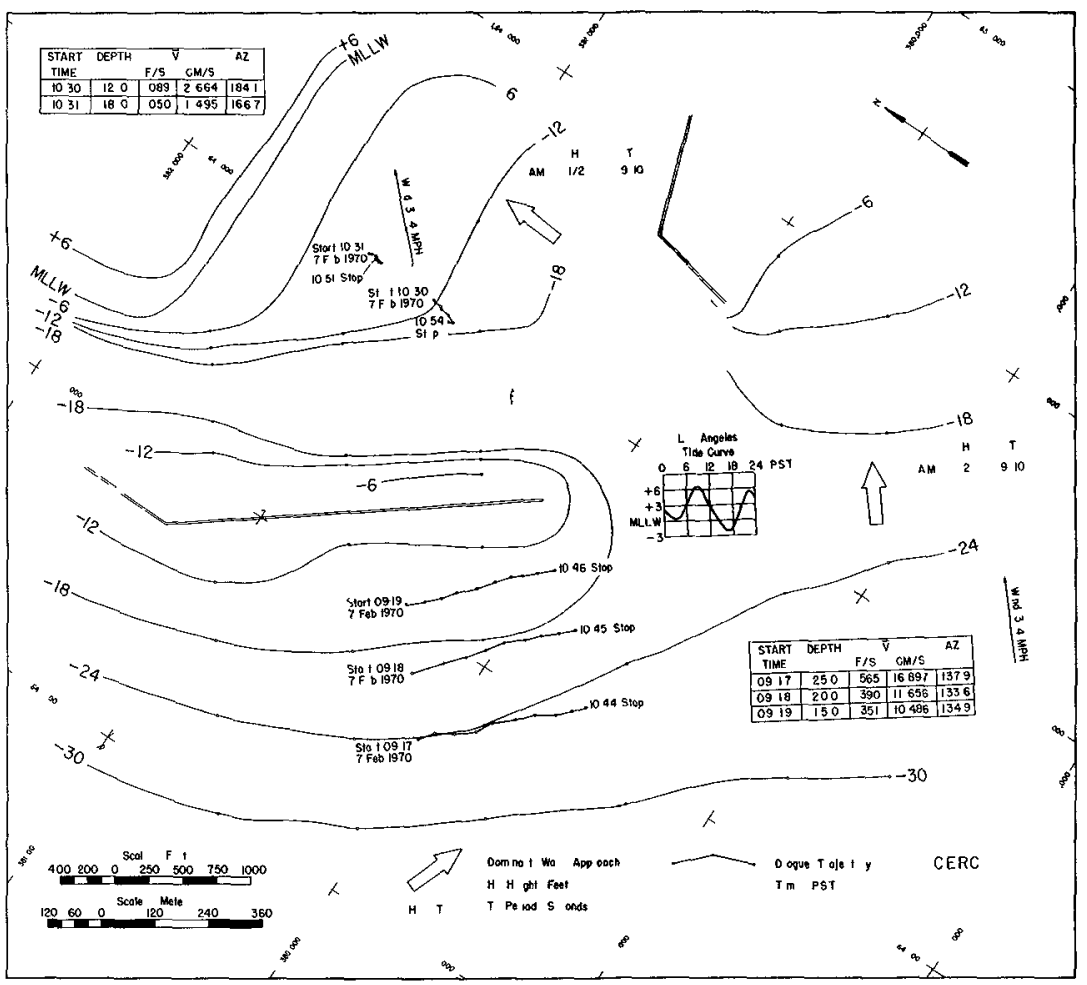

Current drogue patterns, velocitıes, and tide curve representative of conditions during tests 4-8 February 1970

FIGURE 8 
Dispersal patterns of two point source injections of gold-tagged sand observed approxinately 24 hours after injection and just after sea and swell had returned to eastward-directed approach.

FIGURE 9 

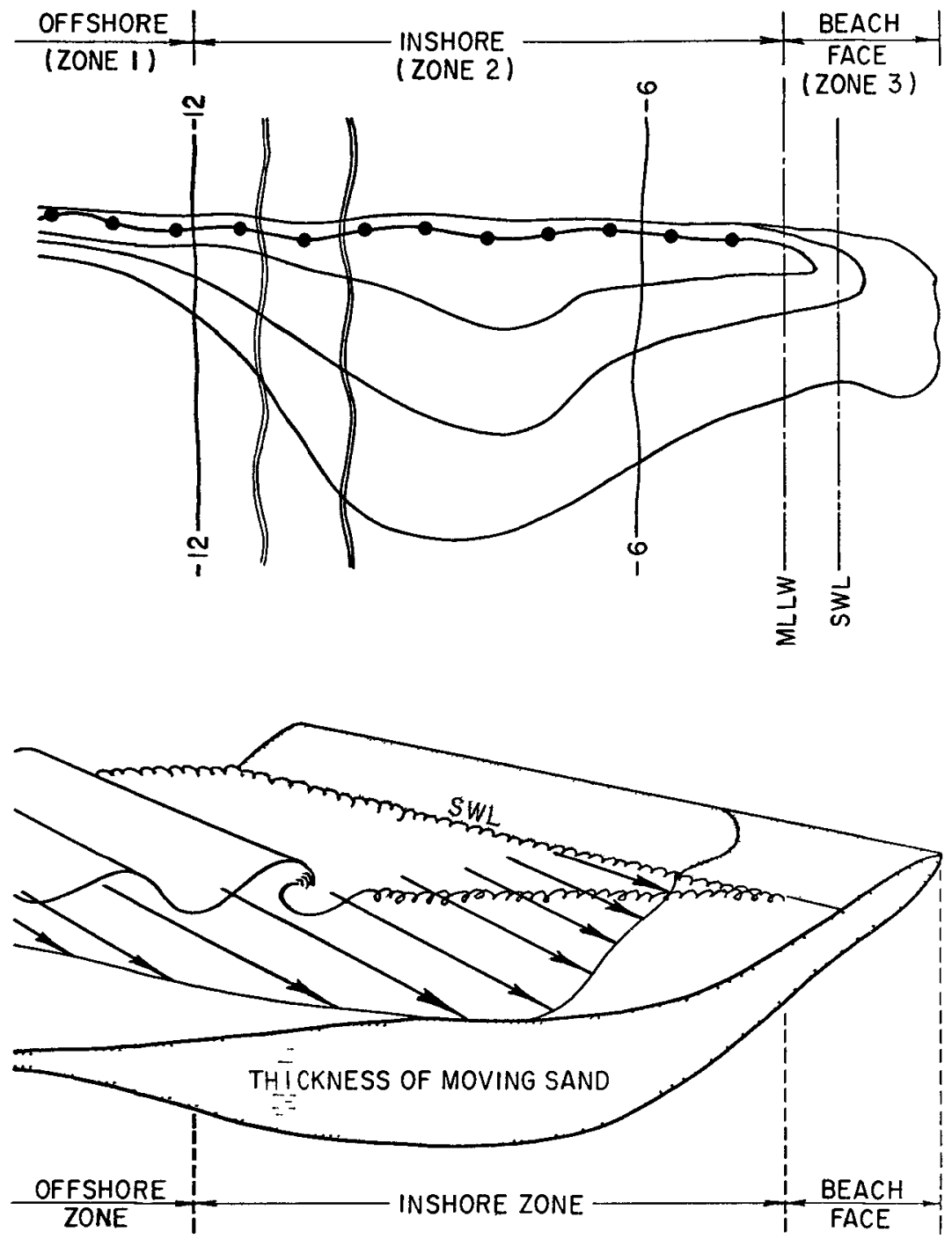

Conceptual model for sediment transport in the 1Ittoral zone, numbers refer to depth of bottom below mean lower low water (MLLW) (A) Schematic map of sand dispersal system showing regions of hIgh, medium, and low level radiation (B) Schematic Isometric projection showing relative thickness of sand layer Involved in transport, length of arrows proportional to rate of transport 



\title{
CHAPTER 50
}

\author{
AN UNDERWATER SURVEY SYSTEM FOR RADIONUCLIDE- PAGGED \\ SEDIMENT TRACING' \\ E H Acree, H R Brashear, and F N Case \\ Oak Rıdge National Laboratory \\ Oak Rıdge, Tennessee
}

\begin{abstract}
Survey equipment for use in tracing sediment tagged with radionuclides has been designed and field tested The system is unique in its ability to operate on the dry beach, in the surf, and in deep water, making it possible to synoptically examne the beach face, surf zone, and off-shore marine environment The ball-shaped radiation detector vehıcle is towed behınd an amphibious vessel Data are collected on punched tape at a rate of one set of data (position, radiation counts, time) every two seconds Gold-198 and xenon-133 have been used for tagging sand indigenous to the survey area under study Operational characteristics and detection sensitivity are discussed
\end{abstract}

This underwater survey system was developed for use in the RIST program which is under the direction of the U S Army Corps of Englneers, Coastal Englneerıng Research Center The survey system was designed and bullt at Oak Ridge Natıonal Laboratory and was sponsored by the U S Atomic Energy Commission, Division of Isotopes Development The design criteria for the instrument were

1 It must be capable of operating as a gamma spectrometer

2 It must automatically correlate radiation counts, position, and time

3 It must be capable of operating on the beach face, in the surf zone, and off shore

Research sponsored by the Division of Isotopes Development, U S Atomic Energy Commission, under contract with the Unıon Carbide Corporation 
The components of the basic detector unit are shown in Fig. 1. The detectors are 2 in. by 2 in. sodium activated cesium iodide crystals

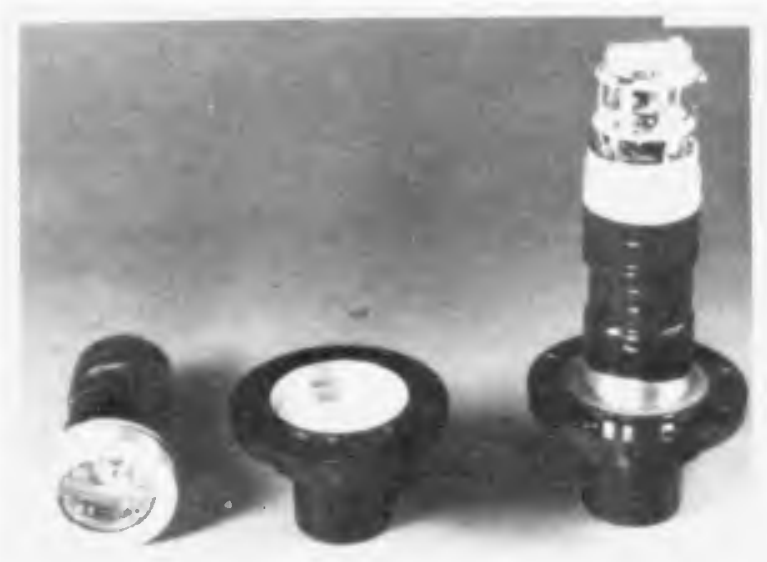

Fig. 1. Underwater Detector Components enclosed in aluminum cans which have 0.030-in.thick walls. Since the detectors are exposed to water pressure, a l/2-in.thick Plexiglas light pipe is used as a pressure barrier between the crystals and the photomultiplier tubes. Four detector assemblies and the preamplifier are mounted in a water-tight chamber suspended from the axle of an open mesh ball-like cylinder (Fig. 2). As the "ball" rotates on the stationary axle, the detectors remain oriented toward the surface over which the ball rolls.

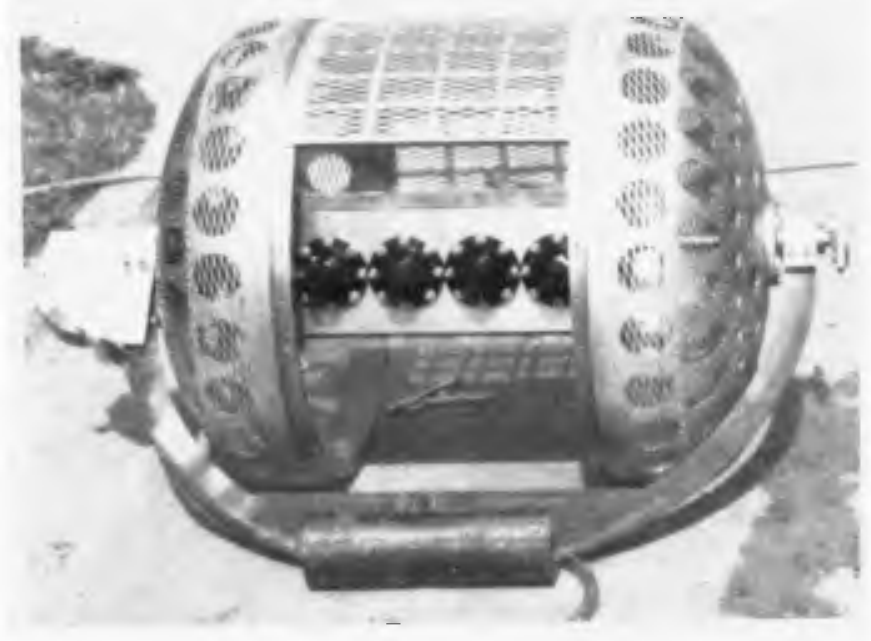

Fig. 2. Radiation - Detector Vehicle 
When designing the underwater survey vehicle, we were concerned with lts ablity to travel over moderately rocky areas that are common to the West coast The ball design, chosen over a sled-type, functions well over rocks that are up to 1 to $2 \mathrm{ft}$ in dlameter and over rock outcrops It is fabricated from rectangular steel bars to form an open lattice with a minimum of shlelding of the detectors The entire device is covered with expanded metal to exclude stones and other debris The detector chamber is welghted with lead to malntain the detectors in a vertical position

The ball was tested for underwater tracking characteristics at the $U$ S Naval Shlp Research and Development Center in Washington to determine hydrodynamic performance The physical characteristics of the vehicle are listed in Table 1

Table 1 Physical Characteristics of Detector Vehıcle

\begin{tabular}{lr}
\hline Overall width, in & 50 \\
Overall diameter, in & 30 \\
Housing width, in & 42 \\
Helght, in & 30 \\
Distance from center shaft to tow polnt, in & 32 \\
Welght in alr, lb & 505 \\
Welght in fresh water, lb & 410 \\
\hline
\end{tabular}

The minimum length of cable required to maintain the detector vehicle In a surveying position on the ocean bottom is shown in Fig 3

We are using approximately $100 \mathrm{ft}$ of cable and travel at a speed of approximately 3 knots The vehıcle is very stable under these conditions, and during an average survey the detector vehicle wlll cover approximately $1 \%$ of the total survey area

A schematic of the overall survey system is shown in Fig 4 


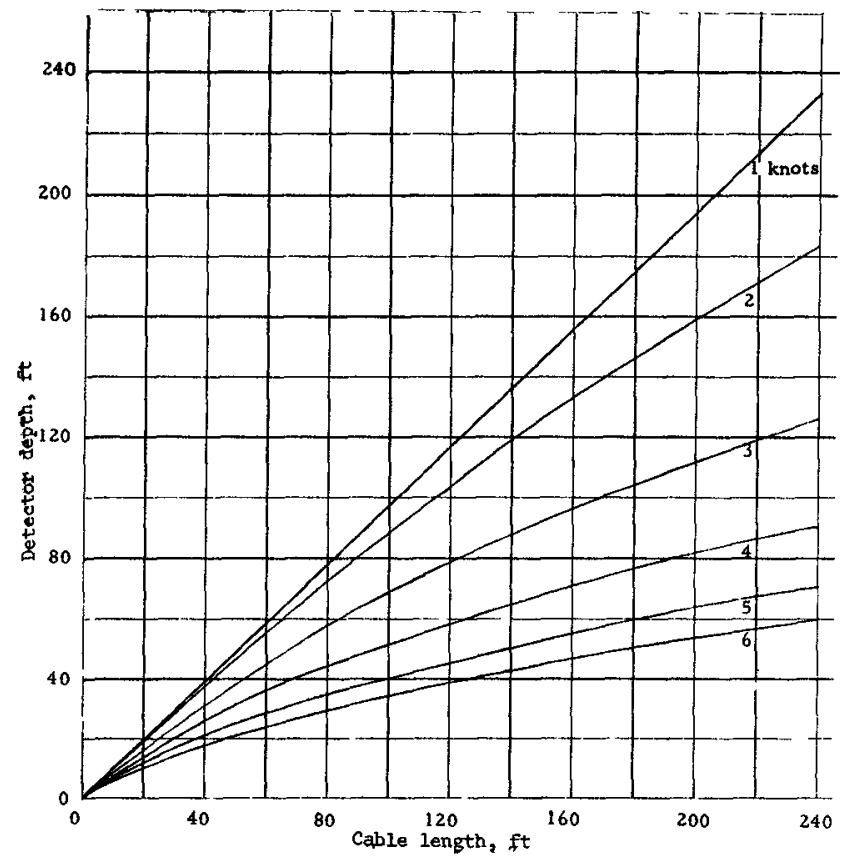

Fig 3 Minimum Required Cable Length as a Function of Detector Depth for Various Speeds

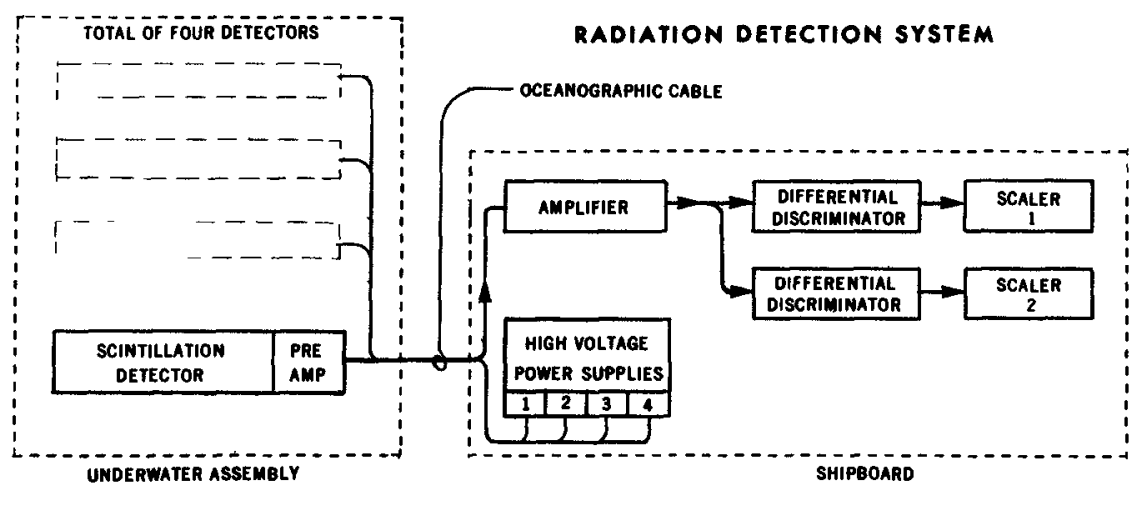

Fig 4 RIST Survey System Schematic 
Signals from the individual detectors are mixed in the preamplifier whose output is matched to a 50-ohm coaxial cable which carries both the positive $24 \mathrm{~V}$ dc power to the preamplifier and the output voltage pulses The coaxial cable is part of a wire bundle that has an additional eleven conductors which provide high voltage to the photomultiplier tubes High voltage to each tube is supplied by individual power supplies which permit gain adjustment of the tubes so that the responses from all four detectors wlll be equal The cable assembly also serves as the tow cable and has a tensile strength of $30,000 \mathrm{lb}$ The preamplifier output is amplified with a linear amplifier and feeds two differential discriminators for choosing which segment of the gamma spectrum is to be recorded The output signals from the discriminators are used to drive two buffered scalers The printout control unit samples each scaler and records the data on an 8-level punched paper tape The printout control unit also samples a scaler which generates a line number, a scaler whlch generates time, and the boat position data The boat position is determined by use of a microwave system which provides two distances from two fixed positions on shore A complete set of data can be collected once a second on in multiples thereof by switch selection on the console The data readout presents a log of line number, time, radiation channel No 1 , radiation channel No 2 , distance 1, distance 2, and water depth

The punched tape is used to provide data input to a computer for interpretation and the preparation of contour maps The detection sensitivity of the detector system submerged in water for gold-198-199 is $23 \times 10^{3}$ counts/sec when the activity concentration is $1 \mu \mathrm{Cl} / \mathrm{ft}^{2}$

The tow vessel that is currently being used for surveying is an amphiblous vessel (LARC-15) which is approxamately $60 \mathrm{ft}$ long This craft can easily operate in 6- to 8-ft breakers

In our early field tests with this equipment, xenon-133 tagged into sand indigenous to the test area was used as the tracing material. While sand injection and health physics problems are greatly simplified with xenon-133, the quantity of activity that can be introduced into a survey area is limited to approximately 40 liters of sand tagged with approximately $800 \mathrm{mCl}$ of xenon-133 This small quantity of activity tends to 
limt the scope of surveys We are currently using a gold-198-199

mixture The amount of activity that we can use is only limited by the equipment used to transport and dispense at our recent experiments were conducted with $10 \mathrm{cl}$ of activity tagged onto approxımately $1 / 211$ ter of sand The use of the survey equmment is not limited to xenon-133 and gold-198-199, because 1t functions as a gamma spectrometer and can be used to monıtor any gamma radiatıon above $30 \mathrm{keV}$ Other possible applicatıons for this equipment are conducting natural background surveys in inland lakes and streams and prospecting for heavy mineral deposits 


\title{
CHAPTER 51
}

PROCESSING AND ANALYSIS OF RADIOISOTOPIC SAND TRACER (RIST) STUDY DATA*

\author{
H $\mathrm{R}$ Brashear
}

Instrumentation and Controls Division

Oak Ridge National Laboratory

$$
\begin{array}{lll}
\text { E } & H & \text { Acree } \\
\text { F } & N & \text { Case }
\end{array}
$$

Isotopes Division

Oak Ridge National Laboratory

P A Turner

D B Duane

Coastal Engineering Research Center

Washington, D C

\section{ABSTRACT}

Data collected during the Radiolsotopic Sand Tracer (R1ST) field tests are proces sed through digltal computers Data treatment requires computing and plotting the detector position and correcting the corresponding radiation count rates for radioactive decay The field data are recorded on punched paper tape which is then edited and transferred to magnetic tape for input to data reduction programs The navigation data, which are in the form of distances to shore-based microwave responder beacons, are tested for spurious values by comparison w1th the theoretical maximum travel distances of the survey vehicle between successive fixes The navigation ranges are then converted to rectangular geographical coordinates

Present emphasis is in the development of computer programs to construct a count rate surface from data collected along track lines This technique facilitates machine contouring and enables numerical integration of the count rate surface The ultimate goal is to obtain an estimate of mean direction and volume of littoral transport and a radiation material balance to be used to check the results Several programs required to accomplish these tasks are operating at the Coastal Engineering Research Center (CERC) and the Oak Ridge National Laboratory (ORNL)

\footnotetext{
* Research sponsored by the Division of 1sotopes Development, U S Atom]
} Energy Commission, under contract with the Union Carbide Corporation 
Data processing for the Radiousotopic Sand Tracer (RIST) Study is computer oriented to handle the large volume of collected data whlch are digltal in form and represent parameters that must be correlated to obtain sand transport rates Figure 1 enumerates the objectives of the computer programs

Fig 1 Objectives of Computer Programs

1 Assembling the data in graphical form
2 Interpolation to establish the count rate
surface within the survey area
3 Computation of the surface rate and direction
of the sand movement
4 Correlation of other data with survey data to
calculate volume rate of sand transport
5 Correlation of the volume rate of sand trans-
port with ocean conditions for fundamental
studies in sand transport

To accomplish the first objective of assembling the data in geographical form, two programs are operational one at the Coastal Englneering Research Center and the other at the Oak Ridge National Laboratory The data collection system ${ }^{1}$ shown in Fig 2 illustrates the automatic correlation of three parameters necessary in assembling the data in graphIcal form

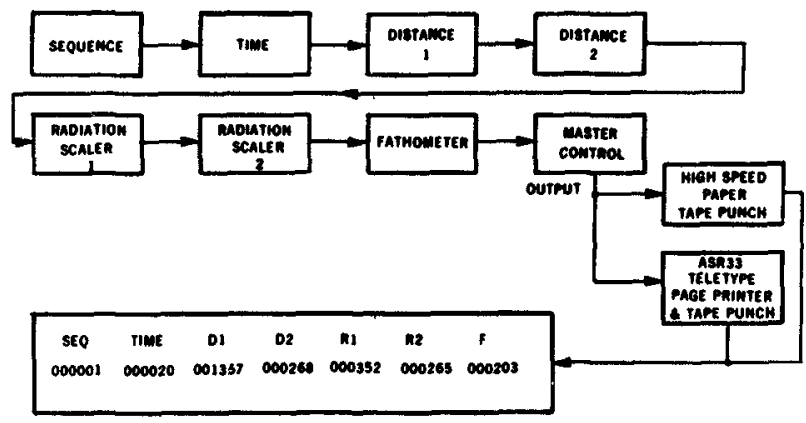

F1g 2 Data Collection System

\footnotetext{
${ }^{1}$ An Underwater Survey System for Radionuclide-Tagged Sediment Tracing

$E$ Acree, $F \mathrm{~N}$ Case, and $\mathrm{H} R$ Brashear
} 
The data are collected on punched paper tape in a serial fashion to form a line of data Each line of data consists of numbers which represent time, tow vehicle position, radiation count rate, and water depth Time and radiation units have buffers so the data can be recorded while new data are being taken These buffers permit data to be taken at 2-sec intervals with no instrument dead time due to readout Tow vehicle position is represented by $D 1$ and $D 2$, the distances between the tow vehicle and two f1xed shore stations The distances are measured at the same time that the time and radiation values are transferred to the buffers for readout Water depth measurements are near the first part of each data cycle, but are not as precisely timed

One survey may have up to 2000 lines of data, which is approximately the capacity of one roll of paper tape A normal survey consists of 1700 1ines of data An average of four surveys are made each day, producing 47,600 six-digit numbers, an average field test y1elds a total of 380,800 six-d1git numbers

Data stored on punched paper tape are copied onto magnetic tape for computer input The tow vehicle positions are calculated from D1 and D2, using either the law of cosines or the intersection of two circles, depending upon which coordinate system is desired The law of cosines is used to place the tow vehicle on a geographical grid, and the intersection of two circles is used for placement on an arbitrary $x-y$ coordinate grid The validity of each location is checked by comparison with the previous location If the separation distance between the two locations is greater than the theoretical distance possible, the location is changed This change is based on the average velocity and direction of the tow vehicle

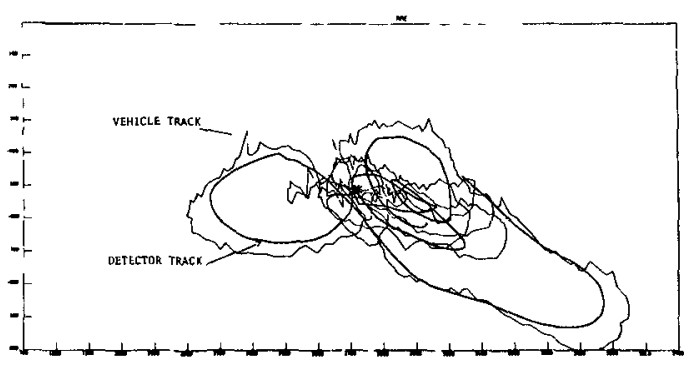
Figure 3 is a computerdirected machine plot of a tow vehicle path and detector vehicle path with no corrections for the tow vehicle locations The irregularities are due to errors from the range-finding equipment Fig 3 Plot of Tow Vehicle and Detector Path in measuring the distances 
from the tow vehicle to each shore station The location of the detector vehicle, which is towed approximately $100 \mathrm{ft}$ behind the tow vessel, must be calculated by performing a series of approximations These approximations are quite accurate due to the frequency of obtaining tow vehicle position The initial detector vehicle position is determined on an arbitrary basis The second position is determined by computing the equation of the line which connects the first detector vehicle position with the second tow vehicle position The actual length of the tow cable, minus any correction due to water depth, is used as the distance from the tow vehicle (along the computed line) to the second detector vehicle position Subsequent detector vehicle positions are determined in a similar manner As each detector vehicle position is calculated, the corresponding time and radiation count rates are assigned to that position In Fig 3, the paths of both vehicles are drawn by straight lines connecting successive calculated positions

Radiation data are scanned to obtain an average radiation background count rate (due to natural radioactive materia1) A11 radiation count rates are adjusted by subtracting the average background, and the adjusted count rates which are equal to or greater than the background are corrected for radioactive decay by the following formula

$$
R_{C D}=\frac{R}{E^{-\lambda t}}
$$

$$
\text { where } \begin{aligned}
\mathrm{R}_{\mathrm{CD}} & =\text { radiation counts corrected for decay (counts/sec) } \\
\mathrm{R} & =\text { radiation counts } \geqq 2 \mathrm{x} \text { average background (counts/sec) } \\
\mathrm{E} & =\text { base of natura } 1 \text { logarıthm } \\
\lambda & =\text { decay constant of isotope used in test } \\
t & =\text { time from tagged sand insertion (unit) }
\end{aligned}
$$

Figure 4 is a machine plot of numbers which represent ranges of corrected count rates calculated from data obtained at Point Mugu, California, in September, 1969 


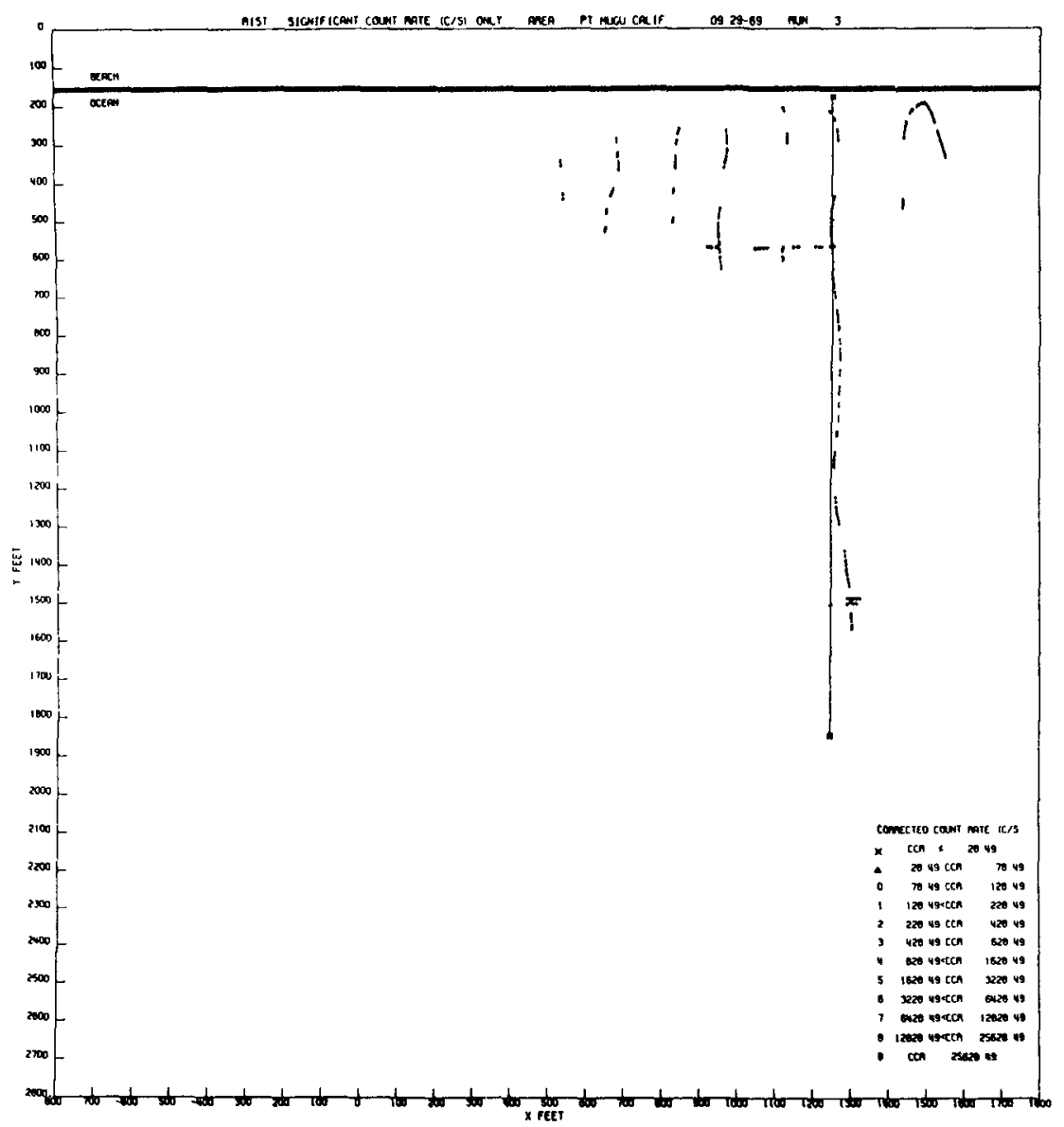

Fig 4 Calculated Radiation Count Rate Ranges Over Detector Track

Figure 5 is an enlargement of F1g 4 in the area of significant radiation count rates All background count rates have been omitted to make the trend of sand movement more apparent The computer programs allow more than one survey to be plotted on a single map to enhance the distribution pattern as it develops 


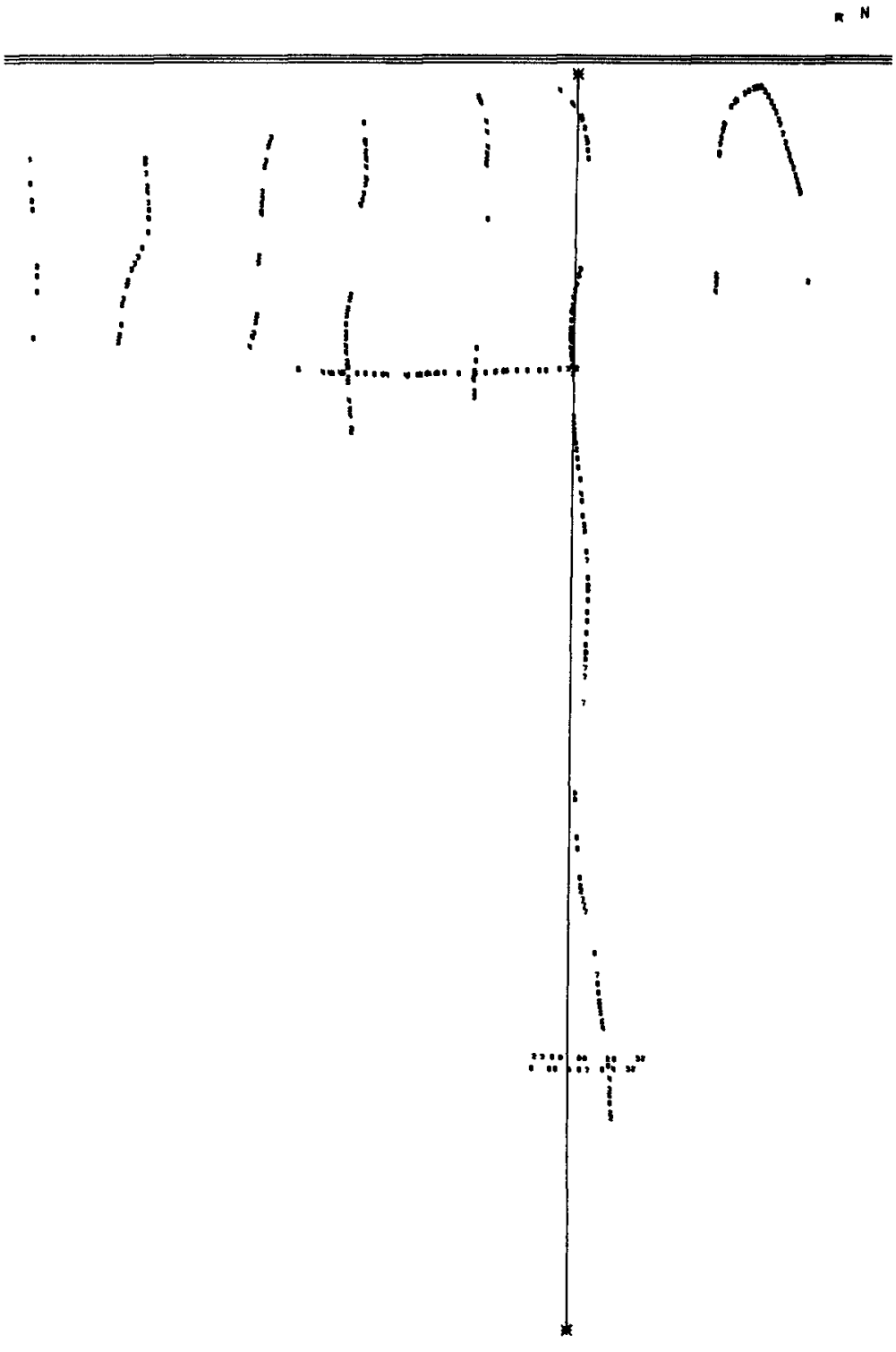

Fig 5 Section of Fig 4, Area of Significant Count Rates 
The second objective, interpolation of data, is required because it is physically impossible to sample every square foot of the study area Several programs are e1ther operational or near operational to complete the count rate surface and machine draw radiation intensity contours one of the programs is a linear interpolation technique This program divides the $x-y$ coordinate system into a grid adjacent to the $x$ and $y$ axes having square areas of $25 \mathrm{ft}$ on a side In general the $x$-axis lies parallel with the beach In the studies to date the predominate directions of sand movement have been parallel or perpendicular to the beach The inter polation is done in either or both directions depending on the preliminary indication of sand movement direction

The second step in this program is to assign all square areas which have Iadiation count rates within an area a single count rate, which is the average of all radiation count rates $w_{1}$ thin that area Figure 6 is the result of the second step of the program The results here are much like the data display of radiation count rates along the detector vehicle track of the first program, but somewhat simpler due to a grouping of numbers

The third step computes radiation count rates for those areas with no count rates assigned Interpolation is made only between areas which have real data The interpolation 15 linear, and the average radiation count rate of two areas is assigned to the area halfway between the two areas The formula used is

$$
R_{1+K, J}=R_{1, u}-\frac{\left(R_{1, J}-R_{1+L, J}\right)}{L}(L-K)
$$

where $R=$ radiation value of the area

$$
\begin{aligned}
& 1=x \text { location of area of larger } R \text { data } \\
& J=y \text { location of area } \\
& K=x \text { location of area for } R \text { interpolation } \\
& L=x \text { location of area of smaller } R \text { data }
\end{aligned}
$$

If interpolation is desired in both the $x$ and $y$ direction, the interpolation is done independently in each direction and the average of the two is used as the final value 


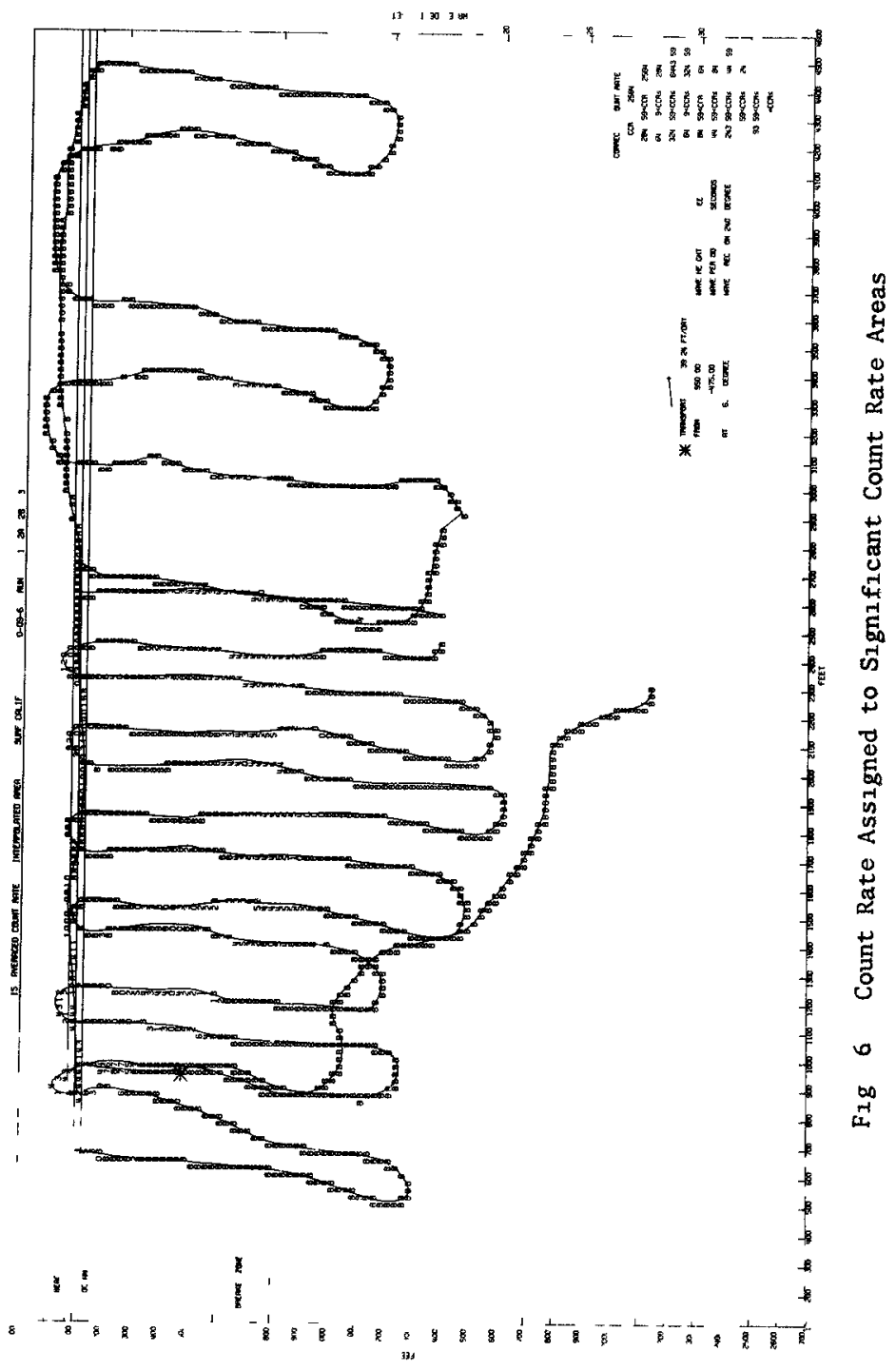




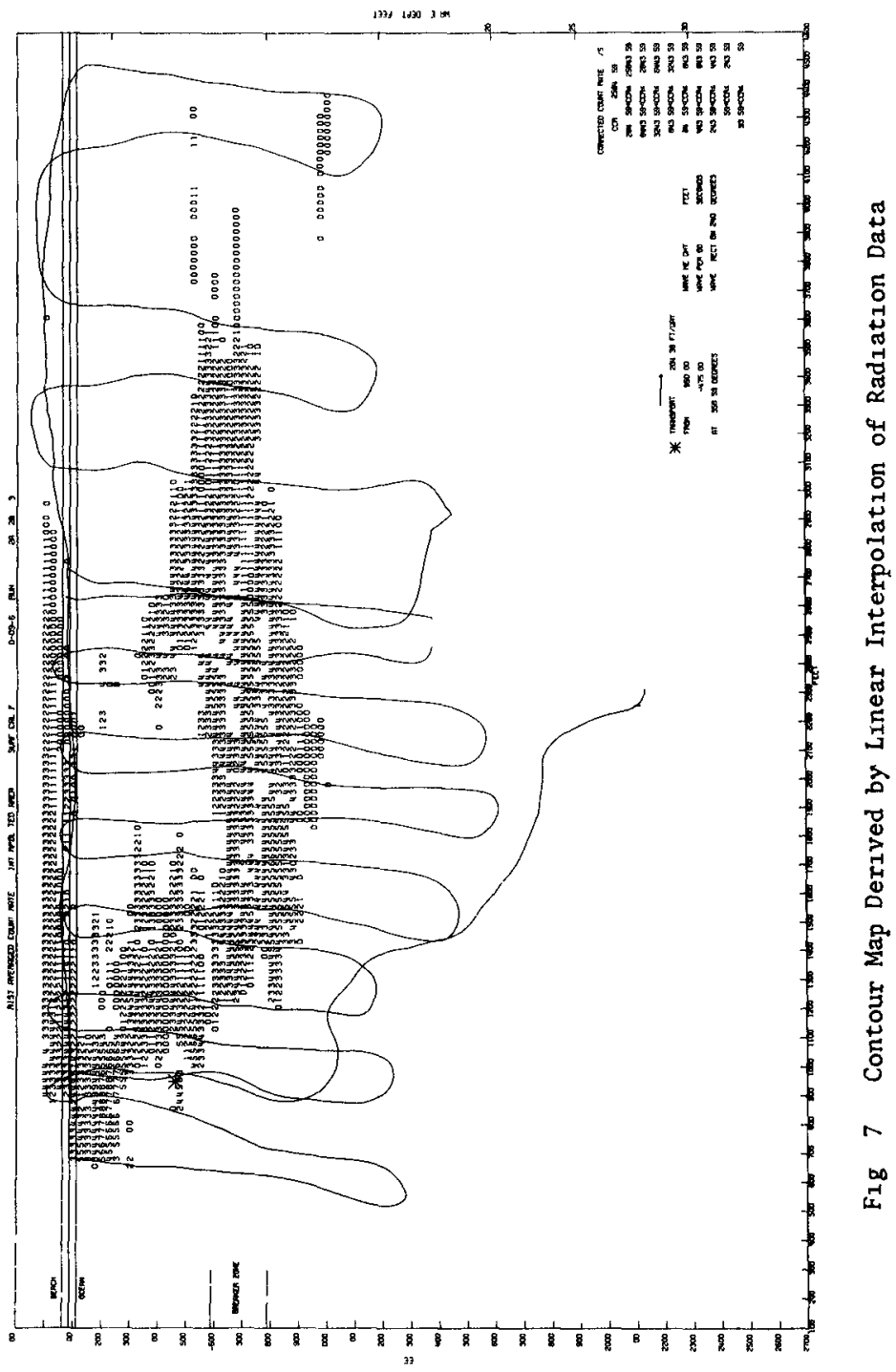


Figure 7 is the completed nap using the linear interpolation method A11 background radiation count rates have been omitted for clarity

Beyond the 1nitial plotting of trackline data, present emphasis at CERC is silghtly different from that at ORNL The procedure being tested at CERC for RIST survey data is to use a we1ghted least-squares fit of a plane through the centrold of each grid cell containing a data point The area surrounding each grid mesh point is divided into octants, and each octant is searched for the nearest data point to the grid centroid Once the search is completed, the nearest point in each octant is used to construct a welghted least-square fit of a plane through the centrold Each data point is assigned a weight which is an inverse function of the distance from the data point to the grid centrold

Th1s procedure has been tested by generating a random surface with a double Fourier series and evaluating 1 t at 100 randomly distributed points in a plane When interpolated over a uniform grid, these points produced a surface that appeared similar to the original surface, but with reduced variance The numerical approximation routine has not been tested yet with a hypothetical trackline superimposed on the random surface It has already been observed that, in certain instances w1th real data, the routine produces grid point values that are artifacts of the numerical approximation procedure Although the result is not as aesthetically appealing as a handdrawn contour map of the data, it does provide a quick first approximation of the survey area Testing and evaluation of the two approaches $1 \mathrm{~s}$ being made at CFRC and ORNL, but at present is incomplete

Correlation of data for fundamental studies and volume-rate of sand transport are in the early stages of development 


\title{
CHAPTER 52
}

A Class of Probability Models for Littoral Drift

\author{
Willıam $R$ James \\ Special Assistant \\ Coastal Engıneerıng Research Center \\ Washington, D C
}

Introduction

The major goal in the development of sediment tracer technology is to produce an accurate method for the field measurement of short term volume littoral rate Many of the technical difficulties involved in tagglng, injecting, and sensing the movement of radiolsotope sand tracers in the 1Ittoral zone have been overcome by the RIST project However, quantitative determination of volume drift rate requires more than knowledge of tracer position in time and space A mathematical model 1 s required to relate the flux of tracer material to the sediment flux

A linear (or average) rate of tracer transport along the coastline can be measured to a falr degree of accuracy with tracers These measurements, when determined from tracers injected along a line source which span the transport zone, can be used to provide an estımate of an areal transport rate However, it is not obvious how to measure the third dimension, depth of transport This, of course, is needed to provide the desired estimate of volume transport rate

This problem arıses, even if the relation of tracer concentration to burial depth is everywhere known without error sediment transport does not occur as a sheet of constant thickness moving at a constant rate If this were so tracer concentration would rapıdly attain a uniform concentration over a fixed depth and no tracers would appear below that depth In fact no observations of the relation between tracer concentration and burlal depth support this model as even a first approximation

Studies such as those of Courtols and Monaco (1969) and Hubble and Sayre (1964) suggest that the concentration of tracers is related to burial depth in a complex fashion The concentration on the surface is finite, but not maximal The concentration increases with depth to some point where a maximum is reached and diminishes in a "long talled" fashion

If sediment transport occurred as a sheet of uniform thickness, and tracers were thus uniformly distributed over the range of that thickness, one measure of depth of movement would be the mean of the maximum observed tracer depth A more stable estimate, if this condition was assumed on $1 y$ to approximate reality, would be to double the observed mean depth of burial 
Yet the observed distribution curves so strongly deny the original hypothesis that neither of these methods can be relied upon What is needed is a model for sediment particle burlal which admits the observations as realizations and directs the form of estlmation This paper at tempts to present a class of such models which lead to a particularly simple form for the calculation of littoral volume drift rate

\section{The Conceptual Model}

Assume a two dimensıonal sediment transport system which includes only depth of burlal and transport direction The motion of sand particles is initiated by the passing of a surge As wave crests pass over a point particles are alternately lifted from the sea floor, transported, and redeposited Inasmuch as wave helght and perıod are random varıables, the depth to which materıal is eroded from the sea floor varıes with each wave

When the wave train impinges obluquely upon the shoreline a net longshore current is superimposed upon the oscillatory motion so that particles suspended by the passing surge are subjected to a net motion along the shoreline Contrary to the situation in uniform flow, it is the individual energy pulses which control the erosive mechanısm and the wave climatology as a whole which controls the transporting mechanism Short perıod high waves do not effect littoral druft if they are directed onshore The longshore current is the transporting mechanism, but is not the erosive mechanism except in extreme cases

\section{The Probabiluty Model}

For the purposes of this development the elevation of a point on the sea floor can then be considered to change instantaneously through a series of erosional and depositional increments Define the thickness of the erosional increment accompanying the passage of the 1 th surge as $\varepsilon$, and the thickness of the following depositional increment as $\delta$ Both $\varepsilon$ and $\delta$ are assumed to be random varıables with equal expectations ${ }^{1}(\mu)$ Particles $\frac{1}{\text { whIch }}$ are eroded are assumed to $\mathrm{mIx}$ in transport so that the depth below the sediment-water interface at which tney are redeposited is independent of the depth from which they were eroded

At time zero tracer particles are injected on the surface Initially all tracer partıcles wı 11 be eroded, transported and redeposited As successive erosional and depositional episodes occur, some of the tracer particles will become burıed below normal erosional depths, thus occupying sites of relatıve stability Ultımately an equilibrıum state will be reached where the concentration of tracer particles with depth of burial will no longer change This state will be that where the probability of erosion of particles buried at a given depth times the proportion of tracer particles burıed at that depth precisely equals the probabılıty that a freshly eroded particle will be deposited at that depth times the total probability that a tracer particle is eroded, for all depths 
Certain characteristics of the equilibrium distribution can be derived without knowledge of the specific probability density functions describing erosional and depositional increment thicknesses

Define

$f_{1+1}(z) d z=$ the probability that after the 1 th surge passes, a tracer particle is buried between depths $z$ and $z+d z$

$f_{\text {new }}(z) d z=$ the probability that a particle which 1 s eroded by the 1 th surge, is redeposited between depths $z$ and $z+d z$

$P_{\text {move }}(z)=$ the probabılity that a partıcle burıed at depth $z$ is eroded by the 1th surge

$\mathrm{P}_{\text {stay }}(\mathrm{z})=$ the probability that a partıcle burıed at depth $\mathrm{z}$ is not eroded by the ith surge

$f_{\varepsilon}(\varepsilon) d \varepsilon=$ the probabılıty that the thickness of an erosional increment 1s between $\varepsilon$ and $\varepsilon+d \varepsilon$ $\mathrm{F}_{\varepsilon}(\varepsilon)=\int_{0}^{E} \mathrm{f}_{\varepsilon}(\mathrm{x}) \mathrm{dx}=$ the probabılity that the thickness of an erosional

Then $\quad P_{\text {stay }}(z)=F_{\varepsilon}(z)$, and $P_{\text {move }}(z)=1-F_{\varepsilon}(z)$

Define $\mathrm{Pm}_{1}=$ the total probability of a tracer particle being eroded by the 1th surge, independent of burial depth

$$
P m_{1}=f_{0}^{\infty}\left[1-F_{E}(z)\right] f_{1}(z) d z
$$

Then

$$
f_{1+1}(z)=F_{\varepsilon}(z) f_{1}(z)+P m_{1} f_{\text {new }}(z)
$$

An equilibrium probabılıty distribution of tracer burial depth will be obtalned when

$$
f_{1+1}(z)=f_{1}(z)
$$

Define this distribution as $\mathrm{f}_{\mathrm{eq}}(\mathrm{z})$

Then

$$
f_{\text {eq }}(z)=F_{\varepsilon}(z) f_{e q}(z)+P m_{e q} f_{n e w}(z)
$$


or,

$$
f_{e q}(z)=\frac{P_{e q} f_{n e w}(z)}{\left[1-F_{\varepsilon}(z)\right]}
$$

It now remains to relate the fresh deposition burlal law ( $f$ to the probability density for depositional increment thicknesses ${ }^{\text {new }}(z)$

Define

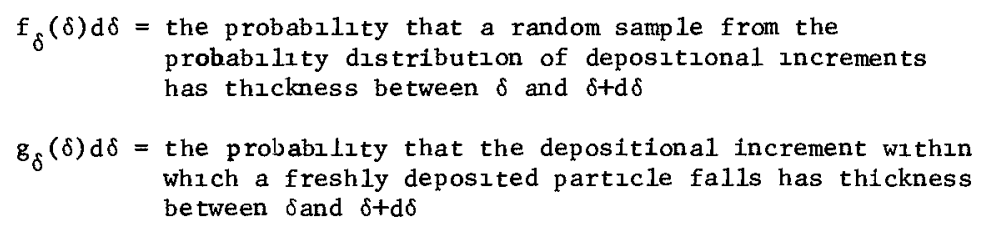

Then in order to maintain a volume balance

$$
g_{\delta}(\delta) d \delta \propto \delta f_{\delta}(\delta) d \delta
$$

$$
\begin{array}{ll}
\text { As, } \quad & \delta_{0}^{\infty} g_{\delta}(\delta) d \delta=1 \\
\text { and, } \quad \delta_{0}^{\infty} f_{\delta}(\delta) d \delta=\mu \\
\text { then, } \quad g_{\delta}(\delta) d \delta=\frac{\delta f_{\delta}(\delta) d \delta}{\mu}
\end{array}
$$

From the mixing in transport assumption, a particle which falls within a depositional increment of size $\delta$ is equally likely to fall at any depth between the surface and the base of the increment

Thus

$$
\begin{aligned}
f_{\text {new }}(z \mid \delta) & =\frac{1}{\delta} & & 0<z<\delta \\
& =0 & & z>\delta
\end{aligned}
$$

Then the joint probability density of depth of burıal and size of depositional increment is given by

$$
\begin{aligned}
f_{\text {new }}(z, \delta) & =f_{\text {new }}(z \mid \delta) g_{\delta}(\delta) \\
& =\frac{(1 / \delta) \delta f_{\delta}(\delta)}{\mu}
\end{aligned}
$$


or,

$$
\begin{aligned}
f_{n e w}(z, \delta) & =\frac{f_{\delta}(\delta)}{\mu} & & 0<z<\delta<\infty \\
& =0 & & z>\delta
\end{aligned}
$$

Then the probabilıty distribution describing burlal depths of freshly deposited particles 1 s given by

$$
\begin{aligned}
f_{\text {new }}(z) & =\int_{z}^{\infty} f_{\text {new }}(z, \delta) d \delta \\
& =\int_{z}^{\infty}(1 / \mu) f_{\delta}(\delta) d \delta
\end{aligned}
$$

or,

$$
f_{\text {new }}(z)=\frac{1-F_{\delta}(z)}{\mu}
$$

where $F_{\delta}(z)=$ the probability that a random sample from the probability distribution of depositional increments has thickness less than $z$

The resulting equilibrium distribution of burlal depths is thus

$$
f_{e q}(z)=\frac{P_{e q}}{\mu} \frac{\left[1-F_{\delta}(z)\right]}{\left[1-F_{\varepsilon}(z)\right]}
$$

A sıgnificant special case being

$$
\mathrm{f}_{\mathrm{eq}}(0)=\left(\mathrm{Pm}_{\mathrm{eq}} / \mu\right)
$$

For this class of probability models, the total surface concentration of tracer material is independent of the specific probability laws governing erosional and depositional increment thicknesses

\section{Volume Littoral Drift Rate}

Even more important 1 s the 1 mplied relation between the rate of trace $r$ transport and the volume rate of littoral drift 
At equilibrium

1 The expected proportion of tracer particles moving with each passing surge is $\mathrm{Pm}$ eq

2 The average distance of movement is defined as $E(\Delta X)$

Thus the average longshore tracer velocity is

$$
\begin{aligned}
& v_{x}=P m_{e q} E(\Delta x) / \Delta t \\
& Q \simeq \mu E(\Delta x) / \Delta t \\
& \mu=P_{e q} / f_{e q}(0) \\
& E(\Delta x) / \Delta t=v_{x} / P m_{e q}
\end{aligned}
$$

Thus

$$
Q=\frac{P m}{f_{e q}(0)} \quad \frac{V_{x}}{P m_{e q}}
$$

Or

$$
\mathrm{Q} \simeq \mathrm{V}_{\mathrm{x}} / \mathrm{f}_{\mathrm{eq}}(0)
$$

Volume drift rate is simply the tracer centrold velocity divided by the total surface concentration of tracer material, both of which are measurable quantities

These results can be applied to the three dimensional case if one assumes that diffusion of material in the shore-normal direction is negliglbly small In this case, for a line injection the littoral drift rate is given by

$$
Q=\int \frac{v_{x}(y)}{f_{e q}(0 / y)} d y
$$

where the integral is taken across the entire zone of transport

Concluding Remarks

Before the above equations can be relied upon, it is necessary to test some of the underlying assumptions both in the laboratory and in the field Experiments are presently being desıgned at CERC and elsewhere for this purpose 
References

Courtols G and Monaco, A (1969), "Radıoactive Methods for the Quantıtatıve Determination of Coastal Drıft Rate", Marıne Geology, v 1, pp 183-206

Hubbe11, D W and Sayre, W W (1964), "Sand Transport Studıes wıth Radıoactıve Tracers", ASCE, vo1 90 , HY 3 , pp 39-68 


$$
\checkmark
$$




\section{CHAPTER 53}

PROPERTIES OF LONGSHORE BARS IN THE GREAT LAKES

by

James $H$ Saylor

Edward B Hands

Natıona1 Oceanıc and Atmospheric Administration

Detrolt, Michigan 48226

Longshore bars are permanent features of nearshore bathymetry along the wrndward coasts of the Great Lakes The stabizty and permanency of these features hove been noted by numerous investrgators, but movements of the bars and troughs in relation to varying lake levels and inoudent wave energies are not fully understood studies of nearshore bathymetry and sediment properties were conducted durng 2967 and 2969 along a forty-five kizometer reach of the easterm coast of Lake Mrahrgan

Results show that the offshore bars mgrate sugnifreantly due to changes in lake level, a mse of one-half meter in the surface of Lake Mrchigan between 2967 and 2969 was accompanied by a shoreward movement of bar crests and troughs over a dustance averaging 30 meters Elevatrons of the crests and troughs are also bunlt upward toward new equiblzbrum levels dumng msing water levels, but elevating of the crests lags the increase in stage Extensive shore erosion occurs because of the reduced effectrveness of longshore bars in dissipating incrdent wave energs The average crest depth was found to increase linearly in the offshore drrection Average dustances between crests increase exponentrally These relationships are preserved during the bar growth and shrfting that accompanes long term changes in lake level

Bar troughs are characternstzcally crescent shaped, with no abrupt changes in slope Fathogroms from several ranges show atypreal trough confrgurations consisting of flat bottoms whth discontrnurties in slope on ascent to adjacent crests Thus unusual trough shape is indrcatrve of an rmobrle stratum exposed along the bottom of the trough

\section{INTRODUCTION}

Longshore bars are permanent features of nearshore bathymetry along the windward coasts of the Great Lakes where an abundant supply of sand-size sediment is present The bars and intervening troughs are oriented essentially parallel to the shoreline Along the eastern coast of Lake Michigan the longshore bars are continuous in coastal reaches exceeding many tens of $k_{1} 1$ ometers, and the structure of these remarkable features has been described by numerous investigators The continuous bars are usually three or four in number in Lake Michigan, although as many as five or six have been observed As a rule, the 
spacing between bars increases in the offshore direction, so that the distance between the first and second bars is less than the distance between the second and third bars as one proceeds toward deep water The trough associated with each bar occurs inshore of the crest, and the height of the crest above the trough also increases going offshore, w1 th the exception that the bar farthest offshore is often of small helght and configured as a long, gently-sloping swell of the lake bottom lying just lakeward of the more typlcal longshore bar structures

The longshore bars of Lake M1chigan were first described by Desor (1851), and more comprehensively investigated by Evans (1940, 1942) The observations of bar structure made by Evans have been widely reported (e g , Shepard (1950) and Zenkovitch (1967)), and constitute the framework of present knowledge There have been relatively few studies concerning movements of the bar structures due to varying incident waves and currents, or due to long-duration variations in water surface elevation Evans (1940) concluded that minor readjustments in the positions of bars and troughs may occur with changes in intensity or direction of incident waves, but that the bars do not migrate in response to significant increases in water surface elevation He believed that an increase in water level would strand the deeper bars as relicts, and that a new set of crests and troughs would be built inside them The only shoreward migration of bars would be associated with a lowering of lake level, which would decrease the water depth over the innermost bar and cause currents or waves of translation across it With these conditions, Evans felt that the innermost bar would move shoreward as a subaqueous dune Davis and McGeary (1965) studied the stability of nearshore topography in southeastern Lake M1chigan during the summer months of 1963 Their results agreed w1th Evans' findings that the offshore bars do not move apprec1ably Bajorunas and Duane (1967) studied nearshore topography in a simslar environment in southeastern Lake Superior and contrary to previous findings, they showed that considerable movement of bars can occur during just a few months in southeastern Lake Michigan, Hawley and Judge (1968) indicated that the bars and troughs do migrate from year to year, but they did not identify a regular and consistent pattern of movement

Th1s paper describes the results of an investigation of bar stability conducted during 1967 and 1969 on the eastern coast of Lake Michigan The reach of coast studied extends about $45 \mathrm{~km}$, centered about Little Sable Point (Figure 1) Twenty-nine range lines were established perpendicular to the shoreline at nearly equally-spaced intervals throughout the study area Topography was monitored along each range line from the back beach to an offshore water depth of about $9 \mathrm{~m}$, which is a depth in excess of that in wh1ch the bar structures occur Sediment samples were collected along each range and grain-size distributions determined in the laboratory wave height and period and wind speed and direction were recorded continuously from an offshore platform The platform was erected at a s1te where the water depth was $6 \mathrm{~m}$, and it was located about $500 \mathrm{~m}$ north of the Pentwater jetties, near the northerly end of the study area, and $400 \mathrm{~m}$ from the strandiine Water surface elevation and current speed and direction were also monitored from the platform during the investigations 


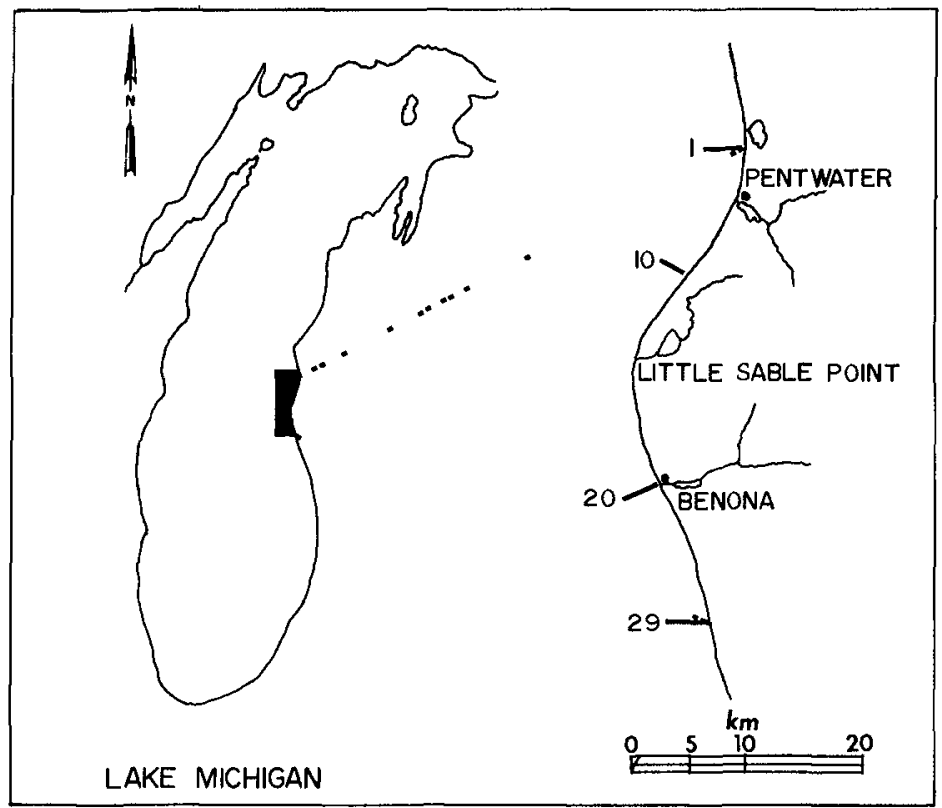

Fig 1 Location of study area and selected ranges

\section{CHARACTERISTICS OF OFFSHORE BARS}

From casual observation of the eastern Lake Michigan coast by either personal visits or inspection of aerial photographs, the continuity and regular spacing of the offshore bars and troughs are striking features However, if the nearshore topography is studied in more detal 1 over a lengthy reach of the coast, the regularities of bar structure become more obscure The spacing and number of bars vary from range to range, as does the depth of water over the crests and the distance of the bars from shore The most pronounced variations occur in shallow water near shore The shallow-water bar, or bars, may not conform to the trend of the shore, as do deeper bars, but will sometimes merge at an angle with the beach face In other reaches, the coastal ridge may appear as an alignment of discontinuous shoals, a crescentric bar (horns and cusps directed toward shore), or simply a perturbance on the outer edge of a bench lying below the swash zone Such coastal bar forms are also more variable in time than the deeper longshore bars They are continually subject to changes in size and position, and may disappear and reform in the course of a few days Just south of the Pentwater jetties the entire sequence of longshore bars is virtually absent The jetties, which extend about $150 \mathrm{~m}$ into the lake, disrupt 1ittoral drift and as a consequence the fullydeveloped sequence of bars is not observed again until one proceeds abouts $1 \mathrm{~km}$ south From this location southward to the vicinity of Little Sable Point, a quartet of well-developed bars persists with 
11ttle variation in geometry At Little Sable Point, the inflection point between a northeasterly and southwesterly trending coast, the regularity of bar-trough configuration is again broken The smooth, regular bar pattern is replaced by an irregular profile which more closely resembles that found on ocean coasts exposed to a wide variety of wave trains A regular sequence of bars is reestablished south of the point and persists throughout the southerly $20 \mathrm{~km}$ of the study area The crests are deeper and more widely spaced in this reach, however

In spite of the variations in bar structure from range to range, the entire reach of Lake Michigan coast studied is typified by the presence of three, and in several subreaches four, essentially-continuous longshore bars The spacing and depth of these bars, while not constant, do fit remarkably simple patterns These bars conform with the classical descriptions, in that the elevation of crest above trough (helght) increases going offshore, as does the spacing between crests The depth of water over successive crests increases linearly in the offshore direction, as 11lustrated in Figure 2, which shows the average values observed during 1969 for ranges 1 through 15, and ranges 18 through 29 The continuous bar nearest shore is labeled in the figure as crest 2 because of the frequent presence of one or more low amplitude bar-11ke structures between 1 and the strandline In all reaches studied, the crest elevations of the three continuous bars exhibit a nearly linear distribution, although the slope of the line connecting crest elevations does vary along the coast as 11lustrated in the figure, and the bars are deeper south of Little Sable Point than they are to the north

Between the continuous bar nearest shore and the strandine, one or more bar-11ke structures are occasionally observed If more than one are present, the depth of water over the crests is nearly the same and averages about $05 \mathrm{~m}$ These shallow-water features show much var1ability from range to range and they are not continuous along the coast for lengthy distances The 1967 investigations were conducted in the northern third of the coastal reach and during the summer months of June through August During this period the shallow-water crests were always present, but they were not as prevalent on the same ranges during the 1969 studies made during the spring and fall months The summer months on Lake Michigan are characterized by moderate winds and waves, with storms occurring infrequently, while during spring and fall the coast is subjected to frequent intervals of high wave stress Thus, the shallow-water features are prominent in summer when the deeper and permanent bars are inactive and lie below the depth of the normal wave forces They represent the reworking of the nearshore sediment due to moderate waves which pass unaltered across the deeper bars Intense storm waves effect the entire bottom structure and disrupt the shallowwater structures built under moderate wave conditions

Much variability in bar structure also occurs offshore from the three continuous crests In the northern parts of the study area, a forth continuous bar is present and the depth of water over 1 ts crest is linearly related to the depth of water over the three shallower continuous crests In this region, the fourth continuous bar conforms w1 th the characteristics of the inner three, as the amplitude and bar 


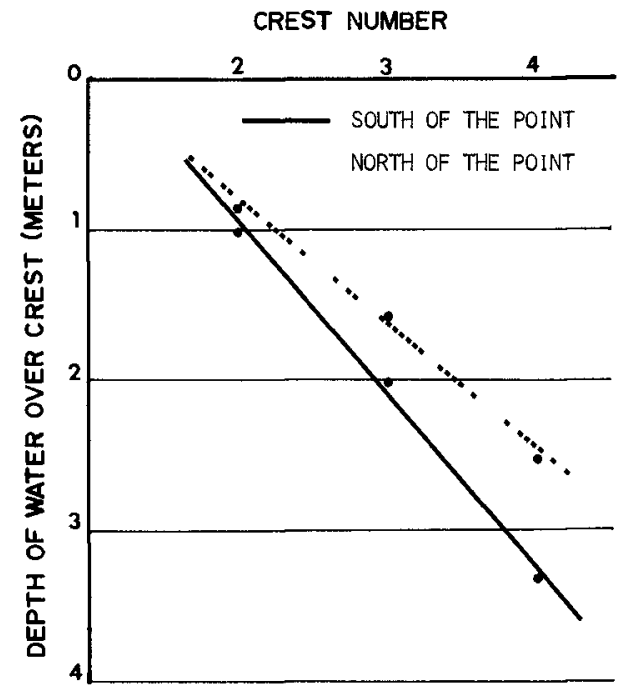

F1g 2 Average depth of water over successive bar crests

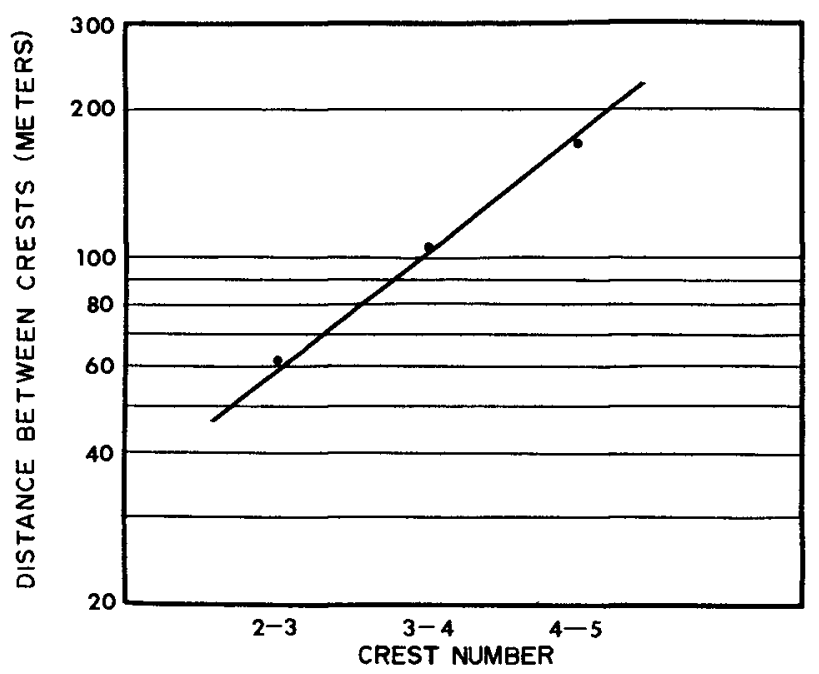

Fig 3 Average distance between bar crests based on measurements at 29 sites over approxımately $45 \mathrm{~km}$ 
spacing increase in a regular fashion going offshore Outside of the quartet of continuous crests, a long-crested, low-amplitude swell of the lake bottom is sporadically present on various ranges The northern half of the reach south of Little Sable Point is also characterized by four continuous crests, while near the southerly end of the coastal reach only the three continuous crests are present Again, the lowamplitude swell occurs lakeward of the continuous features on only several of the range lines

The depth of water over the crests of shallow-water bars and of the deep lake bottom swells does not fit the linear distribution observed for the continuous crests At both ends, the observed water depths are greater than those which would be obtained from interpolation of the linear trend for the persistent triplet If the linear distribution of crest elevations is truely representative of an equilibrium longshore bar structure, as is certainly suggested by the consistent observations, the deep swells of the lake bottom must be representative of bar formation at a depth where there is insufficient energy expended by breaking waves for full bar development This is perhaps to be expected, since the deep crests would cause wave breaking only for the most intense and infrequent Lake Michigan storms The sporadic occurrence of the deep swells, and the development of four continuous bars in two subreaches, would be the result of the variations in wave intensity along the coast due to wave refraction and differences in exposure

The spacing between crests in the offshore direction does not increase in a linear fashion, but rather increases exponentially The spacing between bars shows more variability than the depth of water over the crests, although there are clearly defined distributions Figure 3 shows the bar spacings observed during the spring of 1969 in the entire coastal reach The numbering scheme for the crests shown on the figure is the same as used previously, 1 e, crests $2-4$ refer to the three bars continuous along the coast The spacing between crests varies considerably as the offshore gradient varies from range to range At Little Sable Point, for example, the slope is greater than it is at the northern end of the study area, so that the bars are compressed together even though an exponential increase in spacing is still observed in the offshore direction

The ratio of water depth over the trough (Dt) to depth over the crest (DC) falls between 11 and 21 Keulegan (1948) reported that this parameter remained fixed even as other measures of the bar changed due to varying wave conditions The average value of 15 from our data agrees closely with value 15 (below 11w) given by Shepard (1950) as most typical of Pacific beaches, and with the average of 169 determined by Keulegan in wave tank experiments It should be noted, however, that cholce of reference level (mlw, $11 \mathrm{w}$, etc) and method of calculating the average can significantly alter the results Further, no relationship between the type of bottom material and Dt/Dc could be found even through the bottom material was found to exert a major influence on bar profile configuration, as will be discussed later

In examining bar measurements made on the Pomeranian coast by otto 
and Hartnack (1n Keulegan, 1948) and on Lake M1chigan by Evans (1940), Keulegan felt that there was field evidence that Dt/Dc increases with increasing distance of the bar from shore No significant difference was found in this ratio for the three persistant bars $(2,3$, and 4$)$ in this study A lower value of 13 for the outer bar contributes to the evidence that the outer bar, which 1 s out of the reach of frequent wave disturbance, has not yet been able to build to an equilibrium state

The physical significance of the linear increase in depth of water over successive crests and of the exponential increase in bar spacing is not readily apparent The water depth at which incident wind waves break is a constant fraction of the depth, $1 \mathrm{e}$, the waves break when the wave height is on the order of 08 of the water depth Thus, a linear increase in water depth over the crests going offshore would imply a linear decrease in the height of waves breaking on successive crests in the onshore direction Since wave energy is proportional to the square of the wave helght, the energy dissipated on each bar would not decrease in a linear manner, and the energy dissipated on each crest may play an important role in determining bar spacing However, it is probable that the large wave heights are associated with the longer wave lengths incident on the coast so that the process of breaking across successive bars can filter out wave lengths as well as wave heights The wave length may also be a factor in determining bar spacing

\section{BAR STABILITY}

After reaching record low levels in 1964, the water surface elevation of Lake Mich1gan 1ncreased stead1ly through 1969 (F1gure 4) According to Evans' (1940) hypothesis, the rise in lake level should have stranded the deeper bars as relicts, with a new set of longshore bars built inside them But the observations do not indicate that this has occurred On the contrary, the bars have bullt upward toward the water surface and moved inshore Figure 5 shows bottom elevations measured along two ranges in the northern third of the study area which typify the observations in this coastal reach The profiles were measured during the summer of 1967 and the spring and fall of 1969

An onshore movement of bars and troughs is unmistakable, and for the profiles shown in Figure 5 averages about $30 \mathrm{~m}$ The direction of movement in relation to long-duration changes in lake level is exactly opposite to the movements hypothesized by Evans (1940), who indicated that onshore movement of the shallower continuous bars would be assoclated with falling lake levels During the summer months the deeper bars exhibit much stability, in agreement w1 th summer observations made by Davis and McGeary (1965) Comparison of aerial photographs may also give a misleading interpretation of bar stability unless the cyclical changes with respect to water surface elevation are taken into account

The average depths of water observed over successive crests during the summer of 1967 and spring of 1969 are compared in Figure 6 for those ranges studied both years If the bars were static, fossil fea- 


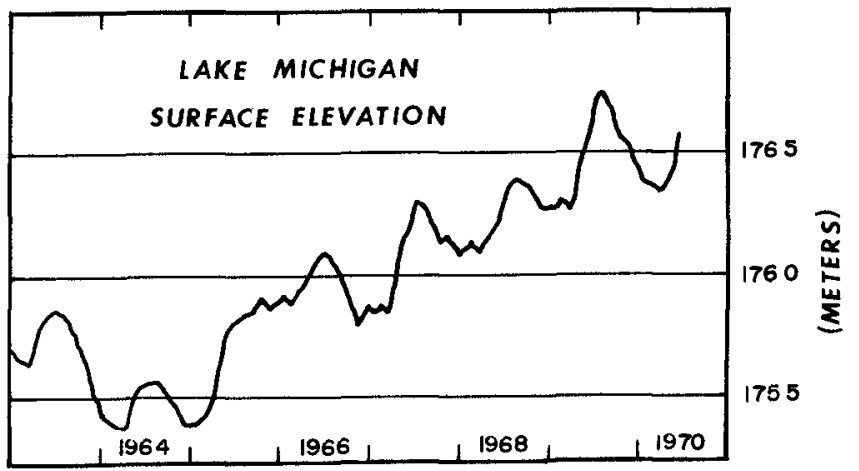

F1g 4 Hydrograph of monthly mean levels of Lake Michigan since the record low of 1964 (IGLD, 1955)

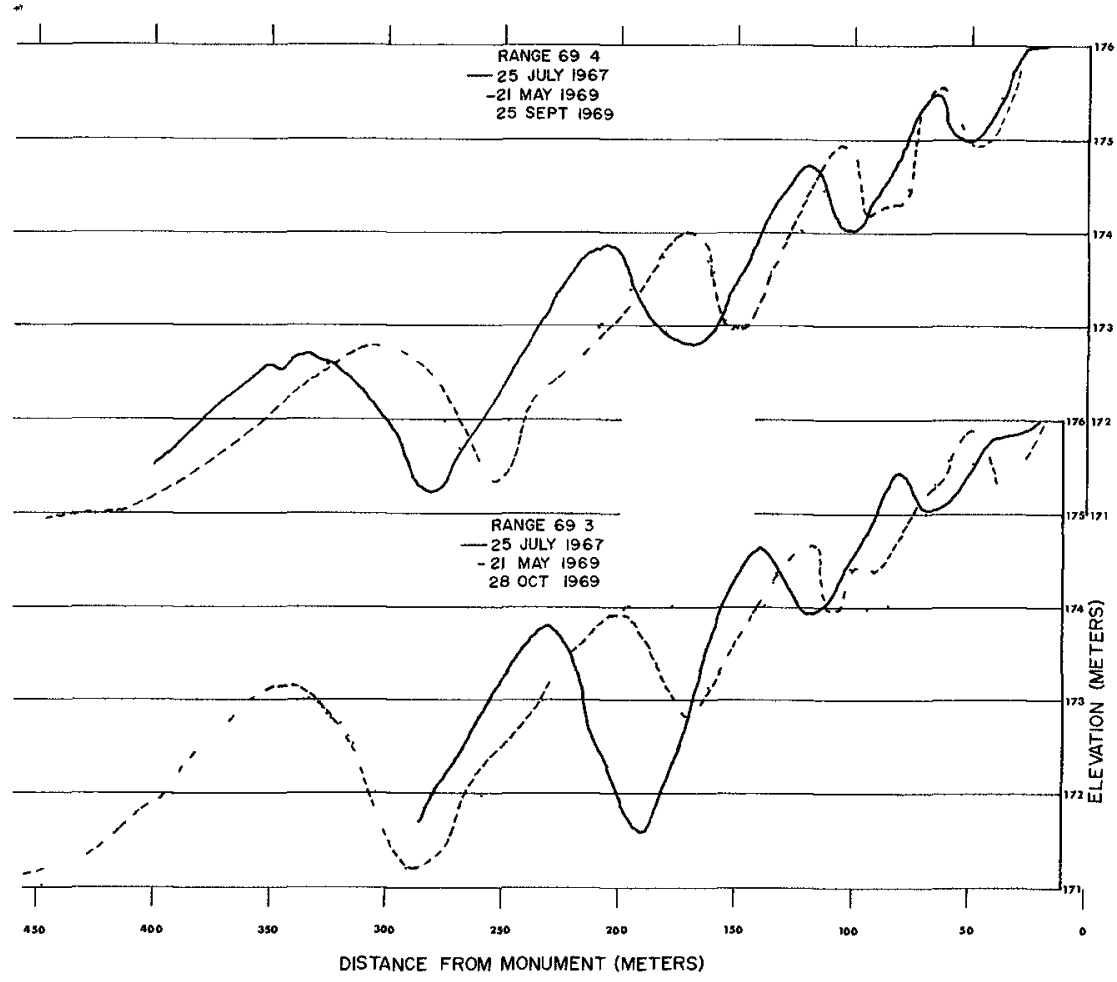

Fig 5 Movement of bars and troughs 
tures, they would simply have been drowned during the subsequent rise In lake level, $1 \mathrm{e}$, the 1969 depths would merely plot $031 \mathrm{~m}$ above the 1967 depths But this is not what occurs The bars have responded to the rising lake level by bullding upward in a manner to preserve fixed depths below the water surface Discrepency between the 67 and 69 depths increases toward deep water (where the response is slower), but even on the deeper bars the difference in depth is less than the change in lake level

The most rapid movements of bottom material are associated with the spring and fall intervals of frequent high wave intensities The fall season is most important because wind across the lakes is accelerated due to unstable air stratification above the warm lake surface, whereas stable stratification occurs in spring and summer over the cold lake surface Thus, lake elevation during the fall months is perhaps the determining factor in the establishment of equilibrium nearshore topography, and in this sense the profiles measured during the summer of 1967 and the spring of 1969 are probably representative, at least for the deep water ends of the profiles, of conditions established during fall of the previous year For a considerable part of the 1 ce-free season, an increase in water surface elevation allows higher amplitude waves to propagate to the shoreline than would be possible if the profiles were fully adjusted to an equilibrium state These non-equilibrium stages are associated with extensive beach erosion and structural damage Conversely, during low lake levels the longshore bars are more effective in dissipating the incident wave energy, and the shallow-water areas are unusually stable Noting the annual cycle of water surface elevation in Lake Michigan as shown in Figure 4, It is to be expected that the beach is most vulnerable to erosion during the early sunmer months of peak water level

\section{TEXTURE OF BOTTOM SEDIMENTS}

A fine grain, very well sorted quartz sand 1 s the typical bottom sediment from the water's edge out to a depth of $9 \mathrm{~m}$ and from range 1 to range $29,45 \mathrm{~km}$ south While diving with the ald of SCUBA, a few pools of colloidal clay (about a meter in diameter and a few centimeters in thickness) were noted, resting gently over scattered depressions in the bottom None of the more than 270 bottom samples had so much as $1 \%$ by weight in the silt-clay size range, 1 e, $<4 \phi$, where $\phi=-\log 2$ diameter in $\mathrm{mm}$ Toward the other end of the size range granules and pebbles, though uncommon, did occur at the strandline along some reaches of the coast and they appeared in a few samples from specific troughs

Sieving the sediment on $1 / 4 \phi$ intervals disclosed a small but clear trend toward finer material on the crests away from shore Superimposed on this classical pattern is a tendency for trough samples to be coarser, more poorly sorted, and more negatively skewed than samples from the adjacent bar (Figure 7) Changes in these three textural parameters can most simply be viewed as expressing the absence, on crests, of some material from the coarse end of the normal size distribution 

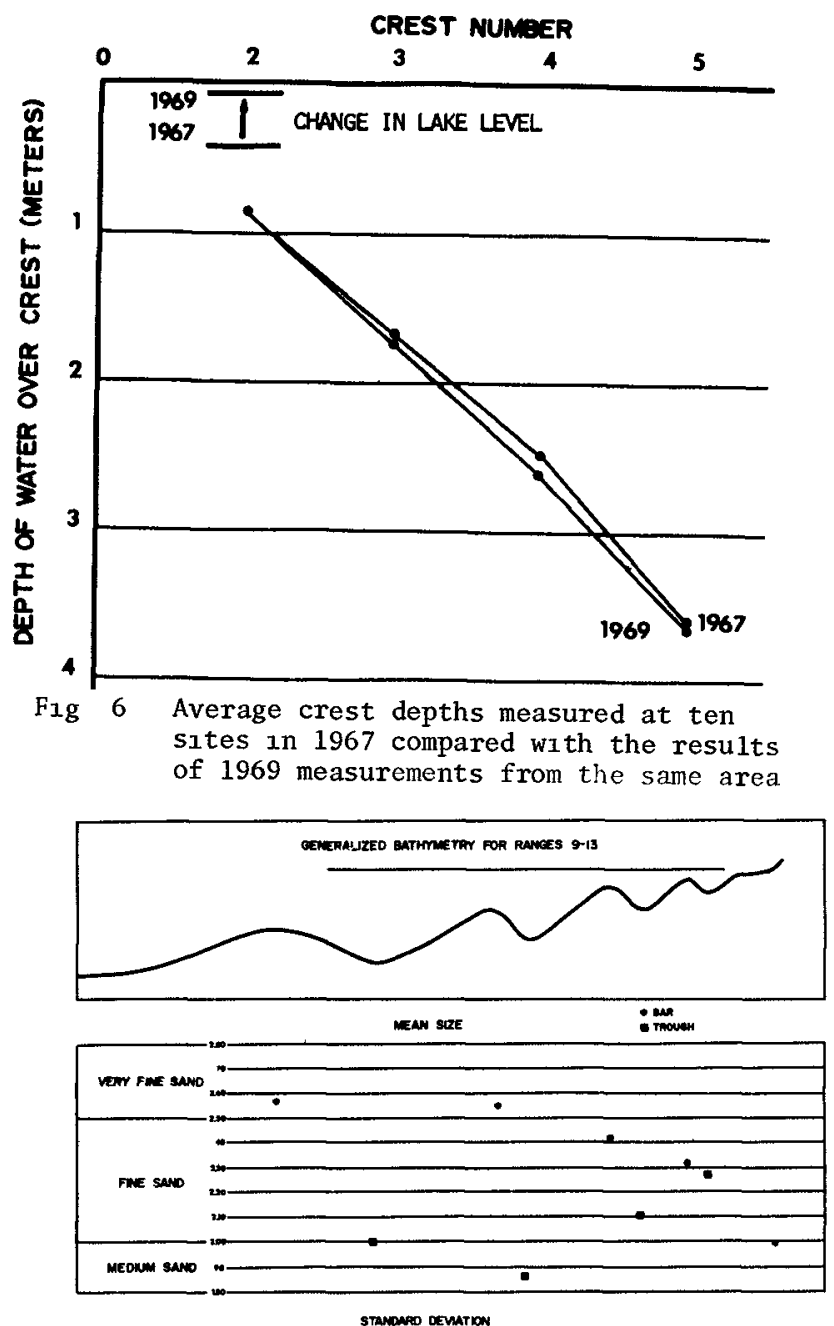

STAMDWFo DEMATION

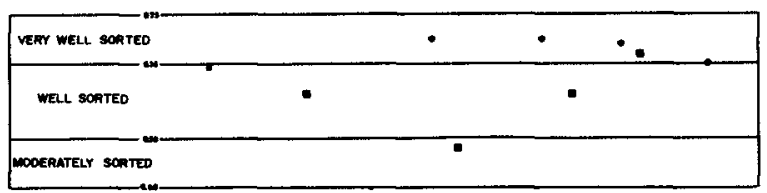

Fig 7 Average textural parameters of crest and trough samples from ranges 9-13 Trough samples are coarser and more poorly sorted than samples from adjacent troughs 
These observations are in accord with the principal theories on bar genesis, in as much as the various theories all envision some winnowing action at trough sites with concomitant deposition on a bar site located immediately lakeward

Figure 7 , 11lustrating textural variations in an offshore direction, is based on the average values from the various bars on ranges 9-13 during 1969 In addition to the accumulation of slightly coarser sand at the troughs, which appears to be a general rule, we found a few troughs floored with a relict, immobile material Therefore, to examine size variations of just the mobile fraction in the longshore direction, the crest samples were averaged for the three persistent bars which occur on each range, and the results are shown in figure 8 A regional trend emerged, the finest material in littoral transit and stable within the surf zone accumulates in the area just north of Little Sable Point (in the vicinity of range 10) This reach of the coast occupies an intermediate position between shore bluffs that mark the intersection of the modern shoreline with glacial moraines A high bluff $(>50 \mathrm{~m})$ to the north is subject to periodic slumping as indicated by the presence of recently uprooted trees in the shallow water at the toe of the bluff and by the testimony of residents This source of new material to the littoral system 1 ies about $5 \mathrm{~km}$ beyond the northern limit of the area sampled, but as shown by the pattern of accretion and recession around the Pentwater jetties the net longshore drift is from the north in this region and the bluff appears to be a source of sediment to the nearshore zone as far south as Little Sable Point Cobbles lie at the toe of the bluff and the finer constituents eroded from the morainal debris are redistributed by 11ttoral drift Clays and silts in suspension evidently move considerable distances alongshore, but are ultimately diffused into deeper water prior to burial The sand load becomes gradually finer in its direction of flow away from the bluff toward Little Sable Point, Figure 8 The transport of some of the coarse sand reaching Pentwater may be arrested by the jetties there South of Little Sable Point the moraine again meets the shoreline, and in the past has contributed fresh material to the shore environment However, because of the configuration of the coast (Figure 1) the northwesterly storms are less important here than southwesterlies which cause littoral drift in this region toward the north The finer sediments in the vicinity of range 10 are protected somewhat from northerly drift by the Point Northwesterly storms 11kewise fall to induce strong littoral flow at this point since the waves approach normal to shore Therefore, it is not unreasonable that moving away from glacial bluffs to the north and south toward this zone of converging littoral currents, increasingly finer and more mobile sediments are encountered It should be pointed out that the spatial variations in texture, (alongshore, offshore, and bar verses trough) are sma11 $(\sim 3 / 4 \phi)$ and would not have been disclosed by the stil1 common practice of sieving at $1 / 2 \phi$ intervals

\section{BAR GEOMETRY AND COMPOSITION}

A relationship between sand size and slope of the beach has been demonstrated by Bascom (1951) Moreover, wave tank experiments at the Coastal Englneering Research Center (Saville and Watts, 1969) have 


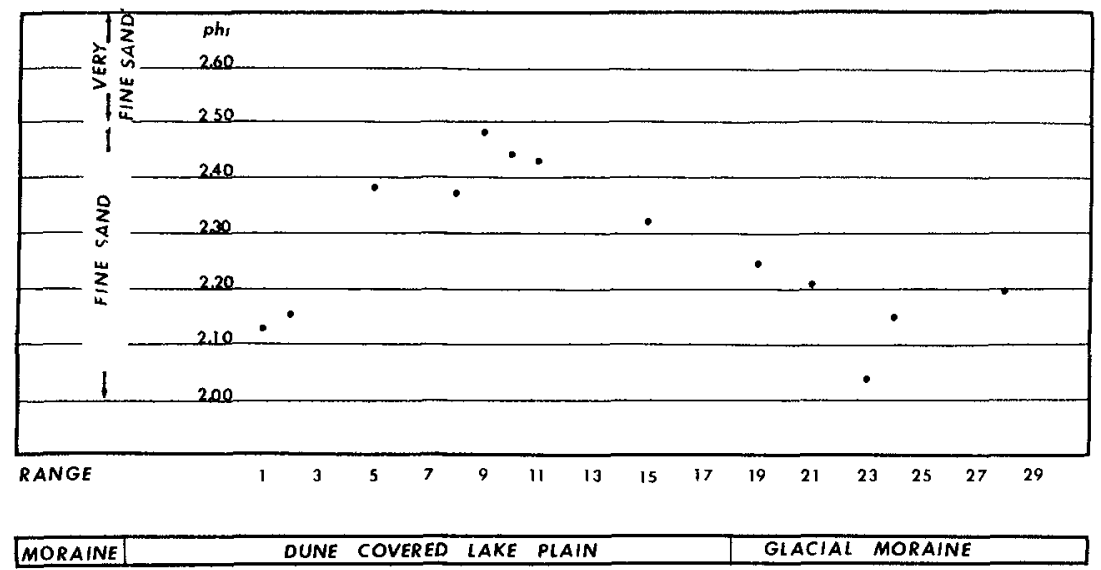

F1g 8 Longshore variation in mean diameter of mobile sediment

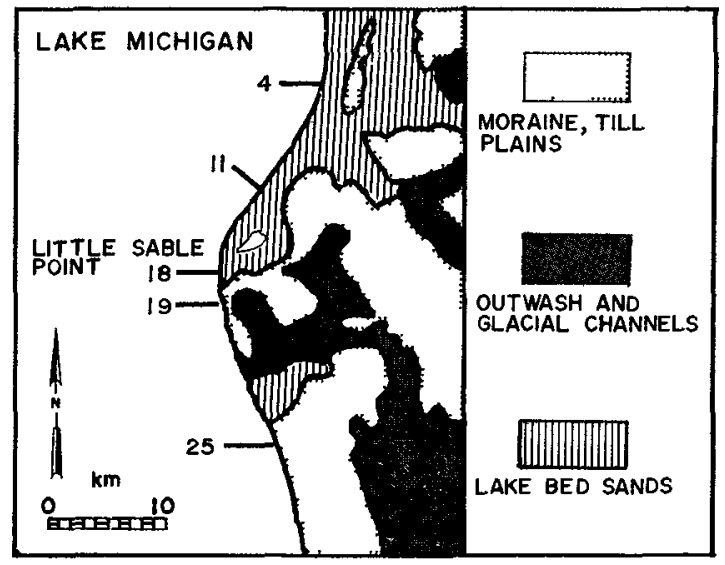

F1g 9 Location of ranges w1th boulder-paved troughs and their relationship with glacial formations onshore (From M1ch1gan Geological Survey, 1955) 
shown a tendency for bars to bu1 1d slightly farther offshore as the grain size of the sediment is decreased The textural variations discussed in the preceding section are, however, too small to relate to changes in bar morphology The response of profile configuration to radical changes in bottom composition, several orders of magnitude greater than the variations revealed by sieve analysis, will be discussed here

Longshore bars occur on t1dal as we 11 as tideless bodies, 1 n1and lakes as we11 as open oceans The resulting forms are quite varıable, reflecting local sediment response to a particular energy environment In spite of this variability, longshore troughs in sandy material are characteristical1y U-shaped It was therefore surprising to find on the fathograms several profiles exhıbiting flat and even convex upward trough floors in each instance investigation using SCUBA revealed the presence of a rock pavement at these sites of anomalously shaped troughs

The rocks were from cobble to boulder size ( $\begin{array}{ll}0 & 1\end{array}$ to several meters in diameter) and ran the gamut of lithologies through sedimentary, 1.gneous, and metamorphic types This indicates the necessity of glacial transport to explain the1 $\mathrm{r}$ occurrence on the shore of lake Michigan Three of the five ranges with flat bottom troughs are indeed directly offshore from truncated glacial moraines The occurrence of boulders on these three southern ranges 15 thus readily explainable as an effect of post glacial coastal erosion Boulders also floor troughs on ranges 4 and 11 , and these are separated by k1 lometers from the nearest boulder clay deposit Evidently the glacial moraines formerly extended considerably farther westward (F1gure 9)

The correlation between atypically shaped troughs and the presence of boulder pavements seems well established by this study The use of grab samplers (VanVeen, 1936, Shipek, 1965), so routınely employed in surveys of shallow-water sediments, fall to reveal the rock pavements due to the large size of individual rocks $(01-2 \mathrm{~m})$ and the elongate, patchy distribution of the deposit Likewise, Hough's method (1952) for identifying bottom composition by interpreting the density and thickness of fathogram traces also falled to reveal these substantial changes in bottom type Rock pavements do however reveal their presence by effecting profile configuration They interrupt normal profile development, causing abrupt changes in slope and flat to convex upward troughs (Figure 10) This previously unrecognized relationship permits the use of fathograms to infer the presence of clay, gravel, boulder, or bedrock substratum and the thickness of mobile sediment 


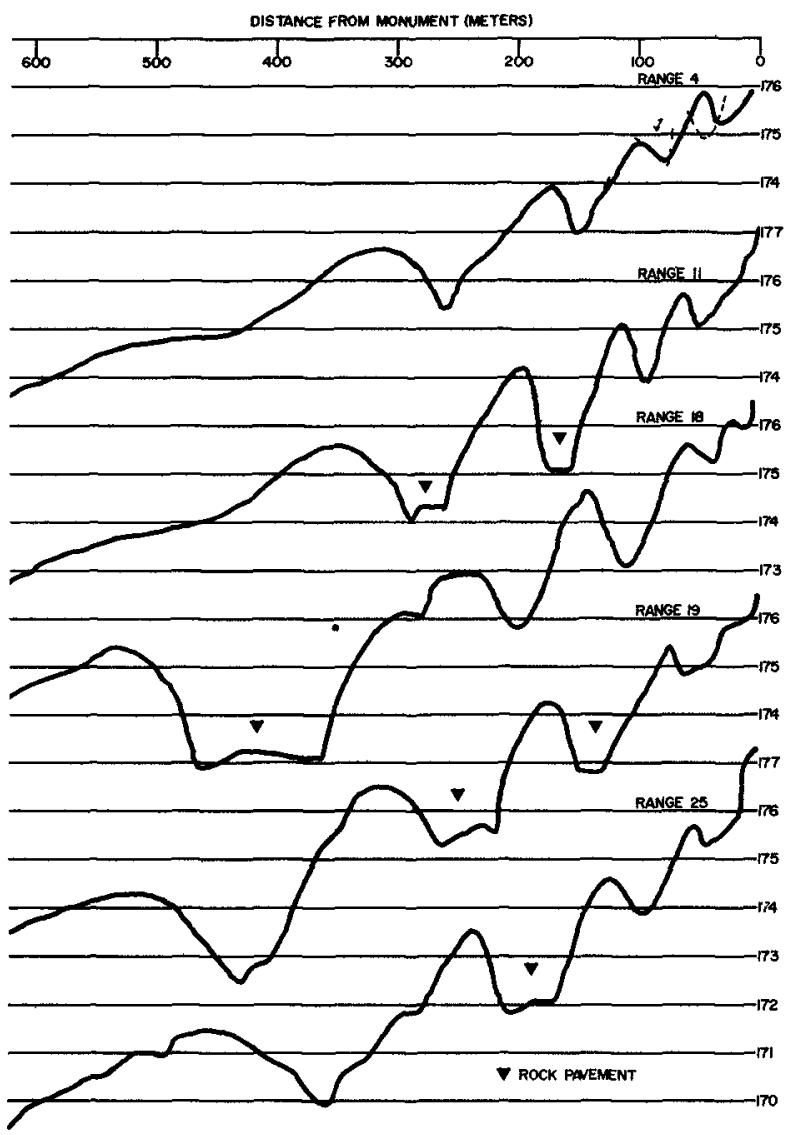

F1g 10 Atyplcal, flat-bottom troughs arise where immobile strata interrupt normal profile development

\section{REFERENCES}

Bajorunas, $L$ and $D$ B Duane

1967 Shifting offshore bars and harbor shoaling Jour Geophysical Res, V 72 , No $24,6195-6205$

Bascom, $W N$

1951 Relationships between sand size and beach face slope Transactions American Geophysical Union, V 32, No 6, 866-874

Dav1s, $R$ A and D F $R$ McGeary

1965 Stability in nearshore bottom topography and sediment distribution, southeastern Lake M1chigan Proc 8th Conf on Great Lakes Res, Pub No 13, Great Lakes Res Div, Univ M1ch, Ann Arbor, M1ch , 222-231 
Desor, E

1851 On the superficial deposits of the district In Foster, J W and J D Whitney, Report on the Geology of the Lake Superior Land District, Part 2, Wash, D C $258 \mathrm{p}$

Evans, $0 \quad F$

1940 The low and ball of the eastern shore of Lake Michlgan Jour Geology, V 48, No 5, 476-511

1942 The origin of spits, bars, and related structures Jour Geology, $\mathrm{V} 50$, No $7,846-865$

Folk, $R \quad L$ and $W$ C Ward

1957 Brazos river bar a study in the significance of grain size parameters Jour of Sed Petrology, V 27, No 1, 3-26

Hawley, E F and C W Judge

1969 Characteristics of Lake Michigan bottom profiles and sediment

from Lakesıde, Mıchigan to Gary, Indiana Proc 12 th Conf on Great

Lakes Res, Internatıonal Associatıon for Great Lakes Research, 198-209

Hough, J L

1952 Fathogram indications of bottom materıals in Lake Michigan

Jour of Sed Petrology, V 22, No 3, 162-172

Keulegan, G H

1948 An experımental study of submarıne sand bars Beach Erosion Board, Tech Rept 3

Martin, $H \quad M$

1955 Map of the surface formations of the southern peninsula of

Michigan Mich Geol Survey Pub 49

Savi1le, $\mathrm{T}$ Jr and $\mathrm{G} W$ Watts

1969 Coastal regime, recent $U S$ experience Proc 22nd International Navigatıon Congress, Reprint 3-70, Coastal Engıneerıng Research Center, Washington, $\mathrm{D}$ C

Shepard, F P

1950 Longshore bars and longshore troughs $U$ S Beach Erosin Board, Tech Mem 15, 32p

Shıpek, C J

1965 A new deep sea oceanographic system in Ocean Scıence and Ocean Engineerıng Trans of the Joint Conf and Exhıbıt, Marıne Tech Soc and Am Soc Limnology and Oceanography, 14-17, V 2, 999-1008

Van Veen, Johann

1936 Onderzoekingen in de Hoffden Landsdrukkerı], The Hague, 252p

Zenkovitch, $V$ P

1967 Processes of Coastal Development Interscience Publishers, New York, $\mathrm{N} Y 738 \mathrm{p}$ 



\title{
CHAPTER 54
}

\section{SOME SAND TRAITSPORT PHENOMENA ON COASTS WITH BARS}

\author{
M. Dyhr-Nielsen, Research Engineer \\ and \\ Torben Sørensen, Director \\ Danish Institute of Applied Hydraulics \\ Copenhagen, Denmark
}

\section{INTRODUCTION}

Longshore wave currents and their influence on the sand transport phenomena in the shore zone have attracted the attention of numerous researchers. Also the existence of transverse, secondary currents, superposing the longshore component, has been known for years, but less attention has been given to analysis of their effect on the sediment movement.

This paper presents some examples of the Influence, these relatively weak transverse currents may have on the processes in the shore zone. They have a parallel in the effect of secondary currents in alluvial streams, which, although weak, give $x\lrcorner$ se to such an Impressive phenomenon as meandering.

All conclusions below are based on simple, qualitative considerations of the physics of the system. A strict mathematical, quantitatıve approach to the problem does not seem possible wath the present knowledge of the fluzd dynamics in the shore zone, and much further research into these complex phenomena remains, of course, necessary.

\section{NET CURRENTS IN THE SHORE ZONE}

\section{Iongshore currents}

It is well known that the breaking of waves in shallow water exerts a force on the water body between the breaker zone and the shore. ThIs Is due to the existence of the wave thrust [Iundgren 19637, found by Integrating the flux of momentum and the wave pressure over the water depth in a wave perıod. The wave thrust has the same direction as the wave orthogonals. 
It is also known that the longshore component of the wave thrust generates longshore currents in the trough between breakers and shore. An equation of equilibrium of the forces in the longshore direction may in principle be established to yield an expression for the longshore velocIty. ThIs approach Is physlcally sound, but lack of sufflclent knowledge of the bed shear stress generated in the complex movement $\mathrm{in}$ and behind the breaker zone renders this approach impracticable at this moment. Furthermore the stochastic aspects of the problem are undoubtedly very important and cannot at present be grasped in mathemathıcal form.

\section{Clrculation currents}

The component of the wave thrust parallel to the shore generates the longshore currents.

Simllarly, the component perpendlcular to the shore generates clrculating currents in the cross section. These currents were first observed by Bagnold [Bagnold 19401 and have since been verlfled by many others. In the following a simple qualitative explanation of the generation of these currents Is given, and thelr occurrence Is related to speciflc properties of the wave motion.

\section{Bottom currents under shoalıng, nearly-breaknng waves}

The deformation of the waves approaching the breaker zone wll Increase the wave thrust I In the shoreward direction, so that the thrust $\mathrm{I}_{2}$ In section 2 is greater that $I_{1}$ In section 1, see $F_{I g}$. 1. Therefore the resulting thrust $I_{R}$ on a control volume from section 1 to section 2 has an offshore direction. This force ls compensated by a decrease in mean water level in onshore directıon, creating a static water pressure reaction $P_{R}$ agalnst $I_{R} \cdot$

The strong deformation of the orbital velocity profile of a wave Immedlately before breaknng causes $I_{R}$ to act at a higher level over the bottom than $\mathbb{P}_{R}$. Hence an equilibrium in the moments can only be obtalned if there Is a resulting force along the bottom, l.e. a resulting shear stress T. T must act on the water body in a seaward direction, and in a shoreward direction on the bottom. The waves will contribute to this, as the resulting shear stress due to wave motion wil have an onshore component, but near the breaker zone this appears not to be sufficlent, and a shoreward bottom current wIll superpose the wave motion.

The existence of shoreward currents under nearly-breaking waves can clearly be observed in a laboratory flume. 
Bottom currents under surfing waves

After breakıng the wave proceeds for some wave lengths as a surfing wave. The heavy energy losses wzll reduce the wave helght, and the wave thrust will decrease in the onshore direction, see Fig. 2. The resulting wave thrust $I_{R}$ acting on the control volume will now have a shoreward direction. A rise in mean water level will give a statıc pressure reaction $\mathrm{P}_{R}$, but due to the high particle velocities at the water surface, $I_{R}$ will attack at a bigher level than $\mathrm{P}_{\mathrm{R}}$.

Equilibrium in the moments again requires the existence of a resulting bottom shear stress $T$, but now acting shoreward on the water volume and seaward on the bottom. Hence under surfing waves the wave movement must be superposed by a resulting bottom current in a seaward direction.

This current can also be observed in a laboratory flume.

\section{Circulating currents}

Under the breaker line, the onshore and offshore bottom currents under the waves will meet. Observations in a flume have shown, that there is only a very smalt transport of water through the breaker zone near the bottom. CIrculating cells are formed as shown in Fig. 3 whereby the return flows for the bottom currents are established. Fig. 3 shows a two-breaker zone where the surfing waves are regenerated and a secondary breaker zone is formed. Two curculating cells are formed between the breaker lines, preventing transport in the bottom zone from the inner to the outer cell.

If the surfing waves reach the shore like in FIg. 4, only one cell is formed, and transport from the shore to the outer breaker line whl occur. This has a very important effect on the coastal sediment transport as will be shown later.

\section{EFFECTS ON SEDIMENT TRANSPORT}

\section{Formation of longshore bars}

It has been shown above that waves breaking on a slopIng plane will generate secondary currents directed towards the breaker line. ThIs will have an accumulating effect on the grains on the bottom, moving them towards the breaker line where they build up a bar.

The shape wll stabılıze when the slope gets so steep that the bottom current cannot transport the sand up over 
it, or when there is equilibrium between the amount of sand carried up by the bottom current and the sand returned in suspension at higher levels. At present it is not possible to tell which of these two conditions is the governing factor.

The position of the bar Is closely connected to the position of the breaker line. Therefore, on tidal shores where this position is changing rapidly with the tidal cycle, distinct bars can only be expected to occur under severe wave conditions. Hence the existence of bars is more likely to occur in the winter as a result of more frequent storms.

The lower waves in the summer will not break over the bar, so the accumulating effect is replaced by an onshore bottom current that wil erode the bar configuration and change the winter-profile to a summer-profile.

\section{Formation of trangverse bars}

In certain cases the situation with a two-breaker zone may change to a one-breaker zone, where the surfing waves from the outer breaker line are not regenerated but extends to the shore as shown in Fig. 4 and 5 . It might for instance happen when the depth over the outer bar is increased by erosion or dredging.

In this case a shoreward bottom current extends from the shore to the outer breaker line, see FIg. 4. This current will carry at least a part of the material transported on the inner bar out to the outer bar. Studies of bottom topographies in the nature have shown, that in connection with this a transverse bar Is formed.

The deficlt in the near shore transport downstream of the transverse bar affects the stability of the beach profile. Erosion of the beach occurs in the downstream region in order to reduce the deflcit and satisfy the transport capacity of waves and currents downstream of the transverse bar.

\section{The stable beach}

It has been shown in laboratory tests that the wave steepness, defined as the ratio between wave height and wave length, has a high influence on the stability of a beach. Waves steeper than a certain value were shown to erode the beach proflle whlle waves below this steepness would build up the beach.

The existence of secondary currents seems to offer a simple explanation of this. Conslder a plane beach with slope 1:n, see Flg. 6. A wave of helght $H$ will break at a depth approximately equal to $H$. Hence the distance from the shore to the breaker line will be approximately $n$ H. 
It is obvious that if this distance is large, surfing waves are formed and seaward bottom currents are generated. But if the breaker line is very close to the shore this is not the case and the breaker will instead form a swash wave on the beach. Surfing waves are able to remove sand from the shore, whereas swash can move sand up on the beach for the following reasons: Swash has the highest velocity and hence the hlghest transport capacity, when the water is moving shoreward, and seepage losses reduce the seaward transport capacity further when the water is running back.

Assuming that the change between surf and swash happens when the breaker line $1 \mathrm{~s}$ distance $\mathrm{k}$ I from the shore, where $I$ Is the wave length. Now a simple similarity consideration gives the limiting wave steepness $H / I$ for the stable beach as $\mathrm{H} / \mathrm{I}=\mathrm{k} / \mathrm{n}$.

It can be seen that the critical steepness depends on the slope of the beach. This may have some implications for model tests with movable bed and a distorted scale. The effect of distortion on the slope of a beach might change an eroding wave in the nature to an accumulating wave in the model.

\section{Meandering in the shore zone}

An interesting observation of some shore line configurations may be mentioned in this connection.

On a $10 \mathrm{~km}$ long reach south of Hvide Sande on the west coast of Denmark it is possible to detect a perlodic fluctuation on the shore line and of the outer bar. The wave length of this fluctuation $1 \mathrm{~s}$ about $3000 \mathrm{~m}$, and the amplitude of the order $100-200 \mathrm{~m}$. Hence with $3000 \mathrm{~m}$ interval the width of the beach is relatively large, and in between It 1 s relatively narrow. This pattern has been remarkably stable in the 9 years for whlch this shore has been studued in detall, although it has been superposed by local erosions and accretions in connection with the formation and passage of transverse bars.

If the area between the bar and the shore 1 s consldered as a "river" carryıng the longshore current, the ratio between the wldth of this river (about $300 \mathrm{~m}$ ) and the length of Its "meander" (about $3000 \mathrm{~m}$ ) is the same as found for meanders in al luvial rivers. From this it seems possible that the longshore current in certain aspects is similar to an alluvial river. The observations of formation of dunes in the trough between bar and shore [Zenkovich 19677 further support this assumption.

A simllar "meander" pattern in a smaller scale has been observed on other Danish coasts. 


\section{THE "HVIDE SANDE" STUDY}

\section{Investigations in the prototype}

Hvide Sande is a small harbour tuwn on the west coast of Denmark. It is located on a narrow sana spit in a tidal outlet whIch is now controlled by sluices. The undisturbed coast proflle at Hvide Sande has two parallel bars and the resulting sana transport along the shore has a southward direction.

To stabllize the outlet two $100 \mathrm{~m}$ long groynes were constructed In 1931, but these had no significant Influence on the outer bar. In the fifties the depth over the outer bar was considered too shallow for safe navigation and in order to improve the conditions a $400 \mathrm{~m}$ long groyne was constructed about $100 \mathrm{~m}$ north of the outlet, see FIg. 7 .

ThIs groyne crossea the outer bar and forced it to move seaward. Furthermore a strong erosion took place on the outer bar at least up to $3 \mathrm{~km}$ south of the new groyne in the following 3-4 years as the supply from north was temporarily cut.

Hence in October 1966 the orlginal bar was significantly reduced over long reaches south of the groyne. In the same period a severe erosion of the beach occurred at a point aoout $3 \mathrm{~km}$ south of Hvide Sande. (SImIlar attacks were reported 6 and $10 \mathrm{~km}$ to the south, In places where the "meandering" had narrowed the beach.) In connection WIth this soundings in April 1967 showed that transverse bars had been formed in the winter of 1967 connecting the near-shore bar with the remnants of the outer bar, see FIg. 8. Just south of these bars the severe erosions were observed. The transverse bars migrated towards south with a velocity of about $600 \mathrm{~m} /$ year, changlng the points of attack on the beach so that the erosion in a point decreased or was substituted by a temporary accretion after the passage of the bar.

South of the transverse bars the outer bar was buIt up again, which Indicates that the outer bar was supplied with sand from the shore zone as mentioned above. The erosion of the outer bar in connection with the groyne construction changed the breaker regime from a two-breaker zone to a one-breaker zone causing the formation of the transverse bar.

It should be mentioned that transverse bars have been observed at many other locations, for example at the upstream side of a groyne where the beach transport has to leave the shore to get around the groyne. They are also found on coasts without manmade interference, but again in connection with local erosion of the beach. 
Model tests

WIth the purpose of studying the effect of the groyne constructions on the wave cur rent patterns during storms three fixed bed models were bullt. The hor zontal scale was 1:300, the vertical scale 1:100. The models Illustrated the situation in 1927 before any groynes were built, In 1961 wath the two small groynes just before construction of the big groyne, and in 19673 years after the big groyne was completed.

As the bottom currents were of major interest they were traced by plastıc balis having a dlameter of $1 \mathrm{~cm}$ and a settling velocity of $7 \mathrm{~cm} / \mathrm{sec}$. These balls were shown to be light enough to fol low the wave current but heavy enough to stay at the bottom.

The movement of the balls showed clearly the existence of resuiting transverse botton currents as mentioned in section 2, as they always had a velocity component towards the breaker zone. But an even more convincing demonstration came from the injection of small ( $1 \mathrm{~mm} \phi$ ) polystyrol gralns whlch were transported seaward under surfing waves and shoreward under nearly-breaking waves. The accumulating effect of the circulation currents concentrated the grains under the breaker lines where they were transported downstream in very narrow bands parallel to the shore.

That something similar happens in the nature has been demonstrated by Ingle in his studies of the movement of dyed grains ox beaches in Calıfornia [Ingle 1966].

The only exception from the pattern above was found $1 y_{1}$ the region where the beach erosion had been reported. Here the relatively low outer bar caused a change from a two-breaker zone to a one-breaker zone, and the granns were transported from the bar near the shore out to the outer bar. In the two other models where the outer bar was undisturbed this did not occur.

Hence it wis concluded that the beavy beach erosions were a secondary effect of the groyne construction. The erosion of the outer bar and the change in the breaker zone reglme, caused by temporary cutting of the sand transport from north, gave rise to the formation of transverse bars, and these in turn were responsible for the erosion of the beach.

\section{REFERENCES}

Bagnold, R. A. (194C). Beach formation by waves; some model experıments in a wave tank. J. Inst. Civ. Engrs., London, $15,27-52$. 
Ingle, J. O. (1966). The movement of beach sand. Elsevier, Amsterdam.

Iundgren, H. (1963). Wave thrust and wave energy level. Internat. Assoc. Hydr. Res., 10th Congr., Iondon, I.20, 147-151.

Zenkovich, V. (1967). Processes of coastal development, Interscience Publishers, New York.

\section{WAVE PROPAGATION}

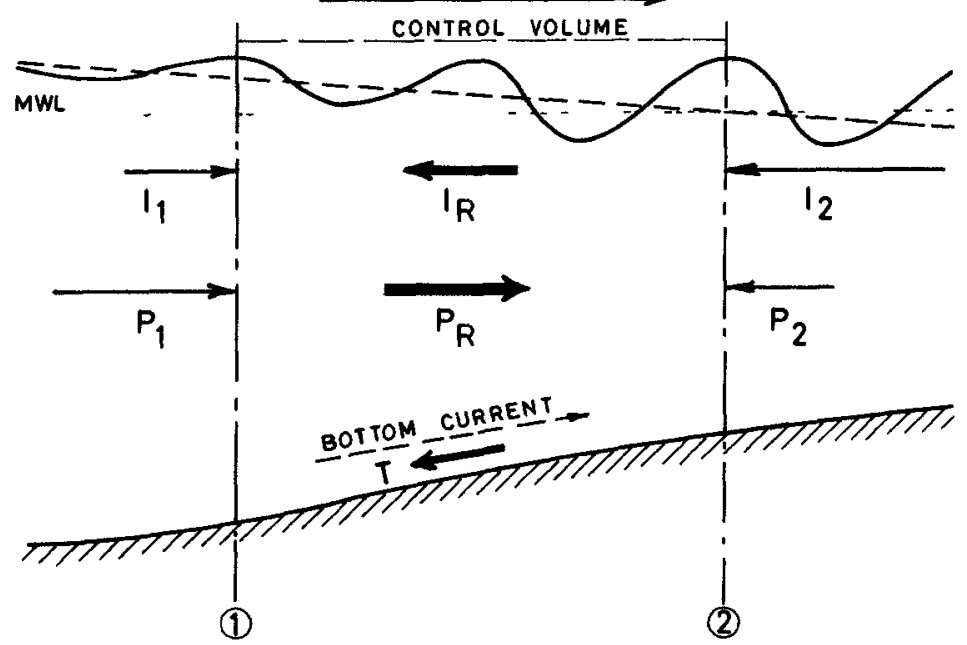

FIG 1 WAVES NEAR BREAKER ZONE 


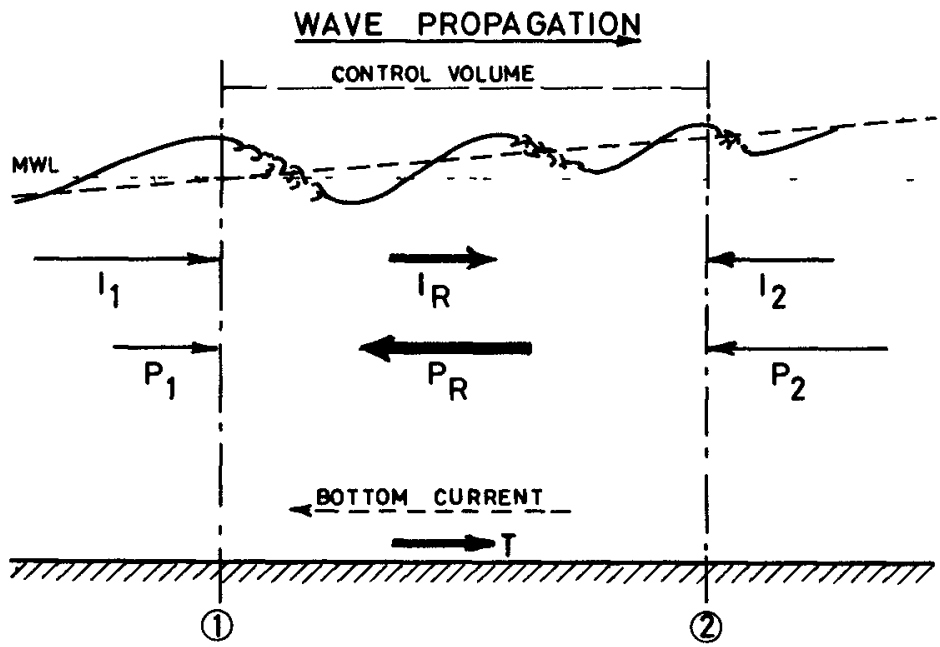

FIG 2 SURFING WAVES

\section{PRIMARY BREAKER LINE}

SECONDARY BREAKER LINE

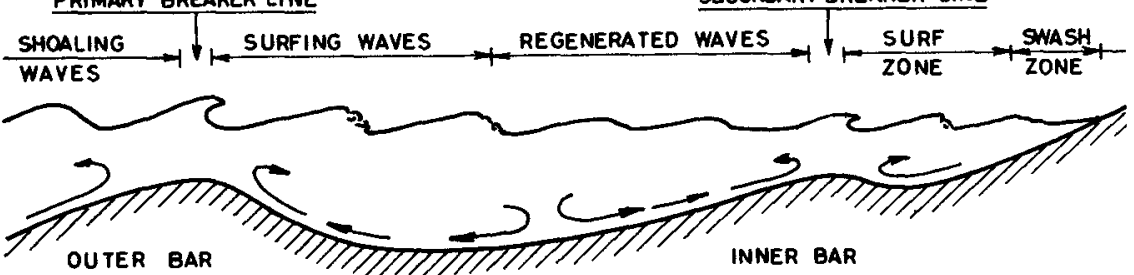

FIG 3 BOTTOM CURRENTS IN A TWO-BREAKER ZONE 
864

COASTAL ENGINEERING

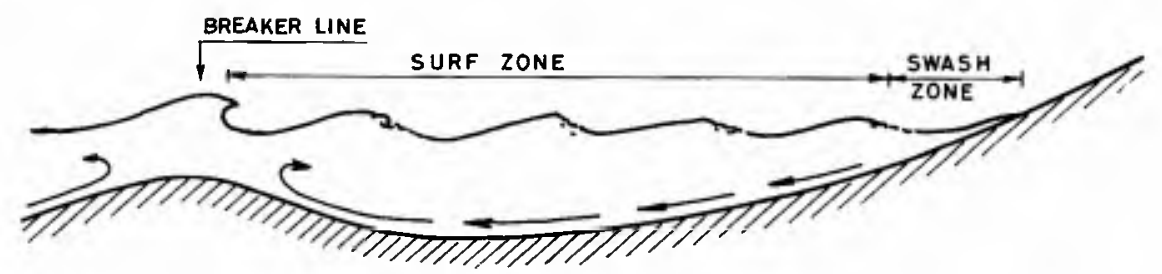

FIG. 4 BOTTOM CURRENTS IN A ONE-BREAKER ZONE

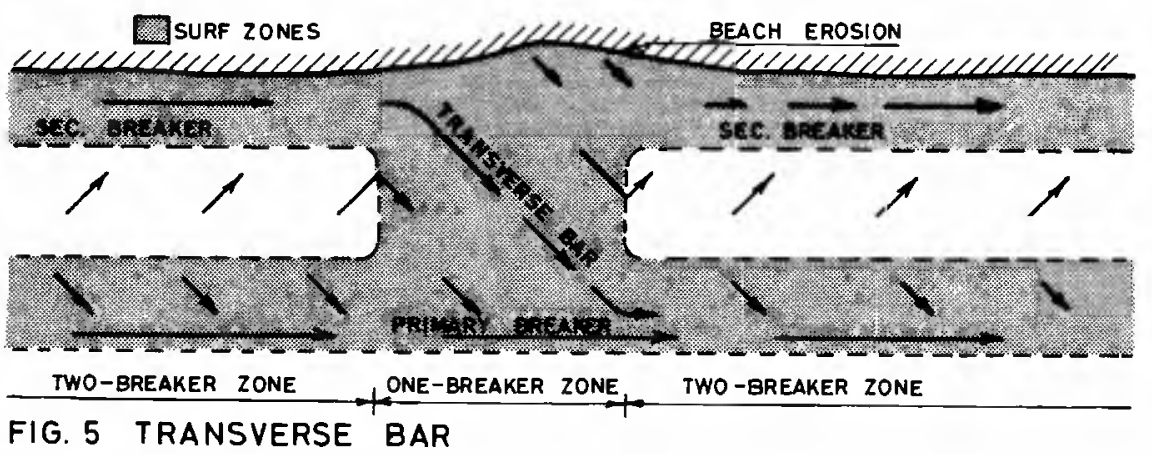

FIG. 5 TRANSVERSE BAR

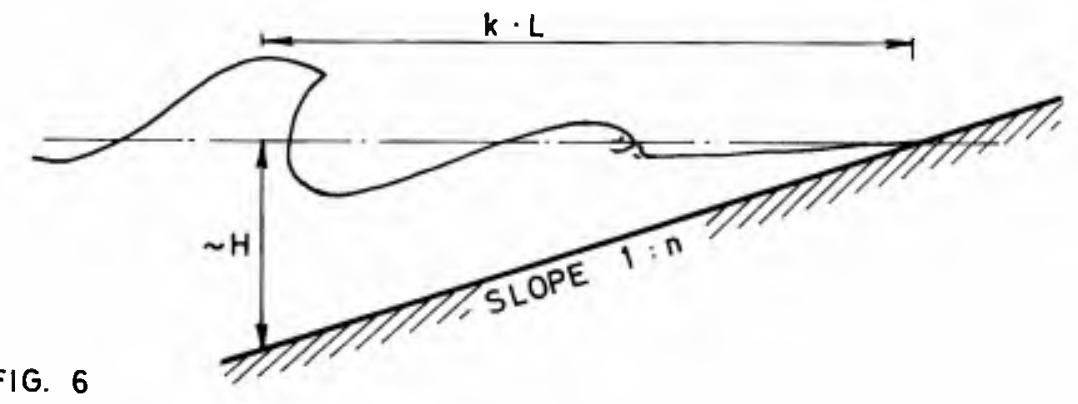



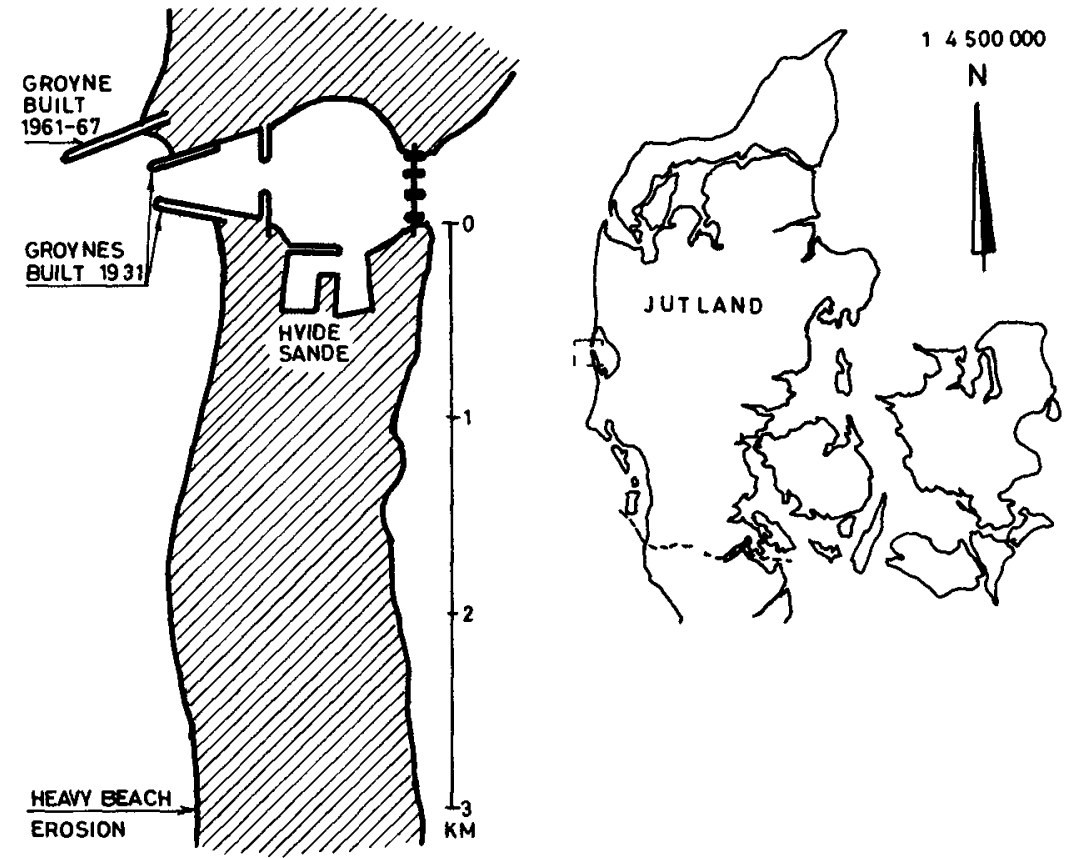

FIG 7 STUDY AREA
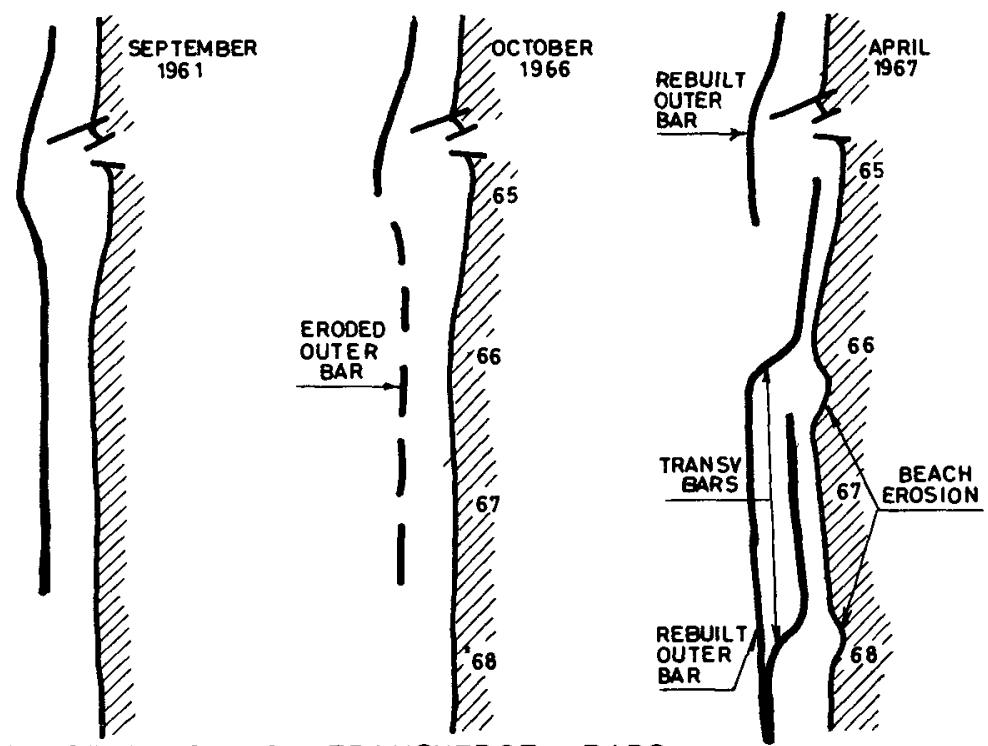

FIG 8 FORMATION OF TRANSVERSE BARS 



\title{
CHAPTER 55
}

\section{LABORATORY TESTS OF LONGSHORE TRANSPORT}

\author{
by \\ John C Faurchild, M, ASCE \\ Research Hydraulic Engineer \\ U S Army Coastal Engineering Research Center \\ Washington, D C
}

\begin{abstract}
Tests were made in CERC's Shore Processes Test Basin with wayes approaching the toe of a test beach at a 30-degree angle Beach material was quartz sand with median diameter of 022 millifmeter which, in most tests, was molded to a 1 on 10 slope before starting a test Long crested waves generated in a constant depth of 233 feet traveled over the beach, shoaled and were refracted before breaking near the shoreline The breaking action caused the sand to be transported along the shore in the direction of the longshore component of the wave energy flux Transport rates of 2 to 170 cubic yards per day were measured, with the lower rate within the range of laboratory rates reported by Savage $(1)$ and the higher rate comparable to field rates reported by Watts $(2)$ for South Lake Worth Inlet, Florida Analysis includes correlation of the measured rates to the longshore wave energy flux, and in some tests, to the longshore current Transport rates, defined by visual fit curve of the data, are about 3 times the rates $2 n-$ dicated by the CERC TR-4 design curve for a longshore energy range of 0016 to $0760 \mathrm{mllli}$ ions of foot pounds per foot of shore per day
\end{abstract}

\section{INTRODUCTION}

\section{Genera1}

Water waves Impinging obliquely on a sandy shore scour and suspend shore materials causing them to move along the shore in the direction of the longshore component of the wave energy flux The amount of material moving depends primarily on the wave breaker angle and the energy of the waves impinging on the shore However, the amount of material moving at a given energy flux is influenced by the wave steepness, breaker type, sand size, and the beach slope, and experience indicates that these factors may act to increase or decrease the longshore transport, where there is little or no change in the wave energy flux

The amount and direction of longshore transport is important in the planning and design of shore improvements Rellable field data on longshore transport is required in the design and economic evaluation of jetties navigation inlets, beach erosion projects, and hurrican protection projects Data, usually of questionable accuracy, is available for a few coastal areas, but present coverage is inadequate and field data is expensive and difficult to get Therefore, CERC has for some time had underway a program to obtain laboratory data whych would define basic relationships and which might be used with field data to more quickly and less expensively provide the relationship between longshore wave energy flux and longshore sand transport 


\section{Laboratory Tests}

This report presents results from laboratory tests of longshore transport $(3,4,5,6)$ made in CERC's Shore Processes Test Basin (SPTB) Tests were made with waves approaching the beach at a 30 degree angle in a constant depth of 233 feet Figure 1 is a plan view typical of the test set-ups used, showing the wave generators and the test beach, with the sand trap at the downdrift end and the feeder beach at the updrift end

Beach material was a unfformly sized sand with a median dzameter of 022 millimeter In most tests the beach was molded to a 1 on 10 slope before starting wave action Several tests, including some groin tests in 1957 (1) and 1958, were started on a "150 hour profile slope", which was an equilibrium slope determined from 150 hours of wave action 0ther tests in 1959 and earlier were started on a 1 on 20 slope

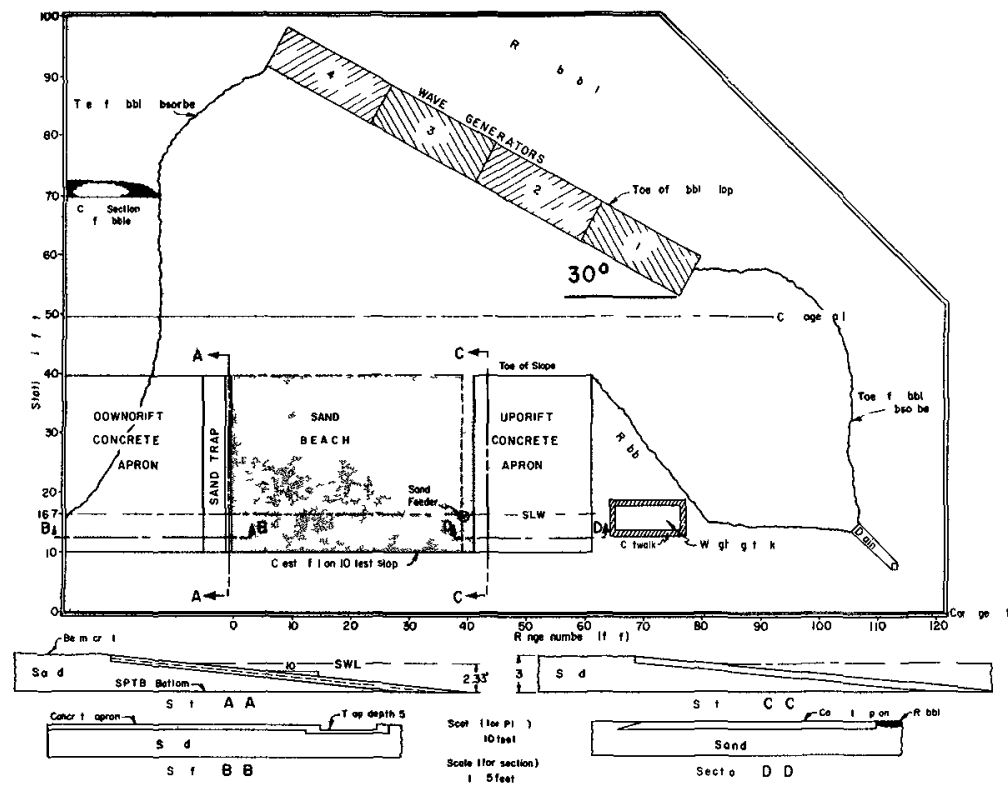

FIGURE I LONGSHORE TRANSPORT TEST LAYOUT IN THE NORTH SECTOR OF SPTB (1966)

Tests were carried out by generating long crested waves which traveled from the wave generator to the toe of the beach slope in a constant water depth As waves continued over the beach they shoaled and were refracted before breaking When the waves broke, part of their energy was dissipated in turbulence, scouring and suspending sand, part was transformed into a longshore current and part was reflected from the beach The wave action scoured the beach sand, forcing it into suspension, to be carried along the shore by the longshore current Also, the swash and backwash of the waves 
caused the sand to move onshore and offshore, with a resultant slantwise movement along the shore

In sumary, the wave action caused the sand to move along the shore in the breaker zone, in the swash zone and in the deeper offshore zone The sand moved onshore and offshore, and with continued wave action, the bottom profile progressed toward an "equilibrium profile" characterized by a reduced transport rate and a reduced onshore-offshore exchange of material

\section{Purpose}

The purpose of the tests was to measure the longshore transport rate for a range of wave characteristics, and to establish a correlation between the measured rates and the corresponding longshore components of wave energy flux Longshore transport rates were measured in tests, wherein sand moving along the shore was deposited in a sand trap from which it was pumped and welghed under water Longshore components of wave energy flux were computed from measured wave heights and calculated wave breaker directions

From an engineering viewpoint, the purpose encompassed the obtention of laboratory data to define basic relationship for use with fleld data, to more quickly and economically 1 mprove and develop previous correlations between wave energy flux and longshore transport rate

\section{OBSERVATION AND MEASUREMENT}

\section{Genera1}

The sand ( $022 \mathrm{~mm}$ quartz), water depth (2 $33 \mathrm{ft}$ at toe of beach), and angle of wave generator with initial shoreline $\left(30^{\circ}\right)$ were constant in the tests

TABLE 1

TEST VARIABLES

Experıment al Varıables Range of Variables Tested

$\begin{array}{lll}1 & \text { Wave period } & 125 \text { to } 375 \text { seconds } \\ 2 & \text { Wave generator stroke } & 20 \text { to } 150 \text { inches } \\ 3 & \text { Depth at beach toe } & 230 \text { to } 233 \text { feet (bottom uneven) } \\ 4 & \text { Initial slope } & 1 \text { on } 10 \text { to } 1 \text { on } 20 \\ 5 & \text { Beach material } & 022 \mathrm{~mm} \text { median diameter quartz sand } \\ 6 & \text { Angle of waves to toe of slope } & 30^{\circ} \text {, constant for all tests } \\ 7 & \text { Test time } & 25 \text { to } 100 \text { hours } \\ 8 & \text { Layout of basin } & \text { training walls or flume to open basin }\end{array}$


TABLE 2

WAVE CONDITIONS AND TEST SET-UP CHANGES

*(total hours in test)

Wave Condtions

\begin{tabular}{|c|c|c|c|c|c|c|c|}
\hline $\begin{array}{c}\text { Test } \\
\text { No }\end{array}$ & & & & $\begin{array}{l}\text { Ecc } \\
\text { aches }\end{array}$ & $\begin{array}{l}\mathrm{H} \\
\mathrm{ft}\end{array}$ & $t *$ & Set-up Changes \\
\hline $1-58$ & 1 & 50 & 1 & 00 & 176 & 60 & $\begin{array}{l}\text { Starting slope } 1 \text { on } 20, T \text { was sequenced in } \\
15 \text { minute intervals at } \mathrm{T}=150,130,150 \text {, } \\
176 \text {, etc, no sand fed on feeder beach } \\
\text { after } 35 \text { hours }\end{array}$ \\
\hline $2-58$ & 1 & 50 & 1 & 00 & 176 & 70 & $\begin{array}{l}\text { Starting slope } 1 \text { on } 20, T \text { was varied as in } \\
\text { test } 1-58 \text {, feeder beach maintained throughout } \\
\text { entire test }\end{array}$ \\
\hline $2 a-59$ & 3 & 00 & 2 & 35 & 192 & 80 & $\begin{array}{l}\text { Starting slope } 1 \text { on } 10, T \text { was sequenced in } 15 \\
\text { minute intervals at } \mathrm{T}=250,300,375,300 \text {, } \\
\text { etc, upbeach training wa } 11 \text { curved for wave } \\
\text { refraction, beach length } 90 \text { feet }\end{array}$ \\
\hline $3 a-59$ & 3 & 00 & 2 & 35 & 192 & 50 & $\begin{array}{l}\text { Beach length reduced to } 30 \text { feet along SWL, } \\
\text { other conditions same as Test } 2 \mathrm{a}-59\end{array}$ \\
\hline $4 a-59$ & 2 & 18 & 1 & 75 & 210 & 50 & $\begin{array}{l}\text { Starting slope } 1 \text { on } 10 \text {, updrift trng wall re- } \\
\text { curved by wave refraction for new } T \text {, sequenced } \\
\text { in } 15 \text { munte Intervals at } T=194,218,250 \text {, } \\
218 \text {, etc }\end{array}$ \\
\hline $1-59$ & 1 & 50 & 1 & 00 & 176 & 25 & $\begin{array}{l}\text { Starting slope based on a } 150 \text { hour "equilibrium } \\
\text { proftle", segment of downbeach training wall } \\
\text { from carriage rail to toe of slope, removed, T } \\
\text { was varied same as in test } 1-58\end{array}$ \\
\hline 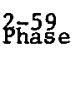 & $1^{1}$ & 50 & 1 & 00 & 176 & 32 & $\begin{array}{l}\text { Same starting slope as } 1-59 \text {, above, } T \text { was } \\
\text { varied same as in test } 1-59 \text {, downdrift train- } \\
\text { Ing wall completely removed }\end{array}$ \\
\hline $\begin{array}{l}2-59 \\
\text { Phase }\end{array}$ & $\begin{array}{r}3 \\
11\end{array}$ & 00 & 2 & 35 & 192 & 80 & $\begin{array}{l}\text { Starting slope was the beacn slope at end of } \\
\text { Phase } 1, T \text { was sequenced as in test } 2 a-59\end{array}$ \\
\hline $3-59$ & 3 & 00 & 2 & 35 & 192 & 75 & $\begin{array}{l}\text { Starting slope } 1 \text { on } 20 \text {, updrift training wall } \\
\text { curved along wave refraction orthogonal, } T \\
\text { was sequenced as in test } 2 a-59\end{array}$ \\
\hline $4-59$ & 3 & 00 & 2 & 35 & 192 & 50 & Starting slope 1 on 20 , wave period constant \\
\hline $5-59$ & 3 & 75 & 2 & 35 & 140 & 50 & Starting slope 1 on 20 , wave period constant \\
\hline $6-59$ & & 50 & 2 & 35 & 246 & 50 & Starting slope 1 on 20 , wave period constant \\
\hline $1-60$ & 2 & 18 & 1 & 75 & 210 & 50 & $\begin{array}{l}\text { Starting slope in this and all subsequent tests, } \\
1 \text { on } 10 \text {, downdrift training wall reinstalled, } \\
\text { and curved for wave refraction, } T \text { was sequenced } \\
\text { as in test } 4 a-59\end{array}$ \\
\hline $2-60$ & 2 & 18 & 3 & 50 & 420 & 26 & $\begin{array}{l}\text { Wave Height increased as shown, } T \text { was sequenced } \\
\text { in test } 4 a-59\end{array}$ \\
\hline
\end{tabular}


TAB́LE 2, cont'd

* (total hours in test)

Wave Conditions

\begin{tabular}{|c|c|c|c|c|c|}
\hline $\begin{array}{c}\text { Test } \\
\text { No }\end{array}$ & $\begin{array}{c}\mathrm{T} \\
\mathrm{sec}\end{array}$ & $\begin{array}{l}\text { Ecc, } \\
\text { Inches }\end{array}$ & $\begin{array}{l}\mathrm{H}, \\
\mathrm{ft}\end{array}$ & $t^{*}$ & Set-up Changes \\
\hline $3-60$ & 300 & 235 & 192 & 28 & $\begin{array}{l}\text { A repeat of test } 3 a-59 \text { condltions to check } \\
\text { transport of suspended sand past the sand } \\
\text { trap }\end{array}$ \\
\hline $4-60$ & 218 & 500 & 614 & 25 & $\begin{array}{l}\text { Test at maxlmum wave height for generators, } \\
\mathrm{T} \text { was sequenced as in test } 4 a-59\end{array}$ \\
\hline $5-60$ & 300 & 470 & 422 & 26 & $\begin{array}{l}\text { Increased wave height as shown, } T \text { was se- } \\
\text { quenced as in test } 2 a-59\end{array}$ \\
\hline $6-60$ & 218 & 250 & 300 & 50 & To test intermediate wave helght value \\
\hline $7-60$ & 136 & 150 & 320 & 50 & $\begin{array}{l}\text { To test maxımum height at minımum perıod, } T \\
\text { was sequenced in } 15 \text { minute intervals at } \\
\mathrm{T}=125,136,150,136 \text {, etc }\end{array}$ \\
\hline $1-61$ & 300 & 235 & 192 & 50 & $\begin{array}{l}\text { Wave period changed every } 5 \text { instead of every } \\
15 \text { minutes, } T \text { was sequenced as in test } 2 a-59\end{array}$ \\
\hline $2-61$ & 300 & 235 & 192 & 50 & $\begin{array}{l}\text { Wave perıod changed at } 1 \text { munute intervals, } T \\
\text { was sequenced as in test } 2 a-59\end{array}$ \\
\hline $3-61$ & 300 & 235 & 192 & 50 & $\begin{array}{l}\text { To compare results with test } 4-59 \text { (started on } \\
\text { a } 1 \text { on } 20 \text { slope) Constant wave period }\end{array}$ \\
\hline $5-61$ & 300 & 235 & 192 & 50 & $\begin{array}{l}\text { Wave period varied continuous } 1 \mathrm{y} \text { from } \mathrm{T}=375 \text { to } \\
\mathrm{T}=250 \text { through the mean, } 300 \text { seconds and } \\
\text { return }\end{array}$ \\
\hline $6-61$ & 250 & 235 & 246 & 50 & $\begin{array}{l}\text { Constant wave perlod, for comparison with re- } \\
\text { sults of test } 6-59 \text { ( } 1 \text { on } 20 \text { slope) }\end{array}$ \\
\hline $7-61$ & 375 & 235 & 140 & 50 & $\begin{array}{l}\text { Constant wave period, for comparison with re- } \\
\text { sults of test } 5-59 \text { ( } 1 \text { on } 20 \text { slope) }\end{array}$ \\
\hline $1-61$ & 375 & 235 & 140 & 50 & $\begin{array}{l}\text { Constant wave period, test of sand feeder, } \\
\text { elevation } 2 \mathrm{ft} \text { above SWL }\end{array}$ \\
\hline $2-62$ & 375 & 235 & 140 & 25 & Same as $1-62$, elevation $01 \mathrm{ft}$ above SWL \\
\hline $3-62$ & 375 & 235 & 140 & 25 & Same as $1-62$, elevation at SWL \\
\hline $4-62$ & 375 & 235 & 140 & 25 & $\begin{array}{l}\text { Constant wave perıod, to Investigate effect of } \\
\text { extraneous wave }\end{array}$ \\
\hline $6-62$ & 150 & 094 & 172 & 48 & $\begin{array}{l}\text { Constant wave period, feasıbılity test of sand } \\
\text { tracers }\end{array}$ \\
\hline $8-62$ & 150 & 094 & 172 & 30 & $\begin{array}{l}\text { Same as } 6-62 \text {, longer half } 1 \text { Ife tracer, } T \text { was } \\
\text { constant up to t=8 haurs and wave varied as } \\
\text { in test } 1-58 \text { after } 8 \text { hours }\end{array}$ \\
\hline $1-64$ & 375 & 235 & 140 & 50 & $\begin{array}{l}\text { Constant wave period, offshore area divided } \\
\text { Into } 8 \text { flumes }\end{array}$ \\
\hline $1-65$ & 375 & 235 & 140 & 40 & $\begin{array}{l}\text { Special constant wave perıod test, open test } \\
\text { basin, rubble around test area }\end{array}$ \\
\hline
\end{tabular}




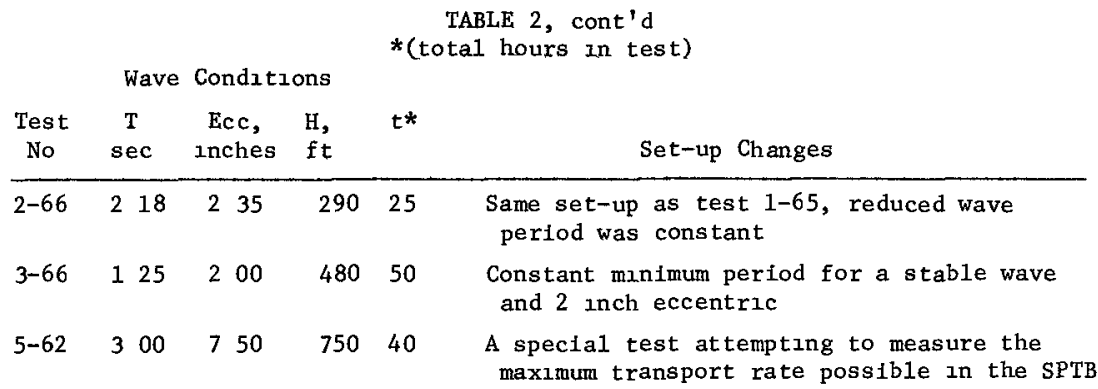

Five other variables under the experimenter's control were wave period, wave height, initial beach slope, test duration and basin geometry Although not as simple a variable as others noted above, basin geometry changes from test to test included, the general conflguration of the test layout, test beach length, arrangement of training and splitter walls, and sand feeding techniques These changes were made after continuing observation and analysis, in the hope that they would improve the quality of the data in the tests

Table 1 outlines the experimental variables and the range of these variables tested This table gives an overview of the test variables within the positive control of the project engineer Table 2 is a more detalled listing of the wave conditions and test sec-up changes by test number, with the last column giving a running commentary of test-to-test changes in set-up The variables 1isted in Table 2 include wave height, wave period and test duration, and each of these plus five other areas of observation and measurement are discussed separately in the following paragraphs

\section{Wave Herght}

Wave recordings using strip chart recorders were made regularly in the tests using parallel wire wave sensors placed along the toe of the beach slope Spot recordings were also made at other locations in the test basin Wave heights were determaned from an analysis of the wave recordings, as the average helght of ten successive waves

As the tests continued, wave helghts were found to vary significantly, from point to point, and with time as at a fixed point special wave measurements tests were made in 1963 and 1964 attempting to Identify the cause or causes of the wave helght variability The measurement results were not conclusive Another series of tests in the SPTB are presently investigating wave reflection as a cause of wave helght varlability in inclosed basins such as the SPTB With continued testing and consideration of wave energy analysis in the tests, the large wave helght varlability (up to and exceeding a factor of 2) made it difficult to confidently specify a causative wave height in relation to a measured transport rate Specifyıng a causative helght - say from reflectionfree waves in the SPTB was difficult because of the short distance for wave travel and the long length of the waves For the longer wave periods only two waves could be measured before wave reflection from the beach began to affect the measurements 
Because of the length limltation in the SPTB, the measurements of waye height used in this report were made in CERC's 72-foot tank under experimental conditions equivalent to a $1 / 2$ scale Froude model of the depth, eccentrics and periods tested in the SPTB When the SPTB wave conditions were reduced to $1 / 2 \mathrm{scale}$, the 72 -foot tank was 1 ong enough to generate sufficient reflection-free waves from which a sound evaluation of the wave helghts was possible

Considerable care was taken in measuring the wave heights in order to have heights as free from reflection effects as practicable only those waves which reached the wave gage before reflected waves returned from the absorber beach were used For example, a wave period of 375 seconds and water depth of 233 feet in the SPTB reduces to a wave period of 265 seconds and 117 feet, respectively, at $1 / 2$ scale At this period and depth the wave length in the 72-foot tank was 156 feet which would allow for measurement of 7 to 8 reflection-free waves in a 60-foot spacing between a wave sensor and an absorber beach These measurements in the 72-foot tank provided wave helghts for 8 wave generator eccentrics, over a range of wave periods The range of wave periods was 125 to 375 seconds with minimum wave heights of the order of 01 foot and maximum wave helghts up to 75 feet Figure 2 is a graph of the wave height measurements made in the $1 / 2$ scale Froude model, plotted as prototype SP'TB values

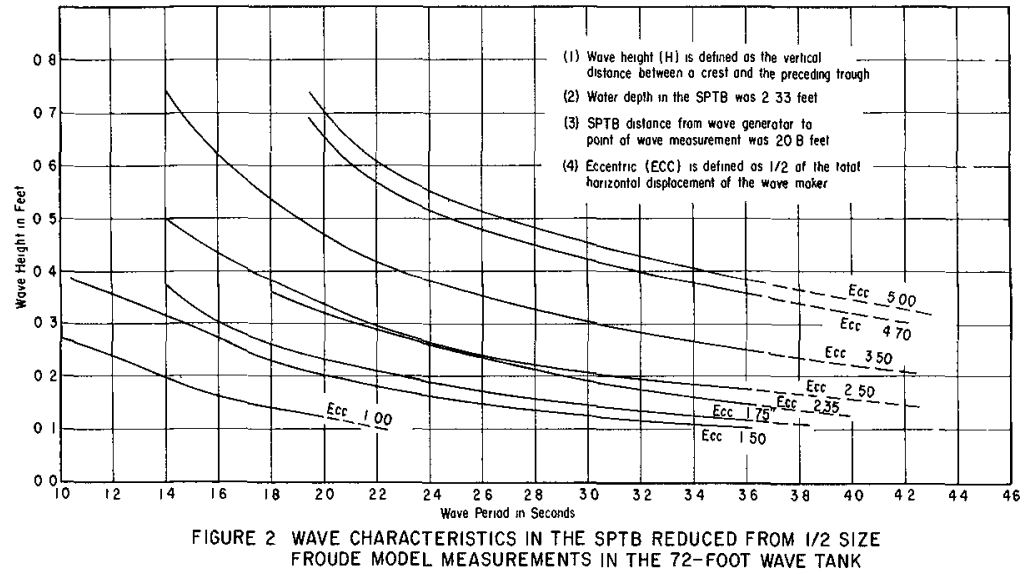

\section{Wave Period}

Wave period, $T$, could be arbitrarıly selected within a range of 1 to 4 seconds on the SPTB wave generators Dial settings, corresponding to speclfic wave periods in seconds, were made on a varidrive motor, which was electronically coupled by remote cable to a $71 / 2$ horsepower A C drive motor for each wave generator Since there was "drift or noise" in the electronic control system, dial settings of the wave periods were callbrated at frequent intervals Also, wave period, once selected, was closely monitored to keep It constant Monitoring was done by visually timing rotations of the wave generator eccentric arm by stop watch Monitored values 
were checked against the varıdrive dial settıngs and when needed, corrections were made promptly Wave period checks were made generally at 15 minute intervals for both variable and constant period tests The variable period tests were varied in sequence above and below the mean wave period and height at a fixed time interval In most of the tests this time interval was 15 minutes but in test 1-61 it was 5 minutes and in test 5-61, period and height variation was continuous The last column in Table 2 gives some detajl on wave perıod sequencing

\section{Longshore Transport}

Longshore transport is the movement of material along the shore in the 11ttoral zone by waves and currents (7) In the laboratory tests under discussion, longshore transport rate is defined operationally as the rate of accumulation of beach material in a sand trap on the downdrift end of the test beach The material accumulated YPropumed sand trap, using eductors and hose line, into a weighing bin where it was weighed while submerged Submerged welghts were converted to their equivalent dry weights (or weights in air) by multiplying them by the factor, $\left.\rho_{s} / \rho_{s}-\rho_{w}\right)$, where $\rho_{s}$ and $\rho_{w}$ are the specific gravities of quartz and water, respectively Using this factor to convert submerged weights to dry weights assumes that the sand is $100 \%$ quartz These dry weights of sand, along with the time between weighings, were used to compute the longshore transport rates Generally, transport rates were computed for the first hour or a lesser time, and in 5-hour intervals to the end of the test In a few cases, rates were computed for periods of 15 minutes

\section{Sand Feeding}

The feeder beach area was a small area at the updrift end of the test beach. where sand was fed into the wave swash in order to maintaln the test beach The sand feeder at the updrift end of the sand beach, shown in Figure 1, is in the feeder beach area In earlier tests, a variety of methods were used to feed sand into the feeder beach, and to maintann a hydrography in the feeder beach area similar to that which develops downdrift of 1 t The initial method was to stockpile sand on the beach, and to shovel sand from the stockpile directly into the wave swash Later methods included dumping from an overhead boom-supported bin, wheelbarrow dumping and the discharge of a water-sand slurry into the wave swash Also, wheelbarrow lots were dumped at the shoreline and then shoveled directly into the wave swash, similar to shoveinng from a stockpile Except for the discharge of sand slurry by hose line, which scoured the beach, sand feeding methods were adequate to keep the tests goung What appeared to be needed was a method of continuous sand feeding, with a minimal influence on the natural action-reaction between wave and beach

In 1962 a method for sand feeding (8) was introduced which enabled an automatic and continuous feeding of wet sand Into the wave swash. The method made use of a vertical cylindrical sand feeder which deposited wet sand continuously and automatically into the wave swash The sand feeder, shown in Figure 3 with functional parts indicated, is basically a vertical cylinder filled with sand and water It had been modified by welding a cone section at the bottom to retard the flow of wet sand through it Another modification was a supply line at the top for keeping the sand feeder supplied with sand

In the absence of waves, the sand in the sand feeder mouth rested directly on the sand beach tending to stabilize the colum of sand in it but with some noticeable oozing of sand about the mouth In the presence of wave action, 
and so long as the sand was maintained at a constant level, the sand feeder was observed to feed at a fairly consistent rate. The two main factors

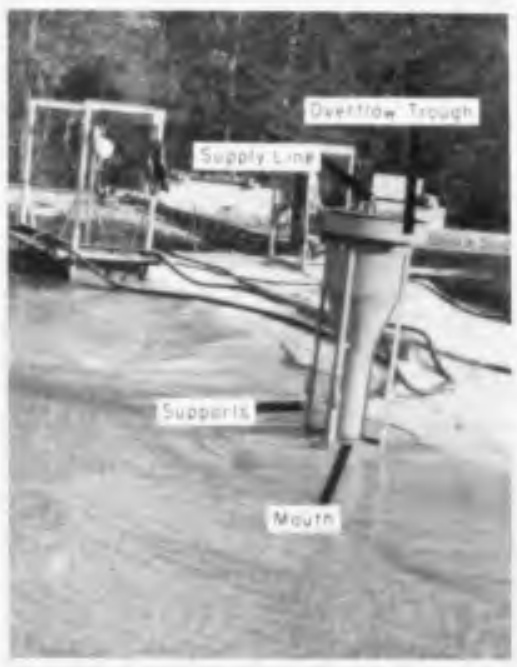
affecting the rate of sand fed through the feeder and on to the beach were the weight of sand and water in the feeder and the scouring action at the mouth of the feeder due to the wave swash and backwash. The weight of the sand and water in the feeder acted to force sand out at the mouth and onto the beach, while the wave swash and backwash scoured the beach under the feeder mouth making it easier for sand to be forced out and onto the beach. The amounts of sand fed into the sand feeder or onto the feeder beach were obtained by weighing the sand while submerged- the same method used to obtain the weight of sand deposited in the sand trap.

FIGURE 3 AUTOMATIC SAND FEEDER

\section{Beach Soundings}

Soundings of the test beach were made regularly. The first sounding was made on a smooth molded beach before any waves acted on $i t$. This inftial sounding was made after submerging the beach for one or two days, to allow time for settling of the material before the start of wave action. Subsequent soundings were made after one hour and five hours of wave action and thence at 5-hour intervals to the end of a test. Soundings were made from a level railing using a telescoping sounding rod with a hinged aluminum foot. Readings were made with respect to a still water level datum to one thousandth of a foot; and recorded areally on a survey sheet, analogous to the method of recording elevations in plane table surveys. Figure 4 is a contour chart of the beach obtained by contouring along points of equal depth as recorded in sounding surveys in test $4-60$ after 25 hours of wave action.

\section{Test Duration}

In the earlier tests, there were no hard and fast rules for deterwining the length of a longshore transport test. However, there were general principles, and the philosophy of these was, that during longshore transport measurement there be a meaningful similarity between the test beach profiles and typical profiles found on natural beaches. Based on this philosophy, tests were run until the beach profiles had reached a condition defined as "equilibrium profile". "Equilibrium profile" is defined as the near constant or minimum change stage of a beach profile under the sustained action of 
constant condition waves (9) When tests neared an equilibrium profile stage, longshore transport rate decreased, becoming faurly constant in some tests

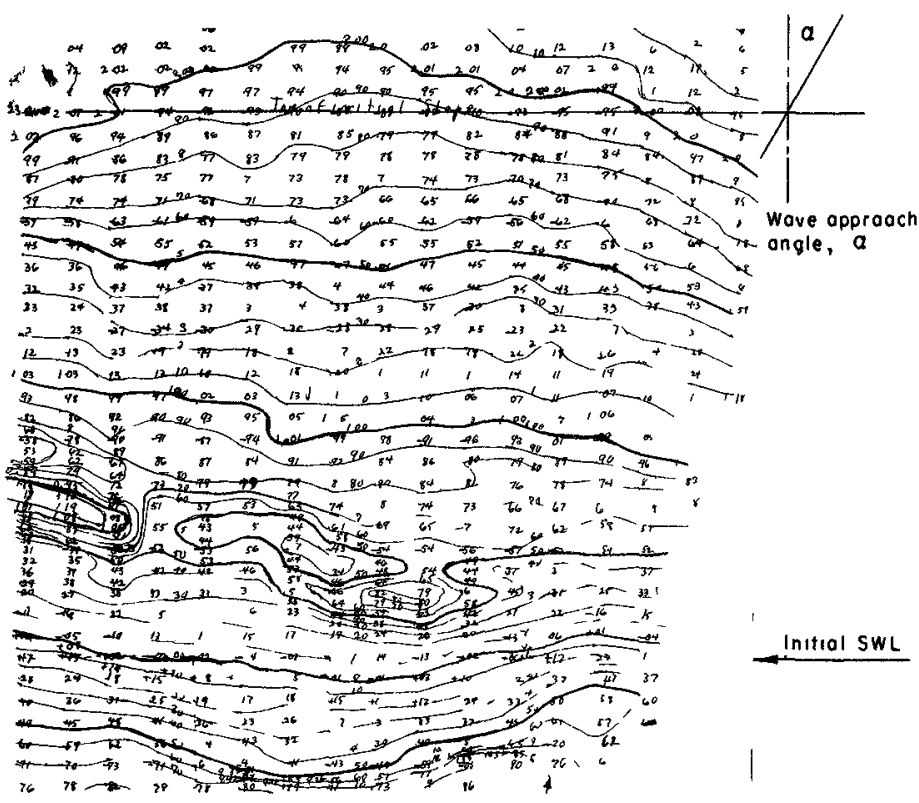

FIGURE 4 BEACH CONTOURS AFTER 25 HOURS IN TEST 4-60

Wave action time required for a test beach to reach an equilibrium profile stage generally ranged from 15 to 30 hours depending on the wave period, wave height and the starting beach slope Tests started on a 1 on 10 slope genexally reached equilibrium in less time than tests started on a 1 on 20 slope While an equilibrium profile stage was observed to occur as early as 15 hours, and as late as 30 hours, typical tests were run for 50 hours and while a large number of the tests were rum 50 hours, a few tests exceeded 100 hours and several repeat tests were run only 25 hours Fifty hours was usually enaugh time for a smooth beach to reach an equilibrium profile stage, with 20 hours or more remaining for observation and measurement under a falrly "steady state" condition, of continuing but reduced, profile adjustment and longshore transport rate

\section{Longshore Current}

Estimates of the longshore current velocity were made from timings of fluorescein dye travel in the surf zone Generally, two estimates were made, one for the updrift and one for the downdrift part of the test beach The dye was squirted into the wave uprush by a plastic "squeeze" bottle Actual clockings observed the travel time of the leading or downdrift edge of a dye trace, and not the travel time of the dye-patch center Since the leading edge of the dye trace was tumed, the rates estimated are probably maximum rates for the wave conditions tested 
Water Temperature

Water temperature was taken hourly and in some tests more frequently Dial type thermometers were used, mounted on a rod, where the dial was visually accessible and the sensing element remanned submerged Water temperature data, although taken regularly, did not seem to be of any direct usefulness in the analyses of the tests However, considerable data on wave induced suspended sediment (10) and also on suspended sediment in rivers (11) have shown that water temperature does effect the quantity of sediment in suspension, and since these tests were made in an outdoor facility, subject to a significant range of water temperature change, it was felt that the temperature measurements, though not of immediate usefulness in the analysis, were justified in terms of their anticipated value in some future analysis The water temperature data observed in the tests is avallable at CERC

TABLE 3

\section{WAVE PARAMETERS}

\begin{tabular}{|c|c|c|c|c|c|c|c|c|}
\hline $\begin{array}{l}\operatorname{Ref} * \\
\text { No }\end{array}$ & $\begin{array}{r}\mathrm{T}, \\
\mathrm{Sec}\end{array}$ & $\begin{array}{l}H \\
f t\end{array}$ & $\underset{\mathrm{ft}}{\mathrm{H}_{\mathrm{b}}}$ & $\mathrm{ft}_{\mathrm{t}}^{\mathrm{d}_{\mathrm{f}}}$ & $\mathrm{E}_{\mathrm{b}}^{* *}$ & $\mathrm{~d}_{\mathrm{b}}{ }^{\circ}$ & $\mathrm{K}_{\mathrm{R}}$ & $\mathrm{E}_{\mathrm{a}}^{\star \star \star}$ \\
\hline $3-66$ & 125 & 048 & 363 & 459 & 417 & 16 & 95 & 108 \\
\hline $7-60$ & 136 & 032 & 310 & 399 & 317 & 14 & 94 & 066 \\
\hline (6) & 150 & 018 & 227 & 294 & 165 & 12 & 93 & 029 \\
\hline (3) & 218 & 021 & 325 & 410 & 586 & 10 & 94 & 086 \\
\hline $6-60$ & 218 & 030 & 410 & 522 & 1045 & 10 & 94 & 158 \\
\hline $2-60$ & 218 & 042 & 507 & 660 & 1794 & 12 & 94 & 322 \\
\hline $4-60$ & 218 & 062 & 642 & 854 & 3248 & 13 & 94 & 629 \\
\hline (2) & 250 & 025 & 386 & 495 & 1046 & 9 & 94 & 143 \\
\hline (11) & 300 & $\begin{array}{ll}0 & 19\end{array}$ & 355 & 452 & 1024 & 7 & 93 & 107 \\
\hline $5-60$ & 300 & 042 & 596 & 760 & 3700 & 9 & 94 & 505 \\
\hline (8) & 375 & 014 & 309 & 388 & 899 & 5 & 93 & 068 \\
\hline
\end{tabular}

\section{ANALYSIS OF TESTS}

A single longshore transport test included the specification of a wave and test set-up condition, test operation, measurements, observations and analysis The first part of Table 2 lists the test number, eccentricity, height, period, and duration The second part of Table 2 contains comments on special aspects of the test set-up

Table 3 lists measured and computed parameters associated with the waves, and Table 4 lists the longshore wave energy flux and the measured transport rates The wave helght, $H$, as listed in both Tables 2 and 3,1 s the wave 
height appropriate to the offshore part of the test area, where the water depth was 233 feet This helght can be read from Figure 2 by taking a wave period and a wave generator eccentric from those listed in Table 2 for a given test number

Thus far this report has considered the tests in general The following paragraphs present a test by test description of four representative tests

\section{Representative Tests}

Tests selected for discussion are representative of the entire series of 36 tests The representative tests are tests numbered 4-60, 4-62, 1-64, and 3-66 The longshore transport in each representative test was measured over 25 or more hours at a specified wave helght and period in the general setup 11lustrated in Figure 1 Wave conditions span the full range of the wave conditions tested, test layouts are typıcal of the different aetups tested, the range of transport rates approximate the total range of all rates tested, and, testing problems are felt to be typical Tests selected for discussion are also special in that each test usually represents a specific type of set-up For example, some of the types of set-up in representative tests were a maximum wave height condition in test $4-60$, dividing the offshore basin area into wave flumes in tests $4-62$ and 1-64, and eliminating training and splitter walls entirely in test $3-66$

\section{Test $4-60$}

Test $4-60$, made in the North Section of the SPTB, had the highest helght and largest transport rate of the five tests run at a 218 second period The period, $T$, was changed at 15-minute intervals through a sequence of wave periods as indicated in Table 2 for test 4a-59 Inltial wave action began on a molded, 1 on 10 beach slope at a wave period of 194 seconds See Tables 2 and 3 and Figure 2 for details of wave conditions

The emphasis in test 4-60 was in measuring the transport rate at a mean period of 218 seconds for the maximum wave height obtalnable at this period This test resulted in an average longshore transport rate of 9,880 pounds of sand per hour, $45 \%$ higher than the immediately preceding test, and nearly 4 times higher than the previous maximums As a result of the higher rate, the test apparatus and personnel were hard-pressed just to keep the test going and to make the observations and measurements One of these tasks was simply feeding enough sand, properly, to keep the test beach from eroding seriously Another task was keepıng the wave generators operating as smoothly and as contınuously as possible, since at a higher rate, smoothness of operation was difficult to maintain and wave machıne stops due to breakdown or run-away were frequent

Longshore transport sand feeding and longshore current results for test 4-60 are summarized in Figure 5 Note the correlations in Figure 5 between longshore transport and downdrift longshore current, and between sand feeding rate and updrift longshore current compilations of the data from all the tests, including that used to plot Figure 5, are available at CERC

Test $4-62$ and $1-64$

Changes in Tests 4-62 and 1-64 included the installation of splitter walls in the offshore portion of the test layout to study wave height variability 
The splitter wall in Test 4-62 was installed parallel to, and 10 feet updruft of the downdrift training wall The splitter wasl was concelyed because repeated visual observations of wave profiles and the results of wave height

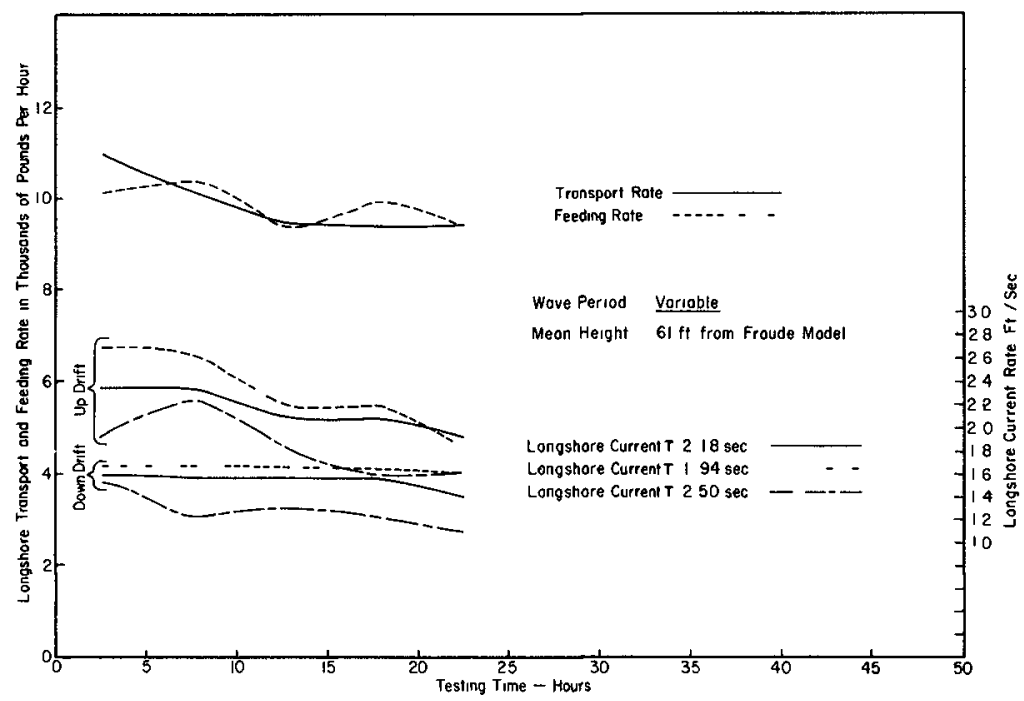

FIGURE 5 TEST NO 4-60, RELATIONSHIP OF LONGSHORE TRANSPORT, SAND FEEDING AND LONGSHORE CURRENT TO TESTING TIME

measurements suggested that a signiflcant variation in wave height with distance across the basin was caused by resonance between training walls The visual observations were views of the water surface profile along a wave crest as outlined against the wave generator blade at the instant of generating the wave crest Figure 6 is a schematic drawing of the modes of profiles observed Mode 1 was observed only for the longer period waves - say 375 seconds or longer Modes 2 and 3 were also observed, with mode 3 generally assoclated with shorter wave perıods

The function of the splitter wall was to change the mode of the cross basin wave It was further hypothesized that placing the splitter wall 10 feet ( $L / 4$ ) updrift of the training wall, placed 1 t at an antinode of a cross basin wave A splitter wall placed at the antinode would impede the cross basin flow and thus force the wave mode to change with a possible lessening of wave helght variability across the basin The two curves in Figure 7 for a 375 second period show that the total range of the wave height varlability was reduced, and that the initial bimodal distribution of wave height was changed

The test set-up for Test 1-64 was a further application of splitter walls in which 7 splitter walls divided the offshore test area into 8 flumes, each 5 feet wide The reasoning, as in Test No 4-62, was that the splitter walls should change the mode of a cross-basin wave hypothesized to be a cause of 
wave height variability across the basin Wave height measurements showed that the 7 splitter walls in Test 1-64 did change the distribution of wave heights, as a single wall did in Test 4-62, but had little effect in reducing the wave height variation In summary, measurement results in Test 1-64, Test 4-62 and a similar four-flume test suggest that the wave height variability is independent of the spacing between training or splitter walls

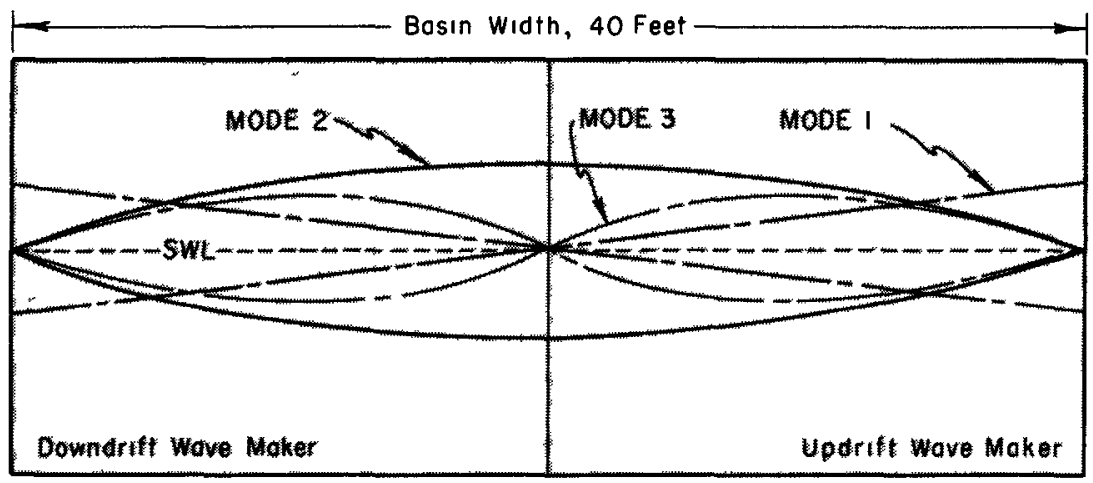

\section{FIGURE 6 SCHEMATIC DRAWING, ILLUSTRATING VISUALLY OBSERVED WAVE MODES IN THE SPTB}

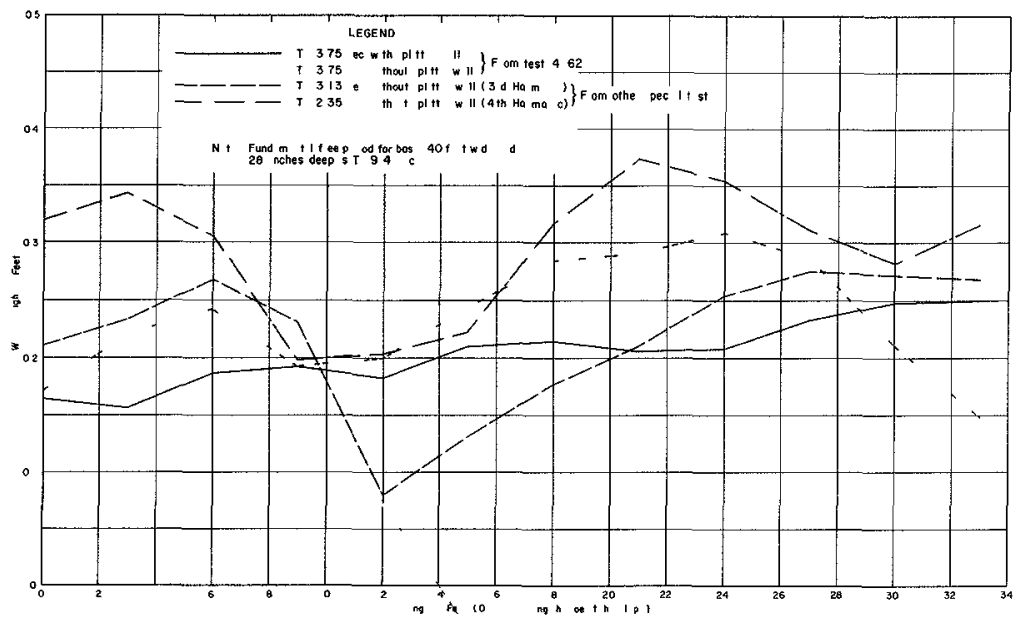

FIGURE 7 COMPARISON OF WAVE HEIGHT BEFORE AND AFTER INSTALLING THE SPLITTER WALL IN TEST NO 4-62 
Longshore transport, sand feedıng and longshore current results for Tests 4-62 and 1-64 are summarized in Figure 8 Note the correlations in Figure 8 between downdrift longshore current and longshore transport rate Also note similar correlations between the sand feeding rate and the updrift longshore current

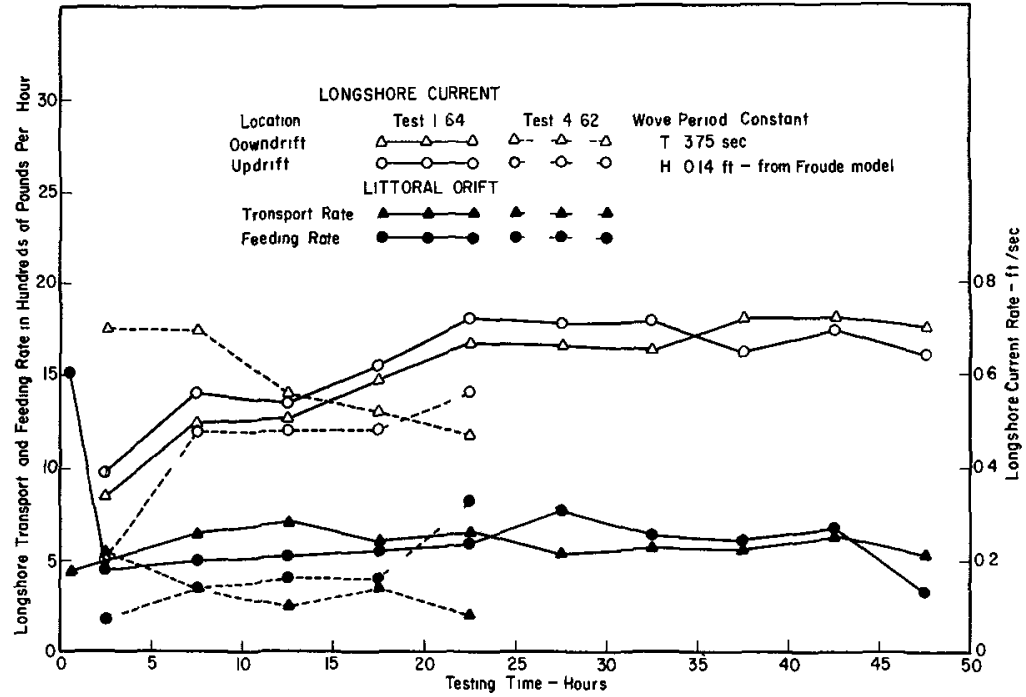

FIGURE 8 TEST NO 4-62 AND 1-64, RELATIONSHIP OF LONGSHORE TRANSPORT, SAND FEEDING AND LONGSHORE CURRENT TO TESTING TIME

Test $3-66$

Test 3-66, completed in October 1966, was the last test completed WIth two important differences, the test set-up was similar to the set-up for Test 1-64 described in the previous section The first difference was the deletion of all training and splitter walls and the use of a longer beach necessitating the generation of a longer crested wave ( 80 feet in contrast to 40 feet in Test 1-64 and other prior tests) The second difference was the installation of a concrete slope, 20 feet wide, Immediately updrift of the feeder beach as shown in Figure 1 The first difference, an open basin set-up, was designed to reduce varlability in wave height, by eliminating some reflective surfaces (the training walls) and the second, an updrift concrete slope, was designed to provide a more natural longshore current It was reasoned that wave reflection could be a significant contributor to the wave height variability problem and that the small distance between the training wall and the feeder beach area in previous tests may have hindered the development of a natural longshore current

Test 3-66 was a constant wave period test w1th a high wave steepness It had a high wave energy relative to the wave energies in many other tests Wave breaker type in the test was spilling to plunging and yet the transport rate seems relatively low for its relative wave energy level among the tests 


\section{TABLE 4}

LONGSHORE WAVE ENERGY FLUX AND LONGSHORE TRANSPORT RATES $\left(E_{a}\right.$ is in millions of $\mathrm{ft}$ lbs/ft of shore/day)*

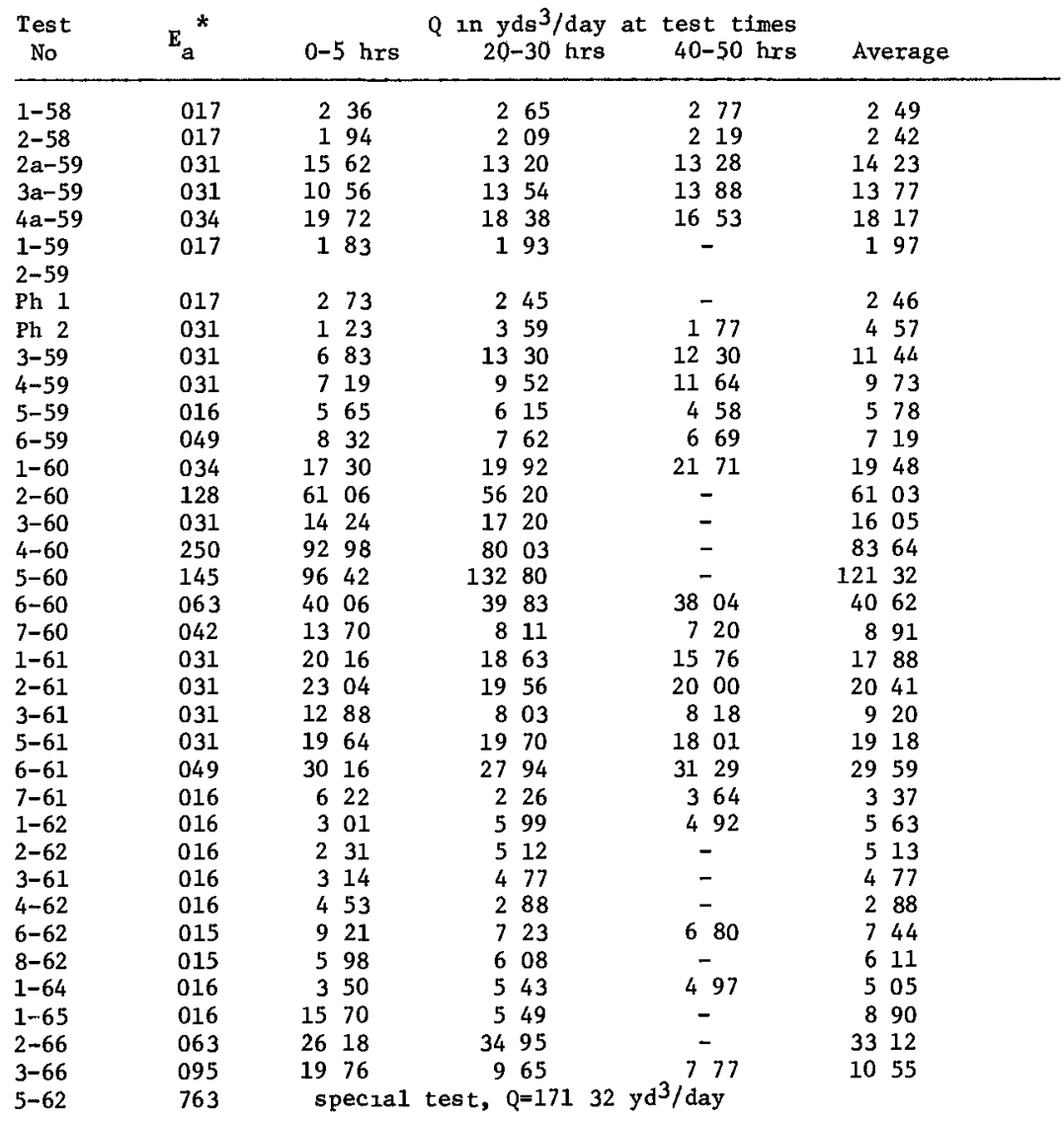

The longshore current rate is quite high and seemed by direct observation to be out of step with the transport rate in the test The wave breaking turbulence appeared to remain very much near the water surface and seemed too weak to really stir up the bottom boundary layer

Longshore transport, sand feeding and longshore current rates for Test 3-66 are summarized in FIgure 9 These tests results also show a correlation between longshore transport and downdrift longshore current rate and between sand feeding rate and the updrfft longshore current rate 
As described earlier, rates of longshore transport, sand feeding and longshore current have been compiled for each of the tests Reduced and compıled rates and associated littoral drift data are available at CERC Some

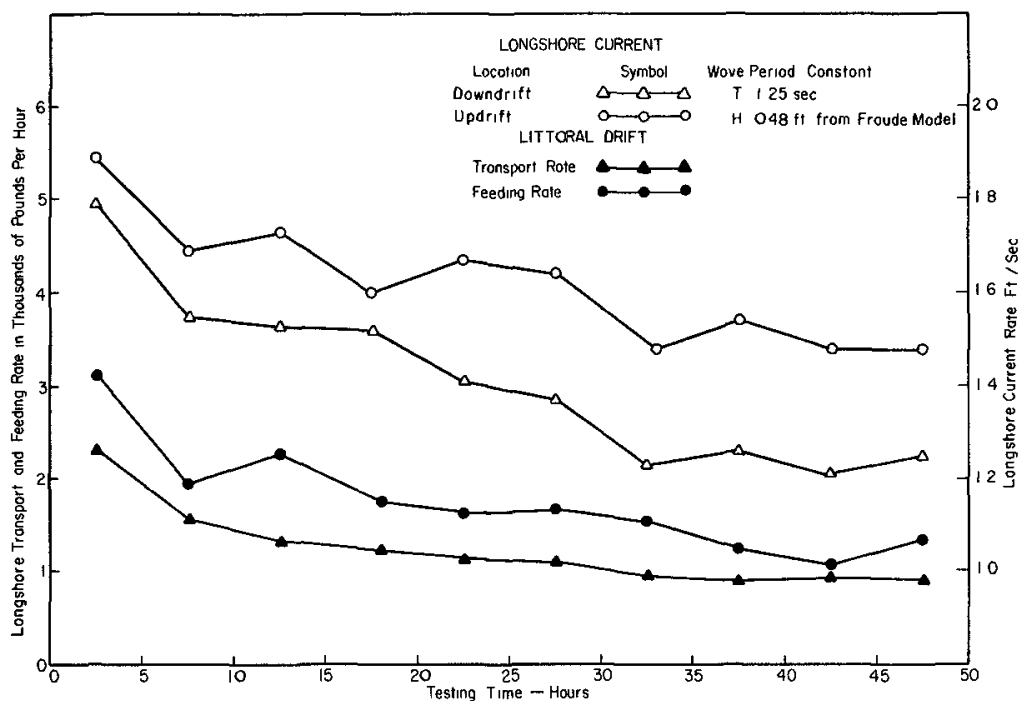

FIGURE 9 TEST NO 3-66, RELATIONSHIP OF LONGSHORE TRANSPORT, LONGSHORE CURRENT AND SAND FEEDING TO TESTHNG TIME

of this data has been summarızed and Is shown In Table 4 The data in Table 4 gives the test number, longshore wave energy, $\mathrm{E}_{\mathrm{a}}$, and the longshore transport rate, $Q$, at test times of $0-5,20-30$ and 40-50 hours The rates listed are volumetric rates based on a conversion factor of 105 pounds per cubic foot (satisfactorily checked by volumetric measurements of the sand) to obtain rates for the dry weight, or welght in air of the sand, as described in the section on "Longshore Transport"

The transport rates vary considerably, as the values in Table 4 show The greatest variation appears to be in the long perıod - low energy tests run at a constant wave period Exght of these tests are listed in Table 4, each with a longshore energy flux, $E_{a}$, of $016 \mathrm{millions}$ of foot pounds per foot of beach per day Figures 10 and 11, respectively, give a graphical comparison of transport rate and longshore current rate varıation with test time for ten selected tests The ten tests include the four representative tests described in the previous section The data on Figures 10 and 11 indicate, that tests with higher transport rates generally have higher longshore current rates and vice versa Several tests corroborate this similarity, but there are two notable exceptions One is Test 3-66 having a very high steepness and another is Test 1-64 with a very low steepness Another similarity is the increases and decreases of longshore transport and longshore current rates whlch appear to be falrly well correlated In most tests, the variatıons in longshore current are 


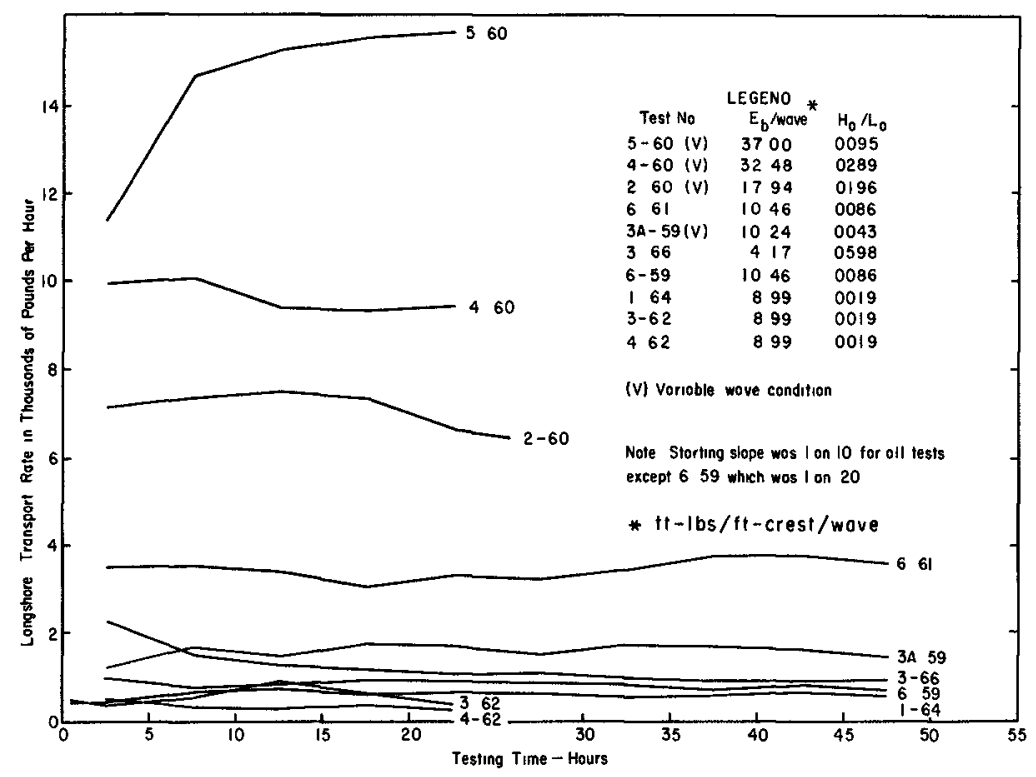

FIGURE IO COMPARISON OF LONGSHORE TRANSPORT RATES IN REPRESENTATIVE TESTS

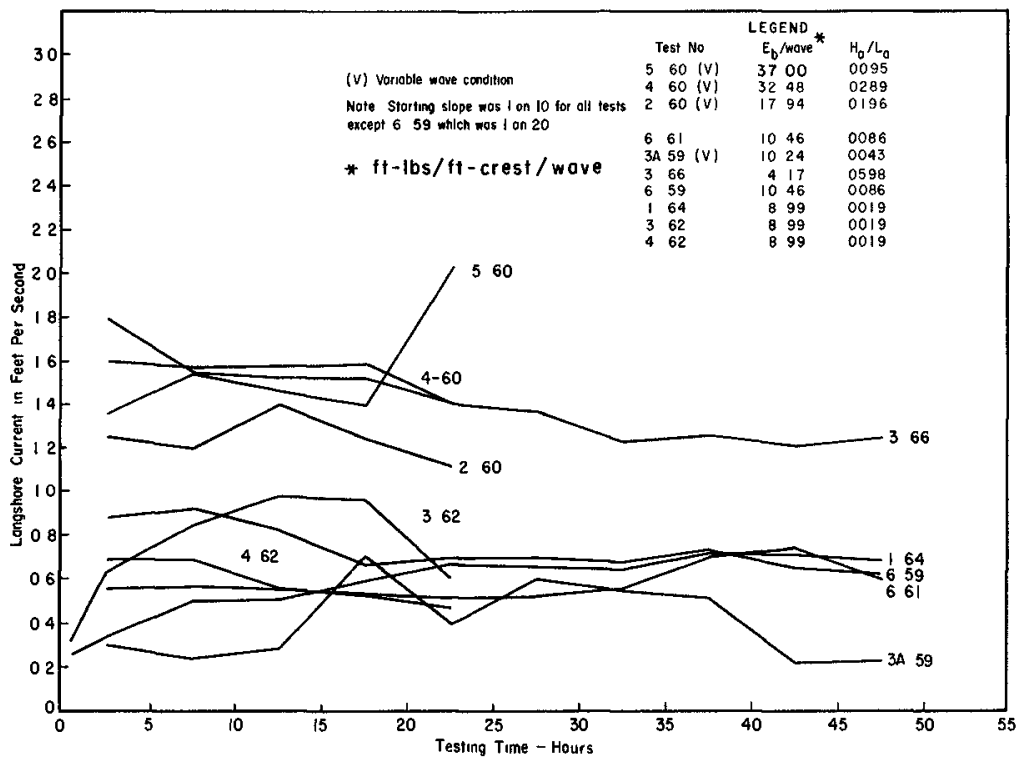

FIGURE II COMPARISON OF LONGSHORE CURRENT RATES IN REPRESENTATIVE TESTS 
noticeably greater than the variations in longshore transport Actually, the transport rates appear steady when compared with the quite varzable longshore current rates

\section{Energy Flux - Longshore Transport Correlation}

The total wave energy per unt crest width in one wave length of an oscillatory wave ${ }^{(7)}$ is given by $E_{t}=\frac{1}{8} \mathrm{pgH}^{2} \mathrm{~L}\left[1-\mathrm{M}-\frac{\mathrm{H}^{2}}{\mathrm{~L}^{2}}\right]$ where $\rho=\mathrm{w} / \mathrm{g}$ is the mass density of water ( $f$ resh water $=194$ slugs $/ \mathrm{ft}^{3}$ ) and $M$ is an energy coefficient defined as $\frac{\pi^{2}}{2 \tanh ^{2}(2 \pi d / L)}$ When considering a single wave at the breaking depth the above formula may be re-written as $\mathrm{E}_{\mathrm{b}} /$ wave $=1 / 8 \rho \mathrm{gH}_{\mathrm{b}}^{2} \mathrm{~L}_{\mathrm{b}}\left[1-\mathrm{M}_{\mathrm{b}} \frac{\mathrm{H}_{\mathrm{b}}^{2}}{\mathrm{I}_{\mathrm{b}}^{2}}\right]$, where the subscrıpt, $\mathrm{b}$, refers to wave breaking conditions This formula was used in these tests to compute the wave breaker energy per wave Calculations were carried out on a desk calculator using the Modifled Solitary Wave Theory - wave breaker indices curves and Welgels Tables in CERC TR-4 Wave breaker height, $\mathrm{Hb}$, breaker depth, $\mathrm{d}_{\mathrm{b}}$, and wave breaker energy, $E_{b}$, are tabulated in Figure 3 for discrete combinations of wave helght and period

Wave breaker angle, $\alpha_{b}$, and wave refractıon coefficlent, $k_{R}$, were obtalned empirically using the analytıc expression, $k_{R}=\sqrt{\frac{\cos \alpha}{\cos \alpha}}$, of Snell's law in a nomograph of $\mathrm{d} / \mathrm{L}_{\mathrm{O}}, \alpha_{\mathrm{O}}$, and $\mathrm{K}_{\mathrm{R}}$ (12) In using the nomograph, it was assumed that $\alpha_{0}$ was $30^{\circ}$ which was the wave approach angle in a constant depth of 233 feet in the test set-up The breaker depth, $d_{b}$, was used in the expression $d_{b} / L_{\mathcal{O}}$, to enter the nomograph and the angle, read from the graph was considered as $\alpha_{b}$, even though it was not based on the theoretical deep water for the respective wave perlods When the wave breaker energy, $E_{b}$, per wave was multiplied by cos $a_{b}$ $\sin \alpha_{b}$ and $K^{2}$ the product was the longshore component of the wave energy flux, $\mathrm{E}_{\mathrm{a}}$, in foot pounds per foot of shore per wave These last three parameters, $\alpha_{\mathrm{b}}$, $\mathrm{K}_{\mathrm{P}}$ and $\mathrm{E}_{\mathrm{a}}$ are tabulated in Table $3 \quad \mathrm{E}_{\mathrm{a}}$ is also tabulated in Table 4, in millions of foot pounds per foot of beach per day

The final results are given in Figure 12 as a scatter plot of longshore wave energy flux versus longshore transport rate at test times of 20-30 hours The visual best fit curve drawn through the points is based only on the 1 on 10 starting-slope tests Figure 12 also includes the suggested design curve of the wave energy-longshore transport relationship excerpted from CERC's TR-4 Results plotted in Figure 12 include a total of 36 points which includes elght points for the relatively low energy - low transport, constant period tests Most of the points falling on or near the CERC TR-4 curve have a 1 on 20 starting slope The maximum point on the curve is from a special test of four hours duration In this test, sand moving past the downdrift end of the beach dropped over a vertical ledge and formed a mound of sand, which was measured volumetrically by a method of successive surveys

Most of the data point scatter in the longshore transport rates is not felt to be just simple data scatter per se, but is more likely meaningful scatter 
For example, the legend in Figure 12, whych categorizes the results into only four discrete classes, cannot adequately account for significant influences on the longshore transport rate caused by waye height yariability, wave breaker type, model effects at the sand trap and at the feeder beach, or wave diffraction All of these causes, admittedly influence longshore transport rates in model basins, and the influence of one, wave diffraction, was tested and demonstrated in the SPTB at CERC (13)

\section{Summary}

A total of 36 tests were completed for the following experimental conditions, wave perlods of 125 to 375 seconds, and wave helghts of 014 to 075 feet in a constant water depth of 233 feet between a wave generator and the beach Waves were generated at a $30^{\circ}$ angle to the beach with portable wave generators, which when used singly, generated a wave crest 20 feet long, or when used - say in groups of five - generated a continuous wave crest 100 feet long Waves, so generated, traveled in the constant depth to a molded sand slope, where they $1 \mathrm{~m}$ pinged along a variable length shoreline from 30 to 95 feet, depending on the length of wave crest generated and the test set-up conditions In addition to variable perlod, height, length of wave crest generated and shoreline length, specific tests were elther variable about some mean perıod and helght or they were constant, with starting slopes of 1 on 10,1 on 20 , or a 1 on 30 equilibrium slope

As waves shoaled and broke along the $022 \mathrm{~mm}$ medlan-diameter sand beach, they caused the sand to move downdrift where it was deposited in a sand trap Amounts of sand deposited in a given time were reduced and complled as longsnore transport rates Longshore wave energy flux, computed from measured wave heights and calculated wave breaker angles is plotted against measured longshore transport rates in Figure 12 The results in the plot are compared with the suggested

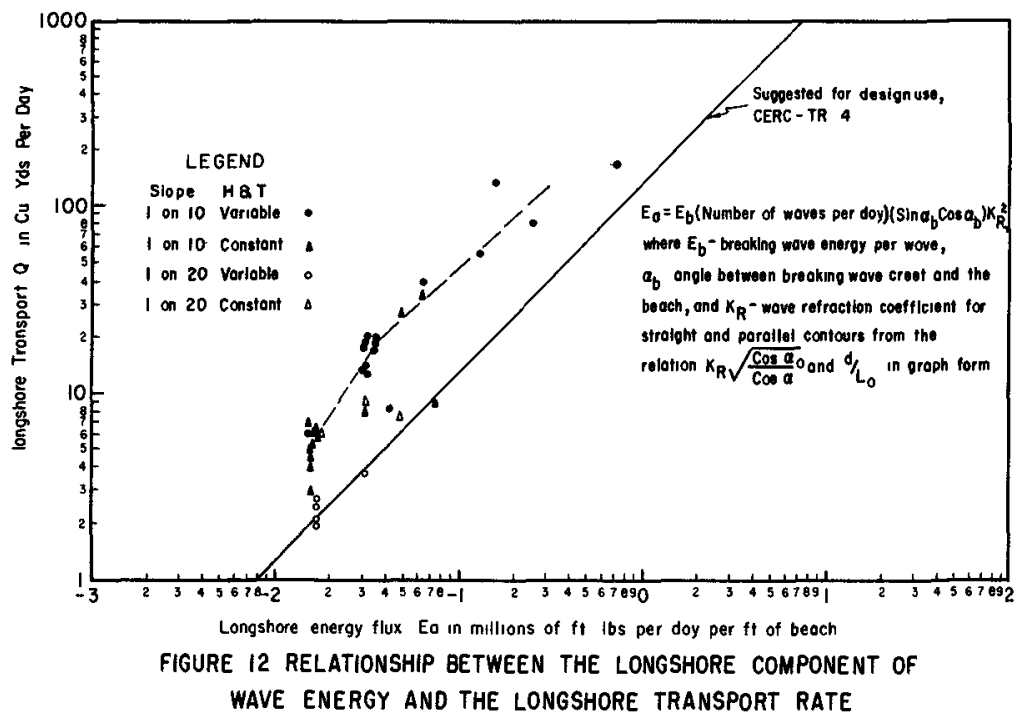


design curve of the longshore wave energy flux - longshore transport relation from CERC TR-4 Transport rates along the best fit curve of the laboratory data range from a low of about $6 \mathrm{yd}^{3}$ per day to a high of $170 \mathrm{yd}^{3}$ per day, and exceed the CERC TR-4 design curve rates by an average factor of 3

\section{Conclusions}

It is concluded that the laboratory rates, noted above as exceeding the CERC design rates by a factor of 3 , are at least as large as indicated in Figure 12, and except for some test difficulties noted above, would be larger than indicated Moreover, transport rates would be expected to be higher if, (1) tests were run continuously, not intermittently, and (2) at a changing water level, simulating a tide, instead of a constant water level

A localized and deep wave scour immediately updrift of the sand trap, restricted the transport to that in suspension in some tests, and thus reduced the transport rate In several tests, bars, cuts and cusps developed along the shoreline, and it is felt that these features reduced the transport rate In addition, accumulation of pebbles armored the beach locally against the waves in some tests, which was judged to have reduced the transport rate

It is also concluded that the actual wave height, defined as the effective wave helght impinging along the test beach, was significantly higher than the scaled-up Froude model height used to compute the wave energy Figure 13 is presented in support of this conclusion, as a typical result from the wave height variability measurements It shows that the average sustained height of the SPTB waves exceeded the Froude model height by 46 percent in a wall compartmented basin and by 71 percent in an open basin It is important to note here, that if these percentages were applied in Figure 12, the plotted points

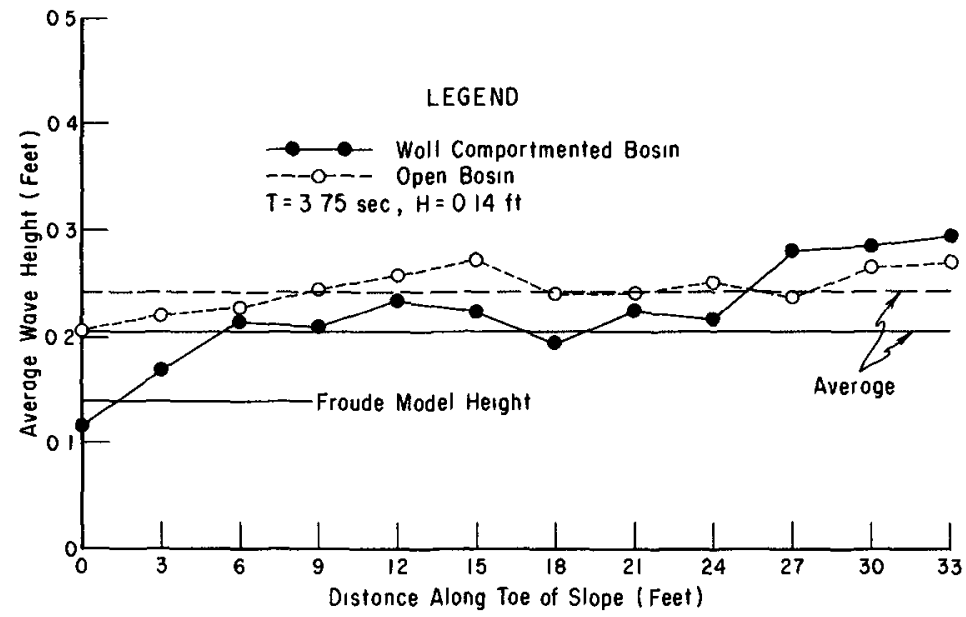

FIGURE I3 COMPARISON OF WAVE HEIGHT VARIABILITY AT A CONSTANT WAVE CONDITION IN A WALL COMPARTMENTED BASIN AND AN OPEN BASIN 
would move to the right by a factor of about 2 to 3 and then fall much nearer, or even cluster about the CERC TR-4 curve Whyle such good agreement would indeed be gratifying, it must be recognized that some part of the increase in height (energy) illustrated in Figure 13 was caused by reflected waves, either from the beach, the wave generator or both

\section{Recommendations}

It is recommended that in future tests, consideration be given to running some tests continuously to completion, with a tide range superposed on the mean water levels It is further recommended that wave height variability, wave breaker type, model effects at trap and feeder beach and wave diffraction effects be monitored and documented in future tests and so become part of the test results

It is also recommended that in future tests, consideration be given to short period tests of the order of 10 minutes to be made - say in the middle of a test beach length - somewhat removed from the influences of trap, feeder beach and wave diffraction Such tests might be made by interfacing sheet plastic along a profile line vertically, and laterally along the bottom for collecting sand drifting past the interface At the same time measurements could be made of the wave height at the toe of the beach profile and at the peak height before wave breaking Wave measurements and analysis should account for the percent of a wave height which is due to wave reflection

\section{ACKNOWLEDGMENT}

Acknowledgment is extended to Thorndike Saville, Jr, Chief, Research Division, who provided general guldance during this work, $R$ P Savage, Assistant Chlef, Research Division, who provided guidance and who, with $C \mathrm{~J}$ Galvin, Jr , Chief, Coastal Processes Branch, reviewed the final draft of this report, and $R \quad P$ Stafford, who reduced, compiled and helped to analyze the data

The work described herein is a portion of the research program of the $U S$ Army Corps of Engıneers and 1 ts Coastal Engineerıng Research Center Permission to publish this information is appreciated

\section{REFERENCES}

1 Savage, R P, "Laboratory Study of the Effects of Groins on the Rate of Littoral Transport Equipment Development and Inıtial Tests," Beach Erosion Board Technical Memorandum No 114, 1959

2 Watts, G M, "A Study of Sand Movenent at South Lake Worth Inlet, Florida" Beach Erosion Board Technical Memorandum No 42, 1953

3 Savage, R P , "Laboratory Determination of Littoral Transport Rates", Journal of Waterways and Harbors Division, ASCE, $v$ 88, No WW2, May 1962

4 Krumbein, W C , "Shore Currents and Sand Movement on a Model Beach", Beach Erosion Board Tech Memo No 7, 1944

5 Shay, E A, and Johnson, J W, "Model Studıes on the Movement of Sand Transported by Wave Action Along a Straight Beach", Issue 7, Series 14, Inst of Engrg, Res, Univ of Calif, 1951 (unpublished) 
6 Sauvage, D, and Vincent, G, "Transport Littoral Formation de Fleches et de Tombolos", Proc of the Fifth Conference on Coastal Engineering, Grenoble, France, 1954

7 "Shore Protection, Planning and Design", Coastal Englneering Research Center Technical Report No 4, ThIrd Edıtion, 1966

8 Savage, R P, "A Sand Feeder for Use in Laboratory Lıttoral Transport Studıes", Bulletin of the Beach Erosion Board, Vo1 15, 1961

9 Rector, R L , "Laboratory Study of Equılibrıum Profıle of Beaches", Beach Erosion Board Technical Memorandum No 41, 1954

10 Fairchild, J C , "Suspended Sediment Sampling in Laboratory Wave Actıon", Beach Erosion Board Technical Memorandum No 115, 1959

11 Straub, L D, "Effect of Water Temperature on Suspended Sediment Load in an Alluvial Stream", Proceedings of the S1xth General Meeting of IAHR, The Hague, 1955

12 U S Navy Hydrographıc Offıce, "Breakers and Surf", Publication Number 234 and Supplement, 1950

13 Faırchild, J C , "Wave Diffraction in a Laboratory Movable-Bed Set-up", Coastal Englneering Research Center, Bulletin and Sumnary of Research Progress, Fiscal Years 1967-69, Vo1 III, 1970 



\section{CHAPTER 56}

\section{INFLUENCE OF GRAIN SIZE ON LITTORAL DRIFT \\ José Castanho*}

Influence of grain size in sediment transport depends on flow con ditions For bed load transport a maximum probably exists for load dis charge as a function of grain size The important parameter seems to be the ratio $\tau_{0} / \tau$ between the threshold shear stress and the flow shear stress

1 It has been pointed out ${ }^{* *}$ that authors interested in littoral drift sometimes reach very different conclusions as to the influence of grain size

In fact, prototype observations made in Calıfornia beaches by Inman have shown that littoral drift was practically independent of grain size

On the other hand, $L$ Bajournas concluded from dimensional ana lysis considerations that littoral drift should increase with the square root of grain size According to this author this result agrees with observations made in prototypes and in models for medium and fine sands Finally, laboratory tests conducted by Larras and Bonnefille at Chatou Laboratory (France) revealed clearly the existence of a maxi-

* - Research Engıneer, Laboratório Macional de Engenharia Civil, LIS boa, Portugal

* - See for instance the General Report S II C 4 of the International Congress on Navigation, Paris - 1969, by $J$ Larras 
mum for littoral drift as a function of grain size

In the present paper an attempt is made to show in a more quall tatıve than quantitatıve manner that the divergence of results obtain ed by different researchers is explained by the fact that the influence of grain size should be variable according to circumstances, namely how far one is from the beginning of the sediment movement

2 - It is commonly accepted that littoral drift is a function of wave and sediment characteristics

$$
Q_{1}=f(\text { wave, sedıment })
$$

As wave characteristics one should consider $H$ (wave height), T (wave period) and $\alpha$ (wave obliquity) As for sediment, the important parameters are the submerged specific weight $\left(\gamma_{s}^{\prime}\right)$ and grain size (D)

Some other factors with possible influence on $Q_{1}$, such as beach slope and bed roughness, wind up being functions either of wave or se diment characteristics or both and so we may ultımately write

$$
Q_{1}=f\left(H, T, \alpha \gamma_{s}^{\prime}, D\right)
$$

3 - Now let us consider, according to some authors, the following schema tic model for littoral drift sediments are moved by the lorgshore cur rent in a direction parallel to the shore line, waves, with their turbu lence, merely "prepare" material to be moved

That being so, to a given wave $(H, T, \alpha)$ on a given beach will correspond a certain longshore current and consequently a certain shear stress $\tau$, so that we may write 


$$
Q_{1}=f\left(\tau, \gamma_{s}^{\prime}, D\right)
$$

In the above formula $D$ means the direct influence of grain size, the indirect influence corresponding to beach slope and bed roughness being implicit in $T$

4 - Equation (1) is formally identic to the equations accepted for un -directional flow, namely Meyer-Peter's and Einstein's formulas if we consider that bed-load transport is predominant relative to the transport in suspension

The direct influence of grain size in load discharge can be easily understood if in equation (1) we put $\tau=$ const, $\gamma^{\prime} s=$ const and com pute $Q_{1}$ as a function of $D$

Meyer-Peter's formula is based on the concept of threshold shear stress and can be written

$$
q_{s}^{\prime}=\theta\left(\frac{g}{\gamma}\right)^{1 / 2}\left(\tau-\tau_{0}\right)^{3 / 2} \quad(*)
$$

In this formula, $q^{\prime} s$ is the load discharge weighed underwater and $\gamma$ is the water specific weight The threshold shear stress $\tau_{0}$ may be computed by

$$
\tau_{0}=\begin{array}{lllll}
0 & 05 & \gamma^{\prime} & 0
\end{array}
$$

Calculations carried out on equation $(2)$ for natural sand $\left(\gamma_{s}^{\prime}=\right.$ $=16)$ and for two distint values of $\tau\left(\tau=01 \mathrm{~kg} / \mathrm{m}^{2}\right.$ and $\tau=1 \mathrm{~kg} /$

* - Rigorously one should write $\tau^{\prime}$ instead of $\tau$, with $\tau=\mu \mu$, whe re $\mu$ is a coefficient which takes into account the relationship between bed form roughness and the roughness due to grain size For the sake of simplicity we will put $\mu=1$ 
$/ m^{2}$ ) led to the results presented in Fig 1 , where the weighed out of water load discharge $\left(q_{s}\right)$ is plotted against the grain size (D)

It can be concluded that after Meyer-Peter's formula load dischar ge is zero for sufficiently great sizes and grows as size gets smaller For sufficiently small sizes, bed-load discharge tends to remain a cons $\operatorname{tant}^{*}$

Einstein's formula for bed-load transport is based on the probability of motion of bottom particles and the load discharge $q_{s}$ is given by means of two parameters $\psi$ and $\emptyset$

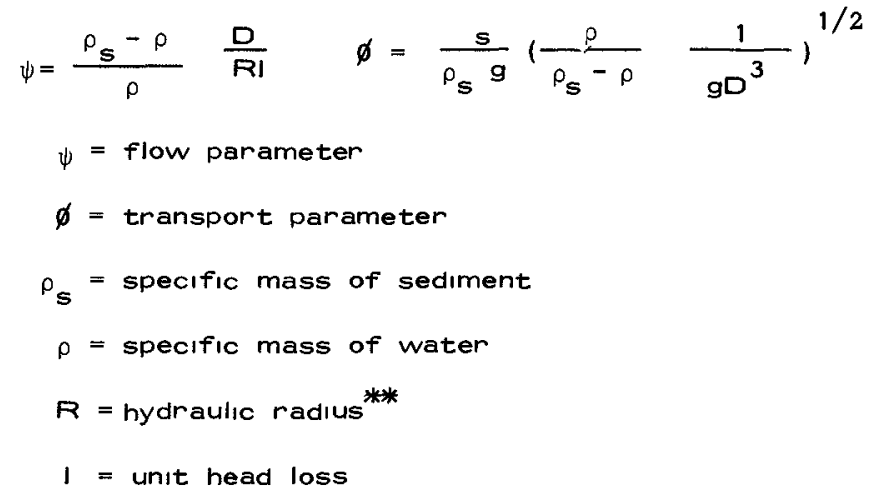


GRAIN SIZE
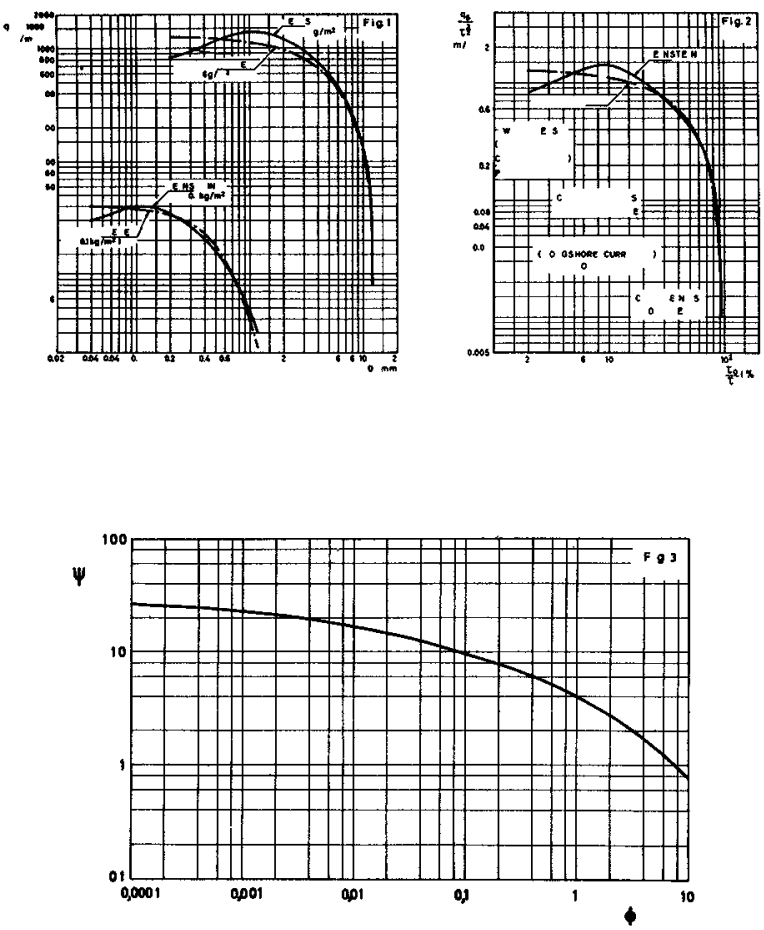


$$
\begin{aligned}
& \psi=1600 \frac{D}{\tau} \\
& \varnothing=10^{-4} \frac{q_{s}}{d^{3 / 2}}
\end{aligned}
$$

Calculations on equations (3) for $\tau=01 \mathrm{~kg} / \mathrm{m}^{2}$ and $\tau=1 \mathrm{~kg} / \mathrm{m}^{2}$ led to the results plotted in Fig 1 it can be seen that in this case a maximum of load discharge exists for a size $D$ varying with $\tau$

These results are in qualitative agreement with Larras and Bonef fille results

Also we may say that they agree with Bajournas results Indeed, If the load discharge increases with the square root of the sediment size for a certain range of diameters, and if, for sufficiently great diameters, the load discharge is zero, then there must exist a maximum of load discharge as a function of grain size

5 - A more suggestive interpretation of the above results may be achiev ed by plotting $Q_{1} / \tau^{3 / 2}$ as a function of $\frac{\tau_{0}}{\tau}\left(F_{1 g} 2\right)$ in this plot the value of $\tau_{0}$ was computed from $\tau_{0}=005 \gamma^{\prime}{ }_{s} D$ which would be true for unidirectional flow

It is the author's impression that the curves in Fig 2 may also be assumed valıd in case of longshore current movement if for $\tau_{0}$ a smaller value than the above is taken ${ }^{*}$, that is, if $\tau_{0}$ is divided by a parameter $W>1$ which would represent the "help" granted by the wa ve itself to the longshore current in the "preparation" of the material

* - The same results would be obtained if a greater value of $\tau$ were taken 
to be moved

According to this reasoning, the relative value of littoral drift Will depend on the zone of Fig 2 plot where one is working The influence of diameter will be represented through the parameter $\tau_{0}$ and the effect of the additional turbulence due to waves will be represent ed by the parameter $\underline{W}$

Situations indicated in Fig 2 for waves and currents should be frequent for the usual materials in prototypes and in models

The above reasoning may explain certain observed facts Let us consider the case of a movable bed model subject to wave and current action in which both waves and current are reproduced in the Froude scale

For a given $\tau$ we may find ourselves in the extreme right of the plot in case of currents, while for waves (lesser $\tau_{0}$ ) we maybe working in the middle zone Hence, load discharge by wave action is comparatively greater than the one due to current action This fact, observed in different laboratories, is one of the great difficulties in the calibration of estuary models, where waves and currents have equal importance These difficulties have been avoided both by enhanc Ing the effect of currents and by reducing the effect of waves

* - River or tidal current 


\section{6 - CONCLUSIONS}

a) Influence of grain size on sediment transport depends on the flow conditions If the transport is mainly in suspension, then load dis charge should decrease when the diameter increases When the transport is mainly through bed load discharge, then discharge will at the begınnıng increase with increasıng diameter reachıng a maxımum and de creasing afterwards

b) The value of the grain size for which the maximum transport is attained depends on the flow shear stress

c) The influence of grain size is expressed through the parameter $\tau_{0} / \tau$ It is very different for conditions near the beginning of the sediment movement (great $\tau_{0} / \tau$ ) and for conditions of fully developed movement (small $\tau_{0} / \tau$ )

d) In the case of currents (tidal or river currents) $\tau_{0}$ may be computed from $\tau_{0}=0.05 \gamma_{s}^{\prime} D$ In the case of transport due to wa ve action (longshore current) $\tau_{0}$ should be divided by a coefficient $W$ which depends on the wave characteristics (It should be noted that dividing $\tau_{0}$ by $W$ is the same as multiplying $\tau$ by $W$ ) 


\title{
CHAPTER 57
}

\section{COMPUTATION OF ALONGSHORE ENERGY}

AND LITTORAL TRANSPORT

\author{
Theodor R Mogel 1 \\ Robert L Street, ${ }^{1}$ M ASCE \\ Byrne Perry
}

\section{ABSTRACT}

This work encompasses a study of the littoral regime of a section of the coastline of the city and county of San Francisco, California The study included a complete refraction analyses of all applicable deep water wave directıons and perıods, the determınatıon of breaker locatıons, and a computation of alongshore energy and potential littoral transport for seven stations located Just offshore along the thirty foot depth contour The waves are refracted from deep water locations to the shorel Ine us Ing the Stanford Wave Refraction computer program Wave breaking is assumed to take place whenever the computed wave height exceeds 078 times the local water depth The effect of limiting the refraction coefficient is explored since the refraction theory, in analogy with its parent theory of geometric optics, falls along caustic curves and predicts unreasonably large values for the refraction coefficient

\section{INTRODUCTION}

The proper design of coastal structures, improvements or controls depends crucially upon an accurate estimate of the amounts of sand supplied to or lost from the shore region The most significant portion of the sand supply is the littoral drift--the material of the beach and nearshore bottom moved by the action of waves and currents The rate and direction of littoral transport or the movement of littoral drift is dependent on many factors, among which are the alongshore components of water-wave energy, currents, avaılability of littoral drift, its size characteristics, shapes and mineral compositions, $t$ Ide range, and beach slope Unfortunately, neither the precıse mechanism of transport nor the interaction of the various factors is clearly understood $(1-5)$ The present work is based on the concepts that the principal supply of energy for the movement of littoral drift comes from water waves Impinging upon the shore and that an emp Irıcal relationship $(1,4)$ between transport and alongshore energy is the most appropriate means for est ımatıng the potential littoral transport

lDepartment of Civil Engineering, Stanford Universıty, Stanford, Calıfornia 94305 


\section{The Purpose and Scope of the Study}

This paper reports on a study (6) of the 1 ittoral regime of a section of the coastline of the City and County of San Francisco, Calıfornia, from the Golden Gate Bridge to Mussel Rock, south of the southerly boundary of the City and County of San Francisco The original study includes a complete refraction analysis of all applicable deep-water wave directions and perıods, determination of the locatıons of wave breaking, application of 12 monthly tables and an annual table of digitized deep-water wave characteristics (7), calculation of alongshore energy and potential littoral transport, and tabulation of shallow-water wave direction and refraction coefficients for the San Francisco coastline Here we review the original results and explore the effect of limiting the maximum value that the refraction coefficient can attaın $(8,11)$

\section{The Plan of the Study}

The basıc Inputs to the study were the hindcast deep-water wave statıstics (7) compiled for a deep-water station (number 3, Ref 7) almost due West from San Francisco By use of a computer program that carried out a refraction analysıs of waves of all applicable periods, heights and directions represented in the wave statistics tables, the deep-water values were carried to seven points distributed in shallow water along the San Francisco shoreline ( $f$ Ig 1) Then a second computer program computed the alongshore energy components of the waves and, utilizing an empirical relation between these energy components and 1 ittoral transport $(1,4)$, computed the monthly and annual potential transport at the seven points on the shoreline All computations were carried out and plots and printed tables were generated by an IBM 360, Model 67 digital computer with an associated CALCOMP 750 plotter All programs were written in FORTRAN IV

The Refraction Program solves the refraction equation and the wave intensity equation along each individual wave ray for arbitrary bottom shapes The theoretical bases for the computer program were given in detail by Dobson (g) and are discussed briefly below (cf, Ref 10) The bottom hydrographic data in digitızed form and contoured grid maps for two study areas were supplied by the San Francisco Engineer District of the $U S$ Army Corps of EngIneers The INNER GRID or study area covered the Immediate vicinity of the San Francisco shorelune from Pirates Cove, north of the Golden Gate, to south of Mussel Rock at the southern boundary of the County with a 215 unit by 150 unit grid ( 33333 feet per grid unit) The OUTER GRID or study area covered the coast from shore to approximately the 300 fathom contour and approximately from the mouth of the Russian RIver in Sonoma County to the City of Santa Cruz in Santa Cruz County with a 303 unit by 199 unit grid (1666.67 feet per grid unit) of much greater sIze The INNER GRID provided the detalled bottom contours needed for an accurate prediction of wave behavior in shallow water near the coast, while the OUTER GRID provided the link to the deep-water wave conditions Figures la and Ib show CALCOMP (computer) contour plots of the hydrography described by the grids of depths waves ranging in period from 4 to 20 seconds and in height from 1 to 25 feet and 
comıng from directions between North and South-Southeast were considered

The Drıft Program utılızes a l ınear relatıon between alongshore energy components and potential littoral transport The alongshore energy components are calculated from output supplied by the Refract Ion Program and the deep-water wave statıstıcs

\section{METHOD OF COMPUTATION}

\section{I Refraction}

\section{Theory and Background}

The theoretical basis of the present refraction computations is well known (10-12) and will only be touched on here In effect the same theory is commonly employed in hand computations and only the implementation for the computer led to differences in detall The original version of the theory comes from the physical science of geometrical optics, the key result of which is Snell's law A detailed discussion of the method was given by Dobson (g)

The main feature that has been added for the computer work is a technique for falring a smooth surface through the known bottom depth data $A$ quadratic surface is constructed to give the best fit in the sense of least squares to the local point and its surrounding neighbors Thus, the bottom is always represented as a smoothly-varyıng surface Starting from a known point on a grid and in a given initial direction, the computer program constructs a single wave ray (or orthogonal) step-by-step across the grid. Because the fitted bottom surface is smooth, and hence differentiable, the equation of wave intensity (equivalently wave height) may be solved at each step in the ray construction process, and the relationship between initial and present wave height carried forward cont inuously along a single ray This is an essential feature of the analysis without which the breaking height location computations, described below, would not have been feasible The refraction technique has proved very sat Isfactory in many test cases, as shown by comparison with exact theory and hand computations (g) Battjes (13) has recently shown that, according to both linear and nonl inear theory, the energy flux in wave propagation in three dimensions is always directed along the wave rays (orthogonals) [our wave intensity equation is based on this fact] regardless of the wave amplitude gradients and that refraction of water waves over bottoms of small slope can indeed be considered as a case of wave propagation through an inhomogeneous (depth varies), two-dimensional medium This is in agreement with the method used in the present work and current practice

The refraction theory, in analogy with its parent theory of geometric optics, falls along caustic curves and predicts unreasonably large values for the refraction coefficient $K_{r}$ near the cauştıc curve Pierson $(8,11)$ gave Just If lcation for the approximate 1 imit $K_{r}{ }^{2} \leqq 2.0$ and we have run test cases
for $K_{r}{ }^{2} \leqq 20, K_{r}{ }^{2} \leqq 40$, and $K_{r}{ }^{2}<\infty$ 


\subsection{Technique}

The refraction program constructed rays on either the OUTER GRID (303 $x$ $199 \mathrm{gr}$ id units) or the INNER GRID $(215 \times 150 \mathrm{grid}$ units) The depth data at the intersection points of the grids was provided by the San Francisco Engineer District and was digitized from their bottom hydrography charts $A$ match line for transfer of computations from one grid to the other was established

The calculation of littoral transport requires the shallow-water wave helght and direction at the point of breaking of every possible wave in the three-dimensional matrix of deep-water statistics (parameterized by deep-water height, direction and period) Accordingly, on an inward run from deep to shallow water, the refraction program punched a data card whenever breaking occurred on the INNER GRID, only those breaking points near the beach were retained For the range of wave heights and periods considered in this study, breakıng was assumed to occur whenever the height of a particular wave exceeded 078 times the local water depth. While more sophisticated criterla are avallable (12), it appears that none is significantly more accurate on the average for prototype waves in our period range Furthermore, in view of the basis of the refraction analysis on linear theory, a more refined breaking criteria does not appear warranted

The objective of the refraction analysis was to bring one ray from deep water to each of the seven target points near the shore for each applicable deep-water direction and period Because local wave height is a linear function of deep-water height, all the results for the heights between 2 and 24 feet were obtained from a single ray computation. In view of the complex hydrography of the San Francisco shore region, it was decided to work outward from each target station with fans of rays, the rays in each fan having a different period New fans were generated unt 11 a set of results were obta Ined in deep water that would permit us to run a complete set of applicable waves in from deep water (corresponding to the wave statistics) it was not possible to generate the necessary data on an outgoing run because the refraction coefficient calculation is not valid for rays started in shallow water (the starting condition of parallel rays is not correct there) Therefore, it remains necessary to retrace outgoing rays from deep water Inward In some cases it was possible to begin with incoming rays of specific period and direction and to hit a target station by using trial-and-error and the information known about adjacent stations

A study of the hindcast data (7) shows that the wave statistics are given for 22 5-degree direction segments, two-second period intervals and two-foot height intervals We used the mid-direction, mid-period and mid-heights in our refraction calculations In light of the difficulty of hitting a given direction with an outward running ray or a given station with an inward ray we established tolerance criteria of 5 degrees on direction when running outward and an $x$-distance tolerance of \pm 4 grid units when running in Tests of the results showed that no large changes in wave characteristics occurred within these tolerances In any case the major 1 ty of the runs are well within the tolerance limits which seem reasonable in view of the expected accuracy of the hindcast deep-water statistics 


\section{Notes on Computer Programmıng}

The results of the present study were obtained on an IBM 360/67 digital computer and an off-l Ine CALCOMP 750 plotter The program is an extension of the Stanford Refraction Program originally developed for the IBM 7090 Dobson (g) has given a very thorough documentation including flow charts for the main program and subrout ines in the original program, except for the CALCOMP subrout Ine calls which are highly installation dependent

The primary changes made in the or Iginal Stanford Program have been related either to differences between the IBM 7090 and IBM 360/67 computers or Improvements in the graphical displays and printed or punched outputs of the program

For the present study the program has been specifically modified to do the following

a Read the Depth data and associated fixed grid and ray constants and ident If Iers from Disc storage in the machine

b Plot wave rays on a map ( $F ı g$ 1) The map Includes labeled $X$ and $Y$ axes, a north direction arrow, sultable contours (stored on Disc also), and Identification block giving relevant parameters and Identification The contours on the INNER GRID are the shoreline and 30-foot contours, while only the shoreline is shown on the OUTER GRID If the ray is to cont Inue to another grid, the map also shows the match line outline of the next grid

c Create a punched card data deck to cont inue wave rays on another grid The $X$ - and $Y$-coordinates in the data deck have been converted to the new coordinate system The data deck is complete with all necessary title and end-of-set cards so that it may be loaded for the next run without further processing

d Create a punched-card data deck givıng coordınates and wave parameters at the 30-foot contour for each wave ray

e Create a punched-card data deck with one card for each deep-water wave height on each ray when the wave breaks $(\mathrm{H} / \mathrm{D}>078)$ for helghts of 2 to 24 feet in increments of 2 feet These cards contained, in addition to the wave height, direction, period and spacial location, the shoaling coefficient, the deep-water height, the water depth and the local angle between the wave ray and the gradient of the bottom topography

f Limit the refraction coefficient to a specific value

Figures la and $1 \mathrm{~b}$ show sample plotter output from the refraction program A ray was started a short distance to each side of each of the actual rays used to better Illustrate the shift Ing of the wave front Tables la and lb are the printouts generated for one of the rays shown in the figures 


\section{Littoral Transport}

\section{Analysis and Hindcast Data}

Nat Ional Marine Consultants compiled deep-water wave statistics based upon meteorological records and charts for the years 1956, 1957 and 1958 for seven deep-water stations along the California coast (7) The wave hindcast data for deep-water sea (generated by local wind) and swell were given as height-period-direction average frequency distributions in percent monthly and annualiy A digitized data deck for Station 3 (Lat Itude 376 degrees $N$, Longitude 1235 degrees $W$ ) was provided the authors by the San Francisco Engineer District This station is due west of San Francisco As none of the other stations were close to this area, Station 3 was the only data source, cf, Fairchild (3) who interpolated between several stations for an east-coast study

The treatment of deep-water wave statistics and littoral transport calculations was based on the methods of Saville (14) and Fairchild (3) In particular, sea and swell energies were added linearly, and the significant wave heights, corrected in accordance with Falrchild (3) and Saville (14), were used to est imate the wave energy at the shore The refraction analysis used the dominant wave perıod, as outlined by Saville (14), associated with the significant wave heights given in the statistical data

Littoral transport was computed from the alongshore energy components derived from the combination of wave statistics and refract ion program output for the seven shallow-water stations shown in Fig lb An empirical littoral transport equation was obtained from Fig 2-22 of CERC Technical Report No 4 (1) According to this figure, the potential littoral transport past a point on shore and caused by a given period wave of given deepwater height and direction is

$$
Q=128 E_{a} \quad 10^{-6} \mathrm{cu} \quad \mathrm{yds} / \text { month }
$$

where

$$
\begin{aligned}
& E_{a}^{l-}=058 E_{a} F_{1 j} k_{l}, \\
& E_{a}=5400 \frac{\gamma H_{b}^{2} L_{o}}{T K_{S}^{2}} \sin \alpha_{c} \cos \alpha_{c}=177 \quad 10^{6} \frac{H_{b}^{2}}{K_{S}^{2}} \sin \alpha_{c} \cos \alpha_{c} \\
& \text { in } \mathrm{ft} \text {-lbs/ft of beach/day, } \\
& \alpha_{c}=\text { angle between wave ray and the gradient of the bottom } \\
& \text { hydrography in degrees, } \\
& \gamma=\text { specific we Ight of sea-water }=64 \mathrm{lbs} / \mathrm{ft}^{3} \text {, }
\end{aligned}
$$




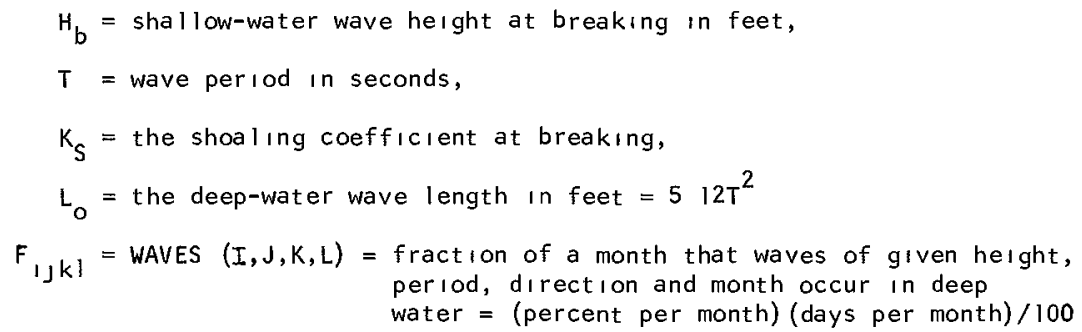

The actual (as opposed to potential) littoral transport may be less than $Q$ If bottom bed material is not avallable at the point in question The factor 058 is required (14) to reduce the given deep-water spectral energy for significant waves to the proper average energy that causes littoral transport Note that in this analysis the littoral transport caused by waves of varyıng helghts will occur where the wave breaks, thus, for each station location our transport results reflect the alongshore transport on the average through the broad-band breaking or surf zone (see Fig L) created by a set of deep-water waves whose common point is that they all pass through (or near) a given station location ( 1 through 7) on the 30-foot contour along the San Francisco shorel ine

The machine calculations were checked by hand for those cases in which only one deep-water wave height for a given period and direction had a nonzero occurrence in a month or for the year

\section{Programming and Output}

The operation of the program that calculates the alongshore energy and potential littoral transport is particularly simple and proceeds as follows

a The program reads the digitized sea data (Table 3 , Ref 7) and constructs WAVES $(I, J, K, L)$ for the sea data for each month

b The program reads the swell data and adds it to the appropriate WAVES $(I, J, K, L)$

c The program reads a refractıon-program-punched input card for a particular deep-water height, direction, perıod and station (always starting with the 24-foot he Ight and working downward) and constructs $\mathrm{E}_{\mathrm{a}}{ }^{\prime \prime}$ for that card Cards are read and energies calculated unt 1 all the heights for a given ray are surveyed, and their energies are added together to find the total energy for that period and direction only the last complete height survey (24 feet to 2 feet) is used to calculate the transport, $1 \mathrm{e}$, only the breakıng zone cont Iguous to the beach is considered to cause transport No allowance has been made for energy losses in prior breakıng zones away from the beach (see Sec 4 2)

d The program repeats $c$ for the remaınıng perıods and directions 
e The littoral transports $Q$ corresponding to the $E_{a}^{-}$for each period and direction are computed

$f$ Tables of $\mathbf{E}_{a}^{-}$and $Q$ are constructed for each station

\section{RESULTS AND DISCUSSION}

\section{Transport and Energy Tables}

The tables of littoral transport and alongshore energy components computed by the Drift Program for the three limits on $K_{r}$ are given in Tables 2 and 3 for each of the seven stations of the study Results are given for each month in the year and the annual total transport as a station $A$ positive sign indicates energy components and transport motion up-coast or generally North

As noted above (Secs 213 and 221 ) the alongshore energy component computation is based upon the local angle $\alpha$ at breaking between the wave ray and the gradient of the bottom hydrography (the perpendicular to the local bottom contour) We considered the use of the mean gradient direc$t$ ion of the beach as a whole near any given station in lieu of the local gradient Tests with this concept had predictable results, namely, the transport and energy components vary widely for small changes in mean beach gradient direction Because of the complex hydrography near the shore ( $F ı g \quad l b)$ and the wide zone of breaking ( $F, g 2$ ) the direction of a ray at the breaking point for a given wave is not correlated to any observable mean beach gradient direction Accordingly, no computations ut Ilızed an $\alpha$ based on mean beach gradients, rather, the ray direction was correlated with the bottom contour at the actual breaking point

As can be seen from the alongshore energy and littoral transport tables (Tables 2, 3, \&4), l Imıtıng the maxımum value of $\mathrm{K}_{r}$ tends to make the calculated energy and transport more uniform The majority of the alongshore energy at each station is the result of a few rays When some of these rays pass through or near a caustic and their $K_{r}$ is not restricted, their dominance is exaggerated and they break further from shore By limiting the maximum value that $K_{r}$ can attain, the dominance of these waves is reduced and they break closer to the beach zone Station I, for example, has a ray from the west which contributes most of the energy and transport When this ray crosses the $30 \mathrm{ft}$ contour $K_{r}=367$ When $K_{r}$ is restricted to a more reasonable value, the dominance of the ray dimınishes and the net transport is brought into line with the transport of station 3 as expected because the bottom hydrography near these two stations is simılar

\section{Unusual Features}

Several features of the bottom hydrography are worth noting The Faralion Islands are the visible portion of a shoaling region which lies mainly between 60 fathoms and the surface Long waves, in particular, are affected by this region. The peaked nature of the region causes bending of the longer waves which is quite unpredictable and led to much of the ted Ium of locating the starting positıons of these longer waves 
The Fourfathom Bar blocks or diverts much of the wave energy of the longer period waves from the Golden Gate area The small energy and transport figures for stations 6 and 7 are a direct result of this blocking effect The Southern and Central portions of the San Francisco Bar also severely bend the rays However this effect is quite predictable

\section{Comparison with other studies}

Kamel (15) made a study of sand transport along the Calıfornia Coast in the Russian River-Point San Pedro reach using, mainly, radioactıve tracer and heavy mineral concentratıons His study predicts a general transport in the southerly direction In the region between the Golden Gate and Merced Lake (our statıons 4 and 2) no predomınant direction of transport was predicted and the radioact Ive tracer samples predicted a Southern transport near our stations 1 and 3 However a close examination of the heavy mineral concentration data for the same region shows a Northern transport for the same region

Adding to the uncertainty Johnson (16) states in his study of the Half Moon Bay-Russian River region that little if any material is transported in this littoral zone our results indicate, with the exception of station 2, a northerly direction of sand transport Johnson (16) also concludes that there is no major source of littoral materlals along the Half Moon Bay-Russian River region indicating that perhaps the experimental results revealed not what is taking place now but what has taken place over geologic time

\section{Conclusions}

\section{Conclusions based on the present results}

In this study we brought together three essent lal ingredients to synthesize the potential littoral transport and alongshore energy The combination of deep-water hindcast wave statıstics, linear refract Ion of wave components and an empirical relation between energy and transport produced energy and transport patterns which clearly show that

1) The average annual transport and energy direction is north

2) The Farallon Islands and their associated shoalıng region both block wave energy from the shore and bend and focus wave rays to zones not otherwise reachable

3) Limiting the maximum value of $K_{r}$ produces energy and transport predictions which are more uniform and realistic

However it must be emphasized that the littoral transport results are obtalned from an empirical energy-transport relation developed for other coastal areas and based on very scattered data $(4,5)$ Accordingly, within the accuracy of the hindcast data and the l Inear refraction analys Is, we consider the alongshore energy distribution to be accurate, but the potential (computed) littoral transport can only be considered as a qualitative indicator of the actual transport 


\section{Recomendations for Modification of the Present Technique}

Three problem areas arise in connection with the present study First, a large effort in terms of man-hours and computer time is expended in selecting the desired wave rays for each deep-water wave direction and period that run to each station second, and more significant, no account is made of energy losses that occur through wave breaking on bars and other shoaling regions far from the beach area Third, the relationship between energy and sand movement rests on little in the way of sound principles and analysis, Thornton (5) has recently made a start in remedying this situation, but his success is limited

Battjes (13) proposes a refraction technique in which the wave characteristics are determined as cont inuous field variables over an ent ire grid This method requires the solution of a pair of non-linear partial differential equations, but would give the necessary data at all points along a shoreline However his method would require large amounts of storage $\left(\sim 10^{6}\right.$ words for our outer Grid) and long run tımes (probably more than 10 minutes per wave direction and perıod). Our present program has none of these prob lems

The greatest need, however, is to find a way to account for energy loss when a wave breaks offshore before comıng into the breaker zone Battjes (13) technique assumes no energy losses from the wave system so would suffer from some of the present inaccuracies

\section{ACKNOWLEDGEMENT}

The basic refraction and littoral drift studies were carried out under Contract No DACW07-68-C-0054 for the U $S$ Army Engineer District, San Francisco, Corps of Engıneers 


\section{REFERENCES}

1 Shore Protectıon, Plannıng and Desıgn, Tech Rep No 4 (3rd ed), U S Army Coastal Eng Ineering Research Center, Dept of the Army, Corps of Engıneers, Washıngton, D C , June 1966

$2 \mathrm{~J} \mathrm{~W}$ Johnson and $\mathrm{P} S \mathrm{~S}$ Eagleson, "Coastal Processes," Estuary and Coastlıne Hydrodynamıcs, McGraw-Hill Book Co, Inc, New York, Chapter 9,1966

3 J C Falrchlld, "Correlation of Littoral Transport with Wave Climate along Shores of New York and New Jersey," Tech Memo No 18, U S Army Coastal Engıneerıng Research Center, Dept of the Army, Corps of Engineers, Washington, D C , November 1966

4 T Saville, Jr, G M Watts," Coastal Regime, Recent U S Experience," XXII Internat Ional Navigation Congress, Parıs, 1969

5 E B Thornton, "Longshore Currents and Sedıment Transport," Tech Rep No 5, Department of Coastal and Oceanographic Engineering, University of Florida, Ga Inesville, Florıda, December, 1969

6 Street, R L, T Mogel and B Perry, "Computation of the Littoral Regime of the Shore of San Francisco County, Cal Ifornia, by Automatic Data Processing Methods," Final Report, Contract No DACW07-68-0054, $U$ S Army Engineer District, San Francisco, Corps of Engineers, I January 1969

7 "Wave Statıstics for Seven Deep Water Statıons Along the Calıforn Ia Coast," Natıonal Marıne Consultants, Santa Barbara, Calıfornı, December 1960

$8 \mathrm{~W} \mathrm{~J}$ Pierson, "The interpretation of Crossed Orthogona is in Wave Refraction Phenomena," Tech Memo No 21, Beach Erosion Board, Corps of Engıneers, January 1951

$9 \mathrm{R} S \mathrm{~S}$ Dobson, "Some Applicatıons of a Dıgıtal Computer to Hydraulıc Engıneerıng Problems," Tech Rep No 80, Dept of Civil Engineerıng, Stanford Unıversity, Stanford, Calıfornı, June 1967, DDC AD No 659309

10 G H Keulegan, J Harrison, "Tsunamı Refraction Dıagrams by Digıtal Computer," J Waterways and Harbor DIvision, Proc ASCE, V96, No WW2, Paper 7261, May 1970

11 W J Pierson, G Neumann and R W James, Practical Methods for Observing and Forecasting Ocean Waves by Means of Wave Spectra and Statıstics, U S Navy Hydrographic Office, Publication No 603, 1955 
$12 \mathrm{R} L$ Wiegel, Oceanographical Engineering, Prentice-Hall, Inc, Englewood Cliffs, New Jersey, 1964

13 J A Battjes, "Refraction of Water Waves," J Waterways and Harbor DIVIsion, Proc ASCE, V 94, No WW4, Paper 6206, November $196 \mathrm{~B}$

$14 \mathrm{~T}$ Saville, Jr, "North At lant Ic Coast Wave Stat Istıcs HIndcast by Bretschneıder-Revised Sverdrup-Munk Method," Beach Erosion Board Tech Memo No 55, Dept of the Army, Corps of Engıneers, Washington, D C , November 1954

15 A M Kamel, "Lıttoral Studies Near San Francısco Usıng Tracer Technıques," Tech Memo No 131, Beach Erosion Board, November 1962

$16 \mathrm{~J} W$ Johnson, "Nearshore Sedıment Movement--Central Calıfornı Coast," Coastal Engıneering, ASCE Santa Barbara Specialty Conference, Chapter 23, 0ct ober 1965 

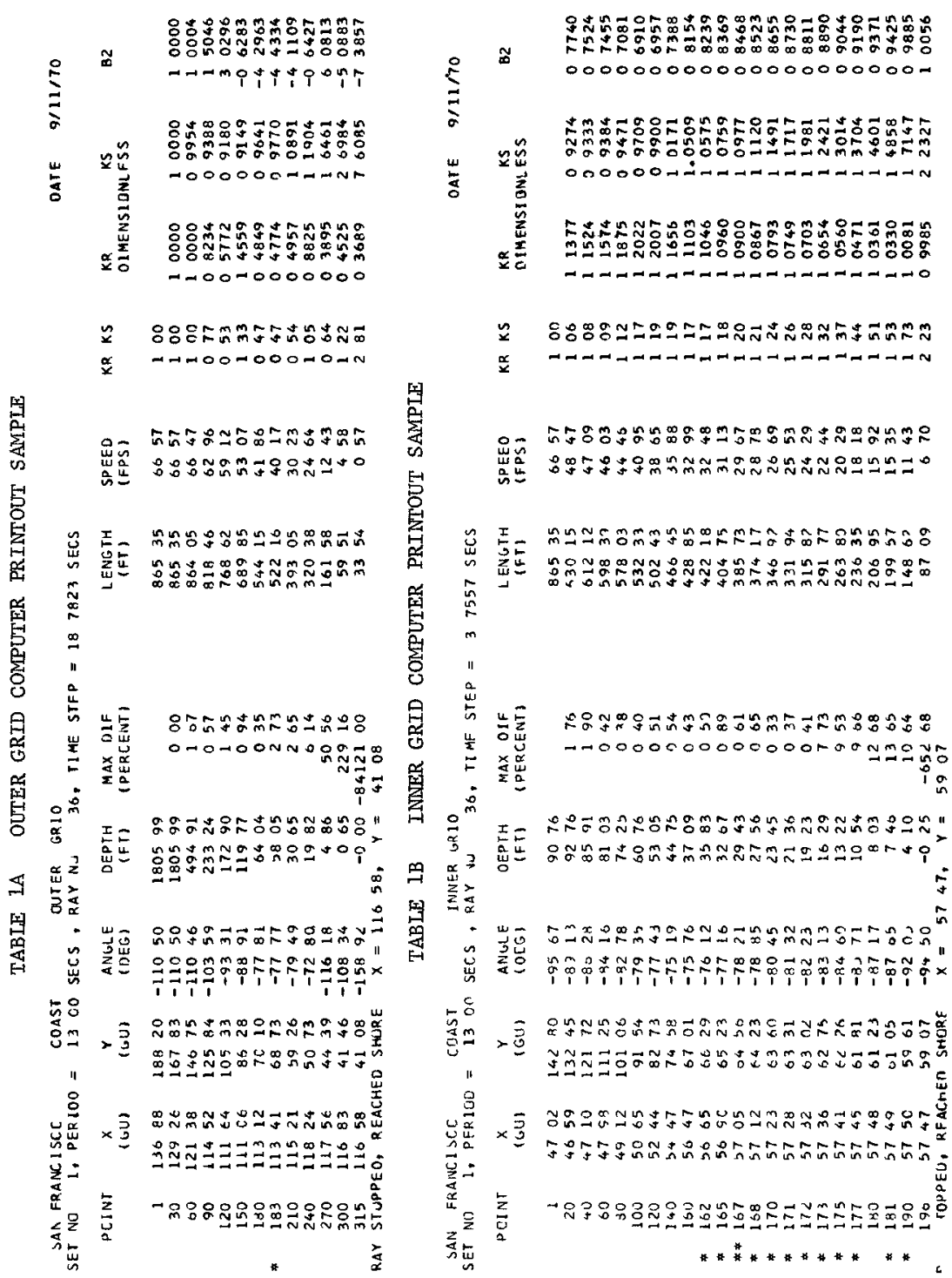
TA8LE 2 ALONGSHORE ENERGY FOR $K_{r}<\infty\left(10^{6} \mathrm{ft}-1 \mathrm{bs} / \mathrm{ft}\right.$ of beach)

$\begin{array}{lrrrrrrr}\text { STAT ION } & 1 & 3 & 2 & 5 & 4 & 6 & 7 \\ \text { JAN } & 3489 & 1452 & 4180 & 22092 & 5031 & 666 & 225 \\ \text { FE8 } & 4625 & 3114 & 8302 & 21418 & 8030 & 244 & 1084 \\ \text { MAR } & 2167 & 1180 & 2705 & 10584 & 2583 & -9 & 4 \\ \text { APR } & 769 & 824 & -39 & 5195 & 2642 & 2617 & 177 \\ \text { MAY } & 104 & 347 & -261 & 1253 & 1271 & -143 & 169 \\ \text { JUN } & -429 & -80 & -247 & -1717 & 75 & -3 & 1 \\ \text { JUL } & -361 & -56 & -90 & -1163 & 34 & -5 & 2 \\ \text { AUG } & -411 & -45 & -182 & -1043 & 35 & 23 & 2 \\ \text { SEP } & -92 & 110 & 152 & -27 & 349 & -20 & 7 \\ \text { OCT } & 305 & 226 & 297 & 1206 & 1049 & 384 & 70 \\ \text { NOV } & 982 & 89 & 88 & 2669 & 839 & 1081 & -5 \\ \text { DEC } & 1866 & 603 & 1009 & 5857 & 1430 & 1171 & -14 \\ \text { TOTAL } & 13014 & 7764 & 15914 & 66324 & 23368 & 6006 & 1722\end{array}$

POTENTIAL LITTORAL TRANSPORT FOR $K_{r}<\infty\left(10^{3}\right.$ cubIC yards)

$\begin{array}{lrrrrrrr}\text { STATION } & 1 & 3 & 2 & 5 & 4 & 6 & 7 \\ \text { JAN } & 447 & 186 & 535 & 2828 & 644 & 86 & 29 \\ \text { FE8 } & 592 & 399 & 1063 & 2741 & 1027 & 31 & 139 \\ \text { MAR } & 277 & 151 & 346 & 1355 & 331 & -1 & 1 \\ \text { APR } & 98 & 105 & -5 & 665 & 338 & 335 & 23 \\ \text { MAY } & 13 & 44 & -33 & 160 & 163 & -18 & 22 \\ \text { JUN } & -55 & -10 & -32 & -220 & 10 & 0 & 0 \\ \text { JUL } & -46 & -7 & -12 & -149 & 4 & 1 & 0 \\ \text { AUG } & -53 & -6 & -23 & -134 & 4 & 3 & 0 \\ \text { SEP } & -12 & 14 & 20 & -4 & 45 & -3 & 1 \\ \text { OCT } & 39 & 29 & 38 & 154 & 134 & 49 & 9 \\ \text { NOV } & 126 & 11 & 11 & 342 & 107 & 138 & -1 \\ \text { DEC } & 239 & 77 & 129 & 750 & 183 & 150 & -2 \\ \text { TOTAL } & 1665 & 993 & 2037 & 8488 & 2990 & 770 & 221\end{array}$


TABLE 3 ALONGSHORE ENERGY FOR $k_{r} \leqq 2\left(10^{6} \mathrm{ft}-1 \mathrm{bs} / \mathrm{ft}\right.$ of beach)

$\begin{array}{lrrrrrrr}\text { STAT ION } & 1 & 3 & 2 & 5 & 4 & 6 & 7 \\ \text { JAN } & 3029 & 1440 & -184 & 6701 & 3708 & 21 & 225 \\ \text { FE8 } & 3719 & 3006 & 199 & 7888 & 6822 & 319 & 1084 \\ \text { MAR } & 1410 & 1097 & -616 & 1963 & 1564 & 35 & 4 \\ \text { APR } & 392 & 789 & 156 & 1420 & 2001 & 579 & 177 \\ \text { MAY } & -196 & 342 & -185 & -806 & 462 & 12 & 169 \\ \text { JUN } & -556 & -80 & -198 & -1722 & 75 & 37 & 1 \\ \text { JUL } & -435 & -56 & -67 & -1162 & 34 & 9 & 2 \\ \text { AUG } & -469 & -45 & -182 & -1044 & 35 & 37 & 2 \\ \text { SEP } & -251 & 107 & -192 & -496 & 166 & 30 & 7 \\ \text { OCT } & 13 & 211 & -594 & -49 & 458 & -18 & 70 \\ \text { NOV } & 179 & 89 & -644 & -304 & 330 & -49 & -5 \\ \text { OEC } & 943 & 562 & -1673 & 925 & 881 & -28 & -14 \\ \text { TOTAL } & 7778 & 7462 & -4179 & 13314 & 16536 & 984 & 1722\end{array}$

POTENT IAL LITTORAL TRANSPORT FOR $K_{r} \leqq 2\left(10^{3}\right.$ cubIC yards)

$\begin{array}{lrrrrrrr}\text { STAT ION } & 1 & 3 & 2 & 5 & 4 & 6 & 7 \\ \text { JAN } & 388 & 184 & -24 & 858 & 475 & 3 & 29 \\ \text { FEB } & 476 & 385 & 26 & 1010 & 873 & 41 & 139 \\ \text { MAR } & 180 & 140 & -79 & 251 & 200 & 4 & 1 \\ \text { APR } & 50 & 101 & 20 & 182 & 256 & 74 & 23 \\ \text { MAY } & -25 & 44 & -24 & -103 & 59 & 2 & 22 \\ \text { JUN } & -71 & -10 & -25 & -221 & 10 & 5 & 0 \\ \text { JUL } & -56 & -7 & -9 & -149 & 4 & 1 & 0 \\ \text { AUG } & -60 & -6 & -23 & -134 & 4 & 5 & 0 \\ \text { SEP } & -32 & 14 & -25 & -63 & 21 & 4 & 1 \\ \text { OCT } & 2 & 27 & -76 & -6 & 59 & -2 & 9 \\ \text { NOV } & 23 & 11 & -82 & -39 & 42 & -6 & -1 \\ \text { OEC } & 121 & 72 & -214 & 118 & 113 & -4 & -2 \\ \text { TOTAL } & 996 & 955 & -535 & 1704 & 2116 & 127 & 221\end{array}$


TABLE 4 ALONG5HORE ENERGY FOR $k_{r} \leqq 141\left(10^{6} \mathrm{ft}-1 \mathrm{bs} / \mathrm{ft}\right.$ of beach)

\begin{tabular}{lrrrrrrr} 
5TATION & 1 & 3 & 2 & 5 & 4 & 6 & \multicolumn{1}{c}{7} \\
JAN & 3200 & 1357 & 6 & 3233 & 2921 & 168 & 128 \\
FEB & 3130 & 2537 & 1477 & 5295 & 4478 & 346 & 625 \\
MAR & 902 & 792 & -496 & 882 & 1229 & 83 & -9 \\
APR & 260 & 583 & -243 & -13 & 1426 & 60 & 101 \\
MAY & -342 & 295 & -171 & -961 & 294 & 61 & 92 \\
JUN & -593 & -80 & -190 & -1712 & 75 & 53 & 1 \\
JUL & -456 & -56 & -63 & -1160 & 34 & 15 & 2 \\
AUG & -486 & -45 & -182 & -1041 & 35 & 45 & 2 \\
SEP & -327 & 67 & -159 & -499 & 133 & 60 & 4 \\
OCT & -237 & 163 & -446 & -334 & 353 & 64 & 34 \\
NOV & -179 & 89 & -578 & -468 & 273 & 62 & -8 \\
DEC & 642 & 434 & -1902 & 228 & 739 & 133 & -19 \\
TOTAL & 5514 & 6136 & -2947 & 3450 & 11990 & 1150 & 953
\end{tabular}

POTENTIAL LITTORAL TRAN5PORT FOR $K_{r} \leqq 141$ (10 $0^{3}$ cubIC yards)

$\begin{array}{lrrrrrrr}\text { STATION } & 1 & 3 & 2 & 5 & 4 & 6 & 7 \\ \text { JAN } & 410 & 174 & 1 & 414 & 374 & 22 & 16 \\ \text { FEB } & 401 & 325 & 189 & 678 & 573 & 44 & 80 \\ \text { MAR } & 115 & 101 & -64 & 113 & 157 & 11 & -1 \\ \text { APR } & 33 & 75 & -31 & -2 & 183 & 8 & 13 \\ \text { MAY } & -44 & 38 & -22 & -123 & 38 & 8 & 12 \\ \text { JUN } & -76 & -10 & -24 & -219 & 10 & 7 & 0 \\ \text { JUL } & -58 & -7 & -8 & -149 & 4 & 2 & 0 \\ \text { AUG } & -62 & -6 & -23 & -133 & 4 & 6 & 0 \\ \text { SEP } & -42 & 9 & -20 & -64 & 17 & 8 & 1 \\ \text { OCT } & -30 & 21 & -57 & -43 & 45 & 8 & 4 \\ \text { NOV } & -23 & 11 & -74 & -60 & 35 & 8 & -1 \\ \text { DEC } & 82 & 56 & -243 & 29 & 95 & 17 & -2 \\ \text { TOTAL } & 706 & 787 & -376 & 441 & 1535 & 149 & 122\end{array}$




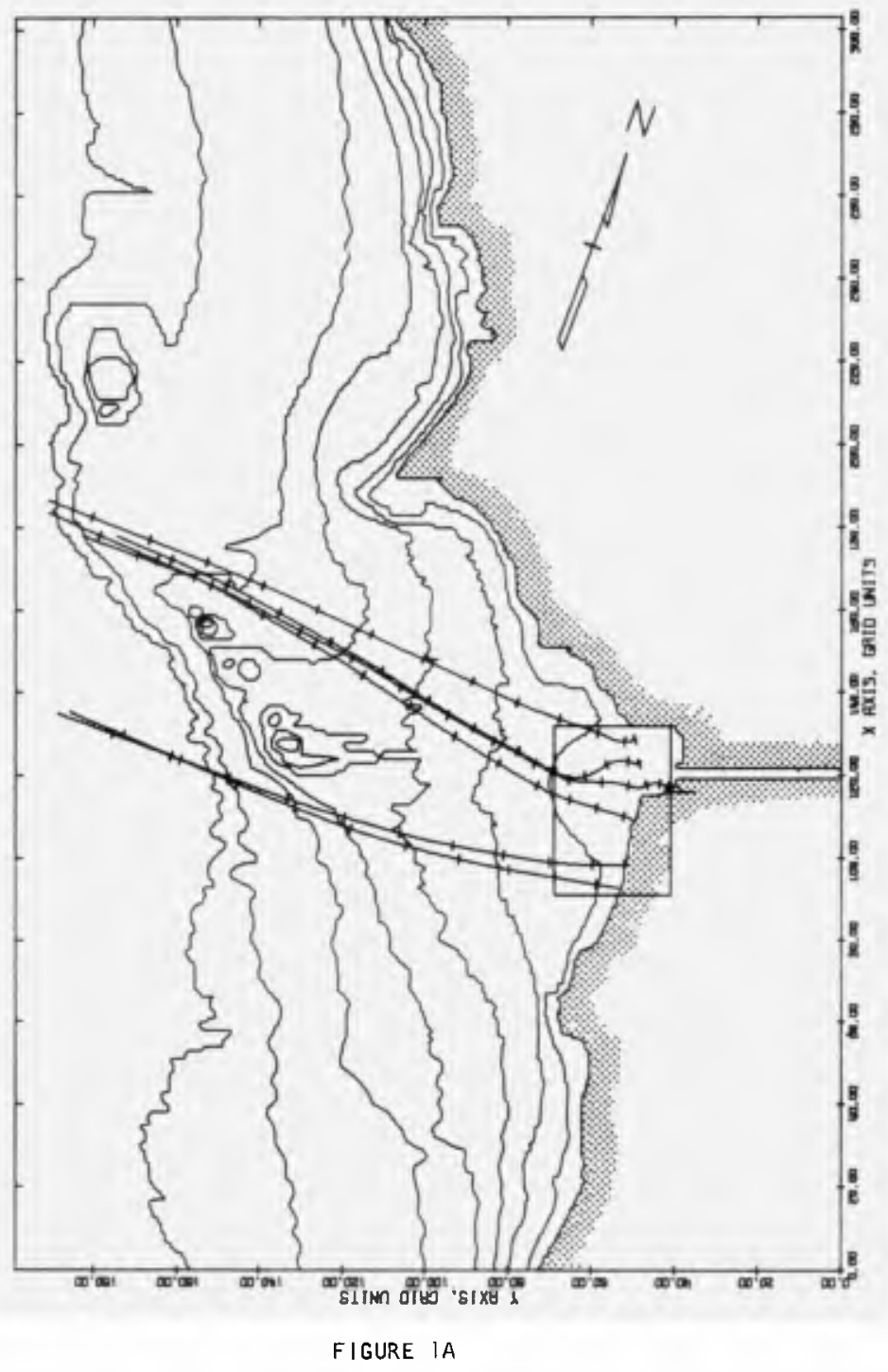

OUTER GRID CONTOUR MAP and

13 SEC. WAVE RAYS TO STAT IONS $1,2,3,4,5,7$ 


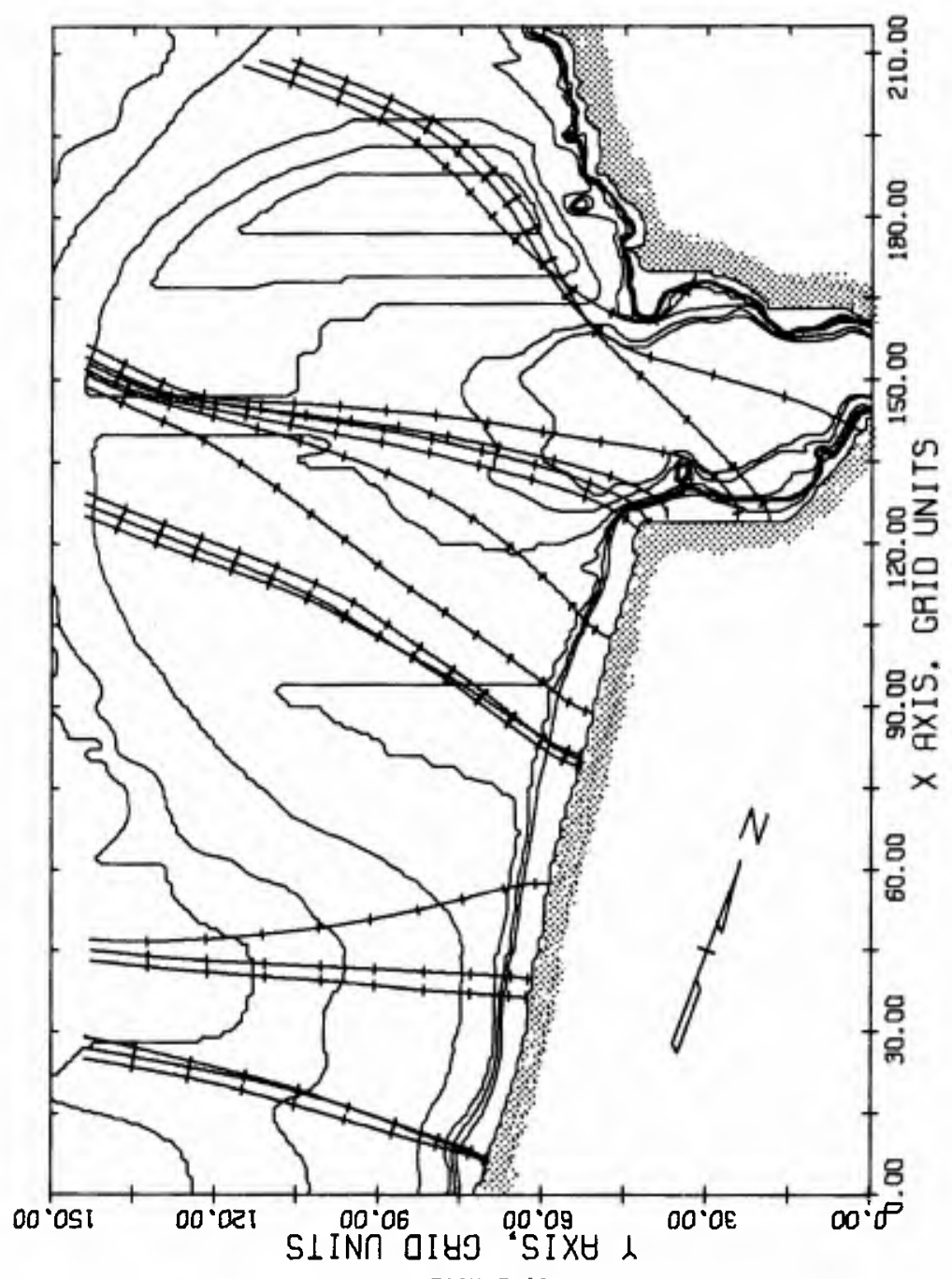

FIGURE IB

INNER GRID CONTOUR MAP and

13 SEC. WAVE RAYS TO STATIONS $1,2,3,4,5,7$ 


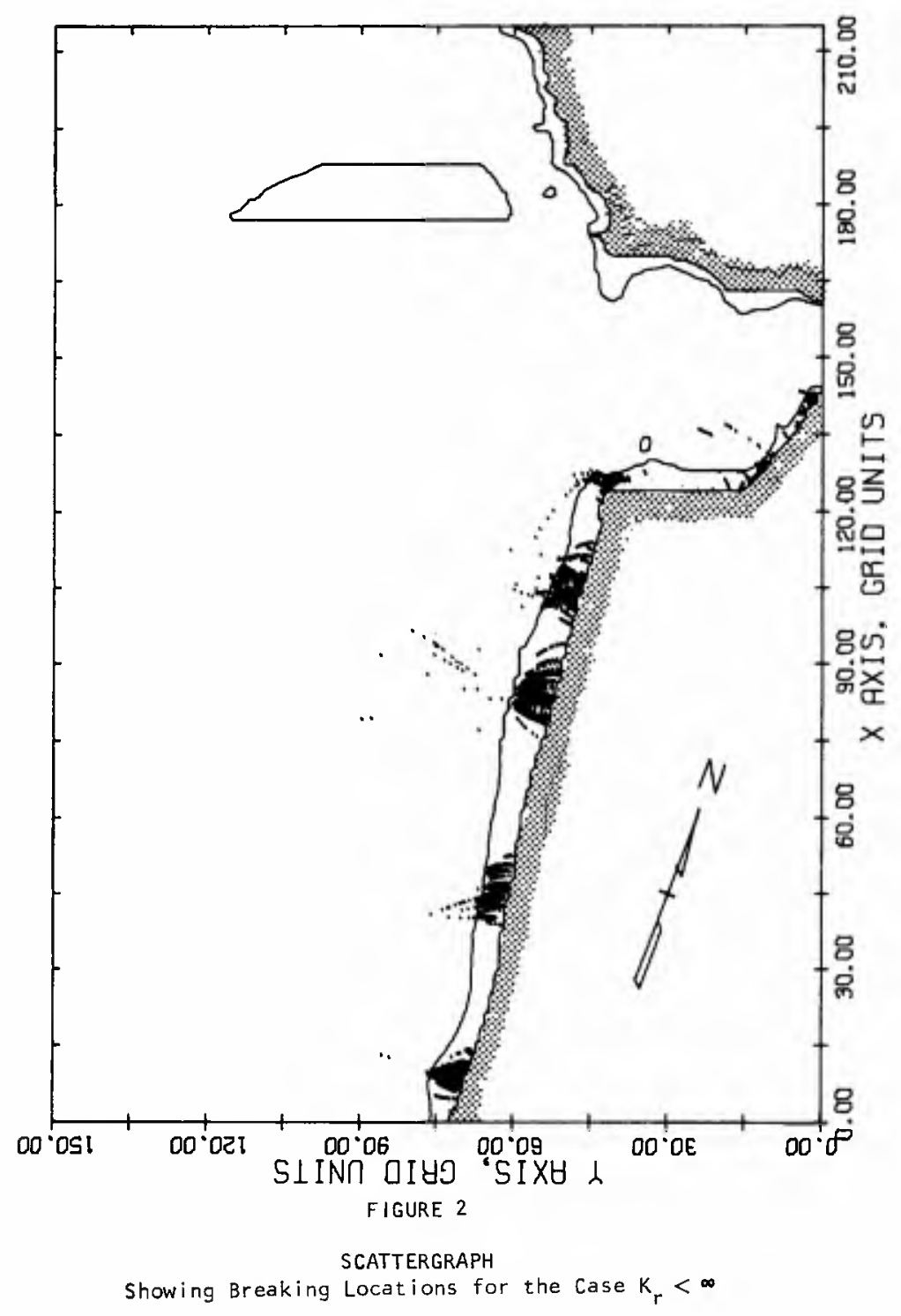





\section{CHAPTER 58}

\section{CRATER-SINK SAND TRANSFER SYSTEM}

by

Douglas L Inman

Scripps Institution of Oceanography, University of California

La Jolla, California 92037

and

Roland W Harris

Dredgıng Engıneer, currently with Leedshıll-Deleuw

Dacca, East Pakıstan

\section{ABSTRACT}

A sand transfer system that requires no surface Impoundıng area and that can be installed and operated at low cost is proposed The system consists of a hydraulic jet assembly operating from the bottom of a sand crater A jet pump and suction mouth are located at the lowest point of a crater-l ike depression dredged into the sea floor The crater acts as a gravity-fed sink for sand and other cohesionless material, thus serving the dual purpose of a mechan Ism for collecting sand and a sub-surface impounding area for the accumulation of sand

\section{INTRODUCTION}

The large volumes of sand transported by waves and currents in the nearshore zone has always presented a serious problem to the coastal engineer (Johnson, 1957) The problem is particularly serious with engineering structures associated with the entrances to harbors and coastal lagoons coastal structures that interrupt the longshore transport of sand produce local areas of accretion on the up-coast side of the structures and corresponding areas of erosion on the down-coast side In time, both the accretion arid the erosion become problems, whose common solutions require that the accreted material be bypassed to the area of erosion Over the years various procedures have been developed to handle the bypassing of sand around coastal structures, (Eaton, 1951, Watts, 1966, Tornberg, 1968)

All of these bypassing procedures require the construct ion of "Impounding" or storage areas where the sand accumulates untll its volume is sufficient to warrant bypassing by suction dredge or other means Impounding requires that the longshore transport of sand be intercepted before it reaches the entrance channel This is usually accomplished by building a suitable structure, which commonly takes one of the forms illustrated in Figure 1 All of these procedures involve expensive construction and maintenance Further, to attain maximum efficiency the impounding areas must be large, thus occupying valuable coastal area 

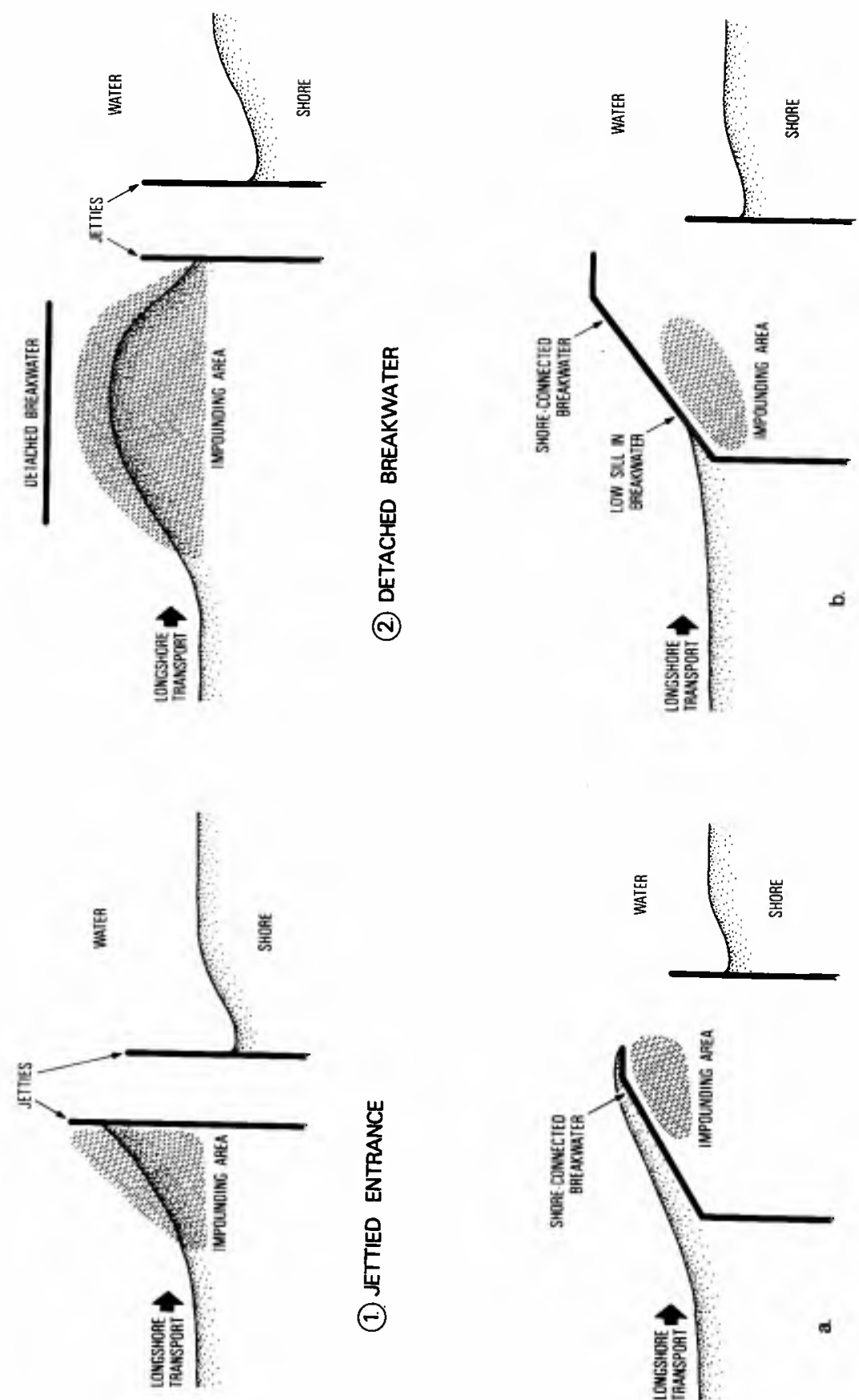
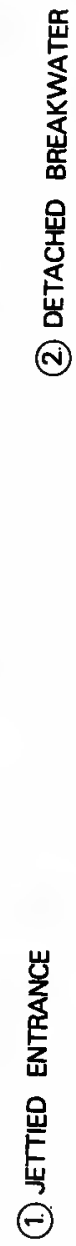

"ก

$8 \overline{0}$ 范 元罾 就文 80 वิ⿵人一

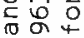

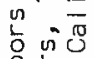
西走 范药

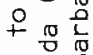
ज: $-\frac{1}{2}$

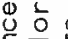
近立至 $+-i$ ब两 드릉요 उᄃ․ 둥 운 $\frac{0}{4} \stackrel{9}{-10}$ in $\begin{array}{ll}0 \\ 0.0\end{array}$

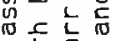
⿰幺 苛蒫 10 兄二远 g) ... ᄂ 독옴모 읃 을그음 든 to 45 요은 呈高范足

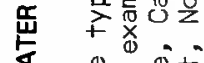
至害京 4 동드 웅고 잉 든온당

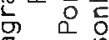

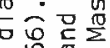
$\because \stackrel{0}{-0}$ to.

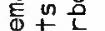

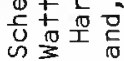
- $\frac{1}{0} \frac{n}{0}$

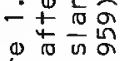
는 
Considerations of the overall budget of sand in the nearshore areas of the world show that the problem has a more basic aspect than just that of sand bypassing Beach sand has become an important natural resource that is diminishing in quantity at an alarming rate This has come about because man has progressively decreased the supply of sand to the beaches by damming rivers and has interrupted its longshore movement with coastal structures on the other hand, the loss of sand from the nearshore zone, either down submar ine canyons or by other means, remains at a high rate (Inman and Frautschy, 1966) Various procedures, such as dredging sand from the continental shelf (Taney, 1965) and pumping sand from coastal impoundment areas (Herron and Harrıs, 1962) have been suggested for supplyıng sand to undernourished beaches

The increasing need for sand bypass systems and for beach nourishment systems makes it apparent that the development of different, more efficient sand transfer systems is an urgent necessity if we are to retain our coastal zone resources Accordingly, a sand transfer system that requires no surface impounding area and that can be installed and operated at low cost is proposed

\section{CRATER-SINK SAND TRANSFER SYSTEM}

The crater-sınk sand transfer system consısts of a hydraulıc jet assembly operating from the bottom of a sand crater The hydraulic jet assembly consists of a suction mouth, a jet pump, a drive-water pipe, and, a delivery pipe that transports the sand-water mixture away from the crater site (Figure 2) Since the suction mouth is located at the lowest point of a crater-like depression in the sea floor, the crater acts as a gravity-fed sink for sand and other cohesionless material Sand transported to the perimeter of the crater will cascade down the sides of the crater to the suction mouth where it becomes avallable for transfer Thus, the crater-sink serves the dual purpose of a mechanism for collecting sand and a sub-surface impounding area for the accumulation of sand When located in the entrances to harbors and inlets, it provides the greatest depth of water in the entrance channel where it is needed Once installed the system is stationary and the only moving parts are in the drive-water pump When required, the jet pump assemblage and delivery pipe can be floated to the surface for maintenance, as discussed in the section on installation

\section{HYDRAULIC JET ASSEMBLY}

Recent developments in jet pump technology now make it practical to use hydraulically driven jet pumps to move sand through pipelines The excessive wear rates caused by the highly abrasive sand-water mixtures have been markedly reduced in recently designed pumps The new designs have resulted from experiments (1) on the optimum shape and angle for the nozzle entry into the throat of the Venturi section of the pump (Figure 3), and, (2) with various types of resistant linings for the 
a PLAN OF ENTRANCE CHANNEL

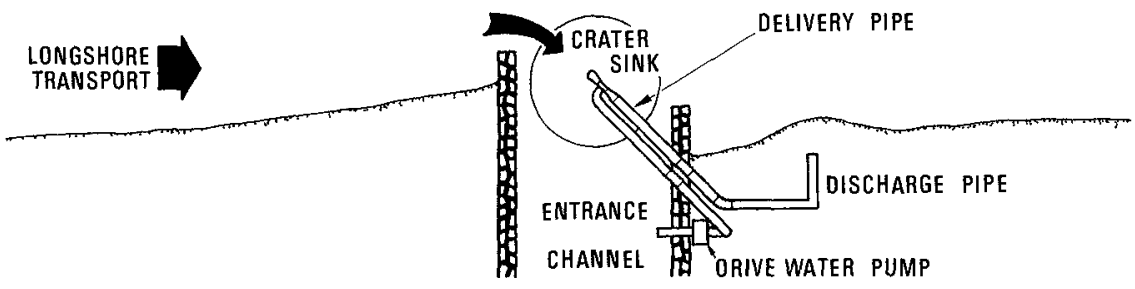

b CROSS SECTION (enlarged)

DISCHARGE PIPE RUBBER SLEEVE

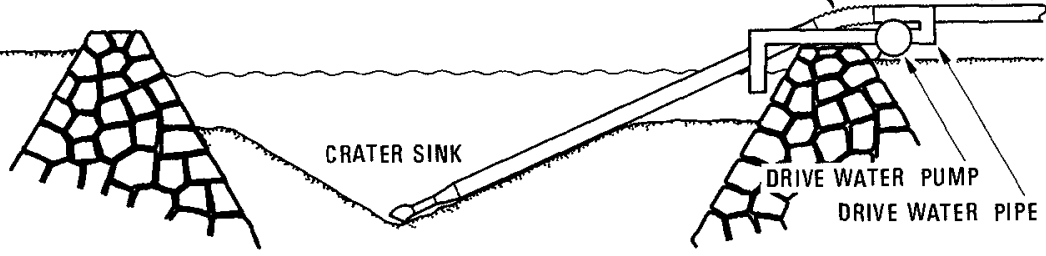

Figure 2 Schematic diagram of crater-sink sand transfer system when used to bypass sand across the entrance to a harbor or lagoon

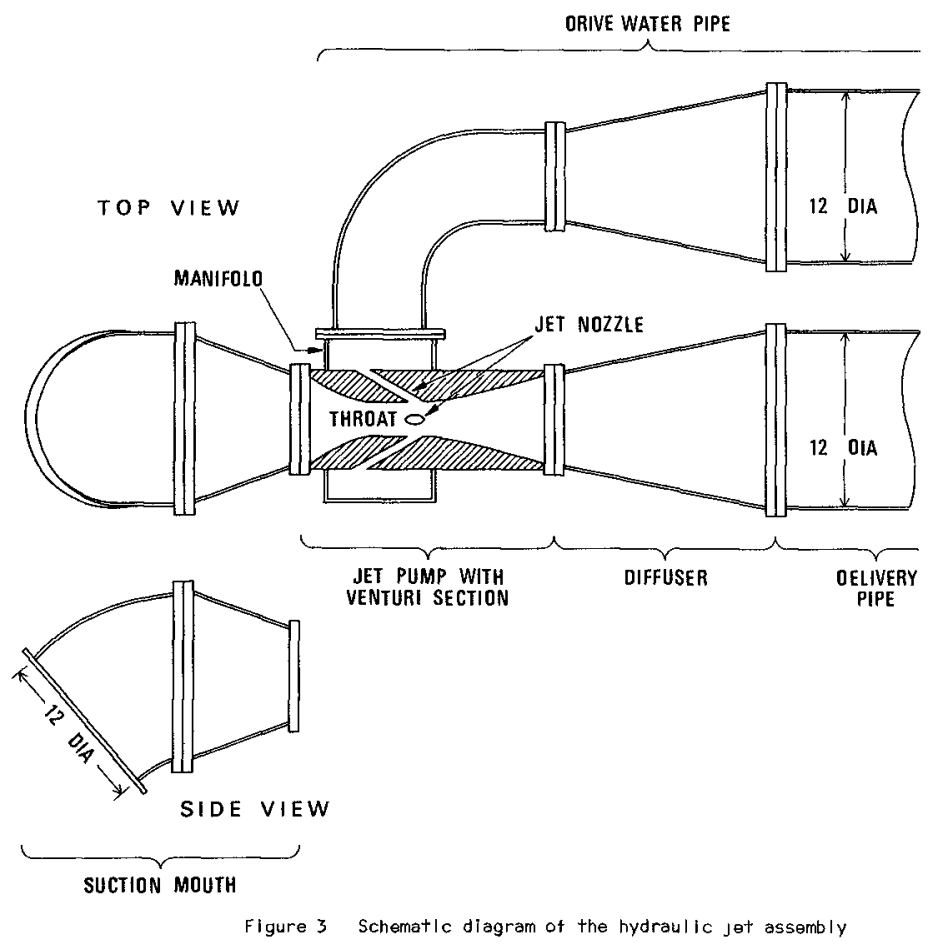


Venturl throat of the pump (D M Frazıer, 1967, W G Fraser, unpubllshed manuscrlpt, J V Barker, 1969)

Improved jet pumps of this type have been in use for several years as suction booster pumps behind the cutter head on suction dredges Experlence by varıous users * ind Icates that the wear life of a jet pump, before requirıng new plastic linıngs, is now measured in millions of cubic yards rather than hundreds of thousands of cubic yards The basic rate of pumping for a jet pump driving a 12-Inch ID delivery pipe would be in excess of one million cubic yards of sand per year This includes approximately $25 \%$ down-tıme for maintenance

The hydraulic jet assembly driving the crater-sınk sand transfer system consists of a suction mouth, jet pump, diffuser, and delivery pipe The jet pump consists of a cylindrical cast steel Venturi section which is fitted with a series of peripheral nozzles (Figure 3) The nozzles are inclined towards the centerl ine of the Venturi throat, and are driven by water supplied to the cyllndrical manifold surrounding the Ventur I section Drive water is supplled to the manifold through a separate pipe which is fed by a water pump

The delivery pipe is coupled to the jet pump by a diffuser which increases the diameter of the Venturi section to that of the delivery pipe The delivery pipe brings the sand to the landing pipe, where it may be discharged at the shorel ine through a short discharge pipe If the sand is to be discharged at a more distant point, then a booster pump driving an extended discharge l Ine must also be installed, as shown in Figure 7

\section{INSTALLATION AND CHARACTERISTICS OF THE CRATER-SINK}

The initial instailation can be made in severai ways if the thickness of cohesionless sand is sufficient to provide a crater of the desired size, the crater can be excavated by the hydraulic jet assembly The assembly could be floated and towed to the site, where it could then be flooded with water and used to excavate itself into place The crater will then be developed as the del ivery pipe is extended from a handling barge or ship (Figure $4 a$ )

If the thickness of cohesionless sand above a consolidated soll horizon is thin, then it will be necessary to excavate a crater of the desired size, using conventional methods such as a suction dredge $w$ th a rotary cutter head once the crater is formed then the jet pump and discharge pipe can be floated over the site, flooded with water and the rig set into position ( $F$ igure $4 b$ )

* For example, jet pumps have been used by Generai Construction co , Portland, Oregon (personal communication), Pacific Dredging Co, Long Beach, Calif (personal communication), Western-Pacific Drédging Co, Portland, Oregon, Utah Dredging Corp, San Francisco, Cailif, and, the $U S$ Corps of Engineers, Portland District (Fraser, unpub(Ished manuscript) Also, a jet pump system for offshore sources of sand has been recommended by Govatos and Zandı (1969) 
In the absence of currents, the crater walls would normally stand at the static angle of repose of the sand which is about 30 degrees Thus, the minimum size of the crater would be that occurring for static conditions, where the diameter would be approximately four times its depth, and the volume of the crater would equal about 42 times the cube of its depth Therefore, a 50 foot deep crater would have a min Imum circular, perimeter with a diameter of about 200 feet, and a volume of $20,000 \mathrm{yd}^{3}$, while a 100 foot deep crater would have a min Imum diameter of 400 feet and a volume of $155,000 \mathrm{yd}^{3}$

The dynamic effects of currents flowing across the sand crater would result in crater slopes that are less than the static angle of repose, and thus increase the periphery and the volume of the crater Also, the effective collecting periphery of the crater-sınk would be extended by the sand transported to the rim of the crater by currents The effect of currents and wave action on crater shape is not clearly understood and should recelve further study

The successful functioning of the crater-sink concept requires that the crater be sited so as to intercept the longshore transport of sand Entrance channels tend to migrate along the coast in the direction of the longshore transport as well as in an on-offshore direction wlth changing wave and current conditions (Inman, 1950, p 15) Thus, the construction of a short up-coast jetty to stabilize the location of the "transport path" of the littoral drift is important in siting the crater-sink (Figure 2) The principal function of a down-coast structure would be the protection of the site for the drive-water pump and the landing and discharge pıpes

\section{Excavation of the Crater Sink with the Jet Unit}

The equipment for this operation is shown schematically in Figure 4a and includes a sultable barge with ground tackle and IIfting gear and a drive-water pump The jet pump end of the assembly of delivery and drivewater pipes is suspended from a barge in such a way as to provide both vertical control and horizontal control of the suction mouth The delivery pipe is fitted with pontoons which support the dellvery pipe for transporting the sand away from the crater site

After the crater is excavated the pipelıne and jet assembly are moved to the beach where the pontoons are removed, the plpel ines are lengthened to suit the installation and stabılıty tanks are attached The shore ends of the drive-water pipe and delivery pipe are blanked off at the landing pipe and air is pumped into both pipes since there is less bouyancy at the jet pump end of the pipes, the suction mouth wlll always float lower than the rest of the plpe, thus effectively preventing a large loss of alr through the suction mouth when the assembly has been floated into the correct position air is vented at the landing pipe position and the jet pump end of the assembly begins to descend, the descent rate being controlled by the residual air in the pipes After 
sinking into position, the discharge pipe is connected to the shore end of the landing pipe, and the drive-water pipe is connected to the drivewater line from the drive-water pump Raising of the hydraulic jet assembly is carried out in the reverse procedure as described above

\section{EXTENSION OF CRATER-SINK BY FLUIDIZATION}

It would appear that the perimeter of the crater-sink could be effectively extended in any desired direction by fluidizing the sand bed $A$ sand body tends to behave as a fluid when the fluid pressure in the pore spaces at any level are equal to or greater than the immersed weight of sand above this level This technique is widely used in industry for the transport and mixing of granular-fluid media (Flood and Lee, 1968) and could be used to extend the effective perimeter of a crater-sınk system without requiring an increase in the depth or width of the crater it would appear that this could be accomplished by installing a length of pipe, fitted with jet holes, that extended from the crater-sink to some distant shoal If the pipe were laid along the bottom with jet holes in the "down-position", then pumping water at a high rate through the pipe would activate the jets and cause the pipe to bury into the sand bed Once in place, a reduced pumping rate could be employed that would fluidize the sand above the pipe, without causing it to jet further into the bed As long as the bottom of the crater is deeper than the shoal the fluidized layers of sand, under the influence of gravity, will flow towards the crater-sink (FIgure 5)

The extension of a crater-sink by long fluidization units appears to be particularly appl icable to long, narrow entrance channels, which commonly result in the formation of shoals outside of the entrance (Figure 6) This application is essentially the reverse of that suggested by Hagyard, et al (1969), which would have employed a one mile long fluidization unit located off Westport Harbor, New Zealand, to transport sand from the entrance channel into deeper water However, the experiments performed by Hagyard, et al (1968) on fluidizing velocities, depth of burial of pipe, and the shape of the fluidized zone above the pipe, should also apply to the crater-sink concept In fact, since the installation proposed here would involve relatively short fluidization units operating over relatively steep slopes and feeding continuous sand sinks, its application should be less critical than that suggested by Hagyard, et al However, the technology for fluidizing natural sand beds has not been perfected, and it is quite apparent that both applications require further study before committing them to prototype conditions (WIIson and Mudie, in preparation) 


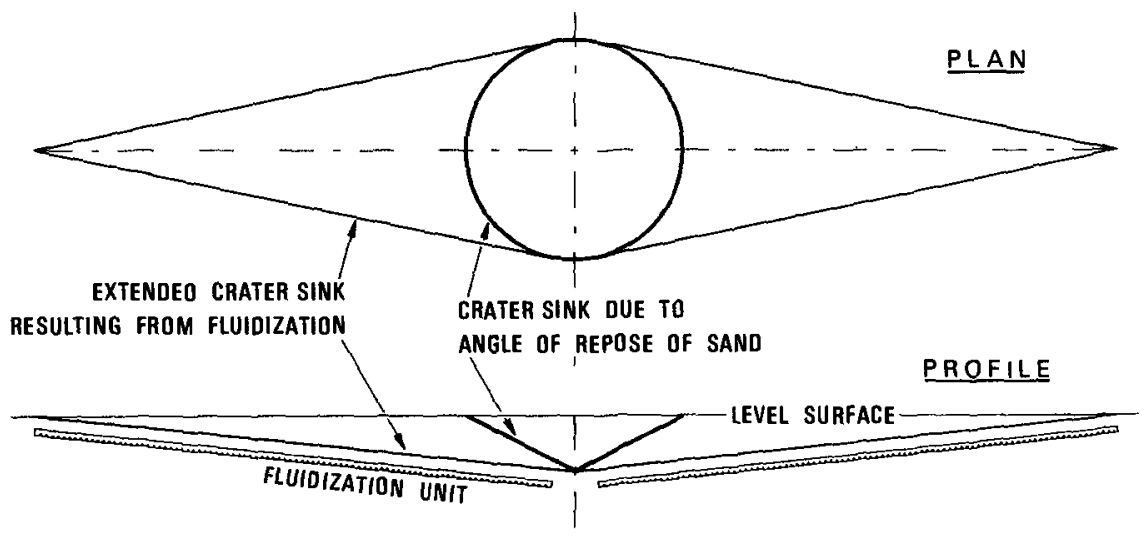

Figure 5 Extension of the periphery of the crater-sink using two fluidization units (Refter to Figure $\theta$ )

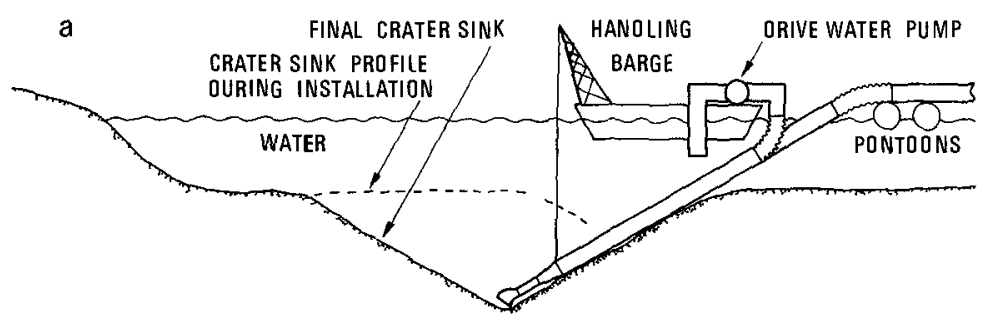

UNCONSDLIOATED SAND

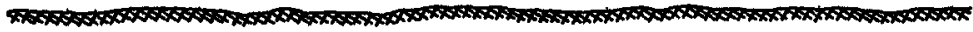
CONSOLIDATEO SOIL

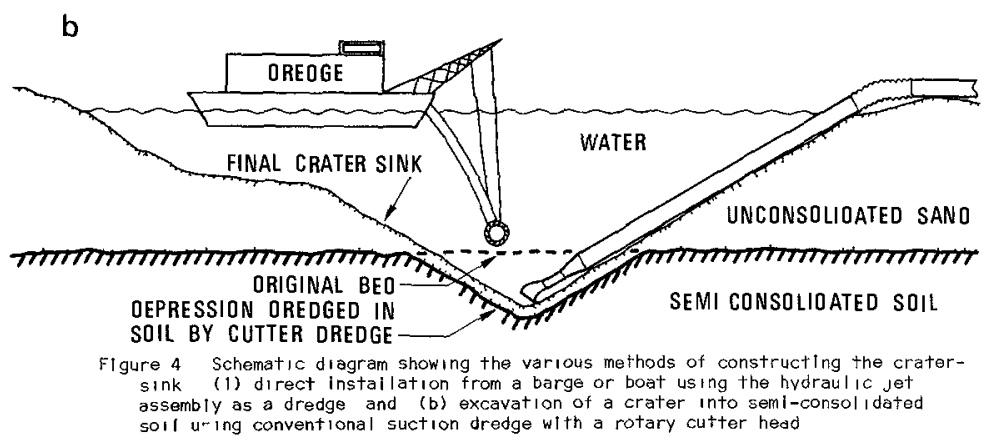


CHARACTERISTICS OF A TYPICAL INSTALLATION AT A SANDY INLET

As stated previously, additional research is warranted before the crater-sink concept and its extension by fluidization is employed in the fleld However, it is instructive to consider the characteristics of such an installation as based on present understanding

Consider the crater-sink sand transfer system that would be required to maintain an entrance channel 20 feet deep and 500 feet wide through an inlet where the net longshore transport is known to be 400,000 cubic yards per year Assume that there are occasional reversals in the direction of transport, amounting to about 100,000 cubic yards, so that the system must be capable of pumping in excess of 500,000 cubic yards per year Further, assume that there is a tendency for a bar to form offshore from the inlet, so that the perimeter of the crater-sınk must be extended for 1000 feet offshore from the end of the up-coast jetty This situation is analogous to that shown schematically in Figure 6 and listed in Table 1, and is a fairly common one along the sandy coastlines of the world

For an inlet of this type a hydraulic jet assembly pumping from the bottom of a 50 foot deep crater would probably provide sufficient perimeter for the basic crater The minimum width of the crater would be about 200 feet as determined by the static angle of repose of the sand, while the dynamic width in the presence of waves and currents would probably be about 300 feet as shown in Figure 6 A vertical lift of 70 feet and a horizontal dellvery distance of about 400 feet is with in the capacity of existing jet pumps If the basic crater can be extended seaward by installation of a 1000 foot long fluidization unit it would then be possible to extend and maintain the channel through the central portion of the offshore bar as shown in Figure 6

The use of turn pipes and rubber sleeves permits accomodation of a variety of bottom profiles, while providing lateral stiffness and maintalnıng alıgnment Life of the equlpment can be extended by exchanging sand delivery and drive-water pipes when the former wear thin

The operating schedule for the jet pump would depend upon demand For example, the pump could be turned on every 24 hours and would then operate until the sand fill in the crater had been removed During times of low wave activity it would operate perhaps one or two hours per day, while during times of pronounced longshore transport it could operate 24 hours per day 
Table 1 (Contınued)

\begin{tabular}{|c|c|c|}
\hline drive-water pump**/ & flow capacity & $\begin{array}{c}2,200 \mathrm{gpm}\left(5 \mathrm{ft}^{3} / \mathrm{sec}\right) \\
250 \text { horsepower }\end{array}$ \\
\hline \multirow[t]{2}{*}{ delivery pipe } & $\begin{array}{l}\text { length (jet pump to } \\
\text { landing pipe }\end{array}$ & $300 \mathrm{ft}$ \\
\hline & diameter I D & $12 \mathrm{in}$ \\
\hline $\begin{array}{l}\text { elevation of discharg } \\
\text { mean water level }\end{array}$ & e point above & $10 \mathrm{ft}$ \\
\hline
\end{tabular}

* For example, jet pump model $12 \mathrm{in}$, manufactured by Pacific Coast Engıneerıng Co, Alameda, Cal ifornia

** For example $2200 \mathrm{gpm}$ at $150 \mathrm{psi}, 250$ horsepower, electric driven water pump, manufactured by Byron-Jackson Pump Co, Los Angeles, Cal I forn Ia 


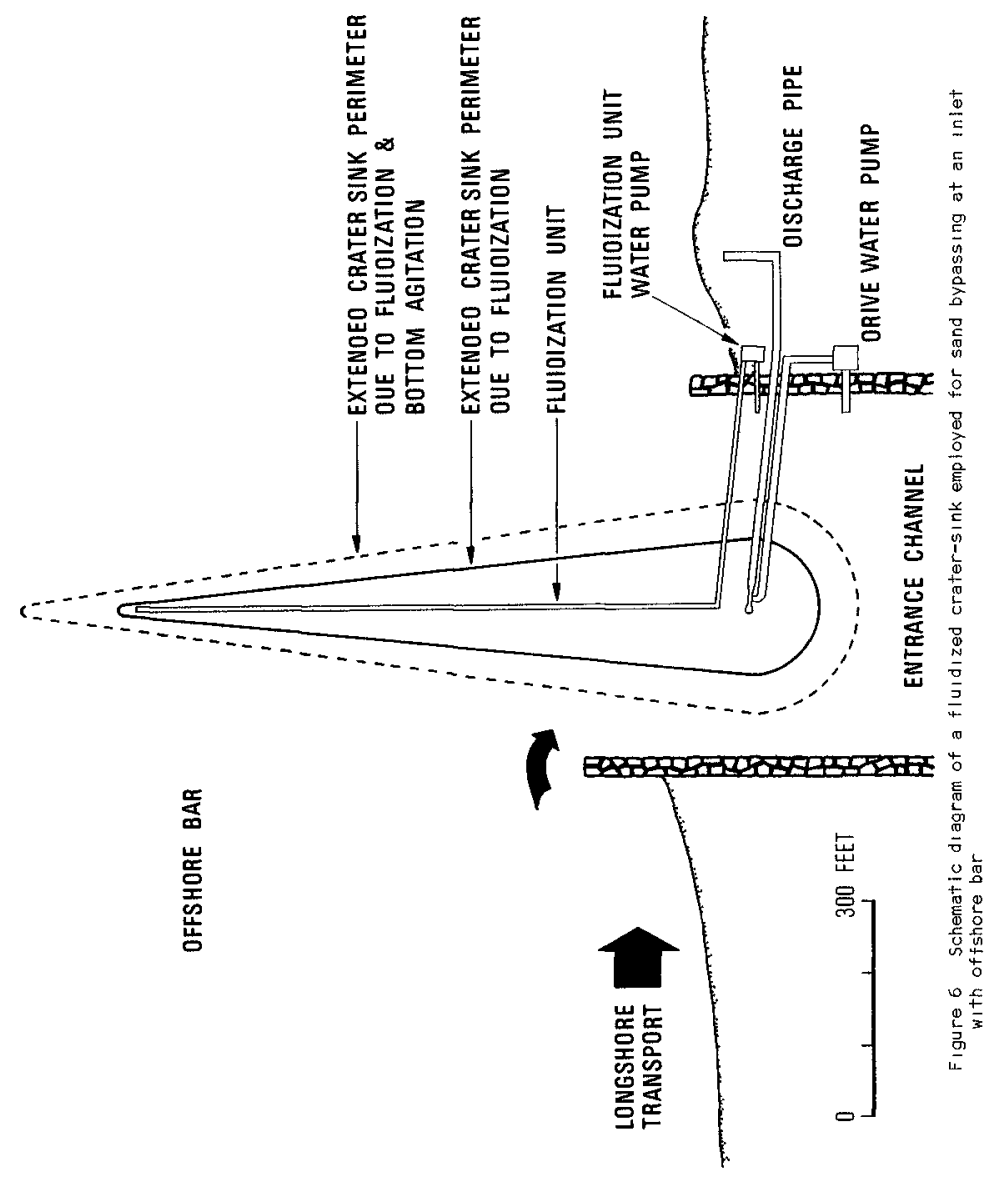




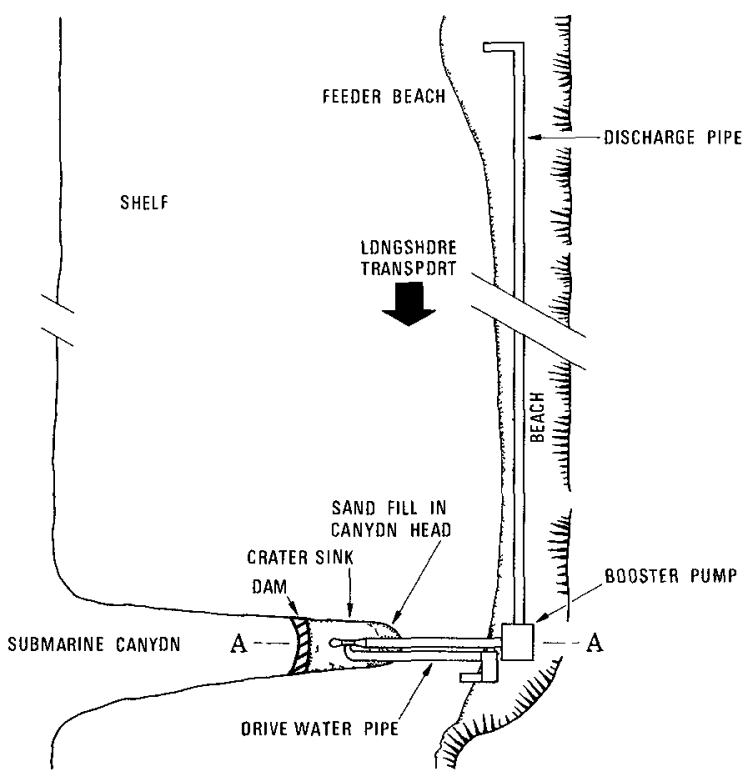

A
BOOSTER PUMP SEA LEVEL

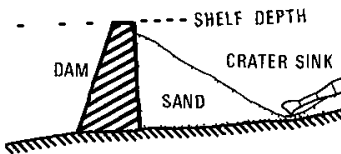

FIguro 7 Schematic diagram of the crater-sink sand transter system used to recycle and in a littoral cell with longshore transport Sand trapped by a sub-surface dam across the head of a submarine canyon is pumped up-coast to a feeder beach where it again becomes aval! able for lonyshore transport 


\author{
CONCLUSIONS
}

The advantages of the crater-sink sand transfer system over conventional dredging appear to include (a) simple, inexpensive equipment requirıng a minimal amount of labor, (b) lower operating costs, (c) no obstruction to navigation, (d) el imination of the up-coast sand impoundIng area, and, $(e)$ the ability to operate throughout the year, thus providing a contınuous bypass operation which maintains the natural drift rates along the coast and eliminates the down-coast zone of erosion

Once perfected, it would appear that a crater-sınk sand transfer system would have a variety of coastal and inland applications in addition to its use at sandy inlets (Figures 2 and 6 ), it would appear to have potential for pumping sediment from dams that have intercepted the supply of sand formerly brought to the coast by streams and rivers Also, the sand transfer system could be used to recycle sand to feeder beaches on a coast with littoral drift Sand trapped on the down-coast end of a beach could be pumped to feeder beaches along the up-coast portions of the beach where it again becomes avallable for longshore transport as III ustrated schematical ly in Figure 7

\title{
ACKNOWLEDGMENTS
}

The authors are indebted to George M Watts, U S Army Corps of Engıneers, Coastal Engıneerıng Research Center, and John D Mudie, Scripps Institution of Oceanography, for valuable suggestions and critical reading of the manuscript This paper represents, in part, application of concepts developed by the University of Cali fornia, San Diego, under contract with the Advanced Research Projects Agency

\section{REFERENCES}

Angas, W M, 1960, "Shark River Inlet sand bypassıng project", Jour Waterways and Harbors Div. Amer Soc Civil Engin, vol 86, WW 3 paper 2599, p 29-47

Barker, J V, 1969, "The PACECO jet stream system", World Dredging and Marine Construction, June p 28-30

Eaton, R W, 1950, "Littoral processes on sandy coasts", Proc First Conf on Coastal Engin, Council on Wave Research, the Engineering Foundation, p 40-54

Flood, H W and B S Lee, 1968, "Fluidizatlon", Scientific American, vol 219 , no 1, p 94-104

Fraser, W G, "Development of a dredge jet pump", Pacific Coast Engineering Company, 5 pp (unpubl Ished) 
Frazıer, D M, 1967, "New dredging techniques usıng an annular jet", Proc of WODCON, World Dredging Conf, p 115-131

Govatos, $G$ and I Zand1, 1969, "Beach nourlshment from offshore sources", Shore and Beach, vol 37, no 2, p 40-49

Hagyard, T, I A GIImour, W D Mottram, 1969, "A proposal to remove sand bars by fluidization", New Zealand Jour ScI, vol 12, p 851-64

Herron, W J Jr, and R L Harrıs, 1966, "Littoral bypassıng and beach restoration in the vicinity of Port Hueneme, Cal if", Proc Tenth Conf Coasta! Engin p 651-675

Inman, D L, 1950, "Beach study in the vicinity of Mugu Lagoon, Cal if", Beach Erosion Board, Corps of Engin, Technical Memo 14, 47 pp

Inman, D $L$ and J D Frautschy, 1966, "Littoral processes and the development of shorel ınes", Coastal Engıneerıng, Santa Barbara Specialty Conference, Amer Soc Civil Engin p $5 \overline{11-36}$

Johnson, J W, 1956, "Dynamics of nearshore sediment movement", Amer Assoc Petrol Geologists Bull, vol 40, p 2211-2232

Johnson, J W, 1957, "The I Ittoral drift problem at shorel ine harbors", Jour Waterways and Harbors Div, Amer Soc Civil Engin, vol 83, WW1, paper 1211, 37 pP

Magnusom, N C and A C Rayner, 1966, "Stabilization of Masonboro Inlet", Shore and Beach, vol 34, no 2, p 36-41

Taney, N E, 1965, "A vanıshing resource found anew", Shore and Beach, vol 33 , no 1, p 22-26

Tornberg, G F, 1968, "Sand bypassıng systems", Shore and Beach, vol 36 , no 2, p 27-33

Watts, G M, 1966, "Trends in sand transfer systems", Coastal Engin Santa Barbara Specialty Conf, Amer Soc Civil Engin, P 799-804

Watts, G M, 1953, "Study of sand movement at South Lake Worth Inlet, Florıda", Beach Erosıon Board, Corps of Engıneers, Technical Memo, 42, $24 \mathrm{PP}$

Weigel, R L, 1959, "Sand bypassing at Santa Barbara, Cal If", Jour Waterways and Harbors Div, Amer Soc Civil Engin, WW 2 paper 2066, $30 \mathrm{pP}$

Wilson, $C R$ and $J$ D Mudie (in preparation), "Comments on the removal of sand bars by fluidızation", New Zealand Jour of Science 



\section{CHAPTER 59}

USE OF VOLCANOES FOR DETERMINATION OF DIRECTION OF LITTORAL DRIFT

By

Per Bruun and Gisli Viggosson

The Technical Unıversity of Norway

Abstract - The title of this paper may sound like a joke Correctly the title ought to be "Determination of Direction of Littoral Drift on the South Coast of Iceland by Geomorphological Approach" In order to check the results of such study based on the movements of river entrances and their geometry the use of an accelerometer buoy to be placed in offshore open waters for collection of wave data combined with the results of meteorological data was discussed Then the volcano Surtsey suddenly emerged from $400 \mathrm{ft}$ depth (Nov 1964) and its huge outpours of volcanic material bullt up an "offshore pole station", where the shoreline development provided some information which supported conclusions from the shoreline study on the mainland computation of wave energy input provided further information

GEOMORPHOLOGICAL APPROACH

OBSERVATIONS OF RIVER OUTLETS

The littoral drift on the Icelandic south coast was investigated by means of topographic surveys and aerial photos including 
Survey by the Danish Geodetic Institute, 1906 Survey by the Danısh Navy, ab 1926

Aerial photography, 1945

Aerial photography, 1960

(Icelandic Survey Dept)

Aerlal photography, 1960 U S Navy

Aerial photography, July 1963 (Icelandic Dept of Lighthouses and Ports)

Aerial photography, Aug 1963 (Icelandic Dept of Lighthouses and Ports)

Most of these surveys were undertaken during the summer period, when the littoral drift because of winds from South East tends to be westward This may have considerable influence on the geographical direction of the outlets of minor rivers on the South Coast whlle the major streams will not change the general orlentation of the outlet which points in the direction of the predominant littoral drift

The results of studies of this material are depicted on Fig 1, indlcating that the littoral drift at Holsa is eastward, that the drift at the shore between Affall until west of Holtsos is neutral, that the drift from west of Jokulsa and up to Dyrholaey probably is eastward although some minor outlets demonstrate westward direction which, as mentioned above, most likely is a seasonal phenomenon Furthermore that the littoral drift just east of Dyrholaey Is westward

Professor Trausta Elnarsson in his article on "Sudurstrond Islands og mundunarsaga hennar" published in Timarit, Verkfrædingafelag Islands (Proceedings of the Icelandic Engineering Association), No 1-2, 1966 in section IV "Radir foksandshola og forsoguleg stała strandarınnar" explains the development of shore and shorelıne configuration west of Dyrholaey from the outlet of Thorsa and up to Reynisfjall towards the East He takes a closer look at the shores at Dyrholaey Based on the development of ancient and recent shorelines it is quite clear that the shore between Klifandi (Fıgs 1 and 3 ) and Skogá (Fıgs 1 and 2) has been a "neutral area", whlch means that the net drift has been relatively small or the drift has taken place in opposite directions 
according to season and in almost equal quantities on a year round basis The sediments whlch washed down to shore by the rivers apparently drifted in part towards the Dyrholaey (Dyrhola-ısland) building up a tombolo (barrier connecting island and main land) and partly westwards towards Vestmannaeyjar (1slands south of Iceland - see Fig 1) which caused the development of another major tombolo inside the wave shadow of these Islands (FIg 2). With enough "patience" and material avallable the Vestmanna Islands would finally become connected to the malnland provided current concentrations between Island and mainland would not make this development impossible

This confirms the results of the observations of direction of outlets mentioned above The orientation of the shoreline west of Dyrholaey is almost constant 27 degrees north of west

OBSERVATIONS OF SHOREIINE DEVELOPMENT OF VOLCANOE SURTSEY

It is in this respect interesting to note the development of shorelines at the volcanoe Surtsey as studied by Thorarınsson (Surtsey Research Progress Reports Nos II and III 1966 and 1967) and by Norrman (Surtsey Research Progress Report No IV, 1968)

Surtsey is a submarine volcanoe, whlch erupted on Nov 14 th, 1963 (Fig 4) at ab 100 meters depth In 6 days an 1sland $600 \mathrm{~m}$ long and almost as wide with top elevation of 60 meters came into existence

Gradually the configuration of the lsland changed to hoof shape, which Immediately after Nov 26 th (FIg 5) normally was open towards the southwest sometımes a barrier blocked the opening, however, but it only lasted, until it was broken down by the surf, or until it was blown away by explosions from the volcanoe After the middle of December the 1sland became nearly circular, later more squared because two sldes developed to be almost parallel as explained below 
Figs 6 and 7 show the development of shorelines at Surtsey during the period from 1964 to 1967 when coarse lava and pebbles normally were avallable in a narrow beach around the island for longs migration by wave action During extreme storms the solid lava could become exposed, however, in certain sections of the shore As it may be seen from the figures, the general trend of shoreline development was towards a rectangular shape with rounded cornes against SW The island has two almost parallel sides running $\mathrm{SW}-\mathrm{NE}$ and an accumulation area on the NE side which developed a lagoon between two beach ridges growing out from SW, typlcal for an "angular foreland". The orientation of the two parallel sldes is given in the figures. It may be seen that the average orlentation of the two parallel sides in 1964 was 27 degrees $\mathrm{E}$ of $\mathrm{N}$, which is identical with the orientation of the shoreline west of Dyrholaey

\section{WAVE ENERGY APPROACH}

An attempt was made to study this situation in a more rational way by evaluating the wave energy input on the south coast of Iceland in order to find the direction of shorelıne with "neutral drıft" No wave energy data were available however. The procedures were based on the Los Angeles formula

$$
Q=\frac{1}{2} k_{1} w e \sin 2 \alpha_{b}
$$

where $Q=$ the total amount of sand moved in littoral arift past a given point per year by waves of given perıod and direction

$\mathrm{w}=$ total work accomplished by all waves of a given period and directıon in deep water durıng an average year

$e=$ wave energy coefficlent at the breaker line for waves of a given period and direction. It is the ratio between the distance between orthogonals in deep water and at the shore line 
$\alpha=$ angle between wave crests at the breaker line and the shore line, or the angle between orthogonals and the normal to the shore line (l e $\alpha=\alpha_{b}$ )

$k_{1}=$ factor depending on dimensional units and empirical relations It varies with beach slope, grain size, and other varıables

The wave energy coefficient may be written $e=\cos \alpha_{0} /$ $\cos \alpha_{b}$ and $\sin 2 \alpha_{b}=2 \sin \alpha_{b} \quad \cos \alpha_{b}$

hence $e \sin 2 \alpha_{b}=2 \cos \alpha_{o} \sin \alpha_{b}$

The relationship between $\sin \alpha_{0}$ and $\sin \alpha_{b}$ for different steepness ratios of the waves is given in Fig 8

Neglecting energy dissipation and reflection the total work may be written

$$
w=\frac{Y{ }_{1 / 3}^{H_{1}} \quad C_{1 / 3}}{16} \mathrm{ft-1bs/sec/ft} \mathrm{of} \mathrm{crest}
$$

Eqs (1), (2) and (3) combined gives

$$
\begin{aligned}
& Q=\frac{1}{2} 6,3 \quad 10^{8} \quad k \quad H_{1 / 3}{ }^{2} \quad T_{1 / 3} \text { e } \sin 2 \alpha_{b} \text { ft-l.bs/ } \\
& \text { year/ft of crest } \\
& \text { (4 a) } \\
& Q=6,3 \quad 10^{8} \quad k \cdot H_{1 / 3} T_{1 / 3} \cos \alpha_{0} \sin \alpha_{b} \\
& \text { ft-lbs/year/ft of crest }
\end{aligned}
$$

where $\mathrm{H}_{1 / 3}$ and $\mathrm{T}_{1 / 3}$ are the slgniflcant wave helght and period

Wind conditions in Iceland are characterized by cyclones moving from sw giving rise to variable wind fields The average duration of a cyclone moving from $\mathrm{SW}$ towards Iceland is 1 to 3 days The predominate direction of wind wave 
propagation is towards NE. Usually the cyclones pass south of Iceland but they may also pass north of Iceland Fig 9 shows the characteristic situation during the winter and sommer seasons Fig 10 demonstrates the characteristic wind direction for the three paths of the cyclones As it may be noted from Fig 10 the cyclones give rise to strong winds from the east when they pass south of Iceland In this situation waves propagate from three directions, SW, $S$ and $E$ Fleld experiments show that high waves from SW occur although the wind has blown from $E$ for some time

Because of the fact that no wave data were avallable and the Los Angeles formula refers to an average year, it was necessary for a preliminary evaluation to use the average wind condltions Avallable wind data are meteorological observations coverıng a perıod of 10 years wind data from three meteorological stations, located in the area between Vestmannaeyjar and Dyrholaey, were statıstıcally evaluated Fig 11 shows frequency dlagram The average wind speed ranged from 12 to 225 knots Hundcastıng was based on the SMB method The problem here, as usual, is to determine the fetch A 225 knots wind generates a fully developed sea at a fetch of about $135 \mathrm{NM}$ (nautical miles) and a duration of about 14 hours. The wave energy $1 \mathrm{~s}$ a function of $\mathrm{H}^{2}$ and $\mathrm{T}$, and the SMB dlagrams indlcate that wind speeds of 12 to 20 knots have no practical influence on the significant wave helght, when the fetch increases from $100 \mathrm{NM}$ to $250 \mathrm{NM}$ However, there is an increase of one second in the significant wave period For waves generated by the cyclones moving from SW, It is therefore realistic to select a fetch of 250 NM for $W$ and $S W$ For the other directions a fetch of 135 NM was selected Thls agrees with results of Danlsh investigations on wave action for the harbour of vestmannaeyjar The results of hindcasting as well as the calculation of the deep water energy is shown in Table 1

Each direction represents a sector of 45 degrees The actual shore boundary conditions lncluding true shore orlentation west of Dyrholaey are shown in Fig 12. In Fig. 13 the shoreline was turned 5 degrees clockwise in order to observe the possible influence of this on the drift direction computed 
on the basis of Input of longshore wave energy

As shown in FIg 12 the $W$ and SE sectors are bounded respectively 39 and 36 degrees, and only half of the $E$ sector is represented west of Dyrholaey Wind direction from the $\mathrm{E}$ tends to concentrate in the area around Dyrholaey, partly due to the Bernoulli effect from the nearby Myrdalsjokull (glacıer) east of Dyrholaey West of Dyrholaey the wind blows along the shore and Increases the longshore wave energy Moreover the wave energy west of Dyrholaey also increases due to a combination of diffraction and refraction at Dyrholaey In this preliminary evaluation, It is difficult to calculate the wave energy from east representing the average year It is possuble however, to estimate roughly the wave energy coefficient " $e$ " In Equation (1)

The maximum input of wave energy is determined approximately by the geometric shadow line whlch gives $e=05^{2}$ The minimum input of wave energy is determined approximately by the 27 degrees diffraction ray which gives a diffraction coefflclent of about 010 approximately $1 \mathrm{~km}$ west of Dyrholaey or $e=01^{2}$ Due to the refraction, one may expect a wave energy coefficlent between $e=05^{2}$ and $e=03^{2}$

Diffracted waves are only of Importance in the area Immediately west of Dyrholaey They break under an angle of approximately 25 degrees with the shoreline Further westwards refraction of waves towards the shore takes place, developing low swells which are superimposed by wind waves corresponding to actual fetches west of Dyrholaey

The numerical calculations carried out in Tables 1-5 with $e=04^{2}$ and ave $H / L=0025$ (Table 1) refer to the area immediately west of Dyrholaey It may be noted that the H/Lratıo plays an important role, and that turning the shoreline 5 degrees clockwise from the actual direction (Fig 13) changes the resultant energy balance from eastward predominance to westward predominance thereby causing westward drift This still refers to the area just west of Dyrholaey Further westward the Importance of $\mathrm{E}$ winds tends to decrease because 
of the shadow by the Dyrholaey headland This in turn would create more tendency to eastward drift Assuming that this is correct, the shoreline should develop slightly convex (turn clockwlse) up towards the Dyrholaey apart from a small area Influenced by leeside erosion just west of the Dyrholapoint As it may be seen from Figures 2 and 3 this is actually the way shoreline configuration developed It is therefore evidenced that the orientation of shoreline of $a b$ 27 degrees $N$ of $W$ is close to the direction which causes neutral drift The correct average direction may be a few degrees more as is in fact also indlcated by the early development of shorelines at surtsey

\section{CONCLUSION}

Although none of the methods used are exact in the true sense of the word, the similarity of the results are noteworthy The development of shorelines of volcanoes popping up from the bottom of the sea, llke Surtsey, may be used to determine the direction of littoral drift on nearby shores As a good luck other methods are avallable, however 
List of figures

\begin{tabular}{|c|c|c|}
\hline $\mathrm{F} \perp \mathrm{g}$ & 1 & $\begin{array}{l}\text { Iceland Iittoral drift investigation on the } \\
\text { Icelandic south coast }\end{array}$ \\
\hline Fig & 2 & From Dyrholaey to Thorsa \\
\hline 19 & 3 & South coast of Iceland The area around Dyrholaey \\
\hline 19 & 4 & Surtsey two days after its eruption, Nov. 16,1963 \\
\hline 19 & 5 & $\begin{array}{l}\text { Surtsey has emerged from the bottom of the sea on } \\
\text { Nov } 26,1963\end{array}$ \\
\hline ig & 6 & Surtsey, Oct 23,1964 \\
\hline Fig & 7 & Outlines of Surtsey during 1965 to 1967 \\
\hline 19 & 8 & $\begin{array}{l}\text { Relationships between } \sin \alpha_{0} \text { and } \sin \alpha_{b} \text { for } \\
\text { different steepness ratios of the waves }\end{array}$ \\
\hline F19 & 9 & $\begin{array}{l}\text { Characteristic cyclones and dominating wind } \\
\text { directions }\end{array}$ \\
\hline Fig & 10 & $\begin{array}{l}\text { Characteristic wind direction for the three paths } \\
\text { of the cyclones }\end{array}$ \\
\hline FIg & 11 & Wind frequency diagrams, the south coast of Iceland \\
\hline Fig & 12 & The shore boundary condıtions west of Dyrholaey \\
\hline FIg & 13 & $\begin{array}{l}\text { The shore boundary conditions west of Dyrholaey } \\
\text { when the shore line is turned } 5 \text { degrees clockwise }\end{array}$ \\
\hline
\end{tabular}

\section{Ilst of tables}

Table 1 Hindcasting

Table 2 e $\sin 2 \alpha_{b}$ corresponds to Fig 12 for various steepness ratios

Table 3 e $\sin 2 \alpha_{b}$ corresponds to Fig 13 for various steepness ratios

Table 4 Littoral drift west of Dyrholaey (Solution of $\mathrm{Eg}(4$ a) and $(4 \mathrm{~b}))$

Table 5 Littoral drift west of Dyrholaey when the shoreline is turned 5 degrees clockwise (Solution of Eq (4 a) and (4 b)) 
Table 1 Hindcasting

\begin{tabular}{|c|c|c|c|c|c|c|c|}
\hline Direction & Wind & Fetch & $\mathrm{H}_{1 / 3}$ & $\mathrm{~T}_{1 / 3}$ & $\mathrm{H}_{1 / 3 / \mathrm{L}_{1 / 3}}$ & Duration & $\mathrm{w}_{\mathrm{Eq}}$ \\
\hline & Knots & NM & ft & sec & & hours & $f t-1 b s / f t /$ year \\
\hline$E$ & 225 & 135 & 85 & 79 & $\begin{array}{ll}0 & 027\end{array}$ & 14 & $360010^{8}$ \\
\hline $\mathrm{SE}$ & 125 & 135 & 36 & 58 & $0 \quad 021$ & 20 & $474 \quad 10^{8}$ \\
\hline $\mathrm{S}$ & 120 & 135 & 3.3 & 57 & 0020 & 21 & $39010^{8}$ \\
\hline SW & 145 & 250 & 50 & 73 & $0 \quad 018$ & 30 & $115010^{8}$ \\
\hline w & 120 & 250 & 35 & 65 & 0019 & 35 & $500 \quad 10^{8}$ \\
\hline
\end{tabular}

Table $2 e \sin 2 \alpha_{b}$ corresponds to Fig 12 for various steepness ratios

\begin{tabular}{|c|c|c|c|c|c|c|c|c|}
\hline \multicolumn{2}{|c|}{ Fıg 12} & \multicolumn{3}{|c|}{ Fig 8} & \multicolumn{4}{|c|}{$e \sin 2 \alpha_{b}=2 \cos \alpha \sin \alpha_{b}$} \\
\hline \multirow{2}{*}{ Direction } & \multirow{2}{*}{$\alpha_{0}$} & \multicolumn{3}{|c|}{$\mathrm{H} / \mathrm{L}$} & \multicolumn{4}{|c|}{$\mathrm{H} / \mathrm{L}$} \\
\hline & & 002 & & 03 & 0 & 02 & 0 & 03 \\
\hline$S-43^{\circ} \mathrm{E}$ & $70^{\circ}$ & $\left.\begin{array}{ll}0 & 3\end{array}\right]$ & & 37 & & 212 & 0 & 253 \\
\hline$S$ & $27^{\circ}$ & 019 & 0 & 23 & & 321 & 0 & 41 \\
\hline SW & $18^{\circ}$ & 013 & 0 & 16 & 0 & 24 & 0 & 304 \\
\hline$w-3^{\circ} \mathrm{s}$ & $60^{\circ}$ & $0 \quad 32$ & 0 & 373 & 0 & 32 & 0 & 37 \\
\hline
\end{tabular}

Table 3 e sin $2 \alpha_{b}$ corresponds to Flg 13 for varıous steepness ratios

\begin{tabular}{|c|c|c|c|c|c|c|c|c|c|}
\hline \multicolumn{2}{|c|}{ Fig 13} & \multirow{2}{*}{\multicolumn{4}{|c|}{$\frac{F \perp g 8}{H / L}$}} & \multicolumn{4}{|c|}{$e \sin 2 \alpha_{b}=2 \cos \alpha \sin \alpha_{b}$} \\
\hline \multirow{2}{*}{ Direction } & \multirow[b]{2}{*}{$\alpha_{b}$} & & & & & \multicolumn{4}{|c|}{$\mathrm{H} / \mathrm{L}$} \\
\hline & & 0 & 02 & & 03 & 0 & 02 & 0 & 03 \\
\hline$S-40^{\circ} \mathrm{E}$ & $72^{\circ}$ & 0 & 31 & & 366 & 0 & 192 & 0 & 229 \\
\hline s & $32^{\circ}$ & 0 & 215 & & 262 & 0 & 365 & 0 & 434 \\
\hline SW & $13^{\circ}$ & 0 & 092 & & 118 & 0 & 179 & 0 & 23 \\
\hline$W-3^{\circ} \mathrm{s}$ & $55^{\circ}$ & 0 & 312 & & 364 & 0 & 358 & 0 & 418 \\
\hline
\end{tabular}


Table 4 - Littoral drift west of Dyrholaey Fig 12 (Solution of Eq $\left(\begin{array}{lll}4 & \mathrm{a}\end{array}\right)$ and $\left(\begin{array}{ll}4 & \mathrm{~b}\end{array}\right)$ )

\begin{tabular}{|c|c|c|c|c|}
\hline \multirow{3}{*}{ Direction } & \multirow{3}{*}{$\alpha_{0}$} & \multirow{3}{*}{$\begin{array}{l}\text { Reduction } \\
\text { of wave energy } \\
\text { from dom direct }\end{array}$} & \multicolumn{2}{|c|}{ Q in cubic yards per year } \\
\hline & & & \multicolumn{2}{|c|}{$\mathrm{H} / \mathrm{L}$} \\
\hline & & & 002 & 003 \\
\hline $\mathrm{E}$ & $\sim$ & $05\left(\begin{array}{ll}0 & 4\end{array}\right)^{2}$ & $-110 \mathrm{k} 10^{8}$ & $-110 k 10^{8}$ \\
\hline$S-43^{\circ} \mathrm{E}$ & $70^{\circ}$ & $220 / 45=0 \quad 89$ & $-43 k 10^{8}$ & $-51 \mathrm{k} 10^{8}$ \\
\hline & $27^{\circ}$ & 10 & $-63 k 10^{8}$ & $-80 k 10^{8}$ \\
\hline & $18^{\circ}$ & 10 & $+142 k 10^{8}$ & $+175 k 10^{8}$ \\
\hline $\mathrm{w}-3^{\circ} \mathrm{s}$ & $60^{\circ}$ & $2195 / 45=087$ & $+69 k 10^{8}$ & $+80 k 10^{8}$ \\
\hline \multirow{2}{*}{\multicolumn{3}{|c|}{$\begin{array}{l}\text { + means eastward drıft } \\
\text { - means westward drıft }\end{array}$}} & $5 \times 10^{8}$ & $+14 k 10^{8}$ \\
\hline & & & \multicolumn{2}{|c|}{ ave $\quad Q=+5 \mathrm{k} 10^{8}$} \\
\hline
\end{tabular}

Table 5 - Iittoral drift west of Dyrholaey when the shoreline is turned 5 degrees clockwlse Fig 13

(Solution of Eq ( 4 a) and ( 4 b)

\begin{tabular}{|c|c|c|c|c|}
\hline \multirow{3}{*}{ Direction } & \multirow{3}{*}{$\alpha_{0}$} & \multirow{3}{*}{$\begin{array}{l}\text { Reduction } \\
\text { of wave energy } \\
\text { from dom direct }\end{array}$} & \multicolumn{2}{|c|}{ Q in cublc yards per year } \\
\hline & & & \multicolumn{2}{|c|}{$\mathrm{H} / \mathrm{L}$} \\
\hline & & & $0 \quad 02$ & 003 \\
\hline $\mathrm{E}$ & $\sim$ & $05\left(\begin{array}{ll}0 & 4\end{array}\right)^{2}$ & $-110 \mathrm{k} 10^{8}$ & $-110 \mathrm{k} 10^{8}$ \\
\hline$S-40^{\circ} \mathrm{E}$ & $72^{\circ}$ & $218 / 145=08$ & $-36 \mathrm{k} 10^{8}$ & $-43 k 10^{8}$ \\
\hline$S$ & $32^{\circ}$ & 10 & $-72 k 10^{8}$ & $-85 k 10^{8}$ \\
\hline & $13^{\circ}$ & 10 & $+103 k 10^{8}$ & $+132 k 10^{8}$ \\
\hline$w-3^{\circ} \mathrm{s}$ & $55^{\circ}$ & $2195 / 45=087$ & $+78 k 10^{8}$ & $+91 k 10^{8}$ \\
\hline \multirow{2}{*}{\multicolumn{3}{|c|}{$\begin{array}{l}\text { + means eastward drıft } \\
\text { - means westward drıft }\end{array}$}} & $-37 \mathrm{k} 10^{8}$ & $-15 \mathrm{k} 10^{8}$ \\
\hline & & & \multicolumn{2}{|c|}{ ave $Q=-26 \mathrm{k} 10^{8}$} \\
\hline
\end{tabular}



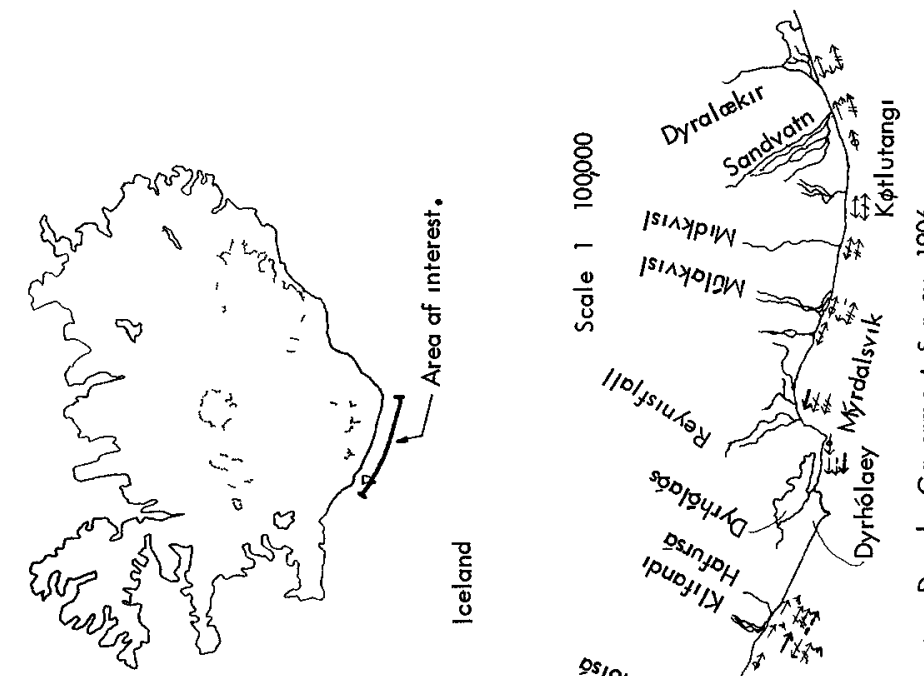

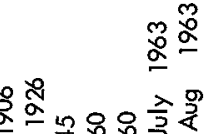

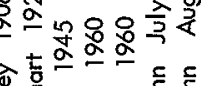

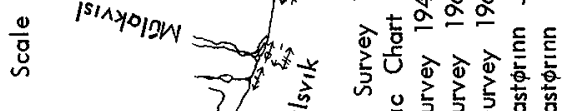

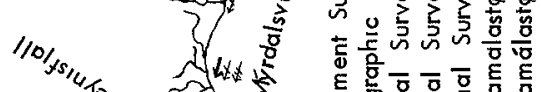
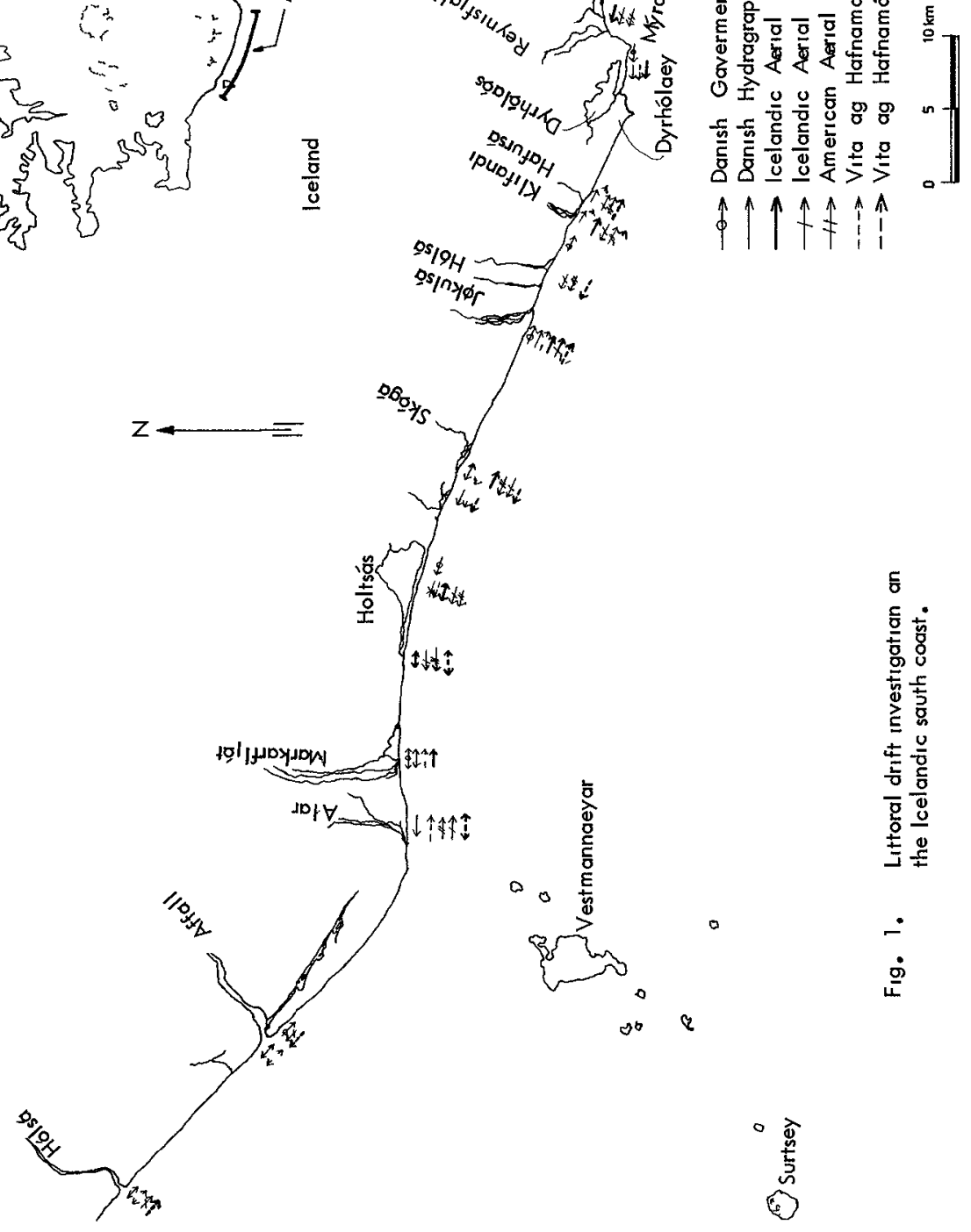

$\hat{\uparrow} \uparrow \uparrow \hat{\imath} \hat{1}$ 


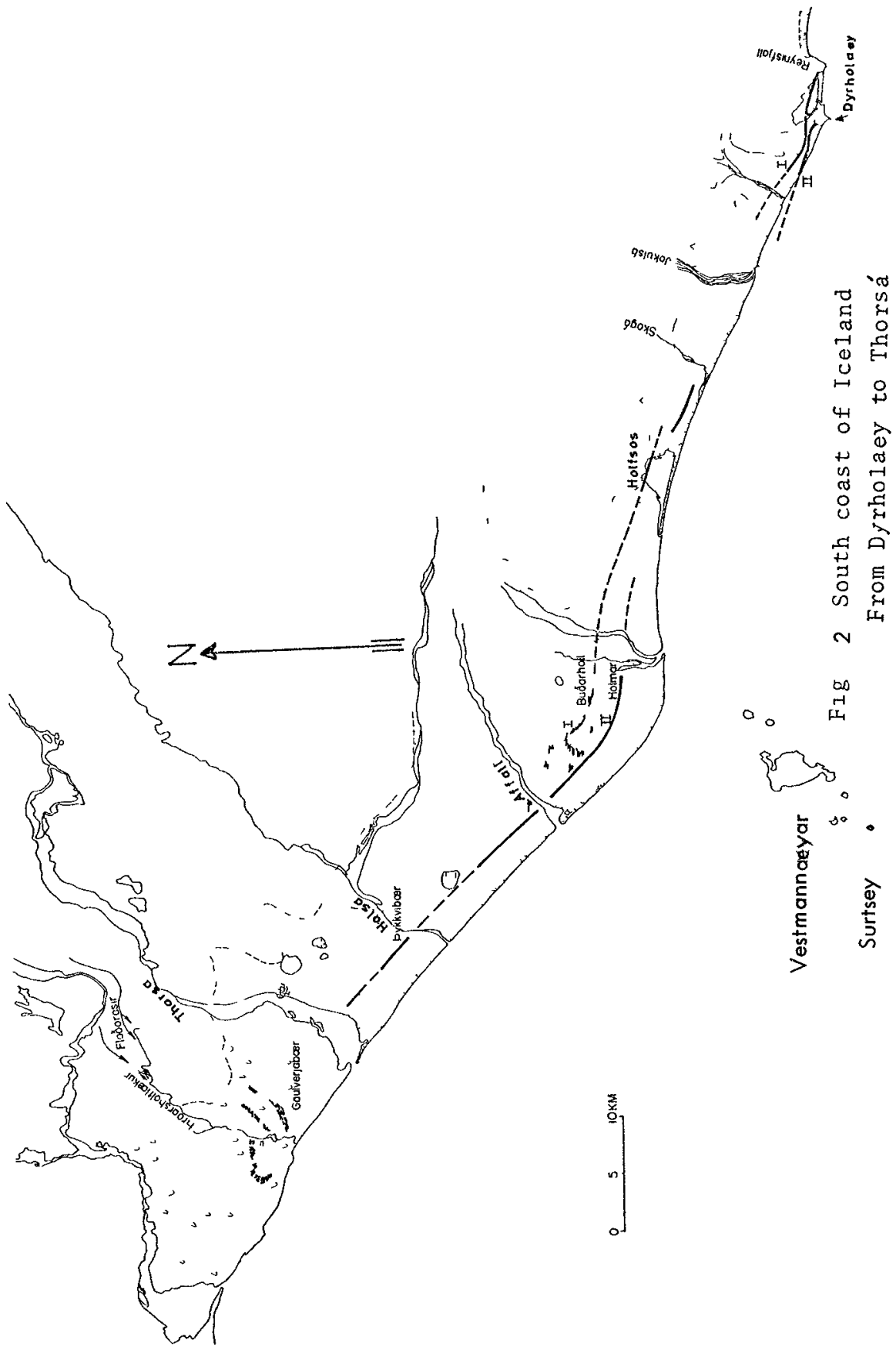




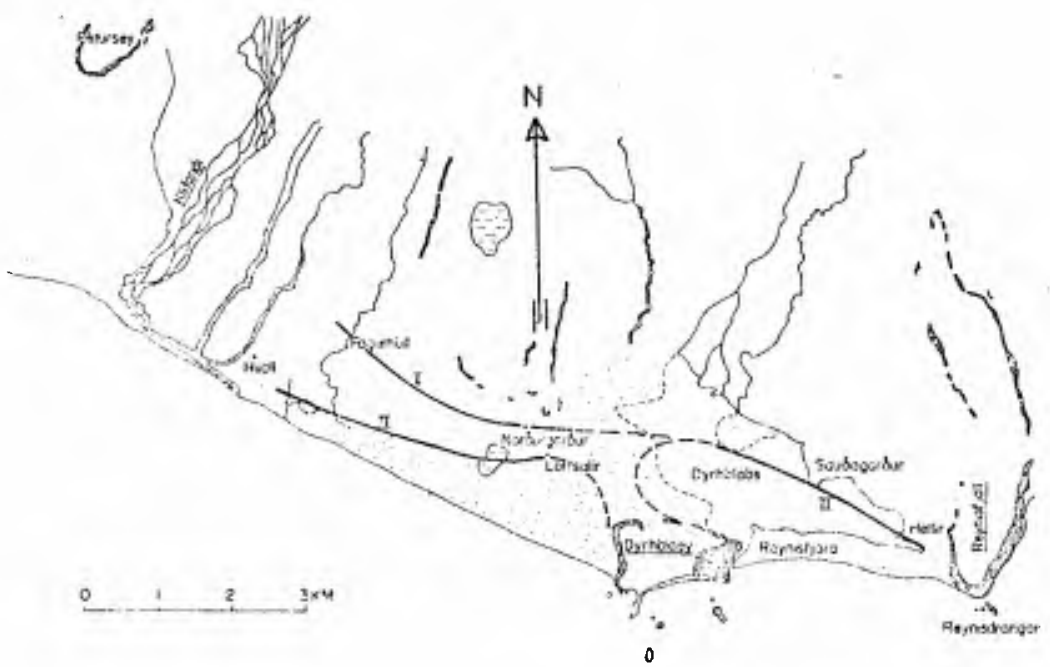

Fig. 3 South coast of Iceland

The area around Dyrholaey

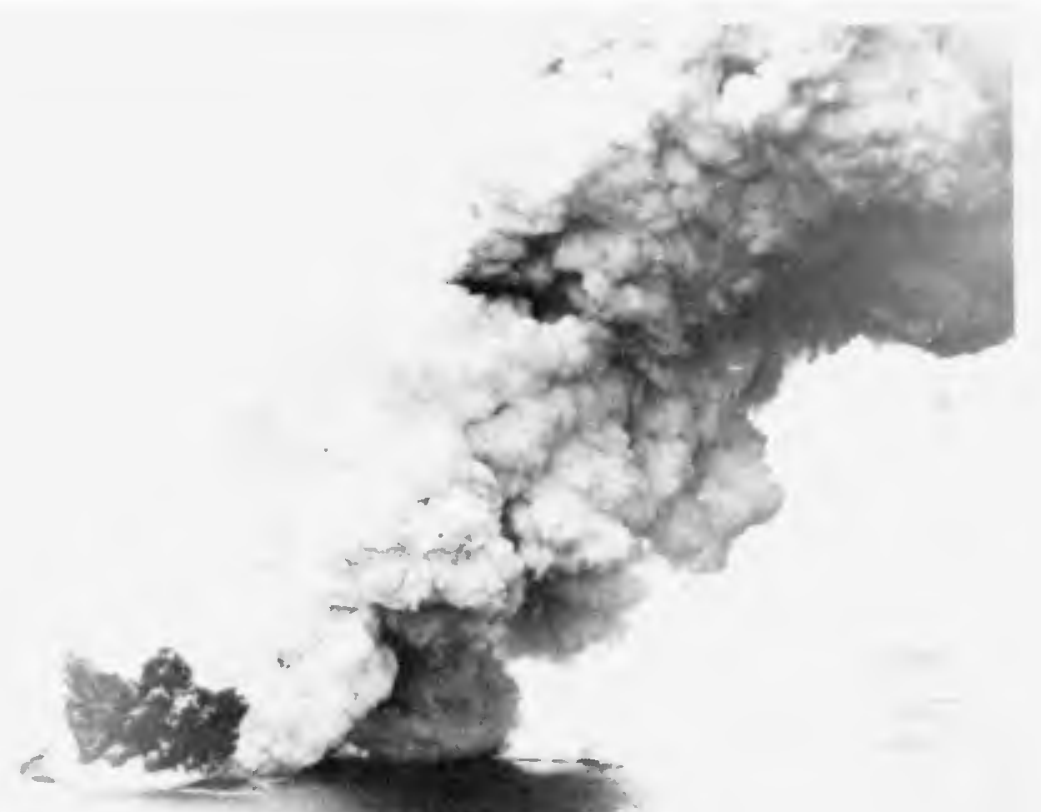

Fig. 4. Surtsey two days after its eruption, Nov. 16, 1963 


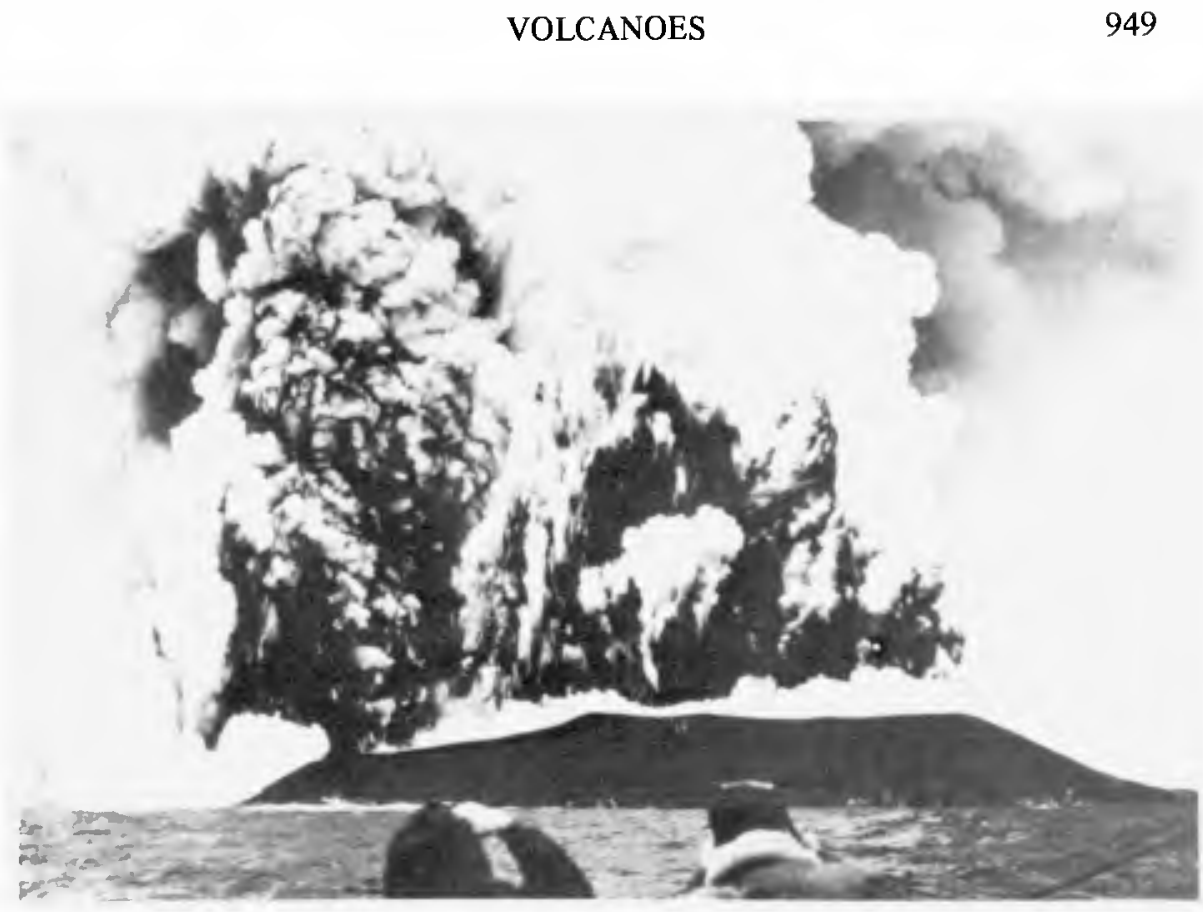

Fig. 5. Surtsey has emerged from the bottom of the sea on Nov. 26, 1963

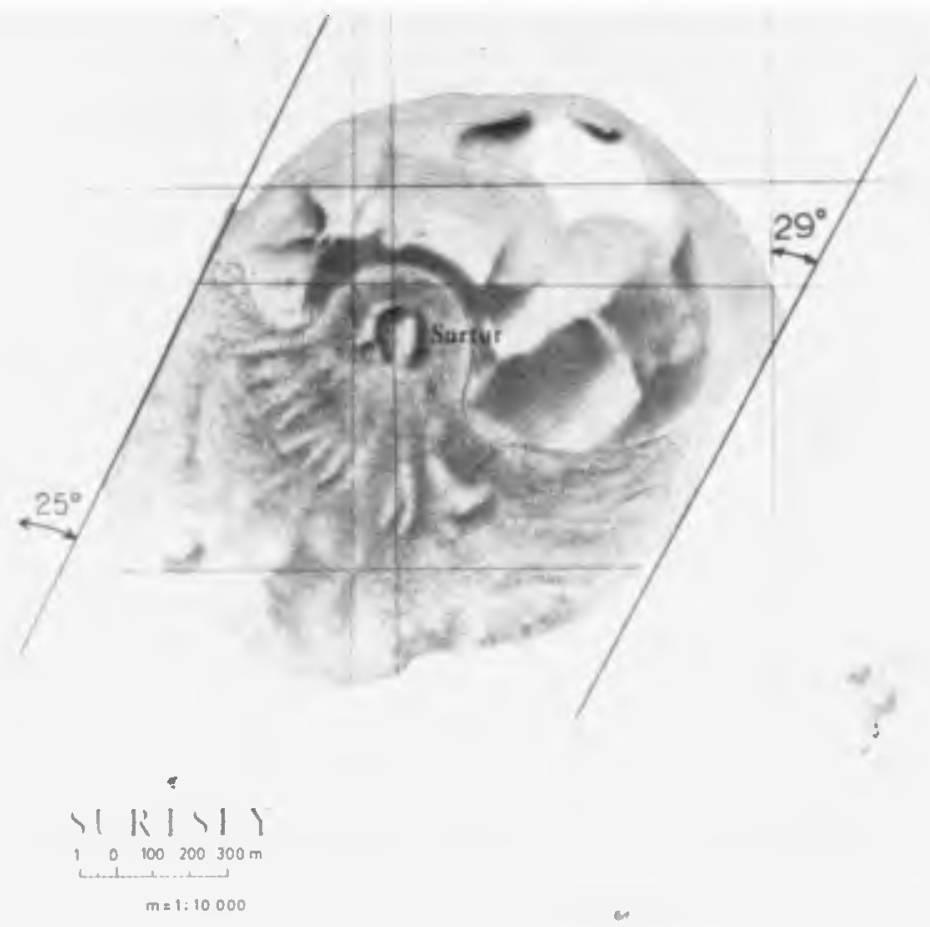

Fig. 6. Surtsey, October 23, 1964 


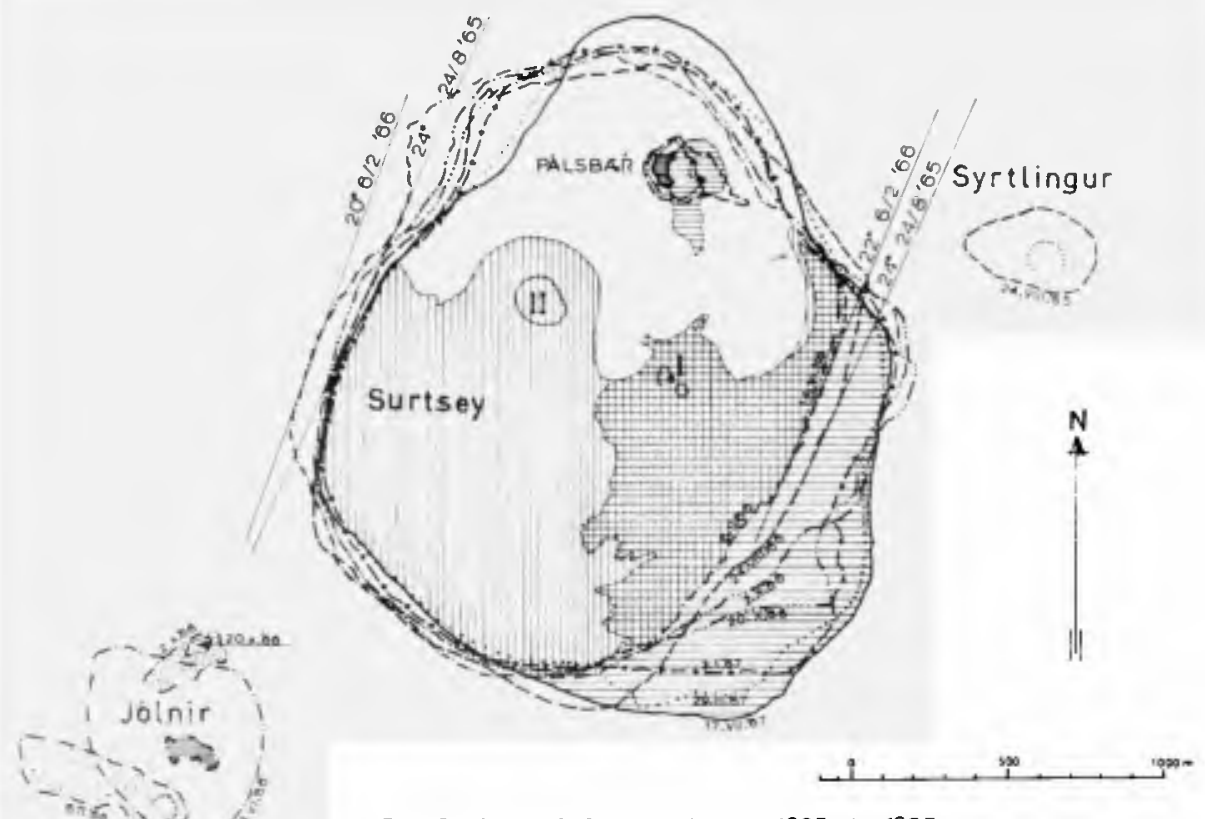

Fig. 7 Outlines of Surtsey during 1965 to 1967.

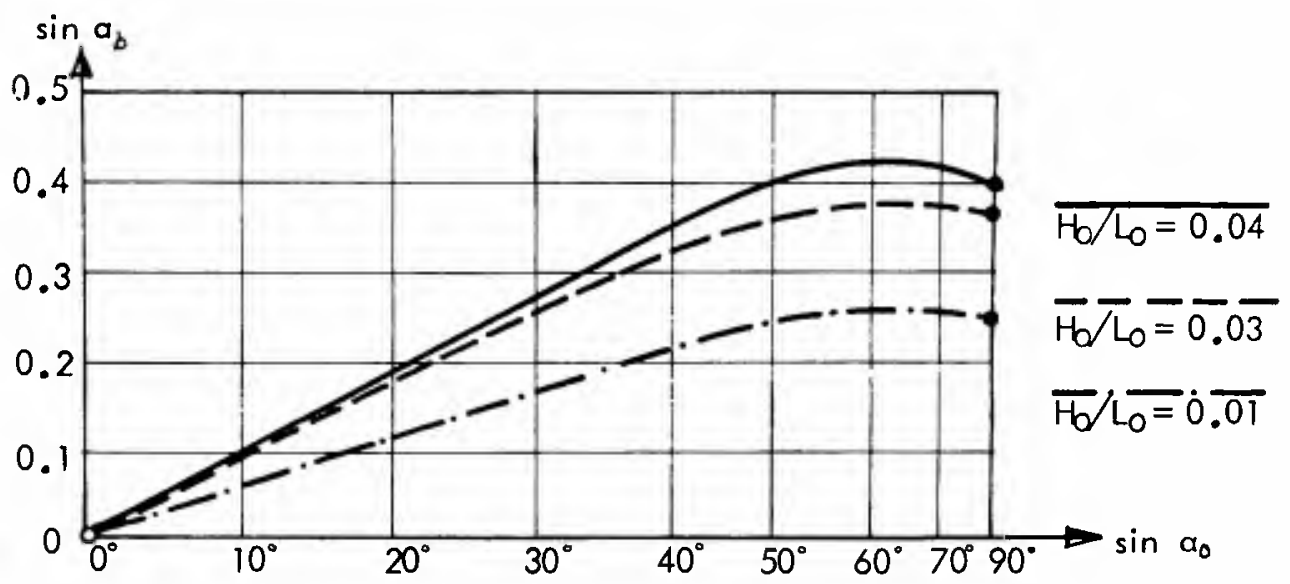

Fig. 8 .

Relationships between $\sin \alpha_{b}$ and $\sin \alpha_{b}$ for different steepness ratios of the waves. 


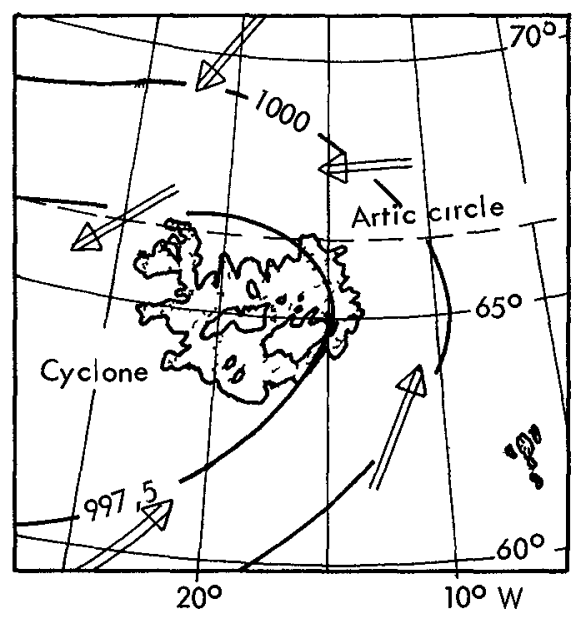

JANUARY

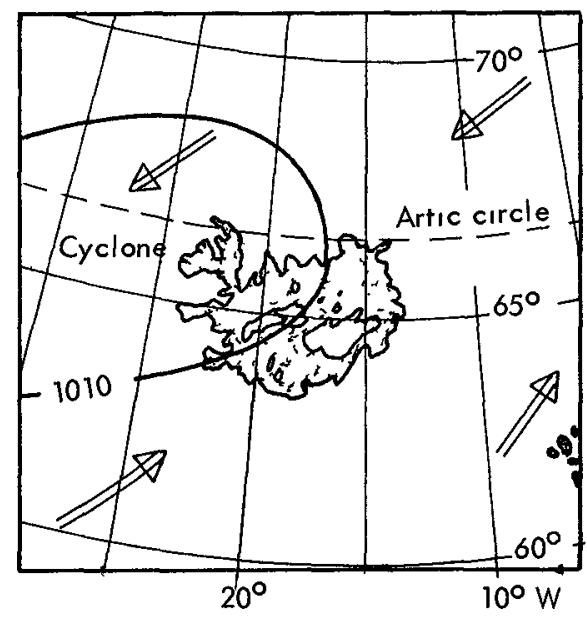

JULY

FIG 9 Choracteristic cyclones ond dominot wind directions
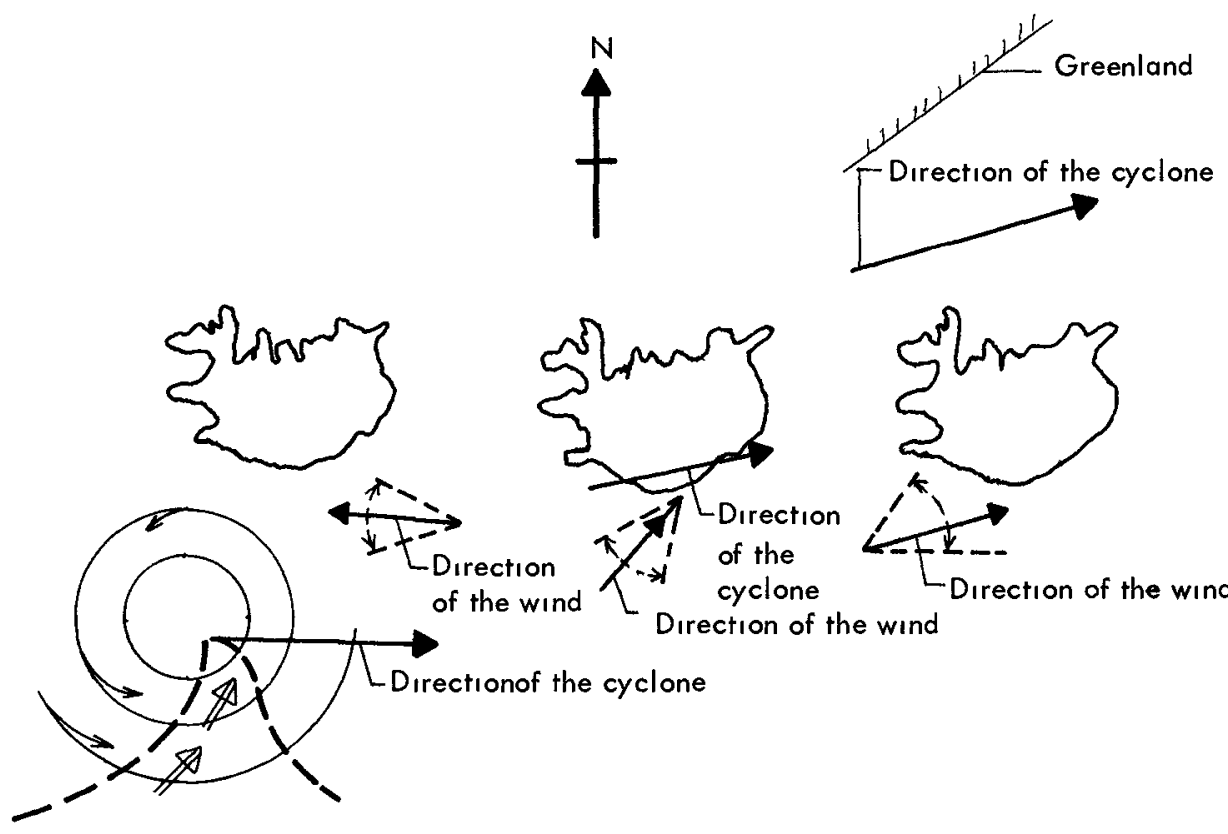

FIG 10 Charocteristic wind direction for the tree paths of the cyclones 


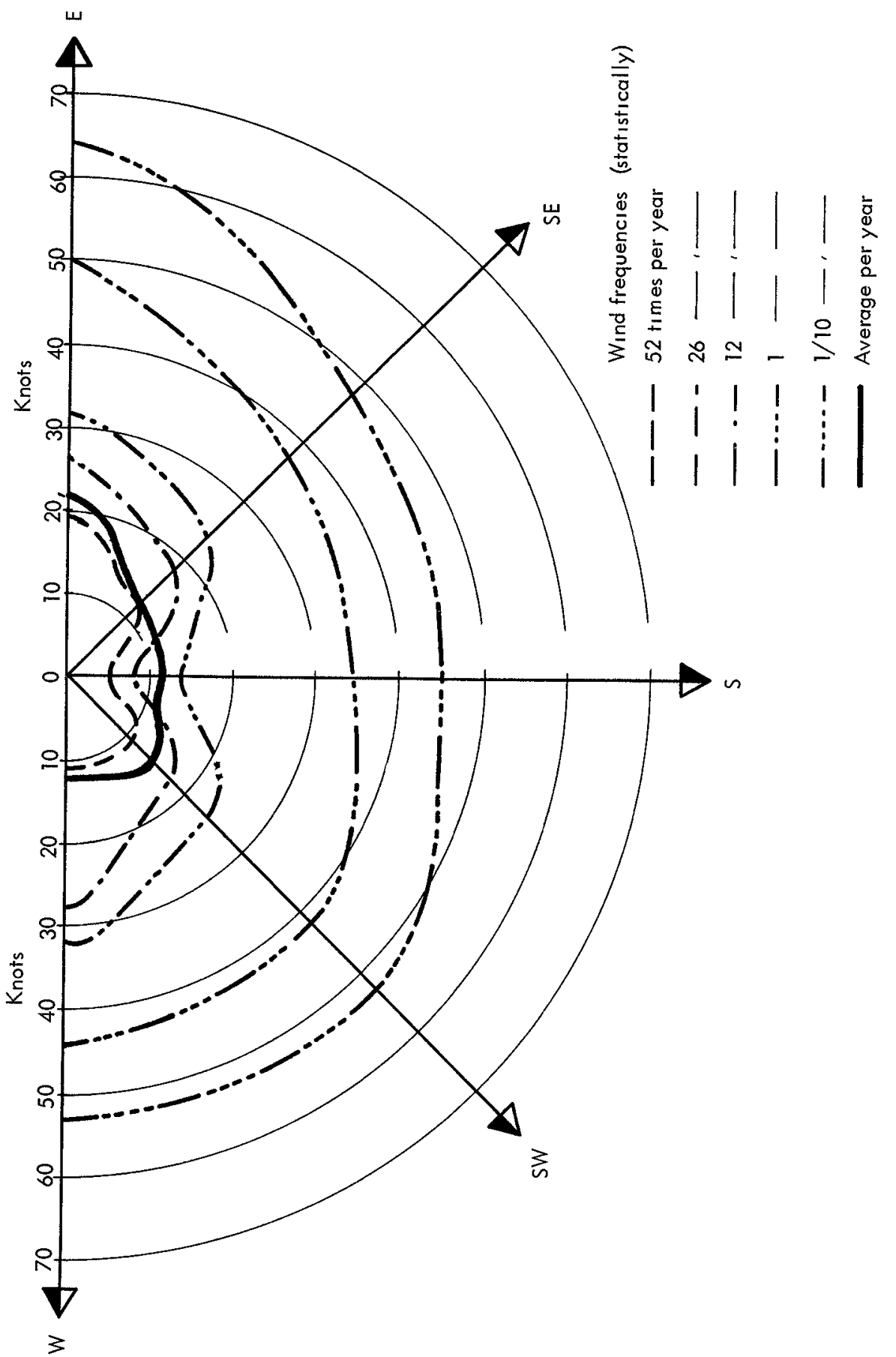

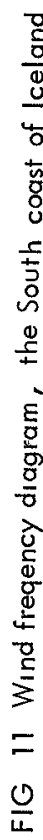




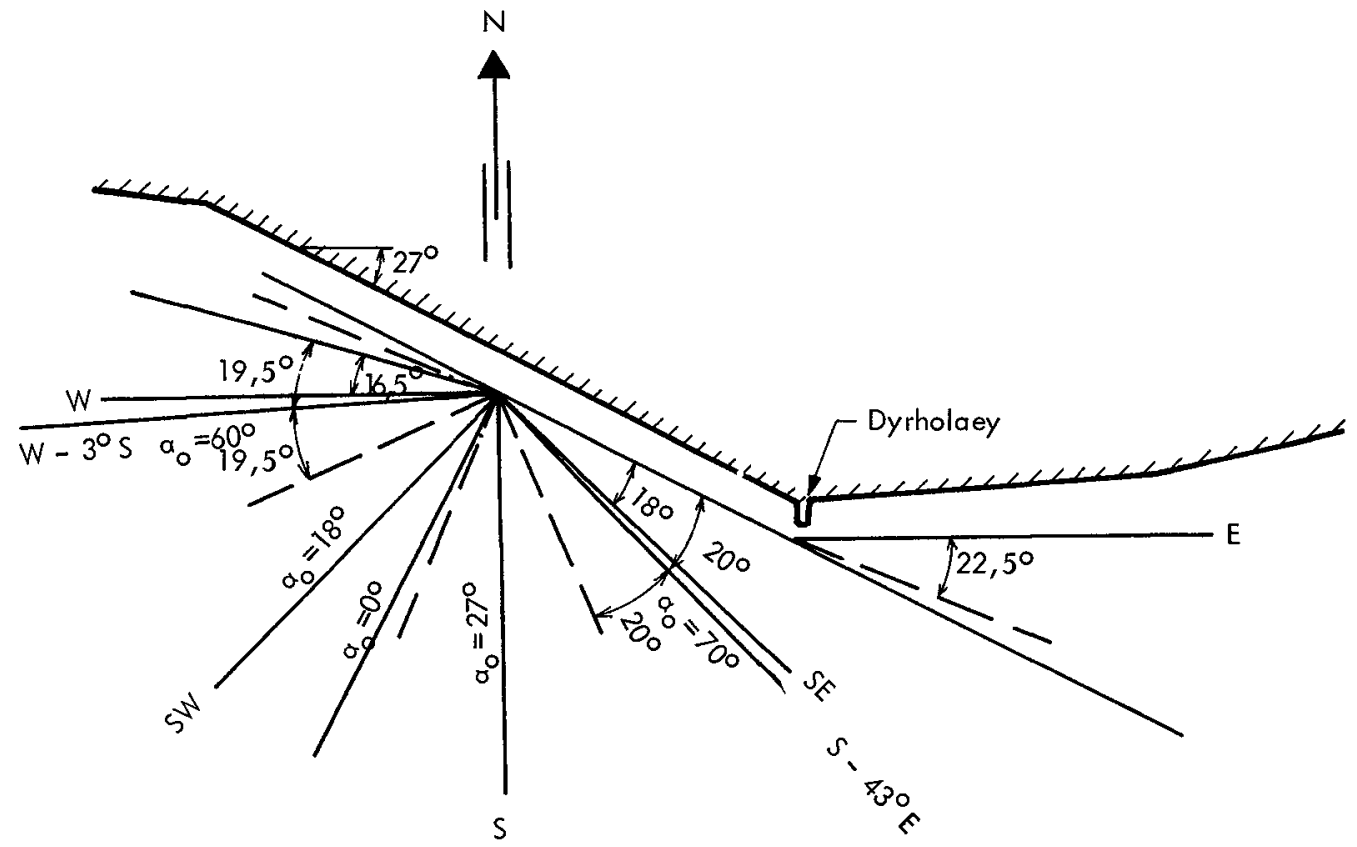

FIG 12 The boundary conditions west of DYRHOLAEY

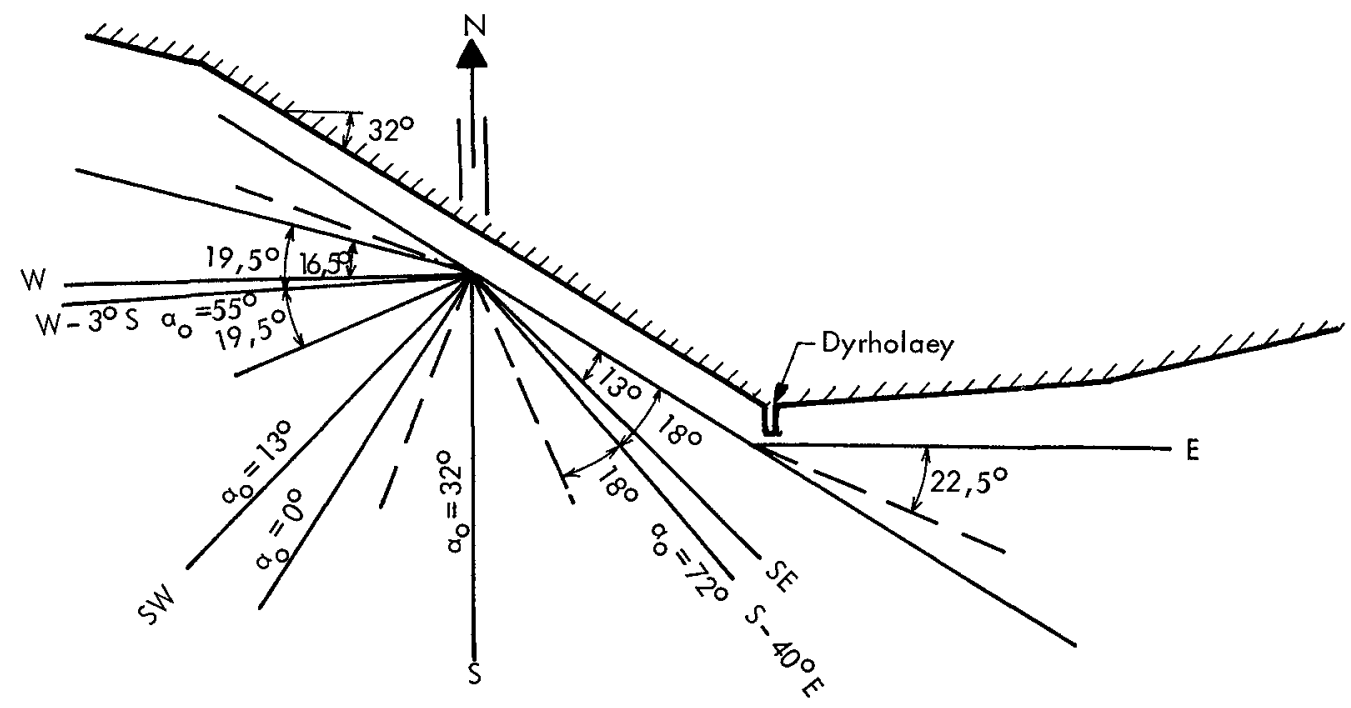

FIG 13 The boundary conditions west of DYRHOLAEY when the shoreline is turned 5 degrees clockwise 



\title{
CHAPTER 60
}

\section{AN OFFSHORE BEACH NOURISHMENT SCHEME}

\author{
by Robert A Dalrymple \\ Department of Coastal and Oceanographıc Engıneerıng \\ University of Florida \\ Gainesville, Florida
}

\begin{abstract}
The University of Florida has conducted a serıes of tests to evaluate the effectiveness of beach nourıshment from offshore sources by a specially designed drag scraper The field work, over a nine-month study period, included hydrographic surveys, fluorescent sand tracing, and a sand sampling program for two scraper sites at Jupıter Island, Florida

The conclusions provide information as to the effect of the borrow pits and the dredged sand on the beach characteristics, the mechanisms of the filling of the pits, and the efficiency of the drag scraper

\section{Introduction}

As beach erosion becomes more of a critical problem, extensive sources of sand are necessary for the artificial replenishing or rebullding of beaches At Jupiter Island, on the east coast of Florida, where erosion has been quite severe due mainly to a large inlet to the north, onshore sources of nourishment sand were being depleted in a beach rehabilitation program begun in 1957, and $1 t$ became necessary to find another readily avallable source

In 1963, Dickerson, Inc of Stuart, Florlda began nourıshing the beaches with a specially deslgned Sauerman drag scraper (See Gee, 1963) The scraper consists of a three-drum holst powered by a 260 horsepower engine, which pulls a bottomless, three cubic yard capacity bucket over a wedge-shaped offshore borrow area, demarcated by two anchored buoys The buoys are 850 feet offshore and about 500 feet apart, floating between them is a mooring float which guides the scraper bucket over the borrow area The scraper is able to make over 300 round-trips of the bucket per day, placing the recovered sand at the base of the winding machine See photographs 1 to 4

During 1967 and 1968, the Department of Coastal and Oceanographic Eng1neering (DCOE) conducted a field study of the two borrow areas (denoted Areas I and II) at which the drag scraper operated The purpose of the study was (1) to determine If the scraper was a viable method of beach nourishment, (2) to determine if spoil placed on the beach returned directly to the borrow area, and (3) to estimate the efficiency of the scraping operation
\end{abstract}

During the first part of the summer of 1967, from May until the end of June, the scraper was at Area I, then It was moved two mules south to Area IJ 
where It operated untIl August 4, when it shut down for the season The scraper was contracted to recover 30,000 cublc yards of sand at each site, the total being the estimated annual erosion at the developed section of Jupiter Island (Coastal Engineering Staff, 1957) The unit cost of the sand was 50 c per cublc yard

\section{Environmenta1 Data}

At both locations, the shoreline is straight and the beach is narrow The offshore profile is shallow The dune lines have been protected by sloping energy absorbing seawalls, and adjustable grolns had been placed on the beach in 1963

Waves in the summer at Jupiter Island approach from the southeast and are generally sma11, as large ocean swe11 are blocked by the Bahama Islands from reaching shore During the winter, however, the wave climate becomes very severe and predomnantly comes from the northeast In 1967, the seasonal shift between the summer and winter wave climate occurred on September 4, with the onset of a period of northeast storms

The 1ittoral drift follows the wave direction It is mild and from the south in the summer, and more intense and from the north in the winter The net quantity of 1 ittoral drift passing Juplter Island inside the $18^{\prime}$ contour has been estimated by the Corps of Engineers as 230,000 cubic yards per year Its direction is north to south

\section{Offshore Effects}

At both areas, when the scraper began operation, pits were dug about 175 feet offshore in about 9 feet of water These lengthened in the offshore direction into oval depressions 500-600 feet long and 300 feet wide, as scraping continued, until maximum recorded depths of 14 and 17 feet were reached at each area

During the scraping operation, the summer littoral current moved large quantities of suspended sand, kicked up by the fast-moving scraper bucket, northward, resulting in the formation of a bar on the north side of the borrow pit This "shoulder" bar became quite large at both sites as scraping continued, covering an area about two-thirds the area of the borrow plt, and a height of 3 feet above the bottom see figure 1

At Area I, after the scraper was stopped and moved to Area II, the offshore borrow pit began to change shape slowly under the action of waves and currents The elevation contours of the pit became more rounded, and the bottom of $1 t$ shoaled about one foot during the first two months The shoulder bar, during this time, was moved shoreward, by wave action concentrated on it by refraction at the hole, and filled the trough that had previously separated it from the beach

Soon after the scraper was shut down for the season at Area II, the wave climate changed Northeast storms during early September brought about the seasonal littoral drift reversal and the larger winter waves This more 
severe wave climate caused rapid shoaling of the borrow pits Calculated shoaling rates for the bottom of the borrow pits from August 31 to September 22 were 22 foot per day at Area II and 1 foot per day at Area I, which was a much shallower pit by this time These fill rates are much higher than rates calculated for borrow pits examined by Watts (1963)

The high fill rate at Area II produced a migration of the borrow pit As seen in Figure 3, the pit had moved southward A simple experiment in a movable bed hydraulic model showed that a hole in a sand bed, under the action of a unidirectional current, was filled from the upstream side, while sand was eroded from the downstream side--the net result being filling of the hole, coupled with translation downstream

The shoulder bar, at Area II, was moved both shoreward and into the borrow pit, by the northeast waves, the shoulder bar here was a more important factor in the fllling of the borrow pit than at Area I, due to the intensity of the littoral current See figure 2

At Area II, 1000 pounds of fluorescent tracer were placed in the spoil plle on the beach in front of the scraper, 20 days after scraping was discontinued The purpose of the test was to estimate the return of dredging spoll to the borrow pit Sand samples taken in the borrow pit subsequently showed some tracer being incorporated offshore into the filling borrow pit However, this recovered tracer was very fine grained, and did not represent a direct return of all the spoil to the borrow pit, but rather the natural action of beach sorting The fill material, including the tracer, had a median diameter of $13 \mathrm{~mm}$ and was well sorted, whereas the natural beach sand and the tracer had a median diameter of $29 \mathrm{~mm}$ Watts (1963), too, has observed fine gralned material in filling borrow pits The sources of the fill material in the plts are longshore current and wave transported material, the shoulder bar, particularly at Area II, and the transversally transported fine-grained dredge material, moved offshore by natural beach sorting processes

By the following summer, both pits had filled entirely and the bottom topography was similar to that of the previous summer

\section{Beach Effects}

During scraper operation, the dry beaches at both areas grew, as the spoil pile was dispersed by wave action and due to the natural onshore movement of sand during the summer The Mean Sea Level (MSL) contour moved seaward an average at each station of 5 feet at Area I and 34 feet at Area II However, by the next summer, June 28, beach surveys showed significant erosion had occurred during the winter, after the scraper was shut down, with an average net loss after the whole year of 20 feet and 42 feet at the beaches

\section{Stability}

No major beach stability problems were observed, however, they may be created if the drag scraper operates too near shore If the borrow pit is dredged very deep, slumping of the foreshore can occur, with the dredged sand returning directly to the borrow pit Also, as pointed out by Rector (1966), 
sand moved offshore by steep waves during storms, will fill the borrow pit and be lost to the beach, as this material will not be returned to the beach by natural action when the wave climate becomes less severe For Jupiter Island, where winter and summer bars exist, it was recommended that the scraper recover sand from an area offshore of the normal location of the winter bars, thus ensuring there would be no sand lost from the beach by natural processes

An additional stability problem may arise if the offshore sand is much finer than the natural beach sand This would also be the case if the scraper returned to the same place for additional spoil The fine sand would probably be quickly removed from the beach by wave action

As mentioned previously, the incidental deposition of the shoulder bar by the drag scraper provides a stablilizing effect When the longshore current changed to southward, the deposited sand was moved on to the beach and into the plt, thus removing some of the aforementioned hazard of normal beach sand being lost to the pit during storms

\section{Efficiency}

The present scraper design was observed in the field by DCOE personnel to be unable to carry its full capacity due to its design Volume estimates of the beach spoil pile and the offshore borrow pit and shoreline monitoring showed that the scraper did not move the quantity of sand necessary to match or exceed the annual erosion

\section{Conclusions}

The drag scraper technique is a viable method of beach nourıshment, provided it recovers sand in a quantity to balance or exceed the annual amount of erosion

Only the fine gralned dredge materlal returns to the borrow pit under normal operation Primary sources of pit fill material are the littoral drift and the shoulder bar, built by suspended sand

Beach stability is ensured if the scraper dredges sand compatible with the natural beach sand and from an area at a sufficlent distance from the shore

\section{Acknowledgements}

Mr James A Purpura, Associate Professor at the Department of Coastal and Oceanographic Englneering, initiated this project and directed the field program The study was funded cooperatively by the Jacksonville District, $U S$ Army Corps of Englneers and the Florlda Bureau of Natural Resources The photographs are courtesy of Dickerson, Incorporated 


\section{References}

1 Coastal Engineering Staff, Coastal Engineerıng Investigation at Jupiter Island, Technical Progress Report No 5, Florida Engineering and Industrial Experiment Station, March, 1957

2 Gee, Herbert C, Beach Nourishment from Offshore Sourcea, ASCE Journal of Waterways and Harbors, Vo1 91, No WW3, August, 1965 Discussion by $R$ L Rector, No WW2, Vo1 92, May, 1966

3 Watts, George $M$, Behavior of Offshore Borrow Zones in Beach Fill Operations, Int Assoc for Hydraulic Research Congress, London, 1963 


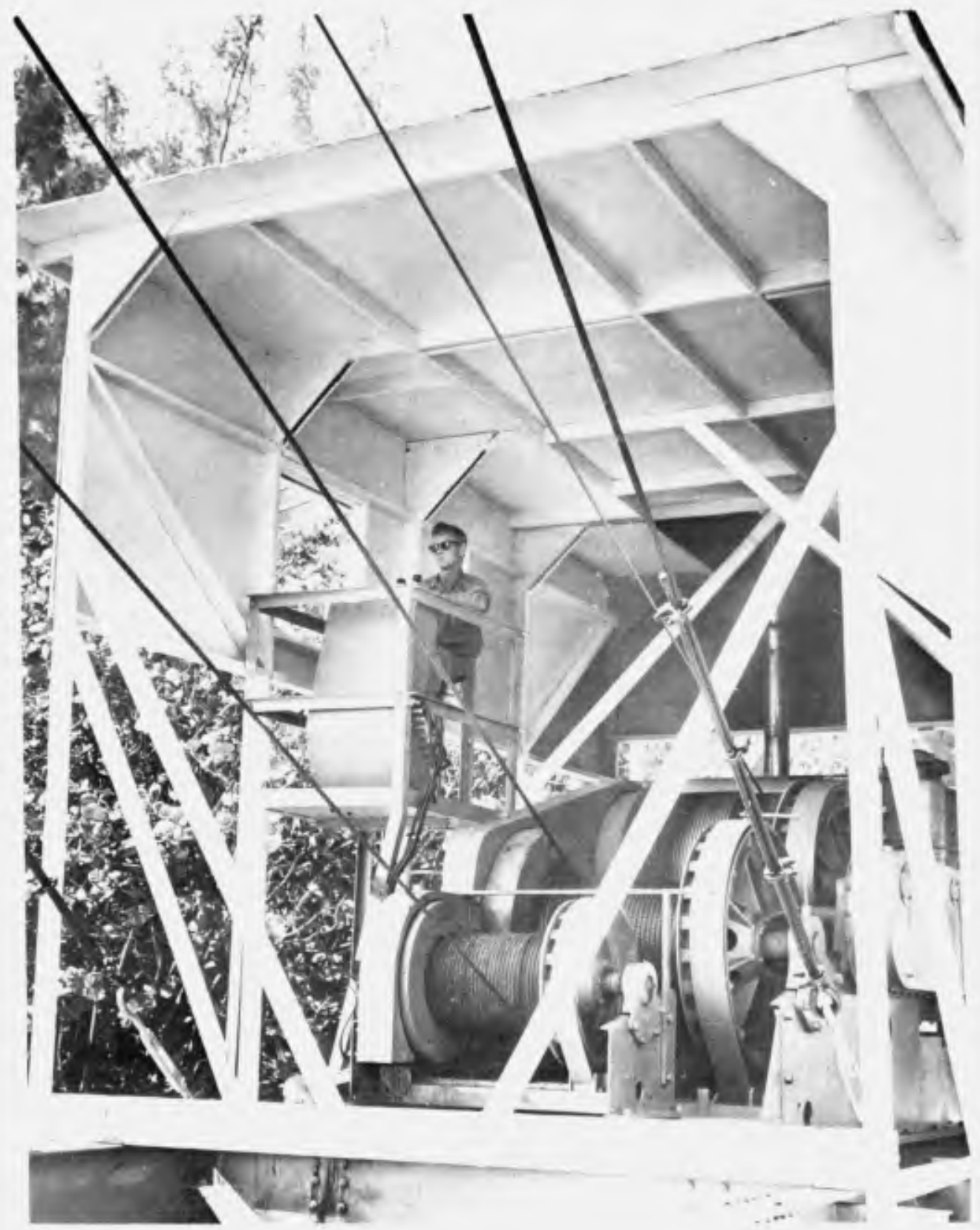

Photograph 1. Winch System for the Sauerman Drag Scraper 


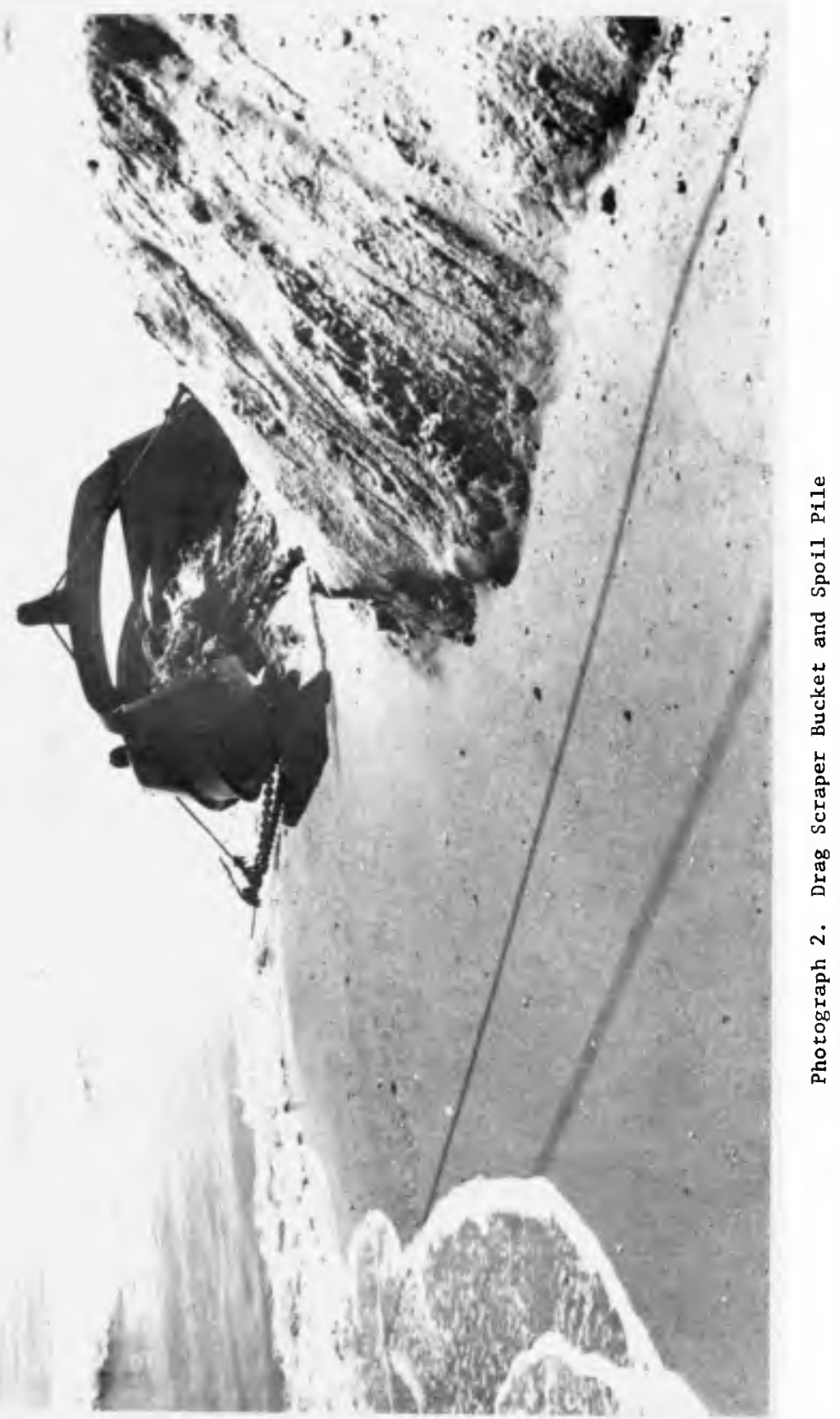




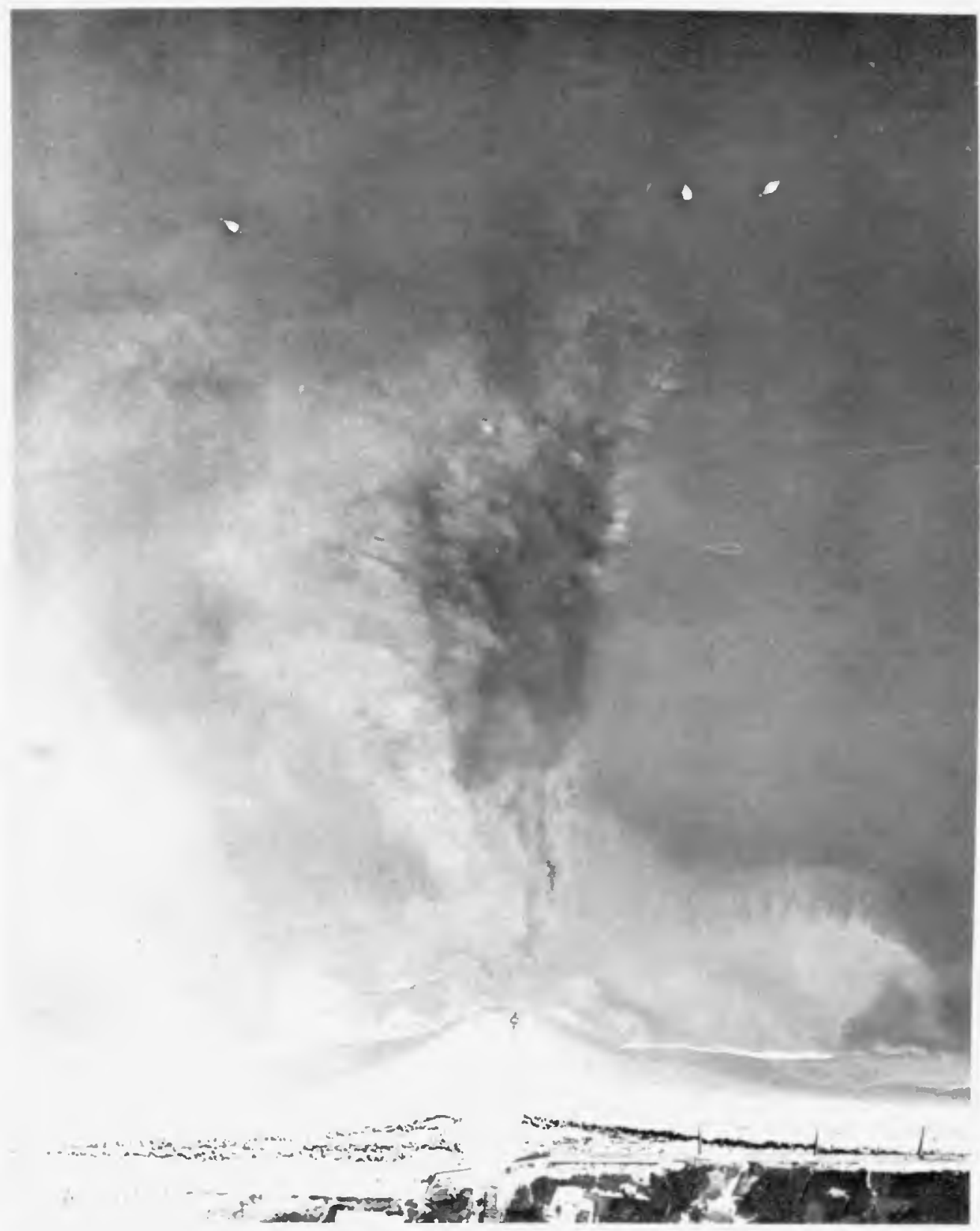

Photograph 3. Drag Scraper in Action. Note the large amount of suspended sand in borrow area. 


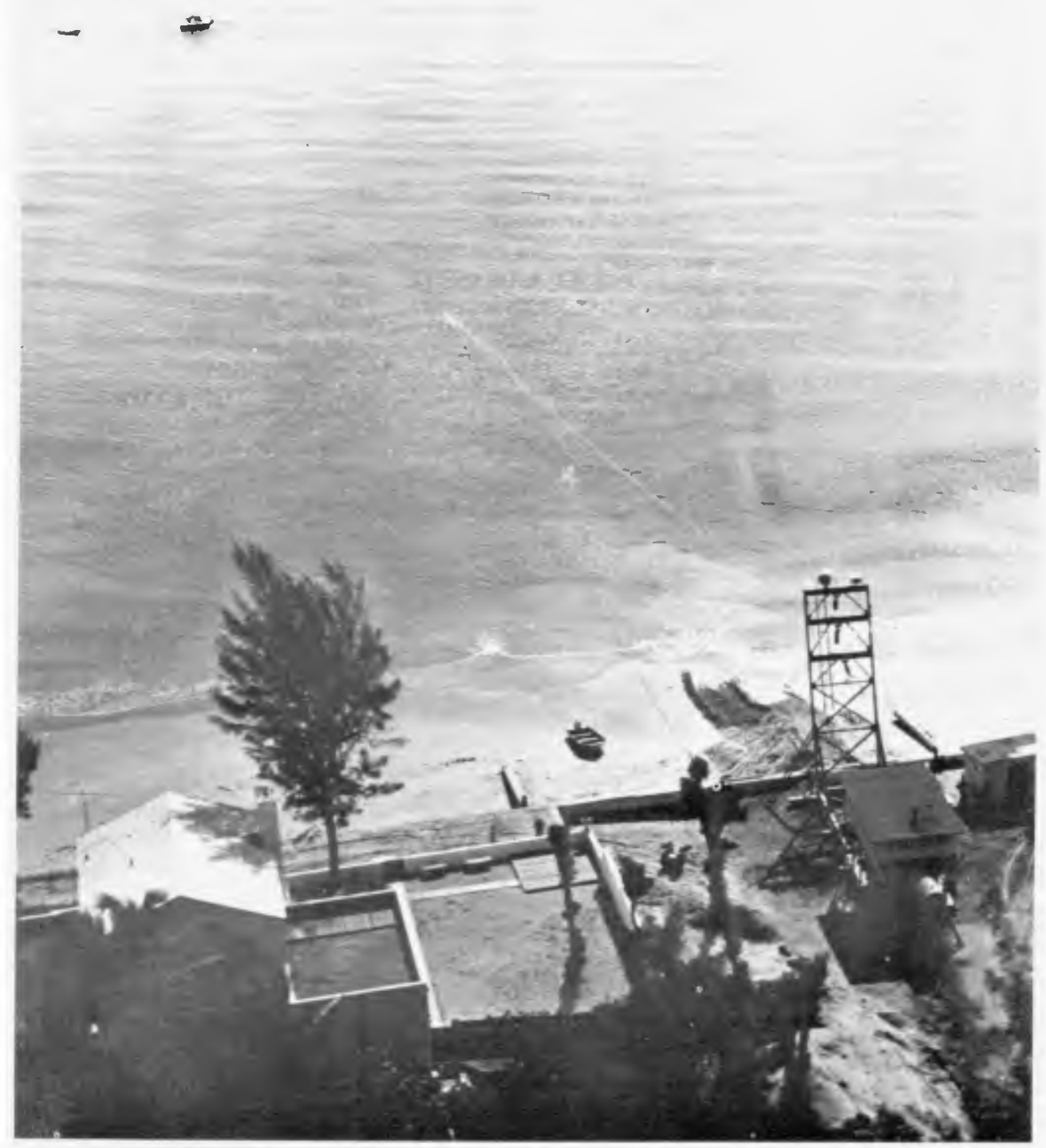

Photograph 4. Aerial View of Drag Scraper 


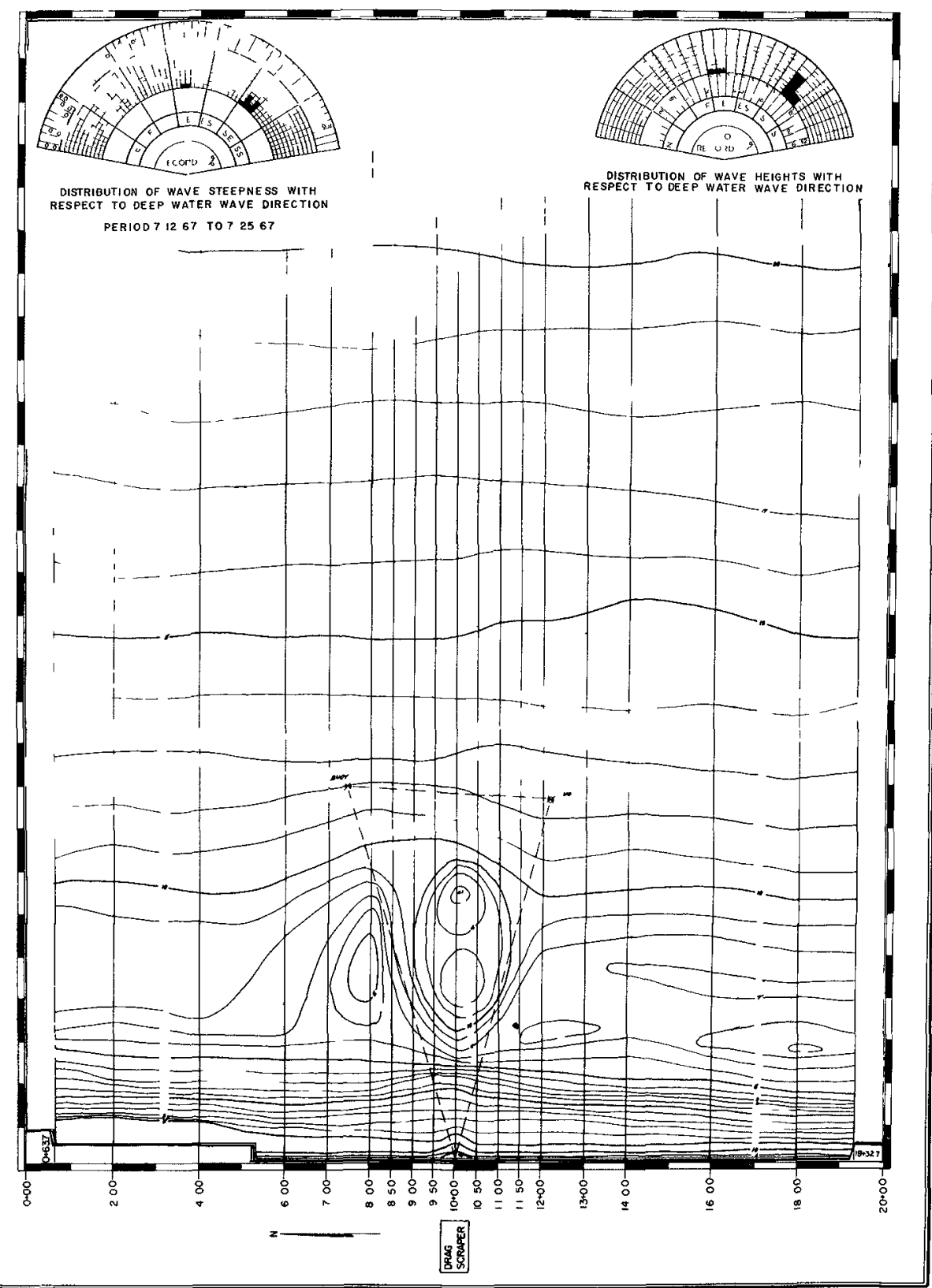

Figure 1 Hydrographic Survey, Area II No 3,7-25-67 


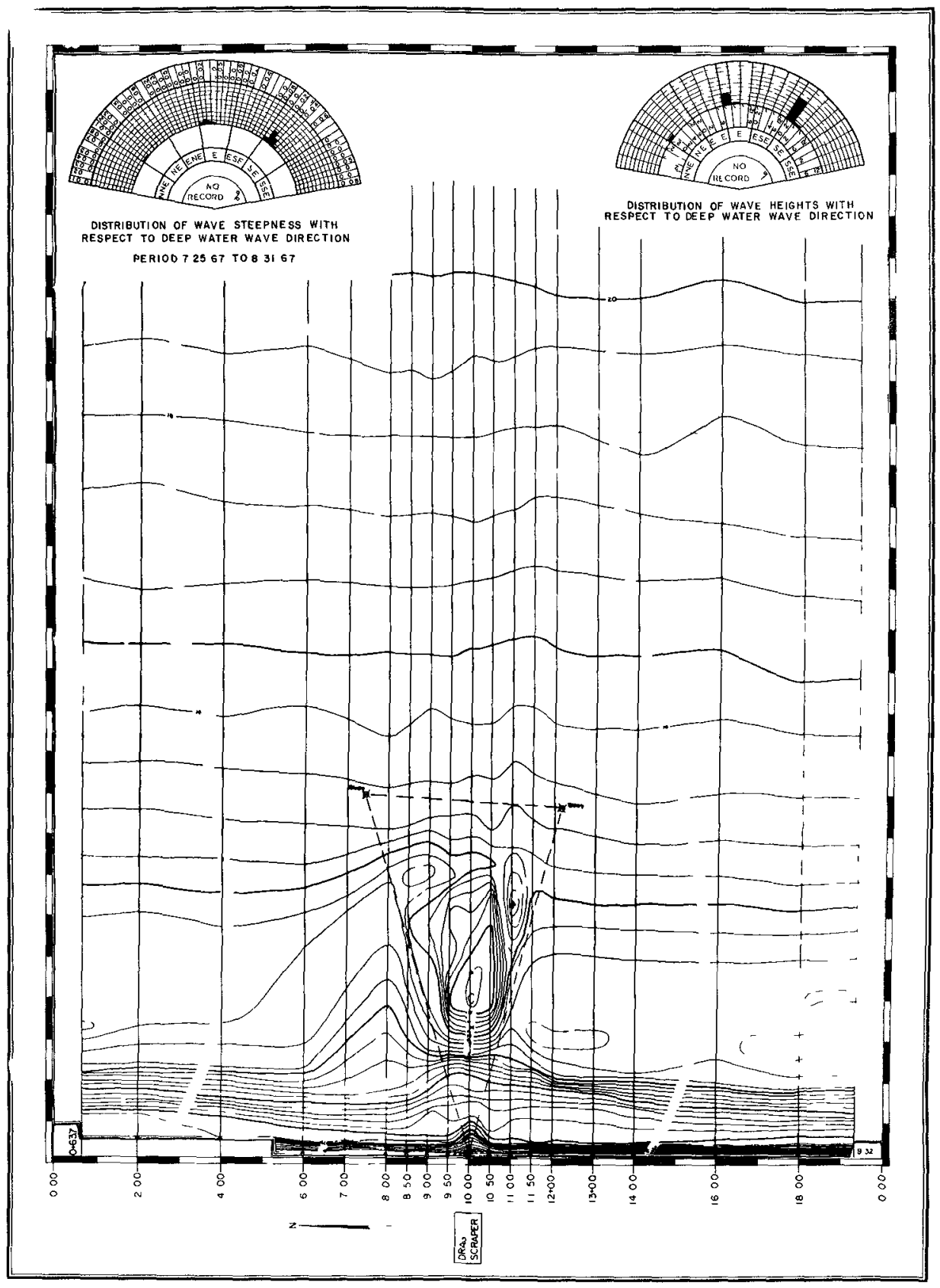

Figure 2 Hydrographic Survey, Area II No 4,8-31-67 


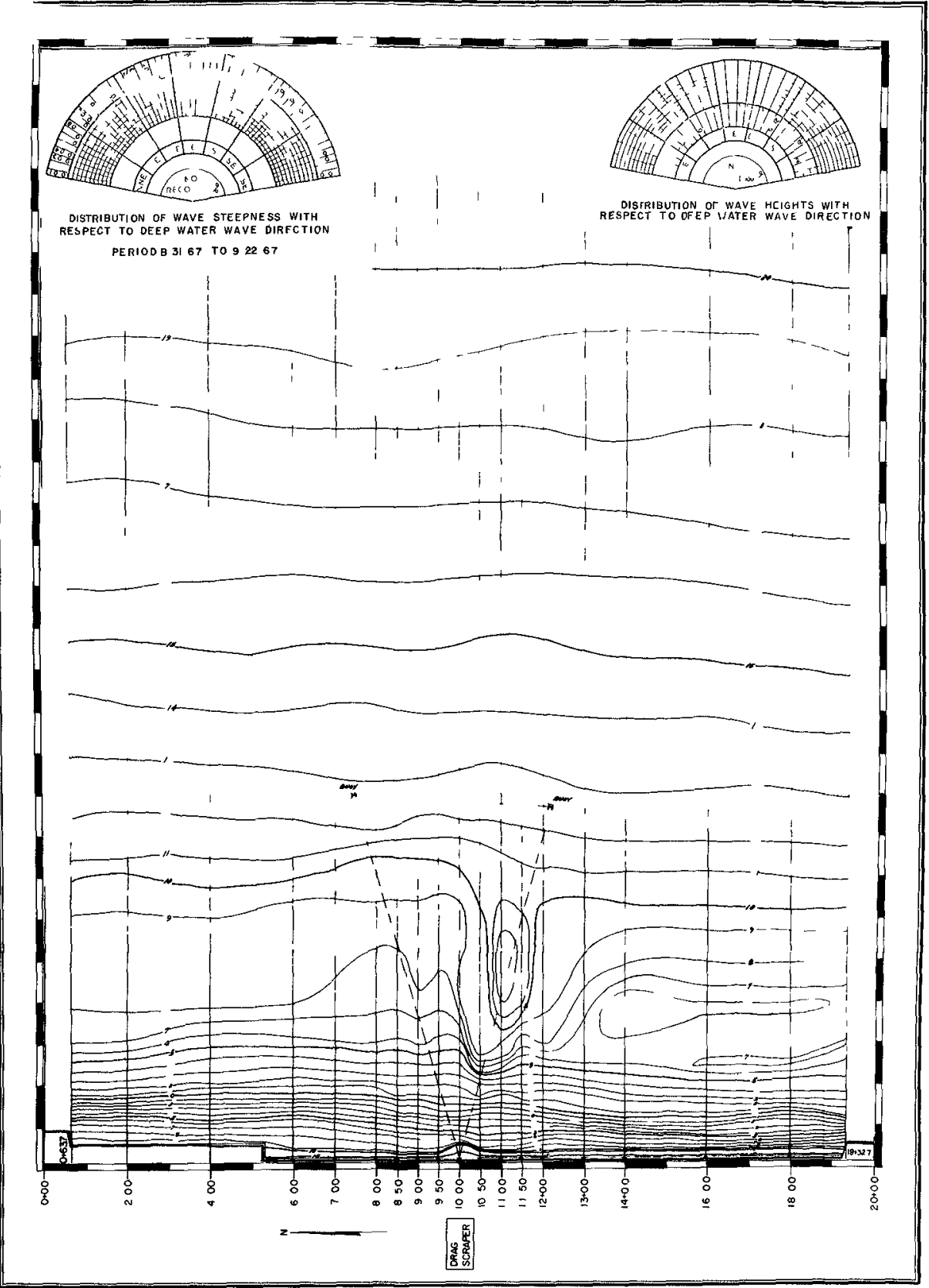

Figure 3 Hydrographic Survey, Area II No 5,9-22-67 


\title{
CHAPTER 61
}

\author{
Beach Nourishment at \\ Vırgınıa Beach, Virgınıa \\ James $W$ Bunch \\ Army Corps of Engineers \\ Norfolk, Virgınıa
}

\begin{abstract}
Aspects of the Federal beach erosion control project at Virginia Beach, Virginia are described with particular emphasis directed to the sources, characteristics, costs, methods of placement and quantities of material periodically placed on the beach following project inception The beach response to nourishment is analyzed on the basis of yearly survey records compiled from data gathered by the sea-sled direct leveling technique The quantity of annual nourıshment material required to maintain present beach dimensions is determined to be approximately 141,000 cubic yards
\end{abstract}

\section{INTRODUCTION}

In the present appraisal, information gathered by a comparison of bathymetric surveys is used as a basis for evaluating the beach response and annual sand nourishment requirement at Virgınia Beach, Virgınıa Survey data gathered by the sea-sled direct leveling technique and collected annually over seventeen identical ranges for a three-year period (1966-1969) were employed in the appralsal

\section{DESCRIPTION OF STUDY AREA}

Virginia Beach, as shown in figure 1, is located on the east coast of Virginla, about 19 miles east of Norfolk, Virginla and 35 miles south of Cape Henry which is the south point of the entrance to Chesapeake Bay The shoreline in the area bears South $12036^{\prime}$ East

The existing Federal beach erosion control project here extends from Rudee Inlet to 49 th street and includes the $33 \mathrm{mules}$ of coastline treated In this paper (figure 2) A concrete bulkhead and promenade, within the limpts of the project, was constructed by local interests in 1927 It extends from 7 th to 35 th street and 1s supported on two rows of prling generally parallel to the shore A wooden bulkhead, constructed by local Interests, extends from 35 th to 49 th Street

Tides at Virgınıa Beach are semidiurnal The mean range of tide is 34 feet and the spring range is 41 feet

Waves reaching the area are predominately from the southeastern quadrant during the summer and from the northeastern quadrant during the winter The greatest yearly percentage of waves arrive from the 
east-northeast and range between 1 and 6 feet CaIms prevall approximately 19 percent of the time Avallable evidence suggests that the predominant direction of the littoral drift in the area is from south to north An analysis of winds and waves reaching Virginia Beach indicates that the predominant energy applied to the shore is from the northeast quadrant However, observations and surveys of the jetties at Rudee Inlet and an experimental groin at Seventh Street clearly suggest a net northerly drift Th1s anomaly with respect to the direction of the littoral movement has been attributed to the possible assistance of a tidal eddy extending for some distance south of the Chesapeake Bay entrance

\section{DESCRIPTION OF EXISTING FEDERAL BEACH EROSION CONTROL PROJECT}

The existing Federal beach erosion control profect at Virginia Beach includes (a) artificıal placement of suitable sand fill on the ocean shore to provide and maintain a beach berm having a width of approximately 100 feet at elevation $54 \mathrm{~m} \mathrm{~s} 1$ with a 1 on 15 foreshore slope extending to the natural bottom and (b) a deferred system of groins to be constructed if experience indicates that it is more economical than periodic sand nourishment The project is now being maintained with dredging equipment owned by the Virginia Beach Erosion Commission, a state agency

Nearly $\$ 200,000$ is being expended annually to maintain the project of which 50 percent is borne by the Federal Government

\section{BORROW AREA}

Material for beach nourishment during the study perıod was dredged from the Ow1 Creek Estuary (Fig 2) The remaining avallable material in this area is estimated to be sufficient to supply the annual nourishment requirements through 1970 For a source of future nourishment material, the Virgınia Beach Erosion Commission has acquired stateowned lands on the south side of 0 w Creek containing approximately 500,000 cubic yards of fine sand This will provide sufficient materlal to nourish the beach for approximately four years, or to early 1975 at the heretofore proposed annual rate of 130,000 cubic yards

The median diameter of nourishment materıal during the period 1964 - 1969 was $26 \mathrm{~mm}$ as compared to $31 \mathrm{~mm}$ for natural material found on the beach

\section{EQUIPMENT USED}

Figure 3 shows the normal layout of dredging plant equipment when operating in the Owl Creek borrow area It includes a 10-inch hydraulic cutterhead dredge, a 10-inch floating booster station at 
the foot of Mediterranean Avenue, and a flxed booster station at Seventh Street The pipeline is 15,000 feet long when discharging at lts farthest point, usually between 21st Street and 22nd Street A 12-inch dredge has just been purchased for the purpose of keeping Rudee Inlet open and wıll also be available to pump sand on Virgınia Beach

\section{$\cos \mathrm{T}$}

For dredging work only, Table 1 indicates the cost per cubic yard of placing material on Virginia Beach durıng the period of investigation

Table I Cost of Beach Nourishment

\begin{tabular}{|c|c|}
\hline FY 1966 & $\$ 1 \quad 41$ \\
\hline FY 1967 & 151 \\
\hline FY 1968 & 291 \\
\hline FY 1969 & 0 \\
\hline
\end{tabular}

QUANTITIES OF MATERIAL DREDGED

Table 2 Indicates the quantities of new source material pumped by the dredge annually during the study period

Table II

Quantities of New Material Pumped on Beach

\begin{tabular}{lc}
\hline FY 1966 & 117,000 cubic yards \\
FY 1967 & 119,000 cubic yards \\
FY 1968 & 6,000 cublc yards \\
FY 1969 & 0 \\
\hline
\end{tabular}

COMPARI SON OF SURVEYS

Comparison of the 1966, 1967, 1968 and 1969 surveys, which were made using an identical method over the nearshore and offshore profiles, yields what is thought to be an accurate indication of beach response in the project area and is summarized in Table III

The nearshore profile over which surveys were made extends from the bulkhead line for a distance of 400 feet oceanward Including berm material which was in excess of design dimensions, the deficiency in the design berm of 150,000 cublc yards that existed in June 1966 was reduced to 110,000 cubic yards in 1967 The deficlency in the design berm for July 1968 was 209,000 cubic yards or an increased deficiency of 99,000 cubic yards over July 1967 Similarly, a deficiency in the design berm for June 1969 of 330,000 cubic yards represents an increase of 121,000 cublc yards over July 1968

The offshore profile, as defined for survey purposes, extends from the bulkhead line to the 25-foot depth contour Including excess berm material, there was an indicated loss of approximately 392,000 cubic 
yards of material frrm the offshore area between 1966 and 1967 This is equivalent to 030 -foot over the entire project Between 1967 and 1968 there was an indicated gain of 7,600 cubic yards which is a negligible gain over the entire project In 1969, the offshore profiles indicated a loss of 221,000 cubic yards of material from the previous year This is equivalent to 020 -foot over the entire project

Overall, during the three-year period, 1966-1969, the net loss of material over the nearshore profile has been 179,000 cubic yards while the net loss over both profiles was 605,000 cubic yards During the same period, a total of approximately 242,000 cubic yards of suitable material has been placed on the beach on the basis of the foregoing figures, there has been an apparent loss of 847,000 cubic yards or an average annual loss of approximately 282,000 cublc yards of material in the problem area Total nearshore losses of 179,000 cubic yards indicate an average annual loss of approximately 141,000 cubic yards in the project area Consequently, a quantity of suitable nourishment material, totaling at least 141,000 cubic yards should be placed in the project area to maintain present beach dimensions

Table III

\begin{tabular}{|c|c|c|c|c|c|}
\hline $\begin{array}{l}\text { Fiscal } \\
\text { Year } \\
\end{array}$ & $\begin{array}{l}\text { Suitable } \\
\text { Material } \\
\text { Pumped } \\
\text { On Beach } \\
\text { (Cubic Yards) }\end{array}$ & $\begin{array}{l}\text { Deficiency } \\
\text { from Design } \\
\text { Berm } \\
\text { (Nearshore) } \\
\text { (Cubıc Yards) } \\
\end{array}$ & $\begin{array}{l}\text { Net Change in } \\
\text { Nearshore } \\
\text { from Previous- } \\
\text { Year } \\
\text { (Cubic Yards) }\end{array}$ & $\begin{array}{l}\text { Net Change } \\
\text { in Nearshore } \\
\& \text { Offshore from } \\
\text { Previous Year } \\
\text { (Cublc Yards) } \\
\end{array}$ & $\begin{array}{l}\text { Net } \\
\text { Change in } \\
\text { Offshore } \\
\text { Area } \\
\text { (Cubic Yard } \\
\end{array}$ \\
\hline 1966 & 117,000 & 150,000 & & & \\
\hline 1967 & 119,000 & 110,000 & $+41,000$ & $-392,000$ & $-433,000$ \\
\hline 1968 & 6,000 & 209,000 & $-99,000$ & $+8,000$ & $+107,000$ \\
\hline 1969 & 0 & 330,000 & $-121,000$ & $-221,000$ & $-100,000$ \\
\hline
\end{tabular}

As indicated in Table III, although a state of erosion or accretion may occur on the beach during any given year, an opposite condition may result in the offshore reaches As more survey data becomes available, the relationship between nearshore and offshore profile changes can possibly be determined 


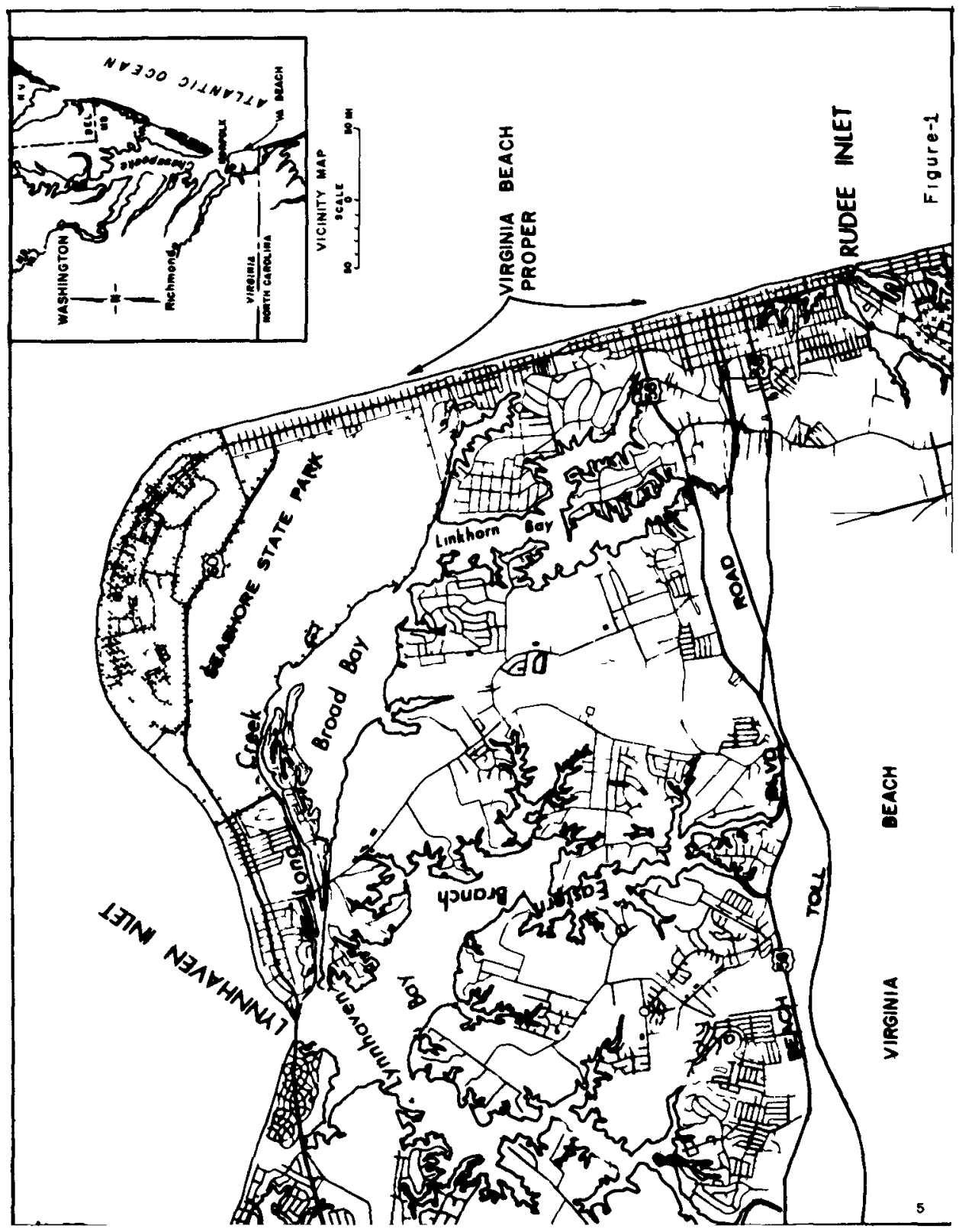




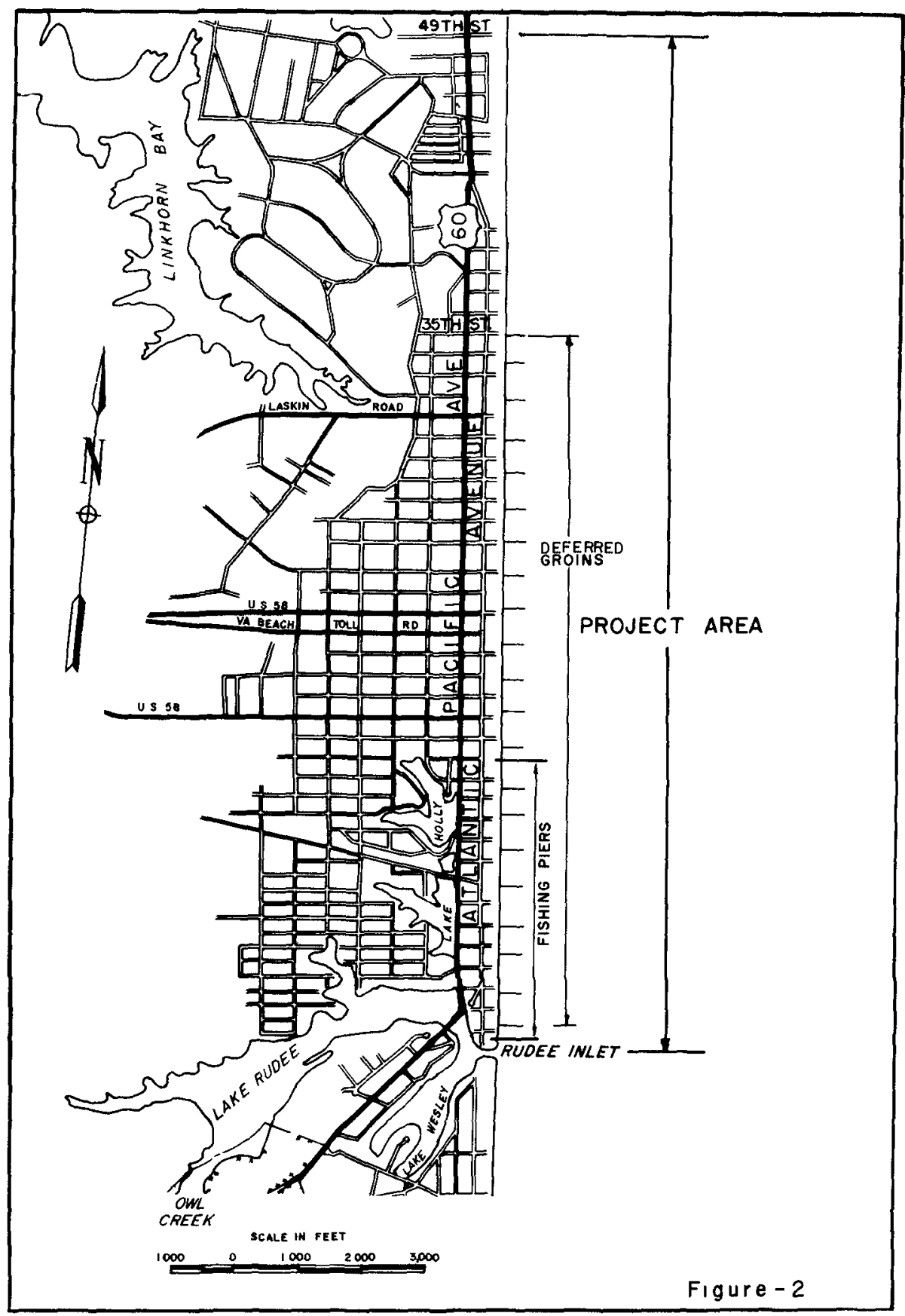




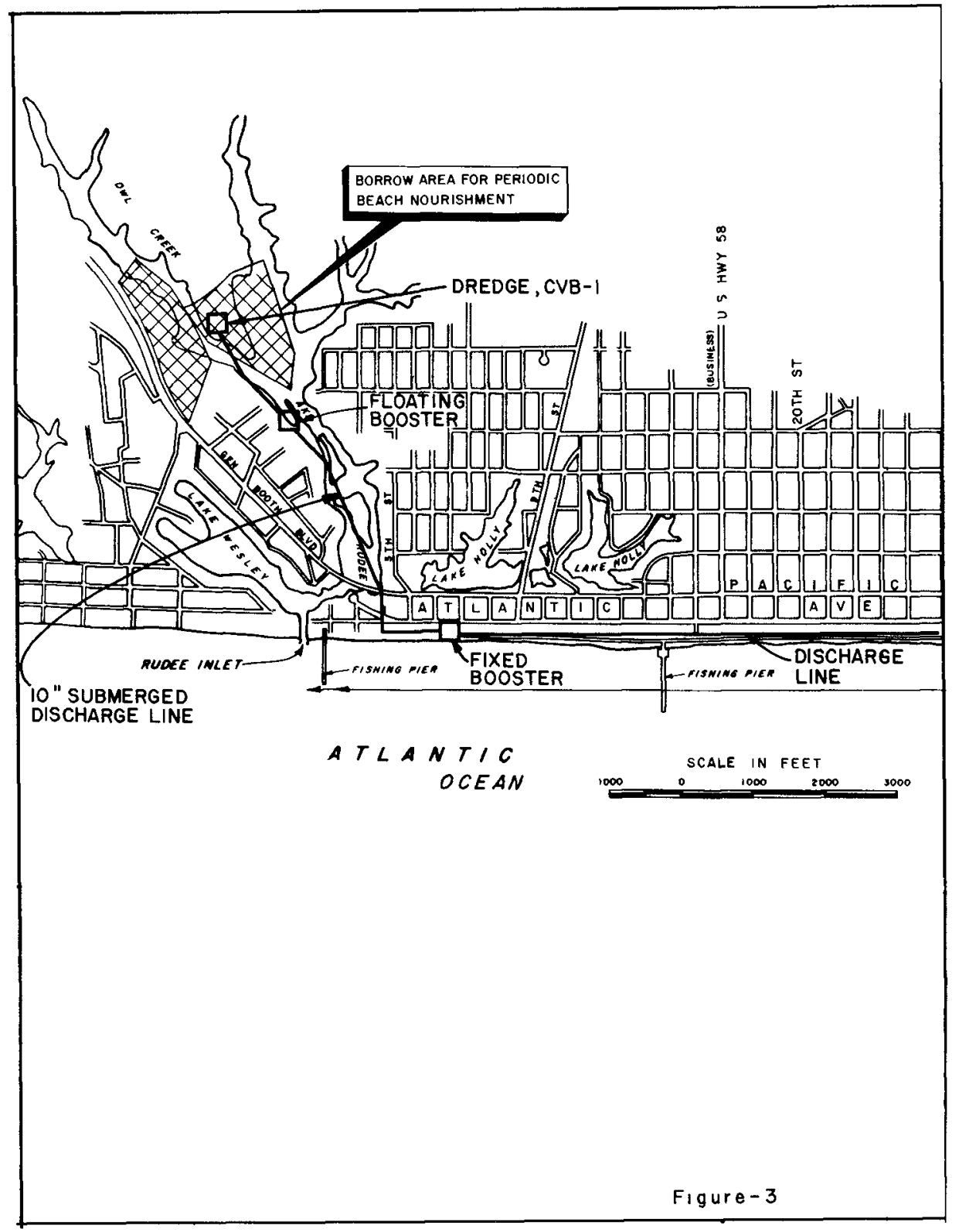





\title{
CHAPTER 62
}

\section{UNDERWATER MOUND FOR THE PROTECTION OF DURBAN'S BEACHES}

\author{
by \\ J A Zwamborn ${ }^{x}$, G A W Fromme ${ }^{x}$, J B FitzPatrick
}

\begin{abstract}
The construction of an underwater mound of sand for the protection and improvement of Durban's beaches has been recommended on the basis of intensive investigations These investigations included prototype measurements of beach changes as related to recorded sea conditions, basic scaling tests in which these beach changes were reproduced to scale in movable bed models and tests of the proposed underwater mound in models, using different scales in order to eliminate possible scale effects
\end{abstract}

The test results showed that, provided the shear-settling velocity similarity criterion is satisfied, beach changes can be reproduced in a movable bed model to a reasonable degree of accuracy Optimum dimensions for the cross section of the mound were determined on the basis of the criterion for erosive and non-erosive wave conditions which was derived from the prototype beach profile changes and confirmed by model tests The resulting dimensions are a mound of sand about $45 \mathrm{~km}$ long, about $1200 \mathrm{~m}$ offshore, reaching to $73 \mathrm{~m}$ below LWOST, with side slopes of 1 in 25 and a crest width of $61 \mathrm{~m}$ of the total quantity required $\left(8000000 \mathrm{~m}^{3}\right)$ some $2500000 \mathrm{~m}^{3}$ of sand, avallable from harbour dredging works in Durban Bay, had been dumped by May, 1970 Model predictions on mound stability and beach improvements were confirmed to a high degree of accuracy by the ful1 scale events

X Head, Chief Research officer and Research Assistant respectively, Hydraulics Research Unit of the National Mechanical Englneering Research Institute, South African Council for Scientific and Industrial Research, Stellenbosch, Republic of South Africa 


\section{INTRODUCTION}

As part of an investigation into the siltation of the entrance to Durban harbour, South Africa's biggest port and the erosion of the adjoining ocean beaches, various possibilities for the protection and Improvement of these beaches, which make Durban South Africa's premier holiday resort, were studied between 1962 and $1964^{1}$ The causes of the deterioration of Durban's beach and conventional methods to improve the situation, e $\mathrm{g}$ groynes, are described by Jordaan in a separate conference paper ${ }^{2}$ Subsequent to Jordaan's work, the senior author developed a possible solution, which emerged from the necessity of finding a suitable dumping site for material dredged from the harbour entrance and from the harbour extension works in Durban Bay ${ }^{3}$ Th1s scheme consisted of dumping the spoil along a line parallel to the beach line some $1200 \mathrm{~m}$ offshore, in an attempt to form a continuous underwater sand ridge eventualiy of some $45 \mathrm{~km}$ long (see Figure 1)

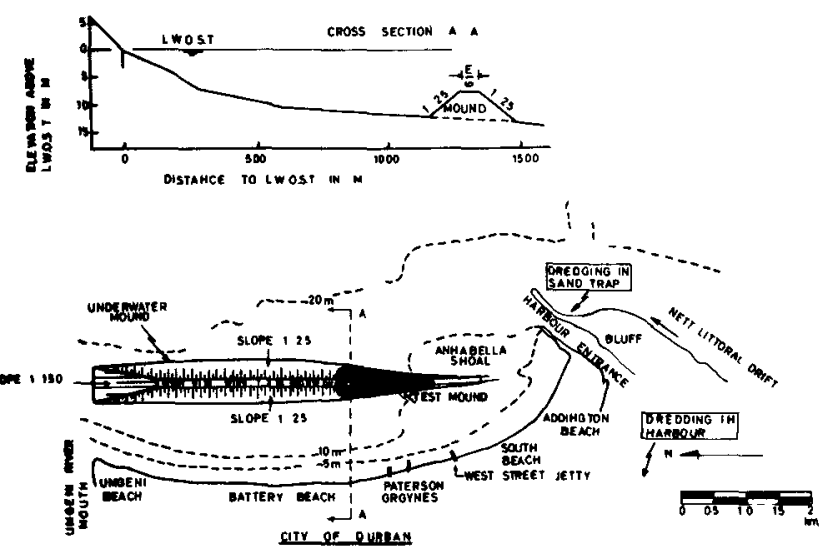

FIGURE 1 SCHEMATIC LAYOUT OF THE DURBAN UNDERWATER MOUND

If such an underwater mound could be built up to a sufficient height and provided it would remain fairly stable, it would act as a selective wave filter, e $g$ low waves would pass unhindered, whereas large erosive waves would break on the mound and thus loose much of their energy As a result, beach building conditions were expected to Improve in the lee of the mound and due to the reduction of the 
incident wave heights, longshore sand bar and trough dimensions were expected to be reduced, resulting in safer bathing conditions

\section{THE UNDERWATER MOUND}

Although underwater breakwaters have been bu11t before for the protection of certain beach areas, particularly in Japan, the underwater mound scheme, when concelved in 1963, is belleved to have been the first application of an artificially placed sand bar to protect the leeward beaches Large amounts of $s$ and are avallable from harbour dredging works at Durban but since trailing-suction dredgers are used, It is impractical to discharge the sand directly onto the beaches Nearshore dumping, in an attempt to feed the beaches, was tried at Long Beach, New Jersey and Santa Barbara, Calıforn1a ${ }^{4,5}$, both attempts were, however, unsuccessful because the sand remanned where 1t was dumped instead of moving onto the beach

This evidence strengthened the 1dea that dredging spoll could be used to build an offshore underwater sand ridge or mound which would be sufficlently stable to form an effective beach protection scheme In this way a solution would be obtained for both the beach problem and the problem of finding a satisfactory dumping site Preliminary tests and calculations based on breaker depth functions ${ }^{3}$ showed that the mound should reach a height of at least $73 \mathrm{~m}$ below LWOST $\mathrm{X}^{\mathbf{X}}$ to be effective. Since this is also the minimum depth in which most of the avallable hopper dredgers could safely operate, this he1ght was accepted for the further investigations In the field it was established that side slope with underwater dumping would not be steeper than about 1 in 25 and this slope was therefore used in the model tests

The final scheme which eventually evolved is shown in Figure 1 The proposed underwater mound runs parallel to the Durban beaches in a water depth varying from 7 to $16 \mathrm{~m}$ Its crest level is $73 \mathrm{~m}$ below LWOST, the crest w1dth $61 \mathrm{~m}$, the length about $45 \mathrm{~km}$ and the total quantity of sand required is about $8000000 \mathrm{~m}^{3} \quad$ A gradual slope of 1 in 150 is included at the northern end of the mound to minimise side effects

x Low Water Ordinary Spring Tide 
Before the above scheme could, however, be recommended to the Durban Corporation, it was necessary to estab11sh whether the underwater mound would remain sufficiently stable and what its effect would be on the beaches. Because of the uniqueness of the problem, it was decided, firstly, to carry out extensive research into basze scaling problems for movable bed mode1s, whereafter optimum dimensions of the mound and the stability of the mound and 1 ts effect on the beaches were determined in movable bed mode1s. It was also decided to supplement the model tests with the construction of a $1200 \mathrm{~m}$ long test section off the Durban beach, to establish mound stability and possible sand migration from the mound under prototype conditions

\section{BASIC SCALING TESTS}

Detailed measurements of beach changes, wave conditions and sand sizes were made for a beach section along West Street Jetty, Durban, during $1965^{6}$ Using these data, tests were carried out in a 023 m wide wave flume, applying the following scale ratio's

$\begin{array}{ll}\text { horizontal scale ratio } & \mathrm{L}_{\mathrm{r}}=200 \\ \text { vertical scale ratio } & \mathrm{h}_{\mathrm{r}}=72 \\ \text { geometric distortion } & \mathrm{S}_{\mathrm{r}}=\mathrm{h}_{\mathrm{r}} / \mathrm{L}_{\mathrm{r}}=1 / 278 \\ \text { hydraulic time ratio (tides) } & \mathrm{t}_{\mathrm{r}}=236^{\text {(based on Froude's 1aw) }} \\ \text { wave period scale } & \mathrm{T}_{\mathrm{r}}=\mathrm{h}_{\mathrm{r}}^{\frac{1}{2}}=847\end{array}$

The average value of the mean grain sizes, $d_{m}$, along West street Jetty was found to be 350 micron Three separate serles of tests were carried out using sand $\left(d_{m}=250\right.$ micron $)$ and anthracite $\left(d_{m}=190\right.$ and 270 micron) with a specific gravity of 135 Wave conditions, as measured in nature including tides, were reproduced to scale in these tests All the wave conditions were tested until equilibrim profiles had been reached

The results of these tests ${ }^{7}$ showed that with the 270 micron anthrac1te, the average prototype beach slope of 5 per cent was correctly reproduced. Moreover, the general beach shapes as well as the quant1tative changes compared remarkably well On the other hand, the results from the tests w1th 250 micron sand and 190 micron anthracite, did not compare with nature at a11, average beach slopes found in the 
model being 8 and 3 per cent respectively

It has been shown ${ }^{8,9}$ that in the case of river models with a movable bed, good similarity between model and prototype is achieved when, apart from the Froude and friction criteria, the shear-setting velocity criterion is also satisfied, $\operatorname{viz}\left(V_{x} / W\right)_{r}=(h S)_{r}^{\frac{1}{2}} / W_{r}=1$, in which $V_{x}$ is the shear velocity, $W$ is the settling velocity of the mean grain size and subscript $\mathbf{r}$ denotes prototype to model ratio To reproduce the 350 micron prototype sand in accordance with the shearsettling velocity criterion either sand with $\mathrm{d}_{\mathrm{m}}=120 \mathrm{micron}$, or anthrac1te with $d_{m}=260$ micron should have been used in the tests The 270 micron model anthracite thus almost satisfied the above criterion and since this material resulted in nearly correct reproduction of prototype events, it is concluded that, for the model scales used for the tests, the shear-setting velooty criterion must be satisfied to correctly reproduce coastal changes ${ }^{9}$

\section{CRITERION FOR EROSIVE AND NON-EROSIVE CONDITIONS}

It has been shown 7,10 that beach deformations relative to an equi 11brium beach slope, 1 , are a function of the deep water wave steepness, Ho/ $\lambda_{0}$ and the parameter $\left(g H_{0}\right)^{\frac{1}{2}} / W$ ( $\mathrm{g}$ is acceleration due to gravity, Ho wave height and $\lambda_{0}$ wave length) The beach profiles measured along West Street Jetty, as related to particular wave conditions, were divided into erosive, non-erosive and equilibrium profiles and from the results the criterion shown in Figure 2 was obtained (put $S_{\mathbf{r}}=1$ ), which defines the conditions for accretion, erosion or a neutral profile in case of the Durban beach with its 5 per cent equilibrium beach slope

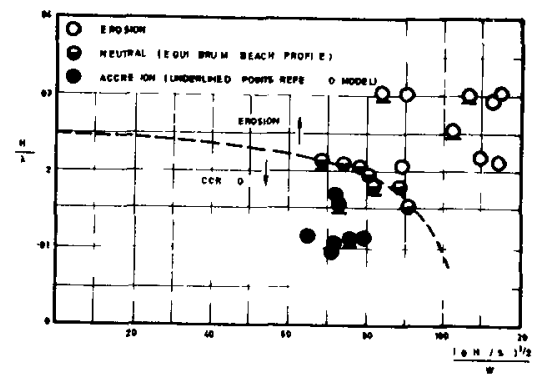

FIGURE 2 CRITERION FOR EROSIVE AND NON-EROSIVE CONDITIONS 
So far only prototype conditions have been considered Assuming now that model tests are made of beach deformations and that the criterion $(\mathrm{hS})_{\mathrm{r}}^{\frac{1}{2}}=\mathrm{W}_{\mathrm{r}}$ is satisfied, the beach deformation wil1 become a function of ${ }^{7}$

$$
\text { deformation }=\mathrm{f}\left(\mathrm{Ho} / \lambda_{\mathrm{O}}, \frac{\left.\left(\mathrm{gHo}_{\mathrm{O}} / \mathrm{s}_{\mathrm{r}}\right)^{\frac{1}{2}}\right)}{\mathrm{W}}\right.
$$

This expression is applicable to both model and prototype $\left(S_{r}=1\right.$ for prototype) In Figure 2 are also shown the test results with the 270 micron anthracite which closely agree with the prototype data This yields further proof of the importance of the shear-settling velocity criterion

Although it is realised that these findings are based on a limited amount of data, it may nevertheless be concluded that a reasonably accurate criterion has been established to differentiate between erosive and non-erosive wave conditions This criterion has proved invaluable for the interpretation of the results of additional scaling tests and for the determination of the optimum dimensions of the mound (see following sections) Moreover, since for points on the dividing line neither erosion nor build up occurs, it is suggested that this line be used to define the conditions which yield the equilibmum beach profile which 1 s of particular value for model tests

The criterion shown in Figure 2 only applies to the Durban cond1tions, viz a slightly protected beach with $350 \mathrm{mlcron}$ sand and 5 per cent beach slope However, using the relationship between beach slope and average grain size given by $W_{1}$ ege $1^{11}$, the Durban results can be extended to cover a range of beach slopes and grain sizes In Figure 3 a generalised criterion is given for sizghtly protected beaches, which clearly shows the influence of beach slope, 1 Using Wiege1's data, similar criteria can be derived for protected and unprotected beaches Extensive tests are at present being undertaken at the University of Stellenbosch to check on the validity of the generalised criterion

\section{ADDITIONAL TESTS ON SCALE EFFECTS}

In the above it was shown that the shear-settling velocity ratio must be satisfied in the case of a mode1, with $L_{r}=200$ and $h_{r}=72$, to ensure proper reproduction of prototype events It remained to establish 
whether this criterion also applied to other scale ratio's to be used for the model tests on the underwater mound Tests on scale effects, as listed in Table $I$, were therefore carried out using a typical section of the Durban beaches w1th $350 \mathrm{mlcron}$ sand as a basis for the tests

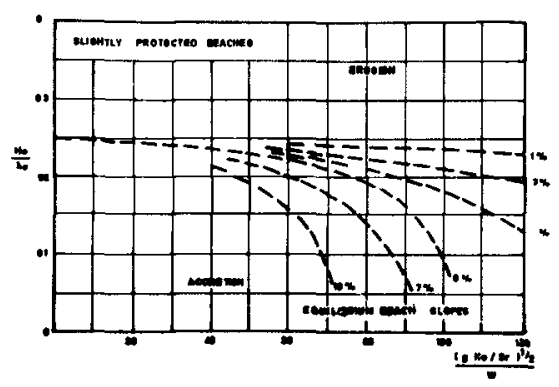

FIGURE 3 GENERALISED CRITERION FOR EROSIVE AND NON-EROSIVE CONDITIONS

TABLE I TESTS ON SCALE EFFECTS

\begin{tabular}{|c|c|c|c|c|c|c|c|}
\hline \multirow{2}{*}{$\begin{array}{l}\text { Test } \\
\text { Series }\end{array}$} & \multirow{2}{*}{$\mathrm{L}_{\mathrm{r}}$} & \multirow{2}{*}{$\mathrm{h}_{\mathrm{r}}$} & \multirow{2}{*}{$\frac{1}{s_{r}}$} & \multirow{2}{*}{ Sediment } & \multicolumn{2}{|c|}{$\begin{array}{l}\text { Mean grain size, } \\
\mathrm{d}_{\mathrm{m}} \text { (m1cron) }\end{array}$} & \multirow{2}{*}{$\begin{array}{l}\text { Width } \\
\text { of flume } \\
(\mathrm{m})\end{array}$} \\
\hline & & & & & $\begin{array}{l}\text { Req for } \\
\left(V_{x} / w\right)_{r}\end{array}=1$ & Aval lab le & \\
\hline 1 & 200 & 200 & 1 & Anthracite & 135 & 150 & 023 \\
\hline 2 & 200 & 100 & 2 & $"$ & 205 & 190 & 023 \\
\hline 3 & 72 & 72 & 1 & $"$ & 180 & 190 & 122 \\
\hline 4 & 72 & 18 & 4 & Mine sand & 200 & 225 & 122 \\
\hline
\end{tabular}

For all the above tests model sediment was used which nearly satisfied the shear-settling velocity ratio and test conditions included both erosive and build up type waves Resulting model beach profiles were compared with those predicted on the basis of Figure 2 and 1 t was found that definite scale effects were present for the smallest model (1 in 200 undistorted) but for all scales equal to or larger than $I_{r}=200$ and $h_{r}=100$ the model results closely agreed with the predictions ${ }^{7}$

Thus it may be concluded that models in the range of scales listed in Table I (excluding the smallest scale) and for which the shear-settling velocity ratio is satisfied, may be relied upon to reproduce prototype conditions to a reasonable degree of accuracy 


\section{OPT IMUM DIMENSIONS OF THE MOUND}

Initially (1964) the dimensions of the mound were rather arbitrarily chosen, 1 e a crest width of $92 \mathrm{~m}$, side slopes of 1 in 9 and reaching to $73 \mathrm{~m}$ below LWOST From early echo sounders made in 1966 over a test section of the mound, it was found that the dumped sand reached an equilibrium underwater slope of 1 in 25 This slope was therefore accepted for all subsequent model tests

Although the underwater mound is a long way from the beach, since its function is to cause the larger waves to break over it, its behaviour could well be simllar to that of a bar in the main breaker zone Keulegan has found that for a nearshore bar to be stable for different wave steepnesses, the depth of crest immersion must be about half the water depth 12,13 As can be seen from Figure 1 the main body of the mound will be in about $15 \mathrm{~m}$ water depth and thus the crest should reach to about $75 \mathrm{~m}$ below LWOST Based on this and considering the required depth for safe manoeuvring of the hopper dredgers, the original crest level of $73 \mathrm{~m}$ below LWOST was mantained for the further tests on the mound To determine an effective crest width for the mound, both fixed bed and movable bed model tests were carried out in the $023 \mathrm{~m}$ and $122 \mathrm{~m}$ wide wave channels

The frxed bed model tests were performed for a beach and mound cross section Just north of the Patterson Groynes (Section A-A, Figure 1) The water depth near the mound in this area is $13 \mathrm{~m}$, side slopes of 1 in 25 and a crest level of $73 \mathrm{~m}$ below LWOST were used The tests were carried out in the $023 \mathrm{~m}$ wide flume at an undistorted scale of 1 in 100, using crest widths of $0,30,61$ and $92 \mathrm{~m}$ respectively Tides were not reproduced, the tests were performed at $\mathrm{MSL}^{\mathrm{x}}$

The results of the tests are shown in Figure 4 where the incident wave heights are plotted against the wave heights in the lee of the mound It should be noted that with no breaking of waves over the mound, the reduction in wave height due to the mound is about 30 per cent but as soon as the waves start to break the reduction increases rapidly From Figure 4 it is clear that the mound is significantly

Mean Sea Level, 1 e $09 \mathrm{~m}$ above LWOST for Durban 
effective only when the crest width 1 s at least $61 \mathrm{~m}$ In this case wave heights behind the mound are 1 imsted, due to breaking, to $25 \mathrm{~m}$ Irrespective of the incident wave height A further rather insignificant reduction to $225 \mathrm{~m}$ is effected by an increase in crest width to $92 \mathrm{~m}$ On the basis of these tests it was therefore concluded that the crest width of the mound should be at least $61 \mathrm{~m}$

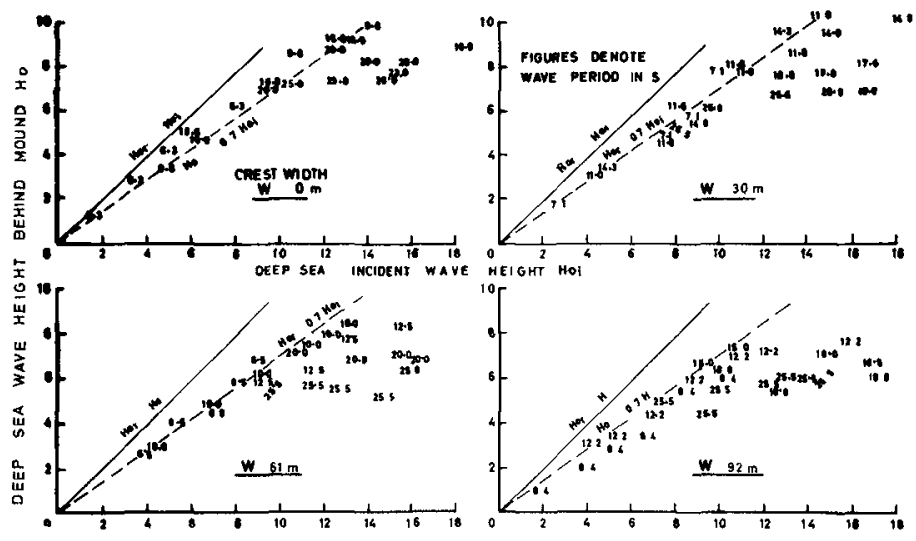

\section{FIGURE 4 RELATIONSHIPS BETWEEN MODEL WAVE HEIGHTS}

SEAWARDS AND BEHIND THE MOUND

In Figure 5 are shown density patterns of waves recorded in Durban with a wave clinometer over the period 22/7/65 to $27 / 10 / 66$, superimposed on the criterion for erosive and non-erosive waves (see Figure 2) The full lines in Figure 5 represent incident wave conditions as recorded In Durban, whereas the dotted lines enclose the reduced wave conditions behind a $61 \mathrm{~m}$ wide underwater mound It Is clear from a comparison of these density patterns that the balance between erosion and build up wil1 be disturbed by the mound and accretion can be expected to take place until a new equilibrium beach profile is established For the Incident waves 30 per cent of the waves 1 ie in the erosion zone and 70 per cent in the buIld up zone (of course the smaller waves in the buIld up zone are too small to cause any sand movement so that the large difference in the percentages is misleading) When a similar percentage division between erosive and non-erosive waves is assumed, the expected new equilibrium beach slope in the lee of the mound would be steeper if the generalised criterion given in Figure 3 would still apply to the 
conditions behind the mound

Although the latter is probably not the case, there is a strong indication that the equilibrium beach slope behind the mound will be somewhat steeper than the beach profile without the mound

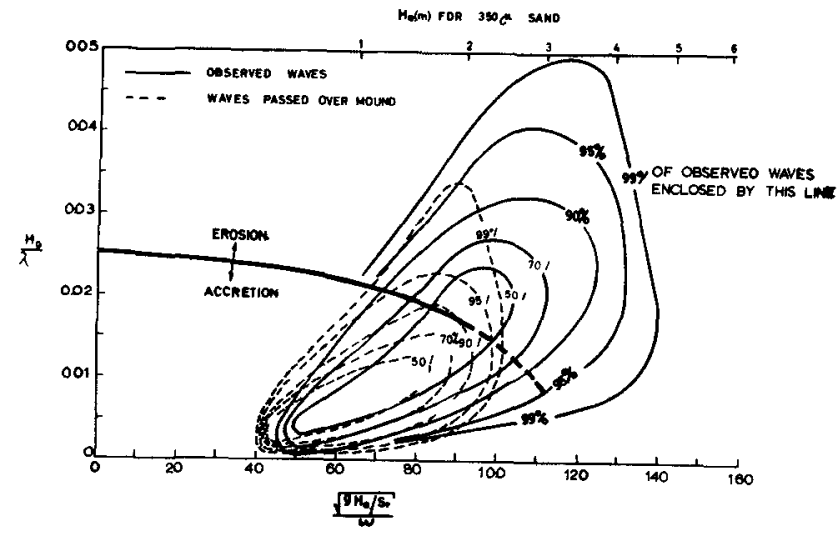

FIGURE 5 DENSITY PATTERN OF OBSERVED WAVES

AND WAVES AFTER PASSING OVER MOUND

The percentages erosive waves behind mounds with different crest widths were calculated in the same way as for the $61 \mathrm{~m}$ wide mound The results, shown in Table II, emphasise the validity of the conclusion reached earlier that the crest width should be $61 \mathrm{~m}$

\section{TABLE II PERCENTAGES EROSIVE WAVES}

\begin{tabular}{|c|cccc|}
\hline \multirow{2}{*}{$\begin{array}{c}\text { Without } \\
\text { mound }\end{array}$} & \multicolumn{4}{|c|}{$\begin{array}{c}\text { With mound with } \\
\text { crest width }(\mathrm{m})\end{array}$} \\
\cline { 2 - 5 } & 0 & 30 & 61 & 92 \\
\hline 30 & 10 & $5 \frac{1}{2}$ & 3 & $2 \frac{1}{2}$ \\
\hline
\end{tabular}

This conclusion was further confirmed by movable bed model studies which showed a significant improvement on the beaches only for a crest width of $61 \mathrm{~m}$ and over ${ }^{7}$ The dimensions of the underwater mound as shown in Figure 1 viz mound crest level at $73 \mathrm{~m}$ below LWOST, side slopes 1 in 25 , crest width $61 \mathrm{~m}$ and total volume about $8000000 \mathrm{~m}^{3}$ were therefore accepted 


\section{WIND-WAVE FLUME TESTS}

In order to study the long term stability of the proposed mound and 1 ts effect on the beaches tests were performed in the CSIR's $120 \mathrm{~m} 1$ long, $3 \mathrm{~m}$ wide wind-wave flume 14,15 For these tests a beach section at Battery Beach was used (see Figure 1) w1th a 1 in 25 beach slope above and 1 in 40 below low water The beach sand had an average diameter of 350 micron whereas the sand in the mound was assumed on average $250 \mathrm{mic}-$ ron The choice of the model sediment was based on the shear-setting velocity criterion The scale factors used are given in Table III

TABLE III SCALES FOR WIND-WAVE FLUME TESTS

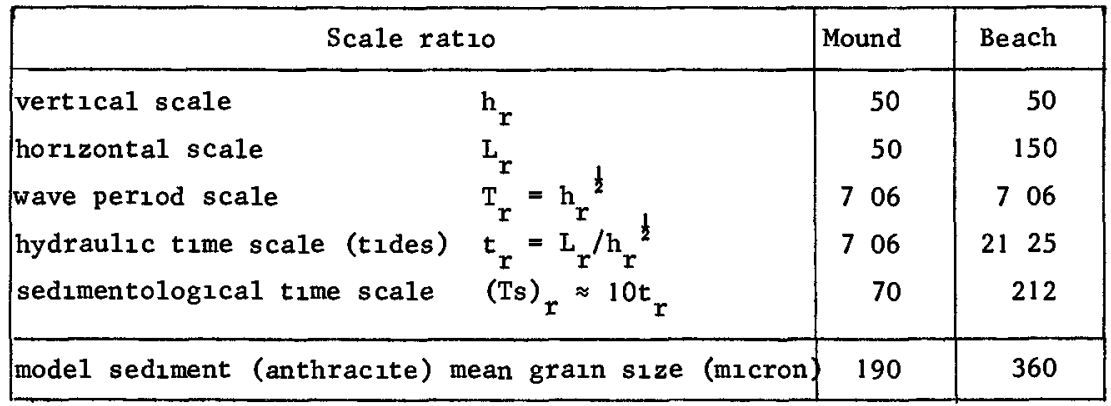

As can be seen from Table III different scales were used to model the mound and the beach although they were tested at the same time This was essential because no distortion could be allowed for the mound, since the wave attenuation for a distorted mound, 1 e with a too small crest width relative to the wave length, would be too small (see Figure 4) Since the whole object of the underwater mound is beach improvement, the time scales pertaining to the beaches were adopted for the entire system

Wave conditions as recorded in Durban from June, 1965 to June, 1967 (2 years) were schematised and scaled down in accordance with the above scale ratio's Recorded wave height spectra were found to agree closely with the Rayleigh distribution of wave heights which could very nearly be reproduced in the flume by a combination of machine and wind generated waves (see Figure 6) 
A continuous test, representing the two years prototype conditions, was carried out whereby two models, one with and the other without the mound were tested simultaneously side by side in the flume Profiles were measured after each wave condition and a typical result is shown in Figure 7 , whlch shows the beach and mound sections after an extremely severe storm condition Even with these large waves the mound remained virtually stable, whereas a considerable improvement, due to the mound, is noted on the beach (2 $5 \mathrm{~m}$ vertical or $90 \mathrm{~m}$ horizontal)

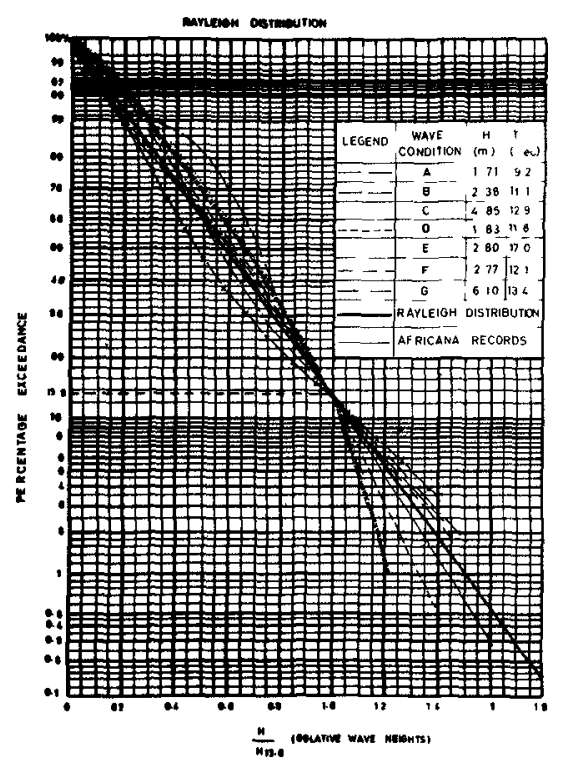

FIGURE 6 WAVE HEIGHT SPECTRA The maximum change of the crest level of the mound was found to be $1 \mathrm{~m}$ and the maximum horizontal movement about $40 \mathrm{~m}$ for the entire period of the test As was expected, a slightly steeper beach slope established under most conditions as a result of the presence of the mound

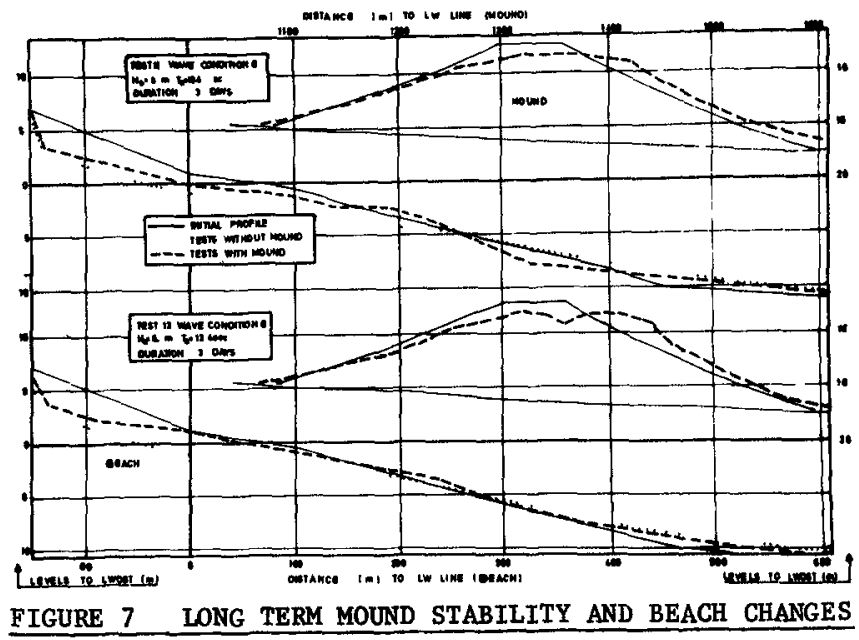


On the basis of thrs extensive test progromme, it was concluded that the underwater mound (as shown in Figure 1) would remann virtually stable, wrth maximum crest level fluctuations of about $1 \mathrm{~m}$ and that the beaches in the lee of the mound would sugnufrcontly improve

\section{CONSTRUCTION OF TEST SECTION}

In order to investigate dumping techniques, stability of the dumped sand, side slopes and possible sand migration from the dumping area towards the nearby harbour entrance or the roadstead, it was decided to supplement the model tests with the construction of a test section of the underwater mound (see Figure 1) Sand dumping started in June, 1966 at the most southern end of the mound and the test mound was virtually completed in November, 1966 when some $700000 \mathrm{~m}^{3}$ of sand, coming from dredging works in Durban Bay, had been dumped (see Figures 8 and 9) By this time the test mound had reached a height of 8 to $85 \mathrm{~m}$ below LWOST over a length of about $1200 \mathrm{~m}$ with side slopes of 1 in 25

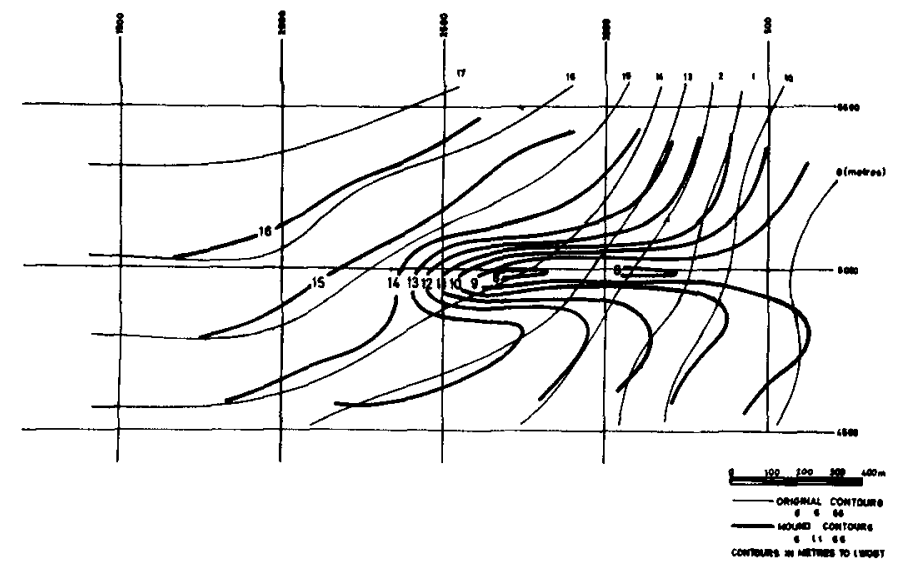

FIGURE 8 BUILD UP OF TEST MOUND AFTER FIVE MONTHS

The test mound provided answers to most of the above questions No difficulties were encountered with dumping with a $1000 \mathrm{~m}^{3}$ capacity hopper dredger in $8 \mathrm{~m}$ water depth, in fact the contractor found it advantageous to dump in this area because of the shorter haulage Weekly echo sounding surveys were made of the area to check on the dumpings and sand quantities calculated from these surveys are compared in 
Figure 9 with the dumping records This comparison shows close agreement and 1 t was thus concluded that there was no loss of sand and that the dumped sand remained in place

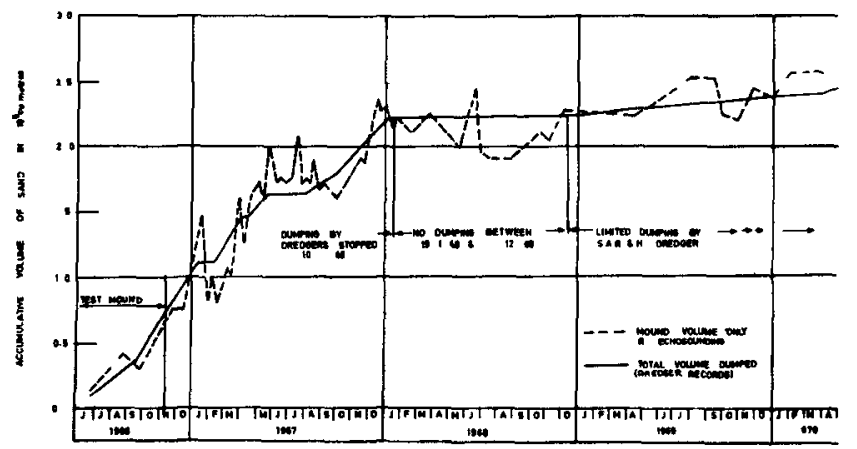

FIGURE 9 ACGEILATIVE QUANTITIES OF SAND IN THE MOUND FROM JUNE 1966 TO APRIL 1970

This was further confirmed by detailed sediment movement studies Sieve analyses of the sand in the mound area were carried out before dumping comnenced The mean grain sizes of the sand on the sea-bottom were found to vary between 212 and $340 \mathrm{micron}$ Samples from the sand in the hopper collected dally by the dredger crew showed fluctuations in mean grain size from 130 to 495 micron Subsequent sampling operations of the sea-bottom at strictly controlled positions provided some evidence of the stability of the dumped sand but this evidence was not conclusive because of these large variations A better check was obtained from the separation of heavy and 11ght minerals by using heavy 11quid (bromoform, s $g=288$ ) The heavy mineral content of the samples was found to be throughout much higher in the original sea bed than in the dumped sand from the dredging site The high content of heavy minerals in the sand on the sea-bottom is due to an abundance of ilmenite, magnetite and garnets, while the dumped sand is rich in light feldspars, calcites and clay minerals (phyllosilicates) Six weeks after dumping started, when $130000 \mathrm{~m}^{3}$ had been dumped, a considerable reduction in the heavy mineral content of the sand collected from the dump area could be discerned, while samples collected from the surrounding areas showed no change at a11 
Finally, tests were performed using fluorescent tracers dumped at the site of the test mound Earlier model results were confirmed by these tests in that sand movements, if any, were found to be inshore Recorded sand grain velocities varied between 5 to $30 \mathrm{~mm} / \mathrm{s}$ for significant wave heights from 1 to $2 \mathrm{~m}$

Based on these results it was concluded that the underwater mound could be expected to remain stable and that no sand from the mound would move towards either the harbour entrance or the roadstead seaward of the mound

\section{FURTHER CONSTRUCTION OF THE MOUND}

Some $1500000 \mathrm{~m}^{3}$ of sand were dumped between November, 1966 and January, 1967 (see Figure 9) The main $1000 \mathrm{~m}^{3}$ hopper capacity dredger ceased operating in August, 1967 and was replaced by three hopper barges with a capacity of $120 \mathrm{~m}^{3}$ each No dumping took place during 1968, which provided an ideal opportunity to study the stability of the completed part of the mound From the end of 1968 onwards limited amounts of sand were dumped by the South African Ra1lway dredgers, carrying out maintenance dredging of the harbour entrance To date (May, 1970) a total of some $2500000 \mathrm{~m}^{3}$ has been dumped

In Figure 9 are shown the cumulative quantities dumped as recorded by the dredger masters, in comparison with quantities calculated from echo sounding surveys These surveys were made weekly or fortnightly during intensive dumping periods to control the dumping and at monthly intervals after January, 1968 It is clear from Figure 9 that no sand is permanently lost from the mound, although fluctuations in quantities do occur as a result of adverse wave conditions For instance, a 20 per cent reduction occurred after a storm in August, 1966 (significant wave height Hs $=24 \mathrm{~m}$, wave period $\mathrm{Ts}=14 \mathrm{~s}$, direction ESE) and a 23 per cent loss occurred, mainly from the incomplete sections of the mound, after a storm in June, 1968 (Hs = $35 \mathrm{~m}$, direction $\mathrm{s}$ ) However, in both cases these losses were regained by natural forces and 1 thus appears that the mound, once it has been built up to the required level of $73 \mathrm{~m}$ below LWOST, is reinstated naturally even after considerable temporary losses of sand 


\section{THE STABILITY OF THE MOUND}

In the above it has been shown that the sand quantities in the completed section of the mound do not change materially as a result of the actions of the sea Figure 10 gives an example of contours of the mound for December, 1969 and comparisons with similar surveys before and after this date showed that no significant displacement of the mound takes place ${ }^{16}$

This is further borne out by a study of some of the cross sections of the mound shown in Figure 11 (for positions of cross sections refer to Figure 12) These cross sections are based on echo sounding surveys made during 1969 when no dumping took place, except at the northern extremity of the mound (sections 1000 to 1500, see Figure 12) The profiles show random variations with a maximum fluctuation in crest levels of $1 \mathrm{~m}$ and maximum lateral movements of the crest of the mound of about $75 \mathrm{~m}$

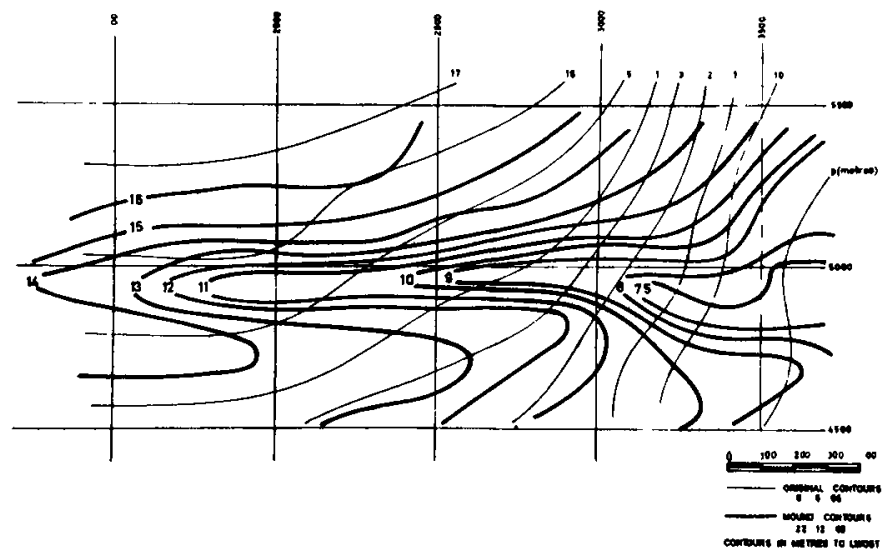

FIGURE 10 DEPTH CONTOURS OF THE UNDERWATER MOUND AS ON 22.1269

Although it can be seen from Figure 11 that, except for section 3300 , the mound did not yet have the required cross section, the agreement between these recorded movements with those obtained in the model tests, viz $1 \mathrm{~m}$ crest level variation and about $60 \mathrm{~m}$ lateral movement, are extremely good 


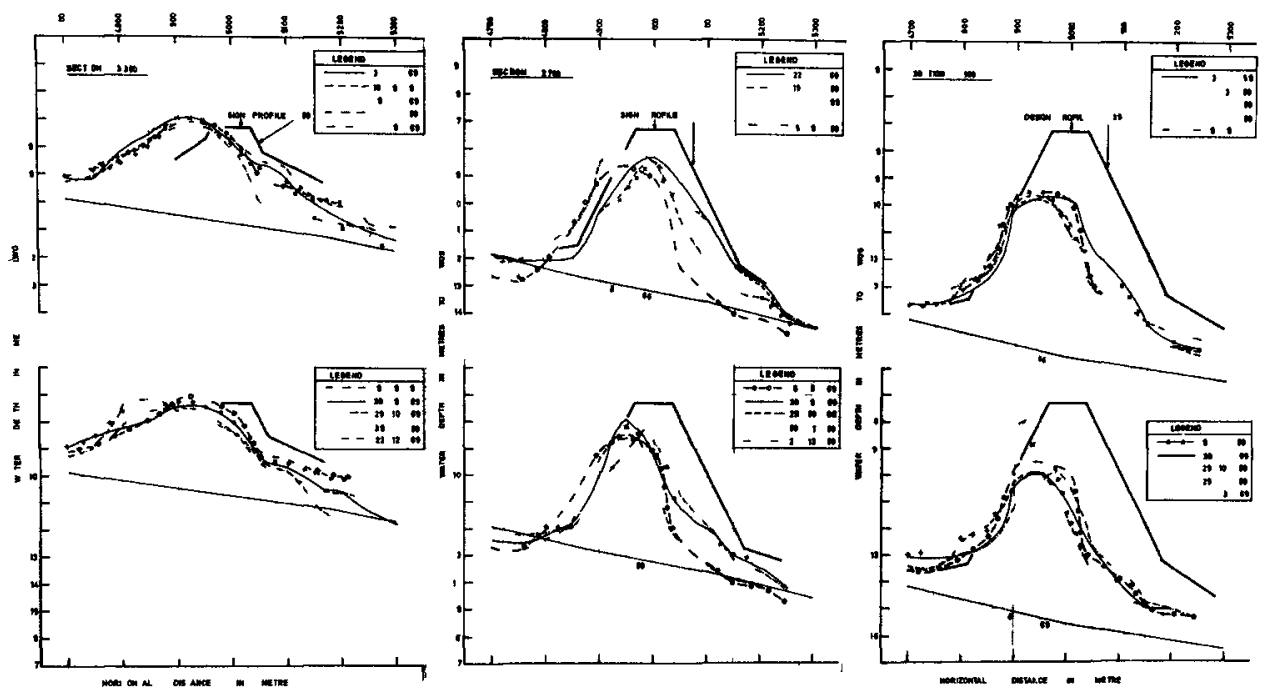

FIGURE 11 BEHAVIOUR OF UNDERWATER MOUND CROSS SECTIONS DURING 1969

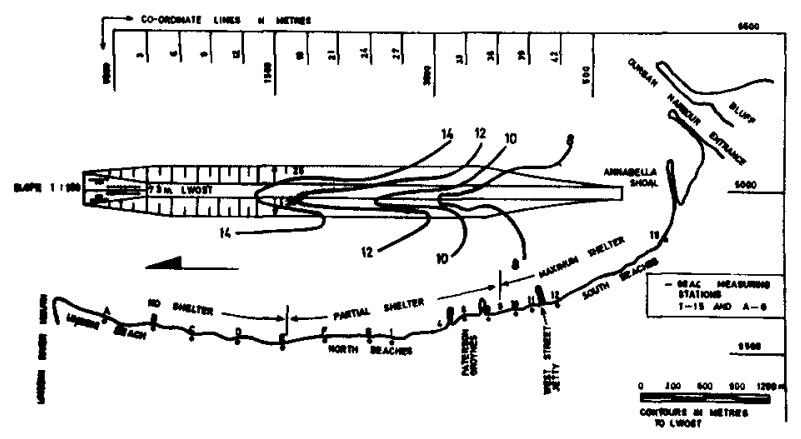

FIGURE 12 SCHEMATIC PLAN OF THE DURBAN

UNDERWATER MOUND AS ON $22 \quad 1269$

EFFECT ON THE BEACHES

As can be seen from Figures 11 and 12, the underwater mound 18 still far from complete On1y $2500000 \mathrm{~m}^{3}$ had been dumped by May, 1970 against the required $8000000 \mathrm{~m}^{3}$ and the crest leve1 is only reasonably close to the required $73 \mathrm{~m}$ below LWOST over a length of some $1500 \mathrm{~m}$, which is about one third of the required length of the mound The most log1- 
cal method in assessing the beaches therefore, is to compare the beaches in terms of the amount of protection they recelve from the partly completed mound A sub-division is made between beaches receiving ful1 protection (south beaches), partial protection (Patterson Groynes area) and zero protection (northern beaches to Umgeni mouth) (see Figure 12)

Figure 13 shows the overa11 effect on the beaches since the construction of the mound started The 100 per cent is based on the average of the measured sand volumes on the beaches above mean sea level for the period November, 1965 to May, 1966 and a11 subsequent month1y measurements were compared with these values

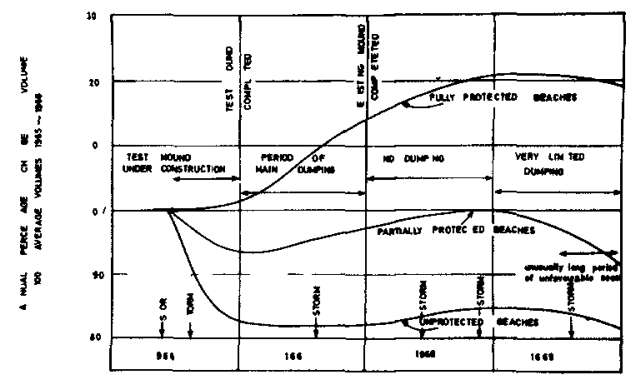

FIGURE 13 EFFECT ON BEACHES FOR VARIOUS DEGREES OF PROTECTION

Although even at the south end the mound has not yet reached its ful1 design crest width, it is clear from Figure 13 that the fully protected beaches greatly benefited from the mound (about 20 per cent 1ncrease in sand quantities) whereas partially protected beaches show less improvement and those unprotected appear to loose sand

The partially protected beaches were close to equilibrium at the end of 1968 but they show a dec1ine in beach volumes during 1969 Th1s was caused by a severe storm with $45 \mathrm{~m}$ high SE'1y waves which occurred on the 22nd August, 1969 Th1s was the most severe storm since the devastating storm in May, 1966 which caused severe erosion of the northern beaches (see Figure 13) and the first major test of the completed section of the mound The mound stood up to the test extremely we11, hardly any loss of sand was recorded and crest levels remained within $05 \mathrm{~m}$ the same as before the storm

The effect of the storm on the beaches was serlous, as can be expected from a storm of such magnitude The northern unprotected 
beaches showed a loss, compared with the condition just before the storm, of 21 per cent against 33 per cent for the May, 1966 storm, the partially protected central beaches 1 ost 16 per cent, which 18 the same as in 1966, whereas the protected beaches lost only 8 per cent agannst 20 per cent in May, 1966

It is thus clear that the completed section of the underwater mound has considerably improved the beaches in 1 ts lee, as was predicted on the basis of the model tests

\section{CONCLUSIONS}

The following conclusions may be drawn from the Durban underwater mound studies

(a) An underwater mound of sand of the correct dimensions offers an effective beach protection scheme

(b) Such a mound of fine to medium sand may be expected to remain virtually stable under most wave conditions and if losses occur during severe storms, natural processes will re-build the depleted mound to its original size

(c) Reliable results, 1 e accurate predictions of prototype events, were obtained from movable bed models which were designed in accordance with the shear-setting velocity criterion

The entire mound w11l have to be built to provide protection to all the beaches between the harbour entrance and the Ungenl mouth It is expected that the mound will be completed in about 2 to 3 years, using sand from future harbour extension works in Durban Bay

\section{ACKNOWLEDGEMENTS}

The continual and very stimulating support of this work by the Durban Corporation and in particular of the then Direction of Special Works, Mr A Kinmont, is gratefully acknowledged

\section{REFERENCES}

1 JORDAAN, J M Durban harbour siltation and beach erosion investigations CSIR Contract Report CMEG 558, Pretoria, South Africa, September, 1963 
JORDAAN, J M Study of Durban harbour silting and beach erosion Proc XIIth Conference on Coastal Englneering, Washington, September, 1970

3 ZWAMBORN, J A Underwater mound for the protection and improvement of Durban's ocean beaches Preliminary investigations CSIR Contract Report CMEG 596, Pretorıa, South Afrıca, July, 1964 HALL, J V Test of nourishment of the shore by offshore deposition of sand Techn Memo No. 17, B E B , June, 1950

5 HALL, J V Artificially nourished and constructed beaches Techn Memo No 29, B E B , December, 1952 ZWAMBORN, J A, VAN WYK, W Durban beach protection Interim Report No 5 CSIR Report MEG 528, Pretoria, South Africa, July, 1964

7 ZWAMBORN, J A, VAN WYK, W Some positive results in reproducing coastal processes in models CSIR Symposium Paper $S 37$, Stellenbosch, South Africa, June, 1969

8 ZWAMBORN, J A Reproducibility in hydraulic models of prototype river morphology La Houllle Blanche, No 3, 1966

9 ZWAMBORN, J A Hydraulic models CSIR Report MEG 795, Stellenbosch, South Africa, July, 1969

10 IWAGAKI, $Y$, NODA, H Laboratory study of scale effects in twodimensional beach processes Proc VIIIth Conference on Coastal Engineering, Mexico City, 1962

11 WIEGEL, R L Oceanographical engineerıng Prentice-Hall, Inc /Eaglewood Cliffs, N Y

12 KEULEGAN, $\mathrm{G} H$ Depths of offshore bars Techn Memo No 8, Beach Erosion Board, Washington, July, 1945

13 B E B Longshore bars and longshore troughs Techn Memo No 15, Beach Erosion Board, Washington, January, 1950

14 VAN WYK, $W$ Durban beach protection Long term mound stability and beach 1mprovement CSIR Report MEG 852, Stellenbosch, September, 1969

15 ZWAMBORN, J A CSIR's new wind-wave flume is amongst world's longest S A Englneer, Vo1 58, No 595, December, 1967

16 FITZPATRICK, J B Stability of underwater mound for the protection of Durban's beaches Symposium Paper No G4, Oceanography, 1970, CSIR, Durban, South Africa 


\title{
CHAPTER 63
}

\section{LABORATORY TESTS ON ARTIFICIAL SEAWEED}

W A PRICE - Senior Principal Scientific Officer

$K W$ TOMLINSON - Senior Scientific Officer

D H. WILLIS - Scientific Officer

Hydraulıcs Research Station, Wallıngford, Great Britain

\author{
ABSTRACT
}

Tests are described in which the effect of artificial seaweed on bed sediment movements due to waves was studied in a model Water velocities near the bed and coal particle velocities on the bed, within the seaweed array, were measured over a range of wave conditions and densities of packing of the seaweed The water velocity measurements were erratic, probably due to additional turbulence caused by the seaweed Coal particle movements on the bed were slowed down in the presence of seaweed

\section{Introduction}

A paper was presented to the 1lth Conference on Coastal Engineering entitled, "The Effect of Artificial Seaweed in Promoting the Bulld-up of Beaches", see Ref 1 This paper described tests conducted in a laboratory wave channel using a beach of crushed coal, in addition to full-scale tests on the beach at Bournemouth, England Anchorage problems stopped the full-scale tests before meaningful results could be obtained although some accretion had occurred inshore of the seaweed array In the model, the presence of artificial seaweed offshore caused a bulld-up of the beach levels an explanation of this build-up was suggested from the theory of wave propagation in a two-layered liquid of differing viscosities

Since that time further laboratory investigations have been carried out to attempt to find an optimum arrangement of seaweed, and to test the applicability of the viscosity theory over a range of wave helghts, perlods and densities of seaweed packing 


\section{Test Procedure and Results}

The artificlal seaweed was a white polypropylene ribbon $3 \mathrm{~mm}$ wide having a specific gravity of 093 Twenty strands of this, $030 \mathrm{~m}$ long, formed a clump whlch was stapled to the plywood floor of a wave channel clump spacing was varied to make three seaweed arrays, each $460 \mathrm{~m}$ long and $061 \mathrm{~m}$ wide

$$
\begin{aligned}
\text { Clumps at } 150 \mathrm{~mm} \times 150 \mathrm{~mm}=120 \text { clumps } \\
\text { Clumps at } 75 \mathrm{~mm} \times 75 \mathrm{~mm}=420 \text { clumps } \\
\text { Clumps at } 50 \mathrm{~mm} \times 50 \mathrm{~mm}=990 \text { clumps }
\end{aligned}
$$

The wave channel was $285 \mathrm{~m}$ long and $061 \mathrm{~m}$ wide, with a mean water depth at the seaweed of $043 \mathrm{~m}$ At the offshore end a paddle generated single-frequency waves The tests were carried out with a fixed bed and landward of the seaweed installation was a pebble spending beach, $10 \mathrm{~m}$ long parabolıc in shape

Fourteen wave conditions, with periods from 15 sec to $25 \mathrm{sec}$ and heights from $50 \mathrm{~mm}$ to $150 \mathrm{~mm}$ were generated for the channel bed without seaweed and for each seaweed installation Observations were made of the wave orbital velocities near the bed and of the movement of coal particles along the bed

1 Wave orbital velocities near the bed A miniature propeller current meter was placed $10 \mathrm{~mm}$ above the bed and connected to an instantaneous ratemeter to glve a continuous record of orbital velocities during one wave period This record was integrated to obtain the net wave induced drift, and divided by the wave period to give the mean drift velocity at the point of measurement It was assumed that an increase in the landward drift velocity offshore might be expected to produce an increased landward sedument transport, and hence a buld-up of levels inshore and on the beach

The results are show in Fig $1 \mathrm{a}, \mathrm{b}$ and $\mathrm{c}$ It $\mathrm{is}$ apparent that there is little correlation between the density of seaweed packing and drift velocity some tests were repeated, and the net drift velocities were found to vary by as much as $\pm 300 \%$ Differences of $200 \%$ were found with only a $75 \mathrm{~mm}$ change in the horizontal position of the propeller The instruments were checked frequently to ensure that they were operating correctly

The conclusion was reached, therefore, that the seaweed created additional turbulence, making veloclty readings at a single point unrepresentative of water movements over the entire array In order to study conditions over the array as a whole, the movement of coal particles along the bed was also measured 

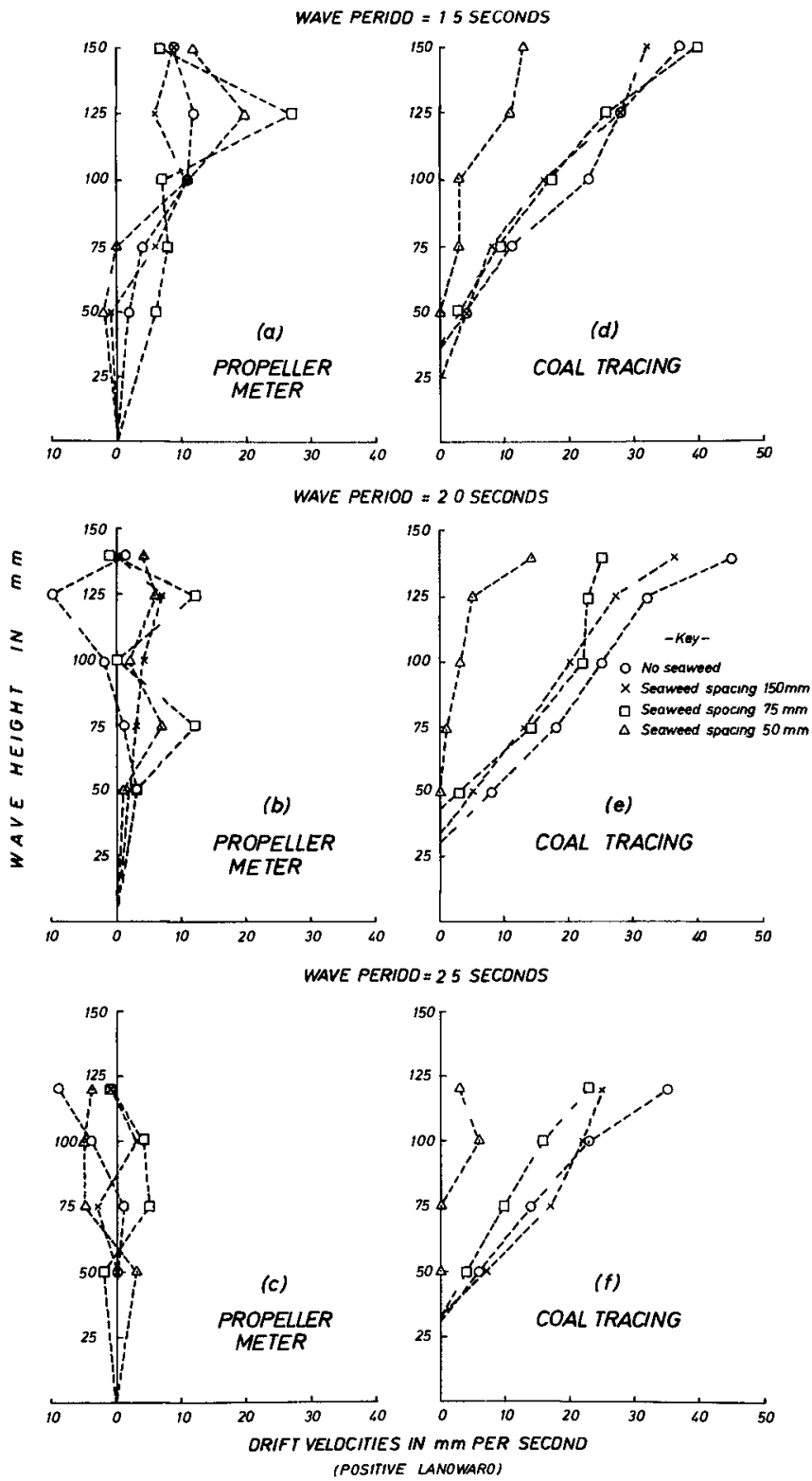

FIGI 
2 Movement of coal particles along the bed coal particles were injected on the bed, and the number of waves required to move a particle over a known distance was counted The net drift velocities found in this way are plotted on Fig $1 d$, e and $\mathbf{f}$

These figures show a trend towards lower net drift velocities with denser seaweed packing, and with the exception of one seaweed installation, the results were repeatable For the exception, seaweed at a clump spacing of $50 \mathrm{~mm}$, turbulence was markedly greater with larger waves even the boundary layer behaved turbulently, and the coal dispersed in all onshore directions rather than parallel to the channel axis, with lower waves, the coal collected at scattered points in the array where bed shear was insufficient to move It

\section{Discussion}

In Interpreting the results of the propeller meter tests, the method of analyzing the data must be considered Wave Induced drifts towards and away from the beach were calculated by integrating the velocity records and these were subtracted to obtain the net drift The net drift so obtained was of the same order as the probable error in the velocity measurements In addition, there were obvious differences In the shape of the velocity record for repeated tests The lack of correlation between drift velocity and seaweed packing was probably due to the combined effects of computational error and turbulence

The coal particle experiments, however, gave some interesting results of the fourteen wave conditions tested, all but three showed drift velocities with seaweed less than or equal to those without seaweed Furthermore, in seven cases there was a definite trend towards lower drift velocities with denser seaweed packing, and only for a clump spacing of $150 \mathrm{~mm}$ with 15 sec waves was there a large incongruity These results are not consistent with those predicted from the theory of wave propagation in a twolayered liquid of differing viscosities

The results of the present serıes of tests suggest that the presence of artificial seaweed inhibits the transport of sand from the offshore seabed towards the beach This appears to contradict the findings of the earlier tests in which a bulld-up of the beach was measured shoreward of the seaweed Installation However, the earlier bulld-up may not have been due to increased shoreward bed-load transport, but caused by a reduction in wave steepness due to attenuation of the wave height by the seaweed In addition, the two series of tests were not truly comparable first, the response of the instruments dictated wave periods for the most recent series greater than the 133 sec of the first series, and second, the use of a coal beach in the 
original tests introduced the factors of beach slope, grain size distribution and suspended sediment load, none of which were present in the tests reported here

The results of the coal tracing indicate that artificial seaweed may have an application in uni-directional flow as a sediment trap, reducing the velocity gradient and bed shear and causing accretion within the array

\section{Reference}

1 PRICE, W.A, TOMLINSON, $\mathrm{K}$ W, and HUNT, J $\mathrm{N}$ The effect of artificial seaweed in promoting the build-up of beaches Proceedings lith Conference on Coastal Englneering, London, 1969, Vol 1 pp 570-578

This report is published by permission of the Director of Hydraulıcs Research, Wallingford, England 



\section{CHAPTER 64}

THE DYNAMTCS OF A COAST WITH A GROYNE SYSTEM

by W.T. Bakker, E.H J. Kleln Breteler, and A. Roos

Coastal Research Department, Rijkswaterstaat

The Hague, Netherlands

\section{ABSTRACT}

This paper is a continuation of the paper with the same name, presented on the XIth Conference on Coastal Engineering by the first author [1], in which a mathematical theory was given about the behaviour of a coast after the construction of a groyne system. Now this paper extends the former paper theoretically and practically.

1. Theoretically a computer program has been made in which the influence of diffraction behind the groyne has been taken into account.

2. Practically the coastal constants used in the theoretical model of the coast will be expressed in terms of wave helght and wave direction, based on the theory of SVASEK [2] .

Results are given of computations with a coastal model in which the coast is schematized to one line (one-line theory) and a model in which the coast is schematized to a beach line and on inshoreline (two-line theory).

The influence of changing wave conditions is investrgated.

\section{INTRODUCTION} (f1g. 1)

The construction of a groyne has the following effects

1. Prevention of the littoral sanddrift in the area between the coastline and the head of the groyne.

2. Prevention of the longshore current in the same area.

3. Formation of a sheltered area at the lee-side of the groyne, caused by the diffraction.

4. Changing the wave helght by reflection

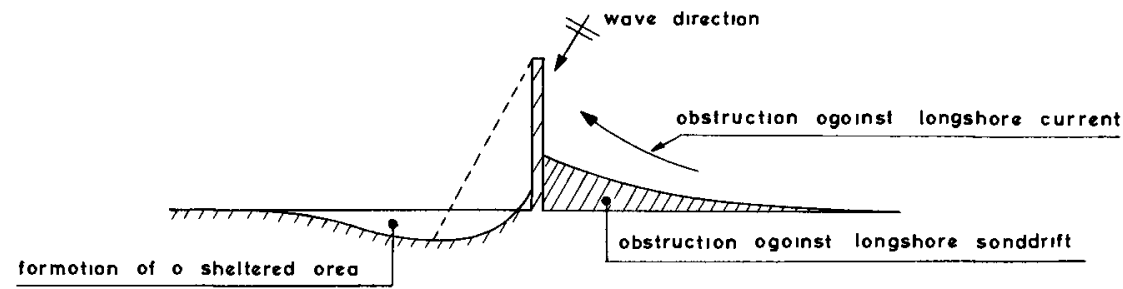

Fig 1 The effects of the construction of a groyne 
The former paper dealt only about the first aspect, now we shall pay attention to the first and the third aspect. The second and fourth one will be investigated in the future.

\section{ONE-LINE THEORY}

The theory given here is an extension of the theory of PELNARD-CONSIDERE [3].

PELNARD-CONSIDERE assumes, that the profile of the coast always remains the equilibrium profile, so that he only needs to consider one coastline, being one of the contourlines. He assumes no currents, constant wave direction, small angle of wave incidence and a linear relation between angle of wave incidence and the littoral drift.

The derivation of his theory is summarized in [1].

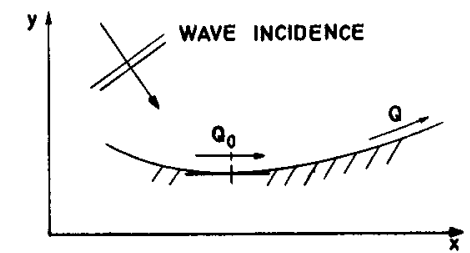

Fig 2 Littoral drift along the coast For the littoral drift he finds $Q=Q_{0}-q \frac{\partial X}{\partial x}$ In which $Q=$ littoral drift.

$Q_{0}=11$ ttoral drift at the point, where

$$
\begin{aligned}
& \frac{\partial y}{\partial x}=0 . \\
q= & \frac{\partial Q}{\partial \varphi}=\text { the derivate } \\
& \text { of the littoral } \\
& \text { drift } Q \text { to the angle } \\
& \text { of wave incidence } \varphi .
\end{aligned}
$$

He finds, that the accretion is proportional to the curvature of the coast

$$
\frac{\partial y}{\partial t}=\frac{q}{D_{\text {tot }}} \frac{\partial^{2} y}{\partial x^{2}}
$$

From this equation the coastline $y$ as a function of $x$ and $t$ can be found for many boundary conditions. Pelnard-Considère gives analytical solutions of his equations. The interrupted line in fig. 5 shows the accretion and erosion near a groyne according to his theory. He assumes that wave height and wave direction are constants along the coast. At the lee-side of the groyne however, the wave helght is less and the waves have an other direction, as a result of the diffraction.

We introduce diffraction in the theory of Pelnard-Considere

- The equations become more complicated, that's why we have to give numericil solutions. The derivation of the one-line theory including diffraction is given in appendix A'1. For the littoral drift the same formula of Pelnard-Considere remains of value

$$
Q=Q_{0}^{*}-q^{*} \cdot \frac{\partial y}{\partial x}
$$

but now $Q^{*}$ and $q^{*}$ are functions of $x$. 
The effect of the diffraction can be splitted in a stationary effect and an instationary effect. This can be made $c^{7}-3 r$ in the following way (fig. 4 ). If everywhere wave helght and wave direction are the same, a straight coastline is stable, the transport is everywhere the same. If the wave height and the wave direction change in $x$-direction, the transport will change also and therefore the coastal shape has to adapt Itself in order to make the transport everywhere the same again and give a stable coastline. In appendix $A 1$ a mathematical formulation of this problem is given. The transport has been taken proportional to the square of the wave helght and to the angle of wave
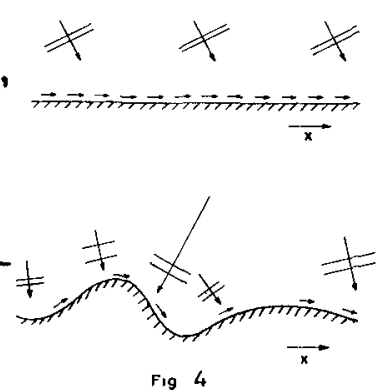
incidence. A possible stable coastilne $y_{0}$ as a function of $x$ is found (appendix AT), ruled by the differential equation

$$
\frac{d y_{0}}{d x}=\varphi_{x}-\frac{\varphi_{\infty}}{h^{2}}
$$

In which $\varphi_{x}$ is the angle of the waves with the $x^{a x 1 s,} \varphi_{\infty}$ the angle of wave incidence far from the groyne and $h$ is the ratio between the wave height at an arbitrary point $(x, 0)$ to the wave helght at $x=\infty, h$ is a function of $x$.

A short analysis of (4) learns, that if the wave height should be everywhere the same $(h=1)$ this would give $\frac{d y_{0}}{d x}=\varphi_{x}-\varphi_{\infty}$, thus the changing of the coastal direction $1 s$ equal to the changing of the wave direction.

However, the problem of diffraction near a harbour mole is more intricate.

As the groyne stops all the transport, and as at $x=\infty$ the transport remains $Q_{O}$, a stable coastline can never be achleved. We split the coastline $y$ into two parts, yo being a stationary effect of the diffraction ${ }^{7}$ and $y^{\prime}$, being an unstationary effect

$$
y(x, t)=y_{0}(x)+y^{\prime}(x, t)
$$

As shown in appendix A1, the equation for the unstationary part $y^{\prime}$ becomes about (2), but with an additional term, because $q^{*}$ is a function of $x$

$$
\frac{\partial y^{\prime}}{\partial t}=\frac{g^{*}}{D_{t o t}} \frac{\partial^{2} y^{\prime}}{\partial x^{2}}+\frac{1}{D_{t o t}} \frac{d g^{*}}{d x} \cdot \frac{\partial y^{\prime}}{\partial x}
$$

in which $q^{*}=A h^{2}$

1)

$y_{0}$ is the stable coastline, which would develop, if an artificial nourishment $Q_{0}$ would be administered on the lee-side of the groyne. 
A is a proportionality constant, being investigated in the chapter "coastal constants".

The amount of $\mathrm{h}$ and $\varphi_{X}$ in (4) and ( 7 ) in the diffraction case 25 found from the simplified theory of PUTNAM and ARTHUR [4].

The unstationary part $y^{\prime}$ can be found by numerical integration of equation (6).

Superposition of $y_{0}$ and $y^{\prime}$, according to (4) gives the coastline $y(x, t)$.

For the calculation of the coastines a computerprogram has been made. Fig. 5 shows the calculated development of a coast with one groyne. Comparison of the interrupted and the solid line gives an impression of the influence of diffraction.

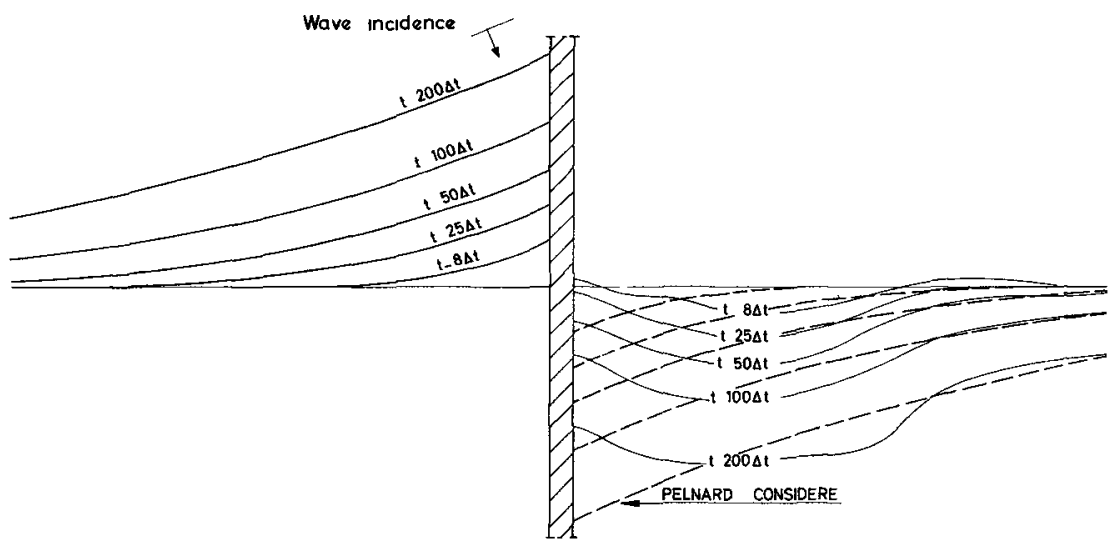

Fig 5 Accretion and erosion near a groyne numerical solution with diffraction (one line theory) The dotted lines at the right hand gives erosion according to Pelnard-Considere

Wh the computerprogram we calculated the behaviour of the coastline between two groynes with the influence of diffraction. The result 18 shown in $\mathrm{fig} \cdot 6$.

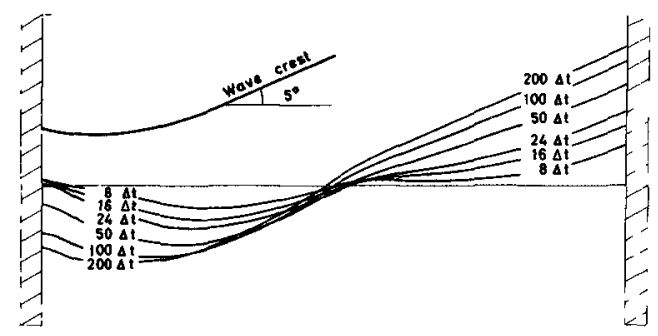

Fig 6 Behavior of the coastline between two groynes (one-line theory) 


\section{TWO-LINE THEORY}

Now we schematize the coastal profile to two lines, the beach $\left(y_{1}\right)$ and the inshore $\left(y_{2}{ }^{\prime}\right)$. This gives the possibility to take the off - and

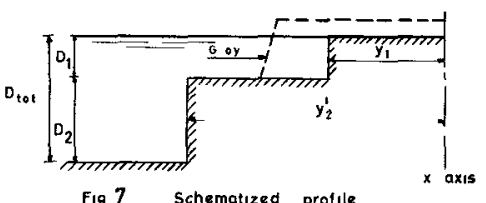
onshore transport into account. In top view one sees two lines at a distance $y_{3}$ and $y_{2}$ ' from the $x$-axis. The "equilibrium distance" is the distance $y_{2}^{\prime}-y^{\prime}$ between beach and Fig 7 schematized profile inshore, when the profile is an equxlibrium profile. The following dynamic equations are

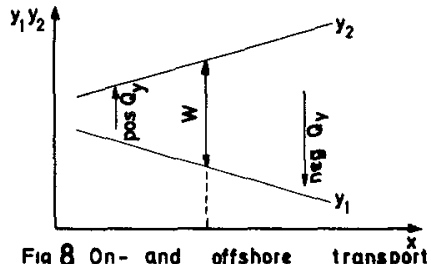
issumed. If the distance $y_{2}^{\prime}-y^{\prime}$ is equal to the equilibrium distance $W$, no interaction is assumed. If the distance $y_{2}{ }^{\prime}-y_{1}$ is less than $W$, the profile is too steep and an offshore transport $\$ 111$ be the result. An onshore transport will occur in the opposite case.

We linearize this relation and take for the offshore transport $Q_{y}$ per unit length

$$
Q_{y}=q_{y}\left\{y_{1}-\left(y_{2} \cdot-w\right)\right\} \quad \cdots \cdot(9)
$$

In which qy 15 a proportional constant with the dimension [1/t]. It 18 a function of $x$. For a simpler notation, we denote

$$
\mathrm{y}_{2}=\mathrm{y}_{2}, \mathrm{~W} \quad \cdot \text {. . (10) }
$$

Then (9) becomes

$$
Q_{y}=q_{y}\left(y_{1}-y_{2}\right)
$$

With respect to the littoral drift, the assumption of PELNARDCONSIDERE is applied, both for beach and inshore, the transport is linearized

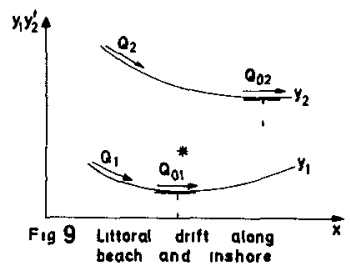

$$
\begin{aligned}
& Q_{1}=Q_{01}^{*}-q_{1}^{*} \frac{\partial y_{1}}{\partial x} \quad \cdots \cdot \cdot \cdot(12 a) \\
& Q_{2}=Q_{02}-q_{2} \frac{\partial y_{2}}{\partial x} \cdot \cdots \cdot \cdot \cdot \cdot(12 b)
\end{aligned}
$$

In whIch $Q_{01}$ and $Q_{02}$ are respectively the transports where $\frac{\partial y_{1}}{\partial x}=0$. $Q_{01}^{*}$, ulction of $x, Q_{02} 15$ a constant. $q_{1}^{*}$ and $q_{2}$ are proportionallity factors, $q^{*}$ is a function of $x$ and $q_{2}$ is a constant. In appendix $A 2$ the derivation of the two-line theory is given. The beach line $y_{1}$ is splitted into two parts, analogue to (4).

$$
y_{1}(x, t)=y_{0}(x)+y_{1}^{\prime}(x, t) \quad \cdots \cdot \cdot \cdot \cdot
$$


In which $y_{0}(x)$ is the same function as given in (8).

For the accretion along the beach and the inshore we find

$$
\begin{aligned}
& \frac{\partial y_{1}^{\prime}}{\partial t}=\frac{q_{1}}{D_{1}} \frac{2}{\partial y_{1}} \frac{1}{\partial x^{2}}+\frac{1}{D_{1}} \frac{\partial q_{1}}{\partial x} \cdot \frac{\partial y_{1}^{\prime}}{\partial x}-\frac{q_{y}}{D_{1}}\left(y_{1}^{\prime}+y_{0}-y_{2}\right) \ldots(14) \\
& \frac{\partial y_{2}}{\partial t}=\frac{q_{2}}{D_{2}} \frac{\partial^{2} y_{2}}{\partial x^{2}}+\frac{q_{y}}{D_{2}}\left(y_{1}^{\prime}+y_{0}-y_{2}\right)
\end{aligned}
$$

These are two simultaneous partial differential equations. For the calculation of the beach line $y_{1}$ and the inshore IIne $y_{2}$ we made a computer program in which the equations are solved numerically (appendix A2). In fig. 10 the development of a coast with one groyne is shown.

Wove incidence
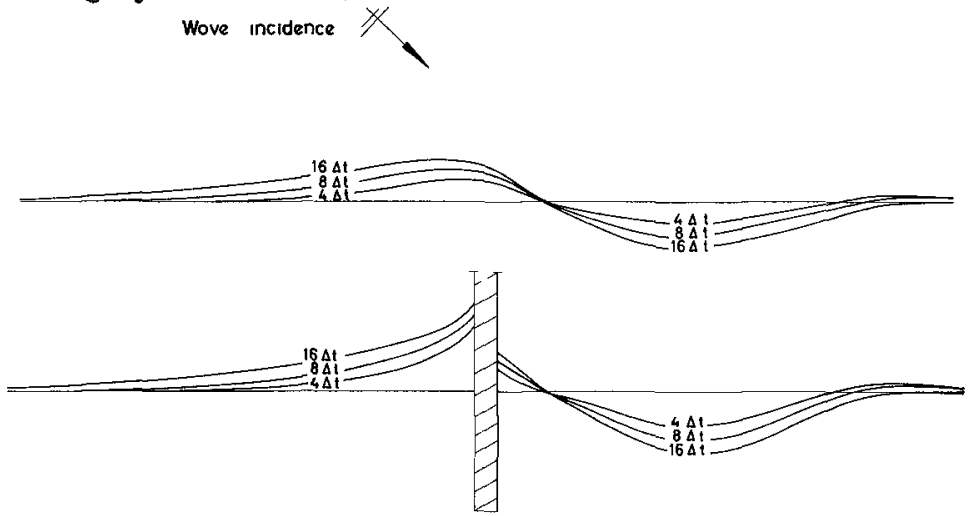

Fig 10 Accretion and erosion neor a groyne numericol solution with diffroction Wove incidence (two-line theory)
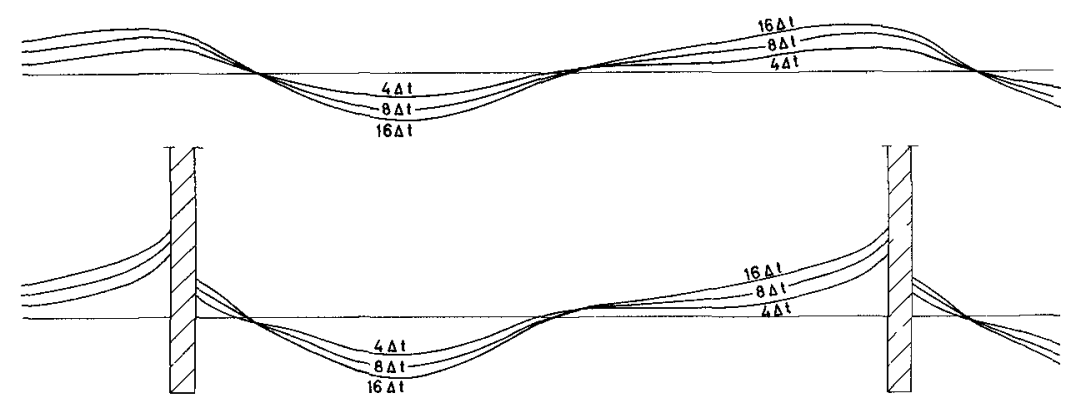

Fig 11 Behaviour of beach and inshore between two groynes (two-line theory) 
From a coast with an infinite row of groynes, we calculated the development of the coastal shape between two groynes. This is shown in fig. 11.

\section{COASTAL CONSTANTS}

In this chapter some expressions will be derived for the coastal constants, respectively using the one-line and the two-line theory. For the one-line theory the CERC-formula will be used, for the two-line theory the SVASEK-variation of this [2]. It is assumed, that the transport is confined to the breaker-zone.

$$
D_{\text {tot }}=D_{b r}
$$

Considering the longshore theory of BOWEN 6 it may be expected that the transport takes place over a distance 1 to 1,5 times the breaker zone, and that most of the transport is confined to the breaker zone. Probably it is better to assume for $D_{\text {tot }}$ the

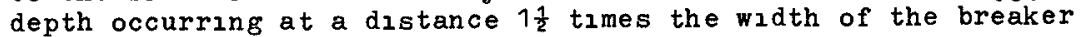
zone. In this case the factor q becomes less, for a concave profile about $100 \%$ to $80 \%$ of the computed value.

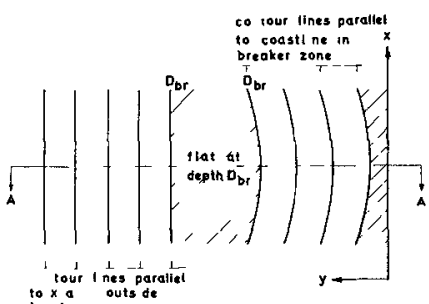

Fig $12^{\circ}$

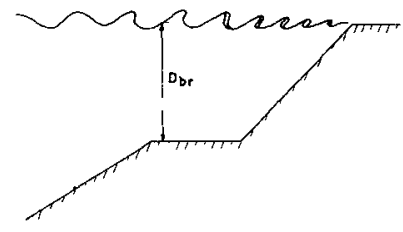

Fig $12^{\mathrm{b}}$ protile A-A

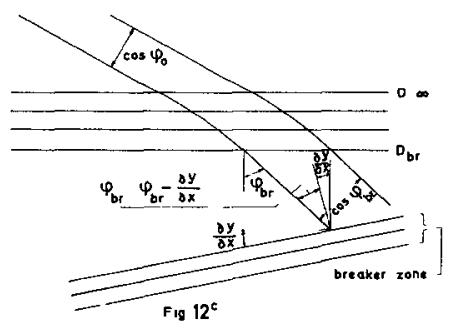

Fig $12^{\circ}$

\section{One-line theory}

We assume the topography and notation according to fig. 12 The CERC-formula relates the longshore transport $Q$ to the longshore 
component of the wave energy flux

$$
Q=1.4 \times 10^{-2} \mathrm{H}_{0}^{2} \mathrm{C}_{\mathrm{o}} \mathrm{K}^{2} \sin \varphi_{\mathrm{br}} \cos \varphi_{\mathrm{br}} \ldots \ldots \text { (17) }
$$

In which $\mathrm{H}_{0}=$ wave helght in deep water

$C_{0}^{0}=$ phase velocity in deep water

$\mathrm{K}=$ refraction coefficient

$\varphi_{b r}=$ angle of wave incidence in the breaker zone

From fig. 12 can be derived

$$
\begin{aligned}
\varphi_{b r} & =\varphi_{b r}-\frac{\partial y}{\partial x}\left(\frac{\partial y}{\partial x} \operatorname{small}\right) \\
Q_{0} & =1.4 \times 10^{2} \mathrm{H}_{0}^{2} c_{0} K^{2} \sin \varphi_{b r}{ }^{\prime} \cos \varphi_{b r}{ }^{\prime} \cdots \\
q & =\frac{d Q}{d\left(\frac{\partial y}{\partial x}\right)}=1.4 \times 10^{-2} \mathrm{H}_{0}^{2} c_{0} K^{2} \cos 2 \varphi_{b r}{ }^{\prime} \cdots
\end{aligned}
$$

One can write

$$
\mathrm{H}_{b r}=A_{2} D_{b r} \quad . \quad(21) \text { and } C_{b r} \sqrt{g D_{b r}} \cdot \text {. }
$$

In which $A_{2}$ and $A_{3}$ can be taken from any wave theory or measurements (for instance, solitary wave theory [?]

$$
A_{2}=0.78 \text { and } A_{3}=2 \times 0.78 \text { ). }
$$

Conservation of wave energy between wave rays gives

$$
\mathrm{H}_{0}^{2} \mathrm{C}_{0} \mathrm{~K}^{2}=\mathrm{H}_{\mathrm{br}}^{2} \mathrm{C}_{\mathrm{br}}=\mathrm{A}_{2}^{2} \mathrm{~A}_{3} \mathrm{~g}^{\frac{1}{2}} \mathrm{D}_{\mathrm{br}}{ }^{5 / 2}
$$

Th1s makes

$$
\begin{aligned}
& Q_{0}=A_{1} A_{2}^{2} A_{3} g^{\frac{1}{2}} D_{b r}{ }^{5 / 2} \sin \varphi_{b r}{ }^{\prime} \cos \varphi_{b r}{ }^{\prime} \cdots \text { (23) } \\
& q=A_{1} A_{2}^{2} A_{3} g^{\frac{1}{2}} D_{b r}{ }^{5 / 2} \cos 2 \varphi_{b r}{ }^{\prime} \ldots \ldots . . . .(24)
\end{aligned}
$$

In which $A_{1}=1.4 \times 10^{2}$

Often cos br can be taken equal to 1 .

Now it is easy to give numerical values to the proportionality constants, used elsewhere in this paper, for instance, in ( 7 )

$$
A=1.4 \times 10^{-2} \mathrm{H}_{0}^{2} C_{0} K^{2}
$$


and in appendix $A 1,(A 10)$

$$
\begin{aligned}
& \Delta t=\frac{D_{\text {tot }}}{A_{\text {max }}^{2}} \frac{(\Delta x)^{2}}{2} \\
& \Delta t=\frac{D_{b r}}{A_{1} A_{2}{ }^{2} A_{3} g^{\frac{1}{2}} D_{b r}^{5 / 2} h^{2}} \frac{(\Delta x)^{2}}{2} \\
& \Delta t=\frac{1}{2 A_{1} A_{2}^{2} A_{3} h^{2}} \frac{(\Delta x)^{2}}{D_{b r} \sqrt{g D_{b r}}}
\end{aligned}
$$

Two-lıne theory

In the two-line theory, the coastal constants mentioned in fig. 13 are of importance. The exact definitions are giver in (9) and (12). The constant $q_{y}$, which defines, how the offshore transport changes, when the profile changes, will be treated in a separate paper in the future. Some investigation about this constant has already been done 8 . The coefficients $Q_{01}, q_{1}, Q_{02}$ and $q_{2}$ w1ll be computed with the SVASEK-theory 2 which only treats the longshore transport. SVASEK neglects the longshore transport outside the breaker-zone We assume, that the profile outside the breaker-zone has reached already its equilibrium profile, and that the on- and offshore transport can be neglected there.

The assumed profile topography can be like given in fig. 14 (see next page), more natural than shown in fig. 7 .

SVASEK assumes, that the littoral drift between two depth contours is proportional to the longshore component of the loss energy between these contourlines ( 2 , formula 5 - 7)

$$
\Delta Q=A_{1}^{\prime} \cdot \Delta\left(\frac{\mathrm{H}^{2} \mathrm{C}}{\mathrm{K}^{2}}\right) \mathrm{K}_{\mathrm{m}}{ }^{2} \sin \varphi_{\mathrm{m}} \cos \varphi_{2} \quad \cdots \cdot
$$

in which $Q=$ littoral drift between two depth contours $D-\frac{1}{2} \Delta D$ 
and $D+\frac{1}{2} \Delta D, \Delta\left(\frac{H^{2} C}{K}\right)=$ difference

between

$$
\begin{aligned}
& \frac{\mathrm{H}^{2} \mathrm{C}}{\mathrm{K}} \text { on both dept contours } \\
& \mathrm{K}_{\mathrm{m}}, \varphi_{\mathrm{m}}=\text { value of refrac- }
\end{aligned}
$$

tion coefficient $K$ and angle of wave incidence $\varphi$ in the midst between the depth contours. It appears (appendix A3), that $\triangle Q$ can be written

$$
\Delta Q=3 A_{1} A_{2}^{2} A_{3} B^{\frac{1}{2}} D^{1 \frac{1}{2}} \Delta D \sin \varphi_{m} \cos \varphi_{m} \ldots(26)
$$

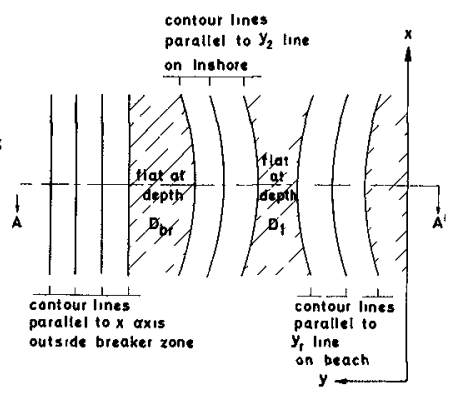

and after some simplifications, treated in appendix $A 3$, the following constants are found for small angle of wave incidence

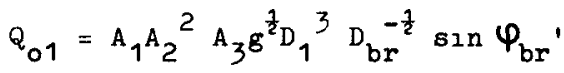

$$
\begin{aligned}
& q_{1}=A_{1} A_{2}^{2} A_{3} B^{\frac{1}{2}} D_{1}^{2 \frac{1}{2}} \\
& Q_{o 2}=A_{1} A_{2}{ }^{2} A_{3} g^{\frac{1}{2}}\left(D_{b r}{ }^{3}-D_{1}{ }^{3}\right) D_{b r}{ }^{-\frac{1}{2}} \sin \varphi_{b r} \\
& q_{2}=A_{1} A_{2}^{2} A_{3} B^{\frac{1}{2}}\left(D_{b r}{ }^{3}-D_{1}{ }^{3}\right) D_{b r} b^{-\frac{1}{2}}
\end{aligned}
$$

Fig $14^{\circ}$ Upper view

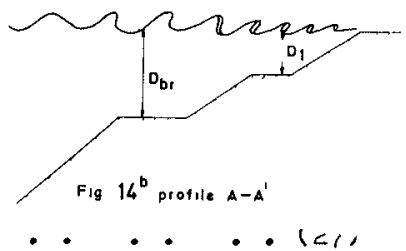

The factor $\mathrm{A}_{1} \mathrm{~A}_{2}{ }^{2} \mathrm{~A}_{3} \mathrm{~g}^{\frac{1}{2}}$ varıes between $2.37 \times 10^{-2}$ and $3.85 \times 10^{-2} \sqrt{\mathrm{m} / \mathrm{sec}}$, dependent of the kind of waves (harmonic or random).

\section{VARIABLE WAVE CONDITIONS}

There has to be distinguished the influence of variable wave conditions on the coastal constants and the influence of the boundary conditions.

\section{Influence varıable wave condı tıons on coastal constants}

The derivation used for the PELNARD-CONSIDERE-formula (2) keeps its validity when the littoral drift $Q$. the stationarv transport $Q_{0}$ and the constant $q$ are averages over a year instead of instantaneous values. However, it will not be directly clear, whlch value has to be taken for $D_{\text {tot. }}$. In order to est1mate $D_{\text {tot }}$ it 15 useful to compute first ${ }^{\text {s }}$ the distribution of the littoral drift perpendicular to the coast. An example of such a distribution gives fig. 15. The yearly littoral drift between two

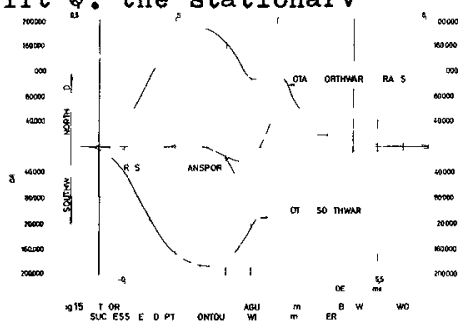


depth contours $D-\frac{1}{2} \Delta D$ and $D+\frac{1}{2} \Delta D$ is computed w1th the ald of (26), which becomes in case of variable wave conditions ( $\cos \varphi_{b}$ has been takenland Snell's law has been applied)

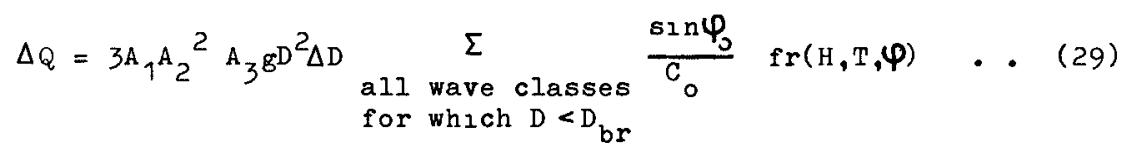

in which $\mathrm{fr}(\mathrm{H}, \mathrm{T}, \varphi)$ denotes the frequency of occurrence of a wave class for which $\mathrm{H}, T$ and $\varphi$ lie between certain values (for instance $\left.\frac{1}{2} \mathrm{~m}<\mathrm{H}<1 \mathrm{~m}, 5 \mathrm{sec}<\mathrm{T}<6 \mathrm{sec}, 30^{\circ}<\varphi<60^{\circ}\right)$. More detalls about the computation are given in [9] From the distribution of the transport $Q_{0} 1$ and $Q_{02}$ are found (f1g. 16)

$$
\begin{aligned}
& Q_{01}=\sum_{O<D<D_{1}} \overline{\Delta Q} \\
& Q_{02}=\sum_{D>D_{1}} \overline{\Delta Q}
\end{aligned}
$$

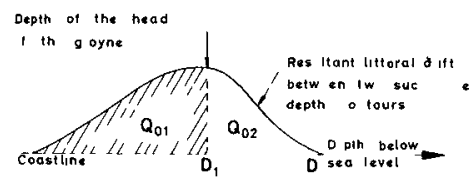

After that, $q_{1}$ and $q_{2}$ can be found by repeating the computation according to (29), but with a "wrong" coastal direction, which has been turned over an angle $\Delta \varphi$, say $15^{\circ}$. This gives the interrupted line in fig. 17, instead of the solid Iine, which represents the transport distribution for the original coastal direction.

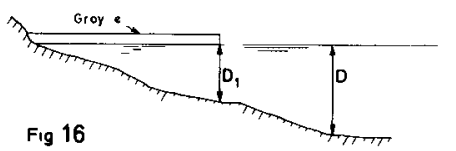

Now $q_{1}$ equals $F_{1} / \Delta \varphi\left(F_{1}\right.$ is the lefthanded hatched area in $\left.f_{1} g .17\right)$ and $q_{2}=F_{2} / \Delta \varphi$. From the transport distribution, also a reasonable guess about $D_{\text {tot }}$ can be made.

Influence variable wave condltıons on the bound

This paper is concentrated on two effects of a groyne prevention of the littoral sand drift and formation of a sheltered area We shall investigate these two effects in case of changing wave conditions. When the wave direction changes periodically for instance according to

$$
\varphi=\hat{\varphi} \sin \omega^{\omega} t_{\quad} \quad . \quad(31)
$$

this generates a sandwave near a groyne (fIg. $15,[10\rfloor$ )
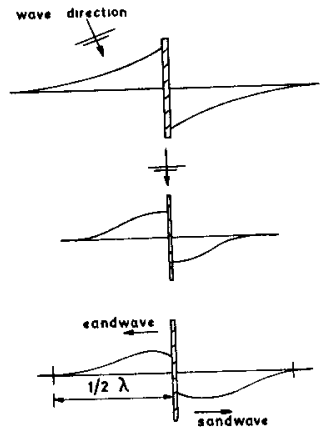

Fig 18 Generat $n$ of eandwavee by variabie wav direct on 


$$
y=\frac{\hat{\varphi} \lambda_{\varphi}}{2 \pi \sqrt{2}} \cdot e^{-k} \varphi^{x} \quad \cos \left(\omega_{\varphi} t-k_{\varphi} x\right) \quad \ldots .
$$

in which

$$
\lambda_{\varphi}=\frac{2 \pi}{k_{\varphi}}=2 \sqrt{\pi_{\frac{1}{D_{\text {tot }}}} \mathrm{T}_{\varphi}} \quad \ldots . . . . . .
$$

T $\varphi$ being the period of the fluctuations of $\varphi$ and $\lambda_{\varphi}$ being the length of the sandwave. this gives

$$
\lambda_{\varphi}=.183 \mathrm{D}_{\mathrm{br}}^{3 / 4}\left(\mathrm{~g} \mathrm{~T}^{2}\right)^{1 / 4} \quad \ldots \ldots \ldots
$$

Taking as an example $T_{\varphi}=1$ week, this makes $\lambda_{\varphi}=324 \mathrm{~m}$. Now the decay of this sandwave 18 very strong within $\frac{1}{2} \lambda_{\varphi}$ it 16 attenuated to $4 \%$. Thus, outside this area, no influence of the stopping of the littoral drift by the groyne will be observed. In case of the two-line theory $\mathrm{D}_{\mathrm{br}}$ in (34) probably can be replaced by $D_{1}, \hat{\varphi}$ has to be replaced by $\varphi_{01}^{\mathrm{br}}$, according to (A 36)

The second influence of the groyne is the wave-shelter. We shall assume first, that the sheltered area is large with respect to $\frac{1}{2} \lambda_{\varphi}$. As in (12a), $Q_{0}$ and $q_{1}$ become functions of $x$, called $Q_{01}{ }^{*}$ and $q_{1}{ }^{*}$. Consider fig. 19. The influence of diffraction will be neglected with respect to the influence of changing wave conditions.

The computation of $Q_{01}$ and $q_{1}$ in area $A$ can be performed according to
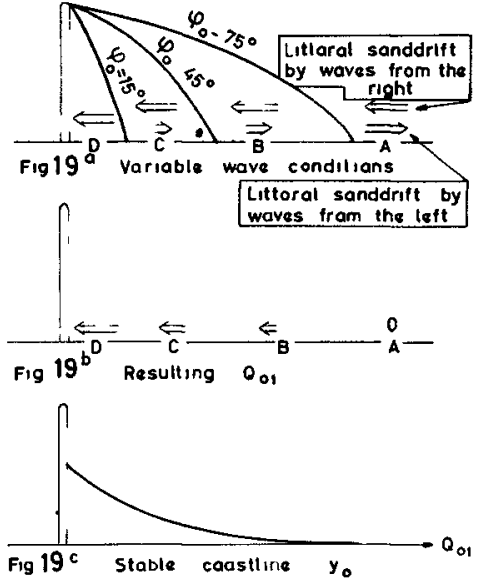
(29), (30) and fig. 14. But applying (44) to area $B$, all wave classes with $\varphi_{0} \geqslant 75^{\circ}$ must be excluded in the summation, in area $C$ all wave classes with $\varphi_{0} \geqslant 45^{\circ}$, and so on. When, for instance, the resulting transport in area $A$ would be zero for a coastline parallel to the $x-a x i s$ (fig. 19a), the transport $Q_{01} *\left(\frac{a y_{1}}{\partial x}=0\right)$ (started because $1 t$ changes in $x$-direction) will be larger and larger (in negative direction) in the areas $B, C$ and $D_{1}$, and also $q_{1}^{*}$ wlll change Now we have returned to the normal computer program, treated in (5) to (7) and in appendix 3, only with other

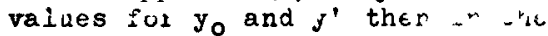
diffraction cases.

The stable coastline $y_{0}$ can be found from continuity for $y_{0}$ the transport 16 everywhere the same 


$$
\begin{aligned}
& {\left[Q_{01}\right]_{x=\infty}=Q_{1} \rightarrow\left[Z_{01}\right]_{x=\infty}=} \\
& =Q_{01} *-q_{1}^{*} \frac{d y_{0}}{d x} \rightarrow \frac{d y_{0}}{d x}=\frac{Q_{01}{ }^{*}-\left[Q_{01}\right]_{x=\infty}}{q_{1}^{*}}
\end{aligned}
$$

From (35) the stable coastline $y_{0}$ can be found, which gives the initial value of the unstationary part $y_{1}^{\prime}$
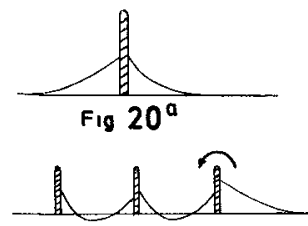

Fig $20^{b}$

In case of changing wave conditions, and no resultant drift, the final coastal shape near one single groyne will become just the stable shape $y_{0}$, because then everywhere the resultant drift is zero. This will give accretion on both sides of the groyne, which will be withdrawn from a very long stretch of coast ( $11 \mathrm{~g} .20 \mathrm{a}$ ). in case of a row of groynes, the sand for the accretion near the groyne is withdrawn from the area in the midst between the groynes, and only near the boundary of the groyne system some real accretion can be expected (fig. 20b). However, after some time this sand will move to the areas between the groynes, and so this shelter effect may give some accretion (in case of no resultant drift), starting from the boundaries of the groyne system

In case $\frac{1}{2} \lambda_{\varphi}$ is not small with respect to the sheltered area, the best way of computation is a kind of "hindcasting", using the oneline or two-line computer program described before, and changing the wave conditions during the program.

Th1s has been done at the Coastal Research Department of Rijkswaterstaat.

N B The vertical scale of fig 5 and 6 is 5 times and of fig. 10 and $1^{11}$ is 10 times exaggerated. 


\section{APPENDIX}

\section{A1. One-lıne theory Assumptions and formulae (diffractıon)}

Assumptions littoral drift proportional to the angle of wave incidence and to the square of the wave height (fig. A1)

$$
Q=\operatorname{Ah}^{2}\left(\varphi_{\mathrm{x}}-\frac{\partial \mathrm{y}}{\partial \mathrm{x}}\right) \quad \cdots . . \cdot \cdot \cdot \cdot \cdot
$$

In which $A$ is a proportionality constant and $h$ is the ratio between the wave helght at $x=x$ and the wave height at $x=\infty \mathrm{Eq}_{\mathrm{q}}$ (A1) is a special case of (3)

Continuity $\frac{\partial y}{\partial t}=-\frac{q}{D_{\text {tot }}}-\frac{\partial Q}{\partial x}$

Using (3) $\quad \frac{\partial y}{\partial t}=\frac{1}{D_{\text {tot }}}\left(q^{*} \frac{\partial^{2} y}{\partial x^{2}}+\frac{\partial q^{*}}{\partial x} \cdot \frac{\partial y}{\partial x}\right)-\frac{1}{D_{\text {tot }}} \frac{d Q o}{d x}$

The stable coastline $y_{0}$ from (5) is a solution of (A2), or (A3) Continuity gives $Q$ is constant for $y_{0}$. The amount of $Q$ can be derived from the condition at infinity

$\mathrm{h}=1, \varphi_{\mathrm{x}}=\varphi_{\infty}, \frac{\partial \mathrm{y}}{\partial \mathrm{x}}=0$, from (A1) follows (4)

$$
A \varphi_{\infty}=A h^{2}\left(\varphi_{x}-\frac{d y_{o}}{d x}\right)
$$

Eq (6) can be derived from (A3) by substituting $y_{0}$ in (A3) and subtracting this equation from (A3).

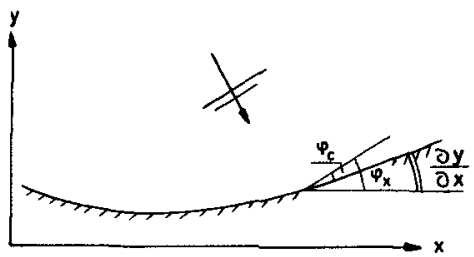

Fig Al

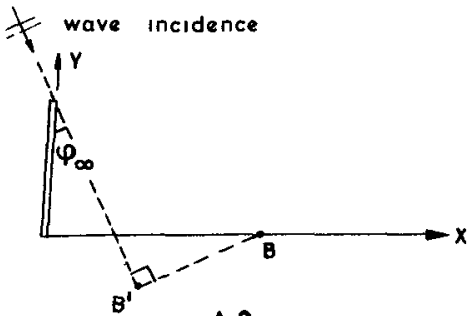

Fig A 2

For the determination of the values of $\varphi_{X}$ and $h$ the simplified diffraction theory of PUTNAM and ARTHUR [ 4 ] is used. It can be shown [11] that $\varphi_{x}$ is about equal to

$$
\varphi_{\mathrm{x}}=\varphi_{\infty}-\frac{\lambda}{2 \pi} \frac{\mathrm{d} \Theta}{\mathrm{dx}}
$$

in which $\lambda$ - wave length diffracted wave and $t=$ phase difference of the waves between $B$ and $B^{\prime}$ (fig. $A 2$ ). $B=$ point on $x$-axis, for which $y_{0}$ is computed. 
Substitution of this result in (4) and integration gives the relation between $y_{0}$ and the basic data of diffraction $h$ and $\theta$ $\mathrm{y}_{0}=-\frac{\lambda}{2 \pi} \theta+\varphi_{\infty} \int \frac{h^{2}-1}{h} d x \ldots . . .(A 6)$

The first term of the right hand side of (A 6 ) is the influence of the turning of the waves, the second term glves the influence of changing the wave helght by diffraction.

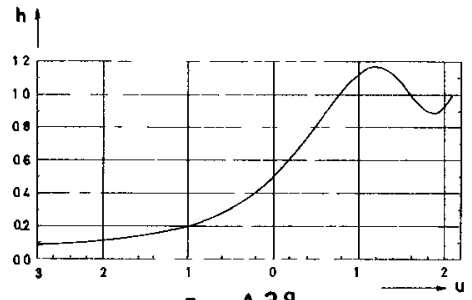

Fig $A 3^{a}$

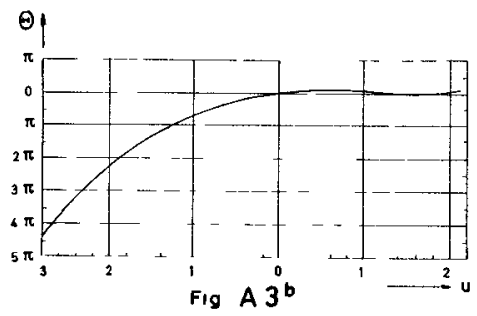

Fig A3 WAVE DIFFRACTION ACCOROING TO PUTNAM ANO ARTHUR [6]

$\theta$ and $h$ as a function of $u=2 \sqrt{\frac{A B-A B^{2}}{\lambda}}$ ac u.uing to NUTNAM AND ARTHUR [4] are shown in fiB. A5.

Unstationary part $y^{\prime}$

(6) has been taken as a difference equation, taking

$$
\begin{aligned}
& q_{\max }=A h_{\max }^{2} \cdot \cdot \cdot \cdot \cdot \cdot \cdot(\mathrm{A} 7) \\
& \Delta t=D_{\text {tot }}(\Delta x)^{2} / 2 q_{\max } \cdot \cdot \cdot \cdot(A 10)
\end{aligned}
$$

Substituting (A7) and (A10) in (6), from three adjacent points of a curve at time $t$ one point of the curve at point $t+\Delta t$ can be found (explicite method)

$$
\begin{aligned}
& y^{\prime}[x, t+\Delta t]= \\
& (\tilde{q}[x+\Delta x] / 8+\tilde{q}[x] / 2-\tilde{q}[x-\Delta x] / 8) \cdot y^{\prime}[x+\Delta x, t]+ \\
& \left(\begin{array}{r}
-\tilde{q}[x]+1 \quad) \cdot y^{\prime}[x, t] \\
(-\tilde{q}[x+\Delta x] / 8+\tilde{q}[x] / 2+\tilde{q}[x-\Delta x] / 8) \cdot y^{\prime}[x-\Delta x, t]
\end{array}\right.
\end{aligned}
$$

In which $\bar{q}=q^{*} / q_{\max }$ has been introduced to avold instability.

Boundary conditions at $x=0$ no transport and therefore

$$
\frac{\partial y}{\partial x}=\frac{d y}{d x}+\frac{\partial y^{\prime}}{\partial x}=\left[\varphi_{x}\right]_{x=0} \cdot . \cdot \cdot \cdot \cdot(\text { A 12) }
$$


Substitution of (4) in (A12) gives

$$
\frac{\partial y^{\prime}}{\partial x}=\varphi_{x}-\left(\varphi_{x}-\frac{\varphi_{\infty}}{h^{2}}\right)=\frac{\varphi_{\infty}}{h^{2}} \ldots . . . . . \text { (A13) }
$$

We can write this equation into differences and express $y^{\prime}[-x]$ in $y^{\prime}[\Delta x]$ This gives for the boundary point at the lee-side

$$
\begin{aligned}
y^{\prime}[0, t+\Delta t]=- & \left(-\bar{q}[\Delta x / 8+\bar{q}[0] / 2+q[-\Delta x] / 8) \cdot 2 \Delta x \cdot \frac{\varphi}{[h]_{x}^{2}}+0\right. \\
& (-q[0]+1) \cdot y^{\prime}[0, t]+\bar{q}[0] \cdot y^{\prime}[\Delta x, t]
\end{aligned}
$$

The expression for the luffside can be found by changing everywhere in (A14) $\Delta x$ by $-\Delta x$.

\section{A2 Two=lıne theory.}

$(74)$ and $(75)$ can be derived by substitution of the dynamic equations $(12 a, b)$ in the continuity equations

$$
\begin{aligned}
& \left.\begin{array}{l}
\frac{\partial Q_{1}}{\partial x}+Q_{y}+D_{1} \frac{\partial y_{1}}{\partial t}=0 \\
\frac{\partial Q_{2}}{\partial x_{2}}-Q_{y}+D_{2} \frac{\partial y_{2}}{\partial t}=0
\end{array}\right\} \\
& \text { We state } \Delta t=c D(\Delta x)^{2} / q_{1 \max } \text { and call } q_{y} \cdot(\Delta x)^{2} / q_{1 \max }=q_{y}^{\prime} \\
& y_{1}^{\prime}[x, t+\Delta t]= \\
& \text { c. }{ }_{D}^{D}\left\{\left(\tilde{q}_{1}[x+\Delta x] / 4+\tilde{q}_{1}[x]-\bar{q},[x-\Delta x] / 4\right) \cdot y_{1}^{1}[x+\Delta x, t]+\right. \\
& +\left(D / C D_{1}-2 q_{1}[x]-q_{y}^{1}\right) \cdot y_{1}^{\prime}[x, t]+ \\
& +\left(-\bar{q}_{1}[x+\Delta x] / 4+q_{1}[x]+q_{1}[x-\Delta x] / 4\right) \cdot y_{1}^{\prime}[x-\Delta x, t] \\
& \left.-q_{y}^{\prime}\left(y_{0}[x]-y_{2}[x, t]\right)\right\} . . . . . . . . .(A 21)
\end{aligned}
$$




$$
\begin{aligned}
& y_{2}\left[x_{1} t+\Delta t\right]- \\
& \text { c. } \frac{D_{2}}{D_{2}}\left\{\left(J_{2}[y+\Delta x, t]+y_{2}[r-\Delta x, t]\right) \cdot q_{2} / q_{1 \max }+\right. \\
& \quad+\left(D_{2} / c D-2 q_{2} / q_{1 \max }-q_{y}^{\prime}\right) y_{2}[x, t]+ \\
& \left.\quad q_{y}^{\prime}\left(y_{1}^{\prime}[x, t]+y_{0}[x]\right)\right\} \ldots
\end{aligned}
$$

The boundary conditions for $y^{\prime}$, can be found by substituting $x=0$ and (lee-side)

$$
y_{1}^{\prime}[-\Delta x]=y_{1}[\Delta x] \quad \Delta x \cdot \frac{\varphi_{\infty}}{\left[h^{2}\right]_{x-0}} \ldots \ldots \text { (A23) }
$$

A3. Coastal constants according to the adapted SVASEK-theory

SVASEK assumes, that the littoral drift between two depth contours is proportional to the longshore component of the loss of energy between these contour lines ( (25))

We assume that in the breaker zone $\cos y=\cos$ for' and we neglect the influence of the refraction factor $K$ inside the breaker zone

$$
Q=A i \cdot \Delta\left(H^{2} C\right) \sin \varphi \cos \varphi \ldots . . .(A 25)
$$

le assume, that the relations between $H$ and $D$ and between $C$ and according to (21) and (22) on the boundary of the breaker zone remain their validity inside the breakerzone (spilling breaker)

$$
\mathrm{H}=\mathrm{A}_{2} \mathrm{D} \cdot \quad \cdot(\mathrm{A} 26) \quad \mathrm{C}=\mathrm{A}_{3} \mathrm{~g}^{\mathrm{D}} \cdot \text {. (A27) }
$$

Thus the difference in $\mathrm{H}^{2} \mathrm{C}$ between two adjacent depth contours equals

$$
\Delta\left(H^{2} C\right)={ }_{d D}^{d}\left(A_{2}^{2} A_{3} B^{\frac{1}{2}} D^{2 \frac{1}{2}}\right) \Delta D=\frac{5}{2} A_{2}^{2} A_{3} B^{\frac{1}{2}} D^{1 \frac{1}{2}} \Delta D \ldots .(A 28)
$$


Now first the stationary transport Qo will be computed, according to SVASEK's theory. In this case all contour lines are parallel and Snell's law is valid

$$
\frac{\sin \varphi}{\sin \varphi_{b r}}=\frac{C}{C_{b r}}=\sqrt{\frac{D}{D_{b r}}} \cdots \cdot \cdots \text { (A29) }
$$

Substitution of (28) and (29) in (25) glves $\Delta Q$, expressed In $D$

$$
\Delta Q=\frac{5}{2} A_{1} A_{2}^{2} A_{3} B \quad D^{2} D_{b r}^{-\frac{1}{2}} \Delta D \sin \varphi_{b r} \cos \varphi_{b r}
$$

We find the total transport by integration over the depth. Again we assume $\cos \varphi=\cos \varphi$.

$$
Q_{0}=\int^{D_{b r}} \Delta Q d D=\frac{5}{6} A_{1} A_{2}^{2} A_{3} g^{\frac{1}{2}} D_{b r}^{2 \frac{1}{2}} \sin \varphi_{b r} \cos \varphi_{b r} \cdot \text { (A31) }
$$

Comparison of (A31) and (23) leads to the conclusion, that for parallel depth contours the relation should exist

$$
\left.A_{1}^{\prime}=\frac{6}{5} A_{1} \cdot . \cdot . \cdot . \cdot \text { ( } A 32\right)
$$

The reason 1s, that SVASEK multiplies the component of the wave energy $w 1$ th $\sin \varphi$ instead of $\sin \varphi_{b r}$ and 2 n the breakerzone $\sin \varphi_{1 s}$ less than $\sin \varphi$

Thus the transport betweeñ two depth contours wall be, in general, using (A25) (A29) and (A32)

$$
\Delta Q=3 A_{1} A_{2}^{2} A_{3} B^{\frac{1}{2}} D^{1 \frac{1}{2}} D \sin \varphi_{m} \cos \varphi_{m} \cdot \cdots \cdot \text { (A33) }
$$

In 12 has been considered in detall how the littoral drift changes when the beach and inshore direction change in case of the topography at fig. A5 (cf fig. 14).

Using SVASEK's assumptions and a proper use of Snell's law, for the littoral drift along the inshore is found

$$
Q_{2}=A_{1} A_{2}^{2} A_{3} B^{\frac{1}{2}}\left(D_{b r}^{3}-D_{1}^{3}\right) D_{b r}^{-\frac{1}{2}} \sin \varphi_{b r} \cos \varphi_{b r} \ldots \text { (A34) }
$$

and for the transport along the beach

$$
\begin{aligned}
Q_{1}=A_{1} A_{2}^{2} A_{3} & g^{\frac{1}{2}} D_{1}^{2 \frac{1}{2}} \sin \varphi_{10} \cos \varphi_{10}-\frac{\partial y_{1}}{\partial x} \cos 2 \varphi_{10} \\
& +\frac{\partial y_{2}}{\partial x}\left(1-\frac{1}{2} \sin 2 \varphi_{10} \cos \varphi_{b r}\right) \frac{\cos ^{2} 2 \varphi_{10}}{\cos ^{2} \varphi_{10}}
\end{aligned}
$$

in which $\varphi_{10}$ is the angle of Incidence of the wave on the beach ( 1 g. A 5 ), which occurs, when the inshore is parallel with the $x$-exis

$$
\varphi_{10}=\arcsin \left(\sqrt{\frac{D_{1}}{D_{b r}}} \sin \varphi_{b r}^{\prime}\right) \cdots \cdots \cdot \cdots
$$




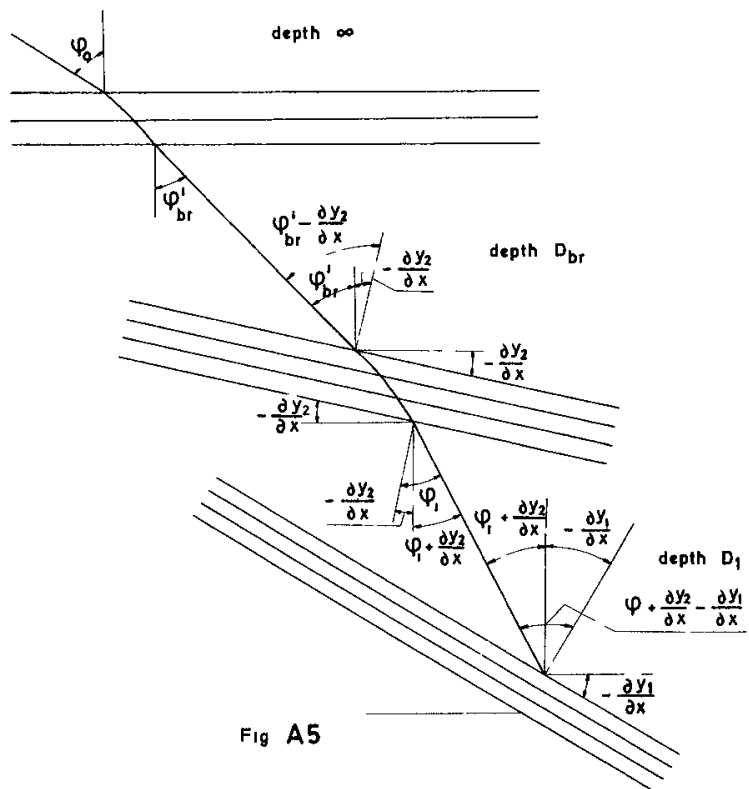

In which $\varphi_{b r}^{\prime}$ is the breaker angle, if the inshore would be parallel to the $x$-axis.

For small angle of wave incidence, (A35) can be written

$$
Q_{1}=A_{1} A_{2}^{2} A_{3} g^{\frac{1}{2}} D^{2 \frac{1}{2}}\left[\sin \varphi_{10}-\frac{\partial y_{1}}{\partial x}+\frac{\partial y_{2}}{\partial x}\left(1-\sqrt{\frac{D_{1}}{D_{b r}}}\right)\right.
$$

As would be expected, there is some

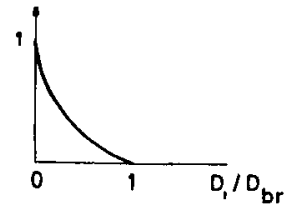

Fig A 6 Influence direction inshore influence direction beach versus $D_{1} / D_{b r}$ influence of refraction on the inshore on the transport on the beach. The dynamic equations $(12$ ) do not account for that. With (A37) we are able to estimate the inaccuracy caused by this neglection. (without taking the curvature of the inshore into account) When the beach and the inshore turn over the same angle, the influence of the direction of the inshore on the transport on the beach is $\left(1-\sqrt{D_{1} / D_{b r}}\right)$ times the influence of the direction of the beach. This function is shown in fig. A6.

the Iudw ulae for $q_{1}$ and $q_{2}$ can be derived by differentiation of $(A 34)$ and $(A 35)$ to $\frac{\partial y_{2}}{\partial x}$ and $\frac{\partial y_{1}}{\partial x}$ respectively. For $\varphi_{b r}$ equals

$$
\varphi_{\mathrm{br}}=\varphi_{\mathrm{br}}^{\prime}-\frac{\partial \mathrm{y}_{2}}{\partial \mathrm{x}}
$$

Thus the derivative to $\frac{\partial \mathrm{y}_{2}}{\partial \mathrm{x}}$ is miru $\mathrm{d} \varphi_{\mathrm{br}}$

The influence of the inshore on the beach has been neglected. 


\section{REFFRENCES}

1. W.T. Bakker

The dynamics of a coast with a groyne system

C.E.C. XT, London 1968, ch. 31.

2. E.W. Bijker and J.N. Svasek

Two methods for determination of morphologlcal changes by coastal structures. XXIInd. Int. Nav. Congress, Sect. II, subj. 4, pp 181-202.

3. R. Pelnard-Considère Essal de théorie de l'évolution des formes de révages en plages de sable et de galets. IVme Jaunnées de I'Hydraulique Paris 13-15 Juin 1954.

4. J.R. Putnam and R S. Arthur

5. E.W. Bijker

6. A.J. Bowen

7. J.Mac. Cowan

8. W. T. Bakker

9. W.T. Bakker

10.W.T. Bakker Diffraction of Water Waves by Bredewaters Trans. A.G.U. Vol. 29, 4, Aug. 1948. Littoral drift as function of waves and current. Publ. 58 Delft Hydr. Lab. The generation of long shore currents on a plane beach J. Mar. Res. Vol. 27,May'69 On the highest wave of permanent type. Ph11. Mag. (V) Vol. 38 pp. 351-357. The influence of dune height on dune erosion.

Rijkswaterstaat, Dept. for Coastal Res. Computation of ilttoral drift with the Svasek-method. Rijkswaterstaat, Dept. for Coastal Res. Rep. W.W.K. 69-7.

The Coastal Dynamics of Sandwaves and the Influence of Brakewaters and Groynes. Rijkswaterstaat, Dapt. for Coastal Res. 11.W.T. Bakker The influence of diffraction near a harbour mole on the coastal shape Rljkswaterstaat, Dept. for Coastal Res. Rep. W.W K 70-2.

12.W.T Bakker The relation between wave climate and coastal constants Rijkswaterstaat, Dept. for Coastal Res. (yet unpublished). 


\title{
CHAPTER 65
}

\section{EXPERIMENTAL STUDY OF THE HYDRAULIC BEHAVIOUR \\ OF INCLINED GROYNE SYSTEMS}

\author{
Jül 10 Patriarca Barceló \\ Research Offlcer Department of Hydraulics \\ Laboratór 10 Nacional de Engenharia Civil Lisboa Portugal
}

SYNOFSIS

\begin{abstract}
Thrs paper presents the results of an experimental study on the behaviour of inclined groynes and a short drscussion on the optrmization of groyne systems It supplements a paper presented at the XI Coastal Engineering Conference (London, 1968) In the present paper both studies are applied to the design of a groyne system located to the south of the Tagus estuary (near Lisbon), where a serious erosion has been under way
\end{abstract}

\section{1 - INTRODUCTION}

Groynes are one of the most applied coast protection structures because, owing to their transverse position, they detain long shore drift, which is the man cause of semous coastal erosion phe nomena (see fig 1) Groynes have practically no influence on trans port normal to the coast but this, in addition to being usually small, tends to balance in the annual wave cycle Groynes can be used to stop longshore drift, which may cause undesirable depositions in cer tain zones (case of beaches in dynamic equihbrium), or to protect beaches where the supply of material is exceeded by the losses. In any case, a single groyne or a group of groynes can be used, the latter - called a groyne system - being particularly important owing to apphcations in long sand shores

The hydraulic optimization of groyne systems (structural ques tions, although influenced by the hydraulic behaviour, belong to the stability of maritime structures) is extremely important because groynes must obey economic requrements and simultaneously must provide beach stretches satisfactory for recreational purposes, 


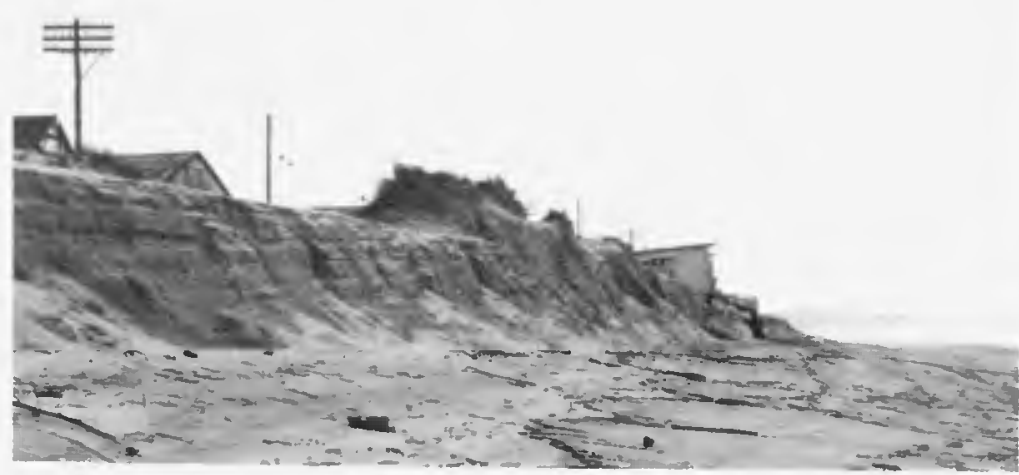

Fig. 1 - Erogion. in Caparica beach, January 1970

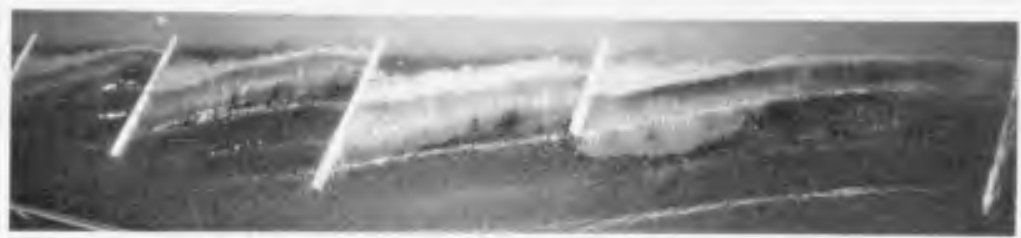

Fig. 2 - Groyne system tested in the Ist stage of the atudy

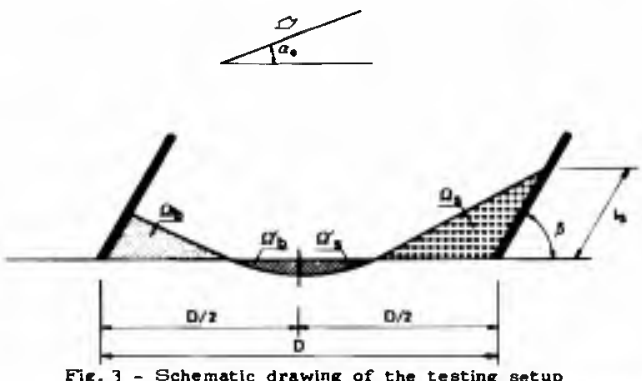

Fig. 3 - Schematic drawing of the testing setup

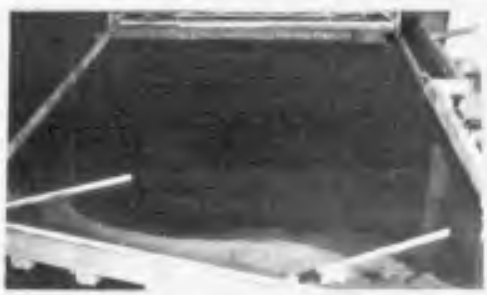

Fig. 4 - Vew of a test $\left(B=50^{\circ} ; a_{0}=20^{\circ}\right)$

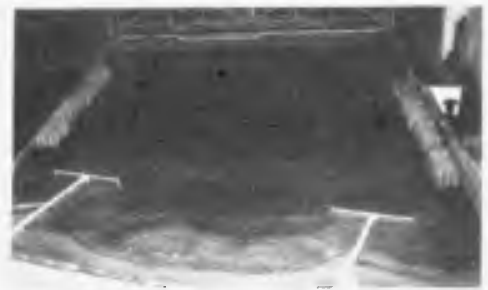

Fig. 5 - Test of T-groynes $\left(a_{0}=20^{\circ}\right)$ 
which, together with coast protection, is their main object This leads to the use of long very spaced groynes, which in addition to affording a satisfactory protection against wave erosion and provid ing wide beach stretches satisfactory for recreational purposes, im proves the landscape of these A groyne system is functional when it meets these requirements

A paper was presented at the XI Coastal Engineering Conference (London,1968) describing an experimental study of the hydraulic behaviour of systems of groynes normal to the bathymetric curves 11 \} (see fig 2) This study is now complemented by the present study on inclined groynes, which also contains some consideration on the optimization of groyne systems based on the results of both studies, and an instance of application to a serious case of erosion in the Portuguese coast (Caparica and Cova do Vapor beaches)

\section{2 - EXPERIMENTAL TECHNIQUE}

The tests were carried out in a tank (with a net area of about $8 \mathrm{~m} \times 18 \mathrm{~m}$ ), fitted with a translation wave machine The plain sine waves generated had periods from 10 to $13 \mathrm{~s}$, heights between $300 \mathrm{~cm}$ and $550 \mathrm{~cm}$ and obliquities of $20^{\circ}, 10^{\circ}$ and $5^{\circ}\left(\alpha_{0}-\right.$ - obliquity of the breaking wave - see $f_{1 g}$ 3) The moblle material used was pumice stone with a unit weight of $146 \mathrm{gf} / \mathrm{cm}^{3}$ and a median diameter of about $15 \mathrm{~mm}$ The tests begun with a beach with a transverse slope of $1=8 \%$ The still water level remaned constant, 1 e tides were not reproduced Fixed, high, and impermeable groynes were tested and the beach stretches between them can be considered as independent physiographic units

The testing setup was as extensive as possible so that the evolution of beach stretches between groynes could be studied in detall (see figs 4 and 5) The interference of the tank borders with the transport of sediments was taken into consideration but it was only in one test of very inclined groynes that it was neces 
sary to reproduce the area near the downdrift groyne with a fixed bed and to remove moble material beyond this groyne The curve of fig 6, plotted from the quantities removed, represents drift at

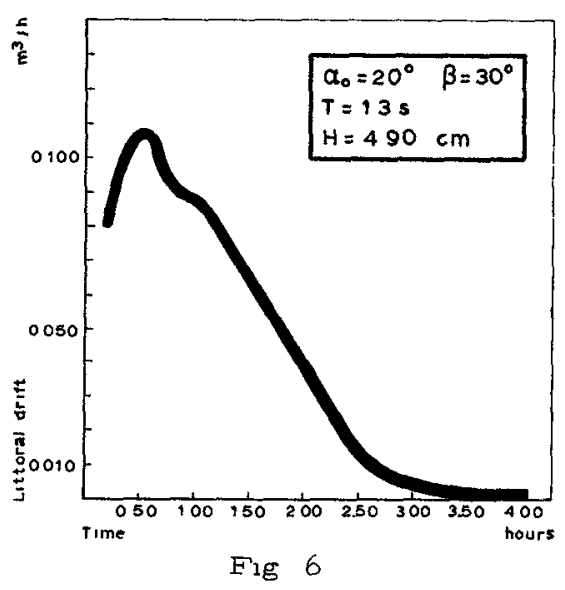

the end of the groyne versus the duration of the test It should be noted that the conclusions drawn are not invalidat ed by the absence of updrift feed, which corresponds to the most adverse conditions in the extreme updrift groyne

The inclinations considered were those affording the best protection to groyne roots, inclinations symmetrical of these were not tested as they obviously are of no practical interest The study was completed with tests of T-groynes for comparison with the former results

The measurement and bottom surveying methods used were those described in $\{1\}$ Fresh water was used and the flow can be considered as turbulent Systematic tests to no particular scale were also carried out as the testing setup represents no specific case

\section{3 - EXPERIMENTAL RESULTS}

On the whole inclined groynes (see $f \mathrm{ig} 3$ ) operate roughly as normal groynes $\left(\beta=90^{\circ}\right), 1$ e beaches initially with a uniformtrans verse slope of $8 \%$ reach final equilibrium conditions essentially depending on the characteristics of the wave for each value of $\beta$ The characteristic parameters of the operation of groynes are those indicated in $\{1\}$, but the following are particularly relevant in the present study accretion areas $\Omega_{s}$ and $\Omega_{b}$, total accretion area $\Omega=\Omega_{s}+\Omega_{b}$, evolution $I_{s}$ of the beach near the downdrift groyne, spacing $D$ between groynes, length $c$ of the groynes, and value 

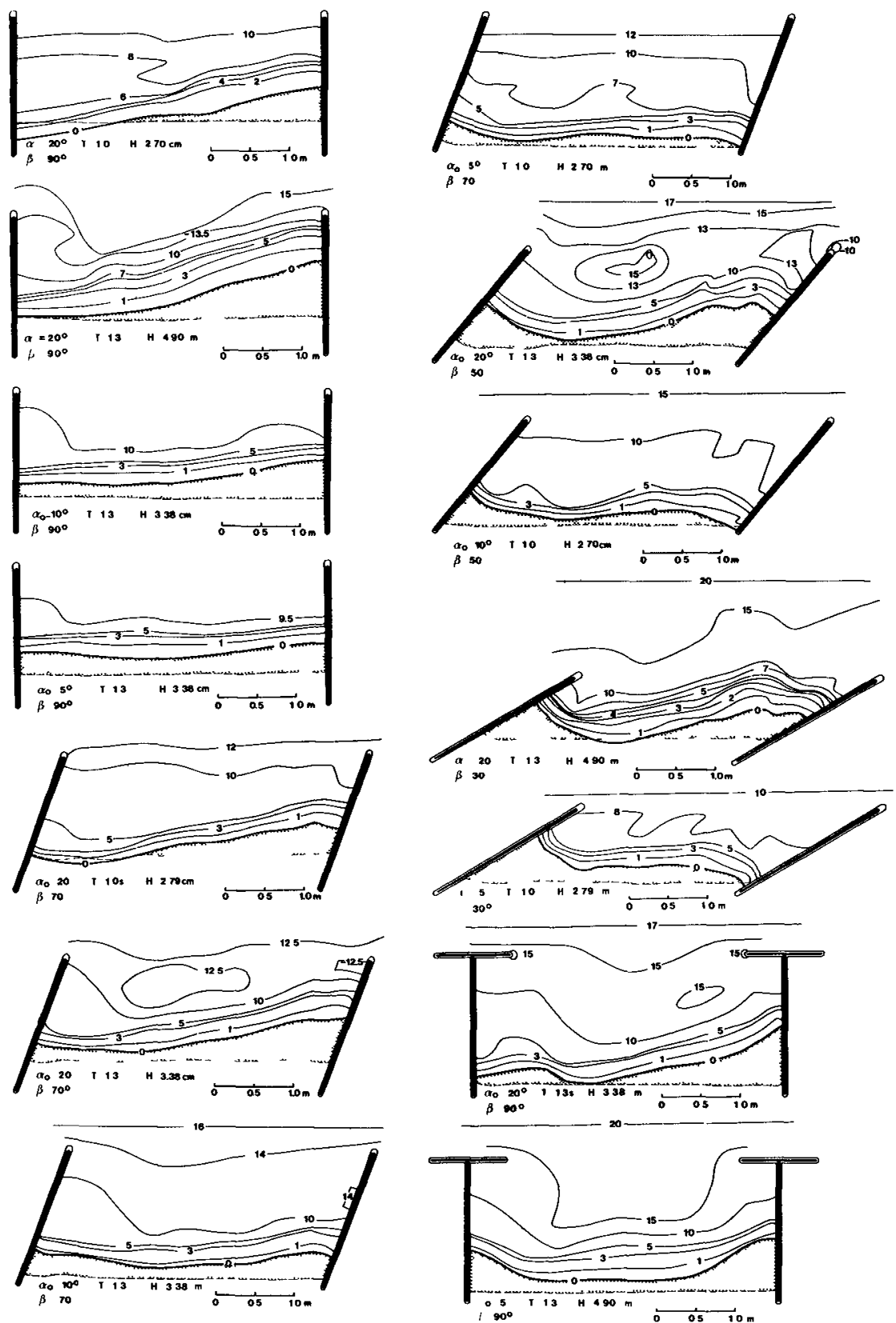

Fig 7 - Typeal plang of final equilibrium conditions of the teated cases 
$\mathrm{H}_{0}^{2} \mathrm{C}_{0}$ proportional to the transmitted wave power $\left(\mathrm{H}_{0}\right.$ and $\mathrm{C}_{0}$ are the wave height and the wave speed in infinite depth, respectively) Detall characteristics of the evolution of beach stretches will be taken into consideration and the efficiency of inclined groynes will be defined in function of these characteristics

The detall characteristics of the evolution of beach stretches are presented in fig 7 , which shows the final equabrum condition for the different typical cases studied, the dotted line represents initial conditions in the beach for a zero water level, the indicated value of the wave helght, $H$, concerns the depth where the wave generator is placed Groynes with $\beta=90^{\circ}$ may cause erosions near the updrift groynes for the maximum obliquities tested, whereas accretions extend throughout the width of the beach stretch for lower obliquties, in which transverse movements predominate For $\beta=70^{\circ}$ the overall behaviour of the groyne system is not changed, but erosion hazards near the updrift groyne decrease, and for obliquities of $\alpha_{0}=20^{\circ}$ there is even deposition throughout the beach stretch, owing to the shelter afforded by the updrift groyne, for smaller obliquities, the beach retreats near the doivndrift groyne and the area $\Omega_{\mathrm{s}}$ decreases For $\beta=50^{\circ}$ the increase in the accretion area $\Omega_{b}$ and in the protection afforded by the root of the up drift groyne grow more marked For smaller obliquities regression increases near the downdrift groyne and the areas $\Omega_{\mathbf{s}}$ decrease For $\beta=30^{\circ}$ the beach stretch can hardly be considered as a phys ographic unit for large obliquities, for smaller obliquities considerable erosions occur in the root of the downdrift groyne and the areas $\Omega_{s}$ are appreciably reduced $T$-groynes operate roughly as normal groynes but the protection afforded to the groyne roots is present in all cases and the symmetry of areas $\Omega_{b}$ and $\Omega_{s}$ is more marked

The two most relevant parameters for the definition of the efficiency of groynes (accretion areas and evolution of the beach near the downdrift groyne) are defined in fig 8 , which represents 

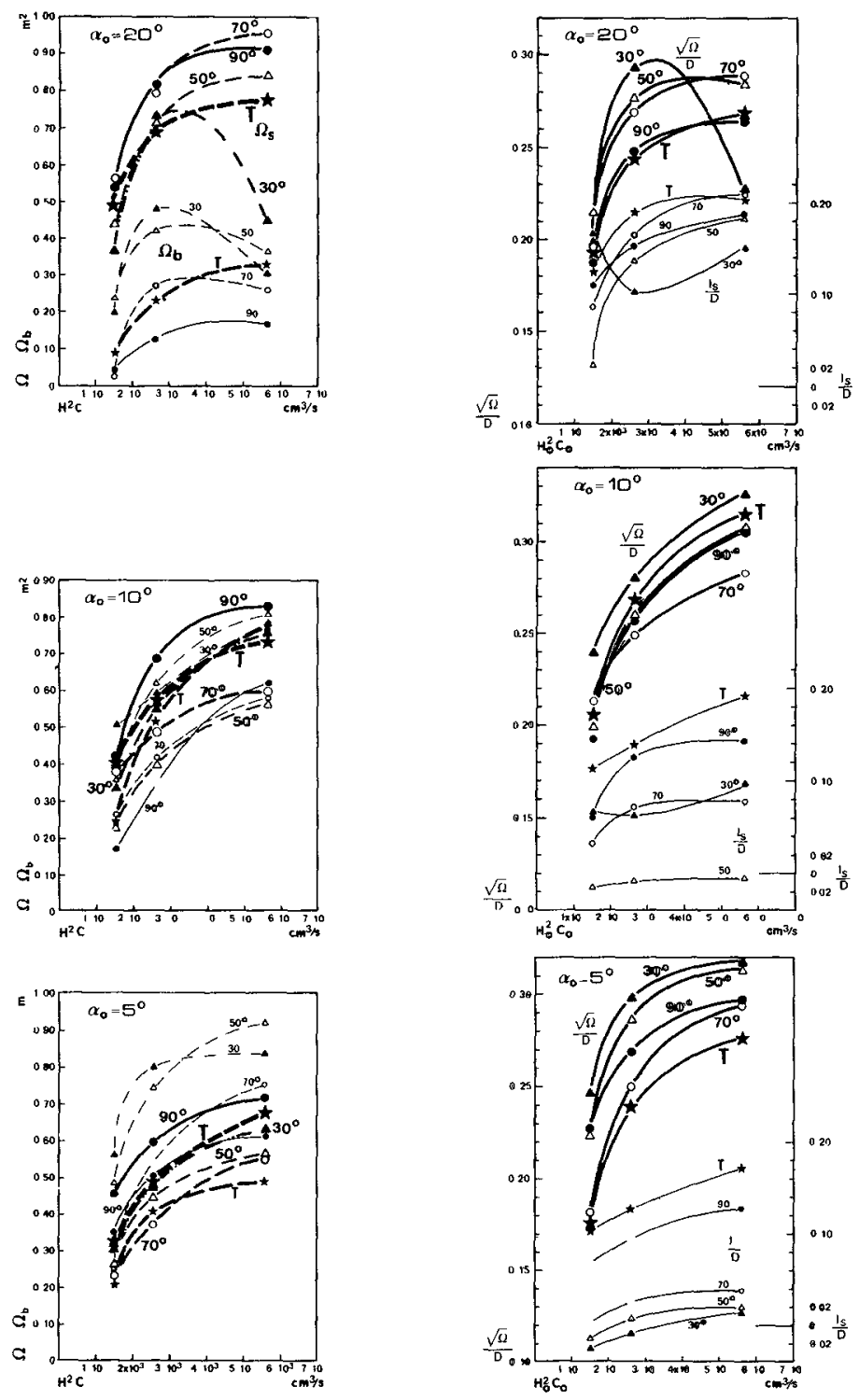

Fig 8 - Curves of the parametero defining the efficiency of groynes in function of the transmitred wave power 
the curves of $\Omega_{\mathrm{s}}$ and $\Omega_{\mathrm{b}}$ (thick and thin lines respectively) with dimensions which give an idea of the values observed in the model tests, and the curves of $\sqrt{\Omega} / D$ together with those of $l_{s} / D$ ( $d_{1}-$ mensionless values sutable for practical applications) The figure also presents the curves of these parameters in function of the value $\mathrm{H}_{0}^{2} \mathrm{C}$ proportional to the transmitted wave power for each obliquity $\alpha_{0}$ of the breaking wave It is from these dimensionless values that the efficiency of the groyne types tested will be defined For $\alpha_{0}=20^{\circ}$ the maximum efficiency corresponds to $\beta=70^{\circ}$, if the increase not only of $\sqrt{\Omega} / D$ but also of $l_{s} / D$ is taken into account For $\alpha_{0}=10^{\circ}$ the groynes with $\beta=90^{\circ}$ are the most efficient taking into account the fact that $1_{s} / D$ is larger for $\beta=90^{\circ}$ although the values of $\sqrt{\Omega} / D$ are higher for $\beta=30^{\circ}$ For $\alpha_{0}=5^{\circ}$, groynes with $\beta<90^{\circ}$ yield values of $l_{s} / D$ which are very low or correspond to erosions, so that the maximum efficiency is obtained for $B=90^{\circ}$ T-groynes are not more efficient than normal groynes $\left(\beta=90^{\circ}\right)$, their only advantage being the absence of erosions in the groyne roots

The efficlency of the different groyne types was defined for the maximum transmitted wave power

These results agree with Prof Nagal's $\{2\}$, as regards both the inclinations of the groynes and the values of the ratio $c / D$

\section{4 - FRACTICAL AFELICATION}

Fortuguese beaches are extremely valuable assets for our economy due to their variety and remarkable natural conditions, so that they must be 1 mproved, and protected against the serious phenomena of erosion which endanger them

One of the most serious erosion phenomena in the Portuguese coast occurred in the beaches of Cova do Vapor and Capar1 ca, a long sand expansion south of the Tagus estuary near Iisbon From the situation presented in fig 9, which prevalled in 1929 , erosion begun due to the collapse of the natural protection afford ed by the sand formation north of the beaches, which ends at the 


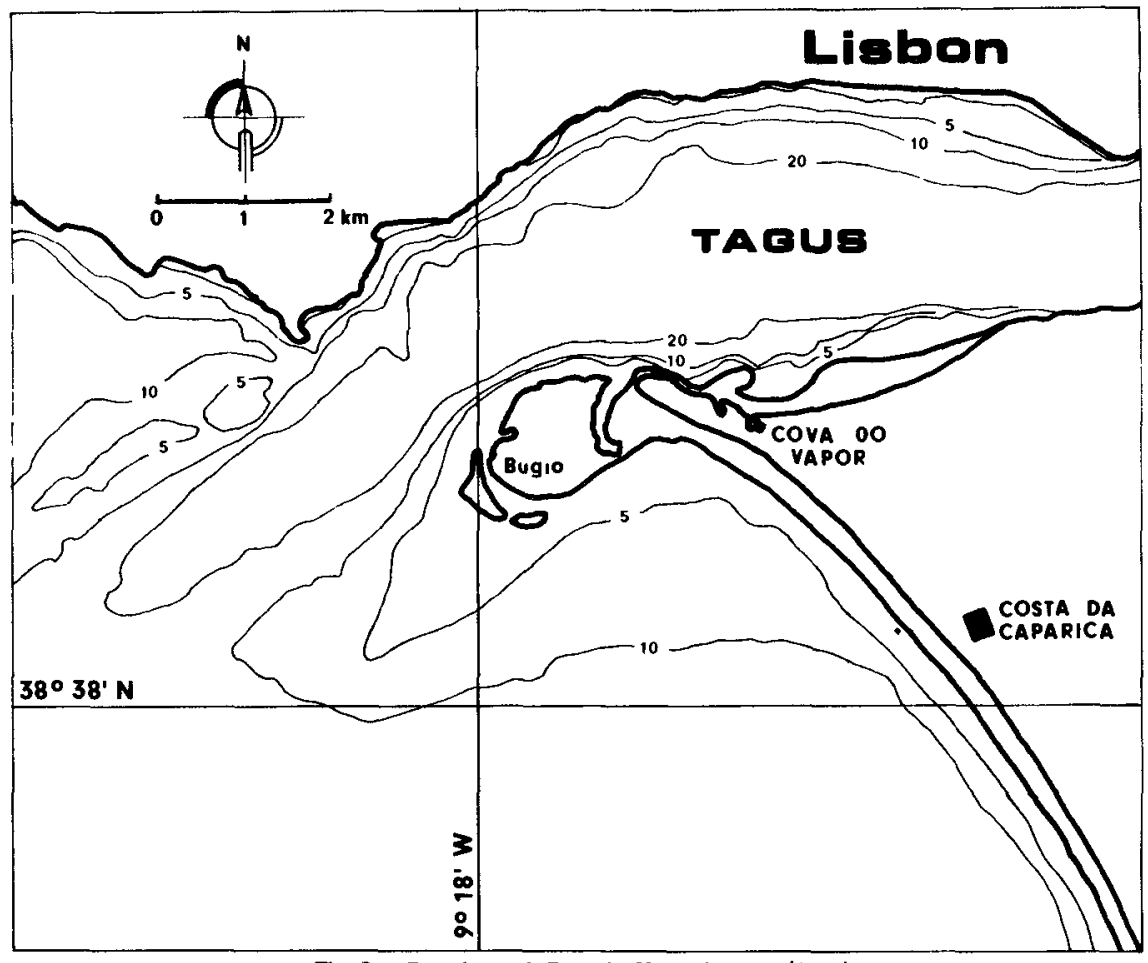

Fig 9 - Caparica and Cova do Vapor beaches (2929)

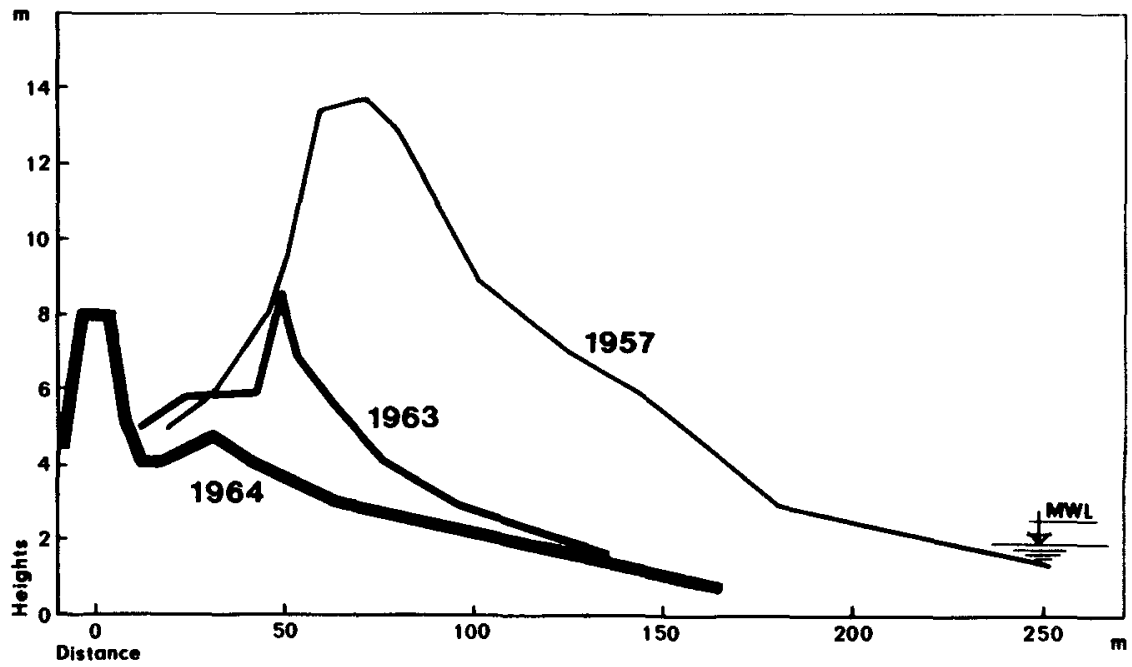

Fig 10 - Evolution of transverse profiles in Caparica (transverse profile in the dike root) 
zone of the Bugio lighthouse The very oblique approach of the waves caused northward sand movements from the central zone of Caparica beach, where the obliquity of the wave action was very reduced or even normal to the coast so that the beach south of Caparica was very stable

When the natural protection to the north collapsed an intense longshore drift begun that formed a spit in the root of the existing formation, this spit was fed in part by the sands carried northward, the remainder being deposited in the channel of the Tagus estuㅡ ary, the great depth of which avolded sermous disturbances This phenomenon begun to affect Caparica practically in 1960 in the form of the erosion mechanismillustrated in fig 10 The first emergency mea sures, taken iff 1959, consisted in the construction of a groyne (see fig 11, E3) and were followed by a frontal protection structure and groynes $\mathrm{E}_{1}(1962)$ and $\mathrm{E}_{2}$ (1963) at Cova do Vapor when the waves cut the sand dunes between Cova do Vapor and Caparica a dike with a light structure was bult (1959) to protect the urban areas in the nelghbourhood, located below the mean level of the sea, against the runup of spring tide waves

The situation grew worse after 1964 when serious destructions occurred in the central zone of Caparica, so that the dike was strengthened and a frontal protection core was bult at its root, together with a short groyne, which, of course, proved entirely useless Obviously the influence zone of the groyne bult at Cova do Vapor was too small to protect the southern beach and the other protection works in the same site merely helped to form a resisting core that prevented the entire destruction of the $z$ one and of the small village in it Other emergency measures were taken after 1964, so that the situation evolved to the state presented in fig 12 (a comparison of which with fig 11 shows the evolution in 1963-1969) after the groyne $E_{2}$ was extended and the beach between Cova do Vapor and Caparica was entirely eroded except for a small zone protected by the first groyne bult, the northern spit moved under the influence of the waves up to the NATO jetty 


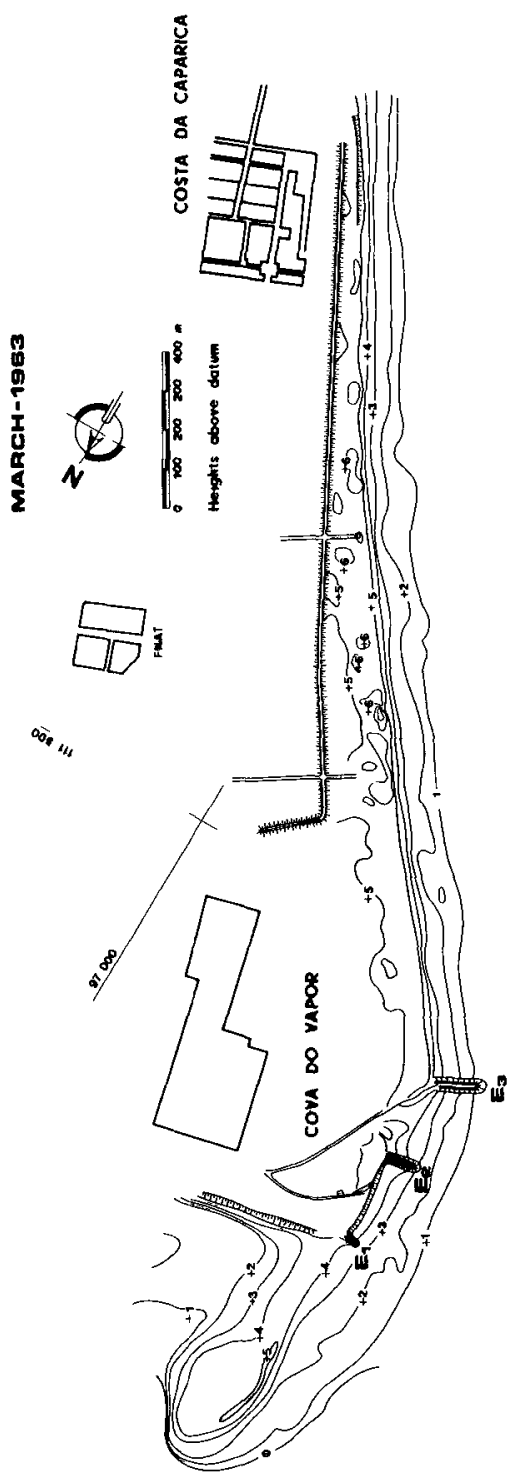




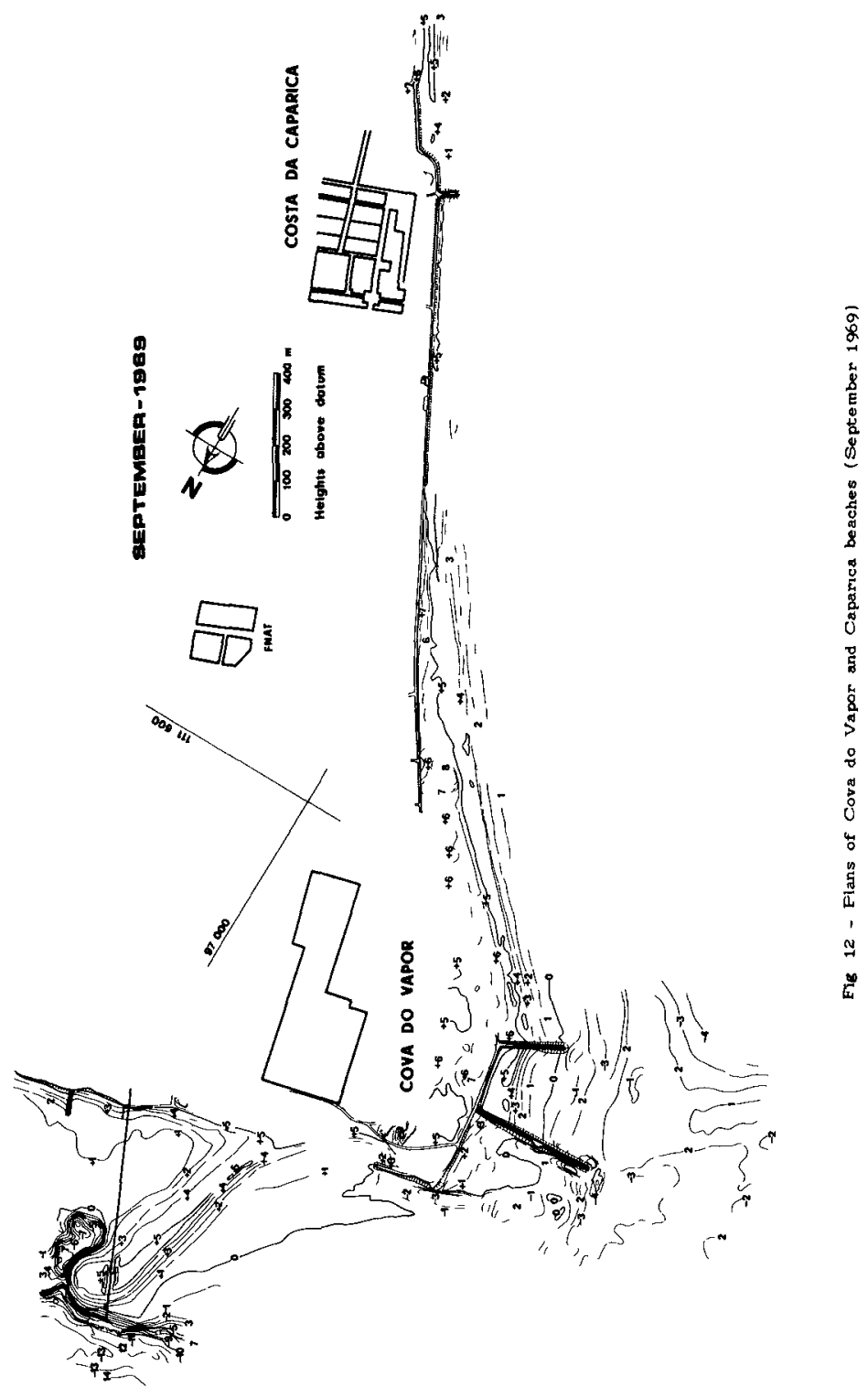


also shown in $f_{1 g} 12$ According to the studies carried out $\{3\}$ the littoral drift in this zone is estimated in $1000000 \mathrm{~m}^{3} /$ year At the same time, owing to changes in the sea-bottom relief and their $\mathrm{m}$ fluence on the wave action, a rapid erosion started in the central zone of Caparica particularly serious in the beaches to the south (see fig 1) Another emergency structure for frontal protection was bult and subsequently strengthened in the central zone of $\mathrm{C}_{\underline{a}}$ parica

The main physiographic characteristics of the beaches of Co va do Vapor and Caparica have been very rapidly presented Many comprehensive studies of this case were carried out by official and private bodies charged with and interested in the development and exploration of marine zones Owing to insufficient means, deficient policies of beach protection and development, and uncoordmated activities of the different official and private bodies involved emer gency structures - some of which entirely unsuited - were resorted to, but did not prevent the loss of one of the best Eortu guese beaches

Figures 13 and 14 give an idea of conditions in Caparica beach before the erosion begun and now (July 1970) The groyne $E_{2}$ (fig 11 and 12) is now being extended, and the construction of a groyne system and the strengthening and extension of the frontal protec tion structure $\{4\}$ are in preparation The groyne system is made up of units which, although very long, have a reduced effective length and a small spacing and are located at very high elevations With such a system, the restoration of the beach is out of question, as the existing groynes to the north cannot operate efficient ly for lack of supply from the south due to the disappearance of the beach On the other hand the southern stretch of the beach will tend to be eroded away up to the extended frontal protection structure, which will have to be exaggeratedly strengthened These measures, planned a long time ago for hydrographic conditions no longer prevaling, are unsulted to the present needs and will never 


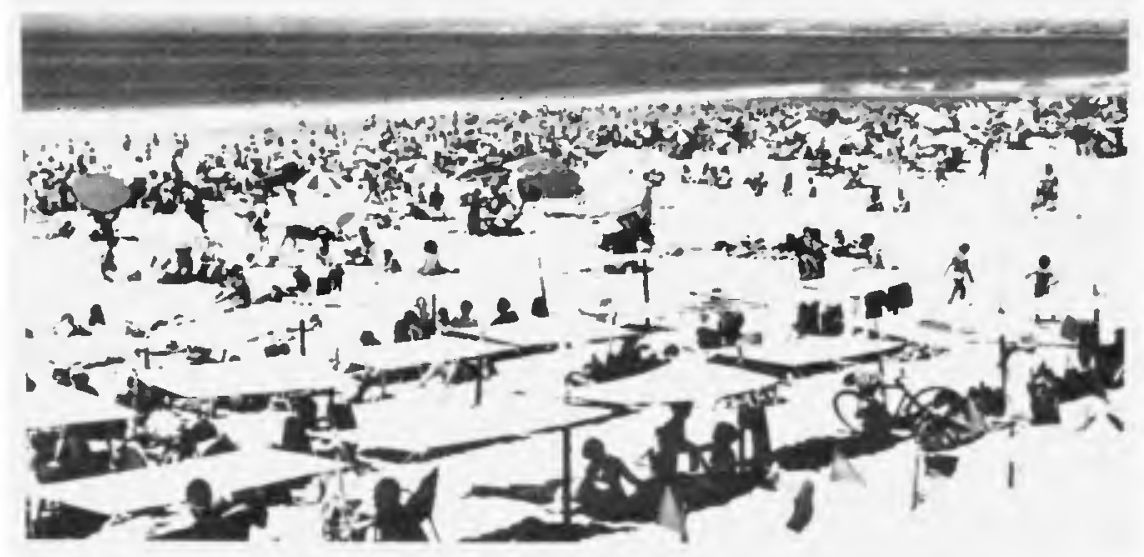

Fig. 13 - Caparlca beach before erosion begun

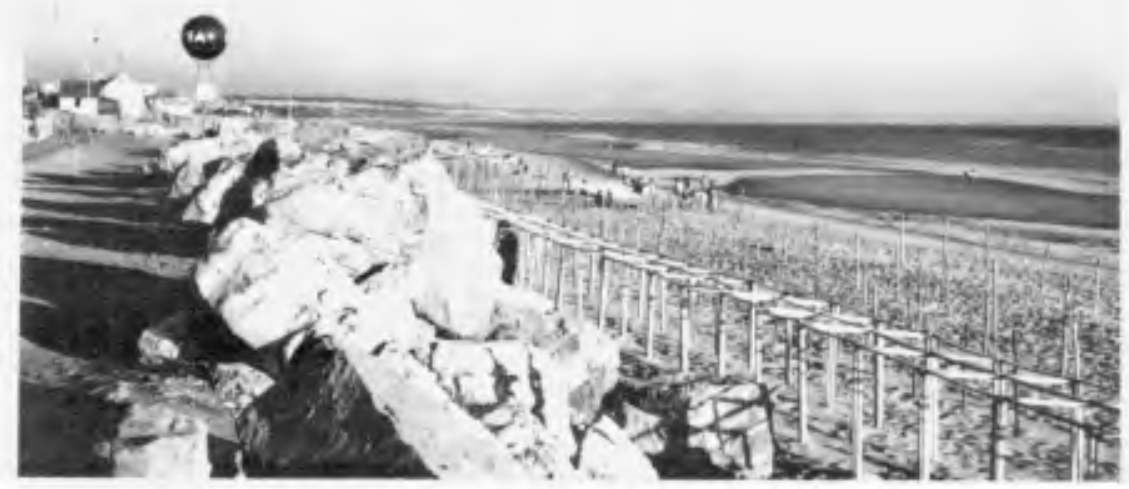

Fig. It - Coparica besch in July 1970 (low water level)

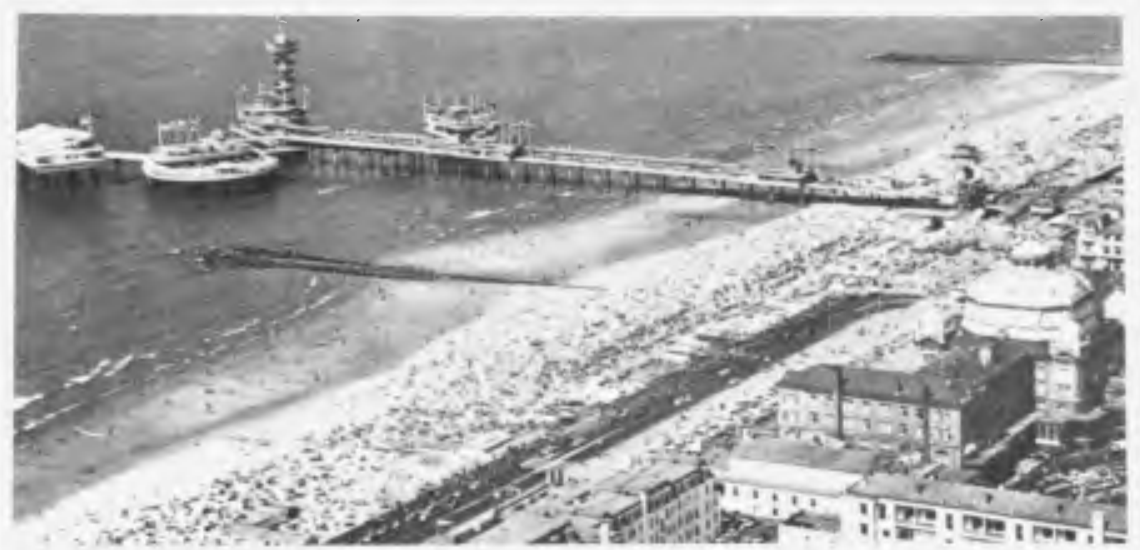

Fig. 15 - Scheveningen beach (The Hague, Netherlands) 
provide a beach with real recreational and touristic interest As an instance of a system of functional groynes, the one bult at Sche veningen beach (The Hague, Holland) is presented, the construction was very expensive owing to the extraordinary structural perfection of the groynes, but the results were excellent (see fig 15)

The restoration and protection of these beaches requires an artificial feeding and a system of functional groynes, 1 e long very spaced groynes The artificial feeding would restore e g. 1963 con ditions, as shown in fig 16 The northern spit is represented in the figure, because studies on the touristic development of Cova do Vapor carried out in 1966 for the TECFINT project \{3\}, recommended the construction of a long groyne in the northern end and the creation of an extensive beach mainly at the cost of sand dredged from the spit, in which a pleasure boat port (represented by the dotted Ine in the figure 16) would also be bult The solution recommended here for the present conditions would require the straightening and protection of the bank, upstream of the root of groyne $\mathrm{E}_{0}$, the artificial restoration of the beach being necessary to the south of $E_{2}$ alone This location of groyne $E_{0}$ is preferable to the one shown in fig 12, which would leave two groynes very near one another disturbing the operation of groyne $\mathrm{E}_{0}$

By means of the plan presented in fig 16 it would be possible not only to recover the beaches and protect the coast to the south of Caparica but also to avold the extension and strengthening of the frontal protection structure Obviously a more detalled study of this plan (which is no more than a typical solution), could yield a better and more economical solution $A$ single very long groyne to restore the natural connexion with Buglo together With a large volume of sand to restore the beach (a natural restoration being impossible owing to lack of supply and the irreversibility of erosion) would leave a long stretch of beach without protection making possible local erosions, so that the solution pre sented is preferable 


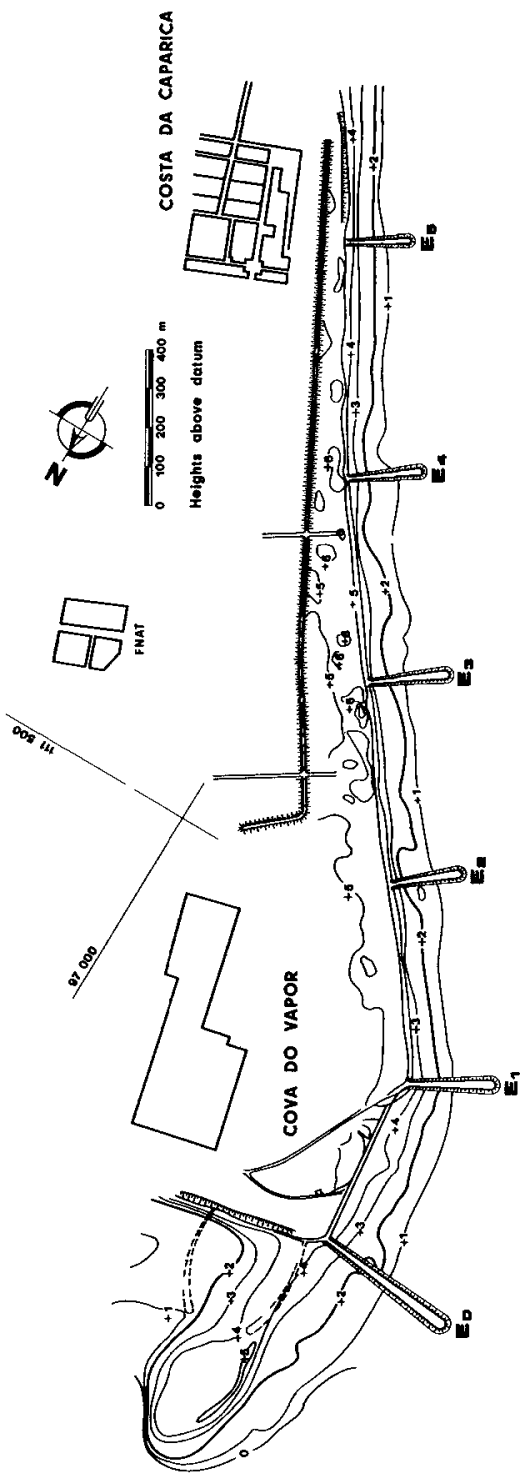


$J$

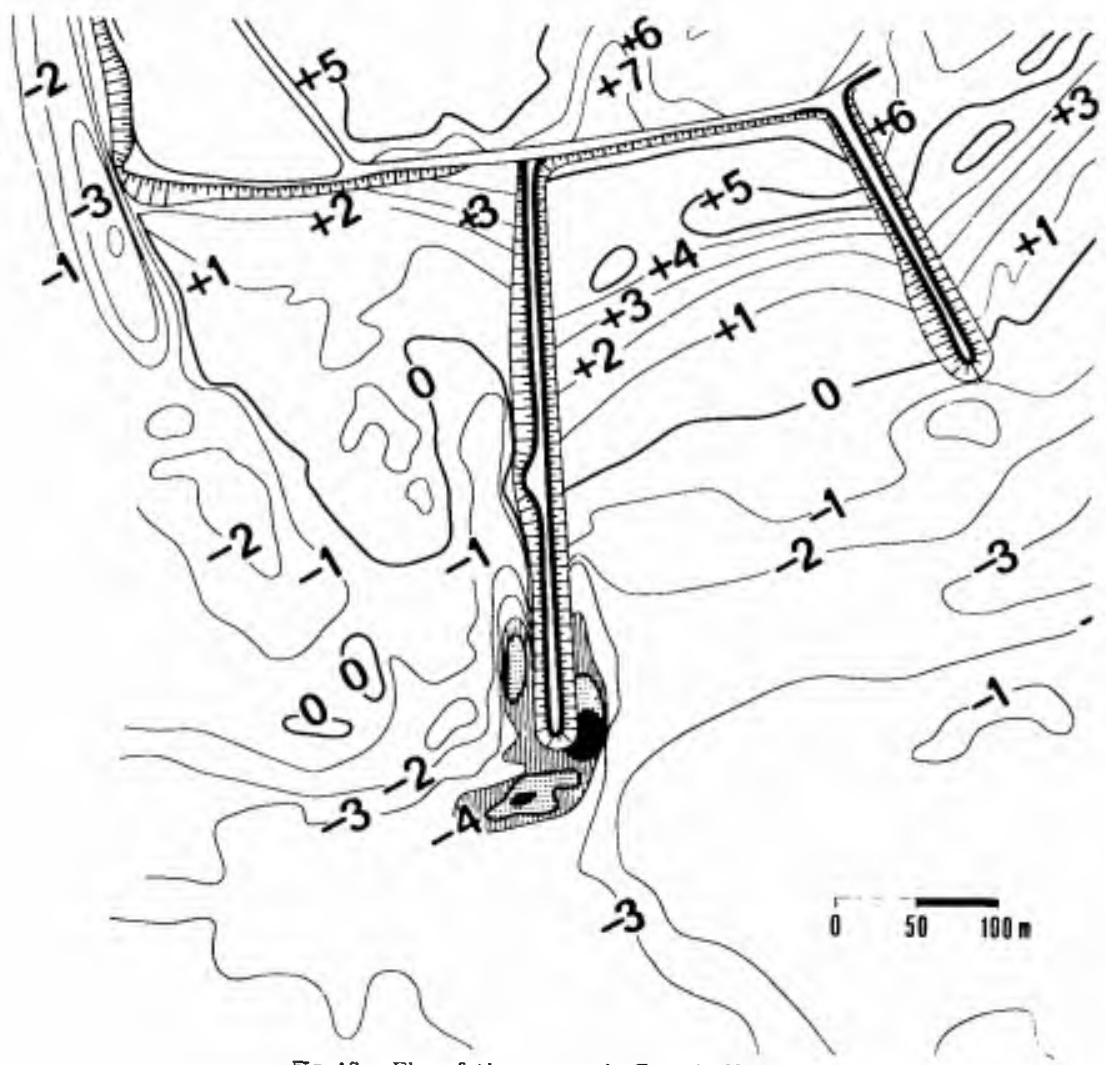

Fig. 17 - Plan of the grognes in Cova do Vapor

(September 1969)

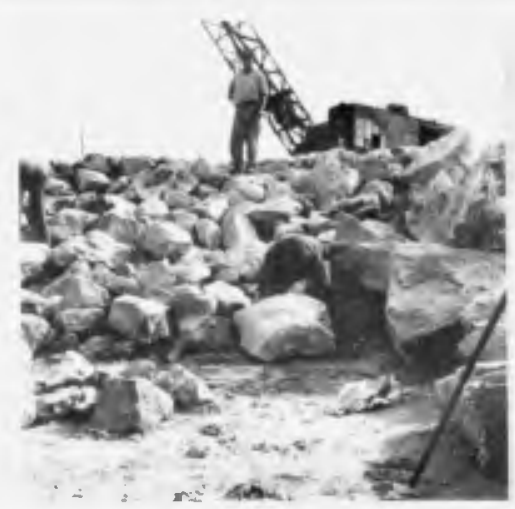

Fig. 18 - Detall of the construction of groynes in Cors do Vapor

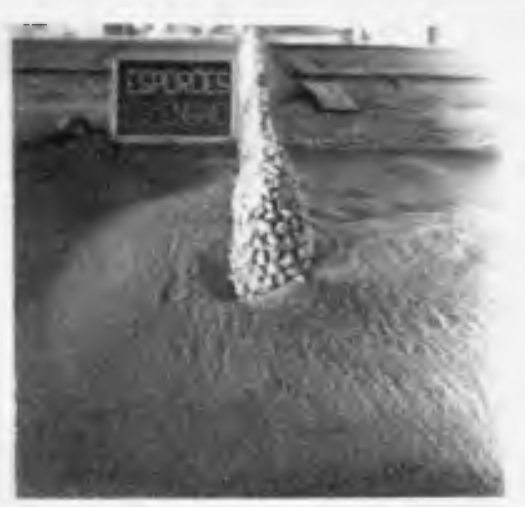

Fyg. 19 - Erosion at the ends of the groynes obtalned in the models 
The typical solution presented for the groyne system takes into account the two studies carried out As wave conditions are variable, groynes with $B=90^{\circ}$ were chosen The obluquities $\alpha_{0}$ considered are $10^{\circ}$ in the zone from Cova do Vapor to the middle of the dike, and $5^{\circ}$ from this point to the central zone of Caparica The reference line for calculating the length of tne groynes will be the bathymetric curve $+200 \mathrm{~m}$ For $\alpha_{0}=10^{\circ}, c / D=35$ and for $c=150 \mathrm{~m}, D=520 \mathrm{~m}$ (groynes $E_{1}$ to $E_{4}$ ), for $\alpha_{0}=5^{\circ}, c / D=40$, which for $c=150 \mathrm{~m}$ yields $D=600 \mathrm{~m}\left(E_{5}\right)$ Groyne $E_{5}$ may be shorter $(\mathrm{c}=100 \mathrm{~m}$, above $+200 \mathrm{~m})$ because the zone updrift of this groyne would be stable and obliquities are very small, an inversion to the south being even possible with small obliquities

It should also be noted that an efficient system does not de pend on the location of the groynes alone Their structure and cer tain constructional detals are also essential As an instance, the case of groyne $E_{2}$ (fig 11 and 12) is presented excavations shown in the fig 17, occurred at its end, in concentric zones at depths $-4,-5,-6$, the darker zones corresponding to the last depth These peculiarities seriously interfered with the construction of the groynes and caused an exaggerated use of rockfill An adequate base of the groynes protecting the adjacent sea floor against erosion is essential but, as shown in fig 18, these groynes lack such a special base As can be seen in fig 19 erosions at the end of the groynes occur even in the model tests, those shown having been observed in tests carried out in the first stage of these studies

\section{5 - CONClUSiONS}

- The angle of the groynes with the shore line is a function of the obliquity $\alpha_{0}$ of the waves For high obliquities $\left(\alpha_{0}=20^{\circ}\right)$ the maximum efficiency corresponds to inclined groynes with $\beta=70^{\circ}$ For intermediate $\left(\alpha_{0}=10^{\circ}\right)$ and low $\left(\alpha_{0}=5^{\circ}\right)$ obliquities, normal $\beta=$ $=90^{\circ}$ ) groynes are preferable to inclined groynes

In variable wave conditions efficiency will be defined in func 
tion of the range of obliquities of the waves present In this case normal groynes $\left(\beta=90^{\circ}\right)$ are recommended

It should not be forgotten that inclined groynes are longer (increased $c$ ) for the same spacing $D$ For $\alpha_{0}=20^{\circ}$, groynes with $\beta=70^{\circ}$ are $30 \%$ longer than normal groynes $\left(\beta=90^{\circ}\right)$, whereas the increase in the area $\Omega$ of the inclined groyne 1 s $20 \%$ only The fact that groynes with $\beta=70^{\circ}$ protect the root of the updrift groyne against erosion should be taken into account.

- T-groynes are not more efficient than normal groynes, except for $\alpha_{0}=10^{\circ}$, and even then the difference is small (see fig 8) Their only advantage les in preventing erosion near the roots of the groynes Nevertheless they are not recommended because, in addition to their construction being more expensive, they disturb the utilization of the beaches for sea baths

- The hydraulic optimization of groyne systems must take into account not only the ratio $\mathrm{c} / \mathrm{D}$, the values of which were defined in $\{1\}$, but also the inclination of the groynes according to the criteria presented above A judicious application of these results must obviously be based on a knowledge as complete as possible of physiographic conditions in the involved zone, and other questions must also be taken into account such as the stability and the crest elevation of the groynes (the latter basically depends on the transverse profile of the beach and its evolution)

Another basic point to be borne in mind when designing systems of functional groynes and which may helpto reduce the volume of the structure is that its object is not, as a rule, entirely to stop the littoral drift occurring in zones of the beach variable under the action of tides, but to preserve a certain area of the beach On the other hand littoral drift is more marked for high tide levels, precisely the conditions in which groynes operate most efficiently This leads to adopting groynes, which although long, do not extend to very low levels 
- The cooperation of the different official and private bod les interested in the exploration of marine zones seems extremely important Frotecting structures should be thoroughly studied and designed, and constructed without delay, emergency or experimental structures are strongly disapproved, all the more so as the present knowledge in this field makes them entirely unnecessary

- In the case of Cova do Vapor and Caparica, the essential solution for the restoration and improvement of the beaches is their artificial feeding and a functional compartimentation of the beaches by means of a system of groynes

\section{LITERATURE}

$\{1\}$ - BARCELó, J F - Experimental Study of the Hydraulic Behaviour of Groyne Systems Memörza no 350 do Labora tórı Nacional de Engenharia Civil Lisboa 1969

$\{2\}$ - NAGAI,S, KUBO,H - Motion of sand particles between groins Journal of the Waterways and Harbors Division. ASCE, vol 84, No WW5 December 1958 Fart 1

\{3\} - TECHINT - Estudo do aprovertamento turistrco da área da Cova do Vapor Lisboa Outubro 1966

$\{4\}$ - DIRECÇÃO DOS SERVIÇOS MARÍTIMOS (MOP-DGSH)- Projecto de defesa e valorzacão turistrca das praras a sul da embocadura do Tejo Lisboa Junho 1966 


\section{CHAPTER 66}

IAND RECLAMATION AND GROIN-BUILDING IN THE PIDAL FIATS

by

Heie Focken Erchinger *)

\section{COASTAL PROTECTION BY RECIAIMING IAND}

Along the North Sea coast of Germany there are two large areas where land reclamation work in the tidal flats is bezng carrled out. One is on the coast of SchleswigHolstein and the other in Ostfriesland, on the coast and along the estuary of the river Emo near the border with the Netherlands. Condltions and working methods for land reclamation in tidal flats as well as the development of new groln constructions on the Ostfriesian coast are described below.

Before the middle of this century, land reclamation was done malnly for reclalming new fertile soil for agricultural purposes on the tidal flats. Nowadays, the aim of reclaiming land $1 \mathrm{~s}$ for coastal protection, for the reclalmed foreland has many favourable effects on the dikes:

*) Oberbaurat, Chlef of the Coastal Protection Department in Lower Saxony, 298 Norden/Ostfriesland, West Germany 
1. The run-up of waves on the dike is reduced as the waves break on the foreland with spilling breakers (FUHRBOTER 1966) and are thus prevented from reaching the slope of the dike with all their vehemence. During the storm surge on Febr. 16 ${ }^{\text {th }}, 1962$ for instance, this was observed distinctly as to be seen in figure 1 .

2. Wavewash on the slope of the dike becomes considerably smaller.

3. The land before the dike, in the case of a dike failure In a storm surge, also prevents a fallure of the base from taking place so that even at mean $h 1$ gh water level the sea cannot enter the land.

4. An expensive rabble slope for the protection of the dike 18 not necessary.

5. For the upkeep of the grass and clay covered dike, it is important to find sods and clay near the dike on the reclaimed land.

For coastal protection a $200 \mathrm{~m}$ whde strip of land in front of the dikes is needed. This was found in research works carried out by Prof. Hensen, Technical University, Hannover. 
WAVE RUN-UP ON THE DIKE

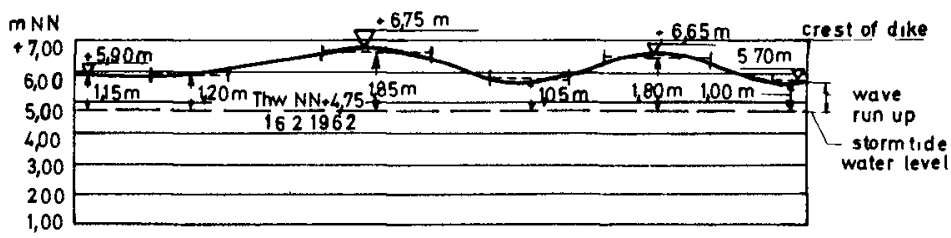

\section{PLAN VIEW}
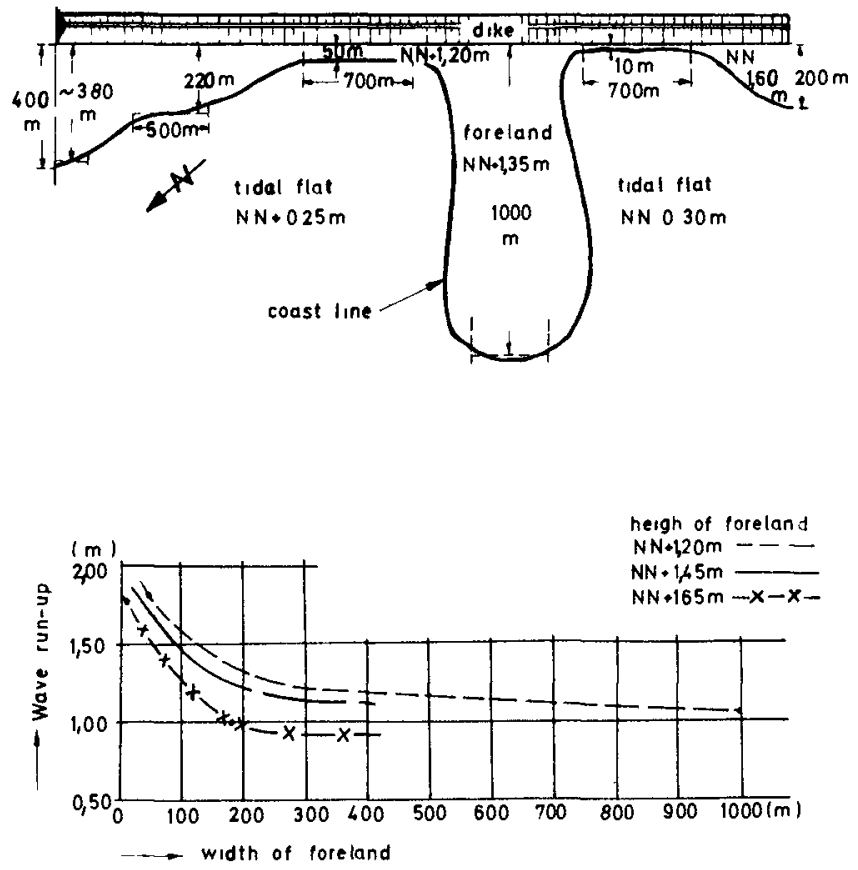

Fig 1 Relation between wave run up and width and height of the foreland (KRAMER, LIESE LUDERS 1962) 
CONDITIONS AND WORKING METHODS FOR LAND RECLAMATION

For reclaiming new land, rectangular sedimentation flelds, measuring about 100 by $200 \mathrm{~m}$, are constructed of grolns. Hence, the flelds are saved from strong currents and wave movements so that the sedimentation of sand and silt can be allowed to take place unhindered. Through the inlet in the groln on the sea-side, called "cross groln", the water can enter and leave the fleld quickly. During the calm period, the settling and a part of the suspended solids preclpitate on the soil.

It is of interest to know something about the origin of the solids which could be classified as Sand, Clay Minerals, Organle Matter and Calcium Carbonate. The composition of the sediment varies considerably along the Ostfriesian coast. Near the brackish water region of the river Ems an the Dollart Bay the clay mineral and organic material contents are relatively high. Along the North coast the sediment contains more sandy material (Figure 2).

The rate of sedimentation and the composition of the sediment depends also on the work of the molluscs, which prepare the fine organic material and clay minerals by bullding small lumps of silt (KAMPS, 1962). The gills, whlch are used by these creatures for breathing, also 


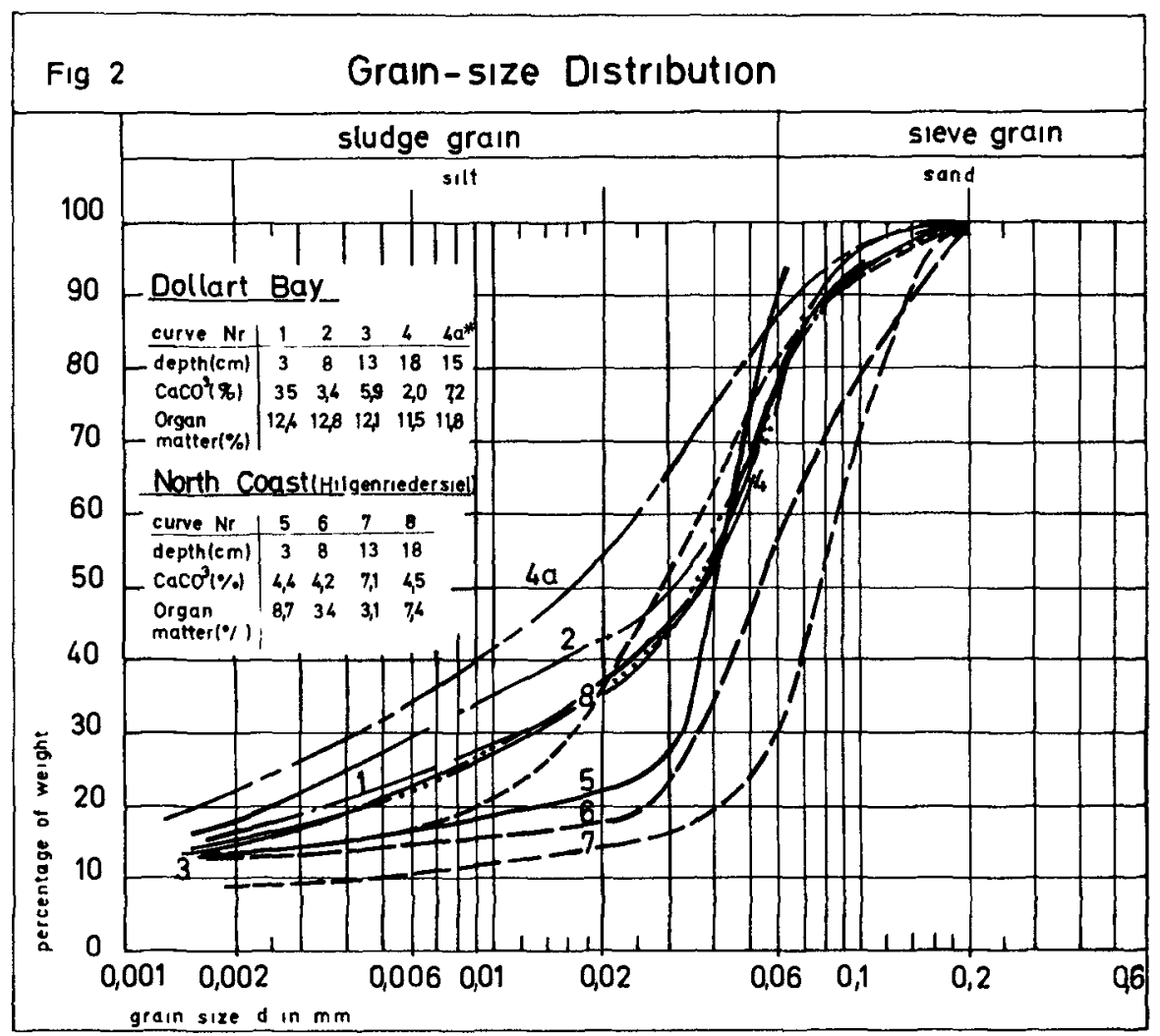

* Sample 4a from the sedimentation field, all the other samples from grass covered foreland

serve the purpose for the collection of food, which they sleve out from water. In this process, sand and mud components, whlch are of no value to the creature are collected and covered with slime, out of which the coarse material, which in this case would be sand and some or- 
ganic matters is ejected at the inlet, while the fine material, including the mud, is discharged together with the other remnants of digestion in the form of tiny lumps, strongly joined together. For reclalming land it 28 very Important that these lumps effect a higher rate of sedimentation which is rich in clay and organic matter. The important molluses on the Ostfriesian tidal flats are Mussels (Mytilus edulis L.), Cockles (Cardium edule I.) and Gapershells (Mya arenaria L.).

Our knowledge in this difficult matter is not complete. For Instance Mr. Raudkivi of New Zealand is of the opinion, that in brackish water the rate of sedimentation is dependent upon the change in the electruc charge of the single solid as they come in from fresh water (RAODKIVI, 1967).

It is relevant to mention some aspects of plant-growth and ditch-bullding in the sedimentation fields. When the terrain in the fields has reached a height, at which plant-growth can be expected, that is about 30 to $40 \mathrm{~cm}$ below mean hlgh water level, ditches are dug at regular intervals by special excavators.

Figure 3 shows the hydralic excavator, mounted on a pontoon, which is cablemoperated and slides on the ground. On the higher fields with vegetation a ditch-millingcutter is used for making ditches. The earliest natural 
plants to take root in

the sedimentation

fields are Glasswort (Salicornia herbacea

I.) and Cordgrass

(Spartina Townsendii).

A rank plant-growth

effects a greater

calmness on the field,

facilitating better

sedimentation and hence

a more clayey soil.

When the terrain has

Fig. 3

reached mean high water level, the first plant of the grass-family to take root is the Sea Poa (Puccinellia maritima Parl.).

THE CONSTRUCTION OF THE GROINS

Although methods of land reclamation are not different on shallow and deeper tidal flats, the construction of the groins depends on the level of the flats. Formally, the reclamation of land for agricultural purposes was done mainly on shallow tidal flats. The groins were built of brushwood and earth. They were constructed of two rows of wooden piles with cornpact brushwood in between and wooden planks at the leeside to prevent permeation. The piles are held together on top with wire (Iigure 4). 


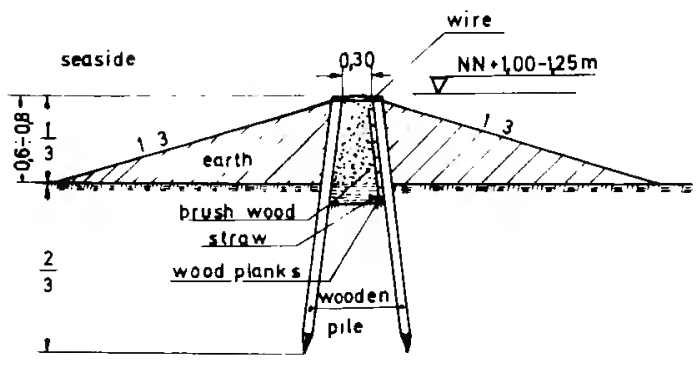

Fig 4 Brushwood groin

Occasionally the cross-groin at the sea-side is strengthened with fascine mattresses (figure 5).

In deeper tidal flats it was necessary to construct the groins

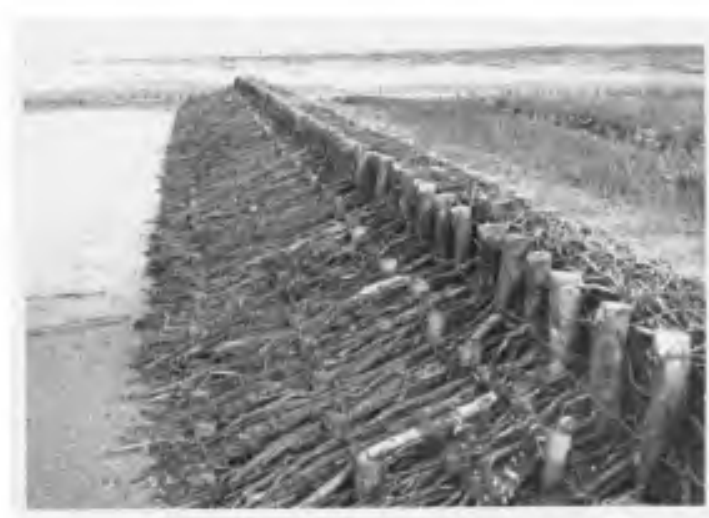

Fig. 5 more solid by using new materials. So far five types of groins have been developed for this purpose:

1. A precast concrete unit with a height of 50 to $90 \mathrm{~cm}$. Each unit has a length of $1 \mathrm{~m}$, the weight is 900,1200 and $1800 \mathrm{~kg}$ (figure 6). They are set on top of a wooden sheeting on either side. The joints between the single units are made close 
with a special joint ribbon of foamed Polyäthylen held in a groove at one side of the unit.

2. A flexible tube of plastic web with a diameter of about $1 \mathrm{~m}$ is filled with sand

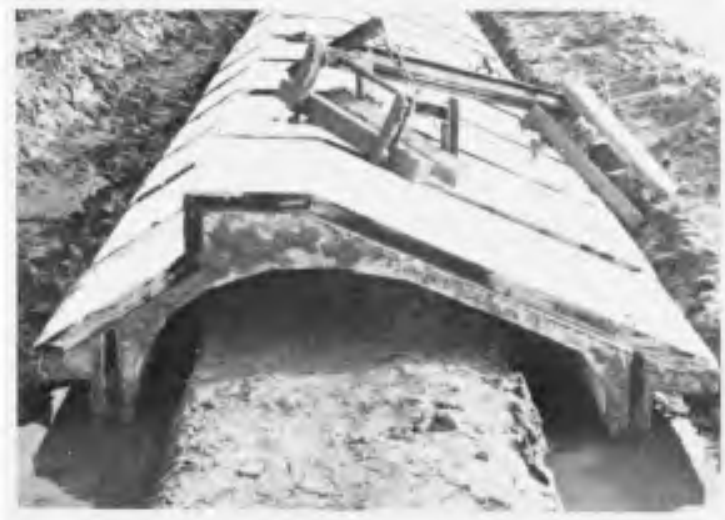
by hydraulic dredging and takes the form of a sausage (figure 7). The plastic web is woven of small strips of a polyäthylen membrane. Normally, the sandy soil can be taken from the ground of the tidal flat at a small distance to the building site. It is an inexpensive method to construct groins. Besides, the following construction works are made use of, when the abovementioned types are not high and heavy enough.

3. A groin with a core of earth, with plastic mem- 
brane (Polyathylen) around this and concrete blocks interlocking horizontally and vertically on top. A wooden sheeting and wooden planks fastened to the wood piles encloses the groin on both sides. A woven mat of reed above the plastic membrane, protects these from getting damaged (figure 8) (ERCHINGER, 1967).

seasıde sedimentation field

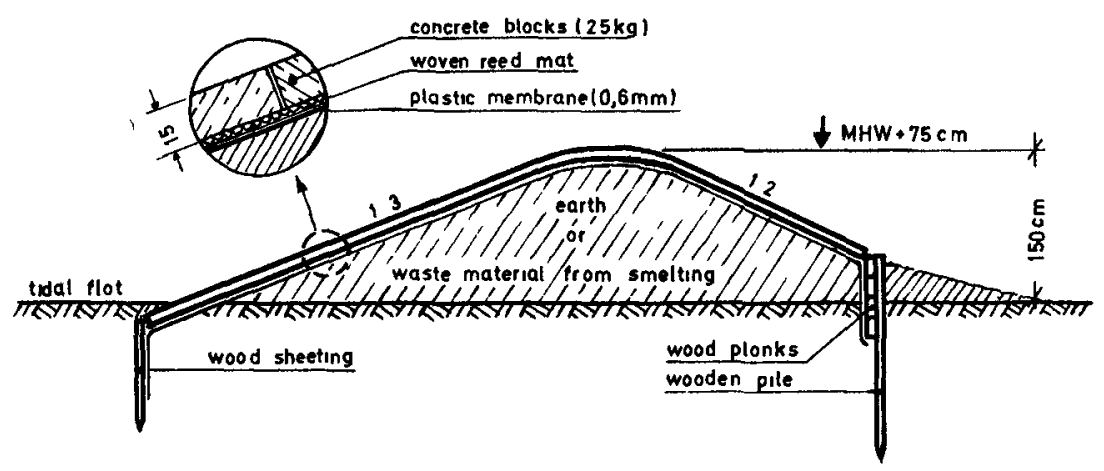

Fig 8 Section through o groin

4. This type is nearly the same as the third, but the surface is covered with quarry stones instead of concrete blocks and with steeper slopes. As the layer of the stones is about $40 \mathrm{~cm}$ thick, this construction needs much more material than the type with concrete blocks, which is to be transported to the building site. Transport on the flats is difficult and expensive.

5. This construction is chosen when the area in front of the dikes is to be filled up by hydraulic dredging and an embankment is to be built at the sea-side. Instead 
of the plastic membrane, we take a plastic web which is woven very close, so that even the finest particles of sand and silt cannot get through (figure 9). However, 1t is necessary for the outlet of the water and for preventing water pressure under the surface.

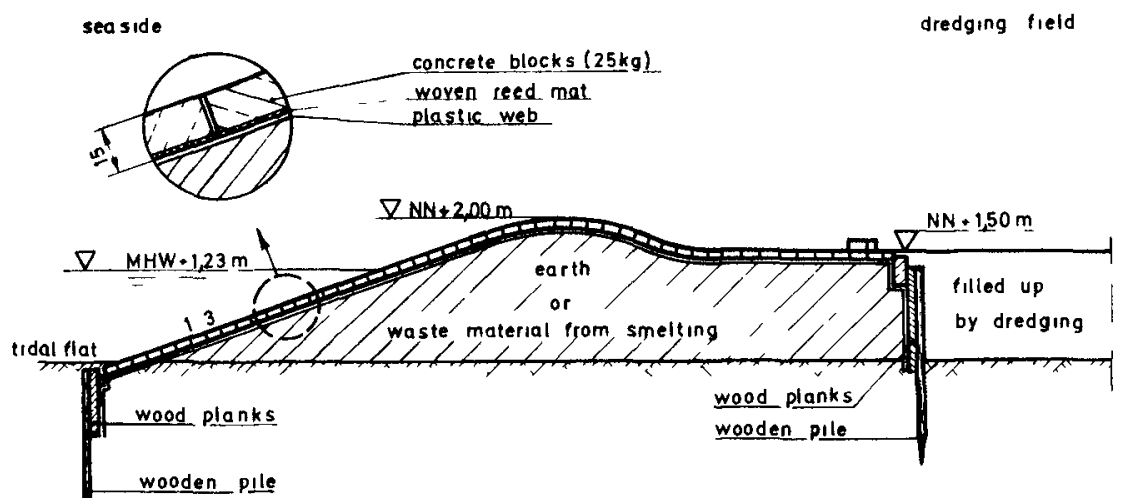

Fig 9 Section through an embankment in front of a filled up foreland

In some cases of groin construction of the last three types, waste material from smelting has been taken for the core of the groin. This material has been found to be very usefull. Its hydraulic substance, which formed a $50 \mathrm{~cm}$ thick and hard layer on the tidal flats was so stable, that even loaded heavy motor vehicles could use this as a road to transport the materlal to the bullding site. This ist a great advantage, considering that it is derıved from waste material. 
In 1965, the smelting industry, the Hoesch Concern, made a test with $100000 \mathrm{t}$ of waste material, containing residues and scrap heaps as well as mud and dust from the smelting process. A forefield in front of a dike was created with this material. This was done in the expectation to make the waste products useful for dike protection and also as a means to get rid of this substance from the vicinity of the foundries, with insufficient storage capacity for thls (HAUKE, 1968). Several scientists have inquired into the chemical and blological consequences of this waste material at the surrounding tidal flat area. No unfavourable effect has been found (KUSTENAUSSCHUSS, 1969).

\section{REFERENCES:}

ERCHINGER, H. F.. FUHRBDTER, $A_{\text {* }}$

HAUKE, $M_{*}$ KAMPS, L. F. KRAMER, J., LIESE, R. LUDERS, $\mathrm{K}$. KUSTENAUSSCHUSS NDRDUND DSTSEE RAUOKIVI, A. J.
Küstenschutz durch Vorlandgewznnung. Wasser und Boden 19, H, 10, 1967 Der Oruckschlag durch Brecher auf Dezchböschungen, Mzttezlung des FranziusaInstztutes fur Grunde und Wasserbau der Technischen Universi tat Hannover, Heft 28, 1966 Dezchsicherung mat Verhüttungsruckstanden. Helgolander wassenschaftl 2che Meeresuntersuchungen 17, 1968 Mud Dzstribution and Land Reclamation in the Eastern Wadden Shallous. Rijkswaterstaat Communzcations Nr. 4, The Hague, 1962 O1e Sturmflut vom 16./17. Februar 1962 1m Naedersächsıschen Küstengebret. Dre Küste 10, 1962, H, 1 Dezchsicherung durch Verhüttungsruckstände. Die Kuste 18, 1969, Hsg.. Küstenausschull Norde und Dstsee, KuelmWak, Feldstrabe 251/253 Loose 8oundary Hydraulics. Perganon Press, Dxford a. 0., 1967 


\title{
CHAPTER 67
}

THE EFFECT OF GROYNES ON ERODED BEACHES

\author{
W A PRICE - Senıor Principal Scientific officer \\ K W TOMLINSON - Senıor Scientific Offıcer
}

Hydraulıcs Research Statıon, Wallıngford, Great Britain

\begin{abstract}
Laboratory tests are described, in which the effect of impermeable groynes on an eroded beach was studıed A beach was allowed to reach equilıbrıum for a partıcular wave climate and supply of littoral material The foreshore was then manually eroded, and the beach allowed to return to equilibrium with and without groynes It was found that the presence of groynes increased the rate of accretion but did not significantly build up the inshore beach beyond the stable levels Bed levels seaward of the groynes were increased
\end{abstract}

\section{Introduction}

As part of the continuing research into the effects of groynes on beaches being carried out at the Hydraulics Research Station, the authors presented a paper to the lith Conference on Coastal Englneering, see Ref 1, describing laboratory tests to study the effect of permeable and impermeable groynes on a beach that was stable for a particular wave climate and a given supply of littoral material These tests showed that on the part of the beach between high water and low water levels the groynes induced little or no build-up However, accretion was found seaward of the impermeable groynes It was concluded that this offshore bulld-up was necessary in order that, at equilibrium, the littoral transport that formerly travelled along the foreshore could pass seaward of the groynes The permeable groynes tested had little effect on elther inshore or offshore levels

Since then, the more practıcal case of groynes installed on an eroded beach has been studied The tests were conducted in the same wave basin as the previous series, see Fig 1 This facility allows the generation of waves at an angle to the beach using the serpent-type wave generator shown plus tides and littoral currents, although the littoral current 
generator was not used during these tests

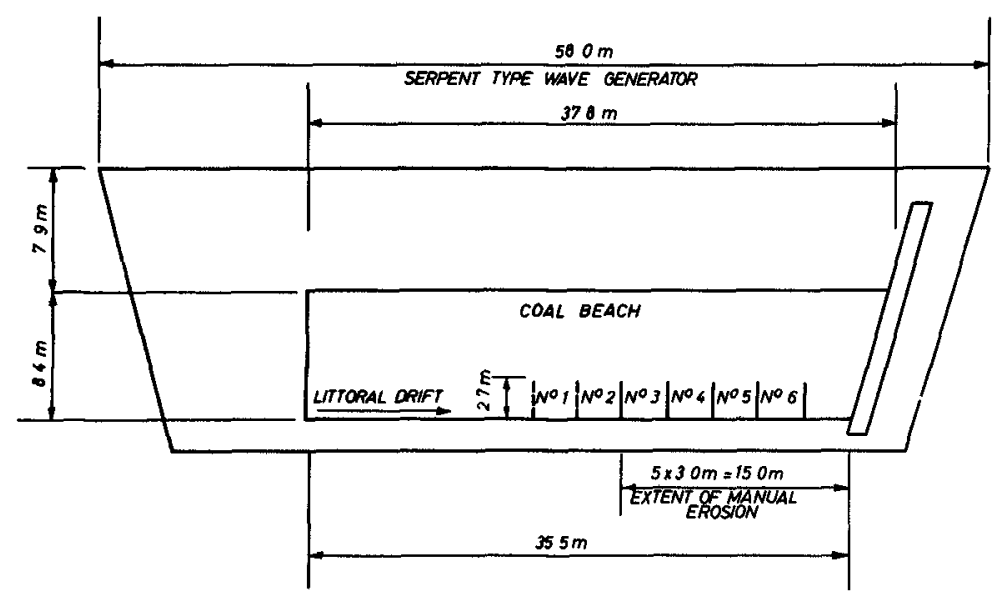

FIG IDETAILS OF WAVE BASIN

\section{Test Procedure}

In the absence of groynes, waves and tides were generated to bring the beach to equilibrium The waves were $65 \mathrm{~mm} \mathrm{high}$ at mean water level, with a period of 115 seconds and an angle of approach of 5 degrees in deep water The tidal period was 75 minutes, with a range of $012 \mathrm{~m}$ Beach material was crushed coal of speciflc gravity 135 and median grain size $08 \mathrm{~mm}$ The experiments were continued untll a comparison of successive beach surveys showed that little change was taking place and the quantity of littoral material trapped at the downdrift end of the beach was the same as that fed in at the updrift end, this normally required at least 25 tides

An eroded beach, $15 \mathrm{~m}$ long, was then created at the downdrift end by removing material between high and low water to an average depth of $25 \mathrm{~mm}$ Five impermeable groynes were placed on the eroded beach The groynes, shown as solid lines in Fig 1, were $27 \mathrm{~m}$ long and about $35 \mathrm{~mm}$ high, spaced at $30 \mathrm{~m}, 1 \frac{1}{2}$ times the horlzontal distance from high water to low water

Again the same waves and tides were generated and the littoral transport measured. Beach material was fed to the updrift beach at the average rate measured on the equilibrium 
beach Beach surveys were made at $1,3,5,10,15$ and 20 tides, at which time the beach had returned to its equilibrium profile

The experiment was then repeated with no groynes on the eroded beach

\section{Calculation of Results}

The parameter selected for the comparison of beaches was the volume of coal within groyne compartments The six compartments for which volumes were calculated are defined in Figs 1 and 2, all compartments were $30 \mathrm{~m}$ long, $27 \mathrm{~m}$ wide and a maxımum of $028 \mathrm{~m}$ deep

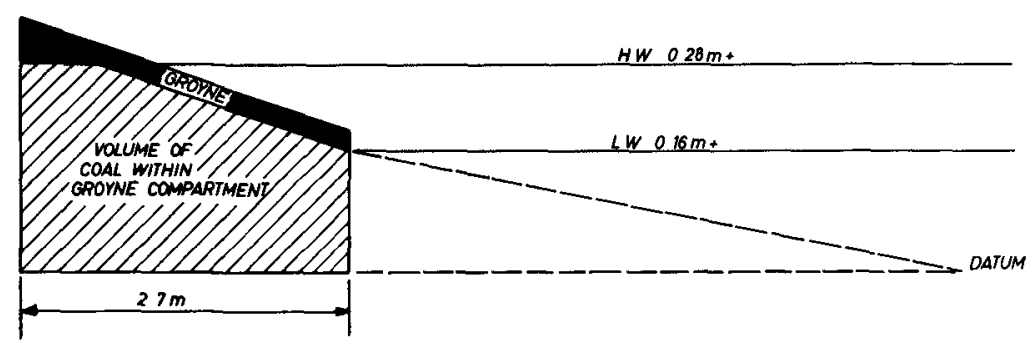

FIG 2 BEACH ZONE REFERRED TO IN CALCULATIONS

Although not bounded on both sides by groynes, compartments 1 and 2 were included to show the effect of groynes on the updrift beach

Three cross-sections were measured in each compartment $015 \mathrm{~m}$ from the groynes or boundaries, and on the compartment centre-line Volumes were then calculated using the trapezoldal rule These volumes were plotted in Fig 3

(1) Stable beach without groynes

(11) Eroded beach without groynes after 1, 3, $5,10,15$ and 20 tides

(1i1) Eroded beach with groynes after 1, 3, 5, 10,15 and 20 tides 

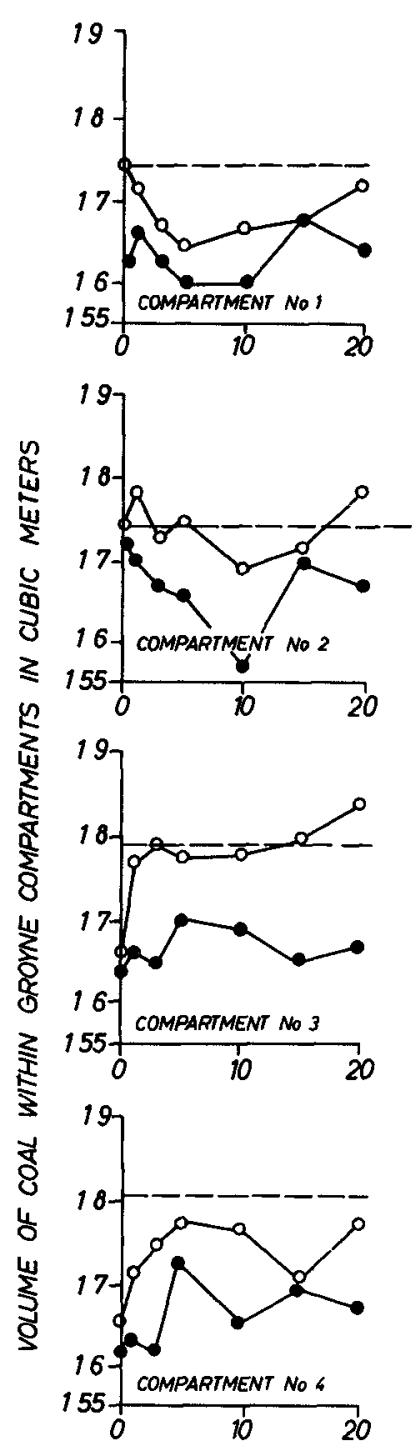

NUMBER OF TIDES TIDAL PERIOD 75 MINUTES
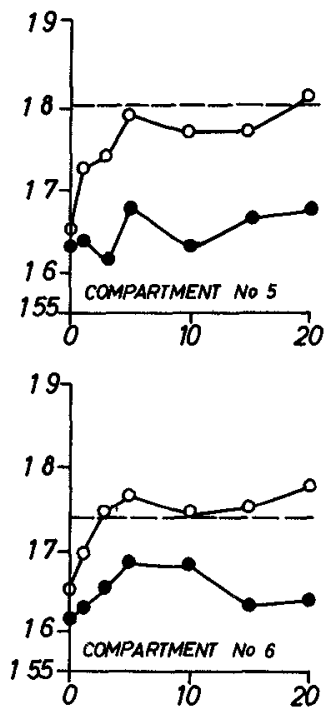
KEY

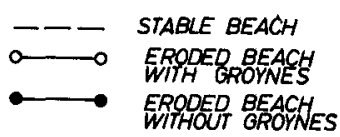

FIG 3 BEACH CHANGES WITH TIME 


\section{Interpretation of Results}

The changes with time in the volumes of material in each compartment with and without groynes can be seen in Fig 3 In addition, the volumes for the stable beach, before manual erosion, are indicated

Clearly, the rate of accretion in the groyne compartments, Nos $3,4,5$ and 6 , was greater in the presence of groynes This increased rate of inshore accretion was accompanied by a bulld-up in bed levels offshore of the groynes, not shown in Fig 3, necessary in order that at equilibrium the littoral transport that formerly travelled along the foreshore might travel seaward of the groynes with groynes, the beach had returned to its equilibrium profile in approximately 20 tides, without groynes, the experiment was terminated at 20 tides since it was clear that the beach was returning to the equilibrium profile, although slowly

No significant bulld-up of the groyned beach was found above the stable levels, with the exception of the accretion already mentioned seaward of the groynes

\section{Conclusions}

The following conclusions might be drawn from the results of the present series of tests

1 Groynes will increase the rate of accretion on an eroded beach if there is a sufficlent supply of littoral material

2 The presence of groynes will not result in a build-up of the inshore beach above the stable open beach levels for the given wave climate and supply of littoral material

3 Bed levels seaward of groynes will be increased in order that a uniform littoral drift may be malntalned along the coast

\section{Acknowledgement}

The authors wish to thank Mr $\mathrm{H}$ Hashimoto who carried out the experimental work at the Hydraulics Research station while on a grant from the Japanese Government 
This report is published by permission of the Director of Hydraulıcs Research, Wallingford, Great Brıtain

\section{$\underline{\text { References }}$}

I PRICE, $W A$ and TOMLINSON, $K \mathrm{~W}$ "The effect of groynes on stable beaches" Proc lith Conf on Coastal Englneering, 1968, Vol 1 


\title{
CHAPTER 68
}

\author{
CHARACTERISTIOS OF SHINGLE BEACHES
}

THE SOLUTION TO SOME PRACTICAL PROBLERS

A. M. Mulr Wood

Partnsr, Sir William Halcrow \& Partners

ABSTRACT

Shingls beaches duffer from eand beaches mainly in the mods of transport of the matemal and in the permeability of the beach. Ths typical beach forms axe in coneequence diffsrent and ths typical problems of beach stabilieation require diffsrent typee of solution.

Ths mechanlem of littoral dxift of shingle 28 controllsd predomnantly by the action of the breaking wave, on a groyned beach a simpls theory is advanced to rslats drift to groyne length and spacing.

Longltudinal sortzng of ebingle eizes ze a specially notabls elgm of a etabls beach. Wsll marksd slzs-sorting transverss to the coaetline is a mors general charaoteristio.

Examples of schemes of management are provided for a bsach whth hagh littoral drift and a beach whooh has to be oontrolled as artzfiolal cells

The accrsting shingls beach may in suztabls circumstancss dsvslop a steep zn-ehore profile - examplee ars given of such.

\section{FORCES CONDROLLING THE BEACH PROFILE}

The extensivs literaturs on the enginesring properties of a natural beach le principally conoemed with funs to medium sand foreshores. In coneequenos, a number of gensralisations havs bsan made concsrning the propertzes of a bsaoh that do not apply, howevsr, to a shagie or evan to a coarse sand bsaoh. The objeot of thrs paper 28 to discuss soms of the propsrties specific to a shingle beaoh and briefly to describs solutions relevant to the typioal problems that arzss wzth them. By definztzon, a ehingls beach 18 one in which the median particle size $D_{50}$ is larger than $10 \mathrm{~mm}$. The mean size is most of tan in the rangs $10-40 \mathrm{~mm}$. 50

South-eaet Britazn is well provided whth natural shingle beaches and Fig. 1 illustratss a number of the lengths of coastlins concsrned. Ths main reason for ths prsdominancs here of shingls ie that ths princlpal constituent, flint psbbles, was originalily formed in the massivs chalk whoh has sincs bsen widsly eroded ovsr thrs arsa. Secondary fluvial and marine deposite have provided copious sources of flint (silica) which havs besn washed Inshors as ths ssa levsl ross followng ths most recent glaciations. Oncs the flunt pebbles bsooms wsll rounded they tend to bo reduced gradually in 612s by attrition rathsr than to be reducsd to sand by fragmentation. 


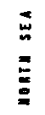

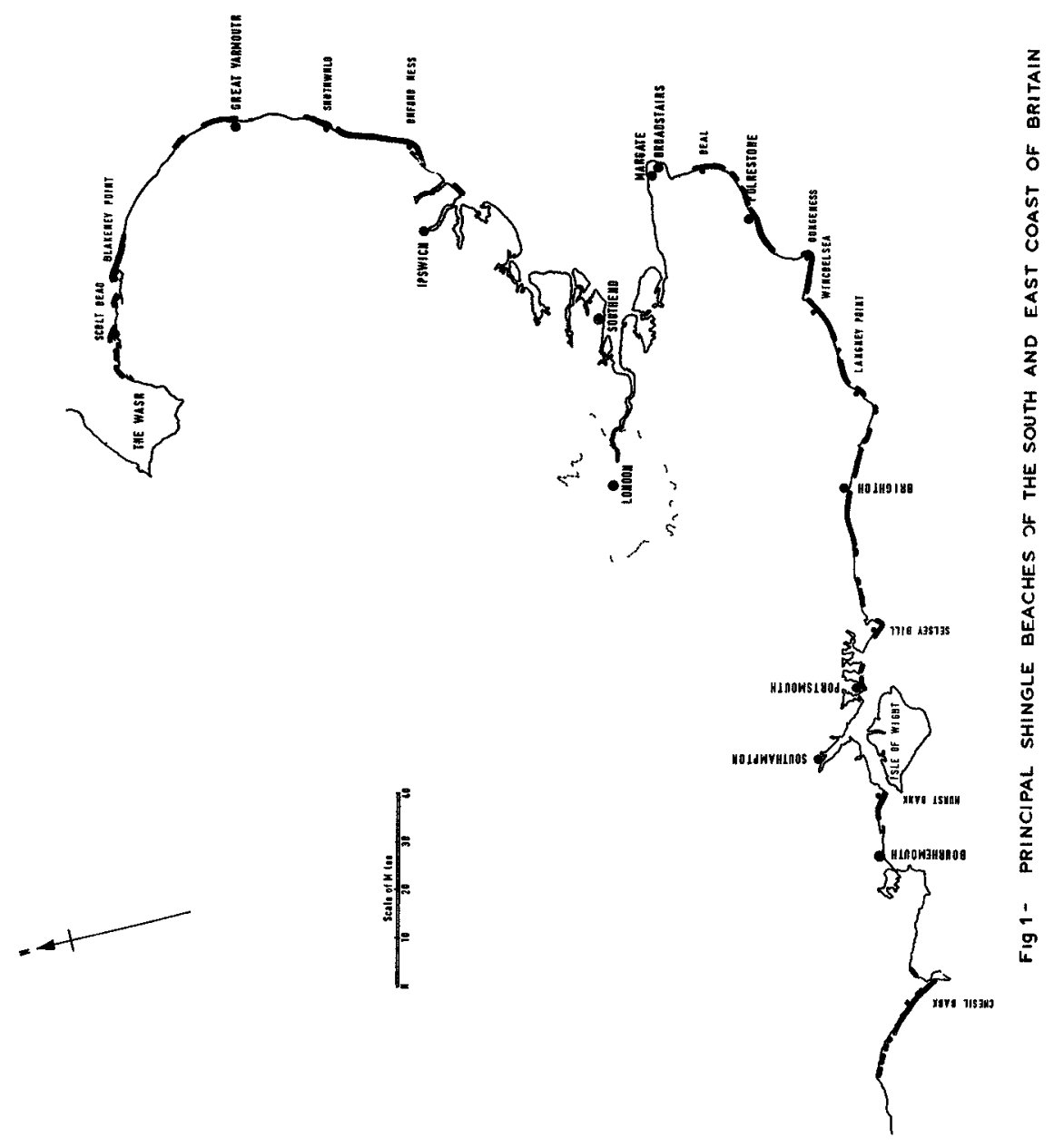


The man differences between the coastal behaviour of shingle and sand are relatsd to the mode of transport and to the permeability of the beach. Whereae eand 19 moved by the sea predominantly in saltation and, near and inshore of ths breaker line, in suspension, shingle is ehifted. by sliding and rolling along the bottom. The slgniflcance of thrs difference is that, whereas sand will tend to be moved in the dareotion of the vector representing reeldual wave velocity plus tidal velocity, shingle is only moved during that part of the wave velocity cycle in which a certain threshold value ie exceeded. Generally this threehold value will be little below maximum velocities at the situation and in consequence the darection of $h$ gh velocity will greatly predominate In addition, the emount of work required to move ehingle on the eeabed in substantial quantitiee ie euch that this movement will generally be confined to areae of high rate of disszpation of wave energy, $1 . \theta$. landward of the breaker line. Shingle zmediately to eeaward of this line, except whers the offshore bsd is wteep, will tend to move ehoreward, If dzeturbed at all, on account of the aeymetrical shape of the wave lsading to higher ahoreward orbital vslocities.

The ehingle beach, whth a typical slope of 110 or steeper, 10 appreciably stseper than the equilibrium sand beaoh and this is largely due to the extent of percolation of the ewash of the breaking wave into the shingle beach, leading to a diminution of the dowawash To treat this phenomenon in a very exmpls instance, we may consider the lower bound of ths velocity of steady percolation of water into a beach at gradzent $S$ to be $k / \frac{\text { Is }}{2}$ which is about 6 om/s for a typucal value of permeablilty, $k$, of $10^{2} \mathrm{~cm} / \mathrm{e}$ for a beach at 110 slope.

To attempt theoretzcally to calculate the shaps of a beach profile, even for a regular train of wavee, would be sxtremely complicated annce. apart from the non-uniformity of the material of the beaoh, we have to be able to oalculate the fluctuating dsgree of eaturation of the beaoh, and the effecte of drag and lift on the surfaoe particles on the beach. A point of intereat is that the peroolation of water into the beaoh will entazl the flow lines of the swash flow and, to a lesser degree, the downwash flow, oonverging towerds the beech and the bed velooltiee at any instant will therefore tend to be greater than they would bs over an impermeable bed of comparable roughness, where the flow linee would be parallsl to the bed. The bed profile will be expectsd generally to be convex upwards since the ratio of return to upward flow will tend to dimunush bugher up the beach.

Referxing to $F_{18}$. 2, the force $P_{1}$ neoeesary to dielodge a eubmerged pebble up a beach of gradzent $\beta$ and limpting angle of repose $\phi$ is given by

$$
P_{1}=m_{g}\left(\rho_{8} / \beta_{w}-1\right) \sin (\phi+\beta)
$$

where $\rho_{s}$ and $\rho_{t}$ are reepectively epecifio weight of pebble and water.

Simlarly the foroe $P_{2}$ neceeeary to dzelodge a pebble down the beach is given by

$$
P_{2}=m_{g}\left(\rho_{e} / \rho_{w}-1\right) \sin (\phi-\beta)
$$

The energy flux of the swash and backwaeh may be repreeented respsctively as

$$
\mathrm{E}_{1}=\frac{1}{2} \boldsymbol{p}_{w} v_{1} \bar{u}_{1}^{2}
$$




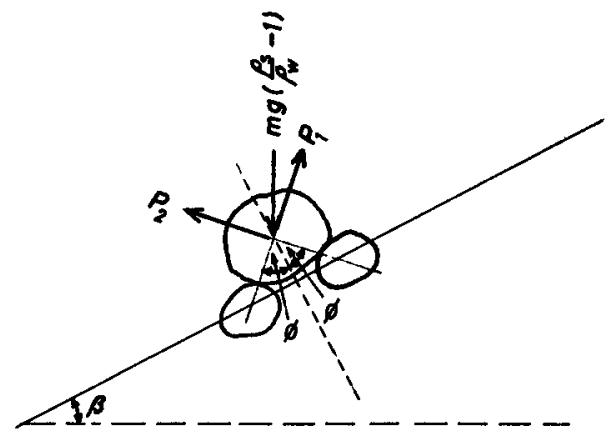

\section{FIg 2 FORCES ACTING ON AN EXPOSED SHINGLE PEBgLE}

and

$$
E_{2}=\frac{1}{2}\left(w v_{2} \bar{u}_{2}^{2}\right.
$$

$v_{1}$ and $v_{2}$ repreeent volumes of water with mean epscific energy $\vec{z}^{2}$.

$P_{1}$ may,be consldersd as rolated to $u$, steady water veloolty parallsl to and up the beach, by

$$
P_{1}=\frac{1}{2} \sim \mathrm{C}_{c} \mathrm{u}^{2} / \mathrm{B}
$$

where $C_{c}$ is a coefficient wath lift and drag components. $P_{2}$ is eimlarly rslated to velocity down ths beach.

The number of uphill and downhill dislodgements may then be considersd by eqns. (3), (4) and (5) to be proportional to $E_{2}$ and $E_{Q}$ rsepectively. For a stabls profila thers must be a balance of dislodgements and, if $\mathrm{E}_{2}=\mathrm{CE}$, from eqne. (1) and (2), considering the thrsshold values of $P_{2}$ and $P_{2}$,

$$
c=\sin (\phi-\beta) / \sin (\phi+\beta)
$$

i.8. $0=(\tan \phi-\tan \beta) /(\tan \phi+\tan \beta)$

a result similar to that obte.jned by Bagnold ${ }^{l}$ by conslderation of the work done by the swash and downwaeh in movang pebblss up and down the beaoh.

A study of natural ehringle beach profiles after drawdown by atorms indicates generally a profile which may be representsd batwesn beach crsst and beach atsp level approximataly as a parabola. Thus, Kemp's records ${ }^{2}$ of three ssctions of the Cheal Bsaoh (A, B and W) fit closely to the sams parabola abovs the levsl of the stap. 
The beach grading given by Kemp ${ }^{2}$ indraates $D_{1}$ sizes as $6 \mathrm{~mm}, 4 \mathrm{~mm}$ and $20 \mathrm{~mm}$ at $A, B$ and $W$ raspeotively. The coefficients of parmeability are to be expeoted therefors to be approximately in the ratios 1.2 , 1.0 and 2.2 raspectively, providad the same beach greading extends to appreciable depth. One faotor to explaun the common profile may be the greater work done in transporting mobile beach material up and down the beach where ths particle sizs if finer and thus oompensating for lower volumetric loss $\left(V_{1}-V_{2}\right)$ by higher specific energy loss, $\left(\frac{1}{2} u_{1}^{2}-\frac{1}{2} \bar{u}_{2}^{2}\right)$

A shrngle beach usuelly exists in the presence of sand in the anshors (and often also offshors) zones, A certain amount of sand 18 therefore generally in suspension, followng breakong of the wave, and thissand tends to percolate into ths beach as a result, apart from the mobils shingls near the surface, the interstaces of the biach will be more or less charged whth sand, affactive permeability, even of a relatively thrck depth of predominant shangle, will be coxxesondingly reduced. The thicioness of mobile abungle mannly controls the critical helght and period of wave that will provoks draw-down of the beach by Ita Incapacity to absorb an adequate fraction of tha water in the swash.

Thers are certain typical profiles to be racognised on a shingls beach. The accreting beach has a profile concave upwards becoming convex upwards as the storm crest 13 reached. On thr erodung beach, the concave upward curve runs into a sharp scarp at the had, with ths slope immeciately bolow the scarp standing at the critical angle of repose of the beach material. During the course of recovernng, one or more secondary crests form at the limit of the swash of the breaking waves but below ths upper crest, the upper orsat represents ths height reached by ths breakang aub-critical wave at a tzme of high mean sea leval, ('crltical' wave rafars to the wave that just bagins to draw the beach down).

\section{LITTORAL DRIFT OF SHINGLE}

Gansrally rif currents play no appreciable rols in longshors movament of shingle inshorg of the breakar zons. This movement is caused predominantly by the drrection of up-rush of the breaking wavs and, though the downwash usually returns falrly directly down the beaoh, thrs contributes to a oertain extent when the breakang wavss approach very obliqualy.

For a natural foreshors, most of tha longshore movement of shugle probably occurs in the upper part of the beach. In particular, an the foreshors slops ia known to vary conslderably with tha state of the tide during perzods of atorm, increased littoral drift is to be expected in an area of hlgh tidal range, other factors rsmainung unchanged.

When a sea wall is present, extrems flattening of the beach occurs whsn storm waves coms into contaot with the well, and yet higher rates of littorel drift may therafors be associated with the consequent change of profile wh th each tide.

Wi th the variations of waathex, tide and mobility of a shangls beach profila it 1a unilkely that any dirset general relationship will be found between longshore energy flux and littoral drift even for the same beach, and no reliable quantitative oolution of general applicability is foresseable, without aeparation of the many parameters. 
The concept of Pelnard-Considere ${ }^{3}$, expressing littoral dxuft for glven wave energy flux as directly proportional to the angle between the crest of the breakang wave and the beaoh line, may be expected to be applicable as a first approxumation where $\sin 2 \alpha \sim 2 \alpha, \alpha$ being the angle of approach of the wave to the foreshore, but the interesting development of thrs theory by Bakkert cannot be applied directly to the typlcal shangle beach. Bakker supposee an equlibriun between variable littoral drift and the consequential onshore and offshore movement between the foreehore and the inshore area. His principle is based upon a conoept of a unzue stable bsach profile but, as Illustrated by Fi8. 3, the shingle profile in the upper beach will adopt an angle so different from that of the sand in the lower beach that it is possibls to have variations in elze of the shangle wedge without resultang onshore and offshore motion. In consequence, where It le required to provide groynes to mantain a shangle beach, the criterion wall maznly depend upon the extent to which the toe of the shrngle bsach extends seaward of the groyne at a time of appreciable littoral drift. It appears to be the general experience, although there are exceptions to thie rule, that the sand in the inshore and offshore zones becomes adapted to the genersl line of the shingle beach. Thus, If the shangle can bs maintained in adequate quantities to provide natural proteotion, the lower beach will adjust itself acoordzagly.

Referring to Fig. 3, if the predominant angle of ayproach of ths wave creet to the natural coaetline is $\alpha_{0}$ and the eystem of groynes is requzred to reducs littoral dxift from do to $\mathrm{Qg}$, then the groynee must provide a beach creet line making an angle of $\alpha_{0}-\alpha_{g}$ w th the original coaetline, where the euffices $O$ and $g$ relate to the original and the groyned conditions and $\alpha_{g}$ to the change in the angle of approach of the wavee to the beaoh crest due to the groynee. At the present tume we do not know how to relate the reduction of ilttoral dxift $\left(Q_{0}-Q_{g}\right)$ to the extsnt of projection of the toe of the beach, at times of storm, beyond the seaward end of the groyne. The follown makes a first attempt to such a relationship.

For a system of groynes bult sufficiently hagh to prevent overtopping, the degree of reduction of longshore motzon of shungle paet each groyne wall depend only on the poeitzon of the toe of the mobile beach, (probably situated at or inshore of the breaker point) relative to the end of the groyns.

If we aseume that littoral dmift

$$
Q=K O
$$

for constant wave characterietics, where $\alpha$ is the angle of approaon of the wave to the beach and $K=$ a constant for the particular sltuation, then, where exoynee cause the angle of approach of the waves to be reduced from $\alpha_{0}$ to $\alpha_{g}$ (eee Fib. 3 )

$$
\begin{aligned}
& Q_{0}=K \alpha_{0} \\
& Q_{g}=K \alpha_{g}
\end{aligned}
$$

But, at a groyne, where the dustance in plan of the mobils beach from crest to toe is $L$ and the projection of the toe beyond a groyne $18 \mathrm{a}$, (Fig. 3), for eteady flow condztions of littoral drift we may poetulate

$$
Q_{g}=K^{\prime} a / L \quad \text { where } K^{\prime}=K f(\alpha)
$$




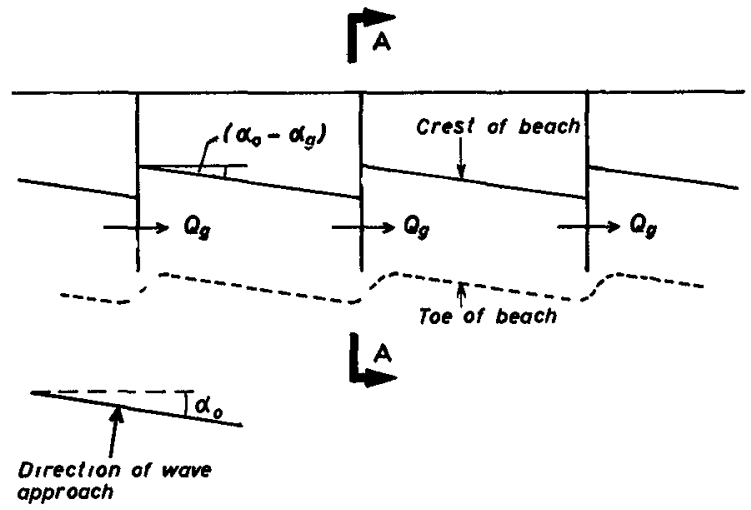

PLAN

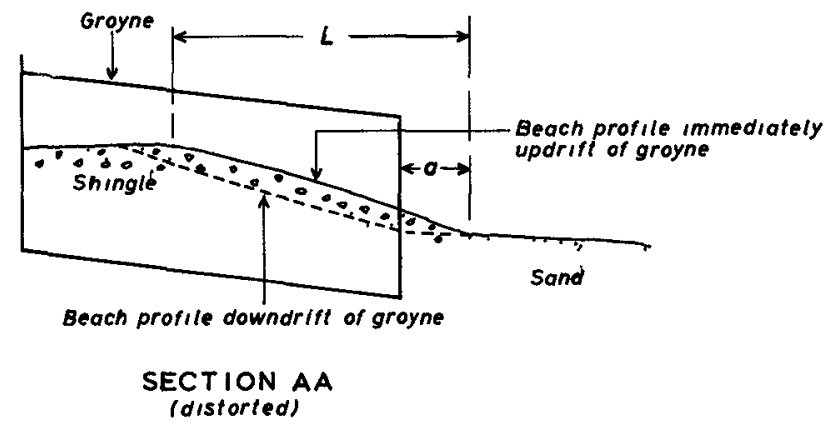

Fig 3 A SHINGLE BEACH IN EOUILIBRIUM ON A GROYNED FORESHORE 
At a groyne, the line of the toe of the beach wall tend to maxcmase drift past the groyne, i.e. localiy $\alpha \rightarrow \pi / 4$ if we suppose that for large values of $\alpha, Q=\frac{K}{2}$ sin $2 \alpha$. Then

$$
K^{\prime}=K \sin \pi / 4 \cos \pi / 4=K / 2
$$

and hence, from eqns. (10), (11) and (12),

$$
Q_{g}\left(=K \alpha_{g}\right)=K a / 2 L \text {. Hence } \alpha_{g}=a / 2 L
$$

whence for given valuee of $a$ and $L, Q_{g}$ may be estimated as a fraction of Qo, from equs. (9) and (10).

LONGITUDINAL SORTING OF SHTWGLE

Many raferences may be found to the sortang of shingle by sea waves to produce longitudinal exze grading along a beaoh. Shingle of the largest sizs tends to movs towards the zone of the highest degree of exposure to the wavss. A olassic exemple of this phenomenon is found at Chsail Beach (ses Fig. 1) where the most slgmifioant featurss are as sst out in Table 1 .

TABLE 1

\begin{tabular}{|c|c|c|c|c|}
\hline \multirow{2}{*}{$\begin{array}{l}\text { Distance from } \\
\text { West Bay (km) }\end{array}$} & \multirow{2}{*}{$\begin{array}{l}\text { Average of fshore } \\
\text { slope }(0-5 \text { fathoms })\end{array}$} & \multicolumn{3}{|c|}{$\begin{array}{l}\text { Average longrtudinal drameter } \\
\text { of pebbles (cm) }\end{array}$} \\
\hline & & $\begin{array}{r}\text { Foreshore } \\
\text { (Bef 12) }\end{array}$ & & $\begin{array}{l}\text { 8eabed } \\
\text { (Ref 12) }\end{array}$ \\
\hline $\begin{array}{c}0 \\
6.1 \\
7.2 \\
11.3 \\
12.6 \\
15.1 \\
17.1 \\
21.4 \\
23.8 \\
25.6 \\
28.0\end{array}$ & $\begin{array}{l}1: 75 \\
1: 50 \\
1: 50 \\
1: 20 \\
1: 20 \\
1: 20 \\
1: 20 \\
1: 20 \\
1: 20 \\
1: 12 \\
1: 25\end{array}$ & $\begin{array}{l}0.86 \\
1.16 \\
1.16 \\
1.56 \\
1.64 \\
\\
3.36 \\
3.64 \\
4.28 \\
5.89\end{array}$ & & $\begin{array}{l}4.5 \\
3.0 \\
2.0 \\
3.5 \\
3.0 \\
4.8 \\
3.2 \\
5.0 \\
6.0\end{array}$ \\
\hline
\end{tabular}

Significant Features of Chesil Beaoh

Jolliffe ${ }^{6}$ describes a number of experiments on beaches at Deal and Winchelsea (see Fag. 1) to reocrd the relative rate of littoral drift by means of tracer pebbles of different slzes matohed to the range of pebble sizss present on ths beaches. He found a signifioant oorrelation between the size of pebble and the rate of littcral drift, the size of grsatest mobility bsing rslated to the wavs height. 
On a beach comprisung an assemblage of shingle sizes, we may consider that a pebble will begin to move when drag and lift cause the pebble to rotate about a line between pounts of contrat with other pebbles. Fig. 2 indicates how, on a beaoh of pebbles of different slzee, this foroe will bear the leaet ratio to the pebble mess for the pebble of the largest dzameter. Moreover, once set in motion by a wave, translational and rotational inertia wll tend to cause a laxge pebble to travel conslderably further than a small one. The pebble of mall size will tend to become rebedded anto the beach with drag from downward peroolation oppoeed to wave lift.

For a given beach and given wave clumate there must be a size of pebble so large that it is only infrequently dislodged. Pebbles below such a size may be expected to undergo some degree of longltudinal sortung if there is a longshore component of wave energy flux. For a beach which is an lang term stability, but which 18 subjected to different directions of littoral drift by different sizes of wavee, the sorting should be a sigmificant feature, and generally it appeare so to be ThIs effect may arlse from the different degree of exposure to prevelling winds and swell or from different degrees of refraction of different types of waves.

The size grading along Chenll Beach might bs explained principally by increasing exposure to the Atlantic as one proceeds along the beaoh towards Its south-east extremity. Thls increase le due not only to differences in shelterng afforded by start point, the weet headland of the bay, but also to the increasing depth of water offshore in the same sense.

Reverse drift of shangle along Chesll Beach, $1 . e$. towards the north-west, will occur under the action of wavee generated withan the English Channel, but these will only be of a height to affect the amaller sizes of shingle. Further studies are warranted here to observe differences in long-shore mobility of shingle of different sizes and gradings subjected to a varying wave climate.

It $2 \theta$ to be noted that for a beaoh not in long-term equilibrium little sign of longtudinal sorting is likely to be observed unless it is subjected to long-term cyclee of reversal of arift. Nor 18 this effect likely to be observed on a groyned foreshore except in individual groyne bays, for the reason of the interference with natural drift caused by the preeence of the groynes.

\section{BEACH REPIEATISHMENT: A SYSTEM OPERATED AT DUNGEAVESS}

Poesibly the finest long-term contunuous records of shingle movement avallabe anywhere up to ths present day are those relatung to Dungenese (se Fig. 1). Here the existence of a pattern of ehrngle ridgee, lookang like a magnifled fingerprint, permits a reconstruction of events over nsarly 2,000 years?.

In recent years the Ness has been bullding out towards the easteouth-east at a rate of 3-4m per year, at the expense of erosion along the south coast of the feature. As andicated in Fig. 4 the changeover from eroezon to accretion occurs at a pount about $700 \mathrm{~m}$ weet of the Hess proper. It will be noted that thas change must be accounted for by the angle of approach becomang super-critzcal 1,e. greater than that for maximum littoral arift, since the degree of sheltering is reduced from west to east and the extent of refraction of the domanant southmwesterly waves is reduced in the same direction. 


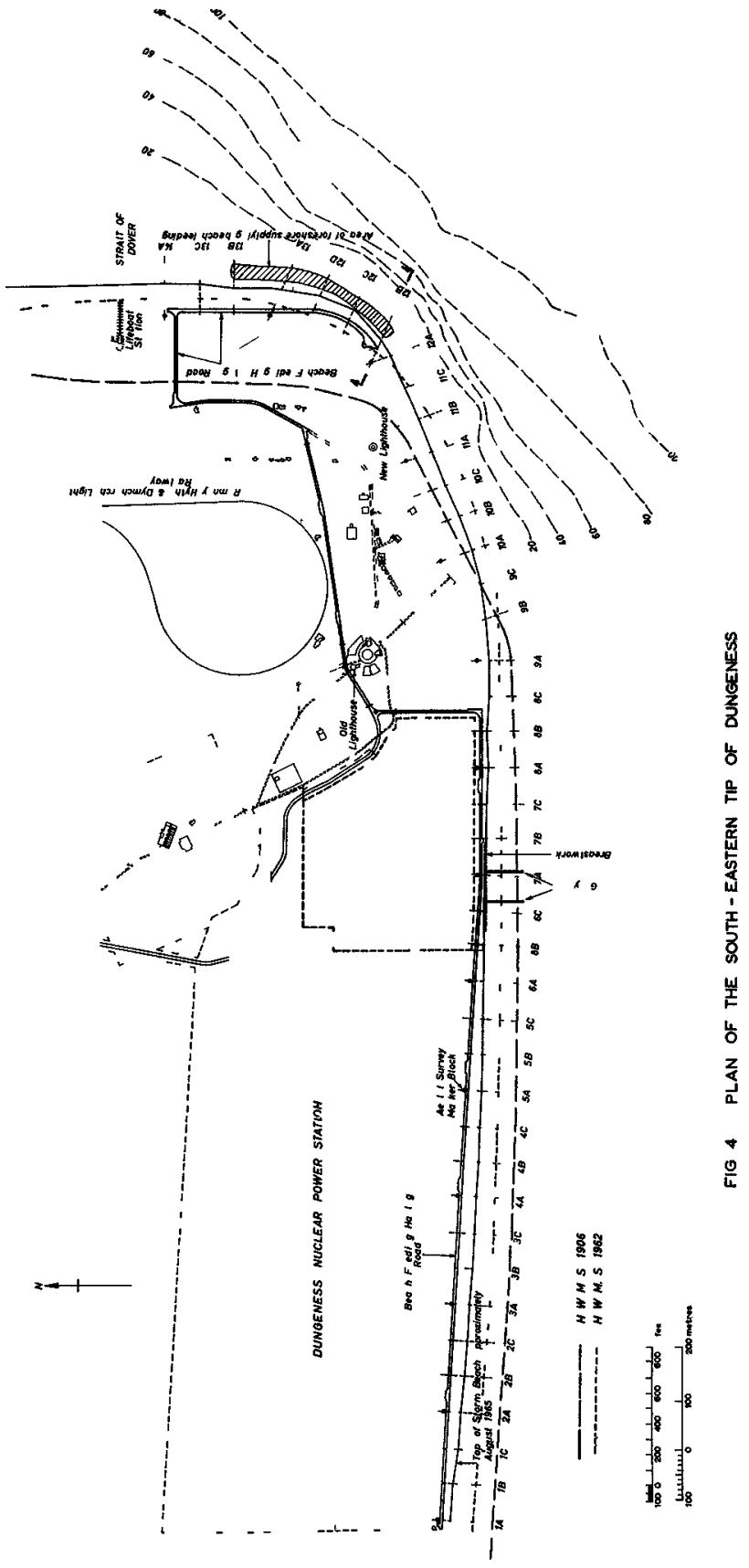


Since 1965 a scheme of beach recharge has boen operated to stabiliss the shoreline between Sections $1 A$ and $8 B$ (F18. 4) whers 1 t forms a frontage to the sxasting and projected Nuclear Powsr Stations. Shingle for this purpose, won from ths foreshore and upper bsach in the vicinlty of ths Ness, has been transported each year in lorries to the Powsr Station frontage and tipped on the beach at rechargs points. These tips bulld out to form shingle breakwaters on ths foreshors and it is to bs expscted that, as they psrsist virtually throughout the winter, they will reduce the erosion updrift from the points of recharge. "Niaking due allowance for the local and short term variations, this effect appear to be supported by the figures set out in Table 2 .

The plan of the bsach has been sub-dzvidsd by section lines about $30 \mathrm{~m}$ apart and fixed points have been selected at intsrvals along these lines seaward from the crest of the beach which is at about $6 \mathrm{~m}$ above $0 \mathrm{D}$. (approximate mean sea level). Vertioal co-ordinates of the shingle level at such points are provided each year from an asrial survey taken at the tume of Low Water Spring Tides (about $3 \mathrm{~m}$ below $0 \mathrm{D}$ ) in August. The volumes of shingle in each sub-division of ths beach are then provided by a computsr program and on these date the bsach rechargs plan for the followang winter is formulated.

It is to be noted, in Tabls 2, that during 1965/66, ths ysar of inception of the schsme, a conszderably blgher degree of bsaoh feeding was undertaken than during subsequent years. Table 2 also indicates the annual variation in the longehoms movsment of shingls and in the quantity arriving at ths Ness. The Powsr Station foreshore is now virtually stable from ysar to year and lt seemsd reasonable to assume that any variations in the beach profile below low water of spring tides could be lgnored, tne volume changes providsd by ths computer data then being actual volums changes on ths beach. Howsvsr, as the lless is still advancing into deep water, allowance has now been made for the volums of shangle accretion on the steep face to the Ness below low water. Ths volumes of shingls arriving at the Ness, shown in Table 2, also include an allowance for accretion north of the northemmost section of the survsyed area.

In view of ths detalisd records avallabls of ths shingle movement over four consecutive years it was decided to study the possible relation bstween wavs energy in the area of ths Nsss and the rates of erosion and accretion along the foreshore. However, thsre are no wavs rsoords avaluable direotly applicable to Dungeness. Ths nearest rscorders ars on ths Varne and Dyck lishtsh2ps, but in the snclossd waters of ths eastsm English Channel differential sheltering is an important faotor. Consequsntly, since thers is no signiflcant long period swell in this part of the Channel, wand records may be ussd to dercvs at least a first order estumate of longshors wavs ensrgy flux and wand rscords wers available from the Dungeness lighthouse. These rscords were analyssd into durations (T) of wind spseds from points of the compass from east through south to wsst for winds of Beaufort Force 8 and bigher.

A wave energy spectrum was then obtained, using the sumplified graphical relationship prepared by Darbyshire and Draper 8 to provids maxumun wavs helghts and thence the corresponding significant wave helghts (Hs). The total annual enerey flux faotors ( $\mathrm{KH}_{\mathrm{S}}{ }^{2} \mathrm{TCs}$ in $2 \alpha$ ) were calculated and these are indicated in the final column in Table 2, ths positive figures indlcating ensrgy from winds west of the south ssctor. Ths comparison of these flux factors, which are proportional to the total longshore energy flux, with the figures in Table 2 sither for nett littoral drift or for materlal arriving at the Ness, shows no general relationship, although the highest flux factor is associated with the highest movement of material. However, ths trend of recharge does ganerally follow the factors and wh a few more ysars experzence it might be possible to uss these calculated factors to prsdict the quantity of recharge necessary, though some form of survey would be necessary to determine the optimal pounts of recharge. 
TABLE 2

Shingle Recharge and Littoral Draft at Dungeneee (In cuble metree)

\begin{tabular}{|c|c|c|c|c|c|c|}
\hline \multirow{2}{*}{\multicolumn{2}{|c|}{$\begin{array}{c}\text { Seotion of } \\
\text { Shore }\end{array}$}} & \multicolumn{5}{|c|}{ Year } \\
\hline & & $1965-66$ & $1966-67$ & $1967-68$ & $1968-69$ & $1969-70$ \\
\hline $1 A-4 A$ & $\begin{array}{l}\mathrm{R} \\
\mathrm{L}\end{array}$ & $-1 \overline{800}$ & $-\overline{-}$ & $\begin{array}{r}20600 \\
-16100\end{array}$ & $\begin{array}{l}12600 \\
-1300\end{array}$ & \\
\hline $4 A-5 C$ & $\begin{array}{l}\text { B } \\
\text { L }\end{array}$ & $\begin{array}{l}8000 \\
-800\end{array}$ & $\begin{array}{l}19000 \\
-1600\end{array}$ & -4100 & $\begin{array}{l}11500 \\
-4900\end{array}$ & \\
\hline $5 C-7 A$ & $\begin{array}{l}\mathbf{R} \\
\mathbf{L}\end{array}$ & $\begin{array}{r}43000 \\
-10100\end{array}$ & 1000 & $\begin{array}{l}7400 \\
-700\end{array}$ & $\begin{array}{r}9500 \\
-3400\end{array}$ & \\
\hline $7 A-8 B$ & $\begin{array}{l}\text { R } \\
\text { L }\end{array}$ & $\begin{array}{r}23000 \\
-900\end{array}$ & $\begin{array}{r}5400 \\
-1000\end{array}$ & $\begin{array}{r}4400 \\
-3000\end{array}$ & $\begin{array}{r}6400 \\
-2200\end{array}$ & \\
\hline $1 A-8 B$ & $\begin{array}{l}\mathbf{R} \\
\mathbf{L}\end{array}$ & $\begin{array}{r}74000 \\
-13600\end{array}$ & $\begin{array}{l}24400 \\
-3900\end{array}$ & $\begin{array}{r}32400 \\
-23900\end{array}$ & $\begin{array}{r}40000 \\
-11800\end{array}$ & \\
\hline $\begin{array}{l}\text { Estimat } \\
\text { quant2 } \\
\text { ehongl } \\
\text { arrivan } \\
\text { the Hes }\end{array}$ & $\begin{array}{l}\text { ed } \\
\text { of at } \\
\text { at }\end{array}$ & 80000 & 30000 & 90000 & 55000 & \\
\hline $\begin{array}{l}\text { Energy } \\
\text { factor }\end{array}$ & flux & 6.7 & 6.5 & 10.1 & 2.2 & \\
\hline
\end{tabular}

$\mathrm{R}=$ Shangle Recharge

$I=$ (IIttoral drift into eection) -(I.ttoral drift out of section)

It wall be noticed in Table 2 that a large volume of recharge at a eection of the shore $1 e$ generally aeeoolated with a high rate of loee. Thie $1 e$ no doubt due to the form of tipping, which le always onto the end of the t2p, and where no recharge is made at the adjacent downdrift section a build up of beach only ocoyre againet the following point of recharge. It ze noticeable that in 1968-69 when a fairly even

dietribution of recharge was made the lose of material wae aloo facrly evenly dietributed along the beach.

Prior to 1965, the average annusl loee of material along the length between Seotions $1 \mathrm{~A}-8 \mathrm{~B}$ amounted to about 25,000 cublo metres per year.

CONTROL OF A BEACH AT SEAFORD WITHOUT EXTERRALAL REPLEHISISHMENT

The coast protection problem at Seaford (eee Flg. 1) le fundamentally one of retaining a ehrngle beach without the benefit of natural recharge. Seaford wae orignally establiehed behind a natural shungle bank and there are many hundrede of yeare of hietory of the variations in the poeztion of the mouth of the RIver Ouse through thie elangle bank. Since the 18th century the river hae boen trained to flow through Newhaven Harboux to the west of Seaford. From 1847 the east harbour breakwater arm hae prevented eaeterly drift of ehingle and hae also affected the wave pattern that arrives around the percmeter of the bay. For the prevalling eouth-weeterly winde thie hae the effect of causing a revereal of drift, 1.e. towarde the west, alons the weeternmoet protected length of foreehore. 
For many years sea walls and conventional groymes have been constructed at Seaford during whych period the eea contunued to encroach, causing considerable damage and the collapse of sea walls. The notaral drift along this foreehore towards the east has not been measured but is probably of the order of $10^{5} \mathrm{ch}$.m of shingle per year. The economics of a beaoh recharge scheme have been examined but thie 28 highily uneconoma in the absence of long term supplies of natural shingle nearby. The only altemative scheme that could continue to place reliance in natural shingle as the principal medium of protection 18 one that would contazn the shingle along the protected length. The object was then to determine the munumum length of groyne to ensure effectuve contaznment of the shangle. An empirical approach is to suppose that a shangle beaoh, being relatively thin over a solid chalk bottom, may at times of storn be dragged out to sea so that 2 ts toe corresponds approximately to the breakex pount of the largest waves. Model studzes carried out by the Hydraulics Research Station generally polnted to a eimilar lumit of shangle movement, although allowance has to be made for the fact that, while the seyeral relevant hydrodynamo dzmensionless factors were satiafied (Yalin, 1963) $10^{\circ}$, the material shape and gradung were very different from the natural shingle.

The form of conetruotion of long groynes deozded upon had to be much more robust than conbentional groymes taken out approxumately to low water. The groynes, erected intially and experimentally as a timber gantry faced wath steel sheet paling, were eubsequently converted into mass concrete groynes buzlt an cellular sheet plled coffer dams. They are taken out to a point at which the bed level le approximately $6 \mathrm{~m}$ below mean sea level $(O D)$ and at the eastern end the principal groyne was constructed to provide, in addition, a sewer outfall.

In view of the anclunation of the beaoh oreet to the shoreline for zero druft, it is necessary to sub-duvide the length into intermediate cells to avold the need for a large amount of contunuous redietribution of shingle from the east end towards the west. A small amount of redistribution can be tolerated and it 18 not necessary for the intermedzate long groynes to be taken out far enough to achieve full cutoff. The tidal range here $2 \mathrm{~s}$ approximately $3.5 \mathrm{~m}$ on Neap Tadee and 6.0m on Sprang Tidss. As a reeult, the redistrabution necessary to maintax an adequate beach in the ares of maximum scour ( $i . e$. near the pount of reversal of draft) amounts to about $5,000 \mathrm{cu} . \mathrm{m}$. per year for storm wavee of about $3 \mathrm{~m}$ helght and $6 / 7$ seoands persod.

Perzodical aerial surveys eetablieh that the overall shingle quantities along the foreshore remann approximately constant and also indicate the volume appropriate for periodical distrubution.

An insidzous cause for fazlure of sea walls on shingle beaches can be attributed to undermining by the sea near the time of High Water, whioh action can then become obscured to view on account of partial reoovery of the beaoh a Low Water. At Seaford it was observed that, whlle the upper layers of ehungle are maintained relatively clean as a result of the mobility of the shingle in stormy weather, the lower shangle tends to become charged with finer shingle, eand and chalk particles, carried by the water percolation through the beach. It $1 \mathrm{~s}$, consequently, possible to determine after heavy storms the depth to whoch the beach has been disturbed. The chalk particlee act as a comentitious binder so that the side of an excavation made after a heavy etorm stands vertical, 1 mmediately below the baes of the moblle beach. The depth to which the beach has been disturbed at valnerable polnts 18 recorded and compared against previous records, against the known levels of the wall fating and of the surface 
chalk in which, generally, at Seaford, the wall 18 founced. Although local avaliability of heavy plant for maintenance for theee works facilitates this simple method of control, in the absence of such plant, some ermple penetrometer device, or a heavy ring around a pile, might be used for the same purpose. At seaford trials of altermative geophysioal methods of locating the boundary were unsucceesful.

\section{REGIME OF A NATURALLY ACCBETING BEACH}

The natural regime of a shingle beach is ueually confueed by tices, by the presence of a well maxked division between the medrum to coarse shingle of the upper beach and fine to medium eand of the lower beaoh, also by haghly variable winds and currents. The natural form of a beach may beet be etudied where such factors do not intervene and the characteristics of a beach in thessalonika help to illuminate this aspect.

The beach in queetion is eituated in a bay on the wouthwest coast of the Sithona Penznsular facing the Gulf of Kaseandra. The beach le contained by rock headlande and ze largely composed of particlee of natural quarzlte graded from fine gravel to coarse eand. The schistoee rocke drop away into deep water and there is little fine material in suspeneion in the eea. The beach material has been carried to the bay by a river which flowe into 1 t. The beach in coneequence repreeents the etable profile of a elowly accreting beach fed from this eource. F1g. 5 andicatee how the gradzng and sorting factors vary around the margan of the bay. It is seen that the material becomes very well sorted for elze as it travels away from the raver mouth, whth the size adjusted to the degree of expoeure to the wavee.

Fig. 6 ehows a typical profile of the shore taken to a depth of about $30 \mathrm{~m}$ below sea level. Contraxy to the normally accepted shingle beach profile, eeaward of the step, situated approximately on the breaker point for the maximum helght of slgmifloant wave of about $1.7 \mathrm{~m}$, the shore drops steadily away into deep water, at a slope of $30^{\circ} \mathrm{m} 32^{\circ}$ around the bay, except locally near the river mouth. The abeence of an appreciable fraction below coarse eand elze, coupled with a low coastel current, permite the bed material to stand at an angle a little below ite naturel angle of repose of about $36^{\circ}$. Further to seaward from thrs eteep slope there 18 a varlable flatter slope at a gradient of about I in 10 in which the material le predominantiy fine sand. In Fig. 5, samples $\mathrm{A}-\mathrm{J}$ are from the foreshore, samples SI, S3, S5 and $\mathrm{S7}$ are from the steep inshore bed and samplee S2, $\$ 4, S 6$ and $\$ 8$ are from near the foot of the steep inshore slope.

It is interesting to compare the profile of the Sithonja beach with that at Dungeness Point (eee F1g. 6) where a shingle beach le acoreting in very different conditions, but neverthelese exhibits the eame eteep inshore feature

In general therefore one can say that a naturally accreting shingle beach $w_{1}$ il tend to develop a profile of a foreehore that le concave upwards, steepening towarde the crest to the angle of repoee, an onshore section flattening to a step at the breaker point, then changing seawards to a convex upward profile, funally arrivang at a steep section seaward of the low water mark whoch assumee a slope a little flatter than the angle of repose. The lower features of thie general profile, however, are frequently obscured by accumulation of eand against the shungle beach. Fine to medrum sand would only accumulate at a steep angle in deep still water, on account of ite eusceptibility of movement by relatively small 


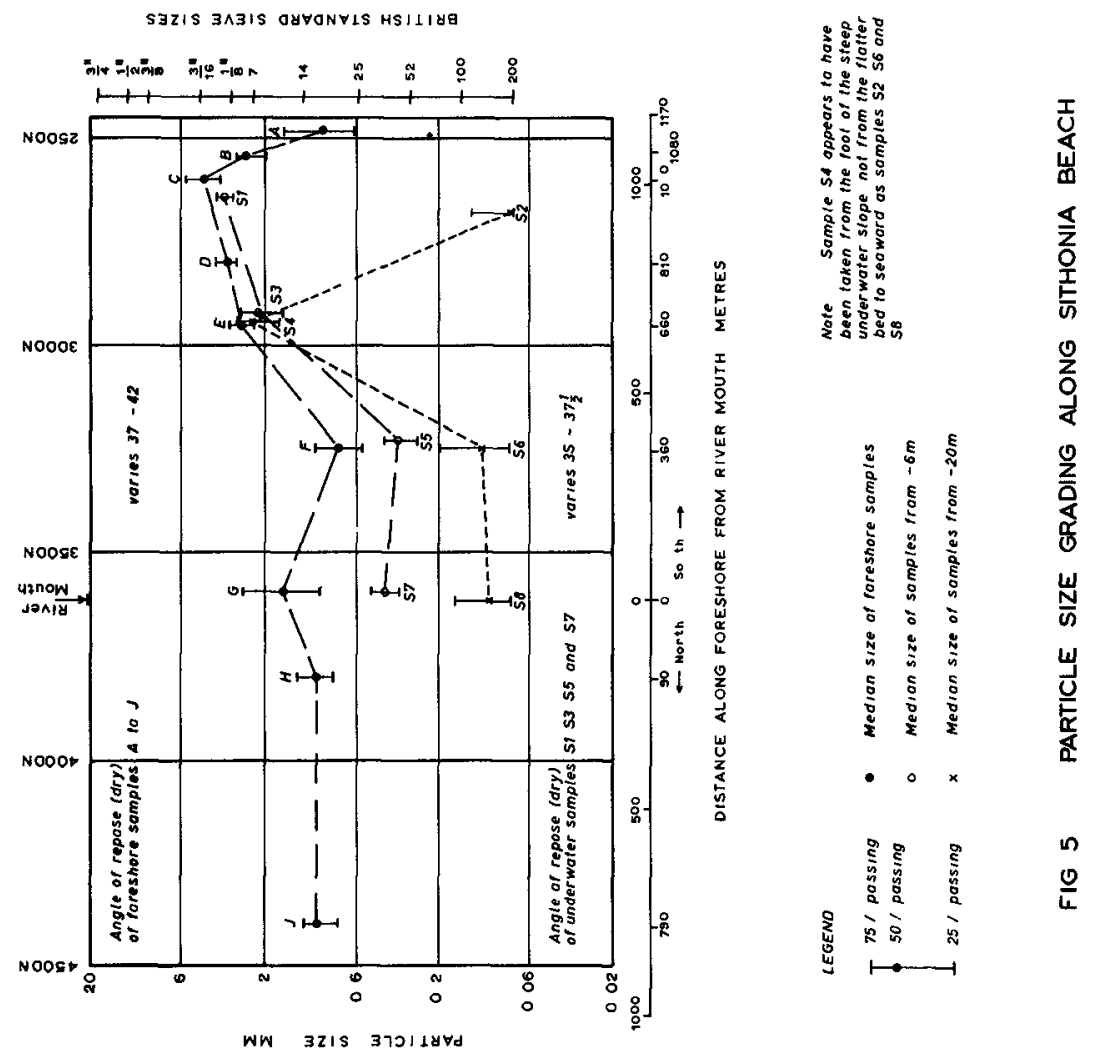



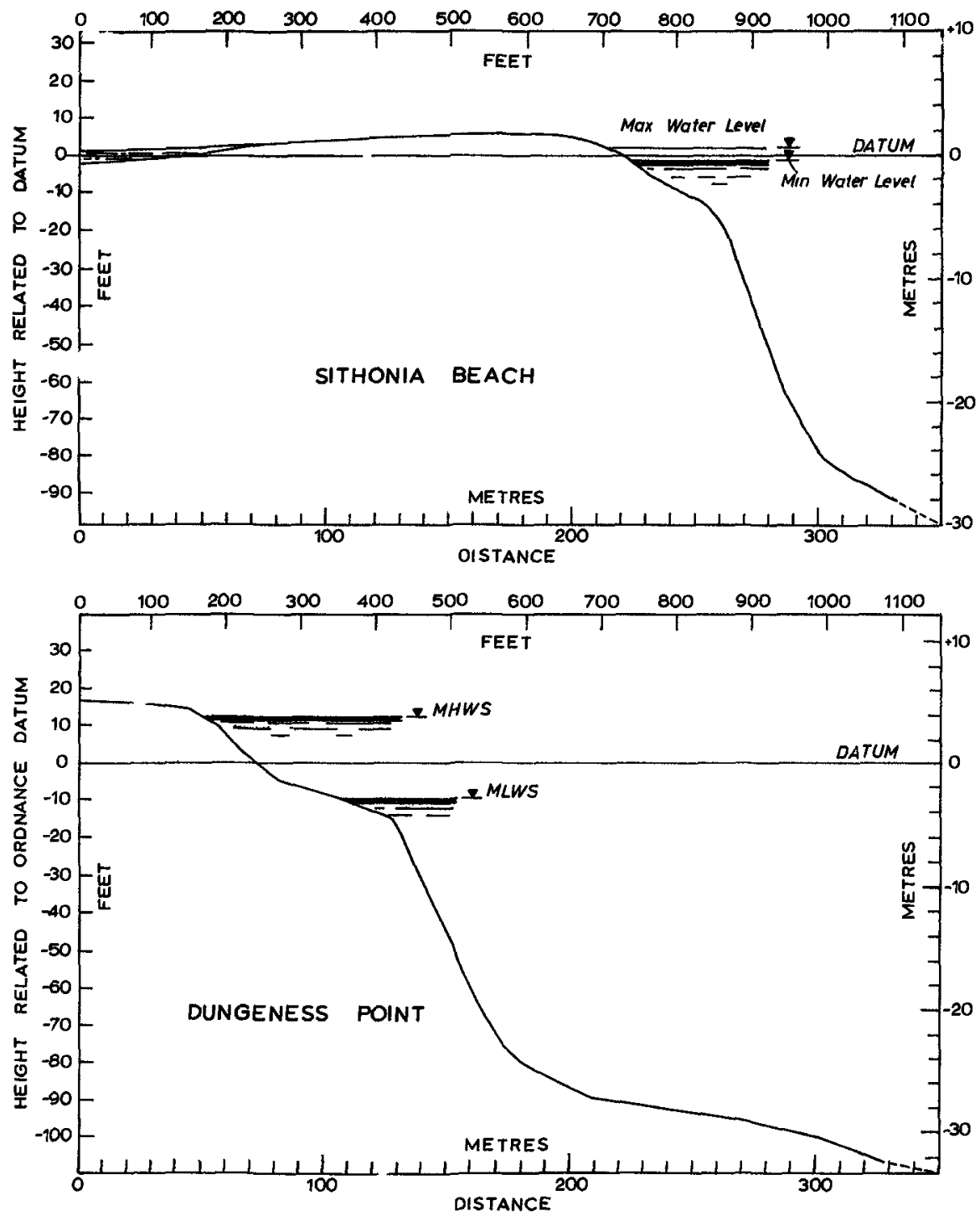

FIg 6-PROFILE OF ACCRETING BEACHES AT SITHONIA AND DUNGENESS. 
oscillatory currente, the eusceptibility being attributabls not only to the grain size of the material but also to 1 ts pronenees to ripplang. An eroding shingle shore, on the other hand, will not dsvelop the steep offshore feature, even in the absenoe of sand.

\section{REAFEROHCES}

1. Bagnold, R.A

2. Kemp, $\mathrm{PH}$

3. Pelnaxd-Conszders k.

4. Bakker, W T

5. Carr, A.P.

6 Jolliffe, I P

7. Lewzs, W.V.

8. Derbyshurs, M. \& Drapsr, L.

9. Hydraulios Ressarch Station

10. Yalin, M.s

11. Nsate, D J M
Mechanzcs of marins ssdumentation, The Sea, 3 , Interscisnce Bublishere Inc., (1963) pp 507-528

A field study of wavs action on natural beachss, I A H.R. Xth Congress, I, Paper 1.18 (1963) pp 131-138

Eseai de theorze de l'evolutzon dss formes de rivage en plage de eable et de galets IV 1 sme Jourméee de L'Hydraulique Question IV (Paris) 1956.

The dynamics of a coast whth a groyne syetem. XIth Conf. on Coastal Enginserzng, London (1968) Vol II pp 492-517

S1ze grading along a pebble beach chesil Baach, England Jnl. of Sedimentary Petrology, Vol 39, No 1 (Mar 69) pp 297-311

Bxperzments designed to compars the relative rates of movement of different sizes of beach pebble. Proc. Geol. Assoc. Vol 75 (1964) pp 67-86.

The formation of Dungenees Foreland. Geogr. Jnl. Vol 60 (1932) pp 309m324.

Forecasting wand-generated ssa waves, Engunsering (5th April 1963) op 482-484.

Seaford Sea Wall: protection by artzficzal fill. Report DXX 209 (May 1963).

A model shangle beach with permeability and drag forces reproduced. XIth Congress Int. Assoc. Hyd. Rss., London (1963).

Underwater pebble grading of cheezl Beach. Proc, Geol. Assoc. Vol 78 (1967) Pr. 3 pp 419-426.

Note Tabls 2 reproduced by permisszon of South Eastern Regzon of the Central Electricity Generding Board 



\title{
CHAPTER 69
}

\section{A COMPARISON OF FLUVIAL AND COASTAL SIMILITUDE}

By Bernard Le Mehaute, D Sc

Vice-President, Tetra Tech, Inc

Pasadena, Calıfornia

\begin{abstract}
A comparison of the conditions of similitude for movable bed scale models of rivers and estuaries on one hand, and beaches and shorelines on the other hand, is presented It is demonstrated that despite the fact that the knowledge in sediment transport by wave action is less advanced than in the case of steady current, the conditions of similitude for beaches are less stringent than for rivers In particular, the effect of relative roughness is comparatively unimportant in the case of beaches, while the necessity of similitude of head loss imposes an imperative condition in the case of scale models of rivers An introduction to a natural law of distortion for beaches is presented in analogy with the Lacey condition for rivers
\end{abstract}

\section{INTRODUCTION}

Belief in movable bed scale model technology is somewhat mystical--God's existence can be proven by $\log 1 \mathrm{c}$, while as many reasons can be proposed for proving the opposite This belief is not a matter of logic--it is a matter of faith Similarly, agnostic specialists in sediment transport can easily find many reasons why similitude of movable bed scale models is not possible On the other hand, hydraulic engineers believe in movable bed scale model technology as a tool for solving practical problems and guiding their intuition Sometimes this belief or disbelief is completely irrational One finds the scientist who does not want to admit that this tool could be of practical value and one also finds the engineer whose faith in his scale model fringes on the rim of superstition, while he should know its limit of validity The analogy can be further prolonged by considering that different scale model practices and technology prevailing in different hydraulic laboratories correspond to slightly different religions But the road to salvation is not unique--despite some disagreement between laboratories on what should be done, none of these methods is "completely wrong" One can only discuss what could be the most universal approach-- the better approach actually being the one which is the best mastered by individual experimenters

A detailed investigation of what can and cannot be done in movable bed scale models dealing with coastal structures has been presented in a previous report (Fan and Le Mehauté, 1969) This presentation will be limited to some reflections which may not necessarily be original, but which are felt pertinent to this convention, which assembles agnostics as well as believers in movable bed scale models One would like to clearly establish that the chance for success of a movable bed scale model dealing with shoreline processes is much higher than a movable bed model of fluvial hydraulics Subsequently, the first kind of model will require less knowledge in the law of sediment transport and less astuteness on the part of the experimenter than the second kind

The reasons which make a coastal model more reliable than a river model are

a) A less imperative choice of the condition of similitude This is discussed at length in the following In general, coastal models do not 
require any condition on bottom roughness, while the necessity of reproducing head loss in similitude in fluvial models imposes an additional condition upon the size of the model material to be used

b) A wider cholce of criteria of similitude in the case of a coastal model One can always satisfactorily reproduce an observed shoreline evolution by adjusting wave height, period, direction, duration, or intensity of tidal current (However, similitude of sand deposit taking place in a diffraction area may be subjected to scale effects)

In fluvial hydraulics, the choice of criteria is limited to typical flood histograms and required fineness of topography (Secondary meandering effect may not need to be reproduced in similitude)

c) The third reason for making coastal models more successful is related to the problem of hydraulic instability A river flowing in its own alluvium is fundamentally unstable in the sense that if there is erosion, more wate $r$ will flow, and, consequently, more erosion will follow The study of a meandering iıver on a scale model is only possible if the banks are fixed A meandering process can be reproduced on a scale model only if it obeys a well defined cycle, such as the Seine estuary

In coastal processes if one excepts cusps and rip currents on long, straight beaches, the flow pattexn is generally well determined by an imposed bottom topography Short coastal engineering scale models always deal with well defined topography and man-made works, such as harbor entrances Consequently, there is a general tendency towards a stable state a dune tends to be flattened out and a channel tends to be filled

d) Finally, the last reason for the success of coastal engineering models is due to the fact that beaches are generally made of relatively uniform noncohesive material, while rivers may present a much wider size distribution of material and have cohesive characteristics The law of similitude for cohesive material cannot be established until more is known about the sediment transport of cohesive material

\section{PRELIMINAR Y REMARKS}

Prior to entering this subject, it is pertinent to briefly recall some of the fundamental principles governing movable bed scale model technology

1) Quotıng L F Vernon-Harcourt, who contınued the work of Reynolds

"If I succeed in demonstrating with the model that the originally existing conditions can be reproduced typically, and if, moreover, by placing regulating works in the model, the same changes can be reproduced that were brought about by the training works actually built, then I am sure that I can take the third and most important step, namely, of investigating, with every promise of success, the probable effect of the projects that have been proposed "

This principle has been the basic guideline ever since

2) A movable bed scale model must fulfill the following conditions

a) It must be exact 1 e, it must reproduce with exactness the natural phenomenon under study 
b) It must be consistent 2 e it must always give the same results under the same conditions

c) It must be sensitive--or more exactly, its sensitivity has to be imposed by the fineness of the topography which needs to be reproduced for the phenomenon under investigation Secondary meandering flow, small benches, and at the limit ripples, will, of course, not need to be reproduced in the scale model

d) It must be economical, of reasonable size, and completed within a reasonable time interval

3) In a movable bed scale model, the basic similitude requirement is the reproduction of bottom evolution observed in the field even if it is not achieved through exact similitude of water motion (the way in which water motion is simulated must be logical so that it can be extended to future conditions) Reproducibility of test results under the same conditions is a general requirement for all experimental studies This requirement of the model also implies stability under random disturbances An unstable phenomenon cannot be studied adequately in a movable bed model

"For example, it would be illusive to study the stability of a river flowing in its own alluvium on a scale model because a simple bush or a local soil a little more cohesive can definitely guide a meander in a way different from the model result Such studies embody by their very nature a risk of complete faslure " (Le Méhauté, 1962 )

4) It is important to distinguish between conditions of similitude and criteria of similitude The conditions of similitude are an ensemble of formulas deduced from the physical laws governing the phenomena under investigation e $g$, the similitude condition governing sediment transport is obtained by an analysis of the mechanics of sediment transport They have an absolute definition which cannot be changed unless an improvement in the knowledge of the physical law $1 \mathrm{~s}$ obtained They are not chosen by the experimenter but are imposed on him Unfortunately, it is known that in the field of sediment transport, many phenomena still remain to be analyzed or clarified Thus, the conditions of similitude are not as well defined as they should be A choice of what is important will have to be based again on the knowledge of these laws obtained by "1nspectional analysis"

In performing a model study, an experimenter must specify certain criteria such as model wave conditions and fineness of model bottom features The criteria of similitude is a free choice of the experimenter to a very large extent For example, sea states vary from day to day and from hour to hour The experimenter will choose, for the sake of simplicity as well as practical necessity, a characteristic wave condition and will only be able to reproduce simplified storm and swell conditions on the scale model He will choose the wave direction and the wave amplitude and the duration guided by his knowledge of natural conditions In particular, the wave generato $\mathbf{r}$ will generate waves at an angle which corresponds to the dominant direction of storm wave energy Even though he 15 guided by his knowledge of wave statistics, his final choice will be determined by a trial and error method which permits him to reproduce the same bottom evolution as observed in the prototype This faithful reproduction of bottom evolution determines 
the choice of the wave characteristics, rather than the strict conditions of similitude of wave motion

Other criteria of similitude will be to what extent he wants to reproduce the fineness of the bottom topography, a typical tide cycle, the currents and their variations with time, and so on In summary, the criteria of similitude are specified by the experimenter as reasonable approximations for simplification of model operation

5) Movable bed scale models are distorted, 1 e, the vertical scale is different from the horizontal scale Distortion is not an engineering trick for reducing the size of the model and the bottom friction, but is the extrapolation of a natural observed phenomenon The method to obtain a satisfactory scale model is first to obey the law of nature, even though this law may not be fully understood For example, a small river flowing in its own alluvium can be considered a distorted model of a large river This means that the ratio depth to width of the small river is comparatively greater than the relative depth of the larger one The ratios of depths $\mu$ and widths $\lambda$ are approximately related by the law of Lacey $\left(\lambda^{2}=\mu^{3}\right)$, in accordance with the "regime theory" Similarly, a beach in a protected area has a relatively steep slope, while a beach in an exposed area tends to have a more gentle slope (Wregel, 1964) The vertical scale being defined by the ratio of incident wave heights, a protected beach can be considered as a distorted scale model of an exposed beach in both the case of the river and the beach, the cholce of distortion becomes a stringent condition to be respected quantitatively, however, the natural law determined by statistical observation of natural phenomena needs to be modified in the case of a river model due to the fact that scale models generally do not use the same material as the prototypes in order to satisfy other conditions of similitude On the other hand, natural distortion based on the use of sand is compatible with other conditions of similitude in the cases of beaches as will be seen in the following However, the use of sand would rather be discarded as leading to too large a distortion and, subsequently, to large scale effects

6) To the old teaching tradition which consists of presenting a parallel between Froude and Reynolds similitude, I would prefer to make a parallel between what we can call "short model" and "long model" In a short scale model, viscous friction is unimportant as compared to gravity and inertia, therefore, it is governed by Froude similitude Also, energy dissipation may result from a fully turbulent condition, as in the case of a hydraulic jump or a wave breaking on a beach Boundary layer effects in both cases are unimportant The dissipative forces are also proportional to the square of velocity like the inertial forces (A small hydraulic jump is a scale model of a large hydraulic jump under proper depths-discharge relationships) This is the generalized Froude similitude

On the other hand, in a long model, friction has a definite influence on the flow pattern therefore, in addition, a similitude of head loss is required This head loss is a function of the Reynolds number, but is not determined by the so-called Reynolds similitude requiring an equality of Reynolds numbers Therefore, similitude for long models requires, in addition to the Froude similitude, another condition which makes long models more difficult, if not impossible, to handle The model of a smooth concretelined gallery cannot provide a "Froudian" discharge under similar pressure head, since the friction coefficient can only be larger at a smaller Reynolds number (Moody diagram) On the other hand, the head loss in the scale model of a rough (rocky) gallery can be adjusted for the same friction factor insuring the Froude similitude to be satisfied 
The magnitude of long shore currents and location of rip currents may, to some extent, depend upon friction characteristics of the beaches, in which case the study of this phenomena would have to be considered as belonging to the categories of long models, and therefore, may not be studied on scale models Nevertheless, most scale model studies have to deal with short coastal structures(like entrance of harbor), and therefore, the water motion is not too dependent upon the friction coefficients The main dissipative mechanism is due to wave breaking If viscous damping is too significant, as in the case where the wave has to travel a long distance in very shallow water, it just means that the model is not properly designed However, very rarely do we have to be concerned with adjustments of roughness for similitude of energy dissipation, and coastal models can be considered as short models On the other hand, as has been pointed out previously, a similitude of head loss is imperative for models of rivers and estuaries - these are long models Despite this adjustment, vertical velocity distribution being a function of the Reynolds number could never be in similitude

7) This relative advantage of a short model is somewhat balanced by the lack of knowledge of sediment transport by wave action The law of sediment transport in rivers is relatively well understood Therefore, the condition of similitude may be established with more certainty than in the case of beaches Boundary layer characteristics do not vary too much from one place to another While on the other hand, in the case of beaches, the boundary layer characteristics vary from off shore to the upsurge of the wave A cholce has to be made concerning which part of the beach we want to have the best similitude requirements In general, it will be in the breaking zone, where the shearing force at the bottom is quadratic However, it is to be realized that because of this variation of boundary layer characteristics from place to place perpendicular to the beach, a total similitude 1 s impossible

8) In designing a movable bed model, there are four basic unknowns namely, horizontal scale $\lambda$ vertical scale $\mu$, sediment size $\delta$, and sediment specif $1 \mathrm{c}$ weight $\gamma_{s}$, which require at the most four basic equations However, the horizontal scale $1 \mathrm{~s}$ generally determined by economic considerations and available space The three remaining unknowns are relatively well determined in the case of a river, by a well accepted unique set of conditions of similitude as summarized in a following section

In the case of beaches, there is a great controversy concerning which condition of similitude should be imperative This controversy is due on one hand to a lack of knowledge of the law of littoral processes but is also due to the fact that the road to a successful coastal model is not unique As a matter of fact, a thorough analysis of the subject matter may only lead to two conditions which give us a free choice for one of the unknowns These conditions are 1) an equality of ratio of shearing force to relative gravity, the shearing force being quadratic as in the case of a turbulent boundary layer, and 2) a second condition is imposed by the law of dis tortion of beaches (equilıbrium profile of beaches) under different wave actions, which embodies globally many misunderstood phenomena In addition to these two, one can choose somewhat arbitrarily another con. dition, such as a dynamic condition $n_{u} / n_{w}=\lambda / \mu$, where $u$ is a horizontal current, $w$ is the free fall velocity of the particles One would rather choose an equality of boundary layer Reynolds number $R *$ The theoret1cal formulation based on these assumptions is presented in the following section, based on the assumption that the scale for the friction factor is the same as in the case of a fully turbulent flow, as in the surf zone 
This relative freedom is partly due to the lack of understanding of the law of sediment transport under wave action But it is also an indication of one of the reasons why coastal models are generally more successful than fluvial models

9) It is pertinent to point out that since the only requirement of a movable bed model is a reproduction of bottom evolution, it is not necessary that this be achieved through exact similitude of water motion Since the model is distorted, a similitude wave refraction and wave breaking only is being searched as a most satisfying condition, susceptible to producing satisfactory reproduction of long shore current and sediment transport distribution This is achieved by keeping the ratio of wave lengths and wave heights like vertical scale $\mu$ Based upon this condition, the following wave characteristics are preserved in the model a) wave steepness, b) refraction pattern and angle or refraction with bottom contours, c) breaking angle of wave crests with shorelines if the distortion is not too large, and d) breaking depth

Also, the scale for long shore current and mass transport velocities is approximately $\mu \frac{1}{2}$ Therefore, the ratio of scales of wave particle velocity to current velocity is approximately unity

\section{A BRIEF REVIEW OF THE HY DRAULIC PROPERTIES OF SEDIMENTS UNDER}

\section{WAVE ACTION AND CURR ENT}

1) It is first recalled that in the case of water waves, the laminar shear velocity $u_{\not<}\left(\right.$ see Appendix for notation) $u=\sqrt{\tau_{0} / \rho}=U_{\delta}{ }^{\frac{1}{2}}\left(\nu_{\pi} / T\right)^{\frac{1}{4}}$

$\mathrm{U}_{\delta}$ is the amplitude wave bottom velocity $\mathrm{U}_{\delta}=\pi \mathrm{T} / \mathrm{sinh} \mathrm{kd}$

For $R_{\delta}>160$, the boundary layer flow is turbulent, then the turbulent bottom shear $\tau_{0}=\rho f U_{\delta}^{2} / 8$ where $f$ is the Darcy-Weisbach friction factor, and $u_{*}=\sqrt{\frac{f}{8}} U_{\delta}$

2) The hydraulic properties of a sediment particle are often represented by its fall velocity $w$ in the water, defined by the equation

$$
133 \frac{\mathrm{gD}}{\mathrm{w}^{2}} \gamma^{\prime}=\mathrm{f}\left(\frac{\mathrm{wD}}{\nu}\right) \text { or } \frac{\mathrm{w}}{\sqrt{\gamma^{\prime} \mathrm{gD}}}=\mathrm{f}\left(\sqrt{\gamma^{\prime} \mathrm{gD}} \mathrm{D} / \nu\right)
$$

In Stokes range, $w D / v<0 \quad 1$

$$
\frac{w}{\sqrt{Y^{\prime} g D}}=\frac{1}{18}\left(\sqrt{\gamma^{\prime} g D} \mathrm{D} / \nu\right)
$$

3) In coastal processes, the sediment motion is caused by wave and tidal current actions In studying such interactions, an important criterion is the critical condition initiation of sediment motion Shields'criterion of the initiation of sediment motion is in the case of a steady current.

$$
\frac{T_{C}}{o g \gamma^{\top} D}=f_{1}\left(R_{{ }^{\prime} C}\right) \text { where } \tau_{C} \text { is the critical shear stress }
$$

$\mathrm{R}_{{ }_{x \mathrm{C}}}=\mathrm{u}_{\varkappa \mathrm{C}} \mathrm{D} / \nu$ is the critical boundary layer Reynolds number based upon grain diameter $D$ 
$u_{*}=\sqrt{T_{C} / \rho}$ is the critical shear velocity If a boundary layer densimetric Froude number $F_{*}$ is defined as $F_{*}=\frac{u_{*}}{\sqrt{g Y^{1} \bar{D}}}$ then Equation (6) can be expressed as $F_{{ }_{x}}=f\left(R_{{ }^{*}}\right)$ where $F_{\ddot{ }} C_{1}$ is the critical boundary layer Froude number For a given size of sediment, a critical velocity ${ }_{C}$ can easily be derived by noting the relation $u=C_{c} u_{y} / \sqrt{g}$ where $C_{c}$ is the Chezy coefficient (9)

Such criterion has also been investigated extensively in the case of sediments under wave action It is remarkable that the criterion is identical with that of the steady current case, although the range of $R * C 1 s$ considerably smaller For initiation of sediment motion due to wave action, the boundary layer Reynolds number $R_{*}$ based on grain diameter is given by

$$
R_{*}=21 \mathrm{D}\left(\frac{\mathrm{H}^{2}}{v^{3} \mathrm{~T}^{3} \sinh ^{2} \mathrm{kd}}\right)^{\frac{1}{4}} \mathrm{C}_{1 \mathrm{n} \text { a laminar boundary layer }}
$$

At present (1970) there is no experimental information on initiation of sediment motion due to combined wave and current actions known to the duthors Based upon dimensional consideration of similar nature as Equation (8), the criterion can be expressed as

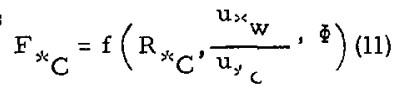

where $F_{* C}$ and $R_{* C}$ are critical boundary layer Froude and Reynolds numbers, based upon either wave or current shear velocities $1 \mathrm{e}$, $u_{* w}$ or $u_{\nu_{c}}$ respectively The parameter $u_{*} / u_{*_{c}}$ shear velocity ratio represents the relative importance of wave and current effects An additional factor is the angle of wave incidence to the current direction $\Phi$

4) The volume transport rate of bed load qs can be expressed in general as a function of boundary layer Reynolds number, $R_{*}$, boundary layer Froude number $F_{*}$ and sediment size distribution

$$
\begin{aligned}
& \frac{\mathrm{q}_{\mathrm{S}}}{\mathrm{u}_{\varkappa} \mathrm{D}}=\mathrm{f}_{1}\left(\mathrm{R}_{\varkappa}, \mathrm{F}_{\psi}, \sigma_{\mathrm{D}}\right) \\
& \frac{\mathrm{q}_{\mathrm{s}}}{\mathrm{u}_{,}\left(\mathrm{u}_{* *}^{2} / \mathrm{g}\right)}=\mathrm{f}_{2} \quad\left(\mathrm{R}, \mathrm{F}_{*,}, \sigma_{\mathrm{D}}\right)
\end{aligned}
$$

where $q_{5}$ is the volume sediment transport rate per unit width The effect of distribution of the sediment size is considered by including the geometric standard deviation $\sigma_{D}$

Sediment transport by waves is mainly due to mass transport and longshore currents On a beach, the onshore and off shore transport of sediments are reflected by the beach profiles The alongshore transport of sediment ( $\mathrm{e}$, littoral drift) is induced by longshore currents

For bed load transport due to combined wave and current action, the volume rate is expected to be affected by two additional parameters, 
namely, 1) the ratio of characteristic velocities between current and wave $u_{c} / u_{w}$, and 2) the incident angle of wave to the current direction $\Phi, 1 \mathrm{e}$,

$$
\frac{q_{s}}{u_{{ }_{n}}\left(u_{s}^{2} / g\right)}=f\left(R_{,}, F, u_{c} / u_{w}, \Phi, \sigma_{D}\right)
$$

5) Beaches are formed by wave and coastal sediment interactions Beaches are said to be in equilibrium when they reach stable profiles under relatively constant wave action This means that the offshore and onshore transport of sediments are in balance In general, the form of equilibrium profile of a beach depends upon the wave characteristics, such as wave height and wave length or wave steepness, sediment specific weight and sizes

There is no well established law on beach equilibrium which can be applied both to offshore and surf zones, or to both natural sand and scale model light material A study is now being conducted at Tetra Tech for this purpose

6) The most commonly accepted relation for littoral drift is approximately

$$
\frac{\gamma_{f} E}{2 g\left(\gamma_{s}-\gamma_{f}\right) D}=K \Omega_{s}\left(\frac{\gamma_{s}-\gamma_{f}}{\gamma_{f}}\right)^{1 / 2}\left(\frac{1}{g D^{3}}\right)^{1 / 2}
$$

where $E$ is the longshore energy and $K$ a constant coefficient (Manohar, 1962)

\section{SIMILITUDE RELATIONS FOR MOVAB LE BED RIVER MODELS}

The similitude relations for movable bed river models are derived based upon similitudes on (Boucher and Le Mehauté, 1957) l) basic flow characteristics and flow patterns, 2) head loss, 3) sediment transport characteristics and 4) kinematic condition on sediment motion

As in a coastal model, there are four basic scale ratios to be determined namely, $\lambda$, the horizontal scale $\mu$, the vertical scale (or distortion, $\Omega$ ), $D$, artificial sediment size, \& $\gamma^{\prime}$ apparent specific weight However, in many river model studies, an extra distortion is allowed for the river slope Here, only the case with one distortion is discussed

For similitude on basic flow characteristics and flow patterns, the model flow conditions are deduced based upon the Froudian law The scale ratio of flow velocity $n_{u}$ is $n_{u}=\mu^{\frac{1}{2}}$ and the time scale $n_{t}{ }^{1 s} n_{t}=\lambda_{\mu}{ }^{-\frac{1}{2}}$

For similitude of head loss, there are two conditions 1) the energy gradient, and 2) the bed configuration Based upon Chezy's formula

$\mathrm{u}=\mathrm{C}_{\mathrm{c}} \sqrt{\mathrm{dS} \mathrm{e}}$ where $\mathrm{C}_{\mathrm{c}}$ is the Chezy coefficient $=\sqrt{8 / \mathrm{f}}$

$d$ is the water depth $\simeq R_{h}$, the hydraulic radius for wide rivers $S_{e}$ is the energy gradient

Since the energy gradient for a distorted model must be exaggerated by a factor of $\Omega$, the scale ratio for the Chezy coefficient ${ }^{n_{C}}{ }_{c}{ }^{i s}{ }^{n} C_{c}=\Omega^{-\frac{1}{2}}$
and $n_{f}=\Omega$ 
This condition is valid for movable bed models as well as for fixed bed models (For the latter, the head loss is set up experimentally without taking into account the relative roughness ) For movable bed models, since the flow is kept fully turbulent, $C_{c}$ can be expressed as $C_{c}=K(d / D) 1 / 6$

where $K$ is a proportionality constant, and $D$ is the sediment size

A necessary condition for this Equation for $C_{c}$ to be applicable is that there should be no ripples or dune formation in the model This condition is $\mathrm{R}_{*}=\frac{\mathrm{u}_{*} \mathrm{D}}{\nu}=116 \frac{\mathrm{D}}{\delta_{\mathrm{v}}}>500$ (in the model), where $\mathrm{R}_{*}$ is a boundary layer

Reynolds number, $u_{2}=\sqrt{g_{d S}}$ is the shear velocity The condition $R_{*}>500$ implies $R>500$ where $R=\underline{u R_{h}}$ (i e , the flow is fully turbulent in the

model) Then the scale ratio for the sediment size ${ }^{n_{D}}$ is given by ${ }^{n} D=\mu \Omega^{3}$

For the similitudes of sediment transport characteristics, there are three basic aspects namely, 1) initiation of sediment motion, 2) regimes of bed configuration and 3) bed load sediment transport characteristics So far, modeling of the suspension transport has not been carried out

The condition of initiation of sediment motion due to current action, as discussed in a previous section, is $F_{{ }^{\prime} C}=f\left(R_{{ }^{\circ} C}\right)$, where $F_{C}$ is the critical boundary layer Froude number, and $R^{\prime} C$ is the critical boundary
layer Reynolds number

Based upon the general formula for bed load transport rate per unit width, $\mathrm{q}_{\mathrm{s}}$ can also be expressed in general functional relationship as

$$
\frac{q_{s}}{u_{*}\left(u_{*}^{2} / g\right)}=f_{2}\left(R_{*}, F_{v}, \sigma_{D}\right)
$$

Based upon the above reasoning the similitudes of sediment transports including condition of initiation of sediment motion, require $n_{F}=1$

$\mathrm{n}_{\mathrm{R}_{*}}=1 \quad$ (26) and $\mathrm{n}_{\sigma_{\mathrm{D}}}=1$

These Equations are entirely similar to those derived for coastal models

They imply $n_{u_{* x}}^{2} n_{\gamma^{\prime}}^{-1} n_{D}^{-1}=1$ and $n_{u_{v}}=n_{U_{\delta}}\left(n_{f}\right)^{\frac{1}{2}}=\mu^{\frac{1}{2}}\left(\frac{\mu}{\lambda}\right)^{\frac{1}{2}}$

or

$$
\mathrm{n}_{\gamma}, \mathrm{n}_{\mathrm{D}}=\mu \Omega
$$

In general, $R_{, t}$ in the field is large For large $R_{*}$, the sediment transport characteristics are approximately independent of $R_{*}$ On the other hand, it is desirable to prevent occurrence of ripples in the model Thus, Equation $n_{R_{y}}=1$ is relaxed in practice except specifying $R_{*}>500$ in the model At lar'ge $\mathrm{R} *$, the sediment transport characteristic $s$ are believed to be dependent primarily on $F_{x}$ On the model bed, it is undesirable to have extensive ripple formation $s$ because the model ripples often contribute too much flow resistance in comparison with the prototype condition and their effect is difficult to control

A kinematic condition of sediment motion is specified here as an additional basic similitude relation, a sediment in suspension travels distances in the 
vertical and the horizontal directions proportional to its fall velocity $w$ and its horizontal velocity $u, l_{1}$ e $x / y=u / w$, where $x$ and $y$ are horizontal and vertical distances of travel This Equation gives an additional condition on the basic unknowns

$$
\Omega=n_{w} / n_{u}=n_{w} \mu^{-\frac{1}{2}}
$$

Considering the value of the fall velocity $w$, the scale ratio $n_{w}$ is then given by $n_{w}=\left(n_{Y^{\prime}} n_{D} / n_{f w}\right)^{\frac{1}{2}}$, where $n_{f w}$ is the scale ratio of the function $f_{w}(w D / \nu)$ This condition cannot be expressed explicitly, because of the inclusion of $n_{f w}$ However, it is interesting to note that if one uses the same sediment in the model as in the prototype, $n_{w}=1$, the above Equation reduces to $\lambda^{2}=\mu^{3}$, which matches the Lacey relation (33) based on river statistics, but $n_{F_{*}}=1$ and $n_{R_{\psi}}=1$ are then not verified

In summary, the similitude relationships of movable bed river scale models are determined from three conditions 1) similarity of head loss $n_{f}=\mu / \lambda$, 2) similarity of sediment transport characteristics $n_{F}=1$ and 3 ) kinematic condition of sediment motion $n_{u}=\lambda$ leading to $F_{x}$ a well defined distortion In addition, there $\quad \frac{u}{n_{w}}=\frac{\lambda}{\mu}$ should be no ripple formation in the model $R_{*}>500$

The bed load transport formula, Equation (24) is used to obtain the time scale of bed evolution $n_{t b} n_{t b}=\lambda \mu / n_{q s}=\lambda^{5 / 2} \mu^{-2}$

The kinematic conditions imply Lacey's law at $n_{1}=1$ The Lacey's law is also compatible with the regime theory of rivers based upon statistical analyses of meandering rivers width $\propto \mathrm{Q}^{\frac{1}{2}}$, depth $\propto \mathrm{Q}^{1 / 3}$ In scale relations, it gives Identical relations as the one obtained by previous considerations, $1 \mathrm{e}$. $\lambda^{2}=\mu^{3}$

which is often used as a guide in choosing $\lambda$ and $\mu$ values However, since this is derived by assuming identical model and prototype sediments, another relationship should be used in actual choice of scale ratios This relationship can easily be verified quantitatively from the previous set of Equations where the particle fall velocity for the light scale model material is taken into account exactly

\section{SIMILITUDE OF SEDIMENT MOTION FOR COASTAL MODELS}

Similitude of sediment motion means homogeneous scaling of sediment transport characteristics in the model, $1 \mathrm{e}$, consistent quantitative relationships between the model and prototype transport quantities For a distorted Froudian model, similitude of wave refraction insures 
approximately identical $\mathrm{u}_{\mathrm{c}} / \mathrm{u}_{\mathrm{w}}$ and $\Phi$ values as in the prototype For constant $q_{s} / u_{\bullet}\left(\frac{u_{x}{ }^{2}}{g}\right)$, it requires

$$
\mathrm{n}_{F_{\%}}=1 \quad \mathrm{n}_{\mathrm{R}_{*}}=1 \quad \text { (36) (37) and } \mathrm{n}_{\sigma_{\mathrm{D}}}=1
$$

(The condition ${ }^{n_{\sigma_{D}}}=1$ is disregarded by most investigators ) These Equations give $\quad n_{u_{*}}^{2}=n_{Y^{\prime}} n_{D}$ and $n_{u_{*}} n_{D}=1$

The scale for $n$ is actually difficult to define, since the boundary layer characteristics vary ${ }^{\text {w }}$ from off shore (viscous case or ripples) to the breaking zone, where it is fully turbulent, without ripples

In the first case (viscous) $\quad \mathrm{n}_{\mathrm{u}_{*}}=\mu 1 / 8$

In the breaking zone

$$
\mathrm{n}_{\mathbf{u}_{x c}}^{\mathrm{u}_{\%}}=\mathrm{n}_{\mathrm{f}}{ }^{1 / 2} \mu^{1 / 2}
$$

Considering the state of the art, it is difficult to assess the value of $n_{f}$ If one assumes that $f$ in the surf zone is a function of the relative roughness, as in the case of a river, then $n_{f}=\mu / \lambda$ Then $n_{u_{*}} \cong \mu \lambda^{-\frac{1}{2}}$

$$
\mathrm{n}_{\mathrm{D}}=\lambda^{\frac{1}{2}} \mu^{-1}
$$

$$
n_{\gamma^{\prime}}=n_{D}-3=\mu^{3} \lambda^{-3 / 2}
$$

The use of natural sand, $n_{\gamma^{\prime}}=1$, would require that $n_{D}=1$ This implies that model sand should be identical to the prototype sand In this case, Equation (40) gives $\lambda^{\frac{1}{2}}=\mu$, which could be considered as the natural distortion law equivalent to Iacey condition $\lambda^{2}=\mu^{3}$ for rivers Since the scale for the slope is equal to distortion $n_{s}=\frac{\mu}{\lambda}, n_{s}=\frac{1}{\lambda^{\frac{1}{2}}} \quad$ (A natural beach subjected to 10 foot, 12 second waves of slope $\frac{1}{50}$ will ${ }^{\lambda^{\frac{1}{2}}}$ have a $\frac{1}{100}$ scale model slope of $1 / 5$ when subjected to a 1 foot, 4 second wave ) However, a lighter material than sand will actually insure less distortion and a more gentle scale model slope, therefore, less scale effects It is interesting to note that Equation $\lambda \frac{1}{2}=\mu$ is compatible with $n_{F_{*}}=1$ and also $n_{R_{*}}=1$ Consequently, sand can be theoretically used on scale models of beaches

Although similitude of $R_{*}$ and $F_{*}$ are obtained based upon the bed load transport formula, the similitude relationships have the following additional implications 1) similitude of initiation of sediment motion, and 2) similitude of regimes of sediment motion

Thus, such similitude relationships imply a similar and homogeneous transport characteristic over the model as in the prototype However, there are several restrictions before the above equations for $n_{D}$ and $n_{\gamma^{\prime}}$ can be used These limitations are

1) Both the prototype and the model boundary layers are turbulent (this is certainly true in the surf zone)

2) The model friction factor is scaled according to distortion of $n_{f}=\frac{\mu}{\lambda}$ model and prototype 
4) Moreover, the analysis here does not apply in the case of a pebble beach In such case, the condition of $\mathrm{n}_{\mathrm{R}_{*}}=1$ is replaced by a limiting value of
$\mathrm{R}_{*}$, say, of 500

Theoretically, relationships between scales, distortion, artificial sediments, etc, are obtained based upon similitude of sediment transport characteristics and equilibrium beach profiles Because of the uncertainties involved, certain prelıminary wave tank experiments are necessary The purpose of such wave tank experiments is to confirm the cholce of distortion and artificial sediments The similitude of sediment transport characteristics by selecting $n_{R}=1$ and $n_{F_{*}}=1$ gives not only the correct scaling of bed load transport, $R_{\rtimes}$ but afso the critical condition of sediment motion

The condition $n_{R}=1$ may then be too stringent and be replaced by a condition such as $R_{*}>R_{x<*}$, where $R_{s}$, is a minimum critical value for the scale model (say 160 implyıng a turbulent boundary layer)

An important scale ratio is the time scale of bottom evolution $n_{t b}$ The value of $\mathrm{n}_{\mathrm{tb}}$ is usually determined in the process of reproduction of bottom evolution Analytically, $n_{t b}$ can be obtained from any sediment transport formula (15) For a coastal movable bed model, the littoral transport formula, is proposed here to be used for determining the value of $n_{t b}$

$$
n_{t b}=\frac{\lambda^{2} \mu}{n_{Q s}}=\lambda^{2} \mu^{-3 / 2} n_{D}^{-1} n_{Y^{\prime}}^{\frac{1}{2}} \text { where } n_{Q s} \text { is the scale ratio of }
$$

the littoral transport rate This Equation is applicable only in the case of coastal problems where the littoral transport is the dominant sediment transport mechanism. For river models, a different formula has already been proposed This formula is useful in predicting time scale of bed evolution in the model At present, there are, to ou $r$ knowledge, two sets of data available for comparison

1) Cobourg Harbor Study (Le Méhaute and Collıns, 1961)

Model conditions $\lambda=1 / 200, \mu=1 / 60$ Artificial sediment Gilsonite, $\mathrm{n}_{\gamma}{ }^{\prime}=00182, \mathrm{n}_{\mathrm{D}}=7$.

Timescale by model reproduction of bottom evolution $n_{t b}=25 \mathrm{~min} \quad 1 \mathrm{yr}$

Timescale from proposed Equation $\mathbf{n}_{t b}=117$ minutes 1 year

In this study, the model wave helght was exaggerated, approximately, by a factor of two A correction of the wave height scaling based upon the littoral drift equation gives $\mathrm{n}_{\mathrm{tb}}=29$ minutes 1 year, which is close to the predicted value

2) Absecon Inlet Study (U $S$ Waterways Experiment Station, 1943)

Model conditions $\lambda=1 / 500, \mu=1 / 100$ Artificial sediment Sand, $\mathrm{n}_{\gamma^{\prime}}=1, \mathrm{n}_{\mathrm{D}}=063$

Timescale by model reproduction of bottom evolution $\mathrm{n}_{\mathrm{tb}}=13 \mathrm{hrs} 1 \mathrm{yr}$

Timescale from proposed Equation $n_{t b}=56$ hours 1 year

Again there is a correction on the exaggeration of the model wave heights (a factor of about two) $n_{t b}=14$ hours 1 year 
An additional correction is to be made due to the fact that 22 percent of time was not reproduced in the model when there was no littoral transport in the field $\mathrm{n}_{\mathrm{tb}}=11$ hours 1 year

Thus, these predicted time scales compared favorably with observed values The accuracy is estimated to be within 30 percent

\section{CONCLUSION}

The success of a movable bed scale model depends upon the proper choice of distortion and material Distortion is a natural observed phenomena, which needs to be strictly adhered to for similitude Its choice can theoretically be justified in the case of a river, based on well defined conditions of similitude These conditions lead to a Lacey type relationship close to $\lambda^{2} \cong \mu^{3}$ The law $\lambda^{2}=\mu^{3}$ prevalls in the case where the same material (sand) is used in both the prototype and the model This is the law of "natural distortion" which is not compatible with other conditions of similitude $\left(n_{F}=1, n_{f}=\Omega\right)$

The choice of scales and material is less well defined in the case of beaches However, for a given material and vertical scale, there is also a well defined rate of distortion based on the equilibrium profile of beaches This approach compensates to a large extent for the lack of understanding of the law of sediment transport by wave action If one assumes that the regime in the boundary layer is turbulent, the friction coefficient can be related to relative roughness (as in the case of a steady flow) and the same material, a law of "natural distortion" is also obtained such as $\lambda^{1 / 2}=\mu$ The first law is approximately verified by compilation of river statistics, while the second law $\left(\lambda^{1 / 2}=\mu\right)$ is obtained from theoretical considerations This law still needs to be proven, improved, or disproven from observation For the time being, one can only insure that it gives a qualitatively observed trend (Wiegel, 1964) Iighter scale model material will insure a smaller rate of distortion Therefore, the use of sand in movable scale models may provide too large a scale effect, even though it is now compatible with $n_{F *}=1$ and $n_{R}=1$ It is hoped that present studies on equilibrium profiles of beaches will solve some of the uncertainties that have been brought out in this paper In the meantime, pre$11 \mathrm{minary} 2 \mathrm{D}$ tests a re still necessary for determining distortion prior to designing any large $3 \mathrm{D}$ model

It 1 s also hoped that the present paper will help to demonstrate that movable bed scale model technology is not "magic witchcraft" after all

\section{ACKNOW LEDGMENTS}

This study was sponsored by the Coastal Engineering Research Center, Washington, D C , under Contract DAC W 72-68-C-0020 The writer is indebted to Drs 1 Collins, E Noda, and L N Fan for many fruitful discussions 


\section{SUMMAR Y}

Conditions of Similitude

\begin{tabular}{|c|c|}
\hline Coastal & Fluvial \\
\hline $\begin{array}{l}\text { 1) Similitude of wave refraction } \\
\text { Time scale } n_{t}=\mu^{\frac{1}{2}} \\
\text { Velocity, current } n_{u} \cong \mu^{\frac{1}{2}} \\
\text { Similitude of wave breaking } \\
n_{H_{b}}=\mu\end{array}$ & $\begin{array}{l}\text { 1) Normal Froude similitude for dis- } \\
\text { torted model } \\
\text { Time scale } n_{t}=\lambda / \mu^{\frac{1}{2}} \\
\text { Velocity } \quad n_{u}=\mu^{\frac{1}{2}}\end{array}$ \\
\hline 2) $n_{F_{*}}=1 \quad n_{u_{*}}^{2}=n_{Y^{\prime}} n_{D}$ (imperative) & 2) $n_{F_{x}}=1 \quad n_{u_{y}}^{2}=n_{\gamma^{\prime}} n_{D}$ (imperative) \\
\hline 3) $n_{R_{*}}=1 \quad \begin{array}{l}\text { (not imperative, } \\
\text { but recommended) }\end{array}$ & 3) $R_{x, 5}>500$ no ripple (imperative) \\
\hline $\begin{array}{l}\text { 4) Short model independent of } \\
\text { relative roughness } \\
\mathrm{n}_{\mathrm{u}_{\nu}} \cong \mu \lambda^{-\frac{1}{2}} \text { if }(?) \mathrm{n}_{\mathrm{f}}=\mu / \lambda \text { (surf zone) }\end{array}$ & 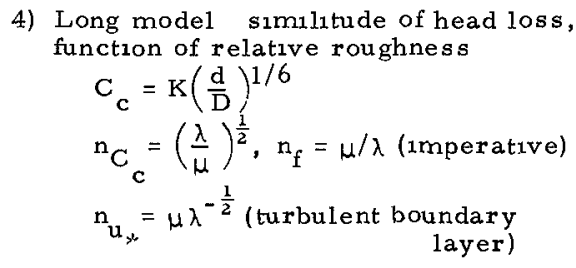 \\
\hline $\begin{array}{l}\text { 5) Suspension } \\
\qquad \frac{n_{u}}{n_{w}}=\frac{\lambda}{\mu} \text { (not imperative?) }\end{array}$ & $\begin{array}{l}\text { 5) Suspension } \\
\qquad \frac{n_{u}}{n_{w}}=\frac{\lambda}{\mu}\end{array}$ \\
\hline $\begin{array}{l}\text { 6) Distortion imposed by equilibrium } \\
\text { profiles of beaches (Still undeter- } \\
\text { mined for light model material) }\end{array}$ & $\begin{array}{l}\text { 6) } 2,4 \text {, \& } 5 \text { combined lead to a dis- } \\
\text { tortion with different material (1mper- } \\
\text { ative) }\end{array}$ \\
\hline $\begin{array}{l}\text { Natural distortion (same material), } \\
\text { and where } \\
\qquad \mathrm{n}_{\mathrm{f}}=\frac{\mu}{\lambda} \lambda^{\frac{1}{2}}=\mu \\
\quad \text { Compatible with } \mathrm{n}_{F_{*}^{*}}=1, \mathrm{n}_{\mathrm{R}_{\text {, }}}=1 \\
\text { but too large distortion(scale effects) }\end{array}$ & $\begin{array}{l}\text { Natural distortion (same material) } \\
\lambda^{2}=\mu^{3} \\
\text { Compatible with "regime theory", but } \\
\text { not with } n_{F,}=1\end{array}$ \\
\hline
\end{tabular}




\begin{tabular}{|c|c|c|c|}
\hline \multirow{2}{*}{\multicolumn{2}{|c|}{$\begin{array}{l}\text { Phenomena and } \\
\text { Important Quantities }\end{array}$}} & \multicolumn{2}{|c|}{ Simulitude Relations } \\
\hline & & Fluvial & Coastal \\
\hline A) & $\begin{array}{l}\text { Geometry } \\
\text { Horizontal length } \\
\text { Vertical length } \\
\text { Distortion } \Omega \\
\text { Slope (river, beaches) S }\end{array}$ & $\begin{array}{c}\lambda \\
\mu / \lambda \\
\mu / \lambda\end{array}$ & $\begin{array}{l}\lambda \\
\mu \\
\mu / \lambda \\
\mu / \lambda\end{array}$ \\
\hline B) & $\begin{array}{l}\text { Water Motion Characteristics } \\
\text { Water depth d, wave breaking depth } \mathrm{d}_{\mathrm{b}} \\
\text { Wave height } \mathrm{H} \text {, breaking wave hgt } \mathrm{H}_{\mathrm{b}} \\
\text { Wave length } \mathrm{L} \\
\text { Wave period } \mathrm{T} \\
\text { Refraction angle \& wave breaking angle } \\
\text { Wave diffraction, reflection } \\
\text { Time scale t } \\
\text { Particle velocity } \\
\text { Mass transport longshore velocity } \\
\text { Friction coefficient } \mathrm{f} \\
\text { Chezy coefficient } \mathrm{C} c \\
\text { Viscous sublayer } \\
\text { Shear velocity } \mathrm{u}_{,} \\
\text {Energy slope } \\
\text { Head loss }\end{array}$ & $\begin{array}{l}\mu \\
\\
\lambda / \mu^{\frac{1}{2}} \\
\mu \frac{1}{2} \\
\Omega \Omega \frac{1}{2} \\
\Omega^{-\frac{1}{2}} \\
(\mu \Omega)^{-\frac{1}{2}} \\
(\mu \Omega)^{\frac{1}{2}} \\
\\
\Omega \\
\mu\end{array}$ & 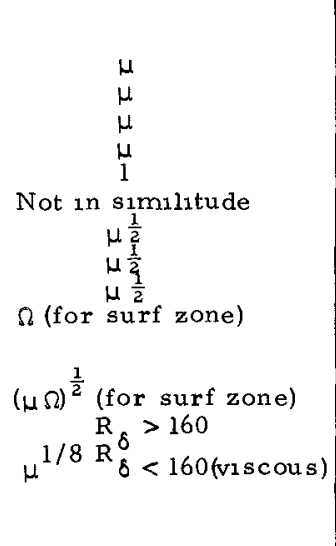 \\
\hline C) & $\begin{array}{l}\text { Sediment Characteristics } \\
\text { Sediment size D } \\
\text { Apparent specific weight } \gamma^{\prime} \\
\text { Size distribution } \sigma_{D}\end{array}$ & $\begin{array}{l}\lambda_{3}^{\frac{1}{2}} \mu^{-1} \\
\mu^{-3 / 2} \\
1\end{array}$ & $\lambda_{3}^{\frac{1}{2}} \mu^{-1}($ for surf zone $)$ \\
\hline D) & $\begin{array}{l}\text { Initiation of Motion } \\
\text { Critical boundary layer } \\
\text { Reynolds number } R_{4} \\
\text { Critical boundary layer } \\
\text { Froude number } F_{3 \times}\end{array}$ & $\begin{array}{c}R_{2 x}>500 \\
1\end{array}$ & 1 \\
\hline E) & $\begin{array}{l}\text { Sediment Transport Characteristics } \\
\text { Boundary ldyer Reynolds number } R_{*} \\
\text { Boundary ldyer Froude number } F_{*} \\
\text { Volume of deposition or erosion } \\
\text { Time of bed evolution }\end{array}$ & $\begin{array}{l}>500 \\
\lambda^{1} \lambda^{2 / 2} \mu \\
\mu-2\end{array}$ & $\begin{array}{l}1 \text { or } n_{u_{*}} n_{D}=1 \\
1 \text { or } n_{u}^{2}=n \gamma^{\prime} n_{D} \\
\lambda^{2} \mu \\
\lambda^{2} \mu^{-3 / 2} n_{D}-1 n_{\gamma^{\prime}} \frac{1}{2}\end{array}$ \\
\hline
\end{tabular}


Comparisón of Various Approaches for Determination of Basic Scale Ratios of A Coastal Movable Bed Model

\begin{tabular}{|c|c|c|}
\hline Authors & Basic Relations & Method of Derivation \\
\hline $\begin{array}{c}\text { Goddet \& Jaffry } \\
(1960)\end{array}$ & $\begin{array}{l}n_{D}=\mu{ }^{17 / 20} 8 / 5 \\
n_{\gamma^{\prime}}=\mu^{3 / 20} \Omega^{-3 / 5}\end{array}$ & $\begin{array}{l}\text { Sediment motion due to } \\
\text { combined action of waves } \\
\text { and currents }\end{array}$ \\
\hline $\begin{array}{l}\text { Valembo1s } \\
(1960)\end{array}$ & $\begin{array}{l}\Omega=n_{\gamma^{\prime}}^{-1} \\
n_{\gamma^{\prime}} n_{D}^{3}=1 \\
\mu=n_{\gamma^{\prime}}^{3} n_{D}\left(n_{H} / \mu\right)^{-4}\end{array}$ & $\begin{array}{l}\text { Kinematics of motion of } \\
\text { suspended sediments } \\
\text { Similitude of D } \\
\text { Modified relation of initia- } \\
\text { tion of sediment motion } \\
D \quad=K R, 8 / 9\end{array}$ \\
\hline Yalin (1963) & $\begin{array}{l}\mathrm{n}_{\mathrm{D}}=\mu^{3 / 4} \lambda^{1 / 2} \\
\mathrm{n}_{\gamma^{\prime}} \mathrm{n}^{3}=1\end{array}$ & Dimensional analysis \\
\hline Bijker (1967) & $\begin{array}{l}n_{\gamma^{\prime}} n_{D} \Omega^{-1}=\mu n \\
\Omega \leftarrow \text { equilibrium beach profiles }\end{array}$ & Similitude of $F_{2}$ \\
\hline $\begin{array}{l}\text { Present } \\
\quad \text { Method(1970) }\end{array}$ & $\begin{array}{c}n_{\gamma^{\prime}} n_{D}^{3}=1 \\
n_{\gamma^{\prime}}=\mu^{3} \lambda^{-3 / 2} \text { or } \\
{ }^{n_{D}}=\lambda^{1 / 2} \mu^{-1} \\
\Omega \text { equilibrium beach profiles }\end{array}$ & $\begin{array}{l}\text { Similitude of sediment } \\
\text { transport characteristics, } \\
1 \mathrm{e}, \mathrm{F} \text { and } \mathrm{R} \text {, }\end{array}$ \\
\hline
\end{tabular}




\section{LIST OF SYMBOLS}

$C_{c} \quad$ Chezy coefficient $\left(L^{\frac{1}{2}} T^{-1}\right)^{x}$

d depth of water

$d_{b} \quad$ depth of water at breaking

D mean sediment size

$D_{\%} \quad$ dimensionless sediment size $=\left(\frac{\left(\gamma_{s}-\gamma_{f}\right) g}{\nu^{2} \gamma_{f}}\right)^{1 / 3} D=\left(R_{,} / F\right)^{2 / 3}$

E wave energy flux per unit length of the crest $\left(\mathrm{MLT}^{-2}\right.$ )

f Darcy-Weisbach friction factor

$F_{*} \quad$ boundary layer densimetric Froude number $=u_{2 \xi} / \sqrt{\gamma^{\prime} g D}$

$F_{*} \quad$ critical boundary layer Froude number on initiation of sediment motion

g acceleration of gravity

$\mathrm{H}$ wave height

k wave number $=2 \pi / \mathrm{L}$

L wave length

$\mathrm{n}_{\text {quantity }}$ scale ratio with subscripts denoting corresponding quantities = (value of model)/(value of prototype) e $g, n_{L}$ is the scale ratio of the wave lengths

$q_{S} \quad$ volumetric sediment transport rate per unit width $\left(\mathrm{L}^{2} \mathrm{~T}^{-1}\right)$

$Q_{\mathrm{s}} \quad$ volumetric sediment transport rate $\left(\mathrm{L}^{3} \mathrm{~T}^{-1}\right)$

$r$ radial coordinate

$R_{h} \quad$ hydraulic radius

$R_{\delta} \quad$ boundary layer Reynolds number $=U_{\delta} \delta / \mathrm{V}$

$R_{\varkappa} \quad$ boundary layer Reynolds number based upon sediment size $=$ $u_{2 c} D / v$

$R_{x_{s}} \quad$ critical boundary layer Reynolds number on initiation of sediment motion

S slope

$S_{e} \quad$ energy gradient

t time

$t_{b} \quad t_{1}$ me of bed evolution

$\mathrm{T}$ wave period

u horizontal velocity

${ }^{u_{C}} \quad$ critical velocity for sediment motion 


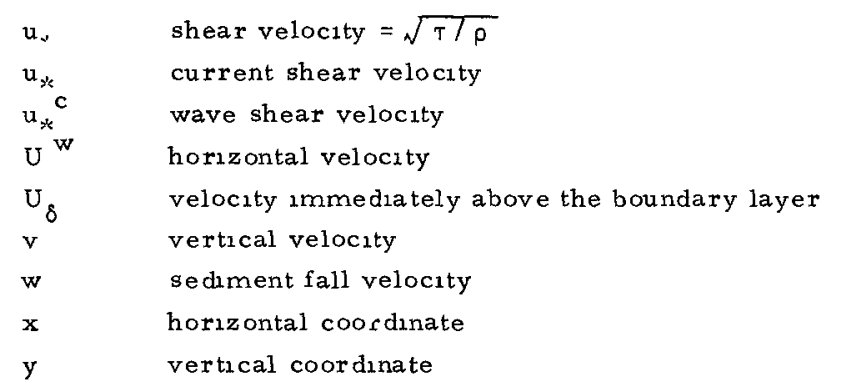

$\delta$ boundary layer thickness

${ }^{\delta} \mathrm{L} \quad$ laminar boundary layer thickness parameter turbulent boundary layer thickness

${ }^{T_{C}} \quad$ critical shear stress on the initiation of sediment motion 


\section{PARTIAL LIST OF REFERENCES}

Bascom, W N (1951), "The Relationship Between Sand Size and BeachFace Slope", Trans AGU, 32, No 6, December, pp 866-874

Bijker, E W (1967), "Some Considerations About Scales for Coastal Models with Movable Bed", Delft Hyd Lab, Pub No 50, Nov, 142 pages

Bonnefille, R, and Pernecker, L (1965), "Note de Synthese Sur Les Mouvements Des Fonds Marıns Sous LAction des Courants et de La Houle", National Hydraulics Lab, Chatou Res and Exp't Center, France 66 pages

Boucher, R, and Le Méhauté, B (1957), "L'etude Experimentale des Problemes de 1'Hydraulique Fluviale", L'ingenieur, Montreal, pp $49-58$

Collıns, J I (1963), 'Inception of Turbulence at the Bed Under Periodic Gravity Waves", J of Geophy, Res, Vol 68, No 21, Nov, pp $6007-6014$

Divoky, D, Le Mehaute, B, and Lin, A (1969), "Breaking Waves on Gentle Slopes", AGU

Eagleson, P S , Glenne, B , and Dracup, J A (1963), 'Equilibrium Characteristics of Sand Beaches", J of Hyd Div, Proc ASCE, Jan, pp 35-57

Fan, L N , and Le Mehauté, B (1969), "Coastal Movable Bed Scale Model Technology", Tetra Tech, Inc No TC-131

Goddet, J (1960), "The Intral Stages of the Transport of Materials Under Wave Action" La Houlle Blanche, No 2, March-April, pp 122135

Goddet, J, and Jaffry, P (1960), "Sımilitude Laws for Sediment Transport Under the Simultaneous Action of Waves and Currents", La Hourlle Blanche, No 2 March-Apr11, pp 136-147

Larras, J (1957), "Effet D'echelle Dans Les Etudes D'erosion du Sable Par La Houle Et Par Le Clapotis", Proc of 7 th Cong If IAHR, Lisbon, Paper No A-5 13 pages

Le Méhauté, B, and Brebner, A (1961), "An Introduction to Coastal Morphology and Littoral Processes", CE Dept, Res Rept 14, Queen's University, Jan, 46 pages

Le Mehauté, B , and Collıns, J I (1961), "A Model Investigation of Cobourg Harbor", CE Dept, Res Rept 17, Queen's University, July

Le Mehaute, B (1962), "Theory, Experiments, A Phılosophy of Hydraulics", J of Hyd Div, Vol 88, HY 1, Proc of ASCE, Jan pp $45-66$ 
Le Mehaute, B and Koh, R C Y (1967), "On the Breaking of Waves Arriving at an Angle to the Shore", J of Hydraulic Research, 5. No 1, pp 67-88

Manohar, M (1962), Discussion of "Laboratory Determination of Littoral Transport Rate", by $R$ P Savage, J of Waterways and Harbors Div, Proc ASCE, $\underline{88}$, No 4, Nov pp 144-147

Savage, R P (1962), "Laboratory Determination of Littoral Transpoxt Rates", Journal of Waterways and Harbors Div, Proc , ASCE, May, pp 69-92

Saville, T Jr (1950), "Model Study of Sand Transport Along an Infinitely Long, Straight Beach", Trans AGU, 31, No 4, Aug pp 555-565

Saville, T Jr (I957), "Scale Effects in Two-Dimensional Beach Studies", Paper No A-3, Proc of 7 th Congress of IAHR, Lisbon

Simmons, H B (1950), "Contribution of Hydraulic Models to Coastal Sedimentation Studies", Proc of lst Conf on Coastal Engrg, Long Beach, 19 pages

Simmons, H B (1966), "Tıdal and Salınity Model Practıce", Ch 18 of Estuary and Coastlıne Hydrodynamics", Ed by $A$ T Ippen, pp $711-731$

Sitarz, J A (1963), "Contribution a l'Etude de L'evolution des Plages a Partir de la Connaissance des Profils D'equilıbre"', Travaux du Center de Recherches et d'Etudes Oceanographiques, Vol 5, Sept

Valembois, J (1960), "Etude Sur Modele Du Transport Littoral Conditions De Similitude", Ch 18, Proc 7th Conf on Coastal Engrg, The Hague, pp 307-317 (English Translation, U S Army Corps of Engineers, Waterways Experiment Station, Translation No 65-7, July 1965)

Vanon1, V A Brooks, N H and Kennedy, J F (1961), "Lecture Notes on Sediment Transportation and Channel Stabılity", W M Keck Lab of Hydraulics and Water Resources, Tech Report KH-R-1, Callf Institute of Technology, Pasadena, California, Jan

Wiegel, R I (1964), "Oceanographical Engineerıng", Prentice-Hall, Inc , Englewood Cliffs, N J , 527 pages (p 359)

Yalin, S (1963), "Method for Selecting Scales for Models with Movable Bed Involving Wave Motion and Tidal Currents", Proc of 10 th Congr of IAHR, Vol 1 , paper I 30, London 
STUDY OF DURBAN HARBOR SILTING AND BEACH EROSION ${ }^{1}$

1)

\author{
Jan Malan Jordaan, Jr., M. ASCE ${ }^{2)}$
}

Abstract

A 1 - 300 vertical, 1 : 100 horizontal scale model of seven miles of coastline, including the major area of the port limits and the inner harbor, was constructed to study combined wave, tide and wind action on transport of sand along the coast.

The effects of three predominant conditions of swell and wind-waves on the state of the ocean front beaches were studied by observing relative changes in shoreline contours. Fluorescentdyed sand tracers were also used in the model. The accumulation and dredging of sand near the harbor entrance was reproduced in the model and varlous dredging and storage proposals were carried out to scale on the model.

The model study enabled the cause of beach erosion to be attributed to the existence of an of fshore shoal produced by the localized dumping of sand dredged from the harbor approaches. This shoal caused selective wave action along the coastline, which was reoroduced to scale in the model. It was found that wind and tidal action had a major effect on the redistribution of sand on the beaches as modeled but a minor effect on the permanence of the harbor entrance channel. Apart from the interaction of dredged sand at times being fed to the beaches, the problem of beach erosion could be studied independently of that of harbor silting on the same model. Sand was fed to maintain a state of equilibrium on the southern aporoach beach to the harbor to simulate the littoral supply.

The northern beach downdrift of the harbor entrance, where the erosion problem existed, was found to be essentially starved of littoral supply, due to maintenance of the harbor entrance by dredging and offshore dumping.

The requirements of the model study also gave rise to a program of field data collection on the governing environmental factors, which in itself alded considerably to the diagnosis of the causes of the state of unbalance in the shoreline. The model study led to several proposed remedial schemes, certain of which have been adopted since completion of the model study. The model study was conducted by the South African Council for Scientific and Industrial Research, for the South African Railways and the City Council of Durban.

1) This work was conducted by the author while a Senfor Research Officer of the Nat. Mech. Engrg. Res. Inst., South African Council for Scientific and Industrial Research, under contract with the South African Rallways and the City Council of Durban.

2) Assistant Chief Engineer (Design), Department of Water Affairs, Pretoria, South Africa. 


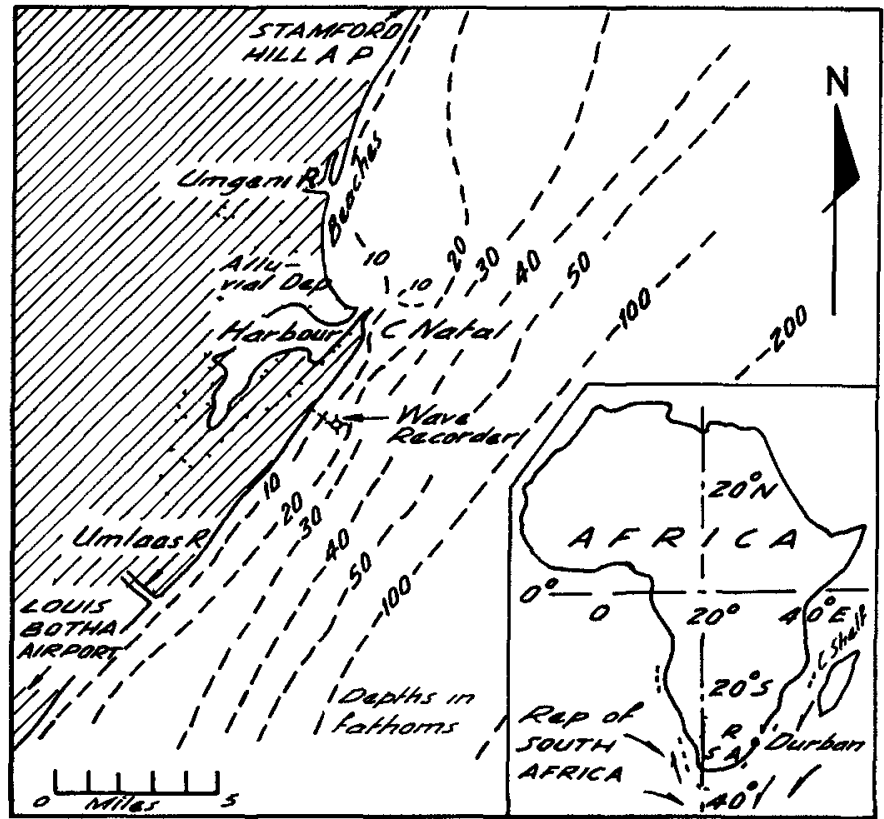

Figure 1 Plan of Vicinity and Locetion.

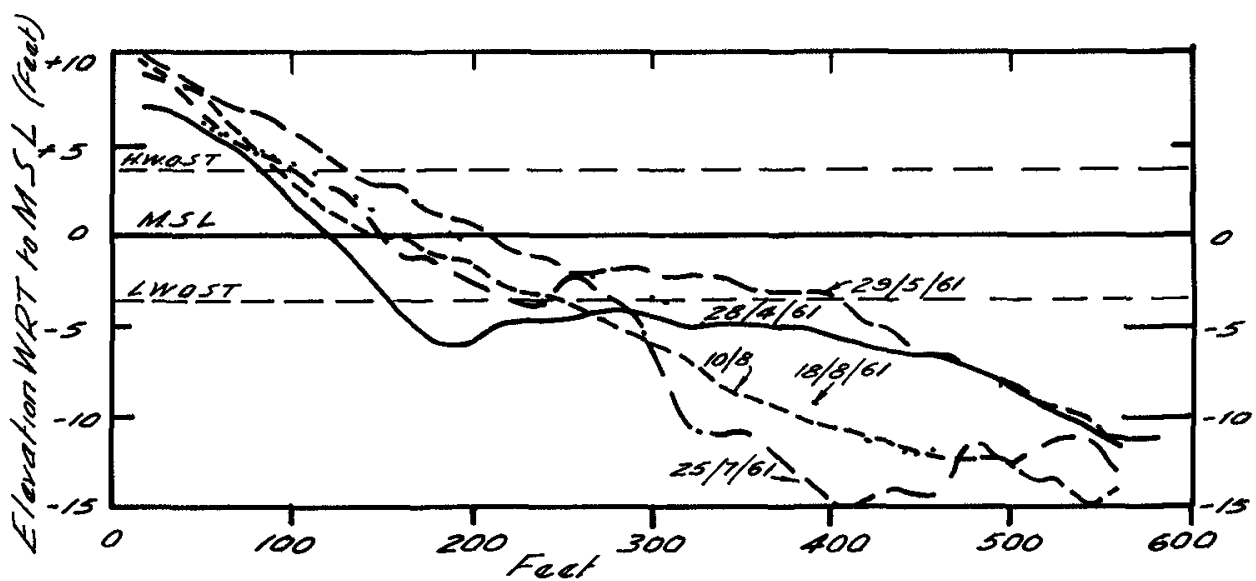

Figure 6. Varlability of beach profiles at Vest Street Jetty, South Beach. 

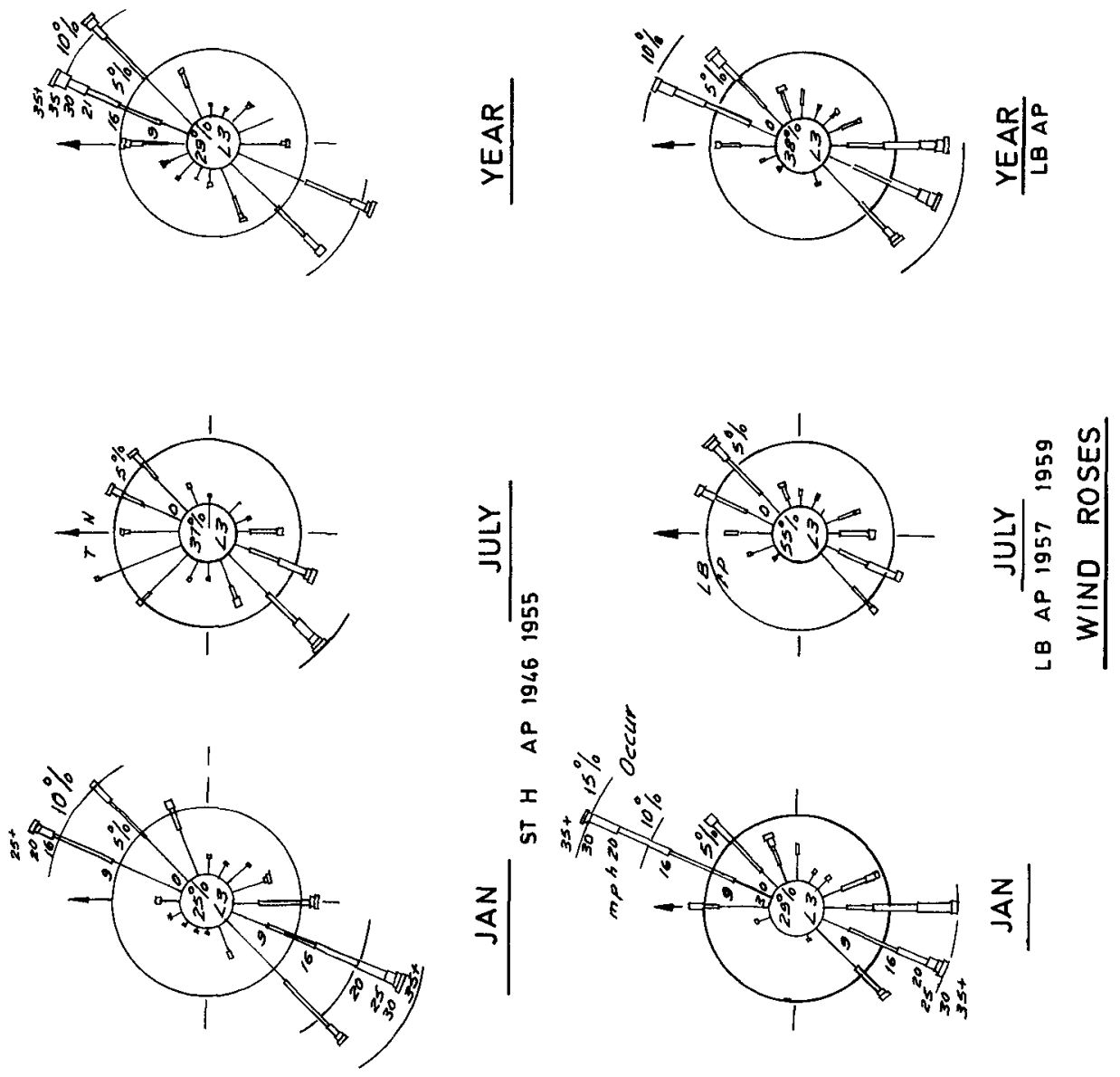

Figure 2. Wind roses for Stamford Hill and Louis Botha Airports, 


\section{Historical resume and statement of the problem}

Durban is situated on South Africa's east coast around a natural bay which was first settled in 1824. It has since grown to become the country's largest port and most popular seaside resort (Fig. 1).

The Durban port is located at a geological irregularity of the otherwise stralght coastline. The harbor entrance channel is formed between the 300 ft high projecting spur "The Bluff" and the 20 ft high estuarine formation tine Point". The nearshore area and the beaches are referred to as the Bluff side and the Bight respectively.

Before 1851 the estuarine channel remalned open naturally, mainly as a result of tidal action and the protection afforded by the Bluff from SW to SE storms. The depth over the bar was, however, only about 6 feet at that time. In 1851 the harbour entrance-channel works were commenced. Breakwaters were built and extended at intervals on both the north and the south sides of the entrance, until the present state was reached in 1952 (Moffatt, 5).

At present the entrance channel is dredged to a width of 650 feet and 18 kept 42 feet deep in the channel proper and 48 feet deep at the bar. Maintenance aredging, which consists mainly of keeping the sand trap just south of the entrance approximately 54 feet deep, has steadily increased and now amounts to approximately $800,000$ cubic yards a year ( $F 1 \mathrm{~g} .5)$. One of the main a ims of the investigation was to find means of reducing this amount.

The material which was dredged was mainly dumped off shore, in the earlier years up to 1938 in an area about $1 \frac{1}{2}$ miles east of the entrance and thereafter at various distances south-east of the entrance. During more recent jears, however, some of the material has been supplied for pumping to the beaches (Fig. $7 a$ ).

The second main task of the investigation was concerned with stabilizing Durban's ocean beaches (Fig. 6). The beaches are located between the Point and the Umgeni River mouth on Durban Bay which forms part of a concave oortion of coast stretching north from cape Natal (F1g. 1). In the past, due to this particular location, a rather stable condition must have existed (4). Due to the harbor entrance improvements the equilibrium of the sand movements was d isturbed (3). During the earlier stages of breakwater building (1851-1903) large deposits, a result of greater protection against SW to $S E$ swells, caused the beaches to progress generally seawards a distance of about 800 feet.

From 1938 until 1949 some 5 million cubic yards of sand was pumped to Vetch's Bight (Fig. 9) by S.A. Railway dredgers moored at the North Pier thro ugh a $42^{\prime \prime}$ dia. pipe (4).

From 1950 to 1953 sand was pumped from a suction plant just south of the South Breakwater directly to Vetch's Bight through a $16^{\prime \prime}$ dia. submarine pipeline across the entrance channel. Because insufficient sand collected near the suction plant in the Cave Rock Bight, after 1953 the orlginal system with the Rallway aredgers delivering sand to Vetch's Bight was resumed. It was presumed that initially 600,000 cubic yards of sand per annum would be pumped and, 


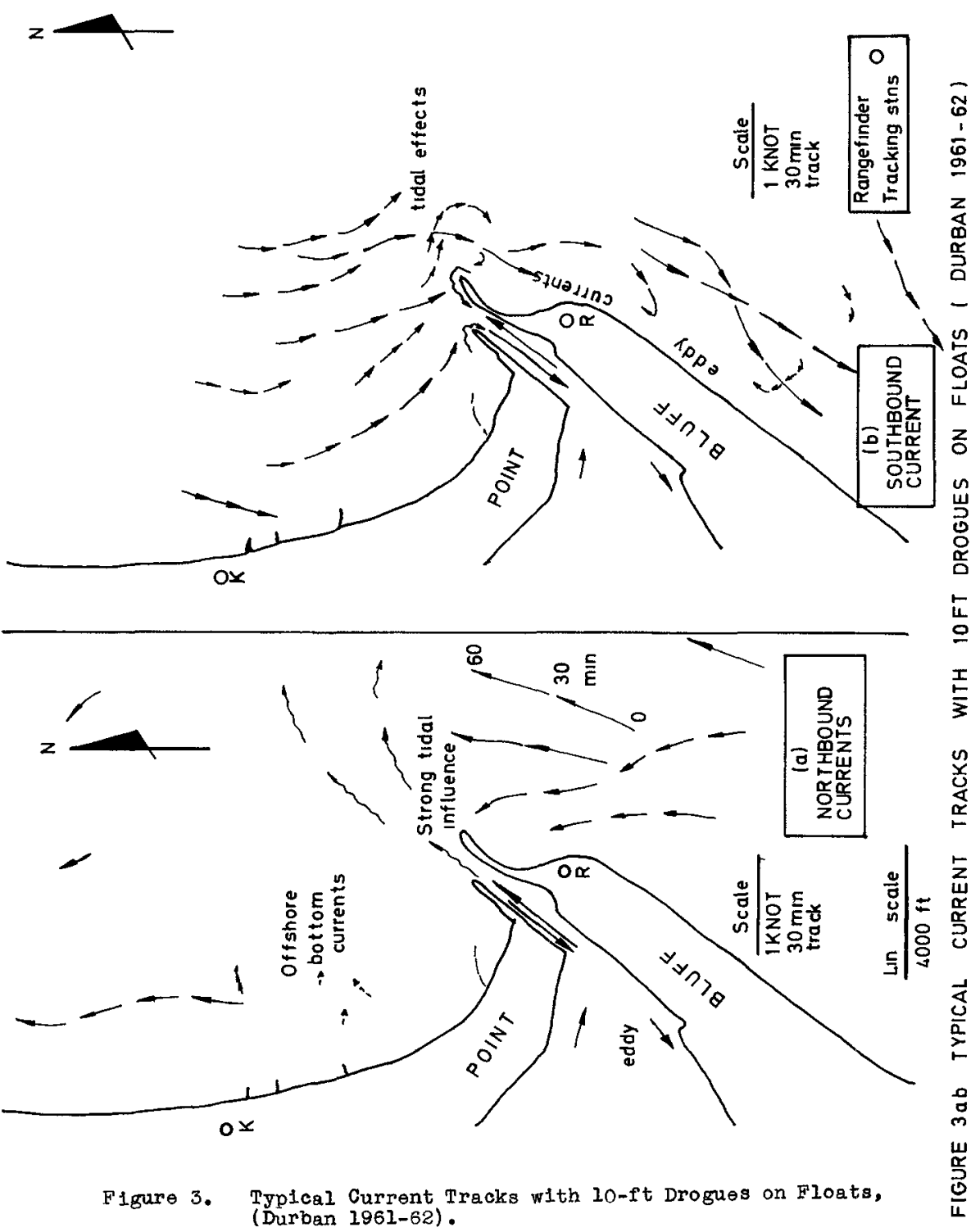


after a period of bullding uo, 300,000 cubic yards of sand would be available for maintenance. Moreover, two groins or breakwaters (Paterson Groynes) were built between 1954 to 1956 in order to stabilize the sand supplied to the Central Beach.

In 1960 , at the start of this investipation, the supply of only one-thind of the annual quantity of san required for mainterance purooses could be realized, so that the Central Beach in particular was badly starved of sand. In adiltion, scour roles developed near the tios of the Paterson croins, causing a steep beach slope and dangerous swimming conditions near the groins.

\section{Data Collection}

In order to successfull. perform a hydraulic model study such as the Durban investigation, a complete set of field dati, covering both oceanographic, topographic and geomorphological aspects must be obtained. Oceanographic data were collected over a period of 26 months from October 1960 until November 1962.

Wind. The result of the analyses of wind reconds tiken over ten consecutive years at Stamford Hill Alrport ank over three years at Louis Botha Airport are shown in Figure 2. The predominance of strong winds from north-easterly and south-westerly alrections is striking. This fact was made use of in the layout of the model.

Waves. Iong period waves (swell) and storm waves cause rapid migration of sand directly an also incirectly by wave-induced currents. Sand movements can be correlated witn incoming wave energy. Proner wave records are therefore essential for the analysis of sand movement. Waves can be fully described by their direction of approach, height and period. Becalase wave natterns are often very complex the above values have to be expressed statistically.

Wave characteristics have been recorded over various periods at the beach front (Iest Street Jetty), at the harbor entrance ind by inverted echo-sounder recorder 1 mile off the Bluff (?). Wave birection, refraction and diffraction were successfully recorded by means of aerial photogranhy for three typical weather conditions. It is clear from Figure 9 that wave direction in the Bight area is very mach affected by wave refraction, while in the Vetch s $P$ ier area, diffraction plays an linortant role.

The six months echo-sounder records have been supplemented and combined with Pilot Boat logs and clinometer readings to give the wave energy roses shown in Figure 4. These wave roses are fully reoresentative for an average yea $r(6)$. A very severe storm occurred from 24th to 30 th August, 1962 when the recorder was inoperative. Stereo-photo pairs of the waves during the peak of the storm were obtained from an aircraft and these, together with visual estimates of rave heights, led to the conclusion that waves up to 20 feet high and of 11 to 12 seconds period occurred near the harbor entrance. The nersistence of swell from any one direction has been determined as normaliy tiree to four days. A persistency of seven days was never exceeded.

Gurrents. Even fairly weqk ar rrents can cause substantil sand 

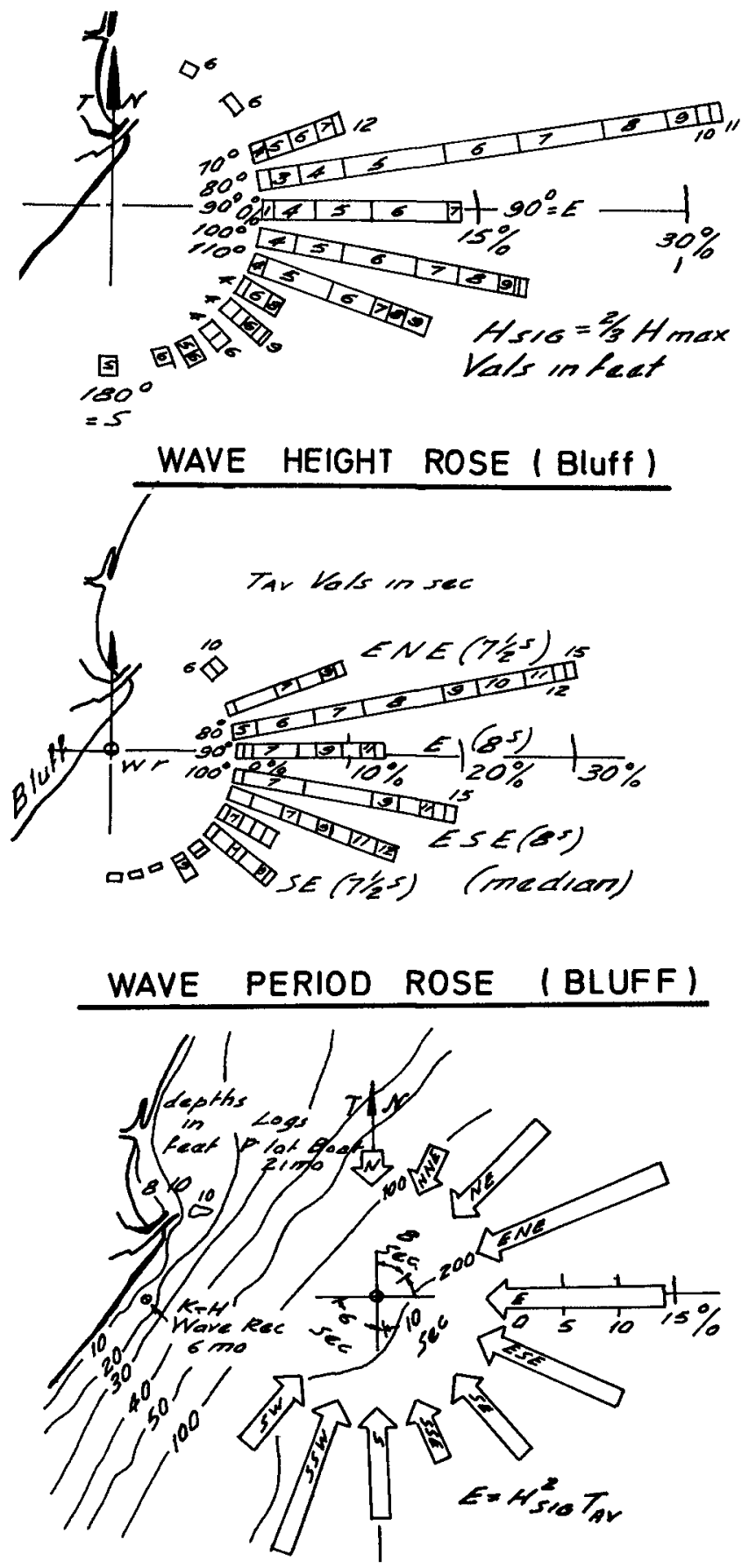

Figure 4. Wave Energy Distribution, offshore locations, Durban. 


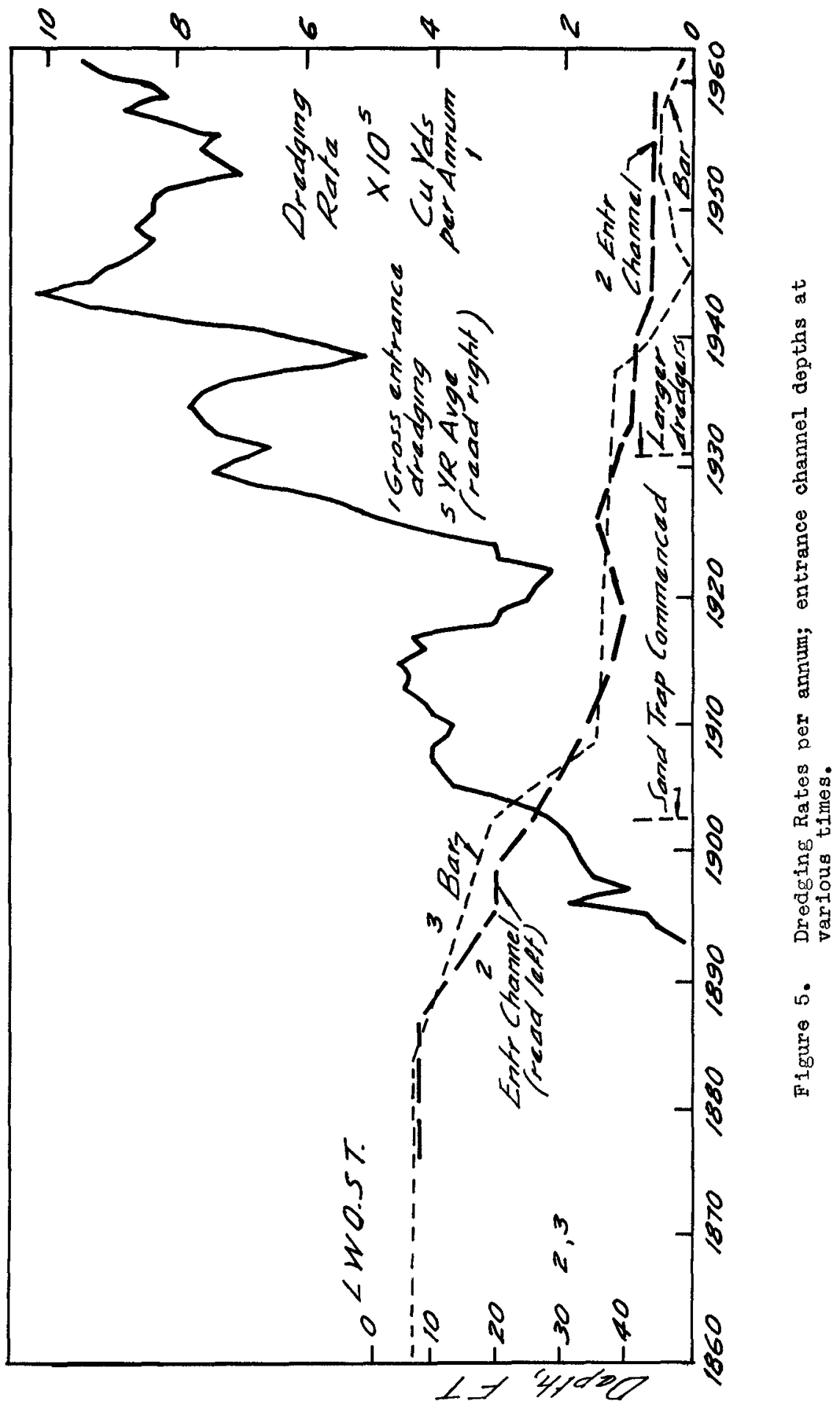




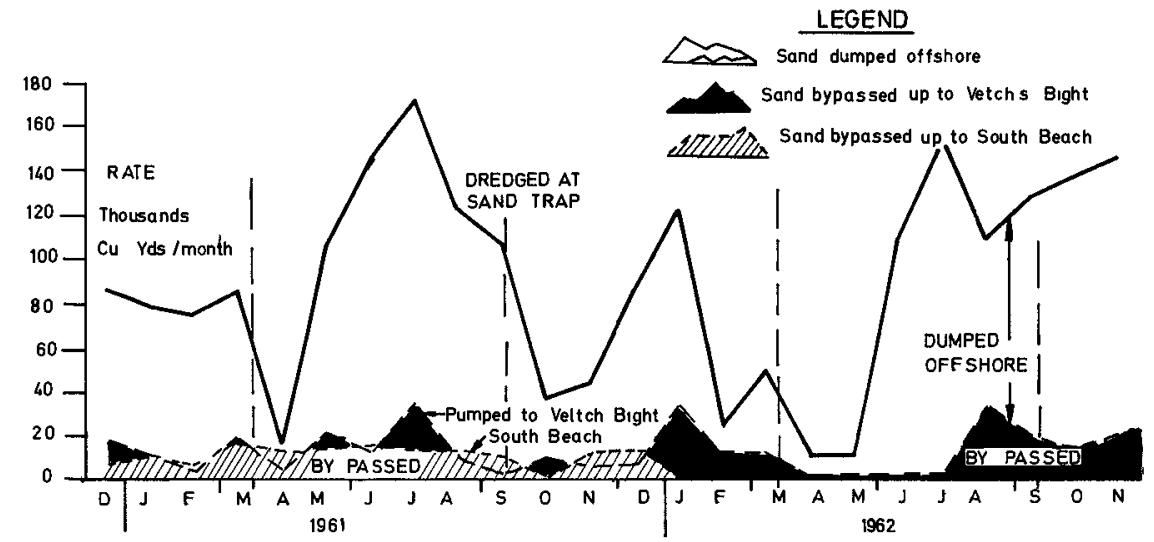

Figure 7a Distribution of Sand dredged, Sand dumbed of fishore and
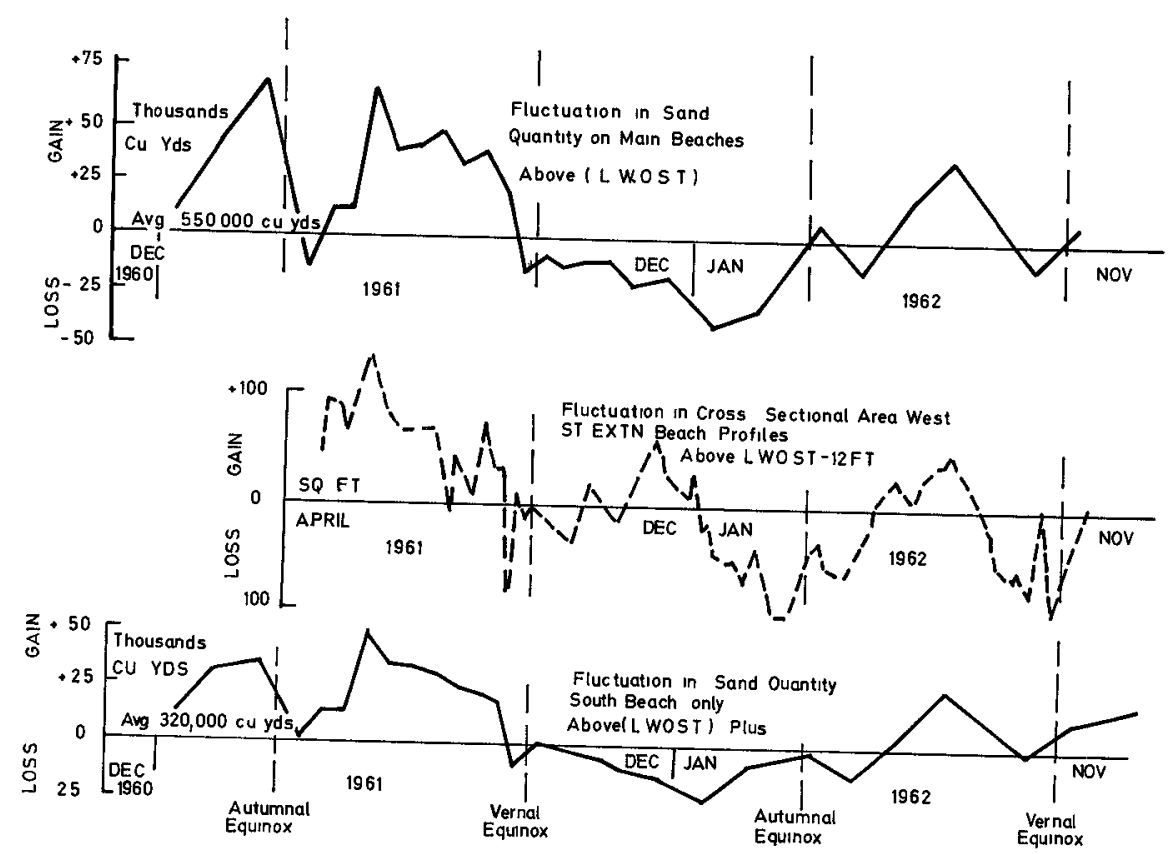

FIGURE $7 \mathrm{~b}$ Corresponding ohanges to beach sand quantitises, maing as affected by segsonal snfluence moving sand on- and 
movements when occurring concurrently with waves of swell which stirs uo the bottom material into susmension. On trie other hand, current velocities have to de about 1.25 knots to be abla to transoort material not already in susbension. The sout 2 bound Mozambique current, which is in general not found within the 100 fathom 1 ine, creates a counter current from south to north along the cosstine at Durban. The extent and strength of this counter current nas been found to be extremely variable.

For correct resroduction of prototyoe conditions in the model 3 better understandine of the current system near Durbin was therefore cilled for. Massurements, spread over a der lod of nearly two years, included exrial photograbhic tracing of surface floats with drogues attzched, propeller current meter measurements from a boat, tracking of floats with two rangefinders and measurements of bottom currents Nith jelly bottles.

\section{Morphology}

The quantities of sand in beaches gbove low water mark t-ken from regular fortnightly surveys made by the Durban Copporation are given in Figure 7b. The total and the South Beacn quantities have zlso been plotted. A significant loss of sand is seen to have occurred at the equinoctizl soring tides. A set of typical extremes in bezch profiles taken along west Strest Jetty over a period of siohteen months reflect the effects of various extreme conditions on unferwater beach formation (Fig. 6). The maximum variation of beach level near mean sea level wis found to be nine feet, corresponding to a bezch width change of approximately 150 feet.

Dredging at Durban harbor started in 1884 in the entrance channel and in 1893 on the bar. From 1903 onwards dredging was concentrated more in the sand tran area. The data for floating fivevear averages of annual dredging outside the harbor basin are plotted in Figure 5. Avallable records of channel and bar depths have been plotted as well. The correlation between dredged quantities, channel and bar depths can readily be seen from the figure. For the past several decades the South Afrlcan Rallways (S.A.R.) have made available to the Durban Corporation (D.C.) a certain quantity of sand ber annum for beach renourishment. This sand is pumoed by the S.A.R. and discharged just north of Vetch's Pier where it is retrieved by the D.C. pumping plant and discharged just north of West street Jetty via booster stations. Records made available by the S.A.R. 3nd D.C. for the two jears 1961 and 1962 are presented in Figure 7a. The various monthly quantities dredged and oumped via the various oive lines are shown.

Sand samoles were taken at various blaces zlong the beaches fnd from the sea bottom in the area reoresented in the model ( 1 ). They showed significant differences in size distribution, hue and texture. The results yielded a basis for classifying the sand by sipe as well as by color and permitted interoretztion to be made of the oattern of sand movement. This made the use of soecial tracer exneriments unnecessary. The sind south of the Umgeni River down to the harbor entrance is rather fine, well-sorted 


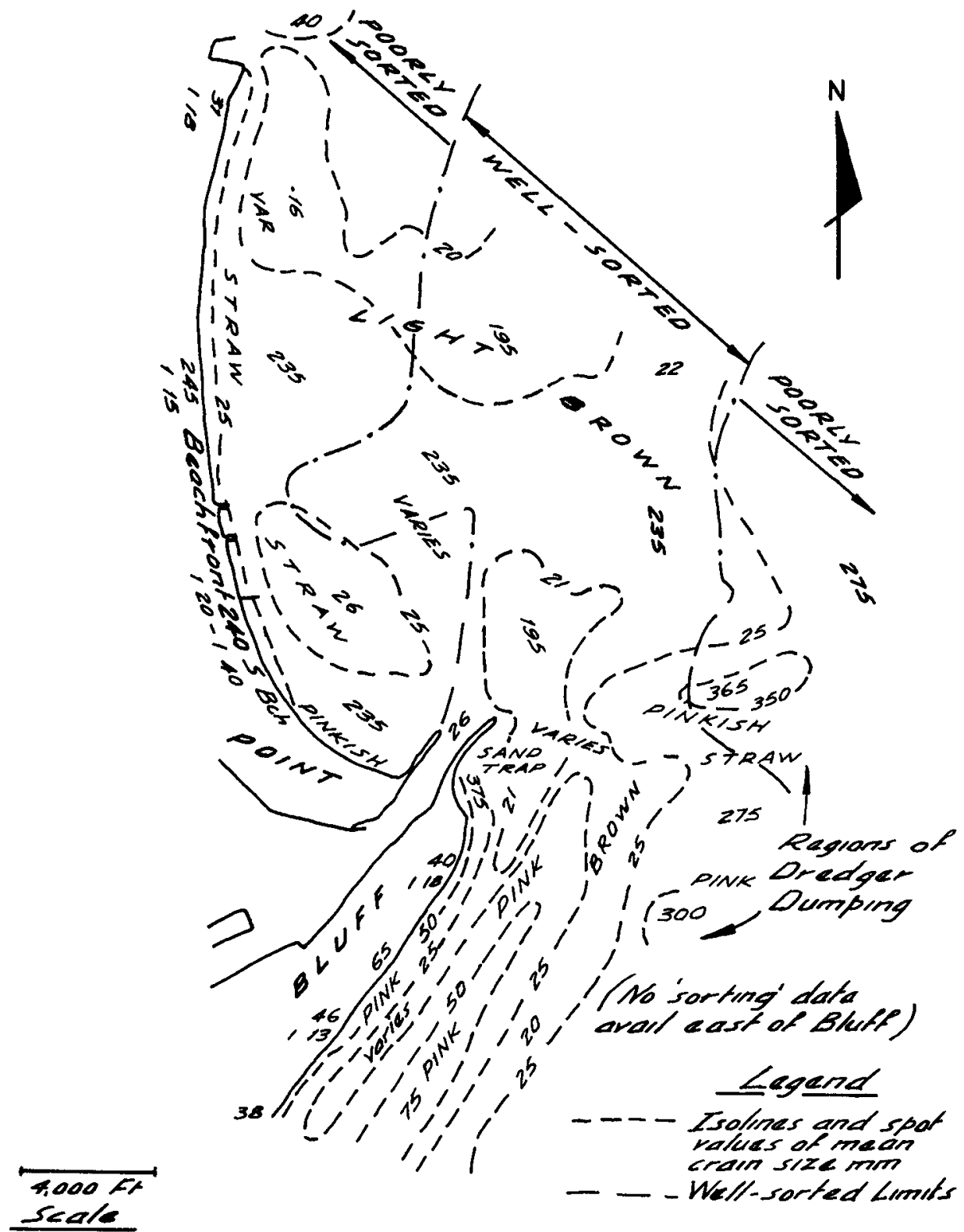

Figure 8. Sand size and color distribution, Durban 1961 - 1962.

DURBAN $1961-62$


(closelr sized) and rounded in shape. South of the harbor entrance on the Bluff beaches, the sand is very coarse, irregular in shape and it occurs in $a$ wide band of sizes (Fi. 9). The most imoortant observation is that the Bluff sands are distinctively colored bink or red - comsared to the Bight sands which are straw-colored. The presence of the pink color points to the fact tit "The Dump" cozsists of Bluff sand dredged from tne sind tido and dumped offshore. A renarkable findine was the close correlation between sand size and color. As a result of this correjation, Bluff sarsio could be identified elsewhere in the sampling area, both by color and by size.

\section{The Model}

Althougn thenretical considerations conbined with an extengive measirino camoaign in nature, can often yield an evajuation of the existing hydrolooical and morphological conditions, changes to the existing status can only be evaluated economical is in a hydraulic model. Nore often than not, a distorted model, with vertical heiehts accentuated, is used as the most expedient solution. The Durhan sand movement oroblem therefore caller for a movable bed model. In order to reprodixe the natural forces in the model is correctlv as possible, besides the usuzl waves, current and tides, wint wa also rebroduced. This was auite unusual in a threedimensional model. The model was built in Durban for Iegsons of convenience and suitable control. A Belman Hangar, belonging to the S.I.R. Was made avallable for this purvose.

Model Sczles. A 6.5 mile stretch of coastline, by 4 mile of shore Tistance had to be reproduced in the avallable space of the hangar120 by 96 feet. Furthermore, a model wave height of about 1 inch minimum is required in order to avoid unscilable influences such as surface tension and viscosity effects. The following scale ratios were consequently selected:

Horizontal scale (except wave length): $I_{r}=300$

Vertical scale (Including wave length): $h_{r}=100$

Further scale ratios follow from the Froude similitude law'-

Velocity scale (currents).

Hyoraulic time ratio (tides):

$\mathrm{V}_{\mathrm{r}}=\sqrt{\mathrm{h}_{\mathrm{r}}}=10$

Celerity soale (shallow water).

$t_{r}=I_{r} / N_{r}=30$

Wave perion scale (for $\lambda_{r}=h_{r}$ )

$c_{r}=\sqrt{h}_{r}=10$

$\mathrm{T}_{r}=\lambda_{r} / C_{r}=10$

Satisfactorv results were obtained with fine beach sand as movable bed material, with specific gravity of 2.65 and a medi in diameter of 250 microns.

Construction. The construction of the model was a joint undertaking of the CSIR, the D.C. and the S.A.R. and took 9 months from October, 1960 to June, 1961. The main model structure consists of level concrete floor approximately 3 inches thick 
and a surrounfing concrete wall $2^{\prime} 3^{\prime \prime}$ hlgh, forming a watertight basin. The motel topography was moulded in concrete using asbestos templates, according to the 1960 offshore survey (F1g. 9).

Wind generation. To be able to reproduce $N$ ind effects from the predominant directions - NNE and SSW - a bank of 20 inch diameter axial flow fans was installed alons the NE and $S$ h boundaries of the model (F1g.9). Each fan produced some 5000 cubic feet per minute free air which was blown horizontally over the model. The opening of the converging duct which covered the fans was 15 inches high. With the aid of guide vanes the air jet could be deflected at will. The maximum air velocity ranged from about $35 \mathrm{ft} / \mathrm{s}$ at the jet outlet to $6 \mathrm{ft} / \mathrm{s}$ at the beach front. More realistic, irregular and steep, wave shapes were obtained when using wind as well, than by the use of the wave generator alone.

Wave generation. Waves were created to scale in the model by three banks of unlque oneumatic wave makers, oriented perpendicular to the three main directions of wave anoroach, viz. NE, ESE and SE (FIg. 9). The wave generators were made up of two-foot air-dome elements, surge chambers and diverging underwater nozzles. Air let into the domes neriodicaliy depresses the water surface, thus creating a nave at the nozzle outlet. The surge chambers were connecter via four-arm rotary valves alternately to the suction and the compression ducts of $a$ common blower. All fourarm valves, fitted on to a common rotating shaft. By turning ezch valve housing through a pre-determined angle, the phase between the individual 2-ft sections of the wave-maker could be set. In this way waves could he produced in any required direction, from 0.4 to 1.2 sec. in period and $u_{p}$ to $2^{\prime \prime} \mathrm{high}$. The two separate drives for the shift allowed the creation of a typical "sea" and swell combination coming from two different directions.

Currents. For the reproduction of currents axial-flow propelier numos of $1 \mathrm{c}^{\prime \prime}$ and $6 "$ d lameter were used to circulate the water. Pide Generation. The tides in the model were created by admitting water to and withdrawing water from the model according to a preset propram controlled by a tidal synthesizer which produced $a$ saw-tooth-shaped tidal curve.

operation. As a result of the analyses of wave records it was found that three basic sea conditions, viz. NE, ESE and SSE and combinations thereof could be used to represent natural conditions. Quantitative measurements of $10 \mathrm{ss}$ and giln were obtained by measuring the change in the depth of sand over the fixed concrete bed, as well as by photographing contour strings from a traversing gantry tower spanning the model. Quantitative measurements of aredging rates were made by continuousiy weighing accretions "dredsed" with a centrifugal pump and suction hose, the $\delta$ ischarge of which was admitted under water into a constant volume sikiming weighing tank.

Sedimentologlcal time scale. Tests showed that the equivalent of the $3 / 4$ miliion cubic yards sand dredged annually can be moved from one area into another with SSE conditions in about 
four hours when the bed material consists of 250 micron sand. In neture SSE stomy conditions onlv occur during about three per cent of the time, $1 . e .260$ hours ver year. An aporoximate vilue of the sedimentological time scale, for SSE conditions is thus about 65 .

Verification. Because a satisfactory correlation was found between monel and prototype phenomena (wavs, current and sand transport patterns), predictions made from the model regarding changes which might result from the coastal eno ineering structures envisaged in this investigation, may be regarded with confidence.

\section{Results of Model Tests.}

Iittoral Drift. Then not affected by dredging, the rorth bound littoril drift will encroach into the harbor entrance and eventually form a bar across, thus creatine much the same conditions $a \mathrm{~s}$ existed in 1851, but shifted 3000 ft out to sea. Dredging is therefore necessary to prevent the siltation of the harbor entrance. It is the reduction of the necessury maintenance dredging which was almed at with the testing in the model of the various remedial schemes.

From the diagnostic studies it was clear that with the present sand trao dredging technique, the gross northerly drift is removed (3). The main alm of the investigation was therefore to provide conditions which would ensure that only the net northerly drift will have to be dredged.

Standard Test Cycle. The conditions producing littoral drict to the north are SSE swell by itself or in combination with ESE swell, south-to-north current and s'ly wind. Under these storm conditions a jearly accrual in the sand trap of some 800,000 cuble yards, could be simulated in the model in about four hours.

Conditions under which a reduction of deposits in the Cave Rock Bight can be expected are NE swell with NE wind. These two storm conditions were anlied in the model in such a way that the energy distribution corresnonded as near as possible to that found in nature.

\begin{tabular}{|c|c|c|c|c|}
\hline Phase & $\frac{\text { Swel1 }}{\text { direction }}$ & $\frac{\text { Period }}{\text { in sec. }}$ & $\frac{\text { Approximate }}{\text { duration, days }}$ & $\frac{\text { Wind }}{\text { direction }}$ \\
\hline$I$ & SSE & 10 & $\begin{array}{c}\text { (storm) } \\
1\end{array}$ & SW \\
\hline & $\begin{array}{l}N E \\
\text { FSE }\end{array}$ & 8 & 1 & $\mathrm{NE}$ \\
\hline II & SSE & 10 & 2.5 & SW \\
\hline & $\begin{array}{l}\text { NE } \\
\text { ESE }\end{array}$ & $\begin{array}{l}8 \\
8\end{array}$ & $\begin{array}{l}4.5 \\
2.5\end{array}$ & $\begin{array}{l}\mathrm{NE} \\
-\end{array}$ \\
\hline III & $\begin{array}{l}\text { SSE } \\
\text { ESE }\end{array}$ & $\begin{array}{l}\text { 10) } \\
7)\end{array}$ & $\begin{array}{l}\text { combined } \\
4.5\end{array}$ & SW \\
\hline IV & $\begin{array}{l}\mathrm{NE} \\
\mathrm{SSE} \\
\mathrm{NE}\end{array}$ & $\begin{array}{c}8 \\
10 \\
8)\end{array}$ & $\begin{array}{c}2.5 \\
2.5 \\
\text { combined }\end{array}$ & $\begin{array}{l}\mathrm{NE} \\
\mathrm{S} N\end{array}$ \\
\hline & $\mathrm{ESE}$ & a) & 4.5 & $\mathrm{NE}$ \\
\hline
\end{tabular}


The foregoino test sequence consisting of four phases was used for most of the remedial scheme tests, the times given being is for nature, the cycle representing anproximately one vear's storm conditions in nature, (6).

\section{H $\rightarrow$ hor Silting Prevention}

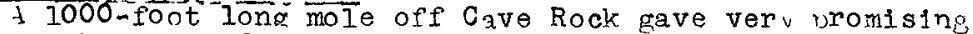
results when tested in the model. To the north of the mole, an oscillation basin is formed between the mole and the South Breakwater which is well protecter for SSE conditions.

Some 0.5 million cubic yards cin be stored in the Cave Rock Bight before broassing of the breakvater starts uncer prolonged SSE swe11. Moreover, some 1.5 million cubic vards can accumult te south of the mole, bringing the total possible accumulation before there is any encroachment into the channel to sorne 4 million cubic virds, or 5 to 7 years storage csoacity for the entre northerly 1 ittoral drift.

Due to NE'Iy conditions, some stind will be moved out of the basin to the south again. Moreover, a large portion of the sand accumulated to the south of the mole is moved south, thus creating new storage capacity for SSE conditions, (Fig. 11), (6).

Beach imnrovement structures tested

A system of $\mathrm{Y}$-groynes with intermediate straight groynes, and the adrition of sours to the Paterson Groynes, yielded positive results in maintaining a beach everywhere exceot at the southern portion of the Lower Marine Parade, where a beach could only be mintained by intermittent sand renourishment or with offshore orotection (Fig. 10).

Offshore breakwaters were investiogted with the object of protoctino existino and artificially created beaches and of building uo new beaches in the lee of the structures. Solid reflecting and semi-solid partip-absorbino structures were tested in the model in a number of positions. A good build-up of the sholtered begches "as generally obtained and the initizl fill was satisfactorily protecter.

The ontimum solution was found to be two 1000 foot long Darallel breakwaters, do roximately 2000 feet offshore. Two flogtin rafts, 900 by 300 feet in the same positions, tested as zlternative measures, resulted in as good a protection of the beaches as the offshore breakvaters woula give.

\section{Summiry of the man conclusions}

a. Although conditions at Durban vary almost daily, neither seasonil nor yearly variations were found to be very significant. The field data collected over a period of two years could be consilered renresentative.

b. Data collected over much longer recording periods than tvo vears have to be orocsssed when analysing geomorinological dita. Nexrshore surveys and dredging records extending many decades, prover an invaluable source of information. Color and size aniljsis and sand samnles from the sea bottom yielded a method for classifyine the sand whicn simolified tne diagnosis of $s \neq n$ i movement 


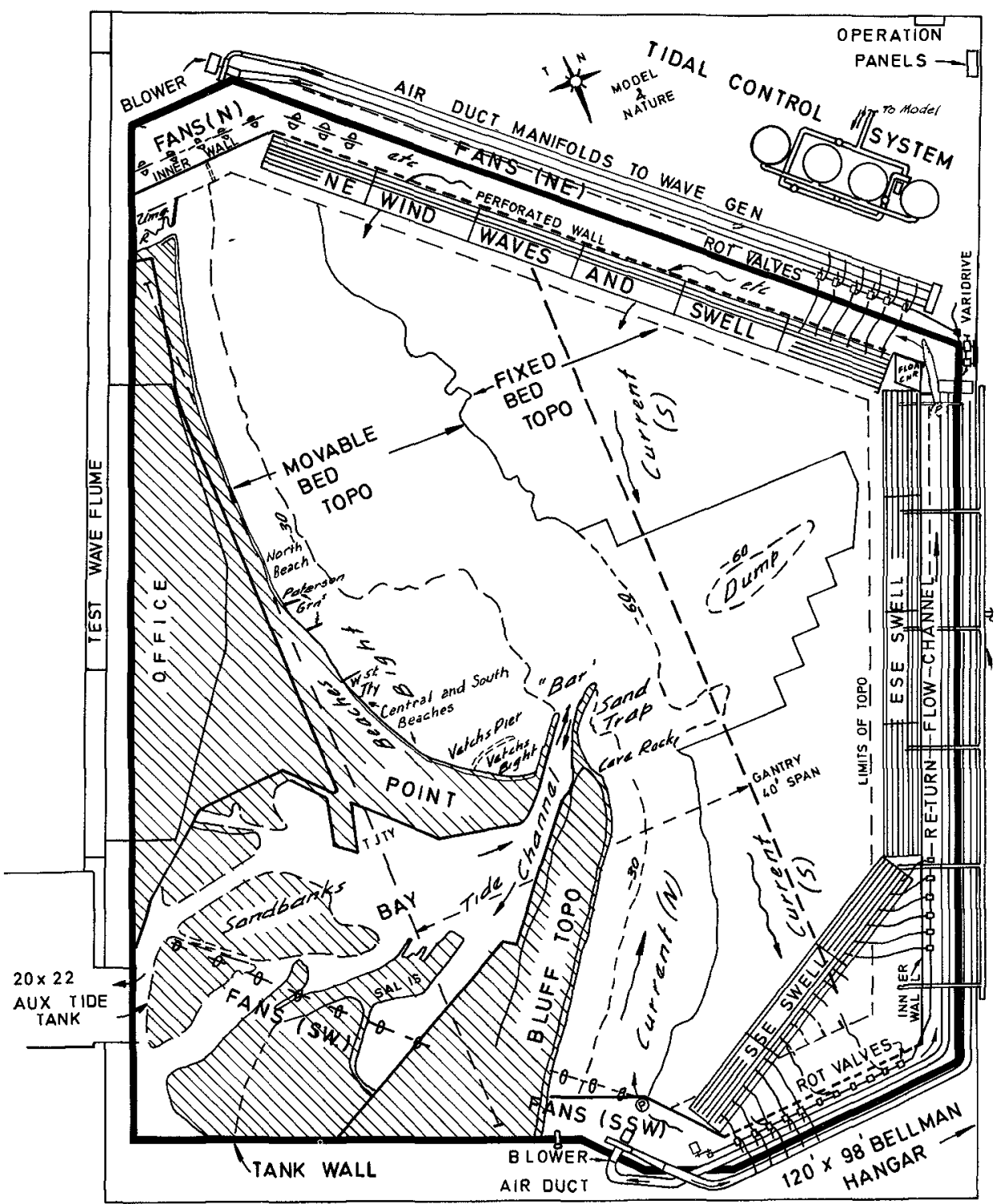

Figure 9 Plan of Durban Coastal Model 


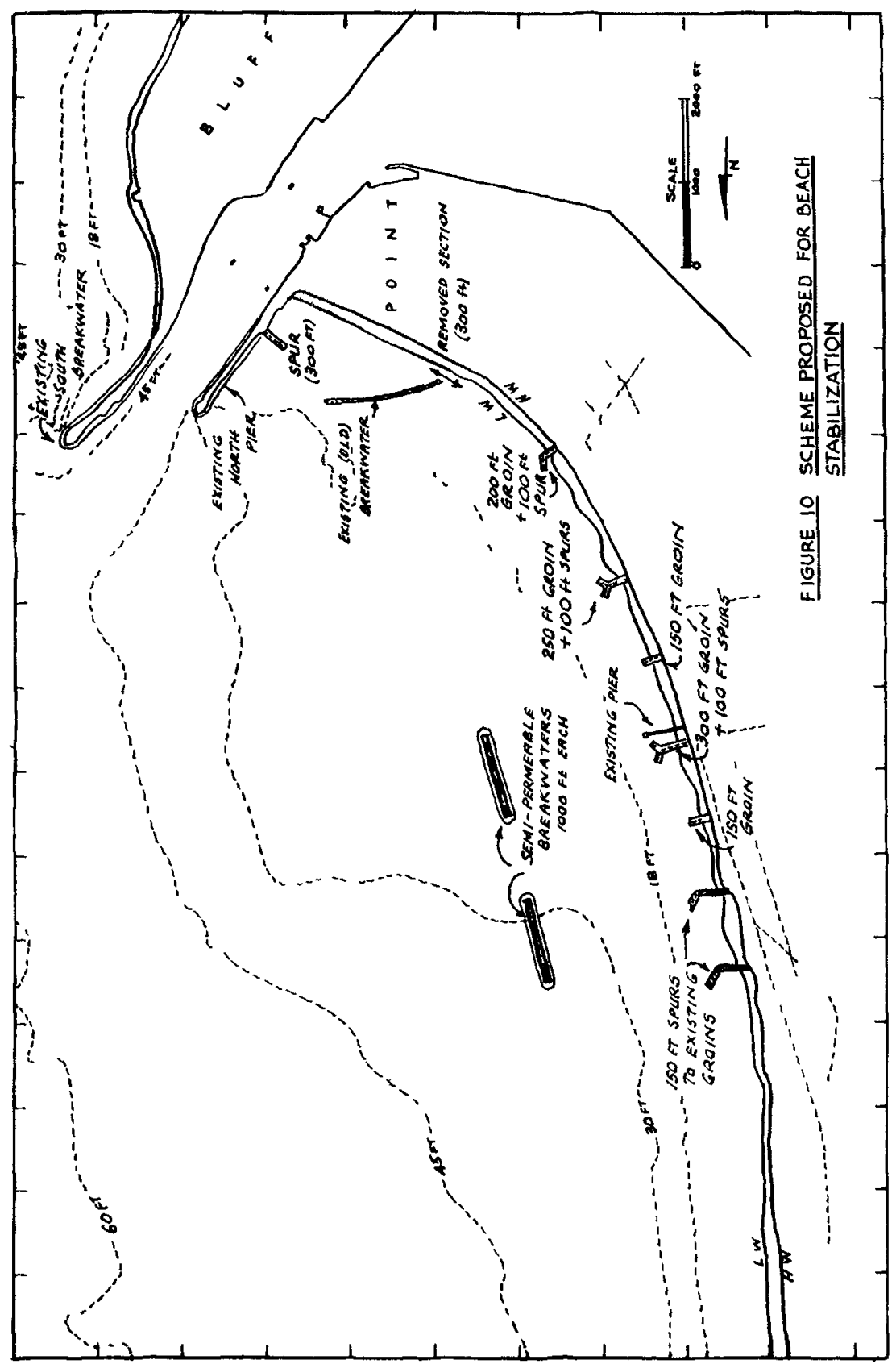

Figure 10. Scheme proposed for beach stabilization. 
c. Prorressive extension of the brejkwiters and deepenins of the hasbor entrance, together nsta the associzted mintenunce tredring, resulte in a cradually incrsising intercention of the northarly littoril irift. The coastal are s nonih and solut of tie entrance coannol can at present be regardel as tvo iniejenast systens with reference to littoral orocesses. Obseivations inficate that the najor movement of sand occurs close to the Soutr PI $r$ tin. Only relatively fine matorial can be carrier in suspension zcross the nirbor channel. The nresent state of the beaches is therefore not mat $\rightarrow$ rally affected by the methor of tredring south of the harbor entrance.

d. Nearshore bottom changes, mainly brovght about ny dumping of sooll, affect wavo refraction in such a way that $a$ concentration of wave ensroy in the Paterson Grovne area resilts for certain sea condjtions(3). Partiy due to this and nartiy due to the prezter sheltering effect caused by the South Brexkline has adantor itsojf into $z$ ne. long-term equillbriun josition, concavo in olan compred with the 1851 conditions. Sezsonal ani shortterm or storm effects can cause a redistribution of bezch material in the bezch and surf zones resultino in possible beach variations of ur to 150 feet in width.

e. If Aredgino is interrupted altogether, the northerly sani drift ovent ially finds its way into the harbor enteance by creevine in, as a narrow bind, around the south Pier tıp. Dredelin is therefore essential. I reduction in maintenance iredgins rate can, however, be achieved when conditions are creater such that only the net northerly littoral druft has to be coned with

It has been found in the model that this can be realised with varlous schemes, of which the one with a 1000 feet lone nole off Cave Rock iroved most advantageous. This scheine orovides for an initial sand storage of some $4 \mathrm{mllilion}$ cublc yards, and anticipated reduction in meintenance dredging rate of some 25 per cent and a reserve storage volume of some 1.5 mililion cubic $y$ ards, $(6)$.

f. As the oresent Bight beaches have been found to be not subject to prorressive erosion, imorovenent schemes were directed towards the formation of beaches in those areas wnere there are none at oresent and the nrotection and stabilisation of newly formed and existing beaches.

A scheme including two 1000-foot long offshore break waters or floating rafts, as well as a system of Y-groynes and additional sroynes has prover to be successful in matntaining a beach everywhere, after the initial fill ( 1 million cubic vards) had been brought in to form a beach in the central and South Bedch areas.

With a groynes-only scheme (Fig. 10) it has been established in the model that, after an initial fill of aporoximately 250,000 cubic yards and with a mintsinance sand pumoing rate of about 10,000 cubic yards ner year, a mean beach widt l above HW of some 55 feet in the Central Bezch area and 250 feet in the Nortl and Sout Beach areas could be mintained. Moreover, the groynes and spurs could successfully reduce seasonal variations to a negligible amount, (5). 


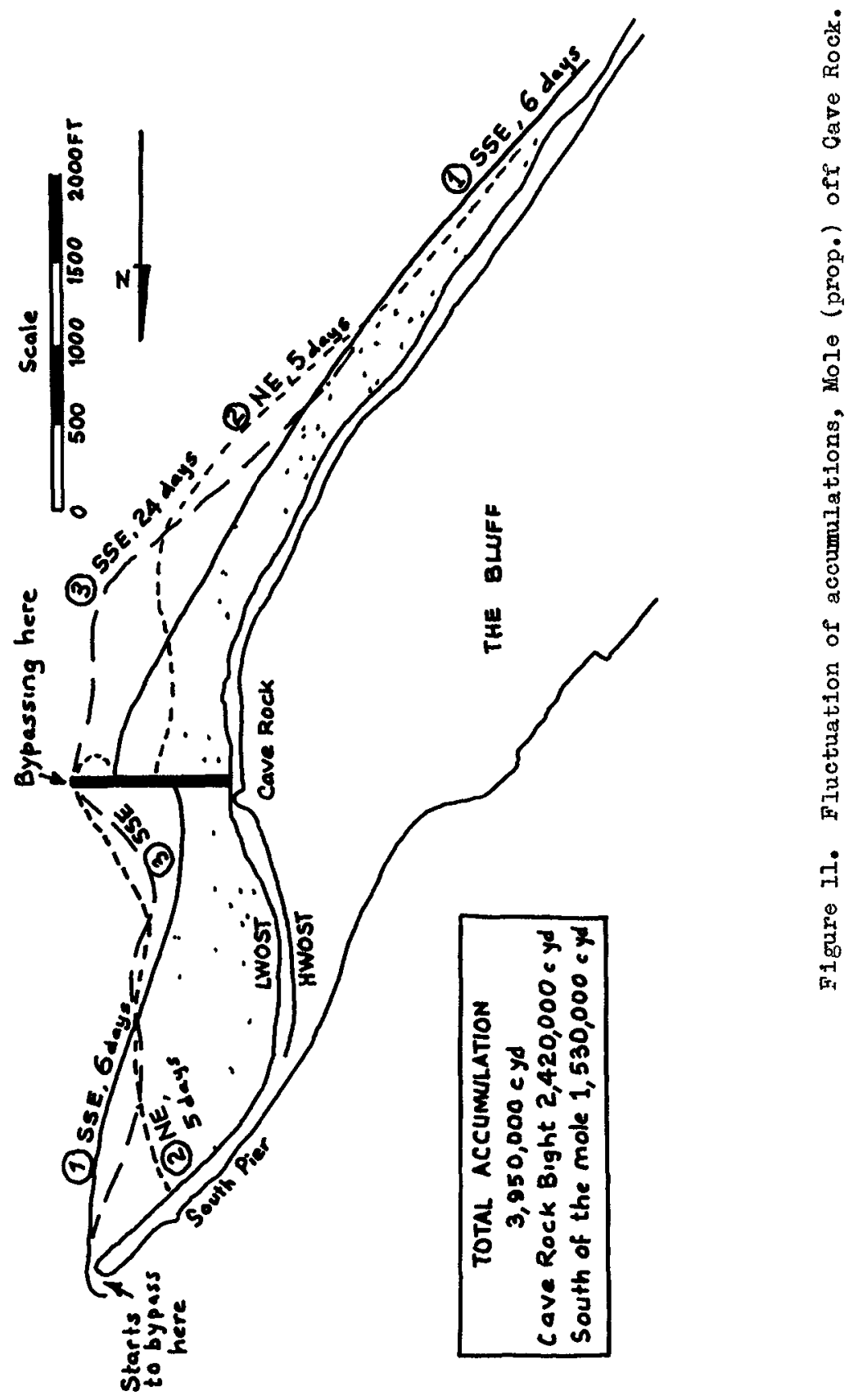


An alternative scheme by which a continuous underwater sand mound be formed some 4000 feet offshore in the Durban Bight seemed to warrant further investigation, and was eventually adopted ( 7$)$.

\section{REFERENCES}

1. Belderson, R.H. Thesis for the degree M.Sc., Geology Department, University of Natal, Durban, 1962.'

2. Jordaan, J.M., Jr. Experience with recording of storm waves, swell and tide using an inverted echo-sounder off Durban (South Africa), International Hydrograohic Review, Monaco, Vol XI, No. 2 , July 1963, po. 125-140.

3. Jordaan, J.M., Jr. Effects of hydrogranhic changes due to near-shore dredger dumping, on wave refraction and littoral san balance. Proc. Ninth Conf. on Coastal Enplineering, Lisbon, Portugal. June 1964, Pub. A.S.C.E., New York, 1964, pp. 310-322.

4. Kinmont, A. The nearshore movement of sediment at Durban. CSIR Symposilum, No. 52, Pretoria, March 1961, pp.46-58.

5. Moffatt, H.R. The develobment of the Harbours of South Africa, Proceedings at the Western Case Regional Convention, 1961, The S. Afr. Inst. of Civil Engineers, pp. 44-52, eso. 49,50 .

6. CSIR Renort CMeg 558 Durban Harbour Siltation and Beach Erosion Investigation, Sept. 1963, Pretoria.

7. Zwamborn, J.A., G.A.W. Fromme and J.B. FitzPatrick. Underwater Mound for the orotection of Durban Beaches. XII Conference on Coastal Engineering, Washington, 1970.

\section{ACKNO NL EDGMENTS}

The author aynreciates the encouragement and assistance from Mr. R.E. Jones, then System Harhour Engineer, Mr. A. Kinmont, then City Engineer, Durban, Dr. H.G. Denkhaus, Director OSIR National Mechanicel Engineering Research Institute and other members of the Technical Committee and their organizations.

Mr. J.A. Zwamborn, Mr.W.G. van Lienden, Mr. J.B. FItzPatrick collaborited over long periods of time on this investigation and their unsparing efforts are greatly appreciated.

The author is indebted to all who contributed their time and efforts to the work herein reported.

Permission to present this paper was granted by the south African Raliways Administration, the city Counc 11 of Durban, and the Council for Scientific and Industrial Research. 


\title{
CHAPTER 71
}

\section{ESTUARY INLET CHANNEL STABILIZATION STUDY USING A HYDRAULIC MODEL}

\author{
Theodore T Lee \\ Associate Researcher \\ Look Laboratory of Oceanographic Engineering \\ University of Hawan \\ Honolulu, Hawan \\ U S A
}

\begin{abstract}
A hydraulic model study was conducted to determine the most feasible means of reducing shoaling into a coastal inlet channel that had been used by loaded Landing Shup Tank (LST) class vessels serving the port of Tan My near Hue, Republic of Vietnam The ultumate objective was to increase the time duning which the channel will be open to navigation by umprovements in physical configuration

An extensive field data acquisition and analysis program was executed to provide basic information for model prototype correlation. Thus incidentally resulted in the re-evaluation of several empincal formulae being used for prediction of littoral transport and of tidal currents The comprehensive field measurements of shoaling in the channel area also permitted the determination of the scale effects on the quantitative results of sediment deposition in the channel and on the time scale of bottom evolution

The experments were conducted systematically in three phases The three-dimensional studies for Phase I and II were conducted using a rigid bed, sand or walnut shell covered (1/250 horizontal and 1/50 vertical scale) hydraulic model of a $15 \mathrm{sq} \mathrm{mi} \mathrm{oceanestuary} \mathrm{land} \mathrm{area} \mathrm{Periodic} \mathrm{long-crested} \mathrm{waves} \mathrm{tides} \mathrm{tide/river} \mathrm{induced} \mathrm{currents} \mathrm{were}$ simulated in the model Phase I covered qualitative evaluation of nine improvement plans, from which a BEST PLAN," an upshore jetty to protect the existing channel, was selected Phase II covered qualitative and quantitative evaluation of four vanants of the "BEST PLAN,' from which the most effective and economical variant was determined To insure rehability of quantitative results, comprehensive experiments were conducted using three different seduments $\left(D_{50}=041 \mathrm{~mm}\right.$ and $090 \mathrm{~mm}, \gamma=135$ for two types of ground walnut shells and $D_{50}=022$ $\mathrm{mm}, \gamma=265$ for sands), and three model scaling criteria (Froude Scaling, "Ideal Velocity" Scaling, and Modified Froude/Ideal Velocity Scaling) Phase III covered two-dimensional model tests, in a 180 foot flume, of the stability characteristics of the proposed rubble mound type jetty on a movable bed

The major conclusions of this study include (1) the jetty, as postulated in the BEST PLAN,' would reduce annual mantenance dredging requirements by 74 to 84 percent, (2) a systematic three phase model study such as used in this investigation is most feasible (from a tume-consumption viewpoint, use of light weight materials such as ground walnut shells may be more economical than use of natural sands as model material), and (3) use of theory alone in predicting a tume scale for bottom evolution should be approached with caution espectally where field data are not readily available
\end{abstract}




\section{INTRODUCTION}

This paper describes the techniques used for a particular coastal inlet stabilization study using a hydraulic model in order to obtain an optumum solution to the excessive shoaling of the existing ship channel The channel in question is located at Tan My, on the South China Sea Coast near Hue Republic of Vietnam The shoaling results from stgnificant storm waves dunng the winter and summer monsoon seasons dominated by wind waves from the Northeast during the period from November to January Sediment contribution from the inland rivers (Huong, Bo and O Lau) is manly deposited in the large estuary area with only negligible wash load diverted into the offshore channel area To maintain a $20 \mathrm{ft}$ deep and $300 \mathrm{ft}$ wide ship channel considerable efforts were expended to keep the channel open by dredging (1 8 million cubic yards per year) Unfortunately, the duration of the relatively safe dredging periods are prohubitively short and infrequent It was therefore considered necessary to construct permanent barner structures in order to protect the Tan My Channel from shoaling so that the channel can be operationally useful for most time with minımum dredging maintenance

The study (Lee, 1970) was sponsored by the Officer in Charge of Construction Republic of Vietnam Naval Facilities Engineering Command Department of the United States Navy

\section{DESCRIPTION OF STUDY AREA}

The subject study covered a small area $(3 \mathrm{mlles}$ by 5 mules) of the coastal line and offshore topography, the inlet, and a portion of the estuary The boundaries of the project area to be modeled were selected on the basis of such criteria as (a) the model should extend to sufficient depth ( 10 fathoms) to obtain correct refraction patterns of waves approaching the shoreline from prevaling directions, including $\mathrm{NE}, \mathrm{N}$, and $\mathrm{NNW}$, around the area of interest, (b) sufficient shore length should be provided to permit cutting a new channel through the spit and to allow a natural development of longshore current by water waves and (c) the boundary condition in regard to the flow should not change as vanous improvement plans for the inlet are studied in the model

\section{OCEAN ENVIRONMENTAL DATA ACQUISITION}

An extensive field data acquisition and analysis program was executed in order to provide basic information for model prototype correlation

Waves and Swell Wave climates simulated in the model were determined from observations by Lyon Associates (1968 1969), NAVFORV Weather, Sa1gon, RVN from wave budget analyses by Marine Advisors (Inman and Harns, 1966), and wave hnd castung by Glenn Associates (1966) The wave rose developed from most recent field observations 1s shown in Fig l(a)

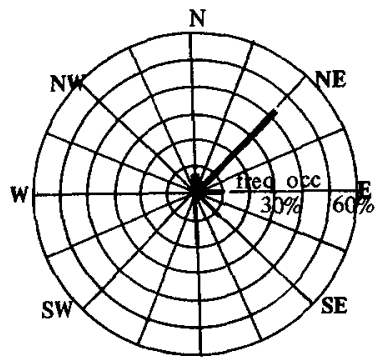

(a) Wave Rose

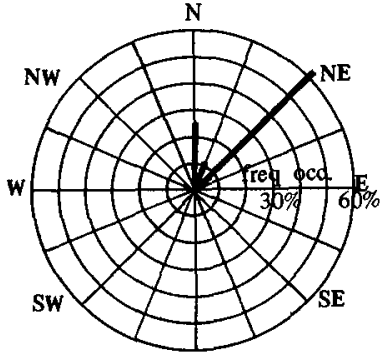

(b) Sand Transport Rose Savage Method Total Annual Sediment Into Channel $1,409000 \mathrm{Cu}$ Yds

Fig 1 Wave and Sediment Transport Distribution

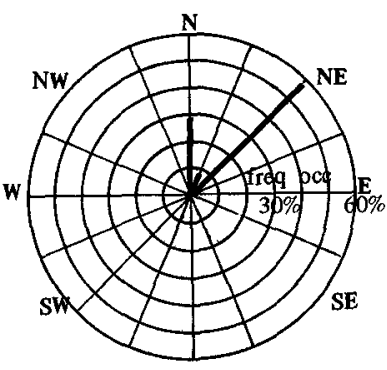

(c) Transport Rose Bowen \& Inman Method Total Annual Sand Transport Into Channel 1 924,000 Cu Yds 
The following "Typical Waves' were used in the model tests for the selection of the "BEST PLAN"

$\begin{array}{lcc}\text { Wave he1ght } & \begin{array}{r}\text { Wave period } \\ \text { (feet) }\end{array} & \begin{array}{r}\text { (seconds) } \\ \text { Severe Storm Wave Condition }\end{array} \\ \text { Normal Storm Wave Condition } & 10 & 8 \\ \end{array}$

Wave directions selected for model tests included NE, N NNW An analys1s of the waves incident on the channel has shown that shoaling in the channel results as follows $60 \%$ from NE $25 \%$ from $N$, and $15 \%$ from NNW and other drections [ $F_{1 g}$ 1(b) and (c)] Furthermore, the wave and swell data, wind information, and weather maps related to the penod of Typhoon Bess $(27$ September 1968) were analyzed It was found that the average wave climate incidentally corresponded to the Normal Storm Wave condition defined above 1 e $5 \mathrm{ft}$ high, 5 second period (from NE)

Tides and Tidal Currents The diurnal tide at Tan My was an average of 20 feet with a tidal period of 25 hours and 35 minutes The semi-diurnal tide was 16 feet high and 12 hours and 47 minutes Tide induced current in the Tan My entrance channel without nver discharge was predicted, using the theoretical method of Keulegan (1967) The maximum velocities predicted compared reasonably well with those measured by Lyon Associates and by the U $S$ Naval Oceanographic Office The field measurements were made of tides and tidal currents at four locations as shown in Fig 2 The Lyon s measurements involved detalled measurements of tidal current velocities covering the whole cross section at 02 and 08 of the water depth, from which the mean velocity through each section can be determined Unfortunately, $t_{1}$ des

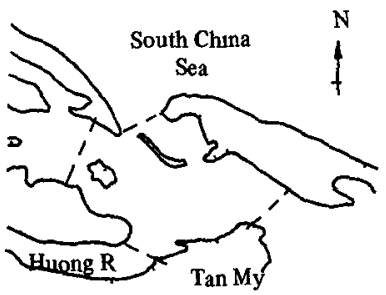

Fig 2 Tide and Current Measurements at Four Statrons were not recorded contunuously and the measurements at the sections were not made simultaneously Ten senes of measurements were made at each station durng the 5 month period from September 1968 to January 1969 The measurements by the Naval Oceanographic Office were made simultaneously at each of the four stations but only currents at one or two points were recorded instead of numerous points at each station as done by Lyon The measurements covered a four month period from December 1968 to March 1969 The field measurements helped verify somewhat the tide and tidal current predictions but it is not feasible to simulate such t1des because the measurements include the effects of waves, nver discharge, and longshore currents Therefore, the tide simulated in the model was based on tide prediction and the tidal currents through the channel according to proper adjustments of tidal prism in the model estuary

The maximum tide prism measured at Tan My was $47 \times 10^{6} \mathrm{~m}^{3} /$ duurnal tide cycle as determined from the maxumum mean tidal discharge of $1500 \mathrm{~m}^{3} / \mathrm{sec}$ measured by Lyon This compared well with the $49 \times 10^{6} \mathrm{~m}^{3} / \mathrm{drurnal}$ trde cycle as estimated by Inman and Harris (1966)

The tide and tidal currents sumulated in the model are considered adequate because the low tide range does not significantly affect the sand transport in to the channel

Hydraulic Regime Offshore Tan My Inlet For calibration of the model and study of the flow conditions in the inlet area, it was necessary to obtain current patterns These patterns were obtained by the Naval Oceanographic Office using infra red techniques

Huong (perfume) River Flow Based on frequency of occurrence of the Huong River flow, $500 \mathrm{~m}^{3} / \mathrm{sec}$ and 1,800 $\mathrm{m}^{3} / \mathrm{sec}$ were selected to simulate the normal and severe flood conditions This seems to be high, but it would compensate for the omission of the contributions from the Bo and $O$ Lau Rivers and reduce the scale effects of the use of sands as bed materals (Flow measurements at Huong River duning a 4 month period showed the maximum discharge was $1,250 \mathrm{~m}^{3} / \mathrm{sec}$ and averaged $300 \mathrm{~m}^{3} / \mathrm{sec}$ )

Longshore Currents Offshore ocean currents upcoast of the Tan My lnlet were measured with float technques but the measurements are not utulized because they were made too far offshore to be within the model limits Subsequently, longshore currents of $20 \mathrm{ft} / \mathrm{sec}$ and $40 \mathrm{ft} / \mathrm{sec}$ were predicted by theory and simulated in the model for normal storm wave ( $5 \mathrm{ft}$ high, 5 second penod) and for severe storm wave ( $10 \mathrm{ft} \mathrm{high,} 8$ second period) conditions respectrvely 
Sediment Properties. Beach samples were taken upcoast of the Tan My Inlet. The typical size distribution of sediment-the median diameter $\left(D_{50}\right)$-was $0.41 \mathrm{~mm}$. The sediment is 100 percent quartz, containing no carbonate, with a density of 2.65 . The size distributions of both prototype and model sediments are shown in Fig. 3 .
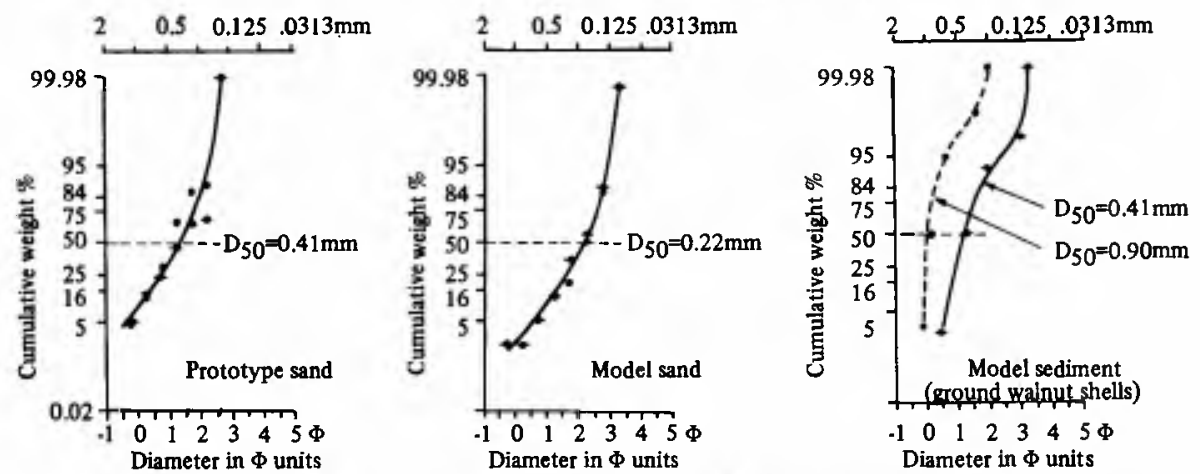

Fig. 3 Size Distribution of both Prototype and Model Sediments

Coastal Morphology. The shoreline evolution around Tan My lnlet from April through December 1968 was studied from the analyses of monthly uncontrolled aerial photographs. These photographs showed the unstable nature of the Tan My Inlet. A comparison of the coastal morphology at Tan My during August, September, and December 1968 is shown in Fig. 4. The stability parameter for Tan My is compared with other similar inlets in Table 1.

Shoaling and Dredging Information. An extensive study was made of the shoaling and dredging aspects at the Tan My existing entrance channel. Included are: annual sediment budget, sediment transport during a special storm (Typhoon Bess), bottom topography changes, and dredging studies. It was found that the total sediment transport into the existing channel amounted to 2.1 million cubic yards (1.6 $\times 10^{6} \mathrm{~m}^{3} /$ year) as compared with 1.4 to 19 cubic yards per year $\left(1.1\right.$ to $1.5 \times 10^{6} \mathrm{~m}^{3}$ /year) predicted by theories of Savage (1959) and Bowen-Inman (1966) respectively [see Fig. 1(b) and (c)]. Based on the estimated inlet stability parameter (Bruun, 1969) the annual sediment transport would be 1.4 million cubic meters or 1.8 million cubic yards. This estimate was the basis for economic evaluation of the improvement plans.

Summary of Ocean Environment Data for Model Tests. The ocean environmental data selected for the model tests are summarized in Table 2.

Fig. 4 Coastal Morphology at Tan My Inlet

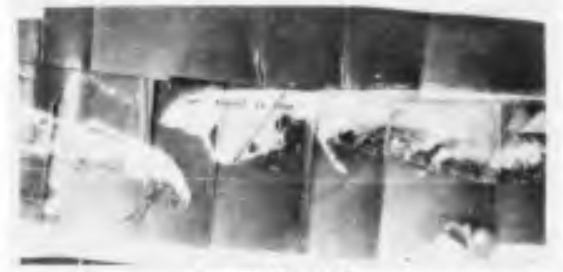

August 22, 1968

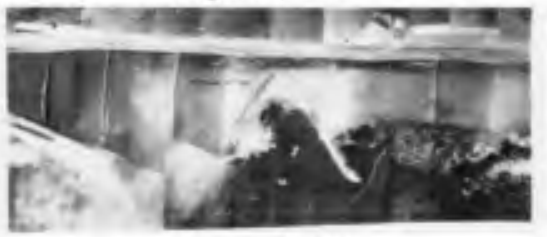

September 29, 1968

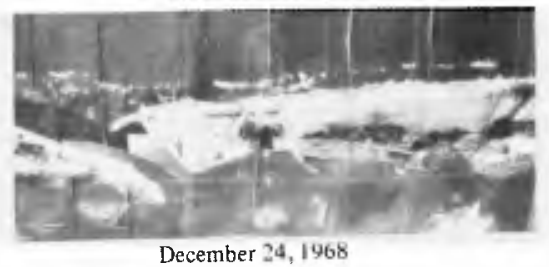

December 24, 1968 
Table 1 INLET STABILITY

\begin{tabular}{|c|c|c|c|c|}
\hline Inlet & $\begin{array}{l}\text { Tide Prism }(\Omega) \\
\left(\mathrm{m}^{3} / \mathrm{t}_{1} \text { de cycle) }\right.\end{array}$ & $\begin{array}{l}\text { Sand Transport }(M) \\
\left(\mathrm{m}^{3} / \text { year }\right)\end{array}$ & $\frac{\Omega}{\mathbf{M}}$ & Stability* \\
\hline $\begin{array}{l}\text { Tan My } \\
\text { Model } \\
\text { Savage } \\
\text { Bowen \& Inm } \\
\text { Dredge Recor }\end{array}$ & $47 \times 10^{6}$ & $\begin{array}{l}150 \times 10^{6} \\
108 \times 10^{6} \\
146 \times 10^{6} \\
161 \times 10^{6}\end{array}$ & $\begin{array}{l}31 \\
43 \\
32 \\
29\end{array}$ & \\
\hline Average & & $140 \times 10^{6}$ & 34 & Very Poor \\
\hline \multicolumn{3}{|c|}{ Masonboro, N C (Bruun, 1966) } & 30 & Very Poor \\
\hline \multicolumn{3}{|c|}{ Ponce de Leon, Fla (Bruun, 1966) } & 40 & Poor \\
\hline \multicolumn{3}{|c|}{ Figueira da Foz, Portugal (Bruun \& Gerritsen, 1960) } & 40 & Poor \\
\hline \multicolumn{3}{|c|}{ Oregon, N C (Bruun \& Gerritsen, 1960) } & 80 & Poor to Falr \\
\hline $\begin{array}{l}\text { * Very Poor } \\
\text { Poor } \\
\text { Poor to Farr } \\
\text { Farr } \\
\text { Good }\end{array}$ & $\begin{array}{l}\text { very much shoaling } \\
\text { much shoaling } \\
\text { significant shoaling } \\
\text { some shoalıng } \\
\text { negligible shoaling, } \frac{\Omega}{M}=100\end{array}$ & & & \\
\hline
\end{tabular}

Table 2 SUMMARY OF OCEAN ENVIRONMENT DATA FOR MODEL TESTS

\begin{tabular}{|c|c|c|}
\hline Envuronment & $\begin{array}{c}\text { Normal } \\
\text { Storm Condition }\end{array}$ & $\begin{array}{c}\text { Severe } \\
\text { Storm Condition }\end{array}$ \\
\hline \multicolumn{3}{|l|}{ Wave and swell } \\
\hline Wave height $(\mathrm{ft})$ & 5 & 10 \\
\hline Wave period (sec) & 5 & 8 \\
\hline Wave direction & NE, N, NNW & NE, N, NNW \\
\hline \multicolumn{3}{|l|}{ Tide } \\
\hline \multicolumn{3}{|l|}{ Diurnal } \\
\hline Tide range $(\mathrm{ft})$ & 2 & 2 \\
\hline T1de period or cycle & $25 \mathrm{hr}, 35 \mathrm{~min}$ & $25 \mathrm{hr}, 35 \mathrm{~min}$ \\
\hline \multicolumn{3}{|l|}{ Semi Diurnal } \\
\hline Tide range $(\mathrm{ft})$ & 16 & 16 \\
\hline Tide period or cycle & $12 \mathrm{hr} \quad 47 \mathrm{~min}$ & $12 \mathrm{hr}, 47 \mathrm{~min}$ \\
\hline Tide Prism & \multicolumn{2}{|c|}{$47 \times 10^{6} \mathrm{~m}^{3} /$ diurnal tide cycle } \\
\hline Huong River Discharge $\left(\mathrm{m}^{3} / \mathrm{sec}\right)$ & 500 & 500 and 1,800 \\
\hline Longshore Current (ft / sec) & 1820 & 3640 \\
\hline \multicolumn{3}{|l|}{ Sediment Property } \\
\hline Medium diameter $\left(\mathrm{d}_{50}\right)$ & $041 \mathrm{~mm}$ & $041 \mathrm{~mm}$ \\
\hline Density & 265 & 265 \\
\hline \multicolumn{3}{|l|}{ Annual Sand Deposition into } \\
\hline Channel (used in study) & \multicolumn{2}{|c|}{$18 \times 10^{6}$ (cu yds /year) } \\
\hline
\end{tabular}




\section{THE TAN MY ESTUARY MODEL}

Design of Model The model (Fig 5) has the following linear model scale ratios
(a) Horizontal Scale
(b) Vertical Scale
$1 / 250=n_{1}=\lambda$
$1 / 50=n_{d}=\mu$
$1 / 5=n_{d} / n_{1}=\mu / \lambda=\Omega$
(c) Model Distortion

$$
\begin{aligned}
& 1 / 50=n_{d}=\mu \\
& 1 / 5=n_{d} / n_{1}=\mu / \lambda=\Omega
\end{aligned}
$$

The vertical scale and model distortion were selected with due consideration of the scale effects, the artificial sediments to be used in the model, and prototype beach slope In general most models of movable bed have a distortion ratio from 13 up to 18 The natural beach slope at Tan My is relatively gentle (1 60) and the equilibrium beach slope is changed with wave characteristics, thereby affecting the decision of choosing a single model distortion to satisfy all equilibrium beach slope conditions, therefore a 15 model distortion was selected The selection of the vertical scale does not materially affect model results because the model has a semi movable bed rather than a movable bed the foreshore and offshore beach slopes were maintaned constant during the tests because the natural beach slope is relatively flat

Simulitude Relationship The model and prototype scale relationshıp for the Tan My Estuary Model for the Phase I and early Phase II tests is summarized by Lee (1970)

The following references were helpful Fan and LeMehaute (1969), Bijker (1967) Bruun, et al (1966)

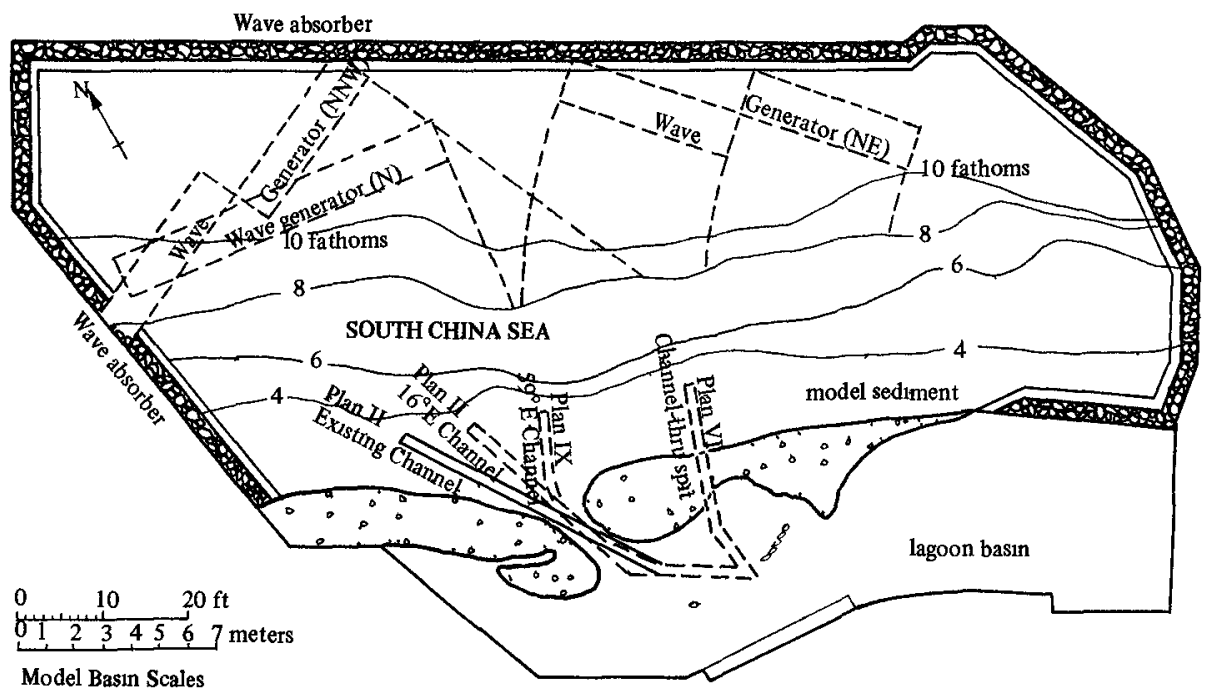

Fig 5 General Layout of Tan My Estuary Model 


\section{EXPERIMENTAL EQUIPMENT AND PROCEDURE}

\section{Ocean Environment Simulation, Measuring and Recording Apparatus}

Wave Generators The wave generator for the three dimensional model tests was of the plunger type, capable of simulatıng long crested, periodic water waves The wave generator for the 180 foot long wave flume was also of the plunger type, with a unique control unit The generator is driven by a high torque, low speed hydrostatic pump motor The hydrostatic drive is infinitely variable in speed in either direction off detent center from 1 to $60 \mathrm{rpm}$ The variable displacement hydraulic pump is driven at a constant speed by means of a $20 \mathrm{hp}, 1750 \mathrm{rpm}$ motor

Tide Generator Tides of durnal and semi-durnal types were sumulated by alternately pumping and dranning water to and from the model basin The outer ocean basin and inner lagoon basin were controlled separately The tide generator was designed to reproduce tidal discharge through the inlet rather than tidal elevation differentials between the ocean and bay lagoon The tide generator had a unique electro pneumatic flow control system which was simple and economical It was calibrated by a cam arrangement to reproduce inlet flow by changing electrical voltage which in turn controlled the valve opening of the pipe lines

River Flow Generator Huong River discharge was simulated by spillıng water over a V weir into the estuary near the river mouth through a pumping system

Other Measuring and Recording Systems Time-exposure photographs were used extensively to provide information on the speed and direction of currents in the inlet area and to llustrate graphically the eddy locations and current patterns This helped evaluate the hydraulic regime of each particular improvement plan tested in the model Current through the channel entrance was measured by timing the travel of a surface float over a known distance Waves were measured by means of optical (Paimer, 1970) and electrode resistance type wave guages Tidal current and tide level variations were measured respectively and intermittently by float and point guage techniques over the entire tide cycle to verify the outputs which had been calibrated River flow was measured by a V shaped weir Motion p1ctures were taken to document the pertinent tests of the entire study

Experimental Procedure The followng experımental procedure was followed

1 Calıbrate the three dimensional modcl after a senes of preliminary tests to determine the most significant parameters which would affect the flow characteristics and the quantity and distribution of sediment transport and deposition in the channel Relative effects of waves, tıdes, river discharge type of model sediment, artificial roughness were thoroughly investigated

2 Conduct Phase 1 tests covernng qualitative evaluation of nine channel improvement plans (Fig 6) from which a 'BEST PLAN" (an upshore jetty to protect the exusting Tan My channel) was selected

3 Conduct Phase II tests covering the qualitative and quantitative evaluation of four variants (Fig 7) of the BEST PLAN" from which the most effective and economical variant was determined

4 Conduct Phase III tests in a large wave flume at a much larger scale to determine the stability characteristics of the rubble mound type jetty of the BEST PLAN' aganst normal and severe storm wave actions

\section{MODEL STUDY AND RESULTS}

Proof of Mode1 The model base line is the quantity and distribution of sand deposited in the existing channel during a 35 day period, 9 August to 13 September 1968, which covered the period of Typhoon Bess, 31 August to 7 September Field data on channel configuration at the beginning and end of the period were also avallable, as well as the quantity and location of dredging during the period Excitation during the period was not measured However, hindcasting procedures indicated that it likely was the Normal Storm Condition as shown in Table 1 Coincidentally, this condition also represents the average wave condition during the NE wave monsoon season Therefore, model prototype correlation was also made of shoaling caused by normal, or long term wave action

Because the orientation of the channel is unclear and the river flow through it during Typhoon Bess is unclear and shoaling is sensitive to these factors proper proof of the model on a quantitative basis was not possible Therefore, only qualitative model prototype correlation was achieved during the Phase I tests Subsequently, comprehensive tests with seven scaling criteria were conducted to relate their relationship quantitatively under Phase II tests which will be discussed later

Furthermore, the favorable reproduciblity of the bottom topography at the inlet [Fig 6(a)] and of the hydraulic regime (current pattern) in the model leads to the belief that the model prototype correlation is considered adequate with the data avalable for the purpose Preliminary viewing of the imagery indicated that the eddy near the mouth of the Tan My Channel in the model appears also in the imagery [Fig 8] The patterns appear to be consistent with a 

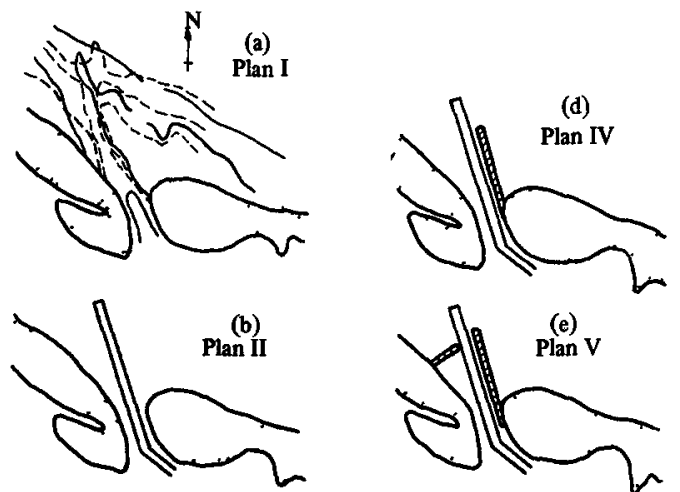

(c)
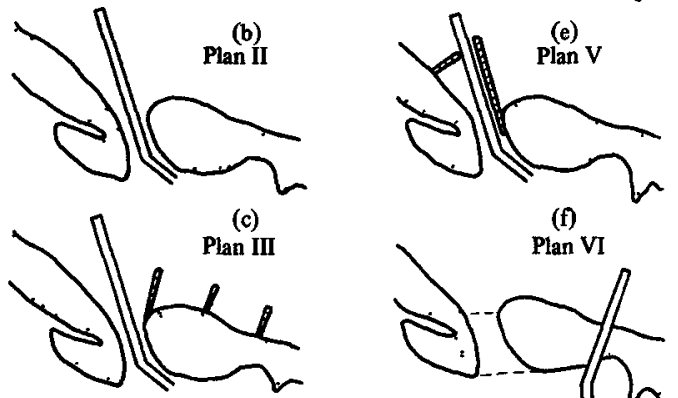

(f)
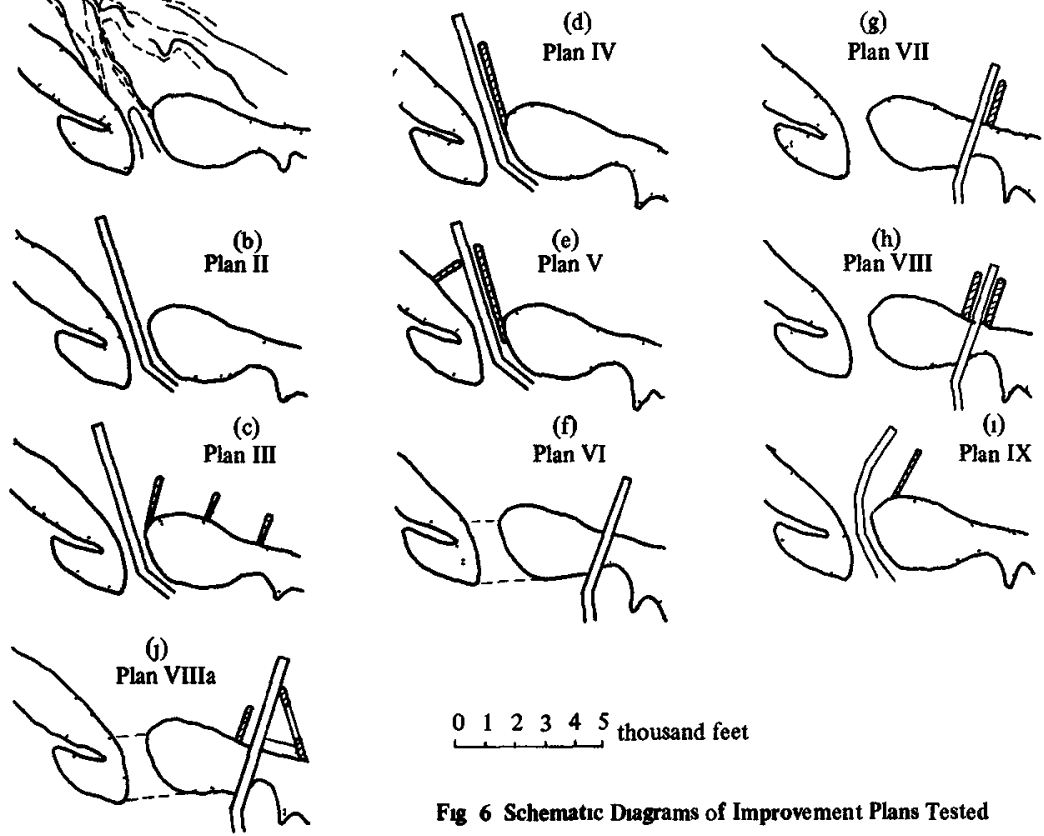

\section{$\begin{array}{llllll}0 & 1 & 2 & 3 & 4 & 5 \\ & & \text { thousand feet }\end{array}$}

Fig 6 Schematic Diagrams of Improvement Plans Tested

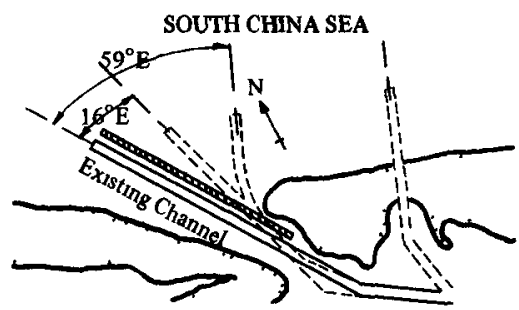

(a) Plan IV a 6000 foot East Jetty

SOUTH CHINA SEA

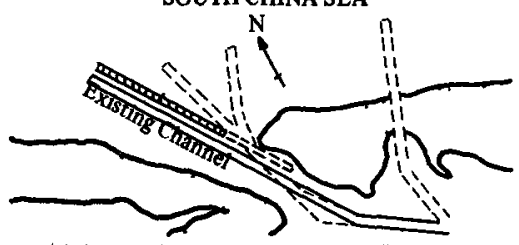

(b) Plan IV b or IV-b 4,000 foot East Jetty
SOUTH CHINA SEA

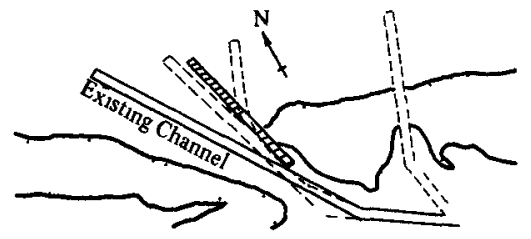

(c) Plan IV-c 4,500 foot Jetty Weir Sand Trap System

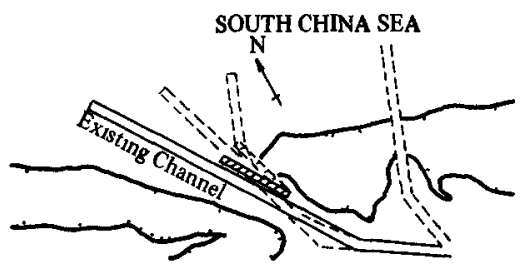

(d) Plan IV -d 2,000 foot East Jetty

Fug 7 Schematic Dragrams of Four Varants of "BEST PLAN Tested 
postulated, relatively small, clock-wise eddy just off the inlet (Wiemat, 1969). This favorable prototype-model relationship helped increase confidence in the model results. The distribution of peak shoaling area is consistent with actual prototype conditions observed over long periods of time under normal conditions. Also, the favorable comparability of the littoral transport (Table 3) by model, prototype, and prediction, and favorable comparability of measured and predicted tidal current (Table 4) have encouraged the preceeding of the model tests for subsequent test and evaluation of a variety of channel improvement plans.

Table 3. COMPARABILITY - LITTORAL TRANSPORT

Method/

Source

Model

measurement

Measured

Prototype

Prediction

",

,

,

\section{Calculated from}

dredging records

Caldwell (1956)

Savage (1959)

Bowen \& Inman (1966)

Bijker (1968)

Littoral Transport (cu. yds./day)

(1) Normal wave (2) Storm wave NE, $5 \mathrm{ft} ., 5$ sec. $\quad$ NE $10 \mathrm{ft}$, 8 sec.

Ratio

(2) $/(1)$

10,500

79,900

9,700

$\begin{array}{rll}10,500 & 53,750 & 5.13 \\ 18,500 & 50,000 & 5.90 \\ 8,800 & 69,000 & 7.85 \\ 6,450 & 48,900 & 7.59\end{array}$

Table 4. COMPARABILITY OF MEASURED \& PREDICTED TIDE CURRENT

$\begin{array}{lcc} & \text { max. mean current vel. } & \text { max. surface current vel. } \\ \text { Measured in model } & 0.093 \mathrm{ft} . / \mathrm{sec} . & 0.210 \mathrm{ft} . / \mathrm{sec} . \\ \text { Predicted by Keulegan (1967) method } & 0.078 & 0.175 \\ \text { Error } & 16 \% & 17 \%\end{array}$

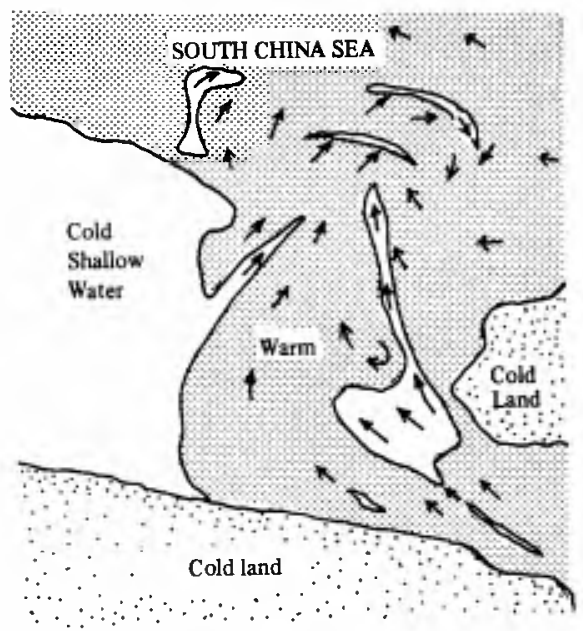

(a) Prototype Circulation Pattem Infrared Imagery June 24, 1969

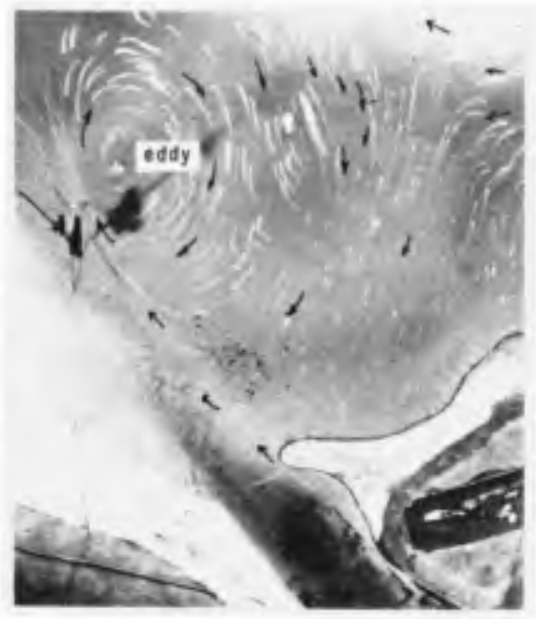

(b) Model Circulation Pattern

Fig. 8 Comparison of current circulation patterns, prototype vs. model 
Phase I Qualitative Evaluation of Improvement Plans Phase I covered qualitative evaluation of nine (9) 1mprovement plans [Fig 6] from which a "BEST PLAN," an upshore jetty to protect the existing channel, was designated These sedumentation reduction plans involved four channel configurations These were the existing channel two channels rotated about $16^{\circ}$ and $59^{\circ}$ East, relative to the existing channel and a new channel relocated through the spit about one mile upshore of the existing entrance All these channel configurations (except the $59^{\circ} \mathrm{E}$ channel) were protected by grouns and/or jetties upshore In some cases downshore jetties were tested as well (Plans tested are shown schematically in Fig 6)

The quantity and distribution of sand transport into the channel for vanous test plans, as measured in the model are compared in Fig 9 It $1 \mathrm{~s}$ shown that Plan IV, V, or VIII provides better protection from shoaling of the navigation channel, $1 \mathrm{e}$ the existing channel or a new channel cut through the spit Therr relative merits will be described below

The four unprotected channels [Fig 5]-two associated with Plan II, existing and $16^{\circ} \mathrm{E}$, and IX and VI-behave simularly as a function of their onentation relative to the direction of the incident wave when the other excitations are constant, 1 e river, Iongshore, and tidal currents

Their behavior is highlighted by a dominant constant river outflow which (both aided and opposed by the wave as well as by tidal and longshore currents) tends to generate eddies up and downcoast of the entrance, this action, in turn, builds a bar on the upcoast side and scours a hole on the downcoast side

At the channel entrance the wave generated current (mass transport plus oscillatory) tends at significant times to transport sediment into and up the channel Thus the quantity of sedıment (Q) deposited in the channel should be a function of the ratio of component (Py) of the wave generated power (P) parallel to the centerline of the channel proportional to $P$ This appears not only to be the normal case, but also rougly a linear case for Test Condition $I$ with NE waves with $\mathrm{H}_{0} / \mathrm{L}_{0}=040$ incident on the four channels as indicated by a plot of Py/P versus $\mathrm{Q} / \mathrm{ft}$ of channel [Fig 10] where $H_{\circ}$ and $L_{\circ}$ refer to deep water wave height and length respectively, even though Johnson (1965) and others indicate transport should be dominantly offshore when $\mathrm{H}_{\mathrm{o}} / \mathrm{L}_{\mathrm{o}}=0025$

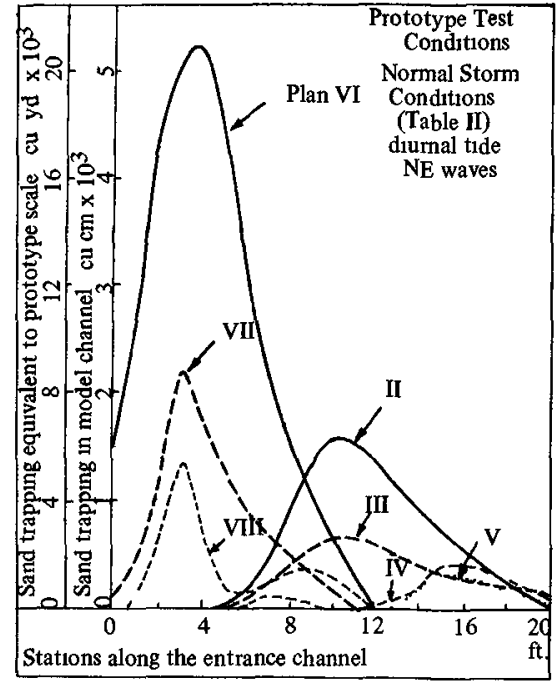

Fig 9 Comparison of Relative Ments of Various Plans for the Existing Entrance and New Entrance through the Spit

Fig 10 Correlation of Sediment Deposition in Four Channels Tested

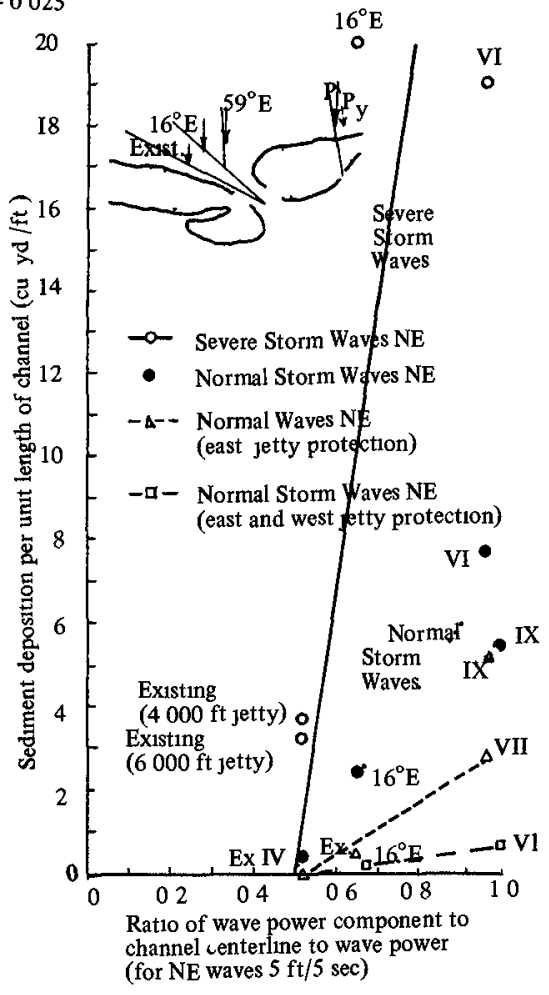


A review of these data indicates that the existing, $16^{\circ}, 1 \mathrm{X}$ and VI channels are sediment retarders in that order of effectiveness this is attributable to the fact that the channel centerlines are $64,49,5$, and 17 degrees, respectively, to the NE direction of the incident wave in deep water That is, the channel (existing) closest to perpendicular to the incident wave crests is the most effective sediment retarder

The four channels (two associated with Plan $1 \mathrm{I}-$-existing and $16^{\circ} \mathrm{E}$-and $1 \mathrm{X}$ and $\mathrm{Vl}$ ), when protected by a full length upshore jetty (Plans IV and VII), and partial length groin well upshore (Plan IX), behave predictably This predictability is, as in their unprotected condition somewhat a function of channel "heading" relative to the direction of incident waves The protected versions in all cases are more effectively self cleaning channels

An upshore jetty is considered entirely adequate as a channel protector The addition of a downshore jetty adds a quite small increase in effectiveness at the expense of inducing undesirable eddies at the entrance and is not recommended

It was found that the unprotected ant shoaling effectiveness of the four channels (existing, $16^{\circ} \mathrm{E}, 59^{\circ} \mathrm{E}$ and through the spit) varies inversely as the heading into the incident waves The existing channel with full upshore jetty is most effective (and the channel through-spit the least effective) as designated "BEST PLAN

An upshore jetty werr sand trap system, such as at Masonboro Sound $\mathrm{N}$ Carolina, is no more effective as a total sedimentation retarder than a conventional system However, it reduces sedumentation into the channel (balance in trap), and hence ments further study only if preservation of downshore configuration is important

The reproducibility of the magnitude and distribution of sand deposited in the channel from run to run is close when the beach is stabilized, $1 \mathrm{e}$ after two tıde cycles for normal waves and four to six cycles of severe storm waves There is a variation of 1 to 12 percent, which is quite acceptable

Sand transport measured in the prototype and model compare well with those predicted theoretically by Savage (1959) and Caldwell (1956) for normal to moderate waves, and Inman (1966) for storm waves The comparison is poor for those predicted by Bijker (1968) ie 40 percent lower than those measured in the model and predicted by other methods (see Table 3)

Phase II Qualitative and Quantitative Evaluation of the BEST PLAN' Phase II covered qualitative and quantitative evaluation of the most effective and economical variant of the "BEST PLAN" This phase of the study involved determination of the optimum length and onentation of the jetty among four variants [Fig 7] To insure reliability of the quantitative results, a series of comprehensive experiments were conducted with sand $\left(D_{50}=022 \mathrm{~mm}, \gamma=265\right.$ ) and two sizes of light weight, ground walnut shell material $\left(D_{50}=041\right.$ and $\left.090 \mathrm{~mm} \quad \gamma=135\right)$ as model sedument Three different model-similitude criteria were used in these tests, 1 e, Froude Law Scalıng 'Ideal Velocity Scaling, as proposed by Bijker (1967) of Delft University, and "Modıfied Froude Law/ldeal Velocity" scalıng, as proposed by the Look Laboratory The time scale of bottom evolution was determined expermentally for each case and compared with theory

Three versions of the "BEST PLAN" included jetties immediately upshore (east) of the existing channel, with lengths of $6,000,4,000$ and 2,000 feet, located along the channel bank a fourth version included a 4,500 foot jetty upshore of the channel bank to form a jetty weir sand trap system [Fig 7]

Three model simulitude critena were employed

Froude Cnteria $\quad n_{v}=n_{u}=n_{d}^{1 / 2}, n_{H}=n_{d} n_{T}=n_{d} 1 / 2$

where $n=$ scale ratio $=$ value of model $/$ value of prototype

$v$ and $u$ designate respectively current (tidal or river) velocity, and orbital velocity (wave)

$\mathrm{d} \mathrm{H}$, and $\mathrm{T}$ designate vertical depth, wave height, and wave period

Ideal Velocity Criteria

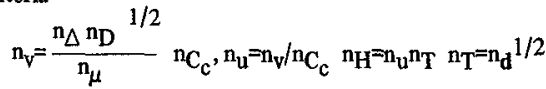

where $\Delta, \mathrm{D}, \mu$ and $\mathrm{C}$ designate respectively relative apparent density of sediment, mean grain diameter, npple coefficient, and resistance coefficient 
Modified Froude/“Ideal Velocity" Critena

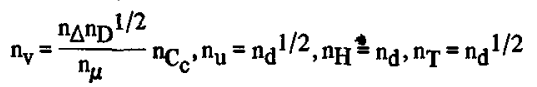

1 use Froude Critena for wave characteristics, and “Ideal Velocity' Critena for current velocity (tidal and river flow)

The primary objectives were to

(a) determine the approprate tume scales of sediment deposition, frequently referred to as time scales of bottom evolution,

(b) determine the jetty effectiveness in each case,

(c) determine annual mantenance dredging requirements after the jetty is bult,

(d) determine the effect of by passing when the jetty is built

Froude Law Scaling tends to distort sediment transportation simularity particularly in areas where oscillatory wave action is absent However, this wave action tends to reduce the critical shear stress and/or velocity necessary for initial movement of material in the zone of littoral dnft over that without wave action For this reason, Froude Law Scaling may stll be reasonable for use in wave induced sediment transportation

Ideal Velocity Scaling relationship is established by Bijker (1967) with due consideration of shear stress sumilitude for both tidal/river currents and wave orbital velocities toward reducing sumilarity distortion likely when Froude velocty scaling is used This criterion requires that both the tidal/river and wave orbital velocities be exaggerated, hence the height of the waves and of the jetty which obstructs them must be exaggerated

Modified Froude Law/Ideal Velocity scaling as established by the Look Laboratory is designed to reduce the wave height and model exaggerations necessary when "Ideal Velocity' scaling is used The tidal/river current velocities required in 'Ideal Velocity" scaling and the waves required in "Froude Law' scaling are used in the 'Modified Froude Law/Ideal Velocity' scaing The simulitude relationshup of these criteria is shown in Table 5

Froude Law scaing criterza were applied to the tests in which sand was used as sediment lack of time and inadequate wave-generator capacities precluded application of two other scalıng criteria to tests with sand However all three critena were applied in tests using ground walnut shells as sediment material (in two grain sizes, D50 $=041 \mathrm{~mm}$ and $090 \mathrm{~mm}$, respectively) However, when larger walnut shell material was used the desirable similitude relationship for "Ideal Velocity' criteria and "Modified Froude Law/Ideal Velocity" criteria were adjusted as shown in Table 5 It was impractical to perform required exaggerations in wave height and tidal/river velocities because of limitations in the wave generator

Test Procedure Excitation was by normal and severe storm waves from the NE, on the existing channel with or without upshore east jetty protection

Because the rate of sediment transportation and deposition of ground walnut shell material is much faster than that of sand, the test was correspondingly shorter, $1 \mathrm{e}$ periods of much less than a complete tide cycle, but including portions of both ebb and flood tides After a view of the tidal/river current pattern obtained previously with sand for Plan II, it was concluded that there are ebb currents in the channel during approxumately two thirds of a tide cycle, and flood currents dunng the remaining one-third penod The sediment deposited in the channel during a portion of tide cycle and during operation of ebb and flood currents (for 15 and 10 minutes respectively) was measured These test results were used to extrapolate the anticipated deposition over a complete tide cycle (on a basis of proportional contributions, two third ebb current and one third flood environment)

The time scale for each case is dependent on the amount of sediment passing over the channel Every eftort was exerted to avoid a condition in which the model channel would be filled to capacity therefore, there was unnecessary by pass of bed load material Tests of short duration have resulted in negligible by passing over the channel during normal and severe storm wave conditions The time scale under no bypassing condition was used to predict sediment deposition in the channel Furthermore, deposition by waves from $\mathbf{N}$ and NNW was estimated using depositions experienced in earlıer tests with sands This is considered feasible because the "BEST PLAN' does not provide significant protectıon against $\mathrm{N}$ and $\mathrm{NNW}$ waves, duration is sıgnificantly short 


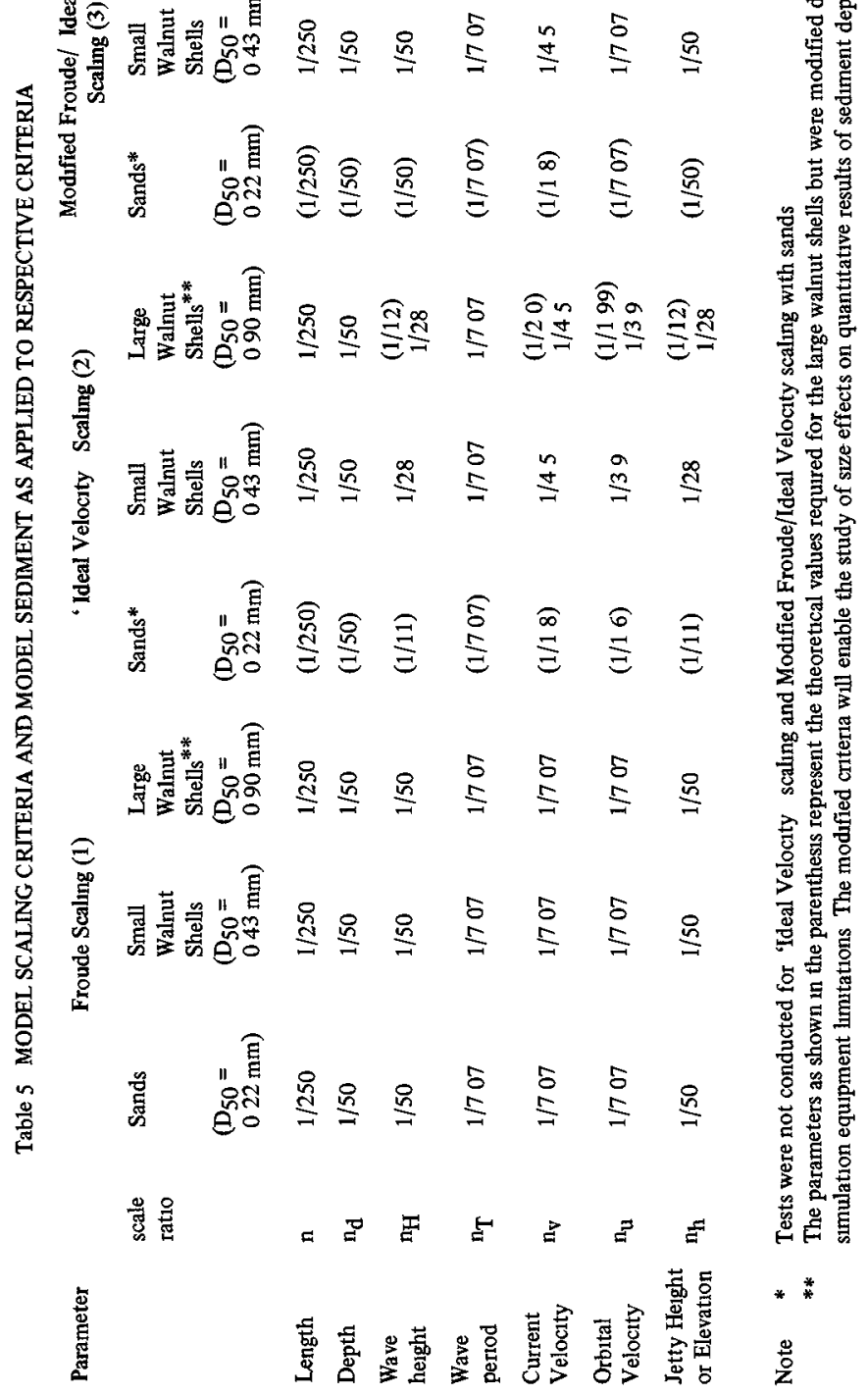

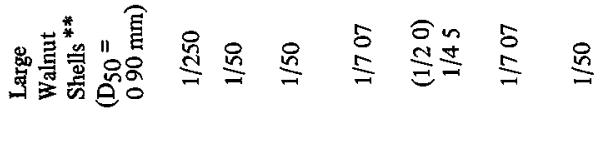

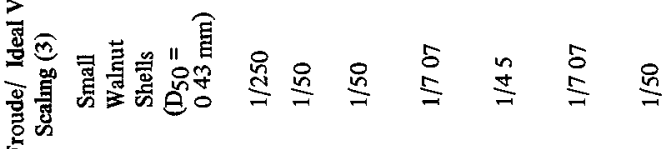

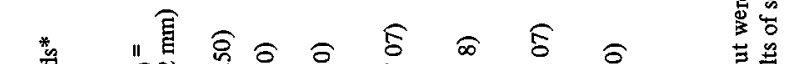

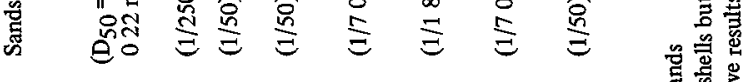

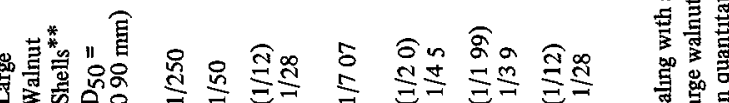

政

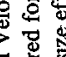

要量

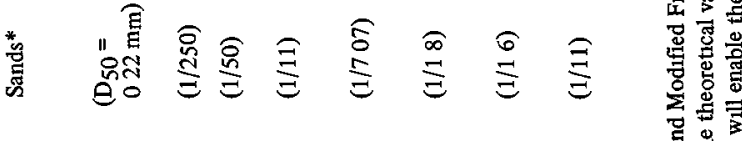

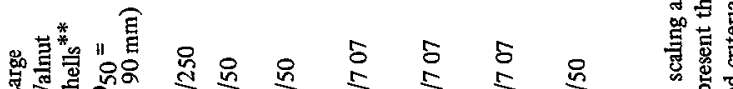

s.

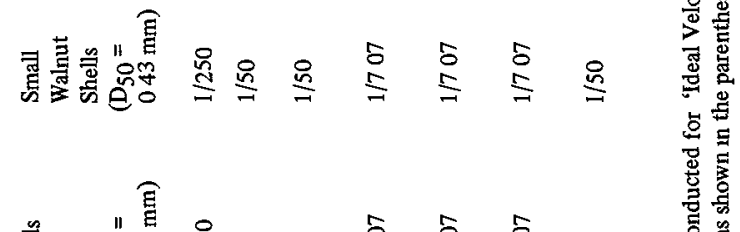

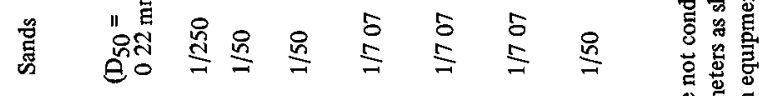

올 올

2
$y$

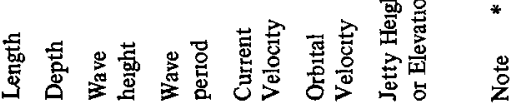


Summary of Phase II Test Results Figure 11 was prepared to facilitate comparison between the distribution of sedment depostion along the existing channel in the model with no jetty protection and related measurements in the actual channel before and after Typhoon Bess All deposition quantities are normalized to represent average one day deposition during Typhoon Bess, considening both volume and respective time scales as undicated It was found that

(a) Distribution predictions made by use of the Froude Law Criteria, based on results with large walnut shell material, tend to be far for the Typhoon Bess period

(b) Distribution predictions tend to be fair, under the normal wave condition over a long period of time (for example, one year), either by use of Froude Law Critena based on results with sands, by use of the "Modified Froude Law/Ideal Velocity" scale criteria or the "Ideal Velocity" scale criteria (as modified) based on results with large walnut shell material, or by use of "Ideal Velocity" scale criteria based on results with small walnut shell material

(c) Quantitative prediction by use of the Froude Law scaling critera based on results with sands, tends to be fair when one considers only comparison of the model time scale of bottom evolution with theoretical value However, it is expected that quantitative prediction should be reasonably good if the appropriate time scale of bottom evolution or deposition factor is applied for each test (based on respective scaling criteria with either sands or walnut shell matenal)

\begin{tabular}{|c|c|c|c|}
\hline$\Delta-$ & $\begin{array}{l}\text { Before 'Bess' } \\
\text { After "Bess" }\end{array}$ & & Prototype \\
\hline $\mathrm{O}$ & $\begin{array}{l}\text { Sands } \\
\text { Small Walnut }\end{array}$ & Froude & \\
\hline $\begin{array}{l}++++ \\
\ldots \\
=-\infty\end{array}$ & $\begin{array}{c}\text { Large WaInut } \\
,\end{array}$ & $\begin{array}{l}\text { "Ideal Velocity" } \\
\text { Modıfied Ideal } \\
\text { Froude } \\
\text { Ideal" } \\
\text { Modified Ideal }\end{array}$ & Model \\
\hline
\end{tabular}

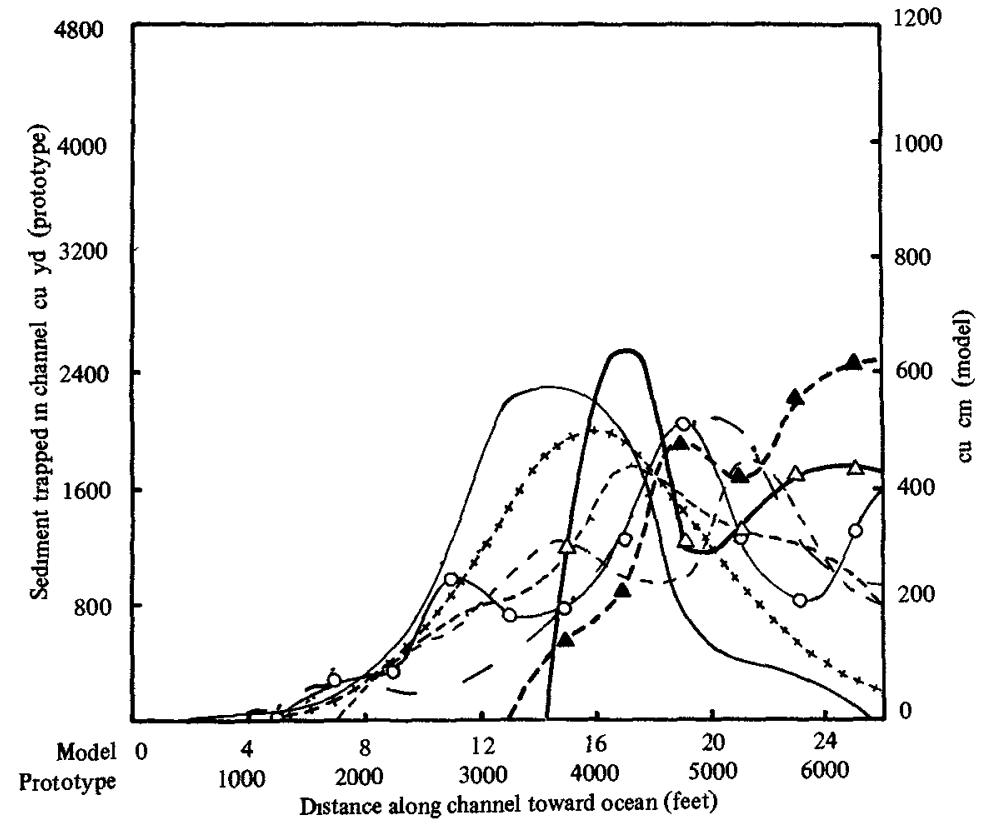

Fig 11 Model-Prototype Correlation 
Tume Scale Determmation Time scales (the ratio of model time to prototype tume for equal prototype sediment transport) were evolved for normal storm wave excitations using the three model scaling criteria and appropriate deposition in the model Measurement of the actual shoaling which occurred during Typhoon Bess is the basis for time scale determination for normal storm wave condition

As shown in Table 6, the time scales of bottom evolution vary significantly because the scale, at munimum is a function of scale factors of grain diameter, apparent density and wave height distortion However, they did not compare well with the theoretical values predicted using methods of either Manohar (1962), or Waterways Experiment Station (Fan and LeMehaute, 1969) The significant differences between theory and model results obtained with walnut-shell material raise the question of whether or not the theory is valid for the model scaling criteria used Further, the theory did not take the tide/river current scale effects into consideration in the formulation Further research is needed to determine the feasibility of using the theoretical time scale of bottom evolution in a movable bed study when no prototype quantitative data are avalable

Table 6 COMPARISON OF TIME SCALE OF BOTTOM EVOLUTION BETWEEN MODEL AND THEORETICAL VALUES

\begin{tabular}{|c|c|c|c|c|c|c|c|}
\hline \multirow{3}{*}{ Time Scale of Bottom Evolution $\mathbf{n}_{\mathbf{t b}}$} & \multirow{3}{*}{$\begin{array}{l}\text { Sand } \\
\text { Froude }\end{array}$} & \multicolumn{6}{|c|}{ Ground Walnut Shells } \\
\hline & & \multicolumn{3}{|c|}{ Small Grains } & \multicolumn{3}{|c|}{ Large Grauns } \\
\hline & & Froude & Ideal & $\begin{array}{l}\text { Modified } \\
\text { Froude/ } \\
\text { Ideal }\end{array}$ & Froude & $\begin{array}{l}\text { Ideal } \\
\text { (Modified) }\end{array}$ & $\begin{array}{l}\text { Modified } \\
\text { Froude/ } \\
\text { Ideal }\end{array}$ \\
\hline Model & $1 / 7 \mathrm{~s}$ & $1 / 4820$ & $1 / 6070$ & $1 / 5400$ & $1 / 3160$ & $1 / 3585$ & $1 / 2450$ \\
\hline \multicolumn{8}{|l|}{ Theory * } \\
\hline Manohar & $1 / 97$ & $1 / 410$ & $1 / 1330$ & $1 / 410$ & $1 / 85$ & $1 / 2750$ & $1 / 2750$ \\
\hline WES (Fan \& LeMehaute 1969) & $1 / 115$ & $1 / 600$ & $1 / 1940$ & $1 / 600$ & $1 / 1030$ & $1 / 3340$ & $1 / 3340$ \\
\hline
\end{tabular}

Effectiveness of the Jetty in Reduction of Mantenance Dredging Requirements it is predicted from the test results that the jetty will significantly reduce sedimentation in the existing channel (Table 7) The predicted reductions vary due to NE waves, from 82 to 99 percent when compared to sediment deposited in the unprotected channel with an average improvement of 91 percent The 87 percent reduction predicted by use of the Froude Law scale criteria (based on results with sands) is relatively conservative

Predictions of annual reduction by the jetty of sedımentation into the channel by waves from NE, N and NNW directions are also included in Table 7 (For greatest rigor, these require considerable prototype data, e $\mathrm{g}$ for calibration of the time scale of bottom evolution) Reductions in sedimentation are predicted on the order of 74 to 84 percent to the unprotected channel with an average value of 79 percent Deviations of about 5 percent may be expected between predictions based on the three scaling criteri It is concluded that the predictions (by use of the Froude Law scale criteria, based on results of tests in the model with sands) of quantities of sand deposits are adequate for engineering purposes

Table 7 PREDICTED "BEST PLAN PERFORMANCE

(Based on Model Tests)

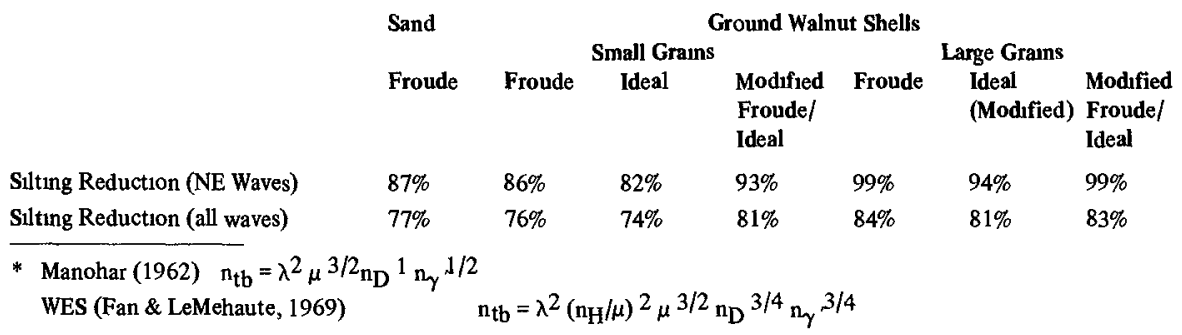


Effect of Bar By Passing Mechanics after Jetty Construction A prolonged test of the existung channel protected by a 6,000 foot long upshore East jetty (Plan IVa, Fig 7) was conducted using ground walnut shell material (small) as sediment The purpose was mainly to study the vitally important process by which sediment bypasses around the jetty causing shoals to develop seaward of the protected channel area These tests determine the usefulness of the improved channel system, including the equlibrium condition of updrift bar formation By passing from the updrift to the downdrift, and bar development outside the channel entrance were also considered The initial shoreline for the coast area updrift (East) of the jetty is considered to be the one formed by, and in equilibrium with excitation by severe NE storm waves

The base for the study of by pass and offentrance shoaling in which the excitation was Test Condition 1 was as follows $\mathrm{NE}$ waves $5 \mathrm{ft} / 5 \mathrm{sec}$, and $500 \mathrm{~m}^{3} / \mathrm{sec}$ river flow, $18 \mathrm{ft} / \mathrm{sec}$ tidal current in one diurnal tide cycle ( 44 minutes in model), increments up to three tide cycles As a result, a new equilibrium shoreline was formed as shown in $F_{1 g} 12$ (a) Bottom profiles were taken through the beach area (Section A A), around the channel entrance (Section B B), and along the existing channel (Section C-C), as shown in Figure 12 (c), (d), and (e), respectively These permit evaluation of the shoaling and by passing process as a function of time It is interesting to note that the new equilibrium shoreline forms parallel to the incident NE wave and establishes after two tide cycles This is equivalent to approximately 280 days in the prototype, using a time scate for bottom development of 14820 based on Froude Law scale criteria for no by passing

Heavy shoaling developed upshore, and near the center of the 6,000 foot long jetty [Fig 12(c)] A significant bar did not develop outside the channel entrance of the protected channel even after one additional tide cycle beyond the time necessary for the beach to attain equilibrum [Fig 12(d)] This is perhaps controlled by the scouring effect of the high currents present offshore of the end of the jetty, and by the onshore component of the wave action which tended to curtal littoral drift, and caused deposition of sediments in other areas as noted in Fig 12(a) However, a bar of about 160,000 cubic yards did develop inside the channel entrance, with a peak at approximately 750 feet upstream from the end of the jetty This bar represents about a year ( 420 days) of accumulation in the protected existing channel, with no dredging It seems unlikely that the large bar would develop should the channel be mantanned reasonably by periodic dredging

Action of the waves, other than the normal NE storm waves, studied in these special tests (1 e waves from $\mathrm{N}$ and NNW and severe NE storm waves) should tend to reduce, by erosion, the sediment trapped upshore of the jetty This would act as a storage area for sediment driven into the area by NE waves, during the normal NE monsoon season Thus, updrift of sediment into the channel should be inhbited

As a result of these tests, it is concluded that

1 The effectiveness of the improved channel system (Plan IVa-6,000 foot long jetty upshore of the existing channel) can be maintained by dredging at a minumum level ( 21 percent of the onginal estımate without jetty protection)

2 Seasonal changes of wave action will help to mantain the effectiveness of the jetty In particular, they will scour the upshore beach and make it a more effective trap during monsoon season Thus, excessive dredging will not be required after the monsoon season Delay in ship operation will be reduced to a minimum

Findings and Conclusions of Phase Il Study A 6,000 foot long jetty along the upshore bank of the exusting channel is most effective and economical, with reasonable initial cost and minimum maintenance dredging requirements

It was found that a jetty as postulated in the "BEST PLAN' would reduce the annual mantenance dredging required in the Tan My Inlet navigation channel (caused by NE wave induced shoaling) by 82 to 99 percent This had been predicted with different model scalıng criteria and types of sedıment materials A 10 percent error (5 percent on annual basts), attributable to scale effects, may be expected No attempt was made to determine the relative merit of conflicting model scalıng criteria By consideration of the appropriate time scale of bottom evolution, one can obtain nearly the same quantitative results of annual dredging requirements The "Ideal Velocity Scaling" with small walnut-shell material tends to predict most conservatively

As far as the distribution of sediment deposition along the channel is concerned, none of the scaling criteria gave comparable distribution to that found in the prototype using Typhoon Bess conditions However, model results (with sands using Froude Law scaling, with small walnut-shell material using 'Ideal Velocity' scaling, and with large walnut-shell material using "Modified Froude/Ideal Velocity" scaling or "Ideal Velocity', as modified) tend to give fair to good comparison of sediment distribution over long periods of time under normal conditions

From the viewpoints of time consumption for a model study, the use of light weight sediment materials (such as the ground walnut shells used in this study) may be more economical than sands 


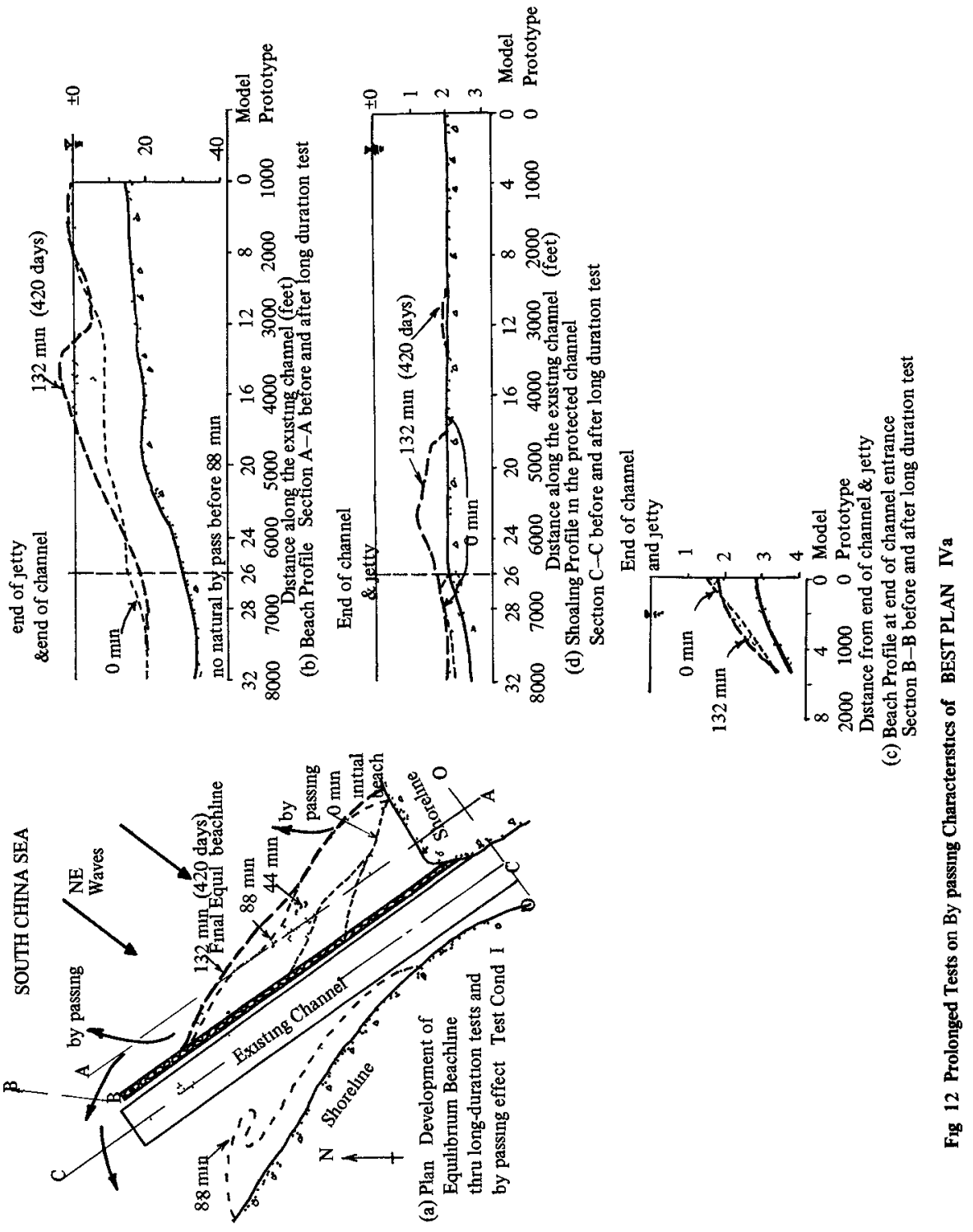


Further research work on the model technology of a movable bed model is needed to clarify the uncertanties encountered in this study Furthermore, an important point on the necessity of by pass operation should be discussed Periodic by pass of the littoral dnft that accumulated upshore of the jetty may be desirable only if the protection of the downdnft beaches from erosion is of vital importance and only when the value of the beaches is sufficient to justify the cost of such a by-pass operation On the other hand, where preservation of the downdrift beach is not of major importance, as in the case of Tan My Inlet, the suggested "BEST PLAN" should be adequate to reduce substantially the maintenance dredging required in the navigation channel by trapping a significant portion of the predomenant littoral drift on the updnft side of the jetty and by encouraging natural by passing of some of the untrapped portion

Phase III Stability Charactenstics of Rubble Mound Type Jetty of the BEST PLAN Phase III covered the two dimensional model studies which were conducted at a much larger scale in a 180 foot long wave flume The objective here was to determine the stablity charactenstics of the rubble mound type jetty of the 'BEST PLAN against storm wave action From these tests, the jetty design was optimized Tests and evaluations were made on the bastc designs of Fredenc $R$ Harris Inc for Jetty Sections IV and V From these

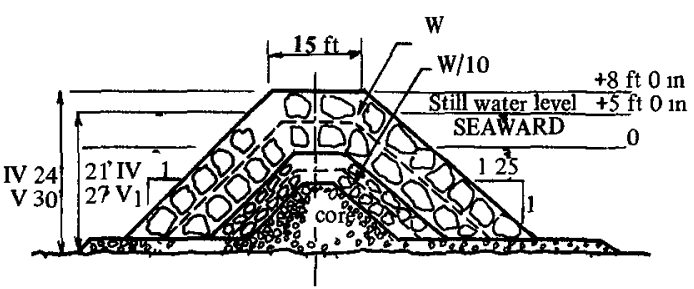

Fred Harris Inc

original design section No IV and V (1968)

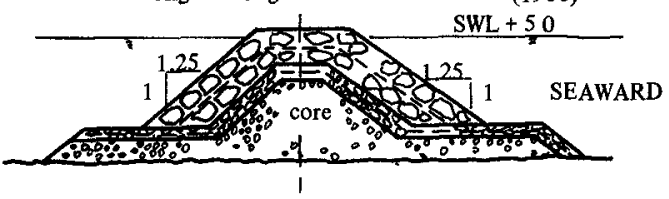

Modified Section (1) based on Model Tests

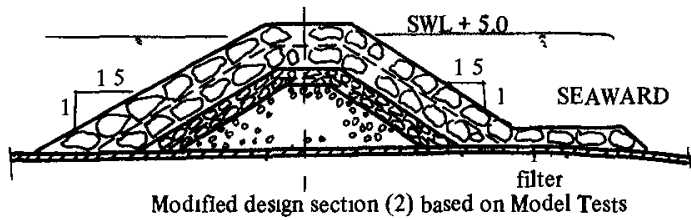

F1g 13 test results, the design was improved for use in this case ( $F_{1} g$ 13)

Scale dimensions for tests were 1 20, 1234 and 1245 The tests were conducted with a 160 beach slope covered with sands (or walnut shells) as an erosible bed Stone weight scaling followed the sumlatude by Hudson (1959) $1 \mathrm{e}$

$$
\begin{aligned}
\frac{\left(\mathrm{W}_{\mathrm{r}}\right) \mathrm{m}}{\left(\mathrm{W}_{\mathrm{r}}\right) \mathrm{p}} & =\frac{\left(\gamma_{\mathrm{r}}\right) \mathrm{m}}{\left(\gamma_{\mathrm{r}}\right) \mathrm{p}}\left(\frac{\mathrm{L}_{\mathrm{m}}}{\mathrm{L}_{\mathrm{p}}}\right)^{3}\left(\frac{\left(\mathrm{S}_{\mathrm{r}}\right) \mathrm{p}-1}{\left(\mathrm{~S}_{\mathrm{r}}\right) \mathrm{m}-1}\right)^{3} \\
\mathrm{~W}_{\mathrm{r}} & =\text { welght of stone } \\
\gamma_{\mathrm{r}} & =\text { specific weight of stone } \\
\mathrm{S}_{\mathrm{r}} & =\frac{\gamma_{\mathrm{r}}}{\gamma_{\mathrm{W}}} \\
\gamma_{\mathrm{W}} & =\text { specific weight of water }
\end{aligned}
$$

The jetty was trapezoldal in cross section with 90 foot base, 15 foot wide crown, 24 feet high, with side slopes of 1 on 125 It rested in water 21 feet deep Design configuration was rubble type rock in double layer at 64 or 103 tons each on the outside (armor), double layer at 03 tons each (as secondary cover), and quarry run of 2 inch size as both core and 4 foot thick base It was intended to be overtopped by the design waves which, face on, were 14 feet high, with an 8 second period However, toward conservatism, the jetty was tested in waves up to 20 feet high with a 10 second period

Major findungs were as follows

1 A slope of 1125 is acceptable for the 14 foot high design waves

2 An armor stone size of 64 tons (for Jetty Section IV) and of 103 tons (for Jetty Section V) is adequate for the $\mathbf{1 4}$ foot high design waves under either low or high tide and storm surge conditions 
3 To prevent scounng under the jetty a layer of core materials should be extended beyond the armor stone toe with two layers of secondary stones placed over this layer of core material for protection An alternate for protection against sand scour is a plastıc filter (such as Poly Filter X) extended in front of the jetty and under the sand with secondary stones above the sand as protection

4 A filter layer, preferably of plastic, is necessary in the prototype between the core material and the sand beach This could not be demonstrated in the model due to the effect of the scale, $1 \mathrm{e}$, larger voids in the prototype than those in the model

5 Further testing of scourng depth at the jetty toe is necessary because of the discrepancies found between test results with sand and those with walnut shell material as erosible bed materials

6 The stability of the rubble mound jetty should be studied in a movable bed model particularly if the jetty is founded in a depth shallower than the critical depth for sand movement Otherwise the designer should specify that the jetty bottom should either extend below scour depth to be determined, or it should rest on a plastic filter

7 The filter system for a jetty should be designed with extreme care, particularly if the translatory currents induced by tidal and river flow along the longitudinal axis of the jetty are significant

\section{CONCLUSIONS}

The major conclusions of this study were

1 A 6,000 foot long rubble mound type jetty along the east bank of the existing channel [Fig 7] should be considered the BEST PLAN' - the most effective and economical solution of the exterior channel improvement at Tan My Inlet The rubble mound type jetty should be constructed of 64 to 103 ton armor stones with two layers of secondary stones and core materials laid underneath it A filter system must be carefully designed to avoid any falures due to erosion of the jetty foundation and toe

2 A sem movable bed model study of the inlet stability improvements, following the three phase experumental procedure reported herein, may be considered a feasible method by which to obtain both qualitative and quantitative results this is particularly so when sufficient prototype data are avalable to relate the time scale of bottom evolution

3 A jetty as postulated in the 'BEST PLAN' would reduce the annual maintenance dredging required in the Tan My Inlet navigation channel (caused by NE N, and NNW wave induced shoaling) by 74 to 84 percent This was predicted with different model scaling criteria and types of sediment materials By consideration of the appropriate time scale of bottom evolution, one obtaned nearly the same quantitative results of annual dredging requirements "1deal Velocity scaling with small walnut shell material tended to predict most conservatively

As far as the distribution of sediment deposition along the channel was concerned none of the scaling criteria gave distribution comparable to that found in the prototype using Typhoon Bess conditions However model results (with sands using Froude Law scaling with small walnut shell material using "ldeal Velocity" scaleng and with large walnut shell material using "Modified Froude/Ideal Velocity scaling or "1deal Velocity,' as modified) tended to give farr to good comparison of sediment distribution over long periods of time under normal conditions

From the viewpoint of time consumption for a model study, the use of light weight sediment materials (such as ground walnut shells) may be more economical than use of sands Further research work on the model technology of a movable bed model is needed to clarify the uncertainties encountered in this study

4 Use of theory alone in predicting the time scale of bottom evolution should be done with caution, especrally in cases where field data are not readily avalable for model prototype correlation This conclusion was quite obvious, at least for this study Further verification is needed

5 The quality of the model study is still greatly dependent on the quality and quantity of environmental data collected When adequate data are avallable to permit the determination of the time scale of bottom evolution the discrepancy between the results obtained with different scaling criteria and model sediment was not too large as one would expect normally of course there are stlll many research problems involved in a movable bed model 


\section{ACKNOWLEDGEMENTS}

The Officer in Charge of Construction, Republic of Vietnam, Naval Facilities Engineernng Command sponsored the model study, the assistance provided by LCDR W H Kay, ROICC, and his staff is appreciated MI J C Kray of NAVFAC furmshed valuable comments Their permission to publish this paper is appreciated

The invaluable support given by the colleagues of this author is acknowledged, particularly that of Dr C $\mathrm{L}$ Bretschneider, Project Manager Mr J T O'Brien Director of Look Laboratory, Mr R Q Palmer Co Principal Investigator Messts A R Fallon and David Lally and other study team members

The Research Corporation of the University of Hawall monitored the contract of this study

\section{REFERENCES}

Byjker, E W (1967), “Some Consıderations about Scales for Coastal Models with Movable Bed,' Pub 50, Delft Hydraulics Laboratory, Nov 1967

Byjker, E K (1968), Littoral Drift as a Function of Waves and Current' Proceedings of the 11th Conference on Coastal Engineernng (London, Sept 1968), Amencan Society of Civil Engineers, 1969

Bowen, A J and D L Inman (1966) Budget of Littoral Sands in the Vicinity of Point Arguello Calif Tech Memo 19 U S Army Coastal Engineering Research Center, Dec 1966

Bruun, Per J A Batties, T Y Chiu and J A Purpura (1966), "Coastal Engneering Model Studies of Three Florida Coastal Inlets" Bull 122 Flonda Eng and Ind Expenment Station, University of Flonda, Gainesvile June 1966

Caldwell, J M (1956), ‘ Wave Action and Sand Movement Near Anahemm Bay, Calif ' BEB TM 68, Feb 1956

Fan, L N and B LeMehaute (1969) 'Coastal Movable Bed Scale Model Technology,' Final Report, US Army Coastal Eng Research Center, (DACW 72 6g-C-0020) by Tetra Tech, 1nc , January 1969

Glenn, A H and associates (1966) Meteorological-Oceanographic Evaluation for Planning, Design, Operation of Marine Facilities Along the Coast of South Vietnam General Report," 19 Aprl 1966

Hudson, R T (1959), "Laboratory Investigation of Rubble Mound Breakwaters," Journal Waterways and Harbors Division, Proceedings, ASCE, vol g5, noWW3, Sept 1959

Inman, D L and R W Harris (1966) Oceanographic and Engineerıng Report on Investigation of Sedimentation Silting, and Dredging Requirements Various Locations, Republic of Vietnam, Contract NBY $79 g 44$ Damel, Mann Johnson, and Mendenhall, Saigon Vietnam, June 30, 1966

Keulegan, G H (1967), “Tidal Flow in Entrance Water Level Fluctuations of Basins in Communications with Seas,' Tech Bull no 14, Committee on Tidal Hydraulics, Corps of Engineers, U S Army, Vicksburg, Miss July 1967

Lee, T T (1970), "Model Investigation to Improve the Stability of Tan My Channel Hue, Republic of Vietnam, Technical Report 10, Look Laboratory University of Hawau, Feb 1970 (Contract N 631g5 69-C-0089)

Lyon Associates, 1nc (1968 1969) "Tan My Channel Study Field Measurements" OICC/RVN Contract P11 o0gg B1 weekly Report No 1 through 10 dated Sept 1968 Jan 1969

Manohar, M (1962), 'Discussion of Laboratory Determination of Littoral Transport Rate ' by R P Savage,' Jour Waterways and Harbors Div Proc ASCE 8g, WW4 Nov 1962

NAVFORV Weather Unit, Sargon, RVN (1968), Wave observations at Tan My dunng 1 Oct 1967 to 30 Sept $196 \mathrm{~g}$

Palmer, R Q (1970) “Optıcal Wave Recorder for Hydraulic Models,” LOOK LAB/HAWAII, vol 1, no 1, Jan, 1970

Savage, R P (1959) "Laboratory Study of the Effect of Groins on the Rate of Littoral Transport Equip Development and Init1al Test,' BEB TM 114, June 1959 (also TR-4, U S Army Coastal Eng Research Center)

Wiemat, D R (1969) Letter to Mr R Steward concerning prelımınary results of the infra red imagery Survey of Tan My area $14 \mathrm{July}, 1969$ 


\title{
CHAPTER 72
}

MODEL TESTS ARD STUDIES FOR PORT RASHID, DUBAI

\section{ERIC LOEWY}

Partner

Sir Willıam Halcrow \& Partners

Consulting Englneers, London

\begin{abstract}
This paper is a factual account of studies carrled out for the design of a new deep water harbour As so often happens construction work had to be begun before many of the conclusions of the study were avallable so that alterations to the inltial designs had to be made while work progressed The studies comprised tidal and wave recordings and analysis, model studies to determine residual wave conditıons at the quays, studies to determine the extent of littoral drift, the effect of the proposed works upon this and possible measures to counter downdrift erosion In addition studies were made of the stability of the adjacent creek channel which had previously been the harbour and a mathematical model study was carried out of the effects on the creek regime of varıous proposed entrance works including the construction of an entirely new creek entrance channel through the new deep water harbour
\end{abstract}

\section{INTRODUCTION}

The State of Drubal Is situated on the southerly coast of the Stralt of Hormuz which forms the seaward entrance of The Guif (sometimes called the Persian or Arabian Gulf - see flg 1) The Capltal Town of Dubal consists essentlally of the twin towns of Dubal proper and Dalra (fig 2) whlch lie astride the mouth of a tidal creek whlch has up to now formed its harbour This harbour was used by country craft (dhows), lighters and other small vessels, with only very minor works on jetties and wharves up to approximately 1958 The combination of littoral drift towards the north east and the creek tidal flow resulted in a gradual migration of the creek entrance in the direction of the drift and, in the years after the last war, to a reduction in the depth of water avallable at the entrance starting in 1959 entrance training works were constructed and the channel dredged out to 8 feet below chart datum These works caused an interruption of the littoral drift with consequent erosion of the downdrift site (figs 3 and 4 ) 


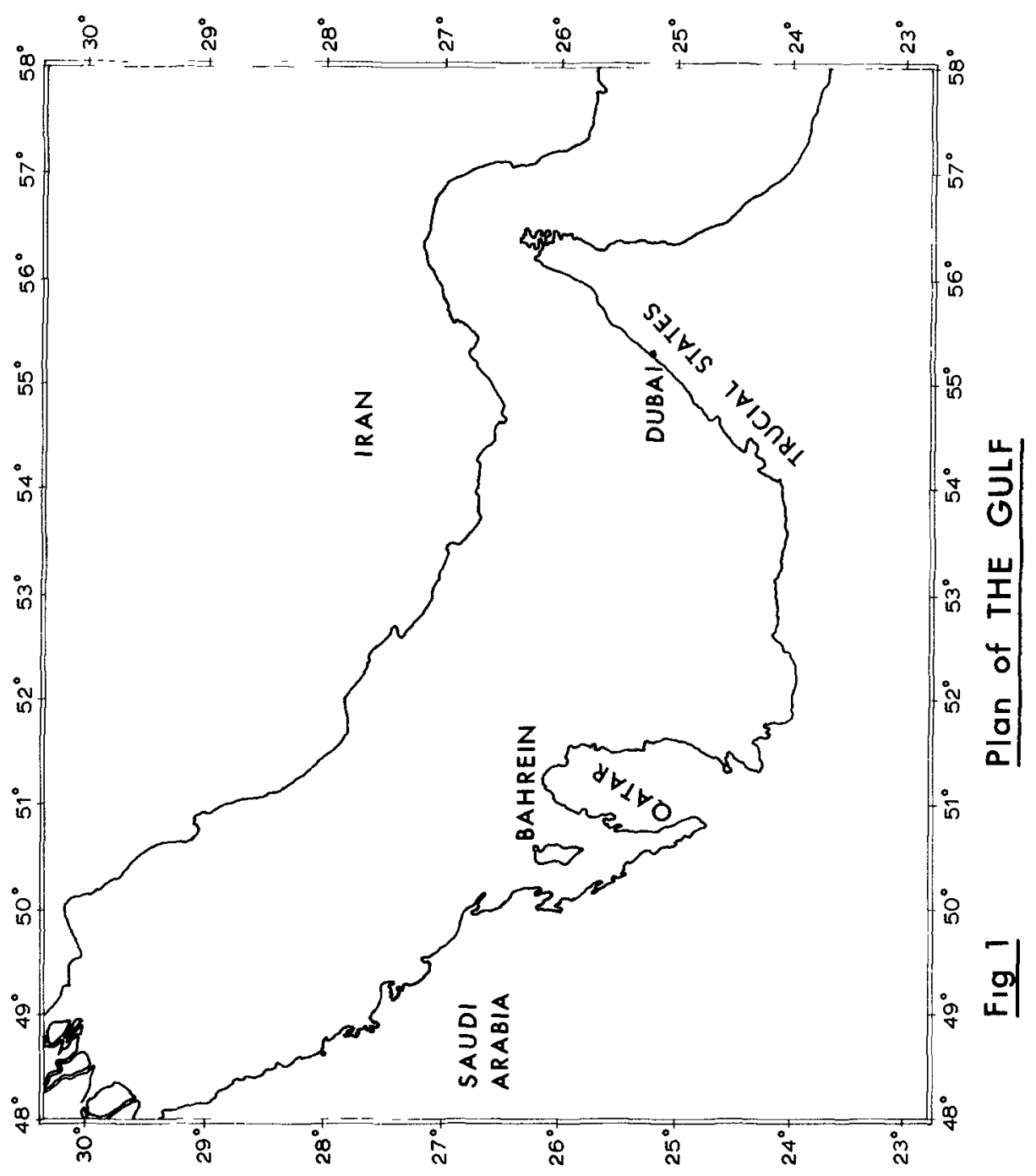




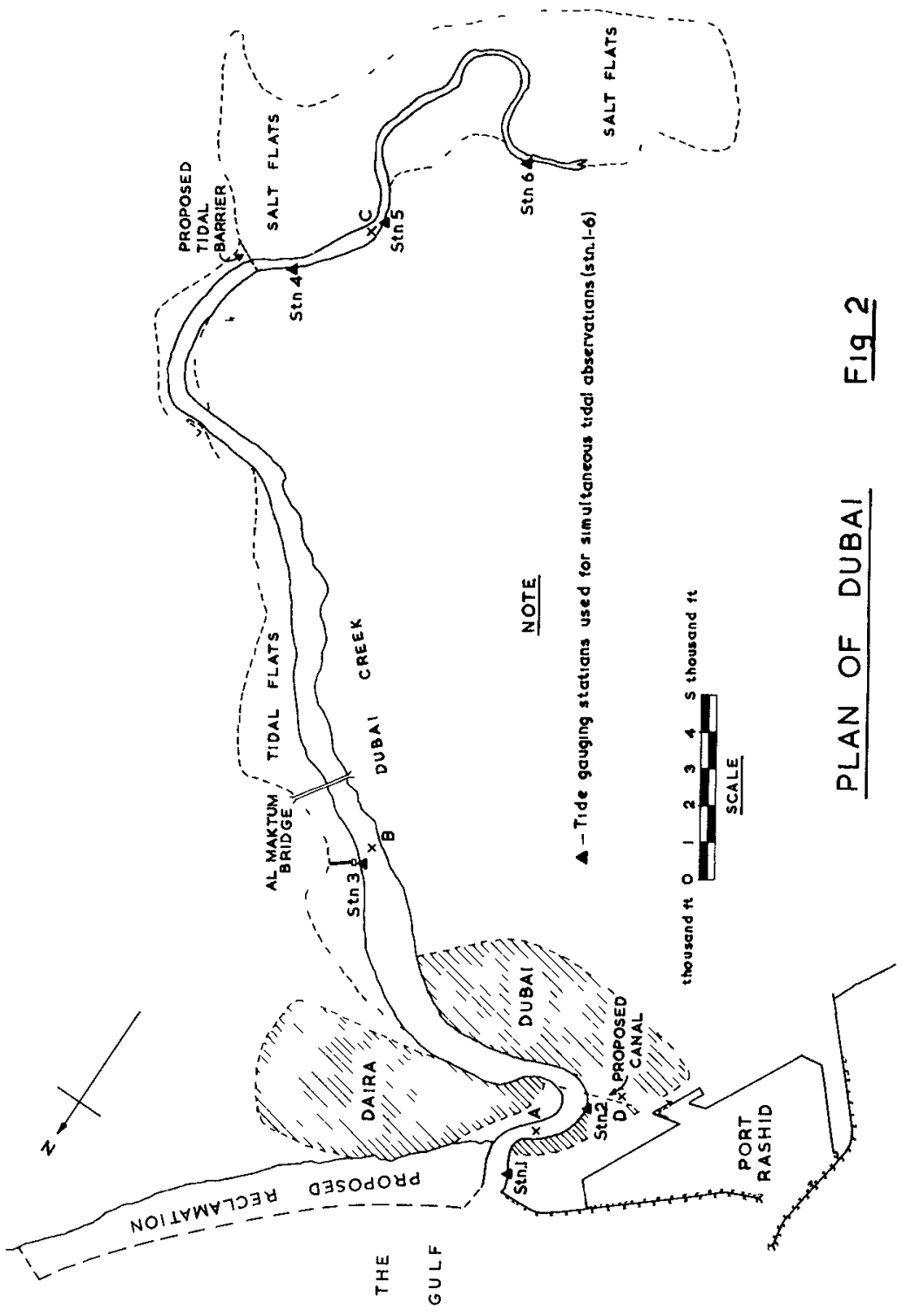




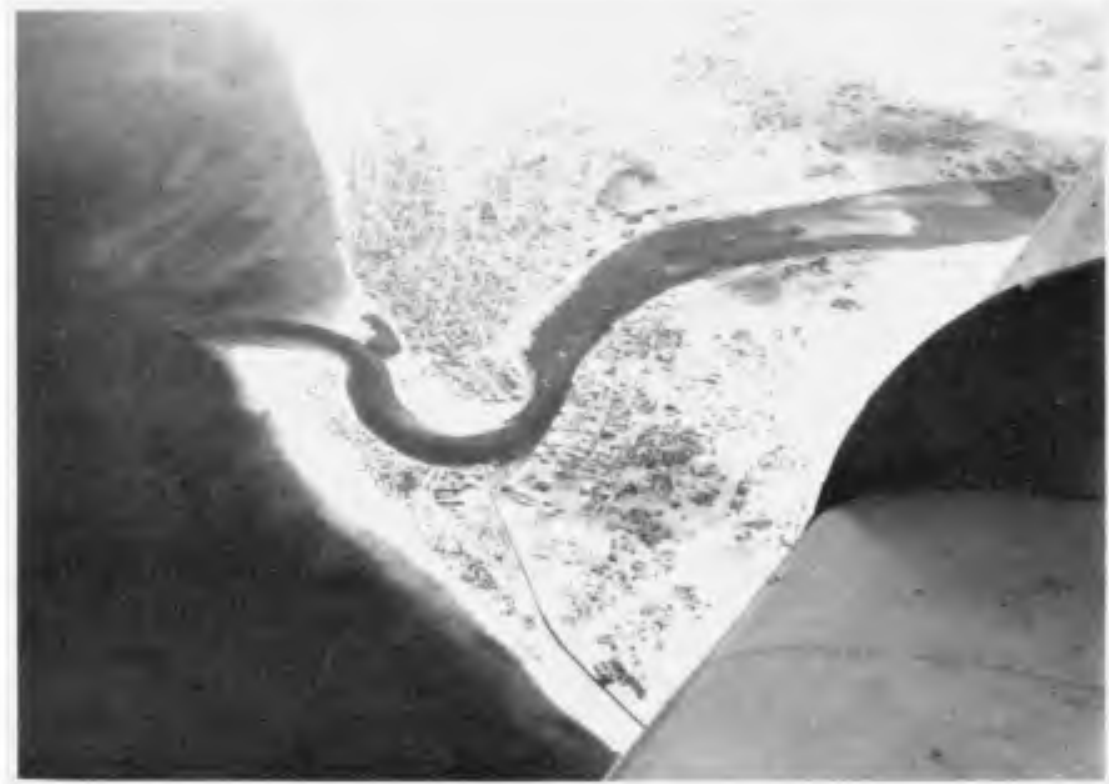

Fig. 3 DUBAI CREFHK

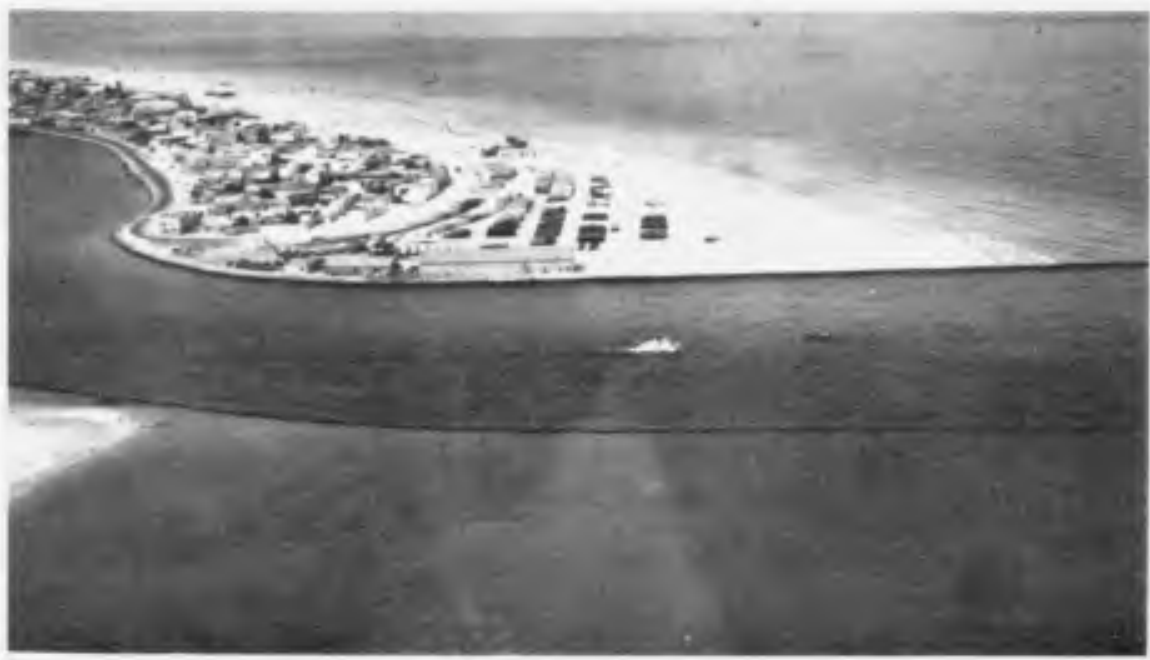

Fig. 4 CREEK ENTRANCE 
In 1966, as a result of oll being found in Dubal waters, His Highness the Ruler was enabled to embark upon the construction of a new deep water harbour, to be known as Port Rashld, ımmedlately south-east of the creek entrance ( $f$ lg 2) The new harbour, whlch wlll enclose a water area of approxımately 350 acres and wll provide 15 deep water berths each approximately $600 \mathrm{ft}$, long, is still in course of construction It will have a bed level at its entrance approximately $36 \mathrm{ft}$ below chart datum and wll be dredged to 305 feet below chart datum throughout since the harbour works will form a solid barrier to the littoral drift for approximately one mile out to sea from the previous shore line it is clear that these are likely to have an even greater effect on the coastal regime than the creek entrance works

There are proposals to close or partly close the existing creek entrance and form a new canal link from the creek to the new harbour There Is also a proposal to reclaim a coastal strıp of land north east (downdrıft) of the new harbour and protect this with a heavy sea wall whlch would have the effect of preventing further erosion there and transferring the erosion to less valuable land further along the coast

Model Tests and Studies were inltiated to consider varıous aspects of the works The majority of these were carried out at the Hydraulics Research Station at Wallingford, Fingland

\section{INVESTIGATIONS}

A tide recorder was set up on the side of a small jetty bullt out acsoss the beach into the sea An analysis of the first year's tidal records was carried out by the Institute of Coastal Oceanography and Tldes at Bırkenhead, Fngland The analysis demonstrated the extent of the "set up" which can occur at the south-east end of the Gulf on 25th January 1969 this was 12-18 inches continuously for a period of 12 hours Shorter period selches up to 17 inches have also been recorded The tides are subject to a large diurnal inequality Their normal range Is between $\mathrm{MHHW}+55 \mathrm{ft}$ (chart datum), NLHW $+44 \mathrm{ft}$, NHLW $+26 \mathrm{ft}$ and MLLW $+13 \mathrm{ft}$

An OS P O S wave recorder was installed This type of self-contained recorder was chosen in place of an instrument which would record on shore to avold the danger of the connecting cable being broken by small vessels achoring offshore For most of the recording period the OS P O S Instrument was anchored approxımately $\frac{3}{4} \mathrm{mlle}$ offshore in $36 \mathrm{ft}$ of water (below chart datum) with the recorder tethered 8 to $10 \mathrm{ft}$ below chart datum so as to be approximately 3 ft below what was anticipated to be lowest wave trough level The relationships and equations used in the analysis of the wave records were reduced to forms sultable for the Ollvettr Programma 101 Desk Top Computer The analysis was carried out in accordance with Draper's procedure (ref 1) using the empirical corrections for attenuation worked out by Draper and Maxted (ref 2) 
Since no commercially-avallable, proven, wave direction recorder of reasonable cost appeared to be avallable wave directıons were estımated visually, normally twlce dally Twice dally observations were the most whlch could be expected from the already overbusy Resident Englneer's Staff but as the helght recordings occurred 8 times per day (every 3 hours) it is obvious that considerable interpolation of wave directions had to be estimated with corresponding risks of error There is no doubt that the development of a reliable, commercially-avallable, reasonably-inexpensive wave direction recorder would be very greatly welcomed

\section{WAVE ANAIYSIS}

To date six month's wave records have been analysed The data has been adequate and sufflclently representative for wave helght frequency prediction graphs to be drawn for "all directions" waves and for waves from $300^{\circ}$, the dominant direction, and from $330^{\circ}$, but not other directions The "all directions" prediction ( $f 1 g$ 5) drawn on probability graph paper, extended upwards to obtain a Once in 100 Years wave value, indicates a maximum deep water wave helght of $345 \mathrm{ft}$ in 100 years, $315 \mathrm{ft}$ in 50 years, and $260 \mathrm{ft}$ in 10 years Using Darbyshire and Draper's relationships (ref 3) between wind speed and duration for the maximum possible fetch of approximately 500 nautıcal miles these maximum wave helghts would require wind speeds of respectively 44,43 and 36 knots for a period in each case of approximately 33 hours, or equivalent combinations of speed and duration

In the restricted area of The Gulf the majority of wave pattems are composed of short-period waves The recording of such waves led to difficulties which were not sufficiently appreciated at the beginning of the analysis The first was locational, in that the record tended to be increasingly flattened the deeper the pressure-actuated instrument was tethered below the water surface This was overcome in large measure but still required an attenuation factor adjustment before feeding the data into the computer The second was instrumental, in that a considerable number of the 12 minute duration records were of waves about 1 foot in helght, show on the trace as $1 \mathrm{~mm}$ The difficulty of classifying these in wave helght steps was such that the analysis proved unreliable in the smaller ranges and in fact only derıved maximum waves over 7 feet in helght were plotted, the linear results being extended to cover predictions for lesser waves and extrapolated for waves beyond the scope of the instrument readings

Estimation of the likely frequency of stated wave helghts has so far been assessed only by reference to the British Manistry of Technology's statıstical survey of wave characteristics estimated visually from Voluntary Observing Shlps on their normal shlpping passages (ref 4) It is interesting to note, however, the confirmation provided by maximum helghts up to 25 feet being estimated at points a little further west in The Gulf during a storm in 1964

The relationshlp between maxımum wave helght and maximum significant wave helght was found during this analysis of slx-months' recordings to be approximately 2 to 1 , for the range of wave periods of between 4 and 9 seconds which was of principal interest and occurrence 
WAVE HEIGHT (FT)

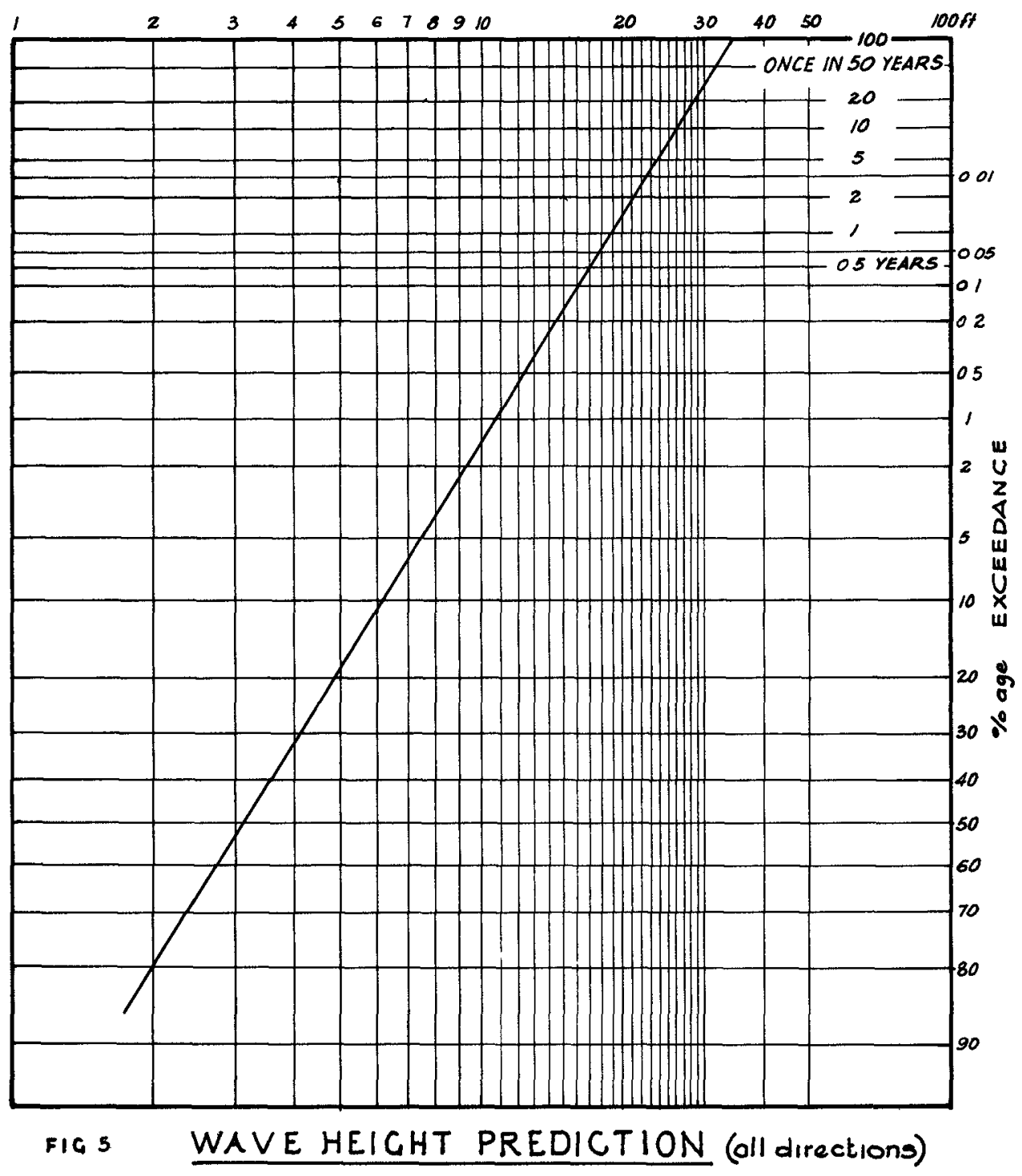




\section{HYDRAULIC MODEL TESTS}

A rigld bed hydraulic model of Port Rashld was constructed to a scale of 1150 Waves of different equivalent helghts, periods and directions were generated and directed on to the harbour and their residual helghts along the quays were measured for a number of harbour layouts $A$ final harbour layout was chosen by this means These model tests had to be carried out before any results could be obtalned from the wave recorder The waves used for the tests were therefore estumated from wave recordings taken further west in the Gulf and an assessment, using synoptic chats, of the corresponding waves off Dubal

Idealısed shlp movement tests, using the same equivalent waves, were also carried out using models representing 10,000 dwt and 4,000 dwt vessels at the quays The results of these model tests were satisfactory, except in the case of two berths when waves were from the north direction This is in fact the direction in which the harbour entrance points so that there is virtually no obstruction to these waves Although no prediction for waves from the north has been possible from wave recordings at Dubal for reasons mentioned previously it has become clear that considerable wave activity from that general direction is rare This conclusion has since been confirmed by an analysis of the wave records from Voluntary Observing Shlps steaming in the vicinity of Dubal These records were kindly supplied by the British Meteorological office

In connection with the wave model a study was carried out of the extent to which waves could be generated within the enclosed harbour area over the greatest fetch of approximately 13 miles by strong winds blowing from approximately south west or north east Although it was appreciated that these conditions would only occur infrequently, experience with the model showed that the resulting waves would not significantly hamper the workıng of shıps alongside the quays

No evidence was obtained that the natural period of oscillation of the harbour basin would glve rise to selches having periods or length of range which could affect moored vessels in Port Rashid, providing of course that such mooring lines and fenders were in appropriate combination of stiffness and softness whlch previous model and full-scale tests had shown to be surtable

\section{LITTORAL DRTFT}

The coastal regime at and in the vicinity of Dubal was studied partlcularly in order to assess the effects of the construction of Port Rashid As has been mentioned, training works were carried out between 1959 and 1962 at the entrance to Dubal Creek These involved the construction of steel sheet-piled training walls to stabilise and maintain the creek channel into the sea out to approximately the seabed contoux $80 \mathrm{ft}$ below ohart datum, this being a sufficient depth of channel for the country craft and lighter traffic at that time ( $f$ g 3 ) 
Since the net littoral drift is south west to north east along the coast the effect of these training walls had been to cause accretion on the south west side and erosion on the north east side By 1968 the downdrift erosion, which affected the densely-populated town of Dalra, had begun to cause serıous concern, though by about that time some natural sand by-passing of the creek had just begun to occur There were therefore naturally fears of the effect of the very much bigger barrier of Port Rashid Comparison of regular measurements of beach and bed levels on a number of lines perpendicular to the coast updrift of the training walls early led to the conclusion that the natural net rate of littoral drift was approximately $70,000 \mathrm{cu}$ yds per year In 1969 a further calculation was made based upon the relationship -

$$
\begin{aligned}
Q_{0}= & 210 \mathrm{E}^{08} \\
\text { where } Q_{0}= & \text { intensity of littoral drift in cu yds per day } \\
\text { and } E= & \text { longshore wave energy in milions of food- } \\
& \text { pounds per foot of beach per day }
\end{aligned}
$$

The net littoral drift per year calculated from this amounted to 79,000 cu yds Again, because wave data from the wave recorder was not avallable when this calculation was made, the same assumptions as have been mentioned before were made regarding the waves However since the two calculations showed such close agreement it was considered justified to assume a net littoral drift of the order of $80,000 \mathrm{cu} y d s$ per year from south west to north east and no further calculations have been undertaken using the results of the analysis of the first 6 months of actual wave records

Based upon such an assessment of annual volume, it will be at least 20 years before any significant by-passing of sand across the entrance of Port Rashid is likely to occur The form which this natural by-passing may take is uncertain and may well be unacceptable During this time corresponding erosion of the downdrift coast of Dalra wlll occur unless remedial measures are taken Since this township is densely populated to the coastline this is obviously not a result which can be accepted and the Ruler is at present considering the adoption of one of several alternative solutions proposed to him Artificial nourlshment is one course, another, more favoured, is to concentrate the erosion length further downdrift, where the coastal strip is devold of use or habitation, by carrying out protection and reclamation works from the creek entrance to beyond the downdrift end of Dasra

\section{DUBAI CREHEK}

The influence of the creek flow upon the situation has not so far been mentioned because it does not appear that there is any significant outflow or inflow of bed material associated with it As far as is known the creek has remained remarkably stable of recent years in these respects though there has undoubtedly been a gradual migxation of the entrance towards the north east 
Normal sand by-passing did not apparently produce the formation of sand bars which cause navigational difficulties But when the channel began to follow a course leading over a coral reef an unacceptable reduction in natural entrance depth resulted The works carried out between 1959 and 1962 successfully relocated the entrance by a system of sheet plled training walls with dredging to restore and deepen slightly the channel linking the creek port with the sea

With the fIrm proposal taken to construct the deep water port on the coast off Dubal, there followed naturally the suggestion of a connection by water between the already-flourishing Creek port, with its winding and. restricted entrance, and the new harbour enabling cargo liners to discharge and export freight in security from wind and sea conditions It was evident that the sediment loads and tidal velocities through the creek port had a large effect on the success of such a scheme to direct the flow into and out of the tidal basin upstream of the port and to close, wholly or partially, what would become the former entrance Examination of this proposal could only be through model analysis, unfortunately time and cost limited the study to a mainly qualative rather than a quantative one, but nevertheless the results were instructive

\section{INVESTIGATION AND MATHEMATICAL MODEL}

The creek is not fed by anysurface streams, even intermittent ones, but there is a small amount of sub-surface seepage of water into the upper reaches and this seepage is very highly saline In addition there are very extensive tidal flats at the head of the oreek which are covered at high water and dry out at low water These large areas of grenerally very shallow water are of course areas where high evaporation leads to increasing salinity For these two reasons, therefore, the water at the head of the creek Is considerably more saline than the sea and the water lower down the creek This reverses the more usual situation in which fresh water flows into the head of a tidal inlet and the salinity decreases as the distance from the sea increases A natural corollary of the situation at Dubal is that the highest salinities occur at low water The result of this salinity pattern is that there is a net drift of water near the bed of the creek towards the sea, but the velocities involved are so low that this is not of significance as regards the transport of bed material

No measurements are avallable to show up any areas of accretion or erosion elther permanent or temporary in the creek or to indicate quantitatively any natural changes in the entrance channel However it seems clear that there is a negligible transport of solids to the creek through sub-surface flows The most likely sources are from wand-blown material and erosion of the bed or banks Erosion does not appear to provide any overall change in the quantity of solid material entering or leaving the creek though there are local movements within it Wind-blown sand undoubtedly does provide an input source, but such calculations as have been possible in this respect suggest that the quantity is small and that It is confined to the upper reaches Due to a large diurmal inequality in the tides at Dubal the maximum ebb flows in the creek are greater than the maximum flood flows so that bed material from the creek tends to be flushed out into the sea rather than the reverse, and oonsequently the entrance channel remains open 
A large number of cross-sections of the oreek were surveyed and tidal observations carrled out simultaneously at six stations ( $f 1 g$ 2) over a high spring tide cycle on $13 / 14$ May 1968, with an extensive serles of current velocity measurements taken during this cycle The mathematical model of tidal flows in the creek, with the view to assessing the effects of various proposed schemes, was proved against this set of tide levels and flows Because of the large diumal inequality, which results in veloclties and discharges on the fall of the major tide being much greater than the velocities and flows during any other period, subsequent analyses were directed towards determining the maximum velocities and volumes of water (tıdal prism) leaving the creek on the ebb of the major tide under the conditions of $13 \mathrm{th} / 14$ th May 1968

Once the model had been satisfactorlly proved the effects of proposed engineering works could be studied These proposed works are indicated on Figure 2 and they comprised -

(a) Construction of a canal link between Port Rashid and the creek and the closure or partial closure of the by-passed seaward end of the latter

(b) Construction of a tidal barrier near tide gauging station 4 to close off the salt flat area at the head of the creek The object of the barrier would be primarily to reduce the tidal flow by eliminating the large storage volume contained in the tidal flats at the head of the creek As a secondary object it would serve as a barrage to increase water level permanently above the barrier in a reach to whlch vessels would have access through a lock such a barrier might indeed be valuable but would probably be better sited further towards the sea so that the locked area would be able to serve the towns and the shlps

\section{RESULIS AND CONCLUSIONS}

The mathematical model quickly demonstrated that the tidal regrme in the creek is at present dominated by two factors These are the throttling effect of the narrow channel through the Town and the presence of the large inter-tidal flats storage volume at the head of the creek As a result schemes to vary the entrance to the creek cannot alone greatly affect the regime except locally The only practical scheme examined which caused an appreciable change in the whole regime was that to constmuct a tidal barrier blocking off the major portion of the inter-tidal flats storage volume

The model was able to calculate water level, velocity and flow at any time at a number of selected points in the creek and proposed canal for the conditons resulting from the varıous schemes under the open-sea ebb tidal 
conditions of the 13/14 May 1968 Volumes of tidal prisms could also be calculated for the various tide levels

Some actual mathematically-calculated figures may be of interest here At present the creek channel tidal maximum velocities vary greatly along its length Peak maxımum velocity is $52 \mathrm{ft} / \mathrm{sec}$ at Section A (flg 2), reducing to $133 \mathrm{ft} / \mathrm{sec}$ at section $B$ and increasing again to $25 \mathrm{ft} / \mathrm{sec}$ at Section $\mathrm{C}$ Incipient sediment movement was calculated to correspond to a water surface velocity of $14 \mathrm{ft} / \mathrm{sec}$ for a $01 \mathrm{~mm}$ sand (taken as representative of the finer fractions of bed material) This means that at present some sediment movement towards the sea probably takes place in the upper reaches but not in the middle reaches In the lower reaches between the towns quite extensive movement would take place were it not for the fact that the bed here mainly consists of coral with limestone boulders and the quantity of sand is small It would appear therefore that there is probably some accumulation of bed material in the middle reaches though there are no indications that this is of serious proportions The bed sand lost in the upper reaches appears to be approxımately made good by the windblown sand previously mentioned

When the proposed canal was introduced into the model, with varying proportions of the existing creek entrance channel remaining as well, It was found that the effect of such new entrance proposals on the tidal prism was limited to a max.mum increase of 66 per cent because of the throttling effect of the reach timough the town A large change in the entrance channel conveyance produced only a small change in the tidal prism As a result it was possible to work out a simple method of calculating the performance of any combination of entrance channels (so long as thelr combined conveyance exceeded the value of the existing entrance channel) This no longer required the use of the mathematical model

Provigionally it was considered that a canal link having a 340 ft wide rectangular section and a bed level $12 \mathrm{ft}$ below chart datum, with the exısting creek entrance completely closed off, would result in a peak maximum velocity of $44 \mathrm{ft} / \mathrm{sec}$ (at Section D, fig 2) This would be acceptable provided that the bed material was found to be sultable or was sultably protected Peak maximum velocities somewhat higher than $14 \mathrm{ft} / \mathrm{sec}$ would also be present within part of Port Rashid under the conditions of this arrangement

The effect of the tidal barrier was to decrease the tidal prism (major $\mathrm{ebb}$ tide $13 \mathrm{th} / 14$ th May 1968) by causing a reduction in the canal peak maximum velocity from the $4.4 \mathrm{ft} / \mathrm{sec}$ quoted above to approximately $34 \mathrm{ft} / \mathrm{second}$ Policy on both these matters remains to be decided, as there are many other factors of a non-hydraulic kind to be taken into account

The Author appreciates the assistance given in preparing this paper by the Hydraulics Research Station Staff, partıcularly Messrs W A Price and $\mathrm{K} W$ Tomlinson, and of his colleague, Mr H E B Frederickson 


\section{REFTERFNCES}

1 I Draper The analysıs and presentation of wave data - A plea for uniformity Proc 10th Conf Coastal Engineering, 1966

2 I Draper and A V Maxted Graphs of attenuation of waves w1th depth Intermal Report A17, National Institute of Oceanography 1962

3 M Darbyshire and I Draper Forecasting W2nd-generated Sea Waves Fingineering 5th April 1963

4 Minıstry of Technology, Unıted Kıngdom Ocean wave statıstıcs Her Majesty's Stationery Office, 1966 



\title{
CHAPTER 73
}

\section{STUDY OF TOPOLOBAMPO'S BAY, MEXICO, ENTRANCE}

\author{
by \\ Héctor López Gutiérrez 1 \\ Daniel Cervantes Castro
}

SUMMARY

Brief examination of the geomorphological background of the Topolobampo's bay inlet is made

A theoretical study of the inlet and spits, based on field data such as wind, waves, tides, currents, bathimetric charts from 1875 to 1965 , was developed in order to determinate the most convenient position of an experimental channel dregged on 1963.

Radioactive tracer studies, photographic and periodical surveys of the inlet and the channel gave the information for the design of the definitive channel, which at present is behaving in accordance with the predicted way in the theoretical study

\section{INTRODUCTION}

The economical needs of the northwest of Mexico have led to the conclusion that a port is required at Topolobampo Bay. 
The main problem to establ ish the port has been the existence of a bar at the entrance of the bay The study of such problem started in 1952 and several solutions have been given, most of them, considering the use of jetties, but these being so long, the solution have always been an economic obstacle for the port

Wih hat thought in mind, this study has been carried out, with the purpose of finding a solution based on a dredged channel across the bar, which has to be hept by small works or maintenance dredging

\section{GEOMORPHOLOGIOAL BACKGROUND}

Topolobampo Bay is located at the northwest of Mexico (fig 1), it has an approximate area of $4885 \mathrm{~km}^{2}$ and also has several shallow zones and secondary inlets It is communicated with the Gulf of Cal ifornia through an inlet with a width of $45 \mathrm{~km}$ which is $\mathrm{l}$ imited at its ends by two sandy spits called "Punta Copas" and "Punta Santa María" Tnere are two perfectly defuned channels, the main one that covers the whole bay with NE-SW direction up to "Punta Copas" where it changes to an E-W direction, up to Santa María where the second channel, called Lechugullas, joins it This letter channel is communicated with San Esteban Bay located in the northern zone From there the inlet channel is formed with a S-SW direction

Topolobampo Bay is communicated with Ohuira Bay by a narrow and deep pass $\left(30 \mathrm{~m}\right.$ ) The letter bay with an extension of 148 o0 $\mathrm{km}^{2}$ is a very important factor in the bar's dynamics due to the great tidal prism generated by it

The present shape is due to an evolution of the "Rio Fuerte" delta, whose first mouth combined with volcanic action, remolded a great part of Topolobampo and Ohuira bays (1)

Latest processes determined an emigration of the mouth towards the North, and its old delta continued as the main source for the litoral barriers that lımic San Esteban, Topolobampo and San Ignaclo bays At the present time the "Rio Fuerte" contınues as the main source of beach material

Due to the wave characteristics acting in the zone as well as the induced currents produced by flood and ebb of the tide, a sandy bar has 
been formed at the bay's entrance, with a length of $12 \mathrm{~km}$, a width of $2 \mathrm{~km}$ and a depth of $23 \mathrm{~m}$ in a shape of a horse shoe, that joins "Punta Santa María" and "Punta Copas"

2 WINDS, WAVES, TIDES AND CURRENTS

21 Winds.

The prevalling and strongest winds come from the WNW, their main influence is the formation of seas which act from October to May, and from June to September there are others that produce swells from the SE The effect of both of them on the wind transport is of a secondary importance (2)

22 Waves.

Table 1

$\begin{array}{lcccc}\text { Type } & \text { Direction } & \begin{array}{c}\mathrm{H}_{1 / 3} \\ (\mathrm{~m})\end{array} & \begin{array}{c}\mathrm{T})^{2} \\ (\mathrm{sec})\end{array} & \begin{array}{c}\text { Acting Time } \\ \text { hr/year }\end{array} \\ \text { Sea } & \text { WNW } & 1.25 & 5 & 3720 \\ \text { Swell } & \text { S30W } & 09 & 12 & 480 \\ \text { Swell } & \text { SSE } & 0.9 & 12 & 840\end{array}$

\subsection{Tides}

They are of the mixed type with a mean range of $0826 \mathrm{~m}$, and a spring tidal range of $180 \mathrm{~m}$ They were recorded simultaneously at four points in order to relate them with the observed currents (fig 1)

\section{Currents}

The $t_{1}$ dal currents are significant at the main chamel ( 3 ) being the mean maximum velocity of $12 \mathrm{~m} / \mathrm{sec}$

From the field measurements it was obtained the following descharges distribution in everyone of the section indicated in fig. 2 and table 2 (4). 
Table 2

\begin{tabular}{|c|c|c|c|c|c|}
\hline $\begin{array}{c}1 \\
\text { Phase }\end{array}$ & $\underset{m}{2}$ & $\begin{array}{c}3 \\
\text { Section I }\end{array}$ & $\begin{array}{c}4 \\
\text { Section II }\end{array}$ & $\begin{array}{l}5 \\
\text { Secton } \\
\text { I+II }\end{array}$ & $100^{6} / 0$ \\
\hline Flood & 125 & $66 \%$ & $338 \%$ & $998 \%$ & $02 \%$ \\
\hline Ebb & 1.31 & $44 \%$ & $31.6 \%$ & $756 \%$ & $24 \quad 4 \%$ \\
\hline Flood & 1.128 & $62 \%$ & $28.7 \%$ & $908 \%$ & $9 \mathbf{3} \%$ \\
\hline Ebb & 1087 & $51 \%$ & $382 \%$ & $892 \%$ & $10.8 \%$ \\
\hline
\end{tabular}

Discharge in Section III $=100 \%$

Column 3 shows that at Lechuguilla Inlet $Q_{f l o o d}=Q_{E b b}$

Column 4 shows that the shallow zones have a considerable regulation effect.

Column 6 gives us an idea of the regulation capacity of the shallow zones.

\section{LITTORAL DRIF†}

The studies of grain sizes carried out in the bay as well as in the bar showed the presence of fine sand with a mean dlameter ranging from $0.25 \mathrm{~mm}$ to $0.125 \mathrm{~mm}$ with a very good distribution

On the bar there are two defined types of sedıment transport

\subsection{Shoreward transport.}

Produced by the swells that with their incidence almost normal to the shoreline and the bar itself as well as their length and period induce a transport towards the bar

\section{Littoral transport}

Due mainly to waves with a short period which effect is located at both 
littoral barriers.

The analysis of these data lead to the computation (4), (5), that the littoral drift along the bar, in the zone in which it was gang to be dredged, the channel was of the order of $360,000 \mathrm{~m}^{3} /$ year. Larras, Pychkine and Manohar criteria were used (fig 3 and 4)

\subsection{Suspended transport}

Suspended transport measurements were made at the different velocity control sections, and it was found that the amount of suspended transport varies from $0 \mathrm{mg} / \mathrm{lt}$ up to $155 \mathrm{mg} / \mathrm{lt}$, depending on the current velocity and the sections of study, being the maximum recorded values taken at the channel zone, finding out that at $6 \mathrm{~m}$. depth and a velocity of $060 \mathrm{~m} / \mathrm{sec}$, the transport was $10 \mathrm{mg} / \mathrm{lt}$, on the other hand, at $1 \mathrm{~m}$ depth in the same zone, it was recorded $5 \mathrm{mg} / \mathrm{lt}$, which leads to the conclusion that its effect is of secondary importance.

\section{BAR EVOLUTION FROM 1875 TO 1962}

The analysis (4) was made considering the variations of the centroidal axis of the bar in order to study its movements in a plan view as well as in elevation ( $F$ ig 5 )

It was observed that

a Between $5^{\circ}$ and $45^{\circ}$ the bar tended to grow seaward, due probably to the channel effect of Punta Copas which dissipates the wave action from the SW. The maximum varlation was 1300 metres for 1967

b Between $45^{\circ}$ and $75^{\circ}$ exists a transition zone, because the bar showed movements towards WNW as well as to the bay, the reason for this behaviour could be the gradual disappearance of high ebb currents, that could appose to the SW waves.

c Between $75^{\circ}$ and 1200 the bar tendency was to grow towards the bay, due to the wave action in that zone in which there does not exist any definite active of tidal currents. Nevertreless, due to the sand transport diffusion and the channels that limit the zone, the movements are, as an average, 4 times smaller than the ones occuring between 50 and $45^{\circ}$ 
d. From 1270 up to $180^{\circ}$ the bar tendency is growing towards the sea in a similar way to the first zone considered above, being the maximum movements between $150^{\circ}$ and 1650 , which are of the order of $1455 \mathrm{~m}$. for 1962. This is due to the fact that some small bars appeared as a result of the littoral drift from the WNW and the veryefficient hydraulic groin formed by the main outlet channel.

\section{Depth variations.}

Generally speaking, the vertical movements of the bar are not excesive, being up wards as well as downwards, these variations being noticible at radiations $5^{\circ}, 15^{\circ}, 75^{\circ}, 90^{\circ}$ and $105^{\circ}$ whose maximum relative values are $1.5 \mathrm{~m} ., 1.55 \mathrm{~m}, 070 \mathrm{~m} ., 135 \mathrm{~m}$ and $1.30 \mathrm{~m}$ respectively.

The rest of the zones tend to keep the same depth that in 1875, having small movements upwards as well as down wards The most stable zones are the $450,60^{\circ}, 127^{\circ}, 130^{\circ}, 1500$ and 1800

The mean depth in the bar varies from $3 \mathrm{~m}$. to $4 \mathrm{~m}$, the $3.90 \mathrm{~m}$ being the most stable depth which is located between $105^{\circ}$ and $120^{\circ}$, the smallest depths are between 150 and $75^{\circ}$ with a value of $3 \mathrm{~m}$.

\section{EXPERIMENTAL CHANNEL}

In 1962, from theoretical results showed in the above pragraphs, the existing zone between $75^{\circ}$ and $120^{\circ}$ was ctosen as the proper place for the dredging of the experımental channel as this had demostrated the maximum stability.

The al ignment of the channel was made following the tendency of the natural channel.

The design dimensions were

$\begin{array}{ll}\text { Bottom width } & 75 \mathrm{~m} \\ \text { Slopes } & 51 \\ \text { Mean depth } & 7 \mathrm{~m}\end{array}$

The experimental channel had as a main function the precise evaluation of the littoral drift and hence its maintenance cost In addition the channel would permit the access of small ships to the port. 
From the date of its construction, periodical soundings were made, and during a four months period, in 1964, the channel dredging was suspended in order to be able to know if its natural tendency would be its accreation, maintanance or erosion Simultaneously, a radioactive tracer study, was carried out (Fig 6)

The results of the experimental channel can be divided into two groups

Channel behaviour

Effects on the bar.

\subsection{Channel behaviour}

The amount of littoral drift that reached the channel, recorded in its initial phase, when its efficiency and discharge were still low, was of the order of $320,000 \mathrm{~m}^{3} /$ year $\left(360,000 \mathrm{~m}^{3} /\right.$ year computed) (fig 7) At that time, the mean currents velocities in the channel was $04 \mathrm{~m} / \mathrm{sec}$. From 1965 in which dredging was practicaly suspended, a tendency to increase the section has been observed In table 3 , the results of control soundings are shown, pointing out that in 1966, the channel velocities, for similar tide conditions were of $08 \mathrm{~m} / \mathrm{sec}$

Table 3

$\begin{array}{lcccc}\text { Date } & \begin{array}{c}\text { Minimum } \\ \text { depth }\end{array} & \begin{array}{c}\text { Maximum } \\ \text { depth }\end{array} & \begin{array}{c}\text { Channel mean } \\ \text { width at } 6 \text { m depth }\end{array} \\ \text { July } 1966 & 615 & 840 & 50 \\ \text { Aug } 1966 & 690 & 865 & 60 \\ \text { Oct } & 1936 & 655 & 865 & 70 \\ \text { Nov. } 1966 & 705 & 8.80 & 90 \\ \text { Dic } & 1966 & 710 & 8.70 & 75 \\ \text { Feb. 1967 } & 6.95 & 900 & 100 \\ \text { Mar. } 1967 & 680 & 875 & 115 \\ \text { Apr. } 1967 & 755 & 9.15 & 150 \\ \text { Jun } 1967 & 7.20 & 920 & 120 \\ \text { Sept. } 1968 & 8.50 & 935 & 150\end{array}$




\section{Effects on the bar}

Inmediately after dredging the experimental channel, a big shallow zone started its formation on the NW side of the bar, which was modified by the changes in wave action, but that in general, showned the tendency to increase in size in such away that at present its distance from Punta Santa María is of only $150 \mathrm{~m}$ Initially that distance was $1200 \mathrm{~m}$ (fig 8)

\section{RADIOACTNE TRACERS INVESTIGATION}

During a period of four months in 1964 a radioactive tracers investigation was programmed using a synthetic glass sample with $\mathrm{Ta}_{2} \mathrm{O}_{5}$ to obtain $T_{a} 182$ with a 250 milicurie of activity The tendencies showed by the tracers checked notably with the results of the soundings in the same period of time (fig 7) The accretion tendency observed at that time disappeared when the hydraulic efficiency of the channel was improved

\section{RESULTS ANALYSIS AND CHANNEL DESIGN}

The analysis of the experimental channel behaviour shows a definite tendency to the increase of tidal currents velocities and hence an increase of the shear stress which represents a channel erosion This effect is favoured by the shallow zones formed, so it can be assumed that the channel tends to an equilibrium state, due to the increase of its hydraulic efficiency when the tidal prism diffision decreases due to the shallow zone and its concentration into the channel This tendency can be natural or be increased in an artificial way by means of dredging in the channel so that it can acquire a stable section

It is convenient to point out that in the analysed period there was a season (January to March 1967) in which the channel showed a tendency to accretion This tendency corresponded to the forecast made by the computations and radioactive tracer studies, nevertheless, spring tides in April eliminated the accretion (7) continuing in such a way the self dredging of the channel From that date up to now all the dredging work has been abandoned

Providing that for navigation requirements the channel must have a minimum width of $150 \mathrm{~m}, 11 \mathrm{~m}$ depth and construction slopes 51 (fig 6), 
at present a dredging volume of $1,300,000 \mathrm{~m}^{3}$ is still required

Considering that the mean diameter of the sandy material that constitutes the bar in the channel zone is $0125 \mathrm{~mm}$, it is required a $=036 \mathrm{Kg} / \mathrm{m}^{2}$ and a velocity of $25 \mathrm{~cm} / \mathrm{sec}(8)$ in order that the bottom particles start their movements and taking irto accourt that at present, mean maximum velocities have been measured with a value of $0.8 \mathrm{~m} / \mathrm{sec}$ it is expectable that for spring tides the mean maximum velocity reaches similar values to that existing in the present main channel with a value of $1 \mathrm{~m} / \mathrm{sec}$ (3)

Therefore, using the mean velocity of $08 \mathrm{~m} / \mathrm{sec}$. the following results in relation to the self dredging capacity of the channel are obtained

$$
\mathbf{Z}=\frac{640}{2020}=0.315 \mathrm{~kg} / \mathrm{m}^{2}
$$

In accordance with Kal inke's Formula

$$
\begin{aligned}
q_{s} & =\frac{10 D \zeta^{5 / 2}}{\rho^{1 / 2} D^{2} \gamma^{2}\left(S_{s}-1\right)^{2}}=\frac{10 \times 0.56}{110 \times 125 \times 10^{-3}(1000)^{2}(16)^{2}}= \\
& =\frac{056}{35 \times 10^{3}}=16 \times 10^{-4} \mathrm{~m}^{4} / \mathrm{seg} / \mathrm{m} . \\
Q_{s} & =16 \times 10^{-4} \times 195=314 \times 10^{-4} \mathrm{~m}^{3} / \mathrm{sec} \\
M_{s} & =495,000 \mathrm{~m} 3 / \text { year } \\
\frac{M_{s}}{M_{e}} & =\frac{495,000}{300,000}=1,66>1
\end{aligned}
$$

So the section will tend to increase (9) diminishing the shear stress, until the equilibrium between the channel capacity for washing out the accretion and the material transported from the bar is reached.

With the increased values of the velocities observed in the channel zone, it is very feasible that it could reach the value of $1 \mathrm{~m} / \mathrm{sec}$, and hence, the required shear stress required for the equilibrium is attained with a much bigger area than the one necessary for navigation purposes. 
In other words, the channel in the way it has been designed, will not have any maintenance problems as depth concerns.

\section{CONCLUSION}

From the above study the following conclusions are obtained

1 The applied theoretical and experimental methods have been checked with nature results.

11 The access channel has a definite tendency to be stable

i11 This stable status will be completely attained when, by navigation requirements, the channel gets at least $150 \mathrm{~m}$. of bottom width and $11 \mathrm{~m}$. depth

IV The maintanance dredgings during certain seasons in the year will be small and very well located. 
Ayala-Castañares A \& F Pnleger - Informe final del Estudio de Geología Marına del Area de la Bahía de Topolobampo, Sinaloa Instıtuto de Geología México 1966

2 Cervantes C D - Nolas sobre Transporte Eól ıco Anál isıs de Dlferentes Criterıos utılızados para tratar de clantıfıcar el acarreo por vierto Departamento de Estudios y Laboratorios, Secretaría de Marina Boletín Técnico No 1, Tomo II México, marzo 1900

3 López G H \& J Aguılar - Análısıs Hıdráulıco del Comportamıento de la Onda de Marea a través de la Bahía deT polobampo y sus Efectos en la Barra Proc of the 8th Conference on Coastal Engineering

4 López G H - Estudio Integral del Azceso a la Bahía de Topolobampo, Sin Depto de Estudios y Laboratorios, Boletín Técnico No 2 y 3, Tomo II Méxlco, Junio-septiembre 1966

5 De Alba $L$ P - Estudio General del Acceso al Puerto de Topolobampo, Sin Aplicación de Trazadores Radioactivos Tesis Profesional Méxıco 1965

6 López G H - Aplıcacıón de Radioısótopos a Problemas de Ingenıería de Costas Estudios en Topolobampo, Sin - Depto de Estudios y Laboratorios, Boletín Técnico No 1, Tomo I Mexico, 1965

7 Cervantes C D - Acceso al Puerto de Topolobampo, Sin - Primera Reunión para el De sarrollo del Puerto de Topolobampo (No publicado) 1909

8 Larras J - Embouchures, Estualres, Lagunes et Deltas Collection du Centre de Recherches et D'Essais de Cnatou Eyrolles, Paris 1964

9 Bruun P - Tidal Inlets and Lirtoral Drıft Vol 2 - Ed Universitets Forlaget, Oslo, 1906 


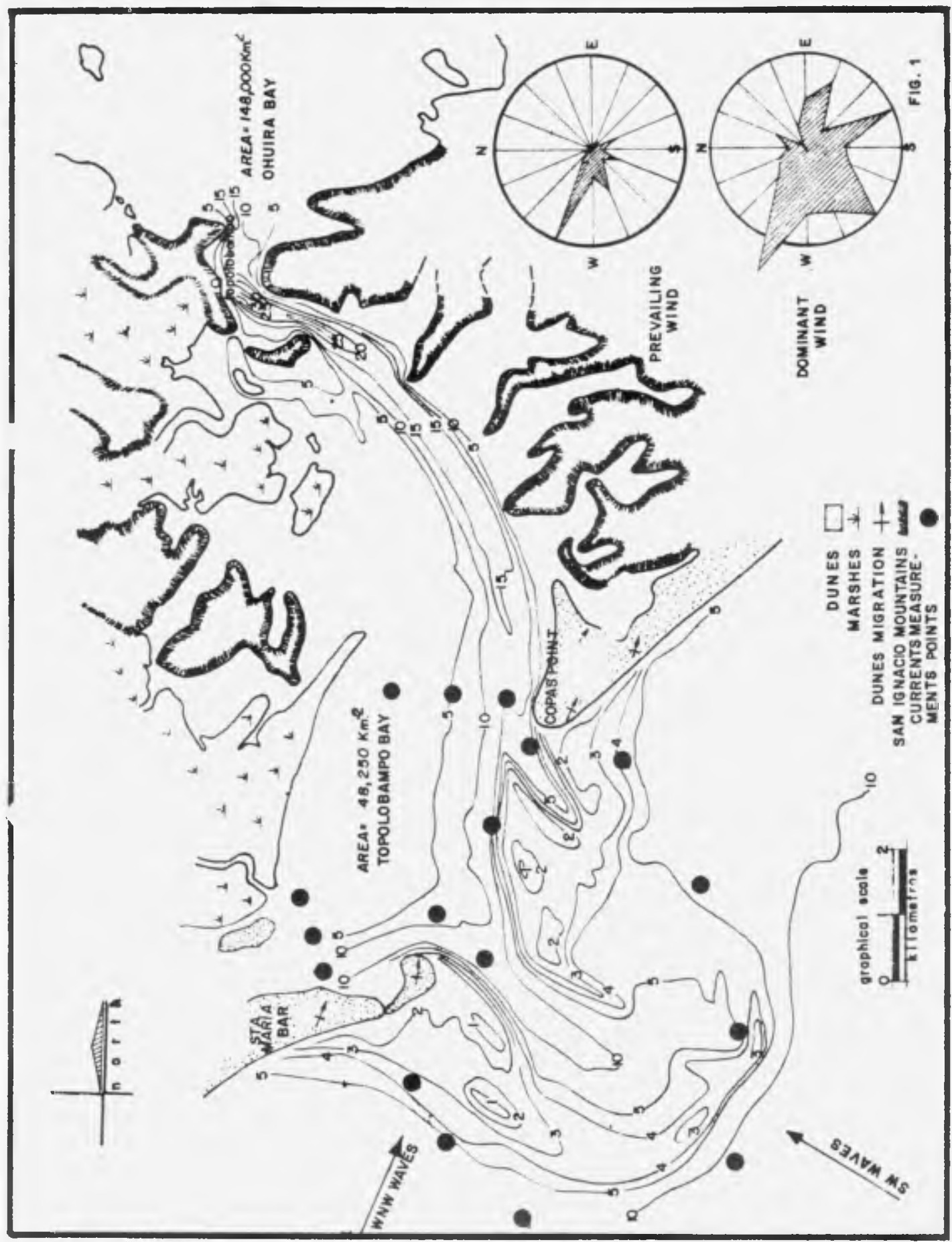


TOPOLOBAMPO'S BAY ENTRANCE

1163

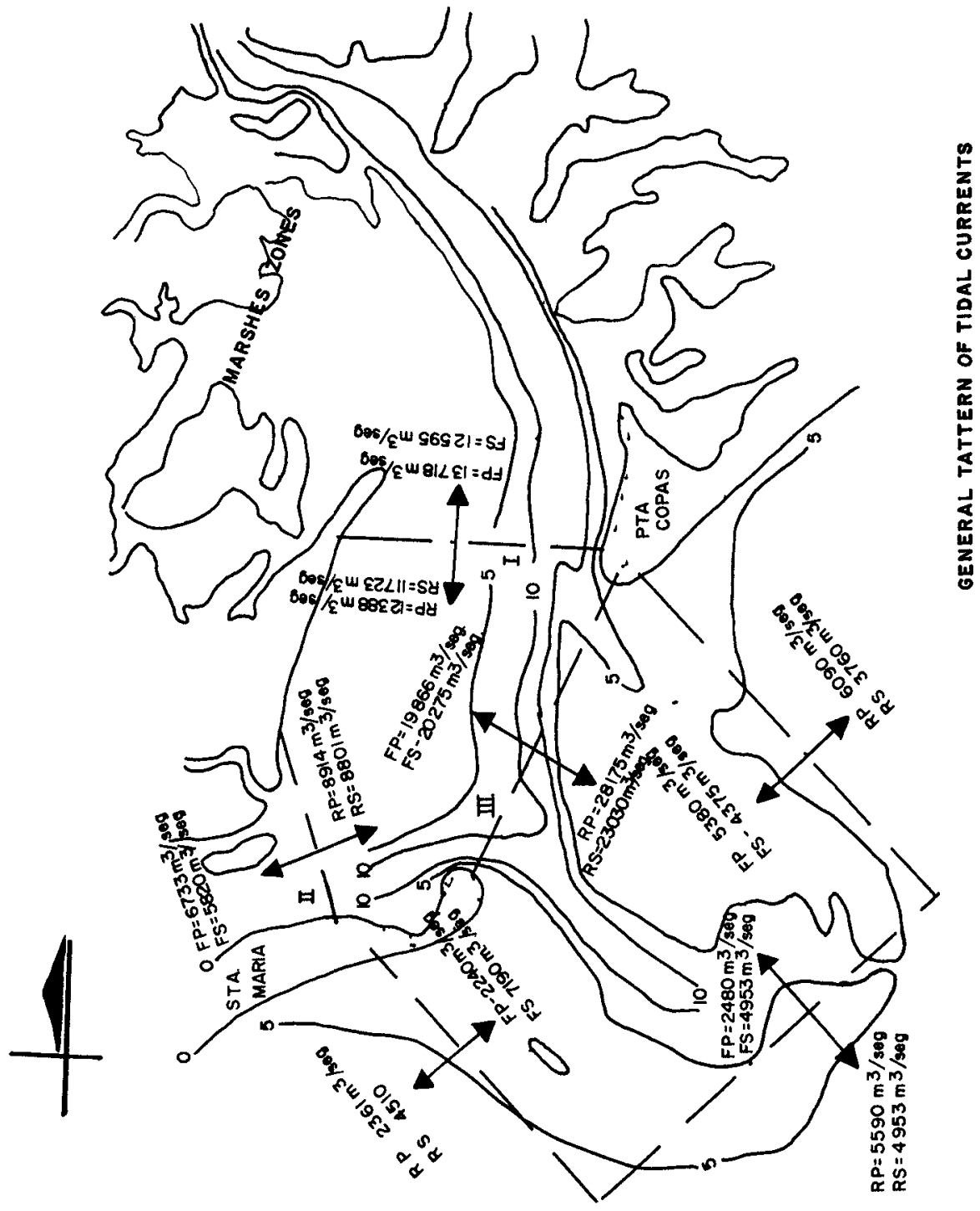

$\omega$

of
2
인 


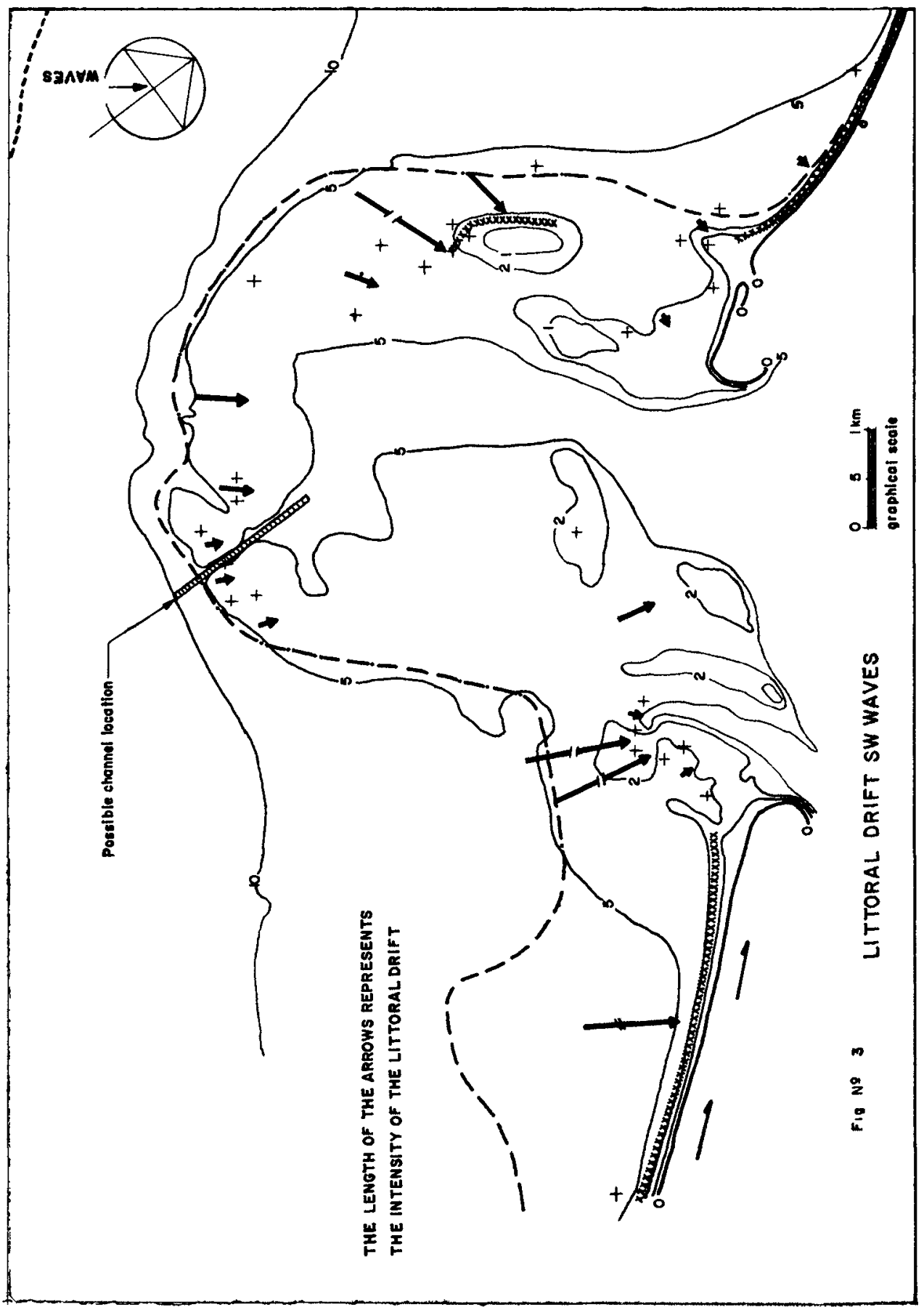




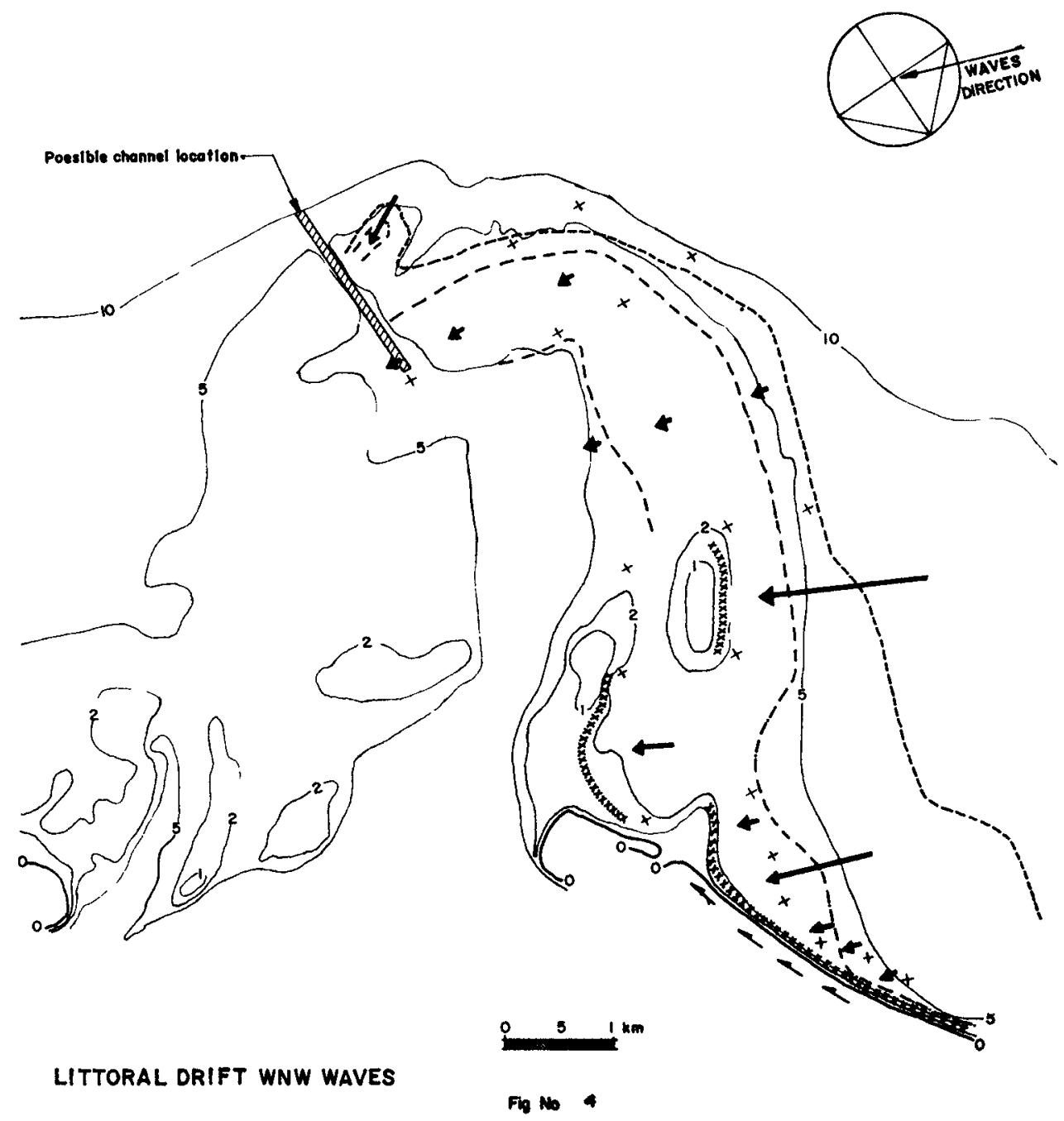




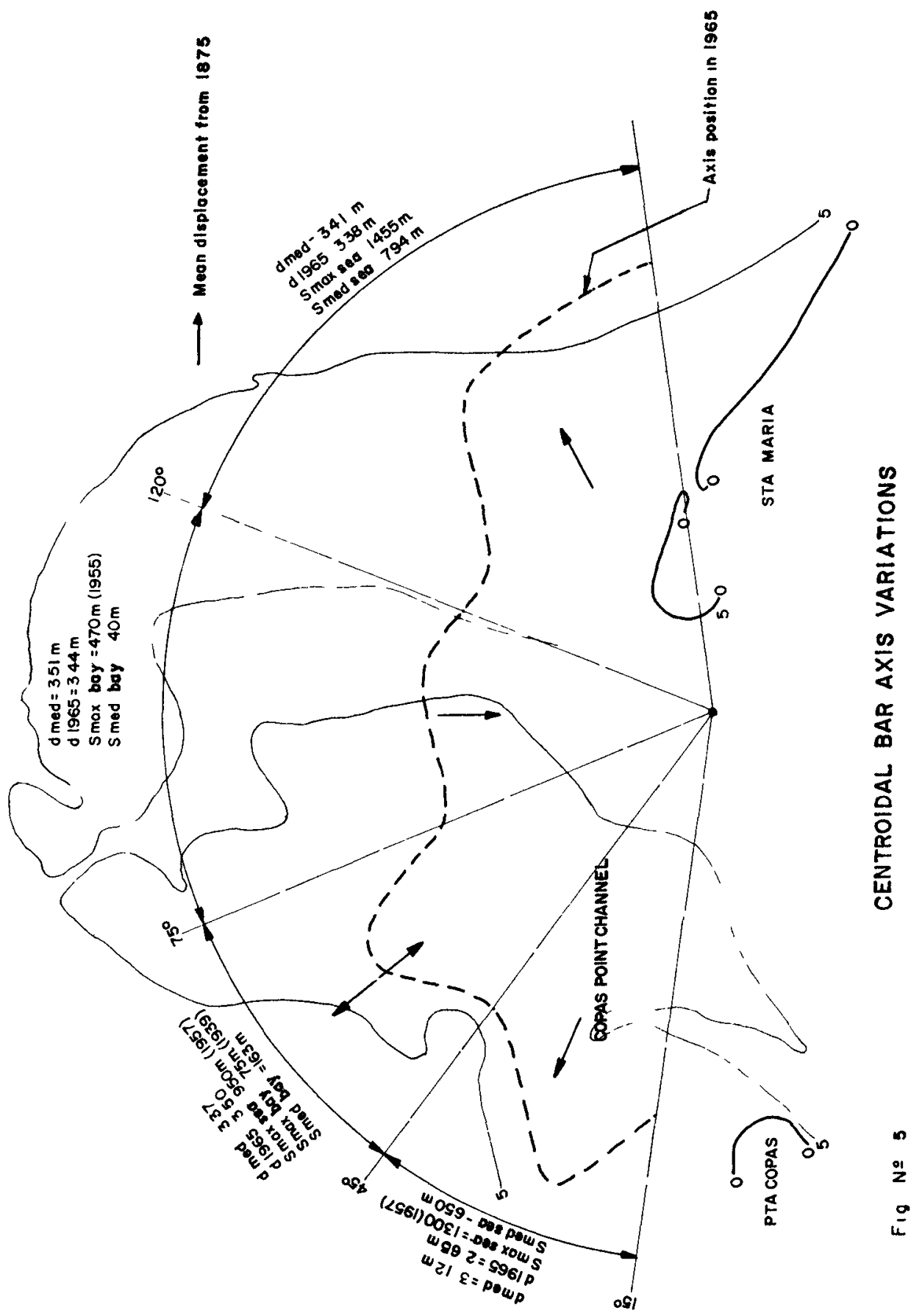




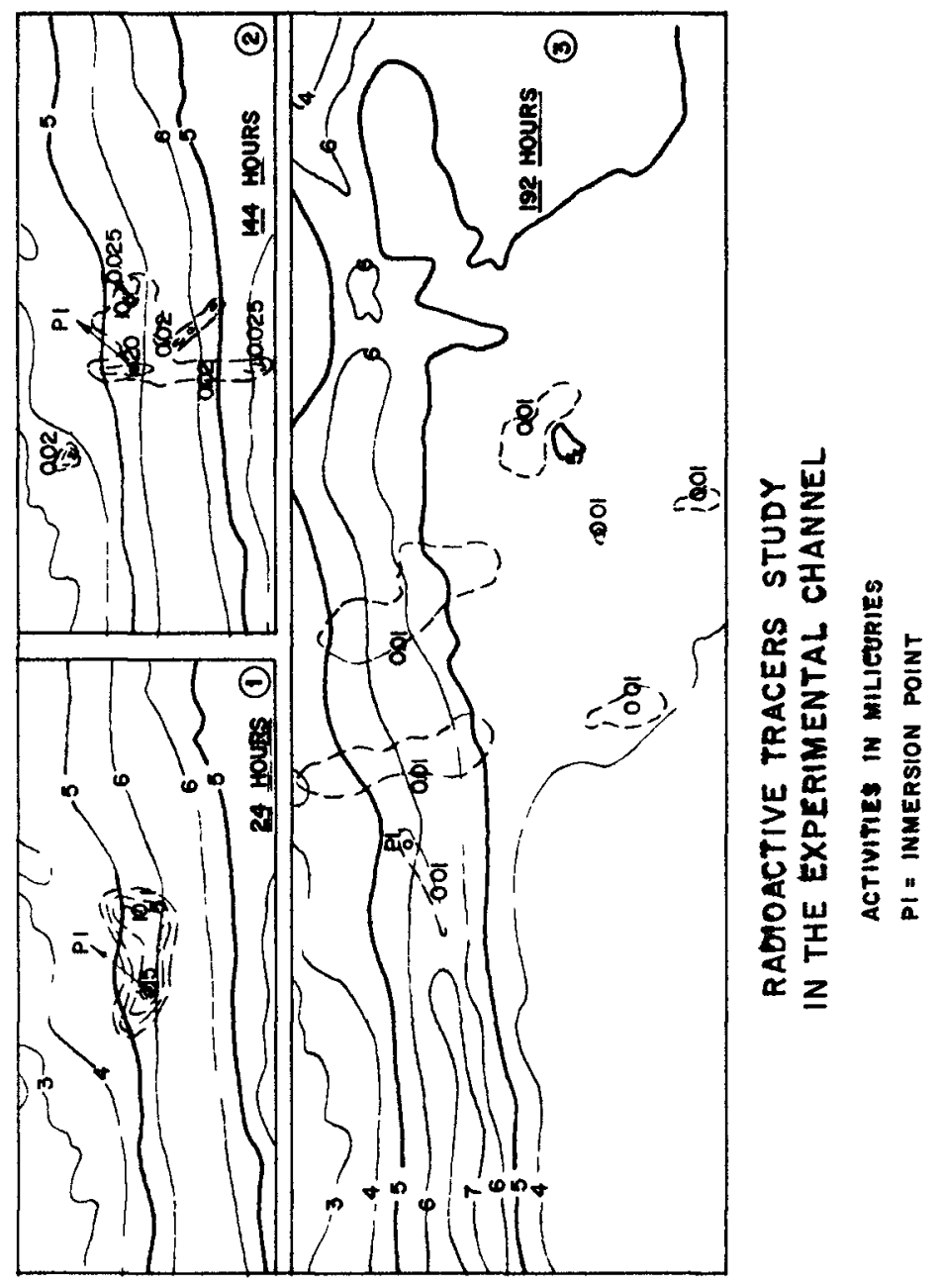




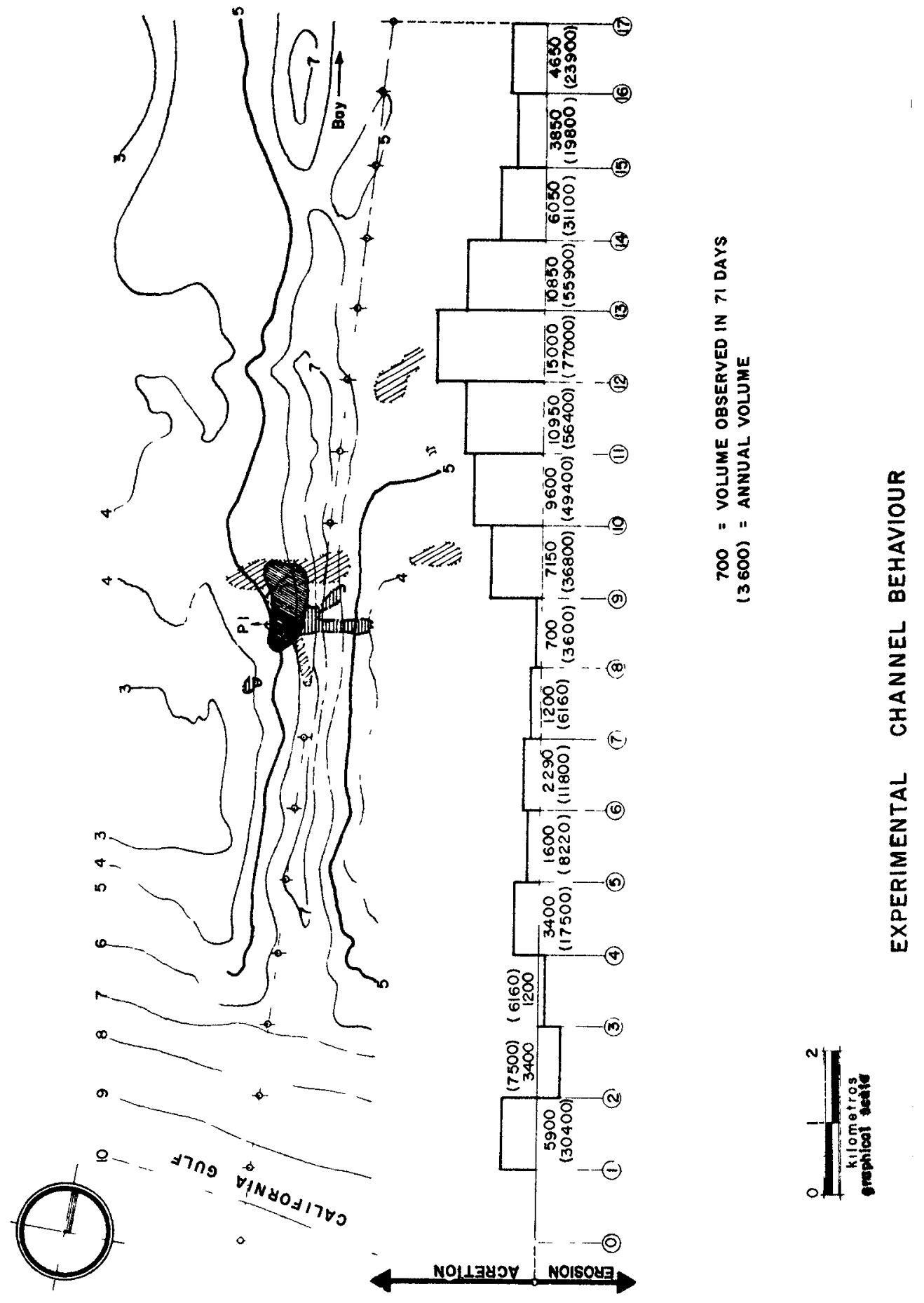




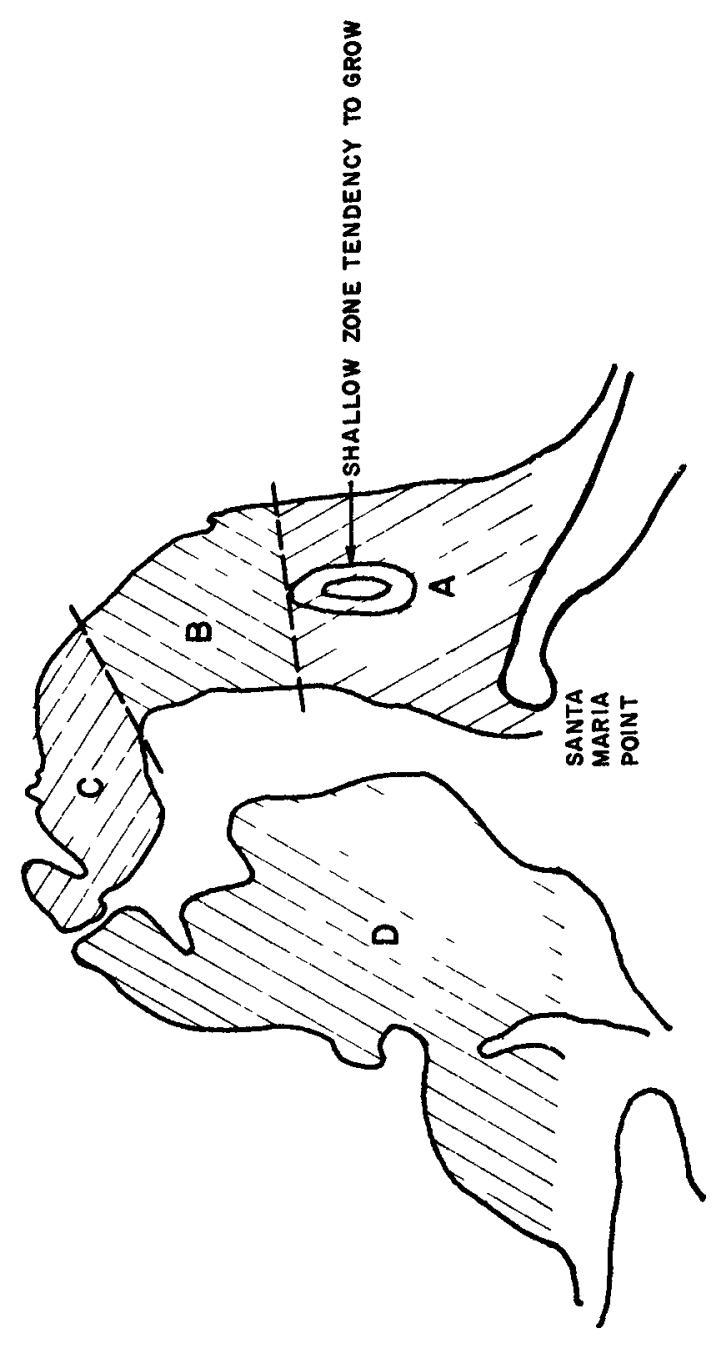

; 



\title{
CHAPTER 74
}

TIDAL INLET PROBLEMS ALONG THE NEW ENGLAND COAST

\author{
Oscar E Arpin \\ United States Army Corps of Engineers, \\ New England Division \\ Waltham, Mas sachus etts
}

\begin{abstract}
The New England Division of the United States Army Corps of Engineers has created and improved many harbors along the New England coast for the benefit of commercial fishing and recreational boating fleets A harbor, to be effective, must provide a protected area for boats and it must have a safe access, that is, a protected and stabilized tidal inlet A coastal inlet has been defined as a waterway connecting a bay, lagoon or similar body of water with a larger body of water The major engineering problems encountered in the development of harbors have involved stabilization of these inlets The problems have been caused by excessive littoral drift resulting from erosion of the shorefront adjacent to the inlets by wave attack Planning, designing, constructing, and modifying these inlets under very dynamic conditions is complex and difficult Each inlet is unique This paper discusses very briefly some of the problems encountered
\end{abstract}

\section{INTRODUCTION}

Two principal factors are involved in producing littoral drift One factor involves storm waves and tides New England is often subjected to severe storms, including hurricanes (See Figure No 1) New England has been battered by a severe hurricane on the average of once every five years during the last 60 years The second factor involves geologic structure Storm wave attack on unconsolidated materials results in severe shoreline recession and an excessive rate of littoral drift The section of the New England coast where tidal inlet problems have been encountered is that extending southerly from Portland, Maine to and including Cape Cod and the offshore islands of Martha's Vineyard and Nantucket

Littoral drift does not pose a problem along the coast of Maine extending between Portland and the Canadian border, as this shorefront is composed largely of massive ledge outcrops which are very resistant to e rosion forces, in spite of severe storm wave attack and normal tide ranges up to 20 feet The shore of Connecticut is composed largely of hard rock Further, it is afforded some protecţıon from severe storm 


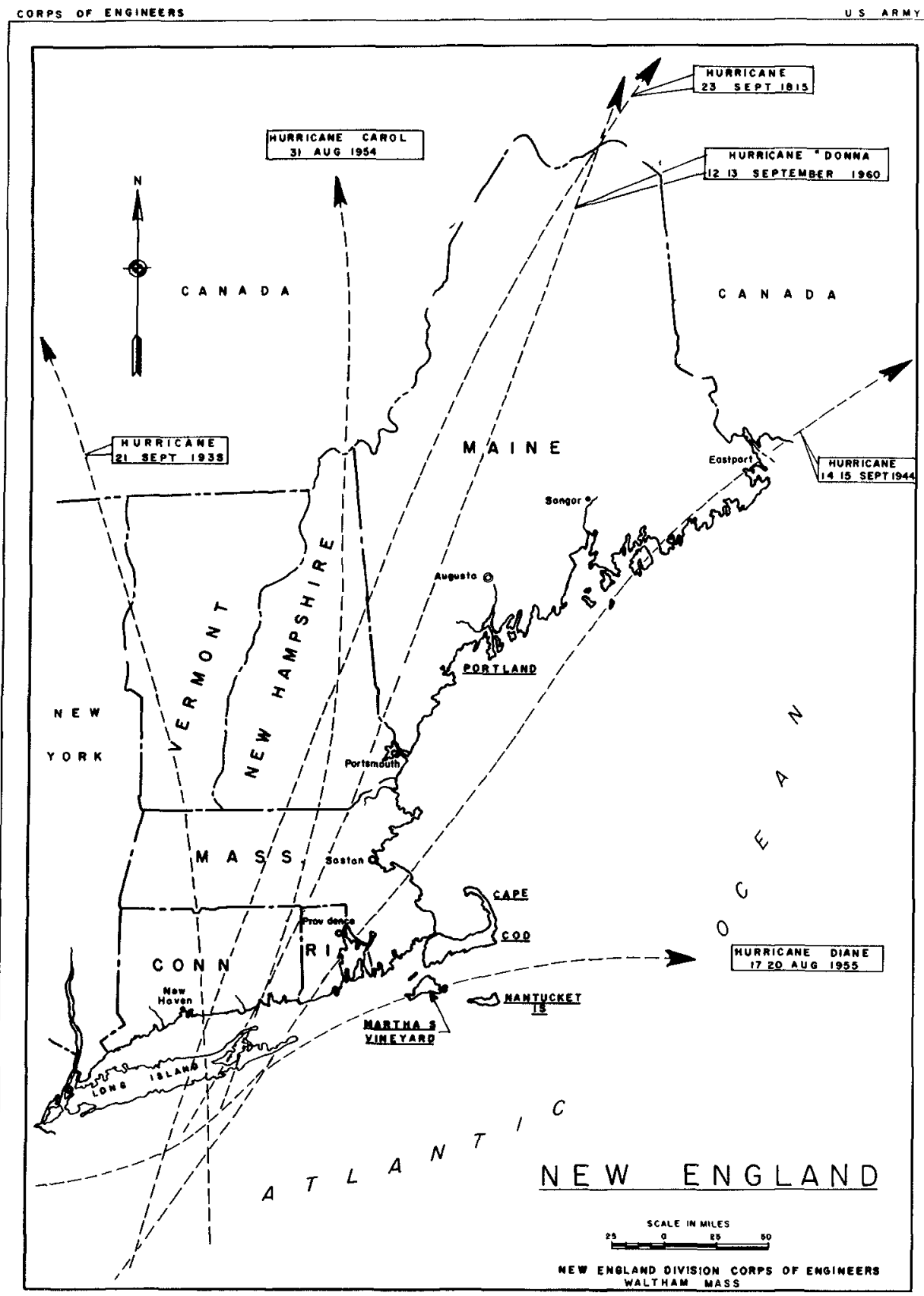


waves by Long Island to the south and it experiences tide ranges of relatively few feet Thus, its coast remains irregular in configuration, whereas the shore of Rhode Is land just to the east, also compos ed of unconsolidated glacial debris like that of Connecticut, is now almost straight with long sand barrier beaches The primary differences are that the Rhode Island shore consists of boulders mixed with clay, uncemented and thus easily eroded, and the shore is directly exposed to severe storm waves from southerly quadrants This coast, much of which is still privately owned, is relatively undeveloped The littoral drift encountered in Narragansett Bay, Rhode Island and in Buzzards Bay, Massachusetts is not of major significance The chief difficulty requiring attention within these bays is tidal flooding due to hurricanes

Continuous battering of the unconsolidated materials of Cape Cod and the offshore is lands of Martha's Vineyard and Nantucket by large waves generated over vast expanses of water, result in rapid and extensive erosion For example, much of the south shore of Martha's Vineyard is estimated to be receding at a rate of 8 feet each year, involving sand losses of 400,000 cubic yards. Some portions of the south side of Nantucket Island and of the southeasterly side of the outer arm of Cape Cod are estimated to be receding at rates up to 15 feet each year

\section{Newburyport Harbor, Mass achus etts}

Man has studied storms and their generated waves and the geologic structure of the coasts for many years He uses all the advanced and sophisticated knowledge avalable concerning coastal development $\mathrm{He}$ conducts wave refraction, diffraction and reflection studies and makes mathematical and hydraulic model studies He does all this and more, only to find that nature interjects a new unforeseen factor An example of this occurred in February 1969 at Newburyport Harbor, Massachusetts, located 55 miles north of Boston, Massachusetts (See Figure No 2) First, a little background The Federal navigation project for this harbor was constructed in the early 1900's to stabilize the badly mig rating inlet at the mouth of the Merrimack River which rises in central New Hampshire and has a drainage basin of about 5,000 square miles The south jetty is nearly 2,500 feet long The north jetty is over 4, 100 feet long Both jetties were built to an elevation of 12 feet above mean low water In February, 1969 three northeast storms struck within a relatively short time of each other The first prevailed during 9 and 10 February, the second from 19 through 21 February, and the third lasted for about 4 days, 24 through 27 February

Storm waves from the northeast overtopped and diffracted around the end of the deteriorated north jetty Waves from the east entered the inlet directly These waves ran generally along the smooth face of the south jetty, then being rehabilitated, and attacked the shore fronting the United States Coast Guard Station located at the inshore end of the south jetty The mean high water line receded 150 feet as a result of these 


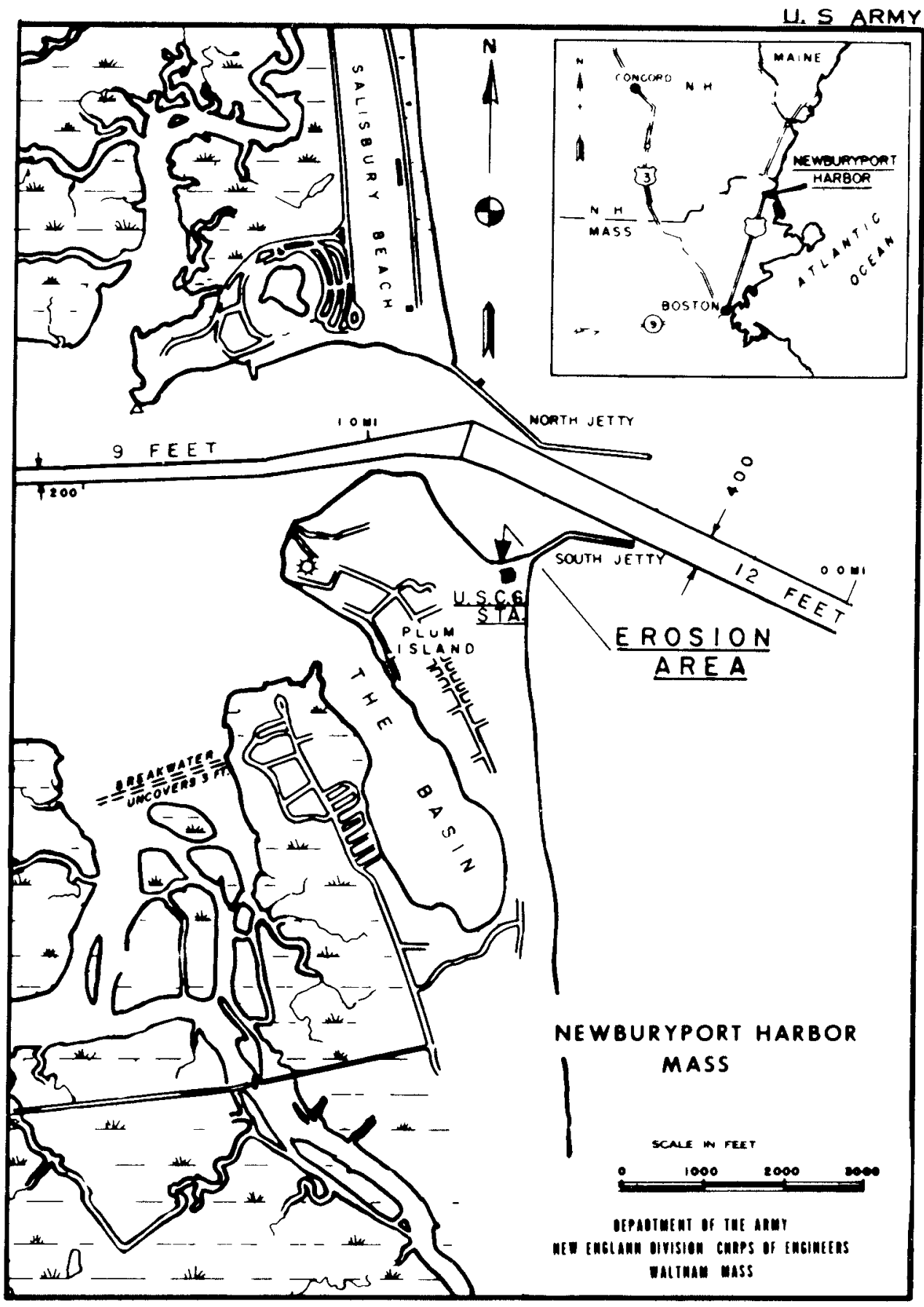


storms The erosion, once triggered, continued at a more gradual rate toward the station A special study was quickly made to determine what corrective measures were necessary to save the station, which was built in 1930 to provide emergency assistance to ships and small boats in distress offshore A stone mound with sandfill, and revetment was recently completed to stabilize that shorefront. Erosion had reached one corner of the station at the time of initiation of the emergency work

\section{Green Harbor, Mass achus etts}

Another inlet problem recently developed at Green Harbor, Massachusetts, located 35 miles south of Boston (See Figure No 3) The Commonwealth of Massachusetts and the Town of Marshfield have provided and maintained navigation improvements at this harbor for over 70 years The jetties were constructed by the Commonwealth in 1898 and 1899 In 1969, the United States government, in financial cooperation with the State, County and Town governments, completed a modification of the existing project The modification provided for sealing and extending the west jetty 200 feet to prevent littoral drift from passing through and around the end of the jetty and into the channel It is interesting to note that the south to north $\mathrm{drift}$ at the inlet constitutes a local reversal of the overall predominant net north to south drift along these shores Project modification also included raising the top of the east jetty from 12 to 14 feet above mean low water and dredging a harbor channel and anchorage

Shortly after completion of these improvements, erosion began along the shore at the inshore end of the east jetty Field observations and wave studies indicate that the erosion is probably the result of reflection of easterly waves off the smooth face of the west jetty extension with little reduction in wave height A stone revetment and sandfill are being prescribed for stopping the erosion

\section{Andrews River, Massachus etts}

In 1968 , a new harbor was created in an area of negligible value marshlands within Andrews River in Harwich, Massachus etts, a small tidal creek on the south side of Cape Cod (See Figure No 4) The project provides for a Federal entrance channel protected by stone jetties and a maneuvering area to serve a marina complex provided jointly by the Commonwealth and the town The harbor was developed to accommodate the fast growing recreational boating fleet in the area Nearby harbors had long been saturated After much study and consultation between the New England Division and the Office of the Chief of Engineers in Washington, D C, it was decided to defer construction of the west jetty until experience and observation showed the need for it It was generally considered that the long jetty protecting and stabilizing the Wychmere Harbor inlet immediately to the west would also provide 


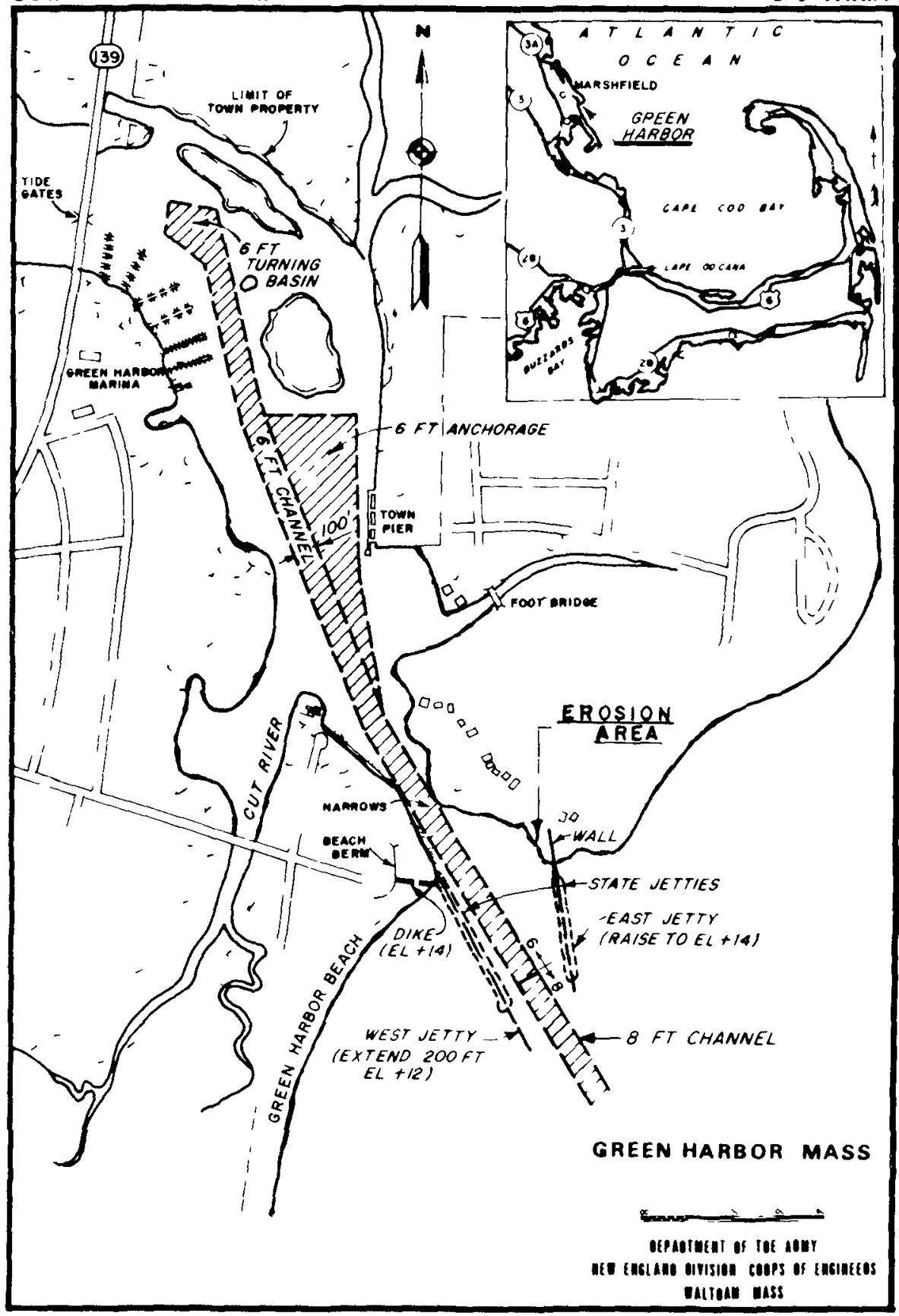

Figure 3 


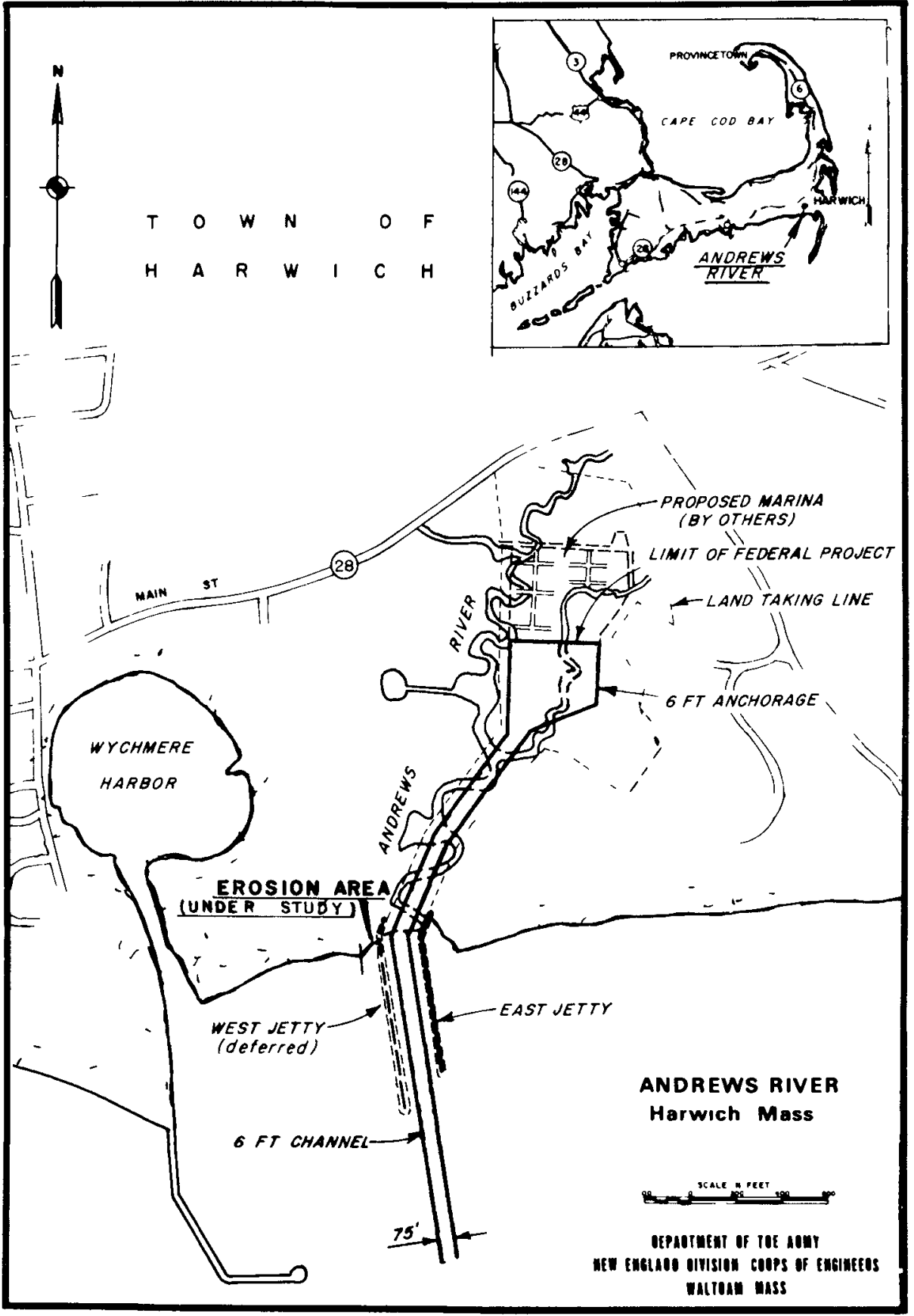

Figure 4 
protection to and prevent the west to east littoral druft from fouling up the Andrews River inlet Since completion of the project, erosion of the shore just west of the new inlet and shoaling of the entrance channel have occurred Field observations and wave studies made to date indicate that south and southeasterly storm waves may be diffracting around the end of the Wychmere Harbor jetty and then moving easterly More detalled studies are being made to determine if the west jetty should now be recommended for construction, or if some other corrective measure is required

\section{Scarboro River, Maine}

In 1960, a problem was encountered during construction of a jetty at Scarboro River, a small shallow stream rising in the marshlands of the town of Scarboro, Manne about 17 miles southeast of Portland, Maine (See Figure No 5) The jetty was being built to impound excessive west to east littoral drift, which was rapidly shoaling the Federal entrance channel constructed several years earlier When the jetty had been constructed 350 feet from the shoreline, severe scour occurred at the seaward end The area of scour extended $100 \mathrm{feet}$ beyond the end of the jetty, beyond which accretion occurred The sea bottom in the area of scour went from 2 feet below mean low water to nearly 19 feet below mean low water. Consultation with known experts in the field of tidal hydraulics revealed that tidal currents were flowing nearly perpendicular to the jetty during both the ebb and flood tides resulting from a shift in tidal flow not anticipated Also, the bottom materials were found to consist of very fine sand A greater thickness of bedding, 3 to 4 feet instead of 18 inches, consisting of $10-150$ pound stone placed at least 50 feet in advance of the core and armor stone, was found to be the answer to the problem

\section{Wells Harbor, Marne}

As a result of the establishment of the Wells Harbor Committee in 1953 to determine what measures were necessary to develop a harbor at Wells, Malne (See Figure No 6), the Federal government constructed two converging stone jetties at the mouth of the Webhannet River to stabilize the migrating inlet The project included dredging a channel and anchorage in the marshlands During construction of the south jetty to its angle point, that is, where it then extends parallel to the channel, accretion of the northerly tip of Wells Beach took place However, as construction of the jetty progressed to 1 ts full length of 940 feet parallel to the channel, erosion of the tip of Wells Beach occurred rapidly Over 400 feet were lost in eight months Concurrently, the north jetty, 640 feet long, was already nearly completely impounded, with littoral materials about to enter the inlet These occurrences meant that the proposed anchorage would be directly exposed to easterly storm waves and to severe shoaling 


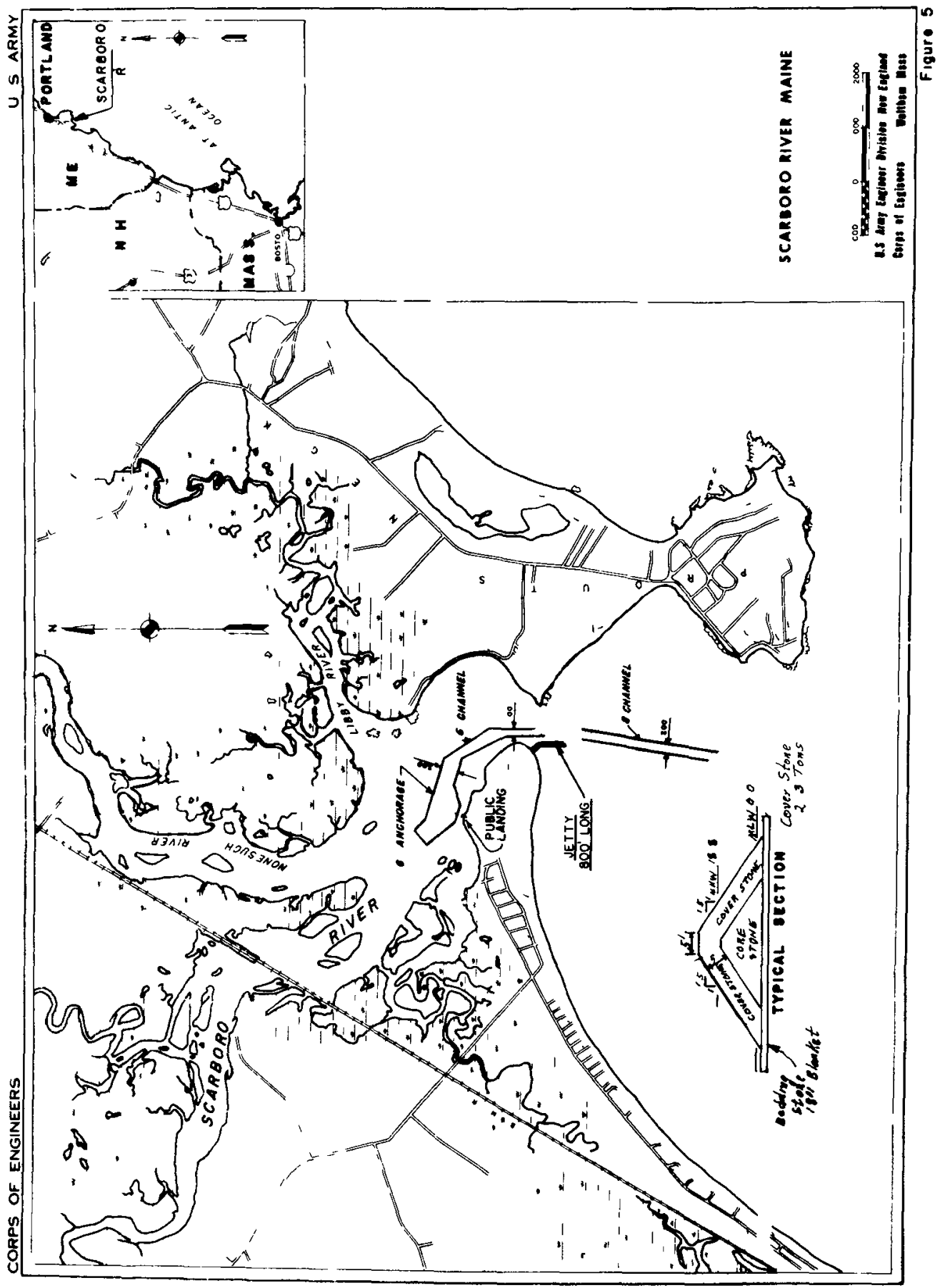




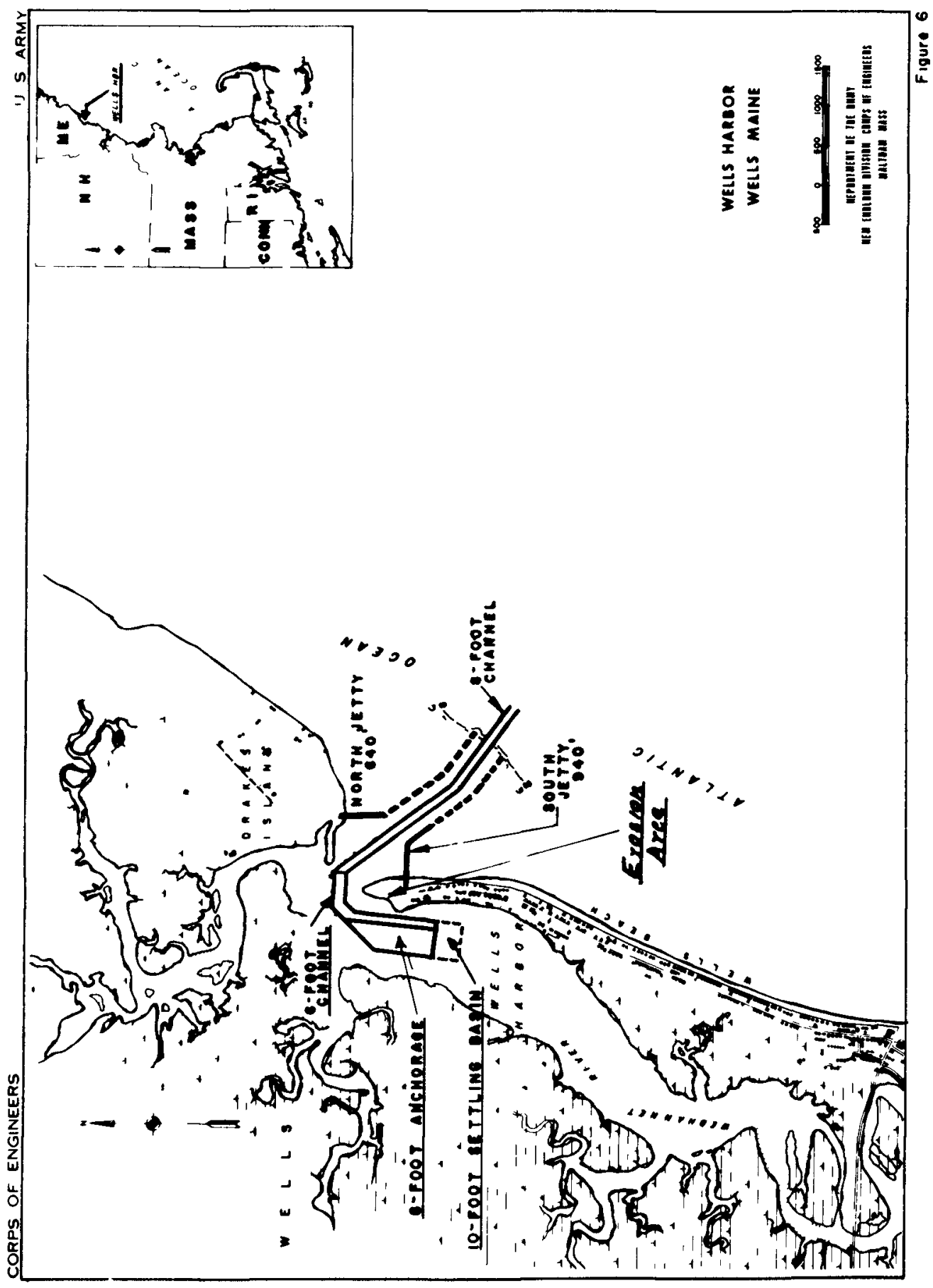


After discussions with the Committee on Tidal Hydraulics and representatives of the Waterways Experiment Station at Vicks burg, Mississippi, it was decided to construct a 100 -foot long wave absorber along the inner end of the south jetty, place stone revetment along 680 feet of the tip of Wells Beach, and extend the north jetty The channel and anchorage were then dredged However, because of a continued high rate of shoaling, both from littoral drift and from sediments carried down the Webhannet River, both jetties were later extended to about the 8 -foot depth contour and a 10-foot deep settling basin dredged at the upstream end of the anchorage to intercept river sediment In spite of numerous problems encountered in the creation of Wells Harbor, it now is overcoming the growing pains and is beginning to function as planned It is a safe harbor for the commercial fishing boats which transferred from nearby inadequate coves and for a new fleet of recreational boats which could not be accommodated at the saturated harbors to the north and south of Wells Harbor

\section{Chatham Harbor, Massachusetts}

Chatham Harbor, Massachusetts is located on the southeasterly corner of Cape Cod in an area of very rapid and continuously changing shoreline conditions (See Figure No 7) The directions, rates, and amounts of littoral drift are extremely complex and variable Planning and designing a modification of the existıng Federal navigation project at Chatham Harbor proved to be difficult under these unique conditions In 1956, Monomoy Beach, a long narrow barrier beach, was connected to Morris Island At that time, it was breached by storm waves The breach widened, deepened and became a continuous waterway By 1961, s ediment moved by the tidal currents through the breach, extended over a wide area and filled the existing Federal channel around the tip of Harding Beach The shoaling continued to advance westward along the south side of Harding Beach toward the Chatham Roads approach channel Concurrently, tidal flows in the vicinity of Harding Beach caus ed its tip to erode rapidly and the deep scour hole at the breach to extend toward Harding Beach and Chatham Harbor Detalled studies found that the most feasible solution was to construct a sand dike between Morris Island and Harding Beach with a timber pile structure seaward of the dike to protect it ag ainst severe tidal currents, and relocate the channel through Harding Beach stabilized by a stone jetty The improvement has proved to be successful

\section{Edgartown Harbor, Massachusetts}

Edgartown Harbor, Massachusetts is located on the easterly end of Martha's Vineyard (See Figure No 8) The natural sand barrier beach extending for 3 miles along the south side of Katama Bay has had a history of breaches caused by storm waves recorded back to 1776 These breaches occur at the west to mid-portion of the barrier beach as the offshore hydraulic grade line lengthens and flattens in this area, the tidal currents diminish, and the beach narrows in width The breaches result in excessive tidal currents at Edgartown Harbor and in shrfting sands 


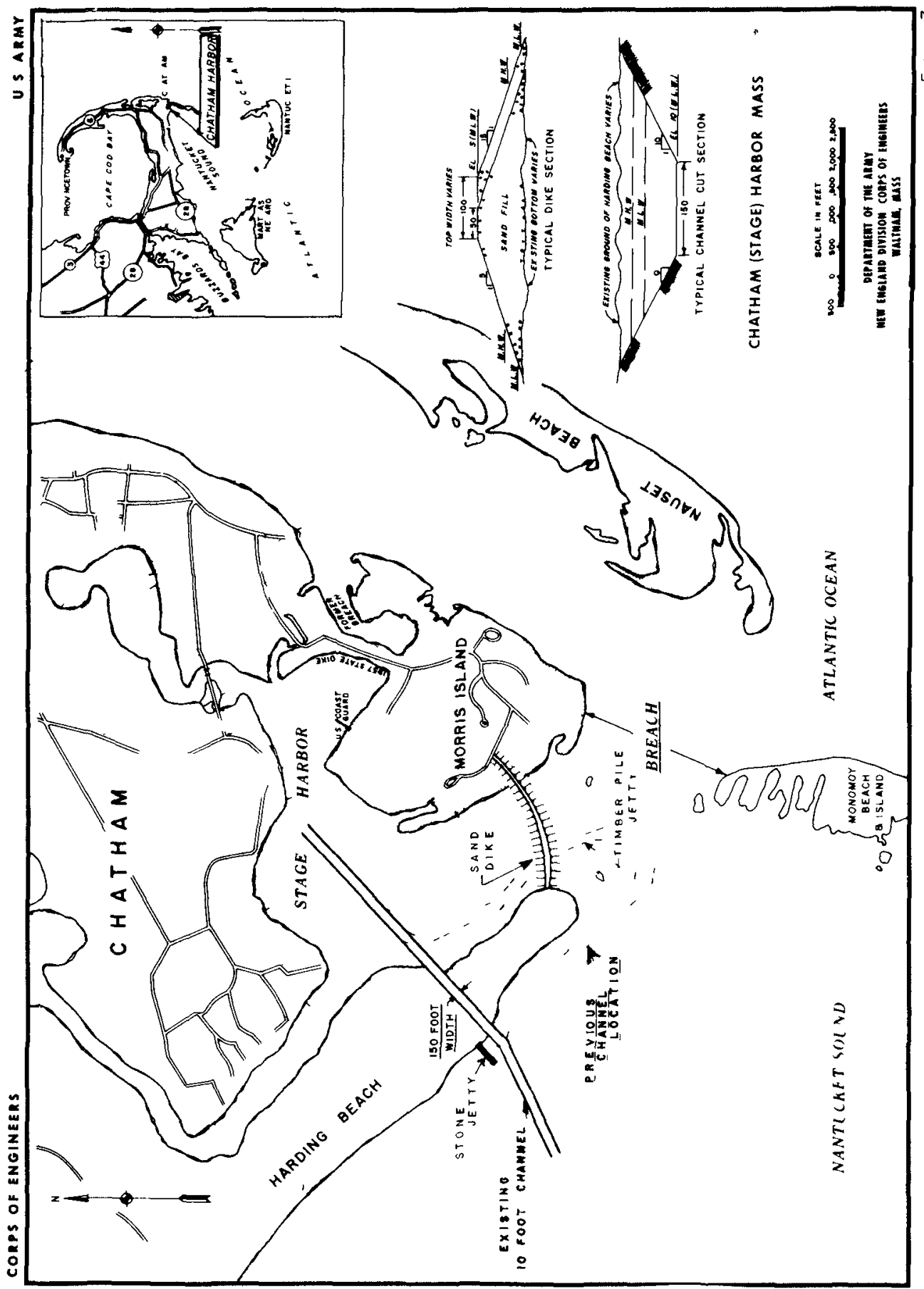




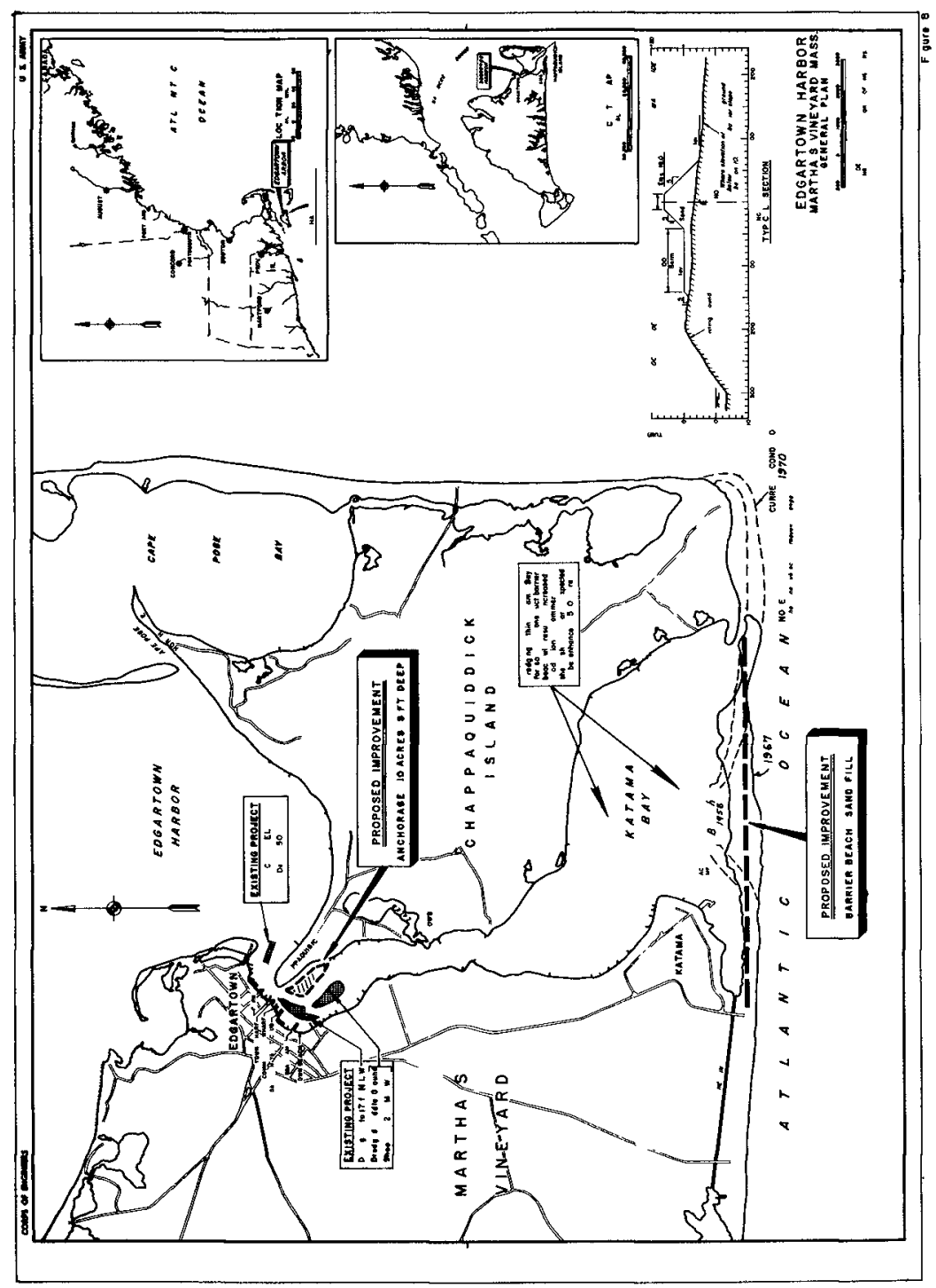


within Katama Bay, which destroys the valuable shellfish crops in the bay The breaches mig rate to the east until they reach Chappaquidick Island at which time the westerly portion of the barrier beach is once again susceptible of a new breach At present, the barrier beach connects Martha's Vineyard to Chappaquidick Island A survey report now being forwarded to Congress for consideration for authorization, recommends that the barrier beach be raised and widened to prevent future breaches The Fish and Wildlife Service of the United States Department of Interior, in cooperation with State and town fish and wildlife agencies, expects the improvement to result in a complete re-establishment of the shellfish industry within Katama Bay involving benefits of nearly two million dollars each year In addition, the recreational boating fleet will be benefited

\section{GONCLUSIONS}

As a result of the inlet problems discussed above, the following observations are offered First, inlet jetties should be studied closely to avold their construction with smooth faces resulting from placed stone, if it appears that this condition could contribute to excessive wave run-up, run-along, or reflection All avallable advanced knowledge concerning inlet development should be utilized and exhaustive wave studies made for each inlet Field observations of inlet sites should be made more often and more intensively Every effort should be made by the coastal engineer to respond to the greatest desire and need of local interests while cooperating and coordinating fully with all affected interests to provide the optimum plan of improvement within the dictates of available funds and environmental controls

It should be recognized that inlet problems can occur at any stage of development It should also be recognuzed that changes in shorelines adjacent to inlet developments are a rather frequent occurrence Congress responded to this recognition by including Section 110 into the River and Harbor Act of 1968, which authorizes the Corps of Engineers to provide corrective measures at shorefront areas shown to be adversely affected by Federal inlet improvements

In summary, the coastal engineer should plan for all predictable factors, but he should be prepared to modify his plans at any time to correct for the unpredictable

\section{ACKNOW LEDGEMENTS}

The information presented herein represents largely a summary of the very extensive and detailed studies made by coastal engineering experts of the New England Division office of the U S Army Corps of Engineers for each of the project areas discussed 


\section{REFERENCES}

Task Committee on Small Craft Harbors (1964), Small Craft Harbors Development, Committee on Ports and Ha rbors, Jou rnal of the Waterways and Harbors Division - Vol 90, No WW3

U S Army Corps of Eng ineers, New England Division, Reports on the seven navigation projects discussed in this paper

Watts, George M (1962), Mechanical Bypassing of Littoral Drift at Inlets, Journal of the Waterways and Harbors Division Proceedings of the American Society of Civil Engineers, Vol 88, No $W W 1$

Coastal Engineering Research Center (1968), Shore Protection Planning and Design, Technical Report No 4, 3rd Edition

Sharp, Henry Staats, A M (1929), State Geological and Natural History Bulletin No 46, Ins tructor in Geology, Columbia University, State of Connecticut

Rhode Island Development Council (1955), Hurricane Tidal Protection in Narragans ett Bay, $R$ I

Chute, Newton E, (1939), Geology of the Coastline Between Point Gammon and Monomoy Point, Cape Cod, Massachusetts

U S Army Corps of Engineers, New England Division(1965) Massachusetts Coastal and Tidal Areas - House Document No 293, 89 th Congress, list Session

U S Army Corps of Engineers, New England Division(1962), Shore of the State of New Hampshire, Beach Erosion Control Study House Document No 416, 87th Congress, 2nd Session 



\title{
CHAPTER 75
}

\section{OFFSET COASTAL INLETS}

\author{
Miles 0 Hayes ${ }^{1}$ \\ Victor Goldsmith 1 \\ Carl H Hobbs III 1 \\ Abstract
}

Offset coastal inlets are common on the coasts of New England and the northern Gulf of Alaska In both areas, the dominant waves approach the shore at an oblique angle, resulting in a strong net littoral drift The most common type of offset on these coasts is a downdrift offset ( $1 \mathrm{e}$, the downdrift side of the inlet protrudes further seaward than the updrift side) Wave refraction around the ebb-tidal deltas at the inlets is an important process in the formation of the downdrift offsets, inasmuch as $1 t$ creates a local reversal in drift direction just downdrift of the inlet, and allows sediment to accumulate there Associated with the offset appears to be a segregation of tidal current flow in the inlets, with the ebb flow being more channelized than the flood

The type of inlet offset at any one location can vary through time Studies of the changes at the Hampton Harbor, New Hampshire, inlet from 1776 to the present show two reversals from updrift to downdrift offsets during that interval

\section{INTRODUCTION}

An examination of coastal charts from almost any area in the world will reveal that coastal inlets are rarely symmetrical with respect to the placement of beaches on either side of the inlet They are usually offset, either updrift or downdrıft As yet, no satisfactory theory or model has been proposed to explain these offsets 2 Inlets with barrier spits that overlap adjacent down$\mathrm{dr}$ ft barrier beaches, such as those of Long Island, $N \mathrm{~V}$, seem in accordance with what might be predicted However, many of the

\footnotetext{
1 Institute of Coastal Research, Department of Geology, University of Massachusetts, Amherst, Massachusetts 01002

2 This problem is under study by Cyril J Galvin, Jr of the Coastal Engineering Research Center, who considers the ratio of net drift to gross drift significant
} 
inlets of the New England and Alaskan coasts are offset in just the opposite direction, that is, the barrier beach downdrift from the inlet protrudes further seaward than the one on the updrift side In fact, our studies indicate that this is by far the most common type of offset inlet on both the Alaskan and the New England coasts

\section{NEW ENGLAND INLETS}

Over the period of the last five years, most of the sandy inlets on the New England coast have been studied by members of the Institute of Coastal Research, Department of Geology of the University of Massachusetts With respect to coastal sedimentation, northeasterly storms, which generate waves that approach most of the shoreline obliquely from the northeast, have a dominant influence on the New England coast These waves set up a net 11ttoral drift to the south in most areas At almost every inlet in New England, the barrier beach downdrift of the inlet ( $1 \mathrm{e}$, on the south side) protrudes further seaward than the one on the updrift side One of the most important porcesses in the accumulation of sand on the downdrift side of these inlets is wave refraction around ebb-tidal deltas In the case of the Merrimack River Inlet, Massachusetts, the refraction of the dominant northeast waves causes waves to approach from the southeast, resulting in a northerly 11 ttoral drift at the south side of the inlet (Figs 1, 2, and 3) This reversal in drift direction on the downdrift side of the inlet serves to promote accumulation of sand to such an extent that the beach builds seaward at that point A wave refraction diagram constructed for the inlet of the Merrimack River illustrates this principle (Fig 1)

\section{ALASKAN INLETS}

The depositional portion of the coastione of southeastern Alaska is similar in many respects to the New England coast Important to the theme of this paper is the fact that most of the coastal inlets on this part of the Alaskan coast have downdrift 


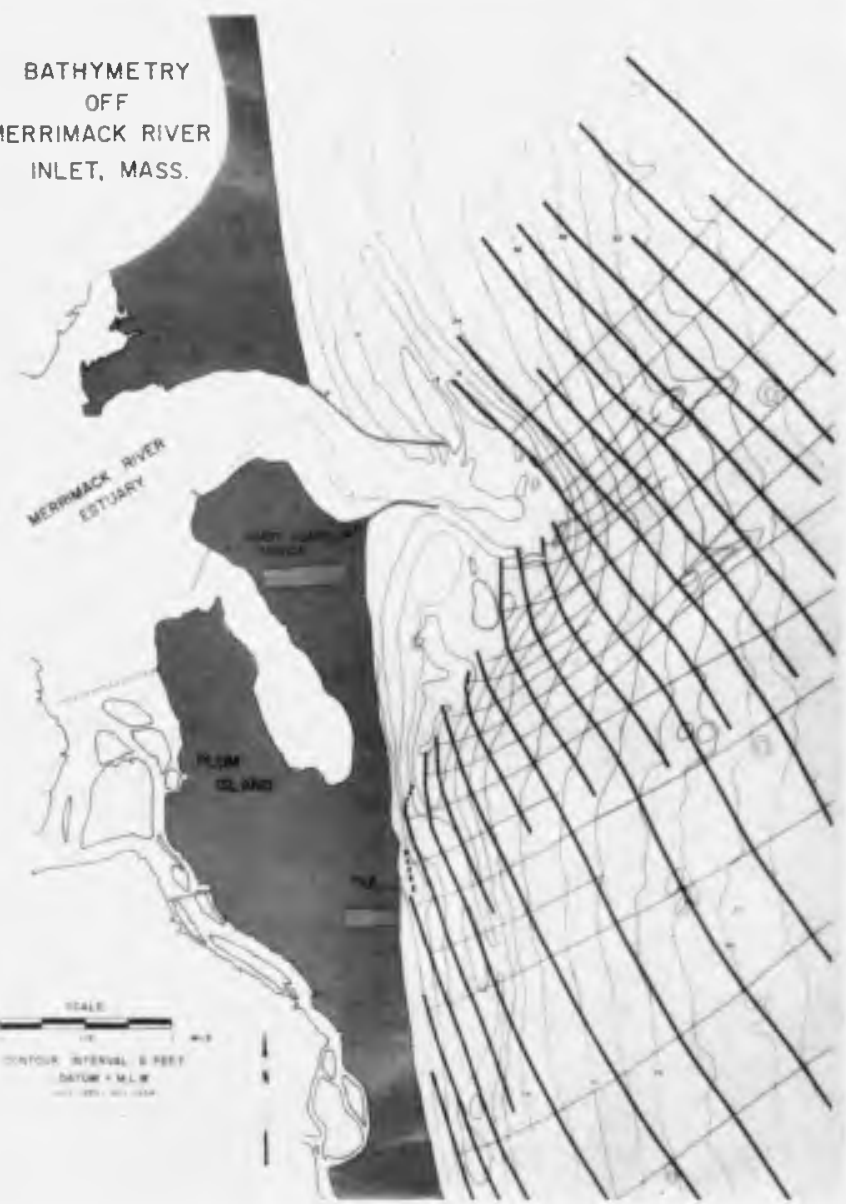

Fig. 1 Wave refraction diagram at the mouth of the Merrimack inlet, Massachusetts. Note that the refraction of the dominant northeast waves around the ebb-tidal delta has resulted in waves approaching from the southeast and thus in accretion at the south (i.e., downdrift) side of the inlet. Compare diagram with photographs in Figures 2 and 3 . Wave fronts are approaching from the northeast with a period of 9 seconds. 


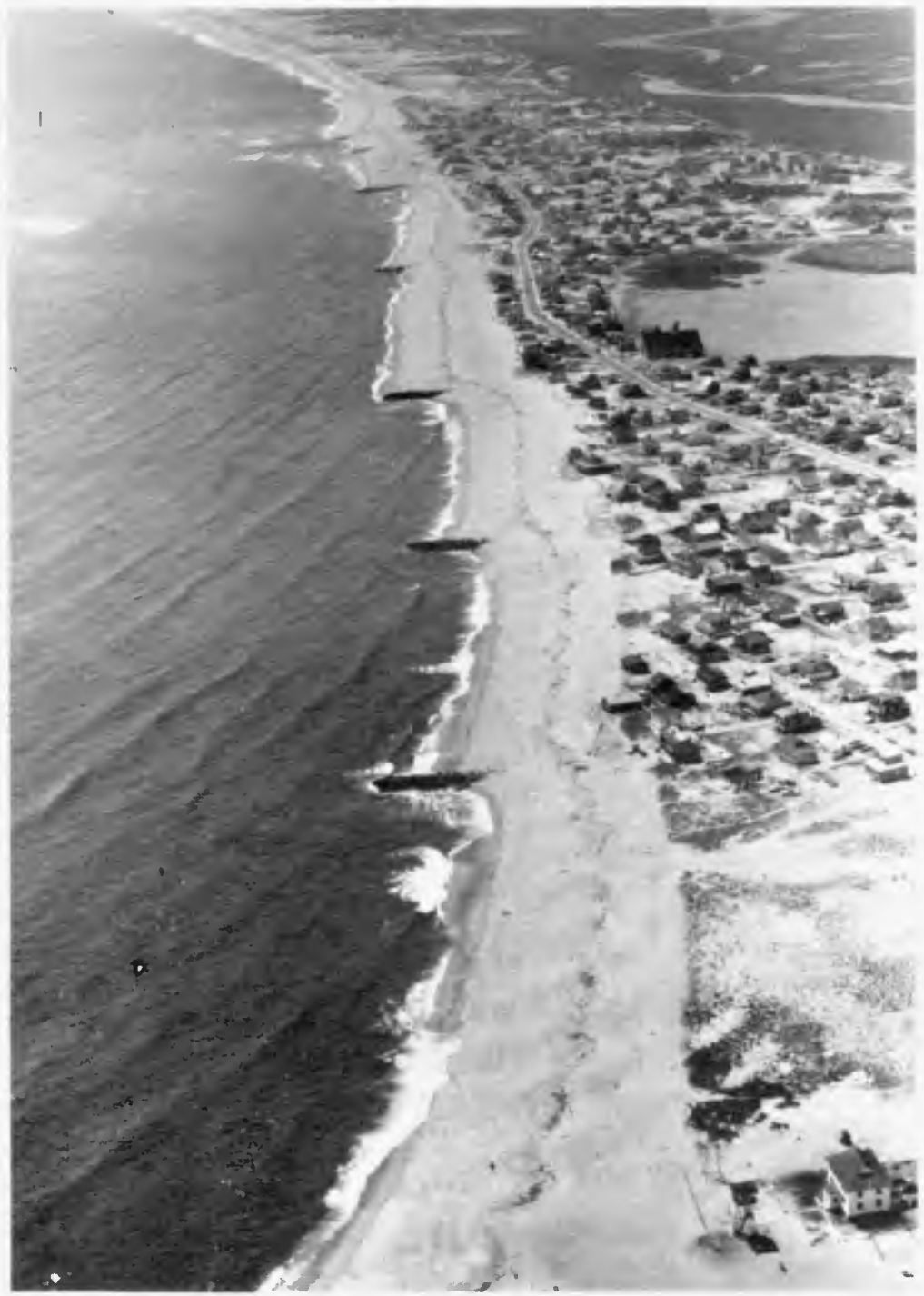

Fig. 2 View looking south along the Plum Island beach, Massachusetts, just south of the Merrimack inlet. Coast Guard station in foreground is located on the wave refraction diagram of Figure 1 . Note accumulation of sand on the south side of the groins and the oblique approach of the wave crests from the southeast. The groin field is located inside the ebb-tidal delta and is in an area of local reversal of longshore drift which results from wave refraction around the ebb-tidal delta (Fig. I). In the far background of the photograph, drift resumes its normal southerly trend. Photograph taken June 1968. 


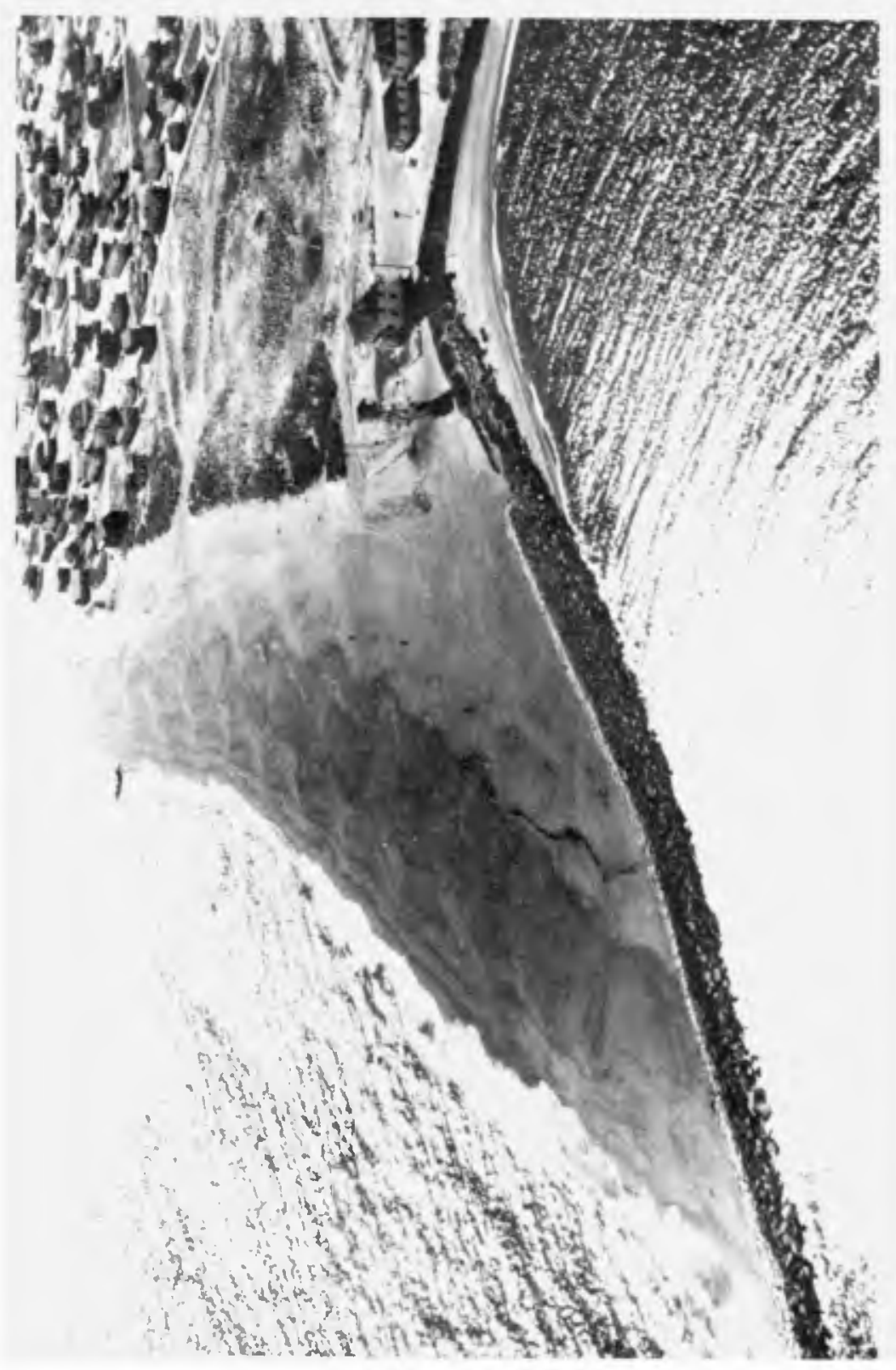

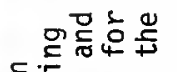

등

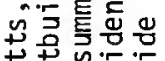

记 的

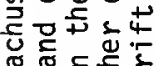

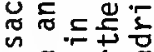

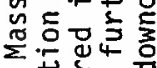

त

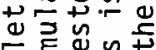

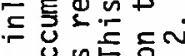

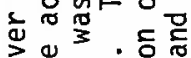

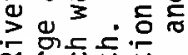
$\propto 5$.

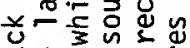

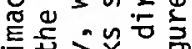

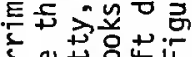

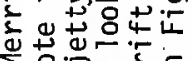

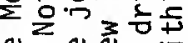

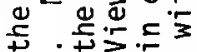
ᄂ 舟

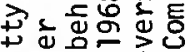
造证 당 부의 애은

m 
offsets Net littoral drift is from east to west, as determined by geomorphologic evidence Figure 4 shows the lineation of coastal sand dunes in response to strong southeast winds These southeast winds are the result of low pressure centers that pass up the Aleutian Island chain and strike the Alaskan coast in the apex of the Gulf of Alaska

The coastal inlets off the Copper River Delta show pronounced downdrift offsets The barrier beaches on the west sides of the inlets have developed large seaward protrusions (Figs 5 and 6) Wave refraction appears to play an important role in the formation of downdrift offsets in this area ( $F_{1 g}$ 6)

\section{DOWNDRIFT-OFFSET MODEL}

From the study of approximately 15 offset coastal inlets, we have derived a simple model that we feel is representative of 1 lets with downdrift offset The model is given in Figure 7 Waves approaching the shore at an oblique angle produce a strong net 11 ttoral drift The supratidal portion of the beach on the downdrift side of the inlet usually protrudes seaward with the beach face of that portion of the barrier beach facing the oncoming waves The barrier beach on the updrift side is usually composed of multiple recurved spits, indicating strong transportation of sediment into the inlet Commonly, the intertidal portion of the inlet is also offset, in that large accumulations of sand on the ebb-tidal delta (usually swash bars) protrude further seaward on the downdrift side than on the updrift side The Barnstable inlet in Massachusetts is an excellent example of this type of offset

Another common feature of these coastal inlets is the segregation of ebb and flood flow Each inlet usually has a main channel that is oriented perpendicular to the shoreline and carries a large portion of the ebb flow The flood flow, on the other hand, tends to be distributed as a sheet with several individual flood channels developed in some cases Usually, the flood channels hug both beaches, flanking the main ebb channel A similar segregation 


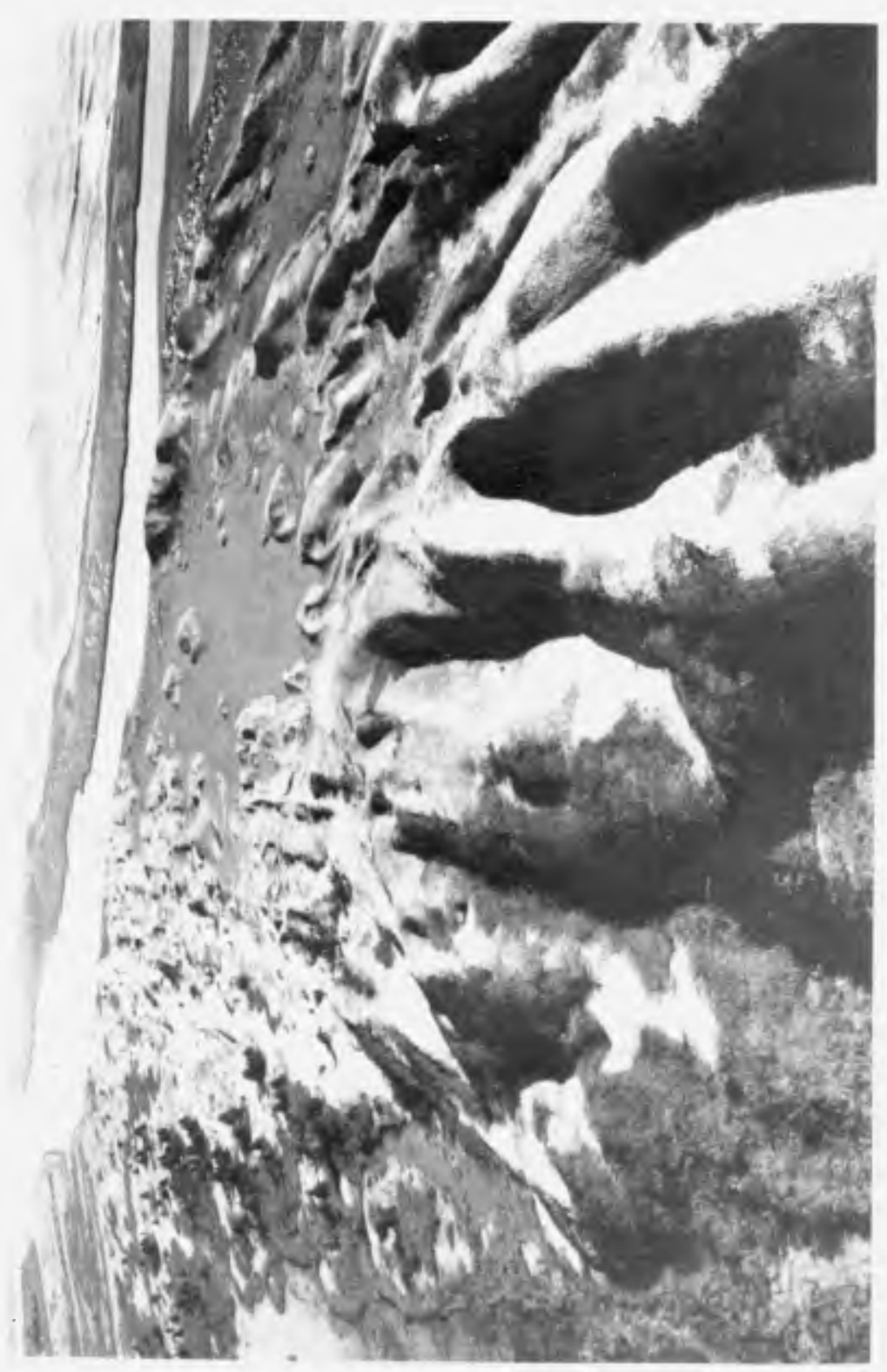

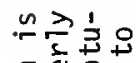

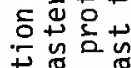
ब。 迄势

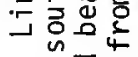
过它 芴方文 文的 멈 d苾贾 可的芒 ज楁

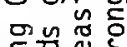

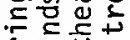

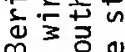

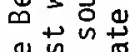

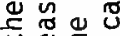
$+\frac{a}{5}$ ๙ ¿

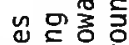
ᄃㅎำ

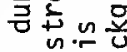
西衣 in 85 氙谣

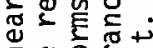

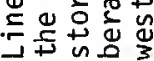
$\nabla$

$\dot{0}$ it 

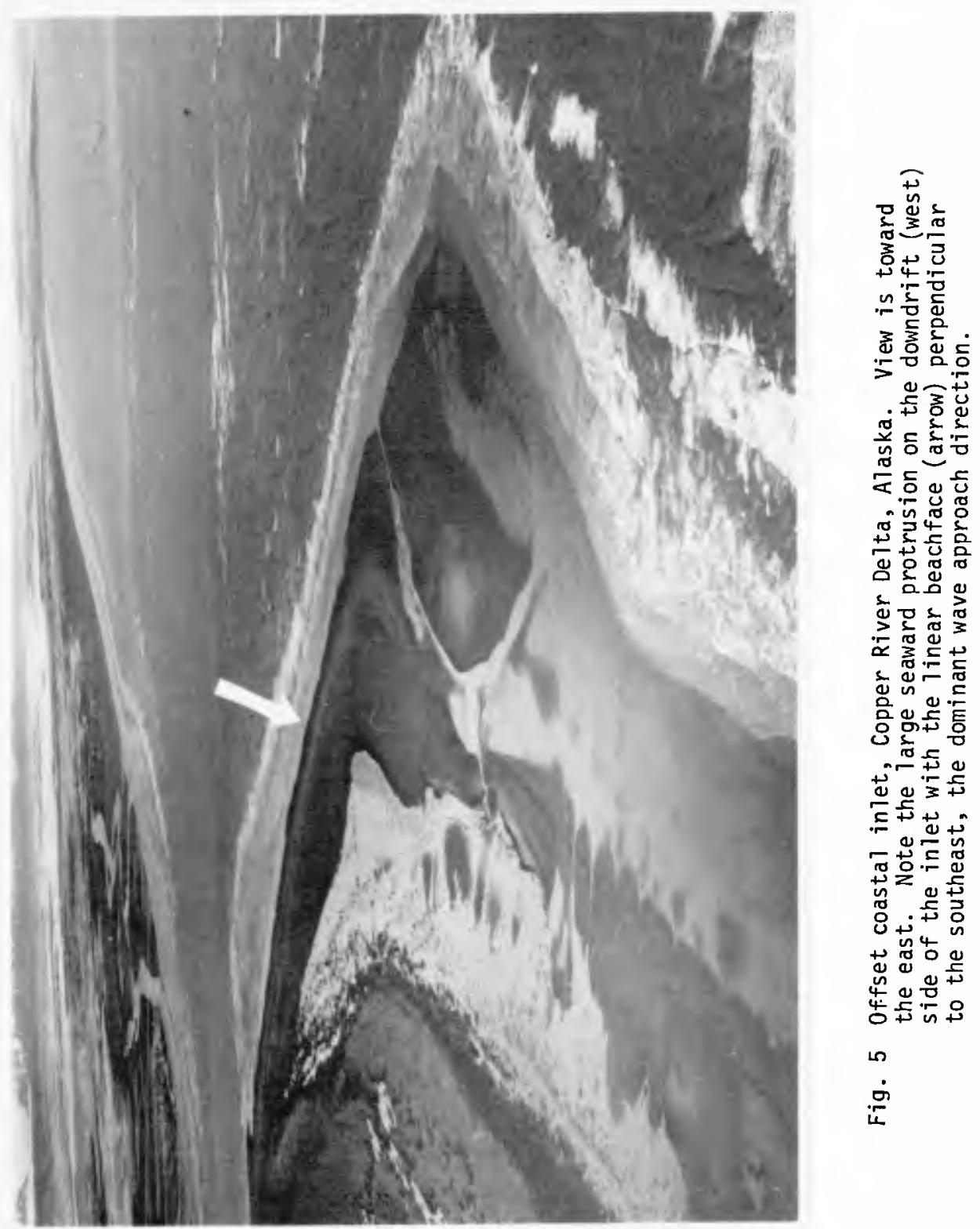


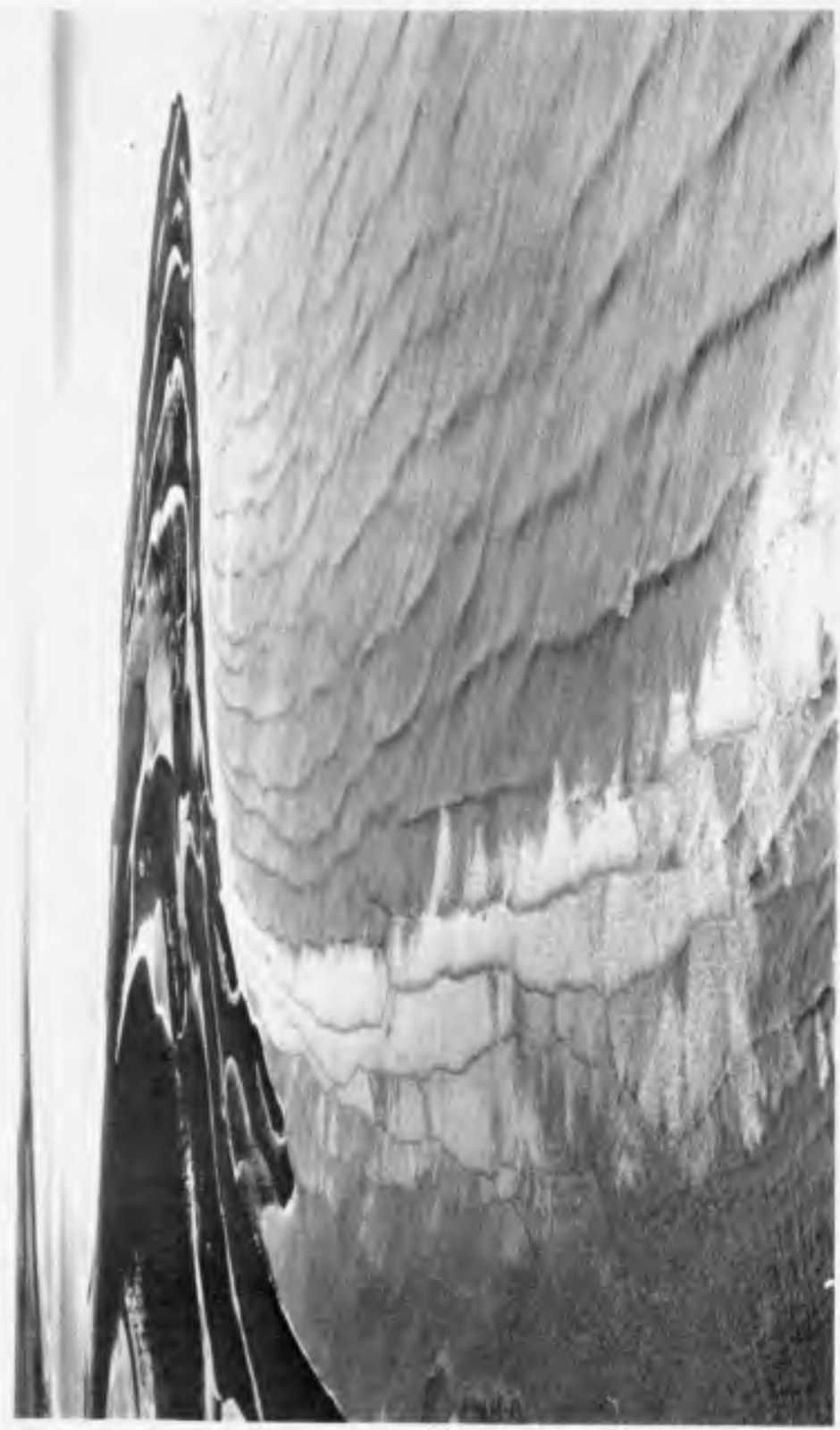

픙유

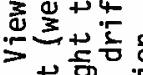
艺可尔

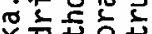

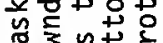

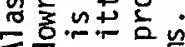

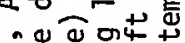

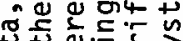

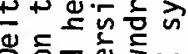

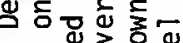

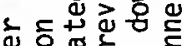

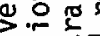
은 는돈 - 京西要

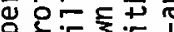
응드응 8 a $ᄃ$ 웜

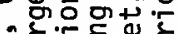
ก

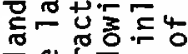

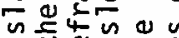

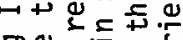

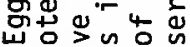
舟要 a ثـ 到话的 过范范 下 芺志范要

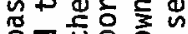

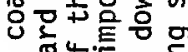

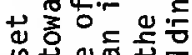

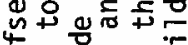
ق $\infty$

ii 


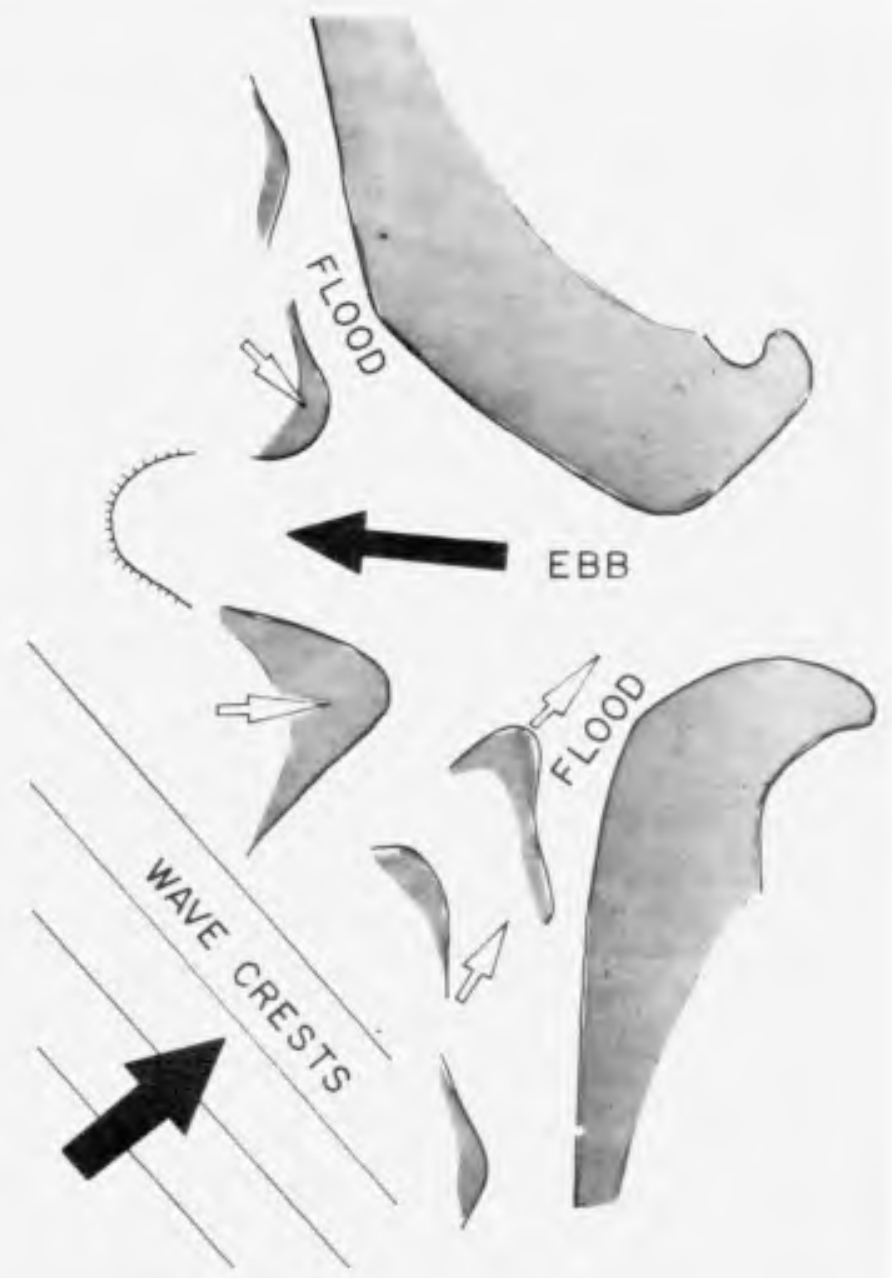

Fig. 7 A simple model for coastal inlets with downdrift offsets on the New England and Alaskan coasts. The downdrift side of the inlet, which is offset seaward, usually has a straight beach face perpendicular to the dominant wave approach direction. The ebb flow is channelized, whereas the flood current tends to develop as sheet flow across the intertidal swash bars and through minor flood channels. 
of flow has been observed in model studies by Henry Simmons and his associates at Vicksburg (personal communication, September 1970) We feel that the reason for this segregation of flow is the occurrence of time-velocity asymmetry of the tidal currents Maximum flood velocities are usually late in the flood-tidal cycle, between mid-tide and high-tide Similarly, the maximum ebb flow is between mid-tide and low-tide, usually quite close to low-tide Thus, the ebb flow tends to be much more channelized than the flood, which flows in as a sheet The exact relationship of this segregation of flow to inlet offset is unknown

A spectacular example of a flood channel located on the downdrift side of an inlet, flanking the main channel, is shown in Figure 8 This figure, a photograph looking downdrift, shows large sand waves in this shallow flood channel which indicate transport toward the inlet An inspection of the bedforms in the channel (on the ground) shows a complete predominance of flood flow in this channel

\section{HISTORIC CHANGES IN INLET OFFSET}

Our studies of historical changes on the New England coast indicate that inlet offsets can change through time A study of the inlet at Hampton Harbor, New Hampshire, shows that the offset of the inlet has passed through two cycles since 1776 (Fig 9) In 1776, the northern beach overlapped the southern beach By 1885 this pattern had reversed with the southern beach protruding further seaward than the northern beach This cycle repeated again, with the inlet attaining 1 ts present configuration, with a southern protrusion, in 1931 The Merrimack River entrance, the next inlet to the south, shows a similar pattern of migration This long term variation in inlet offset needs to be investigated in much greater detal1 


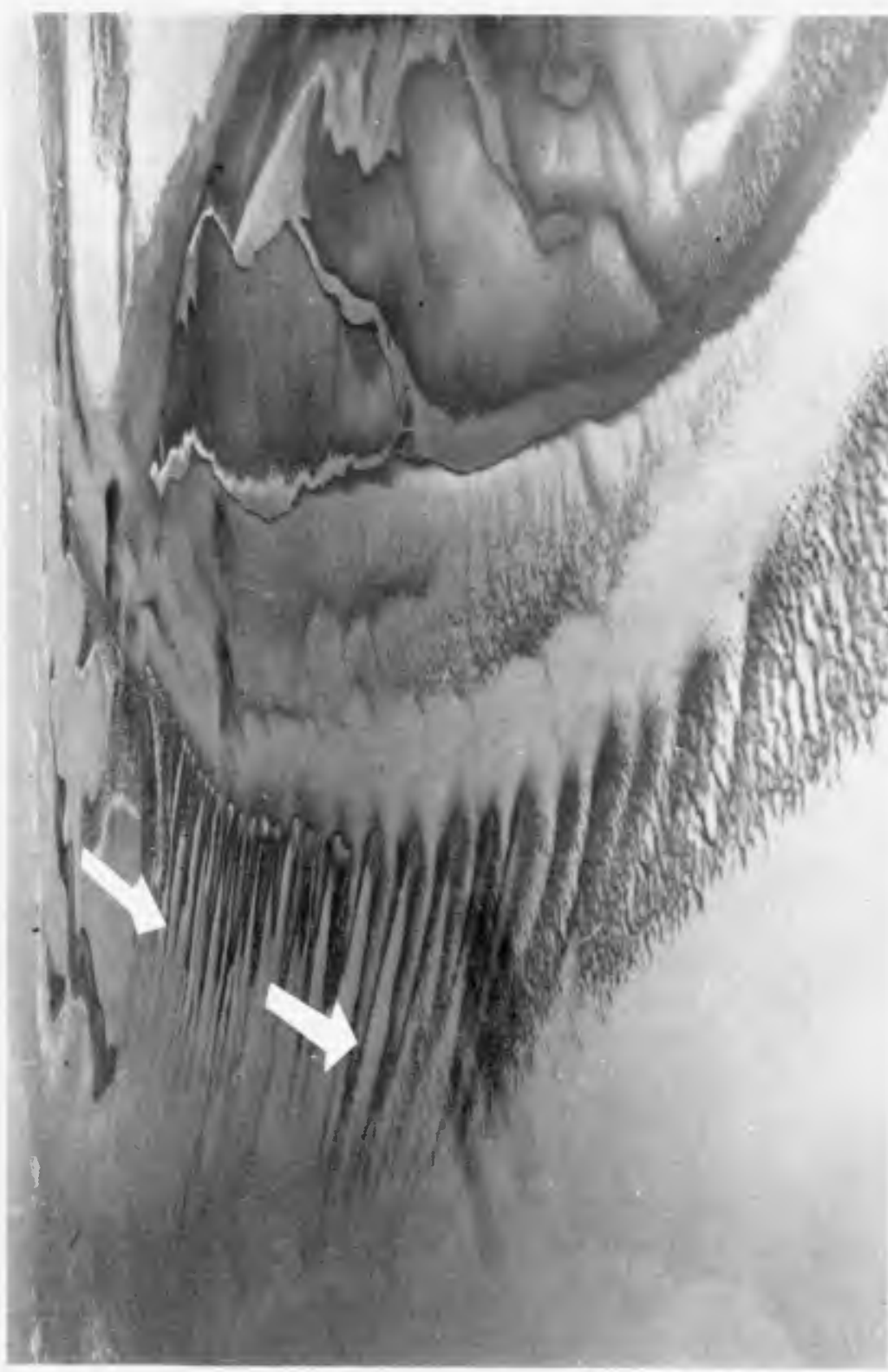

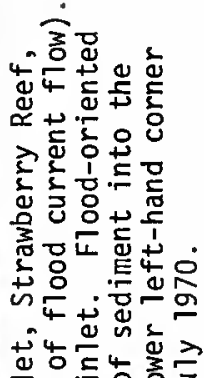

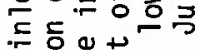

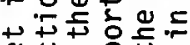
Q y

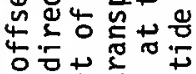

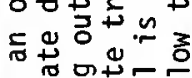
4 资. 它羊宁

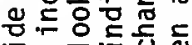
in n-

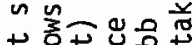
女

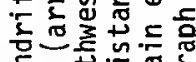

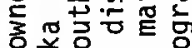

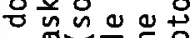
은 †

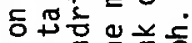
-

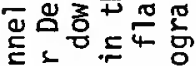

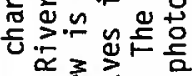

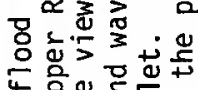
4 응웡 운 <品 品.

i. 


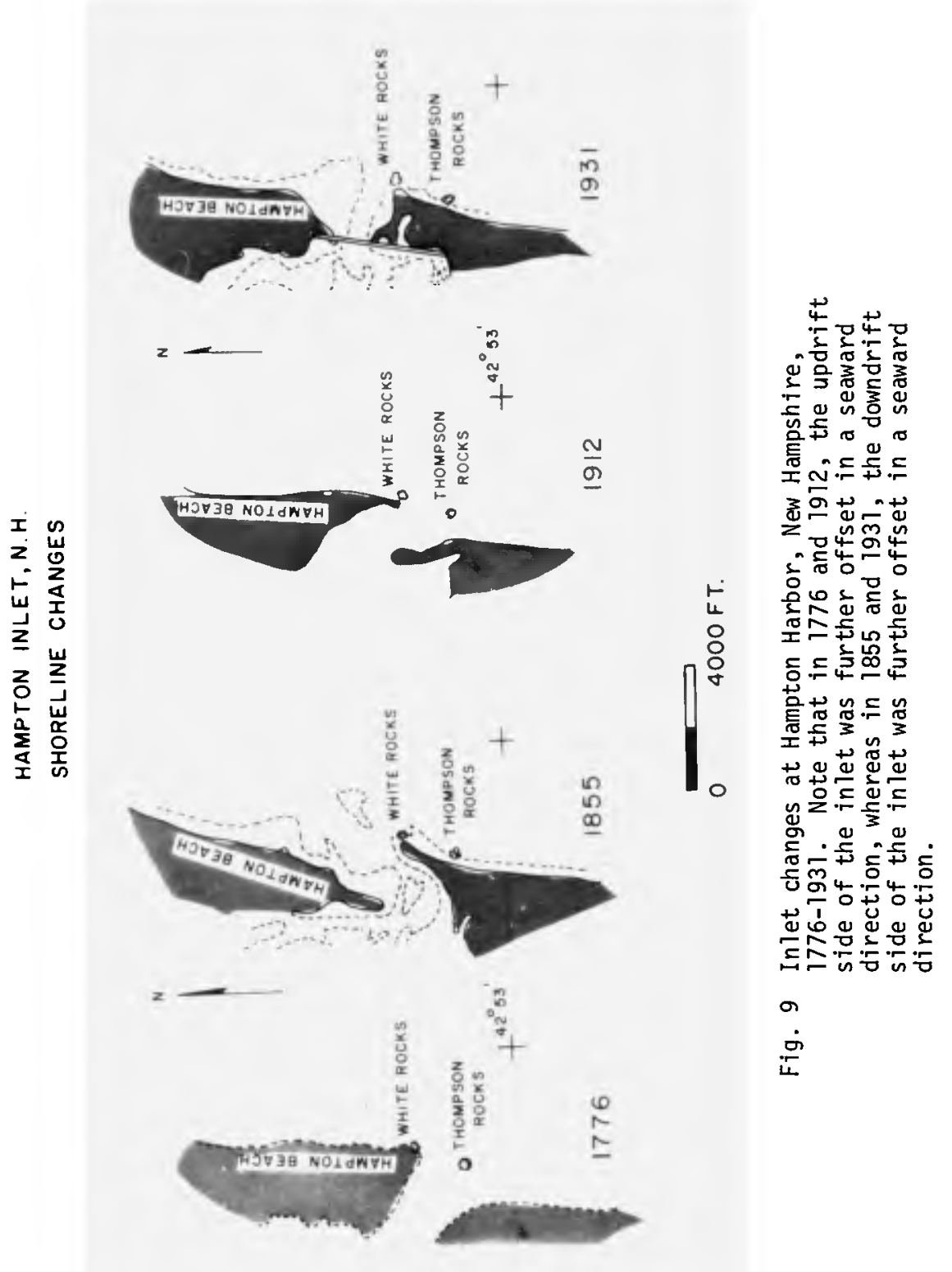


SUMMARY

Coastal inlets of the shoreline of New England and the northern Gulf of Alaska show some striking similarities Both of these areas are characterized by dominant waves that approach the shoreline obliquely, producing a large net 11ttoral drift offset coastal inlets are common in both areas, the prevalent type being inlets with downdrift offset We have presented a simple downdrift-offset model that we feel is typical of most of the inlets studied

Associated with the downdrift offsets is a segregation of tidal current flow in the inlets, with the ebb flow being more channelized than the flood How the flow segregation relates to inlet migration is one of the most important questions raised by our s tudy

In this paper we have dwelt on what we consider to be the most common type of offset in the area of study The type of offset can and does vary from inlet to inlet, and, as indicated by our historic studies, can vary even at the same inlet through time

\section{ACKNOWLEDGMENTS}

The observations reported in this paper were incidental to research being conducted in New England under contract DACW 72-67-0004 with the Coastal Engineering Research Center, U S Army Corps of Engineers, and in ATaska under contract N00014-67-A-0230-0001 with the 0ff1ce of Naval Research (Geography Branch) 


\section{CHAPTER 76}

FIELD STUDY OF A TIDAL INIET, BIMINI, BAHAMAS ${ }^{\perp}$

W HARRISON, $R$ J BYRNE, J $D$ BOON, III, and $R$ W MONCURE

Division of Physical, Chemzcal, and Geologscal Oceanography Vırginza Instıtute of Marıne Science, Gloucester Point, Virginia 23062

\section{ABSTRACT}

The inlet bathymetry was mapped by standard photogrammetric technıques after photographing the bottom directly through the water column The channel bottom is bare beachrock or is floored with bloclastic sands and gravels $(S \mathrm{G}=284$ )

A nine-day time series of observations of current speed and direction, and water temperature and salinity was obtained at two depths at each of elght stations using tripod-mounted, telemetering sensor packages Twelve-to-18-minute pulsations in the flow were often observed Spectral analysis of near-surface current speeds shows significant peaks at 24 , $32,43,67$, and $123 \mathrm{hrs}$

Evolution of sand ripples and dunes was monctored over a 200-ft distance during one tidal cycle The pattern of growth illustrates the differences in bedform geometry which may be expected to influence friction coefficients Evidence is presented for a bottom jet that is induced by flow over the crests of sand waves

Tracer sand, sorted into two size groups and color-coded for identification, was released on a flood current sampling of color-coded tracer sand was conducted by divers using strips of grease-coated plastic tape Spatial distribution of tracers in relation to the bedforms, and the amportance to grain erosion of a natural mucold coating, are noted

\section{INTRODUCTION}

This study was designed to examine the hydrography and the flucdsediment interactions in an inlet floored with carbonate sand The approach adopted involved 1) describing the basic inlet geometry and the sem--permanent bedforms, 2) documenting the general flow field, 3) documenting the response of relatively small-scale bedforms to ebb and flood currents, and 4) conducting specıfic sand-tracer experiments

Morphologically speaknng, the inlet studied is a specialized tidal channel that connects waters of the Florlda Stralts with a shallow lagoon about elght square mlles in area (Fig I) The lagoon is not enclosed, but opens to the Great Bahama Bank across a broad area of flats

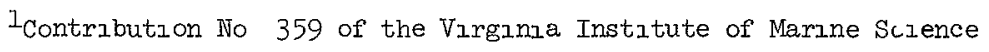




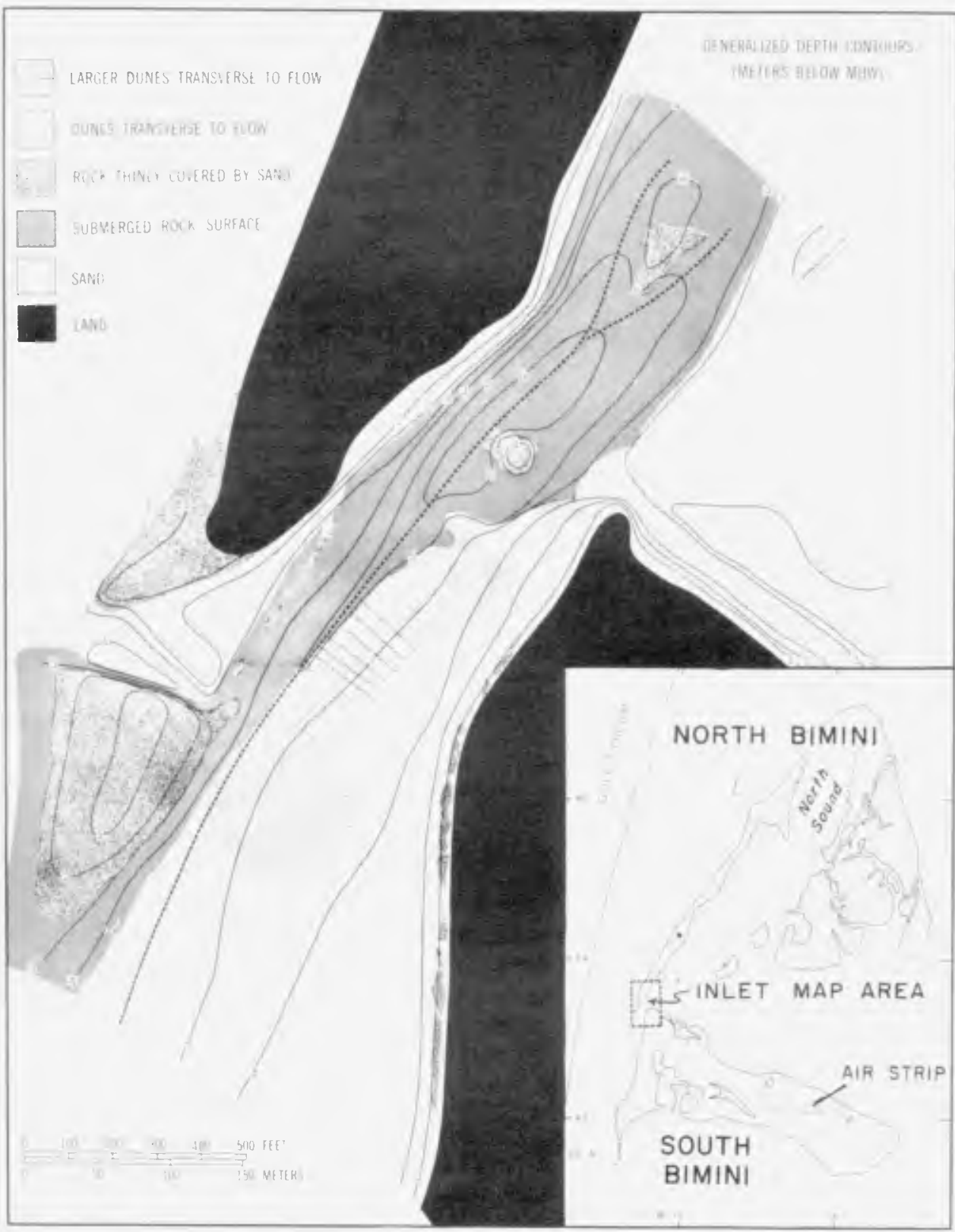

Figure 1.- Location, generalized bathymetry, and geology of Bimini Inlet channel. 
Turekıan (1957) felt that the lagoon was "host to three distınct water masses i) an Indlgenous mass that moves in and out of the North Sound with the tides, 2) a mass entering from the Florida stralts through Entrance Pount, and 3) a mass enterang from the Bank" to the east

The tıde range at Bımını varıes between about 25 to $30 \mathrm{ft}$ and sea level tends to rise or fall together at both Bımını and Mlaml (Wunsch, et al , 1969) Tides are of the semldiurnal type with a slight diurnal. inequality Propagation of the tide wave through the open lagoon is such that the ebb flow (to the southwest) through the inlet is about 30 percent longer than the flood

Current speeds in the northern, constricted part of the inlet channel (FIg 1) are high enough to sweep sediment from the beachrock floor Some of the larger dunes in the central portion are composed of fragmented shell gravel ThIs glves way to sand with median graln diameters of 06 to $08 \mathrm{~mm}$ in the southern portion of the channel Most of the grain surfaces in sand samples of shell hash taken from the central and southern portions of the inlet exhıbıt a glazed appearance when viewed under the bınocular mecroscope Sand-graln surfaces become more chalky, however, as one moves lagoonward (Bathurst, 1967) The specıfıc gravity of sand graıns from elght samples in the area of "dunes transverse to flow" (FIg l) was 284

During the period of study, November 5-13, 1967, water temperature in the inlet channel varied between $20^{\circ}$ and $27^{\circ} \mathrm{C}$, salinity between 34 and $38 \%$, and density between 10201 and $10267 \mathrm{~g} / \mathrm{cm}^{3}$ In May, 1955, Turekıan (1957) found that salinity in the inlet varied between 360 and $370 \%$ and temperature ranged between 263 and $286^{\circ} \mathrm{C}$ over one tidal cycle

\section{DETERMTNATION OF INLET GEOMETRY}

Existing nautical charts of the Bimini Islands (British Admiralty Surveys of the $1840^{\circ} \mathrm{s}$ ) inadequately define the inlet geometry Considering the general clarity of water at the inlet, it was decided to attempt bottom contouring by standard photogrammetric techniques

To provide horlzontal control for aerotriangulation, a base line 2319 ft in length was established by electrotape Each end of the base line was pre-marked with a 4-x-4-ft, red plywood panel, the north end of the base being on North Blminl and the south end on South Bimln

Five vertical control pounts on land were also established and pre-marked in the same manner The levels were run with a Zelss Opton level and were based on a tidal bench mark on North Biminl

The photography as planned placed some photo centers over water wath land areas on only one side To facllitate the clearing of models during both aerotriangulation and compllation, seven floating targets (4-x-4-ft plywood) were anchored offshore to provide photographic images 


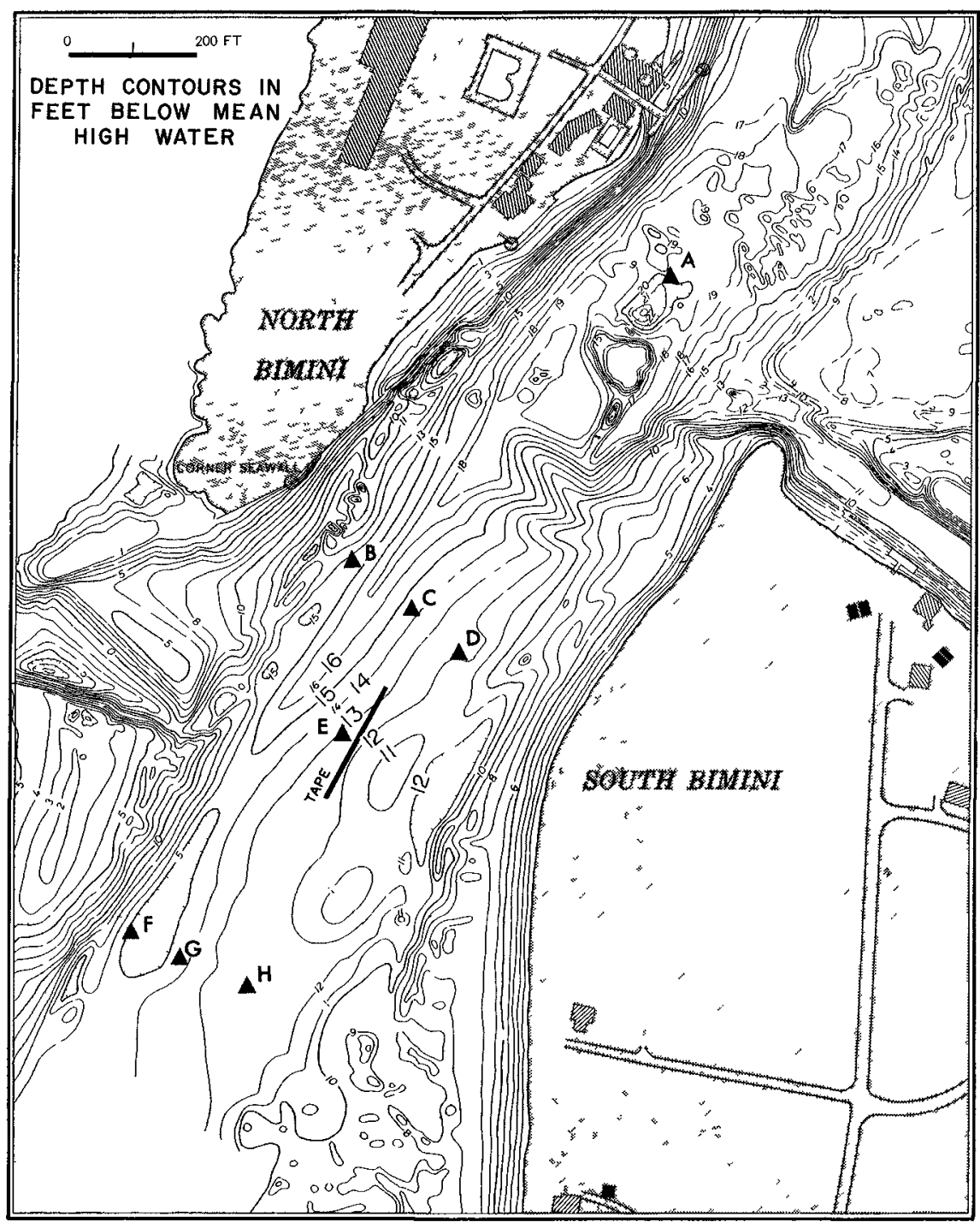

Figure 2 - Detalled bathymetry of anlet, locations of bottom-mounted sensor packages, and position of reference tape 
A WIId RC-8 camera with a focal length of $15229 \mathrm{~mm}$, mounted in an Aerocommander alreraft, was used to obtain the photography on September 8 , 1967 Fl.m was Anscochrome D-200 with an ASA rating of 165 The flylng helght was $2000 \mathrm{ft}$ above mean sea level, resulting in a photograph scale of approximately 14,000

Aerotriangulation was by stereoplangraphic methods using drilled glass plates The contouring of the bottom (Fig 2) was accomplished on a Wild B-8 Plotter at 12,400 scale with a contour interval of one ft The average correction for the refraction index of water for the models contoured was 14

Hydrographic check Innes run 45 days later indlcated that the depth contours ( $F_{2 g}$ 2) were whthin \pm 025 to 0 5-ft for the shallow-water areas of the inlet The "larger dunes transverse to flow" (FIg l) were adequately brought out in the bathymetric contours and the hydrographic check lines showed them to be essentialiy stationary over the 45 days that had elapsed since they were photographed

\section{DOCUMENTATION OF FLUID PROPERTIES, WATER IEVEL, AND FLOW}

Two trupod-mounted, "Geodyne" sensor packages were installed at each of the elght stations $(\mathrm{A}-\mathrm{H})$ shown on Figure 2 Each sensor package contained a platınum thermometer for measuring temperature, a torroldal cell for conductivity, a Savonzus rotor for current speed, and a vane for current direction A given pair of instrument packages were mounted in the tripod so that the Savonus rotor of one was one ft above the bottom whlle that of Its companion was elght ft above the bottom The mounting configuration was such that the flow pattern around a given rotor or vane was unaffected by the tripod legs or by the adjacent sensor package

Each tripod was placed on a level part of the bottom and the sensor packages were hardlıned to one of four shore-based stations Each shore station contained a magnetıc tape recorder and a radio transmitter $A$ given sensor was interrogated every six minutes, each interrogation involving five separate readings of current direction and speed and one each of temperature, conductivity, and reference

Data from the four shore-based transmitters were sent by an RF IInk to a master station at the Lerner Marine Laboratory on North Bumini At the master station, all data from the 16 sensor packages were recorded sequentially on computer-compatıble magnetıc tape The entire system of sensors was continuously operated from 5 November through 13 November, 1967

A bubbler-type tide gage was installed on North Biminl, the orifice was attached to the tripod at Station B 


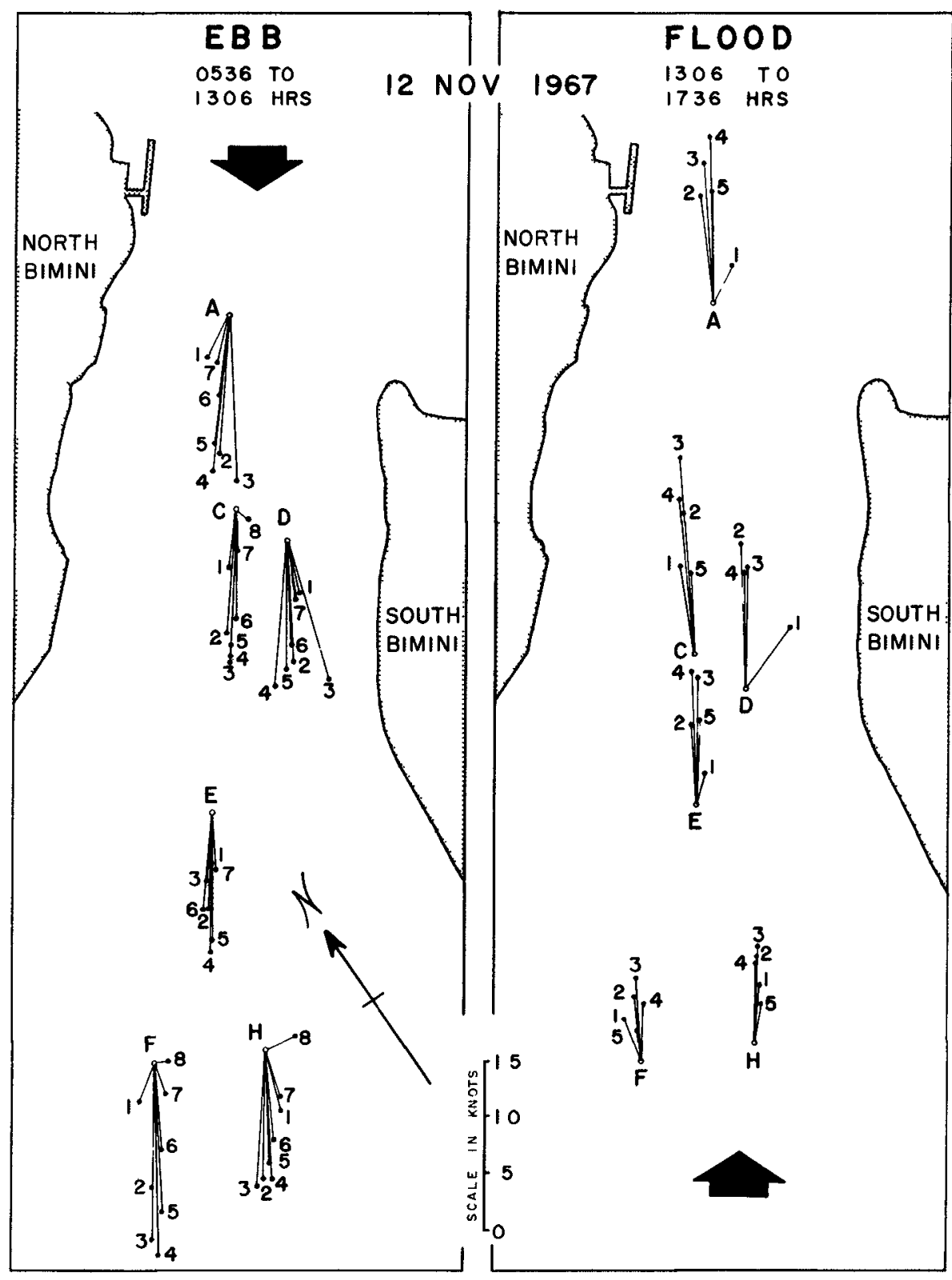

Flgure 3 - Tidal-current vectors for a neap-tıdal cycle ( In hours after high-slack or low-slack water) 
Data on maximum flow elght ft above the bottom are presented in Table 1 The absolute peak speed measured does not appear in Table 1 because it occurred prior to having all elght stations operational This peak speed was 41 knots and was measured eight ft above the bottom at Station A The time of measurement was during a maximum ebb flow that colncided with spring tides and a strong wind from the northeast that had been blowning for several hours

TABIE 1 - NEAR-SURFACE VEIOCTTIES AT SELECTED STATIONS (5 Nov 67, through 13 Nov 67) ( $F=$ flood current, toward $N E$ into lagoon, $\mathrm{E}=\mathrm{ebb}$ current)

\begin{tabular}{|c|c|c|c|c|c|}
\hline $\begin{array}{l}\text { SENSOR } \\
\text { STATION } \\
\left(\begin{array}{ll}\text { fIg } & 2\end{array}\right)\end{array}$ & $\begin{array}{c}\text { MAXIMUM } \\
\text { VEIOCITY } \\
\text { (kts, flood or ebb) } \\
\text { (drrection, true) }\end{array}$ & $\begin{array}{l}\text { TIME } \\
\text { (date) } \\
\text { (hours, } \\
\text { EST) }\end{array}$ & $\begin{array}{l}\text { AVERAGE } \\
\text { VEIOCIT } \\
\text { flood } \\
\end{array}$ & $\begin{array}{l}\text { AXIMUM } \\
(\mathrm{kts}) \\
\mathrm{ebb}\end{array}$ & $\begin{array}{l}\text { AVERAGE DEPTH BEIOW } \\
\text { SUREACE TO ROTOR ( } f t \text { ) } \\
\text { (AIl rotors elght } \\
\text { ft above bottom) }\end{array}$ \\
\hline A & $\begin{array}{c}231^{\circ} \\
3\end{array}$ & $\begin{array}{l}5 X I \quad 67 \\
2142 \text { hrs }\end{array}$ & 17 & 17 & 105 \\
\hline$c$ & $\begin{array}{l}28 \mathrm{E} \\
190^{\circ}\end{array}$ & $\begin{array}{ll}17 \times I & 67 \\
0824 & \text { hrs }\end{array}$ & 17 & 19 & 40 \\
\hline$D$ & $2 \underset{04^{\circ}}{1} \mathrm{~F}$ & $\begin{array}{l}6 \mathrm{XI} \quad 67 \\
0936 \mathrm{hrs}\end{array}$ & 13 & 15 & 27 \\
\hline $\mathrm{E}$ & $\begin{array}{l}22 E \\
213^{\circ}\end{array}$ & $\begin{array}{rl}11 \times I & 67 \\
0618 & \text { hrs }\end{array}$ & 13 & 17 & 33 \\
\hline $\mathrm{F}$ & $\begin{array}{l}33 \mathrm{E} \\
195^{\circ}\end{array}$ & $\begin{array}{ll}11 \text { XI } & 67 \\
0824 & \text { hrs }\end{array}$ & 10 & 20 & 57 \\
\hline $\mathrm{H}$ & $\begin{array}{l}26 \mathrm{E} \\
234^{\circ}\end{array}$ & $\begin{array}{l}6 \times 1 \quad 67 \\
0142 \mathrm{hrs}\end{array}$ & 16 & 14 & 40 \\
\hline
\end{tabular}

An 1 dea of tidal-current speeds and directions during a neap-tide flood and ebb cycle may be obtalned from the vectors of Figure 3 It is interesting to note that the directional spread is less than $21^{\circ}$ (Station D, ebb) at all stations during relatıvely strong flows

An example of pulsations in the flow, for the upper rotor at Station $\mathrm{E}$, is shown in Figure 4 Pulsations of this type were observed at all sensors and generally were of 12 or 18-minute frequency spectral analysis with a boxcar window falled to reveal any obvious power at these relatively high frequencies Significant power was found at the tidal frequencies (6 7 and 123 hours) and at the sub-tidal frequency of 43 hours Power peaks of possible significance were also noted at all stations at frequencies of 24 and 32 hours 


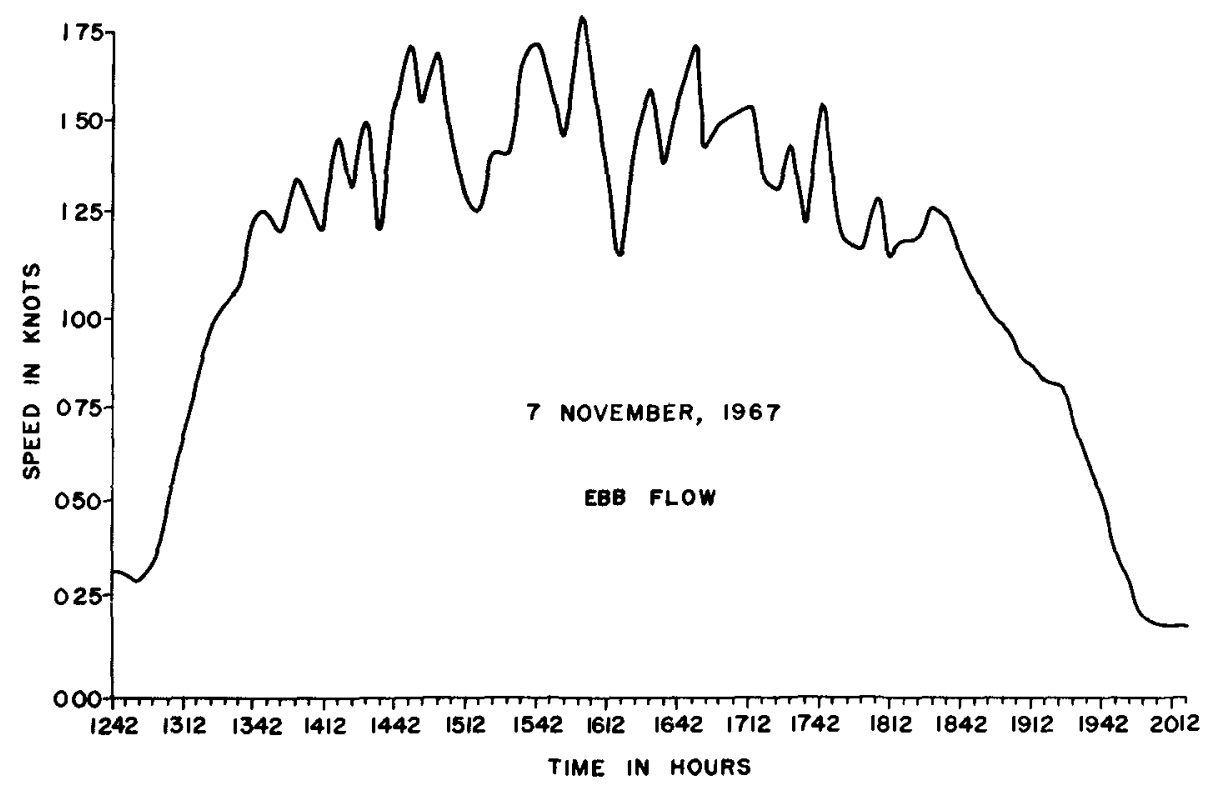

Flgure 4 - Flow pulsations at upper rotor (elght feet off bottom) at Station $E$ (F1g 2), during ebb flow

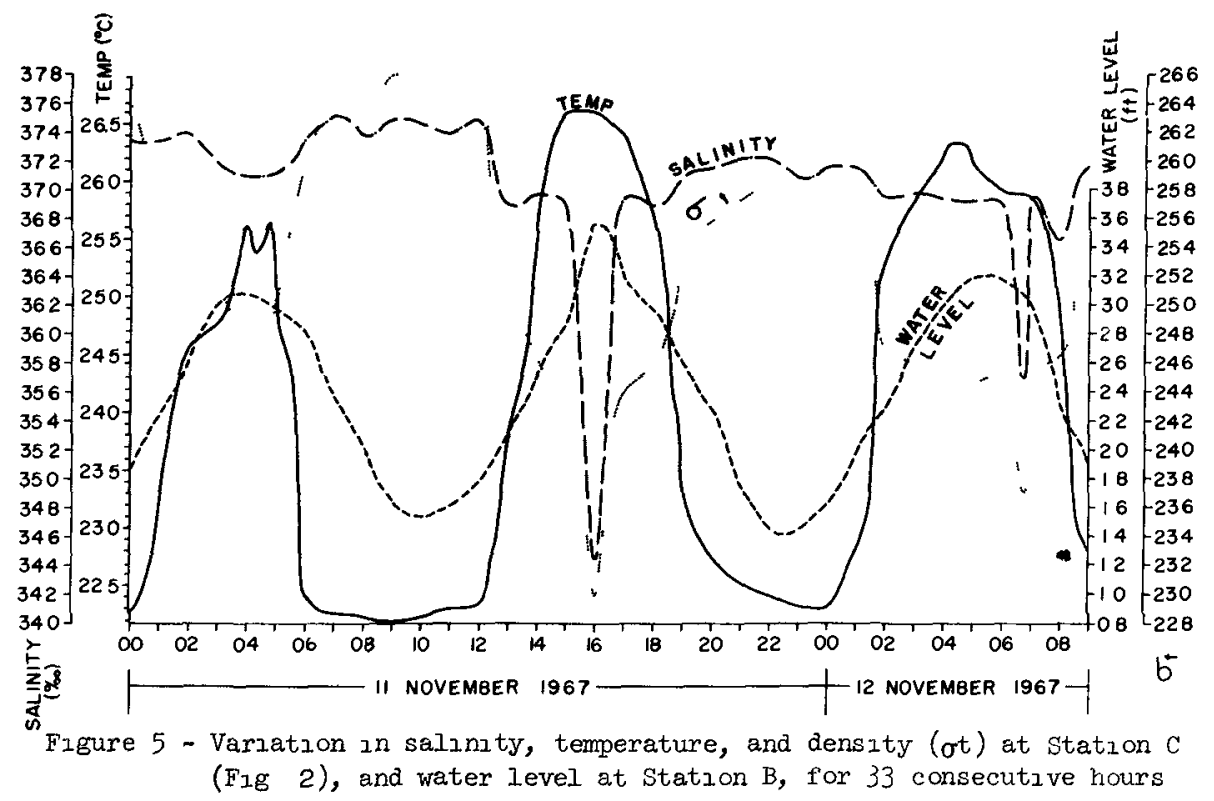


A portion of the curves for variations in temperature, salinity, and sigma-tee at Station $\mathrm{C}$ and water level at Station B are plotted in Figure 5 The inverse relationship between sigma-tee and water level is due to the fact that in November, cooler, more-saline, high-density water flows out of the lagoon on ebb tide This water is completely replaced at high tide by warmer, lower-salınıty, less-dense Gulf Stream watex No density stratification was observed in this shallow channel of well-mixed waters

\section{BEDFORM MORPHOLOGY}

\section{Measurement Procedures}

The goal of the bedform montoring program was to measure changes in geometry and position of the various forms through the reversing fllow of one tidal cycle A falrly complete run was made on 13 November 1967 It started near the beginning of ebb flow and continued through one-half of the flood

Photographic technuques were the prime tools in maknng the bedform measurements Early in the survey a 200-ft, rigld tape measure (graduated in I-ft intervals) was installed about one ft above the Irregular bottom (Fig 2) About $15 \mathrm{ft}$ to the west of the tape, and parallel to It, a wire gulde-line was installed five ft above the bottom The gulde-line served to mantain the diver-photographer at a uniform distance from the tape and allowed hym to control his progress in the rather swift flow A second diver positioned hamself behind the 200 $\rightarrow \mathrm{ft}$ tape The second diver pushed a fork-like device into the sediment so that the elevation of the bed relative to the tape measure could be photographed The fork device was composed of a serıes of $1 / 4-1 n$ rods welded at a 0 5-ft spacing to a rigld cross-member Each rod was graduated in 0 l-ft intervals The rigid cross-member had a bubble level cemented to it so that the upper member was horizontal, which in turn insured that the rods were vertical Two forks were used, each was six ft long and three ft hlgh

A typical run was executed as follows Two divers would descend at the upstream end of the tape one diver would take the two forks to the tape and insert them, end to end, into the bed The photographer-diver then took a stıli plcture from the gulde-line position The other diver would then remove the upstream fork and place it in the downstream position for another photo, and so on A single-photograph sequence of the bedforms generally took thrrty munutes Immediately before or after the still-camera sequence a run was made with a 16-mm movie camera From the developed stıll-camera sequence, the configuration of sand bed relatıve to horizontal was reconstructed 

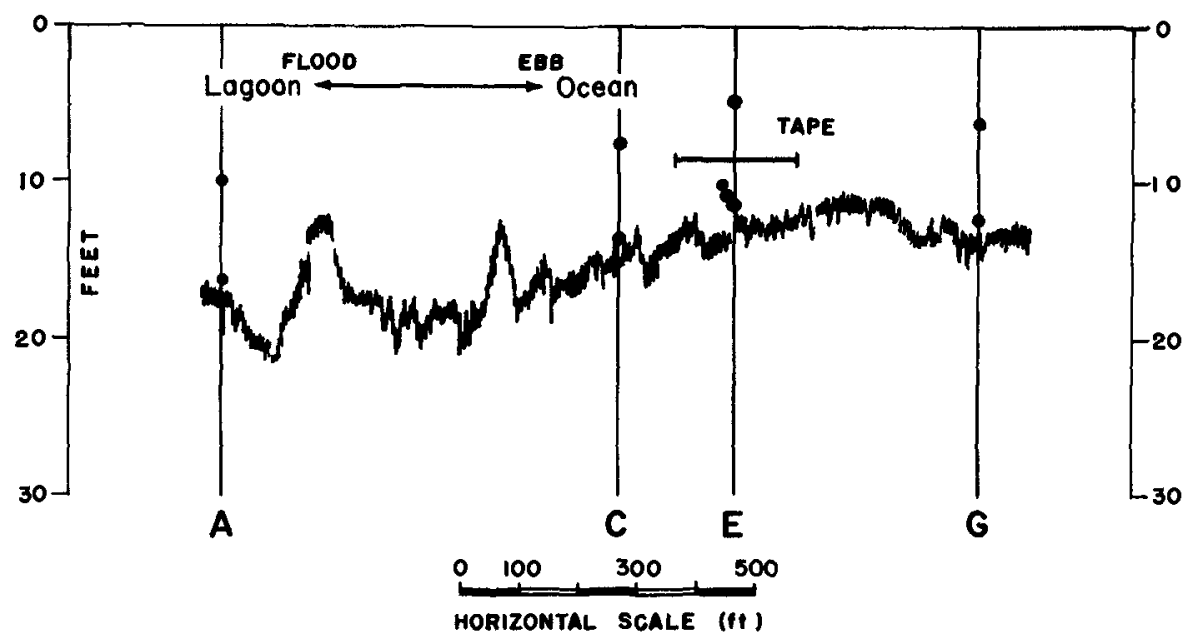

- CURRENT METERS

Figure 6 - Fathometer profile from sensor Stations A to $C$ to $E$ to $G$ and vertical position of current meters

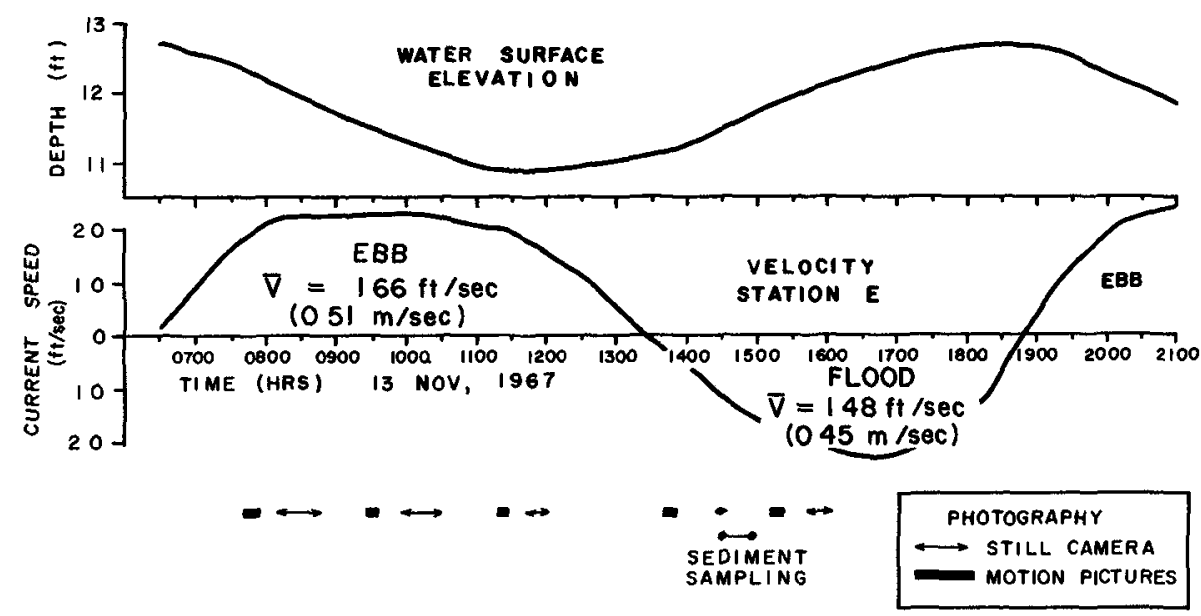

Fugure 7 Flow history and sampling times at Station E, 13 Nov, 1967 


\section{Field Setting}

At any given time during the study three bedform length scales could be observed sand waves dunes, and ripples Inspection of Figure 1 shows that the sand waves exist only on the easterm side of the channel The dunes were long crested, the crest length being 10 to 15 times the wave length A fathometer run from stations $A$ to $C$ to $E$ to $G$ (FIg 2) Is shown in Figure 6 There is a large rock outcrop at the northern end of the throat of the Inlet, about $150 \mathrm{ft}$ south of station A A series of five sand waves had an average wave length of approx.mately 90 feet The fathometer profile shown was obtalned durlng the slack water following a flood flow The fact that the ebb flow asymmetry still remalns suggests that these bedforms are not strongly influenced by the flood flow Superımposed on these larger features were dunes and rupples that were modified by the reversing flow The position of the 200-ft tape is shown also, Its northerm end rests on the crest of the last sand wave Thus, the bedforms montored included those in the lee of the sand wave crest, as well as those exposed to ebb flows of a converging nature Convergence was due to decreasing water depth in the direction of the southern end of the tape

The water surface time history and the velocity history at Station $E$ on 13 November are shown in Figure 7 Also shown are the times when photographlc sequences of the bedforms were obtalned The velocity history lliustrates the strong asymmetry in duration of the ebb relative to flood flow, which was found to be characteristic for this inlet The velocity history is at a mean relative depth $(\mathrm{z} / \overline{\mathrm{d}}$ ) of 066 (where $\mathrm{z}$ is the helght of the rotor above the bottom and $\bar{d}$ is the mean water depth) The mean velocities (Integrating over the flow duration) for the ebb and flood are, respectively, $166 \mathrm{ft} / \mathrm{sec}$ and $148 \mathrm{ft} / \mathrm{sec}$ In summary, we have a condition in which the ebb flow is generally stronger and of appreciably longer duration Net sediment transport may then be expected to be seaward

\section{Evolution of Bedforms}

The sequence of events in bedform change is shown schematically in Figure 8 where, for descriptive purposes, the cycle Is started at the end of flood ( $F 1 g$ 8a) WIth the reversal of fllow (FIg 8b), the bed sediment is transported up the steep gravity face of the dune to form what dimensionally might be considered the new dune crest A trann of ripples also occurs on the dune flank These mpples are all of smaller amplitude than the one derived from the old edge of the dune crest The dominant ripple length is approximately 1 to $15 \mathrm{ft}$

With continued ebb flow the primary crest overtakes some of the other ripples in Its lee There is a consequent increase in amplitude and the growning ripple slowly becomes a dune (FIg 8c) As the new dune crest advances ( $1 n$ the ebb flow direction, Fig 8d), the ripples in its lee become increasıngly less actıve due to shelterıng action other ripples are then observed translating up the upcurrent flank of the dune These are of small amplitude and approximity 2 to 2 5mft in length (Fıg $8 e, f$ ) 
FLOOD
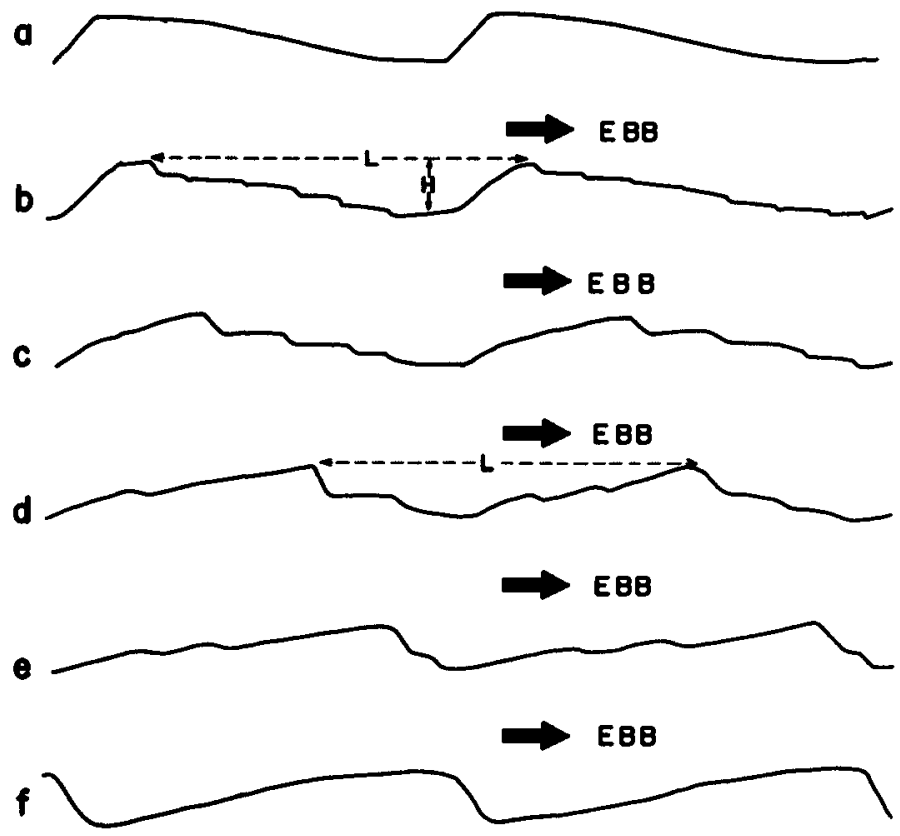

Figure 8 - Schematıc bedform evolution

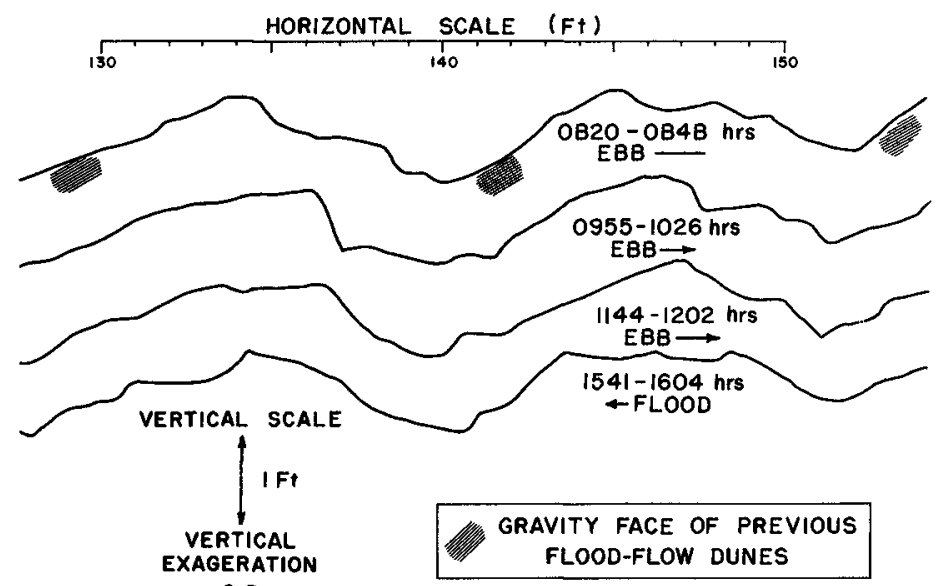

Fıgure 9 - Fxample of observed bedform changes during a tidal cycle 
As the velocity of the ebb flow diminishes the dune crest slows its advance until bed transport stops, at which point the bedforms become dormant until the new Ilood flow is intense enough for grain transport Some of the measured bedform changes are shown in Figure 9 The flow conditions for the various sampling times may be noted in Figure 7

Throughout the study period visual observations indicated most of the sediment transport occured va dune advance wlth no perceptible suspended load Given the aforementioned asymetry in strength and duration of the flow (FIg 7) it migh be expected that the ebb flow dune advance would be greater than the flood flow dune retreat This was observed to be the case over a three day perıod 10 November to 13 November Successive measurements of ebb-flow, dune-crest positions indicated a mean net advance out of the inlet on the order of 1 foot per tidal cycle This value is a very rough estimate as large variability was noted both in time and space

The evolving nature of the dunes presents some problems in determining length characteristics, however, as a flow cycle starts a reversal, a distinctive crest is generated on each dune This crest is the highest point above the downstream trough and It probably exerts a dominant influence on the gross wake characteristics for the dune The dune wave length ( $I$ ) Is defined as the distance between a given domnant crest and the next downstream crest Dune helght $(H)$ Is defined as the vertical distance between the dommant crest elevation and the downstream trough

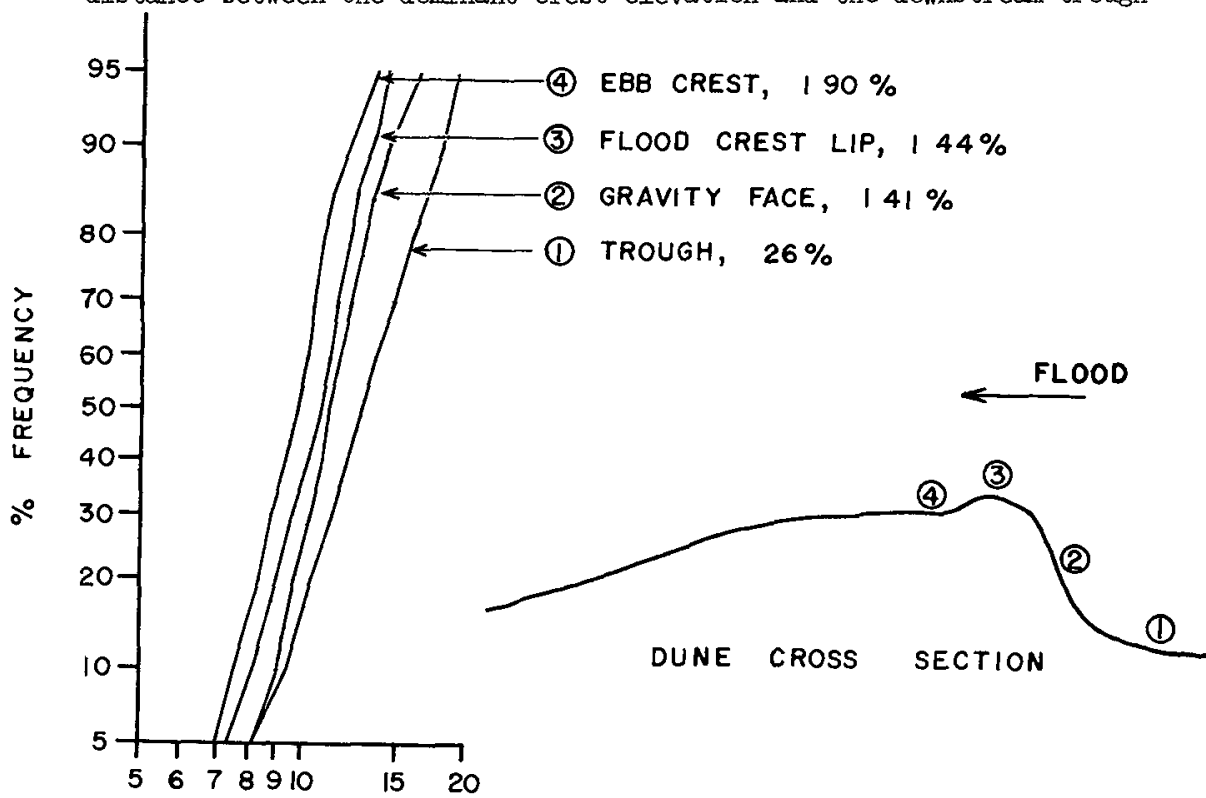

FALL VELOCITY $\mathrm{cm} / \mathrm{sec}$

Figure 10 - Sediment fall velocity distributions welght of material larger than $2 \mathrm{~mm}$ 
Inspection of the bedform profiles over the 200-ft distance indicated that the wave lengths and helghts were signiflcantly different for the northern half (115 ft) of the tape as opposed to the southern half This Is perhaps to be expected, as the first half encompasses those dunes which are formed in the lee of the large sand wave (see Fig 6) The results, cast as averages, are shown in Table 2 Two facts deserve specufic coment First of all, it should be noted that during the ebb flow the dunes in the lee of the large sand wave have longer wave lengths and smaller heights than those beyond the direct influence of the sand wave There Is only scanty evidence for the flood flow, but that avallable (154l-1604 hrs), suggests a uniformity $I n$ dune helght The second point of Interest Is the relative stabllity of wave length and helght (and, therefore, steepness) during the various phases of the reversing flow

TABIE 2 - SIZE PARAMETERS OF DUNES

\begin{tabular}{|c|c|c|c|c|c|}
\hline \multirow[t]{2}{*}{ Parameter } & \multirow[t]{2}{*}{$\begin{array}{l}\text { Position } \\
\text { on Tape (Ft ) }\end{array}$} & \multicolumn{3}{|c|}{ Trme } & \multirow[b]{2}{*}{$\begin{array}{l}1541- \\
1604 \mathrm{hr}\end{array}$} \\
\hline & & $\begin{array}{l}0820- \\
0848 \mathrm{hrs}\end{array}$ & $\begin{array}{l}0955- \\
1022 \text { hrs. }\end{array}$ & $\begin{array}{l}1144- \\
1202 \mathrm{hrs}\end{array}$ & \\
\hline \multirow[t]{2}{*}{$\begin{array}{l}\bar{I}(f t) \\
\text { Range of I }\end{array}$} & $0-110$ & $\begin{array}{c}143 \\
95-185\end{array}$ & $9 \begin{array}{l}140 \\
9-200\end{array}$ & $\begin{array}{cc}143 \\
10 & 0-200\end{array}$ & $\begin{array}{c}156 \\
100-18 c\end{array}$ \\
\hline & $110-200$ & $9 \stackrel{107}{5-125}$ & $\begin{array}{l}109 \\
90-120\end{array}$ & 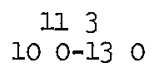 & $\begin{array}{c}105 \\
90-13 c\end{array}$ \\
\hline \multirow[t]{2}{*}{$\begin{array}{l}\bar{H}\left(f^{\prime} t\right) \\
\text { Range of } H\end{array}$} & $0-110$ & $\begin{array}{cc}0 & 56 \\
0 & 27-1 \quad 10\end{array}$ & $\begin{array}{c}063 \\
025-14\end{array}$ & $\begin{array}{c}053 \\
025-120\end{array}$ & $\begin{array}{c}082 \\
025-1=\end{array}$ \\
\hline & $110-200$ & $\begin{array}{c}075 \\
0 \quad 50-100\end{array}$ & $\begin{array}{c}081 \\
0 \quad 60-100\end{array}$ & $\begin{array}{ll} & 081 \\
0 & 5-100\end{array}$ & $\begin{array}{l}080 \\
050-1 c\end{array}$ \\
\hline \multirow[t]{2}{*}{$\overline{\mathrm{I} / \mathrm{H}}$} & $0-110$ & $\begin{array}{c}30 \\
8-41\end{array}$ & $\begin{array}{l}33 \\
9-68\end{array}$ & $\begin{array}{c}32 \\
11-50\end{array}$ & $\begin{array}{c}25 \\
12-64\end{array}$ \\
\hline & $110-200$ & $\begin{array}{c}16 \\
12-26\end{array}$ & $\begin{array}{c}14 \\
11-20\end{array}$ & $\begin{array}{c}14 \\
10-23\end{array}$ & $\begin{array}{c}15 \\
11-25\end{array}$ \\
\hline
\end{tabular}

\section{Dune Freld Sediments}

Sedument samples were obtanned on 13 November durang flood flow, about one hour after flow reversal Samples were taken of the upper half-inch of the bed material on the dune flanks, crests, and troughs The trough samples are biased, as large fragments of the conch Strombis glgas were not collected Laboratory treatment consisted of a wet separation of the material greater than 2-mm, followed by a settling analysis of the finer fraction 
A.I of the grain-size distributzons from the settling analyses closely approxımated a log-normal distribution with very little variation in the geometric standard deviation, $\sigma 8=W_{84} 1-W_{50}$, which had an average value of about 12 The average median fall velocity of the trough material was about $126 \mathrm{~cm} / \mathrm{sec}$ and at least 25 percent of the material (by welght) Is larger than $2 \mathrm{~mm}$ The seduments composing the dune flank and crest had a somewhat smallex median settling speed $(10-11 \mathrm{~cm} / \mathrm{sec})$ and were generally less than 7 percent greater than $2 \mathrm{~mm}$ in mean size

An example of these results is shown in Figure 10 The sequence shown was obtanned during the flood flow when the flood crest was becoming established Diver observations suggest that the small flood-flow crest is composed of material swept up the ebb-flow gravity face

\section{Flow Profile--Station E}

In an attempt to obtan an estimate of the velocity profile over the dunes an auxiliary current-meter tripod contalning two additional Savonius rotors was placed near Station $\mathrm{E}$ Sampling of the rotors was synchronized with the two tripod-mounted Savonzus-rotors and a sample of 46-seconds: duration was taken every six minutes The relative positions of the four rotors are shown in Figure 6, the lowest rotor was about 1 5-ft above the sand interface, the remaining rotors were 21,32 , and $83 \mathrm{ft}$ above the bottom

During the ebb flow the rotor outputs indicated a larger speed near the bottom than that registered at the uppermost rotor (F1g 6) ThIs pattern persisted throughout the ebb flow until one-half hour before flow reversal at whlch time a normal profile was present Durıng the flood flow the enhancement of flow speed near the bottom was not present, instead, the flow was essentially unform with depth

It seems likely that the flow maximum near the bottom is a jet due to the sand wave field The nature and development of jet flow over sand waves is being studied by $J$ Dungan Smith (Univ of Washington) Smzth (personal communcation) has formulated a model predicting the jet and has documented jet occurrences in tidal flows His early results indicate that the jet amplitude is a maximum when the sand wave height is about $20 \%$ of the water depth and that the jet is fully developed at the fifth wave in the field The bedform conditions of the Biminl Inlet case described here are simlar to those found by smith During flood flow the approach toward unlformaty of velocity wath depth is consistent wath the fact the depth is then divergent downstream

\section{Discussion of Bedform Changes}

Although past studies have delineated the significant varıables that control bedform characteristics in unidirectional. flow, there is no satisfactory formulation to predict the length and amplitude of bedforms found in the fleld It may be anticipated that the problem is even more 
complex in the case of reversing tidal flow There are two man differences between the two cases In the case of tidal flows the time variations in stage (and discharge) for a given flow direction occur in a few hours and bedforms may not come into equilibrium with the maximum flow speeds Carey and Keller (1957), in a study of Mississippl River bedforms noted a bedform size dependence on discharge and postulate a lag between bedform change and change in stage Secondly, with the change in flow direction the flow may encounter (as in this study) an established bedform morphology of reverse symmetry The role of such inltial conditions needs detalled attention

Aside from flow-acceleration effects, it may be anticipated that friction factors during a flow cycle will be somewhat different for reversing flows as opposed to a undirectional flow of equivalent maximum velocity, because the character and position of the separation zone in the lee of the crest changes in time Model studies by Bayazit (1969) for rupples generated by reversing flows indicated fmetion factors less than those found for corresponding unldirectional flows The difference between the two cases was attributed to a possıble lack of bedform equilibrium with the reversing flow In order to avold the effects of acceleration, Bayazit used the maximum velocity in formulating a friction factor

In the absence of detalled studies it has frequently been assumed that bedform development in an inlet may not differ significantly from a corresponding steady flow (Bruun, 1966) If such was the case one might expect to find a progression from mpples to dunes as the velocity increases and the reverse order as the velocity decreases The present study indicates this view is probably oversmplified For this case, the baslc bedform sizes are stable through time with the major changes being simply a reversal of dune geometry It is of interest to compare the dune lengths and helghts observed here whth those observed in unldirectional flow Analysis of field data by Allen (1968) showed that the characteristic lengths of dunes were strongly correlated with flow depth although it was recognzzed that dune size is not a unique function of depth Allen's relationships are, for lengths in meters

$$
\begin{aligned}
& \text { 1) } \mathrm{H}_{\mathrm{D}}=0 \quad 086 \mathrm{~d} \perp 19 \text { and } \\
& \text { 2) } \mathrm{I}_{\mathrm{D}}=116 \mathrm{~d} \perp 55
\end{aligned}
$$

where $H_{D}$ is dune helght, $d$ Is flow depth and $I_{D}$ Is dune wave length Because there was relative stability in the average dune length and helght during the observation period (Table 2), the mean depths over the two tape segments were used to calculate the expected values from equations $I$ and 2 For the lagoon side of the tape the computed wave length is $356 \mathrm{ft}$ whereas the observed mean value is approximately $145 \mathrm{ft}$ The computed dune height is $16 \mathrm{ft}$ and the observed mean is about $06 \mathrm{ft}$ For the oceanside segment of the tape the computed wave length is $282 \mathrm{ft}$ and the observed is about $110 \mathrm{ft}$, for dune helghts we find $132 \mathrm{ft}$ versus an observed value of about $080 \mathrm{ft}$ The expected values from equations $I$ and 2 are thus significantly larger than the observed mean values 
Gencrally it can be sad that the resulting scour is strongly influenced by the pile and the wave characteristics In most of the runs, very little to no bed movement could be observed away from the pile $I$ he pile scrved as a catalyst to start the scoul activity and once started around the pilc it spredd over a large area and extended in some cascs great distances from the pile Figs 9 and 10 show somt typical scour patterns obtaned from the expeiments

\section{CONCLUSIONS AND REMARKS}

1 The critical velocity necessary to cause incipient motion in oscillatory flow appcas to be lowel thin that for steady state flow

2 The idto of the maximum velocity on the pile boundary and the initid free stream velocity, is less than the value of 20 for potential flow theory

3 Incipient motion on the ple boundary appears to be independent of $\frac{\mathrm{H}}{\mathrm{h}}$ and directly dependent on the parameters $\frac{\mathrm{h}}{\mathrm{gT}^{2}}$ and $\frac{\mathrm{d}}{\mathrm{h}}$

$4 \quad \frac{\overrightarrow{\mathrm{Su}}}{\mathrm{H}}$ appears to be dix ectly related to the sediment number $\mathrm{N}_{\mathrm{s}}$ and the pile Reynold s Num

be1 $\mathrm{N}_{\mathrm{RP}}$

5 A maximum of only 6000 waves are required to redch an ultimate scour depth and in most cases 3000 waves are sufficient

6 The rclative ultımate significant scoul depth increases veıy rapidly at tisst, reaching thrce fourths of its ultimate depth in the first 1000 waves, and increases more slowly after that until it reaches its ultimate depth

7 Eddy toices, although initially influcncing the scour patteins, do not appcar to be of signifi cance in the find scoul pattern

8 The scour pattern rosulting is pimarly influenced by the pile and the wive chaldeteistics

9 In all the scour experiments, the pile acted as a catalyst causing scour of the bed particles to be initiated whereas if the pile was not present little to no scour would have iesulted

Io try and predict scour depths for a prototype case or relate these unconclusive results to a pro totype would be presumptuous To piedict happenings or occuriences of a phenomenon in a proto type requires that there be similitude, both geometric and dynamic, between the model and proto type This requiles that similitude exists between the orbital velocities and orbital lengths (1 $c$ wave chal icteistics ac similar), gidm size and grin size distribution in the bed, roughness of the beds, and translation of the oibit duc to drift Without these similitudes ciloneous conclusions could be leached in attempting to piedict piototype conditions The difficultics in acquiring similitude be tween prototype and model were pointed out by Poscy and Sybert 18 in the 11 studics of scour around piles on olfshore platiorms It required scveral years of study and expcimentation beforc actual prototype conditions werc duplicated in the model 


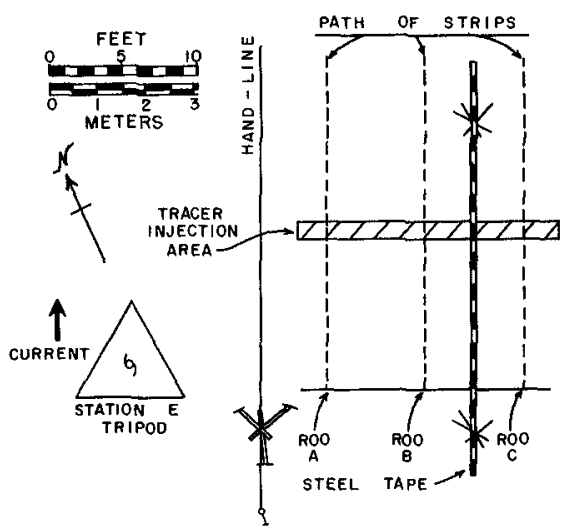

Fızure 11 - Map View of Tracer Experzment Layout

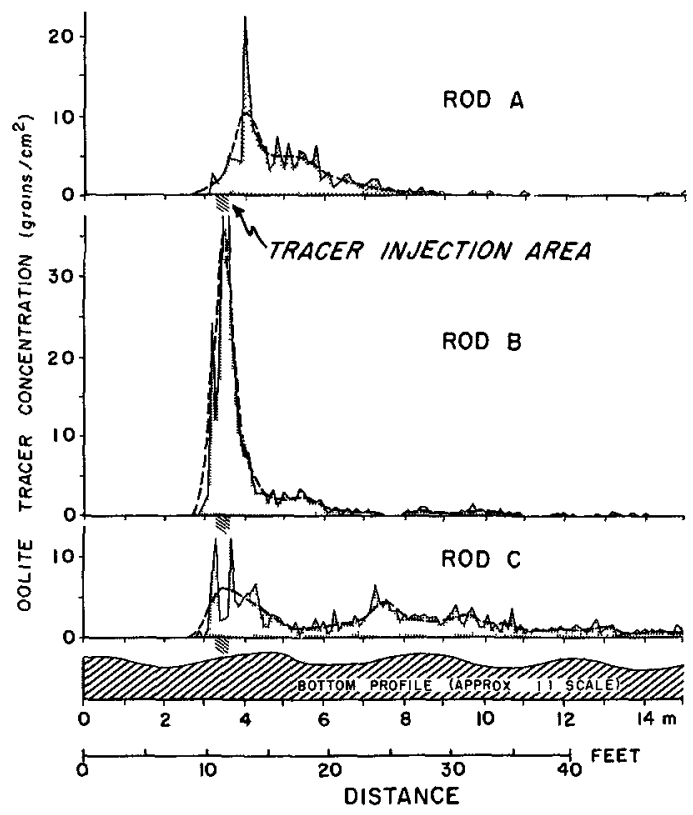

Figure 12 - Plot of Oolıte Tracer Concentration versus Distance for Rods A, B, and $C$ after the First Sampling Run 
Plastic bags contanning the tracers were placed within the anjection area (IIg 11) by a diver who eut them open with a knlfe at the designated time of release Recovery of released tracers was accomplished using $015 \times 18 \mathrm{~m}$ strıps of $\mathrm{K}$ \& E draftıng fIIm (Herculene) Loated with DowCorning 1.03 compound These strups were unrolled on the bottom in a northerly direction parallel to a graduated steel tape Three strips, fixed at one end by steel rods were unrolled simultaneously by divers according to the configuration shown on Figure 11. Four sampling runs were made at 30-manute intervals begnnning at 1307 hours

The recovered sampling strıps were examned in the laboratory under ultraviolet light Tracers were counted withln a $1.0 \mathrm{~cm}^{2}$ grid placed over the strips at $01 \mathrm{~m}$ increments for the full length of the tape Tracer concentrations, expressed as the number of gralns per $\mathrm{cm}^{2}$, were then plotted agannst distance from the holding rods in meters Figure 12 is a plot of the inztial. sampling run for rods $A, B$, and $C$ The dashed curves, intended for smoothing of the data, were obtalned by dividing distance $ı$ to $05 \mathrm{~m}$ class $\mathrm{n}$ tervals and averaging grain concentratıons therein

In examning the data, It was immediately apparent that the quartz grains (0 59-0 $84 \mathrm{~mm}$ ) were present in smaller amounts than the oolite (0 30-0 $59 \mathrm{~mm}$ ) and that their distribution was more limited A few quartz grans traveled as far as $15 \mathrm{~m}$ downstream $\mathrm{n}$ the first 30 munutes after the first release Most of the quartz tracers, however, assumed an almost normal distribution about a point less than $10 \mathrm{~m}$ downstream from the release zone The quartz tracers also appeared to be distrubuted uniformly in a lateral sense as evidenced by simultaneous comparisons between adjacent strips

oolite tracers were recovered in greater numbers and their distribution appears to have been more widespread than that of the quartz materzal Following their release, significant concentrations of oolite $\left(\sim 1\right.$ graln $/ \mathrm{cm}^{2}$ ) were found at maxımum distances from the release zone after 30 minutes, also the greater mass of these tracers, whlle remalning some l-2 $\mathrm{m}$ downstream from the release zone, showed markedly skewed distributions toward the downstream side (F1g 12 , Rods $A$ and $B$ ) with pronounced $b_{2}-$ or trumodallty 1 many cases (Fig 12, Rod C) Moreover, the oolite tracers often showed great lateral varıability, as seen through the simultaneous comparisons of adjacent strips, and temporal. variability, as seen in tume-sequential comparisons along each of the strip paths

A bedform profile, obtanned from photographs at the end of the flood current, is included in Figure 12 to show the relation of tracer concentration to bedforms

In order to quantify the observed lateral and temporal variabılıty in ollte tracer distribution, a two-way analysis of variance (Rods versus Runs) was conducted using the oolite data of the four runs for rods $A, B$, and $\mathrm{C}$ The variable used in the analysis was tape distance in meters with 
TABIE 3 - IWO-WAY AIALIYSIS OF VARIAITCE OF TRACER RECOVERY DATA (Varıable Is Tape Djstance in Meters Tracer oolite)

\begin{tabular}{|c|c|c|c|c|c|}
\hline$\overline{\text { ROD }}$ & SAMPLE & $\begin{array}{c}\text { RUN } \\
1\end{array}$ & $\begin{array}{c}\text { RUII } \\
2 \\
\end{array}$ & $\begin{array}{c}\mathrm{RUN} \\
3 \\
\end{array}$ & $\begin{array}{c}\text { RUN } \\
4 \\
\end{array}$ \\
\hline A & $\begin{array}{l}\text { Mean } \\
\text { Var } \\
\text { Size }\end{array}$ & $\begin{array}{l}492 \\
131 \\
188\end{array}$ & $\begin{array}{ll}3 & 63 \\
0 & 17 \\
180\end{array}$ & $\begin{array}{c}408 \\
0221 \\
48\end{array}$ & $\begin{array}{c}459 \\
066 \\
47\end{array}$ \\
\hline B & $\begin{array}{l}\text { Mean } \\
\text { Var } \\
\text { Size }\end{array}$ & $\begin{array}{ll}3 & 83 \\
0 & 52 \\
302\end{array}$ & $\begin{array}{c}390 \\
018 \\
81\end{array}$ & $\begin{array}{l}459 \\
112 \\
461\end{array}$ & $\begin{array}{ll}4 & 43 \\
0 & 55 \\
155\end{array}$ \\
\hline $\mathrm{C}$ & $\begin{array}{l}\text { Mean } \\
\text { Var } \\
\text { Slze }\end{array}$ & $\begin{array}{l}606 \\
464 \\
200\end{array}$ & $\begin{array}{cc}3 & 58 \\
0223 \\
55\end{array}$ & $\begin{array}{c}429 \\
021 \\
21\end{array}$ & $\begin{array}{cc}400 \\
048 \\
97\end{array}$ \\
\hline
\end{tabular}

\begin{tabular}{|c|c|c|c|c|c|}
\hline $\begin{array}{l}\text { VARTATION } \\
\text { SOURCE }\end{array}$ & $\begin{array}{c}\text { SUMS } \\
\text { of } \\
\text { SQUARES }\end{array}$ & $\begin{array}{l}\text { DEGREES } \\
\text { of } \\
\text { FREEDOM }\end{array}$ & $\begin{array}{c}\text { MEAN } \\
\text { SQUARE }\end{array}$ & $\begin{array}{c}F \\
\text { VAIUE }\end{array}$ & $\begin{array}{c}\text { CONNIDENCE } \\
\text { LEVEI } \\
\text { (PERCENTAGE) }\end{array}$ \\
\hline TOTAL & 297763 & 1834 & & & \\
\hline INTERACTION & 45905 & 6 & 7651 & 674 & $>99$ \\
\hline ERROR & 207400 & 1828 & 113 & & \\
\hline ROD & 17852 & 2 & 8926 & & \\
\hline RUN & 26529 & 3 & 8843 & & \\
\hline
\end{tabular}

the number of gralns recovered at a particular distance serving as a frequency Index for that distance Each sample of tape distances was therefore an indicator of the distribution of the tracer population for which a mean and a varıance could be estimated

The object of the analysis was to determine whether or not tape distances could be pooled for all three rods and a separation of tape distance means effected between runs After the analysis was run, significant interaction indicated that pooling could not be done and therefore separation of the means was not possible Table 3 gives the results of this analysis 
A sımlar analysıs was attempted for the quartz tracers However, by using a one-way analysıs of varıance inltually, it was learned that none of the means for runs could be separated due to their close simlarity in value and the slgniflcant varlance within each run This result only confirmed the visual impression that the quartz tracers evidenced very lıttle bulk movement

\section{DISCUSSION}

The results obtanned suggest that the oollte tracer exhybited a greater tendency toward transport than did the quartz tracer Probably the oolite tracer was "undersized" for the existing flow regime in the inlet whlle the quartz tracer was close to the prevaling grain sizes on dune crests Compared wath the greater ebb current speed obtalned at Station E (Av 17 knots, $\max 22$ knots) It is not surprising that the flood current seemed to transport only a few quart $z$ grains and that the distrıbutions of these were less skewed Caution must be used in this interpretation, however, because the effect of burlal of tracer gralns is unknown due to the sampling method employed It has been Instructive to the authors that an inlet such as the one at Bimini represents an extremely complex environment and even detalled measurements are inadequate for a clear picture of the way in which sand is transported For example, lateral variablilty of apparent grain motion was much more evident than anticipated, bottom transport is truly a three-dımensional problem

Although the results of the experiment are not conclusive, it seems evident that dunes play a major role in the distribution of tracer sands, and the modes of the concentration curves indicate points of grain accumulation which move slowly downcurrent with time In some cases, grains apparently travel very quickly over a succession of dunes, but a majority of samples indicate that most of them are fixed by the dune topography and therefore travel at much slower rates This slow transport is in keeping with the fact that the dunes themselves migrate very little durıng any given tidal cycle

The Blminl Inlet sedıments appear to possess an organic coating (Bathurst, 1966) which did not allow most of them to adhere to the greased sampling strıps All tracer grains were thoroughly dried and rewetted with detergent before release, they adhered very well to the strips Although mucold coatings or gelatinous mats are not cormon to the active sands typlcal of the Bıminl Inlet (Bathurst, 1966, p 90), there was clearly a difference in the surfaces of the in-situ and tracer grains This then leaves the question of whether or not the in-situ grains experience a binding effect that is not otherwise apparent 


\section{ACKNOWLEDGMENTS}

All of the fleld work for this study was supported by the Atlantic Oceanographlc and Meteorologlcal Laboratories, of the Environmental Science Services Administration (ESSA), Miami, Florida Logistical support was obtalned from the Lerner Marıne Laboratory (American Museum of Natural History) on North BIm工nI Aerial photography and contour mapplng was accomplished by the Photogrametry Division, Coast and Geodetic Survey, ESSA

This study could not have been accomplished without the concerted efforts of many indlviduals We wish to express our great appreciation to Messrs $F$ Beugnet, $S$ Cofer, J Falkenhoff, B G Grant, C Kıersh, $M \quad P$ Lynch, $E$ W Rayfield, $G$ Reynolds, and $D G$ Tyler for their efforts in the field $R$ W Mathewson and his staff at the Lernex Laboratory were also most helpful in the field effort

\section{REFERENCES}

Allen, $J \quad R$ I 1968 The nature and origin of bedform hierarchies Sedımentology, $10161-182$

Bathurst, $R$ G C 1967 Dolite fllms on low energy carbonate sand graıns, Bımın lagoon, Bahamas Marıne Geology, 5 89-109

Bayazıt, M 1969 Resistance to reversing flows over movable beds Proc ASCE, Jour Hydraulics Div, 95(July 1109-1127)

Bruun, P 1966 Tidal Inlets and Inttoral Drzft, $v 2$, Skıpnes offsettrykkerı, Trondhe.m, Norway, $p$ 1-193

Carey, W C and H D Keller 1957 Systematic changes in the beds of alluvidal rivers Proc ASCE, Jour Hydraulic DIv, 85(August 1-24)

Turekıan, K K 1957 Salınıty varıatıons in sea water in the vicınıty of BımıI, Bahamas, B W I, Amer Museum Novitates, No 1822 , $12 \mathrm{pp}$

Wunsch, C, D V Hansen, and B D Zetler 1969 Fluctuations of the Florida current inferred from sea level records Deep Sea Research (SuppI) $16447-470$

Yasso, W E 1962 Fluorescent coatıngs on coarse sediments, an integrated system Office Naval Research, Geog Branch, T'ech Rep I, $48 \mathrm{pp}$ 


\section{CHAPTER 77}

RECENT HISTORY OF EROSION AT CAROLINA BEACH, N $\mathrm{C}$

Limberios Vallianos, Chlef, Coastal Englneering Studies Section, Department of the Army, Wilmington District, and Member ASCE

ABSTRACT

The reaction of shores adjacent to sallent features which interrupt alongshore processes has long been recognized as an important consideration in connection with the investigation of englneering works to be undertaken on shores characterized by a littoral drift regimen particular emphasis has been placed on the evaluation of shore changes related to major control structures at nav1gation entrances, however, manmade interruptions of small scope, which inftially appear innocuous, can produce costly damage to the adjacent shores located on the downdrift side of the interruption

The town of Carolina Beach, a seaside resort on the Atlantic ocean in southeastern North Carolina, is a classic example of an area experlencing inordinate and costly erosion associated with an inftially small manmade interruption on the updrift shore In 1952, local boating interests excavated a channel through the updrift barrier beach to connect the Atlantic Ocean and a lagoonal area traversed by the Atlantic Intracoastal waterway The channel, located 8,000 feet north of the Carolina Beach town $11 \mathrm{mlts}$, soon developed into a small, permanent coastal inlet having a width of approximately 550 feet and a depth of 15 feet In the ensuing 17-year period, 1952-1969, this inlet entrapped over 4 million cublc yards of littoral material, resulting in a concomitant downdrift erosion which progressed southward to the town of Carolina Beach A protective beach fill placed along the town's ocean front in 1965 has suffered considerable erosion damage

Th1s paper develops and quantifies the cause and effect relationships of the problem generally in terms of the alongshore processes and, in so doing, also furnishes basic information in regard to the performance of the largescale artificial beach fill placed alons the ocean front of Carolina Beach in 1965

\section{INTRODUCTION}

In october 1962, Congress authorlzed the construction of a beach f1ll, for the purpose of hurricane protection and beach erosion control, to extend from the northern town limits of Carolina Beach, $\mathrm{N} C$, to the southern town limits of Kure Beach, $N$ C, a distance of about 26,000 lineal feet In April 1965, a portion of the project was completed by the placement of approximately 2,632,000 cubic yards of f1ll material along the 14,000 lineal feet of shore fronting the town of Carolina Beach (see FIGURE I) The geometric configuration of the fill consisted of a dune having a crest width of 25 feet, at an elevation of 15 feet above mean low water, fronted by a 50-foot-wide storm berm at an elevation of 12 feet above mean low water (see FIGURE II) Along the northernmost 3,700 lineal feet of constructed project, the storm berm was widened to 70 feet to provide an advanced beach-nourlshment stockplle construction of the authorlzed project south of the town limlts of Carolina Beach was deferred due to the inability of local interests to f1nance a portion of the non-Federal share of the project costs Herein, reference to the authorized project applies only to the constructed section, specifically, that portion of the project fronting the town of carolina Beach, $\mathrm{N} C$ 


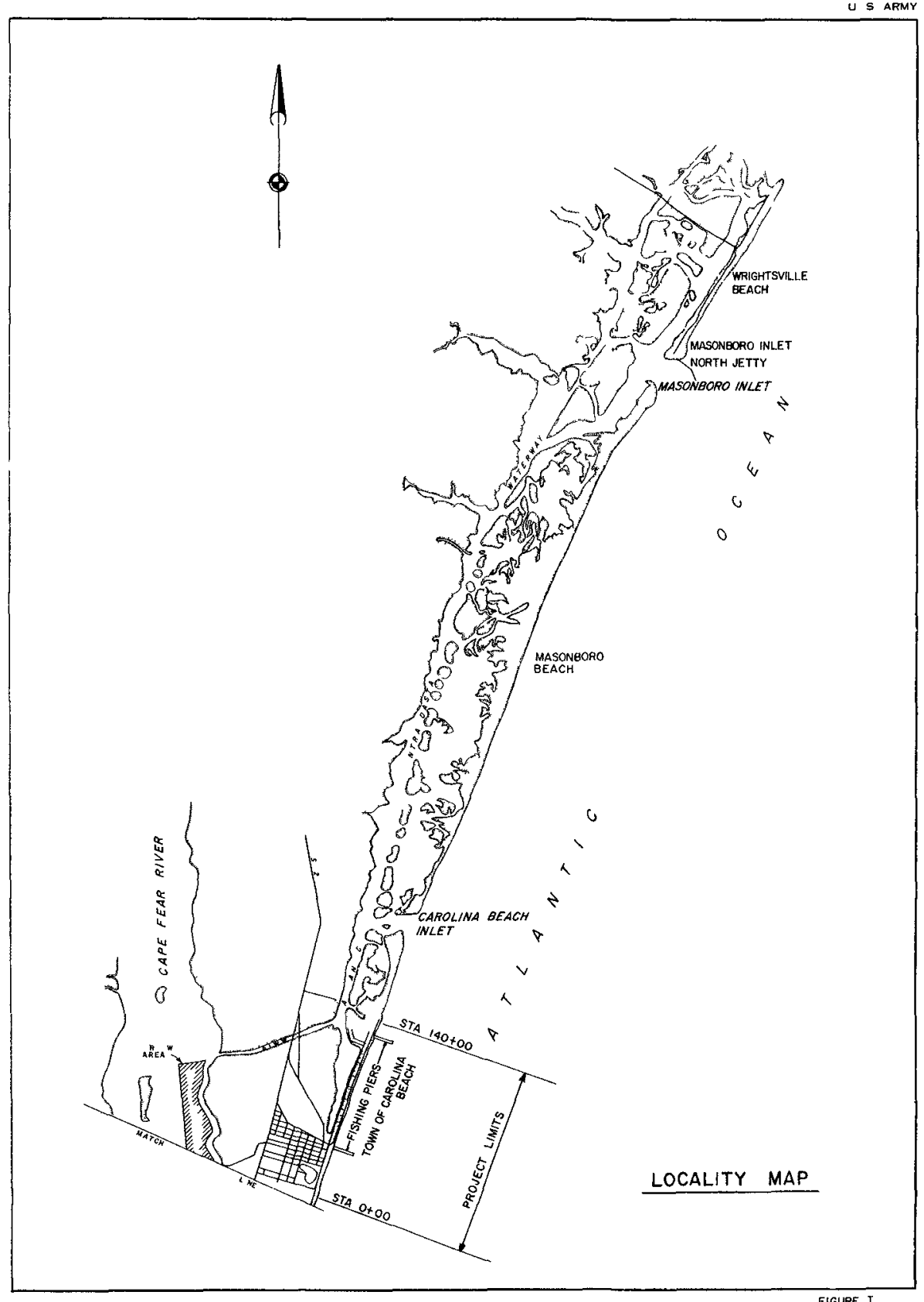

FIGURE I 


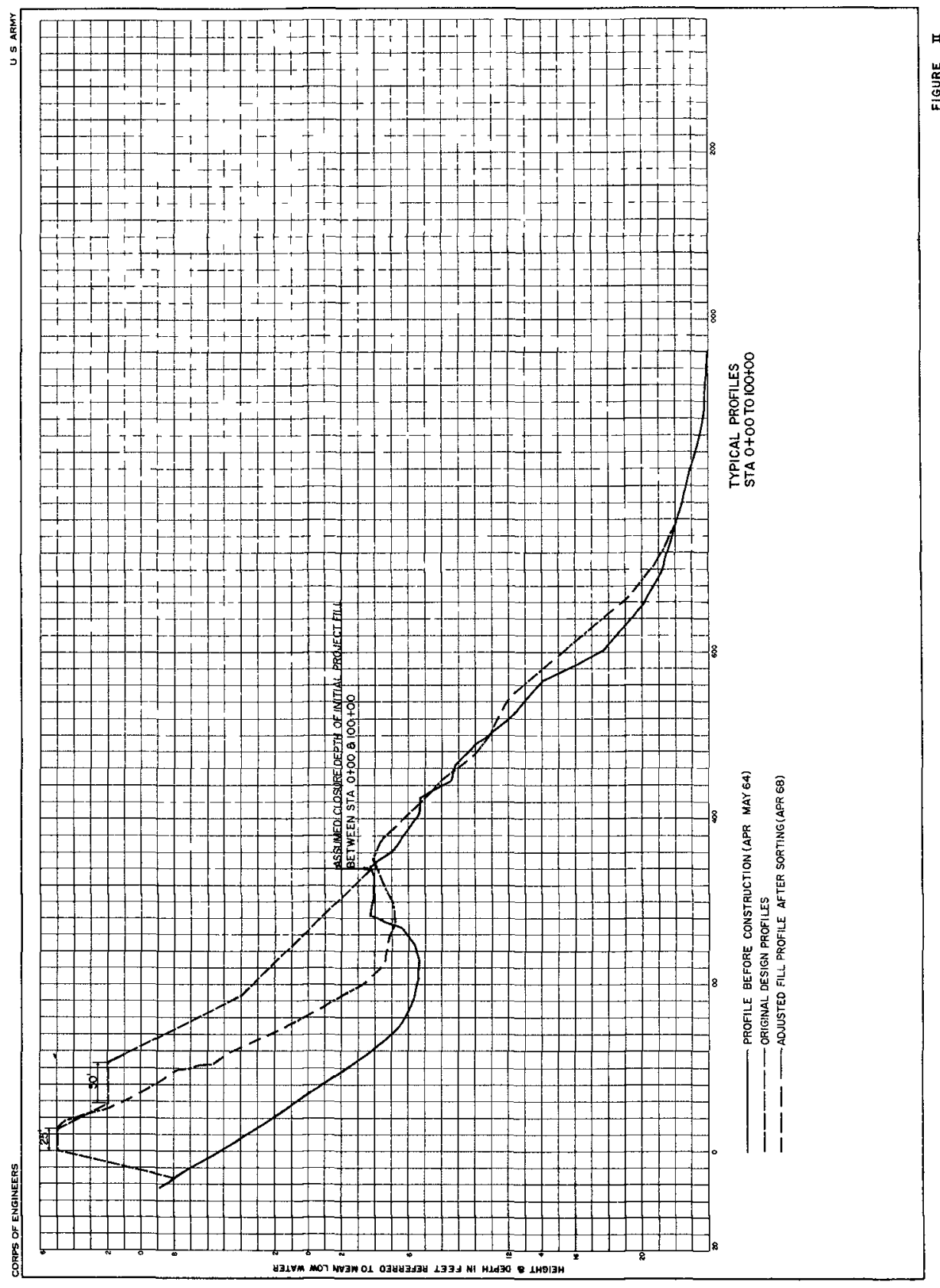


Immediately following the construction of the Carolina Beach project, rapid erosion was manifest along the entire length of the fill structure Though inftial adjustments were expected, the actual changes, particularly those evidenced along the onshore section of the project, were much greater than anticipated during the planning and design phases of the project in the first 2 years of project operation, erosion was a persistent phenomenon along the entire length of $f 111$, however, the rate of erosion along the southern 10,000 feet of project was considerably smaller than that experienced along the northern 4,000 feet

During the Initial 2-year period, approximately 712,000 cubic yards of f1ll were lost from the entire profile, to seaward depths beyond the $22-\mathrm{foot}$ mean-low-water contour, within the southern section of the project This volumetric loss represents about 43 percent of the total in-place fill inftially placed along that section of the project, therefore, in terms of fill, the degree of project protection was reduced by 43 percent The attendant onshore changes resulted in an 82-foot recession of the high-water line in the initial 2-year period and the destruction of the horizontal storm berm of the design profile By the end of the second year of operation, the southern 10,000 lineal feet of project stabilized and have remained in more or less the same condition to the present

In the first 2 years of project operation, erosion along the northernmost 4,000 Iineal feet of project was even more extensive than that which had occured in the 10,000-foot southern section within the intilal 2-year period, approximately 550,000 cublc yards of f1ll were lost from the active profile along this section of the project, which amounted to a 56-percent reduction in the total in-place fill By March 1967, 2 years after inftial construction, the highwater line along this section of project receded 140 feet, resulting in the total destruction of 1,500 lineal feet of dune and storm berm and the severe deterioration of an additional 1,200 feet of onshore f1ll section This erosion was progressing rapidly in a southward direction and threatenting the more stable southern section of the profect Therefore, in March 1967, emergency measures were implemented to alleviate the problem These emergency measures involved restoration of the north end of the project by the placement of approxImately 360,000 cublc yards of beach f11l and the construction of a 405-rootlong groin near the north terminal of the project The groin was considered necessary, as there appeared to be a reversal in the predominant direction of littoral transport at the north end of the project In the year following the Implementation of the emergency measures, approximately 203,000 cubic yards of emergency fill were lost to erosion and the major portion of the shoreline returned to about the same position it had prior to the emergency work The shoreline immediately south of the groin, for a distance of about 400 feet, has remained relatively stable, and the rate of loss of emergency fill along this small segment of shore was about 42 percent less than that experienced along the remaining emergency section

\section{ENVIRONMENTAL FACTORS}

Beach-profile characteristics Within the area of interest, the qualities of the normal beach profile are characterized by conditions existing along the southern 10,000 lineal feet of project Along this area, sufficient time has now elapsed for the borrow material, placed in 1965, to be sorted and to estabIIsh itself on the profile in more or less a condition of dynamic equilibrium, as discussed below in connection with shore processes Bottom-material characterfstics are defined by the grain-size analysis of surficlal sediment samples taken from two representative range lines along the active profile, contained 
between the backshore and the 30-foot-depth contour (see TABLE 1) It w1Il be noted that the resulting composite phi mean diameter $1 \mathrm{~s} 169(031 \mathrm{~mm})$ and that the ph1 standard deviation 1 s 0.91

\section{TABLE 1}

Characteristics of material on profile at Carolina Beach (May 1967)

\begin{tabular}{|c|c|c|c|c|}
\hline Stat1on & Sample & $\begin{array}{c}\text { Ph1 mean } \\
(\mathrm{M} \phi)\end{array}$ & $\begin{array}{c}\text { Ph1 standard } \\
\text { deviation } \\
(S \phi) \\
\end{array}$ & $\begin{array}{c}\text { Ph1 vartance } \\
\left(S \phi^{2}\right) \\
\end{array}$ \\
\hline $10+00$ & $\begin{array}{l}\text { Top of berm } \\
\text { Mean h1gh water } \\
\text { Mean sea level } \\
\text { Mean low water } \\
-6 \\
-12 \\
-18 \\
-24 \\
-30\end{array}$ & $\begin{array}{ll}1 & 30 \\
0 & 85 \\
1 & 46 \\
0 & 76 \\
2 & 39 \\
2 & 16 \\
1 & 87 \\
\text { No sample } \\
1 & 57\end{array}$ & $\begin{array}{ll}0 & 51 \\
0 & 58 \\
0 & 40 \\
0 & 47 \\
0 & 48 \\
0 & 59 \\
0 & 59 \\
0 & 61\end{array}$ & $\begin{array}{ll}0 & 260 \\
0 & 336 \\
0 & 160 \\
0 & 221 \\
0 & 230 \\
0 & 348 \\
0 & 348 \\
0 \\
0 & 372\end{array}$ \\
\hline $70+00$ & $\begin{array}{l}\text { Top of berm } \\
\text { Mean h1gh water } \\
\text { Mean sea level } \\
\text { Mean low water } \\
-6 \\
-12 \\
-18 \\
-24 \\
-30\end{array}$ & $\begin{array}{ll}1 & 03 \\
1 & 00 \\
1 & 25 \\
0 & 38 \\
2 & 49 \\
2 & 66 \\
2 & 91 \\
3 & 13 \\
1 & 51\end{array}$ & $\begin{array}{ll}0 & 76 \\
0 & 54 \\
0 & 39 \\
0 & 54 \\
0 & 86 \\
0 & 45 \\
0 & 47 \\
0 & 52 \\
1 & 13\end{array}$ & $\begin{array}{ll}0 & 578 \\
0 & 292 \\
0 & 152 \\
0 & 292 \\
0 & 740 \\
0 & 203 \\
0 & 221 \\
0 & 270 \\
1 & 277\end{array}$ \\
\hline & $M_{\phi}=169$ & 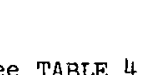 & 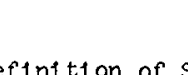 & $=037$ \\
\hline
\end{tabular}

The assoclated average proflle conflguration, in terms of slope, is as follows

\begin{tabular}{l|c|c|c|c|}
\hline Depth range below mean low water & $\begin{array}{c}4 \text { feet } \\
\text { to } \\
12 \text { feet }\end{array}$ & $\begin{array}{c}12 \text { feet } \\
\text { to } \\
18 \text { feet }\end{array}$ & $\begin{array}{c}18 \text { feet } \\
\text { to } \\
24 \text { feet }\end{array}$ & $\begin{array}{c}24 \text { feet } \\
\text { to } \\
30 \text { feet }\end{array}$ \\
\hline Average slope & 122 & 129 & 150 & 1167 \\
\hline
\end{tabular}

The natural berm elevation 1s located at an elevation of about 8 feet above mean low water The foreshore slope, extending from the berm crest to a depth of about -2 feet mean low water, averages 1 on 8 Between the -2 and -4 -foot contours, the profile is characterlzed by an alongshore trough and submerged bar Typical profiles are shown on FIGURE II

Winds on an annual basis, winds blow onshore 388 percent of the time and offshore 500 percent of the time, w1th 112 percent representing calm conditions W1th reference to onshore winds, 507 percent occur from the northeast and east Normal wind speeds range up to 20 knots

Waves During the fall and winter months, waves approach the area more frequently from the northeast and east, producing north to south littoral currents Durling the spring (March, April, and May), a transition pertod is observed during which waves attack the shore w1th almost equal frequency from 
all directions, resulting in frequent reversals in the direction of littoral transport During the summer, waves are more likely to come from the southeast and south and produce northward drift on an annual basis, the predom1nant direction of wave attack, in terms of energy level, is from the northeast and east, producing a net drift to the south The most frequent waves affectIng the area have helghts ranging from 1 to 5 feet and perlods of from 5 to 10 seconds

Littoral currents Littoral current observations have indicated that during northeast and east wave attack, littoral currents north of $s$ tation $140+00$ are less than those south there of During southeasterly and southerly attack, currents north of station $140+00$ were observed to be stronger than those south of that location

Tides The normal tidal range in the vicinity of Carolina Beach is about 40 feet The average spring range is 47 feet Storms, particularly hurr1canes, can cause considerable varlation in these normal tides for example Hurricane Hazel (15 october 1954) generated a tide of 127 feet above mean-lowwater datum, which is the highest recorded tide in the study area

\section{SHORE PROCESSES - CAUSE AND EFFECT RELATTONSHIPS}

General Prior to 1952, the shoreline between Masonboro Inlet, located 11 miles north-northeast of Carolina Beach, and New Inlet, located 9 miles south-southeast of Carolina Beach, constituted a continuous physiographic unit This shoreline reach was characteristic of the general coastline of North Carolina, being comprised of plain, unobstructed sandy beach areas subject to reversals in the direction of littoral transport, but with a predominant southward movement of littoral materlal The long-term average annual recession of the waterline was estimated to be of the order of magnitude of 1 foot

In September 1952, local interests excavated a tidal inlet at a point approximately 7,500 feet north of the town limits of Carolina Beach, thus providing for the Immediate area a direct connection between the Atlantic Intracoastal Waterway (ATW) and the Atlantic ocean (see FraURE I) This inlet, later to be known as Carolina Beach inlet, rapidly developed as a permanent coastal feature having a low-waterline width of 550 feet and an associated cross-sectional area of 6,500 square feet The ebb and flood flows are $300 \times 10^{6}$ and $450 \times 10^{6}$ cublc feet, respectively Immediately following the development of this inlet, severe erosion was evidenced at the south shoulder of the inlet and has, over the 17-year period of the 1nlet's existence, progressed southward to the extent of serlously affecting the performance of the beach-restoration and hurricane-protection project constructed in 1965 along the 14,000 lineal feet of shoreline fronting the town of Carolina Beach Though the effect, of the inlet on the downdrift shore have been recognized for some time, in a broad qualitative sense, the full assessment of the shore processes involved and the quantification of the phenomena required the detalled synthesis of data accumulated in the 17-year perfod, 1952-1969 (the bulk of wh1ch were obtained between 1965 and 1969), In connection with a survey monitoring program involving the shores of Carolina Beach northward to and including the inlet complex It has been through the collection of these data, as well as other recent information related to nearby shores, that a rational appraisal of the general problem can be made

The total length of shoreline germane to this discussion extends 21,100 lineal feet between Carolina Beach Inlet and station $0+00$ at the southern 
terminal of the Carolina Beach project This reach of shoreline can be divided into three units, each of which has responded to shore processes in a distinctly different manner, though the causative factors are interrelated These units are designated herein as (a) Segment I - the 10000 lineal feet of shore northward from station $0+00$ at the south end of the Carolina Beach project to project station $100+00$, (b) segment II - the 4,000 lineal feet of shore extending northward from project station $100+00$ to station $140+00$ at the north end of the carolina Beach project, and (c) Segment III - the 7,100 lineal feet of undeveloped shoreline between the Carolina Beach town limits and Carolina Beach Inlet

Segment I Survey records for the 19-year period, 1938 to 1957, reported In the orlginal Carolina Beach project report show that the average annual recession rate was approximately 1 foot per year with a short-term maximum rate of 28 feet belng observed in the pertod 1952-1957, during which the area had been exposed to four major hurricanes The volumetric loss of material for the entire active beach profile was estimated to be approximately 4 cubic yards per lineal foot of beach annually

Following the placement of artificial f1ll along Segment $I$, in connection with the construction of the Carolina Beach project, the effects of shore processes in this area were radically different during the first 2 years of project operation than those determined from historical records During these first and second years of project operation, April 1965-April 1966 and April 1966April 1967, the shoreline receded at an average annual rate of 67 feet and 15 feet, respectively, with corresponding volumetric losses of fill material amounting to 370,000 cubic yards and 342,000 cubic yards In the third year of operation, April 1967-April 1968, a marked change occurred in the response of the artificlal fill to shore processes, as the rate of shoreline recession decelerated to 5 feet per year and the volumetric change of material amounted to a slight accretion of about 17,000 cublc yards Shoreline movements and volumetric changes following project construction are given in FIGURE III and TABLE 2, respectively Surveys in 1969 indicate that the project is in essentially the same condition as observed in 1968 Full verification of the present project condition will depend on the results of surveys conducted in the summer of 1969 and winter period of 1970 However, on the basis of existing information, it can be assumad that the section of the Carolina Beach project within Segment $I$ of the study area required 2 years of exposure to reach a state of dynamic equilibrium with the prevaliling environment

The rapid recessions of the waterline in Segment I during the first 2 years of project operation were a result of profile adjustment along the active profile which terminates at depths between -22 and -30 feet mean low water, as well as net losses in material volume resulting from natural sorting action alsplacing fine material, which was incompatible with energy levels on the active profile, to depths seaward of the active profile Reference is made to FIGURE II, which shows the typical beach profile in Segment I prior to construction of the Carolina Beach project and the ortginal design project profile It will be noted that the foreshore and of fshore design profile slope of 1 on 20 terminates at a depth of 4 feet below menn low water and deviates from the natural profile by the exclusion of the offshore bar and trough situated at a depth of 4 to 6 feet below mean low water The adjusted project profile of April 1968 also given on FIGURE II, shows the actual profile closing at a depth of about 22 feet below mean low water, as well as the characteristic bar and trough Thus, displacement of the initial fill with the concomitant reduction of the onshore design section, was an inevitable eventuality of normal sorting action and the reestablishment of the normal profile configuration Note that the actual profiles shown on FIGURE II are for the month of April, during which average annual profile conditions obtain in the vicinity of Carolina Beach 


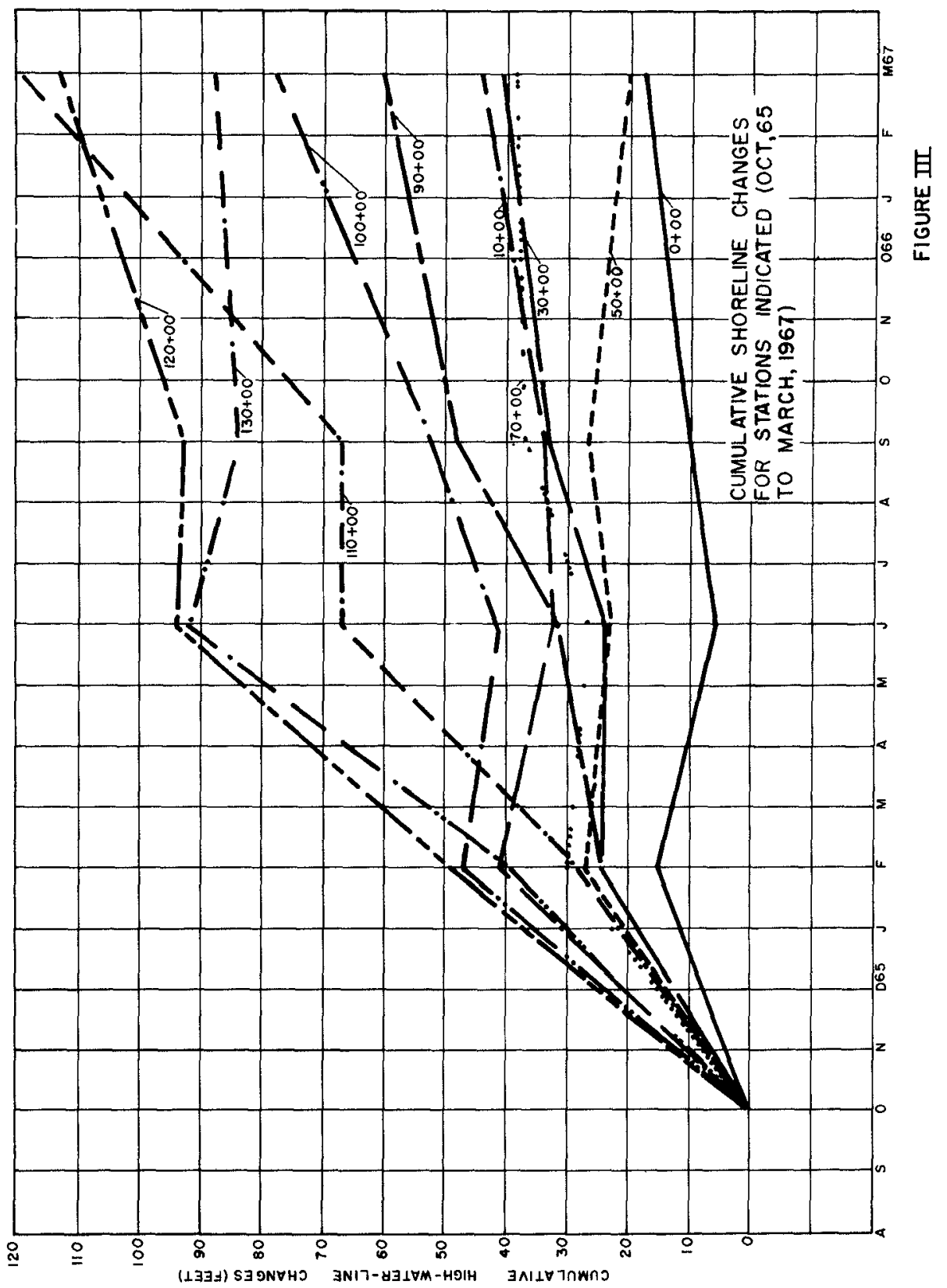


TABLE 2

Total volumetric change since construction of the project (SEGMENT I - station $0+00$ to station 100+00)

\begin{tabular}{l|c|c}
\hline \multicolumn{1}{c|}{ Period } & $\begin{array}{r}\text { Volumetric change } \\
\text { during period } \\
\text { (cubic yards) }\end{array}$ & $\begin{array}{c}\text { Cumulative volumetric } \\
\text { change by end of period } \\
\text { (cubic yards) }\end{array}$ \\
\hline As bu11t to June 1966 & $-435,300$ & $-435,300$ \\
June 1966 to September 1966 & $-143,800$ & $-579,100$ \\
September 1966 to December 1966 & $-7,100$ & $-586,200$ \\
December 1966 to May 1967 & $-172,000$ & $-758,200$ \\
May 1967 to June 1967 & $+257,800$ & $-500,400$ \\
June 1967 to 0ctober 1967 & $-29,000$ & $-529,400$ \\
October 1967 to February 1968 & $-326,400$ & $-855,800$ \\
February 1968 to Apri1 1968 & +156700 & $-699,100$ \\
Apri1 1968 to August 1968 & $+75,400$ & $-623,700$ \\
\hline
\end{tabular}

NOTE $+=$ accretion, - = erosion

Two computations were made in an attempt to determine the degree to which sorting of the original borrow material affected the rate of erosion of the project fill The first computation consisted of a mathematical comparison of the size characteristics of the original borrow material to the size characteristics of the material composing the beach profile The results of this computation indicated that for every cubic yard of borrow material remaining on the profile, 21 cubic yards of this material had to be sorted The ratio of the total material sorted to the amount remaining on the profile after sorting action has occurred, as defined by $W \quad C$ Krumbein and $W R$ James ${ }^{l}$, is referred to as the "critical ratio" (Rфcrit), where

$$
\begin{aligned}
& R \phi c r i t=\left[\frac{S \phi_{b}}{S \phi_{n}}\right]\left[e^{-\frac{\left(M_{\phi n}-M_{\phi b}\right)^{2}}{2\left(S \phi_{n}{ }^{2}-S \phi_{b} b^{2}\right.}}\right] \\
& S \phi_{\mathrm{b}}=\text { standard deviation or borrow material, in phi } \\
& \text { units ( } 128 \text { in this case, see TABLE 3) } \\
& S_{\phi n}=\text { standard deviation of native material, in phi } \\
& \text { units (0 } 91 \text { in this case, see TABLE 1) } \\
& M \phi_{\mathrm{b}}=\text { phi mean of borrow material }(088 \text { in this case, } \\
& \text { see TABLE 3) } \\
& M \phi_{n}=\text { phi mean of native material ( } 169 \text { in this case, }
\end{aligned}
$$$$
\text { in which }
$$

\footnotetext{
${ }^{1}$ See U S Army Coastal Engineering Research Center Technical Memorandum No 16, "A Lognormal Size Distribution Model for Estimating Stability of Beach F1ll Material "
} 
TABLE 3

characteristics of original fill material placed between stations $0+00$ and $100+00-$ segment I

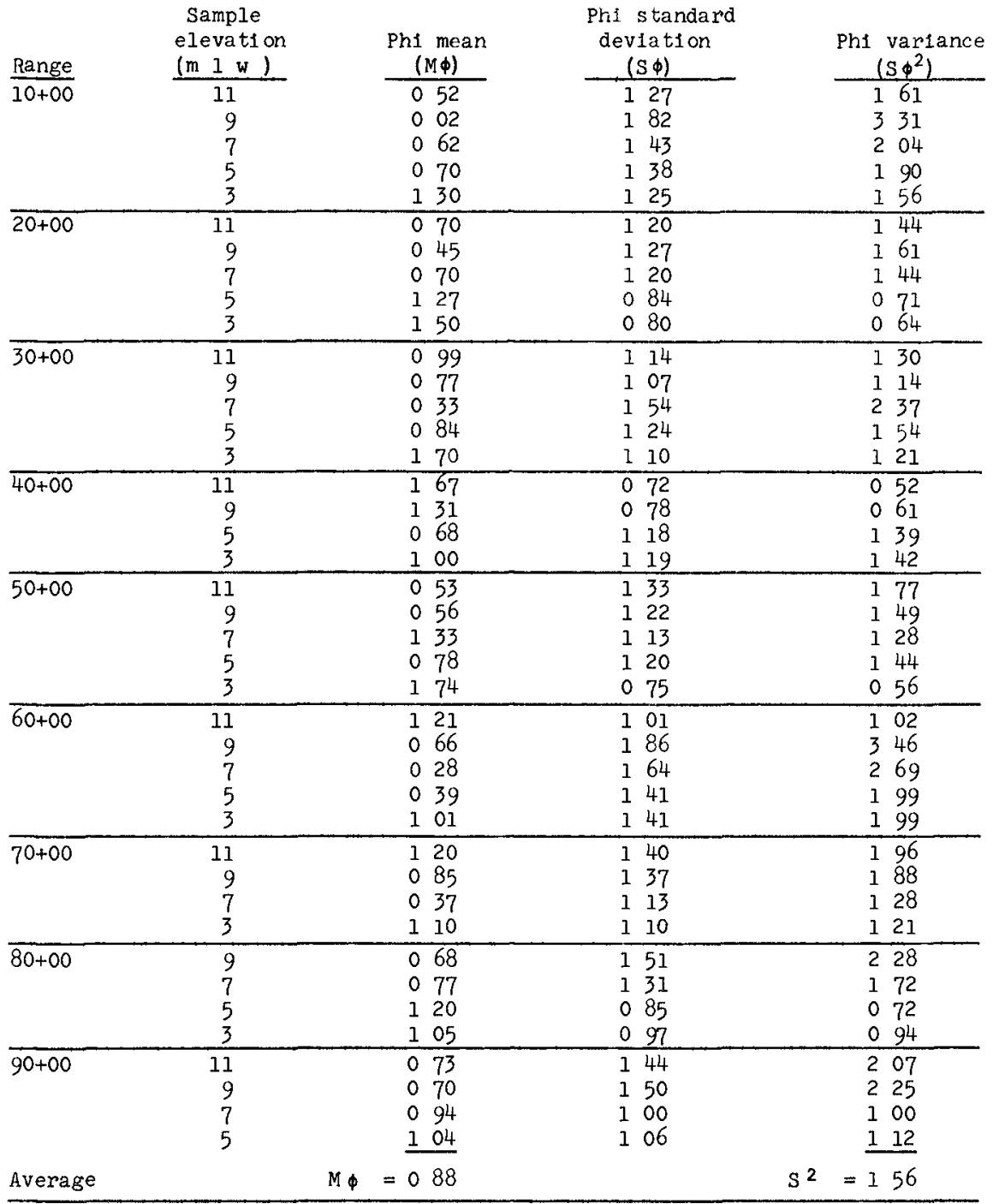

The composite phi variance $\left(S \phi^{2} \mathrm{comp}\right)$ and standard deviation (S申comp) are computed by

$$
S \phi^{2} \text { comp }=S^{2}+\frac{(B-A)^{2}}{12}=156+\frac{(0 g 2)^{2}}{12}=163
$$


Sфc omp $=128$

Where $A$ is the average of the minimum phi-mean values obtalned along the various ranges The value $B$ 1s the average of the maximum phi-mean values

The second computation consistea of a detemination of the ratio of borrow material placed within segment $I$, which was subjected to worting action, to the amount of that material which remalned on the profile at the end of approximately 2 years of project operation The ratio thus determined was 23 , which compares extremely favorably with the computed "critical ratio" Since the two independent checks of this ratio resuited in essentially the same value, the implication is that most of the material lost from the project in segment I was the result of sorting action displacing fine-grain materlal to depths seaward of the active profile It is remarked that the entire artificial fill was not exposed to hydraulic action during the 4 years of project operition and this fact was accounted for in the sorting computations Had the artificial fill been exposed to a storm of hurricane intensity with the attendant severe churning of the beach profile cover, larger juantities of fill would have been exposed to sorting action and the net losses of materlal would have, doubtless, been greater It is worthy of mention that the analytical procedure lsed in arriving at the value of the "critical ratio" was not developed during the design phase of the Carolina Beach project Had this procedure been avallable, the material losses experienced by the project in segment I could have Deen predicted with a remarkably high degree of accuracy

Insofar as the present conditions are concemed, the relative stability of the project in segment I is apparently due to the balance of alongshore transport into and out of segment $I$, as shown in Dlagram 1

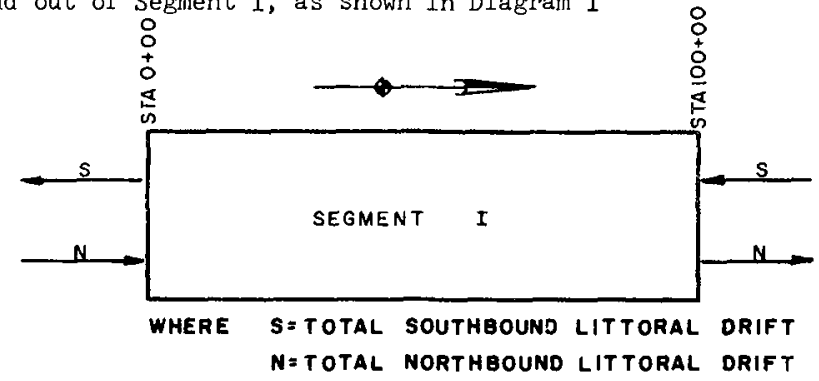

PRESENT ALONGSHORE PROGESSES IN SEGMENT I DIAGRAM 1

Segment II and Segment III Prior to the opening of Carolina Beach Inlet In 1952, the shorelines of segments II and III were continuous with, and on the same alignment as, segment I Therefore, the behavior of the entire shore area was more or less uniform However, Immediately following the opening of the inlet, dramatic changes began occurring in segment III and, with time, progressed southward into segment II In the period 1952-1963, prior to the construction of the Carolina Beach project, the high-water line receded 1,135 feet at the shoulder of Carolina Beach Inlet, the north end of Segment III, and 37 feet at station $100+00$, the south end of Segment II The difference in the extent of recession near the inlet and at station $100+00$ resulted in a change of alignment of the combined shoreline of Segments II and III with respect to Segment I This change of alignment, which was later to have a prominent role 
in the behavoir of the artificial fill in Segment II, was a natural development resulting from a deficit of littoral drift from the north, caused by material entrapment in the inlet shoal system By way of a brief qualitative analysis of this phenomenon, the following explanation $1 \mathrm{~s}$ given The alongshore movement of littoral material results from the existence of an alongshore current generated by the obliquity of the wave crests attacking the shore, in other words, it can be assumed that, if the breaking-wave crests are parallel to the shore, little or no alongshore current exists and, consequently, there is no alongshore movement of beach material When there is a substantial reduction in the quantity of Iittoral material to a segment of shore, that shore w1ll erode to the extent of reaching some new state of equilibrium with the eroding forces This was accomplished in Segment III, and to a smaller degree in Segment II, by the shore retrograding to an alignment approaching parall $\in 11$ sm with the general approach of wave crests from the northeast sector of attack As any given section of shore attains near parallelism with the attacking wave crests, it ceases to supply large quantities of material to the adjacent downdrift section, resulting in a downdrift progression of the erosion, as shown in Dlagram 2

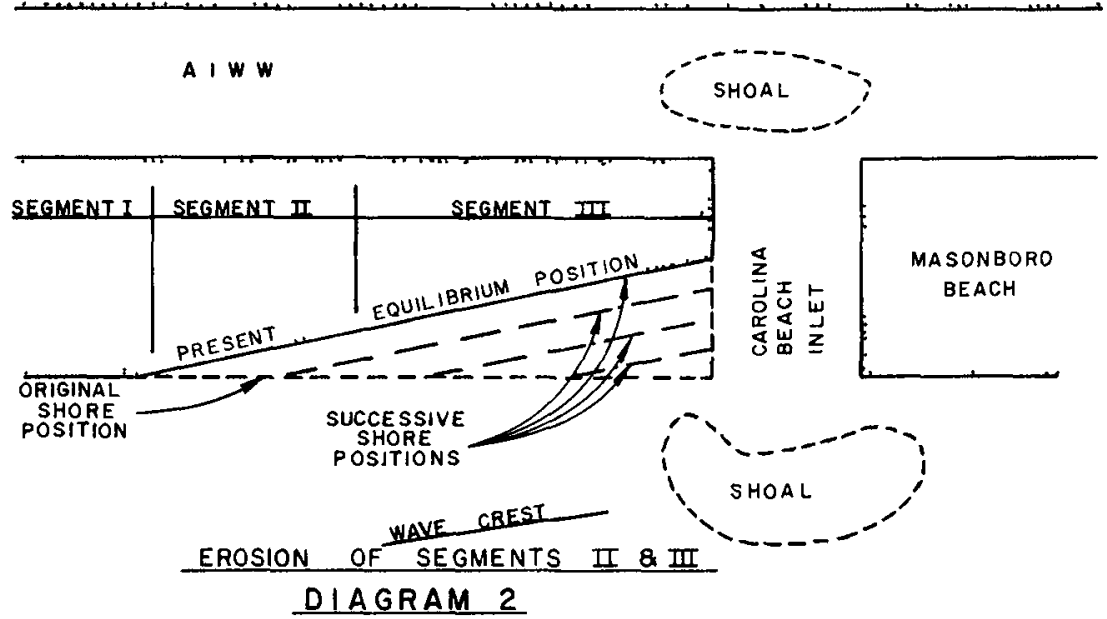

of course, wave attack along the area of interest is nelther uniform nor unidirectional and, moreover, there is some natural bypassing of material across Carolina Beach Inlet However, the generalization outlined above is not only rational, in a theoretical sense, but validated by the time - space relationships of the erosion in Segments II and III, presented in TABLE 4 PHOTOGRAPH 1 clearly shows the change of shore alignment

Immediately following the placement of artificlal fill along Segment II In March 1965, severe erosion began and continued at an intense rate to March 1967 The cumulative shore recession in the 2-year period, March 1965-March 1967, amounted to 140 feet, with an attendant loss of 550,000 cublc yards of material, or an average annual loss in the 2-year period of 275,000 cubic yards As a result of this severe erosion, emergency measures were implemented for Segment II in March 1967 by the construction of a groin at station $136+75$ and the addition of 321,000 cubic yards of f111, of wh1ch 284,000 cuble yards were placed south of the groin and approximately 37,000 cubic yards were placed north of the groin The emergency work was completed in May 1967 By May 1968, 


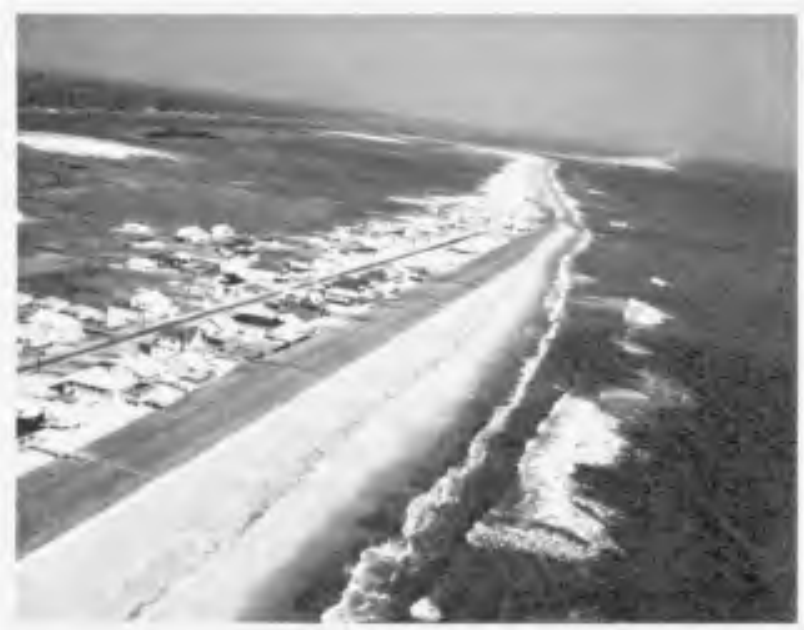

PHOTOGRAPH 1 - VIEW NORTHWARO OVER CAROLINA BEACH PROJECT IN 1965. NOTE CHANGE OF SHDRE ALIGN. MENT CENTER BACKGROUNO OF PHOTOGRAPH.

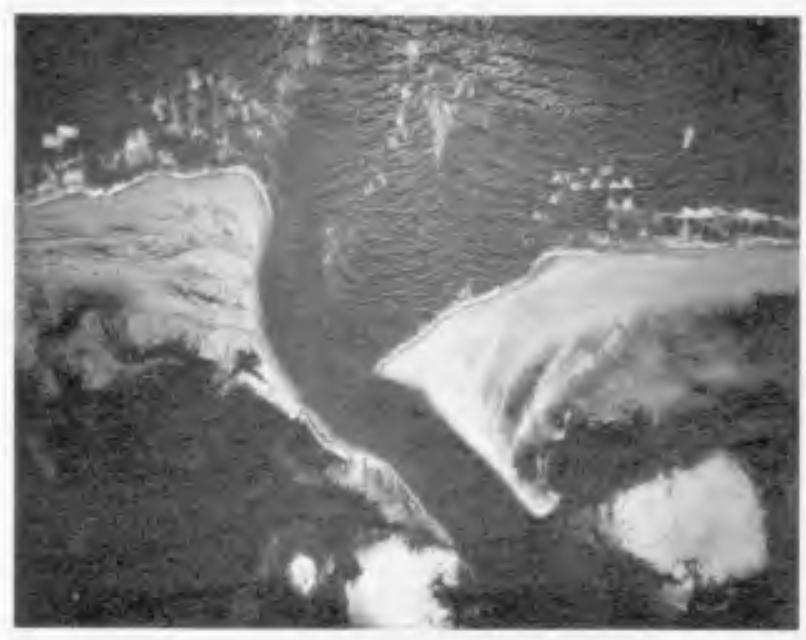

PHOTOG RAPH 2 - VIEW OF CARDLINA BEACH INLET IN 1969. NOTE OFFSET BETWEEN INLET SHOULDERS, AND WAVE REFRACTION PATTERN. 
1 year after the placement of emergency fill, the net loss of this material amounted to 203,000 cublc yards, of which 175,600 were lost between stations $100+00$ and $132+50$ It is noted that, notwithstanding the groin, losses of emergency beach material in segment II were extremely high, particularly in view of the fact that conditions observed in segment I showed that the general area was experiencing some natural accretion in 1968 The rates of shoreline movements in Segment II, shown in comparison with shore-movement rates in segment $I$, are presented on FIGURE III Volumetric changes in segment II are gIven in TABLE 5

TABLE 4

Rate of shoreline movement at specified stations within segment II and Segment III (1952-1963) (rate in feet per year)

\begin{tabular}{l|r|r|r|r}
\hline \multirow{2}{*}{$\begin{array}{c}\text { Segment } \\
\text { and station }\end{array}$} & \multicolumn{3}{|c}{ T1me per10 } \\
\cline { 2 - 5 } SEGMENT II & $1952-55$ & $1955-57$ & $1957-63$ & $1952-63$ \\
\hline $100+00$ & & & & \\
$110+00$ & +30 & -70 & -53 & -33 \\
$120+00$ & -37 & -35 & -78 & -5 \\
$130+00$ & -97 & -55 & -90 & -8 \\
$140+00$ & -120 & -60 & -130 & -105 \\
SEGMENT III & -140 & -190 & -430 & -267 \\
\hline $150+00$ & -136 & -250 & -570 & -394 \\
$160+00$ & -103 & -600 & -687 & -510 \\
$170+00$ & -100 & -1070 & -765 & -639 \\
$180+00$ & -83 & -1390 & -912 & -772 \\
$190+00$ & -67 & -1560 & -803 & -740 \\
$200+00$ & -533 & -1000 & -1291 & -1032 \\
\hline
\end{tabular}

NOTE $+=$ accretion, $-=$ erosion

TABIE 5

Total volumetric change since construction of the project (SEGMENT II - station 100+00 to station 140+00)

\begin{tabular}{l|c|c}
\hline \multicolumn{1}{c|}{ Period } & $\begin{array}{c}\text { Volumetric change } \\
\text { during period } \\
\text { (cubic yards) }\end{array}$ & $\begin{array}{c}\text { Cumulative volumetric } \\
\text { change by end of period } \\
\text { (cubic yards) }\end{array}$ \\
\hline As built to June 1966 & $-412,200$ & $-412,200$ \\
June 1966 to September 1966 & $-64,300$ & $-476,500$ \\
September 1966 to December 1966 & $-58,300$ & $-534,800$ \\
December 1966 to March 1967 & $-15,200$ & $-550,000$ \\
March 1967 to May 1967 & $1+284,000$ & $(\mathrm{Fl11})$ \\
May 1967 to June 1967 & $-54,400$ & $-604,400$ \\
June 1967 to 0ctober 1967 & $-60,100$ & $-664,500$ \\
October 1967 to February 1968 & $-158,000$ & $-822,500$ \\
February 1968 to April 1968 & $+67,100$ & $-755,400$ \\
April 1968 to August 1968 & $-9,300$ & $-746,100$ \\
\hline
\end{tabular}

\footnotetext{
$I_{\text {Not included in cumulative total }}$ NOTE $+=$ accretion, - = erosion
} 
The quality of fill material placed in Segment II with the Initial construction of the Carolina Beach project and later as part of the emergency measures was compared with the quality of borrow material used in segment $I$, and It was found that all of the materlal was essentially of the same quality Therefore, it can be assumed that the "critical ratio" for all the material placed in segment II was of the order of magnitude of 2 Considering that the total Ioss of material in segment II during the 3-year period, May 1965-May 1968, amounted to 753,000 cublc yards, the average annual loss of material from this area can be taken as 251,000 cublc yards of the total annual loss, a portion is allocated to sorting-action displacement to depths seaward of the active profile and the remaining portion is allocated to a deficit in the alongshore transport into segment II Th1s defic1t in alongshore transport, which in fact is the defic1t in material transport imposed by Carolina Beach Inlet, is easily computed by applying the "critical rat1o" to the total average annual loss of material experienced in segment II, that is

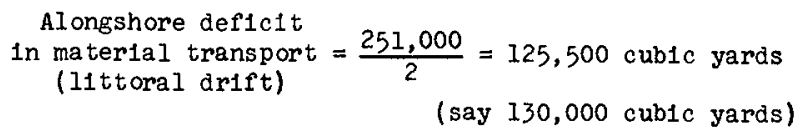

The shore processes in terms of annual alongshore movements in segment II are represented schematically in Diagram 3 It is evident from Diagram 3 that the total annual alongshore transport phenomenon cannot be described without the development of two other conditions through which the values of $B$ and $N$ can be computed Such conditions can be developed, as demonstrated below, by making certain assumptions, one of which is that all observed annual values of I1ttoral transport represent average annual values over a relatively long pertod, specti1cally, the 17-year period, 1952-1969, In which Carolina Beach Inlet has been in exis tence

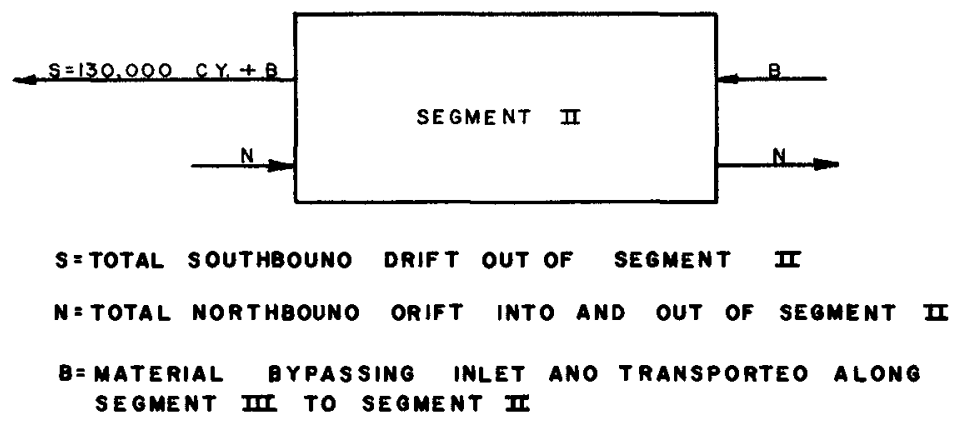

$\frac{\text { ALONGSHORE MOVEMENTS IN SEGMENT II }}{\text { DIAGRAM } 3}$


In order to obtain a relatively accurate value of $B$, it is necessary to have a measured value of $S$, the total southbound drift such a value is ava1lable for the pertod June 1966-June 1967 for the shore of Wrightsville Beach, located 9 miles north of Carolina Beach (see FIGURE I) It is remarked that Wrightsville and Carolina Beaches are exposed to the same wave climate and are composed of essentlally the same material In June 1966, a 3,600-footlong welr-type fetty was constructed on the south end of Wrightsville Beach, at Masonboro Inlet During the period June 1966-June 1967, approximately 150,000 cublc yards of 11 ttoral material were transported by natural forces across the weir section of the jetty and deposited in the lee-side deposition basin In addition, an estimated 70,000 cubic yards of material accumulated on the updrift side of the jetty in the form of an accretion fillet Therefore, the total southbound drift, $S$, was approximately 220,000 cub1c yards, presuming that the accretion fillet, being small during the first year of jetty operation and in the shadow $z$ one of the jetty, was not exposed to attack by waves from the southeast sector Referring to Dlagram 3, $B=220,000$ cublc yards minus 130,000 cublc yards, or $B=90,000$ cuble yards

At this point, only the value of the northbound drift, $N$, remains to be computed For the determination of this value, conditions at Carolina Beach Inlet are used to develop a continuity relationship for the total alongshore movement of material Here, the assumption 1s made that, contrary to the natural southward bypassing of 90,000 cublc yards of material at carolina Beach Inlet, there has been no signiflcant northward bypassing at the inlet The rationale on which this assumption is based is as follows First, bypassing of material at an inlet is highly dependerit on storm activity, as wave crests, encroaching on the sea shoal during normal sea conditions, are refracted to such an extent that they split and approach the inlet throat from both the updrift and downdrift directions, making bypassing difficult This phenomenon is easily observed in the fleld or from aerial protographs and, in fact, is the reason for the multiple, confused chop existing on shoals Moreover, the refraction phenomenon can, as at Carolina Beach Inlet, create permanent, but shifting, nodal $z$ ones on the shores near and adjacent to the inlet However, during storm activity with attendant storm tides, high-energy levels, and short-perlod waves which are not too susceptible to refraction, natural bypassing can be accomplished Therefore, since most storm activity to wh1ch Carolina Beach Inlet is subjected comes from the nor theast sector of exposure, 1t can be assumed that the largest proportion of natural bypassing is in a southward direction The second reason that bypassing in a northward direction is assumed small relative to southward bypassing is that the ocean shoreline on the north shoulder of the inlet is of set seaward from the shoreline on the south slde by a distance of approximately 1,500 feet (see PHOTOGRAPH 2) This offset doubtless restrains the northward movement of material across the inlet The phenomenon discussed above, insofar as normal sea conditions are concerned, is schematized in Diagram 4 Note that there would be no change in the general refraction pattern for normal conditions if the deepwater wave crest approached from the northeast quandrant under normal sea conditions The actual refraction phenomenon is readily discemible in PHOTOGRAPH 2 


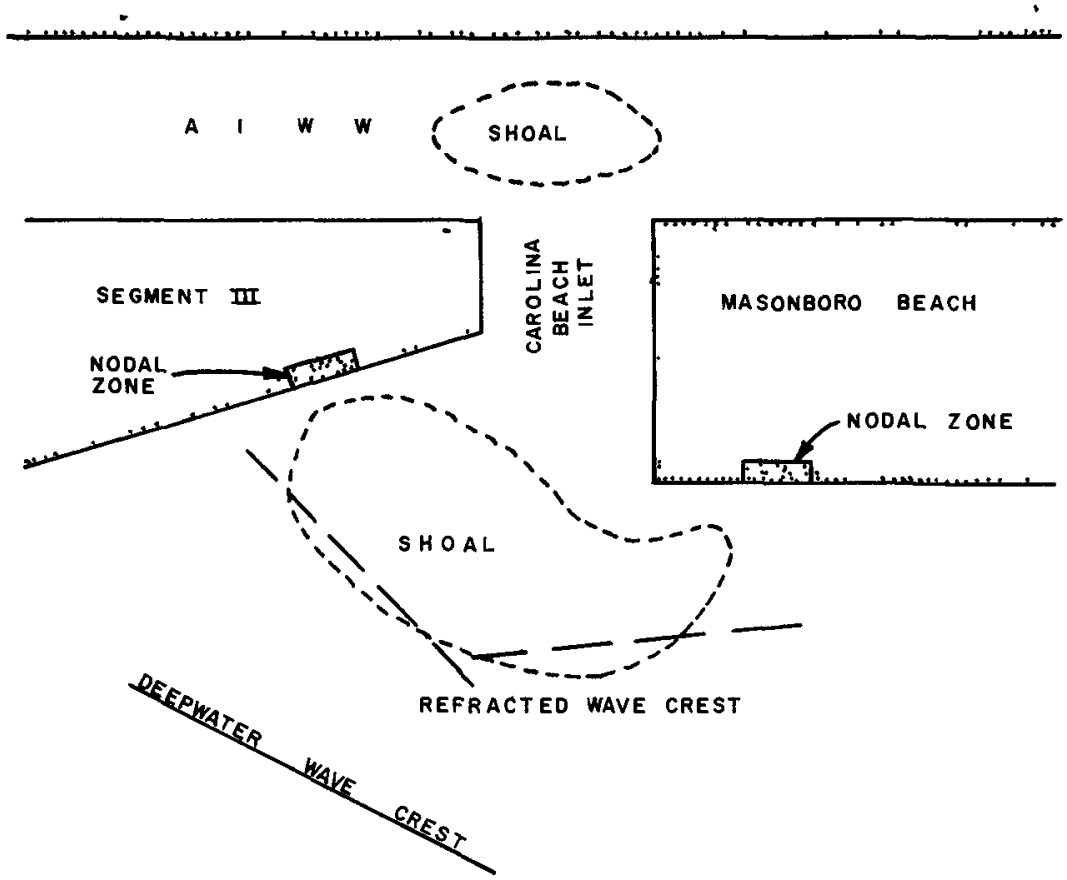

DIAGRAM 4

With acceptance of the assumption that northward bypassing at the inlet is Insignificant, the value of the total northbound drift, $N$, can be computed by determining the total volume of material accumulated in the inlet complex during 1 ts 17 years (1952-1969) of existence The total accumulation was determined from hydrographic surveys, dredging records in the AIW, at the inlet throat, and from aerlal photographs This accumulation amounted to $4,160,000$ cub1c yards, of which $3,250,000$ cublc yards were stored on the sea shoal, 680,000 cub1c yards were deposited and removed from the AIWw, at the throat of the inlet, and 230,000 cublc yards were flushed into the marshes of Masonboro Beach at a polnt approximately 2,000 feet north of Carolina Beach Inlet as a result of a breakthrough wh1ch occurred during Hurricane Hazel in 1954 Th1s breakthrough remalned open as a small inlet for approximately 4 years, and was closed by natural forces W1th a total storage of $4,160,000$ cub1c yards in a 17-year period, the average annual accumulation rate is 245,000 cublc yards The value of $\mathrm{N}$ is determined from the continuity relationship

$$
\begin{aligned}
N+S-B=A c c u m u l a t i o n & \text { in the inlet } \\
\text { or } & \\
N+220,000-90,000 & =245,000 \\
N & =115,000 \text { cublc yards } \\
\text { (say } \quad \mathrm{N} & =120,000 \text { cublc yards) }
\end{aligned}
$$


A schematic flow diagram of the computed average annual littoral process at the inlet is given in Dlagram 5

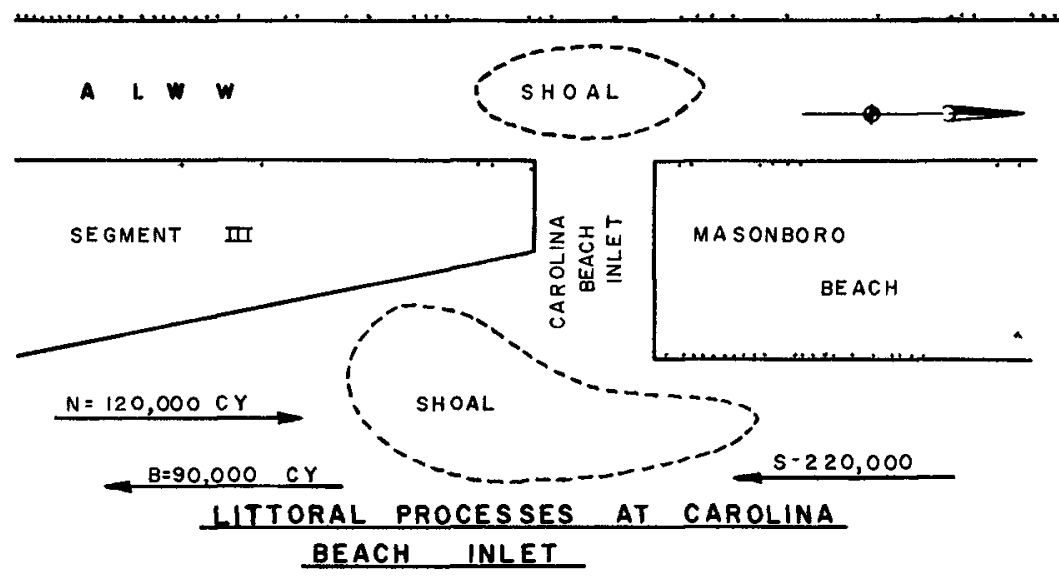

\section{DIAGRAM 5}

To formulate a complete shore-processes scenar1o for the Carolina Beach area, conditions along segment III of the study area were also investigated Surveys indicate that the total quantity of material eroded from Segment III durling the 17-year period, 1952-1969, amounted to approximately 3,670,000 cub1c yards, which results in an average annual loss of about 220,000 cubic yards, however, 1 was shown previously that the average annual defic1t of material imposed by the inlet amounted to only 130,000 cublc yards Thus, the remainIng 90,000 cubic yards must be accounted for These losses can, with reasonable assurance, be attributed to material lost from the beach profile through wave and/or tidal overtopping of Segment III and the quantity of material displaced seaward of the active beach profile as a result of sorting action In connection w1th wave and tidal overtopping, Segment III is a low barrier ridge w1th a general maximum elevation between 4 and 5 feet above mean sea level Therefore, wave and/or tidal overtopping is a frequent occurrence in this area during any given year, however, major overtopping losses are associated with hurricane events From aerlal photographs and existing topography, 1t was determined that the landward side of Segment III accreted westward into the adjacent marsh at a rate of approximately 20,000 cublc yards per year Thus, there remains a value of 70,000 cublc yards lost through sorting action In view of the severe erosion of the landmass in Segment III, it can be assumed that sorting losses were substantial The assumed sorting action loss of 70,000 cublc yaras results in a "critical ratio" of $[220,000 /(220,000-70,000) 7=$ 147 , which is not at all an unreasonable value It is also noted that the quantity of material attributed to sorting losses beyond the active profile represents approximately 32 percent of the total loss Th1s corresponds closely to the "rule-of-thumb" value of 30 percent used, in the vicinity of segment III, in determining the quantities of fine s1lt, peat, and clay lost in the placement of dredged fill It is remarked that exposed peat laminae are found along much of the Segment III shoreline The shore-processes scheme for Segment III is 1llustrated schematically in Dlagram 6 It should be noted that the shore processes described above for Segment III relate to average conditions prevalling over the 17-year period that this section of shore was adjusting to a new 
equilibrium condition as a result of changes produced in the IIttoral regime by Carolina Beach Inlet As mentioned above, Segment III has generally attained a state of equilibrium through erosion and a reortentation of the shoreline At present, the major effects of the Itttoral material deficit imposed by the inlet have been transferred to Segment II

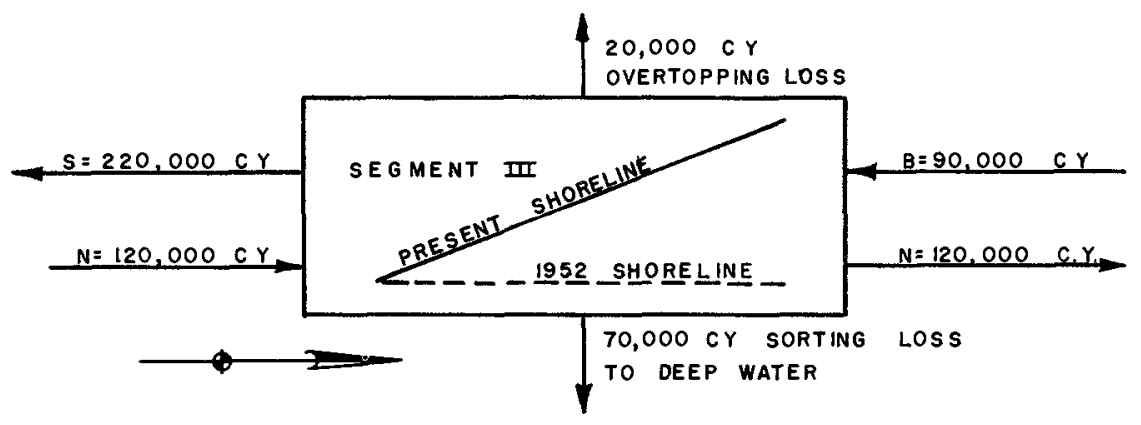

AVERAGE ANNUAL PROCESSES 1952 TO 1969
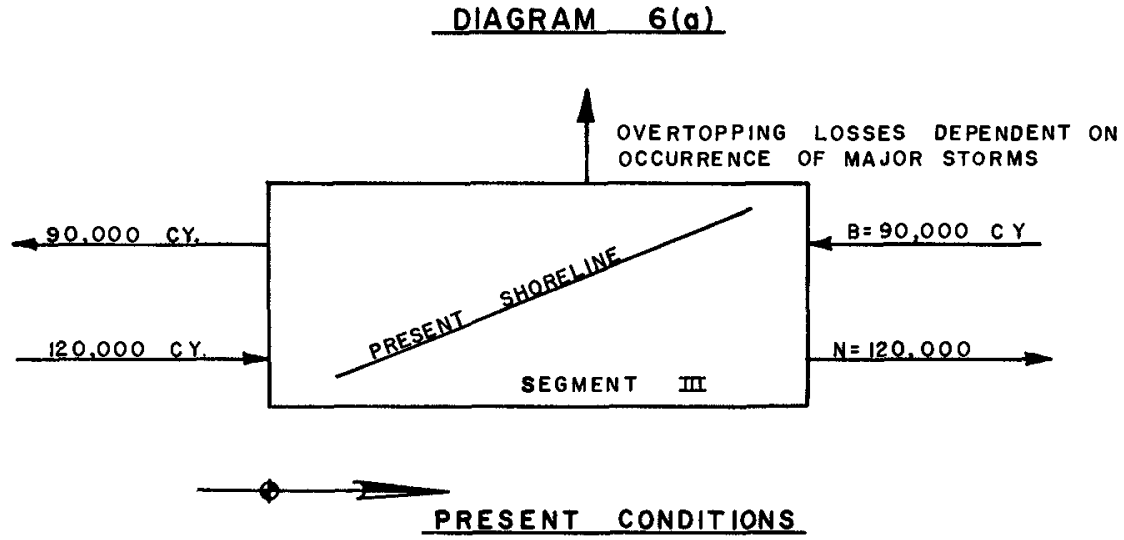

DIAGRAM $6(b)$ 
Verification of computed values A condition used to verify the computed values, specifically $\mathrm{N}$ and $\mathrm{S}$, is taken at Masonboro Inlet, which is exposed to the same gross littoral drift as Carolina Beach Inlet In the 4-year interval, 1965-1969, two hydrographic surveys show that approximately 900,000 cubic yards of material have been deposited on the outer and inner shoals of Masonboro Inlet In addition, approximately 170,000 cublc yards were removed from the inlet by hopper dredges in the period 1965-1969 Therefore, the total accumulation within the inlet during a 4-year period amounted to approximately 1,070,000 cubic yards, resulting from the intrusion of southbound and northbound drift on an average annual basis, the deposition rate is about 270,000 cubic yards Furthermore, the growth of the accretion fillet along Wrightsville Beach, updrift of the Masonboro Inlet Jetty, has an accretion rate of about 60,000 cubic yards per year for the 2-year period 1966 to 1968 Construction of the jetty began August 1965 and was completed June 1966 If 1 t is assumed that the average of the 2year fillet growth rate is representative of an average annual growth rate for the 4-year period of 1965-1969, the gross drift toward the inlet would be 270,000 cubic yards $+60,000$ cublc yards $=330,000$ cubic yards, which compares extremely well with the value $N+S=340,000$ cublc yards Note that natural inlet bypassing was not considered, as the inlet shoals are well shadowed by the jetty, also, the 1965-1969 period was free of major storm activity

Conclusion The analysis presented above results in a rational understanding of shore processes in the study area from 1952 to the present It is remarked that the quantitative values determined in the analysis represent only the average annual conditions for the data period of record used, and, moreover, insofar as shore processes are extremely nonuniform, wide variations from average annual values can occur in any given short-term period

In closing, it is remarked that detalled plans for solving the erosion problem described herein have been formulated and are presently under review 


\title{
CHAPTER 78
}

\section{RIO GRANDE BAR - THE CASE HISTORY OF A LAGOON OUTLET INTO A TIDELESS SEA}

\author{
V. F. Motta \\ Head of the Coastal Divigion. \\ Hydraulics Research Institute \\ of the Federal University of \\ Rio Grande do Sul (Pôrto Ale- \\ gre, Brazil).
}

\begin{abstract}
Rio Grande harbour is situated Inside the outlet of Lagoa dos patos (9910 km2) and Lagoa Mirim ( $3770 \mathrm{~km} 2)$. Most of the rivers in the state of Rio Grande do Sul, that are not tributaries of river Uruguay, flow into the two lagoons and the latter discharge through Canal do Norte into a tideless sea. Flood flowe can exceed 20000 cumecs. The outlet is on a sandy coast with littoral drift in both shoreline directions.

The time history of the outlet can be divided into three periode. Before breakwater construction, the bar was extremely ungtable In lay-out, position and controling depth. The latter ranged from 2.5 to $7 \mathrm{~m}$. From 1911 to 1916 two $4 \mathrm{~km}$ long rubble mound breakwaters were built. They deepened the entrance channel to 8 - $10 \mathrm{~m}$ but a new bar came about further offshore entaling objectionable navigation conditions in the approach channel. No previous dredging was carried out as the breakwater construction proceeded out to sea, and as result a total of 14 mililon cublc metres was scoured out between the two breakwaters and discharged offshore to build up the new bar. Some improvement by dredging was not achieved unt11 1962 .

The Improvement of the approach channel lay-out as well as deepening the entrance to $14 \mathrm{~m}$ below datum are under consideration. Field and model investigations have been designed for this purpose.
\end{abstract}

I) The Coastal Environment and Hydraulic Conditions, Rio Grande harbour ig situated inside the outlet of Patos Lagoon $(9910 \mathrm{~km} 2)$ and MIrim Lagoon $(3770 \mathrm{~km} 2)$. The key map of Fig.1 shows the area discussed in this paper. It lies near the outhern border of Brazil, Rio Grande belng the main port in the state of Rio Grande do sul. 
1244

COASTAL ENGINEERING

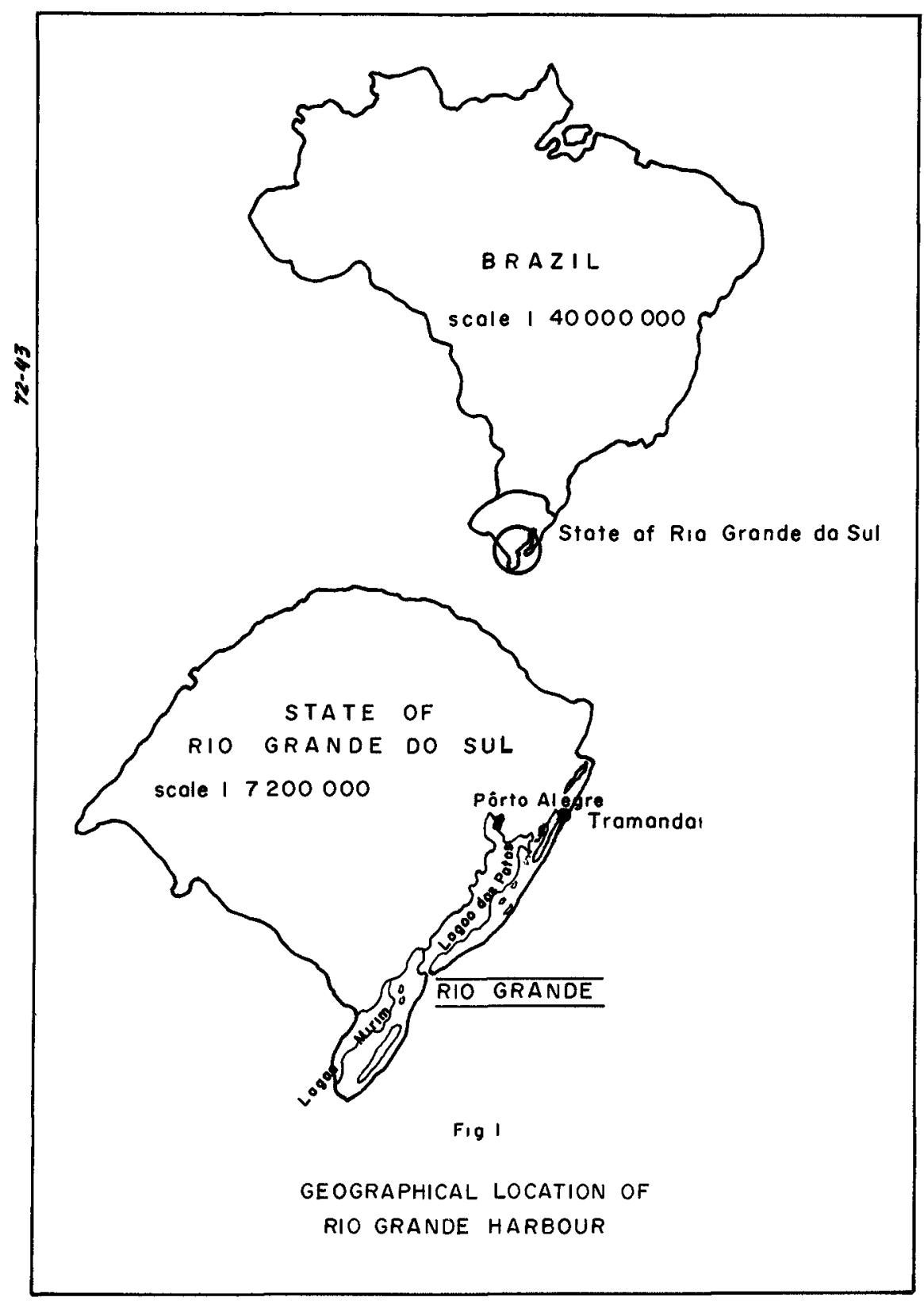


The coast of this state is a long barrier beach dividing eeveral lagoone from the sea. Patos and Mirim lagoons are the two most important bodies of water. They are connected by a $70 \mathrm{~km}$ long channel, Canal São Gonçalo, and they discharge into the sea through a common outlet (Canal do Norte) on whose western bank the port of Rio Grande has been built. Most of the rivers in the state of Rio Grande do Sul, that are not tributaries of River Uruguay, flow into theee two lagoons. As a result, the upland flow in Canal do Norte drains a basin with an area of $162000 \mathrm{~km} 2$, and peak flowe can exceed 20 ooo cumecs. Canal do Norte is quite etable in position, width, depth and length. 1 ts width varies from $1.5 \mathrm{~km}$ at the mouth to about $3 \mathrm{~km}$ at the northern end, a major portion of its shipping channel being over $15 \mathrm{~m}$ deep.

The lagoon outlet ie on a eandy coaet with littoral drift in both ehoreline directions. The coaet is farrly straight, flat, and uniform in profile, the offshore slope from the -1 to the $-15 \mathrm{~m}$ contour being about $1 \%$. The beach material is fine sand, a representative mean diameter being about $0.20 \mathrm{~mm}$. The shoreline runs on a SW to NE alignment but the axie of Canal do Norte nearly colncidee with a meridian, which accounts for its name the Portuguese for Northerly Channel.

Accoraing to $|1|$ the main characteristice of the deepmater wave climate are the same from Punta del Este (in Uruguay) to Tramandai, some $320 \mathrm{~km}$ north of Rio Grande, where wave characterietics were recorded at a $20 \mathrm{~m}$ depth for more than a year, in 1962-63, in connection with the design of an offshore oil terminal. The main characterietic of thie wave climate ie that swell comes mostly from $S E$ whereae the dominant and prevailing winds blow from NE. As a result, there is a marked correlation between wave periods and directions as shown is Fig. 2. The waves with the greateet energy (Fig. 3) and least eteepnese - therefore with the greatest transport capacity - come from SE which cauees the northweetward littoral drift to be dominant over the eouthweetward drift. The latter is induced by the waves, mostly locally generated sea, from the NE quadrant.

The only indication available as to the intensitiee of this two-way littoral drift is that the average dominant 11 toral drift would be 1.5 timee ae great as the secondary drift. The average total annual volume of the dominant littoral drift would amount to eome $100000 \mathrm{~m} 3$ per year. Theee figures were arrived at from the results of a mobile-bed model investigation into the control of Tramandal inlet which was carried out in 1964 at the Hydraulics Reeearch Inetitute of the Federal Univereity of Rio Grande do Sul |2|. A time scale for bed movement was worked out by comparing model and prototype durations of the northward inlet migration when Tramandai inlet was uncontrolled, and the transport capacity of the wavee reproduced in the model wae measured by trapping eand at the downdrift end of the model. The total volume of littoral drift in a model year wae then scaled up. The amount of $100000 \mathrm{~m} 3 /$ year for the dominant drift seeme rather lower than valuee reported for simlar conditions in other parts of the world and may be due to the model improperly eimulating suspended transport in the eurf zone. On the other hand, it should be noted that the angle of attack of the dominant wavee is usually small about $10^{\circ}$ at a $20 \mathrm{~m}$ depth. Whatever the accuracy 


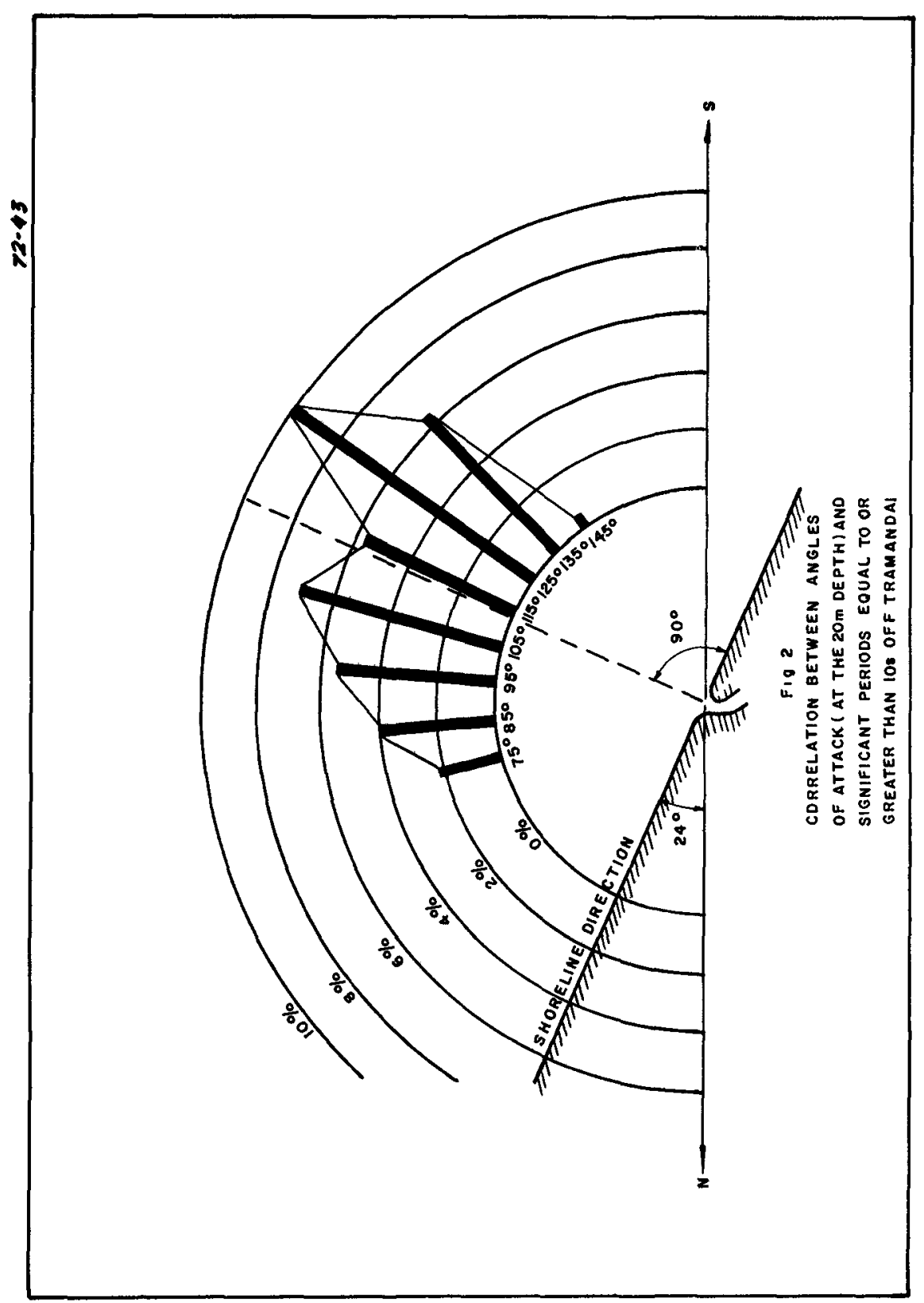




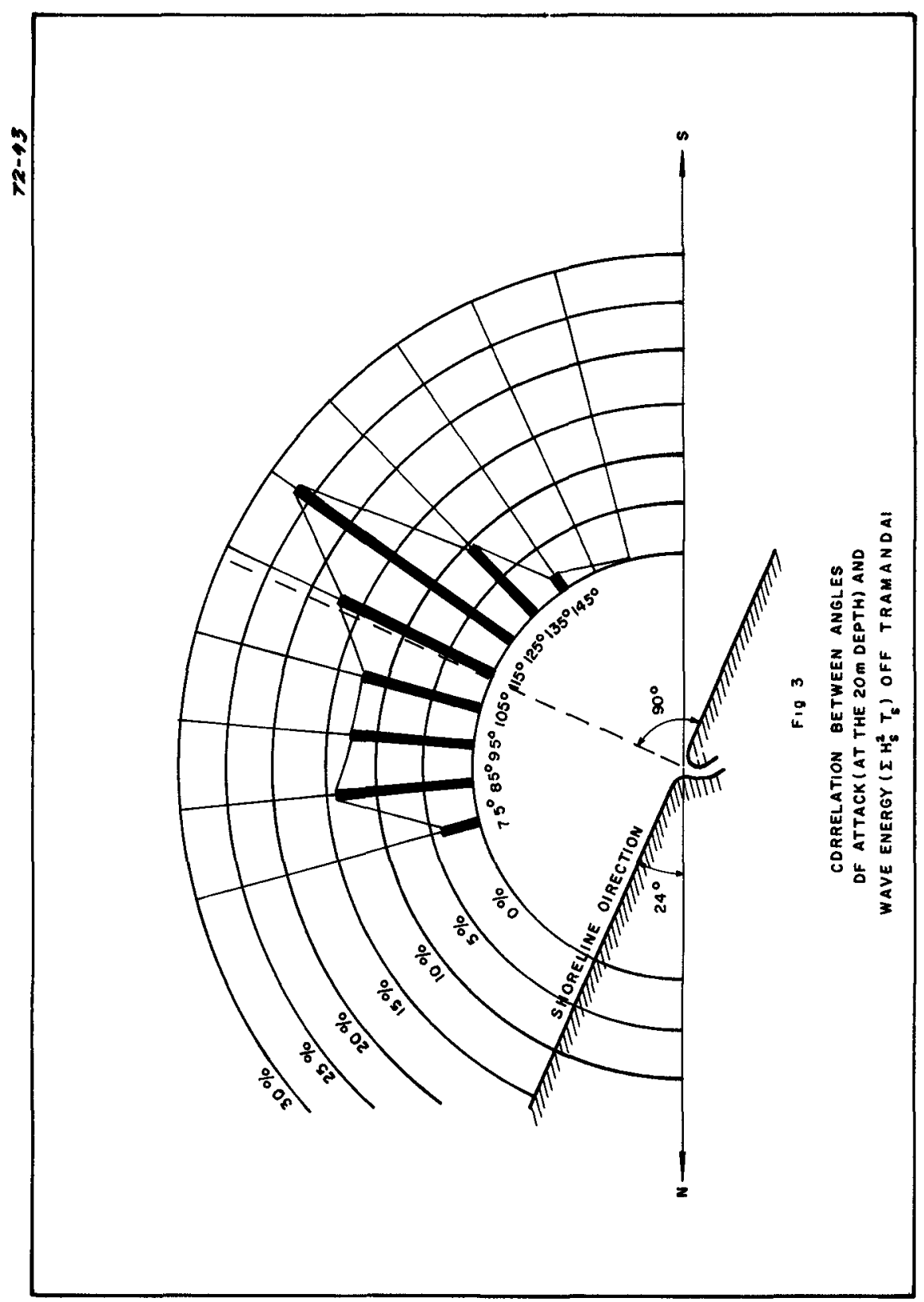


of the above estimate for littoral drift on the coast of Rio Grande do Sul may be, what is actually known is that the most frequent significant wave height is $1.5 \mathrm{~m}$ at the $20 \mathrm{~m} \mathrm{depth}$, and the most frequent signjficant wave period is $9 \mathrm{~s}$. Wave heights of $4 \mathrm{~m}$ at the $20 \mathrm{~m}$ depth occur at least once in a year.

The most striking feature of Rio Grande harbour entrance is that fresh-water flow largely domnates over tidal flow in determining its hydraulic and morphological characteristics. This is the reason why the present paper refers to Canal do Norte as a "lagoon outlet" instead of adapting the usual expression "coastal inlet". While six sizable rivers flow into Patos lagoon and five into Mirim Lagoon the sea off the harbour entrance is practically tideless. The area of Patos Lagoon alone is about three times as large as that of the Ijsselmeer, the dammed Zuiderzee, in the Netherlands. On the other hand, the mean monthly tidal range is usually below half a metre. The overall result is that waterlevels as well as velocities, directions and durations of flow through Canal do Norte are mainly determined by a complex interaction between wind effects at sea and in the lagoon system, and fresh-water flow in the latter. Instead of periodical reversals of ebb and flood in Canal do Norte, the flow may be out to sea for several days on end in the rainy season if no strong winds blow from the south whereas strong winds from the south can bring about flow from the sea into the lagoons for several days in the dry season.

Increase in upland flow or northerly to northeasterly winds tend to cause ebb flow in Canal do Norte. Decrease in upland flow or winds from the south (SE to W) tend to cause flood flow. The greatest ebb discharges obviously take place when upland floods occur together with winds from $N$ to NE. The greatest flood discharges are brought about by wind action from the south in the dry season.

The values of the upland discharges as well as the pattern of seasonal changes of ralnfall and wind characteristics are such as to make ebb flows in Canal do Norte largely dominant over floods both in discharge values and durations throughout the year. Rainfall reaches a maximum in late winter, August and September, the southern Hemisphere winter and spring being the seasons with greater rainfall Summer, December to March, is the dry season. Although northeasterly winds prevall over the year, they are more frequent from september to March. Winds from the southern quadrant are more frequent during the winter, from June to September. As a result, the greater upland discharges in winter counteract the flood-producing effect of the southerly winds. In the summer the effects of the northeasterly winds offset the decrease in upland flow to some extent.

One of the French engineers who built the breakwaters at the entrance in 1908-1916, M. B. Malaval |3|, made an analysis of discharge values and durations of $\mathrm{ebb}$ and flood in Canal do Norte for s1x years $(1908,1911,1912,1913,1914,1915)$. The water surface profile was determined by two water level recorders $8250 \mathrm{~m}$ apart, one near the port and the other near the entrance. Discharges were calculated with the ald of the Chezy formula, the assumptions on the value of the chezy coefficient being controlled by some velocity measurements. The average results of Malaval's analysis for the six 
years under consideration were as follows:

1) 2604 hours of flood per year, or 108 days in a year, with a mean discharge of $6767 \mathrm{~m} 3 / \mathrm{s}, 9$ mean velocity of $0.8 \mathrm{~m} / \mathrm{s}$, and an annual volume of $63 \times 10^{9} / \mathrm{m} 3$,

2) 4925 hours of ebb per year, or 205 days in a year, with a mean discharge of $8650 \mathrm{~m} 3 / \mathrm{s}$, a mean velocity of $1.0 \mathrm{~m} / \mathrm{s}$, and an annual volume of $149 \times 10^{9} \mathrm{~m} 3$,

3) 1239 hours of slack water, or 52 days in a year, for which no flow was assumed.

Malaval's analysis did not take salinity effects into account.

Malaval also drew a list of ebb and flood periods with durations from 18 to 24 consecutive hours. He found an ebb period lasting for 19 consecutive days in July 1915 and a flood period lasting for 9 days in December 1915. On the average in a year he found for the ebb

$\begin{array}{rcccccccc}2 & \text { periods } & \text { lasting } & \text { for } & 10 & \text { to } & 19 & \text { consecutive days } \\ 5 & n & n & n & 5 & n & 10 & n & n \\ 18 & n & n & n & 2 & n & 4 & n & n \\ 21 & n & n & n & 1 & \text { whole day } & \end{array}$

and for the flood

1 period lasting for 5 to 9 consecutive days

6 periods lasting for 2 to 4 consecutive days

21 " " 1 whole day

Analysis of flood and ebb durations in Canal do Norte more refined than Malaval's were not carried out to this day.

The two lagoons act both as large storage basins for the upland flow and sediment traps for the bed load brought down by the rivers. As a result, the sand in and around the harbour entrance is brought in by wave action on the continental shelf. The bed material is sand at the entrance and in the outer stretch of canal do Norte. The Inner stretch and the harbour basin have a muddy bed. Coastal currents are wind induced and can only cause sand transport in conjunction with waves.

II) The Uncontrolled Outlet and the Effects of Breakwater Construction.

The main object of this paper is the time history of the bar that came into being off the entrance to the lagoon outlet as a result of the interaction between the transport capacity of the waves and the flushing action of the upland flow. This time history can be divided into three periods

1) The situation of uncontrolied outlet, before breakwater construction in 1908-1916,

2) the changes which breakwater construction brought about,

$3)$ the present situation that prevails since the bar, the shoreline and the outer stretch of Canal do Norte adapted themselves to the new conditions.

The time history of the bar is fairly well known as from 
1883, annual surveys being avallable from that year to $1956|4|$. Surveys have been less frequent since 1956. prior to 1883 the information available is poor.

Before breakwater construction the bar was extremely unstable in controlling depth, distance to the shoreline and pattern of the channels, Between 1883 - when the first comprehensive, rellable survey was carried out - and 1914 - when the influence of the breakwaters then under construction was brought to bear for the first time - the controliing depth varied in an erractic manner from a minimum of $2,5 \mathrm{~m}(1883)$ to a maximum of $7 \mathrm{~m}(1894)$. However, the most frequent values ranged from 4 to $5 \mathrm{~m}$.

Figs. 4, 5 and 6 show the time hietory of the bar as taken from $|4|$. In $F i g .4$ the annual values of the controlling depth are plotted from 1883 to 1956. Fig. 5 correlates the controliing depth to distances to a reference alignement joining two triangulation vertices on the shore. Fig. 6 is a plot of the variation in time of the least depths over the bar outside the shipping channel.

The distances from the reference alignement to the contour standing for the controlling depth ranged from a minimum of $2.8 \mathrm{~km}$ (in 1885, 1898 and 1899) to a maximum of $5.0 \mathrm{~km}$ (in 1892). The average distance of the controlling depth to the reference alignement was therefore $3.9 \mathrm{~km}$ (actual positions in 1886 and 1894 ). Very different values of controlling depth could be associated to a given value of dietance to the reference alignement. For instance, the $4.0 \mathrm{~km}$ distance wae associated to a controliing depth of $2.5 \mathrm{~m}$ (1883), $5.0 \mathrm{~m} \mathrm{(1902)} \mathrm{and} 6.0 \mathrm{~m}(1893)$. The $3.9 \mathrm{~km}$ distance was associated with values of $4.0 \mathrm{~m}(1886)$ and $7.0 \mathrm{~m}(1894)$. The minimum $2.8 \mathrm{~km}$ distance was aseociated with $3.0 \mathrm{~m} \mathrm{(1885)}$ and $6.0 \mathrm{~m}$ (1898 and 1899). The maximum $5.0 \mathrm{~km}$ distance, which occurred only once (1892), was associated with $5.0 \mathrm{~m}$.

Lack of reliable data for rainfall or upland diecharges at the time under consideration in all the river basine involved preeludes any attempt at correlating bar characteristics with upland flow values.

The lay-out of the main channel accross the bar used to swing from $S W$ to $S$ and $S E$, although a sW alignement was most frequent. The instability of natural-channel pattern can also be eeen in the different breakwater lay-outs proposed by different engineers at different times. Each proposed design was baed on the most recent available survey.

While the bar underwent the changes discuesed above the outlet itself, Canal do Norte, remained remarkably stable in position, width and depth. It is interesting to point out that the other lagoon outlet (Tramandai) and the mouths of small rivers(Chui, Mampituba and Axarangua) on the same stretch of the Brazilian coast migrate northwards, in the direction of the dominant littoral drift. It appears that the stability of Canal do Norte was due to its much greater upland flow which flushed to considerable distances out to sea the sand brought in by the waves from the sides and in frontal action. At the other lagoon outlet and river mouths mentioned above the bar builds up much nearer the shoreline, and the interaction between littoral drift and the transport capacity of the upland flow in this tideless sea cause inlet migration. 


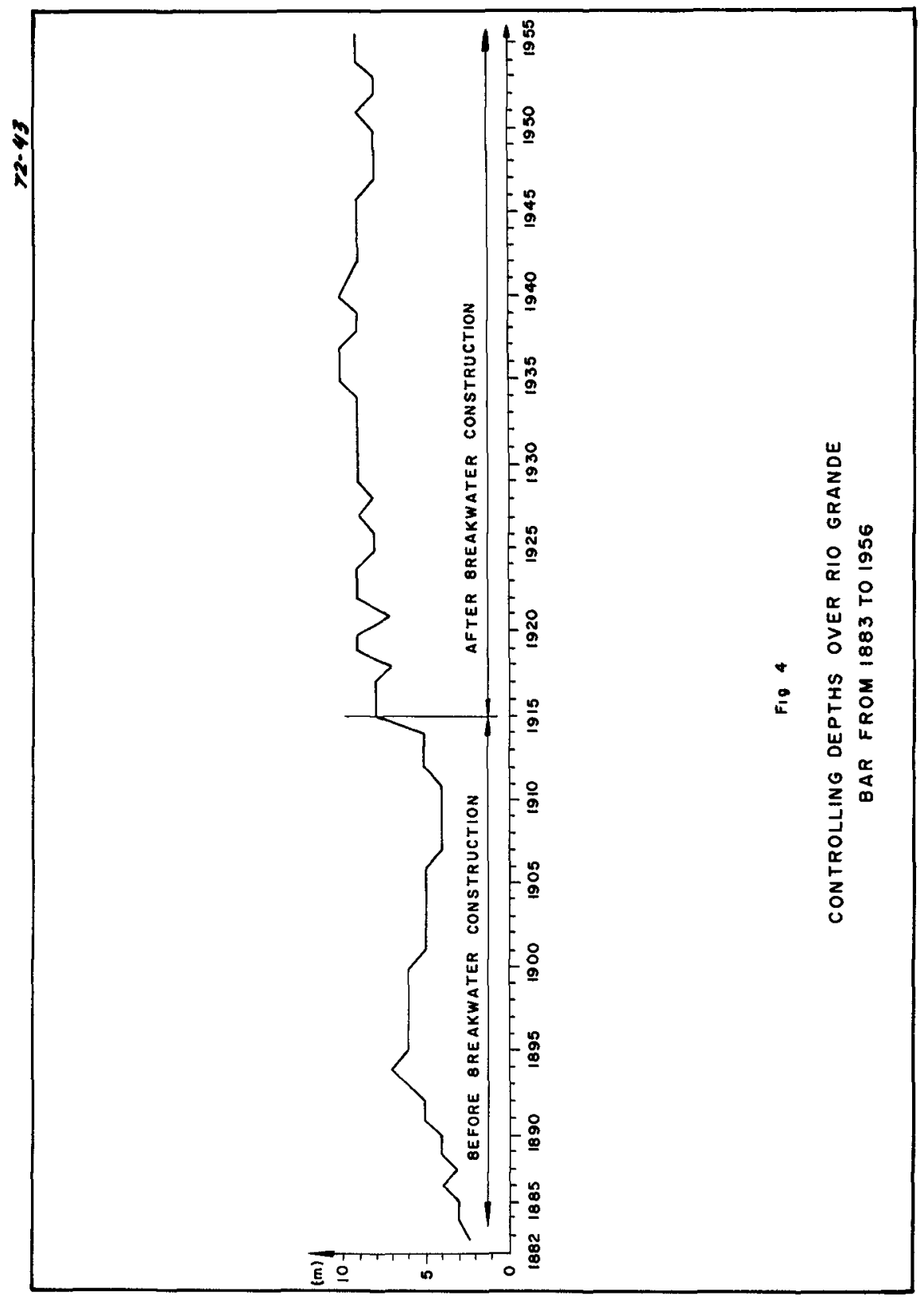


<smiles>C1=CC2CCC=C1C2</smiles> 


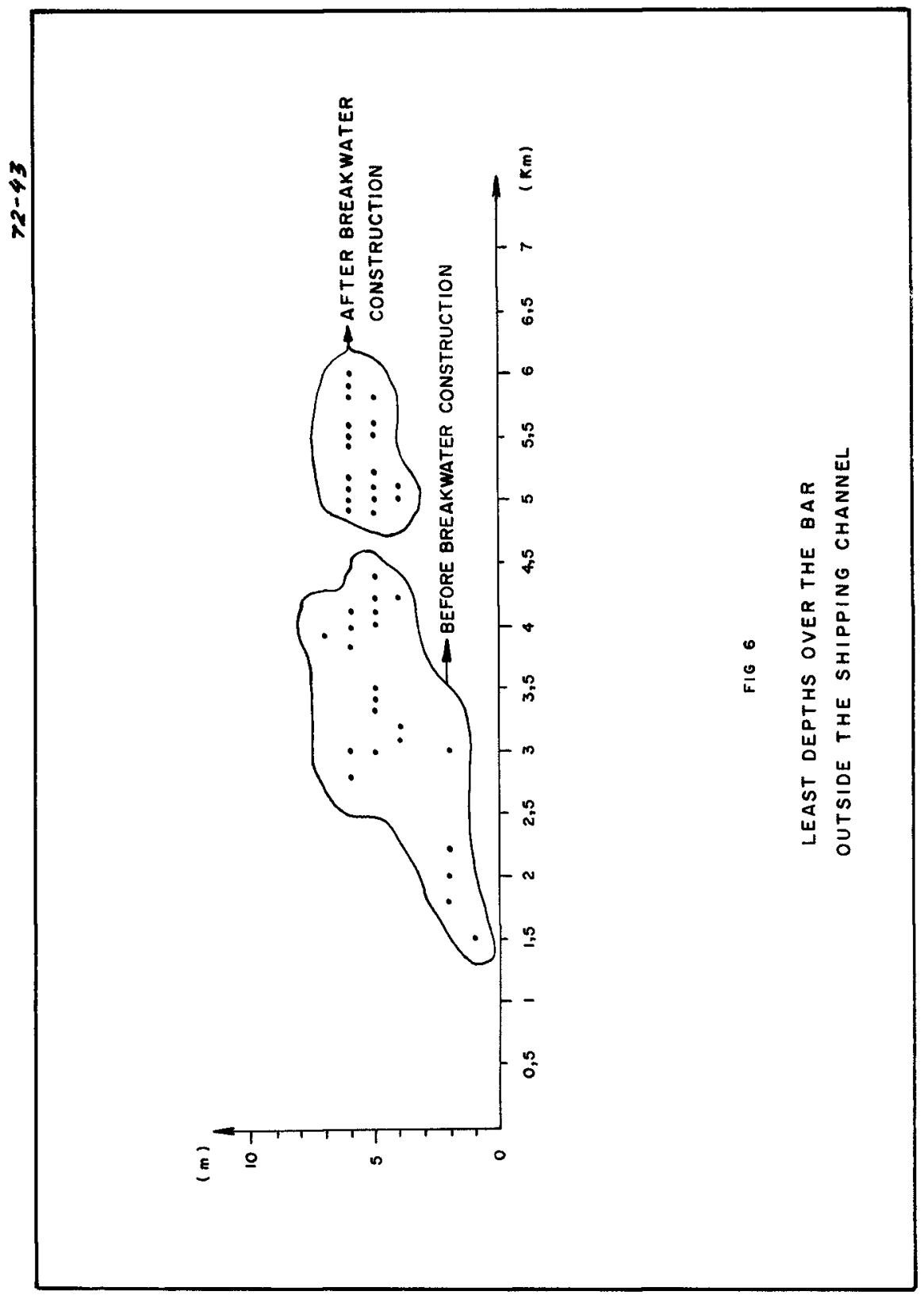


On the other hand, the great instability of Rio Grande bar eeems to be largely accounted for by the fact that upland flow, and not tidal flow, ie the natural agent whose flushing action tends to preserve depths over the bar. Upland flow discharges are much lese repeatable from year to year than would be tidal flow dischargee in a place with a regular tide.

As far ae it can be ascertained from the available surveye the shorelines on both sides of the lagoon outlet were fairly stable ae long as it remained uncontrolled.

The earliest propoeal for breakwater construction at Rio Grande wae put forward by a Britieh engineer, sir John Hawkshaw, who was commiseioned by the Brazilian Imperial Government in 1872 to report on methode to provide for a eafe ehipping channel accroes the bar. However, in his report |5| lawkehaw pointed out that the size and cost of the required breakwaters would be such as to make the construction of a ehoreline harbour at Torres, near the northern boundary of the state of Rio Grande do Sul, more advisable.

In 1883 the Brazilian Imperial Government entrusted a Brazilian engineer, H. Bicalho, with the taek of carrying out the field surveys and design work to improve Rio Grande entrance. Bicalho $|6|$ propoeed the conetruction of two $4 \mathrm{~km} 10 \mathrm{ng}$ breakwaters and requeeted the Government to seek the advice of a European or American engineer with a great experience in harbour entrance training to pase judgement on his propoeal. The Governinent choice fell on the Dutch englneer, P.Caland, who had been in charge of the works for Rotterdam harbour entrance. Caland $|7|$ approved of Bicalho'e proposal with some minor changee in laymout.

Hawkshaw, Bicalho and Caland were the first men to graep the behaviour of Rio Grande bar and lay down rules for its improvement al though the lack of the eea and swell concept in their time misled them ae to the direction of the dominant littoral drift on the coast of Rio Grande do Sul becauee of the prevailing and dominant winds from NE.

Caland was prophetical in his recommendation that, when the breakwatere would be built, previous dredging should be carried out as the construction work proceeded out to sea in order to forestall ecour caused by the increaeed traneport capacity of the upland flow between the breakwatere, of which the formation of a new bar further offehore would be the inevitable result.

Breakwater construction wae not started until 1908 when a French company wae awarded a concession to build the breakwaters and dock faclintiee. Work in the breakwaters themeelves wae carried out from 1911 to late 1915, and major effects on the bar cane about in 1914 which was a year with great rainfall and long, strong ebb flows in Canal do Norte. The Rio Grande breakwaters were the biggeet hydraulic-engineering etructures built in Brazil until some fifteen years ago, and at the time they ranked among the largest coaetal works in the world. From 1922 to 1928, already under Brazilian administration, the outer $288 \mathrm{~m}$ of the eastern breakwater, which had been left in 1916 as a submerged dyke, were brought to the same top level (+3.00 m) ae the remainder of the etructure.

M. B. Malaval, one of the French engineers in charge of the conetruction work, left a detailed account $|3|$ of its progrese and 
effects on the bed configuration. Malaval'e paper followed up of deepening process from 1913 to 1919 in great detail.

Fag. 4, 5 and 6 bring out the effects of the breakwaters on the bar. Fig. 5 shows that the deepening of the former bar was quite eudden* from 1914 to 1915 the plot jumps from 5 to $8 \mathrm{~m}$. Ever since that occasion

1) the leaet controlling depth has always been above $7 \mathrm{~m}$ and thie minimum only occurred in two years (1918 and 1921) in the time interval from 1915 to 1956 , the most frequent value being $9 \mathrm{~m}$ (which occurred in 23 yeare), a value of $8 \mathrm{~m}$ occurring in 11 yeare, and the maximum between 9 and $10 \mathrm{~m}$ occurring in 4 years $(1935,1936,1937$ and 1940),

2) the range of the controlling-depth variation has decreaeed from $2.5-7.0 \mathrm{~m}$ before breakwater construction to $8-10 \mathrm{~m}$ as from 1922 .

Fig. 5 proves that, in addition to increasing the contralling depth, breakwater construction increaed ite dietance to the ehore12ne.

However, it can be seen from Fig. 6 that, although the breakwaters did increase the depths at the harbour entrance, the scheme wae not entirely successful. The former bar wae destroyed but a new bar built up further offehore. Thie fact entaled objectionable conditions for navigation at the entrance becaues the shipe had to follow tight $S$ - curvee between the breakwater tipe and the new bar. The radius of curvature did not exceed $500 \mathrm{~m}$.

The obvioue reason for this unhappy outcome was the fact that Caland's recommendation for previoue dredging ae breakwater construction proceeded out to eea went unheeded. No previoue dredging wae carried out, and as much as 14 million cubic metree of sand was scoured out between the breakwatere from January 1913 to January 1919 of which 10 militon in 1914 alone. Such a huge volume was diecharged offshore, and sinceit far exceeded the transport capacity of wavee and currents offehore, a new bar came into being. The bed levels were irreversibly raised off the breakwater tips, and the interaction between the transport capacity of wavee and upland flow has gone on ever eince over the eubmarine mound thus created.

Previoue dredging has proved euccessul in foretalling the formation of a new bar at other harbour entrances $|8|$.

The firet approach channel to deepen was the one leading to the seaward end of the eastern breakwater. This channel wae the main route to negotiate the entrance until 1962. The eastern channel was the first to deepen because construction of the eastern breakwater went ahead of that of the western breakwater. Another unfortunate featuie of the conetruction work was the fact that advance of both breakwatere wae not kept at the same dastance to the shoreline. This seems to have been mainly due to difficulties in access to the weetern breakwater ae a result of the 1914 upland floods which caueed damage in a railway bridge. The western channel did not deepen completely until 1917 but in 1919 it wae nearly 10 m deep. Adaptation of the bar and channels to the new conditions went on until 1922 
when the new configuration became stable in its main features. Fig.7 reproduces the 1922 survey and shows the shipping lanes to negotiate the entrance.

Breakwater construction at Rio Grande harbour entrance had two other noteworthy effects. A deep scour hole developed around the eastern breakwater tip which eventually reached depths above $20 \mathrm{~m}$. At the same time a ehoal came about in the middie of the entrance which considerably reduced the avallable width between the breakwaters for shipping. The shoal developed in step with the scour hole, and it le believed to be formed by materlal from the latter. Velocity measurements carried out with floats in the late twenties sketched out the flow pattern and showed that, whereas ebb flows use the full width between the breakwaters, flood flows separate from the seaward end of the western breakwater and hug the eastern one. This flow concentration along the seaward end of the eastern breakwater is believed to be the man cause of the scour hole However, both the scour hole and the mudale shoal eventually reached equilibrium. To this day the shipping lane in the shelter of the breakwaters runs between the middle shoal and the western breakwater

The other outstanding effect was beach accretion on both sides of the breakwaters although accretion on the western side was much greater than on the eastern slde. Accretion on the weetern side was very fast shortly after breakwater construction. It amounted to a 200 in shoreline advance over a great $I$ ength and went as far as Cassino beach some $6 \mathrm{~km}$ west of the western breakwater. Later on, the accretion slowed down and the shoreline on both sides seems to have reached a new equilibrium. Fig. 8 ehowe the shoreline position on both sidee of the breakwaters in 1911, 1919, 1922, 1950 and 1956. Lack of adequate data on beach profilee and boundariee in plan and elevation precludes a cubature of this accretion

The outer portion of Canal do Norte was also considerably deepened by breakwater construction.

\section{III) The Situation after Breakwater Construction.}

The maln outlines of the bed configuration that came about as a result of breakwater construction are falriy stable since the early twentiee. The main features are the outer bar, the two 8 to $10 \mathrm{~m}$ deep approach channels between the bar and the breakwater tips compeling the ships to follow tight curves around the latter, the scour hole around the eastern breakwater seaward end, the middle shoal and the deep. wide Canal do Norte.

As already seen the controlling depth in the two outer approach channels fluctuates between 8 and $10 \mathrm{~m}$. The depth over the top of the outer bar fluctuates between 5 and $6 \mathrm{~m}$. However, in terms of volume the outer bar is far from stable, annual fluctuatione of over 1 mililon cubic metres in a year having been detected There is evidence to the effect that the volume of the bar is very sensitive to yearly changes of the natural forces at play. On the other hand, no correlation can be found between the volume of the bar and the controlling depth in elther of the two approach channels.

The annual changes in volume of the bar have ranged from 


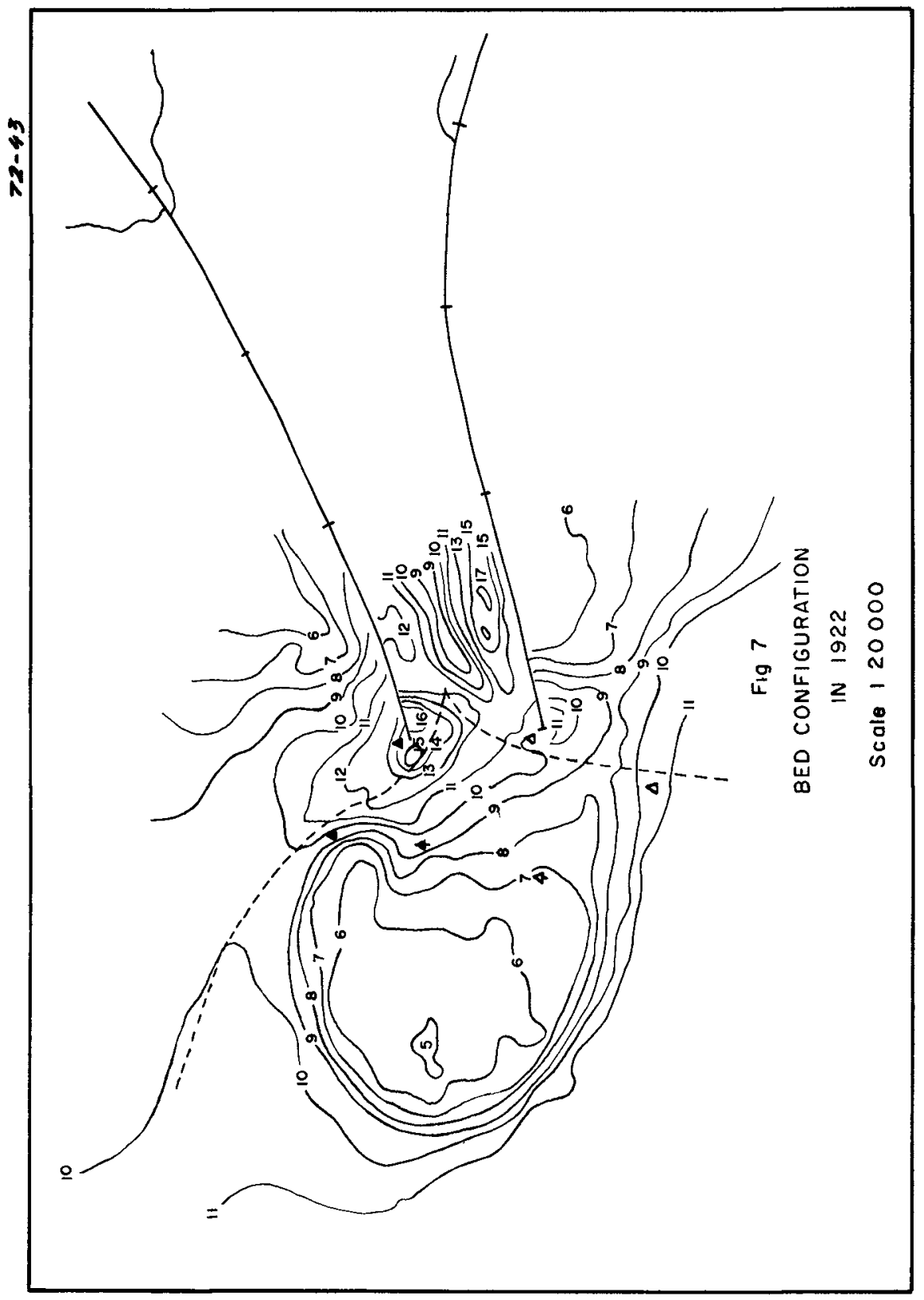




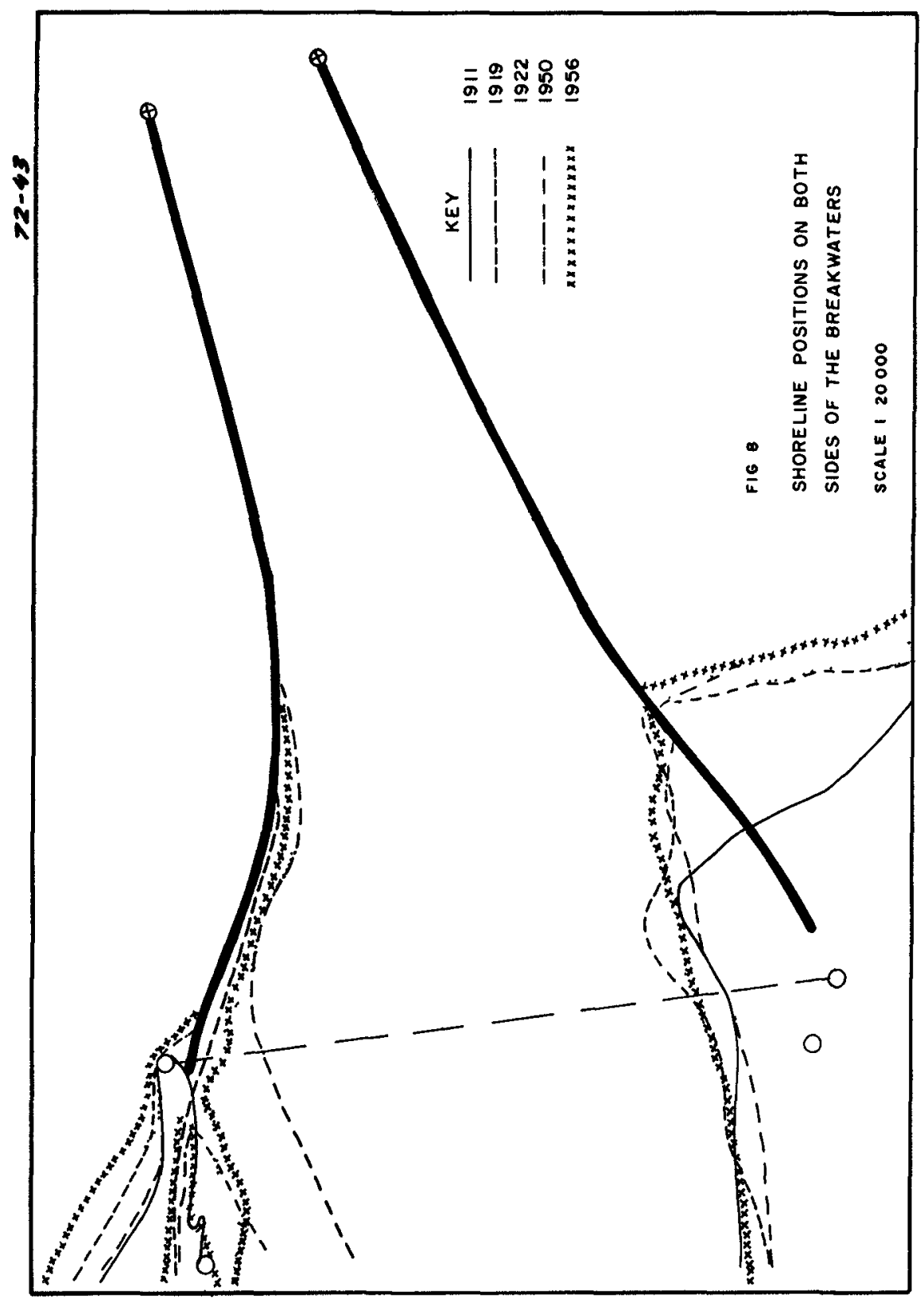


$2.000 \mathrm{~m} 3(1952-53)$ and $1264000 \mathrm{~m} 3$ (1929-1930) for accretion, and from $58000 \mathrm{~m} 3(1931-32)$ to $1238000 \mathrm{~m} 3(1926-27)$ for erosion.

An important event that took place after breakwater construction was the great upland flow in Apri1-Nay 1941, the greatest on record. The table below lists estimated mean monthly discharges in Canal do Norte for several months in $1941|9|$.

\begin{tabular}{l|c} 
Month & $\begin{array}{c}\text { Mean discharges } \\
\left(\mathrm{m}^{3} / \mathrm{s}\right)\end{array}$ \\
\hline January & 3100 \\
February & 7800 \\
March & 1600 \\
Apri1 & 21600 \\
May & 22700 \\
June & 6000 \\
July & 6800
\end{tabular}

Surveys of Canal do Norte and the outer bar were made before and after the peak flows. Therefore, the effects of the high upland flow in April and lay on the bed configuration could be readily assessed. The outer bar was displaced offshore, the -6 m contour having moved nearly 500 th out to sea, the middle shoal was topped off, and the scour hole around the seaward end of the eastern breakwater filled up to some extent, the $18 \mathrm{~m}$ depth being reduced to $12 \mathrm{~m}$. However, the effects of this powerful flushing action vanished in a few months.

Endless discussions wore held in Brazil during the twenties and the thirties about remedial moasures to improve shipping conditions at Rio Grande entrance but no action was taken. Severai proposals were put forward with a view to extending the breakwatera or changing their top levels. Many modern coastal engineering concepts were not avaliable at the time, and knowledgo of the wave characteristice at the site was poor. As a result, in the light of modern ideas several of those proposals now seem ill-founded. One particular proposal | 10 | was bound to have disastrous effects, had it been implemented, because it was still implicitly based on the wrong assumption thet, if the provaling and dominant winds blow from NE, the dominant sand transport must be towards SW. It consisted of keeping the western breakwater as it is and extending the eastern breakwater in a curved shape.

No improvement was achieved until $1961-62$ when a $12 \mathrm{~m}$ deep eastern approach channel was dredged in the outer bar in order to eliminate the $S$ - shaped curves in the entrance route and provide for a greater radius of curvature which was increased to $1400 \mathrm{~m}$. No maintenance dredging was done but the total deposit in the dredged channel from January 1963 to October 1907 did not exceed $120000 \mathrm{~m} 3$. In the most recent available survey (1965) the $12 \mathrm{~m}$ dredged depth had been reduced to $10.5 \mathrm{~m}$ The maximum draught presently allowed by the harbour authority is $8.8 \mathrm{~m}$ (29 $\mathrm{ft}$ ). Maintenance dredgine in this channel is under way at the time of writing. 
The Federal and State harbour authorities now contemplate improving Rio Grande larbour entrance and deepening it to $14 \mathrm{~m}$. The Coastal Division of the Hydraulics Research Institute of the Federal University of Rio Grande do Sul (IPH da UFliGS) has acted as consulting engineer to the state dock and harbour authority by Giving advice as to the problems to be solved and by making recommendations. In the light of the progress in dredging technology extension of the two $4 \mathrm{~km}$ long breakwaters is not deemed an economical solution. A new approach channel must be set up and maintained by dredging. The radius of curvature of the edstern approach channel is to be increased to $2.500 \mathrm{~m}$, and the widh at the bottom to $250 \mathrm{~m}$. IPH da UFRGS has proposed to carry out a fixed-bed model investigation using radio-controlled model ships to determine the most suitable lay-out of the approach channel to be dredged outside the breakwaters. However, the state dock and harbour authority has preferred not to increase the $2.500 \mathrm{~m}$ value so as to keep the amount of capital dredging to a minimum.

In addition to dealing with the outer-bar problems the improvement of Rio Grande harbour entrance has to cope with the middle shoal between the breakwaters. A $14 \mathrm{~m}$ deep shipping channel with a $250 \mathrm{~m}$ widh at the bottom cannot be accomodated between the middle shoal and the western breakwater. A mobile-bed model

investigation is to be carried out into the means to eliminate the middle shoal or reduce the amount of maintenance dredging in a channel that cuts into 1 t.

Dredged spoils in Canal do Norte are to be pumped ashore or dumped in dyked-in disposal areas. A radioactive tracer experiment is to be carried out to check on the suitability of the dumping grounds of the material presently dredged in the harbour basin. Deepening the approach channel to Rio Grande is inseparable from bullding new dock facilities on the west bank of Canal do Norte. Depths along the quays of the present port to not exceed $10 \mathrm{~m}$ and cannot be increased because of structural reasons.

\section{IV) Biblıographical References.}

1) USNHO. "Sea and Swell Charts for the South Atlantic Ocean". Washington, 1 st edition, 1948.

2) v. F. Motta. "The Model Investigation into the control of Tramandal Inlet" (in portuguese). proceedings of the 2nd Brazilian Conference on Maritime Transport and Naval Engineering. Rio de Janezro, 1967 .

3) M. B. Malaval. "Travaux du port et de la Barre de Rio Grande, Brésil". Ed. Eyrolles, Paris, 1922.

4) DNPVN. "Rio Grande Bar Surveys, 1883-1956" (in Portuguese). Compiled by C. M. Vassäo, Porto Alegre, 1959.

5) Sir John Hawkshaw. "Report on Rio Grande do Sul Harbour". In "Rio Grande do Sul Bar and Harbour Works", Vol. IIl (in Portuguese), a compliation of several reports, Porto Alegre, 1924.

6) H. Bicalho. "Report of Rlo Grande Bar Improvement Commission, 1883" (in portuguese). Idem.

7) p. Caland. "Amélioration de la Barre du Rio Grande do Sul. 
Rapport Presenté au Gouvernement Brésilien". Rio de Janeıro, 1886

8) G. H. Farleigh, H. C. Coode, A. F, Dickson, J, Green. British Report to the 20th International Navigation Congress. Baltimore, 1961.

9) F. Duprat da Silva. "Effects of the 1941 Flood" (in Portuguese). Rio Grande, 1941.

10) H. C. Ripley. "Some Notes on the Rio Grande do Sul Bar Improvement". New York, 1925.

11) V. F. Motta. "Report on Improving and Deepening Rio Grande Harbour Entrance" (in Portuguese). Submitted by IPH da UFRGS to Departamento Estadual de Portos, Rios e Canais, Porto Alegre, 1969. 



\title{
CHAPTER 79
}

\section{SCOUR AROUND A CIRCULAR GYLINDER DUE TO WAVE MOTION}

Donald R WELLS, LCDR, Civil Engineer Corps, U S Navy, Instructor of Ocean Engineering Naval School, Civil Engineer Corps Otficers, Port Hueneme, Calıfornı

and

Robert M SORENSeN, Associate Professor of Givil Engineerıng, Texas A\&M Unuversity, College Station, Texas

\begin{abstract}
ABSTRACI
A vertical circular cylinder to simulate a pile was installed in the Texas A\&M Hydrodynamics Lab two dimensional wave tank along with a built up section contaning a horizontal bed of fine sand This was subjected to monochromatic waves of diftering characteristics and conditions for incipi ent motion were observed for edch of three sands Also, the magnitude and pattern of ultimate scour and the time interval required to reach this state were measured for six different conditions of wave steepness and relative depth
\end{abstract}

These results were related to influentıal parameters, includıng wave, pile and sedıment character istics, and developed by dimensional analysis with consideration of the literature pertaining to past work on the movement of sediment by oscillatory flow Conclusions regarding the critical flow velocity for incipient motion, the effect of the above parameters on incipient motion and ultimate scour depth, the time required tor maximum scour, the significance of eddies generated by the pile, and the catalytic action of the pile in causing the initiation of scour are presented

The above conclusions are also generally discussed in light of the difficulties involved in extend ing the results to prototype conditions

\section{INTRODUCTION}

The scour of bed particles adjacent to an obstacle begins when the velocities and accelerations of the water particles cause hydrodynamic forces sufficient to overcome gravity and cause the bed particles to move When the bed particles begin to tip from their angle of 1 epose is defined as incipi ent motion and is the point where any study of scour must hegin

Incipient motion and scour have been studied extensively with regard to steady open channel flow but it has only been in the last two decades that research has been carried out in oscillatory motion It is extremely difficult to formulate mathematical equations that represent accurately the phenomena of incipient motion, scour, and ultimate scour depth Because of this difficulty no tormulation of mathematical equations was attempted However, the interrelationships and inter dependency $f$ the parameters were experimentally studied using terms derived by dimensional analysis 
The studies were conducted in a two dimensional wave tank using three uniformly gr ided com mcicial sands and monochromatic waves of various steepness and relative depth to produce the incipient motion and scour results

\section{BACKGROUND}

Incipient Motion It becomes obvious when reading the literature that theie is no unveisally excepted definition for incipient motion Because of this it is sometimes difficult to compare the re sults obtained by various authors For the purposes of this study the definition put forth by Eagleson and Dean, 1 is best suited They defined incipient motion as an instantaneous condition reached when the resultant of all the active forces on the particle intersects the line connecting the bed particle contact points" The term "active" means all the forces due to water particle motion and gravity

The first major work on incipient motion caused by oscillatory flow was done in 1954 by $\mathrm{Li}_{1} 2$ Using a oscillating bed in a still fluid, he found that the transition point from a laminar to a turbu lent boundary layer occurred at a Reynolds number of 800 for a hydrodynamically smooth bound ary Several years later Vincent ${ }^{3}$ carried out similar experiments using a wave flume and tound the Reynolds number for the transition point to be much less than that 1 eported by $\mathbf{L}$ Both concluded that the transition point was a function of roughness and would vary depending on the character istics of the bed material

Eagleson and Dean ${ }^{1}$ continuing work initiated by Ippen and Eagleson ${ }^{4}$ made a rigorous mathe matical analyses of incipient motion and sediment transport and piesented equations for both Several otber authors have presented equations for incipient motion notably $\mathrm{Ko}^{5}$, Vincent ${ }^{3}$, and Chepil ${ }^{6}$ All of the equations presented are accurate within certain limits but none of them will piedict the exact occurrence of incipient motion This is primanly due to the inability to evaluate the coefficients of drag and lift and the influences of the angle of repose and bed particle geometry Raudkıvi ${ }^{7}$ presents a very good discussion of these problems

Coleman $^{8}$ recently has developed relationships between the drag coefficient, $\mathrm{C}_{\mathrm{D}}$, the lift factor, $\mathrm{K}$, which is similar to the lift coetficient, $\mathrm{C}_{\mathbf{L}}$, and the Reynolds numbei His equations dithough for steady state conditions, do give a representation for the lift on a bed particle Reterence 9 presents an excellent discussion on incipient motion including an analysis and comparisons of the results of several authors It also presents equations for incipient motion from various authors

Scour Very little experiment work has been done on scour due to oscillatoly wave motion However, theie exists a wealth of knowledge on scour in open channel flow Since the forces that cause scour are somewhat similar for oscillatory flow as for open channel (steady state) flow, the hnowledge gained from experiments in open channel flow can be applied with ieservations to oscil latory motion Tbe majority of the work done on scour in oscillatory motion has been concerned primarily with scour of beacbis and littoral sediment transpoi $\mathrm{t}$

Murphy, ${ }^{10}$ Van Weele 11 and $\mathrm{Ko}^{5}$ studied scour in front of seawalls of various angles, and therr results are summarized by Herbich et al 12 They found that the ultimate depth of scour is ifunc tion of wave characteristics as well as the number of waves passing a given point where scour occurs and scour approaches its maximum value asymptotically after initially incieasing veiy xapidly 
Roper, Schneidei and Shen ${ }^{13}$ have shown that for steady statc conditions in open channel flow the depth of scour is a function of the pier Reynolds number, defined as

$$
\mathrm{N}_{\mathrm{RP}}=\frac{\mathrm{UD}}{\nu}
$$

where

$$
\begin{aligned}
& \mathrm{U}=\text { hoilzontal free stream velocity } \\
& \mathrm{D}=\text { pile diamettr, and } \\
& \nu=\text { kincmatic viscosity }
\end{aligned}
$$

They have fulther shown that the scoul is influenced by the type of vortex system cuused hy the pier For a cncular piei a horseshoe vortex system is most generally formed For nonsteady state conditions (oscillatoiy motion) this hoiseshoe vortev system may not have time to build up to such an intensity that it is shed and therefore the vortex system formed by oscillatory wave motion may not influence the scour The influence of the bed palticle size on scoul is not generally known, how ever, studics conducted by Roper, Schneide and Shen ${ }^{13}$ show that when the bed particle stze is greate than 052 millimeter, the particle size influences scour depth and when the particle size is less than 052 millimeter scour depth is independent of particle size

Carstens ${ }^{14}$ has made cxtensive studies of the scour associated witb different types of obstacles From his study he has shown that the rate of scour caused by an object in the flow path is a func tion of the sediment number $\mathrm{N}_{\mathrm{S}}$ sedment grain geometry, and the ratio of the scoul depth to the obstacle size The sedimcnt number is defined as

$$
\mathrm{N}_{\mathrm{s}}=\frac{\mathrm{U}}{\sqrt{\left(\mathrm{S}_{\mathrm{s}} 1\right) \mathrm{gd}}}
$$

where

$$
\begin{aligned}
& \mathrm{U}=\text { free stream velocity } \\
& \mathrm{S}_{\mathrm{S}}=\text { specifıc gravity of sedıment, } \\
& \mathrm{g} \quad=\text { acceleratıon of gravity, and } \\
& \mathrm{d} \quad=\text { mean sediment particle didmeteı }
\end{aligned}
$$

His studies were primarly conducted in steady flow He piesints equations for the ultimate scouı depth associated with a vertical cylinder and for the relative scour depth as a function of the sedi ment number However, all his equations are based on the supposition that the scour hole formed will have the appearance and form of an inverted frustum of a right circula1 cone having a base di ameter equal to the pile diameter and a side slope equal to the angle of repose

\section{THEORETICAL CONSIDERATIONS}

In this investigation the watei particle motions, velocities and accelerations, and the forces tbey in turn produce were calculated using Stokes third oider wave theoly This theory was chosen to be used after studyıng papers by Dean ${ }^{15}$ and Le Mehaute, Divoky and Lin ${ }^{16}$ and compan ing the wave characte11stics with the results published by these authors

The forces causing bed particle motion are hydiodynamic and consist of the forces of drag lift and inertia However, since the force due to inertia is a function of $\mathrm{d}^{3}$ whereas the force due to diag 
is a function of $d^{2}$ and thus meiti fotces will nerei picdommite due to the sm ill puticle si/e, the force of mertid will be neglected The tot il hydiodyn imic losce will thustor be the combinition of the lift force and the drag force The hydiodrnamic forces ne opposed by the force of gl ivity and influenced by bed paiticle geometiy

Drag The drag force is the combination of the folm diag duc to picssuc differcntill ind the viscous drag due to skin function The point through which the di ig tolce tets is not necess ully the center of gravity of the bed purticle but depends on the iclatic m ignitude of the lift and di ig force components which ale functions of bed particle geometis, locition and loc al Rcynolds numbu The steady force due to drag as developed an any clement uy fluid mech unics text $c$ in be shown to be

$$
\mathrm{F}_{\mathrm{D}}=\frac{\mathrm{C}_{\mathrm{D}}}{2} \rho \mathrm{A} \mathrm{U}^{2}
$$

where

$$
\begin{aligned}
& \rho=\text { fluid density } \\
& \mathrm{A}=\text { piojected ared of object noimal to flow diection, and } \\
& \mathrm{C}_{\mathrm{D}}=\text { drag coelficient }
\end{aligned}
$$

The coefficient of diag is a function of Reynolds numbei and bed particle geometry and is also in fluenced to some unknown extent by adjacent particles ciusmg anomalics in the flow patteins

Lift The relationship for the folce due to lift is similar to that for form drag and is given by

$$
\mathrm{F}_{\mathrm{L}}=\frac{\mathrm{C}_{\mathrm{L}}}{2} \rho \mathrm{A} \mathrm{U}^{2}
$$

where

$$
\begin{aligned}
& \mathbf{C}_{\mathrm{L}}=\text { coefficient of lift and } \\
& \mathbf{A}^{\prime}=\text { projected area peipendicular to llow direction }
\end{aligned}
$$

The lift force is the resultant due to the piessuse difieicntial above and below the particle The pics sure diffeiential is caused when the flund velocity is mcieased as it passes over the top of the particle thereby decreasing the pressure Since the pressuse below the particle iemans fanly static theie is a pressure differential or lift force A significant number of the studies conducted on forces related to particle movement have neglected the lift force, however, the proof that it does exist and is signifi cant has been reported $8 \& 9$

The coefficient of lift has not been studied as cxtcnsively as the cocfficient of di ig primaily due to the difficulty in evaluating it Coleman's 8 work appears to give the best indications of ats value

Gravity The hydrodynamic forces are opposed by the weight of the particle, friction and the in tergranular reactions The friction and intergranular ieaction are difficult to cvaluate but the gravity force can be represented by the equation

$$
\mathrm{F}_{\mathrm{g}}=\frac{\pi \mathrm{d}^{3}}{6}\left(\begin{array}{ll}
\gamma_{\mathrm{s}} & \gamma_{\mathrm{f}}
\end{array}\right)
$$


where

$\gamma_{\mathrm{S}}=$ specific weight of the sand, and
$\gamma_{\mathrm{f}}=$ specific weight of the fluid

Mechanics of Motion It is very difficult to study the motion of sand grains primarily due to their valymg sizes, angularity, and distribution in a bed Therefore, the problem must be simplified This can be done by considering the sand grains to be spheres of uniform size Referring to Fig 1 , it can be seen that the total hydrodynamic force $\mathrm{F}_{\mathrm{T}}$, is the combination of the lift ind drag force

For motion to cccur the sum of the moments about point $R$ must be zero or in other words, $\mathrm{F}_{\mathrm{T}}$ times its moment arm, $d$, must equal $F_{g}$ times its moment arm, $d$ sin $\phi$ When this condition exists incipient motion can occur As pointed out by Coleman ${ }^{8}$, the lift force car be negative if the Reyn olds number is below 100 It is therefore possible for the particle to be pushed into the bed rathcr than be lifted out or rolled along it

The velocity, $U$, used in evaluating the results of these experiments will be the maximum water particle velocity that occurs at the bed and is the velocity associated with the wave crest for shallow water waves

Flow around the pile and its relationship to scour Any obstacle inserted into the region of flow will cause tha flow to be diverted around the obstacle The flow velocity will increase as the flow deflects around the obstacle with a consequential reduction of pressure Depending on surface roughness on the boundary, local Reynolds number, boundary shape and boundary layer character istics, the flow can separate from the boundary cousing a wake to occur behind the pile

From potential flow theory, it can be shown that for flow around a cylinder the velocity of the flow at points on the cylinder ninety degrees from the initial direction of flow will be twice the in tial velocity of flow Because of the periodic disection changes of the flow and boundary layer de velopment it is doubtful that the velocity of flow at the ninety degree points will become twice the initial velocity In oscillatory flow the separation condition might not occui unless the distance the water particle moves is several pile diameters long From observations it is felt that if the distance the water paticle moves is approximately five or more pile diameters then separation should occur and eddies should torm and be shed periodically from the pile

In studies conducted by Roper Schneider, and Shen ${ }^{13}$, it was shown that the vortex system formed by flow around an obstacle was related to the shape and size of the obstacle They con cluded that the eddy structure formed is the basic mechanism of scour and that the depth of scour was a function of the pier or pile Reynolds number, $N_{R P}$

Because of the difficulties in evalu ttion lift and drag coefticients and intergranular iedctions, a mathematical inalysis of scour is beyond achievement

Dimensional Analysis The significant variables influencing incipient motion are still watei depth, h wive height, $H$, wave peiıd, $T$ viscosity, $\mu$, accelcration of gidvity $g$, densities of fluid and bed partictes, $\rho$ and $\rho_{\mathrm{s}}$ respectivcly, mean bed puticle diameter, $\mathrm{d}$, and angle of repose $\phi$ Using the Buckangham pi theorem the functional equation for incipient motion can be derived as

$$
\mathrm{f}\left(\frac{\mathrm{d}}{\mathrm{h}}, \phi \frac{\mathrm{H}}{\mathrm{h}} \frac{\mathrm{h}}{\mathrm{gT}^{2}} \frac{\mathrm{h}^{2}}{\mu \mathrm{T}}\left(\rho_{\mathrm{S}} \rho\right)\right)=0
$$




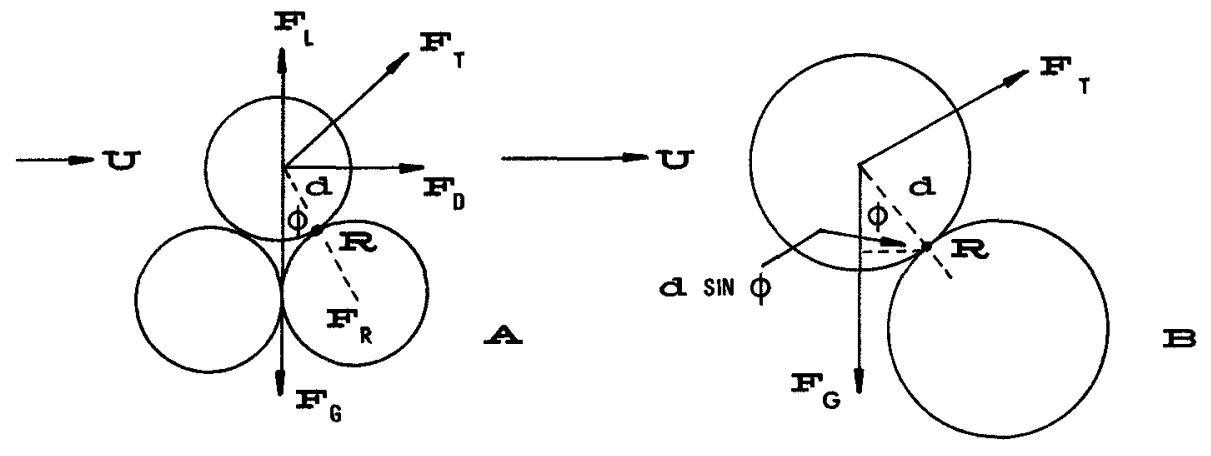

Fig 1 MECHANICS OF MOTION

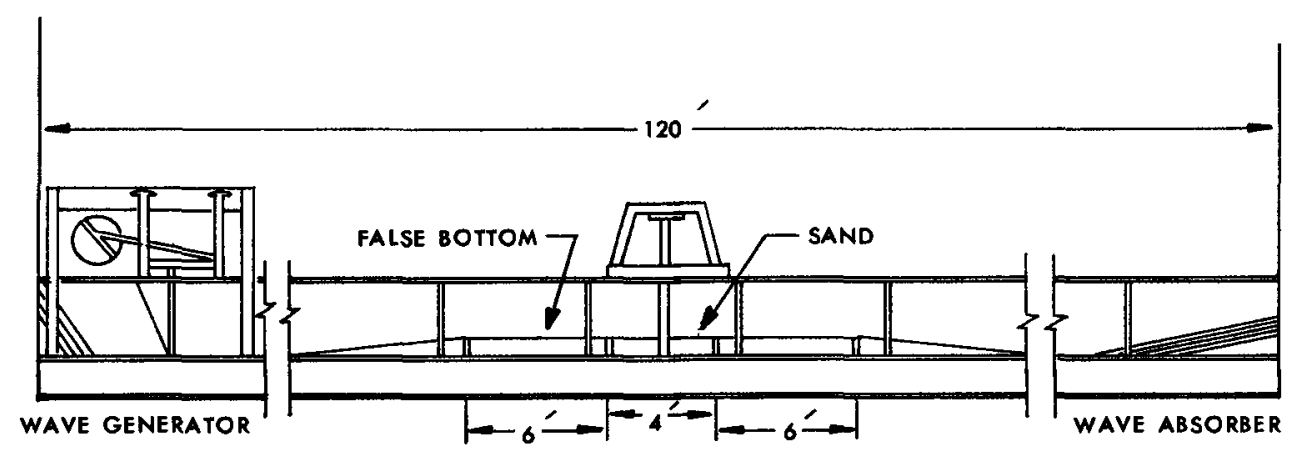

Fig 2 TEST APPARATUS IN THE WIND-WAVE CHANNEL 
Since these experiments will use sand as a bed material the density of sand $\rho_{\mathrm{S}}$ will not change, also the density and viscosity of water will reman constant Therefore the parameter $\frac{\mathrm{h}^{2}}{\mu^{\mathrm{T}}}\left(\rho_{\mathrm{s}} \quad \rho\right)$ will vary as $\frac{h^{2}}{T}$ varies Since $h$ and $T$ are included in the $\frac{h}{g T^{2}}$ parameter the $\frac{h^{2}}{\mu \mathrm{T}}\left(\rho_{\mathrm{S}} \rho\right)$ parameter can be dropped Also it is doubtful that the angle of repose will be a significant parameter in these ex periments, therefore, it will be discarded That leaves the function relationship

$$
\frac{\mathrm{d}}{\mathrm{h}}=\mathrm{F}\left(\frac{\mathrm{h}}{\mathrm{gT}^{2}}, \frac{\mathrm{H}}{\mathrm{h}}\right)
$$

which is to say that for incipıent motion the relative particle size is a function of relative depth and relative wave height

The variables considered to be significant for scour contam those considered for incipient motion and the additional variables of pile diameter, D, U, ultimate significant scour depth $S_{\mathrm{u}}$ and elapsed time $t$

The orbital particle velocity, U, although not independent of those IIsted above was included so that the parameter $\mathrm{N}_{\mathrm{s}}$ could be defined in its normally accepted form

Again using the Buckingham pi theorem, the functional equation detining scour around a circu lar pile in oscillatory motion is

$$
\frac{\overline{\mathrm{S}}_{\mathrm{u}}}{\mathrm{H}}=\mathrm{f}\left(\frac{\mathrm{H}}{\mathrm{gT}^{2}}, \frac{\mathrm{h}}{\mathrm{gT}^{2}}, \mathrm{~N}_{\mathrm{RP}}, \mathrm{N}_{\mathrm{s}}, \frac{\overline{\mathrm{S}} \mathrm{d}}{\mathrm{d}}, \frac{\mathrm{H}}{\mathrm{h}}, \frac{\mathrm{t}}{\mathrm{T}}, \phi\right)
$$

\section{EXPERIMENT AL APPARATUS AND EQUIPMENT}

The experiments on incipient motion and scour were conducted in a 120 foot long, 3 foot deep and 2 foot wide two dimensional wave channel (Fig 2) A false bottom 6 inches deep and 16 feet long was constructed in the channel At each end of the false bottom was a gradual slope to bring the wave up to the new depth at the top of the false bottom The false bottom was split into three scctions, two 6 foot sections at cach end with 4 feet of the test sand in the center between the 6 foot sections The $1 \frac{1}{2}$ inch diameter stecl pile was placed in the center of the 4 foot sand test sec tion and anchored to an aluminum frame above the wave tank to keep the pile vertical and stable

Scour deptb measurements were mide using a depth probe that was attached to a device that could be rotated around the pile 360 degrecs and extended up to 8 inches from the outer edge of the ple The rotating ring was marked in degrees and the extended arm was marked in inches so that any scour measurcment could be identificd in polar coordinates

The wave generator was an oscillating pendulum type whose stroke and consequently wave height $c$ in be varied by adjusting the excentricity of the paddlc arm on the flywheel 1 he period was varied through a variable rheostat that controls the specd of the flywheel $W_{a v e}$ heights and periods were moasured by 1 capacitance wave gagc connected to d Hewlett Packard Dual Channel Carrier amplifı cr rccorde (Model No 321) A michanical counter was attached to the wave generator so that the number ol waves generated could be determined 


\section{EXPERIMENTAL PROGEDURES}

Incipient Motion Prior to the start of each incipient motion run, the sand bed was leveled The wave generator, set for a particular wave height and peilod, was started and the sand along the boundary of the plle was observed The wave period was adjusted untıl several sand grans were observed to tip out of their position of rest and the wave charactcristics weie then recorded for that run The wave period was then further adjusted to attempt to observe incipient motion on the bed far enough away from the pile so that the pile had no influence on the sand grams These experı ments were run fol all three sands at depths of 15 inches and 8 inches and for approximately 15 runs per sand Several paddle positions were used in making the runs in oider to observe incipient motion for intermediate and shallow water waves The bottom water paiticle velocity for each run was calculated using Stokes thrrd order wave theory The incipient motion data can be found tab ulated in Apperadix I of reference 17

Scour For the experiments on scour, three experımental waves were selected of varyıng charac teristics for each experimental wave at each depth are shown in Table 1 Runs were made for each sand at each depth for each wave for a total of 18 runs At the start of each run the sand bed was leveled and measurements were made to ascertam the level of tbe bed

The wave generator adjusted for a particular experımental wave, was then started and the wave period and height were recorded Measurements of scour depth wele made after each 200, 400 800, $1200,2000,3000$, etc waves until there appeared to be no increase in scour depth aftei two succes sive measurements This procedure was adjusted occasionally when it was felt that the run should be continued to observe scour pattern changes although there was no increase in scour depth The scour depth measurements were made on a random basis, measuring the deepest scour holes and trying to use the same holes for each measurement as a control basis This could not always be done because when ripples formed on the bed a scour hole would occasionally be filled in The ielative significant scour depth and the relative ultimate significant scour depth were calculated by aver aging the scour depths for the deepest one third scour measurements and tbe latter being divided by the wave height For each sequence point in a particular run, a collection of at least six data points was attempted but occasionally this could not be done due to the lack of scou holes For each data point, the angle, distance from the ple and scour deptb were recorded At the completion of each run a number of data points were taken so as to be able to construct a contour map of the scour pattern The data for the scour runs are tabulated in Appendix I of reference 17

Three sands were selected for use in the experiments The sands were all standard Ottawa sands that are produced with a controlled size distribution Each sand was subjected to a standard ASTM sieve analysis to determine mean particle diameters The data are shown in Table 2 
TABLE 1 EXPERIMENTAL WAVE CHARACTERISTICS

\begin{tabular}{|c|c|c|c|c|c|c|}
\hline $\begin{array}{c}\text { Test } \\
\text { Wave } \\
\text { (1) }\end{array}$ & $\begin{array}{c}\text { Depth, } \\
\text { h in } \\
\text { feet } \\
\\
(2)\end{array}$ & $\begin{array}{c}\text { Average } \\
\text { wave } \\
\text { herght, } \\
\mathbf{H} \text {, in } \\
\text { feet } \\
(3)\end{array}$ & $\begin{array}{c}\text { Average } \\
\text { wave } \\
\text { period, } \\
T, \text { in } \\
\text { seconds } \\
(4)\end{array}$ & $\begin{array}{c}\text { Average } \\
\text { wave } \\
\text { length, } \\
\text { L, in } \\
\text { feet } \\
(5)\end{array}$ & $\begin{array}{c}\text { Relative } \\
\text { depth } \\
\frac{\mathbf{h}}{\mathrm{L}} \\
(6)\end{array}$ & $\begin{array}{c}\text { Wave } \\
\text { steepness } \\
\frac{\text { H }}{\mathbf{L}} \\
\text { (7) }\end{array}$ \\
\hline \multirow{2}{*}{1} & 125 & 016 & 38 & 2474 & 00506 & 000647 \\
\hline & 0666 & 019 & 38 & 2001 & 00333 & 000950 \\
\hline \multirow{2}{*}{2} & 125 & 0187 & 308 & 1942 & 00644 & 000963 \\
\hline & 0666 & 013 & 308 & 1514 & 00440 & 000858 \\
\hline \multirow{2}{*}{3} & 125 & 0275 & 1875 & 1085 & 01152 & 002530 \\
\hline & 0666 & 022 & 1875 & 923 & 00721 & 002380 \\
\hline
\end{tabular}

TABLE 2 EXPERIMENTAL SAND GHARACTERISTICS

\begin{tabular}{|c|c|c|c|c|}
\hline $\begin{array}{r}\text { Test } \\
\text { Sand } \\
(1)\end{array}$ & $\begin{array}{c}\text { Manufacture } \\
\text { trade name } \\
\text { (2) }\end{array}$ & $\begin{array}{l}\text { Mean grain } \\
\text { drameter, } \\
\text { d in } \\
\text { millimeters } \\
\text { (3) }\end{array}$ & $\begin{array}{l}\text { Average weight } \\
\text { per grain } \\
\text { in grams } \\
\text { (4) }\end{array}$ & $\begin{array}{l}\text { Density un grams } \\
\text { per cubic } \\
\text { centimeter } \\
(5)\end{array}$ \\
\hline 1 & Sawing sand & 062 & $2314 \times 10^{4}$ & 267 \\
\hline 2 & Crystal sand & 0325 & $1310 \times 10^{4}$ & 266 \\
\hline 3 & Bond sind & 030 & $6854 \times 10^{5}$ & 2665 \\
\hline
\end{tabular}




\section{PRESEN IATION AND DISCUSSION OF RESULTS}

Incipient Motion The occurrence of incipient motion w is obseived at the pile and on the bud and the maximum undistuibed bottom velocity for each case was calculated from the measured wave height and period These were compased with the theoretical potential flow of two The dver age velocity $\mathrm{r}$ tio for 11 expeimental runs was 169 Furthei experimentation should be done for various pile sizes and roughnesses betore any conclusions can be made ts to what the velocity ratio will be ind how it is influenced by roughness and pile size

It was assumed that incipient motion was a function of the relative depth, the relative wave height, and the dimensionless particle size These parameters were plotted on a log log plot and are shown in Fig 3 for incipient motion occurring at the pile boundary Referring to Fig 3, it can be seen that incipient motion appears to be influenced only slightly by the paramcter $\frac{\mathrm{H}}{\mathrm{h}}$ and appcars to be dil ectly related by the parameters $\frac{h}{g T^{2}}$ and $\frac{d}{h}$ However in the case of incipient motion on the bed, it ippears to be independent of $\frac{\mathrm{H}}{\mathrm{h}}$ and $\frac{\mathrm{h}}{\mathrm{gT}^{2}}$ More data collection will be necessdry betore any conclusion can be diawn regarding incipient motion on a pile boundary except to siy that the inı tiation of motion appears to a function of the relative depth and dimensionless particle size This, of course, is only true for sands since these cxperiments did not investigate non cohesive matelidls of other specific gravities

Reference 9 presents a collection of data regdiding the mcipient velocities for various mateirals for steady state conditions A plot of these data along with the velocities calculated for incipient motion on the bed for the three experimental sands is shown in Fig 4 As can be seen from the graph, the incipient velocities for the three sands fall on the lower boundary and below the. region of data piesented by reference 9 The ieason for the lower values is not known except to say that for ascillatory motion, mcipient motion appears to occur at a lower velocity Howevel as pointed out by Vanoni ${ }^{9} \mathrm{~m}$ his discussion of the incipient velocity data, the curve to Shields (1936) data gave cubstantially higher incipient velocities than did that of Mivis and Laushey (1949) ind the data of Hulstrom (1935) did not comp ire to either of the other two curves Becuse of the incon sistencies in the data for incipient velocities Vanoni therefore iecommends that critical shear stress be used ts the parameter for comparing incipient motion rather than incipient velocity

\section{SCOUR}

The dimensionless parameters developed for scour were calculated and their in terdependency wa, studied by plotting the parameters The parameter $\frac{\bar{S} u}{H}$ was plotted tgainst the wave steepness $\frac{\mathrm{H}}{\mathrm{gT}^{2}}$ for varıous values of relative steepriess $\frac{H}{h}$ however no conclusive relationship could be diawn fiom a study of the plot It is, however, telt that as the wave steepness increases from a point of incipient motion the relative ultimate significant scour depth incieases until d point is ie iched where toi fur thei increases in wave steepness a rapid deciease in scour depth occurs The rapid decrease in scoul depth is associated with the phenomena of ripple formation It is conjectured that after the ilpple formation becomes stable or well defined there will be no further significant inciease or deciease in scour depth for further increases in wave steepness 


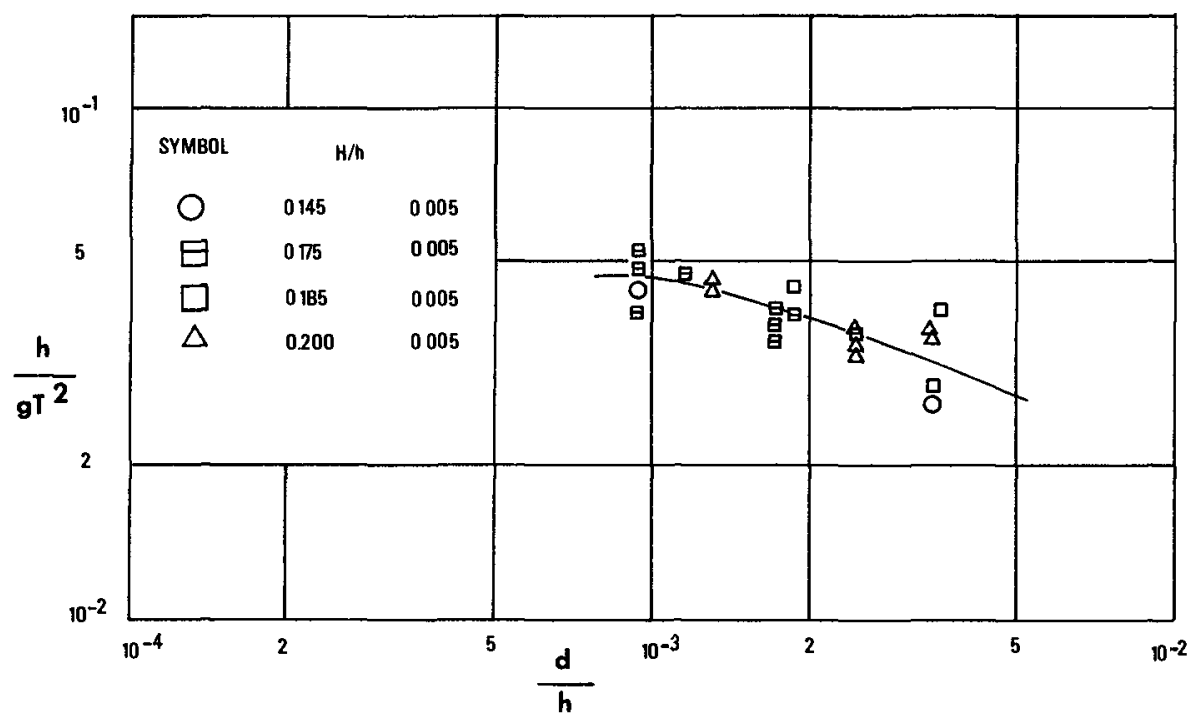

FIg 3 INCIPIENT MOTION OCCURING ON THE PILE BOUNDARY FOR VARЮUS VALUES OF RELATIVE WAVE HEIGHT

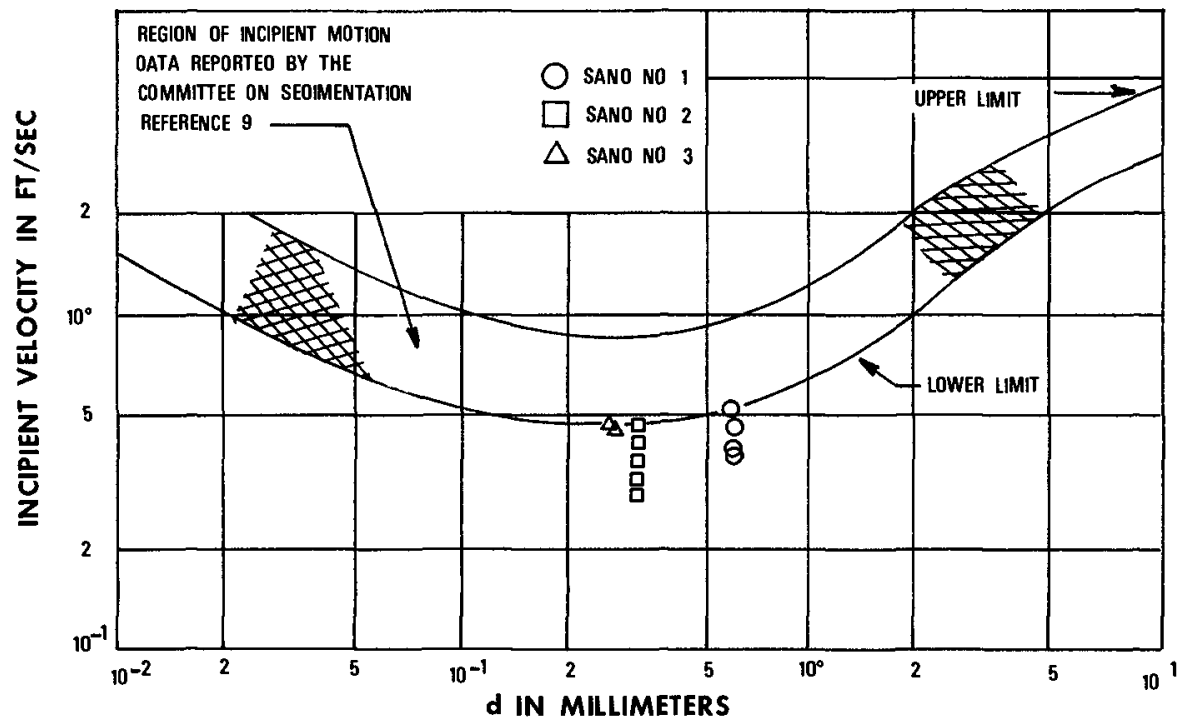

Fig 4 INCIPIENT VELOCITY AS A FUNCTION OF GRAIN SIZE 
The parameters $\frac{\overrightarrow{\mathrm{S}} \mathrm{u}}{\mathrm{d}}$ and $\frac{\mathrm{h}}{\mathrm{gT}^{2}}$ were graphically studied to determine their relationship to each other It was found that the relative ultimate significant scour depth is a function of relative depth and bed particle size As the relative depth decreases for each bed particle size the relative scouı depth increases slowly at first untl a relative depth of approximately $15 \times 10^{3}$ is reached where the relative scour depth increases rapidly for decreases in bed particle size Although relative ultı mate significant scour depth is a function of relative depth and possibly a function of wave steep ness, it appears to be primarily influenced by the sediment number $\mathrm{N}_{\mathrm{s}}$ and the pile Reynold's Number NRP

Figs 5 and 6 show the functional relationship betwetn the relative ultimate significant scour depth and the stdiment number, $\mathrm{N}_{\mathrm{S}}$, and pile Reynold's number, $\mathrm{N}_{\mathrm{RP}}$, respectively The incipient values of $\mathrm{N}_{\mathrm{s}}$ and $\mathrm{N}_{\mathrm{RP}}$ for each of the sands were calculated and included on $\mathrm{F}_{1 \mathrm{gs}} 5$ and 6 to show that tbe curves actually have a rapid initial increase in $\frac{\overline{\text { Su}}}{\mathbf{H}}$ The functional ielationship appers to be similar in both cases in that relative scour depth increases very rapidly trom the point where incip ient motion occurs to a maximum relative scour depth Any furthei increase in $N_{S}$ or $N_{R P}$ results in a rapid decrease in ultimate scour depth reaching a point where the relative ultimate significant scour depth becomes independent of $\mathrm{N}_{\mathrm{S}}$ and $\mathrm{N}_{\mathrm{RP}}$ but not of bed particle size It is unknown why the number 2 sand, which has a smaller mean diameter than the number 1 sand, has the maximum ultimate scour depth and also levels off at a higher relative scour depth than the number 1 sand One possible answer could be, as was pointed out by Roper, Schne1de1, and Shea1 13, that when the bed paiticle size is less than 052 millimeter, the scour depth is independent of the bed particle sizc

Figs 7 and 8 show the relationship between the ielative significant scour depth and the pala meter $\mathrm{t} / \mathrm{T}$ which is the number of waves These are typical cuives and the remainder of the plots for all the runs can be found in Reference 17 The parameter $\frac{\overrightarrow{\mathrm{S}}}{\mathrm{H}}$ for each of the three sands is plotted versus the number of waves for a particular experımental wave so as to compare the relative signifi cant scour for the three sands The majority of the curves have a characteistic initial lapid increase in the relatively significant scouı depth Most of the curves reach their approximate ultimate condi tion after 2000 waves Also the majority of curves reach chasacteristic platedus where the scour activity is dormant for a period of time and then it starts to increase again For the i uns where ripple activity was dominant (Fig 8), the curves seem to reach a peak value 1apidiy followed by a deciease in relative scour depth for further increases in number of waves and then findly level otf at the ultımate scour depth

Intuitively one would think that Sand number 1 would have the laigest relative significant scour followed by Sands number 2 and 3 However, this is not always the case and the reasons for it are unexplamable except for the reason pointed out by Roper, Schneider and Shen For all the runs, the ultımate scour conditions are reached after 6000 waves

\section{SCOUR PA ITERN OBSFRVATIONS}

The resulting scour patterns for each run were studied to determine the similurities or diffelences that might be attributed to wave characteristics or sand sizes In almost all cases, scour mitially started around the pile perifery and when eddies were formed, two relatively deep scour holes formed at the rear of the pile approximately 1 to 2 inches from the pile and 30 to 40 degrees from a normal to the wave direction The two eddy influenced scour holes normally convelged toward each other forming a ripple front The rapidity of the formation of the ripples is dependent on the water depth, the wave characteristics and the mean particle diameter 


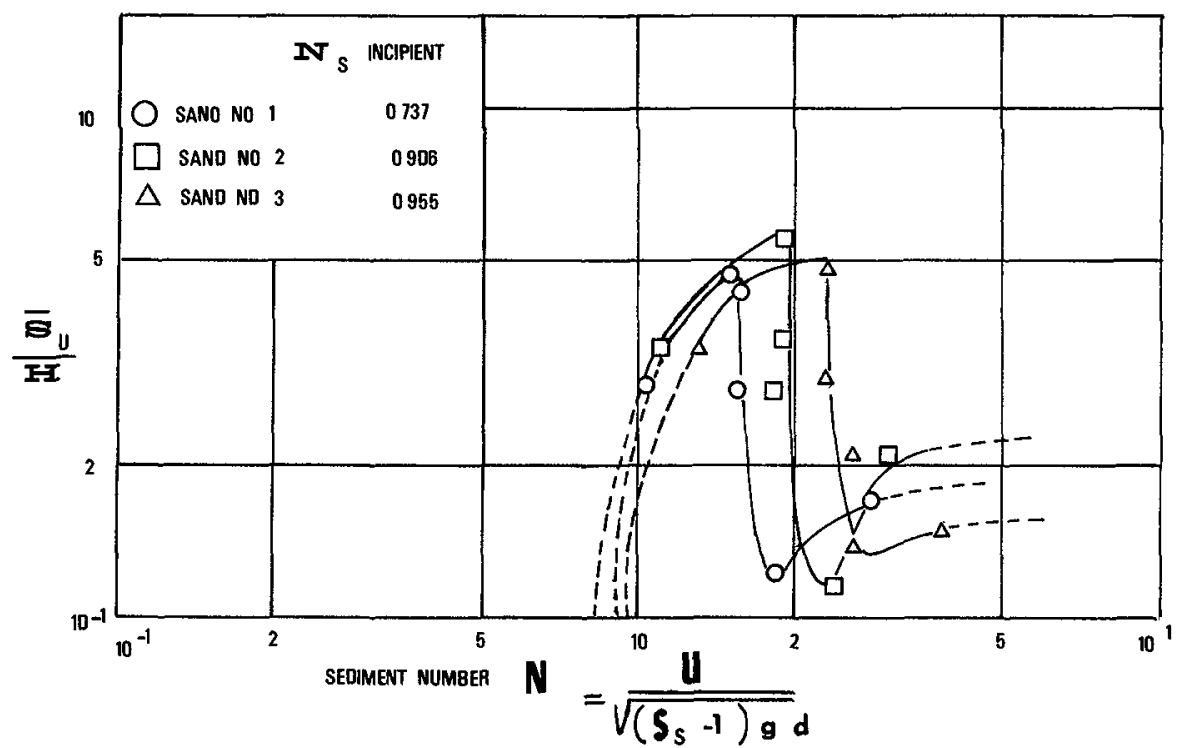

Fig 5 RELATIVE ULTIMATE SIGNIFICANT SCOUR DEPTH AS A FUNCTION OF THE SEDIMENT NUMBER

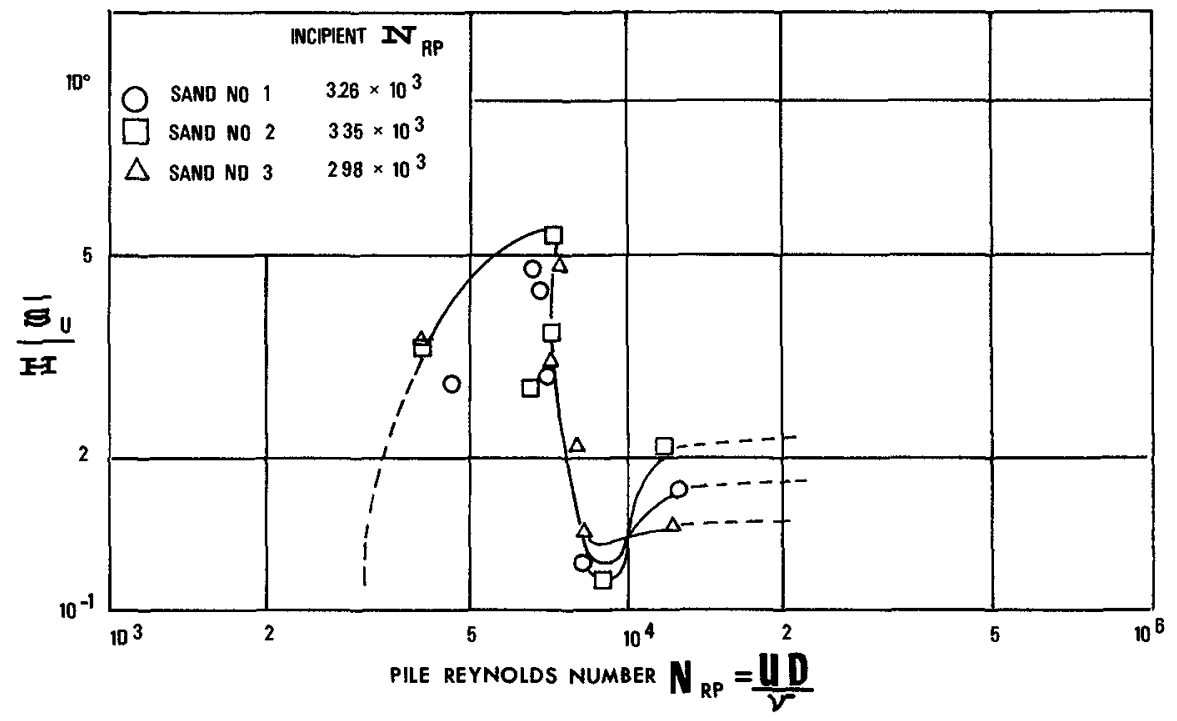

FIg 6 RELATIVE ULTIMATE SIGNIFICANT SCOUR DEPTH AS A FUNCTION OF THE PILE REYNOLDS NUMBER 


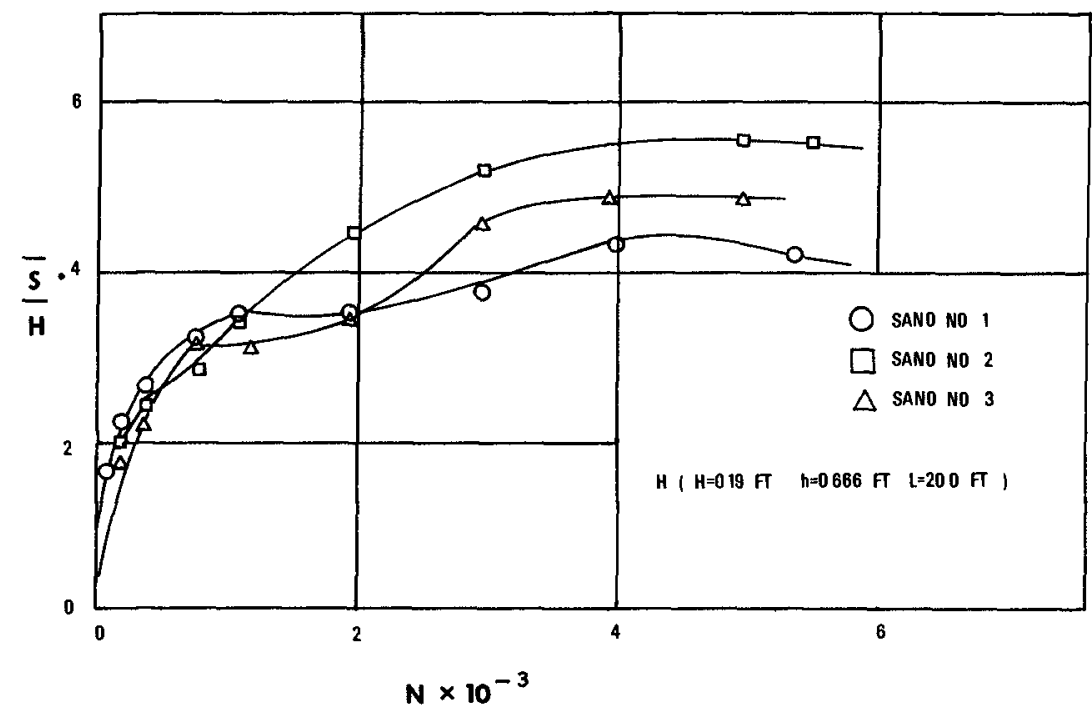

Fig 7 RELATIVE DEPTH OF SCOUR AS A FUNCTION OF NUMBER OF WAVES

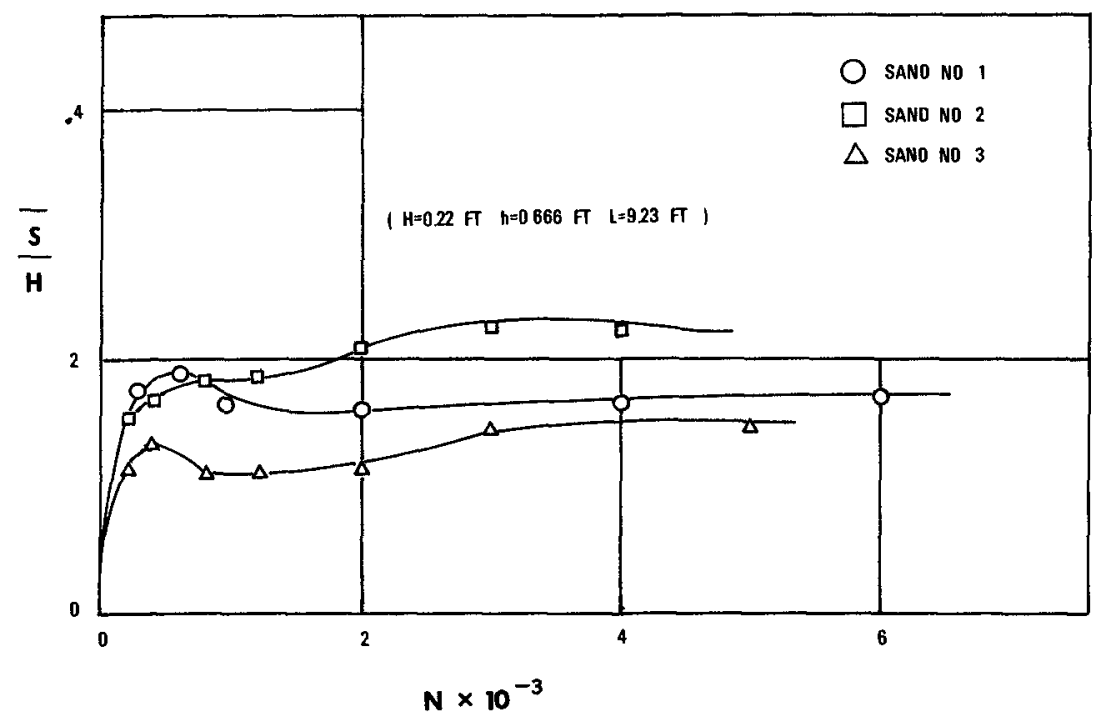

Fig 8 RELATIVE DEPTH OF SCOUR AS A FUNCTION OF NUMBER OF WAVES 
Generally it can be said that the resulting scour is strongly influenced by the pile and the wave characteristics In most of the runs, very little to no bed movement could be observed away from the pile The pile served as a catalyst to start the scour activity and once started around the pile it spread over a large area and extended in some cases great distances from the pile Figs 9 and 10 show some typical scour patterns obtained from the experiments

\section{CONCLUSIONS AND REMARKS}

1 The critical velocity necessary to cause incipient motion in oscillatory flow appears to be lower than that for steady state flow

2 The ratio of the maximum velocity on the ple boundary and the initial tree stream velocity, is less than the value of 20 for potential flow theory

3 Incipient motion on the pile boundary appears to be independent of $\frac{\mathrm{H}}{\mathrm{h}}$ and directly dependent on the parameters $\frac{\mathrm{h}}{\mathrm{gT}^{2}}$ and $\frac{\mathrm{d}}{\mathrm{h}}$

$4 \quad \frac{\overrightarrow{\mathrm{Su}}}{\mathrm{H}}$ appears to be directly related to the sediment number $\mathrm{N}_{\mathrm{S}}$ and the pile Reynold's Num ber $\mathrm{N}_{\mathrm{RP}}$

5 A maximum of only 6000 waves are required to reach an ultimate scour depth and in most cases 3000 waves are sufficient

6 The relative ultimate significant scour depth increases very rapidly at first, reaching three fourths of its ultimate depth in the first 1000 waves, and increases more slowly after that untll it reaches its ultimate depth

7 Eddy forces, although initially influencing the scour patterns, do not appear to be of signifi cance in the final scour pattern

8 The scour pattern resulting is primarily influenced by the pile and the wave characteristics

9 In all the scour experiments, the pile acted as a catalyst causing scour of the bed particles to be initiated whereas if the pile was not present httle to no scour would have resulted

To try and predict scour depths for a prototype case or relate these unconclusive results to a pro totype would be presumptuous To predict happenings or occurrences of a phenomenon in a proto type requires that there be similitude, both geometric and dynamic, between the model and proto type This requires that similitude exists between the orbital velocities and orbital lengths ( $\mathrm{e}$, wave characteristics are similar), grain size and grain size distribution in the bed, roughness of the beds, and translation of the orbit due to drift Without these similitudes, erroneous conclusions could be reached in attempting to predict prototype conditions The difficulties in acquiring similitude be tween prototype and model were pointed out by Posey and Sybert 18 in their studies of scour around ples on offshore platforms It required several years of study and experimentation before actual prototype conditions were duplicated in the model 


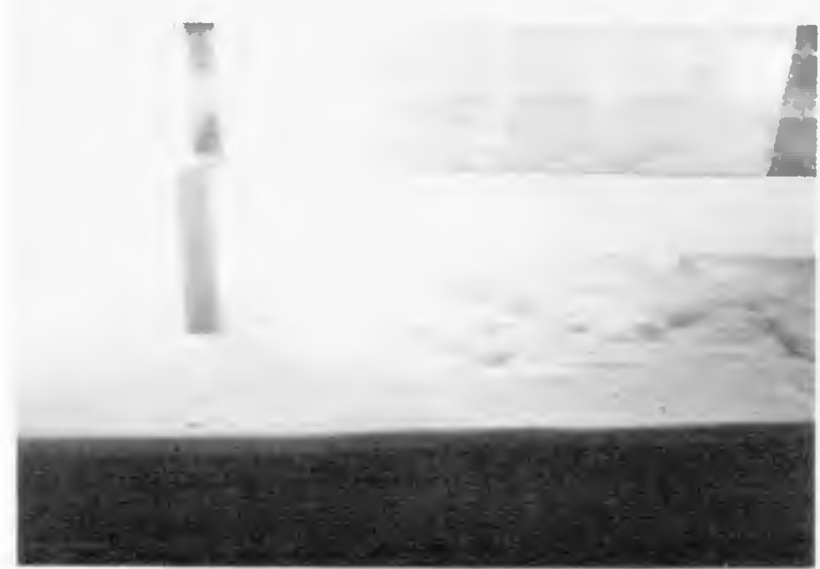

Fig. 9. SCOUR PATTERN WAVE NO. 1 - 8 inch depth, Sand No. 2

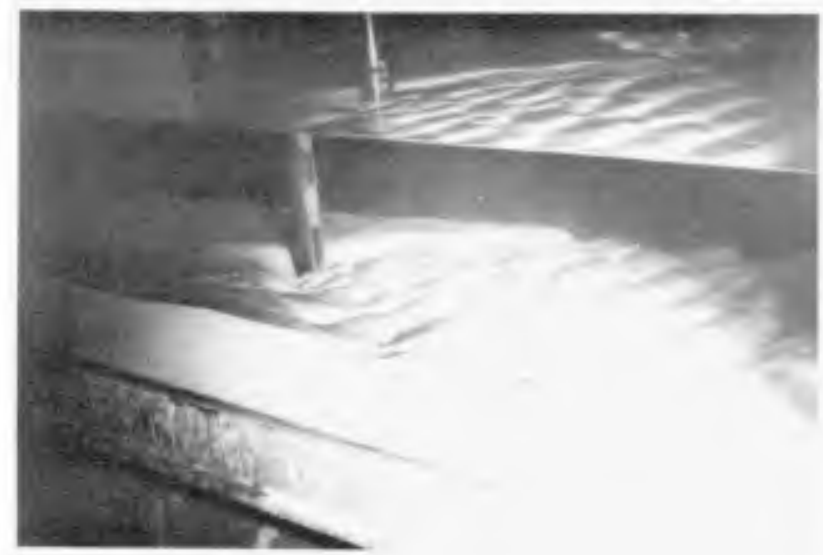

Fig. 10. SCOUR PATTERN WAVE NO. 1 - 8 inch depth, Sand No. 3 
However from the experiments conducted on scour it is Ielt that cert in conjecturcs on proto type conditions can be in ide The maximum scour medsurcd in the cxpcriments w is ipproxim itcly onc pile diameter It, therefore, is conjectuicd that the maximum scour ohserv tble in a prototype would be approximately cqual to one pile diameter, which for a typical off shore pile ol 4 to 6 lect would be approxim itcly 5 fect $\operatorname{Kreig}^{19}$ has reported observed scour depths on ollshore pl itlorms in the Gulf of Mcxico of 8 to 10 fect ind Poscy and Sybert ${ }^{18}$ me istured in iximum scour depths of 13 fect with average scour depths of 8 to 10 lect loi ofishore plitforms in linc sinds oll Pidic Island, $\Gamma$ cxas $T$ he average pile diameter associsted with the scour measurements made by Poscy and Sybert ${ }^{18}$ was approximately 3 feet and there was a farly significant litior il current prescht lt is important to notc from the above discussion that cxact similitude is very import int Without cvery condition duplicated betwecn model and prototype $(1 \mathrm{c}$, the littoral current) croncous results will be had The scour patterns for the Padre Isl ind platforms had a dish or slucer appe in ince, that w is much larger in shape than the plitform Scour patterns such as these would not normally be cxpected

The conclusion that the scour is very I ipid at first and decicases theicalter $h$ is been verified by Poscy and Sybert ${ }^{18}$ who obscrved th th the scoul $\mathrm{r}$ itc is high during the first yc ir or two, and de cicascs thercafter

\section{REFERT NGIS}

1 Eagleson, P S, Dean R G, "Wave Inducted Motion of Bottom Scdimcnt Pirtıcles" Iransac tıons ASCL, Vol 126, 1961, P trt 1, pp 1 I 621189

2 Huon Li, "Stability of Oscillitory Laminar Flow Along a Wall, Icehnecal Mcmorandum No 47 Bcich Fiosion Board, 1954

3 Vincent G E, "Contribution to the Study of Scdiment Iransport on a Horizont al Bed Duc to Wave Action," Proceeding, Sixth Conference on Co ist il Enginceing, $\Lambda$ SCF, Dec 1957, pp 326335

4 Ippen, A I , Eagleson, P S, "A Study of Sediment Sorting by Waves Shoding on a Planc Beach," MII Hydrodynamics Laboratory Report No 181955

5 Ko, S C, Scour of Flat Sand Bcaches in Fiont of Scawalls," Fritz I ngmecring Iaboratory Report No 2935 Lchigh Universıly, March 1967

6 Cheprl, W S , "Equilibrium of Soll Gr uns at the Thrcshold of Movement by Wind," Procecding" of Soil Scrence Socicty of Amcilcd, Vol 23, pp 422428

7 Raudkivi, A J , Loose Boundary IIydraulacs Pergamon Prcss I ID I ondon 1967

8 Colcman, N I , A Theorcucal and Expcrimental Study of Drag and I ift Forces $\Lambda$ cting on a Spherc Resting on a Hypothetical Strcam Bcd," Proccedings Iwclfth Congress, Intern ition 1 Associdion for Hydraulic Rescarch, Vol 3, Section C18, 19, 1967

9 Vanoni, V ct il , "Scdiment Transportation Mcch inics Initiation of Motion, Progrcss Report of the Task Committee on Preparation of Sedimentation Manual, Committee on Sedimenta 11on,' Journal of the Hydraulics Division ASCI, Vol 92, No HIY2, Proc P iper 4738, M irch 1966 
10 Murphy, H D , "Scour of Flat Sand Beaches Due to Wave Action," Fritz Engineernng Labora tory Report No 293 3, Lehigh Univeisity, June 1964

11 Van Weele, B , "Beach Scour Due to Wave Action on Seawalls," Fritz Engincermg Laboratory Report No 2933 Lehigh University, Apıl 1965

12 Herbich, J B , Murphy, H D , Van Weele, B , "Scour of Flat Sand Beaches Due to Wave Action in Front of Seawalls Proceedings Codstal Engineering Santa Barbara Specialty Conference, Chapter 28, Oct 1965, pp 703726

13 Roper, A T , Schneider, U R, Shen, H W , "Analytical Approach to Local Scour,” Proceed ings Twelfth Congress, International Association for Hydraulic Research, Vol 3, Section C18, 19,1967

14 Carstens, M R , "Similarity Laws for Localized Scour," Journal of the Hydraulics Division ASCE, Vol 92, No HY3, Proc Paper 4818, May 1966

15 Dean, R G, "Relative Validitıes of Water Wave Theorıes," Proceedings, Conference on Civil Engincering in the Oceans, ASCE, San Francisco, 1967

16 Le Me'haute', B , Divoky, D, Lin A, 'Shallow Water Waves A Comparison of Theories and Experiments," Proceedings Eleventh Conference on Coastal Engineerıng, London, 1968

17 Wells, D R, "Scouı Around a Circular Psle Due to Oscillatory Wave Motion," a M S thesis, Texas A\&M University, January 1970, unpublished

18 Posey, C J , Sybert, J H , “Erosion Protection of Production Structures,' Proccedengs Ninth Congress, International Association for Hydraulic Research 1961, pp 11571162

19 Kreig, J L, "Criteria foi Planning an Offshore Pipeline," Journal of the Pipeline Division ASCE, Vol 91, No PL 1, July 1965, pp 1537 


\title{
CHAPTER 80
}

\section{COMPARISON OF MODEL AND BEACH SCOUR PATTERNS}

\author{
by \\ John B Herb1ch, Ph D, P E \\ Professor and Head \\ Coastal and Ocean Engineering Division \\ Texas A\&M University \\ College Station, Texas 77843
}

ABSTRACT

Artificial or natural barmers may be divided into two classes, those from which waves are reflected and those on which waves break In general, any intermediate type that gives a combination of reflection and breaking may set up severe erosive action of the beach in front of barriers When the reflected waves are superimposed on the incident waves a stat ionary spatial envelope of the combined incident and reflected waves is produced Previous laboratory studies Indlcated that the crests of the $s$ and bed appear fairly closely under the nodes of the envelope and troughs of the scoured sand bed under the loops of the envelope The predominant scouring pattern had a spacing between crests equal to one-half the wave length

Other studies by Keulegan and Shepard established characteristıc parameters for bar and trough depth for laboratory conditions and for several field locations Their studies were compared with beach profiles taken along the Texas Gulf Coast

Relationships between (a) scour depth and sand crest wave length, (b) between trough depth and sand bar depth and between wave characteristics and beach scour were established for selected locations along the Texas Coast 


\author{
INTRODUCT ION
}

Natural or artificıal barrıers may be divıded in two classes, those from which waves are reflected and those on which waves break In general, any intermediate type that gives a combination of reflection and breaking may cause excessive erosive action seaward from the barrier

One laboratory study was confined to non-breaking waves at the seawall and the main objective was to investigate the nature of scour of a flat, horizontal sand beach in front of a seawall due to wave action Some of the results were presented at the Eleventh Conference on Coastal Englneering (1)* Since then field data were acquired and analyzed for several locations along the Gulf of Mexico Texas coast Another study by Sato et al, also in laboratory, principally dealt with waves breaking at the seawali The principal objective of that investigation was to determine the basıc characteristics and to determine possible measures to prevent erosion around coastal structures (2) The study was followed up by field investigations which indicated that beach scour depends not only on wave characteristıcs just in front of structures and wave reflection from the structures but also on the currents set up by waves around the structures

Studies by Otto (3), Hartnack (4), Keulegan (5) and Shepard (6) dealt with laboratory or field studies on characteristics of offshore sand bars formed by waves

In recent years a beach profile and jetty condition survey along the Texas Gulf Coast was undertaken (7) and prelimunary analysis indicates that scour patterns and other characteristics resemble those obtained in laboratories and at other field locations

Unfortunately data for waves which produced these patterns are very scarce, If at all avallable, so that any comparisons between laboratory and field conditions can only be very approximate

\title{
LITERATURE REVIEW
}

Underwater sand bars are found in all parts of the world along the coastlines of lakes, seas and oceans There may be only one distinct bar consisting of a crest, or ridge on the seaward side and a trough on the landward side, or there may be a series of bars with related crests and troughs

The bar formations have been studied by geologists and geographers for over a century and considerable field data of the form, number of bars and dimensions are avaliable However, only a few laboratory

* Numbers in parenthesis refer to references at the end of paper 
studies were made and only limited explanations of the hydromechanics process have been put forward

Field observations of form and dimensions of natural, underwater beach profiles were made along the Baltic Coast, Lake Michıgan, Calıfornıa and the Texas Coast

Otto and Hartnack $(3,4)$ reported on measurements along the Pomeranian Baltıc Coast, while Evans (8) reported field measurements of the bars along Lake Mchigan There were essentially three distinct bars measured on the Eastern shore of Lake Michigan

Keulegan (5) indicated that the form of experimental bars varies considerably from that of the natural bars In general the natural bars are flatter and longer than the bars formed in a laboratory wave channel This may be due in large part to the fact that one cannot scale down the size of sand used, as most of the laboratory investigations employed natural sands of approximately the same size as the prototype sands It should also be noted that the laboratory beach profiles are usually subject to monochromatic waves while the prototype waves are usually irregular and contain a spectrum of wave helghts and wave lengths However, Keulegan also noted that the ratio of depth of crest $\left(h_{c}\right)$ to the depth of the bar $\left(h_{T}\right)$ was similar in the model and prototype

\section{DISTANCE FROM SHORELINE}

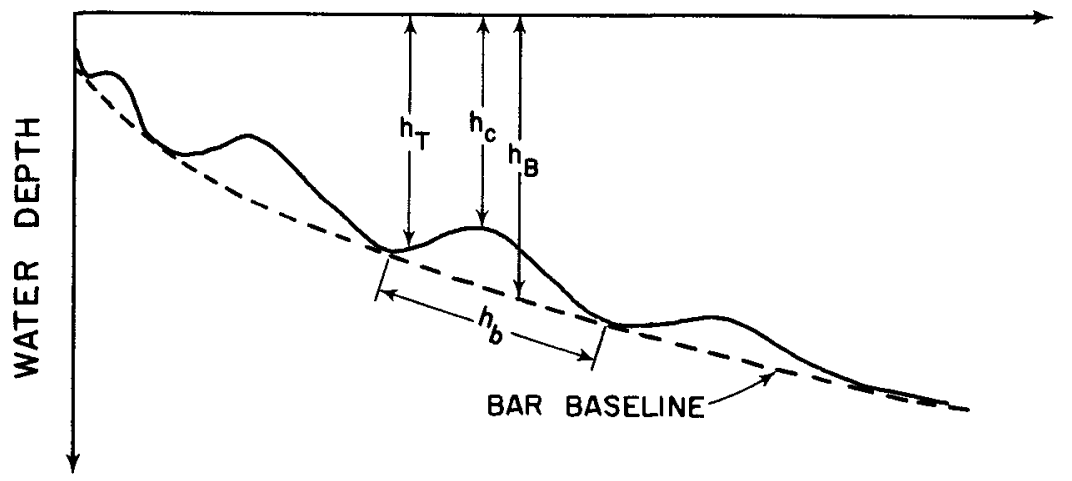

\section{FIG I DEFINITION SKETCH}

Shepard (6) examined thousands of beach profiles taken along the ocean plers in California and indicated that the troughs which lie between the shoreline and the submerged beach bars are formed by the plunging breakers and by the longshore currents which feed the rip currents He also found that there is a relationship between the depth of the bars and troughs and the wave height 


\section{SAND SCOUR ON SLOPING SURFACES}

\section{Preveous Investegations}

Both Keulegan (5) and Shepard (6) conducted Investigations conceming the characteristics of beach scour patterns in response to breaking waves Keulegan conducted a series of laboratory experiments and found that the scour trough forms at the breaker plunge point, with a corresponding accumulation of sand, or the sand bar, forming just seaward of this location It was found that after initiation of uniform wave patterns over the sloping bottom, a trough-bar complex developed rapidly, migrating onshore until an equilibrium position was reached Thereafter only small changes in the scour pattern occurred Similar results were reported by Herbich and Ko (1), Indicating that the scour depth limit is approached asymptotically

Shepard (6) summarized the results of three hundred and fifty three beach profiles taken at Scripps Pier at La Jolla, Califorma

Both Keulegan and Shepard stress the Importance of wave characteristics as influencing the scour pattern Keulegan's results show that the ratio of $\mathrm{H} / \mathrm{h}_{\mathrm{B}}$

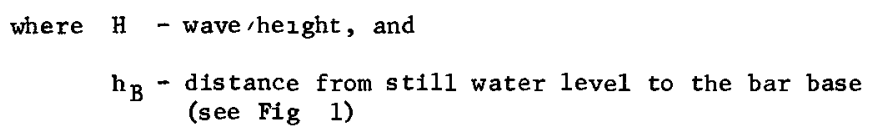

increases with wave steepness $(\mathrm{H} / \mathrm{L})$ up to a steepness of 004 For greater values of steepness the ratio of $\mathrm{H} / \mathrm{h}_{\mathrm{B}}$ is independent of wave steepness and remains constant at about 083 This relationship was found to be independent of beach slopes for slopes between 115 to 170

Keulegan and Shepard also examned the ratio of $\mathrm{h}_{\mathrm{T}} / \mathrm{h}_{\mathrm{c}}$ where

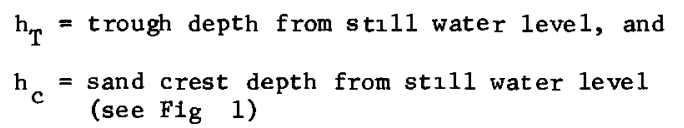

Keulegan noted that the $\mathrm{h}_{\mathrm{T}} / \mathrm{h}_{\mathrm{c}}$ ratıo was practıcally independent of wave steepness and beach slope and computed an average value of 169 for his laboratory experiments

Shepard also determined a relatively uniform relationship of $\mathrm{h}_{\mathrm{T}} / \mathrm{h}_{\mathrm{C}}$ with an average value of 116 at Scripps pier (using mean sea leve 1 as datum)

\section{Current Investigations}

A survey report prepared by U S Army Corps of Engl neers (7) presents beach profiles taken at selected locations along the Texas Gulf Coast during the past several years Analysis of these profiles was made to determine the characteristics of natural scour pattern along the coast, and to compare with previous laboratory and field studies The data were analyzed for the following locations 


\author{
(1) East Beach, Galveston, Texas (Fig 2) \\ (2) Groln Area, Galveston, Texas \\ (3) West Beach, Galveston, Texas \\ (4) Panther Point, Matagorda - Port Arans as, Texas \\ (5) Yarborough Pass, Padre Island, Texas
}

Since space limutation prevents reproduction of all tabulated data, only average values and selected sample data are glven

Table I gives the average values of distances between $s$ and crests (sand wave lengths) and average depth of scour for East Beach at Galveston Tables II through $V$ gives similar information for Groin Area-Galveston, West Beach-Galveston, Panther Polnt-Matagorda - Port Arans as and Yarborough Pass, Padre Island

Fig 3 presents sample beach profile for East Beach at Galveston The crests of bars were jouned to indlcate the crest pattern along the beach section Fig 4 is for Panther Point and Fig 5 is for Yarborough Pass location

\title{
Relationshep Between Scour Depth and Sand Crest wave Length
}

Scour depth readings as well as scour wave length measurements were taken for all locations and a sample location is given in Fig 6 A1though the scatter is considerable (as may be expected) the relationship may be approximated by a straight line having a slope of 0004

\section{Pelatconshep Between Trough Depth and Sand Bar Depth}

Values of trough depth to sand bar depth $\left(h_{\mathrm{T}} / h_{\mathrm{c}}\right)$ were determined at each location along the Texas Coast for various times of the year

The ratio of trough depth to sand bar depth was plotted for three locations along the Texas Coast as a function of time (Figure 7) It will be noted that there is some annual variation in this ratio which, of course, may be caused by major storms, or hurricanes, but the variation is within falrly narrow limits of $h_{\mathrm{T}} / \mathrm{h}_{\mathrm{c}}$ For example for Matagorda $B$ ay the variation is between 115 and 142 , with an average value of about 125

Figure 8 shows the relationship for other locations in addition to locations shown in Figure 7 The data indicate that this ratio tends toward an equilubrium value characteristıc of each location, but at times a significant deviation from this value may occur

Depth of trough is plotted in Figure 9, against the depth of bar for several field locations along the Texas Coast Field data from Washington and Oregon coast and data from Calıfornua are also plotted as well as Keulegan's laboratory data Remarkably consistent results are obtalned for all locations with field data ranging between $h_{\mathrm{T}} / \mathrm{h}_{\mathrm{c}}$ values of 116 and 160 The laboratory data gives a value of $169^{c}$ 


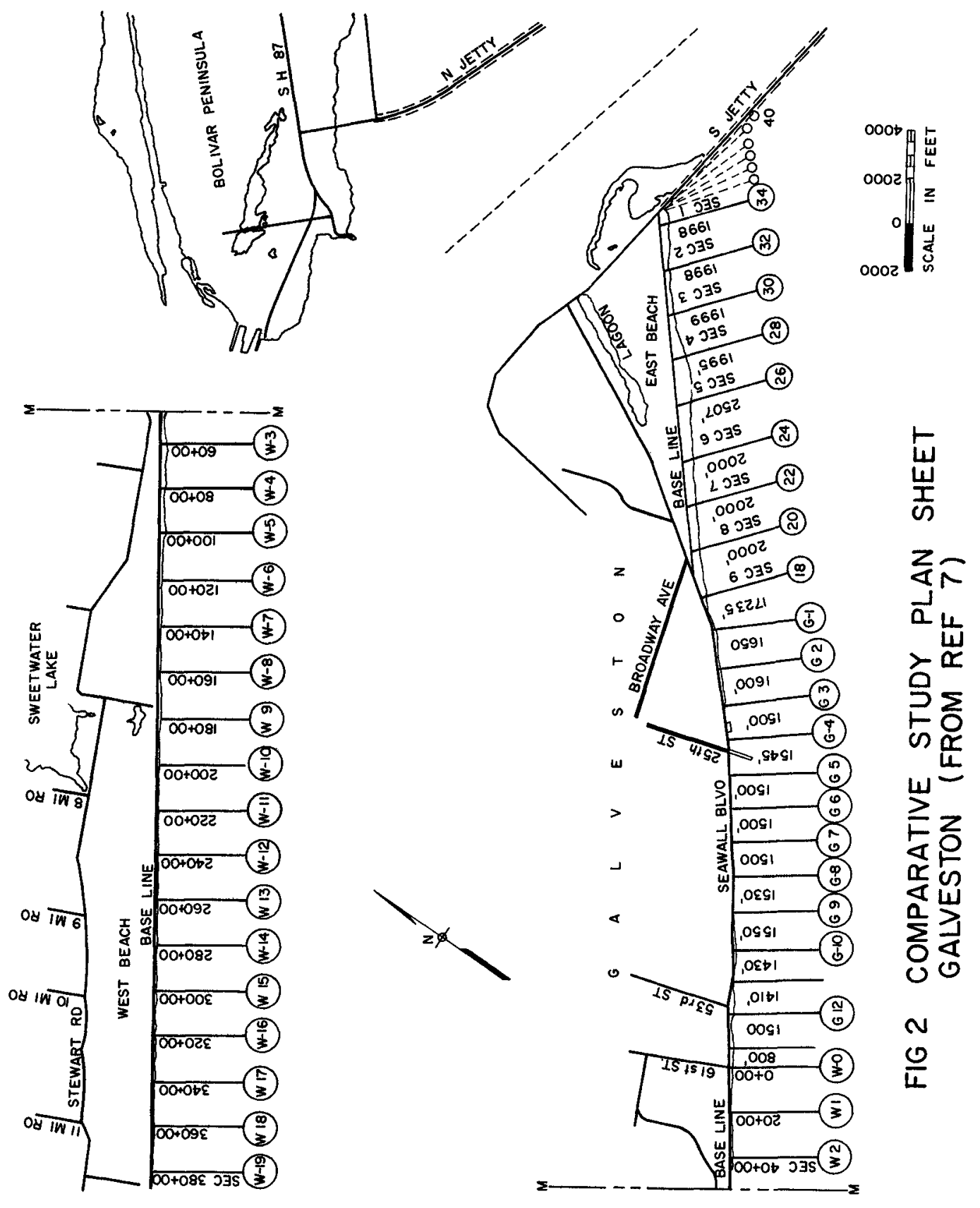


SCOUR PATTERNS

EAST BEACH

GALVESTON, TEXAS

\begin{tabular}{rrrr} 
Section & Period & $\begin{array}{c}\text { Average Distance Between } \\
\text { Sand Crests (ft) }\end{array}$ & $\begin{array}{c}\text { Average Depth of } \\
\text { Scour (ft) }\end{array}$ \\
\hline 1 & $1934-68$ & 301 & 76 \\
2 & $1934-68$ & 257 & 68 \\
3 & $1934-68$ & 354 & 99 \\
4 & $1934-68$ & 327 & 93 \\
5 & $1934-68$ & 298 & 97 \\
6 & $1934-68$ & 354 & 87 \\
7 & $1934-68$ & 277 & 23 \\
8 & $1934-68$ & 310 & 124 \\
9 & $1934-68$ & 272 & 73 \\
10 & $1949-68$ & 240 & 107 \\
11 & $1949-69$ & 314 & 70 \\
12 & $1949-69$ & 345 & 77 \\
13 & $1949-69$ & 252 & 89 \\
\hline
\end{tabular}

TABLE I 
GROIN AREA

GALVESTON, TEXAS

\begin{tabular}{|c|c|c|c|}
\hline Section & Perıod & $\begin{array}{l}\text { Average Distance Between } \\
\text { Sand Crests (ft) }\end{array}$ & $\begin{array}{l}\text { Average Depth of } \\
\text { Scour (ft) }\end{array}$ \\
\hline 14 & $1949-69$ & 280 & 70 \\
\hline 15 & $1949-69$ & 202 & 48 \\
\hline 16 & $1949-69$ & 180 & 45 \\
\hline 17 & $1949-69$ & 286 & 88 \\
\hline 18 & $1949-69$ & 232 & 87 \\
\hline 19 & $1949-69$ & 247 & 67 \\
\hline 20 & $1949-69$ & 227 & 48 \\
\hline 21 & $1949-68$ & 305 & 138 \\
\hline 22 & $1949-69$ & 192 & 45 \\
\hline 23 & $1949-69$ & 206 & 44 \\
\hline 24 & $1949-69$ & 230 & 118 \\
\hline 25 & $1949-69$ & 207 & 26 \\
\hline 26 & $1949-69$ & 212 & 35 \\
\hline 27 & $1949-69$ & 187 & 49 \\
\hline 28 & $1949-69$ & 178 & 36 \\
\hline 29 & $1949-69$ & 165 & 48 \\
\hline 30 & $1949-69$ & 202 & 91 \\
\hline 31 & $1949-69$ & 242 & 35 \\
\hline 32 & $1949-69$ & 189 & 43 \\
\hline 33 & $1949-69$ & 186 & 37 \\
\hline 34 & $1949-69$ & 178 & 46 \\
\hline 35 & $1949-69$ & 178 & 68 \\
\hline 36 & $1949-69$ & 227 & 48 \\
\hline 37 & $1949-69$ & 181 & 58 \\
\hline 38 & $1949-69$ & 276 & 61 \\
\hline 39 & $1949-69$ & 221 & 47 \\
\hline 40 & $1949-69$ & 194 & 64 \\
\hline 41 & $1949-69$ & 220 & 77 \\
\hline 42 & $1949-69$ & 262 & 61 \\
\hline 43 & $1949-69$ & 279 & 62 \\
\hline 44 & $1949-69$ & 209 & 50 \\
\hline 45 & $1949-69$ & 195 & 37 \\
\hline 46 & $1949-69$ & 341 & 60 \\
\hline 47 & $1949-69$ & 230 & 61 \\
\hline & & verage & 59 \\
\hline
\end{tabular}

TABLE II 
WEST BEACH

GALVESTON, TEXAS

\begin{tabular}{|c|c|c|c|}
\hline Section & Period & $\begin{array}{c}\text { Average Distance Between } \\
\text { Sand Crests (ft) }\end{array}$ & $\begin{array}{l}\text { Average Depth of } \\
\text { Scour (ft) }\end{array}$ \\
\hline $0+00$ & $1949-69$ & 231 & 70 \\
\hline $20+00$ & $1949-68$ & 200 & 80 \\
\hline $40+00$ & $1949-68$ & 210 & 10 \\
\hline $60+00$ & $1949-68$ & 215 & 95 \\
\hline $80+00$ & $1949-68$ & 181 & 103 \\
\hline $100+00$ & $1949-68$ & 304 & 75 \\
\hline $120+00$ & $1949-68$ & 273 & 117 \\
\hline $140+00$ & $1949-68$ & 178 & 13 \\
\hline $160+00$ & $1949-68$ & 198 & 10 \\
\hline $180+00$ & $1949-68$ & 363 & 78 \\
\hline $200+00$ & $1949-68$ & 231 & 73 \\
\hline $220+00$ & $1949-68$ & 244 & 83 \\
\hline $240+00$ & $1961-68$ & 187 & 83 \\
\hline $260+00$ & $1961-68$ & 218 & 107 \\
\hline $280+00$ & $1961-68$ & 373 & 77 \\
\hline $300+00$ & $1961-68$ & 311 & 87 \\
\hline $320+00$ & $1961-68$ & 423 & 103 \\
\hline $340+00$ & $1961-68$ & 184 & 70 \\
\hline $360+00$ & $1961-68$ & 221 & 73 \\
\hline $380+00$ & $1961-68$ & 184 & 67 \\
\hline $400+00$ & $1961-68$ & 226 & 90 \\
\hline $420+00$ & $1961-68$ & 202 & 60 \\
\hline $440+00$ & $1961-68$ & 310 & 73 \\
\hline $460+00$ & $1961-68$ & 333 & 67 \\
\hline $470+00$ & $1961-68$ & 279 & 60 \\
\hline
\end{tabular}




\section{PANTHER POINT}

MATAGORDA - PORT ARANSAS, TEXAS

\begin{tabular}{|c|c|c|c|c|c|}
\hline Section & Date & $\begin{array}{c}\text { Distance Between Sand Crests* } \\
\text { (In Feet) }\end{array}$ & Avg & $\begin{array}{l}\text { Depth of } \\
\text { Scour, Ft }\end{array}$ & Avg \\
\hline $\begin{array}{l}0+00 \\
0+00 \\
0+00 \\
0+00 \\
0+00 \\
0+00 \\
0+00 \\
0+00\end{array}$ & $\begin{array}{l}5 / 23 / 1967 \\
11 / 5 / 1967 \\
7 / 26 / 1968 \\
10 / 10 / 1968 \\
1 / 9 / 1969 \\
3 / 10 / 1969 \\
7 / 15 / 1969 \\
1 / 21 / 1970\end{array}$ & $\begin{array}{c}85,40,50,100,70,140,100,65 \\
45,100,150,150,110,85 \\
104,160 \\
47,57 \\
150 \\
80 \\
165 \\
100\end{array}$ & $\begin{array}{r}81 \\
107 \\
132 \\
52 \\
150 \\
80 \\
165 \\
100\end{array}$ & $\begin{array}{llll}0 & 2, & 0 & 5 \\
0 & 4, & 0 \\
0 & 3 & \\
0 & 25 & \\
0 & 8 & \\
0 & 3\end{array}$ & $\begin{array}{ll}0 & 35 \\
0 & 2 \\
0 & 3 \\
0 & 25 \\
0 & 8 \\
0 & 3\end{array}$ \\
\hline $\begin{array}{l}10+00 \\
10+00 \\
10+00 \\
10+00 \\
10+00\end{array}$ & $\begin{array}{l}5 / 23 / 1967 \\
11 / 5 / 1967 \\
7 / 26 / 1968 \\
3 / 10 / 1969 \\
7 / 15 / 1969\end{array}$ & $\begin{array}{c}45,85,70,115,95,100,70,70 \\
60,90,140,160,90,100 \\
152,95 \\
110,40 \\
95,105,90\end{array}$ & $\begin{array}{r}81 \\
107 \\
123 \\
75 \\
97\end{array}$ & $\begin{array}{cccc} & 04,06 \\
& 0, & \\
0 & 4,0 & 2,0 & 15\end{array}$ & $\begin{array}{ll}0 & 5 \\
0 & \\
0 & 25\end{array}$ \\
\hline $\begin{array}{l}20+00 \\
20+00 \\
20+00 \\
20+00 \\
20+00 \\
20+00 \\
20+00\end{array}$ & $\begin{array}{l}5 / 23 / 1967 \\
11 / 5 / 1967 \\
7 / 25 / 1968 \\
7 / 9 / 1969 \\
3 / 10 / 1969 \\
7 / 15 / 1969 \\
1 / 21 / 1970\end{array}$ & $\begin{array}{c}165,70,90,100,75,95,65,125 \\
70,105,180,125,45,140 \\
165 \\
283 \\
95 \\
190,97 \\
300\end{array}$ & $\begin{array}{r}98 \\
111 \\
165 \\
283 \\
95 \\
143 \\
300\end{array}$ & $\begin{array}{lllll}0 & 9 & & \\
0 & 8 & & \\
0 & 2 & & \\
0 & 3 & 0 & 8 \\
0 & 5 & & \end{array}$ & $\begin{array}{ll}0 & 9 \\
0 & 8 \\
0 & 2 \\
0 & 55 \\
0 & 5\end{array}$ \\
\hline $\begin{array}{l}30+00 \\
30+00 \\
30+00 \\
30+00 \\
30+00 \\
30+00 \\
0+00\end{array}$ & $\begin{array}{l}5 / 23 / 1967 \\
11 / 5 / 1967 \\
7 / 26 / 1968 \\
1 / 9 / 1969 \\
3 / 10 / 1969 \\
7 / 15 / 1969 \\
1 / 21 / 1970\end{array}$ & $\begin{array}{c}100,70,60,165,75,85,80,125 \\
50,115,195,125,40,180 \\
235 \\
210 \\
95,45,75 \\
115,207 \\
153,45\end{array}$ & $\begin{array}{r}95 \\
118 \\
235 \\
210 \\
72 \\
161 \\
100\end{array}$ & $\begin{array}{llll}0 & 6 \\
0 & 7 & & \\
0 & 4,0 & 2,0 & 1 \\
0 & 4, & 0 & 5 \\
0 & 5, & 0 & 4\end{array}$ & $\begin{array}{ll}0 & 6 \\
0 & 7 \\
0 & 22 \\
0 & 45 \\
0 & 45\end{array}$ \\
\hline $\begin{array}{l}11 \text { Sections } \\
11 \text { Sections } \\
11 \text { Sections } \\
11 \text { Sections } \\
11 \text { Sections } \\
11 \text { Sections } \\
11 \text { Sections } \\
11 \text { Sections }\end{array}$ & $\begin{array}{l}5 / 23 / 1967 \\
11 / 5 / 1967 \\
7 / 26 / 1968 \\
10 / 10 / 1968 \\
1 / 9 / 1969 \\
3 / 10 / 1969 \\
7 / 15 / 1969 \\
1 / 21 / 1970\end{array}$ & $\begin{array}{l}\text { Average } \\
\text { Average }\end{array}$ & $\begin{array}{r}89 \\
111 \\
152 \\
52 \\
214 \\
77 \\
133 \\
150\end{array}$ & & $\begin{array}{ll}0 & 33 \\
0 & 20 \\
0 & 6 \\
& 16 \\
& 44 \\
& 43\end{array}$ \\
\hline $\begin{array}{l}0+00 \\
0+00 \\
0+00 \\
30+00\end{array}$ & & Average & $\begin{array}{r}121 \\
97 \\
171 \\
142\end{array}$ & Average & $\begin{array}{l}46 \\
25 \\
59 \\
48\end{array}$ \\
\hline
\end{tabular}

* Some of the data relate to sand bars formed during the hurricanes which are now above water surface 
YARBOROUGH PASS

PADRE ISLAND, TEXAS

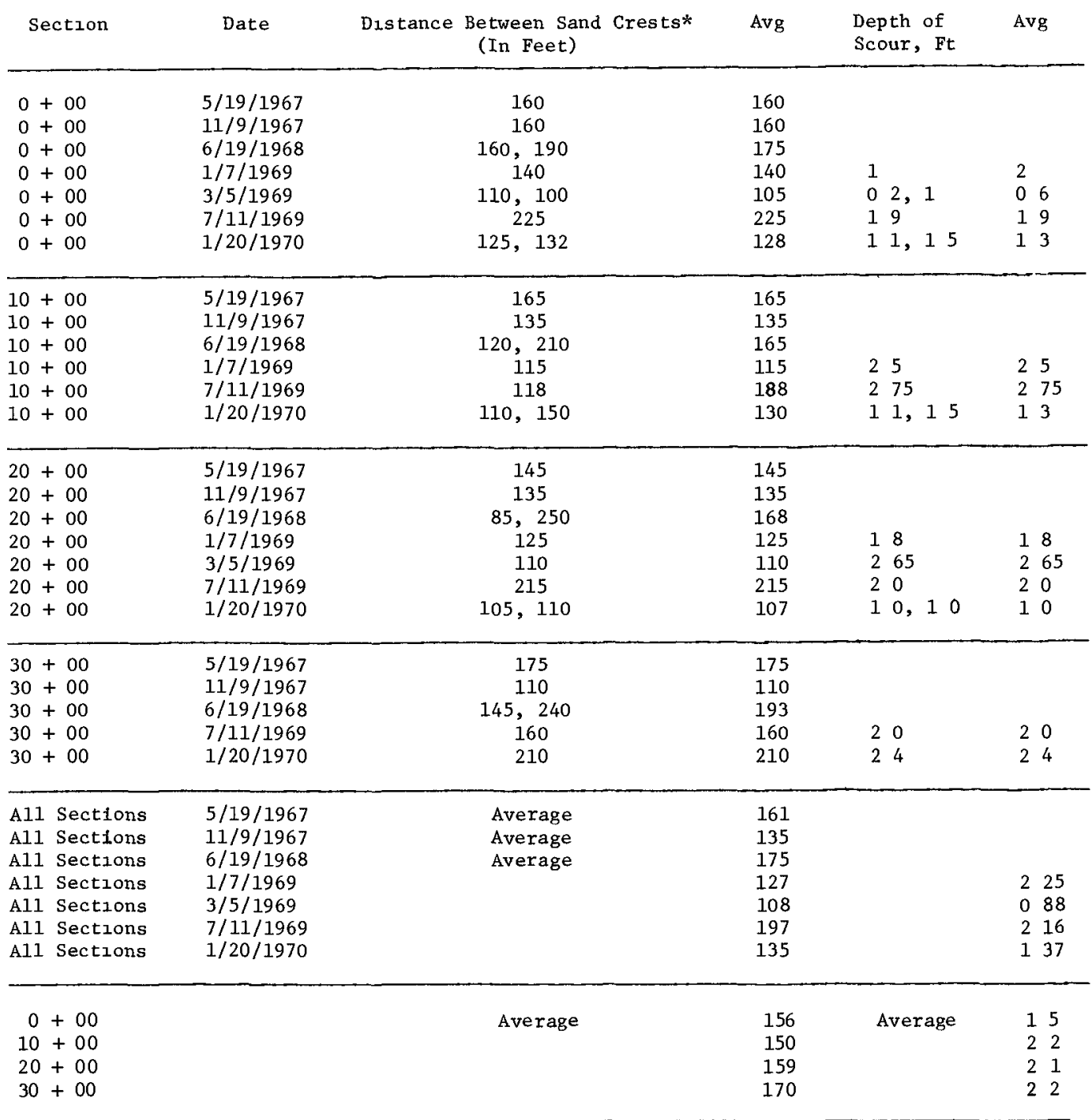

* Some of the data relate to sand bars formed during the hurricanes which are now above water surface 


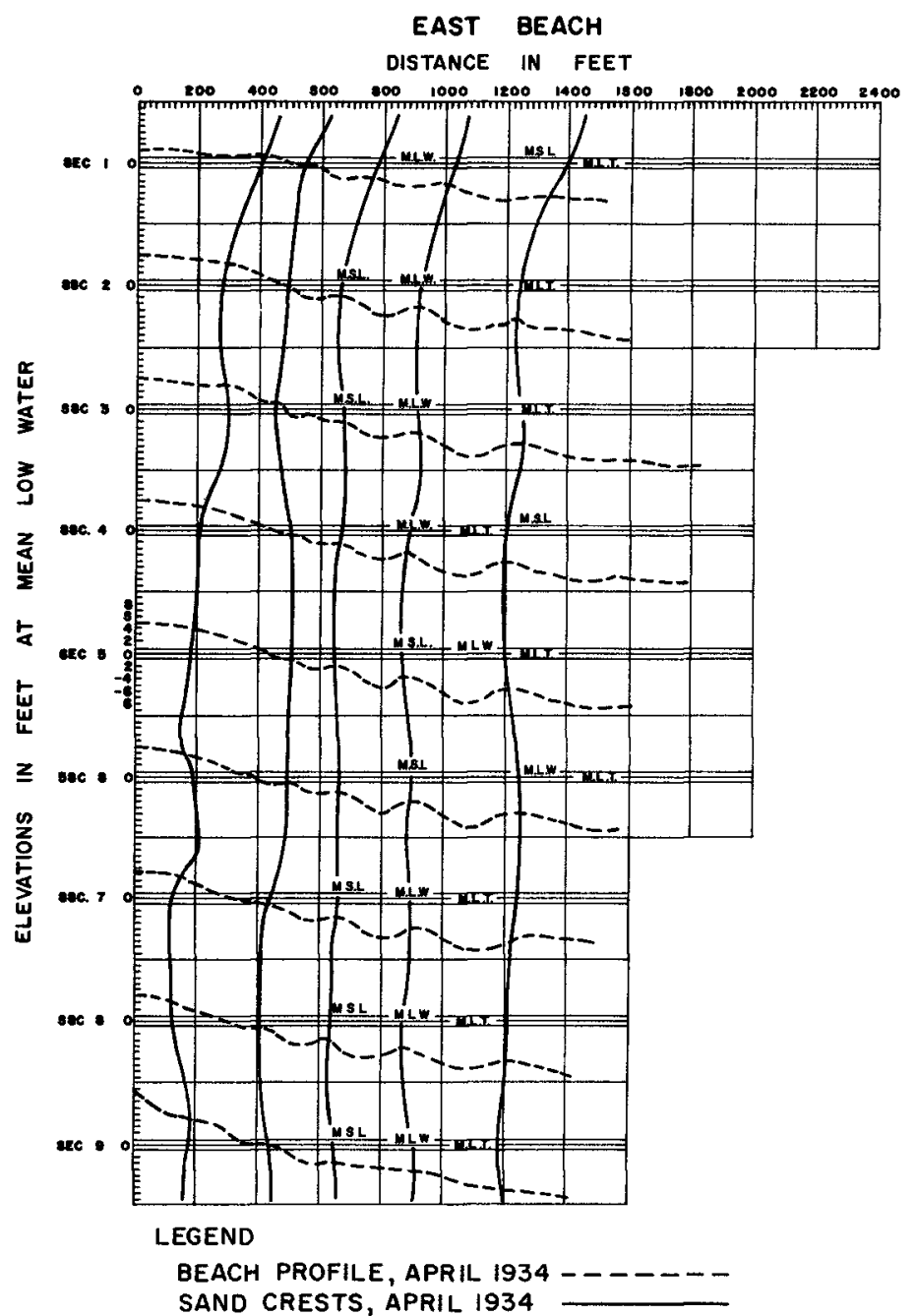

FIG 3 BEACH PROFILE AND SAND CRESTS AT EAST BEACH, GALVESTON (FROM REF 7) 

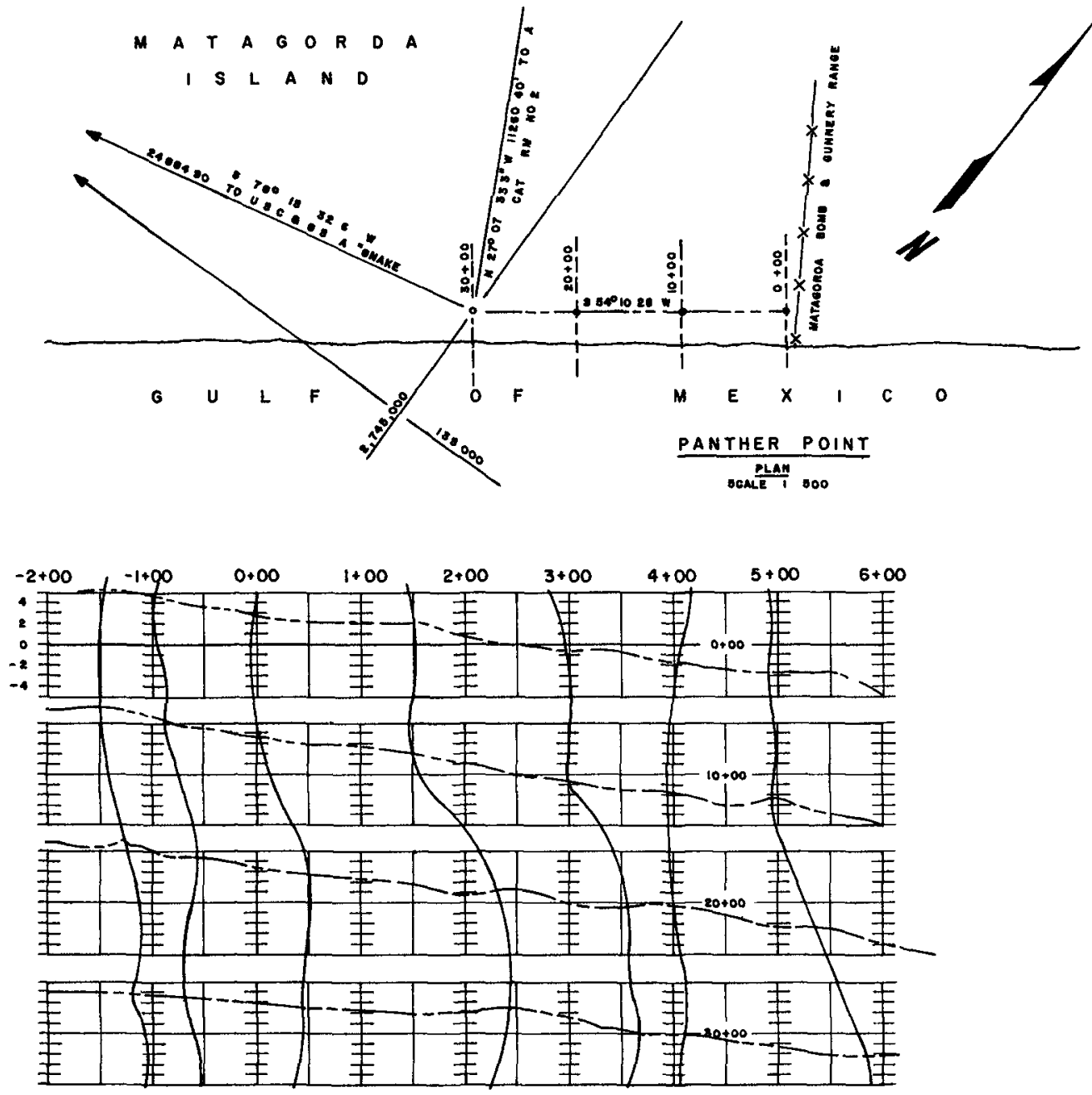

\section{LEGEND}

BEACH PROFILE, 5 NOV $1967---$

SAND CREST, 5 NOV 1967

FIG 4 BEACH PROFILE AND SAND CRESTS AT PANTHER POINT (FROM REF 7) 

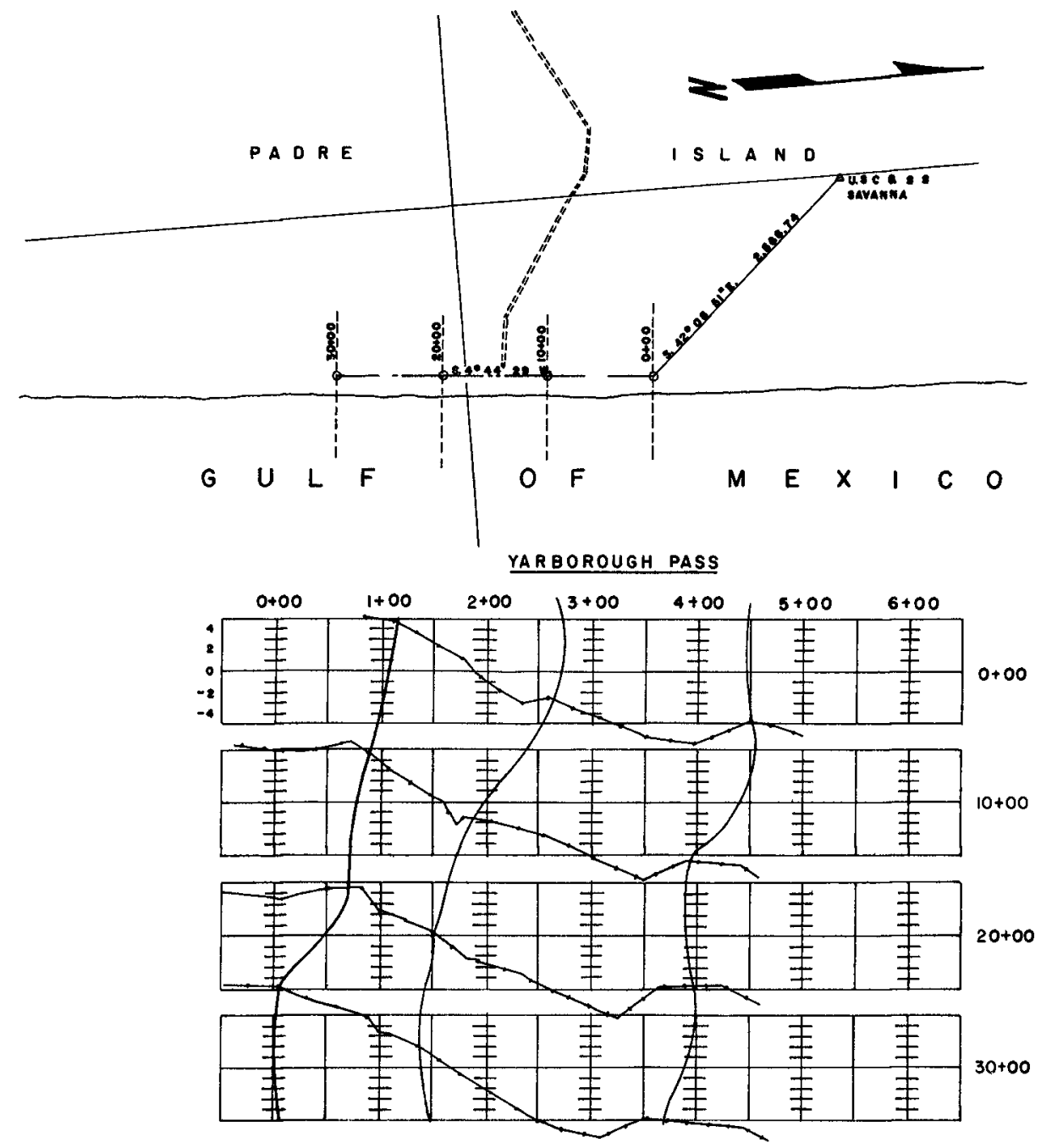

LEGEND

BEACH PROFILE, 19 JUNE 1968

SAND CREST, 19 JUNE 1968

FIG 5 BEACH PROFILE AND SAND CRESTS AT YARBOROUGH PASS (FROM REF 7) 


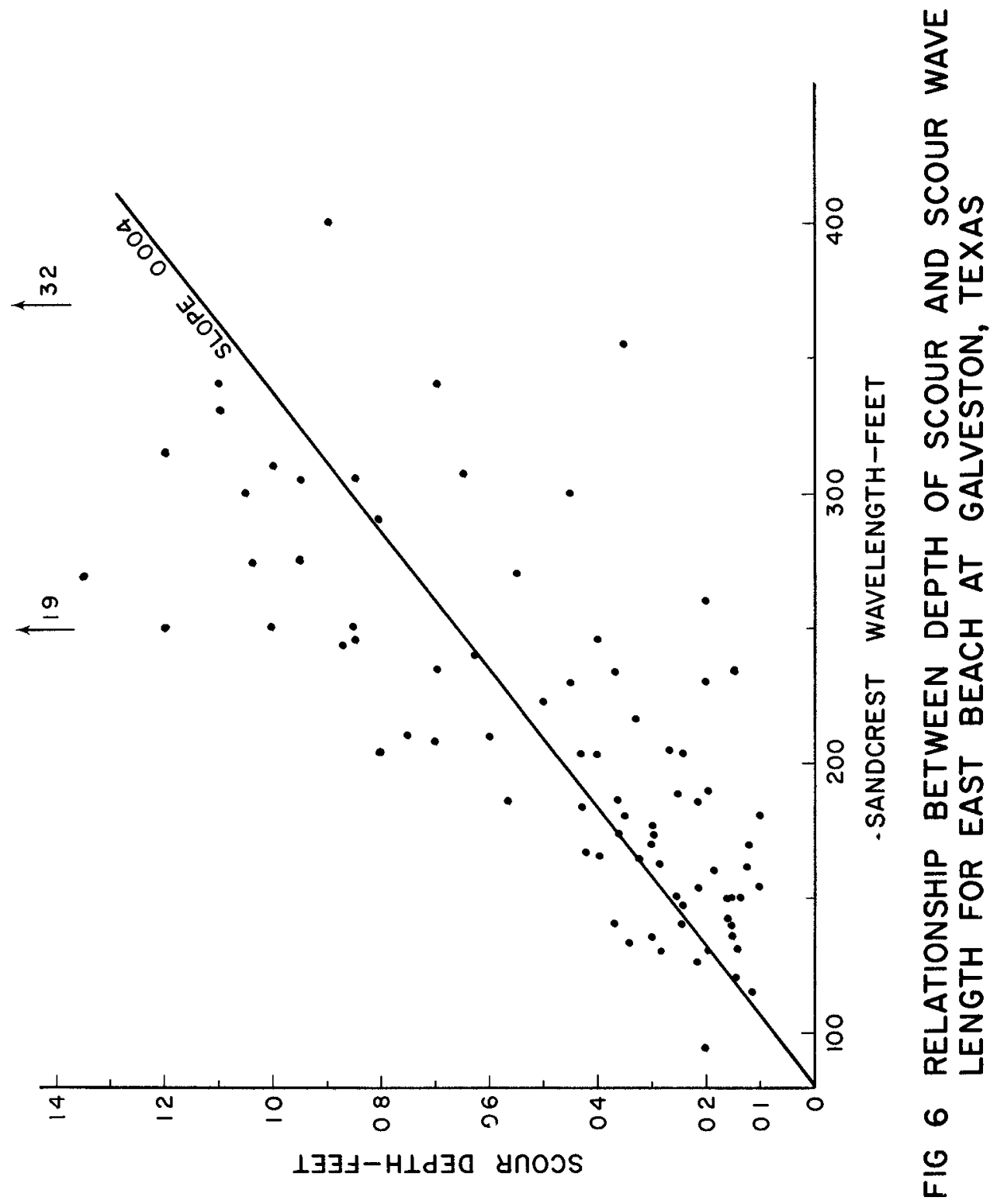




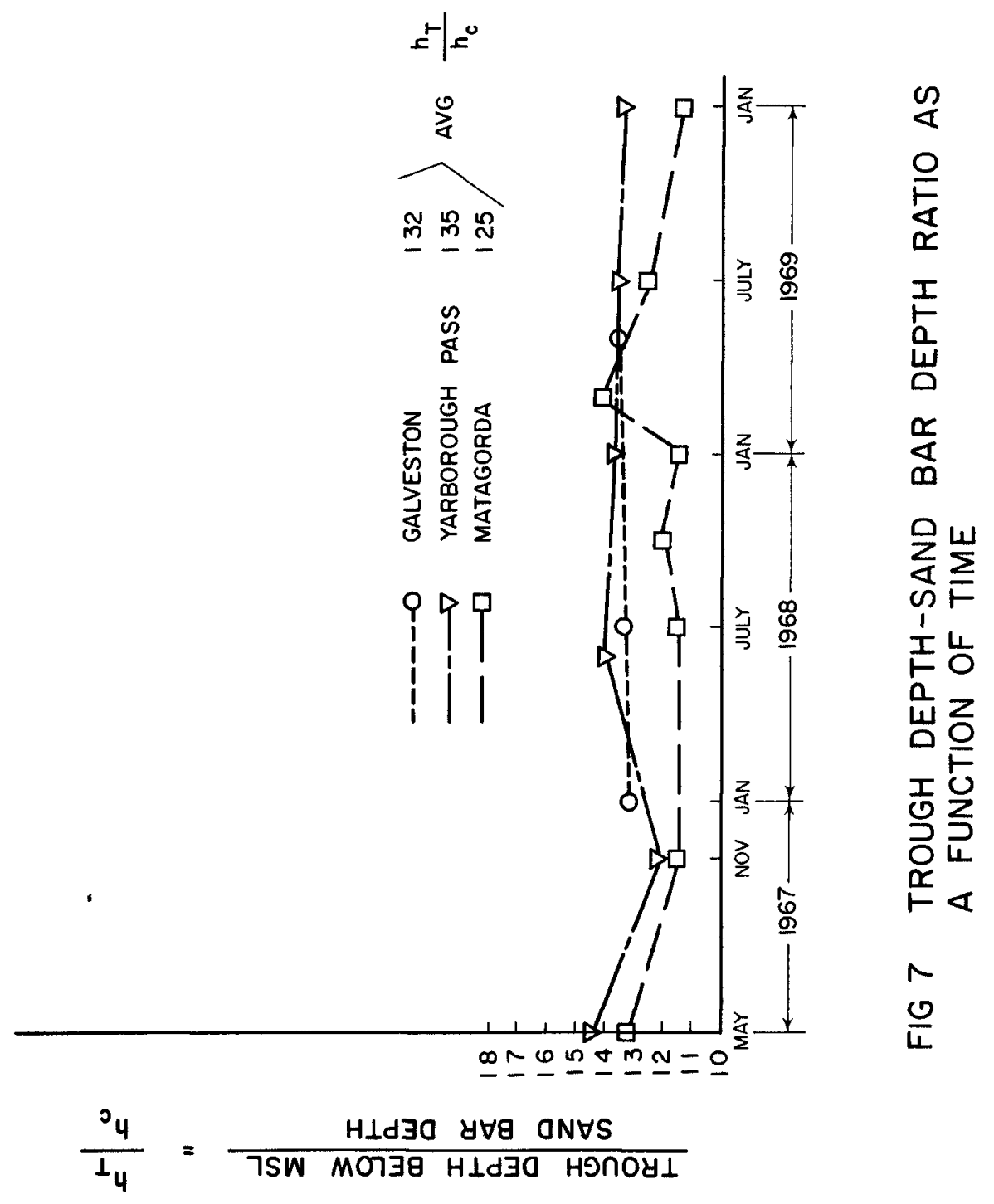




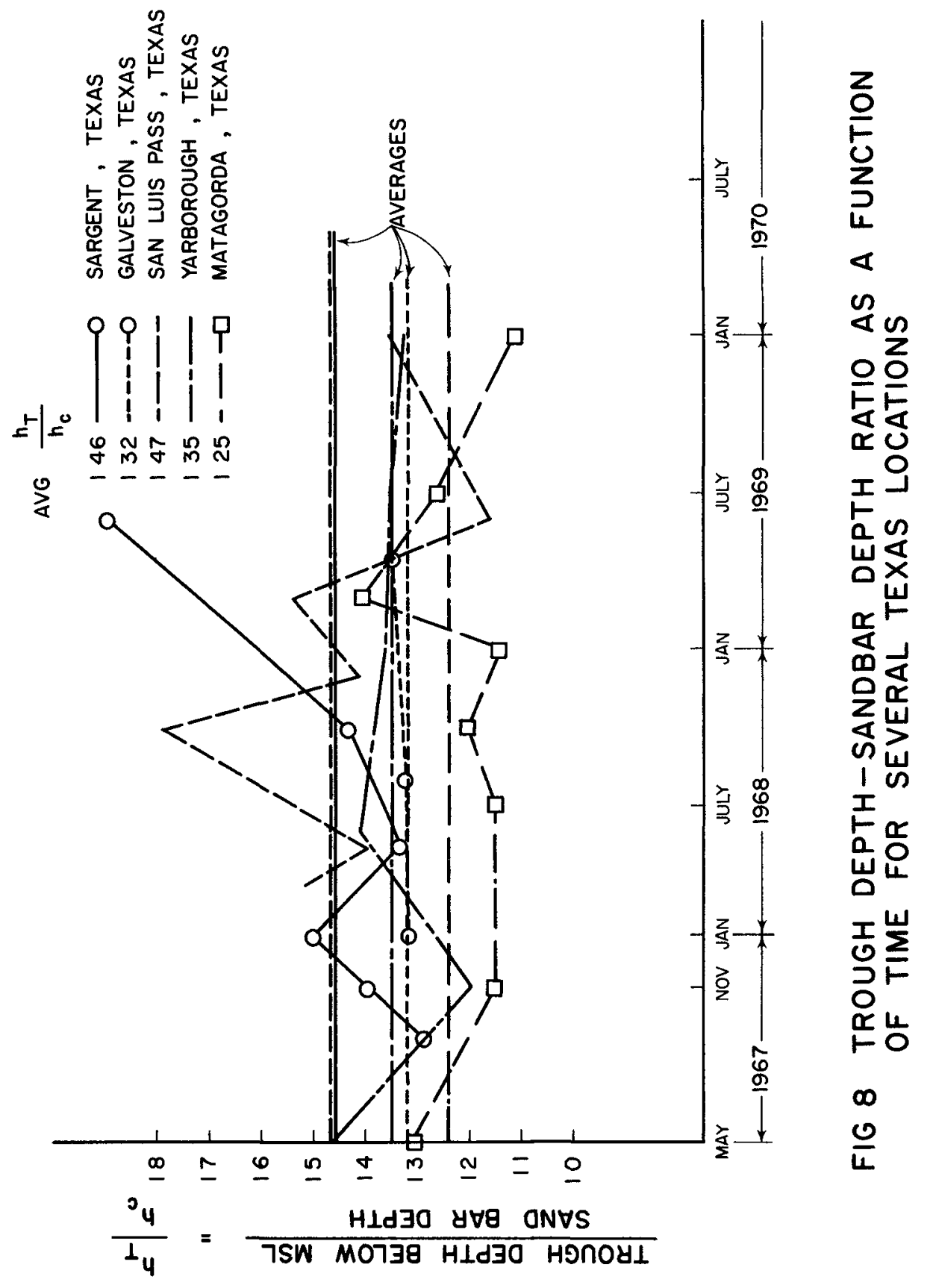




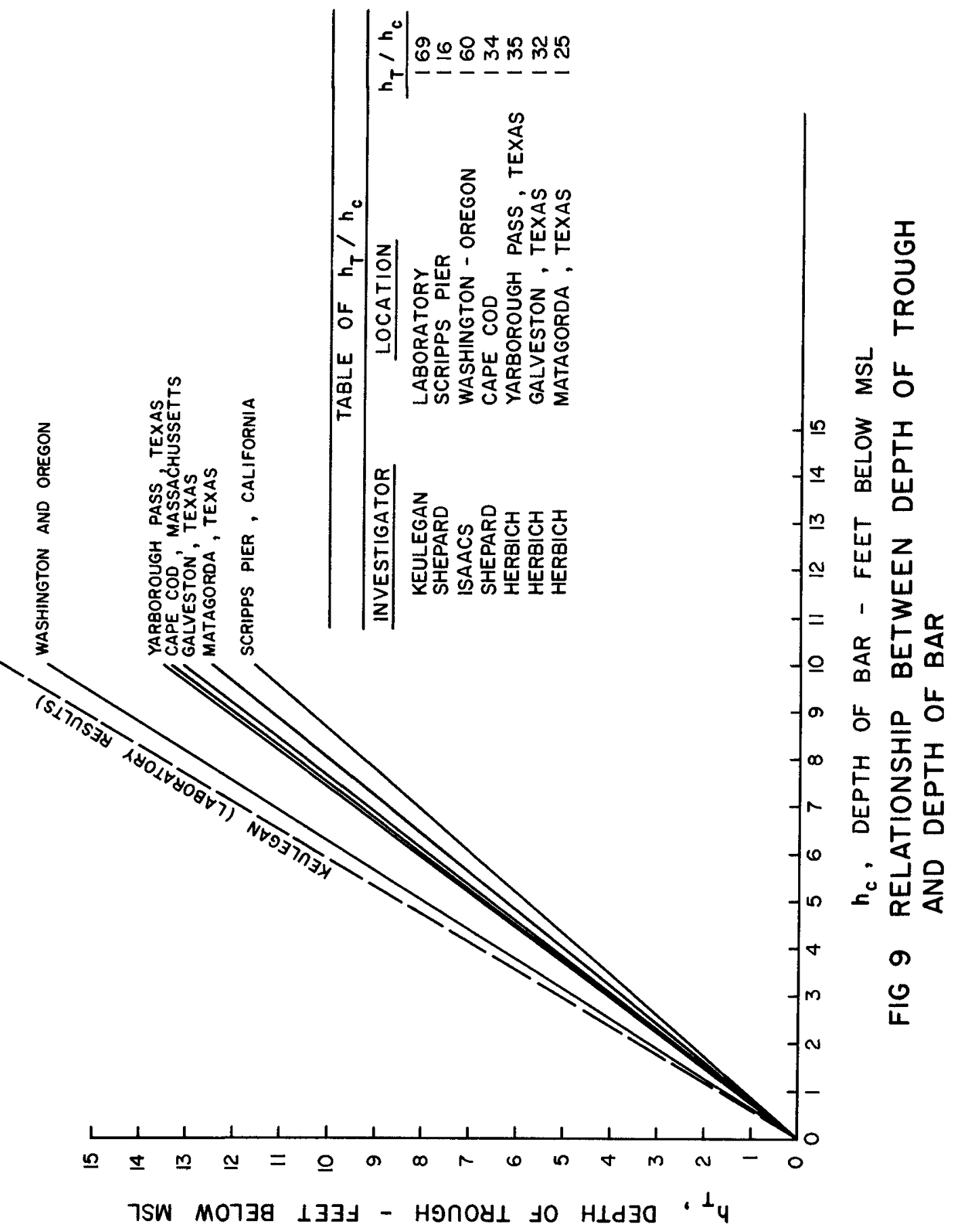




\section{Relatconshep Between Waves and Beach Scour}

Analyses of Texas beach profiles indicate that in most cases more than one bar-trough complex formation was created by wave action Laboratory experiments by Herb1ch and Ko (1) and by Sato et al (2) have revealed that the distance between suceeding bar crests is a function of wave length, and was generally equal to one half the wave 1 ength generated, for several sand sizes employed

In general wave data for locations where beach profiles are measured are very scarce, or difficult to obtain For the purpose of this analysis wave data taken during hurricane Beulah (October 1967) at Galveston were obtalned from the Coastal Engineering Research Center (9) The wave data were analyzed, using spectral methods, and equivalent wave helght and period at maximum spectral density were obtained

Beach profiles taken in January, 1968 were also analyzed The results of the analyses are as follows

\begin{tabular}{lccccc} 
Location & $\begin{array}{c}\text { Average Length } \\
\text { Between Crests } \\
(\mathrm{ft})\end{array}$ & $\begin{array}{l}\text { Average Deep** } \\
\text { Water Wave } \\
\text { Length } \\
(\mathrm{ft})\end{array}$ & $\begin{array}{l}\text { Average* } \\
\text { Wave Len } \\
\text { at } 18^{\prime} \text { Depth }\end{array}$ & $\begin{array}{c}\text { Average } \\
\text { Scour Depth } \\
(\mathrm{ft})\end{array}$ & $\begin{array}{c}\text { Equivalent** } \\
\text { Wave Helght } \\
\text { (ft) }\end{array}$ \\
\hline East Beach & 330 & 261 & 159 & 086 & 536 \\
Groin Area & 312 & 261 & 159 & 076 & 536
\end{tabular}

* Wave gauge was located at $18 \mathrm{ft}$ depth

**Analyzed using power spectrum method

Comparison of the beach profiles at Galveston 1ndicates that the average spacing between crests was greater than the deep water wave length and about twice as long as the average wave length at $18 \mathrm{ft}$ depth, which does not agree with laboratory studies The relationship between average scour depth and the ave rage wave height is approximately equal to 0160 for the East Beach area and equal to 0142 for the Groin area

\section{CONCLUSIONS}

\section{Definıte relationship exıst between}

(1) trough depth and sand crest depth

(2) average length between sand crests and the average wave length

(3) average scour depth and average wave helght

(4) additional studies, oarticularly in the fleld, should be conducted 


\author{
ACKNOWLEDGEMENT
}

Cooperation of Galvest on District of $U$ S Army Engineers in providing beach profile dat a along the Texas Coast $1 \mathrm{~s}$ appreciated Assistance of Miss Rosella Duke, a civil englneering student and Messrs Ch Chestnutt, C McClenan and C Mason, graduate students in coastal and ocean engineering in dat a reduction 18 acknowledged

\title{
REFFRENCES
}

1 Herbich, J B and S C Ko, "Scour of Sand Beaches $1 n$ Front of Seawa11s", Proceedings of the Eleventh Conference on Coastal Engineering, London, England, Chapter 40, pp 622-643, September 1968

2 Sato, S, M Tanaka and I Irie, "Study on Scouring at the Feet of Coastal Structures", Proceedings of the Eleventh Conference on Coastal Engineering, London, England, Chapter 37, pp 579-598, September 1968

3 Otto, T, "Der Darss und Zingst", Jahresber Geogr Ges Grelsswald, Vo1 12, 1911-12

4 Hartnack, W, "Uber Sandriffe", Jahresber Geogr Ges Greisswald, Vo1 $40-42,1924$

5 Keulegan, G H, "An Experımental Study of Submarine Sand Bars", Technical Report No 3, Beach Erosion Board, Corps of Engineers, U S Army, $40 \mathrm{pp}, 1948$

6 Shepard, F P, "Longshore-Bars and Longshore-Troughs", Technical Memorandum No 15, Beach Erosion Board, Corps of Englneers, U S Army, $31 \mathrm{pp}$, January 1950

7 "Texas Coast Inlet Studies", Galveston District, Corps of Engineers, U S Army, 1968, 1969

8 Evans, O F, "The Low and Ball of the Eastern Shore of Lake Michigan", Journal of Geology, Vo1 48, p 476,1940

9 Personal communication from Dr Harrıs, Coastal Engıneerıng Research Center, U S Army Corps of Engineers, August 1970 


\title{
CHAPTER 81
}

\section{VARIATION OF TOPOGRAPHY OF SEA-BED CAUSED BY THE CONSTRUCTION OF BREAKWATERS}

\author{
Shoj1 Sato and Isao Irle \\ Hydraulics Division, Port and Harbour Research Institute \\ Ministry of Transport \\ 1-1, 3 Chome, Nagase, Yokosuka, Japan
}

\section{INTRODUCTION}

In Japan, many breakwaters or jetties have been constructed in the sandy beach from the past decade for new ports to cope with the development of industry It is needless to say that the construction and prolongation of breakwaters or jetties cause the change of bottomtopography in their vicinity, but many points remain indistinct on this change of bottom-topography

In this paper, some general properties on the change of the topography of sea-bed caused by the construction of breakwaters are discussed on the basis of the hydraulic sounding maps of several ports and the results of model tests The terminology used in this paper is given in Figure 1

\section{VARIATION OF SEA-BED TOPOGRAPHY AT KASHIMA PORT}

Port Kashima is located on the coast of Kashimanada facing the Pacific Ocean, as shown in Figure 2 Waves of the coast of Port Kashima approach from the direction almost perpendicular to the shore line, though the southerly waves exceed a little than the northerly waves The maximum significant wave observed in the period from 1961 to 1968 is $5 \mathrm{~m}$ in helght and $103 \mathrm{sec}$ in period The mean diameter of bottom material is about $015 \mathrm{~mm}$ in the offshore and $06-02 \mathrm{~mm} 1 \mathrm{n}$ the inshore The alongshore littoral transport per year is estimated to be the order of 600,000 cubic meters both in the southerly and northerly directions on the basis of the hydraulic sounding data and the calculation of wave energy, though the littoral transport from the south exceeds a little that from the north in the sum of several years

Figure 3 and 4 shows the position of equi-depth lines for each summer from 1963 to 1968

In July 1963, the down-side breakwater and the jetty of the working basin were constructed as far as the alongshore bar The shore line advanced upside of the jetty and severe scouring was seen in the vicinity of the tip of the jetty

In July 1964, the working basin was completed and the up-side breakwater was constructed for nearly $200 \mathrm{~m}$ These parts of the breakwater was constructed with rouble stones The equi-depth lines of 0 to $3 \mathrm{~m}$ advanced near the jetty of the working basin on the up-side, 


\section{Direction of Littoral Transport}

(1) OBLIQUE PART

(2) BENO - POINT

(3) RECTANGULAR PART

(4) PARALLEL BREAKWATER

(5) JETTY
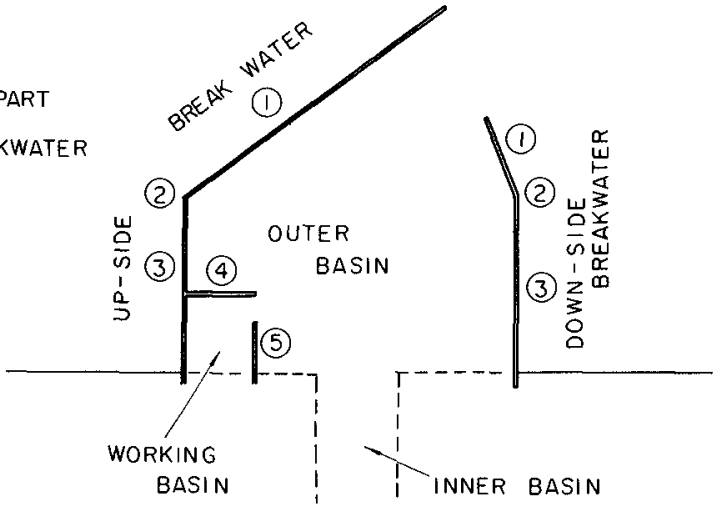

Fipure 1 Terminology of each part.

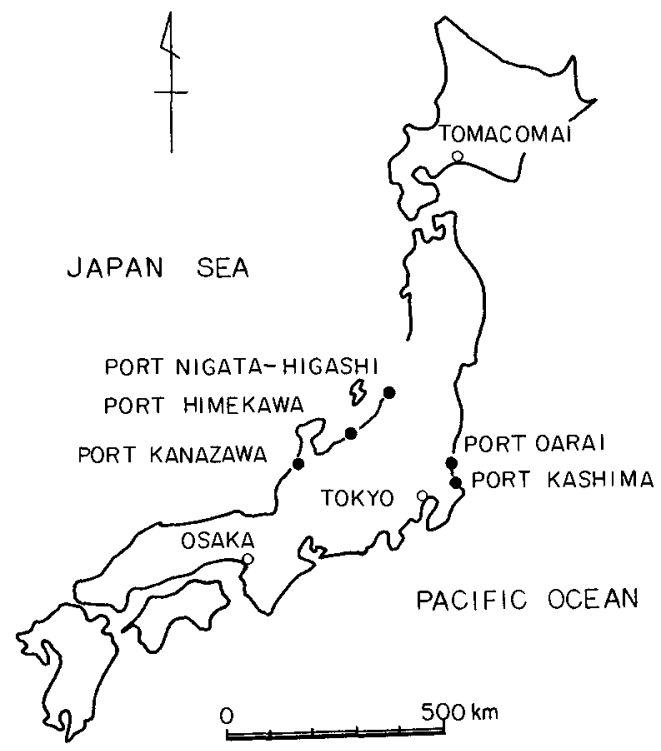

Figure 2 Position of ports 


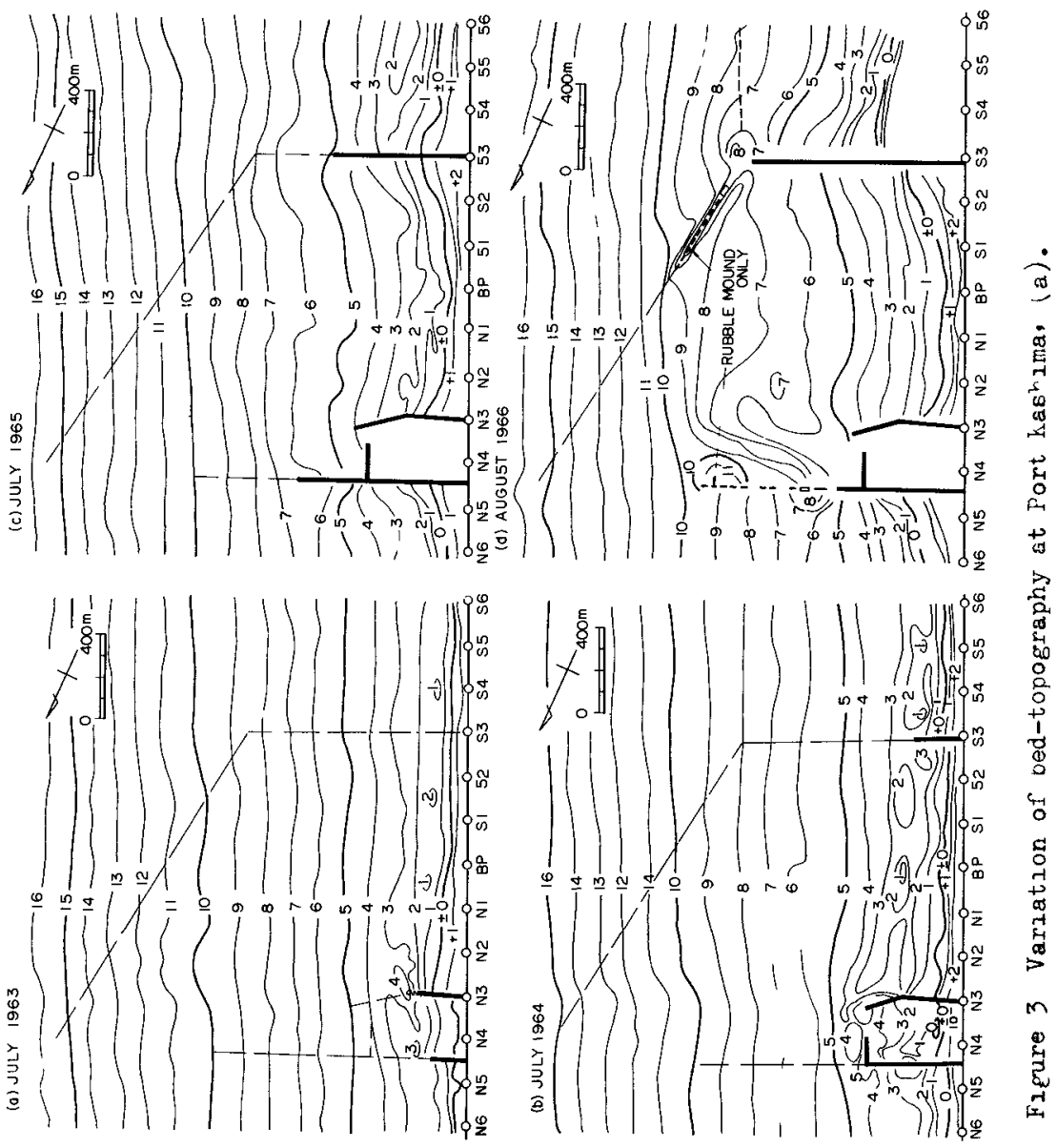



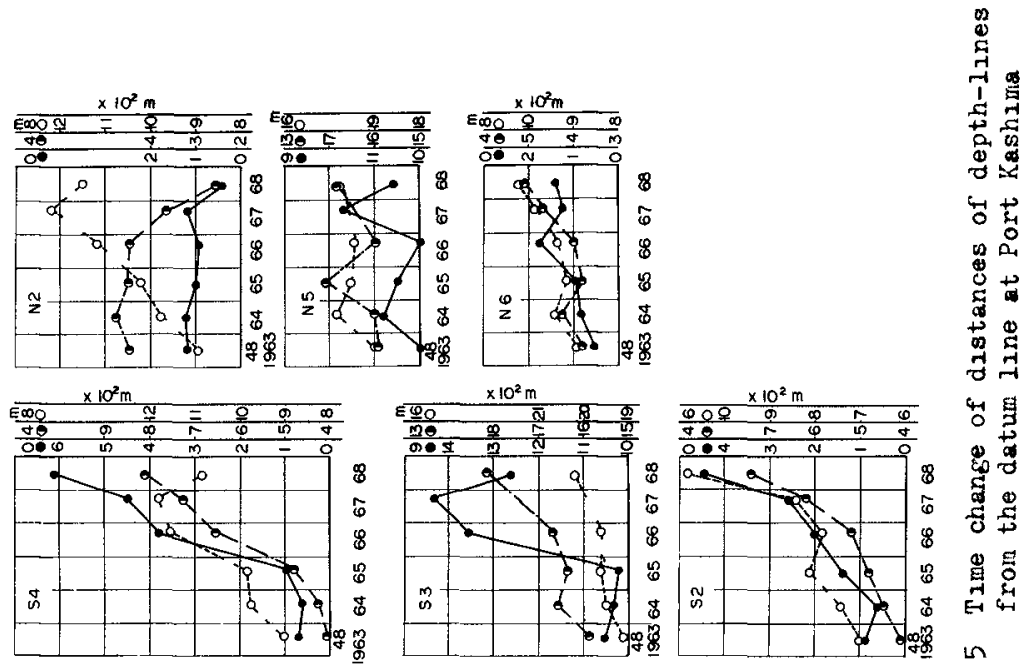

है

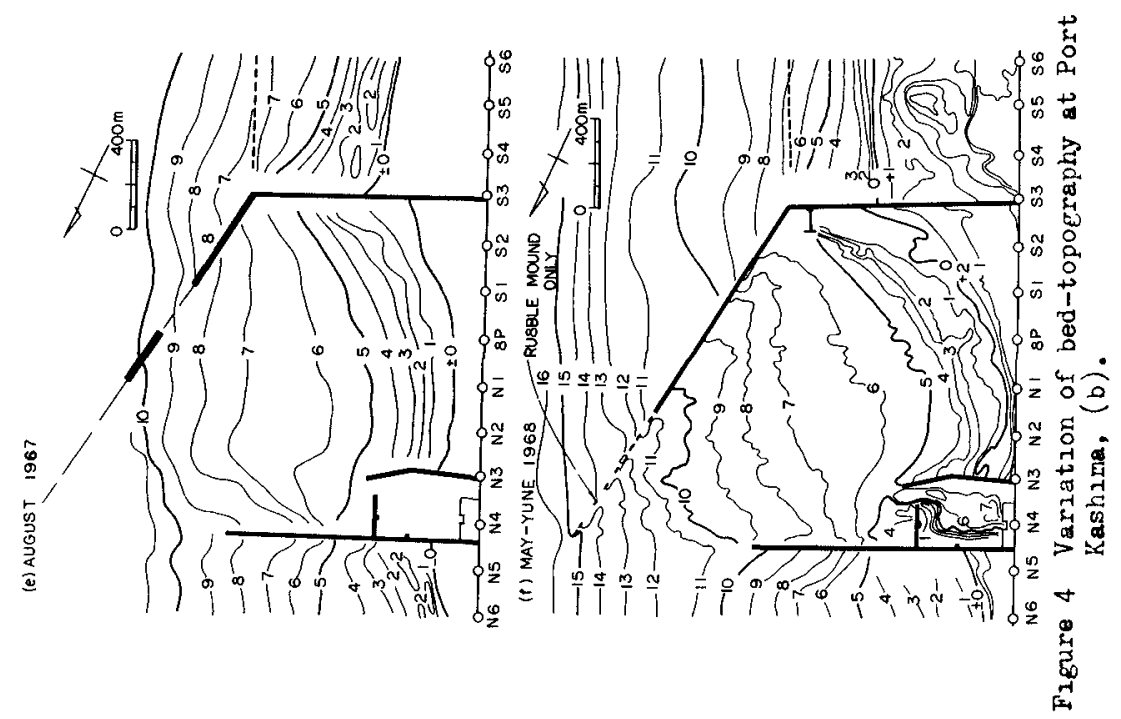


and alongshore bars advanced up-side of both jetty and breakwater and became indistinct down-side of them

In July 1965, the equi-depth lines shallower than $5 \mathrm{~m}$ in the outer basin move landward, especially at the center, near to the datum point BP The longshore bar remained distinct on the out-side of the up-side breakwater, but is indistinct in the outer basin and on the down-side of the down-side breakwater The advancement of shoreline out-side of the down-side breakwater is due to the discharge of materials dredged in the working basin

In August 1966, the portion between the t1p 1n July 1965 and the bend point of the up-side breakwater was constructed with concrete blocks Thick dotted lines indicate the portion where only the rubble mound base of calssons is constructed Scour along this rubble mound was more severe on the inside than on the out-side of it Especially, the inside portion along that of the down-side breakwater is scoured remarkably and remarkable shoaling is seen within this scoured portion The central portion of the outer-basin was shoaled and the equi-depth lines shallower than 5 m moved further landward than in 1965 The remarkable shoaling out-side of the up-side breakwater is due to the discharge of the materials dredged from the inner basin The dredging of inner basin started in June 1965 by dredgers which entered in the part of the inner basin dredging from the working basin through the broken line shown in Figure 6 Therefore, the shoreline between the

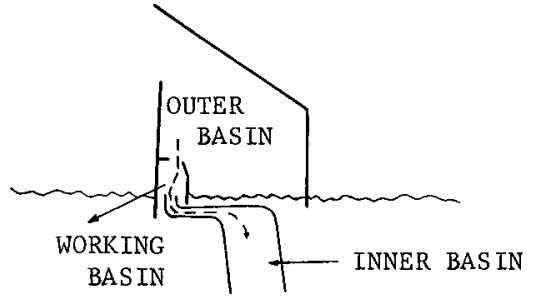
jetty and downside breakwater (between datum points $\mathrm{S} 3$ and N3) was not disturbed by the dredging

FIG 6

In August 1967 and May - June 1968, the equi-depth lines of 6 meters and less became still more landward-convex in shape and that of $7 \mathrm{~m}$ and more became seaward-convex in shape with the prolongation of the breakwaters Morever, he most receded point of shoreline was shifting towards the working basin The equi-depth lines deeper than 7 meters outside of the up-side breakwater bulged offshoreward, on the offshore of the oblique part of the breakwater The remarkable shoaling outside of the rectangular part of the up-side breakwater was mainly due to the discharged materials mentioned above, but some part of them passed through the breakwater into the basin and deposited in the vicinity of datum points S1 to S3

Figure 5 shows time change of the distances of $0 \mathrm{~m}, 4 \mathrm{~m}, 8 \mathrm{~m}$ and other depth-lines from the datum line $\mathrm{S} 6$ to $\mathrm{N} 6$ at the points $\mathrm{S} 4, \mathrm{~S} 3$, S2, N2, N5 and N6 The time change graph serves to make more clear the characteristics of the bottom changes described above For example, at $\mathrm{S} 2$, the line of $0 \mathrm{~m}$ receded in July 1964 when the up-side breakwater was about $200 \mathrm{~m}$ in length and then advanced steadily and remarkably until 1968, the depth-11nes of 4 and $6 \mathrm{~m}$ 

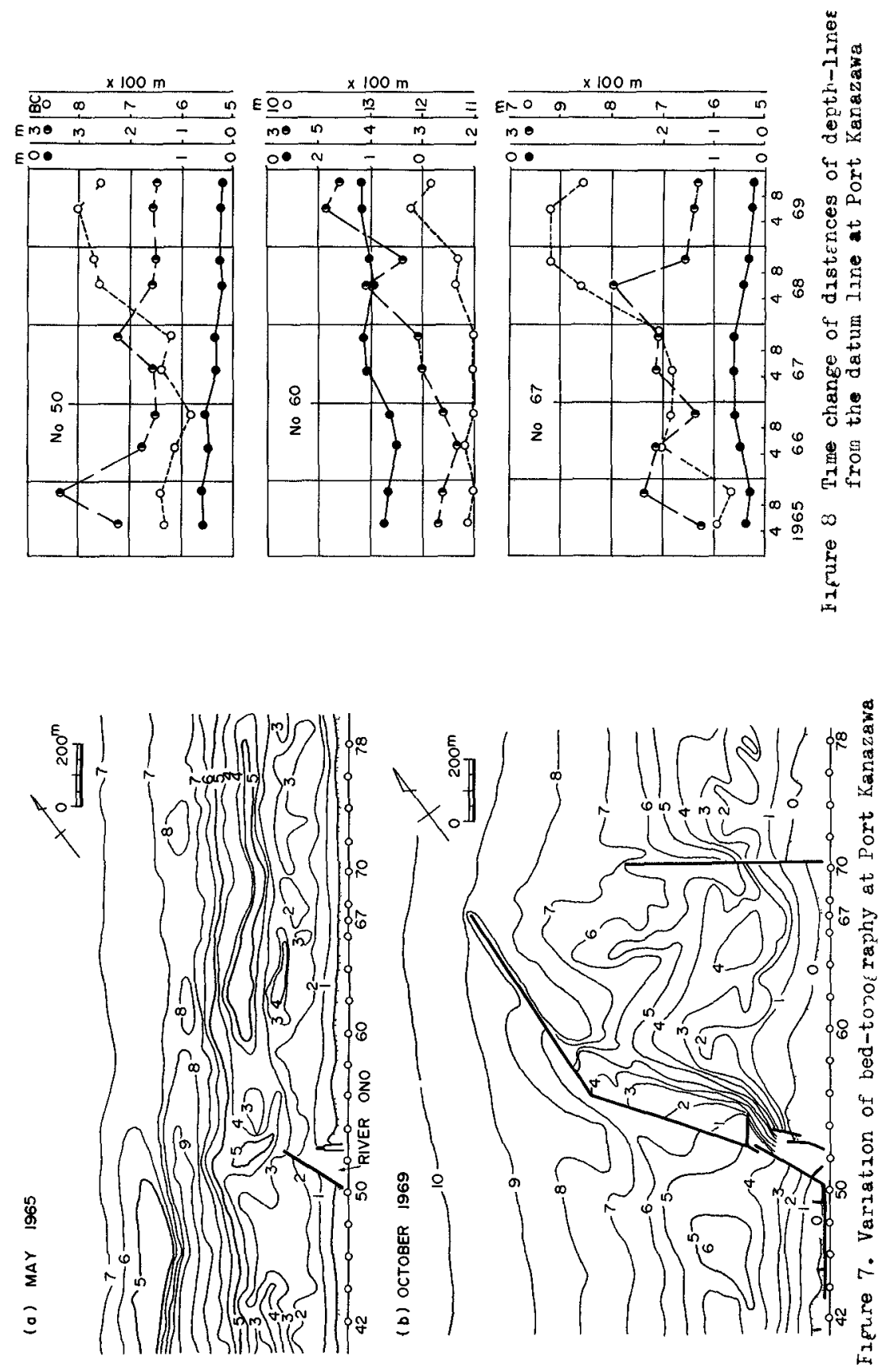
continuing to advance through this period except for $6 \mathrm{~m}$ 1ine in August 1966 These advances are remarkable in 1967 and 1968 when the oblique part of the up-side breakwater was being extended Also, at N2, the depth lines of 0 and $4 \mathrm{~m}$ advanced in 1964 and then continued to recede, especially remarkably in 1967 and 1968 on the other hand, the line of $8 \mathrm{~m}$ at the same datum point advanced steadily except in 1968

\section{VARIATION OF SEA-BED TOPOGRAPHY AT KANAZAWA PORT}

Port Kanazawa is located on the coast of Japan Sea as shown in Figure 2 The wave approach in a direction almost perpendicular to the shoreline, though the southerly waves exceed the northerly waves a littile similar to Port Kashima Waves of 3 to $5 \mathrm{~m}$ in significant wave height and $7-10$ sec in perıod very often attack during winter But the sea is very calm in summer The mean diameter of bottom materials is $015-03 \mathrm{~mm}$ in offshore zone The littoral transport from the south is slightly more than that from the north

Figure 7 shows the position of equi-depth lines in May 1965 and October 1969, and Figure 8 shows the time change of the distances of some equi-depth lines at the datum points Nos 50, 60 and 67 from the datum line The construction of the up-side breakwater began with prolonging the jetty which had existed at the mouth of River Ono, as shown in the sounding map of May 1965 of Figure 7

From these figures, the pattern of change of bottom topography is found to be similar to that of Port Kashima except the severe recession of the shoreline outside of the up-side breakwater This recession appeared in 1967 and 1968, as can be seen from the time change of $0 \mathrm{~m}$ at No $50 \mathrm{in}$ Figure 8 , and hence a sea wall was constructed along the shore-line between the datum points 50 and 44 in the summer of 1968 The recession seems to be caused by the extension of the up-side breakwater in the direction oblique to the shoreline

The line of $\mathrm{BC}$ of No 50 in figure 8 shows the time change of the longshore bar which existed about $630 \mathrm{~m}$ seaward of the datum line In May 1965 This longshore bar advanced with the prolongation of the breakwater though it became indistinct since 1968 The down-side breakwater began to be constructed in the spring of 1968 , which caused the recession of shoreline near and inside of that breakwater as seen from the time change of $0 \mathrm{~m}$ line on the datum point No 67 in Figure 8 The bottom material dredged in the channel extending from the mouth of River Ono were discharged on the outside of the down-side breakwater, which advanced the equi-depth lines there as seen in Figure 7

\section{VARIATION OF SEA-BED TOPOGRAPHY AT NIIGATA-HIGASHI}

Port Nilgata-higashi is located on the coast of Japan Sea, a. shown in Figure 2 The predominant wave direction is NNW, almost perpendicular to the shoreline, but the westerly waves are sightly predominant than the easterly waves, and hence the direction of the net alongshore transport is from west to east along the shore The other wave condition and the mean diameter of bottom materials are 

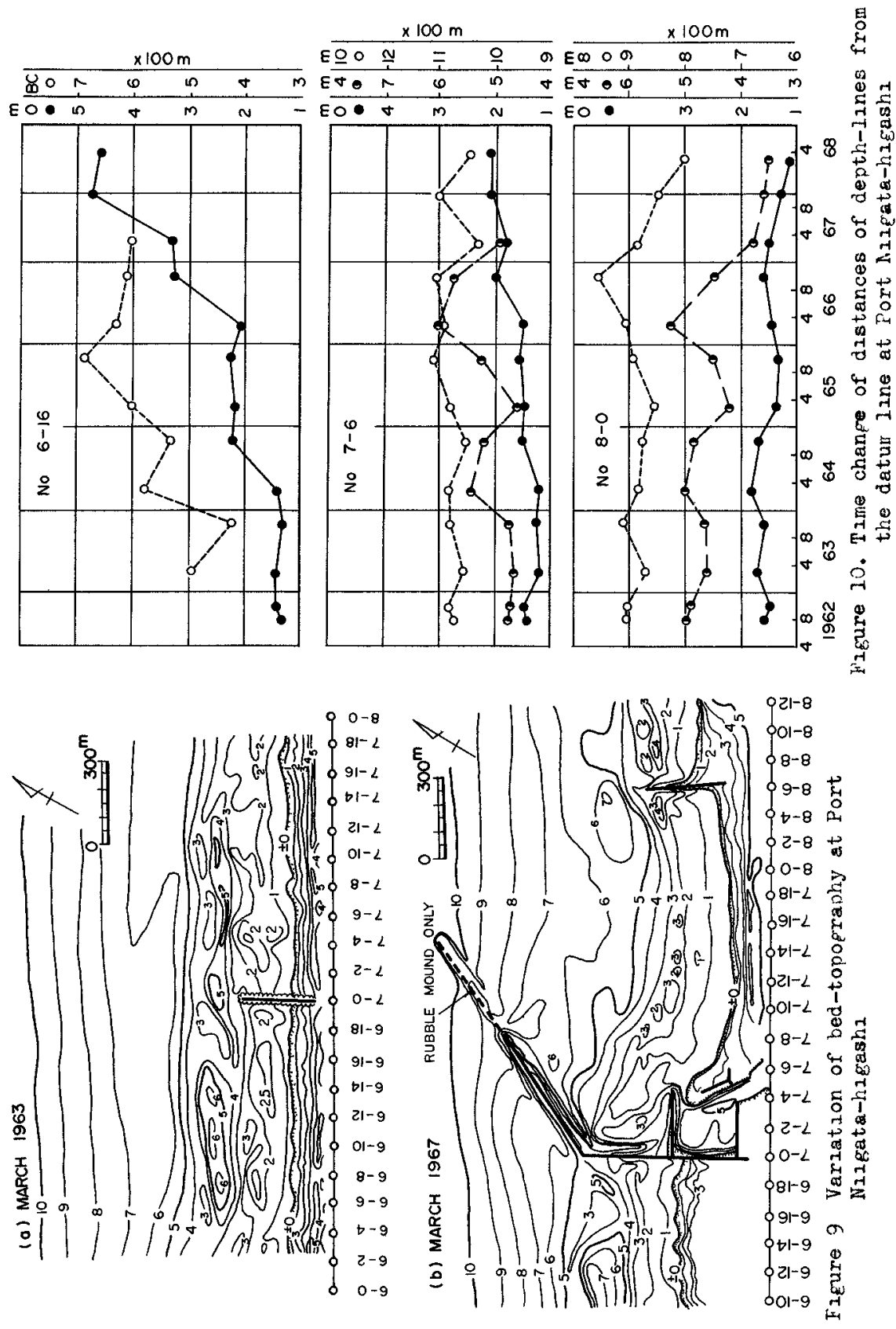
nearly the same as those at Port Kanazawa The construction works of breakwaters is stopped in winter due to severe waves similar to Port Kanazawa Figure 9 shows the sounding maps of March 1963 and March 1967 and Figure 10 the time change of the distance from the datum line of a few equi-depth lines at the datum point Nos 8-0, 6-16 and 7-6

The above figures show the same tendency as Port Kashima on the bottom-change due to the construction of breakwater, though the deeper equi-depth lines did not become seaward-convex in shape in the map of March 1967 This is due to the down-side breakwater being still short The down-side breakwater began to be constructed in 1966

The inner basin was dredged by dredgers which entered from the workıng basın similarly to Port Kashima, and the dredged material was discharged on the outside of the up and down-side breakwaters It caused the abrupt advancement of shoreline out-side of the breakwaters since 1964, as seen in the time change of $O \mathrm{~m}$ line on No 6-16 in Figure 10 As seen also in Figure 10, the alongshore bar advanced unt 1 October 1965, receded ti11 March 1967, and then disappeared due to the above-mentioned discharged materials 0,4 and $8 \mathrm{~m} 1$ ines on No 8-0 receded abruptly from 1966 when the down-side breakwater began to be constructed

\section{TOPOGRAPHY OF PORT OARAI AND HIMEKAWA}

Port Oaral is located near to Port Kashima, and Port Himekawa between Port Kanazawa and Nilgata-higashi as shown in Figure 2

Figure 11 shows the sounding maps of Port Oaral in 1967 In this case, the bulge of equi-depth lines is not seen offshore of the oblique part of the up-side breakwater, but the shoaling is remarkable inside of the tip of breakwater This seems to be due to the extension of the oblique part of the up-side breakwater at a small angle to the shoreline

Figure 12 is for Port Himekawa in 1969 In this case, the rectangular part of the up-side breakwater is very short, and so the shoreline has advanced remarkably on the down-side to form a tombolo and has receded on the up-side of the breakwater

\section{MODEL TEST ON CHANGE OF SEA-BED TOPOGRAPHY CAUSED BY A JETTY}

Some model tests were conducted on the change of sea-bed topography caused by a jetty in a basin, $30 \mathrm{~m}$ wide, $50 \mathrm{~m}$ long and $08 \mathrm{~m}$ deep At first, a model beach of $18 \mathrm{~m}$ long in the direction of shoreline and of $1 \quad 15$ bed slope was made with fine sand of about $02 \mathrm{~mm}$ in mean diameter The model beach was attacked by waves which were $8 \mathrm{~cm} 1 \mathrm{n}$ helght, $12 \mathrm{sec}$ in period and 15 degree against the shoreline in wave direction Morever, sand was fed in the shoreline of up-side end so that the shore line there did not change during the wave action

Change of bed-topography caused by a long jetty

Figure 13(a) shows the bed topography after two hours of wave 


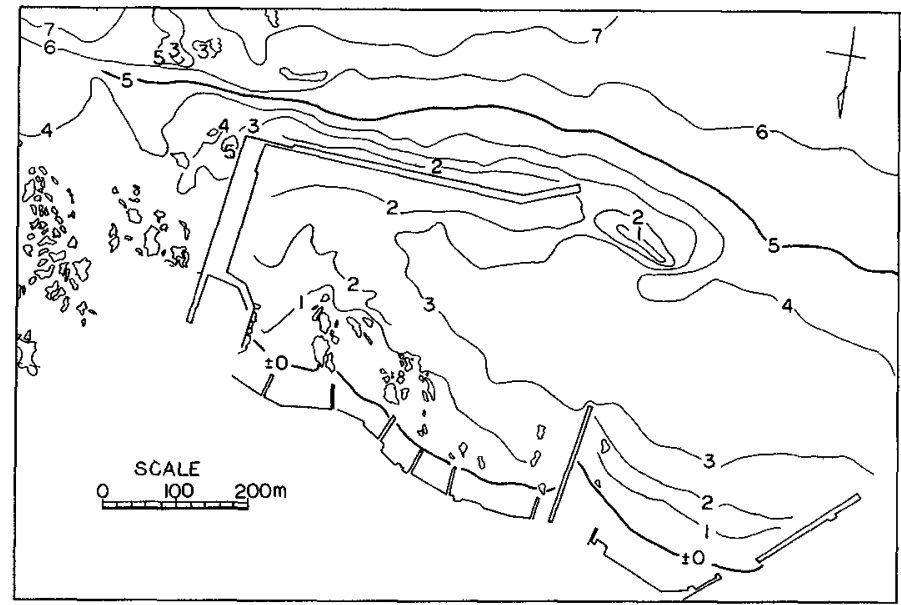

Fipure 11 Port Oaral an 1967

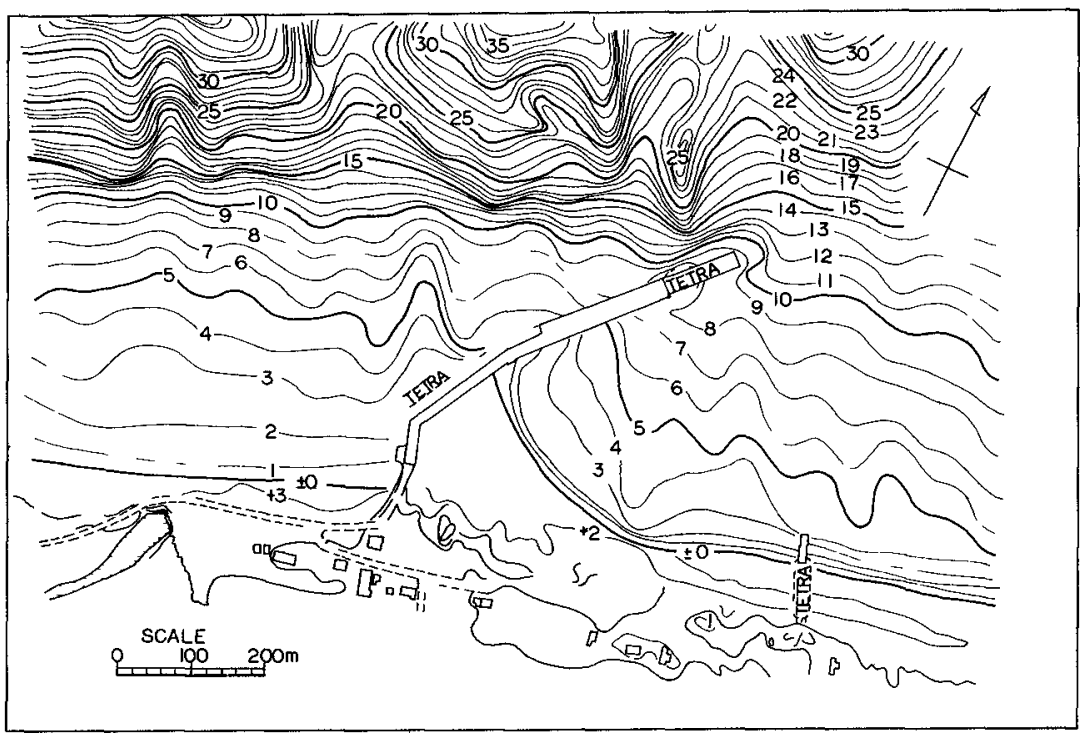

Figure 12 Port Hznekawe in 1969 
(o) BEO TOPOGRAPHY IN TWO HOURS OF WAVE ACTION

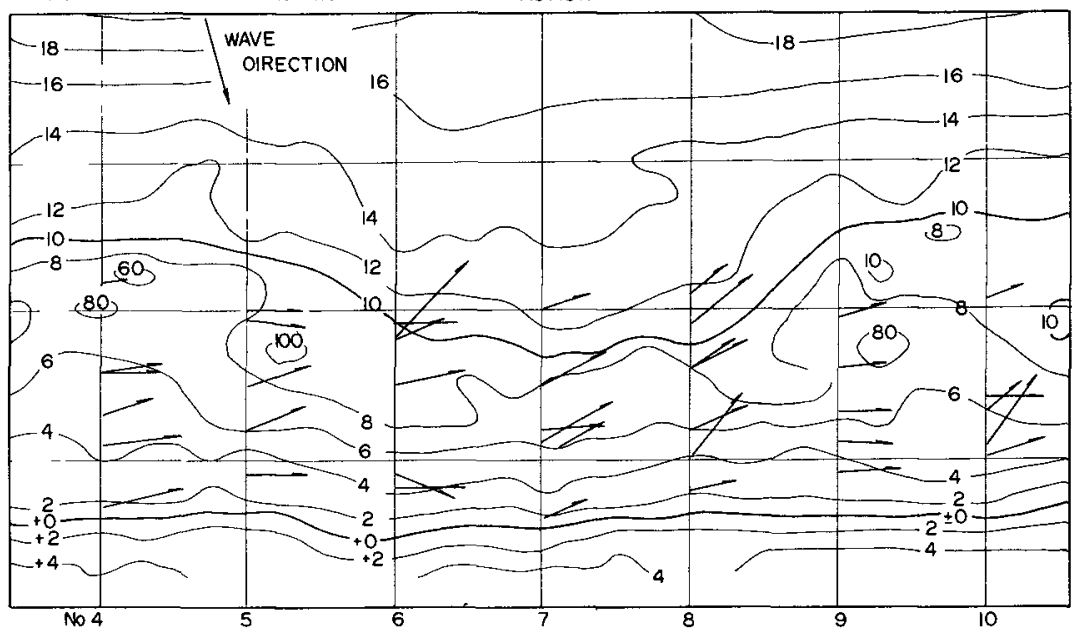

(b) 8EO TOPOGRAPHY IN MORE TWO HOURS OF WAVE ACTION AFTER A LONGE GETTY WAS INSGT IN (o)

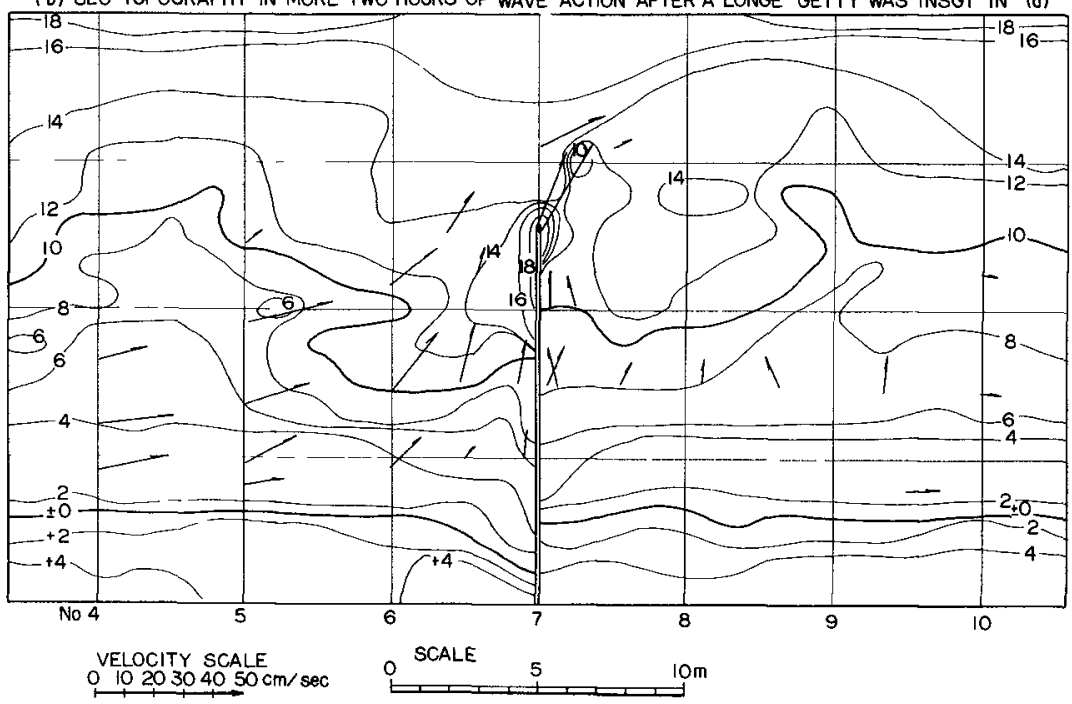

Fierure 13 Chinpes of equi-depth lines and bottom currents oy a long jetty (model test) 
action on the above-mentioned initial model beach and Figure 13(b) shows the bed after an additional two hours of wave action, after a jetty was inset in the bed of (a) The arrow marks show bottom currents measured using a plastic ball of $15 \mathrm{~cm}$ in diameter filled with water, which moves along the bottom The jetty was made with a plastic plate having smooth surfaces

The longshore bar is seen between the equi-depth 11nes of 6 to 10 $\mathrm{cm}$ in (a), and $1 \mathrm{t}$ advances offshoreward in front of the datum point No 3 to 6 and 8 to 11 The bottom currents are flowing nearly parallel with the shoreline mostly, except that they go obliquely offshoreward on the up-side of the advancing part of the longshore bar

In (b) with a jetty, there are seen severe scouring at the tip of jetty, scour along the jetty and erosion of the shoreline on the up-side of the jetty The alongshore currents on the up-side of the jetty change their direction towards the tip of the jetty as they approach the jetty Morever, seaward currents exist along the up-side of the jetty from the foreshore These alongshore currents and seaward currents join to become a strong current in the vicinity of the tip of the jetty, which cause the above-mentioned severe scouring there, together with the breaking wave along the jetty The wave height is higher along the jetty than in the area away from the jetty, because the wave comes obliquely to the jetty, which results in the iricrease of disturbance of bottom materials and the rise of the water level at the foreshore near to the jetty The rise of the water level causes the above-mentioned seaward bottom currents The other hand, on the down-side of jetty, the area near to the jetty is by some degree sheltered by the jetty from waves, which results in the advancement of shoreline and the disappearance of alongshore bars

\section{Relation between length of a jetty and erosion of up-side foreshore}

Figure 14 shows the change of bed topography for jettres of 20 , 15 and $125 \mathrm{~m}$ long from the datum line with the same surface as the above-mentioned jetty of figure 13 and also for a jetty of $25 \mathrm{~m}$ long with the surface of rouble stones In the figure, equi-depth 1ines are drawn by dotted lines for after two hours of wave action on the initial model beach of 115 in bed slope and by full lines for after an additional two hours of wave action after a jetty was inset

The up-side foreshore near the jetty was eroded $1 \mathrm{n}$ (a) of $2 \mathrm{~m}$ long jetty as well as in Figure 13, changing 1 ittle in (b) of $15 \mathrm{~m}$ long jetty and being accreted in (c) of $125 \mathrm{~m}$ long jetty In (d) of $25 \mathrm{~m}$ long and rough surface jetty, the up-side foreshore was accreted The rough surface, as well as the decrease of length, of the jetty serves in the decrease of wave height and the decrease of the offshoreward current velocity along the jetty, which results in the decrease of erosion and scour along the up-side of the jetty

MODEL TEST ON CHANGE OF SEA-BED TOPOGRAPHY CAUSED BY TWO BREAKWATERS

Prior to the prolongation of the oblique part of the up-side breakwater at Port Kashima, model tests were conducted for different stages 
(a) $20 \mathrm{~m}$ LONG WITH SMOOTH SURFACE

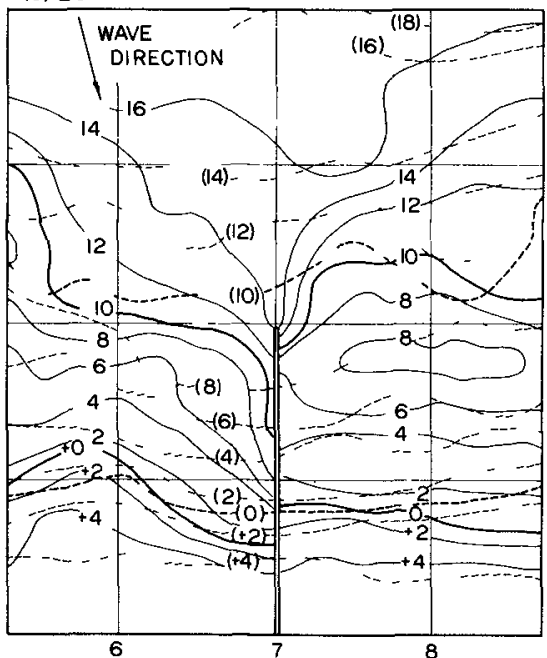

(c) $125 \mathrm{~m}$ LONG WITH SMOOTH SURFACE

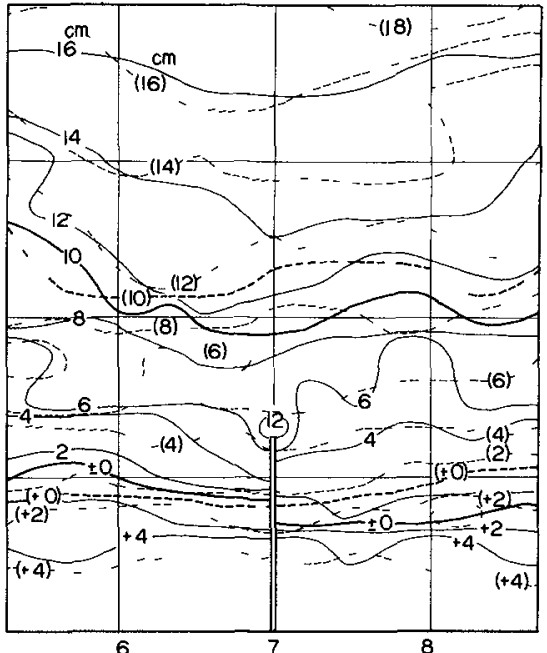

$(+4)$ (b) $15 \mathrm{~m}$ LONG WITH SMOOTH SURFACE

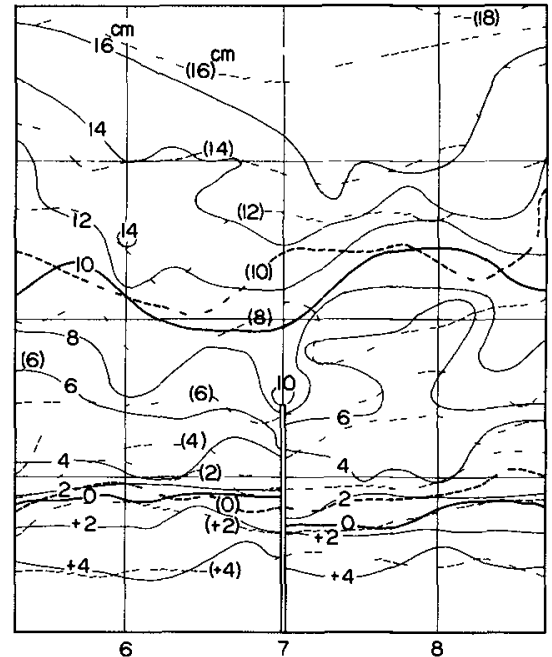

(d) $25 \mathrm{~m}$ LONG WITH ROUGH SURFACE
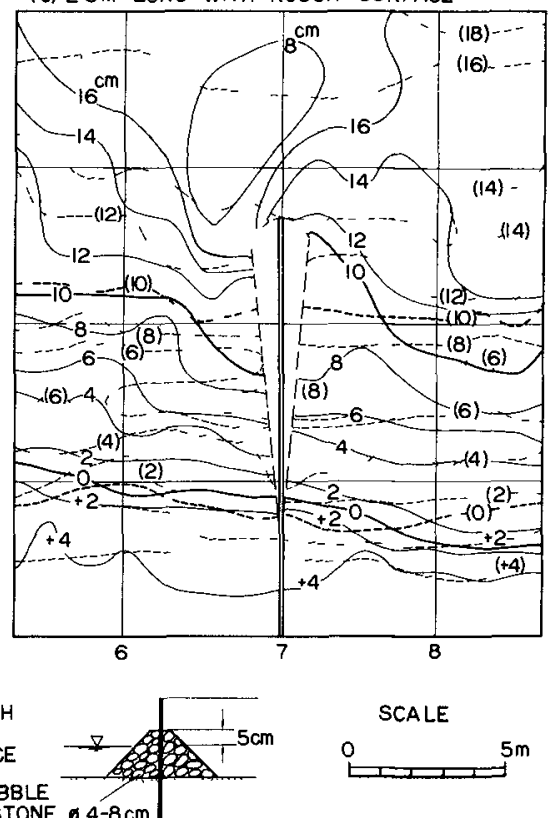

Figure 14 Relation between length of a jetty and erosion of up-side foreshore (modol test) 
of the construction of breakwaters in the same basin as the abovementioned test Figure 15 shows a part of the model tests The up and down-side breakwaters were prolonged from the state in upper figure (a) to the state in the lower figure (b) and then waves were acted for four hours The result by the wave action is shown in the lower figure In this case, model waves in the offshore were $14 \mathrm{~cm}$ in height, 14 sec in period and $I N E$ in direction Model bed materials were sand of 022 mm in mean diameter and model scale was 1200 in horizontal and 140 in vertical Also, the model of breakwater was made by concrete blocks which was impermeable against water Figure 16 shows the conditions of bottom currents and wave-heights for the bed condition in Figure 15(b)

\section{Comparison of change of bottom topography between model and prototype}

The length of breakwaters is not so much different between Figure 15 (a) and Figure 3(d) though the latter has a little more extended breakwater than the former, and so also between Figure 15(b) and Figure $4(\mathrm{f})$ Therefore, the change of bottom topography from the above (a) to (b) of the model is compared with that from the above (d) to (f) of the prototype

The offshoreward bulge of equi-depth lines near the oblique part of the up-side breakwater on the out-side, the advancement of all equi-depth lines near the up-side breakwater on the in-side, the shift of the most receded point of the shoreline towards the jetty of working basin and the shoaling of the center part in the outer basin are seen both in the model and the prototype It is seen also in the model the tendency for equi-depth lines to become of landward-convex shape in the shallower area and of offshoreward-convex shape in the deeper area in the outer basin The tendency of change of bed-topography coincides between the model and the prototype, though there is some difference on the intensity or rate of the change

\section{Distribution of waves and bottom currents}

It is seen from (a) of Figure 16 that the wave height in the outer basin is larger in the portion extending from the mouth to the datum points $\mathrm{N} 1$ to $\mathrm{N} 2$ and smaller in the portion near the up-side breakwater It is also seen from (b) of Figure 16 that there is currents circulating in the ant1-clockwise direction in the outer basin The velocity of the circulating current is smaller in the center of the outer basin and in the portion near the up-side breakwater This indicates that the pre-described most eroded point of the shoreline have the higher wave and more rapid bottom current, and the pre-discribed shoaling portion in the center of the basin has the slower current Also, the accumulation seen in the area near the up-side breakwater seems to be caused by the condition of current velocity and wave helght which are smaller in the area near the upside breakwater than in the area near the down-side breakwater

\section{CONCLUSION}

The general characterıstics of bottom topography change caused by the construction of breakwaters on a sandy beach are concluded from the foregoing chapters as follows Figure 17 is the illustration of the 

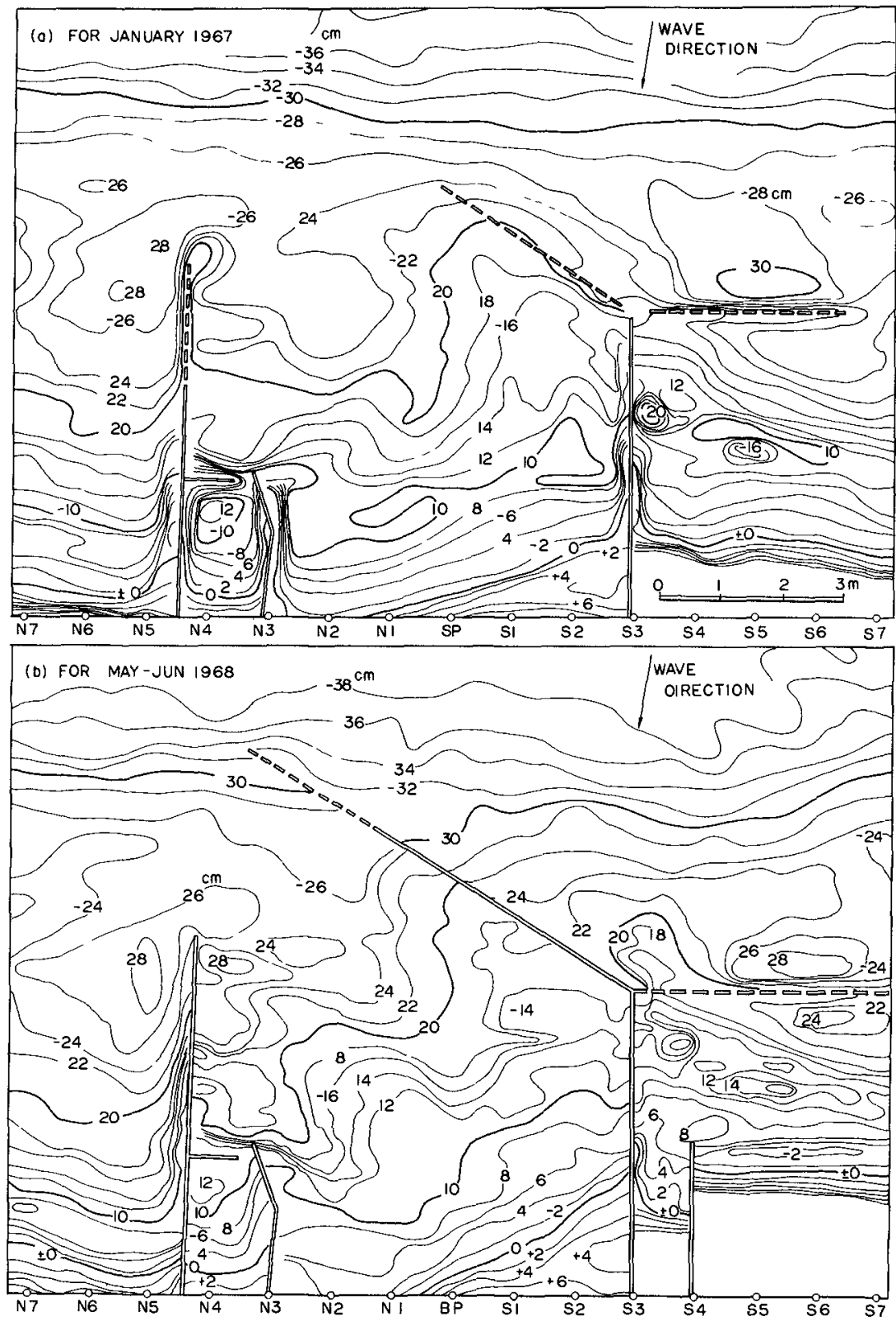

Figure 15 (hature of bottor tonography caused by the prolongation of oreakwaters (model test of Port Kashnma) 


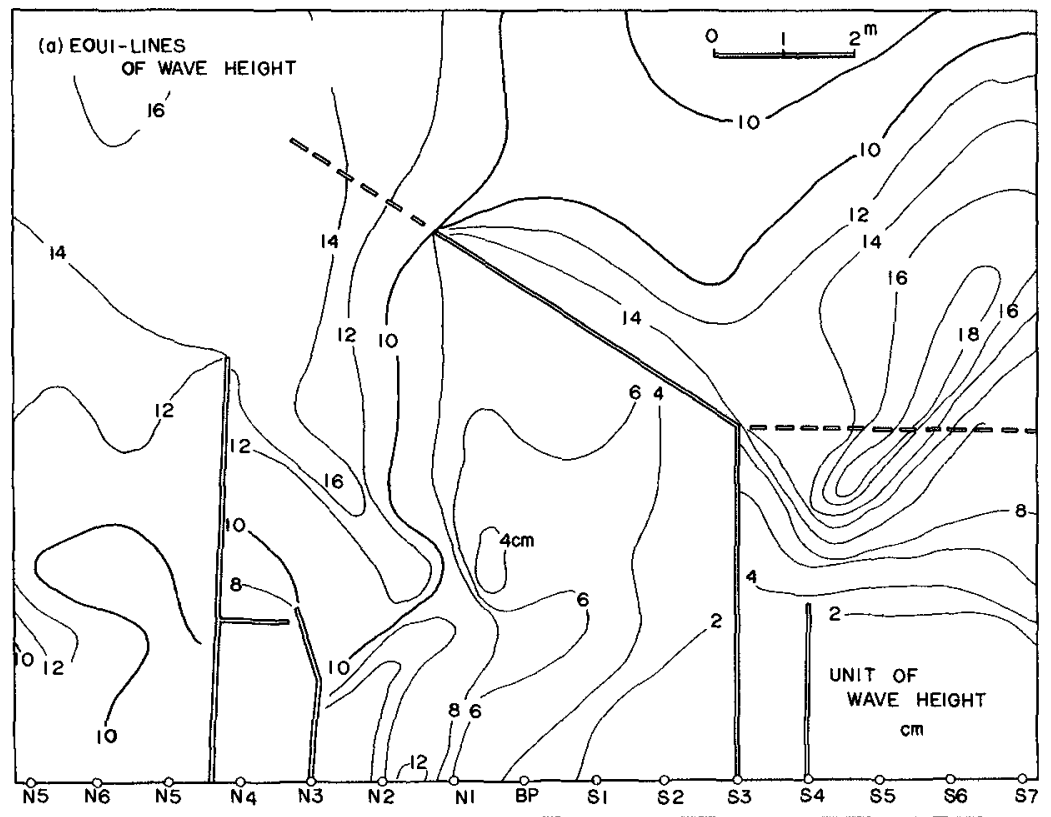

(b) BOTTOM CURRENT
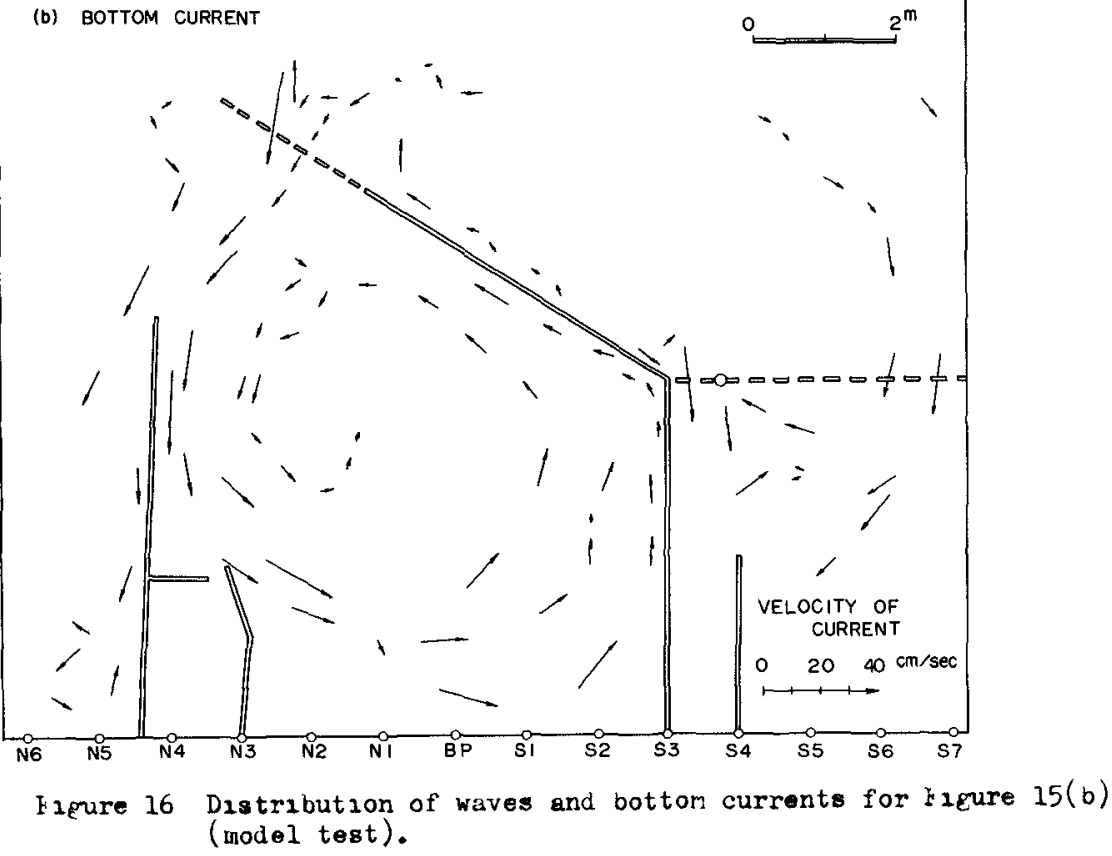
same

Out-side of the up-side breakwater

(1) The shore-1ine near to the breakwater advances offshoreward first and then stops or recedes according to the prolongation of the breakwater In this case, the more oblique the direction of a breakwater is, the more severe the recession of shoreline is Such recession of shoreline is less in the breakwater with rough sidesurface than that w1th smooth surface as shown in the model test

(2) The alongshore bar near to the breakwater advances offshoreward becoming distinct in shape with the prolongation of the rectangular part of the breakwater and stops or recedes becoming indistinct with the construction of the oblique part of the breakwater

(3) The equi-depth lines near to the oblique part of the breakwater bulge offshoreward, as most of 11 ttoral drift carried along the shore moved seaward along the rectangular part to deposit offshore of the bend-point without moving directly along the oblique part of the breakwater However, in the case where the rectangular part is short and the bending angle of the oblique part is large, such bulge of equi-depth lines does not occour but the shoaling tends to be remarkable inside the tip of the breakwater, as in Port Oarad.

Inside of breakwater (outer basın)

(1) According to the construction of an up-side breakwater, the shoreline advances in the part near to the breakwater and recedes in 1ts down-side, namely, having a shape of landward-convex The most receded point in this case moves towards the down-side with the increase of length of the breakwater Such advancement of a shore 1ine near to an up-side breakwater becomes larger when the direction of breakwater is oblique to the shoreline and is closely related to the diffraction of waves by the breakwater

(2) The alongshore bar in the outer basin becomes indistinct or disappears with the prolongation of breakwaters

(3) The scouring at the toe of the rouble mound is more severe inside of it than outside of it during the period before calsons are set on it This seems to be due to the swirl formed inside the rouble mound by waves passing over it

(4) When both up and down-side breakwaters are prolonged, equidepth lines move landwards in the shallower zone and offshorewards in the deeper zone, namely, having a shape of landward-convex in the former and a shape of offshoreward-convex in the latter

(5) Waves entering from the mouth progress near the down-side breakwater so that they erode the foreshore near to the down-side breakwater and produce circulating currents flowing from the downside breakwater to the up-side breakwater and then to the center of the outer basin

(6) In the shallower area, the bottom is eroded or scoured where 
OUTSIDE OF THE UP-SIDE BREAKWATER

(1) and (2)

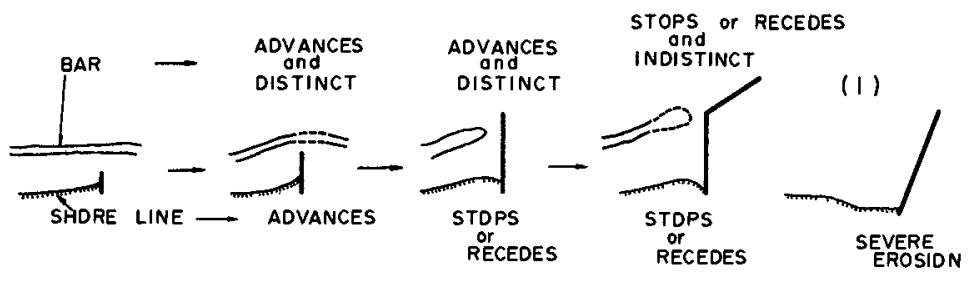

(3)

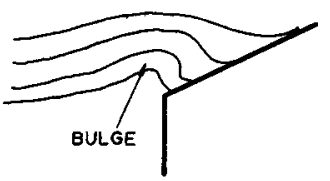

(3')

(4)

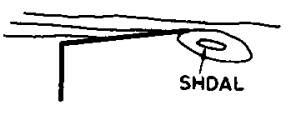

4) SEVERE ERDSION

\section{INSIDE OF BREAKWATERS (OUTER BASIN)}

(1) and (2)

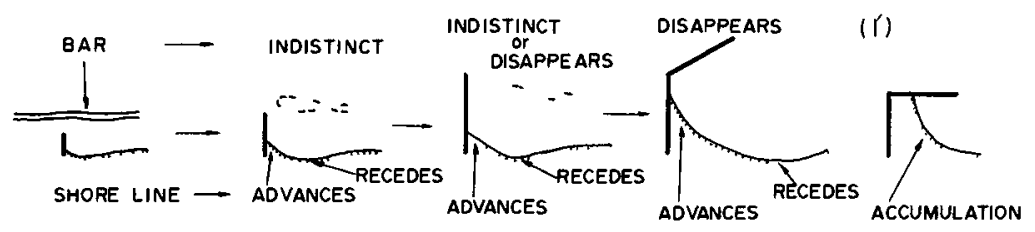

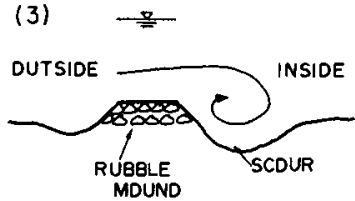

(5)

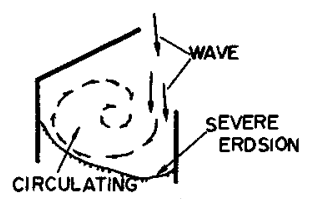

(4)

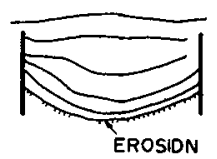

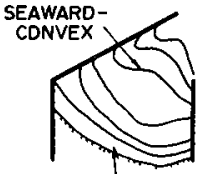

LANDWARD - CONVEX

\section{BDTTOM CURRENT}

Hequre 17 Tynzcal pattern of oottor topor raphy caused by the construction of wrokwaters 
waves and bottom currents are more severe than its surroundings and is shoaled where they become weak and slow down on the other hand, in the deeper area, the shoaling of bottom happens where bottom currents slow down rather than waves

\section{Outside of the down-side breakwater}

The change of a shoreline to landward-convex in shape, the disappearance of alongshore bars, and the scour of bottom along the breakwater are seen often in the area near to the breakwater 


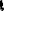




\section{CHAPTER 82}

\section{FQUILTBRTUM PROFTLES OF MODEL B'ACHLS}

By Irvathur Vasudeva Nayak ${ }^{1}$ A.M. ASCE.

\section{ABSTRACT}

The investigation reported herein covers two aspects of equilibrium beach profiles, namely, (a) the criterion eovcrning their type and (b) their refltction characteristics The problems are first andysed from dimensional considerations and then stualed experimentally in laboratory wave flumes using different sizts of quartz, ground walnut shell and ground plastic as movable bed material. tmpirical relations between pertinent parameters governing the beach prucess are given.

\section{Introduction.}

The wave and hydrologic climate at a beach location is everchanging and the coastal engl netrs have always been interested in the deformation of the natural beach caused by wave action over a short or long interval of time. The resulting changes in beach characteristics, such as the type of profile, beach width, berm level, location and size of offshore bars, he ight of run-up, the rate and mode of sediment transport, areas of depositicn and scour and the amount of energy absorbed or reflected from the beach, are often rapid. Coastal management requires a knowledge of the fundamental principles involved in the behaviour of the

1 Professor and Head of Department of Applied Mechanics, \& Hydraulics, K.R. sngl neering College, Surathkal, INDIA Formerly Graduate student, University of California, Berkeley, Calıfornia. 
beach material ana research at tempts have, long since, been directed to obtain analytical and empirical relaticns between the different pertinent variables involved in the extremely complicated bedch process.

The study reported herein covers two aspects of equilibrium beach proflles, namely, criterion Eoverning thei $r$ type and reflection characteristics. The experiments relating to the criterion for the type of profile have been performed as series $A$ and those of the latter as series $B$ in two separate experimental units in the laboratcry.

Part 4. Criterion for type of profile.

Almost all the investigations that have been conducted to date have 1 nd 1 cated that deep-water wave steepness is an important parameter related to the beach orocess and a critical value of this parameter characterises various elements of the phenomenon like the type of profile, mode and rate of transport and the type of breakers etc. In this part of the study an attempt is made to relate the variation in the critical value of deep-water wave steepness concerning the type of beach profile, "storm" or "summer", to the characteristic size and specific gravity of the beach material.

The type of equilibrium profile, storm or summer, that results from the detion of waves in a two dimensional flume can be assumed to be governed by the following variables.

(1) deep-water wave height, $\mathrm{H}_{\mathrm{O}}$ (11) wave period, $\mathrm{I}$

(111) specific gravity of sediment in water, $s$ (iv) median diameter of sediment, $D$ 
(v) depth of water in the channel, d

(vi) acceleration due to gravity, $g$

(v11) initial slope of beach, $1_{0}$

(vili) standard deviation of grain size, $\sigma$

$1 \mathrm{e}$

$$
\begin{aligned}
\text { type of profile } & =f_{1}\left(H_{0}, I, S, D, \sigma, 1_{0}, 8, d\right) \\
& =f_{2}\left(H_{0}, L_{0}, 0, D, 1_{0}, E, d\right)
\end{aligned}
$$

The role of viscosity of the fluid is of minor importance if we assume that the keynolds number is sufficiently high and the entire phenomenon takes place well with in the turbulent range. If the zone of sand movement is limited on the beach slope, the effect of depth of water can be neglected. The gravitational acceleration is constant. The standard deviation of grain size is of minor importance and from earlier studies one can find that the initial beach slope plays no major role in the phenomena Thus considering the equilibrium condition which is independent of duration,

$$
\begin{aligned}
\text { Type of profile } & =f_{3}\left(H_{0} / L_{0}, H_{0} / D, S\right) \\
\text { or } & =f_{4}\left(H_{0} / L_{0}, H_{0} / S D, i\right)
\end{aligned}
$$

The problem can be also viewed as

$$
\begin{aligned}
\text { Type of profile } & =f\left(H_{0}, I_{0}, S, L\right) \\
& =f\left(H_{0}, L_{0}, V_{f}\right)
\end{aligned}
$$

considering that the fall velocity in water, $v_{f}$, characterises the sediment,

i.e., type of profile $=f\left(H_{0} / L_{0}, \frac{\sqrt{g H O}}{V_{f}}\right)$

The fall velocity can be taken to be proportional to the quant $t y\left(\frac{S g d}{C_{D}}\right)^{\frac{t}{2}}$

$$
\begin{aligned}
\therefore \text { Type of profile } & =f\left(H_{0} / L_{0}, \frac{\sqrt{g} H_{0}}{S^{\frac{1}{2}} g^{z} D^{2}} C_{D^{2}}{ }^{2}\right) \\
& =f_{5}\left(H_{0} / L_{0}, H_{0} / S D\right) \text {, disregarding } \\
\text { the effect of } C_{D^{*}} &
\end{aligned}
$$


The parameter $\mathrm{H}_{0} / \mathrm{SD}$ can also be taken as the ratio of a typical unit force exerted by the wave to unit resistance offered by the beach sediment, because the former is proporticnal to

$$
\left.P_{f} \times \text { (a characteristic velocity of flow pattern }\right)^{2} \times D^{2}
$$

and the latter to the submerged weight of the grain of bea materiel, 1.e., $g\left(P_{s}-P_{f}\right) D^{3}$. If the characteristic velocity is taken as $\sqrt{\mathrm{gH}_{\mathrm{o}}}$, the ratio becomes

$$
\frac{\rho_{f} \cdot u_{c}^{2} \cdot D^{2}}{g\left(\rho_{s}-\rho_{f}\right) D^{3}}=\frac{\rho_{f} u_{c}^{2} / g}{\left(\rho_{s}-\rho_{f}\right) D}=\frac{H_{O}}{S D}
$$

The rat $10 \mathrm{H}_{\mathrm{o}} / \mathrm{SD}$ is also comparable to the rat 10 $1 / \psi$ where $\psi$ is the intensity of flow given by the expression

$$
\psi=\frac{\rho_{\mathrm{s}}-\rho_{\mathrm{f}}}{\rho_{\mathrm{f}}} \cdot \frac{\mathrm{Dg}}{\bar{v}^{2}},
$$

an important quantity used in sediment flow problems.

Thus the ratio of wave helght to the product of specific gravity in water and the median diameter of the sediment can be taken to be a very important parameter in the beach process.

The importance of the size of the beach material has been pointed out by kector (1954) and the ratio of wave hel ght to median diameter by Twagaki (1962) (Fig.1). The present experiments were carried out in a wave flume shown in $\mathrm{k} 1 \mathrm{g.2}$. I'he wave helght was noted with the help of a point gause placed Just of fshore from the toe of the beach and was taken as twlce the alstance recorded between the crest of the wave and the still water level. This is correct in deep water. The beach profile was recorded by measuring the horizontal and vertical coordinates on a rectangular gria fixea to the side of the flume. The first four materials whose size alstribution is given in Fig. 4, were usea as beach material in 


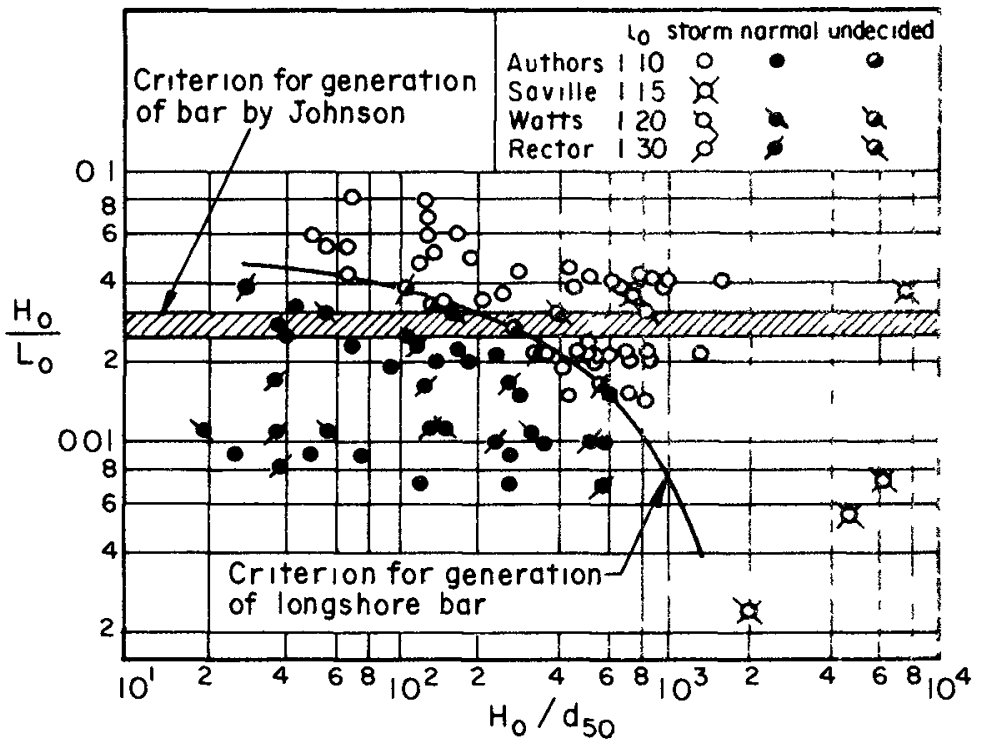

FIG 1 CRITERION OF BAR GENERATION (IWAGAKI)

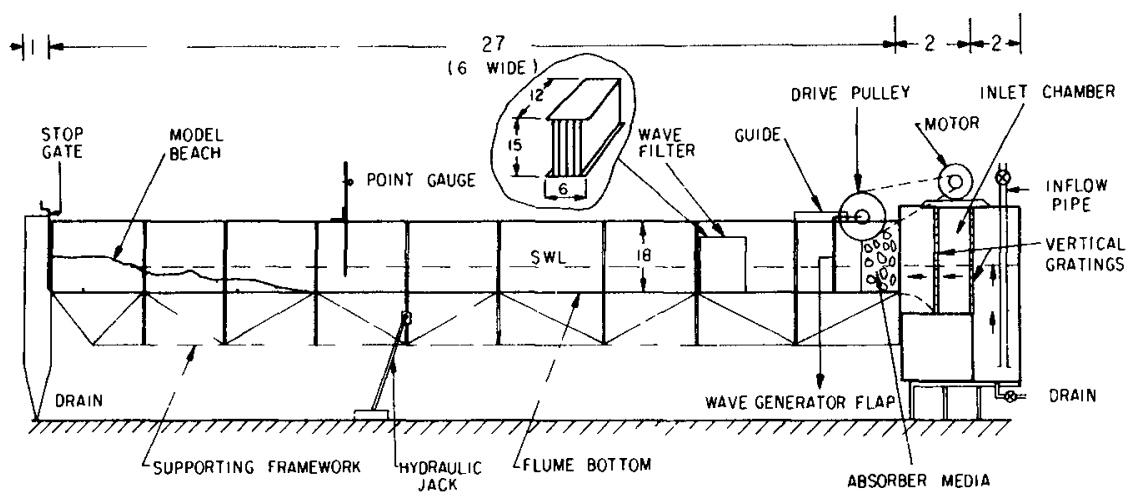

FIG 2 WAVE FLUME USED IN SERIES A 

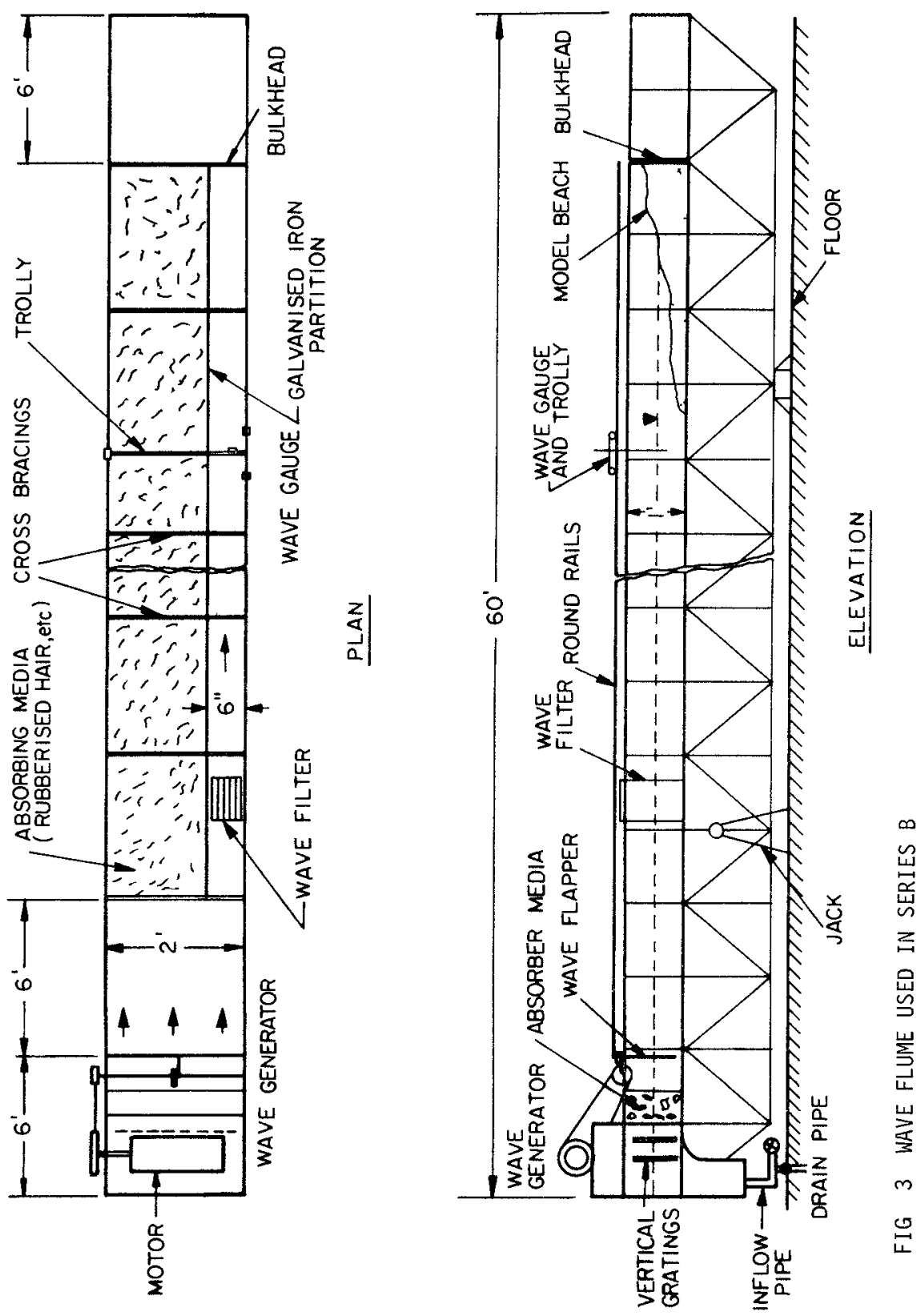

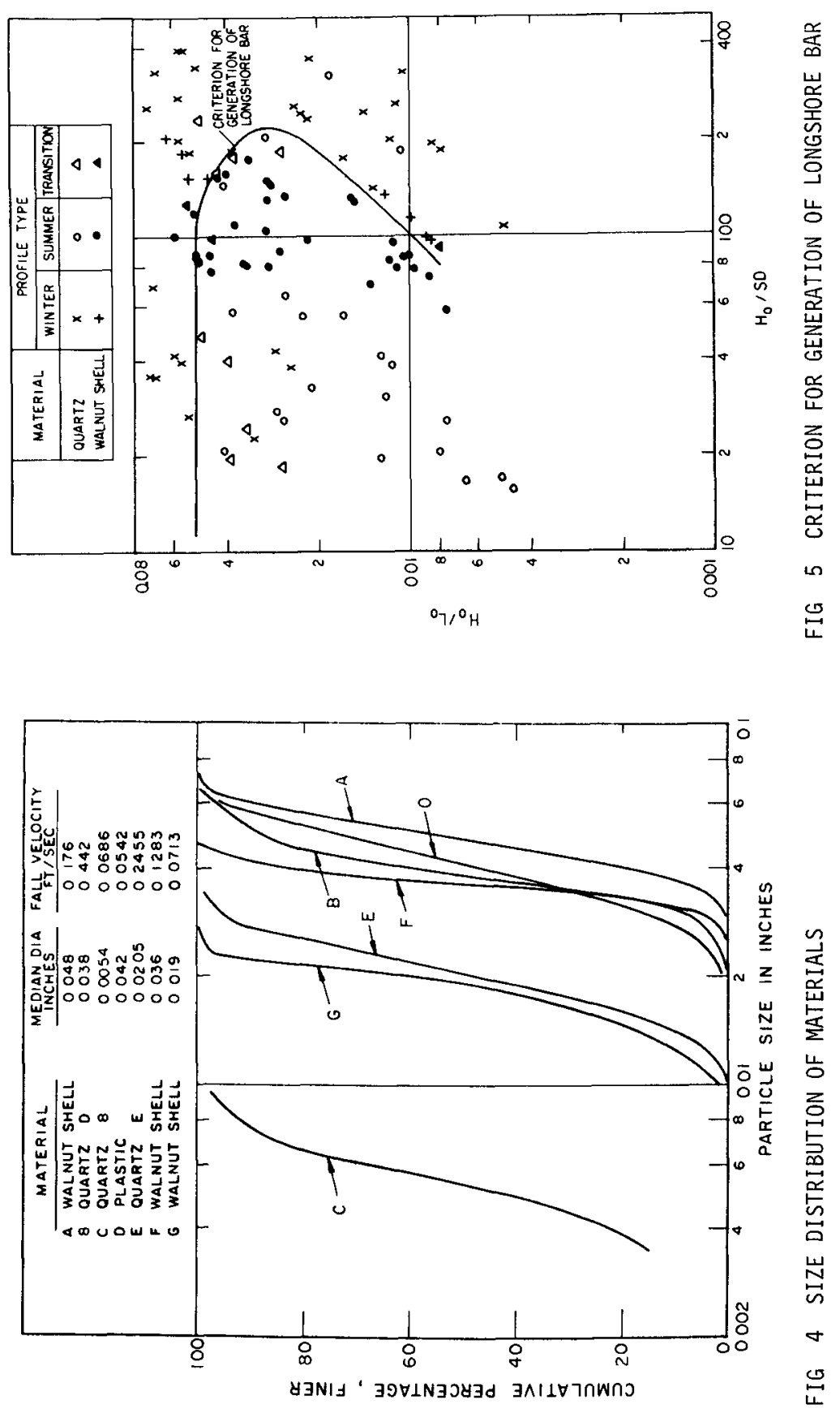
series 4. In the case of each material, the aepths of water used were $030,035,0.40,645$ and $050 \mathrm{ft}$. For each depth the following wave perlous were used 1.86 , $160,1.26,1 \mathrm{c} 1, C 845$ and 0.72 secs, At the beglnning of each group of experiments just onough materidl was placed in the flume such that the beach crest was generally located 1 to $2 \mathrm{ft}$, from the end of the flurae. No particular initial slope was used. The beach was allowed to shape 1 tself under wave action until equilibrium conditions were reached.

The criterion for tat type of profile, summer or winter, based on this study has been shown in Fig.5 in terms of two parameters, namely, atep-water wave steepness, $H_{0} / L_{0}$, and the ratio of deep-water wave helght to the product of specific gravity of the bcach material in water and 1 ts median $d i a m e t \in r, H_{0} / S D$. Tn this plot the resul's of experiments with the two sizes of quartz sands and the walnut shell have been used.

The behaviour of the ground plastic was quite different in none of the runs with this material were ripnles observed or were the waves found to break. The material appearea "soupy" and proved a very efficient absorber of energy Under wave action most of the material went into thick suspension and oscillated to the entire depth of the movable bea. For this reason the results of the ground plastic were not included in the plot of Fig. 5 Tt is also to be nutea that no correction has been applied to the obsurved wave helghts to take into account the reflected wave component.

The curve separating the region of summer profiles from that of winter profiles in Fig.5 is a riolng stralght line in the range of wave steepnesses from c.008 to 0.02 . For the higher values of wave steepness, 

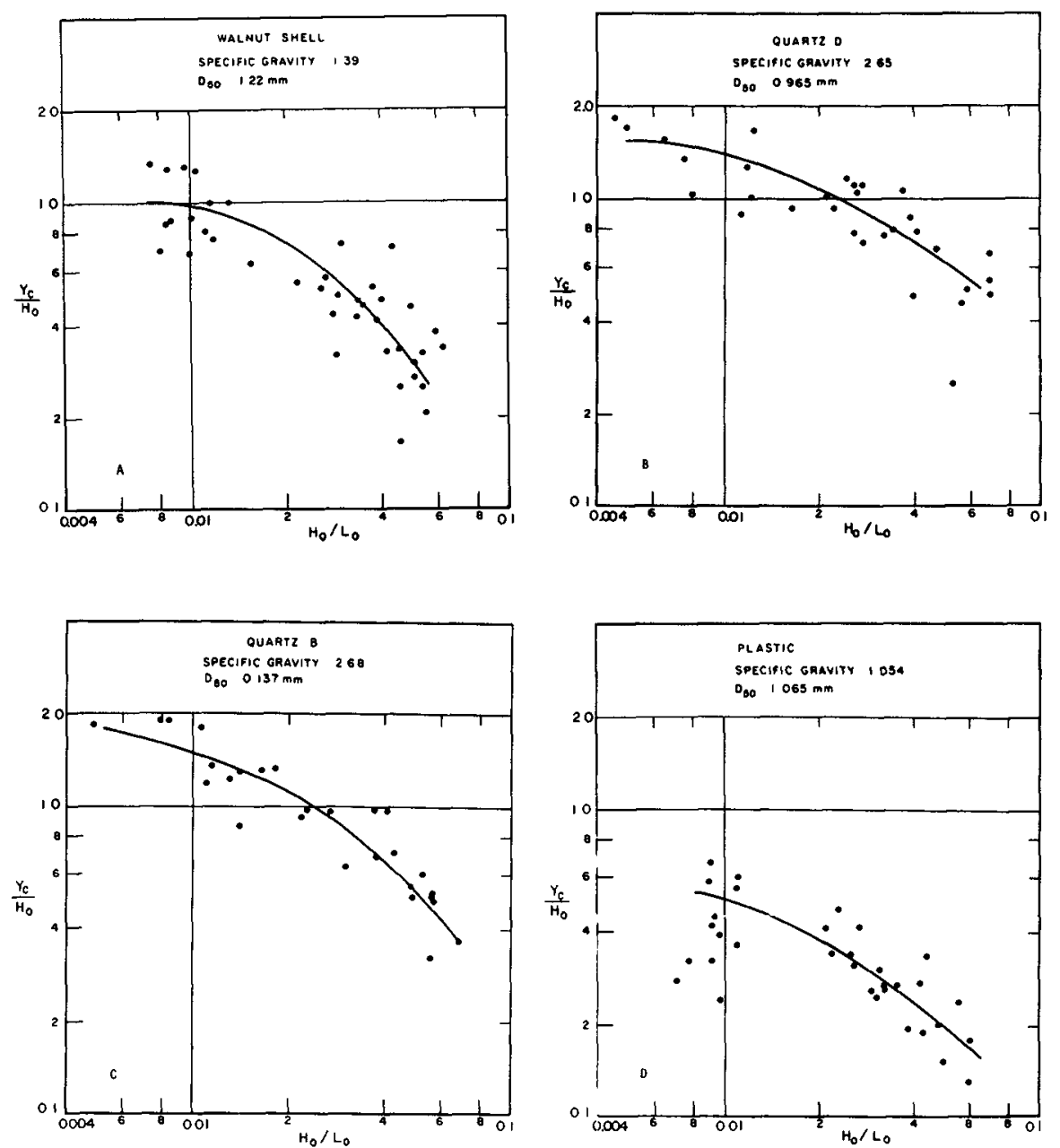

FIG 6 PLOT OF $Y_{C} / H_{0}$ VS $H_{0} / L_{0}$ FOR VARIOUS MATERIALS 
the curve is a curved line from $H_{0} / L_{0} \approx .05$ to .03 , the value of the parameter $H_{0} / O D$ increasing from 24 to abuut 220, where it turns round to join the straight line from the lower range. The curved line separating the two regions is, at the beginning, nearly horizontal for the range $\mathrm{H}_{\mathrm{O}} / \mathrm{SD} \approx 20$ to $1 \mathrm{CO}$. For the range of $\mathrm{H}_{0} / \mathrm{NL}-30$ to 260 it was possible to get a storm profile fur high values of wave oteepness as well as low values with an intermediate range in which the profiles were of summer type without any bar When compared with Fig. 1 , the critical wave steepnesses obtalned in this study are slightly higher probably due to the reflected energy not being taken into account. The results indicate that with certain combination of size and specific gravity it is possible to get "winter" profiles at low wave steepnesses as well as high ones with an intermediate range for which the profiles will be of "summer" type. This fict can be taken advantage of in achieving similarity in the type of profile in model studies.

Flg. 6 shows the almensionless wave run-up observed for different materials plotted against the deep-water wave steepness In each case the wave run-up decreases with increase in wave steepness because the steeper wavts break farther orfshure in greater depths. Plastic material gave the least uprush and the quartz sands the maximum while for walnut shell the value was intermediate to the other two. Irrespective of the size difference the value of the uprush was almost same for the quartz sands.

\section{Part B Reflection characteristics}

In this second part of the article the reflection characteristics of two-dimensional equilibrium profiles are considered $T_{t}$ is assumed that the action of a 
given wave condition on a certaln sediment results in a unique equilibrium profile when the waves are allowed to shape the brach for a sufflciently lone time. The variables involved in the reflection pnenomenon in that case wlll be

(1) percentage of energy reflected from the profile, $\mathrm{E}_{\mathrm{r}}$

(11) wave helght, $\mathrm{H}_{\mathrm{O}}$

(111) wave perlod, $T$

(iv) median size of grains, D

(v) mass density of sediment, $\rho_{s}$

(vi) mass density of fluid, $\rho_{f}$

(vil) depth of water, d

ine can, then, write

$I_{\mathrm{r}}=\mathrm{f}\left(\mathrm{H}_{\mathrm{O}}, \mathrm{T}, \mathrm{D}, \rho_{\mathrm{s}}, \rho_{\mathrm{f}}, \mathrm{d}\right)$

the assumptions regarding viscosity of the fluid and standard deviation of the grain size being same as in Part $A$ 'f the fall velocity, $V_{f}$, of the sediment is taken to characterise the sediment size and its aensity ana the density of the fluid, then one can write

Now

$$
\mathrm{E}_{\mathrm{r}}=\mathrm{f}_{1}\left(\mathrm{H}_{\mathrm{o}}, \mathrm{T}, \mathrm{V}_{\mathrm{f}}, \mathrm{d}\right)
$$

${ }^{+} \propto \mathrm{K}_{r}^{2}$

$\because \mathrm{K}_{\mathrm{r}}=\mathrm{f}_{2}\left(\mathrm{H}_{0}, 1, \mathrm{~V}_{\mathrm{f}}, \mathrm{a}\right)$

or

$$
f_{3}\left(K_{r}, \frac{H_{0}}{V_{f} T}, \frac{d}{V_{f}{ }^{T}}\right)=0
$$

in which $V_{f}$ and $I$ are rakun as repeating varlables.

This can also bc written as

$\mathrm{f}_{4}\left(\mathrm{~K}_{\mathrm{r}}, \frac{\mathrm{H}_{\mathrm{O}}}{\mathrm{V}_{\mathrm{f}} \mathrm{T}}, \mathrm{d} / \mathrm{h}_{\mathrm{o}}\right)=0$ if $\mathrm{V}_{\mathrm{f}}$ and $\mathrm{H}_{\mathrm{o}}$ are taken as the repeating variables. The third parameter in these relations is important if the reflection coefficient, $\mathbb{K}_{r}$, is measured in shallow water. Otherwise, the relation $F\left(\mathrm{~F}_{\mathrm{r}}, \mathrm{H}_{\mathrm{d}} \mathrm{V}_{\mathrm{f}} \mathrm{T}\right)=\mathrm{O}$ should apply and it is this relation 
which is empirically determined.

The experiments related to the second part of the study were conductea in a wave flume ( $1 \mathrm{~g} .3$ ) 2' wade, $60^{\prime}$ long and $1^{\prime}$ deep. Wlth the help of a longltudinal partition, only a narrow 6" wide section of the flume was used in the experiments. This ensured that the results were very little influenced by the secondary reflection takıng place from the wave generator. Five different materials, 1.e., all excepting the first and the third one in the list given in Fig.4, were used in this serles of runs. The following depths of water and wave perlods were utllised

\begin{tabular}{ccc}
\hline $\begin{array}{c}\mathrm{d} \\
\mathrm{ft}\end{array}$ & $\begin{array}{c}\mathrm{I} \\
\text { secs. }\end{array}$ & $\mathrm{d} / \mathrm{L}_{\mathrm{o}}$ \\
\hline 0.50 & 1.26 & $\mathrm{C} 0678$ \\
0.45 & 1.20 & 00610 \\
0.45 & 1.00 & 00878 \\
040 & 1.00 & 0.0543 \\
0.40 & 1.20 & 0.0781 \\
0.30 & 1.00 & 0.0586 \\
\hline
\end{tabular}

For each depth three alfierent wave heights were applied by changing the stroke of the wave generator. The duration of each run was on an avtrage two hours so that the beach attained substantial equilibrium betore observations were made. The coefficient of reflection, $\mathrm{K}_{r}$, was then found by recording the envelope of the wave system in front of the beach by a movable parallel - wirt resistance type wave gauge and noting the wave helghts at the node and ant1-node of the wave system and using these values in the Keulegan formula, If $\mathrm{H}_{\mathrm{n}}$ and $\mathrm{H}_{1}$ are the wave helghts registered at the $n c$ de and anti node respectively, then according to the linear wave theory 


$$
R_{r}=\frac{h_{1}-H_{n}}{h_{1}+H_{n}}
$$

The resulting error in using this expressior for shallow water waves in the calculation of the reflection coefficient is consiaered negligible.

The empirical ralationship between the reflection coefficient, $\mathrm{K}_{r}$, and the parameter, $\mathrm{H}_{\mathrm{O}_{1}} / \mathrm{V}_{\mathrm{f}} \mathrm{T}$, as developed from dimensional considerations above is shown in Fig.9. This plot using ordinary scales along the two axes was found to be the but form of representing this relation. The reflection cofficient reduces sharply from a high value of approximately 0.55 to about 0.10 as $\mathrm{H}_{\mathrm{ol}} / \mathrm{V}_{\mathrm{f}} \mathrm{T}$ Increases from 0.1 to 0.5 and thereafter $\mathrm{K}_{\mathrm{r}}$ shows $\mathrm{l}_{1}$ thle variation even when $H_{O I} / V_{f}$ is increased up to 2.0 The results of the two quartz sands fell in the range of $\mathrm{H}_{\mathrm{oI}} \mathrm{N}_{\mathrm{f}} \mathrm{T}-0.10$ to $\mathrm{C.37}$ and those of walnut shell and plastic in the range - 02 to 2.0

Figs.7 and 3 show the same relation for the bed material of grain size 1 and $0.5 \mathrm{~mm}$ respectively, the results for these dlagrams be ing taken from those of f1g.9. These figures have been arawn to observe the effect of size and specific gravity on the reflection coefficient. Within the same type of searment, the smaller size shuwed a higher trena of values of $K_{r}$. This is due to the reduced permcability and roughness magnitude. However op posing this tenaency is the fact that a larger quantity of the smaller size should be in movement ano suspension for the same wave conaltion and this should lead to a reduction in the value of $\mathbb{R}_{r}$ indicating increased absorption of energy. The resulting trend noticed in the experiments shows, however, that the effect on reflection of the latter factor is not so oignificant at least in the range involved. A change in 


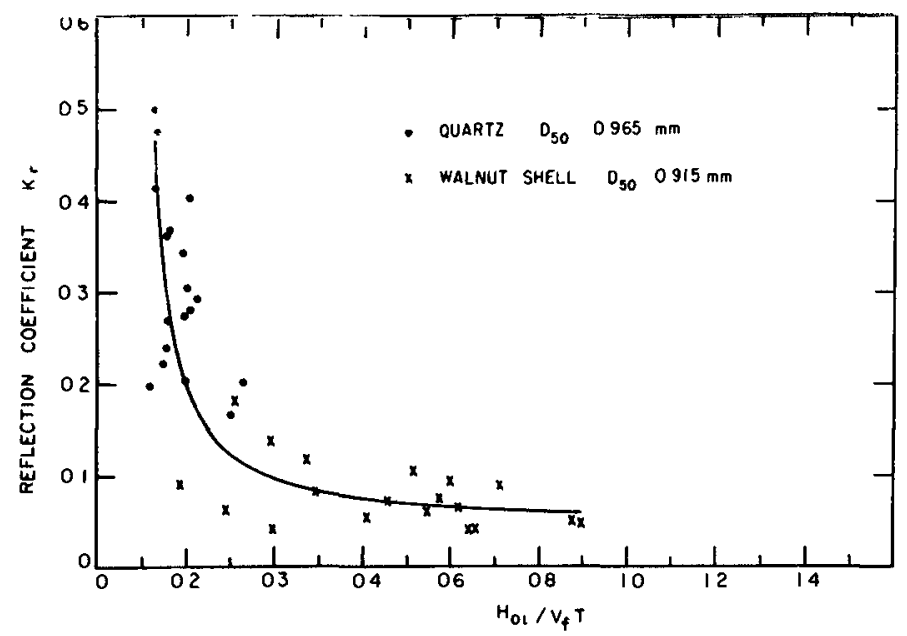

FIG 7 PLOT OF $K_{r}$ VS $H_{O I} / V_{f} T^{\prime}(D \approx 1 \mathrm{~mm})$

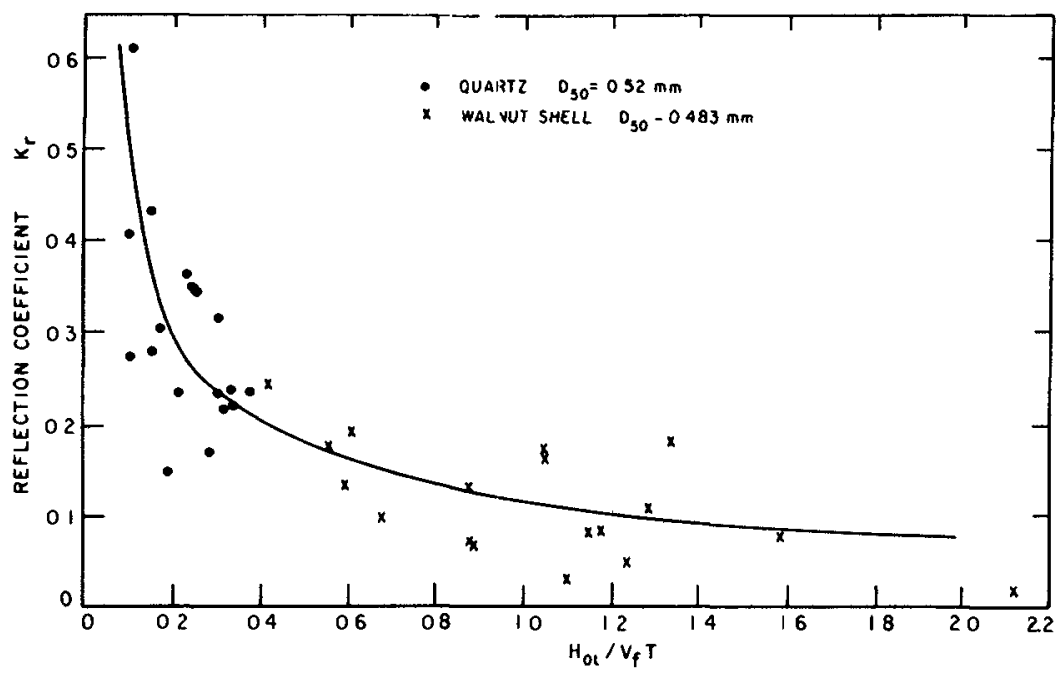

FIG 8 PLOT OF $K_{r}$ VS $H_{01} / V_{f}^{\top} \quad(D \approx 05 \mathrm{~mm})$ 


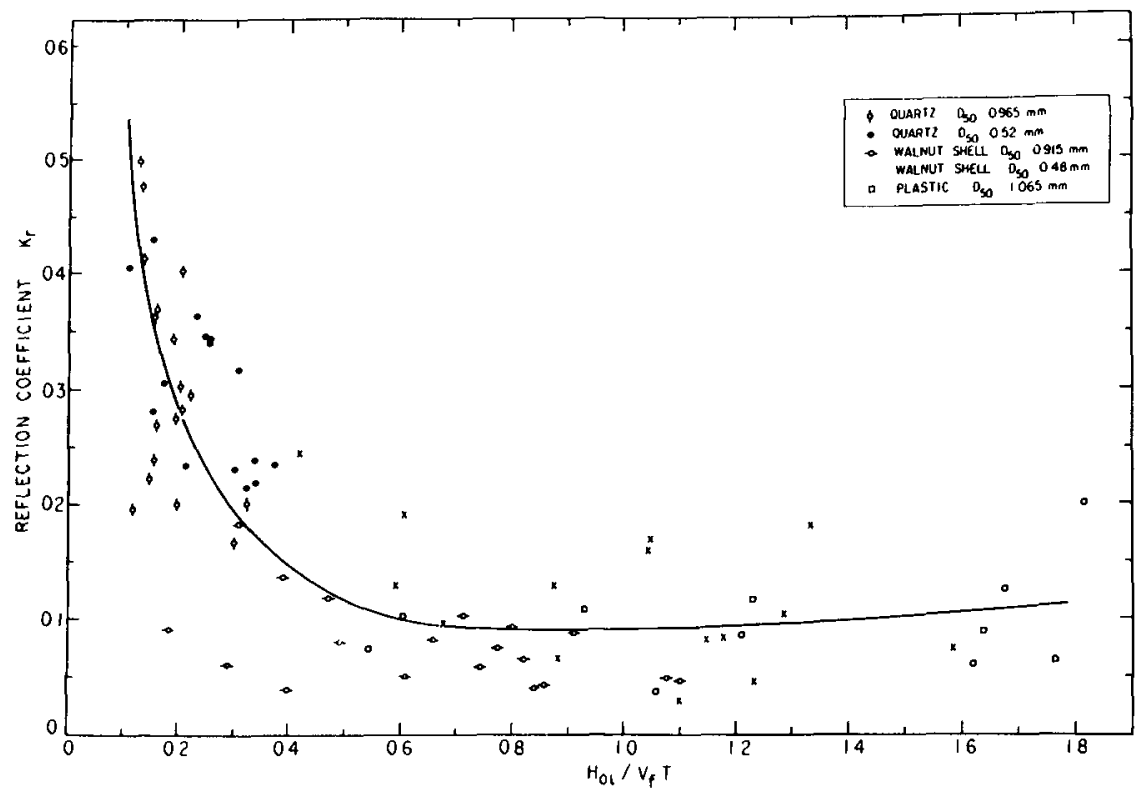

FIG 9 REFLECTION COEFFICIENT $K_{r}$ VS $H_{01} / V_{f}^{\top}$

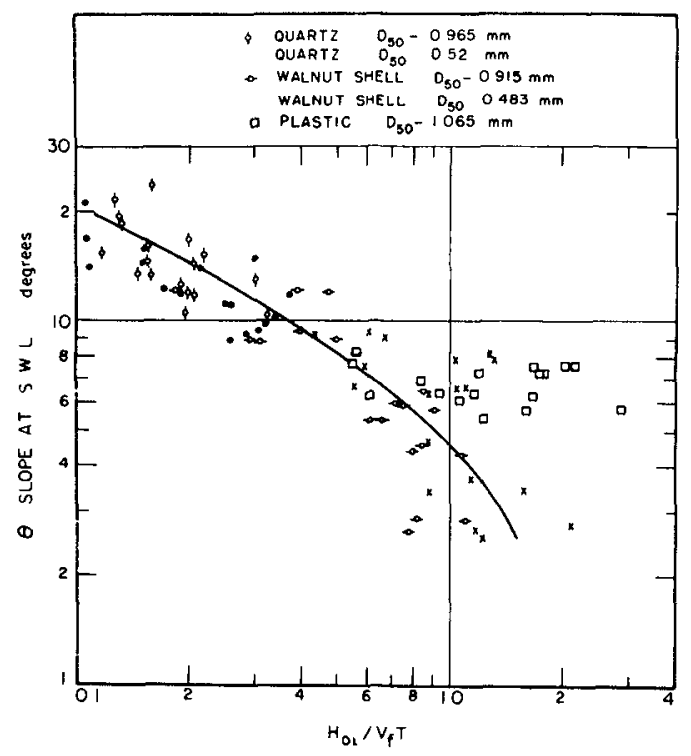

FIG 10 PLOT OF $\theta$ VS $\mathrm{H}_{01} / \mathrm{V}_{\mathrm{f}}^{\top}$ 
specific gravity, however, markealy effects the value of $\mathrm{K}_{r}$. If one assumes that the median dlameter cnaracterises the roughness matnitude as well as permeablilty, the effect of absorption of energy by the particles in motion at the bottom and those in suwpension can be estimated by looking at the variation of $\mathrm{I}_{\mathrm{r}} \mathrm{w}$ wh $\mathrm{H}_{\mathrm{O}} / \mathrm{V}_{\mathrm{f}} \mathrm{I}$ for nearly the same size but different type of material. Ihis can be done with the help of Figs. 7 and 8 .

Figure 10 shows the variation of the slope of the profile at the still water level, $\theta$, with the dimensionless parameter $\mathrm{H}_{\mathrm{O}_{1}} / \mathrm{V}_{\mathrm{f}} \mathrm{T}$ and $\mathrm{F}_{\mathrm{g}} .11$, the variation of $\mathrm{K}_{\mathrm{r}}$ with $\theta$. The use of a less dense artificial sand leads to a lowering of the slope, $\theta$. If the slope at still water level is taken to represent the slope of the profile, the effect of greater ouantity of material being in motion and suspension at higner values of $\mathrm{H}_{\mathrm{O}_{1}} / \mathrm{V}_{\mathrm{f}} \mathrm{T}$ is enhanced by that due to the reduction in slope. The slope decreases regularly from about $22^{\circ}$ to $2.5^{\circ}$ as $\mathrm{H}_{\text {ol }} / \mathrm{N}_{\mathrm{f}} \mathrm{I}$ is increased from 0.1 to about 2.C. For tie plastic material, however, the slope remalns nearly the same with an average value of about $6.5^{\circ}$. The behaviour of this material was characteristically different from the rest as in series'.

Fig. 1< is a logarithmic plot of slope at still water level versus incident wave steepness for al t the materials. In all cases except the plastic the slope is found to increase as the steepness is decreased.

Fig. 13 is a comparison plot of the results of this study using a movable bed with Miche's theoretical values as well as those of Greslou and Mahe, empirically obtained, for smooth impermeable slopes at constant vilues of deep-water wave steepness. Ihe range of values for wave steepness in all cases is .005 to .03 Although 
EQUILIBRIUM PROFILES
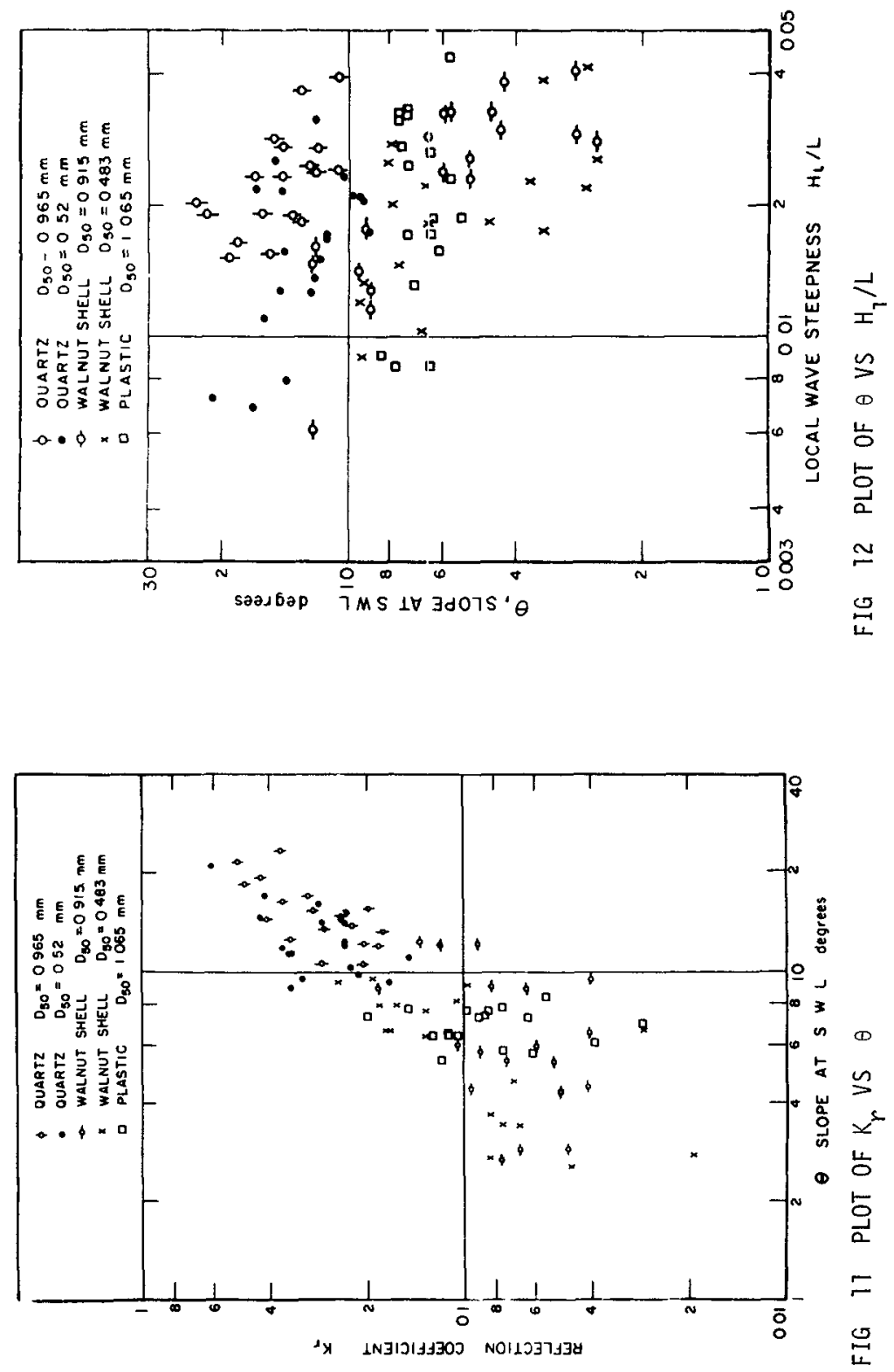


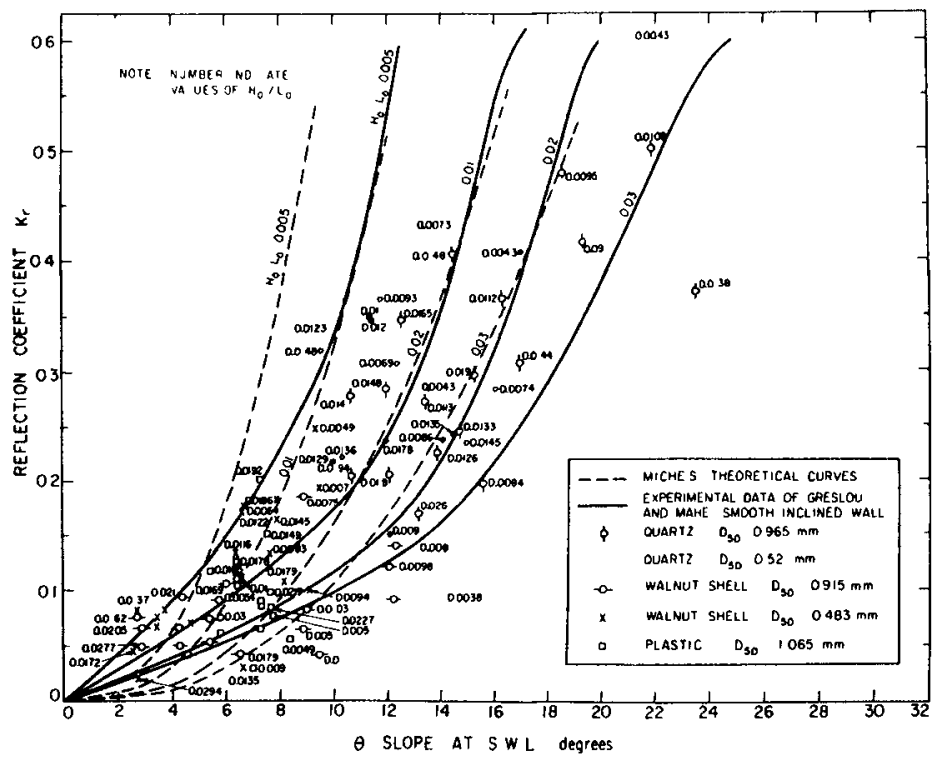

FIG 13 COMPARISON PLOT OF $K_{r}$ VS $\theta$

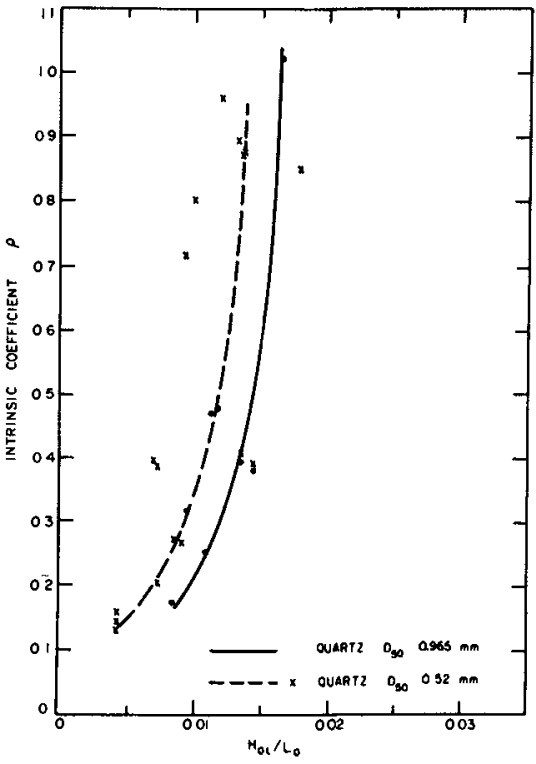

FIG 14 PLOT OF INTRINSIC COEFFICIENT, $\rho$ VS $\mathrm{H}_{01} / \mathrm{L}_{0}$ 
the total spread of the results agreed very closely with Mche's and Greslou's curves, no clear pattern of distribution of wave steepness as a third parameter could be noticed in the case of the movable bed which includes the effects of roughness, purmeability and suspension.

I $1 \mathrm{~g} .14$ gives the values of Miche's intrinsic coefficient, $P$, plottea as a function of the aeep-water wave steepness for the two sizes of quartz sands. The average value of $\rho$ was found to be $c .54$ and 0.49 for the larger and smallex size of sand respcctively. The values of the intrinsic coefficicnt for walnut shell ana plastic were found to be higher than 1.00 on an averate as thc slope, $\boldsymbol{\theta}$, produced by thesc materials were much lower than $10^{\circ}$ and Miche's theory was not applicable the variation of $P$ with $\mathrm{H}_{\mathrm{O} 1} / \mathrm{L}_{\mathrm{O}}$ follows the same pattern a that found in the studies at Mnnesota for artificial wave absorbers.

\section{Conclusions}

1. The criterion for generation of a longshore bar can be described in terms of deep-wcter wave steepness and the term $H_{0} / S n$ Certain combination of size ana specific Eravity may produce storm-type proflles at high wave cteepnesses as well as low ones with an intcrmealate range where the profiles are of summer type.

2 The parameter $\mathrm{H}_{\mathrm{O}} / \mathrm{V}_{\mathrm{f}} \mathrm{I}$ is very significant in determining the reflection coefticient of model beaches rcrmed of noncohtsive, muvable bed material of falrly uniform given size and density

3. The reflection coefficient rapidiy decreases as the parameter $\mathrm{H}_{\mathrm{Ol}} / \mathrm{V}_{\mathrm{f}}^{\mathrm{T}}$ increases and appears to attaln a cunstant value of abcut 0.07 over the range of the present experiments it does not appcar to have any 
alstinct relationship with deep-water wave steepness.

4. The slope at still-water level is relatea to the term $\mathrm{H}_{\mathrm{ol}} / \mathrm{V}_{\mathrm{f}} \mathrm{T}$ in a well aefined fashion. Ihe slope is affected to a greater extent by specific gravity than grain size of sediment. It is also influenced by the incident wave s teepness.

5. The value of Hiche's intrinsic coefficient for beaches composed of natural quartz is founa to be 0.5

\section{ACKNOWLEDGEMEN IS}

The work reported herein was carried out towards the requirements of the $\mathrm{Ph} . \mathrm{D}$. degree at the University of Calıfornı, Berkeley and the author would like to express his grateful thanks to Prof. J.W. Johnson for the advice and guldance received during the course of this study.

References

1. Nayak, I.V., "Fquilibrium Profiles of Model Beaches", Hydraulic ingl neering Laboratory Report No.HHL-2-25, University of Calıfornia, Berkeley, May, 1970. 


\title{
CHAPTER 83
}

\section{STOCHASTIC ANALYSIS OF BEACH PROFILE DATA}

By Choule $J$ Sonu and Myron $H$ Young

Coastal Studies Institute

Loulsiana State University

Baton Rouge, Louısıana

\begin{abstract}
Stochastic prediction of beach changes by means of a Ilnear leastsquares transfer function requires a knowledge of power spectra Since most field data are too short to ensure stable analysis, an attempt was made to generate data artifically by a Monte Carlo simulation A beach profile transition model which considers the beach profile as a dynamic system allows beach width, sediment storage, and surface configuration to be determined in successive profiles and simulates beach cycles assoclated with random waves whlch are in sufflclent agreement with the actual observation The simulated data are amenable to standard stochastic analysis to yleld power spectra, cross spectra, coherence functions, and phase lags Comparison of the results with those derived from actual data shows reasonable agreement It appears that the process of beach sediment storage involves a combination of classes of Markov Gaussian random processes, whereas that of beach width resembles a white nolse Coupling between these two parameters occurs in the lower frequency range with perlodiclties longer than about 8 days Moreover, the beach width shows phase advance before sediment storage Although the beach profile transition model requires further refinement, especially in regard to quantitative response to waves of various magnitudes and characteristics, the basic concept of the model is sound and will probably explain beach changes in various types of world coasts
\end{abstract}

\section{INTRODUCTION}

The process of beach change is stochastic in nature It is also a Markovian process in the sense that the resulting beach profile is partly a function of the preceding profile Stochastic analysis is a well-established method in many areas of geophysıcs (see, e $g$, Blackman and Tukey, 1958) but has seldom been applied to the study of beach changes An attractive feature of this approach is the possible design of an optimum transfer kernel function which will allow Iinear prediction of beach changes as extrapolated time serıes (Wiener, 1956, Lee, 1960)

A difficulty in the application of stochastic analysis to the process of beach change is the need for data with sufficient length To 1 llustrate the length of time required to gather a meaningful data set, consider semidiurnal sampling intervals and let the normalized standard error in a power spectrum be $\varepsilon$ This is calculated by (see, 
e $g$, Bendat and Plersol, 1966)

$$
\varepsilon=\sqrt{\mathrm{m} / \mathrm{N}}
$$

in which $m$ is the maximum correlation lag value and $N$ is the total sample size The relationship between the sampling interval, $\Delta t$, the maximum correlation lag, $\mathrm{m}$, and the equivalent resolution band width, $\mathrm{B}_{e}$, is

$$
\Delta t=1 /\left(B_{e} \quad m\right)
$$

Assuming a modest 20 percent for $\varepsilon$ and $2 \times 10^{-3}$ cycles per hour (CPH) for $B_{e}$, the required total sample length is

$$
\begin{aligned}
\Delta t \times N & =12 \times 10^{3} \text { hours } \\
& =500 \text { days } \\
& =1 \text { year } 4 \text { months }
\end{aligned}
$$

A continuous field operation requiring such a long period of time is impractical, if not impossible

To the writers' knowledge, the longest avallable beach profile data set is one representing 180 days of successive measurements by the Coasta1 Studies Institute, Lou1siana State University, on an Outer Banks beach, North Carolina (Dolan et al, 1969) However, it is evident that the data are still far short of the desired length

The purpose of this paper is to explore the possibility of generating beach profile data of desired length by means of stochastic simulation In the first step, a beach profile transition model is established which describes the sequence of profile changes under accretive or erosional wave excltation The beach profile is defined as a system which essentially incorporates three parameters beach width, sediment storage (or profile cross section), and surface configuration The pathways of transition of the surface configuration are dictated by the accretive and erosional wave actions, which are simulated by random numbers The actual values for the remaining two variables to assume are determined by the Monte Carlo method Time serıes of beach profiles thus generated are subjected to stochastic analysis The resulting power spectrum, cross spectrum, coherence function, and phase lag between the beach width and the sediment storage are then compared with the results obtained with actual data

\section{DATA ACQUISITION}

The field site was located at Nags Head, the Outer Banks, North Carolina (Fig 1) The coastline was relatively smooth, trended in the north-south direction, and faced the Atlantic Ocean on the east Rhythmic 


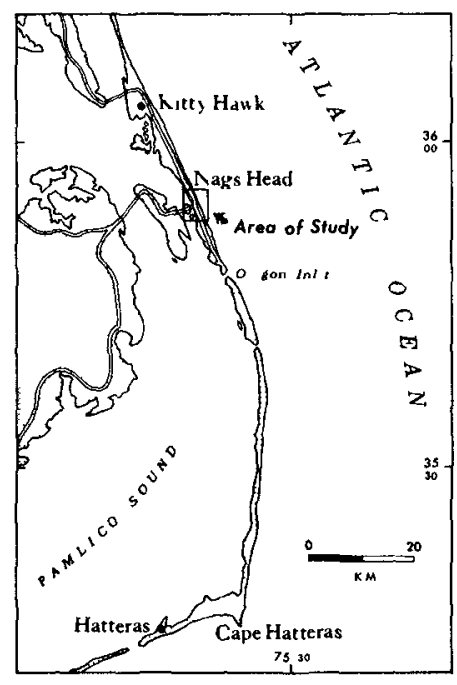

Fig 1 Location of Nags Head, site of field investigation beach features such as lunate bars and cuspate shorelines occurring on this coast have a1ready been discussed (Sonu and Russe11, 1966, Sonu et a1 , 1966)

Five traverses perpendlcular to the shoreline were set up 30 feet apart Stakes were set at 10-foot intervals on each traverse over the entire length of approximately 200 feet between the dune and the shoreline The crests of the stakes were initially surveyed in reference to a permanent datum, so that the successive profile surveys read in rapid sequence the exposed lengths of the stakes The accuracy of measurement of beach elevation was on the order of 001 foot

The field investigation was conducted between October 1963 and May 1964 The profiles were measured at successive sem1diurnal low tides at intervals of approximately 12 hours 25 minutes A stepresistance wave gage of the Coastal Eng1neering Research Center, placed 400 feet from the shore alongside a fishing pier, provided data

Figure 2 shows the cumulative distribution of these wave data, which were processed from 2-minute strip chart records at 4-hour intervals for about 6 months Waves higher than about 45 feet, representing a 25 percent probability of occurrence, broke over the outer bar, while those higher than about 25 feet, representing a 65 percent probability of occurrence, broke only over the inner bar The median wave height was slightly above 3 feet, and the standard deviation was 18 feet Wave periods occurred in a wide range, between 4 and 20 seconds

Figure 3 shows the mean profile and histograms of semidiurnal elevation changes Though there was an indication of net erosion on the upper beach level and of net accretion on the lower beach leve1, net equilibrium of sediment load was maintalned over the entire profile The higher intensity of elevation changes closer to the shoreline is indicated by the increase in the standard deviation with proximity to the shoreline Elevation changes were particularly active in an 80-foot width immediately next to the shoreline where the beach was washed by most of the ordinary waves Only storm waves coinclding with the flood tides sent swashes beyond this limit

\section{DERIVATION OF PROFILE TRANSITION MODEL}

In many of the past studies on beach change, it was customary to represent the beach profile by single parameters Thus, such parameters as beach width (or shoreline position relative to a fixed base line), beach face slope, and elevations at selected stations were singled out 


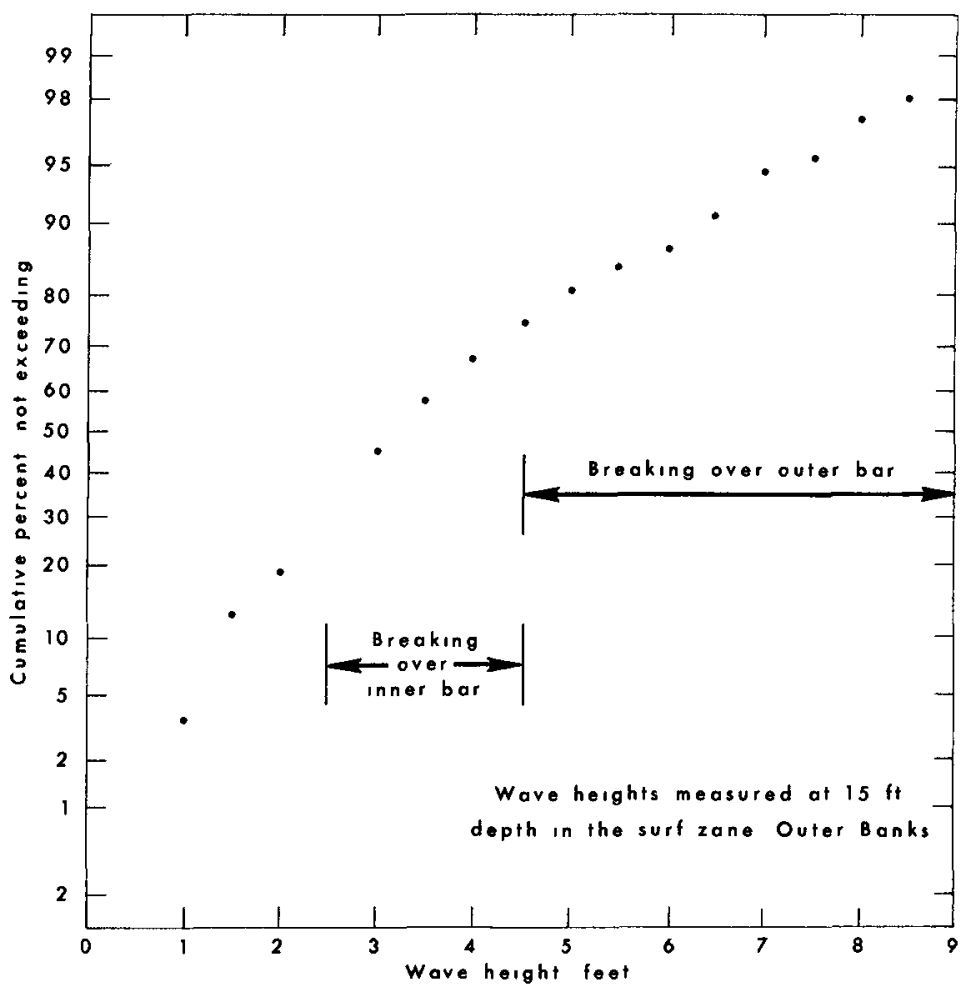

Fig 2 Cumulative distribution of wave helghts from 6month records between December 1963 and May 1964 at Jennette's Pier, Nags Head, North Carolina

and dealt with independently However, such an approach falls to treat the beach profile as a dynamic system

Sonu and van Beek (In press) introduced the Idea that the beach profile may be represented as a system in which varying amounts of sediment would assume varying distribution patterns within the varying subaerial spaces To describe this system, obviously, three parameters were needed the beach width $(X)$, which represented the subaerial space in a two-dimensional profile, the profile cross section ( $Q$ ), which represented the amount of sediment accommodated in the subaerıal beach space, and the surface configuration $(\Omega)$, which represented the manner by which the sediment was distributed For brevity, Q may be referred to as "sediment storage"

The predominant occurrence of sıx major profile configurations $(\Omega)$ was noted, as follows (see Fig 4) 


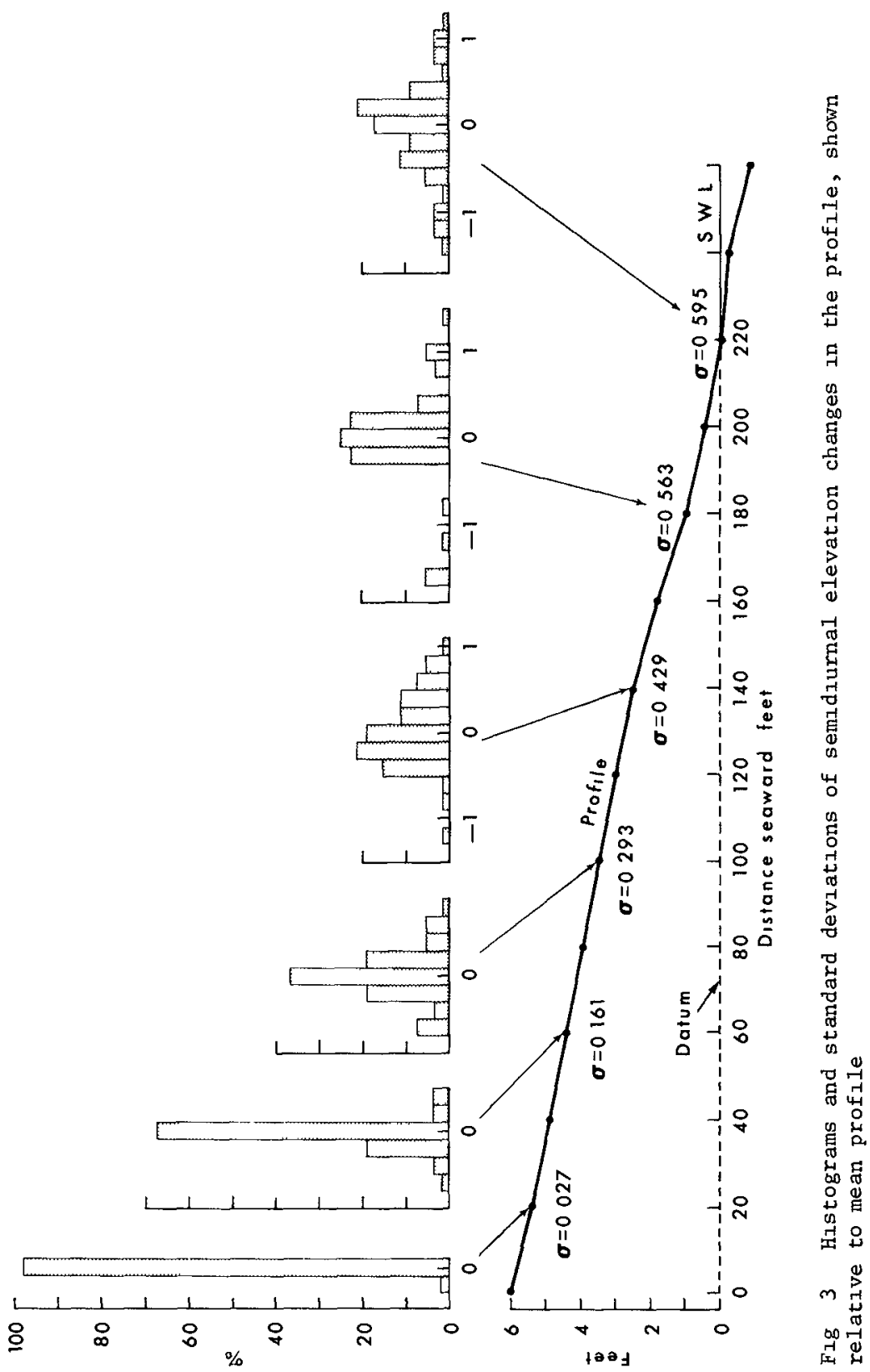


A smooth concave profile,

$A^{\prime}$ concave profile having a berm at the lower beach elevation,

B smooth linear profile,

$B^{\prime}$ linear profile having a berm at the intermediate beach elevation,

C smooth convex profile,

C' convex profile having a berm at the upper beach elevation

The relationship among the three beach profile parameters is illustrated schematıcally in Figure 4 According to this diagram, accretion of the profile (e $\mathrm{g}$, increase in sediment storage Q) is accomplished through elther growth in beach width (X) or transformation of surface configuration from concave to linear or from linear to convex profiles, in elther case involving a profile with a berm The pathways of profile transition are indicated by arrows Since the profile $C^{\prime}$ represents the maximum state of accretion, no further climb of a berm, hence no further accretion, would occur once this profile was realized

On the other hand, erosion of the profile (e $g$, decrease in sediment storage Q) is accomplished through elther decrease in beach width (X) or transformation of surface configurations from $C$ to $B$ or from $B$ to $A$, the process involving only the smooth profiles Note that profile A represents the maximum state of erosion, therefore, no further erosion would occur once this profile was formed

Another interesting characteristic of the profile transition model herein described is that it explains the occurrence of beach cycles by random wave excitation, e $g$, without considering cyclic energy input such as tides Because of the unidirectional accretive transitions through $A^{\prime}$, $B^{\prime}, C^{\prime}$, and also because of the unidirectional erosional transitions through

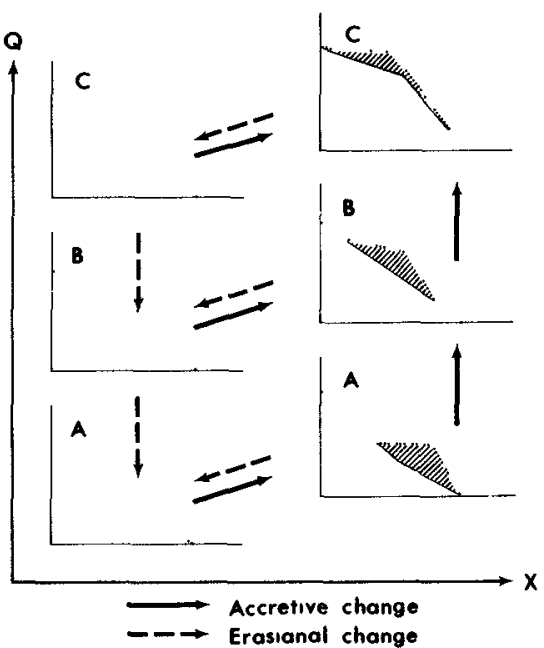

Fig 4 Beach profile transition model
$C, B$, and $A$, the beach changes in the long run are bound to produce a net loop of pathways connecting $A$, $\mathrm{A}^{\prime}, \mathrm{B}^{\prime}, \mathrm{C}^{\prime}, \mathrm{C}, \mathrm{B}$, and back to $\mathrm{A}$ A simulated analysis to test this cyclic characteristic was performed Uniformly distributed random numbers were used to simulate random wave excitation, and the number of transition steps needed to complete a cycle at each trial was counted Random numbers between 0 and 05 were consldered to represent accretive excitation, those between 05 and 1 were considered to represent erosional excitation A total of 3,000,000 trials were carried out on an IBM 360 Mode 165 computer, and the result is presented as a histogram in Figure 5 The mode, which represented the most frequent number of steps required to complete a cycl $\epsilon$ was located at 18 The actual observed cycle had 20 steps, showing close agreement with the experiment 


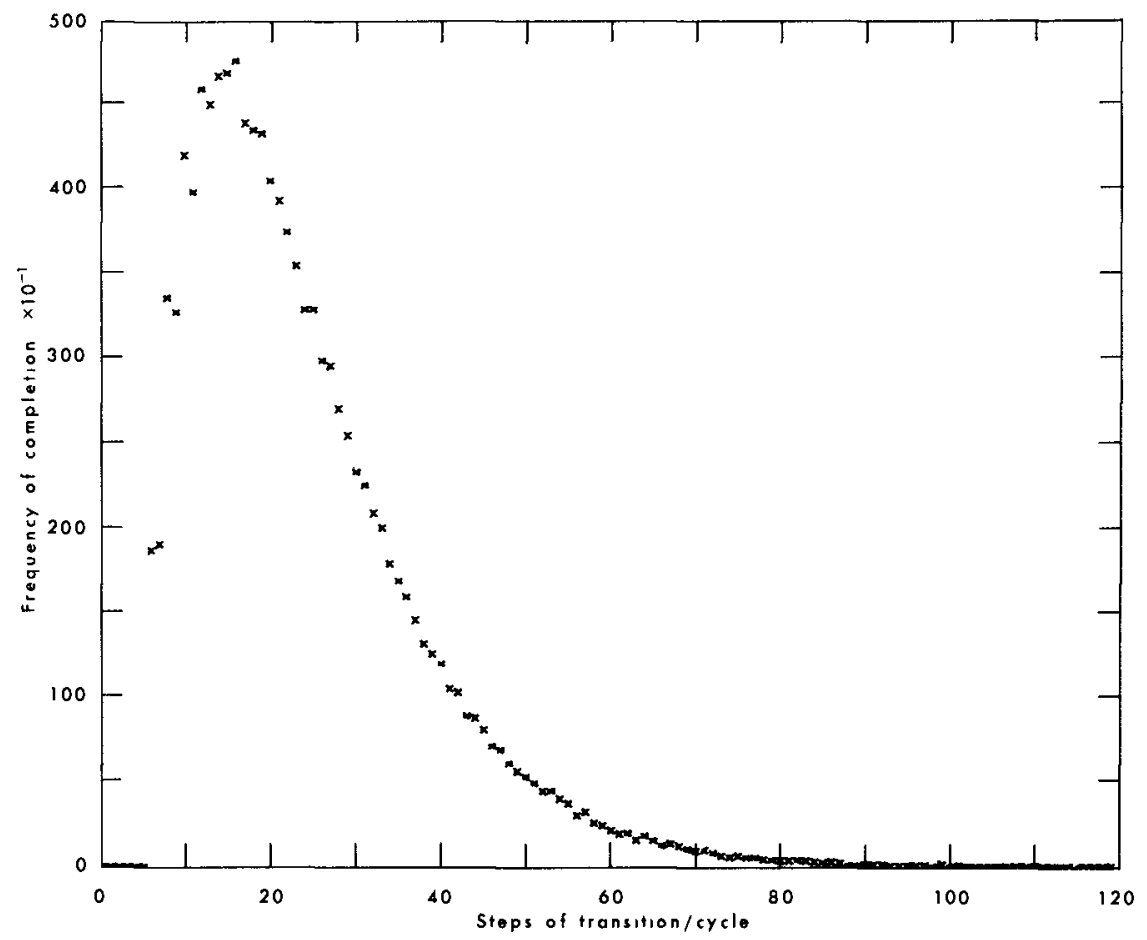

Fig 5 Histogram of the number of transition steps to complete a beach cycle upon $3,000,000$ trials

\section{DATA GENERATION}

Each of the surface configurations, $A, A^{\prime}, B, B^{\prime}, C$, and $C^{\prime}$, was represented by a linear regression relationship between $Q$ and $X$, as follows (see Fig 6)

For profiles $A$ and $A^{\prime}$

$$
\begin{array}{ll}
Q=045 X \\
A \quad 32<X<46, & 29<Q<41 \\
A^{\prime} \quad 46<X<55, & 41<Q<49
\end{array}
$$

For profiles $B$ and $B^{\prime}$

$$
\begin{aligned}
& Q=100 \mathrm{X} \\
& \mathrm{B} \quad 32<\mathrm{X}<43, \quad 32<\mathrm{Q}<43
\end{aligned}
$$




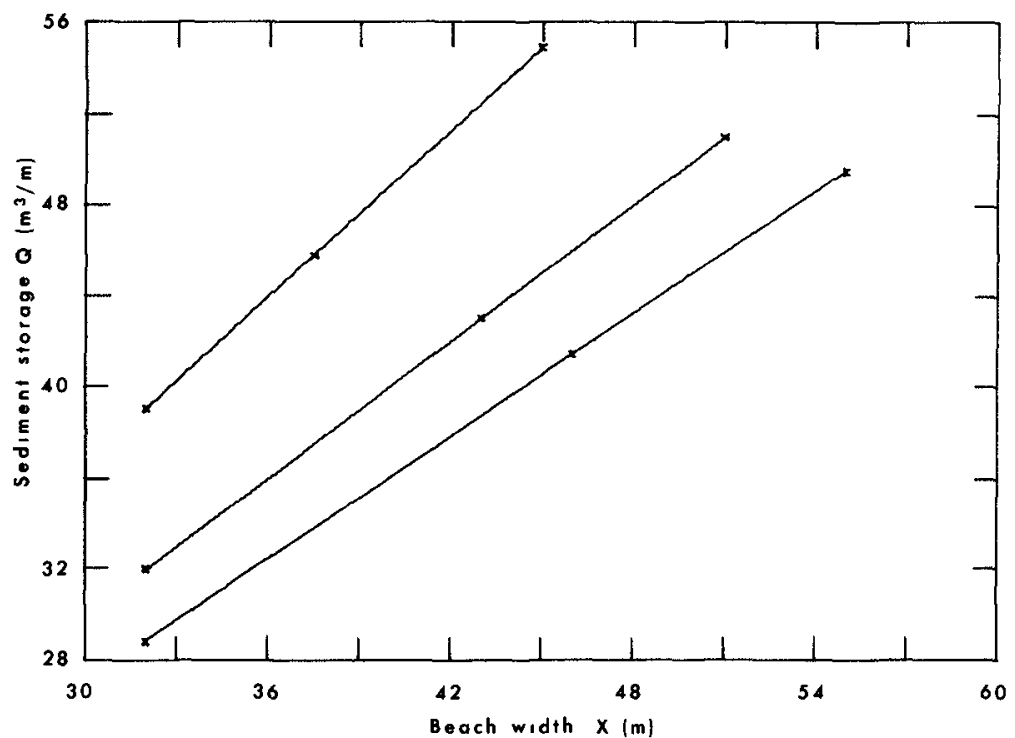

F1g 6 Representation of the beach profile transition model through linear regression relationships

$$
\text { B }^{\prime} \quad 43<\mathrm{X}<51, \quad 43<\mathrm{Q}<51
$$

For profiles $C^{\prime}$ and $C^{\prime}$

$$
\begin{array}{ll}
Q=122 X \\
C \quad 32<X<37, & 39<Q<46 \\
C^{\prime} \quad 37<X<45, & 46<Q<55
\end{array}
$$

The derivation of these relationships has been given elsewhere (Sonu and van Beek, in press)

The first step of data generation by the Monte Carlo technique is to draw a random number to induce a profile transition A number less than 05 meant accretive transition, and those larger than 05 , erosional transition In the next step, another random number was drawn to determine quantıtative measures of the resulting profile, e $g$, beach width $X$ and sediment storage $Q$

The quantitative profile was determined by using a random number as the proportional length in the corresponding regressive curve A point thus located in the curve ylelded a beach width $(X)$ and sediment storage $(Q)$ palr in accordance w1th F1gure 6 The random number generator was provided by the "RANDU" subroutine of the IBM scientific sub- 
routıne package A unıform distribution-type generator was used in view of the nature of the problem These steps were repeated to generate a succession of data in regard to $X$ and $Q$ The total sample size was 6,000 , It was limited by the computer storage capacity when spectral analysis was performed at the same time Figures 7 and 8 show part of the generated data

It was necessary to filter out low-frequency oscillations in order to eliminate the underlying trend This was done by smoothing with weighted running means

$$
\tilde{X}(k)=\sum_{1=-n}^{n} X(k+1) \quad W_{k+1}
$$

in which $1=-n,-n+1, \quad n-1, n$

$$
\{\mathrm{X}(\mathrm{J})\}, \mathrm{J}=\mathrm{k}+\mathrm{I}=1,2, \quad \mathrm{~N}
$$

are the original data, and

$$
\{\tilde{x}(k)\}, k=n+1, n+2, \quad N-n
$$

are the smoothed data The welghting function $W_{3}$ serves as a low-pass filter Therefore, the desired time series after removal of low frequencies is obtalned as

$$
\begin{aligned}
x^{\prime}(k) & =x(k)-\tilde{X}(k) \\
k & =n+1, n+2, \quad N-\mathfrak{n}
\end{aligned}
$$

Note that the sample size has now reduced to $N-2 n$

Figure 9 shows the response characteristics of the weighting functions An equal-welght smoothing function is a constant for all $\mathrm{J}$ 's, $e \mathrm{~g}$,

$$
\begin{aligned}
\mathrm{w}_{\mathrm{J}} & =1 /(2 \mathrm{n}+1) \\
\mathrm{J} & =\mathrm{n}+1, \mathrm{n}+2, \quad \mathrm{~N}-\mathrm{n}
\end{aligned}
$$

However, the range of summing, $\mathrm{n}$, may vary, $\mathrm{e} g, \mathfrak{n}=10$ and 30 As shown in Figure 9, the equal-welght filter with a larger range of summation $\mathrm{n}=$ 30 gives a cutoff at lower frequencies, thus saving a greater portion of the higher frequencies for analysis than the filter using $n=10$ This advantage is gained only at the expense of a larger amount of data, lost because the data length is reduced to $N-2 n(N-60)$ Note also the prominent peaks, which exceed unity, followed by rippling effects, which 

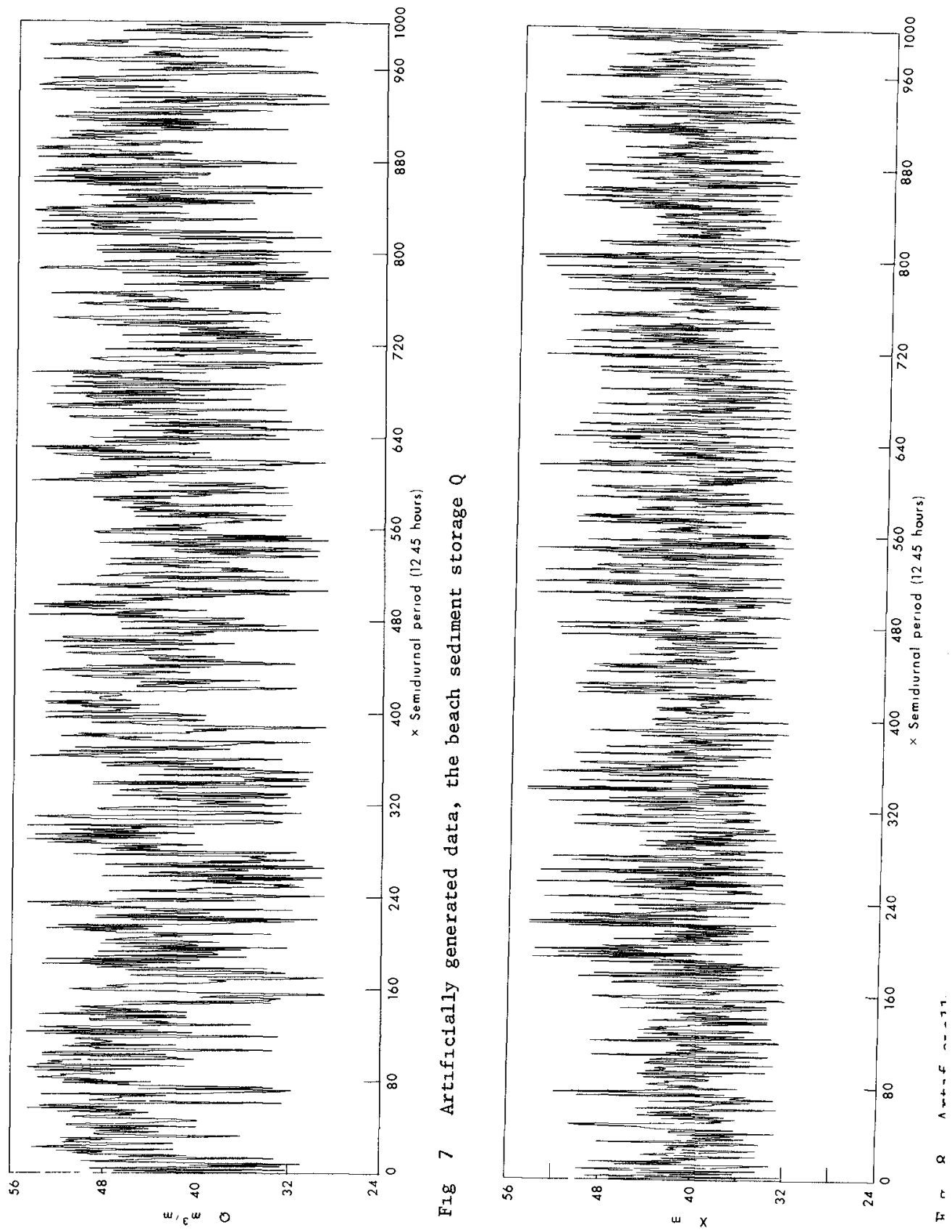


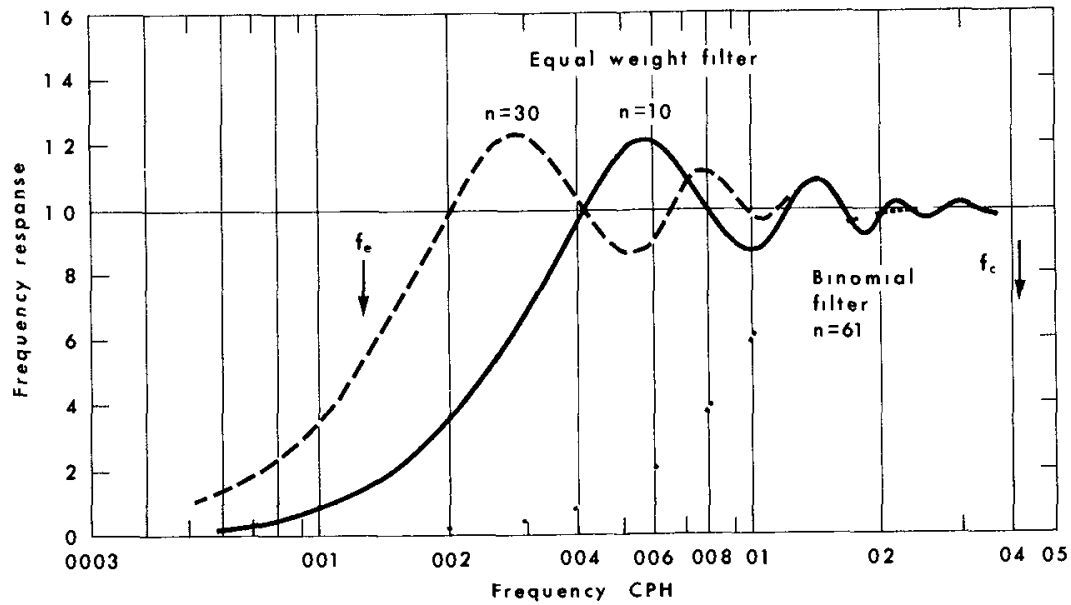

F1g 9 Frequency response characterıstics of various high-pass (smoothing) filters

may produce corresponding false peaks in the power spectrum It is also known that the equal-weight function produces polarity reversal in the filtered data (Holloway, 1958) These problems may be overcome by using a binomial weighting function But, as seen in Figure 9, a cutoff at a sufficiently low frequency cannot be expected without a large $n$, hence a considerable reduction in the data size After repeated trial-and-error calculations it was decided to use an equal-weight filter with $n=10$ and a binomial filter with $\mathrm{n}=61$ Figures 10 and 11 show the real data and the filtered data, respectively, using the binomial filter for sediment storage

\section{DATA ANALYSIS}

Table 1 shows the frequency resolution $f_{e}$ and the Nyquist frequency $f_{c}$ for the real and the simulated data

Table 1 Frequency Resolution $F_{e}$ and Nyquist Frequency $f_{c}$ for the Real and Simulated Data

\begin{tabular}{|l|c|c|}
\hline & $f_{e}$ & $f_{c}$ \\
\hline Real data & 00156 & 00402 \\
\hline S1mulated data & 00003 & 00402 \\
\hline
\end{tabular}

The power spectrum, cross spectrum, coherence function, and phase 


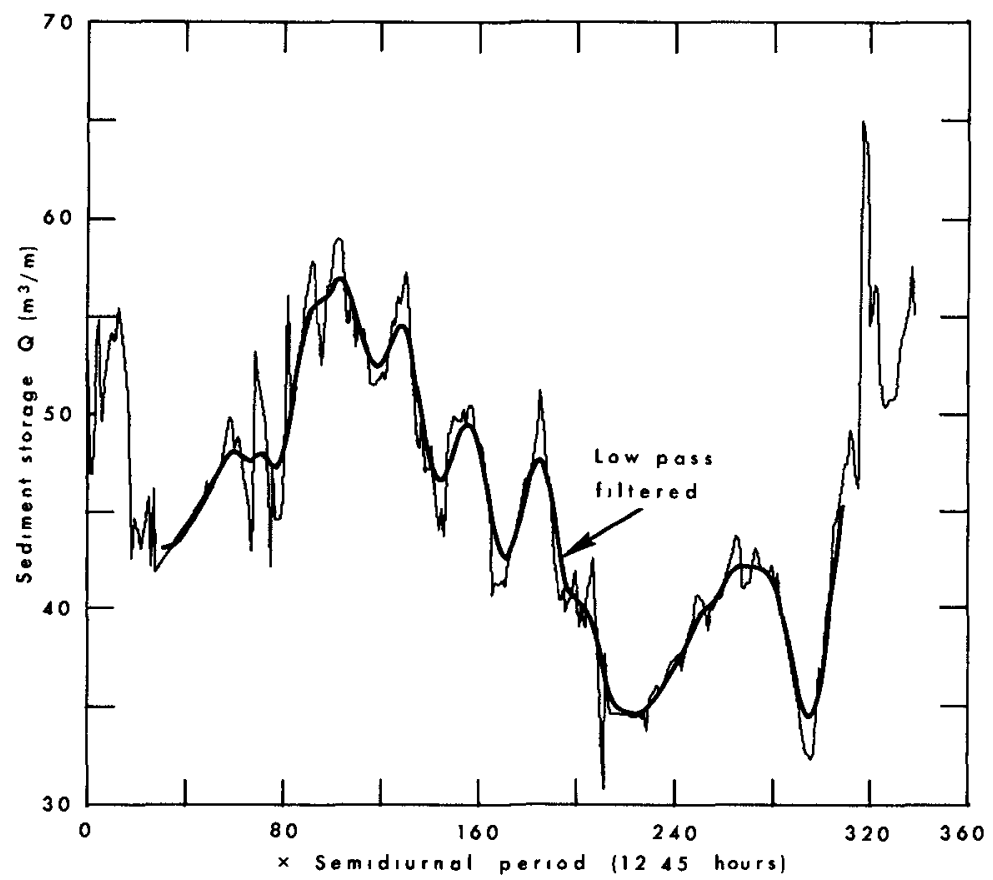

Fig 10 Original real data, the beach sediment storage $Q$, shown with the binomial low-pass filtered data in thick line

lag calculations in the present study are essentially the same as described by Blackman and Tukey (1958) and Bendat and Piersol (1966)

However, the Fast Fourler Transform (FFT) program by Cooley and Tukey (1965) is used to improve the accuracy of calculation as well as to reduce computational time (see also Rothman, 1968)

The autocovariance function at the $k$-th lag is given as

$$
R(k)=\frac{1}{N-k} \sum_{1=1}^{N-k} X(1) \quad X(1+k) / R(0)
$$

The one-side power spectrum can be calculated as a Fourier transform of the autocovariance function,

$$
G\left(\frac{k f}{m}\right)=2 \Delta t\left[R(0)+2 \sum_{1=1}^{m-1} R(k) \cos \left(\frac{\pi 1 k}{m}\right)-(-1)^{k} R(m)\right]
$$

The FFT subroutine computes the values within the brackets For 


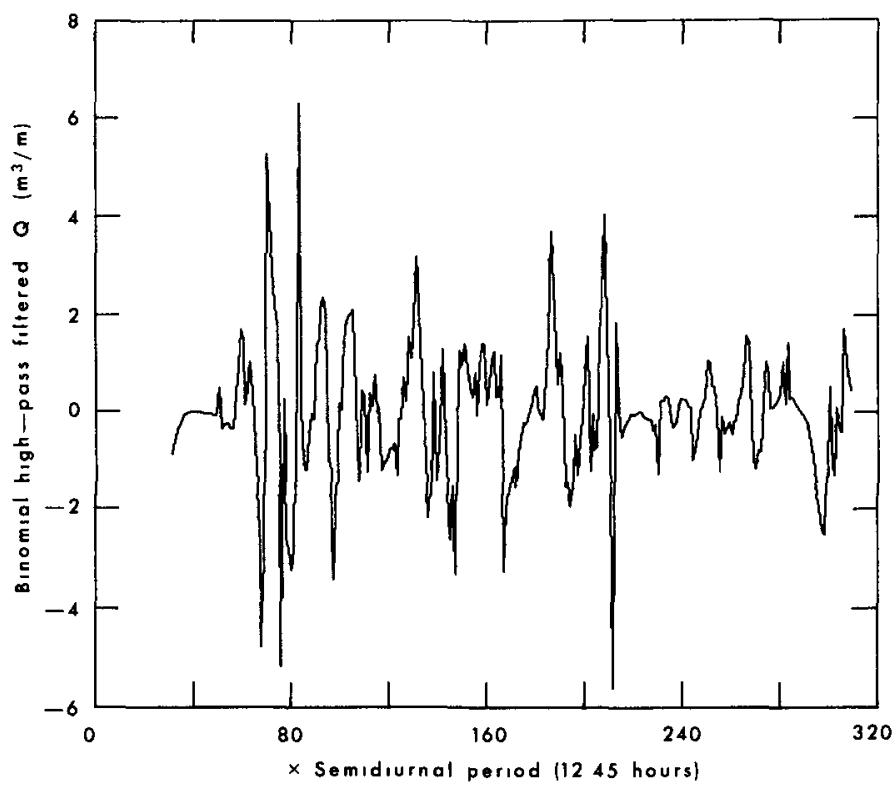

F1g 11 Real data upon subtraction of the binomial low-pass filtered data from the original data ( $n=61$ )

stochastic representation, the final estımates are smoothed by Hannıng's equation (see Blackman and Tukey, 1958)

The coherence function and phase lag of beach width and sediment storage are calculated by first obtaining the cross covariance functions between the time series data of $X$ and $Q$

$$
\begin{aligned}
& R_{X Q}(k \Delta t)=\frac{1}{N-k} \sum_{n=1}^{N-k} x_{n} Q_{n}+k \\
& R_{Q X}(k \Delta t)=\frac{1}{N-k} \sum_{n=1}^{N-k} Q_{n} X_{n}+k
\end{aligned}
$$

The cross spectral function $G_{X Q}$ (f) is then obtained from the cospectrum and quadrature spectrum, which are the Fourier transform of the modified functions of the above cross covariance functions RXQ and $\mathrm{R}_{\mathrm{QX}}$ These relations are given in equations (18) through (22)

$$
G_{X Q}(f)=C_{X Q}(f)-J D_{X Q}(f)
$$


In wh1ch $C_{X Q}(f)$ is the cospectrum, $D_{X Q}(f)$ is the quadrature spectrum, and $J=\sqrt{-1}$ They are related to the cross covariance functions as

$$
\begin{aligned}
& \mathrm{C}_{\mathrm{XQ}}\left(\frac{\left.\mathrm{kf} \mathrm{f}_{\mathrm{c}}\right)}{\mathrm{m}}=2 \Delta \mathrm{t}\left[\mathrm{A}_{0}+2 \sum_{1=1}^{\mathrm{m}-1} \mathrm{~A}_{1} \cos \left(\frac{\pi 1 \mathrm{k}}{\mathrm{m}}\right)+(-1)^{\mathrm{k}} \mathrm{A}_{\mathrm{m}}\right]\right. \\
& \mathrm{D}_{\mathrm{XQ}}\left(\frac{\mathrm{kf} \mathrm{f}_{\mathrm{c}}}{\mathrm{m}}\right)=4 \Delta t \sum_{1=1}^{\mathrm{m}-1} \mathrm{~B}_{1} \sin \left(\frac{\pi 1 \mathrm{k}}{\mathrm{m}}\right)
\end{aligned}
$$

where $A_{1}$ and $B_{1}$ are the modified cross covarı ance functions

$$
\begin{aligned}
& A_{1}=1 / 2\left(R_{X Q}(1 \Delta t)+R_{Q X}(1 \Delta t)\right) \\
& B_{1}=1 / 2\left(R_{X Q}(1 \Delta t)-R_{Q X}(1 \Delta t)\right)
\end{aligned}
$$
be obtained

Finally, the coherence function $\gamma_{k}^{2}$ and the phase angle $\phi_{k}$ can

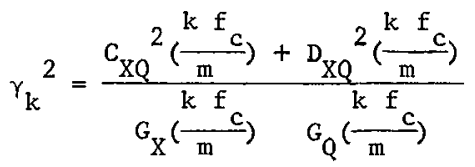

$$
\begin{aligned}
& \left.\phi_{k}=\tan ^{-1} \frac{D_{X Q}\left(\frac{k f c}{m}\right)}{c_{X Q}\left(\frac{k f}{m}\right)}\right)
\end{aligned}
$$

The Monte Carlo method requires repeated trials of data generation and analysis so that the end product may be evaluated through the central limit theorem In the present study, the results of the initial several trials were so simılar to each other that no further calculation was pursued

\section{INTERPRETATION}

Figure 12 shows the power spectrum for Q The real-data spectrum using an equal-welght filter and one using a binomial filter show peaks in the spectral density at 0003 and $0008 \mathrm{CPH}$, respectively A gross peak is also located near $0010 \mathrm{CPH}$ in the simulated power spectrum using a binomial filter However, all these peaks are spurious since 


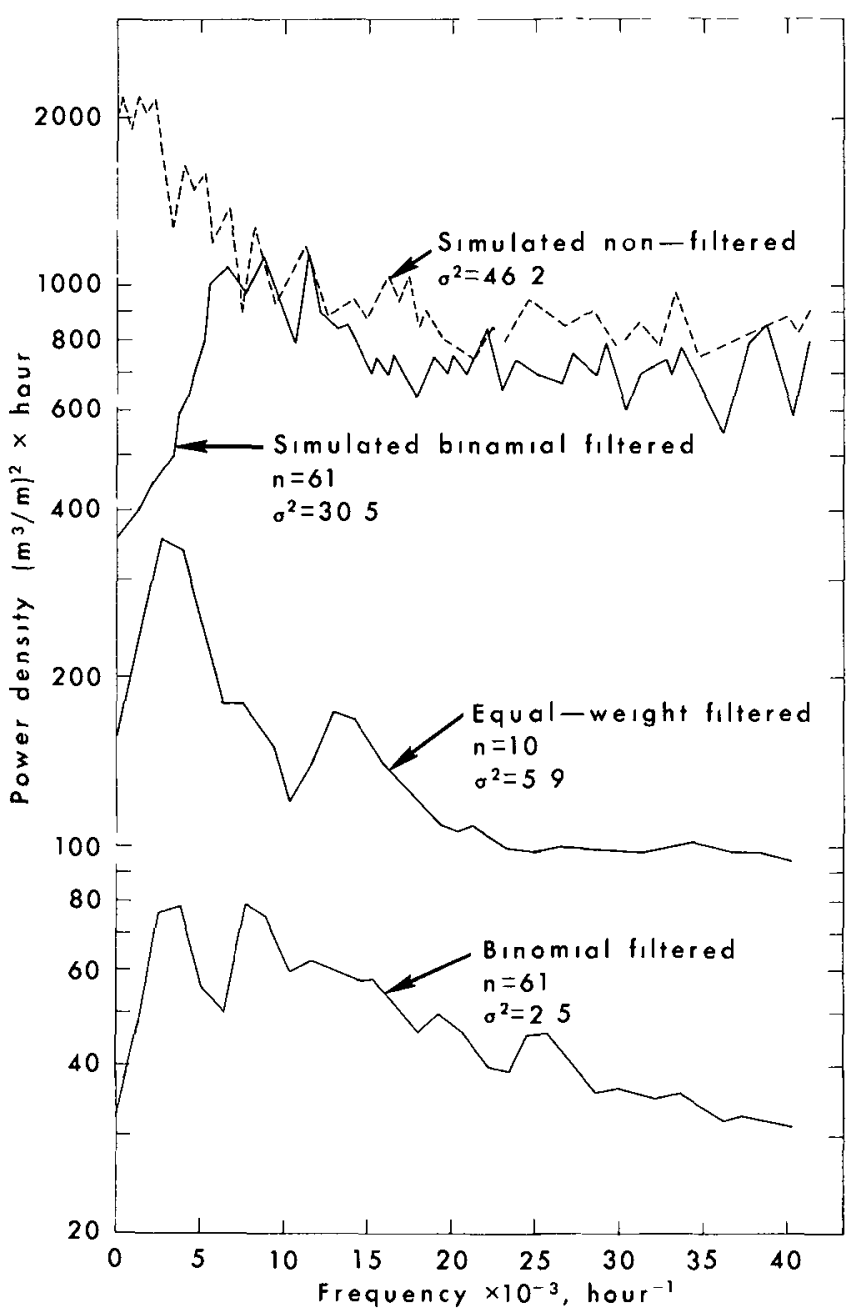

Fig 12 Power spectrum of Q (sediment storage) Data smoothed with equal-we1ght filter $(n=10)$, with binomal filter $(\mathrm{n}=61)$, simulated data using binomial filter, and simulated data with no smoothing

they represent the effect of $10 \mathrm{w}$-frequency cutoff by the high-pass filter used (see F1g 9) The true spectrum for the actual sediment storage Q is very likely to be a smooth curve which has no prominent peak but attenuates monotonically toward high frequencies (see Fig 17, case 1) This type power spectrum was indeed obtained from the simulated data in 
which no high-pass filter was applied (The ripples are probably the result of intrinsic noise in the simulation process)

The minımum number of transition steps necessary to complete a beach cycle is six, representing a frequency of $0013 \mathrm{CPH}$ The majority of beach cycles take more steps than $s i x$, which corresponds to frequencles shorter than $0013 \mathrm{CPH}$ The most probable number of steps is 18, which gives a frequency of $00067 \mathrm{CPH}$ In Fıgure 12 beach cycles shorter than $0013 \mathrm{CPH}$ indeed constitute the major part of the simulated power density Note particularly that a peak appears at $0005 \mathrm{CPH}$, which is equivalent to a 16-step simulated beach cycle and hence approximates the observed 20-step beach cycle of the real data reasonably well This peak is especially noteworthy because of 1 ts occurrence in the frequency range where the response has been considerably reduced by the high-pass filter

There are smaller beach cycles in the analysis For example, the four-step cycles through $A, A^{\prime}, B^{\prime}, B$, and $A$ or through $B, B^{\prime}, C^{\prime}, C$, and $B$ represent a frequency of $00201 \mathrm{CPH}$ The two-step cycles through $A$, $A^{\prime}$, and $A$, through $B, B^{\prime}$, and $B$, or through $C, C^{\prime}$, and $C$ represent a frequency of $0040 \mathrm{CPH}$ However, they fall to comprise a significant level of power spectral density

The discrepancy between the actual and the simulated power spectra of $Q$ is due mainly to the fact that in the simulation the profile transition was only allowed to take either erosional or accretive pathways, disregarding the third possibility, in which the profiles would remain unchanged As a result, the variability was exaggerated in the simulation, leading to a larger value of power density $\left(\sigma^{2}\right)$ This discrepancy may be adjusted as the interaction between the profile and wave characteristics is better established

Power spectra for the real-data beach width $X$ are shown in Figure 13 Both an equal-welght filter and a binomial filter are used in the analysis The density concentration is found in the frequencies below $0015 \mathrm{CPH}$, which corresponds to a period of 60 hours or a five-step transition period Although the binomial filter allows only 40 percent or less response for this frequency range, the filtered spectra reveal peaks at $0012 \mathrm{CPH}$ as we11 as at $0005 \mathrm{CPH}$ The $0012 \mathrm{CPH}$ frequency corresponds to a period of about 83 hours or a 6 7-step transition period

The simulated power spectrum for $X$, on the other hand, is quite inconsistent Repeated calculations using different random numbers showed that individual peaks in this power spectrum were fortuitous Therefore, they may average out only if a long record can be simulated in the analysis The general trend of the simulated power spectrum for X strongly suggests a similarity to a white nolse spectrum, which characterizes a consistent power density level for all frequencies It is not immediately clear why the beach width should behave so differently from the sediment storage

Figure 14 shows coherence functions between the beach width $X$ and the sediment storage $Q$ The real-data coherence functions are obtained by using both equal-welght filter and binomial filter Both curves show a hıgh level of coherence About 50 percent coherence is found for 


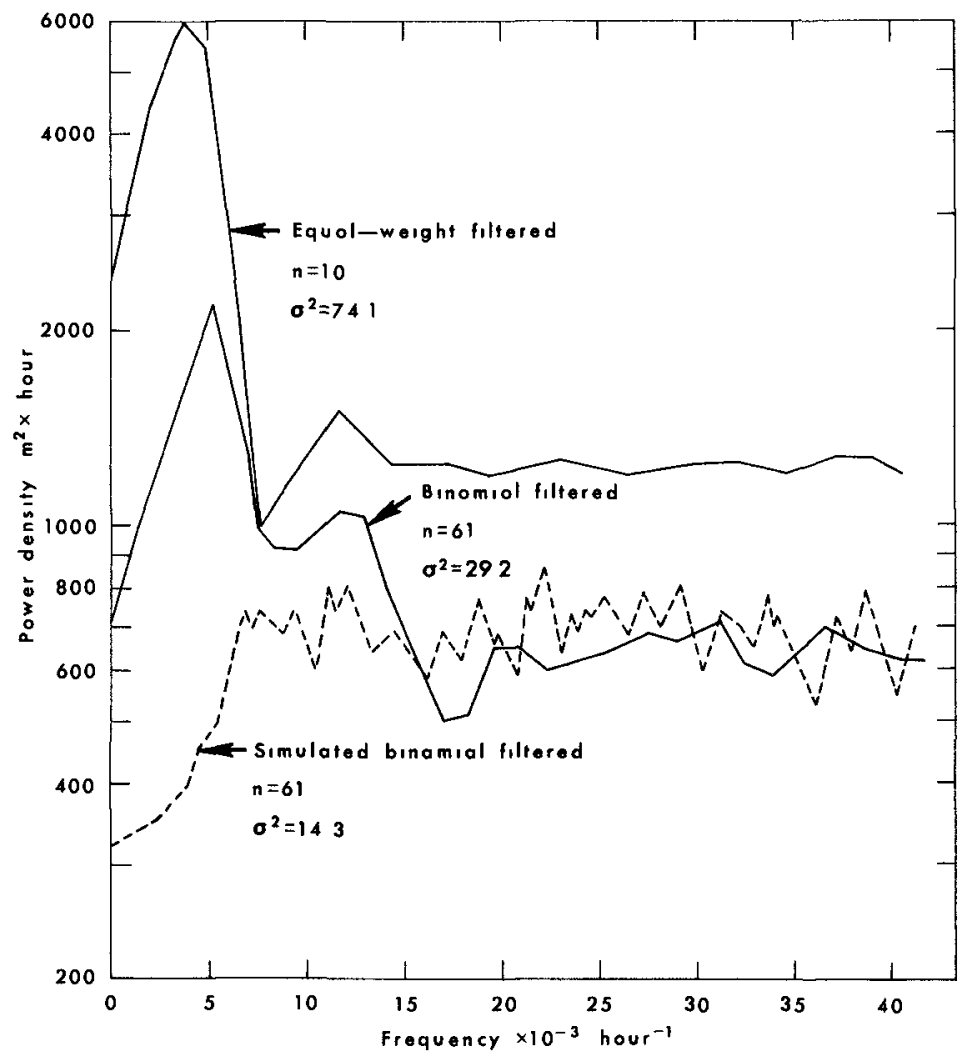

Fig 13 Power spectrum of $X$ (beach width) Data smoothed with equal-welght filter $(n=10$ ), data smoothed with b1nomial filter $(n=61)$, and simulated data

frequencies below about $0005 \mathrm{CPH}$, which is a region where the beach width power spectrum displays a peak density The coherence drops sharply to less than 20 percent at higher frequencles, which means that the coupling between the beach width and the sediment storage is basically a low-frequency phenomenon with perlodiclties of 8 days or longer

A discrepancy with the above results is noted in the simulated coherence function Here, the coherence is low at low frequencies and high at high frequencies A probable explanation for this may be revealed from the following considerations The beach process is basically a response to a weather regime which will change gradually with perlodiclties of several days Consequently, following "persistence" In natural processes, the beach width change will essentially be a gradual process in which the effect of the preceding profiles 


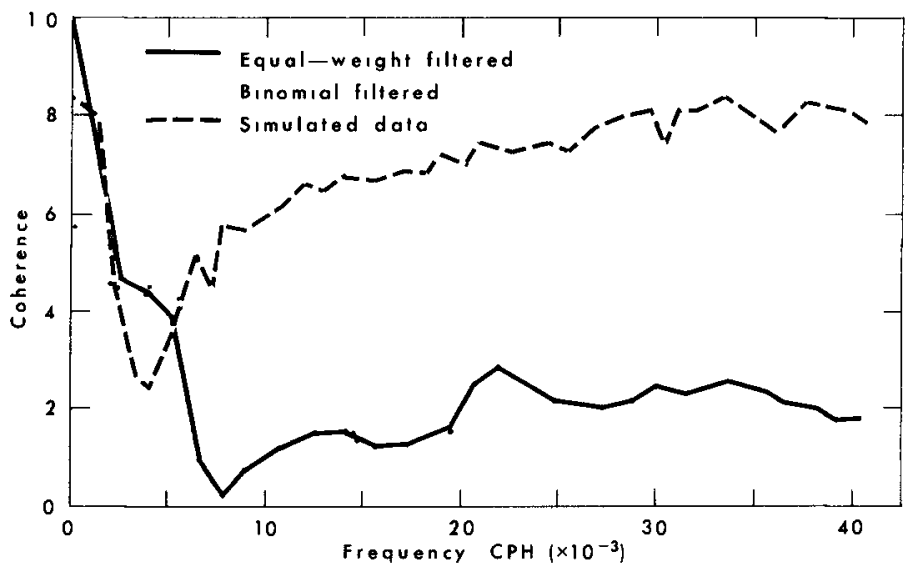

Fig 14 Coherence function for $X$ and $Q$ Data smoothed with equal-welght filter $(n=10)$, smoothed with binomial filter $(n=61)$, and simulated data

W1 11 persist for some time In the data simulation, the effect of persistence was taken into consideration in the transition of surface configurations but not in the successive values of beach width and sediment storage These parameters were determined by random numbers not influenced by their preceding values Consequently, the coupling effect with periodiclties on the order of the weather influence may not be expected The high coherence at high frequencies in the simulated coherence function is not important because the power density in the corresponding frequency range is extremely low

Figure 15 shows phase lags in the correlation between the beach width and the sediment storage In the real data, the sediment storage lags behind the beach width in the low-frequency region, where a coherence coupling between the two parameters exists At somewhat higher frequencles, however, this relationship is reversed, and eventually the phase difference disappears at frequencies higher than about $0020 \mathrm{CPH}$, which corresponds to 2 days or less in period In the simulated data, the phase lag of the sediment storage also lags behind the beach width However, it appears at a much lower frequency than that of the real data It is then followed by a reversed relationship and eventually reduces to a zero phase difference at frequencles higher than $0020 \mathrm{CPH}$

\section{DISCUSSION}

Dynamic beach transitions are Gaussian processes in nature Therefore, these random processes deserve special attention for two reasons

1 They approximate reasonably well a number of experimental data involving nolse and random phenomena 


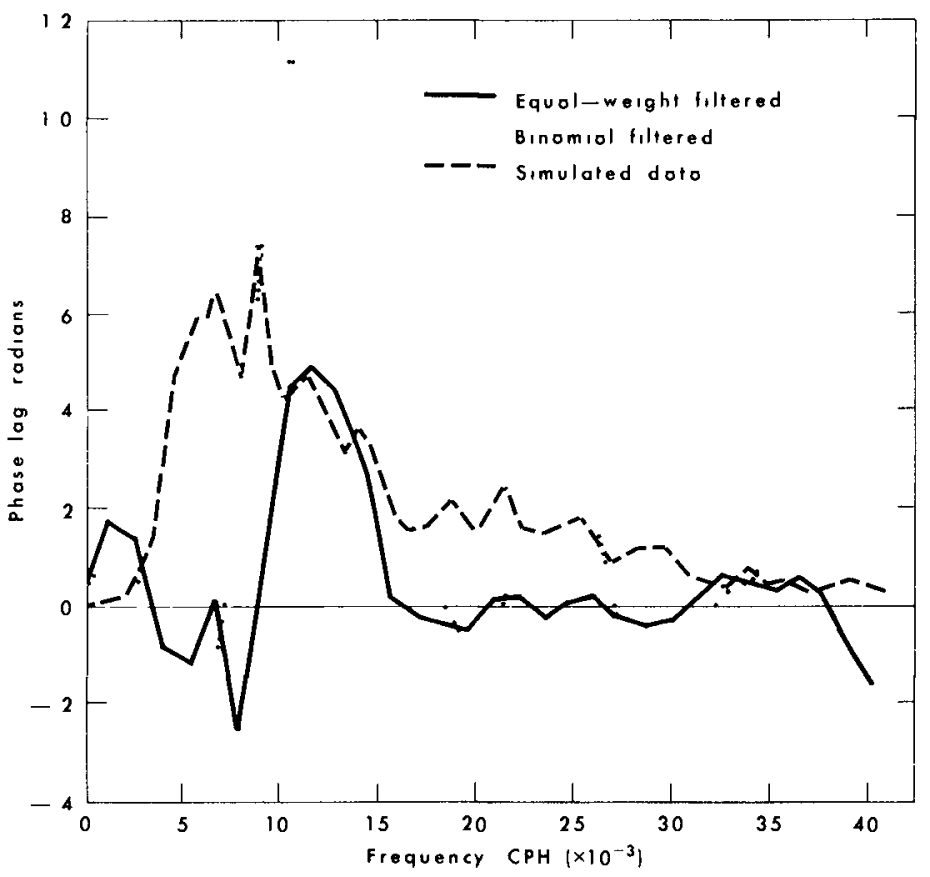

F1g 15 Phase lag for $X$ and $Q$ Data smoothed w1th equal-weight filter $(n=10)$, smoothed with binomial filter $(n=61)$, and simulated data

2 They arise from "multidimensional central limit theorem and, therefore, [are] of theoretical significance as an Idealization of the superposition of small effects" (Bendat, 1958)

In the present case, a Markovian feature must also be considered, since according to the profile transition model the successive profiles are products not only of elther accretive or erosional wave excitation but also of the preceding profile Thus, a Markov Gaussian random process may possibly explain beach profile changes According to Doob (1953), the stationary Markov Gaussian random process requires an exponential autocovariance function and hence necessarily a power spectrum of the form

$$
G(f)=\frac{2 k}{\pi} \frac{f^{2}+\left(k^{2}+c^{2}\right)}{f^{4}+2\left(k^{2}-c^{2}\right) f^{2}+\left(k^{2}+c^{2}\right)^{2}}
$$

In which parameters $k$ and $c$ are obtained by a curve fitting the autocovariance function in the low lag region, e $g$, 
$R(\tau)=e^{-k|\tau|} \cos c \tau$

Figure 16 shows the approximation of the real data autocovariance function fitted by equation (24), in which $c=0048 \mathrm{CPH}$ and $\mathrm{k}=0024 \mathrm{CPH}$ AccordIng to Bendat (1958), two types of power spectrum may result, depending on the relative size of $3 c^{2}$ and $k^{2}$ (see Fig 17) With the above values for $c$ and $k$, it is obvious that $3 c^{2}>k^{2}$ for the sediment storage, hence the power spectrum of $Q$ must have a peak density, contrary to the actual case Moreover, for the theoretical power spectrum the power density is inversely proportional to $\mathrm{f}^{2}$, e $\mathrm{g}$,

$$
G(f) \propto f^{-2}
$$

whereas in the present case

$$
G(f) \propto f^{-1 / 3}
$$

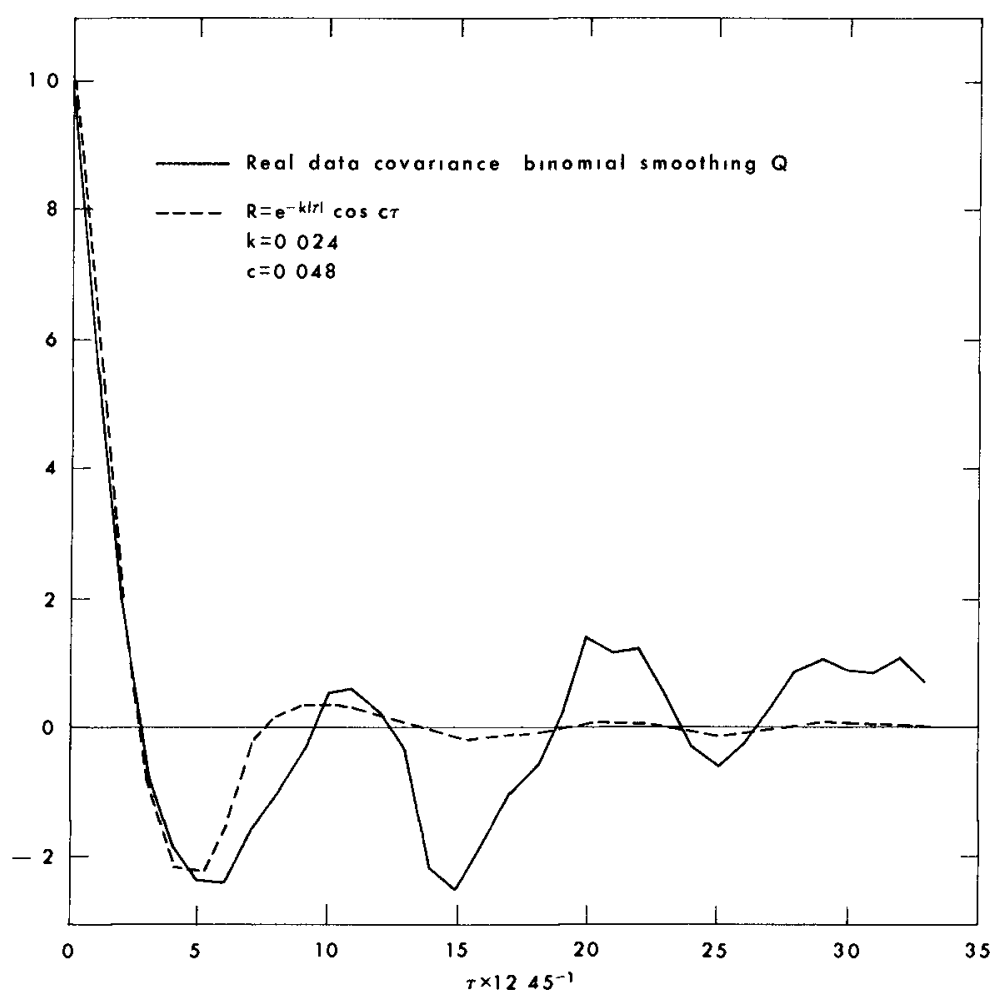

Fig 16 Autocovariance function of the real data $Q$ compared with the theoretical covariance function for Markov Gaussian ranđom process 

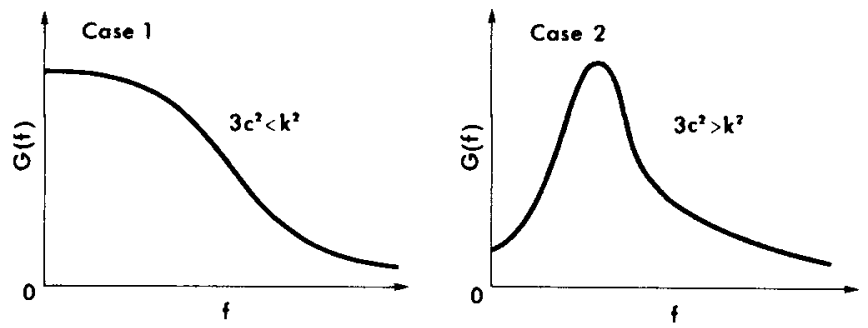

F1g 17 Two cases of power spectrum for Markov Gaussian random process (after Bendat, 1958)

The reason for this discrepancy is not lmmediately clear, but it seems possible that the actual example represents the superposition of two or more case 1 and/or case 2 situations

The time series simulation by means of a Monte Carlo technique, as demonstrated in this paper, appears to be reasonably successful Areas of needed improvement are the interactions between waves and beach profiles "Accretive" and "erosional" wave excitations, which are simulated by random numbers in the present study, need further clarification According to Sonu and van Beek (in press), erosion of the beach profile is associated with a period of growth of waves, while accretion is associated with a period of wave decay This relationship is more pronounced than the generally acknowledged effect of wave steepness In fact, it is noted that waves of the same steepness could cause either erosion or accretion, depending on whether they occurred during growth or decay of a wave field The basic mechanism controlling this relationship is not known Also unknown is the extent of beach change as a function of wave energy In the simulation study, it is assumed that each wave excitation would cause only one step of profile transition More precisely, the number of steps per semidiurnal period (the speed of beach change) should vary by types of profiles as well as by waves

The beach profile transition model, while requiring further refinement, appears to be basically sound It is likely that various regional coasts may be represented by different regression areas in the Q-X plane As indicated schematically in Figure 18, a type I coast may be found in an embayed coastline with a flat slope receiving little lateral supply of sediment, a small change in sediment balance may seriously affect the shoreline positions A type III coast may be, on the other hand, a steep beach sufficlently close to the source of sediment supply so that it requires a large amount of sediment movement to cause appreciable dislocation of the shoreline A type II coast is a typical ocean-exposed sandy coast, while type $I^{\prime}$ ' Is exposed to limited fetch, such as on a lake or an inland sea With accumulation of data, It is hoped that a generalized picture of beach profile transition model for various regions may be constructed in the ent1re Q-X plane 


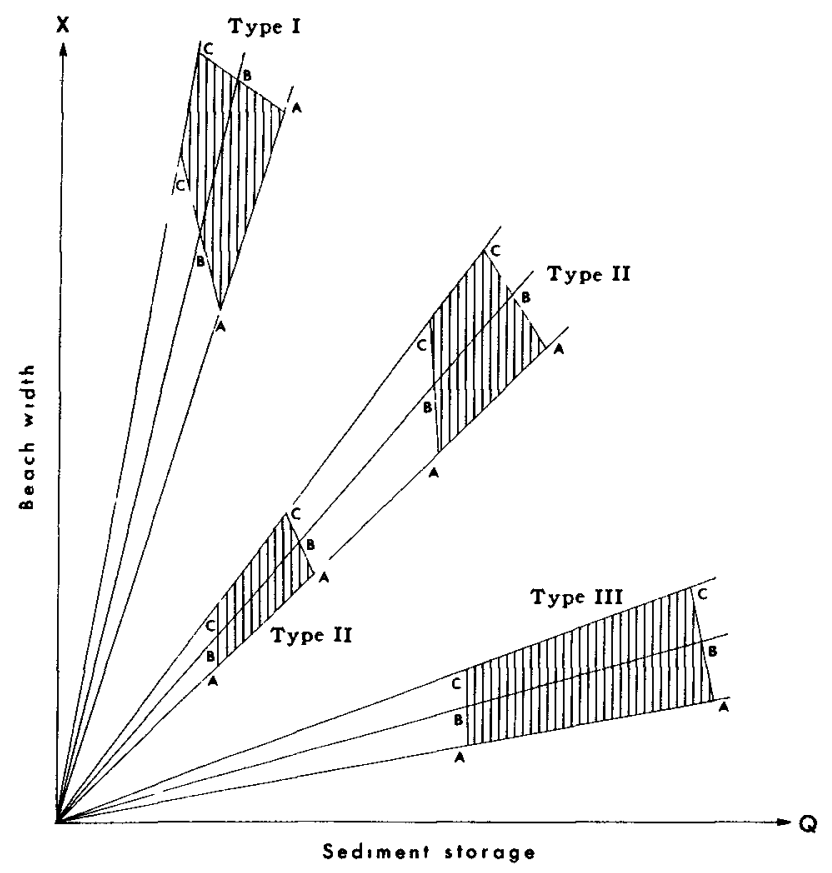

FIg 18 Suggestion for a generalized beach profile transition model for different types of coasts

\section{ACKNOWLEDGMENTS}

This study was supported financially by the Office of Naval Research, Geography Programs, under contract N00014-69-A-0211-0003, NR 388002 , with the Coastal Studies Institute, LouIsiana State University Miss Sharon Dunn did the drafting 


\section{REFERENCES}

Bendat, J S , 1958, Principles and applications of random nolse theory New York (John Wiley and Sons, Inc)

, and A C Piersol, 1966, Measurement and analysis of random data New York (John Wiley and Sons, Inc )

Blackman, $R$ B , and J W Tukey, 1958, The measurement of power spectra from the point of view of communications englneering New York (Dover Publications, Inc)

Cooley, J W, and J W Tukey, 1965, An algorlthm for the machine calculation of complex Fourier series Mathematics of Computers, $19297-$ 301

Dolan, R, J C Ferm, and D S McArthur, 1969, Measurements of beach process varıables, Outer Banks, North Carolina La State Unıv, Coastal Studies Instıtute Tech Report No 64

Doob, J L, 1953, Stochastıc processes New York (John Wiley and Sons, Inc )

Holloway, J L , Jr, 1958, Smoothing and filtering of time serıes and space fields Advances in Ceophysics, vol 4, New York (Academic Press, Inc)

Lee, Y W , 1960, Statistical theory of communication New York (John Wiley and Sons, Inc )

Rothman, J E, 1968, The Fast Fourler Transform and 1ts implementation Decuscope, Dig1tal Equipment Computer Users Soc1ety, vo1 7, no 3

Sonu, C J , 1969, Collective movement of sediment in littoral environment Proceedings, XIth Coastal Englneering Conference, Councll on Wave Research, London

, J M McCloy, and D S McArthur, 1966, Longshore currents and nearshore topographies Proceedings, Xth Coastal Englneering Conference, Council on Wave Research, Tokyo

, and $\mathrm{R} J$ Russe11, 1966, Topographic changes in the surf zone profile Proceedings, Xth Coastal Englneering Conference, Council on Wave Research, Tokyo

, and $\mathrm{J} I$ van Beek, in press, Systematıc beach changes on the Outer Banks, North Carolina J Ceo1

Wiener, N, 1956, The theory of prediction In (E F Beckenbach, ed ) Modern mathematıcs for the engıneer New York (McCraw-Hıl1 Book Company, Inc 


\section{.}




\title{
CHAPTER 84
}

FIELD SIUDY OF SURATHKAI BEACH

P. Suryaprakasa Rao* \& P. Kassim **

\begin{abstract}
ABSIRACT
Beach profile observations and sediment sampling were carriea out for $s 1 x$ months at frequent intervals to study the seasonal changes of the beach. The study is helpful in bringing out the qualitative and quantitative changes of the beach under varying wave and tide conditions. Useful information is obtained and presented regarding i) the maximum rate of erosion 11) the total quantity of material eroded iil) the critical wave steepness values which changed from depositing to eroding nature and iv) the sediment characteristics of the beach.

\section{INTRODUCIION}

Ihis paper presents the results of a six month (February to August 1969) field study of the seasonal profile changes and sediment characteristics of a natural sand beach at Surathkal.
\end{abstract}

* Assistant Professor of Civil Engineering, Karnataka Regl onal Engineering College, Surathkal (Ind 1a)

* Lecturer, I.K.M. College of Engineering, Quilon (Ind1a) Formerly post graduate student at Karnataka Reglonal Engineerıng College, Surathkal (India) 
Surathkal beach is on a straight reach of the west Coast of India facing the Arabian sea at a distance of $20 \mathrm{~km}$ north of Mangalore. The beach material is well sorted sand having median diameters in the range of 0.2 to $0.4 \mathrm{~mm}$. The climatic conditions on the coast repeat annually with remarkable regularity and uniformity. The beach is exposed to relatively uniform swells of low steepness arriving with crests parallel or nearly parallel to shore during fair weather season when the beach is built up. During the monsoon period, the wave helght and the wave steepness are increased and the beach is eroded. The average tidal range is about 1 metre.

Beach erosion problems exist along some stretches of West Coast of India and the information obtained from this study could be useful in planning shore protection measures.

\section{FIELD MEASUREMENTS ALD OBSERVATIONS}

\section{1) Beach profiles:-}

A stretch of beach of 200 metres length was selected for study and observation. Three cross sections, 100 metres apart, were marked by constructing two pillars for each section. The pillars were constructed on the storm berm where the waves do not normally reach. The line joining the three pillars nearer the water line was taken as the base IIne.

Levelling was done using a precise level along the three cross sections on the portion of the beach exposed at 
low tide between the base line and the water line. The beach elevations given in the various figures are with respect to an arbitrary datum. Profile measurements were done at intervals of 3 to 9 days, the interval depending on the magnitude of the changes that occured. A total of 33 measurements were made during six months. Surface sediment samples at various points along the profiles were collected during some of the observations.

\section{1i) Wave and Irde data:}

Wave data was obtalned from the wave records of the offshore subsurface pressure type wave rtcorder installed by the Mangalore Harbour Project authorities at Panambur about $10 \mathrm{Km}$. south of Surathkal beach. The waves were recorded for 15 minutes at 3 hour intervals during fair weather season and at closer intervals during monsoon period.

The maximum recorded wave helghts and the corresponding perlods for each day were avallable from the records. The maxımum surface wave heights for each day were computed by using the corresponding pressure response factors.

For the periods of considerable changes in the beach profiles, the continuous wave records for the days were studied and representative strips, each of 15 minute duration, were analysed to find out the mean wave heights and the average periods. The surface mean wave helght was computed and the corresponding deepwater mean wave height 
had the same value as this because the shoaling and refraction coefficients were both nearly equal to unity for the prevailing conditions. The deepwater mean wave steepness was computed using the deepwater mean wave height and the deepwater wave length corresponding to the average period. Visual observations were also made to study the type and location of the breakers and width of surf zone.

The tide data was obtalned from the tide recorder installed by the Mangalore Port authorities at Mangalore. The tidal range can be taken to be the same at Mangalore and Surathkal.

\section{RESULIS OF STUDY}

\section{i) Seasonal beach profile changes:-}

The period of study covered two distinct portions of the year. One was the premonsoon fair weather season upto middle of May when the beach was still being bullt up by processes of deposition which commenced at the end of the previous year's monsoon. The second one was the monsoon period from middle of May to August when the beach was eroded. After August the beach would again slowly start building up. The climatic conditions on the Coast repeat annually with remarkable regularity and hence the corresponding beach processes are also more or less repetitive in nature. Thus the study included the period when the beach process changed from depositional to eroding nature and the period when the total erosion 
occured.

Typical beach profile changes at one of the three cross sections are shown in figure 1. upto about the middle of May, the beach was subjected to long perlod waves of low steepness and was being bullt up. The maximum wave helght was less than one metre and the mean wave steepness was less than 0.002. The height and widh of the summer berm increased to a maximum and the berm had a slope towards the land.

Fig. 2 shows the beach profile changes during the period of erosion from middle of May to AuElust. It is not as if there was continuous erosion during the period. There were periods of deposition in between but the net effect was one of erosion. The complete summer berm was progressively eroded.

During the latter half of Nay there was a graqual increase in the maximum wave helght from less than one metre to about 1.75 metres. The mean wave steepness also increased from about 0.002 to 0.003 and above as shown in fig.4. This is the period when the beach erosion has set in as can be seen from the beach profiles on 20 th and 28 th May in fig.2. Therefore the critical deepwater mean wave steepness value which changed the wave action on the beach from depositing to eroding nature lies between 0.002 and 0.003 .

The maximum rate of erosion occured during the period 28 th May to 5 th June. The beach profiles at one 
of the sections are shown in fig.3. Similar profile changes occured at all the three cross sections. During this period the maximum wave height increased to about 2 metres; the mean wave steepness 1 ncreased to 0.004 ; and this happened to be the spring tide period with an increased tidal range. It was computed from the three cross sections that, on an average, 15.8 metres wadth of berm was removed and $18.65 \mathrm{cu}$, metres of material per metre length of beach was eroded during this period. This gives a maximum rate of erosion of 1.18 cu.metres of material per sq.metre change in beach surface area in one week (i.e. $0.143 \mathrm{cu} \mathrm{yds} / \mathrm{ft}^{2} /$ week)

Fig. 5 shows two profiles at one of the cross sections. One profile corresponds to the stage of maximum deposition and the other one to that of maximum erosion. From similar profile changes at the three sections, the total quantity of material eroded during the season was an average of $74 \mathrm{cu}$. metres per metre length of the beach and the average width of beach eroded was 31 metres. One sq.metre of change in beach surface area equals 2.39 cu. metres of beach material eroded (i.e. 1 sq.ft. of change in beach area equals 0.3 cu.yds.of materzal eroded). This, it may be observed, 13 a much smaller value compared to other exposed beaches in the world.

From such profile changes as shown in fig.5 it was also found that the active zone of the beach, the material of which partook in the beach processes, was confined to 
a depth of about 3 metres.

Figs. 6 and $6(\mathrm{a})$ show the profile changes at two of the cross sections. The beach profiles appeared to oscillate in response to a tidal cycle of about a month's duration. The profiles did not repeat exactly as the wave characteristics had not remained the same throughout the period.

Cusps were found to form on the beach during periods of deposition as well as erosion. They always formed during the neap tide periods and disappeared during the subsequent spring tides. The spacing of the cusps along the beach was regular and varied from 27 to 45 metres. The spacing of the cusps and the level difference between the valley and the ridge of the cusp formation increased with increase in wave height.

\section{ii) Sediment Characteristics:}

The median diameter of the beach sand was between 0.2 and $0.4 \mathrm{~mm}$. The dune sand was finer than that on the berm. The sands on the dumes, berms and the foreshore were all well sorted with the sorting coefficlent lying between 1.1 and 1.4. The grann size distributions were nearly symmetric with the skewness values lying between 0.9 and 1.1. There was no regular variation of median diameter with depth at a point on the beach and also along the beach at various points.

Relationship between grain size (median diameter in $\mathrm{mm}$ ) and the foreshore slope on which it was resting is shown in fig.7. The curve is inserted in a simlar plot given in 
reference number 1 for comparison wh th other coasts. Sand of a given size within the range of 0.2 to $0.4 \mathrm{~mm} s$ tands on a steeper slope on this beach.

\section{Acknowledgements}

The work reported here was done by the junior author, while at the College, for his thesis submitted in partial fulfilment of the requirements of the M.Tech. degree. Grateful acknowledgment is made to the Mangalore Harbour Project authorities for the wave data, to the Mangalore Port for the tide data and to Prof. Evald Nielsen, Unesco visiting Professor of Coastal Engineering at the College for his encouragement and overall gurdance. Permission of the College authorities to publish this paper is appreciated. REFERENCES

1. U.S. Army, CERC (1966). Technical Report No.4, Shore Protection, Planning and design.

2. Kassim P. (1969). A field atudy of seasonal profile changes and sediment characteristics of Surathkal beach. M.Tech. Thesis, K.R. Engg. College, Surat hkal.

3. Shepard, F.P.(1963). Submarı ne Geology (2nd Edition): Harper \& Raw, Publ., New York.

4. King C.A.M. (1966) Beaches and Coasts (2nd reprint) Edwin Aknold Publ. Itd., London.

5. John M. Darling (1964). Seasonal changes in beaches of the north Atlantic Coast of the Uni ted States. Prof., 9 th Conference, Coastal ingineering. 
6. Bascom, Willard J., (1954) Characteristics of natural beaches, Proc, 4th Conf. Coastal Engg., Berkeley, Calıf. 


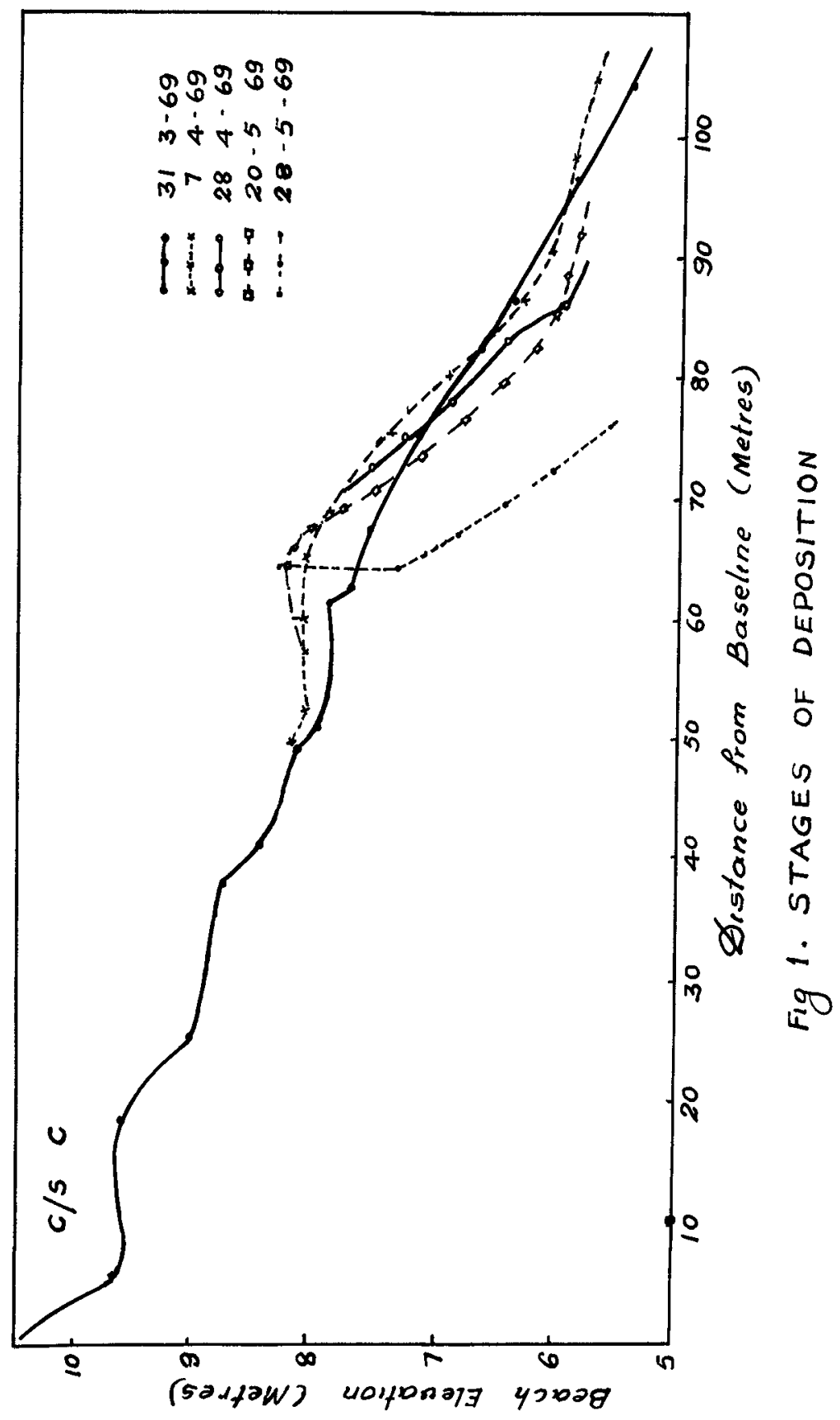




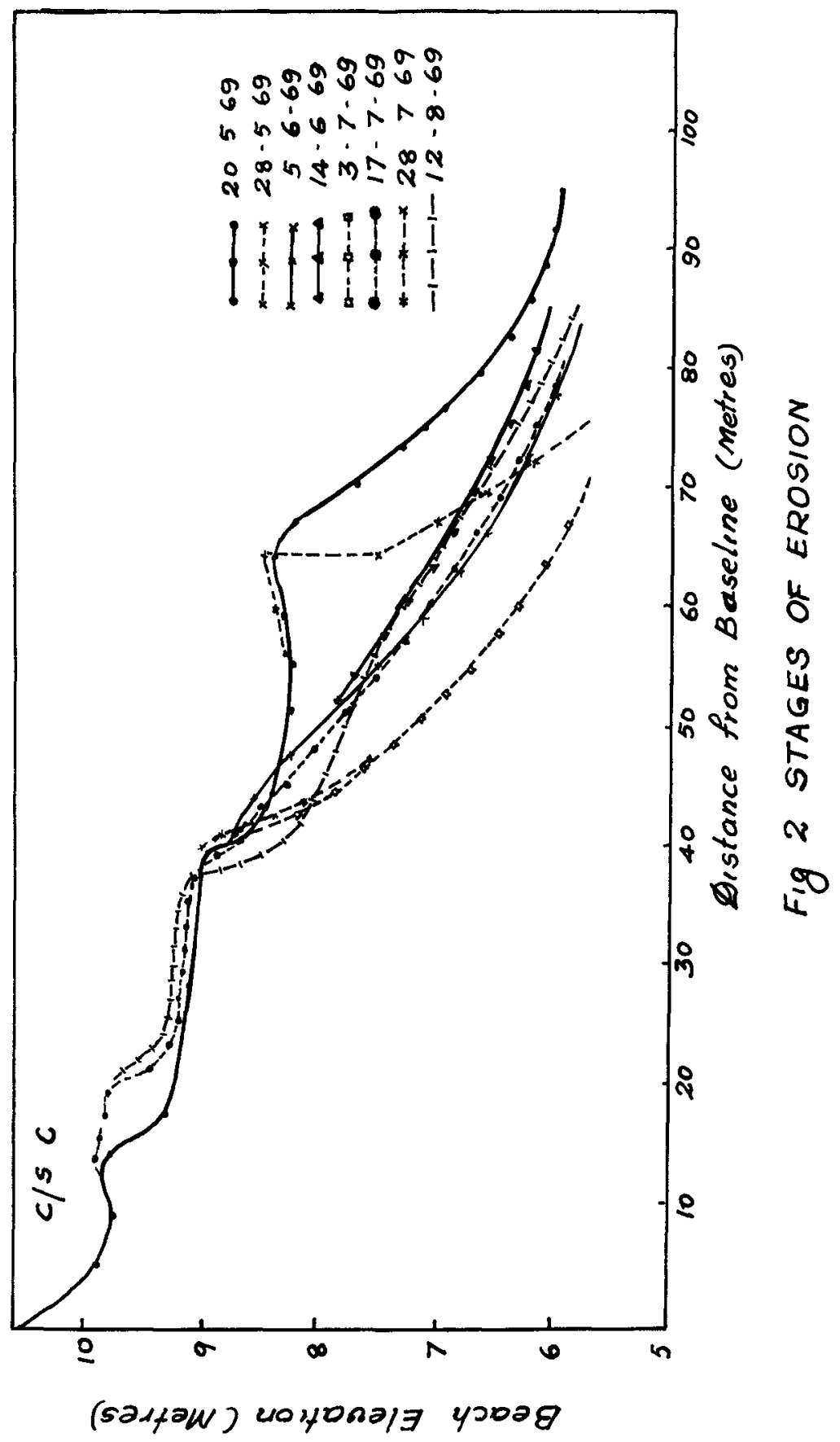




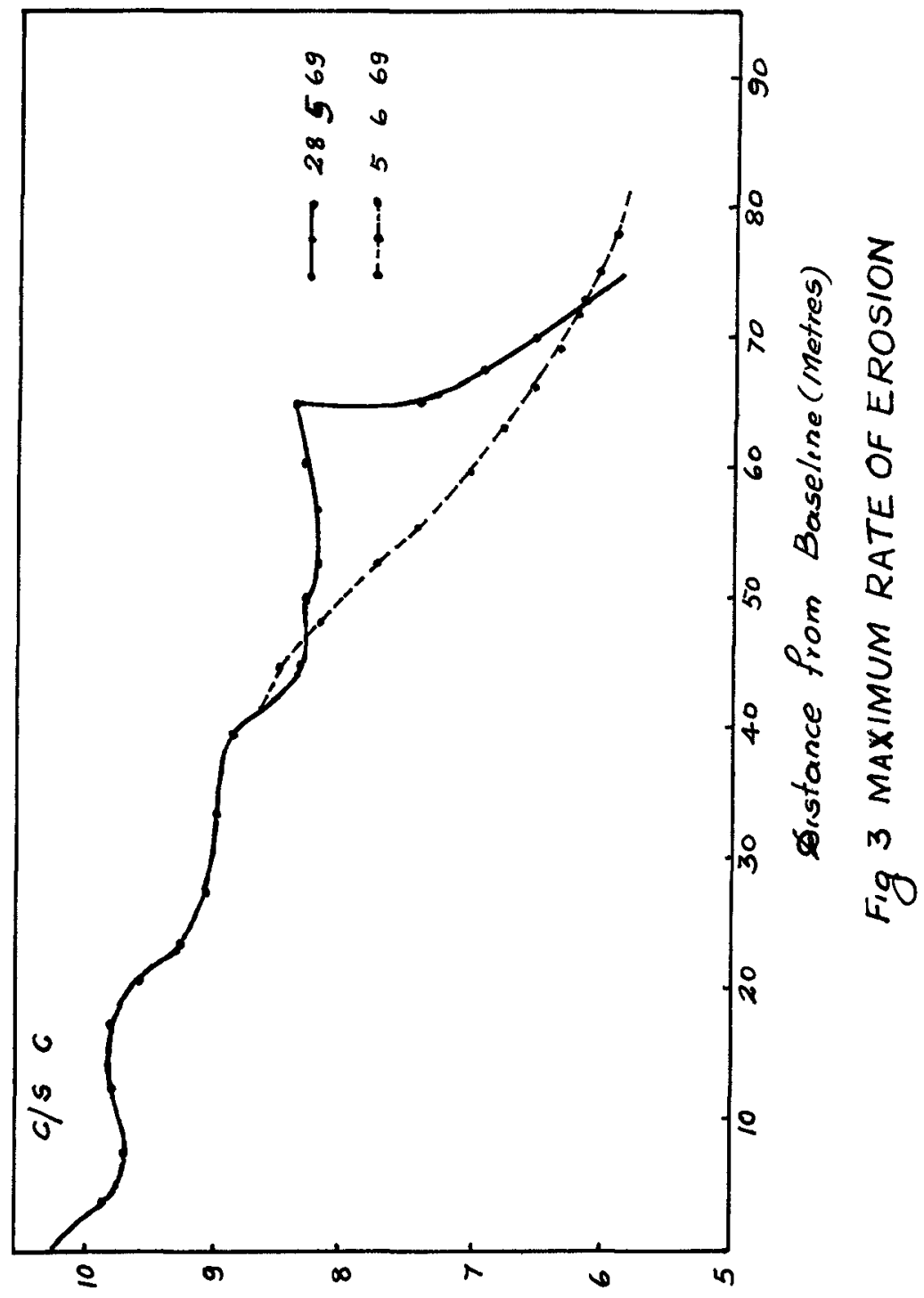

(s2lfw) noxfora/ 


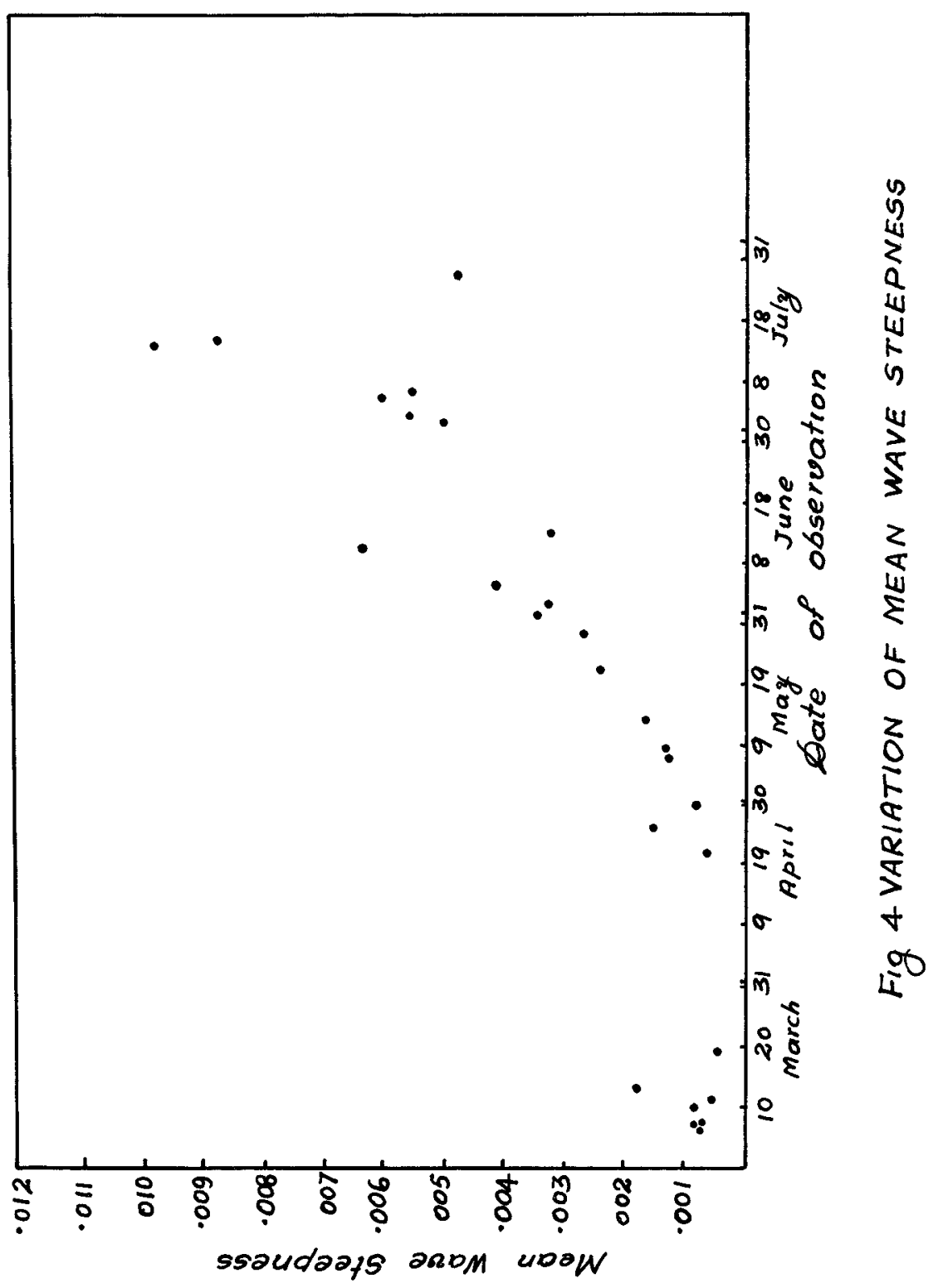




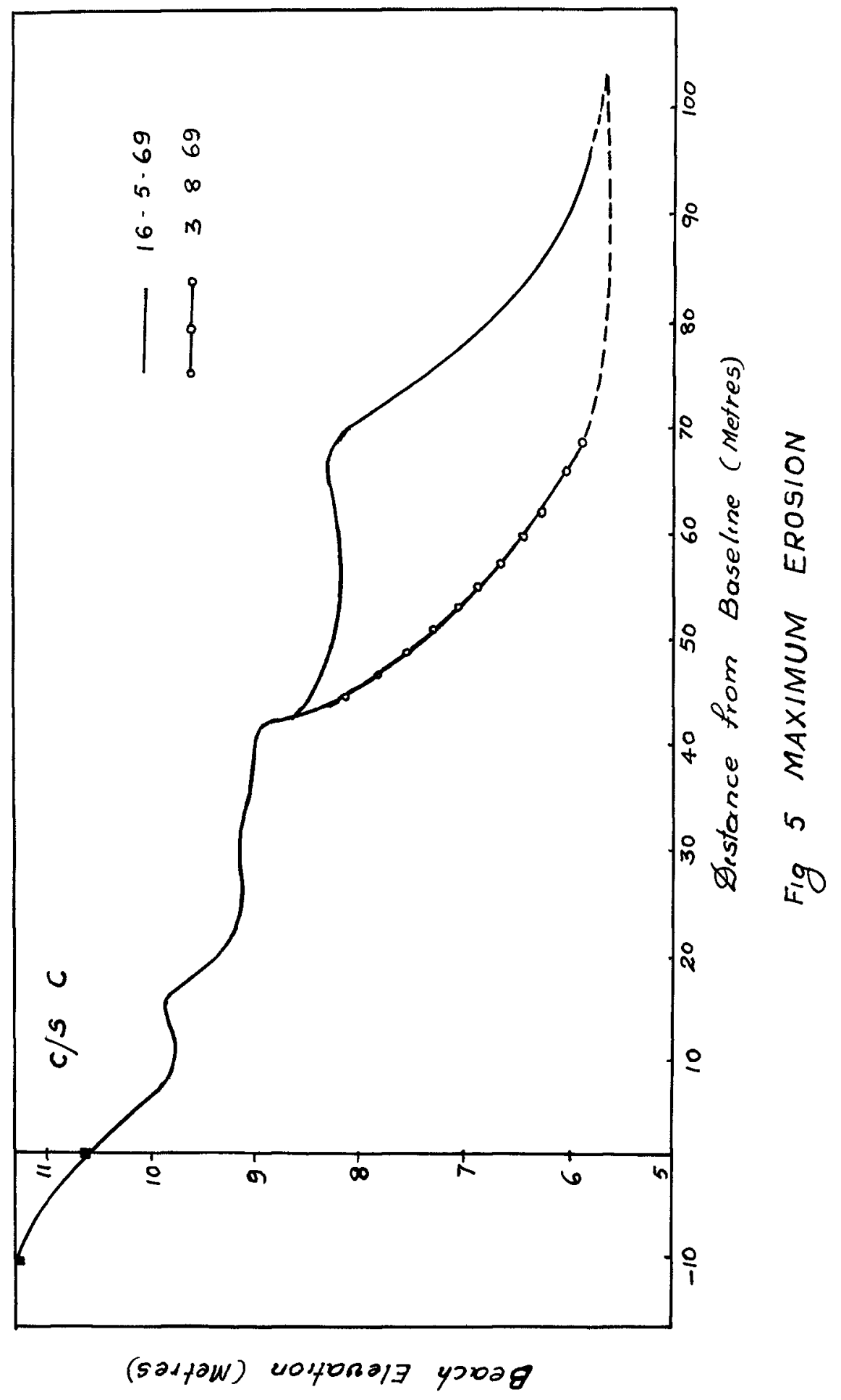




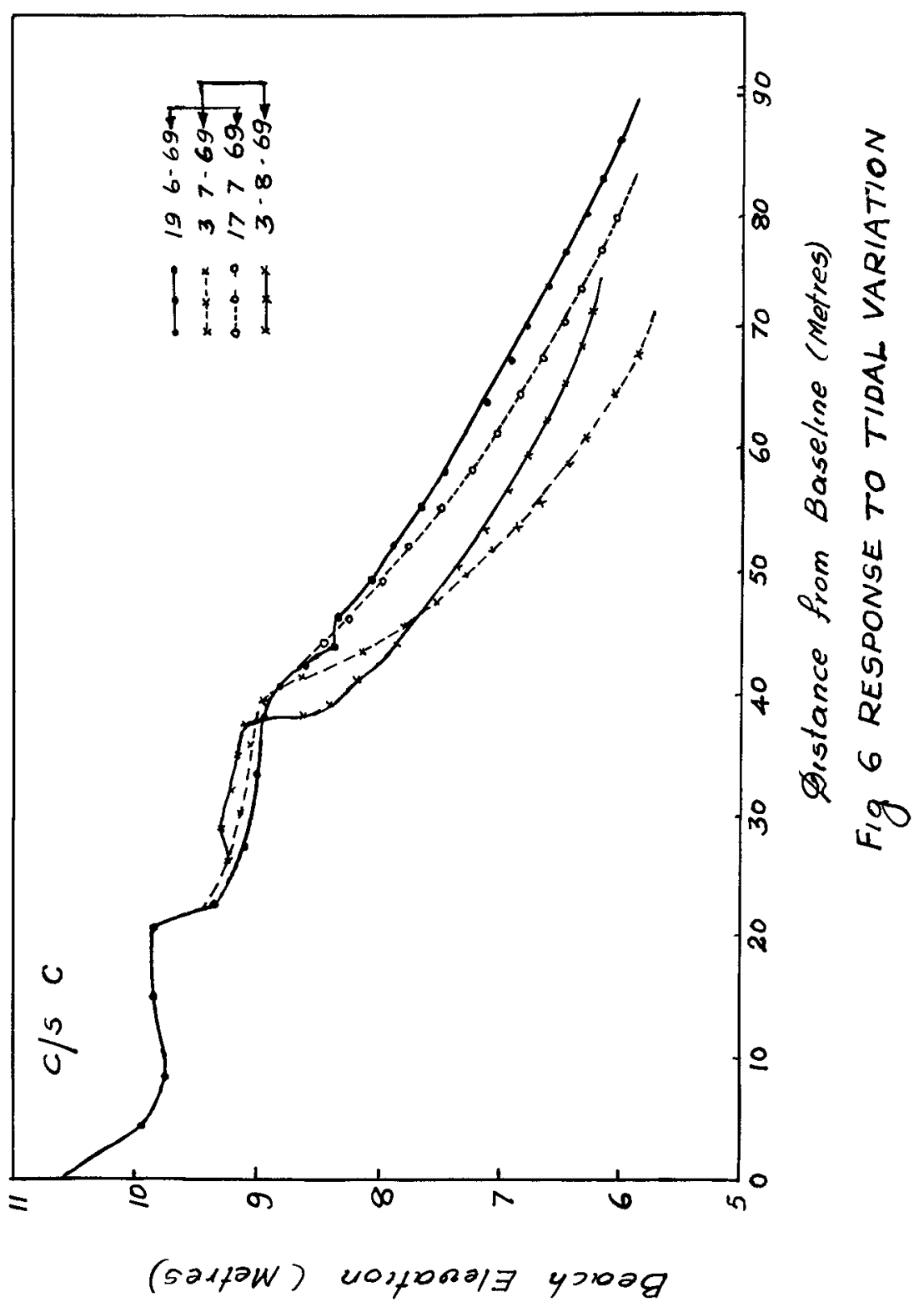




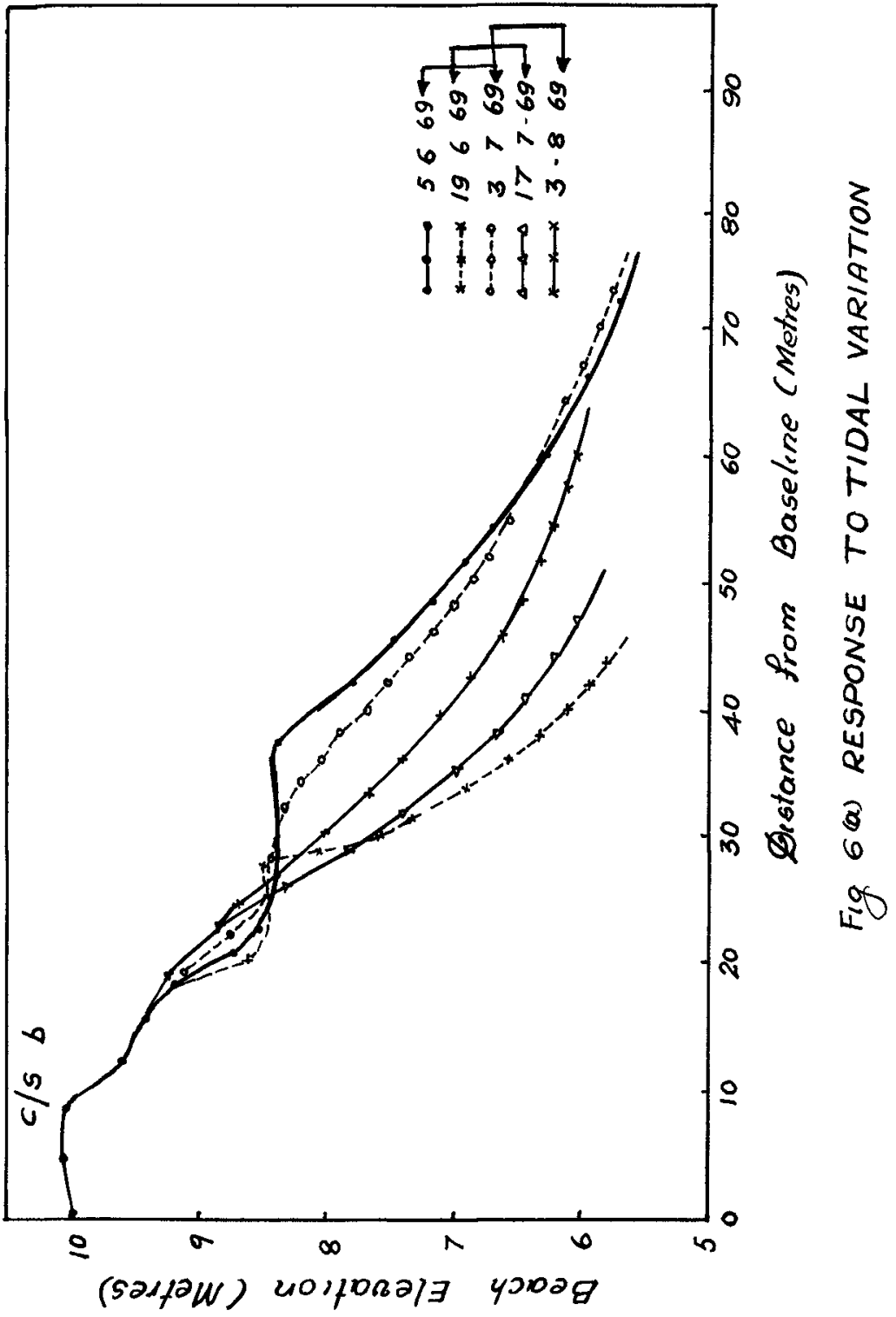




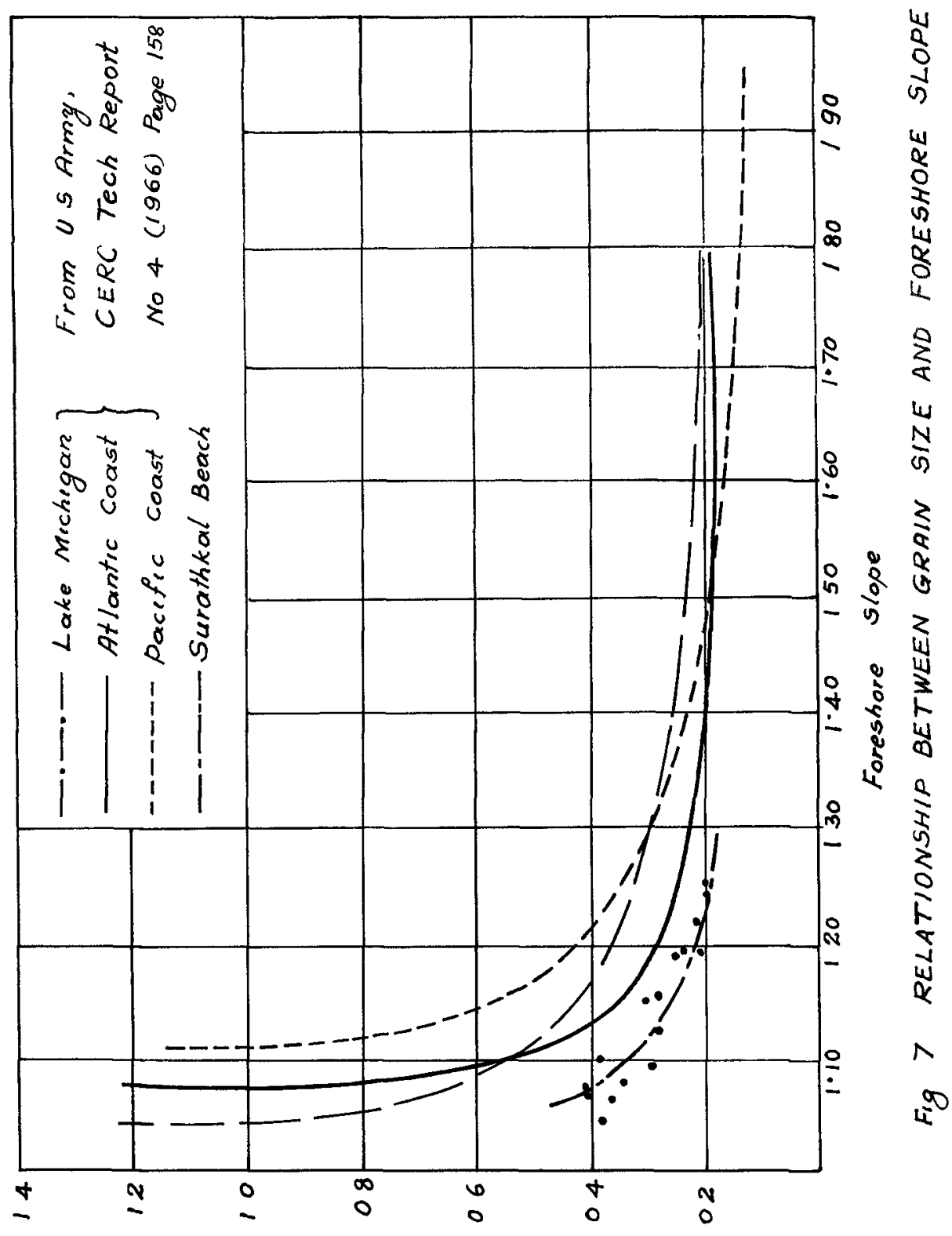

(ими) rafamoiф unorg-pung unipaw 



\title{
CHAPTER 85
}

\author{
SEASONAL BOTTOM CHANGES \\ BOLINAS BAY, CALIFORNIA \\ J W Johnson \\ Unıversity of Calıfornia \\ Berkeley, Californıa
}

\begin{abstract}
Five bottom surveys over a period of a year were made in the north portion of Bolınas Bay, California Comparisons between successive surveys permitted an evaluation of the areas and depths of scour and fill with the seasons and were correlated with 1ittoral current measurements Data for a 22-year period also were available on the position of the mean high-tide line over a limited length of beach These data showed a cyclic variation of the position of the high-tide line with the season with the most variation from year to year occurring during the spring months
\end{abstract}

\section{INTRODUCTION}

In connection with a comprehensive study of the environment of a tidal lagoon, the inlet, and the adjacent ocean area at Bolinas, California (1)* numerous beach and bottom surveys were made at various times in the northern part of Bolinas Bay The portion of Bolinas Bay in which repeat surveys of the bottom were made is shown in Figure 1 These surveys covering a year were made in May, August, and December in 1968, and in April and May in 1969 Five beach ranges were established along Stinson Beach by the Corps of Engineers in 1961 and profiles were determined in March and August of that year (F1g 1) They were resurveyed in March and April of 1969 by the Callformia State Lands Commission The State also established the location of the mean high tide line and several beach profiles along a $600 \mathrm{ft}$ length of the beach on numerous occasions since 1948 (F1g 1) Twenty-seven surveys whlch were made during the years 19481970, inclusive, provide important information on seasonal profile changes on this semi-exposed beach Wave data, observed simultaneously with the bottom and beach surveys, were obtalned from a bottom pressuretype wave gage as well as visual observations of wave helght and period, 1ittoral currents, and beach characteristics at Bolinas and Stinson Beach

\section{WAVE CONDITIONS}

The most Important factors in the movement of sand in the nearshore area are wave action and tidal currents Information on these factors

\footnotetext{
${ }^{*}$ See References
} 


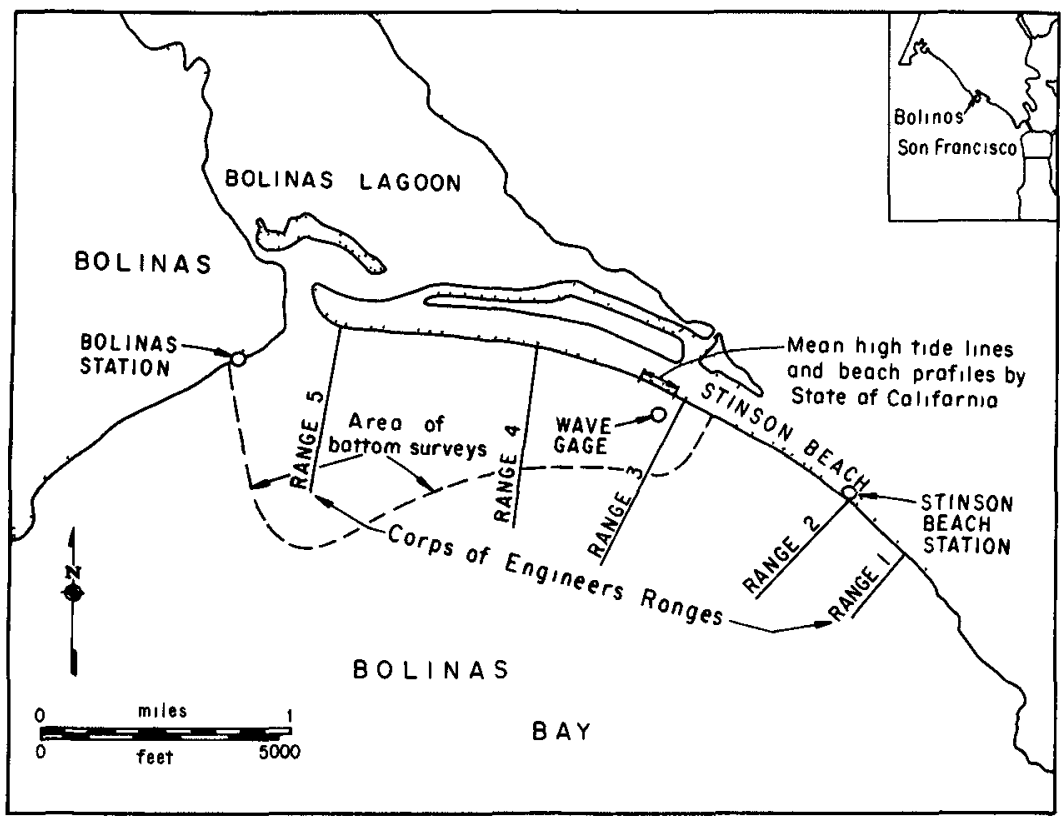

Figure 1 Location map, Bolinas Bay

In Bolinas Bay is provided by data obtained from a wave gage operated by the Bolinas Harbor District and visual surf observations made at the Stinson Beach State Park and at Bolinas for the Coastal Engineering Research Center (2) Figure 1 shows the locations of these stations The visual observations are of course not as accurate as the wave gage data on height and period, but there is considerable additional information such as wave direction, wave type, wind data, direction and strength of 1ıttoral current, beach-face characteristics, tide conditıons, etc These data are of Importance in the discussion below on the character and extent of sediment movement in the nearshore area of Bolinas Bay For ready accessibility, the avallable data from the Coastal Engineering Research Center on wave perıod and the direction and strength of the littoral current have been plotted In Figures 2 and 3 for the Stinson Beach and Bolinas stations, respectively It is evident from these two figures that there are extended periods of time when the littoral current prevalled in one direction For example, during the period of February to June, Inclusive, In 1969 the littoral current at Stinson Beach was generally in a southeast direction with a reversal in direction occurring only on about 15 days

\section{BOTTOM SURVEYS}

To obtain a measure of the seasonal bottom changes in the offshore area each survey made between May 17, 1968 and May 16, 1969, Inclusive, 


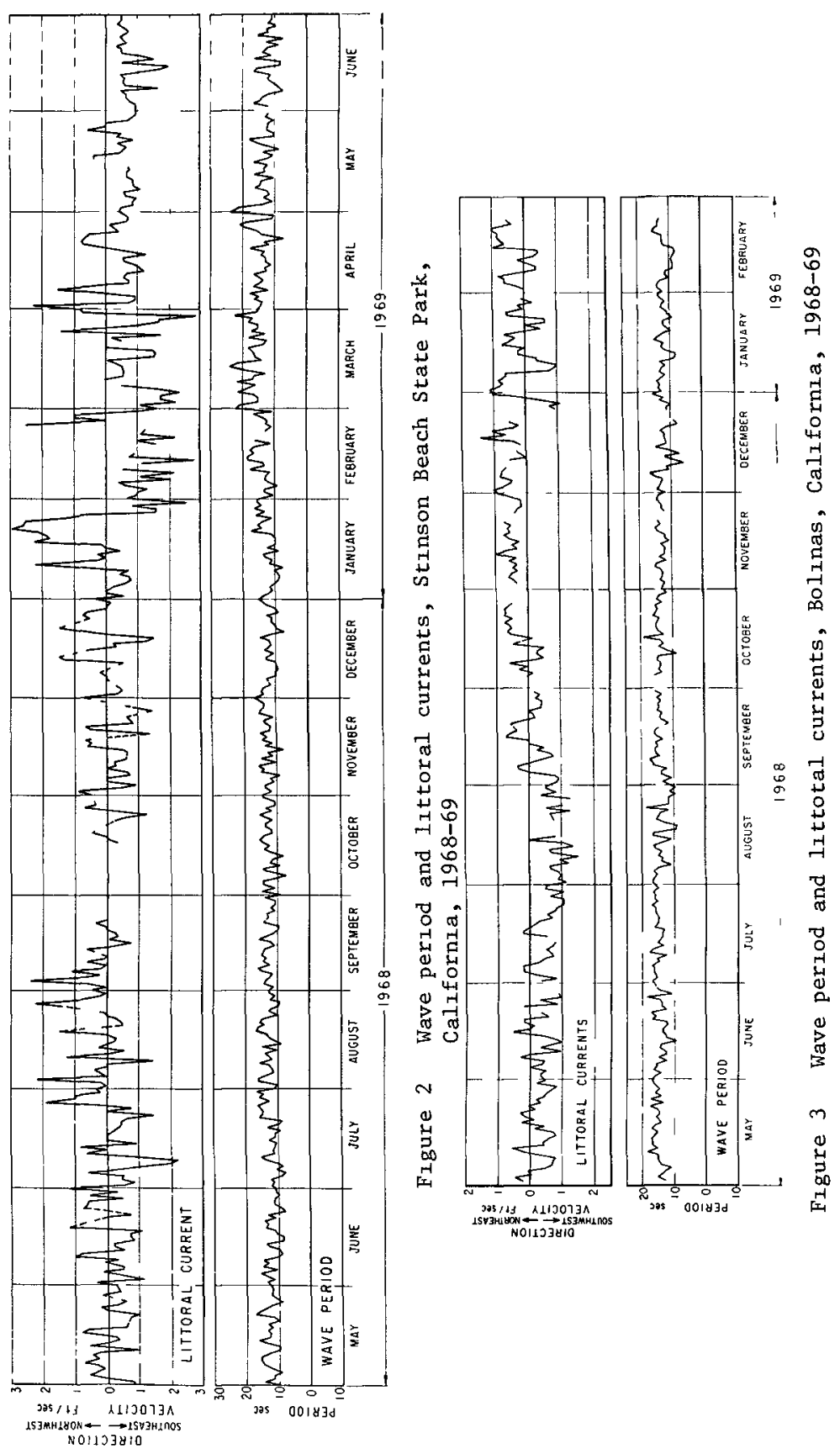


was compared with the next following survey and the depth of scour or fill was noted on an overlay These overlays were then contoured to give areas and depth of scour and fill occurring between the two dates These comparison maps are shown in Figures 4-7, inclusive Thus, Figure 4 is the result of comparing a survey made toward the end of the winter season (May 17, 1968) and a survey well into the summer season (August 21, 1968) The differences in depths between surveys are a11 minus, that 1s, depths in August were all deeper than in May As will be shown below in connection with beach changes this indicates that sand 1s moved generally from the offshore area to create a fill on the beach face during the summer This onshore movement 18 also lllustrated by the fact that the $30 \mathrm{ft}$ depth contour is closer inshore in August than in May

F1gure 5 shows a comparison between the summer survey of August 21 , 1968 and the survey of December 19, 1968 which 18 at the beginning of the winter season An area of scour occurred near the entrance of Bolinas Lagoon, but a general fill occurred over the remainder of the area surveyed, with the largest fill occurring near the surf zone-probably such material was removed from the beach face and deposited immediately offshore by early winter storms As a result of this general fill the position of the $30 \mathrm{ft}$ depth contour in December was further offshore than in August

Figure 6 shows a comparison between the winter survey of December 19 , 1968 with a survey taken on April 15, 1969 which was toward the end of the winter season This map shows a general scour of one to two feet in April compared with December A deep area of scour occurred immediately offshore of the lagoon entrance Areas of fill occurred on the west and northeast sides of the area mapped As a result of the general scour the position of the $30 \mathrm{ft}$ contour in April is slightly further inshore than in December

Figure 7 shows a comparison between two surveys taken a month apart (April 15 and May 16, 1969) in the transition perıod from winter to summer conditions A slight fllling occurs in the offshore area, a considerable fill at the lagoon entrance, and a scour area on the westerly part of the area surveyed Because of the limited coverage by the April survey, it is not possible to determine the source of the sand which filled much of the area represented by this map, however, it is possible that this sand has been shifted laterally from the west to the east as a result of the change in wave direction from the southwesterly direction in winter (April) to the more northwesterly direction at the beginning of summer (May)

That sand may be shıfted laterally withın Bolınas Bay is evident in Figure 8 which shows a comparison of the positions of several depth contours as they existed on May 17, 1968 and on May 16, 1969 Examination of this figure shows that the area opposite the entrance to Bolinas Lagoon has been filled generally by May 1969 compared with the May 1968, that 1s, over most of the area the May 1969 contours are seaward of the positions of the contours in 1968 On the other hand, at the easterly end of the map shown in Figure 8, a region of scour has occurred, that 1s, the 1969 contours are generally shoreward of the 1968 positions 


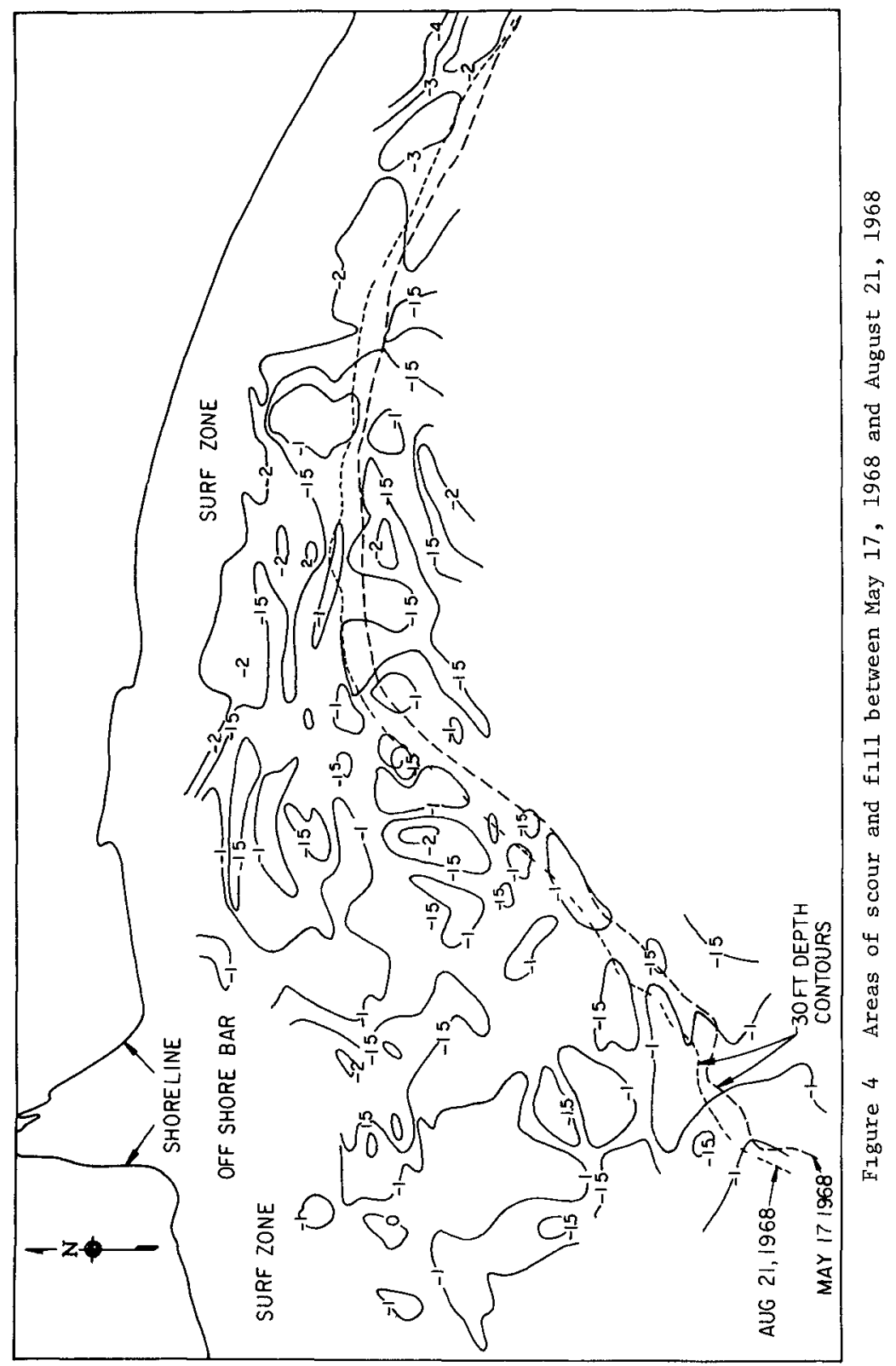




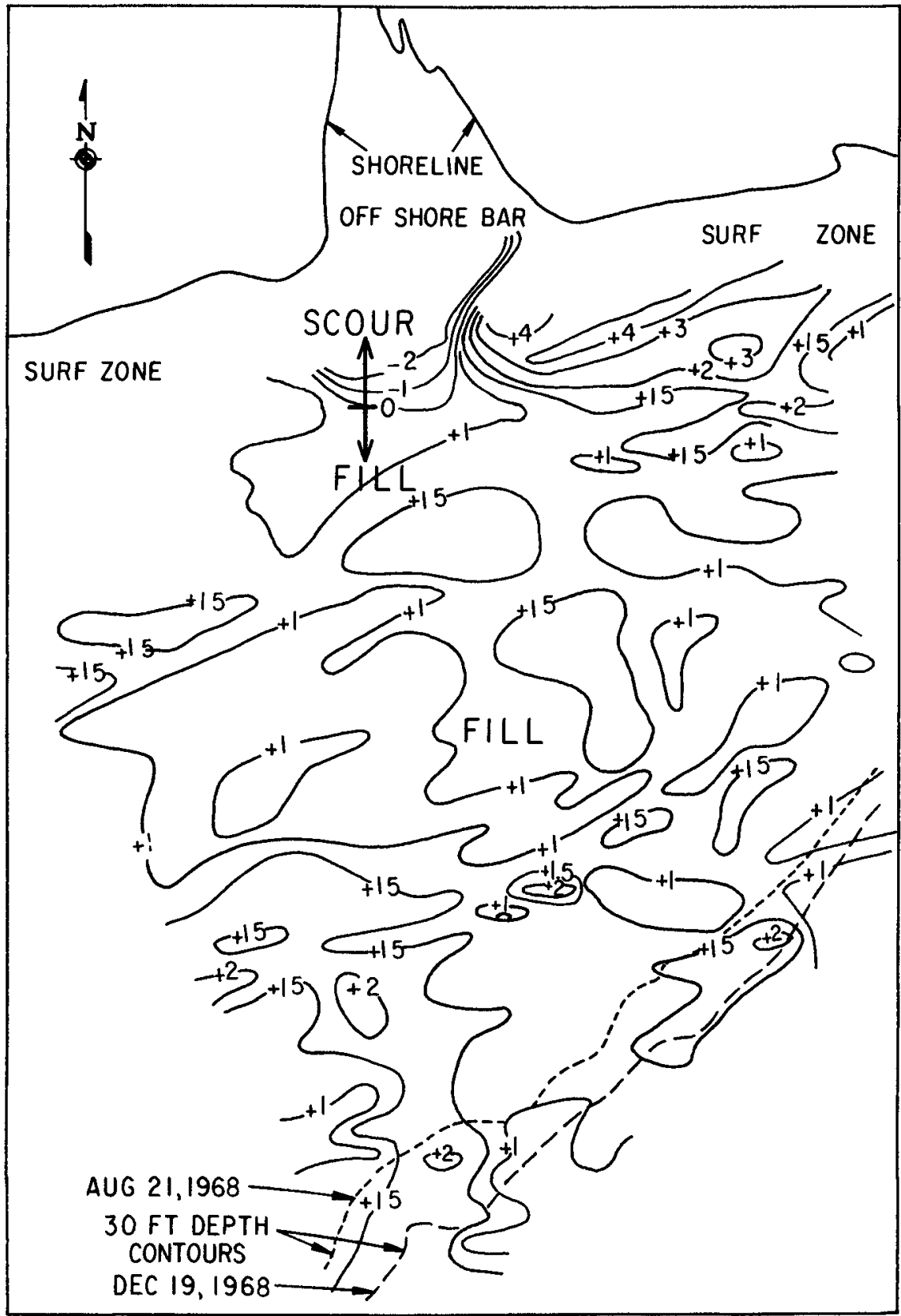

Figure 5 Areas of scour and $f_{111}$ between August 21, 1968 and December 19, 1968 


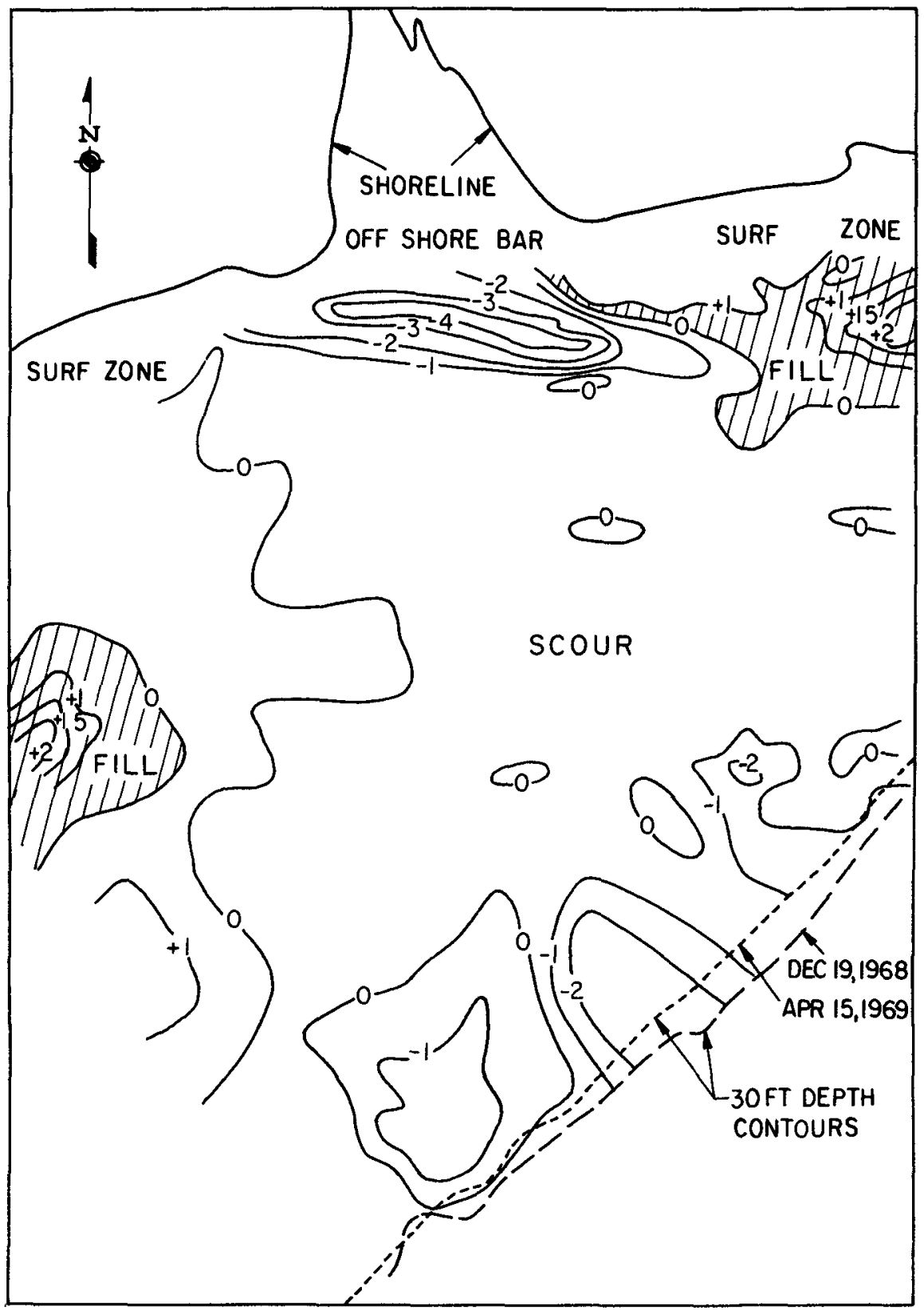

F1gure 6 Areas of scour and fill between December 19, 1968 and April 15, 1969 


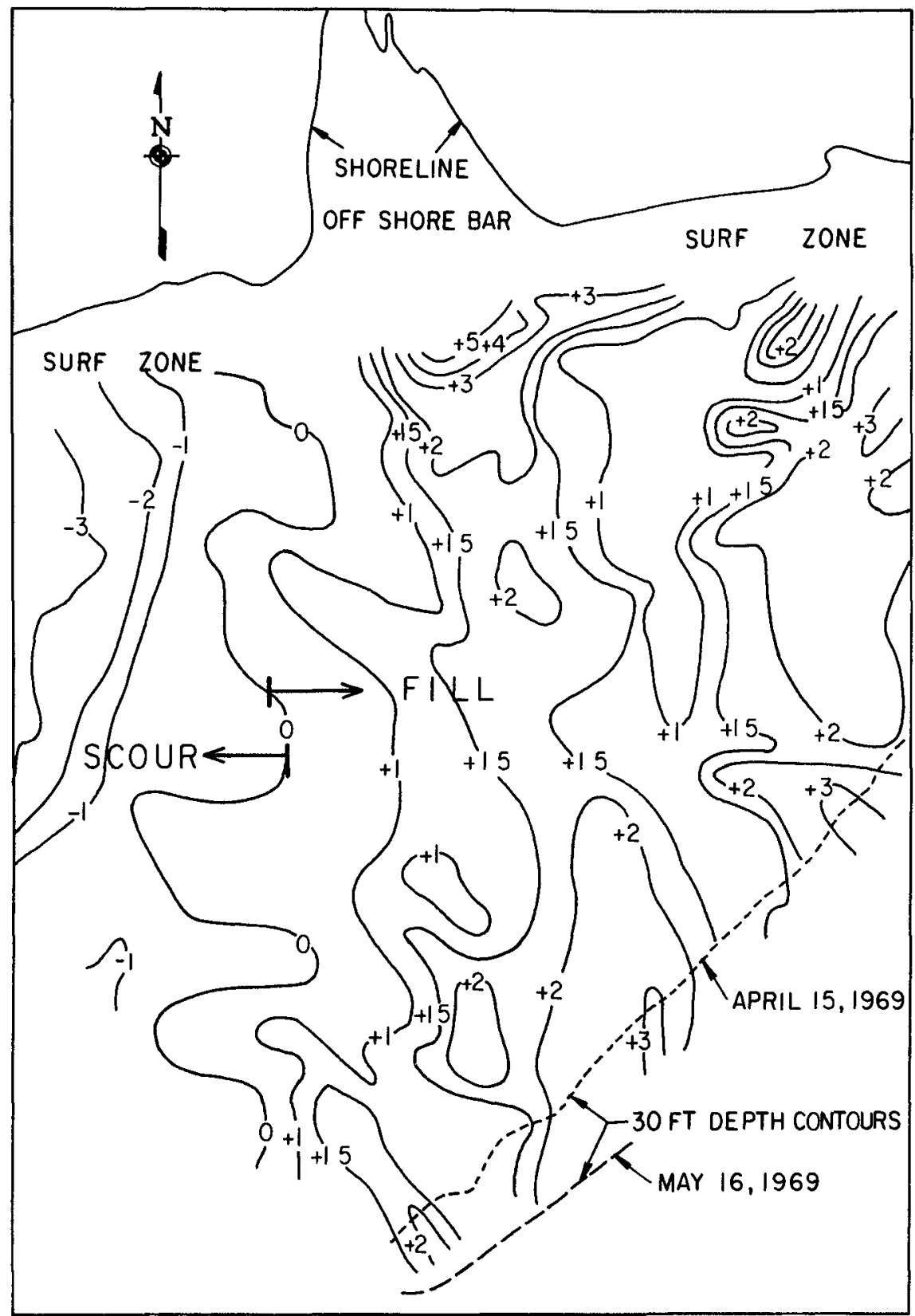

Figure 7 Areas of scour and f111 between Apri1 15, 1969 and May 16, 1969 


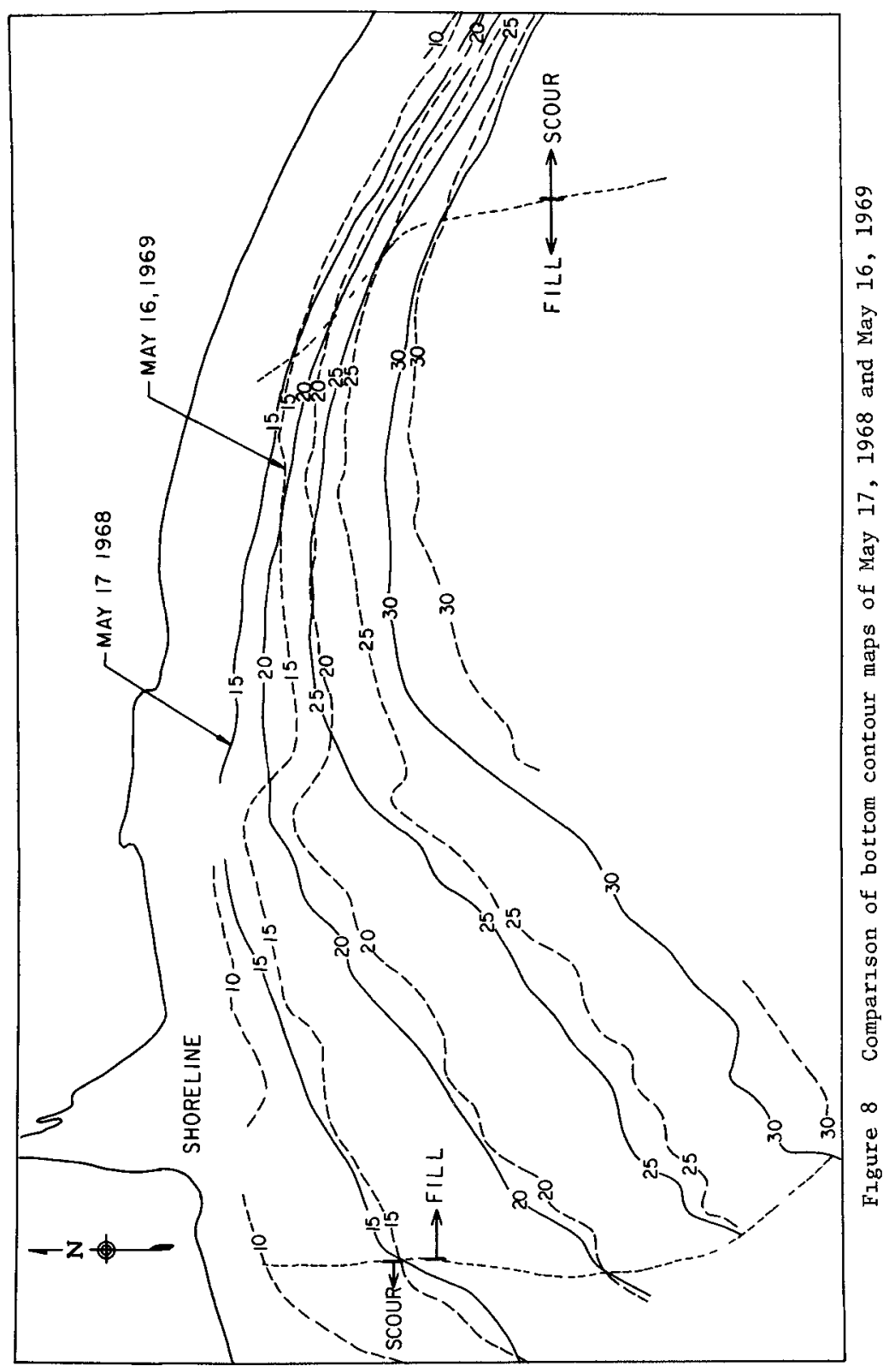


This indicates that, between the 1968 and 1969 surveys, sand appears to have been shifted northward from Stinson Beach into the north end of Bolınas Bay Wave conditions and tidal currents in the coming year could possibly be of a character that the material would be shifted back in an east and southerly direction It should be recognized in the comparison maps discussed above (Figs 4-8) that the actual depths of scour and fill are subject to some sounding error, but the areas of scour and fill show no randomness and are consistent with seasonal changes as generally observed elsewhere

Other data which provide information on the general movement of sediment in the entıre Bolinas Bay are presented in the following chapter by Wilde and Yancey in which heavy minerals are utilized as tracers The reader is referred to that paper for details on procedures and conclusions Other supplementary data on sediment movement in Bolinas Bay resulted from sand tracing studies along the beach face as obtained by the U S Geological Survey in connection with investigations of sedimentation and hydrology in Bolinas Lagoon (3) This study involved the placement of fluorescent-dyed sand on the beach at both the town of Bolinas (Brighton Avenue) and the Stinson Beach State Park and then periodically detecting the direction of movement away from the source by use of an ultraviolet lamp Placement of sand at both localities was made on July 23-24, 1968 and then periodically sampled untıl late October 1968 when the dyed material either was completely scattered and burled or the dye strength had been greatly reduced

The results of these limited and qualitative tests showed that at Bolinas the movement of sand was always eastward toward the entrance of Bolinas Lagoon, but no material ever crossed the lagoon entrance to the Stinson splt Although the surf observations summarized by the Coastal Englneering Research Center showed a general westerly littoral current in this same locality during the period of the sand tracing studies (Fig 2), it is possible that the tidal currents into Bolinas Lagoon rather than wave generated littoral currents were instrumental in generally moving sand eastward along the beach at Bolinas

In the case of the Stinson Beach State Park studies it was found that the dyed sands were moved in both the southeasterly and northwesterly directions, with some material being detected about one and onehalf miles northwesterly up the beach two months after initial placement of the dyed sand This might be expected upon examination of Figure 2 which shows that a northwesterly littoral current prevailed over most of the period, (August and September), following the start of the tracer tests

\section{BEACH CHANGES}

It has long been established that beaches exposed to wave action undergo changes to their profiles throughout the year because of the seasonal changes in wave characteristics $(4,5$, and 6$)$ One of the most important factors in determining the character of a beach profile is the ratio of wave helght to wave length--a factor commonly referred to as the "wave steepness" Durıng extended perıods of low wave steepness, 
low berms usually are built on the foreshore with very steep profiles on the beach face, and bars and underwater berms 1mmediately offshore tend to disappear or become discontinuous Durıng periods of high wave steepness (storm or winter conditions) the beach face becomes less steep and the offshore underwater bars become more pronounced

In the case of the beaches along Bolinas Bay the seasonal changes in their characteristics are less well defined than in the offshore area This lack of beach data is primarily due to the difficulties and hazards of making accurate bottom surveys in the surf zone--especially during winter storms It is to be noted that the hydrographic surveys used in preparing Figures 4-7, inclusive, were not made closer to shore than about the $8 \mathrm{ft}$ depth contour The data on seasonal beach changes are therefore confined to the beach profiles made by (a) the University of California (6) and (b) the Corps of Engineers in 1961 (7) and repeat surveys of these ranges by the Calıfornia State Lands Commission in 1969 The location of the Corps' ranges is shown in Figure 1 Surveys by the State Lands Commission to determine the position of the mean high-tide lines over a limited length of the Stinson spit for the period 1948 to 1970 are of importance in evaluating beach changes in Bolinas Bay

To provide an accurate measure of the seasonal fluctuations of the width of the beach at Stinson spit the data on the position of the mean h1gh-tide line, as determined by the State Lands Commission and other sources on twenty-seven occasions from 1948 to 1970 , are of considerable value To obtain information on the position of the mean high-tide line the distance from a base line to the high-tide line was measured and plotted as shown in Figure 9 for the appropriate day of the month There is an obvious cyclic pattern with the seasons as to the position of the high-tide line, but no precise relationship obviously exists because of the variation of intensity of wave attack that undoubtedly occurred from year to year An upper and lower envelope has been drawn on Figure 9 to enclose the plotted points Inspection of this plot shows that the position of the mean high-tide lines is more variable from year to year during the spring months than during late summer and fall The distance between envelopes during the spring may amount to as much as $50 \mathrm{ft}$, and the beach at the mean high-tide line is approximately $150 \mathrm{ft}$ wider in summer than in winter The surveys of the five Corps of Engineers ranges showed an average difference in width of about 100 feet between winter and summer conditions at the mean high-tide level (7)

of importance in connection with Figure 9 is the magnitude of the mean monthly wave helght for the California coast as shown in Figure 10 This figure, plotted from data compıled by Galvin et al (1969), shows that the time of occurrence of the lowest mean monthly wave height occurs at about the same time (August) as does the time when the beach on Stinson spit (F1g 9) 1s the widest, that 1s, the beach is widest when the wave steepness is the lowest 


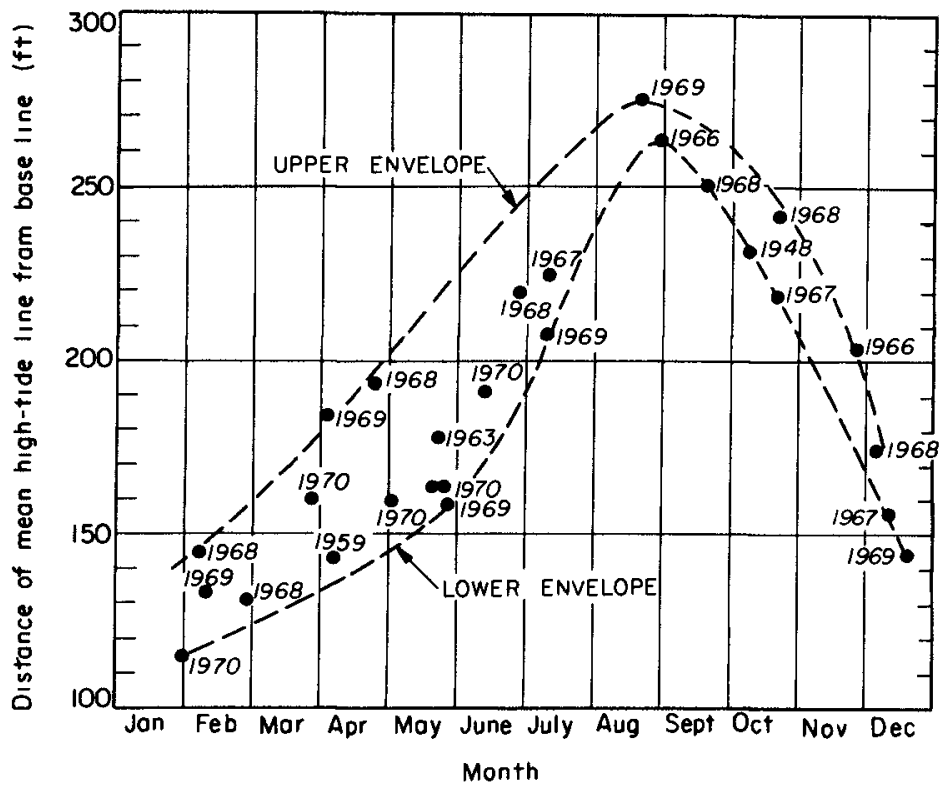

Figure 9 Seasonal variation of the position of the mean hightıde line, Stınson Beach sp1t 1948-1970 (Data compiled by California State Lands Commission)

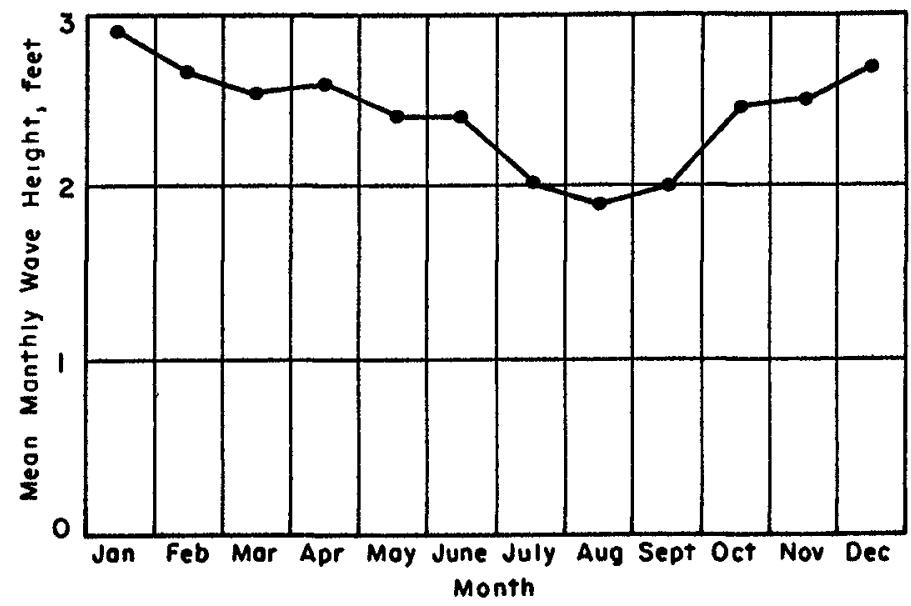

F1gure 10 Mean monthly wave heights for the California coast From data for 31 station years compiled by C J Galvin, Jr, et a1 (1969) 
SUMMARY

1 Five bottom surveys made in 1968-69 of the northern part of Bolinas Bay show an offshore-onshore movement in the depths between $8 \mathrm{ft}$ and $35 \mathrm{ft}$ below MSL as a result of varying wave conditions with the seasons A survey in May 1969, when compared with a survey one year earlier in May 1968, shows an accretion in the northern part of Bolinas Bay and a scouring of the shoreline southward along the Stinson spit

2 Repeat surveys at five ranges located along the Stinson spit show a seasonal change with the seasons, that is, the beach averages about $100 \mathrm{ft}$ greater in width during the summer compared with the winter Repeat surveys of the location of the mean high-tide line at one location on Stinson spit over a period of several years (1948-1970) shows a cyclic position of the high-tide line with the season with the most variation from year to year occurring during the spring months

\section{ACKNOWLEDGMENTS}

This study was conducted for the Bolinas Harbor District in which Mr Norman T Gilroy was project coordinator and consultant planner Data on the position of the mean high-tide line as provided by the Calıfornia State Lands Commission is gratefully acknowledged

\section{REFERENCES}

1 Gilroy, Norman T (1970) Bolınas Lagoon Its system of environmental dynamics and recommendations for 1 ts future preservation and utilization, Mill Valley, Calıf

2 Corps of Engıneers (1970) Littoral environment observation program in Calıfornia, Preliminary report, February-December 1968, Misc Paper No 2-70, Coastal Engineerıng Research Center, Wash, D C

3 Ritter, John (1969) Prelıminary studies of sedimentation and hydrology in Bolınas Lagoon, Marın County, Calıfornia, May 1967June 1968, U S G S Open-File Report, Apri1 4, 1969

4 Johnson, J W (1949) Scale effects in hydraulic models involving wave motıon, Transactions, Amer Geophysical Unıon, Vo1 30 , No 4 , pp 517-525

5 Shepard, Francis P (1950) Beach cycles in Southern California, Beach Erosion Board Tech Memo No 20, Wash, D C

6 Trask, Parker D (1959) Beaches near San Francısco, Calıfornıa, 1956-57, Beach Erosion Board Tech Memo No 110, Wash, D C

7 Corps of Englneers (1965) Cooperative beach erosion study of coast of Northern Calıfornia, Point Delgado to Point Ano Nuevo, San Francisco District, June 1965 
8 Galvin, C J , Jr, D G Dumm, Jr , B R Sims, and $L$ W Tenny (1969) Nearshore visual wave observations for United States' coastlines, U S Army Coastal Engineerıng Research Center (unpublished) 


\title{
CHAPTER 86
}

\author{
SEDIMENT DISTRIBUTION AND ITS RELATIONS \\ TO CIRCULATION PATTERNS IN BOLINAS BAY, CALIFORNIA
}

P Wllde ${ }^{1}$ and $T$ Yancey ${ }^{2}$

ABSTRACT

Grain size and heavy mineral analyses of $6 \mathrm{cliff}, 12$ beach, and 44 marıne sediment and rock samples from Bolinas Bay and its surrounding drainage area were done as part of a long term study of sediment transport on the continental shelf off Central California

Sediments in the bay are predominately very fine sands Some samples, particularly adjacent to Duxbury Reef on the west, have a coarse sand to pebble component The primary mode of the marine samples is in the range 088 to $125 \mathrm{~mm}$, whereas, the primary mode for beach material 1s from 175 to $25 \mathrm{~mm}$ The range of median diameters of the marine samples is from 07 to $14 \mathrm{~mm}$ The median diameters show a trend of decreasing grain size seaward parallel to the depth contours except opposite the entrance to Bolinas Lagoon where a tongue of relatively coarser material cuts across the depth contours The range of other statistical parameters are (1) sorting coefficient 110 to 141 , (2) skewness 083 to 118 , and (3) kurtosis 015 to 032

our sediment studies indicate

(1) The heavy mineral assemblage is predominantly green hornblende with secondary amounts of hypersthene and augite Glaucophane and jadeite occur in locally high concentrations near shore

(2) The pattern of distribution of the heavy minerals shows (a) a tongue of high concentrations of minerals with a granitic source extending northwest from the San Francisco Bar, (b) flanked on the north and northeast by increasing landward concentrations of Franciscan metamorphic minerals

(3) The major source of heavy minerals is the San Francisco Bar Secondary contributions come from Bolinas Lagoon and the adjacent cliffs

(4) The circulation in the Bay is primarily counterclockwise, produced by a combination of wave refraction around Duxbury Reef and the tidal Coast Eddy Current The tidal influence, however, of Bolinas Lagoon is restricted to about one mile from the lagoon mouth circulation

\footnotetext{
1 p Wilde, Department of Civil Engineering, University of California, Berkeley, California

$2 \mathrm{~T}$ Yancey, Department of Paleontology, University of California, Be- 'reley, California
} 
patterns in the Bay greatly influence the sediment distribution

(5) The annual sediment flux in Bolinas Bay is about 300,000 cubic yards The bottom sediments in the Bay are apparently in quasiequilibrium 
This report summarizes a comprehensive study of Bolinas Bay sediments given in Isselhardt and others, 1968, 1969, and Wilde and others, 1969 Bolinas Bay is a parabolic shaped embayment of the pacific Ocean at the southern tip of the Point Reyes Penınsula, ten miles north of the entrance to San Francisco Bay (FIg 1) Bolinas Bay is bounded (1) on the north by the Bolinas Cliffs, (2) on the northeast by sea Drift Splt, the barrier bar at the entrance to Bolinas Lagoon, (3) on the east by the Marin County Coast Ranges, (4) on the south by the Pacific ocean, and (5) on the west by the shoals of Duxbury Reef The long axis of the bay points towards Bolinas Lagoon and is oriented about $N 40^{\circ} \mathrm{W}$ along the submarine trace of the San Andreas Fault

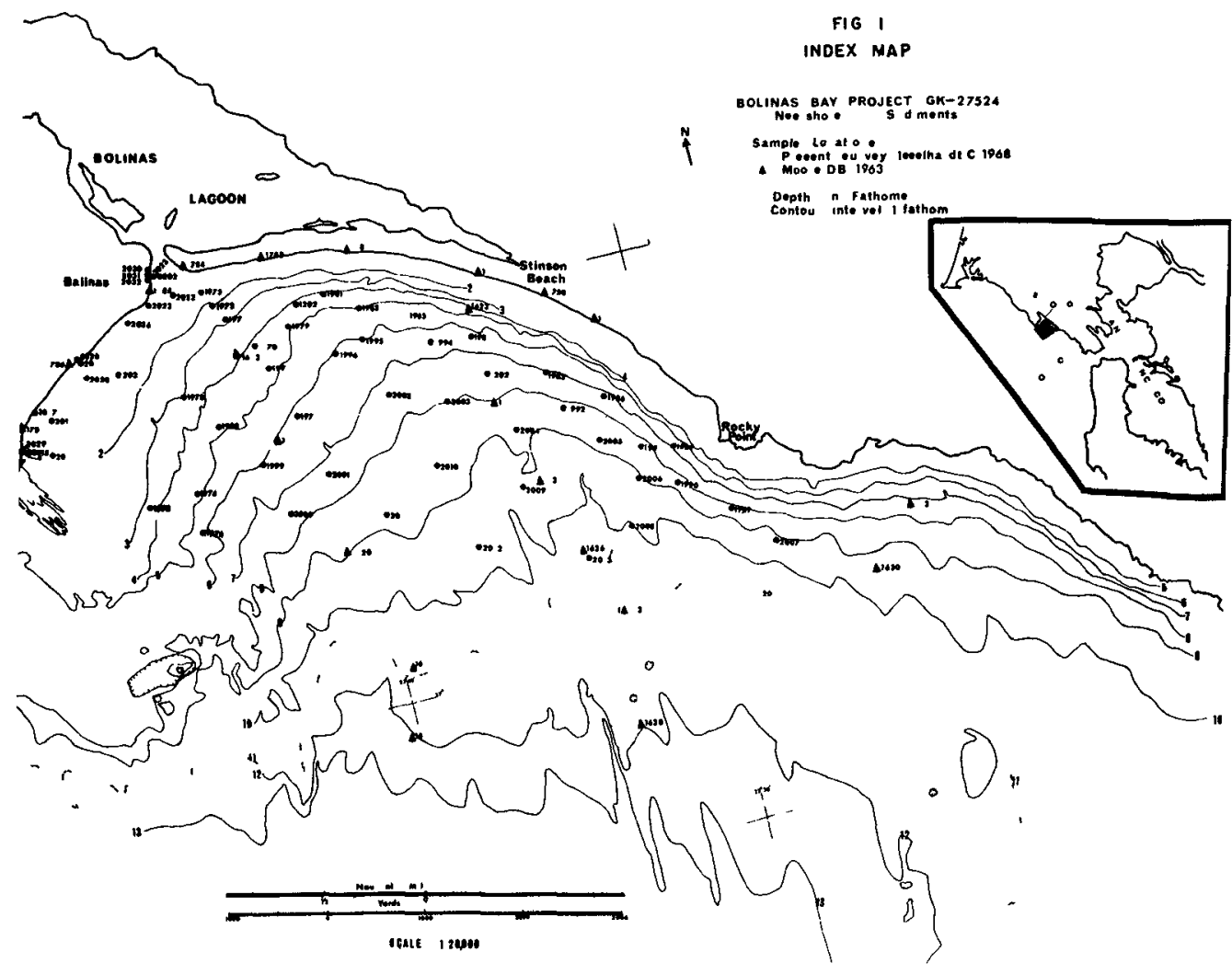


The land area in the drainage adjacent to Bolinas Bay can be divided, east to west, respectively, Into three topographic regions, (1) Bolinas Ridge which extends northeastward from Mount Tamalpais to Tomales Bay, (2) the San Andreas rift zone of rolling hills, sag ponds, and Bolinas Lagoon, and (3) the Bolinas Plateau with Duxbury Reef, a wave cut bench at the base of the western edge of the plateau (U S G S $7 \frac{1}{2}$ minute quadrangle Bolinas sheet) The main feature of the Bolinas region is the conspicuous rift valley of the San Andreas Fault, which is prominently expressed by Bolinas Lagoon In Bolinas Bay it can be traced, less conspicuously, in the submarine contours This rift separates structural blocks of greatly different geological composition, as well as acting as a focus for drainage within the region

Depth contours in Bolinas Bay generally are sub-parallel to the shore line (Fig 1) Bay bottom gradients are steepest on the eastern side near Rocky Point where the 10 fathom line lies one-half mile offshore The contours diverge to the west where adjacent to Duxbury Reef the 10 fathom line is one and three-quarters miles offshore

Two submarine channels heading southeast downslope can be traced seaward of 7 fathoms These channels are aligned with the northwestsoutheast trend of the San Andreas Fault The channels empty into a depression lying between Bolinas Bay and the north rim of the San Francisco Bar (Potato Patch Shoal)

Changes in bathymetry with tidal fluctuations apparently are limited to a small region with an offshore bar just seaward of the entrance to Bolınas Lagoon (Ritter, 1969) Seasonal depth changes are being studied now by comparison of various precision depth surveys of the bay Preliminary results (Johnson, 1969) indicate that seasonal depth changes also are limited to shallow nearshore regions near the entrance to the lagoon

\section{Tides}

The Bolinas a rea has a mixed tide like San Francisco Bay, with a maximum range of 8 feet from higher high water to lower low water (Ritter, 1969, p 13)

\section{Currents}

Open Ocean Currents The major offshore current off the central California coast is the southwesterly flowing California Current which is the eastern return gyre of the clockwise circulation pattern of the North Pacific (Reid and others, 1958, U S Hydro, 1947) During the winter months a coastal northward flowing current called the Davidson Current (Reid and Schwartzlose, 1962) interposes itself between the coast and the California Current The Davidson Current flows during the periods of maximum water runoff and erosion (Hendricks, 1964) on the adjacent land areas Thus, the Davidson current probably carries a significant load of suspended sediment in a northward direction along the coast 
Tidal Currents The most apparent water movements in Bolinas Bay are tidal, complicated by the configuration of the coastline and the inputs of the tidal prisms of both Bolinas Lagoon and San Francisco Bay Strong non-surging currents have been encountered by scuba divers near station 1627 ( $R$ Zelwer, 1968, personal communication) in about 70 feet of water, which indicates tidal rather than wave produced currents The Coast Eddy Current ( $U \mathrm{~S}$ Coast Pllot, no 7, 1968, p 152), a feature of the San Francisco Bay tide, flows north and counterclockwise north of the San Francisco Bar into Bolinas Bay

Longshore Currents Longshore currents develop in response to the prevalling west northwest swell (Fig 2) Refraction of the waves about Duxbury Reef would produce a northerly drift north of the Golden Gate Northwesterly longshore currents within Bolinas Bay is suggested by the orientation of Sea Drift Spit across the mouth of Bolinas Lagoon Drift measurements by dye drops (rhodamine B) and floating rubber balls by the U S Geological Survey (RItter, 1969, p 30), and by milk bottle caps (Brown and Caldwell Consulting Eng, 1961) Indicate counterclockwise drift in the eastern portion of Bolinas Bay

\section{Geology}

The Bolinas Bay drainage area is at the southern edge of the Northern Coast Ranges and is divided by the San Andreas rift zone into two distinct geological provinces (Fig 3) The eastern section noted topographically as Bolinas Ridge consists entirely of Franciscan Formation rocks except for recent alluvium in stream valleys In the Bolinas Bay drainage area the Franciscan consists of undifferentiated sandstones, chert, serpentine, and diabases Feldspathic sandstone is the most abundant rock type (Gluskoter, 1962)

The area to the west of the San Andreas fault is in the Salinian Quartz Diorite province, although no granitic type rocks crop out in the Bolınas Bay drainage, such rocks are found nearby at point Reyes, Tomales Point, and Bodega, and presumably are the basement rocks in the Bolinas area Two Cenozozc formations, the Monterey and the Merced, form the surface exposures, except for patches of recent alluvium, over the entire Bolinas Peninsula The Merced Formation, found as a thin band parallel to the fault, consists of fine grained, friable sandstones and siltstones with small amounts of fossiliferous sandstone and pebble conglomerates The Monterey Formation is the most extensive unit found on the Bolinas Peninsula and forms the rapidly eroding cliffs extending from Duxbury Reef almost to the Bolınas Lagoon entrance The Monterey rocks are predominately tan to gray mudstones, silty mudstones, and siltstones with occasional lenses of sandstones

The San Andreas rift zone is a jumbled mass of rock composed of slivers from formations on both sides of the zone The fault is active and displacements of one foot vertically and 8-14 feet horizontally were recorded in the Bolinas area from the 1906 earthquake (Lawson, 1908, p 70, 84) In fact, the eplcenter of the 1906 earthquake was located near Bolınas Bay on the Pt Reyes Peninsula 


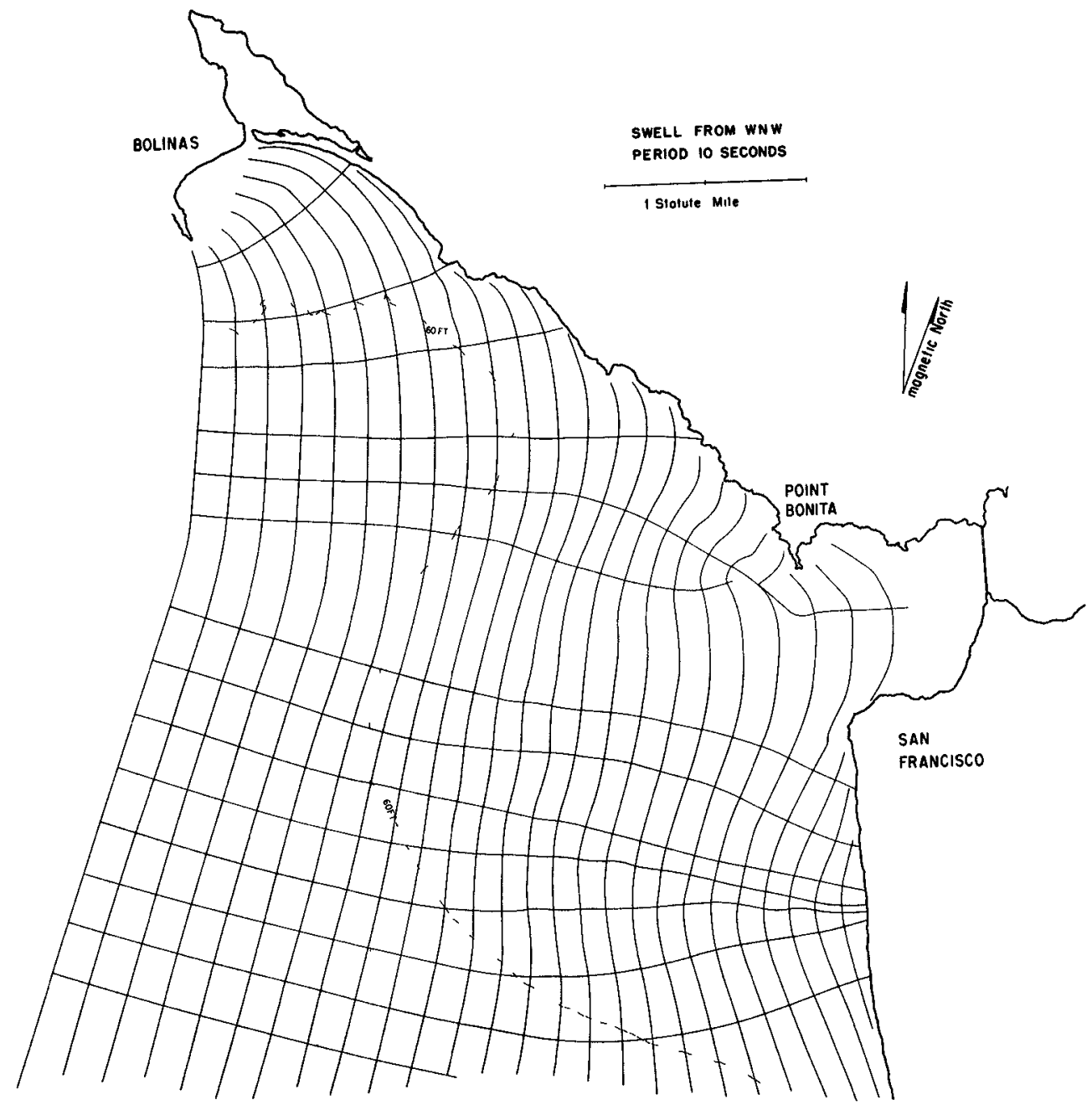

FIG 2 WAVE REFRACTION DIAGRAM (WNW, 10 SEC) FOR MARIN-SAN FRANCISCO AREA 


\section{GEOLOGY OF THE BOLINAS BAY REGION} EXPLANATION

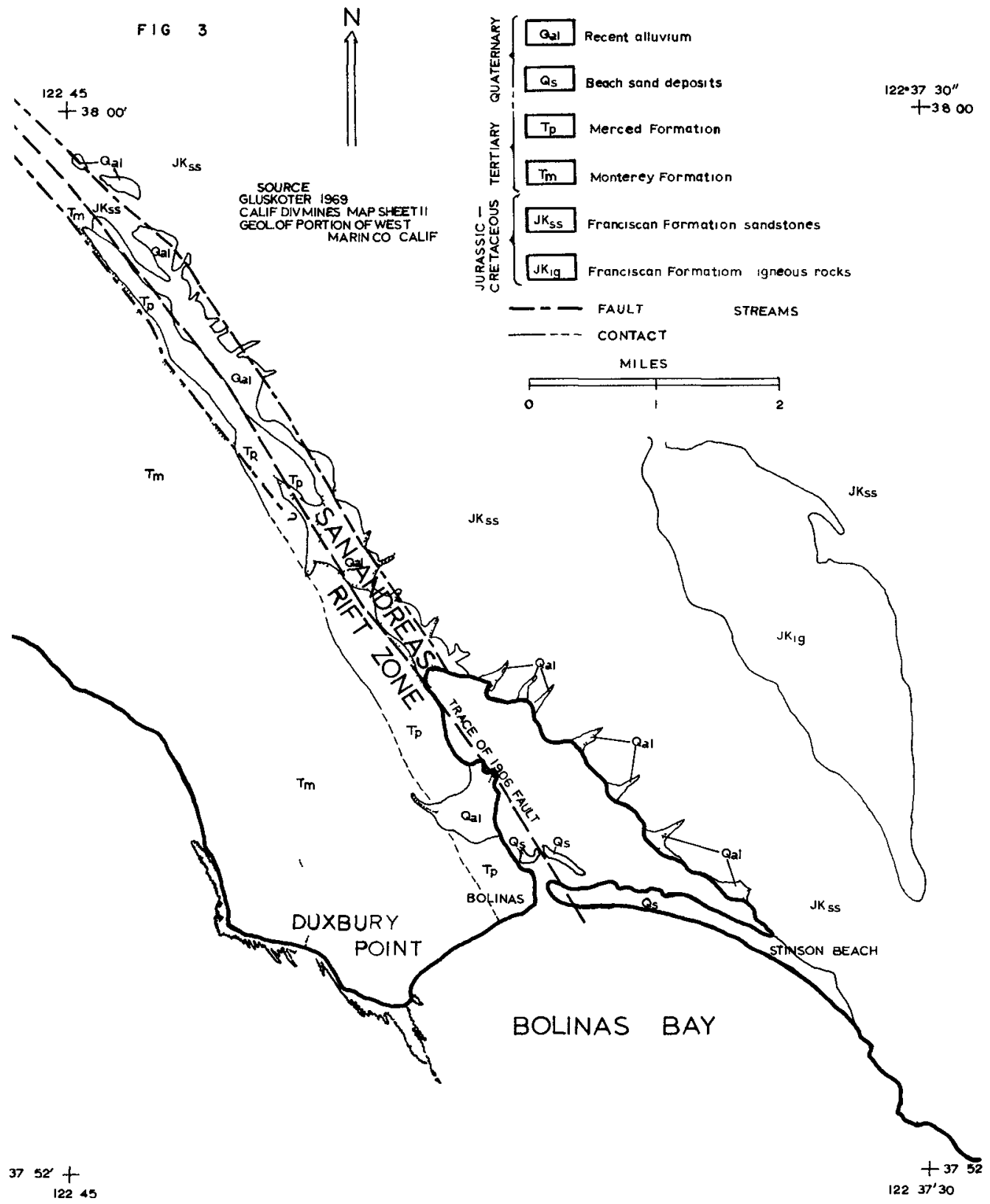


For more detalled information on the geology of the Bolinas area, see Weaver (1949), Gluskoter (1962, 1969), Galloway (1966), and Balley (1964)

\section{Grain Size Properties}

The statistical parameters, median grain size, and sorting coefficient are derived from data presented in Isselhardt and others, 1968 The average of median grain size for the bay sediments $1 \mathrm{~s}$ about $010 \mathrm{~mm}$ or fine sand, with extremes at $040 \mathrm{~mm}$ medium sand to $007 \mathrm{~mm}$ very fine sand The median grain size generally decreases seaward paralleling the depth contours Figure 4 shows variations of this trend as two lobes of coarser sediment extending (1) seaward from Rocky Point and (2) from the mouth of Bolinas Lagoon, and a band of finer grained sediment on the western margin of the bay next to Duxbury Reef Topographic and bathymetric irregularities produce the Rocky Point and Duxbury Reef anomalies Wave agitation around Rocky point prevents fine material from depositing While on the protected lee side of Duxbury Reef, finer particles may settle out The lobe of coarser material near the mouth of Bolinas Lagoon presumably is a product of tidal action Coarser sediment is carried seaward by the strong tidal currents measured by Ritter (1969) exiting the lagoon Winnowing by tidal reversals prevents settling of finer material here

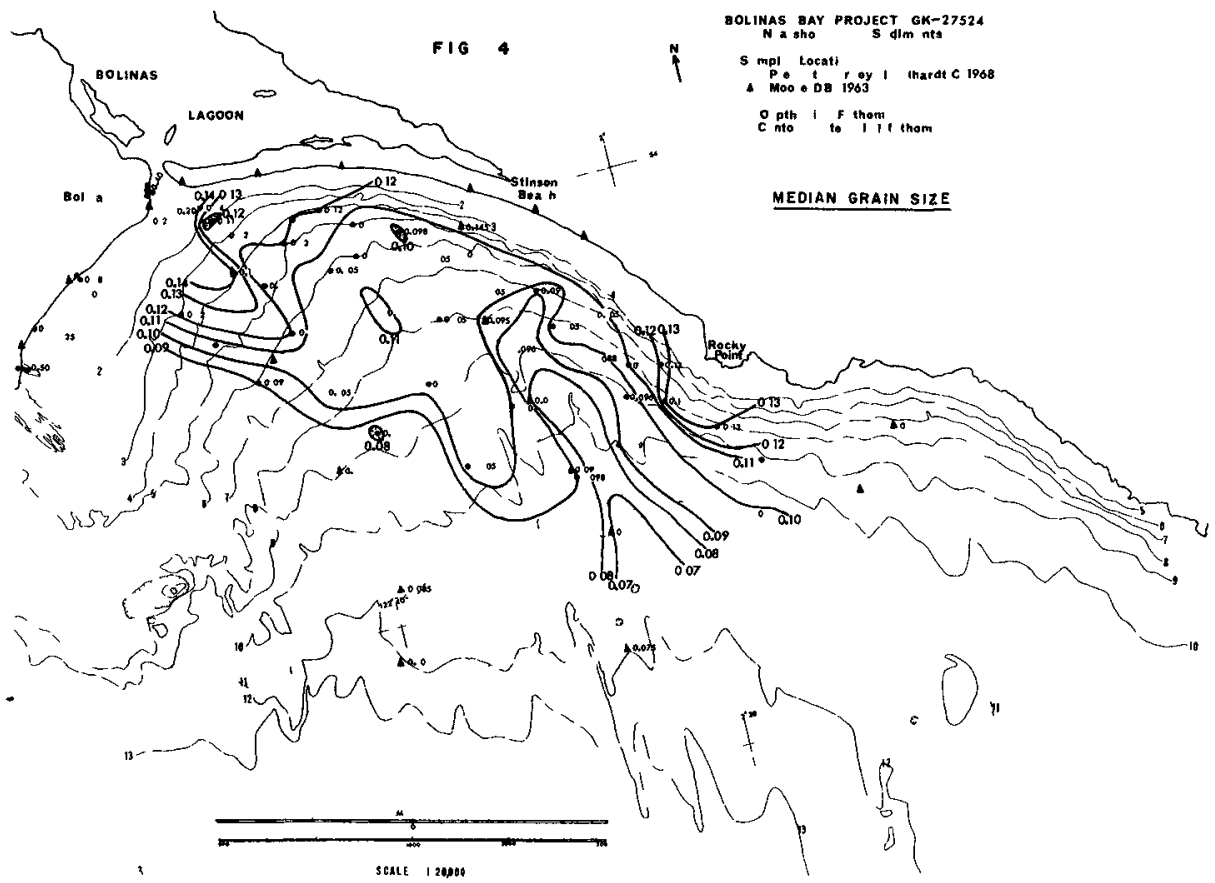


Values of the sorting coefficient indicate all the samples are well sorted, with the expected gradient of poorer sorting seaward The gradient is greatly masked by sample to sample variation, but deep samples have values of 120 or greater whlle shallow samples have values usually less than 120 The best sorting in the bay is associated as expected with the entrance to Bolinas Lagoon and with Rocky point The sorting roughly shows the same pattern as the median grain size, but much less clearly In Bolinas Bay the sorting of the sediments is primarily a function of the grain size of the sediments, in both deep and shallow water

Contours of weight percent heavy minerals (F1g 5) also gives strong indication of bottom currents flowing between the lagoon entrance and deeper water The major trend is a linear concentration of heavy minerals aligned from the entrance to Bolinas Lagoon to the center of the bay It extends between and connects the two tongues of coarse sediment noted on the median grain size chart, and apparently is caused by tidal currents

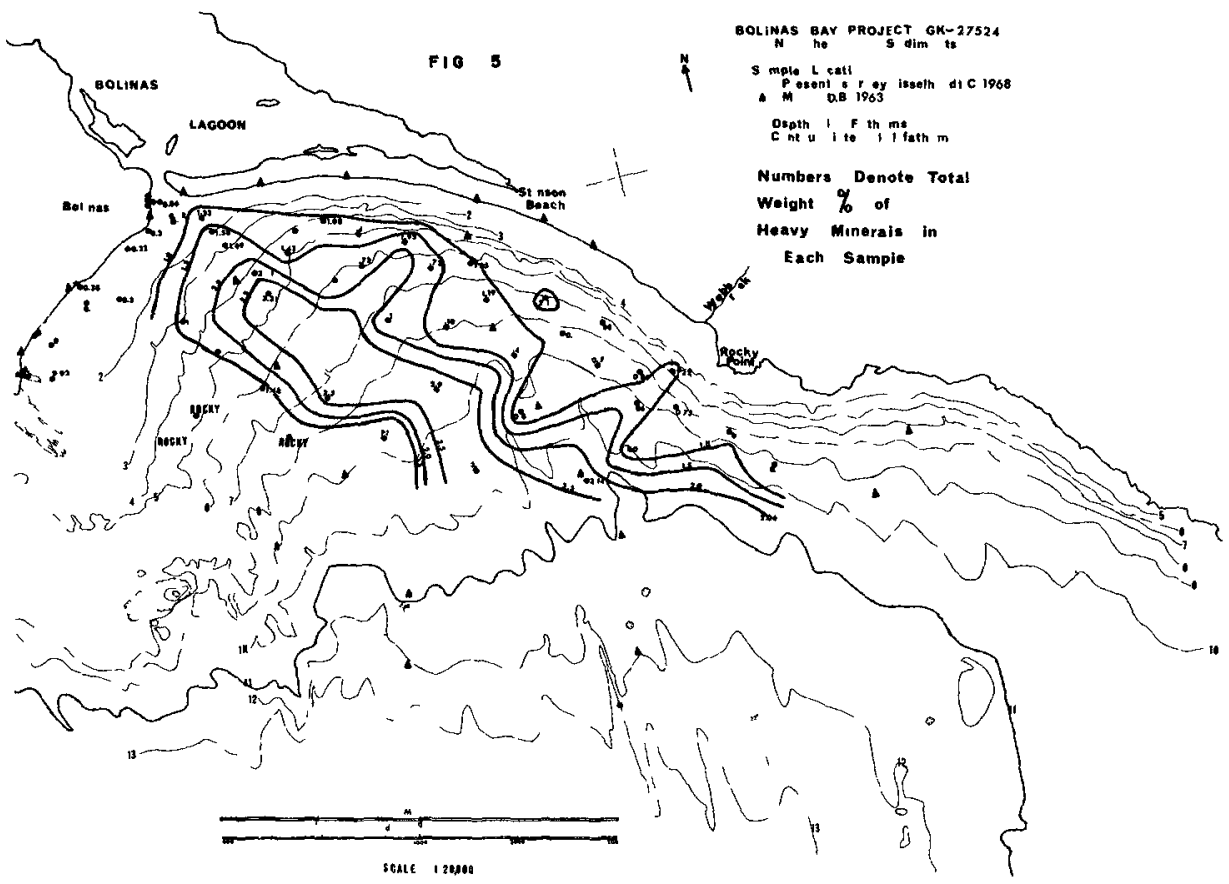




\section{Mineralogy}

The heavy mineral assemblages reveal two discrete suites (1) in a mid-bay tongue typified by green hornblende and hypersthene, and (2) along the shore characterized by glaucophane and Jadeite Figure 6 shows the relative proportion of the two mineral sultes in each sample plotted as a function of distance from shore These data plotted on a map (F1g 7) describe three zones (1) off shore, for sediments of the hornblende + hypersthene sulte, (2) near shore, for samples with a glaucophane + jadeite assemblage, and (3) at intermediate distance from shore where the two sultes are mixed

\section{Provenance}

Potential sources for the sediments of Bolinas Bay are

(1) Webb Creek dralnage, whlch empties directly into Bolınas Bay,

(2) draınage area of Bolınas Lagoon, with sediment transported from the lagoon into Bolinas Bay by tidal action,

(3) sea cliff erosion of cliffs between Duxbury Reef and the town of Bolinas,

(4) San Francisco Bar, with sediments transported into Bolinas Bay by northward flowing bottom currents,

(5) rocks north and south of Bolınas Bay - sediments brought into the bay by longshore drift

Webb Creek, draining Franciscan terrain, is the only stream that empties directly into Bolınas Bay Sediments in Bolınas Bay adjacent to the mouth of Webb Creek contain high concentrations of glaucophane and jadeite, a typical Franciscan mineralogy, indicating a flow of Webb Creek sediments into the bay

The mineralogy of bottom sediments in the lagoon (Helley, in Ritter, 1969) Indicates that Pine Gulch Creek, the largest creek, principally contributes Monterey shale sediments to the lagoon, which is mixed with the contribution from the smaller streams with exclusively Franciscan drainage As a result, Bolinas Lagoon sediments are dominantly of Monterey shale fragments, whlle containing a heavy mineralogy characteristic of Franciscan Formation sediments

The Bolinas Cliffs are actively eroding and supply large amounts of sediment to Bolınas Bay annually Helley (In Ritter, 1969) cites an annual rate of cliff erosion of 23 feet per year (determined by $A$ J Galloway) The sea cliffs fronting on Bolinas Bay are 7000 feet long and are at least 120 feet $h 1 g h$, and would glve an annual yleld of sediment of 72,000 cublc yards The cliffs are composed mostly of Monterey shale, with lesser exposures of Merced Formation near the town of Bolinas 


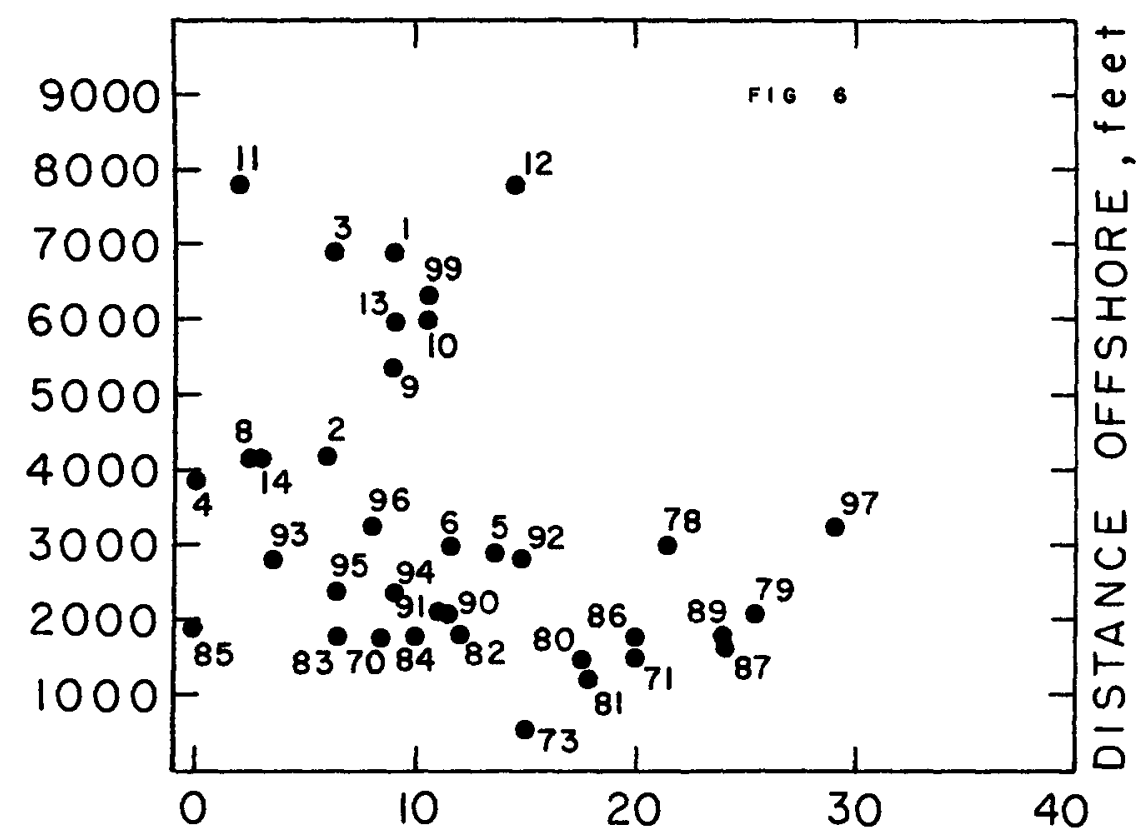

$\%$ gLAUCOPHANE + JADITE FRANCISCAN MINERALS

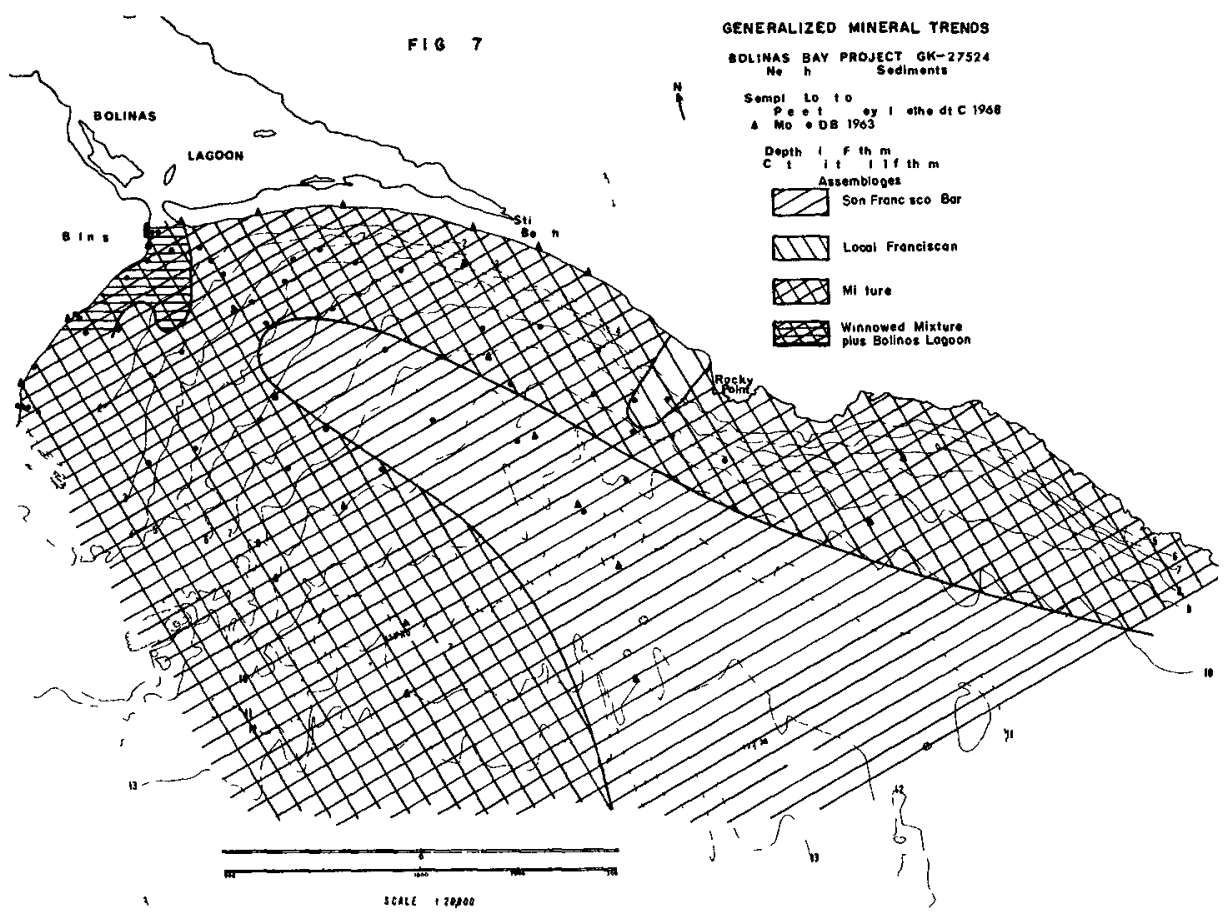


The San Francisco Bar, to the south of Bolınas Bay, contains a very large reservolr of unconsolidated sediment, part of which is brought into Bolinas Bay by tidal currents Sediments of the San Francisco Bar are characterized by higher concentrations of augite and hypersthene than are found in marine sediments to the northwest or south (Moore, 1965) The source of the hypersthene is the volcanic rocks and volcanic sediment of the Central Valley drainage area (Hall, 1965) Part of the sediments in Bolinas Bay are characterized by similar high concentrations of auglte and hypersthene and thus are probably derived directly from the San Francisco Bar, carried by the northward flowing Coast Eddy Current ( $U$ S Coast Pllot, No $7,1968, p$ 152)

The San Francisco Bar is formed of sediment brought to the Pacific through the Golden Gate from the Central Valley drainage area $U S$ Geological Survey estimates (in Homnn and Schultz, 1963, p 4) of the sediment entering San Francisco Bay are from 72 to $96 \mathrm{million}$ cubic yards per year Table 1 shows the rates of sedimentation of San Francisco Bay and Bar for the past 101 years (Homan and Schultz, 1963) With the present rate of sedimentation, 29 to 53 milion cubic yards of sediment per year passes through the bay and off the Bar A portion of this volume must move north into Bolinas Bay by northward flowing currents

The sea cliffs south of Bolinas Bay, between Rocky Point and Point Bonita, are a possible source for sediments in Bolinas Bay However, sediment entering Bolinas Bay from this source would be carried noxthward by longshore drift, which would be blocked or directed seaward by the irregular shoreline, and its character would be masked by mixing with the San Francisco Bar sediments Duxbury Reef acts as a barrier to sediment movement into the bay from sources north of Bolinas Bay The reef forms a continuous barrier on the sea floor extending about two miles out into the ocean and acts as a baffle which traps sediment, or as a barrier to deflect it to the south The reef completely blocks sediment movement into the shallow part of Bolinas Bay from the coastline to the northwest, and probably keeps it out of the bay entirely Figure 7 shows these mineralogic trends

\section{Sediment Regime}

Sediment Sources The data from mineralogical studies presented in this report permit us to distinguish two major and one minor source(s) of sediment for Bolınas Bay A fourth source, of nondiagnostic mineral composition, is known from studies of sea cliff erosion The first source is Bolinas Lagoon, which supplies sediment of a distinctive heavy mineral composition characterized by glaucophane and jadeite Bolinas Bay sediments near the entrance to Bolinas Lagoon are of this composition, and there is a limited eastward dispersal of sediment from this source

The other major source is the unconsolidated sediments of San Francisco Bar, south of Bolinas Bay, characterized by hornblende and hypersthene Sediments derived from this source cover a large area in the southeast and central portions of the bay, in areas of the bay closest 
to the bar sediment from the bar enters Bolınas Bay from the southeast, and is mixed with sediment from other sources This sediment is moved into the bay by bottom currents in the deeper parts of the bay, in depths probably of 50-80 feet, where topographzc gradients are low This sediment does not enter the bay by longshore drift, and does not enter depths that lie within the regime of the surf zone (approx 0-30 feet)

A third, and minor, source of sediment is Webb Creek near Rocky point Sediment with a Franciscan glaucophane-jadeite mineral composition enters Bolinas Bay at this place, and is spread a short distance to the north, west, and south of the point The limited and equal distribution of sediment from Webb Creek shows that sediment bypassing around Rocky point, from either the north or the south, is relatively unimportant As a result, longshore drift on a long term basis is unimportant in sediment movement in the vicinity of Rocky point This supplies further evidence that sediment transported into Bolinas Bay from the San Francisco Bar enters through the deeper portions of the bay, outside the surf zone

A fourth source of sediment, although indistinguishable by heavy mineral content, is present in the northwest corner of the bay The sea cliffs between the town of Bolinas and Duxbury Reef are rapidly eroding at the present and deliver sediment directly into the bay The relative importance of this source cannot be determined on the basis of heavy mineral data, but estimates of 72,000 cublc yards of erosion per year indicate that this is a major source of sediment

Sediment Transport The sedimentary budget of Bolinas Bay is a function of (1) the volume, size distribution, and entry point of material introduced into the bay, and (2) the volume, size distribution, and exit areas for material leaving the bay wathin the bay these sediment fluxes are governed by (3) bottom currents which distribute the sediment, and (4) the bottom configuration which modifies the hydraulic regime causing transportation or deposition of sediment in a given area The above four factors also, at least $1 \mathrm{n}$ part, are time dependent, some seasonal like the volume of stream discharge, or semi-diurnal like tidal fluctuations of the bottom currents However, Johnson (1969) has shown that the changes in bottom configuration are limited to shallow areas near the mouth of the lagoon Therefore, the mineralogic distributions shown in this report probably are valid for the generalized annual picture for the entire bay

Sediment Budget As noted in the section on provenance, the major source of the sedimentary cover is San Francisco Bar Secondary to this is the sediment from Bolinas Lagoon, and of least importance is Webb Creek For the Bolinas Cliffs the rate of retreat of the cliffs is known, and volumes of sediment can be roughly determined

As cited earlier in this report, the Bolinas Cliffs are presently eroding at a rate of 23 feet per year computing the area of cliff face subject to erosion, the resultant sediment yleld to the ocean is determined to be approximately 72,000 cubic yards per year 
Ritter (1969) made measurements of the amount of sediment transported through the mouth of Bolinas Lagoon for one complete tidal day, a period of 25 hours, on October 24-25, 1967 Most of the sediment was moved during the major ebbtide, and the resultant of all portions of the tidal cycle was a net removal of about 330 tons of sediment from the lagoon for a 25 hour period projecting this over a full year, and using a density of 79 pounds per cublc foot, which Ritter (1969) determined to be the average density of sediments in Bolinas Lagoon, the resultant is a sediment yield of about 32,500 cubic yards per year This figure is used as a minimum value of yearly sediment yleld The measurements were made at the end of the California dry summer season, when sediment yield is low High sediment ylelds are associated with perlods of high water runoff during the winter, which our calculations do not allow for, although it may be counterbalanced to a small degree by dry summer months with a lower sediment yield A safe estimate would be an average of about 50,000 cublc yards per year

No data on the amount of water flow, or suspended sediment content is avallable for Webb Creek, so an estimate of the sediment yield is made on the basis of the areal distribution of sediments derived from this source in the sediment cover of Bolinas Bay A rough estimate of between $1,000-10,000$ cubıc yards per year can be made on this basis Sediment yield of Webb Creek is augmented by sea cliff erosion of Rocky Point, which may provide as much, or more, sediment as Webb Creek proper In any event, this source is not a major source of sediment to Bolinas Bay

An estimate of the volume of sediment entering Bolinas Bay from San Francisco Bar can be made from the computed volume of sediment passing over San Francisco Bar Sediment is transported over the bar at a rate of 2 9-5 $3 \mathrm{million}$ cubic yards per year Assuming a falrly even radial dispersal off the bar, about $20 \%$ of this total will move towards Bolinas Bay, or about 800,000 cublc yards per year Assuming that $25 \%$ of this amount will actually reach Bolinas Bay, with the remainder moving into deeper water, the resultant total is 200,000 cubic yards of sediment per year comparing the relative areal distribution of sediment in Bolinas Bay from each source, with a standard of about 50,000 cublc yards of sediment per year from Bolinas Lagoon, 200,000 cubic yards per year is a reasonable estimate of sediment yield to Bolinas Bay from San Francisco Bar

Estimates of sedimentary inputs into Bolınas Bay are summarızed in Table I

Figure 8 shows postulated sediment flux and non-tidal currents for the end of the winter season in Bolinas Bay As seen in Fig 7 , the orientation of the heavy mineral provinces is not adequately explained by the non-tidal currents in Bolinas Bay This is particularly true for the lobe of high hornblende-hypersthene emanating from the Potato Patch Shoals of the San Francisco Bar The influence of the tides in governing the sediment distribution in Bolinas Bay is postulated as a two step process 
Table 1 Estımated Annual Sedıment Influx into Bolinas Bay

Source

Bolinas Cliffs

Bolinas Lagoon

Webb Creek

San Francisco Bay

TOTAL
Volume

$72,000 \mathrm{cu}$ yards

$32,500 \mathrm{cu}$ yards

$1-10,000 \mathrm{cu}$ yards

$200,000 \mathrm{cu}$ yards

314,500

315,000 cubıc yards/year
Authority

rate from Galloway

Ritter

this report

estimation Holman \& Schutz

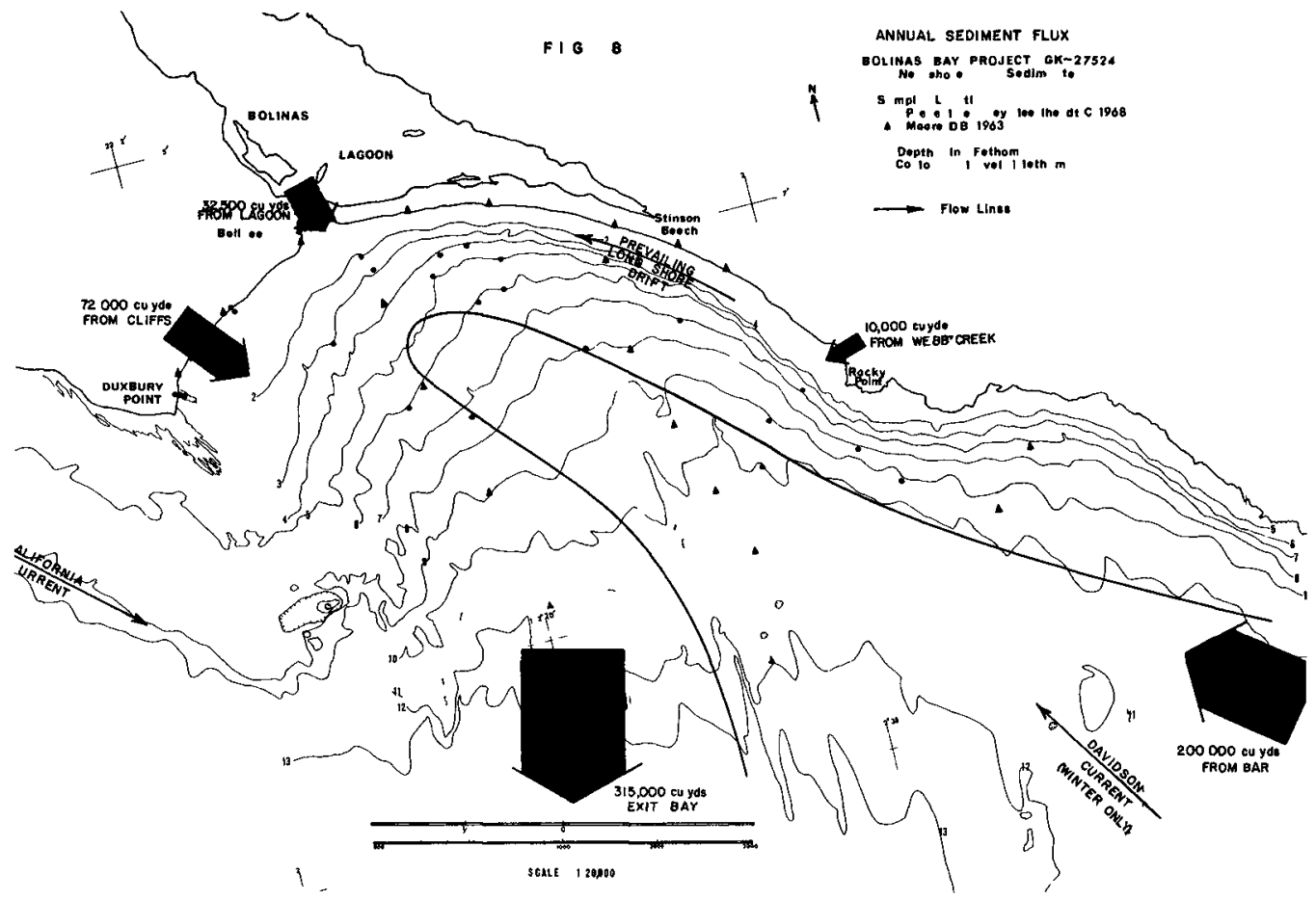


1 During flood tide in the lagoon (Fig 9), the flow of water and entrained sediment to the northwest is at a maximum as (a) the Coast Eddy Current, (b) the Davidson Current, (c) longshore drift by wave refraction around Duxbury Reef, all act in consort with the incoming tide in the lagoon Apparently the axis of transport is directed approximately along the 50 foot (8-9 fathom) line as shown by the orlentation of the hornblende-hypersthene lobe

II During ebb tide in the lagoon (F1g 10), the outflow from the lagoon combines with or produces the southwest return gyre of the coast Eddy Current, which is reinforced to the southwest off the tip of Duxbury Reef by the California Current The leading edge of the counterclockwise gyre, (a) entrains material from local sources which mixes with lagoonal material from the ebb tide on the southwest side, but (b) maintains on the inner side of the gyre the compositional integrity of the sediment brought from the san Francisco Bar The gyre thus produces a northwest oriented lobe of San Francisco Bar material surrounded on three sides by sediments from local source areas

The above models are based on an input from various sources of 315,000 cubic yards annually to Bolinas Bay (Table 1) Johnson (1969) has shown that the bottom configuration in deeper water does not change throughout the year Thus, there is no net aggradation-degradation in Bolinas Bay This implies quasi-equilibrium with a balancing outflow of approximately 315,000 cublc yards annually with sediment added along the counterclockwise gyre (a) from the southeast from the san Francisco Bar, (b) from the northeast from Webb Creek, and (c) from the northwest from the lagoon and cliff exosion, the logical exit is to the southwest to deeper water on the shelf This is indicated on the bathymetry map by (1) the steep seaward gradients in the channel adjacent to Duxbury Reef, and (2) two small channels emptying into the embayment which separates the bay from Potato Patch shoals

\section{Acknow ledgments}

This work was sponsored by the Coastal Englneering Research Center, $U$ S Army Corps of Engineers, by contract 72-67-C-0015, through the University of California 


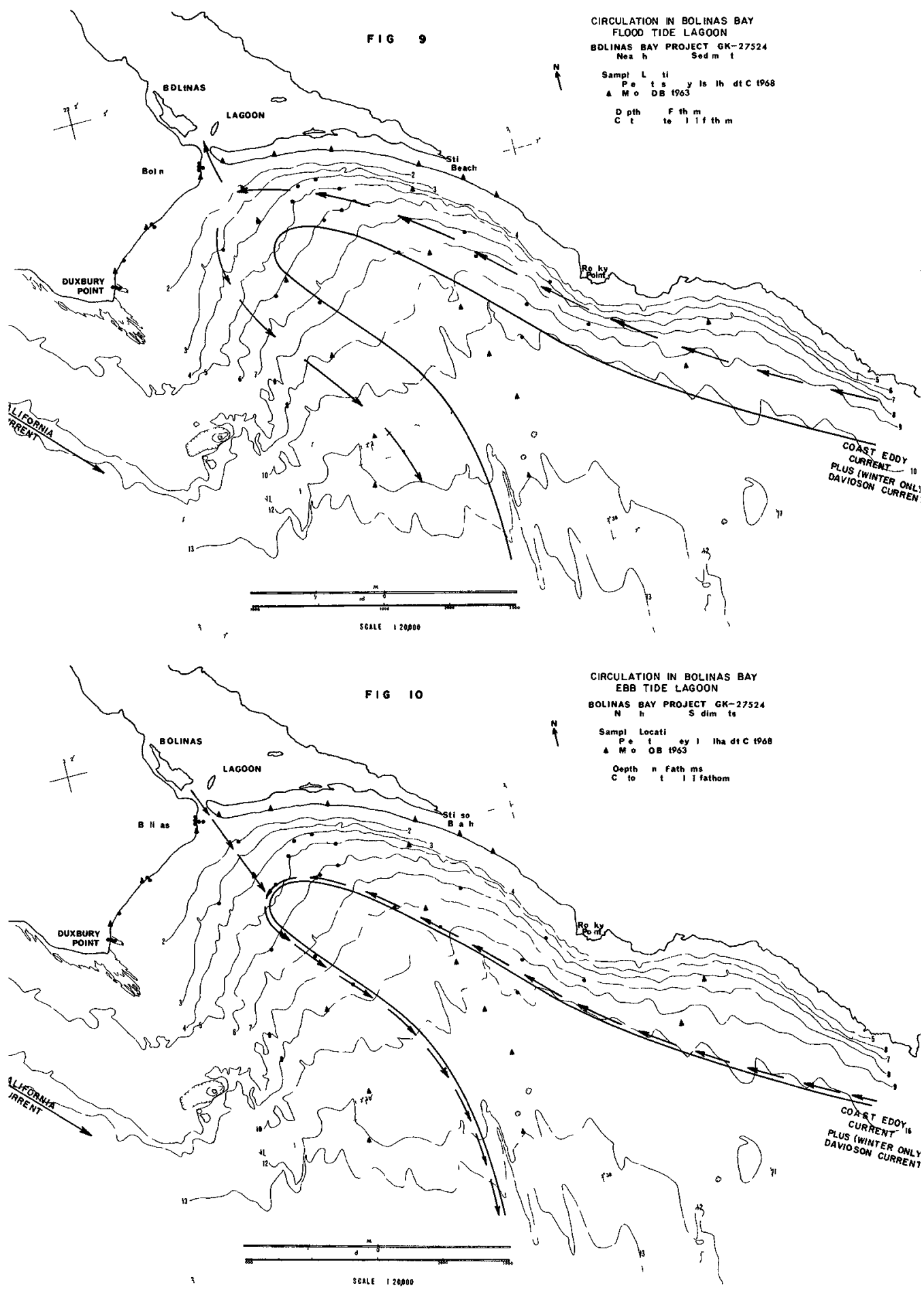




\section{References}

Balley, $\mathbf{E}$ H, Irwin, W P, and Jones, D L, 1964, Franciscan and related rocks, and their significance in the geology of western California Calif Div Mines and Geology Bu11 183, $177 \mathrm{p}$

Brown and Caldwell, 1967, Interim report on the Bolinas watershed Brown and Caldwell Consulting Englneers

Cherry, J A, 1964, Sand movement along a portion of the northern California coast Univ of Calif, Inst of Eng Res Tech Report Series HEL-4-3, $150 \mathrm{p}$

Cherry, J A, 1966, Sand movement along equilıbrium beaches north of San Francisco Jour of Sed Petrology, v 36, no 2, p 341-357

Galloway, A J, 1966, Geology of Northern California Calif Div Mines, Bull 190, p 429-440

Gluskoter, H J , 1962, Geology of a portion of western Marın County, Calif unpub $\mathrm{Ph} D$ thesis, Univ of Calif, Berkeley, $184 \mathrm{p}$

Gluskoter, H J , 1969, Geology of a portion of western Marın County, Calif Calif Div Mines and Geology, Map sheet 11

Hall, N T, 1965, Petrology of the type Merced Group, San Francisco Peninsula, Calıfornia Univ of Calif, Berkeley, unpublished M A thesis, Geology, $127 \mathrm{p}$

Hendricks, H L , ed, Compilation of records of surface waters of the Unıted States, October 1950 to September 1960, part 11, Pacific slope basins in California U $S$ Geol Survey Water-Supply Paper 1735, $715 \mathrm{p}$

Homan, W J, and Schultz, E A, 1963, Model tests of shoaling and of dredge spoil disposal in San Francisco Bay Jackson, Miss, Federal Interagency Sedimentation Conf, $30 \mathrm{p}$

Isselhardt, C, Osuch, $L$, and Wllde, P, 1968, Recent sediments of Bolinas Bay, Calif, Part A Introduction and grain size analysis, Univ of Calif, Hydraulic Engineering Lab, Tech Rept HEL-2-19, $55 \mathrm{p}$

Isselhard t, C, Osuch, L, Yancey, T, and W1lde, P, 1969, Recent sedıments of Bolınas Bay, Calıf, Part B Mineralogical data Univ of Calif, Hydraulic Engineering Lab, Tech Rept HEL-2-22, $155 \mathrm{p}$

Johnson, J W, 1969, Stabilization of the Bolinas Lagoon Inlet Report to Bolinas Harbor District, $40 \mathrm{p}$ 
Minard, $C \mathrm{R}, 1964$, The erosional and depositional history of the coast of northern California Univ of Calif, Inst of EngineerIng Research, Tech Rept HEL-2-10, $63 \mathrm{p}$

Moore, D B , 1965, Recent coastal sediments Double Point to Point San Pedro, Calif Unıv of Calif, Hydraulic Engineering Lab, Tech Rept HEL-2-14, $86 \mathrm{p}$

Reid, J L , Roden, G I , and Wyllie, J G, 1958, Studies of the California current system Calif Co-op Ocean Fish Invest Progress Reports 1 July 1956 - 1 January 1958, p 27-56

Reid, J L , and Schwartzlose, $R A$, 1962, Direct measurement of the Davidson Current off central California Jour Geophysical Research, $v$ 67, p 2491-2497

Ritter, J R, 1969, Preliminary studies of sedimentation and hydrology in Bolınas Lagoon, Marın County, Calıf , May 1967June 1968 U $S$ Geol Survey Open File Report, Menlo Park, $68 \mathrm{p}$

U S Coast and Geodetıc Survey, 1947, Tide Chart, San Francisco Bay

U S Coast and Geodetıc Survey, 1968, Unıted States Coast Pılot 7, Pacıfıc Coast, Calıf, Oregon, Washıngton, and Hawaı1, Fenthedition, $380 \mathrm{p}$

U S Hydrographic Office, 1947, Atlas of surface currents, northeastern Pacıfıc Ocean

Weaver, C E, 1949, Geology of the Coast Ranges immediately north of the San Francisco Bay region, Calif Geol Soc of America Memozr $35,242 \mathrm{p}$

WIlde, P, Isselhard t, C, Osuch, L, and Yancey, T, 1969, Recent Sediments of Bolinas Bay, Part C, Interpretation and Summary of Results Univ of Calif, Hydraulic Engineering Research Lab, Tech Rept HEL-2-23, $86 \mathrm{p}$

Yancey, T E, 1968, Recent seduments of Monterey Bay, Calif Unıv of Calif, Hydraulic Engineering Lab, Tech Rept HEL-2-18, 145 p 



\title{
CHAPTER 87
}

\section{SUCCESSIVE SALTATION OF A SAND GRAIN BY WIND}

\author{
Yoshito Tsuchiya \\ Professor of Coastal Enguneerung \\ Disaster Prevention Research Institute \\ Kyoto Unı versity, Kyoto, Japan
}

\begin{abstract}
In order to establish the mechances of sand transport in an alr or water stream, the mechanics of saltation of sand grains should first be considered In sand storms, most of the saltating sand grains on a granular bed have successively continued the saltation motion In this paper, such a saltation motion is defined as successive saltation A theoretical approach to the saltation of a sungle sand grain on a fixed granular bed $1 \mathrm{~s}$ proposed on the basis of the equations of motion for the saltation and the dynamic characterustics of collision between a saltatung sand grain and bed sand grauns Some experıments of the successive saltation of a single sand grain on a fixed granular bed were carried out to compare with the theoretical relationships It was verified from the comparison that the theoretical relationships of the height and distance of saltation of a sand grain are in faurly good agreement in substance with the results of experiment
\end{abstract}

\section{INIRODUCTION}

One of the crucial problems in the mechanics of sediment transport by wind is to establish the mechanics of the motion of sand grains near the bed In 1941, Bagnold published a famous book entitled "The physics of blown sand and desert dunes", and investigated the motion of sand by wind defined as saltation and surface creep Although many investigations have been conducted sunce then, the mechanics of the motion of sand gralns have not yet been established completely In 1951, Kawamura proposed an excellent theory of sand movement by wind based on the equation of motion of a sand grain by applying the drag force acting on the sand grain to the equation of motion but neglecting the virtual mass force since the force is usually very small compared with the drag force Recently, Owen studied the mechanlsm of saltation of sand grains by wind to discover the velocity proflie in a saltation layer and the rate of sediment transport

On the other hand, in the case of water streams, in 1964 Yalin first established a theory of saltation of a sand gral $n$ by taking into consideration the upluft force actung on a sand grain, as measured by Elnsteln and Samm and by Chepl 1 and proposed a tormula for the rate of sedument transport And after that $\mathrm{Kl}$ shl and Fukuoka recently carried out a baslc experiment on the first saltation of a single spherical particle from the beginning of movement in a turbulent stream and modıfied Yalın's theory of saltatıon by takıng into consıderation the virtual mass force The authors also conducted the same experiments as those done by $\mathrm{K}_{1} \mathrm{sh}$. and Fukuoka to make clear the mechanısm of successıve 
saltation of a single sand grain from the beginning of motion and other baslc experiments on the motion of sand grains in bed load In addition the authors proposed a theoretical approach to the motion of sand grains, namely the sliding or rolling motion and the saltation motion, based on a different concept of the motion of a sand grain from the theorles of Yalin and of $K_{1}$ shl and Fukuoka In the theory there are two types of motion of a sand grain which has begun to move from the rest condition The first one is defined as the rolling motion includlng the sliding one and the second the so-called saltation motion which the grain skips for a distance With regard to the transition from the rolling motion to saltation it was pointed out from the photographs and the direct observations of motion that the grain always begins saltation after rolling for a certain distance And it was concluded from the theory that the rolling distance is a function of the flow intensity and the ratio of the density of grain to that of fluxd and that the distance dereases rapidly with the increase of flow intensity and of the density ratio With regard to the hydrodynamic forces acting on a sand grain of which the slze is large, both the drag and virtual mass forces were applied to the establishment of the equation of motion of a sand grain, because the so-called uplift force is consldered to be very small compared whth the drag force as measured by Chepı 1 and calculated by Iwagakı

In this paper, a modification of the theory is made based on the fact that the rolling and sliding motion do not exist in the case where sand grains are transported by whd and an application of the theory to the saltation of sand grains by wind is presented in comparison with some results of experiments on the successive saltation of a sand grain on a fixed granular bed

\section{THEORY OF THE SALTATION OF A SAND GRAIN}

\section{(1) Equation of Motıon}

Since the Reynolds number becomes very high in the motion of a sand grain in general, the quadratic law for drag forces is applicable to the equation of motion It is assumed that the slze of the grain is so large that the effect of turbulence on the motion is not taken into consideration Although the hodograph space can be used in establishing the equation of motion, the equations are assumed to be established in the vertical and horizontal directions respectively, because the saltation helght is assumed not to be very high compared with the saltation distance Neglecting the Basset term which is one of the virtual mass forces, the equation of motion of a sand grain can be written as

$$
\left.\begin{array}{l}
d W / d t=\mp(3 / 4) C_{D} W^{2} /(\sigma / \rho+1 / 2) d-(\sigma / \rho-1) g /(\sigma / \rho+1 / 2) \\
d U / d t=(3 / 4) C_{D}(u-U)^{2} /(\sigma / \rho+1 / 2) d
\end{array}\right\}
$$

In the vertical and horizontal directions respectively, in which $W$ is the vertical velocity component of the sand grain, $U$ the horlzontal component, $C_{D}$ the drag coefflclent, $d$ the diameter of the grain, $g$ the acceleration of gravity, $t$ the time and $\sigma$ and $\rho$ the densities of the grain and fluzd respectively And $u$ in Eq (1) denotes the velocity in a saltation layer which is a function of the ordinate

Let the following dimensionless quantities be introduced into $\mathrm{Eq}$ 
$\left.\begin{array}{l}U=U / u^{*}, \quad \bar{W}=W / u^{*} \quad K^{2}=(4 / 3)\left\{(\alpha / \rho-1) g d / u^{*} C_{D}\right\} \\ \tau=\left\{C_{D} /(\sigma / p+1 / 2)\right\}\left(u^{*} t / d\right)\end{array}\right\}$

in which $u_{*}$ is the shear velocity and the solution under the inutial condition that $\overline{\mathrm{W}}=\overline{\mathrm{W}}_{0}^{*}$ and $\overline{\mathrm{U}}=\overline{\mathrm{U}}_{0}$ at $c=0$ becomes

$$
\begin{aligned}
& \vec{W}=K\left\{\left(\bar{W}_{0} / K\right)-\tan K_{\tau}\right\} /\left\{1+\left(\bar{W}_{0} / K\right) \tan K_{\tau}\right. \\
& \bar{U}=u-\left(u-\bar{U}_{0}\right) /\left\{1+\left(u-\bar{U}_{n}\right) *\right\}
\end{aligned}
$$

in the upward motıon of the sand grain And the solution for $W$ in the downward motion under the initial condition that $=0$ at $\tau^{\prime}=0$ becomes

$$
\bar{W}=-K \tan \left(K^{*}\right)
$$

In which $c$ is the same expression as $c$ Therefore, the velocity components $\bar{W}_{1}$ and $\vec{U}_{1}$ just before arriving at the bed can approximately

$$
\bar{W}_{1}=\bar{W}_{0}, \quad \bar{U}_{1}=u-\left(u-\bar{U}_{0}\right) /\left(1+2\left(u-U_{0}\right) \bar{W}_{0} / K^{2}\right\}
$$

In the case where the velocity in a saltation layer u is assumed to be constant because the saltation helght is very small, further integration of Eq (3) under the initial condition that $\xi(=x / d)=\eta(=z / d)=0$ at $\tau=0$ yields

$$
\begin{aligned}
& \bar{H}=(2 / 3)\left\{(\sigma / \rho+1 / 2) / C_{D}\right\} \log \left\{1+\left(\bar{W}_{0} / K\right)\right\}-(2 / 3)\left\{(\sigma / \rho+1 / 2) / C_{D}\right\}\left(\bar{W}_{0} / K\right) \\
& L=(4 / 3)\left\{(\sigma / \rho+1 / 2) / C_{D}\right\}\left\{2 u \bar{W}_{0} / K-\log \left\{2\left(u-\bar{U}_{0}\right) \bar{W}_{0} / K^{2}+1 \mid\right\}\right.
\end{aligned}
$$

in which $\mathrm{H}$ is the saltation height, $\mathrm{L}$ the distance, $\widetilde{\mathrm{H}}=\mathrm{H} / \mathrm{d}$ and $\overline{\mathrm{L}}=\mathrm{L} / \mathrm{d}$

\section{(2) Collision and Rebound of a Saltating Sand Grain on Bed Grains}

Fig 1 shows a schematic diagram for the collision and rebound of a sand grain in which $V_{1}$ and $V_{2}$ denote the velocity vectors of saltating sand grain and the other notations are shown in the flgure Making some assumptions, the conservation law of momentum in the vertical and horlzontal directions yelds

$$
\begin{aligned}
-e V_{1} \cos (\gamma-\alpha) & =V \cos (\pi-\beta-\gamma) \\
V_{1} \sin (\gamma-\alpha) & =V_{z} \sin (\pi-\beta-\gamma)
\end{aligned}
$$

in which e is the coefficient of rebound of a saltating sand grain Introducing the quantities

$$
\begin{array}{ll}
V_{1} \cos \alpha=U_{1} & V_{1} \sin \alpha=W_{1} \quad V \cos \beta=U_{0} \\
V \sin _{1} s=W_{1} & U_{1}=-W_{1} \cot \alpha=0_{1} W_{1}\left(\delta_{1}<0\right) \\
U_{0}=W_{0} \cot \beta & \delta W_{0}(\delta>0) \\
W_{1}=W / u_{*} & \bar{U}_{1}=U_{1} / u_{*} \quad W_{0}=W_{0} / u_{*} \\
\bar{U}_{0}=U_{0} / u_{*} & \bar{U}_{1}=\delta_{1} W_{1} \quad \bar{U}_{0}=0 \bar{W}_{0}
\end{array}
$$

the relationships between the velocity components just before and after the

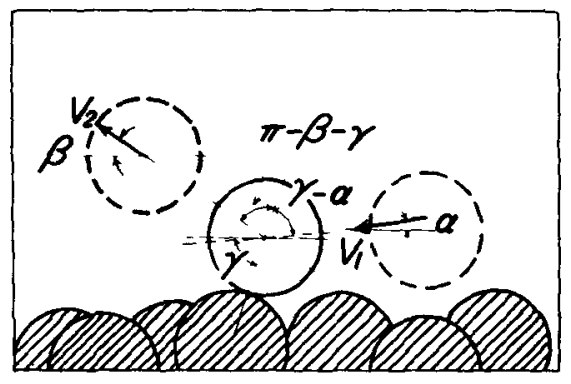

F1g 1 Schematic diagram for collision of sand grain 
collusion can be expressed approximately as

$$
\left.\begin{array}{l}
\bar{U}_{0}=e \bar{U}_{1} \\
\bar{W}_{0}=e\left\{b_{1} \bar{U}_{1}+b_{2} \bar{W}_{1}\right\} /\left\{b_{3}+b_{1}\left(\bar{W}_{1} / \bar{U}_{1}\right)\right\}
\end{array}\right\}
$$

In whrch $b_{1}=(1+e) \tan \gamma \quad b_{2}=\left(1-e \tan ^{2} \gamma\right)$ and $b_{3}=\left(\tan ^{2} \gamma-e\right)$

(3) First Saltation

In this paper the first jumping motion of a sand grain from the rest condition is defined as first saltation In the sand movement by wind the rolling or sliding motion scarcely ever occurs The friction force in the equation of motion can be neglected because the sand grain always begins to saltate just after the collusion on a nelghboring sand grann Therefore the equation of motion of a sand grain for the motion from the rest condition to the saltation can be wrutten as

$$
d U^{\prime} / d t=(3 / 4)\left[C_{b} /(\alpha / \rho+1 / 2) d\right](u-U)^{2}
$$

In whlch $U^{\prime}$ is the horlzontal velocity of the grain and $u^{\prime}$ the velocity near the graln which may be affected by the velocity of fluctuation and assumed to be $\bar{u}=$ $\mathbf{u}^{\prime} / \mathrm{u}_{*}$ The integration of $\mathrm{Eq}$ (10) with the inztial condition that $U^{\prime}=0$ at $t=$ 0 yıelds

$$
U / u^{*}=(3 / 4) u^{2} \tau /\{1+(3 / 4) u \tau\}
$$

From Eq (11) the relationship for the change of velocity with the distance under the assumption that $\tau$ is very small can be expressed approximately as

$$
U / u^{*}-u \sqrt{2}(\bar{x} / d) \bar{s} N /\{1+\sqrt{ } 2(x / d) N\}
$$

in which $N=(3 / 4)\left\{C_{D} /(\sigma / \rho+1 / 2)\right\}$

As described already, makıng the assumption that the sand grain begins to saltate just after the collision on a nelghborıng sand grain from the rest condition, the value of $x / d$ in $\mathrm{Eq}$ (12) is assumed to be that $x / d$ $\approx 1$ Since the initial velocity of the grain for the first saltation can be estimated, putting the velocity into an approximate expression of $\mathrm{Eq}(6)$ for $\left(W_{0} / K\right)^{2}$ $\ll 1$, the saltation height can be expressed approximately as

$$
\begin{aligned}
& \bar{H}_{L=}(1 / 2) \beta^{2} A r^{2}\{(\sigma / \rho+1 / 2)\} \\
& \left\{2 N /(1+V 2 N)^{2}\right\}\left\{u^{* 2} /(\sigma / \rho-1) g d\right\}
\end{aligned}
$$

in which $\beta=W_{0} / U_{0}$ and $A_{T}=\bar{u}$ By the same means the cafculation for the saltation distance can also be made

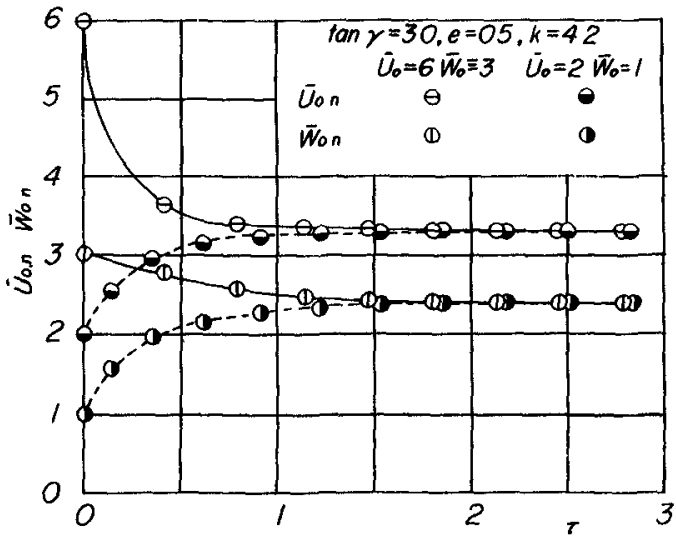

Fig 2 Changes of ind tial velocity of sand grain in successive saltation 
saltation can be calculated by Eqs (5), (6) and (9) as seen in Fig 2, the veloc1 ty components of the saltating sand grain become constant after several saltations Such a saltation is defined as the stationary saltation of which the velocity components in the vertical and horizontal directions are expressed by $W_{s}$ and $\mathrm{U}_{\mathrm{s}}$ respectively

Assuming that the sand grain has alternately taken saltation and rebound keeps the stationary velocity at the $k$-th step in saltation, the relationship can be obtanned as

$$
U_{k 0}=P u \quad \bar{W}_{k 0}=Q u \quad U_{k 10}=U_{k_{0}}=P u \quad \bar{W}_{k 10}=W_{k_{0}}=Q u
$$

Transformation of Eq (14) using Eqs (5), (6) and (9) yields approximately

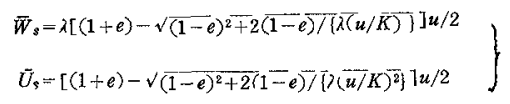

According to $\mathrm{Eq}$ (15), it is seen that the values of $W_{s}$ and $U_{s}$ are real, since $e=1$ in general, and then the roots in the equation also are real As seen from $\mathrm{Eq}_{\mathrm{q}}$ (15), the condiction that $\overline{\mathrm{W}}_{\mathrm{S}}=\overline{\mathrm{U}}_{\mathrm{S}}=0$ can be written as

$$
u_{*}{ }^{2} /(\sigma / o-1) g d=(2 / 3)\left(1 / C_{D}\right)\left\{(1-e) / e \lambda u^{2}\right\}
$$

which is generally different from the so-called critical flow intensity

It is concluded from the above description that the sand grain moving downstream, repeating saltation and rebound alternately, reaches a certain stationary velocity after several steps of successive saltation in the case where the flow intensity is larger than the critical one expressed by $\mathrm{Eq}$ (16) and that the initial velocity components in the vertical and horizontal directions in stationary saltation are expressed by $\mathrm{Eq}$ (15)

Although the helght and distance of saltation of a sand grain are formulated by $\mathrm{Eq}$ (6) in connection with the inctial velocities, wathout loss of generality, for simplicity, the following relationships can be used under the assumptions that $\left(\overline{\mathrm{w}}_{\mathrm{O}} / \mathrm{K}\right)^{2} \ll 1$ and $2\left(\overline{\mathrm{u}}-\overline{\mathrm{U}}_{\mathrm{O}}\right) \mathrm{W}_{\mathrm{O}} / \mathrm{K}^{2} \ll 1$

$$
\left.\begin{array}{l}
\bar{H}=(2 / 3)\left\{(\sigma / \rho+1 / 2) / C_{D}\right\}\left(\bar{W}_{0} / K\right)^{2} \\
\bar{L}=(8 / 3)\left\{(\sigma / \rho+1 / 2) / C_{D}\right\}\left(\bar{U}_{0} \bar{W}_{0} / K^{2}\right)
\end{array}\right\}
$$

Putting Eq (15) Into Eq (16), the relationship of the helght and distance of saltation with the flow intensity and the condition of the granular bed can be written approximately as

$$
\left.\begin{array}{l}
\bar{H}_{m}=(1 / 6)\left\{(\sigma / \rho+1 / 2) / C_{D}\right\} \lambda\left[(1+e)-\sqrt{ }(1-e)^{2}+2(1-e) /\{\lambda(u / K)\}\right]^{2}(u / K) \\
\bar{L}_{m}=(2 / 3)\left\{(\sigma / \rho+1 / 2) / C_{D}\right] \lambda\left[(1+e)-\sqrt{ }(1-e)^{2}+2(1-e) /\left\{\lambda(u / K)^{2}\right\}\right]^{2}(u / K)^{2}
\end{array}\right\}
$$

in which $H_{m}$ denotes the mean values of saltation height and $I_{m}$ the mean values of saltation distance From Eq (18), the value of $\lambda$ can be expressed as

$$
\lambda=4\left(\bar{H}_{m} / L_{m}\right)
$$


which is an empirical constant to be determined by the experimental results for the mean values of helght and distance of saltation

\section{(5) Distributions of Height and Distance of Saltation}

Although the distribution characteristlcs of the saltation helght and distance of a sand grain generally depend upon the characteristics of the velocity fluctuation of a sand grain just before and after its collision with bed grains, the dispersion characteristics of the angle of collision and the effect of turbulence on the motion of the grain, it is assumed that the distribution of height and distance of saltation is affected only by the characteristics of the velocity fluctuation

From this point of view, assuming that the density functions of the horlzontal velocity of a sand grain both in flist and stationary saltations $f_{1}(\bar{u})$ and $f_{1}(\bar{U})$ can be expressed respectively by the Gaussian distribution in the form

$$
f_{1}(u)=\left(1 / \sqrt{ } 2 \bar{\pi} \sigma_{0}\right) \exp \left\{-\left(u-u_{m}\right)^{2} / 2 \sigma_{0}^{2}\right\}
$$

for first saltation and

$$
f_{2}(\bar{U})=(1 / v 2 r)\left(1 / \sigma_{\ell}\right) \exp \left\{-\left(\bar{U}-U_{3}\right)^{2} / 2 \sigma_{\iota}{ }^{2}\right\}
$$

for stationary saltation, in which $\sigma_{0}$ and $\sigma_{\bar{U}}$ are the standard deviations of the dimensionless horızontal velocities $\overline{\mathrm{u}}$ and $\widetilde{U}$ respectıvely and are assumed to be

$$
\sigma_{0}=\boldsymbol{u} \mathrm{m} \quad \sigma_{L}=\epsilon U \quad \epsilon=\mathrm{const}
$$

and $\bar{u}_{m}$ is the mean value of $\mathfrak{u}$, assuming that the relation between the horlzontal and vertical velocitıes can be expressed generally by $\bar{W}=\lambda \bar{U}$, the density function of the saltation helght can finally be written after some transformations as

$$
f(\bar{H})=(1 / 2 \sqrt{ } 2 r)\left(1 / \epsilon \sqrt{\bar{H} \bar{H}_{m}}\right) \exp \left\{-\left(\sqrt{ } \bar{H}^{-} \sqrt{ } \bar{H}_{m}^{-}\right)^{2} / 2 \epsilon^{2} \bar{H}_{m}\right\}
$$

for both first and stationary saltation Similarly the density function of the saltation distance can finally be expressed as

$$
f(\bar{L})=(1 / 2 \sqrt{2} r)\left(1 / c \sqrt{ } \bar{L} \bar{L}_{m} \exp \left\{-\left(\sqrt{ } \bar{L}-\sqrt{L}_{m}\right)^{2} / \iota^{2} \bar{L}_{m}\right\}\right.
$$

\section{EXPERIMENTS OF SUCCESSIVE SALTATION OF A SAND GRAIN}

\section{(1) Experımental Apparatus and Procedure}

A wind tunnel, $216 \mathrm{~m}$ long, $075 \mathrm{~m}$ wide and $10 \mathrm{~m}$ deep was used in order to make clear the characteristics of successive saltation of a sand grain on a flxed granular bed Properties of grains used in the experiments are shown in Table 1 , in which $\rho_{0}$ is the density of water Paths of saltation of a grain from the rest condition were photographed with a $16 \mathrm{~mm}$ hlgh speed camera under various conditions of wind and the film was analyzed with a film motion analyzer Wind velocity profiles in the wind tunnel were measured with a hot wire anemometer and the shear velocity was estimated by the wind velocity profiles measured based on the logarithmic law of velocity profile 
Table 1 Properties of grains used in the experıment

\begin{tabular}{|c|c|c|}
\hline KInd of grains & Diameter d cm & Speciflc gravity $\sigma / \rho_{0}$ \\
\hline sand & 0225 & 2624 \\
& 0184 & 2523 \\
& 0144 & 2474 \\
\hline seed & 0184 & 1155 \\
& 0144 & 1155 \\
\hline
\end{tabular}

\section{(2) Results of Experiments}

Fig 3 shows some examples of the successive saltation of sand grains and seeds respectively obtained in the experiment From the results of the experiment the saltation angle $\beta$ shown In Fig $I$ were measured Fig 4 describes the relationship between the angles in each step of saltation and the flow intensity in which $\beta_{1}, \beta_{2}$ and $\beta_{3}$ are the saltation angles in the first, second and third saltations respectively It can be found that the angle of the first saltation is mostly independent of the flow intensity and is approximately 40 degrees

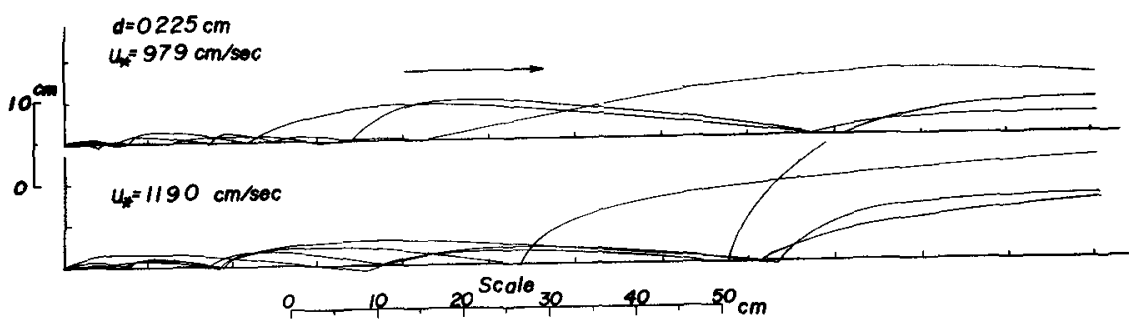

(a) In the case where sand gralns were used

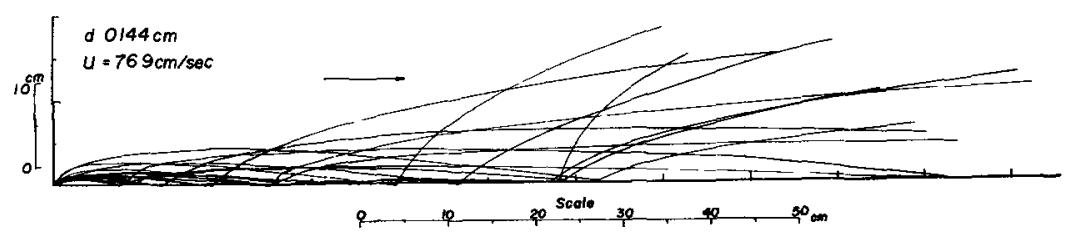

(b) In the case where seeds were used

Fig 3 Some examples of paths of saltating grains in successive saltation

Fig 5 shows a comparison between the experimental values of the height of first saltation and the theoretical relationship for the first saltation obtained by $\mathrm{Eq}$ (13) In which $\mathrm{A}_{r}$ is assumed to be 105 including the effect of turbulence 


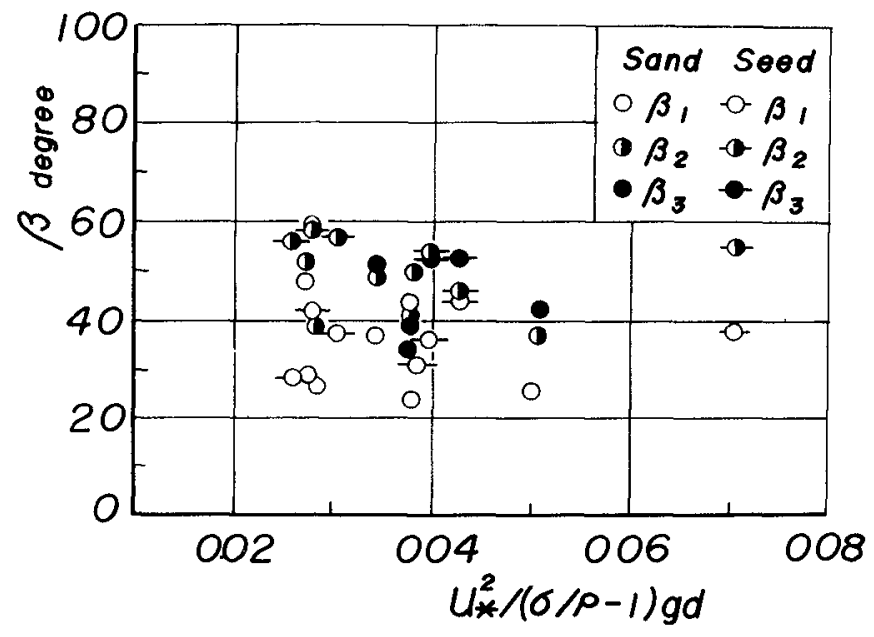

Fig 4 Variations of saltation angles with flow intensity

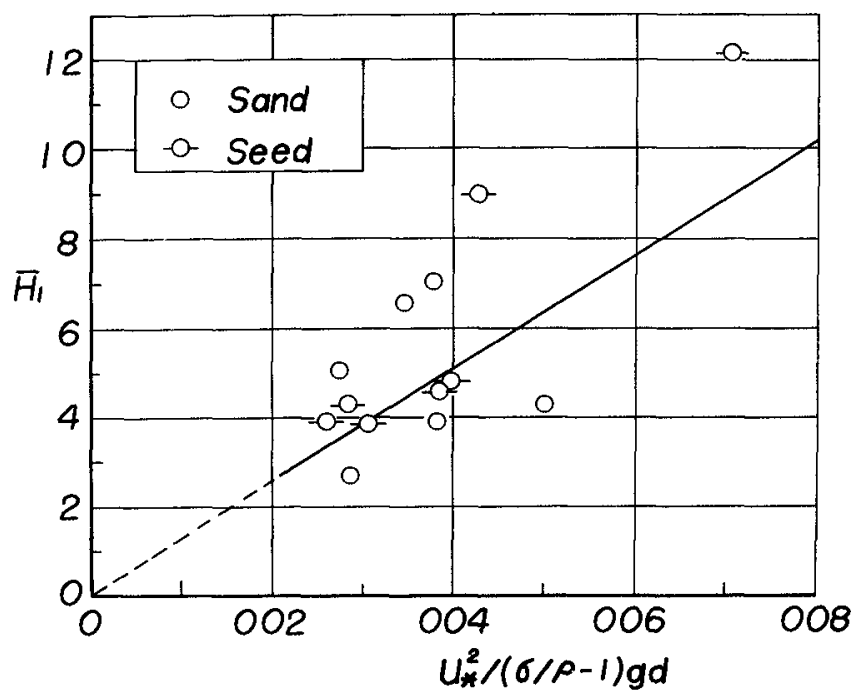

Fig 5 Comparison between theoretical relationship for the first saltation and experimental values 
and $\beta$ Is determined by the best fit to the values of experiment which Is estimated to be nearly 08 It Is concluded from the comparison that the theoretical relationship for the first saltation is in good agreement with the experimental values though there 1 s a large scatter

Fig 6 describes the variation of the value of $\lambda$ which is the ratio of the saltation helght to the distance with the increase of flow intensity It is seen that the value of $\lambda$ Is nearly constant and Is estimated to be 023 The value is different from that in a water stream because of the difference of col-

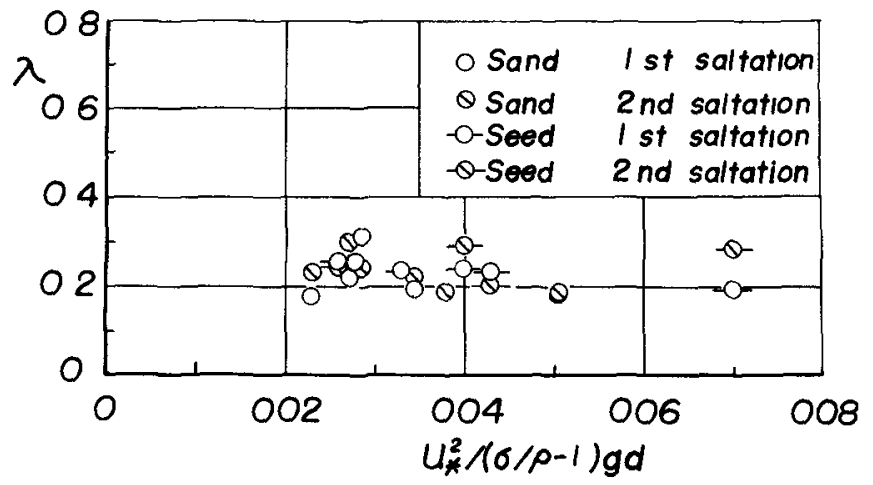

Fig 6 Variation of value of $\lambda$ with flow intensity

lision characteristies

Figs 7 and 8 show the comparisons between the theoretical curves of the saltation height and distance in stationary saltation and the experimental values for the first, second and third saltations Experiments of more successive saltation than the third saltation could not be conducted due to the limatation of the experimental apparatus Therefore a complete comparison between the theory for the stationary successive saltation and the experiment cannot be made It Is seen from the comparisons however that the experimental values in successive saltation tend to approach the theoretical curves for stationary saltation in which the value of $A$ is assumed to be the value corresponding to $H=100$ because the saltation height is not small and the effect of velocity profile on the saltation should be taken into consideration It is concluded that this theoretical approach to the saltation of a single sand grain by wind is in fairly good agreement with the results of experiments

\section{CONCLUSION}

Although the phenomena of saltation of a sand grain by wand are very complicated, there generally exists some kinds of saltation such as first saltation, successive saltation and stationary saltation as defined in this paper

A theory of successive saltation is established, based on the equations of motion of a sand grain and the dynamic relationship of the collision of a saltat- 


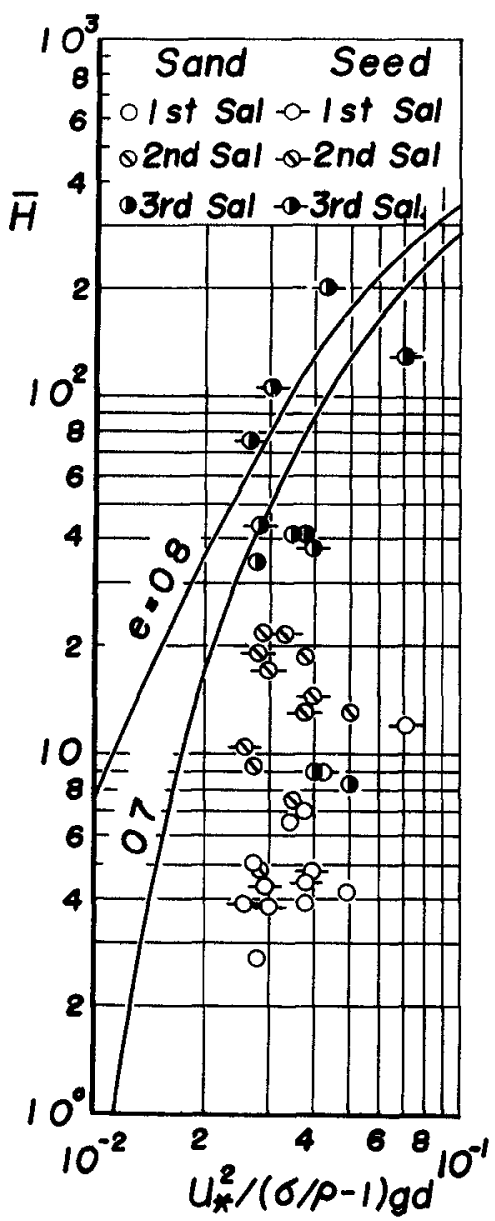

F1g 7 Comparison between theoretical curves of saltation helght in stationary saltation and experimental values for flrst, second and thr.rd saltations

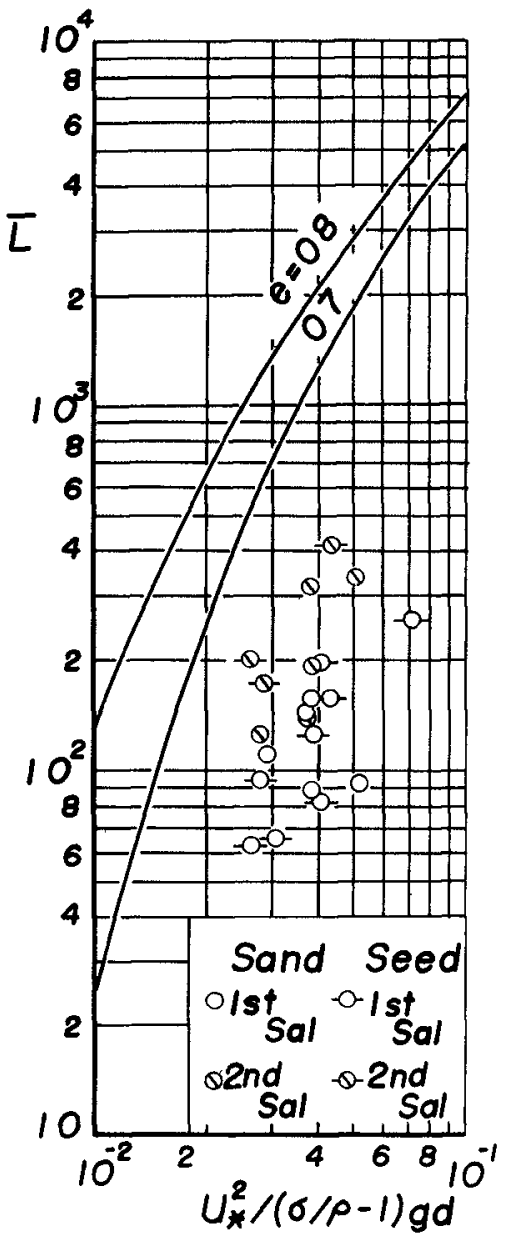

Fig 8 Comparison between theoretical curves of saltation distance in stationary saltation and experimental values for first, second and thrord saltations 
Ing sand graln with bed grains It is concluded that the theoretical relationships for the saltation are in farrly good agreement with the results of the experiment although further comparisons should be made

Further investigations on the saltation of sand grains will be conducted studying the saltation characteristics of sand grains in a water stream and comparing the results with the data of field observations in sand storms

\section{ACKNOWLEDGEMENT}

The author wishes to acknowledge his sicere thanks to Mr T Shıbano, Technıcal officer, for his kınd help in preparing this paper and to $\mathrm{Mr} \mathrm{Y}$ Kawata, Graduate Student, for his kind help in conducting this investigation

\section{REFTERENCES}

Bagnold, R A (1941) The physucs of blown sand and desert dunes Methuen \& Co Ltd, London

Chepıl, W S (1961) The use of spheres to measure lift and drag on wind-eroded soll gralns Soll Sc1 Soc Amerıcan Proc, Vol 25, pp 343-345

Elnstein, H A and Sammi, E L (1949) Hydrodynamic forces on a rough wall Reviews of Modern Phys, Vol 21, No 3, pp 520-524

Iwagakı, Y (1956) Hydrodynamıcal study on the critıcal tractive force Trans JSCE, No 41, pp 1-21 (in Japane se)

Kawamura, R (1951) Study of sand movement by wind Report of Tech Res Inst, Unuv of Tokyo, Vol 5, pp 95-112 (1n Japanese)

K1sh1, T and Fukuoka, S (1966) The mechanısm of saltation of sand grains and the rate of sediment transport Proc loth Conf on Hydraulics, JSCE, pp 59-64 (in Japanese)

Owen, P R (1964) Saltation of unform gralns in alr Jour Fluıd Mech, Vol 20, Part 2, pp 225-242

Tsuchıya, $Y$ and Sumıno, M (1967) Experıments on the mechanısm of movement of a spherical sand particle on a granular bed in turbulent streams, Annuals, Disas Prev Res Inst, Kyoto Unuv, No 10, pp 97-107 (In Japanese)

Tsuchiya, $\mathrm{Y}$, Watado, $\mathrm{K}$ and Aoyama, $\mathrm{T}$ (1969) On the mechan sm of saltation of a sand particle in a turbulent stream (1), Annuals, Disas Prev Res Inst Kyoto Unlv, No 12B, pp 475-490 (in Japanese)

Tsuchiya, $Y$ and Aoyama, $T$ (1970) On the mechanı sm of saltation of a sand particle in a turbulent stream (2) -- On a theory of the successive saltation, Annuals, Disas Prev Res Inst, Kyoto Unıv No 13B, pp 199-216 (1n Japane se)

Yalın, M S (1963) An expression for bed load transportation, Proc ASCE, Vol 89 , HY 3 , pp 221-250 




\title{
CHAPTER 88
}

\section{RESEARCHES ON SEA-WALLS}

\author{
5 Nagai \\ Professor of Hydraulics, \\ Dean of Faculty of Englneering, \\ Osaka Cıty Unıversıty, Osaka, Japan
}

\section{ABSTRACT}

In the recent decade, very wide areas of sea where the depths of water are from several meters to ten meters or more during storms have been reclaimed for industrial firms and port facilities in many places in Japan As the incident wave energy in such cases is very large at the sea-walls, the protection of the reclaimed lands from wave overtopping by the conventional sea-walls of vertical type or composite-slope-and-berm type is generally impossible from an economical point of view In Japan a special type of sea-wall, which is of such a type that a rubble-mound covered with specially shaped precast concrete armor blocks is built in front of the sea-wall to absorb most of the incldent wave energy, has been constructed to protect the reclaimed lands from wave overtopping Most of the seawalls have been proved satisfactory after passing of typhoons over or near the sea-walls The design of the sea-walls is presented here in by showing the comparisons between the experıments and prototypes during typhoons

\section{INTRODUCTION}

Until about fifteen years ago most of sea-walls had been bullt on the shore line or in shallow water for the protection of low lands or coastal areas from the attack of high tides and storm waves In such cases incoming wave energy is generally not large and the sea-walls can be designed only with the estimations of the change of the incoming wave characteristics before the waves reach the sea-walls and of the wave run-up on the sea-walls In a recent decade, however, very wide areas of sea where the depths of water are several meters to ten meters or more during typhoons or storms have been forced to reclaim at many place in Japan, because of rapid expanding industries and increasing population In the latter cases the incident wave energy at the sea-walls is much larger than that in the former cases, and the protection of the reclalmed land from wave overtopping during typhoons is so difficult that a conventional sea-wall of vertical type would need very high crowns with heights of two to several times the design wave height above the design sea level or a seawall of composite-slope and berm type would require a very large crosssection Therefore the sea-walls of these two types are seldom possible to be designed from an economical point of view

In Japan a special type of sea-wall has been constructed since around 1961 to protect reclaimed lands and coastal areas from wave overtopping It is of such a type that a rubble-mound covered with specially shaped precast concrete armor blocks is built in front of the sea-wall in order to absorb most of the incoming wave energy

The comprehensive experiments of the sea-walls of this type have been performed since 1960 in wind channels with a wind blower in Osaka City University, and numerous sea-walls have been designed and constructed in seas since 1961 by the use of the experimental results Most of the 
sea-walls have proved after attacks of storm waves during typhoons that the results obtained in the experiments were in a good agreement with prototype

\section{PARAMETERS REIATED WITH WAVE OVERTOPPING AND CLASSIFICATION OF OVERTOPPING}

In the investigation to determine the quantity of wave overtopping from the sea-walls of special type mentioned above the following variables should be considered

$q$ = quantity of overtopplng over the unit length of the sea-wall for a period,

$H=$ helght of the incoming wave,

$I=$ length of the lncoming wave,

$h_{1}=$ depth of water at the toe of the rubble-mound,

$\mathrm{H}_{\mathrm{C}}=$ height of the crown of the sea-wall above the design sea level,

$\mathrm{H}_{\boldsymbol{r}}=$ helght of the crown of the rubble-mound above the design sea level,

1 = slope of the sea bottom

$\tan \alpha=$ slope of the rubble-mound,

B = width of the crown of the rubble-mound, and

$\mathrm{V}=$ wind velocity

These symbols are shown in Fig 1

If $q_{0}$ defines the volume of water transported shoreward by a shallow water wave for a period, it is given by the small amplitude wave theory

$$
\begin{aligned}
q_{0} & =\int_{0}^{T / 2} \int_{h_{1}}^{0} u \text { dzdt } \\
& =\int_{0}^{T / 2} \int_{h_{1}}^{0} \frac{H}{2} \frac{2 \pi}{T} \frac{\cosh \frac{2 \pi}{L}\left(h_{1}+Z\right)}{\sinh \frac{2 \pi}{L} h_{1}} \sin \left(\frac{2 \pi}{L} \times-\frac{2 \pi}{T} t\right) d z d t \\
& =\frac{H L}{2 \pi}
\end{aligned}
$$

If $q / q_{0}$ is used as a dimensionless parameter of wave overtopping, it is a function of the following dimensionless parameters

$$
q / q_{0}=f\left(h_{1} / H, h_{1} / L, H_{C} / H, H_{r} / H, B / H, 1, V / \sqrt{g H}, \tan \alpha\right) \cdots \cdots \cdot(1)
$$

when the permeabllity and roughness of the rubble-mound are kept constant

The dissipation of the energy of a wave striking the sea-wall with a rubble-mound covered with specially shaped precast concrete armors depends, to a considerable extent, on the characteristics of the armor biocks, that 1s, the permeability, the distribution of the volds of the armor layers and the roughness of the armors, as well as the slope of the rubble-mound, $\tan \alpha$, and the crown width, B In practical designs the value of tan $\alpha$ has mostly been taken 115 or 12 from an economlcal polnt of view and the stablilty of the armor blocks used on the slope The crown wldth, $B$, of the rubble-mound also has usually been taken the width of two to three rows of the armor blocks from the same reasons as mentioned above 
Therefore $\tan \alpha$ was kept 115 in our experiments, and most of $B$ were taken four to six meters in prototype-scale The velocity of wind has a great effect on the quantity of overtopping of a sea-wall when it exceeds about ten meters per second, but $1 t$ was kept constant $v=20$ to 25 meters per second in the experiments According to the results of the experiments, in which $\mathrm{B} / \mathrm{H}$ ranged from 10 to 40 , and $\mathrm{V} / \sqrt{\mathrm{gH}}$ were proved to have a negligibly small effect on overtopping Finally the relative overtopping was shown as a function of the following five major dimensionless parameters

$$
q / q_{o}=f\left(h_{1} / H, H / L, H_{C} / H, H_{r} / H, 1\right), \ldots \ldots \ldots \ldots \ldots \ldots \ldots \ldots
$$

when the shape and hydraulic characterlstics of the rubble-mound is kept constant

When the depth of water at the toe of the rubble-mound, $h_{l}$, is so small that the incoming wave breaks offshore the rubble-mound, it is rather easy to construct a sea-wall whlch can completely prevent the overtopping even during windy storms However, when $h_{1}$ is equal to or larger than the depth of breaking of the incoming wave, it is generally seldom possible from anc economical point of view to design a sea-wall of no-overtopping As $h_{1}$ increases compared with $H$, the difficulty increases much more, and it cannot be helped to permit some quantity of overtopping from the seawall The quantity of overtopping to be allowed depends upon the economical value of the land to be protected, the purpose of use of the area, the stabllity of the sea-wall and the scale of the drainage channel. According to the results of the experiments, the state and quantity of the overtopping can be classified into the four cases shown in Table 1 by the value of $q / q_{0}$

TABLE 1 CLASSIFICATION OF BEHAVIORS OF OVERTOPPING

\begin{tabular}{|c|c|c|c|}
\hline $\begin{array}{l}\text { Classifi- } \\
\text { cation }\end{array}$ & Behavior of overtopping & $q / q_{0}$ & $\begin{array}{l}\text { Propr1- } \\
\text { ety }\end{array}$ \\
\hline I & $\begin{array}{c}\text { Only spray overtops } \\
\text { (very well absorption of wave) }\end{array}$ & $10^{-4}$ & \multirow{2}{*}{$\begin{array}{l}\text { Ade- } \\
\text { quate } \\
\text { for a } \\
\text { sea-wall }\end{array}$} \\
\hline II & $\begin{array}{l}\text { Lumps of water overtop (hlgher } \\
\text { limlt applicable to a sea-wall) }\end{array}$ & $\begin{array}{l}10^{-4} \text { to } \\
5 \times 10^{-3}\end{array}$ & \\
\hline III & $\begin{array}{l}\text { A substantial part of wave over- } \\
\text { tops (1mperfect absorption of wave) }\end{array}$ & $\begin{array}{l}5 \times 10^{-3} \\
\text { to } 10^{-2}\end{array}$ & \multirow{2}{*}{$\begin{array}{l}\text { Inad- } \\
\text { equate } \\
\text { for a } \\
\text { sea-wall }\end{array}$} \\
\hline IV & $\begin{array}{l}\text { Large volume of wave overflows } \\
\text { (poor absorption of wave) }\end{array}$ & $\begin{array}{c}10^{-2} \text { to } \\
10^{-1}\end{array}$ & \\
\hline
\end{tabular}




\section{EXPERIMENTAL EQUIPMENT AND PROCEDURES}

The experiments were performed by dividing into two groups, one was concerned with sea-walls constructed in comparatively shallow waters with depths less than several meters, and the other concerned with seawalls bullt in deeper waters with depths from about 8 meters to 14 meters The former group of the experiments were conducted in 1963 and 1964, and the latter in 1966 and 1967 The wave channel used for both groups of the experiments is $50 \mathrm{~m}$ long, $1 \mathrm{~m}$ wide, and $165 \mathrm{~m} \mathrm{~h} 1 \mathrm{gh}$, and has a wind blower by which winds of velocities up to $60 \mathrm{~m}$ per sec can be blown over the water waves generated by a wave-generator of flutter-type The scale of the experiments used in both the groups 1 s $1 / 20$ horizontally and vertically The characteristics of the waves tested and other conditions used in the experiments are summerized in Table 2 The $10 \mathrm{~m}-$ length of the bottom in front of the sea-wall has a slope of 1 , and the remaining part of the bottom is flat

TABLE 2 CONDITIONS USED IN EXPERIMENTS

\begin{tabular}{|c|c|c|c|c|c|c|c|c|c|}
\hline \multirow{3}{*}{$\begin{array}{l}\text { Group } \\
\text { of } \\
\text { exper- } \\
\text { Iment }\end{array}$} & \multicolumn{2}{|c|}{ water depth } & \multicolumn{4}{|c|}{$\begin{array}{c}\text { Wave } \\
\text { characteristics }\end{array}$} & \multirow{2}{*}{$\begin{array}{c}\text { slope } \\
\text { of } \\
\text { bottom }\end{array}$} & \multicolumn{2}{|c|}{ Wind velocity } \\
\hline & model & $\begin{array}{l}\text { proto- } \\
\text { type }\end{array}$ & & del & $\begin{array}{r}\text { pro } \\
\text { ty }\end{array}$ & co- & & model & $\begin{array}{l}\text { proto- } \\
\text { type }\end{array}$ \\
\hline & $\underset{\mathrm{cm}}{\left(\mathrm{h}_{1}\right) \mathrm{m}}$ & $\underset{m}{\left(h_{1}\right) p}$ & $\mathrm{Hm}$ & $\begin{array}{l}\text { Tm } \\
\text { sec }\end{array}$ & $\begin{array}{r}\text { Hp } \\
\text { m }\end{array}$ & $\begin{array}{l}\text { Tp } \\
\text { sec }\end{array}$ & 1 & $\begin{array}{c}\mathrm{Vm} \\
\mathrm{cm} / \mathrm{sec}\end{array}$ & $\begin{array}{l}\mathrm{Vp} \\
\mathrm{m} / \mathrm{sec}\end{array}$ \\
\hline $\begin{array}{l}\text { Shal- } \\
\text { lower } \\
\text { waters }\end{array}$ & $\begin{array}{r}2 \\
\text { to } \\
33\end{array}$ & $\begin{array}{rl}0 & 4 \\
\text { to } & \\
6 & 6\end{array}$ & $\begin{array}{r}3 \\
\text { to } \\
22\end{array}$ & $\begin{array}{l}14 \\
\text { to } \\
30\end{array}$ & $\begin{array}{l}06 \\
\text { to } \\
44\end{array}$ & $\begin{array}{ll}6 & 3 \\
\text { to } & \\
13 & 4\end{array}$ & $\begin{array}{l}1 / 10 \\
\text { and } \\
1 / 40\end{array}$ & 45 & 20 \\
\hline $\begin{array}{l}\text { Deeper } \\
\text { waters }\end{array}$ & $\begin{array}{l}40 \\
\text { to } \\
70\end{array}$ & $\begin{array}{ll}8 & 0 \\
\text { to } & \\
14 & 0\end{array}$ & $\begin{array}{l}10 \\
\text { to } \\
25\end{array}$ & $\begin{array}{l}112 \\
\text { to } \\
28\end{array}$ & $\begin{array}{l}20 \\
\text { to } \\
50\end{array}$ & $\begin{array}{ll}5 & 0 \\
\text { to } & \\
13 & 0\end{array}$ & $1 / 100$ & 45 & 20 \\
\hline
\end{tabular}

The rubble-mound was made of quarry stones with dlameters of about $2 \mathrm{~cm}$ to $35 \mathrm{~cm}$ ( $40 \mathrm{~cm}$ to $70 \mathrm{~cm}$ in prototype), and covered with two layers of precast concrete armor blocks such as hollow square, hollow tetrahedron, and $N$-shape blocks with dead weights of 250 grams and 750 grams (2 tons and 6 tons in prototype) The slope of the rubble-mound was kept 115 
as shown in Fig 1 The characteristics of the armor blocks used in the experiments are summerized in Table 3

TABLE 3 CHARACTERISTICS OF ARMOR BLOCKS USED IN EXPERIMENTS

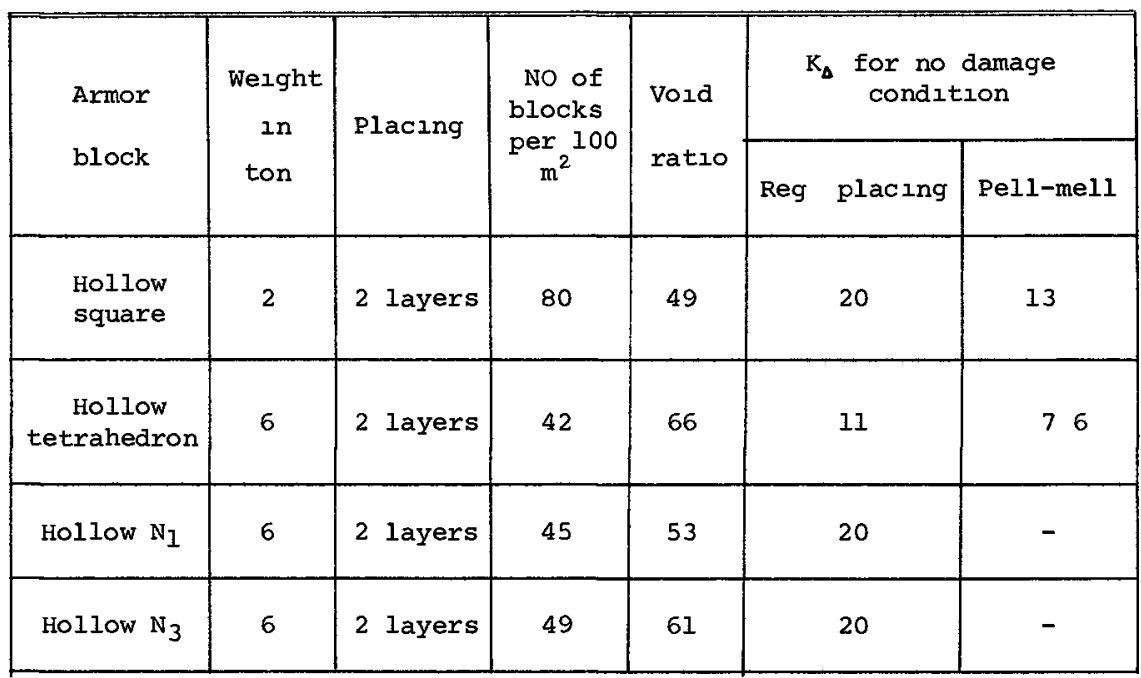

The crown width of the rubble-mound, B, was taken the width required for placing 25 or 3 rows of the armor blocks used, from the experiences in seas of the stability and absorption of wave energy of the armor blocks

\section{EXPERIMENTAL RESULTS}

Effect of Water Depth at the Toe of the Rubble-Mound of the Sea-Wall

The volume of wave overtopping is greatly affected by the point of breaking of the incident wave, whlch can be divided into the following three cases

1 When the incident wave breaks offshore from the toe of the rubblemound This case may be termed "Offshore Breaking", in which the over. topping is the minimum of the three cases

2 When the incldent wave breaks at or near the toe of the rubble-mound This case may be termed "Breaking at Toe" The relative overtopping and relative run-up are the maximum of the three cases as seen in Fig 3 3 When the incident wave breaks on the slope of the rubble-mound Thıs case 15 termed "Breaking on slope" This case occurs when the rubblemound is located in larger depth of water than the depth of breaking of the incident wave 
The effect of $h_{1} / L$ on $q / q_{0}$ is shown in Fig 3 in the cases when the relative crown helghts of the sea-wall and rubble-mound, $\mathrm{H}_{C} / \mathrm{H}$ and $\mathrm{H}_{r} / \mathrm{H}$, as well as the steepness of the Incident wave, $H / L$, are kept constant Fig 3 shows that $q / q_{0}$ is the maximum when the toe of the rubble-mound is located at a little larger depth of water than or near the point of breakıng, 1 e $h_{b}=128 \mathrm{Hb}_{\mathrm{b}}$ whlch $1 \mathrm{~s}$ the breaking depth of solitary wave The reason is attributed to the fact that the point of breaking of the incldent wave somewhat moves toward offshore due to the existence of the rubble-mound

However, when $h_{1} / H \geqq 17$, In whlch the Incldent wave always breaks on the slope of the rubble-mound, the parameters of $h_{1} / H$ and $h_{1} / L$ have little effect of overtopping and run-up The experimental results obtaıned at the Waterways Experıment Station (1) and Coastal Englneerıng Research Center (2) also showed roughly the fact that when the ratio of $h_{1} / H$ is between 218 and 312 or as long as the waves break on the slope of a structure, $h_{1} / H$ had little effect on wave run-up

Effect of the Steepness of the Inczdent Wave

Fig 4 shows the effect of $\mathrm{H} / \mathrm{L}$ of the incident wave on $\mathrm{q} / q_{0}$ when $\mathrm{H}_{\mathrm{C}} / \mathrm{H}, \mathrm{H}_{\mathrm{r}} / \mathrm{H}$, and $\mathrm{h}_{1} / \mathrm{H}$ are kept constant Though the relative overtopping seems the maximum near $H / L$ of about 0.025 , sea-walls in general are designed by the characterıstics of desıgn waves decided from storm conditions at the site

Effect of the Bottom Slope

According to comparisons of the results of the experiments, Figs 5 and 6 , In whlch the bottom slope of 1 he wave channel was changed $1 / 10$ and $1 / 40$, It was noted that the cases of $1=1 / 10$ generally caused larger overtopping than those of $1=1 / 40$, but the effect of the bottom slope on the overtopping was smaller than that of the other parameters

Effects of the Relative Crown Helghts of the Sea-Wall and Rubble-Mound

The effect of the relative crown helght of the sea-wall, $\mathrm{H}_{\mathrm{C}} / \mathrm{H}, \mathrm{Is}$ shown in Figs 7 and 8 for the varlous values of the relative crown helghts of the rubble-mound, $\mathrm{H}_{r} / \mathrm{H}$ It may be seen in Figs 7 and 8 that the value of $\mathrm{H}_{\mathrm{C}} / \mathrm{H}$ must be taken larger than 10 at least in order to be $q /$ qo less than $5 \times 10^{-3}$ which will be the higher limit applicable to the sea-walls Figs $g$ and 10 show the effect of $H_{r} / H$ on $q / q_{0}$ for the scope of $10 \leq \mathrm{H}_{\mathrm{C}} / \mathrm{H}<13$, and also the effect of the bottom slope on $\mathrm{q} / \mathrm{qo}$ It may $\overrightarrow{b e}$ understood in Figs 9 and 10 that in order to be $q /$ qo less than $5 \times 10^{-3}, \mathrm{H}_{r} / \mathrm{H}$ must be taken larger than 07 for the scope of the "Breaking at Toe" and $I=1 / 40$, and $\mathrm{H}_{r} / \mathrm{H}>11$ for the same scope of $1=1 / 10$

Relatıonshıps among $\mathrm{q} / \mathrm{q}_{0}, \mathrm{H}_{\mathrm{C}} / \mathrm{H}$ and $\mathrm{H}_{\mathrm{r}} / \mathrm{H}$ for the Case of "Breakıng on slope"

As has been mentioned, when the value of $h_{1} / H$ exceeds 17 the effect of $h_{1} / H$ or $h_{1} / t$ on $q / q_{o}$ is negliglbly smaller than the other parameters, and in the scope of the "Breaking on slope" the following relationship was found 


$$
\frac{\mathrm{H}_{C}}{\mathrm{H}}+\frac{\mathrm{H}_{\mathrm{r}}}{\mathrm{H}}=\mathrm{C}
$$

in which $\mathrm{C}$ is a constant for a value of $\mathrm{q} / \mathrm{q}_{0}$ and $\mathrm{a}$ kind of armor block used on the slope of the rubble-mound Figs 11 and 12 show the relationships in the scope of $\mathrm{h} / \mathrm{L}=0090$ to 0450 for the various values of $q / q_{0}$ and the two kinds of armor block such as $\mathrm{N}_{1}$ - and $\mathrm{N}_{2}$ - blocks Tables 4 and 5 show the values of $\mathrm{C}$

TABLE 4 VALUES OF $C$ FOR $\mathrm{N}_{1}$-BLOCK

\begin{tabular}{|c|c|c|c|c|}
\hline$q / q_{0}$ & $10^{-4}$ & $10^{-3}$ & $5 \times 10^{-3}$ & $10^{-2}$ \\
\hline$C$ & 25 & 20 & 175 & 16 \\
\hline
\end{tabular}

TABLE 5 VALUES OF $C$ FOR $\mathrm{N}_{3}-\mathrm{BLOCK}$

\begin{tabular}{|c|c|c|c|c|}
\hline $\mathrm{q} / \mathrm{qo}$ & $10^{-4}$ & $10^{-3}$ & $5 \times 10^{-3}$ & $10^{-2}$ \\
\hline $\mathrm{C}$ & 23 & 18 & 155 & 14 \\
\hline
\end{tabular}

\section{Effect of the Permeability of the Rubble-Mound}

It has been well realized that the permeability and the shape of volds of rubble-mounds play a great role of the absorption of waves ruming up the slope of the rubble-mound In order to prove the effect of the permeability and the shape of volds of the rubble-mound on the overtopping of sea-wall, the three kinds of $\mathrm{N}$-shape armor block, shown In Fig 13, which have a same shape but different vold ratioes of 53 per cent for $\mathrm{N}_{1}, 55$ per cent for $\mathrm{N}_{2}$, and 61 per cent for $\mathrm{N}_{3}$, were used as the armor block of the rubble-mound, and tetrahedron blocks which have a different shape of volds and a vold ratio of 66 per cent were also used The experimental results are shown in Fig $14 \quad$ Fig 14 shows that for a same shape of volds of armor layers the capacity to absorb waves increases as the vold ratio increases, but for different shapes of volds of armor layers the capacity to absorb wave energy is not always proportional to the vold ratio 
EFFECT OF RECURVATURE OF A SEA-WAIL AND SIMILARITY ON OVERTOPPING

Taking the origin of the rectangular co-ordinates at the top of the recurvature of a sea-wall, as shown in Fig 15, the x-axls as positlve toward the offshore direction, and the z-axis vertically upward, the equation of motion of a water mass, $m$, exerted by wind force are given by

$$
\begin{aligned}
& m \frac{d^{2} x}{d t^{2}}=-P \quad \ldots \ldots \ldots \ldots \ldots \ldots \ldots \ldots \ldots \ldots \ldots \ldots \ldots \ldots \ldots \ldots \ldots \ldots \ldots \ldots \ldots \ldots \ldots \ldots \ldots \ldots \ldots \ldots \ldots \ldots \ldots \\
& m \frac{d^{2} z}{d t^{2}}=-m g+P_{u} \quad \ldots \ldots \ldots \ldots \ldots \ldots \ldots \ldots
\end{aligned}
$$

In whlch $P$ denotes the horlzontal component of the wind pressure acting from the offshore side, $P_{u}$ is the vertical component of the wind pressure, and $\mathrm{g}$ defines the acceleration of gravity $\mathrm{P}$ is given by

$$
P=\zeta \cdot w_{a} \cdot A \cdot \frac{V^{2}}{2 g}
$$

In whlch $\mathrm{V}$ denotes the wind velocity, A is the area of the water mass exerted by $P, w_{a}=\rho_{a} \cdot g=$ unlt welght of $a l r$, and $\zeta$ defines the coefflclent of drag which is a function of Reynolds number and the shape of the water mass

(1) A water mass of sphere

Iutting the water mass be a sphere with a diameter of $d$, and let us calculate Reynolds number for $d=001$ to $010 \mathrm{~m}$, a wind velocity of 20 meters per second and at temperature of 20 degrees in centigrade

$$
R_{e}=\frac{V d}{V}=133 \times 10^{-4} \text { to } 133 \times 10^{-5}
$$

The drag coefficient is nearly constant for the Reynolds number, $1 e, \zeta=04$ to 05

Taklng $\zeta=05$, and $m=(4 / 3) \rho \pi r^{3}$ in whlch $\rho$ is the density of water, and $r$ is the radius of the water sphere,

$$
\frac{d^{2} x}{d t^{2}}=-\frac{3}{16} \cdot \frac{\rho_{a}}{\rho} \cdot \frac{v^{2}}{r}
$$

Let the velocity of the water mass be $v_{0}$ at $t=0$ and $x=0$, and the angle between the direction and the $x$-axis be $\theta_{0}$,

$$
\frac{d x}{d t}=-\frac{3}{16} \quad \frac{\rho_{a}}{\rho} \cdot \frac{v^{2}}{r} t+v_{0} \cos \theta_{0} \ldots \ldots \ldots \ldots \ldots . .
$$

and

$$
x=-\frac{3}{32} \cdot \frac{\rho_{a}}{\rho} \cdot \frac{v^{2}}{r} t^{2}+v_{0} t \cos \theta_{0}
$$


The time, $t_{0}$, which a water mass spends untll it comes back again on the z-axis after leaving the top, 0 , of the sea-wall, is obtalned from $\mathrm{Eq} 9$

$$
t_{0}=\frac{v_{0} \cos \theta_{0}}{\frac{3}{32} \cdot \frac{\rho a}{\rho} \cdot \frac{v^{2}}{r}}
$$

If $v_{u}$ represents the upward component of wand veloclty at the top of the sea-wall, $P_{u}$ is glven by

$$
\mathrm{P}_{\mathrm{u}}=\zeta \cdot \rho_{\mathrm{a}} \cdot \mathrm{g} \cdot \mathrm{A} \cdot \frac{\mathrm{Vu}^{2}}{2 \mathrm{~g}}=\frac{1}{4} \rho_{\mathrm{a}} \cdot \pi \mathrm{r}^{2} \cdot \mathrm{Vu}^{2}
$$

Assuming $v_{u}=\frac{1}{3} v$,

$$
\mathrm{Pu}_{\mathrm{u}}=\frac{1}{36} \rho_{\mathrm{a}} \cdot \pi \mathrm{r}^{2} \cdot \mathrm{v}^{2}
$$

Substituting Eq 12 into Eq 5,

$$
\frac{d^{2} z}{d t^{2}}=-g+\frac{1}{48} \frac{\rho_{a}}{\rho} \frac{v^{2}}{r}
$$

as

$$
\frac{d z}{d t}=v_{0} \sin \theta_{0}, \text { for } t=0
$$

\section{Integrating}

$$
z=v_{0} t \sin \theta_{0}+\frac{1}{2}\left(-g+\frac{1}{48} \cdot \frac{\rho_{a}}{\rho} \cdot \frac{v^{2}}{r}\right) t^{2}
$$

If the third term of the right hand side of Eq 14 is neglected, since it is approximately 20 per cent of the second term,

$$
z=v_{0} t \sin \theta_{0}-\frac{1}{2} g t^{2}
$$

If $t_{z}$ represents the time whlch a water mass spends untıl it falls agaln onto the $x$-axis by the gravity force after leaving the top of the sea-wall,

$$
t_{z}=\frac{2 v_{0} \sin \theta_{0}}{q}
$$

Denoting by $t$ the time which the water mass spends until it falls down lnto the land over the sea-wall after leaving the top of the seawal1,

$$
t_{z} \geqq t>t_{0}
$$


The values of $t_{0}, t_{z}$, and $(1 / 2) t_{0} v_{0} \cos \theta_{0}$, whlch represents the horizontal flying distance of the water mass in the time of $t_{0}$ are tabulated in Table 6 for $\mathrm{V}=10,15$ and $20 \mathrm{~m} / \mathrm{sec}, \mathrm{V}_{0}=4$ meters per second, $r=05,10$ and $50 \mathrm{~cm}$, and $\theta_{0}=50^{\circ}$ and $60^{\circ}$

TABLE 6 VALUES OF $t_{0}, t_{z}$, and $\left.\frac{1}{2} t_{0} v_{0} \cos \theta_{0}\left(\rho_{a} \chi \rho=1 / 827\right)\right)$

\begin{tabular}{|c|c|c|c|c|c|c|c|c|c|c|}
\hline \multirow{2}{*}{$\begin{array}{c}\mathrm{V} \\
(\mathrm{m} / \mathrm{sec})\end{array}$} & \multirow[b]{2}{*}{$\theta_{0}$} & \multicolumn{3}{|c|}{$r=05 \mathrm{~cm}$} & \multicolumn{3}{|c|}{$r=1 \mathrm{~cm}$} & \multicolumn{3}{|c|}{$r=5 \mathrm{~cm}$} \\
\hline & & $\begin{array}{c}t_{0} \\
\text { (sec) }\end{array}$ & $\begin{array}{c}t_{z} \\
\text { (sec) }\end{array}$ & $\begin{array}{c}\frac{1}{2} t_{0} v_{0} \\
x \cos \theta_{0} \\
(m)\end{array}$ & $\begin{array}{c}t_{0} \\
(\sec )\end{array}$ & $\begin{array}{c}t_{z} \\
(s e c)\end{array}$ & $\begin{array}{c}\frac{1}{2} t_{0} v_{0} \\
x \cos \theta_{0} \\
\text { (m) }\end{array}$ & $\begin{array}{c}t_{0} \\
(\mathrm{sec})\end{array}$ & $\begin{array}{c}t_{0} \\
(\mathrm{sec})\end{array}$ & $\begin{array}{c}\frac{1}{2} t_{0} v_{0} \\
x \cos \theta_{0} \\
(\mathrm{~m})\end{array}$ \\
\hline 10 & $\begin{array}{l}50^{\circ} \\
60\end{array}$ & $\mid \begin{array}{ll}1 & 14 \\
0 & 88\end{array}$ & $\begin{array}{ll}0 & 63 \\
0 & 71\end{array}$ & $\begin{array}{ll}1 & 47 \\
0 & 88\end{array}$ & $\begin{array}{ll}2 & 27 \\
1 & 76\end{array}$ & $\begin{array}{ll}0 & 63 \\
0 & 71\end{array}$ & $\begin{array}{ll}2 & 93 \\
1 & 76\end{array}$ & $\begin{array}{rl}11 & 4 \\
8 & 80\end{array}$ & $\begin{array}{ll}0 & 63 \\
0 & 71\end{array}$ & $\begin{array}{rl}14 & 7 \\
8 & 80\end{array}$ \\
\hline 15 & $\begin{array}{l}50 \\
60\end{array}$ & $\mid \begin{array}{ll}0 & 50 \\
0 & 39\end{array}$ & $\begin{array}{ll}0 & 63 \\
0 & 71\end{array}$ & $\begin{array}{ll}0 & 65 \\
0 & 39\end{array}$ & $\begin{array}{ll}1 & 01 \\
0 & 78\end{array}$ & $\begin{array}{ll}0 & 63 \\
0 & 71\end{array}$ & $\begin{array}{ll}1 & 30 \\
0 & 78\end{array}$ & $\begin{array}{ll}5 & 04 \\
3 & 92\end{array}$ & $\begin{array}{ll}0 & 63 \\
0 & 71\end{array}$ & $\begin{array}{ll}6 & 48 \\
3 & 92\end{array}$ \\
\hline 20 & $\begin{array}{l}50 \\
60\end{array}$ & $\mid \begin{array}{ll}0 & 28 \\
0 & 22\end{array}$ & $\begin{array}{ll}0 & 63 \\
0 & 71\end{array}$ & $\begin{array}{ll}0 & 36 \\
0 & 22\end{array}$ & $\begin{array}{ll}0 & 57 \\
0 & 44\end{array}$ & $\begin{array}{ll}0 & 63 \\
0 & 71\end{array}$ & $\begin{array}{ll}0 & 72 \\
0 & 44\end{array}$ & $\begin{array}{ll}2 & 85 \\
2 & 20\end{array}$ & $\begin{array}{l}0.63 \\
0.71\end{array}$ & $\begin{array}{ll}3 & 68 \\
2 & 20\end{array}$ \\
\hline
\end{tabular}

Since $t_{0}$ is larger than $t_{z}$ in the scope surrounded by a thick line, the water mass does not jump into the land over the sea-wall This means that if the water mass is assumed a sphere with a diameter of $d$, the water mass with $d \leq 1 \mathrm{~cm}$ wlll jump into the land over the sea-wall when $\mathrm{y} \geq 15 \mathrm{~m} / \mathrm{sec}$, the water mass w1th $\mathrm{d} \leqq 2 \mathrm{~cm}$ will jump into the land when $\mathrm{y} \geq 20 \mathrm{~m} / \mathrm{sec}$

(2) A wall of water

Let us consider that the water spray over the top of the sea-wall is a wall of water with a thickness of $b$ since the drag coefficient of the water wall is taken $\zeta=2$ for Reynolds numbers $\operatorname{Re}=5 \times 10^{3}$ to $10^{6}$, the horizontal component of the wind pressure per unit area is

$$
\mathrm{P}=\rho_{\mathrm{a}} \cdot \mathrm{V}^{2}
$$

Since $m=\rho \cdot b, E q \quad 4$ may be written

$$
\frac{d^{2} x}{d t^{2}}=-\frac{\rho_{a}}{\rho} \cdot \frac{v^{2}}{b}
$$


Using the boundary condltions $d x / d t=v_{0} \cos \theta_{0}$ and $x=0$ for $t=0$, $x=-\frac{1}{2} \cdot \frac{\rho_{a}}{\rho} \cdot \frac{v^{2}}{b} t^{2}+v_{0} t \cos \theta_{0}$

$t=t_{0}$ for $x=0$ Is given by

$$
t_{0}=\frac{v_{0} \cos \theta_{0}}{\frac{1}{2} \cdot \frac{\rho_{a}}{\rho}: \frac{v^{2}}{b}}
$$

If $P_{u}$ is neglected, $t_{z}$ is given by Eq 16 As previously mentioned, only when the condition $t_{z} \geqq t>t_{0}$ is satisfied, the water wall can fall Into the land over the sea-wall Table 7 is shown the values of $t_{0}, t_{z}$, and $(1 / 2)$ tovo $\cos \theta_{0}$

TABLE 7 VALUES OF $t_{0}, t_{z}$, and $\frac{1}{2} t_{o} v_{0} \cos \theta_{0}$

\begin{tabular}{|c|c|c|c|c|c|c|c|c|c|c|}
\hline \multirow{2}{*}{$\begin{array}{c}V \\
(m / s e c)\end{array}$} & \multirow[b]{2}{*}{$\theta_{0}$} & \multicolumn{3}{|c|}{$\mathrm{b}=1 \mathrm{~cm}$} & \multicolumn{3}{|c|}{$\mathrm{b}=5 \mathrm{~cm}$} & \multicolumn{3}{|c|}{$\mathrm{b}=10 \mathrm{~cm}$} \\
\hline & & $\begin{array}{c}t_{0} \\
(\mathrm{sec})\end{array}$ & $\begin{array}{c}t_{z} \\
\text { (sec) }\end{array}$ & $\begin{array}{l}\frac{1}{2} t_{0} v_{0} \\
x_{\cos \theta_{0}} \\
\text { (m) }\end{array}$ & $\begin{array}{c}t_{0} \\
(\mathrm{sec})\end{array}$ & $\begin{array}{c}t_{z} \\
\text { (sec) }\end{array}$ & $\begin{array}{c}\frac{1}{2} t_{0} v_{0} \\
x_{\cos \theta_{0}} \\
(m)\end{array}$ & $\begin{array}{c}t_{0} \\
(\sec )\end{array}$ & $\left\{\begin{array}{c}\tau_{z} \\
(\mathrm{sec})\end{array}\right.$ & $\begin{array}{c}\frac{1}{2} t_{0} v_{0} \\
\cos \theta_{0} \\
(m)\end{array}$ \\
\hline 10 & $\begin{array}{l}50^{\circ} \\
60\end{array}$ & $\begin{array}{ll}0 & 43 \\
0 & 33\end{array}$ & $\begin{array}{ll}0 & 63 \\
0 & 71\end{array}$ & $\begin{array}{ll}0 & 55 \\
0 & 33\end{array}$ & $\begin{array}{ll}2 & 13 \\
1 & 65\end{array}$ & $\begin{array}{ll}0 & 63 \\
0 & 71\end{array}$ & $\begin{array}{ll}2 & 88 \\
1 & 65\end{array}$ & $\begin{array}{ll}4 & 25 \\
3 & 31\end{array}$ & $\begin{array}{ll}0 & 63 \\
0 & 71\end{array}$ & $\begin{array}{ll}5 & 46 \\
3 & 31\end{array}$ \\
\hline 15 & $\begin{array}{l}50 \\
60\end{array}$ & $\begin{array}{ll}0 & 19 \\
0 & 15\end{array}$ & $\begin{array}{ll}0 & 63 \\
0 & 71\end{array}$ & $\begin{array}{ll}\text { o } & 24 \\
\text { o } & 15\end{array}$ & $\begin{array}{ll}0 & 95 \\
0 & 74\end{array}$ & $\begin{array}{ll}0 & 63 \\
0 & 71\end{array}$ & $\begin{array}{ll}1 & 22 \\
0 & 74\end{array}$ & $\begin{array}{ll}1 & 89 \\
1 & 47\end{array}$ & $\begin{array}{ll}0 & 63 \\
0 & 71\end{array}$ & $\begin{array}{ll}2 & 43 \\
1 & 47\end{array}$ \\
\hline 20 & $\begin{array}{l}50 \\
60\end{array}$ & $\begin{array}{ll}0 & 11 \\
0 & 08\end{array}$ & $\begin{array}{ll}0 & 63 \\
0 & 71\end{array}$ & $\begin{array}{ll}0 & 14 \\
0 & 08\end{array}$ & $\begin{array}{ll}0 & 53 \\
0 & 41\end{array}$ & $\begin{array}{ll}0 & 63 \\
0 & 71\end{array}$ & $\begin{array}{ll}0 & 68 \\
0 & 41\end{array}$ & $\begin{array}{ll}1 & 06 \\
0 & 83\end{array}$ & $\begin{array}{ll}0 & 63 \\
0 & 71\end{array}$ & $\begin{array}{ll}1 & 36 \\
0 & 83\end{array}$ \\
\hline
\end{tabular}

In Table 7 the scope enclosed by a thick line shows the cases of $t_{0}>t_{z}$ According to Table 7, It may be known that a wall of overtopping with a thicknessof one $\mathrm{cm}$ is blown down within one second into the land over the sea-wall by winds with veloclties equal to or larger than $10 \mathrm{~m} / \mathrm{sec}$, and a wall of overtopping with a thickness of $5 \mathrm{~cm}$ by winds with velocities equal to or larger than $20 \mathrm{~m} / \mathrm{sec}$ 
Similarity on Overtopping

(1) A water mass of sphere

Let us consider of a water mass of sphere with a diameter $d$ overtopping a sea-wall Assuming $d_{p}$, the diameter in prototype, is $3 \mathrm{~cm}$ to $10 \mathrm{~cm}, d_{m}$, the diameter in model, the scale of which is 120 to prototype, is 15 to $5 \mathrm{~mm}$ $\mathrm{m} / \mathrm{sec} 1 \mathrm{~s}$

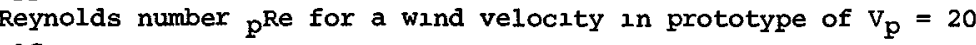

$$
p^{R e}=\frac{V_{p} \cdot d_{p}}{v}=40 \times 10^{4} \text { to } 133 \times 10^{5}
$$

In the model

$$
\mathrm{m}^{\mathrm{Re}}=\frac{\mathrm{Vm} \cdot \mathrm{dm}}{\nu}=45 \times 10^{2} \text { to } 147 \times 10^{3}
$$

Drag coefficlents for the spheres are

$$
\zeta_{p} \neq \sim 045 \text { to } 05 \text { in prototype, }
$$

and

$$
\zeta_{p}=\sim 060 \text { to } 045 \text { in model, }
$$

thus, it may be assumed approximately $\zeta_{\mathrm{p}}=\zeta_{\mathrm{m}}$

This means that if a mass of water overtopping a sea-wall is a sphere with a dlameter $d \geqq 3 \mathrm{~cm}$, the motion of the water mass exerted by a wind of $v_{p}=20 \mathrm{~m} / \mathrm{sec}$ may be stated to be approximately followed by Froude law of similarity, 1 e the results of the model experiment may be stated to ne approximately similar to the results of the nature However, if the dlameter of the water mass, $d_{p}$, is smaller than about $3 \mathrm{~cm}, \zeta_{\mathrm{p}}=\sim 04$ to 05 for $\mathrm{p}$ Re $=\sim 10^{4}$, as against $\zeta_{\mathrm{m}}=\sim 06$ to

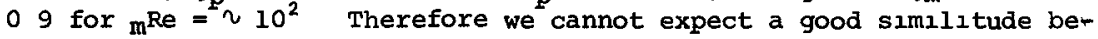
tween model and prototype But from a practical point of view on the water quantıty of wave overtopping, the volume of such spray of water would be consıdered to be neglı'glble small

(2) A wall of water

Let us consider the overtopping as a wall of water This may be the case when large overtopping 15 seen in prototype and model, as seen in Figs 17, 18 and 19, therefore, this case would be the most important in wave overtopping over sea-walls

If the overtopping of wave is assumed as a wall of water, the drag coefficlent of the wall, $\zeta$, is constantly two for all Reynolds numbers larger than $10^{2}$ It, therefore, may be stated that the results of experiments conducted by Froude law are similar to the results in the nature Field observations of overtopping at sea-walls during typhoons have proved that this assumption is correct

It may be concluded from the theoretical considerations described above that the recurvature of a sea-wall would have little effect on wave overtopping when wind velocity exceeds about $15 \mathrm{~m} / \mathrm{sec}$ 


\section{APPLICATION TO DESIGN OF SEA-WALLS AND VERIFICATION BY TYPHOONS}

The results studied in our laboratory have been applied to the design of sea-walls in Japan since 1960 and those sea-walls have been tested by severe typhoons All of the sea-walls whlch have ungone the natural tests have proved that the desıgns were satısfactory and there were generally a falrly good agreement between the experıments and prototypes Some examples are presented herein

(1) Sea-wall in the Port of Wakayama

This sea-wall was constructed in 1958 at a water depth of $7 \mathrm{~m}$ to $8 \mathrm{~m}$ below the Datum IIne offshore a long sandy beach exposed to an open sea in the North Harbor of the Port of Wakayama, Wakayama Prefecture, which is one of the biggest industrial harbors for steel firms in Japan This was the first big sea-wall that harbor eng to protect a reclaimed industrial land located offshore from wave overtopping Fig 16 shows a cross-section of the sea-wall

After completion it was severely hit three times consecutively in September of 1959, 1960 and 1961 Figs 17 and 18 show huge overtoppings of waves whlch were taken when the typhoon was located still far offshore from the harbor

After calculations and experiments, it was recommended that the seawall should have a rubble-mound covered with precast concrete armors in front of the wall and a large drainage channel with a width of $20 \mathrm{~m}$ at the land-side of the sea-wall, as shown in Fig 19

(2) Another Sea-Wall in the Port of Wakayama

The sea-wall, the cross-section of which is shown in Fig 20, was constructed in 1965 and 1966 to protect the industrial area of $18 \mathrm{mill}$ ion $\mathrm{m}^{2}$ reclaimed at the northern part of the Wakayama North Harbor

(a) When the helght and period of the desıgn wave are taken $H_{p}=50 \mathrm{~m}$ and $T_{p}=90$ sec

Since $h_{1} / H=874 / 5=175>17$, thls case 1 s "Breaking on slope" For $\mathrm{H}_{\mathrm{C}} / \mathrm{H}=476 / 5=095=\sim 10$ and $\mathrm{H}_{r} / \mathrm{H}=376 / 5=075$, we obtain $q / q_{0}=5 \times 10^{-3}$ from $F 1 g \quad 11$

(b) When $\mathrm{H}_{\mathrm{p}}=55 \mathrm{~m}$ and $\mathrm{T}_{\mathrm{p}}=130 \mathrm{sec}$

Since $H / L=0041$ and $h_{1} / L=0065$, this case is on the critical condition between "Breaking at Toe" and "Breaking on slope" Using $H_{C} / H=087$ and $H_{r} / H=376 / 55=068$, we obtain $q / q_{0}=5 \times$ $10^{-3}$ for "Breakıng at Toe", and from Fig $11 q / q_{0}=8 \times 10^{-3}$ for "BreakIng on slope", whlch necessitate a large drainage channel as shown in F1g 20

Decision of the Width and Depth of the Dralnage Channel

The design conditions of the drainage channel

$\Delta z=$ helght from the bottom of the dralnage channel to the $x$-axis

$=(D \mathrm{~L}+670 \mathrm{~m})-(\mathrm{DL}+400 \mathrm{~m})=270 \mathrm{~m}$

$\theta_{0}=$ angle of the parapet wall to the $x$-axis $=70^{\circ}$,

$\mathrm{V}=$ wind velocity $=20 \mathrm{~m} / \mathrm{sec}$,

The wave run-ups, $R_{u}$, for the desıgn waves with helghts of $50 \mathrm{~m}$ and $55 \mathrm{~m}$ and periods of $9 \mathrm{sec}$ and $13 \mathrm{sec}$, respectively, were approximately $12 \mathrm{H}$ according to the experiments carried out by using the model of the sea-wall shown in Fig 21 
Let us consider about the design wave with a helght of $5 \mathrm{~m}$ and a period of $9 \mathrm{sec}$, and take the design sea level $\mathrm{D} \mathrm{L}+224 \mathrm{~m}$ The velocity of a water mass of sphere with a dlameter of d at the origin of the co-ordinates, 0 , is obtained by $v_{O}=\sqrt{2 \times 98(60-446)}=55 \mathrm{~m} / \mathrm{sec}$

$t_{z}$ whlch denotes the time that the water mass spends until it falls down on the bottom of the dralnage channel over the sea-wall after leaving the origin 0 is given by

$$
t_{z}=\frac{v_{0} \sin \theta_{0}+\sqrt{v_{0}^{2} \sin ^{2} \theta_{0}+2 g \Delta z}}{g}
$$

By substitution of Eq 22 into $\mathrm{Eq} 9$, the horizontal distance $1_{\mathbf{X}}$ whlch the water mass reaches at the time $t_{z}$ from the origin 0 onto the channel bottom is obtained by

$$
l_{x}=\frac{3}{32} \cdot \frac{\rho_{a}}{\rho} \cdot \frac{v^{2}}{r} t_{z}-v_{o} t \cos \theta_{0} \quad, \ldots . \ldots \ldots \ldots \ldots
$$

From Eqs 22 and $23 t_{z}=144 \mathrm{sec}$ and $1_{x}=160 \mathrm{~m}$ for $\mathrm{d}=001 \mathrm{~m}, 1_{x}=$ $67 \mathrm{~m}$ for $\mathrm{d}=002 \mathrm{~m}$

Consldering a wall of water with a thickness of $b$, the horizontal distance $l_{x}$ is obtalned by substitution of $\mathrm{Eq} 22$ into $\mathrm{Eq} 20, l_{x}=$ $474 \mathrm{~m}$ for $\mathrm{b}=001 \mathrm{~m}, 1_{\mathrm{x}}=224 \mathrm{~m}$ for $\mathrm{b}=002 \mathrm{~m}, 1_{\mathrm{x}}=140 \mathrm{~m}$ for $\mathrm{b}=$ $003 \mathrm{~m}$, and $l_{x}=98 \mathrm{~m}$ for $\mathrm{b}=004 \mathrm{~m}$

The locl of those walls of water are shown in Fig 21 According to Fig 21, if the side wall with a helght of $2 \mathrm{~m}$ from the bottom of the channel is constructed at a distance of $l_{x}=175 \mathrm{~m}$ from the origin 0 , the walls of water with thlcknesses of more than $b=2 \mathrm{~cm}$ and the spheres of water with diameters more than one $\mathrm{cm}$ could be taken into the drainage channel

The length of the sea-wall over which the design wave overtops simultaneously was decided about $350 \mathrm{~m}$ by the experiments, and the factor of safety for the maximum relative overtopping was taken two, therefore the maximum discharge of water for the drainage channel, Qmax, was

$$
\begin{aligned}
& \mathrm{q}=8 \times 10^{-3} \mathrm{q}_{0}=8 \times 10^{-3} \times 5 \times 88 / 2 \pi=056 \mathrm{~m}^{3} / 9 \mathrm{sec} / \mathrm{m} \\
& Q_{\max }=2 \mathrm{q} \times 350=393 \mathrm{~m}^{3} / 9 \mathrm{sec} \\
& =437 \mathrm{~m}^{3} / \mathrm{sec}=\sim 45 \mathrm{~m}^{3} / \mathrm{sec}
\end{aligned}
$$

If the slope of the channel bottom is taken $1 / 1000$, the width $20 \mathrm{~m}$, and the depth $2 \mathrm{~m}$, the channel can discharge $Q_{\max }$ safely

After completion of the drainage channel, the sea-wall has undergone severe typhoons several times, and it has been reported due to the visual observations during the typhoons that the behaviors of the overtopping were quite similar to those of the experiments and almost all overtoppings were assembled into the channels and flowed down safely into the harbor basin

(3) Sea-Wall of the Kansal Electric Power Co Ltd

The sea-wall was constructed in an open sea with a water depth of D I $-250 \mathrm{~m}$ to $300 \mathrm{~m}$ to protect a reclalmed land of about 38 acres whlch was used for an electric power plant of the Kansal Electric Power Co Ltd The sea-wall was requested to be designed no-overtopping even 
during the heaviest typhoon ever experienced there After the calculations shown here and model experiments carrıed out on a scale of 120 in the wave channel shown in Fig 2, the sea-wall shown in Figs 22 and 23 was designed

\section{Estimation of the Overtopping during the Heaviest Typhoon}

The signiflcant wave helght and period of the design wave were taken $\mathrm{H}_{1 / 3}=250 \mathrm{~m}$, and $\mathrm{T}_{1 / 3}=63 \mathrm{sec}$ The hlghest hlgh tide averaged for two hours including the highest tide ever recorded in the harbor was estimated $D L+380 \mathrm{~m}$ Since $h_{1} / H=580 / 25=23>17$, the case $2 \mathrm{~s}$ "Breaking on Slope" Using $\mathrm{H}_{\mathrm{C}} / \mathrm{H}=395 / 250=158$ and $\mathrm{H}_{\mathrm{r}} / \mathrm{H}=175 / 25$ $=070$, we obtain from $F 1 g$ s 11 and $12, q / q_{0}=5 \times 10^{-4}$ and $2 \times 10^{-4}$, respectively

Since the tetrahedron blocks of two tons were used as the armor block of the rubble-mound, $q / q_{0}$ is estimated approximately $3 \times 10^{-4}$ from Fig 14 According to the experiments in which two high tides of $D$ I + $380 \mathrm{~m}$ and $\mathrm{D} \mathrm{I}+330 \mathrm{~m}$ were used and the wind velocity used was always $20 \mathrm{~m} / \mathrm{sec}$, a few overtopping was seen for a tide of $\mathrm{D} \mathrm{L}+380 \mathrm{~m}$ and only a few spray was observed for a $t$ lde of $D \mathrm{~L}+330 \mathrm{~m}$

Shortly after the completion of the sea-wall, it was hit by one of the most severe typhoons ever observed in the harbor, the second Muroto typhoon, whlch passed near over the location of the sea-wall on september 16, 1961 The highest tide during the typhoon was D L $+400 \mathrm{~m}$ and the average highest high tide for two hours was estimated approximately $D$ I $+360 \mathrm{~m}$ The maximum wave helght was assumed $250 \mathrm{~m}$ or $3 \mathrm{Om}$, and strong winds of from $20 \mathrm{~m} / \mathrm{sec}$ to $35 \mathrm{~m} / \mathrm{sec}$ blew from offshore for about $3 \mathrm{hr}$ Pictures taken with the 8-mm movie camera near the top of the seawall during the typhoon showed that comparatively small volume of wave overtopping occursed sometimes over the top of the rubble-mound covered with the two layers of the hollow tetrahedron armors However, there was no overtopping over the top of the sea-wall, as seen in Fig 23 It was proved that there was a good agreement between the experiment and prototype

\section{APPENDIX - REFERENCES}

1 "Wave Run-up and overtopping, Levee Sectıons, Lake Okeechobee, Florlda", Corps of Engrs, Waterways Experıment sta, Tech Report NO 2-449, January, 1959

2 Savılle, Thorndıke, "Wave Run-up on Shore Structures", Proc ASCE, Vol 82, No WW2, April, 1956 


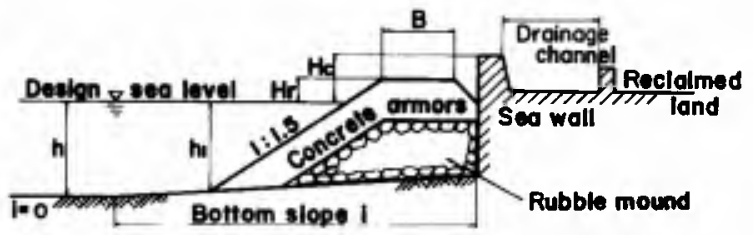

FIG. 1. - CROSS-SECTIONS TYPICAL TYPES OF SEAWALL IN JAPAN UPPER SECTION USED IN DEEPER WATERS LOWER SECTION USED IN SHALLOW BEACH
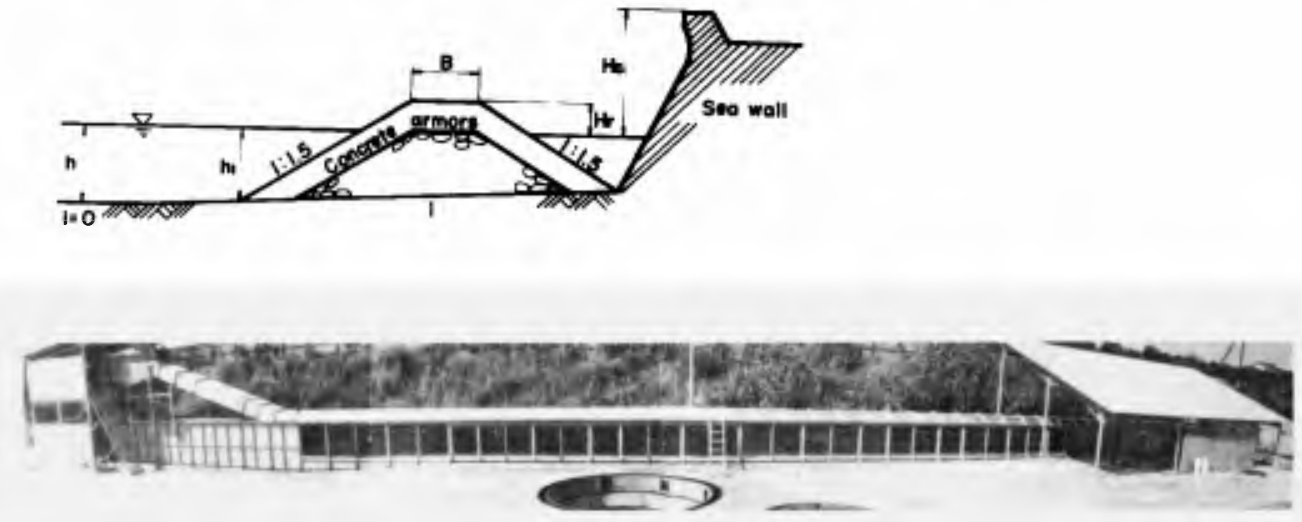

FIG. 2. - WAVE CHANNEL WITH A WIND BLOWER

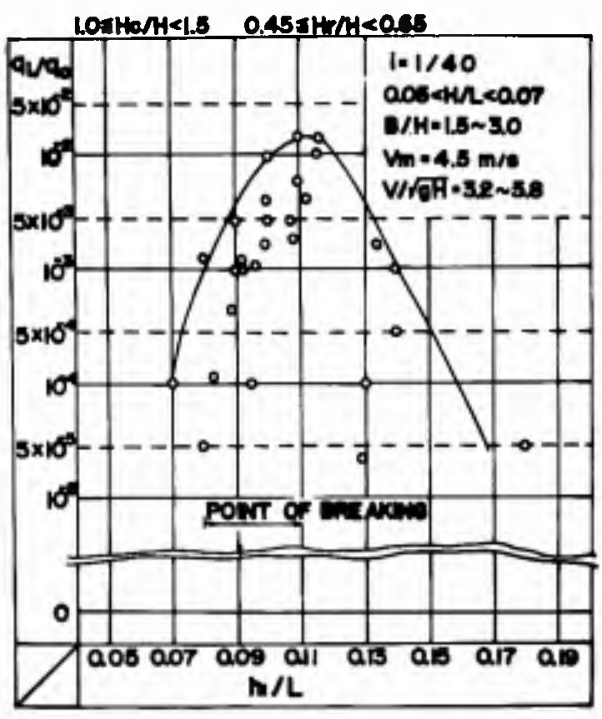

FIG. 3. - RELATIONSHIP BETWEEN RELATIVE OVERTOPPING AND $h_{1} / L$

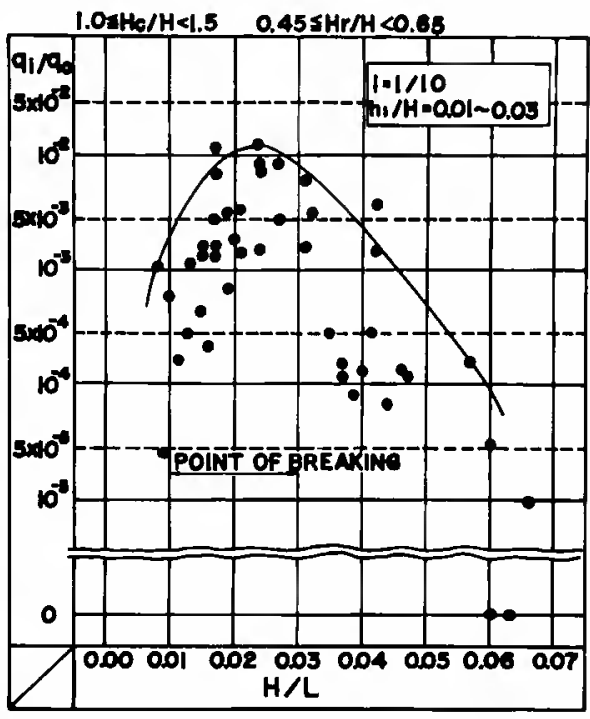

FIG. 4. - RELATIONSHIP BETWEEN $q / q_{0}$ AND $\mathrm{H} / \mathrm{L}$ 


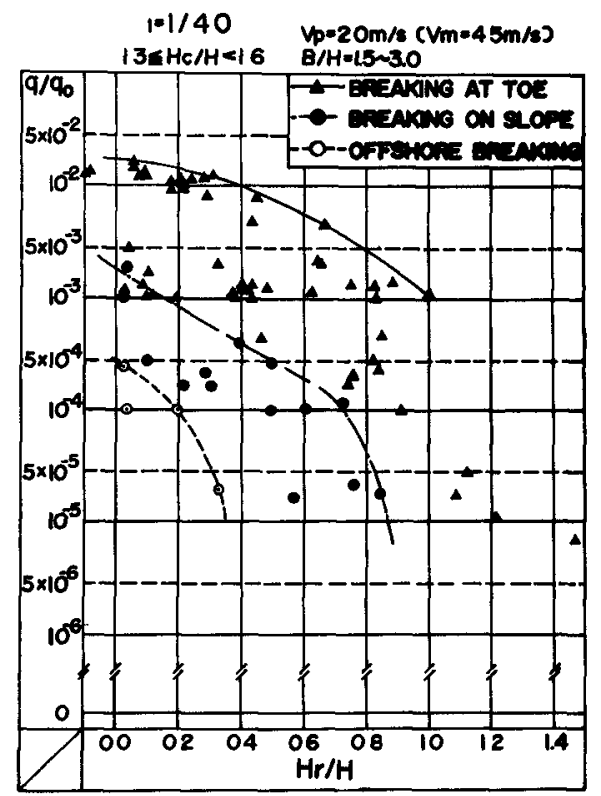

FIG 5 - RELATIONSHIP BETWEEN $q / q_{0}$ AND $H_{x} / H, I=1 / 40$

$1 * 1 / 40$ B/Hil 5 3O V/NA-3.2 58

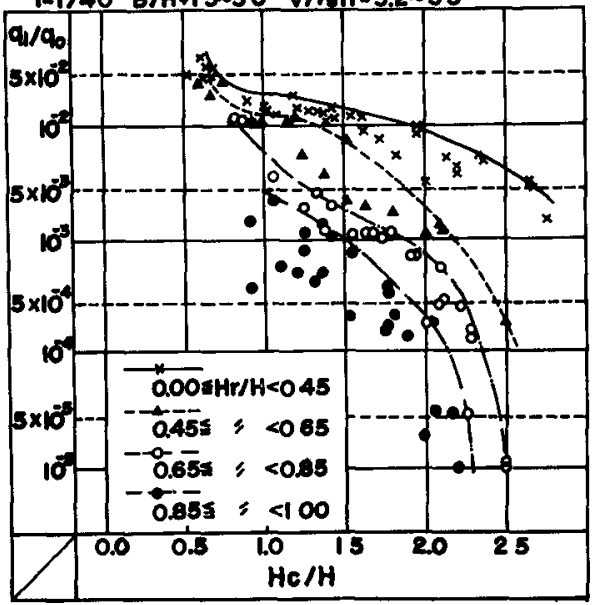

FIG 7 - RELATIONSHIP AMONG $q / q_{0}, \mathrm{H}_{\mathrm{C}} / \mathrm{H}$ AND $\mathrm{H}_{\Upsilon} / \mathrm{H}$

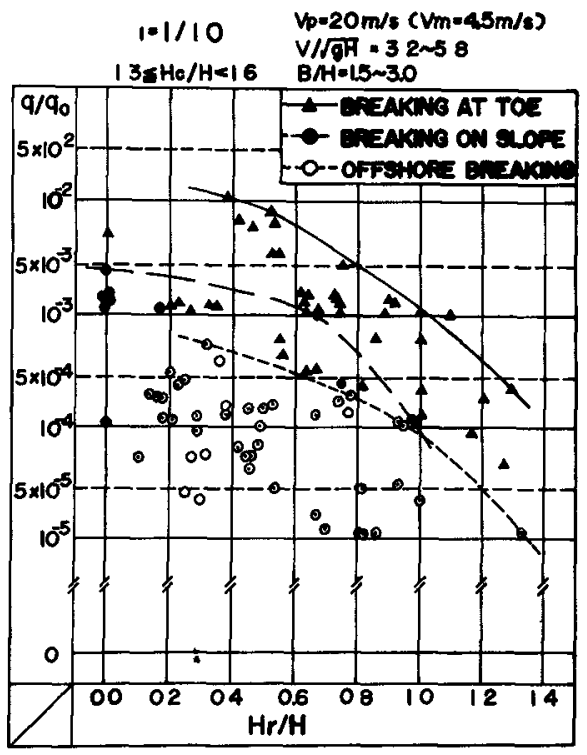

FIG 6 - RELATIONSHIP BETWEEN $q / q_{0}$ AND $H_{K} / H, l=1 / 10$

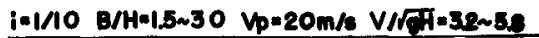

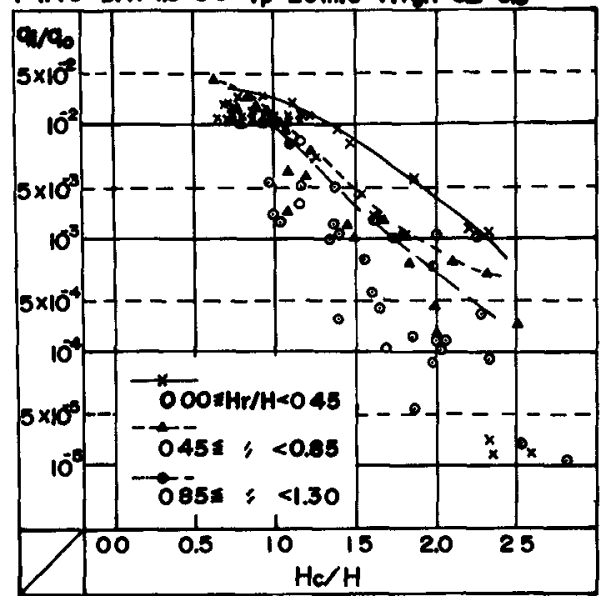

FIG 8 - RELATIONSHIP AMONG $q / q_{O}, H_{C} \mathrm{H}$ AND $\mathrm{H}_{\boldsymbol{x}} / \mathrm{H}$ 


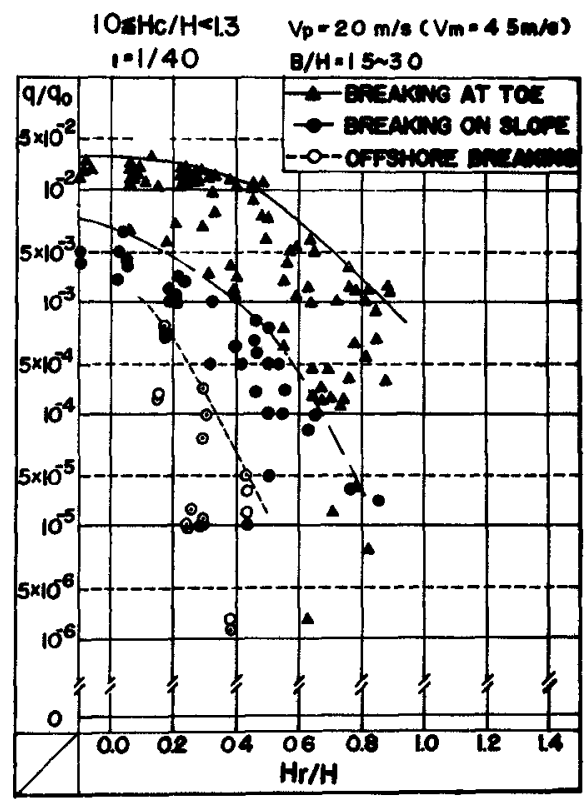

FIG 9 - RELATIONSHIP BETWEEN $\mathrm{q} / \mathrm{q}_{\mathrm{O}}$ AND $\mathrm{H}_{\mathrm{r}} / \mathrm{H}, \mathrm{I}=1 / 40$

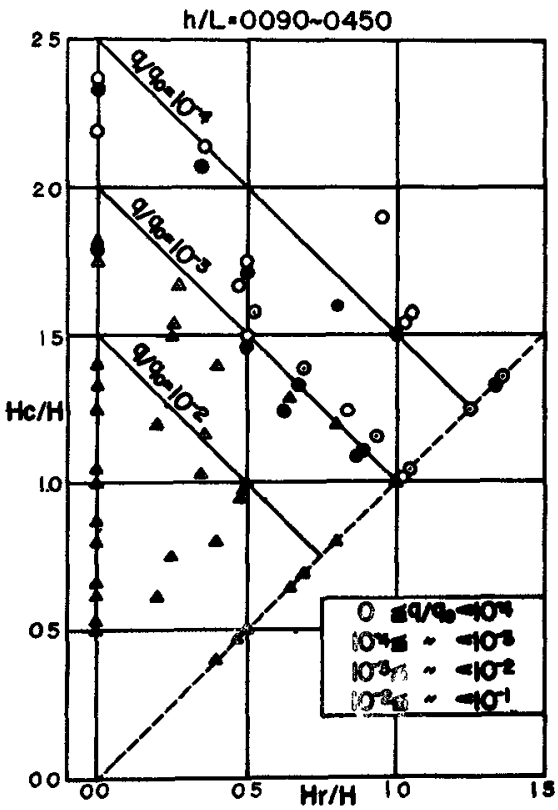

FIG 11 - RELATIONSHIP AMONG $q / q_{0}$, $\mathrm{H}_{\mathrm{C}} / \mathrm{H}$, AND $\mathrm{H}_{\mathrm{r}} / \mathrm{H}$ FOR $\mathrm{N}_{1}-\mathrm{BLOCK}$
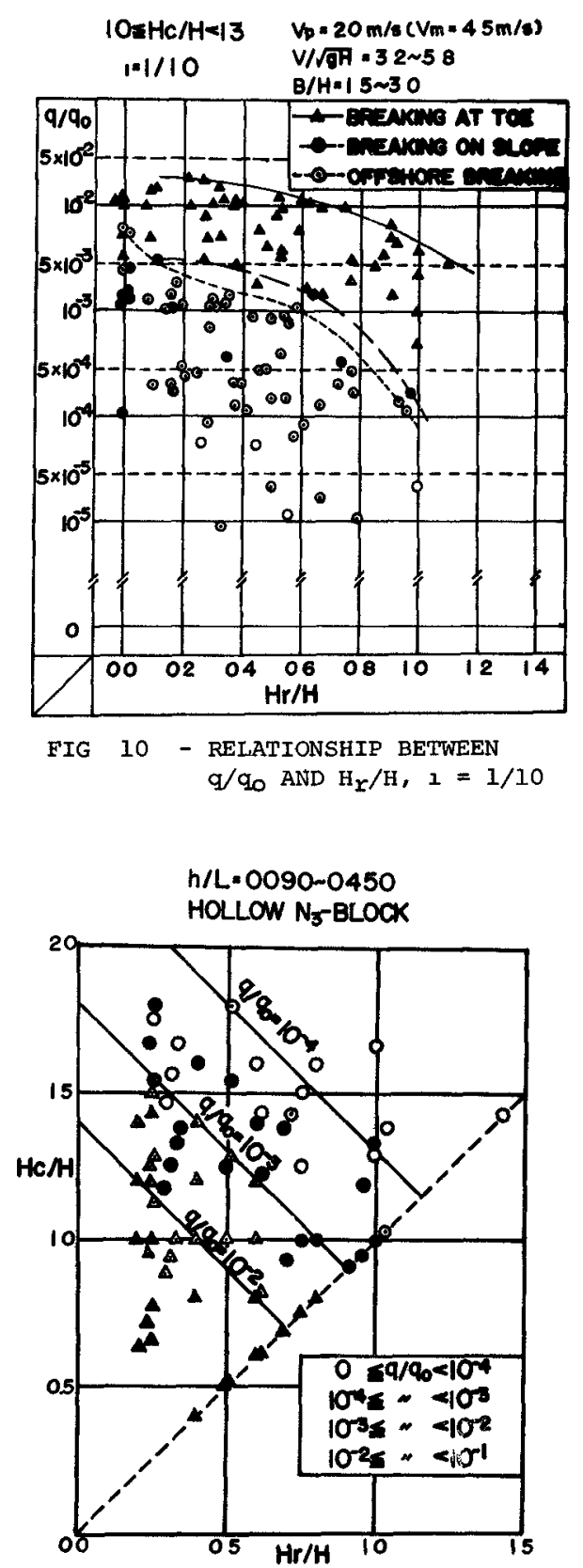

FIG 12 - RELATIONSHIP AMONG $q / q_{0}$, $\mathrm{H}_{\mathrm{C}} / \mathrm{H}$, AND $\mathrm{H}_{r} / \mathrm{H}$ FOR $\mathrm{N}_{3}$-BLOCK 


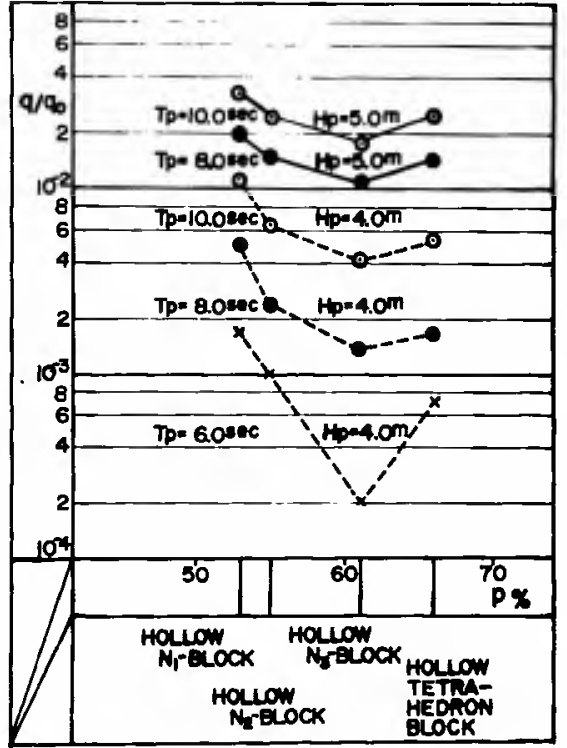

FIG. 14. - RELATIONSHIPS BETWEEN $q / q_{0}$ AND THE VOID RATIO AND SHAPE OF VOID OF ARMOR LAYORS

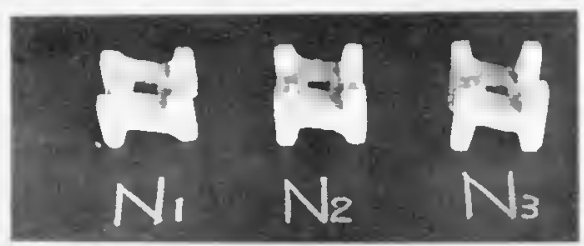

FIG. 13. - HOLLOW N-BLOCK

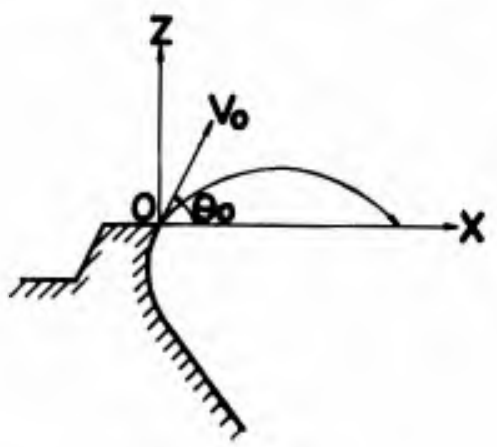

FIG. 15

SEAWARD SIDD

$\nabla=8.4-00000000$

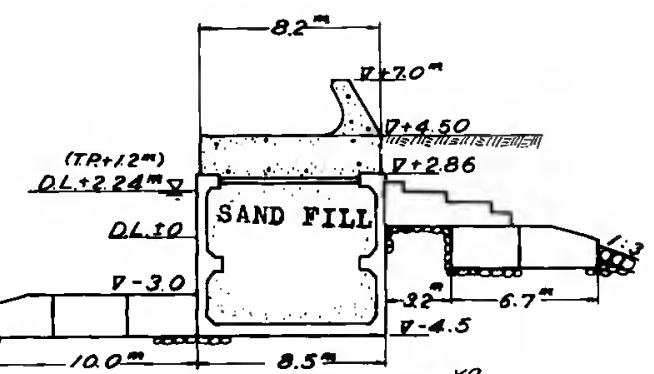

RUBBLE-MOUND $20-60^{\mathrm{Kg}}$

FIG. 16. - CROSS-SECTION OF THE SEA-WALL IN WAKAYAMA HARBOR BEFORE 1961

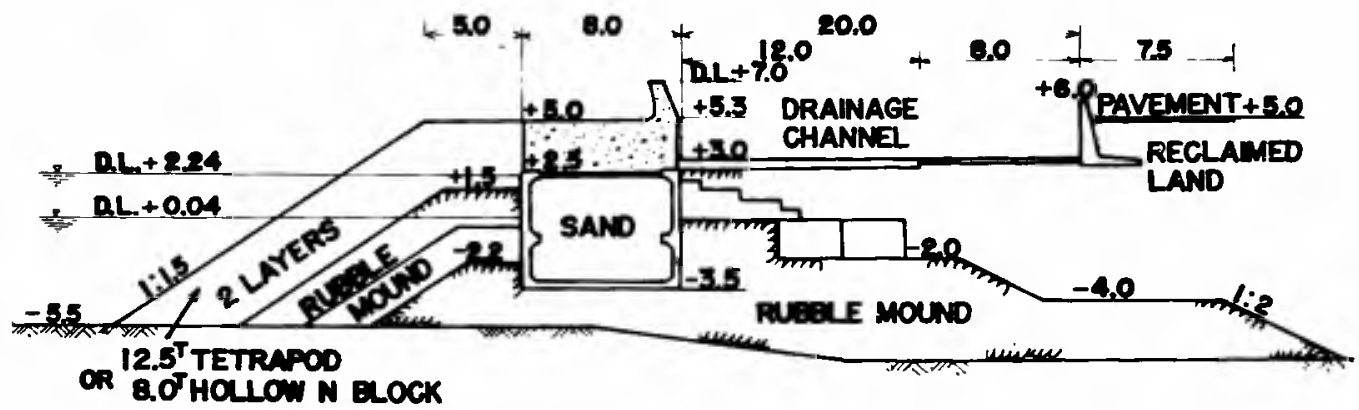

FIG. 19. - CROSS-SECTION OF THE SEA-WALL IMPROVED AFTER THE EXPERIMENTS 


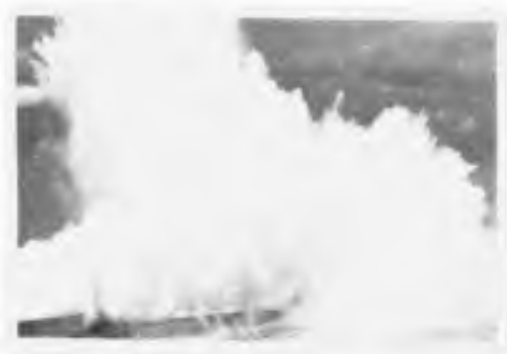

FIG. 17. - LARGE WAVE OVERTOPPINGS AT SEA-WALLS OF WAKAYAMA HARBOR

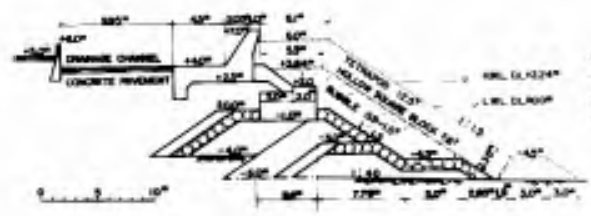

FIG. 20. - CROSS-SECTION OF THE SEAWALI IN WAKAYAMA HARBOR

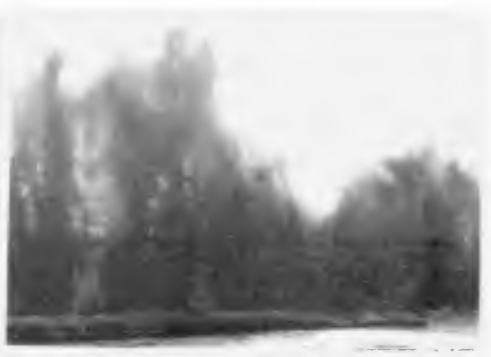

FIG. 18.

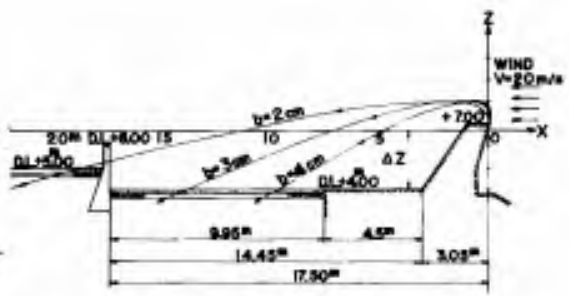

FIG. 21. - LOCI OF THE WALLS OF WATER OVERTOPPING THE SEA-WALL IN FIG. 20

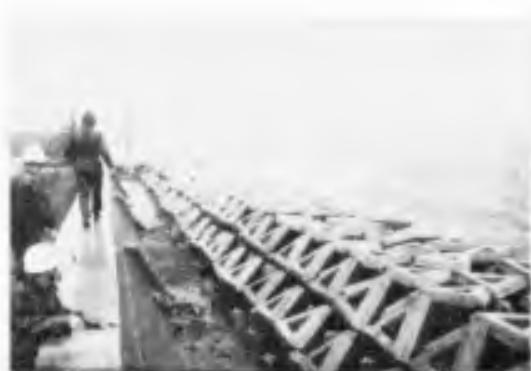

FIG. 23. - SEA-WAIL OF THE KANSAI E. P. CO.LTD. DURING A TYPHOON, 1961 
PRESSURE OF WAVES AGAINST VERTICAL WALLS

\author{
by M.E.Plakida) M.Sc. \\ Docent, Chief of wave Laboratory of Moscow \\ Branch of Water Trangport Institute \\ Moscow, U.S.S.R.
}

SUMACARY

This paper is conoerned with the atudy of the breaking wave pressure exerted upon the breakwater of the vertical type. The method of oalculation of the wave pressure, naned the quasiatatioal method, is based with the theory of the inpact of the water jet on the vertical plane and some new results of the experimental data.

The formulas for the calculation of the preasure distribution of the breaking wave and the ourf wave on the vertical wall are given.

\title{
INTRODUCTION
}

The following byabols are used in this paper.

$$
\begin{aligned}
& \mathbf{h} \text { - wave height; } \\
& \lambda \text { - wave length; } \\
& \tau \text { - wave period, } \\
& \text { c - wave celerity; }
\end{aligned}
$$

*) U.S.S.R. Moscow. K-1, Sadovokudrinskaya street 14-16, Ap.11. 


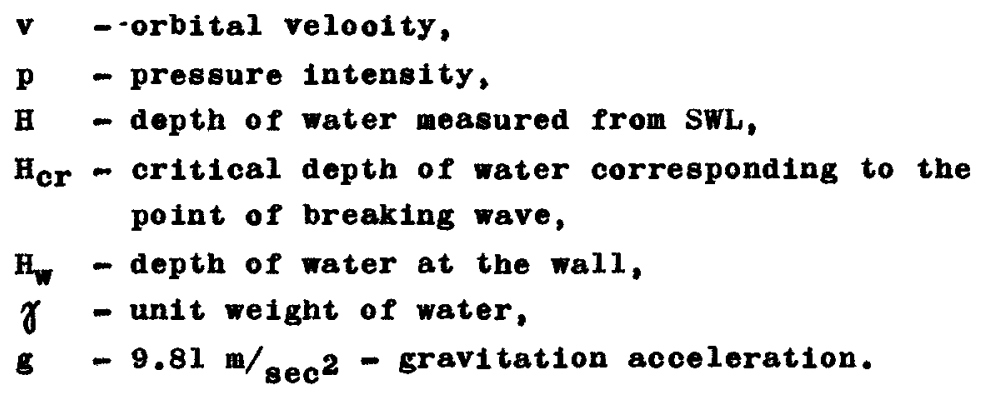

Up to the present time evaluation of the breaking wave force upon a breakwater of the vertical type prosents a complex problem. For many decads this problom has requested very much attention from the harbour engineer, who has to deal with design and construction of breakwaters of the vertical type at a sallow sea.

There are several solutions of this problem, one of them makes use of the conception of the theory of the inpact of a water jet upon a vertical plane. However, in spite of the fact that these investigations have been carried out during a long time the possibility of the conception of the impact of a water jet upon a vertical plane in full measure 18 not used.

This expression is given

$$
p=k \gamma \frac{u^{2}}{2 g}
$$

where

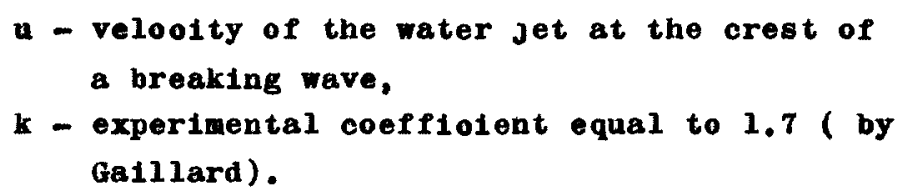
Gaillard).

In their time D. Gaillard, D. Molitor and V. Trenubin developed this direction auggesting to take into account $\mathbf{u}=\mathbf{c}+\mathbf{v}$. 
In $1940 \mathrm{~N} . \mathrm{Dj}$ ounkovekiy $[\mathrm{l}]$ corrected this to $\mathrm{u}=0.75 \mathrm{c}+\mathrm{v}$. In 1958 M.Plakida [2] suggested the new corrections to the distribution of the surf wave pressure exerted upon the vertical wall, based upon the experimental investifation. As a consequence the resultant value of the wave pressure on the vertical wall could be decreased up to 20 per cent in comparison with the value given by N.Djounkovakiy $[3]$.

It is necessary to note that suming up $c$ and $v$ makes no physical cense, this 1 explained by the absence of surfloient information about the kinematics of the breaking wave structure.

In this paper we have given as far as it was possible the development of the caloulation method of the presauro distribution from the breaking wave and the surf wave on the vertical wall. Remaining at the conception of the impact of the water jet for the calculation of the wave pressure at the still water eurface, we have used the standing wave theory of the first approximation and some experimental data for the calculation of the wave pressure at the loot of the vertical wall.

This expression is given

$$
p_{b}=\frac{\gamma_{h}}{\operatorname{ch} \frac{2 \pi H_{y}}{\lambda}}
$$

where

$$
\begin{aligned}
& \mathrm{pb}_{\mathrm{b}} \text { - wave pressure (above hydrostatic) at the } \\
& \text { foot of the vertical wall. }
\end{aligned}
$$

We note that it was about twenty years ago, when the mothod of calculation of the breaking wave pressure, based on the use of the impact impulse of the breaking wave, was suggested. It is the second direction in the solution of this problem. However, the experimental data is unsufficient for the full solution at the present time. 
KINEMATICS AND DYNAMICS OF BREAKING WAVES

The breaking wave or the surf wave is developed when the deep water wave comes up to a shallow water and reaches the oritical depth. The value of the critical depth variea in a very wide range $(1.0+2.5) h$. The critical depth within the linita $(1.5+1.8)$ h are accepted for the ateepness of the deep water waves within $1: 9+1: 25$.

When the water depth in front of the vertical wall is $H>\frac{\lambda}{3}$ or at least $H \geqslant 3 h$ and the depth on the berme at the foot of the wall is $\mathbf{H}_{W} \leqslant H_{c r}$ then the deep water, wave ia broken on the berme at the wall surfaoe; in this case the vertical wall is subjected to by the breaking wave action.

When in front of the vertical wall the sea bed is horizontal or gently oloping at least within $\frac{\lambda}{2}$ before the wall and the water depth is $\mathrm{H}_{1} \leqslant \mathrm{H}_{\mathrm{cr}}$ then the deep water wave is broken before the wall, in this case the vertical wall is subjected to the action of the surf wave.

It is seen that from the condition of development of the breaking wave and the surf wave the former exerts a greater wave pressure on the vertioal wall than the latter for the same height and length of the deep water wave.

The experimental data show that the velocity of the water particles at the crest of the breaking and surf waves may be nearly equal to the wave celerity and even exceed it. The value of this velocity is given as

$$
\mathbf{u}=\sqrt{\mathbf{g H}}
$$

The formation of the front steep lope of the wave, whioh is near to breaking, is the result of dragging of the foot of the wave, while the orest of the wave passes ahead beoause of different wave velocities of the particles in the crest and in the trough. The velocitios of the water par- 
tioles in the orest of the wave are greater and the velooities of the water particles in the trough of the wave are less than the average velocity of the wave at the still water surface. The kinematies of this phenomenon in detail is described by V.Shuleykin [4] based on the olassioal hydrodynamics formula

$$
c=\sqrt{\frac{g \lambda}{2 \pi} \operatorname{th} \frac{2 \pi H}{\lambda}},
$$

taking in (4) $H=H_{1}+0,5 h$ for the wave crest, and $H=H_{1}-0.5 h$ for the wave trough (where $H_{1}$ - water depth from the sea-bed to the otill water surface).

Fron the onergetical point of view in the wave near to breaking the concentration of the potential energy occurs, as the deep water wave approohing challow water decreases in length. In addition the wave energy is carried into shallow water with the full wave velooity as can be seen from the formula (5), when at $H \ll \lambda$ the second item $\longrightarrow$ to 1 .

$$
u_{0}=\frac{c}{2}\left(1+\frac{2 a H}{\text { sh2aH }}\right)
$$

where

$$
\begin{aligned}
& u_{0} \text { - veloeity of the transportation of the wave } \\
& \text { energy; } \\
& a=\frac{2 \pi}{\lambda} .
\end{aligned}
$$

Phenomenon of the breaking of the ware proceeds very rapidly and during very short time. It is accompanied by the transition of the potential energy of the dragged wave into the kinetic energy of the transitional movement of the water stream. When this stream reachea the vertical wall the phenomenon of the impact of the water jet directed to the wall and the wave reflection from the wall are observed. 
The maximum wove pressure on the wall appears earlier than the maxima sovition of the water level at the wall. When the uplift of the water lovel is maximum the wave pressure decreases. The save pressure does not exceed the value $(0.6-1.0) \gamma \mathrm{h}$ at the otill water surface.

EQUIPLENT AND EXPERIMRENS

The laboratory atudy of tho action of the breaking and the surt waves the rertical wall has been carried out in a llume of reotangular oross-section 23 in length, $0,50 \mathrm{~m}$ wide. The water dopth was $75 \mathrm{~cm}$ in the flume and the water depth at the loot oi the wall was $15 \mathrm{~cm}$. The ver tical wall model was placed on the top of the prism, which was 60 on above the flume bottom. The prism olope was 1:2.

The position. of the vertical plane of the wall were 25 on and 82 on from the priem edge (FIg.1). In the first case the wall 28 exposed to the action of the breaking wave and in the second case - to the action of the surf wave.

The waves were reproduced by the wave generator of the type of the flat paddle.

The wave pressures were measured by tensemetric ganges. The wave heights were measured by electrical gauges. A aanple of the osclllograph record is given on rig.2.

Phases of breaking, wave deformation detalle and the monent of amall ball indicatora (prepared with bitumen and parafin, their specific weight is equal to that of watex) were recorded on $35 \mathrm{~mm}$ IIIm at 24 frames per sec. A sample of film record is shown on Fig.3. It is seen that the indicator I between frames 15-17 has moved to the wall with naximun velocity before the impact.

Ware conditions of our experiments are given in table 1. 
Table 1

Wave Conditions

\begin{tabular}{|c|c|c|c|c|c|c|c|c|c|}
\hline \multirow{2}{*}{$\begin{array}{l}\text { Designation } \\
\text { of wave } \\
\text { conditions }\end{array}$} & \multicolumn{5}{|c|}{$\mathrm{H}=75 \mathrm{~cm}$} & \multicolumn{4}{|c|}{$\mathrm{H}_{1}=15 \mathrm{~cm}$} \\
\hline & $\begin{array}{l}\mathrm{h} \\
\mathbf{c m}\end{array}$ & $\underset{\text { om }}{\lambda}$ & $\underset{\mathrm{sec}}{\tau}$ & $\frac{\text { H }}{\lambda}$ & $\frac{h}{h}$ & $\begin{array}{c}\mathbf{h}_{1} \\
\mathbf{c m}\end{array}$ & $\lambda_{\text {com }}$ & $\frac{\mathbf{H}_{1}}{\mathbf{h}_{1}}$ & $\frac{\mathbf{h}_{1}}{\lambda_{1}}$ \\
\hline
\end{tabular}

$\begin{array}{lllllllllll}A-6-220-20 & 8.8 & 175 & 1.06 & 0.43 & 0.050 & 8.8 & 115 & 0.13 & 0.077\end{array}$

$\begin{array}{llllllllllll}A-7-220-20 & 11.5 & 177 & 1.07 & 0.42 & 0.067 & 0.8 & 115 & 0.13 & 0.085\end{array}$

$\begin{array}{lllllllllll}A-8-180-20 & 15.7 & 210 & 1.17 & 0.36 & 0.077 & 9.8 & 130 & 0.12 & 0.076\end{array}$

$\begin{array}{llllllllll}A-8-220-20 & 17.7 & 177 & 1.07 & 0.42 & 0.10 & 9.8 & 115 & 0.13 & 0.085\end{array}$

\section{WAVE PRESSURE}

After giving a short discription of the kinematica and dynamics of the breaking wave and our experinent, wo can begin to ovelve the formulas for the caloulation of the breaking wave pressure and the surf wave pressure exested upon the vertical wall.

Oux position is based on the formulas (1) - (3) and our experimental data. The following caloulation mothod and formulas for the determination of the value of the preasure on the vertical wall frow the breaking and suri wares 18 suggested.

Pressure of breaking waves. It is atter os somo difficulty to assune the value of the water depth H th sormula (3). As solution tha complex question astar gon considerations we suppose posalbie to take a afo ralus of

$$
\mathbf{H}=\mathbf{H}_{\mathrm{er}}=1,8 \mathrm{~h}
$$


Substituting (6) and (3) in Eq. (1) we arrive at the expression for the maximum pressure of the breaking wave, which occurs at or in the vicinity of still water surface

$$
p_{0}=1.5 \gamma h
$$

where

$$
\begin{aligned}
& \text { po - the maximum pressure of the breaking wave } \\
& \text { at the still water surface. }
\end{aligned}
$$

At the foot of the vertical wall rather calm wave conditions are observed (see the pressure fluctuation on the record of $\mathbf{B}_{10}$ on Fig.2).

There are traced (see Fig.4) two curves showing the fluctuation of the relative wave pressure at the foot of the wall. One of them is the experimental curve indicating the pressure of the breaking wave and the second is the theoretical curve showing the pressure of the standing wav. It is seen by the comparison of these two curves that the maximux preseure of the breaking wave at the foot of the wall appears carlier than the maximun pressure of the standing wave, calculated by the wave theory of the first approximation. As a result of this comparison of the two curvea, we propese to base the calculation of breaking wave prossure at the foot of the vertical wall upon the formula (2).

The relationship between $\frac{p_{c}}{\gamma h}$ and $\frac{H_{w}}{h}$ is given on

Fig.5 by the experimental data. This relationship is not linear as it may be expected. The greatest values of the relative wave pressure are found within $\frac{\mathrm{L}}{\mathrm{w}}=(1.2-1.5)$. The curve accepted by the construction rules acting in the USSR (SN -92-60) is also shown. 
The maximum values of the breaking wave pressure measured and calculated are given in Table 2. These data were used for the experimental curve on Fig.5.

\section{Table 2}

Values of wave pressure at the botton of a vertical wall ( gr/ome

\begin{tabular}{|c|c|c|c|c|c|}
\hline \multirow{2}{*}{$\begin{array}{l}\text { h } \\
\text { cn }\end{array}$} & $\underline{\mathbf{H}_{\mathbf{L}}}$ & Calculated & Messured & $\mathbf{p}_{15}$ & $\mathbf{p}_{\mathbf{1 5}}^{\mathbf{n}}$ \\
\hline & $\mathbf{h}$ & $p_{15}^{c}$ & $p_{15}$ & $p_{15}^{D}$ & $\gamma_{h}$ \\
\hline 8.8 & 1.70 & 7.6 & 5.7 & 0.76 & 0.86 \\
\hline 11.5 & 1.30 & 9.9 & 10.9 & 1.10 & 0.94 \\
\hline 15.7 & 0.96 & 14.0 & 12.8 & 0.92 & 0.82 \\
\hline \multirow[t]{2}{*}{17.7} & 0.85 & 14.6 & 13.0 & 0.93 & 0.74 \\
\hline & & & mean & 0.93 & 0.84 \\
\hline
\end{tabular}

where:

$$
\begin{aligned}
& p_{15}^{m} \text { - significant ware preseure that for statis- } \\
& \text { tical purposes is defined as the average } \\
& \text { preseure of the highest one-third of all } \\
& \text { measured breaking wave pressures at the foot } \\
& \text { of the vertical wall; }
\end{aligned}
$$

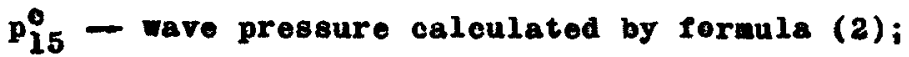

$$
\begin{aligned}
& \text { H } 15 \mathrm{~cm} \text {, - water depth at the wall. }
\end{aligned}
$$

The elevation of water surface above the till water level at the vertical wall is observed within $(0.5-0.8)$ h when the wave preseure is aximum. raking inte consideration somo fluctuation of this value we propose to take

$$
\mathbf{z}=\mathbf{h}
$$


The breaking wave pressure exerted upon the foundation of the vertical wall is given as

$$
=1 / 2 M_{b} p_{b} b
$$

where.

$$
\begin{aligned}
& M_{b}=0.9-\text { experinental coefficient; } \\
& b=\text { width of the wall. }
\end{aligned}
$$

Pressure of gurf waves. The breaking wave and the surf wave possess the vane physics of the breaking phenomenon. This allows us to use, In the case of the surf wave, the same formulas, that were mentioned above in the case of the breaking wave with following replacenents:

1. h - the height of the deep water wave (which is equal to the breaking wave) is replaced by the helght of the wurf wave $h_{1}$, calculated by the formula

$$
h_{1}=0,65 \mathrm{I}
$$

where

$$
\begin{aligned}
& \text { H }=H_{w} \text { - In the case when the sea-bed } 1 \text { s horizental } \\
& \text { in front of the wall, and } \\
& H=H_{0}=H_{w}+0,5 \lambda_{1} 1-\text { In the case, when the } \\
& \text { sea-bed is gently sloped; }
\end{aligned}
$$

where 1 - ser-bed slope.

2. $\lambda$ - the length of the deep water wave is replaced by the length of the surf wave $\lambda_{1}$. caloulated by the rornule

$$
\lambda_{1}=\lambda \operatorname{th} \frac{2 \pi \text { t }}{\lambda_{1}}
$$


The maximun pressure of the surf wave exerted upon a vertical wall occurs at $1 / 3 \mathrm{~h}_{1}$ above till water level according to our experimental data.

The elevation of water surface above the still water level in the case of the surf wave may be also taken as in the case of the breaking wave by the formula (8).

The surf wave pressure exerted upon the foundation of the rertical wall is given as

$$
w=1 / 2 M_{s} p_{b}^{b}
$$

where:

$$
M_{s}=0.7-\text { experimental coeffeient. }
$$

The distribution of the pressure of the breaking and gurt waves is ghown on Fig. 6 .

\section{CONCLUS ION}

1. The action of the breaking and surf waves upon the vertical wall was sudied in the wave laboratory from the point of view of the kinematics and the dynamics. It was found possible to advance somewhat a quasistatical method of the calculation of the wave pressure at the depth conditions

$$
h \leqslant H_{\mathbf{w}} \leqslant \mathbf{H}_{\text {or }}=1.8 \mathrm{~h}
$$

This suggestion is based on the conception of the impact of a water jet upon a vertical plane.

2. Our experimental data lies within of the following values:

- the wave steepness $\frac{h}{\lambda}$ from 0.05 to 0.10 ; and

- the relative water depth at the wall $\frac{B_{W}}{h}$ from 0.85 to 1.71 . 
3. The list of the formulas is given in the table 3 .

Table 3

\section{Formulas}

\begin{tabular}{ccc}
\hline Denomination & $\begin{array}{c}\text { Breaking } \\
\text { wave }\end{array}$ & $\begin{array}{c}\text { Surp } \\
\text { wave }\end{array}$ \\
\hline
\end{tabular}

1. Wave helght and wave

length

h, $\lambda$

$n_{1}, \lambda_{1}$

2. Maximun wave pressure $p_{0}$

$1.5 \gamma \mathrm{h}$

$1.5 \gamma \mathrm{h}_{1}$

3. Maximun wave pressure ia found

At the S.W.L. At $1 / 3^{n_{1}}$ the S.W.L. above

4. Maximun wave preseure at the root of the wall $\mathrm{P}_{b}$..

$\frac{\gamma_{h}}{\operatorname{ch} \frac{2 \pi H_{I}}{\lambda}}$ $\frac{\gamma h_{1}}{\operatorname{ch} \frac{2 \pi H_{I}}{\lambda_{1}}}$

5. Blevation of the water surface above the otill water lerel $=\ldots \ldots \ldots$.....

6. Wave pressure exerted upon the foundation of the wa11 $\ldots \ldots \ldots \ldots \ldots \ldots \ldots$ h

$\mathbf{h}_{\mathbf{1}}$ 


\section{REPERENCES}

1. М.Э.Плапида. Цавление от прибойньх и разбитьх водн на оградительние соорухения типа вертикальной стенки. Гидротехническое строительство юе 8, I965.

M.E.Plakida. Breaking wave and surf wave pressures against the vertical breakwaters. Hydrotechnieal Construction No.8, 1965.

2. И.З.Плакида. Исследование прибойной волни и еө воздөйствие на оградительное соорухение вертикального типа. Труды цнинэт'а. Вопросу гидротехнаки. Вип. Москва, I958.

M.R.Plakida. Investigation of surf wares and its action on the vertical breakwatera. Proo. CNIEwT.Vol.XV, Moscot, 1958.

3. П.К.Бохич и Н.Н.Дхунковский Морское волнение и его действие на соорухения и берега. Мапстройиздат.Москва, I948.

P.K.Bojitoh and N.N.Djounkorakiy. Ses waves and its action on the construotions and the shores. Machiadat. Moscov, 1948.

4. В.В.Пулейкин. Теория морских волн. Изв. А.Н. СССР, I956. V.V.Shuleykin, Sea wave theory. IzV. A.S, of U.S.S.R., 1956. 

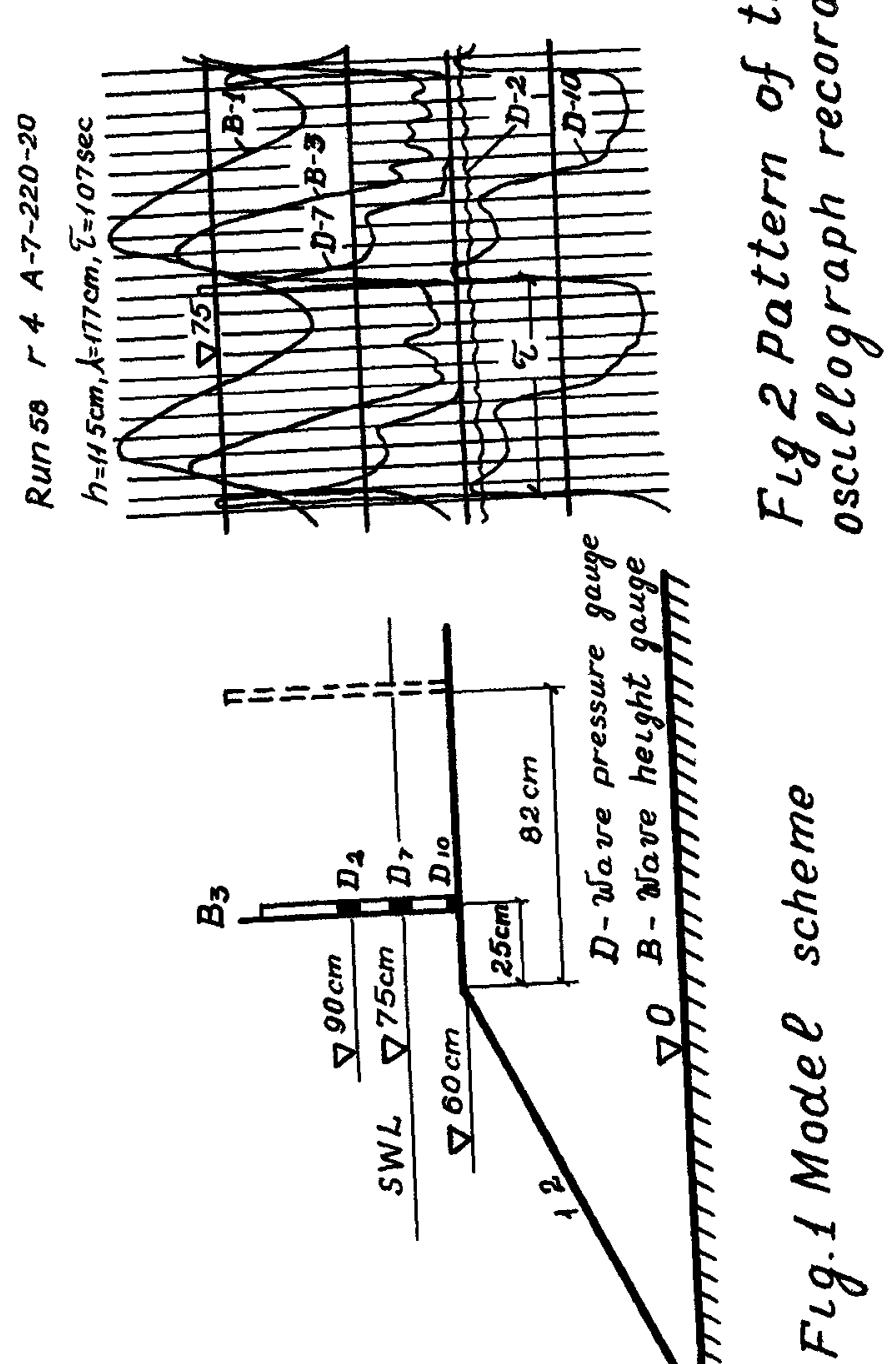


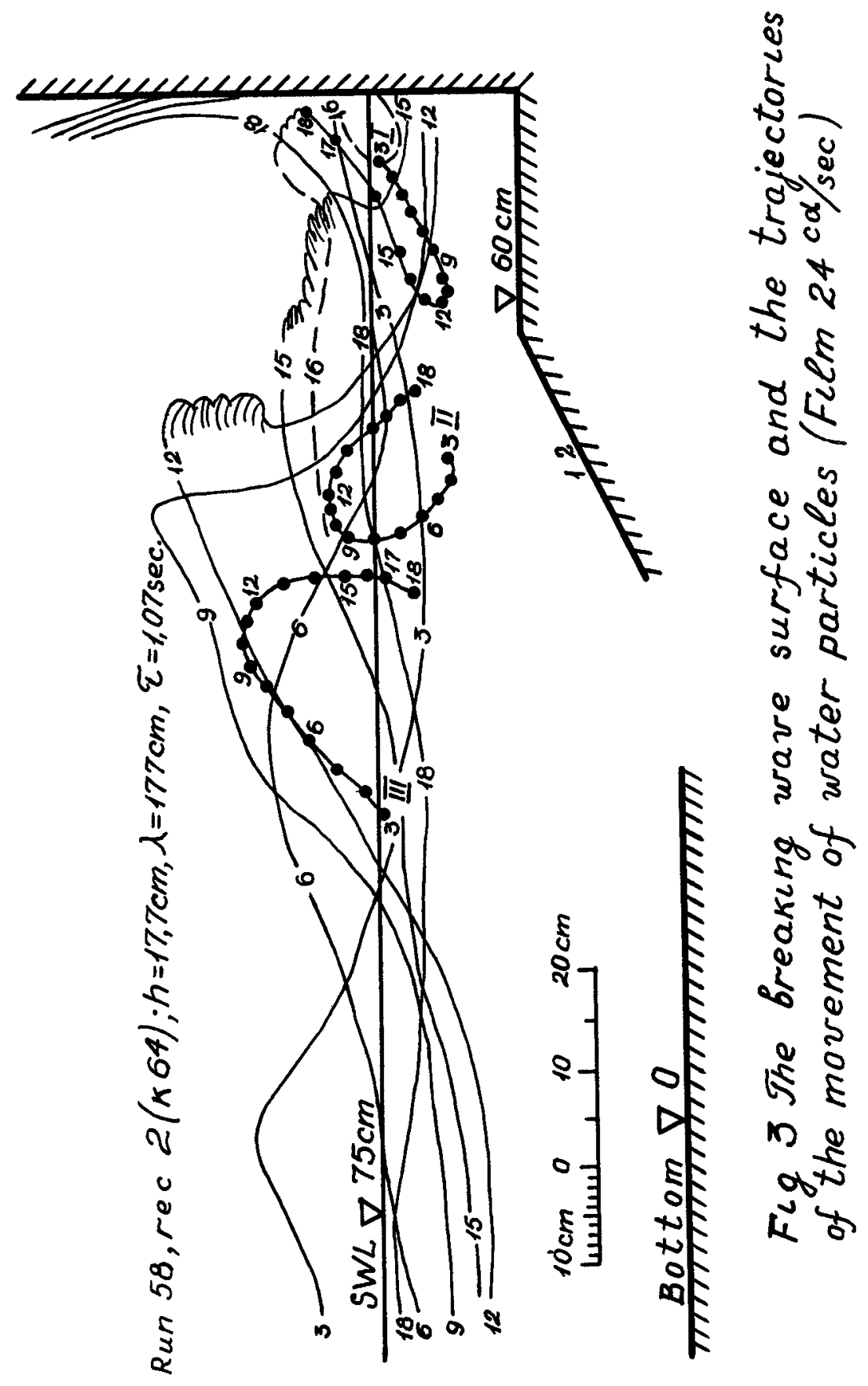




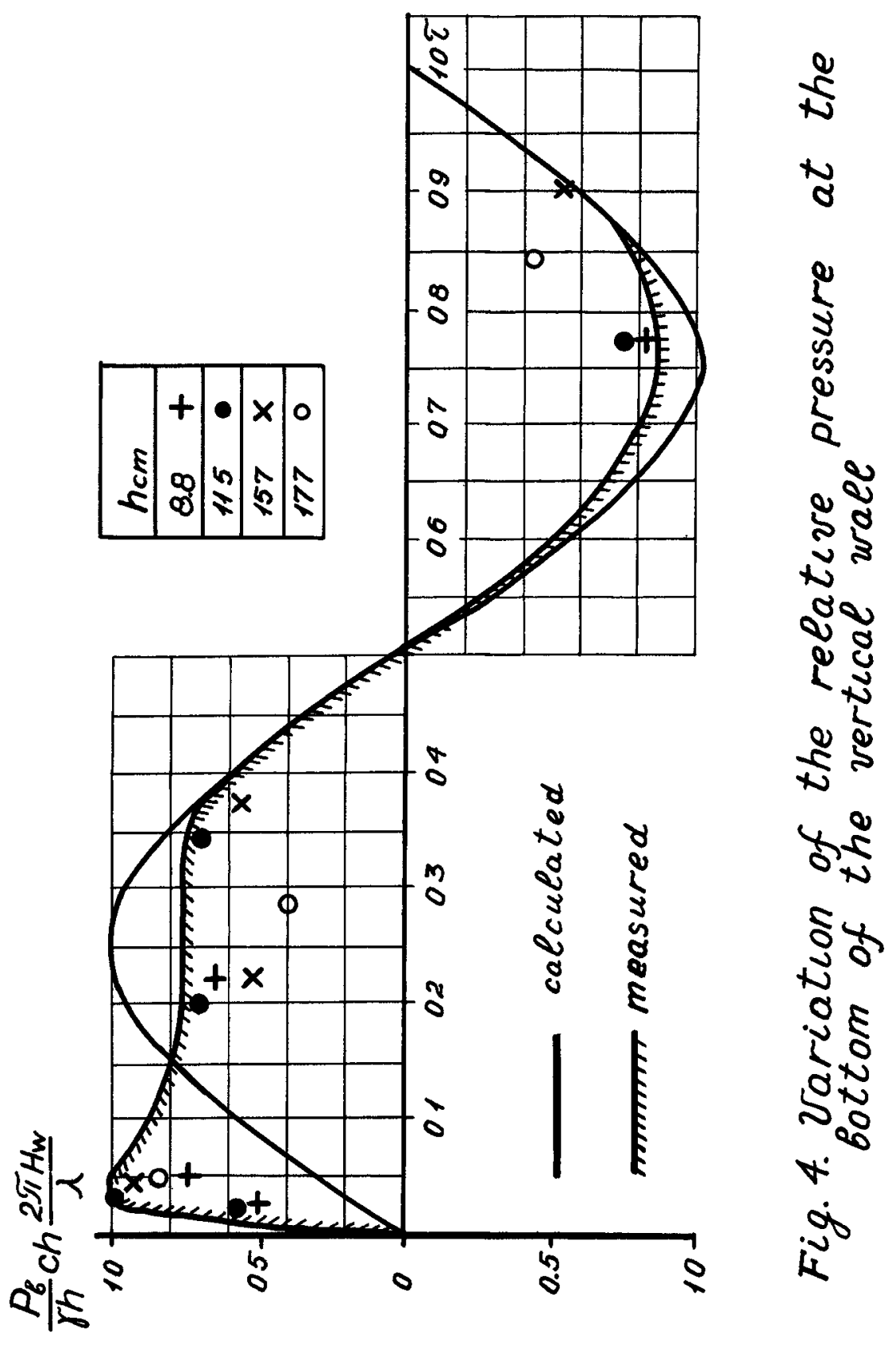




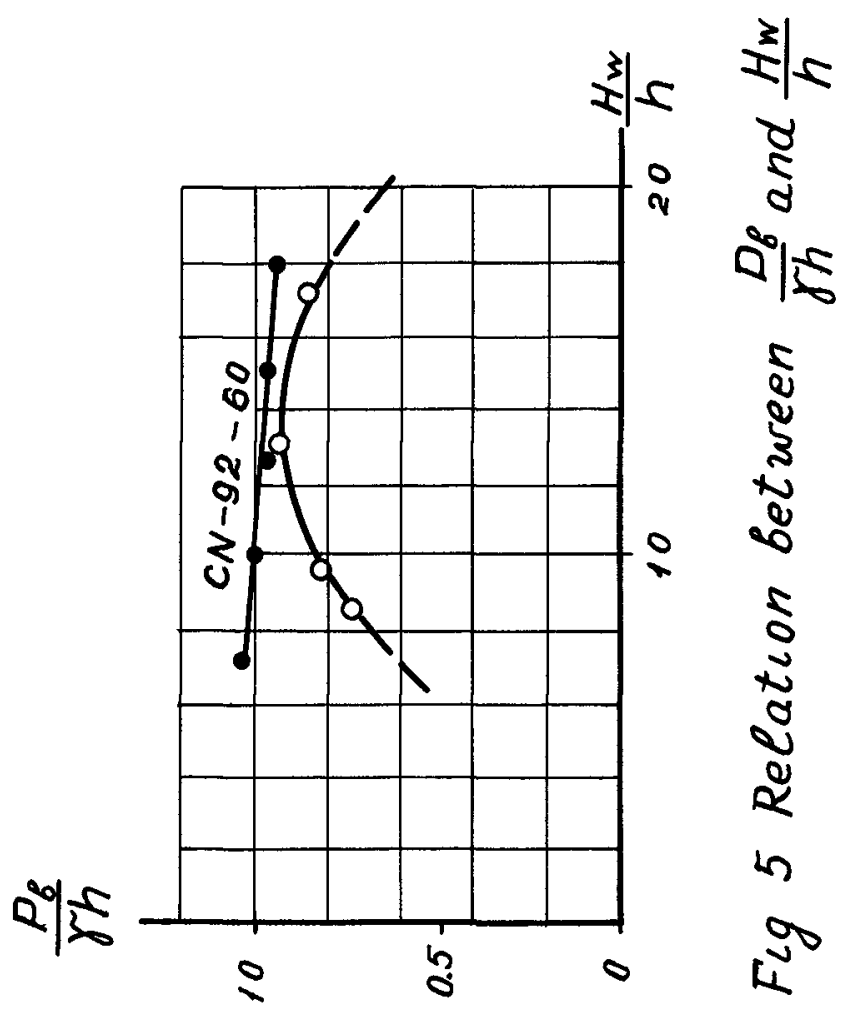




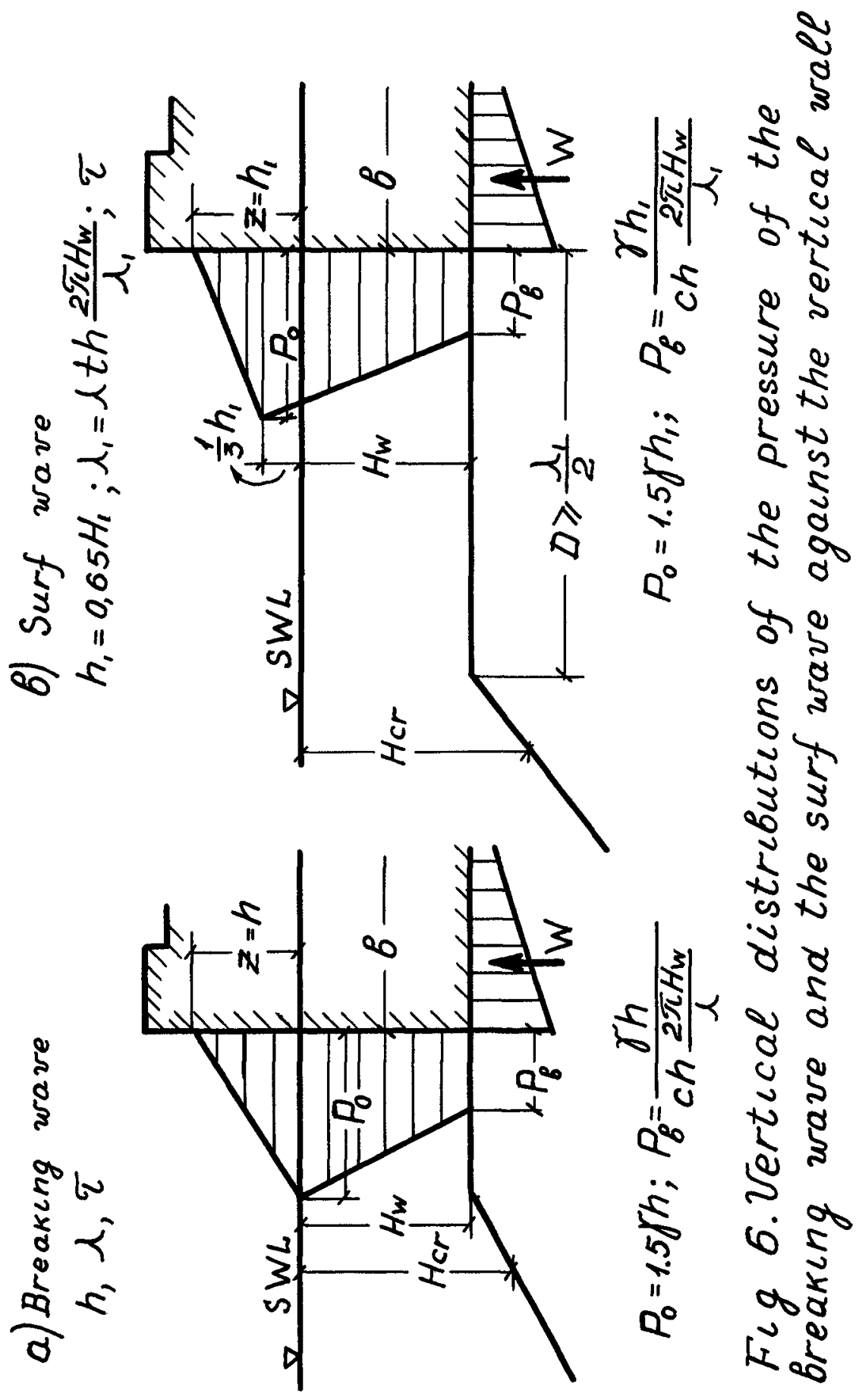




\title{
CHAPTER 90
}

\section{PLUNGING WAVE PRESSURES ON A SEMI-CYLINDRICAL TUBE}

\author{
by \\ Yuan Jen, Assistant Professor \\ Pang-Mou Lin, Graduate Student \\ Department of Coastal and Oceanographlc Englneering \\ University of Florıda-Gainesville, Florida \\ USA
}

\begin{abstract}
Laboratory tests were made on a 6-1nch diameter semi-cylindrical tube simulating a prototype concrete structure designed to be placed parallel to the coastline in the surf zone to intercept of fshore transport of beach materials and thus preserving the beach The model beach was of fixed bed type with a slope of 1 to 15 The crest elevation of the tube was set at the still water level for most of the test runs and the sloping beach intersected a flat channel bottom at a depth of $2075^{\prime \prime}$
\end{abstract}

The test parameters used may be summarized as follows

$$
\begin{aligned}
\mathrm{H} / \mathrm{L} & =0005 \text { to } 0052 \\
\mathrm{~d} / \mathrm{L}_{\mathrm{O}} & =0027 \text { to } 0111 \\
\mathrm{H} / \mathrm{D}= & 026 \text { to } 118
\end{aligned}
$$

where $\mathrm{H}$ is the wave height measured at the toe of the beach, $\mathrm{d}$ is the water depth In the flat portion of the channel, $D$ is the tube diameter, $L$ is the wave length in the flat channel and $L_{0}$ is the equivalent deep water wave length

The above test conditions resulted in plunging breakers on the beach except for a few test cases

Pressure measurements were made around the tube circumference, at an interval of 30 degrees azımuth angle, by using a high frequency response pressure transducer It was found that the pressure distribution could be treated in two parts

Part I During the wave uprush, an impact pressure of short duration was recorded at the front of the tube, followed by smoother pressure varlations in time

Part II During the wave downrush, the volume of water transported across the tube flowed back under gravity The pressure distribution on the beach side of the tube was relatively steady during the flow reversing process 
Solitary wave theory was used to compute the breaking wave height and wave celerity The validity of analytical treatments were evaluated by comparison of measured and observed data The impact pressures at the front of the tube occurred in somewhat random nature The average impact pressure in dimensionless form $\mathrm{P}_{1} / \gamma \mathrm{H}$ was found dependent upon wave steepness The maximum lmpact pressure decreases as the plunging wave steepness increases Waves with $H / L<0$ exert little impact The impulse-momentum theory was applied to investigate the behavio of impact pressures The pressure distribution on the tube surface during the wave downrush was treated as quasi-steady case in which the Bernoulli princlple for steady state condition applies

\section{INTRODUCTION}

The phenomenon of wave and structure interaction has long been a subject of vital interest to coastal engineers Considerable information related to the forces acting on coastal structures due to the action of non-breaking waves can be found in the engineering literature, and dependable methods of predicting wave forces are available However, despite the fact that a large number of published works also deal with breaking waves, few conclusions can be drawn in order to establish confidence in the design of coastal structures to be located in the surf zone Analytical means of evaluating wave-structure interactions in the surf zone are difficult to derive because

1 Presently available wave theories are inadequate in describing the wave characteristics in the surf zone

2 Forces or pressures exerted by traveling breakers differ in magnitude from wave to wave, therefore the analysis of such forces must rely upon statistical means

The Coastal Engineering Laboratory, University of Florida, conducted a two phase study on a semi-cylindrical concrete tube structure, designed to be placed parallel to the beach at or near the mean water line to intercept the offshore transport of beach materials The study includes

\footnotetext{
Phase I - Movable Bed Model Study - To evaluate the stability of the structure under wave action.

Phase II - Fixed Bed Model Study - To measure the pressure distrubution around the tube surface and to determin the wave loading
}

Only the results of Phase II will be presented in this paper, since the results of Phase I are mannly qualitative in nature The effectiveness of the structure in trapping beach materials is beyond the scope of this paper

\section{MODEL SET-UP AND INSTRUMENTATION}

The model study on wave pressures was performed in a $100 \mathrm{ft} x 10 \mathrm{ft} x$ $25 \mathrm{ft}$ wave channel A semi-cylindrical tube, 6 inches in diameter, was mounted on a fixed slope of 1 to 15 at a distance of 85 feet from the wave generator (see Figure 1) The water depths were 175 inches at low water and 2075 inches a 
high water The high water represents a case when the tube is barely submerged and most test runs were conducted at this water level Variable wave heights and periods were recorded by a parallel wire resistance-type wave gage located 22 feet in front of the test structure at the point where the channe1 bottom starts to flatten out A high sensitivity pressure transducer was mounted on a circular test section which was free to rotate about its center so that the pressure sensor could be moved to any desired location along the tube circumference When performing the tests, the transducer was rotated at every $30^{\circ}$ interval and the same test condition was repeated for every transducer position The records showed that the wave inputs could be accurately reproduced and the pressure response at each location could be synchronized by using the wave records as references The calibration of the transducer was performed statically This method of calibration was considered to be sufficiently accurate since the transducer has a natural frequency of $10,000 \mathrm{~Hz}$ which is much higher than the input frequencies, and the natural period of the transducer was much shorter than the duration of shock pressure impulse

\section{CHARACTERISTICS OF MODEL WAVES}

Referring to the model set-up as shown in Figure 1, the model wave characteristics are adequately described at the gage location as

$$
\begin{aligned}
\mathrm{H} / \mathrm{L} & =0005 \text { to } 0052 \\
\mathrm{~d} / \mathrm{L}_{\mathrm{o}} & =0027 \text { to } 0111 \\
\mathrm{H} / \mathrm{D}= & 026 \text { to } 118
\end{aligned}
$$

where $H$ is the wave height at the gage location, $d$ is the water depth in the flat channel, $D$ is the tube diameter, $L$ is the wave length in the flat channel, and $L_{0}$ is the equivalent deep water wave length The measured wave heights range from ${ }^{\circ}$ 0131 to 0589 feet and the wave periods range from 173 to 350 seconds Wiegel (Ref 1) suggested that the waves in shoaling water just prior to breaking might be treated as a solitary wave as an approximation Wilson, Webb and Hendricksom (Ref 2) recommended the range of Ursell's parameter $\mathrm{HL}^{2} / \mathrm{d}^{3}$ within which the solitary wave theory is applicable The calculated Ursell's parameters for this study are between 15 and 36 , well within their recommended range of 10 to 40 Therefore, in this study, the solitary wave theory will be used to describe the mode 1 waves measured at the wave gage During the tests, most waves broke on the 115 beach and the locations where white caps were first found as the wave advanced toward the tube were observed and recorded, so that the breaking depth $d_{b}$, measured from the still water level to the beach bottom, can be computed Figure 2 shows the relationships between $\mathrm{H} / \mathrm{T}^{2}$ and $\mathrm{d}_{\mathrm{b}} / \mathrm{T}^{2}$ as compared to the usually referred breaking index curve A surprising result of the wave breaking data was that $\mathrm{H} \approx \mathrm{H}_{\mathrm{b}}$, where $\mathrm{H}_{\mathrm{b}}$ is the wave height at breaking (not measured) Galvin (Ref 3 ) found that

$$
\frac{d_{b}}{H_{b}}=140-685 m
$$

where $m$ is the beach slope 
Using Eq (1), we obtain, for the present slope of $1 / 15$

$$
\frac{H_{b}}{d_{b}}=104
$$

while Figure 2 suggests that $H / d_{h}=11$, confirming the previous statement of $\mathrm{H} \approx \mathrm{H}_{\mathrm{b}}$ The wave forms fit the description of "plunging breakers" by observation Iversen (Ref 4) found that the plunging breakers occurred when $\mathrm{H} / \mathrm{T}^{2}=005$ to 030 for 110 beach slope and $H_{0} / T^{2}=002$ to 020 for 120 beach slope Galvin (Ref 3) defined the occurrence of plunging breakers as

$$
H_{0} / L_{0} m^{2}=09 \text { to } 48
$$

For this study, $\mathrm{H}_{0} / \mathrm{T}^{2}=001$ to 022 and $\mathrm{H} / \mathrm{L}_{\mathrm{o}} \mathrm{m}^{2}=044$ to 88 Reasonable agreement to suggestions by previous investigators was found in defining plunging breakers

\section{TEST RESULTS AND ANALYSIS}

\section{Description of Pressure Time History}

The variation of pressure in time as the plunging breakers passed through the tube structure can best be described by referring to Figure 3 in which the pressure time histories around the tube curcumference are shown for a typical test condition " $\theta$ " values shown in the figure are defined in Figure 7 During the wave uprush, as the wave front contacts the front face of the tube $(\theta=0$ to $\left.90^{\circ}\right)$, pressures of high intensity and short duration were registered at the pount of impact, this pressure will be referred to as "impact pressure", $P_{i}$, throughout this paper Immediately after the impact, the pressures acting upon the front of the tube undergo a smooth continuous variation, normally decreasing in intensity, as the uprushing waves gradually lose their momentum due to the counteracting gravitational force The secondary peak pressure registered immediately after the impact will be designated as $P_{s}$

As a result of wave uprush, a certain volume of water is transported across the crest of the tube The maximum run-up is attained when all the available kinetic energy is converted into potential energy Under the influence of gravity this volume of water flows back across the tube and the wave downrush begins The behavior of reversing flow resembles the case of flow across a spillway, excep that the avallable head decreases with time The pressure time histories recorded on the beach side of the tube $\left(\theta=90^{\circ}\right.$ to $\left.180^{\circ}\right)$ were quite steady during the flow reversing process The reverse flow is suddenly interrupted by the impact accompanying the next onconing wave, and the wave uprush is again repeated In Fig 4, the pressure variation with respect to time is shown for two test cases The times $t=0$ and $t=T$ represent times of two consecutive impacts during which a high pressure intensities were found at the front of the tube for a short duration Therefore, at $t=0$ and $t=T$, the net resultant force on the tube is 
definitely acting shoreward At $t=3 T / 4$, the tube is under the action of reversed flow, the front portion $\left(\theta<90^{\circ}\right)$ of the tube is subjected to pressures less than the hydrostatic pressure at still water level, while the back of the tube $\left(\theta>90^{\circ}\right)$ is subjected to some dynamic pressure in addition to the initial hydrostatic pressure The net resultant force during the wave downrush is thus acting seaward Repetitive wave actions wlll cause the tube to loosen on a beach Additional instantaneous pressure distributions during impact are shown in Fig 5

The Impact Pressure $\mathrm{P}_{i}$

Test results showed that the impact pressure intensity varied with every wave, even though the wave form at the toe of the sloping beach appeared to be rather uniform and periodic Therefore, an appropriate evaluation of the pressure intensity should be based upon a statistical analysis However, the number of waves available to analysis was limited because, after a certain length of time, the wave form became distorted by the reflection from the wave generator Because of this restriction, only eight pressure peaks were averaged, and the dimensionless parameter $\mathrm{P}_{i} / \gamma \mathrm{H}$ is correlated with the wave steepness $\mathrm{H} / \mathrm{L}$ in Fig 6 " $\mathrm{P}_{i}$ " was the pressure difference between the pressure peak measured during the impact and the pressure just prior to the moment of impact The maximum impact occurred as $T=35$ seconds and $H=0282$ feet when the waves broke right in front of the tube The wave steepness for this test case is 001 Fig 6 clearly indicates that steeper waves exert a relatively lower impact as expressed in dimensionless form, because they break at a distance from the tube For the few cases when $H / L<001$, no appreciable breaking of the waves was observed, therefore the impact is relatively low

In the following, an impact-momentum model is postulated to gain further insight to the behavior of impact pressures

Refer to Fig 7 As breakıng waves impinge upon a portion of the tube surface, the normal force exerted on an element of the circumference $\Delta l$ is equal to the rate of change of momentum in the radial direction Thus we may write

$$
-d\left(M_{e} U_{r}\right) / d t=P_{i} \Delta l
$$

where " $M$ " Is the effective mass of the portion of water causing the momentum reaction on the element $\Delta l$ and " $U_{r}$ " is the radial velocity of the mass $M_{e}$ The "-" sign is inserted here to represent a case of decreasing momentum The effective mass $\mathrm{M}_{\mathrm{e}}$ is considered independent of time during the impact when the velocity of the mass is retarded, and may be expressed as

$$
M_{e}=\rho b * \Delta l \cos \alpha
$$

providing that $\Delta l$ is small It is apparently unrealistic to treat the whole horizontal element of width " $b$ " as solid, therefore the effective width is defined as " $b *$ " where $b$ * is much less than b Substituting Eq (4) Into (3), we obtain 


$$
P_{1}=-\rho b * \cos \alpha \frac{d U_{r}}{d t}
$$

If the time variations of " $P_{i}$ " and that of " $\mathrm{U}_{r}$ " are linear, as that assumed by Weggel (Ref 5), then Eq 5 can be integrated as

$$
\int_{0}^{t_{i}} P d t=-\int_{U_{0} \cos \alpha}^{0} \rho b^{*} \cos \alpha d U r
$$

where $t_{i}$ is the duration of impact and $U_{0}$ is the horizontal water particle velocity just before impact (see Figure 7) After integration, we obtain

$$
P_{i}=\rho U_{0}\left(\frac{2 b * \cos ^{2} \alpha}{t_{i}}\right)
$$

or $P_{i}=\rho U_{0} U *$

where $U^{*}$ is a characteristic velocity which has an upper limat equal to the sonic speed in water

Eq (7) is reduced to dimensionless form through the following procedures

(1) The celerity at breaking is computed from solitary wave theory as

$$
c_{b}=\sqrt{g\left(H_{b}+h_{b}\right)}
$$

where $h_{h}$ is the breaking depth measured to the trough of the waves and is related to the still water depth $d_{b}$ as

$$
h_{b}=d_{b}-c_{h} H
$$

where $C_{h}=02$ to 04 from previous experimental studies

(2) Recalling the previous discussion that

$$
\mathrm{H} \approx \mathrm{H}_{\mathrm{b}} \text { and } \frac{\mathrm{d}_{\mathrm{b}}}{\mathrm{H}}=\frac{1}{11}=091 \text {, }
$$

we may write

$$
P_{i} / \gamma H=\left(U_{0} U * / C_{b}^{2}\right)\left(1-C_{h}+d_{b} / H\right)
$$


or

$$
\mathrm{P}_{\mathrm{i}} / \gamma \mathrm{H}=(151 \text { to } 171) \mathrm{U}_{\mathrm{o}} \mathrm{U} * / \mathrm{C}_{\mathrm{b}}^{2}
$$

Referring again to Figure 6 which shows $P_{i} / \gamma \mathrm{H}=19$ when the waves break right in front of the tube $\left(C_{b} \approx U_{o}\right)$, Equation (al)implies

$$
\mathrm{U} * / \mathrm{C}_{\mathrm{b}}=111 \text { to } 126
$$

which shows $U *$ and $C_{b}$ are in the same order of magnitude, much less than the sonic speed in water The impact pressures shown in Figure 6 are much less than those observed by previous investigators who have dealt with wave pressure acting on vertical walls (Ref 6 to 10) The curvature of the tube, which 15 not as conducive to "shock" as plane surfaces, is mainly responsible for the reduction of impact pressure

\section{The Secondary Pressure $P_{s}$}

After the initial impact, a sudden drop of pressure occurs and this pressure is termed "secondary" as was referred to previously The concept of fixed hypothetical mass no longer applies here since there exists a continuous flux of mass toward the tube The kinematics of flow around the tube surface are beyond a theoretical prediction, therefore an accurate estimate of $P$ is difficult to obtain Figure 8 shows the distribution of $\mathrm{P}_{\mathrm{s}} / \gamma \mathrm{H}$ with respect to $\theta$ for different values of $H / D$ For simplicity, if one assumes that the horizontal velocity of water mass in front of the tube remains constant, then the mass flux in the radial direction is $\rho U_{0} \Delta l \cos \alpha$ and the rate of change of momentum in the same direction becomes $\rho U_{0}^{2} \Delta l \cos ^{2} \alpha$, so the application of momentum principle glves

$$
P_{s}=\rho U_{0}^{2} \cos ^{2} \alpha
$$

A similar analysis to that made on the impact pressure gives

$$
P_{s} / \gamma H=\left(U_{o} / C_{b}\right)^{2} \cos ^{2} \alpha\left(1-c_{h}+d_{b} / H\right)
$$

or

$$
\mathrm{P}_{\mathrm{s}} / \gamma \mathrm{H}=(151 \sim 171)\left(\mathrm{U}_{\mathrm{o}} / \mathrm{C}_{\mathrm{b}}\right)^{2} \cos ^{2} \alpha
$$

The upper 1lmit of $\mathrm{P}_{\mathrm{s}} / \mathrm{\gamma H}$ must occur when $\mathrm{U}_{0}=\mathrm{C}_{\mathrm{b}}$, thus

$$
\left(\mathrm{P}_{\mathrm{s}} / \gamma \mathrm{H}\right)_{\max }=(151 \sim 171) \cos ^{2} \alpha
$$


Eq (15) is plotted in Figure 8, the curve is shown to fit the envelope of all data $\mathrm{P}_{\mathrm{S}} / \gamma \mathrm{H}$ decreases as $\mathrm{H} / \mathrm{D}$ increases The trend is reasonable since $\mathrm{U}_{\mathrm{o}} / \mathrm{C}_{\mathrm{b}}$ values are smaller for large $\mathrm{H} / \mathrm{D}$ values, and $\mathrm{Eq}$ (14) shows that " $\mathrm{P}_{\mathrm{s}} / \gamma \mathrm{H}$ is proportional to $\left(\mathrm{U}_{\mathrm{o}} / \mathrm{C}_{\mathrm{b}}\right)^{2}$

\section{Pressures due to Reversed Flow}

During the wave downrush, the flow reverses its direction under the influence of gravitational force The flow patterns resemble those found across a spillway The pressure distribution at the beginning and the end of the reversing flow are shown for six test conditions in Fig 9 At the beginning of wave downrush, the pressure distribution is believed to be hydrostatic since the motion of water particles was negligible at that instant, while at the end of wave downrush, the flow over the tube approaches a steady state so that the pressure distribution on the beach side of the tube $\left(\theta>90^{\circ}\right)$ remains unchanged for a short period of time The overflow along the ocean side of the tube $\left(\theta<90^{\circ}\right)$ is curvilinear with relatively high speed Negative gage pressures were often recorded for $\theta=30^{\circ}$ and $\theta=60^{\circ}$ The total resultant force during the wave downrush is therefore acting seaward, in contrast to the case of wave uprush

\section{SUMMARY AND CONCLUSIONS}

It is found in this study that the uprush of plunging breakers exert little impact on a semi-cylindrical tube as compared to the "shock" pressure caused by waves breaking on a vertical wall The " $\mathrm{P} / \gamma \mathrm{H}$ " value as defined in the paper is at maximum when waves break right in front of the tube (H/L $=0$ 01) Waves steeper than H/L (>0 01) break at a distance away from the tube and the resulting $P_{i} / \gamma H$ decrease as wave steepness increases No noticeable Impact was observed for waves with $\mathrm{H} / \mathrm{L}<001$ An Impulse momentum model gives

$$
P_{1}=\rho U_{0} U *
$$

where

$$
U^{*}=\frac{2 b^{*} \cos ^{2} a}{t_{i}}
$$

and

$$
\mathrm{P}_{i} / \gamma H=(151 \text { to } 171) \mathrm{U}_{\mathrm{o}} \mathrm{U} * / \mathrm{C}_{\mathrm{b}}^{2}
$$

The characteristic velocity $U^{*}$ is in the same order of magnitude as the wave celerity at breaking, $c_{b}$ Therefore, the impact pressure is much lower than the pressure of the water hammer type Detafled characteristics of U* could be studied by fast speed photography along with fast speed pressure recording systems The "secondary pressure" as defined in the paper has a maximum value

$$
\left(\mathrm{P}_{\mathrm{s}} / \gamma H\right)_{\max }=\left(\begin{array}{llll}
1 & 51 \text { to } 171
\end{array}\right) \cos ^{2} \alpha
$$

when $\mathrm{U}_{\mathrm{o}}=\mathrm{C}_{\mathrm{b}}$ 
The above equation represents the envelope of the data $\mathrm{P}_{\mathbf{s}} / \gamma \mathrm{H}$ becomes smaller as $H / D$ increases The validity of the equation

$$
\mathrm{P}_{\mathrm{s}} / \gamma \mathrm{H}=(151 \text { to } 171)\left(\mathrm{U}_{\mathrm{o}} / \mathrm{C}_{\mathrm{b}}\right)^{2} \cos ^{2} \alpha
$$

could be evaluated by measuring $\mathrm{U}_{0}$ During the wave downrush, wave pressures on the beach side of the tube seldomly exceed the net hydrostatic pressure caused by the wave run-up The high speed curvilinear overflow on the ocean side of the tube results in some negative gage pressures so that the resultant force during the wave downrush is acting seaward, in contrast to the case of wave uprush during which the impact force is acting shoreward The oscillatory behavior of the net resultant force creates a stability problem on the structure When designing this type of structure, cut-off walls extending downward into the soil must be provided to resist sliding and overturning of the structure, and also, reducing the uplift pressure on the tube foundation

\section{ACKNOWLEDGEMENTS}

This work was supported in part by the Gray Tech Industries, Incorporated, Mohnton, Pennsylvania Dr $R$ G Dean kindly reviewed the paper and offerred constructive suggestions 
1 Wiege1, R L, Oceanographical Engineering, Prentice-Ha1I, Inc 1964

2 Wilson, B W, Webb, L $M$ and Hendrickson, J A, The Nature of Tsunamis, Their Generation and Dissipation in Water of Finite Depth Natıonal Englneerıng Sclence Company, Technical Report, August, 1962

3 Galvin, C J, Jr , Breaker Travel and Cholce of Design Wave Height, Journal of Waterways and Harbors Div, Proc ASCE, May, 1969

4 Iversen, $H W$, Waves and Breakers in Shoaling Water, Proc Third Conf on Coastal Engineering, 1952, pp 1-12

5 Wegge1, J R and Maxwe11, H C, Numerical Model for Wave Pressure Distributions, Journal of Waterways and Harbors Div, Proc ASCE, August, 1970

6 Wegge1, J R and Maxwe11, H C, Experımental Study of Breaking Wave Pressures, Offshore Technology Conference, Paper No OTC 1244, Apri1, 1970

7 Kame1, A M , Shock Pressure on Coastal Structures, Journal of Waterways, Harbors and Coastal Engineering Div, Proc ASCE, August, 1970

8 Rundgren, L , Water Wave Forces - A Theoretical and Laboratory Study, Bu11 No 54, Institution of Hydraulics, Royal Institute of Technology, Stockholm, Sweden, 1958

9 Ross, C W, Laboratory Study of Shock Pressures of Breaking Waves, Beach Erosion Board Tech Memo No 59, U S Army Corps of Engineers, February, 1955

10 Nagai, S "Shock Pressure Exerted on Breakıng Waves on Breakwaters" Journal of Waterways and Harbors Div , ASCE, Vo1 86, No WW2, Proc Paper 2504, June, 1960 


\section{NOTATION}

The following symbols are used in this paper

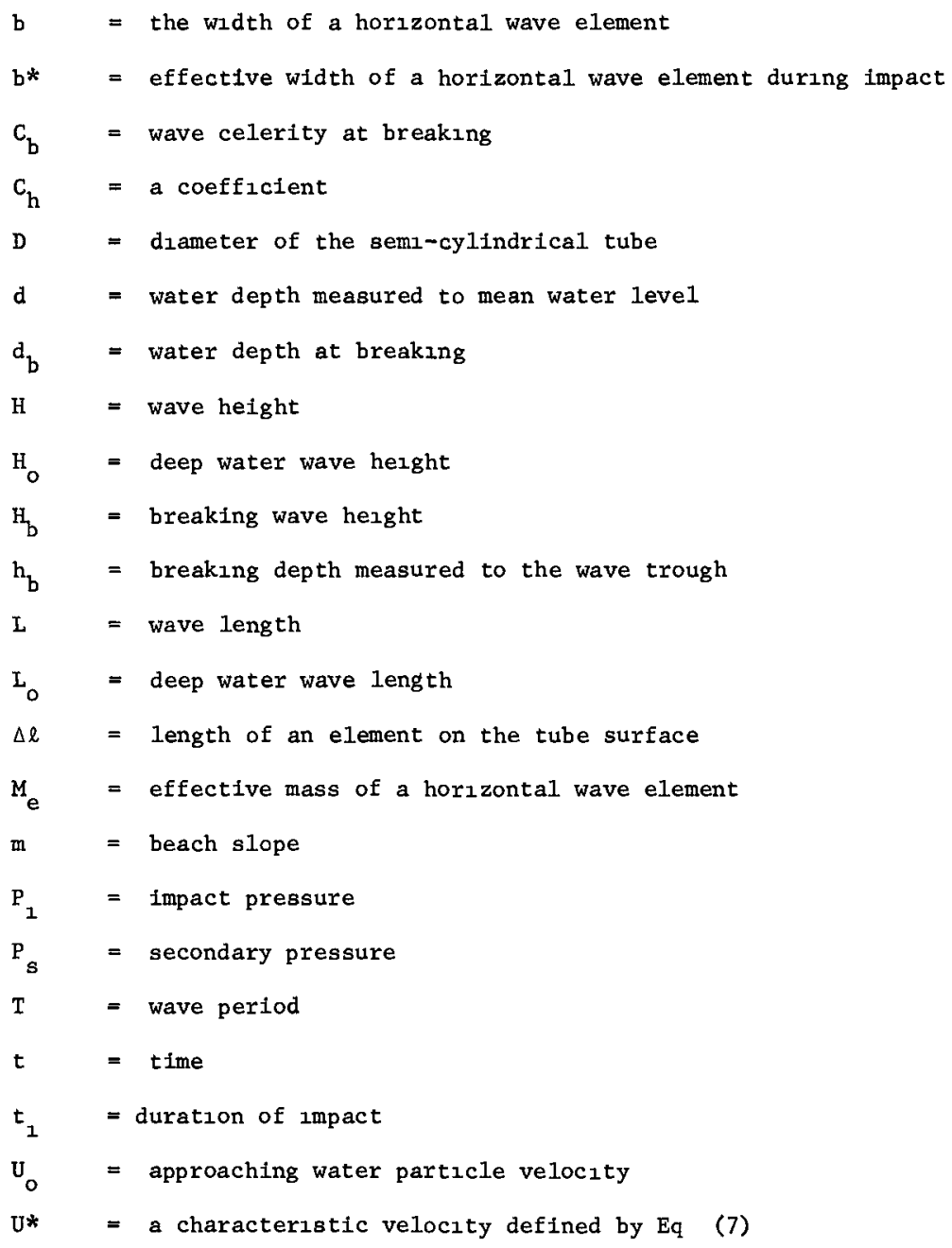




$$
\begin{array}{ll}
U_{r} & =\text { radial velocity component } \\
\rho & =\text { mass density of water } \\
\theta, \alpha & =\text { as defined in Fig } 7 \\
\gamma & =\text { specific density of water }
\end{array}
$$




\title{
LIST OF FIGURES
}

\author{
Figure 1 Model set-up for the pressure test \\ Figure 2 The relationship between $H / T^{2}$ and $d_{b} / T^{2}$ \\ Figure 3 Samples of pressure recordings \\ Figure 4 Typical pressure varlations with respect to time \\ Figure 5 Instantaneous pressure distributions when the pressure \\ intensity at $\theta=0$ is maximum \\ Figure 6 Relationship between dimensionless impact pressure and the \\ wave steepness \\ Figure 7 Wave parameters during impact \\ Figure 8 Distribution of dimensionless secondary pressure around \\ tube surface \\ Figure 9 Pressure variation during wave downrush
}



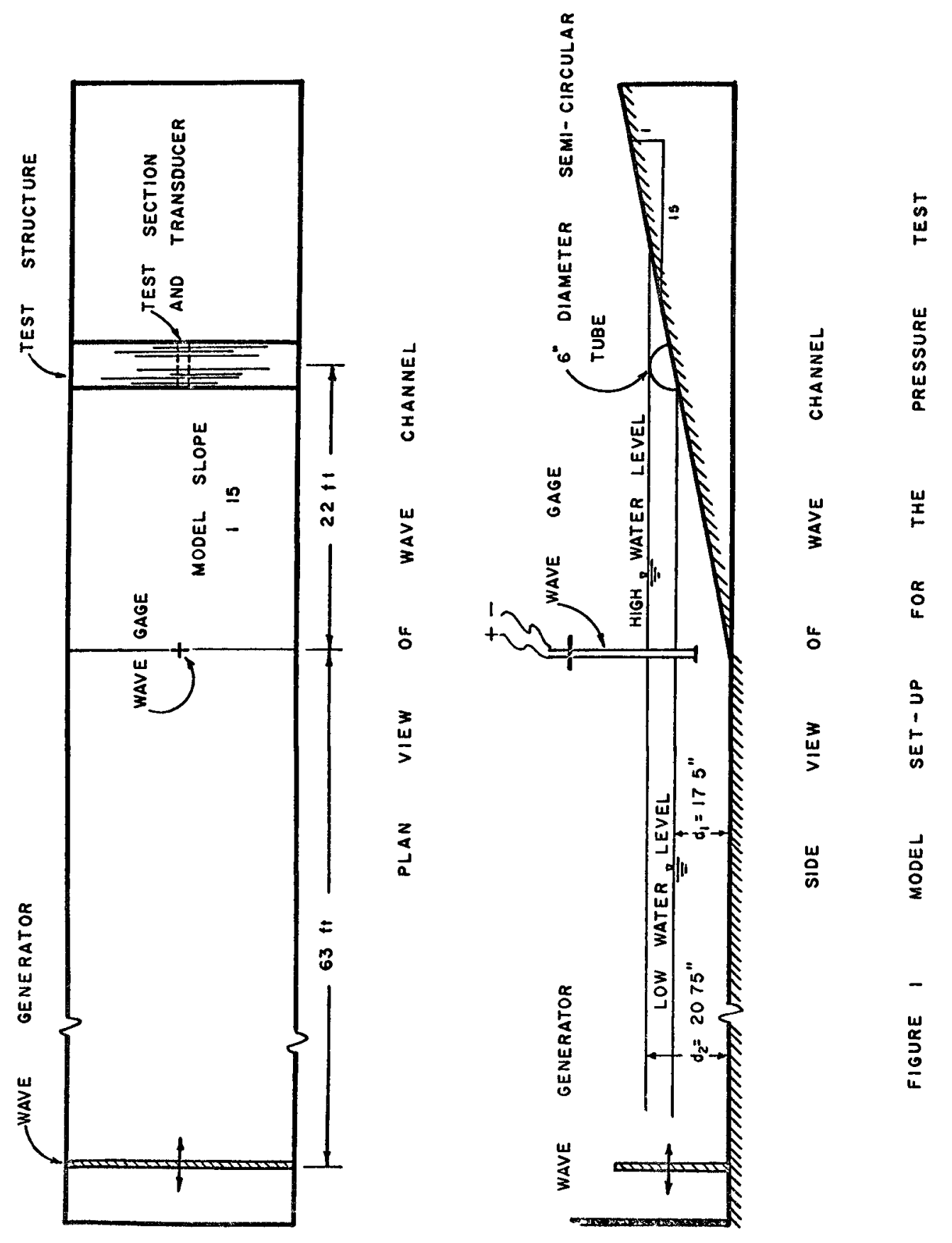


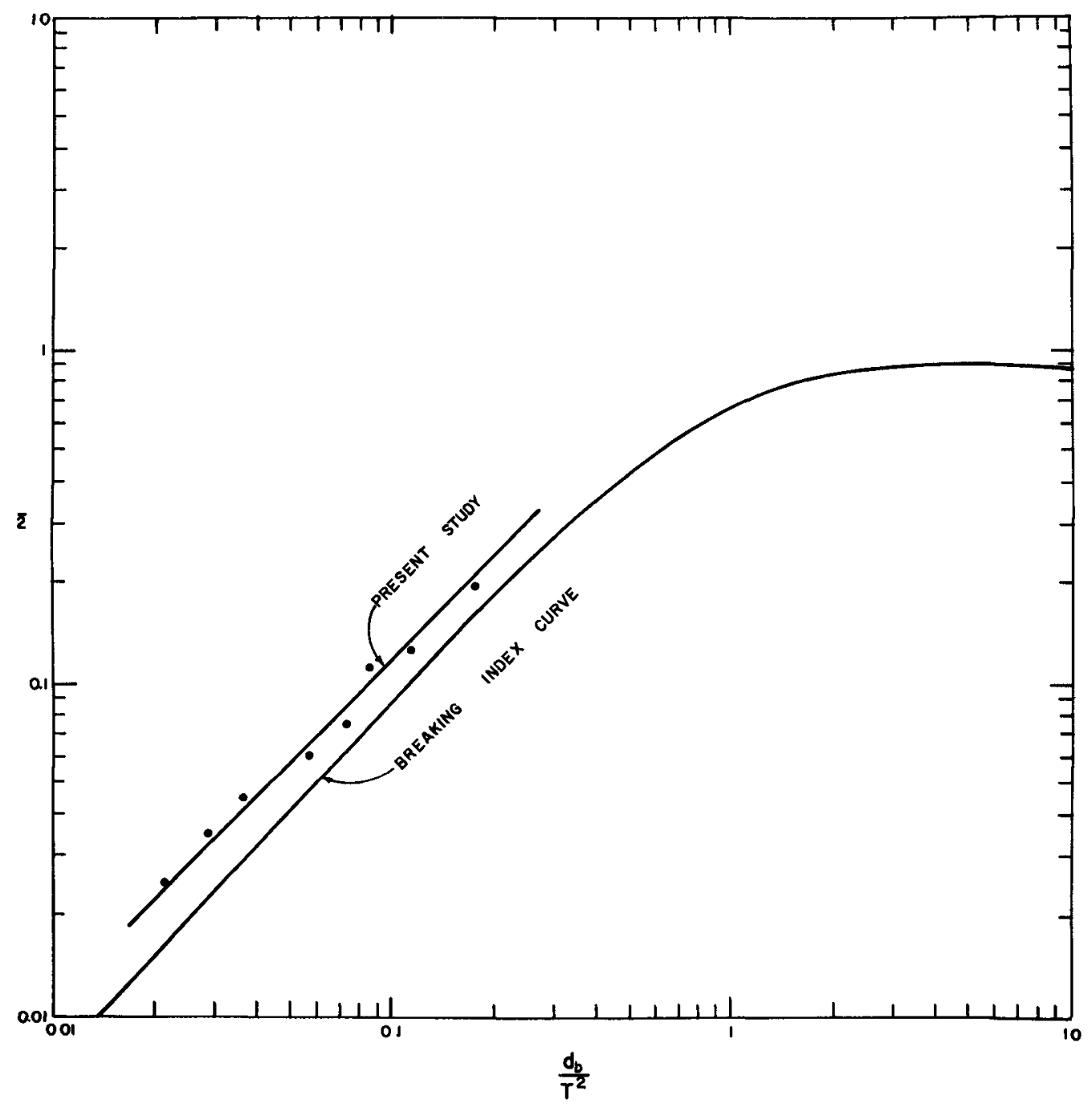

FIGURE 2 RELATIONSHIP BETWEEN $H / T^{2} \& \quad d_{b} / T^{2}$ 
PRESSURE SCALE IN PS I

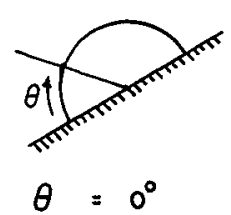

$\theta=30^{\circ}$

$\theta=60^{\circ}$

$\theta=90^{\circ}$

$\theta=120^{\circ}$

$\theta=150^{\circ}$

$\theta=180^{\circ}$

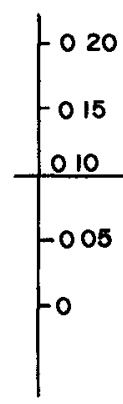

POINT OF IMPACT

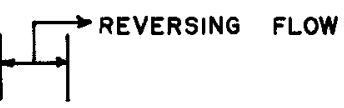

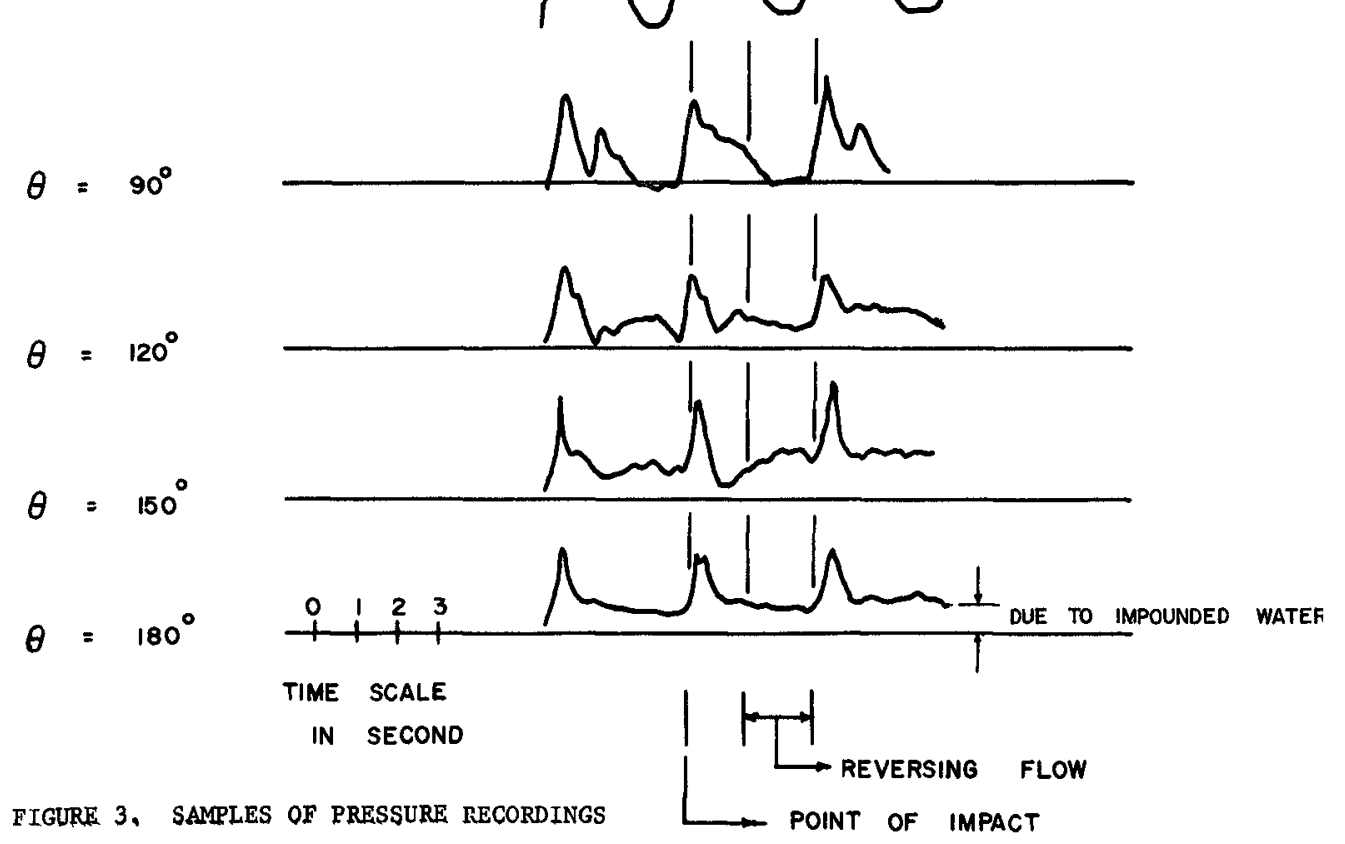


$\theta$

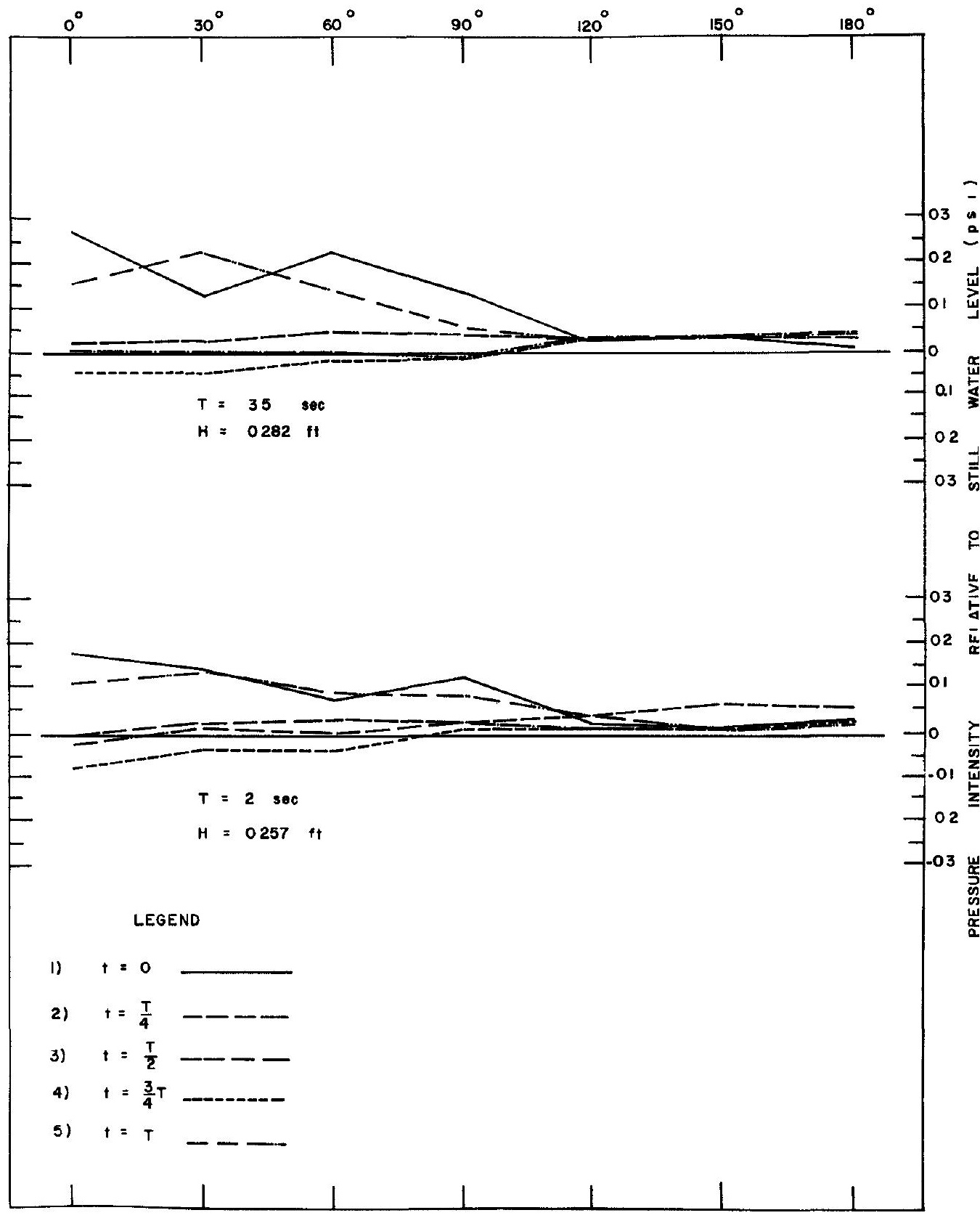

FIGURE 4 TYPICAL PRESSURE VARIATIONS WITH RESPECT TO TIME 


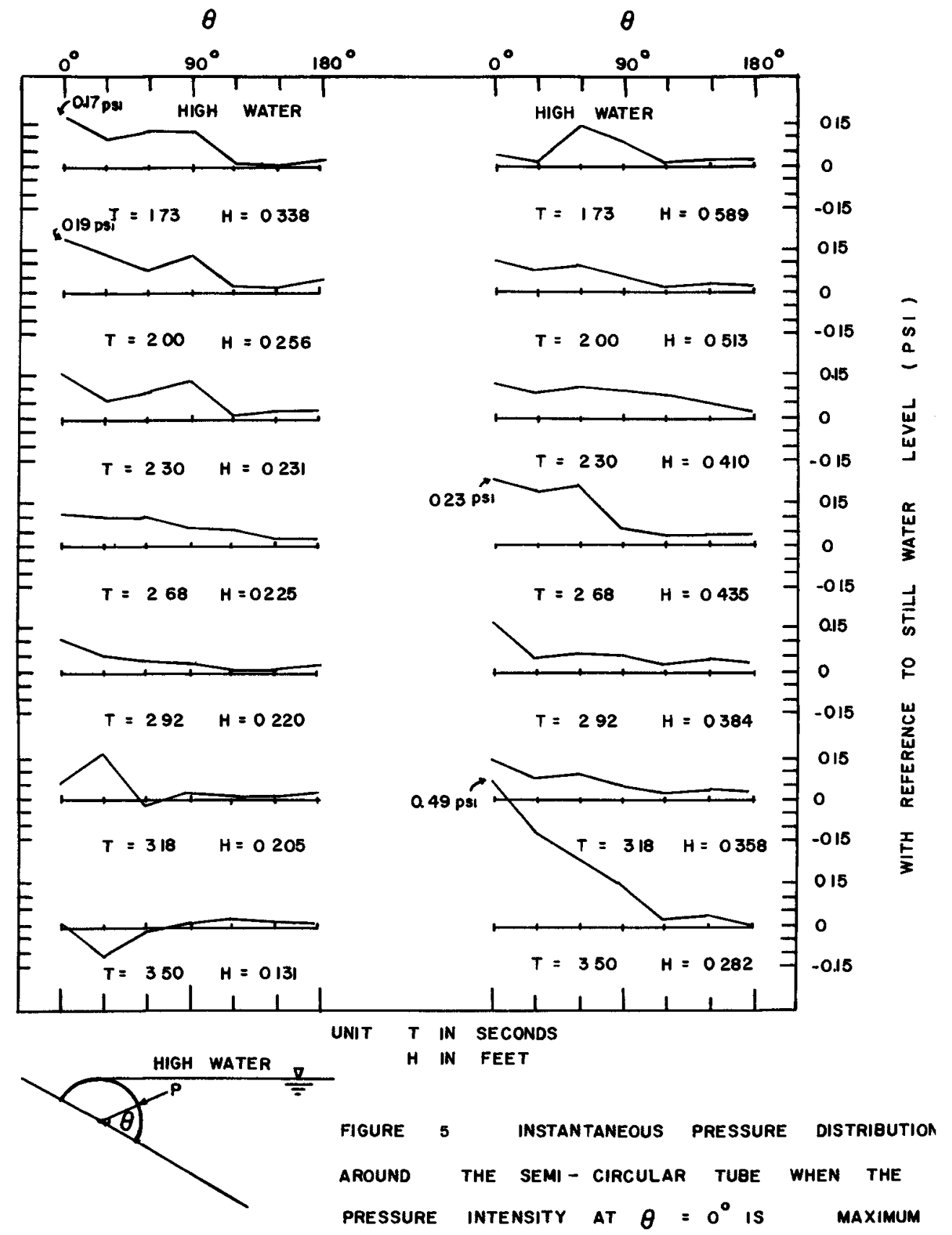




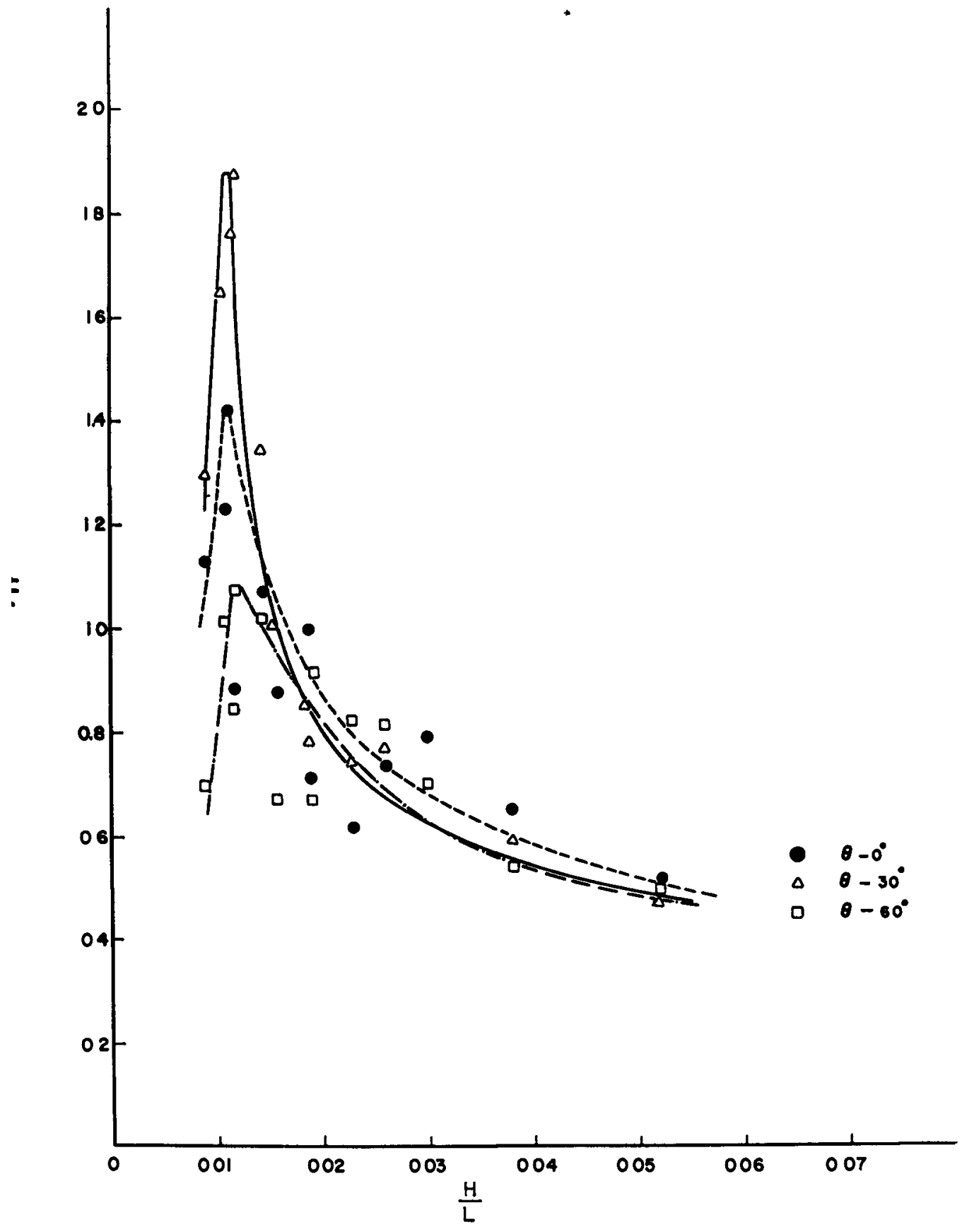

FIGURE 6 RELATIONSHIP BETWEEN DIMENSIONLESS IMPACT PRESSURE AND THE WAVE STEEPNESS 
1488

COASTAL ENGINEERING

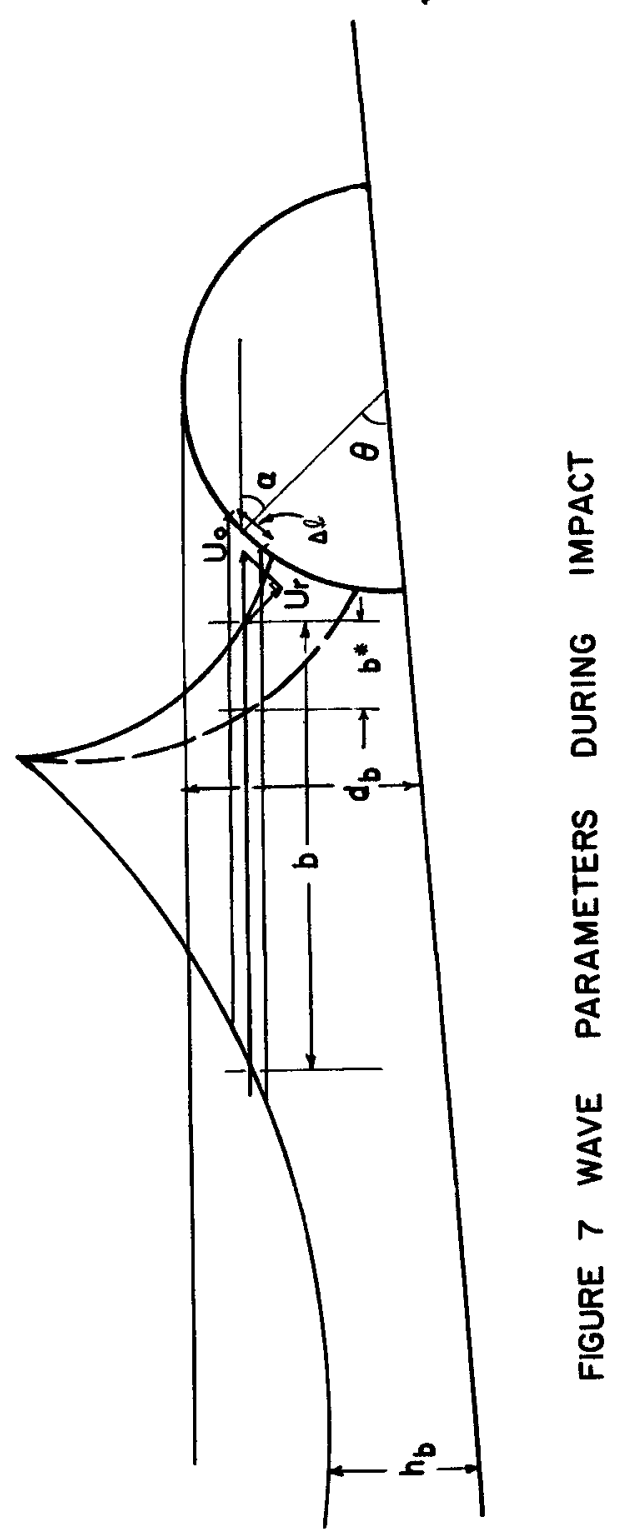




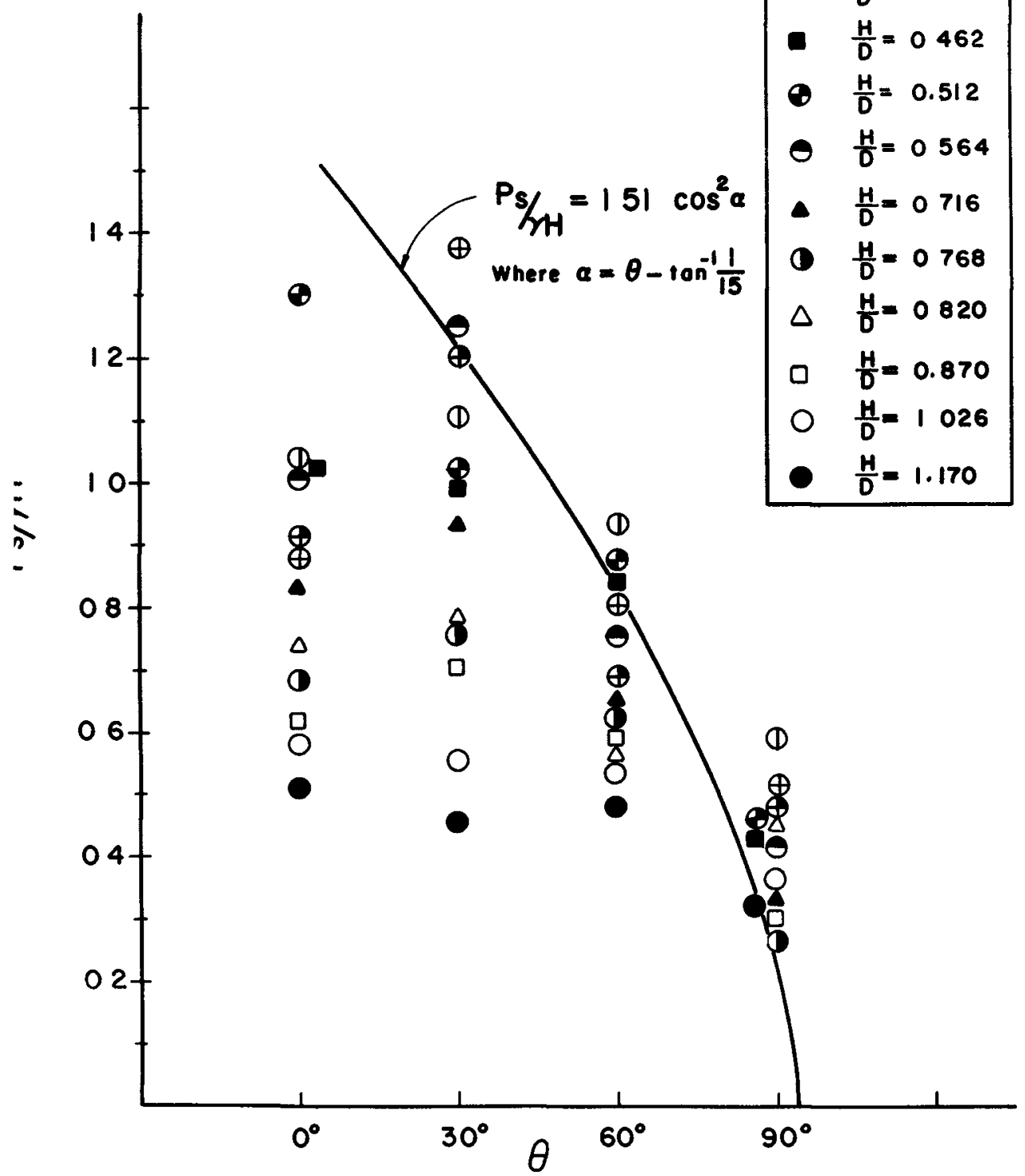

FIGURE 8 DISTRIBUTION OF DIMENSIONLESS SECONDARY PRESSURE AROUND TUBE SURFACE 


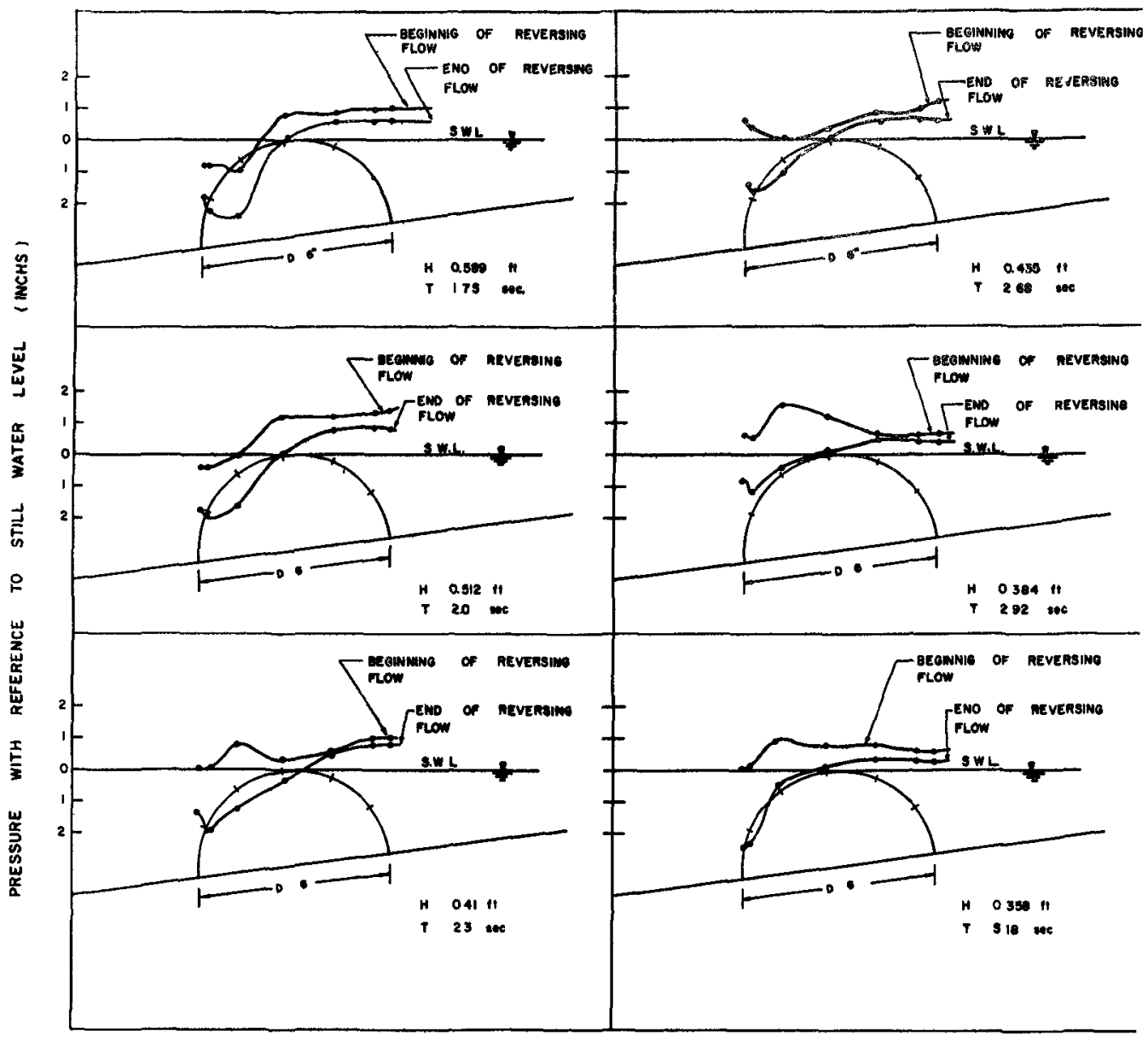

FIGURE 9 PREsSURE VARIATION DURING THE WAVE DOWNRUSH 


\title{
CHAPTER 91
}

LOADINGS ON LARGE PIERS IN WAVES AND CURRENTS

by

Hsıang Wang

Unıversıty of Delaware, Newark, Delaware

\begin{abstract}
Through scale-model tests, the forces, moments, and the pressure distributions, including impact pressures, were determined for large diameter plers extending through large-amplitude wave system in which current strengths vary Tests were performed at $1 / 100$ scale, simulating plers of the bridge for the Northumberland stralt Crossing, Canada. These plers are partially cylindrical with a base diameter of 100 feet Close to the surface, where the wave action is greatest, they are conical Waves up to 25 feet and current up to 4 knots have been reproduced in the laboratory, according to Froude similitude The tests results fully support the known concept that, when the pler diameter is large in comparison with the wave length, Inertial force becomes dominant Generally, the presence of current, elther with or agalnst the wave traln, results in a decreasing wave force semiempirical relations were developed for force predictions that require the experimental determination of a singular coefficient
\end{abstract}

\section{Introduction}

The problems of predicting wave forces on a cylindrical-shaped structure are often categorlzed according to the magnitude of the linear dimensions of the object and of the wave fleld Past advancement had been concerned mainly with the category of small-diameter cylinder under the action of small-amplitude waves (Morison et al, 1949, Goda, 1964, Bretschneider, 1955, 1957) Some attention has been given to the analytical treatment of the problem of a large-diameter plle (in comparison to the wave length) with small-amplitude wave (McCamy and Fuchs, 1954, Bonnefille and Germalne, 1963) These theorles still remain to be verified by experiment When 1 t comes to the problem of determining forces on a large-diameter cylinder protruding through a large-amplitude wave system, one quickly finds the lack of adequate information and reliable guldance The difficulty lay in general the lack of an adequate mathematical model on the one hand and the scarcity of experimental data on the other Therefore, the design of such a marıne structure often presents a unique task of lts own

The present study dealt with the prediction of wave forces on the plers of the bridge for the Northumberland Stralt Crossing These plers are partially cylindrical with base diameter equal to 100 feet, close to the surface, where the wave action is greatest they are conıcal According to the information provided by the Northumberland Consultants, 
Ltd, the design storm is based on a 1 percent risk of occurrence once in 100 years resulting in wind of $95 \mathrm{mph}$ on the northwest and southeast side of the crossing, respectively significant wave of 14 foot helght and 70 to 77 seconds is to be expected The extreme wave can be as high as 25 feet The design problem becomes unconventional Actually, this problem is further complicated because tidal current ranging from 0 to 4 knots is expected in the stralt This alters the wave kinematics and hence wave-induced forces Therefore, to obtain an eng ineering solution of this problem, once has little cholce but to rely on the outcome of a scaled model test

This experimental investigation is intended to provide sufficient information to meet the immediate need of the specific design problem in hand and at the same time to serve as foundation and verification for the development of a possible theory It is realized that few model setups portray perfect dynamic similarity with their prototypes, and that, for all practical purposes, only the pertinent part of the phenomenon need be closely approximated As a result of dimensional analysis, it is not the viscous action, but wave inertia that is to be evaluated in the present instance Therefore, in addition to the absolute magnitude of the force and moment exerted on the structure, it is the above postulation with which the present investigation is concerned

Dimensional Analysis and Scaling Laws

The problem in hand is that of determining from experiments on a model of reduced scale the dynamic forces imposed by shallow water waves of relatively high amplitude upon a system of plers of partly cylindrical, partly conical shape An important aspect is that of determining the scaling laws to be applied

When an obstacle, such as a pier, is placed in the channel, it experlences a hydrodynamic force This force can be considered as consisting of two components respectively related to wave velocity and acceleration The velocity related force in the horizontal direction is given by

$F(v, u)=\frac{1}{2} \pi \rho \int_{-d}^{n} c_{d}[D(z)][v(z)+u(z)]^{2} d z$

where $v$ is the current velocity, $u$ is the horizontal component of the wave orbital velocity, $\rho$ is the density of the water, $d$ is the water depth, $\eta$ is the wave ordinate, $D$ is the diameter of the plex, $z$ is vertical ordinate and $\mathrm{C}_{\mathrm{d}}$ is a drag coefficient The drag coefficient is a function of, among other parameters, Reynold's number which is defined as

$$
R=\frac{V D}{V}
$$

where $v$ is the kinematic viscosity The acceleration related force is given by

$$
F(u)=\frac{\pi \rho}{4} \int_{-d}^{\pi} c_{1}[D(z)]^{2} \quad[\dot{u}(z)] d z
$$


where $C_{1}$ is an Inertia coefficlent which depends mainly on the Ursell number

$$
U_{n}(z)=\frac{4 \pi^{2} \mathrm{D}(z)}{g T^{2}}=\frac{D(z)}{L}
$$

The Ursell number is actually an Inversed Froude number preferred in wave mechanics

The foregoing argument leads to the point that dynamic similarity requires that both Froude and Reynold's numbers be preserved Since simultaneous Froude and Reynolds scaling of reduced model is impossible, a cholce must be made To this end it is brought out that the ratio of the acceleration force to the velocity force at any level of the pler is

$$
\frac{\pi C_{1} \quad D(z)}{C_{d} H(z)}
$$

and since the Inertia and drag coefficients are of approximately equal value, the ratio reduces to $\pi D(z) / H(z)$ This ratio is always greater than unity even for the waves of extreme height and in the cylindrical portion of the pier much greater than unity, so that the acceleration force dominates the velocity force and the resultant hydrodynamic force is close to the acceleration components The consequence of this is that the scaling should follow the froude law

The integration of Eq (2), which represents the acceleration component of the hydrodynamic force, cannot be carried out unless explicit expressions of $\mathrm{C}_{1}$ and $\mathrm{u}$ can be obtalned The other alternative is to establish the functional relationship through dimensional analysis between this force component and other pertinent fluld, flow, and geometrical variables. By the latter method the following relation is obtalned

$$
\frac{F /\left(D^{2} d\right)}{\rho H / T^{2}}=E_{n}\left(\frac{D}{L}, \frac{V T}{D}, \frac{H}{d}, \frac{V}{(g d)^{1 / 2}}\right)
$$

Where $E_{n}$ is a modifled Euler number whlch represents a force coefficlent, and $T$ is the wave period, $H$ is the wave helght, and $g$ is the gravitational acceleration

Evidently the number of parameters involved makes a thorough investigation burdensome, further simplification is desirable McCamy and Fuchs (1955) obtained analytically the amplitude of the wave force per unit height acting on a cylinder at depth $z$ below the surface as

$$
F(z)=\frac{\rho H L}{\pi} \frac{\cosh [2 \pi(z+d) / L}{\cosh 2 \pi d / L} f_{A}
$$


where

$$
f_{A}=\frac{1}{\left[J_{1}^{\prime}(\pi D / L)\right]^{2}+\left[Y_{i}^{\prime}(\pi D / L)\right]^{2}}
$$

and $J_{1}$ and $Y_{1}$ are Bessel functions of the first and the second kinds, respectively, and the prime denotes first derıvative Accordingly the total force is

$$
F=\int_{-d}^{\eta} F(z) d z=\frac{\rho H}{\pi} \quad \frac{L^{3}}{T^{2}} \quad f_{A}
$$

When $\pi D / L$ is small,

$$
\text { and } F \sim \frac{\pi^{2}}{4} \quad \frac{\rho H}{T^{2}} \quad L \quad\left(\frac{\pi}{2}\right) \quad(\pi D / L)^{2}
$$

On the other hand, when $\pi D / L$ 1s large,

$$
f_{A} \sim\left(\frac{1}{2}\right)^{1 / 2} \pi \quad\left(\frac{D}{L}\right)^{1 / 2}
$$

and

$$
F \sim \sqrt{1 / 2} \frac{\rho \mathrm{H}}{\mathrm{T}^{2}} \quad \mathrm{~L}^{5 / 2} \mathrm{D}^{1 / 2}
$$

Phvsically, the last two equations imply that when $D / L$ is small, the pier diameter is the controlling factor, whereas, when D/L is large, the effect of wave length becomes predominant The present pler-diameter-to-wave-length ration ( $\pi \mathrm{D} / \mathrm{L}$ ranges from 1 to 15 ) is believed to fall into the latter category The force coefficient is redefined as

$$
\frac{F /\left(L^{5 / 2} D^{1 / 2}\right)}{\rho H / T^{2}}=C_{m}=C_{m}\left(\frac{V T}{D,} \frac{H}{d,} \frac{V}{(g d)^{1 / 2}}\right)
$$

For the case of shallow water, this equation simplfied further to

$$
\frac{F}{g^{5 / 4} \rho \mathrm{HT}^{1 / 2} \mathrm{D}^{1 / 2} \mathrm{~d}^{5 / 4}}=C_{\mathrm{ms}}=C_{\mathrm{m}}\left(\frac{\mathrm{VT}}{\mathrm{D}}, \frac{\mathrm{H}}{\mathrm{d}}, \frac{\mathrm{v}}{(g \mathrm{~d})^{1 / 2}}\right)
$$

The parameter VT/D is a Strouhal number written in the form preferred by Keulegan and Carpenter (1958), In the case of combined wave and current the more appropriate expression is $\left[V+U_{m}\right] T / D$, where $U_{m}$ is the maximum horizontal particle velocity in the wave $T$ this parameter characterizes the wake and eddy formations and is thus more directly related to viscous forces It has been shown that, in the present case, the viscosity plays a minor role Therefore, it is expected that the effect of the parameter 
on the force coefficlent is insıgnificant In fact, the value of $\left[\mathrm{V}+\mathrm{U}_{\mathrm{m}}\right] \mathrm{T} / \mathrm{D}$ varles from 1 to 3 for the range of varlables in the present experiment Keulegan and Carpenter were able to show that for the parameter as small as such no eddy shedding occurs consequently, the force coefficient remains practically constant Finally, the force relation is simplified to

$$
\frac{F}{g^{5 / 4} \rho H T^{1 / 2} D^{1 / 2} d^{5 / 4}}=C_{m s}\left(\frac{H}{d}, \frac{V}{(g d)^{1 / 2}}\right)
$$

Experumental Apparatus and Procedure

\section{Facllity}

The experımental study was carrıed out in the wave tank shown in Fig 1 Stable waves of steep proflle and of perıod ranging from 074 to 4 seconds for water depths as shallow as 6 Inches can be generated at the test section Current corresponding to controlled rates ranging from 500 to $4000 \mathrm{gpm}$ can be developed elther in or agalnst the direction of wave propagation A current deflector is fitted in way of the ports in the converging section to reduce the up-swelling action of the flow entering from the ports A spending beach of 120 slope is installed at the end opposite from the wave generating section, to reduce wave reflection and to smooth the velocity distribution when the current is made to flow opposite the wave direction

\section{Pler Model}

The pier model (Fig 2) is made of aluminum at a scale of $1 / 100$ that of the prototype It consists of four detachable sections so that, as water depth is varied, the position of the calm waterline remains relatively unchanged and approximately 15 to 2 inches above the top of the cylincrical portion of the pier The pier model is fitted with three adaptors for dlaphragm-type pressure transducers, two in the conlcal section and one in the uppermost section of the cylindrical body Each cylindrical section of the model is also tapped for cavity-type pressure transducers Generally, pressure transducers are sensitive to temperature fluctuations When the pressure fluctuations to be measured are small, as in the present tests, temperature fluctuations can have a pronounced effect on the results To minimize the possible error so introduced, the temperature of the transducers is matched to that of the water in the tank by continuously running a thin fllm of tank water over the transducers

Two force gages are mounted to a 2-1nch by 1/4-inch flat bar which is fixed on the side wall of the wave tank and protrudes into the pier through the opening at Its top These gages are for the measurement of the magnitude and point of application of the horizontal hydrodynamic force exerted on the pler The lower gage is fixed to its flat-bar support and allowed to float in a force-transmitting adaptor which is fixed 

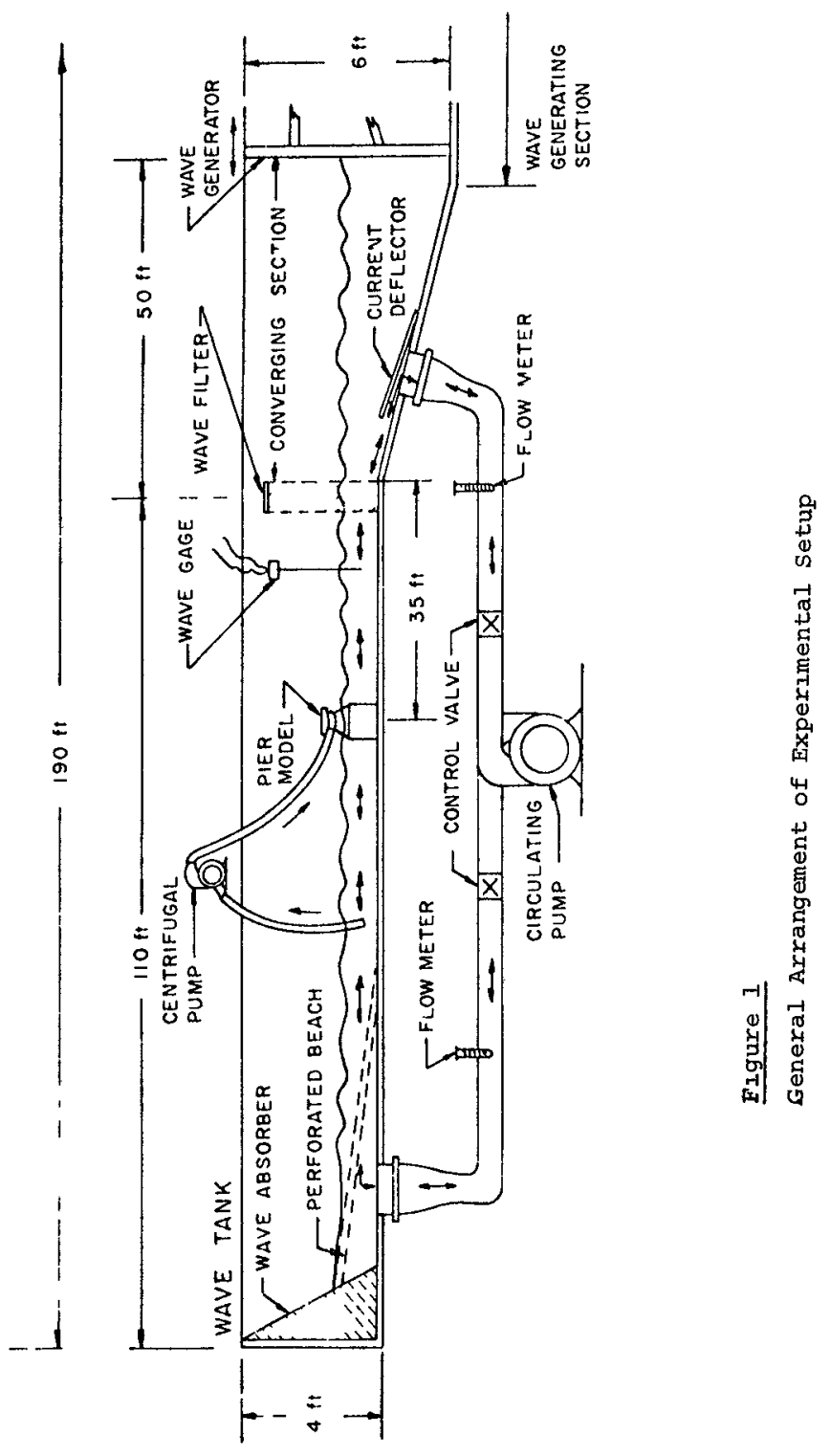


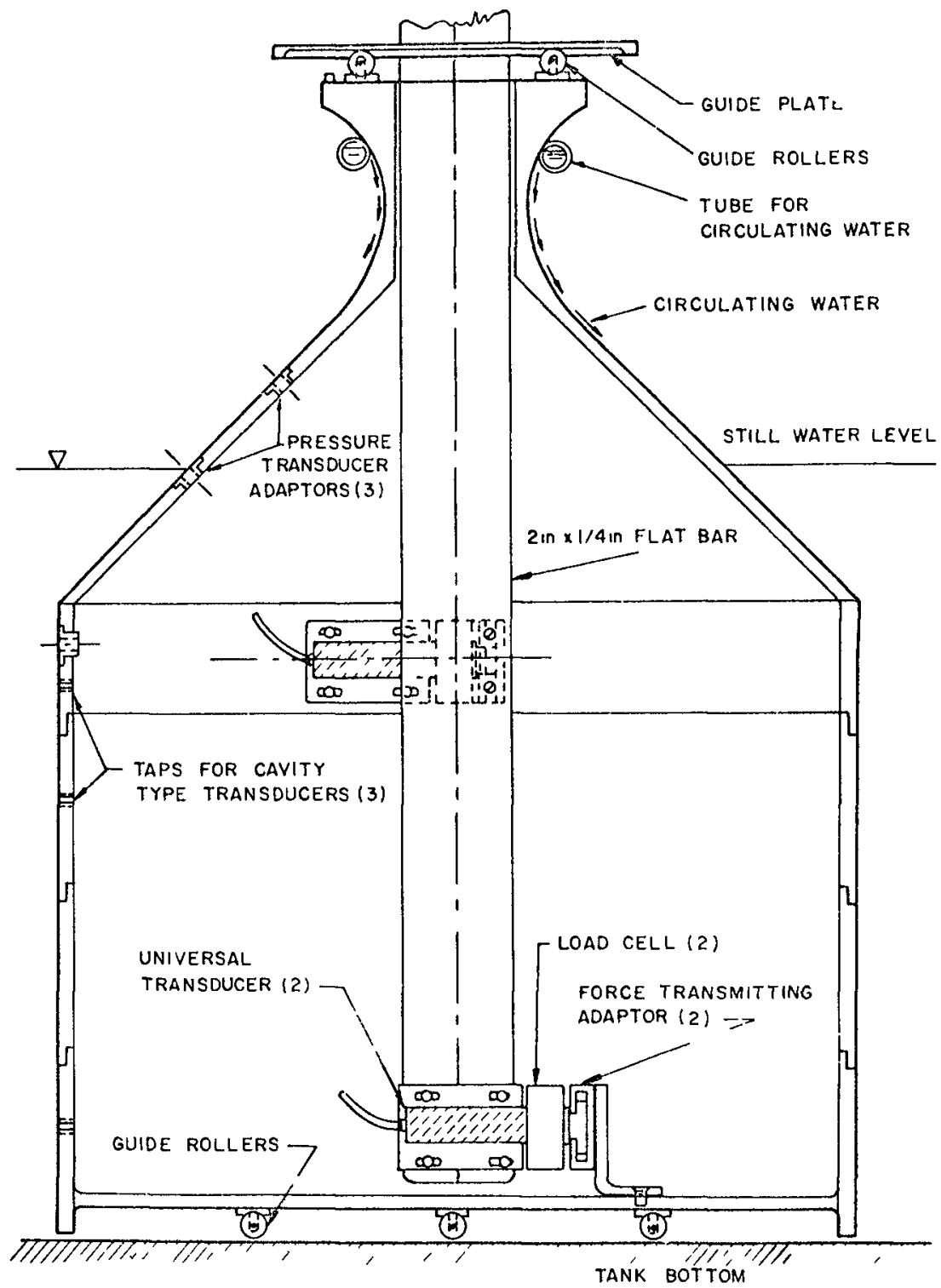

Figure 2

Pler Model 
to the pler and designed to transmit only tension or compression in the horlzontal direction The upper gage is mounted in a reverse fashion, It is flxed to the pler and allowed to float in an adaptor fixed to the flat bar (Fig 2)

\section{Instrument}

The flush dlaphragm pressure transducers and the cavity pressure transducers are both of the resistive type and manufactured by Statham Instruments, Inc The former, whth a pressure range of 0 to $5 \mathrm{psig}$ and a natural frequency of $35 \mathrm{KC}$, are used to measure the wave pressure on the pier in the region where waterline fluctuates and impact is likely to happen, whereas the latter, whth a pressure range of 05 psi differentlal pressure, are used in the region that is always fully submerged

The force gage consists of a Statham Unlversal Transducing cell of Model UC3 and a load cell adaptor with a load range of 50 pounds The Universal Transducing Cell is ltself a basıc sensing element made from strain gages and is capable of measuring a varlety of physical parameters by using different adaptors The load cell adapter 1s, of course, as its name implies, an accessory for transmitting forces

The water surface elevatıons were measured by resistance type gage consisting of a pair of surface-plercling parallel wires, across which an excitation voltage was impressed The corresponding voltage variations caused by fluctuations of the wetted line on the gage were recorded in wave-forms on a paper by a Sanborn Recorder

The current-velocity distributions across the test section were measured by conventional 0 25-inch Pltot tubes in conjunction with precision manometer whlch can be read to 0001 inch

\section{Procedure}

The magnitude and point of application of the horlzontal force were determined for the various combinations of wave and current characterlstics of Table 1 In these tests, the model of the pler was first made quasi-neutrally buoyant by ballasting The model was then suppressed into the water by a horlzontal bar mounted fixed to the side wall of the tank, but clear of the tank bottom Gulde rollers between the model and the bar confined the pler model to move in the horlzontal direction The force gages were mechanlcally blased to one-half of their full range so that they would respond in both tension and compression to a 25-pound maximum value These gages were callbrated by applying through a set of pulleys known horlzontal forces at the midpolnt between gages

The hydrodynamic pressure distrıbutıon was also determined for the combination of wave and current characteristics of Table 1 and for water depths of 6 inches and 12 inches corresponding to 50 and 100 foot full scale In these tests, the model of the pler was mounted so as to be 


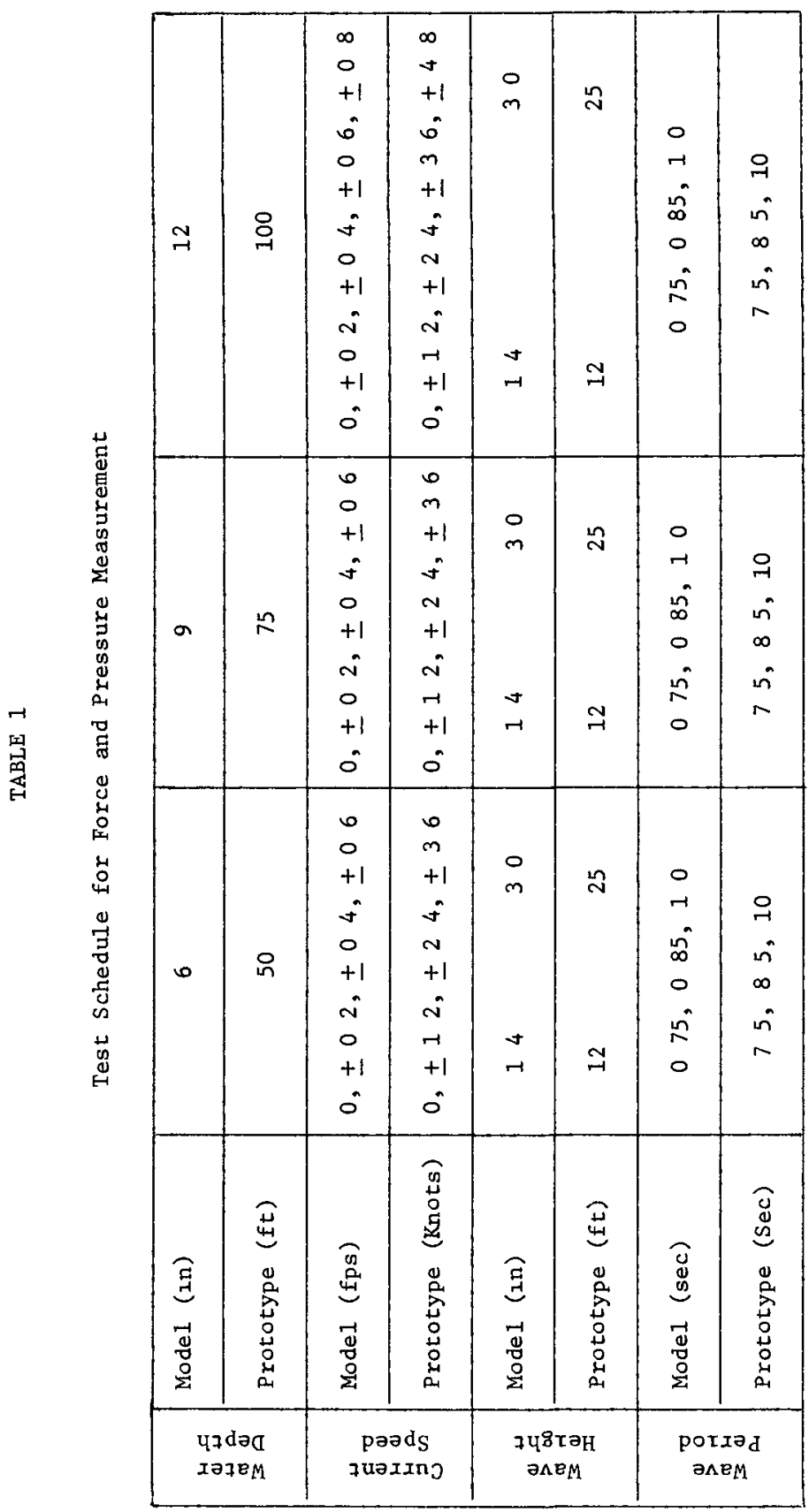


fixed to the tank The pressure was measured at five points along a merldan Measurements were made for 30-degree increments in polar angle with respect to current and wave direction

The impact pressure was sensed by transducers of the flush diaphragm type since in the region of impact the transducer is alternately wet and dry Because of the short duration of the impact, the signal transmltted by the transducer, after belng amplified, was displayed on an oscliloscope and photographically recorded

\section{Test Results and Discussion}

\section{Wave Force}

A typlcal time record of the wave force exerted on the pler along with the history of the wave measured alongside the pler is shown in Fig 3 From these records it is possible to determine the phase of the wave, with reference to the centerline of the pler, at which the wave force is a maximum This maximum occurs when the wave node coincldes with such centerline, for whlch condition the velocity-dependent force is a minimum Thus, the maximum wave force is almost solely of acceleration-induced loading This result supports the argument made under Dimensional Analysis and Scaling Laws

It has been shown that the force coefficlent defined in Eq (2) is a function of the $\mathrm{H} / \mathrm{d}$ and $\mathrm{V} /(\mathrm{gd}) \mathrm{I} / 2$ The effect of wave steepness $(\mathrm{H} / \mathrm{d})$ on the force coefficlent is found to be insignificant, at least in the tested range of $\mathrm{H} / \mathrm{d}(\mathrm{O} 08-04)$

In the case of shallow water, in the present experiment, the parameter $\mathrm{V} /(\mathrm{gd}) 1 / 2$, whlch 1 s the determining the influence of current on wave kinematics, although just how this parameter is related to wave dynamics 1s, as yet, somewhat unexplained The experimental results of $C_{\mathrm{ms}}$ plotted versus $\mathrm{V} /(\mathrm{gd}) 1 / 2$, are shown In Figs 4,5 , and 6 for water depths of 6,9 , and 12 inches respectively The heavy solid lines in these flgures are based on averaged values, The force coefficlent fluctuates in the region of positive $V /(\mathrm{gd}) 1 / 2$ A reverse current of increasing strength, however, always results in smaller force The values of $\mathrm{V} /(\mathrm{gd})^{1 / 2}$ whlch corresponds to maximum $\mathrm{C}_{\mathrm{ms}}$ fall in the reglon of 0 to 025

The foregoing arguments lead to the point that the wave force acting on the (full scale) pler can be calculated from

$$
F=C_{m s} \rho g^{5 / 4} H_{T}^{1 / 2} D^{1 / 2} a^{5 / 4}
$$

where $C_{m s}$ has to be determined by model test at the corresponding Froude number The wave height $H$ is the local wave helght with the absence of the pler and the current 
LOADINGS ON LARGE PIERS

1501

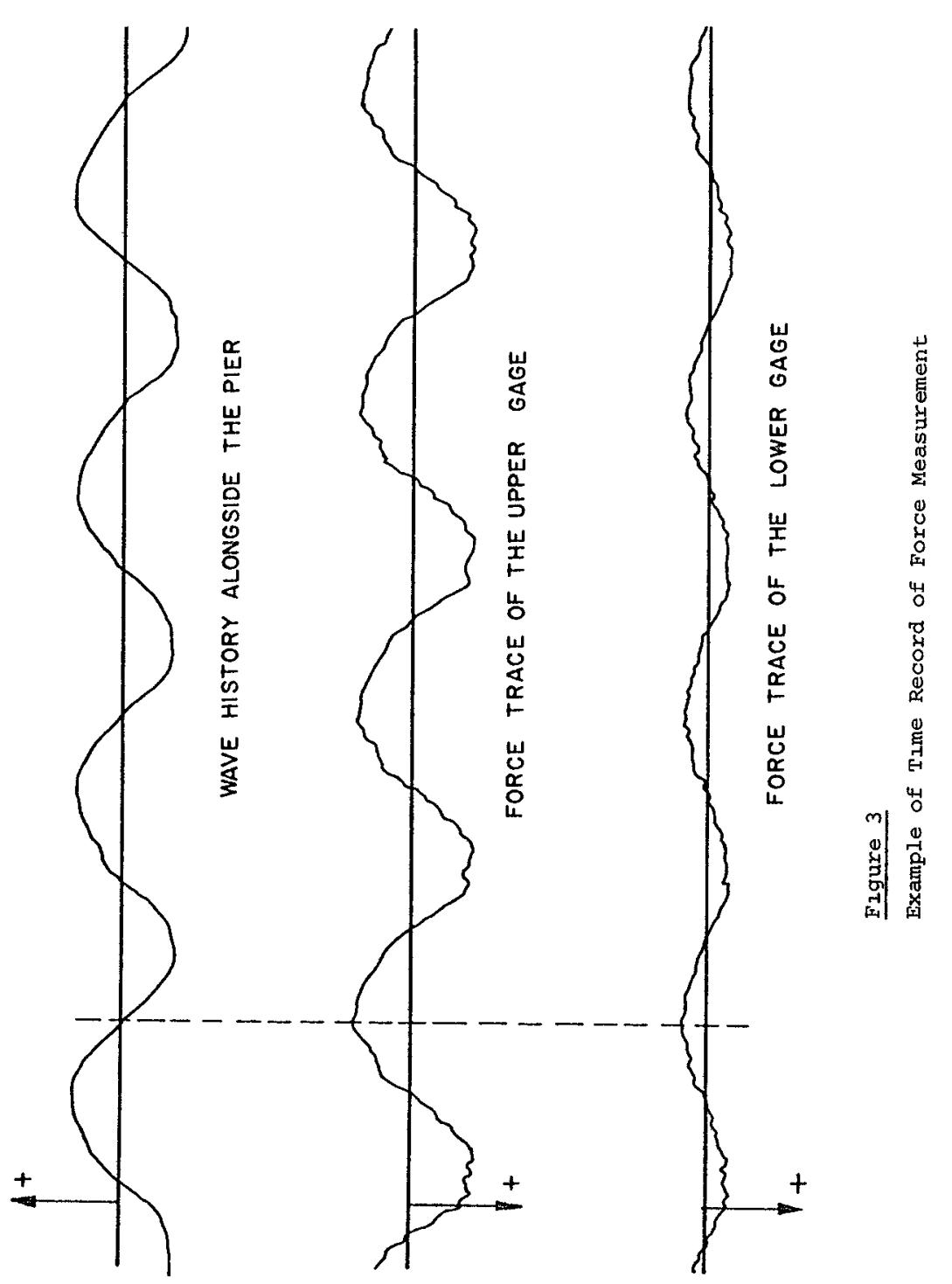




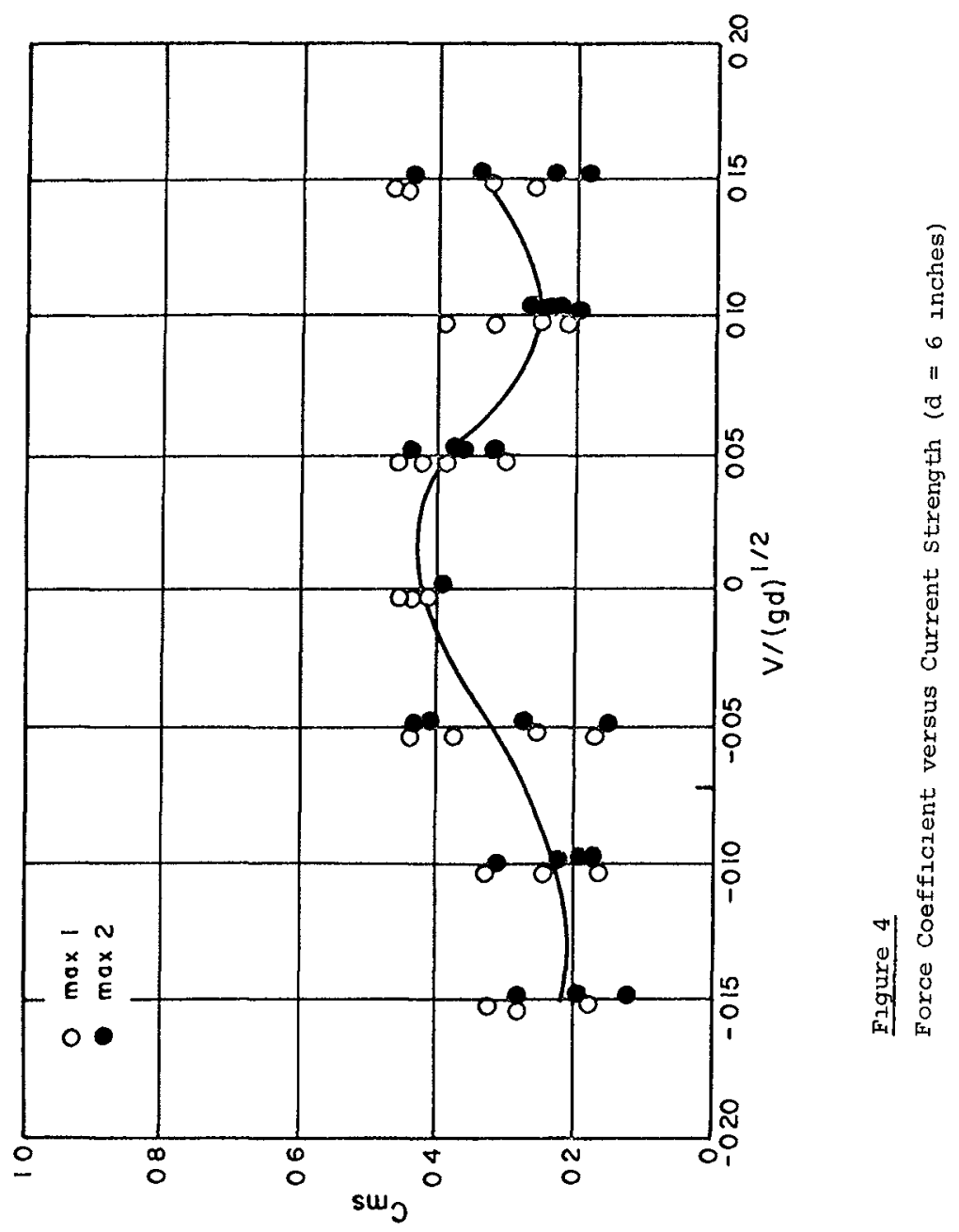




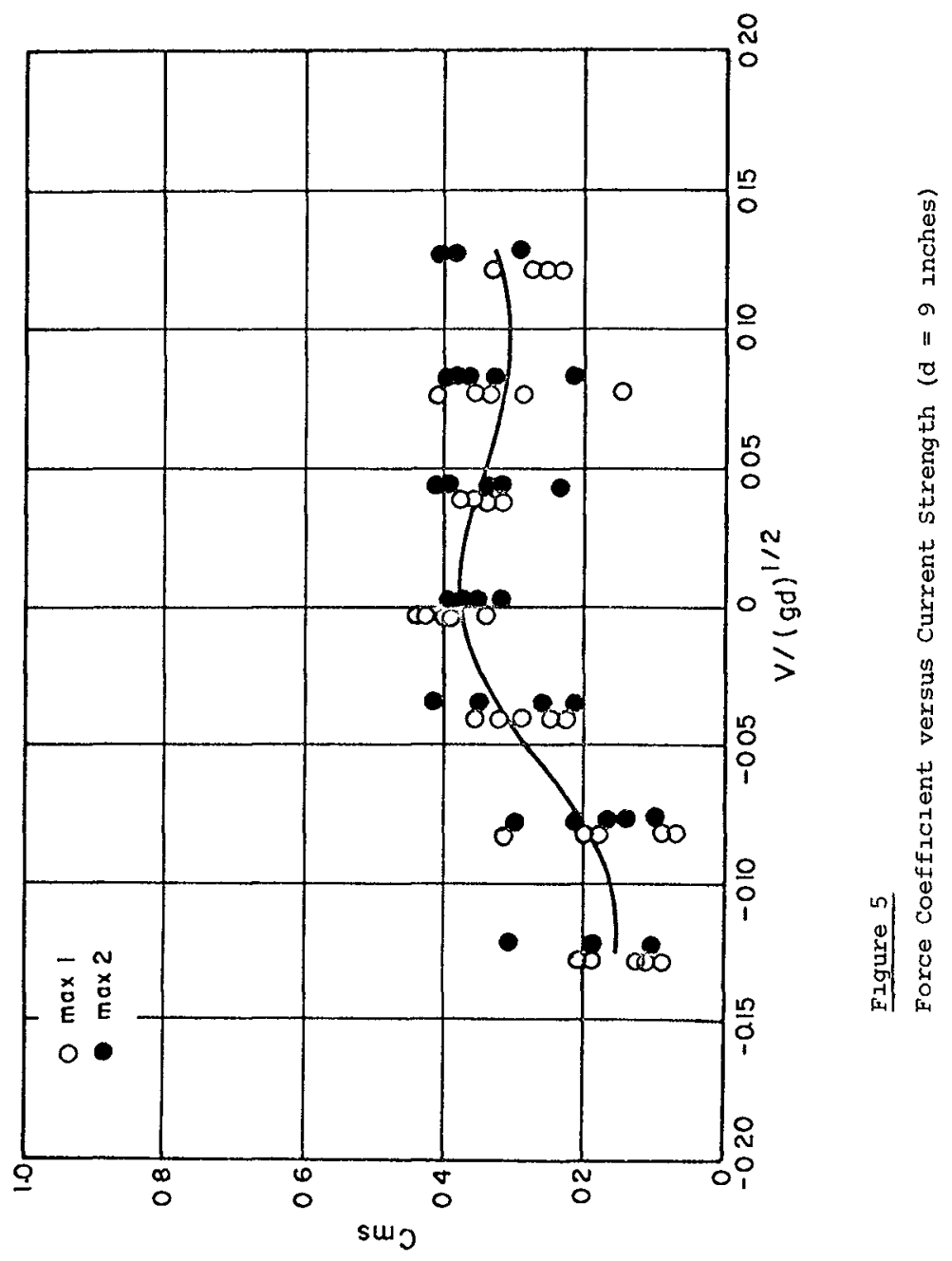




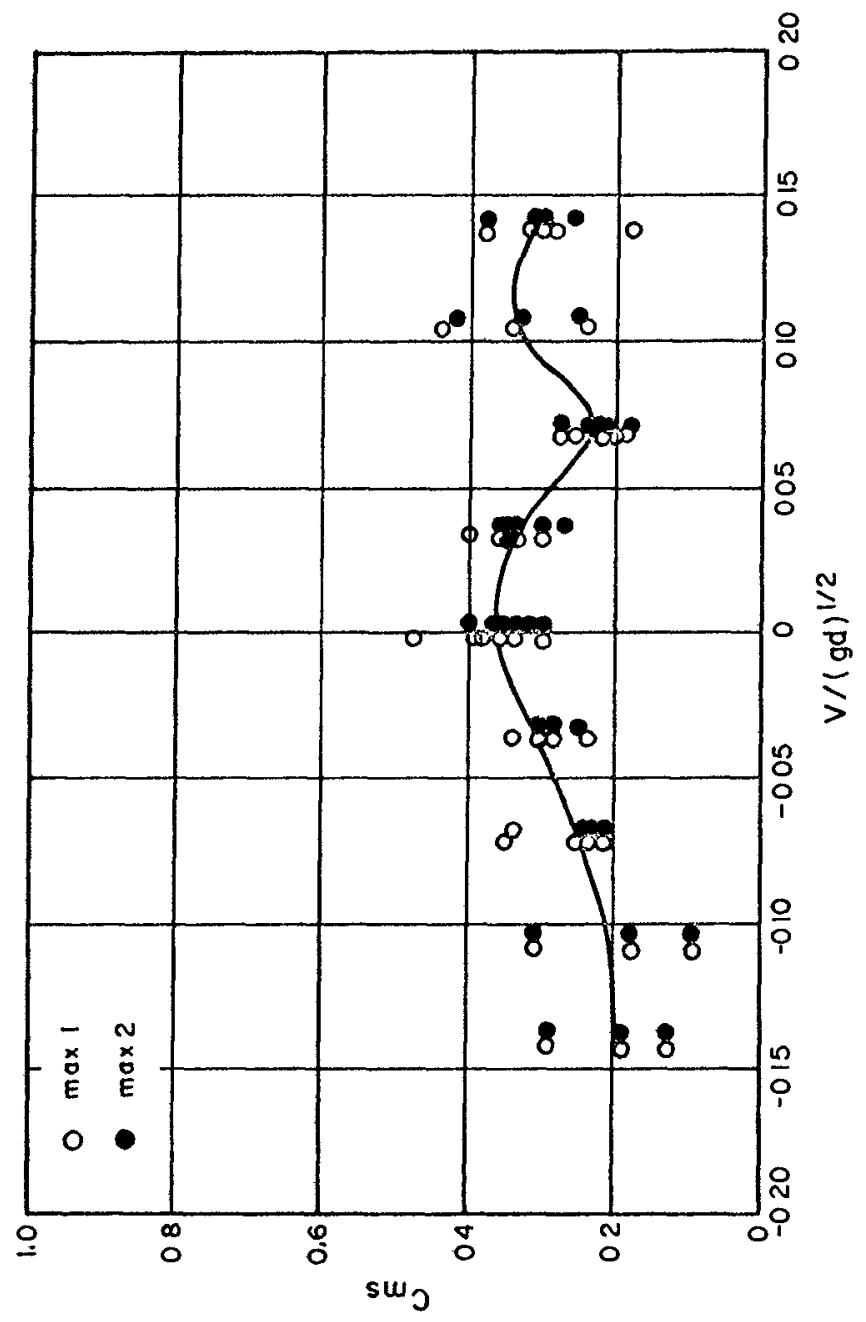


The wave force so obtained were generally larger than those computed by using the linear diffraction theory developed by McCamy and Fuchs (of the order of 20 - 30 per cent) It is also noted here that the experimental study dealt with a case of two-dimensional simulation in that the pler model was placed and tested in the middle of a stralght channel which is approximately 40 feet away from either end of the current inlets Thls arrangement discounted the effects of channel constriction near which wave and current encounters and the flow is el ther accelerated or decelerated depending on the flow directions Experimental results reveal that the wave height at the test section with the absence of the pler model is always diminished by the effect of the current As a consequence, the greatest hydrodynamlc loading exerted on the pler 15 obtalned when the current is null, since the wave force is directly proportional to the wave height

However, one must be cautious to extend this conclusion to cases where three-dimensional effects mentioned above might be pronounced In fact, addilional experiments were conducted in the tank and demonstrated that for long waves in shallow water the wave height was augmented by an oppose current

The point of application of the maximum horizontal force does not vary appreciably with change in test condltion The mean values of the point of application measured from the mud line and their standard deviatıons are listed below

\begin{tabular}{|c|c|c|c|c|c|c|c|c|}
\hline \multirow{2}{*}{$\begin{array}{c}\text { Water depth (d) } \\
\text { (Inch) }\end{array}$} & \multicolumn{4}{|c|}{ Mean $\left(\frac{z}{d}\right)$} & \multicolumn{4}{|c|}{ Standard deviation } \\
\hline & Max & 1 & $\operatorname{Max}$ & 2 & $\operatorname{Max}$ & II & $\operatorname{Max}$ & 2 \\
\hline 12 & 0 & 535 & 0 & 525 & 0 & 049 & 0 & 055 \\
\hline 9 & 0 & 585 & 0 & 555 & 0 & 053 & 0 & 073 \\
\hline 6 & 0 & 575 & 0 & 550 & 0 & 085 & 0 & 098 \\
\hline
\end{tabular}

The Max 1 and Max 2 are defined as in Fig 8

Wave Pressure

Pressure distribution about the pler was measured for water depths of 6 and 12 inches The pressure characteristics for both cases are quite similar Experimental results for the case of 12-inch depth only are presented here for the purpose of discussion Figure 7 1llustrates the instantaneous pressure about the pler at a certain vertical position Figure 8 summarizes the results of maximum pressures as a function of angular and vertical position

The gross characteristics of the pressure about the pler model does not differ signiflcantly from the case of a stralght circular cylindrical calsson in the absence of current (Lalrd, 1955) $=1 \mathrm{e}$, nelther the shape of the distribution curve nor the magnitude of the peak value ap- 


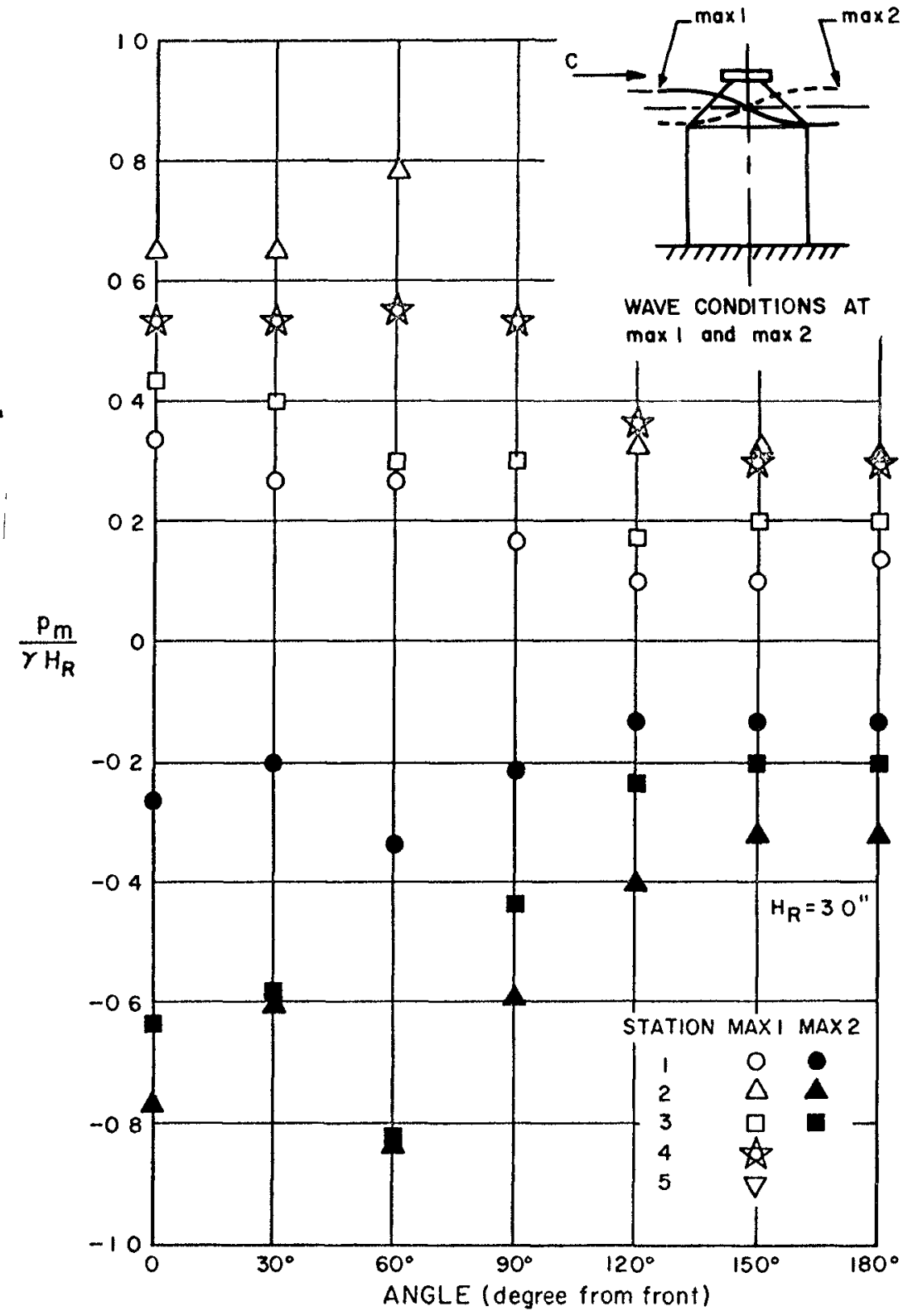

Flgure 7

Pressure About the Pler at a Vertical Section 


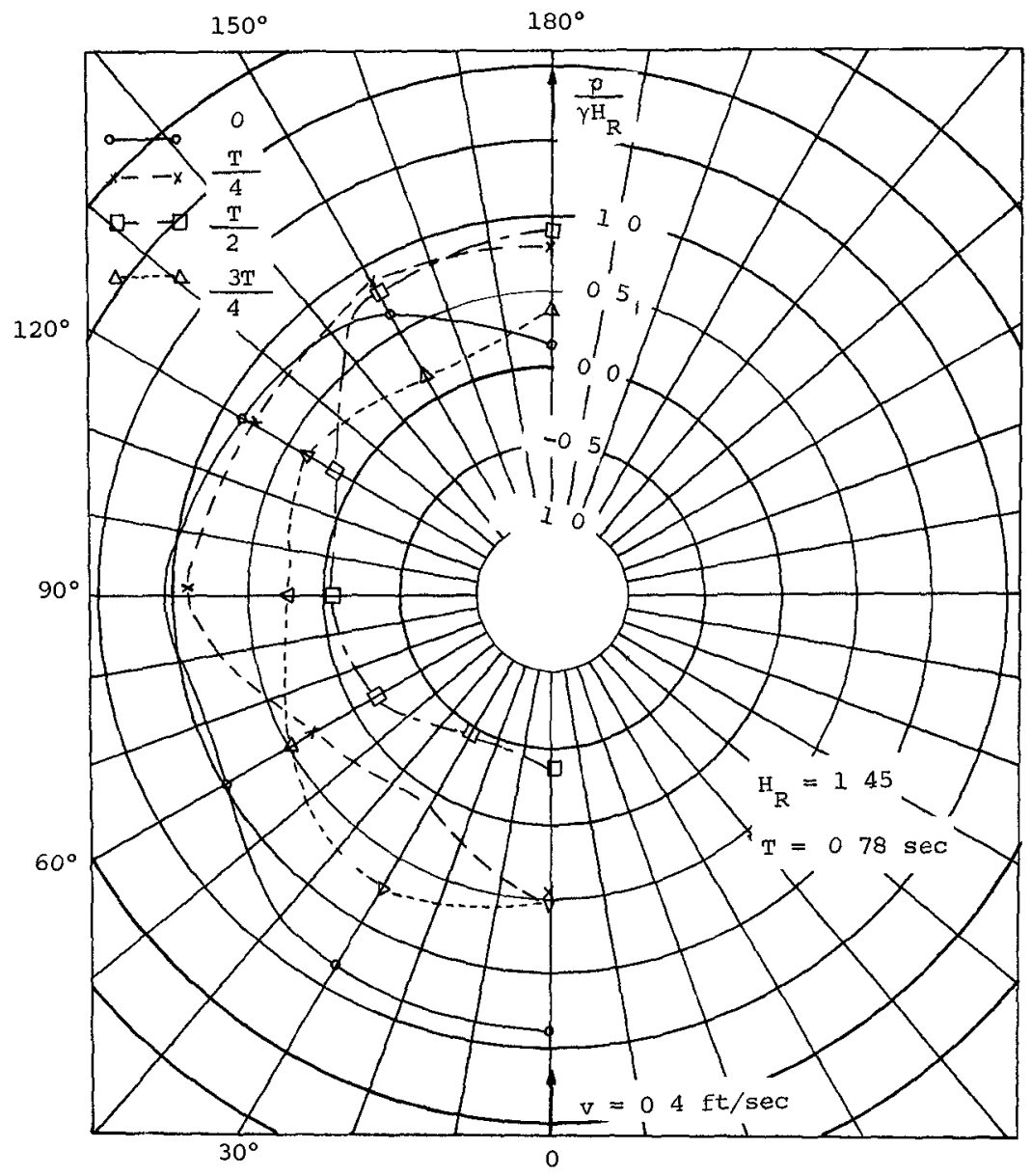

Figure 8 Maximum Pressures as a Function of Angular and Vertical Position
$(V=-02 \mathrm{ft} / \mathrm{sec})$ 
peared abnormal with respect to the case of stralght calsson The effects of the conlcal upper section of the pier model are two fold The wave pressure on this section itself is reduced considerably in comparison with that on a circular cylinder of comparable base diameter (2) The pressure on the cylindrical portion, immediately below the tapered section is also reduced, possibly due to the greatly reduced reflection by the conical section

Note that the maximum pressure amplitude occurs elther at or close to the forward stagnation point (facing waving and current) and is slightly smaller, or at most equal to, than that corresponding to a change in level equal to the wave height This means that the wave is almost completely reflected by the cylindrical portion of pier

The current affects the pressure distribution on the pier in the same manner as it affects the horlzontal force, the magnitude of the maximum pressure fluctuates for increasing strength of forward current but decreases for increasing strength of opposed current To a certain extend the presence of the current tends to deflect the wave front when waves approach the pier This effect causes the location of the maximum pressure to shift away from the stagnation point

The wave force exerted on the pier in the horizontal direction can also be obtained by integrating the pressure around the pler according to the following equation

$$
F=\int_{0}^{n(\theta)} d z \int_{0}^{2 \pi} \frac{D(z)}{2} p(\theta, z) \cos \theta \cos \beta d \theta
$$

or

$$
F=\gamma D H_{R} \int_{0}^{n(\theta)} d z \int_{0}^{\pi} \frac{D(z)}{D} \frac{p(\theta, z)}{\gamma H_{R}} \cos \theta \cos \beta d \theta
$$

where $\theta$ is the azimuth angle and $\beta$ is the angle of normal of the surface measured from mud line As an example, the maximum horizontal force for a specific case of $H_{R}=145 \mathrm{lnch}$ and $\mathrm{V}=0 \mathrm{ft} / \mathrm{sec}$ was computed graphically and compared with the result of direct measurement Upon graphical integration values of

$$
\int_{0}^{\pi} \frac{D(z)}{D} \frac{p(\theta, z)}{\gamma H_{R}} \cos \theta \cos \beta d \theta
$$

are obtalned These values when plotted versus the helght of pler (F1g 9) can be used to construct the horlzontal load distribution diagram on the 


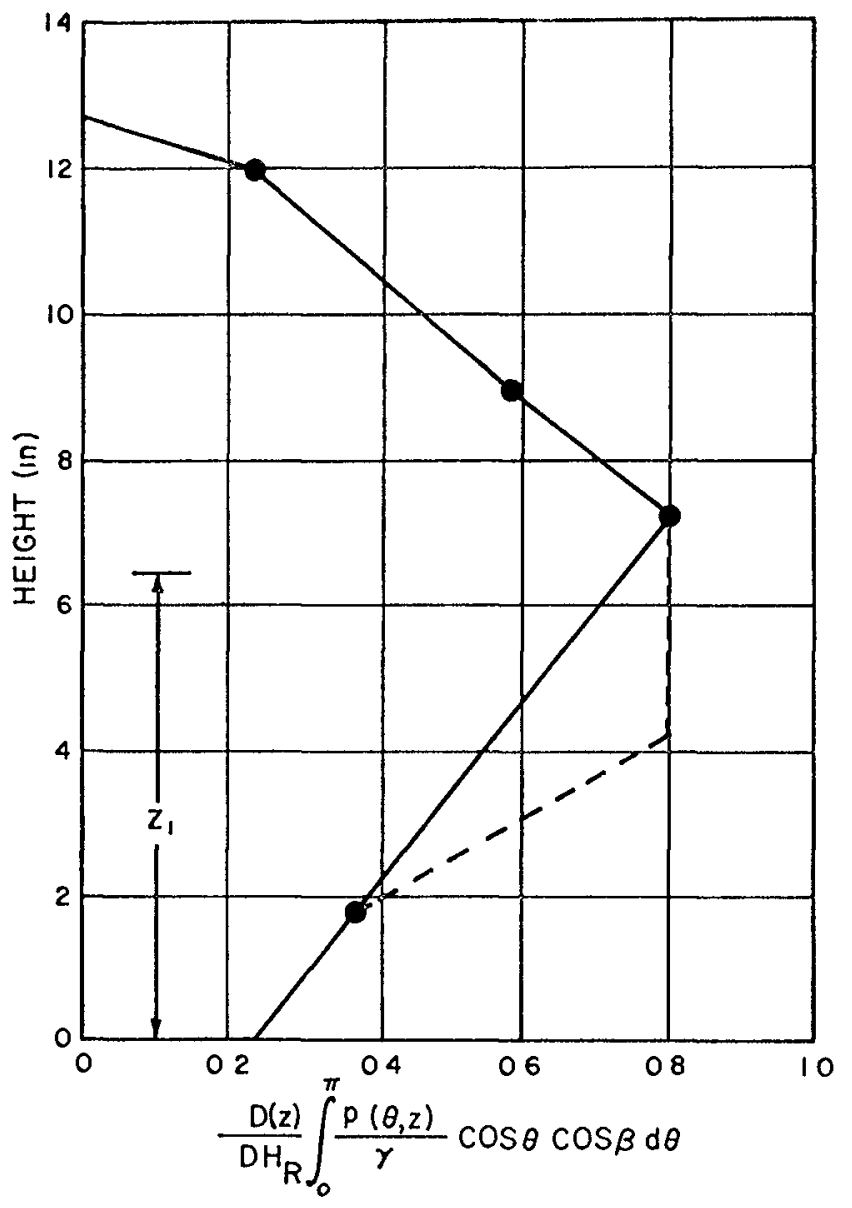

Figure 9

Load Diagram on Pler 
pler The magnitude and the point of application of the total force calculated according to the force diagram which connects the data polnts by straight lines are respectuvely, 485 pounds and 64 inches The corresponding values in the direct measurement are 54 pounds and 63 inches The force magnitude obtalned from pressure integration 1515 per cent smaller than directly measured This exror results from the over simplified load diagram A more realistic, yet still simply constructed, load diagram is proposed and shown by the dotted line According to this modifled load diagram, the magnitude and the points of application of the resultant force become 54 pounds and 61 inches

The load dlagram also reveals two facts (1) A predominate portion of the total load applies on the midale section of pier Thus the point of application becomes insensitive to the variation of load magnitude This fact was observed and noted in the direct-force measurement (2) The horlzontal load on the conlcal section contributes merely 10 per cent to the total load This leads to the explanation that the point of application of the horizontal force in the present geometrical configuration is considerably lower than that of a straight cylindrical cassion

\section{Impact Pressure}

With regard to the impact pressure measurement, Fig 10 shows a typical pressure traces displayed on the oscliloscope In the region of the upper tapered section, no impact was ever observed In the upper portion of the cylindrical section, impact of moderate magnitude (generally smaller than the maximum dynamic pressure) was observed occasionally when the backwash from the tapered section met the oncoming wave It was concluded that, for the present test conditions, the waves are neither steep enough or fast enough to Induce significant impact loadings

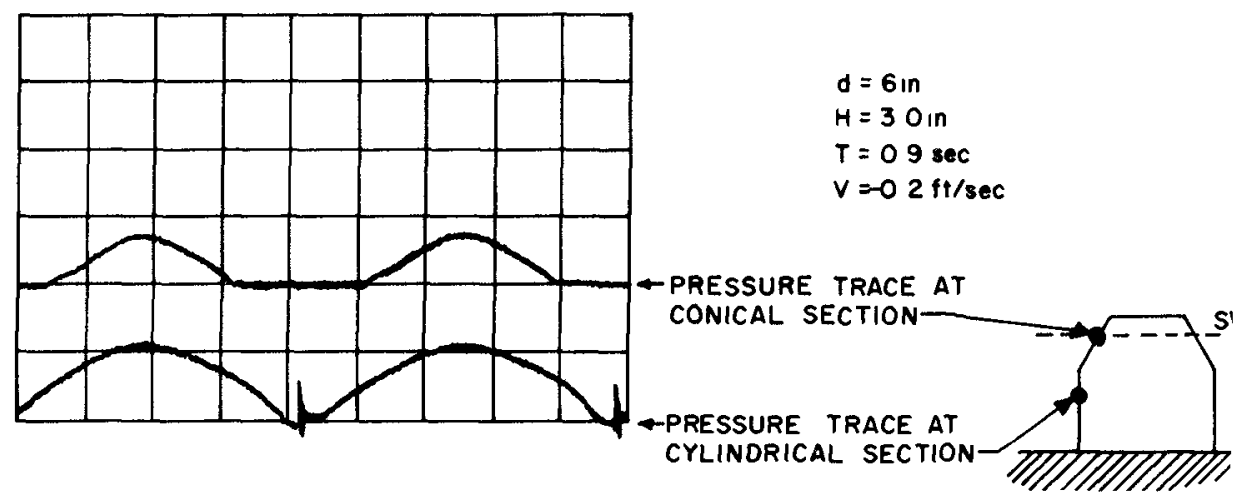

Figure 10

Examples of Tume Record of Impact Pressure Measurement 


\section{Conclusions}

Through scaled-model tests, the forces, moments, and the pressure distributions on the piers of the bridge for the Northumberland strait Crossing have been determined Geometrical simulations were partially fulfilled by placing the pier model in a wave channel that has a width of 4 feet The test results fully support the known concept that when the pler diameter is large in comparison with the wave length, wave inertial force becomes predominant Generally, the presence of current in either direction results in a decreasing in wave force unless the pler is located at the channel entrance where a relatively long wave encounters a strong current in a shallow water

As far as the wave force is concerned, the conical pier section serves three functions the total horizontal force is considerably reduced, the point of application of the horizontal force is lowered and becomes insensitive to the wave height, and the danger of wave impact is removed

Measurements conducted in the wave tank provided the single experimental coefficient required to complete the result of dimensional ana1ysis for this case Tnis coefficient is reasonably well behaved for a range of current strength and wave parameters Consequently, all results reported herein are considered applicable for design purposes to bridge piers and to structures of similar geometry

\section{Acknowledgement}

The program was sponsored by the Northumberland, Itd of Canada The permission of publication by the Department of Public Works of Canada is gratefully acknowledged The author is also indebted to Dr Manley St Denis for his contribution and Drs Thomas Monti, and Ean Ma Calg, and Messrs Carlo Pentassuglia and James Cowley for their fruitful discussion 
References

Bonnefille, $R$ and Germain, $P$, "wave Action on Isolated Vertical Cylinders of Large Dimensions," I A H R Congress, London, 1963

Bretschnelder, C L (1955), "An Evaluation of Inertia Coefficients in Wave Forces Experiments," Texas A \& M Research Foundation Tech Report No 55-2

Bretschneıder, C L (1957), "Evaluation of Drag and Inertıa Coefflclents for Maxımum Range of Total Wave Forces," Texas A \& M Research Foundation Report No 55-5

Goda, Y (1964), "Wave Forces on a Vertıcal Circular Cylınder," Port and Harbour Technical Research Institute, Japan, Report No 8

Keulegan, Garkis H, and Carpenter, Lloyd H (1958), "Forces on Cylinders and Plates in an Oscillating Fluid," Journal of Research, National Bureau of Standards, 60, 5, May 1958

Laird, A D K (1955), "A Model study of Wave Action on a Cylındrical Island," Transition American Geophysical Union, 36, 2 April 1955

Longuet-Hıggıns, $M \quad S$ and Stewart, $R$ W (1961), "The Changes in Amplitude of Short Gravity Waves on Steady Non-uniform Current," Journal of Fluid Mechanics, 10, part 4, June 1961

MacCamy, R C, and Fuch, R A (1954), "wave Force on Plles, a Diffraction Theory," U S Army Corps of Engıneers, Beach Erosion Board, Technical Memorandum No 69, December 1954

Morison, $J$, Johnson, $J W$ and O'Brine, $M$ P (1953), "Experimental studies on Forces on Plles," Proceedings of Fourth Conference on Coastal Englneering 


\section{CHAPTER 92}

SOME EXPERIMENTS WITH SAND-FILLED FLEXIBLE TUBES

by

Per Roed Jakobsen, M sc, Danısh Board of Maritime Works

and

Arne Hasle Nıelsen, M Sc, Danısh Instıtute of Applıed Hydraulıcs

\section{ABSTRACT}

Experiments with the use of long sand-fllled flexible plastlc tubes ("Sand sausages") have been carried out for coastal protection purposes at several exposed locations along the Danish coasts since 1967 In general the results have been encouraging

Several methods have been used to flll the tubes with sand A simple inexpensive hydraulic method for easy fllling of almost impermeable tubes on site has been developed by model and prototype experiments with promising results

As a result of the experiments it seems likely that sand-filled tubes may be used whth advantage as temporary stmetures, 1 e for full-scale pllot investigations and to solve acute problems, or they may be incorporated in more permanent structures

\section{INTRODUCTION}

Flexible sand bags have been used in hydraulic englneering practice for many years, but due to the poor quality of avallable materlals the use was malnly restricted until recent times to projects of non-permanent nature such as emergency flood control and protection of dikes

However, with the development of fabrics of durable, strong synthetic fibres of nylon, polypropylene, etc, new possibilities have arisen for more permanent use of large sand bags in more exposed locations than could previously be considered

The paper describes some preluminary experiments with long flexible sand tubes in coastal protection works that have been carried out in Denmark since 1967 by model and prototype experiments

\section{FILIING METHODS}

One of the major problems in the economical use of long flexible sand tubes is fllling the skin

Several Ingenlous devices and fllling methods have been developed for the filling of skins of permeable fabrics The sand is usually transported into the tube by hydraulic pumping and the water excapes through the permeable fabric, leaving the sand grains in the tube

In most cases the discharge tube is drawn backwards inside the tube with a speed corresponding to the rate of filling leaving the fllled tube behind ( $F 1 \mathrm{~g} \quad 1)$

A much simpler and more economical hydraulic filling method which allows filling of sand tubes to almost any length above or below the water surface from the "front" end of the tube has been developed sumply by using Impermeable instead of permeable fabrics 
When the mixture of sand and water flows into the impermeable flexlble tube, the sand settles out near the inlet until the cross-section has decreased so much that a small "rıver" is formed in the tube on top of the accreted material

\section{PERMEABLE TUBE}

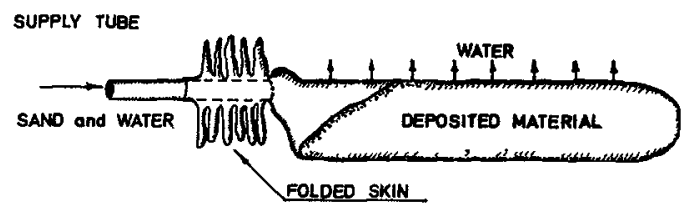

IMPERMEABLE TUBE

SUPPLY TUPE

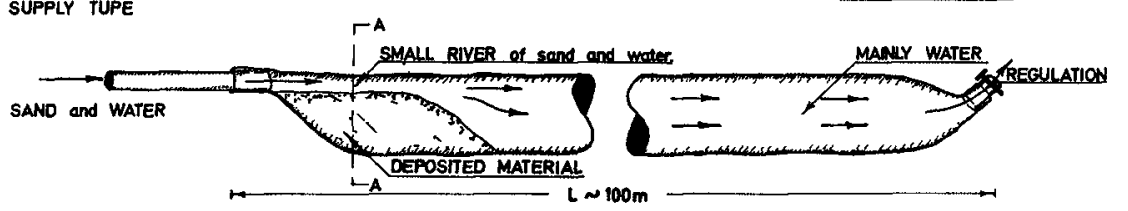

FIG 1 PRINCIPLES OF FILLING OF FLEXIBLE TUBES

The muxture of water and sand is now transported foreward in the tube and the sand grains settle out in front of the already accreted material, whlle the water leaves the tube through an opening at the far end By decreasing the outlet opening, the skin may be expanded by water pressure whereby an almost curcular, filled cross-section can be obtained

In laboratory tests the sand/watex mixture was injected by sandpumping, whlle in experiments in the field a different method was primarlly employed water was fed under high pressure through a type of diffusor into whlch the fllling material was added directly

The impression has been procured from the experiments that in principle almost infinitely long, large cross-section tubes can be filled by hydraulic pumping provided the skin is impermeable at the time of filling

\section{EXPERIMENTS IN NATURE}

While the filling method was developed by model experiments the resistance of fabrics to atmospheric conditions, ultraviolet sun rays, 1ce, human activity, etc, was investigated by full scale experiment in nature where various types of constructions were also tested under different conditions

Experıments were conducted in about 10 different locatıons, includIng sites on the exposed coasts of Jutland and on the more sheltered $15-$ land coasts of Funen and Zealand

The type of structures employed in natural locations so far has in most cases been groins, in some cases however revements and embankments have been bullt

Cross-sections of the various structures have been (1) a single 
tube, (2) one tube resting on two tubes, forming a triangular crosssection, and (3) two tubes placed at the sides of an interjacent sheet covering a sand-prism. Future plans call for experiments with multicellular cross-sections.

Tubes of a diameter of up to $100 \mathrm{~cm}$ have been filled to lengths of up to 100 meters, and at water depths of up to 3 meters.

Various methods have been tried to make the rubes in the structures behave as units. In some cases tubes have been lashed together by plastic ropes, in other cases tubes have been wrapped in filter sheets. Later experiments have involved weaving together the lower tubes in prism structures.

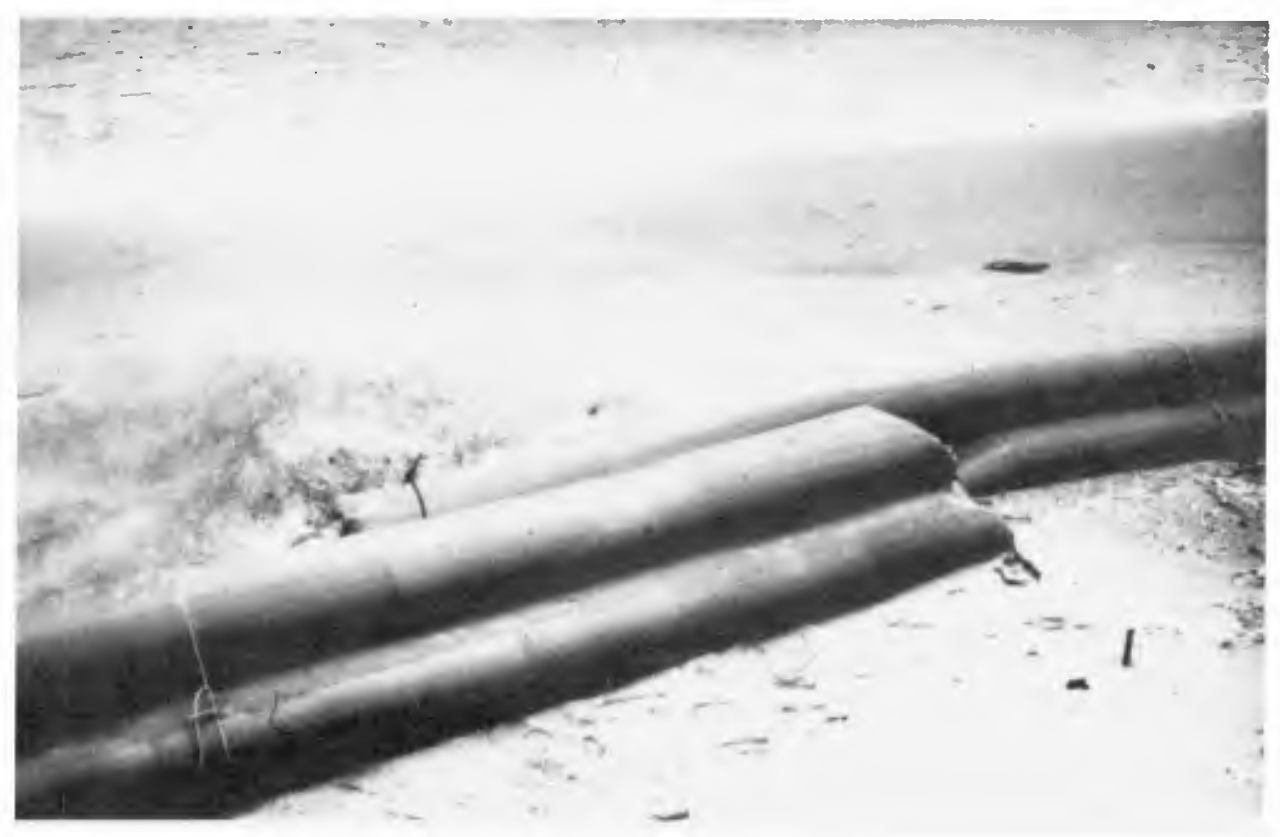

Fig. 2. Structures composed of sand tubes with diameter $0.7 \mathrm{~m}^{-}$( 1 tube on top of 2)

In almost all the structures installed on sandy beaches there has been a problem of bottom protection. (The authors are aware that in autumn 1970 sand-tube structures with a new form of bottom protection of filter sheeting have been erected on the coast of South Carolina, U.S.A.).

On shingle beaches there have been no problems of bottom protection.

In all the experiments tubes have been entirely unprotected; in less exposed locations such structures may have a working life of several years. For permanent use of tubes above the water surface, tubes should be protected by a covering layer of rocks or similar material.

The first sand-tube experiments conducted in Denmark took place in spring 1967. The only point worthy of mention in this connection is that one of the filled tubes, laid at a depth of water of approx. $100 \mathrm{~cm}$ and 


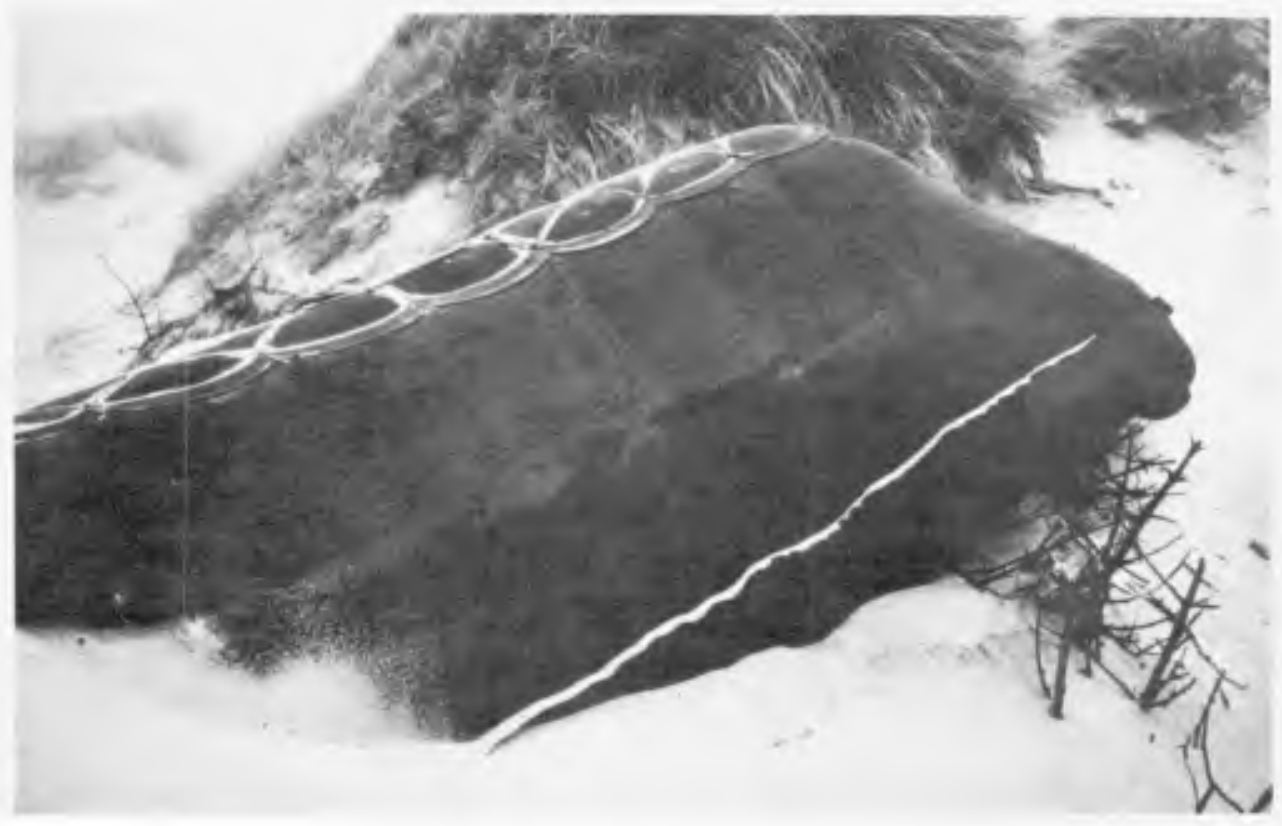

Fig. 3. Tubes wrapped into a filter sheet.

thus protected against sunlight, is still intact, covered with barnacles, cockles and other sea-life.

Some of the experiments are illustrated and described below.

Fig 4 shows the small fishing town of Lonstrup on the west coast of Jutland. Tidal range is about $0.3 \mathrm{~m}$ but wind set-up may be about $1.5 \mathrm{~m}$ combined with heavy wave action.

The littoral South-North drift is estimated at approx. $500,000 \mathrm{~m}^{3}$ p.a. On account of the poor condition of the existing high wooden groin, which was no longer able to ensure the necessary build-up of the beach, three sand tubes were laid out in 1967 along the foot of the groin. As anticipated, this caused a considerable build-up of the foreshore.

The tubes sank somewhat during the winter of 1967-68 although they had been given bottom protection in the form of filter sheeting - but they stopped sinking when they came to rest on an old stone layer, the remains of the foot protection of the wooden groin. Consequently another three tubes were added in 1968.

In autumn 1969 the structure was severely damaged during a military landing exercise.

The structure was repaired in 1970 as shown in the illustration, with a prism groin comprising three hydraulic filled tubes, diameter $0.7 \mathrm{~m}$, lashed together with plastic ropes, the lower tubes woven together. In spring 1969 an experiment began on the barrier beach south of the harbour at Evide Sande on the west coast of Jutland (See Fig. 5). The experiment served three main purposes:

1. A further development of sand tube constructions. 


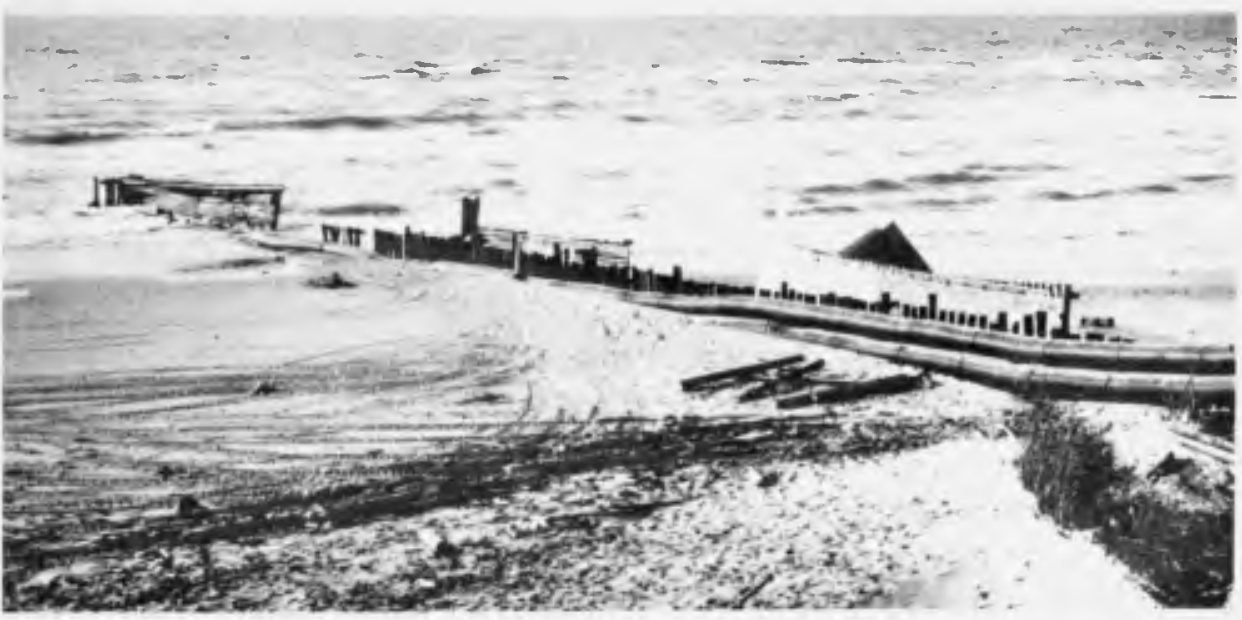

Fig. 4. Sand tube groin (Lønstrup at the Danish North Sea Coast).

2. To obtain better knowledge of the applicability of sand tubes in coastal protection works.

3. Measures against a downdrift recession caused by a mole built to protect the Hvide Sande inlet. dunes.

The barrier is only about $1 \mathrm{~km}$ wide but has a natural protection of

The coast is exposed towards the west, prevailing winds from $W-N W$, and the N-S littoral drift is estimated at $400,000 \mathrm{~m}^{3} \mathrm{p} . \mathrm{a}$. Tidal range less than $0.8 \mathrm{~m}$, wind set up may be up to $+3.0 \mathrm{~m}$.

The constructions described in the following were with few exceptions made of three tubes laid out to form a prism. The tubes were permeable made of double layers of black polypropylene monofilament.

Group 1: Four beach groins. (Fig. 5).

$35 \mathrm{~m}$ long, starting on the dune at a level of $+6-7 \mathrm{~m}$, ending on the beach at level $+2 \mathrm{~m}$.

Group 2: Two artificial beach ridges.

One half-moon shaped with a shingle drain under the middle section. Total length $70 \mathrm{~m}$.

Laid out with top level - $0.2 \mathrm{~m}$ at the front and the ends at top level appr. $+1.0 \mathrm{~m}$.

The construction never really worked because the permeable tubes over the drain were washed out by a gale during the construction period. 


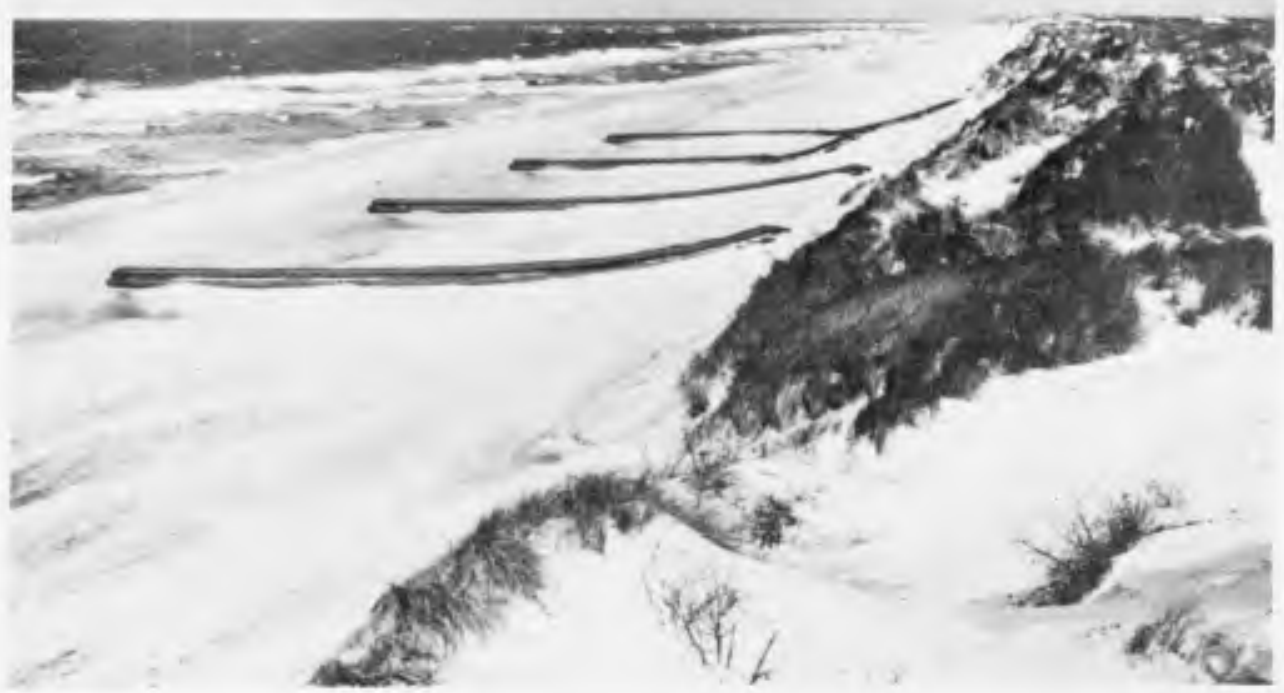

Fig. 5. Beach groins (Hvide Sande. The danish North Sea Coast).

The other ridge boomerang shaped.

Total length $70 \mathrm{~m}$. Laid out on the beach at level $+1 \mathrm{~m}$. After a short while the structure sank and is now buried in the sand.

Group 3: Two groins going from the dune foot to the still water line, spaced $120 \mathrm{~m}$ apart and at an angle of $20^{\circ}$ north to the coast normal.

Group 4: As group three, now showing $20^{\circ}$ south.

Finally two constructions in the form of dozed sand prisms normal and parallel to the coast covered with a filter sheet stitched on both sides to individual tubes; unfortunately the stitching was not strong enough and the structures failed after a short while.

\section{Experimences:}

The material has shown good resistance to the forces acting upon it. The tube structures must be held together by some means: It is essential to pay careful attention to the problem of bottom protection. Some attemps were tried with filter sheet but were not properly executed. Thus the "groins" and "ridges" followed the seasonal beach fluctuations down - but of course never up again. 
A extensive measurement program following the experiments will be finished and finally evaluated in the summer of 1971 so far it can be stated that in the period summer 1969 to spring 1970 stabilisation of the beach has been noted.

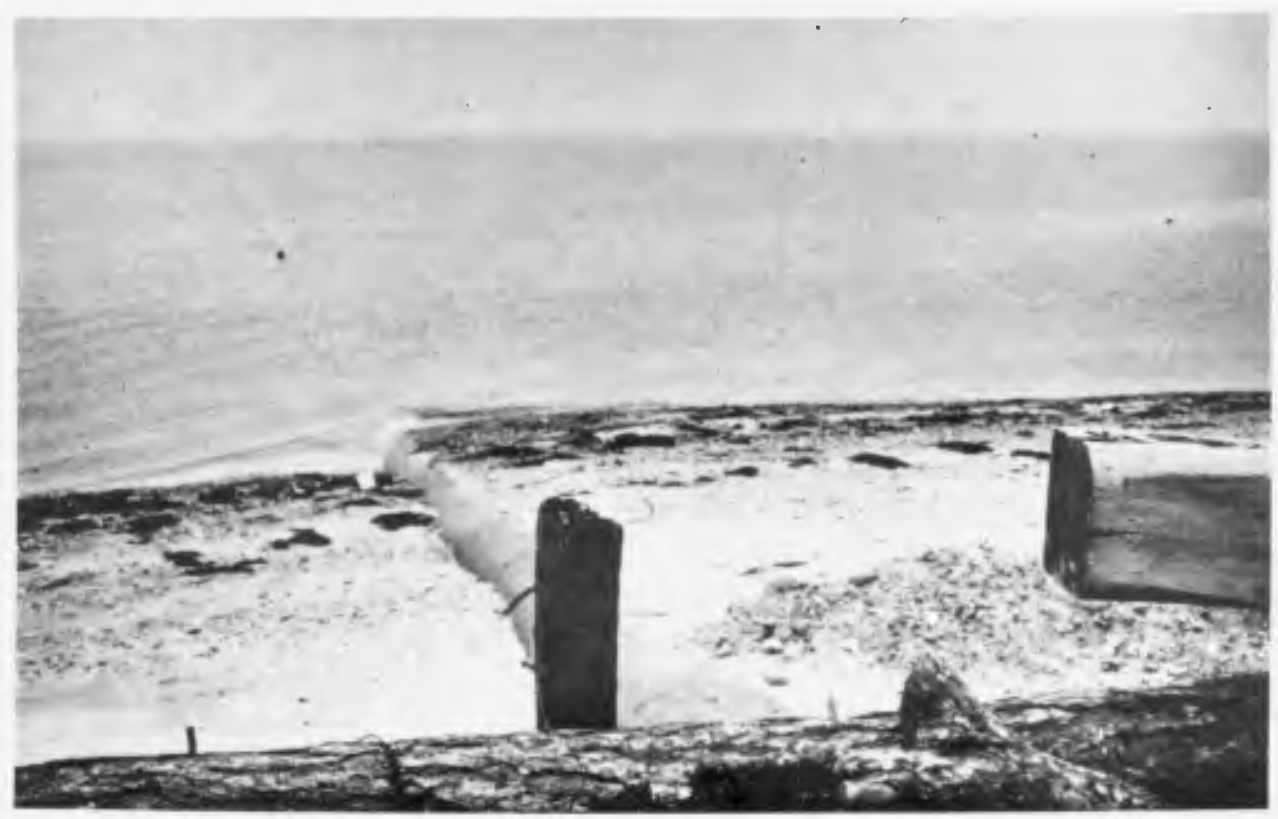

Fig. 6. Enebærodde (Funen).

On the Enebærodde spit on the north coast of Funen a contractor has been conducting some experiments, that began in autumn 1969.

There was a minor erosion problem in the test field. $0.70 \mathrm{~m}$.

The tubes used were made of polypropylene lined with plastic $D=$

There have been some interesting features in these experiments especially concerning some groins made of single tubes going to a water depth of approx. - $1.0 \mathrm{~m}$. (Fig. 6).

1. The sand-tube groins in this relatively calm area had a significant traditional groin effect.

2. The groins did not show any damages in the breaker zone.

3. During the severe winter 1969-70 the tubes remained intact, although subjects to rather hard ice attacks.

4. The spit consists mainly of rather coarse material (shingle) but this has not apparently caused extra wear on the tubes,

5. but keeps them from sinking.

Lately - spring 1970 - sand tubes have been used for dune foot protection on an artificial spit along the Lime Inlet on the west coast of Denmark. 


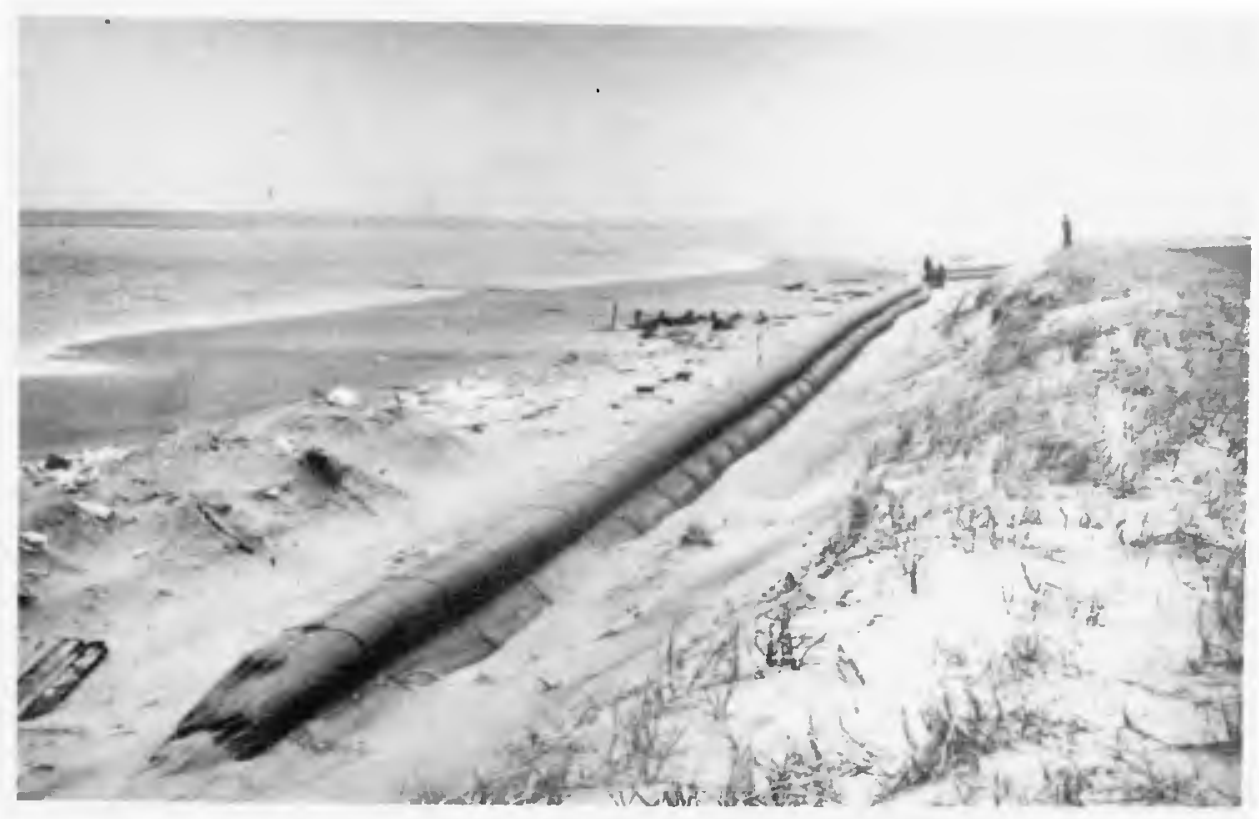

Fig. 7. Sydhalen (Thybor $\phi n$ ).

The tubes were laid out on the west beach of the spit and thus face the east part of the southern Thybor $\phi \mathrm{n}$ barrier; the construction is not therefore in a very exposed position. (Fig. T).

The structure is a $2 \times 100 \mathrm{~m}$ prism arrangement with three $70 \mathrm{~cm}$ diameter tubes made of polypropylene tubes limed with impermeable plastic foil.

On the southern section the tubes are lashed together with ropes while on the northern section the two lower tubes are woven together. Later sand will be dozed up between the eroded dune and the structure. The tubes were filled very succesfully by the hydraulic principle and had a very high filling degree, a level of about $90 \%$ of the theoretical diameter being obtained.

In June 1970 the first 1 m diameter tube was laid out north of the harbour at the Skaw.

The effective height obtained was approx. $90 \mathrm{~cm}$, the far end of the tube was positioned at a water depth of approx. $3.0 \mathrm{~m}$.

It was build in an old landing stage, and accreted in a short while sand to a height varying from $0.20 \mathrm{~cm}$ at depth $1.50 \mathrm{~cm}$ to $1 \mathrm{~m}$ at the shore end. ration.

Other projects have been carried out, and new are under prepa- 


\section{MATERIALS}

Several types of polypropylene fabrics have been used for the skin of the tubes

White polypropylene very soon appeared to have an unsatisfactory resistance to the influence of ultraviolet sun rays, and the fibres of a black polypropylene monofilament had an unfortunate tendency to slide so the sand gralns could be washed out

A woven black fabric, multıplex polypropylene splitfibre, seems to have solved most of these problems and has furthermore proved very resistant to lcy conditions and to the effect of human activity, at bathing beaches for more than 1 year when the hydraulic filling method is used the skin is lined with an inner tube of plastic foll to obtaln impermeability

Tubes with diameters of up to $10 \mathrm{~m}$ have been fllled to lengths of up to $100 \mathrm{~m}$ and at water depts of up to $-3 \mathrm{~m}$ The filling materlal has been natural beach sand with mean graln diameters of about $025-050 \mathrm{~mm}$ A recent small test with hydraulic filling of a tube with a mixture of 1 part cement to 3 parts of sand has worked out well

\section{CONCLUSIONS}

On the basis of preliminary experiments conducted hitherto the following conclusions may be drawn

(1) Impermeable sand tubes can be filled above and under the water to almost any length by hydraulic pumping

(2) The selected type of fabric has demonstrated relatively good resistance to the forces acting in coastal environment

(3) The experiments have shown that sand tubes may be used with advantage to solve minor coast problems, as temporary structures and for prototype pilot tests because the sand tubes can be easily removed

(4) If sand tubes are protected from sunlight and from human activity their use may be of a permanent nature

(5) Many problems are still unsolved and the use of tubes is still in the development stage Work is in progress towards development of larger and more economical sizes of tubes 



\section{CHAPTER 93}

"YIJ HRESSURE OT FLOATINC ICE-FIELDS ON PILES by JoachIm SCHWAFZ ${ }^{1}$ )

\section{S NOPSIS}

In order to determine the maximum ice forces agalnst structures, the compressive strength was investlgated by laboratory tests on cubes of several ice species. The results contain the influence of temperature, velocity of deformation and direction of pressure on the cublc strength.

In oraer to employ these laboratory results for the calculation of structures, a relationship between the strength in laboratory tests and in nature was derlved by measuring the pressure of floating lce-flelds on a plle of a bridge, which crosses the tidal estuary of the EIDER RIver.

The investigation leads to an equation, which allows the calculation of 1 ce pressure against piles.

\section{INTRODUCTION}

In cold regions the pressure of Ice is declsive for the calculation of hydraulic structures. This pressure, however, is still unknown or just in development. It is therefore not surprising that in severe winters hydraulıc structures wll be destroyed by 1 ce run.

In rivers the danger of lce pressure decreases with time, because the ice run in spring will be controlled by lce-breaker-

1) Dr.-InE., Sclentiflc Asslstent of the FRANZIUS-INSTIPUT, Technlcal Unıversity of Hannover, Germany

A sumlar report was given by the author at the 1 . IceSymposium of IAHR in Reykjavik, September 1970 
shlps and because the lce formation wlll be reduced by the heated water of power-stations.

In coastal reglons there Is no way of keeplng the lce forces from structures and just in these locations the question of lce pressure becomes more and more Important, for example by the offshore-construction of deep-water harbors, transloadingpouts for oll, light-houses and bridges.

Intenslve lce research was started after World-War II., especially in USA, Canada and Russla with the Investrgation of fundamental properties of 1 ce (6).

The problem of Ice forces on structures has plcked up during the last 10 years: KORZHAVIN $(3,1962)$ developed an equation to calculate the pressure of river lce in spring. Thls formula Is based upon assumptions, whlch are only derlved through laboratory tests. PEYTON $(4,1966)$ measured the 1 ce pressure on the plles of a drilling-platform in Cook Inlet, Alaska. His qualıtatıve results are in agreement with the Investigations of the author. Some experimental work, carrıed out by oul companies (CROASDALE, 2, 1970) has not yet been published.

A general view about the present sltuation of research of lce pressure on structures was glven by ASSUR $(1,1970)$ at the 1. Ice Symposıum of IAHR, 8 - 10 September 1970 in Reykjavik.

\section{GENERAI CONSIDERATION}

The authors investigation $(5,1970)$ of the pressure of floating lce-fields on plies has been based on the assumption that the maximum pressure of lce ls limlted by lts compressive strength. ThIs strength was first of all ascertalned in compression tests on cubes in order to determine systematically the different influences, such as temperature, velocity of deformation and direction of pressure. The recelved cublc strength can't be Immediately employed for designing structures, because in nature the rupture of Ice occurs in another way than in our laboratory tests. In nature the contact between Ice and structure 
Is, for example, smaller than in the experiments between lce cube and pressure plate. Moreover the shape, the wadth of the structure and the thickness of Ice has an influence upon the strength.

Because the fundamental strength properties nevertheless should be utilized for calculating lce forces, It was necessary to derive a relationship between the strength in laboratory tests and in nature. Thls was done by measuring the Ice-forces on a plle of a bridge.

\section{IABORATORY TESTS}

Strength properties were investlgated by compression tests on lce-cubes from river, lake and harbor (fresh-water-lce) and from the North-Sea, Baltic-Sea and bracklsh-water (saltwater-ıce).

The edge lengths of the cubes were $10 \mathrm{~cm}$. The tests were performed at ice temperatures of $0^{\circ},-10^{\circ}$ and $-20^{\circ} \mathrm{C}$ in two different directions (perpendlcular and parallel to the growth-direction). The veloclty of deformation was varied from $s=3 \cdot 10^{-3} \frac{1}{\mathrm{sec}}$ to $s=3 \cdot 10^{\circ} \frac{1}{\mathrm{sec}} \cdot$ Plywood panels were placed between the cube area and the pressure plate, In order to average out the unevenness on the cube surface, so that the test results scattered only up to $\pm 5 \%$.

\section{RESULTS}

1. By lowering the temperature, the strength of lce Increases at a rate of about.

$$
\begin{aligned}
& \alpha=4,5 \mathrm{kp} / \mathrm{cm}^{2}{ }^{\circ} \mathrm{C} \text { whth fresh-water-ıce and } \\
& \alpha=2,5 \mathrm{kp} / \mathrm{cm}^{2} \mathrm{C} \mathrm{w} \text { th salt-water-1ce. }
\end{aligned}
$$

Thls strengthening is nearly linear down to $-20^{\circ} \mathrm{C}$. The lesser strength of salt-water-ice is attributed to the liquid brine cells whthin the $1 c e$. 


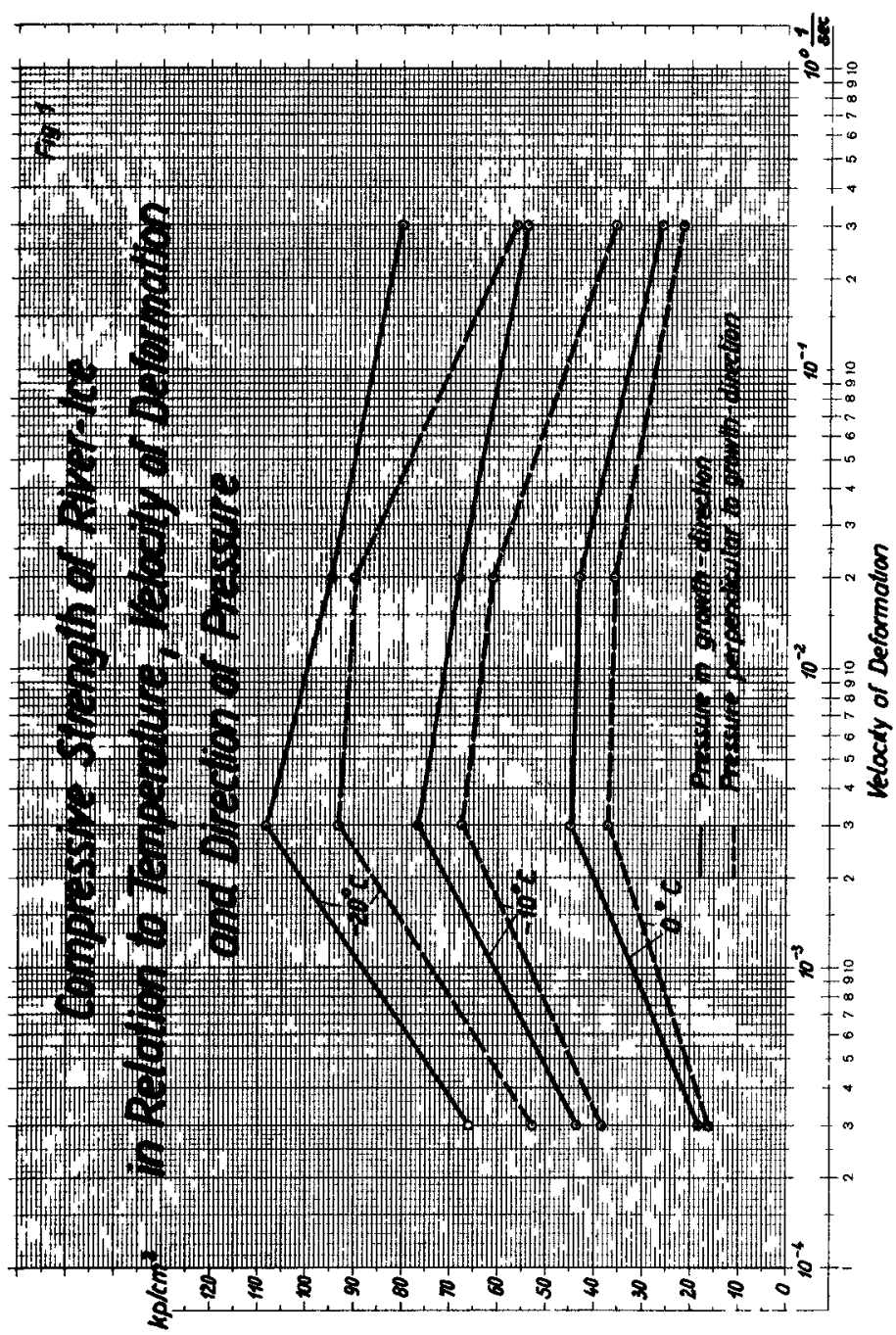




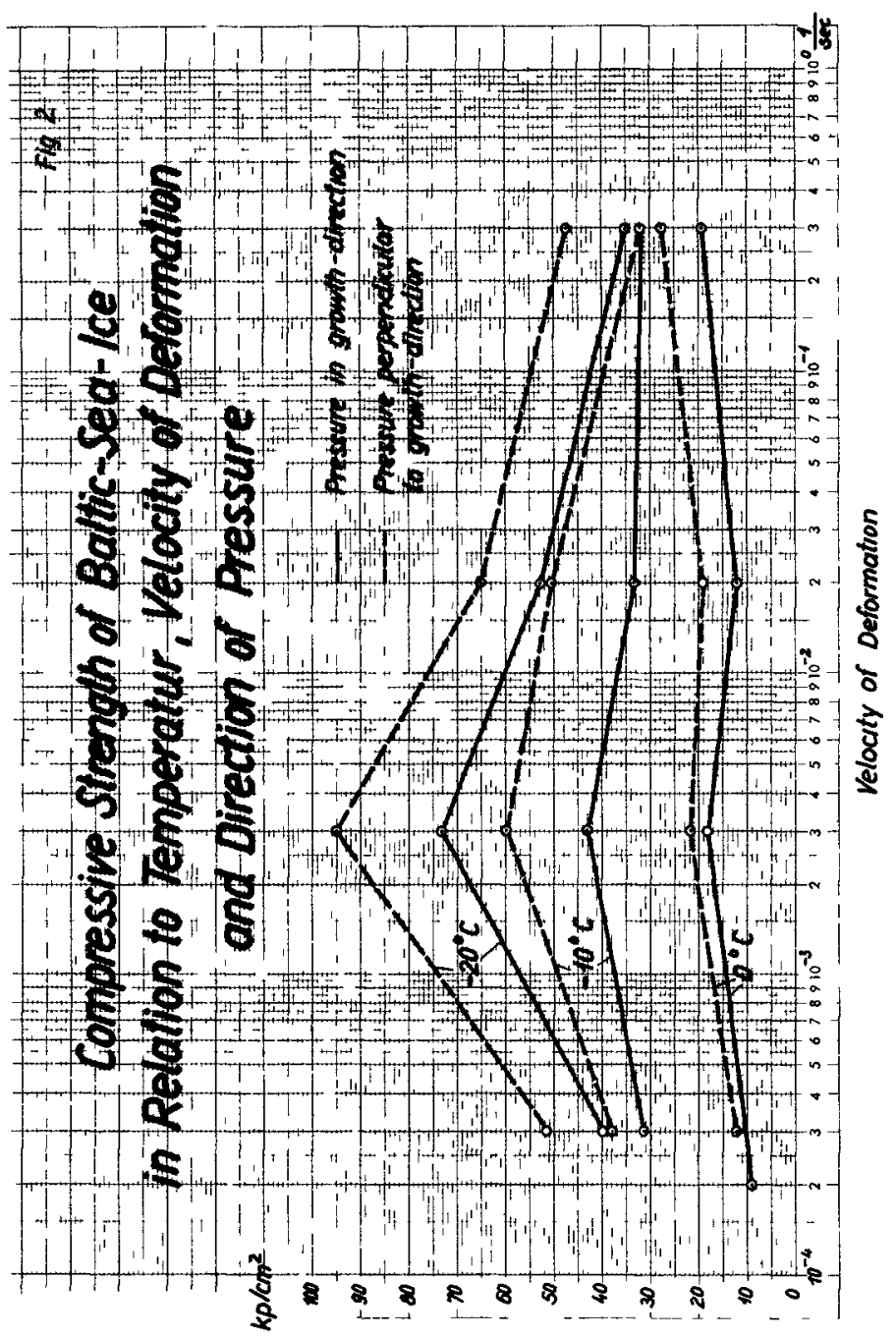


2. At a deformation velocity of $S=0,003 \frac{1}{\text { sec }}$ there is a maximum in strength (FIg. 1,2). This result Is explainable from the deflection-time-curve, show in Fig. 3. The maximum appears at all lce species at the same straln rate and is more evident, the colder the Ice 1s. The deformation velocity of $S=0,003 \frac{1}{\mathrm{sec}}$ correspords to an lce sheet velocity of only a few $\mathrm{cm} / \mathrm{sec}$. That means, If the lce temperature Is low, the maximum lce pressure in nature is to be expected Just before the lce sheet stops.

3. If the pressure acts parallel to the growth direction, the strength of fresh-water-ice is $20 \%$ higher (Fig. 1) than if the pressure direction is perpendlcular to the growth direction. With salt-water-ice these relations are just the reverse.

4. Between alr-content whthin the lce and strength exists a nearly linear relation.

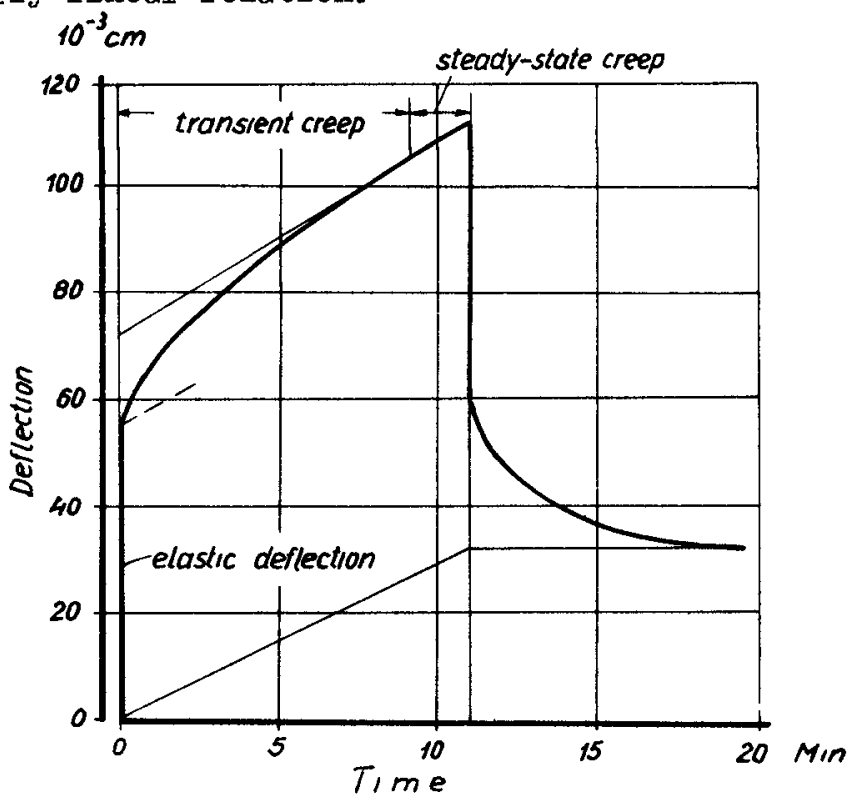

FIG. 3 DEFLECTION OF ICE VS TIME (TABATA, 7) 
MEASURENENT IN NATURE

The compressive strength of sea ice, as occurs in nature, was measured in winter $1967 / 68$ and $1968 / 69$ at a pile of a bridge, which crosses the tidal estuary of the Eider during the construction of a tidal barrier.

Along the entire German coast of the North Sea and al so just outside the estuary of the Eider lie large flat areas (wadden ground), where ice fields can grow very quickly. These ice fields float up only at higher tides and then drift with the tidal current against the bridge, where the ice fields are cut up by the piles. In the hereby occuring state of stress the ice strength has maximum values.

The testing instrument consists of a shield with 50 pressure cells (Fig. 4), 5 in each altitude level halfway encompassing the pile $(\varnothing 60 \mathrm{~cm})$. The area of the pressure cells was

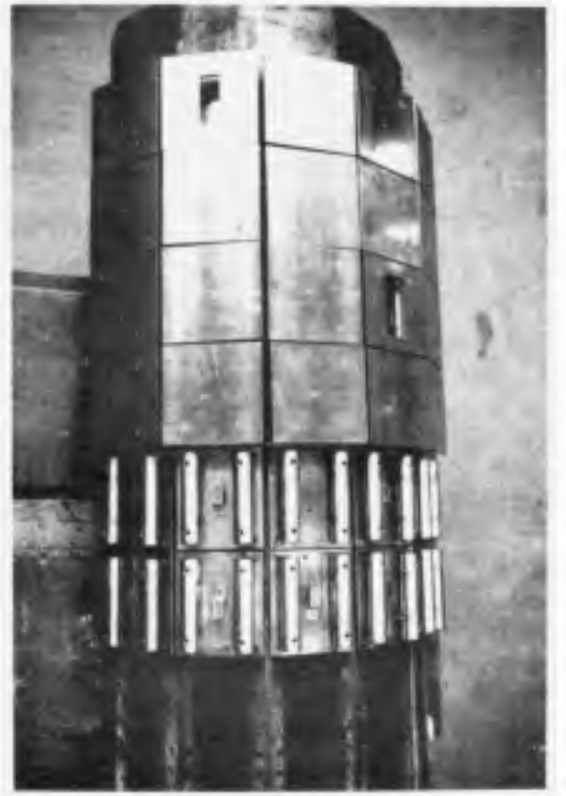

EIG. 4 SHIELD WITH 50 PRESSURE CELLS
$15 \mathrm{~cm} \times 15 \mathrm{~cm}$. In some of these pressure cells were situated smaller ones with areas of $25 \mathrm{~cm}^{2}$ an $50 \mathrm{~cm}^{2}$ in order to determine the relationship between strength and area of pressure. The shield was fixed on the seaside of the pile (Fig. 5).

Insulating the electronic part of the pressure cell against salt water presented a particular problem. It was solved with BOSTIC-NEOSEAL and SILICON-CAOUICHOUC. 


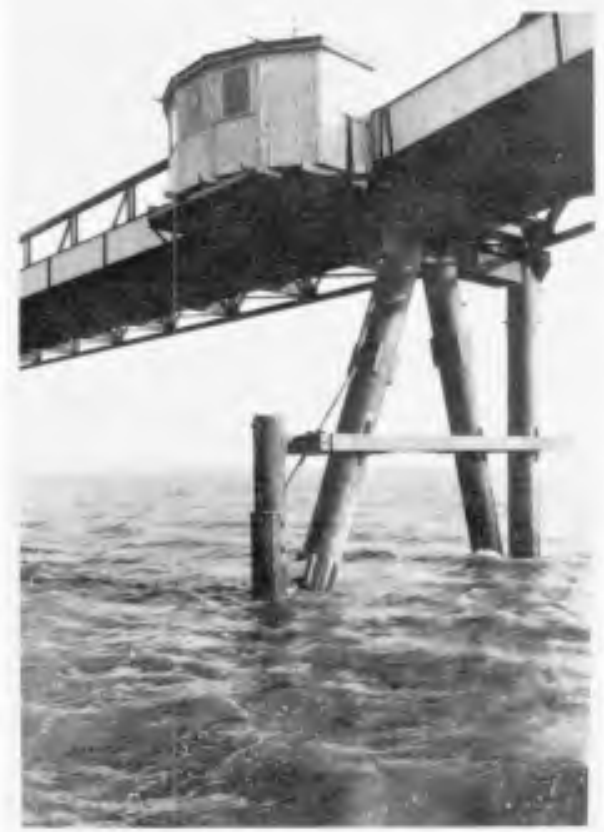

RESULT

In a paper of probability

(Fig. 6) the strength of seaice in nature, related to several areas of pressure $\left(\sigma_{\mathrm{f}} \Rightarrow 25 \mathrm{~cm}^{2}, \sigma_{\mathrm{F}^{\prime}} \Rightarrow 200 \mathrm{~cm}^{2}\right.$, $\sigma_{\varnothing} \Longrightarrow 840 \mathrm{~cm}^{2} \Longrightarrow$ the whole width of the pile at an ice thickness of $14 \mathrm{~cm}$ ) was compared with the cubic strength from laboratory tests with the same ice.

FIG. 5 POINT FOR MEASURING ICE PRESSURE ON A PIIE OF A BRIDGE

1. If the pressure is related to an area of $\mathrm{F}^{\prime}=200 \mathrm{~cm}^{2}$ the compressive strength in nature $\left(\sigma_{F},\right)$ is only half of the cubic strength $\left(\sigma_{W B}\right)$

$$
\frac{\sigma_{F^{\prime} 99}}{\sigma_{W_{50}}}=K_{F^{\prime}}=0.5
$$

This reduction of the cubic strength is attributed to the incomplete contact between ice and structure. Therefore $X$ is called contact coefficient, although this value includes the different state of stress in the cube pressure experiment and in nature.

2. If the area of pressure is only $f=25 \mathrm{~cm}^{2}$, the coefficient of contact increases to $k_{f}=0.56$. 


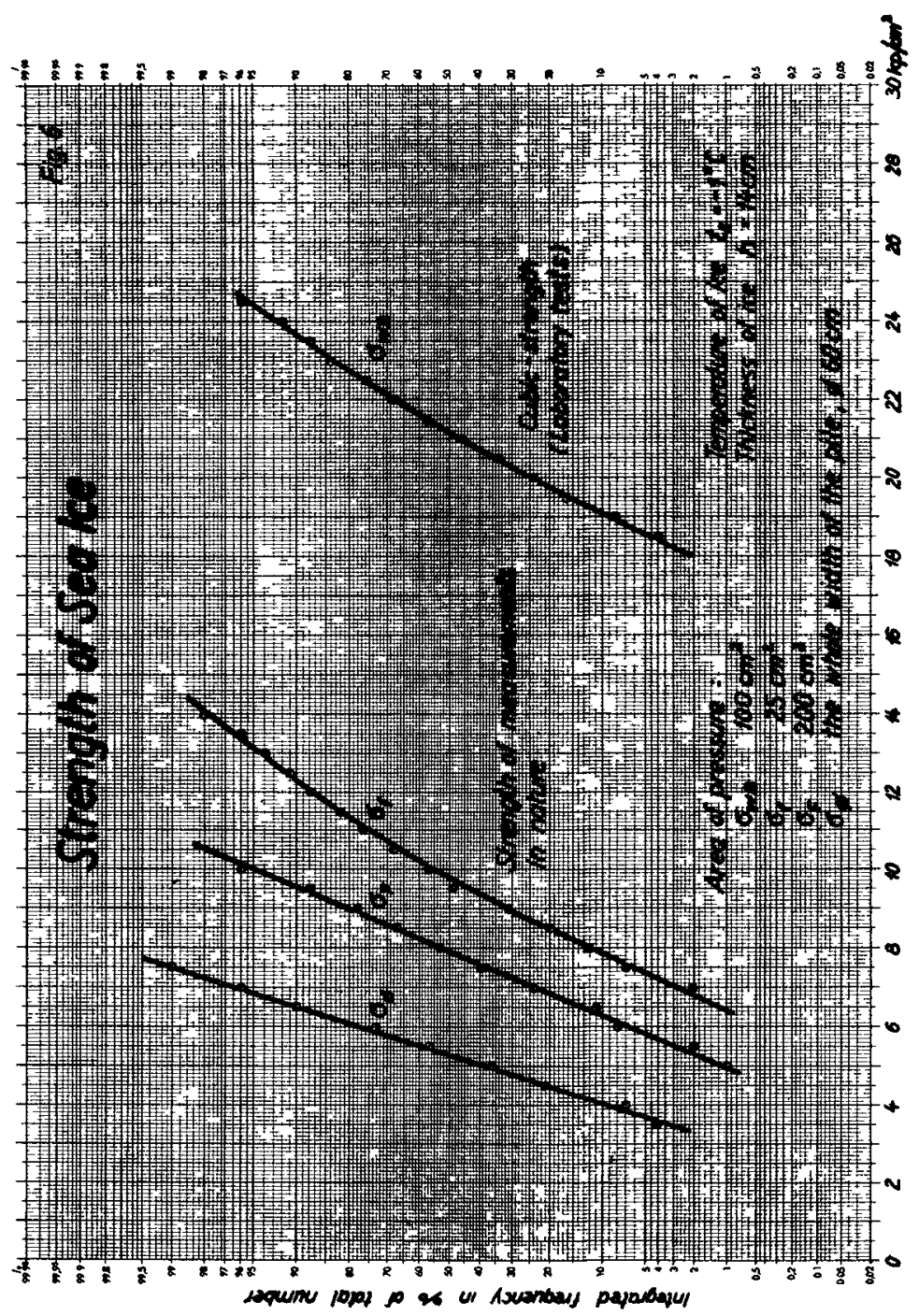




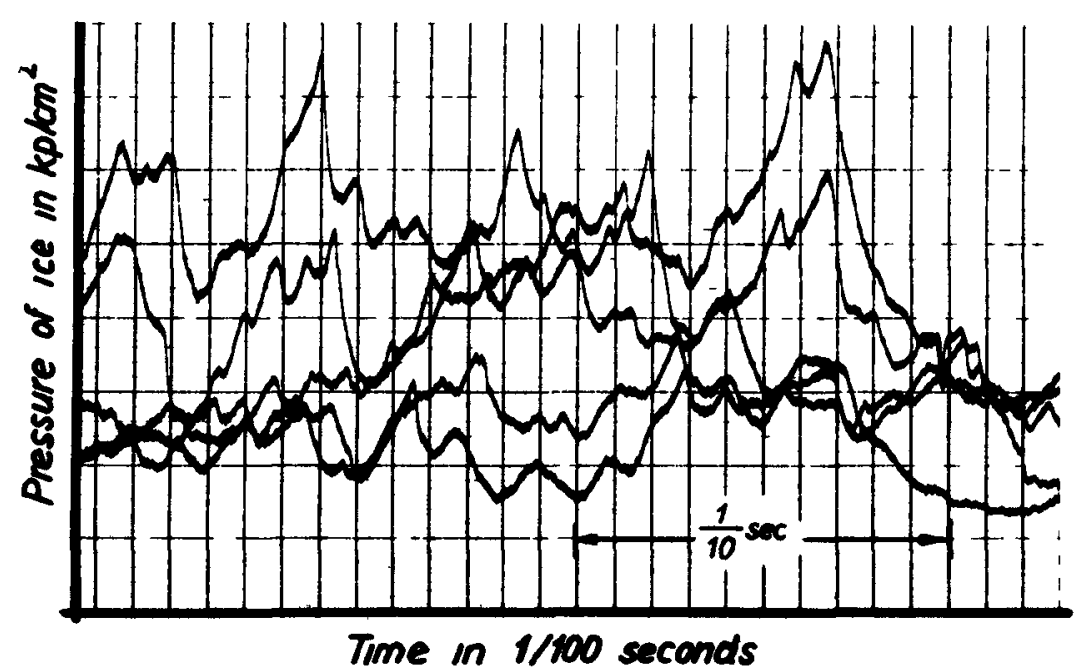

FIG. 7 ICE PRESSURE ON 5 SIDE BY SIDE PRESSURE CELLS

3. Because the peaks of pressure (FIg. 7) occur simultaneously only on 1 or 2 of the 5 slde by slde load cells - the others being largely unpressured - a second reduction factor from the proportion

$$
\frac{\sigma_{\phi}}{\sigma_{F}}=0,66 \text { was ascertanned. }
$$

$\sigma_{\emptyset}$ Is the mean pressure over the whole wldth of the pile $\times$ thickness of lce, projected in the direction of floating. 0.66 takes into account first of all the shape of the structure, but also the increase of the area of pressure from $200 \mathrm{~cm}^{2}$ to $840 \mathrm{~cm}^{2}$.

4. From measurements of different thlcknesses of lce follows, that the strength of Ice increases, if the ratio thlckness of 1 ce to widh of pile becomes greater (FIg. 8). This Is caused by the increase of the 


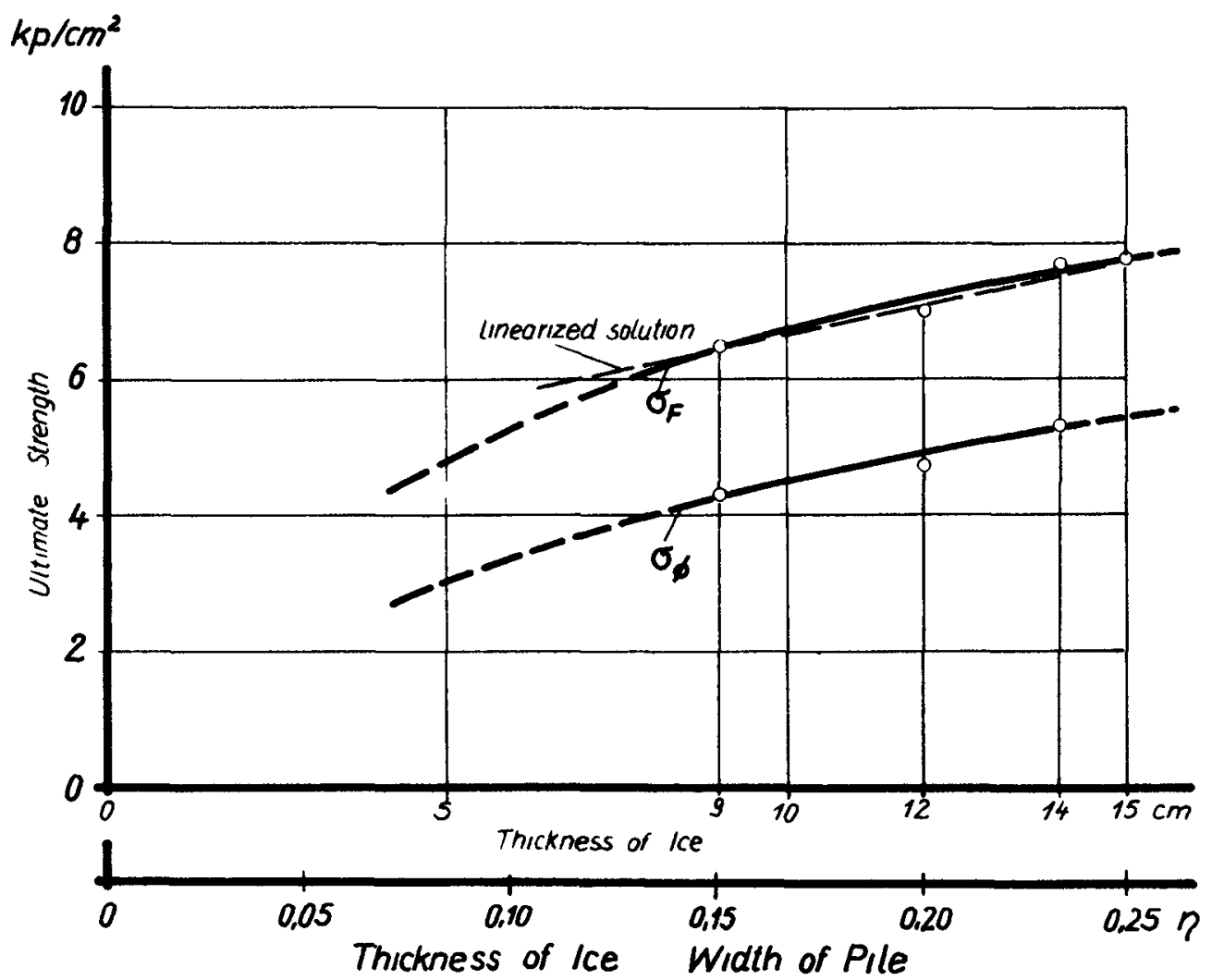

FIG. 8 RELATION BETWEEN ULTIMATE STRENGTH AND THE RATIO THICKNESS OF ICE TO WIDTH OF PILE

threedimensional stress. If the lce sheet grows thicker, the number of planes of shear increases linearly, but also the extension of the planes of shear is lengthend, so that the strength increases exponentially.

5. The pressure of brittle lce with low cublc strength was nearly the same as the pressure of new 1 ce, because of the closer contact between brittle ice and structure. It can be assumed, that the coefficient of contact decreases with lowering temperatures. 
From the results of the laboratory tests and the measurements in nature the following equation is derived in order to determine the maximum pressure of floating lce-flelds on pules

$$
\begin{aligned}
& P=\left[0,5 \cdot 0,66\left(\sigma_{w B}\left(0^{0} \mathrm{C}\right)+0,35 \cdot \alpha\left(t_{\mathrm{L}}-t_{\mathrm{w}}\right)\right)+12,5(n-0,15)\right] \mathrm{h} \cdot \mathrm{b} \\
& 0,5=\text { Coefficient of contact } \\
& 0,66=\text { Coefficient of form (plle } \varnothing 60 \mathrm{~cm} \text { ) } \\
& \sigma_{W 3}\left(0^{\circ} \mathrm{C}\right)=\text { Cublc strength of } 1 \mathrm{ce} \text { at } 0^{\circ} \mathrm{C} \text { and a } \\
& \text { deformation velocity of } S=0,003 \frac{1}{\mathrm{sec}} \\
& 0,35 \cdot \alpha\left(t_{I}-t_{w}\right)=\text { Influence of temperature } \\
& \alpha=\text { Temperature factor } \\
& t_{\mathrm{L}}=\text { Alr-temperature during the last } 24 \\
& \text { hours } \\
& t_{\mathrm{w}}=\text { Water-temperature } \\
& 0,35=\text { Factor to get the mean temperature } \\
& \text { of the Ice-sheet (after KORZHAVIN, 3) } \\
& 12,5(\eta-0,15)=\text { Influence of thlckness in proportion } \\
& \text { to the wadth of plie } \\
& h=\text { Thlckness of Ice } \\
& b=\text { Width of pile } \\
& \eta=\text { Thlckness of lce wldth of structure }
\end{aligned}
$$

This equation should be extended to hlgher values of $\eta$. 
PEFEKENCES

1. ASSUR, A.

2. CROASDAIE, K.R.

3. KORZHAVIN, K.N.

4. PEYTUN, H.R.

5. SCHWARZ, J.

6. WEEKS, W. ASSUR, A.
Forces Exerted by Ice on Marıne

Structures. Proceeding of 1. Ice

Symposium of IAHR in ReykJavik,

8 - 10 September 1970,

wlll be published ir sommer 1971.

The Nutcracker Ice Strength

Tester and Its Operation in the

Beaufort Sea. Preprints of papers

of the 1. Ice Symposium of IAHR in

Reykjavik, 8 - 10 September 1970,

Reykjavik Julı 1970.

The Action of Ice on Englneering

Structures. Publication of the

Siberian Department of the Academy

of Sclence of USSR, Novosibirsk,

1962 (In Russian).

Sea Ice Fiorces. Ice Pressure agannt

Structures.

Proceedings of Conference at Laval

Unlversity Quepec, 1966,

Ottawa 1968.

Trejdelsdruck auf ifahle.

Mittellungen des Franzlus-Instituts

fur Grund- und Wasserbau der Tech-

nlschen Unıversitat Hannover,

Hannover 1970.

The Mechanical froperties of Sea Ice. Ice pressures aganst structures. Proceedings of Conference at Laval Unıversity Quebec, November 1966, ottawa 1968. 
7. TABATA, T. Studies of Visco-Elastic Properties of Sea Ice. Arctic Sea Ice. U.S. National Academy of Sclence. National Research Council. Publication 598. Washington 1968. 


\title{
CHAPTER 94
}

Breakweter and cuay Well by Horzzontel Plates

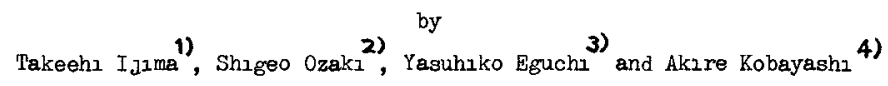

\section{Abstract}

Wave action to breakwater and quay wall by fixed horizontal plates as shown in fig 4 and 5 are studied theoretically and experimentally Transmiseion and reflection coefficients for breakwater are calculated as shown in Fig 6(a)(b), which suggest that only fixing the water surface by rigld plete is effective to reduce the transmitted weves for appropriate wave conditions Pressure distributione to plates ere calculated as shown In Fig 7 and total pressures are in Fig 8 Pressure distributions to norizontal plate and vertical wall of quay wall are shown in Fig 9 and 10, which show thet the plate makes the pressure distribution to vertical wall more uniform than the one whthout plete and also eubrerged plates of breakwater and quay wall make the pressure distributions smooth

Wave action in case when the region under plates is filled by permeable material are also calculated The resulte suggest that the vold of the material has the effect to elongate the plate and the fluzd resistance acte as wave energy absorber

1 Introduction

Pling up the franged-column blocks as shown in Fig I into three or four layers, permeable breakwater and quay wall with vertical sides ere constructed as shown in Fig 2 and 3 Photo 1 and 2 are actually constructed ones of F1g.2 and 3, respectively, In Japan, as wave absorbing structuree Hydrodynamically, they are regerded es composed of horzzontal slabs supported by vertical piles Accordingly, wave actions to these structures are studied by investreating the effect of horizontal plates and then of the permeable material filled uniformely under the plates instead of piles

2 Theory of breakwater by horzontal plete (wathout permeable material)

Suppose thet a rigld horizontal plete of length $2 l 1 s$ fized on water surface with constant water depth $h$ and the water is divided at $x= \pm l$ Into three regions $I, I l$ and III as shom in Fig 4 Assuming small amplitude waves in perfect liquid and velocity potentiel in each region to be $\phi_{1}, \phi_{2}$ and $\phi_{3}$, we have the following Laplace's equations

$$
\partial^{2} \phi_{y} / \partial x^{2}+\partial^{2} \phi_{y} / \partial z^{2}=0 \quad(t=123)
$$

(I) Professor, Faculty of Engineerıng, Kyushu Unıversıty, Fukuoka, Japan (2) Director, the Fourth Harbour Construction Offlce, Mnzatry of Transportation (3) (4) Research Assistant, Kyushu Unıversıty 
Bottom and surface conditions in regzon $I$ and III are as follows

$$
\frac{\partial \phi_{L}}{\partial z}=0 \quad \text { at } \mathrm{z}=-\mathrm{h}, \quad \frac{\partial^{2} \phi_{4}}{\partial t^{z}}+g \frac{\partial \phi_{4}}{\partial z}=0 \quad \text { at } \mathrm{z}=0 \quad(\mathrm{l}=1,3)
$$

In region $I I$, we have

$$
\frac{\partial \phi_{2}}{\partial z}=0 \quad \text { at } z=0 \text { and } z=-h
$$

Fluid pressure in each region and surface profiles in regions I and III are as follows

$$
\frac{p_{L}}{\rho}=-\frac{\partial \phi_{L}}{\partial t}-g z(=1,23) \quad, \quad S_{L}=-\frac{1}{g}\left(\frac{\partial \phi_{L}}{\partial t}\right)_{z=0} \quad(v=1,3)
$$

where $g$ is gravity acceleration and $\int$ is fluzd density

Supposing the inczdent wave of frequency $\sigma(\sigma=2 \pi / T, \mathrm{~T}$ is wave period $)$ and reflected wave in region $I$ and transmutted wave in region III, the velocity potential which satisfies Laplace's equation and bottom and surface boundary conditions in each regzon is given as follows

$$
\begin{aligned}
& \phi_{1}(x, z, t)=e^{i \pi t}\left[\left(A e^{i k x}+B e^{-i k x}\right) \frac{\cosh k(z+h)}{\cosh k h}+\sum_{n=1}^{\infty} C_{n} e^{-k_{n} x} \frac{\cos k_{n}(z+h)}{\cos k n h}\right],(n=1,2,3) \\
& \phi_{2}(x z t)=e^{\cot } \sum_{r=0}^{\infty}\left(D_{r} \cosh \frac{r \pi x}{h}+E_{r} \sinh \frac{r \pi x}{h}\right) \cos \frac{r \pi z}{h}, \quad(r=0,1 z) \\
& \phi_{3}(x, z, t)=e^{t \sigma t}\left[F e^{i k x} \frac{\cosh k(z+h)}{\cosh k h}+\sum_{n=1}^{\infty} G_{n} e^{k_{n} x} \frac{\cos k_{n}(z+h)}{\cos k_{n} h}\right] \quad(n=1,23)
\end{aligned}
$$

where $k$ and $k$ rare eigenvalues determned by the following relations

$$
\frac{\sigma^{2} h}{g}=k h \tanh _{k h}=-k_{n} h \tan k_{n} h, \quad(n=1,2,3)
$$

$A, B$ and $F$ are complex constants which represent the incident, reflected and $t_{\perp}$ ansmlited waves, respectively, and $C_{n}$ and $G_{n}$ are standing waves which vanısh at $|X| \rightarrow \infty$ $D_{r}$ and $E_{r}$ are complex constants to be determaned from the following boundary condztions

At the boundaries $x= \pm l$, continuztzes of horzzontal fluzd velocities and fluctuating wave pressures due to continuzties of mass and energy flux through the boundaries require the following conditions

$$
\begin{aligned}
& \frac{\partial \phi_{1}}{\partial x}=\frac{\partial \phi_{2}}{\partial x}, \quad \phi_{1}=\phi_{2} \quad \text { at } x=l \\
& \frac{\partial \phi_{2}}{\partial x}=\frac{\partial \phi_{3}}{\partial x}, \quad \phi_{2}=\phi_{3} \quad \text { at } x=-l
\end{aligned}
$$

Substatuting eq 5,6,7 into eq 9,10 and adding and subtracting, we obtain next 
relations

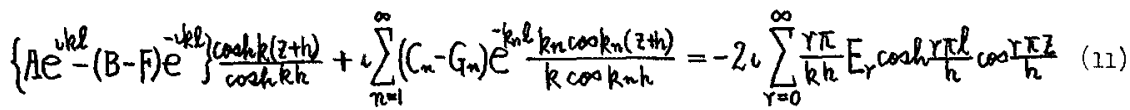

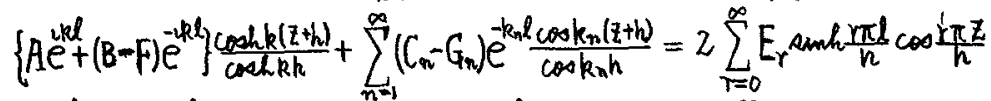

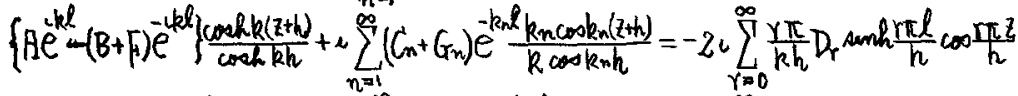
$\left\{A e^{h k l}+(B+F) e^{-k \ell_{1}} \mid \frac{\operatorname{coch} h(z+h)}{\cosh k h}+\sum_{n=1}^{\infty}\left(C_{n}+G_{n}\right) e^{-k_{n} l_{n} \frac{\cos k_{n}(z+h)}{\cos k_{n} h}}=2 \sum_{r=0}^{\infty} D_{r} \cosh \frac{h \pi l}{h} \cos \frac{r \pi l}{h}\right.$

Thultiplying each teras of above equations by $\cos (\mathrm{r} \pi \mathrm{z} / \mathrm{h})$, integrating from $\mathrm{z}=-\mathrm{h}$ to $z=0$ and using the following relations

$\int_{h}^{0} \cos \frac{r \pi z}{h} \cos \frac{s \pi z}{h} d z=0(s \neq r) ;=\frac{h}{2}(r=s \neq 0),=h \quad(r=s=0)$
$\int_{-h}^{0} \cosh k(z+h) \cos \frac{r \pi z}{h} d z=\frac{1}{k} \frac{\operatorname{senh} k h}{1+(r \pi / k h)^{2}}, \int_{-h}^{0} \cos k_{n}(z+h) \cos \frac{r \pi z}{h} d z=\frac{1}{k_{n}} \frac{\sin k_{n} h}{1-\left(\gamma \pi / k_{n} h\right)^{2}}$

we have following equations

$\left\{A e^{k k l}-(B-F) e^{-k l l}\right\} \frac{\operatorname{tanhkh}}{1+(r \pi / k h)^{2}}+i \sum_{n=1}^{\infty}\left(C_{n}-G_{n}\right) e^{-k_{n} l} \frac{\tan k_{n} h}{1-\left(r \pi / k_{n} h\right)^{2}}=-v r \pi E_{r} \cosh \frac{r \pi l}{h}$

$\left\{A e^{k p l}+(B-F) e^{-k l}\right\} \frac{\tanh k h}{1+(r \pi / k h)^{2}}+\sum_{n=1}^{\infty}\left(C_{m}-G_{m}\right) e^{-k_{n} l} \frac{k}{k_{m}} \frac{\tan k k_{n} h}{1-\left(r / k_{n} h\right)^{2}}=k h E_{r} a m h \frac{r \pi l}{h}$

$\left\{A e^{-k l}-(B+F) e^{-i k l}\right\} \frac{\tanh k h}{1+(r \pi / k h)^{2}}+i \sum_{n=1}^{\infty}\left(C_{n}+G_{n}\right) e^{-h_{n} l} \frac{\tan k_{n} h}{1-\left(\pi / k_{n} h\right)^{2}}=-i r \pi D_{r} \operatorname{senh} \frac{r n l}{h}$

$\left\{A e^{-k l}+(B+F) e^{-k k l}\right\} \frac{\tanh k h}{1+\left(T \pi / k_{n}\right)^{2}}+\sum_{n=1}^{\infty}\left(C_{n}+G_{m}\right) e^{-k_{n} l} \frac{k}{k_{n}} \frac{\tan k_{n} h}{1-\left(T / k_{n} h\right)^{2}}=k h D_{r} \cosh \frac{m l l}{h}$

Elimznating $D_{Y}$ and $D_{r}$ in above equations and putting

$A e^{i k l}=a, \quad B e^{-i k l}=b \quad F e^{-i k l}=f, \quad C_{n} e^{-k k_{n} l}=c_{n}$

$G_{n} e^{-k_{n} l}=g_{n} \quad \pi l / h=H \quad \mathrm{kh} / \pi=\lambda_{0} \quad k_{n} h / \pi=\lambda_{n}$

$F_{r, n}=\frac{\lambda_{0}^{2}+r^{2}}{\lambda_{n}^{2}-r^{2}} \frac{\lambda_{n}+r \tanh r H}{\sqrt{\lambda_{0}^{2}+(r \tanh r H)^{2}}}, \phi_{r n}=\frac{\lambda_{0}^{2}+r^{2}}{\lambda_{n}^{2}-r^{2}} \frac{\lambda_{n}+r \operatorname{coth} r H}{\sqrt{\lambda_{n}^{2}+(r \operatorname{coth} r H)^{2}}}$

$\theta_{r}=\tan ^{-1}\left(\frac{r}{\lambda_{0}} \tanh r H\right) \quad \varphi_{r}=\tan ^{-1}\left(\frac{r}{\lambda_{0}} \operatorname{coth} r H\right)$ 
1540

COASTAL ENGINEERING

the following two systems of linear simultaneous equations are provided

$$
\begin{aligned}
& \frac{b+f}{a} e^{-i \theta r}+\downarrow \sum_{n=1}^{\infty} F_{r n} \frac{c_{n}+g_{n}}{a}=e^{i \theta_{r}} \quad(r=0,12) \\
& \frac{b-f}{a} e^{-l g_{r}}+\imath \sum_{n=1}^{\infty} \phi_{r, n} \frac{c_{n}-g_{n}}{a}=e^{l \varphi_{r}} \quad(r=0.12)
\end{aligned}
$$

where

$$
\begin{aligned}
& F_{0, n}=\frac{\lambda_{0}}{\lambda_{n}}, \quad \phi_{0, n}=\lim _{r \rightarrow 0} \frac{\lambda_{0}^{2}+r^{2}}{\lambda_{n}^{2}-r^{2}} \frac{\lambda_{n}+r \operatorname{coth} r H}{\sqrt{\lambda_{n}^{2}+(r \operatorname{coth} r H)^{2}}}=\frac{\lambda_{0}^{2}}{\lambda_{n}^{2}} \frac{1+\lambda_{n} H}{\sqrt{1+\left(\lambda_{n} H\right)^{2}}} \\
& \theta_{0}=0, \quad \varphi_{0}=\lim _{r \rightarrow 0} \tan ^{-1}\left(r H \operatorname{coth} r H / \lambda_{0} H\right)=\tan ^{-1}\left(1 / \lambda_{0} H\right)
\end{aligned}
$$

Eq 20 and 21 are regarded as equations for determining the unknowns $(b+f) / a$, $\left(c_{n}+g_{n}\right) / a$ and $(b-f) / a$ and $\left(c_{n}-g_{n}\right) / a$, respectively, so that we obtain $b / a, f / a$, $\mathrm{c}_{n} / \mathrm{a}$ and $\mathrm{g}_{w} / \mathrm{a}$ from $\mathrm{eq}_{2} 20$ and 21 . Thus, from $\mathrm{eq}_{1} 16$ and $18, D_{r}$ and $\mathrm{E}_{\mathrm{r}}$ are determined as follows

$$
\begin{aligned}
& D_{1}=d_{0} / 2=\frac{a}{2 \pi^{2}} \frac{\sigma^{2} h}{g}\left[\left(1+\frac{b+f}{a}\right) \frac{1}{\lambda_{0}^{2}}-\sum_{n=1}^{\infty} \frac{c_{n}+g_{n}}{a} \frac{1}{\lambda_{n}^{2}}\right] \\
& D_{r} \cosh r H=d_{r}=\frac{a}{\pi^{2}} \frac{\sigma^{2} h}{g}\left[\left(1+\frac{b+f}{a}\right) \frac{1}{\lambda_{i}^{2}+r^{2}}-\sum_{n=1}^{\infty} \frac{c_{n}+g_{n}}{a} \frac{1}{\lambda_{n}^{2}-r^{2}}\right] \\
& E_{r} \operatorname{minh} r H=e_{r}=\frac{a}{\pi^{2}} \frac{\sigma^{2} h}{g}\left[\left(1+\frac{b-f}{a}\right) \frac{1}{\lambda_{0}^{2}+r^{2}}-\sum_{n=1}^{\infty} \frac{c_{n}-g_{n}}{a} \frac{1}{\lambda_{n}^{2}-r^{2}}\right]
\end{aligned}
$$

Letting the incident wave be $S_{0}=\cos (k x+a t)$, we have

$$
a=\imath \alpha g e^{i k l} / \sigma
$$

Thus, fromm eq 5,6 and 7 , the velocity potentials are determined as follows

$\phi_{1}(x, z, t)=a e^{\cot t}\left[\left\{e^{k k(x-l)}+\frac{t}{a} e^{-2 k(x-l)}\right\} \frac{\cosh k(z+h)}{\cosh h h}+\sum_{n=1}^{\infty} \frac{c_{n}}{a} e^{-k_{n}(x-l)} \frac{\cos k_{n}(z+h)}{\cos k n h}\right]$

$\phi_{2}(x, z, t)=a e^{i a t}\left[\frac{d_{0}}{2 a}+\sum_{r=1}^{\infty}\left(\frac{d_{r}}{a} \frac{\cosh r H x / l}{\operatorname{coshrH}}+\frac{e_{r}}{a} \frac{\sinh r H x / l}{\sinh r H}\right) \cos \frac{r \pi z}{h}\right]$

$\phi_{3}(x, z, t)=a e^{i n t}\left[\frac{f}{a} e^{i k(x+l)} \frac{\cos h k(z+h)}{\cosh k h}+\sum_{n=1}^{\infty} \frac{g_{n}}{a} e^{k_{n}(x+L)} \frac{\cos k n(z+h)}{\cos k n h}\right]$

Surface profiles in regions I and III are given as follows

$$
S_{1}(x, t)=\alpha e^{i(k l+a t)}\left[e^{l k(x-l)}+\frac{b}{a} e^{-l k(x-l)}+\sum_{n=1}^{\infty} \frac{c_{n}}{a} e^{-k_{n}(x-l)}\right]
$$




$$
S_{3}(x, t)=\alpha e^{l(k l+a t)}\left[\frac{f}{a} e^{l k(x+l)}+\sum_{n=1}^{\infty} \frac{g_{n}}{a} e^{k_{n}(x+l)}\right]
$$

Reflection and transmission coefficlents are as follows

$$
K_{r}=|b / a|, \quad K_{t}=|f / a|
$$

Pressure distribution $P_{2(z=0)}$ and the total pressure $P_{u}$ to the horizontal plate are as follows

$$
\begin{aligned}
& p_{z(z=0) / \rho g \alpha}=e^{(k l+a t)}\left[\frac{d_{0}}{2 a}+\sum_{r=1}^{\infty}\left(\frac{d r}{a} \frac{\cosh r H x / \ell}{\cosh r H}+\frac{e_{r}}{a} \frac{\operatorname{sunh} r H x / \ell}{\sinh r H}\right)\right] \\
& \left|P_{u} / 2 \rho g \alpha l\right|=\left|\int_{-\ell}^{l} \frac{p_{z(z=0)}}{2 \rho g \alpha l} d x\right|=\left|\frac{d_{0}}{2 a}+\sum_{r=1}^{\infty} \frac{d_{r}}{a} \frac{\tanh r H}{r H}\right|
\end{aligned}
$$

3 Theory of quay wall with horizontal plate (w1 thout permeable material)

Suppose the vertical wall with horzontal plate of length $\ell$ as show in Fig 5 Boundary conditions for fluzd motion in region I are just the same as the previous section and velocity potential $\phi_{1}$ is given by eq 5 In region II, the condition $\partial \phi_{2} / \partial x=0$ at $X=0$ is to be added, so that the velocity potential $\phi_{2}$ becomes, putting $\mathrm{E}_{\boldsymbol{r}}=0$, as follows

$$
\phi_{x}(x, z, t)=e^{i \sigma t} \sum_{r=0}^{\infty} D_{r} \cosh \frac{r \pi x}{h} \cos \frac{r \pi z}{h},(r=0 \quad 1 \quad z)
$$

Boundary condztions at $x=l$ is the same as eq 9

following to the same process as previoue section and using the same symbols as Bq 19, simultaneous equations for $b / a$ and $c_{n} / a$ are provided in the same form as eq 20 as follows

$$
\frac{b}{a} e^{-i \theta r}+i \sum_{n=1}^{\infty} F_{r n} \frac{c_{n}}{a}=e^{i \theta r} \quad(r=012)
$$

Thus, the numerical results of eq 20 for breakwater are used as $2 t$ is and Do and DY are given as follows

$$
\begin{aligned}
& D_{0}=d_{0} / 2=\frac{a}{\pi^{2}} \frac{\sigma^{2} h}{g}\left[\left(1+\frac{b}{a}\right) \frac{1}{\lambda_{0}^{3}}-\sum_{n=1}^{\infty} \frac{c_{n}}{a} \frac{1}{\lambda_{n}^{2}}\right] \\
& D_{r} \cosh r H=d_{r}=2 \frac{a}{\pi^{2}} \frac{\sigma^{2} h}{g}\left[\left(1+\frac{b}{a}\right) \frac{1}{\lambda_{0}^{2}+r^{2}}-\sum_{n=1}^{\infty} \frac{c_{n}}{a} \frac{1}{\lambda_{n}^{2}-r^{2}}\right]
\end{aligned}
$$

For the incident wave $S_{0}=\alpha \cos (k x+a t)$, the velocity potential $\phi_{1}$ is the same as eq 25 and $\phi_{2} 2 \mathrm{~s}$ as follows 


$$
\phi_{2}(x, z, t)=a e^{\cot }\left[\frac{d_{0}}{2 a}+\sum_{r=1}^{\infty} \frac{d_{r}}{a} \frac{\cosh r H x / l}{\cosh r H} \cos \frac{r \pi z}{h}\right]
$$

Reflection coefficient $\mathrm{K}_{r}=|\mathrm{b} / \mathrm{a}|$ is Identically equal to unity Pressure distribution $p_{2(z=0)}$ and $p_{2(x=0)}$, and the total pressure $P_{u}$ and $P_{H}$ to horizontal plate and vertzcal wall are given as follows

$$
\begin{aligned}
& p_{2(z=0) / \rho g \alpha}=e^{l(k l+a t)}\left[\frac{\alpha_{0}}{2 a}+\sum_{r=1}^{\infty} \frac{d_{r}}{a} \frac{\cosh r H x / l}{\cosh r H}\right] \\
& \left|P_{u l} / \rho g \alpha l\right|=\left|\int_{0}^{l} \frac{p_{2(z=0)}}{\rho g \alpha l} d x\right|=\left|\frac{d_{0}}{2 a}+\sum_{r=1}^{\infty} \frac{d_{r}}{a} \frac{\tanh r H}{r H}\right| \\
& p_{2}(x=0) / \rho g \alpha=e^{l(k l+a t)}\left[\frac{d_{0}}{2 a}+\sum_{r=1}^{\infty} \frac{d_{r}}{a} \frac{\cosh r \pi z / h}{\cosh r H}\right] \\
& \left|P_{H} / \rho g \alpha h\right|=\left|\int_{-h}^{0} \frac{p_{2}(x=0)}{\rho g \alpha h} d Z\right|=\left|\alpha_{0} / a\right|
\end{aligned}
$$

4 Breakwater and quay wall with double platee (without permeable material)

In cases of breakwater and quay wall wl th double plates at $\mathrm{z}=0$ and $\mathrm{z}=-\mathrm{h} / 2$ as shown by dotted lines in F1g 4 and 5, velocity potential in region II must satisfy one nore condition $\partial \phi_{3} / \partial z=0$ at $z=-h / 2$ This ze done by taking even numbers for integers $r$ in eq 6 and 33 (that is $r=0,2,4,6$, )

\section{Calculations}

For various values of $\sigma^{2} h / g=2 \pi h / L_{0}$ ( $I_{0}$.s deep water wave length for period $T$ ), $\lambda_{0}$ and $\lambda_{n}$ defined by eq 19 are calculated by eq 8 as shown in Table 1 And then, eq 20 and 21 are solved by means of electronic computor For example, b/a, f/a, cn/a etc for the case of $\sigma^{2} \mathrm{~h} / g=10$ and $l / \mathrm{h}=10$ are as shown in Table 2

\section{Calculated results for horzontal plate breakwater}

Transmission and reflection coefficients $K_{t}$ and $K_{r}$ by eq 30 are shown in F 1 g 6 $6(a)$ (b) for single and double plates For the case of single plate with $l / \mathrm{h}=20, \mathrm{~K}_{\mathrm{t}}=$ 053,038 and 028 for $A^{2} h / g=05,10$ and 15 , respectively, and so only $28 \%, 14 \%$ and $8 \%$ of Incldent wave energy are trnswitted through the plate Comparnng Fig 6(a) with Fig $6(b)$, it is seen that if the total length of plates are equal in both cases (that $1 s,(l / h)_{1}=20$ corresponds to $\left.(l / h)_{2}=10\right),\left(k_{r}\right)_{1}$ is always larger than $\left(k_{\gamma}\right)_{2}$ and $\left(\mathrm{K}_{\mathrm{t}}\right)_{1}$ 1s smaller then $\left(\mathrm{K}_{\mathrm{t}}\right)_{2}$ for all $\mathrm{N}^{2} \mathrm{~h} / \mathrm{g}$, where suff $\mathrm{fx}_{\mathrm{x}} 1$ and 2 show the cases of single plate and double plates, respectively These properties are interpretted ae 
the result that when the water surface is fixed by horizontal plate, the fluid under the plate is constrained in motion, increes its inertial resistance to motion and behaves like as semu-rigld breakwater for short waves

Preseure distribution to plate by eq 31 are shown in Fig $7(a)(b)$, which show that the distrubution for double plates is remarkably uniform compared with that of single plate The total pressure by eq 32 is shown in Fig 8, from which $1 t$ is seen that the averaged pressure per unt length of plate and per unct amplitude of incident wave for double plates is almost independent of $l / h$ and approaches to the value for $l / h=05$ of single plate These properties of preesure to the plate suggest that the submerged plate has the effect of making the pressure distribution uniform

7 Calculated results for horizontal plate quay wall (wn thout permeable material)

The pressure distribution to horizontal plate by eq 37 is shown in Fig 9 for single plate quay wall As was shown for breakwater, the distribution for double plates is remarkably uniform compared with that of single plate Pressure distribution to the vertical wall is in Fig 10 compared with the one without plate (that $1 \mathrm{~s}, \ell / h=0$ ), the distribution is smooth even for single plate wall and the tendency is much clearer for double plates wall Averaged total pressure to horizontal plate and vertical wall by eq 38 and 40 are in Fig 11 and 12, respectively Comparing both flgures, it is seen that for single plate wall the averaged pressure to horlzontal direction becomes equal to that of vertical direction for larger $l / h$, and it is clearer for double plates wall Ihis means that the water in region II is constraned in motion by horizontal plate and vertical wall and the wave pressures are equalized to all directions

8 Theory of horızontal plate breakwater and quay wall. with permeable material

When permeable material is filled under horizontal plate, it causee resistance to the motion of fluid flowing through the vold For simpliclty, we assume that the resistance is proportional to fluid velocity and the coefficient of resistance per unit fluid mass is $\mu$ and the vold ratio is $v$ If $u$, $w$ and $p$ are actual velocities and pressure of fluzd, equations of motion and continuzty are as follows

$$
\frac{\partial u}{\partial t}=-\frac{1}{\rho} \frac{\partial p}{\partial x}-\mu u, \frac{\partial w}{\partial t}=-\frac{1}{\rho} \frac{\partial p}{\partial t}-g-\mu w, \frac{\partial u}{\partial x}+\frac{\partial w}{\partial z}=0
$$

Letting the averare velocities and pressure per unct volume of this region be $\bar{u}, \vec{w}$ and $\bar{p}$, respectivel, we have next relations

$$
\bar{u}=\nabla u \quad \bar{w}=v w \quad \bar{p}=\nabla p
$$


Then, eq 41 are rewritten as follows

$$
\frac{\partial \vec{u}}{\partial t}=-\frac{1}{\rho} \frac{\partial \bar{p}}{\partial x}-\mu \bar{u} \quad \frac{\partial \bar{w}}{\partial t}=-\frac{1}{\rho} \frac{\partial \bar{p}}{\partial z}-g \nabla-\mu \bar{w} \quad \frac{\partial \bar{u}}{\partial x}+\frac{\partial \bar{w}}{\partial z}=0
$$

Thus average motion has velocity potential $\bar{\phi}$ which satisfies Laplace's equation Putting

$$
\bar{u}=\partial \Phi / \partial x, \bar{w}=\partial \Phi / \partial z
$$

fluid pressure $\bar{p}$ is gaven by the following equation

$$
\frac{\bar{p}}{\rho}=-\frac{\partial \Phi}{\partial t}-\mu \bar{\phi}-g V z
$$

The boundary condition for potential $\bar{\phi}$ in regzon II of $F_{2 g} 4$ are $\partial \Phi / \partial z=0$ at $\mathrm{z}=0$ and $\mathrm{z}=-\mathrm{h}$, and from the contanuzty of mass flux at $x= \pm l$,

$$
\frac{\partial \phi_{1}}{\partial x}=\frac{\partial \Phi}{\partial x} \quad \text { at } x=l, \quad \frac{\partial \phi_{3}}{\partial x}=\frac{\partial \bar{\Phi}}{\partial x} \quad \text { at } \quad \dot{x}=-l
$$

and also from the contenuxty of energy flux $p_{1}=\bar{p} / v$ at $x=\ell, p_{3}=\bar{p} / v$ at $x=-l$, so that

$$
\begin{array}{ll}
\frac{\partial \phi_{1}}{\partial t}=\frac{1}{\nabla}\left(\frac{\partial \bar{\phi}}{\partial t}+\mu \bar{\phi}\right) & \text { at } \quad x=l \\
\frac{\partial \phi_{3}}{\partial t}=\frac{1}{\nabla}\left(\frac{\partial \Phi}{\partial t}+\mu \bar{\phi}\right) & \text { at } \quad x=-l
\end{array}
$$

Since the velocity potential in each region is given by eq 5,6 and 7 for breakwater, we obtain next relations corresponding to eq. 20 and 21 , using above conditions and the same symbols as eq 19

$$
\begin{aligned}
& \frac{b+f}{a}+\imath \sum_{n=1}^{\infty} \frac{\lambda_{0}^{2}+r^{2}}{\lambda_{n}^{2}-r^{2}} \frac{\lambda_{n}+P_{r} e^{\omega \nu}}{\lambda_{0}+P_{r} e^{l(\nu-\pi / 2)}} \frac{c_{n}+g_{n}}{a}=\frac{\lambda_{0}+P_{r} e^{u(\nu+\pi / 2)}}{\lambda_{0}+P_{r} e^{(\nu-\pi / 2)}} \quad(r=012)(49) \\
& \frac{b-f}{a}+\iota \sum_{n=1}^{\infty} \frac{\lambda_{p}^{2}+r^{2}}{\lambda_{n}^{2}-r^{2}} \frac{\lambda_{n}+Q_{r} e^{(\nu)}}{\lambda_{0}+Q_{r} e^{(\nu-\pi / 2)}} \frac{c_{n}-g_{n}}{a}=\frac{\lambda_{0}+P_{r} e^{u(\nu+\pi / 2)}}{\lambda_{0}+Q_{r} e^{l(\nu-\pi / 2)}} \quad(r=012)(50)
\end{aligned}
$$

where

$$
\begin{aligned}
& P_{r}=\frac{r \nabla \tanh r H}{\beta} \quad Q_{r}=\frac{r \nabla \operatorname{coth} r H}{\beta} \quad P_{0}=0 \\
& Q_{0}=\lim _{r \rightarrow 0} r H \operatorname{coth} r H / \beta H=V / \beta H, \quad \beta=\sqrt{1+(\mu / \sigma)^{2}}, \quad V=\tan ^{-1}(\mu / \sigma)
\end{aligned}
$$

Solvang eq 49 and 50, we obtain b/a, f/a, $c_{x} / a$ and $g_{x} / a$ for breakwater, and then $D_{r}$ and $\mathrm{E}_{r}$ are given as follows

$$
D_{0}=\frac{d_{0}}{2}=\frac{\eta}{2 \beta} e^{\omega^{\nu} \frac{\sigma^{2} h}{g}} \frac{a}{\pi^{2}}\left[\left(1+\frac{b+f}{a}\right) \frac{1}{\lambda_{0}^{2}}-\sum_{n=1}^{\infty} \frac{1}{\lambda_{n}^{2}} \frac{c_{n}+g_{n}}{a}\right]
$$




$$
\begin{aligned}
& D_{r} \text { coshrH }=d r=\frac{\nabla}{\beta} e^{\frac{\nu \sigma^{2} h}{g}} \frac{a}{\pi^{2}}\left[\left(1+\frac{b+f}{a}\right) \frac{1}{\lambda_{0}^{2}+r^{2}}-\sum_{n=1}^{\infty} \frac{1}{\lambda_{n}^{2}-r^{2}} \frac{c_{n}+g_{n}}{a}\right] \\
& E_{r} \text { sumhrH }=e_{r}=\frac{\nabla}{\beta} e^{2 \frac{\nu \sigma^{2} h}{g}} \frac{a}{\pi^{2}}\left[\left(1+\frac{b-f}{a}\right) \frac{1}{\lambda_{0}^{2}+r^{2}}-\sum_{n=1}^{\infty} \frac{1}{\lambda_{n}^{2}-r^{2}} \frac{c_{n}-g_{n}}{a}\right]
\end{aligned}
$$

Surface wave profiles, reflection and transmission coefficients etc are given In the same form of eq 28, 29 and 30 etc, using b/a, f/a, cN/a and $g_{n} / a$ by eq 49 and 50 The fluld pressure in the region II is given by eq 45 for the incident wave $S_{0}=\alpha \cos (k x+\sigma t)$ as follows

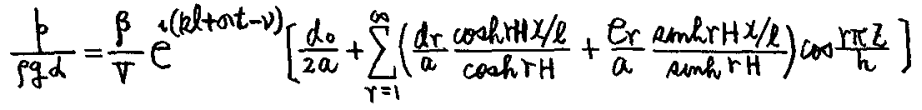

Pressure distribution and the total pressure to horlzontal plate are given as follows

$$
\begin{aligned}
& p_{3(z=0) / \rho g \alpha}=\frac{\beta}{T} e^{l(k l+r t-\nu)}\left[\frac{d_{0}}{2 a}+\sum_{r=1}^{\infty g}\left(\frac{d_{r}}{a} \frac{\cosh r H x / l}{\cosh r H}+\frac{e_{r}}{a} \frac{\sinh r H x / l}{\sinh r H}\right)\right. \\
& \left|P_{u} / 2 \rho g d l\right|=\frac{\beta}{V}\left|\left(\frac{d_{0}}{2 a}+\sum_{r=1}^{\infty} \frac{d_{r}}{a} \frac{\tan \ell r H}{r H}\right)\right|
\end{aligned}
$$

Above-mentıoned results are for single plate breakwater For quay wall, simllar results are obtained by the same process as in section 3

9 Calculated results for horlzontal plate breakwater with permeable material

As an example, b/a, f/a, $c_{n} / a, g_{w} / a$ and $a_{r} / a, e_{r} / a$ by eq 49 and 50 for $\Omega^{2} \mathrm{~h} / \mathrm{g}=10, \quad l / \mathrm{h}=10, \quad \mathrm{~V}=05$ and $\mu / \sigma=10$ are shown in rable 3

Transmassion and reflection coefficients for $l / h=10, v=05$ are shown in

F1g 13 for parameter $\mu_{\sigma}$ When $\mu / \sigma=0, K_{r}$ and $K_{t}$ are equal to those for $\ell / h=20$ in Fig 6(a) for the case without permeable material when $\mu / \sigma \neq 0, \mathrm{~K}_{r}$ becomes smaller than that for $\mu / \sigma=0$ and then 1 increases $w 1$ th the increase of $\mu / \sigma$ on the contrary, $K_{t}$ decreases steadlly with increase of $\mu / \sigma$ This means that the vold has the effect to elongate the length $\ell$ of the plate to $l / V$ and the fluid resistance has the effect to resist to the incident wave and to cause energy dissipation in breakwater

l'he pressure distribution to plate by eq 54 is shown in Fig 14 Compared with Fig $7(a)$, it is seen that the distribution is steeper than the case wh thout permeable materıal and the tendency is remarkable for large $\sigma^{2} \mathrm{~h} / \mathrm{g}$ This colncides with the fact that the vold has the effect to elongate the plate length

It may be sald that the submerged plate has the effect to make the pressure 
distribution uniform but the submerged permeable material has the eftect to make the distribution steep Ihe averaged total pressure by eq 55 is nearly equal to the case without permeable material.

10 Galculated results for horizontal plate quay wall whth permeable material

for the same conditions as previous section, reflection coefficient $K_{\gamma}$ for quay wall is calculated as shown $1 n$ big $15 \quad K_{r}$ decreases steadily witn the increase of $\sigma^{2} h / g$, but not so with $M / \sigma$ for large values of $\sigma^{2} h / g$. Kr 1 s manmum for $\mu / a$ between 10 and 20 That 1 , reflection coefficient 1 s the least for a particular value of $M / \sigma$ dependung on $\sigma^{2} h / \xi$ and becomes $\operatorname{lar}_{\mathrm{t}}$ er for other values of $\mu / \alpha$ As seen from $41 \mathrm{~g} 15, \mathrm{~K}_{\mathrm{r}} \mathrm{is}$ larger than 094 for $\mathrm{a}^{2} \mathrm{~h} / \mathrm{g}<15$ and also by calculations for various values of $l / h$, it is found that $K_{r}$ ls almost constant for $l / h>10$, that is, $\mathrm{Kr}$ is independent on the lencth of plate for $\ell / \mathrm{h}>10$ l'he fact that the reflection coefficient of quay wall does not decrease even for large values of $\mu / \alpha$ and $l / h$ is interpreted to be due to the small fluid velocity and the energy dissipation inside the permeable materıal under the horizontal plate

\section{Comparisons with the experiment}

Using the wave channel of leneth 22 meters, width 10 meters and depth 06 meters with flap type wave generater, experımental measurerent was carrıed out for single plate breakwater of $h=40 \mathrm{~cm}$ and $\ell=40 \mathrm{~cm} \quad(l / h=10)$ without permeable materlal, for Incldent wave anplitude $\alpha$ nearly constant of $3 \mathrm{~cm}$ Pransmission and reflection coefficlents are shown in Fig 16, in whıch each plotted daturn is the mean value of 10 times measurements lleasured trmsmission coefficients agree well with theoretical values but reflection coefficients are lower than theory the measured pressure distribution to plate is shown in Fig 17, which shows that the measured distributions are remarkably steeper than those of theoretıcal ones Phese discrepancies might be due to wave overtopping and eddies generated at the front edge of plate ileasured average total pressure to the plate axe shown in Fig 18, which shows good agreenent with theory for the case with permeable materlal, experinents are now under taken

\section{Conclusions and remarks}

Theory of single plate breakwaver for long waves are introduced by $J$ stoker (1957) and the mathematical study of dock problems are presented by $K O$ Friedrichs and H Levy (1948) and others In this paper we have tried the extension of Stoker's result to the case of general wave conditions by different method from these authors 
llan results of our study are sumperzzed as follows

(1) For the case of breakwater without permeable naterial, a horızontal plate fixed $r l g l d l y$ t water surface behaves lıke as a semı-rıgld breatwater for short surtace waves and more incident wave energy is reflected for shorter waves and longer plate Ihe lonper the plate Is, the more steeply distributes the pressure along the plate In case of double plates, the pressure distribution becomes remarkably smooth (11) For the case of quay wall, the pressure distribution to vertıcal wall is remarkably unjform by the effect of horizontal plate, compared to the case without plate Ihe pressure distribution to plate is simlar to that of breakwater, and the longer the plate 1s, the more equally becomes the averaged pressures to vertical wall and to horizontal plate In other words, the horizontal olate at water surface of quay wall plays the role of pressure equalızer to horizontal and vertıcal directions In case of double plates, pressure distributions to both directions becomes more smooth than the case of single plate

(1.1) When the region under the horizontal plate is tilled by permeable material, the vold has the effect to elongate the plate, so that for breakwater the transmission coefficient decreases and the reflection coefficient increases and for both of breakwater and quay wall the distrioution of pressure along the plate becomes steeper than the case without permeable material the effect of fluid resistance is partly to reflect the incident wave and partly to absorb wave energy, so that for breakwater the reflection coefficient increases and the transmission coefficient decreases and quay wall the decrease of reflection coefficient is not so remarkable The pressure distribution along the plate and the vertical wall is not changed remarkably by the effect of fluid resistance

\section{Leferences}

Stoker, J J (1957) " Water Waves " pp 450 436, Interscience Publıshers "riedrichs, $K O$ and "Levy(1948) "The Dock vroblem" Comunzcations on Pure and Applied Mathematics, Vol 1, pp $135 \quad 148$ 
Table $1 \lambda_{0}$ and $\lambda_{n}$ for $a^{2} h / g$

\begin{tabular}{|c|c|c|c|c|c|}
\hline$a^{2} h$ & 01 & 05 & 10 & 15 & 20 \\
\hline$\lambda_{0}$ & 010237 & 024564 & O 38187 & 051624 & 065742 \\
\hline$\lambda_{1}$ & o 98977 & 094700 & 089075 & o 83459 & 078263 \\
\hline$\lambda_{2}$ & 199492 & 197440 & 194845 & 192251 & 189699 \\
\hline$\lambda_{3}$ & 299662 & 298303 & 296597 & 298489 & 293194 \\
\hline$\lambda_{\Delta}$ & 399747 & 398730 & 397456 & 396182 & 394913 \\
\hline$\lambda 5$ & 499794 & 498985 & 497968 & 496951 & 495953 \\
\hline & 599831 & 599155 & 598308 & 597462 & 596622 \\
\hline
\end{tabular}

Table 2 (a) $\sigma^{2} \mathrm{~h} / g=10, \quad l / \mathrm{h}=10$ (single plate)

$\mathrm{b} / \mathrm{a}=05621+05434 \mathrm{f} \quad \mathrm{f} / \mathrm{a}=04334-04482 \mathrm{I}$

\begin{tabular}{|c|c|c|c|}
\hline $\mathrm{n}$ & & $c_{n} / a$ & $g_{n} / a$ \\
\hline $\begin{array}{l}1 \\
2 \\
3 \\
4 \\
5 \\
6 \\
7\end{array}$ & $\begin{array}{ll}-0 & 1340 \\
\text { - } 0 & 03114 \\
-0 & 01392 \\
\text { - } 0 & 007891 \\
-0 & 005077 \\
-0 & 003532 \\
-0 & 002582\end{array}$ & $\begin{array}{l}-0037531 \\
-0010061 \\
-00046581 \\
-00026831 \\
-00017421 \\
-00012201 \\
-000087511\end{array}$ & $\begin{array}{l}-005896+0028371 \\
-001050+00080751 \\
-0004308+00037881 \\
-0002340+00021951 \\
-0001467+00014301 \\
-0001003+00010031 \\
-00007238+000073741\end{array}$ \\
\hline$x$ & & $\mathrm{~d} r / \mathrm{a}$ & $e_{r} / a$ \\
\hline $\begin{array}{l}0 \\
1 \\
2 \\
3 \\
4 \\
5 \\
6 \\
7\end{array}$ & $\begin{array}{ll}0 & 7063 \\
0 & 08366 \\
0 & 02246 \\
0 & 01004 \\
0 & 005635 \\
0 & 003594 \\
0 & 002488 \\
0 & 001823\end{array}$ & $\begin{array}{l}+0033961 \\
+00039901 \\
+00010711 \\
+000047881 \\
+000026871 \\
+000017141 \\
+000011871 \\
+0000086991\end{array}$ & $\begin{array}{l}006392+0056161 \\
001522+0013371 \\
0006499+00057101 \\
0003563+00031311 \\
0002242+00019691 \\
0001537+00013501 \\
0001118+000098271\end{array}$ \\
\hline
\end{tabular}


Table $2(b) \cdot \sigma^{2} \mathrm{~h} / \mathrm{g}=10, l / \mathrm{h}=10$ (double plate)

$b / a=0001452+08682 I \quad f / a=04963-00008301$ I

\begin{tabular}{|c|c|c|c|c|}
\hline $\mathrm{n}$ & \multicolumn{2}{|r|}{$\mathrm{c}_{n} / \mathrm{a}$} & \multicolumn{2}{|c|}{$\mathrm{En} / \mathrm{a}$} \\
\hline $\begin{array}{l}1 \\
2 \\
3 \\
4 \\
5 \\
6 \\
7\end{array}$ & 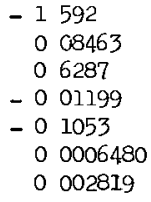 & $\begin{array}{l}-14221 \\
+0078881 \\
+059251 \\
-0011361 \\
-010011 \\
+000061711 \\
+00026881\end{array}$ & $\begin{array}{rl}-0 & 7140 \\
0 & 03229 \\
0 & 2284 \\
-0 & 004250 \\
-0 & 03680 \\
0 & 0002243 \\
0 & 0009689\end{array}$ & $\begin{array}{l}+008723 \text { I } \\
\text { - } 001117 \text { I } \\
\text { - } 009617 \text { I } \\
+00019531 \\
+0017781 \\
\text { - } 000011191 \\
-000049461\end{array}$ \\
\hline$x$ & \multicolumn{2}{|r|}{$d r / a$} & \multicolumn{2}{|c|}{${ }^{e} r / a$} \\
\hline $\begin{array}{l}0 \\
2 \\
4 \\
6 \\
8 \\
10 \\
12 \\
14\end{array}$ & $\begin{array}{ll}0 & 6614 \\
0 & 004674 \\
0 & 0005754 \\
0 & 0001629 \\
0 & 00006688 \\
0 & 00003387 \\
0 & 00001962 \\
0 & 00001247\end{array}$ & $\begin{array}{l}\text { + } 038301 \\
\text { + } 00027071 \\
\text { + } 000033321 \\
\text { + } 0000094341 \\
\text { + } 0000038731 \\
\text { + } 0000019621 \\
\text { + } 0000011361 \\
\text { + } 00000072191\end{array}$ & $\begin{array}{ll}0 & 002614 \\
0 & 0003059 \\
0 & 00008513 \\
0 & 00003464 \\
0 & 00001745 \\
0 & 00001007 \\
0 & 000006383\end{array}$ & $\begin{array}{l}+00044961 \\
+000052621 \\
+000014641 \\
+0000059591 \\
+0000030021 \\
+0000017321 \\
+000001098\end{array}$ \\
\hline
\end{tabular}

Table 3

$\sigma^{2} h / g=10, \quad l / h=10, \quad v=05, \quad \mu / \sigma=10$

$\mathrm{b} / \mathrm{a}=07769+01685 \mathrm{x} \quad \mathrm{f} / \mathrm{a}=01930-012392$

\begin{tabular}{|c|c|c|c|c|}
\hline$n$ & \multicolumn{2}{|c|}{$c_{n} / a$} & \multicolumn{2}{|c|}{$g_{n} / a$} \\
\hline $\begin{array}{l}I \\
2 \\
3 \\
4 \\
5 \\
6 \\
7\end{array}$ & 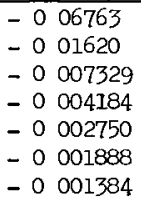 & 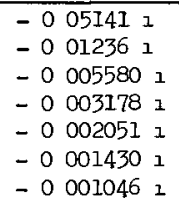 & $\begin{array}{ll}-0 & 02255 \\
\text { - } 0 & 003733 \\
\text { - } 0 & 001490 \\
\text { - } 0 & 0007981 \\
\text { - } 0 & 0004964 \\
\text { - } 0 & 0003377 \\
\text { - } 0 & 0002429\end{array}$ & $\begin{array}{l}\text { - } 00094551 \\
\text { - } 000080741 \\
\text { - } 000019131 \\
\text { - } 0000062911 \\
\text { - } 0000023321 \\
\text { - } 00000083491 \\
\text { - } 00000020151\end{array}$ \\
\hline$r$ & \multicolumn{2}{|r|}{$a_{r} / a$} & \multicolumn{2}{|c|}{ er/a } \\
\hline $\begin{array}{l}0 \\
1 \\
2 \\
3 \\
4 \\
5 \\
6 \\
7\end{array}$ & $\begin{array}{ll}0 & 16771 \\
0 & 03904 \\
0 & 01071 \\
0 & 004838 \\
0 & 002734 \\
0 & 001753 \\
0 & 001218 \\
0 & 0008950\end{array}$ & $\begin{array}{l}\text { - } 0177531 \\
\text { - } 0026381 \\
\text { - } 00071011 \\
\text { - } 00031761 \\
\text { - } 00017841 \\
\text { - } 00011381 \\
\text { - } 000078881 \\
\text { - } 000057821\end{array}$ & $\begin{array}{ll}0 & 028177 \\
0 & 007753 \\
0 & 003508 \\
0 & 001984 \\
0 & 001273 \\
0 & 0008849 \\
0 & 0006505\end{array}$ & $\begin{array}{l}-0031081 \\
\text { - } 00078681 \\
-00034451 \\
-0.0019151 \\
-00012151 \\
-000083821 \\
-000061261\end{array}$ \\
\hline
\end{tabular}




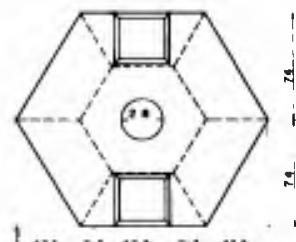

Las 33-40 -301-40

-s

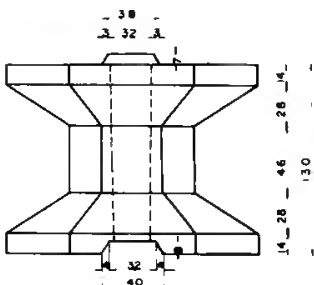

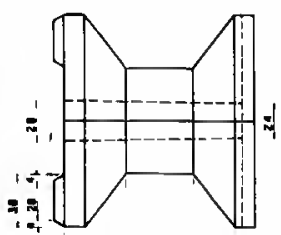

$14-20+\frac{16}{130}-20+14$

Vslume $1.310 \mathrm{~m}$ Weight 30 : .Dimension in $\mathrm{cm}$

Fig 1 Franged-Column Block

\section{Photo.1}

Dimension in Meters

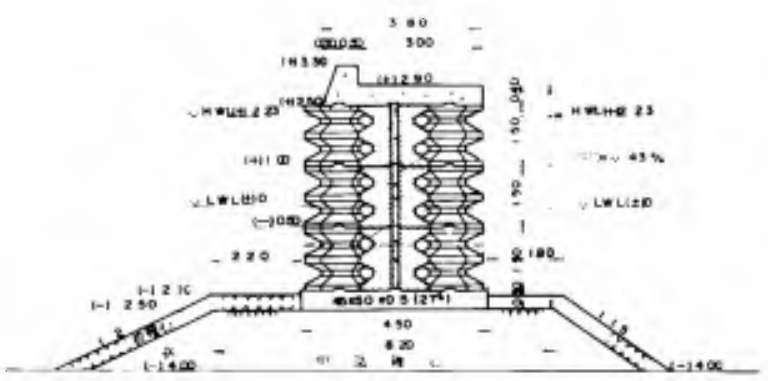

Fig.2 Breakwater by Franged-Column Blocks (Port of Hakata) 


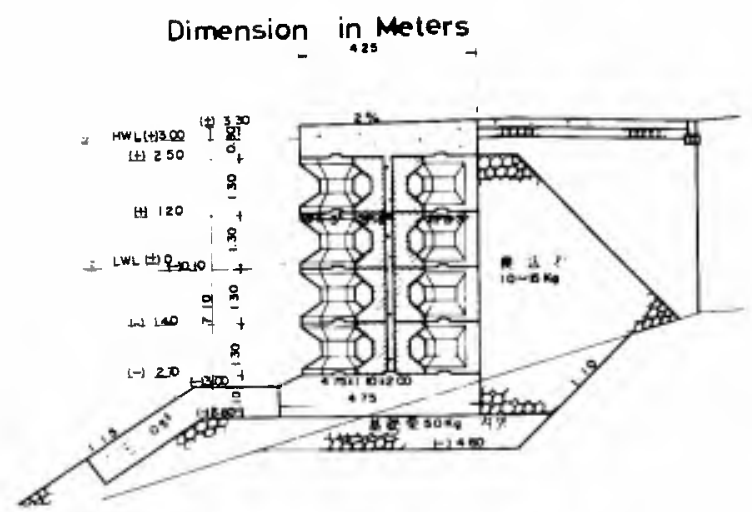

Fig.3 Quay Wall by Franged-Column Block (Tabira Harbour)

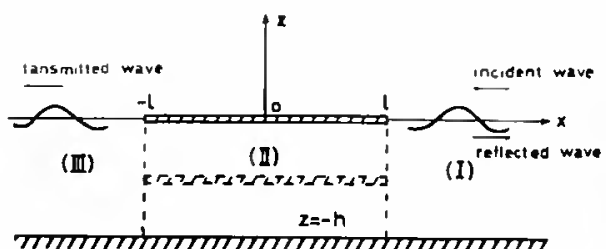

Fig- 4 Bredkwater by Horizontal Plate

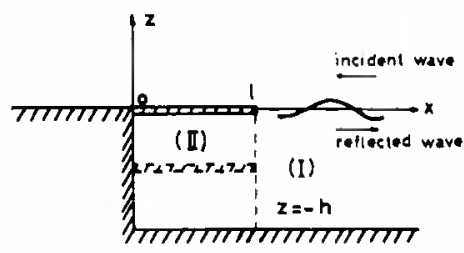

Fig. 5 Quay wall with Horizontal Plate
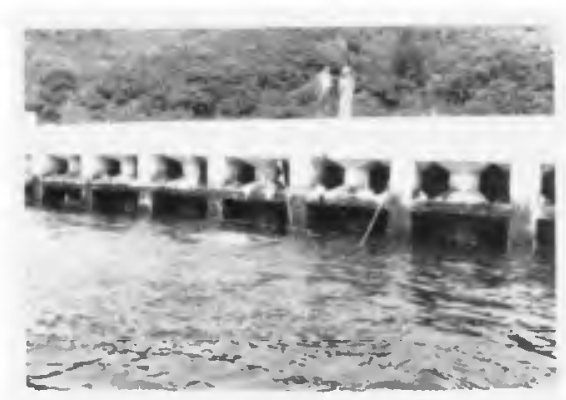

Photo. 2 

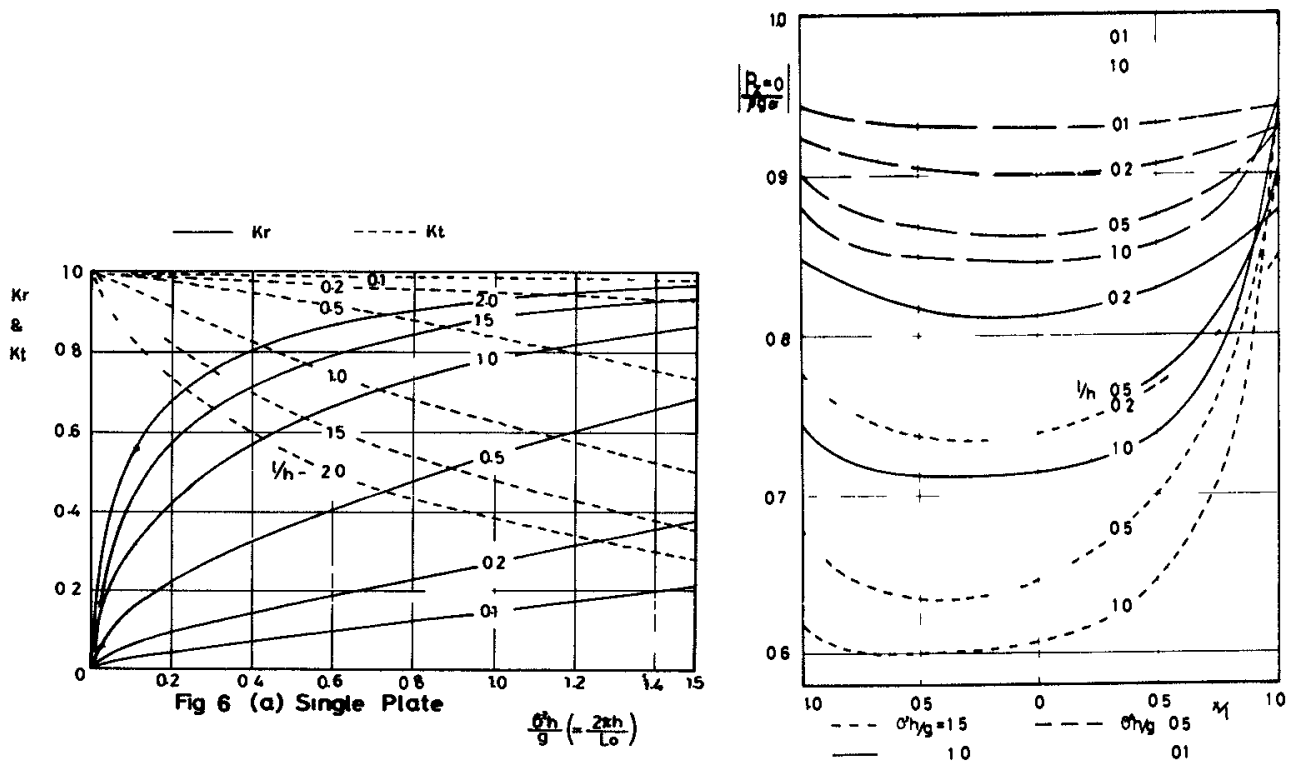

fig 7 (a) Pressure Oistribution To Plate (Single Plate Breakwater)
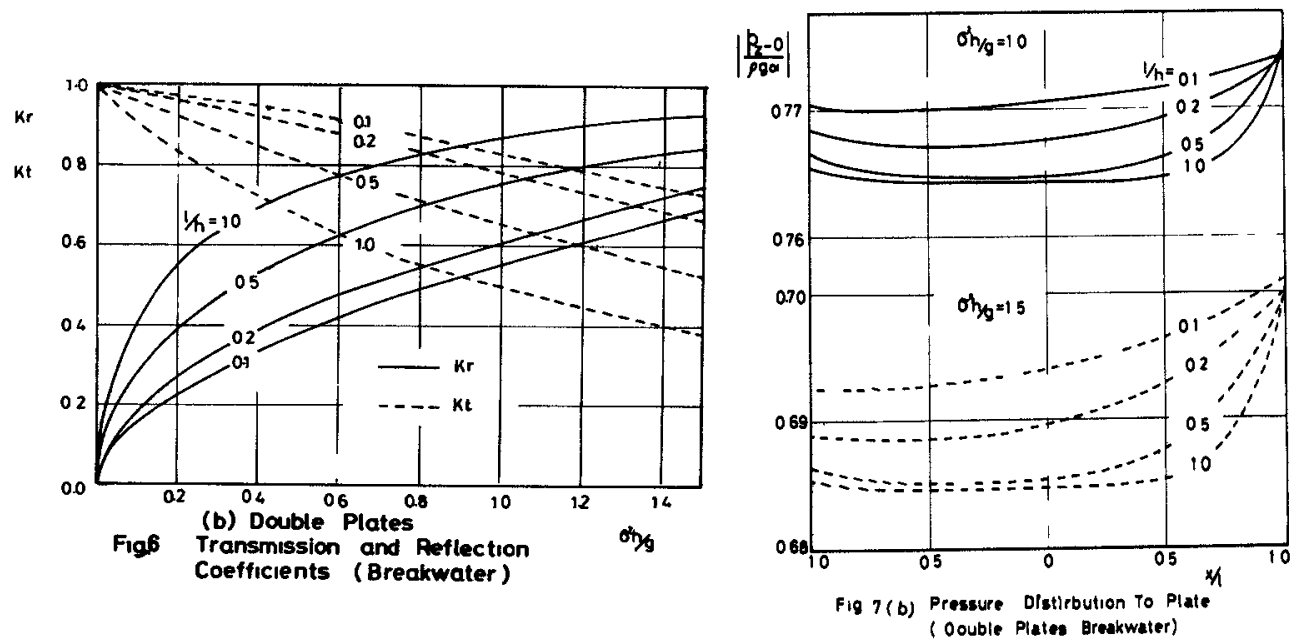

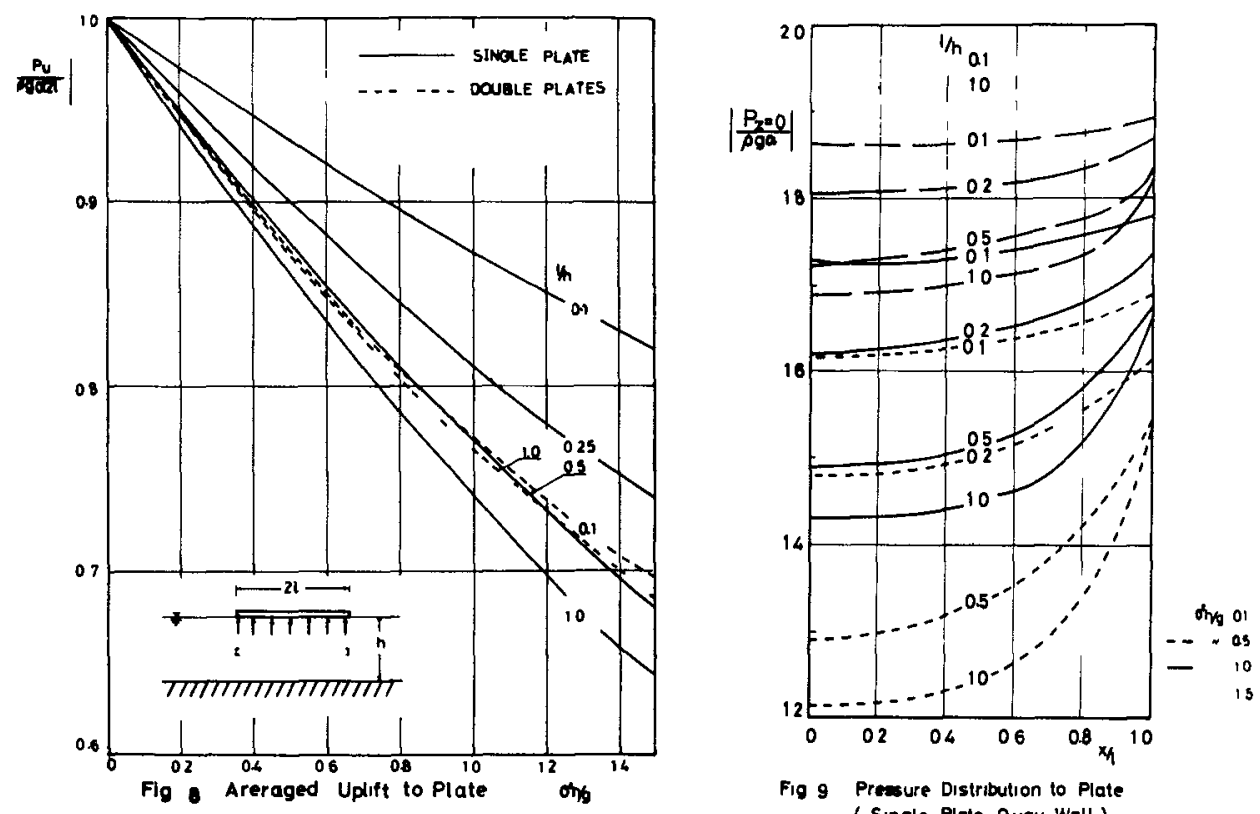

Fig 9 Prescure Distribution to Plate (Single Plate Quay Wall)

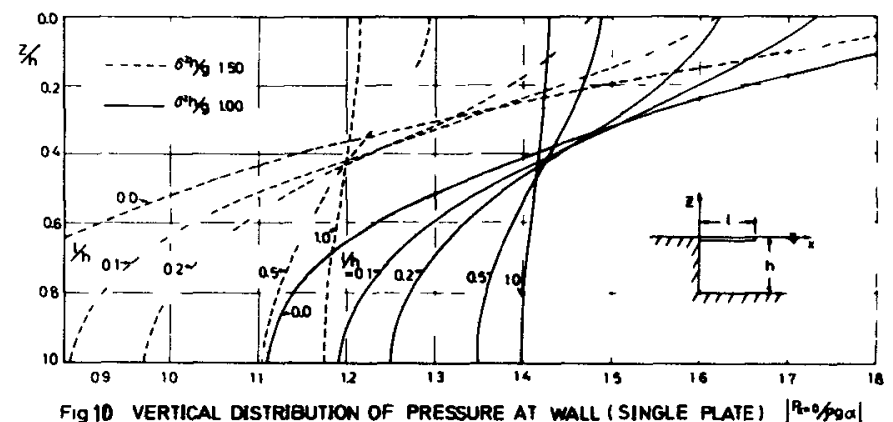

Fig 10 VERTICAL DISTRIBUTION OF PRESSURE AT WALL (SINGLE PLATE) |R-opgal 


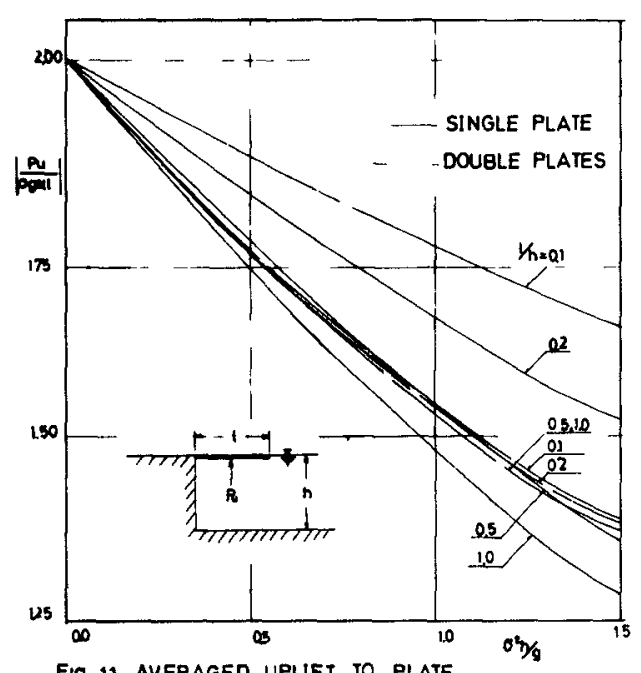

Fig 11 aVERAGED UPLIFT TO PLATE

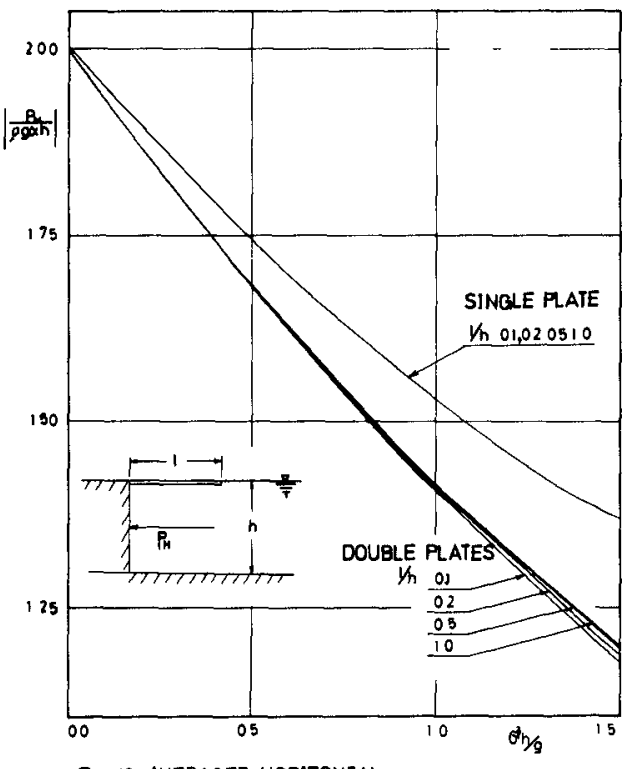

FIg 12 AVERAGED HORIZONTAL

PRESSURE AT WALL

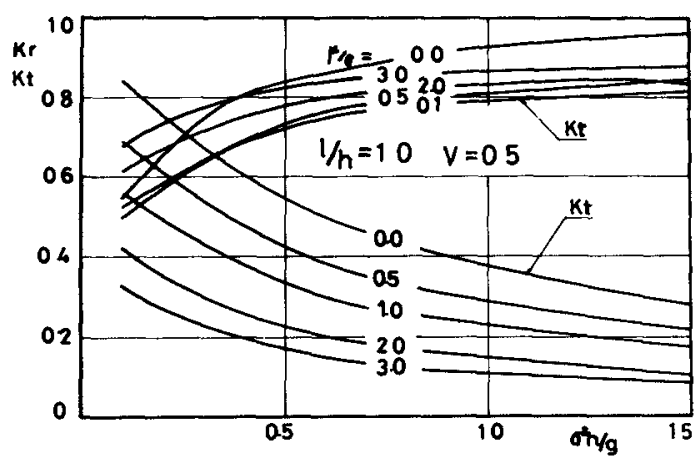

Fig $13 \quad K r$ and $K t$ of Breakwater 

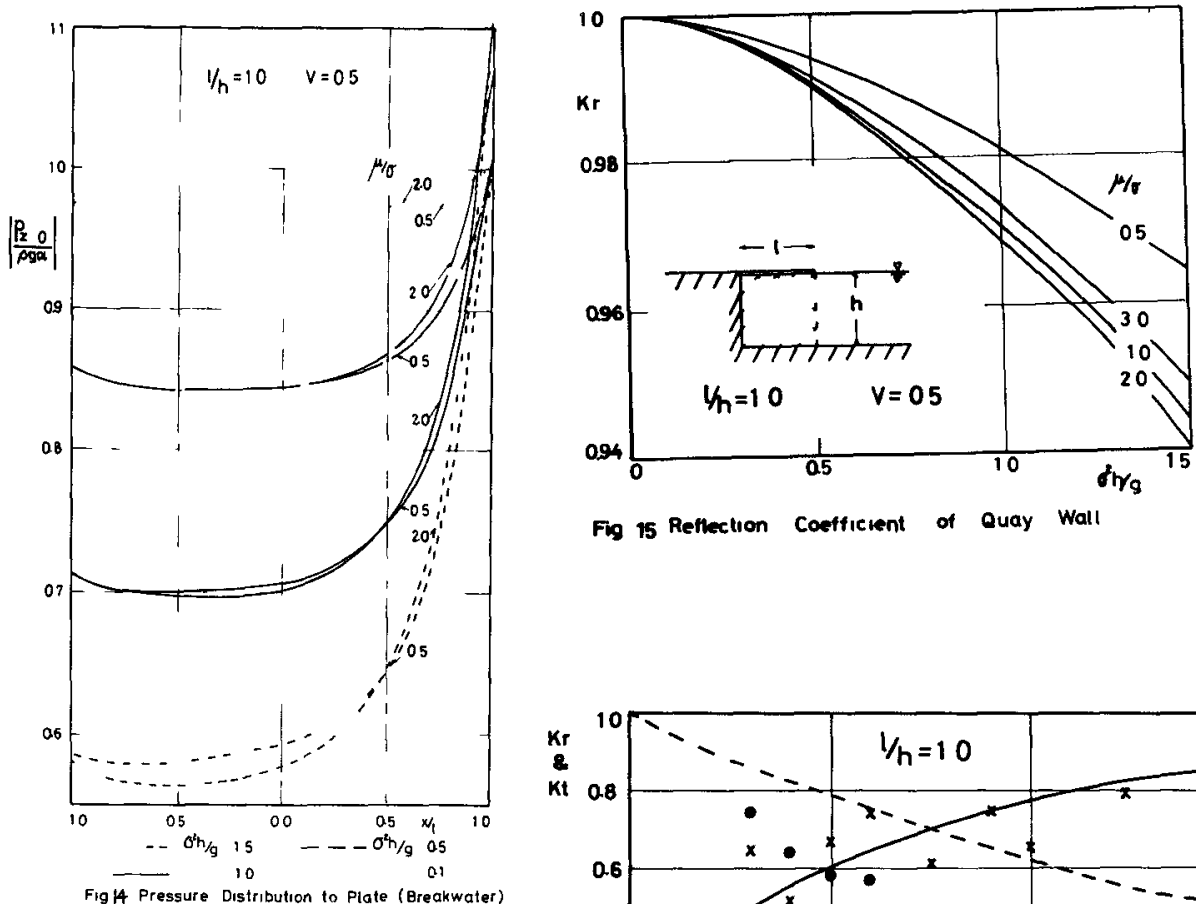

Fig 15 Reflection Coefficient of Quay Wall

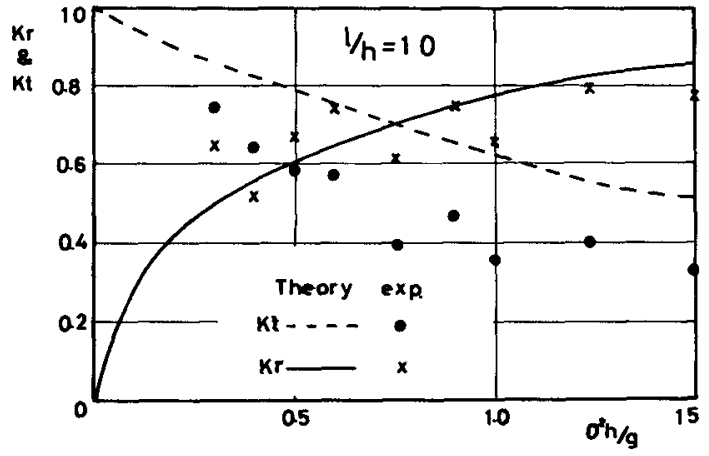

Fig $16 \mathrm{Kr}$ and Kt Single Plate Breakwater 


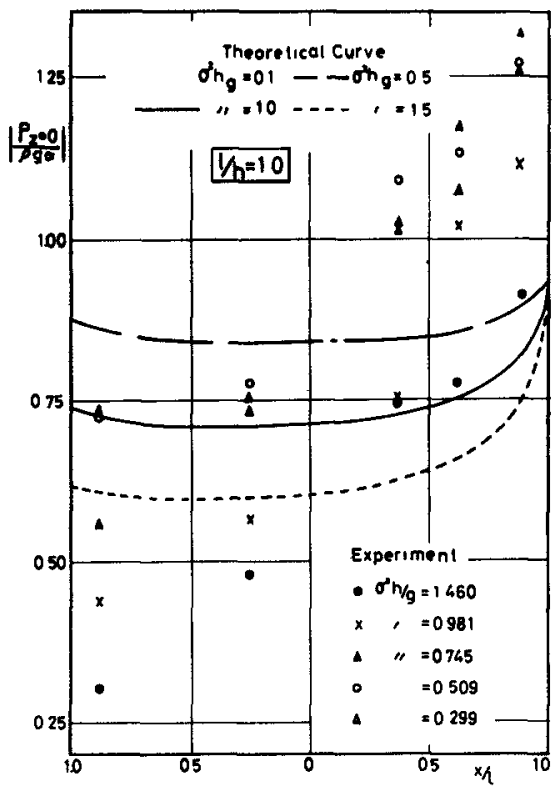

Fig 17 Pressure Distrlbution to Plate Theory and Experimenl( Single Plate Breakwater)

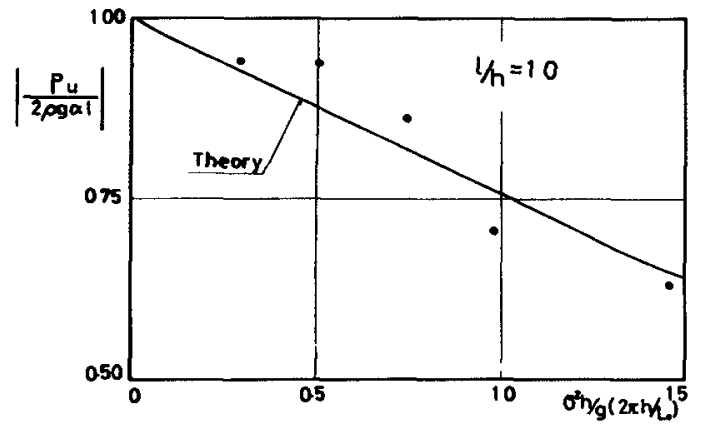

Fig. 18 Total Pressure to Plate Theory and Experiment ( Single Plate Breakwater) 


\title{
CHAPTER 95
}

\section{THE INFLUENCE OF BREAKER TYPE ON RIPRAP STABILITY}

\author{
by \\ John P Ahrens, A M , ASCE \\ Oceanographer \\ U S Army Coastal Englneerıng Research Center \\ Washington, D C
}

\begin{abstract}
Test data related to the stability of dumped quarry stone riprap under wave action is presented The tests were conducted in the large 635-foot wave tank at the Coastal Englneering Research Center at near prototype scale The data indicate that the stability of the riprap is strongly influenced by the type of breaker The lowest riprap stability is associated with a breaker type intermediate between plunging and surgıng, sometımes referred to as a collapsing breaker
\end{abstract}

\section{INTRODUCTION}

From February 1969 to June 1970, tests were conducted in the large 635-foot wave tank at the Coastal Engineering Research Center (CERC) to determine the relation of stability of dumped quarry stone riprap to wave action During this period sixteen riprap protected earth embankments were subjected to progressively higher waves untll the riprap falled The embankments were all constructed to have an inltial slope of 1 on 25 , with a medlan armor stone welght of 28 pounds for ten tests and 79 or 80 pounds for $s+x$ tests The purpose of this study was to collect data related to riprap stability at nearly prototype scale so that current design criteria can be improved The scope of the study includes riprap stability for waves in reservolrs and coastal waters The sixteen tests described here represent all of the tests planned for a slope of 1 on 25 , future tests w11 be conducted with slopes flatter than 1 on 25

\section{TEST CONDITIONS}

All of the sixteen tests described here were conducted in the 635foot wave tank at CERC The width of this tank is 15 feet, the depth is 20 feet, the water depth used for all tests was 15 feet and the distance from the toe of the slope to the mean blade position was about 440 feet S1deboards were placed along the top of the tank wall near the crest of the embankment to allow the crest of the embankment to be bullt to a helght of 24 feet above the bottom of the tank

The core of the embankment was composed of compacted earth, graded to a smooth 1 on 25 slope Between the core and the armor there was a layer of filter stone about half a foot thick The riprap was dumped 
on the filter bed from a skip, no water was in the wave tank durıng the dumping The riprap stone was quarry stone granite from near Occoquan, Virginia, with a speciflc gravity of 271 The median weight of the riprap stone was 28 pounds for tests SPL- 1 through 10 and 79 or 80 pounds for tests SPL-11 through 16 The gradation of the riprap was measured by the ratio of the diameter of the $85 \%$ stone divided by the diameter of the $15 \%$ stone, $D(85) / D(15)$, this ratio was about 17 for a11 tests The range in welghts of the riprap stone was from about 4 times the medlan welght down to about $1 / 8$ of the median welght The general shape of the stones could be descrubed as blocky, almost none of the stones had a ratio of their longest axis divided by their shortest axis greater than 4 The porosity of the riprap was $374 \%$ for the 28 pound stone and $387 \%$ for the $79-80$ pound stone

In order to include waves with characteristics similar to those observed on both reservolrs and in coastal waters, five wave periods were chosen for testing riprap stability Each of the tests in this study were run at one of the following wave periods $28,42,57$, 85 , and 113 seconds

\section{TEST PROCEDURES}

Waves were run in short bursts during the stability tests so that the generator would be shut off just before the wave energy reflected from the slope could reach the generator blade Between wave bursts there were brief interludes to allow reflected wave energy to dampen out Prior to the stability tests there was a calibration phase of this study to determine the proper wave helght to assign to a particular combination of generator stroke and wave period During calibration waves were run in bursts with a wave absorber beach in the tank and the heights were recorded at several locations in the tank The wave heights used in this study were all obtained during calıbration and, therefore, include almost no influence of reflection

A test was started by surveying the newly constructed slope on a square, 20 by 20 -foot grid in the horlzontal plane The inltial survey was then used as a reference wlth which to compare subsequent surveys The slope was surveyed with a heavy, rigid sounding rod with a ball and socket foot, the foot was clrcular with a diameter of 05 feet The helght of the first waves run on the newly constructed slope was chosen to be about $30 \%$ lower than the helght which was expected to dislodge armor stones Waves were run, in bursts, on the embankment until it appeared that no further stones would be moved by waves of this helght, in no case would less than 500 waves be run at a particular wave helght and often over 1500 waves would be run before the slope was considered stable After the riprap was demonstrated to be stable at a particular helght the slope would be resurveyed and the wave generator would be adjusted to generate waves approximately $10 \%$ higher The test procedure can then be summarızed as, running enough waves at a glven helght until it appears that the slope is stable, then survey the slope and Increase the wave helght about $10 \%$ and repeat the cycle The cycle was repeated until enough armor stones were removed to constitute 
fallure Fallure, for these tests, was defined as having occurred when enough riprap stones were displaced so that the filter Iayer was exposed to wave action and core material was actually being removed through the filter layer

\section{DISCUSSION}

The purpose of these tests was to determine the relationship of the stability of dumped quarry stone riprap to wave action on slopes of 1 on 25 The primary measure of stability for these tests was the zero damage wave height The zero damage wave helght was estimated on the basis of the written notes made by observers of their impression of the damage occurring to the riprap during the tests, and from damage calculations obtained from the frequent surveys of the slope By comparing a survey associated with some wave height with the initial survey, the volume of armor displaced and the maximum penetration of damage into the riprap layer were calculated and used to help estimate the zero damage wave he1ght TabIe I gives the estimated zero damage wave height for the various tests and also some other observed data Appendix II explains the meaning of aII symboIs used in this paper

Table I gives an average value of the runup ratio, $\mathrm{R} / \mathrm{H}$, for each test The runup ratio is the average of a number of observations made for waves Iower than the zero damage wave height, while the sIope was still in good condition Within the limited range of wave heights considered the runup ratio was approximately constant for each test The initial slope, shown in Table I, was calculated by fitting a least squares stralght line through the initial survey points The initial slope was used as an estimate of how well the construction conformed to the planned I on 25 slope Table I also shows the average riprap Iayer thickness, $\bar{r}$, for each test

As the test series proceeded, the scatter in the zero damage wave height was greater than expected and appeared to be at least partly dependent on the wave period This was not completely unforeseen, however, since a previous study on riprap stability conducted at CLRC seemed to show some infIuence of wave period The effect of the wave period was confused since these earIier tests were run in three different wave tanks at modeI scale ratios of from 1 to 30 to prototype In add $\perp t i o n$, the previous riprap tests did not generally test a wide range of depth to wave Iength ratios, $d / L$, as they were oriented towards some specific riprap problems on reservoirs

In Table II the stability number $N_{S}$, is defined and tabulated for each test by wave period and median armor weight The stability number shown in TabIe II is usefuI for intercomparing riprap tests, having the same slope but different median weights (Hudson (2)) TabIe II shows that the Iowest stability is associated with the four tests with a wave period of 42 seconds and the two tests at a period of 57 seconds with the larger median weight There is relatively high stability for both the short period waves, $T=28$ seconds and for the long waves, $T=85$ and 113 seconds Table II suggests that the breaker type may influence the riprap stability since it is related to the incident wave height and period 


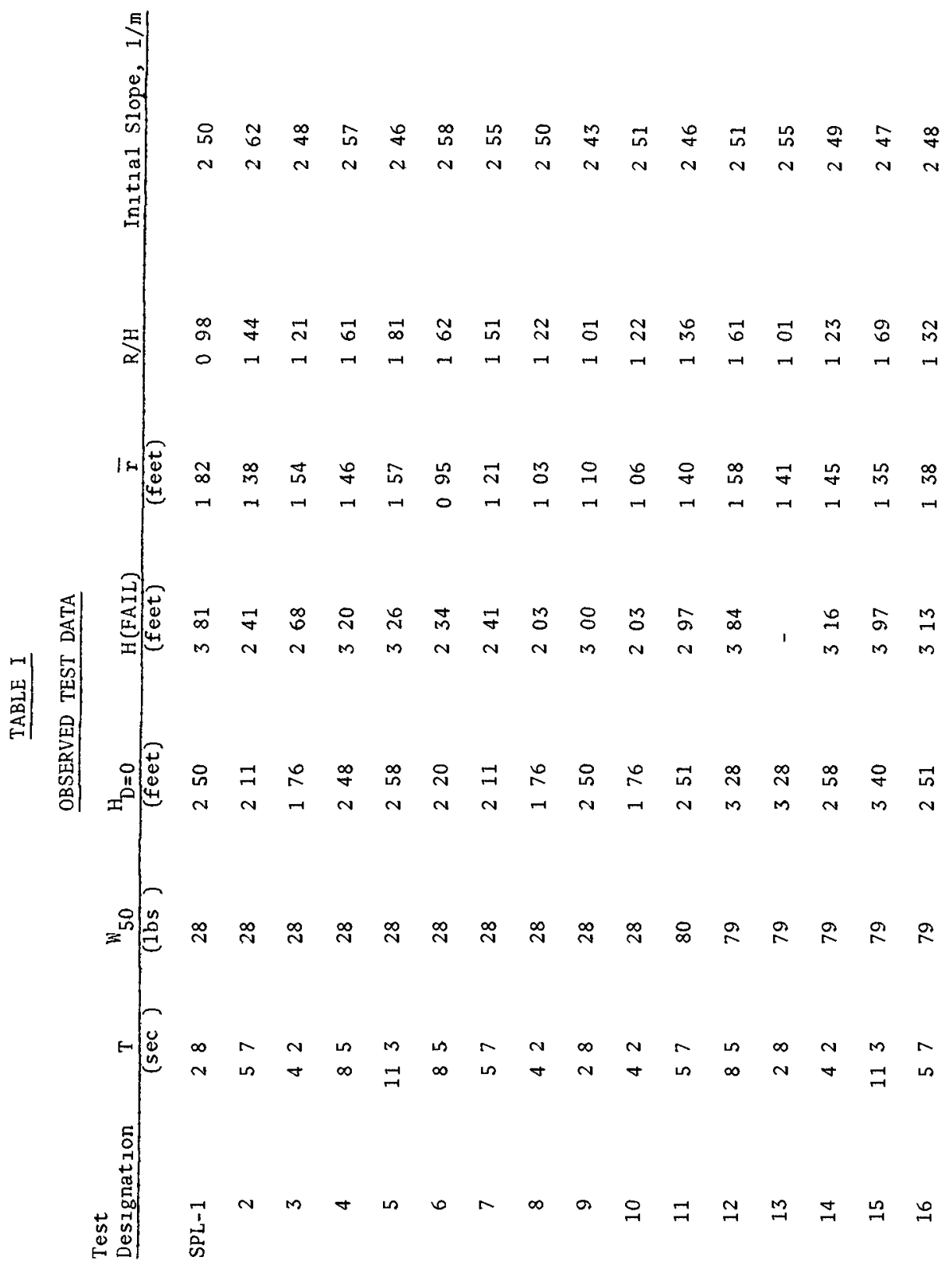


TABLE I

STABILITY NLMBERS BASED ON ZERO DAMAGE WAVE HEIGHTS

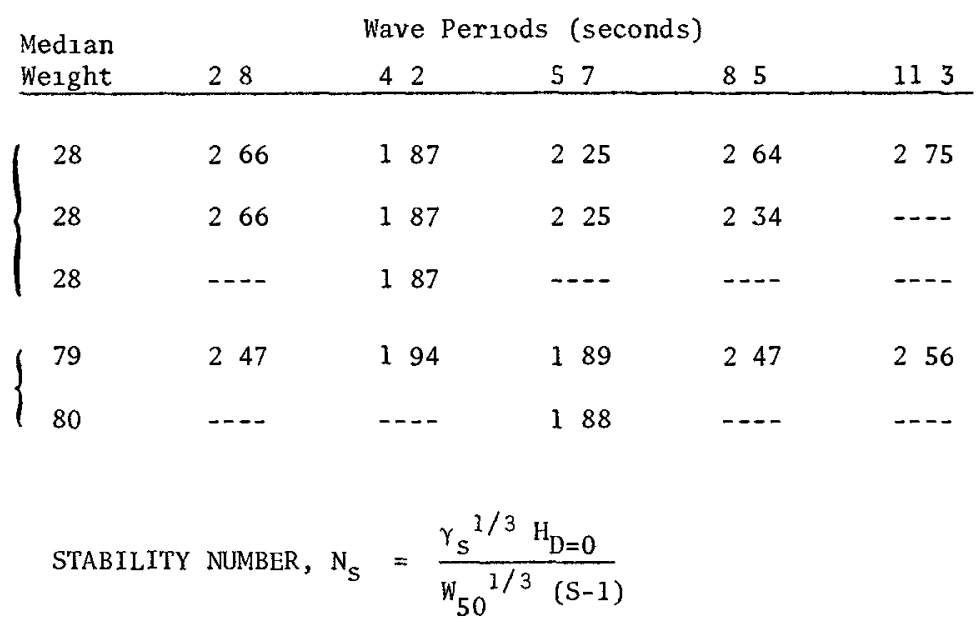


Table II also suggests that for the tests with the lowest stability the median armor weight is proportional to the cube of the zero damage wave helght given by Hudson's (2) formula

The breaker types for the six tests with low stability noted above (tests SPL-3, 8, 10, 11, 14 and 16) were all intermediate between surging and plunging Galvin (1) calls this transitional type of breaker a collapsing breaker Apparently collapsing breakers have characteristics which yıeld low riprap stability Table 111 places each test into a breaker category based on the wave conditions observed for the test before and just after the zero damage wave height Table lII shows that the average stability number for the tests with collapsing waves is considerably lower than tests in the plunging or surging breaker category Galvin's definitions of breaker types have been used throughout this study

In order to more clearly show the effect of breaker type on riprap stability Figure 1 shows the riprap stability coefficient, $K_{R R}$, plotted versus the offshore breaker parameter $\mathrm{H}_{\mathrm{O}} / \mathrm{L}_{\mathrm{o}} \mathrm{m}^{2}$ The riprap stability coefficient is equal to the product of the cube of the stability number and the tangent of the slope angle, it is similar to the stability coefficient, $K_{\Delta}$, which is frequently used in evaluating the stability of armor units in rubble-mound breakwaters The offshore breaker parameter is useful for predicting the breaker type on a known slope for waves with known deep water steepness The offshore breaker parameter for Figure 1 was computed by converting the zero damage wave height for each test to their equivalent deep water height Figure 1 clearly shows the low stability associated with collapsing breakers for these tests the transition between surging and collapsing breakers occurred between values of 008 and 010 of the offshore breaker parameter and the transition between collapsing and plunging occurred between values of 020 and 025 of the offshore breaker parameter

\section{CONCLUSION}

lntuitively it seems reasonable that the manner in which the waves break on a slope will influence the stability of the armor protecting the slope When a plunging breaker hits the armor stones with great impact, the impact has only a small component which is tangential to the slope, so that it takes a surprisingly large wave to dislodge armor stones The uprush following the plunge is a turbulent, spongy mass of water with a great quantity of entrained air and has little impact against the stones The return flow of the uprush appears to lack the volume, elevation and time to develop the strength to overturn the armor stones In the case of a surging breaker the runup travels rather gently up the slope and the impact against the armor stones is slight When riprap stones are removed by surging waves, it is by the return flow of the runup through, out of, and over the armor layer The flow out of the armor layex tends to $11 \mathrm{ft}$ the stones and the return flow over the surface tends to overturn them The breaker type transitional between plunging and surging, referred to as a collapsing breaker, has an uprush which seems to have significant impact against the stones and is directed approximately tangential to the slope The uprush of the collapsing wave 


\section{TABLE 11}

INFLUENCE OF BREAKER TYPE ON STAB1LITY NUMBER, $\mathrm{N}_{5}$, FOR DUMPED QUARRY STONE RIPRAP ON A SLOPE OF 1 ON 25

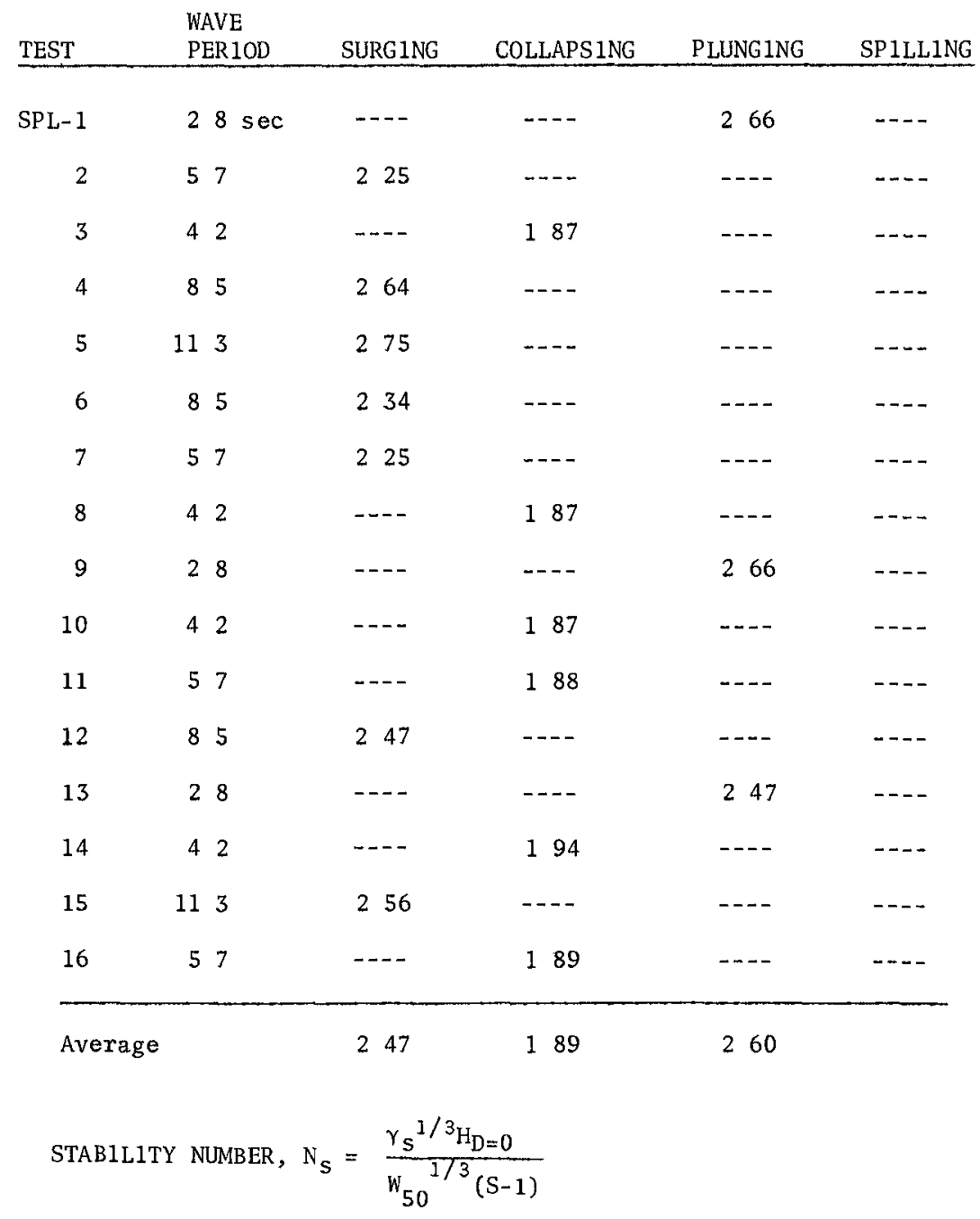




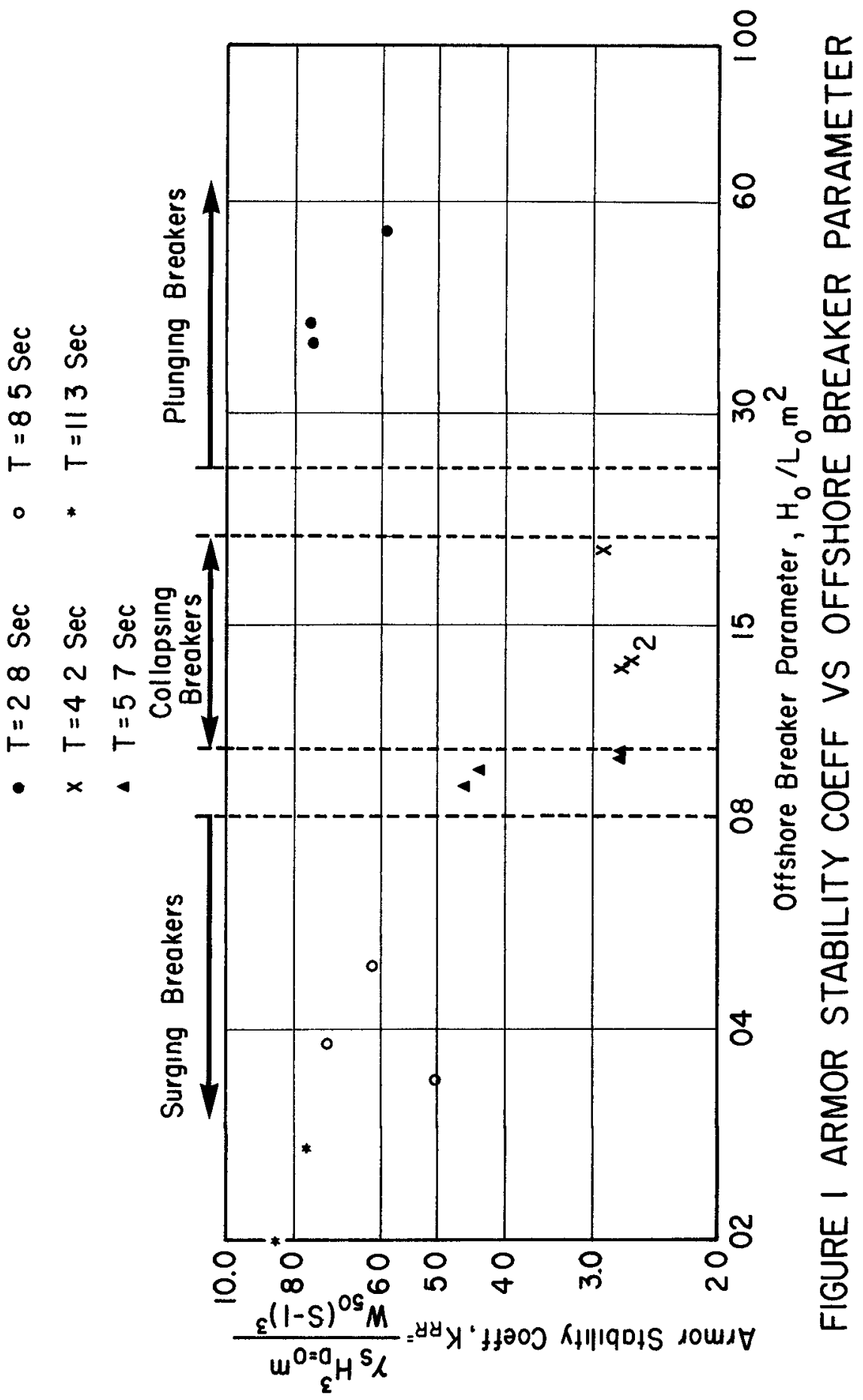


also has dynamic properties which tend to $11 \mathrm{ft}$ the stones The return flow of the runup can then dislodge or overturn stones which have been momentarily placed in a less stable position by the uprush Since the return flow of the collapsing breaker seems to be significantly stronger than that of the plunging breaker it is possible that even stones which were not made unstable by the uprush could be dislodged by the downrush It seems as if the physical characteristics of collapsing breakers have combined in an optimum way to yield low riprap stability

\section{ACKNOWLEDGEMENTS}

The author is indebted to the numerous suggestions of $\mathrm{Mr}$ Thorndike Saville, $\mathrm{Jr}$, Chief of the Research Division, CERC, and $\mathrm{Mr}$ George Simmons, Chief Technician, Research Division, CERC, which have materially improved this study The study was supported jointly by the office of Chief of Engineers and CERC of the U S Army Corps of Engineers, permission to publish this paper is gratefully acknowledged

\section{APPENDIX 1 - REFERENCES}

1 Galvin, Cyrı1 J, Jr, "Breaker Type Classification on Three Laboratory Beaches", Journal of Geophysical Research, Vol 73 , No 12 , June $15,1968, \mathrm{p}$ 3651-3659

2 Hudson, $\mathrm{R} Y$, "Laboratory lnvestigations of Rubble-Mound Breakwaters", ASCE, Journal of the Waterways and Harbors Division, Vo1 85, No WW3, Paper 2171, September, 1959 
The following symbols are used in this paper

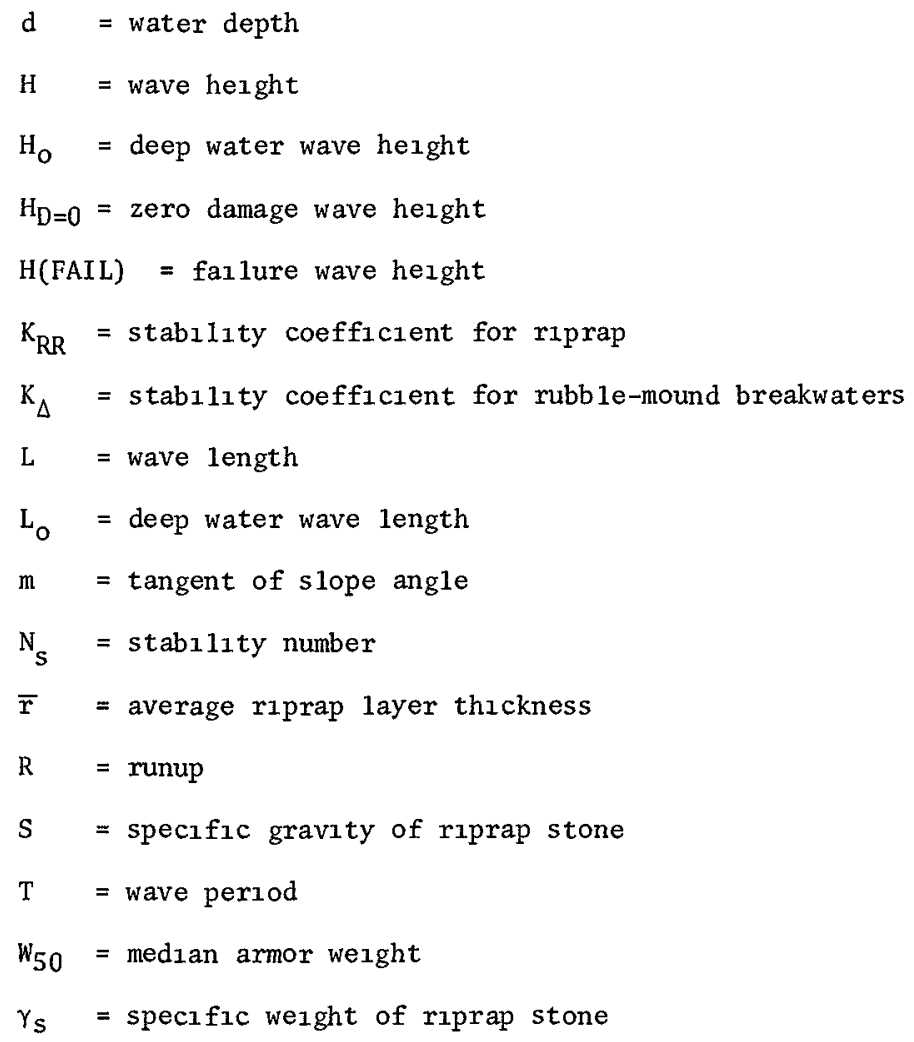




\title{
CHAPTER 96
}

\section{DAMAGE FUNCTIONS FCR A RUBBLE-MOUND BREAKWATER}

UNDER THE EFFECT OF SWELLS

\author{
Juan B Font \\ Assistant Professor \\ College of Englneering, Central University \\ Caracas, Venezuela
}

ABETRACT

In this paper experimental data are given to ald in the design of a rubble-mound breakwater The use of armor damage functions is supported rather than the use of the wave height for the no damage condition Damage curves defined expermentally are proposed, for both rocks and tetrapods, for different wave storm durations and for different placing tech niques

A determinant influence of storm duration is found for advanced damage of the armor layers

The experiments with different placing techniques showed that stability coefficlents based upon the no damage criteria, do not give a reliable picture of the ultimare strength of a rubble-mound breakwater

\section{INTRCDUCTION}

The experiments on which this paper is based have been made in a series of research works Hernández, Pastor and Suárez (1968), Loreto (1969), Ibarra and Blumentals (1969) Neri and Santeliz (1970) These researches were accomplished in the Hyaraulic Laboratory of the central University of Vene zueld, in partial fullfiliment of their Civil Engineer Degree, under the writer's direction

A preliminary paper on the influence of wave storm duration was submitted by the writer to the Eleventh Conferen ce on Coastal Engineering (1968) In that paper were given damage functions por different storm durations with the number of waves as the only parameter A strong dispersion of the 
experimental data showed the importance of the ratio $\mathrm{H}_{1 \%} / \mathrm{H}_{1 / 3}$, $\mathrm{H}_{1} \%$ being the wave height that caused one percent damage and $\mathrm{H} / / 3$ the so called significant wave height or mean of the larger one third In this paper new data on wave storm duration is submitted, in which this ratio is also taken as a parameter, thus obtaining a better fitting of the experimental points

The Hudson's design wave helght

Since the no damage criteria for rubble-mound breakwater design were defined (Hudson, 1959), most research work on this sub ject has focused its attention on the initial damage However, it is generally accepted that rubble-mound breakwaters are expected to withstand the design storm with some damage, elther reaching equilibrium or being repaired before next storm hits

The deffinition of the design conaltion for the one percent damage has the advantage of being equivalent to the no damage situation and thus numerically well defined However, the stabilicy of the one percent of the armor rocks that are first displaced, depends largely on random placing and geometrical fac tors, rather than on the armor capacity as a whole to resist waves Rogan (1968) points out the fact, also observed by the writer, that the filter layers uncovering is simpler to observe and more significant than the number of displaced rocks, specially at the inclpient stages of damage In this sense, also the late Iribarren ( 1965) defined the design criteria based on the total fallure of the breakwater Iribarren proposed his most recent formula consi dering that the fallure of the armour depended largely on the siope stability of about six rows of that could slide at the same time Nevertheless, although this type of fallure is often present at the final stages of fallure, it has been largely induced by the continuous weakening of the armor in the maner considered by Hudson, it est rocks rolling or being lifted one by one

The design using the damage functions

The damage functions, rather than the no damage or total fallure conditions alone, should be given to the design engineer, summarizing the afore discussion in the following reasons

1 Economic considerations in one hand and safety factor in the other, usually lead to a design damage different from either the no damage or total fallure conditions

2 Neither the no damage or total failure conditions are solely determinant of the safety factor for a given design damage 
Both Hudson and Iribarren, in the writings above mentioned, have presented experimental data in the form of damage functions in which the percentage of damage is related to parameters defined by themselves The writer (1968) has proposed to relate the per centage of damage $\Delta \%$ to the ratio $\mathrm{H} / \mathrm{Hl} \%$, $\mathrm{H}$ being the wave height that causes the $\Delta \%$ damage, since technical literature is plenty of data for the $\mathrm{H}_{1 \%}$ selection. This representation also simplifies visual comparison of damage functions for different armor blocks and placing techniques

The experimental data and curves given in this paper are believed to apply specially to the case of decayed swelis, since the waves used in the laboratory are periodic waves of intermediate steepness To the writer knowledge, the first refe rence given to the frecuency spectrum shape influence apeared in a paper by Carstens et Al (1966) Ic could be said that the shape of the frecuency spectrum is related to the tendency of higher waves to break in front of the breakwater, therefore It is suggested that in future experimentation, related with the non-uniform waves effect on breakwaters, the relation between the significant wave height and the breaker height for the mean wave period at the depth of the structure be considered as a significant parameter In the present experiments this relation was

$$
03<\frac{H_{1 / 3}}{H_{6}}<04
$$

$\mathrm{H}_{\mathrm{b}}$ being the breaker height corresponding to the wave period and water depth of the tests as computed after the experiments of Danel (1952)

The influence of duration of locally wind generated waves was studied in the laboratory by Rogan (1968) The main conclusion of the latter is that the effect of a local wave storm is simllar to that of periodic waves with helght equal to the storm significant wave height

\section{INFLUENCE OF SWELI DURATION}

Fig 1 Shows the damage functions for rocks and tetrapods in which $\Delta \%$, the percentage of displaced elements, has been related to $\mathrm{H} / \mathrm{HI} \%$, $\mathrm{H}$ being the height of the wave that causes the damage and $\mathrm{H}_{1 \%}$ the heinght of the wave for the $1 \%$ damage, usually definedas the limit of the no damage condition The symbol $\mathrm{n}$ refers to the number of waves of the swell The simbol $\mathrm{H} / \mathrm{J}$, refers to the significant wave helght of swell

Fig 2 Shows the total damage caused by swells of different durations with $\mathrm{H}_{1 \%} / \mathrm{H}_{\mathrm{I}} / 3$ as parameter $\mathrm{H}_{\text {max }}$ reffers to the maximum vave helght expected during the storm for storms with more than 3 00o waves the $n=\infty$ curve could be used 

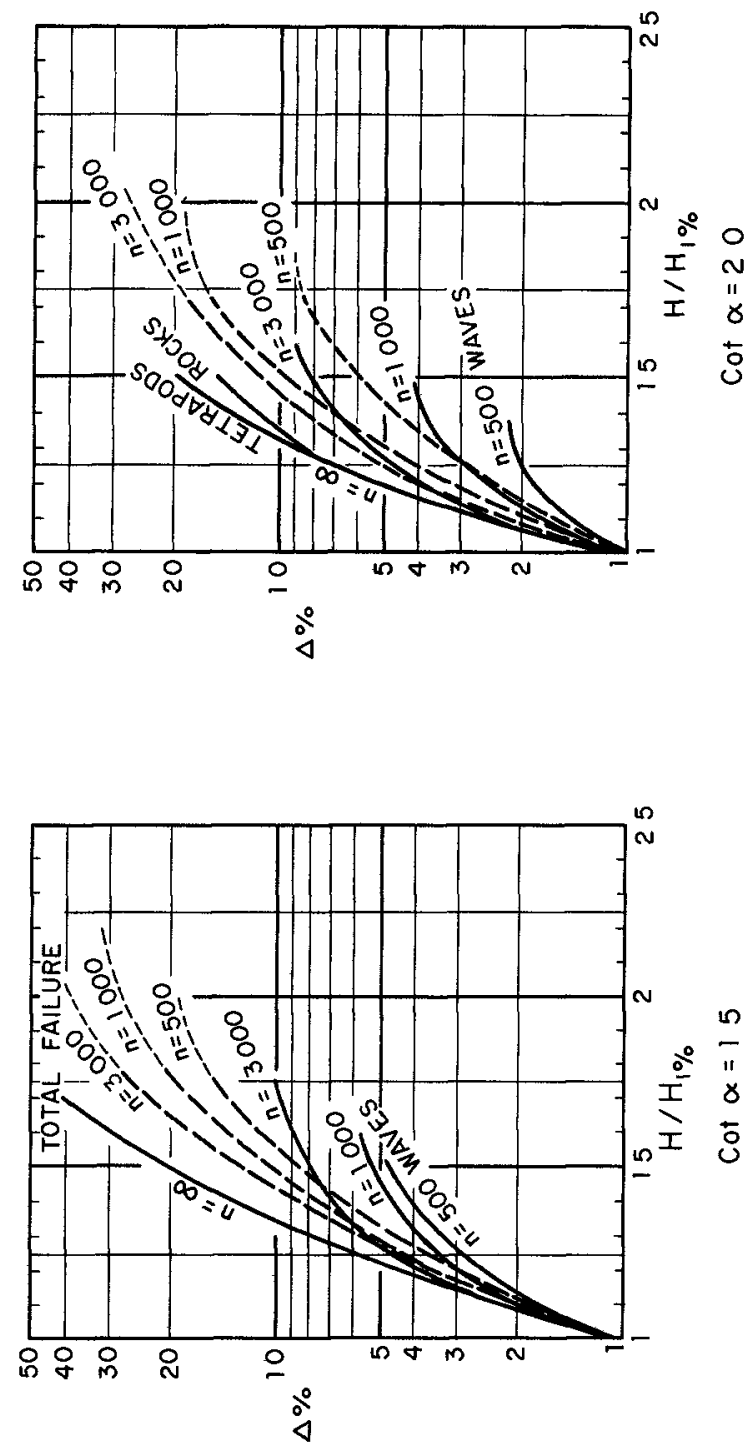

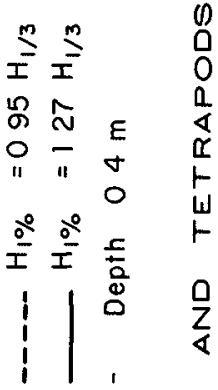

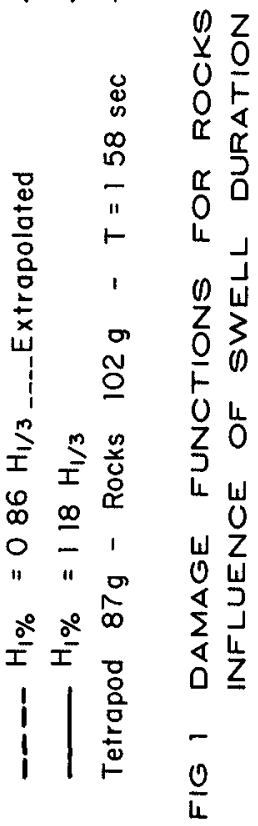



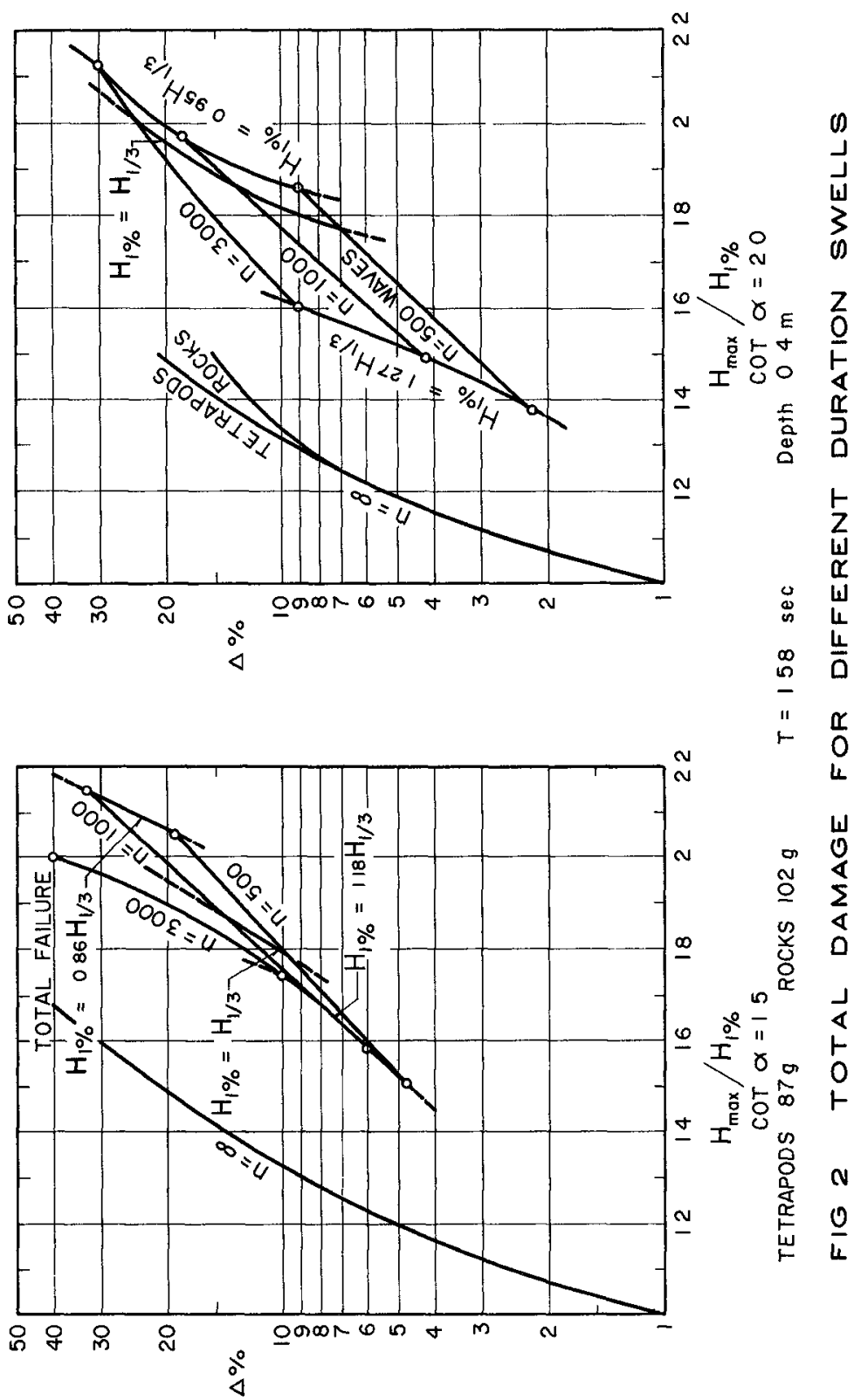
Although a subject for future research, it is believed that design in shallow water, where the largest waves break far from the structure, could be carried using the curves in Fig 1 with $H$ being the maximum active wave height Galvin (1969) has made experiments that may help in the selection of the maximum active wave height

\section{INFLUENCE OF PIACING TECHNIQUES OF ARMOR ELEMENTS}

Fig 3 shows the damage functions for rocks and tetra pods using different placing techniques Dashed curves correspoñd to careless random placing Full curves stand for careful placing, interlocking elements as much as possible

As 1t is shown by the experimental results, placing makes a big difference for the initial damage, but is less relevant for advanced damage, when the armor porosity and "dynamic" stabi lity are essential

\section{DAMAGE DISTRIBUTION ALONG BREAKWATER SLOPE}

Fig 4 Shows the average damage distribution curve of four sets of experiments, with the 115 slope for both rocks and tetrapods It is seen that while at the beginning the the da mage mostly occurs below the still surface level, for larger waves the portion inmediately above that level is also strongly affected As a matter of fact it is in this region where uncovered filter first appears

\section{THE ARMOR DENSITY COEFFICIENT}

Usually the kind of armor block to be used in a breakwa ter (rock, tetrapod, trubar, tetrahedron, dolos, stabit, etc), as well as the constructive method (dumping, placed by crane, pla ced with special techniques, etc), are selected taking into account economic and functional considerations It is sometimes difficult, however, to make economic comparisons since authors do not give enough data, restricting it usually to the Hudson:s coefficien $\mathrm{K}_{\mathrm{d}}$, whlch is only indlcative of the block weight and not of the volume required to cover a given breakwater slope area

In order to adopt a standard terminology and to simplify the economic design, the following coefficients and expresions are proposea to one block, then

If we call At the area of the breakwater slope tributary

$$
A_{t}=\frac{\text { Area }}{\text { Number of blocks }}
$$

In order to give this area in a dimensionless manner, 

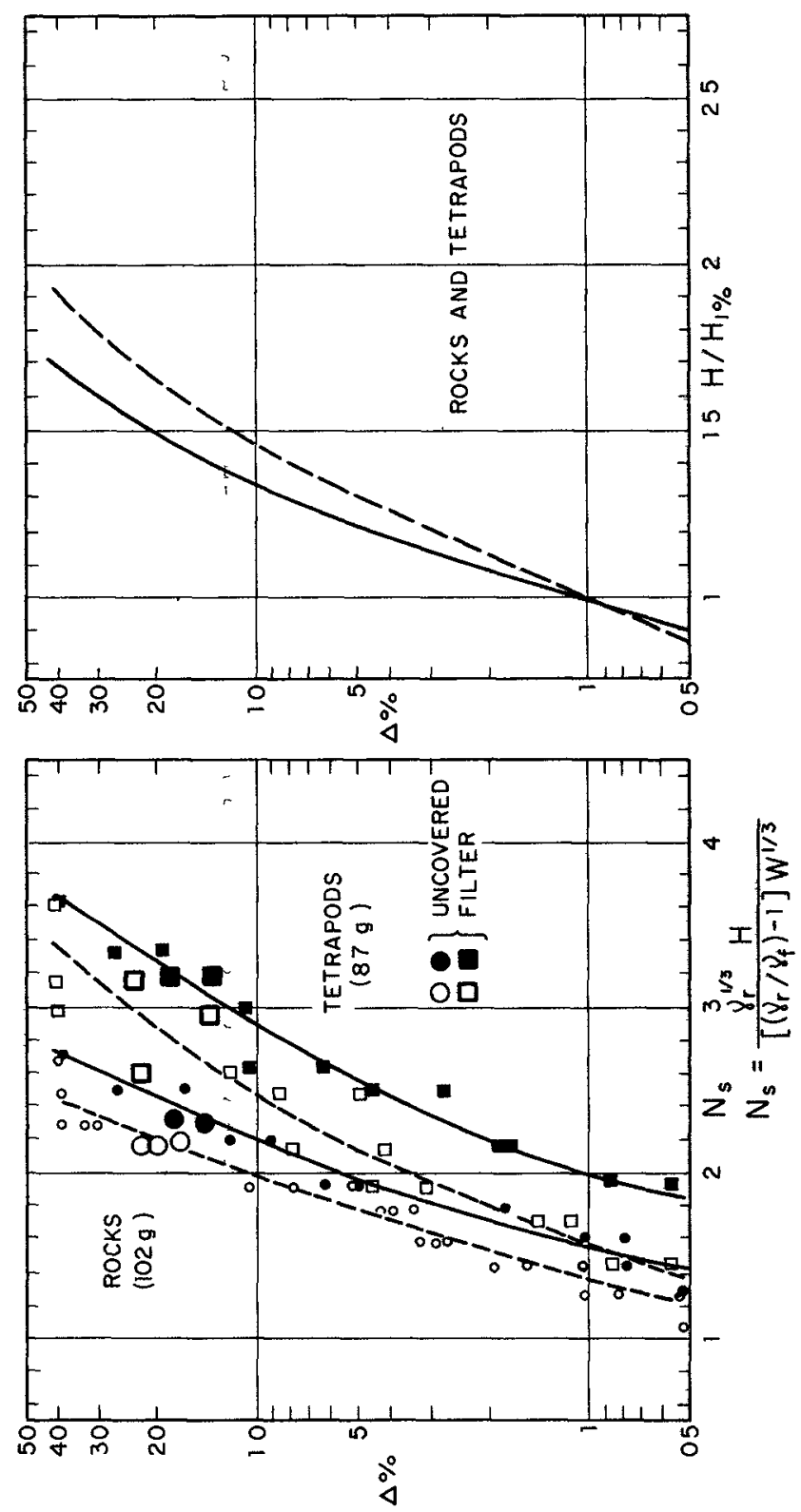

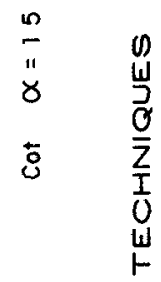

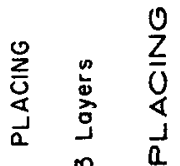

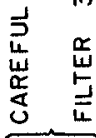

r

o w

on $\frac{0}{a} \quad \frac{4}{a}$

记品

-

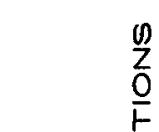

난 


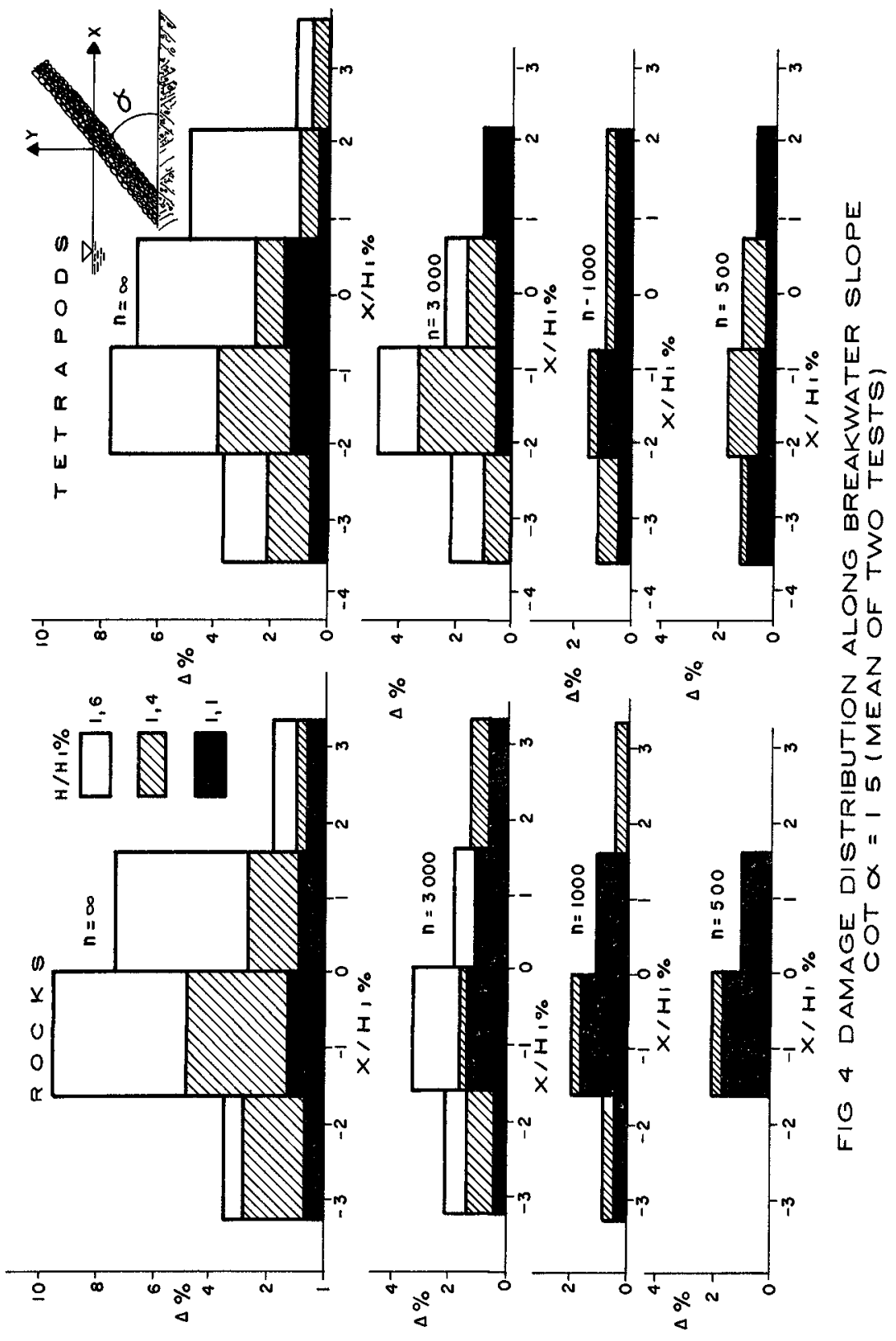


It may be defined the "revetment unit " $U_{r}$ such that

$$
U_{r}=\frac{A_{t}}{V^{2 / 3}}=\frac{A_{t}}{\left(W / V_{r}\right)^{2 / 3}}
$$

Where $V$ Is the solid volume of one block, W its weight and Ur its specific welght

In the experiments reported in this paper, corres ponding to the carefull placing technique, the mean values for $U_{r}$ were

$$
\text { Tetrapods } 1,00 \quad \text { Rocks } 095
$$

Since the specific gravity of rocks is usually 27 and that of concrete 22 , it may be defined a coefficient $\mathrm{K}_{\mathrm{S}}$ such that

$$
\frac{W}{A_{t}}=\frac{1}{K_{s}} \frac{Y_{f} H}{\operatorname{cotg} \alpha}
$$

Where $f_{f}$ is the water spesific weight In this manner the required weight per unit area would be inversely proportional to $\mathrm{K}_{\mathrm{S}}$ for a given breakwater slope and wave height

$$
\text { If } \mathrm{K}_{\mathrm{S}} \text { Is related with Hudson's formula }
$$

$$
W=\frac{\operatorname{lr}_{r} H^{3}}{K_{D}\left[\left(l_{r} / V_{t}\right)-1\right]^{3} \operatorname{cotg} \alpha}
$$

Then the following expressions result

$$
\begin{aligned}
& K_{s}=\frac{\gamma_{f} H U_{r}}{\gamma_{r}^{2 / 3} W^{2 / 3} \operatorname{cotg} \alpha}=\frac{K_{0} \gamma_{f}\left[\left(\ell_{r} / \gamma_{f}\right)-1\right]}{Y_{r}^{5 / 3}} \frac{U_{r} W^{2 / 3}}{H^{2}} \\
& \mathrm{~K}_{\mathrm{S}} \text { could be named "Armor density coefficient "r } \\
& \text { In Fig } 5 \text { the "armor density coefficient " has }
\end{aligned}
$$




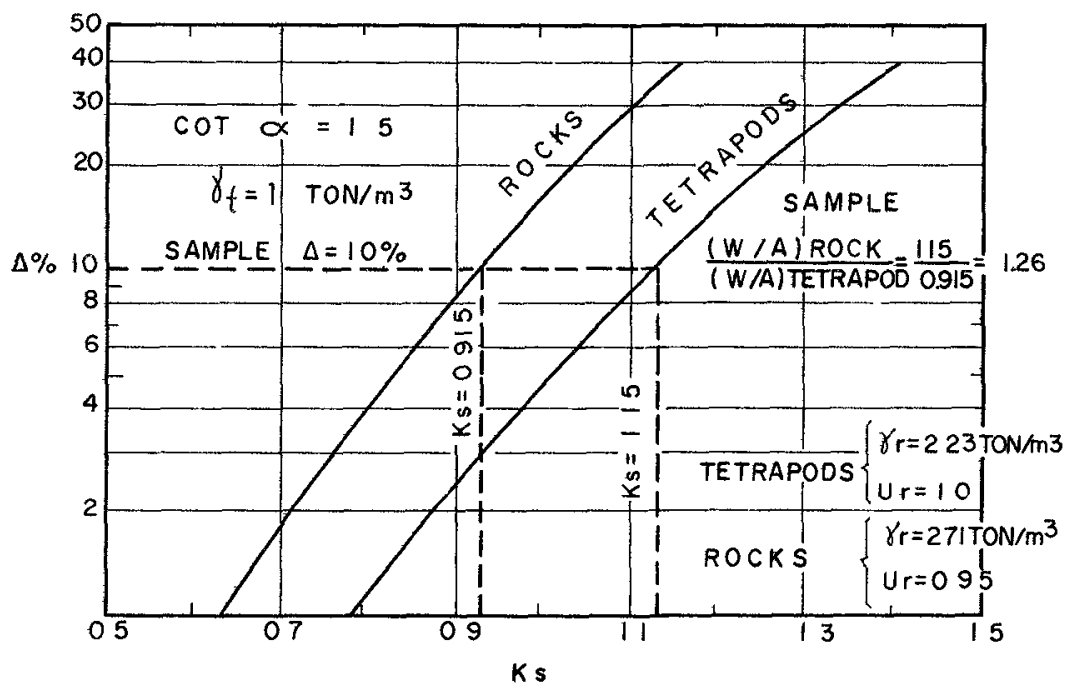

FIg 5 STABLE DeNSITY COEFFICIENT (CAREFul placing)

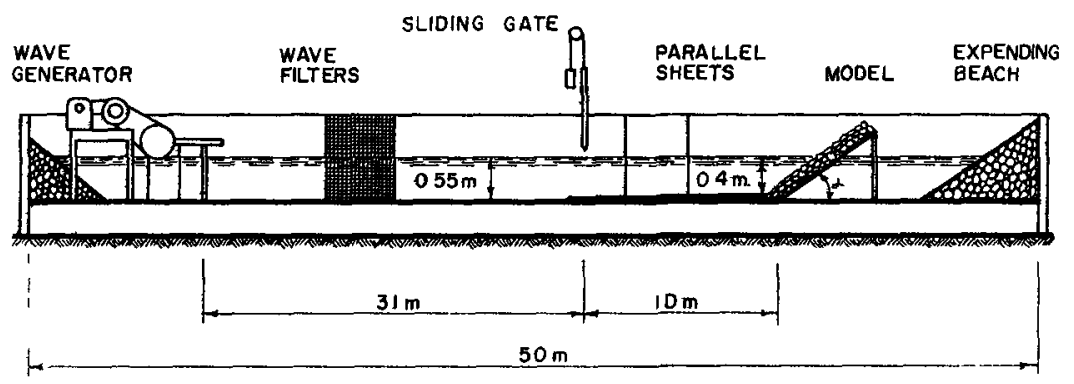

FIG $G$ WAVE CHANNEL AND EXPERIMENTAL $S E T-U P$ 

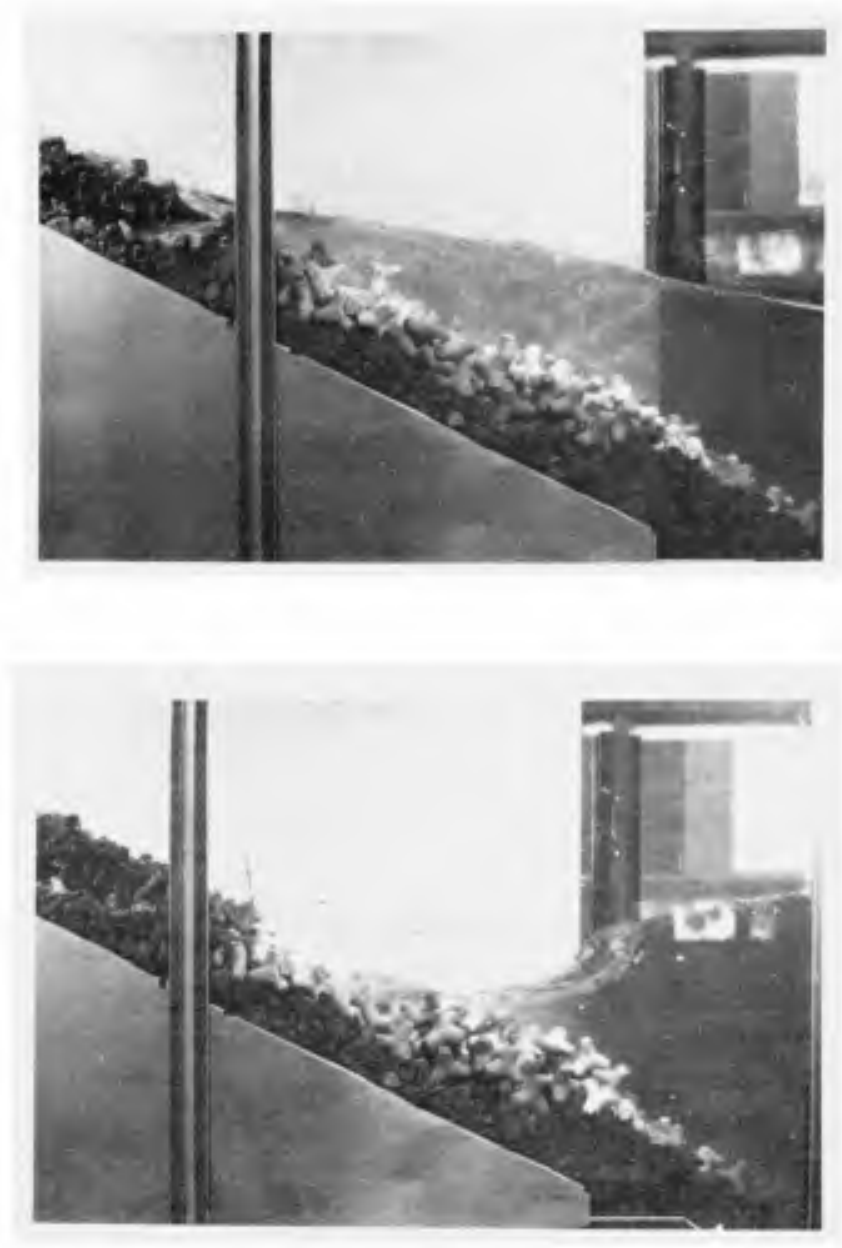

FIG. 7 WAVE ACTION ON THE MODEL BREAKWATER 
DESCRIPTION OF THE EXPERIMENTAL WORK

The tests

For the limited duration storms the model breakwater was subjected to the attack of perlodic waves of helght and in number according with the known statistical distribution for actual swells (Putz, 1954) See in Fig 13 the wave helght his tograph actualiy used in the experiments In Fig 12 are shown records of a typical uniform wave train and a train of "three waves ", It est a train with three larger waves of mean height 27 times the mean wave height, as specified in the 1000 waves histograph

For the infinite duration swells, waves of a given height attacked the breakwater until equilibrium was reached Then the displaced blocks were counted and, without rebuilding the slope, the experiment followed increasing the wave height in steps of about one centimeter The test were conducted in bursts such that reflection from the breakwater would not inter fere with wave generation; furthermore, a slide gate, close to the model, was used to interrupt the "last wave" of the train that is up to $20 \%$ larger than the preceeding waves In terms of Hudson's formula, a $10 \%$ difference in wave helght means $33 \%$ difference in the stability coefficient Kd

The core of the breakwater was considered to be imper vious and, as such, a board with stripes was used completely different results are expected if a core with significant porosity were used

It was observed ( See Fig 7 ) that all waves that caused some damage broke on the breakwater slope in the manner of a collapsing breaker as defined by Galvin (1969)

The Experimental set - up

Set - up

See fig 6 for a description of the experimental

\section{CONCLUS IONS}

1 For the initial movement of rocks and tetrapods It seems that the duration of the swell is not important The duration becomes relevant for advanced damage (See Figs $\mathrm{N}^{\mathrm{C}} \mathrm{I}$ and 2)

2 Economic considerations in one hand and safety factor in the other, usually lead to a design damage different from either the no damage or total fallure conditions Therefore the damage functions, rather than those criteria, should be given to the design engineer. 
3 The experiments show that placing techniques of the armor blocks make a big difference for the initial damage, but are less relevant for advanced damage, when the armor poro sity and "dynamic" stability are essential (See Fig 3.)

4 Uncovering of the filter layers in holes of diame ter equal to two pleces ocured for armor damage percentages between $10 \%$ and $20 \%$ Usually, in the next wave height step, total failure would follow for damage between $30 \%$ and $40 \%$ (See Fig 3)

5 It was observed in the experiments that while at the beginning the damage mostly occurs below the still surface level, for larger waves the portion inmediately above that level is also strongly affected (See Fig 4 )

\section{ACKNOLEDGMENTS}

Financial support for this research was provided by the "Consejo de Desarrollo Científico y Humanlstico" of the Central University of Venezuela through the research project $N^{\circ} 273$ The author is also grateful for the assistance given by the Ministry of Public Works, Ports and Alrports Division, in the preparation of this report

\section{REFERENCES}

Carstens, T, A Torum and A Traetteberg, "The stability of rubble mound breakwaters against irregular waves", Pcoc Tenth Conference on Coastal Indinearnis, A S C E, 1966, Volume II,pp $958-971$

Danel,Pierre, "On the limiting clapotis", Gravity Waves, National Bureau of Standards Circular $\mathrm{N}^{\circ} 521$ (November, 1952), 35-38

Font, Juan B. "The effect of storm duration on rubble-mound break water stability", Proc 11 th Conference on Coastal Engineeriñg, A S C E., 1968, pp 779-786

Galvin, Cyril J "Breaker Travel and Choice of design wave height", proceedings, Journal of the Waterways and Harbors Division, ASCB," Vol $95, \mathrm{~N}^{\circ}$ WW 2, May, 1969, pp 175-200

Hernández, Pastor and Suárez "Estabilidad de rompeolas de roca," Graduation Research, Hydraulic Laboratory, Universidad Central de Venezuela, Aug, 1968

Hudson, Robert $Y$, "Iaboratory investigation of rubble-mound break waters", J Waterways Harbors DIV ASCE, 85, WW 3, paper $\mathrm{N}^{\circ} 2171$ (September, 1959) 93-121 
Iribarren, $R$, "Formule pour le calcul des diques en enroche ments Naturels ou clements artificiels" XXlst Internatiōnal Navigation Congress - Stokholm, 1965, P I A.N C,

Ibarra $E$ and $T$ Blumentals, "Estabilidad de Rompeolas ", Graduation Research, Hyaraulic Laboratory, Universidad Central de Venezuela, Dec, 1969.

Loreto R., Alberto "Estabilidad del Talud de un Rompeolas de Roca,"Graduation Research, Hydraulic Laboratory, Universidad Central de Venezuela, May, 1969

Neri, Lucila and Yandira Santelız "Estabilidad de la Coraza de Rompeolas de Roca y Elementos de Concreto ", Graduation Research Hydraulic Laboratory, Universidad Central de Vene zuela, July, 1970

Putz, $R R$, "Statistical analysis of wave records" Proc Conference on Coastal Engineering, Berkeley, Calif , The Eng Foundation Council on wave Res, 1954 pp 13-24

\footnotetext{
Rogan, Adelkıs J "Destruction Criteria for rubble-mound breakwaters "Proc. 1lth Conference on Coastal Engineering, ASCE, 1968, pp 761-778
} 

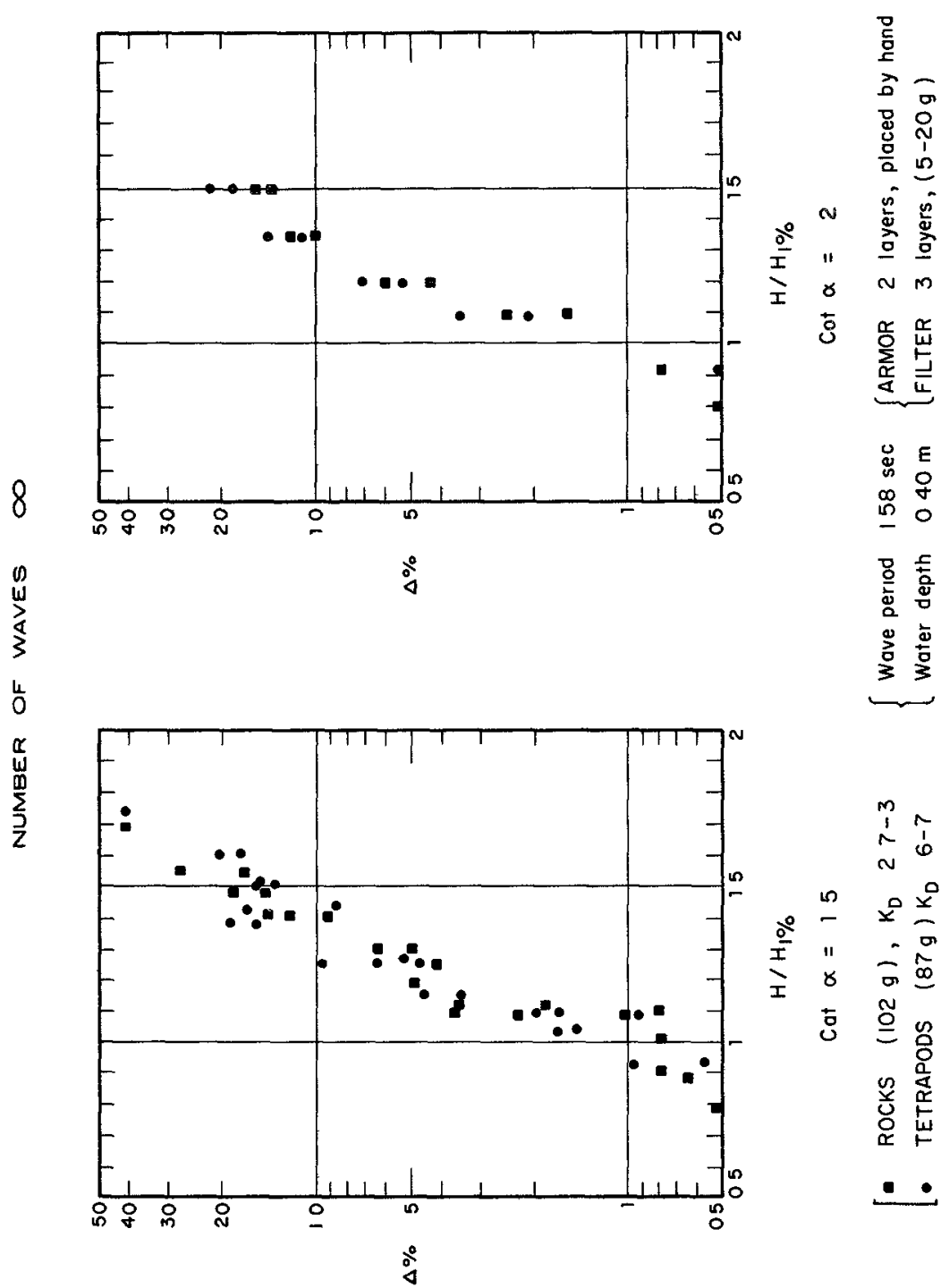

$\frac{4}{4}$

$\stackrel{m}{n} \hat{1}$

$\begin{array}{ll} & 0 \\ \frac{0}{1} & - \\ \text { I } & 6 \\ & 5\end{array}$

م

.

б。

응

n

桴

딴

$\therefore \quad \frac{O}{L}$

$\stackrel{8}{4}$ 


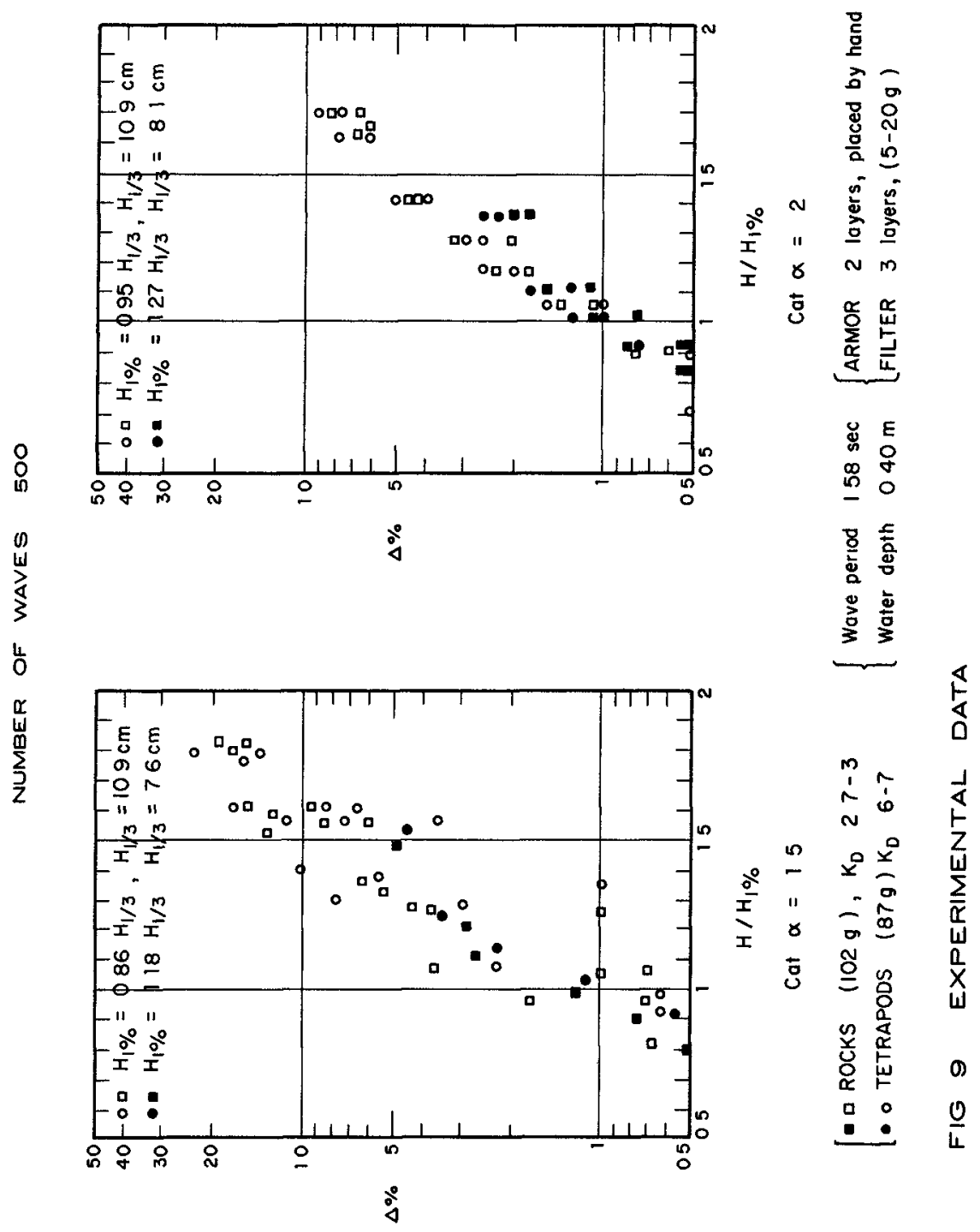




\section{RUBBLE-MOUND BREAKWATER}

1583

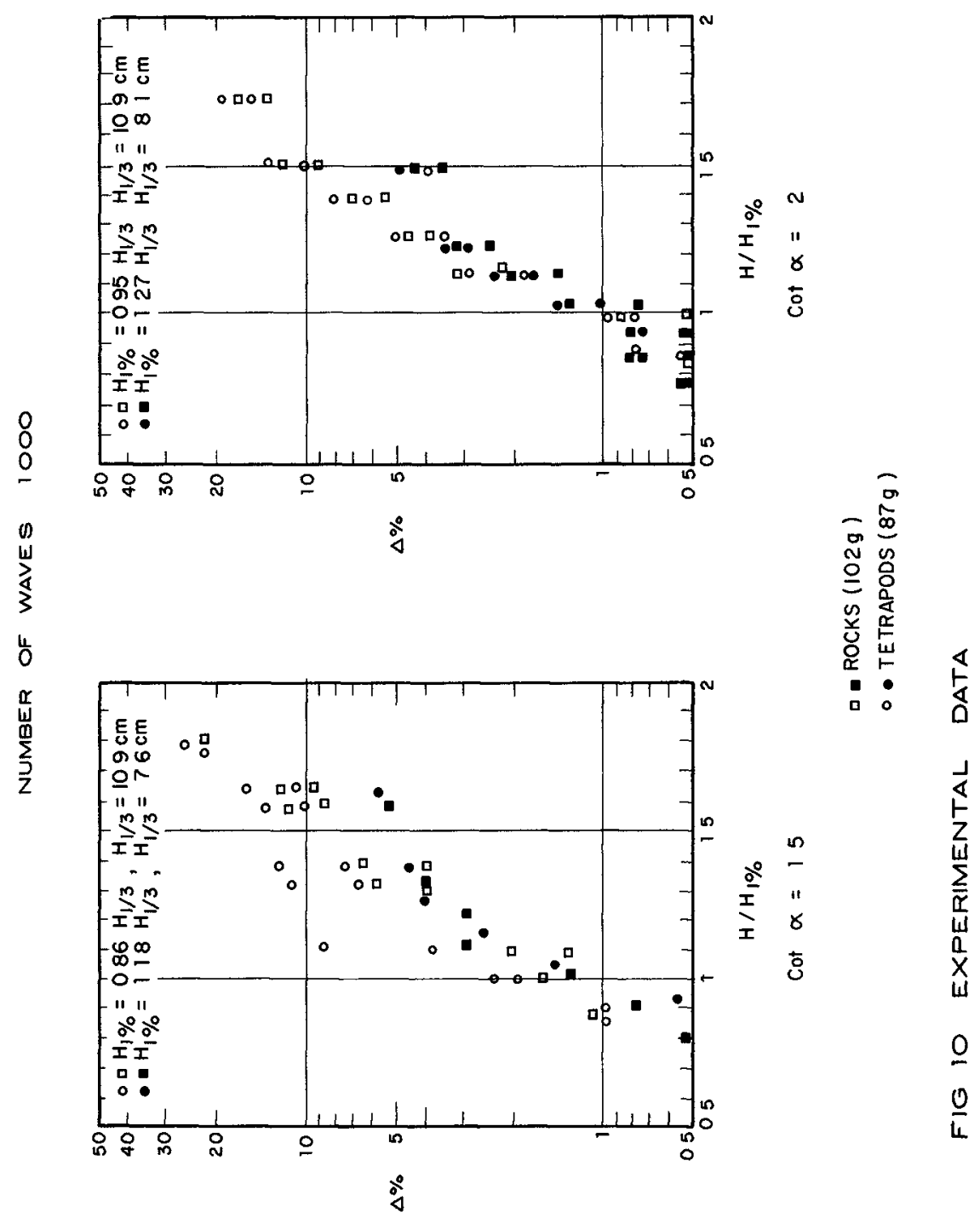




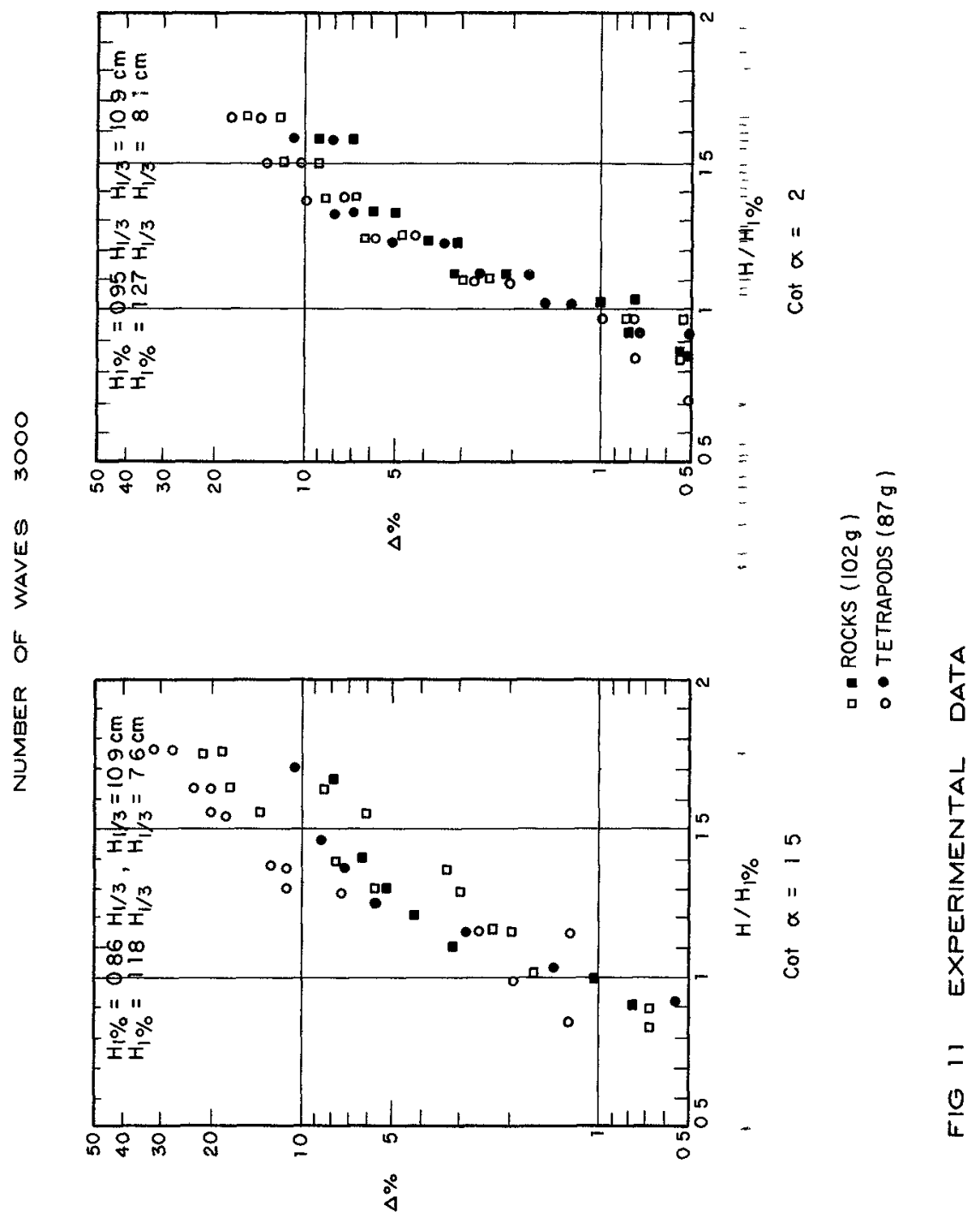



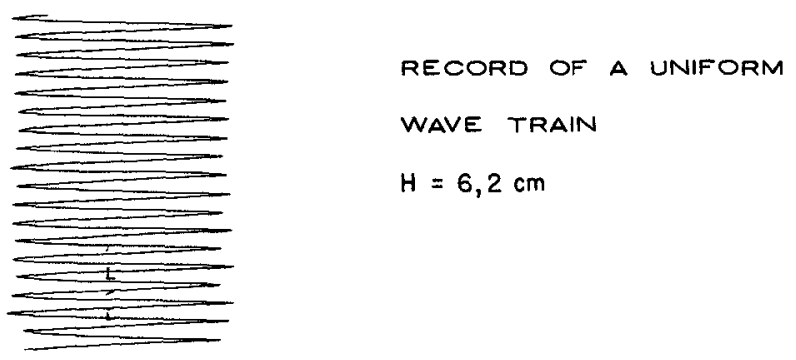

$H=6,2 \mathrm{~cm}$

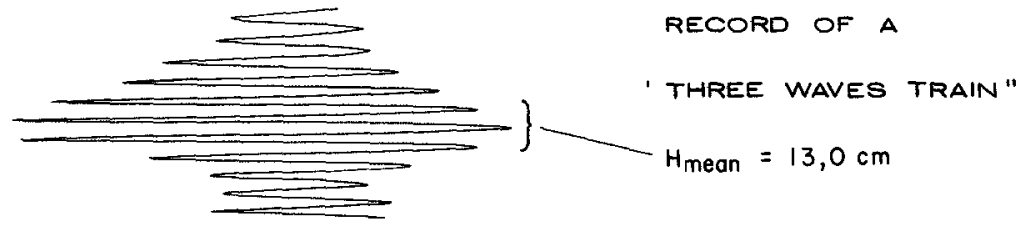

fig 12 sample wave records

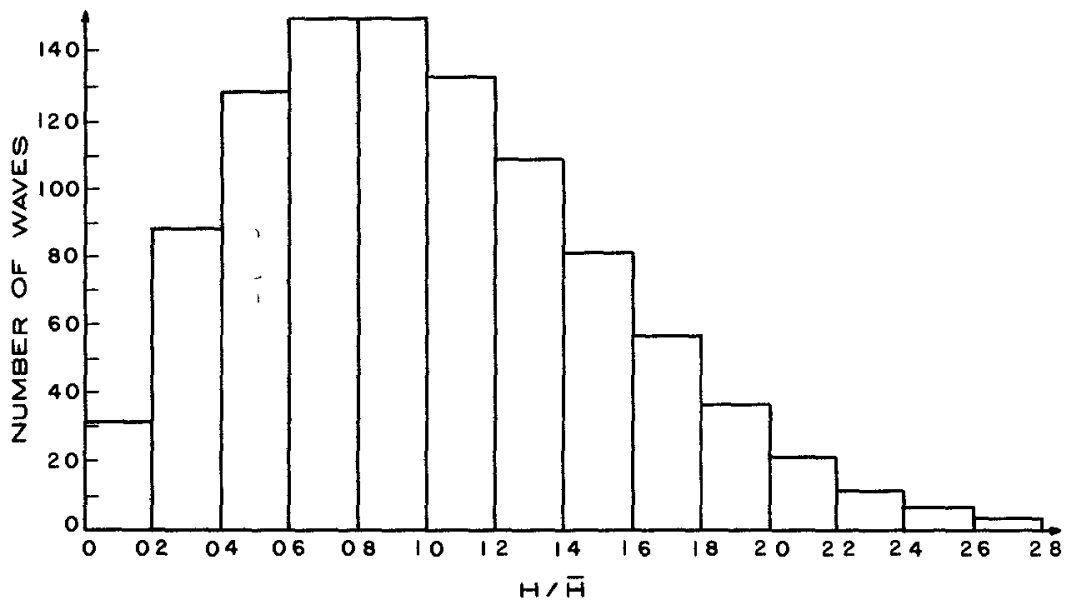

FIg 13 WAVE hEIGHT hISTOGRAM fOR IOOO WAVES 



\title{
CHAPTER 97
}

\section{HYDRAULIC RESISTANCE OF ARTIFICIAL CONCRETE BLOCKS}

\author{
by
}

Nobuo Shuto

Associate Professor, Chuo University, Tokyo, Japan

and

Hıroshı Hashımoto

Senior Research Engıneer, Public Works Research Institute, Munistry of Construction, Japan

\section{Abstract}

Theoretical analysis and experiments are made to clarify the mechanism of reducing the wave energy by the block structures In order to express the hydraulic reistance of three different kinds of blocks, three different models are proposed The hydraulic radius and the porosity of the block structures are essentually important factors in the expression of the hydraulic characteristics of the block structures

It is recomended, from experimental results, to carry out the hydraulic experiments by using blocks heavier than at leact $500 \mathrm{gr}$

Resistance coefficients obtained in the steady and oscillatory flows show almost no difference

Several types of artificially made concrete blocks have been used in coastal and harbour areas Main purposes of using block structures are

(1) to reduce the wave forces acting on coastal structures,

(2) to reduce the wave reflection from coastal structures,

(3) to reduce the height of transmitted waves,

(4) to reduce the ware run-up height,

(5) to reduce the quantity of wave overtopping, and

(6) to protect the toe of coastal structures against erosion

Although the design of the block structures is usually based on hydraulic experiments, the simalitude between model and prototype is not yet clearly understood

In order to fulfill the above mentioned purposes, block structures must be able to effectively reduce wave energy Thus, the knowledge of the mecharism of reducing the wave energy by the block structures is of essential importance Naturally this mechanism should be considered by taking into account the effect of the scale of model

The alm of the present study is to clarify the mechanssm of energy reduction, and to express the hydraulic resistance law of the block structures in terms of their characteristic quantities When this is once achieved, factors such as the reflection and transmission coefficients of block structures become computable and a part of the design may be satisfactorily done without conducting hydraulic experiments In addition if the similitude is once established, the hydraulic experiment will give more reliable 


\section{design data}

First, authors intend to explain the loss coefficient of block structures in terms of the characteristic quantities such as the porosity or the hydraulic radius by using the experimental results obtained in the steady flow

Secondly, authors try to determine whether the flow through the block structures is turbulent or laminar Since a hydraulic model using small blocks does not produce a fully developed turbulent flow through its pores, the experimental results obtained from such a model are not likely to give any reliable design data Therefore, the minimum allowable size of the blocks in the hydraulic experiment must be determined by taking into account the flow characteristics through the pores

Thirdly, comparisons are made between the resistance law obtalned by assumıng the steady flow and that obtanned by assuming the oscillatory flow Naturally, the wave motion actually attacking the block structures in the field is not steady but unsteady However, it is desired to determine the hydraulic resistance law of concrete blocks by the experiments in the steady flow which is simpler and more convenient than those in the oscillatory flow Thus, it may be very useful to find a relationship between the resistance laws obtained in the steady and unsteady flow experiments

\section{Theoretical analysis}

\section{2-1 Steady flow}

\section{2-1-1 Resistance-plpe model}

Figure 1 shows this model The block structure is considered to have a number of fictious pipes in it The total volume of the fictious pipes is set equal to the total volume of the pore in the block structure The total area of the inner surface of the fictious pipes is assumed equal to the total wetted area of the blocks

Then, we have

$$
\begin{aligned}
\operatorname{nd}^{2} \mathrm{~L} & =\epsilon \mathrm{BLH} \\
4 \mathrm{nLd} & =\mathrm{S},
\end{aligned}
$$

where $n$ is the number of the pipes, $d$ the diameter of the plpe, $L$ the length of the block structure, B the width of the block structure, $h$ the average water depth in the block structure, $\varepsilon$ the porosity of the block structure, and $s$ the surface area of the blocks

From the above equations, we have

$$
\begin{aligned}
& \mathrm{d}=4 \frac{\varepsilon \mathrm{BLh}}{\mathrm{S}}=4 \mathrm{R} \\
& \mathrm{n}=\frac{\mathrm{S}}{16 \mathrm{RL}}
\end{aligned}
$$

The hydraulic radius, $\mathrm{R}$, is defined as $\varepsilon \mathrm{BLh} / \mathrm{S}$ and $1 \mathrm{~s}$ considered as a measure of the average size of the pore

When the water flows down through these fictious pipes, loss in energy occurs due to the friction along the inner surface of the pipe By using the average velocity, $v_{p}$, in the pipe, the head loss, $h_{f}$, can be expressed as follows,

$$
h_{f}=f \frac{L}{d} \frac{v_{p}^{2}}{2 g}=f \frac{L}{4 R} \frac{1}{2 g} \frac{Q^{2}}{\varepsilon^{2} B^{2} h^{2}}
$$


in which the average velocity is given as

$$
v_{p}=\frac{Q}{n d}=\frac{Q}{\varepsilon \overline{B h}}
$$

and $Q$ is the total discharge of water

In the experiment, the head loss is obtained as the dufference between the energy heads in front of and behind the block structures

$$
\mathbf{h}_{\mathbf{f}}=\mathrm{h}_{1}+\frac{\mathrm{v}_{1}^{2}}{2 \mathrm{~g}}-\left(\mathrm{h}_{2}+\frac{\mathrm{v}_{2}^{2}}{2 \mathrm{~g}}\right)
$$

By using eqs (5) and (7), the loss coefficient $f$ is determined

\section{2-1-2 Fictious pipe model with sudden expansion and contraction}

In the model shown in Fig 2, the characteristic sizes of the pore and the block are considered equal to $R_{1}$ and $D$, respectively The total number of pores in the block structure $1 \mathrm{~s} \mathrm{~m}_{\mathrm{p}}$ and that of blocks is $\mathrm{m}$. The shapes of the pore and the block are assumed as cubes Then, we have

$$
\begin{aligned}
\mathrm{m}_{\mathrm{p}} \mathrm{R}_{1}^{3}+\mathrm{mb}_{\mathrm{b}} \mathrm{D}^{3}=\mathrm{V}=\mathrm{BLh} \\
\mathrm{m}_{\mathrm{p}} \mathrm{R}_{1}^{3}=\varepsilon \mathrm{V} \\
\mathrm{m}_{\mathrm{b}} \mathrm{D}^{3}=(1-\varepsilon) \mathrm{V} \\
6 \mathrm{~m}_{\mathrm{b}} \mathrm{D}^{2}=\mathrm{S} \\
\mathrm{R}=\frac{\mathrm{V}}{\mathrm{S}}
\end{aligned}
$$

Solving these equations, we can obtajn a relation between a characteristic radius $R_{1}$ and the hydraulic radius $R$ defined previously The relation is, on neglecting the coefficient of proportionality,

$$
\mathrm{R} \propto\left(\frac{1-\varepsilon}{\varepsilon}\right)^{\frac{2}{3}} \mathrm{R}
$$

Now, let us assume a homogeneous distribution of pores within the block structures, and let $n_{1}, n_{2}$, and $n_{3}$ be the numbers of the pores in the directions paraIlel to the length, width and helght of the block structure, respectively The assumption of the homogeneity gives the following relation

$$
\mathbf{n}_{1} \quad \mathbf{n}_{2} \quad \mathbf{n}_{3}=\mathbf{L} \quad \text { B } \quad \mathbf{h}
$$

The average total sectional area of the pore through which the water can flow down 1 s

$$
\mathbf{n}_{2} \mathbf{n}_{3} \quad \mathbf{R}_{1}^{2}=\varepsilon^{\frac{2}{3}} \mathrm{Bh} \text {, }
$$

where the equations (8) to (12) and $\mathrm{m}_{\mathbf{p}}=\mathbf{n}_{\mathrm{t}} \mathbf{n}_{2} \mathrm{n}_{3}$ axe used

Average velocity, $v_{p}$, in the pore is, thus, given as

$$
v_{p}=Q / \varepsilon^{-} B h
$$


Head loss which occurs when the water passes through a pore is expressed as

$$
\text { f } \frac{1}{2 g} v_{p}^{2}
$$

Since the number of pores along the direction of flow is $n$, the total loss in energy is given as

$$
n+f \frac{v_{p}^{2}}{2 g}
$$

The number $n_{1}$ is equal to $\varepsilon^{\frac{2}{3}} L / R_{1}$, because

$$
\mathrm{m}_{\mathrm{p}} \mathrm{R}_{1}^{3}=\mathbf{n}_{1} \mathbf{n}_{2} \mathbf{n}_{3} \mathrm{R}_{1}^{3}=\mathbf{n}_{1}^{3} \frac{\mathrm{Bh}}{\mathrm{L}^{2}}=\varepsilon \mathrm{BhL}
$$

Finally, the total loss in energy is given as

$$
n_{1} f \frac{v_{p}^{2}}{2 g}=f \frac{f}{R_{1}} \epsilon^{-3} \frac{l}{2 g}\left(\frac{g}{\epsilon \frac{2}{3} B h}\right)^{2}
$$

\section{2-1-3 Resistance body model}

If a body is placed in a flow, the force acting on the body changes the momentum of the flow Total resistance, $F$, of the body in the flow is expressed as

$$
\mathrm{F}=\frac{\mathrm{w}_{0}}{\mathrm{~g}} \mathrm{~B}\left\{\mathrm{gh}-\mathrm{v}_{1} \mathrm{v}_{2}\{\Delta \mathrm{h},\right.
$$

where $v_{1}$ and $v_{2}$ are the flow velocities in front of and behind the block structure, and $\Delta h$ is the difference in heads other terms are as defined previously We assume there are a number of rectangular piles in the block structure The helght of a pile is $h$, the sectional area of a pile is $d x d$, and the number of piles in the block structure is $\mathrm{m}$ This model is shown in Fig 3

The total volume and surface area of the fictious piles are assumed equal to those of the actual blocks Thus, we have

$$
\begin{aligned}
& m d^{2} h=(1-\varepsilon) L B h \\
& 4 m d h=S
\end{aligned}
$$

From these equations, the following expressions for $d$ and $m$ can be obtalned

$$
\begin{aligned}
& \mathrm{d}=\frac{4 \operatorname{LBh}(1-\varepsilon)}{\mathrm{S}} \\
& \mathrm{m}=\frac{\mathrm{S}^{2}}{16 \mathrm{BLh}^{2}(1-\varepsilon)}
\end{aligned}
$$

If a single fictious pile is placed in the flow, the resistance $F_{B 1}$ may be expressed as follows by using the conventional expression $C_{D}$ for a resistance coefficient,

$$
\mathbf{F}_{\mathrm{B}_{1}}=\mathrm{C}_{\mathrm{D}} \frac{\mathrm{v}_{1}^{2}}{2 \mathrm{~g}} \mathrm{dh}
$$

When all the pales are placed in the flow whout mutual interaction, the total resistance of the piles is m-times larger than $F_{B 1}$ piles per unit width of the channel is then given as 


$$
\mathrm{m} \frac{\mathrm{FB}_{\mathrm{H}}}{\mathrm{B}}=\mathrm{C}_{D} \frac{\mathrm{dh}}{\mathrm{B}} \mathrm{m} \frac{\mathrm{v}_{1}^{2}}{2 \mathrm{~g}}=\frac{\mathrm{CD}_{\mathrm{D}}}{4} \frac{\mathrm{S}}{\mathrm{B}} \frac{\mathrm{v}_{1}^{2}}{2 \mathrm{~g}} \frac{\mathrm{F}}{\mathrm{B}}
$$

In an actual block structure, the adjacent piles interact each other because of the small spacings of the piles The total drag coefficient may be a function of the number of the piles in the channel The number of piles per unit width may be considered proportional to the surface area of the blocks per unit width of the channel Thus, we have

$$
\frac{F}{B}=f(s / B) \frac{v_{f}^{2}}{2 g}
$$

From this expression, it may be seen that the resistance coefficient is a function of the term $\mathrm{s} / \mathrm{B}$

\section{2-2 Unsteady flow}

The motion of the water column in a pipe without the block structure is given by the following equation,

$$
\frac{d^{2} z}{d t^{2}}+\frac{f}{2 D} \frac{d z}{d t}\left|\frac{d z}{d t}\right|+\frac{2 g}{L} z=0,
$$

where $z$ is the water level, $D$ and $L$ are the drameter and the length of the water column, respectively, $g$ is the gravitational acceleration, and $f$ is the loss coefficient to be determined by the experiment

The motion of the water column wath the block structure placed in it is expressed by the following equations,

$$
\begin{aligned}
& \frac{1}{g} \frac{d v}{d t}+10+\frac{f}{D} \frac{1}{2 g} v|v|=0, \\
& \frac{1}{g} \frac{d v}{d t}+1 v+\frac{f_{1}}{d} \frac{1}{2 g} v|v|=0, \\
& 1(L-1)+1_{1} 1=-2 z, \\
& v=\frac{a}{A} v=-\frac{d z}{d t},
\end{aligned}
$$

where $V$ is the velocity of the water in the tank without the block structure, $v$ the velocity of the water in the block structure, 10 and $l_{1}$ the hydraulic gradients in the part of the tank without the block structure and in the block structure, respectively, L the total length of the water column, 1 the length of the block structure, A the sectional area of the tank, a the average sectional area of the pore in the block structure, $f_{1}$ the loss coefficient of the block structure, and other terms have the same meanings as defined previously

These four equations can be reduced to

$$
\frac{d^{2} z}{d t^{2}}+\frac{\left[\frac{f}{D}\left(1-\frac{1}{L}\right)+\frac{1}{L}\left(\frac{A}{a}\right)^{2} \frac{f_{1}}{d}\right]}{2\left[1+\frac{1}{L}\left(\frac{A}{a}-1\right)\right]} \frac{d g}{d t}\left|\frac{d z}{d t}\right|+\frac{2 g}{L\left[1+\frac{1}{L}\left(\frac{A}{a}-1\right)\right]} z=0
$$


Comparing eq (27) with (32), we can determine the loss coefficient, $f_{1}$, of the block structure in the unsteady 11 ow

\section{Experimental procedure}

Tbree kinds of blocks were used in the experiment they are the tetrapod, the hollow tetrahedron and the bexaleg

Experiments in the steady flow were carried out by using an open channel, $12 \mathrm{~m}$ long, $07 \mathrm{~m}$ wide and $03 \mathrm{~m}$ deep The water discharge was measured with a triangular weir placed at the end of the channel The water depth in front of and behind the block structure were measured to glve the change in the energy gradient During the experiment, the bottom of the channel was kept horizontal

Experıments in the unsteady $f l$ ow were carried out by using an oscillatory tank shown in Fig 4 The length of the tank is variable to give different period of the free oscillation induced in the tank The period of the unsteady flow used in the experiment varied from 34 sec to 49 sec The tank was covered with a lid to form a chamber, which was vacuumized by a pump A sudden removal of the lid induced the free osciliation of tbe water column The amplitude of the free oscillation was damped partly due to the resistance of the block structure and partly due to tbe surface friction and loss at bends of tbe tank

Tbe tank has a square cross-section of $30 \mathrm{~cm} \times 30 \mathrm{~cm}$ In the midale part of the tank, the block structure was placed compactly to restrict their motion perpendicular to the motion of the water column

Comparing the time history of the free oscillations with and without blocks, the effect of the blocks is determined The rate of decay in the amplitude of the oscillation yields data for the determination of the resistance coefficient Figure 5 lllustrates this procedure

Experimental result

Figure 6 shows the experimental results for the tetrapod Anong three models proposed by the authors, the resistance-pipe model was found the most adequate one for the tetrapod Loss coefficients of the tetrapod structure are sufficlently well explained by this model The weight of the tetrapods used in the experiment varied from $55 \mathrm{gr}$ to $8 \mathrm{~kg}$ The flow through the blocks heavier than Ikg is estimated fully developed turbulent This fact is more clearly shown in Fig 7 , in which a relation between the loss coefficient and the Reymolds number is shown The Reynolds number here is defined by using the hydraulic radius and the current velocity through the pore When the Reynolds number becomes greater than about 1,000 , the loss coefficient does not vary with the Reynolds number and is about 05 Judging from this result, an experiment should be carried out with the blocks heavier than at least $500 \mathrm{gr}$ or under the condition of the Reynolds number higher than 1,000 Such an experiment will give us a satisfactory result

The pipe model just mentioned was found only adequate for the tetrapod, but is not applicable to the other blocks A hollow tetrahedron has six members which enclose and occupy one big space within Itself In the structure made with the bollow tetrahedron, the water flows down as if it flows down through the pipe which has the sudden expansions and contractions A new hydraulic radius defined for this model. is expressed by eq (18) Figure 8 shows the experimental results The ordinate of the figure is the new hydraulic radius divided by the one-third power of the porosity The weight of the blocks used in the experiment varied from $125 \mathrm{gr}$ to $1,000 \mathrm{gr}$

Figure 9 shows the variation of the loss coefficient with the Reynolds number As the Reynolds number exceeds 1,000, the loss coefficlent seems to tend to a constant which is equal to 06 Therefore, it seems desirable to carry out hydraulic experi- 
ments, for the hollow tetrahedron, by using blocks heavier than $500 \mathrm{gr}$

In order to express the resistance of the hexaleg, assumption quite different from the other two, had to be made, because pipe models did not give any clear explanation Figure 10 shows a relation between the drag coefficient and the surface area of the blocks per unit width of the channel Except for the experimental results for $200 \mathrm{gr}$, a relation can be established If the blocks heavier than $500 \mathrm{gr}$ are used in the experiment, we will have the reliable design data

In Figs 6 and 8 , experimental results for the unsteady flow are also shown The square marks correspond to the unsteady flow the perlod of the unsteady flow used in the experiment varied from $34 \mathrm{sec}$ to $49 \mathrm{sec}$ Within this range of the period, no remarkable differences axe found between two loss coefficients for the tetrapod (Fig 6) The resistance coefficient in the unsteady flow 1s, however, a little smaller than that in the steady flow This slight difference seems to be caused by the fact that blocks were not completely fixed in unsteady flow The flow velocity relative to the motion of the blocks was a little lower than the velocity estimated from the motion of the water column Thus, the resistance coeficient in the unsteady flow was a little smaller than that in the steady flow

Figure 8 is for the hollow tetrahedron Except for two points corresponding to the blocks with weight of $250 \mathrm{gr}$, the experimental results in the unsteady flow show the same tendency as in the lesults for the tetrapod

\section{Conclusions}

On summarizing experimertal results so far mentioned, the following conclusions were drawn

1 In order to express the hydraulic resistance of blocks in terms of their characteristic quantities, different theoretical models should be used for different types of blocks

2 For the tetrapod, the adequate model is the pipe model No 1 in which loss in energy will be given as a result of the wall friction of the fictious pipe

3 For the hollow tetrahedron, the pipe model with sudden expansions and contractions is adequate

4 For hexaleg, rectangular pile model is suitable

5 For these three kinds of blocks, blocks heavier thar at least 500 gr should be used in the hydraulic experiment

6 Resistance coefficients obtained in the steady and oscillatory flows show almost no difference for the period of the oscillatory flow longer than 3 sec

Acknowledgements

The work reported herein was partly supported by the Science Research Expense of Ministry of Education in Japan

\section{References}

1) Lean, G H A simplified theory of pexmeable wave absorbers, J Hydraulic Res, No $1,5,1967$ 
2) Le Méhauté, B Perméabilıté des digues en enrochements aux ondes de gravité pérıodiques, La Hollle Blanche, No 6, 1957 et No 2, 1958

3) Murota, $A$ and $K$ Sato Basic study on the resistarice law of the ground water Plow, Memoir 21 st Annual Conv, J S C E, 1966 (In Japanese)

4) Ozakı, A, H Sawamura, and $Y$ Aral Basic study on the effect of pores in the rubble mound structures, Memoir 23rd Annual Conv, J S C E, 1968 (in Japanese)

5) Scheidegger, A E The physics of flow through porous media, Univ Toronto Press, 1957

6) Shuto, N Hydrallic resistance of concrete blocks, Proc l6th Conf on Coastal Eng in Japan, 1969 (1n Japanese)

7) Tompnaga, $\mathrm{M}, \mathrm{N}$ Shuto, and H Hashimoto Hydraulic characteristics of concrete blocks, Proc 14th Conf on Coastal Eng in Japan, 1967 (in Japanese) 


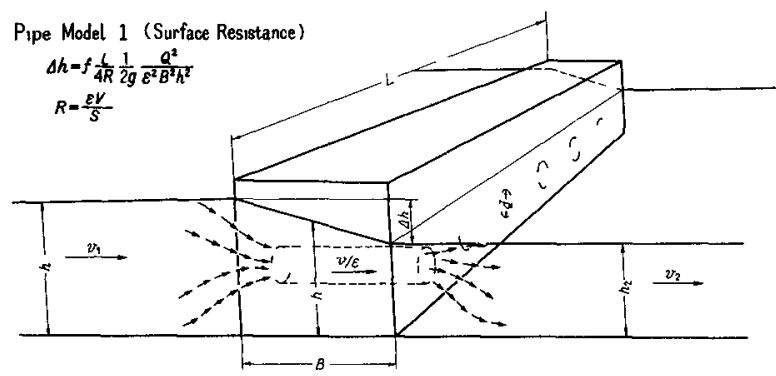

Fig 1 Resistance-pipe model

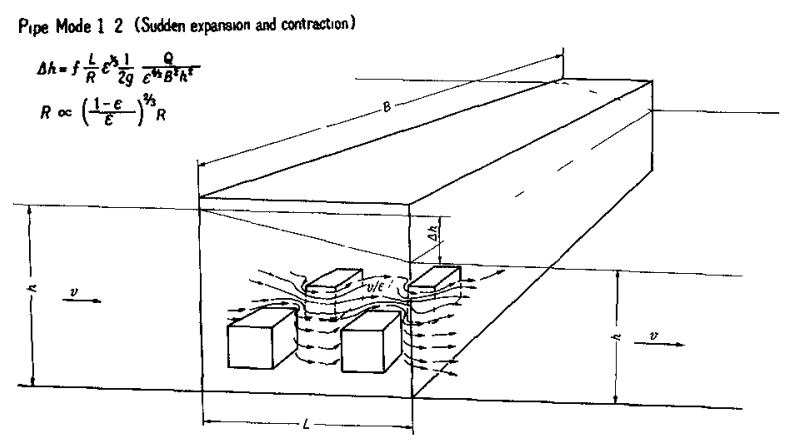

Fig 2 Pipe model with sudden expansions and contractions

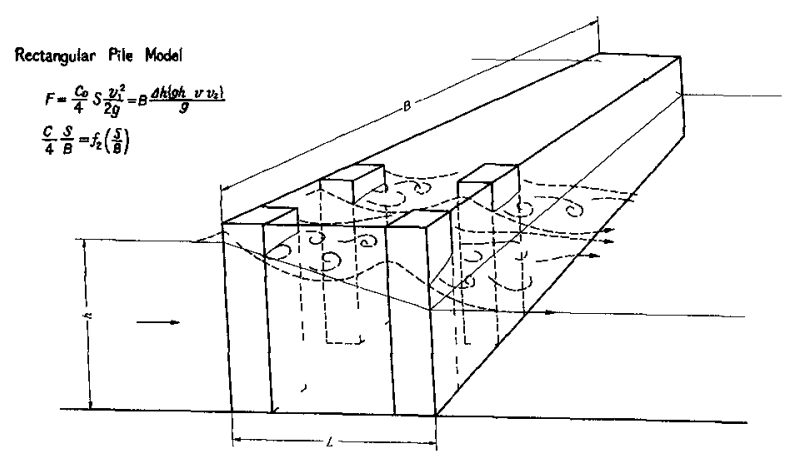

Flg 3 Resistance body model 


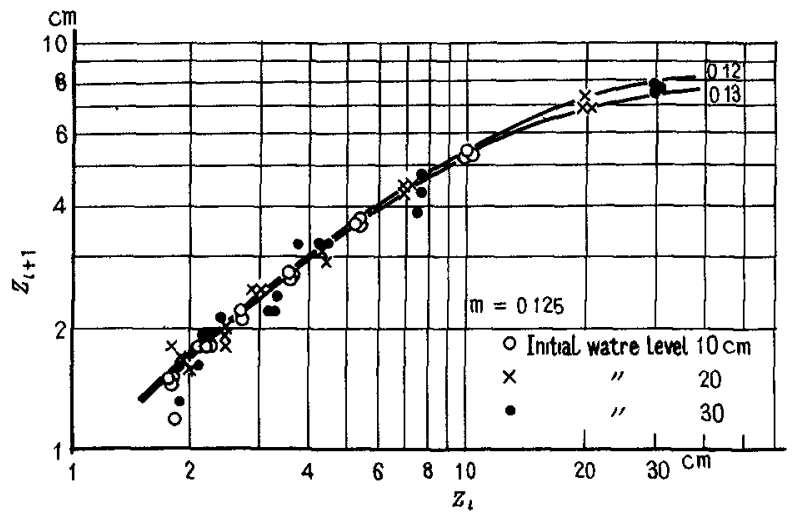

Fig 5 An example of the determination of the resistance coefficient

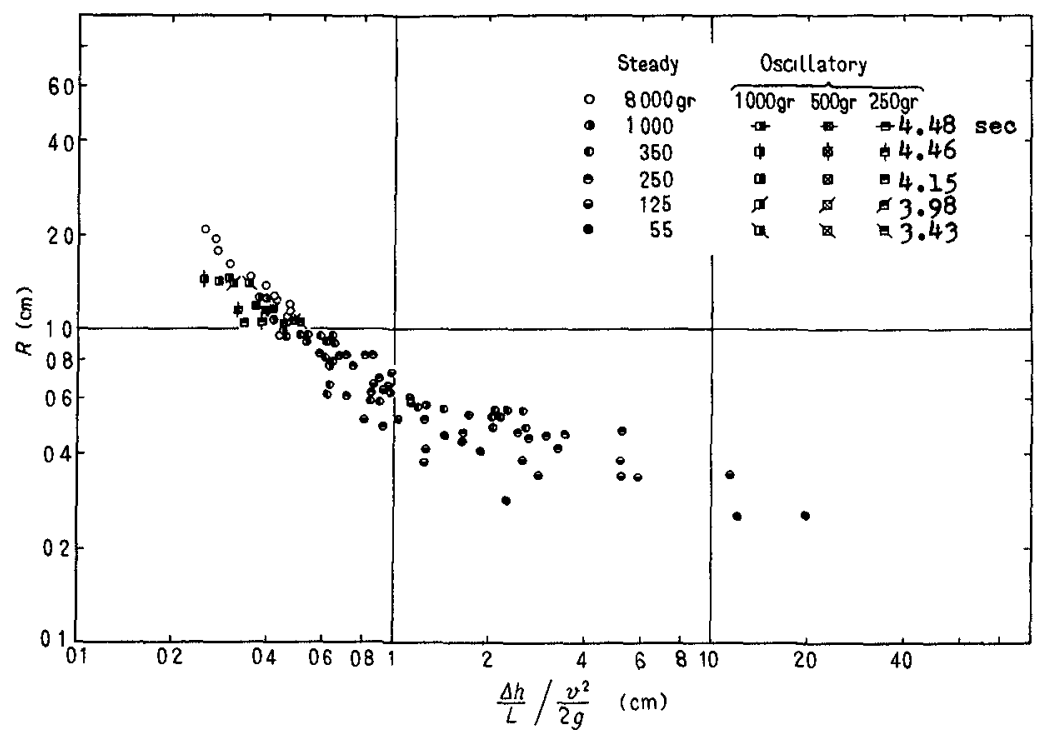

Pig 6 Loss coefficient vs hydraulic redius in rase of the tetrapod 


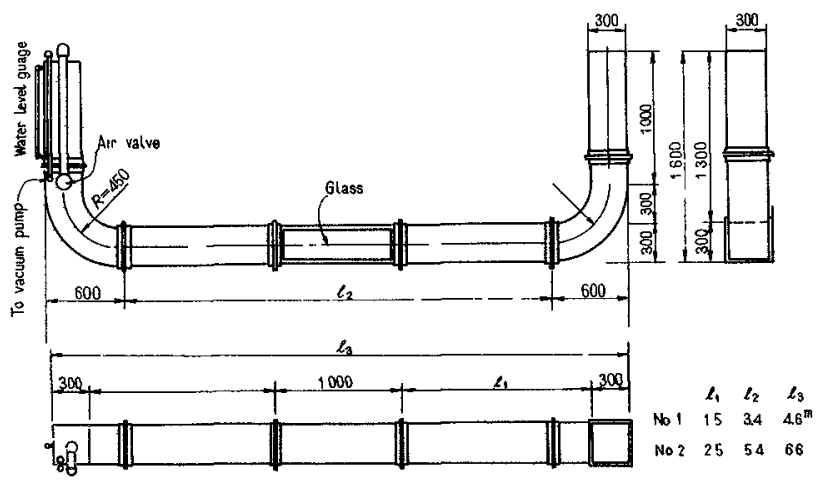

Fig 4 Oscillatory fl ow tank

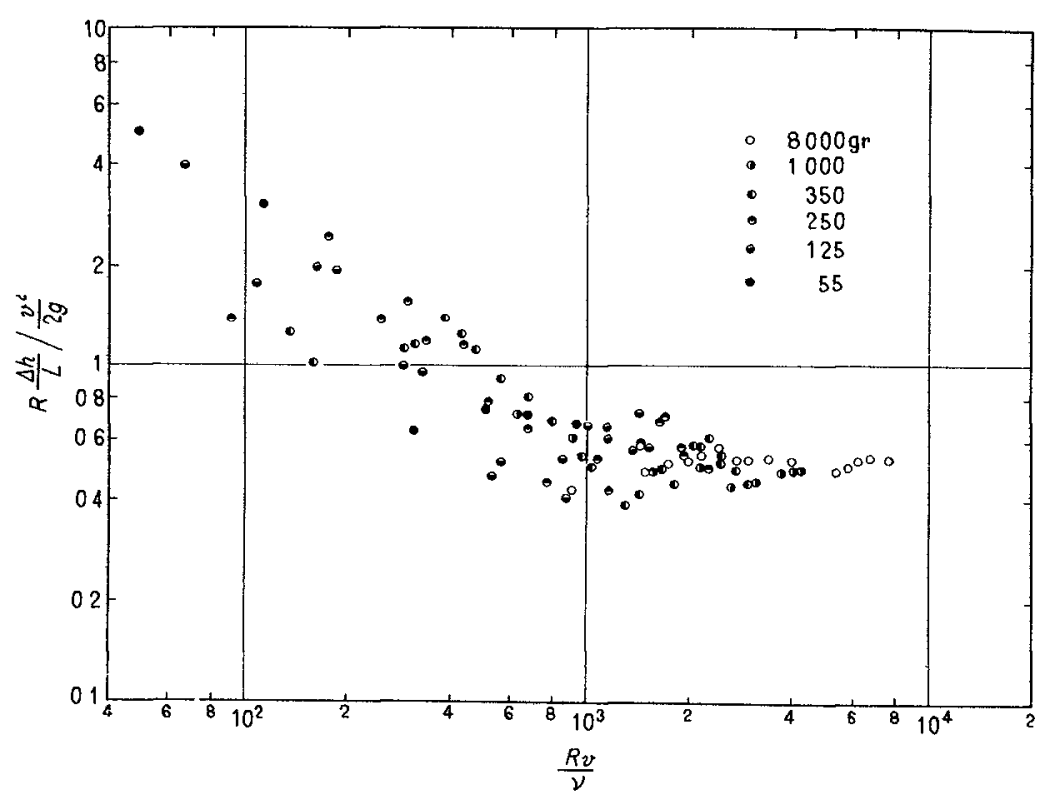

Fig 7 Loss coefficient vs Reynolds number in case of tbe tetrapod 


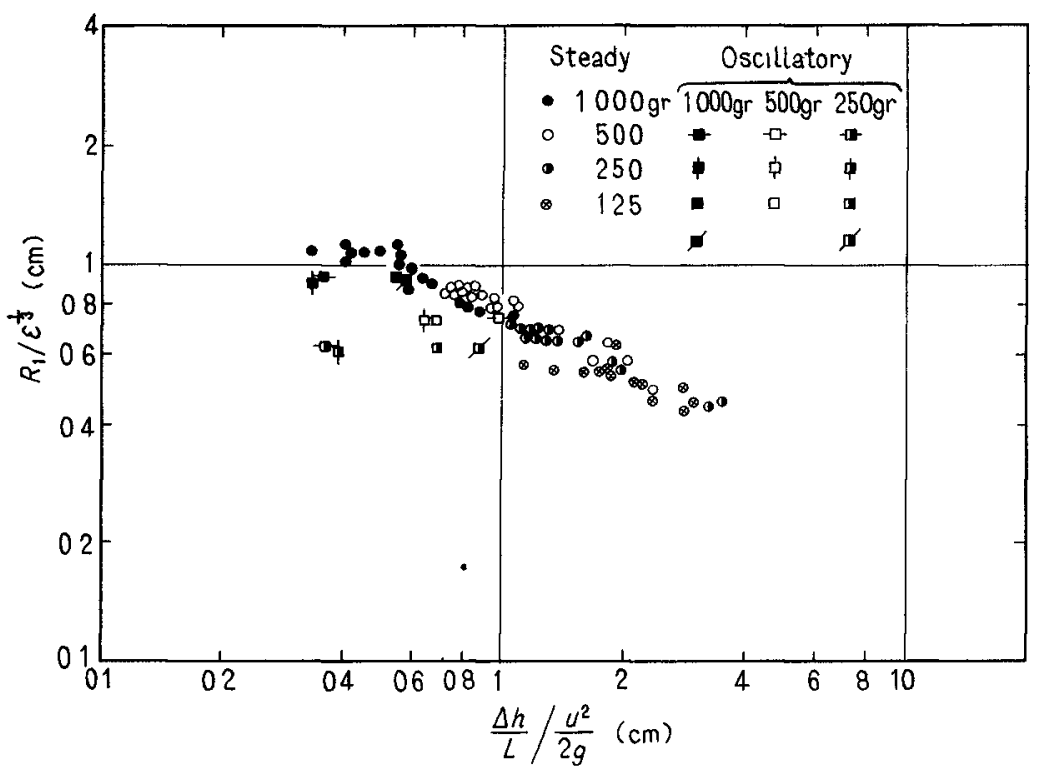

Fig 8 Loss coefficlent vs hydraulic

radius in case of the hollow tetrahedron

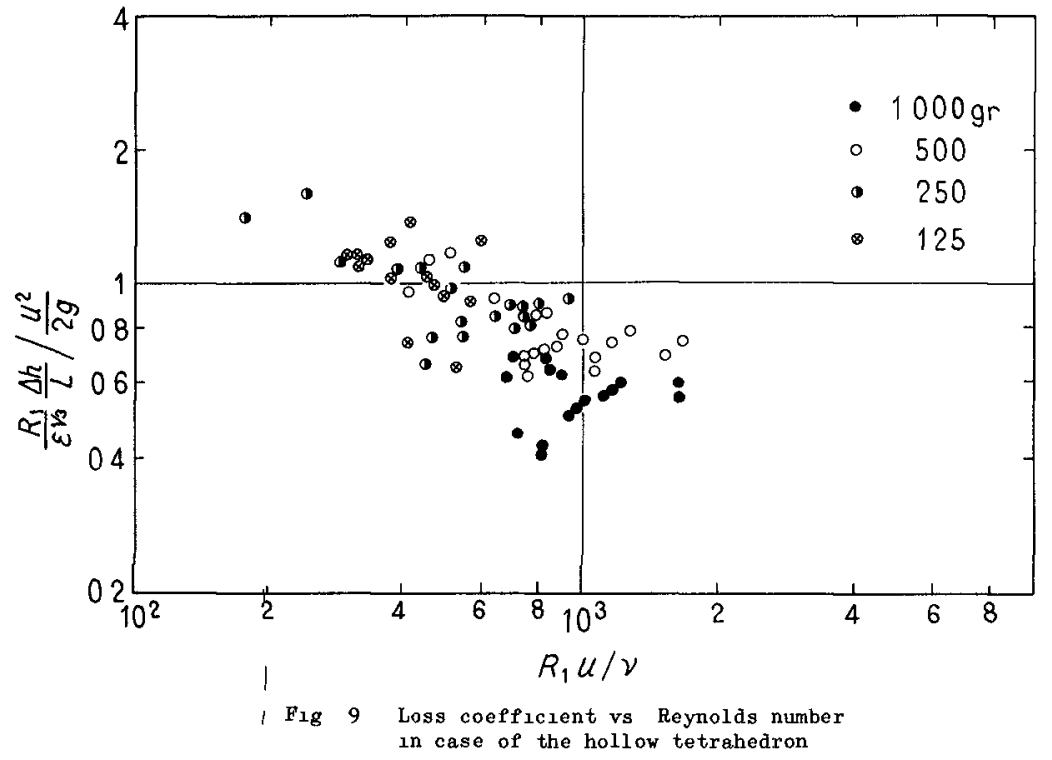




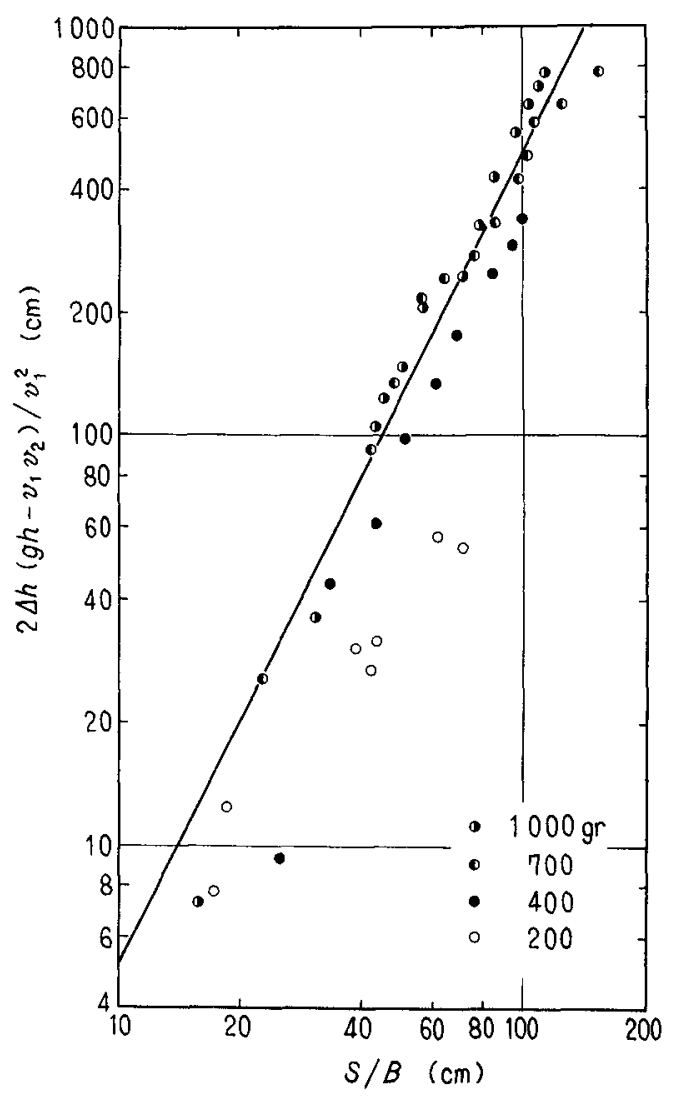

Fig 10 Drag coeffzclent vs surface area of blocks per unit width of the channel in case of the hexaleg 



\section{CHAPTER 98}

\section{USE OF ASPHALT IN BREAKWATER CONSTRUCTION}

\section{by}

$K$ d'Angremond, Delft Hydraulics Lobaratary

H J Th Span, Royal Road Building Cy

J van der Weide, Delft Hydroulics Laboratory

A J Waestenenk, Bitumarin

\section{SUMMARY}

Amang the mony types of breakwater canstructions the sa-called "rubblemaund" type is widely used For the canstruction af expased rubble-maund breakwaters relatively large units are necessory to creote o stoble structure In many ploces in the world rack af the required size is nat avoilable at reasanoble cast, which gave rise to the develapment af a great voriety of armour units

Lately alsa the use of asphalt in breakwater canstruction hos praved feasible

The experience gained during the canstructian af severol projects in the Netherlands resulted in a special method af the use of stone-asphalt in breakwater canstructian Severol cross-sections based on this cancept were subjected ta madel tests to compare their behaviaur under wave-ottack with that af canventionol crosssectians It appeared thot the increase in stobility can be expressed in terms af an "upgrading factar" Attentian was alsa poid to wave run-up

Finally, examples of other applicotions will be presented which incorparate both practical experience and basic reseorch 


\section{INTRODUCTION}

The use of ospholt in road-building is well known, but in the post few decodes the hydroulic uses of ospholt hove olso become common knowledge to civil engineers oll over the world Among the many publicotions in this field, van Asbeck's encyclopaedic work (Reference 1) should be mentioned

An importont contribution to the development of hydroulic opplicotions of ospholt hos been made by the Netherlonds os o result of the interest shown in the subject by Riןkswoterstaot (Stote Troffic ond Woterwoys Department) supported by investigations set up of the Delft Hydroulics Loborotory

In 1960 two mojor Dutch road-bulling firms poined their efforts in the field by estoblishing BITUMARIN, on offiliote company speciolizing in the development ond use of bitumen in hydroulic engineering Close cooperotion was ostoblished with the ospholt loborotory of the Royol Dutch/Shell Group Kerkhoven, one of their leoding engineers, reported on the point ochievements reached together to the Americon Associotion of Aspholt Poving Technologists during its 1965 meeting of Philodelphio (2)

Now thot the development of the vorious ospholt uses in hydroulic engineering hos exponded it seems useful to outline specific developments like the use of ospholt in breakwoter construction, which is the subject of this Paper

In chopter 3 o short historicol review of ospholt techniques in breokwoter construction is given, leoding to 0 discussion of the pottern-grouting technique, which is believed to be most promising for the further development of ospholt uses in breakwater construction As the overoge hydroulic engineer will not be fomilior with the lotest developments of ospholt technology, this Chopter ends with o summory of recently developed theories pertoining to the grouting of stones with ospholt mixes

Chopter 4 is devoted to model investigotions on the hydroulic properties of the constructions described in Chopter 3, introducing on "upgroding foctor" for pattern-grouted slopes

In Chopter 5 the recent construction of the Separoting Jetty ot the Hoek of Hollond is discussed, illustroting the vorious techniques mentioned in this Poper 

IN THE NETHERLANDS

\section{I Eorly morıne uses}

In the Netherlands the use of ospholt in seo defence works in the tidal zone started immediotely ofter Warld War II Examples are the grauting with mastic-osphalt of graynes at the North Sea Coast between the Hoak af Hallond and The Hague, and of the breakwoters of the Hack of Halland The purpase af these repoir works was ta stobilize mounds ond loyers of discrete stanes ogainst heavy wave-ottock by pauring hot mastic osphalt between the stones, thus keeping the stanes in ofixed positian

Asphaltıc grauting proved ta be very effective far two reasans

(1) after hoving cooled dawn ta ombient temperatures mastic-ospholt behoves like o salid moss with high elosticity madulus under short loading times such as wave-attack, and

(ii) as o plastic moteriol af very high viscasity under pralonged loading tumes, thus being able ta fallow subsoil settlements

In due time it was recagnized by the Autharities thot the asphaltic grouting technique was suttable ta replace the traditianal pitching af stanes, ond thus, when the Delto Plan came into executian, the aspholtic grouting technique was adopted as a standard methad of pratecting the slapes af the dikes in the tidal zone Exomples af this use can be faund in the cross-sections of the Veersegat Dam, the Grevelingen Dam, the Horıngvliet Dam and the Brauwershavense Gat Dam (figure I)

The first applicatians were "in the dry", even thaugh in o tidol area Befare long, hawever, methads were develaped for use under water As a result of this development, the asphalt-ship "Jan Heıımans" was built, able to apply mastic-asphalt far grauting underwater sills or plainly for sea-bed protectian in coastol inlets

\section{I Jmuiden breakwaters}

The first importont use of osphalt in "full sıze" breakwoter canstructian con be faund in the IJmuiden breakwaters (1963 - 1967) The old southern and northern breakwater hod ta be extended inta deeper water by 2,100 and $1,200 \mathrm{~m}$ respectively, os a result of which the sauthern breokwater would project $3 \mathrm{~km}$ (abave 2 miles) into the open sea 


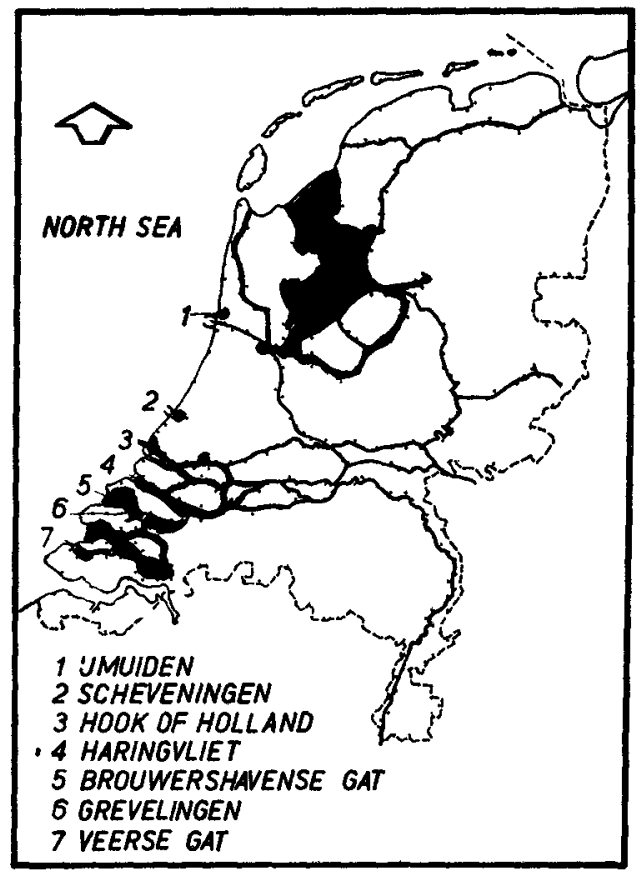

FIG 1 
In principle the design of these breakwaters is of the "rubble-mound" type crowned with a prefab concrete crest-element The core is made of 300 - $1,000 \mathrm{~kg}$ stone to obtain a reasonable degree of core-stability during construction Nevertheless, frequent re-handling of the stone still appeared to be necessary because of the continuous bad weather conditions in the North Sea In fact, the $300-1,000 \mathrm{~kg}$ stones showed considerable lack of stability during construction phases above a level of $M S L-4 m$

A substantial increase in core-stability was achieved by grouting the discrete stones with light stone-asphalt before receiving their final armour The grouting material was designed in such a way that only the upper two layers of the core were penetrated, and that no further "cold" flow into the core took place With mastic-asphalt, as was hitherto in use, such a controlled flow would have been impossible, but by the use in the mixture of stones up to $10 \mathrm{~kg}$ a kınd of "clogging" effect was introduced, enabling control of flow of the grouting material

Instead of the conventional armour layer of discrete though more or less interlocking elements, an impervious monolithic layer of stone-asphalt was adopted The thickness of the layer was dictated by the internal water pressures caused by wave-action in the open rock-core against the impervious armour The thickness of the armour was chosen to be $225 \mathrm{~m}$ (Figure 3)

The construction of asphaltic layers of such a thickness at the steepest slope possible constituted a problem in itself By the time the breakwaters were designed the grouting techniques had not developed to the extent that controlled grouting of stone-layers of several thicknesses at water depths of 5 to 10 metres could be considered feasible

Therefore a premixed product had to be used Conventional asphaltic concrete with its aggregate-size limited to 6 - 8 centımeters lacked too much stabilıty in the hot phase to enable the construction of thick layers as steep slopes The solution to this problem was the development of a new mixture with aggregate-sizes up to $60 \mathrm{~kg}$, called stone-asphalt, which has already been mentioned in this Paper With this material slopes of 1 in 175 under water and 1 in 2 above water were found to be feasible

The experience gained with the IJmuiden breakwaters after three years' service is satisfactory in general, but nevertheless continuous creep of the stoneasphalt layer is causing cracks, especially in summertime (temperatures of both water and air) However, the damage is decreasing every year as a result of the formation of an internal skeleton in the stone-asphalt aggregate 


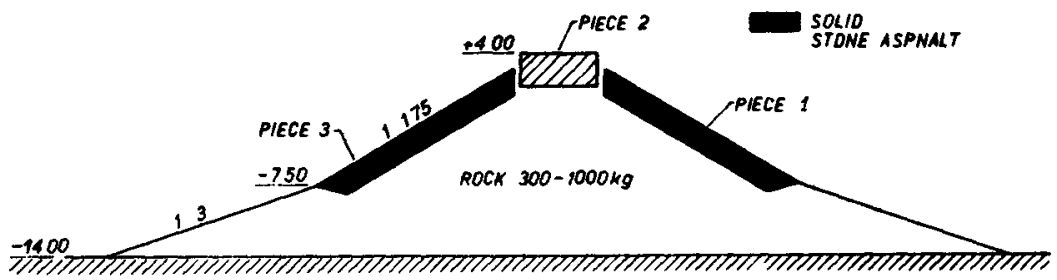

FIG 2

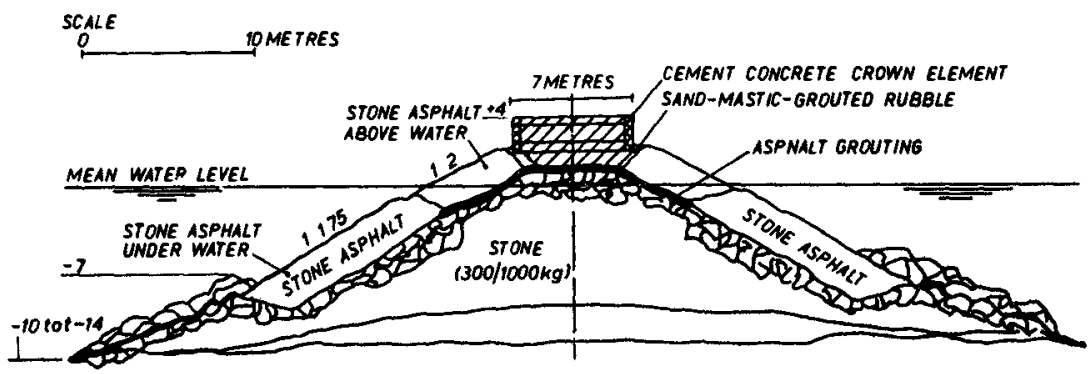

CROSS SECTION OF NEW OREAKWATERS UNDER CONSTRUCTION AT JMUIDEN (FROM DATA SUPPLIED BY RUKSWATERSTAAT)

FIG 3 
As hos just been stoted, the IJmuiden works gove new impulses to the development of grouting techniques The grouting of the core-stone $300-1000$ $\mathrm{kg}$ with light stone-ospholt hos alreody been mentioned os stondord procedure, but moreover o test-section wos corried out succesfully by grouting rock of $300-1000 \mathrm{~kg}$ with horizontol loyers of 15 to $2 \mathrm{~m}$ thickness constructing o monolithic ond stoble cop of heovy stone grouted mossively with light stoneospholt

In considering the first importont use of ospholt in breokwoter construction embodied by the IJmuiden breokwoters, it con be stoted thot its performonce is sotisfoctory in generol, but thot for future works the following two drowbacks will hove to be deolt with

a The concept of on impervious loyer covering o highly permeoble rock-core should be obandoned to ovoid the effect of internol woter-pressures due to wove-oction

b Creep ond extended settıng-time of thick ospholtic loyers should be ovoided by replocing the use of premixed loyers by the grouting of loyers of discrete stones, which hove olready developed o skeleton of their own ond ore therefore no longer susceptible to setting

The experience goined in the IJmuiden works made the solutions to these problems possible, os will be seen in the next parogroph

\section{Pottern grouting}

To ovoid the problem of internol woter pressures originoting from the impermeobility of the stone-ospholt ormour loyer o new concept wos introduced by the ideo of increasing the stobility of on olreody fairly stable rock slope by local groutıng with stone-ospholt in o regulor pattern, thus maintoining the permeoble chorocter of the slope By using a grouting method the problem of creep ond extended setting-tıme would olso be coped with This system of "Internol ormour" was mode technicolly possible by the development of controlled grouting techniques during the IJmuiden works

In working out the ideo of pattern-grouting it was reolized thot in filling up more thon obout $70 \%$ the interstices between the rocks no guorontee could be given for the overoll permeobility of the construction Prelıminory model tests executed in the Delft Hydroulics Loborotory showed on increase in stobility os 0 function of on increosing degree of filling up the interstices, with o relotively slow increase beyond $50 \%$ So the filling up to 50 to $70 \%$ of the interstices seemed to be optımal

For the colculotion of the required weights of ormour-elements, loyer thickness, etc reference is mode to Shore Protection, Plonning ond Design of the U S Corps of Engineers (Reference 7) 
As to the size and spacing of the plots the following can be said An individual plot will penetrate to a depth of $2 d$, $d$ being one layerthickness The shape depends on the local conditions (shape and direction of interstices between the stones) but it can be idealized to the slope of a cube with contents $8 \mathrm{~d}^{3}$, of which approximately $60 \%$ is solid rock and $40 \%$ grouting material So the contents of one plot is $0,48 d^{3}=32 d^{3}$ and its weight

$$
P=32 \quad \gamma g d^{3}
$$

The rock weight $w=\gamma_{s} d^{3}$

Thus

$$
P=32 \quad \gamma_{g} / \gamma_{s} \quad W
$$

in which $P=$ weight of plot in tons

$$
\begin{aligned}
& p=\text { weight of plot in tons } \\
& \gamma_{g}=\text { spec weight of grout in tons } / \mathrm{m}^{3} \\
& \gamma_{s}=\text { spec weight of rock in tons } / \mathrm{m}^{3} \\
& W=\text { weight of rock in tons }
\end{aligned}
$$
is covered

The spacing of the plots should be such that 50 to $70 \%$ of the surface

If placed in such a pattern the plots (each fixating 5 to 10 stones) will touch each other at the edges This leaves stones uncovered at some places, which, however, are "keyed" between the others For reasons of safety it is recommended to use three layers instead of two, only grouting the top two layers A few loose stones will probably be washed away by the waves, which is not dangerous at all, but even if a whole plot were washed away for one reason or another, a third layer would still provide protection to the core, because it would be "keyed" to the surrounding plots

As to the stability under wave-attack, a so-called "factor of upgrading" $F$ Could be attributed to the pattern-grouted system This means that patterngrouted rubble in the $X$-ton-class has the same stability as discrete rubble in the $\mathrm{F} X$-ton class

From prelımınary model tests a value of $F=5$ seemed a conservative estımate, which has been confirmed by the more elaborate tests discussed in the next Chapter This reduction of the required maximum stone-size has a favourable effect on the area of the cross-section of the breakwater because of the reduction in layer-thickness and the absence of secondary layers in most cases

Ease of construction is obtained by the reduction of both crane-reach and crane-load, or by the possibility of working on more gentle slopes 
It seems thot the tıme-proven ospholt groutıng-technıque hos grown into 0 real competitor in rubble-mound breokwoter design With increasing demonds on stobility under wove-ottock, quorries ore proving steadily unoble to produce heovy ormour stone, ond this hos coused the development of o series of ortificiol ormourblocks All these blocks hove in common thot increosed stobility con only be obtoined by increosed weight, which is necessorily occompanied by increosed surfoce for wave-ottock

As distinct from these externol ormours, the internol ormour presented in this porogroph hos the odvontoge of dimınishing the wove-ottock on the discrete ormour elements by partly filling up the interstices between them, while on the other hond their stobility is increased os o result of the "keying" effect of the grouting moteriol

\section{Aspholt mixtures for patch-grouting}

The generol principles for design ond properties of ospholtic mixtures for hydroulic opplication, os developed in the Netherlonds, ore described by Kerkhoven (Reference 2)

For pottern grouting of the ormour loyer of lorge sized stones, some odditionol principles ore necessory

The mix-design of the patches must be reloted to the lorge size of the stones and the shope ond weight of the patches in this connection it is importont whether the grouting is executed under woter or not, os in the first cose the hot stoge of the mixture is rother short ond, consequently, olso the time for settlement

Experiments hove shown thot for grouting with potches of limited size $\circ$ relotion exists os o blocking criterium between the smoll-sized stones in the ormour loyer, defined os $\mathrm{d}_{15} \mathrm{l}$ ), ond the lorge-sized stones in the ospholt mixture, defined os $\mathrm{d}_{85} \mathrm{~b}^{1)}$ For underwoter grouting the relotion $d_{15} / d_{85}=$ co 10 wos found ond for obove-wotergrouting $d_{15} d_{85}=\operatorname{co} 5$

The six design for smoll-sized stones, ond sond inside the grouting moteriol, depends on the working circumstonces during execution ond on the ploce of use in the totol breakwoter construction it is common thot for patch-grouting under woter level, on ospholtic mixture in grop-groding is odopted ond for potch grouting obove woter level on ospholtic mixture in concrete-groding

The flow in the hot stoge ond the viscous creep in the cold stoge, in relotion to the size ond slope of the ormour lorge-sized stone loyer, depend on the percentoge ond type of filler ond bitumen in the grouting mixture

1) $d_{15} c q d 85$ ore the equivolent diometers $d$ of stone size-distribution, possing in percentoge of weight for $15 \mathrm{c} \mathrm{q} 85 \%$ 


\section{MODEL INVESTIGATIONS}

\section{Introduction}

Since 1964 breakwaters with the use af asphalt have been the regular subject of madel investigatians in the Delft Hydraulics Labaratary in the beginning the knawledge of the material was insufficient ta repraduce the properties of the material on model scale Therefore the "asphalt-design" far the IJmuiden breakwaters was ariginally not tested in a madel When, already during the execution of the warks, again discussion arase on the required thickness af the stone-asphalt, it was decided to start simplified tests In these tests the flexible structure was schematized applying rigid cancrete martar in the madel, instead af stone-asphalt, which means that investigations into the mechanic and elastic behaviour af stone-asphalt were prevented

The cap af cancrete and stane-asphalt was reproduced as 3 independant rigid and relatively strang pieces of concrete (compare Figures 2 and 3 )

It was shawn visually in these tests that the averall stability af the slope cap was insufficient due ta water pressures under the caver layer Ta solve this prablem the toe of the slope was loaded with rubble and cancrete blacks to a level of $-4 \mathrm{~m}$ for the exposed sections

When, thereafter, Bitumarin propased an application of stone-asphalt to prevent the uplift pressures by keeping the outer layer permeable, it was decided to carry aut further model tests, by replacing asphalt by cancrete grouting

Fram a comparisan between cross-sectians with and without patch-grauting It appeared that the stability number $k_{D}$ increased cansiderably It must be noted, hawever, that the schematizatian of the tests was such that the elasticity and the strength of the patches were not ta scale

\section{Reproductian af asphalt}

Since pattern grouting proved to be a feasible methad af stabılızıng rubble-maund structures, a number af additional tests have been carried out ta study the behaviour of grauted structures in more detail

When pattern-grouting is used, the principle of the rubble-maund structure is maintained but the stability is increased cansiderably Due ta the effect of the grout, the surface of the structure is smaothed, resulting in a larger amount af uprush and overtapping 
Attention was paid, therefore, to the wave uprush and the stobility of the armour layer, under various conditions using different cross-sections

Due regard was paid to the proper reproduction of asphalt to obtain both geometrical and dynamic similarity between model and prototype

Geometrical similarity was obtained by using the appropriate grading of the armour stone and a low viscosity of the grout to arrive at a depth and width of penetrotion comparable to those found on the site in question

Dynamic similarity wos obtained by composing the grout in the model in such a woy that the density of the mix, os well os its stiffness ond strength, wos reproduced correctly

Asphaltic bitumen is characterized in terms of the penetration and the ring and ball softening point (see Reference 1) Starting from these dota, the stiffness modulus of the material, defined as the ratio between stress ond stroin

$$
\left(S=\frac{\sigma}{\varepsilon}\right) \text {, }
$$

can be determined for a given temperoture and a given frequency of loading

When oggregotes are added, the dynamic properties of the mix chonge, the stiffness of the mix being 0 function of the stiffness modulus of the asphalt and the concentration by volume $\left(C_{v}\right)$ of the mineral aggregate

In the octuol propect o grouting mixture will be used which consists of (by weight) $94 \%$ stone, sand and filler

$6 \%$ bitumen $80 / 100$ pen

So the volume concentration of minerals in the mixture $C_{V}=080$ to 082 and the density $=2300 \mathrm{~kg} / \mathrm{m}^{3}$ Two mixtures were tried out to give both the required density and stiffness

The compositions of the mixes used in the model were

Mixture No 1

(by weight) $40 \%$ dune sond

$40 \%$ barium sulphate filler

$20 \%$ bitumen $80 / 100$ pen

The volume concentration of minerals in the $\operatorname{mix} C_{v}=055$, the density $=2,300 \mathrm{~kg} / \mathrm{m}^{3}$

Mixture No 2

(by weight) $60 \%$ barıum sulphate filler

$40 \%$ bitumen $280 / 320$ pen

The volume concentration of minerals in the $\operatorname{mix}$ is $C_{v}=025$ and the density $=1,850 \mathrm{~kg} / \mathrm{m}^{3}$ 
The stiffness of the mixtures wos calculated with the help of the monographs mentioned in References 3 ond 4 The ombient temperature in nature is $T=5-15^{\circ} \mathrm{C}$, in the model it was $25^{\circ} \mathrm{C}$ The results are plotted in Figure 4

It is seen thot mixture No l, which is on scole as the density is concerned, is too stiff, while mixture No $I I$ is too light ond also on the supple side os the stiffness-modulus is concerned The strength of the material both in noture ond in model is in the order of 1 to $510^{6} \mathrm{~N} / \mathrm{m}^{2}$ (Reference 4), meaning thot the grout in the model is too strong

From the above it follows that the density ond the stiffness con be brought to scole (though not in one $\mathrm{mix}$ ), while the tensile strength is alwoys too high in the model This means that in the event that the collapse of the ormour loyer would be caused by the cracking of the patches, the model would give too fovourable results

Therefore also some tests hove been run in which the patches were already artificially broken beforehond, in order to eliminote ony fovourable effect of tensile strength of the grouting mixture, thus exoggerating the effect in the opposite direction

For this purpose the pattern-grouted armour was frozen ond then deliberotely "demolished" by hitting with o bar, in such a way that the potches broke down internolly, only leaving a three-dimensional "chinese puzzle" In the latter tests a situation is represented in which only the "keying" effect of the grout con be called upon, while the patches themselves have lost all internol bond

To eliminote the odhesion between stone and aspholt, the stone in the model wos covered with lime before grouting This was done to represent the situation in nature, where due to the wet environment little or no odhesion of the grout to the stones can be expected

\section{Stability of grouted slopes}

The results of stobility tests for rubble-mound breakwoters are generolly expressed in terms of the dimensionless stability number

$$
K_{D}=\frac{H^{3}}{W \Delta^{3} \operatorname{cotg} a}
$$

Vorıous outhors hove determıned volues for $K_{D}$ in order to obtaın a stoble structure 


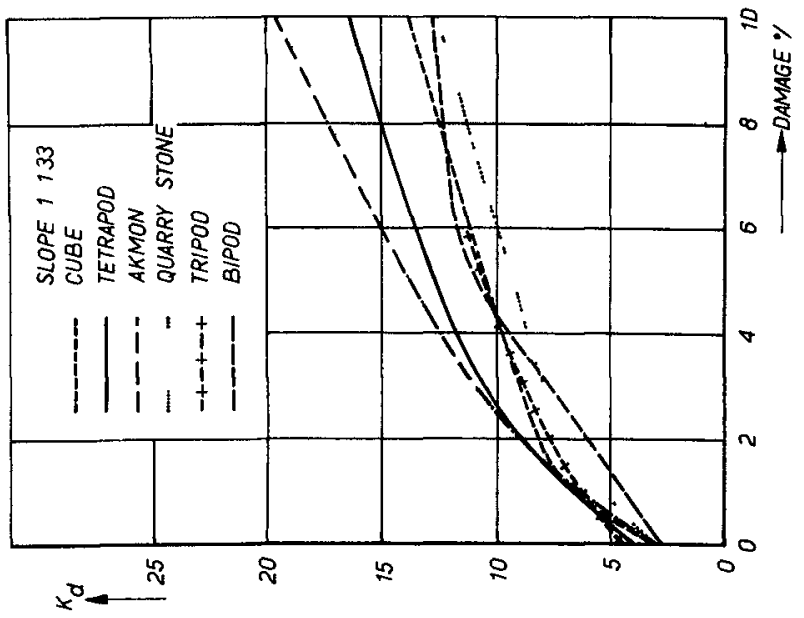

$\frac{n}{4}$
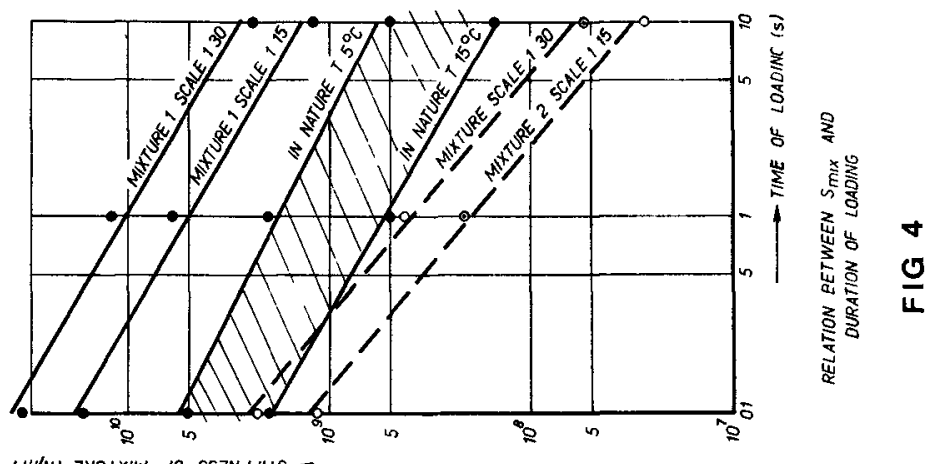

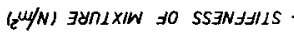


A relation between the $K_{D}$ value and the percentage of damage is given in Figure 5 (Reference 5)

Numerous references are present in literature describing artificıal blocks which have a higher value of $K_{D}$ for the no-damage criteria, due to the special interlocking effect The results of the model tests indicate that the critical value of $K_{D}$ can be increased by a factor 2 to 3 in this way Pattern-grouting also increases the interlock between the various units, because of the three-dimensional effect, thus creating more or less irregular artificial units Moreover, the wave-attack is reduced as a result of the filled voids Obviously these aspects result in an increase in the stability of the structure which manifests itself in a higher $K_{D}$ value

The upgrading-factor $F$ is defined as the ratio

$$
F=\frac{K_{D} \text { for pattern grouted armour layer }}{K_{D} \text { for non-grouted armour layer }}
$$

\section{Test results}

\section{Cement grouting}

The first series of stability tests on grouted slopes was performed on a crosssection as indicated in Figure 6 As already mentioned, the cement grouting was used in these early tests

The patch-grouted test section was situated between - 85 ad $-3 \mathrm{~m}$ It has slopes of 12,13 and consisted of rubble material from several weight classes during the various tests

The dimensions of the patches and the pattern of grouting was also varied under the following conditions

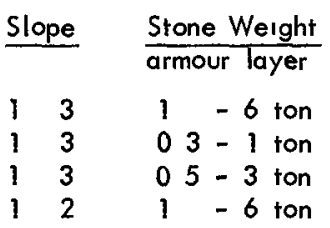

The cross-section was subjected to regular waves with a period of $95 \mathrm{sec}$ The wave height was increased from 3 to $8 \mathrm{~m}$ in steps of $1 \mathrm{~m}$ The duration of each step was 3 hours prototype After each step the damage was determıned by counting the displaced stones and expressing this number in a percentage of the 


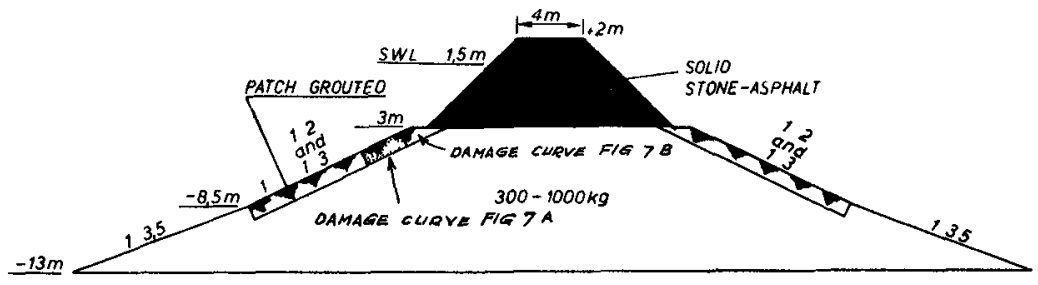

FIG 6

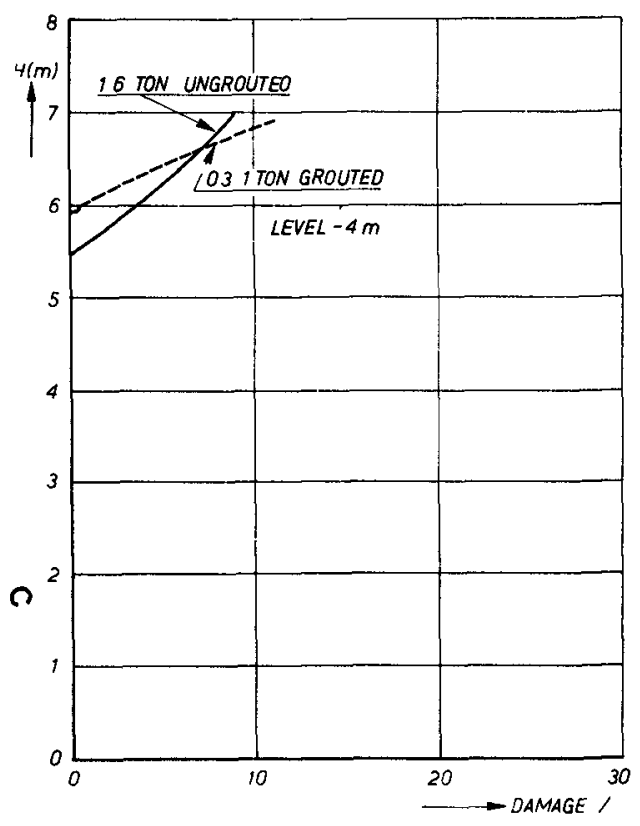

FIG $7 \mathrm{~A}$

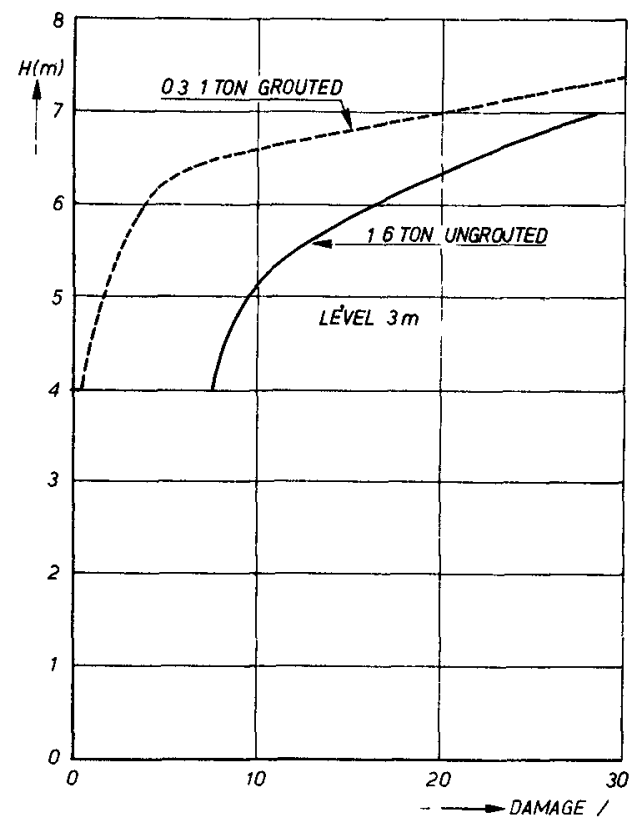

FIG 7B 


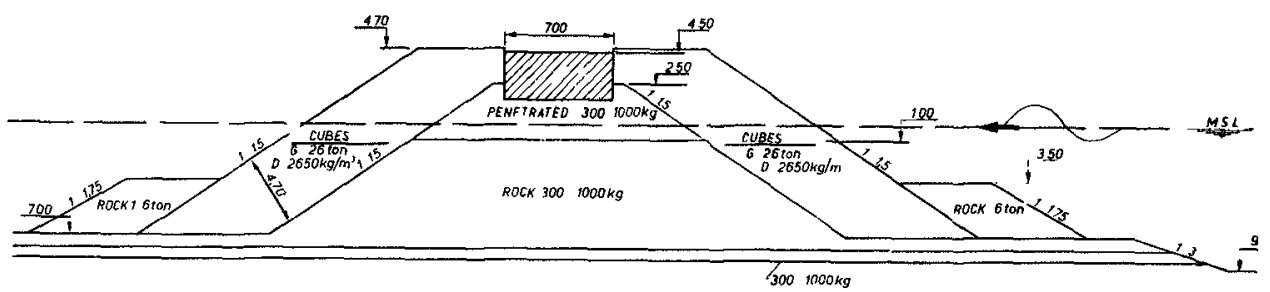

FIG 8 A

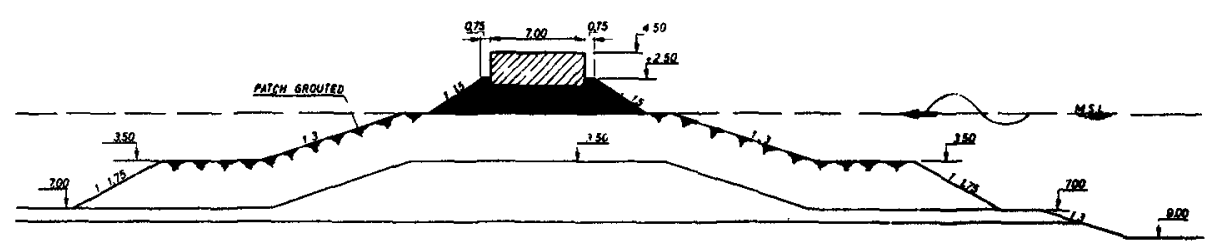

FIG 8 B
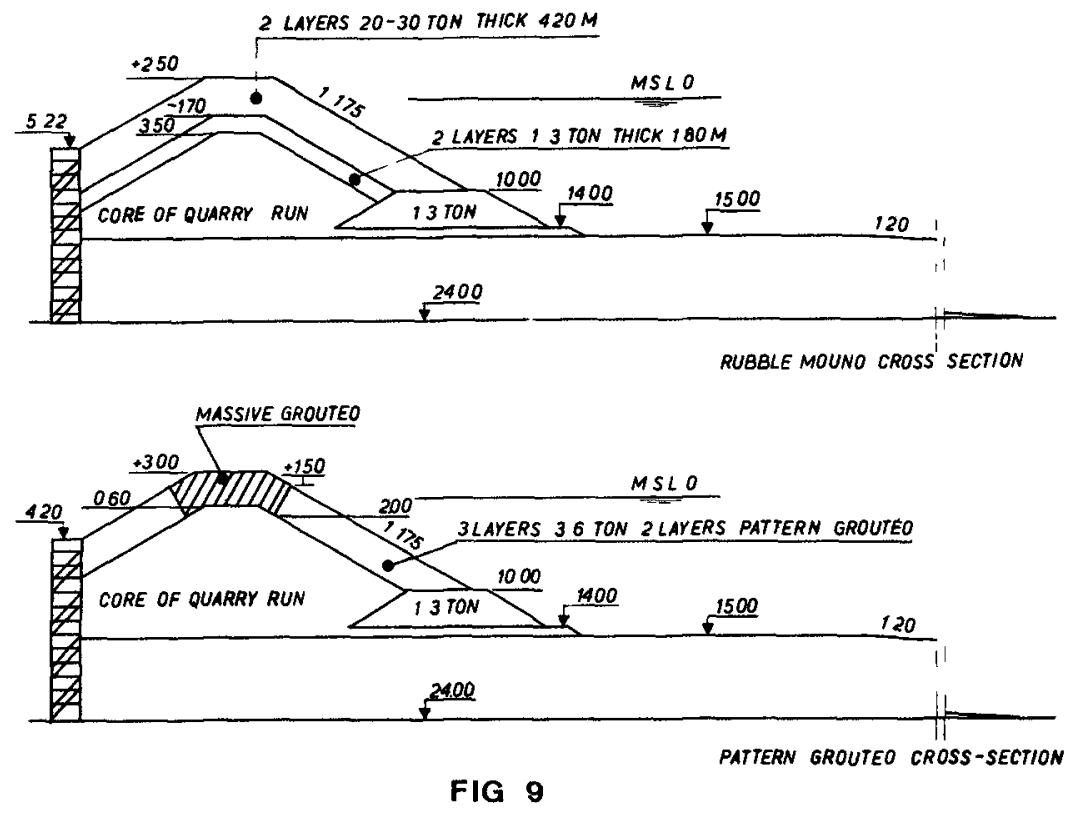

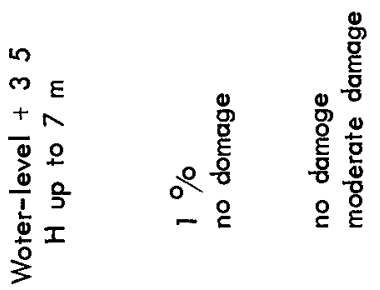

นn

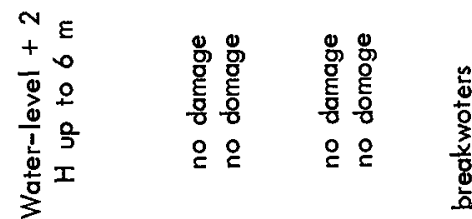

n $E$

on

in

$\frac{3}{1} \stackrel{0}{2}$

交I

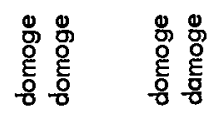

으을

요

3

n

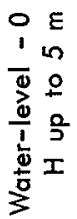

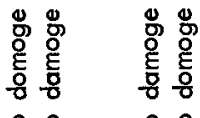

을 울

옫

$\frac{5}{5}$



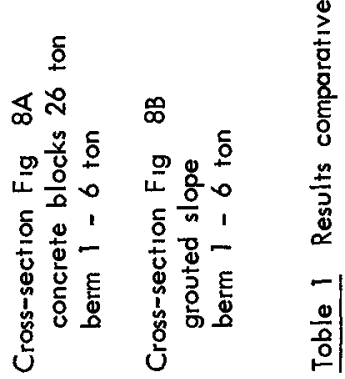


tatal number of stones in the zone cancerned In this way it was possible ta establish a relationship between damage percentage and wave height

A comparison of the relatian between damage and wave height for grouted slapes with stones of $03-1$ ton and non-grauted slopes consisting af 1 - 6 ton quarry (Figures $7 \mathrm{~A}$ and $7 \mathrm{~B}$ ) shows that these situations are comparable for damage less than $10 \%$ This leads ta the canclusion that far a practical range af damage percentage the upgrading factor can be assumed ta be about 5

This canclusion has been verified far an alternative design af the Schevenıngen breakwater, where a cross-section in stone asphalt was campared with a method using cancrete blacks (Figures 8A and B) These tests were also performed in regular waves Data on the damage are presented in Table 1

In all conditions the grouted slope was equally as stable as the rubblemound breakwater with cubes of 26 tans Only in the most severe conditions did the smoath surface of the grouted slope increase the downrush in such a way that damage occurred to the non-grouted berms This draw-back could be remedied by making the grouting slightly deeper The stone-asphalt was also reproduced by a cement mortar in these tests

\section{Asphalt grouting}

Since groutıng praved ta be a useful methad for stabılizing rubble-maund structures, some additianal tests were carried aut to study the stability of grouted rubble-mounds in waves Far that purpose a number of grauted sectians were campared with an equivalent number of traditional rubble-mound sectians consisting of stanes 5 times heavier than those used in the pattern-grauted section Using the formula of Hudson, the rubble-mound section was sa designed that no apprecıable damage should accur under maxımum wave attack

As stated in Sectian 42 special care was taken to reproduce both the geometrical and dynamical properties of the asphalt grout Since the strength af the material cauld not be reproduced carrectly, the tests were repeated with broken patches

Wave heights were increased step by step until maximum wave height were reached A review of cross-sections tested in the model is given in Figures 9 to 11 , whilst a summary of test conditions and the test results is given in Table 2 
Table 2 Test Conditions and Results for Varıous Grouted Sections

$\begin{array}{crccccc}\text { Profile } & \text { Fig } & \begin{array}{c}\text { Scole of } \\ \text { Test }\end{array} & \text { Mixture } & \begin{array}{c}T \\ (\mathrm{sec})\end{array} & \begin{array}{c}H_{\operatorname{mox}} \\ (\mathrm{m})\end{array} & \begin{array}{c}\text { Damoge } \\ (\%)\end{array} \\ \text { A } & 9 & 30 & 1 & 10 & 85 & \text { NONE } \\ \text { B } & 10 & 30 & & 10 & 85 & \text { NONE } \\ \text { B } & 10 & 30 & & 8 & 6-7 & \text { NONE } \\ \text { C } & 11 & 30 & & 8 & 6-7 & \text { NONE } \\ \text { B } & 10 & 30 & 11 & 10 & 7 & \text { NONE } \\ \text { B } & 10 & 30 & 11^{2} & 10 & 7 & \text { NONE }\end{array}$

1) Domoge expressed as $\%$ of stone removed

2) Patches broken

Test results indicoted thot both the traditionol ond the grouted sections showed little or no domoge, even when the patches were broken This proved the volidity of an upgrading foctor of 5

\section{Run-up on grouted slopes}

In many coses the crest level of a breakwoter or o sea wall is determined on the basis of on acceptoble omount of overtopping under extreme conditions, olthough sometimes the acceptable wove run-up is also used os a criterion

The level of wave run-up $z$ above MS L has been investigoted extensively for smooth impermeable slopes by severol authors (See a 0 Reference 6, Reference 7 ond Reference 8) An extract of these results is presented in Figures 12 ond 13 for $d / H>3$

For slopes covered with rip-rap and for rubble-mounds the run-up is much less becouse of the roughness of the surface and the porosity of the outer layers The reduction in run-up due to these effects is expressed by a reduction foctor $r$, indicating the ratio between the run-up on the rough surfoce ond the run-up on a smooth impermeable surfoce under the same conditions Though the scatter of the measured figures is considerable, authors from different origin indicate for $r$ a value of 05 to 06 (References 9, 10, 11)

With a patch-grouted slope, porosity ond roughness are reduced in comparison with rubble slopes Consequently the run-up must be expected to be greater This may lead to o higher crest level of patch grouted breokwoters which involves o higher cost 


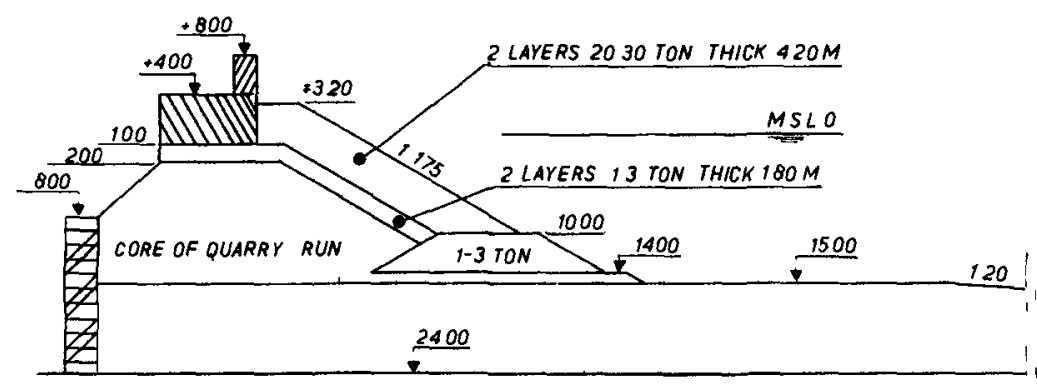

SECTION

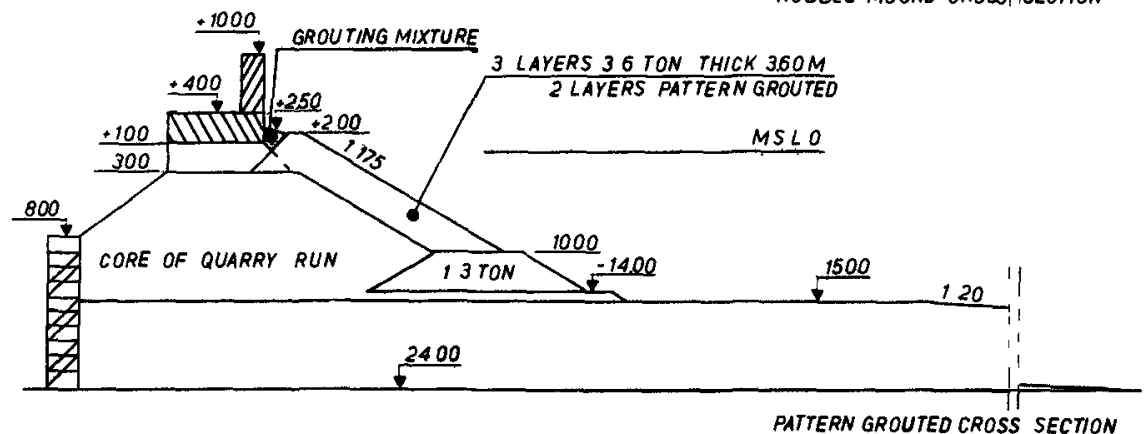

FIG 10
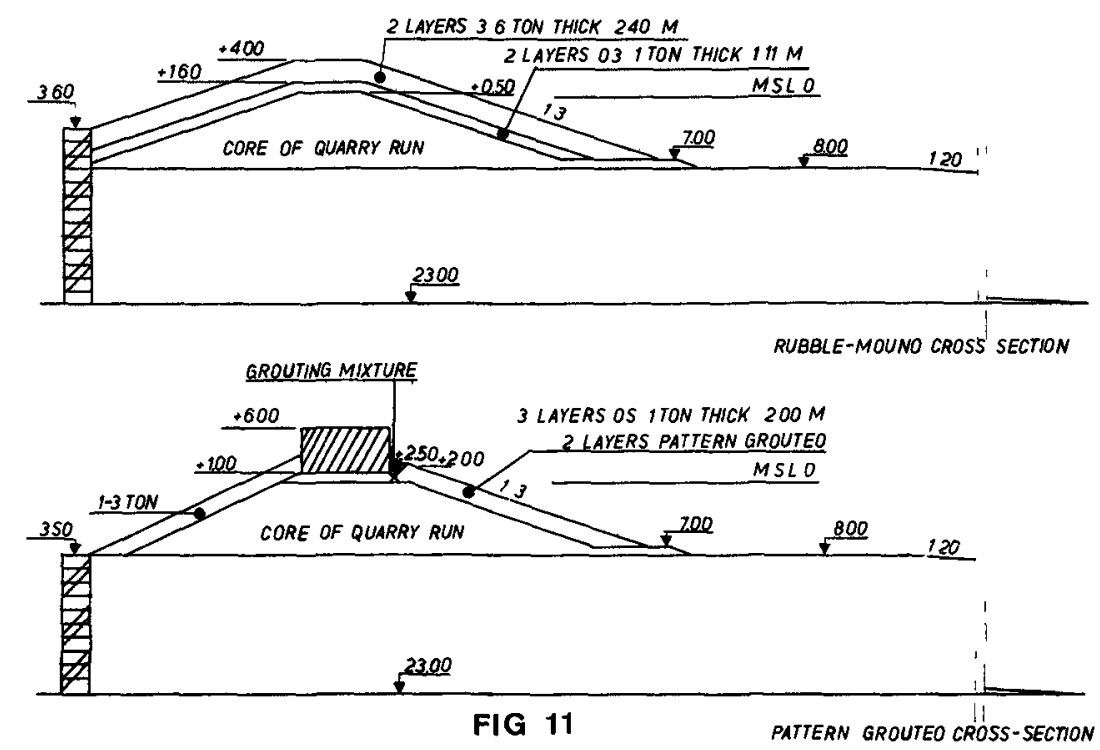
Model tests have been corried out on fully grouted slopes, elımınotıng porosity completely Any reduction in run-up was due to surface roughness

The run-up $z$ depends on the following foctors

$$
z=f(H, L, T, d, v, \rho, g, k, p \text { and } o)
$$

in which $\mathrm{H}=$ wove height

$$
\begin{aligned}
L & =\text { wave length } \\
T & =\text { wave period } \\
d & =\text { water depth } \\
\gamma & =\text { kinematic viscosity } \\
p & =\text { density } \\
g & =\text { grovity } \\
k & =\text { roughness } \\
p & =\text { porosity } \\
\operatorname{cotg} a & =\text { slope }
\end{aligned}
$$

Becouse $k$ ond $p$ were kept constont during the tests $(p=0)$ the relative run-up can be expressed as

$$
z / H=f\left(H / g T^{2}, d / g T^{2}, \operatorname{cotg} a\right)
$$

$\mathrm{H} / \mathrm{gT}^{2}$ and $\mathrm{d} / \mathrm{gT}^{2}$ were varied from 00004 to 001 and from 0009 to 0095 respectively

The slope cotg $\circ$ wos 175 ond 225 The grouted quorry stones weighed from 100 - $250 \mathrm{~kg}$ (prototype) As o check olso tests hove been mode on o smooth

\begin{tabular}{|c|c|c|}
\hline Slope & $\mathbf{r}$ & Source \\
\hline smooth impermeable & 10 & - \\
\hline $\begin{array}{l}\text { rip-rap covered } \\
\text { rubble-mounds } \\
100 \% \text { grouted } \\
\text { patch grouted }\end{array}$ & 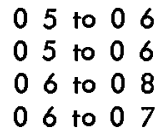 & $\begin{array}{l}\text { model tests + literature } \\
\text { model tests + literature } \\
\text { model tests } \\
\text { interpolation }\end{array}$ \\
\hline
\end{tabular}
impermeoble slope ond on o non-grouted slope covered with quarry stone

As the plot of $\mathrm{z} / \mathrm{H}$ versus $\mathrm{H} / \mathrm{gT}^{2}$ showed o scatter which could not be explained by the differences of $d / g^{2}$ only, the maximum values of $z / H$ hove been plotted as a function of $H^{\prime} g T 2$, for both slopes separately (Figures 12 and 13)

To compare the actual model tests with the results of others, Figures 13 and 14 show also dota obtained from References 7,9 ond 10

From the Figures it can be concluded thot reduction coefficients for the wove run-up con be used os indicoted in Toble 3

\section{Toble 3 Reduction Coefficients}




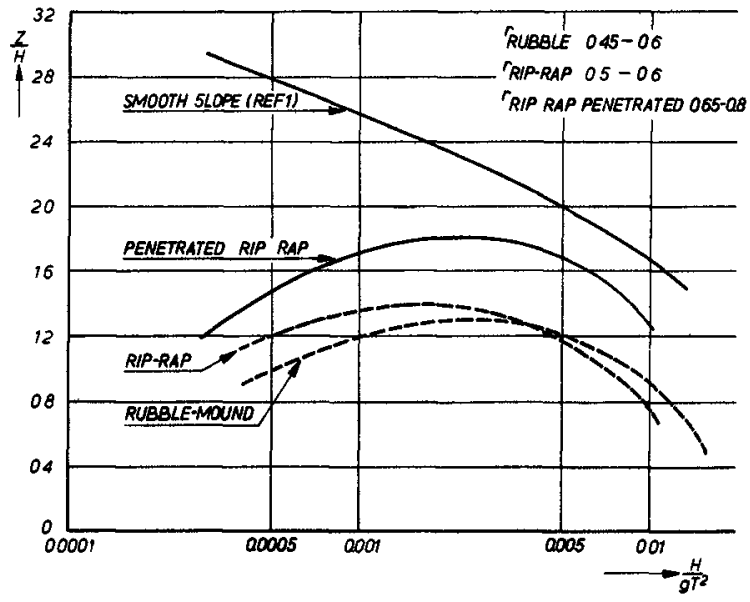

FIG 12

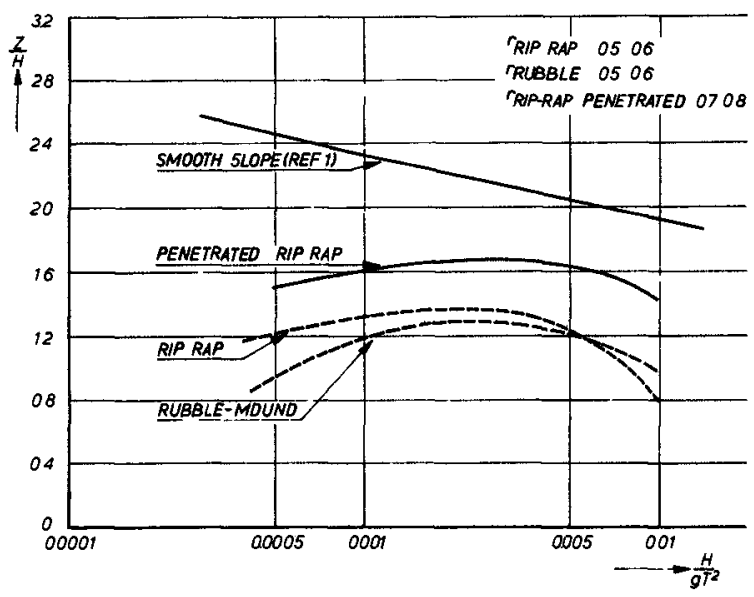

FIG 13 


\section{SEPARATING JETTY HOOK OF HOLLAND}

\section{Design conditions}

A recent example of the use of asphalt in breakwater canstructian can be found in the so-called "Separating Jetty" at the Hook of Holland

This Separating Jetty (Figure 14) is situated in the new entrance being canstructed far Rotterdam Harbour, separating bath traffic and the tidal density currents af the Ratterdam Waterway leading ta the inner harbaurs af Ratterdam, and the Caland Canal leading to the Eurapaart harbaurs far mammath tankers So its main objective is of a nautical nature, but by virtue of its exposed situatian perpendicular wave-attack up to $H_{\text {sign }}=6 \mathrm{~m}$ can be expected at the head, and oblique attack along the trunk

From the inner and going seaward the subsal is descending from above water down to approx $M S L$ - $5 \mathrm{~m}$ Nevertheless it can hardly be regarded as a shallow water breakwater, due to the fact that the underwater banks will be dredged to 1 an 4 slapes gaing down beyand M S L - $20 \mathrm{~m}$

\section{Standard design}

For the deeper part of the dam an embankment has been constructed cansisting of fascine mattresses pratecting the dam footing, and dumped stone and other waste materials from an ancient jetty to be cleared away, up to a level of $M S L-2 \mathrm{~m}$

From an econamical point of view two materials came inta cansideratian for the dam core constructian abave water mine-waste and lean sand asphalt The latter was chosen because of its low permeability, which will be explained later

In the standard design the armaur was designed as an almost traditianal fully grauted stane-layer, which is to be regarded as impermeable Fram investigations in an electric analogue, it appeared that in the lean sand asphalt dam care, being af the same permeability as the sand it is made of, pressure gradients develap during the tidal cycle which resulting in lower water pressures under the armaur than the dam care were made of mine-waste

This is why lean sand asphalt was chosen as the dam care material

In view of the geametry of the dam and the maderate permeability of the dam core, the influence of wave action an the water pressures cauld be neglected 
ํํㄴ

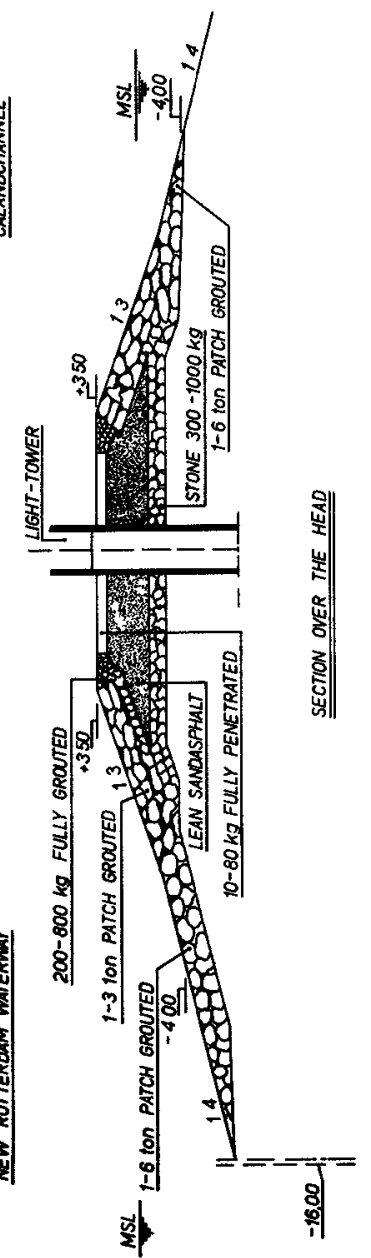

ज़

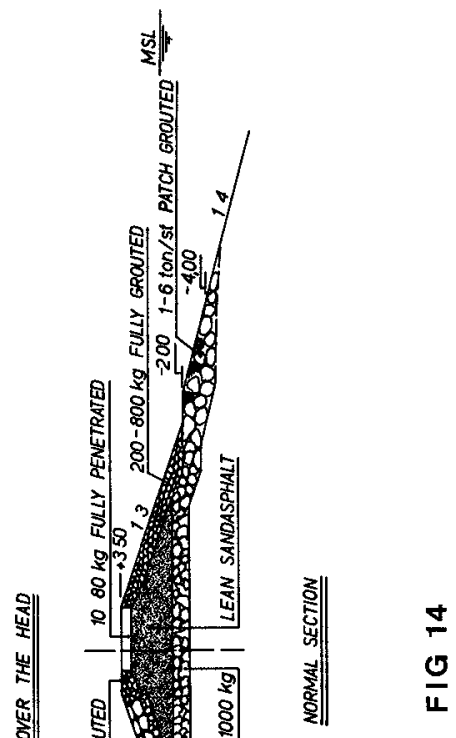


During the actual construction of the dam, which started in December 1969, the amplitude of lean sand asphalt for the dam core proved to be succesful also as far as the stability under wave action during the execution of the works is concerned Negligible losses of dam core material were suffered, in spite of several heavy storms encountered during construction time

\section{Test sections}

As the Authorities ( 1 e, Rijkswaterstaat) are very much interested in the recently-developed pattern-grouting system as described in Paragraph 33 two test sections will be constructed in the near future to investigate the merits of this method actually These sections are situated at the most exposed part of the dam, namely, the head and the adjacent part of the trunk

Of these two wave attack on the head is assumed to be frontal with a significant wave heıght of $H_{s}=6 \mathrm{~m}$, whereas the trunk is mainly attacked by oblique waves of the same height

Using Hudson's formula with $K_{D}=29$ for angular quarry stone as the head and an upgrading factor $F=5,1-3$ ton stones are used for the armour, to be pattern-grouted with 6 ton patches Model investigations have confirmed the stability of this armour, and from these it could also be deduced that the pattern-grouting had to be used down to a level of $M S L-4 \mathrm{~m}$ Below this level stone I - 6 ton is sufficiently stable without pattern-grouting

For economical reasons the recommended third layer is made of stone of a somewhat lighter class $200 / 800 \mathrm{~kg}$ This layer is applied on a layer of light permeable stone-asphalt, which in its turn protects the lean sand asphalt core

Wave attack on the trunk will be less than on the head, as it consists mainly of oblique waves Therefore the trunk armour is designed assuming $K_{D}=35$ Because trunk sections are also considered as test sections and upgrading factor $F=10$ has been applied which possibly may lead to some damage within a few years Together with the extensive wave measuring system of Ri,kswaterstaat in the Europoort area it will be possible in this way to obtain insight in the behaviour of this type of structure under prototype conditions 


\section{CONCLUSIONS}

Considering the development of the use of asphalt in breakwater construction it appears that in view of its favourable properties and its peculiarities, the most profitable application in this part of hydraulic engineering is the use as a groutingagent

As a matter of fact, asphalt grouting already constituted the beginning of the development of fixating unstable slopes New technıques and working methods, however, grouting is developing into a system of internal armour protecting rubblemound breakwaters and sea walls against the heaviest wave attack

In the pattern-grouting technique described in this Paper a new tool is given to the designer of a rubble-mound breakwater In practice it often happens that with the available rock-size from the quarry an armour-layer can be designed which is stable enough under wave attack of "normal" frequency, while just lackıng stability in the exceptional design-storm Pattern-grouting provides that extra "upgrading" which is needed for the exceptional design wave, at relatively reasonable costs For design purposes an upgrading of 5 can be safely accepted 


\section{REFERENCES}

1 Boron W F von Asbeck, Bıtumen in Hydroulıc Engıneerıng Vol 1 ond 2 Elsevier Publishing Company, 1964

2 R E Kerkhoven, Recent Developments in Asphalt Technıques for Hydroulic Applicotions in the Netherlonds Paper to Annual Meeting, Association of Asphalt Poving Technologists, Philodelphio, 1965

3 The testing of bituminous moteriols Kon -Shell Loborotorium, Amsterdam, april 1969

$4 \mathrm{~W}$ Heukelom, Observotions on the rheology ond frocture of bitumens ond aspholt mixes Shell-Bitumen Reprint no 19, Febr 1966

5 A Poope ond A W Wolther, Akmon Armour Unit for cover layers of rubble-mound breakwoters

Proc VIIIth Conf on Coastal Engineering, Berkeley Colıf, 1963, Chapter 25, pp 430-443

6 Soville, Th, Wove run-up on shore structures, Proc A S C E $82 \mathrm{WW} 2$, opril 1956

7 Shore protection, Planning ond Design, CERC, Techn Memorondum no 4, 1966

8 Fronzius, L, Wirkung und Wirtschaftlıgkeıt von Ronkdeckwerken im Hinblick ouf den Wellenouflouf

Mitt des Franzius Instituts 25, Honnover 1965

9 Hudson, R Y , Loborotory Investıgatıons of rubble Mound Breokwoters, Proc A S CE , 85 W W 3, sept 1959

10 Hydroulıcs Reseorch Stotion, Wollıngford, Hydraulics Reseorch, 1966

11 Savoge, R P, Wave run-up on rough ond permeable slopes, Proc A S CE 84 WW 3, may 1958 



\title{
CHAPTER 99
}

\author{
HONOLULU REEF RUNWAY DIKE \\ Robert $Q$ Palmer, $F$ ASCE \\ As sociate Researcher of Ocean Engineering \\ Unıversity of Hawalı \\ Honolulu, Hawanı \\ James R Walker, M S \\ Assistant Researcher \\ University of Hawall \\ Honolulu, Hawall
}

\begin{abstract}
Criterıa for design of a wave barrier to protect the proposed Honolulu International Airport Reef Runway from breaking waves were developed in wave flume model tests Structures with tribar and quarrystone armor units placed in single and multiple layers, on homogeneous and composite slopes, were subjected to both overtopping and non-overtopping breaking waves Data on wave runup, armor unit stability, quantities of overtopping water, and transmitted wave helghts were obtained using a 150 bottom slope, which modeled the irregular coral bathymetry seaward of the proposed structure The model to prototype scale ratios ranged from 15 to 135

Model Tests indicated that the weight of armor units placed below one-third the water depth may be three-fourths that of the units located near the water surface It was noted that the maximum wave runup was 18 times the water depth fronting the structure Data were obtained concerning quantities of overtopping water and transmitted wave helghts over the low barriers The study augments avallable criteria for economical design of structures subjected to breaking waves

\section{INTRODUCTION}

The anticıpated greater volume of future alr traffic and larger aircraft imposes the need for an additional runway at the Honolulu International Airport, Oahu, Hawall The most practical site for the runway, shown in Figure 1, is on a coral reef, adjacent to the existing airport The proposed runway location and alignment will not conflict with use of existing runways, but it will eliminate hazardous takeoffs and landings over the city, and will alleviate the present noise problem in urban areas
\end{abstract}




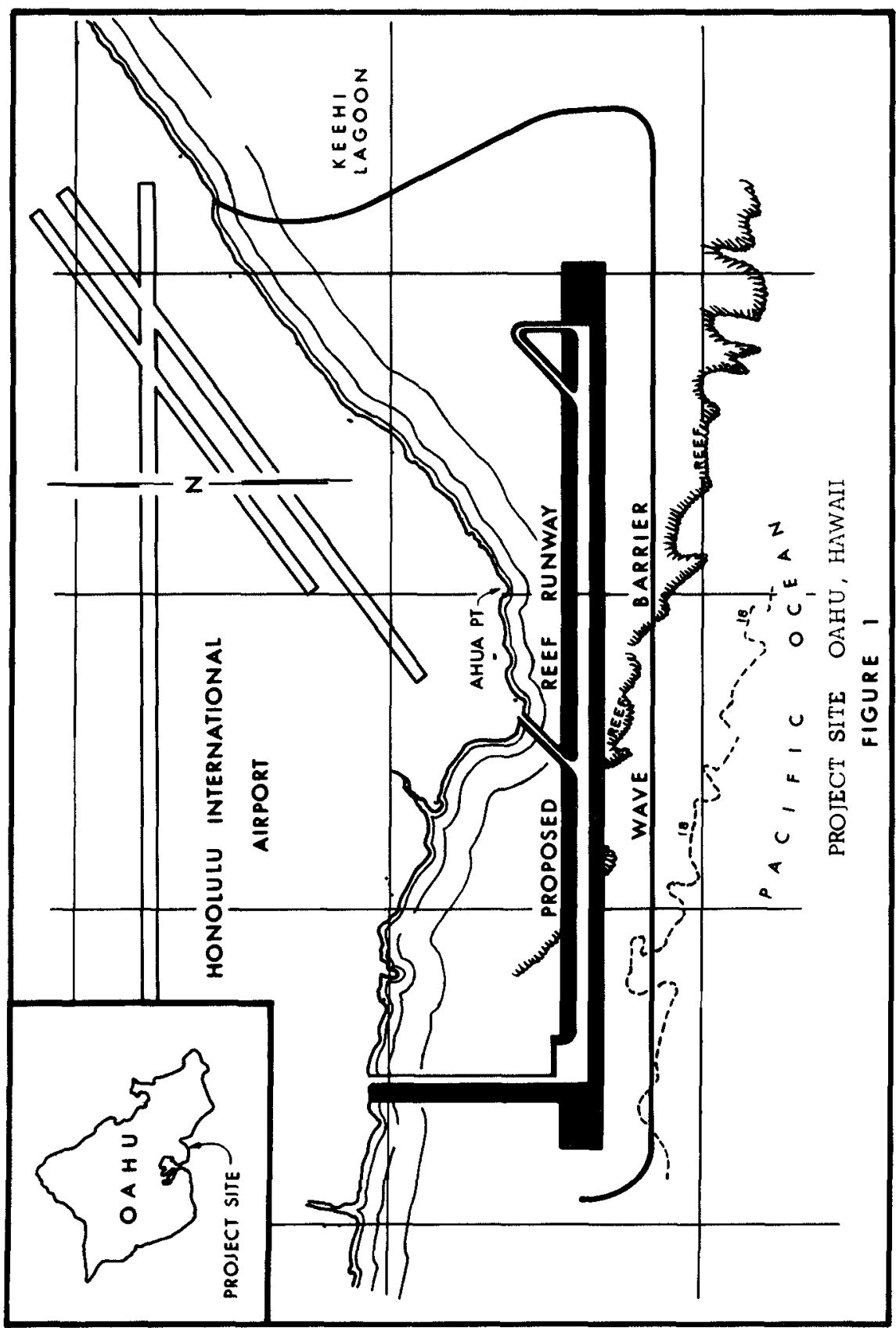


The proposed wave protection for the reef runway would be situated in water ranging in depth from about one to twenty-five feet below mean low lower water (MLLW) Approximately one-third of the site would be located on a wide, flat coral reef, one to five feet deep and only 200 feet of the deeper portion would be in water greater than 25 feet deep The bottom slope is approximately 150 to seaward The design water surface of +35 feet MLLW allows for concurrence of a fairly high tide and storm surge Southern hemisphere storms generate waves that approach the site with deep-water wave helghts up to 15 feet, with 16-second periods On rare occasions, local storms generate about 25-foot waves with shorter periods A refraction analysis and a threedimensional hydraulic model study ${ }^{7}$ indicated that waves are sufficiently high to break seaward of all reaches of the site at least ten hours per year

The purpose of this study was to develop the most economical system to protect the runway from storm waves The runway, with 1,000foot overruns, will be 14,000 feet long, 200 feet wide with 200 -foot shoulders, and will have a centerline elevation of about seven feet above mean sea level (MSL) Alr-s pace criteria require that there be no obstacles higher than the runway centerline for a distance of 1,000 feet from the centerline Beyond 1,000 feet there is to be a side-clearance zone slope of one vertical to seven horizontal

The apparent dearth of design data for structures subjected to breaking waves prompted the model investigation described herein The testıng objectives were divided into four categorıes, they were to determine wave runup, armor unit stability, quantities of overtopping water, and transmitted wave helghts over low structures in order to provide a basis for correlation of results with previous work, preliminary tests and procedures used by other investigators $3,8,11$ were made with nonbreaking waves Procedures were then modified as necessary to obtain the design breaking wave conditions

The first three of six general concepts considered for the reef runway wave protection system are shown in Figure 2 These concepts are a) a seaward wave barrier which will contain future fill to provide access for emergency vehicles, b) a wide berm with flat armored slopes, and c) a wide berm with a flat beach slope similar to the Sand Dam of Europort in the Netherlands Figure 3 shows the three other concepts which are d) a combination of a low seaward barrıer and an artıficial beach along the runway shoulder, e) a combination of a low seaward barrier and an armored levee along the runway, and f) a detached nonovertopped breakwater

\section{THE MODEL}

The wave flume used for the two-dimensional tests was 180 feet long, four feet wide, and six feet deep The walls were made of Transite and glass paneling The glass panels were arranged to allow viewing 


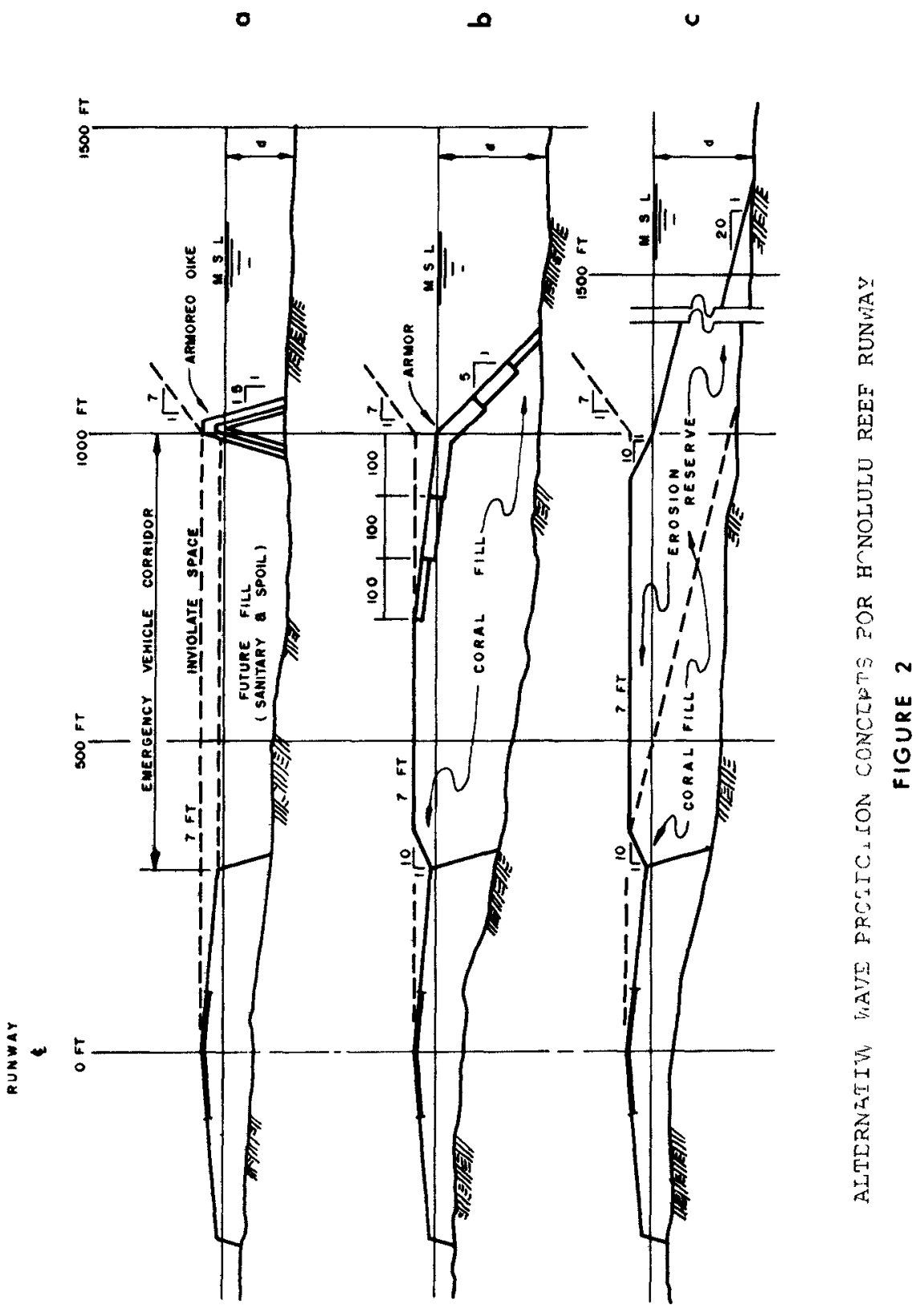




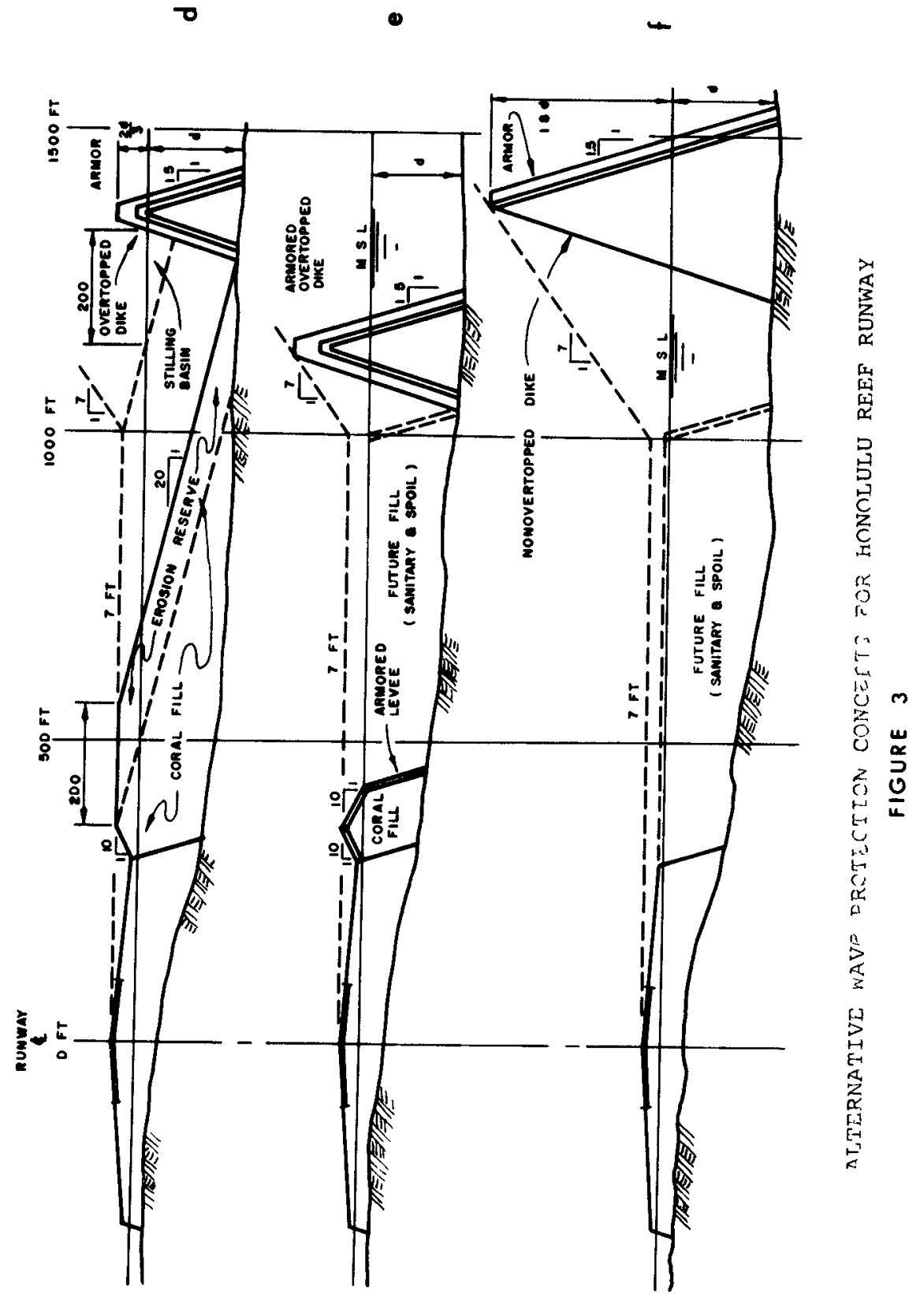


of the test section from one side of the flume A plywood floor on a 150 slope simulated the offshore bathymetry This slope extended approximately 65 feet seaward from the model test section

The test sections were subjected to waves generated by a plunger-type wave generator The wave generator was powered by a 20-HP electric motor coupled to a hydraulic pump The hydraulic pump drove a high-torque, vanable-speed hydraulic motor, which rotated an adjustable crank attached by a connecting rod to the plunger

Wave heights were measured by resistance wave gauges, the signals were displayed on a light beam oscillograph recorder Wave runup elevations on structures were measured visually Overtopping quantities were trapped in a one-foot wide box located in the center of the test section, at the core level, on the basin side of the structure The trap door on top of the box opened and closed to capture the overtopping water of a selected series of waves

The model breakwater sections had stone and tribar armor units The armor stone was hand-shaped to simulate the type of stone that would be used in the prototype The mean weight (saturated and surfacedried) of these units was $1043 \mathrm{lb}$, with a standard deviation of 0045 ib The mean specific welght was $17703 \mathrm{lb} / \mathrm{cu} \mathrm{ft}$, with a standard deviation of $283 \mathrm{lb} / \mathrm{cu}$ ft However, the specific weight of the prototype stone may be as low as $165 \mathrm{lb} / \mathrm{cu}$ ft The applicable model scales were determined by the WES (Hudson) ${ }^{1}$ formula to range from 15 to 125 for these stones

The model tribars were made of a mixture of concrete and barite sealed in a resin coating The mean weight of these units was $210 \mathrm{lb}$, with a standard deviation of $0023 \mathrm{lb}$ The specific weight of these units was $14574 \mathrm{lb} / \mathrm{cu} \mathrm{ft}$, with a standard deviation of $105 \mathrm{lb} / \mathrm{cu} \mathrm{ft}$

The armor stone and tribar underlayer stone mean weights were $0055 \mathrm{lb}$ and $0189 \mathrm{lb}$, respectively, with specific gravities of 169 $\mathrm{lb} / \mathrm{cu} \mathrm{ft}$ The impermeable core was made of a mixture of fines to gravel, 100 percent of the material passed a three-fourth inch sieve

Test procedures were based upon the precedent set by related model studies $3,8,9,11$ and were modified as necessary to simulate prototype breaking waves The incldent wave helghts were measured by a wave gauge located five feet seaward of the toe of the 150 bottom slope (70 feet from the test section) The incident wave heights were measured before reflections from the structure set up a standing wave This wave height was used to estimate the deep water height, $\mathrm{H}_{0}$, by application of a shoaling coefficient Another gauge was located one to five feet from the toe of the structure to measure the breaking-wave height In order to generate a consistent wave of known height, the wave generator was stopped before wave reflections from the structure returned to the generator The water was allowed to still before the generator was restarted 
Test sections were subjected to breakıng waves to determıne their stability under design conditions The general procedure was to subject the structure to a series of small, non-breaking waves for a short period of time to allow the units to settle Then the structure was subjected to the highest wave obtainable with the given depth to toe The duration represented five to six hours of prototype wave attack If the structure remained stable, the water depth was increased and a larger wave was allowed to attack the structure This was done to determine if fallure would occur with a slightly larger wave Thus, a degree of safety was indicated

Two types of armor stone placement were used in the models in a single layer with the long axis perpendicular to the slope, and in multiple layers in a random fashion Tribar armor units were elther placed in a single layer arrangement or in multıple, random layers Placement in the model was done to simulate, as closely as feasible, the placement in the prototype For the majority of the tests, tribars were randomiy placed in two layers below minus ten foot elevation This placement was adopted in the model because of difficulties anticipated in prototype placement in murky water on an irregular bottom Above minus ten feet, the units were placed upright with bars in contact with adjacent units

The wave runup, $R_{u}$, as a function of deep-water wave height, $\mathrm{H}_{\mathrm{O}}$, wave period, $\mathrm{I}$, and water depth $\mathrm{d}$, was determined by visual observations Runup, the elevation above the still-water surface to which a wave rises on a structure, was tested by increasing by increments wave helght and period untll the range of prototype conditions was covered For a constant period, the wave height was increased from a small, non-breaking wave to a wave that broke sufficiently seaward of the structure to ensure that the maximum runup was observed The wave attack was allowed to stabilize on the structure, and then the average runup of the next six to 15 waves was recorded The number of waves used in the average runup was limited to the time it took for the first wave to reflect off the generator and return to the test section When waves broke on or seaward of the structure, there was a large variation in the runup, therefore, the maximum runup value was recorded In order to obtain a clear definition of the effect of location of the breaking wave, the generated wave helght was uncreased by small increments when the wave started to break at the toe of the structure

The quantity of water overtopping structures was trapped to determine the rate of over-swash The procedure was to allow the wave attack to stabilize,then the over-swash of several waves was trapped and the quantity was measured The procedures followed in obtaining the design wave in the overtopping tests were the same as described in the runup tests 
The water swashing over the top of the structure generated a transmitted wave helght, $\mathrm{H}_{\mathrm{T}}$ This wave was measured by a gauge on the basin side, located five feet from the heel of the structure The levees protecting the runway, as shown in Figures $3 d$ and $3 e$, were modeled and also acted as wave absorbers for the transmitted waves

\section{TESTS AND RESULTS}

Runup on a structure subjected to breaking waves is a prımary concern when considering a non-overtopping structure For this reason, a typical dike section was designed and tested to determine the maximum runup The inset in Figure 4 shows the test section The core was impermeable and the material used has been described previously Two layers of underlayer stone were randomly placed over the core, then a double layer of 1 04-1b stone was randomly placed on a 12 slope from the floor to -05 feet From this point up to +35 feet, a single layer of fitted stone was placed on a 115 slope The water depth was -10 foot at the toe of the structure

One hundred and seventy-six tests were run on this structure to determine the maximum runup under prototype conditions The results are shown in Figure 4 as a plot of the runup divided by depth, $\mathrm{R}_{\mathrm{u}} / \mathrm{d}$ vs $\mathrm{d} / \mathrm{T}^{2}$ for isolines of deep-water wave steepness, $\mathrm{H}_{\mathrm{o}} / \mathrm{T}^{2}$ These isolines are lines fitted through data points of non-breakers (solid line) and breakers (dashed line) The range of breaking waves is outlined by a line drawn approximately where the waves start to break at the toe of the structure An increase in wave steepness approaches an upper limit of runup which is also drawn in Figure 4 This plot shows a rapid increase in runup as the wave period is increased unt $1 \mathrm{~d} / \mathrm{T}^{2}=015$ and $\mathrm{H}_{\mathrm{O}} / \mathrm{T}^{2}=0 \quad 1$

Saville ${ }^{8}$ previously conducted tests on a similar structure fronted by a 110 beach slope Saville's results were plotted with the Reef Runway data for comparison it was noted that the primary difference between the results of the two sets of data was that the runup Saville measured was about 45 percent greater than that of the Reef Runway data Apparently, the steeper beach slope in Saville's experıment allowed a nearly 50 percent larger wave to break near the structure This increase in breaker helght, attributed to the difference in the bottom slope, was noted in the experimental work of lversen ${ }^{2}$ et al The effect of the 12 slope at the toe of the Reef Runway test section does not account for such an increase in $\mathrm{R} / \mathrm{d}$ when comparing the two structures using the composite slope method ${ }^{9}$ These runup data provide the designer of the Reef Runway project with an upper limit to which breakıng waves will run up on a single-layer, stone-armor structure with a 115 slope

Stability coefficients, $\mathbf{K}_{D}$, were computed for each test section subjected to the breaking waves When damage occurred to the armor, the degree was noted The WES (Hudson) ${ }^{1}$ formula was used in the analysis The results indicate that a) published values of $\mathrm{K}_{\mathrm{D}}$ for 


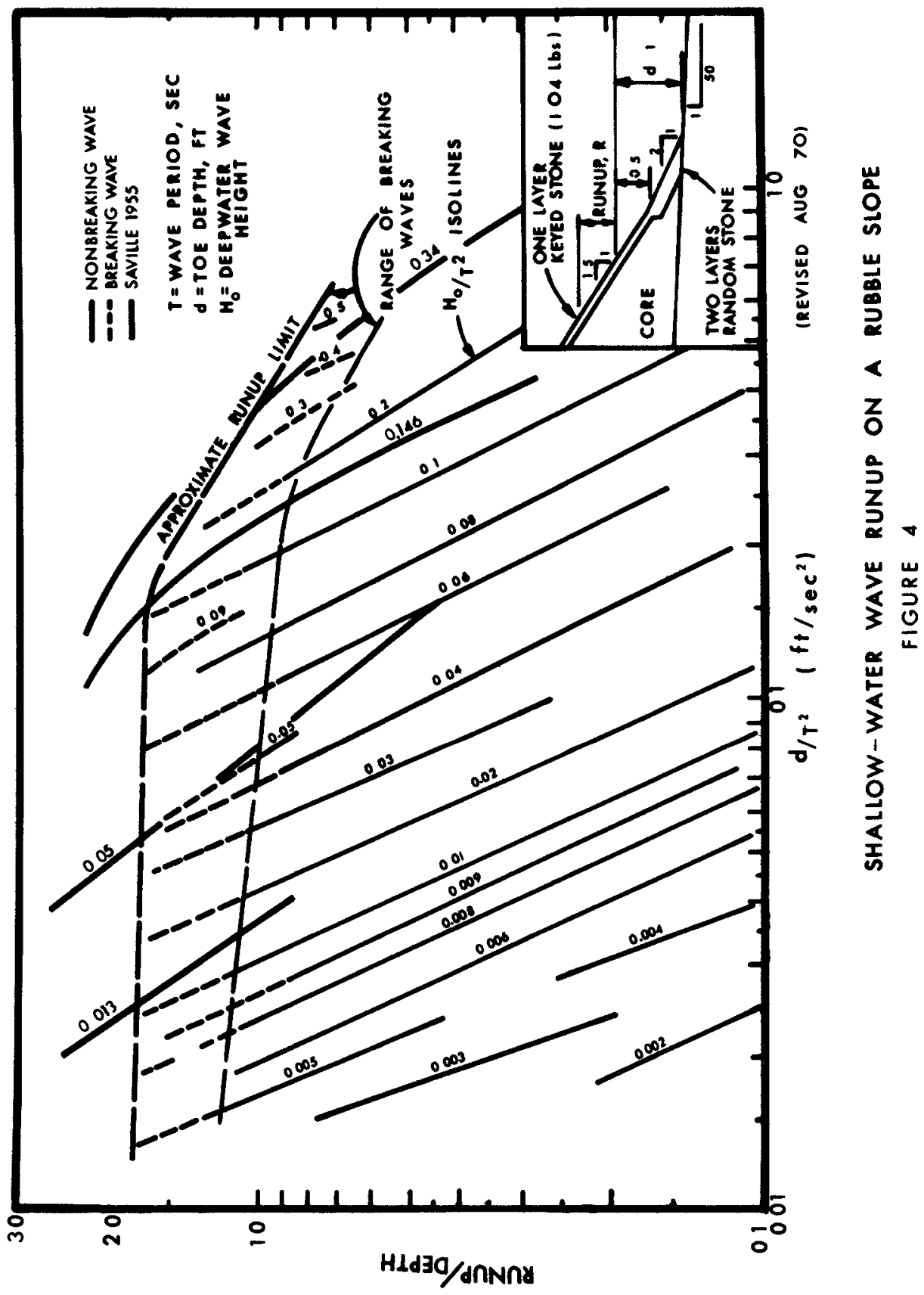


breaking waves are conservative, b) keyed and fitted armor is several times more stable than loosely-placed armor, c) displacement of one armor unit does not lead to sudden massive fallure, in fact, the armor tends to heal unless it is grossly underweight, and d) the weight of armor may be reduced by one-fourth at depths below one-third of the toe depth and by three-fourths below the two-thirds depth as shown in Figure 5 A summation of stability coefficients for breaking waves is shown in Figure 6 The ordinate of the author's recommended design values are identified However, it has been the general practice to design on the borderline of damage or even for a percentage of damage from infrequent waves

Economically, the runway elevation should be kept as low as possible A rise in water level behind the wave protection structure could affect the operation of the runway In order to evaluate the effect of crest elevation upon the rate of overtopping water, series of tests were made on typical sections with varıous crest elevations Results from two representative tests are given in Figures 7 and 8 The data is given in prototype dimensions and the test sections are shown in the insets The linear model scale for these data was 1152 The plots of these data are shown in Figure 7 for a crest elevation of 1380 feet and in Figure 8 for a crest elevation of 935 feet The rate of overtopping water per linear foot of crest, $Q$ (cublc feet per second per foot of crest), is given as a function of deepwater wave height for isolines of wave perlods For a given wave period, the rate of overtopping rises rapidly with an increase in $\mathrm{H}_{\mathrm{O}}$ until the wave breaks at the toe of the structure An increase in $\mathrm{H}_{\mathrm{O}}$ causes the wave to break seaward of the toe and the rate of overtopping approaches a maximum for a given wave period The plots also indicate the effect of wave steepness It was noted that it was inversly proportional to the amount of overtopping

Wave swash over a structure generated waves in the basin behind the structure When waves break just seaward of the structure, the maximum wave is generated in the basin The relation of the effect of crest elevation is shown in Figure 9 as a plot of transmitted wave height, $\mathrm{H}_{\mathrm{T}}$, as a function of deep water wave helght, $\mathrm{H}_{\mathrm{O}}$, for two wave periods The linear model scale was 1152 , and the test section in prototype dimensions is shown in Figure 9 The plots indicate that once the wave has broken, an increase in incident wave height does not produce an increase in transmitted wave helght An important observation noted during the testing program concerned the period of the transmitted wave, overswash of the incident wave generated a number of transmitted waves This had a significant effect upon the runup on the runway levees The size and depth of stilling basins also produced noticeable effects upon the transmitted wave Due to these and other complicatıng factors, no general relations were developed for runup on the runway levees 


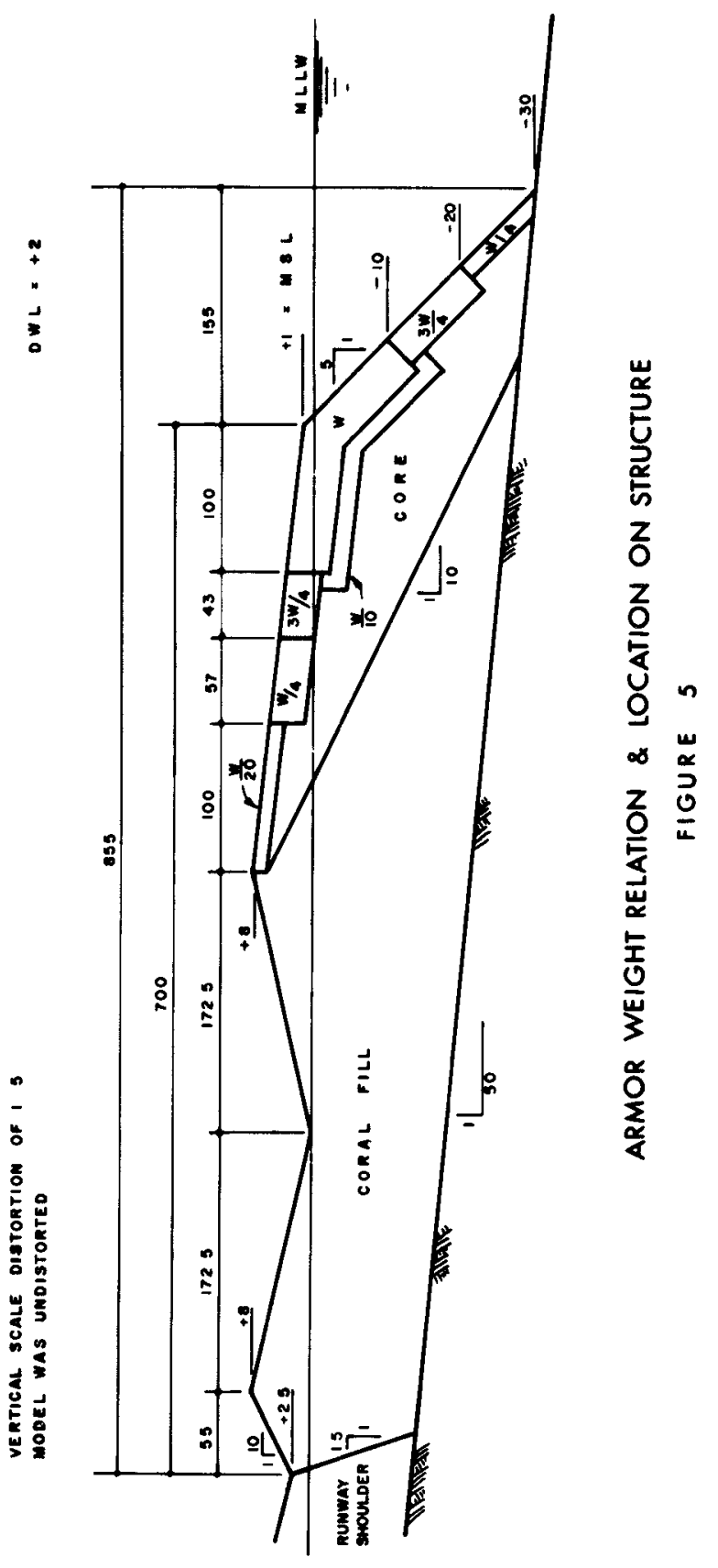




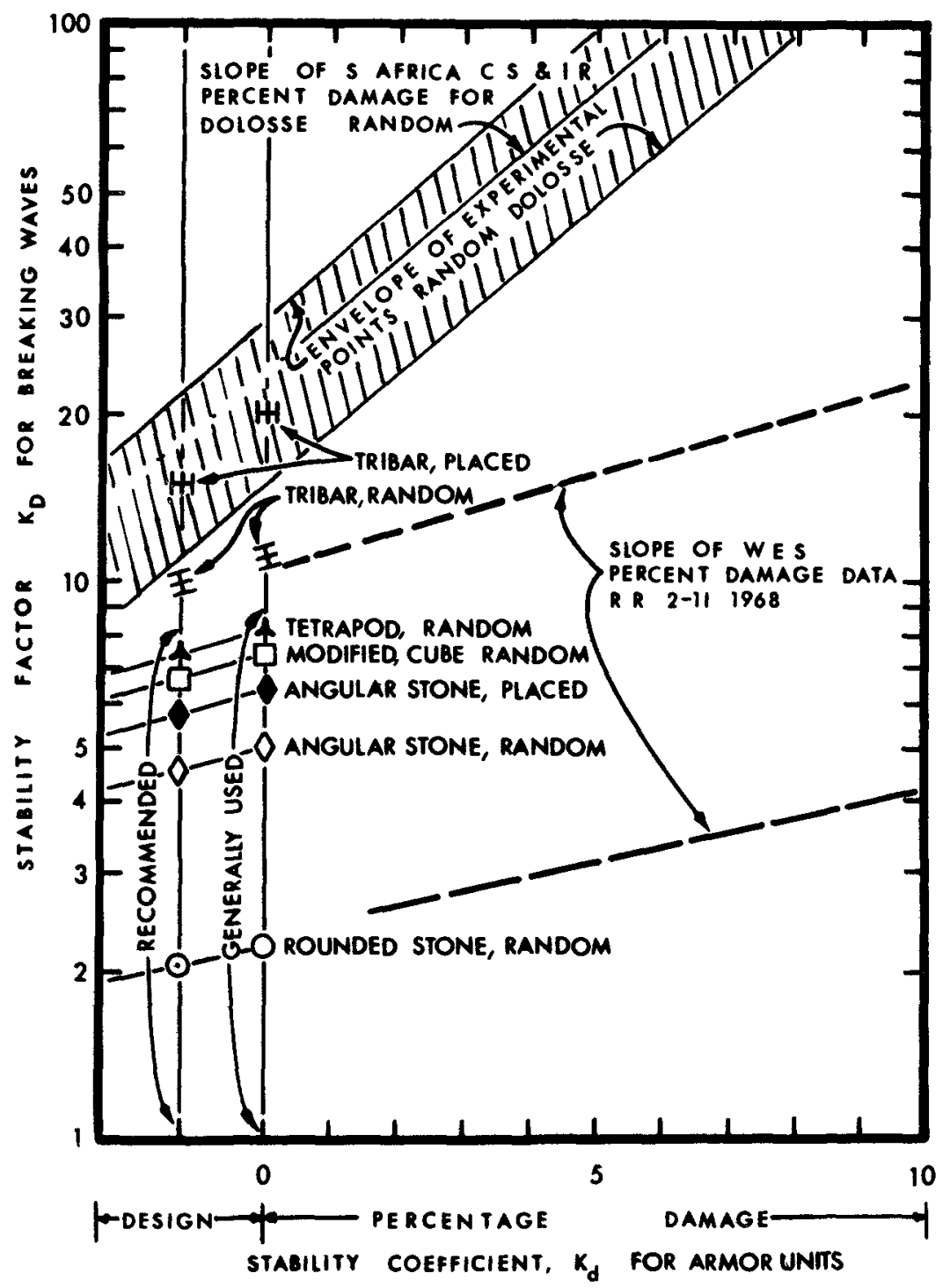

Note See reforences $4,5,9,11$ 


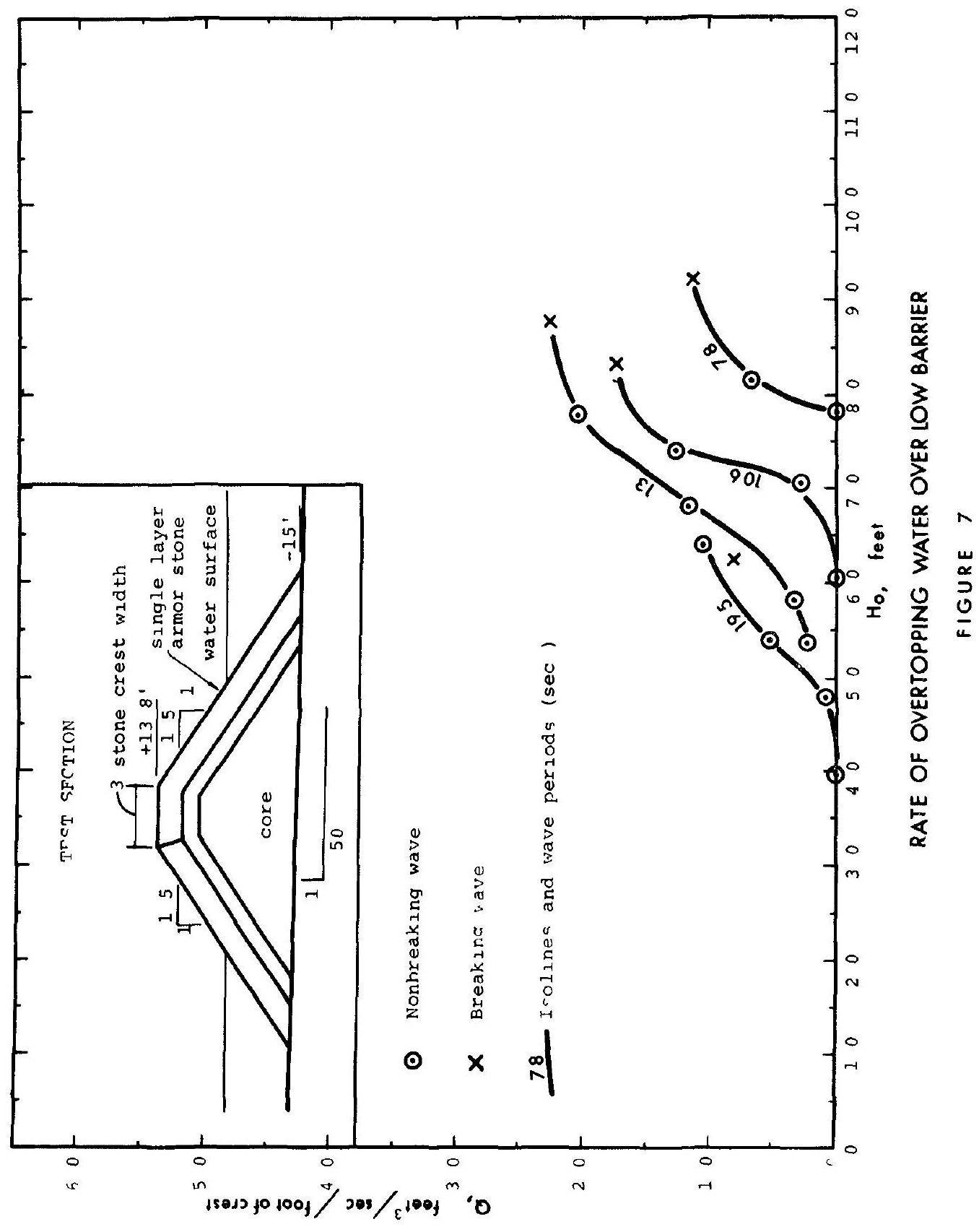




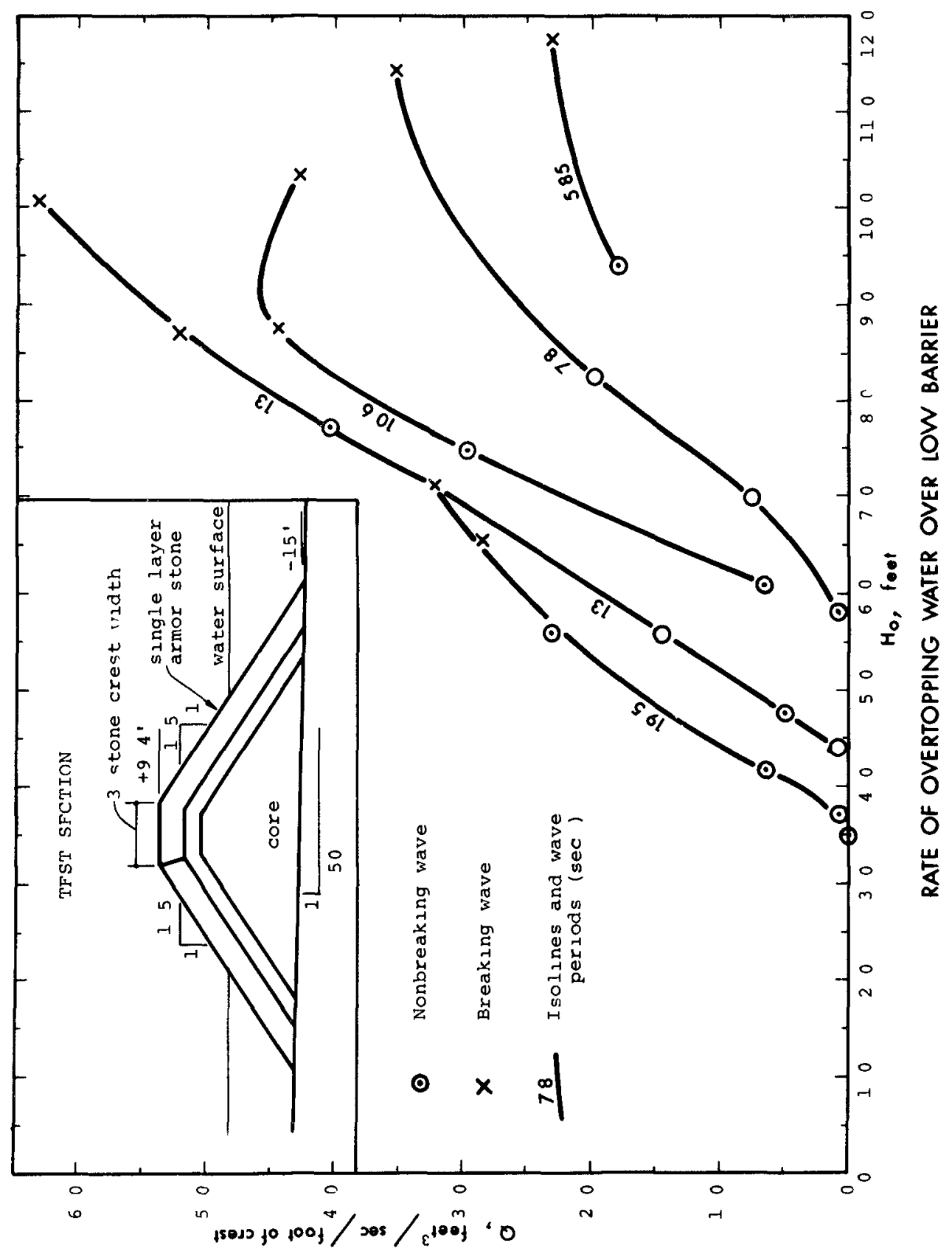




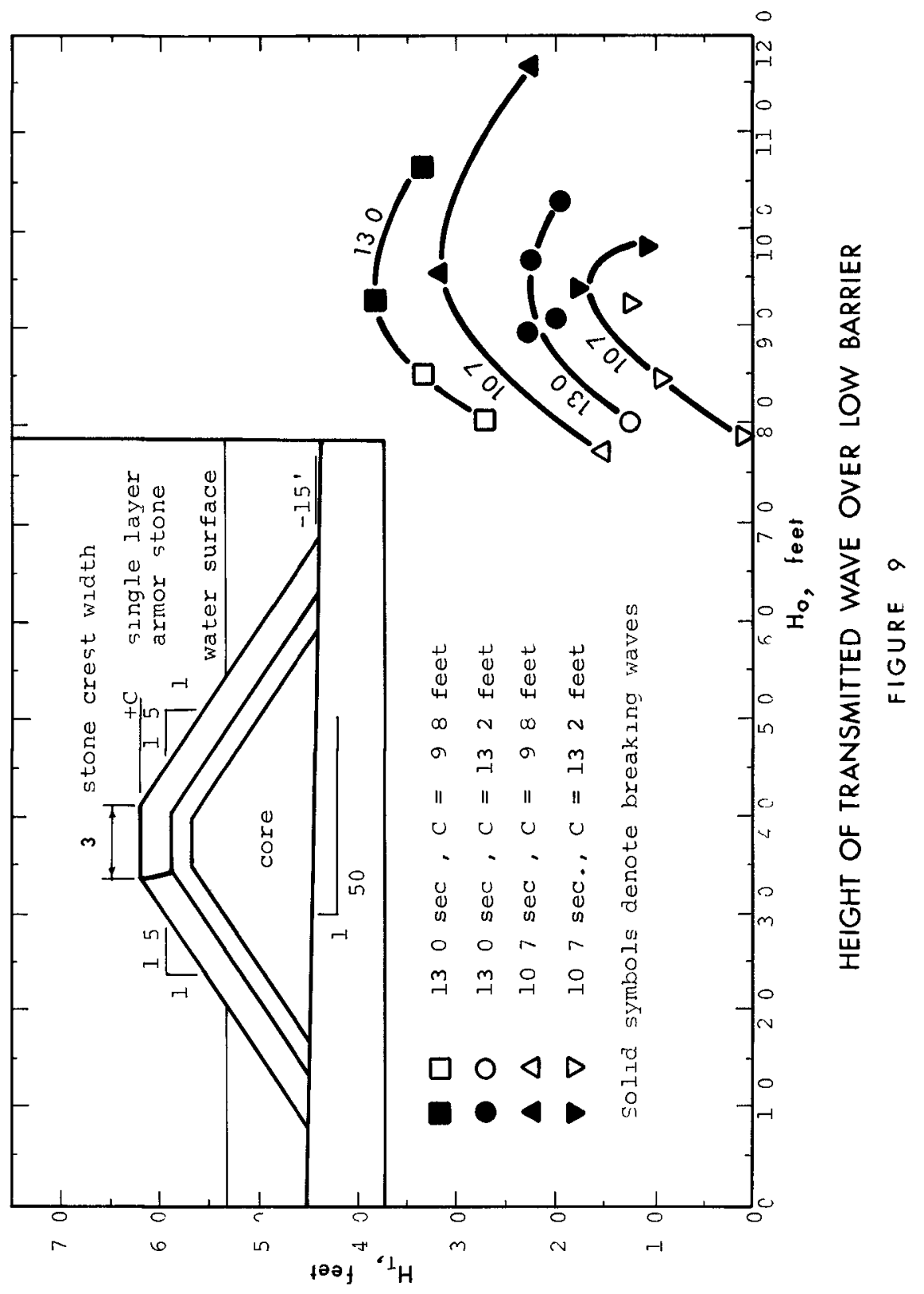


Forty-five sections were tested in this study The results of these tests and comparative costs will be the subject of another paper An example of one of the sections developed to attenuate runup is shown in Figure 10 Preliminary tests indicated that this high-vold structure is stable and reduces runup

\section{CONCLUSIONS}

A large scatter in the data was observed in the model testing of rubble structure with breaking waves Even though a reasonably consistent wave train was generated, each wave broke in a different location, thus changing the effect of the wave upon the test Since prototype waves are not consistent the location of the breaking point varies even more For this reason, maximum data points for the breaking waves were observed in the model tests

Tests indicate that the maximum runup of a breaking wave for conditions shown on Figure 4 is 18 times the water depth fronting the structure This was valid for the range of $\mathrm{d} / \mathrm{T}^{2}$ and $\mathrm{H}_{\mathrm{O}} / \mathrm{T}^{2}$ tested

The tests confirmed the findings of previous investigations that the type of placement of armor is a major variable Only skilled and experienced technicians can build rubble models which will yield fairly consistent results Also, the designer and construction inspectors should be fully aware of the relationshıp between armor stability and placement The tests confirmed that placement is as important a factor as the weight of the armor units Specifically, the required weight of loosely placed stone may be twice that of a well-placed stone

It is apparent that there is an overwhelming number of variables involved in analyzing breaking waves on complex rubble-mound structures General desıgn criteria can be developed for only a few unıque condıtions It therefore appears that model tests should be made for all important rubble structures subject to breaking waves

Scale effects ${ }^{10}$ should be considered when applying these data to prototype design

\section{ACKNOWLEDGEMENTS}

The Honolulu International Aurport Reef Runway model study was funded by the State of Hawall This work was performed by the University of Hawall, Department of Ocean Engineering, Look Laboratory for Ralph M Parsons Corporation representatives for the State of Hawalı Department of Transportation The Department of Transportation's permission to publish this paper is appreciated 


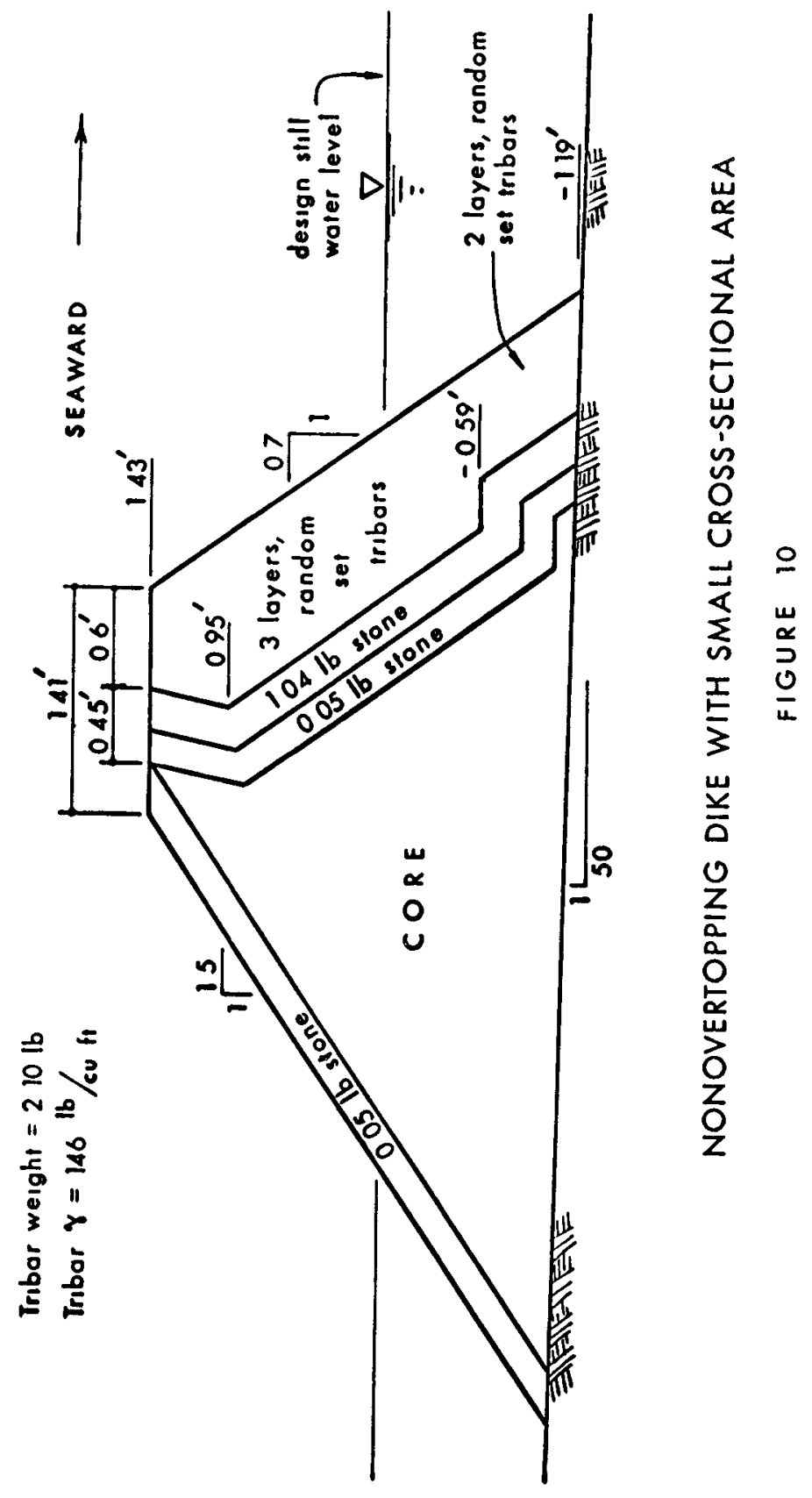




\section{REFERENCES}

I Hudson, R Y , Laboratory Investigations of Rubble-Mound BreakWaters Proc, American Soclety of Civil Englneers, Waterways and Harbors Division, Vol 85, No WW3, Paper No 2171, September 1959

2 Iversen, $\mathrm{H}$ W "Discussion of Results from Studies of Wave Transformation in Shoaling Water " Institute of Engıneerıng Research, Unıv of California, Iech Report Series 3, No 331, March 1952

3 Jackson, R A , "Design of Cover Layers for Rubble-Mound Breakwaters Subjected to Nonbreaking Waves " U S Army Engineer Waterways Experiment Station, Research Report No 2-11, June 1968

4 MerrifieId, Erıc M , "DoIos Concrete Armor Protection," Civil Engineer, ASCE, December 1968

"Desıgns for Rubble-Mound Breakwater Repalr, Morro Bay Harbor, CaIıf ," TR 2-567, WES, May 1961

6 Palmer, $\mathrm{R} Q \mathrm{Q}$, "Breakwaters in the Hawallan Islands," Proc ASCE, WW1 and WW2, 1961

7 Palmer, $R \quad Q$ and $J$ R WaIker, "Three-Dimensional Storm Wave Study," Reef Runway HydrauIıc Model Study, HonoIulu International Aurport, HonoIulu, Hawall, September 1969

8 Saville, $T$, Jr , "Laboratory Data on Wave Run-up and Overtopping on Shore Structures " U S Army Corps of Engıneers, Beach Erosion Board, Tech Memo No 64, October I955

9 Saville, T , Jr , "Wave Run-up on Composite Slopes," Proc of the 6th Conference on CoastaI Engineering, Council on Wave Research, Univ of CaIıfornia, 1958

"Shore Protection Planning and Desıgn," Beach Erosion Board, Technical Report No 4, Revised I966

11 Tetra Tech, Inc , "Oceanographıc Engineerıng For Reef Runway," Honolulu, International Airport (Final Report), Contract No TC-143, Pasadena, Calıfornia, September 1968

12 Vanonı, Vito $A$ and Fredrıc Raıchlen, "Laboratory Design-Studies of the Effect of Waves on a Proposed Island Site for a Combined Nuclear Power and Desalting PIant," Report No KH-R-I4, CaIıfornıa Instıtute of Technology, July 1966 


\title{
CHAPTER 100
}

FLOATING BREAKWATER FOR RESERVOTR MARINAS

\author{
by \\ Kenneth Chen, Research Assistant \\ University of California \\ and \\ R L Wiegel, Professor of Civil Englneering \\ University of California
}

\begin{abstract}
The concept of multiple use of reservolrs is resulting in the construction of marinas for recreational bodting requiring breakwaters that can function for a large range of water levels A typical set of design criteria Is an average water depth of 20 to 25 feet, wave lengths from 5 to 60 feet (wave periods of from 1 to 4 seconds) and wave heights from $\frac{1}{2}$ to 5 feet Calculations based on Bulson's results showed a pneumatic breakwater to be too expensive An extensive literature search revealed that floating structures based upon the concepts of large effective mass or moment of inertia resulting from "entrained" water, or structures which can dissipate energy might be more effective than one of the floating bag types of breakwaters Several new-types of moored floating structures which combined two or more of the concepts mentioned above were tested in a wave tank, and several of the devices appear to have merit in that they were reasonably small compared with the 1 ongest design wave length and could reduce the highest design incident wave helght to less than one foot, prototype, in the 1 ee of the breakwater
\end{abstract}

\section{INTRODUCTION}

Owing in part to the development of multiple purpose reservoir and inland lake recreation areas in recent years, the number of small craft used in these bodies of water has increased rapidly in the design of small craft harbors for the protection of boats from storms, consideration must be given to the variation of the water level This is of special importance for reservolrs because of draw-down during the summer and fall seasons It appears that mobıle breakwaters might be the best solution for such a condition

Research on mobıle breakwaters has been done in the past, but very few have been bullt One purpose of this paper is to present the conclusions reached by the authors from a literature review One can categorize moblle breakwaters into three main types 1) pneumatic and hydraulic breakwaters, 2) flexible structures, and 3) rigid floating structures

Based upon the conclusions derived from the literature review, three rigid floating breakwaters were designed, each making use of a different mechanism or combination of mechanisms of wave energy dissipation and reflection The models were designated A, B and C Later Mr J $\mathrm{S}$ Habel, Supervisor of Engineering of the Department of Harbors and Watercraft, State of California, and Professor $J$ V Wehausen each suggested a concept of wave energy dissipation and reflection which resulted in the design and construction of Models 
$\mathrm{D}$ and $\mathbf{E}$

Almost no information was discovered on the maximum height and period of waves that are considered to be acceptable within a small craft marina The Task Committee on Small Craft Harbors of the American Society of Civil Engineers (1969) state on page 57 that in general, wave heights should be reduced to approximately $\frac{1}{2}$ to $1 \mathrm{ft}$ for small boat harbors Some data on maximum wave helghts in which different classes of working barges and vessels can operate have been given by Glenn (1950) and Santema (1955)

The problem of mooring so as to prevent damage to boat, mooring lines or dock is extremely complex, depending upon wave heights, periods and direction, and upon the weight, shape, natural periods of the boat and upon the characteristics of its moorings A theoretical study was made by Raichlen (1968) for the simplified case of the surging motion of several classes of small boats (ranging from 2 to 8 tons - 20 to $40 \mathrm{ft}$ in length) subjected to uniform periodic standing waves with crests normal to the longitudinal axis of the moored boats, with two bow lines and two stern lines The restoring force versus displacement of the moorings were non-linear, as is apparently the normal case A detalled anilyses was made for one of the boats (Harbor Boat No 3), which had a length of $26 \mathrm{ft}$, beam of $9 \mathrm{ft}-2 \mathrm{in}$, maximum draft of $2 \mathrm{ft}-4$ in and an approximate displacement (unloaded) of $52001 \mathrm{bs}$ Details of the mooring configuration and mooring line characteristics were also presented Measurements of the period of free osciliation of surge for three mooring line conditions (zero slack, $4 \mathrm{in}$ and $8 \mathrm{in}$ slack) for several different initial displacements were made and compared with theory The comparisons, shown in Fig 1 , are quite good

Some of the complexities of the problem can be seen from Fig 2 which compares the maximum motion (in one direction only) of the boat as a function of wave period and the forcing function $\zeta$ for taut mooring 1 ines and for 8 in slack $\zeta$ is a rather complicated function, and Rachlen describes lt as the maximum with respect to time of the water particle velocity averaged over the displaced volume of the moored body All other things being equal, $\zeta$ is directly proportional to the standing wave emplitude It is evident that a boat moored with slack lines at one tide stage may have taut lines at another stage of the tide, so that its response will vary with tide stage, all other conditions being equal

Two other examples have been chosen from Ralchlen's report, and are shown in Fig 3 The maximum positive displacement from rest is shown as a function of wave period and $\zeta$ for two boats, one of $37001 \mathrm{bs}$ with a length of $22 \mathrm{ft}-5 \mathrm{in}$, and the other of $17,0001 \mathrm{bs}$ and a length of $38 \mathrm{ft}$ First, it appears peculiar that the smalier boat should have larger "natural periods" than the larger boat The reason for this was that the mooring lines of the larger boat were much stiffer compared with its weight than was the case for the smaller boat This emphasizes again that the moorings are extremely $1 \mathrm{~m}-$ portant to the problem and there can be no simple wave height criteria for a harbor

\section{PREVIOUS WORK}

Pneumatic and Hydraulic Breakwaters

An artificial surface current can be produced by air bubbles released from a comprised air manifold on the sea bed, or by means of horizontal water jets from a pipe floating on the water surface If the surface current is of sufficient magnitude, and is directed towards the oncoming waves, the wave lengths are reduced and their heights increased until instability occurs, and the waves either breaks or are reflected 


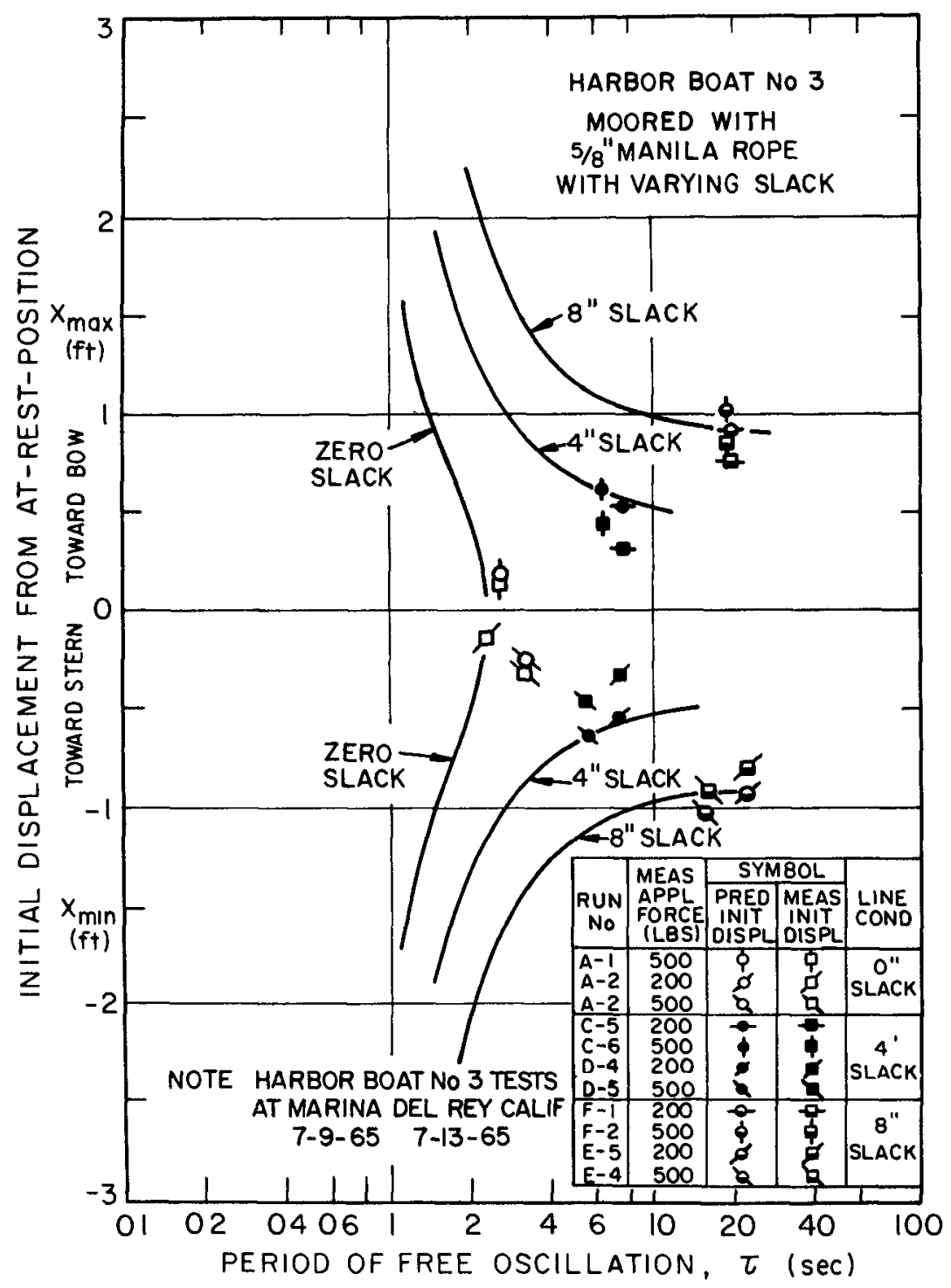

FIG I MEASURED AND PREDICTED PERIODS OF FREE OSCILLATION HARBOR BOAT NO 3

(FROM RAICHLEN, 1968) 

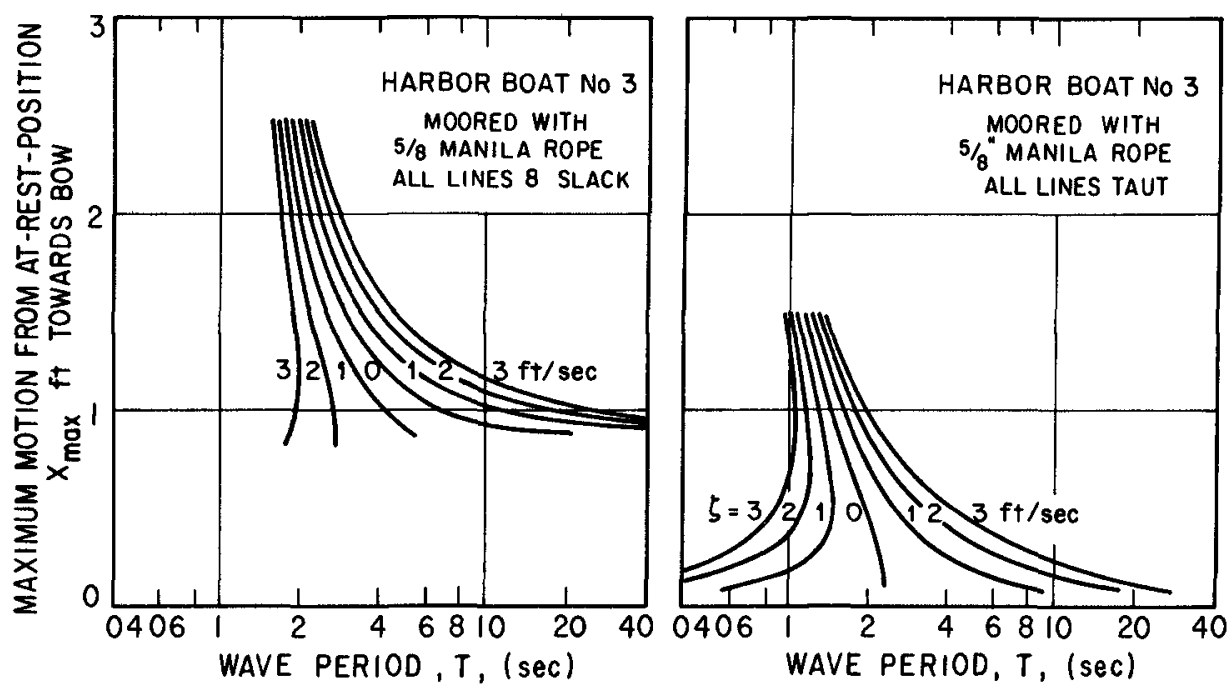

FIG 2 RESPONSE CURVES HARBOR BOAT NO 3 (FROM RAICHLEN, 1968)
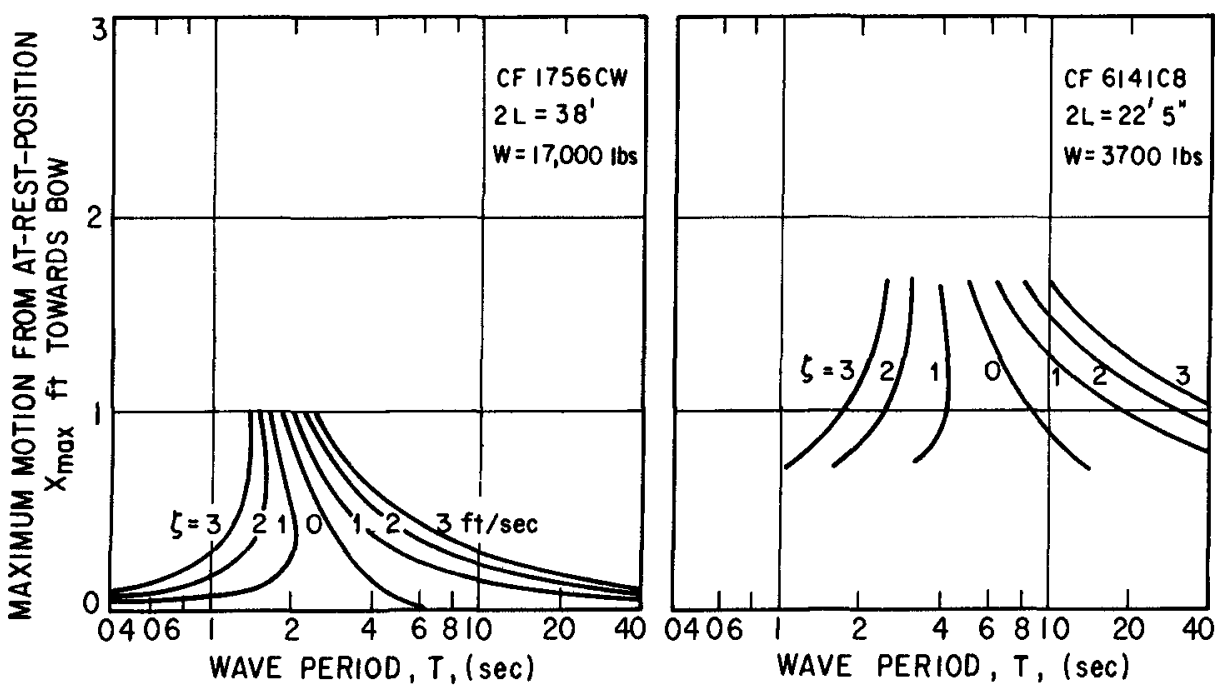

FIG 3 RESPONSE CURVES. 17,000 Ib BOAT AND 3700 ib BOAT (FROM RAICHLEN, 1968) 
This concept was first employed by $\mathrm{P}$ Brasher (1915) in 1907 It was used by the Standard Oll Company in 1915 at El Segundo, Calıfornia, apparently with little success In 1936 Professor Thysse of Delft University showed that the surface currents produced by the bubbles were the main mechanism The theoretical work was continued by White (1943) and Taylor (1955) in England during 1939-1945 As a result of their work, it became possible for the first time to predict the quantity of air required to produce a given surface current as well as the speed of current required to dissipate (and/or reflect) the energy of waves of known length After World War II, a large amount of research was done in connection with this subject

Williams and Wiegel (1963) generated waves in a tank by blowing alr over the water surface and subjected them to a horizontal current of water created by horizontal water jets issuing from a manifold at the water surface (hydraulic breakwater) The energy spectra of the waves were computed for condltions before and after the hydraulic breakwater was turned on It was found that the shorter, steeper wave components were attenuated to a much greater extent than were the longer wave components They concluded that although a large portion of the wave energy could get past such a breakwater, the waves in the lee of the breakwater looked considerably lower to the observer, so that the claims made for the effectiveness of this type of breakwater were probably impressions rather than reality

Both experimental and analytical studies have been carried out by Bulson (1963, 1967, 1969), who concluded

"The experimental and theoretical studies during the past 25 years have made it possible for a reasonably accurate estimate to be made of the air quantity required to operate a bubble breakwater The quantity is astronomical and costly to supply The practical difficulties of operating a full scale system are immense It is doubtful whether any novel ideas of bubble formation and size can produce economices, and $h 1$ gh cost is bound to be the basic feature of any apparatus of this type which is designed to combat the energy of the sea"

It was thought that perhaps a pneumatic breakwater might be a reasonable solution for the relatively short waves expected to be encountered in a reservolr Calculations were made for the following conditions water depth of $20 \mathrm{ft}$, wave lengths from 5 to $60 \mathrm{ft}$ (wave perlods from 1 to 4 seconds), and wave heights from $\frac{1}{2}$ to $5 \mathrm{ft}$ Based on Bulson's results, one can calculate the quantity of air required to suppress waves of length $L$, height of $H$, in water depth, $d$

$$
\mathrm{V}_{\mathrm{m}}=146\left[\frac{\mathrm{gQOP}}{\mathrm{P}+\mathrm{d}}\right]^{1 / 3}
$$

where $V_{m}=$ the surface velocity of the current, feet per second

$Q_{0}=$ the quantity of free alr delivered by compressors, cubic feet per second per foot

$\mathrm{P}=$ atmospheric pressure expressed as a head of water, feet of water

In this case the air supply manifold is placed on the bottom

The current velocity $V$ diminishes approximately linearly with depth, until It equals zero at a depth $D$ below the surface

$$
D=032 P \log _{e}\left[\frac{P+d}{P}\right] \text { feet }
$$

When the same quantity of alr passes through a variety of orifice diameters and spacings, there is no significant difference in $V_{m}$, furthermore, results 
for a single manifold at depth $d$ are not noticeably different from those when two or more adjacent manifolds deliver the same total quantity of air The critical current velocity at the surface to suppress completely deep water waves, $V_{m}$, is given by

$$
\bar{v}_{m}=\frac{1}{\alpha_{m}}\left[\frac{\lambda g}{2 \pi}\right]^{\frac{1}{2}}
$$

where

$$
\begin{aligned}
c^{2}=\mathrm{gL} / 2 \pi & \text { for deep water } \\
\alpha_{\mathrm{m}}^{2} / z & =\mathrm{L} / 2 \pi \mathrm{D} \\
\mathrm{z} & =\mathrm{Dg} / \overline{\mathrm{V}}_{\mathrm{m}}^{2}
\end{aligned}
$$

in which $C$ is the wave speed in feet per second $\alpha_{m}$ can be obtained from Fig 5 of Bulson's 1969 paper Combining Eqs (1) and (3)

$$
Q_{c r}=\left[\frac{P+d}{P g}\right]\left[\frac{g L}{2 \pi}\right]^{3 / 2}\left[\frac{1}{146 \alpha_{m}}\right]^{3}
$$

According to Eq (6), $\mathrm{Q}_{\mathrm{Cr}}$ is Independent of wave height, but experiments show that when waves are neither truly sinusoldal nor of infinitesimal height, the quintity of air necessary to produce complete damping can exceed $\mathrm{Q}_{\mathrm{cr}}$ It has been suggested by Bulson that $Q_{\max } c$ in be represented by 1 inear relationship between $Q_{\max } / Q_{c r}$ and the wave steepness, $H / L$

$$
\mathrm{Q}_{\max } / \mathrm{Q}_{\mathrm{cr}}=06+(20 \mathrm{H} / \mathrm{L})
$$

Suppose one wishes to determine the quantity of air required to suppress waves $60 \mathrm{ft}$ long, $5 \mathrm{ft} \mathrm{h}$ igh in a water depth of $20 \mathrm{ft}$ From $\mathrm{Eq}$ (2), $\mathrm{D}=032 \times 33 \times \log _{\mathrm{e}}(63 / 33)=683 \mathrm{ft} \quad$ Also, $\frac{\alpha_{\mathrm{m}}^{2}}{\mathrm{z}}=60 /(2 \pi \times 683)=14$, and from Fig 5 of Bulson's paper, $\alpha_{m}=305$ Substitution in Eq (6) gives $Q_{c r}=(33+20) /(33 \times 322) \times(g \times 60 / 2 \pi)^{3 / 2} \times(1 / 146 \times 305)^{3}=359 \mathrm{cfs} / \mathrm{ft}$ Finally, $Q_{\max } / Q_{c r}=06+(20 \mathrm{H} / \mathrm{L})=227$, and $Q_{\max }=815 \mathrm{cfs} / \mathrm{ft}$ Therefore, the quantity of free alr required is $815 \mathrm{cfs}$ per ft This represents an air power at the pipe of 26 horsepower per foot Thus, a 300 yard long breakwater would require a total of $23,400 \mathrm{Hp}$ The operation alone is very costly, even if installation costs were not considered

Flexible Breakwaters

In general a floating structure of relatively light weight will only be able to reflect a small amount of wave energy If, however, the structure has sufficient length, a larger amount of wave damping and wave reflection will occur

Model tests performed by Whegel et al (1959), showed that floating sheets of plastic material will have a wave damping effect if the length of the plastic sheets, $\lambda$, is equal to many times the wave length, $L$ For a value of $\lambda / \mathrm{L}=5$, the wave height behind the structure appeared to be equal to $\mathrm{H}_{\mathrm{T}}=08 \mathrm{H}_{\mathrm{T}}$, for $\lambda / \mathrm{L}=10, \mathrm{H}_{\mathrm{T}}=04$ to $05 \mathrm{H}_{\mathrm{I}}$, and for $\lambda / \mathrm{L}=20, \mathrm{H}_{\mathrm{T}}=02 \mathrm{H}_{\mathrm{I}}$ From a similar series of tests 1 t appears that with a layer of plywood a wave reduction up to $50 \%$ could be obtained with $\lambda / L=2$ to 3 Other studies were made of the wave damping properties of waterfilled bags, ("hovering breakwater") floating in the water with their top at the water surface The dimensions of the bags were $10^{\prime} \times 10^{\prime} \times 4^{\prime \prime}$ The results showed that within the 
range of $L / \lambda$ from 05 to 08 , the ratio of the wave height in the lee of the "hovering breakwater" to that of the incident wave $\left(H_{T} / H_{I}\right)$ was approximately equal to the ratio $\mathrm{L} / \lambda$ This means that an effective damping requires a relatively wide structure

Tests made with prototype waves in San Francisco Bay, with a hovering breakwater $20^{\prime} \times 24^{\prime} \times 4^{\prime}$ deep in water $7^{\prime}$ deep (below MLLW) by Wiegel, Shen and Cumming (1962) showed that $\mathrm{H}_{\mathrm{T}} / \mathrm{H}_{\mathrm{I}}$ was one-half of the value obtained $\mathrm{fn}$ the laboratory for the same value of L/ $\lambda$, using the "significant wave length" computed from the measured "zero upcrossing period" of about 17 seconds The reason why the "prototype" was more effective than the "model" was not determined

Other extensive experiments were made by Ripken (1960) These experiments deal with the wave damping properties of cylindrical bags, filled with alr or liquid, which are placed just below the water surface with their longitudinal axis parallel to the direction of wave propagation It was found that a satisfactory attenuation requires a big length of about 15 to 2 times the wave length A diameter of $20 \%$ of the water depth was recommended for the cylindrical bags, although the influence of the relative depth appeared to be small A fill ing of about $95 \%$ seems to be the most effective filling percentage for the bags Use of fluids in the bag of a greater viscosity than water did not substantially increase the amount of wave attenuation Ripken stated that the wave attenuation provided by water filled bags was associated with a progressive pressure wave in the bag Thus pressure wave was found to be slightly out of phase with the wave motion As a consequence of this, the material used for the bags must be strong

Other studies were carried out by Ripken (1960) for two different types of wave absorbers a blanket and a shallow moored floating structure Ripken concluded

The degree of attenuation achieved increases as the ratio of wave length to blanket length decreases and as the ratio of blanket thickness to water depth increases 'The blanket thlckness should be of the order of 15 percent or more of the water depth For a thin blanket the length should be several times as 1 ong as the wave length A multiple of about three or more is ind lcated depending on the attenuation desired "

A similar conclusion was reached in regard to the wave trap, a considerable length is needed to damp the oncoming waves

An experimental study of fascine mattress composed of willow twigs and reeds has been made by Vinje (1966) It dppeared that the wave attenuation was nearly $45-50 \%$ when the ratio of the length of mattress to the length of wave was larger than 1

There are some other studies which have been made, and the general conclusion appears to be that the length of breakwater in the direction of wave propagatıon should be much larger than the wave length

\section{Floating Rigid Structures}

Floating structures have three modes of oscillation due to the restoring force of gravity rolling, pltching, and heaving A moored floating structure has three additional modes of oscillation owing to the restoring force of the moorings A floating structure which is to reflect wave energy must have the requisite long natural periods in each of these three modes of oscillation compared with the wave periods To obtain a long natural period, it is necessary to combine a large mass with small "elasticity" In a floating structure the 'elasticity" is represented by its change in buoyancy as it heaves, rolls, and pitches A solution of this problem is the enclosure of a laxge 
mass of water within a relatively light enclosing structure in such a way that the restoring force was reduced to a minimum This was the principle of Bombardon floating breakwater designed for and used in the Normandy invasion of World War II (Lochner, Faber and Penney (1948) In the official report on the operation of Bombardon floating breakwater the following statement was made

"A full scale breakwater, assembled off the Dorset coast in April 1944, successfully withstood an on-shore gale of force 7 (30 $\mathrm{m} \mathrm{p} \mathrm{h}$ ) with gusts up to force 8 (39 $\mathrm{m} \mathrm{h})$ "

"Both breakwaters we re moored in 11-13 fathoms, giving sufficient depth inshore for Liberty ships to anchor In this depth they reduced the height of the waves by the measured amount of $50 \%$, which represents a $75 \%$ reduction in wave energy These measurements were carefully made at the British harbour on the $16 \mathrm{th}$ June, $1944 \mathrm{with}$ a wind blowing force 5-6 Unloading operations and small boat work were going on inside the breakwater at that time which would not have been possible outside the breakwater"

The requirement of 1 arge mass may be usefully replaced by large moment of inertia of mass in the development of floating breakwaters This concept has been applied by Brebner and Ofuya (1969) in developing the "A" frame breakwater The "A" Frame breakwater consists essentially of a central rigid curtain of wood, and two aluminum cylinders symmetrically located and rigidly connected to the curtain at intervals The mass radius of gyration of the structure about a lateral axis through its center of gravity (axis parallel to wave crest) may be varied by altering the cylinder spacing Laboratory experiments showed that an effective floating breakwater system can be developed in which a large moment of inertia of mass is the dominant factor rather than the mass The reduction of wave heights is effected through the processes of wave reflection, dissipation, and wave interference The best wave damping was obtained when the distance between the cylinders was nearly equal to the wave length

The third concept is that of a perforated breakwater which was originally developed by Jarlan (1965) This study is concerned with the application of that breakwater as a mobile system and for possible operation in the floatingmoored or fixed to the bottom A recent study has been done by Marks (1967) who states

"The dynamic processes that result from the incidence of waves on the perforated breakwater can best be visualized by considering [Fig 3] * As the wave impinges on the porous front wall, part of its energy is reflected and the remainder passes through the perforations The potential energy in the wave is converted to kinetic energy in the form of a jet, upon passage through the perforation, which then bends to be partially dissipated by viscosity in the channel and partially by turbulence in the fluid chamber behind the perforated wall As the water in the fluid chamber flows back out of the holes, it encounters the next oncoming wave and partial energy destruction is accomplished even before that wave reaches the breakwater If the walls were not perforated (e g a calsson), total reflection would occur on the face of the wall with resultant high impact forces and scouring on the base, if it is 
fixed to the bottom If the breakwater were floating and anchored, part of the incident wave force would be transmitted to the mooring cables and part would be directed to oscillating the breakwater thus inducing it to make waves on the shoreward side In the case of the perforated breakwater, that part of the incident wave energy which is dissipated internally in the form of heat and eddies is not avalable for such deleterious activity Hence, it is expected that less force would be felt in the mooring lines, and/or that smaller waves would be produced shoreward of the breakwater

It is clear that wave attenuation is most effective at low periods and least at high periods The perforated unit is better up to ibout 9 seconds of wave period and worse beyond compared with calsson type

"The breakwater geometry specifying 4-foot diameter holes, 4-foot wall thickness, and 40 feet between front and back wall was found to be most effective, as predicted by theory The mooring lines in the perforated breakwater experienced less force by about a factor of 2 compared with calsson type Wave reduction by the solid floating-breakwate $r$ varied from about 02 to 06 For the perforated floating breakwater, wave reduction varied from about 02 to 08 As expected, the perforated breakwater was far effective in reducung wave height for shorter waves ( 01 to 03 ) than for longer waves $\left(\begin{array}{llllll}0 & 6 & \text { to } & 0 & 7\end{array}\right)^{\prime \prime}$

\section{NEW MODEL FLOATING BREAKWATERS}

\section{Int roduction}

It was decided to design three types of rigid structure breakwaters for laboratory testing, combining the principle of the use of a large entrained moment of inertia with the principle of partially absorbing the wave energy by a perforated wall or by a sloping board The three types have been designated Type A, B, and $C$

The experiments were performed in a $106 \mathrm{ft}$ long by $1 \mathrm{ft}$ wide by $3 \mathrm{ft}$ deep wave channel The water depth in the wave channel was set at 25 in, corresponding to a "prototype" depth of $25 \mathrm{ft}$ The floating breakwater model was placed about seventy feet from the wave generator An energy absorbing beach was located at the other end of the channel Wave measurements were obtained at a position about $15 \mathrm{ft}$ 'seaward" of the floating breakwater model and at a location about $8 \mathrm{ft}$ to the "lee" of the model, using parallel wire resistance wave gages (Wiegel, 1956)

Two wave helghts were used for each wave length tested, one low and one high, to check approximately the effect of wave steepness (also, called the wave slope) on the phenomenon The wave length, L, is related to the wave period, $T$, and water depth, $d$, by the equation

$$
L=\frac{\mathrm{gT}^{2}}{2 \pi} \tanh \frac{2 \pi \mathrm{d}}{\mathrm{L}}
$$

in which $g$ is the acceleration of gravity For the water depth and wave periods tested, this equation could be approximated by

$$
\mathrm{L} \approx \mathrm{gT}^{2} / 2 \pi
$$


A portion of the wave energy was transmitted to the lee of each breakwater as a train of waves, a part of the energy was dissipated, and a part of the energy was reflected as a train of waves LIttle wave energy was reflected by Model A breakwater, while Models B, C, D and E reflected a considerable amount of wave energy No attempt was made to measure the amount of wave energy reflected

The most important pardmeter of the study is the transmission coefficient, defined as

$$
\text { Wave Transmission Coefficient }=\frac{\text { Transmitted Wave Height }}{\text { Incident Wave Height }}=\frac{\mathrm{H}_{\mathrm{T}}}{\mathrm{H}_{\mathrm{I}}}
$$

A typical example of the record of both the incident and transmitted wave heights is shown in Fig 4 It can be seen that the reflected waves interfered with the incident waves in a complex manner Reported values of the wave transmission coefficient were based on the largest transmitted wave helght and the average incident wave helght
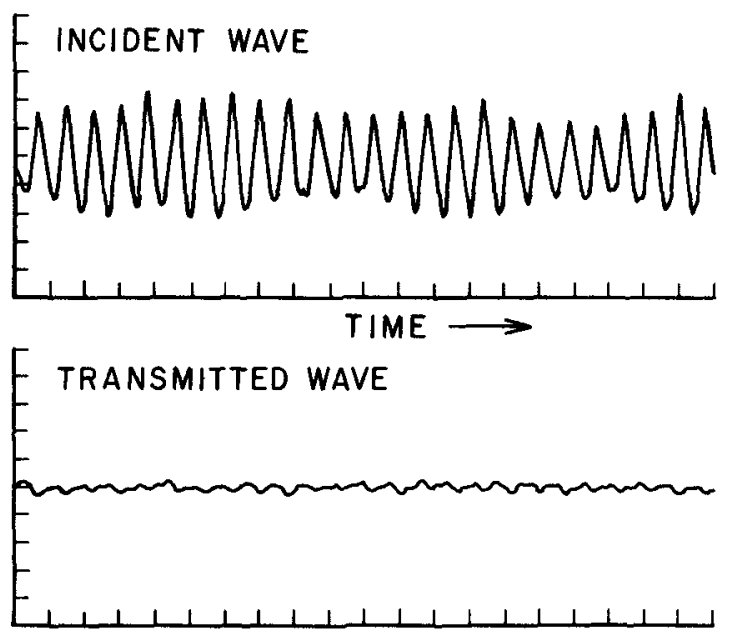

\section{FIG 4 RECORD OF INCIDENT WAVE AND TRANSMITTED WAVE}

One important factor that was not studied at this stage was the mooring line system and the mooring forces The mooring line system forms an umportant feature of a floating breakwater since breakwater performance depends on the type of restraints imposed on lts motion by the mooring lines Three additional natural periods result from the mooring lines yaw, sway and surge Further studies must be made on the mooring system Different types of floating breahwaters moorings need to be studied to find out the best mooring system 
Mode1 A

The basic concept of this design was the dissipation of wave energy by waves breaking on a sloping board "beach"

Model A, shown in Fig 5, consisted of a sloping board "beach," with the seaward end attached to a rectangular alx chamber (pontoon) which provided buoyancy The "lee side" of the sloping board was connected to a vertical wall ( $14^{\mathrm{t}} \mathrm{hlgh}$ ) by steel frames A "lee side" floating box (pontoon) was connected to the vertical wall by a steel frame The total length, $\lambda$, of this model was $435 \mathrm{ln}$ ( $1 \mathrm{e}, 435 \mathrm{ft}$, "prototype" as the ratio between the model and the contemplated bxeakwater for use in reservours is 1 12)

in order to chtck the effect of the height of the vertical wall on the wave transmission coefficient, the model was modifled by increasing the

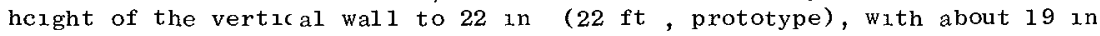
(19 ft, prototype) of 1t being submerged

It was found that when the incident waves moved over the sloping board "beach," they started to break as if they were moving ove $r$ a sand beach The small amplitude waves broke completely on the board The large amplitude waves did not break completely on the board "beach" owing to the limited length of the board For waves which were about as long as the dimension $\lambda$ of the breakwater, or shorter, a substantial amount of wave energy was dissipated in this brcaking process The top of the board 'beach" was designed to have a saw tooth shape The large amplitude waves partially broke on the board "beach,' then ran over the top of the board, dropping through the space into the water on the lee side of the "beach' No water went over the top of the vertical wall Considerable alr entrainment and muxing occurred during the process This periodic impact of the wave run-up dropping on the water surface eventually created a pressure fluctuation in the water undes the board "beach," and in front of the vertical wall The water moved up and down in this region This resulted in a pressure fluctuation in the region between the bottom of the vertical wall and the bottom of the tank, which in turn caused a heaving motion of the water surface in the section between the vertical wall and the lee side pontoon It appeared that the distance between the vertical wall and the lee side pontoon would be importint, but lack of time prevented a study of varıous spacings

A further observation was made by permitting the model drift (e $g$, the mooring lines were removed from the model) in the waves It was found that Model A drifted much more slowly than Model B It was believed that the slow rate of dxift indicated there would not be too great a problem in mooring the structure

The results of the wave transmission coefficient vs the ratio of wave length to breakwatex length $(\mathrm{L} / \lambda)$, and vs the wave length (given in "prototype' scale) are shown in Fig 6 The results show an Irregular curve In the range of wave lengths from $30 \mathrm{ft}$ to $55 \mathrm{ft}$ (prototype), the steep waves (wave slope $=0$ 055-0 075) had a higher transmission coefficient than did the waves of relatively small steepness (slope $=0$ 022-0 030) The term wave steepness refers to the ratio of the incident wave height to the wave length $\left(\mathrm{H}_{\mathrm{I}} / \mathrm{L}\right)$ The two curves crossed at a wave length of about $60 \mathrm{ft}$, and $\mathrm{in}$ the range of wave lengths from $65 \mathrm{ft}$ to $90 \mathrm{ft}$, the relationship was opposite to that for the smaller wave lengths

When the model was modified with the $22 \mathrm{in}$ (22 ft, prototype) deep vertical wall, there was less difference in the transmission coefficients for the relatively flat waves thin the steep wave There was about a 10 percent improvement in the wave transmission coefficient for the range of wave lengths between $60 \mathrm{ft}$ and $80 \mathrm{ft}$ (prototype) 


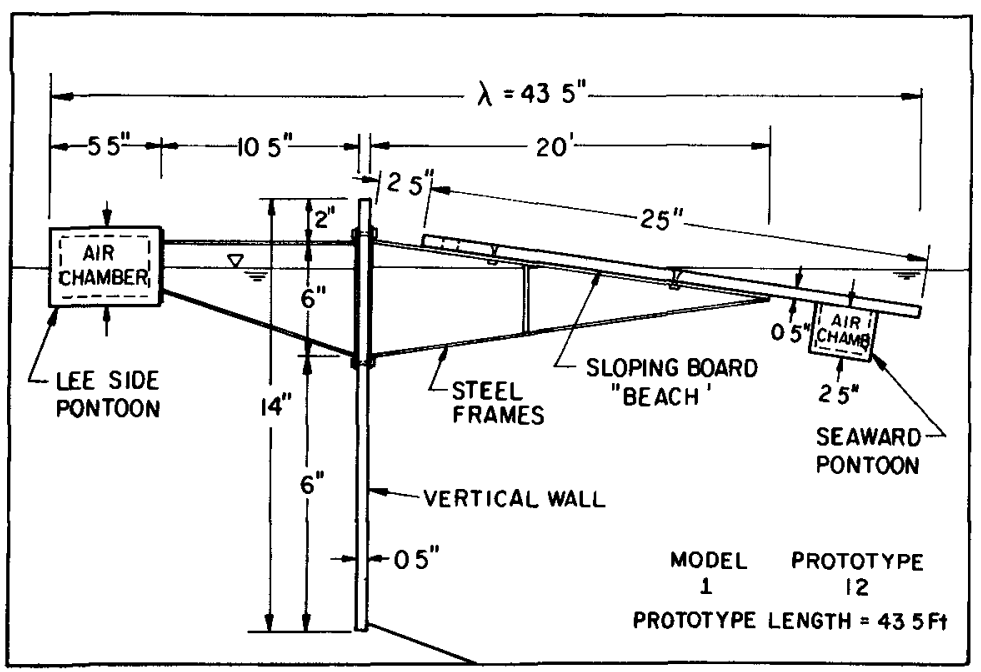

FIG 5 FLOATING BREAKWATER "TYPE A"

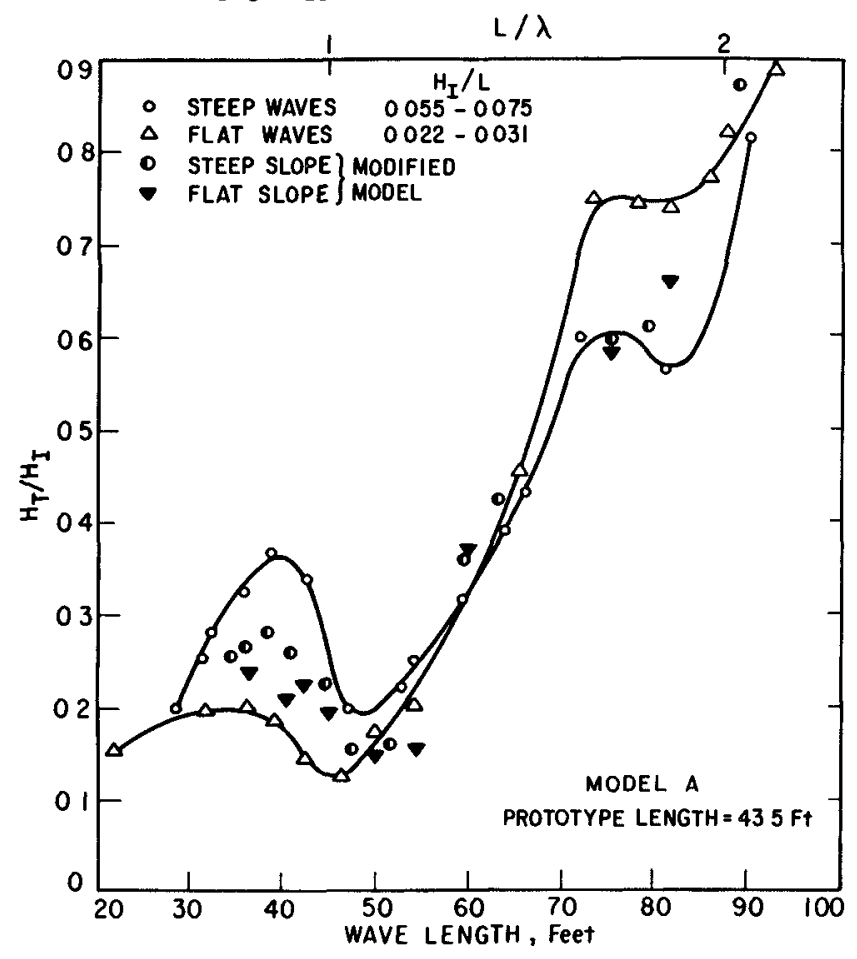

FIG 6 TRANSMISSION COEFFICIENT VS WAVE LENGTH (ALSO L/A) 


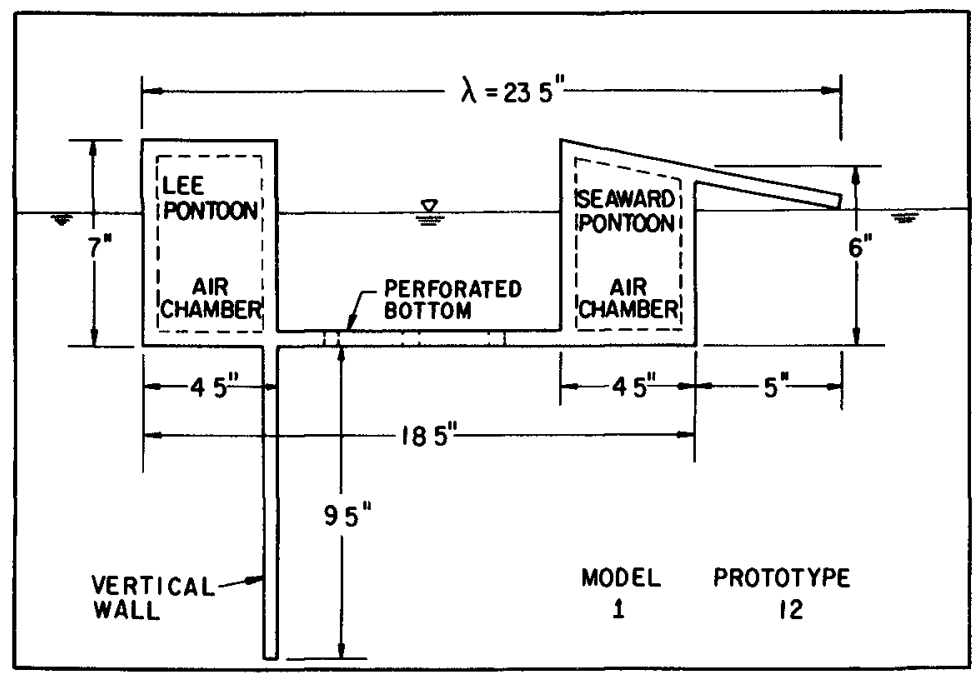

FIG 7 FLOATING BREAKWATER "TYPE B"

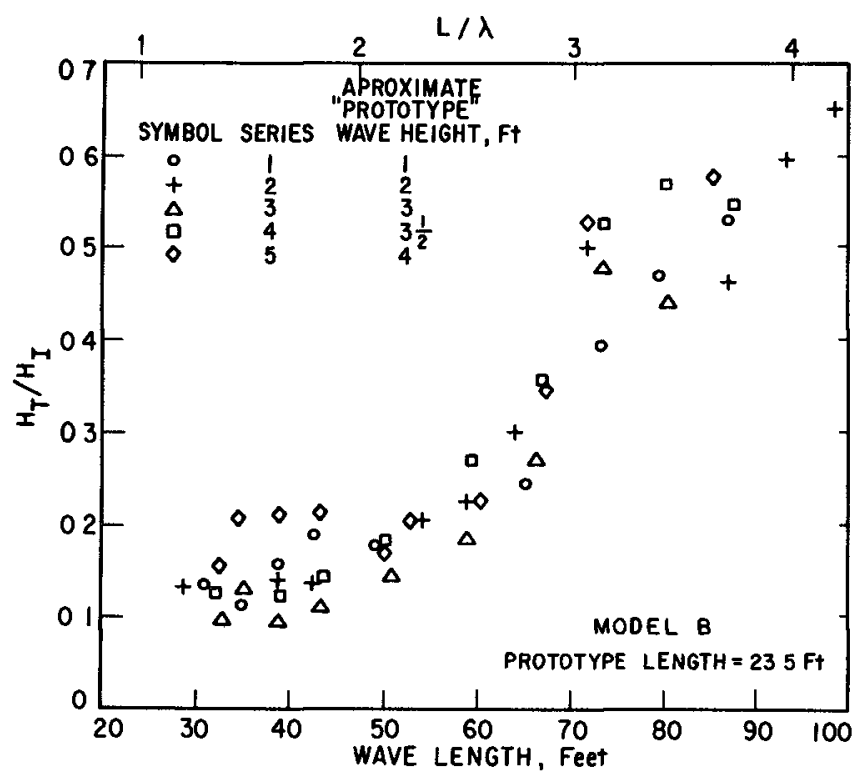

FIG 8 TRANSMISSION COEFFICIENT vs WAVE LENGTH (ALSO L/ $\mathrm{A}$ ) 


\section{Model B}

Floating breakwater Model B, shown in Fíg 7 , consisted of two pontoons separated by a perforated bottom The length, $\lambda$, of the structure is 185 in (18 $5 \mathrm{ft}$, prototype), with an additional 5 in (5 ft, prototype) of sloping "beach" extending seaward above the water surface for a total length

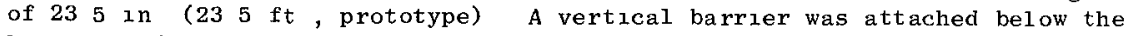
lee side of the perforated section The pontoon on the lee side had a rectangular cross section The pontoon on the weather side has unequal vertical sides, with a sloping top extending outwards a distance of 5 in ( $5 \mathrm{ft}$, prototype) towards the weather side

The results of the wave transmission coefficient vs wave length and vs $\mathrm{L} / \lambda$ are shown in Fig 8 for five series of runs, each with a different wave height For wave lengths less than $60 \mathrm{ft}$, prototype, ( $\mathrm{L} / \lambda$ less than 27 ), the transmission coefficient is less than about 020 Considering the expected range of wave lengths in a reservoir, this is probably a satisfactory attenuation For wave lengths between $65 \mathrm{ft}$ and $80 \mathrm{ft}$ (prototype), the transmission coefficient rises rapidly from about 03 to 05 For wave lengths of about $90 \mathrm{ft}(\mathrm{L} / \lambda$ of $3 \mathrm{8})$, the transmission coefficient rises to 0 60-0 65 Under the latter circumstances, a substantial portion of the wave energy is transmitted past the floating breakwater

It appeared that more than half of the energy of the incident waves was reflected by the breakwater as a wave train, with an "apparent higher frequen$c^{\prime \prime}$ than the frequency of the incident waves The breaking up of the oncoming waves into a series of smaller reflecting waves appeared to result in smaller forces acting on the mooring system The action of the water and the structure is shown schematically in Fig 9 In Fig 9 the oncoming wave crest is shown striking surfaces $A, B$ and $C$, it then reflects at different times in the form of a series of reflecting waves with a smaller amplitude compared with the incident waves, and with different phases

Owing to the vertical barrier and the overall geometric arrangements, this floating breakwater had a rather large moment of inertia with respect to rolling motion Also, the center "water channel" with the perforated bottom worked as a damping device, in some ways similar to an antirolling tank on a ship For oncoming waves with large amplitude, the crest washed over the sloping top of the weather side pontoon, flowed into the center channel and then flowed through the perforated bottom Part of the energy apparently was dissipated by water rolling over the top of the slope and by the eddies that formed in the water channel Some alr trapped under the surface of the extended portion of the sloping top of the seaward pontoon was compressed, and mixed with the water This process was too complicated for analysis, but it appeared to be a good mechanism for dissipating some of the wave energy

The tension in the mooring line consisted in general of two components, one caused by the rolling motion of the breakwater, and one caused by the wave crests striking the structure However, the model was designed so that the two components would not cause maximum forces in the mooring line at the same time Since the axis of rolling of the system is above the mooring point, when the incident waves strike on the system, the rolling motion of the body tended to release the tension in the mooring line When the incident wave troughs reached the floating breakwater, the tension in the mooring line was caused only by the rolling motion 


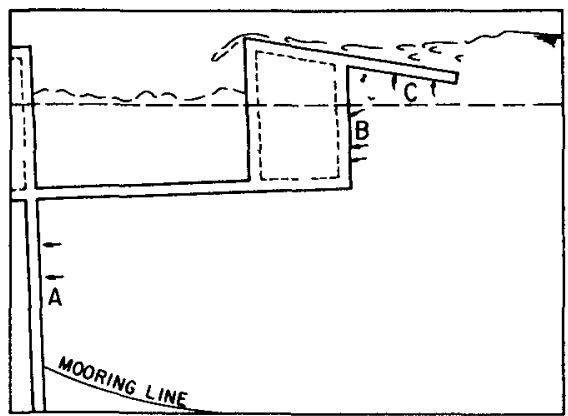

(a) WAVE CREST AT BREAKWATER

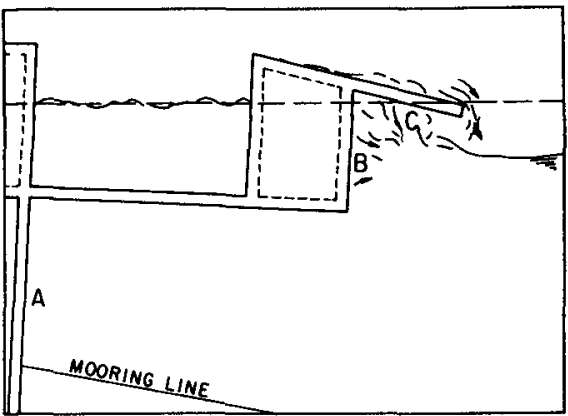

(b) WAVE TROUGH AT BREAKWATER

Model $C$

FIG 9 SKETCH SHOWING WAVE ACTION ON MOOEL $B$

Model C (Fig 10) is a type of perforated breakwater the purpose of the perforated wall being to decrease the direct striking force by decreasing the retlecting ared and to dissipate wave energy by the flow of water through the holes The "water chamber" has two perforated walls Tht back will extends downward as a vertical barrier to provide sufficient moment of ineitia of added mass The air chamber (pontoon) serves as a walkway along the breakwater During the model test, ballast was used to obtain proper balance of the structure This model was the shortest of all five models, being only 14 in $(\lambda=14 \mathrm{ft}$, prototype) long

Values of $\mathrm{H}_{\mathrm{T}} / \mathrm{H}_{\mathrm{T}} \mathrm{vs}$ wave length ind vs $\mathrm{L} \lambda$ are shown in Fig 11 For uave lengths up to about $40 \mathrm{ft}$, the result appears to be reasonably satisfactory The curve of the wave transmission coefficient vs wave length (and vs $L, \lambda$ ) has a rather steep slope

The expected amount of energy dissipation did not occur the rest of the energy was either reflected or transmited past the breakwater olther through the motion of the breakwatcr which acted as a wave generator, or by wave energy passing under the structure

\section{Model D}

The side and top $v i$, of Model D are snow in Fig 12 Ihe platform, 32 in ( $\lambda=32 \mathrm{ft}$ prototype) in length, was ballasted sufficiently to cause $1 \mathrm{t}$ to be immersed with its bottom 6 in ( $6 \mathrm{ft}$ prototype) beneath the water surface A series of gates were suspended vertically upwards by their own buoyancy Each gate was connectea to the platform by a rubber sheet hinge A string was used to resirain the motion of the gates to only one side from the vertical The gates were designed so that the rolling motion of each row of gates could move in only one direction in an alternative manner the model was carefully ballasted so that each gate emerged 05 in (0 5 ft, prototype) above the water surface It was designed so that any water current could bypass the scries of gates with a rather small striking force, and to dissipate the energy through turbulence The restrained motion of the gates may interrupt the orbital motion of the waves and energy dissipation occurs The motions of the water current and the gates are shown in Fig 13

The experimental results are shown in Fig 14 The high wave transmission coefficients are due to the following two reasons first, the joint between the gates and the platform was a one-inch wide (model dimension) rubber sheet instead of a simple hinge Because of the inflexibility, the motion of the gates was not confined to a simple rolling motion, but also had a parallel displacement which regenerated the wave and transmitted the wave energy to the lee of the breakwater In addition, the simple flat platform provided a smaller moment of inertia (largely due to added mass) than a structure with a vertical barrier 


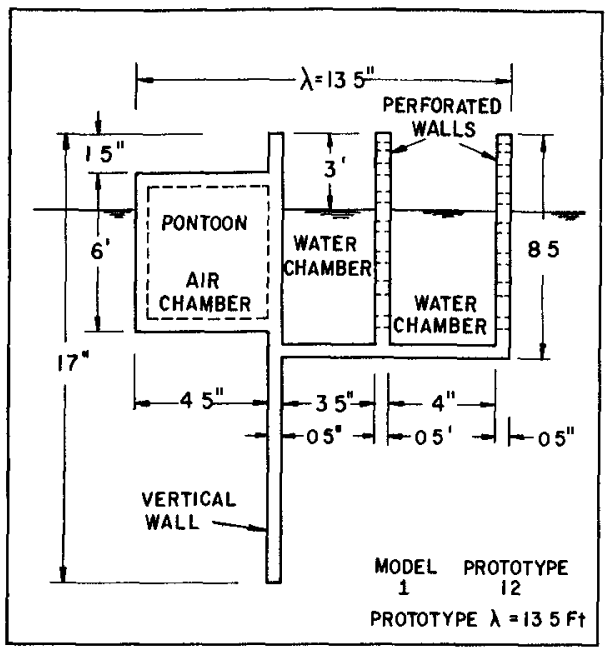

FIG IO FLOATING BREAKWATER TYPE " $c$ "

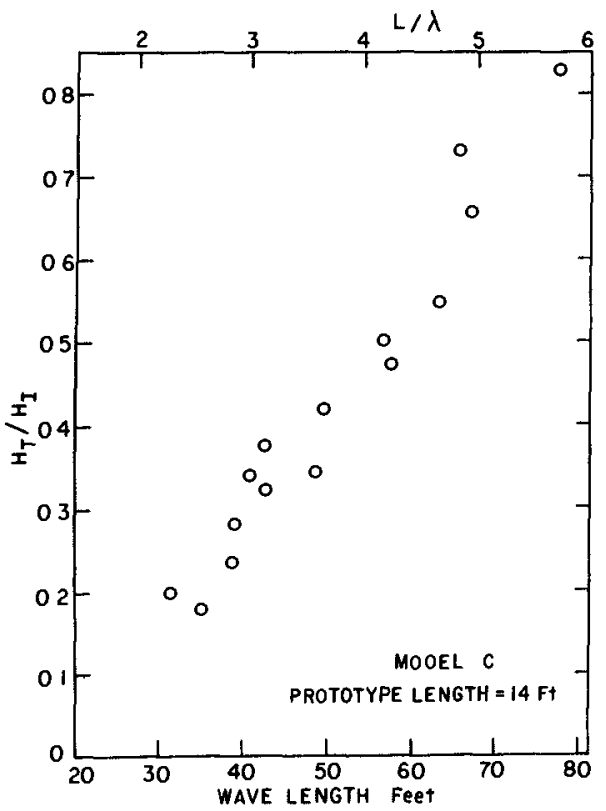

FIG II TRANSMISSION COEFFICIENT VS WAVE LENGTH (ALSO $L / A$ ) 


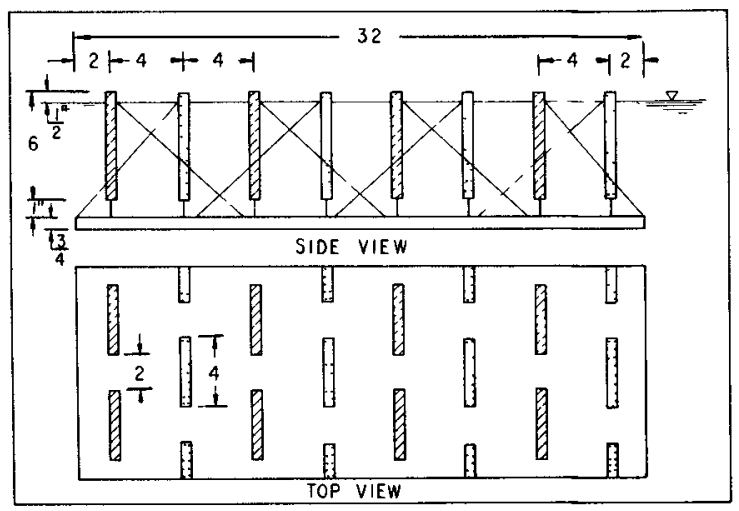

FIG I2 FLOATING BREAKWATER TYPE D

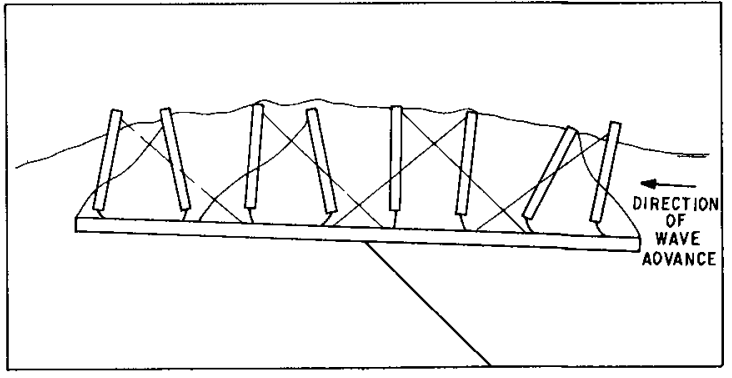

FIG I 3 WAVE PASSING BREAKWATER TYPE D

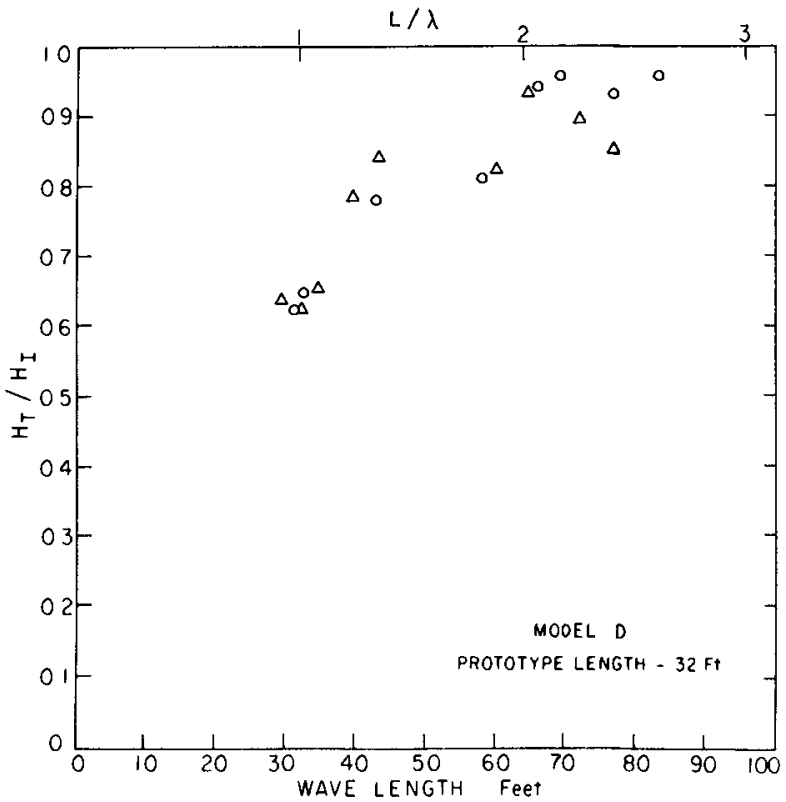

FIG 14 TRANSMISSION COEFFICIENT US WAVE LENGTH (ALSO L $/ \lambda$ ) 


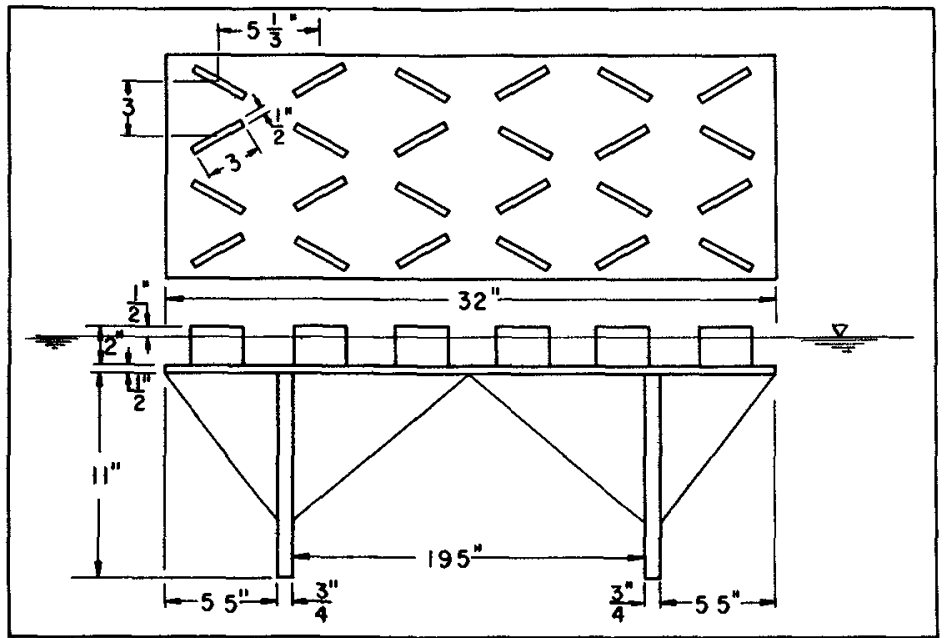

FIG 15 FLOATING BREAKWATER TYPE 'E'

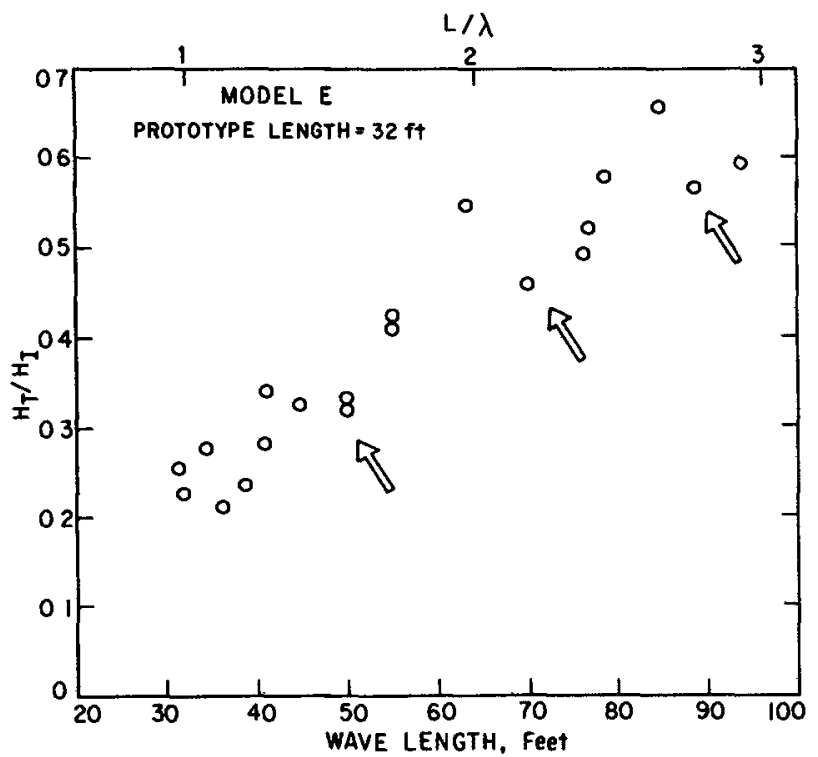

FIG 16 TRANSMISSION COEFFICIENT VS WAVE LENGTH (ALSO L/ $\lambda$ ) 
Mode1 E

In order to avold the transmission of energy through the gates, another model was adopted, based upon the concept of a fixed energy dissipator similar to those used at the foot of a spillwdy, rather than using the flapping gates The vertical walis and the platform formed a $\pi$ shape (Fig 15), which provided a large moment of inertia ( 1 argely due to added mass), and minimized the transmission of wave energy under the platform The length, $\lambda$, of the structure was $32 \mathrm{ft}$, prototype The vertical sections mounted on the top side of the horizontal submerged platform consisted of six rows of blocks Each block was set at an angle of 30 degrees to the direction of wave propdgation in an alternating pattern

Fnergy dissipation occurred as the waves washed through the maze of vertical blocks, and formed eddies and turbulence It was observed that the waves collided with the platform This collision may have been caused by the combination of the motion of the platform and the wave motion This occurred for wave lengths of about $50 \mathrm{ft}, 70 \mathrm{ft}$, and $90 \mathrm{ft}$ prototype The impact of this wave collision apparently resulted in the dissipation of some of the wave energy, this can be seen in the plot of wave transmission coefficient vs the wave length (and $\mathrm{L} / \lambda$ ) for wave lengths of $50 \mathrm{ft}, 70 \mathrm{ft}$, and $90 \mathrm{ft}$ (see arrows in Fig 16)

As can be seen in Fig 16 the transmission coefficient was about 045 for a wave length of about $60 \mathrm{ft}$, prototype

\section{ACKNOWLEDGEMENT S}

The work described herein was performed at the Unıversity of California, Berkeley, Californid, under contract to the Department of Harbors and Watercraft of the State of California The authors wish to acknowledge the valuable advice of $M r J S$ Habel of the Department and Professor $J \mathrm{~V}$ Wehausen of the University during the course of this study

\section{REFERENCES}

1 Amerlcan Society of Civil Englneers, Task Committee on Smal1 Craft Harbors, 'Report on Small Craft Harbors," ASCE-Manuals and Reports on Englneering Practice-No 50 1969, 139 pages

2 Brasher, P, Compressed Air Magazıne, Vo1 20, 1915

3 Brebner, A and A O Ofuya, "Floating Breakwaters," Proceeding of Eleventh Conference on Coastal Englneering, London, 1968, Vol II, ASCE 1969, pp 1055-1094

4 Bulson, $\mathrm{P}$ S , 'Large Scale Bubble Breakwater Experiments "The Dock and Harbour Authority, Vo1 XLIV, No 516, Oct 1963, pp 191-197

5 Bulson, P S, "Transportable Bredkwater," The Dock and Harbour Authority, Vo1 XLVIII, No 560, June 1967, pp 41-46

6 Bulson, P S, "The Theory and Design of Bubble Breakwaters," Proceedings of the Eleventh Conference on Coastal Englneering London, 1968, Vol II, American Soczety of Civil Englneers, 1969, pp 995-1015 
7 Glenn, A H, 'Progress Report on Solution of Wave, Tude, Current, and Hurricane Problems in Coastal Opcrations," Oxl and Gas Journal, Vol 49, No 7,22 June 1950 pp 174-177

8 Jarlan, G E, "The Application of Acoustic Theory to the Reflective Properties of Coastal Engineering Structures," National Research Council of Canada, DME/NAE Quarterly Bulletın No 1965(1)

9 Lochner, R, Faber, O, \& Penny, W G, "The Bombardon Floating Breakwater," Ihe Civil Engineer in War, Vol 2, Dochs and Harbors, The Institution of Civil Englneers, London, 1948, pp 256-290

10 Raxchlen, Frederıc, "Motıons of Small Boats Moored in Standing Waves," California Institute of Technology $W$ M Keck Laboratory of Hydiaulics and Water Resources, Report No KH-R-17, August 1968, 158 pages

11 Rupken $J \mathrm{~F}$, 'An Experımental Study of Flexible Floating Breakwaters," Unxversity of Minnesota, St Anthony Falls Hydraul ic Laboratory, Tech Paper No 31 , Series B $1960,72 \mathrm{pp}$

12 Ripken, J F , "Evaluation Studies of the Unıted States Rubber Comprny Wave Blanket and Wave Trap,' University of Minnesota, St Anthony Falls Hydraul tc Laboratory, Project Report No 62, 1960, 77 pp

13 Santema, P 'About the Estimation of the Number of Days with Favorable Meteorological and Oceanographical Conditions for Enguneering Operations on the Sea Coast and in Estuaries,' Proceedings of the Fifth Conference on Coastal Englneering, Council on Wave Research, The Fngineering Foundation Berkeley, Cal ifornia, 1955 , pp 405-410

14 Taylor, Sir Geoffrey "The Action of a Surface Current Used as a Breakwater," Proc Roy Soc (London), Ser A, Vol 231, No 1187, September 155, pp 466-478

15 Vinje, $J J$, "Increase of Effectuve Working Tume Durıng Operations at Sea by Means of Movable Structures," Delft Hydraulucs Laboratory, Publication No $42,1966,38 \mathrm{pp}$

16 Wregel, R L "Parallel Wire Resistance Wave Meter," Proceedings of the First Conference on Coastal Engineering Instruments, Council on Wave Research, The Engineering Foundation, Berkeley, California, 1956, pp 39-43

17 Wlegel, $R$ L, Shen, $H W$ and Cumming $J$ D, "Final Report on Hovering Breakwater,' Unıversity of Calıfornia Berkeley Hydraulic Engıneering Laboratory, Wave Research Projects Serıes 140, Issue 5, 1959

18 Wregel, R L , Shen, $\mathrm{H} W$ and Cummıng, J D, "Hoverıng Breakwater," Journal Waterways and Harbors Div, Proc ASCF, Vol 88, No WW 2, May 1962 , pp 23-50

19 Williams John $A$ and $R$ L Wregel, 'Attenuation of Wind Waves by a Hydraulic Breakwater, Proceedings of the Elghth Conference on Coastal Figineering, Council on Wave Research, The Engineering Foundation, Berkeley, Calıfornia, 1963 , pp 500-520 


\section{CHAPTER 101}

FORCES ON A PONTOON IN THREE DIIENSIONAL WAVES

by

J. EIE, Research Enganeer $\mathrm{x}$

A TRATTEBERG, Senjor Research Engineer $\mathbf{x}$

A. TøRUM, Senior Research Engineer $x$

1. INTPODUCTTON

Wave forces on a long pontoon (floating breakwater, floatzng bridges etc.) depend to a large extent on the three dimenszonal wave pattern. There is no determanistic method for calculating wave forces for such structures in a three dimensional sea and laboratory equipment for testing long structures in irregular trree dimensional waves does hardly exist

Forces, bending moments etc on floating structures may in principle be calculated on basis of a transfer functzon $K(f, \theta)$ and the two dimenszonal wave power spectrum $E(f, \theta)$ giving the one dimensional force power spectrum $\phi(f)$ according to

where

$$
\phi(f)=\int_{\theta} K^{2}(f, \theta) E(f, \theta) d \theta
$$

$$
\begin{aligned}
f= & \text { frequency } \\
\theta= & \text { angle between mean wave direction and direc- } \\
& \text { tion of the individual wave component }
\end{aligned}
$$

\footnotetext{
$x_{\text {River and harbour Laboratory }}$

Technzcal Unzversity of lorway, Trondhezm, Norway
} 
This concept, which is being used to a certaln extent in ships hydrodynamics, is based on a lincar relationship between wave helght and forces, bending moments etc.

The force distribution is calculated from the relationship derived by Longuet-Higgins (2) and is freouently used in wave statistics. This relationship is the following

$$
F=k \sqrt{\int_{f} \emptyset(f) d f}
$$

where

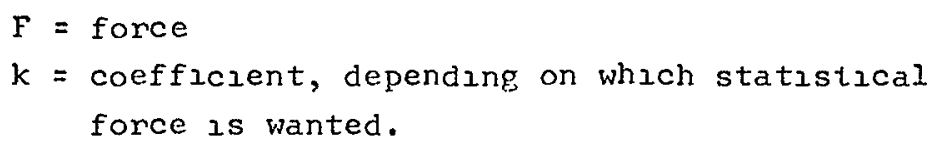

The force $F$ is the zero-upcrossing force as defined in Fig. 7. (see chapter $3 \mathrm{l}$ ). For the significant force $F_{1 / 3}, k=2,83,(2),(3)$.

The whole concept is shown in Fig. I.

The transfer function $K(f, \theta)$ can be found analytically or by model tests.

The purpose of the investigation described in this paper was to obtaln some experimental data on the valıdity of the concept applied to a pontoon of rectangular cross-section. As the transfer function for such a pontoon, partly reflecting and partly transmitting the waves, is not so easily obtalned by calculations, the force transfer function was found experimentally in regular waves. The wave forces in three dimonsional waves were obtalned in a wand wave flume. The scalar wave 
power spectrum has been measured, but unfortunately we have not had the opportunity to measure the directional power spectrum of the waves. We have therefore in the calculations had to make assumtion of the directional spread of the spectrum.

\section{MODEL TEST ARRANGEMENT}

The tested pontoon had a rectangular cross-section with width, draft an length of $0,44 \mathrm{~m}, 0,12 \mathrm{~m}$ and 3,00 meters respectively.

The two dimensional transfer function was obtained in tests using reqular waves in a test arrangement as shown in Fig. 2 Fig. 3 shows detalls of the pontoon and its instrumentation. The total lateral forces were measured by use of strasn gauges placed at both ends of the pontoon Except for the small motions necessary to obtain a response of the strain gauges the pontoon was fixed.

The tests in three dimensional waves were carried out in a wind/wave channel This channel is 78 meters long, and 3,8 meters wide. The water depth at the pontoon was 0,37 meters, whlle the depth in most parts of the channel was approximately $1,0 \mathrm{~m}$. The test setup in the wave channel is shown in Fig. 4.

The waves were generated by wind with a velocity of approximately $10 \mathrm{~m} / \mathrm{sec}$. The ratıo between und velocity = and wave celerity in such wind wave flumes is generally hIgh, and the waves tend to have a steepness which is higher than normally found in fjords and in the ocean In order to reduce the wave steepness a wave filter was placed some distance in fromt of the pontoon as shom In FIg. 4. 


\section{$3 \quad$ TEST RESULTS}

\subsection{Transfer function}

The transfer function was obtained by running tests with different wave perlods, wave helghts and wave directions.

The wave pattern in the test basin was as indicatcd in Fig 2. All measurements were made before the secondary reilected waves from the walls of the test basin reached the pontoon.

Fig 5 shows samples of the test results The diagrams show wave force vs wave helght The angle between the direction of the wave propagation and the direction of force is indicated.

The relation between wavc force and wave holght is falrly IInear and "best-fit" IIncs showing the lincar relation is drawn by eye. This relation is given by

$$
F^{*}=K^{*}(\theta, f) H
$$

where

$$
\begin{aligned}
F^{*}= & \text { force } \\
H= & \text { wave helght } \\
K^{*}(\theta, \Gamma)= & \text { coerficicnt dcpending on wave frequency and } \\
& \text { wave direction }
\end{aligned}
$$

It 13 seen that the forces towards the "Ice" direction of the waves are slightly largcr than towands the "windward" side. This is also revcalcd in the force recordings, a sample of wrich is shown in Fig. 6 .

The reason for the difference betwecn the forces in the two directions is belleved to bc higher order effects 
However, it is not possible within the surnle lincar concept we arc dealing "zth to ancluae these righer order effects lie have thercfore combuned the two darectzons by defining another cocfficzent $K(\theta$,f) as

$$
K(\theta, I)=K^{*}(\theta, f)_{l e e^{+K^{*}}(\theta, f)_{\text {wandward }}}
$$

The following relationship is then obtained

$$
F=K(\theta, f) H
$$

where $F$ is the double amplatude force

I is comparable to $H$ as indicatcd in F 28

The $K(\theta, f)$ valucs are shorn in the diagram of Eig 8

Based on the diagram of Fag 8 we have made a contour "map" of the transfer function as shown in Fig 9

\section{Dircctional spectrum}

As mentioned in the introduction we have not had the opportunity to measure the dircctional spectrum of the waves in the ward wave ilume. howcver, the scalar spectrum has becn obtained. The raves were measurea at the pontoon site in the flumic wren the pontoon was taten away $F$ Ig 10 shows a sample of a paper reconc of the wdves.

The waves vere recordcd on a mapnetzc tape This record was thcil diglizcd with a time intcrval betwecn samples of $0,128 \mathrm{sec}$ and the poner spectrum was calculated. from a sample of 200 consccutzve vaves The "raw" spectrum was smoothed by the mcthod of hanning (3) The calculatce spectrum is shoin in Fir 11 
It has been ucual in theory on rave directional snectra to assume a spectrum directionality function

$$
[a(f, 0)]^{2}=[a(f)]^{2} y\left(\theta, \eta_{s}\right)
$$

where

$$
y\left(\theta, \eta_{s}\right)=\frac{(\cos \theta)^{\eta_{3}}}{K\left(\eta_{3}\right)}
$$

and

$$
k\left(\eta_{s}\right)=\int_{-\frac{\pi}{2}}^{+\frac{\pi}{2}}(\cos 0)^{\eta s} d \theta
$$

FIg. 12 shows y vs $\theta$ for different values of $\eta_{s}$

We have estimatcd the dircctional power spectrum bascd on $a \cos ^{4} \theta-1 a w$ The estinate of the directional spectrum 15 shom as a contour map in Ilp 13 The Irplication of using a $\cos ^{4} \theta-1 a w$ instead of, say, a $\cos ^{2} \theta-$ law will be discussed later.

\section{Calculated force spectra and force distributzons}

Based on the transfer functzon and the estimated directional wave power spectrum, the force porer spectrum was calculated according to equation (1) The numerical calculation was carried out by applying $\Delta f=0,1 \mathrm{scc}$ and $\Delta \theta=\pi / 96$. The result of the calculation is shown in $\Gamma$ Ig 14

The force distribution was calculated according to ecuation (2) and is shown in $\Gamma$ ig. 15.

\section{4. Mercured forces and force distrilution}

The forces were recorded on both a paper and a marnetıc tape A sample of a force record is shown in Fin 16 
The ineasurca force onwer epectrum is shown in Fop 14 together wath the calculated spectrum

The measured force distribution is shom in Fig 15 The measured force as well as the ealeulated foree is the zero-uperossing force as defined in Fig $7 \mathrm{~d}$

$4 \quad$ COI\}'FIJTS

There is apparently a good agreement betreen the measured force distribution and the calculatcd force distribution as shown in Fig 15 however, the calculated foree spectrum and the calculated foree distribution is based on assumptions on the srectrum directionality function The form of the transfer-function is such thot a $\cos ^{2} \theta$ - alrectionality law wall give lower calculated forees than a $\cos ^{4} \theta-1 a w$

It is therefore deemed necessary that the dircetional wave spectrum should be measured

\section{$5 \quad$ CONCLUGTONS}

The work deseribed in this paper pives an indieation that the concedt of cquation (1) is a useful tool for enpaneering purposes when dealung with forces on long floating pontoons like floating bridges, floating breakwaters ete 
LITIA IURE

1. TRRITLELRG, A The cffect of wave cicsts on lave forces Proc of Llcventh Coastal Enguncerang Conference, London, Lnpland 1968

2. LONGUET-HIGGINS, 11 On the statistical dastmabilion of the hejghrs of sca raves Journal of liar Research. Vol 1], 1952, no 3, pp, 246-266

3 KORVIN KROIKOVSKY, B V. Thcory of seateeping Sink1', New York, 1962 

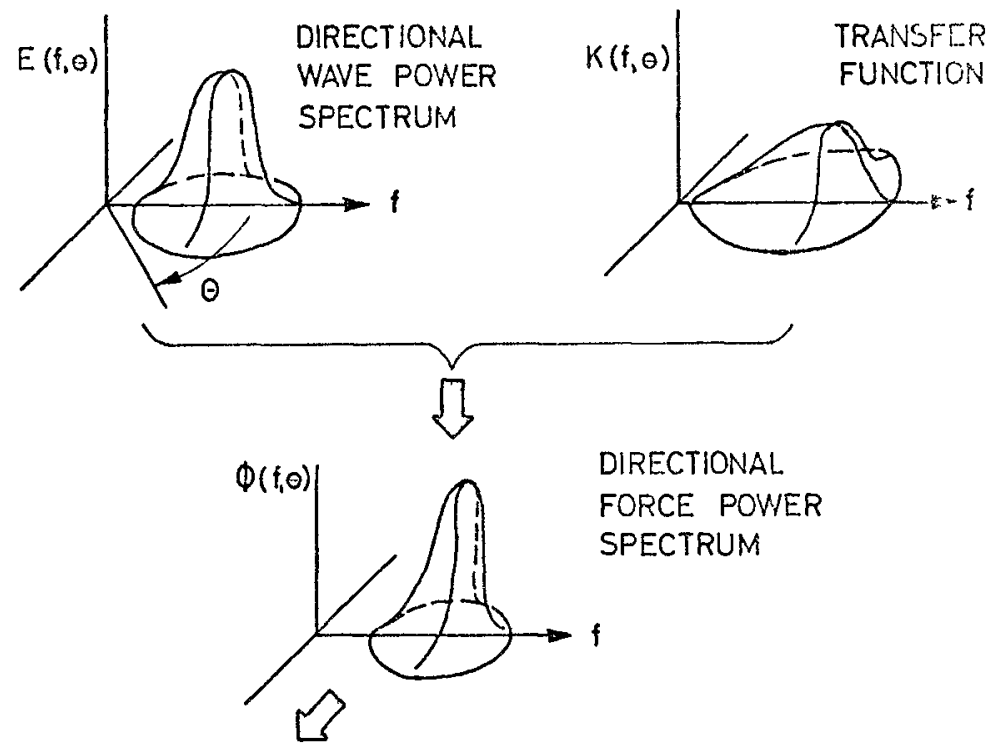

DIRECTIONAL

FORCE POWER

SPECTRUM
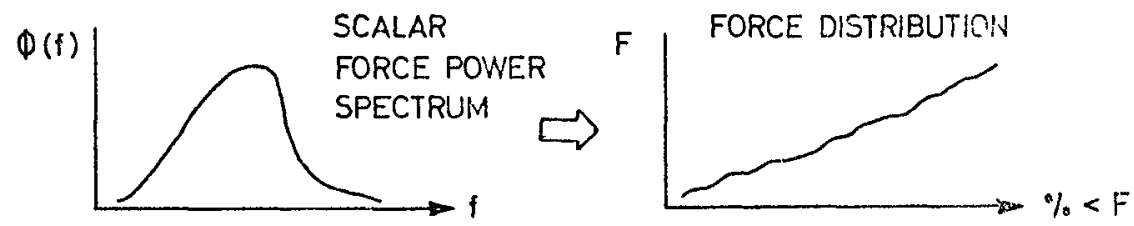

FIG 1 


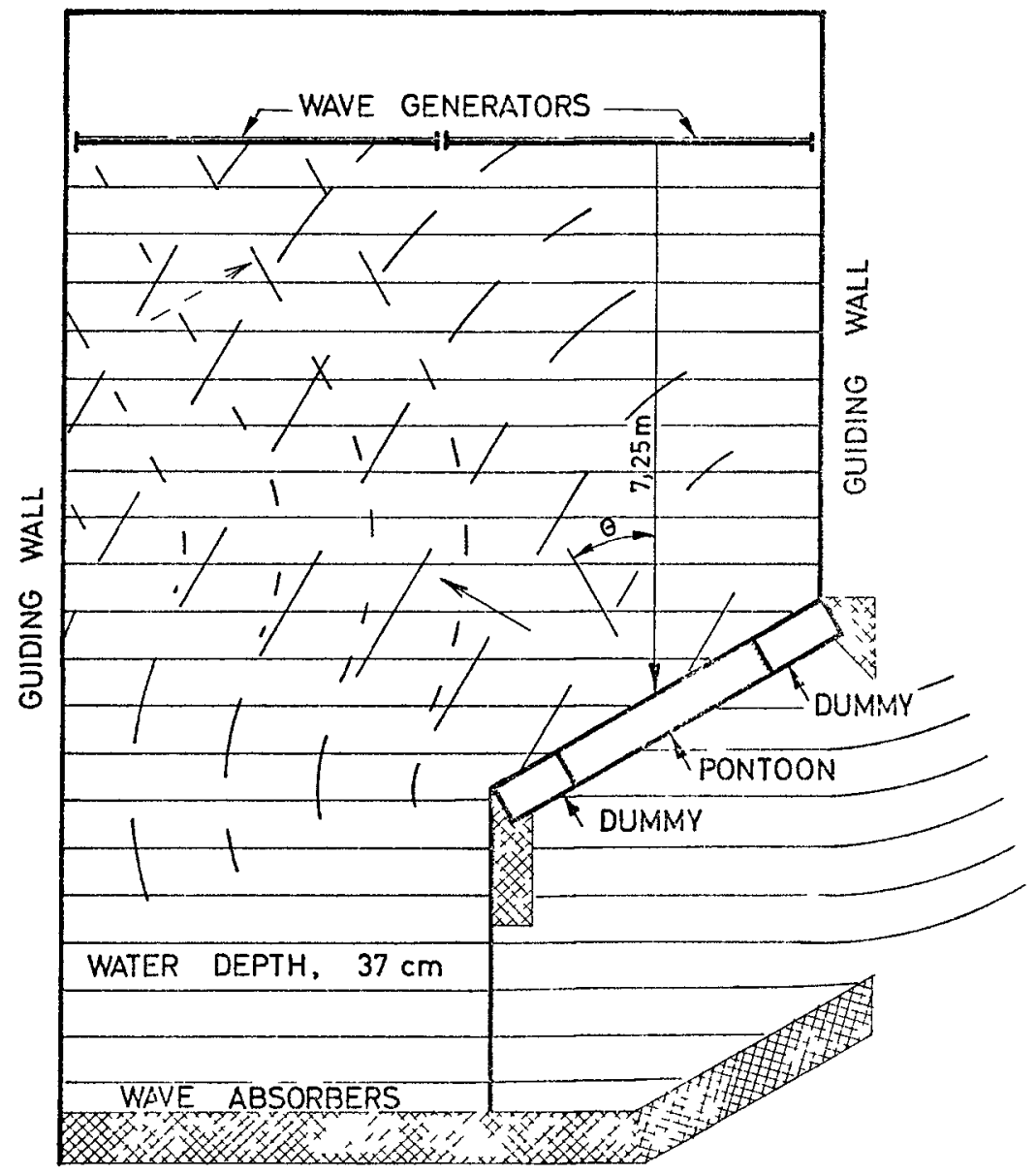

FIG 2 TEST ARRANGEMENT 


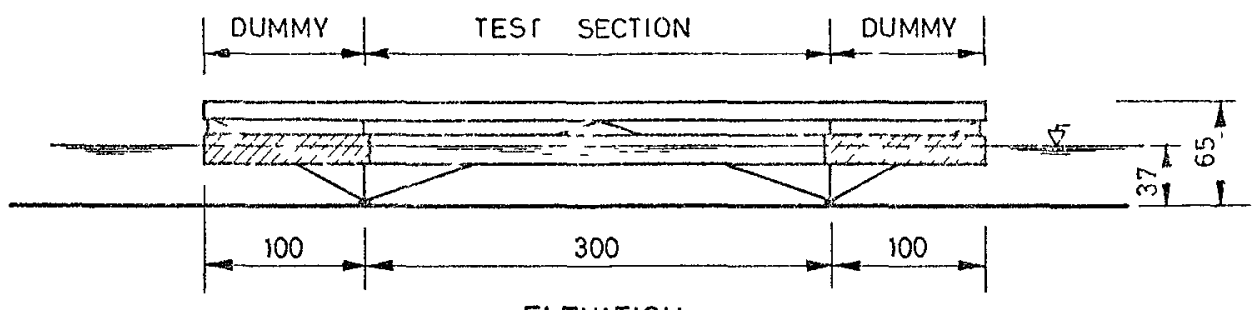

ELEVATION
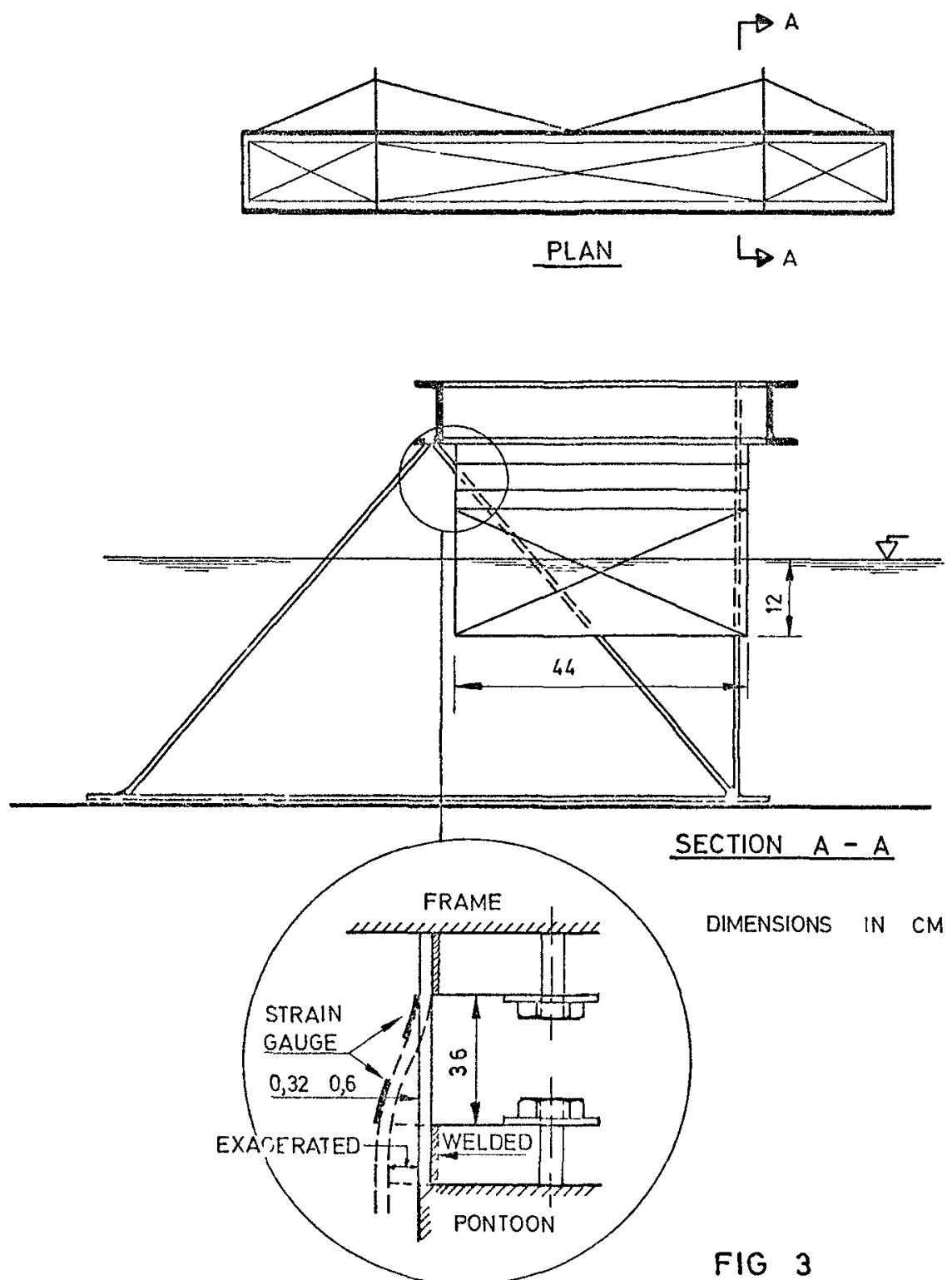


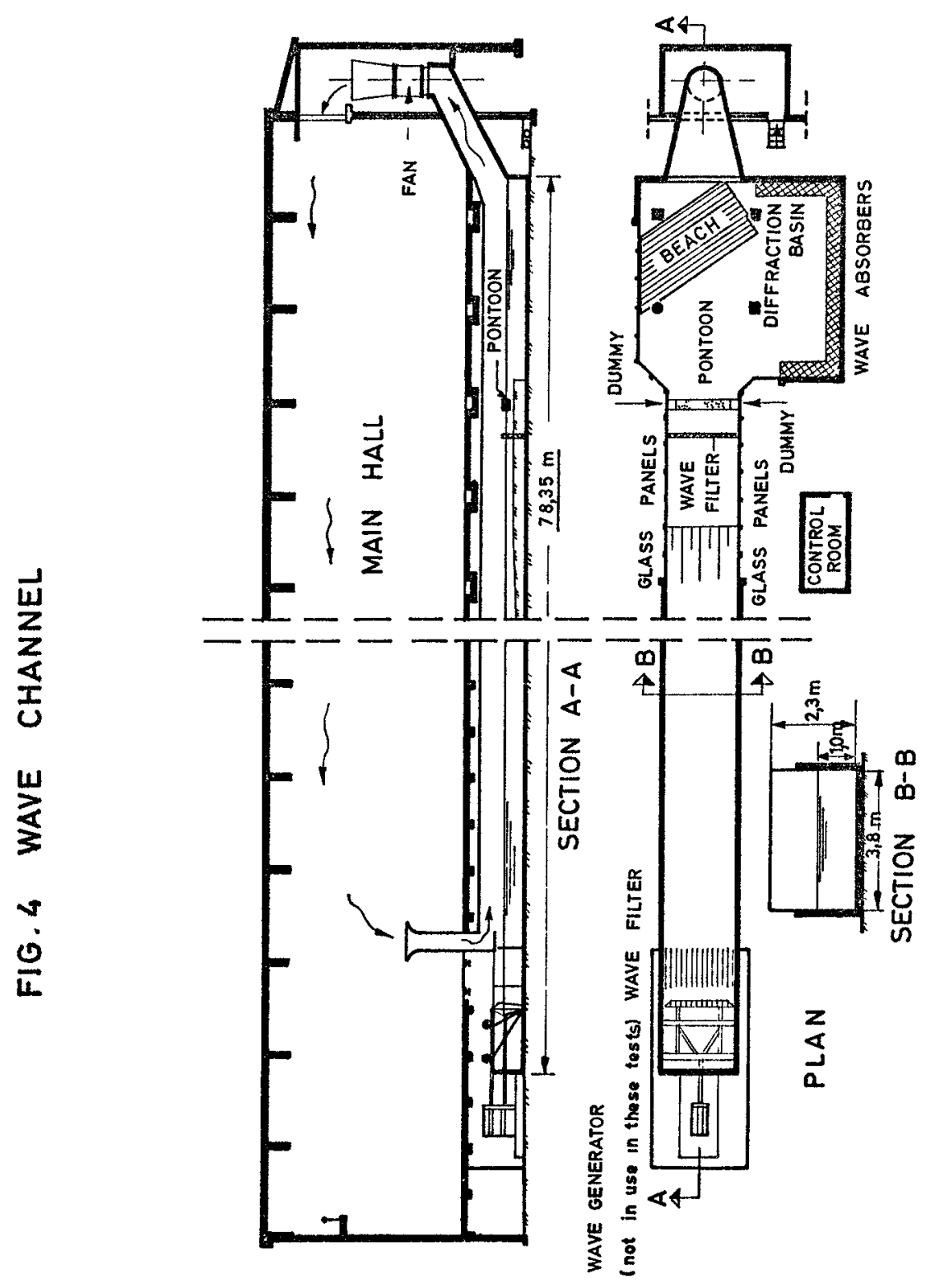



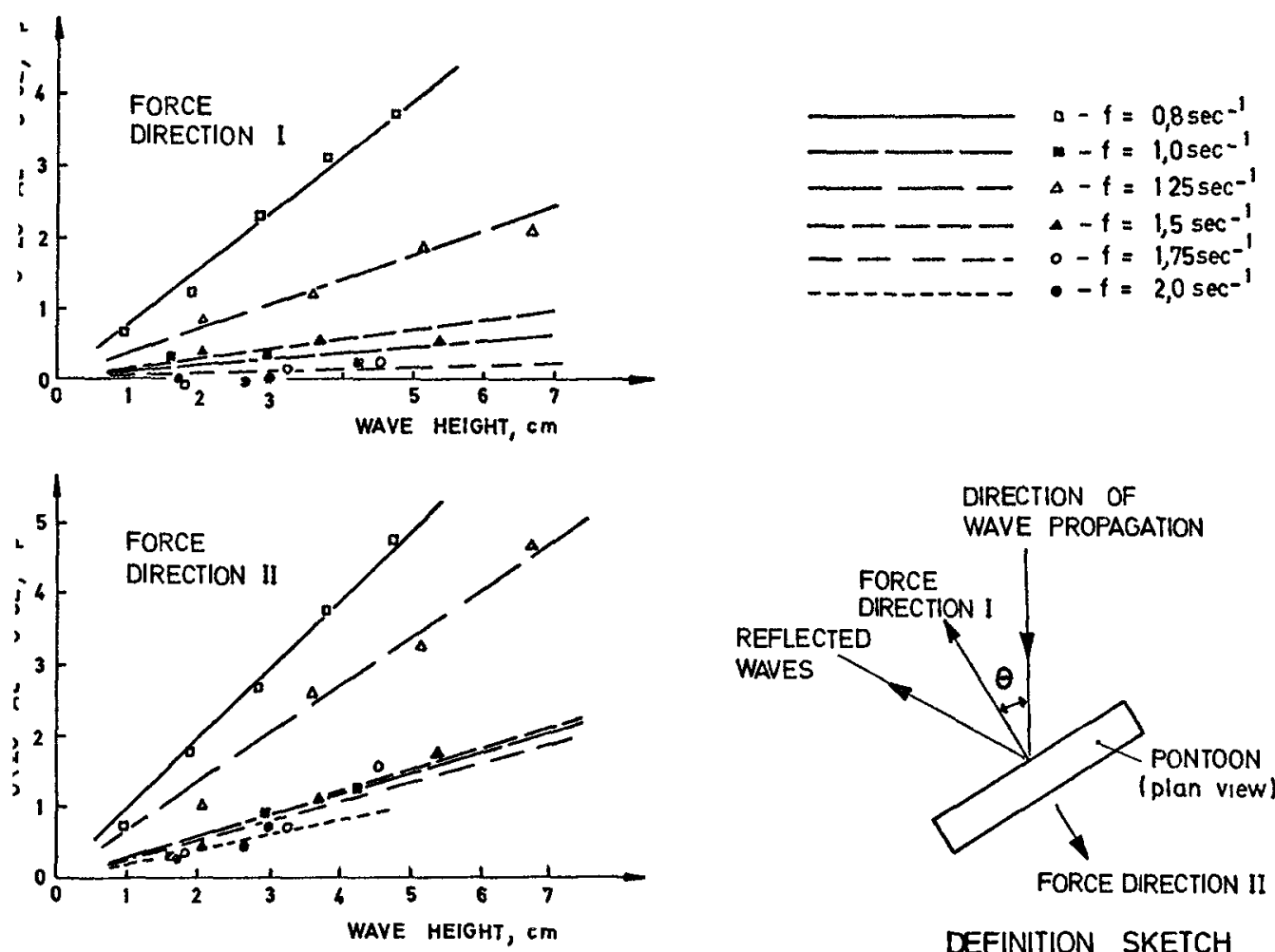

DEFINITION SKETCH

FIG 5 FORCE VS WAVEHEIGHT FOR $\theta=30^{\circ}$

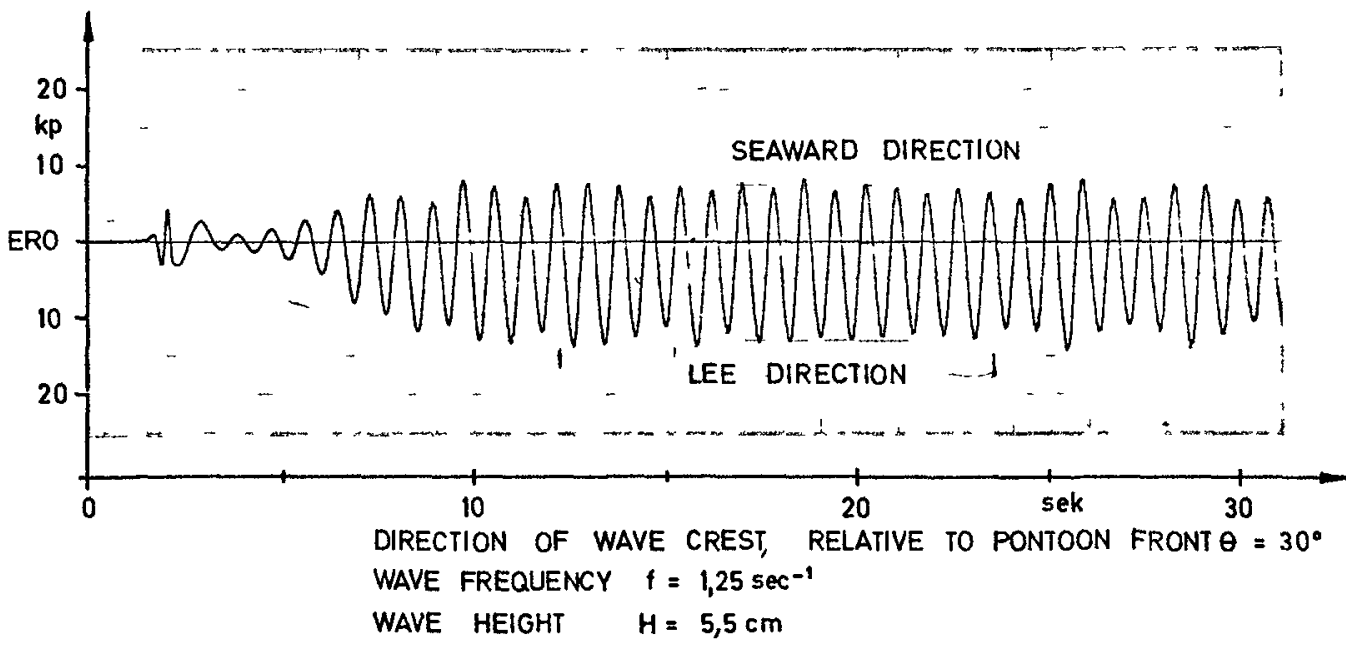

FIG 6 SAMPLE OF FORCE RECORD (regular waves) 
a

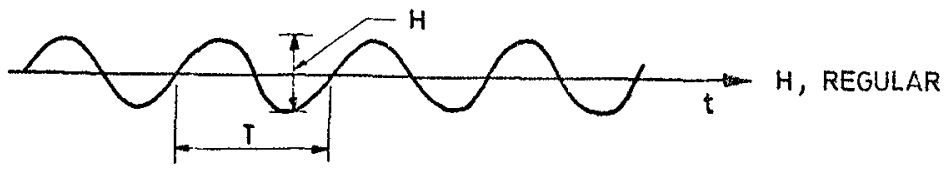

b

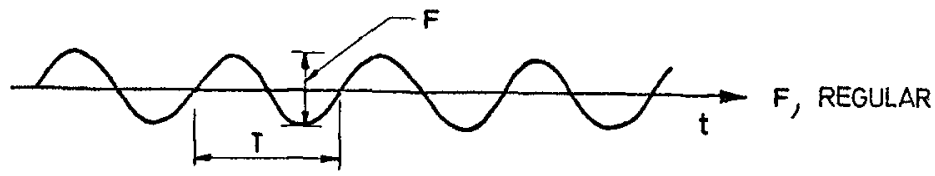

C
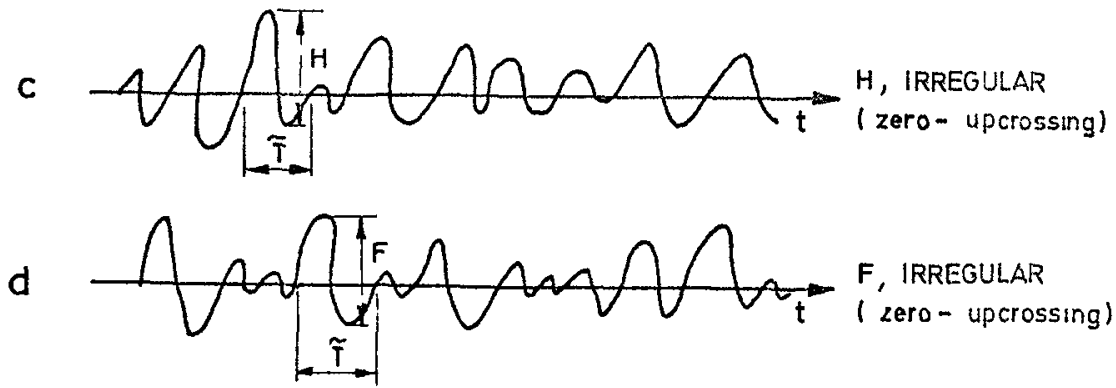

$H=$ WAVE HEIGHT
$F=$ FORCE

$T=$ PERIOD

$\tilde{T}=$ APPARENT PERIOD

FIG 7 DEFINITION SKETCHES

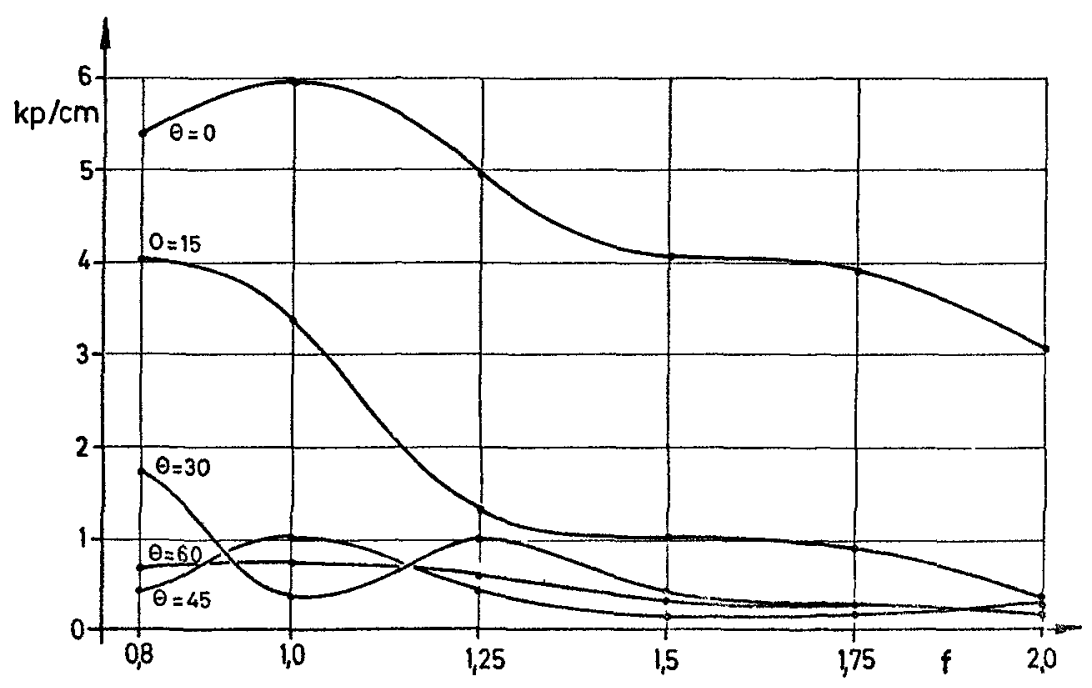

FIG 8 THE TRANSFER FUNCTION $K(\theta, f)$ 


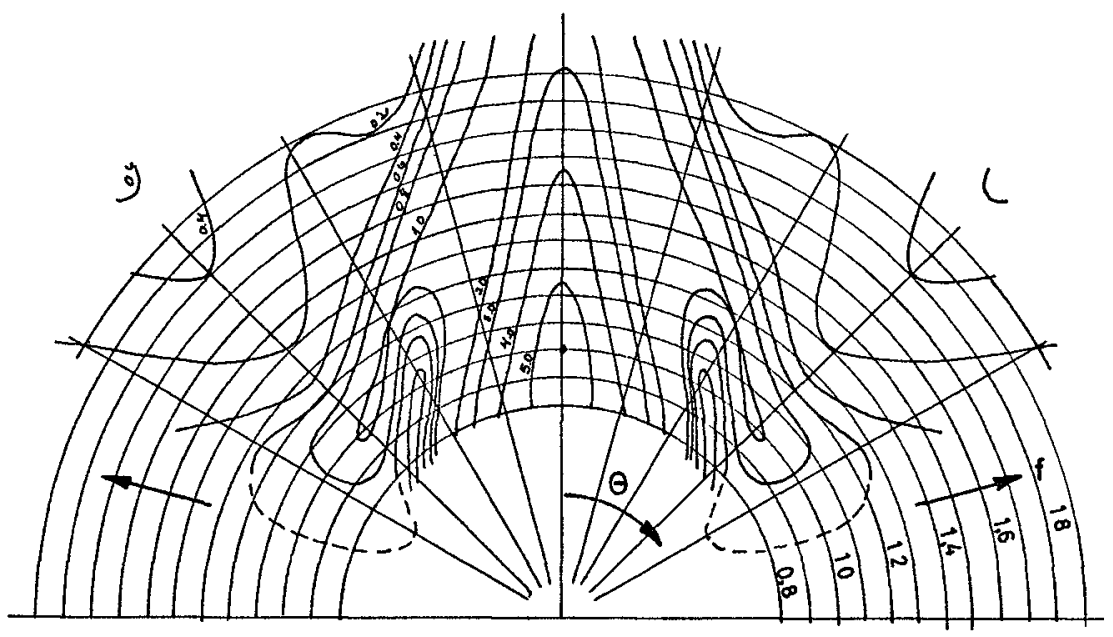

FIG 9 THE TRANSFER FUNCTION $K(\theta, f), \mathrm{kg} / \mathrm{cm}$, AS A CONTOUR MAP

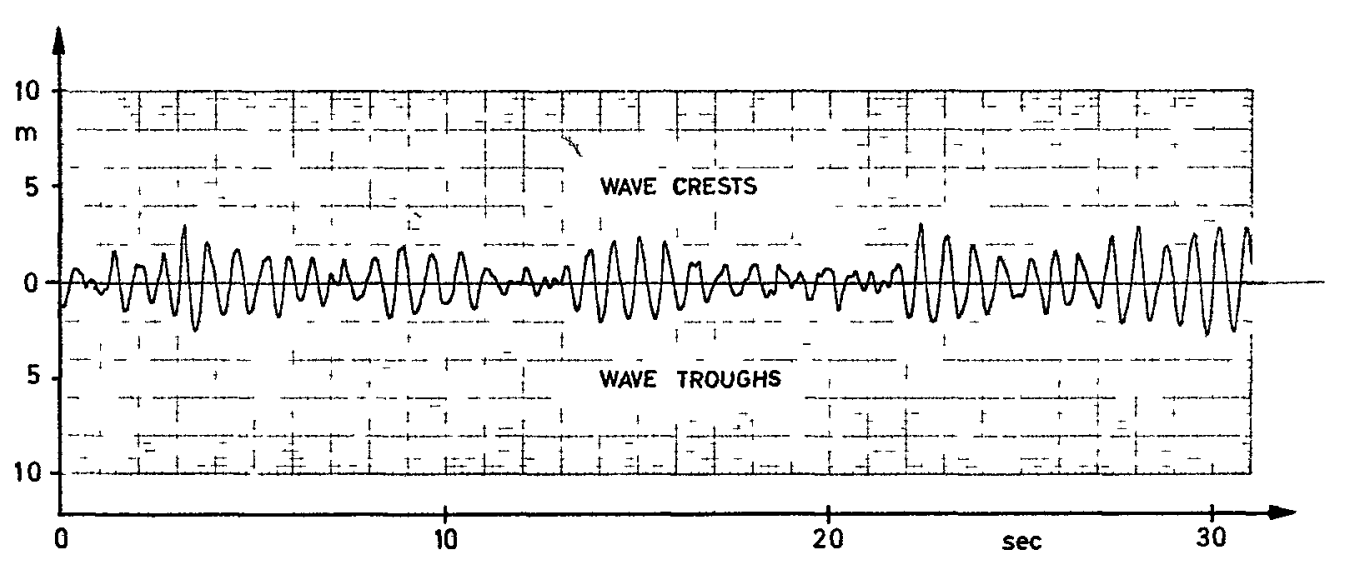

FIG 10 SAMPLE OF IRREGULAR WAVES 


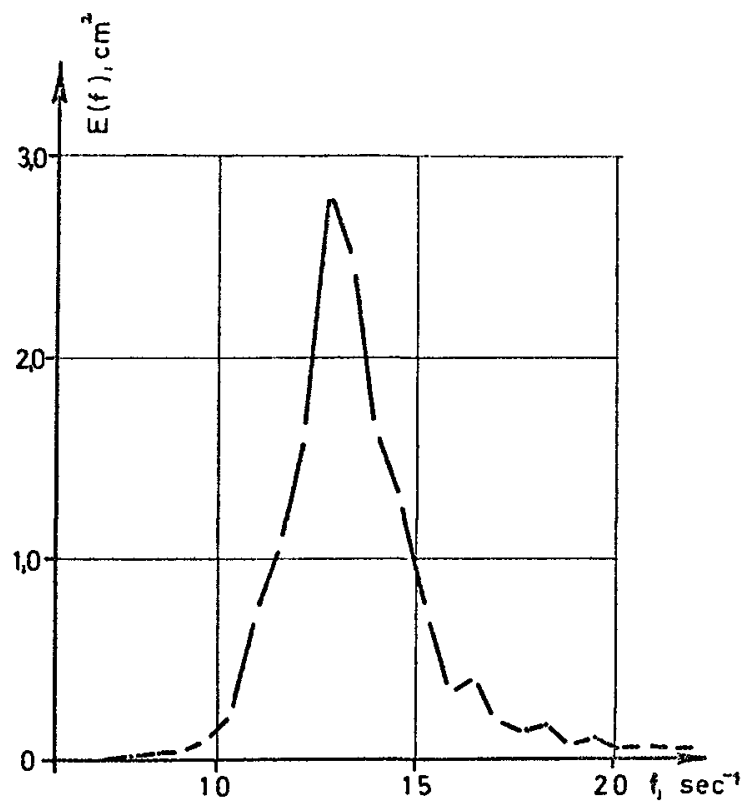

FIG 11 WAVE POWER SPECTRUM

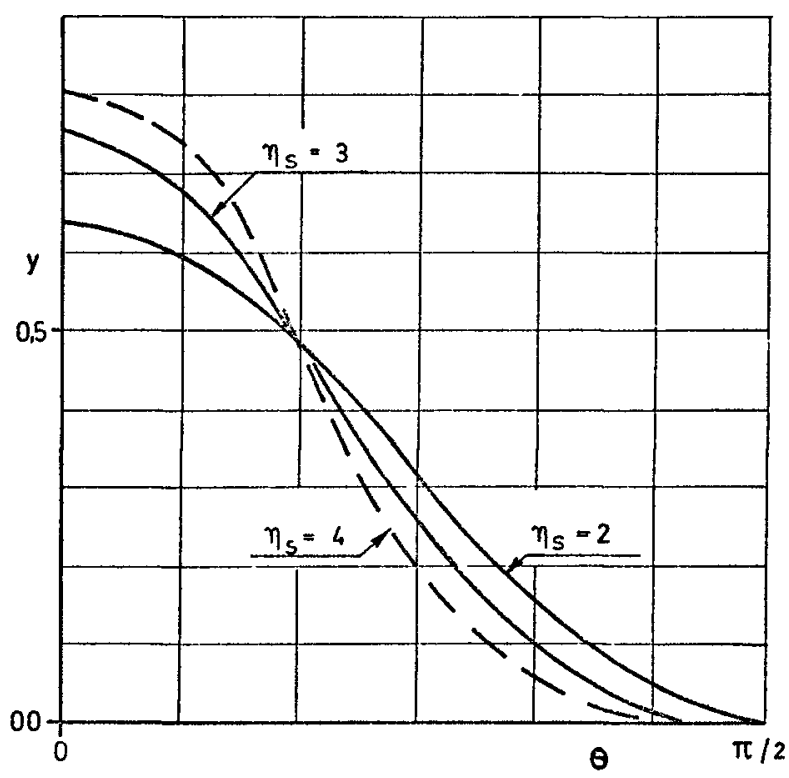

FIG 12 SPECTRUMA DIRECTIONALITY FUNCTION 


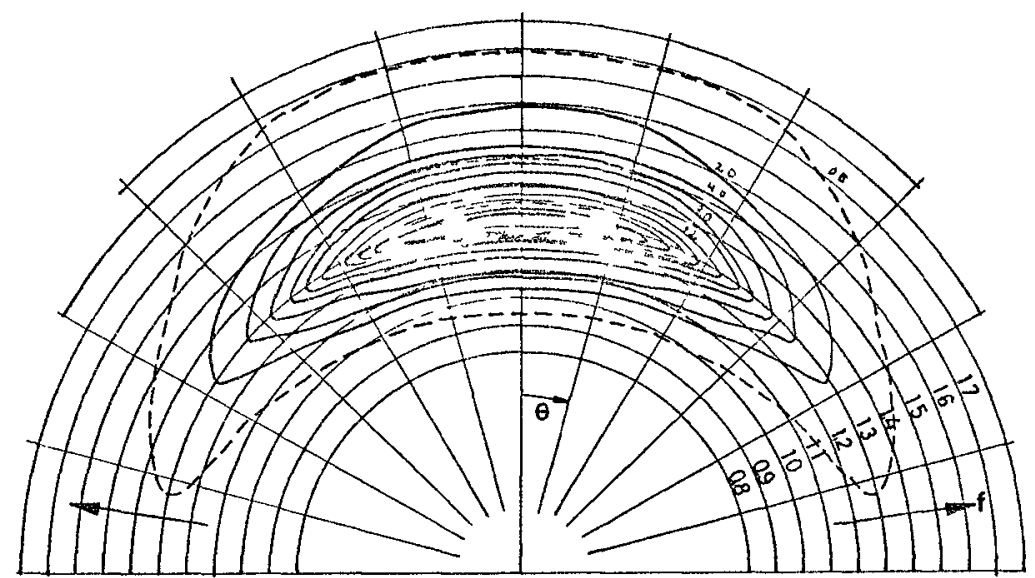

FIG 13 ESTIMATED DIRECTIONAL WAVE POWER SPECTRUM $\left(\cos ^{4} \theta-L A W\right)$

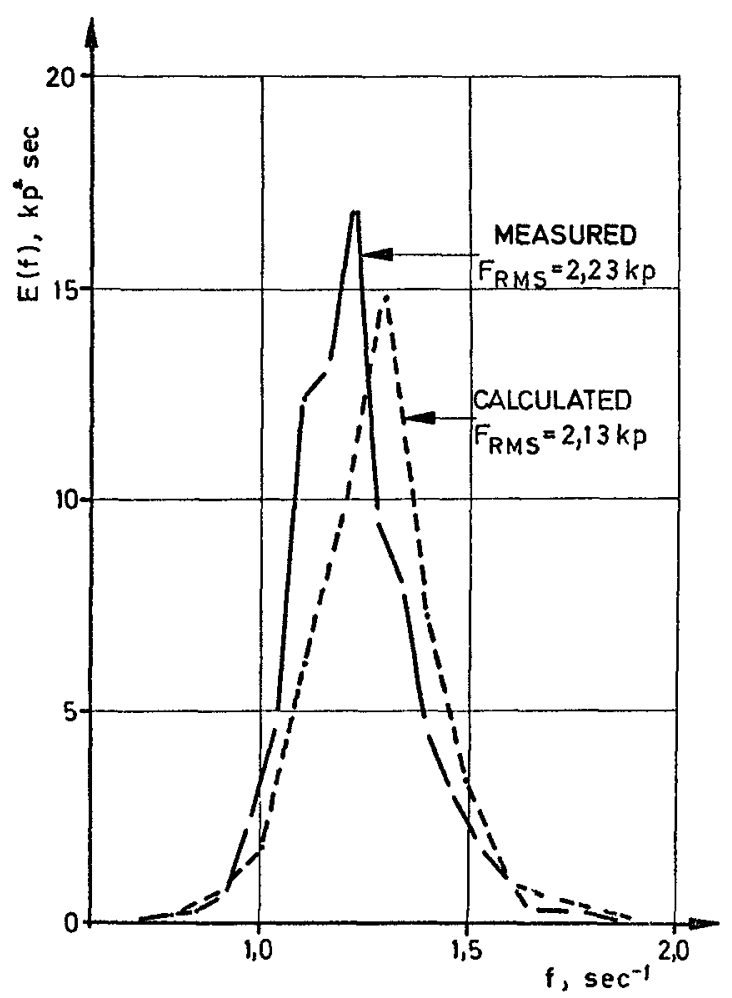

FIG 14 FORCE POWER SPECTRA 


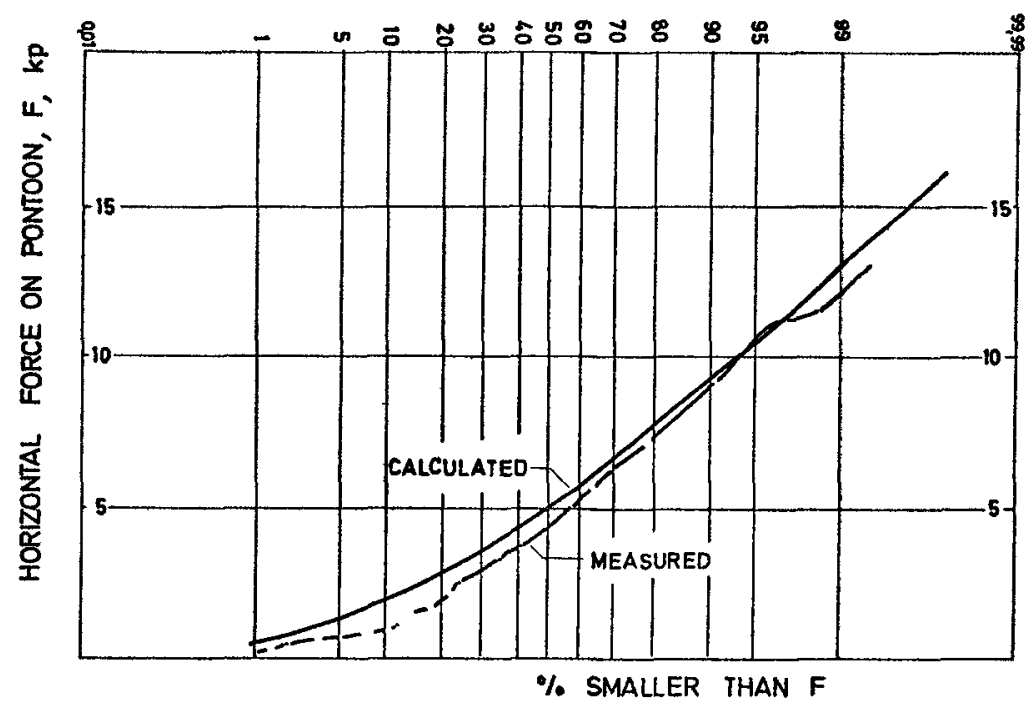

FIG 15 WAVE FORCE PROBABILITY DISTRIBUTIONS

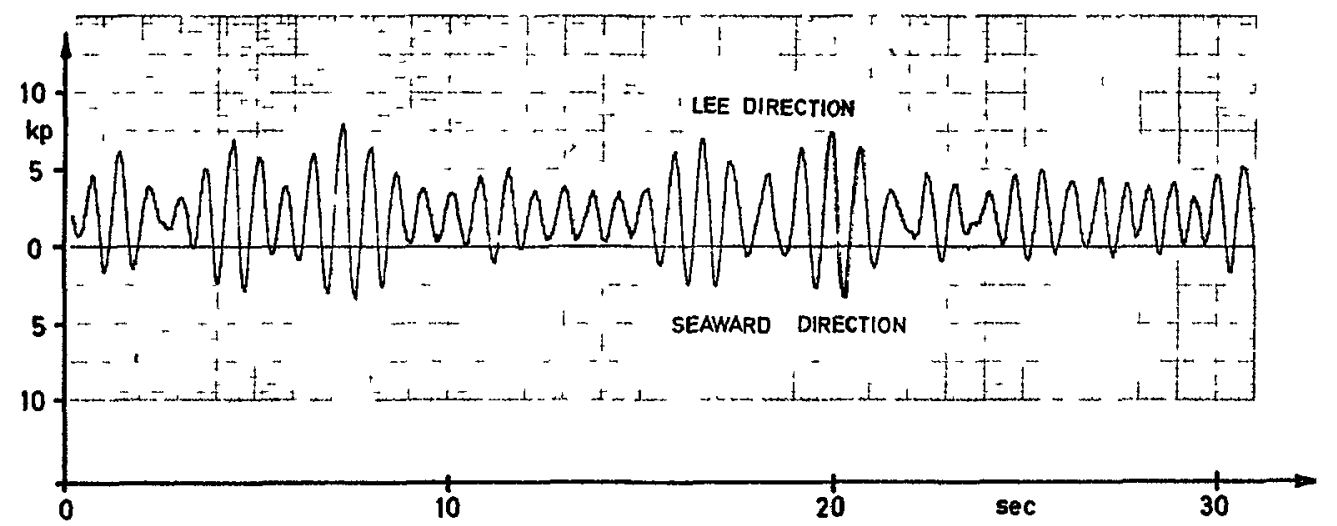

FIG 16 SAMPLE OF FORCE REGISTRATION IN IRREGULAR WAVES 
RESONANCE OF MOORED OBJECTS IN WAVE TRAINS

by

\author{
A.J. Hermans ${ }^{x}$ \\ Q.F.M. Remery
}

\title{
Abstract
}

In thls paper the resonance of moored object in wave trains is discussed and demonstrated with the help of some results obtalned from model tests with a right angled barge The measured results correspond well with a simple theory which calculates the slowly varying drifting force of regular wave trains.

I Delft Technical University Woriking at the Courant Institute of Mathematical Sciences Adviser of the N S.M.B

ix Head of the Wave and Current Laboratory of the N S.M.B. 


\section{Introduction}

Due to the enormous increase of tanker size during the last years, a lot of harbours could not be adapted quick enough to recelve these big ships.

That was, besides other reasons, why it became necessary to load and discharge large tankers in open sea near the coast Th1s caused a lot of problems, because now, the loading or unloading ship was fully exposed to wind, waves and current In the course of time many different mooring systems have been developed and tested, such as.

- single buoy mooring

- spread mooring

- turret mooring

- ships moored to plers both rotating and flxed

of these systems the single buoy mooring is probably the most used.

W1th this system the ship is moored by means of a bow hawser to a flosting buoy.

The buoy itself is anchored to the sea bottom by means of one or more anchor legs.

At the Netherlands ShIp Model Basin, extensive studies and model test programs have been executed concerning mooring of ships at sea.

A range of systems have been investigated and tested both as feasibility and as design studies

Normally model tests with moored vessels are conducted in Irregular waves

From the results of this type of tests 1t was found that high peak forces can occur also in light weather conditions.

It appeared that these high forces of ten occurred after a group of higher waves had passed the ship 
These waves gave the moored vessel a horizontal excursion which induced a long periodical oscillating motion. The period of this oscillating excursion equalled the natural period of the horizontal motion of the moored vessel in still water.

Superimposed on the long perlodical excursion, the moored object normally performed a h1gh frequency osclilating sway, surge and yaw motion.

The periods of these motions corresponded to the period of the waves.

Especially when the relationship between the elongation and the tension of a mooring line is progressively non-linear, the resultant horizontal motion could induce rather high peak rorces in the mooring lines.

A lot of ilterature is avallable concerning the vessel motions due to the higher frequency osclilating wave motion However, only little information is avallable about the lons periodical phenomena, which are important in studying mooring problems.

Therefore this paper is concerned with that problem

\section{The wave drifting force}

Considering the moored vessel as a mass spring system with a natural period $T_{n}$, 1t 18 known that b1s excursions w1ll be induced by oscillating forces with a period near that natural period.

For the large ahtps under consideration the natural period of the horizontal motion will be 50 to 200 seconds or more This indicates that the high frequency exciting forces due to the wave cannot induce important oscillating motions So there has to be another source of forces From the theory of Maruo [1] and the experiments of Ogawa [2] the phenomenon $1 \mathrm{~s}$ known of the steady drifting force on bodies in regular waves 
Th1s steady drifting foroe per unit length equals:

$$
\frac{1}{2} \rho g a^{2}
$$

In which:

$$
\begin{aligned}
\rho= & \text { specific mass density of water } \\
\mathbf{g}= & \text { acceleration due to gravity } \\
= & \text { amplitude of the wave reflected and } \\
& \text { scattered by the body }
\end{aligned}
$$

For the case that the body is restrained, no waves can be generated by the motions of the body, which means that there is only wave reflection

When the direction of the reflected waves is just opposite to the direction of the lncident waves the amplitude of the refleoted wave can be written, according to Haskind [3]. for deep water as:

$$
\mathbf{a}=\zeta_{\mathbf{a}} \mathrm{R}
$$

in which.

$$
\begin{aligned}
\zeta_{\mathrm{a}}= & \text { amplitude of incldent wave } \\
\mathrm{R}= & \text { reflection ooefflelent depending an } \\
& \mathbf{k} \mathrm{T} \text { (see F1gure } 5 \text { ) } \\
k= & \text { wave number }=\frac{2 \pi}{\lambda} \\
\lambda= & \text { wave length } \\
T= & \text { draft of body }
\end{aligned}
$$

This expression is exact for deep water and a vertical barrier of infintte length extending a distance $T(=$ draft) below the st11l water surface

From this it will be olear that when the wave height is not constant but varies slowly with a certain period, also the drifting force will vary with that period. 
In other words, when in an irregular sea wave groups are present which enccunter a moored body with a frequency in the nefghbourhood of the natural period of the mooring system, resonance phenomena may induce large slowly oscillating horizontal motions

In order to check this conception some model tests have been carried out with a simplified single point mooring system

\section{Description of the tests}

A vessel was moored by means of a single bow hawser to a fixed point

Generally the external conditions may be current, wind and waves, while the ship may have propulsion and steering capacity However, from the point of simpliclty only waves have been taken into consideration

The model represented a right angled barge of 107,000 metric tons displacement to a scale 180 (see Figure 1), in which also the main particulars are given for the full size barge

The bow hawser consisted of a linear spring with spring constant $C$ To avold that the bow hawser became slack, a counter mass $M$ was used as can be seen in Figure 1

The tests were executed in the Wave and Current Laboratory of the N S.M B which has a length of $60 \mathrm{~m}$ and a breadth of $40 \mathrm{~m}$ The water depth was $0915 \mathrm{~m}$, corresponding to 7320 meters for the full scale

The barge was positioned with its centre line parallel to the direction of propagation of the waves

In Figure 2 an example is given of the wave trains generated The perlod of the wave groups was kept constant during all tests and amounted to about 100 sec

By changing the spring constant of the bow hawser, the natural perlod of the surge motion of the moored barge could be varied 
Tests have been executed with 5 different springs, inducing natural surge pertods of $63,88,103,113$ and 134 seconds, in a number of wave trains different with regard to the mean pertod and the signiflcant wave helght

The wave train depleted in Figure 2 has a mean period of about 9 sec. and a signiflcant wave helght (double amplitude) of about $5 \mathrm{~m}$.

The wave spectrum and the distribution of the wave elevation is given in Figure 4.

During the tests the force in the bow hawser was measured and recorded on magnetic tape and paper chart,

In Figure 2 an example is given of the measured force in the bow hawser for two different springs

From this it will be clear that the forces are much higher for the spring which gives the system a natural period $T_{n}$ which almost equals the period of the wave groups

It is also easy to distingulsh the slowly varying force component on which a high frequency component has been superimposed The amplitude of the low frequency force has been plotted for each wave train as a function of the ratio

$$
\Lambda=\frac{\text { natural pertod of vessel's surge motion }}{\text { perlod of wave groups }=100 \mathrm{sec}}
$$

An example is given in Pigure 3 for two wave trains with a mean period of 9 seconds and a signiflcant helght of 5 and 8 meters The same type of resonance curves were found for wave trains with mean pertods of $7,11,13$ and 15 seconds 


\section{Theoretical considerations}

The generated wave trains can be considered to be bullt-up by two regular waves with different amplitudes and with only a small difference $\Delta \omega$ in frequency

Then the wave elevation $\zeta$ can be expressed as.

$$
\zeta=b_{1} \cos (k x-\omega t)+b_{2} \cos (k x+\Delta k x-(\omega+\Delta \omega) t+\Delta \varepsilon)
$$

This can be written in a slowly varying form.

$$
\zeta=a \cos (k x-\omega t)
$$

with $a=b_{1}+b_{2} e^{1(\Delta k x-\Delta \omega t+\Delta \varepsilon)}$

In which a is the envelope of the wave elevation or the slowly varying amplitude It was already shown that the drifting force is a function of the square of the wave amplitude a

$$
a^{2}=b_{1}^{2}+b_{2}^{2}+2 b_{1} b_{2} \cos (\Delta k x-\Delta \omega t+\Delta \varepsilon)
$$

Also notice that taking the square of the wave motion $\zeta$, one finds

$$
\begin{aligned}
\zeta^{2}=\frac{1}{2}\left(b_{1}{ }^{2}+b_{2}{ }^{2}\right) & +b_{1} b_{2} \cos (\Delta k x-\Delta \omega t+\Delta \varepsilon)+ \\
& + \text { high frequency components }
\end{aligned}
$$


Assuming that the heave and pitch motion of the barge can be neglected and that the waves will be reflected by the flat bow in a direction parallel to the direction of the incident waves, the drifting force $F_{d}$ on the barge, according to Haskind [3], can be expressed as

$$
\begin{aligned}
F_{d} & =\frac{1}{2} \rho g a^{2} R^{2} B \\
B & =\text { breadth of barge }
\end{aligned}
$$

In which:

$$
a^{2}=b_{1}^{2}+b_{2}^{2}+2 b_{1} b_{2} \cos (\Delta k x-\Delta \omega t+\Delta \varepsilon)
$$

From this expression it follows that there is a slowly oscillating drift force component with frequency $\Delta \omega$ and amplitude $\mathrm{F}_{\mathrm{da}}$.

$$
F_{d a}=\rho g R^{2} b_{1} b_{2} B
$$

The equation of motion of the mass spring system representing the moored ship can be written as

$$
M_{x} \quad x+N_{x} \quad x+C x=F_{d}
$$

in wh1ch

$$
\begin{aligned}
M_{\mathbf{x}}(\Delta \omega) & =\text { total mass of system } \\
& =M_{\text {ship }}+M_{\text {added }}+M_{\text {we ight }} \\
N_{\mathbf{x}}(\Delta \omega) & =\text { damping coefficient } \\
\mathrm{C} & =\text { spring constant } \\
\mathrm{F}_{\mathbf{d}} & =\text { drift force }
\end{aligned}
$$

$\mathrm{M}_{\mathrm{x}}$ and $\mathrm{N}_{\mathrm{x}}$ are functions of the frequency $\Delta \omega$ of the surge motion and have been determined from extinction tests 
Becauge the force in the bow hawser $F_{b}$ equals $C \times$ the amplitude $\mathrm{F}_{\mathrm{ba}}$ of the bow hawser force due to the slowly oscillating drifting force component $F_{d a} \cos (\Delta k x-\Delta \omega t+\Delta \varepsilon)$ can be calculated from the solution of the equation of motion

$$
F_{b a}=\frac{F_{d a} c}{\sqrt{\left(C-M_{x} \Delta \omega^{2}\right)^{2}+B^{2} \Delta \omega^{2}}}
$$

In Figure 3 the results of the calculations for two wave trains, with mean periods of about 9 seconds, have been plotted as a function of the non-dimensional frequency $\Lambda$

$$
\begin{aligned}
& \Lambda=\frac{2 \pi \sqrt{\frac{{ }_{x}{ }_{x}}{C_{x}}}}{T_{g}} \\
& T_{g}=\text { period of wave groups }=100 \mathrm{sec}
\end{aligned}
$$

The results of the calculations appear to be in a good agreement with the measured ones

For the wave trains with perlods of 11 and 13 seconds the difference between measurement and calculation appeared to be larger

\section{Discussion of the results}

From the results of the tests 1 is is clear that wave groups can induce resonance phenomena at moored ships

It has been 1llustrated by some simple calculations that the slowly varying drifting force can be determined from the square of the wave motion 
Also for 1rregular waves the square of the wave motion gives information about the square of the irregular slowly varying wave envelop, which is a measure for the drifting force Therefore, to obtain information about the occurrence of slowly varying drifting forces, one has to calculate the spectral density of the record representing the square of the wave motion

From the distribution of this spectral density in the low frequency range it can be seen whether there will be 1mportant drifting force components near the natural perlod of the moored vessel

This means that describing the sea state only by the spectral density distribution of the wave motion is not sufficlent to predict what may happen with a moored vessel at a particular location

What one really needs is the wave motion record 1 tself or the spectral density distribution of the square of the wave motion.

What has been mentioned up to now are the results of a not yet finished study

There are still a lot of 1 tems which have to be taken into consideration, 1ike.

- vessel's pitch and heave motions

- Influence of bow form

- Influence of restricted water depth

However, at this stage of the study two important conclusions have been obtained

- Wave groups can induce resonance phenomena on moored ships

- The normal wave helght spectrum does not provide sufficlent information to predict the behaviour of the moored vessel. 


\section{Literature}

[1] Maruo, $\mathrm{H}$, "The drift of a body floating on waves" Journal of Ship Research, Vol 4 Number 3, December 1960

[2] Ogawa, A, "The drifting force and moment on a ship in oblique regular waves" Publication no 31 of the Delft Shipbuilding Laboratory, Holland

[3] Hask1nd, M D , "The pressure of waves on a barrler" Inzhen $S$ b. 4, no 2, 147-160 (1948) see also "Encyclopedia of Phystes" Springer Verlag, Berlin 1960 "Surface waves" of Wehausen and Laltone sect 17 


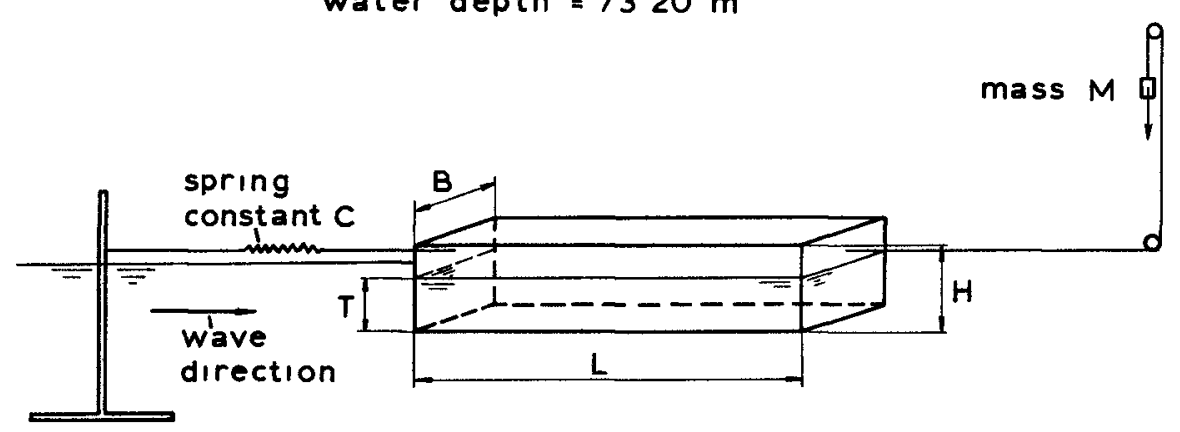

$\begin{array}{lll}\text { length } & L=18240 & \mathrm{~m} \\ \text { breadth } & B=4896 & \mathrm{~m} \\ \text { draft } & T=1200 \mathrm{~m} \\ \text { depth } & H=1920 & \mathrm{~m} \\ \text { displacement } & \nabla=107,163 \mathrm{~m}^{3} \\ \text { counter mass } & M=819 & \text { ton }\end{array}$

natural period of heave motion $\approx 1100 \mathrm{sec}$ natural period of pitch motion $\approx 1300 \mathrm{sec}$ natural period of roll motion $\approx 1135 \mathrm{sec}$ 


$$
\begin{aligned}
& \tilde{H}_{1 / 3}=\text { significant wave height }=5 \mathrm{~m} \\
& \tilde{f}^{1 / 3}=\text { mean wave period }=9 \mathrm{sec}
\end{aligned}
$$
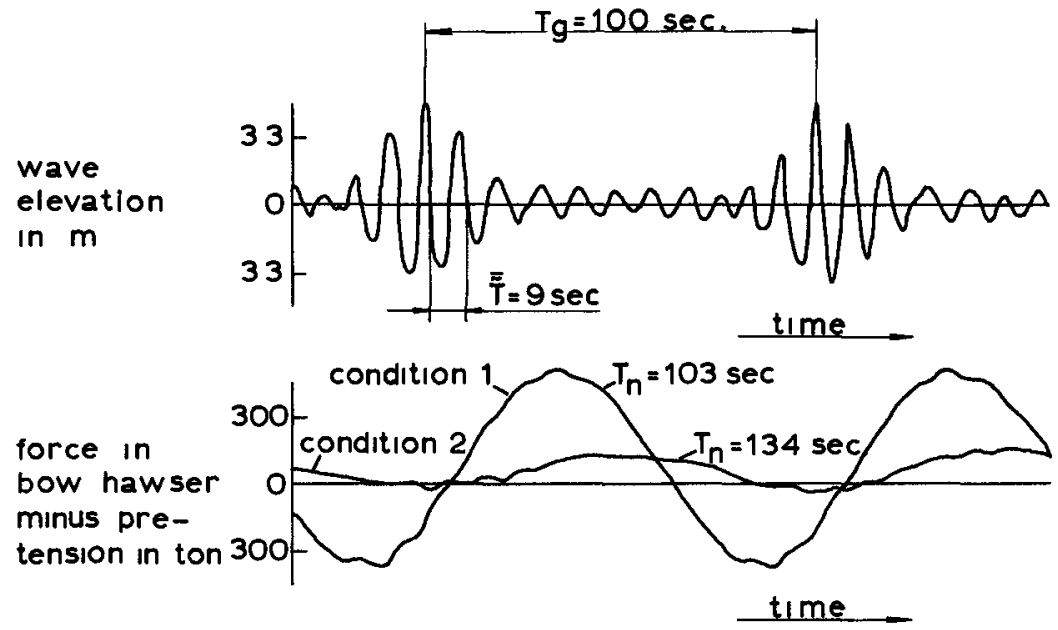

$T_{n}=$ natural period of vessel's surge motion

FIG 2 


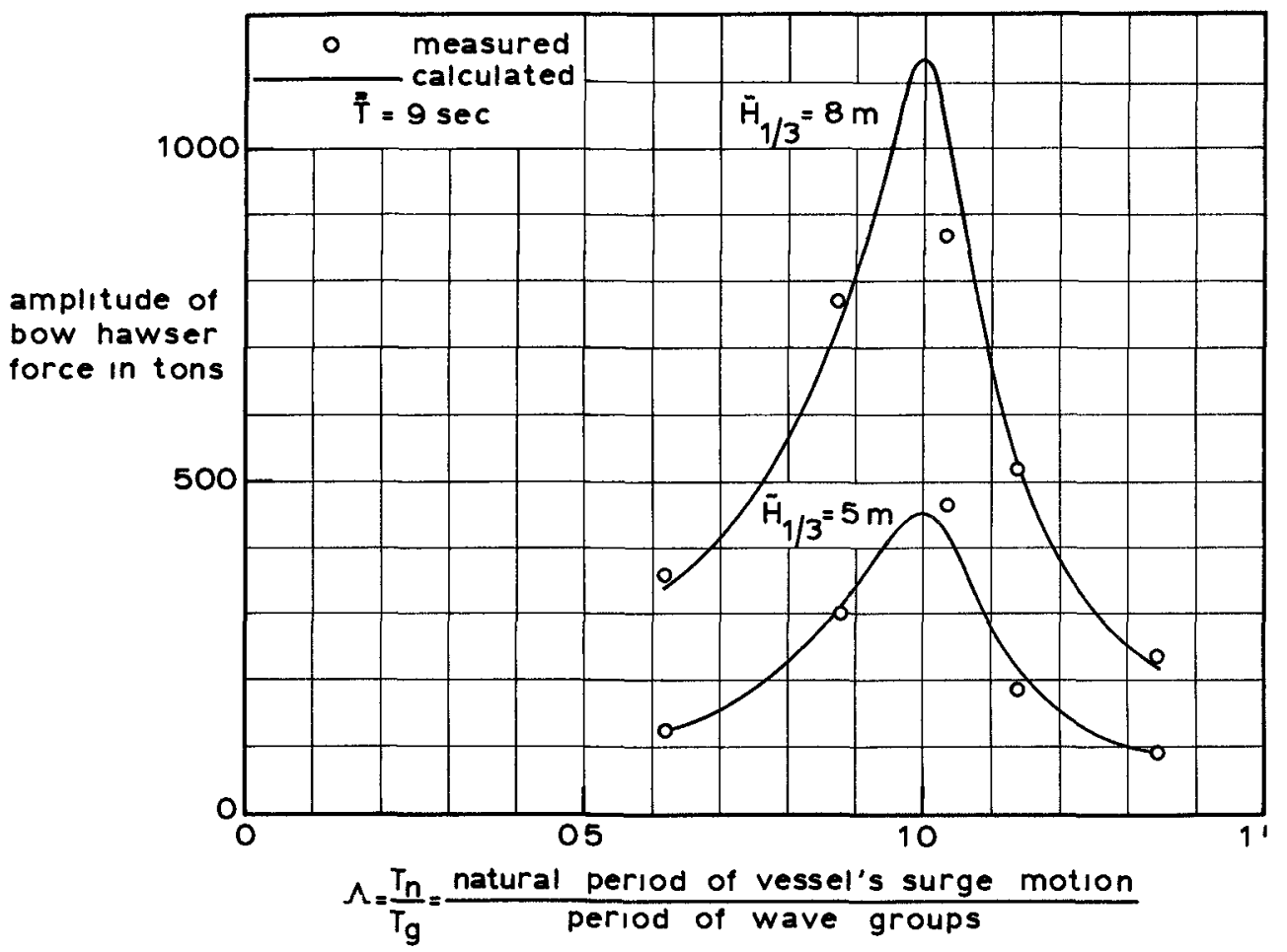

FIG 3 

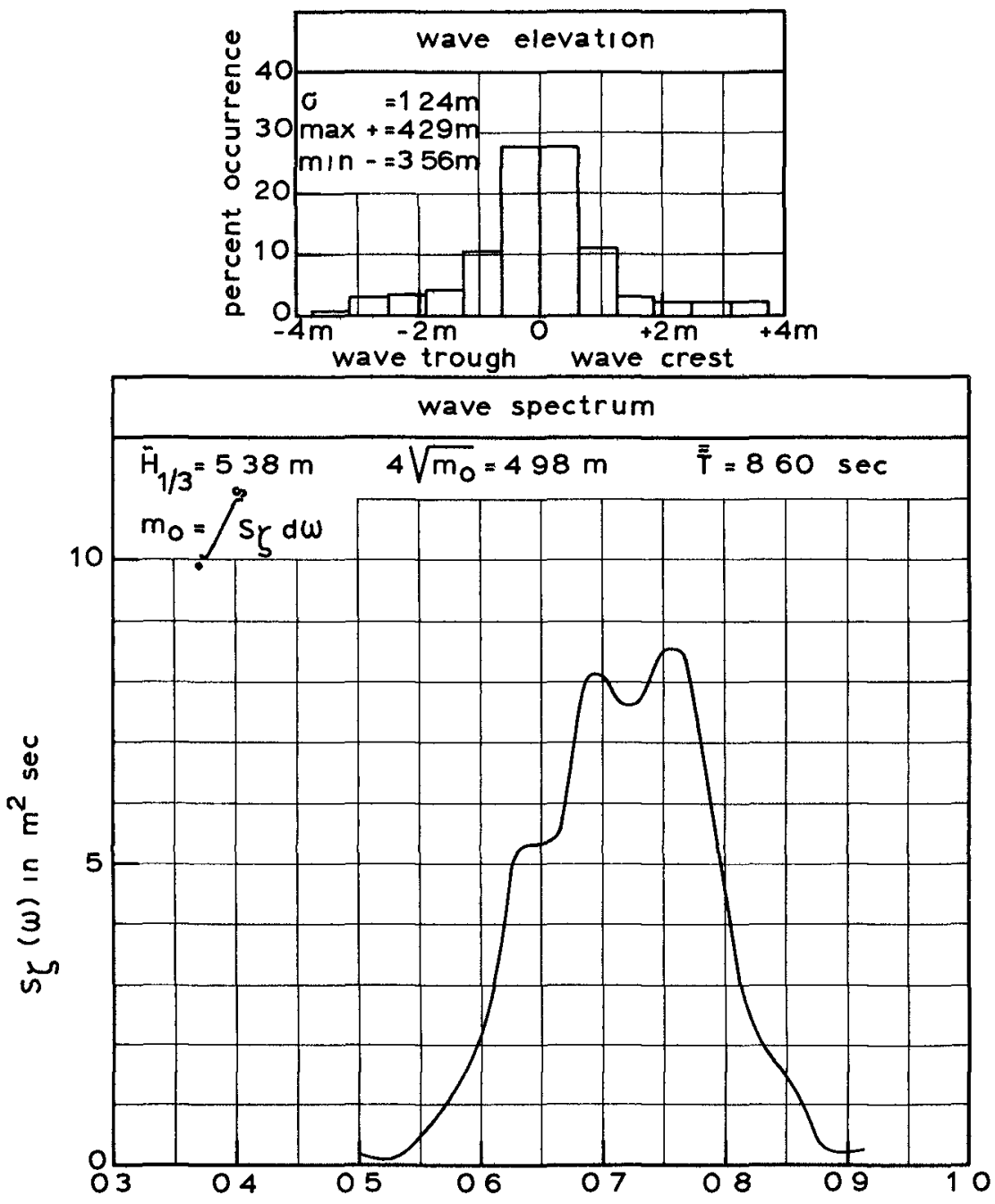

$\omega$ in rad $\sec ^{-1}$

FIG 4 
Reflection coefficient $R$ as a function of $k T$

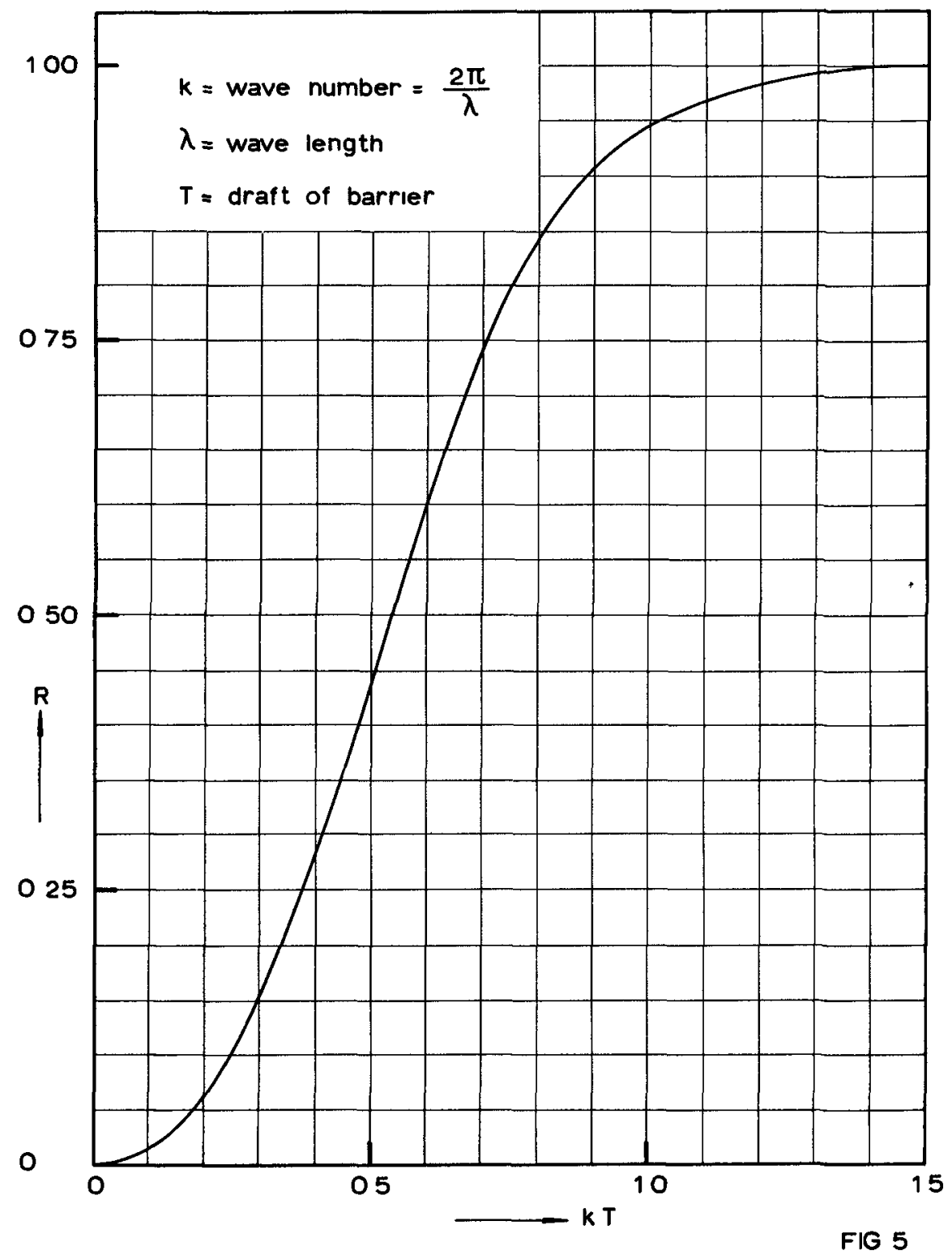




\title{
CHAPTER 103
}

\section{ON THE HYDRODYNAMIC FOR CES}

OF TWIN-HULL VESSELS

\author{
Shen Wang \\ Tetra Tech Inc \\ Pasadena, Calıforna
}

\begin{abstract}
A BSTRAC'T
The added mass and damping coefficients for semi- and fullysubmerged twin cylinders in vertical motion are determined as functions of the oscillation frequency, the cylinder spacing ratio, and the cylinder submergence ratio It has been found that resonance may occur in particular combinations of cylinder spacing and oscillation frequency at which the hydrodynamic inertial and damping characteristics deviate from the trend curves for the case of a single cylinder Justification of using the two-dimensional results to calculate motions of threedimensional twin-hull vessels is discussed It is suggested that, by means of strip theory approach, these results can be used to estumate the hydrodynamic forces for catamaran type vessels in pitch and heave motions
\end{abstract}

\section{INTRODUCTION}

This paper presents a method to estimate the vertical hydrodynamic forces for twin-hull vessels, including the catamaran type surface ship which has two hulls floating on the free surface and a certain type of floating platforms which have two parallel and closely spaced hulls submerged under the free surface

Analytical calculation of the mono-hull ship motions generally follows the method of strip theory approach The validity of using the strip theory approach to calculate motions of twin-hull vessels has not been completely established Objections certainly may raise, if one considers the overall width of the vessel as the beam, which is relatively too large for the strip theory to apply However one may feel different if he considers it as though there were only one hull plus a wall effect 
It is not the purpose of this paper to validify the strip theory for twinhull vessels, but through the availability of the twordimensional results for the twin-hull model, obtained from this and the previous analyses, investigations on motions of catamaran type vessels by means of strip theory approach may proceed

According to the strip theory, the hydrodynamic quantities, such as the added mass and damping coefficients, are estimated by making use of two-dimensional data of long cylinders For the present purpose, we consider a body having two identical, rigidly connected, circular cylinders As to the added mass and damping of the catamaran type surface vessels, the two cylinders are considered semi-submerged initially in the free surface Theoretical and experimental investigation of this problem has been given by Wang and Wahab [1] To complete the analysis, the present work considers the two cylinders being fully submerged The problem is formulated as a linearized boundary value problem in the theory of small amplitude waves Within this framework, a potential function is constructed by superimposing a series of various order singularities, and the solution is obtained through determining the singularity strength by means of satisfying the boundary conditions

Numerical results of the added mass and damping coefficients are presented For the convenience of discussion, the results of the sem1submerged case are also summarized and reviewed Finally, applications of these results to calculating forces on catamaran type vessels are discussed

\section{SUBMER GED TWIN CYLINDERS}

\section{Formulation of the Problem}

We consider two identical circular cylınders, each of radius a, rigidly connected with a spacing distance of $2 \mathrm{~b}$ between their center-line axes They are fully submerged and are forced to make small vertical harmonic oscillations about a mean level $f(>a)$ under the free surface The problem reduces to the special case of semi-submerged cylinders oscillation if $f=0$ The two cylinders are assumed to be infinitely long, and the resulting motion is two-dimensional

Taking the undisturbed free surface as the $x$-axis, a Cartesian coordinate system is defined as shown in Figure 1 The center line axes of the two cylinders are then $(b, f)$ and $(-b, f)$ Two sets of polar coordinates are employed with their origins located at the two cylinder centers, they are related to the Cartesian coordinates as follows

$$
\begin{aligned}
& x=b+r \sin \theta=-b+r^{\prime} \sin \theta^{\prime} \\
& y=f+r \cos \theta=f+r^{\prime} \cos \theta^{\prime} \\
& r=\left[(x-b)^{2}+(y-f)^{2}\right]^{1 / 2} \\
& r^{\prime}=\left[(x+b)^{2}+(y-f)^{2}\right]^{1 / 2}
\end{aligned}
$$


TWIN-HULL VESSELS

1703

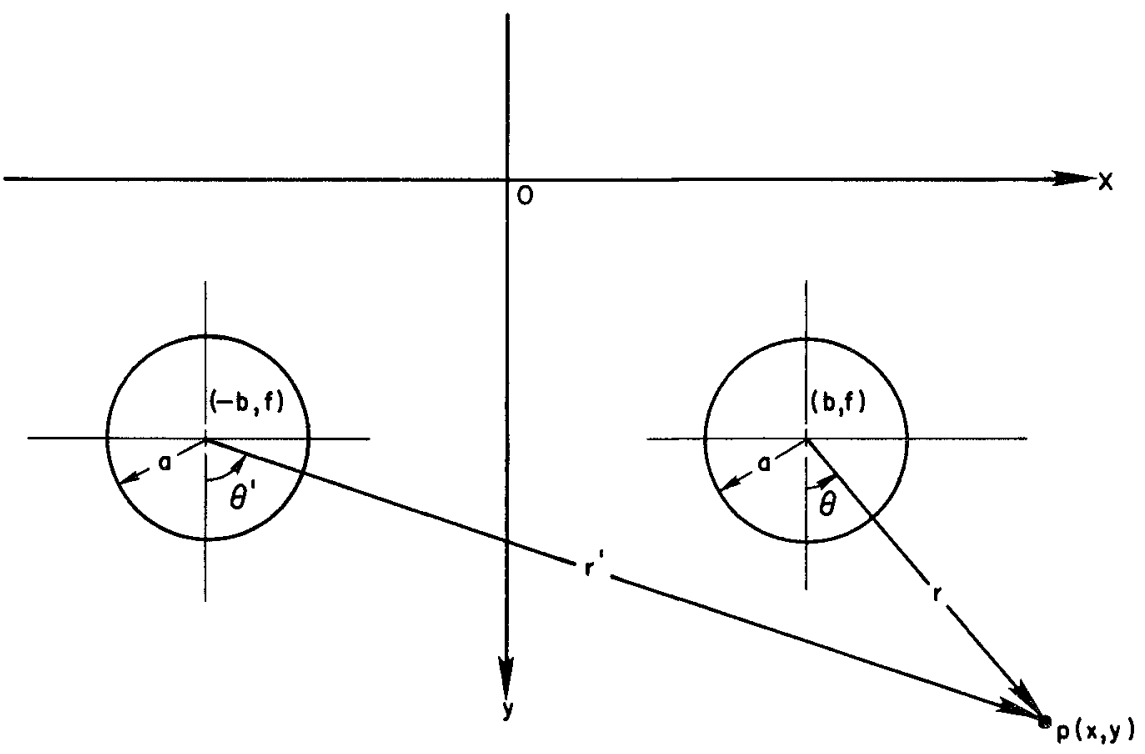

Figure 1 Coordinate systems 
The fluid is assumed invisid and incompressible, then the problem reduces to seeking a potential function for the described motion Let the motion be simple harmonic of period $2 \pi / \omega$ When the motion amplitude $\eta_{0}$ is sufficiently small, the velocity potential $\Phi(x, y, t)$ may be described by a series of singular function $\varphi_{n}(x, y)$, linearly superimposed in the following form

$$
\Phi(x, y, t)=a \omega \eta_{0} \operatorname{Re}\left[\sum_{r=0}^{\infty} A_{n} \omega_{n}(x, y) e^{-1 \omega t}\right]
$$

The function $\varphi_{n}(x, y)$ satisfies

$$
\begin{aligned}
& \text { (A) } \nabla^{2} \varphi_{n}=0 \text { in the fluid } \\
& \text { (B) } K \varphi_{n}+\frac{\partial \varphi_{n}}{\partial y}=0 \text { on the free surface }\left(K=\frac{\omega^{2}}{g}\right) \\
& \text { (C) } \frac{\partial \varphi_{n}}{\partial x}=0 \text { at } x=0 \\
& \text { (D) } L_{1 m}\left(\frac{\partial \varphi_{n}}{\partial x} \mp 1 K \varphi_{n}\right)=0 \text { as } x \rightarrow \pm \infty \\
& \text { (E) } \varphi_{n}=0 \text { as } y \rightarrow \infty
\end{aligned}
$$

The coefficient $A_{n}$ is a complex-valued number corres ponding to the strength of the $n^{\text {th }}$ order singularity it is a function of the cylinder spacing $b$, submergence $f$ and frequency $w$ These coefficients are to be determined by satisfying the kinematic boundary conditions on the cylinder surface, which will be described later

\section{Method of Solution}

The singular solution of the two dimensional Laplace equation (3) satisfying the boundary conditions (4), (6) and (7) may be written as a source function or its derivatives of any assigned order For a singularity located at (o f) the solutions have been given by Thorne [2] as follows

$$
\begin{aligned}
G_{0}(x, y)= & \log \frac{\rho}{\rho_{1}}+\oint_{0}^{\infty} \frac{e^{-k(y+f)}}{K-k} \cos k x d k \\
& -12 \pi e^{-K(y+f)} \cos K x
\end{aligned}
$$




$$
\begin{aligned}
G_{n c}(x, y)= & \frac{\cos n \sigma}{\rho n}+\frac{(-1)^{n-1}}{(n-1)^{\prime}} \oint_{0}^{\infty} \frac{K+k}{K-k} k^{n-1} e^{-k(y+f)} \cos k x d k \\
& +1 \frac{(-1)^{n}}{(n-1)^{1}} 2 \pi K^{n} e^{-K(y+f)} \cos K x \\
G_{n s}(x, y)= & \frac{\sin n \sigma}{\rho n}+\frac{(-1)^{n}}{(n-1)^{1}} \oiint_{0}^{\infty} \frac{K+k}{K-k} k^{n-1} e^{-k(y+f)} \sin k x d k \\
& +1 \frac{(-1)^{n-1}}{(n-1)^{\prime}} 2 \pi K^{n} e^{-K(y+f)} \sin K x
\end{aligned}
$$

Equations (8) and (9) are solutions for describing symmetrical motions, and Equation (10) for anti-symmetrical motion In these equations, $(\rho, \sigma)$ are the polar coordinates, measured from origin $(o, f)$ and $\rho_{1}$ is the radial distance from the image of $(o, f), 1$ e,$(o,-f)$

By expanding the integrals in Equations (9) and (10), one may show that any of the higher order potentials, $G_{n c}$ and $G_{n s}$, can be constructed by the following functions

$$
\begin{aligned}
& G_{1 c}(x, y)=\frac{\cos \sigma}{\rho}+K \oint_{0}^{\infty} \frac{e^{-k(v+f)}}{K-k} \cos k x d k \\
& -12 \pi \mathrm{Ke}^{-\mathrm{K}(\mathrm{y}+\mathrm{f})} \cos \mathrm{Kx} \\
& G_{1 s}(x, y)=\frac{\sin o}{\rho}-K \int_{0}^{\infty} \frac{e^{-k(y+f)}}{K-k} \sin k x d k \\
& +12 \pi K e^{-K(y+f)} \sin K x \\
& G_{n c}^{\prime}(x, y)=\frac{\cos n_{\sigma}}{\rho n}+\frac{K}{n-1} \frac{\cos (n-1)_{\sigma}}{\rho^{n-1}} \\
& \text { \} } \mathrm{n}=2,3 \\
& G_{n s}^{1}(x, v)=\frac{\sin (n+1) \sigma}{\rho n+1}+\frac{K}{n} \frac{\sin n_{0}}{\rho n}
\end{aligned}
$$

The functions $G_{l c}$ and $G_{l s}$ represent, respectively, the vertical and the horizontal dipoles, combining with appropriate wave function to satisfy the free surface condition (4) and the wave radiation condition (6) Each $t \in r m$ in these equations satisfies the infinite depth condition (7) The functions $G_{n c}^{\prime}$ and $G_{n s}^{\prime}$ are wave-free potentials, they represent only local fluid motion which decays rapidiy at a distance and yields no waves at infinity 
To derive the potential function $\varphi_{n}(x, y)$, one may construct it by using singular solutions corresponding to singularities located along the two cylinder axes, $(b, f)$ and $(-b, f)$, so as to satisfy the symmetry condition, Equation (5) This may be obtained as follows

$$
\begin{aligned}
& \omega_{o}(x, y)=G_{0}(x-b, y)+G_{o}(x+b, y) \\
& \omega_{1 c}(x, y)=G_{1 c}(x-b, y)+G_{1 c}(x+b, y) \\
& \omega_{1 s}(x, y)=G_{1 s}(x-b, y)-G_{1 s}(x+b, y) \\
& \omega_{n c}(x, y)=G^{1}{ }_{n c}(x-b, y)+G^{1}{ }_{n c}(x+b, y) \\
& \omega_{n s}(x, y)=G_{n s}^{\prime}(x-b, y)-G^{\prime}{ }_{n s}(x+b, y)
\end{aligned}
$$

Substituting Equations (8), (11), (12), (13) and (14) 1nto (15) through (19) and invoking the following identities [3]

$$
\begin{aligned}
& \frac{\cos n \theta}{r^{n}}=\frac{1}{(n-1)^{1}} \int_{0}^{\infty} k^{n-1} e^{-k(y-f)} \cos (x-b) a h \\
& \frac{\sin n \theta}{r^{n}}=\frac{1}{(n-1)^{\prime}} \int_{0}^{\infty} k^{n-1} e^{-k(y-f)} \sin (x-b) d k
\end{aligned}
$$

we obtain

$$
\begin{aligned}
& D_{0}(x, y)=\log \frac{r r^{\prime}}{r_{1} r^{\prime}}+4 \oint_{0}^{\infty} \frac{e^{-k(y+f)}}{K-k} \cos k b \cos k x d k \\
& -14 \pi e^{-K(y+f)} \cos K b \cos K x \\
& D_{1 C}(x, y)=4\left\{\int_{0}^{\infty} e^{-k y} \sinh k f \cos k b \cos k x d k\right. \\
& \left.+K \oiint_{0}^{\infty} \frac{e^{-k(y+f)}}{K-k} \cos k b \cos k x d k\right\} \\
& -14 \mathrm{TT} \mathrm{e}^{-K(\mathrm{y}+\mathrm{f})} \cos \mathrm{Kb} \cos K \mathrm{X} \\
& \omega_{1 s}(x, y)=-4\left\{\int_{0}^{\infty} e^{-k y} \cosh k f \sin k b \cos k x d k\right. \\
& -K \int_{0}^{\infty} \frac{e^{-k(y+f)}}{K-k} \sin k b \cos k x d k j \\
& -14 \pi K e^{-K(y+f)} \sin K b \sin K x
\end{aligned}
$$


$\omega_{n c}(x, y)=\frac{4}{(n-1)^{1}} j_{0}^{\infty}(k+K) k^{n-2} e^{-k y} \begin{aligned} & \cosh k f \\ & \sinh k f\end{aligned} \cos k b \cos k x d k, n=\begin{gathered}e v e n \\ \text { odd }\end{gathered}$

$$
\omega_{n s}(x, y)=-\frac{4}{(n-1)^{\prime}} \int_{0}^{\infty}(k+K) h^{n-2} e^{-k y} \begin{aligned}
& \cosh k f \\
& \sinh k f
\end{aligned} \quad \sin k b \cos k x d k, \quad n=\begin{gathered}
\text { odd } \\
\text { even }
\end{gathered}
$$

If we put $A_{n} \omega_{n}=A_{n c} \varphi_{n c}+A_{n s} \varphi_{n s}$, we may write Equation (2) in a different form as follows

$$
\Phi(x, y, t)=a_{\omega} \eta_{0} \operatorname{Re}\left[A_{0} \omega_{0} e^{-1 \omega t}+\sum_{n=1}^{\infty}\left(A_{n c} \omega_{n c}+A_{n s} \omega_{n s}\right) e^{-1 \omega t}\right]
$$

To determine the expansion coefficients the velocity potential shall be forced to satisfy the normal velocity on the body surface

$$
\begin{array}{ll}
\frac{\partial \Phi}{\partial r}=\omega \eta_{0} \cos \omega t \cos \theta & \text { on } r=a \\
\frac{\partial \Phi}{\partial r^{\prime}}=\omega \eta_{0} \cos \omega t \cos \theta^{\prime} & \text { on } r^{\prime}=a
\end{array}
$$

assuming that the vertical velocity of the body is $\omega \eta_{0} \cos \omega t$

Since the two cylinders are identical, the boundary condition (28b) is equivalent to $(28 \mathrm{a})$ and need not be considered For the convenience of computation in this particular case, the potential functions (Equations (22) through (26)) are expanded into series about the cylinder axis (b f) The numerical computation then proceeds by formulating Equation (28a) 1nto a set of simultaneous algebraic equations, and the expansion coefficients can be determined by a collocation technique

\section{Added Mass and Damping Coefficients}

The steady state vertical force on a unit length of the cylinders is

$$
\left.F_{v}=-2 \rho a\right\rfloor_{0}^{2 \pi} \frac{\partial \Phi}{\partial t} \cos \theta d \theta
$$

One may write $F_{v}$ in terms of $\eta(t)\left(=\eta_{0} \sin w t\right)$ as

$$
F_{v}=-M_{v} \eta(t)-N_{V} \eta(t)
$$

then the added mass coefficient $\alpha$ and the nondimensional damping coefficient $\delta$ is defined as follows 


$$
\begin{aligned}
& \alpha\left(K a \frac{b}{a} \frac{f}{a}\right)=\frac{M_{v}}{2 \pi \rho a^{2}} \\
& \delta\left(K a \frac{b}{a}, \frac{f}{a}\right)=\frac{N_{v}}{2 \pi \rho \omega a^{2}}
\end{aligned}
$$

These coefficients are directly related to the potential function $\Phi$, using the expression given in Equation (2), one obtains

$$
\begin{aligned}
& \left.\alpha=\frac{1}{\pi} \operatorname{Re}-\sum_{n=0}^{\infty} \int_{0}^{2 \pi} A_{n}\left(K a, \frac{b}{a}, \frac{f}{a}\right) m_{n}\left(K a \frac{b}{a}, \frac{f}{a} \theta\right) \cos \theta d \theta\right\rfloor \\
& 0=\frac{1}{\pi} \operatorname{Im}\left[\sum_{n=0}^{\infty} J_{0}^{2 \pi} A_{n}\left(K a, \frac{b}{a}, \frac{f}{a}\right) \omega_{n}\left(K a, \frac{b}{a}, \frac{f}{a}, \theta\right) \cos \theta d \theta\right\rfloor
\end{aligned}
$$

\section{The Horizontal Force}

Because of the unsymmetrical flow over the cylinder surface, there is a horizontal force component induced between the two cylinders, exciting sideway oscillations For a unit length of the cylinder, this force is

$$
F_{h}=-\rho a \int_{0}^{2 \pi} \frac{\partial \Phi}{\partial t} \sin \theta d \theta
$$

or

$$
I_{h}=\sqrt{M_{h}^{2}+N_{h}^{2}} \sin (\omega t-\varepsilon)
$$

where

$$
\begin{aligned}
& M_{h}=\rho \omega^{2} \eta_{0} a \operatorname{Re}\left[\sum_{n=0}^{\infty} \int_{0}^{2 \pi} A_{n}\left(K a \frac{b}{a}, \frac{f}{d}\right) r_{n}\left(K a, \frac{b}{a}, \frac{f}{a}, \theta\right) \sin \theta d \theta\right] \\
& N_{h}=\rho \omega^{2} \eta_{0} a \operatorname{Im}\left[\sum_{n=0}^{-\infty} j_{0}^{2-} A_{n}\left(K a, \frac{b}{a}, \frac{f}{a}\right) \omega_{n}\left(K a, \frac{b}{a}, \frac{f}{a}, \theta\right) \sin \theta d \theta\right]
\end{aligned}
$$


In terms of the maximum hydrostatic force variation during the oscillation a horizontal force coefficient is defined as

$$
h=\frac{\sqrt{M_{h}^{2}+N_{h}^{2}}}{2 o g a \eta o}
$$

and the phase angle by which the inward horizontal force falls behind the oscillating displacement is therefore

$$
e=\tan ^{-1} \frac{N_{h}}{M_{h}}
$$

\section{RESULTS AND DISCUSSION}

\section{Added Mass and Damping Coefficients}

(1) Semi-submerged twin cylinders

Before entering into discussion of the results for the fully submerged case, we shall first review and summarize the results for the semi-submerged case, which has been obtained in [1] The theoretical results of the added mass and damping coefficients for this case are shown in Figures 2 and 3 These results are presented as a function of nondimensional frequency $\mathrm{Ka}$ with the hull spacing ratio b/a as a parameter

It is important to note that there exists a discrete set of characteristic frequencies at which the motion of the fluid between the two cylinders is strongly excited by the forcing oscillations These characteristic frequencies can be obtained from the following equation

$$
\mathrm{K}(\mathrm{b}-\mathrm{a})=\mathrm{n} \pi \mathrm{n}=12,
$$

They bear a correspondence to the natural modes of the motion of fluid between two vertical walls of $2(b-a)$ apart

The first few characteristic numbers of $\mathrm{Ka}$ in accordance with the normal modes for four cylinder space ratios are listed below

\begin{tabular}{|cc|c|c|c|c|}
\hline $\mathrm{b} / \mathrm{a}$ & $\mathrm{n}$ & 2 & 3 & 4 \\
\hline \hline 1 & 5 & $2 \pi$ & $4 \pi$ & $6 \pi$ & $8 \pi$ \\
2 & 0 & $\pi$ & $2 \pi$ & $3 \pi$ & $4 \pi$ \\
3 & 0 & $\pi / 2$ & $\pi$ & $3 \pi / 2$ & $2 \pi$ \\
4 & 0 & $\pi / 3$ & $2 \pi / 3$ & $\pi$ & $4 \pi / 3$ \\
\hline
\end{tabular}

Table I Characteristic frcquencies as a function of hull spacing ratio 


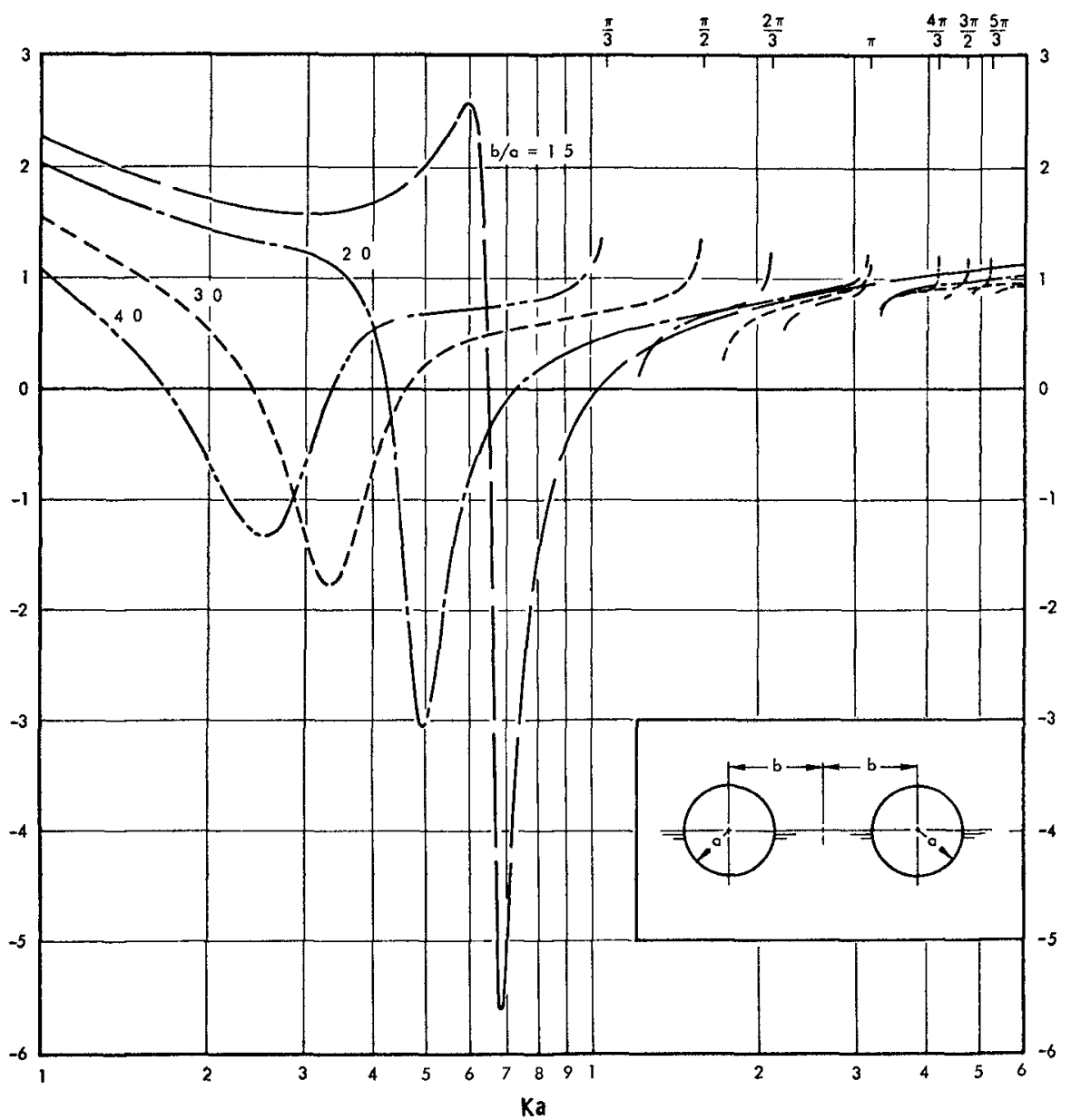

Figure 2 Added Mass Coefficient $\alpha$ as a Function of Ka Sem1-submerged Twin-cylinders, f/a $=0$ 


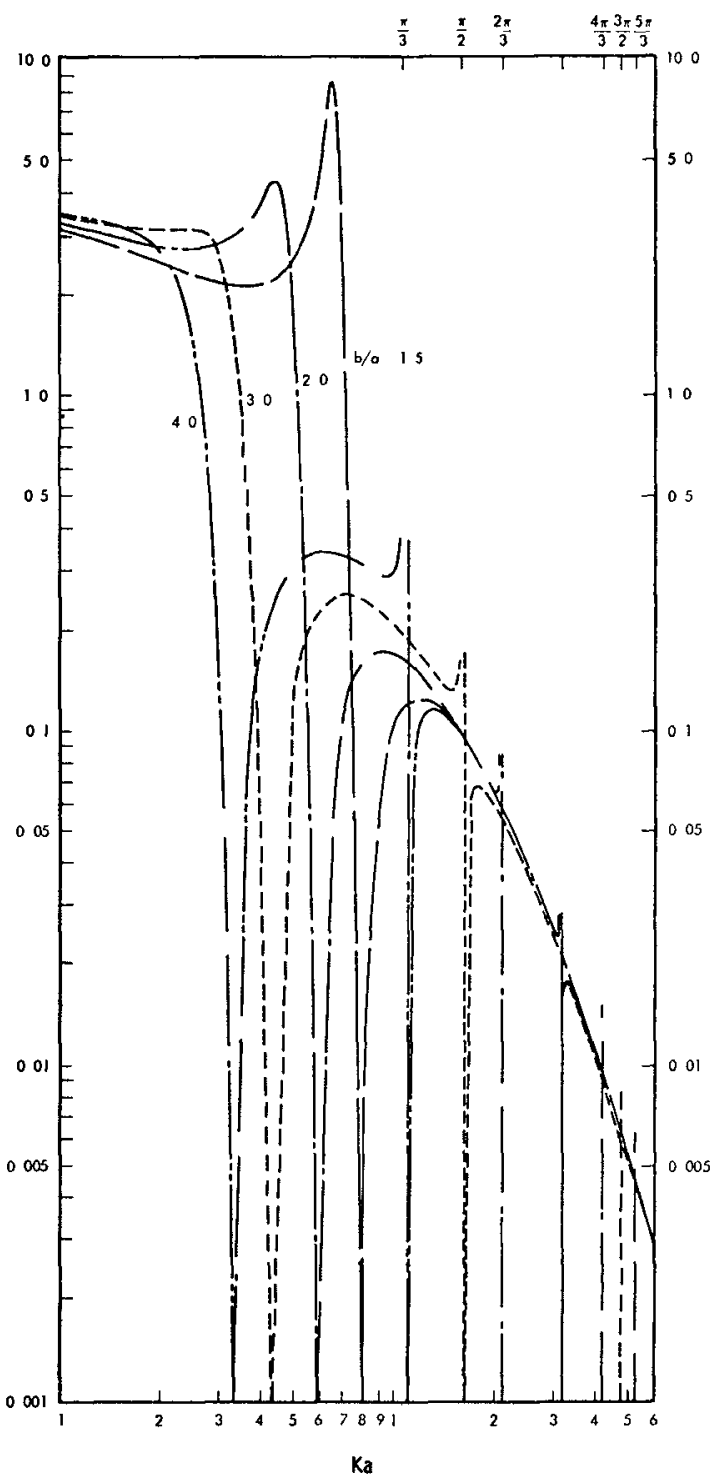

Figure 3 Nondimensional Damping Parameter $\delta$ as a Function of Ka Semi-submerged Twin-cylinders, $f / a=0$ 
From the graphs, it can be seen that the added mass and damping coefficients are markedly peaked at values of $\mathrm{Ka}_{\mathrm{a}} \mathrm{close}$ to those specified above, and as the oscillation passes across the characteristic frequency (a phase change taking place in the surface waves), the added mass becomes negative in a very narrow band of frequencies and the damping coefficient falls down to zero at a certain frequency

Besides these normal modes of resonance, one may find, from Figures 2 and 3 that there is another peak occurring between $\mathrm{Ka}=0$ and the first characteristic frequency This peak corresponds to the degenerate mode (or zero'th mode) at which the fluid displacement between the cylinders is approximately uniform and $180^{\circ}$ out of phase with that immediately outside the cylinders This peak occupies a rather wide range of frequencies, as compared to those which occur at highex modes

In regard to the resonance phenomena discussed above, it must be noted however that they are strictly two-dimensional characteristics All the resonance peaks, except that of the zero'th mode, would disappear if the hull beams vary along the length (regular catamaran hull) or if the band width of the input oscillation is large For a detailed discussion, one is referred to [1]

$$
\text { Fully-submerged twin cylinders }
$$

The computation has been done for four cylinder spacing ratios, $\mathrm{b} / \mathrm{a}=15,2025$ and 30 and four submergence ratios, $\mathrm{f} / \mathrm{a}=15,20$, 25 and 30 To demonstrate the spacing effect which arises from the interference between the two cylinders values of $\alpha$ and $\delta$ for different spacing ratios are superimposed and presented in Figures 4 through 11

Each plot is given for one given submergence ratio As the frequency of interest for practical application is mostly in the neighborhood of $\mathrm{Ka}=$ 10 , the presentation is limited to a frequency range up to $\mathrm{Ka}=20$

As discussed in the preceding case when the two cylinders are semisubmerged, there are certain characteristic frequencies, around which in a very narrow frequency band width the added mass deviates from its normal trend and the damping coefficient falls to zero while the radiated wave changes phase When the two cylinders are fully submerged, although the spacing distance still has a tendency to amplify the surface waves between the two cylinders around those characteristic frequencies as given in Table I, there is no physical boundary on the free surface to characterize the wave length so that there is no equivalent resonant phenomenon as described for the semi-submerged case except that the radiated wave does change 1ts phase around the neighborhood of those frequencies and that the damping falls to cero

Similar to the semi-submerged case, there is a peaked added mass and a zero damping occurring somewhere between $\mathrm{Ka}=0$ and the first characteristic frequency It must be noted that, for both semi- and fullysubmerged cases, these peaked added mass and deviated damping occupy a wide band of frequencies This is very important to the twin-hull vessels, as these peaks and deviations will not be completely removed either by the effect of three-dimensional hull form or by random input oscillations 


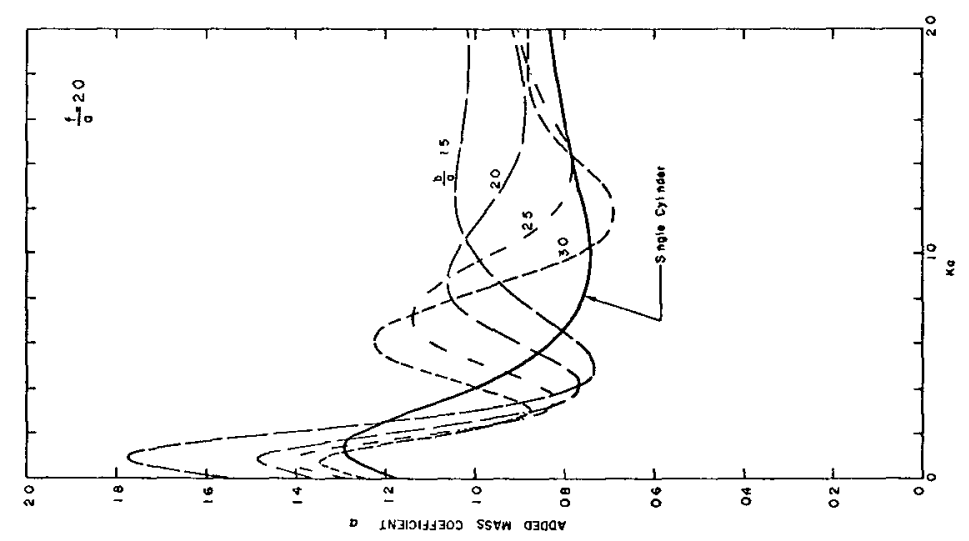

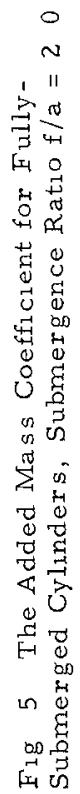

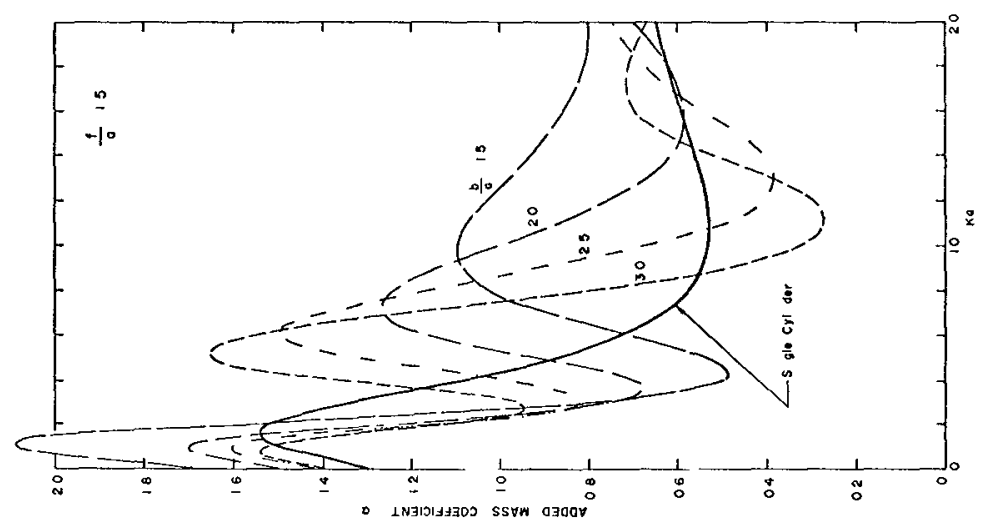

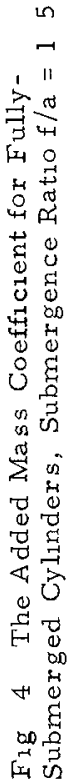




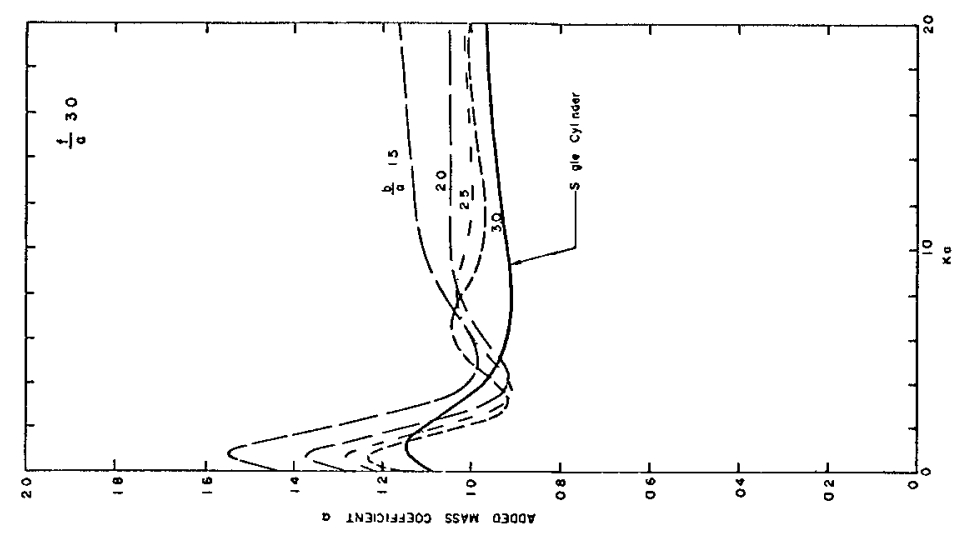

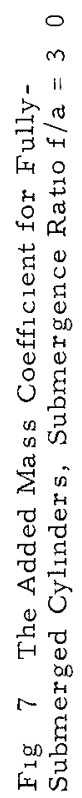

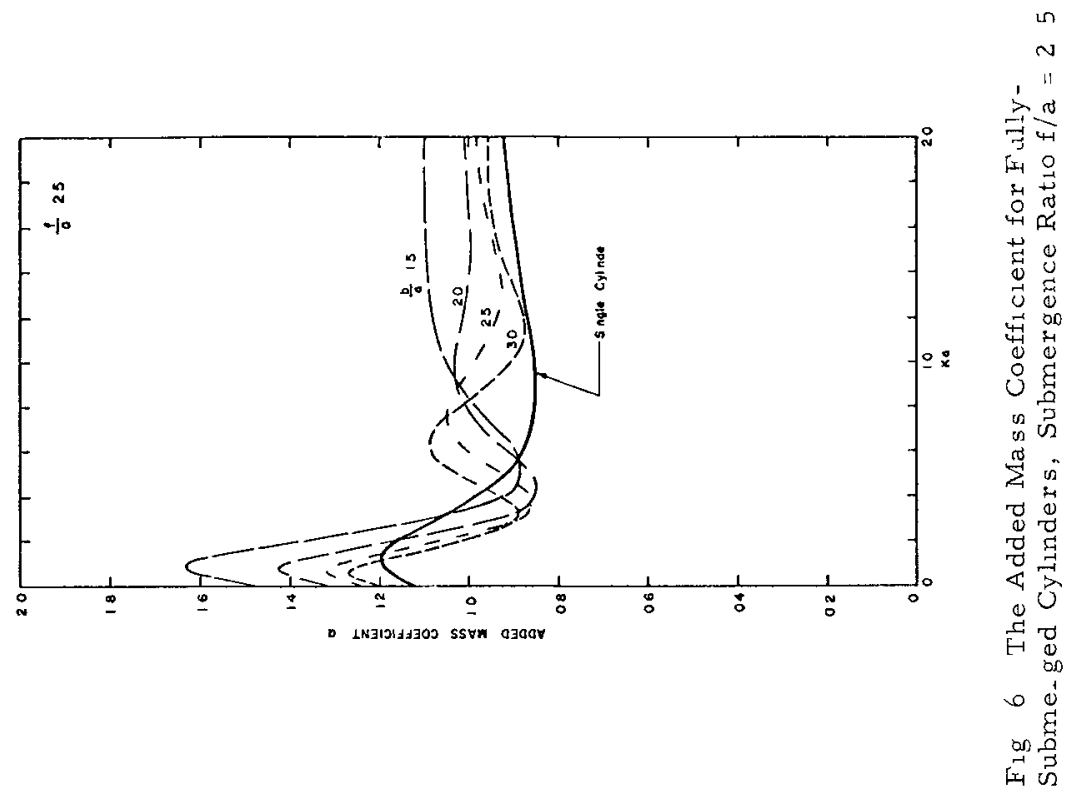




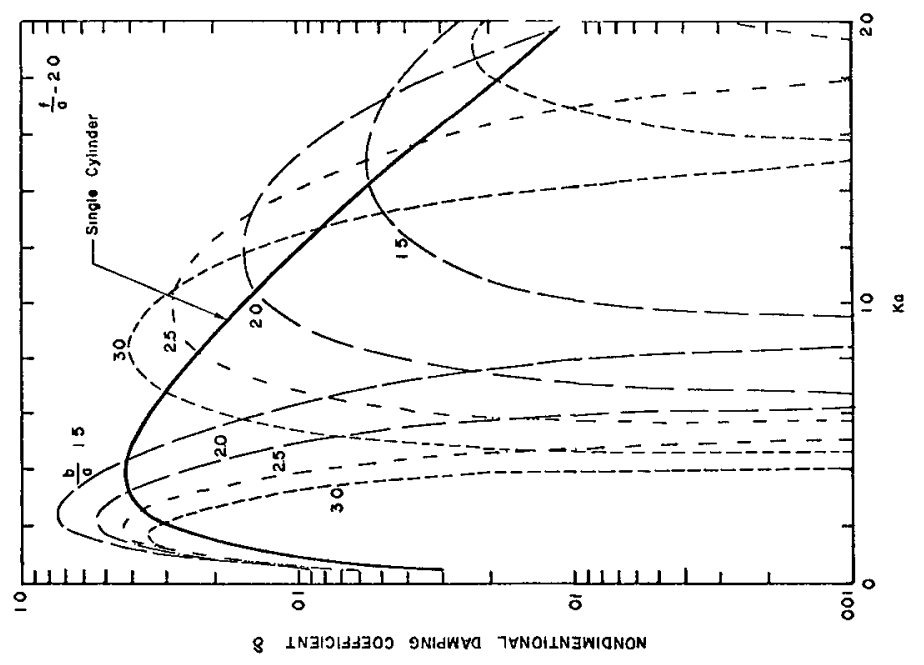

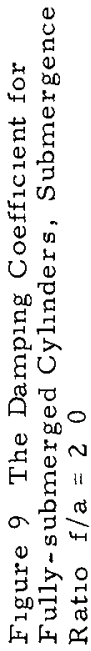

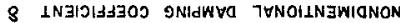

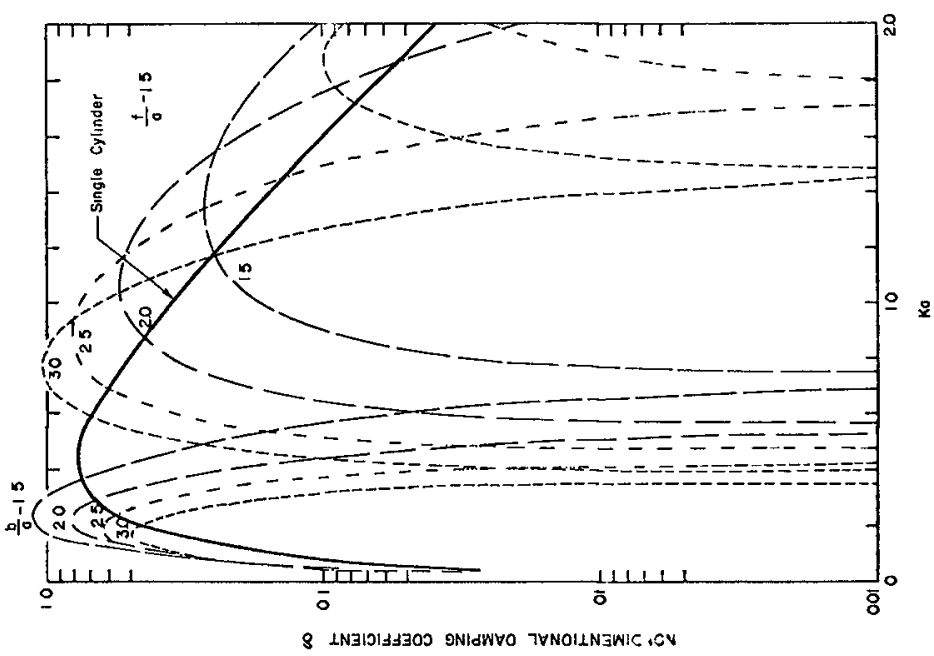

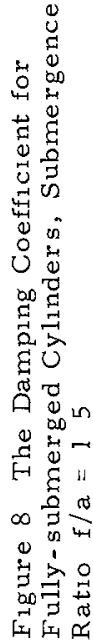

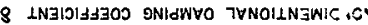




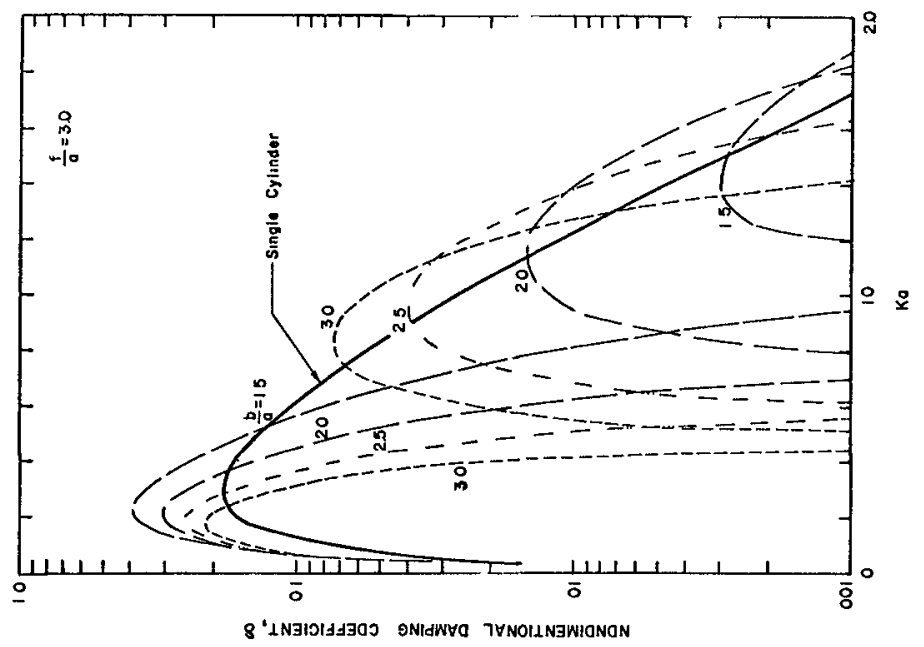

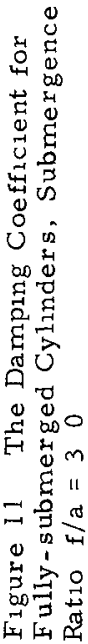

8'INGIOIJA30J gNIAWYG TYNOILNGWIONON

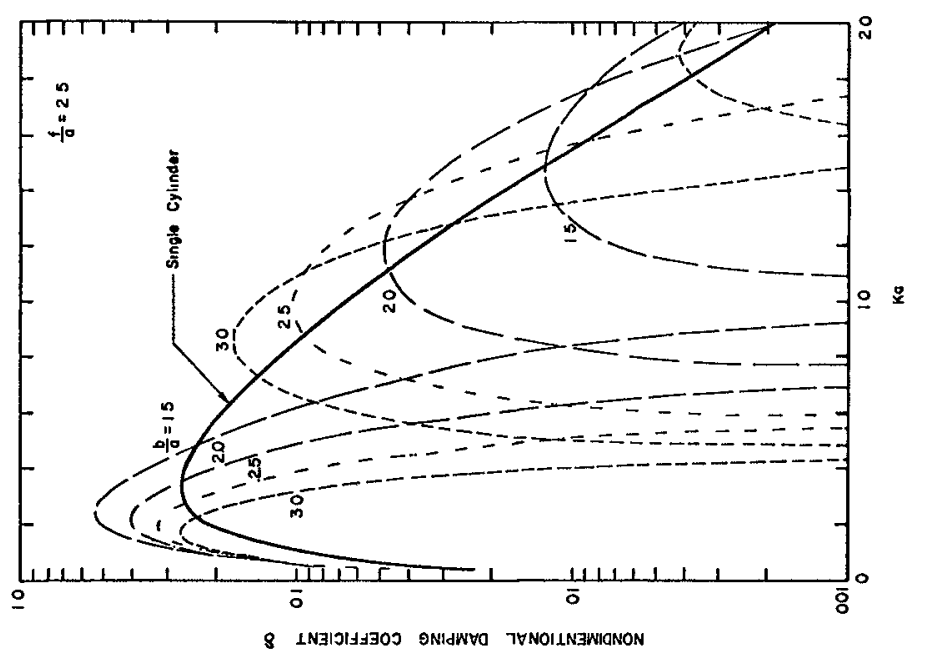

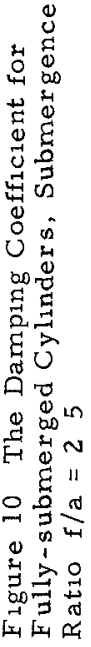




\section{The Horizontal Force}

The horizontal force, as well as the vertical component, added mass and damping oscillates in the neighborhood of the characteristic frequencies It can be shown however, all the high frequency peaks are not larger than the first one But this is not the case, when $\mathrm{f} / \mathrm{a}=0$ (semi-submerged) The high frequency peaks for the sem1-submerged case may be much larger because of the surface wave resonance Nevertheless, this is strictly a two-dimensional phenomenon as discussed before and it occupies only a very narrow band of frequencies, the high frequency peaks, therefore, are of little importance to the practical applications

In Figure 12, the dimensionless peak amplitude of the horizontal force are given as a function of cylinder spacing All these values including those for the case $\mathrm{f} / \mathrm{a}=0$, refer to the lowest frequency peaks The hull spacing effect is clearly demonstrated in this figure for the semi-submerged case, the maximum side force can increase by ten times when the hull spacing ratio $\mathrm{b} / \mathrm{a}$ is reduced from 30 to 15 For the fully submerged cases, the hull spacing effect is not as strong as that for the semi-submerged case, however, the submergence effect seems rather evident The maximum side force may reduce approximately $70 \%$ when the submergence depth is increased by one cylinder radius Based on the computed data, the maximum force coefficient $h$ for the fully submerged case, can be interpolated in terms of the hull spacing ratio b/a and submergence ratio f/a as follows

$$
h=e^{2-0} 2 \frac{b}{a}-114 \frac{f}{a} \quad\left(\begin{array}{lll}
1 & 5<\frac{b}{a}<3 & 0 \\
1 & 5<\frac{\mathrm{f}}{\mathrm{a}}<3 & 0
\end{array}\right)
$$

A group of floating platforms (such as the Mohole) and a certain type of the novel high speed vessels (such as the Trisec [4]) consist of two parallel cylindrical type hulls to facilitate good maneuverability, as well as to provide buoyancy As to these kinds of vessels, the results of the present analysis on the fully submerged twin cylinders are to provide good hydrodynamic information and can be used directly for the vessel motion response estimation

As to the catamaran type surface vessels, the results obtained from the semi-submerged twin cylinders analysis can be used to approximate the added mass and damping in both heaving and pitching by means of strip integration technique As an example, the heave motion coefficients of an ASR (submarine rescue) catamaran are estimated The procedure of this estimation begins with calculating the two-dimensional section added mass and damping of one hull of the catamaran by considering it independent from the interaction of the other hull Then these values are corrected in considering the twin-hull interation effect by multiplying the following ratio 
$1718 \quad$ COASTAL ENGINEERING

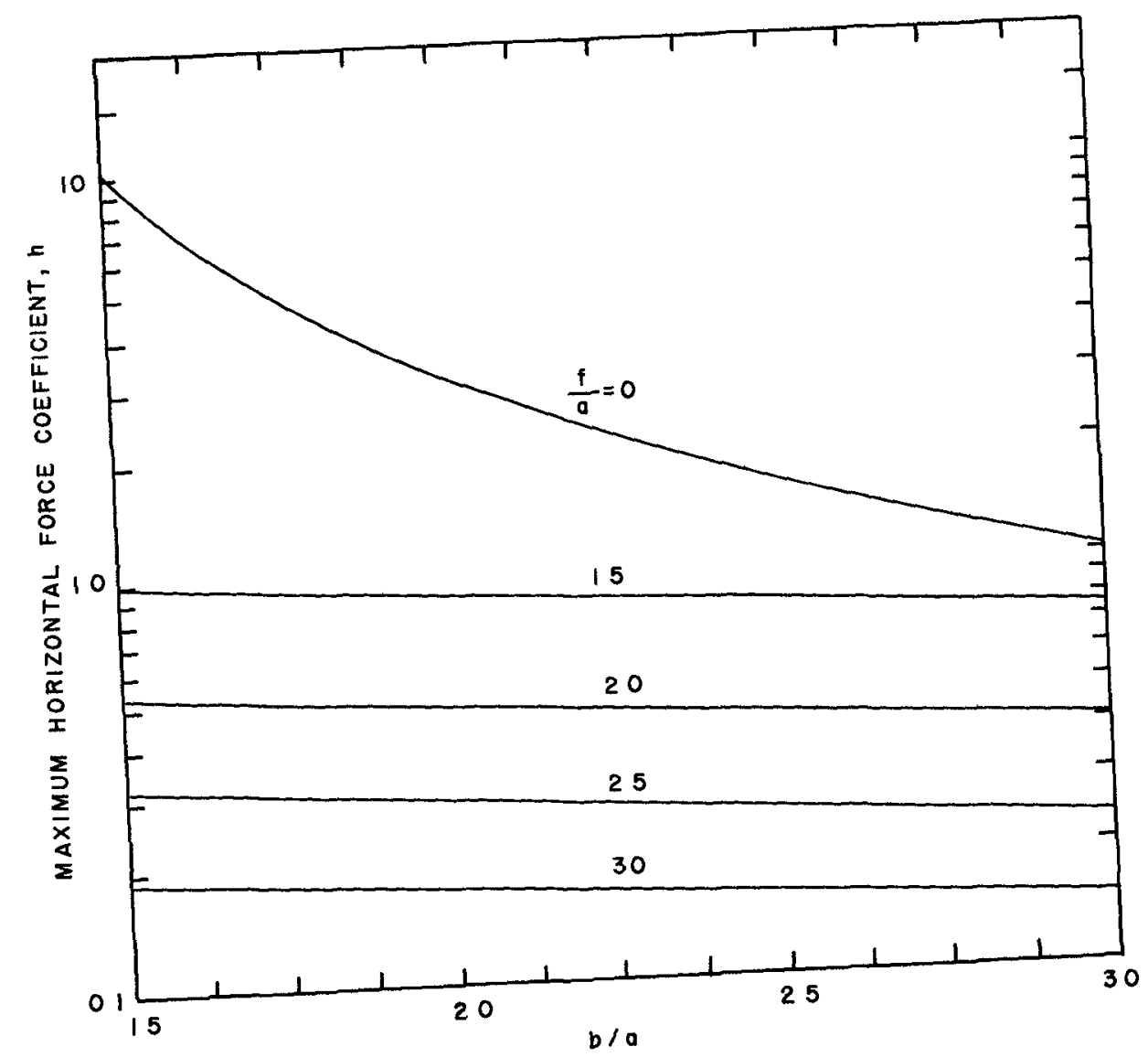
Figure 12 Effect of the Cylinder Spacing Ratio on the Maximum
Horisontal Force Coefficient 


$$
R=\frac{a_{s}\left(\frac{b}{a}, K a\right)}{a_{0}(K a)}
$$

where

$$
\begin{aligned}
a_{s}= & \text { added mass or damping coefficient (whichever applies) } \\
& \text { for twin-cylinder oscillation } \\
a_{0}= & \text { added mass or damping coefficient (whichever applies) } \\
& \text { for single-cylinder oscillation }
\end{aligned}
$$

The ratio $R$ is a function of the cylinder spacing ratio $\frac{b}{a}$ and the frequency parameter $\mathrm{Ka}$, where the cylinder radius a here is assumed equivalent to and substituted by the local half-beam of one hull, and $b$ is one-half of the spacing between center to center of the two hulls This approximation implies that the effect of twin-hull interaction is not sensitive to the hull shape details

The principal dimensions and the hull lines of this catamaran are shown in Figure 13 A $1 / 1689$ scale model of this catamaran was tested by NSRDC for the purpose of determining the added mass and damping coefficients in both pitching and heaving oscillations [5] The model tests included investigations of the speed effects, covering a speed range up to Froude No $=0316$ The estimated results of the added mass and damping coefficients are compared with the experimental results for the catamaran at zero speed and shown in Figure 14 The agreement is fairly good in regard to the negative added mass in the neighborhood of $\mu=25$, where $\mu$ is a nondimensional frequency defined in Figure 14 The experimental results of damping are rather scattered This is essentially due to the effect of resonance, which occurs in the neighborhood of $\mu=25$

[1] Wang, S \& Wahab, R "Heaving Oscillations of Twin Cylinders in a Free Surface " Journal of Ship Research (1n press)

[2] Thorne, R C , "Multiple Expansions in the Theory of Surface Waves, " Proc Comb Phil Soc 49, 1953

[3] Whittaker, E T \& Watson, G , "A Course of Modern Analys1s, ' Cambridge University Press, 1927

[4] Leopold, R, "A New Hull Form for High-Speed Volume Limited Displacement-Type Shıps, " SNAME Spring Meeting, 1969

[5] Jones, H D, "Experimental Determination of Coupled Catamaran Pitch and Heave Motion Coefficients " NSR DC T\&E Report No 348-H-03, June, 1970

The author wishes to express his appieciation to Dr Li-San Hwang for his interest and support throughout this investigation 

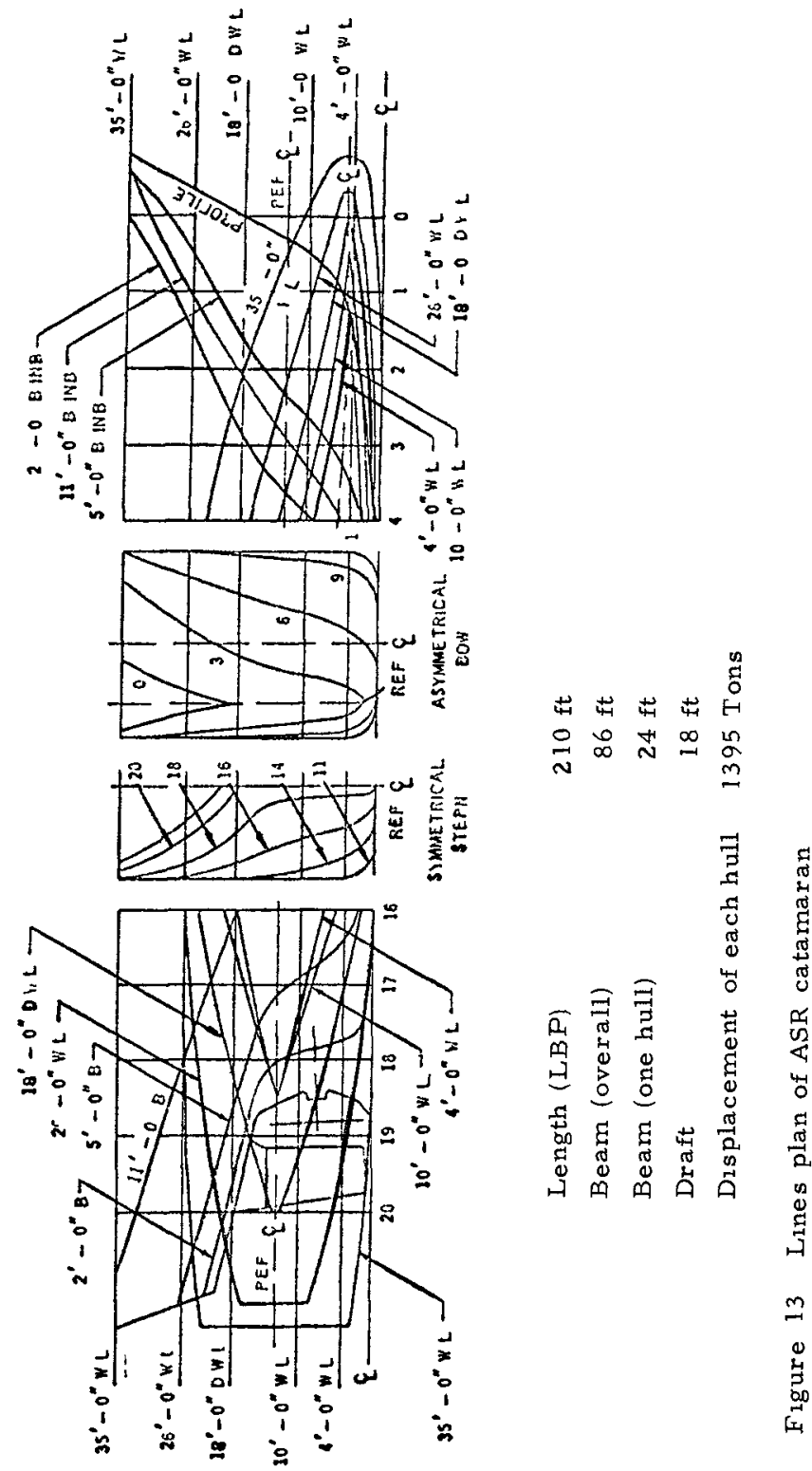
TWIN-HULL VESSELS
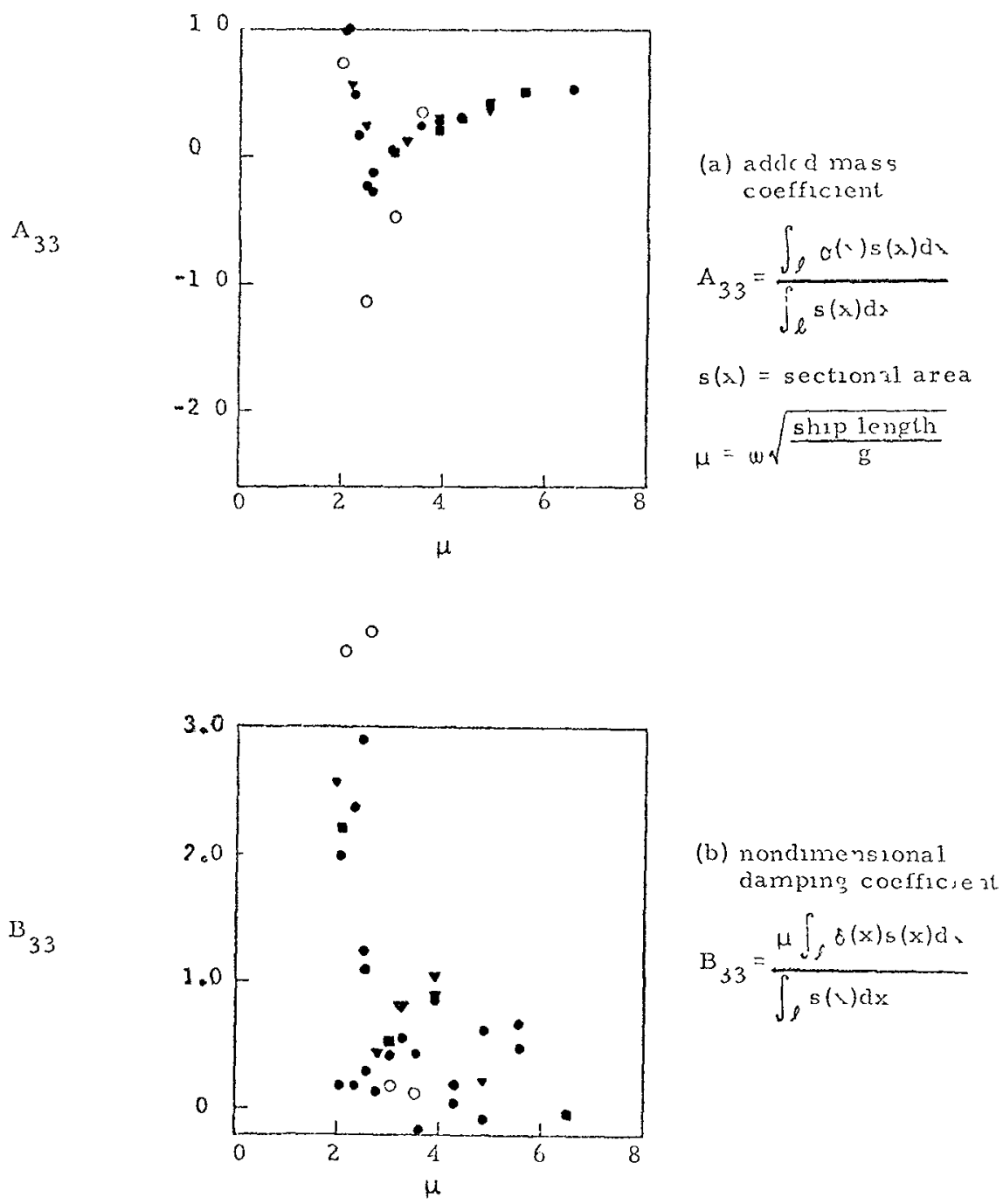

Thgue 14 Hydrodyn $\mathrm{m}$ c cocfficients of ASR cotamax m un heiving motion

I. perincital (Reference [5])

Computed
- $0125^{1}$ motion ruplituctc

- $0250^{\prime \prime}$ motion ri plactc

- $0375^{11}$ motion implatude 



\section{CHAPTER 104}

\section{BEHAVIOR OF A SLENDER BODY IN SHALLOW-WATER WAVES}

by

Hsiang Wang*

and

L1-S an Hwang $* x$

\section{Abstract}

The unsteady-state response of a slender body in nonlinear shallowwater wave environment was studied Numerical scheme has been developed which permits rapid calculation of the following, which describe the motion of an arbitrarily shaped body in three degrees of freedom anywhere within such an environment
a Unsteady-state response
b Centroid locus
c Forces and moments

Sample calculations are given for a typical submersible Results are expressed in generalized parameters, defining the circumstances wherein varıous dısplacements, velocities, acceleratıons, etc, would occur

\section{Introduction}

The primary objective of the present work was to study the unsteady state response of a slender body in nonlinear shallow-water wave environment Consideration was restricted to the wave-induced motions of a rigid body confined to one plane, hence, involving only three degrees of freedom--either surge, pitch, and heave, or sway, heave, and roll This would correspond to the case when the wave 15 long-crested and is incidental along the body in the former case and 15 incidental to the broad side in the latter case

The hydrodynamic forces under consideration consist of four parts pressure and inertial forces that can be derived from velocity potential, drag force that is proportional to the square of the relative velocity, restoring force due to the relative position and orientation of the body in the fluid, and thrust and uprighting moment due to the body

\footnotetext{
* Unıversity of Delaware, Newark, Delaware 19711

** Tetra Tech, Inc , Pasadena, Calıforn1a 91107
} 
The seaway which enters as the input to the system, is derived from the following wave theories

a Cnoldal wave theory of Keulegan and Patterson for high, long, near-breakıng and breakıng waves

b Alry linear theory for short perıod waves

c McCowan solitary wave theory of matching period for very long waves

These theorxes are chosen on the basis that they provide the best approximation to internal wave characteristics as obtained experimentally

A numerical scheme has been developed which permits rapld calculation of the following, which describe the motion of an arbitrarily shaped body in three degrees of freedom anywhere within such an environment

a Unsteady-state response

b Centroid locus

c Forces and moments

Results are expressed in generalized parameters, defining the circumstance wherein varıous displacements, velocities, accelerations, etc, would occur

\section{The Equations of Motion of a Submerged Body}

Consideration was restricted to the wave-induced motions of a rigid submerged body confined to one plane, hence involving only three degrees of freedom--either surge, pitch, and heave or sway, heave, and rol1, as defined on Figure 1 This would correspond to the case when the wave is long-crested and is incident to along the body in the former case and incident to the broad side in the latter Although, in principle, these two cases are the same hydrodynamically, they differ somewhat in the method of obtaining an engineering solution

Two sets of coordinate systems were employed in analyzing the responses of a submerged body, they are

a Fixed coordinate system used to describe the sea conditions and the position of the body, the origin is arbitrary and was chosen here at the sea bottom with the $x$-axis parallel to the longltudinal axis of the body, the y-axis pointing upward vertically, and the $z$-axis in the transverse direction

b Body coordinate system used to describe the oscillations of the body The body had three principal axes, hence six degrees of freedom, corresponding to a translation and a rotation for each axis Symbol definitions are shown in Figure 1 The body axes have their origin at the center of gravity of the body and are coincident with the intersections of the principal planes of inertia 
The problem of body response in a wave environment is treated in four steps

a Derivation of flow environment

$b$ Derivation of hydrodynamic excitation

c Derivation of body response and tracing of locus of body motion

d Derivation of forces exerted on the body

Incident Wave Parallel to the Longltudinal Axis

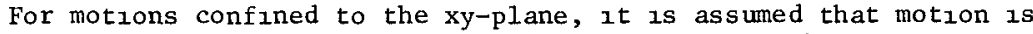
described by three functions of time $X(t), Y(t)$, and $\theta(t)$, which are such that the location of the center of gravity of the body is $(X, Y)$ at time $t$, and the angle of inclination of the body is $\theta$ (Figure 1) Then the equations of motion of the body are

$$
\begin{aligned}
& M \ddot{X}=F_{x} \\
& M Y=F_{y} \\
& I_{\theta} \ddot{\theta}=F_{\theta}
\end{aligned}
$$

where $M$ is the natural mass, $I_{\theta}$ is the pitching moment of inertia, and $F_{x}, F_{y}$, and $F_{\theta}$ are the total hydrodynamic forces and moments on the body The main problem is, of course, the estimation of these hydrodynamic forces

It is convenient to separate the liydrodynamic forces into four parts pressure and acceleration forces that can be derived from velocity potential, velocity force that has to be estimated using empirical drag coefficient, restoring force due to the relative position and orientation of the body in the fluid, and thrust or uprighting moment provided by the body

For a body which 1 s slender ( $1 \mathrm{e}$, has small cross-section relative to its length and to a typical wave length), the pressure forces, or Froude-Krylov forces (Korvin-Kroukovsky, 1961), are relatively easy to estimate Suppose the given incident pressure field is $p(x, y, t)$ and the local horizontal and vertical pressure gradients at a station $\xi$ of the body are calculated

$$
\begin{aligned}
& P(\xi, t)=\frac{\partial P}{\partial x}(X+\xi \cos \theta, Y+\xi \sin \theta, t) \\
& Q(\xi, t)=\frac{\partial P}{\partial y}(X+\xi \cos \theta, Y+\xi \sin \theta, t)
\end{aligned}
$$




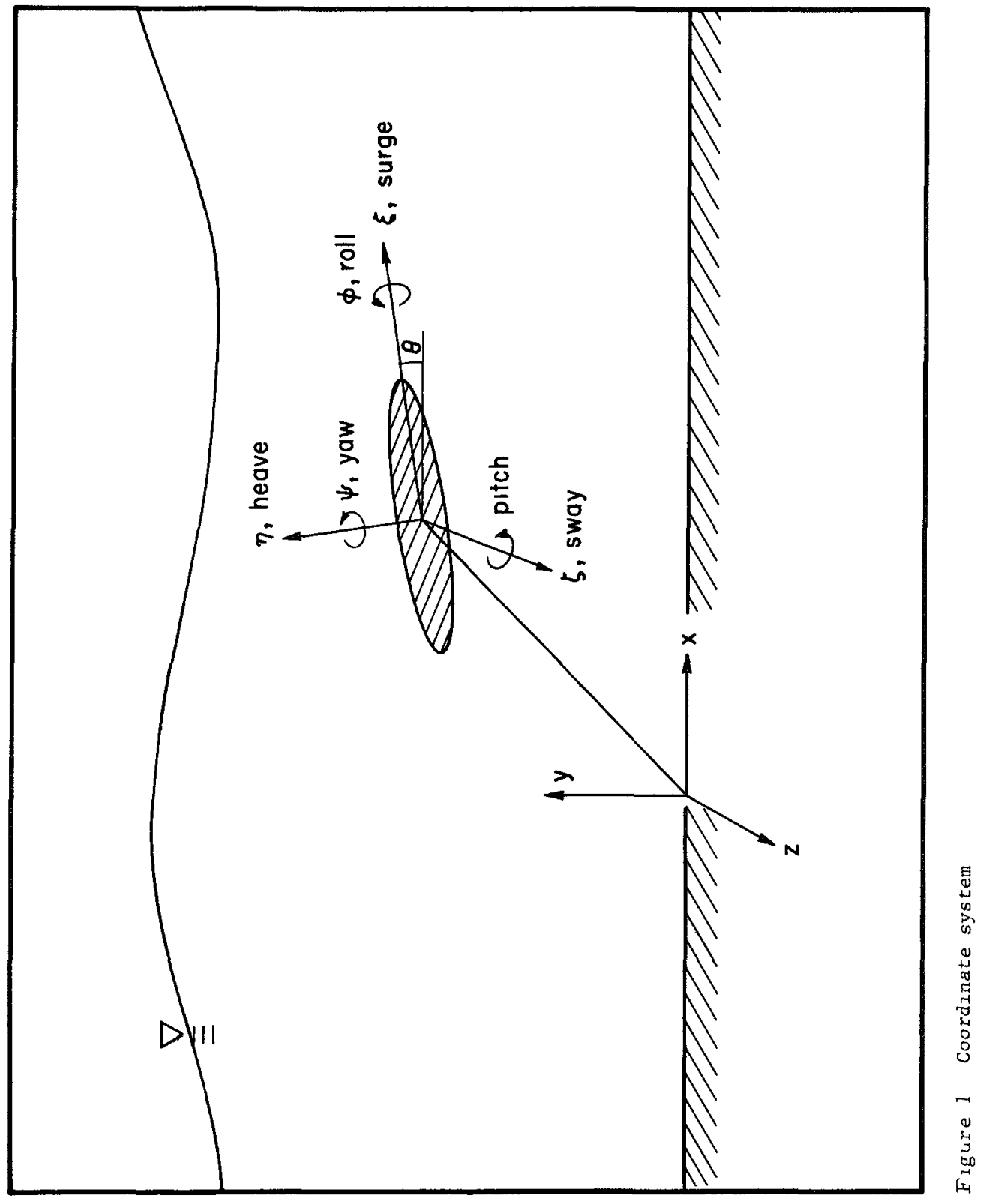


then the pressure forces are easily seen to be

$$
\begin{aligned}
& F_{x}^{F K}=-\int_{l} P(\xi, t) S(\xi) d \xi \\
& F_{y}^{F K}=-\int_{l} Q(5, t) S(\xi) d \xi
\end{aligned}
$$

and the pitching moment is

$$
F_{\theta}^{\Gamma K}=-\cos \theta \int_{l} \xi Q(\xi, t) S(\xi) d \xi+\sin \theta \int_{l} \xi P(\xi, t) S(\xi) d \xi
$$

Here $S(\xi)$ Is the area of the cross-section of the body at station $\xi$, and the integrations extend over the length of the body The acceleration forces include that due to the motion of the body and that due to the diffraction of the wave field by the body If the body is slender, Its effect on the fluid is sensible only when there is a relative motion across its axis Thus, the longitudinal added mass of a spherold with a thickness ratıo 1 in 10 is only 2 percent of the displaced mass (Lamb, 1932), whereas the lateral added mass is nearly equal to the displaced mass Hence, "strip" methods may be used to obtain the added inertia effects by considering only the cross flow at each station $\xi$ The fluid in the nelghborhood of station $\xi$ has an acceleration

$$
\frac{1}{\rho} P(\xi, t) \sin \theta-\frac{1}{\rho} Q(\xi, t) \cos \theta
$$

normal to the body axis, whereas the body itself has acceleration

$$
-\ddot{X} \sin \theta+\ddot{Y} \cos \theta+\ddot{s} \ddot{\theta}
$$

Hence, there is a relative acceleration of the section at $\xi$ of

$$
a(\xi)=-\left(\ddot{X}+\frac{1}{\rho} P\right) \sin \theta+\left(\ddot{Y}+\frac{1}{\rho} Q\right) \cos \theta+\ddot{\xi} \ddot{\theta}
$$


The cross flow, or slender-body hypothesis, now asserts that the hydrodynamic effect of this relative motion is an opposing force $\mu(\xi) \mathrm{a}(\xi) \mathrm{d} \xi$ on a section of thickness $\mathrm{d} \xi$, where $\mu(\xi)$ is the added mass of the section, calculated as if the flow were two dimensional, Irrotational, and infinite in extent Resolving horizontally and vertically and taking moments, we have inertia terms

$$
\begin{aligned}
F_{x}^{A} & =-\int_{\ell} \mu(\xi) a(\xi) d^{-} \sin \theta \\
F_{y}^{A} & =\int_{l} \mu(\xi) a(\xi) d \xi \cos \theta \\
A^{A} & =\int_{f} \xi u(\xi) a(\xi) d \xi
\end{aligned}
$$

The force generated through the relative velocity between the body and the flurd flow is associated with the momentum defect of the flurd due to the body This force is generally expressed in the form

$$
F_{D}=\rho \frac{C_{D}}{2} A|V| V
$$

where

$$
\begin{aligned}
& C_{D}=\text { drag coefficient } \\
& A=\text { frontal area (normal to flow) } \\
& V=\text { relative flow velocity }
\end{aligned}
$$

The drag coefficient is a function of Reynolds number and differs for different body geometry The velocity force in the cross-flow direction and longltudinal-flow direction can be written separately as

$$
\begin{aligned}
F_{D \eta} & =\rho \frac{1}{2} \int_{\ell} C_{D}\left|v_{R \eta}\right| v_{R \eta} d A \\
& =\rho \frac{C_{D C}}{2} \int_{\ell}\left|v_{R \eta}\right| v_{R \eta} d A
\end{aligned}
$$


and

$$
\begin{aligned}
F_{D \xi} & =\rho \frac{1}{2} \int_{B} C_{D}\left|V_{R \xi}\right| V_{R \xi} d A \\
& =\rho \frac{C_{D L}}{2} \int_{B}\left|V_{R}\right|_{R} V_{R} d A
\end{aligned}
$$

where

$$
\begin{aligned}
& \mathrm{F}_{\mathrm{D} \eta}=\text { drag force in the cross-flow direction } \\
& \mathrm{F}_{\mathrm{D} \xi}=\text { drag force in the longltudinal-flow direction } \\
& \mathrm{F}_{\mathrm{DC}}=\text { average drag coefficient in the cross-flow direction } \\
& \mathrm{F}_{\mathrm{DI}}=\text { average drag coefficient in the longltudinal-flow direction }
\end{aligned}
$$

and the integral limits $\ell$ and $B$ denote that the integrations are performed along the longitudinal axis and along the vertical axis of the body

The relative velocities in the cross-flow direction $V_{R n}$ and in the longltudinal direction $V_{R \xi}$ are, respectively,

$$
\begin{gathered}
V_{R \eta}=-U_{R} \sin \theta+V_{R} \cos \theta+\xi \dot{\theta} \\
V_{R \xi}=U_{R} \cos \theta+V_{R} \sin \theta
\end{gathered}
$$

with $U_{R}$ and $V_{R}$ defined as

$$
\begin{aligned}
& U_{R}=X-u \\
& V_{R}-Y-v
\end{aligned}
$$

where $X$ and $Y$ are the velocity components of the body in the $x-$ and $y-$ directions and $u$ and $v$ are the velocity components of the fluid in the $x-$ and $y$-directions

Agaın, like Inertıa terms, resolving horızontally and vertıcally and taking moments to ohtaln the drag forces 


$$
\begin{aligned}
& F_{x}^{D}--F_{D} \xi^{\cos \theta}+\Gamma_{D \eta} \sin \theta \\
& F_{y}^{D}=-F_{D}-\sin \theta-F_{D \eta} \cos \theta \\
& r_{\theta}^{D}=-\rho \frac{C_{D C}}{2} \int_{l} \xi\left|V_{R \eta}\right| V_{R \eta} d A
\end{aligned}
$$

The restoring forces are simply

$$
\begin{array}{rlrl}
F_{x}^{R} & =0 & \\
F_{y}^{R} & =-w_{s} & & \text { for partial submergence } \\
& =0 & & \text { for full submergence }
\end{array}
$$

where $w_{s}$ is the partial weight of the body that is surfaced

The restoring moments are

$$
F_{\theta}^{R}=-\rho g \forall_{s} \overline{\operatorname{ee}} \sin \theta-\cos \theta \int_{l_{1}} \rho_{s} g \xi d V
$$

for partıal submergence and

$$
F_{\theta}^{R}=-\rho g \forall_{S} \overline{c e} \sin \theta
$$

for full submergence

where

$$
\begin{aligned}
& \forall_{S}=\text { volume of displaced water } \\
& \text { ee }=\text { metacentric helght of body } \\
& \rho=\text { density of sea water } \\
& \rho S=\text { density of body } \\
& l 1=\text { body length above free surface }
\end{aligned}
$$


Finally, the thrust and righting moments produced by the body when resolved into $x-, y-$, and $\theta$-directions, are

$$
\begin{aligned}
& \Gamma_{x}^{T}=I_{t} \cos \theta \\
& \Gamma_{y}^{T}=I_{t} \sin \theta \\
& F_{\theta}^{T}=-M_{r} \theta
\end{aligned}
$$

where $T$, is the thrust, and $\mathrm{M}_{r} \theta$ is the righting moment, which $1 \mathrm{~s}$ assumed to be proportional to the pitch angle

Thus, we have completed the disposition of the total forces acting on the body, and the equations of motion become

$$
\begin{gathered}
M X=F_{x}^{F K}+F_{x}^{A}+F_{x}^{D}+F_{x}^{I} \\
M Y=F_{y}^{F K}+F_{y}^{A}+F_{y}^{D}+F_{y}^{R}+F_{y}^{T} \\
I_{\theta} \dot{\theta}=F_{\theta}^{F K}+F_{\theta}^{A}+F_{\theta}^{D}+F_{\theta}^{R}+F_{\theta}^{T}
\end{gathered}
$$

When expressed explicitly, the equations of motion are

$$
\begin{aligned}
& M \ddot{X}=-\int_{\ell} P(\xi, i) S(\xi) d \xi-\int_{\ell} \mu(\xi) a(\xi) d \xi \sin \theta-p \frac{C L}{2} \int_{B}\left|V_{R \xi}\right| V_{R \xi} d \wedge \cos \theta \\
& +\rho \frac{C_{D C}}{2} \int_{l}\left|v_{\text {IR } \eta}\right| V_{R \eta} d \Lambda \sin \theta+T_{t} \cos \theta
\end{aligned}
$$

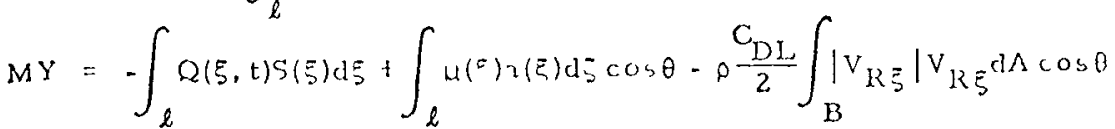

$$
\begin{aligned}
& -\rho \frac{C_{D C}}{2} \int_{\ell}\left|V_{I \eta}\right| V_{K \eta} d A \cos \theta+T_{t} \sin \theta+F_{y}^{R} \\
& I_{\theta} \theta=-\cos \theta \int_{\ell} \xi Q(\xi, t) \mathrm{S}(\xi) \mathrm{d} \xi+\sin \theta \int_{\ell} \xi P(\xi \mathrm{t}) \mathrm{S}(\xi) \mathrm{d} \xi+\int_{\ell} \xi \mu(\xi) \mathrm{a}(\xi) \mathrm{d} \xi \\
& -\frac{C_{D C}}{2} \int_{\ell}\left|V_{R \eta}\right| V_{R \eta} d A-M_{r} \theta+F_{\theta}^{R}
\end{aligned}
$$


where $F_{y}^{R}$ and $F_{\theta}^{R}$ are defined in Eqs 22 and 23, respectively once the flow field has been described, these three simultaneous equations can be solved using a high-speed computer

The surging force, heaving force, and pitching moment are evaluated, respectively, according to the following equations

$$
\begin{gathered}
F_{\xi}=M(X \cos \theta+Y \sin \theta)-I_{\theta}(\theta)^{2} \\
F_{\eta}=M(X \sin \theta+Y \cos \theta)-I_{\theta} \theta \\
M_{T}=I_{\theta} \dot{\theta}
\end{gathered}
$$

where

$$
\begin{aligned}
& F_{\xi_{3}}=\text { surging force } \\
& F_{n}=\text { heaving force } \\
& M_{T}=\text { pitching moment }
\end{aligned}
$$

Incident Wave Perpendicular to the Longltudinal Axis

The equations of motion are similar to the previous case, except the evaluation of some forces were different In the determination of the mass coefficients, the main body was treated as a cylindrical body of variable diameter The drag term was calculated in a much similar way as the acceleration term, with due consideration in choosing drag coefficients for different parts

The calculation of pressure force, and restoring force, remains the same as in the case of parallel waves The complete calculations of motion for sway, heave, and roll, when expressed in force components, are, respectively,

$$
\begin{gathered}
M Z=\Gamma_{z}^{\Gamma K}+\Gamma_{z}^{A}+\Gamma_{z}^{D} \\
M \dot{Y}=\Gamma_{y}^{\Gamma K}+F_{y}^{A}+\Gamma_{y}^{D}+F_{y}^{T}+\Gamma_{y}^{R} \\
I_{\varphi} \dot{\varphi}=\Gamma_{\varphi}^{F K}+\Gamma_{\varphi}^{A}+F_{\varphi}^{D}+\Gamma_{\varphi}^{T}+\Gamma_{\varphi}^{R}
\end{gathered}
$$




\section{Wave Environment and Flow Field}

Three wave theorıes were used for evaluating exc1tation forces They are

a Cnoldal wave theory of Keulegan and Patterson for high, long, near-breakıng, and breakıng waves

b Alry linear theory for short perıod waves

c McCowan solıtary wave theory for very long waves

The cnoldal wave has wave profile (Wlegel, 1964)

$$
y_{s}=y_{t}+\operatorname{Hcn}^{2}\left[2 K(k)\left(\frac{x}{L}-\frac{t}{T}\right) k\right]
$$

with wave perıod to the first order

$$
T=\frac{4 d}{\sqrt{3 \mathrm{gH}}}\left\{\frac{k K(k)}{\sqrt{1+\frac{\mathrm{H}}{\mathrm{d}}\left[-1+\frac{1}{\mathrm{k}^{2}}\left(2-3 \frac{\mathrm{K}(\mathrm{k})}{\mathrm{K}(\mathrm{k})}\right)\right]}}=\right.
$$

The corresponding wave length is

$$
L=\sqrt{\frac{16 \mathrm{~d}^{3}}{3 \mathrm{H}}} \mathrm{kK}(\mathrm{k})
$$

where

$$
\begin{aligned}
& \mathrm{y}_{\mathrm{s}}=\text { water surface elevation measured from sea bottom } \\
& H \text { = wave height } \\
& \mathrm{cn}=\text { one of the Jacobian ellıtıc functions } \\
& k=a \text { real number varied from } 0 \text { to } 1 \\
& K(k)=\text { ellıptıc Integral of first kind } \\
& E(k)=\text { elliptic integral of second kind } \\
& y_{t}=H\left((d / H)-I+\left(16 d^{3} / 3 L^{2} H\right)\{k(k)[K(k) \quad E(k)]\}\right)
\end{aligned}
$$


In Eq 34, when $T$ is plotted as a function of $k$ for fixed $d$ and $H$, It takes a form shown in Fig 2 Thus, if one starts at point $A$ on the curve for increasing value of $T$, the corresponding $k$ can increase or decrease depending upon which branch one follows The left branch should be discarded because it corresponds to increasing values of $T$ with decreasing values of $\mathrm{L}$, which is physically meaningless For waves of periods shorter than $T_{m}$, the Alry theory is to be applied By differentiating $\mathrm{Eq} 34 \mathrm{with}$ respect to $k$ and equating the result to zero,

$$
\frac{d}{\tilde{H}}=1-\frac{1}{2 k^{2}}\left[4-9 \frac{E(k)}{K(k)}+\left(1-k^{2}\right) \frac{K(k)}{E(k)}\right]
$$

for $T \sqrt{g / d}=$ minimum

Thus, the value of $(T \sqrt{g / d})_{m 1 n}$ versus $d / H$ so obtained defines the matching line between the cnoldal wave and linear wave It is also evident from Fig 5 that, when the elliptic parameter approaches unity, the period approaches infinity rapıdly For instance, when the $k$ values are changed from 1 to 09999 , the period $4 \mathrm{~K}(\mathrm{k})$ is decreased from $1 \mathrm{n}-$ finity to about $7 \pi$ In the numerical calculation, the wave period (or length) is specified, and the value of $k$ is found by Eq 34 through Iteration For very long waves, the value of $k$ is very nearly equal to 1 , and it becomes impractical to obtain numerically the value of $k$ through iteration In this case, the solitary wave, which is the 1imiting case of the cnoldal wave, can be treated as having a finite period for many practical purposes The upper 11mit of elliptic parameter has been chosen as equal to 09999 in the present study Figure 3 shows the regions where the different wave theorles apply

\section{Method of Computation}

\section{Numerıcal Analysıs}

The differential equations to be solved are a set of three simultaneous, nonlinear second-order equations The fourth-order formula of Runge-Kutta (HIldebrand, 1956) is used to perform the numerical evaluation This method, which extends forward the solution of differential equations from known conditions by an increment of the independent variable without using information outside this increment, has been applied extensively in solving initial value problems In essence, the fourth-order formula evaluates the slope of the wave at the initial point, the $1 / 4$ point, the $1 / 2$ point, and the $3 / 4$ point of the interval of increment The numerical solution is then obtained in agreement with the Taylor series solution through terms of the fourth order of the interval $h$ The local truncated error is then of the order of $h^{5}$, where $h$ is the size of the increment In the present case, the independent variable is the nondimensional time, which is equal to $t / T$, where $t$ is real time, and $T$ is the wave period 


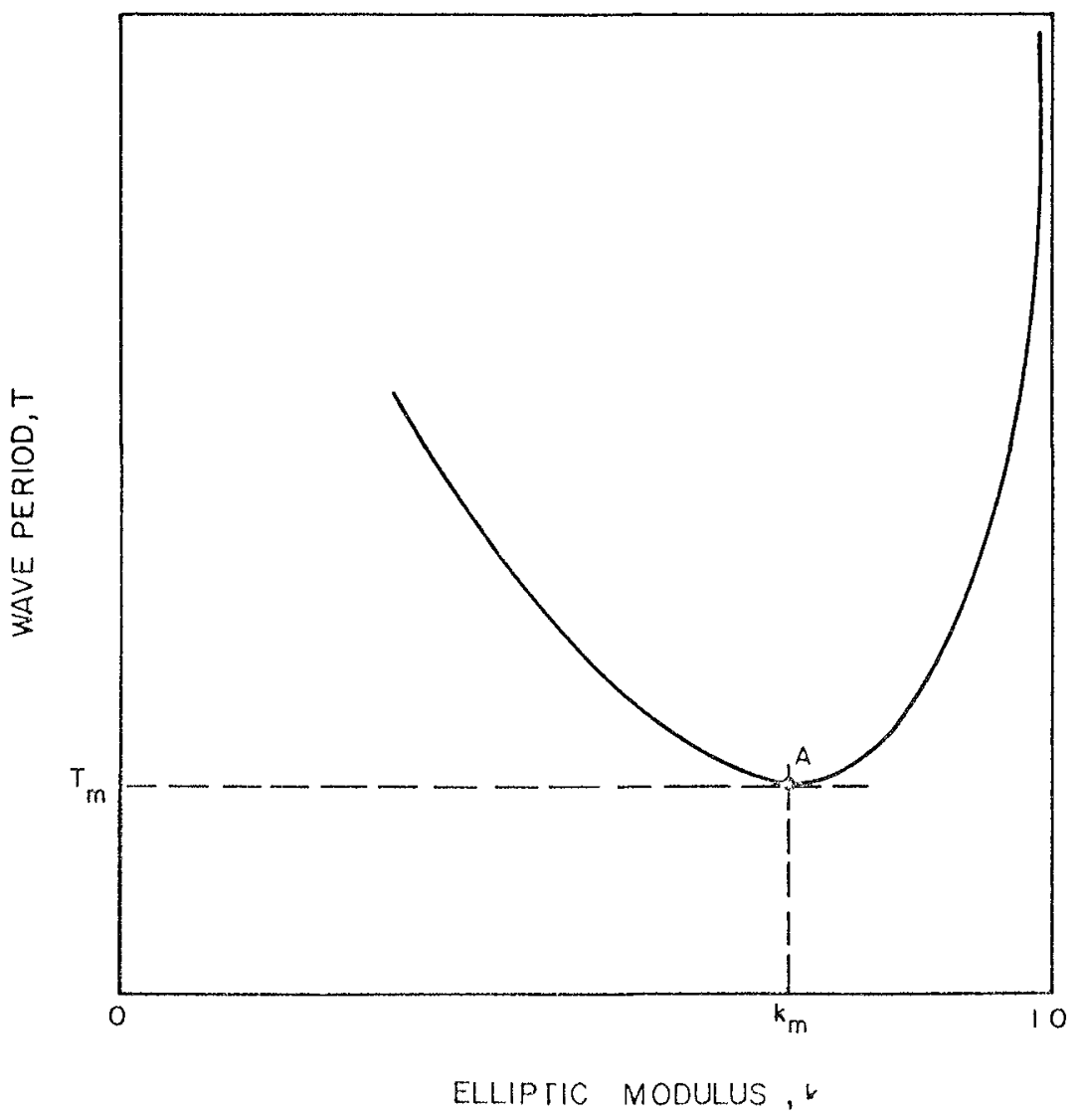

Figue 2 Wave perodversus cllipte modulus for cnordal wive 


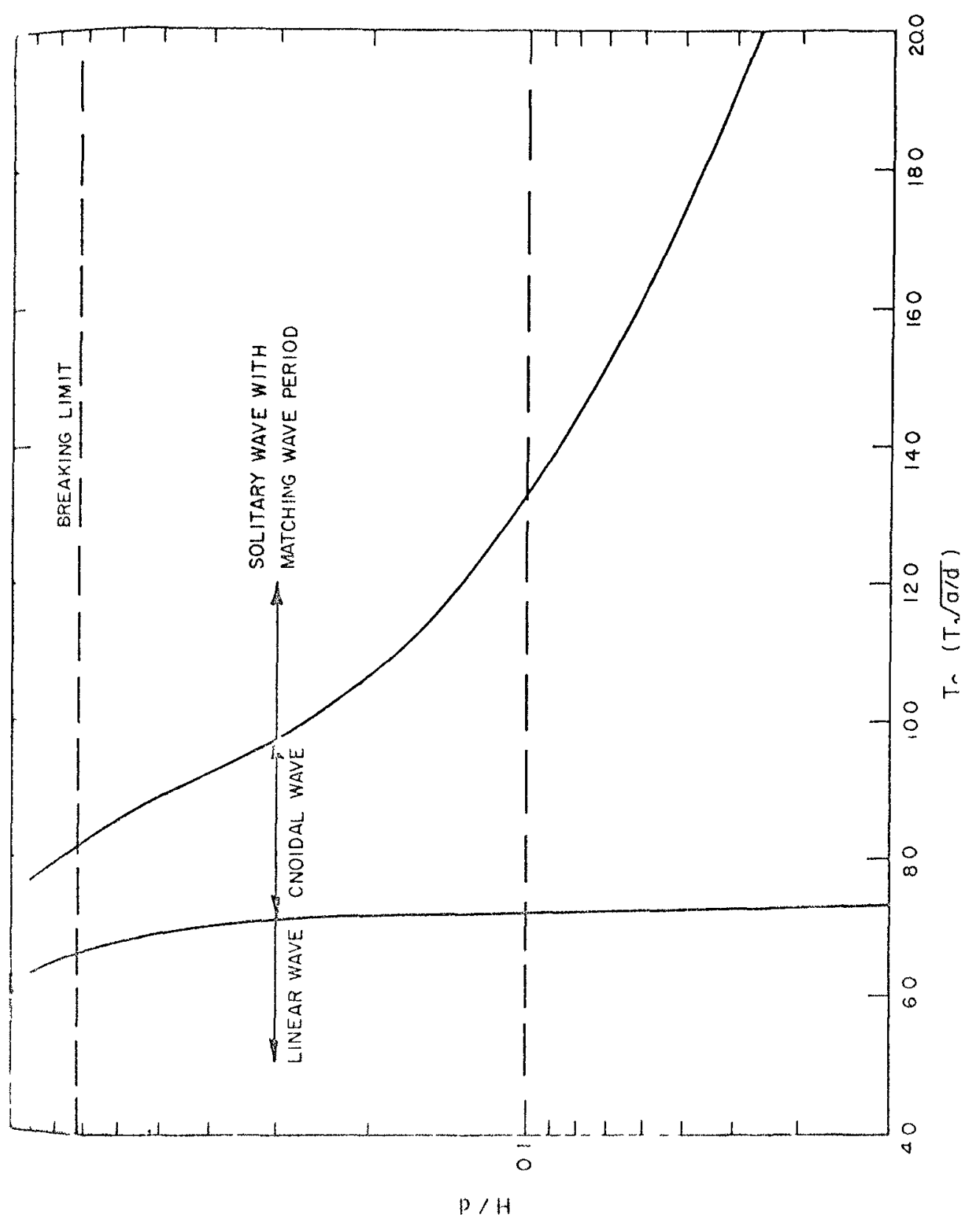

Fighre 3 Region of wave theortes 
After several tests, the incremental interval $h=\Delta t / T$ was selected at $1 / 64$ throughout the computation

\section{Input Conditions}

The independent elements which affect the behavior of the body are

1 Environment

Wave herght $\mathrm{H}$

Wave period $\mathrm{T}$

Water depth $d$

Gravıty $g$

2 Flund properties

Fluid density $p=20$

Viscosity $\mu$ (Not explicitly involved, appears in terms of drag coefficients)

3 Submerged body

Length $\ell$

Cross sectional areas along the lonitudinal coordinate $S$ $(1=1$ to $\mathrm{m}$ number of station)

Longitudinal moment of inertia $I_{\theta}$

Transverse moment of inertia I

Weight $W$

Metacentric helght ee

Righting moment $M_{0}$

Added mass coefficients $\mathrm{C}_{\mathrm{m}}$ 's

4 Inltial conditions

Depth of submergence $d_{s}$

Velocity $\mathrm{V}_{1}$

Angle of attack $\alpha_{1}$

Form of first effective wave $F$

Orlentating submerged body 0

A11 of these factors are required inputs in the computer program

\section{Nume rical results}

Figures 4 and 5 show, respectively, the vertical and horizontal displacements of the center of the body in a wave environment In these figures, the free surface variation is drawn with respect to che gravity center of the submerged body In the case of Figure 4, che body was initially placed at the middle water depth The body has a tendency to surface In the case of Figure 5, the body was placed right beneath the free surface, rose partially above the surface, and then dived down to hit the bottom, partially due to the additional downward force imposed on the body from the reduction of buoyancy force resulting from the surfacing 


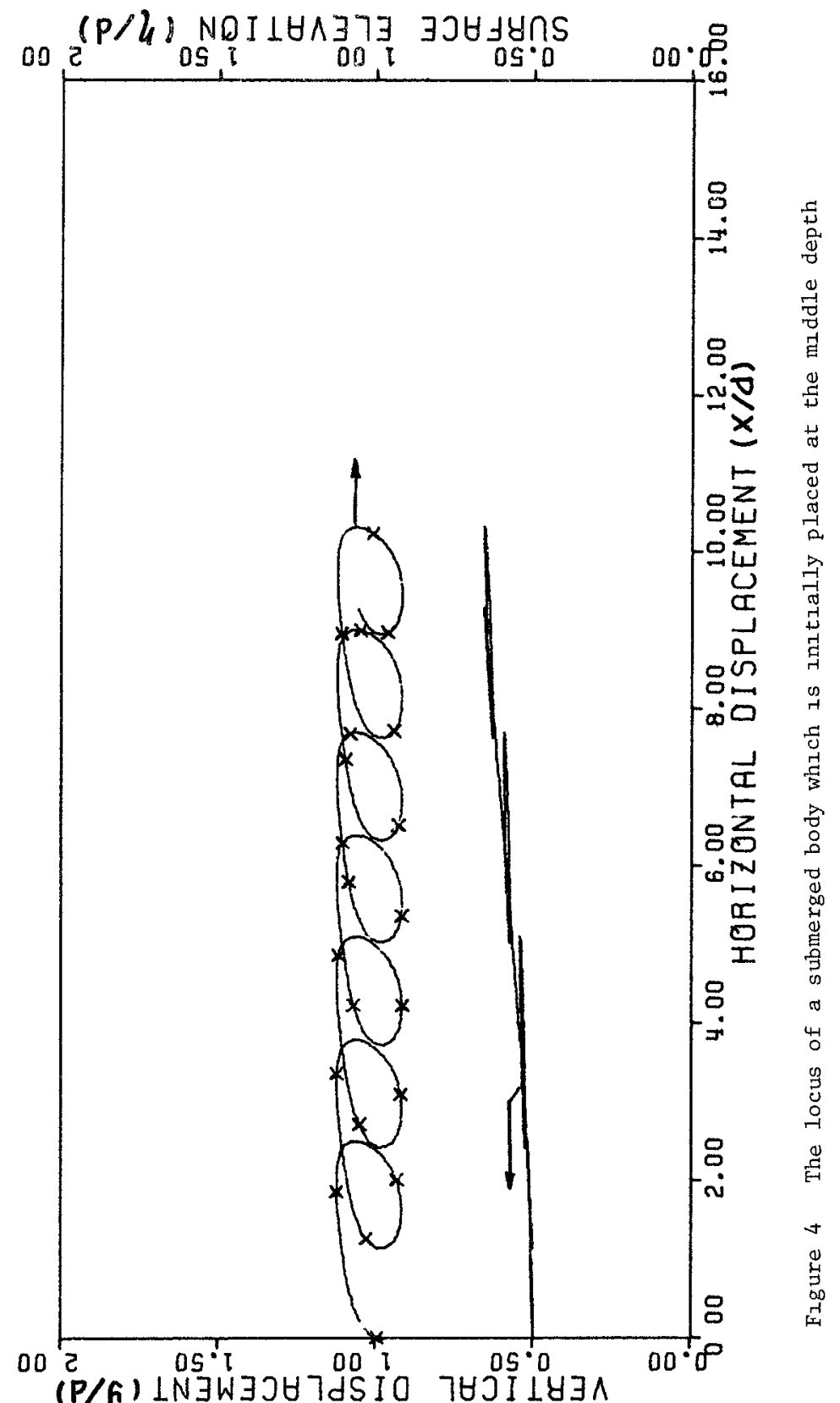




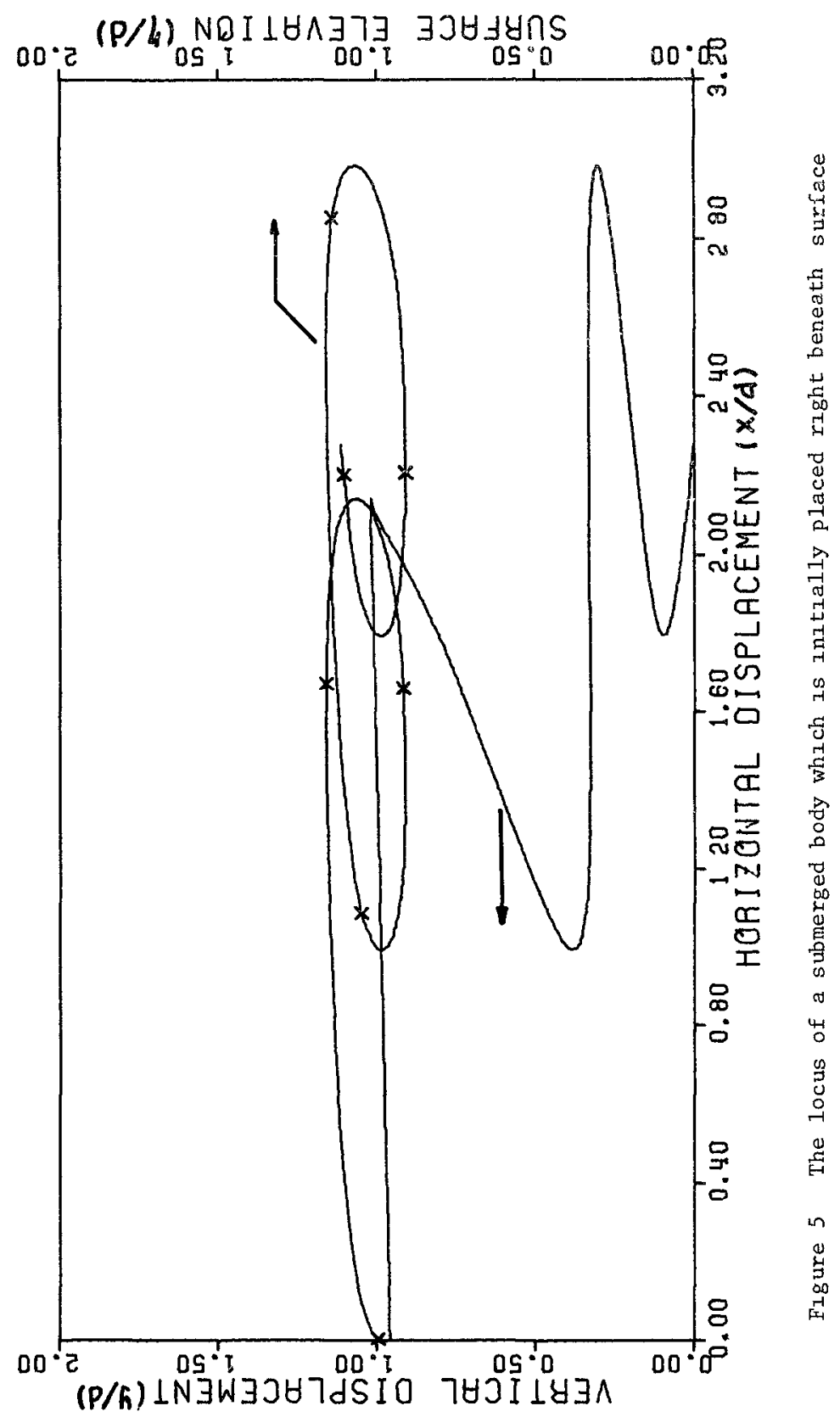


Figures 6 and 7 show the differences of the acceleration pattern of the body for different initial positions The oscillation was more regular when the body was initially placed at mid-depth The amplitude of the oscillation grew with time as the depth of the submergence decreased, as shown in Figure 4 Figure 7 shows the acceleration pattern for the case where the body was placed right beneath the free surface The sudden increase of acceleration results from surfacing Similar patterns were found for the heaving forces, which are closely related to these accelerations A typical pitching motion is shown in Figure 8

\section{Discussion}

The present work is to provide an analytıcal tool to examine the dynamic behavior of a submerged body when it is exposed in a shallowwater wave environment A thorough investigation, considering every variable as 1isted in the previous section, though desirable, would be very cumbersome Therefore, the consideration was restricted to specific hull configurations Attempts were then made to examine the influence of environmental variables on the response of the structure Even with such restriction, only qualitative evaluations can be made

The wave height and the water depth were found to be the most influentıal variables Dynamic stability, $1 \mathrm{e}$, chance of capsize, depends significantly on them Wave period is less important for the unsteadystate case considered Original altitude of the body is also found to be of secondary importance, partially because the wave theories, even to the second order, yleld hydrostatic pressure distribution in the vertical direction This conclusion can not, however, be extended to the region where the body is close to the surface as illustrated in Figures 4 and 5

The water inertia force, better known as the added mass effects, was found to vary with the altitude of the body Correction was made by using experimentally determined added mass coefficients in the numerical computation This coefficient, being approximately equal to 098 when the submergent depth is equal to or larger than six times the height of the body, decreases monotonically with the decreasing of submergence to a value of approximately 075 when the body is barely submerged Further decrease in submergence w111 resuli in slgnificant surface disturbance and was not considered Because of the high waves used in the computation, it was found that the velocity-related force is no longer negligible Entirely different results were obtained for the cases in which the velocity-related force was neglected, linearized and left to be proportional to the velocity square

Also worth mentioning is the effect of the form of the first wave that encounters the body Since waves are oscillatory in nature, the unsteady response of the body depends strongly on when the body is released in the wave cycle In general, the body has a net translation in the wave direction when the first wave is in the form of a crest, whereas the net translation is opposite to the wave direction if the first wave is a trough This phenomenon can easily be demonstrated experimentally 


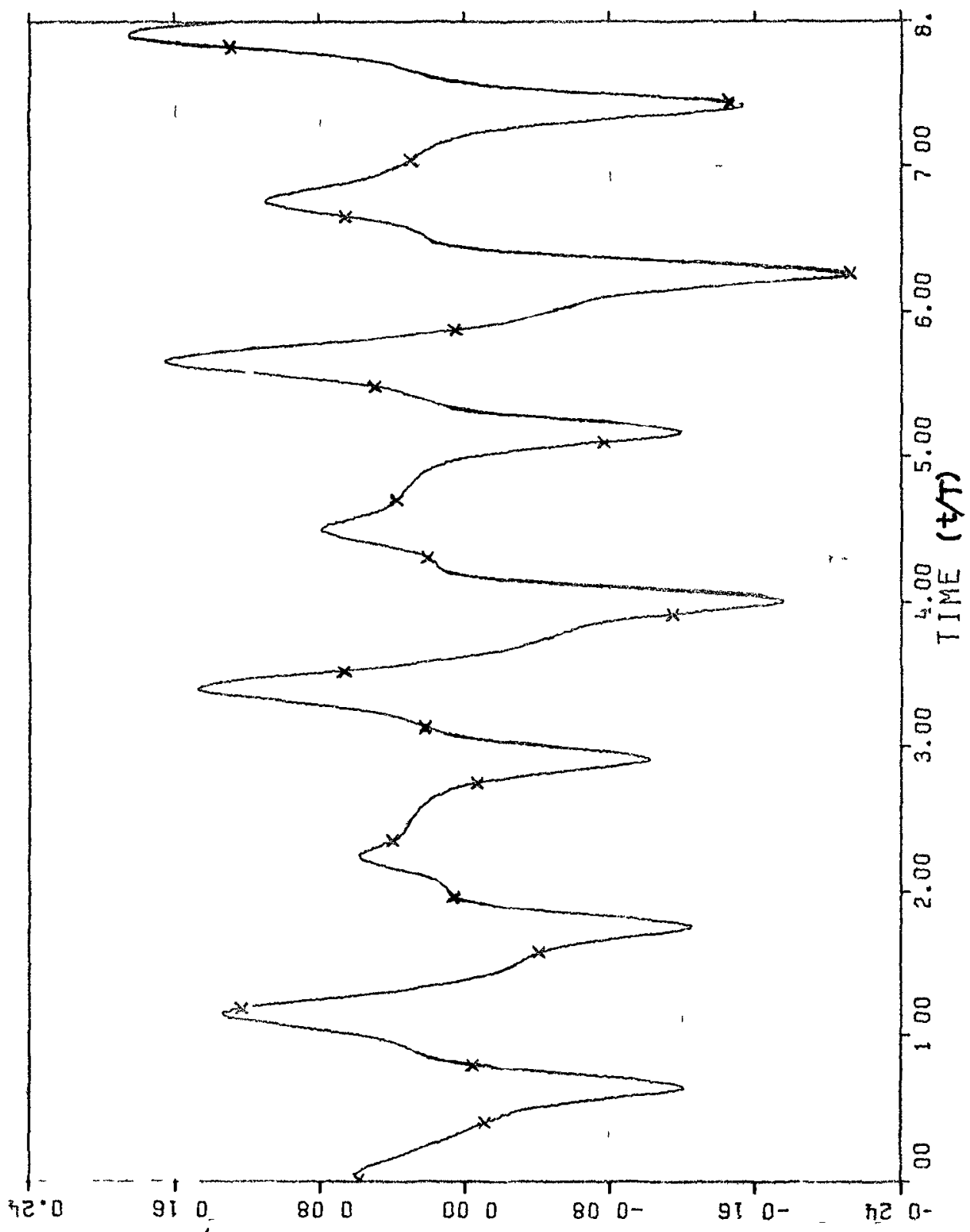

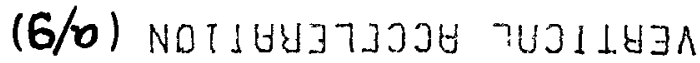

Figure 6 Vertical acceleration (mid-depth) 


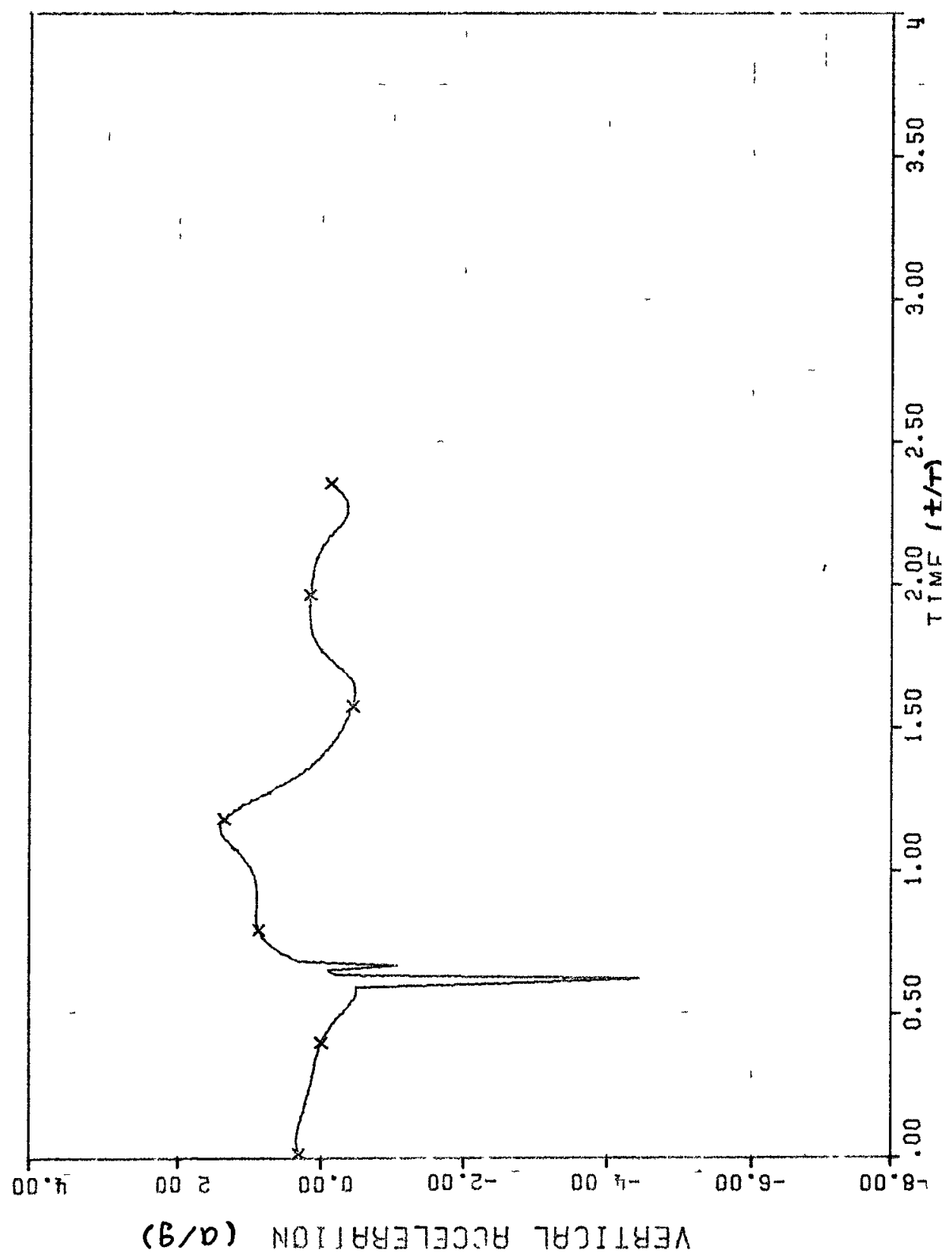

Figure 7 Vertical acceleration (beneath the free surface) 


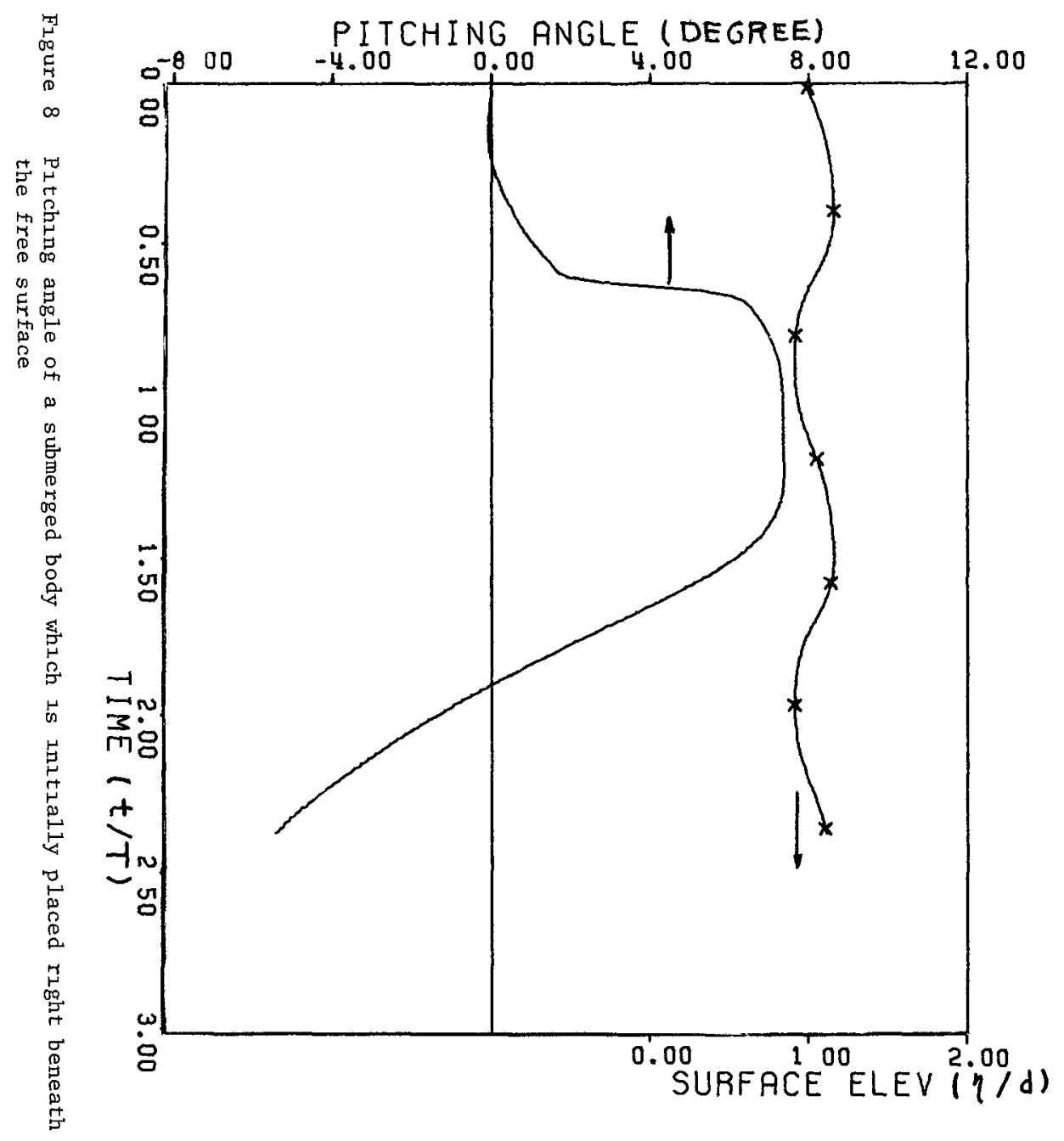




\section{Conclusion}

Through analytical consideration, a numerical method was developed to sult the englneering purpose of quick assessment of the dynamic behavior of submerged slender body in high amplitude shallow water waves To serve this purpose, as many varıables as possible of engineering interest were included In exchange, rigor in mathematics was compromised Approximations such as strip theory, and empirical relations such as drag coefficient were used Much desired are the future 1mprovements of wave theory in shallow water region and a better understanding of velocity-related forces in oscillating fluid flows

\section{Acknowledgement}

This work was performed under the sponsorship of ONR, and the authors would like to express their gratitude to Dr B LeMéhauté for his contribution

\section{References}

Hı1debrand, F B (1956), Introductıon to Numerıcal Analysıs, McGrawHı11 Book Company, New York

Keulegan, C H and $G$ W Pattersen (1940), "Mathematical Theory of Irrotational Translation Waves," National Bureau of Standards Paper RP 1272

Korvın-Kroukovsky, B V (1961), Theory of Seakeepıng, Soc1ety of Naval Architects and Marıne Englneers, New York

Lamb, H (1932), Hydrodynamics, 6th ed 


\title{
CHAPTER 105
}

\author{
OIL BOOMS IN TIDAL CURRENTS
}

by

Ralph H Cross $^{1}$ and David P Hoult ${ }^{2}$

\section{INTRODUCTION}

The classic, and most effective way to prevent the spread of oul spilled in harbors is by surrounding the spill with a floating barrier, or boom In calm water, with no currents, early devices made from telephone poles and canvas were more or less effective In the presence of currents, however, and particularly with larger spi11s, the problem rapidly becomes more complex, and the rational design of ol 1 booms requires an understanding of the behavior of the oll and the boom in the current

This paper presents the results of an investigation into the oll holding capacity of a boom in a steady current, and the forces and moments acting on such a boom

\section{OLL CAPACITY}

A floatıng oll boom anchored at each end in a current to form a Ushaped pocket, with the opening in the $U$ facıng upstream, can gather and hold slgniflcant volumes of olI under proper condltions There are two maln features which determine whether such a boom w111 hold o11, and how much oll can be held The first is the existance of a critical Froude number, above whlch the boom holds no o11, and the second is the shape and behavior of the pool of oll held by the barrier

\section{A Critıcal Froude Number}

A quick estımate of whether or not a boom will hold oll at a11 can be made by comparing the forces acting to draw a small column of oll under the barrier with the buoyancy of such a column of oll, for a section of barrier perpendicular to the current For a column of oll of depth equal to the barrier depth, $d$, and unit cross-sectional area (1n the horlzontal plane), the force driving the o1 1 downward 1 s the difference between the stagnation pressure where the free surface of the water intersects the boom and the free-stream pressure near the bottom of the boom Neglecting hydrostatic pressures (included in the buoyancy term), this difference $15 \mathrm{U}^{2} / 2 \mathrm{~g}$, where $\mathrm{U}$ is the current velocity and $\mathrm{g}$ is the acceleration due to gravity The resulting downward force is, thus $1 / 2 \rho_{\mathrm{w}} \mathrm{U}^{2}$ The net buoyancy is given by $\rho_{\mathrm{W}} \mathrm{g} \Delta \mathrm{d}$, where $\rho_{\mathrm{W}}$ is the mass density of the water and $\Delta$ is the fractional density difference between the oll and the water, convenıently given by $\Delta=$ (1-specıfc gravity of oll) At inclpient fallure of the boom to hold any oll, these two expressions can be equated, ylelding a densimetric Froude number

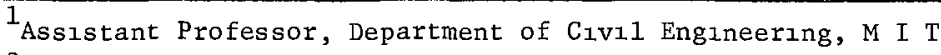

${ }^{2}$ Assoclate Professor, Department of Mechanical Englneering, M I T 


$$
F^{\prime}=\frac{U}{\sqrt{g \Delta d}}=2^{1 / 2}
$$

At Froude numbers above $\sqrt{2}$, the barrier will hold no o11, while below this figure, some quantity of oil will be held

Since the stagnation pressure depends on the component of the velocity normal to the barrier, this analysis also shows that fallure will occur most readily in the apex of the boom (bottom of the U)

\section{B Capacity of the Boom}

Given conditions, then, under which a barrier will hold oil, the remaining question concerns the amount of oll held This question can be approached by considering a two-dimensional pool of oll held up against a barrier by a current (Fig 1) If the oll is significantly more viscous than the water, motions in the oll itself are small, and the configuration of the pool, $h(x)$ vs $x$ (see Fig 1), is determined by a balance between the shear stresses on the underside of the $011, \tau$, and the horizontal hydrostatic pressure gradient corresponding to an increase in thickness of the pool of 011

$$
\rho_{w} g \Delta h \frac{d h}{d x}=\tau=\frac{1}{2} \rho_{w} c_{f} U^{2}
$$

where $C_{f}$ is the friction coefficient Rearranging and integrating,

$$
h^{2}=\frac{U^{2}}{g \Delta} \int_{0}^{x} c_{f} d x
$$

which shows that the shape of the pool depends on the distribution of the shear stress coefficient along the underside of the slick

Near the leading edge of the pool $(x=0)$, the above analysis does not always apply For velocities above 075 to $10 \mathrm{ft} / \mathrm{sec}$, a "head wave" forms, as described in Wick (1969) While the detalls of this phenomena are not well understood, it has been noted that leakage of the barrier can occur by entrainment of ol 1 droplets from the head wave into the floor, and, moreover, it is believed that the head wave does not scale according to the densimetric Froude number

Near the barrier, the slick can become slightly thinner, due to stagnation pressures against the barrier This, however, does not materially affect the volume of oil held

\section{Experimental Results}

A serıes of experıments were performed in the glass-walled sedimentation flume in the Ralph M Parsons Laboratory for Water Resources and Hydrodynamics of the Department of $C_{\perp V I l}$ Engineering at M I T This flume is 
$25 \mathrm{ft}$ wide, $10 \mathrm{ft}$ deep, and $40 \mathrm{ft}$ long, and is equipped with a selfcontained recirculating flow system capable of discharges up to $175 \mathrm{ft}^{3} / \mathrm{sec}$

The barrier used consisted of a masonite panel extending across the channe1, and mounted from above so that 1 ts depth of 1mmersion, or draft, $d$, could be adjusted For given flow conditions, o11 was added upstream, and a1lowed to collect against the barrier untıl leakage was imminent Slight leakage at either end of the barrier, where it met the glass tank walls, was 1gnored Measurements were then taken of the pool thickness, $h$, at varıous distances from the barrier, using a scale on the glass tank walls Two types of oll were used in these experiments soybean oll and No 2 fuel o11, with the following properties

$\begin{array}{lll}011 & \frac{\Delta}{1 / \mu} & \frac{\mu \text { water }}{30} \\ \text { No } 2 \text { fuel } & 077 & 2 \\ \text { Soybean } & 138 & \end{array}$

The profiles so obtained are shown dimensionlessly as g $\Delta \mathrm{h} / \mathrm{U}^{2}$ vs $\mathrm{g} \Delta \mathrm{x} / \mathrm{U}^{2}$ in Fig 2, taken from work done by Robbins (1970) The scatter can be attributed to several sources

1 As the water flow velocity was not constant across the tank, the position of the leading edge varied, the point chosen was an "eyeball average" of 1 ts position across the tank

2 The presence of interfacial waves on the o11-water interface made an accurate measurement of thickness difficult At higher flow rates, these waves were $1 / 8^{\prime \prime}-1 / 4^{\prime \prime}$ h1gh and an inch or two in length Near the boom, especially, reflections from the boom acted to make the waves higher Superposed on the interfacial waves were longer surface waves of simllar helght generated by the turbulence at the upstream end of the tank

The data of Fig 2 gives $h \propto x^{1 / 2}$, Implyıng that $C_{f}$ is independent of $\mathrm{x}, \mathrm{Eq} 3$ then becomes

or

$$
\mathrm{h}^{2}=\frac{\mathrm{U}^{2}}{\mathrm{~g} \Delta} \mathrm{C}_{\mathrm{f}} \mathrm{x}
$$

$$
\frac{g \Delta}{u^{2}} h=C_{f}{ }^{1 / 2}\left(\frac{g \Delta}{u^{2}} x\right)^{1 / 2}
$$

For the fuel o11, $\mathrm{C}_{f}=005$, while for the soybean $011, \mathrm{C}_{f}=008$ for $\mathrm{g} \Delta \mathrm{x} / \mathrm{U}^{2}>120$ (The deviation for low $\mathrm{g} \Delta \mathrm{x} / \mathrm{U}^{2}$ for soybean o11 is believed due to low Reynolds' numbers) 
The difference between the values of $C_{f}$ observed is probably due in part to the scatter in the data, however, weak viscosity or surface tension effects cannot be ruled out

The arguement for a constant $C_{f}$ at sufficiently high Reynolds' number (based on $x$, the distance from the leading edge), has been made by Robbins and Hoult by analogy w1th a sand roughened flat plate For low Reynolds numbers, the plate looks smooth, and $C_{f}$ decreases with increasing $x$ For high Reynolds numbers, w1th the sand grain size, $k_{s}$, increasing with increasing $x$ such that the ratio $\mathrm{k}_{\mathrm{s}} / \mathrm{x}_{1 \mathrm{~s}}$ constant, $\mathrm{C}_{\mathrm{f}}$ is constant (Schlichting 1960) In the 011 slick, the sand roughness corresponds to the growing interfacial waves

Using a value of 008 for $\mathrm{C}_{\mathrm{f}} \mathrm{Eq} 4$ can be written

$$
\frac{g \Delta}{U^{2}} \mathrm{~h}=009\left(\frac{\mathrm{g} \Delta}{\mathrm{U}^{2}} \mathrm{x}\right)^{1 / 2}
$$

or

$$
h=009\left(\frac{\mathrm{U}^{2}}{\mathrm{~g} \Delta}\right)^{1 / 2} \mathrm{x}^{1 / 2}
$$

By inserting the effective boom draft, $d$, for $h$, and the slick length, $l$, for $\mathrm{x}$ in $\mathrm{Eq}$, a relation between slick length and boom draft is obtained

$$
\ell=\frac{d^{2}}{(009)^{2}\left(U^{2} / g \Delta\right)}
$$

To obtain the volume stored per foot width, $h$ can be integrated against $x$ to give

$$
v=\int_{0}^{l} h(x) d x=\frac{2}{3}\left[\frac{U^{2}}{g \Delta} c_{f}\right]^{1 / 2} e^{3 / 2}
$$

or, in dimensionless form,

$$
\frac{\mathrm{V}}{\mathrm{d}^{2}}=82 / \mathrm{F}^{1 / 2}
$$

The profile data was integrated numerically to find the volume stored, the results are shown in Fig 3 (Included in Fig 3 are data from preliminary runs using other o1ls The scatter in this data is generally worse) It can be seen from Fig 3 that Eq 7 gives good engineering prediction of oll retention capacity, and that the critical Froude number is approximately 13 
This section treats the forces on a vertical flat plate oriented normal to the current, a geometry typlcal of most barriers Two cases will be considered the barrier alone, without ol1, and the barrier full of oll to lts depth, d

\section{A Two-Dimensional Forces}

In the absence of 011 , the barrier is simply a vertical flat plate, at hıgh enough Reynolds numbers, the drag coefficlent, $C_{D}\left(D=1 / 2 \rho_{\mathrm{w}} \mathrm{U}^{2} \mathrm{C}_{\mathrm{D}} \mathrm{dL}\right.$, where $D$ is the drag force and $L$ is the barrier length) is independent of Reynolds number With a free surface present, however, one should anticlpate a dependence on the Froude number, $F=U / \sqrt{g d}$, and a simllar dependence for the location of the resultant force

With the barrier full of ol1, the densimetric Froude number must be less than 13 For a typlcal value of $\Delta$ of 010 , the regular Froude number is thus less than 04 Since the Froude number squared represents the ratio of dynamıc $\left(1 / 2 \rho \mathrm{U}^{2}\right)$ to hydrostatıc ( $\left.\rho \mathrm{gd}\right)$ forces, and since this number 1.5 small, a balance of hydrostatic forces from the water on one side and the oll on the other can be made, recognizing that the free surface of the oll lies $\mathrm{d} \Delta$ above the water surface

$$
D / L=\rho_{o} g\left(\frac{\rho_{w}}{\rho_{o}} \frac{d}{2}\right)^{2}-\rho_{w} g \frac{d^{2}}{2}=g \rho_{w} \Delta \frac{d^{2}}{2}
$$

Note that $D$ is independent of the velocity, $U$ The location of the resultant force can be shown by a similar calculation to be approximately $2 / 3 \mathrm{~d}$ above the bottom of the barrier For convenience, the force expressed by Eq 8 can be converted to a drag coefficient, as

$$
\frac{1}{2} \rho \mathrm{U}^{2} \mathrm{~d} \mathrm{~L} \mathrm{C}_{\mathrm{D}}=\frac{1}{2} \mathrm{~L} \rho \mathrm{g} \Delta \mathrm{d}^{2}
$$

Thus,

$$
C_{D}=1 / F^{\prime}{ }^{2}
$$

A serıes of experıments was performed in the tank described above, but with a barrier hung vertically from long wires, and constralned horlzontally only by three LVDT-type force transducers, connected to an operational manıfold and a digıtal voltmeter, arranged and adjusted to give direct readout of force and moment data Dead-welght calıbratıons were used throughout 
Figs 4 and 5, from Robbins' paper, show values of $C_{D}$ and the helght, $z$, of the resultant force, both as functions of the Froude number Without o11, the drag coefficient varies from about 16 at low Froude numbers to 12 at higher Froude numbers With oll, the behavior predicted by Eq 9 appears, verifying the hydrostatic assumptions

The moment data, shown as $z / d$, the relative helght of the resultant force, also support the assumptions, partıcularly for lower froude numbers With oll, z/d Is approximately 055 to 065 , and without o11, 045 to 055 It is important to note that a variation of approximately $02 \mathrm{~d} \mathrm{wl} 11$ be encountered in the depth of the resultant force, so any barrier design has to have adequate roll stability to resist this varying moment

\section{THE DEPLOYED BOOM}

To find the total oil held by a boom anchored by its ends in a current (Fig 6), the shape taken must be found Since the momentum of the flow against the barrier depends on the velocity component normal to the barrier, as $\rho U^{2} \cos ^{2} \theta$, one can assume for simplicity that the drag coefficient for a section of boom at an angle $\theta$ is

$$
C_{D}(\theta)=C_{D}(\theta=0) \cos ^{2} \theta
$$

Assuming that tangential forces on the boom are negligible, and that the boom has zero bending stiffness, an analysis of a differential section of the boom shows that the tension is constant throughout, and that the normal force on the boom is balanced by the tension divided by the local radius of curvature of the boom This can be expressed as

$$
L \frac{d^{2} x}{d y^{2}}=\frac{1}{\lambda}\left[1+\left(\frac{d x}{d y}\right)^{2}\right]^{1 / 2}
$$

where $L$ is the total length of the boom $\lambda$ is a tension factor which relates the tension in the boom, $T$, to the normal force on a "stretchedout" boom,

$$
\lambda=\frac{T}{\frac{1}{2} \rho U^{2} d L C_{D}(\theta=0)}
$$

Assuming $d$ and $C_{D}(\theta=0)$ to be constant, equation 11 can be integrated, using the boundary conditions

$$
\begin{aligned}
& x(0)=x^{\prime}(0)=0 \\
& \frac{L}{2}=\int_{0}^{y_{\max }} \frac{d y}{\cos \theta}
\end{aligned}
$$


The first boundary condition states that the slope of the barrier ( $d x / d y$ ) is zero at the apex of the boom, and the second, that the boom has length $\mathrm{L}$

The solution to Eq 11 gives the boom shape,

$$
\frac{x}{L}=\lambda\left(\cosh \frac{\mathrm{y}}{\mathrm{L} \lambda}-1\right)
$$

where $\lambda$ is obtained as a function of $y_{\max } / L$ (see F1g 6) from

$$
\frac{1}{2}=\lambda \sinh \frac{y_{\max }}{L \lambda}
$$

\begin{tabular}{|c|c|c|c|}
\hline $\mathrm{y}_{\max } / \mathrm{L}$ & $\lambda$ & $\mathrm{y}_{\max } / \mathrm{L}$ & $\lambda$ \\
\hline 10 & 028 & 30 & 163 \\
\hline 15 & 050 & 35 & 231 \\
\hline 20 & 078 & 40 & 336 \\
\hline 25 & 115 & 45 & 560 \\
\hline
\end{tabular}

The table below gives values of $\lambda$ for different values of $y_{\max } / \mathrm{L}$

Note that the ratio of opening width to boom length is $2 \mathrm{y}_{\max } / \mathrm{L}$

The approximate spread of the mooring lines can be computed from the barrier angle at $\mathrm{y}=\mathrm{y}_{\max }$, from

$$
\frac{d x}{d y}=\sin \frac{y_{\max }}{\lambda L}
$$

For $\mathrm{y}_{\max } / \lambda \mathrm{L}<1$, Eq 13 can be approximated by

$$
\frac{x}{L}=\frac{1}{2 \lambda}\left(\frac{y}{L}\right)^{2}
$$

In fact, $y_{\max } / \lambda \mathrm{L}$ is less than 1 only for $\mathrm{y}_{\max } / \mathrm{L}>042$, however, this parabolic approximation is useful over a much wider range of values

A final calculation of the total amount of o1l held can now be made, using Eqs 5 and 16

$$
\text { Volume }=19 \times 10^{3} \mathrm{~L} \mathrm{~d}^{2}\left(\frac{\lambda \mathrm{d}}{\mathrm{L}}\right)^{1 / 2}\left(\frac{\mathrm{g \Delta \Delta} \mathrm{o}}{\mathrm{U}^{2}}\right)^{3}
$$


Here $h_{0}$ is the depth of the oll in the apex of the boom, this must be less than the draft, $d$ - Moreover, $F=U / \sqrt{g \Delta h}<12$, and $\ell<x_{\max }$

CONCLUSION

Using the above information on oll holding capacity, barrier shape, and forces on barriers, it is possible to design and operate floating barriers to capture o11 spills in rivers and tidal currents, at least at modest velocitıes At higher velocities, but sub-critical Froude numbers, some leakage from head wave entralnment can be expected The concentration of 011 in such a pool w111 greatly simplify the collection and removal of 011 from the water surface

\section{REFERENCES}

Hoult, D P , 1970, "Contalnmented 011 Spı11s by Physical and Alr Barriers", Joint Project sponsored by Department of Mechanıcal Engıneering, Department of CIVI1 Engineering and Department of Naval Architecture and Marine Engineering, Massachusetts Institute of Technology

Robbins, R E., 1970, "The 011 Boom in a Current", M S Thesis, Department of Electrical Engineerıng, Massachusetts Institute of Technology

Schlichting, H, 1960, Boundary Layer Theory

W1cks, M, 1969, "Flu1d Dynamics of Floatıng O11 Contalnment by Mechanıca1 Barriers in the Presence of Water Currents", Shel1 Pipe Line Corporatic Research and Development Laboratory, Houston, Texas 
OIL BOOMS
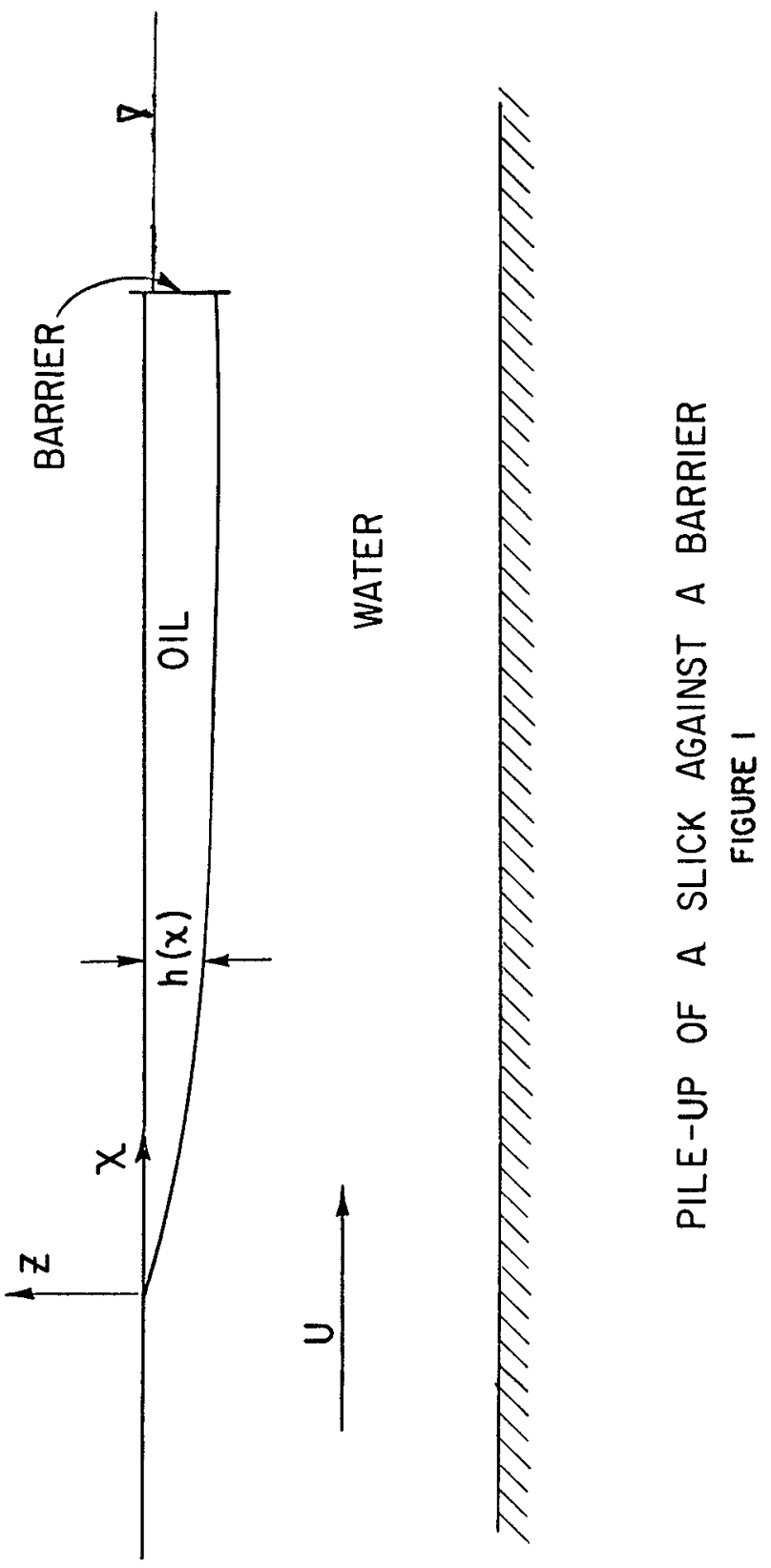


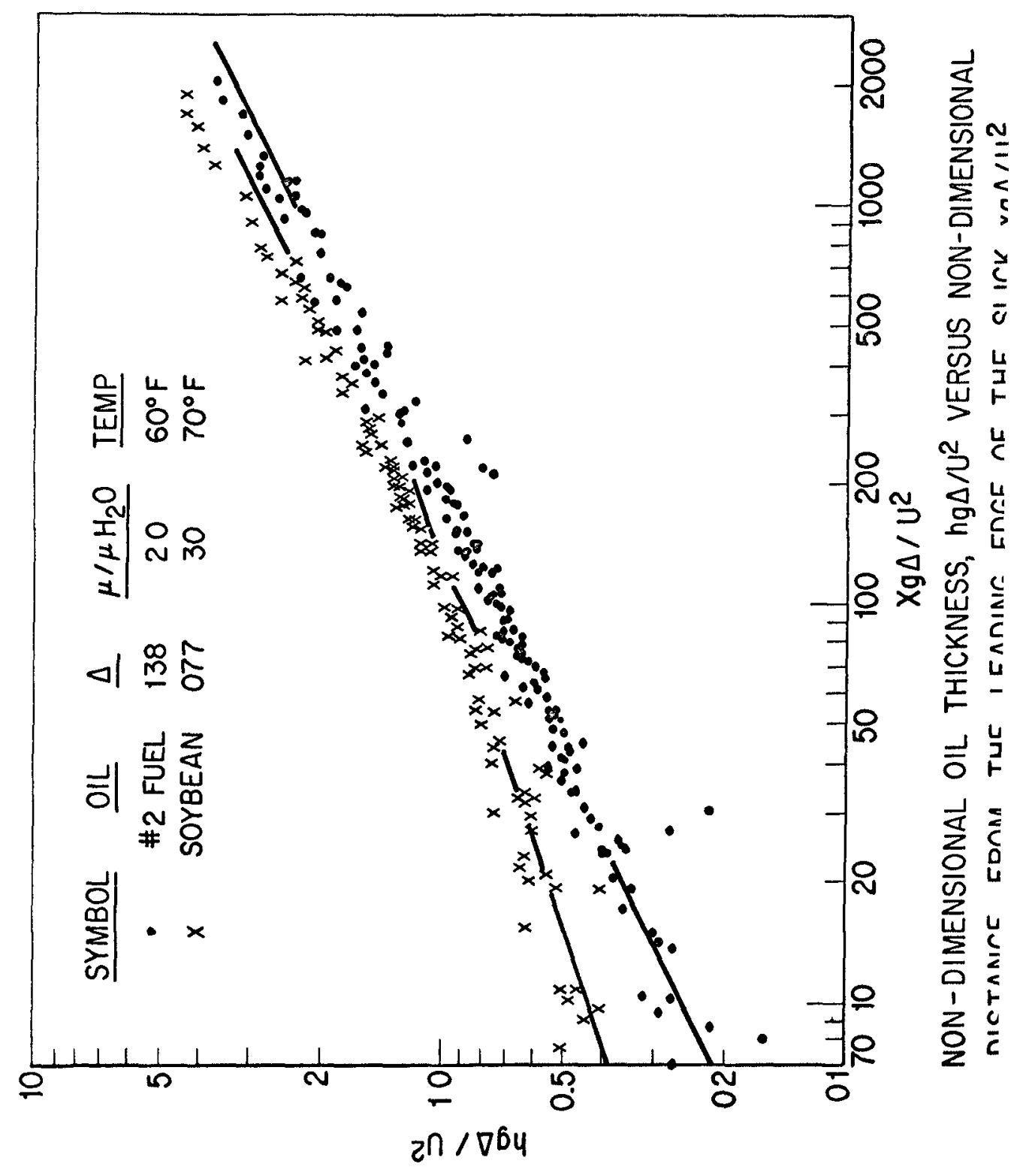

FIGURE 2 


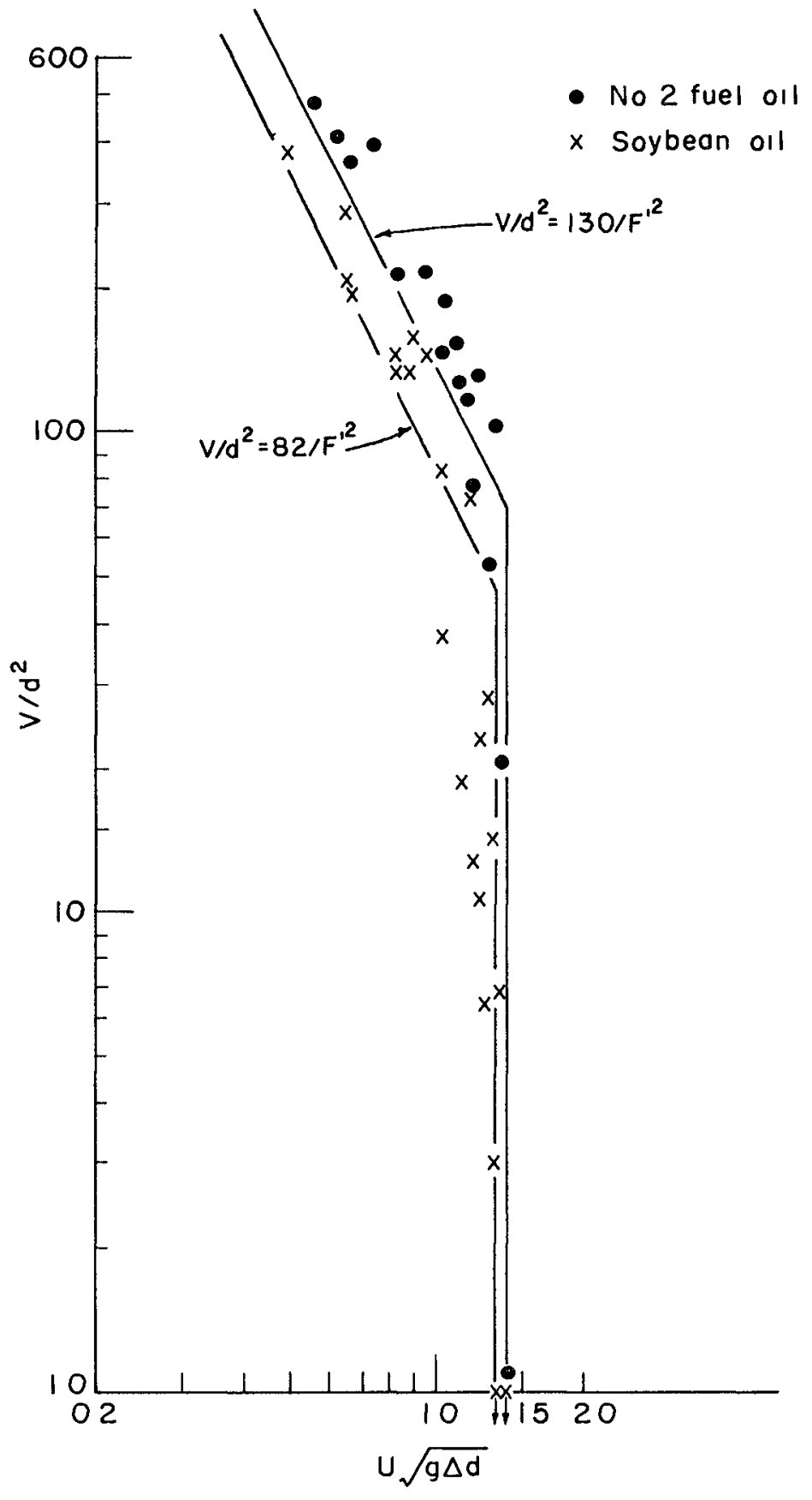

FIGURE 3 Non dimensional oll volume held, $V / d^{2}$, versus densımetric Froude number, $U \sqrt{g \Delta d}$ 


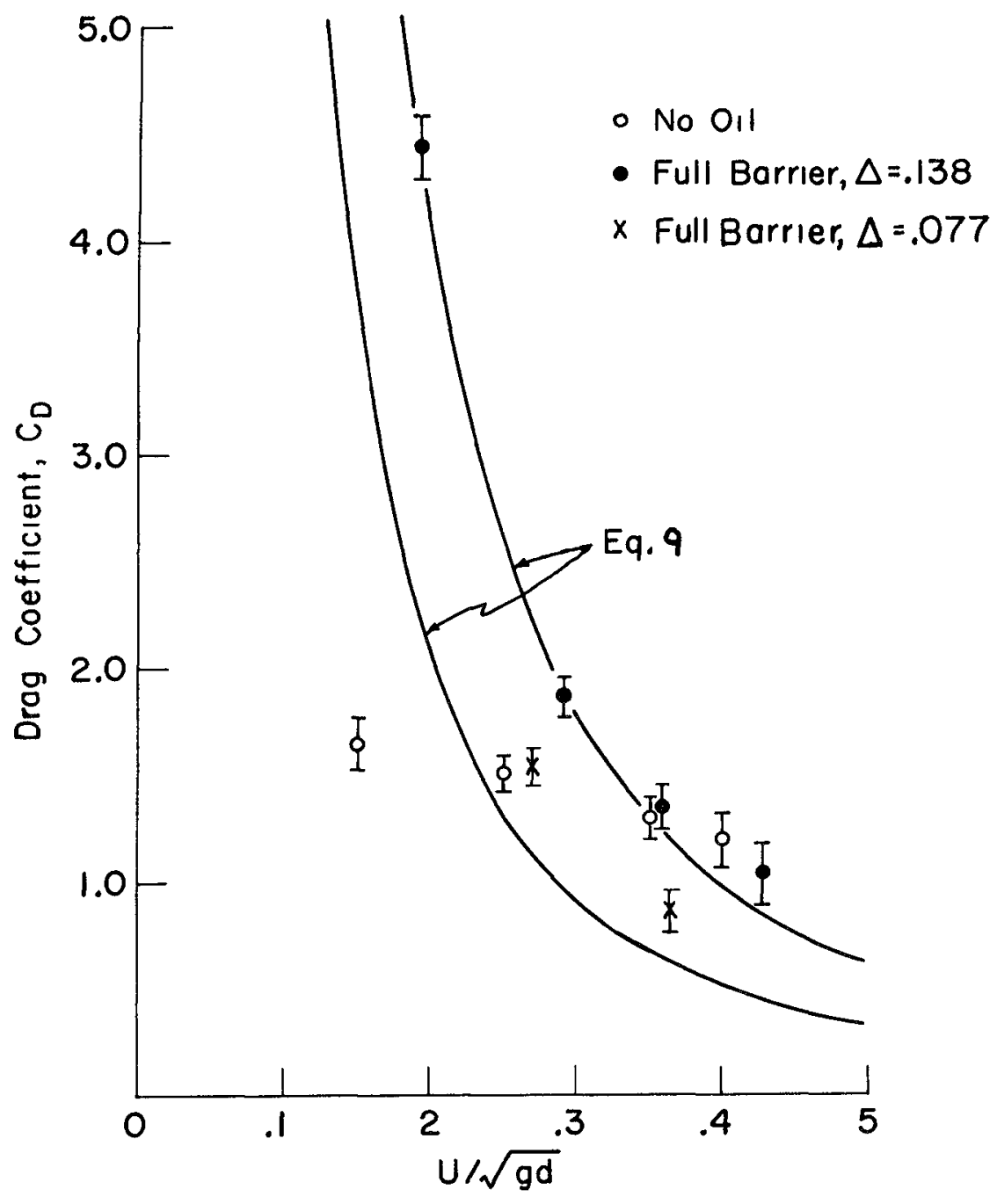

FIGURE 4 Drag coefficient, $C_{D}$, versus $U / \sqrt{g d}$ 


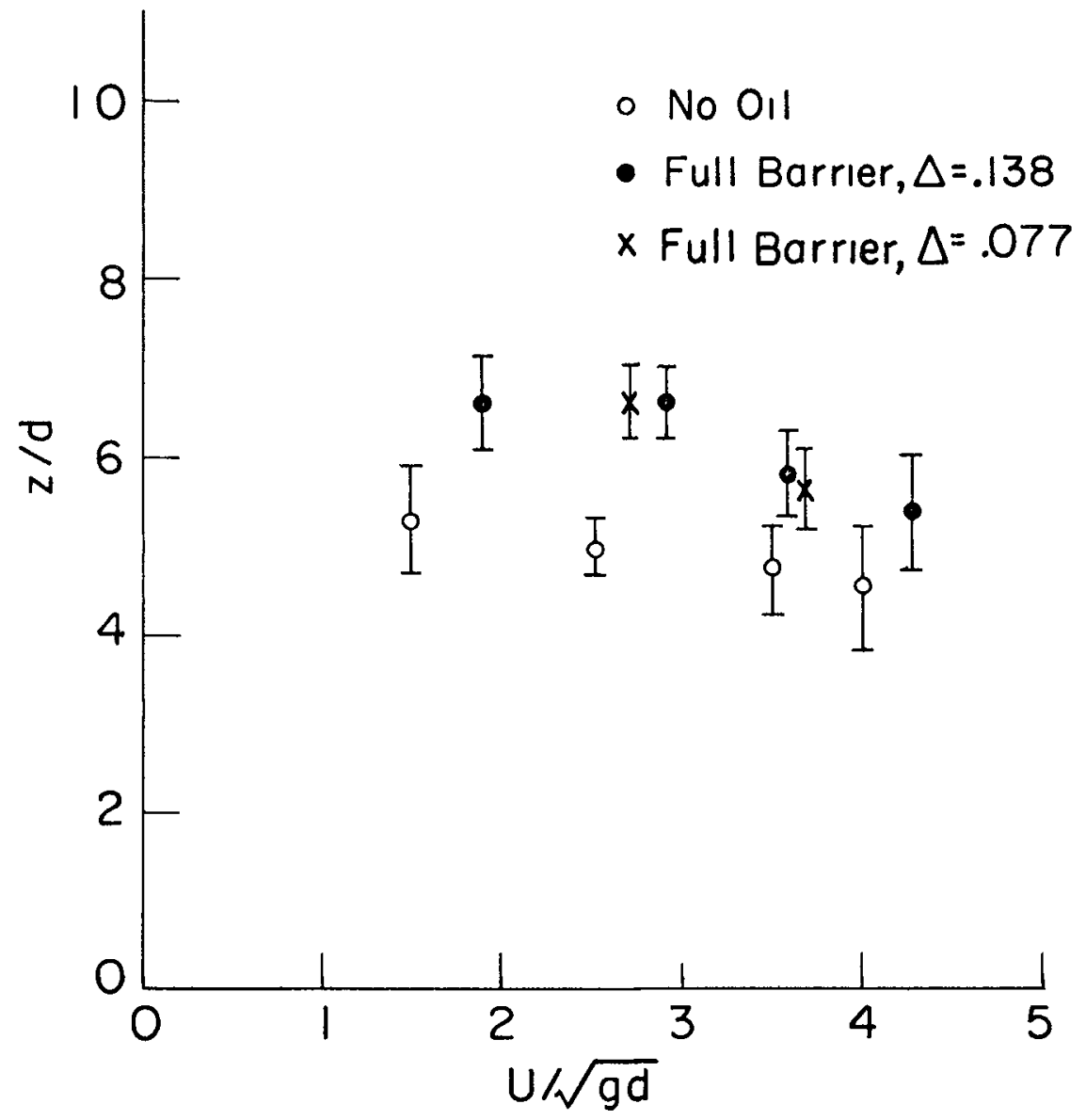

FIGURE 5 Location of drag force from lower edge of boom, z/d, versus $U / \sqrt{g d}$ 


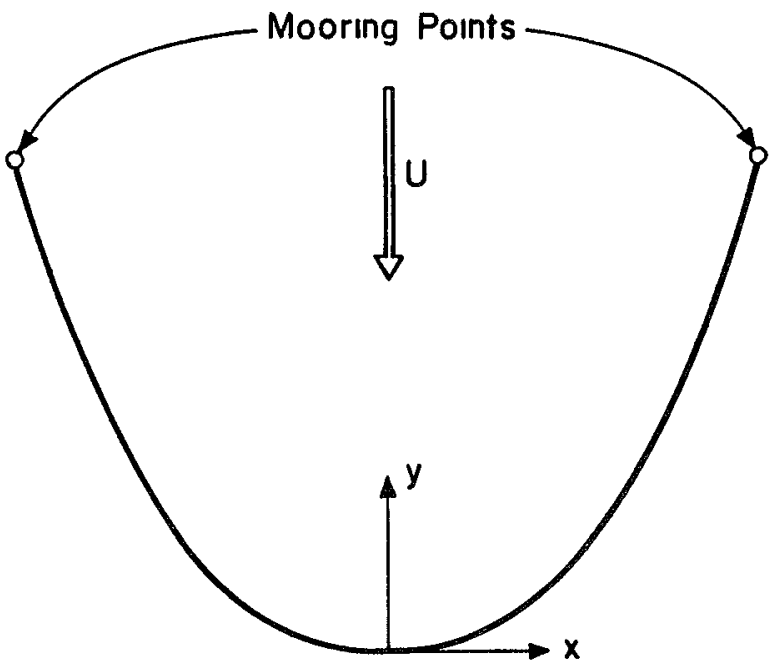

Figure 6 Sketch of planform of a boom in a current showing coordinates 


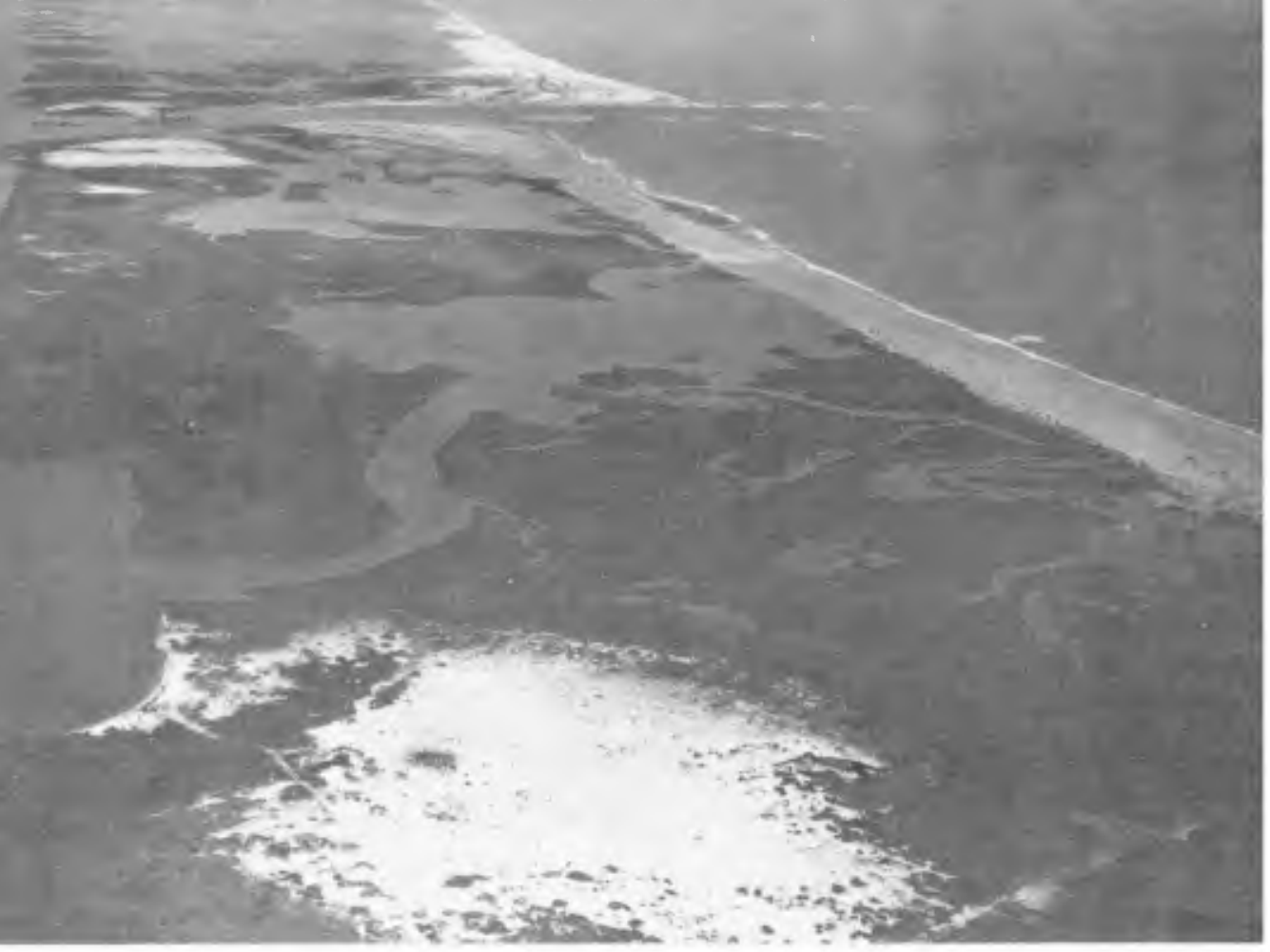

Carolina Beach Inlet, North Carolina

PART 4. COASTAL, ESTUARINE, AND ENVIRONMENTAL PROBLEMS

Treasure Island, St. Petersburg, Florida

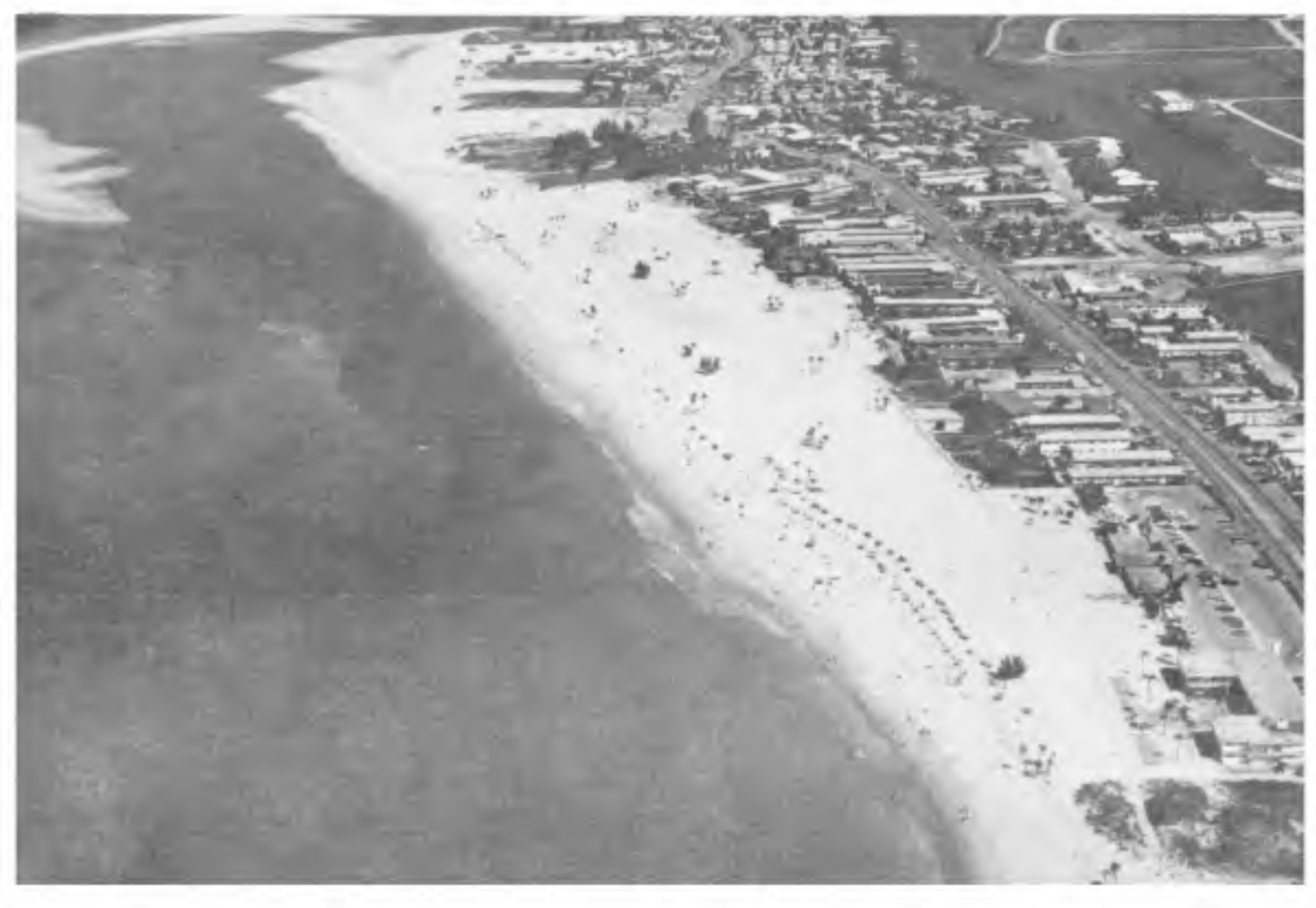





\title{
CHAPTER 106
}

\section{ENVIRONMENTAL PLANNING OF A BAY AND COASTAL LAGOON SYSTEM}

\author{
by \\ Norman $T$ Gilroy \\ Architect and Environmental Planner \\ Norman $T$ Gl lroy and Associates \\ Mill Valley, Calıfornia
}

The 1970s are, it seems, the beginning of an "age of environmental awareness " Never before has our nation been so conscious of the necessity for protection of the quality of 1 ts waters, 1 ts air, 1 ts aesthetic environment, and the productivity of 1 ts earth, and never has 1 t been so aware of the potential, within man's actions for progress for the pollution of his environment or the creation of $1 \mathrm{mbal}$ ance within the natural dynami $c$ system The realization 15 growing that the system of natural dynamics, the ecosystem and the natural aesthetic environment in which we live are, in fact, part of a complete interrelated, interdependent and synergistic "Environmental System" Man made modification of any part of the System carries with it the potential of effects extending throughout the rest of the system, often resulting in major, and at times catastrophic, modification of the natural balance of the physical environment as we know it today

Though the principles of environmental sensitivity are only newly being adopted by our media, our community leaders and our elected off1clals, there are some who have been advocating and practicing the principles of environmental planning for some time It has long seemed logical that major engineering or planning programs, capable of generating extensive modifications to the environment into which they are interposed, should be considered, throughout all their phases in terms of the entire spectrum of environmental factors which make up the site and 1 ts surroundings-yet rarely is this the case

An area of particular environmental sensitivity lies in our ocean and along our coastline Here nature's forces are at their most dynamic, while her ecological delicacy 15 often at 1 ts most vulnerable The ocean's waters frequently act as a transfer medium, permitting the impact of man's modification at a specific location to be felt over great distances We need only to remember any of numerous examples of the loss of beaches, radical modification of coastline characteristics, extensive changes or virtual annihilation of segments of our coastal ecosystem, and the effects of heated waste water emitted from power plant cooling systems upon adjacent ecosystems, to be aware of the potential effects of man's change upon downcoast areas within the ocean system

Nature is not always capable of coping with her own problems, however Frequently the natural sediment movement processes at work in our coastal waters or heavy growth of natural plant $11 \mathrm{fe}$ can be as instrumental in smothering a portion of an ecosystem as any actions taken by man Such 15 the case in many of the intertidal lagoons along the coast11 ne, whose fate it is to disappear, transformed by sedimentation into a muddy meadow, if nature is allowed to run her course without man's intervention 


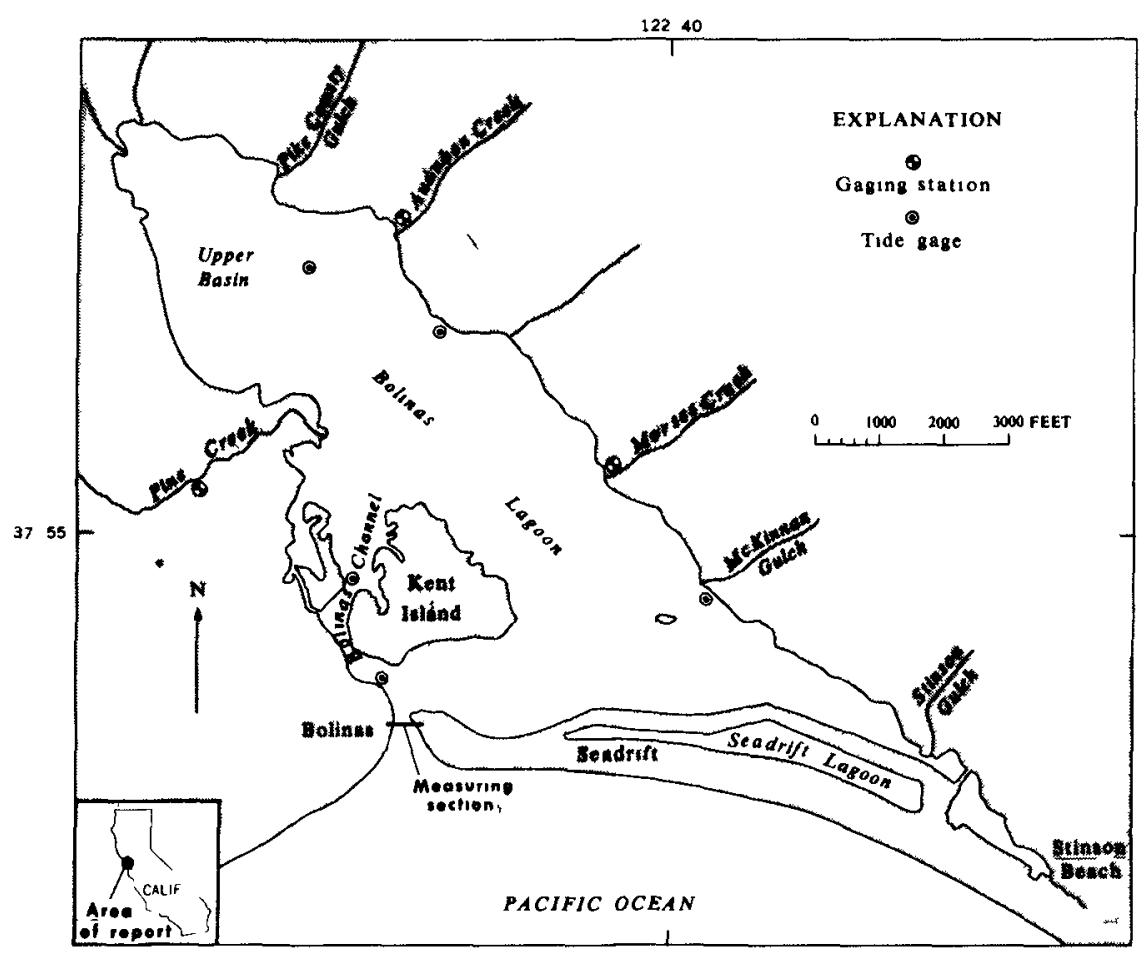

Figure 1 Bolınas Lagoon

Few more 1 llustrative examples of such conditions, combined with the complete range of ocean environmental systems, exists than at Bolınas Lagoon on the west coast of California, twelve miles north of San Francisco (Figure 1) Bolinas Lagoon is a triangular, intertidal coastal lagoon three miles long and one and one-half moles wide, which lies in the rift valley of the San Andreas Earthquake fault which runs the length of the State of California The Lagoon is separated from the Pacific Ocean by a curving sandspit, which, together with the high cliffs of the Bolinas Headland and Duxbury Reef to the west, form a typlcal hooked bay configuration The main channel, the only link between the Lagoon and the ocean, lies between the sandspit and the beginning of the headiand at the southwesterly corner of the Lagoon, adjacent to the historical town of Bolinas Through a geological phenomenon, radically different geological formations flank the Lagoon on el ther side of the fault, contrasting the softly rolling, steep yet stable, Franciscan formation hills of the coastal range to the east, with the highly erodible miocene marine terraces of the Bolinas Mesa Headlands and the beginnings of the Pornt Reyes Peninsula to the west Sedimentation has continued unchecked for centuries, wi th some encouragement by man's logging operations in the 1870s when the watershed lands were denuded to serve the need for timber for the building of San Francisco Today the Lagoon's circulatory pattern is virtually 
inoperative as a self-flushing, self-maintaining, dynamic system, and an offshore bar has grown across the channel mouth further minimizing the flow of oxygenated tidal water to the Lagoon The extremely productive ecosystem of the Lagoon shows signs of faltering, with die-off occurr, ng in areas no longer adequately supplied with tidal circulation Clam populations, subsurface $11 \mathrm{fe}$ systems, Juvenile fish nurseries which serve the plentiful offshore fishery at the Farrallon Islands, bird feeding grounds, and harbor seal habitats all face the prospect of certain death if the process of nature continues unchecked, and with them will disappear many of man's recreational and sports opportunities, as well as a significant water resource of national and statewide importance

Man has added to the complexity of the problem The State of Calıfornia has, for many years, designated Bolınas Lagoon as a priority site for a primary Harbor of Refuge to serve ocean sailors and fishermen from San Francisco Bay in times of fog, storm and emergency The Lagoon is heavily polluted due to a continuous inflow of untreated effluent from an antiquated sewage collection system serving the town of Bolinas The pressures for development of the watershed, as an extension of the growing metropolitan area of San Francisco, are mounting, and the growing flow of tourist visitors to the beaches of the area and to the newlyestablished Pornt Reyes National Seashore is placing new stress upon the small beach communities of the area, and upon the Lagoon 1 tself, through a growing demand for use as a recreational resource

There are still some who advocate inaction in the name of conservation, fearing, apparently, that man's modification of the natural processes may result in catastrophe rather than survival, apparently mindless of the fact that nature is on a sulcide course of her own in Bolinas Lagoon, which can only be reversed by an act of God or by man's 1ntervention Fortunately there are others who recognize the potential for the survival and in the utilization of the Lagoon through a plan of action, created by man and based upon a deep understanding of the environmental systems which are Bolinas Lagoon

In 1966 Noman T Gilroy and Associates, Planners, Architects and Consultants in Environmental Design of, Mill Valley, California, were engaged by local agencies to coordinate, implement and report on a multidisciplinary program of study and research into the natural environmental systems of Bolinas Lagoon and 1 ts watershed, designed to act as the first step for planning the long-term conservation and use of the region and the Lagoon From a virtually nonexistent economic base, thirty-six Federal, State, local, private and university research and funding programs were assembled into an integrated program of investigation valued at over $\$ 208,000$

The natural environment of Bolinas Lagoon was analyzed in its three fundamental segments $(1)^{*}$

a) The Watershed - Field investigations of the mineralogic and sol 1 characteristics of sediment sources in the watershed lands, of the flow, velocity, and sediment transport characteristics of tributary water courses, and of local surface drainage systems and regional rainfall

` See References 
characteristics were undertaken by the $U S$ Geological Survey (2) and the U S Soll Conservation Service over a 3-year period An innovative graphic analysis system was utilized to define the sensitivity of the watershed to both erosion and change through development Remedial measures to control and lessen natural sedimentation, and guidelines to guard against future acceleration of sedimentation through development in the watershed were defined for inclusion in local regulatory ordinances Strong indications linked massive erosion of the Bolinas Headland, which has caused a 125-foot retreat in the 7,000 foot long, 120 foot high cliff11 ne over the past 56 years (and is caused by the combined action of groundwater-generated slides in the cliff face and the battering of heavy ocean storm waves), with the choking inundation of the Lagoon itself with light sediments A combined buffer beach, intercept drainage gallery and surface drainage system are proposed to combat at source a problem which evidently pervades the entire dynamic system of Bolinas Lagoon

b) The system of natural dynamics of Bolinas Lagoon and Bolinas Bay - A broad range of studies under the guidance of the U $S$ Geological Survey was undertaken in the Lagoon itself The Lagoon studies were supplemented by other studies in Bolinas Bay by the USGS (2) and by the University of Cal1 fornia under the guidance of $J W$ Johnson Following a detailed hydrographic and topographic survey of Bolinas Lagoon utilizing laser surveying techniques, periodic monitoring programs of flow velocities and sediment loads in the main channel to the ocean and in the circulatory network within the Lagoon were completed The mineralogical characteristics of the bottom sediments of the Lagoon were analyzed and compared with the characteristics of sediment sources Time lapse photography over a 15-month period monitored the changes in configuration of the sandspit and the main channel, and graphic comparisons were made between a series of quarterly bathymetric surveys of Bolinas Bay to plot the quantitative transport of sediments within the Bay itself These studies were coupled with a mineralogical analysis of the bottom sediments of Bol inas Bay by $P$ Wilde, a series of field observation studies and measurements of wave characteristics, a beach profile monitoring program, and mathematical analysis of the refraction of deep water storm waves as they impinge upon the shoreline of the sandspit Much of this work $1 \mathrm{~s}$ described in greater detail in companion papers by $J W$ Johnson and $P$ W1 lde (See Chapters 85 and 86 )

The system of natural dynamics of Bolinas Lagoon and Bolinas Bay was thus defined Contrary to previous assumptions, it was found that the 11 ttoral system of Bolinas Bay 15 largely self-contained, with sand moving onshore and offshore on a seasonal basis This fact is confirmed by ecological studies of the bottom sediments of Bolinas Bay, which reveal a host ecosystem incapable of surviving in the everchanging conditions which would be consistent with an extensive downcoast 11 ttoral transport system An arrowhead jetty channel stabilization system is recommended at a cost of $\$ 2,500,000--a$ considerable reduct 1 on from the $\$ 11,000,000$ structures recommended previously using "worst case" design criteria without benefit of field research 
c) The Ecosystem - A series of 14 studies of the ecosystem of Bolınas Lagoon was completed by a team led by Joel Gustafs on of Resources and Ecology Projects, Inc (3) Elghteen months of field studies mapped vegetation, fish nurseries, mollusk populations and defined key index species and the trophic food chain A graphic "sensitivity analysis" of the Lagoon was completed, "grading" the Lagoon by degree of 7 mportance or sensitivity in the four major elements of the ecosystem, $1 \mathrm{e}$, subsurface $17 \mathrm{fe}$ (clams, worms, shrimp, etc), bird wading and feeding areas, fish and marine animal habitats, and marine aquatic and terrestrial plant $1 \mathrm{fe}$ A composite analysis of the four resulting charts indicates clearly the areas of greatest sensitivity in most need of preservation through establishment of a wildi ife refuge, and those areas most able to cope with man's intrusion without damage to the ecosystem as a whole Recommended restoration of the circulatory system of the Lagoon will renew the flow of oxygenated water to nourish the brota, while permitting flushing of pollutants and use of the Lagoon by man for recreation and harbor purposes The study resulted in the moving of the primary harbor site location which had been deemed suitable for years, yet which, when scientifically examined, proved to be at the heart of the most delicate, ecologically important, area of the Lagoon system A computer model of the circulatory system was built and tested, and $1 \mathrm{~s}$ a medium for evaluation of all proposed physical change within the Lagoon for the future

Having defined the natural systems of Bolinas Lagoon and evaluated their areas of sensitivity, decisions for the future of Bolinas Lagoon can be made in an atmosphere truly sensitive to the host environment Examination of the future demands of man in the watershed have resulted in various recommendations which will permit nature and man's activities to co-exist, in some cases the improvements that will accompany man's uses will themselves be beneficlal to the system as a whole Not the least of these $w_{1} 17$ be the marntenance of an open artery between the ocean and the Lagoon Separation of the specific functions which the Harbor of Refuge must serve has prompted the innovative concept of a summer harbor located outside of the Lagoon behind the breakwater to cope With the maximum need for shelter in times of summer fog or emergency, and a small basin within the Lagoon to serve the lesser winter storm refuge need at a location protective of the overall ecological system The quality of the natural biotic system has been recognized, and is utilized in a proposed marine biological research and education center and in various conservation-oriented recreation and park functions on the perimeter of the Lagoon Large portions of the land necessary are already passing into the public domain through acquisition utilizing State and Federal funds The Town of Bolinas is proposed for preservation and restoration as a historical landmark, with 1 ts beaches expanded through the cliff erosion solution recommended, and with its traffic problems largely solved as a part of the plan Channel stabilization programs, cliff erosion solutions, harbor of refuge facilities, sewage treatment facilities and public use concepts have all been designed as multipurpose facilities, permitting funding through a number of State and Federal programs and enabling a relatively small community to implement solutions of considerable magnitude through proper structuring at the design concept stage 


\section{REFERENCES}

1 Gilroy, Norman $T$ (1970) Bolinas Lagoon Its system of environmental dynamics and recommendations for $i$ ts future preservation and utilization Prepared for the Advisory Commission on Navigation and 0cean Development, State of California, the Board of Harbor Commissioners of the Bolinas Harbor District and the County of Marin

2 Ritter, John R (1969) Preliminary studies of sedimentation and hydrology in Bolinas Lagoon, Marin County, California, May 1967June 1968, U S G S Open-File Report, Apri1 4, 1969

3 Gustafson, Joel F (1968-69) Ecological studies of Bolinas Lagoon, Parts 1, 2 and 3 (also see appendices of Reference 1) 


\title{
CHAPTER 107
}

\section{DETERIORATION AND RESTORATION OF COASTAL WETLANDS}

\author{
By Sherwood M Gagliano \\ Hyuck $\mathrm{J}$ kwon \\ Johannes $\mathrm{L}$ van Beek \\ Coastal Studies Institute \\ Loulsiana State University \\ Baton Rouge, Loulsiana 70803
}

\begin{abstract}
Coastal Loulslana wetlands are a product of Mississippl Rıver delta bullding that has occurred over a period of 5,000 years The building process was a gradual one, for ruverune and marıne processes were very nearly balanced In modern times man's use of the area (flood control, navigation improvement, exploitation of petroleum and other minerals, road bullding, etc ) has serıously altered the natural balance As a result, overbank flooding has been virtually elıminated and river flow is confined to channels discharging into the outer shelf area Most transported sediment is now deposited in the deep Gulf of Mexico or along the continental shelf Saltwater encroachment in the deltalc estuaries has been detrimental to fauna and flora Even though considerable sediment deposition has resulted from the historic Atchafalaya River diversion and growth of subdeltas, comparatıve map studies indıcate a net land loss rate of 165 miles 2 year during the last 25 to 30 years Land loss is only one symptom of general environmental deterioration
\end{abstract}

A dynamic management plan 1s proposed for better utilization of combined freshwater discharge - dissolved solid and transported sediment input from the Mississippl River Controlled flow into estuaries will reduce salınity encroachment and supply badly needed nutrients Large areas of new marshland and estuarine habitat can be bullt by controlled subdelta diversion Studies of natural subdeltas indicate that these systems are amenable to environmental management, salınitıes and sediment deposition may be manipulated to enhance desired conditions

\section{INTRODUCTION}

Southern Loulsiana 1 s a 300-mlle coastal lowland consisting of large tracts of marshes and swamps and innumerable lakes and bays This extensıve near-sea-level area, estimated at 4,000,000 acres (0'Ne11, 1949), makes up the deltaic plain and marginal components of the Mississippl River delta system and is the result of deposition of river sediment during the past 5,000 years (FIg 1) Like all deltas, that of the Mississippl is a zone of interactions between fluvial and marine processes and constitutes one of the most dynamic situations in nature The interaction of these processes through time results in a dynamically changing complex of environments within delta regions Deposition of sediments vies with subsidence and erosion in a never-ending exchange of land and 


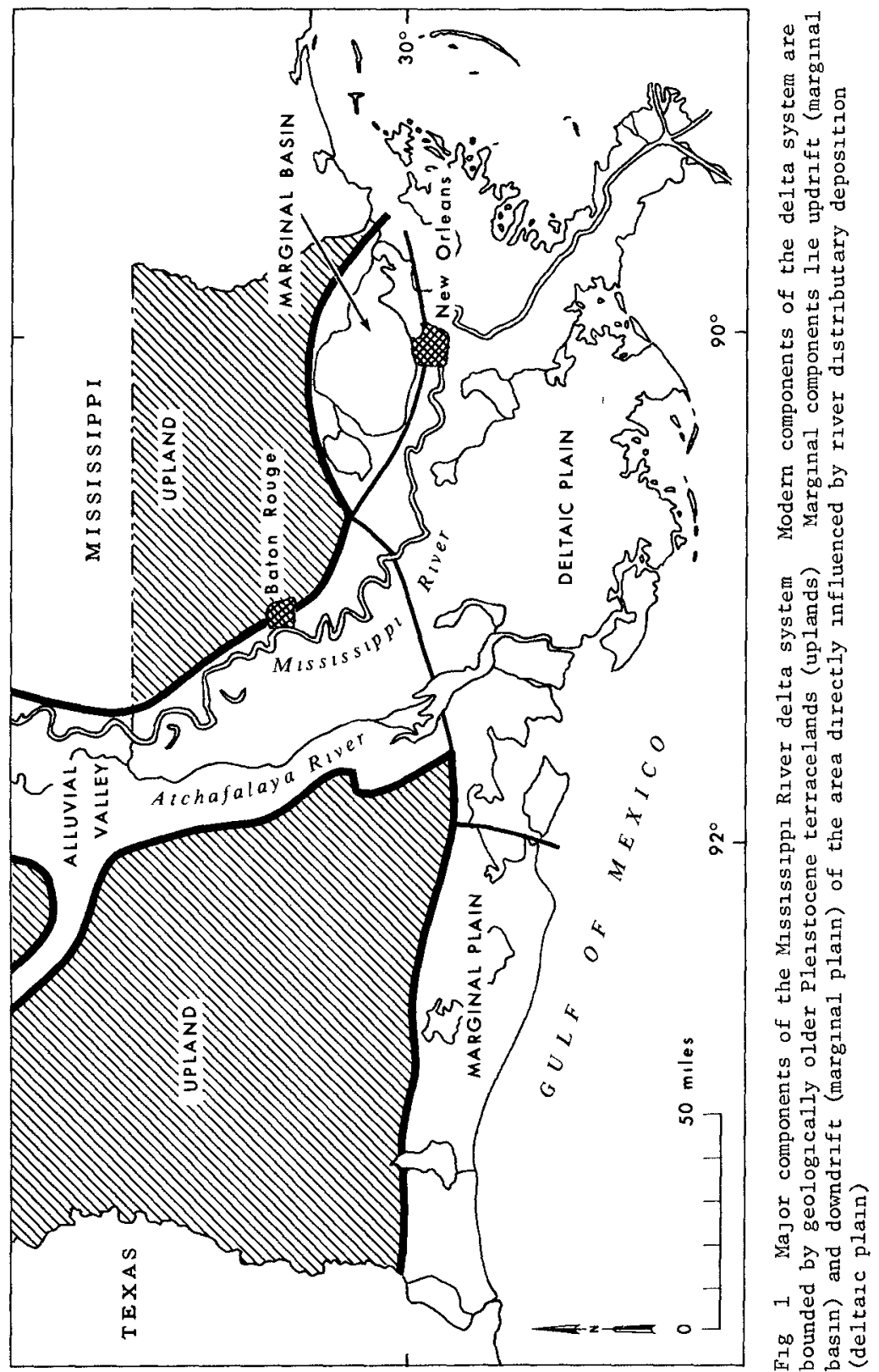


water areas The balance between rates of deposition and the combined effects of subsidence and erosion by the sea causes shorelines of deltas alternately to advance seaward and to retreat Seaward growth occurs at the mouths of active streams, whereas erosion results near the mouths of Inactive streams which no longer transport sufficlent sediment to sustaln thelr seaward advance This is the reason that delta bullding is so often depicted as a contest between the river and the sea If the river deposits sediment faster than the sea $1 \mathrm{~s}$ able to remove $1 \mathrm{t}$, new land is added to the shore, and the delta is said to prograde As the delta is extended, $1 t$ gradually builds upward or aggrades by processes associated with lateral shiftıng of channels, by sediment depositıon during overbank floodıng, and by accumulation of plant and anımal remains

\section{DETERIORATION OF THE DELTAIC COAST}

\section{Under Natural Conditıons}

Deterioration of the delta occurs if all or part of it is deprived of the necessary supply of river-borne sediment for its continued outward growth This deprivation results in the reworking and/or removal of the seaward edge by wave attack, and the combined effects of compaction, consolidation, and subsidence lower the surface below sea level

Under natural conditions progradation along any given segment of the deltalc shoreline is cyclic During Recent geologic times southern Loulsiana has witnessed development of numerous lobate extensions of the delta in areas of active discharge and sedimentation After bullding one delta lobe, the Mississippi channel usually has shifted as a result of upstream. diversion, and a new lobe has been built Many repetitions of this process have produced a deltaic plain that is an aggregate of abandoned or inactive delta lobes, each of which has undergone a degree of deterioration dependant on its relative age

Before the Mississippl occupled its present course, the river emptied 1 ts water and sediment discharge into shallow waters of the inner continental shelf, bullding lobes rapidly and efficiently Under these circumstances the rate of new land bullding was always higher than that of land loss occurring concurrently in abandoned delta lobes

From the preceding discussion it might be concluded that when viewed through geologic time a delta system is always in delicate balance-that is, on one side of the fulcrum there is an input of discharge and transported sediment and on the other side there are such factors as coastal erosion and subsidence, which cause shoreline retreat The very existence of south Loulsiana bears witness to the fact that there has been net progradation over the past 5,000 years

\section{Man's Effect on the System}

Man's intervention in coastal Louisiana has seriously upset the natural balance in the delta system In modern years it has been necessary to alter natural processes in order to prevent flooding and to improve navigation As a result, virtually all overbank flow has been eliminated Furthermore, the modern birdfoot delta is nearing the edge of the continental shelf, 
and most transported sediments are now disappearing into the abys The river's attempt to divert to a new course that would allow development of a shallow-water delta lobe was aborted The we11-known attempted capture of the Mississippi by the Atchafalaya RIver has now been arrested by control structures, and the Atchatalaya is restricted to only 30 percent of the total Mississippi flow (U S Army Corps of Engineers, 1951, Fisk, 1952) As a result of these control measures the amount of land bullding has been sharply reduced, but the rate of land loss in abandoned portions of the deltaic plain contsnues

These restrictions in themselves would have serious repercussions, but other developments have caused further imbalance in the delta system Diking and drainage of marshland for agricultural purposes has been widespread and in many instances unsuccessful After drainage organic marsh solls oxldize and shrink, reducing the dralned land surface to below-sealevel elevatıons Inundation by hurricane-generated storm surge has often resulted in abandonment, numerous rectangular lakes being left as mute testimony to land reclamation fallure (Harrison, 1961) Equally serious has been the dredging of Innumerable canals to provide access to 011 well drilling sites and pipeline right-of-ways These invariably alter circulation patterns in the estuaries, resulting in a general saltwater encroachment of the brackish swamps and marshes

\section{Map Studies of Land Loss}

A fundamental question posed for this investigation, therefore, was, "Is the delta, and for that matter the coastal area on the whole, building or retreating"" The question has been approached by a number of workers (Morgan and Larımore, 1957, Treadwe11, 1955, Kwon, 1969, Saucier, 1963, Russe11, 1936, Peyronnin, 1962, Welder, 1959), who c1ted specific instances of shoreline advance or retreat However, it has long been a common misconception that erosion occurring along some parts of the Louisiana coast is more than offset by the bullding of new land in other areas We have attempted to reevaluate this problem by quantitative map studies, we used the ratio of land to water in a given sample area as an index of net loss or gain of land

Fortunately, systematic planimetrıc mapping of coastal Loulsiana was inltiated in the $1890^{\prime} \mathrm{s}$ by the U S Geological Survey In the $1930^{\prime} \mathrm{s}$ this area was remapped, and use was made of controlled aerial photomosalcs Mapping and remapping have continued since the $1940^{\prime} \mathrm{s}$, so that at present most of the 15-minute quadrangle areas have been covered at least twice An example of changes that occurred in one small area between 1935 and 1953 is shown in Figure 2 Th1s careful periodic remapping has made $1 t$ possible to determine the ratio of land to water for a particular area and mapping interval, these values in turn can easily be converted into land loss or gain in acres per year

The map shown in Figure 3 was constructed by contouring the land loss or gain rate obtaned for each $71 / 2-$ m nute quadrangle From the map several things are ımmediately apparent As indicated by the patterns, most of the deltalc plain is in a serious condition of deterioration During the last 30 to 40 years land gain has been signiflcant in only a few areas (notably in the lacustrine deltas of the Atchafalaya Basin) Areas of maximum loss generally occur inland from the Gulf shore, where brackish 


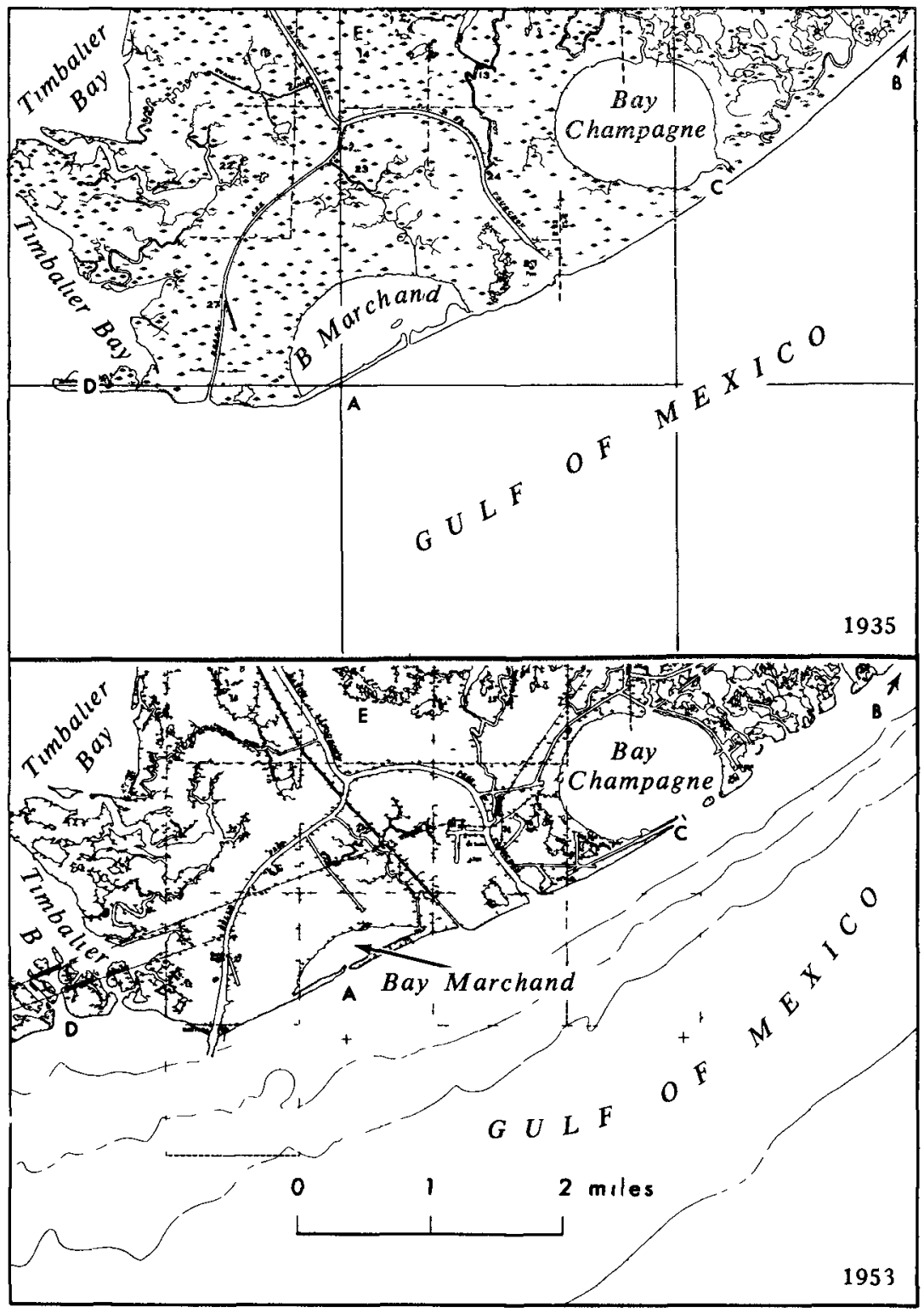

Fig 2 Land loss and shoreline change in the area covered by the Belle Pass quadrangle, south central Louisiana, 1935-1953 Several types of changes have occurred $A, B$, and $C$, Gulf shoreline retreat, $D$, washover fan development downdrift of retreating shoreline, $E$, marsh opening 


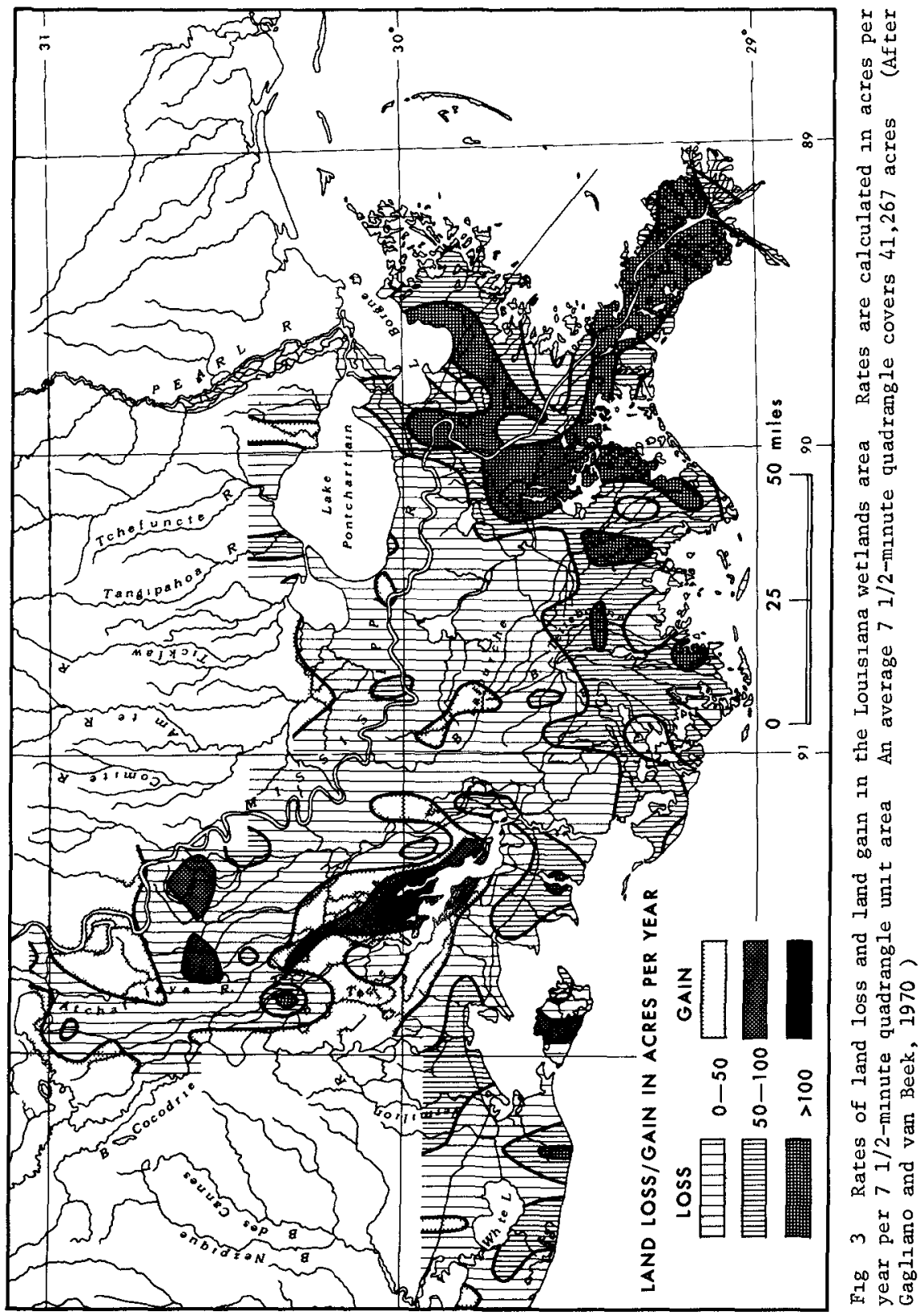


and fresh marshes are being subjected to saltwater intrusion (Chabreck, 1970, Palmisano, 1970) Subsidence is also undoubtedly a factor, as radiocarbon datings of burled marsh peats indlcate that these are also areas of high subsidence rates (Coleman and Smith, 1964, Frazier and Osanık, 1969)

The net land loss figures are most impressive For the coastal Loulslana wetlands the land loss amounts to approximately $161 / 2$ square miles per year (Gagliano and van Beek, 1970) This 1 ) an average for the last 25 to 30 years

The rates of change of land-to-water ratios established for each 7 1/2-minute quadrangle map can be analyzed in a number of ways One technique involves projection of the rates to establish land-water ratios for specific years within and beyond the period of map coverage Although such projections are based on the assumption that rates of change remain constant, the approach has some valıdity as a tool for prediction The map presented in Figure 4 depicts successive positions of the 50 percent land-water 1sopleth in the Mississipp1 deltanc plain for the years 1930 , 1970 , and 2000 The lines were constructed by determining land-water ratios for each 7 1/2-minute quadrangle for each year indicated Values were plotted at quadrangle center points, and contours were drawn on the basis of the points The 50 percent land-water line was selected as an index for analyzing the rate of land deterloration along the highly indented and irregular deltaic coast

The map indicates a progressive landward march of this line across the area The predicted position of the line in the year 2000 alds in ldentifylng the most critical areas of deterioration Major estuaries are clearly undergoing rapıd and drastic changes Within the estuarıes the landward retreat of the line implies increases in both salinity and volume of the tidal prism

\section{PROSPECTS FOR COASTAL RESTORATION}

\section{Areas of Active Sediment Deposition}

The earlier discussion has attempted to define the symptoms of a disease, what are the prospects of a cure?

The key is obviously to be found in those areas where land gain is occurring For example, the Atchafalaya River--the Mississippi's only major distributary--1s actively bullding a lacustrine delta (U S Army Corps of Englneers, 1951, Fisk, 1952) During the past 50 years Atchafalaya sediments have filled a series of large lakes, and the river is expected to construct a delta lobe into the Gulf within the next few decades

In the active delta, where one might antlcipate the highest rates of land bullding, an anomalous situation exists As shown in Figure 5, approximately 75 percent of the total river flow and an equal proportion of transported sediment are discharged through four major outlets or passes As previously mentioned, and as shown in the figure, the mouths of these major passes lie in close proximity to deep water Consequently, most of the sediment transported by the Mississippi is simply dumped Into the deep 


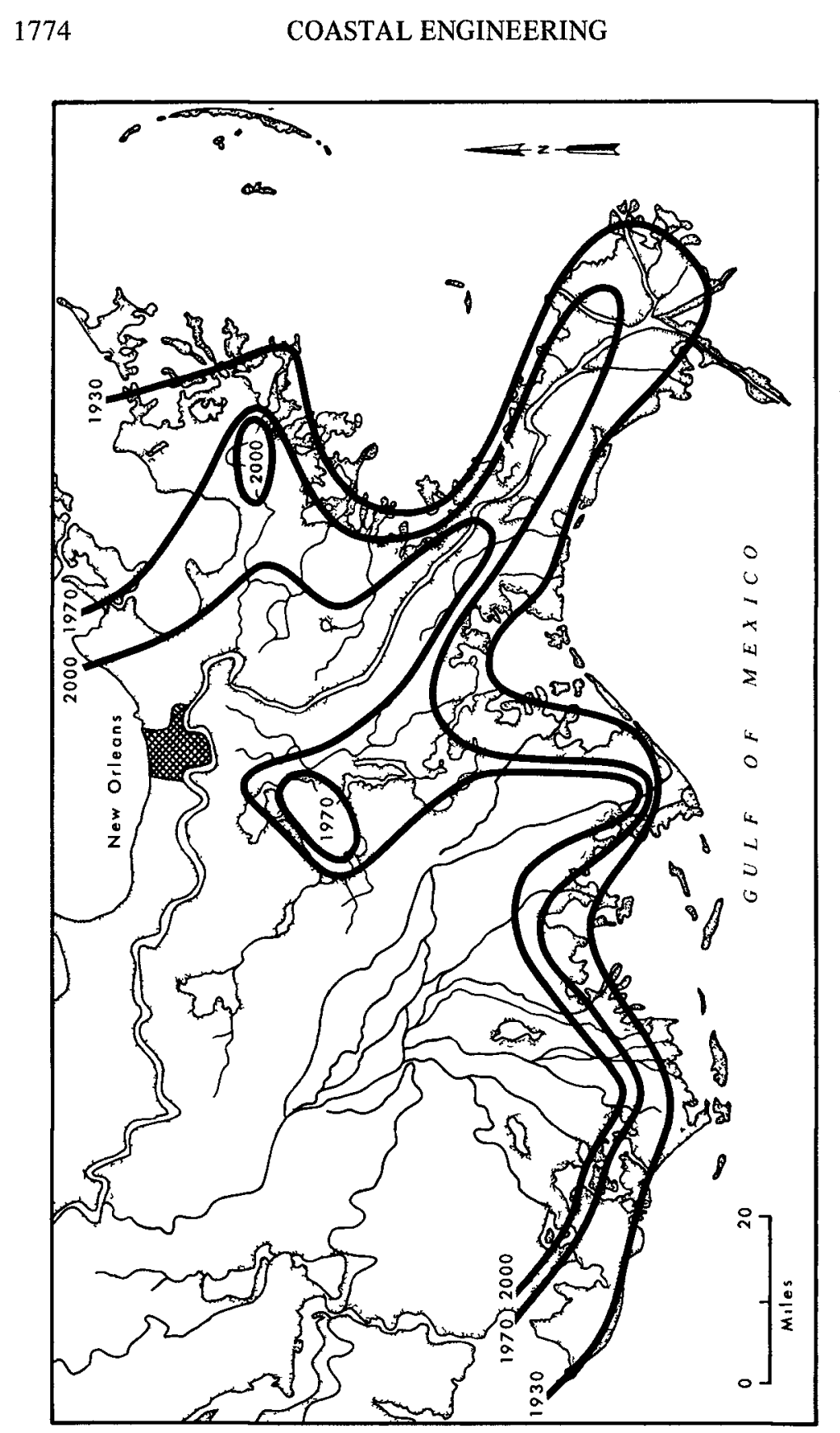




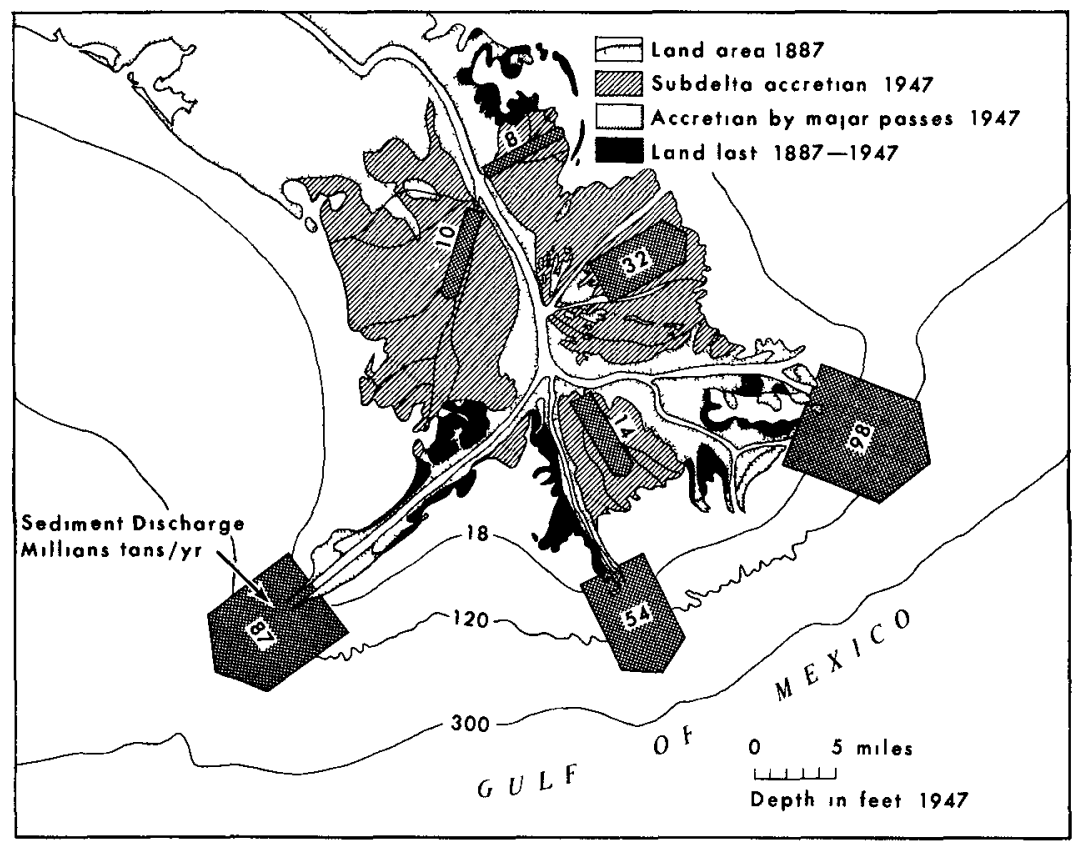

Fig 5 Relative sediment discharge and land accretion in the active birdfoot delta of the Mississippl River

Gulf, and there is little accretion of new land

In sharp contrast to the major passes, the 25 percent of river flow and transported sediment discharged through minor outlets has bullt a considerable landmass during historlc times This landmass is shown as subdelta accretion in Figure 5 From the standpoint of land building, then, these minor outlets, related to subdeltas, are of primary interest

Subdeltas, whlch are appendages of major delta lobes, are usually active for periods of 50 to 100 years As Illustrated in Figures 5 and 6 , much of the landmass of the lower delta has been constructed by such subdeltas during historic times The dates shown in Figure 6 indicate the time of subdelta initiation Prior to their development these areas were occupled by embayments Comparative map studies reveal that the subdeltas account for over 80 percent of the new land bullt in the active delta during historlc times

One of the most interesting aspects of subdeltas is their cyclic nature--that 1s, during a period of 50 to 100 years they progress through a sequence of stages dictated by interaction of such factors as stream gradient, subsidence, and vegetation change (Welder, 1959, Coleman and Gaglıano, 1964, Morgan, 1970) Figure 7 traces the IIfe cycle of the 


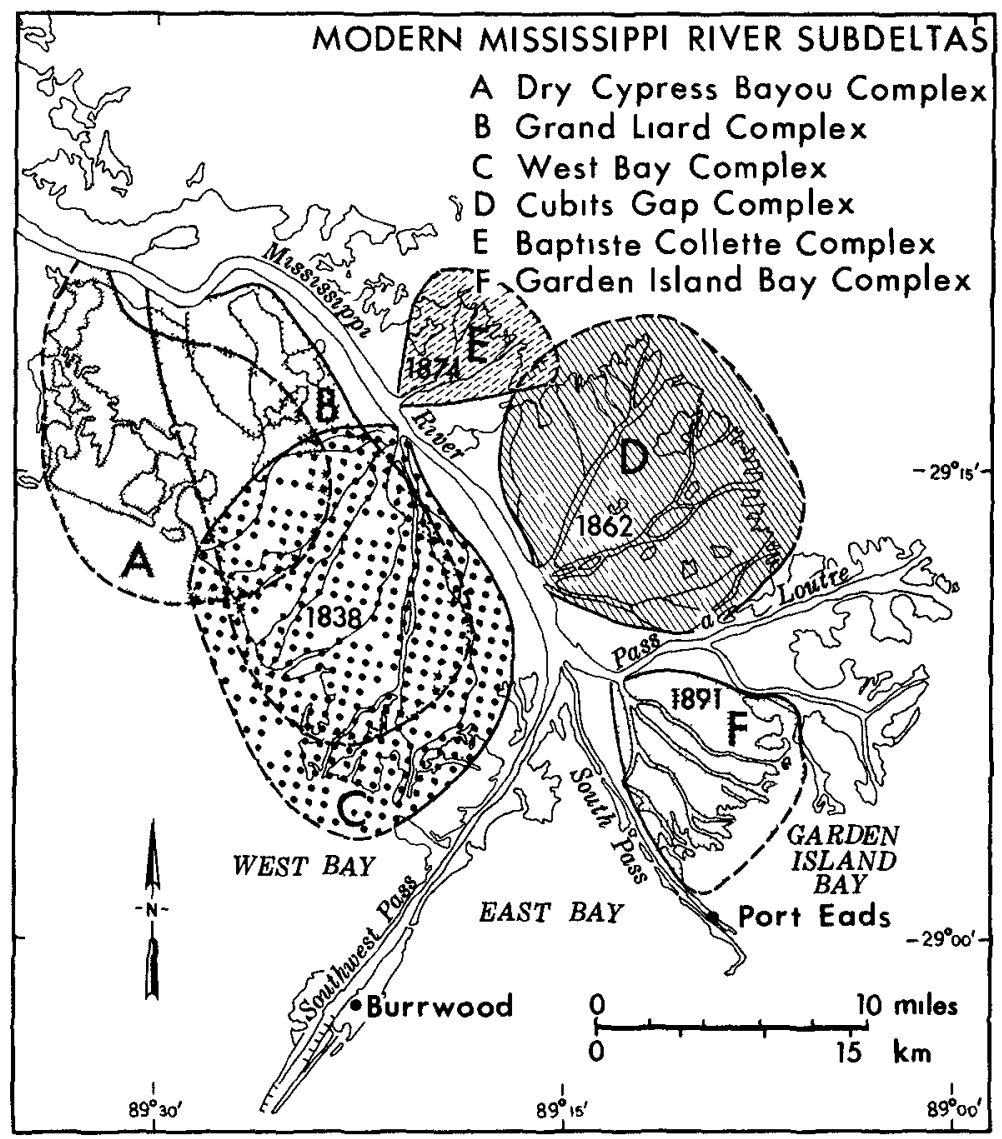

Fig 6 Subdeltas of the modern birdfoot delta Dates indicate year of crevasse breakthrough (After Coleman and Gagliano, 1964)

Garden Island Bay subdelta, as expressed by the gradual gain and loss of land area Note that this subdelta was initiated by a crevasse or break in the natural levee of one of the major distrubutaries in 1891 It went through a short initial stage of subaqueous development until around 1900, when it started to increase in area rapidly During this stage of fast subaerlal growth land was added at an average rate of $075 \mathrm{miles} 2 /$ year Map measurements indicate that by 1940 the subdelta had built more than 30 square miles of land, utilizing less than 3 percent of the total flow of the river

Since 1940 the Garden Island Bay subdelta has been in a stage of 


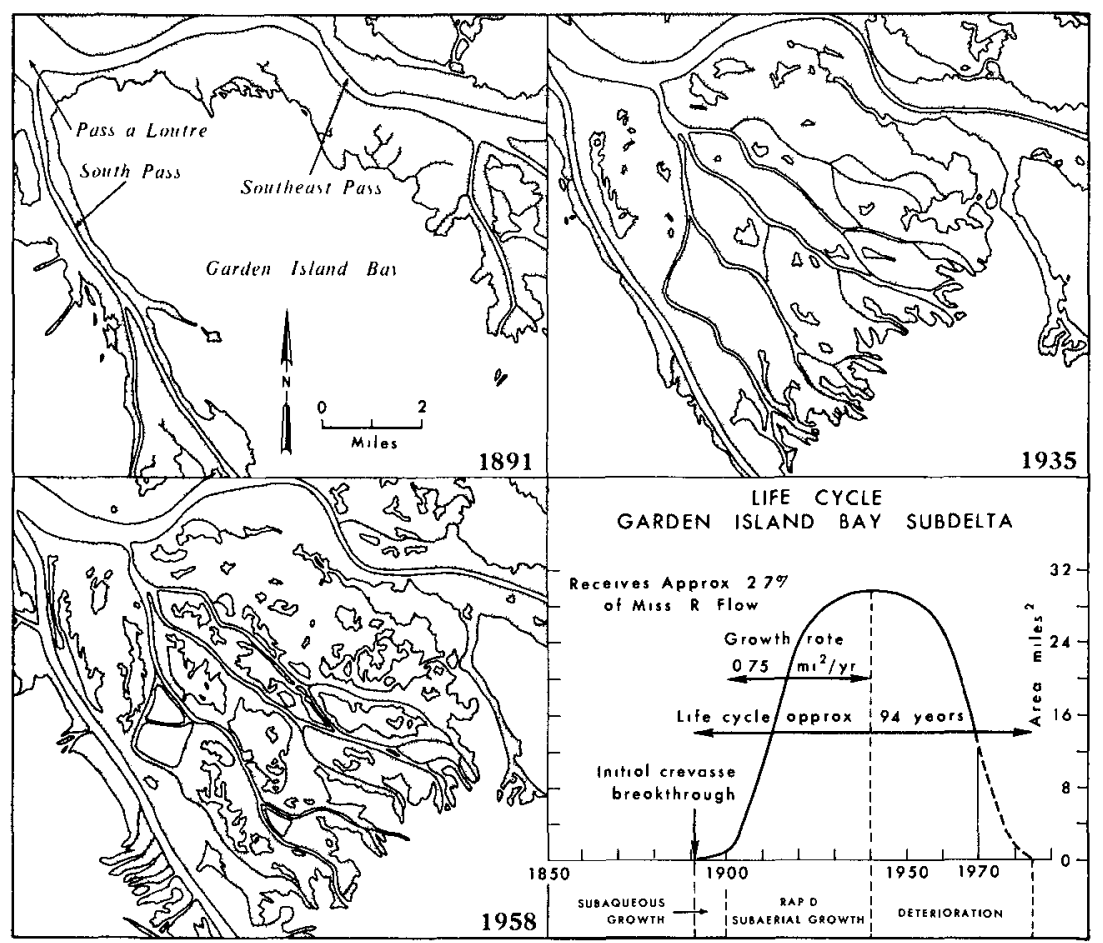

Fig 7 Progressive development of the Garden Island Bay subdelta after the crevasse breakthrough in 1891 Maps from U S C G S navigation charts

deterioration and has progressively decreased in land area as a consequence of subsidence and coastal erosion This example clearly illustrates the relative rapidity of geological processes associated with subdelta bullding and deterioration and suggests that subdeltas are highly amenable to manıpulatıon Controlled diversions which will create new subdeltas are believed to be part of the solution to the environmental problem in coastal Loulsiana

A study correlating subdelta growth with sediment input has been conducted to provide a basis for estimating the 11kely effectiveness of controlled diversions of river flow in creating new land This study centered on the four major historic subdeltas (indicated as C, D, E, and $F$ in Fig 6) The average growth rate for the four subdeltas was found to be 07 square mile per year, using 5 percent of the total flow (Sporadic discharge measurements have been made in the major subdelta channels by the New Orleans District, US Army Corps of Engineers) The efficiency of sediment retention ranged from about 50 to over 90 
percent, and the average rate of retention was 70 percent

\section{Controlled Diversions}

Seven potential sites for the creation of new subdeltas have also been Identified and evaluated three east of the Mississippl and four to the west (FIg 8) Assuming a configuration and size similar to one of the smaller modern subdeltas, average depth and volume for each of the proposed diversion sites have been determined

Long-term measurements indicate that the average sediment load of the Mississippl is $300 \mathrm{mlllion}$ tons per year If a 70 percent sediment retention efficiency figure for subdelta deposition is used, our data show that the river would be capable of bullding 123 square miles of new land per year if diversions were to be inltiated along the lower reaches This is about 75 percent of the current net annual land loss of 165 square miles per year Thus it might be concluded that reestablishment of dynamic equilibrium in the Mississipp deltalc plain can only be approached However, other aspects of our studies indicate that relatively small volumes

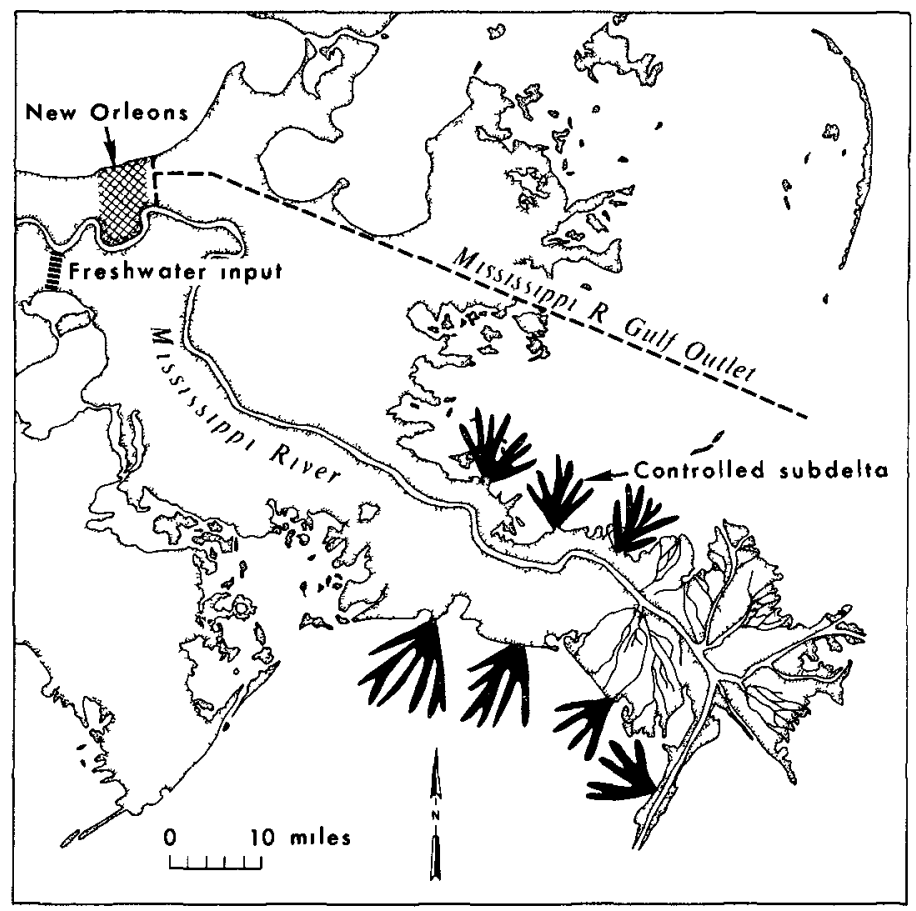

FIg 8 Suggested locations for controlled subdeltas and freshwater input canal in southeastern Loulsiana 
of fresh water introduced into the upper ends of interdistrıbutary estuary systems could be used to offset salinity intrusion and introduce badiy needed nutrients, both of which would offset conditions leading to rapid deterioration of brackish and fresh marshes (see Fig 8)

Such a dynamlc management plan would have a number of benefits

1 The trend of land loss could be reversed,

2 Extension of the landmass would provide a valuable buffer zone for reducing hurricane-generated storm surges,

3 A highly 1rregular subdelta coast with maximum length of 1 andsea interface could be created, which would enhance productivity of fisheries and wild life,

4 Judicious spacing of controlled subdeltas could create new estuaries and increase the total areas of existing ones

During early settlement and initial utilization of the Mississippi valley and delta it was absolutely essential to prevent annual flooding and to improve navigation Accomplishment of these tasks has made possible the unprecedented growth and development that south Loulsiana is experiencing today However, we have entered an era of total utilization of the Mississ1pp1 delta system Because of the delicate natural balance associated w1th the delta, $1 \mathrm{t}$ is mandatory that a new long-term dynamic management plan be devised for orderly development and use of the area It is the a1m of our present studies to contribute to that goal

\section{ACKNOWLEDGMENTS}

The Coastal Studies Institute, Loulsiana State University, is supported by the Geography Programs, Office of Naval Research, under Contract N00014-69-A-0211-0003, NR 388 002 Th1s study was funded by the Department of the Army, New Orleans District, Corps of Englneers, Contract No DACW 29-69-C-0092 The authors gratefully acknowledge the cooperation of personnel of the New Orleans District Office in providing data and guidance 
REFERENCES

Chabreck, $\mathrm{R} \mathrm{H}, 1970$, Marsh zones and vegetative types in the Loulsiana coastal marshes $\mathrm{Ph} D$ Dissertation, Dept Botany and Plant Pathology, La State Univ, Baton Rouge

Coleman, J M, and S M Gaglıano, 1964, Cyclic sedimentation in the Mississippl River deltalc plain Trans Gulf Coast Assoc Geol Soc , $1467-80$

Coleman, J M , and W G Smlth, 1964, Late Recent rise of sea leve1 Bu11 Geol Soc Am, 75833

Fisk, H N, 1952, Geologıcal investigatıon of the Atchafalaya basin and the problem of Mississippi River diversion U S Corps of Engineers Waterways Experiment Station, Vicksburg, Miss, $145 \mathrm{pp}$

Frazier, D E, and A Osanik, 1969, Recent peat deposits--Louisiana coastal plain In (E C Dapples and M E Hopkıns, eds) Environments of coal deposition, Geol Soc Am Spec Paper 114, pp $\quad 63-83$

Gagliano, S M, and $J$ L van Beek, 1970, Geologic and geomorphic aspects of deltalc processes, Mississippi delta system In (S M Gagliano, $R$ Muller, $P$ Light, and $M$ Al-Awady) Hydrologic and geologic studies of coastal Loulsiana, Ia State Univ, Coastal Studies Instrtute and Dept of Marıne Sciences, vol I, $140 \mathrm{pp}$

Harrison, R W, 1961, Alluvial empire Arkansas (Pioneer Press)

Kwon, H J , 1969, Barrier islands of the northern Gulf of Mexico coast sediment source and development La State Univ, Coastal Studies Series No 24,51 pp

Morgan, J P, 1970, Deltas--A résumé J Geolog1c Education, XVIII(3) $107-117$

, and $\mathrm{P}$ B Larimore, 1957, Changes in the Louisiana shoreline Trans Gulf Coast Assoc Geol Soc, $7303-310$

O'Neil, Ted, 1949, The muskrat in the Loulsiana coastal marshes La Dept Wildlife and Fisheries Commission Tech Report, 28 pp

Palmisano, A W, 1970, Plant communlty-soll relationships in Loulsiana coastal marshes $\mathrm{Ph} \mathrm{D}$ dissertation, Dept Botany and Plant Pathology, La State Univ, Baton Rouge

Peyronnin, C A, Jr, 1962, Erosion of Isles Dernieres and Timbalier Islands $\mathrm{J}$ Waterways and Harbors Div, Am Soc Civil Engrs, No WW1, pp 57-69 
Russe11, R J, 1936, Physlography of the Lower Mississippl River Delta La Dept Conservation Geol Bul1 8, pp 3-199

Saucier, R T, 1963, Recent geomorphic history of the Pontchartrain basin, Louisıana La State Unıv, Coastal Studies Serıes No 9, 114 pp

Treadwe11, R C, 1955, Sedimentology and ecology of southeast coasta1 Louisiana La State Univ, Coastal Studies Institute Tech Report No $6,78 \mathrm{pp}$

U S Army Corps of Englneers, 1951, The Atchafalaya River study Mississippi River Commission, Vicksburg, Miss, vo1 1, $47 \mathrm{pp}$

Welder, F A, 1959, Processes of deltalc sedimentation in the Lower Mississippi River La State Univ, Coastal Studies Institute Tech Report No $12,90 \mathrm{pp}$ 



\title{
CHAPTER 108
}

\author{
RESEARCH FOR THE COASTAL AREA OF THE DELTA REGION OF THE \\ NETHERLANDS \\ by $\mathbf{J} \mathbf{J}$ Dronkers \\ Hydraulics Division, Delta Works, The Hague, Netherlands
}

\section{ABSTRACT}

Tidal computations, wave studies, refraction computations, and morphological studies are discussed and practical results are mentioned Theoretical investigations on refraction computations, in particular the accuracy, are dealt with in detall, because difficulties occurred in the practical applications A former study of Morra, based on Kalinske's work on sand transport, has also been discussed in some detall in the section on morphological studies

\section{Introduction}

The closing of the various sea-arms in the Deltaplan, the bulding of sluices, and the construction of Europort Harbour will cause great changes in the hydraulic and the morphologic situation along the south western part of the Netherlands The practical purpose of these studies is the determination of the future conditions for the beaches and dunes, the discharge of the sluices, and the navigation A big hydraulic model has been built at the Hydraulic Laboratory at Delft for the planning of the construction of Europort Harbour The length scales are 1640 in the horizontal plane, and 164 in the vertical

The changes in the tidal motion can be computed in a satisfactory way, based on Leendertse's computational scheme Difficulties do not occur with respect to the stability of this scheme The accuracy of the method depends on the gridsize and the timestep

In section 3 , a discussion $1 \mathrm{~s}$ given about wave research and practical determination of the energy wave spectrum A relation of depth and breaking of waves is obtained from the wave observations Informations are also obtained about the changes of the directions of the waves by means of refraction computations These computations are based on the first order wave theory, which is an approximation of the irregular wave motion Usually an iteration process has been applied for the determination of the celerity of the shallow water wave In section 4 a direct formula for the celerity is derived by means of which the accuracy of the refraction computation is discussed

The future depths must be determined in the coastal area An iteration process must be carried out, because the changes in the currents and waves determine the morphologlcal changes and reversely The determination of the morphological changes is a difficult affair The studies describe the physical factors in a general way Simplifications must be accepted and the empirical coefficients in these formulae have to be determined from 
observations in nature

The relation between the sediment transport and the currents is much better known than for the wave action The methods of the determining of these relations will be discussed in section 5 The application of a physical scale model of the Haringvilet area for these studies has been considered, but rejected, because the sand in the Haringvilet mouths is very fine and the relation between sand transport along the bottom and that in suspension is unrepresentative in the scale model as compared to nature

For the future a great research program has been set up for getting observations from nature, during the period in which the great changes in the coastal area will take place By means of the use of radio-active tracers, the direction and the relative significance of the sand transport can be determined The most important tool is however the study of the changes in the shape of the bottom obtained from perlodically repeated soundings after every three months The programs for the computations on the electronic computer of the Rijkswaterstaat (Elliott 503) are composed in Algol by the Mathematical Physical Division

2 Tidal computations in the coastal areas of the Delta region

21 The effect of the Delta works on the tidal motion in the North Sea

The total quantity of water flowing to and from the sea-arms to be enclosed amounts about $2,000 \mathrm{mill}$ ion $\mathrm{m}^{3}$ during normal tide, while the flood volume passing through the strait of Dover is about 19,000 million $\mathrm{m}^{3}$ and the ebb volume $17,000 \mathrm{million} \mathrm{m}^{3}$ The flood volume passing through a section crossing the North Sea from the isle of Walcheren to Harwich amounts about $50,000 \mathrm{mill}$ on $\mathrm{m}^{3}$, so that the tide in the southern part of the North Sea comes mainly from the north and the effect of the tide passing through thestrait of Dover is limited From these figures it is obvious that the closing of the sea-arms of the Delta-Works must have a small effect on the tidal motion in the North sea and that the studies can be limited to the coastal area

\section{The tide along the Delta coast}

The vertical tide changes considerably along the Dutch coast at Flushing (south of the Delta area) the mean tidal range $1 \mathrm{~s}$ $38 \mathrm{~m}$ and at the Hook of Holland (north of the Delta area) $16 \mathrm{~m}$, where the tide arrives 25 hours later

The shoals along the coast of the Delta region are about 8 km wide The transition from the 10 to $20 \mathrm{~m}$ contour line of depth can be considered as the boundary of the "underwater" delta The greatest depth in the North Sea opposite the coast is $30 \mathrm{~m}$ The bottom topography in the mouths of the sea-arms shows many gullies and shoals

The pattern of the tidal currents in the mouths of the estuaries is complex because of the interference provided by currents flowing in the direction of the estuaries and tidal currents running parallel to the coastline caused by the prevailing tidal motion in the North Sea The shape of the tidal current diagram changes from elongated in the direction of the coast to elliptical and then again to elongated, more or less perpendicular to those of the 
North Sea currents

For the tidal studies in the coastal areas an extensive measuring programme has been set up in order to obtain a detalled insight into the vertical and horizontal tide in a strip $120 \mathrm{~km}$ along the Delta coast The boundaries of the strip are shown in figure 1 Such programs were carried out on 27 and 28 June 1967 and on 22 May and 9 September 1969

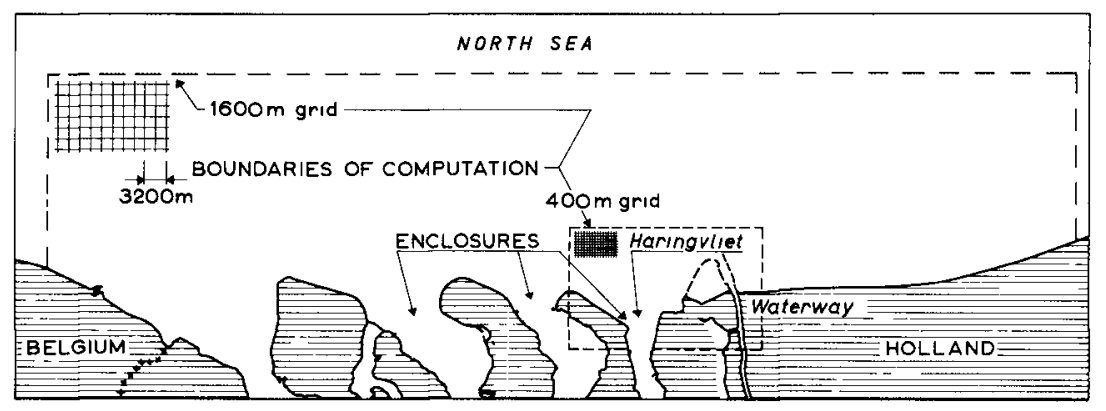

Fig 1 Coast Delta region and boundaries of the grid net

\section{The tidal computations}

The method, developed by Leendertse (1) has been used for the tidal computations Some practical remarks are mentioned hereafter

The vertical tides as measured at the seaward boundaries by pressure meters put on the bottom are used as a boundary condition for the tidal computations The velocities in the sea, which depend more on local depth conditions than does the vertical tide, are not used as boundary conditions

In the mouths of the sea-arms the vertical tides measured by means of tidal gauges or the velocities can be considered as boundary conditions obviously the components of the velocity perpendicular to the coastline of the islands are zero

For computational reasons it is desirable to locate the boundaries so that the values of the second order terms in the tidal equations are small in the neighbourhood of the boundary This is so at the boundary of the strip, but it must be expected that the results of the computation of the velocity vectors near the boundary will be less accurate than in the inner region

There are however other factors which may cause more serious inaccuracies in the computation the values of the resistance coefficient and the schematization of the depths Tests for the accuracy of the computations are necessary

Tidal computations are carried out for a square net of which the gridsize is $1600 \mathrm{~m}$ This grid is too rough for adequately taking into account depth variations in the nelghbourhood of the coast and in the sea-arms In this region a $400 \mathrm{~m}$ square net is used 
(see fig 1) The boundary conditions (vertical tides) of the second net can be computed by means of the results of the coarser net, provided the tide is not influenced by the circumstances near the coast otherwise, a separate measuring programme is necessary for the determination of the boundary conditions

The vertical tides at the various grid points along the seaward and land boundaries are determined by interpolation between the water levels at the locations where the vertical tide has been measured, as follows The shapes of the measured vertical tides are represented by Fourier series with a basic period of $12 \mathrm{~h} 50 \mathrm{~m}$ and the Fourier coefficients of the vertical tides in between are obtained by interpolation These computations are performed by the computer The velocities at a great number of locations were also measured in the program $\mathrm{e}$ at locations in between the places where the vertical tides were measured As the vertical and horizontal tides depend on each other a check can be performed by means of the equation of motion applied to the boundary line

$$
\frac{S u}{S t}+u \frac{S u}{S x}+v \frac{S u}{S y}-\Omega v=-g \frac{S h}{S x}-g \frac{v u}{c^{2} a},
$$

in which the $x$ axis is directed along a part of the boundary, $V$ is the magnitude of the velocity vector of which $u$ and $v$ are the components in $x$ and $y$ directions (counter clock wise) and $h$ is the helght of the water level with respect to a datum plane By replacing the differentials by difference quotients, a relation is found for the differences in water levels between the verticaltides at both sides of the location where the velocity has been measured However, the term $v \frac{S u}{S y}$ could not be determined from the observations The values of the Chézy coefficient, $c$, has been checked by means of these computations and from former research in the coastal area The values of $C$ depend also on the depths, e $g$ according to the well-known logarithmic law However such a relation could not be derived from the observations in the coastal area

The equation of continuity has also been applied to subregions of the coastal strip Furthermore the importance of the variousterms in equation (1) and the similar equation for the y-direction, has been determined, see Dronkers (2)

after preliminary computations detalled tidal computations were applied to the strip along the coast The relative changes of the vertical and horizontal tide due to the enclosure of the sea-arms could then be determined

Calculations were carried out after the Haringvliet was enclosed of which the flood volume was about $300 \mathrm{milli}^{\circ} \mathrm{m}^{3}$ The future Europort Harbour was also included in the computations obviously the changes are maximal in the intermediate neighbourhood of this region at a distance of $8 \mathrm{~km}$ from the coastline the changes are negligeable In fig 2 the results are shown for maximum flood and ebb The directions of the velocities are denoted by arrows A smaller gridsize, $400 \mathrm{~m}$, has been used for these computations (see fig 1)

Computations were also performed for discharges passing through the sluice during the low water period

During this last case the timestep has been shortened in order to provide the necessary accuracy For the $1600 \mathrm{~m}$ gridsize the time step was 10 minutes and the computed vertical tidal curves appeared 
to have a smooth shape Irregularities in the shape of thesecurves occurred in the case of a net with $400 \mathrm{~m}$ gridsize Therefore in thrs case the time step has been taken $2 \frac{1}{2}$ minutes obviously the propagation time in such a square is about 4 times shorter than in the case of $1600 \mathrm{~m} \mathrm{gridsize}$
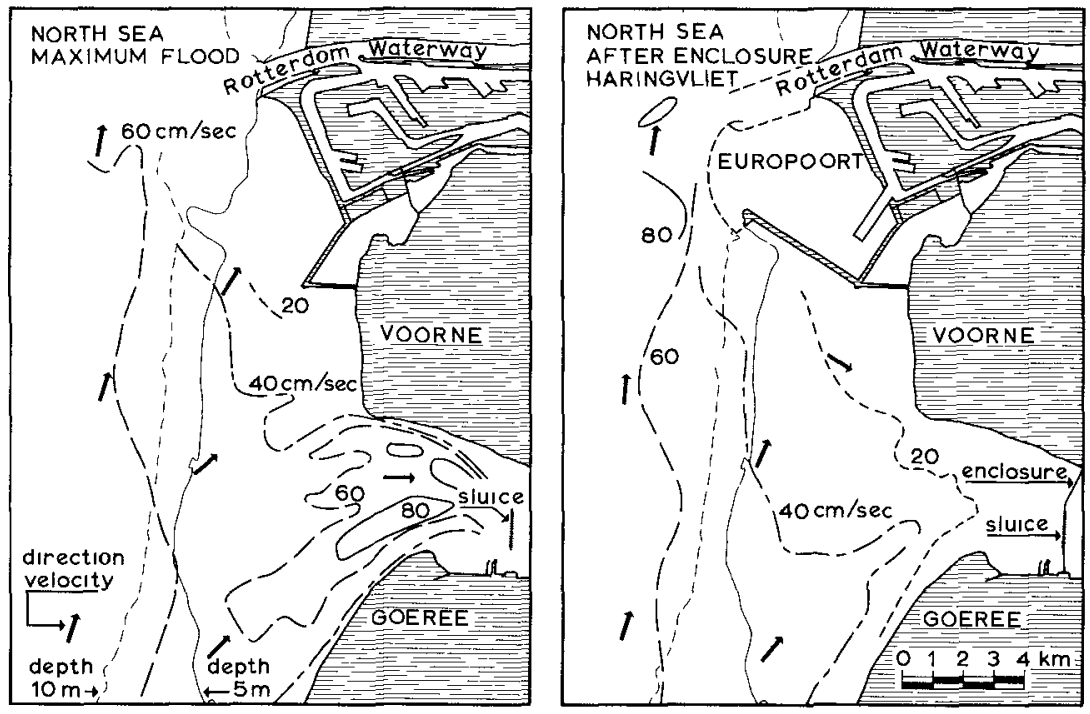

Fig 2 Limes of equal velocities in the mouth of the Haringviıet

\section{Practical wave investigations}

For getting insight into the wave characteristics in the coastal area, a great number of wave observation piles are placed in the coastal area The placing of heavy piles in the seabed is usually a costly affarr and therefore the depth at these locations is limited to $10 \mathrm{~m}$ Furthermore a wave instrument is put on a platform, which is at a location where the waterdepth is about $15 \mathrm{~m}$ At some locations observations are taken for several years, maximal 5 years, usually they are observed for a shorter period, minimal one year, and then the piles are relocated The instrument on the pile (which is an electrical step capacity gauge wave recorder with wireless transmission) does not measure wave helghts smaller than $4 \mathrm{~cm}$ The irregular waves and eventually the vertical tide observations are transmitted by radio to a coastal station where they can be observed on a pen recorder By means of the so-called wave-rider, which is a wave buoy on which instruments are placed, the vertical accelerations of the movement of the buoy are measured They can be used in the deeper parts of the coastal area The directions of the waves cannot be measured by the instruments mentioned above Radar 
observations can be used for this purpose

Frequency and exceedance distributions of wave heights, distributions of periods, the wave period by zero crossing method and the energy spectral density function (energy spectrum) are determined for time intervals of about half an hour, during which the mean water level, due to the tide, does not change considerably in comparison to the depth The significant wave height is one of the most important parameters for the practical applications The largest and smallest value of the periods to be considered depend on the length of the measuring interval of the waves Wave perlods longer than 30 seconds or shorter than 1 second are eliminated by means of a filter procedure For modern theories on waves, and in particular the energy spectrum, reference is made to Kindsman (3a) and Benda and Piersol (3b)

The mean wave period in the coastal areas of the Delta is about 5 seconds, although high waves generated by high winds, may have perlods up to 10 seconds or more A maximum wave height of $10 \mathrm{~m}$ has been measured at a water depth of $16 \mathrm{~m}$ The significant wave height was about $7 \mathrm{~m}$ Generally speaking the waves in the coastal area and even in a great part of the southern North Sea are of intermediate wave type between a long wave and a deep water wave (see section 41 )

It has been found by Koele (4) that the distribution of the wave helghts (trough to crest) over a sufficiently long measuring interval can approximately be represented by the well-known Rayle1gh distribution even in rather shallow water, provided that small disturbances are omitted In particular cases deviations from this distribution has been found

From the wave observations in the coastal area of the Delta 1 t has been found that the product of wind velocity and the frequency which occurs at maximum energy density is about 18 This 1 s more than mentioned by Freudenthal (5) The definition of the wind velocity also effects the product mentioned above

Waves may develop higher harmonics during their propagation in shallow water of which periods depend on the period of the original wave developed in deeper water Consequently more waves of shorter period may be expected in such shallow reglons than in deeper water under the same windfield

It has also been found by Koele and de Bruyn (6) that in the breaker zone the significant wave height of irregular waves does not exceed about 05 of the local depth. The breaking of waves also depends on the steepness of the wave In the example of fig 3 , the bound is denoted by $H_{S}=04 a$, in which $H_{s}$ is the significant wave

helght and a the depth Particular circumstances may also have some influence on the coefficient, and therefore values between 04 and 05 occur

Regular shaped wave profiles e $g$ a trochoide and a cnoidal wave will break when the wave height is maximal about 08 of the local depth During the propagation in a shallow region the regular wavedeforms 


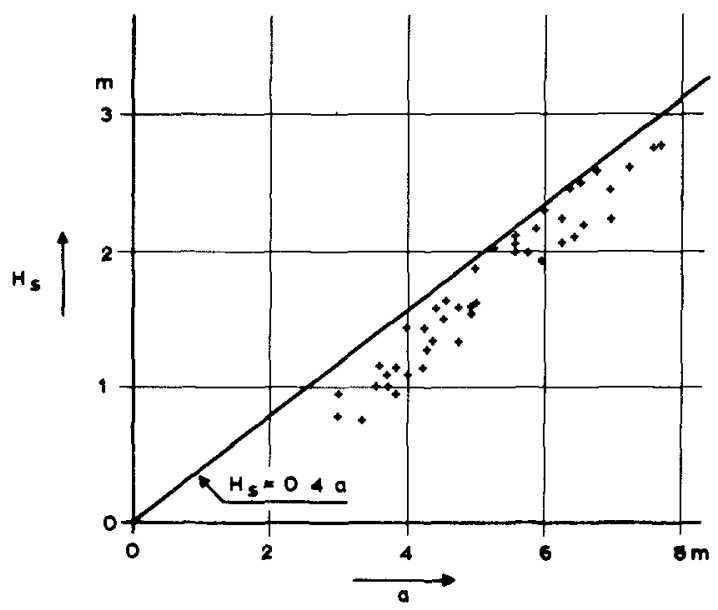

Fig 3 The relation between the significant wave height and the depth

For the statistical evaluation of wave conditions in reference to coastal engineering problems we may refer to Svasek (7) The relation between the significant wave height, $H_{S}$, and the area of the energy spectrum $m_{o}$, is $H_{S}=4 m_{o}^{\frac{1}{2}}$ This relation is based on the Rayleigh distribution and has also been verified by means of wave observations The deviation in the factor 4 is about 02

The calculation of the energy spectrum is based on the calculation of the well-known auto-covariance function $R(\tau)$, applied to the wave helghts $h(t)$

$$
R(\tau)=\frac{1}{P-\tau} \int_{0}^{P-\tau} h(t) h(t+\tau) d t=\int_{2 \pi / 30}^{2 \pi} S^{\prime}(w) \cos (w \tau) d w,
$$

where $S^{\prime}(w)$ is the energy density and $w$ is the frequency of a wave, while in practical applications the observational period $P$ is usually 30 minutes ( 1800 seconds) The integral is replaced by a summation formula, in which $\Delta t=02 \mathrm{sec}$ and $R(\tau)$ is calculated for successive values of $\tau$ at intervals of 02 seconds, from $\tau=0$ up to $\tau_{m}=30 \mathrm{sec}$ Thus if the mean wave period equals 5 seconds about 350 wave periods are considered for each observation period of 30 minutes The energy density $S^{\prime}(w)$ is determined by

$$
S^{\prime}(w)=\frac{2}{\pi} \int_{0}^{\tau_{m}} R(\tau) \cos w \tau d \tau
$$

(In practical applications $S(w)=\frac{1}{2} S^{\prime}(w)$ has been considered) 
41 Formula for the celerity of the wave

The purpose of the computation of wave refraction in the coastal areas of the Delta is to provide data on changes in the local wave characteristics that result from the morphological changes e $g$ for the determination of the attack on coastal structures Numerical refraction calculations on a computer for the case of a regular reference wave have been worked out by $G$ M Griswold ( 8 ) The limitation for the accuracy of the wave ray computation is the accuracy of the depths which determine the celerity of the waves, c A first approximation, based on linear wave theory, is applied

$$
c=\frac{g T}{2 \pi} \tanh \frac{2 a \pi}{c T} \text {, }
$$

In which $T$ is the wave period and ' $a$ ' the depth This formulaholds if $2 \pi H / L \ll 1$ and $\pi H / L \ll(2 \pi a)^{3} / L^{3}$, in whlch $H$ is the wave hesght. For the computation of c from (1) an iteration procedure may be applied (see e $g$ Duthler (9)) Here a direct approximate formula for c as a function of depth and period is deduced This formula is useful for the determination of the accuracy of the refraction computation (see 42 ) The derivation will be included here because the author has not found this method in the literature Equation (1) may be written in the alternative form

$\mathrm{u} \tanh u=P$, where $u=\frac{2 \pi}{\mathrm{cT}} \mathrm{a}, \mathrm{P}=\frac{4 \pi 2}{\mathrm{gT}^{2}} \mathrm{a}=\frac{40255}{\mathrm{~T}^{2}} \mathrm{a}$

Let a be defined such that for a<aT the celerity c depends on the depth For $a>a_{T}$ the wave propagates as a deep water wave For deep water waves tanh $u$ will be approxamated by 1 , e $g$ if $u>27$, tanh $u$ does not depend on depth Then for $a>a_{T}$,

$$
c=\frac{\mathbf{g}}{2 \pi}=156 \mathbf{T}
$$

From equation (1a) an approximate solution for c can be determined for $a<a_{T}$ in the following way After introducing the well-known series for $\tanh u$, $\tanh u=u-\frac{2 u^{3}}{3^{\prime}}+\frac{2^{4} u^{5}}{5^{\prime}}-\frac{2^{4} 17 u^{7}}{7^{\prime}}+$ in (1a), which converges for $|u|<\frac{1}{2} \pi$, and after reversing the series of $u$ tanh $u$ in (1a) in a series of terms of $P$ and by considering three terms of the reversed series, it is found that the approximation for $c$, c', is obtained

$$
\left(c^{\prime}\right)^{2}\left(1+\frac{1}{3} P+\frac{4}{45} P^{2}\right)=a g
$$

This formula has an accuracy of $99 \%$ if $u<15$ It appears that after the addition of a term, $0007 \mathrm{p}^{3}$, a better approximation is obtained, applicable now up to $u \approx 27$, with an accuracy of $98 \%$

$$
\left(c^{\prime}\right)^{2}=\frac{a g}{1+0333 P+0089 p^{2}+0007 p^{3}}
$$

Then 1 t follows from (1a) and (4)

$$
\frac{c^{2}}{\left(c^{1}\right)^{2}}=\frac{p}{u^{2}}\left(1+0333 P+0089 p^{2}+0007 P^{3}\right)
$$


The transition depth, $a_{T}$, is defined by $a_{T}=\frac{c^{\prime} T}{2 \pi} u$ and $u=27$ (see (1a)) Then $\left(c^{\prime}\right)^{2}=037 g_{T}$ and $L^{\prime}=c^{\prime} T=235$ aT (see (4), and $P=27 \tanh 27 \approx 27$ ) The line $\left(c^{\prime}\right)^{2}=037$ ga is shown in fig 4

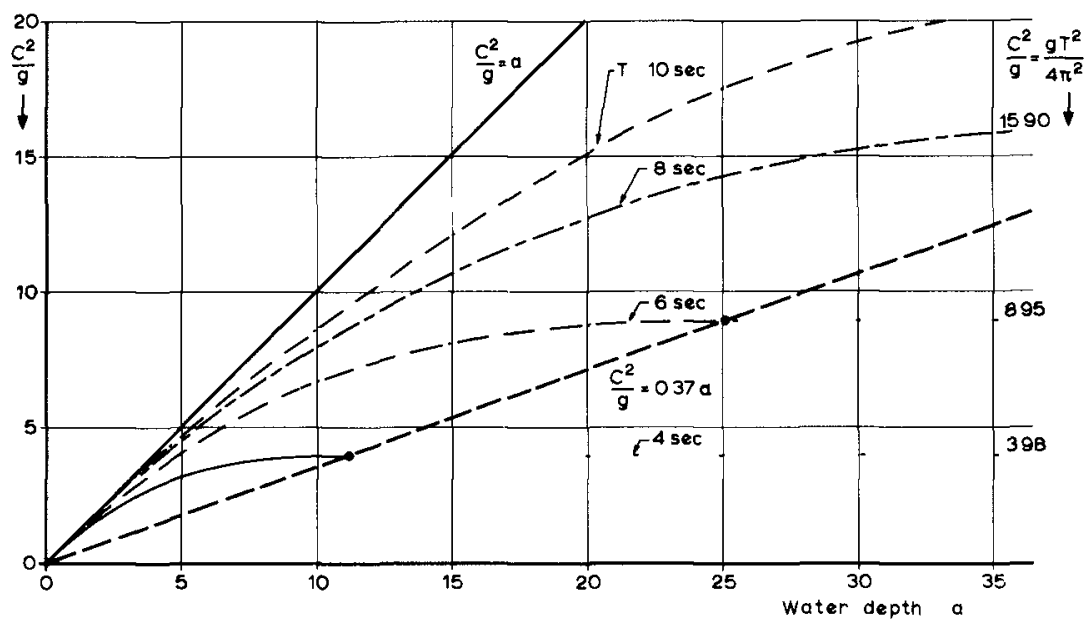

Fig 4 Relation between the celerity of the wave and the depth, according to first order wave theory

In fig 4 the relations (2) and (4) are represented for some periods $T$ The wave propagates as a deep water wave on the right side of the line, $c^{2}=037 \mathrm{ag}$ The derivation mentioned above is based on the required accuracy for the determination of $c$ and $a_{T}$ The criterion for the boundary between shallow and deep water waves, given in the literature, is $L=2 a_{T}$ (see Kindsman (3a))

\section{The formulae for the computation of the refraction and considerations on the accuracy}

Difficulties concerning the accuracy of the computation are often met in the practical applications The computational method will be discussed in detall in connection with this

During the propagation the wave front will change due to the change of the wave celerity, c

Let $x$ and $y$ be a perpendicular coordinate system and $\alpha$ the angle of the tangent to the ray at a point $(x, y)$ with the $x$ axis Furthermore let $s$ be the distance from a fixed point $\left(x_{0}, y_{0}\right)$ to $(x, y)$ along the ray, and $n$ the distance along the wave front at $(x, y)$ perpendicular to the ray

The basic formulae for the computation of the wave refraction are

$$
\frac{d s}{d t}=c, \quad \frac{S \alpha}{S t}=-\frac{S c}{S n} \text {, and } \frac{1}{\bar{p}}=\frac{S \alpha}{S s}=-\frac{1}{c} \frac{S c}{S n}
$$


in which $\rho$ is the curvature radius

The transformation formulae between the coordinate systems ( $s, n)$ and $(x, y)$ are

$$
\frac{S}{\delta n}=-\sin \alpha \frac{5}{\delta x}+\cos a \frac{5}{5 y}, \frac{5}{\delta s}=\cos a \frac{5}{\delta x}+\sin \alpha \frac{s}{5 y}
$$

It follows that $\alpha$ satisfies the first order partial differential equation $\sin \alpha \frac{S c}{\delta x}-\cos \alpha \frac{S c}{S y}=c \cos \alpha \frac{S}{\delta x}+c \sin \alpha \frac{\delta}{\delta y}=\frac{c}{\rho}$ Knowng the values of $c, \frac{S c}{S x}, \frac{S c}{S y}$ to obtain the path of the ray, $\rho(x, y)$ must be solved in a numerical way

Assume that the path of the ray has been computed up to $\left(x_{1}, y_{1}\right)$ Then the point $\left(x_{1+1}, y_{1+1}\right)$ of the wave ray can be computed, after time $(\Delta t)=p T$, where $p=1$, or 2 , etc is the number of wave lengths to be considered successively along the wave ray The following numerical relations are considered

$$
\begin{aligned}
& \Delta s_{1}=c_{1, m} \Delta t,(a), c_{1, m}=\frac{1}{2}\left(c_{1}+c_{1+1}\right),(b), \Delta \alpha_{1}=\left(\frac{1}{\rho_{1}}+\frac{1}{\rho_{1+1}}\right) \frac{\Delta s_{1}}{2},(c) \\
& \frac{c_{1+1}}{p_{1+1}}=\sin \left(\alpha_{1}+\Delta a_{1}\right) \frac{S c_{1+1}}{S x}-\cos \left(\alpha_{1}+\Delta a_{1}\right) \frac{S c_{1+1}}{S y},(d) \\
& \Delta x=\Delta s_{1} \cos \left(\alpha_{1}+\frac{1}{2} \Delta \alpha_{1}\right), \Delta y=\Delta s_{1} \sin \left(a_{1}+\frac{1}{2} \Delta \alpha_{1}\right),(e) \\
& c_{1+1}=c_{1}+\frac{S c_{1}}{S x} \Delta x+\frac{S c_{1}}{S y} \Delta y+\frac{1}{2} \frac{S^{2} c_{1}}{S x^{2}}(\Delta x)^{2}+\frac{S^{2} c_{1}}{S x S_{y}} \Delta x \Delta y+ \\
& +\frac{1}{2} \frac{S^{2} c_{1}}{5 y^{2}}(\Delta y)^{2}, \text { (f) }
\end{aligned}
$$

The functions $c_{1}, \frac{S c_{1}}{S x}$ etc occurring in $(7,(f))$ can be expressed in terms of a, $\frac{S a}{S x}$, and, $\frac{S a}{S y}$, by means of (3), if $a<a_{T}$ (see

For $a>a_{T}, \quad \frac{\delta c_{1}}{S_{x}}=0$ etc

For a $<a_{T}$, 1 t may be put

$$
\frac{S c}{S x}=s(a, T) \frac{S a}{S x} \text { and } \frac{S c}{S y}=S(a, T) \frac{S a}{S y}
$$

In $f i g ~ 5$ the function $S(a, T)$ is represented as a function of ' $a$ ' for $T=4,6$ and 8 seconds The coefficient $S$ is positive for shallow water Then $c^{2}<037 \mathrm{ag}$ (see fig 4) For $\mathrm{c}^{2}=037 \mathrm{ag}, \frac{5 \mathrm{c}}{5 \mathrm{x}}=0$ and $S=0$ In case $c^{2}>037$ ag the value of $s$ must be equal to zero Then the wave is considered as a deep water wave

The solution of the set of non-linear equations, (7), depends on the variations in the depths, so that the density of the sounding net is a very important factor for the accuracy of the solution A second important factor is the accuracy of the numerical solution In this respect great care is necessary Assumptions must be made on the values of the derivatives of the celerity, $c$, and therefore on the depth 


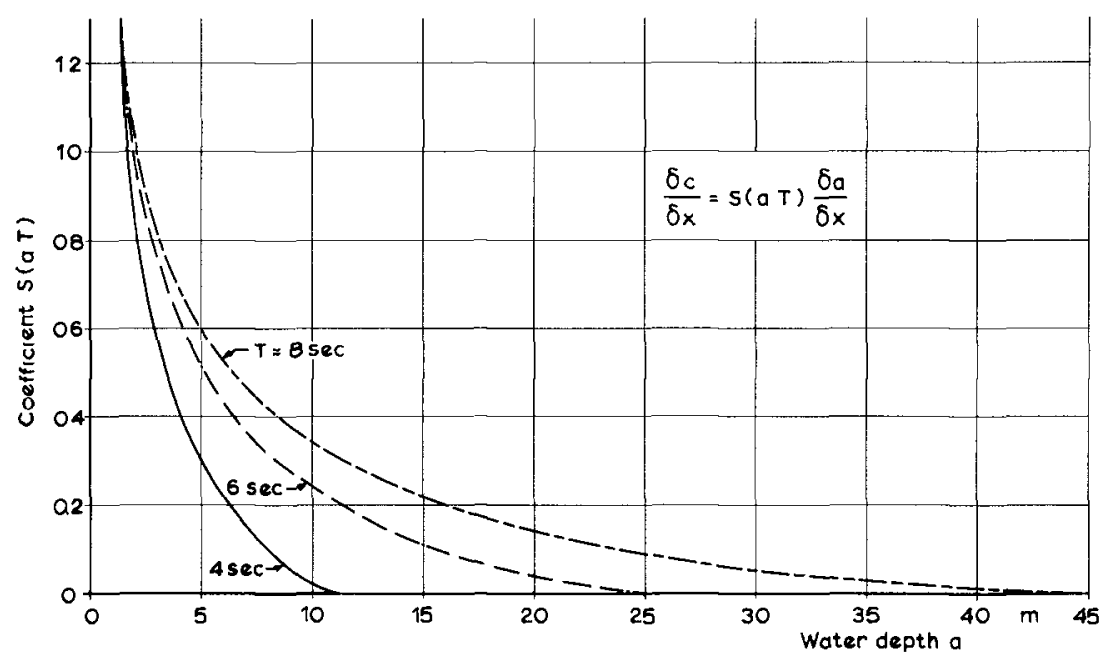

Fig 5 Graphical representation of the coefficient $S(a, T)$ as a function of depth In the practical application is $S(a, T)>0$ for $c^{2}<037$ ag and $S(a, T)=0$ for $c^{2} \geqslant 037$ ag

It 1 s mentioned in the literature that $\frac{S a}{S x}$, or $\frac{S a}{S y}$, may not exceed the value, 01 A detalled research of the accuracy of the solution shows that this value is too great however for obtaining accurate results The maximum value, 001 , is recommended for the slopes $\frac{5 a}{5 x}$ and $\frac{S a}{5 y}$

The following example shows that in case $\frac{S a}{S x}=\frac{S a}{S y}=01$, the computed value of $\Delta \alpha_{1}$ is much too large Let be the value of $\sin \left(\alpha_{1}+\Delta \alpha_{1}\right)=1$ Then 1 t follows from $(7 d)$ and (8) that

$$
\frac{c_{1+1}}{\rho_{1+1}}=\frac{S c_{1+1}}{S x}=S(a, T) \frac{5 a_{1+1}}{S x} \text {, }
$$

and from $(7, a c)$, that $\Delta \alpha_{1}$ is of the order of $\mathrm{pT} \frac{\mathrm{c}}{\rho}$, and $\frac{5 \mathrm{a}}{5 \mathrm{x}}=01$ If $p=1, T=5 \mathrm{sec}$ and the depth $1 \mathrm{~s}$ about $2 \mathrm{~m}$, then $\Delta \alpha_{1} \approx 5 \frac{\mathrm{c}}{\rho} \approx 5 \frac{5 \mathrm{a}}{\mathrm{Sx}} \approx 05$ This value, 05 , is much too large for an accurate numerical computation of $\Delta \alpha_{1}$ In the practical applications $\Delta \alpha_{1}$ may not exceed the value 005 otherwise equation $(7 \mathrm{c})$ and the relation sin $a_{1}=\Delta \alpha_{1}$ etc are not accurate enough, unless $p<1$ It is not desirable to consider parts of waves lengths from physical point of view Therefore the value of $p$ must be an integer

Moreover restrictions must be made with respect to the values of the second derivatives $\frac{S 2 a}{S_{x^{2}}}$ etc The maximum values of these 
second derivatives of 'a' have also to be $11 \mathrm{mlted}$ to $0005 / \Delta \mathrm{s}$ at the most In that case the values of the terms which contain the second derivatives in the formula for $\Delta \alpha_{1}$ e $g$ the terms $\frac{1}{2 c_{1}+1}\left(\sin q_{1}\right) \frac{s^{2} c_{1}}{5 x^{2}} \Delta x$ are smaller than 0025

The complete formula for $\Delta \alpha_{1}$, derived from $(7 \mathrm{c})$ and $(7 \mathrm{f})$, is

$\frac{\Delta \alpha_{1}}{\Delta s}=\frac{1}{\rho_{1}}+\frac{1}{2 c_{1+1}}\left\{\Delta \alpha\left[\left(\cos \alpha_{1} \frac{\delta c_{1}}{\delta x}+\sin \alpha \frac{\delta c_{1}}{\delta y}\right)+\right.\right.$

$\left.+\left(\Delta x \cos \alpha_{1} \frac{5^{2} c_{1}}{5 x^{2}}-\Delta y \sin \alpha_{1} \frac{5^{2} c_{1}}{5 y^{2}}\right)+\left(\Delta x \sin a_{1}+\Delta y \cos \alpha_{1}\right) \frac{\delta^{2} c_{1}}{\delta x \delta y}\right]$

$+\left(\Delta x \sin \alpha_{1} \frac{5^{2} c_{1}}{\delta x^{2}}-\Delta y \cos \alpha_{1} \frac{\delta^{2} c_{1}}{\delta y^{2}}\right)+\left(-\Delta x \cos \alpha_{1}+\right.$

$\left.\left.+\Delta y \sin \alpha_{1}\right) \frac{\delta^{2} c_{1}}{\delta x \delta y}\right\}$

It must be stressed that the accuracy of the refraction computation also depend on the wave length and thus on the period of the wave

(see fig 5) The accuracy is greater for shorter waves, and in that case the maximum steepness of the slope of the bottom may be larger than in the case of longer waves

Finaliy a remark follows on the practical determination of $\frac{5 a}{5 x}$ and $\frac{S a}{\delta y}$ etc They are derived from the contour lines of the depth determined in intervals of $05 \mathrm{~m}$ The location of the contour lines is usually irregular Therefore it is often not possible to determine accurate values for the second derivatives

A grid net for the computation must be constructed on the map The grid size depends on the accuracy of the contour lines Various procedures can be followed for the numerical presentation of $\frac{S a}{S x}$, $\frac{S_{a}}{\delta y}$ etc, e $g$

$$
2 \Delta x \frac{S a}{S x}=a_{p+1, q+1}+a_{q+1, q}-a_{p, q}-a_{p, q+1},
$$

and a similar equation for $\frac{S a}{S y}$

It is noted that the points $\left(x_{1}, y_{1}\right)$ on the wave ray will usually not coincide with the grid points $(p, q)$ of the grid net The study of the accuracy of the refraction computation will be continued 


\section{General remarks}

This section deals with the methods to be applied for the determination of the morphological changes along the Delta coast It was not possible to make satisfactory scientific forecast for these changes because of the uncertainty in the values of the various coefficients in the equations of the phenonena It is intented that after the closing of the Haringvliet these coefficients will be determined based on extensive measurements in the new circumstances that wlll prevall along the coast The sediment transport In the Delta estuaries and coastal regions occurs mainly in suspended form and bed load sediment is negligeable This is the reason that a hydraulic model has not been bullt for this research In the preceding sections the basic factors influencing morphological studies have been mentioned These factors are better defined from a physical and mathematical point of view then are the morphologlcal changes In this respect distinction must be made between the final shape of the seabed that will result when conditions have stabilized and the speed at which the change takes place In particular this speed can be approached more theoretically It is determined by the net sedimentation and erosion and is therefore related to the sand transport However, simplifications must be accepted, and empirical quantities introduced which have to be evaluated by means of measurements in nature Consequently only tendencies for the morphological changes can be given Svasek has discussed these problems for the Delta area in his publications (10) and likewise Terwindt (11)

The concentration of the sand is determined by the currents in combination with the wave motion The concentration can increase considerably by the wave action, because of the increased turbulence of the water, especially in the case of breaking waves Tidal currents and wind waves vary with astronomical conditions and meteorological circumstances, especially during stormsurges Reversely the tidal currents and the wave motion which is statistically distributed, are also affected by the bottom morphology

The instruments for the measurement of sand in suspension that are currently in use are not accurate enough for all circutastances Their application is very limited and is only possible during quiet weather By means of radio-active tracers, the direction and the relative significance of the sand transport can be determined as a mean value over longer time periods Quantitative evaluation of the sand transport directly related to the fluid motion, and of the net transport, still remains uncertain

The most important tool for the determination of the net-sand transport is the study of the changes in the shape of the bottom, as obtained from periodically repeated soundings, combined with a knowledge about tidal currents and wave-action The results of the soundings also give information for the estimation of the final situation of the bottom morphology Along the Delta coast the soundings are repeated every three months, or at shorter intervals in regions unere sand movement is consideravle The study of de Vries (12), on the applicability of tracer techniques for rivers must be mentioned A discussion on sand transport processes is also given 
52 An empirical method for the determination of a cross= section area in the mouth of an enclosed estuary

An important problem is the estimation of the final crosssection area in the mouths of the enclosed estuaries, $\mathrm{g}$ in the mouth of the Haringvliet, where the sluice is about $5 \mathrm{~km}$ inland and a gully must be maintained for the discharge of the sluices It has been found that a relation exists between the quotient of the tidal volume $(e b b+f l o o d)$ and the total profile area of the inlet mouth for the various estuaries of the Delta region This amount divided by the tidal period $(T=44,700$ sec $)$ determines a velocity $\vec{v}$ over the tide, that appears to have a relation with the morphological conditions Its value is $55 \mathrm{~cm} / \mathrm{sec}$ for all the estuaries The diameter of the sand is of the same order of magnitude for all the mouths, $015 \mathrm{~mm}$ to $02 \mathrm{~mm}$

It must be expected that in the future silt will be deposited in the mouth of Haringvliet and consequently the value of the scouring velocity will become somewhat higher By means of laboratory tests it has been estimated as about $70 \mathrm{~cm} / \mathrm{sec}$ in nature

The future discharges in the mouth can be determined from the discharges of the sluices and from tidal calculations Then the future cross-section area can be computed The time scale of the development must be determined from detalled knowledge about the sand transport and from comparative examples in nature e $g$ from the morphological changes after the closure of previous sea arms like Brielse Maas (1951) (see fig 6) and Veerse Gat in the Delta region of which the mean grain-size at the bottom is the same

The mean value of the depth in the future gullies can also be estimated in an empirical way by comparison with these examples Then the same relation between mean and maximum depth can be applied to the future gullies of the Haringviset mouth, provided the widths of the gullies are fixed by banks The preceding studies for the determination of the final cross-section have been applied by Terwindt in his morphological study of the Haringvliet mouth Reference is also made to Bruun and Gerritsen (13)
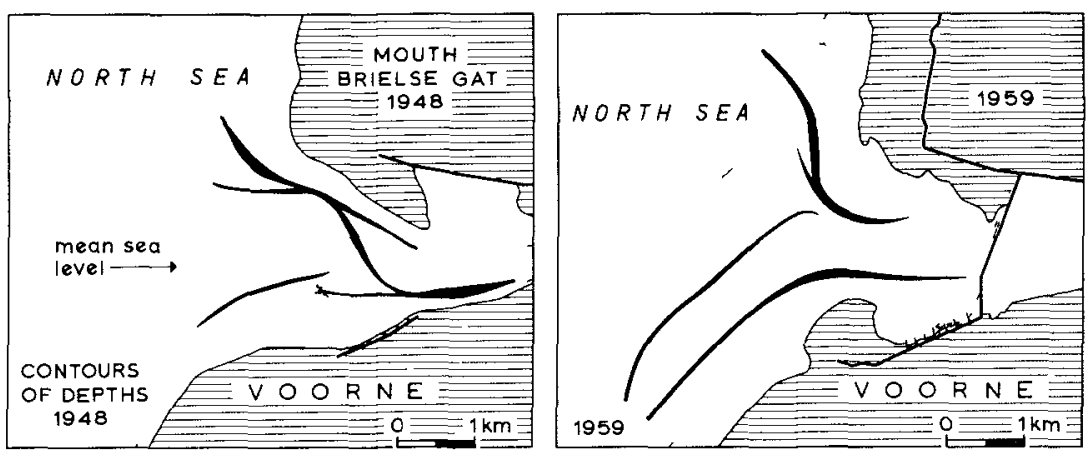

Fig 6 Changes in the contours of depths due to the enclosing of the Brielse Maas 
Extensive shoals have been formed with gullies at both sides after the enclosing of this sea-arm

53 A more theoretical method for the determination of the sand transport in a river

For the computation of the sediment transport due to the tidal currents in the Delta region, various sediment transport formulae can be used They are however generally based on laboratory tests, trials in nature are limited Various bed-load formulae are summarized in the book of A $J$ Raudkivi (14) The formulae for the computation of suspended sediment movement, which is related to diffusion processes, are still more limited The vertical distribution of the velocity in turbulent water is one of the determining factors, however the formulae for such distributions in nature are also more or less based on an empirical basis

The various quantities which are defined in the formulae under 1 dealized circumstances must be redefined for the more irregular conditions in nature in an empirical way by adapting the formulae to the observations obtained from measurements in nature After that the formula can be applied for different conditions and engineering purposes e g higher upland river discharges, or changes in the profiles of the river Then the formula may give indications for changes in scour and sedimentation The method has been applied by Morra (15) In the tidal region of the Delta, where the sand is fine, 015 $\mathrm{mm}$ to $02 \mathrm{~mm}$ The calculation of the suspended sand transport is based on a formula for the distribution of the suspended sand concentration in the vertical, combined with the formula of Kalinske (16) The major feature of the method will be described

The exchange of sand over the vertical is determined by the equilibrium condition per unit of width,

$$
c w=\varepsilon \frac{\mathrm{d} w}{\mathrm{dh}}
$$

in which $w$ is the local sediment concentration (the volume of sand per unit of volume water), h is the helght above the bottom, c is the fall velocity of particles in the fluid at rest, and $\varepsilon$ is an exchange coefficient It is assumed to be equal to the momentum transfer coefficient in the relation for the shear stress $\tau$, $\tau=p \varepsilon \frac{d v}{d h}$, in which $v$ is the velocity at height $h$, determined according to the parabolic formula

$$
v=v_{1} h^{1 / q},
$$

and $\rho$ is the density, $q$ is of the order 5 to 7 Then

$$
\frac{d v}{d h}=\frac{1}{q} v_{1} h(1-q) / q
$$

It is well-known that the shear stress $\tau$ at the helght h is defined by $P$ gal $(1-z)$, in which $z=h / a$, a $1 s$ the total depth, and 1 is the slope of the water surface in the case of steady flow In the case of unsteady flow it is the difference in head, determined by the friction term, $1=v_{m}^{2} / c^{2} a,(4)$, in which $v_{m}$ is the mean velocity at
time $t$ in a cross-section, and $c$ is the coefficient of chezy After integration and some calculation it is found from (1) and (3) that the vertical distribution of the sand concentration is 


$$
\frac{w_{z}}{w_{z_{0}}}=\left[\frac{(1-z) z^{1 / q} e^{-(q+1)} z^{1 / q}}{\left(1-z_{0}\right)^{z_{0}^{1 / q}} e^{-(q+1) z_{0}^{1 / q}}}\right],
$$

in which $z_{0}=h_{0} / a$, and $h_{0} 1 s$ a reference helght above the bottom For $h_{0}=0$, the formula is

$$
\frac{w_{z}}{w_{0}}=\left[(1-z)^{z^{1 / q}} e^{-(q+1)} z^{1 / q}\right]^{p_{c}},
$$

in which $p_{c}=\frac{c c^{2}}{g q v_{m}}, v_{m}$ is the mean velocity over the vertical, defined by $\frac{q}{q+1} v_{1} a^{1 / q}$ (see (4)), and $w_{0} 1 s$ the suspended sand concentration near the bottom The formula of Kalinske determines the relation between $w_{0}$ and the bottom material A brief explanation of this formula follows Let $v^{\prime}$ be the velocity component in the vertical at the height $h$ above the bottom and c the fall velocity in still water of a particle, and let the statistical distribution of $v^{\prime}$ be determined by the formula of Gausz Then the formula

$$
\Delta F(c) \int_{c}^{\sim}\left(v^{\prime}-c\right) \frac{1}{2\left(\overline{\left(v^{\prime}\right)^{2}}\right)^{\frac{1}{2}}} \exp \frac{-v^{\prime 2}}{2\left(\left(v^{\prime}\right)^{2}\right)^{\frac{1}{2}}} d v^{\prime} \text {, }
$$

in which $\triangle F(c)$ is an interval with equal diameters of bottom material with fall velocity c (expressed in percentages), determines the transport of sand from the bottom into suspension in course of time The horlzontal velocity component may not change considerably In the equilibrium situation the transport in vertical direction per unit of time equals the transport downwards, cwo

It may be put for turbulent flow $\left(\overline{\left(v^{1}\right)^{2}}\right)^{\frac{1}{2}}=k(\operatorname{gal})^{\frac{1}{2}}$, in which $k$ is an empirical constant The transport of sand from the bottom, cwo, must be proportional to the expression (7) After the transformation, $v^{\prime}=k(2 \text { gal })^{\frac{1}{2}} u$, it is found

$$
c w_{0}=A c \Delta F(c) \int_{u(c)}^{\sim}\left(\frac{u}{u(c)}-1\right) e^{-u^{2}} d u \text {, }
$$

In which $A$ is the proportional factor and $u(c)=c / k(2 g a l)^{\frac{1}{2}}$ The equation (9) may be rewritten in the form

$\frac{w_{o}}{\Delta F(c)}=\frac{A}{(2 \pi)^{\frac{1}{2}}}\left\{\frac{1}{2 u(c)} e^{-u^{2}(c)}-\frac{\pi^{\frac{1}{2}}}{2}\left(1-\frac{2}{\pi^{\frac{1}{2}}} \int_{0}^{u(c)} e^{-u^{2}} d u\right)\right\}_{1}(10)$

A and $k$ are empirical constants in (8) and (10) They depend on the local circumstances

The following remark must be made for the application of the equation (10) in a tidal river At a location in a tidal region the value of 1 , which is determined by the friction term is usually different from the lope of the water surface caused by the tide at time $t$ This slope equals the algebralc sum of the friction term 
and the inertia terms

It must be assumed in the practical applications that the changes of the tidal velocities are small enough, so that the equilibrium condition of the sand transport, defined above, is satisfied more or less

The bottom material can be considered as homogeneous in tidal retions with a standard grain diameter of $\mathrm{d}_{60}$ for this special purpose In that case $\Delta F(c)=100$ The practical determination of the empirical parameter, $p_{c}$, in formulae (5), and (6) is as follows The relation between $w_{z}$ and $w_{z_{0}}$ is found from the measurements of the sand concentration $w_{z}$ in the vertical, and the exponent $q$ of the parabolic formule (2) ls determined from velocitymeasurements Then the exponent $p_{c}$ in formula (5) can be computed The value of $p_{c}$ varies in the tidal regions of the Delta area between 025 and 25

It is evident that due to the great varlations of the sand transport in the course of time, a great number of measurements must be taken Then the least square rule can be applied for the best fit of the sand concentration in the vertical

After that $w_{0}$ can be calculated from (6) and introduced into the left member of equation (10) The values of $A$ and $k$ can be determined such that equation (10) is satisfied A trial and error procedure is usually necessary It is difficult to obtain reliable values for $A$ and $\mathbf{k}$

It is also possible to determine $A$ and $k$ from the results of the measurements mentioned in Kalinske (10) However Kalinske applies the logarithmic formula for the vertical velocity distribution, and $\varepsilon m$, being the mean value for the exchange coefficient over the vertical Kalinske found by this procedure $A \approx 39$ and $k \approx 075$

\section{Sand transport in the coastal area}

The morphological conditions at the coast of the Delta region will change in the future as a consequence of the enclosing of the Harlingvilet and the works which are in execution The future situation depends on the new equilibrium of the sand transports, due to the combined effect of currents and waves The new equilibrium in the channels, and seaward of the breaker zone is determined considerably by the changes in the tidal currents and in particular by the velocity components towards the coast line A complicated sand transport process exists in the region of the sand banks, in front of the coast line between the existing gullies Here the sand transport depends on the combined effect of currents and waves Breaking waves produce very high sand concentrations in these regions The combined effect has been studied by Bl jker (17) on the basis of laboratory tests He determines the combined shear stress at the bottom of currents and waves in the two dimensional case Vertical velocity components and accelerations are not considered in his study In the breaker zone where tidal currents are negligeable a wellknown procedure is to assume that the total long-shore transport rate is proportional to the loss of long-shore energy flux per unit of length Reference is made to Bl Jker and Svasek (18) The basis of all the morphological studies are the tidal and refraction computations which determine the changes in the velocity 
components and the directions of the waves These morphological studies are continued An example of the changes in the coastal area is shown in fig 7 Some contours of depths in 1960 and 1969 are represented It appears that the 2 and $5 \mathrm{~m}$ lines near the coast changed considerably, due to the wave action and the changes in the currents
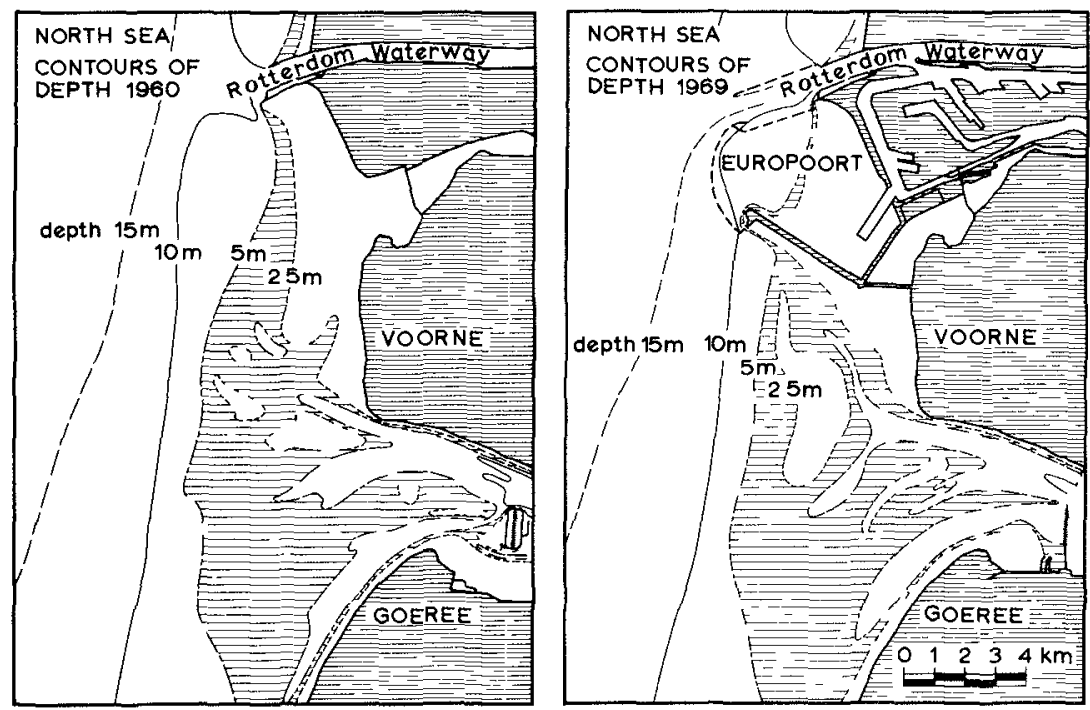

Fig 7 A comparison between the contours of depths in 1960 and 1969 in the mouth of Haringvliet and near Europort

\section{REFERENCES}

1, Leendertse, $J, 1967$, Aspects of a computational model for longperiod water wave propagation, Thesis, The Rand Corporation, Santa Monica, Calıfornia

2a, Dronkers, J J , 1969, Tidal computations for rivers, coastal areas and seas, J Hydraulics Div, Proc Am Soc Civ Eng, New York

2b, Dronkers, $J J, 1969$, Some practical aspects of tidal computations, Thirteenth Congr Int Ass Hydr Res, Proc vol 3, Tokyo

3a, Kındsman, B, 1965, Wind Waves, Prentice-Hall, Ine, Englewood Cliffs, New Yersey

3b, Benda, S J and Plersol, A G, 1966, Measurements and analysis of random data, John Wiley, New York 
4, Koelé, L A, 1962, Statistıcal distribution of wave helghts in shallow water, (Dutch text) Hydraulles Div^, Delta works, The Hague

5, Freudenthal, A M, 1969, Determination sur base aleatorre des criteres pour le calcul des ouvrages maritimes, Ass Internat Perm des Congres de Navigation, Brussels

6, Koele, L A and de Bruyn, P A , 1964, Statistical distribution of wave helghts in correlation with energy spectrum and water depth Ninth Confer on Coast Engin, Lisbon

7, Svašsek, J N, 1969, Statıstıcal evaluatıon of wave conditıons in a deltalc area, Symposium on wave actıon, Delft, Hydraulics Lab

8, Griswold, G M, 1963, Numerical calculation of wave refraction, $J$ of Geophys Res vol 68, no 6

9, Duthler, $J W, 1968$, Refraction computation by means of a digital computer, (Dutch text), Hydraulics Div, Delta works, The Hague

10, Svašek, J N, 1968, Influence of breakıng waves on the stability of sandy coasts, (Dutch text), Hydraulics Div, Delta works, The Hague

11, Terwindt, J H J, 1970, Future depths in the gullies of the mouth of the enclosed Haringvliet, (Dutch text), Hydraulics Div, Delta works, The Hague

12, de Vries, M, 1966, Applications of luminophores in sand transport studies, Thesis 1966, Delft, Hydraulics Lab

13, Bruun, $P$ and Gerritsen, F, 1953, Stability of coastal inlets, North-Holland Publ Comp, Amsterdam

14, Raudkıvı, A J , 1967, Loose boundary hydraulıcs, Pergamon Press, Oxford

15, Morra, $\mathrm{R} H \mathrm{~J}$, 1952, The sand movement in a tidal region, (Dutch text), Hydraulics Div, Delta works, The Hague

16, Kalınske, A A 1939, Relations of suspended to bed materlals, Nat Res Council, Transact Am Geoph Union

17, Bijker, EW, 1967, Some considerations about scales for coastal models with movable bed, Thesis, Delft, Hydraulics Lab

18, B1 jker, $\mathrm{EW}$ and Svašek, J N, 1969, Two methods for determination of morphological changes included by coastal structures, XXIlnd Internat Nav Congr, Paris 



\title{
CHAPTER 109
}

\section{SEDIMENT POLLUTION IN COASTAL WATERS}

\author{
J van de Kreeke*
}

ABSTRACT

Sediment pollution can be defined as the increase in sediment concentration, due to artificial infusion of sediment, to a level harmful to fish and plant $11 \mathrm{fe}$

The coastal engineer may be called upon to evaluate the result of an artificial sediment influx in terms of characteristic parameters 11ke concentration and turbidity and to determine eventual deposition areas of the sediment

This paper describes the field investigations carried out to evaluate a case of sediment infusion in one of the estuaries on the US east coast The sediment was discharged via a pipeline at a rate of approximately $10 \mathrm{lbs} / \mathrm{sec}$ Nearly all the released sediment was in the silt range In addition to the results of the field measurements a simple mathematical mode 1 is presented, describing the temporal and spatial distribution of the sediment in the resulting sediment plume

\section{DESCRIPTION OF THE STUDY AREA}

The study area is located in a bend of the estuary, see Figure 1 The depth contours in this figure show a deep channel at the west side and a relative shallow plateau at the east side of the estuary The hydraulic environment in the study area is governed by the tide and the freshwater inflow The tide has a semidiurnal character $w 1$ th a mean tidal range of $21 \mathrm{ft}$ and a mean spring tidal range of $24 \mathrm{ft}$ Maximum currents are on the order of $1 \mathrm{ft} / \mathrm{sec}$, both for ebb and flood, but the ebb currents can be considerably higher during periods of high freshwater inflow An order of magnitude of the freshwater discharge might be obtained from the following figures During $50 \%$ of the time the average daily freshwater discharge is higher than $6,500 \mathrm{cfs}$ and during $1 \%$ of the time exceeds $70,000 \mathrm{cfs}$ For comparison, the maximum tidal discharge in the study area is on the order of 100,000 cfs Salinities in the shallow part, the area where the sediment is released, can be as high as $2 \%$ o after long periods of low freshwater inflow and as low as $01 \%$ o after periods of high freshwater inflow The water 15 well mixed, at least for the shallow depths

In order to gather additional information on the hydraulic environment in the study area, current measurements were carried out with a stationary and a movable current meter Both instruments were equipped with a Savonius rotor In addition, the stationary meter had a direction device The readout of the movable current meter provided a record of instantaneous velocities

*Research Associate, Dept of Coastal and 0ceanographic Engineering, University of Florida 


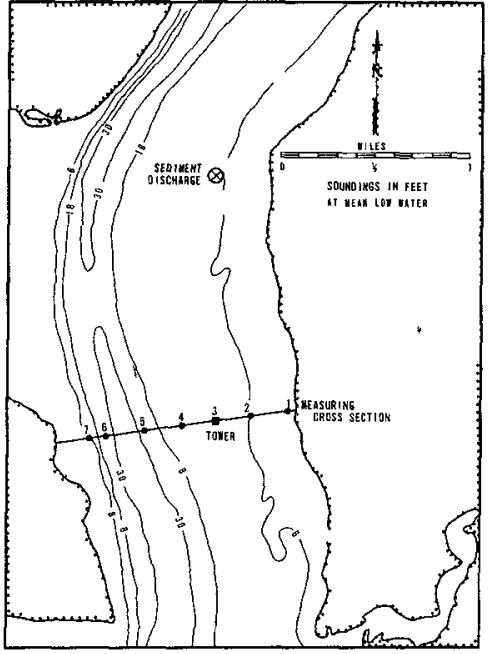

FIGURE 1 - STUOY AREA AND MEASURING STATIONS

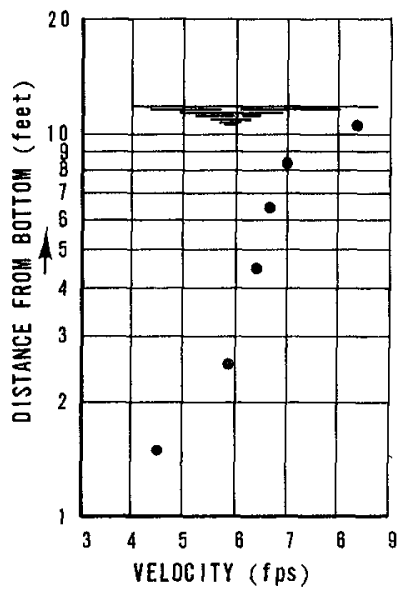

FIGURE 2 - MEASUREO VELOCITY PROFILE IN STATION 3
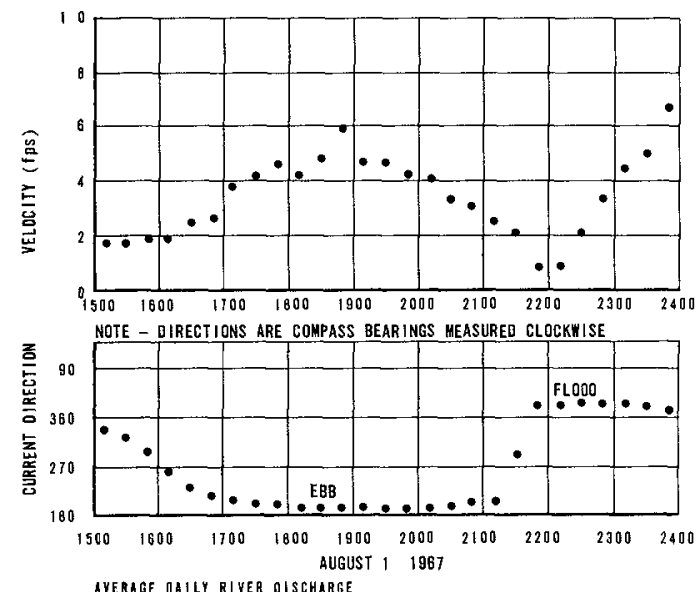

AUGUST $1-5240 \mathrm{cfs}$

FiguRE 3 - CURRENT VeldCITY aNd OIRECTION MEASUREd IN STATION 3 
The output of the stationary current meter consisted of velocities and directions averaged over time periods of 19 minutes Calibration threshold for the rotor and direction device was approximately $01 \mathrm{ft} / \mathrm{sec}$ The stationary meter was placed in a tower, for location see Figure 1, with the center of the rotor $15 \mathrm{ft}$ and the center of the vane $3 \mathrm{ft}$ above the bottom The movable meter was used to determine the velocities at the stations 1-7, indicated in Figure 1 The measurements with the movable current meter were carried out during periods of definite ebb currents It was hoped that by comparing measured and theoretical velocity profiles, an estimate could be made of the eddy diffusivity coefficient This idea had to be abandoned, however, since the measured profiles did not comply with the theoretical logarithmic velocity distribution as may be seen from Figure 2

Typical curves showing the variation in current velocity and direction during a tidal cycle are presented in Figure 3 Attention is drawn to the magnitudes and direction of the currents during slack tide The transverse currents during these periods are one of the reasons why the sediment plume seems to disappear during periods of slack tide, as will be described later

\section{NATURAL SEDIMENT CONTENT IN THE ESTUARY}

To determine the background sediment load in the study area, suspended load samples were taken when no sediment infusion took place The samples were taken with a device consisting of two bottles connected by a short piece of pipe, see Figure 4 The lower bottle contains the sample while the displaced air is contained in the upper bottle The whole system is buoyant even when the lower bottle is filled A weight connected with the sampler by an anchor line is used to lower the sampler When the weight reaches the bottom, the tension in the retrieval inne is released, which automatically opens the intake The positioning of the sampler above the bottom 15 controlled by the length of the anchor line

The sedtment samples were analyzed using the following procedure The suspension was we $11 \mathrm{~m} 7 x e d$ and $500 \mathrm{~m} 7$ was poured into a cylinder The suspended solids were then collected on filter paper The filter paper was dried in an oven When dry, the paper was burned and the ashes weighed

The sediment samples were taken at three different depths at the stations used for the current measurements The various sampling series were carried out for different freshwater discharges in order to study the influence of the freshwater inflow on the sediment content All samples were taken during periods of definite ebb current As an example, the results of one of the sampling series is plotted in Figure 5 It is noted that the data for the bottom samples are probably not very reliable, chances are that the sampler was in the mud layer covering the bottom instead of just above this layer

In Figure 6 , the average sediment concentration for each sampling series, that 15 the average of all 7 sampling stations, 15 plotted versus the average dally freshwater inflow In the averaging procedure, bottom samples were neglected for the reason mentioned in the previous paragraph Although only five data points are avallable, it may be safely concluded that a definite relation exists between sediment content and freshwater inflow Because of the 


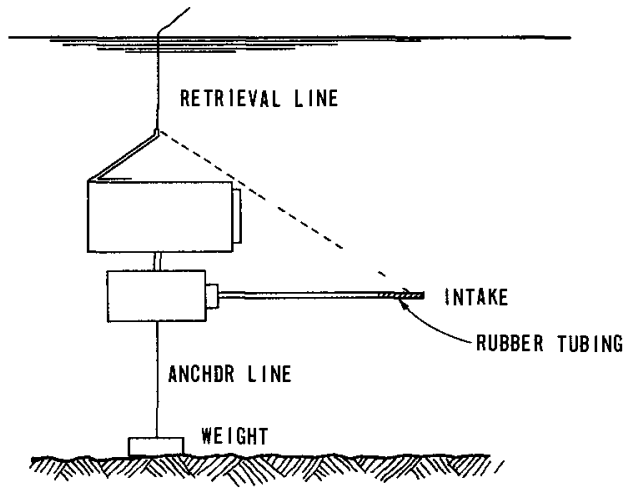

FIGURE 4 - SUSPENDED LDAD SAMPLER

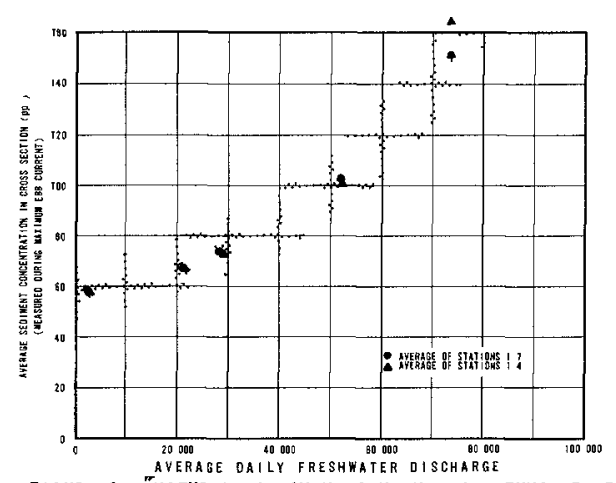

FIGURE 6 -"NATURAL SEDIMENT CDNTENT AS A FUNCTIDN DF FRESHWATER INFLDW

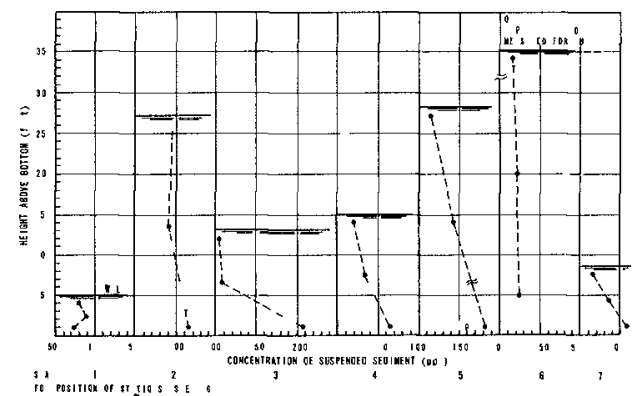

FIGURE 5 - NATURAL SUSPENDED SEDIMENT CDNCENTRATIDN IN DIFFERENT MEASURING STATIDNS

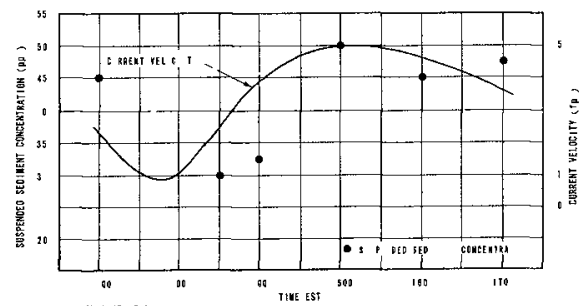

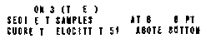

FIGURE 7 - VARIATIDN IN SUSPENDED SEDIMENT CONCENTRATIDN DURING A TIDAL CYCLE 
special importance of the natural sediment in the area of sediment infusion, the average sediment concentration for stations 1 through 4 on ly, neglecting again the bottom samples, were also plotted versus the freshwater inflow in the same figure No significant differences exist between the average sediment concentration in the shallow area (stations 1-4) and the average sediment concentration when regarding the total estuary cross-section (stations 1-7)

To study the variation in sediment concentration during a tidal cycle, a series of samples was taken at station No 3 (tower) at a depth of $6 \mathrm{ft}$ each hour for a period of six hours The results are shown in Figure $7 \mathrm{~A}$ definite variation in suspended sediment content with the tidal currents seems to exist. This agrees with observations by other investigators, see Nichols and Poor [3] At the time of the measurements, $1 t$ was observed that during periods of high velocity the water looked cloudy while during slack tide the water had a less turbid appearance

\section{CHANGES IN THE \\ SEDIMENT FIELD DUE TO THE SEDIMENT INFLUX}

The sediment was discharged via a pipeline on the shallow plateau, see Figure 1 The discharge point was approximately $10 \mathrm{ft}$ below the water surface The total discharge was on the order of $107 \mathrm{bs}$ of solids/sec Most of the discharged material appeared to be $1 n$ the silt range, $90 \%$ of the particles are smaller than $85 \mu$, the median particle diameter $1545 \mu$ and $10 \%$ of the particles are smaller than $10 \mu$ The sediment influx resulted in a plume visible during periods of relative low turbidity of the receiving waters $V_{1}$ sual observations, which are of course restricted to what happens at the surface, indicated that the end of the plume moved approximately with the speed of the current The maximum length of the plume, just before slack tide, was approximately 3 miles The width of the plume remained practically constant At slack tide, the discoloring disappeared indicating that the sediment had settled or was dispersed by the reversing tide

Sediment samples were taken to determine more accurately the effect of the sediment influx The samples were taken in the measuring stations 1-7 and along the center line of the sediment plume As an example, the distribution of suspended sediment across the estuary 15 presented in Figure 8 This figure clearly shows an increase in suspended load at the stations $2 A$ and 3 The influence of the sediment infusion still is restricted to a narrow band at this distance

Sediment concentrations in the axis of the plume are indicated in Figure 9 , the concentrations decrease rapidly with downstream distance The concentration near the discharge point varied between $1000 \mathrm{ppm}$ and $1500 \mathrm{ppm}$ and is already decreased to 200-500 ppm at a distance of $400 \mathrm{ft}$ downstream of the discharge point

It $1 \mathrm{~s}$ clear from Figure $g$ that large amounts of sediment settle close to the point of release Fluorescent tracer was used to gain insight into the deposition areas of the remainder of the sediment The range of particle sizes of the tracer material covered approximately that of the released sediment 


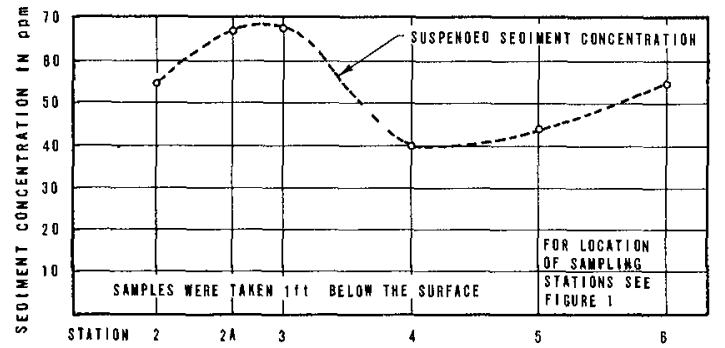

FIGURE 8 - HORIZONTAL DISTRIBUTIDN DF SUSPENDEO SEDIMENT

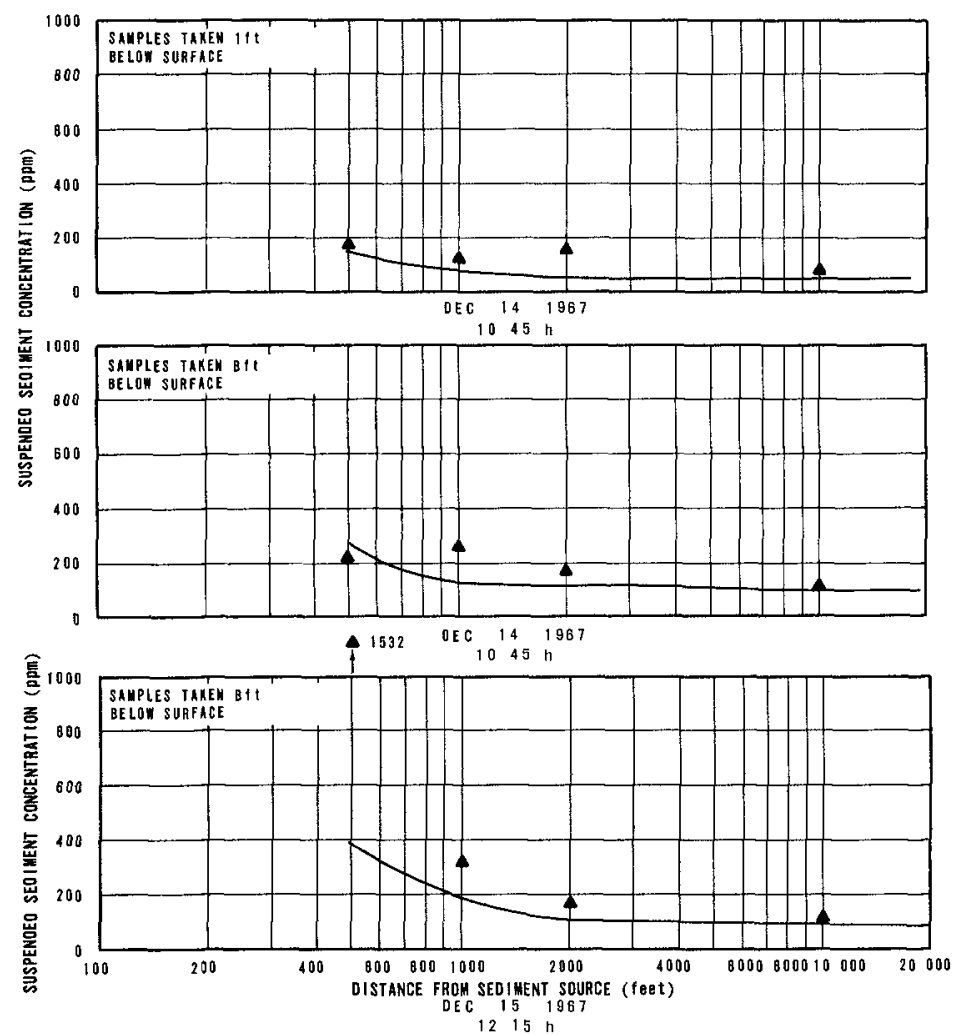

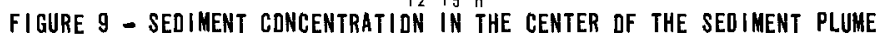


The tracer was released at the location of the sediment discharge point, at a rate of approximately $081 \mathrm{bs} / \mathrm{min}$ for a period of two hours, leading to a total amount of released tracer of $100 \mathrm{lbs}$ The same day and the next day, suspended load samples were taken at different depths in the study area The results were somewhat meager, probably because of the relative small quantity of tracer material, but some tracer grains were found as far away as stations 1 and 5 of the measuring cross-section

\section{MATHEMATICAL DESCRIPTION}

Assuming that lateral and longitudinal diffusion are negligible (as suggested by the results of the measurements), and furthermore assuming a gradient type diffusion, the conservation of matter equation may be written as

$$
\frac{\partial \phi}{\partial t}=\frac{\partial\left(\varepsilon \frac{\partial \phi}{\partial y}\right)}{\partial y}-u \frac{\partial \phi}{\partial x}+w \frac{\partial \phi}{\partial y}
$$

in which $\phi=$ sediment concentration

$$
\begin{aligned}
\varepsilon & =\text { diffusion coefficient } \\
u & =\text { current velocity } \\
W & =\text { fall velocity of a sediment particle }
\end{aligned}
$$

The coordinate system has its origin at the bottom, the horizontal $x$-axis is positive in the current direction, the vertical $y$-axis is positive upward Attempts to arrive at a computer solution for Equation (1) indicated that, with conventional numerical methods and for the dimensions of the computational field encountered in this study (water depth $10 \mathrm{ft}$, length of the sediment plume 20,000 ft), it is questionable whether even the larger computers can handle this problem Therefore, a simplified and admittedly more crude model is presented here to describe the sediment concentration in the plume

It is assumed that a block of polluted water travels at the average current velocity $u(t)$, and thus the position $x$, of the block at time $t$ is $x=u t$ Only vertical diffusion is considered It is also assumed that all particles reaching the bottom stick to $1 t$, while a steady sediment source at the bottom of the moving block of polluted water simulates the stir-up capacity of the flow Only periods of definite ebb and flood current are considered It is assumed that during periods of slack tide, the sediment particles in the plume settle and/or are distributed over the width of the estuary

In deriving the equations, it is assumed that

- The convective velocity $u(t)$ is constant over the depth

- The eddy diffusivity coefficient $\varepsilon$ is constant 
- The fluid properties are not altered by the suspended sediment

- The diffusion is proporicional to the gradient of the concentration

- The sediment concentration in the vertical at the dredging site is constant

- No flocculation occurs

The coordinate system is the same as the one described in the previous paragraph Time $(t)$ is zero at the moment the block of polluted water leaves the dredge For the above assumptions the equations relating the sediment concentration $\phi(y, t)$ and the independent variables $y$ and $t$ are

Field equation

$$
\frac{\delta \phi}{\delta t}=\varepsilon \frac{\delta^{2} \phi}{\delta y^{2}}+W \frac{\delta \phi}{\delta y}
$$

Boundary conditions

$$
y=0 \quad \varepsilon \frac{\delta \phi}{\delta y}=-\lambda
$$

in which $\lambda=$ rate of erosion

$$
y=h \quad \varepsilon \frac{\delta \phi}{\delta y}=-W \phi
$$

in which $h=$ average depth

For a more detailed discussion of the boundary condition, see Partheniades [2]

Initial condition

$$
\mathrm{t}=0 \quad \phi=\phi_{0}
$$

The solution of the above system of equations is

$$
\phi=\frac{\lambda}{W} e^{-2 a y}+\sum_{n=1}^{\infty} B(n) e^{-a y}\left[\cos \alpha_{n} y+\frac{a}{\alpha_{n}} \sin \alpha_{n} y\right] e^{-\left(\alpha_{n}{ }^{2}+a^{2}\right) \varepsilon t}
$$

in which

$$
\alpha_{n}{ }^{\prime} s \text { are the solution of } \operatorname{tg} \alpha_{n} h=\frac{2 \alpha_{n}{ }^{a}}{\alpha_{n}^{2}-a^{2}} \quad \alpha_{n}>0
$$

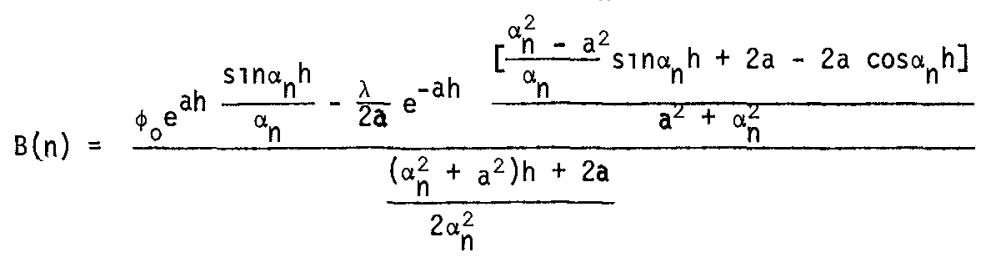

$a=\frac{W}{2 \varepsilon}$ 
Note that the second term in the solution goes to zero for large values of $t$, the first term represents the natural sediment concentration

To check the validity of the model a comparison is made between measured and computed concentrations In the computations, $\phi=1250 \mathrm{ppm}$ The value of $\lambda$ was obtained by assuming a natural sediment concentration $\phi=100 \mathrm{ppm}$ at $y=4 \mathrm{ft}$ The average depth $h=10 \mathrm{ft}$ An estimate of the diffusion coefficient was made by using Elders [1] expression

$$
\varepsilon=0068 \mathrm{hu}_{*}
$$

in which $u_{*}=$ shear velocity

In the computations, $\varepsilon=005 \mathrm{ft}{ }^{2} / \mathrm{sec}$ is used The value $W=0008 \mathrm{ft} / \mathrm{sec}$ was taken as a representative value of the fall velocity of the particle distribution Computed and measured concentrations are plotted in Figure 9 The results agree reasonably well in spite of the many assumptions made in deriving the mathematical description and the $11 \mathrm{~m}$ ted accuracy of the measurements

\section{CONCLUSIONS}

The artificial sediment infusion results in a sediment plume visible during periods of low turbidity of the recelving waters The end of the plume moves approximately with the speed of the current The width of the plume remains practically constant The sediment concentration in the plume decreases rapidiy with increasing distance from the point of sediment influx During periods of slack tide, the plume disappears, the sediment settles and at the same time is dispersed by secondary currents

A crude mathematical model is presented describing the sediment concentration in the plume In 7 ts present form, it should be mainly regarded as a first step to a rational and universal solution

Finally, it is noted that the title of the paper is somewhat of a misnomer as the case of sediment infusion discussed here has never been proven to be harmful

\section{ACKNOWLEDGEMENT}

The study described here was carried out by the author while employed with Sclence Engineering Associates, San Marino, California The guidance of $\mathrm{Dr}$ Per Bruun during the initial stages of the project is gratefully acknowledged

\section{REFERENCES}

1 Elder, J, "The Dispersion of Marked Fluid in Turbulent Shear Flow", J Fluid Mech, Vol 5, Part 5, 1959 
2 Partheniades, E, "A Summary of the Present Knowledge of the Behavior of Fine Sediments in Estuaries", Technical Note No 8, M I T , Hydrodynamics Laboratory, June, 1964

3 Nichols, M $M$ and Poor, $G$, "Sediment Transport in a Coastal Plain Estuary," Journal of the Waterways and Harbors Division, ASCE, Vol 93, No WW4, November, 1967 


\section{CHAPTER 110}

RECIRCULATION IN SHALLOW BAYS AND RIVERS

by

R K Price, Visiting Assistant Professor

$R$ A Dalrymple, Assistant in Engineering

R G Dean, Professorvand chairman

Department of Coastal and Oceanographic Engineerıng

University of Florida, Gainesville, Florida

\section{ABSTRACT}

A theoretical model is presented to provide an approximation of the water and temperature recirculation in a shallow bay, lake or river, between the outlet and inlet canals of the cooling system for a power plant In particular, a temperature recirculation factor relating the outlet and inlet temperatures is derived

\section{INTRODUCTION}

Since the efficiency of a power plant depends critically on a low intake water temperature, an injudicious siting of the discharge canal terminus may cause recirculation of the discharge water, with a consequent increase in the temperature of the intake water Thus a prior knowledge of the effect of particular locations for the terminus of the discharge canal is important

The quantitative evaluation of the siting of the outlet and inlet canals, taking into account tides, meteorological conditions, the diffusion and convection of momentum and temperature, and the stratıfication due to temperature and salinity, is a difficult problem The complexity of the boundary conditions and governing equations for the system requires the use of numerical modeling The recent work of Wada $(1966,1967,1968)$ has clarlfied many of the problems involved and given some solutions to particular models

The following work presupposes that steady conditions prevail and that, to the first order, temperature variations have no effect on the flow This rather crude assumption can be partially strengthened by considering the receiving water system to be shallow and by assuming that the turbulence in the flow is sufficient to ensure that the mixing of the water is complete throughout the water column These assumptions and conditions thus avoid the more difficult problems of varıations in time, and vertical stratification of temperature

To simplify the analysis, the equations governing the flow in the bay are Innearized This procedure is reasonable except near the outlet for the plant cooling system where the non-linear convection of momentum terms may well be important We assume that the flow in the neighborhood of the outlet can be treated as a source rather than as a jet, whence the convection terms can be neglected 
For steady flow, the equations of momentum and continuity can be written as

$$
\begin{aligned}
-f v & =-g \frac{\partial \eta}{\partial x}+\frac{\tau^{s x}}{\rho h}-\frac{k \tilde{U}}{h} u \\
f u & =-g \frac{\partial \eta}{\partial y}+\frac{\tau^{s y}}{\rho h}-\frac{k \tilde{U}}{h} v
\end{aligned}
$$

and

$$
\frac{\partial}{\partial x}(h u)+\frac{\partial}{\partial y}(h v)=0
$$

Here $\mathrm{u}$ and $\mathrm{v}$ are the water partıcle velocitıes in the $\mathrm{x}$ and $\mathrm{y}$ directions respectively, $n$ is the displacement of the free surface from its mean level, $\tau^{s x}$ and $\tau s y$ are the stresses exerted by the wind on the flow, $h$ is the mean depth, $f$ is the Coriolis parameter, $k$ is related to the Chezy bottom stress coefficient, $\mathrm{C}$, by $K=g / \mathrm{C}^{2}, \tilde{\mathrm{U}}$ is a scale velocity and $\mathrm{g}$ is the acceleration due to gravity

The linearization of equations (1) and (2) involved firstly the neglect of the non-linear inertia terms, as commented above, and also the adoption of a linear form for the bottom stress terms The linearization of these latter terms has been shown to be reasonable for oscillating flows such as tides in estuaries However, the use of the linear form for steady flows has not been so readily confirmed It is used here because the basic equations can be written in a particularly useful form and also, the resulting flows appear to give a good representation of the physical situation Equations (1), (2) and (3) also require $\eta \ll h$

From equation (3), a stream function $\psi$ can be defined such that

$$
\mathfrak{u}=-\frac{1}{h} \frac{\partial \psi}{\partial y} \text { and } \mathbf{v}=\frac{1}{h} \frac{\partial \psi}{\partial x}
$$

By eliminating $\eta$ between (1) and (2),

$\frac{\partial^{2} \psi}{\partial x^{2}}+\frac{\partial^{2} \psi}{\partial y^{2}}=\frac{2}{h}\left(\frac{\partial \psi}{\partial x} \frac{\partial h}{\partial x}+\frac{\partial \psi}{\partial y} \frac{\partial h}{\partial y}\right)+\frac{f}{k \tilde{U}}\left(\frac{\partial \psi}{\partial x} \frac{\partial h}{\partial y}-\frac{\partial \psi}{\partial y} \frac{\partial h}{\partial x}\right)-\frac{h^{2}}{\rho \kappa \tilde{U}}\left\{\frac{\partial}{\partial y}\left(\frac{\tau}{h}\right)-\frac{\partial}{\partial x}\left(\frac{\tau}{h}\right)\right\}$

Equation (5) takes on a partıcularly simple form if $h$ is uniform and $\tau^{s x}=\tau^{\text {sy }}=0$

$$
\frac{\partial^{2} \psi}{\partial x^{2}}+\frac{\partial^{2} \psi}{\partial y^{2}}=0
$$

In this case the streamlines are given by potential flow theory, whence sources and sinks can readily be modeled This method is used as the basis for the study of recirculation of the water between the outlet and inlet canals The extension of this type of model in the case of a variable depth and a nonzero wind stress requires the use of numerzcal solutions of (5) 


\section{WATER RECIRCULATION}

Consider inlet and outlet canals embedded in a straight coastline bordering a recelving water system whlch covers the half-plane and has a uniform depth If the distance between the outlet and inlet canals is $2 a$, then the complex velocity potential for the flow in the receiving system is

$$
w \equiv \phi+1 \psi=-\mu \log \frac{z-a}{z+a}-\bar{U} z
$$

where $\mu$ is the strength of the source - sink flow, $z \equiv x+1 y$, the source is at $\mathrm{z}=\mathrm{a}$ and the sink at $\mathrm{z}=-\mathrm{a}$, and $\bar{U}$ is the speed of a uniform steady current along the coast, cf Figures 1 and 2 Th1s current can be regarded as a steady river flow, or a "steady state" tıdal current In exther case, $\bar{U}$ may be a function of time $t$, subject to the condition that the time scale $T$ for changes in $\overline{\mathrm{U}}$ are large compared with $\mathrm{h} / \mathrm{kU}$ and $2 a / \tilde{U}$ If $\tilde{\mathrm{U}}=10 \mathrm{ft} / \mathrm{sec}$, the (varying) tidal amplitude is $15 \mathrm{ft}$ and $k=002$, then for $T=12$ hrs, it can be shown that $\mathrm{h}$ must be less than approximately $14 \mathrm{ft}$ Further, a must be less than 33,000 ft These are fairly strict condltions on $h$ and $a$ The conditions may be realistic only for a few situations

From equation (7), the non-dimensional form for the streamlines is

$$
x^{-2}=1-y^{-2}-2 y^{\prime} \cot \left(\bar{U}^{-} y^{\prime}+\psi^{\prime}\right)
$$

where $\bar{U}^{-} \equiv \bar{U}_{a} / \mu, \psi^{-} \equiv \psi / \mu$ is the dimensionless value for the streamline, and $x^{\prime}=x / a, y^{\prime}=y / a$ It can be shown from equation (8) that the percentage recirculation of water is $100 \%$ for a zero current $\left(\bar{U}^{\circ}=0\right)$ or for a current aided recirculation $\left(\overline{\mathrm{U}}^{\prime}<0\right)$ However, for an opposing current $\left(\mathrm{U}^{\circ}>0\right)$, the percentage circulation is reduced Figure 3 shows the percentage recirculation of water for aiding and opposing non-dimensionalized currents It can be seen that for an opposing current $\overline{\mathrm{U}}$ greater than 20 , there wl11 be no recirculating water

Other elementary shaped coastlines can be treated by the technique of comformal mapping In the general situation, equation (5) can readily be solved numerically Further detalls on this technique w111 be discussed by Price (1971) Figure 4 gives the streamlines in an Idealized model of a bay connected to the ocean This model includes variations in the depth and coastline configuration and permits an inflow of water across two of the boundaries

It is of interest to point out that uniform depth flows can be modeled experimentally using a Hele-Shaw apparatus A Hele-Shaw model has been used at the University of Florida to determine the streamline patterns for flow into a bay

\section{THERMAL RECIRCULATION}

The temperature difference between the outlet and inlet canal of a cooling system depends, in part, on the decay of temperature in the recirculating fluld with distance from the outlet It should be noted that mixing effects are not 
included in this treatment Under the assumption that there is no heat flow through the bottom or the fixed sxde boundaries, heat can only be lost or gained by the recirculating water in exchange with the surrounding receiving water or with the atmosphere We assume that the diffusion of temperature in the receiving system is negligible compared with the convection of temperature Thus the primary mechanism for extracting heat from the water is assumed to be via the atmosphere include

The components of heat loss (or gain) from the surface of a body of water

$Q_{B} \quad$ solar radiation by the sun

$Q_{b}$ - back radiation from the water to the atmosphere

$Q_{c}$ heat loss due to convection

$Q_{e} \quad$ heat loss due to evaporation

$Q_{r} \quad$ heat advection by rain or other inflows

The " "s refer to differentiation with respect to time $t$ Consider the anomalous heat flux due to the heated water, that is

$$
\begin{aligned}
& Q_{\text {net }}=Q_{E}+Q^{\prime} \\
& T_{w}=T_{w_{E}}+T_{w}
\end{aligned}
$$

where the primes denote incremental heat fluxes due to the increased water temperature $T_{w}^{*}$ The quantitıes $Q_{E}$ and $T_{W_{E}}$ are defined such that the water body is in equilibrium (in the average sense) ${ }^{W_{E}}$ for $\mathrm{T}_{\mathrm{w}}=\mathrm{T}_{\mathrm{w}_{E}}$ This implies that $Q_{E}=0$

The heat flux budget can be written as

$$
Q^{\prime}=Q_{s}-Q_{b}-Q_{c}-Q_{e}+Q_{r}
$$

where the various components of heat flux are derined in the following equations

$$
\begin{aligned}
& Q_{s}=(1-r) Q_{m} \\
& \dot{Q}_{b}=097 \circ\left(\theta_{w}^{4}-B \theta_{a}{ }^{4}\right) \\
& Q_{c}=000407 \mathrm{PW}\left(T_{w}-T_{a}\right) \\
& Q_{e}=12 W\left(e_{w}-e_{a}\right)
\end{aligned}
$$




$$
Q_{r}=M_{r} C_{r}\left(T_{r}-T_{w}\right)
$$

here

and

$$
\begin{aligned}
& Q_{\mathrm{m}}=\text { the solar radiation on a horızontal surface, } \\
& r=\text { the reflectivity, assumed to be } 005 \text {, } \\
& \sigma=\text { the Stefan-Boltzmann radiation constant }=1714 \times 10^{-9} \\
& \mathrm{BTU} / \mathrm{hr} / \mathrm{ft}^{2} /{ }^{\circ} \mathrm{R}^{4} \text {, } \\
& \theta_{\mathrm{w}}=\text { absolute water temperature in }{ }^{\circ} \mathrm{R}\left(\mathrm{T}\left({ }^{\circ} \mathrm{F}\right)+45969\right) \text {, } \\
& \theta_{a}=\text { absolute air temperature, } \\
& \beta=\text { radlation factor, depending on cloud cover, vapor pressure, } \\
& \text { etc An average value of } \beta \text { is } 085 \text {, } \\
& W=\text { wind speed in knots, } \\
& P=\text { atmospheric pressure in Inches of mercury, } \\
& e_{w}=\text { vapor pressure of water in saturated air at the temperature }
\end{aligned}
$$

We assume for the purposes of this paper, that $Q_{r}=0$ By utilizing the various definitions for the $Q^{\prime} s$ from (10) and substituting in equation (9), then the incremental heat flux $Q^{*}$, due to the water temperature increased an amount $T_{w}^{\prime \prime}$ above the equilibrium temperature, is approximately

$$
Q^{*}\left(\mathrm{BTU} / \mathrm{ft}^{2} / \mathrm{hr}\right)=\{104+066 \mathrm{~W}\} \mathrm{T}_{\mathrm{W}}^{*}
$$

Thus if $Q$ is the amount of heat above ambient in a volume of water with unit surface area

$$
\begin{aligned}
\frac{\mathrm{dQ}}{\mathrm{dt}} & =Q^{-} \\
& =-\mathrm{KT}^{\circ} \mathrm{W}
\end{aligned}
$$


where $K=104+066 \mathrm{~W} \quad K$ can be called the heat transfer coefficient, and as such includes the effects of radiation, convection and evaporation ds outlined above

$$
Q=\gamma h c_{p} T^{\prime}
$$
where $\gamma$ 1s the specific welght of water with heat capacity $c_{p}$ Thus from
equation (13)

$$
\frac{d Q}{d t}=\gamma h c_{p} \frac{d T_{w}^{\prime}}{d t}=-K T_{w}^{\prime}
$$

To examine the relation between the flow and temperature fields, consuder Figure 5 If the flow is divided into $N$ streamtubes of equal flow $\Delta q$, then it can be seen that for the flow between two particular streamlines is

$$
\Delta \mathrm{q}=\mathrm{h \ell} \mathrm{ds} / \mathrm{dt}
$$

where $\ell$ is the spacing normal to the streamlines, and ds/dt is the local water particle velocity parallel to the local streamline Using equations (14) and (15)

$$
\frac{\mathrm{dT}_{\mathrm{w}}^{\prime}}{\mathrm{ds}}=-\frac{\mathrm{KlT}_{\mathrm{w}}^{\prime}}{\gamma \mathrm{c}_{\mathrm{p}} \Delta \mathrm{q}}
$$

The integration of equation (16) gives

$$
\ln \mathrm{T}_{\mathrm{w}_{1}}=-\frac{\mathrm{KA}}{\gamma \mathrm{j}_{\mathrm{p}} \Delta \mathrm{q}}+\ln \mathrm{T}_{\mathrm{w}_{0}}
$$

where $\mathrm{T}_{w_{i}}$ and $\mathrm{T}_{w_{0}}$ are the inlet and outlet temperatures respectively for the flow between the $(j-1)^{\text {th }}$ and the $j^{\text {th }}$ streamlines, and $A$ is the surface area between the two streamlines By summing over the $\mathrm{N}$ streamlines and averaging the thermal recirculation is expressed by

$$
c_{R}=\frac{\overline{w_{1}}}{T_{w_{0}}}=\frac{1}{N} \sum_{j=1}^{N} e^{-B A_{j} N}
$$

where

$$
B=\frac{\mathrm{Ka}^{2}}{\gamma \mathrm{c}_{\mathrm{p}} \mathrm{q}}
$$


Tne $1^{-8} s$ are the non-dimens $\perp$ onal areas between the streamlines ( 1 e $A_{j}^{-}=A_{j} / a^{2}$ ), where $2 \mathrm{a}$ is the distance between the outlet and inlet), and $\mathrm{q}$ is the total volume recirculating $(q=\pi \mu h) \quad C_{R}$ can be termed the temperature recirculation Cactor It can be seen that $\mathrm{C}_{\mathrm{R}}$ depends on the sum of exponential terms, and of course must be less than unity

A numeical evaluation of $\mathrm{C}_{\mathrm{R}}$ in the case when the equations of the stream1 ines are known can be carried out on a computer In the case of the source and sink embedded in the boundary of the half plane, with $\beta=078$ and $\bar{U}=-375$, (an aıding current and $100 \%$ water recirculation), $C_{R}=43 /$, with on 1 y $50 \%$ recirculation of the outlet water $\left(\mathrm{U}^{\prime}=0326\right)$ and $B=078, \mathrm{C}_{\mathrm{R}}=12 \%$ Ir Lis exanple $N=10$, cf Figure 6

The extension of the technique to more complicated flows as considered briefly in Figure \& is straightforward For example, one can divide the flow from the cutlet using 9 interior streamilnes, whence $N=10$ The areas $A_{j}$ can readily be fouad from graph paper when the streamlines have been plotted For a representative value of $K$ and the proper dimensions and discharge, $\beta$ can be calculated (equation 18), and the temperature recirculation determined from equation (17)

\section{CONCLUUSTON}

The analysis presented here can readily be used to obtain an estımate of the temperature circulation for particular locations of the inlet and outlet canals for a power plant located by a shallow bay, lake or river which is being used in the cooling system A numerical solution of equation (5) can be used for particular cases, including the effects of prevalling wind stresses as well as an arbitrary bottom topography

The limitations of the above technique are obvious due to neglecting temperature gradients, diffusion, and any unsteady feature of the flow Also there may be some doubt about the validity of the linear form for the bottom stress in the case of large variations in the velocity of the water in the receljing system However, despite these limitations, the authors have found that the technique provides a realistic approximation of the temperature recirculation

\section{REFERENCES}

1 Wada, A, "A Study on Phenomena of Flow and Thermal Diffusion Caused by Outfall of Cooling Wates", Proc 10th Conf on Coastal Engr , Tokyo, 1966

2 Wada, A, "Studies of Recırculatıon of Coolıng Water in a Bay", Proc 11th Conf on Coastal Engr , London, 1968

3 Wada, A, "Nunerıcal Analysis of Distribution of Flow and Thermal Diffusion Caused by Outfall of Cooling Water", 13th Congress I A H R, Tokyo, 1969

4 Price, $\mathrm{R} \mathrm{K}$, "Wind Generated Coastal Currents" - in preparation 


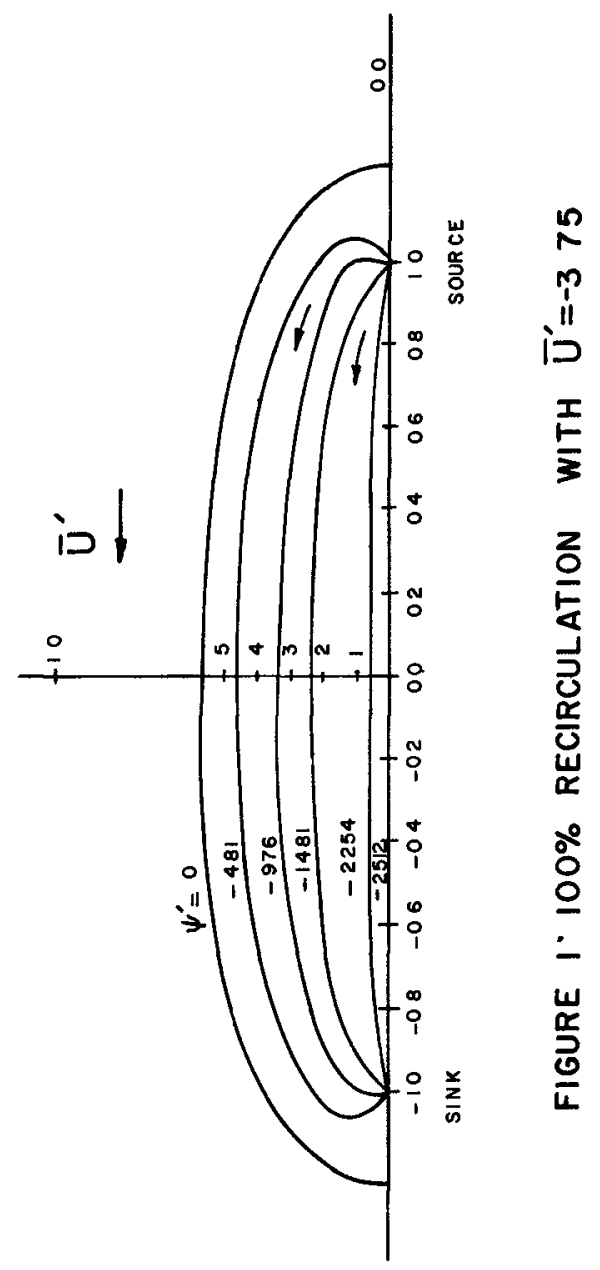




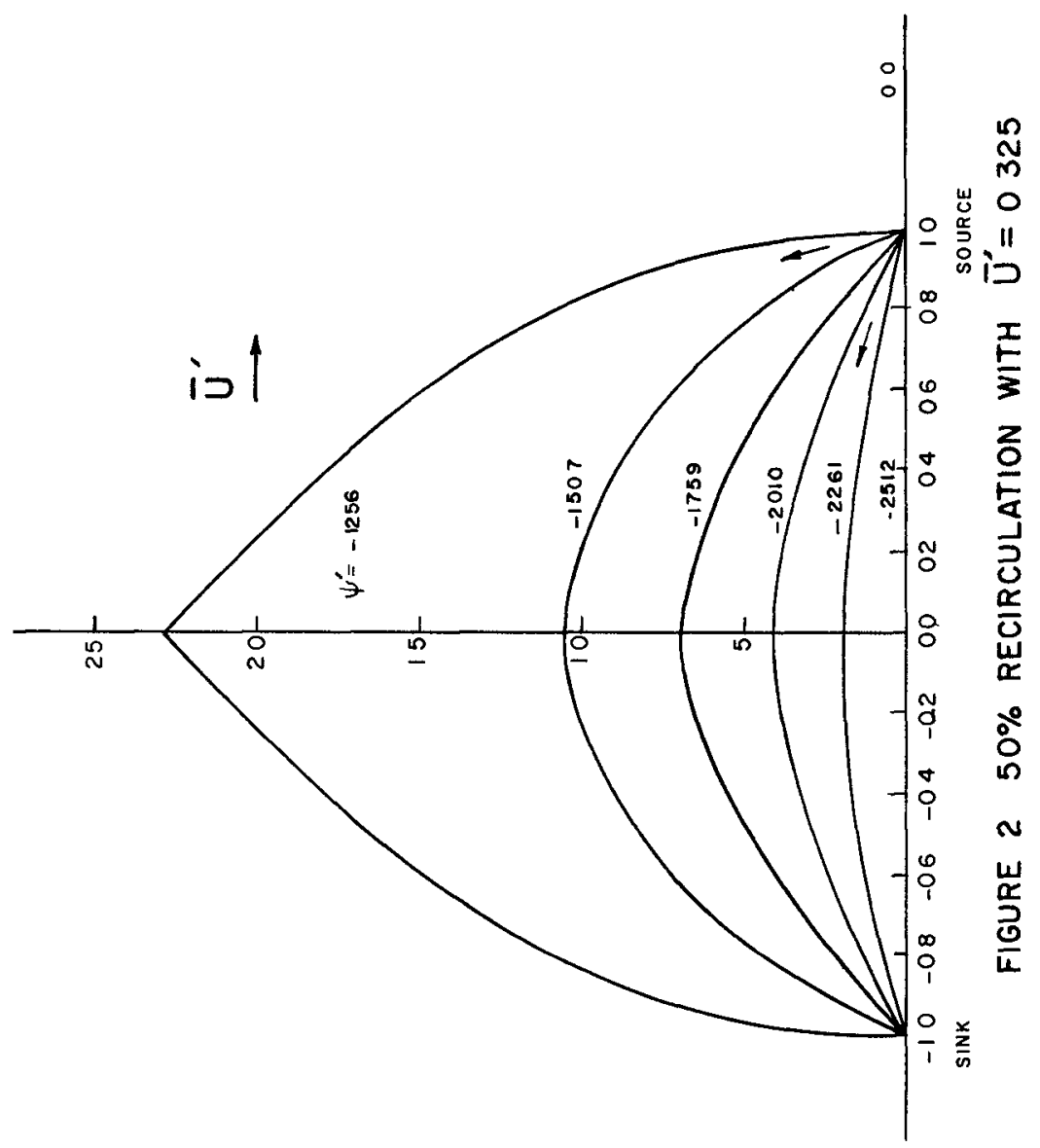




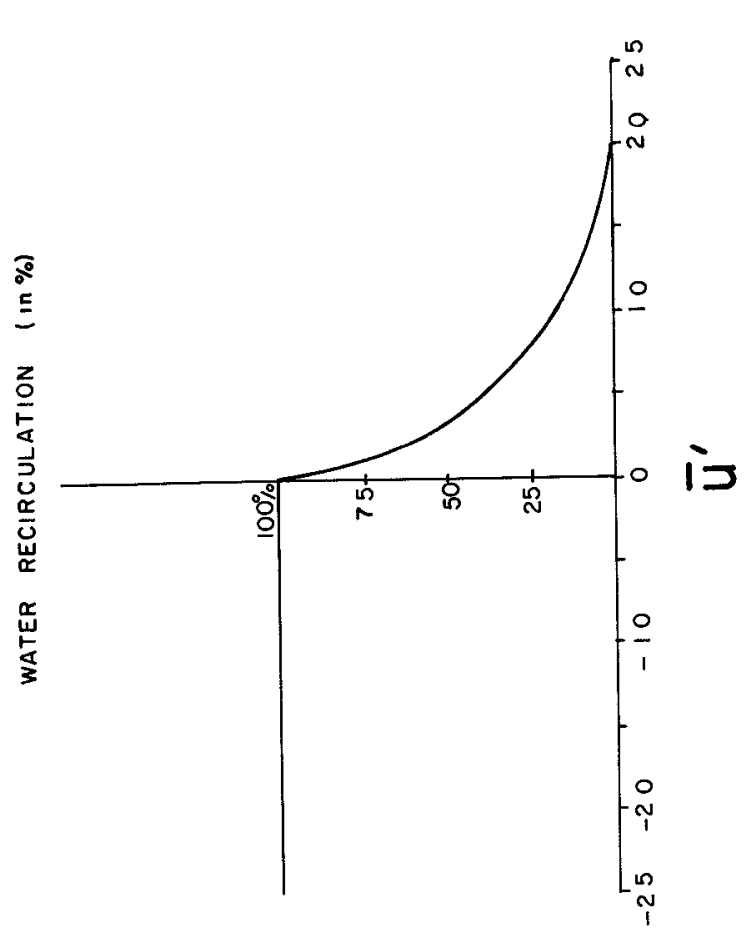

0
$\frac{2}{3}$
0
$\frac{0}{0}$
0

z

$\frac{2}{2}$

인

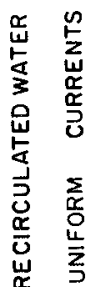

ப

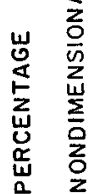

m

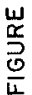




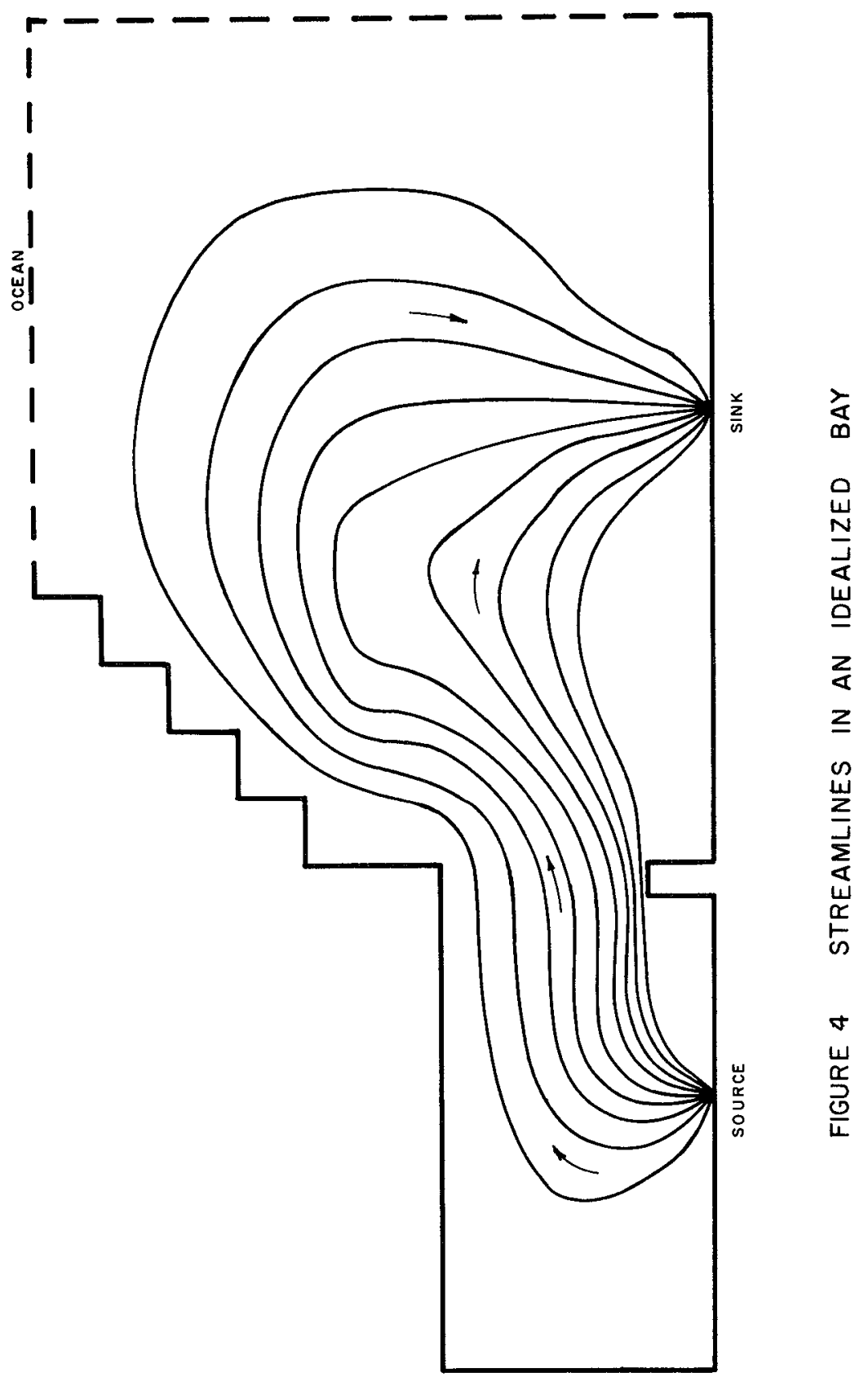




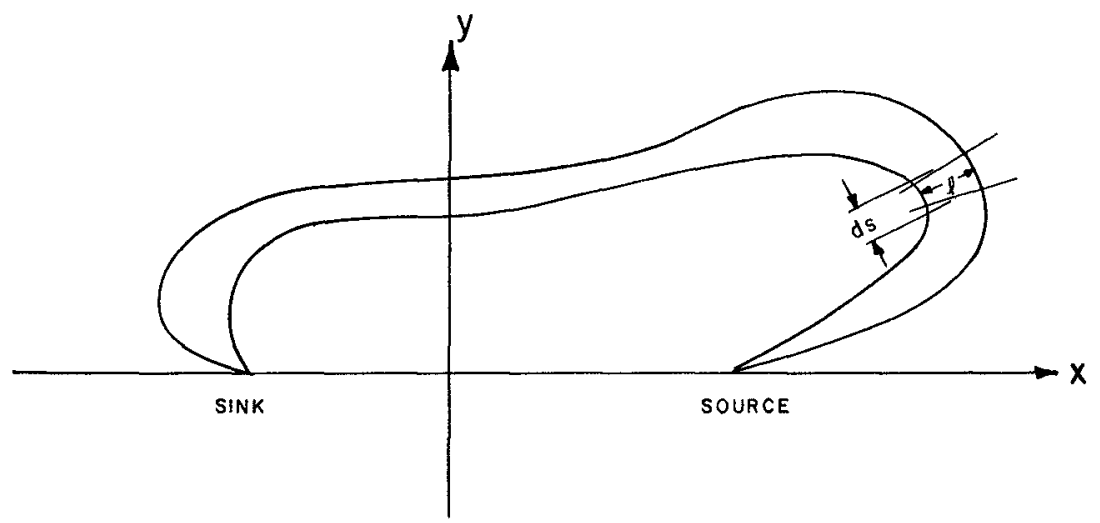

FIGURE 5 DEFINITION SKETCH 


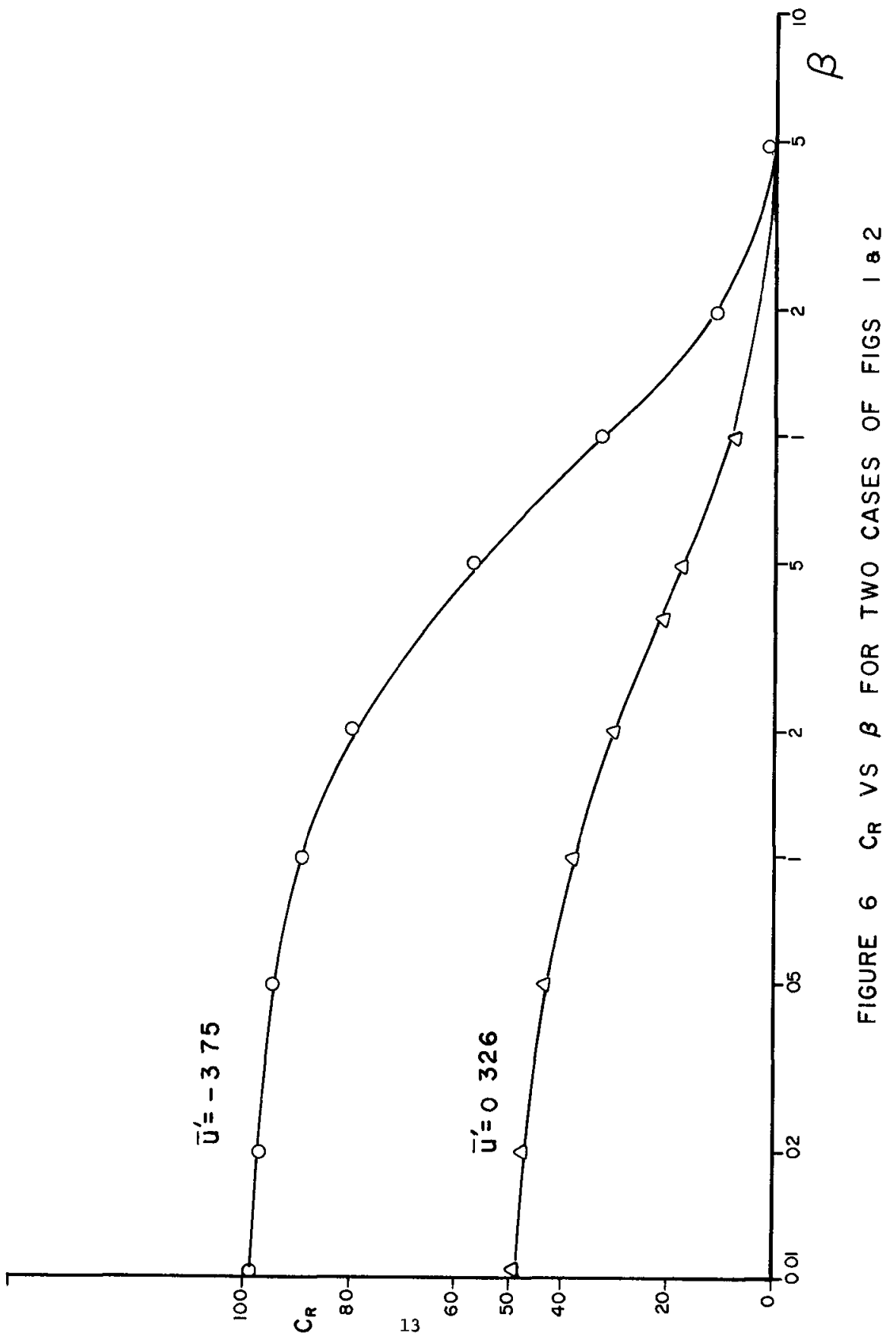





\section{CXIAPTER 111}

NATURAL FL IHITIO ABILITY AN TIDA- N S - is Mct, Oiverrs*

\section{1 - $A E S T F A C T$}

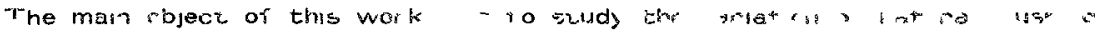

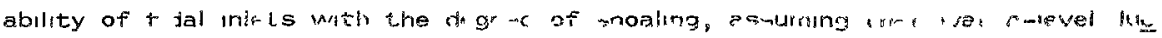
tuations in tha locioon are unifor:, over

It was assismed ther iztural $i^{\prime}-r$ ig ar ity is ploportie to i peduluad capacity of tidal currents end "ai $c_{i} s$ capacily $c_{n}$ be cliaracze at bow" ry the hydrait c power consumed in ine connecting chanre' and by ro 3rd or 6th power of the mean velocity influenre o she slope of thi 7 is tarls ana of the inner head losses was also analysed

\section{2 - INTPODLICTION}

In $1951 \mathrm{EH}$ Keuleyan [1] unicrtcok the snalytic in ieud f the hydra-ynamic behaviour of the in'et-lagoon sj biem, but some restricting hypctneses were assumed which diminish the pracuical interest of the rosults obtained for ins tance, he assumed the relationship satween the deptn of the inlet channel and the tidal range to be very great (and therefore he considered the flow section constant during a tidal cycle) Aiso the banks of the layoon were considered ver tical, and the level variation law tre same for all points of the lagoon

The hydrodynamic behaviour of the system could thiss be characterized by the equations

$$
\begin{aligned}
& \frac{d h_{1}}{d \theta}=k \sqrt{h_{2}-h_{1}} \text { when } h_{2}>h_{1}(\text { floor) } \quad \text { and } \\
& \frac{d h_{1}}{d \theta}=K \sqrt{h_{1}-h_{2}} \text { when } h_{1}>h_{2} \quad(e b b)
\end{aligned}
$$

where (see Fig 1),

$$
h_{1}=\frac{H_{1}}{H}, h_{2}=\frac{H_{2}}{H}, H \text { being the orp m tide armpra sie, } H_{1} \text { and }
$$
$H_{2}$ sea ant lagoon levels respecivei $r$ eferred to the meari sea level, $\theta=\frac{2}{1} t$,

* - Trainee Rusearch Ofticer - L. acu aturio Nacionu du Enger tiun "ia LIVIl, Lisbon, Portugal 
$T$ the tidal period, $t$ the time variable, $K$ a dimensionless parameter which the author has called repletion coefficient, and which condenses the influence of se veral parameters, namely, lagoon and channel dimensions, channel roughness, and sea tide amplitude and period

Owing to the simplifying hypotheses made, the coefficient $K$ is constant all along the tidal cycle and, consequently, the mean level of the lagoon is equal to the mean sea level, the behaviour of the level law in the lagoon is symmetrical with respect to that level, and the flood velocities and rates of flow are symmetrical to those at the ebb In the study of natural flushing ability of a tidal inlet we cannot make use of the results of such a simplified scheme, since the most interesting cases to be analysed are obviously those which are characterlz ed by faulty inlets needing correction, which present small values for the channel depth/tidal range ratio

In the first studies it was sought to analyse the influence of the channel flow section variation and that of the lagoon area during a tidal cycle, subsequently, the influences of head losses in the lagoon were also analysed for a very schematıc case

3 - INFLUENCE OF FLOW SECTION VARIATION OF INLET CHANNEL AND OF BANK SLOPE

\section{1 - Characteristic equation of the system}

Assuming the inlet channel to be well shaped, to have constant width and depth over its entire length, and the level varıation in the lagoon to be uniform, we obtain similarly to Keulegan

$$
\frac{d H_{1}}{d t}=\frac{a}{A} \sqrt{\frac{c^{2} r}{L+\frac{c^{2} r}{2 g}}} \quad \sqrt{H_{2}-H_{1}}
$$

where
A - lagoon area
a - flow section area of the inlet
$L$ - length of inlet channel
$r$ - hydraulic radius of inlet channel 
$C$ - Chezy coefficient $\left(c^{2}=\frac{r^{1 / 3}}{n^{2}}\right)$

n - Strickler coefficent

Assuming further that the hydraulic radius $(r)$ is equal to the depth of the channel, that this depth corresponds to the arithmetic mean of sea and lagoon levels, and that the lagoon area varies linearly with its level, we get

$r=d+\frac{H_{1}+H_{2}}{2}$, where $d$ is the depth referred to the mean sea level (FIg 1)

$a=b r \quad, b$ being the width of the channel,

$A=A_{0}\left(1+N \frac{H_{1}}{H}\right), A_{0}$ being the area of the basin corresponding to the mean sea level (Fig 2)

Parameters $H_{2}, H_{1} r$ and $d$ were reduced to a dimensionless form by relating them to the sea tidal amplitude

$h_{2}=\frac{H_{2}}{H}=\sin \theta, h_{1}=\frac{H_{1}}{H}, r_{0}=\frac{r}{H}=d_{0}+\frac{h_{1}+\sin \theta}{2}$

Besides, $\theta=\frac{2 \pi}{T} t$, so that equation (1) will be

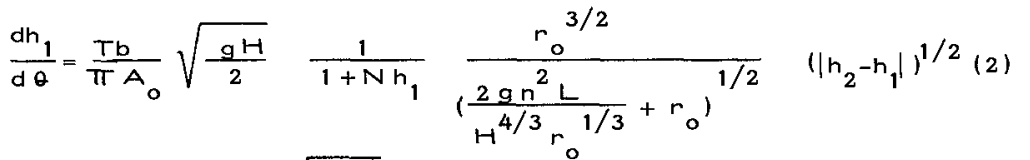

Makıng $D=\frac{T b}{\pi A_{0}} \sqrt{\frac{g H}{2}}$

$E=\frac{2 g n^{2}}{H^{4 / 3}} L$

we get $\frac{d h_{1}}{d \theta}=, \frac{D}{1+N h_{1}} \frac{r_{0}^{3 / 2}}{\left(\frac{E}{r_{0}^{1 / 3}}+r_{0}\right)^{1 / 2}}\left(\left|\sin \theta-h_{1}\right|\right)^{1 / 2}$

$$
\begin{aligned}
& 1=1 \text { for } \sin \theta>h_{1}(\text { flood }) \\
& 1=-1 \text { for } \sin \theta \leqslant h_{1}(\text { ebb) }
\end{aligned}
$$

Since $r_{0}=d_{0}+\frac{h_{1}+\sin \theta}{2}$, it follows that the inlet-lagoon system is well characterized by the dimensionless parameters $D, E, d_{0}$ and $N$ On the other hand, it is easy to see that Keulegan repletion coefficient is related to these parameters by the expression 


$$
K=\frac{D d 0^{3 / 2}}{\left(\frac{E}{\mathrm{do}^{1 / 3}}+\mathrm{do}\right)} \frac{1 / 2}{N=0}
$$

The numerical integration of equation (5) was made in the NCR-Elliott 4100 computer of the Laboratório Nacional de Engenharı Civil, using one of its library programs An integration step $\Delta \theta=01$ radians was adopted, to which corresponds, in the case of a semi-diurnal lunar type tide $(T=12 \mathrm{~h} 25 \mathrm{~min}), \Delta \mathrm{t} \simeq 11$ min $52 \mathrm{~s}$ The numerical integration of equation (5) was effected starting from a situation in which the level inside and outside the basin were equal to the mean sea level After some attempts it was concluded that the second tidal cycle no longer depended on initial conditions, so that integration was made to comprehend two tidal cycles, of which the first was discarded While integrating equation (5) the computer also calculated the other parameters necessary for the study of the natural flushing capacity of the inlet, namely, rate of fiow, velocity, 3 rd and 6 th power of the velocity and consumed power

\section{2 - Rate of flow}

From the continuity equation

$$
Q=A \frac{d H}{d t}=\frac{2 \pi H A O}{T}(1+N h 1) \frac{d h 1}{d \theta}
$$

we get

$$
Q_{a}=\frac{Q}{\frac{2 \pi H A o}{T}}=(1+N h 1) \frac{d h 1}{d \theta}
$$

where $Q a$ is the dimensionless rate of fiow, whose term of comparison

$$
\frac{2 \pi H A_{0}}{T}=\frac{2 H A_{0}}{T / 2} \quad \frac{\pi}{2}
$$

represents the peak discharge corresponding to the sinusoidal flow of the lagoon's maximum admissible prism ( $\left.2 H A_{0}\right)$

33 - Mean velocity

$$
\text { From } \begin{array}{rl}
Q=a & V=A \frac{d H 1}{d t} \text { there results } \\
\qquad V_{a} & =\frac{V}{\frac{T A_{0}}{T b}}=\frac{1+N h_{1}}{r_{0}} \quad \frac{d h_{1}}{d \theta}
\end{array}
$$

where $V a$ is the dimensionless velocity whose comparison term 


$$
\frac{2 \pi A_{0}}{T b}=\frac{2 H A_{0}}{T / 2} \quad \frac{\pi}{2} \frac{1}{b H}
$$

represents the mean velocity of flow, through section $b H$, of the peak sinusoidal discharge corresponding to the lagoon's maximum admissible prism ( $2 H$

Ao)

Another dimensionless velocity ( $V^{\prime}$ ) was further considered, in which the comparison term adopted was $\sqrt{2 \mathrm{gH}}$ it is easy to prove that

$$
V_{a}^{\prime}=\frac{V}{\sqrt{2 g H}}=\frac{1+N h_{1}}{D r_{0}} \frac{d h_{1}}{d \theta}=\frac{V a}{D}
$$

In Keulegan's study a coefficient $C$ was determined, which relates the maximum flow with the one that would occur if the effective prism Pr land not the maximum possible one $\left.2 \mathrm{H} A_{0}\right)$ would flow out sinusoidally $A s$ this author considers the flow section (bd) invariable, constant $C$ also gives the relationship between the maximum velocity and the peak velocity corresponding to the sinusoidal flow of the effective prism Pr in our case, as we take into account the variation of the flow section, there are reasons for the determination of a value $C_{1}$ for the rates of flow and of a value $c_{2}$ for the veloci ties, adopting as term of comparison for them the maximum velocity corresponding to the sinusoidal flow of the effective prism Pr through the mean sea level section

We will then have

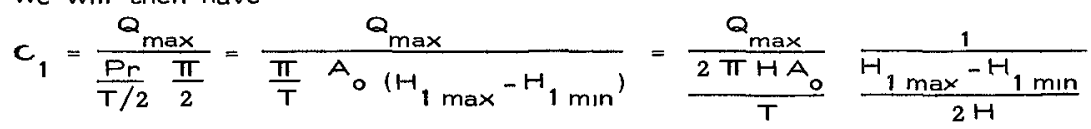

From expression (7) we will thus get, for $N=0$ (vertical banks)

$$
C_{1}=\frac{2}{\Delta h_{1}} \quad Q_{a \max }
$$

where $\Delta h_{1}$ represents the lagoon tidal range in the dimensionless form In the same way

$$
c_{2}=\frac{V \max }{\frac{P r}{\frac{P r}{2} \frac{\pi}{2}}}=\frac{V \max }{\frac{H_{b d}}{T b} A_{0} \frac{1}{d_{0}} \frac{1 \max -H_{\text {min }}}{2 H}}
$$

Thus, from expression (8) there results for $N=0$ (vertıcal banks)

$$
c_{2}=\frac{2}{\Delta h_{1}} \quad d_{0} v_{\max }
$$


We must say that the comparison of $c_{1}$ with the value of $c$ obtained by Keulegan seems to be more logical than the comparison of $\mathrm{C}_{2}$ In fact $\mathrm{C}_{2}$ relates the maximum effective velocity which, as a rule, will occur for a level other than the mean one, with the peak velocity corresponding to the sinusol dal flow through the section fixed arbitrarly for the mean level On the other hand, in the calculation of $c_{1}$, and since we are relating rates of flow, it is not necessary to take into account the value of the flow section This comes close to the Keulegan scheme, in which this section was considered invariable $34-3$ rd and 6th powers of the mean velocity

According to Colby [3], "the relationship of bed-material discharge to mean velocity is the most convenient to apply The computations are simple, and the energy gradient is not required The relationship may be as accurate as any of the other three/which relate bed load capacity, respectively, with shear velocity $\sqrt{\frac{T}{\rho}}$, shear velocity relative to the particles $\sqrt{\frac{\tau}{\rho}}$ and stream power] unless antidunes extend across much of the flow"

From the curves presented by this author it can be concluded that bed-load capacity of an unidirectional current varies almost linearly with a very high power of the mean velocity In the present work it was assumed that for the velocity range occuring in a given inlet, this variation was in fact linear with the 3rd or 6th power of the mean velocity and that natural flushing ability of the inlet was proportional to the integral value, during the ebb and flood periods, of that bed load capacity Again, it was logically assumed that for a given natural flushing abllity the more the integral ebb bed load capacity exceeds that of the flood, the better would be conditions offered by the inlet

While integrating equation (5) the computer therefore calculated the values of function $v^{3}(\theta)$ and $v_{a}^{6}(\theta)$ (see expression $(\theta)$ ) and then obtained the integral value of these parameter by the trapezoidal rule

35 - Hydraulic power consumed in the inlet channel

It was sought to relate natural flushing ability of the inlet with the hydraulic energy consumed in it during a tidal cycle

To achieve this, and in accordance with the hypothesis made when deduc ing the expression (5), it was assumed that the kinetic energy of flow $\frac{V^{2}}{2 \mathrm{~g}}$ is totally dissipated in the sea or lagoon, in a turbulent expansion process, and 
that, therefore, the hydraulic power consumed in the channel can be calculated through the expression

$$
W=\gamma Q\left(\left|H_{2}-H_{1}\right|-\frac{V^{2}}{2 g}\right)
$$

It was deemed unnecessary to present here the deduction of the consumed power in its dimensionless form, which is as follows

$$
W a=\frac{\gamma Q\left(\left|H_{2}-H_{1}\right|-\frac{V^{2}}{2 \theta}\right)}{\frac{2 \pi \gamma A_{0} H^{2}}{T}}=Q\left[\left|\sin \theta-h_{1}\right|-\left(V_{a}\right)^{2}\right]
$$

where the comparison term, which may be written,

$$
\frac{2 \pi \gamma A_{0} H^{2}}{T}=\frac{\gamma_{2} H A_{0}}{T / 2} \frac{\pi}{2} H
$$

represents the power developed, under a difference o levels equal to the sea tide amplitude, by the peak discharge corresponding to the sinusoidal flow of the maximum prism admissible in the lagoon

The expression of $W_{a}$ may also be given as

$$
w_{a}=\frac{E}{D^{2}} \quad \frac{v^{3}}{r_{0}^{1 / 3}}
$$

from which it is concluded that the behaviour of the $w_{a}(\theta)$ function should be analogous to that of the 3rd power of the dimensionless velocity ( $V_{a}$ ) This similarity will increase with the mean depth of the channel, considering the smaller relative fluctuation of the dimensionless hydraulic radius $\left(r_{0}\right)$ during the tidal cycle

\section{6 - Results obtained by the computer}

Through its plotter out put and for each of the cases studied, the computer gave in graphic form all the functions just mentioned $\sin (\theta), h_{1}(\theta)$, $Q_{a}(\theta), V_{a}(\theta), V_{a}^{3}(\theta), V_{a}^{6}(\theta)$ and $w_{a}(\theta)$ Fig 4 shows the results concerning a lagoon-inlet system characterized by $D=02 E=100 \quad d_{0}=6 \quad 0 \quad N=0 \quad 0$ as given by the plotter, the curves $v_{a}^{3}(\theta)$ and $v_{a}^{6}(\theta)$ regarding the system characterized by

$$
D=02 E=10 \quad 0 \quad d_{0}=6 \quad 0 \quad N=02
$$

being added only to illustrate the effect of the lagoon bank slope, which orly differs from the previous system in so far as parameter $N$ is concerned, which charac 
terises the 3 que referred to (F:g a)

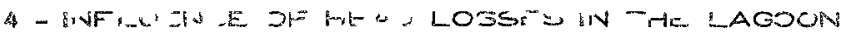

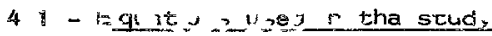

ye cal be coigluded, by mere energetic cinsideratio a to t had lonser molgh fristir in a lagoon will bring about a decrease in au al ru ning

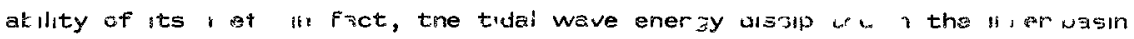
will cease to be ar alsble to remove the litenral drift matei il whic tw wou io sostruct it

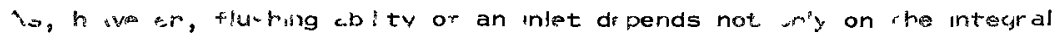

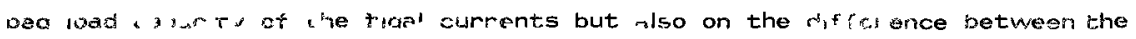
flood and wb conccity, and considering that the friction biu ing effcct of the tiaal wave, iside the lagoon causes the oisplarement of the discharge cume

with r siecs to that of the lavels in the inlet, so that for the same $r^{-}-$ charge tre abb flow section will be smaller than that for the flood, ore cannot a priori and in a general way state that haad losses in the lagoon inpair the natural flushing ability of its inlet

A very simple mathematical model was therefore prepaled, mainly to eva luate the change in the relation between the ebb bed load capacity and that of the flood, as a result of interior head losses

Let us imagine a lagoon constituted by two basins, in both of which levels vary uniformly uver their entıre area, and which are connected to one another by a channel with well defined morphological characteristics (section, length and roughness) These basins are connected to the sea by a singla inlet in which the bed load capacities of tidal currents will be studied The channel connacting the two basins is the energy dissipating factor (F ig 3 ) in this srudy the lagoon banks are considered to be vertical

Index 2 wIl denota the sea, Irdex 1 the basin directly connected to the sea and the inlet, and index 3 the inner basin and the channel connecting the two basıns

The equations characceristic of the system were derived from the equations ralative to flow in the inlet and inner channel, and from the continuity equa tions relative to the inner basin any the entire lagoon, that is 


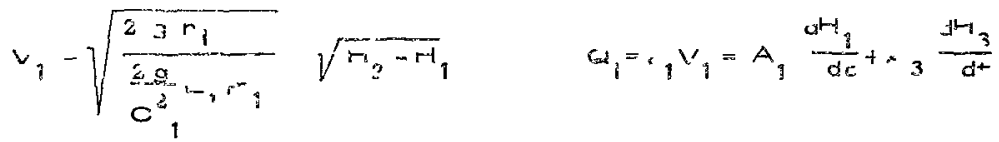

$$
\begin{aligned}
& v_{3}=\sqrt{\frac{2 g r_{3}}{c_{3}^{2} L_{3} r r_{3}}} \sqrt{H_{1}-H_{3}} \quad Q_{3}=a_{3} v_{3}=\Delta_{3} \frac{d H_{3}}{d t}
\end{aligned}
$$

After some manipulation - whose presentation can be disoensed with, a system of two diff rential equations of the ist order, identical in form to equation (5), is obtared, whicn char acteriass the hydrodynanic behaviour of the inlet-lagoon sys-am, namely

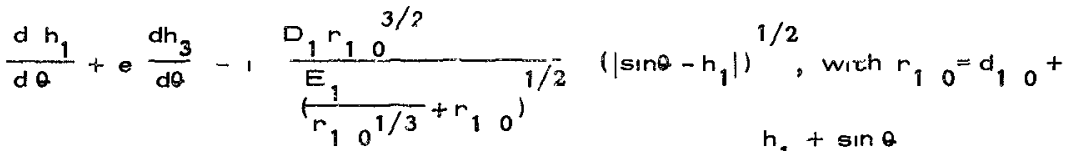

$$
\begin{aligned}
& +\frac{h_{1}+\sin \theta}{2}
\end{aligned}
$$

$$
\frac{d h_{3}}{d \theta}=\frac{D_{3} r_{3} 0^{3 / 2}}{\left(\frac{E_{3}}{\left.r_{30}{ }^{1 / 2}+r_{3}\right)^{1 / 2}}\right.}\left(\left|h_{1}-h_{3}\right|\right)^{1 / 2}, w i t h r_{2}=d_{3}+\frac{h_{1}+h_{3}}{2}
$$

where

$$
\begin{aligned}
& 1=1 \text { for } \sin \theta>h_{1} \quad 1=1 \text { for } h_{1}>h_{3} \quad e=\frac{A_{3}}{A_{1}} \\
& 1=-1 \text { for } \sin \theta \leqslant h_{1} \quad J=-1 \text { for } h_{1} \leqslant h_{3} \quad A=A_{1}+A_{3}=\text { Total area } \\
& \text { of the lagoon }
\end{aligned}
$$

and

$$
h_{1}=\frac{H_{1}}{H}, h_{3}=\frac{H_{3}}{H}, r_{10}=\frac{r_{1}}{H} \text {, where } H \text { is the sea tide amplitude }
$$

The numerical integration of system (14) was made in the NCR Elliot 4100 computer of the Laboratorio Nacional de Engenharia Civil, using one of th library programs [2]

The integration step was $\Delta \theta=01$ radians, the initial situation was cha- 
racterized by equal levels in the sea and inner basins, so that to obtain results independent of initial conditions, the ist calculation cycle, corresponding to a tidal period, had to be discarded

Together with the numerical integration of system (14) the computer also calculated other quantities with interest for the study of the natural flushing capacity of the inlet

As in item 32 and following, these quantities, in their dimensionsless form, were calculated by means of the following expressions which, for the sake of brevity, will not be deduced here

- Flow $Q_{1}=\frac{Q_{1}}{\frac{2 \pi+A}{T}}=-\frac{1}{1+e}-\frac{d h_{1}}{d \theta}+\frac{e}{1+e} \frac{d h_{3}}{d \theta}$

- Velocity

$$
V_{1 a}=\frac{V_{1}}{\frac{2 T T_{A}}{T b_{1}}}=\frac{1}{r_{10}}\left(\frac{1}{1+e} \frac{d h_{1}}{d \theta}+\frac{e}{1+e} \frac{d h_{3}}{d \theta}\right.
$$

- 3rd and 6th power of the velocity

$$
v_{1 a}^{3}=v_{1 a}^{3}(\theta), \quad v_{1 a}^{6}=v_{1 a}^{6}(\theta)
$$

- Power consumed in inlet channel

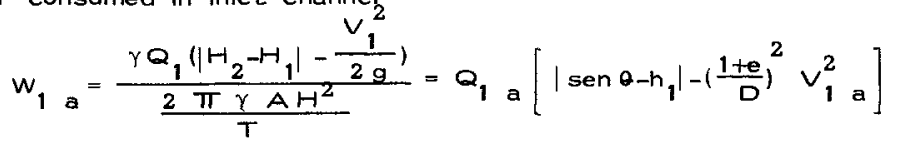

which may be written

$$
w_{1 a}=\frac{E_{1}}{D^{2}} \frac{v_{1}^{3} a}{r_{1} \frac{1 / 3}{0}}
$$

Where $D$ is the dimensionsless parameter relative to the total lagoon basin with area $A\left(A=A_{1}+A_{3}\right)$ and its inlet width $b_{1}$

\section{2 - Results obtained in the computer}

Fig 5 shows, as an example, the dlfferent curves studied for a system characterized by

$$
\begin{aligned}
& D_{1}=04 \quad E_{1}=100 \quad d_{10}=60 \\
& D_{3}=04 \quad E_{3}=10 \quad 0 \quad d_{30}=30
\end{aligned}
$$

which can be compared with the system shown in Fig 4 
In view of expressions (3) and (4) defining parameters $D$ and $E$, and moreover assuming that one considers the same outside tide (equal values of $T$ and $H$ ) and an inlet channel with the same width and roughness (equal values of $b$ and $n$ ), one may in fact conclude that the systems to which Figs 4 and 5 refer have lagoons with the same total area Nevertheless these la goons differ in that the lagoon of the latter system is formed by two basins with the same area $(e=10)$ connected by a channel half as deep as the channel of the outer inlet, with reference to the mean sea level

\section{5 - ANALYSIS OF THE RESULTS}

The studied cases were characterized by the set of the following parameters (in order to make clearer the prototype cases referred to, it is convenient to enclose in parentheses the lagoon area $A_{0}$, the length $L$ and the depth $d$ of the inlet channel referred to the mean sea level, that are compatible with these parameters and with the following conditions semi-diur nal lunar tide with an external amplitude $H=10 \mathrm{~m}$, inlet $b=200$ wide and $n=00226$ rough)

$$
\begin{aligned}
& N=0 \quad 0 \\
& 1-D=05\left(A_{0}=126 \mathrm{~km}^{2}\right), \quad E=19(L=1900 \mathrm{~m}), d_{0}=2,3,4,5,6,8,10 \\
& (\mathrm{~d}=2,3,4,5,6,8,10 \mathrm{~m}) \\
& 11-D=02\left(A_{0}=316 \mathrm{~km}^{2}\right), E=10(L=1000 \mathrm{~m}), d_{0}=1 \mathrm{dem} \\
& 111-D=02\left(A_{0}=316 \mathrm{~km}^{2}\right), E=19(L=1900 \mathrm{~m}), d_{0}=1 \mathrm{dem} \\
& N=0,1,02 \text { for } D=05, \quad E=19, \quad d_{0}=40 \\
& D=02, \quad E=10, \quad d_{0}=60 \\
& D=02, \quad E=19, \quad d_{0}=60
\end{aligned}
$$

Although this work was mainly directed to the study of the bed load ca pacity of tidal currents in the inlet channel other results were obtained that should be compared with those obtained by Keulegan

1t has been shown that an inlet-lagoon system can be well defined only by the set of parameters $D, E, d_{0}$ and $N$ (expression (5)) Hence, the great va riety of possible cases As an attempt to reduce such a variety of cases, it was tried to make use of the Keulegan's coefficient of repletion which, in 
some way, condenses the influence of the first three parameters This parameter has proved satisfactory for the interpretation of some of the studied quantities

Thus Fig 6 gives the variation of the tidal prism against the parameter $K$ in a vertical bank lagoon

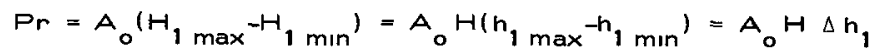

the following function is represented in that figure

$$
\Delta h_{1}=\Delta h_{1}(K)
$$

and compared with Keulegan's results (upper continuous curve) The effective prism is found to be well defined by the parameter $K$, although it is slightly superior to the one obtained by that author The same figure also shows the functions $h_{1} \max =f_{1}(K)$ and $\left|h_{1} \min \right|=f_{2}(K)$ which are simılarly compared with Keulegan's results (lower continuous curve), it being conclud ed that both the parameter $K$ and Keulegan's scheme are less satisfactory in this case in fact since the connecting channel is not "many times deeper than the tidal range" (Kewlegan's hypothesis) a rise of the lagoon mean level results, which is better explained in Fig 7

Fig 8 illustrates the variation of the flood and ebb times against the coefficient $K$ It may be concluded that ebb is always longer than flood and the difference between them increases as the entrance conditions of the tIdal inlet grow worse (lower $K$ values)

Fig 9 illustrates the variation of $C_{1}$ and $C_{2}$ with the coefficient $K$ This variation is compared with the results obtained by Keulegan As was shown in $33, C_{1}$ and $C_{2}$ respectively relate rates of flow and maximum velocity values with those of the sinusoidal flow of the effective prism From the figure it may be derived that in any case the function $C_{2}$ (K) satisfacto rily agrees with the function $C(K)$ obtained by Keulegan, that is not the case with the function $C_{1}(K)$, concerning the rates , f flow, which is clearly different from $C(K)$ In the zone of small $K$ values, the behaviour of the curves obtained seems rather anomalous and, thus, it is intended to carry out laboratory experiments in order to verify them

Fig 10 presents, for the case of a lagoon having vertical banks ( $N=0 \quad 0$ ), 
the variation of the integral bed load capacities of the flood and ebb currents against the coefficient $K$, which are assumed to be proportional to $V^{3}$ and $V^{6}$, It should be noticed firstly, that the general conclusions to be derived from this figure are practically independent from the power that affects mean velo city in its relation with bed load capacity

The most evident indication given by the figure is that the bed load capa city of tidal currents (which we assimilate to natural flushing ability in the ti dal inlet) reaches a maximum for values of the coefficient of repletion $k w i t h i n$ the range

$$
06<K<08
$$

Then it may be stated that a tidal inlet characterized by a K value great er than 08 has an extra natural flushing ability that will allow it to overcome an occasional increase of the littoral drift In fact, as the entrance conditions of the tidal inlet worsen as a consequence of that increase, the coefficient of repletion $K$ decreases and then the natural flushing ability of the tidal inlet improves In other words, it can be said that a tidal inlet characterized by a coefficient of repletion $K>08$ is in a condition of steady alluvial equilibrium On the other hand, following a similar reasoning, we can state that a tidal inlet characterized by a coefficient $k<06$ is in a condition of non-steady allu vial equilibrium, which means that shoaling may be in progress there

The same Fig 10 supplies further information that may be useful to the interpretation of the evolution of such tidal inlets In fact, for $k$ values great er than 08 bed load capacity is found to be higher in the flood than in the ebb which may contribute to introducing littoral drift into the lagoon and to the corresponding formation of shoals and inner bars Both facts would bring about a continuous reduction of the coefficient of repletion Conversely, in a tidal inlet where $k<06$, though the inlet is located in a zone of non-steady alluvial equilibrium, ebb currents overcome the flood ones as regards bed load capacity, which may represent the last resort of the tidal inlet in order to fight agaınst its increasing obstruction

It should be emphasized that the above remarks concern lagoons with vertical banks and in which the fluctuation of levels is uniform over their entire area Nevertheless actual lagoons always deviate more or less from this theoretical condition In fact banks are never truly vertical, sometımes the water 
successively overflows and withdraws from the surrounding land in accordance with the tidal cycle, the extent of the lagoon and the head losses inside it do not allow the hypothesis of a uniform law of levels to be valıd

In Figs 11 and 12 it is tried to evaluate the influence of bank slopes and of the internal head losses over the bed load capacity of tidal currents

Fig 11 shows that the slope of banks, given by the parameter $N$ (see

Fig 2), and thus the existence of large zones that the tide overflows or uncovers, improves the ebb bed load capacity in detriment of that of the flood

In Fig 12 the most significant results of the study relative to lagoons with internal head losses are condensed in 41 it has been shown that these head losses are artificially introduced by considering an inner channel that con nects the two basins in which the lagoon is divided This inner channel is cha racterized as an energy dissipating factor by means of the parameter

$$
F=\frac{E_{3}}{d_{30}}=\frac{2 g L_{3}}{d_{3} c_{3}^{2}}
$$

If there are no head losses in the lagoon $\left(L_{3}=0, d_{3}\right.$ or $\left.c_{3}=\infty\right), F$ equals 0 The variation of $F$ was obtained only by varying $d_{3} 0$, that is, a study was made of four different cases characterized by an inner channel with progressively lower depth

$$
d_{30}=6,5,4,3
$$

Fig 12 shows the general decrease of the bed load capacity of the tidal currents in the outer inlet as the internal head losses increase, which is the result logically expected Nevertheless the same figure also shows the increase of the relative importance of the ebb bed load capacity as compared with that of the flood This figure also shows the percentage with which a capacity overcomes the other for each case considered Such a percentage is found to reach very significant values in some cases which will favourably influence the natural flushing ability of the inlet

Summing up the main conclusions drawn from this work, we have

- The natural flushing ability of the inlet of a vertical bank lagoon where the law of levels can be assumed uniform, reaches a maximum for values of the coefficient of repletion of about 06 to 08 , 
- The slope of the lagoon banks or the existence of areas overflowed and uncovered during tidal cycle, increase the bed load capacity of the ebb currents as compared to the flood ones, and thus improve the na tural flushing ability of the inlet,

- The existence of head losses in the lagoon or the effect of propagation of the tidal wave decrease the tidal prism and the integral bed load capacity of the tidal currents, but improve the ebb capacity as compared to that of the flood which has a favourable influence on the natural flushing conditions of the inlet

\section{REFERENCES}

[1] - Keulegan, $G H$, "Water level fluctuations of basıns in communicatıon with seas", Third progress report on tidal flow in intrances, Beach Erosion Board, 1951

[2]-Sieck, A N, "Numerical integration of ordinary differential equatıons", Matmatics of Computation, Jan 1962

[3] - Colby, B R, "Practical computations of bed-material discharge" Proc ASCE, $90($ Hy2), March 1964 
SEA

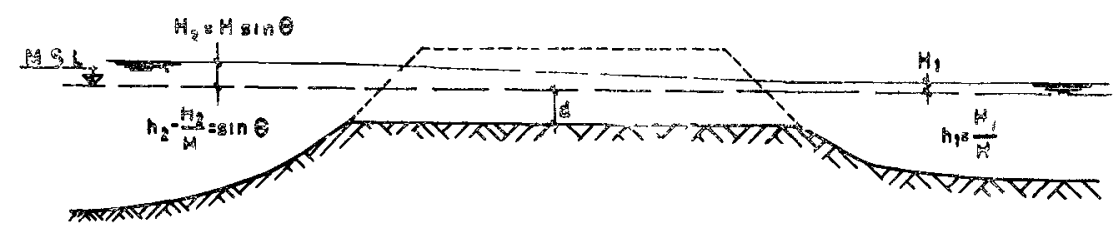

Tig I - SECHEMATIC LONGTTUDINAI SECI ION OF INLLT CHANNEL

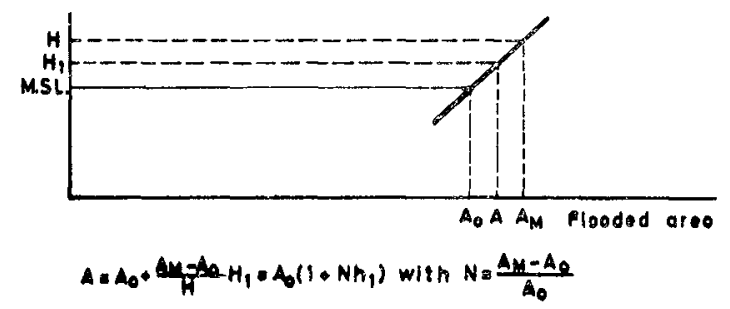

Fig 2 - LINEAR VARIATION OF FLOODED AREA IN LAGOON BASIN

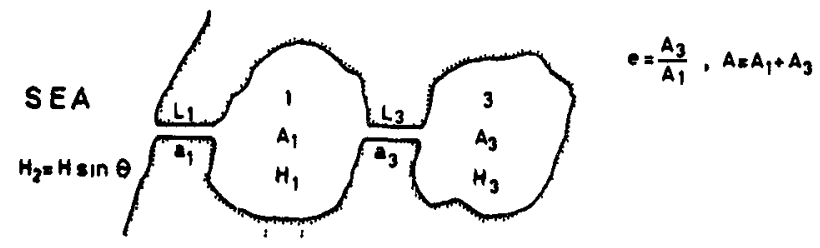

FiB 3 - TWO CHANNEL-LINKED BASINS FORMTNG A LAGOO 


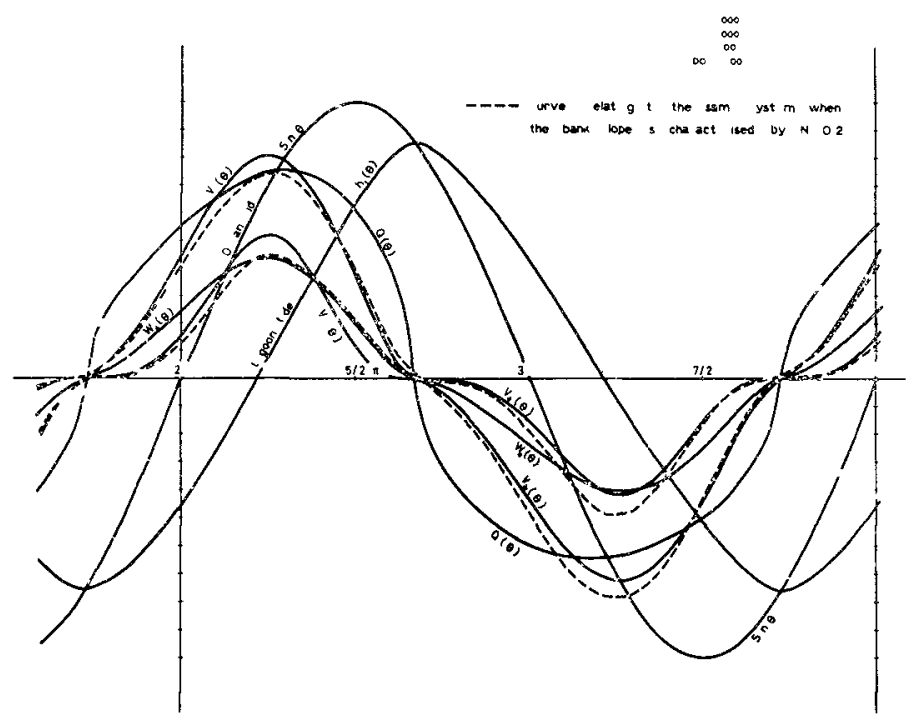

$F_{18} 4$ - CHARACTERISTIC CURVES OF AN INLET-LAGOON SYSTEM WITH VERTICAL BANKS, AS OBTAINED FROM THE COMPUTER

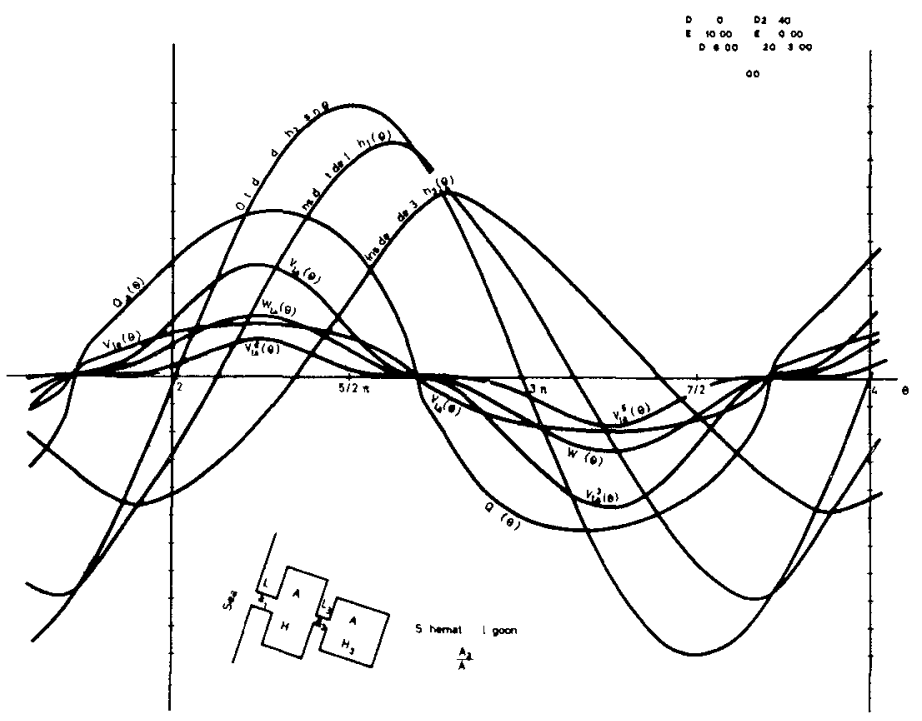

FIg 5 - CHARACTERISTI CURVES OF A TWO-BASIN LAGOON INLFT 

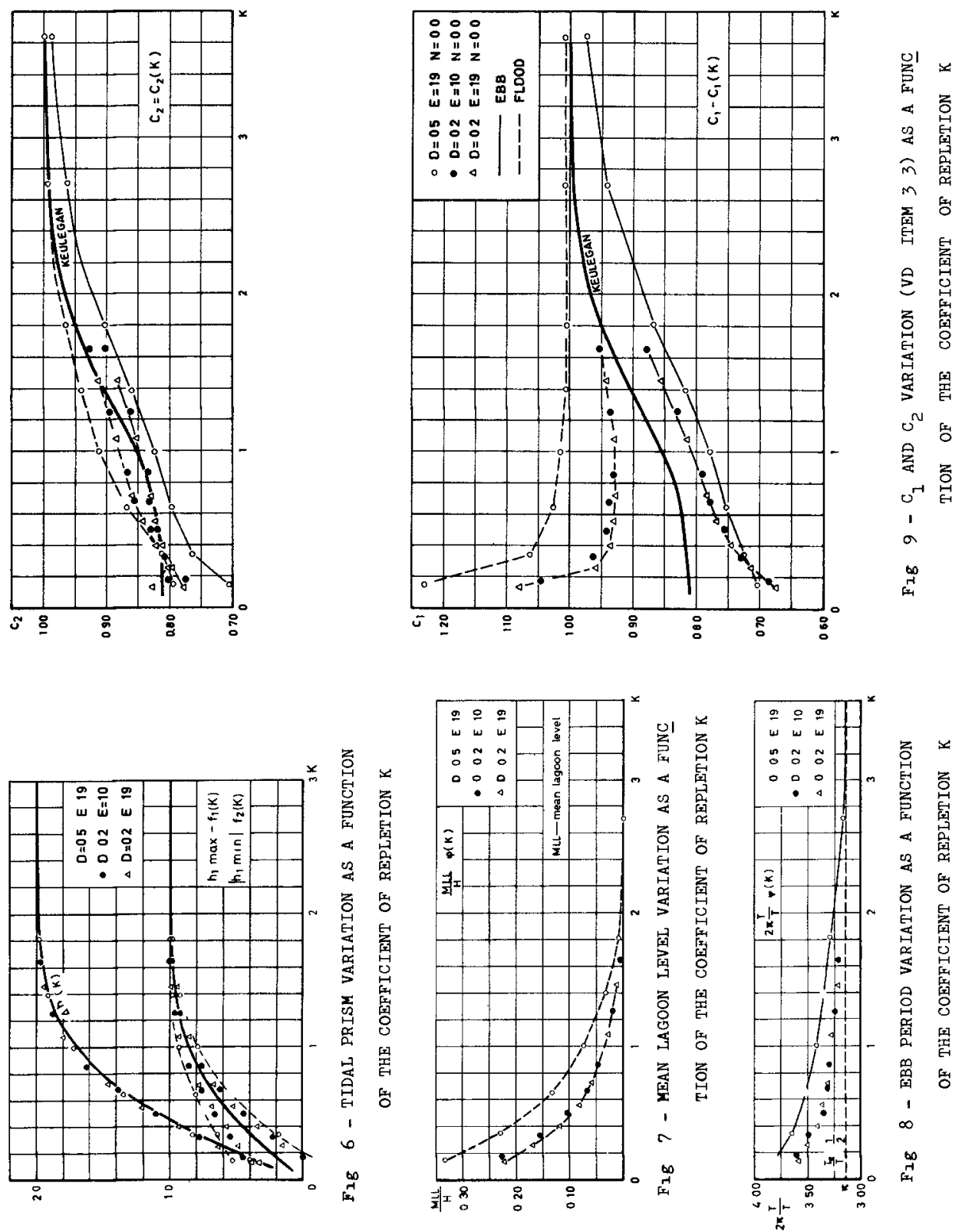

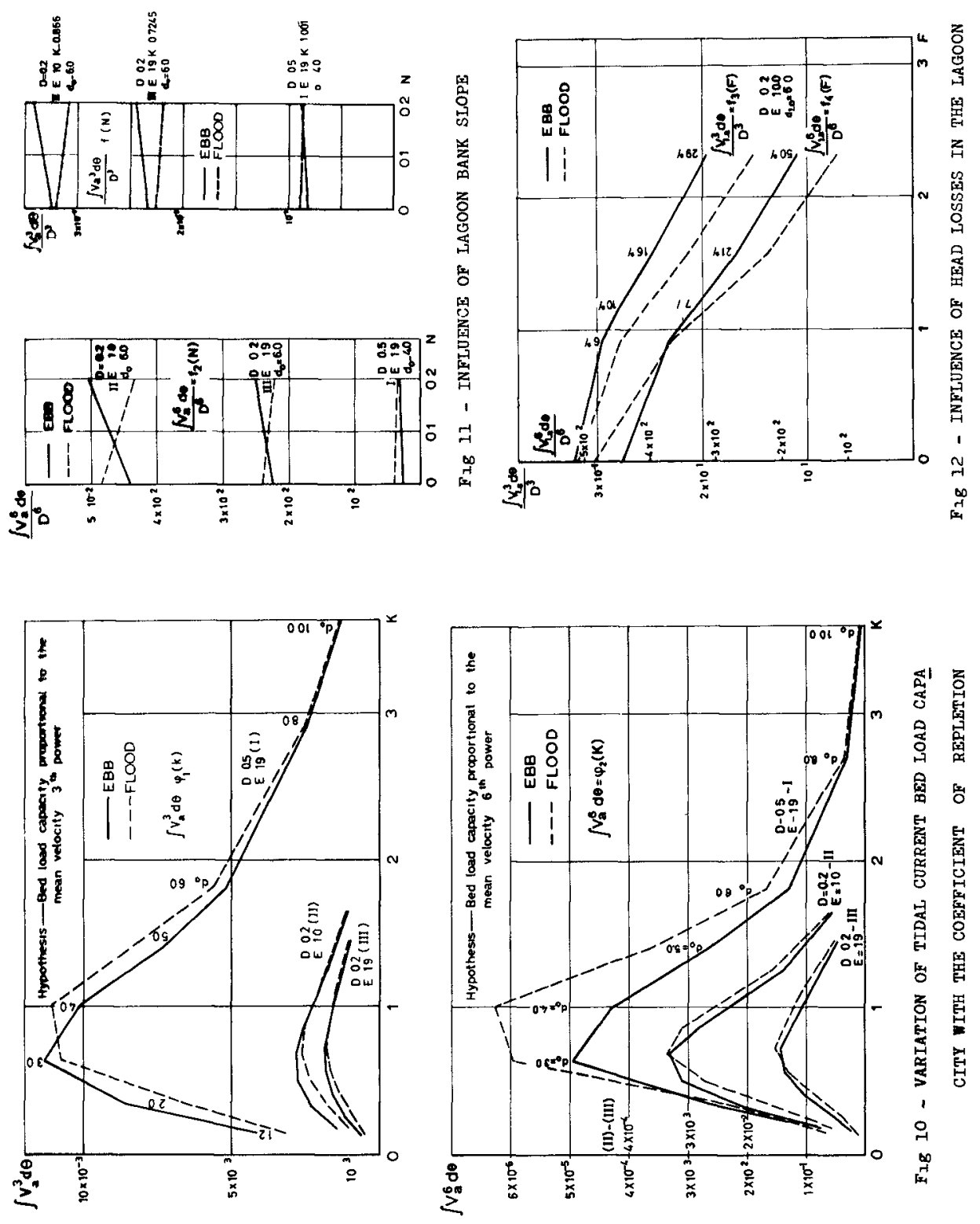



\title{
CHAPTER 112
}

\section{MATHFMATICAL MODEL OF MIXING IN NEW HAVEN HARBOR}

\author{
by
}

Michael D. Diskol

Robert A. Norr1s

Francis C. Lutzl

\begin{abstract}
A mathematical model of New Haven Harbor, a shallow embayment with approximately 8 square nautical miles of water surface within boundaries established by Long Island sound and the mouths of the Quinniplac, West and Mill Rivers, has been developed. The Harbor has extensive tidal flats and dredged channels which help to produce large lateral variations of velocity and mixing over a tidal cycle.

In order to adequately consider these lateral varlations, a two-dimensional model of mixing, dispersion, pollutant reactions, and reaeration is made by linking toqether 28 segments of the Harbor, using a series of mass-balance equations. Mixing, or dispersion, is a complex function of reversing tidal currents, salinity-induced circulation patterns, fresh-water inflow, and the physical boundaries of the Harbor. Field measurements of salinity, dissolved oxygen, $B O D$, and tidal and hydraulic factors are used, in conjunction with laboratory studies, to evaluate coefficients and rate constants for the model. The linked system of equations is solved by matrix inversion procedures on a large computer.
\end{abstract}

After verification, the model was used to predict the effect of treatment levels, outfall locations, and hydrologic parameters on oxygen levels and water quality in the Harbor. An important aspect of the work is that it presents a rational evaluation of estuarine water quality as a function of tidal mixing, outfall location, and the degree of BOD removal by proposed treatment plants, rather than the acceptance of arbitrary treatment standards.

\section{INTRODUCTION}

A critical problem confronting engineers and scientists working In the field of water-quality control is the evaluation of the required degree of treatment necessary for waste effluent discharges into river or estuarine waters. Some typical sources of

1 Consulting Engineers, M. Disko Associates, West Orange, N. J.

2 Associate, Quirk, Lawler \& Matusky Engineers, NYC 
waste effluents are industrial and domestıc discharges, perıodıc purgings of accumulated solids from combined storm and sanitary sewerage systems, surface runoff contalning quantities of nutrients and pesticides, and seepage from sanltary landfill operations in marshlands.

Each recelving water body has the capacity to assimilate a certain cuantity of introduced waste materials as a direct result of naturally occurring physical, chemical, and blological Interactions and conversion processes. Evaluation of the assimilation capacity for a particular river or estuary is a complex and difflcult task.

Typlcal methods used to determine the required degree of waste treatment necessary to malntaln oxygen levels and other water quality parameters, within the framework of the natural assimilation capacity, include field measurements and testing in the recelving water body, experimentation in verified hydraulic models, and formulation of a rational mathematical model. Each of these methods is only approximate. A number of baslc research questions still remain to be answered before any englneer or scientist can predict, with complete certalnty, the response of a recelving water body to a waste effluent discharge.

The object of this paper is to describe the formulation and use of a mathematical model to determine the relationships between the degree of waste treatment prior to effluent discharge and the resulting level of dissolved oxygen in the recelving water body which 15 New Haven Harbor. The mathematical model described, herein, is a two-dimensional system which considers pollutant variations in the lateral and longltudinal direction of the Harbor.

\begin{abstract}
Municipal sewage wastes are discharged into the Harbor from three existing primary sewage treatment plants, 1. $\theta$. the Boulevard Sewage Treatment Plant, the East street Sewage Treatment plant, and the East shore sewage Treatment plant, and from a secondary plant located in west Haven. It was desired to evaluate the degree of BOD treatment required to insure water quality standards, for a number of alternate design schemes under consideration in a sewage treatment plant modification proqram.
\end{abstract}

\title{
2. MATHEMATICAL FUNDAMENTALS FOR TWO-DIMENSIONAL MODELLING
}

Transport of any substance in an estuary is qoverned by the Law of Conservation of Mass. Figure 1 lliustrates the application of this law in a one-dimensional estuary. Waste particles discharged to an estuary are transported from the discharge polnt by convection and by dispersion. The rate of convective mass transport across any river section is equal to the product of fresh-water runoff, and the contaminant concentration.

Mixing, or dispersion, is a complex function of reversing tidal currents, salinity-1nduced circulation patterns, fresh-water inflow, and the physical boundarles of the Harbor. Dispersive 
MASS TRANSPORT RELATIONSHIPS IN A ONE-DIMENSIONAL ESTUARY

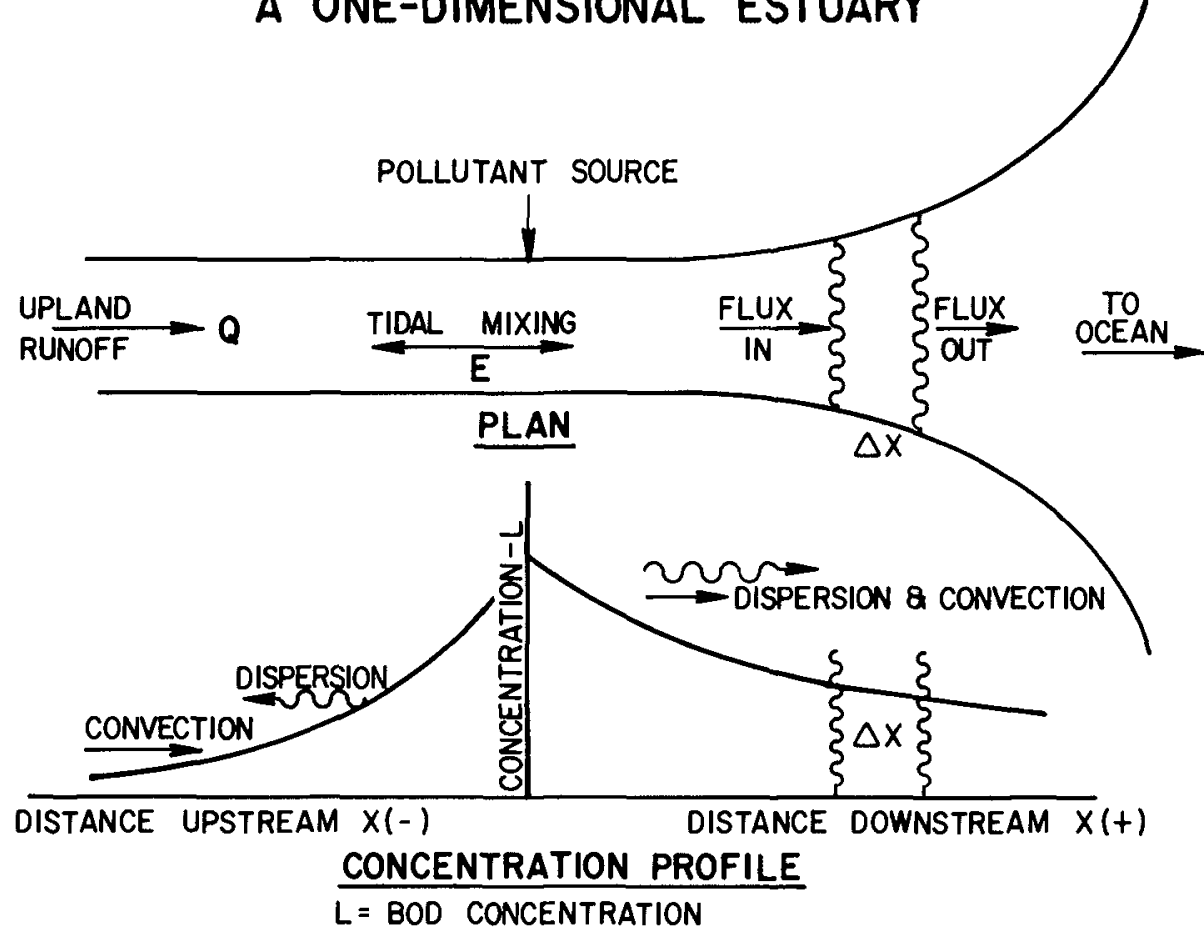

MASS BALANCE OVER VOLUME ELEMENT, A $\triangle X$

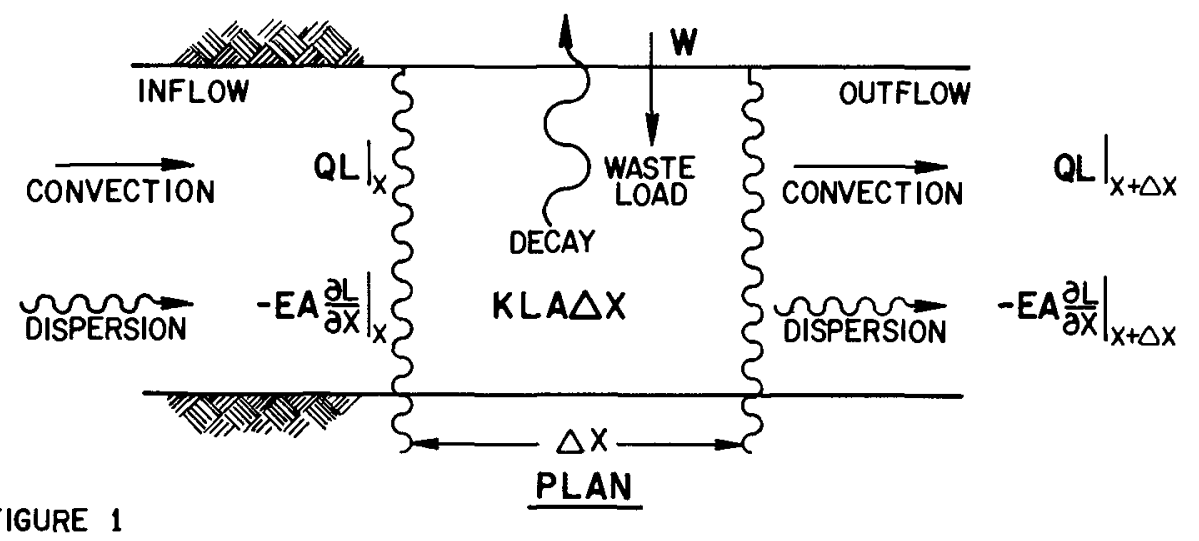

FIGURE 1 
transport occurs only in the presence of a concentration gradient of the materlal belng transported. The rate of dispersive transport is equal to the product of a dispersion coefficient, $E$, and the negative of the longltudinal concentration gradient, $\mathrm{dL} / \mathrm{dx}$. The dispersion coefficient, $E$, is a measure of the estuary's ability to transport material in the direction of a concentration gradient, regardless of the direction of net water movement.

The biochemical oxygen demand, BOD, is a measure of the oxygen required to reduce waste products by blochemlcal oxidation. The rate of $B O D$ decay is equal to the product of the first order decay constant, the $B O D$, and the volume within which the reactions are occurring.

For the case of an estuary with both longltudinal and lateral mixing and dispersion, linked volume segments in a mathematical model can be used to represent the physical system. In such a model, system parameters such as BOD are assumed to be approximately constant with depth. Each volume segment can be considered a regular polyhedron. The top and bottom of each polyhedron represent the water surface and harbor bottom, respectively. Figure 2 shows how each volume segment is directly linked to a number of other segments.

A mass balance over any volume segment shown in Figure 2 is written

INFLOW - OUTFLOW + PRODUCTION - LOSSES = ACCUMULATION $\ldots \ldots(1)$

For a steady-state case, the ACCUMULATION of BOD within any particular volume segment is zero, and EQUATION (1) gives

INFLOW - OUTFLOW + PRODUCTION - LOSSES $=0 \ldots \ldots \ldots \ldots \ldots$ (2)

Each of the terms are described in detail below.

The INFLOW and OUTFLOW terms are the sums of convective and dispersive transport across the faces of the volume segments. A direction 19 assigned for convective and dispersive transport, and, using this sign convention, a consistent material balance is developed for each linked segment. The convectlve components are the product of each flowrate, $O$, and each respective BOD concentration, L. The dispersive component is the term, EA(dL/dS), where $E$ is the average dispersion coefficlent at the interface of each segment linkage, $A$ is the interfacial area, and $S$ is a distance in the direction normal to the area, $A$.

The PRODUCTION of BOD in a volume segment represents the sum of the inputs of waste effluents, surface runoff, and additions of BOD from the scour, by currents, of bottom deposits in the benthal layer of partly decomposed organic products. The net PRODUCTION 1s, therefore, estimated as a BOD load of $W$ pounds per day. 
TWO-DIMENSIONAL MSSS TRANSPORT RELATIONSHIPS

- seuiment midooint \.

-..- HAFBOR BOUNDARY

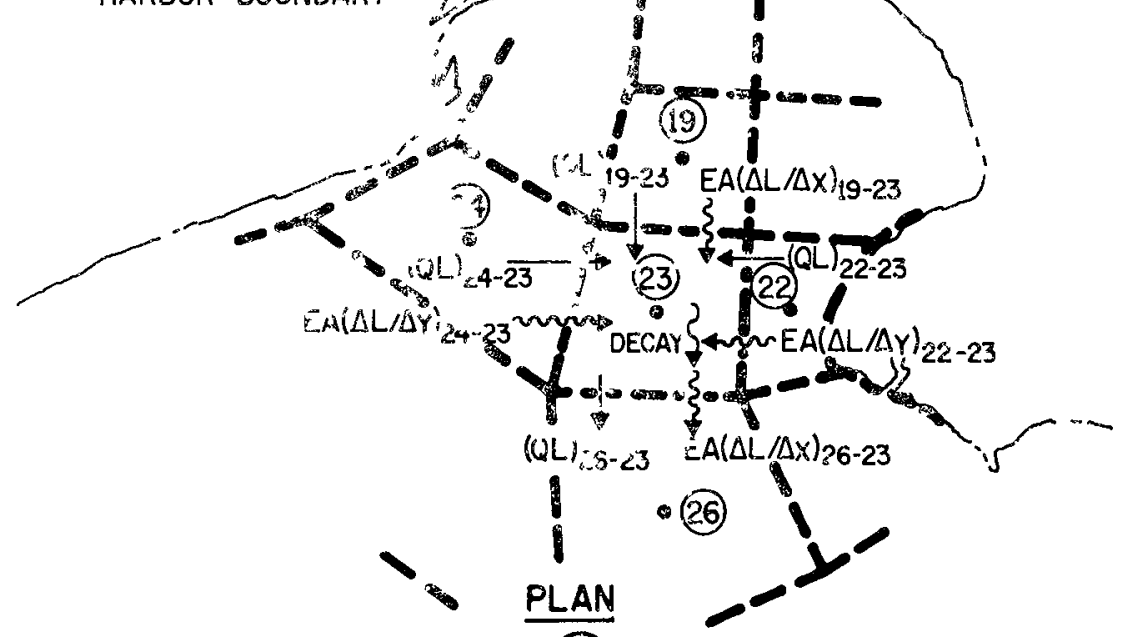

LINKAGE BETWEEN SEGMENT (23) AND ADJOINING SEGMENTS

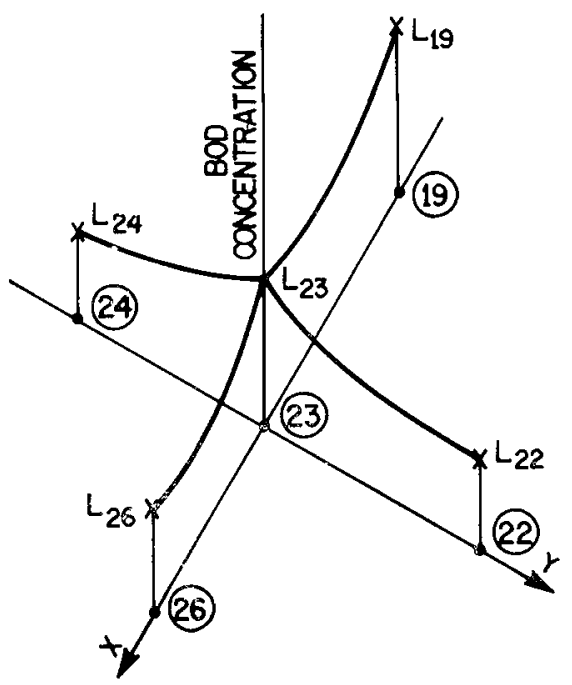

BOU CONCENTRATIONS IN ADJACENT SEGMENTS

FIGURE 2 
The LOSSES of $B O D$ in a segment would include the removal of $B O D$ by sedimentation or absorption, and decay. The BOD reduction, by sedimentation or absorption, may be assumed proportional to the amount of insoluble BOD in the water. The decay of BOD is assumed to be a first-order reaction, proportional to the concentration of BOD. The net decay of BOD in any segment is given by $K_{r} L V$, where $K_{r}$ is the composite BOD decay coefficient (to the base e) due to decay and sedimentation, $L$ is the average BOD concentration in the segment, and $V$ represents the segment volume.

In New Haven Harbor, losses of BOD due to sedimentation and absorption were small because of the high tidal flushing rate. If, however, these effects were substantial in any particular volume segment representing a portion of the Harbor, allowance could be made to reflect these parameters in the coefficients of that segment's mass balance equation.

An algebralc substitution of the individual contributions into Equation 2, for each volume segment, gives

$$
\Sigma O L-\Sigma E A(d L / d S)+W-K_{r} L V=0 \ldots \ldots \ldots \ldots \ldots \ldots
$$

Equations for each segment are developed and linked, noting the relative interconnection, the advective and dispersive transport conventions assumed, and appropriate coefficients such as $\mathrm{K}, \mathrm{E}$, and $\mathrm{A}$.

Dissolved oxygen concentration is used as a principal index of organic waste pollution in a waterway. As the level of Do concentration is decreased, the capacity of a waterway to assimilate waste discharges is reduced, requiring upgrading of treatment facilities for waste flows. The important source of replenishment of DO is atmospheric oxygen transferred to the waterway in proportion to the currents and other estuarine parameters.

Application of Equation 2 to oxygen transport follows the approach outlined above for $B O D$ and yields an equation analogous to Equation 3. The INFLOW and OUTFLOW terms are similar to the BOD terms. The convective term is the product of $O$ and $D$, the dissolved oxygen deficit or the difference between oxygen saturation and actual oxygen concentration in the water. The dispersive term is the product, EA(dD/dS).

The PRODUCTION of DO in a volume segment is the external input of oxygen reaeration from the atmosphere through the water surface, and the addition of oxygen by photosynthesis. The mechanism of reaeration is represented by $K_{A} D V$, where $K_{A}$ is the coefficient of oxygen transfer from the atmosphere 1ntô the seament. $\mathrm{K}_{\mathrm{A}}{ }^{\mathrm{A}}$, of course, a parameter that varies as a function of water depth and current velocity in various sections of the Harbor.

The LOSSES of DO in a volume segment would include the removal of oxygen from the water by diffusion into the benthal layer to 
satisfy the oxygen demand in the aerobic zone, by the purging action of gases rising from the benthal deposits, and by the respiration of plankton, diatoms, and other life. The rate of removal of oxygen, or deoxygenation, is represented as $\mathrm{KLV}$, where $\mathrm{K}$ is the coefficient of deoxygenation or rate of loss of oxygen associated with satisfying the BOD.

The production of oxygen by photosynthesis, the removal of oxygen by respiration and benthic effects were al1 judged to be minor effects that tended to balance one another in New Haven Harbor over long-term periods.

An algebralc substitution of the individual contributions into Equation 2, for each volume segment, gives

$$
\Sigma Q D-\Sigma E A(d D / d S)-K_{A} D V+K L V=0 \ldots \ldots \ldots \ldots \ldots
$$

The mass balance technique employed to develop Equations 3 and 4 is used to formulate a series of equations for BOD and dissolved oxygen deficit, $D$, in each segment. After solution of these equations, the corresponding DO in each segment is then obtained by subtraction of the dissolved oxygen deficit, $D$, from the saturation oxygen concentration.

\section{GENERAL DESCRIPTION OF NEW HAVEN HARBOR}

New Haven Harbor is a shallow embayment with approximately 8 square nautical miles of water surface within boundarles established by the Long Island Sound breakwaters and the mouths of the Quinniplac, West, and Mlll Rivers. The Harbor width varies from less than a thousand feet just downstream of the juncture of the Mill and Quinniplac Rivers to approximately two miles at its mouth. Extensive tidal flats exist on the west shoreline of the Harbor, while the east shore is generally irregular.

The existence of extensive areas of tidal flats, variable harbor width, shore irreqularities, deep navigational channels, breakwaters, and localized fresh-water inflows in a tidal waterway, all require that the mathematical modelling consider both lateral and longltudinal directions. The generally accepted one-dimensional estuarine model leaves lateral mixing effects unaccountable. such a scheme would be forced to employ a cross-sectional average velocity in its development, and would result in potentially substantial errors in contaminant concentration predictions.

The mean depth of the Harbor below mean low water is about 11.5 ft. The mean tidal range for the Harbor is about $6.2 \mathrm{ft}$. The tidal prism, which is the mean volume of water that is exchanged from the Harbor between mean high and mean low water, 1 s approximately $1.87 \times 10^{9}$ ft. ${ }^{3}$. The volume of the Harbor is about $4.4 \times 10^{9} \mathrm{ft}^{3}$ at mean water level. The ratio of the tidal prism to the mean volume of the Harbor is a measure of the flushing that occurs. It should be noted that this exchange occurs twice a day, since the period of the tide is about 12.4 hours. The 
total tidal flushing is equivalent to the replacement of 848 of the Harbor volume each day.

The large variation in Harbor width controls the relative magnitudes of fresh-water and ocean-water velocities. The shore irregularitıes, breakwaters, and the deep navigational channels produce localized currents. Current data was obtaıned from "A Hydrographlc Survey of New Haven Harbor, 1962-1963", by Alyn Crandall Duxbury of the Bingham Oceanographic Laboratory of Yale Unlversity. The survey is published as connectlcut water Resources Bulletin No. 3A.

Shellfish breeding areas are malntalned within the breakwaters, and extensive shellfish harvesting grounds are malntalned beyond the breakwaters. In addition, portions of the Harbor are also reserved for recreational purposes. Bathing beaches line both the east and west shores at the Harbor entrance.

\section{APPIICATION OF MODELLING TECHNIQUES TO NEW HAVEN HARBOR}

In order to apply the material balance approach described in the previous section of this report, the Harbor was divided into 28 volume segments as shown in Figure 3. Segments 1 through 5 are associated with the oulnniplac River, segment 6 with the Mill Rlver and segments 13 and 14 with the West Rlver.

Segmentation was made on the basis of current and depth patterns. Current patterns were determined from composite Bingham Oceanographic Laboratory hydrographic measurements of ebb and flood tide conditions. Depth patterns were obtained by constructing over 60 profile sections of the Harbor.

Each segment was linked to the adjolning segments by applying the material balance, defined by Equations 3 and 4 , for BOD and Do conditions. Many of the segments are linked to four other segments. Evaluation of Equations 3 and 4 often required consideration of ten or more separate coefficients for each segment.

The model could have been divided into a larger number of segments if comprehensive field surveys and measurements were avallable to justify the additional complexity involved. The amount of total complexity increases as an exponential function when the number of segments increases. Division of the Harbor into 28 segments was judged to be the optimum segmentation for the avallable field data on DO, currents, BOD, etc.

Evaluation of the many parameters required for Equations 3 and 4 was a time-consuming process. The following parameters had to be computed for each segment: the BOD load per unit of time; cross-sectional areas at each boundary; fresh-water flow into and out of each segment; volume of waste effluent into each segment; the decay rate of $B O D$; the oxygen saturation concentration; the oxygen transfer from the atmosphere to water, the prevalling currents, and the mean water volumes. 


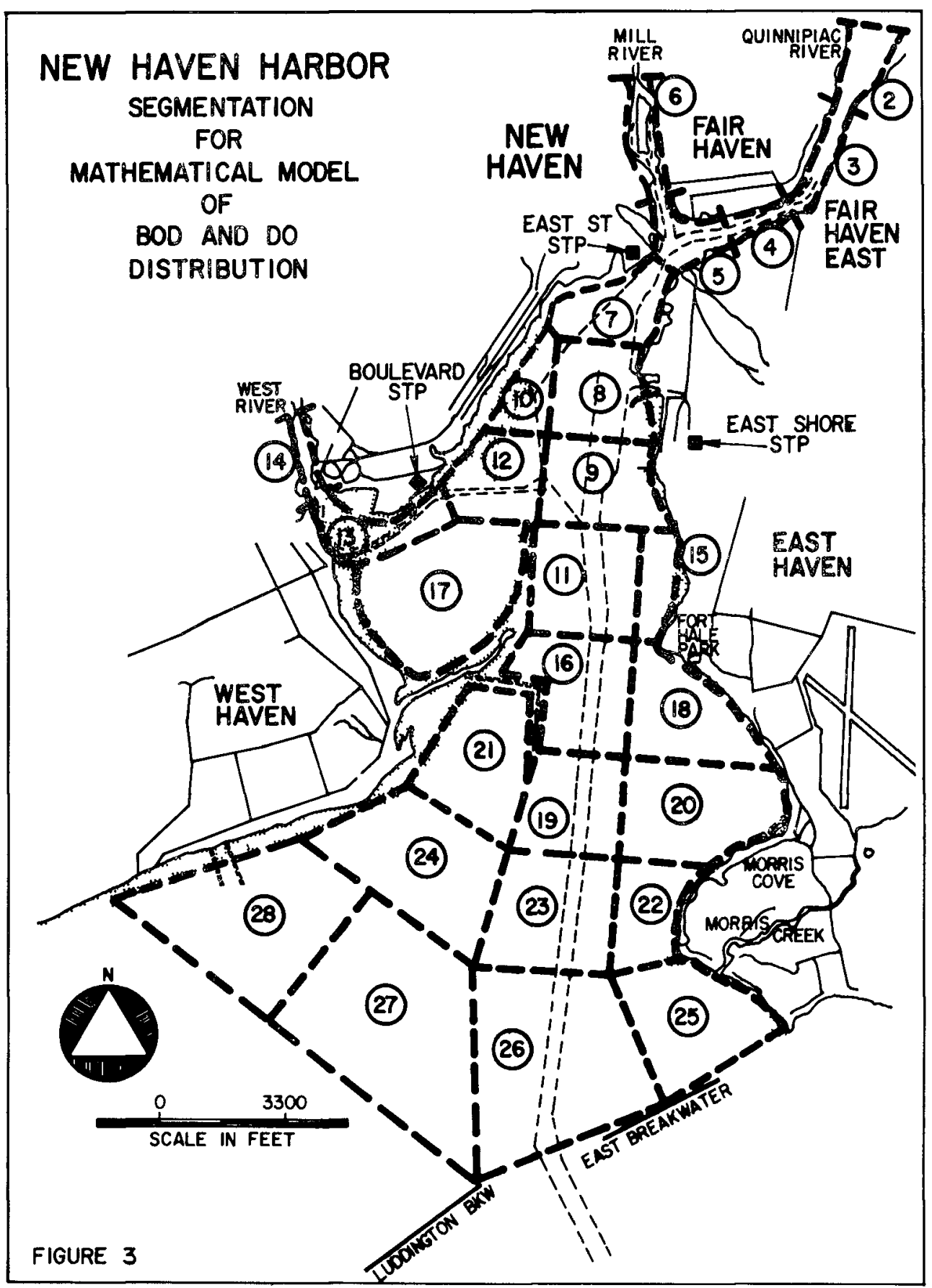


Information required for cross-sectional areas, segment volumes, depths, and tidal range were obtalned from U. S. Geologlcal Survey maps, U. S. Coast and Geodetıc Survey maps, and other published Harbor data.

Fresh-water flowrates for the oulnnlplac Rlver Basin at Wallingford, Connectlcut were obtalned from Geologlcal survey records. Flowrates for the Mlll Rlver and the West River were estimated from a study of watershed areas, and watershed characteristics. Critical flowrates from all three rivers into the Harbor were computed.

Tidal currents were obtalned from composite Blngham survey measurements for ebb and flood condltions. Average current values were computed, based on tidal cycle relationships and the ebb and flood value.

The longltudinal dispersion coefficient at each segment boundary was computed from a knowledge of tidal currents, fresh-water flow, and salinity measurements obtalned by the BIngham hydrographic survey. Longltudinal dispersion can be directly computed from the mean salinlty concentration, the salinlty gradients, and the average current at the segment boundary. In several non-critical areas of the Harbor, Blngham velocity data was not avallable. In these cases, the longltudinal dispersion coefflclents were estlmated from a study of the overall patterns of the computed longltudinal dispersion coefficients and the measured currents in the Harbor.

The first-order, BOD decay rates, or coefflclents, were determined from a laboratory analysis of Harbor water samples. Seven representative water samples were obtalned from predetermined Harbor locations on April 9, 1969. Long-term BOD evaluations were performed to determine the present $K_{r}$ decay coefficient of Harbor waters. The $\mathrm{K}_{\mathrm{r}}$ rate was determined to be about $0.31 / \mathrm{day}$. This measured value is normal for primary effluent and was used for model verification and to estimate the future decay rates of secondary effluent. All seven samples showed $k_{r}$ rates that were similar. An estimated decay rate of 0.20 /day was used for the 1990 secondary effluent.

The coefficlent of atmospheric reaeration, $K_{A}$, was computed, using the well-known $O^{\prime C}$ Connor-Dobbins formula, and another equation developed by U. S. Geologlcal Survey personnel. Atmospherlc reaeration in streams is a function of velocity and depth. The expressions developed for streams are commonly adopted for estuarıne reaeration.

Application of the mass balance approach to each segment, including all the above computed parameters, results in 28 equations for $B O D$ and another 28 equations for the dissolved oxygen deficlt, D. A system of equations, based on Fquations 3 and 4 , can be written in terms of the midpoint concentrations, in the following form 


$$
\begin{gathered}
a_{1-1} L_{1}+a_{1-2} L_{2}+\ldots+a_{1-28} L_{28}=w_{1} \\
\vdots \\
a_{28-1} I_{1}+a_{28-2} L_{2}+\ldots+a_{28-28} L_{28}=w_{28} \ldots \ldots \ldots \ldots
\end{gathered}
$$

and

$$
\begin{gathered}
\mathrm{b}_{1-1} \mathrm{D}_{1}+\mathrm{b}_{1-2} \mathrm{D}_{2}+\ldots+\mathrm{b}_{1-28} \mathrm{D}_{28}=\mathrm{C}_{1} \mathrm{~L}_{1} \\
\vdots \\
\mathrm{b}_{28-1} \mathrm{D}_{1}+\mathrm{b}_{28-2} \mathrm{D}_{2}+\ldots+\mathrm{b}_{28-28} \mathrm{D}_{28}=\mathrm{C}_{28} \mathrm{~L}_{28} \ldots \ldots \ldots
\end{gathered}
$$

In the above system of equations, the $a$ and $b$ coefficlents are obtalned by summation of all the terms pertaining to a respective subscripted $L$ or $D$ parameter. The $C$ coefficients are equivalent to the respective segment values of $\mathrm{KV}$. Many of the $a$ and $b$ coefficients have zero values.

Equations 5 and 6 are sets of simultaneous linear algebralc equations with unique solutions, which are readily solved by any number of standard numerical techniques. of those avallable, matrix inversion was chosen because of its ease of application, and directness for the system studied.

Using matrix algebra, the BOD waste loads into each segment become a column matrix. This column matrix is equated to a coefficient matrix which has 28 rows and 28 columns, and a column matrix which consists of the unknown BOD concentrations in each model segment. Each element of the 28 by 28 coefficient matrix is computed from the varlous parameters described above.

The coefficient matrix is inverted using standard numerical techniques on a computer. After matrix multiplication of the inverted coefficient matrix and the waste load column matrix, the unknown BOD concentration column matrix can be computed. A similar procedure is performed on the 28 equations for D. However, the computed BOD concentrations are required in order to solve the $D$ equations.

The solution of the BOD and oxygen deficit system, Equations 5 and 6, was programmed in FORTRAN $V$ for processing by a UNIVAC 1108 computer. One main program was constructed with a subroutine for matrix inversion. The program can be converted to most FORTRAN IV systems with few changes. An overall flowchart of the program is shown in Figure 4. 


\section{FLOW CHART FOR COMPUTER PROGRAMMING}

READ IN HARBOR PARAMETESTS, TERAP, OXYGEN SATURATION, FLOW S BOD INPUTS

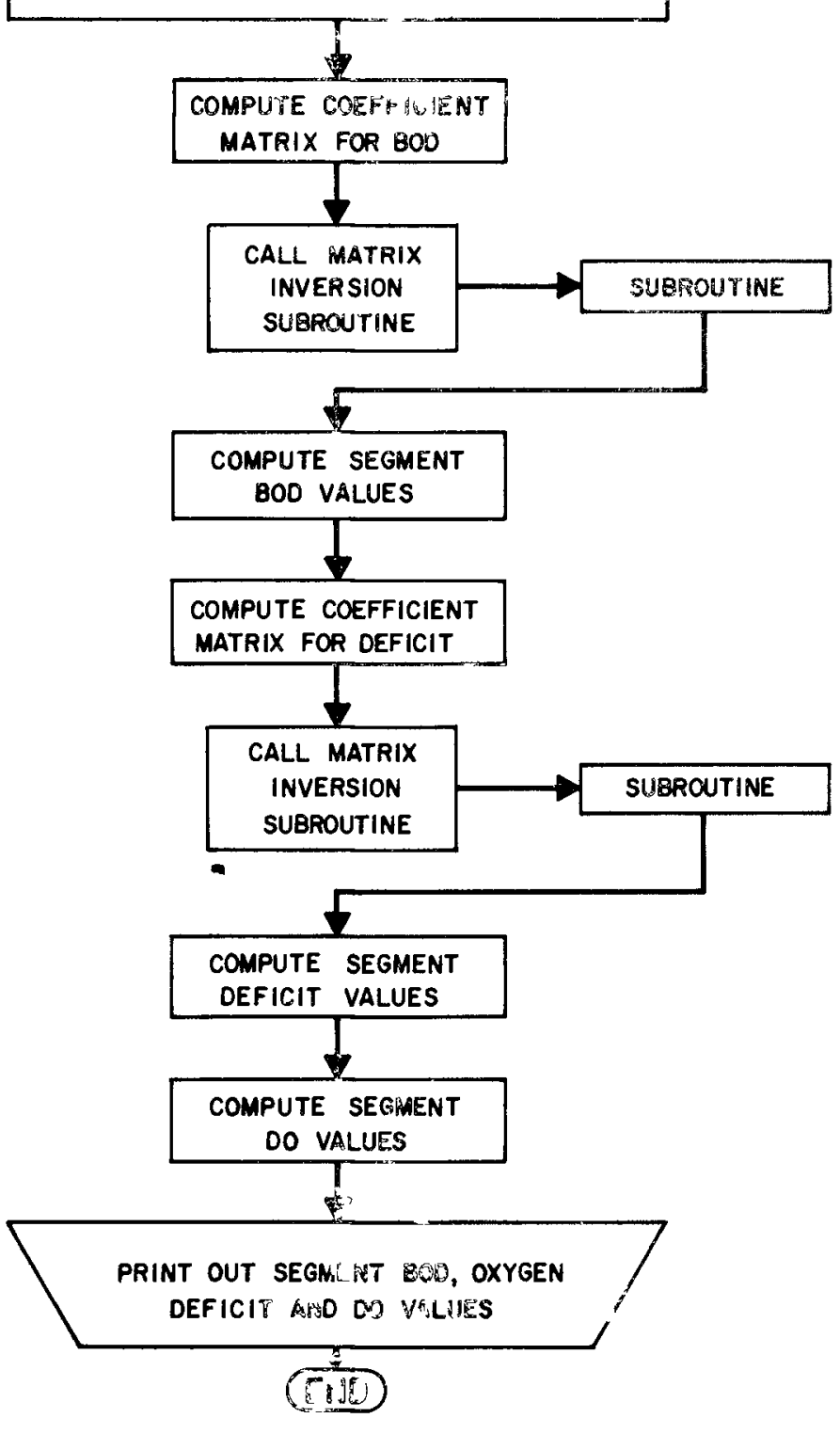

FIGURE 4 
The computer program and mathematical nosel can be adopted sid modified to compute colıform patterns, raduoactivity distrıbu= tions, temperature distributions, and contaminant patterns resulting from various input loadings. program and model modifications would require additional field measurements for the particular type of parameter simulated.

\section{MODEL VERIFICATION IN NEW HAVEN HARBOR}

Model verlfication is accomplished by comparing predicted DO concentrations to measured Do concentrations. Measurements of Do in the Harbor are limited. The only survey giving a reasonable representation of the DO patterns of the Harbor was performed by the Bingham oceanographlc Laboratory from october 1962 through september 1963. In this survey, DO measurements were made approximately once a week, at nine stations in the Harbor and at three depths at each station.

The Do measurements showed significant varlation, depending on the tidal phase of the survey and the cumulative rainfall for the perlod immediately prior to the survey date. The mathematical model, whlch was developed for steady-state conditions, is not applicable to measurements of a duration less than a full tidal cycle. Thus, for valid comparisons of model values to fleld data, predicted DO concentrations must be compared to measured DO concentrations that represent the average Do for a complete tidal cycle. Since numerous measurements within a tidal cycle are not avallable, the exact tidal cycle average Do must be estımated.

Absolute verlfication of the mathematical model requires complete and up-to-date fleld surveys to measure prescribed parameters. These types of field surveys must be designed with the mathematical model in mind so that the data obtalned may be optimally employed. To accomplish this goal, the data must be collected at specific times and locations as predescribed by the model.

Model analysis indicated that the model Do predictions were relatively insensitive to local varlations in $E, K_{r}$, and $Q$ when compared to lts sensitivity to varlations in waste loads. Changes in waste loads are directly related to changes in DO. Consequently, model verlfication depends upon accurate determination of the waste loads that constituted an input to the Harbor colncldent with the survey selected for verification purposes. All surveys that followed periods of excessive rainfall would be unsultable for verification purposes because the actual waste loads during these perlods are unknown. During the Bingham hydrographlc surveys, the waste loads from the existing sewage treatment plants were determined from flow and BOD measurements made on the effluents.

Industrlal waste loads were computed based on discharge flow estimates as reported to the Water Resources Commission by each industry. BOD measurements of the dlscharge flows were avallable for the largest flows. Where measurements were not avallable, reasonable estımates were used. 
The following waste loads were estimated for dry weather conditions during the Bingham hydrographic surveys of 1962-1963:

\begin{tabular}{lr}
\multicolumn{1}{c}{ Source } & Load. Lb. BOD ${ }_{5} /$ Day \\
\cline { 2 - 2 } Boulevard STP & 13,900 \\
East Street STP & 14,500 \\
East Shore STP & 3,940 \\
West Haven STP & 9,900 \\
Central Business District & 6,550 \\
(temporary during this period) & 3,800 \\
Industrles & Total
\end{tabular}

The Bingham survey of April 17, 1963 can be selected to 1llustrate the problems involved in verification of the model. For a six-day period prior to April 17, 1963, the cumulative rainfall was 0.07 inches.

Measurements of oxygen were made on a rising tide. The hydrographlc survey started at the breakwaters in the outer Harbor and proceeded inward to the Quinnlplac River. Bingham station OH2 is recorded as sampled within 2 hours after low water slack. Bingham stations $\mathrm{MC} 3, \mathrm{MCl}, \mathrm{FH} 2, \mathrm{OP} 3, \mathrm{OP} 2$, and $\mathrm{OP} 1$ were sampled within one hour of mean tidal stage. Stations $\mathrm{Hl}$ and $\mathrm{Ql}$ were sampled within one hour after mean tidal stage. In general, surveys will show a lower value of DO at low water conditions, and a higher Do at high water.

Measurements from several of the nine stations are not sultable for direct comparison to the model output. Analysis of measured data at a station showed considerable variations in oxygen concentrations, temperatures, and salinities for the three grab samples taken at the surface, 10-foot and 20-foot depths. The following listing shows a comparison between April 17, 1963 survey estimates of oxygen concentrations and model predictions at several stations:

\begin{tabular}{|c|c|c|}
\hline $\begin{array}{l}\text { Blngham } \\
\text { Survey } \\
\text { Station }\end{array}$ & $\begin{array}{c}\text { Range of Oxygen } \\
\text { Deficit from } \\
\text { Survey Data } \\
\end{array}$ & $\begin{array}{c}\text { Oxygen Deficit } \\
\text { from Model } \\
\end{array}$ \\
\hline & (ppm) & (ppm) \\
\hline $\begin{array}{l}\text { OP I } \\
\text { OP2 } \\
\text { OP } 3 \\
\text { FH2 } \\
\text { MC1 } \\
\text { OH2 }\end{array}$ & $\begin{array}{l}1.1-0.8 \\
0.8 \\
1.1-0.8 \\
0.4-0.1 \\
1.1-0.6 \\
0.7-0.6\end{array}$ & $\begin{array}{l}0.9 \\
0.9 \\
0.9 \\
0.8 \\
0.6 \\
0.5\end{array}$ \\
\hline
\end{tabular}


In general, the model output closely matches the oxygen deficits measured in the hydrographlc survey. Stations OPI, OP 3 , and OH 2 are within $0.2 \mathrm{ppm}$. Other stations are within the survey range or relatively close to 1 t.

\section{USE OF THE VERIFIED MATHEMATICAL MODEL FOR WATER QUALITY PREDICTION}

The mathematical model of New Haven Harbor was used to predict the effect of the degree of BOD removal during effluent treatment on the dissolved oxygen concentration in the waters, and also to evaluate the water quality resulting from the use of long outfalls to discharge waste effluent into deeper sections of the Harbor with higher mixing characteristics. The estimates of future waste loads, during the maximum month in 1990, were used for model predictions.

The major waste contributions to the Harbor are discharged from the Boulevard, East street, East shore, and West Haven Sewage Treatment plants. Additional waste sources are direct industrial. dlscharges and overflows from the munlclpal sewage system.

At present, more than two-thirds of the tributary drainage areas of the Boulevard, East street, East Shore, and West Haven sewage Treatment plants are served by a combined sewer system. consequently, sewage that settles and forms deposits in sewers during dry periods is flushed from the system during significant rainfalls, resulting in an instantaneous load several times larger than the normal BOD discharge. These irregular, instantaneous loads are a direct cause of the low Do condltions that sometimes occur. Future plans for New Haven include modifying the present combined system into a completely separate system.

The following is a list of the 5-day BOD loads for the maxlmum month in 1990

$\begin{array}{lc}\text { Sewage Treatment Plant } & \text { Load, Lb. BOD } 5 / \text { Day } \\ \text { Boulevard } & 52,500 \\ \text { East Street } & 63,500 \\ \text { East Shore } & 64,000 \\ \text { West Haven } & 37,500 \\ & \frac{217,500}{}\end{array}$

The Boulevard, East street, and East shore operations are older, primary treatment plants. The West Haven operation is a secondary treatment plant.

Since the primary treatment plants were under conslderation for renovation, the model was used to evaluate whether any substantial water quality benefit could result from combining the flow in one or more larger, rebullt operations. Five alternate treat- 
ment schemes were proposed as follows

\begin{tabular}{|c|c|c|c|}
\hline \multirow[b]{2}{*}{ Scheme } & $\mathrm{BOD}_{5} \underset{1 \mathrm{nto}}{1 \mathrm{~b} / \mathrm{Day}}$ & $\mathrm{BOD}_{5} \underset{1 \mathrm{lb} / \mathrm{DaY}}{\mathrm{db}}$ & $\mathrm{BOD}_{5} \underset{1 \mathrm{ln} \text { to }}{1 \mathrm{Day}}$ \\
\hline & Boulevard site & East st site & East Shore site \\
\hline 1 & 52,500 & 63,500 & 64,000 \\
\hline 2 & $\begin{array}{r}52,500 \\
63,500 \\
116,000\end{array}$ & - & 64,000 \\
\hline 3 & 52,500 & -- & $\begin{array}{r}63,500 \\
64,000 \\
127,500\end{array}$ \\
\hline 4 & -- & $\begin{array}{r}52,500 \\
63,500 \\
16,000\end{array}$ & 64,000 \\
\hline 5 & - & -- & $\begin{array}{r}64,000 \\
63,500 \\
52,500 \\
180,000\end{array}$ \\
\hline
\end{tabular}

The estumated average monthly BOD loads for 1990 are about elghty percent of the maximum monthly loads above. The west Haven secondary treatment plant wlll, under all schemes, discharge 37,500 lbs. of 5 -day BOD per day

One positive result that the computer model showed was that no particular alternate scheme offered significant advantages over the others in terms of average BOD and DO levels resulting in the Harbor The basic reason for the essentialiy similar water quality patterns produced by the 5 schemes was that the Boulevard, East Street and East Shore plants are all located in the inner section of the Harbor within a distance of 8,000 feet from each other. Hence, the high degree of tidal flushing that occurs effectively spreads and mixes the BOD waste loads throughout the inner Harbor, whth sumilar dissolved oxygen concentration patterns resulting.

The conclusion drawn from these computer model results is that selection of an alternate scheme, at a given BOD removal rate, should be governed by the economics of construction and operation of the rebullt plant or plants in terms of the resulting water quality, and protection of the beach areas, outputs from all the schemes are essentially equal This conclusion 1 s predicted on the condition that discharge at or near the shoreline will be into tidal waters and will not be allowed to spill onto tidal mud flats where BOD concentrations can bulld up.

In order to evaluate the relationship between the degree of effluent treatment and resulting dissolved oxygen in the Harbor, 
a series of computer runs was made for each of the 5 alternate schemes at BOD removal rates of $258,50 \%, 758,858$, and $90 \%$. Printout 1 shows typical BOD output. Table l lists a typical result for the minimum dissolved oxygen in the Harbor, versus the percent of BOD removal.

The type of output displayed in the above Printout and Table 1 is an example of the type of rational englneering analysis that can be obtalned from a predictive mathematical model. Instead of designing a waste treatment operation to an arbitrary set of $B O D$ removal standards or effluent concentration levels, a verified mathematical model can be constructed to determine the relationship between the degree of BOD removal and the approximate distribution of dissolved oxygen, longltudinally and laterally, in the receiving waterbody. For example, if a minimum tidal average of 6 ppm of dissoived oxygen was desired in the Harbor, approximately 85 \% BOD removal would be required for the projected effluent discharges.

TABLE 1

EFFECT OF DEGREE OF BOD REMOVAL ON MINIMUM DISSOLVED OXYGEN IN NEW HAVEN HARBOR

$\&$ BOD

Removal

Durıng

Treatment
TIDAL AVERAGED

NEW HAVEN HARBOR MINIMUM DO, PPM

\begin{tabular}{c} 
Scheme 1 \\
Separate Effluent \\
Discharges at \\
Boulevard, \\
East St., and \\
East Shore STP \\
\hline
\end{tabular}

Scheme 5

All Effluent Directed to East Shore STP

$\begin{array}{llr}25 & 3.8 & 4.1 \\ 50 & 4.7 & 4.9 \\ 75 & 5.6 & 5.7 \\ 85 & 6.0 & 6.0 \\ 90 & 6.1 & 6.2\end{array}$

Another important predictive feature of the verified mathematical model is the ablilty to evaluate the effect of the use of outfalls to dilute the discharged effluent and, hence, to effectively reduce the level of BOD removal required. Table 2 shows a typical summary of computer runs at various percentages of BOD removal. Inspection of the table shows that extension of the outfall into deeper water of the Harbor allows reduction of the degree of sewage treatment, but maintains the desired minimum dissolved oxygen concentration. The economic feasibility of using outfall extensions must be evaluated after detalled construction cost estimates. 


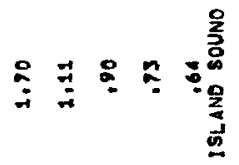

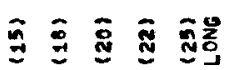
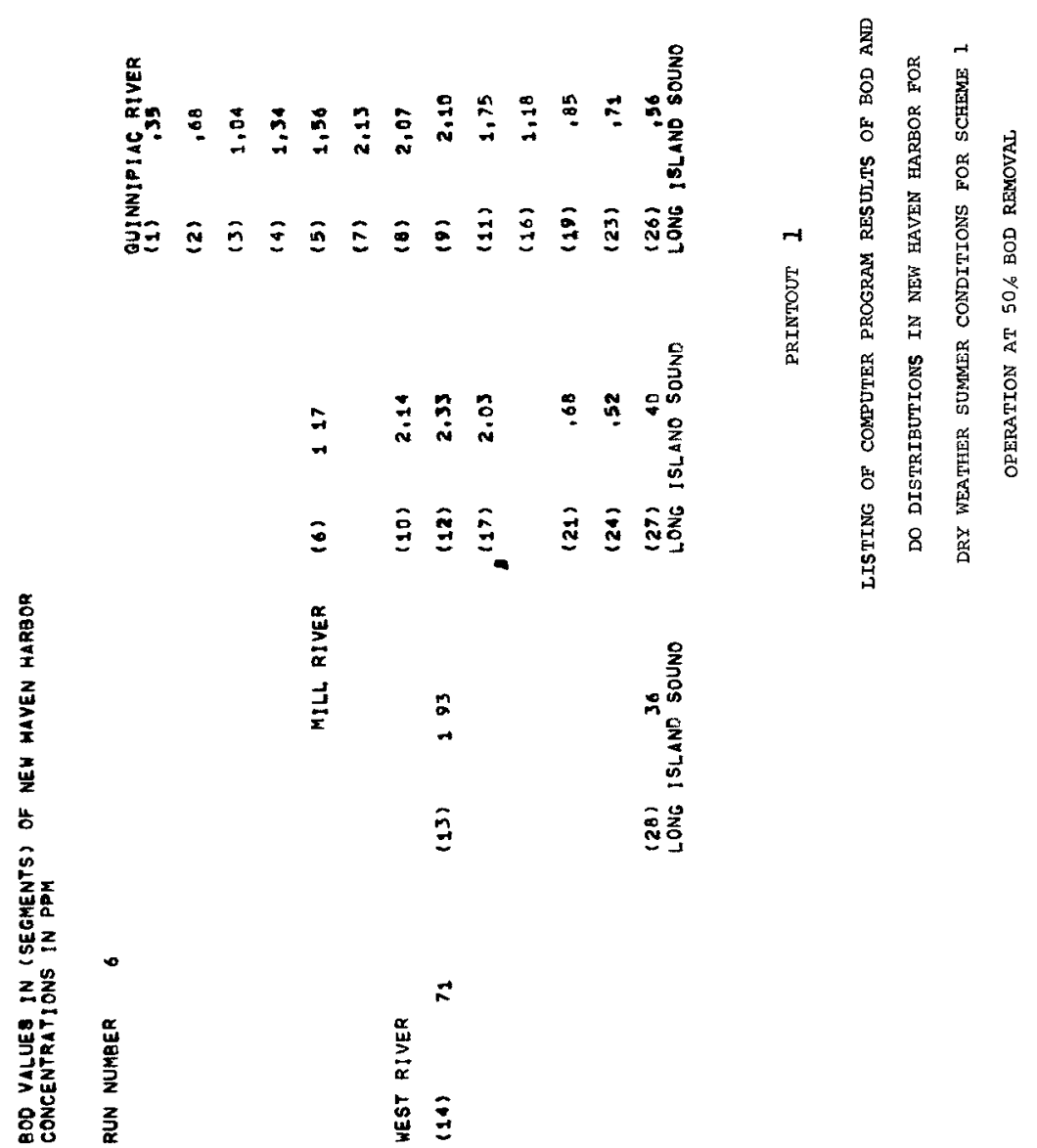


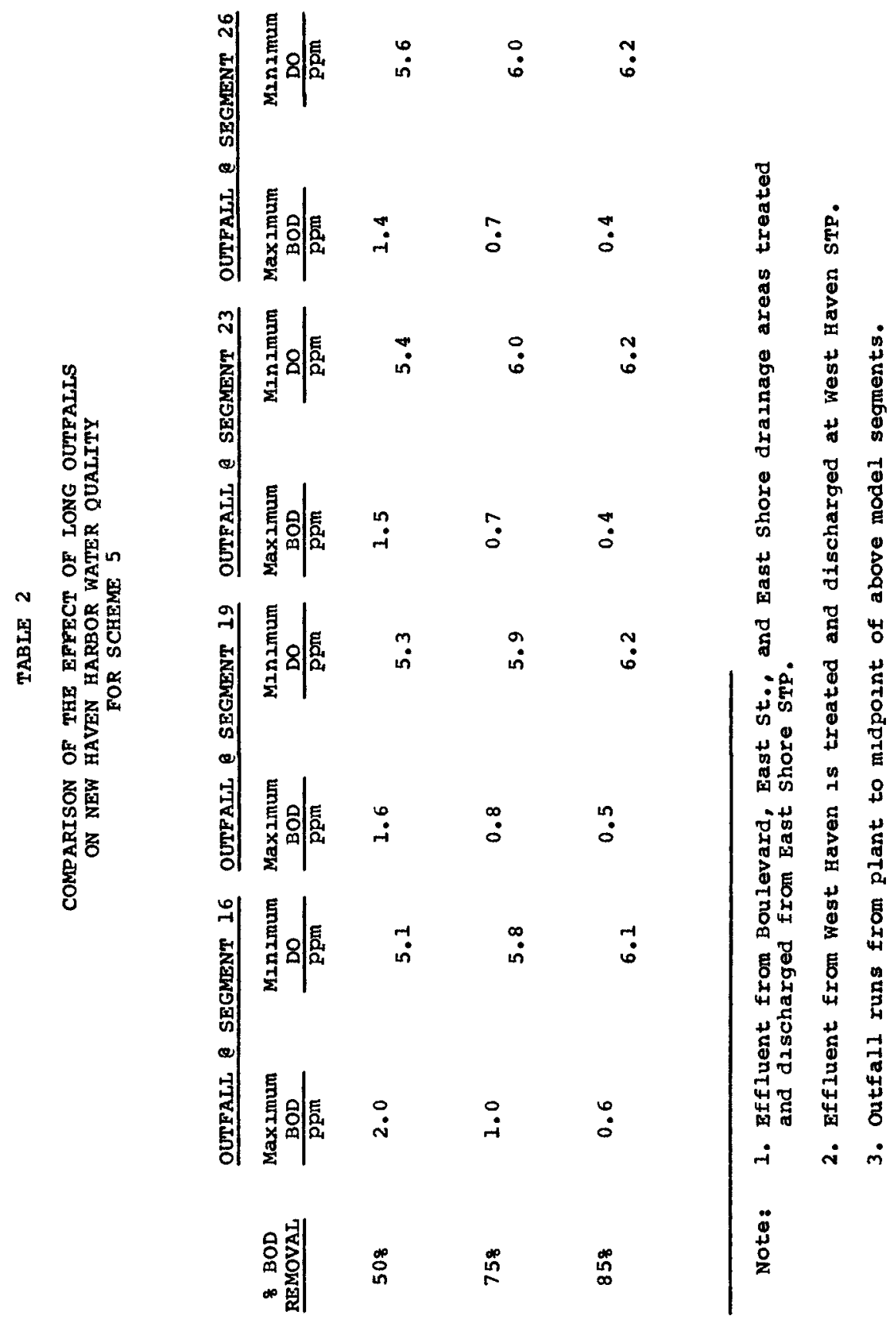




\section{ACRNOWLEDGEMENTS}

This work was performed under the sponsorship of the Water Resources Commission of the state of Connecticut, and was performed by Quirk, Lawler and Matusky Engineers, NYC. 
CHF.PTER 113

FLUSHING PATTERN OF NON-REACTIVE EFFLUENTS

by

Adel M. Kamel, AM.ASCE

Associate Professor of Civil Engineering

Louısiana State Unıversıty, Baton Rouge, La.USA.

\section{ABSTRACT}

In south central Loulsiana non-reactive liquid effluents are introduced into man-made relatively straight prismatic canals which are comparatively narrow and have brackish water To study the flushing pattern of liquid effluents introduced into those canals (or estuaries), a one-dumensional nimerical model is considered for a simplified system consisting of a long stralght gently sloping reach with a sinusoldal trdal variation at the mouth of the estuary, sinusoidal tidal virition with a phase lag and an attenuated amplitude at the upstream end, a variable inflow hydrograph, and a variable inflow or outflow. For this system, the conti nuity $a$ nd momentum equations are solved numericaliy by an explucit funite difference scheme. The output of the model describes the spatial and temporal variations in flow velocity (also in water depth and discharge) from which the flushing pattern is obtained for a liquid effluent introduced at any time during the tidal cycle at any section along the estuary. The numerical model is applied to Charenton drainage canal in south central Loussiana and good agreement is obtalned between the velocities and stage elevations predicted from the model and recorded in the field. An IBN $360 / 65$ computer is utilized.

\section{INTRODUCTTON}

In south central Ioulsiana non-reactive liquid effluents mainly from sugar mills are introduced into man-made relatively straight prismatic canals which are comparatively narrow and have brackish water. Effluents introduced oscilate up and down the canals with tidal movement and are carried slowly to the Gulf of Mexico with the net flow. 
The study of the flushing pattern of effluents introduced into those estuaries is of interest in the selection of the location of new outfalls and the time of release of effluents The flushing pattern would depend on the flow velocity and the density of the effluent. The presence of tidal movement in the estuaries under consideration (for a $1.5 \mathrm{ft}$ tide at the mouth of estuary, Gulf of Mexico, tidal variation of more than $1.0 \mathrm{ft} 1 \mathrm{~s}$ obtained about 6 miles upstream) result in unsteady flow which makes the evaluation of flow velocity based on steady state conditions no longer feasible. Resort to the evaluation of the flow by either empirical methods or continuous field measurements are also not feasible because of the lack of generality in the first and the incredible amount of work and expense in the latter.

Hydraulıc and analog studies could be employed for predicting the flushing pattern in estuaries. However, such models provide results that are unique to the particular tidal reach for which they are applied. Application of those models to another tidal reach would require the construction of new hydraulic or analog model. On the other hand a general purpose numerical model would be applicable to any tidal reach in the area as long as the characteristics of the reach under consideration (such as length, roughness coefficient, shape of cross section of flow, tidal amplitude and phase angle, .. .. etc.) are provided as input to the numerical model.

Techniques for one-dimensional numerical modeling of both natural and artificially induced transient flows in rivers and estuaries have been developed by investigators such as $(1,2,4,5,8,10,11,12$, and 18)*. Recently work has begun on two-dimensional numerical modeling of tidal flows such as the work of $(3,6,7,13$, and 17). Those techniques involve the solution of the shallow water equations for the appropriate initial and boundary conditions.

This paper constitutes the results of a pllot study on the numerical simulation of the flushing pattern of a non-reactive liquid effluent (no decay or absorption of effluent across flow boundaries) which has a density similar to that of the estuarine water. The estuaries considered are

* Numerals in brackets refer to similarly numbered items in

Appendix I-References. 
comparatively narrow and stralght with brackish waters. The numerical model is applied to Charenton drainage canal (figure l) in south central Loussiana and good agreement is obtalned between predicted and measured water stage elevations and velocities in the canal.

\section{ANALYSIS}

Simulation of Spatial and Temporal Velocity Changes.- The estuaries considered are sectionally homogeneous making a one-dimensional presentation adequate for the study of the flushing pattern and concentration distribution of liquid effluents having density similar to that of the receiving waters. The hydromechanics of the estuaries considered could be studied by developing a one-dimensional numerical model for a simplified system consisting of a long stralght gently sloping reach with a sinusoldal tidal variation at mouth of estuary, a sinusoldal tidal variation with phase lag and amplitude attenuation at the upstream end, an inflow hydrograph, and lateral inflow or outflow.

The equations of continuzty and of motion for one-dimensional unsteary flow of homogeneors density in a straight gently sloping prismatic tidal reach are expressed as.

$\frac{\star \partial E}{\partial t}+F \frac{\partial u}{\partial x}+u \frac{\partial H}{\partial x}+u a_{0}-\frac{\sigma}{B}=0 \quad-1-$

$\frac{\partial u}{\partial t}+u \frac{\partial u}{\partial x}+\sigma \frac{\partial H}{\partial x}+\alpha s_{e}-\frac{q u}{A}=0 \quad-2-$

Equations 1 and 2 are the famlitar forms of the shallow water equations and are valud under the follow 7 ? assimptions 1 the effect of the coriolis force and the wind stresc term are neglicable, 2. In the direction normal to the axis of the channel the surface particles lie transversely on a horizontal line since the reach section is prismatic in form, it follows that the width $B$ would be a function of depth only, 3. hydrostatic pressure orevalls at any point and a uniform velocity distribution exists over any cross section.

* Symbols used are defined in Appendix II-Notation. 
Further assuming that the coefficlent of roughness for unsteady flow is the same as that for steady flow and can be expressed by the Manning's equation, the friction slope term in equation 2 could be expressed as

$$
g s_{e}=\frac{9 k}{(R)^{4 / 3}} \quad u|u|
$$

Subistituting equation 3 into 2, the later becomes,

$$
\frac{\partial u}{\partial t}+u \frac{\partial u}{\partial x}+g \frac{\partial E}{\partial x}+\frac{g k}{(R)^{4 / 3}} u|u|+\frac{g u}{A}=0-4-
$$

Equations 1 and 4 are two simultaneous quasi-linear partial differential equations with two decendent variables $u$ and $E$ and two independent variables $x$ and $t$. To solve equations 1 and 4 numerically three methods were considered namely, characteristics, explicit, and implicit. Results obtained from the explicit method only are reported herein.

The computational scheme employed in this study is based on operating with finite differences by using a fixed rectangular net in the $x-t$ plane In this case it is not necessary to calculate the values of the coordinates $x, t$ of the net points themselves The reach of length $L$ is divided into $N$ equal sections of length $\Delta x$. The time interval $\Delta t$ is selected to satisfy the courant condition for empirical stability,

$$
\Delta t=\frac{\Delta x}{Y(|u|+c)}
$$

The boundary conditions of water surface elevation were selected since in canals considered gaging stations are usually operated by the Corps of Englneers and supply continuous record of $\mathrm{E}$. The computational procedure starts by assuming the initial condition (values of $U$ and $E$ at $t=t$, along the reach length and advancing the solution one step to time $t_{1}=\Delta t$. Now that all values of $u$ and $E$ are known at each point in the grid at time $t_{1}=\Delta t$, the same procedure is used to advance the solution from $t_{1}=$ $\Delta t$ to $t_{2}=2 \Delta t$. In this case the values of $u$ and $E$ 
obtalned at $t_{l}=\Delta t$ are considered as initial conditions.

Simulation of Flushing pattern of Index Particles.- The differential equationsdescribing the movement of index partlcles released at any time at any location along the reach is expressed as

$\frac{d x}{d t}=u(X, t)$ $-6-$

A particle released at $x_{0}$ at $t_{0}$ would be located at $x_{j}+1$ at time $t=t_{0}+n \cdot \Delta t$. where:

$x_{\jmath+1}=x_{\jmath}+u_{x \jmath} \cdot n \cdot \Delta t,(n, j=0,1,2,3 \ldots)-7-$

To obtain the flushing pattern of an effluent introduced at any time $t$ at any section $x$ along the reach, the value of $u$ at that Section at that time is read and multiplied by the time increment $\Delta t$ to give the increment $\Delta x^{\prime}$ which the effluent has advanced. $\Delta x^{\prime}$ is added algebrically to $x$ to yield the new location $x$ of the effluent at time $t+\Delta t$. At this new location $x$, the value of $u$ is obtained by linear interpolation from the corresponding values of $u$ in the mesh of length $\Delta x$ to the right and to the left of point $X$. The same procedure is repeated to obtain the flushing pattern for any length of time.

\section{APPI ICATION}

Simulation of spatial and Temporal Velocity Changes.- To establish the range of conditions under which the computational scheme would yield satisfactory results, the following factors are considered

1. effect of assumed inltial conditions, 2. effect of Manning's coefficient of roughness, 3 . stability of the scheme.

Effect of Initial Condition.- The initial conditions employed are the water level elevation $I$ and the flow velocity $u$ at $t=0$. Since the water level is known at the two ends of the reach from gaging stations, an elevation of the water level at different sections along the reach by linear interpolation would be satisfactory for obtaining the initial values of $E$. The corresponding values of $u$ could be computed 
from Manning's formula, in this case a value of $u_{0}=1.45$ $\mathrm{ft} / \mathrm{sec}$ is obtained and is assumed to be the same along the entire reach at $t=0$. To establish the effect of $u_{0}$ on the convergence of the solution, computations were carried out with $u_{0}=1.45,0.725$, and $0.00 \mathrm{ft} / \mathrm{sec}$. It was noted that all values computed converged to a unique value in less than 5 hours (figure 2). Five hours would seem as a short duration in the stidy of the velocity variation during a whole tidal cycle. However, in the study of the flushing pattern during periods of large velocities, the variation in the velocity obtained from the computational scheme during the first hours could result in an appreciable error in evaluating the flushing pattern. This suggests that $\approx$ careful estimate of the initial velocity $u_{0}$ would be necessary If accurate flushing patterns are to be oblained.

Effect of Manning's Coefficlent of Roughness - The effect of the value of $n$ selected for the calculations on the snatial and temporal variation of the velocity wae unvestigated for $\mathrm{n}$ values of $0.0,0.02,0.03$, and 0.10 . Other narameters are held constant. The resilts are ohown in figure 3 and indicate that convergence does not nccur and a variation in the masnjtude of the velocity of as much as 80\% occurs by rrangsng the value of $\mathrm{n}$ from 0.02 to 0.03 . Although the computationa] scheme is sensetive to variations in the value of $n$ employed in the calculations, this should not be of great concern since the value of $\mathrm{n}$ can be estimated with a reaconable iegree of accuracy for elther an existina canal or for one which is in the olanning stage.

Stability.- Questions regarding the stability of the explicit scheme has been raised. For instance (14) showed that the method is unstable when $\Delta x \rightarrow 0$ even though $\Delta t \rightarrow 0$ in such a manner as to satisfy equation 5 . It was reporetd by (19) that satisfying equation 5 does not guarantee that the equation of continuity would be satisfied. According to the same author, over a long period of time the inflow might be greater or less than the outflow plus the accumulated storage. In his study of pverland flow (15) noticed a tendency of the hydrograph to ovshoot at equilibrium flow. In the present study instability was observed when the rate of change of discharge with respect to time is large. This was corrected for by selecting a value of $Y$ variable in equation 5 to ensure stability in convenient means since a rigorous stability criteria for nonlinear equations cannot be established (16). 
After exploring the effect of the initial conditions, the coefficlent of roughness, and the stabllity of the method on results obtained by the computational scheme, it would be necessary to examine the effect of the different hydraulic parameters (such as tidal amplitude and inflow hydrograph) on the spatial and temporal variations of the flow velocity. This is summarized in the following paragraphs.

Effect of Tidal Amplitude.- The effect of tidal amplitude on the velocity, discharge, and water level for two semiamplitudes of $\mathrm{a}=4.00 \mathrm{ft}, \mathrm{al}=3.5 \mathrm{ft}$, and $\mathrm{a}=1.50 \mathrm{ft}$, al $=1.25 \mathrm{ft}$ Indicated minor spatial variation in the values of $u, Q$, and $E$ obtained for the same tIdal amplitude. This is belleved to be due to the small coefficlent of roughness $(n=0.002)$ employed in the numerical model.

Effect of Inflow Hydrograph.- The four inflow hydrographs tested have a sinusoldal form with a peak amplitude $(\mathrm{h})$ of $7.5 \mathrm{ft}$ above existing water level at upstream end of reach. The duration of the inflow hydrograph $(\tau)$ varled from $\mathrm{T} / 16$ to $\mathrm{T} / 4$. The results are shown in figure 4 and indlcate ar equal peak velocity for different values of $\tau$ and a longes duration of high flows for larger values of $\tau$.

Simulation of Flushing Pattern of Index Particles.- After examining the effect of the different hydraulic parameters on the spatial and temporal variation of the flow velocity, two typical examples are selected for the study of the flushing pattern. The first situation is representive of conditions during unusual weather activities. The following hydraulic parameters are selected: $a=4$. Oft, $a l=3.5 \mathrm{ft}, q=$ $0.10 \mathrm{ft} / \mathrm{sec}, u_{0}=1.45 \mathrm{ft} / \mathrm{sec}, \mathrm{h}=4.0 \mathrm{ft}, \tau=\mathrm{T} / 2, \mathrm{~T}=24.83 \mathrm{hrs}$., and $n=0$ 02. The flushing pattern of an effluent introduced at time to $=\mathrm{T} / 2$ at section 1 through 12 of the reach under study is shown in figure 5. The second situation is representative of conditions that prevall during the dry season with normal tidal activity in the Gulf of Mexico. The following hydraulic parameters are selected: $a=0.75 \mathrm{ft}, \mathrm{al}=0.50 \mathrm{ft}$, $q=0.0, u_{0}=0.0, h=0.0, \tau=0.0, T=24.834 h r s$, and $n=0.02$. The flushing pattern for an effluent introduced at time $t_{0}=T / 2$ at sections 1 through 12 of the reach under study is shown in figure 6 . 


\section{VERIFICATION OF NUMERICAL MODEL}

For verifyung the sigas and velocities predicted from the numerical model the stage elevations recorded on three Stevens tide recorders were utilized. Tide charts were obtalned for stations No.1 (at Southern Pacific Raılroad bridge), No.2 ( 1 mile downstream from station No.1) and No.I3 $(0.25$ miles below junction with IWW 6 miles downstream fxom station No.1), figure 1 . The tide stages from stations No. 1 and 13 were digitized and provided boundary condition input to the model. A comparison between the predicted and measured stage elevations for station No.2 is shown in figure 7 for $n=0.045$. The agreement between the predicted and measured stage elevations is good and could be improved by optimizing for the value of $n$. The predicted and measured temporal changes of the average velocity for station No.I is shown in figure 8 and indicates good agreement. The velocity measurements were made from the Railroad bridge utilizing a cub current meter. Again it is believed that better agreement between predicted and measured velocities could be obtaıned by optimizing for the value of $n$.

\section{CONCLUSIONS}

1. The numerical model is sensetive to input values assumed for starting the computations such as the initial velocity, and the Manning's coefficient of roughness. This would call for a careful selection of these values but does not pose a great concern as to the validity of the model.

2. The computational scheme has a tendency to become unstable when the rate of change of discharge with respect to time is large. This difficulty is overcome by taking smaller time increments $\Delta t$, in equation 5 .

3. The flushing pattern depends on the prevalling hydraulic conditions and on the time and location of release of effluent. For instance during normal conditions where a tide amplitude at mouth of estuary is about $1.5 \mathrm{ft}$ and about $1.0 \mathrm{ft}$ at a distance 6 miles upstream of the mouth, with no lateral inflow or outflow and no inflow hydrograph, the flushing pattern shown in figure 5 is predicted for effluents released at low water slack $\left(t_{0}=0.0\right)$. It can be seen from figure 5 that an effluent released at the Southern Paciflc Rallroad bridge 6 miles upstream of the IWW WIll be flushed in a downstream direction to a distance of about $14,700 \mathrm{ft}$ after 5 hours. Fıgure 6 shows typical flushing pattern during unusual weather activities which may prevall during the hurricane season where the variation in water level at the mouth of the estuary may reach as high as $8.0 \mathrm{ft}$ with an inflow hydrograph at the 
upstream end of the reach and lateral inflow caused by rainstorms. It can be seen from figure 6 that effiuents released at the upstream end of the reach at $t=t_{0}+15.30$ hours (1.e. 15. 30 hrs after high water slack) would be flushed in only 1.39 hrs into the mouth of the estuary. The same figure indicates that effluents released during high water slack $\left(t_{0}=T / 2\right)$ as far downstream as 0.5 miles from mouth of estuary would reach the upstream end of reach in 3.89 hrs. 4. Computer time is an important factor in the appraisal of simulation techniques. In the scheme represented herein, for a time interval $\Delta t=1.0$ minute and a section length $\Delta \mathrm{x}=0.5 \mathrm{miles}$, a complete run required 2.67 minutes on a IBM $360 / 65$ computer. A complete run involved predicting the spatial and temporal variations in water level elevation, flow velocity, discharge, and flushıng pattern for a tıdal reach 6 miles long during a tidal period of $24.834 \mathrm{hrs}$. Time required for plotting or printing out of the results is not included in the 2.67 minutes.

5. Agreement between predicted and measured temporal variatlons of water stage elevations and velocities is good and could be improved by optimizing for the value of $n$. 6. Development of two-dimensional stochastic models for predicting the flushing pattern and concentration distribution for non-reactive liquid effluents dlscharged into large water bodies such as rivers and lakes would seem to be the loglcal extension of this work.

\section{ACKNOWLEDGMENT}

The study was sponsored by the Iouisiana Water Resources Research Institute. The continued financial support of the National science Foundation for the operation of the computer Facility at ISU is acknowledged.

\section{APPENDIX I- REFERENCES}

1-Abbott, M.B., and Ionesca, F.,"On the Numerical Computation of Nearly Horızontal Flows," Journal of Hyaraulıc Research, IAHR, Vol.5, No.2, 1967.

2- Amien, M.,"streamflow Routıng on computer by Characterıstics," Water Resources Research, Vol. 2, No.1, 1966.

3. Argelo, S.M., "Tidal Computation in Two-Dimensions", Report R1 Jkweterstaat, 1965.

4. Ballofet, A., and Kupferman, A., "Hydraulıc Studıes in Jamaica Bay", Journal of the Hydraulics Division, AsCE, Vol.90, No.HY6, NOV. 1964. 
5- Collins, J.I.,"Mixed Techniques for Computing Surges in Channels", Journal of the Hydraulics Division, ASCE, Vol.94, No. HY2, March 1968.

6-Daubert, A., and Graffe, O., "Mathematıcal Model of Tidal Propagation in a Coastal Area," Proceedings 12 Congress, IAHR, Vol.4, Part II, Colorado state University, Fort Collins, Col., 1967.

7- Dronkers, J.J.,"Tidal Computatıons for Rivers, Coastal Areas and Seas", Journal of Hydraulics Division, ASCE, Vol.95, No. HYI, Jan. 1969.

8- Einstein, H.A., and Harder, J.A, "Analytıcal and Numerical Methods for Prediction of Tidal Elevations and Tidal Flows in Canals and Estuaries", Report to the Committee on Tidal Hydraulics, Corps of Engineers, U.S Army, 1960.

9- Harris, D.L., and Yelismarski,C.P. "Some Problems Involved in the Numerical Solution of Tidal Hydraulics Equations", Monthly Weather Review, Vol.92, 1964.

10- Isaacson, E., Stoker,J.J, and Troash,A., "Numerical solution of Flood Prediction and River Regulation Problems", Report No. IMM-235, Institute of Mathematical Sclence, New York University, New York, 1956.

Il- Kamphius, J.w., "Mathematical Tidal Study of St. Lawrence River," Journal of the Hyaraulics Division, ASCE, Vol.96, No. HY3, March 1970.

12-Ial, Chintu, "Computation of Transient Flows in Rivers and Estuaries by Multiple-Reach Method of Characteristics", U.S. Geological Survey Prof. Paper 575-D, pp. D273-D280, 1967. 13-Leendertsee, $\mathrm{J} J$, "Aspects of the Computational Model for Iong-Period Water Wave Propagation," Rand Memorandum R.M.5294-P.R., Delft, 1967.

14- Iiggett,J.J., and Woolhiser,D.A., "Difference Solutions of the Shallow-Water Equations", Journal of Engineering Mechanjcs, ASCE., Vol. 93, No.EM2, April 1967.

15- Morgali, J.R , "Laminar and Turbulrnt Overland Flow Hydrographs", Journal of the Hydraulıcs Division, ASCE, Vol.96, No.HY2, February 1970.

16-Richtmeyer, R.D.,"Difference Methods for Initial value Problems", Interscience Publishers, New York, 1957.

I7- Schonfeld, J.C.,"The Boundary Value Problem for Two-Dimensional Long waves", Report RI jkswaterstaat, 1965.

18- Stoker,J.J., "Numerical Solution of Flood Predictions and River Regulations Problems, Report I:Derivation of Basic Theory and Formulation of Numerical Method of Attack, "Report No. IMM-200, Institute of Mathematical Sclence, New York University, New York, 1953.

19- Strelkoff,T., and Amarocho, J., "Gradually Varıed Unsteady Flow in a Controled canal system", Proceedings lith Congress, IAHR, Leningrad, USSR, Paper 3-16, 1965. 


\section{APPENDIX II - NOTATION}

The following symbols are used in this paper

$A==$ flow cross sectional area, $\left(I^{2}\right)$,

$a=$ seml-amplitude of tide at downstream end of estuary, (L),

al= semi-amplitude of tide at upstream end of estuary, (I),

$B=$ surface $w i d t h,(L)$,

$c=$ celerity of gravity wave, $(\mathrm{L} / \mathrm{T})$;

$E=$ elevation of water surface, $(\mathrm{I})$;

$g=$ gravity acceleration, $\left(\mathrm{L} / \mathrm{T}^{2}\right)$;

$\mathrm{H}=$ average depth of flow in cross section, (L),

$\mathrm{h}=$ height of inflow hydrograph above pre-existing water level at upstream end of reach, (L),

$k=a$ function of Manning's coefficient of resistance, $\left(T^{2} / \mathrm{L}^{2 / 3}\right)$;

$\mathrm{L}=$ length of reach, $(\mathrm{L})$;

$m=$ number of time intervals of duration $\Delta t$.

$\mathrm{n}=$ Manning's coefficient of resistance, $(\mathrm{T} / \mathrm{i} I / 3)$,

$N=$ number of reach sections having a length $\Delta x$,

$q=$ lateral inflow per unit length of reach, $\left(\mathrm{L}^{2} / \mathrm{T}\right)$,

$Q=$ discharge, $\left(\mathrm{I}^{3} / \mathrm{T}\right)$,

$\mathrm{R}=$ hydraul $1 \mathrm{c}$ radius, $(\mathrm{I})$;

$\mathrm{S}_{\mathrm{e}}=$ friction slope,

$\mathrm{S}_{\mathrm{O}}=$ bottom slope,

$T=$ tidal perlod, $(T)$;

$t=t i m e,(T)$;

$\tau=$ duration of inflow hydrograph, $(T)$,

$\Delta t=$ time interval, $(T)$,

$\mathrm{u}=$ flow velocity in longitudinal direction, $(\mathrm{L} / \mathrm{T})$,

$u_{0}=$ Inltial velocity in longitudinal direction, ( $(\mathrm{T} / \mathrm{T})$,

$\mathrm{x}=$ distance measured in longitudinal direction, (L),

$\mathrm{Y}=$ variable $\geqslant 1$,

$\Delta x=$ length of equal section of reach, (L), and

$\varepsilon=$ phase angle between tides at downstream and upstream ends of reach. 


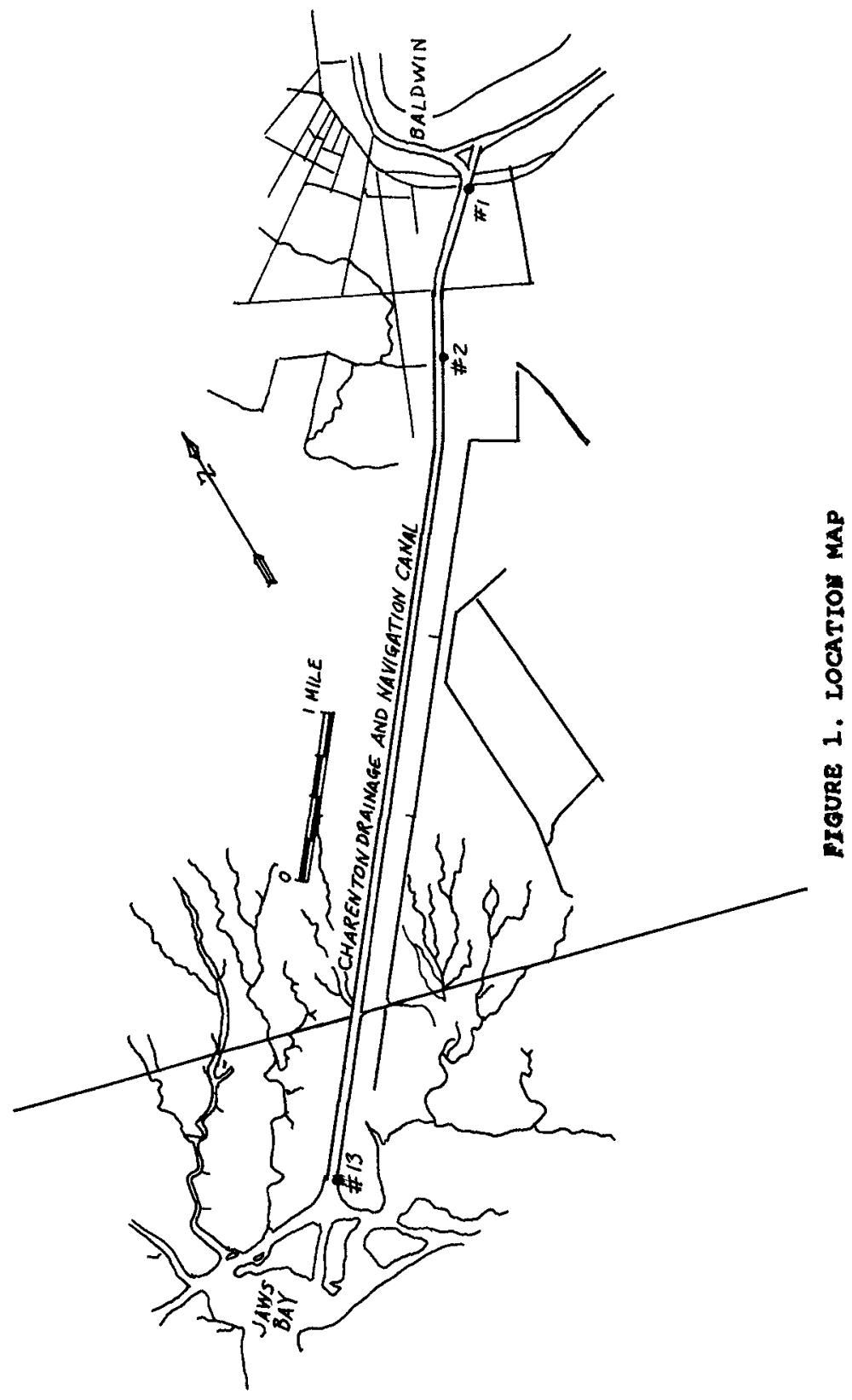




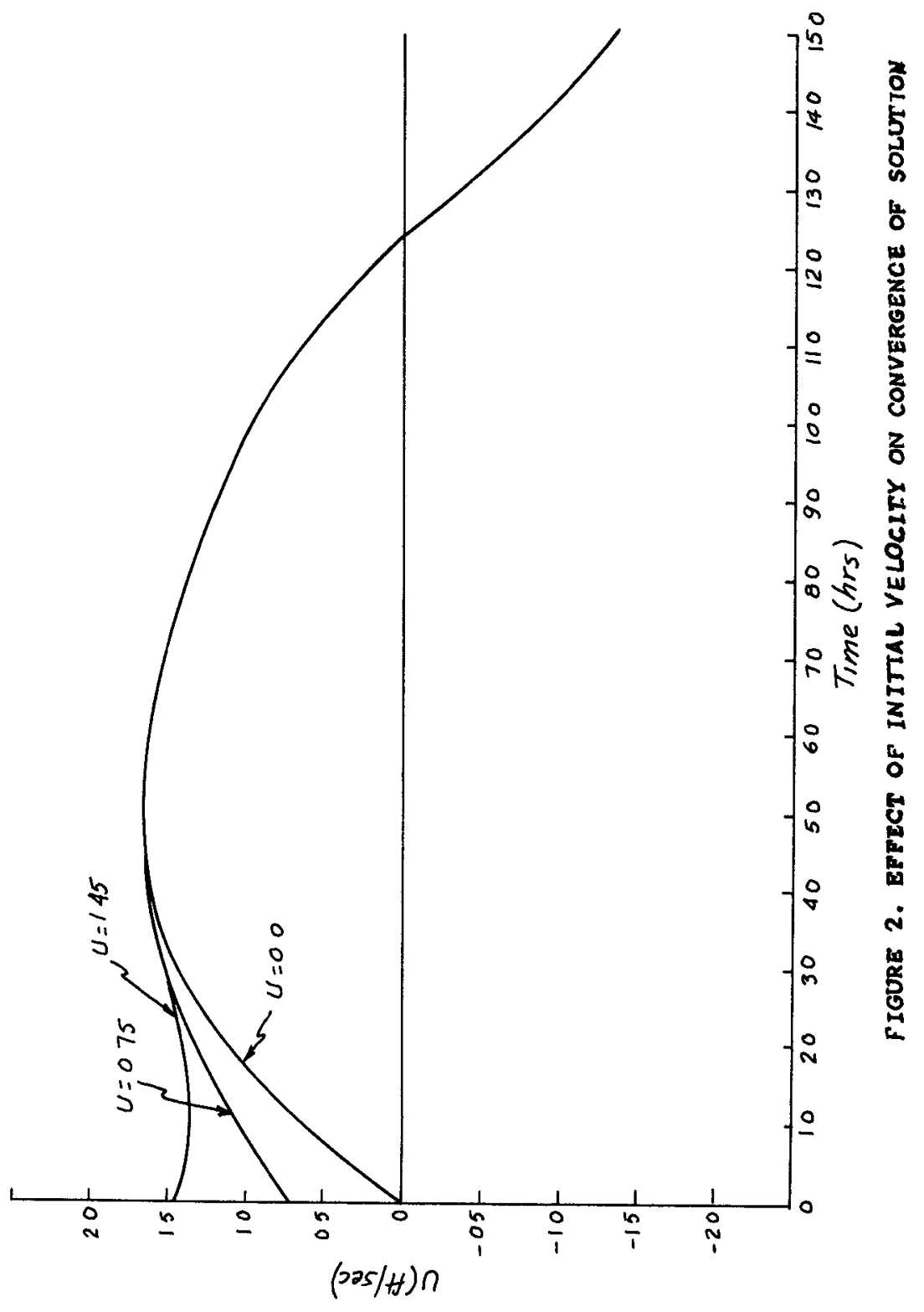




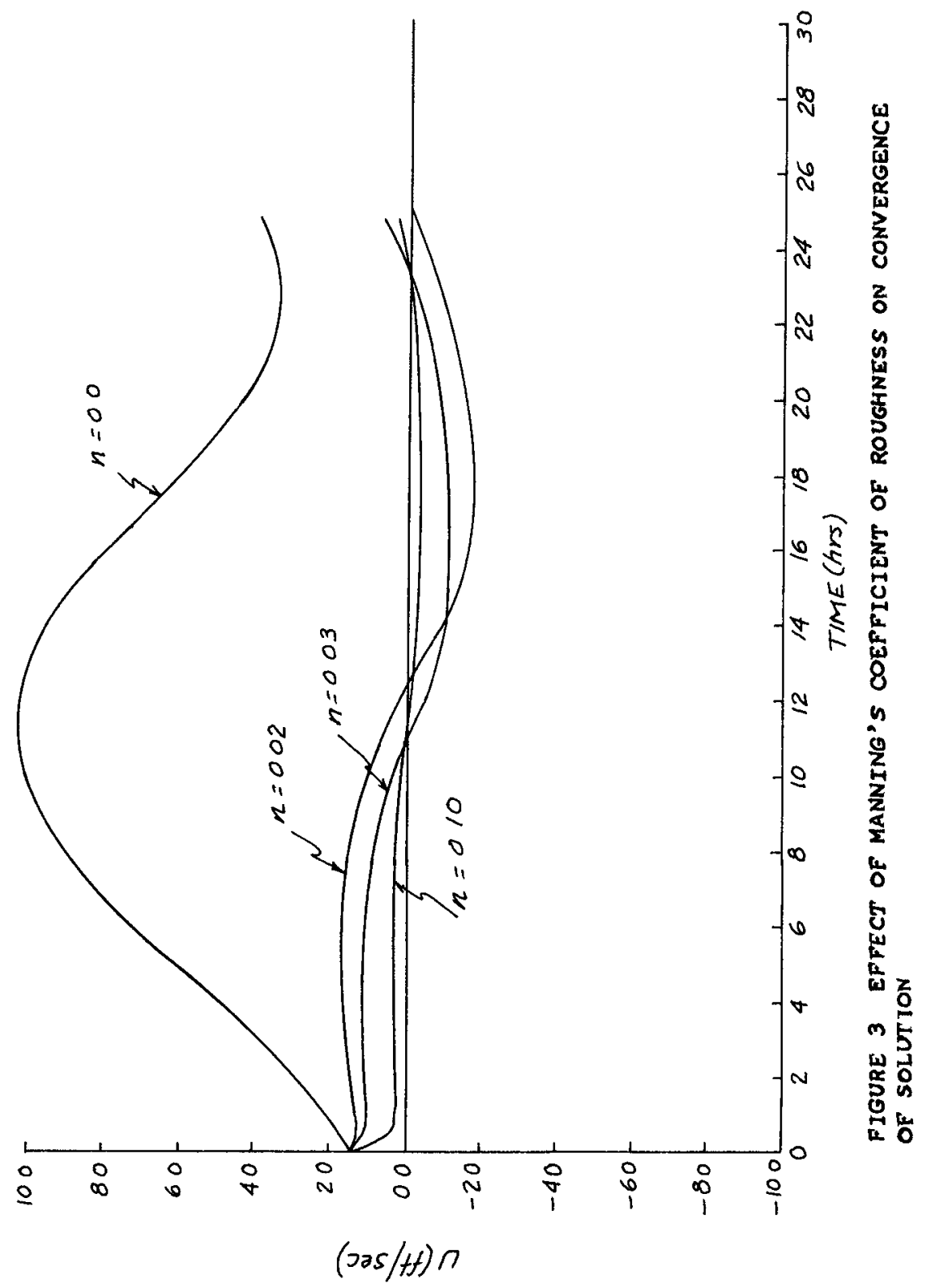




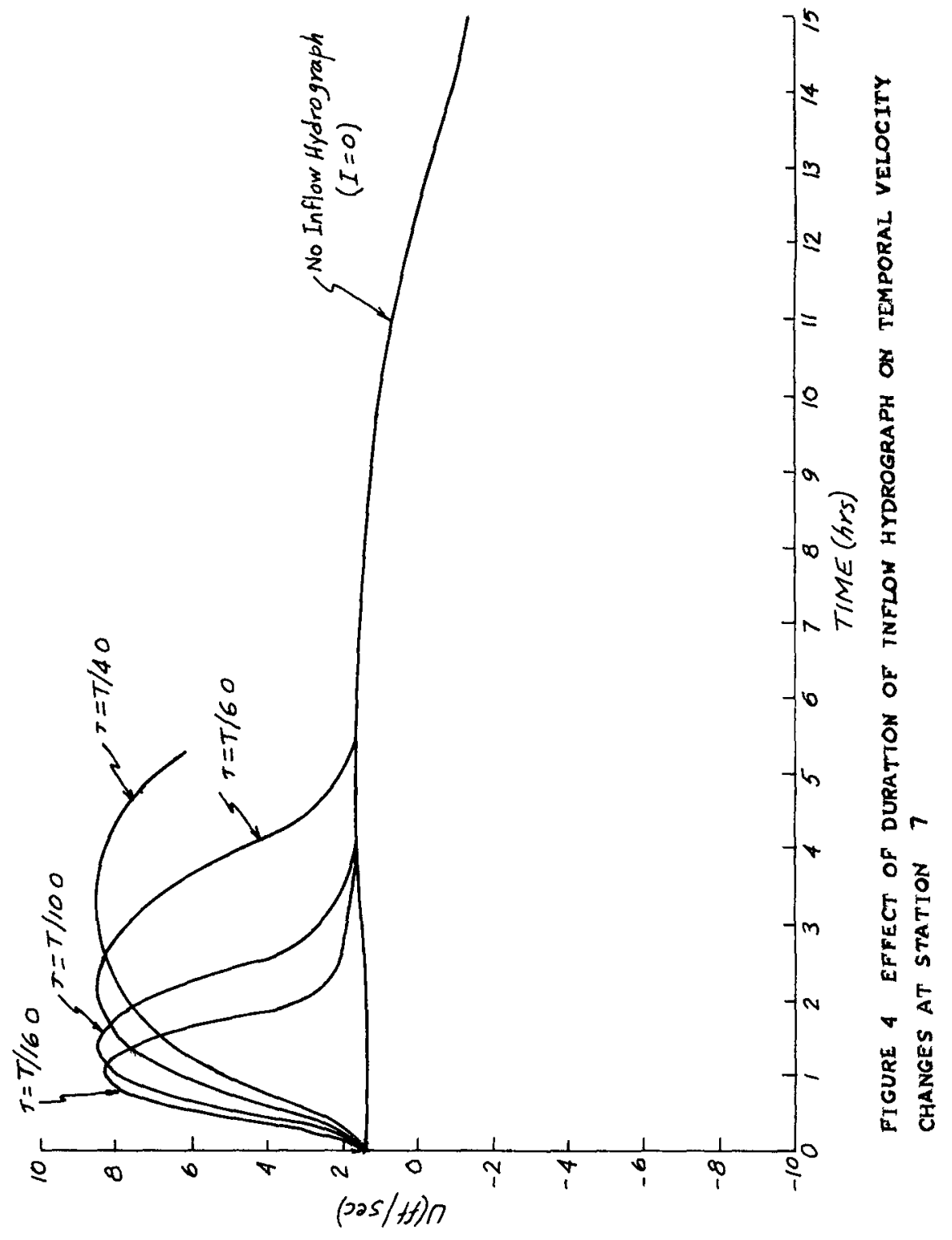




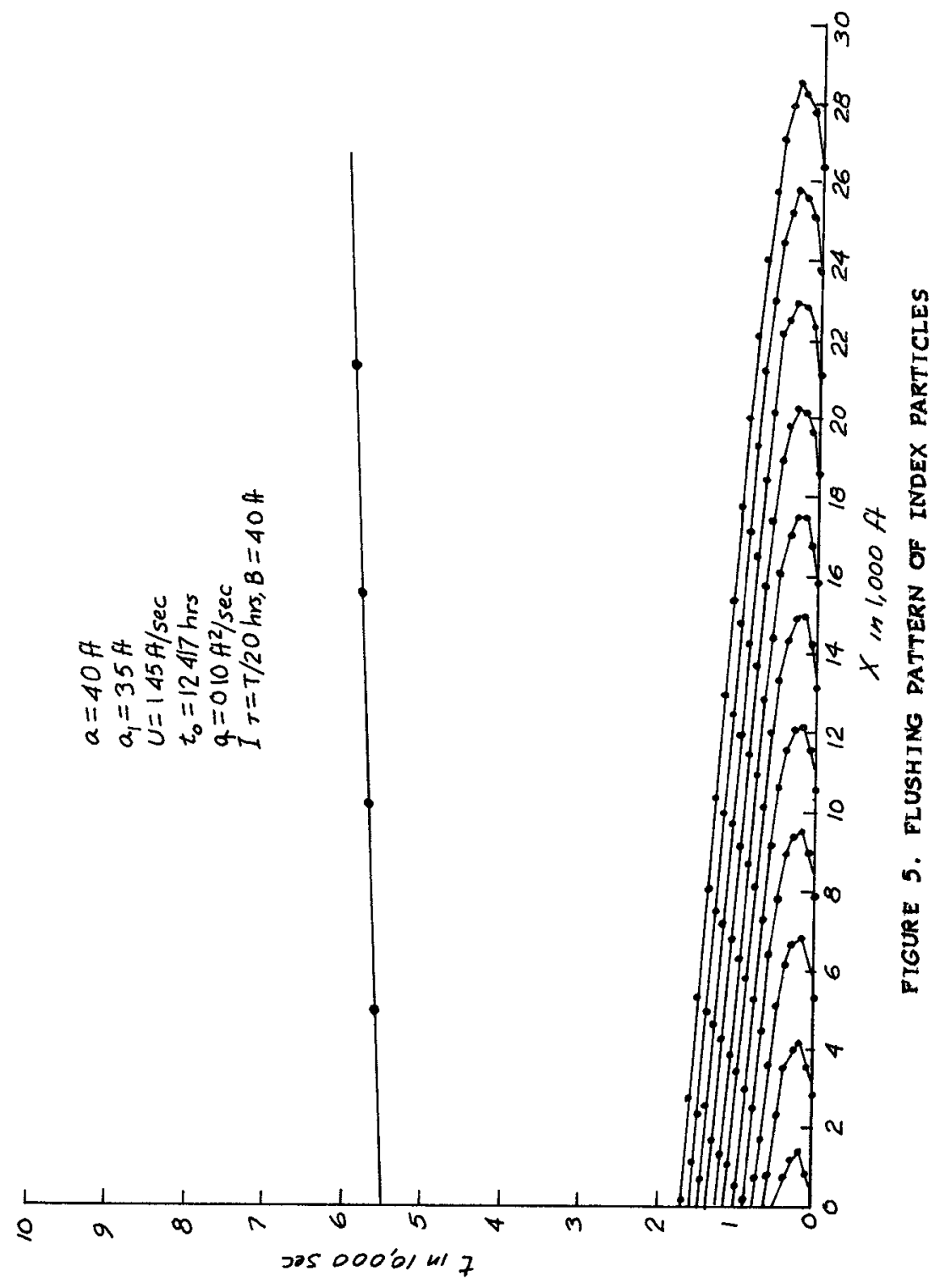


$+5$

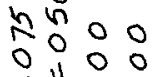

"1 0 "

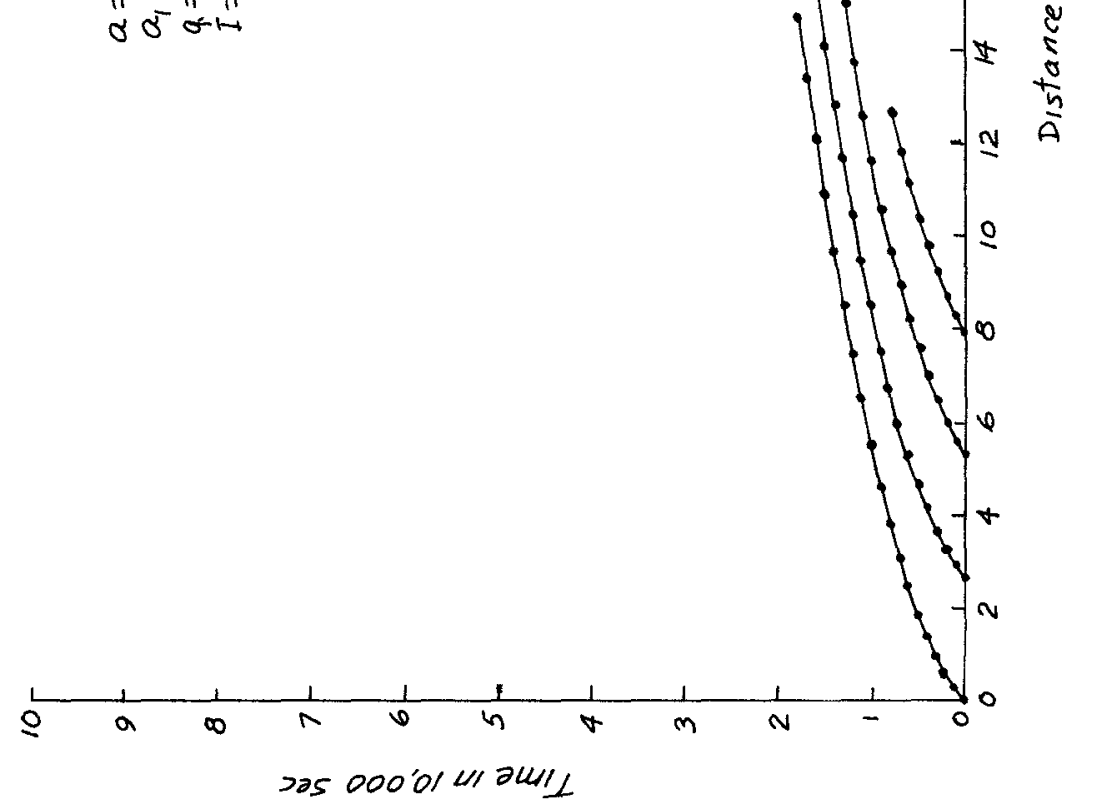




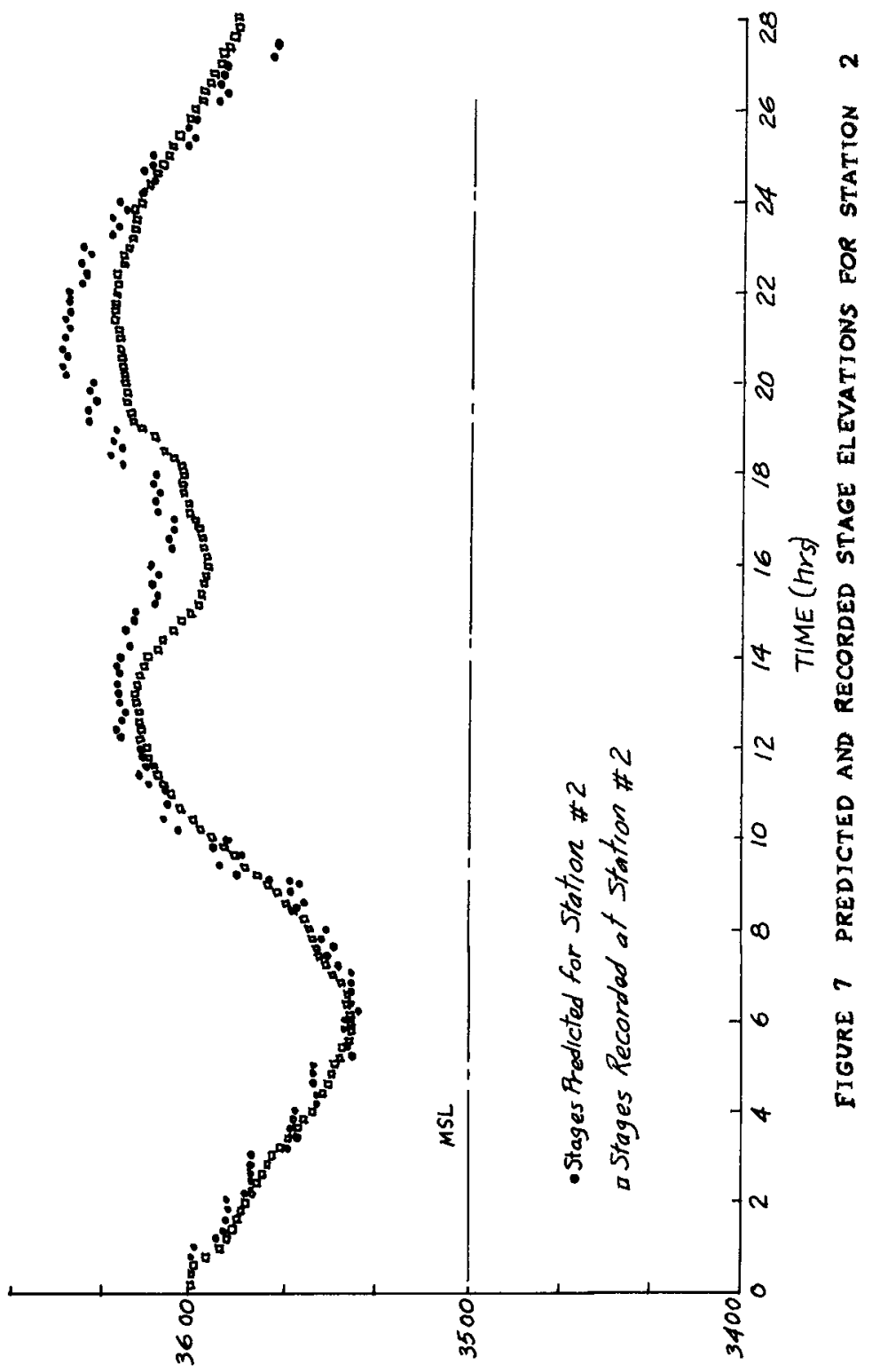

(75w't) 3 


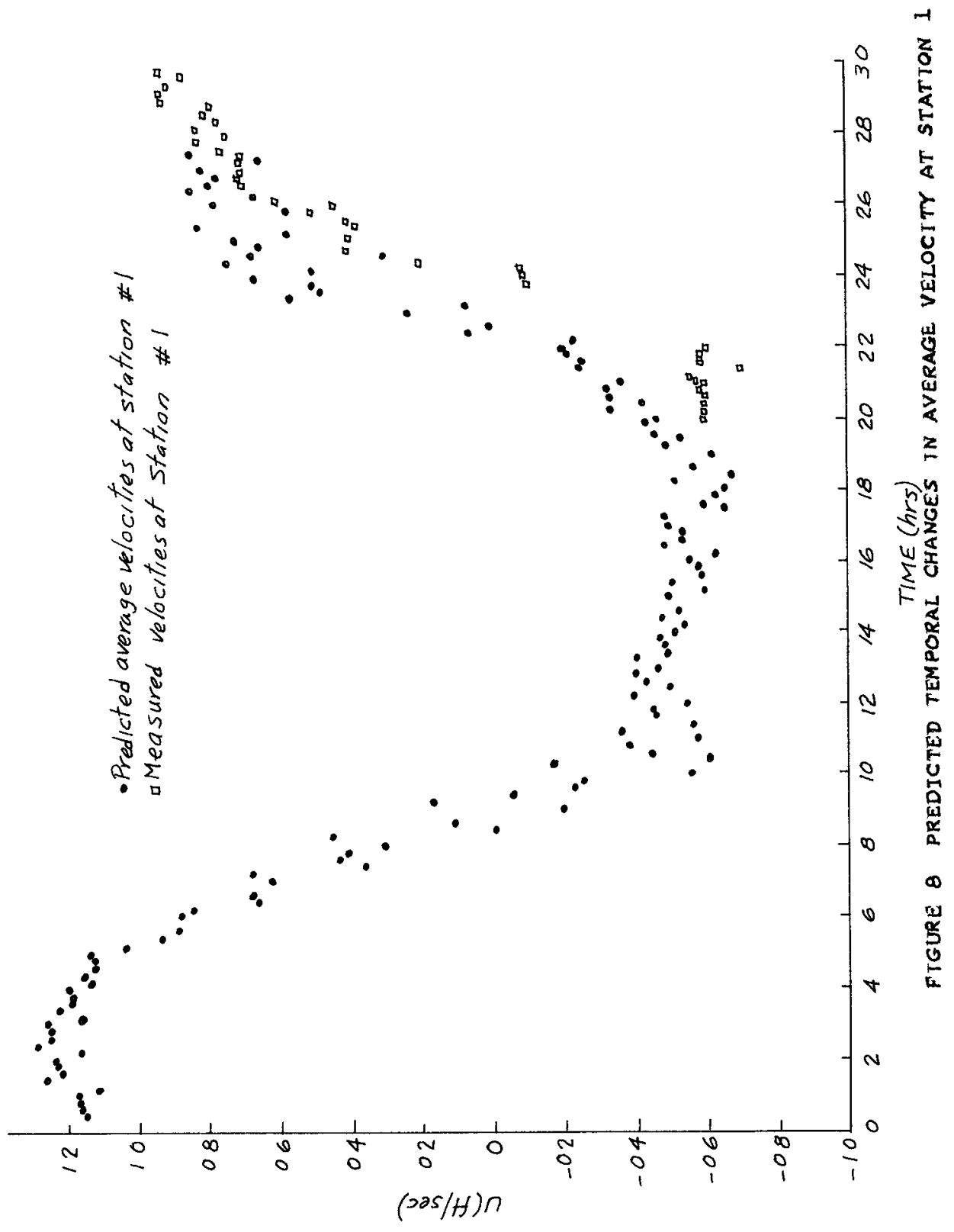





\title{
CHAPTER 114
}

\section{THE MEASUREMENT OF TIDAI WATER TRANSPORT IN CHANNELS}

\author{
R.E. KleIn*
}

\begin{abstract}
Summary
For the electromagnetic flow measurement in straits, channels etc use is made of the electric potential difference between two points in the bottom, near the moving water. The potential difference can be put down to natural causes the water is an electric conductor and moves in the magnetic field of the earth There are a number of quantities and variables that are of importance for the measured signal Beside this, the measurement is above all a matter of eliminating some disturbances Information about the two measuring systems as applied in The Netherlands is given in this article
\end{abstract}

\section{Introduction}

The principle of the electromagnetic flow measurement in rivers, straits etc. has been known now for about 140 years ever since Faraday concelved the Idea in 1832. But it is only in the last 30 years that it has more or less successfully been used at several places in the world $[2,3,4,5,6]$ In The Netherlands it has now been adapted to far smaller flows (the measurements in Washington excepted [4])

In 1964 Schumm started the investigations $[7,8]$

Several difficulties had to be overcome, some of them may be of importance to those who want to apply the method themselves At present there are two measuring systems in use This research work has been commissioned by the RiJkswaterstaat

* Technisch Physische Dienst TNO-TH (Institute of Applied Physics) Delft, The Netherlands. 
The physical background

The method makes use of a natural phenomenon Throughout the world, water moves in the magnetic field of the earth Because water is an electric conductor, an electric potential fleld occurs in the water and, as a result, also in the surrounding ground, fig 1 The potentlal difference measured between the electrodes in $P$ and $Q$ or $P$ and $R$ is a function of all velocities in the channel, and depends also on other quantities and variables such as "the" conductivity of the water and the bottom, the shape and size of the channel, relative changes of the depth by changes of the waterlevel, the vertical component of the magnetic fleld B etc The theoretical basis was lald by M.S. Ionguett-Higgens in 1949 [1] and in 1964 extended by Schumm [7], but the problem is very complicated, so that our knowledge remains relatıvely limited The proposition is now, that this potential difference depends only little on the velocity distribution (except in very shallow, wide stralts), so that in many cases it is allowed to callbrate agalnst mean veloclty, discharge etc

The influence of the vertical component of the magnetic induction $B$ on the calibration is obvious Fortunately in most cases it is constant enough when taken over a few years to neglect its variations. The magnetic disturbances are very small relatively and they are only an addltional effect that can be compensated The effect of the other quantities and variables is very complicated Therefore the systems are callbrated with a number of simultaneous velocity measurements, for instance once a year The measured potentlal difference is highly dependent on the conductive proporties of the bottom The higher It is, the lower the potential difference measured wlll be and the higher the influence of changes of the conductivity of the water and the waterlevel (tIdal range) In The Netherlands the conductivity of the bottom is very high up to a depth of $1 \mathrm{~km}$ it $1 \mathrm{~s}$ about one tenth of that of seawater It causes a loss of 80 percent and It is necessary to take into account the influence of the temperature on the conductivity of the water (knowledge within $3^{\circ} \mathrm{C}$ is adequate). 
In the two cases with whlch we are concerned lt was found that the influence of the waterlevel is such that the system does not glve the mean velocity but the discharge It may be expected that the accuracy wlll range from 5 to $10 \%$

\section{The problem of the disturbances}

The elimination of all kinds of disturbances is an important problem, especially in the Netherlands where the level of the signals is very low.

Four kinds are mentioned here

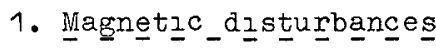

Along the surface of the earth potentlal gradients are generated by the fluctuating magnetic field (especially the horizontal component) of the earth As already stated they give additional sipnals They are eliminated by compensation by means of two signals, measured far away from any flow, in two directions perpendicular to each other, between electrodes in the ground in the same way as the measurement of the flow slgnal fig. 2 Thus

$$
v_{\text {flow }}=V_{P Q}-\alpha v_{A B}-\beta v_{B C}
$$

Is formed,

where $\alpha$ and $\beta$ are sultable constants

In our cases, where the distance between $P$ and $B$ is for example $15 \mathrm{~km}$, the elimination $\mathrm{is}$ very satisfactory

2 Earth currents_caused by_the_electric DC_rallway_trafflcc They may cause heavy disturbances, depending on the distance to the rallway system, the intensity of the traffic, the conductivity of the top layers of the bottom etc 
In most cases the only thing to do is to avold them, for Instance by choosing great distances $(10,20 \mathrm{~km})$ to the disturbing rallway In one case the influence could be reduced by comnensation with a signal measured between two points in the nelghbourhood of the rallway and also by choosing an adequate direction of the line between the measuring points

3 lㅣ ectro-chemical_dısturbances in_the_bottom and In_the electrodes

There are always falrly constant potentlal differences between two points in the earth They are caused by local and extended differences in the composition of the ground water and the bottom The variations in the compositions reault in a drift of the zero level It can be a long-term drift, but drift with the tidal frequency is also possible for example if the electrode 19 placed in the nelghbourhood of the boundary between the fresh and the salt water in the shore This boundary may move under the influence of the tidal changes of the water oressure, glving a slight mixing each time

The electrodes are now placed in 5 to $10 \mathrm{~m}$ deep plpes, as far as posaible from moving or fluctuating ground water This also has the advantage of a sufficiently constant temperature

We developed special purpose Ag-AgCl-electrodes They are constant within $\pm 0,5 \mathrm{mV}$ for a period of one year ( In the laboratory) We found that many kinds of electrodes (and also ours) are extremely sensitive to electric currents, giving a long-term drift Therefore lt was necessary to place a voltage follower between the electrode and the long Ine that connects the measuring point with the mV-meter In one place we found a drift of the zero level as low as $0,5 \mathrm{mV}$ in a week, but at other places it is greater 
4. No1se

The nolse that Is plcked up by the wires used in the telephone cables to connect the measuring points with the apparatus malnly consists of pulses and hum

Sometimes the peak value is 1000 times the slgnal to be measured

It is eliminated by normal filtering after the compenation of the magnetıc and other disturbances has taken place, this to avold differences between the compensating and the compensated signals

\section{The measuring systems in The Netherlands}

At present there are two meacuring systems in use in the north of The Netherlands, fig. 3, for measuring the tidal flow in the Borndlep and the Marsdiep A third, in the Eyerlandse Gat, is belng prepared

\section{The Borndlep system}

The Borndlep system, fig 4, has three measurements for the flow (between WD and WM, $O D$ and WM and ZD and ZM) The measurement for the magnetic compensation 1 s between $W M$ and $O M$ and $W M$ and $Z M$ There is a level gauge for additional data on the "Vrijheldsplaat" An example of the measurement between $\mathrm{ZD}$ and $\mathrm{ZM}$, the two magnetic signals and the result Is glven in fig 5 The amplitude of the flow signal is $3 \mathrm{mV}$, It is the lowest signal we can measure (low electro-chemical disturbances)

Fig 6 gives an example of the flow according to "ZD - ZM" and the water level measured on the "Vrijheldsplaat" In the madie the effect of a storm in the far distance the region between the Atlantıc Ocean and the North Sea 
The Marsdiep oystem, fig 7

The potential difference according to the flow has been measured between ZD and ND, between the electrodes In ZM, NM and oN the two magnetic signals are measured

The electric DC rallway trafflc causes heavy disturbances Fortunately lt is an uncomplicated case In this case compensation is possible We use the (large) signal measured between $Z D$ and $T$ Due to the extensive clay +ayers deep in the bottom pronounced induced polarisation influence occurs It causes differences in the shape of the disturbances meacured between the several points and these differences frow with the distance to the rallway The ZD-T-signal must therefore be filtered so that it gets the rught forms before it is used for compensation

Fig $8 \mathrm{ls}$ an example the disturbed flow slgnal, the three compensation signals and finally the result

The compensation of the rallway disturbances is ratisfactory, but not of the sare quality as that of the masnetic disturbances If necessary, It Is possuble to make improvements by digltal filtering or the extension of the compensation system.

The only calibration avallable at the moment is that of a former experimental system at the Marsdiep, fis 9

It did not work with $Z D$ and ND but it did at two other points, one of them with more than normal electro-chemical disturbances, as will be seen on the right

We are now bullding a third system for measuring the flow in the Eyerlandse Gat It measures the flow slgnal between $Z D^{\prime}$ and $N$ and uses the magnetic compensation signals of the Marcdiep system A fourth svstem may be bullt next year in the stralts east of Ameland

\section{Other applications}

The method ia also sultable to obtain information on the flow along a coast The most simple set-up makes use of one electrode near the coaat and one far inland 
The slgnal glves a welghted average of all velocities, the welghting factor depending on the distances to the two electrodes Unfortunately we know only little about these factors theoretical research 15 necessary

The flow slgnals of rivers are usually too small to be measured by applyzng the present method, due to the relatively small discharges

We are now working on a modified method for falrly big rivers This method makes use of an artiflcial alternating magnetic fleld in order to separate the signal from the nolse

\section{References}

$1 \mathrm{MS}$ Longuett-Hıggins The electrical and magnetic effects of tIdal streams

Monthly Not Roy Astr Soc, Geoph Suppl 1949, 2, 8, 285-307

2 G I Bloom Water transport and temperature measurement in the eastern Berıng Stralt, 1953-1958

J Geoph Res, Aug 1964, 69, 16, 3335-3354

3. K F. Bowden The flow of water through the Straits of Dover related to wind and differences in sea level

Phll Trans. Roy Soc. of London, serles A, no 953. Feb 1956, 248, 517-551.

4 R M Morse, M Rattray Jr, R G Paguette, C A Barnes The measurement of transports and currents in small tidal streams by an electromagnetic mothod Univ of Vashunoton, Dep of Oceanography, Techn Rep 57, 1958

5 B $H$ Olsson The electrical effects of tidal streams in Cook Strazt, New Zeal and Deep-Sea Res 1955, 2, 204-212

5 G K Werthelm Studies of the electric potential between Key West, Florlda and Havana, Cuba Woods Hole Oceanogr Inst Ref 53-95, 1954. 
7 C Schumm Elektromagnetische debletmetıngen in het Marsdiep (Electromagnetic flow measurements in the Narsdiep) Delft Techn. Unıv, Lab for Techn Phys, 1964.

$8 \mathrm{C}$ Schumm. Potentiaalmetingen in het Marsdiep. TNO-nIeuws 1966, 21, 110-121. 

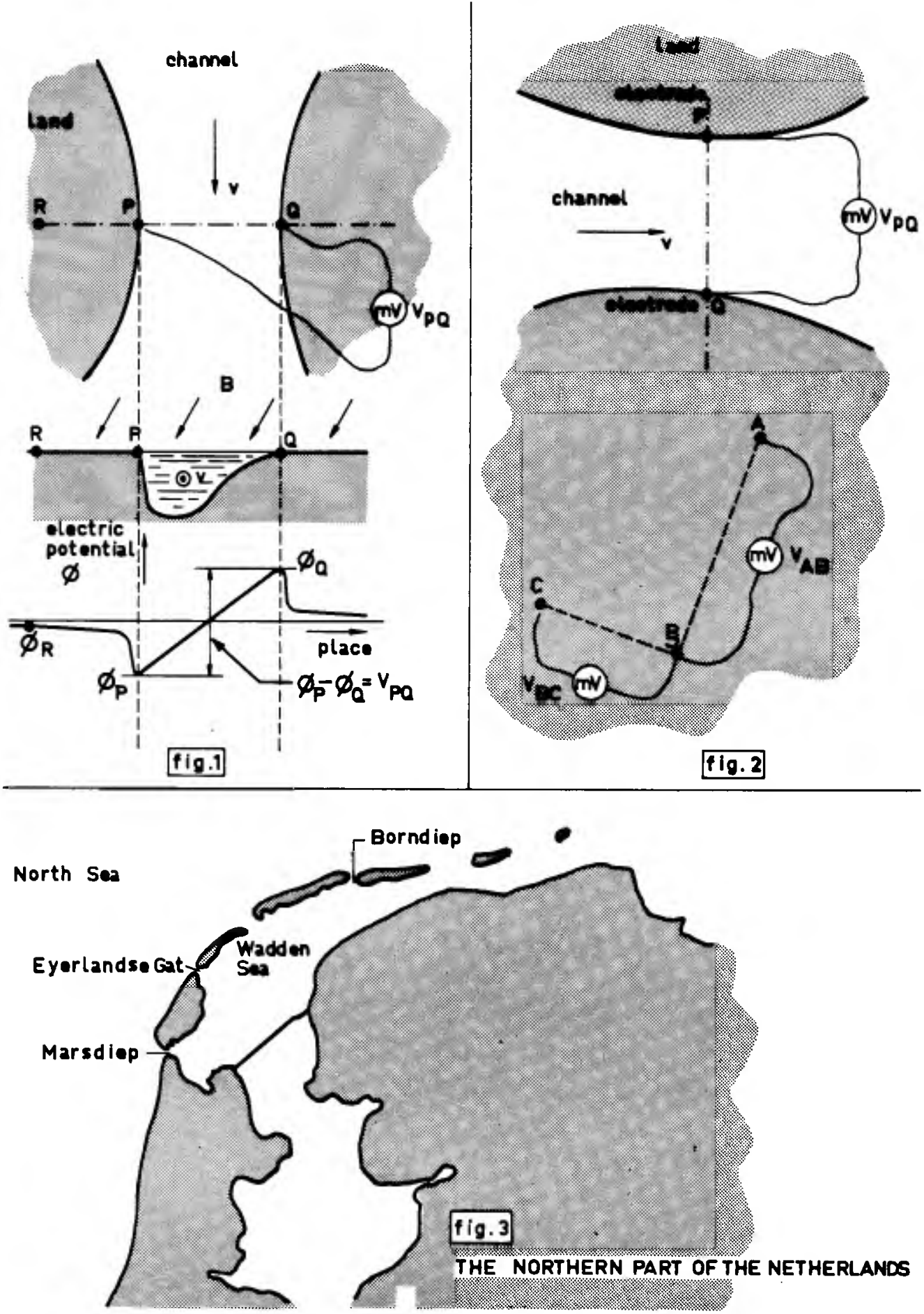


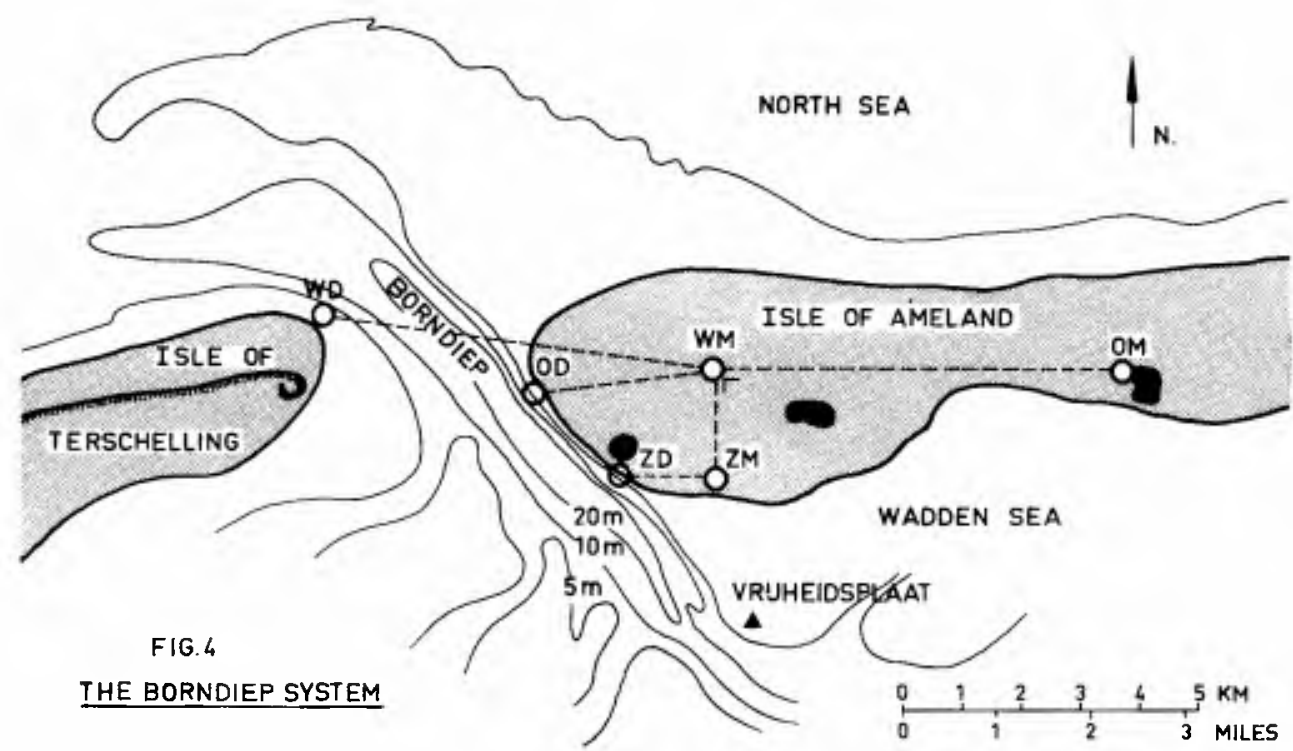




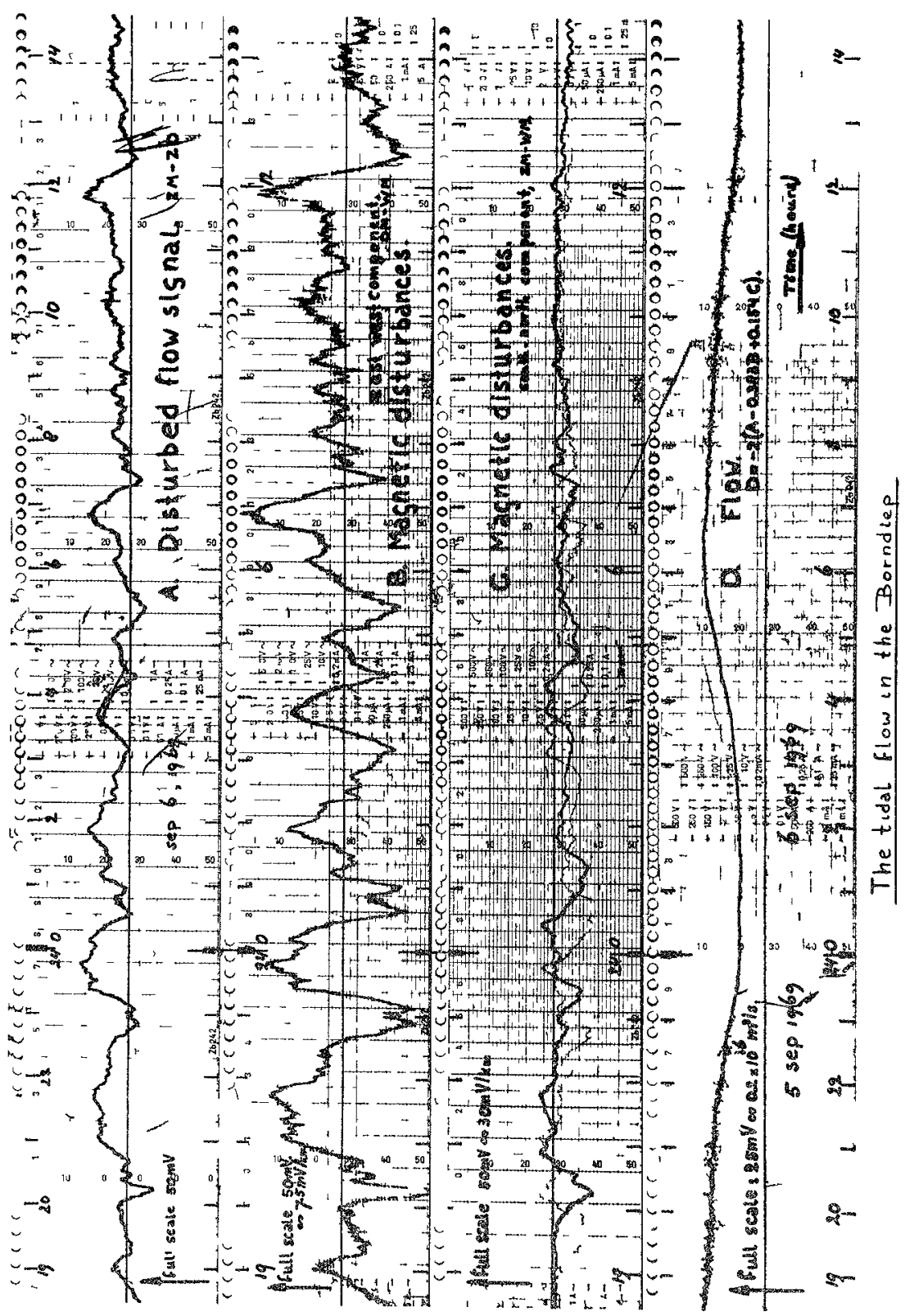




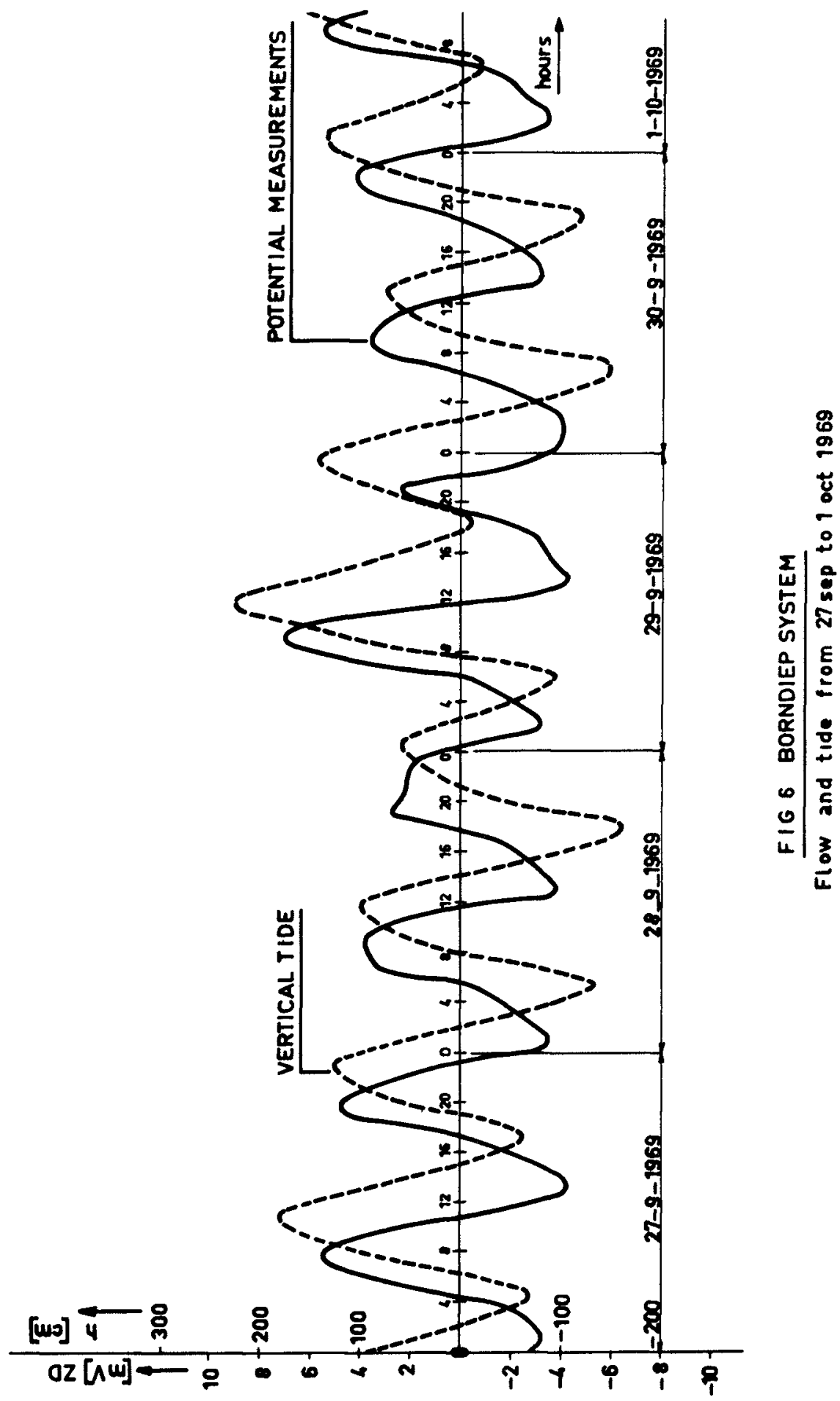




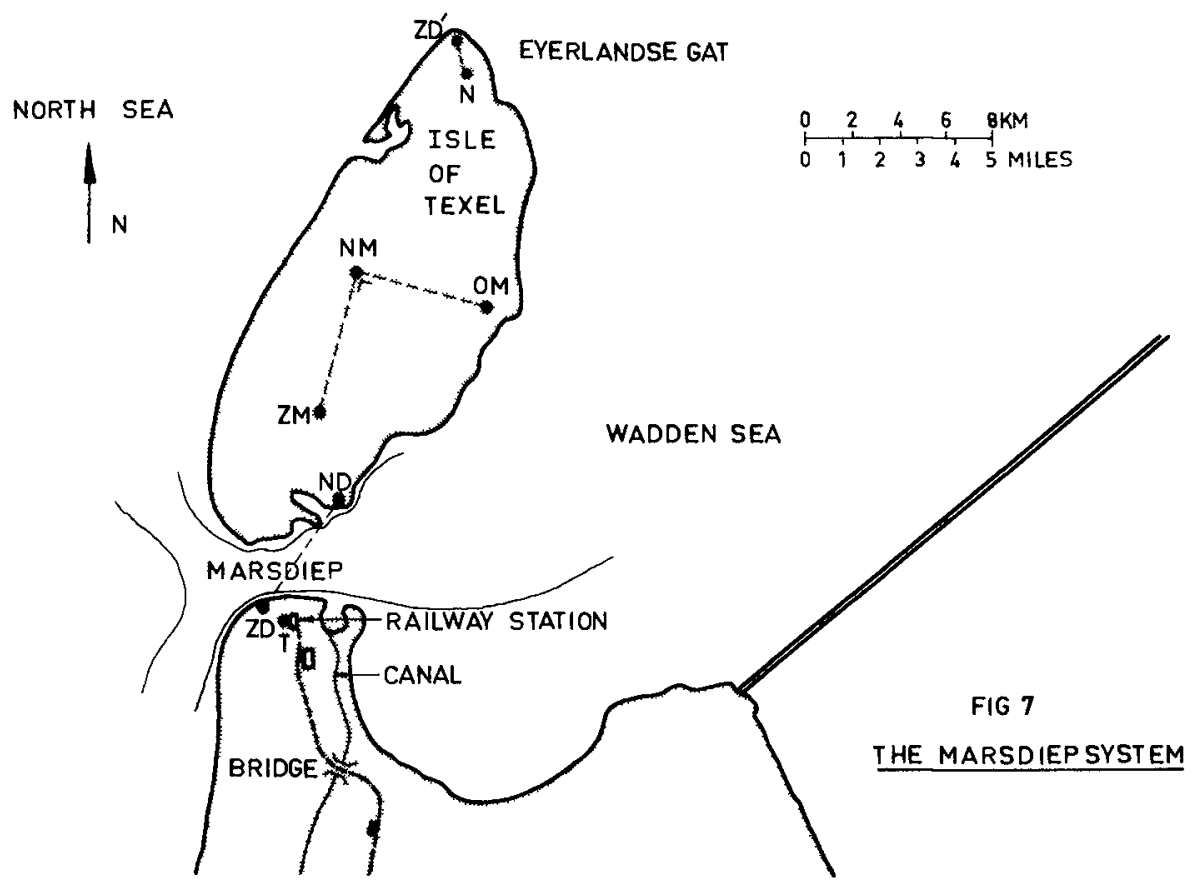




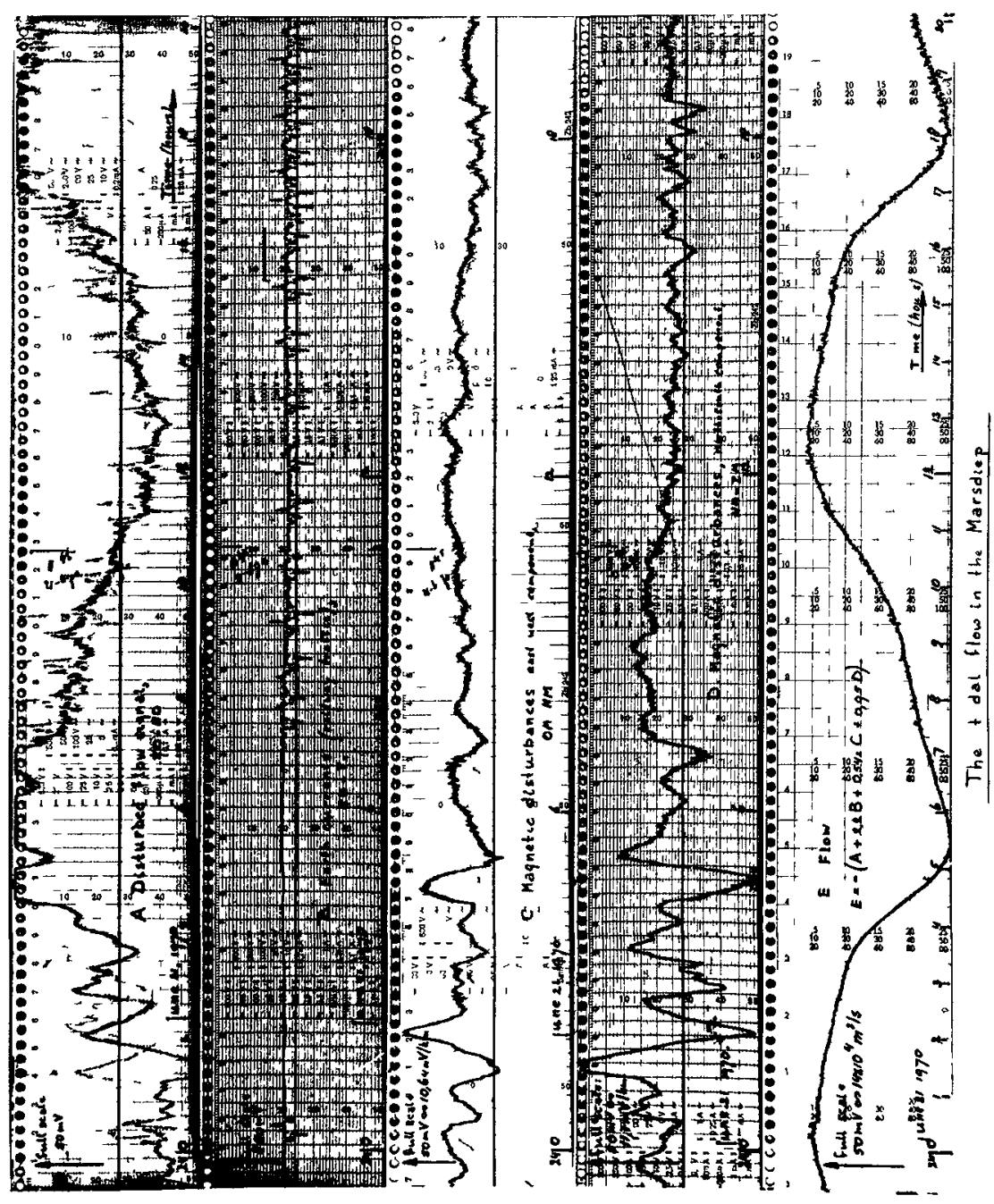

FIG 8 


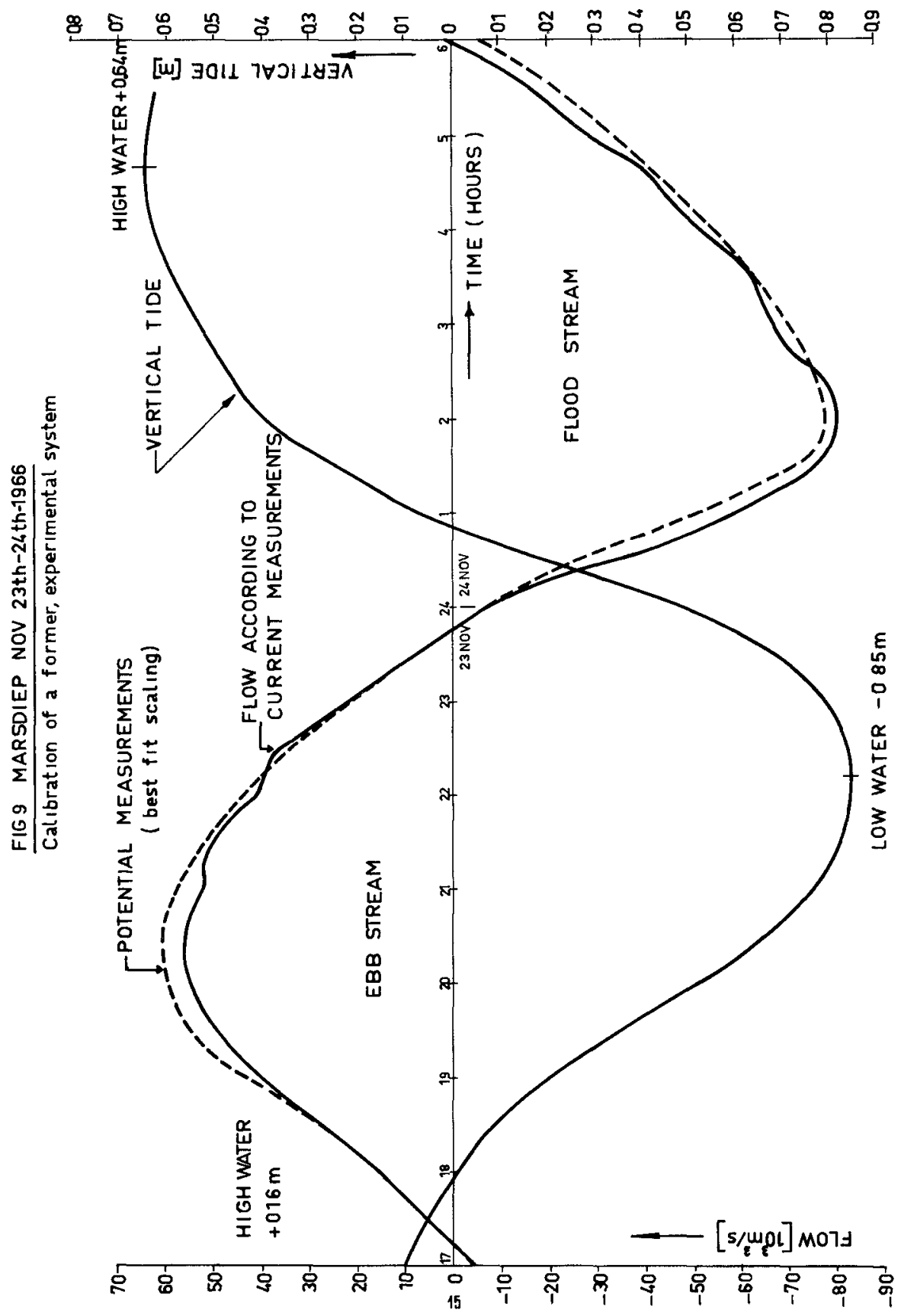





\section{CHAPTER 115}

WATER CURRENT METER FOR MEAN FLOW MEASUREMENTS ${ }^{\perp}$

DAVID SMITH

Instrument Research Division

NASA, Langley Research Center, Hampton, Va 23365

W HARRISON

Division of Physical, Chemrcal, and Geological Oceanography Virginia Institute of Marine Science, Gloucester Point, Va 23062

\section{ABSTRACT}

A drag-sphere water current meter with a 0 to $6 \mathrm{ft} / \mathrm{sec}$ range has been developed for velocity measurements of relatively steady flows A two-component strain-gage type force transducer is mounted within a 3 7-inch-diameter perforated drag sphere Drag force measurements are related to the flow velocity around the meter Several drag sphere configurations were tested in selecting the 336-hole pattern

Frequency response data have been recorded and evaluation tests were made with current meters deployed in an estuary Use of these current meters in studies of steady-state fluid processes and sediment responses is feasible They are simple, relatively inexpensive, and well suited to applications where several simultaneous measurements are needed to determine velocity profiles or map flow distributions 


\section{INT RODUCTION}

This water current meter was developed to satisfy the need for a relatively simple, Inexpensive device to measure generally steady flows characterize certain nearshore waters" The design is compatible with automatic data acquisition and reduction equipment in order that several meters can be deployed and monitored simultaneously This capability makes it feasible to determine and map the velocit distribution and/or profiles in the nearshore areas under study The meter (fig 1) employs strain-gage techniques to measure the force exerted on a submerged drag sphere over a flow velocity range of 0 to $6 \mathrm{ft} / \mathrm{sec}$ This drag force is then related to the flow velocity around the meter

Inman and Nasu [1] were perhaps the first to develop a drag force meter for measuring fluid flow in the nearshore environment Their device consisted of "a small flexible berylitum-copper rod with a sphere mounted on one end and the other mounted rigidly to support "Other efforts toward developing this type current meter are noted $[2,3,4]$ in which the drag sphere or cylinders used were found to be subject to both an acceleration-dependent force and a fluid drag force Consequently, this type of current meter is suitable for use only if fluid acce1erations such as those in oscillatory flow are of low magnitude relative to the mean flow velocity

\section{DESCRIPTION OF THE CURRENT METER}

The current meter ( $f 1 g$ I) consists of a two-component strain-gage force balance designed to fit within a 3 7-1nch-diameter polyethylene drag sphere, similar to the anemometer developed by Reed and Lynch [5] The force balance is constructed of Armco 17-4 PH Steel, with a 1/2-inch-diameter mounting "sting" extending approximately 145 inches from the sphere A machined flat is located on the "sting" for alignment purposes

Each of two perpendicular force components is sensed by 4 active foll-type strain gages The meter is designed to be insensitive to moments or couples and detects only the forces exerted on the drag sphere The output signal is approximately $24 \mathrm{mv}$ per volt for a one pound force The 0 to $\pm 6 \mathrm{ft} / \mathrm{sec}$ velocity $\mathrm{range}$ of interest corresponds to an electrical signal of about 0 to $\pm 18 \mathrm{mv}$ for each component Support equipment for each meter consists of a regulated power supply for the 40 to $60 \mathrm{v}$ input to each straln-gage bridge, and a recorder with two channels Strain-gage bridges used in this application are compensated for temperature variations over a suitable $100^{\circ} \mathrm{F}$ range A $3 \mathrm{M}$ product designated EC 870 is used to waterproof the strain gages and wiring on these devices 


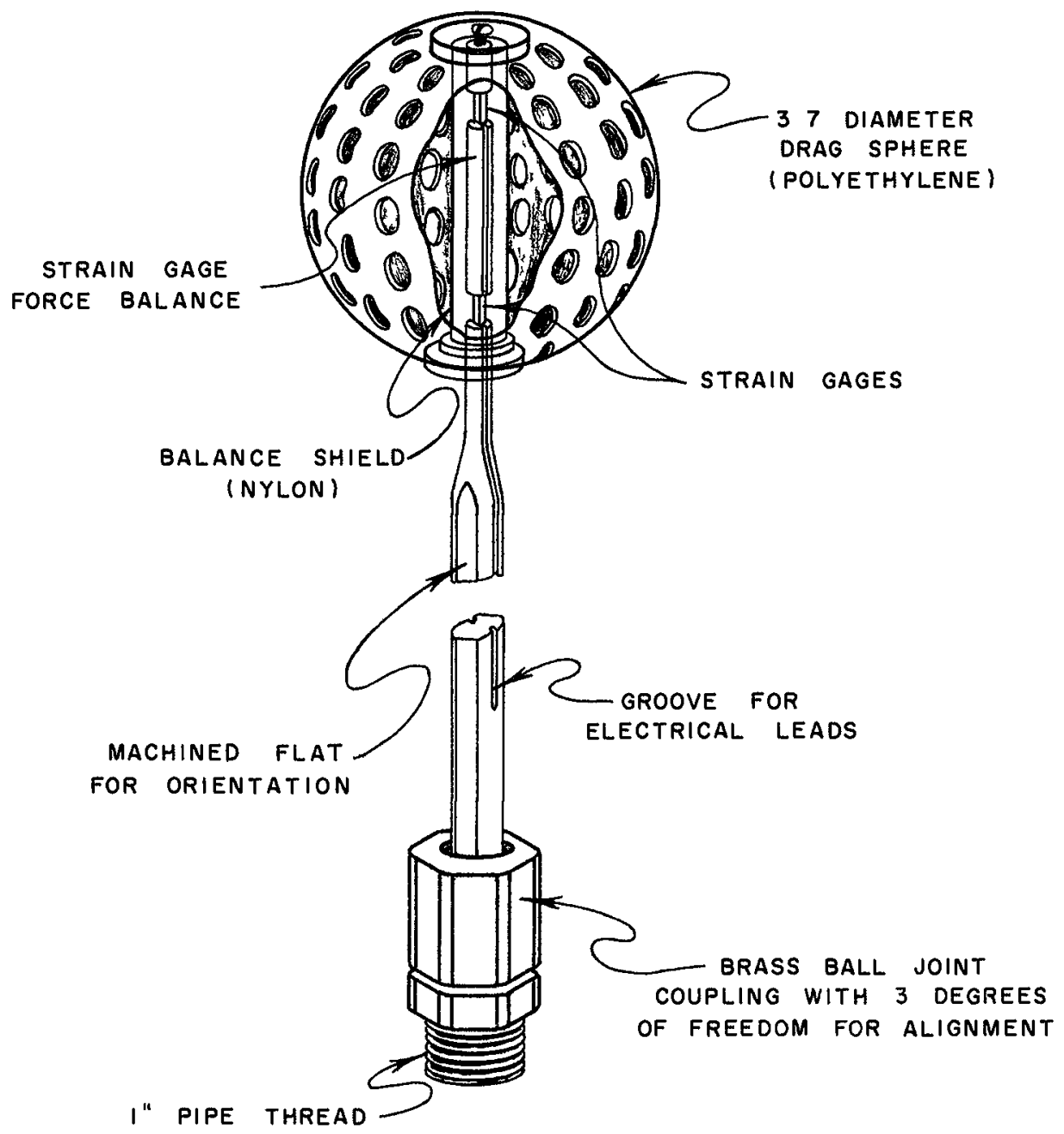

FIG I SCHEMATIC OF DRAG-SPHERE CURRENT METER 


\section{SELECTION OF THE DRAG SPHERE}

Inıtial tow tank experiments with drag spheres were made in the Virginia Polytechnical Institute's towing basin at Blacksburg, Virginia Smooth spheres were found to be very unstable in the flow velocity range of interest (o to 6 $\mathrm{ft} / \mathrm{sec}$ ) The smooth spheres osclllated very erratically in the lateral direction of perpendicular to the flow past the sphere, as observed in other studies [6, 7] Several methods of stabilızıng sphere response were investigated, including various hole patterns, dimples, and bumps on the surface of the sphere, as shown in figure 2

Several significant observations were made in the tow-tank tests Figure 3 shows the variation of force with velocity for three spheres the dimpled sphere, the smooth sphere, and the 336-hole-pattern sphere All of the data points 1ie on or very near the theoretical lines for the appropriate drag coefficients $C_{D}$, showlng that the force exerted on the sphere is a function of the velocity squared Differences in the drag coefficients of the three sphere configurations are quite significant Roughening sphere surfaces by dimples or bumps tends to reduce drag, as also noted by early golfers Smooth golf balls do not go as far and as straight as old balls that are battered or have a roughened surface on the other hand, placing holes in the surface of the sphere increases the drag coefficient in the 0 to $\pm 6 \mathrm{ft} / \mathrm{sec}$ velocity range Larger holes or more holes of the same size increased the drag coefficients

Based on these experimental results, the 3 7-inch-diameter sphere with the 336-hole pattern was selected for use on this water current meter The drag coeffi cient of this sphere configuration was determined experimentally to be 069 , with a standard deviation of 0017 over the Reynolds Number range considered $(35,000$ to 211,000 ) All the surface alterations discussed increased the stability of the spheres, but the 336-hole pattern had the most stabilizing effect, as shown in current meter outputs in figure 4 ("component perpendicular to flow")

\section{RELATION OF FORCE TO FLOW VELOCITY}

\section{Steady Flow}

In steady flow conditions, the drag force exerted on a submerged object is related to the flow velocity by the following equation

$$
\mathrm{F}=\mathrm{C}_{\mathrm{D}} \mathrm{QA}
$$




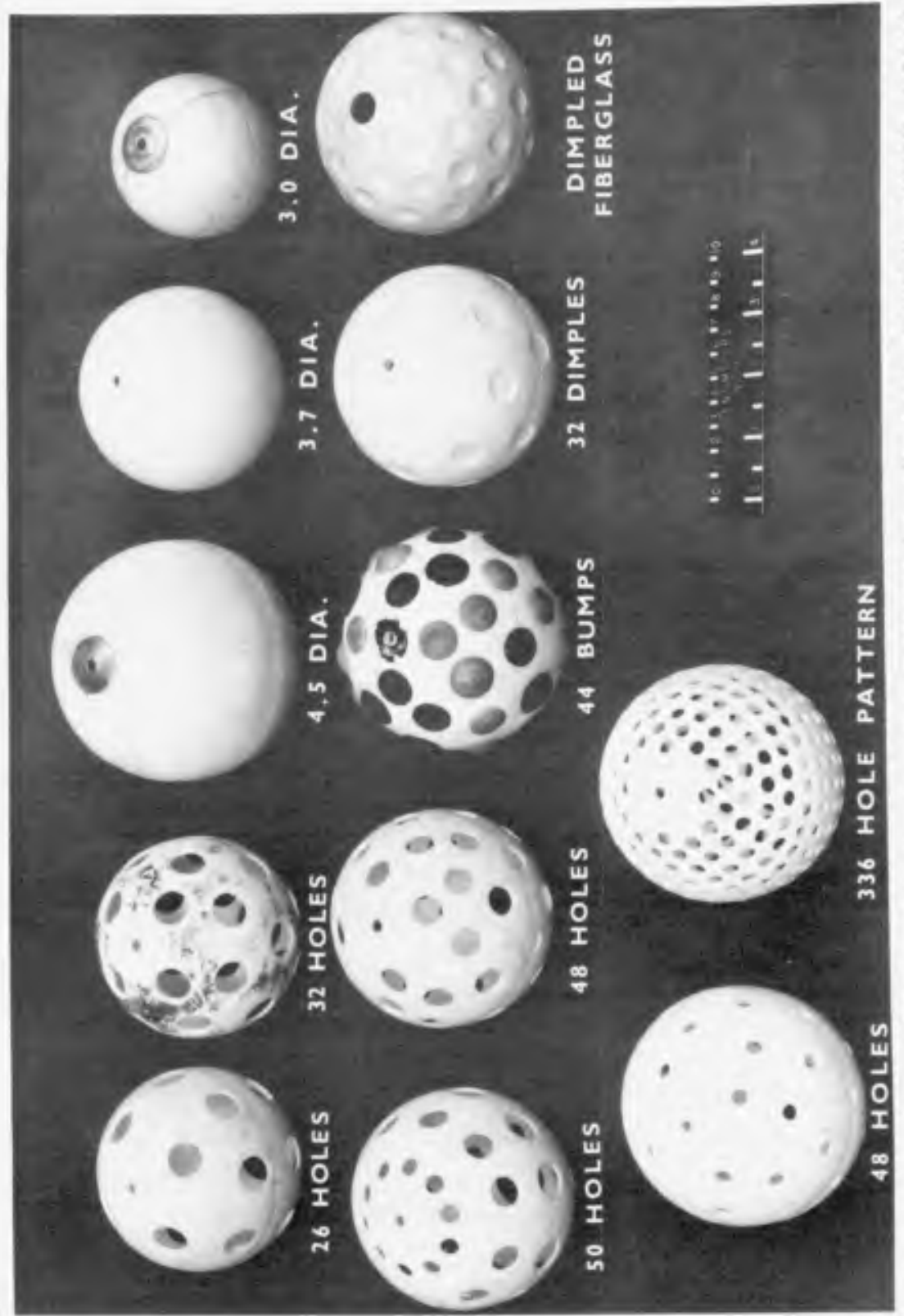

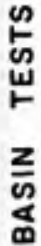

을

$\underline{z}$

岂

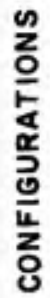

w

親

工

क

这

N

ธำ 


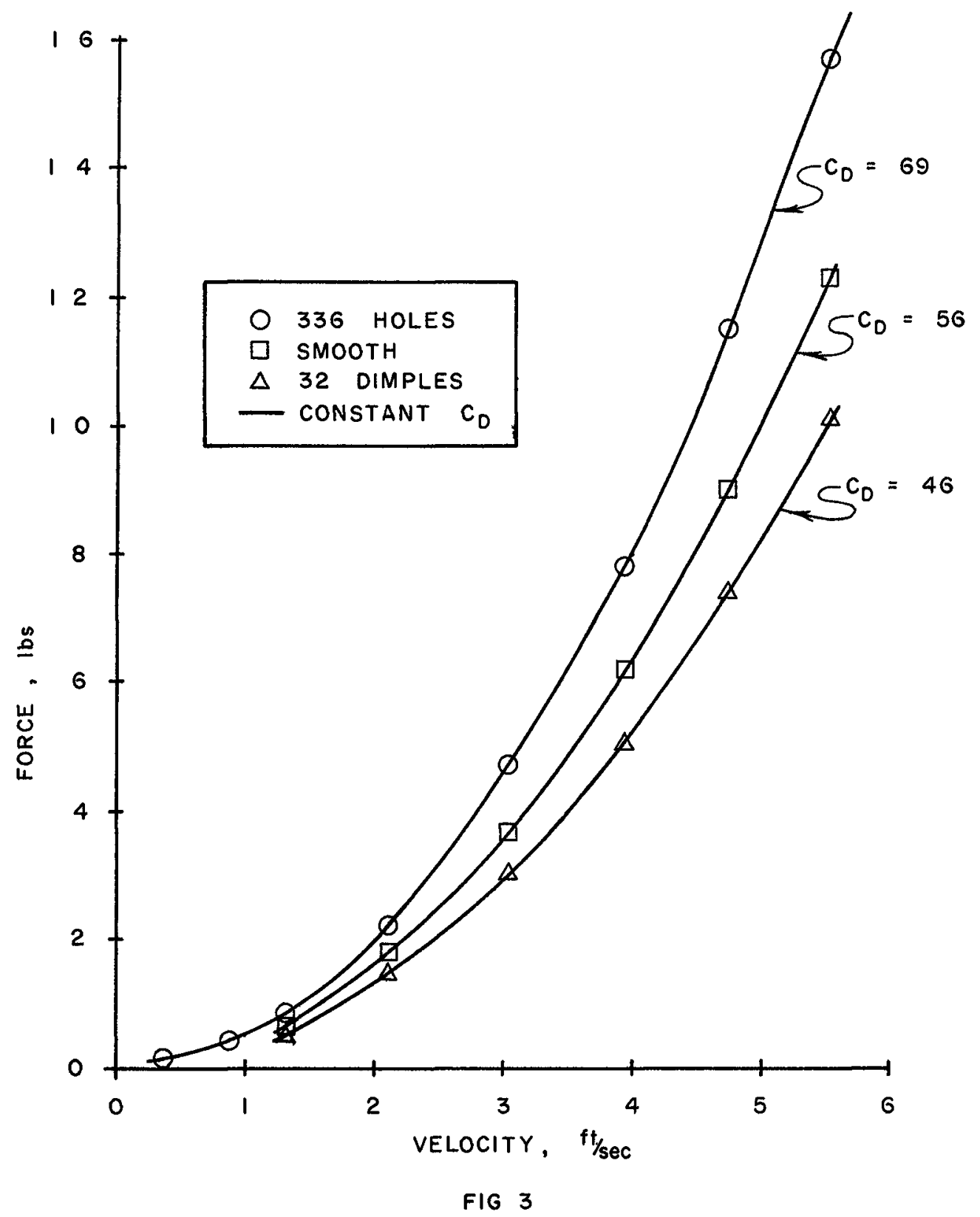

FORCE VS VELOCITY FOR THREE SPHERE-ROUGHNESS CONFIGURATIONS 


\section{SMOOTH BALL, $\quad V=56$ FT/SEC}

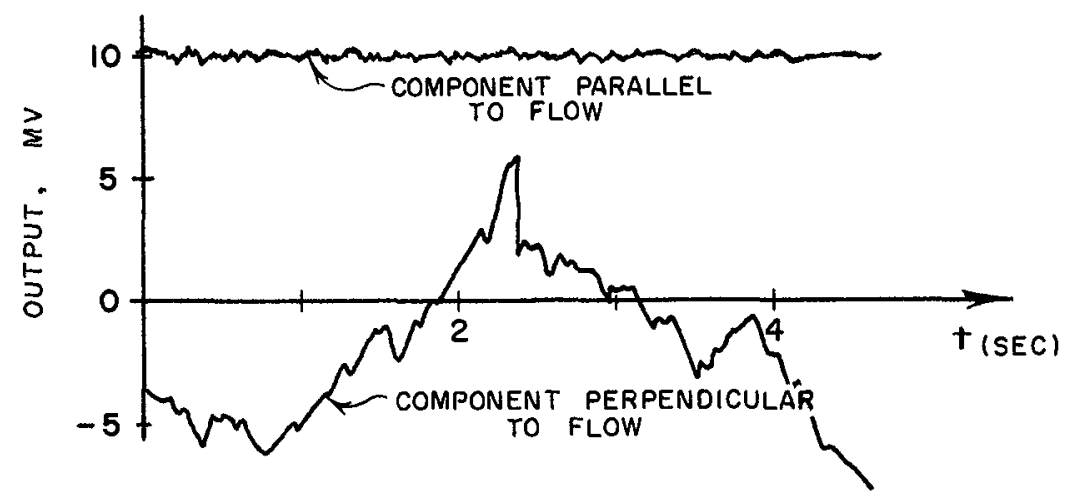

336-HOLE-PATTERN CONFIGURATION ,

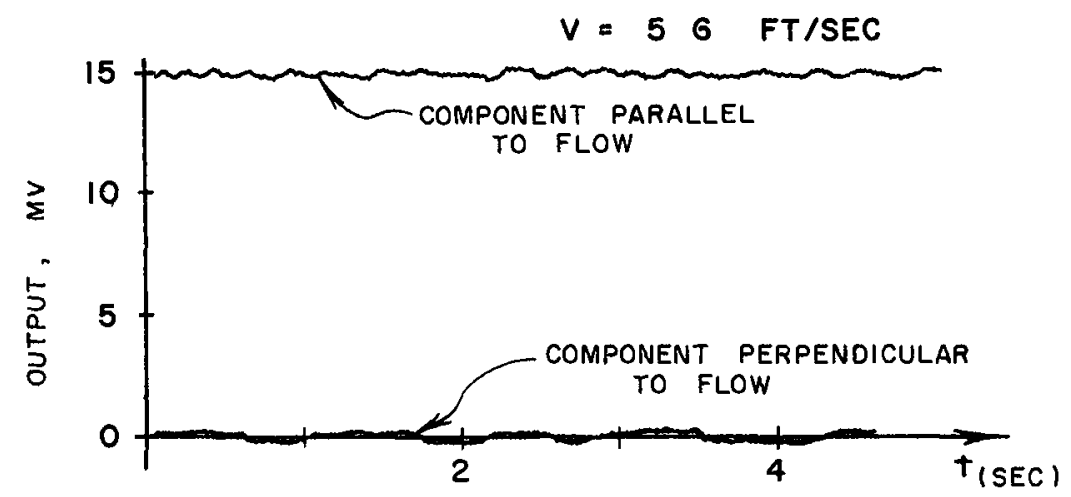

FIG 4 STABILIZING EFFECT OF 336 HOLE PATTERN COMPARED TO SMOOTH SPHERE'S OSCILLATION 
Substituting $Q=\frac{1}{2} \rho U^{2}$, and solving for $U$ yields

$$
\mathrm{U}=\left(\frac{2 \mathrm{~F}}{\rho \mathrm{AC}_{\mathrm{D}}}\right)^{1 / 2}
$$

where $U=$ flow velocity, ft/sec

$\mathrm{F}=\mathrm{drag}$ force, $\mathbf{1 b}$

$\mathrm{C}_{\mathrm{D}}=$ drag coefficient

$p=$ mass density, slugs $/ \mathrm{ft}^{3}$

$A=$ frontal area of drag sphere, $\mathrm{ft}^{2}$

The velocity vector has the same direction as the force vector

By having a well defined, constant $C_{D}$ In the velocity range of interest, and knowing the density of the water as well as the projected area of the drag sphere, measuring the drag force vector enables the determination of the flow vector A significant point is that the total force vector must be used in calculating the velocity, 1 e the individual force components using equation (2)

The velocity range of 0 to $60 \mathrm{ft} / \mathrm{sec}$ corresponds to $\mathrm{drag}$ forces on the order of 0 to $17 \mathrm{lb}$ for the 336-hole-pattern drag sphere used

\section{Accelerating flow}

In unsteady or accelerating flow conditions, the forces exerted on the drag sphere are a function of both the flow velocity and the acceleration of the water [3,8] The general expression for the force exerted on a submerged object in an accelerating flow is glven as

$$
\overrightarrow{\mathrm{F}}=\mathrm{C}_{\mathrm{m}}\left(\mathrm{U}, \frac{\mathrm{dU}}{\mathrm{dt}}\right) \rho \mathrm{V} \frac{\overrightarrow{\mathrm{dU}}}{\mathrm{dt}}+\frac{1}{2} \mathrm{C}_{\mathrm{D}} \rho \mathrm{A}|\mathrm{U}| \overrightarrow{\mathrm{U}}
$$

where $\mathrm{V}=\mathrm{drag}$ sphere volume, $\mathrm{ft}^{3}$, and $\mathrm{C}_{\mathrm{m}}=\mathrm{C}_{\mathrm{m}}\left(\mathrm{U}, \frac{\mathrm{dU}}{\mathrm{dt}}\right)=$ coefficient of mass

Since $C_{m}$ is a function of both the flow velocity and any fluld acceleration in the flow region near the drag sphere $[9,10]$, solving equation (3) for the velocity becomes very impractical, if not impossible Also, experimental results show that $C_{m}$ is the same order of magnitude as $C_{D}$ (3) Consequent1y, forcesensitive current meters must be used where the fluid acceleration is of very low magnitude and the $\frac{\mathrm{dU}}{\mathrm{dt}}$ term of equation (3) can be neglected 


\section{ERROR ANALYSIS}

Error is velocity measurements can be obtarned form a Taylor's expansion of equation [2], which yields

$$
\frac{\Delta \mathrm{U}}{\mathrm{U}}=\frac{1}{2}\left(\frac{\Delta \mathrm{F}}{\mathrm{F}}+\frac{\Delta \rho}{\rho}+\frac{\Delta \mathrm{A}}{\mathrm{A}}+\frac{\Delta \mathrm{C}_{\mathrm{D}}}{\mathrm{C}_{\mathrm{D}}}\right)
$$

The following maximum percent errors were determined

$$
\begin{aligned}
& \frac{\Delta \mathrm{F}}{\mathrm{F}}=05 \% \\
& \frac{\Delta \rho}{\rho}=05 \% \\
& \frac{\Delta \mathrm{A}}{\mathrm{A}}=10 \% \\
& \frac{\Delta \mathrm{C}_{\mathrm{D}}}{\mathrm{C}_{\mathrm{D}}}=40 \%
\end{aligned}
$$

Substituting these values into equation [4] shows that the maximum overall error of the velocity magnitude is 3 percent of full scale A similar analysis of the vector direction shows that it is determined within $03^{\circ}$ of the true value $A$ significant point here is that only the $\frac{\Delta \mathrm{F}}{\mathrm{F}}$ term changes at lower range velocities, e $\underline{g}$, at half scale, the inaccuracy of $F$ the curtent meter would be 325 percent of the reading, instead of 6 percent as would normally be expected

\section{FIELD TESTS}

Four drag-sphere current meters were deployed simultaneously in the York River estuary in an investigation of the tidal current regime near Gloucester Point, Virginia The meters were deployed by mounting them to rigid frames as shown in figure 5 Orlentations of the current sensors were established by relating the location and position of the mounting frames to fixed landmarks

The sensors were orlented to detect two horzontal drag force components, from which the flow vector in the horizontal plane was determined at each meter Because four meters were used simultaneously, the drag-force data were acquired by a portable digital data acquisıtion system and recorded on computer-compatible magnetic tape Inıtial zeroing and force calıbrations of each meter in the $X$ and $Y$ directions were made just prior to placing the current meters in the water 


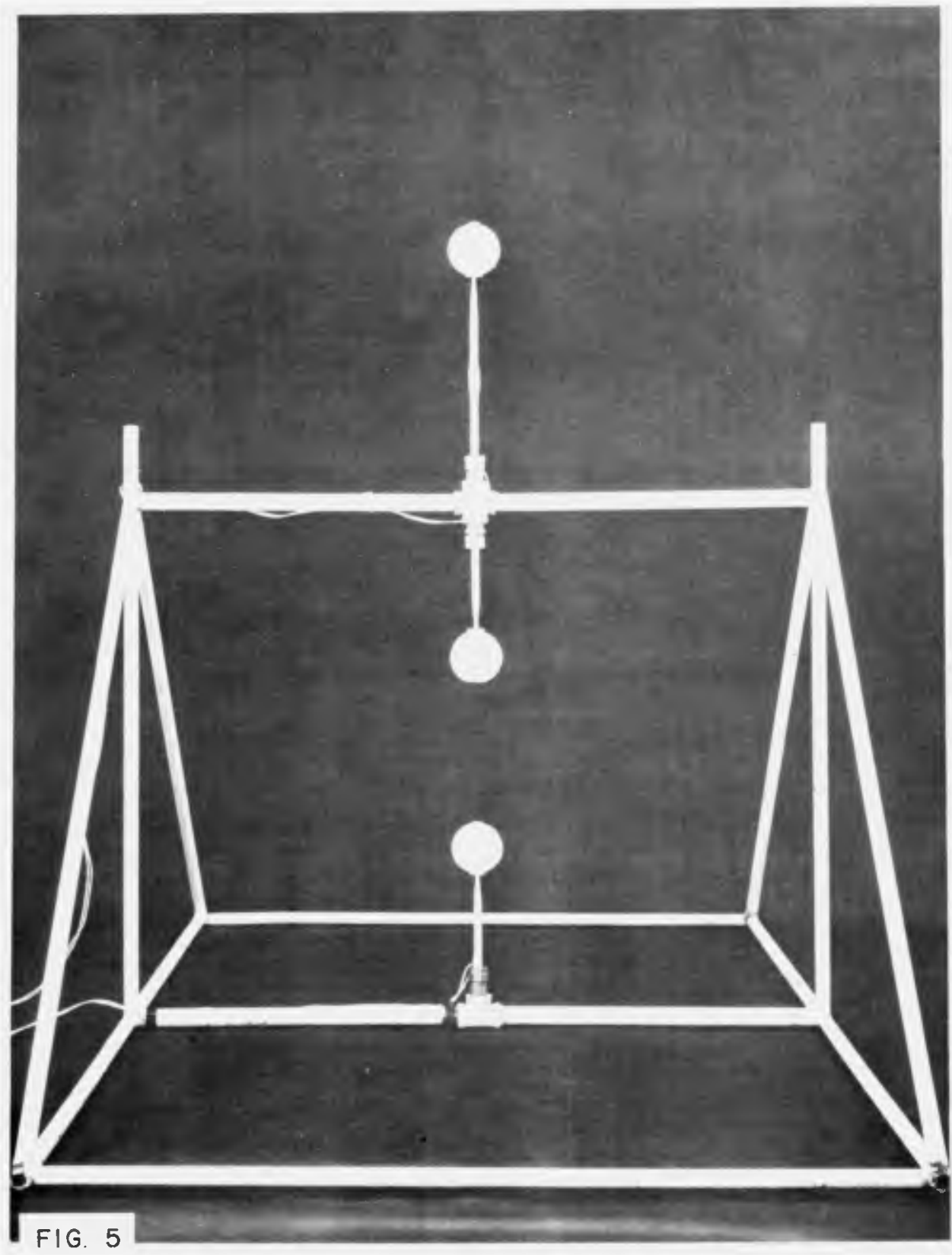

RIGID FRAME FOR DEPLOYING DRAG-SPHERE CURRENT METERS 

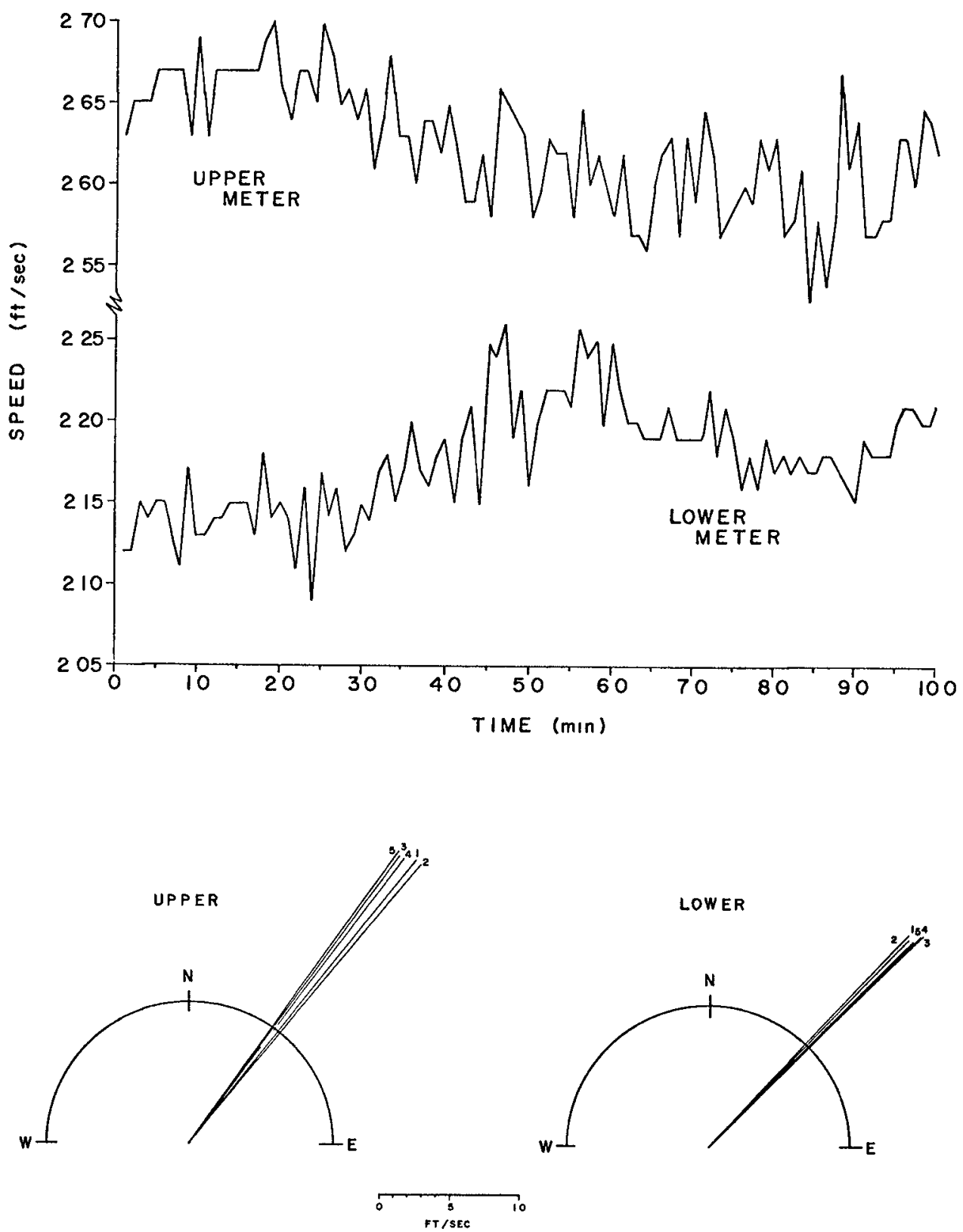

FIG 6 TYPICAL DATA FROM DRAG SPHERE CURREIVT METERS VECTORS REPRESENT TWO-MINUTE AVERAGES OF SPEED AND DIRECTIONAI, DATA EQUUATOR OF UPPER DRAG SPHERE 46 IN ABOVE BOTTOM, LOWER SPHERE 16 IN ABOVE BOTTOM 
At the end of one week, the meters were removed from the water, and the force calibrations were checked Only small descrepancies were noted for three of the meters The fourth meter had developed an electrical short-circult within a few hours after deployment and was disconnected for the duration of the test This short-circuit appeared to result from a breakdown of the bond between the waterproofing compound and the force transducer

$X$ and $Y$ force-component date were then resolved to flow velocity vectors via a computer program Analyzed data (fig 6) revealed vertical differences in the flow consistent with expectable velocity profiles, and directions compatible with those of the known tidal-current field The net drift for various time intervals of interest was easily calculated

\section{CONCLUDING REMARKS}

These evaluations have shown that use of the drag-force water current meters in fluld-process and sediment-response studies is feasible in the 0 to $6 \mathrm{ft} / \mathrm{sec}$ range The net velocity vector at several points in a plane parallel to the sea floor may be obtained with these current meters over periods of a few minutes to several weeks

Rigid frames are convenient for deployıng the current meters, or they can be mounted to a pile or permanent structure

The meter can be oriented to sense elther the horizontal or the vertical flow vector

If the meters are deployed unattended for extended perıods during seasons of significant marine fouling, it will be necessary to impregnate the polyethylene drag spheres with an antifouling compound

Since drag-force type current meters sense both acceleration-dependent forces and velocity-dependent forces, application of this meter is restricted to basically steady flows

The drag-sphere velocity meters are simple, relatıvely ınexpensive, and when combined with an appropriate data acquisition system, they are well suited to applications where a large number of simultaneous measurements are needed

\section{ACKNOWLEDGMENTS}

We are indebted to Carl Roberts, IRD, NASA, for assistance in meter development Thanks are due to the Givil Englneering Department, VPI, for assistance in tow tank tests and to Dr R L Miller, University of Chicago, for use of his calíbration gear and wave channe 1 
REFERENCES

1 Inman, $D$ I and Nasu, N 1956 Orbıtal velocıty associated with wave action near the breaker zone Corps of Eng, Beach Erosion Board, Tech Memo No 79 1-43

2 Beardsley, G F, Jr, Knollman, G C, Hamamoto, A S and E1sler, J D 1963 A device for measuring orbital velocıtıes in an underwater wave field Rev Scl Insts $34 \quad 516-519$

3 Olson, J R 1967 Flowmeters in shallow-water oceanography Naval Undersea Warfare Center, U S Navy, San Diego, Calıf, NUWC TP 5 1-44

4 Earl, M D and Beardsley, G F 1969 A three component drag probe for use in wave fields and tidal channels Abstract Vol, Amer Geophy Unıon, Washington, D C, meetıng, p 631

5 Reed, Wilmer H, III, and Lynch, J W 1963 A simple fast response anemometer Jour App1 Meteoro1 2 412-416

6 Hirsch, P 1924 Motion of spheres in stıll fluıds NACA Technıcal Memo No 257

7 Shapıro, Ascher H 1961 Shape and Flow, the Fluid Dynamıcs of Drag Doubleday and Company, Inc, F1g 13

8 O'Brien, M P and Morison, J R 1952 The forces exerted by waves on objects Amer Geophy Union Trans $33 \quad 32-38$

9 Boussinesq, J 1885 Sur 1a resistance d'une sphere solıde Compt rend 100935

10 Basset, A B 1888 On the motion of a sphere in a viscous liquid Phıl Trans Roy Soc, London $1791-43$ 



\section{CHAPTER 116}

\section{TKAPPING UE OUTFALL CUNSTIIUENTS DEHIND SILLS}

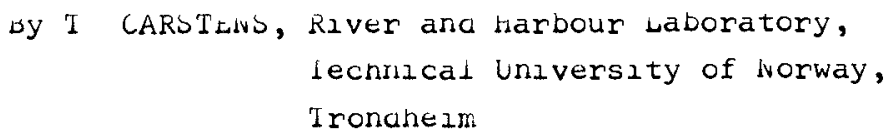

anc A SJUDLRG, nyaraulic Division, Cnalmers Institute of Iechnology, Gotnenburg

\section{ADOTRACI}

Conmunication detween sucn coastal resiplents of waste water as Days and fjords and the ocean is often througn an outlet of small cross section

The combineu effects of a topography with transverse rlages ana a brackisn surface layer creates a rather staglant wody of water. The ridge or sill deflects the traal currents away from the bottom so that only the upper part of the water columin is continually flushea The stabllity of the pycnocline prevents surfacegeneratea turbulence from penetrating auwnwaras, so the turbulerice $2 \mathrm{n}$ the ceep pool is weak.

A scneme nas deen proposea to store the nutrients of sewage outfalls Denzna the sill at vrpdak in the Oslofjord Ine storage capabllity of that fjora was estrmatec for a conservatıve outfall constituent by means of a one almensional asspersion equation from the analysis the galis of deep outfalls through ulffusors collparea witn surface outfalls can de estimated for various perioas between deep water infiows, wnich are observed to occur.

\section{INLRODUCILON}

\section{Topokraphic traps}

The effect of a glven waste water release on the coastal 
enviromient cepenos to a large extent on the coastal morpnology Indentations as well as promontorles tend to create shelterea water bodies with long resiaence times in lárge scàle circulations This tencency is often ennancea by the constricted outlet of many bays ana fjoras

A very efficient kind of constriction is the transverse Dottom riages or silis which are characteristic of the coastal irilets carvea by glaciers ana known as fjoras. A Iongltualnal section of a fjora (Oslofjora) is shown In Flg. $\perp$ several silds alviae the deep fjord into silaller sub-basins of varying size It is olvious tnat tnls type of topography prevents large Docies of water from participating in the nomal movements of coasta 1 waters. In fact, only two significant transport processes seem to exist in the aeep Dasins one, the vertical alffusion, is continuous, while the other is a alscrete process of floud-like iriflows.

Luflcws, Occasional renewal of the water occurs whenevtr the water density at the sill level exceeds the aensity of the vottom water insiae the sill Ine water mass occulylne the Lasin is then forced ufwaras by weislmetric alsplacement, and a conplete or partial rerewal of the basin water takes place witnin a relatively snort perioc of tine The inflowlng volume cepenas on the aensity surpius and its auration and is governea primarily by heteorological variables sucn as wha and atmospherlc pressure (upwelling).

Vertical alffusion Ine density surplus responsidie for tne initial inflow is caused by a salinity surplus and a temperature cefect, comparea witn the overlylng layers Tnus a sailnity gralent is establisnea for a sluw alffusive transport of salt upwares, ana a temperature sraalent for a slullar transport of heat 
downwaras. With time tris alfusion depletes the aensity surplus of the basin water, and the stage is set for a repeat performance, beginning with a new inflow.

Fig 2 snows observed salinities in the two innermost Dasins of the Oslofjora durlind 1962-65, aemonstrating the two processes aescribea above, ana first by GADE (5).

Ine cychocline IId

Ever on open coasts pycnoclines develop near river moutns, tut tne fjoras are particularly prone to stratification, for topograpilc and climatic reasons A regutar feature of any fjord is therefore a pycnocline whicn is usually very stable in the sumrer nalf of tne year

The pycnocline acts as a lia on the deep pool. Vertical circulation that woulu go right to the Dottom in water of constant density, is replacea by a layered flow The shear notion in the pycnocline filters out rost of the norlzontal momentum, so that only very weak flows are set up below the pycnocline

The effect of the aensity graalent on the surfacegeneratea turbulence is equaliy strong. When entralning or eroalng water of higher density, some of the turoulent energy is converted into potential energy in tnis way the pycnocline acts as a sink of turbulence

The comolnea effects of topography ano layering thus create Doales of water witn a rather special flow regline, characterlzed by long residence times and a low exchange witn the surface layer 
Sewage disposal Eenind silis

The sneltered deep basins inslae the sill at Drфbak (Flg 1 ) nave Deen proposed for alsposal of sewage from oslo $(6,7)$. The reasoning is that nutrients can be stored in these basins without causing algal blooms, in contrast to the alsposal through shallow outfalls presently in use. A hign proauction of algae is uncesirable for two reasons, first because it decreases the esthetic and recreational value of the fjord, and seconoly because the organic debris exhausts the oxygen supply at the lower depths.

The usefulness of this scheme ninges on the inflows alscusseu above. A renewal of the deep water must take place before the steady state of maxisum storage has been reached, for which the supply tnrough the sewers equals tne vertical diffusion of a nutrient

\section{A MOUEL FOR CIRCULATION AND DIFFUSION OF DISCHARGLD}

DLWAGL

\section{tartler work}

A nodel for the mixing and the convection in a confined region inauced by a source of buoyancy has been advancec by DAlives and TURiveR (1) veglecting the effect of turvulence in the environment on the rising plume, they obtained asynptotic solutions of the density alstridution valia at large times. The model proposea dy CEDtRWALL (2) for mixing and convection inducea by alschargea sewage follows closely that of DAIivbs and IURNER, Dut is mostly concerned with the alstribution of disposed pollutants 
The simpilfying assumptions made by CLDLRwALL agree well witn those first introduced by the autnors of this paper ( 3 ), nowever, ne neglects tne diffusion of constituents througn the ralocline into the surface layer

\section{Ine present mogel}

A cross stction of the fjorc is snown in Fig 3 The source of the constituent is the effluent outfall at level $z=U$, ana lts strengtn is $Q_{0} c_{0}$, where $Q_{0}$ is the volume flux ana $c_{0}$ the concentration. Because of lts buoyancy, the effluent jet will rise to the level h in the pycnocline rere the censity of the surrounding fluia equals tnat of the jet, and the allutea effluent is trapped in a subnerged fiela referred to as the cloud.

Assumptions In oruer to simplify the analysis we introcuce the following assumptions

1 Inere is no exchange in the deep waters after the complete renewal tnat we cnoose as initial conaltron

2. The density profile is not significantly affectea Dy the induced circulation of sewage

3 The vertical constituent fluy is given by

$$
-D_{z} \frac{G C}{(\sigma z)} A_{z}
$$

wrere $D_{z}$ is the ulffusion coefficient, dc/az the concentration gradient and $A_{z}$ the horizontal area 
4. The effluent is trappeo just below the nalocline and instantaneously spread into a thin layer which will be successively convected and alffused downwarus as new sewage reaches the trapping level

5. Any two scalar components are transported by the same mecnanism. Thus the concentration alstribution of the constituent across the halocline in steacystate condition is just a scaling of the salinity alstribution, or

$$
\frac{a c}{d z}+=\frac{\Delta c}{\Delta s}\left[\frac{d s}{d z}\right]_{h}+
$$

where $\Delta c$ ara $\Delta s$ are the alfference in concentration of the constituent. $z=n$ is the trapping level ano $\mathrm{h}^{+}$refers to "just aoove" ana accoraingly $\mathrm{n}^{-}$to "Just below" The tune lag to reach staoy-state from inltial conditions is of the orcer of one week and may be neglected ( 3 ). The vertical constituent flux upwaras to the surface layer is then given by

$$
-\left[D_{z} \frac{I}{\Delta s} \cdot \frac{\delta s}{\delta z} \cdot A_{z}\right]_{h}+c(h, t)=Q_{v} c(h, t)(3)
$$

For simplicity the concentration of the constituent in the surface is assumeu to be zero

6. The amount of sewage alscharged to the fjord during a year is small comparea with the fjord water avaliable for dilution.

7. Ine freshwater runoff is sufflclent to malntaln a aensity stratification, Lut does not inauce appreciadle upwara entrainment (CARSTens (4)) 


\section{The aispersion equation}

The convection and mixing within the trapped cloud outside the plume is now described by the one dimenslonal alspersion model

$$
\frac{\delta c}{\delta t}-U \frac{\delta c}{\delta z}=\frac{\partial}{\delta z}\left(D_{z} \frac{\delta c}{\delta z}\right)
$$

where $U=Q(z) / A(z)$ is the Induced convective velocity and $Q(z)$ the plume flow rate at level $z$. Eq. (4) can be solved numerically together with the continulty equation for the constituent within the cloud

$$
\begin{aligned}
& Q_{0} c_{0}+\int_{0}^{z} c a Q-Q_{v} c(h, t)+U(z) A_{z}(z) c(z, t) \\
& -D_{z} \frac{\delta C}{\delta z} A_{z}=\int_{z}^{h} \frac{\delta c}{\delta t} A_{z} d z
\end{aligned}
$$

which for $z=n$ has the following form

$$
\begin{aligned}
& Q_{0} c_{0}+\int_{0}^{z} c d Q-Q_{v} c(h, t)+U(z) A_{z}(z) c(z, t) \\
& -\left[D_{z} \frac{\delta c(h, t)}{\delta z} A_{z}\right]_{h}=0
\end{aligned}
$$

Ine first term is the source, the second term accounts for the recirculation of the constituent, and the thira term is the constituent flux into the surface layer. The fourth and the fifth terms, respectively, represent the convective ana the diffusive downward flux.

A straight forward numerical treatment of tqs. (4) and (6) is not feasible at present. We have not completely solvea the problem of supporting convenient mathematical 
models with sound physical arguments. We shall, however, use Eqs. (5) and (6) to estimate the time history $c(h, t)$ of the cloud concentration at the trapping level. we feel our solutions for small and for large values of $t$ are acceptable, but we do not yet have a solution for intermealate values of $t$.

Solution for small $t$ For small $t$ the thlckness of the cloud is small compared with the neight $h$. Hence, the recirculation of the constituent may de neglected

$$
\int_{0}^{n} \operatorname{caQ} \approx 0, \text { and } U(h) \cdot A_{2}(h) \approx Q_{0} S_{0}
$$

where $s_{0}$ is the dilution at the trapping level.

For smill $t$ we may also assune the vertical distrioution of the constituent concentration above the trapping level to be a mirror image of tnat below. Tnus, the upward diffusive flux equals the downward flux ana

$$
-\left[D_{z} \frac{\delta c(z, t)}{\delta z} A_{z}\right]_{n^{-}} \cdot c(h, t)=Q_{v} c(h, t)
$$

With these assumptions Eq ( 6 ) has the solution

$$
\frac{c(n, t)}{c_{0}}=\frac{Q_{0}}{(I+\alpha) Q_{v}+Q_{0} S_{0}}
$$

where $\alpha \approx 2$ for small $t$ As the concentration in the surface layer is assumed to be constant $=0$, a decreases witn growing t. Flg. 4 shows Eq. (9) for a particular case in tre oslofjora.

Solution for large $t$. The effluent cloud is successively convected and diffused aownwaras. In order to get a rough estimate of the transport velocity we consiaer only the convection and neglect the diffusion. We then nave to fina the rate of advance of the first 
front. If we take $U_{0}$ as the front velocity and $z_{0}$ as the front level, it follows from continusty tnat

$$
U_{0}=\frac{Q\left(z_{0}\right)}{A_{z}\left(z_{0}\right)}
$$

and the travel time $T$ is given by

$$
T=\int_{z_{0}}^{h} \frac{d z}{U_{0}}=\int_{z_{0}}^{h} \frac{A_{z}}{Q} d z
$$

For a particular diffusor arrangement $\left(S_{0}=50\right)$ in the oslofjord with $Q_{0}=10 \mathrm{~m}^{3} / \mathrm{s}$ and $h=60$, T required for $z_{0}=0.1 \mathrm{~h}$ may be calculated to about 14 months

For t>14 months most of the deep water has recycled once anc the effluent may be assumed to be approximately uniformly mixed into the deep water In this case of ldeal mixing we have

$$
\begin{aligned}
& c(h, t)=c(z, t)=c(t) \\
& \int_{0}^{h} \frac{\delta c}{\delta t} A_{z} a z=\frac{\delta c}{\delta t} \int_{0}^{h} A_{z} d z=\frac{\delta c}{\delta t} V
\end{aligned}
$$

where $V$ is the deep water volume between the levels $z=0$ and $z=n$. We also have $U(0)=0$. With $z=0 \mathrm{kq}$. (5) then takes the form

$$
Q_{0} c_{0}-Q_{v} c(t)=\frac{\partial c}{\delta t} V
$$

which has the solution

$$
\begin{aligned}
& \text { solution } \\
& c_{0}
\end{aligned}=\frac{Q_{0}}{Q_{v}}\left(1-e^{-\frac{Q_{v} t}{v}}\right)
$$

Flg. 4 shows Eq. (14) for a particular case in the Oslof jora

$$
Q_{0}=10 \mathrm{~m}^{3} / \mathrm{s}, S_{0}=50, D_{z}=3 \cdot 10^{-5} \mathrm{~m}^{2} / \mathrm{s}
$$




$$
\begin{aligned}
& \frac{l}{\Delta s} \frac{d s}{d z}=0,15 / m, A_{z}(h)=1,1 \cdot 10^{8} \mathrm{~m}^{2}, \\
& V=4,2 \cdot 10^{9} \mathrm{~m}^{3}
\end{aligned}
$$

\section{CONCLUSIONS}

A transverse ridge or sill on the bottom shelters the water mass below the sill depth from tidal flushing. A pycnocline proviaes adaltional sheltering from surface-generated turbulence, and so the body of water behind a sill and below a pycnocline is characterized by long residence times.

The Dullaup of the concentration of an outfall constituent released in such a stagnant body of water was investrgated for the special case of the Oslofjord, based on a set of assumptions leading to a general onealmensional dispersion equation.

Solutions were obtalned for small and large periods of constituent release, respectively. The solution for small $t$ assumes a rapld horlzontal spreaa compared with the vertical transport The solution for large $t$ assumes laeal mixing. A tentative "probable curve" has been inserted for intermediate tımes.

From the analysis the gains of deep outfalls through diffusors compared with surface outfalls can be estimated for varlous perlods between deepwater inflows. 


\section{RLELRLIVCES}

1. BAINES, W.D. and TURNER, J.S. Turbulent buoyant Convection from a Source in a Confined Region. - J. Fluzd Mech. Vol. 37 Part I, 1969

2. CEDERWALL, $K$. The Oslo Fjord - A Model for Circulation and Diffusion of Discharged Sewage. - Technlcal Memorandum 70-I. W.M. Keck Laboratory of hydraulics and Water Resources, Cal. Inst. of Techn. 1970.

3 CARSTENS, T. and SJфbERG, A. Osiofjorden II An evaluation of sewage outfalis inside Drøbak. - NIVA, Oslo 1969. (In Norweglan).

4 CARSTENS, T. Turbulent diffusion and entrainment in two-layer flow - ASCE VWI, 77-104, Feb 1970 .

5. GADE, H. Oslofjordens hydrografl. Delrapport nr. 2. - NIVA, Osio 1967. (In Norweglan).

6. The Oslofjord and its poliution problems Samierapport I. NIVA, Oslo, 1968. (In Norweglan).

7. The OsLofjord and 1 ts poliution problems Samlerapport II. INIVA, OsIo, 1970. (In Norweglan). 

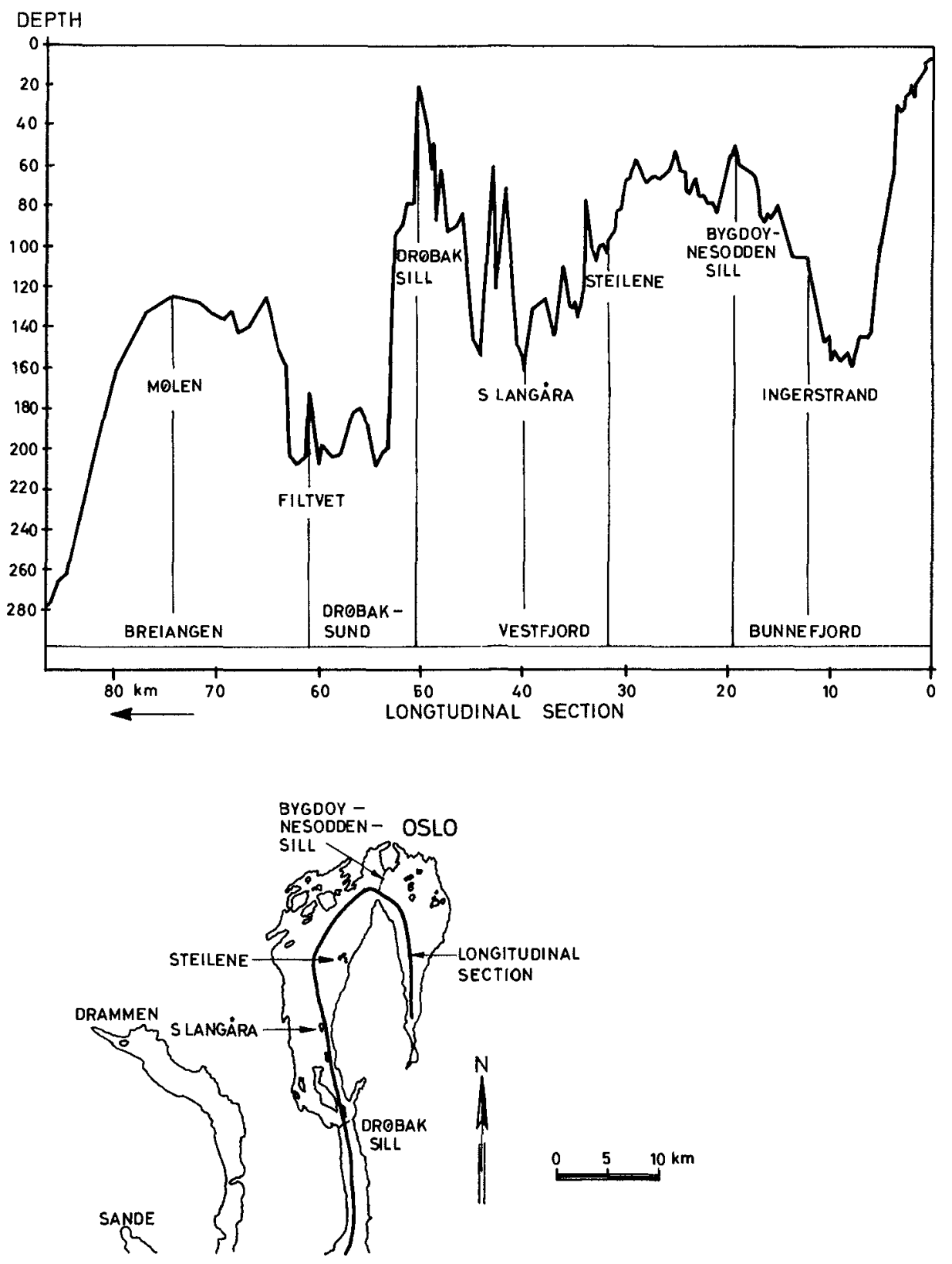

FIG 1 THE OSLOFJORD, PLAN AND SECTION 


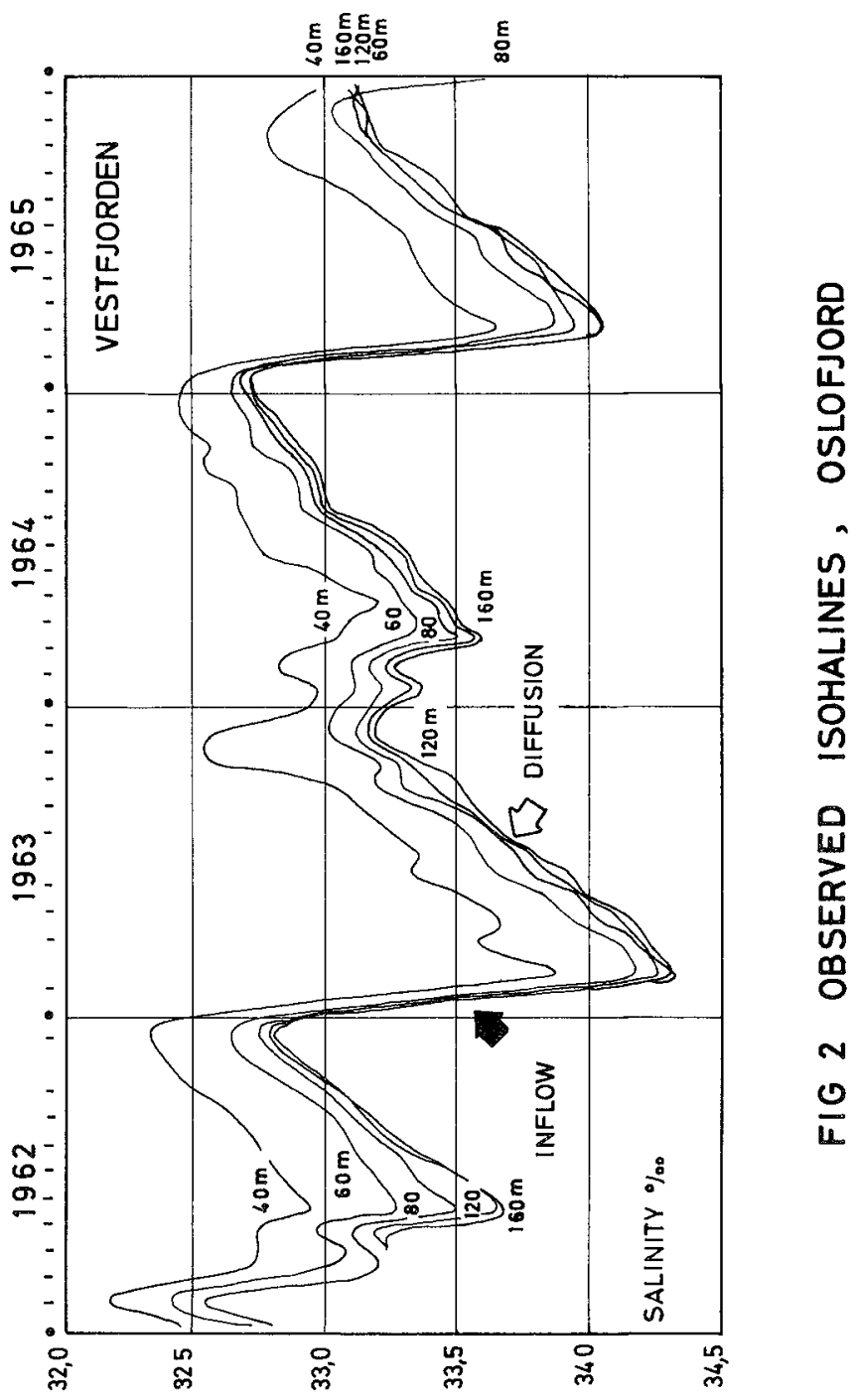




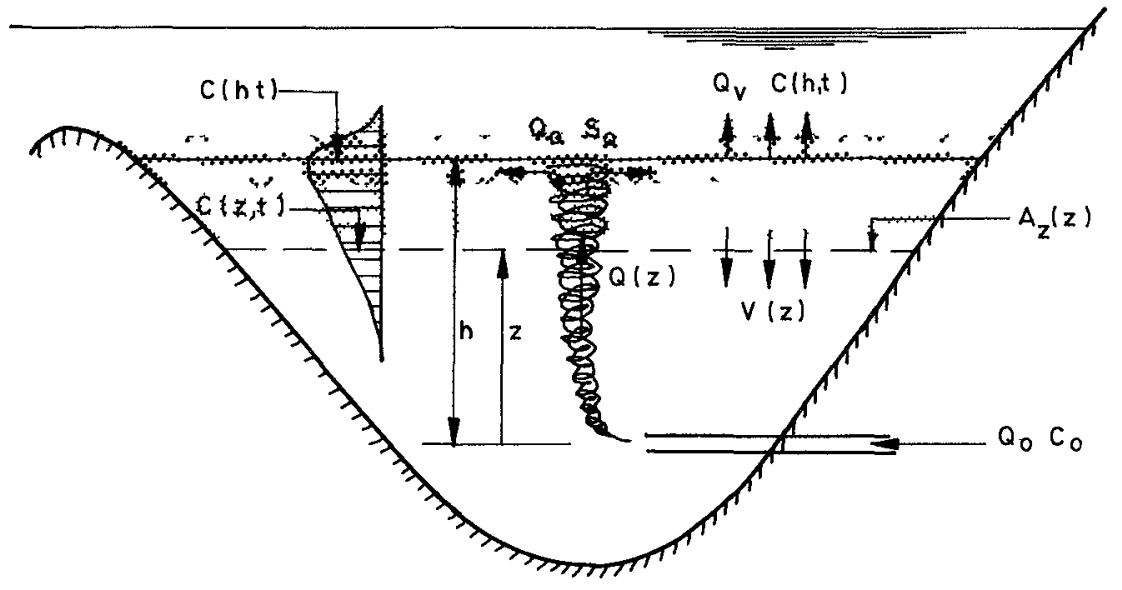

FIG 3 SCHEMATIC OF A SECTION THROUGH THE FJORD

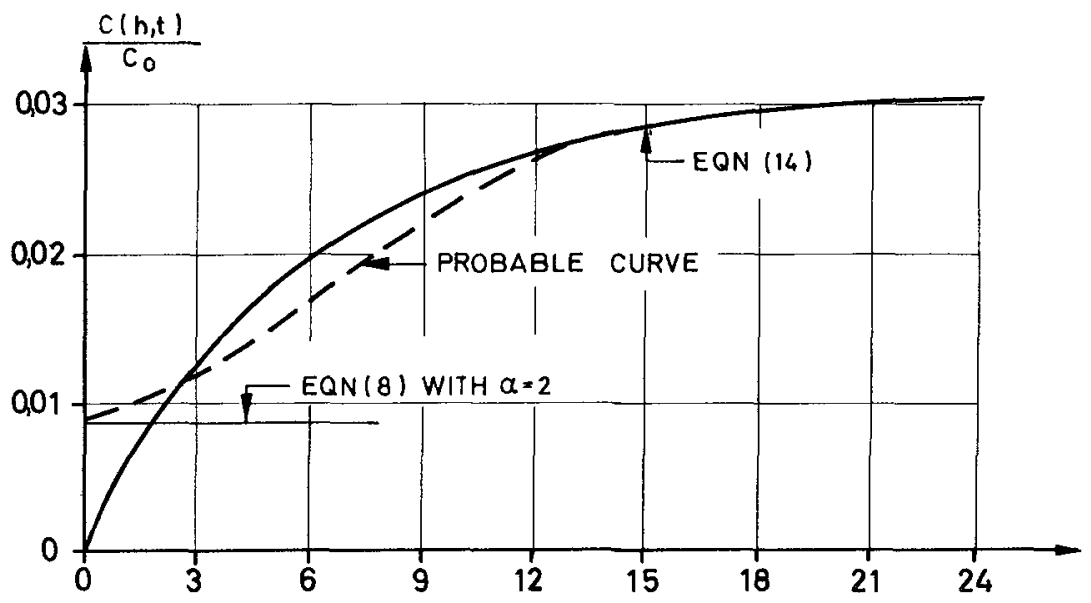

FIG 4 TIME HISTORY OF CLOUD CONCENTRATION AT TRAPPING LEVEL (OSLOFJORD) 


\section{CHAPTER 117}

\section{SUSPENDED LOAD CALCULATIONS IN A TIDAL ESTUARY}

BY BRIAN A O'CONNOR

Lecturer, Department of Civil Englneering, University of Manchester, Manchester, England

\section{ABSTRACT}

The present work describes the derivation of equations which can represent the vertical distribution of sediment in a well mixed tidal estuary The starting point for the analysis is the two dimensional longitudinal equation of motion, including the longitudinal salinity gradient term Equations are given which represent a steady state sediment profile and it is shown that these are similar to the expression used in uni-directional flow e g Rouse's equation

It is argued that the derived equations could be applied to a real estuary, subject to certain restrictions on sediment size and estuary type An application of the theoretical equations to the Mersey Estuary indicates reasonable agreement between observed and predicted sediment quantities for medium and coarse sand particles Agreement is shown to be worst for fine grained sediments and it is concluded that much better results can be obtained by using the non-steady one dimensional sediment distribution equation in discrete steps throughout the tidal cycle

\section{INTRODUCTION}

The calculation of the distribution of sediment in a real estuary is an exceedingly difficult task All the problems of uni-directional flow are present together with the added complications of unsteady flow, varying bed resistance and salt/freshwater density currents

The present paper endeavours to show how the distribution of certain sediment may be estimated in a particular type of well mixed estuary it is not 
intended to be the panacea for tidal sediment problems, but represents a comparatively simple engineering solution to certain tidal sediment distributions METHOD

In uni-directional flow problems, the distribution of sediment is specified by assuming that a steady state is reached between sediment eroded from the river bed and sediment depositing on the river bed clearly, if no sediment is present in the water and erosion is started then a finite time is required for a steady state to be reached The time will be dependent upon particle size, water depth, turbulence level and initial sediment distribution and will thus be expected to be small for large sized particles, whose equilibrium profile is close to the river bed and large for fine sediment, whose equilibrium is more uniformly distributed throughout the water depth A first look at the sediment distribution in a tidal environment would thus be to assume that the sediment particles reached the steady state distribution in a short time, during which the tidal flow characteristics were unaltered 1 e the tide is considered to be a succession of uni-directional flows The sediment distribution for the large sized particles would thus be given by the steady state profile appropriate for each elemental uni-directional flow

The effects of the salt/freshwater density profile and the inertia of the flow system must also be incorporated into the problem This can be done by assuming the tidal flow is frozen at each instant of tome and then using the resultant shear stress distribution to predict the distribution of sediment

\section{ANALYSIS}

The flow of water in a tidal estuary is governed in two-dimensions by the equation 1

$$
A+F+2 D(1-n)-\frac{1}{\rho g H} \frac{\partial \tau}{\partial n}=I
$$

Where $A=\frac{1}{g} \frac{\partial U}{\partial t}, U=$ horizontal water velocity in the horizontal direction $(x)$, 
$t=t_{1} m e, g=$ acceleration due to gravity, $F=\frac{\partial}{\partial x}\left(\frac{u^{2}}{2 g}\right), D=\frac{H}{2 \rho} \frac{\partial p}{\partial x}, \rho=$ water density, $\eta=\frac{y}{H}, y=$ elevation above the bed, $H=$ water depth, $\tau=$ horizontal shear stress, $I$ = water surface slope (positive in the ebb direction)

Equation (1) also assumes that the term $\frac{\mathrm{U}^{2}}{\mathrm{gH}}$ is small and the vertical velocity term is small

The shear stress distribution with depth is obtained from equation (1) by integration $w r t n$, after first specifying the distributions of $A$ and $F$ with depth It has been shown ${ }^{1}$ that simple linear distributers for $A$ and $F$ are not vastly different than logarithmic or parabolic distributions Thus the variation of $A$ and $F$ are taken as

$$
\begin{aligned}
& A=A_{b}+\left(A_{s}-A_{b}\right) \\
& F=F_{b}+\left(F_{s}-F_{b}\right)
\end{aligned}
$$

where $b$ and $s$ refer to bed and surface values respectively Equation (2) and (3) uses known or estımated values of $A$ and $F$ at small distances above the estuary bed and below the estuary surface

Inserting equations (2) and (3) in equation (1) and integrating $w r t n$ gives the expression for the shear stress distribution with depth as

$$
\begin{aligned}
& \frac{\tau}{\rho g H}=I^{\prime}(1-n)-B\left(1-n^{2}\right)-D(1-n)^{2} \\
& \frac{\tau}{\rho g H}=S^{\prime}-2 P^{\prime} n-D^{\prime} n^{2}
\end{aligned}
$$

where $I^{\prime}=I-A_{b}-F_{b}, B=\frac{1}{2}(\Delta A+\Delta F), \Delta A=A_{s}-A_{b}, \Delta F=F_{s}-F_{b}, S^{\prime}=I^{\prime}-B-D$, $P^{\prime}=\frac{1}{2}\left(S^{\prime}-D^{\prime}\right), D^{\prime}=D-B$

The solution of equation (4) requires the knowledge of six prototype quantities at each instant of time However, equation (5) is a function of only the two quantities $S^{\prime}$ and $D^{\prime}$ These quantities can be determined by considering the velocity distribution with depth, as follows 
The shear stress $(\tau)$ is first related to the velocity gradient by Prandt]'s mixing length concept ? e

$$
\tau=\rho l^{2}\left|\frac{d u}{d y}\right| \frac{d u}{d y}
$$

where $\mathfrak{l}=$ a mixing length, $\|=$ absolute value

A suitable mixing length distribution to use in equation (6) is that found by Agnew ${ }^{2}$ in the Haringvijet (Holland) 1 e

$$
\begin{aligned}
& l=k y\left[\frac{1-n}{1-\delta}\right]^{2} \text { for the region } 7>n>\delta \\
& \text { and } \quad l=k y \text { for the region } \delta>n>0
\end{aligned}
$$

where $K=$ Von Karmans Constant, $\delta=\frac{h}{H}, h=$ height of position of zero nett motion of the residual salinity circulation

Equation (5), (6), (7), and (8) thus read to the following expressions for the variation of velocity (U) with depth $(n)$

$$
\begin{aligned}
& \frac{K\left(U-U_{g}\right)}{\sqrt{g H^{\prime}}}=\sqrt{1-\delta} \int_{\delta}^{n} \frac{\left(I S^{\prime}+D^{\prime} n I\right)^{\frac{1}{2}}}{n} d n \text { for } 1>n>\delta \\
& \frac{K U}{\sqrt{g H}}=\int_{n_{0}}^{n} \frac{\left(J S^{\prime}-2 P^{\prime} n-D^{\prime} n^{2} J\right)}{n} d_{n} \text { for } \delta>n>n_{0} \\
& \text { where } n_{0}=y_{0 / H}, y_{0}=\text { height at which } U=0
\end{aligned}
$$

The solutions to equations (9) and (10) depend on the magnitude and sign of the terms $S^{\prime}$ and $D^{\prime}$ If the sign convention, ebb slopes are positive is used', then $S^{\prime}$ is a maximum (tve) about mid-ebb and mid-flood (-ve) while $D^{\prime}$ is negative near high water, and greater than $S^{\prime}$, and positive at low water and greater than $S^{\prime}$ The latter is to be expected as the flow reverses at the bed first at low water due to the density circulation. Values of $S^{\prime}$ and $D^{\prime}$ can be found by fitting the prototype velocity observations to equations (9) and (10) viz table 3

The sediment distribution may now be found from the generai sediment 
diffusion equation 7 e in two dimensions

$$
\frac{\partial C}{\partial t}=\frac{\partial}{\partial x}\left(\varepsilon_{x} \frac{\partial C}{\partial x}-U C\right)+\frac{\partial}{\partial y}\left(\varepsilon_{y} \frac{\partial C}{\partial y}+\omega C\right)
$$

where $\varepsilon_{x}, \varepsilon_{y}$ are diffusion equations in the $x, y$ directions $w=$ particle fall velocity

If attention is confined to a particular type of estuary in which the varration of $U$ with distance $(x)$ is small and the sediment is equally distributed along the estuary then $\partial c / \partial x=0$ and equations (11) wh 11 reduce to

$$
\frac{\partial C}{\partial t}=\frac{\partial}{\partial y}\left(\varepsilon y \frac{\partial C}{\partial y}+\omega C\right)
$$

An analytical solution of equation (12) is unknown for $\varepsilon_{y}=f(y, t)$ Finite Difference methods can be used and are being studied at present However, if only large particles are considered then a steady state profile will be quickly reached and a mathematical expression for the distribution of the sediment can be obtained from equation (12) with $\partial C / \partial t=01 \mathrm{e}$

$$
\ln \left(\frac{c}{C_{a}}\right)=-\omega \int_{a}^{y} \frac{d y}{\varepsilon_{y}}
$$

where $c_{a}=$ a reference concentration at level "a" above the bed The distribution curve specified by equation (13), could also be used to describe the distribution of medium to fine sand particles by replacing $C_{a}$ with an observed prototype concentration value $\left(C_{t}\right) \quad c l e a r l y, C_{t}<C_{a}$ and the concentration given by the modified equation (13) will be greater than in prototype, the error being greatest for the finest sized sediments

The steady state solution is obtained from equation (13) by integration on specifying the variation of $\varepsilon_{y}$ with depth This is done by relating $\varepsilon_{y}$ to the momentum transfer coefficient $\varepsilon_{m} 1$ e

$$
\varepsilon_{y}=\beta \varepsilon_{m}
$$




$$
\begin{aligned}
& \text { and } \varepsilon_{m} \text { is obtained from the equation } \\
& \tau=\rho \varepsilon_{m} \frac{\partial u}{\partial y} \\
& T e \varepsilon_{m}=l^{2}\left|\frac{\partial u}{\partial y}\right| \\
& \text { Thus } \varepsilon_{y}=\beta l \sqrt{\frac{\tau}{\rho}} \\
& 1 e \varepsilon_{y}=\beta K y\left[g H\left|S^{\prime}-2 P^{\prime} \eta-D^{\prime} \eta^{2}\right|\right]^{\frac{1}{2}} \text { for } 0<n<\delta \\
& \varepsilon_{y}=\beta K y \quad \frac{(1-n)}{(1-\delta) \frac{1}{2}}\left[g H \mid S^{\prime}+D^{\prime} \eta\right]^{\frac{1}{2}} \text { for } \delta<\eta<1
\end{aligned}
$$

The variation of $\varepsilon_{y}$ with depth as given by equations (18) and (19) is shown in Figure 1 for mean tide level $\left(S^{\prime}>D^{\prime}\right)$ and near low water $\left(S^{\prime}=D^{\prime}\right.$ and $S^{\prime}$ $=0$ ) conditions, together $w i$ th field data (average tidal cycle values) taken by Bowden ${ }^{3}$ and Sharaf ${ }^{4}$ in the Narrows Area of the Mersey Estuary, England

Bowden's data is seen from Figure 1 to indicate smalier values in the upper half of the vertical profile and indeed it was found that the velocity equations ( 9 ) and (10) gave better answers over the low water period if the following expressions were used for $\varepsilon_{y} 1$ e

$$
\begin{gathered}
\varepsilon_{y}=2 \beta \quad k h(1-n)^{3 / 2}\left[\mathrm{gH} \mid S^{\prime}+D_{n}^{\prime} n\right]^{\frac{2}{2}} \\
\varepsilon_{y}=2 \sqrt{2_{\beta}^{\prime}} k h(1-n)^{2}\left[g H \mid S^{\prime}+D_{n}^{\prime}\right]^{\frac{1}{2}} \\
\quad \text { for the region } \delta<n<1
\end{gathered}
$$

Equations (20) and (21) are also shown in Figure 1 and give a better fit to Bowden's data

The solution of equation (13) is thus dependent upon the sign and magnitude of $S^{\prime}$ and $D^{\prime}$, as were equations (g) and (10) The various solutions to equation (13) have been presented eisewhere ${ }^{5}$ The solutions for the case $\left|S^{\prime}\right|>\left|0^{\prime}\right|>0$, which apply for the majority of the tidal cycle are given below $1 \mathrm{e}$ 
$\frac{c}{c_{a}}=\left[\frac{\left(1 / n-\alpha_{0}\right)+\left[\left(1 / n-\alpha_{0}\right)^{2}-\left(1-\alpha_{0}\right)^{2}\right]^{\frac{1}{2}}}{\left(1 / \eta_{a}-\alpha_{0}\right)+\left[\left(1 / n_{a}-\alpha_{0}\right)^{2}-\left(1-\alpha_{0}\right)^{2}\right]^{\frac{1}{2}}}\right]^{z}$ for $n_{a}<n<\delta \quad \ldots$

where $Z=\frac{\omega}{\beta K U_{*}}$ and $U_{*}^{2}=\tau_{b} / \rho$ where $\tau_{b}=\rho g H S^{\prime}$

$\stackrel{c}{c}_{\delta}=\left[\frac{\left(1+\sqrt{1+n\left(1-2 \alpha_{0}\right)} \times\left(1-\sqrt{1+\delta\left(1-2 \alpha_{0}\right)}\right)\right.}{\left(1-\sqrt{1+\eta\left(1-2 \alpha_{0}\right)}\right)\left(1+\sqrt{\left.1+\delta\left(1-2 \alpha_{0}\right)^{1}\right)}\right.}\right] \times z(1-\delta)^{\frac{1}{2}}$
$\left[\frac{\left(\sqrt{2\left(1-\alpha_{0}\right)}-\sqrt{1+n\left(1-2 \alpha_{0}\right)}\right)}{\left(\sqrt{2\left(1-\alpha_{0}\right)}+\sqrt{1+n\left(1-2 \alpha_{0}\right)}\right)} \times \frac{\left(\sqrt{2\left(1-\alpha_{0}\right)}+\sqrt{1+\delta\left(1-2 \alpha_{0}\right)}\right)}{\left(\sqrt{2\left(1-\alpha_{0}\right)}-\sqrt{1+\delta\left(1-2 \alpha_{0}\right)}\right)}\right]^{\frac{z(1-\delta)^{\frac{1}{2}}}{\sqrt{2\left(1-\alpha_{0}\right)}}}$

for $\delta<n<1$

where $\alpha_{0}=\frac{P^{\prime}}{S^{\prime}}=\frac{1}{2}\left(1-D^{\prime} / S^{\prime}\right), C_{\delta}=$ concentration at height $\delta$

Equation (23) is seen to be indeterminate for the case $\left.\alpha_{0}=\frac{1}{2}\right] \in D^{\prime}=0$ If this condition is substituted in equation (18), (19) and (13), the expression for the variation of concentration with depth is

$$
\frac{c}{c}=\left[\frac{(1+\sqrt{1-n})}{(1-\sqrt{1-n})} \times \frac{\left(1-\sqrt{1-n_{a}}\right)}{\left(1+\sqrt{1-n_{a}}\right)}\right]^{z} \text { for } n_{a}<n<\delta
$$

and

$$
\frac{c}{c_{\delta}}=\left[\frac{1-\eta}{n} \times \frac{\delta}{1-\delta}\right]^{Z(1-\delta)^{\frac{1}{2}}} \quad \text { for } \delta<n<1
$$

Equation (24) is the same as that derived by Tanaka and Sugrmoto ${ }^{6}$ for uni-directional flow 1 e $D=O, A=F=0$ and $S^{\prime}=I$ Thus equations (22) and (23) are aiso similar to the expressions for uni-directional flow, except that the density circuiation and inertia and kinetic terms are included It should be noted that equation (22) reduces to Rouse's equation ${ }^{7}$ for uni-directional flow for $\alpha_{0}=10$ However, equations (21-23) predict a greater quantity of sediment in suspension than found by Rouse's equation This is shown in Fig 2 It will be noticed that both Rouse's equation and that of Tanaka and Sugrmoto give values 
almost identical to that of equation (22) below $\alpha_{0}=050$ However, the different mixing length distribution contained in equation (19) leads to smaller sediment values in the upper half of the water flow at $\alpha_{0}=10$ and 050 respectively

Application of theory to field data

The theory was applied to the Mersey Estuary in order to predict the quantity of sediment in suspension at position $H$ (Figure 3 ) The latter satisfies the requirements, on the flood tide, of complete sediment cover and little velocity variation with distance Prototype sediment data was also available at position $H$ in sufficient detail to be usable The sediment data avarlable is shown in Figure 4

In order to apply the theory, values of $C_{a}$ or $C_{t}, \alpha_{0}, S^{\prime}$ or $U_{*} \omega, \beta$ and $K$ must be avallable or capable of being calculated from the field data The determination of these quantities is now considered in detail below

(1) Determination of $u_{*}$ values

This could be calculated from equation (5), with $n=0$, provided sufficient velocity and tide gauge data was avarlable A lack of data prohibits the use of this method An alternative method is to use semi-log plots of the horizontal water velocity and equate the slope of the graph to $U_{*} / K$, where $K=040$ This is likely to over-estimate $U_{*}$ on the ebb tide and under-estimate it on the flood tide A further method is to take $U_{*}=K \bar{U}^{-1}$ where $\bar{U}$ is the depth mean velocity and $K$ has the value $52 \times 10^{-2}$ The method used eventually was to make semi-log plots of velocity and adjust the answer if necessary by the third method The results are shown in Table 2

Table 2 Calculated $U_{*}$ values from Prototype Velocity Data - 25th Nov 1965 Mersey Estuary Position $H$

$\begin{array}{ccccccc}\text { Time (GMT) } & 0801 & 0845 & 0924 & 1003 & 1044 & 1115 \\ \mathrm{U}_{*} \text { (fps) } & 0.099 & 0288 & 0344 & 0342 & 0261 & 0209\end{array}$


(2) Chaice of $\beta$

Flume tests by Vanoni ${ }^{8}$, Laursen ${ }^{9}$ and River observations by Anderson ${ }^{10}$ indicate values of $\beta$ between $063-410$ Einstein ${ }^{11}$ uses a value of $\beta=101 \mathrm{e}$ the momentum $\left(\varepsilon_{\mathrm{m}}\right)$ and sediment $\left(\varepsilon_{\mathrm{s}}\right)$ transfer coefficients are equal In a tidal estuary, it is to be expected that $\beta<10$ due to the salinity circulation It is also probable that the value of $U_{*}$ to be used in the suspension exponent (Z) will also be reduced (to $U_{*}^{\prime}$ ) due to the presence of sand waves on the estuary bed The product $\beta U_{*}^{\prime}$ will thus be expected to be less than $U_{*}$

In view of the lack of theory from which to estrmate $U_{*}^{\prime}$, the quantity $\left(\beta U_{*}^{\prime} / U_{*}\right)$ was determined from two simultaneous sand samples taken at a station in the Irish Sea The samples were taken at maximum velocities from mid-depth and approximately $1 \mathrm{ft}$ above the bottom The value of $\left(\beta U_{*}^{\prime} / U_{*}\right)$ for sand sizes between $63 \mu-355 \mu$ was found to be 062 This implies a value of $\beta=062$ for no reduction in $U_{*}$ In the present work a value of $\left(B U_{*}^{\prime} / U_{*}\right)$ of 0658 has been used

It is interesting to note that Tofalettı's work ${ }^{15}$ indicates a value of $U_{*}^{\prime}$ of $021 \mathrm{fps}$, which implies a $\beta$ value of 1065 for a $U_{*}$ value of 034 Engalund's work $^{16}$, however, would suggest that in deep water (68ft) the reduction in shear due to sand waves is small $(<2 \%)$, this implies a value of $\beta$ closer to 0658

The value of $Z=\omega / \beta K U_{*}^{\prime}$ is thus known at all points during the tidal cycle, w was taken from standard tables ${ }^{12}$ while a value of $k=040$ was used since the concentration values are relatively low

(3) Determination of $\alpha_{0}$ values

The circulation of $\alpha_{0}$ requires a knowledge of both $S^{\prime}$ and $D^{\prime}$ These could be determined if all the terms in equation (5) are known There are, however, no adequate tide gauge readings to the North of position $H \quad \alpha_{0}$ values are thus computed for position $\mathrm{C}$ (Figure 4 ) and used for the calculation at position $\mathrm{H}$ Velocity observations from positions $\mathrm{H}, \mathrm{C}$ and $\mathrm{N}$ were used, together with the tide gauge information from Princes Pier and Gladstone Lock ${ }^{1}$ to determine approximate 
values of $S^{\prime}$ and $D^{\prime}$ The velocity expressions ( $g$ ) and (10) were then used to give a better estimate of $S^{\prime}$ and $D^{\prime}$ The values found at $C$ are shown in Table 3 in terms of the density term (D) $D=386 \times 10^{-6}$

\section{$\underline{T a b l e ~} 3$}

$S^{\prime}$ and $D^{\prime}$ values calculated for Position C Mersey Estuary for a Spring Tide (28ft HW above Liverpool Datum)

\begin{tabular}{|c|c|c|c|c|c|c|c|c|c|}
\hline $\begin{array}{l}\text { TIME } \\
\text { ReI to } \\
\text { H W } \\
\text { Princes } \\
\text { Prer }\end{array}$ & 0 & $-\frac{1}{2}$ & -1 & -2 & $-2 \frac{1}{2}$ & $-3 \frac{1}{2}$ & -4 & -5 & -53 \\
\hline$s^{\prime}$ & -339 & -758 & $\begin{array}{ll}-13 & 51\end{array}$ & -1888 & -1786 & -100 & -50 & -0885 & -022 \\
\hline$D^{\prime}$ & -24 & -178 & 14 & 05 & 038 & 615 & 527 & 177 & 173 \\
\hline$\alpha_{0}$ & 0146 & 0383 & 0552 & 0513 & 0511 & 0808 & 1027 & 150 & 443 \\
\hline
\end{tabular}

The $\alpha_{0}$ values for position $H$ were found by super-imposing the time scale for the prototype observations (25th November 1965) on the values shown in Table 3 and simplifying the results - viz table 5

\section{(4) Determination of sediment concentrations}

The control concentration $\left(C_{t}\right)$ was determined from the prototype data Sediment samples were taken over successive half hourly periods from a fixed distance of 18 inches above the estuary bed using a pump sampler The half hourly sampling trial was necessary in order to obtain sufficient sand for analysis

The samples were washed, dried and welghed in the laboratory and the concentration determined A grain size analysis was performed for each sample using a sedimentation tube ${ }^{13}$, where sufficient sediment existed, and sieves where the sample size was small The results are shown in Table 4 


\section{Table 4}

Mean concentration (ppm) of sand at $18^{\prime \prime}$ above the estuary bed at Position H Mersey Estuary 25th November 1965

\begin{tabular}{|c|c|c|c|c|c|c|c|c|c|}
\hline \multirow[t]{2}{*}{ Sample } & \multicolumn{2}{|c|}{$\begin{array}{l}\text { Mean Sample } \\
\text { Trme (GMT) }\end{array}$} & \multicolumn{5}{|c|}{ Mean Grain Size $(\mu)$} & \multicolumn{2}{|c|}{$\begin{array}{c}\text { l'ater } \\
\text { Depth (ft) }\end{array}$} \\
\hline & & 76 & 106 & 138 & 165 & 195 & 227 & 298 & \\
\hline - & 0810 & 0 & 0 & 0 & 0 & 0 & 0 & 0 & \\
\hline A & 0825 & 176 & 329 & 217 & 113 & 103 & 004 & 005 & 60 \\
\hline B & 0856 & 620 & 163 & 94 & 836 & 54 & 408 & 071 & 65 \\
\hline 0 & 0925 & 99 & 246 & 301 & 338 & 206 & 151 & 246 & 68 \\
\hline $\mathbf{E}$ & 0955 & 398 & 164 & 215 & 173 & 363 & 188 & 188 & 72 \\
\hline$G$ & 1022 & 787 & 1257 & 2019 & 2604 & 4039 & 1715 & 038 & 73 \\
\hline I & 1050 & 255 & 95 & 95 & 1025 & 143 & 444 & 026 & 75 \\
\hline J & 1120 & 22 & 77 & 93 & 104 & 139 & 145 & 0 & 76 \\
\hline K & 1148 & 126 & 206 & 24 & 175 & 089 & 034 & 0 & 77 \\
\hline- & 1200 & 0 & 0 & 0 & 0 & 0 & 0 & 0 & \\
\hline
\end{tabular}

The usefulness of the preceeding theory is checked by computing the quantity of sand that should have been caught in a suspended Oelft Bottle This was kept at approximately loft below the water surface during the period 08300930 (Sample C) and 0934 - 1030 (Sample F) HRS GMT

In order to use the theoretical distribution curves, the $c_{t}$ values should be instantaneous values A correction procedure was thus adopted to allow for the finite sampling time

The method consisted of first calculating the quantity of sediment that would be collected over each sampling period, assuming that the variation of $c_{t}$ with time was proportional to $\left(U_{*}^{2} U^{2}{ }_{*_{C}}^{-1}\right)$, where $U_{*_{C}}$ is the value of $U_{*}$ at threshold conditions The calculated quantity was then compared with the quantity that would be collected if conditions operating at the mean sampling time had prevalled over the full sampling period Clearly, the ratio of these two terms 
should be unity for sniall time intervals or for little variation of $C_{t}$ with time only those samples collected near maximum velocities showed ratios of about unity, all others indicated greater values

The correction procedure thus made the above ratio equal to unity by finding an equivalent mean time, which was a function of grain size, during the sampling interval The observed concentrations were then acredited to this equivalent mean time The concentration at the actual sample mean time was then obtained by interpolation between the equivalent mean times of all the samples

The position of the Oelft Bottle varied between 5-25\% of the depth during the two sampling periods ( $C$ and $F$ ) The concentration at $25 \%$ and $5 \%$ was determined using the appropriate formula (equations 22-25) The correction procedure was then used and the concentration at the Oelft Bottle level found by interpolation The corrected concentration values are shown in Table 5

The quantity of sediment collected in the 0elft Bottle was then determined by summing up the product of the theoretical concentration values and the observed water velocity at the oelft Bottle level The latter quantity was found by mounting an Ott Mark $V$ Arkansas current mater on the 0elft Bottle framework

Table 5

Estimated Theoretical Concentration at 0elft Bottle Leve]

\begin{tabular}{|c|c|c|c|c|c|c|c|c|c|}
\hline \multicolumn{2}{|c|}{ Time (GMT) } & \multicolumn{6}{|c|}{ Mean Grain Size (H) } & \multicolumn{2}{|l|}{$\%$ Level } \\
\hline HRS & 76 & 106 & 138 & 165 & 195 & 227 & 298 & & \\
\hline $\begin{array}{c}0825 \\
\alpha_{0}=070\end{array}$ & $\begin{array}{ll}0 & 293 \\
& 0 \\
0 & 173\end{array}$ & $\begin{array}{l}027 \\
0 \\
0 \quad 117\end{array}$ & $\begin{array}{ll}0 & 017 \\
0 \\
0 & 01\end{array}$ & $\begin{array}{l}0 \\
0 \\
0\end{array}$ & $\begin{array}{l}0 \\
0 \\
0\end{array}$ & $\begin{array}{l}0 \\
0 \\
0\end{array}$ & $\begin{array}{l}0 \\
0 \\
0\end{array}$ & $\begin{array}{r}25 \\
5 \\
167 *\end{array}$ & \\
\hline $\begin{array}{c}0856 \\
\alpha_{0}=0 \quad 60\end{array}$ & $\begin{array}{ll}2 & 05 \\
1 & 24 \\
1 & 66\end{array}$ & $\begin{array}{ll}3 & 2 \\
1 & 45 \\
2 & 36\end{array}$ & $\begin{array}{ll}0 & 64 \\
0 & 20 \\
0 & 43\end{array}$ & $\begin{array}{ll}0 & 20 \\
0 & 036 \\
0 & 1215\end{array}$ & $\begin{array}{ll}0 & 025 \\
0 & 004 \\
0 & 015\end{array}$ & $\begin{array}{ll}0 & 015 \\
0 \\
0 \\
0 & 0078\end{array}$ & $\begin{array}{l}0 \\
0 \\
0\end{array}$ & $\begin{array}{r}25 \\
5 \\
154^{*}\end{array}$ & \\
\hline $\begin{array}{c}0925 \\
\alpha_{0}=050\end{array}$ & $\begin{array}{ll}3 & 96 \\
2 & 89 \\
3 & 29\end{array}$ & $\begin{array}{ll}6 & 22 \\
3 & 93 \\
4 & 79\end{array}$ & $\begin{array}{ll}3 & 5 \\
1 & 69 \\
2 & 37\end{array}$ & $\begin{array}{ll}1 & 9 \\
0 & 712 \\
1 & 157\end{array}$ & $\begin{array}{ll}0 & 589 \\
0 & 775 \\
0 & 33\end{array}$ & $\begin{array}{ll}0 & 216 \\
0 & 051 \\
0 & 113\end{array}$ & $\begin{array}{l}0 \\
0 \\
0\end{array}$ & $\begin{array}{r}25 \\
5 \\
125 *\end{array}$ & $\begin{array}{l}\text { *Delft } \\
\text { Bottle } \\
\text { Level }\end{array}$ \\
\hline $\begin{array}{c}0955 \\
\alpha_{0}=050\end{array}$ & $\begin{array}{ll}1 & 61 \\
1 & 14 \\
1 & 184\end{array}$ & $\begin{array}{ll}4 & 21 \\
2 & 67 \\
2 & 81\end{array}$ & $\begin{array}{ll}2 & 56 \\
1 & 25 \\
1 & 37\end{array}$ & $\begin{array}{ll}0 & 99 \\
0 & 38 \\
0 & 436\end{array}$ & $\begin{array}{ll}1 & 04 \\
0 & 33 \\
0 & 396\end{array}$ & $\begin{array}{ll}0 & 28 \\
0 & 07 \\
0 & 0894\end{array}$ & $\begin{array}{l}0 \\
0 \\
0\end{array}$ & $\begin{array}{c}25 \\
5 \\
6 \stackrel{95}{ }{ }^{*}\end{array}$ & \\
\hline $\begin{array}{c}1022 \\
\alpha_{0}=050\end{array}$ & $\begin{array}{ll}2 & 79 \\
1 & 985 \\
2 & 06\end{array}$ & $\begin{array}{ll}2 & 617 \\
1 & 559 \\
1 & 657\end{array}$ & $\begin{array}{ll}1 & 716 \\
0 & 7664 \\
0 & 8544\end{array}$ & $\begin{array}{ll}0 & 97 \\
0 & 3264 \\
0 & 3859\end{array}$ & $\begin{array}{ll}0 & 684 \\
0 & 1757 \\
0 & 2221\end{array}$ & $\begin{array}{ll}0 & 133 \\
0 & 027 \\
0 & 037\end{array}$ & $\begin{array}{l}0 \\
0 \\
0\end{array}$ & $\begin{array}{c}25 \\
5 \\
685 *\end{array}$ & \\
\hline $\begin{array}{c}1050 \\
a_{0}=050\end{array}$ & $\begin{array}{ll}0 & 70 \\
0 & 46 \\
0 & 526\end{array}$ & $\begin{array}{ll}1 & 344 \\
0 & 7024 \\
0 & 9044\end{array}$ & $\begin{array}{ll}0 & 447 \\
0 & 1635 \\
0 & 2525\end{array}$ & $\begin{array}{ll}0 & 1755 \\
0 & 0464 \\
0 & 087\end{array}$ & $\begin{array}{ll}0 & 092 \\
0 & 0175 \\
0 & 0411\end{array}$ & $\begin{array}{ll}0 & 0121 \\
& 0 \\
0 & 0038\end{array}$ & $\begin{array}{l}0 \\
0 \\
0\end{array}$ & $\begin{array}{c}25 \\
5 \\
113^{*}\end{array}$ & \\
\hline
\end{tabular}


The results of the calculation for sampling periods $C$ and $F$ are shown in Table 6 This also includes an estrmated quantity which is the measured prototype values, corrected $^{14}$ for sediment loss due to high water velocities within the 0elft Bottle

Table 6

Comparison of Theoretical and Observed Sediment Quantities

\begin{tabular}{|c|c|c|c|c|c|c|c|c|}
\hline \multirow[t]{2}{*}{ Sample } & Grain size $(\mu)$ & 75 & 106 & 138 & 165 & 195 & 227 & 298 \\
\hline & Theory (gms) & 406 & 589 & 211 & 0925 & 0261 & 0094 & 0 \\
\hline \multirow[t]{3}{*}{ c } & Measured (gms) & 161 & 374 & 218 & 218 & 130 & 040 & 009 \\
\hline & Estimated (gms) & 248 & 520 & 284 & 270 & 156 & 0465 & 0095 \\
\hline & Theory (gms) & 403 & 60 & 256 & 115 & 072 & 0164 & 0 \\
\hline \multirow[t]{2}{*}{$F$} & Measured (gms) & 072 & 126 & 086 & 063 & 040 & 0116 & 0012 \\
\hline & Estimated (gms) & 204 & 256 & 144 & 097 & 059 & 016 & 0015 \\
\hline
\end{tabular}

Comments

It would appear, from Table 6 , that the theory predicts the sediment quantities reasonably well for grain sizes in excess of $138_{\mu}$, but less well for sizes below this value It is particularly noticeable that the $75 \mu$ sand is predicted to be approximately double the observed value in both cases The lack of agreement of the theory for small sized sediment is undoubtedly due to the longer time required for the fine sediments to reach an equilibrium profile as compared with the larger sized sediments, e $g$ the majority of the steady state profile for $195 \mu$ particles is much closer to the estuary than that of the $75 \mu$ particles

A better method of prediction, particularly for the fine sand size would be to solve equation (12) and use this solution in discrete time steps to determine the concentrations throughout the tidal cycle Preliminary work on this method indicates that the difference between the non-steady state distribution and the steady state distritution is the order of $10 \%$ for $165 \mu$ particles at a level of $10 \%$ above the estuary bed This increases to approximately $35 \%$ at the same level 
for $75 \mu$ particles If the method is applied to the $75 \mu$ particles and adjusted for the observed sediment concentrations, then values of $19 \mathrm{gms}$ and $34 \mathrm{gms}$ are obtained for samples $C$ and $F$ respectively These qualities are much closer to the observed values than the steady state solution

A further improvement, which is being developed at present, is to allow for variations in sedir. concentration with distance and for diffusion in the longitudinal direction

All three methods mentioned above are still dependent upon the values of $\beta$ and $U_{*}$ which were used in the present theory to determine the suspension exponent $Z$ Clearly, the shape of the distribution curve assumes less significance in view of the uncertainty of $\beta, U_{*}$ and to a lesser extent $\omega$ and $K$ The present results do, however, suggest that in the Mersey Estuary reasonable quantitative results can be obtained by using standard values of $\omega$ and $K$, together with $U_{*}$ values calculated from the velocity data ${ }^{l}$ and a $\beta$ value of 066

\section{Conclusions}

The present work leads to expressions for the vertical distribution of sediment which are similar to those of uni-directional flow The derived equations indicate the vertical distribution of sediment in a real estuary subject to restrictions on grain size and estuary type Preliminary work also indicates that the prediction of fine sediment concentrations is better represented by using the non-steady state equation in discrete steps throughout the tidal cycle 
NOTATION

$a$

A

8

$c$

D

D

F

H

$\Delta A$

${ }^{\Delta} F$

$n$

a distance above the estuary bed inertia term

$=\frac{1}{2}(\Delta A+\Delta F)$, a composite slope term concentration density slope effective density slope $=D-B$ kinetic energy term water depth herght of zero nett motion water surface slope (positive when sloping downwards from land to sea) the slope term I $-A_{b}-F_{b}$ Von Karmans Constant $(=040)$

a mixing length the slope term $\frac{1}{2}\left(S^{1}-D^{\prime}\right)$ 


$\begin{array}{ll}\rho & =\text { density } \\ \tau & =\text { shear stress } \\ \omega & \quad=\text { sediment fall velocity }\end{array}$

Subscripts

$b \quad$ bed and near bed

$S$ near surface

$t$ referring to tıme $t$

$y \quad$ level $y$

- level at which $U=0$ 


\section{REFERENCES}

1 A R Halliwell and B A O'Connor "Shear Velocity in a Tidal Estuary" Proc 11 th Conference on Coastal Engineering Chapter 88 pp $1377-1396$ September 1968

2 R Agnew "Estuarine Currents and Tidal Streams" Proc 8th Conf on Coastal Engineering September 1960 Chapter 28 pp 510-535

3 KF Bowden "The Mixing Processes in a Tidal Estuary" Int $J$ Air and Water Pollution Vol 7 p $343-356,1963$

$4 \quad K F$ Bowden and S H Sharaf El Din "Circulation, Salinity and River Discharge in the Mersey Estuary" Geophysics J ig65 Vol 10

5 B A O'Connor "Sediment Movement in a Tidal Estuary" Ph D Thesis Liverpool University April 1969

6 S Tanaka and $S$ Sugimoto on the Distribution of Suspended Sediment in Experimental Flume Flow No 5 Memoirs of Faculty of Engineering Kyoto Japan 1958

$7 \quad H$ Rouse Experiments on the Mechanics of Sediment Suspension Proc 5th International cong for App Mechanics Vol $55 \mathrm{~N}$ Y 1938

8 V A Vanoni "Transportation of Suspended Sediment by Water" Trans ASCE Vol 1111946 pp 67-133

9 E M Laursen "The Total Sediment Load of Streams" Journal Hyd Div ProC ASCE Vol 84 No HY F Feb 1958

10 A G Anderson "Distribution of Sediment in a Natural Stream" Trans A G V pt II 1942 pp 678-683

11 HA Einstein "The Bed Load Function for Sediment Transportation in Open Channel Flows" United States Department of Agriculture Tech Bull No 1026 September 1950

12 WW Rubey "Settling Velocities of Gravel, Sand and Silt Amer J Soc No 251933 p $325-338$

13 P Sly Marıne Geological Studies in the Eastern Irish Sea and Adjacent Estuaries with Special Reference to Sedimentation in Liverpool Bay and River Mersey Ph D thesis Liverpool University 1966

14 Delft Bottle- Directrons for use - Delft Hydraulics Laboratory Pub No 8 November 1963

15 F B Toffaleti Computation of Sand Loads and its Vertical Distribution Nat Meeting on Wat Res Eng New York 1967

16 F Engelund - Hydraulic Resistance of Alluvial Streams Journal Hyd Div Proc ASCE Vol g2 No NY2 March 1966 pp 315-328 


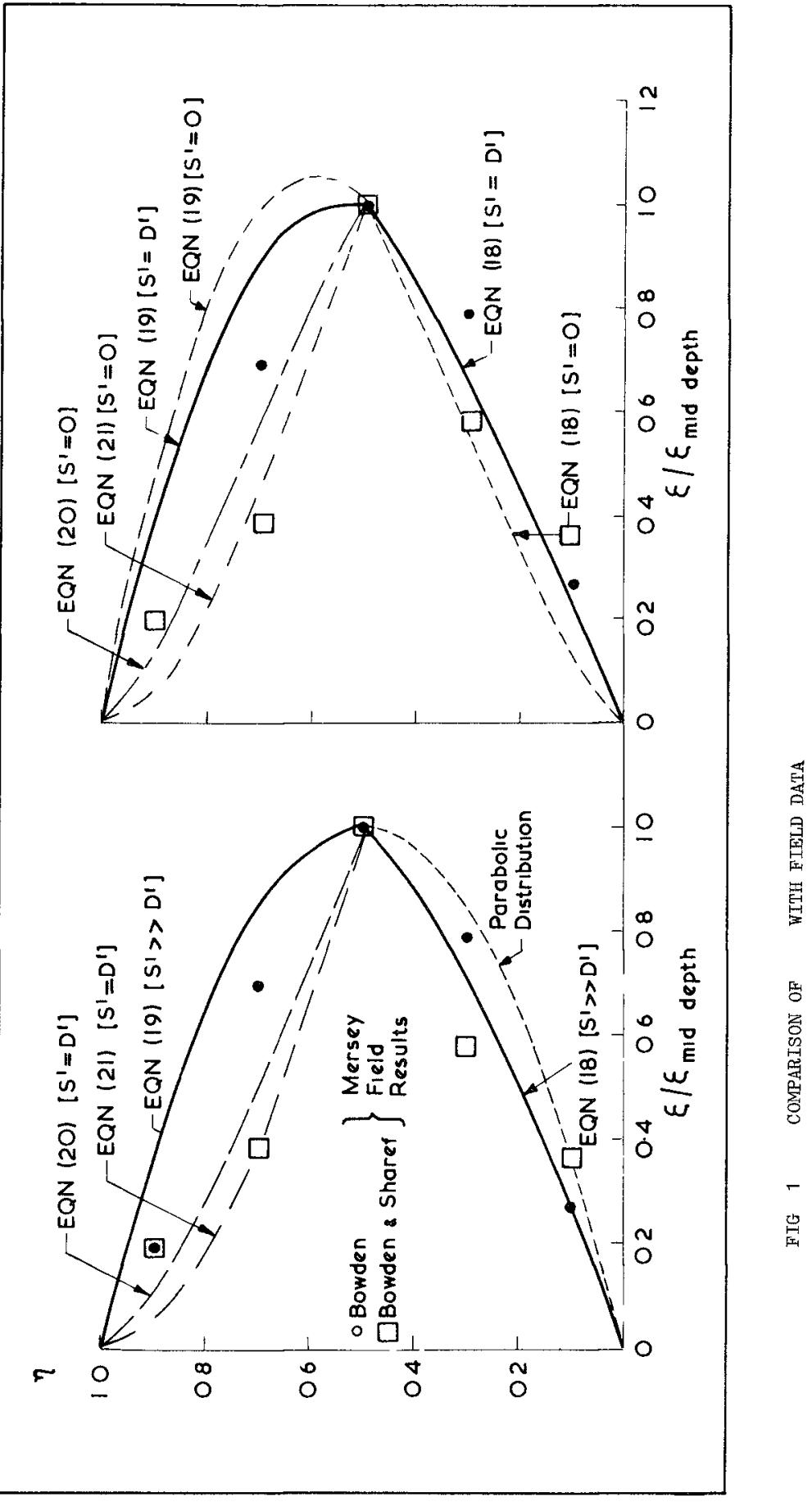




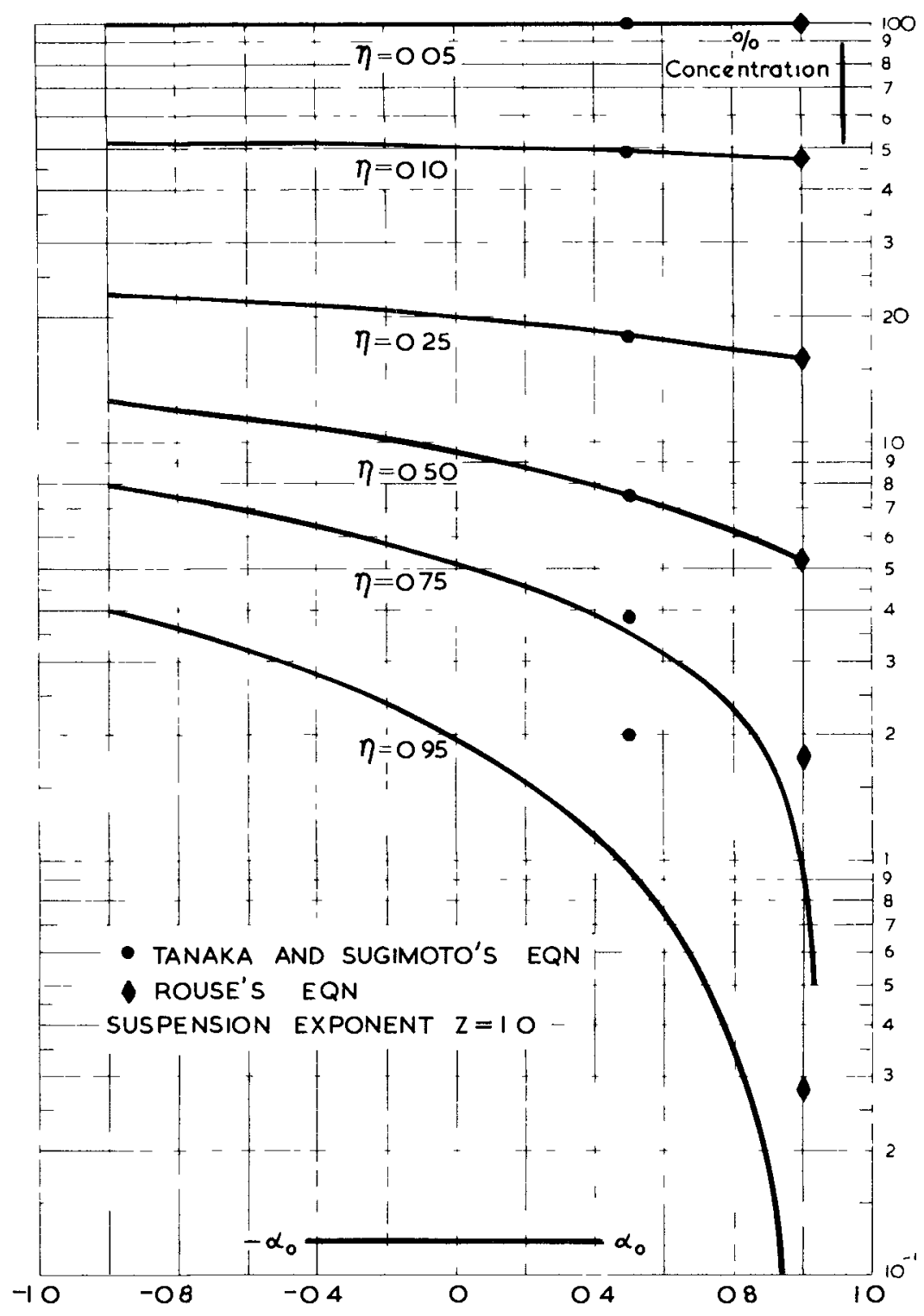

FIG 2 VARIATION OF CONCENTRATION WITH THE PARAMETER 


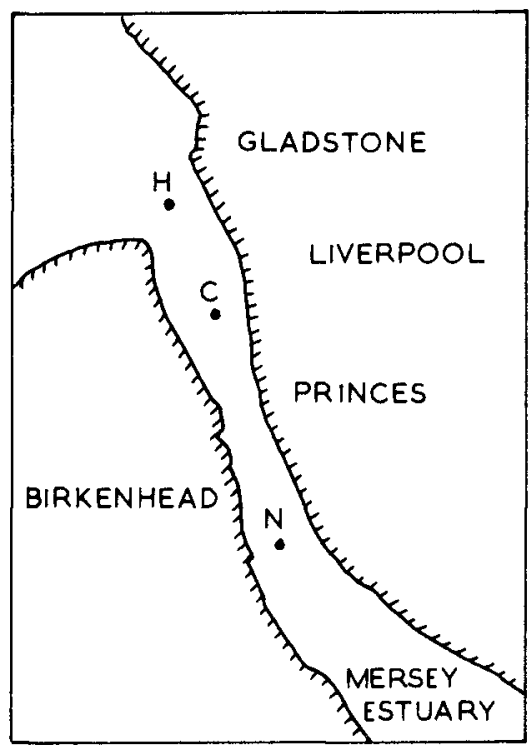

FIG 3 SELECTED OBSERVATION STATIONS - MERSEY ESTUARY

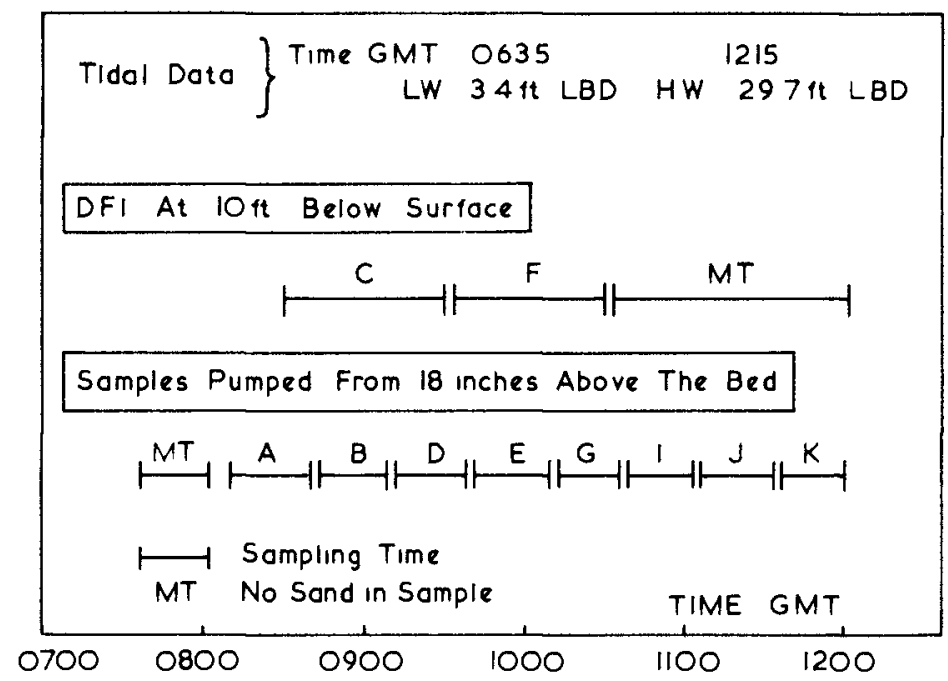

FIG 4 SEDIMENT SAMPLES AT POSITION H - MERSEY ESTUARY (25.11.65) 


\title{
CHAPTER 118
}

\section{PARTICLE VELOCITY REASUREMENTS WITH A LASER DEVICE.}

\author{
by \\ C.A. Greated ${ }^{1}$ and N.B. Webber ${ }^{2}$ M.A.S.C.E.
}

\begin{abstract}
A laser velocimeter has been used to measure particle velocities in a turbulent suspension in open channel flow.

Both mean and fluctuating velocity components can be determined and the system may also be used to glve records of Instantaneous fluld velocities.
\end{abstract}

\section{INTRODUCTION}

Fundamental to the study of those coastal engineering problems which involve diffusion processes or transport of sedimentary material by tidal currents and save action, is the measurement of particle velocities in turbulent flow. Particles of neutral buoyancy which are small compared with the largest wave number eddies wil follow the flow pattern precisely and can therefore be thought of as flud markers In diffusion and sedimentation problems, however, the particles of interest are generally of non-neutral buoyancy and the gravity effect causes them to migrate vertically carrying them across streamlines of the mean flow. In addition to this, the fact that the inertial response of the particles will be different from that of an equal volume of fluid, will cause the particles to move relative to the flur in accelerating turbulent eddies. Thus detalled studies must involve the statistical properties of both the fluid motion and the particle velocities.

Experimental research on these complex motions has hitnerto been handlcapped by lack of satisfactory instrumentation. Photographic metnods have been used to measure particle movements in a Lagrangian manner but necessarily entall tne laborıous analysis of exhaustive data and tend to be very inaccurate Hot film probes and other conventional turbulence measuring systems, on the other hand, are extremely difflcult to use in water and further do not give any indication of particle velocities

In the optical system described here, particles moving within a prescribed measuring volume cause a slgnal to be produced which has a frequency directly proportional to their velocity and thus the Eulerian

1. Lecturer, Mathematics Dept., Southampton University, England.

2. Lecturer, Civil Englneerıng Dept, Southampton Unıversity, England 
statistics of thel motion can be determined Flund velocities can be measured by us $\perp \mathrm{ng}$ extremely fine grain neutral buoyancy tracer particles, assumed to have a negliglble influence on the flow.

\section{OPTICAL MEASURING SYSTEM}

Optical Conflguration Light from a small laser (here lmW helium-neon) is spread and collimated by two cylindrical lenses $L_{1}$ and $L_{2}$ and the parallel beam is then split by a mask $M$ containing two small square apertures of size $a$ and spacing $b$ (figure 1 )
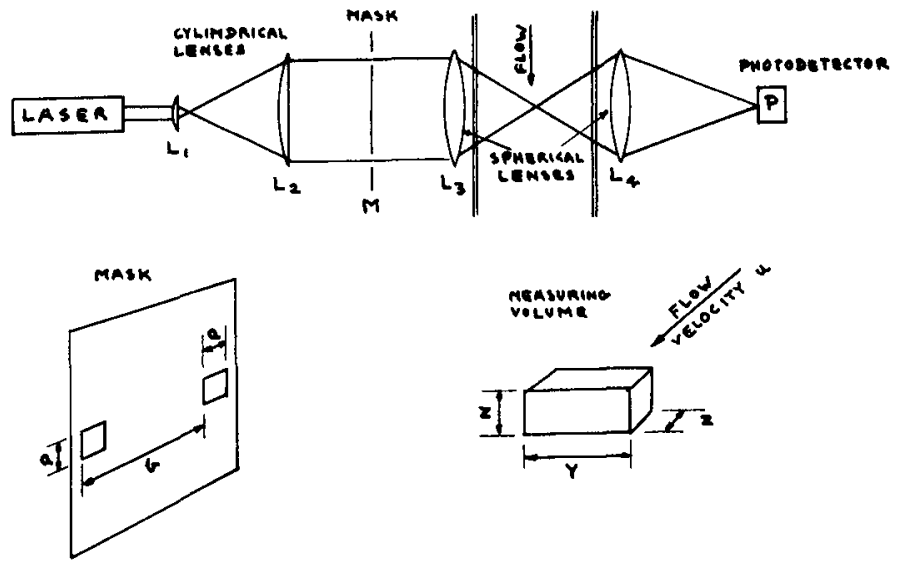

\section{Figure 1. Optical layout.}

A spherical lens $L_{3}$ is then used to bring the two beams to a focus at the requared measuring point in the flow, a further spherical lens $L_{4}$ being used to lmage to focus on to a photodetector $P$.

Interference of the two beams causes a frunge pattern to be formed within a small volume at the focus and as particles move through this volume with velocity $u$, small patches wlll move across the image observed by the photodetector causing a signal of frequency

$$
f_{1}=\frac{b}{\lambda L} u \quad \text { where }\left\{\begin{array}{l}
\lambda=\text { wavelength of laser light } \\
L=\text { focal length of lens } L_{3}
\end{array}\right.
$$

1.e. proportional to velocity. 
Measuring Volume Although the fringes die away gradually to infinity, the measuring volume at the focus from whlch slgniflcant slgnals are produced can be taken as being approximately of size $Z X Z X Y$ where

$$
Z=\frac{\lambda L}{a} \text { and } Y=\frac{\lambda L^{2}}{a b}
$$

This should be chosen so that largest particles. is greater than the dlameter of the

\section{SIGNAL ANALYSIS}

Frequency Domain The simplest method of analysis, lliustrated in figure 2, is to feed the photodetector output directly into an analogue spectrum analyser and integrate the output with an R-C circult The spectrum, whlch can be recorded on an $X-Y$ plotter, then represents the probability distribution of velocitv for particles in the measuring volume

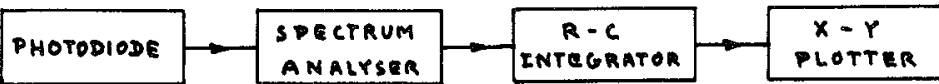

Figure 2. Frequency domain system

Ambigulty in the frequency measurement will occur here due to the finite number of fringes, but the magnitude of this can be predicted by taking the Fourler transforn of the input signal glving

$$
r m s . \text { emblguity }=\frac{a f_{1}}{\sqrt{6} b} .
$$

This should normally be of order $1 \%$ of $f_{1}$ for negligible error in velocity measurement i.e $\frac{b}{a}=40$

The detalls of this analysis have already been published (ref ?) but it is assumed here that the spectrum analyser used gives the modulus of 
the Fourier components of the signal 1.e. the frequency spectrum, rather than the power spectrum This is the case with nearly all commercial spectrum analysers It is also worth noting that the band width of the spectrum analyser should be small and the sweep rate long.

Figure 3 shows a tynlcal spectrum obtalned in a small flume.

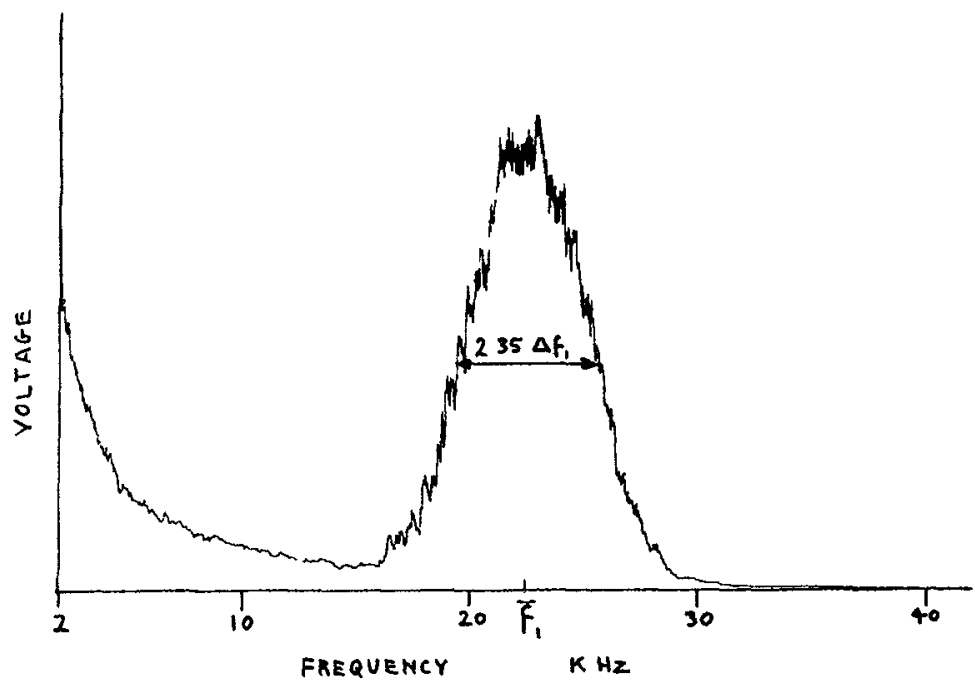

Figure 3. Spectrum of signal from photodetector.

Velocity Cross-Correlations The technique of rotating a hot-wi re probe relative to the mean flow direction, or alternatively using an ' $X$ ' probe, has been used extensively for the measurement of Reynolds stresses in wind tunnels In the same way the fringe pattern in the laser flowmeter can be rotated relative to the optical axis to give cross-correlations of particle velocities Referring to figure 4, let us consider a mean velocity $\bar{u}$ with fluctuating component $u$ ' in that direction, the fluctuating component perpendicular to $\vec{u}$ being $v^{\prime}$.

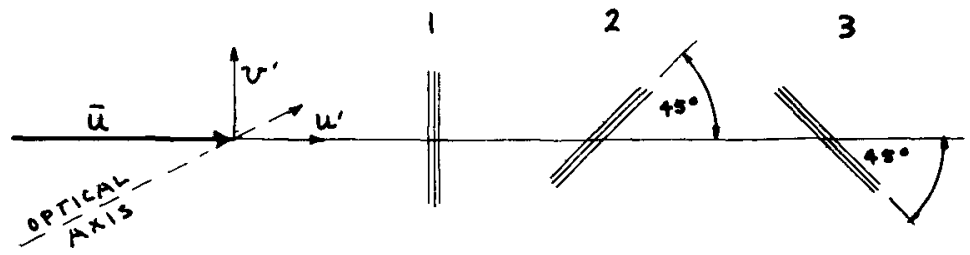

Figure 4. Alignment positions of the fringes. 
Take the constant of proportionality for conversion from frequency to velocity as $K I \mathrm{e}$ with the fringes in position 1 the mean frequency (see flgure 3) will be

$$
\bar{f}_{1}=K \bar{u}
$$
If the r.m.s. width of the spectrum is $\Delta f_{1}$ then the $r m s$ velocity
fluctuation is

$$
\sqrt{\left(\overline{u^{2}}\right)}=\frac{1}{K} \Delta f_{1}
$$

It can then be shown (Ref 2) that by measuring tne $r \mathrm{~m} s$ spectral widths $\Delta f_{2}$ and $\Delta f$ from recordings taken in positions 2 and 3 $1 \mathrm{e}$ at $+45^{\circ}$ and ${ }^{2}-45^{\circ}$ to the mean flow direction, the transverse

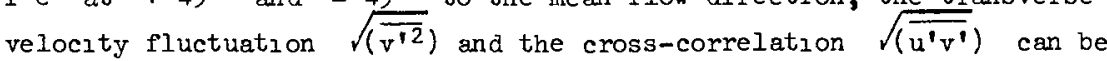
found from

and

$$
\sqrt{\left(\overline{v^{12}}\right)}=\frac{I}{\bar{k}} \sqrt{\left(\Delta \mathrm{f}_{2}^{2}+\Delta \mathrm{f}_{3}^{2}-\Delta \mathrm{f}_{1}^{2}\right)}
$$

$$
\sqrt{\left(\overline{u^{\top} v^{\top}}\right)}=\frac{1}{K} \sqrt{\left(\Delta f_{3}^{2}-\Delta f_{2}^{2}\right)}
$$

For most practical purposes it will be satisfactory to assume that the velocity probability distributions are Gaussian whence the widtl at half the helght is equal to $235 \Delta f$, as indrcated in figure 3

Time Domain Analysis If the system is to be used to record velocities of tracer particles in the fluid then it is possible to construct the electronics in such a way that a continuous signal, proportional to the instantaneous velocity is obtained This will be called the 'time domain' method and the essential elements of the circultry are shown in figure 5 More letaj $^{\top}$ s are $p$ iven in referenco 1

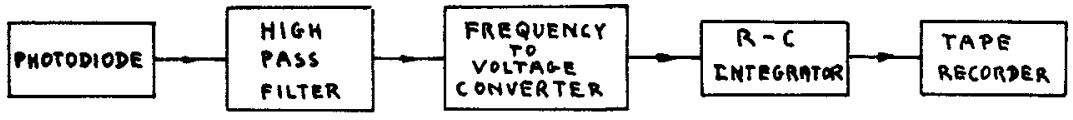

Figure 5. Trme doman system 
Firstly, the signal produced by the photodrode contalns unwanted low frequencles produced by the total amount of 11 ght cut off as particles move across the observation volume, together with the nolse caused by the passage of particles across points in the beam outside the observation volume. These frequencles are eliminated with a hlgh-pass fliter and a frequency-voltage converter is used to glve an output signal proportional to the rate of zero crosings

Even if the fluid velocity were absolutely constant, the signal from the frequency-voltage converter would contaln small fluctuations due to the finite number of fringes, the r.m.s. value of this fluctuation being equal to $a /(\sqrt{6} \mathrm{~b})$ times the mean value. These can be damped before recording by using an $\mathrm{R}-\mathrm{C}$ integrator, thus improving the resolution, but the integrating time should not be so long as to damp fluctuations due to velocity changes

\section{PRELIMINARY RESULTS}

Some preliminary results using the 'frequency domain' method are lllustrated in flgure 6 for the motion of glass spheres of diameter about $0.075 \mathrm{~mm}$ in steady free surface flow. The velocities in the direction of mean flow were recorded in a channel $5 \mathrm{~cm}$ wl de with a measuring volume of size $z=04 \mathrm{~mm}, Y=1 \mathrm{~cm}$ These show the characteristic fall off in mean velocitv and increase in $r \mathrm{m.s}$. velocity fluctuation towards the bed

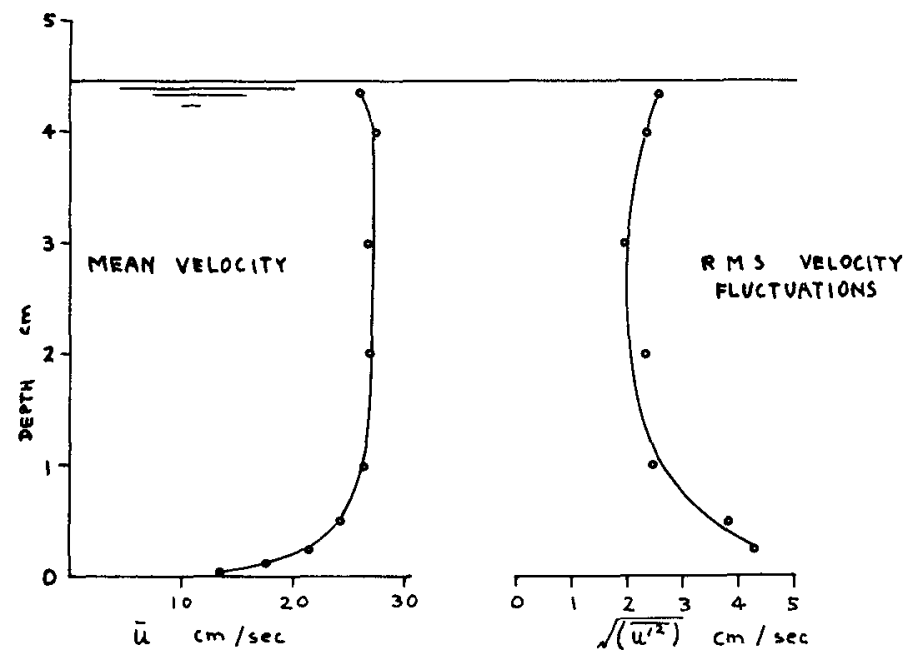

Figure 6 Particle velocities in open channel flow 
At present the system is Deins adanted for measurements in a flume of $\mathrm{lm}$ vidth and $1 \mathrm{t}$ is hoped that 1 t wlll be possible to make simultaneous measurements of velocities and particle concentrations

\section{POTENTIALITIES OF PFASURI.NG SYSTEM}

The system described would seem to fulfill the main requirements of a coastal englneering laboratory both fluid velocities and velocities of larger suspended particles can be meisured with precision and to within a fraction of a milizmeter of a wall The instrument is directionally sensitive and rotation of the fringe pattern wlll give transverse velocity components and cross-correlations

RETDRENCLS

1 Greated C, 1970 Journal of Phvsies $\mathrm{I}$ sel. Instrum Vol 3, $D_{p} 158-160$

2 Greated C, 1970 Journal of Paysies I ScI Instrum Vol 3, Pp 753-756 

CHAPTER 119

DENSITY CURRENTS AND TURBULENT DIFFUSION IN LOCKS

by

A Roy Halliwell* and Martın $0^{\prime}$ Dell ${ }^{+}$

ABSTRACT

The paper describes a study of density-currents in locks where there 15 a net inflow of water into the dock The velocity profiles occurring are different than might be expected by simply superimposing the net flow onto a normal density-current profile The differences are shown to be due to the non-uniform salinity profiles occurring in the docks and a semp-theoretical study is presented which 111 ustrates the importance of this salinity profile

The accretion in the docks is explained by the transport of material into the docks during the levelling period The quantitative agreement between estimates based on measurements during the levelling period and the dredged quantities from the dock are good The locking operation is also shown to be an important factor in certain cases

An attempt to describe the flow of silt into the dock using the onedimensional diffusion equation has shown that allowance must be made for the pick up of silt off the bed of the lock and the settling of silt (as the velocities drop) if quantitative agreement is to be obtained

* Senior Lecturer, Department of Civil Engineerıng, Liverpool University, Liverpool, England

+ Research Student, Department of Civil Engineering, Liverpool University, Liverpool, England 


\section{INTRODUCTION}

It is common practice in some docks on the Mersey Estuary (and elsewhere) to have a "levelling" period of about one hour (before high water) This is achieved, of course, by opening the gates at both ends of the lock communicating with the estuary, the water level in the dock having been run down (if necessary) in order to meet the rising tide level in the estuary Thus, water can be impounded in the dock by inward flow, vessels can enter or leave without delay, and those longer than the lock itself can come and go at this time

In the last few years, the problem of siltation in some of the Mersey docks has been investigated Velocities, silt-concentrations and salinities have all been measured during the levelling periods During this period, there is an influx of water into the dock, as the tide rises, but there is also a density-current between the estuary and the less-dense dock water

The observations in two particular docks are discussed - Gladstone and Bromborough In the case of Gladstone the water is impounded into the dock system by means of the levelling process and also by pumping At Bromborough dock there is a fresh water discharge into the dock from a small river, which along with the levelling process provides the impounded water Another important difference between the two docks is that the lock at Gladstone is long enough to cope with all the ships using the dock and therefore the levelling process is not essential (although of course it may be desirable) whereas the Bromborough lock is relatively small and the levelling period provides the only means for some ships to use the dock

\section{MEASUREMENTS}

The measurements made in each of the locks have been similar in type, but partly because the sizes of the locks are very different the detall 
execution of these measurements has been different at Bromborough than at Gladstone In each case vertical traverses have been made of velocity, silt, salinity and temperature using in-situ measurement with directreading instruments These traverses have been made throughout the levelling period and also for different tides ( 1 e spring/neap) and different season ( 1 e summer/winter) In addition to these, measurements of the $517 t$ in suspension near to the river entrance of the lock were made These were made using the same type of silt-meter as for the traverses, based on the 11ght-extinction method, along with a recorder These allowed a continuous record of $511 t$ in suspension near the lock entrance to be made

Bromborough dock is relatively small, the lock is some $70 \mathrm{ft}$ wide, $160 \mathrm{ft}$ long and $30 \mathrm{ft}$ deep The vertical traverses in this case were made using a cantilever truss fixed to a dock transport bogie, the observation bogie was driven up to the edge of the lock, the truss being of sufficient length to reach the centre of the lock Two sets of instruments were suspended from the truss so that traverses could be made at above $25 \mathrm{ft}$ and loft from the lock wall Each unit consisted of silt and salinity/ temperature probes attached to a current meter and the unit was lowered and raised by hand Readings were made at approximately six or seven levels and each traverse took about twelve minutes to complete In addition to these, measurements were sometimes taken at fixed levels above the bed so that an almost continuous record of velocity etc was obtained throughout the leveling period at these positions This particular rig had the advantage of cheapness and more important still, it allowed the instruments to be removed from the lock when necessary, for example the passage of ships in and out of the lock

Gladstone lock is some $130 \mathrm{ft}$ wide, $600 \mathrm{ft}$ long and $50 \mathrm{ft}$ deep and this meant that measurements were somewhat more difficult in this situation 
than at Bromborough In genera 1, traverses were made by lowering the instruments from a vessel in the lock However, some traverses were made at about the middle of the lock and on the centre line of the lock by raising and lowering the instruments from a swinging foot-bridge across the lock

\section{DENSITY-CURRENT PROFILES}

Although there is a considerable amount of literature published on density exchange flow the authors are not aware of any which deal with the situation where there is a net influx of water across the section In the case of the Gladstone lock the influx of velocity at the start of the level (when the tide is still rising at approximately $5 \mathrm{ft}$ per hour) is considerably greater than the density current At high water, of course, there is no net influx of water and the velocities are entirely due to the differences in density In Bromborough lock the density differences are greater than at Gladstone, while the influx velocities are about the same as those at Gladstone

When the lock gates are opened to the estuary, at say $1 \frac{1}{2}$ hours before high water, water enters the dock through the lock due to the rising tide this velocity is termed continuity velocity and is defined as

$$
\bar{V}=\frac{\text { plan area of dock } \times \text { rate of rise of } t>d e}{\text { cross-sectional area of lock }}
$$

Superimposed upon this continuity velocity there is a density-current between the estuary and the less-dense lock water which produces an outward flow at the surface and an inward flow near the bed figure 1 shows some examples of the vertical velocity profile occurring at the centre of the Bromborough lock during the levelling period on a spring tide The importance of the continurty velocity at the start of the level can be seen by comparing figure 1 (a) with $1(c)$ 
The average iriflux velocity is defincd as

$$
\frac{1}{H} \int_{0}^{H} u d y
$$

where $u$ is velocity into dock at depth $y$,

$H$ is the depth of water

The velocity profile due to density differences alone 15 seen around the high water period in figure 1 (c) when the average influx velocity is almost zero (and the contrnuity velocity is zero) The zero velocity point occurs at a level above the mid-depth position (approximately 40\% depth) and the velocities out of the dock (in the upper surface layers) are considerably greater than those entering the dock (in the lower layers) It is immediately apparent therefore that the velocity profile due to the density-current alone is not that occurring in the situation 111 ustrated in case (A), Figure 2, where the zero velocity point occurs at $50 \%$ depth and the overflow velocity is approximately $125 \times$ the underflow velocity (see refs 1 and 2 )

Measurements of salinity within the Bromborough dock on a number of occasions have shown that the salinity profile within the dock is in fact far from uniform Two examples are given in Figure (3), and it is clear that the starting conditions are more likely to be similar to the situation 111 ustrated in case (B) of Figure (2) than those of case (A) Some model tests have been made of both cases $A$ and $B$ and these have indrcated the velocity profiles for the two cases are indeed different as illustrated in Figure (2) The model tests are still continuing and the results cannot therefore be discussed in detail in this paper but the profile indicated for case (B) in Figure (2) is clearly very close to that measured in the docks on a number of occasions and in particular to that given in Figure 1 (c) 
The underflow velocity occurring in the situation illustrated in case A (fig 2) is given theoreticaliy by

$$
v_{u}=05 v_{\Delta}
$$

and has been assessed experimentally by a number of researchers (e g ref 1 ) as

$$
v_{u}=0465 V_{\Delta}
$$

where

$$
V_{\Delta}=\left(\frac{\Delta \rho}{\rho_{m}} g H\right)^{\frac{1}{2}}
$$

$g=$ the acceleration due to gravity

$P_{m}=$ the average density of the two 11quids

$\Delta p=$ the density difference between the two liquids

$H=$ the depth of the lock

Consider the general case for the situation illustrated in case $B$ (fig 2), let the freshwater occupy the depth aH before the exchange flow starts Assume that the exchange flow is rectangular block flow as 11 lustrated in the sketch below and that the crossover point (or zero velocity) is at depth $\mathrm{KH}$ Let the underflow and overflow velocities be denoted by $v_{u}$ and $v_{0}$ respectively Then at time $\Delta t$ after the barrier is removed the fronts $w 11$ be in the positions 111 ustrated in the sketch

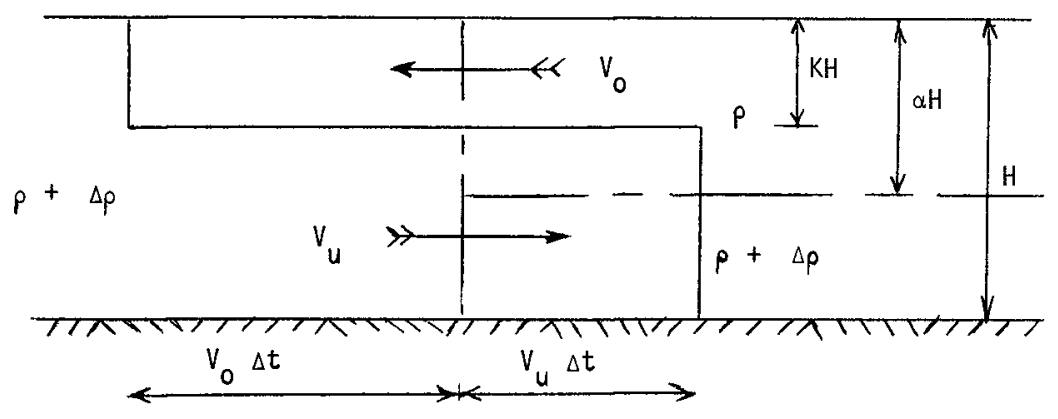


The continurty equation gives

$$
v_{0}=\left(\frac{l-k}{K}\right) v_{u}
$$

The gain in kinetic energy is

$\frac{1}{2} \rho\left\{K H\left(V_{0}+V_{u}\right) \Delta t\right\} V_{0}^{2}+\frac{1}{2}(\rho+\Delta \rho)\left\{(1-K) H\left(V_{0}+V_{u}\right) \Delta t\right\} V_{u}^{2}$

so substituting equation (5) into equation (6) and assuming $\Delta \rho$ is small compared to $\rho$

$$
\text { gain in kinetic energy }=\frac{1}{2} \rho_{m} V_{u}^{3} \Delta t \quad H \quad\left(\frac{1-k}{k^{2}}\right)
$$

The loss of potential energy is

$$
\begin{aligned}
\Delta p\left(V_{0} \Delta t\right) g K H & (\alpha-05 K) H \\
& -\Delta \rho\left(V_{u} \Delta t\right) g(\alpha-K) H \begin{cases}0 & 5(\alpha-K) H\}\end{cases}
\end{aligned}
$$

or

$$
\Delta p g \Delta t H^{2} v_{u}\left[\alpha-05 \alpha^{2}-05 k\right]
$$

Equating the gain in kinetic energy to the loss in potential energy gives

$$
v_{u}^{2}=\left\{\frac{2 K^{2}\left(\alpha-05 \alpha^{2}-05 K\right)}{(1-K)}\right\} v_{\Delta}^{2}
$$

Case 1 $\alpha=10$ and $K=05$ equation (10) and (5) yields

$$
v_{u}=05 \quad v_{\Delta} \text { and } v_{0}=v_{u}
$$

which is the theoretical result for Case $A$ and confirms equation (3)

Case 2 $\alpha=05$ and $K=04$ equations (10) and (5) yield

$$
v_{u}=033 v_{\Delta} \text { and } v_{0}=15 v_{u}
$$

and this represents the model test results of Case B ( $f 1 g$ 2)

Case 3 $\alpha=02$ and $K=015$ equations (10) and (5) yield

$$
v_{u}=0075 v_{\Delta} \text { and } v_{0}=567 \mathrm{~V}
$$


Equation (10) shows the importance of the salinity profiles occurring within the docks. The overflow velocity may not alter very much with changes in salinity profile within the dock, but the underflow velocity is greatly reduced compared with that given by equations (3) or (4)

If the density difference is expressed in terms of a difference of salinity then for the situations in Bromborough lock ( $H=32 \mathrm{ft}$ approximately) and Gladstone lock ( $H=50 f t$ approximately), the underflow velocity according to equation (4) can be expressed as

$$
\begin{aligned}
& v_{u}=0403(\Delta S)^{\frac{1}{2}} \text { at 'Bromborough } \\
& v_{u}=0507(\Delta S)^{\frac{1}{2}} \text { at Gladstone }
\end{aligned}
$$

Using equation (12) the corresponding relationships are

$$
\begin{aligned}
& v_{u}=027(\Delta S)^{\frac{1}{2}} \\
& v_{u}=034(\Delta S)^{\frac{1}{2}}
\end{aligned}
$$

(allowing for the theoretical value to be reduced by 093 in a s imilar way to equation (3))

The velocities measured at Bromborough and Gladstone compare very well with those obtained using equation (15) whereas the values obtained using equation (14) which is based on equation (4) are considerabiy in error The profile is also more closely described by the modified block flow lllustrated in the sketch and the surface velocity is close to the value given in equation (12)

Fig (4) shows the velocities measured at a fixed level above the bed These confirm that the resulting velocity during the leveling process is the simple addition of the average influx velocity (or continuity velocity) and the density-current The density-current is almost constant throughout the level period and equal in this case to $075 \mathrm{ft} / \mathrm{sec}$ The maximum underflow velocity due to the density difference 15 about $10 \mathrm{ft} / \mathrm{sec}$ (occurring at a depth of about 65\%) and compares very closely with the value obtained using equation (15) - the value of $\Delta S$ being approximately $12 \%$ 


\section{ACCRETION IN THE DOCKS}

The main practical interest of the work was centred on the dredging requirements for the docks and the possible means of reducing them The transport of silt through the lock into the dock was determined from the measurements of velocity and silt For example the variation of silt concentration with depth for the traverse corresponding to Fig $T$ (a) is given in Fig 6(a) and the resultant transport of silt is proportional to (velocity $x$ silt concentration) This figure immediately shows the silt burdened estuary water entering the dock in the lower layers and the relatively clear dock water leaving in the upper surfaces By integrating traverses such as these the varration throughout the level period of silt transport into the docks has been determıned (see for example Fig 6(a)) and thence the total amount of material entering during the level period

In the case of Bromborough, measurements on the 10th December 1969 a spring tide - showed that a total amount of 350 tons dry weight of silt entered the dock during the levelling period The amount of solids in the material dredged from the docks depends on the type of material and the consolidation occurring The density of the material in the hoppers of the dredgers has been measured on a number of occasions and has indicated that the percentage of solids is usually less than $40 \%$ and that a reasonable figure is $36 \%$ so that

dredged tonnage $=28 \times$ (tons of dry silt transported into the dock)

This figure also implies that one cubic yard of dredged material weighs approximately one ton, assuming that the specific gravity of the solids is 27 Using this value the amount of material entering the dock in the level period during a spring tide in winter conditions is approximately 1000 dredged tons

Before any attempt can be made to calculate the total amount of silt 
entering the docks throughout the year the variation with tide and season of the silt in suspension in the estuary near the entrance is required The silt concentration varies with tide range and with season and the silt pattern at any position can be very different from that occurring relatively short distances away (see ref 3 ) Continuous silt measurements were therefore made near the entrance to the locks and the results from these instruments were used to allow for the variations throughout the year

When this was done for Bromborough oock the amount entering the dock during the levelling processes was estimated as 270,000 dredged tons (or cubic yards) per annum The average amount of material dredged during the period 1964-68 was 300,000 dredged tons per annum The estimated figure therefore compares very well with the actual amount, however there is another mechanism by which a significant amount of material can enter the dock - the locking procedure or operation

During a locking operation the water in the lock is almost always of different density than that in the estuary At Bromborough the water is usually less saline in the lock (due to the freshwater inflow into the dock) and the estuary water is also carrying a high suspended load which further increases the density difference When the outer gates are opened therefore a density exchange flow starts with the heavier estuary water intruding into the lock near the bed and eventually, if the gates are open long enough, replacing almost all the lock water (ref 2) If the water in the lock is more dense than that in the estuary, for example near the period of low water, then the heavier lock water leaves the lock in the lower layers bringing the estuary water into the lock in the upper layers Whichever mechanism occurs silt is brought into the lock from the estuary during the locking operation when the inner gates (into the dock) are next opened, then a similar mechanısm carries the water and 
silt from the lock into the dock so that all the silt carried into the lock during the locking procedure is eventualiy deposited on the bed of the dock

The relatively small size of Bromborough lock and the large density differences mean that the time needed for say $80 \%$ of the lock water to be replaced by estuary water is not very great (of the order of 10 minutes) At Gladstone the lock is relatively large and the density differences are small so that the time needed for $80 \%$ of the lock water to be replaced by estuary water may be quite large (of the order of an hour) obviously, therefore, the locking procedure may be a significant factor for Bromborough, but is unlikely to be so in the case of Gladstone Estrmates for Bromborough, allowing for half the volume of water in the lock to be exchanged during each locking operation, indicate that approximately 75,000 dredged tons per annum enter the dock through this mechanism The estimated total quantity of material entering the Bromborough dock is therefore approximately 350,000 dredged tons per annum and this, considering the various assumptions made, compares very well with the actual figure of 300,000 dredged tons per annum (averaged)

A number of estimates have been made for the Gladstone lock and these have all shown that the average annual dredging figure of 265,000 hopper tons (1966-68) is accounted for to within a few percent by the leveling process (ref 4) and that the locking procedure does not contribute significantly in this situation

As a result of this work the levelling process at Glads tone dock has been stopped - the water now being impounded entirely by pumping - and at Bromborough (where the levelling period provides the only means of some ships using the dock) the levelling period has been reduced to a minimum There has been immediate reductions in the quantities dredged at both docks 
The one-dimensional convective diffusion equation for a soluble substance in turbulent flow is (see ref 5)

$$
\frac{\partial c}{\partial t}+u \frac{\partial c}{d x}=\frac{\partial}{\delta x}\left(D_{t x} \frac{\partial c}{\delta x}\right)
$$

where $U$ is the average flow velocity across the section $D_{t x}$ is the turbulent diffusion coefficient

c is the concentration of the soluble substance

If a point injection of material is made into the flow at position $x=0$ and time $t=0$ then the concentration at time $t$ and position $x$ is given by Taylor as

$$
c=\frac{M}{A \rho\left(4 \pi D_{t} t\right)^{\frac{1}{2}}} e^{-\frac{(x-U t)^{2}}{4 D_{t} t}}
$$

where $\quad M$ is the mass of material introduced

A is the cross-sectional area of flow

$\rho$ is the density of the flurd

The solution for a series of point injections can obviously be obtained by superimposing the solutions for each separate injection This type of solution is well suited to the digital computer and a computer program has been written (ref 4) incorporating the Taylor solution for finite source injections The program allows for the possibility of injections at various points along the $x$-axis ( 1 e at points other than $x=0$ ) and this allows various initial boundary conditions to be imposed upon the system, 1 e at $t=0, c=c(x)$ is simulated by a series of injections of different strengths at various points along the $x$-axis

Silt measurements in the estuary at the entrance to the Gladstone lock presented the attractive possibility of comparing the measured dis- 
tributions of silt at stations along the lock with computed values using the computer program The vertical distribution of silt was simulated by dividing the lock into five layers with injections being made into each layer, the magnitude of each injection being arranged to produce the measured vertical distribution of silt occurring at the time near the river entrance to the lock The technique for determining a suitable dispersion coefficient for each layer was empirical and to some extent arbitary (ref 4) Eventually after consulting the various 17 terature a value of approximately $100 \mathrm{ft}^{2} / \mathrm{sec}$ was used

Comparison of the field measurements taken with the results obtained from the computer solution showed that there were large differences in magnitude between the two sets of curves However, the basic patterns exhibited by corresponding curves were similar The computed concentration results showed that the times of the peaks occurring in the $90 \%$ and $70 \%$ layers agreed reasonably with the observed results The average difference in concentration over the levelling period between the computed and observed results were determined for each layer and these suggested that material was being picked up from the bed and being diffused upwards into each layer, the quantity becoming successively smaller towards the surface layer This process is almost certain to be occurring since on the day in question a layer of fluid mud was present on the lock sill at most times during the levelling period

Close to high water the observed concentration in the lock decreased especially in the $70 \%$ and $90 \%$ layers, whereas the computed concentrations were still slowly increasing This is thought to be due to the occurrence of flocculation which in turn causes rapid settling of the suspended silt If quantitative agreement is to be obtained therefore it would seem that allowance must be made for both prck-up and settling of silt 
CONCLUSIONS

(1) When there is a net influx of water superimposed upon a densitycurrent situation the resulting velocity distribution is given (to a first approximation) by superimposing a uniform velocity throughout the depth(equal to the influx flow) upon the density-current profile

(2) The density-current profile is considerably influenced by the salinity profiles occurring within the docks

(3) The practice of levelling 15 responsible for most of the accretion occurring in the docks examined in the Mersey Estuary The locking operations account for some material and of course if water is impounded by pumping this may also be an important contribution

(4) Comparison of field measurements of suspended solids in the lock, with results obtained using a computer solution incorporating the Taylor solution for a finite source injection (and dividing the depth of flow in the lock into layers), show that the general pattern of silt transport into the dock can be qualitively described by the semp-theoretical results However, quantitative agreement is poor and this is considered to be due to

(a) the prck-up of silt off the bed of the lock

(b) the settling of silt (when flocculation occurs)

neither of these processes being allowed for in the computer solution

\section{ACKNOWLEDGEMENTS}

The field measurements described were sponsored by the Mersey Docks and Harbour Board and Unilever Merseyside Ltd, and the helpful cooperation of both these organisations is acknowledged The authors are particularly grateful to Mr J D Littler of the University Civil Engineering Department, for his considerable help with many of the measurements taken 


\section{REFERENCES}

(1) DI H Barr Aspects of density surge phenomena

Educational Fluid Mechanics No 31968 Arnfield Engineering Ltd

(2) GH Keulegan An experimental study of the motion of saline water from locks into fresh water channels

U S Dept of Commerce, Nat Bureau of Standards, Report No 5168,1957

(3) A R Halliwell and MO Dell Differences in silt patterns across an estuary

The Dock and Harbour Authority Vol L, No 585, July 1969

(4) MO Dell Silt distributions and s1ltation processes (with particular reference to the Mersey Estuary and Dock systems)

Ph D Thesis Liverpool University Dec 1969

(5) A T Ippen Estuary and coastline hydrodynamıcs published by McGraw $\mathrm{H}_{1} 11$ 
surface

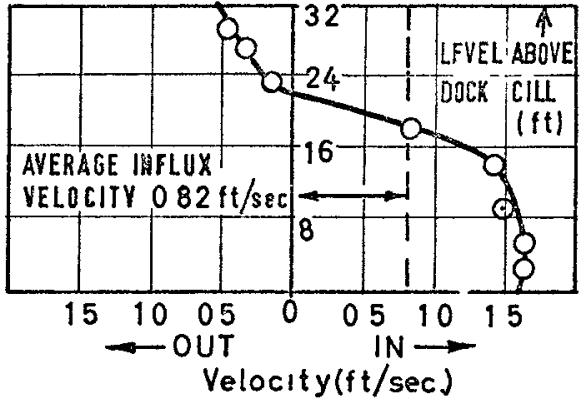

(a) AT APPROX 47 MINS BEFORE HW

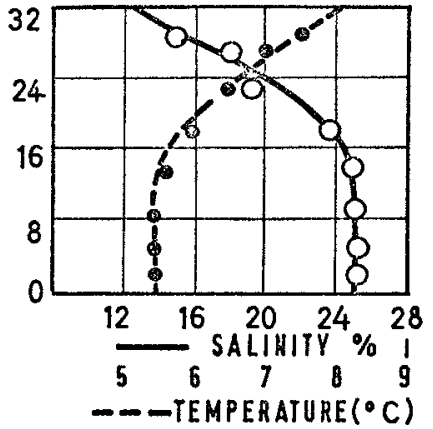

surface

(b) AT APPROX 30 MINS BEFORE HW
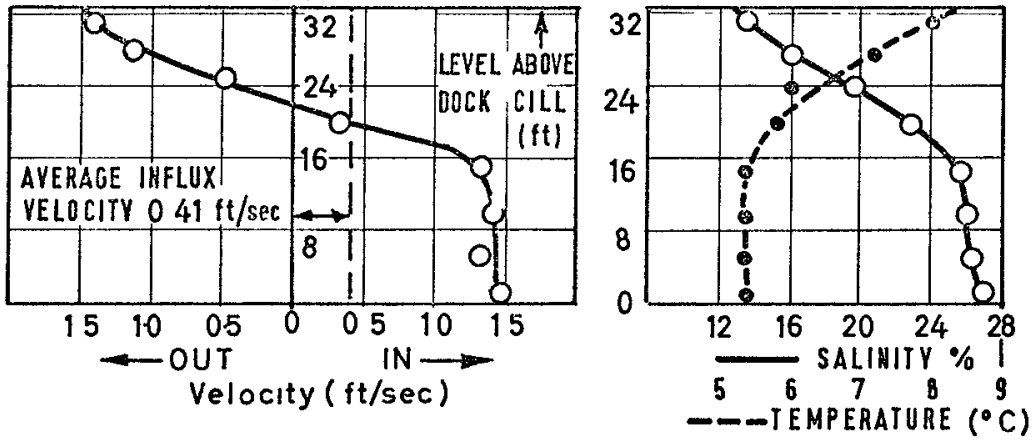

surface (c) AT APPROX HW
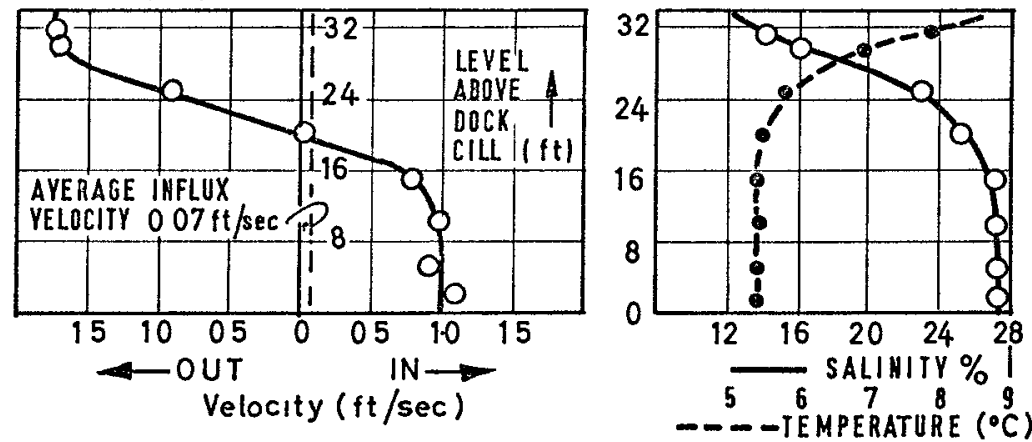

FIG 1 DENSITY-CURRENT PROFILES WITH NET INFLUX-

VELOCITIES (Bromborough Lock 10 12 69) 


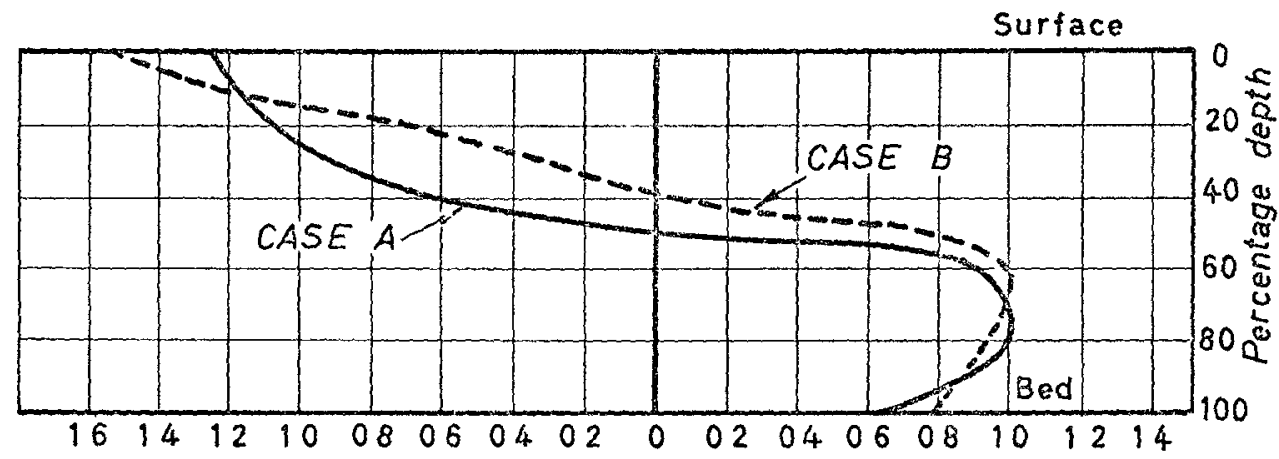

NON-DIMENSIONAL VELOC'TY (Velocity/max underflow velocity)
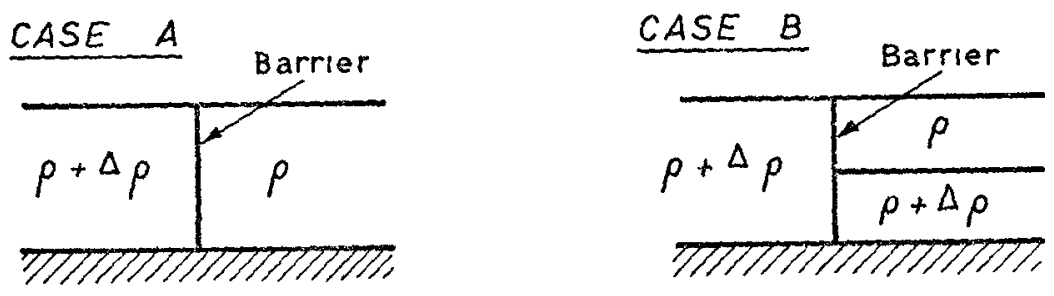

STARTING CONDITIONS

FIG 2 COMPARISON OF VELOCITY PROFILES DURING EXCHANGE FLOW FOR DIFFERENT STARTING CONDITIONS (MODEL TESTS)
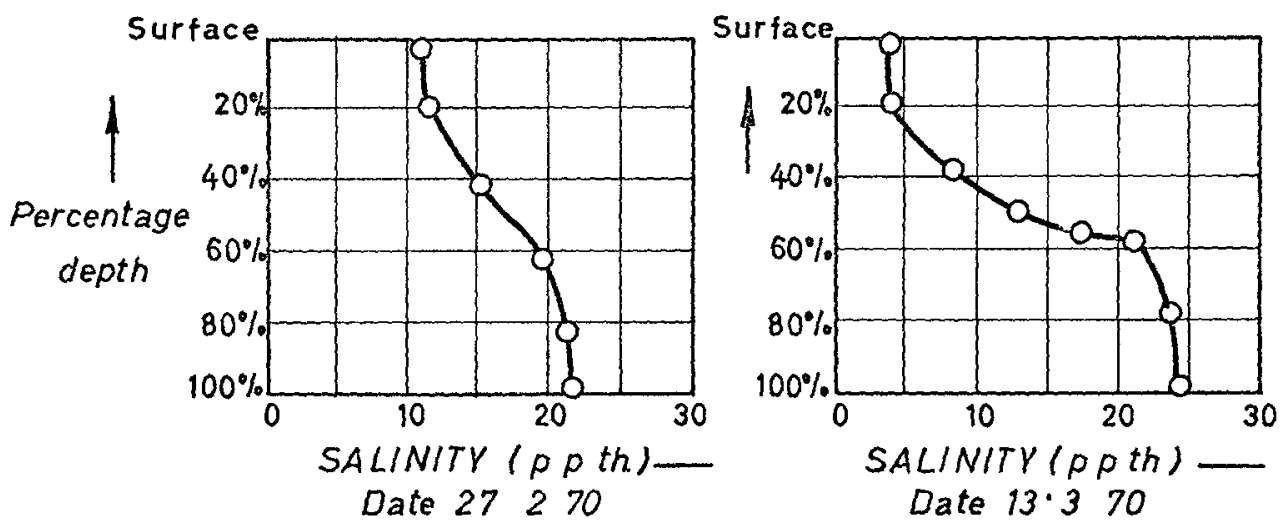

FIG 3 EXAMPLES OF SALINITY PROFILES IN BROMBOROUGH DOCK (MERSEY ESTUARY) 


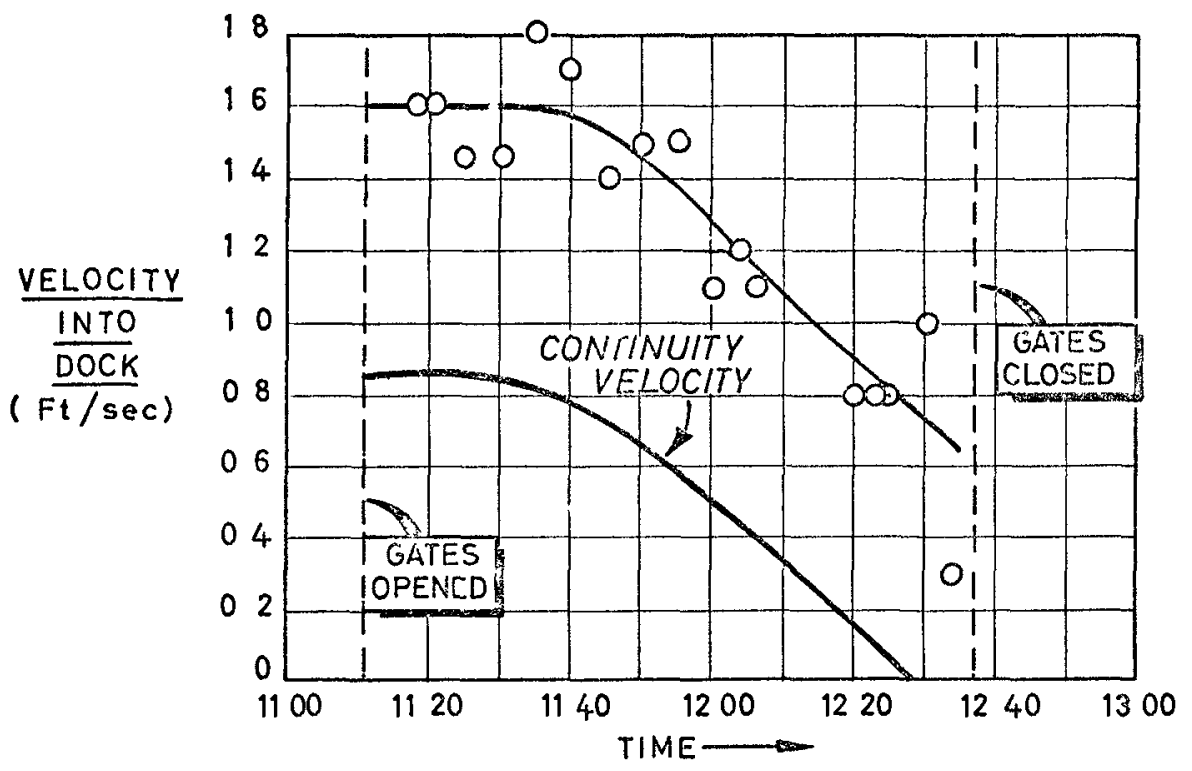

FIG 4 VELOCITY INTO DOCK MEASURED AT A POSITION 1\%.FT ABOVE DOCK CILL ( 1 e at approx $56 \%$ depth) BROMBOROUGH LOCK $10-12 \sim 69$

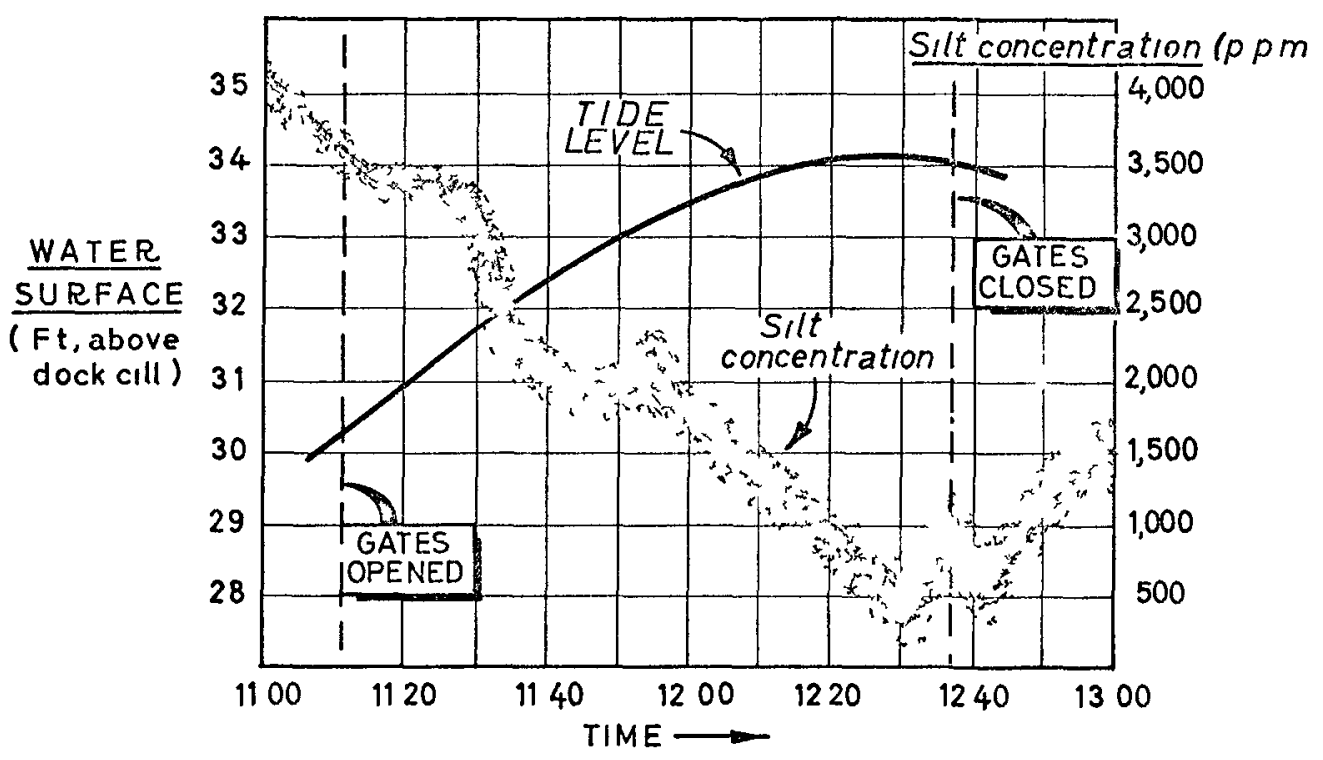

FIG 5 VARIATION OF SILT CONCENTRATION AT JETTY (NEAR BED) AND TIDE LEVEL

BROMBOROUGH LOCK $\quad 10 \sim 12 \sim 69$ 


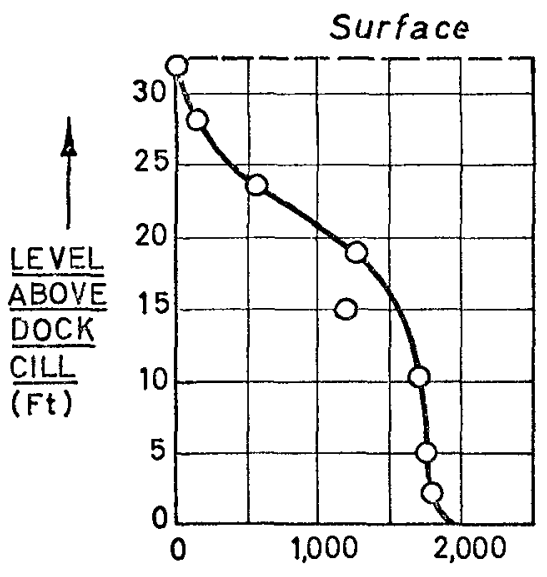

SILT CONCENTRATION (p pm)

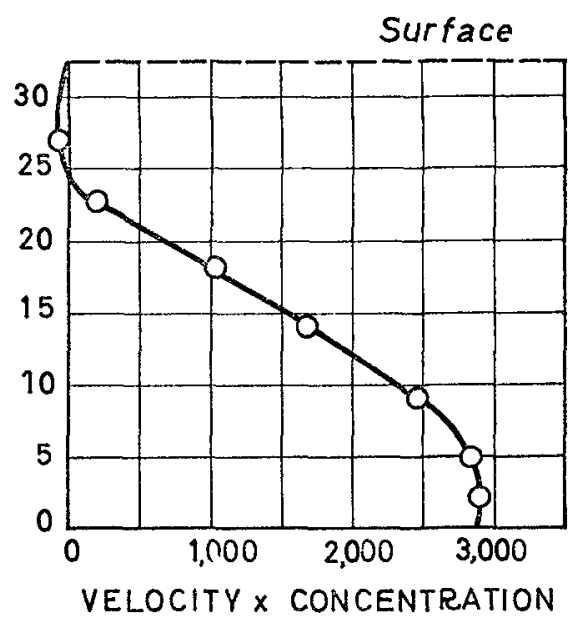

Surface

FIG 6(a) VARIATION WITH DEPTH OF SILT CONCENTRATION AND INFLUX OF SILT INTO THE DOCK AT APPROX 47 MINS BEFORE HIGH WATER (Corresponding velocity distribution is given in Fig 1 (a))

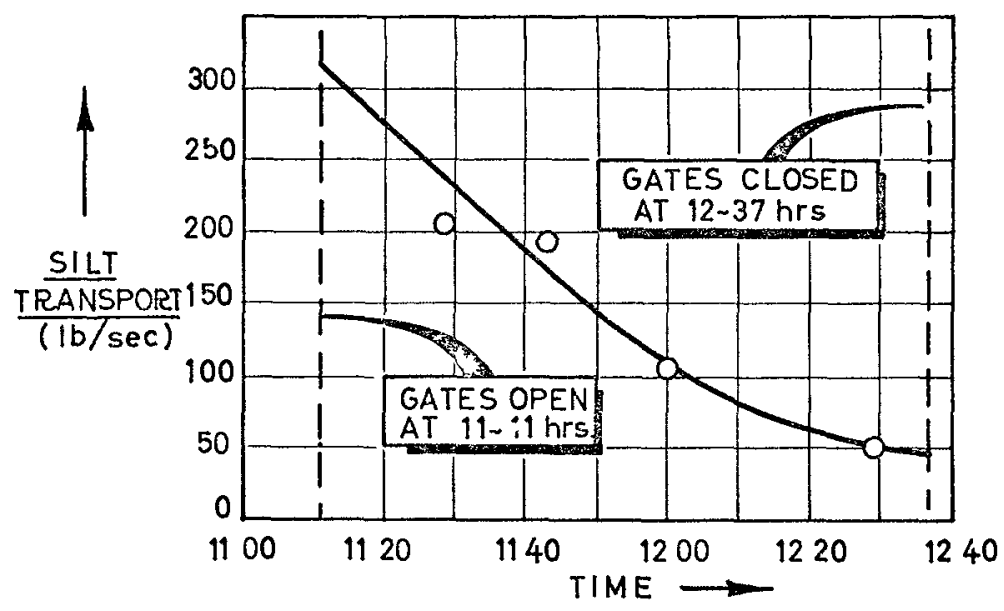

FIG 6(b) $\frac{\text { TRANSPORT OF SILT INTO DOCK }}{\text { (BROMBOROUGH DOCK 10-12-69) }}$ 



\section{CHAPTER 120}

MEASUREMENT OF DENSITY CURRENTS IN AN IDEALIZED MODEL By TImm STUCKRATH ${ }^{1)}$

\section{INTRODUCTION}

The mixing of salt and fresh water in estuaries of tidal rivers is related to two major phenomenons turbulent diffusion and density currents

The turbulent diffusion can be read off from the horizontal and vertical salinity distribution, the density current can be determined from the velocity distribution on the vertical axes

The physical description of tidal mixing in a mathematically closed system is not possible, because turbulent diffusion and gravitational convection are varied by such different influences as tidal action, fresh water flow, river bed form and roughnes, and gravitational and CORIOLIS forces

Most research in this field has been done on turbulent diffusion, especially to predict the mean horlzontal (longltudinal) salinity distribution IPPEN, HARLEMAN (ref 11, 12), and others have found out by various model tests that FICK's law of diffusion used in one-dimensional form is a good physical description for the longltudinal salinity distribution and that the tidal energy dissipation, the cross section, and the fresh water flow are a good means of classifying tidal estuaries from the viewpoint of stratification

1) Diplom-Engineer, Scientific Assistent of the FRANZIUSINSTITUT, Technical University of Hannover, Germany 


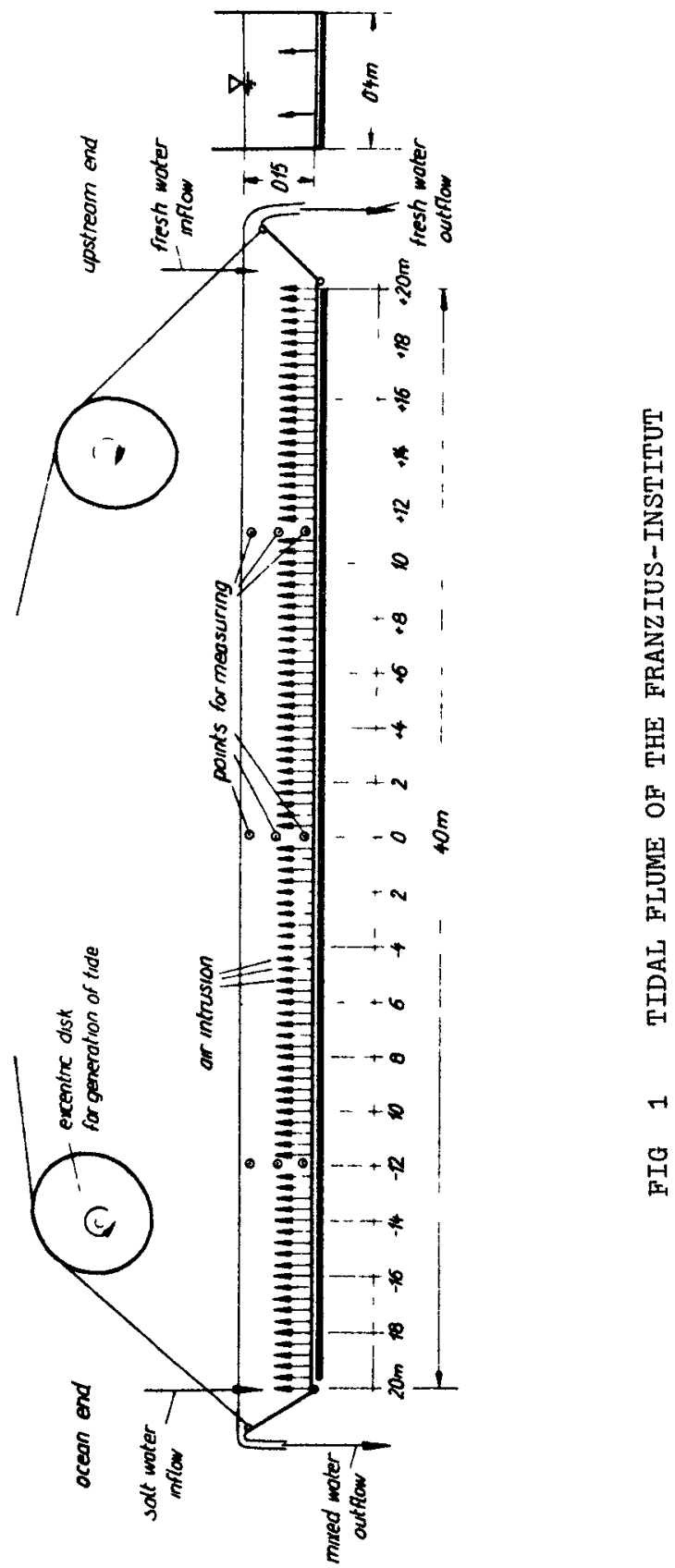


Much research is still needed to predict the density currents in tidal estuaries Most theoretical and practical research on density currents is still limited to rivers or model tests without tide and with high stratification In these channels salt and fresh water are seperated into layers The model tests which were carried out with sponsorship of the DEUTSCHE FORSCHUNGSGEMEINSCHAFT in the FRANZIUS-INSTITUT in Hannover, were assigned to obtain informations about density currents in mixed estuaries

\section{EXPERIMENTAL EQUIPMENT}

The flume that was used, Is shown in $\mathrm{fIg} 1$ It was $40 \mathrm{~m}$ long, $04 \mathrm{~m}$ wlde and $03 \mathrm{~m}$ deep The flume was supplied with fresh water from the upstream end and with salt water from the ocean end The tide was generated on both sides with tidal weirs whlch were moved by excentric disks The flume should represent the zone of brackish water in an estuary Due to the water supply the upstream end of the brackish zone during the whole tidal circle was within the flume, whlle the ocean end of the brackish zone could move downstream past the ocean end of the flume ( $\begin{array}{lll}f^{\prime} & \text { 2) }\end{array}$

As the Intensity of turbulence in the model was nearly zero, artificlal turbulence was generated by an air supply from the bottom of the flume ( $f 1 g$ 1) Constant supply with alr gives constant energy dissipation per unit of mass, even if there are great differences in water depth due to the tidal amplitude or changes in bed level An artificial air supply is therefore a good means of achleving a definite turbulent energy dissipation in tidal models

About 70 model tests were carried out with variation of

fresh water velocity from 020 to $135 \mathrm{~cm} / \mathrm{sec}$ ( 4 serles), ocean salinity from 0 to $35 \%$, air supply from 4 to $85 \mathrm{~cm}^{3} / \mathrm{sec} \mathrm{m}^{2}$ 


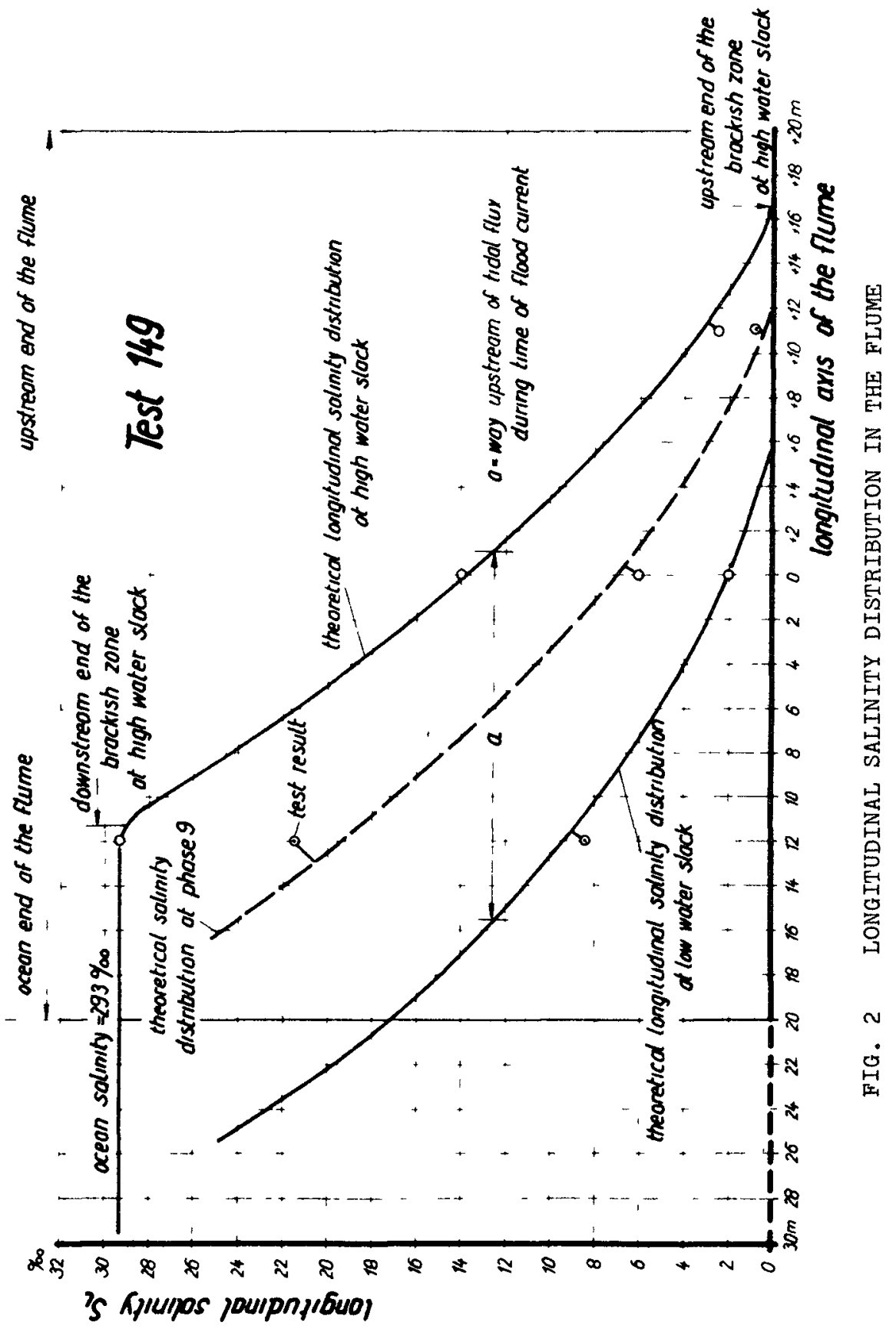




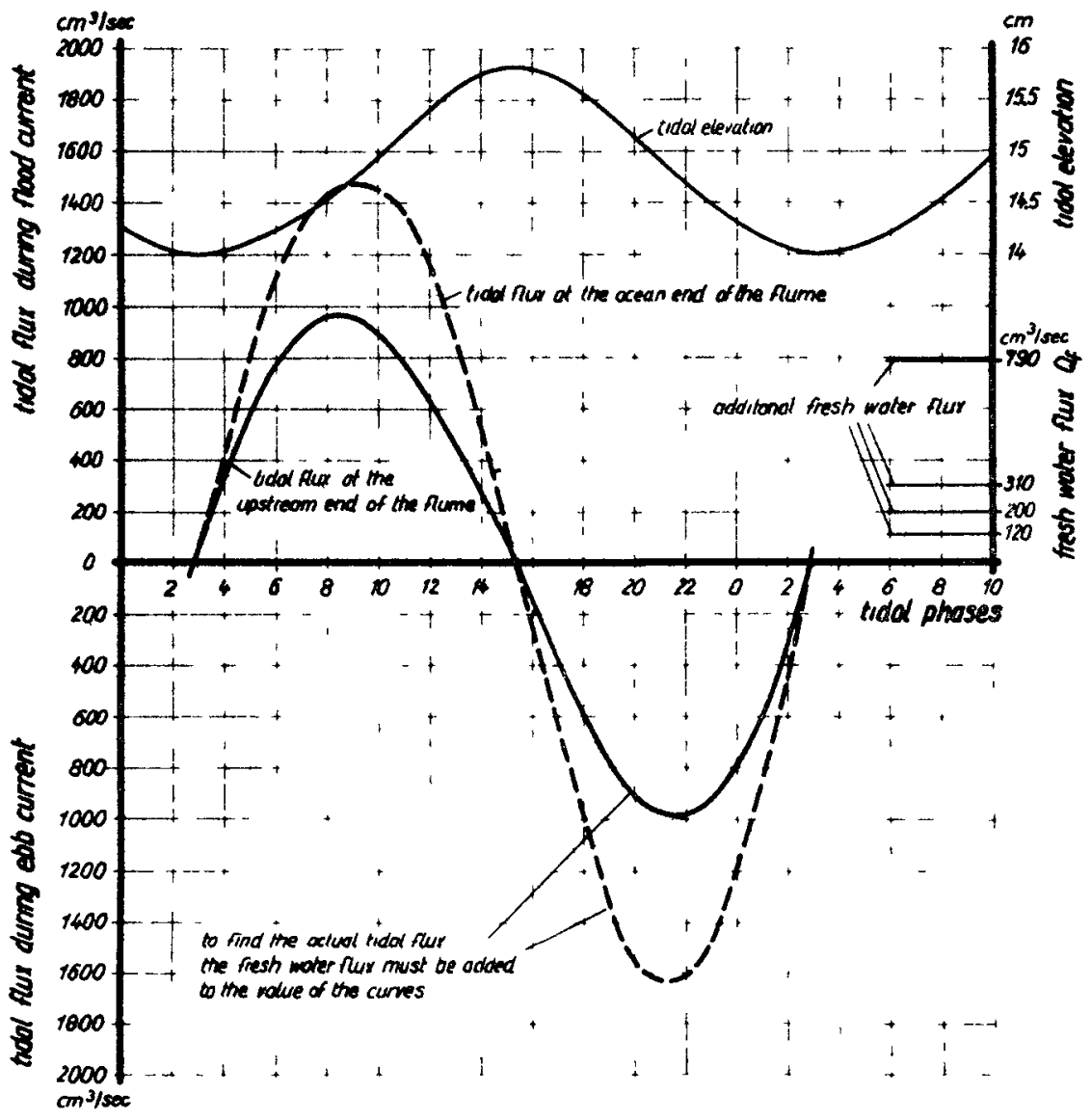

FIG 3 TIDAL AND FRESH WATER FLUX 
Since the tide in the model had nearly no influence on turbulence, the experiments were carried out with a single tide which changed the depth from $14 \mathrm{~cm}$ (low water) to $158 \mathrm{~cm}$ (hlgh water) The time for one tidal circle was 30 minutes The tidal flux on both ends of the flume during one tidal circle ( 24 tidal phases) and the fresh water flux of the 4 serles conducted, can be read from fig 3

The salinity was measured by the electrical conductivity of salt water at 9 fixed points ( 3 vertical axes with 3 levels) (fig 1) The low spead currents were measured in the vecimity of these points with elastic pendulums as shown on fig 4

The elastic flexion of the pendulum is restricted to a short spring plate and recorded by strain gauges which were attached to both sides of the spring Currents of $02 \mathrm{~cm} / \mathrm{sec}$ up to $10 \mathrm{~cm} / \mathrm{sec}$ could be measured with this technique with good accuracy

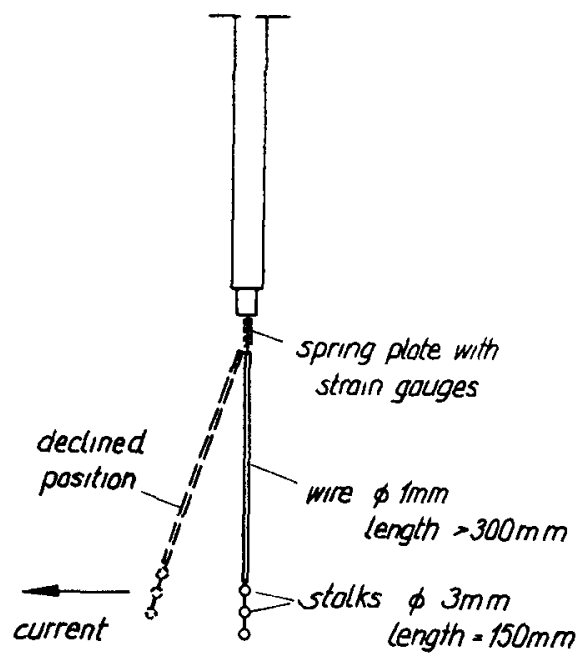

FIG 4 INSTRUMENT FOR MEASURING LOW SPEED CURRENTS

\section{SALINITY DISTRIBUTION}

TIdal research has shown that FICK's law of diffusion can be applied to rivers of varıous conditions of mixing (ref 5 , $8,9,14,15)$ It was therefore also Important to verify the model tests of the FRANZIUSINSTITUT with this theory since the applied theoretical developments and their agreements with the test results cannot be shortened 
appreclably without harming their understanding, they shall not be explained here It was worthwlle to know that all test results could be approximated with this theory closely (flg. 2) and that the longltudinal and vertical salinity distribution in the model could be expressed by physical parameters

Instead of the tidal energy dissipation - which is the major cause for mixing in prototype estuaries - the energy dissipation of the air introduced was used, similar to the experiments which were carried out at the MASSACHUSETTS INSTITUTE OF TECHNOLOGY with occillating grids ( $\operatorname{ref} 6,7,10$ )

For the analysis of density currents it is not neccessary to have a theoretical prediction of the salinity distribution It is however presumed that the salinity at any point and at any time is known, because its distribution and changing is the cause for density currents

\section{DENSITY CURRENTS}

One classical problem of density current was to determine the initial velocity $v_{1}$ which occurs, when a vertical seperation between two liquids of different density ( $\rho_{1}$ and $\left.\rho_{2}\right)$ is suddenly removed ( $f$ ig 5)

The equation which describes this velocity is

$$
v_{I}=K \sqrt{\frac{\rho_{1}-\rho_{2}}{\rho} g h}
$$

$$
\begin{aligned}
& K=\text { constant } \\
& \rho_{1}=\text { density of the water with greater density } \\
& \rho_{2}=\text { density of the water with lesser density } \\
& \rho=\frac{\rho_{1}+\rho_{2}}{2} \\
& g=\text { acceleration of gravity }
\end{aligned}
$$



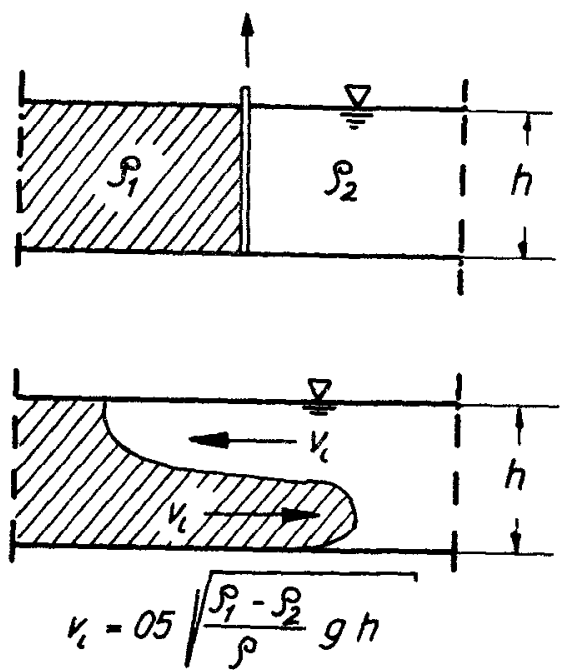

FIG 5 THE INITIAL VELOCITY $\mathrm{v}_{1}$ $h=$ water depth

Many model experiments have been carried out to determine the initial velocity. O'BRIEN and CHERNO (ref. 13), KEULEGAN (ref 4), YIH (ref. 3), BARR (ref 4), and others have proved that the constant before the root, which can also be computed by theoretical considerations as ABRAHAM and $\mathrm{v} d$. BURGH (ref. 2) have shown, has nearly the value of

$$
K=0,5 \text {. }
$$

ALLEN and PRICE (ref. 3) ascertalned this value at shlp locks.

After the vertical seperation is removed ( $f 1 g$ 5) the density current is

$$
\Delta v=2 v_{1} \text {. }
$$

So the equation of the inctial velocity can be rewritten

$$
\begin{aligned}
& \Delta v=2 K \sqrt{\frac{\rho_{1}-\rho_{2}}{\rho} \cdot g \cdot h} \\
& F_{d}=2 K=\frac{\Delta V}{\sqrt{\frac{\rho_{1}-\rho_{2}}{\rho} \cdot g \cdot h}}
\end{aligned}
$$

$F_{d}$ Is called the densimetric FROUDE number For the initial velocity ( $f_{1 \mathrm{~B}}$ 5) $18 \mathrm{~F}_{\mathrm{d}}=1$. 
In a river mouth the density current can be determined as in fig 6 as the difference between the measured velocity and the tidal velocity without density.

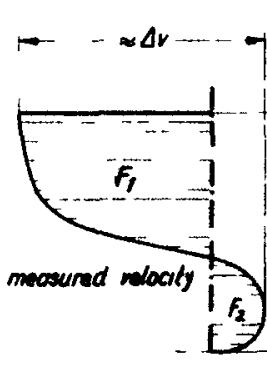

meosured wookty

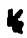

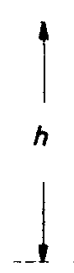

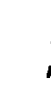

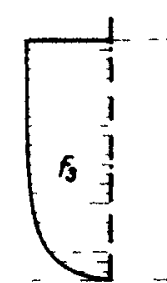

tridal velocity whout density

$v_{0}$

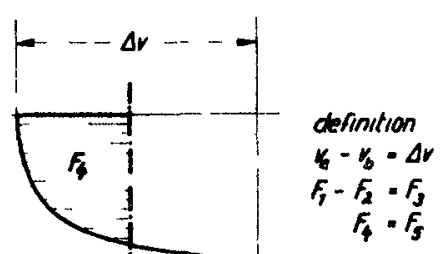

5

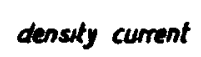

$\Delta v$

FIG. 6 DEFINITION OF DENSITY CURRENT

The experiments in the flume of the FRANZIUS-INSTITUT have shown that the densimetric FROUDE number is a good parameter for approximating the density currents in mixed channels also. In one vertical axis $\rho_{1}$ is the density on the bottom and $\mathrm{P}_{2}$ is the density on the surface of the flume

Fig 7 compares the average density differences (which are linear to the salinity differences) over the whole tidal circle in the three vertical axes of $\mathrm{flg} 1 \mathrm{w}$ th the average density current Fig 7 shows that all experiments give an average densimetric FROUDE number smaller than $F_{d}=1$

The compensating straight line gives

$$
\mathrm{F}_{\mathrm{d}}=06
$$

The computed densimetric FROUDE numbers for every test result of fig. 7 are shown on f1g. 8 with the average hori- 


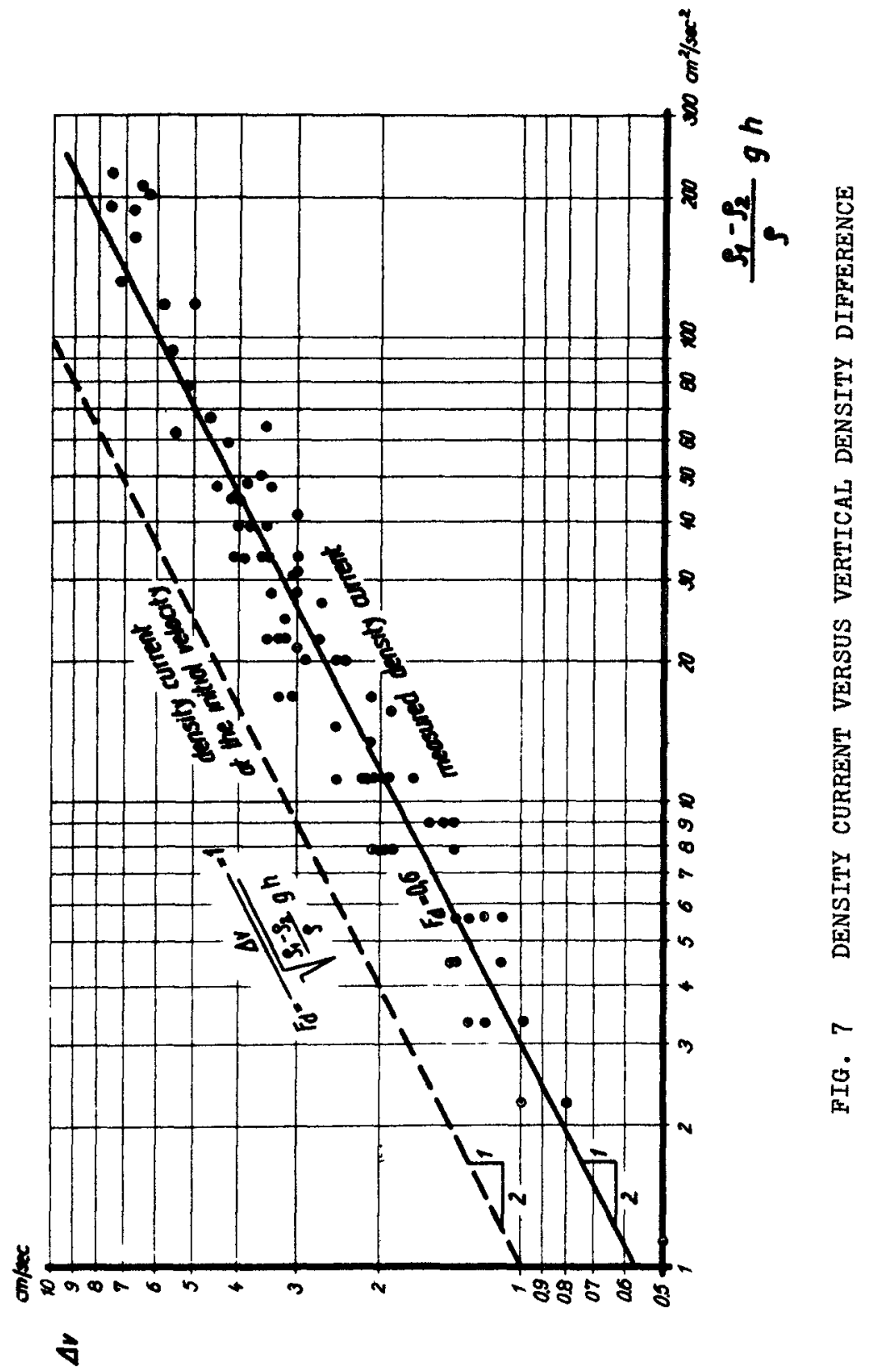




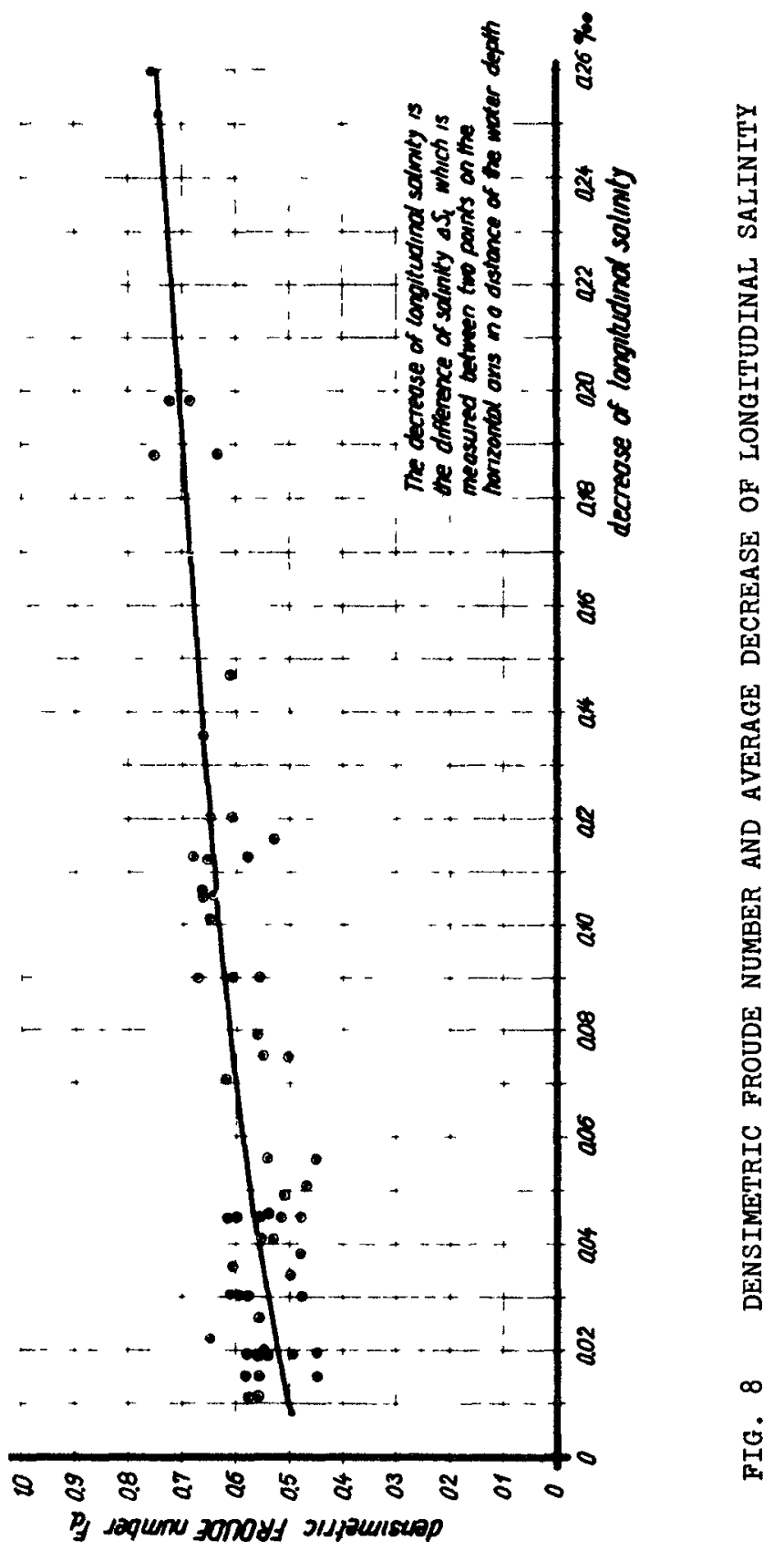


zontal salinity gradient of the same experiment The average horizontal salinity gradient here is explained as the decrease of longltudinal salinity in a horlzontal distance of the same length as the water depth

From fig 8 can be derived what ABBOTT (ref 1) has proposed In a different form from prototype observation If two estuaries have the same vertical salinity distribution and the same depth but unequal lengths of the salinity intrusion, the estuary with the shorter intrusion length has higher density currents than the longer one

Flg 8 cannot show the dependence of density currents on the change of the salinity distribution, because it is derived from time-averaged measurements This dependence can be read from flg 9 which shows the maximum density currents in the flume the maximum density currents occured shortly before the highest density on the bottom was reached on fig 9 the densimetric FROUDE number is compared with the change of density difference during the time of two tidal phases (2/24 of one tidal circle)

$\Delta \rho_{n}$ denotes the vertical density difference at the tidal phase of maximum density current, $\Delta \rho_{n-2}$ denotes the vertical density difference measured 2 tidal phases before If the change of density difference is zero, the value on the abszissa is

$$
\frac{\Delta \rho_{n}}{\Delta \rho_{n-2}}=\frac{\Delta S_{n}}{\Delta S_{n-2}}=1
$$

At this point fig 9 gives $F_{d}=$ about 0,5 so the maximum density current in an estuary where the salinity differences do not change quickly, can be computed as the average density current in fig 8

If

$$
\frac{\Delta \rho_{n}}{\Delta \rho_{n-2}}=\frac{\Delta S_{n}}{\Delta S_{n-2}} \text { tends to } \infty, F_{d} \text { tends to } 1
$$




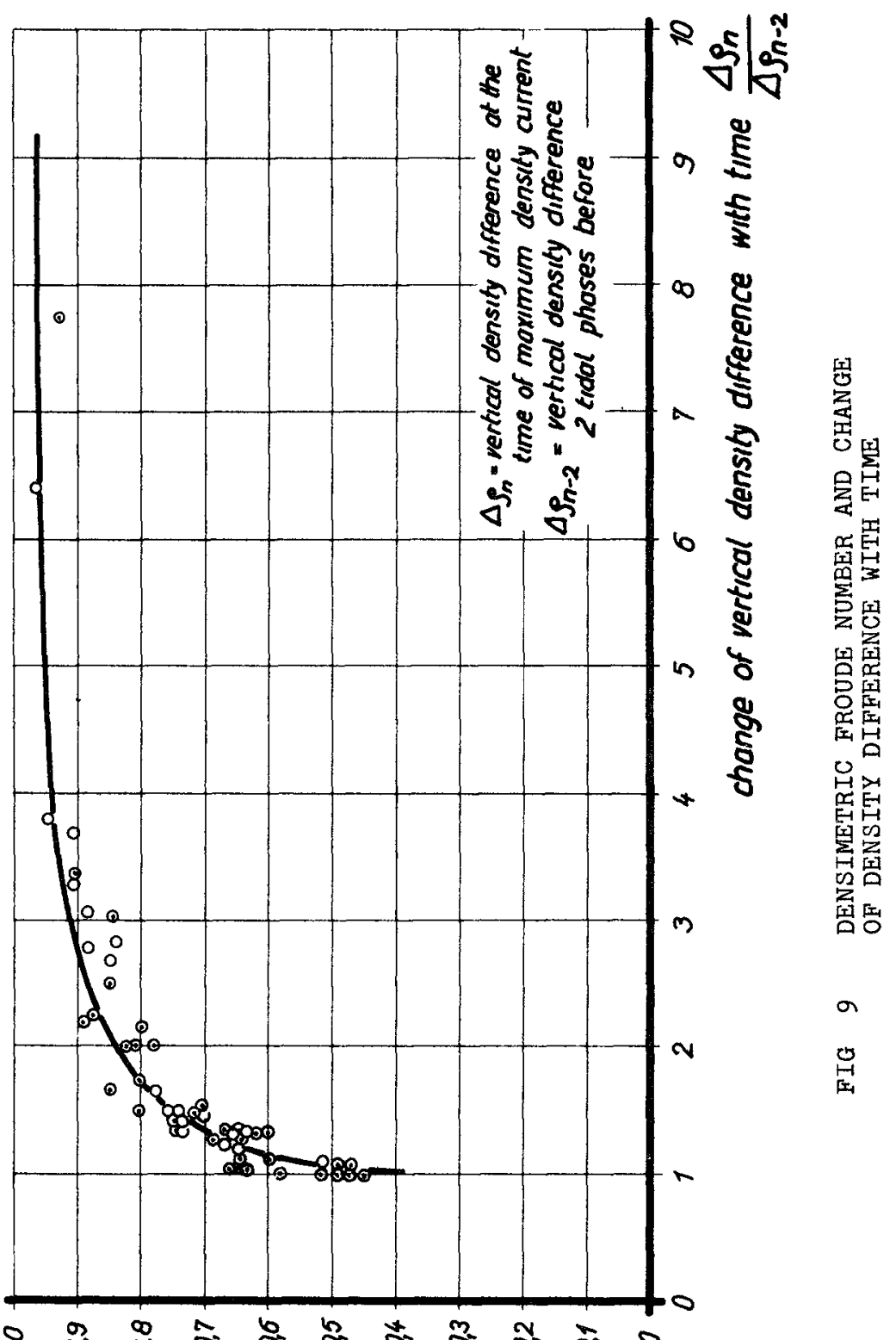

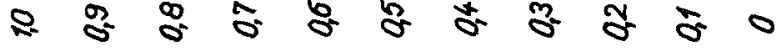

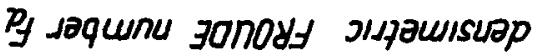


This is evident, because estuaries in which density differences change abruptly, approach the state of the initial velocity as in the experiment of fig 5

\section{CONCLUSIONS}

The model experiments of the FRANZIUS-INSTITUT represented the salinity distribution in an ldealized estuary of various mixing stages The density currents show a dependence on the vertical salinity distribution (densimetric FROUDE number), on the longltudinal salinity distribution, and on the change of salinity with time

\section{REFERENCES}

1 АВвотT, $M$ B

Salinity effects in estuaries. Sears Foundation, Journal of Marine Research, Vol 18, No. 2, October 1960

2 ABRAHAM, G and $v$ d BURGH, $P$.

Pneumatic reduction of salt intrusion through locks Proceedings ASCE, Journal of the Hydraulics Division, January 1964

3 ALIEN, $\mathrm{FH}$ and PRICE, W.A

Density currents and siltation in docks and tidal basins The Dock and Harbour Authorıty, July 1959

4 BARR, D. I H

Densimetric exchange flow in rectangular channels. La Houllle Blanche, November 1963

5 GOLE, $\mathrm{CV}$ and THAKER, V S

Progressive salinity intrusion during dry season in the Hooghly estuary. Proceedings, 13th Congress IAHR, Vol 3, SubJ C, Kyoto 1969

6 HARLEMAN, D.R F , The diffusion of two flulds of diffeJORDAN, J.M , and rent density in a homogenous turbulent fleld Massachusetts Institute LIN, J D of Technology, Hydrodynamics Laboratory, Techn Report No 31, 1959 
7 HARLEMAN, D R.F., MCDOUGALL, $D W$, GALVIN, $\mathrm{C} \mathrm{J}$, and HOOPES, J.A.

8 HARLEMAN, D.R.F and HOOPES, J.A

9 HARLEMAN, D.R F and ABRAHAM, $G$

10 IPPEN, A T, HARLEMAN, D.R.F and LIN, J D.

11 IPPEN, $A T$ and HARLEMAN, D R F

12 IPPEN, A T. ed

13 O'BRIEN, $M P$ and CHERNO, J

14 PARTENSCKY, $\mathrm{H} \mathrm{W}$ and LOUCHARD, L

15 PARTHENIADES, E
An analysis on one-dimensional convective diffusion phenomena in an ldealized estuary Massachusetts Institute of Technology, Hydrodynamics Laboratory, Techn Report No 42 , 1961

The prediction of salinity intrusion changes in partially mixed estuarys. 10th Congress IAHR, Vol 1, 1 15, London 1963

One dimensional analysis of salinity intrusion in the Rotterdam Waterway. Delft Hydraulics Laboratory, Publ No 44, 1966

Turbulent diffusion and gravitational convection in an idealized estuary Massachusetts Institute of Technology, Hydrodynamics Laboratory, Techn Report No 38, 1960

One dimensional analysis of salinity intrusion in estuaries Corps of Engineers, U.S Army, Committee on Tidal Hydraulics, Techn Bull No 5, January 1961

Estuary and coastline hydrodynamics McGraw-Hill, 1966

Model law for motion of salt water through fresh Transactions ASCE, No 99,1934

Etude sur le vartion cyclique de la salınıté moyenne dans l'estualre du Salnt-Laurent Université de Montréal, École Polytechnique, D1vision d'Hydraulique, Sep. 1967

Field investigations to determine sediment sources and salinity intrusion in the Maracalbo estuary, Venezuela Massachusetts Institute of Technology, Hydrodynamics Laboratory, Report No 94, June 1966 



\title{
CHAPTER 121
}

\author{
Computation of Storm Surge
}

RICHARD SIIVESTER

Professor of Coastal Englneering

As1an Inst 1tute of Technology, Bangkok, Tha1land

\section{ABSTRACT}

From knowledge of wind resistance coefficients measured over the sea, it is possible to compute the shear stress of a wind field on its surface Where a body of water is relatively shallow, such a shear stress will transport water to the downwind end and so create a high water level or surge Graphs are presented for ready application of the relevant formulae to cases of lakes and Continental Shelves The wind fields of either uniform or triangular horizontal distribution in velocity are included, as well as conditions of stationary or moving fetches Surges from seven typhoons travelling towards Hong Kong are computed and compared to actual records

\section{INTRODUCTION}

Wind stress on a water surface is a function of its roughness, particularly in respect to the shorter period waves in the spectrum Hence an enclosed body of water can be hydrodynamically rougher than the open sea where the shorter waves are dissipated with the assistance of the longer ones Formulae avallable can be put in graphical form for the computation of surge helghts These apply to lakes or Continental Shelves With the latter the ratio of fetch length to Shelf width assumes importance, as well as velocity of approach of the fetch and the horizontal distribution of the wind velocity Application of the graphs to a number of typhoons approaching Hong Kong indicates the accuracy of the procedure

\section{WIND STRESS}

The shear stress applied by the wind to a water surface depends upon its smaller roughnesses and hence the wave conditions Wu(1) has shown that waves in the order of 07 seconds period and 01 metre height are the main roughness element The stress thus varies with the wind velocity and the fetch avallable For this reason it is understandable that optimum values can be reached which are different for enclosed bodies of water of limited size and the open sea The former contain a larger proportion of short waves that are reaching, or are at, their 1 imiting steepness The latter, however, when they approach the fully arisen state, contain less of the short period components and more of the longer period waves (2) For this reason the 
sea is slightly smoother as far as wind stress is concerned It can be presumed therefore that the stress of a glven steady wind can also vary with duration

The shear stress is given by

$$
\tau=\rho_{\mathrm{a}} \mathrm{U}^{2}
$$

where $\rho=$ density of alr

$\mathrm{U}^{\mathrm{a}}=$ shear velocity near the water surface

this can be expressed as

$$
\tau=\rho_{a} C_{y} U_{y}^{2}
$$

where $\mathrm{C}_{\mathrm{H}}=$ resistance coefficient varying with $\mathrm{U}$

$\mathrm{U}_{\mathrm{y}}^{\mathrm{y}}=$ wind velocity at $\mathrm{y}$ metres above water surface

Many workers $(3)(4)(5)$ have evolved relationships for $C$ and $U$ from measurements over lakes and the sea Wu $(6)$ has summarlsed the data and discussed their significance, from which Figure 1 has been prepared This shows $\mathrm{C}_{10}$ for a range of $\mathrm{U}_{10}$, as most wind measurements have been made at the 10 metre helght Within the boundary of the experimental values the relationships presented by various workers is also shown, including the stepped curve of Wu It 1 s now generally accepted that a limiting value of $C_{10}=26 \times 10^{-3}$ can be applied to limited water bodies when the 10 metre high wind exceeds 10 metres/sec or 30 knots The optimum for the ocean is $C_{10}=24 \times 10^{-3}$ For winds less than this a relationship of

$$
\mathrm{C}_{10}=065 \quad 10^{-3} \mathrm{U}_{10}^{\frac{1}{2}}
$$

would follow Sheppard's curve reasonably well to the aforesald limits To convert wind velocities from other levels to the 10 metre height, graphs have been provided elsewhere (7)

In storm surge calculations it is more appropriate to use the relationship

$$
\tau=k \rho U_{10}^{2}
$$

where $\rho=$ density of the water

so that $k=\frac{\rho a}{\rho} c_{y}$, which for seawater $=\frac{1}{800} C_{10}$

the $11 \mathrm{~m} 1 \mathrm{t}$ ing values of $\mathrm{k}=33 \times 10^{-6}$ and $30 \times 10^{-6}$ apply to 1 akes and ocean areas respectively

A scale for $k$ is included in Figure 1, together with scales for $\mathrm{U}_{10}$ in knots and designations of Beaufort Number and Sea State 


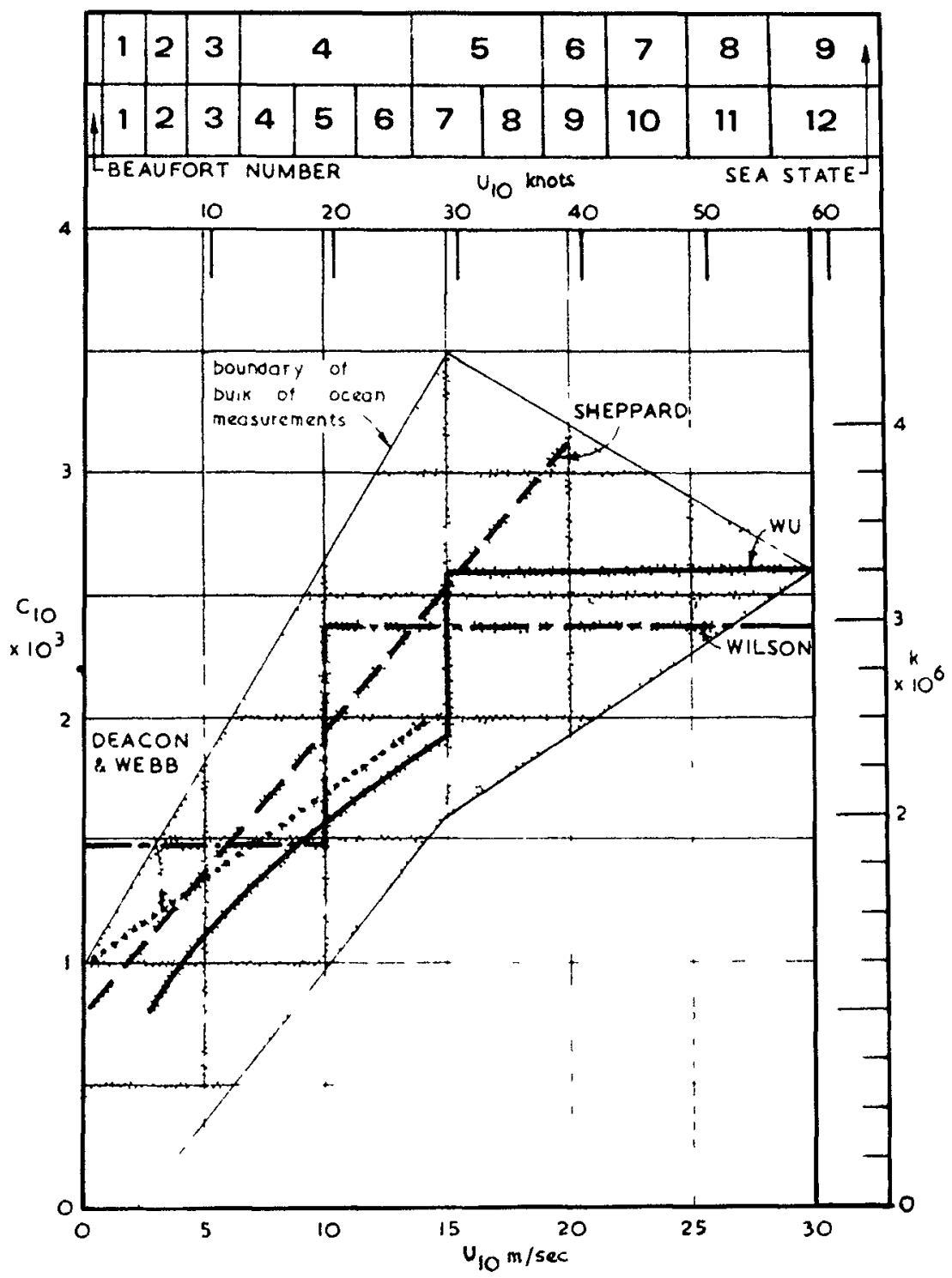

Fig 1 Variation of wind resistance coefficient with wind velocity 


\section{ENCLOSED BODIES OF WATER}

Considering a lake of rectangular proportions in plan, its longItudinal cross-section can be equated to a rectangular one of equal area, giving an equivalent depth $d$ and length or fetch $L$ The equation for the water surface profile(8)( $(9)(10)$ when a steady wind velocity $\mathrm{U}_{10}$ is applied to it is

$$
\frac{S}{d}=\frac{k_{10} U_{10^{2}}^{L}}{2 g d(d+S)}=\frac{k_{10} U_{10^{2}}^{2}}{2 g d^{2}}
$$

where $S=$ surge helght at the down wind end The last form of the equation assumes $S$ to be small in respect to d Equation (5) also approximates the nodal point at the centre of the length $L$

The variables in this dimensionless equation must be chosen consistently The values of the fall and rise of the water surface at the upwind and downwind ends of the lake are presented in graphical form in Figures 2 and 3, for the two conditions of bottom exposure at the upwind end The surface passes through the mean depth plane around the centre of the water body $(8)$ even when part of the bed is exposed Lakes of Irregular depth can be treated similarly by determining an equivalent rectangular section

In the case of narrow bodies of water such as canals the same value of $\mathrm{k}_{10}=33 \times 10^{-6}$ is applicable since the optimum roughness is due to waves of about 1 second period $(11)$ reaching their maximum steepness of $1 / 7$

For the case of non-rectangular planar shapes, of uniform depth or sloping bottoms, Keulegan (11) has derived some form factors $\mathrm{N}$ by which the values of $\mathrm{S} / \mathrm{d}$ in equation (5) should be multiplied These are Illustrated in Figure 4, where the exponential form analysed by Langhaar for constant depth $d$, and trapezoldal shapes with uniform depth or uniformly sloping bottom are presented The high values of $\mathrm{N}$ resulting from the downwind depth decreasing should be noted The same order of magnification will be observed for similar shoaling on the continental Shelf

\section{CONTINENTAL SHELF - STATIC WIND FIELD}

Where a wind is applied to the Continental Shelf blowing towards the shore, the Shelf width can be taken as half the length of the lake considered previously The water feeding the surge comes from the deep ocean The major difference from the previous situation is the decreasing depth towards the shore For the purposes of surge calculation it is reasonable to assume a uniform variation from a depth $d_{1}$ at the Shelf edge to $d_{2}$ near the coast (see inset of Figure 5)

Surge problems assume Importance on reasonably wide Continental Shelves The majority of these have been constructed by sediment deposition for which waves are the predominant distributing factor 
It is not surprising, therefore, that most of these shelf edges occur at around 65 fathoms $(12)$, or the reach of the $12-14$ second waves, which are the most persistent swe11 waves of the oceans (13) Thus, unless more specific information is avallable the depth $d_{1}$ may be taken as 400 feet (120 metres) On sandy shorelines the beach profile is parabolic from the breaker line, which produces depths of 5 fathoms very close to shore It is such depths, rather than zero values at the beach, that are more effective in the surge phenomenon Where a large tidal range occurs, or sediment of silt craracter exists, large tidal flats will necessitate the use of much smaller values of $\mathbf{d}_{2}$

The width of the Shelf will be designated as $L$, which can be different from the fetch length $F$ of the wind zone As seen in Figure 5 the depth ratio $d_{1} / d_{2}$ can be equally expressed by $L / x$ where $x$ is distance inland where the plane of the bed meets the mean water level

Where a storm zone has a fetch length (F) in excess of the Shelf width ( $\mathrm{L}$ ), only that portion across the relatively shallow zone is effective in producing surge, thus $F=L$ This is 1ikely to be the case in extra-troplcal cyclones, where wide expanses of ocean can suffer winds of unlform speed and direction In troplcal cyclones the fetch lengths are smaller and are more likely to be less than the shelf width This case is discussed later, together with the problem of velocity of advance (V) of the wind field

\section{(a) Uniform Wind Velocity $(V=0)$}

For a wind of steady and uniform speed applied to a Continental Shelf the following formula has been derived(14)

$$
S=\frac{k u^{2} L}{g\left(d_{1}-d_{2}-S\right)} \ln \left(\frac{d_{1}}{d_{2}+S}\right)
$$

Since $S$ is small compared to $d_{2}$ equation (6) can be rewritten

$$
\frac{s}{d_{1}}=\frac{k U^{2}}{g d_{1}}\left(\frac{L}{d_{1}-d_{2}}\right) \ln \frac{d_{1}}{d_{2}}
$$

so that $\frac{\mathrm{s}}{\mathrm{d}_{1}}=\frac{\mathrm{k} \mathrm{U}^{2} \mathrm{~L}}{\mathrm{gd}_{1}^{2}\left(1-\mathrm{d}_{2} / \mathrm{d}_{1}\right)} \ln \frac{\mathrm{d}_{1}}{\mathrm{~d}_{2}}$

Values of $\mathrm{S} / \mathrm{d}_{1}$ versus $\mathrm{k} \mathrm{U}^{2} \mathrm{~L} / \mathrm{gd}_{1}^{2}$ have been graphed in Figure 5 for the range of $\mathrm{L} / \mathrm{x}=\mathrm{F} / \mathrm{x}=001$ to 1000 or $\mathrm{d}_{1} / \mathrm{d}_{2}=101$ to 1001 (the latter approximating $\mathrm{d}_{2}=0$ )

\section{(b) Trlangular Wind Velocity $(V=0)$}

The wind in a tropical cyclone is circular in character, but is deflected towards the centre such that it is around $45^{\circ}$ to the radil 


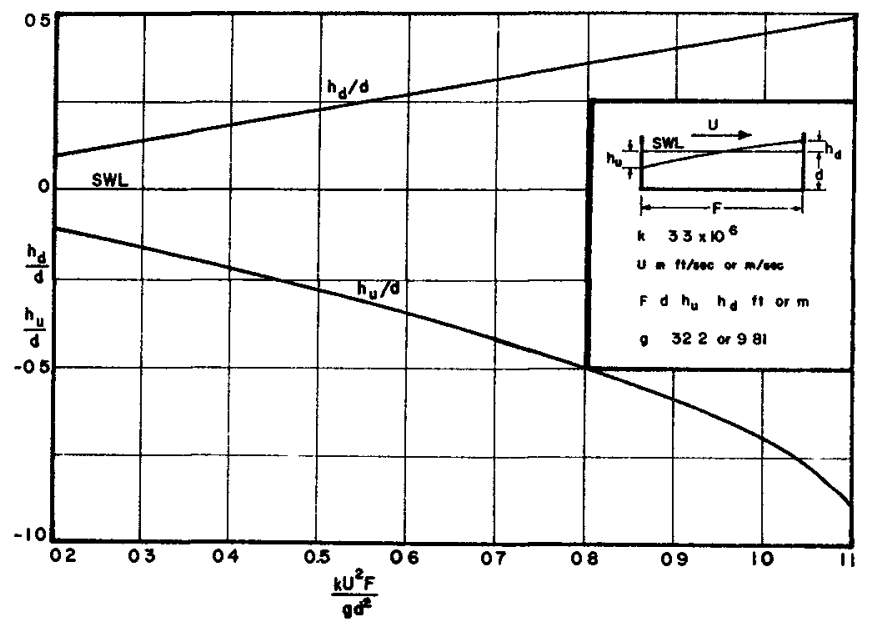

Fig 2 Upwind and downwind surge levels in a lake of uniform depth

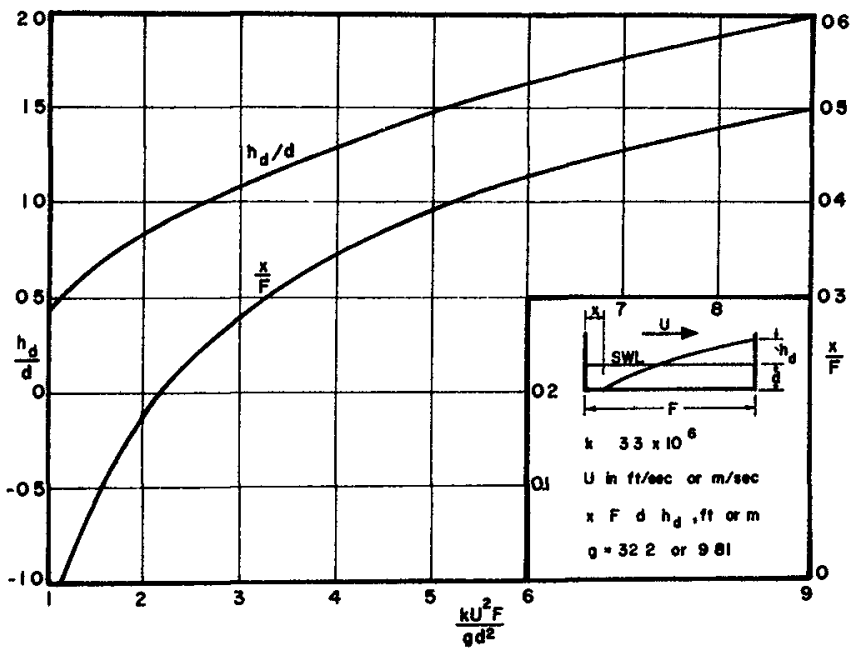

Fig 3 Surge leve1s in a lake when the upwind bed is exposed 

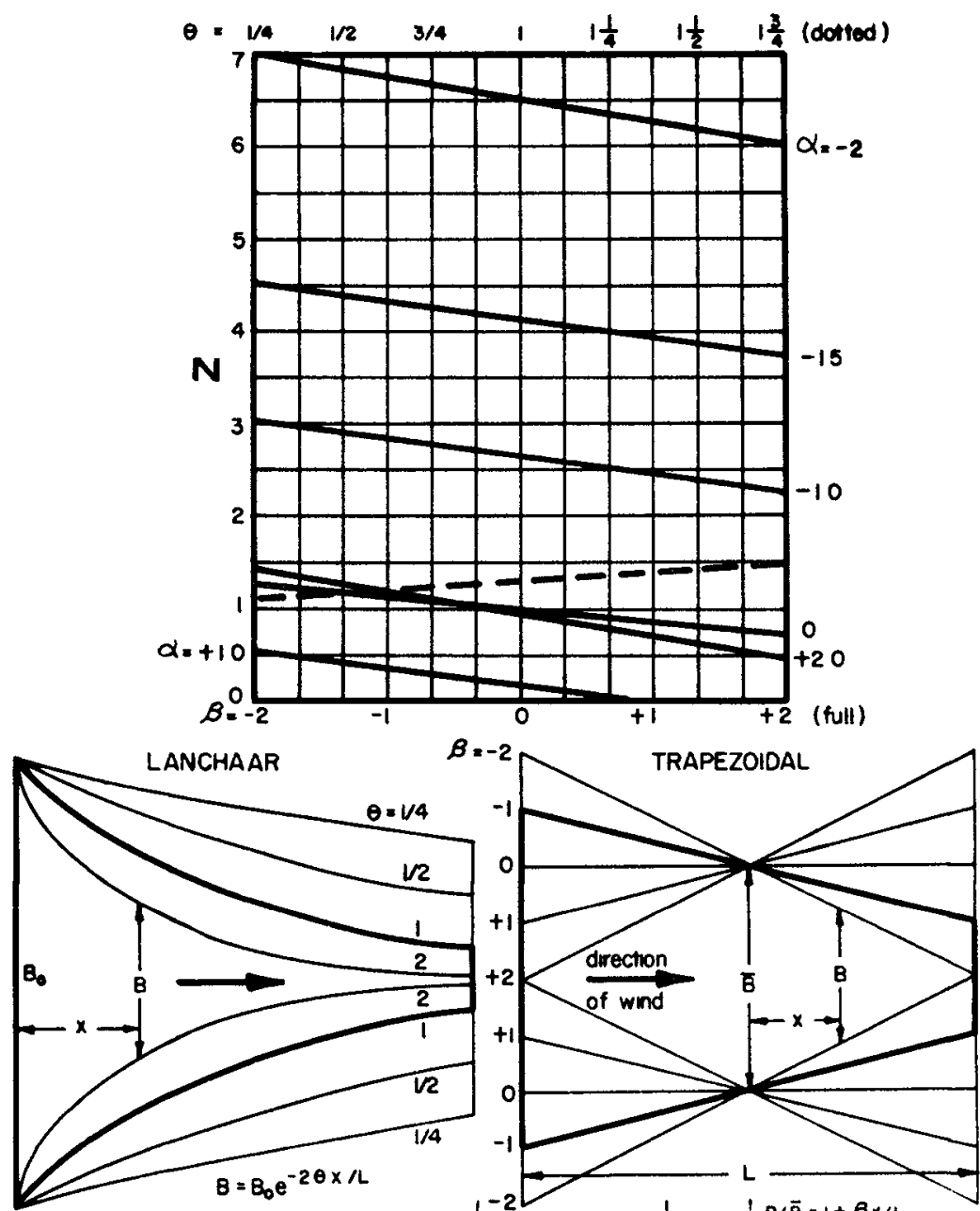
L
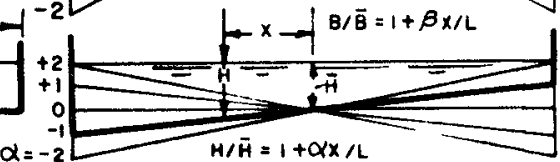

F1g 4 Amplification factor $N$ for Langhaar shaped and trapezoldal 1akes 


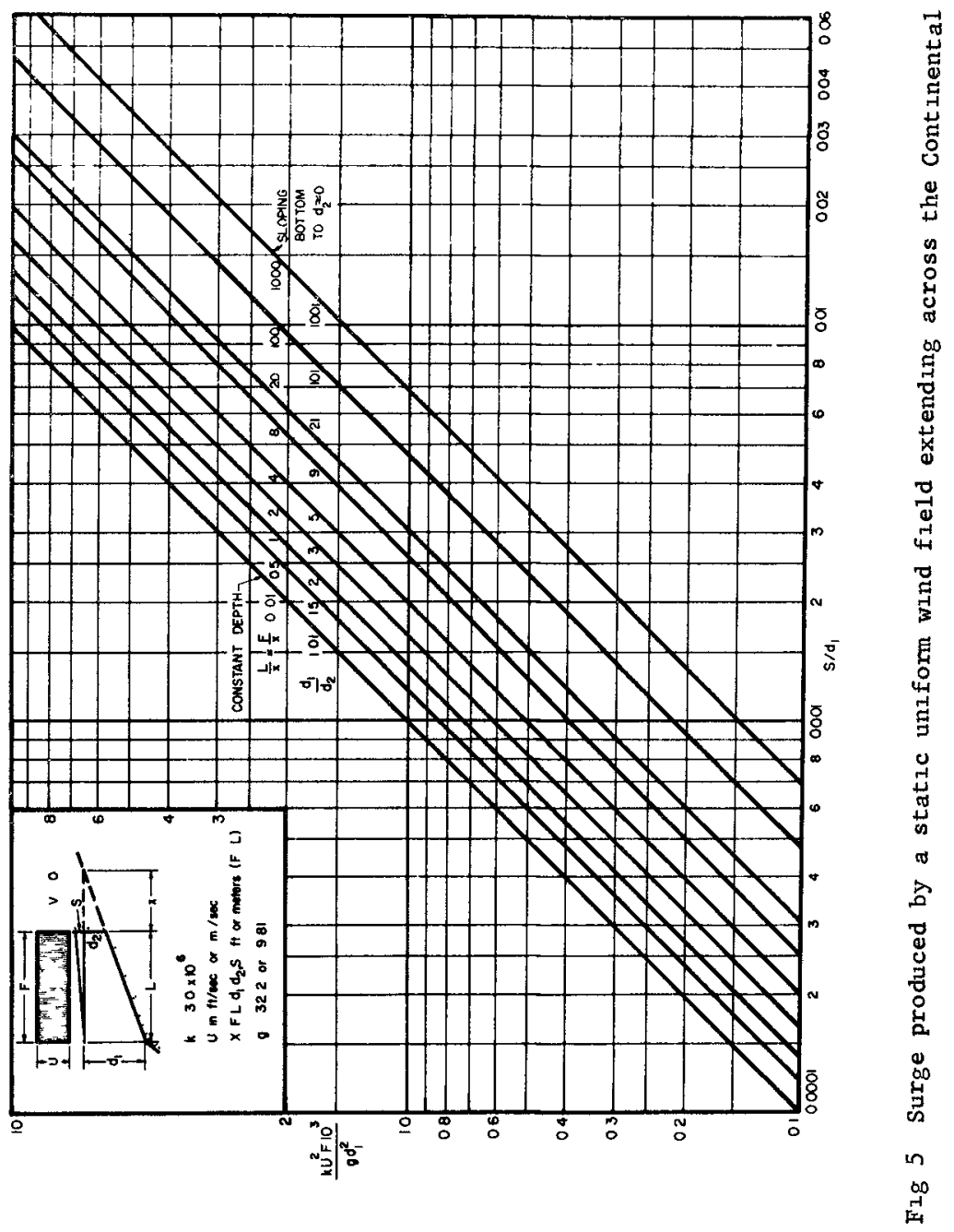




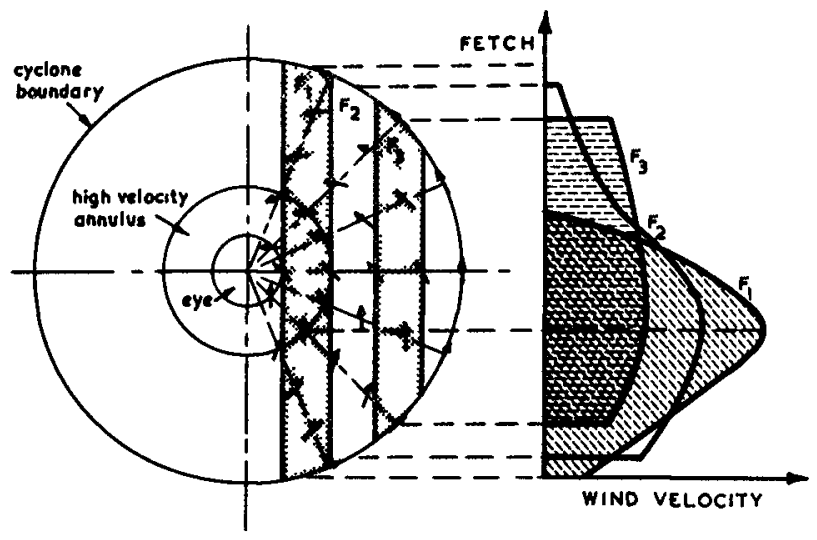

Fig 6 Typical surface wind structure inside a tropical cyclone

(See Figure 6) As the cyclonic centre moves forward the critical conditions as far as surge as well as wave generation is concerned are contained in the quadrant for which the centre and wind vectors are in same direction The cyclone depicted in Figure 6 represents alr circulation for the northern hemisphere in which the right rear quadrant contains the critical conditions for surge generation The worst affected coastal zone will be that in line with fetch $F_{1}$ passing through the high velocity annulus The assessment of wind components along this alignment will result in a triangular distribution of velocity (See Figure 6), the shape of which depends greatly on the radial distribution of the wind around the centre Reid $(15)$ a triangular wind distribution of more-or-less isosceles shape
has derived the following surge formula

$$
\mathrm{S}=\frac{\mathrm{k} \mathrm{U}^{2} \mathrm{~T}}{\mathrm{C}_{1}}\left(\frac{\mathrm{d}}{\mathrm{d}_{2}}\right)^{\frac{1}{4}} \mathrm{z}
$$

where $T=$ mean $t$ ime for surge wave to traverse the Shelf

$\begin{aligned} C_{1} & =\text { celerity of the surge wave at the Shelf edge }\left(=\sqrt{g d_{1}}\right) \\ 2 & =\text { factor, }(=056 \text { for } F=L \text { and } V=0)\end{aligned}$

Substituting $T=\frac{L}{\frac{1}{2}\left(\sqrt{g d_{1}}+\sqrt{g d_{2}}\right)}$ into equation (9) gives

$$
\frac{s}{d_{1}}=\frac{k u_{\max }^{2} L}{g d_{1}^{2}}\left[\frac{112}{1+\sqrt{d_{2} / d_{1}}}\left(\frac{d_{1}}{d_{2}}\right)^{\frac{3}{4}}\right]
$$


Equation (1) has been graphed in Figure 7 in a simılar manner to Figure 5 for a similar range of $\frac{d_{1}}{d_{2}}$ or $\frac{L}{x}+1$.

\section{CONTINENTAL SHELF - MOVING WIND FIELD}

In the case of the storm centre travelling towards the coast the initial surge wave is being reflected as later portions are still approaching The interaction of these establishes a new surge system which Reid $(15)$ has analysed for varlous ratios of $\mathrm{F} / \mathrm{L}$ and $\mathrm{V} / \overline{\mathrm{C}}$

where

$$
\vec{C}=I / T=\frac{1}{2}\left(\sqrt{g d_{1}}+\sqrt{g d_{2}}\right)
$$

From the graphs so presented the ratıo $R$ of maximun surge ( $S_{\text {max }}$ ) to that for static storm conditions ( $S$ ) ( 1 e $F=L$ and $V=0$ ) has beent plotted as in Figure 8, for both uniform and trlangular wind distributions To find $S_{\max }$ it is necessary first to compute $S$ for a hypothetical shelf whose width $L$ equals the fetch length $F$ (not the reverse) With this noted, then $R$ is obtalned from the combination of $F / L$ and $V / C$ It is seen that the influence of the storm-centre speed differs in the two wind distributions Reld's analysis(15) also provides information on the timing of the maximum surge in respect to the location of the fetch, but this has not been included

The absolute resultant is determined also by the depression of atmospheric pressure, which may precede or be concurrent with $S_{\max }$ from the wind alone The surge due to air pressure is given by

$$
\mathrm{s}_{\mathrm{a}}=\left(\frac{1013-\mathrm{p}_{\mathrm{c}}}{34}\right) \frac{1359}{121003}=\left(1013-\mathrm{p}_{\mathrm{c}}\right) 0033
$$

where $S_{2}=$ storm surge in feet of seawater and $h_{c}^{a}=$ pressure at the storm centre in millibars ( 34 millibars $=$ 1 ' mercury) This value should be added to that obtained in the previous equations

\section{EXAMPLES OF HONG KONG TYPHOONS}

Since 1962 the Department of Public Works in Hong Kong has collated some valuable data on typhoons approaching the vicinity of the island These have been summarised in a data sheet for each event, in which rainfall, wind speed, wind direction, atmospheric pressure, and sealevel are plotted against time in hours on the sealevel graph is traced the normal tide curve as preducted in tide tables, from which the surge level can be obtalned The chronologlcal relationship between the variables listed provides sufficient information to draw a plan of the cyclone and to determine its diameter A geographic plan of the ocean area with the typhoon path traced on 1t with time markings, permits the speed of travel to be assessed The distance across the Continental Shelf that the typhoon has traversed can also be measured The wind speed trace indicates whether a triangular or rectangular distribution has produced the surge A modified reproduction of one PWD data sheet is illustrated in Figure 9 


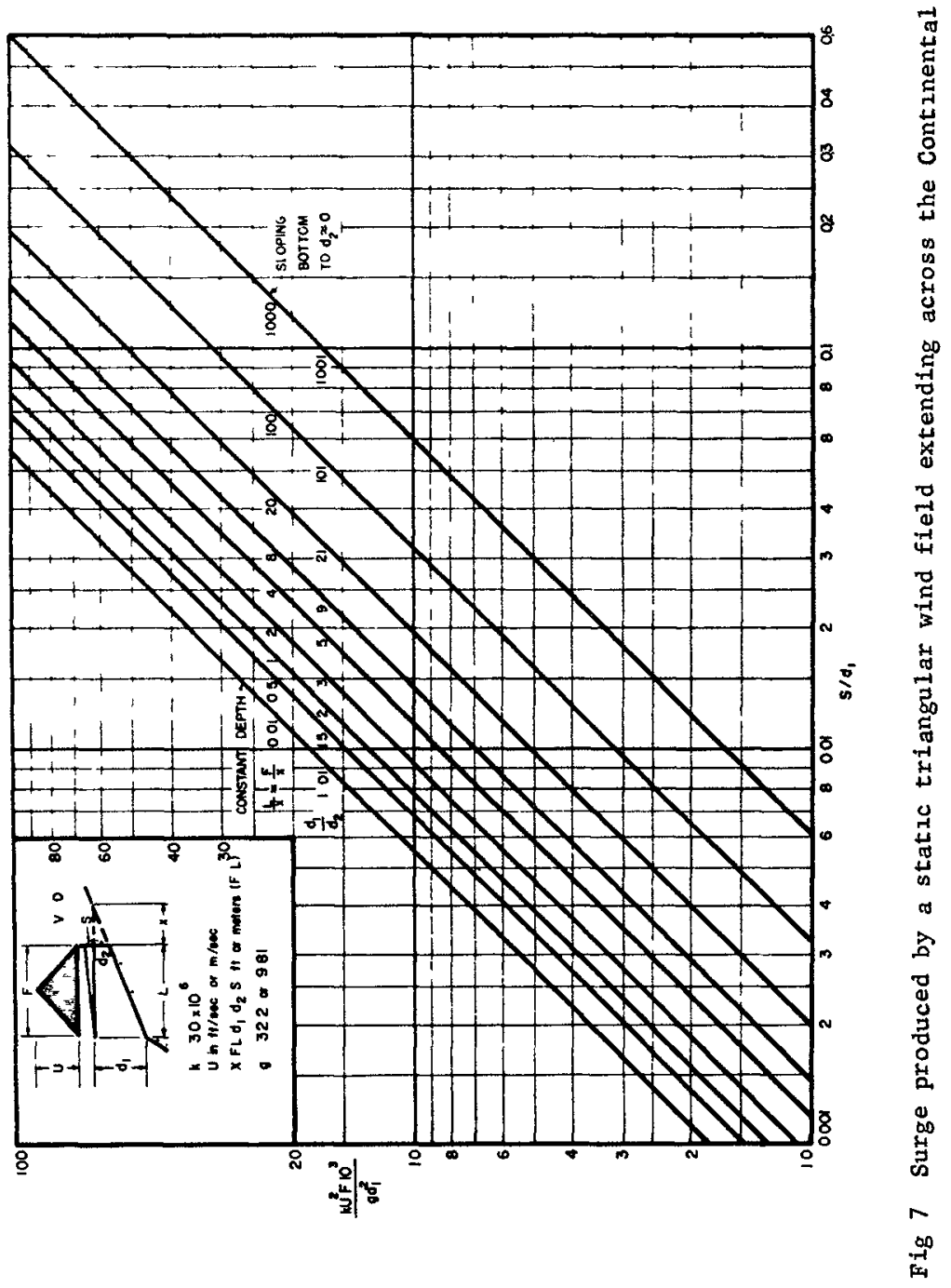




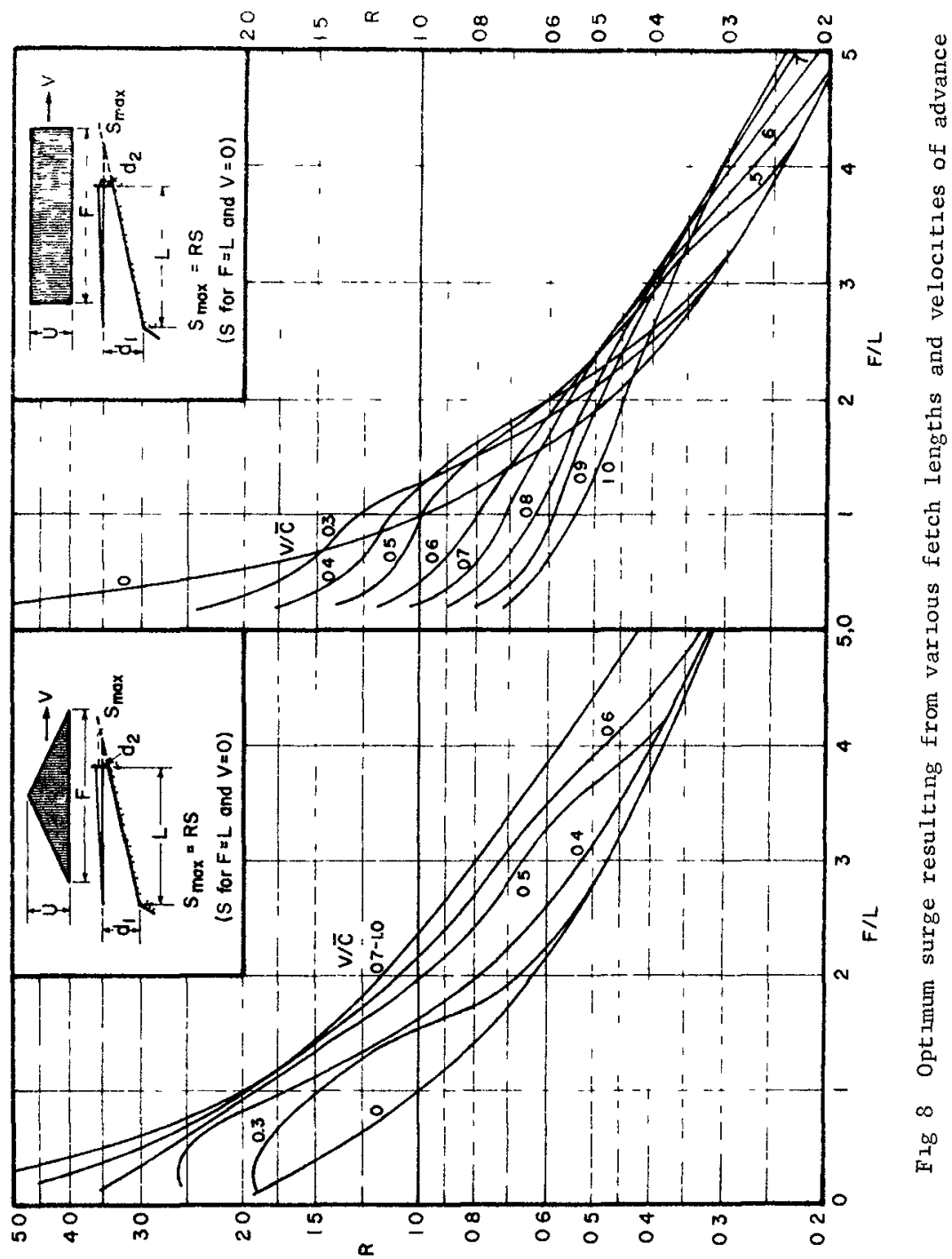




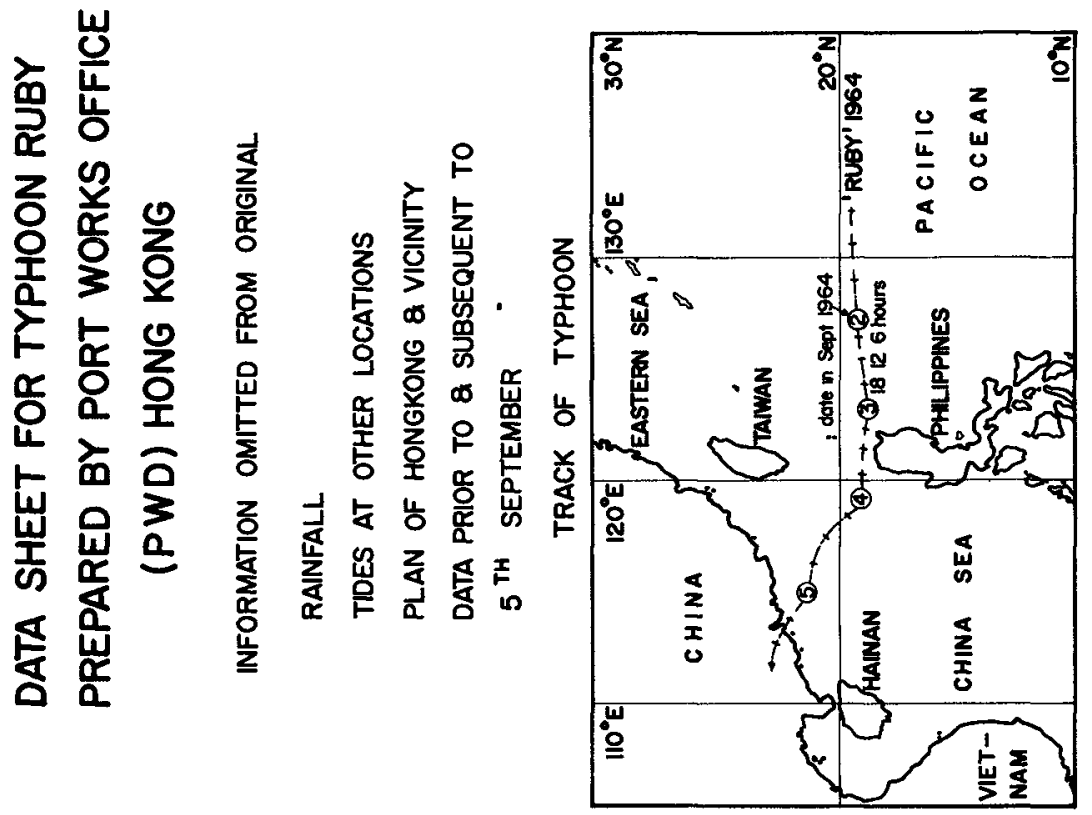

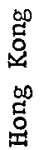

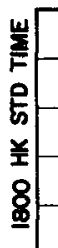
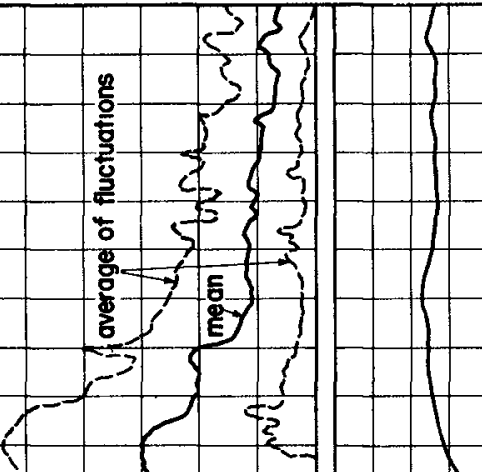

\&

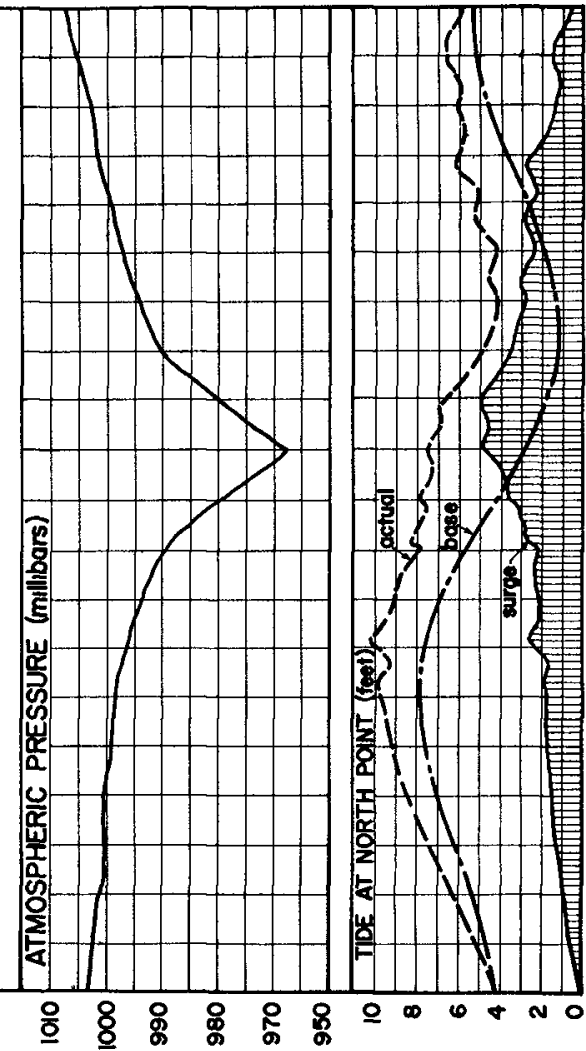


Data as above are avallable for seven typhoons, as 11sted in Table $I$, where relevant parameters leading to the final surge $\left(S_{t}\right)$ are included Comparison of the computed and measured values indicates the accuracy of the procedure presented

To determine a surge prior to 1ts arrival, the possible path in respect to the site must be assessed The maximum wind speed at the boundary of the eye is a function of the atmospheric depression at the centre Kraft $(16)$ provides an equation for Atlantic hurricanes as follows

$$
U_{\max }=14 \sqrt{1013-P_{c}}
$$

where $U_{\max }$ is expressed in knots for $p_{c}$ in millibars

Equation (13) may not be applicable to typhoons or more modest tropica1 cyclones

From table I the only typhoon which was centered on Hong Kong, and for which values could be used for verification, is that of Wanda when $U_{\text {max }}=80$ knots for $1013-952=61$ millibars This would indicate a relationship of

$$
U_{\max }=103 \sqrt{1013-\mathrm{p}_{\mathrm{c}}}
$$

The overall diameter of typhoons in the Western Pacific is around half of those normally recorded for Atlantic hurricanes It is not unreasonable, therefore, to expect lower wind velocities for any given atmospheric depression Such data should be complled for a reglon under study, when optimum storm surges are required to be calculated

The optimum water level to be expected can be computed from a knowledge of the most severe tropical cyclones known to exist in the area The fetch for this should then be traversed across the continental Shelf at the critical speed, to hit the coast just to the left (northern hemisphere) or right (southern hemisphere) of the port under study This maximum surge should then be added to MHWS level which can occur during the cyclone period of the year should the harbour be located near the head of a funnel shaped inlet, or one that has a shoaling approach channel, an amplification factor(17) may have to be determined for the surge level computed for the mouth

\section{ACKNOWLEDGEMENTS}

Appreciation is expressed to the Department of Public Works, Hong Kong, who supplied coples of their typhoon data sheets and gave permission for publication of this material 
Table I Comparison of computed and measured surges at North Point, Hong Kong harbour

\begin{tabular}{|c|c|c|c|c|c|c|c|}
\hline $\begin{array}{l}\text { Name } \\
\text { Date }\end{array}$ & $\begin{array}{l}\text { Wanda } \\
\text { Sept } 62\end{array}$ & $\begin{array}{l}\text { Faye } \\
\text { Sept } 63\end{array}$ & $\mid \begin{array}{l}\text { Viola } \\
\text { May 64 }\end{array}$ & \begin{tabular}{|ll} 
Idal & \\
Aug & 64
\end{tabular} & $\begin{array}{l}\text { Ruby } \\
\text { Sept } 64\end{array}$ & $\begin{array}{l}\text { Sa1ly } \\
\text { Sept } 64\end{array}$ & $\begin{array}{l}\text { Shirley } \\
\text { Aug } 68\end{array}$ \\
\hline $\begin{array}{l}\Delta \mathrm{p}(\mathrm{mb}) \\
\mathrm{s}_{\mathrm{a}}(\text { feet })\end{array}$ & $\begin{array}{rl}61 & \\
2 & 02\end{array}$ & $\begin{array}{l}17 \\
0 \quad 57\end{array}$ & \begin{tabular}{rr|r|}
21 & \\
0 & 70
\end{tabular} & \begin{tabular}{rr|}
38 & \\
1 & 27
\end{tabular} & $\begin{array}{r}45 \\
150\end{array}$ & $\begin{array}{rl}24 & \\
0 & 80\end{array}$ & $\begin{array}{r}44 \\
147\end{array}$ \\
\hline $\begin{array}{l}U_{\max } \text { (knots) } \\
\text { D1stribution } \\
F \text { (NM's) }\end{array}$ & $\begin{array}{r}80 \\
\Delta \\
55\end{array}$ & $\int_{220}^{25}$ & $\mid \begin{array}{c}30 \\
\text { 데 } \\
110\end{array}$ & $\begin{array}{r}45 \\
\Delta \\
210\end{array}$ & $\begin{array}{r}60 \\
\Delta \\
166\end{array}$ & $\begin{array}{r}35 \\
\Delta \\
107\end{array}$ & $\begin{array}{r}42 \\
\Delta \\
90\end{array}$ \\
\hline $\begin{array}{l}\mathrm{d}_{1} \text { (feet) } \\
\mathrm{k} \mathrm{U}^{2} \mathrm{~F} 10^{3} / \mathrm{gd}_{1}^{2} \\
\left.\left.\mathrm{~s} / \mathrm{d}_{1} \times 10^{3} \text { (F1gs } 5 \& f\right)^{2}\right) \\
\mathrm{s} \text { (feet) }\end{array}$ & $\mid \begin{array}{rl}400 & \\
3 & 55 \\
5 & 5 \\
2 & 2\end{array}$ & $\begin{array}{rl}400 & \\
1 & 4 \\
3 & 8 \\
1 & 52\end{array}$ & $\left|\begin{array}{rl}400 & \\
1 & 0 \\
2 & 7 \\
1 & 08\end{array}\right|$ & $\mid \begin{array}{rr}400 & \\
4 & 3 \\
6 & 5 \\
2 & 6\end{array}$ & $\begin{array}{rr}270^{(1)} \\
13 & 7 \\
20 & 0 \\
5 & 4\end{array}$ & $\begin{array}{rl}400 & \\
1 & 32 \\
2 & 0 \\
0 & 8\end{array}$ & $\begin{array}{rr}400 & \\
1 & 6 \\
2 & 5 \\
1 & 0\end{array}$ \\
\hline $\begin{array}{l}\mathrm{L} \text { (NM's) } \\
F / L \\
V(k n o t s) \\
V / \vec{C} \\
R(F 1 g \quad 8) \\
S_{\max }(=R S)\end{array}$ & $\begin{array}{rl}180^{(3)} & \\
0 & 3 \\
12 & \\
0 & 280 \\
1 & 9 \\
4 & 18\end{array}$ & \begin{tabular}{|cl}
240 & $(4)$ \\
0 & 92 \\
10 & \\
0 & 234 \\
1 & 3 \\
1 & 98
\end{tabular} & $\left|\begin{array}{rl}120 & \\
0 & 92 \\
8 & \\
0 & 187 \\
1 & 4 \\
1 & 51\end{array}\right|$ & $\mid \begin{array}{rl}120 & \\
1.75 \\
15 \\
0 & 350 \\
0 & 9 \\
2 & 34\end{array}$ & \begin{tabular}{|cl}
$80^{(1)}$ \\
2 & 08 \\
12 & 8 \\
0 & 348 \\
0 & 7 \\
3 & 78
\end{tabular} & $\begin{array}{rl}120 & \\
0 & 89 \\
13 & 3 \\
0 & 312 \\
1 & 6 \\
1 & 28\end{array}$ & $\begin{array}{rl}120 & \\
0 & 75 \\
9 & 1 \\
0 & 213 \\
1 & 5 \\
1 & 5\end{array}$ \\
\hline $\begin{array}{l}s_{t}\left(=s_{a}+s_{\text {max }} \text { ) }\right. \\
s \text { (measured) }\end{array}$ & $\begin{array}{ll}6 & 20 \\
4 & 6\end{array}$ & $\begin{array}{ll}2 & 55 \\
2 & 8\end{array}$ & $\begin{array}{ll}2 & 21 \\
3 & 0\end{array}$ & $\begin{array}{ll}3 & 61 \\
4 & 0\end{array}$ & $\begin{array}{ll}5 & 28 \\
5 & 0\end{array}$ & $\begin{array}{ll}2 & 08 \\
1 & 8\end{array}$ & $\begin{array}{ll}2 & 97 \\
3 & 0\end{array}$ \\
\hline
\end{tabular}

(1) Typhoon changed direction part way across Shelf where $d_{1}=270$ feet and $\mathrm{L}=80 \mathrm{NM}^{\prime} \mathrm{s}$

(2) Assuming $d_{2}=30$ feet

(3) Approach was from ESE, equivalent $I=180 \mathrm{NM}$ 's

(4) Approach was from east, equivalent $L=240 \mathrm{NM}^{\prime} \mathrm{s}$

(5) Tide gauge out, water mark observations only 


\section{REFERENCES}

(1) Wu J "A criterion for determining alr-flow separation from wind waves", Tellus 21, 1969, 707-714

(2) Barnett T P and A J Sutherland "A note on an overshod effect In wind generated waves" J Geoph Res, 1968, 6879-6885

(3) Sheppard P A "Transfer across the earth's surface and through the alr above", Quart J Roy Met Soc , 84, 1958, 205-224

(4) Deacon E L and E K Webb "Sma11 scale interaction" The Sea (Ed M N H111) Interscience $\mathbb{N} \times 1062$

(5) W11son B W "Note on surface wind stress over water at low and hıgh w1nd speeds", J Geoph Res 65, 1960, 3377-3382

(6) Wu $\mathrm{J}$ "Wind stress and surface roughness at sea interface" J Geoph Res 74, 1969, 444-455

(7) Silvester $R$ and $S$ Vongvisessomja1 "Energy distribution curves of developing and fully arısen seas" J Hyd Res (In Press)

(8) He1lstrom B "Wind effect on 1akes and rivers" Roy Inst of Tech Stockholm, Bul1 41, 1941

(9) Langhaar H L "Wind t1des in inland waters" Proc M1d Western Conf on Flu1d Mechs , 1951, p 278

(10) Keulegan G H "Wind tides in small closed channels" $J$ Res of Nat Bur Stds, 46,1951

(11) Keulegan GH "The form factor in wind-t1de formulas", Nat Bur Stds Rep No 1835,1952

(12) Shepard F D Submarine Geology (2nd Ed ) Harper \& Row N Y , 1963

(13) Silvester $\mathrm{R}$ and $\mathrm{S}$ Vongvisessomja1 "Computation of Storm Waves and Swel1" Proc I.C E (In Press)

(14) Bretschneıder C L "Englneerıng aspects of hurricane surge" Estuary and Coast11ne Hydrodynamics (Ed A T Ippen), McGraw-H111, 1966, 231-256

(15) Reid R O "Approximate response of water level on a sloping shelf to a wind fetch whlch moves towards shore" Beach Erosion Board, Tech Mem No 83, 1956

(16) Kraft $\mathrm{R} \mathrm{H}$ "The hurricane's central pressure and h1ghest winds" Mar1ners Weather Log $\underline{5}(5), 1961$

(17) Silvester $R$ "Tides and long period waves on continental margins" Proc 13th Congress I A H R , 3, 1969, 113-121 
CHAPTER 122

\author{
STATISTICAL PREDICTION OF \\ HURRICANE STORM SURGE \\ by \\ C Y Yang, A $M$ Parisl, and $W$ S Galther*
}

\begin{abstract}
High water assoclated with a hurricane is an important design parameter in coastal engineering Long range rational predictions can be made on the basis of Gumbel's theory of extremes and Wemelsfelder's theory respectively Fundamentals and underlying assumptions of the two theories are investigated and predictions are made for Breakwater Harbor, Lewes, Delaware, and Atlantic City, New Jersey Gumbel's theory is found satisfactory according to a criterion A crucial point, the ground rule of counting exceedances, is found to be vague in wemelsfelder's method The ground rule must be made definite in a meaningful prediction
\end{abstract}

\title{
Introduction
}

In coastal protection projects, marine operations in harbors, and marine structural design, a reliable prediction of the extreme sea state associated with a hurricape is of utmost importance because of damages to lives and property 1 The engineer would want to know the answers to the following simple questions How high will the sea level rise? How often do the high water levels occur in the future? How long does each high water level last when it occurs? How high will the highest wave crest be? How often does the high waves occur and how long do they last? What are the oscillatory characteristics of the extremely strong waves? In answering these questions a large amount of research work has been conducted generally in two categories -- deterministic and probablilstic approaches In 1966 Harris investigated the characterıstics of observed hurricane surges in the stategs 2 Bretschnelder in 1959 studied local surges for the Delaware Bay He made predictions of water level and waves in the bay for a given hurricane storm nearshore by a deterministic approach Such a study and similar ones as by LaSeur and Moore in 1966, provide useful information for short-time (days) forecast and warning systems

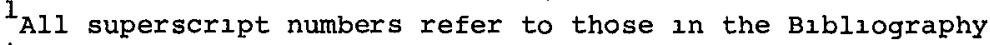

*Assoclate professor of Civil Englneering, Research Fellow of Civil Englneering and Dean of the College of Marine studies, respectively, Unıversity of Delaware, Newark, Delaware 19711
} 
For long-tıme (50 years) predictions, a deterministıc approach based on laws of mechanics is too complicated and consequently the only rational approach is one based on laws of probability and statistics of past records Indeed this was recognized for quite sometime and Important researgh work was published by Wemelsfelder in 1961, on high yater levels, by Pierson and Marks in 1952 on wave spectrum analysis, and by Cartwright and Lonquet-Higgins in 1956 on high wave crests More recently Frendenthal in 1969 outlined the gany important aspects of probabilistic design of marine structures He and Galther further investigated the economic aspects in desłgn 10 In a serles of papers Borgman investigated yave force on piles and spectral density for ocean wave forces

In this paper we are concerned with the prediction of extreme mean sea levels generally associated with hurricanes near the Delaware coast by a statistical apptoach The term hurricane surge is used as by Welgel to indicate a general association between extreme high water and a hurrlcane storm, though the analysts is not restricted to those caused by hurricanes This study is almed at answering the question of how high the extreme water level will be and how often each extreme water level will occur near the Delaware coast in the future years and decades It is based on a rational analysis by Gumbel and Wemelsfelder, respectively

\section{Gumbel's Theory}

The well known statistical theory of extremes now associated with Gumbel's name was originated in the early twentieth century In the $1930^{\prime}$ 's in a series of papers Gumbel presented important developments of the theory and its applications in a variety of problems -- breaking strength of materlals, floods and gust winds and stock market trends ${ }^{4}$ The fundamentals may be introduced by its application to our title problem

At Breakwater Harbor, Lewes, Delaware mean sea-level records have been obtalned glving monthly maximum levels for the period 1953-1969 Our problem is to predict the occurance of high water levels in the future based on these avallable records and Gumbel's theory First we concerntrate our attention on the maximum height in each year of 365 days clearly the annual maximum level is a random varlable whlch we can not predict definitely rather only with probability statements Let the random annual maximum be denoted by $x$ The probability that $x$ be less or equal to a certain quantity $x$ is the same as the probability that all 365 dally water levels are less or equal to $x$ Furthermore, if we assume that random dally levels are independent to one another and have a common probability distribution, then

$$
F_{X}(x)=\left[F_{X}(x)\right]^{n}
$$


where $x$ odenotes the random dally level and $n=365$ Therefore, If the probability distribution $\mathrm{F}_{\mathrm{x}}(\mathrm{x})$ of the random dally level $X$ is known, the above simple relation gives the desired probability distribution of the annual maxima since $F_{X}(x)$ is derived from $F_{x_{0}}(x)$, the latter 15 known as the parent distribution Now clearly the parent distribution is not known Fortunately, lt has been shown that if we can assume that the number of random daily levels can be very high, then the distribution

$$
F_{X}(x)=e^{-\alpha(x-\mu)}
$$

for large $\mathrm{n}$, in which $\alpha$ and $\mu$ are parameters This is known as the first asyimptote of the extreme distribution The problem is reduced to one of determination of the parameters $\alpha$ and $\mu$ sultable to our particular case This may be accomplished by plotting all avallable data pounts on an extreme probability paper and then fitting the points by a straight line The two independent paramcters of the line then correspond to the desired parameters $\alpha$ and $\mu$ respectively so if all goes well we now find what we are looking for, namely, the probability distribution of the annual maximum water levels, $F_{x}(x)$ whether all things go well or not must be polnted out so that reliable predictions can be made and the direction of future improvement can be understood These are the basic assumptions of independence among daily random levels, common parent distribution, the ground rule of plotting data points on the probability paper and the quality of fitting a stralght line to these points In addition, the assumption of time invarient of the parent distribution must also be valid

Wemelsfelder's Theory

The essentıal dıfference between Gumbel's and Wemelsfelder's theory lies in the initial manipulation of avarlable data and in the final presentation of results Instead of picking annual maximum out of 365 dally records in a year, Wemelsfelder proposed to count the number of exceedances of high water levels in the entire period of observations Thus in any one year the number of exceedance of a level may range from 0 up to 5 or 10 , and all high water occurances are to be counted in a year This is a clear distinction from the data collection method under Gumbel's theory in which one and only one high water level, the annual maximum, is consldered in one year Having counted the number of exceedances of high water levels, Wemelsfelder proposed to plot the yearly rate or the number per year $v$ versus the corresponding level $\mathrm{H}$ and called such a plot a frequency curve Assuming that the high water levels are rare and independent events, he then applied the Poisson probability law law we can state that the probability $p$ of no exceedance of a level in a specifled period of $T$ years is

$$
p=e^{-v T}
$$


where the parameter $v$ is the mean rate of exceedance parameter can be estımated from the frequency curve Thls the probability of exceeding that level is

$$
q=1-e^{-v T}
$$

This probabllity implies a chance of fallure and is therefore, defined as the risk In summary, an analytic relation is thus established among three variables, namely, the risk $q$, the desıgn perlod $T$ and the design wacer helght $H$ Recall that the mean rate $\nu$ is a function of the helght $H$ as established by the frequency curve Wemelsfelder presented the theory in a two dimenslonal plot, with ordinates $H$, abscissa $T$ for varlous risks $q$ This presentation is very appealing and different from that by Gumbel as wlll be shown later in applications

\section{Applications and Results}

All data used in this paper was recorded by the coast and Geodetic Survey, Environmental Sclence Services Administration, U S Department of Commerce through tide gauges located at Breakwater Harbor, Lewes, Delaware, and at Atlantıc City, New Jersey Highest tides above the 1929 Mean Sea-Level Datum in each month were tabulated by the Coast and Geodetic Survey for Lewes from 1953-1969, and for Atlantic City from 1923-69 These are reproduced here as Tables 1 and 2 respectively the number in parenthesis indicate estimations and the blanks indicate missing data Based on some of this data, the Corp of Engineers in 1964 made studies on hurricanes afgng the Atlantic coast DelawareMaryland line to Virginia, 6 , and presented frequency curves* with extrapolations In our study the same frequency curves were used but were extrapolated by means of linear reqression computer program where a polynomial was fitted to the curve 18 The output of the program ylelds a stralght line for Breakwater Harbor, Lewes, Delaware, and a curve for Atlantic Clty These extrapolated frequency curves are shown as Figures 1 and 2

Figures 3 and 4 show the predictions of high water levels for Breakwater Harbor, Lewes, Delaware and Atlantlc City, New Jersey, respectively by Gumbel's theory and with the data obtalned from Tables 1 and 2 The ordinate is water helght in feet the abscissa on the bottom is probability distribution of the asumptote of the annual extremes and on the top is the return period in years A fourth scale is a linear transformation

\footnotetext{
'The references used there is Mean Low water whlch is $25^{\prime}$ and $453^{\prime}$ above the datum of 1929, Mean Sea-Level for Lewes, Delaware, and Atlantic City, New Jersey, respectively
} 
variable which is not important in physical interpretations The plotted points are data points The theoretical prediction is represented by the central straight line A point on the line in Figure 3 for example, indicates that the helght of 11 feet has a chance of 9 of not belng exceeded in any one future year, and on the average it will be exceeded once in every ten years Looking at the upper portion of the line for Breakwater Harbor, Lewes, Delaware, in Figure 3, we find that a helght of $122^{\prime}$ has a chance of 98 of not being exceeded in one year and on an average of once in 50 years The remalning feature in Figure 3 is the two curves forming a band along the theoretical line These curves are called control curves, indlcating an allowable deviation of data points from the line When all data point fall within this band, the theory is considered valid according to Gumbel Fortunately, this is the case for both Breakwater and Atlantic City Furthermore, the control curves supplement the stralght line predictions For example, in Figure 3, in addition to the statement made for the polnt $122^{\prime}$ helght, we can make two more statements First, the helght $122^{\prime}$ ' will be exceeded within the interval of 18 to 160 years on the average with probability of 68 Secondly, in 50 years on the average the helght w1 11 exceed 114 ' and not exceed 13 1', with a probability of 68 This fixed probability corresponds to a one standard deviation from the straight line consequently, different control curves can be plotted for different probabilities, and standard deviations Figure 5 shows a comparative prediction for Atlantıc Clty, New Jersey Important computations are presented in Tables 3 and 4

Now consider applying Wemelsfelder's theory to avallable data, monthly extremes in Tables 1 and 2 , and frequency curves in Figures 1 and 2 By the method previously outlined risk curves of Breakwater Harbor, Lewes, Delaware, are constructed and shown in Figure 5 and for Atlantic City, New Jersey in Figure 6 In Figure 5 the ordinate is helght of the water level and abscissa design period in years The curves indicate, for example, in 50 years a helght of $135^{\prime}$ will be exceeded with probability or risk of $108,153^{\prime}$, with a risk of $1 \%$ and $172^{\prime}$ ' with a risk of 0 lo at Breakwater Harbor, Lewes, Delaware

\section{Conclusions and Discussions}

Through the course of study, we feel that both Gumbel's and Wemelsfelder's theorles are founded on rational basis and are useful in practical predictions However, it is very important to be cautious about the validity of assumptions when applied to a particular location Unfortunately, no rational method is known to the authors in case any assumption is violated consequently, an educated guess must be made to modify the theoretical prediction in those cases As for the questions of whlch of these two theories is more reliable, our investigation at this point does not permit us 
to answer it satisfactorily However, we did find out one cruclal polnt which must be resolved before any meaningful comparison can be made That is in the Wemelsfelaer's theory, we could not find a clear ground rule for making the count of exceedances suppose that a severe hurricane storm hits a coastal area for three days with highest water levels on the first and third day such an event may be counted as two if the unit of time intervals is a day, counted 1 if lt is a month, or counted as 1 if the entire hurricane event is considered a unit This ambigulty must be removed by a clearly defined ground rule since we have not been able to find out the precise ground rule in Wemelsfelder's paper nor that used by the Corp of Englneers in their frequency curves, we can only assume that the count was made in a more or less arbitrary manner depending on the engineers As a result it is proposed here that a universal ground rule be establıshed, say a dally unit so that the ambigulty can be removed and precise communication among englneers can be established

Finally, we wish to polnt out that nelther of the two theorles take into account the important parameter of the time-span cf an cccurance of high water since this parameter is obviously significant in the characterization of the damaging effect of a stcrm, even a crude modjfication of the two theories for this effect is extremely useful Also, we wish to mention that during the conference presentation of the paper Lee harris rassed an important question he argued that the astronomical tides should be substracted from the total water helghts to get that part of the height due to hurricane surge alone Thıs question was responded by both $J$ B Schijf and $C$ L Bretschneider The general agreement is that in a location where the water reights are governed by a non-linear law and sufficiently large quantity of records are avallable, then the separation of the astronomical tide and hurricane surge is neither possible nor useful however, If a linear law prevalls then the separation is justifiable

\section{Acknowledgement}

This work is supported in part by the office of Naval Research under the contract, 2-97-815, No0014-69-A-0407, with the University of Delaware Data used in this study was obtained through Lee Harris of the Coastal Englneering kesearch Center, Corp of Englneers, Washingtor, D C, Martha Winn of the coast and Geodetic survey, Environmental Science Service Administration, Rockville, Marylana, and Vance Myers of the Weather Bureau, Environmental Science Service Administration, Silver Springs, Maryland 


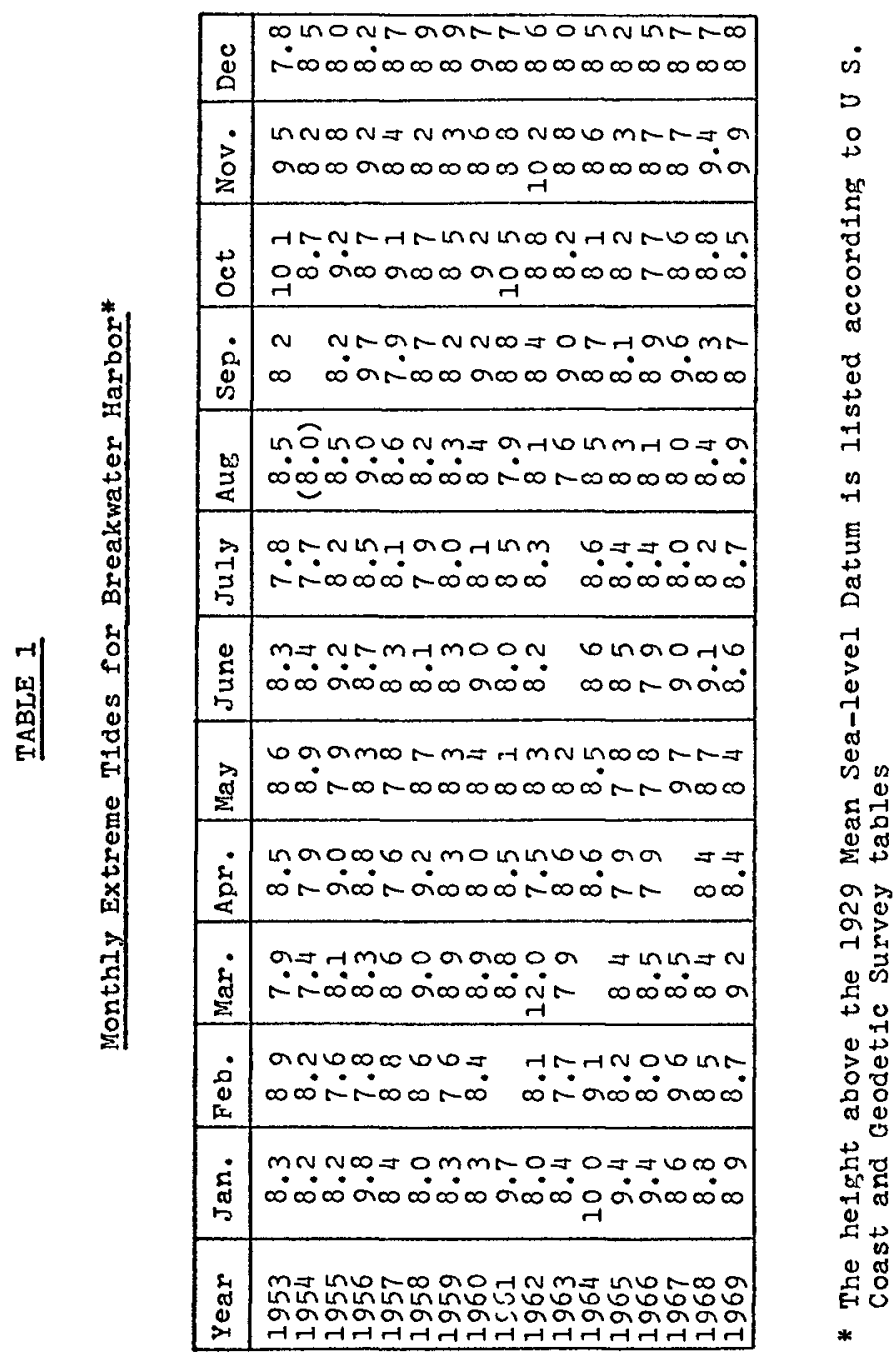




\begin{tabular}{|c|c|c|}
\hline \multirow{13}{*}{ 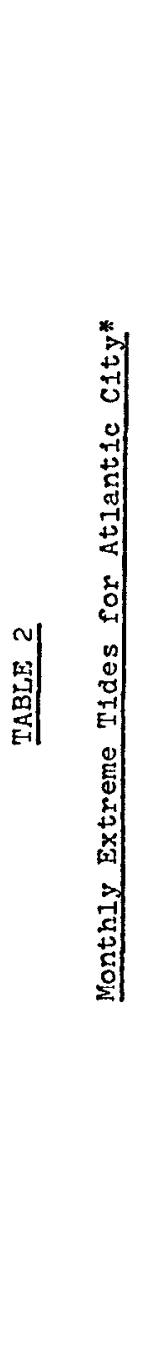 } & $\ddot{\dot{y}}$ & 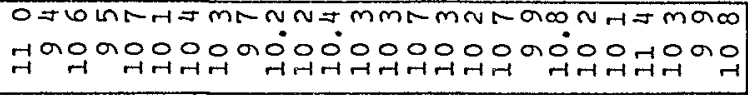 \\
\hline & $\begin{array}{l}3 \\
0 \\
z\end{array}$ & 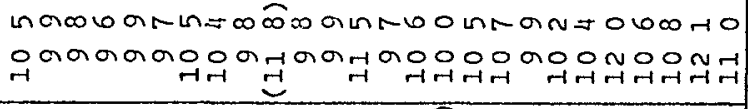 \\
\hline & 苍 & 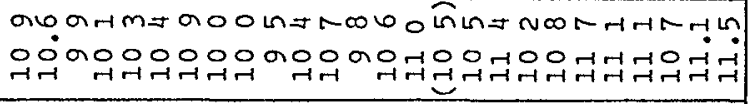 \\
\hline & $\begin{array}{l}0 \\
0 \\
0 \\
0\end{array}$ & 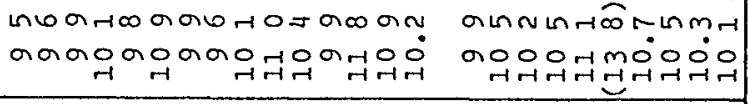 \\
\hline & $\dot{8}$ & 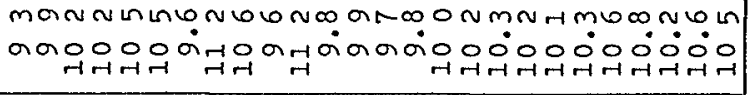 \\
\hline & ra & 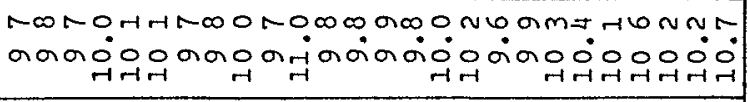 \\
\hline & 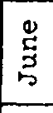 & 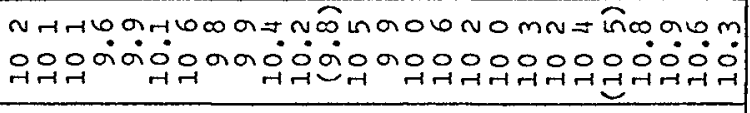 \\
\hline & 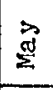 & 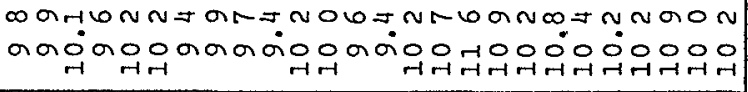 \\
\hline & $\stackrel{\dot{2}}{\dot{2}}$ & 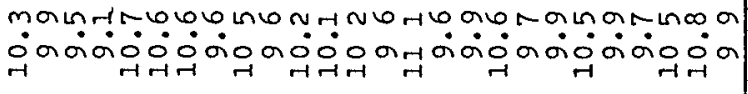 \\
\hline & $\stackrel{\substack{j \\
\Sigma}}{ }$ & 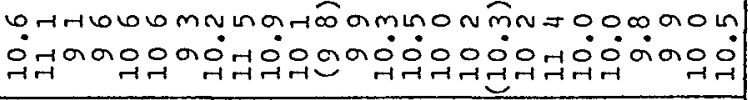 \\
\hline & $\stackrel{\dot{0}}{0}$ & 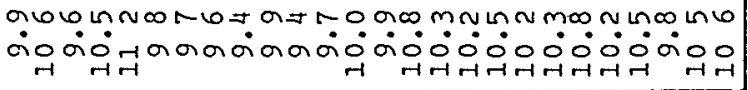 \\
\hline & 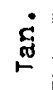 & 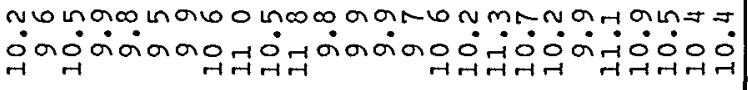 \\
\hline & 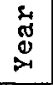 & 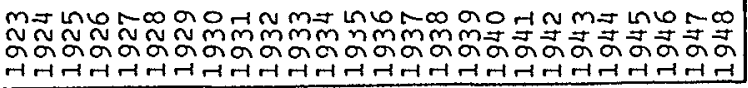 \\
\hline
\end{tabular}




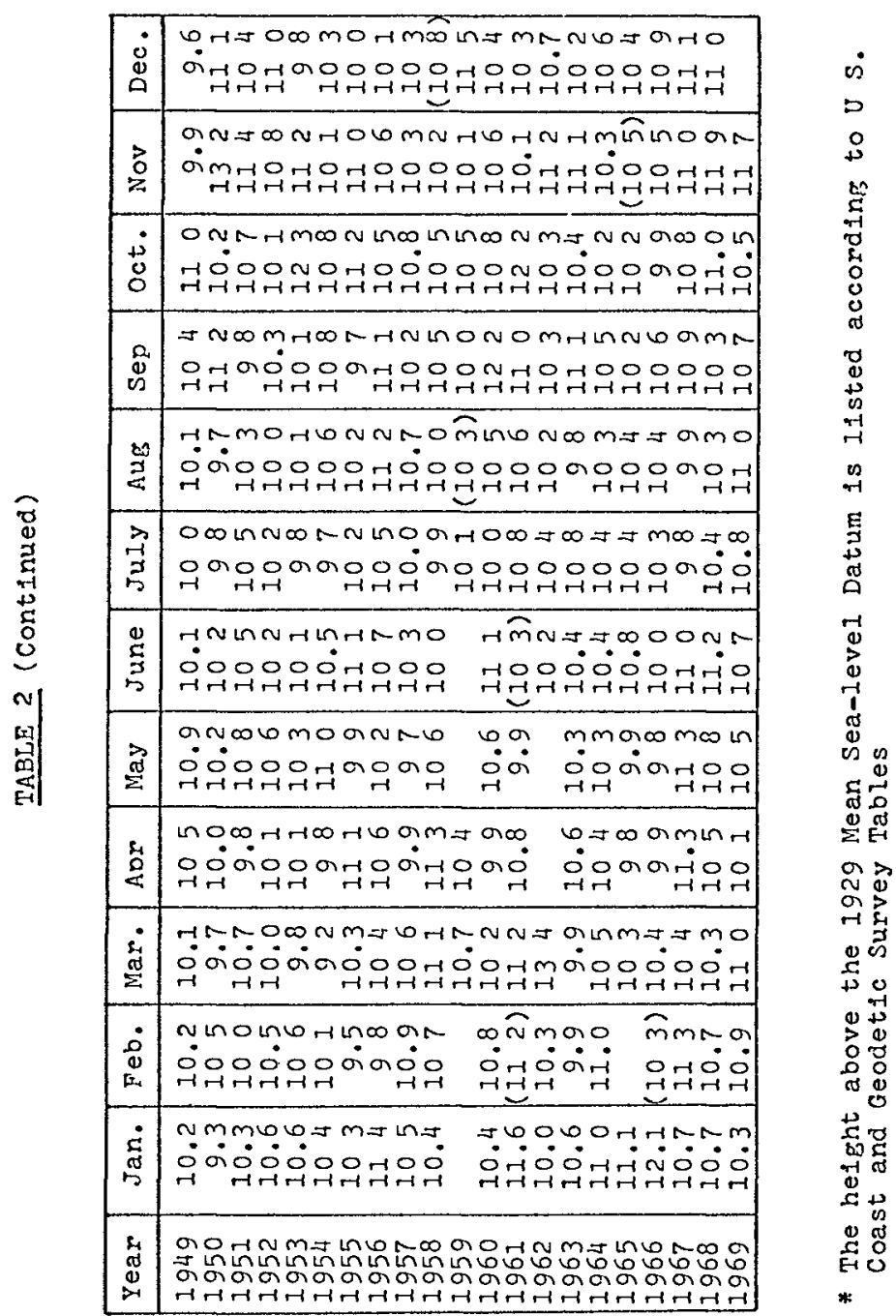




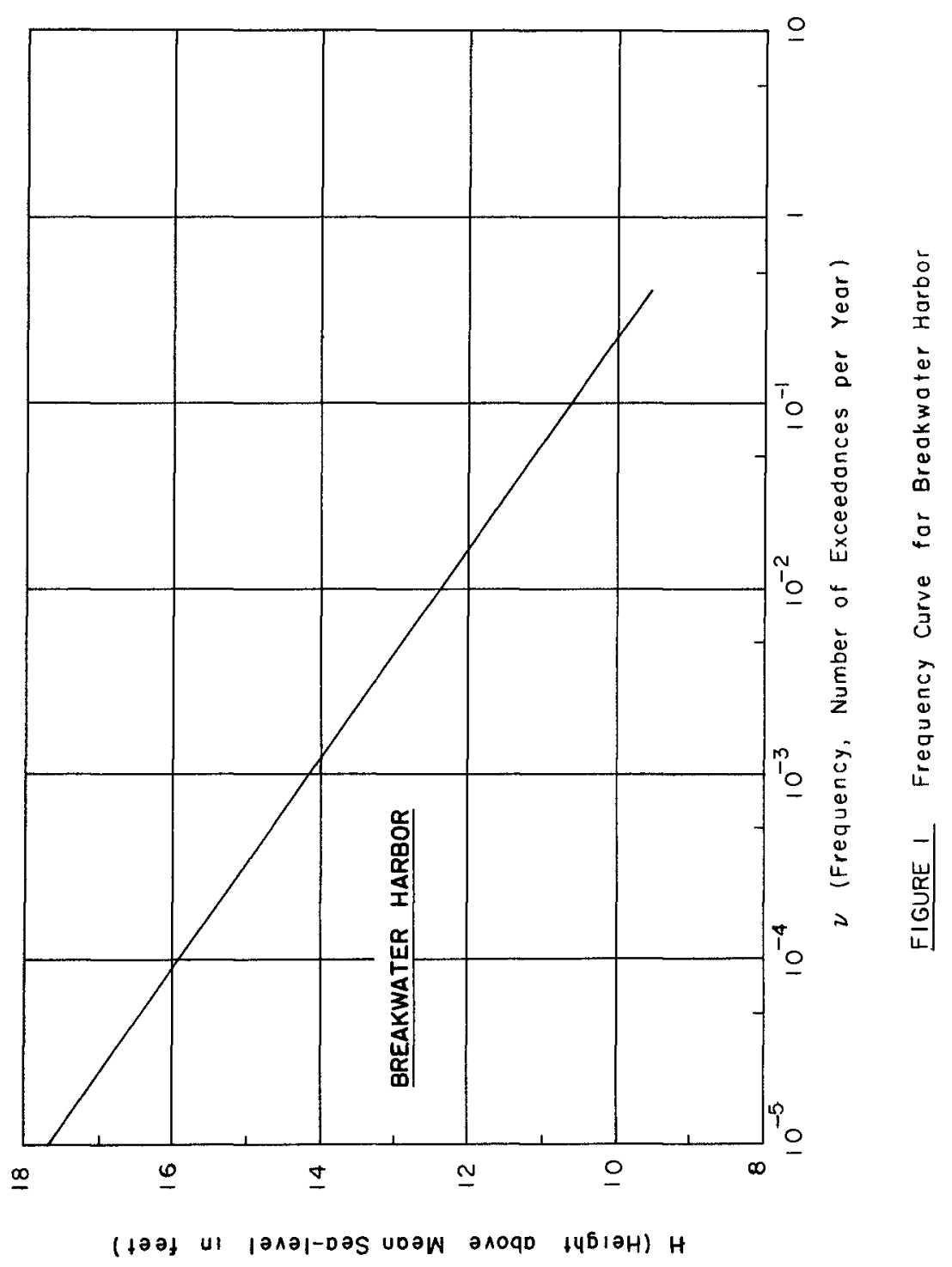


HURRICANE STORM SURGE

2021

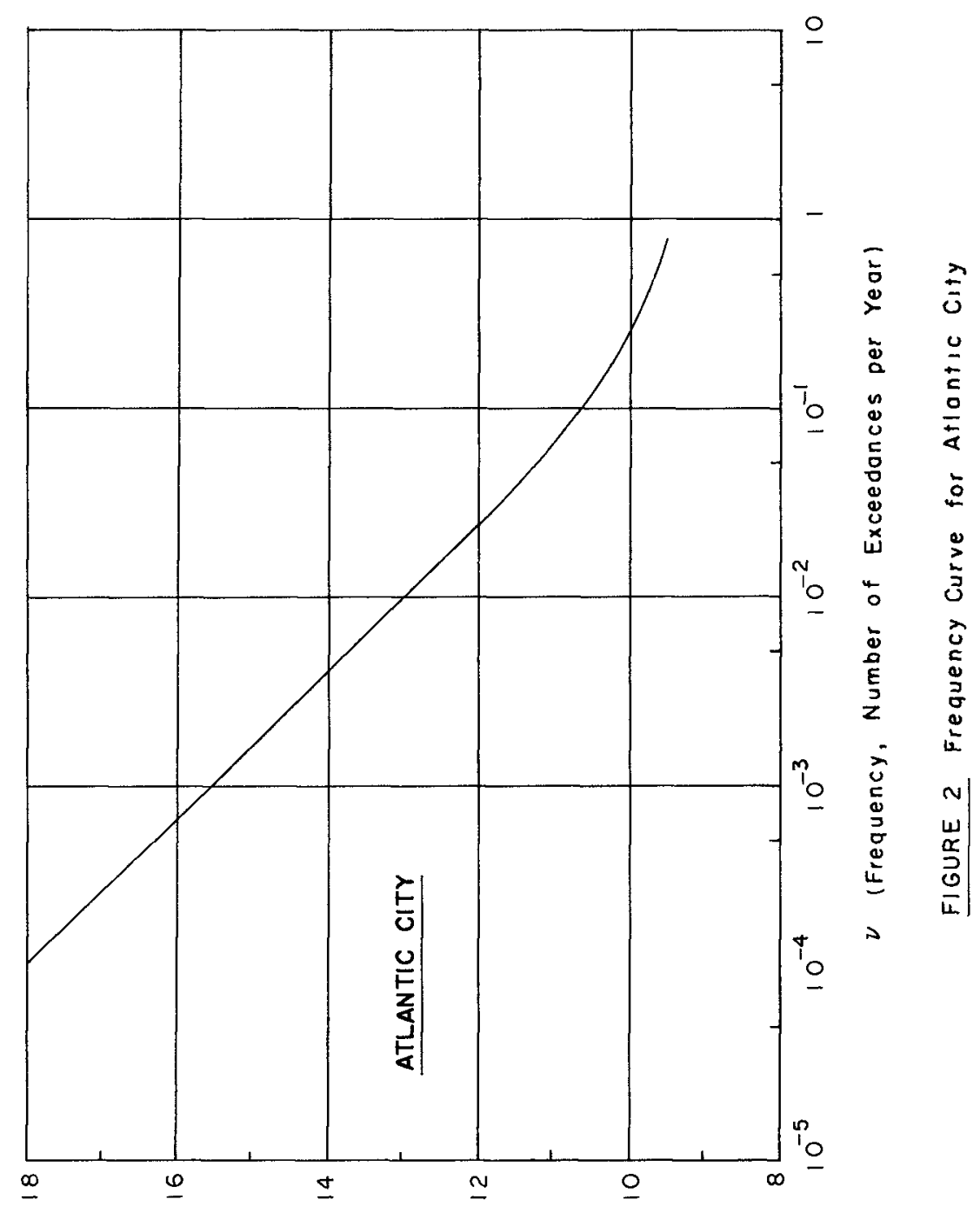

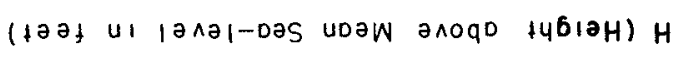




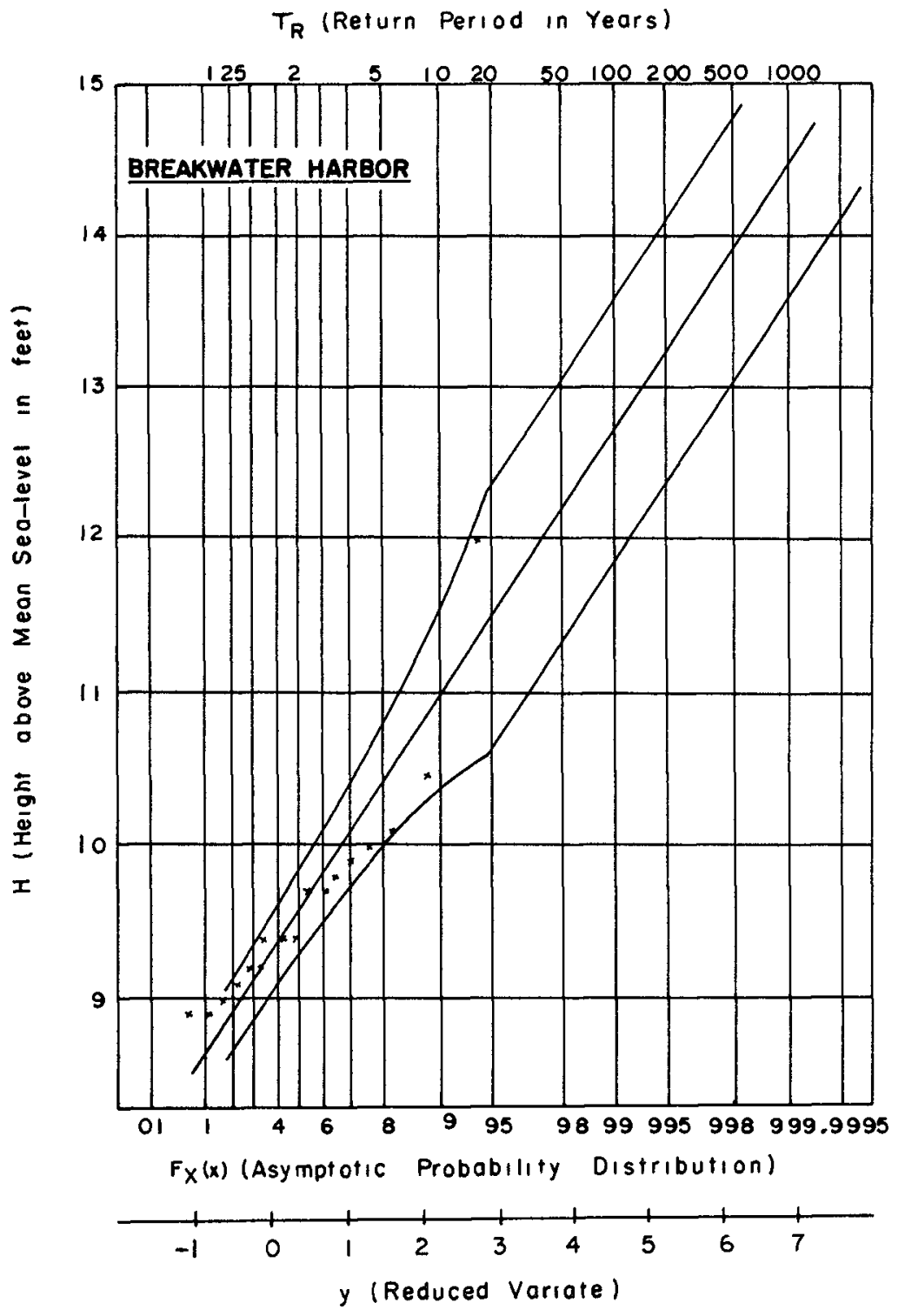

FIGURE 7 Gumbel Analysis for Breakwater Harbor 
$T_{R}$ (Return Period in Years)

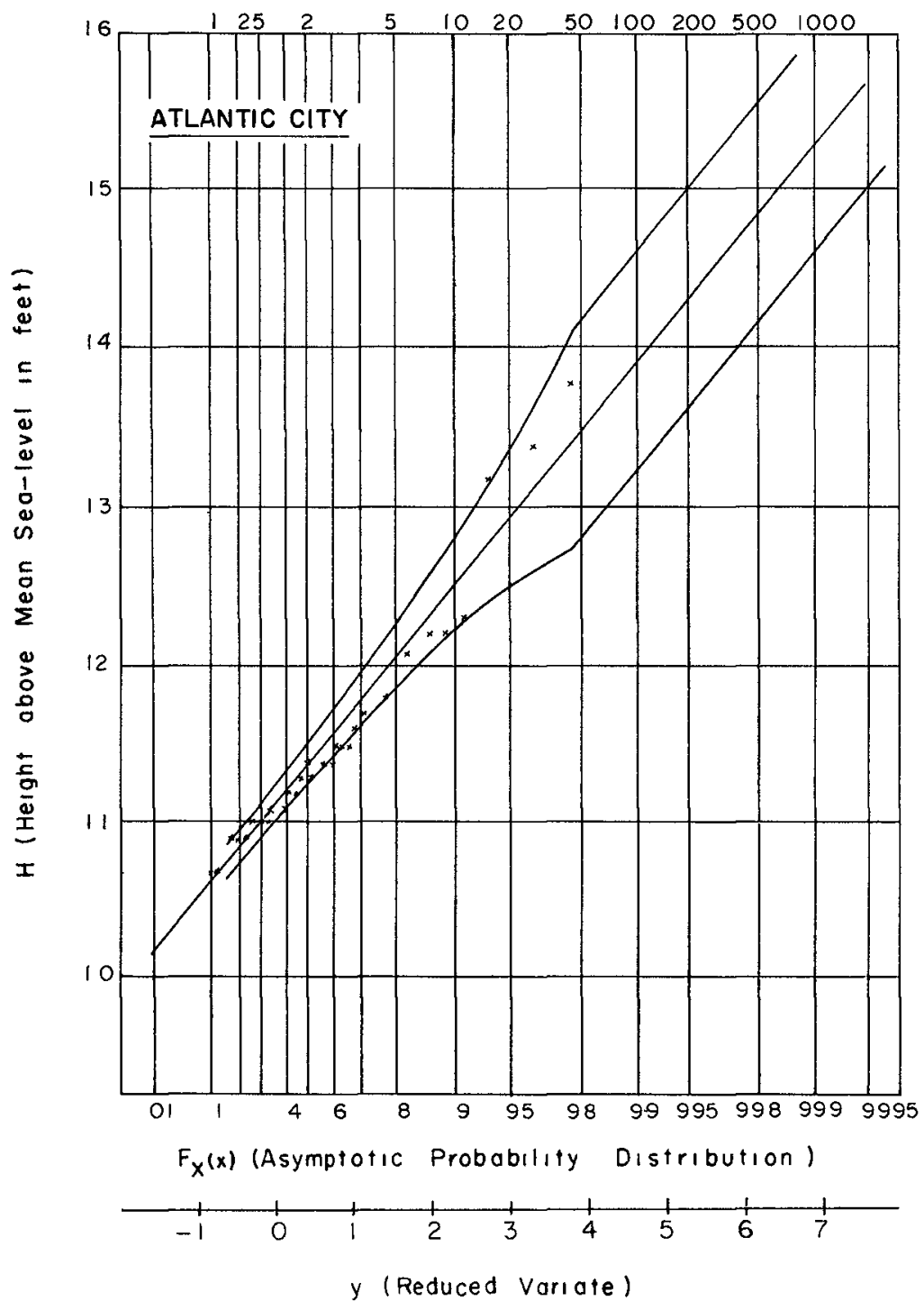

FIGURE 8 Gumbel Analysis for Atlontic City 


\section{TABLE 7}

Gumbel Analysis of Breakwater Harbor Data

\begin{tabular}{|c|c|c|c|c|}
\hline 1 & $\mathrm{H}^{*}$ & $\mathrm{H}^{2}$ & $\frac{1}{\mathrm{~N}+1}$ & $\mathrm{y}$ \\
\hline 1 & 8.9 & 79.21 & 0.056 & -1.059 \\
2 & 8.9 & 79.21 & 0.111 & -0.788 \\
3 & 9.0 & 81.00 & 0.167 & -0.582 \\
4 & 9.1 & 82.81 & 0.222 & -0.409 \\
5 & 9.2 & 84.64 & 0.278 & -0.247 \\
6 & 9.2 & 84.64 & 0.333 & -0.095 \\
7 & 9.4 & 88.36 & 0.389 & -0.057 \\
8 & 9.4 & 88.36 & 0.444 & +0.208 \\
9 & 9.4 & 88.36 & 0.500 & 0.366 \\
10 & 9.7 & 94.09 & 0.556 & 0.533 \\
11 & 9.7 & 94.09 & 0.611 & 0.708 \\
12 & 9.8 & 9604 & 0.667 & 0.904 \\
13 & 9.9 & 98.01 & 0.722 & 1.122 \\
14 & 10.0 & 100.00 & 0.778 & 1.382 \\
15 & 10.1 & 102.01 & 0.833 & 1.700 \\
16 & 10.5 & 110.25 & 0.889 & 2.140 \\
17 & 12.0 & 144.00 & 0.944 & 2854 \\
\hline
\end{tabular}

$\Sigma H=164.2 \Sigma H^{2}=1595.08$

$$
\begin{array}{ll}
\bar{y}_{N}=0.5181 & \bar{H}=9.65 \quad \overline{H^{2}}=93.828 \\
\sigma_{N}=1.0411 & \bar{H}^{2}=93.292 \\
& s_{H}^{2}=0.535 \quad s_{H}=0.731 \\
1 / \alpha_{N}=0.761 & u_{N}=\vec{H}-\left(1 / \alpha_{N}\right) \bar{y}_{N}=9.264
\end{array}
$$

\begin{tabular}{|c|c|c|c|c|c|c|c|c|c|}
\hline$\Phi$ & & & & 400 & 10 & 0 & 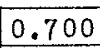 & 0 & \\
\hline$(y) \sqrt{n}$ & & & & & 3 & & 835 & & 2 \\
\hline 11 & & & 34 & & 7 & 7 & & .414 & 0 \\
\hline
\end{tabular}

$$
\Delta_{\mathrm{HN}}=0.869 \quad \Delta_{\mathrm{H}, \mathrm{N}-1}=0.578
$$

* Yearly Extremes from Table 1 
TABLE 8

Gumbel Analysis of Atlantic City Data

\begin{tabular}{|c|c|c|c|c|}
\hline 1 & $\mathrm{H}^{*}$ & $\mathrm{H}^{2}$ & $\frac{i}{N+I}$ & $y$ \\
\hline $\begin{array}{l}1 \\
2 \\
3 \\
4 \\
5 \\
6 \\
7 \\
8 \\
9 \\
10 \\
11 \\
12 \\
13 \\
14 \\
15 \\
16 \\
17 \\
18 \\
19 \\
20 \\
21 \\
22 \\
23 \\
24 \\
25 \\
26 \\
27 \\
28 \\
29 \\
30 \\
31 \\
32 \\
33 \\
34 \\
35 \\
36 \\
37 \\
38\end{array}$ & $\begin{array}{l}10.5 \\
10.6 \\
10.7 \\
10.7 \\
10.7 \\
10.9 \\
10.9 \\
10.9 \\
10.9 \\
10.9 \\
11.0 \\
11.0 \\
11.0 \\
11.0 \\
11.0 \\
11.1 \\
11.1 \\
11.1 \\
11.1 \\
11.2 \\
11.2 \\
11.2 \\
11.3 \\
11.3 \\
11.4 \\
11.4 \\
11.4 \\
11.4 \\
11.4 \\
11.5 \\
11.5 \\
11.5 \\
11.6 \\
11.7 \\
11.8 \\
11.8 \\
11.8 \\
11.9\end{array}$ & $\begin{array}{l}11025 \\
112.36 \\
114.49 \\
114.49 \\
114.49 \\
118.81 \\
118.81 \\
118.81 \\
118.81 \\
118.81 \\
121.00 \\
121.00 \\
121.00 \\
121.00 \\
121.00 \\
123.21 \\
123.21 \\
123.21 \\
123.21 \\
125.44 \\
125.44 \\
125.44 \\
127.69 \\
127.69 \\
129.96 \\
12996 \\
129.96 \\
129.96 \\
129.96 \\
132.25 \\
132.25 \\
132.25 \\
134.56 \\
136.89 \\
139.24 \\
139.24 \\
139.24 \\
141.61\end{array}$ & $\begin{array}{l}0.021 \\
0.043 \\
0.064 \\
0.085 \\
0.106 \\
0.128 \\
0.149 \\
0.170 \\
0.191 \\
0.213 \\
0.2314 \\
0.255 \\
0.277 \\
0.298 \\
0.319 \\
0.340 \\
0.362 \\
0.383 \\
0.404 \\
0.426 \\
0.447 \\
0.468 \\
0.489 \\
0.512 \\
0.532 \\
0.553 \\
0.574 \\
0.596 \\
0.617 \\
0.638 \\
0.650 \\
0.681 \\
0.702 \\
0.723 \\
0.745 \\
0.766 \\
0.787 \\
0.809\end{array}$ & $\begin{array}{l}-1.35 \\
-1.14 \\
-1.01 \\
-0.90 \\
-0.80 \\
-0.72 \\
-0.64 \\
-0.57 \\
-0.50 \\
-0.44 \\
-0.37 \\
-0.31 \\
-0.25 \\
-0.19 \\
-0.13 \\
-0.08 \\
-0.02 \\
+0.04 \\
0.10 \\
0.16 \\
0.22 \\
0.28 \\
0.33 \\
0.40 \\
0.46 \\
0.52 \\
0.59 \\
0.66 \\
0.73 \\
0.80 \\
0.88 \\
0.96 \\
1.04 \\
1113 \\
1.22 \\
1.32 \\
1.43 \\
1.55\end{array}$ \\
\hline
\end{tabular}


TABLL 8 (Continued)

\begin{tabular}{|c|c|c|c|c|}
\hline 1 & $\mathrm{H}^{*}$ & $\mathrm{H}^{2}$ & $\frac{1}{\mathrm{~N}+1}$ & $\mathrm{y}$ \\
\hline 39 & 12.1 & 146.41 & 0830 & 1.68 \\
40 & 12.1 & 146.41 & 0.851 & 1.82 \\
41 & 12.2 & 148.84 & 0.872 & 1.99 \\
42 & 12.2 & 148.84 & 0.894 & 2.19 \\
43 & 12.3 & 151.29 & 0.915 & 2.42 \\
44 & 13.2 & 174.24 & 0.936 & 2.72 \\
45 & 13.4 & 179.56 & 0.957 & 3.12 \\
46 & 13.8 & 190.44 & 0.979 & 385 \\
\hline
\end{tabular}

$\Sigma H=526.7 \quad \Sigma H^{2}=6053.03$

$$
\begin{array}{llr}
\nabla_{N}=0.5468 & \bar{H}=11.45 & \bar{H}^{2}=131.103 \\
\sigma_{N}=1.1538 & \overline{H^{2}}=131.588 & \\
& s_{H}^{2}=0.485 \quad s_{H}=0.697 \\
1 / \alpha_{N}=0.604 & u_{N}=\bar{H}-\left(1 / \alpha_{N}\right) \bar{y}_{N}=11.120
\end{array}
$$

\begin{tabular}{|c|c|c|c|c|c|c|c|c|c|}
\hline$\Phi$ & 0.150 & 0.200 & 0.300 & 0.400 & 0.500 & 0.600 & 0700 & 0.800 & 0 \\
\hline$\sigma(y) \sqrt{\mathrm{N}}$ & 1.255 & 1.243 & 1.268 & 1337 & 1.443 & 1.598 & 1.835 & 2.241 & 2.585 \\
\hline$\sigma(H)$ & 0.112 & 0.111 & 0.113 & 0.119 & 0.128 & 0.142 & 0.163 & 0.199 & 0.230 \\
\hline
\end{tabular}

$$
\Delta_{H, N}=0.689 \quad \Delta_{H, N-1}=0.458
$$

* Yearly Extremes from Table 2 


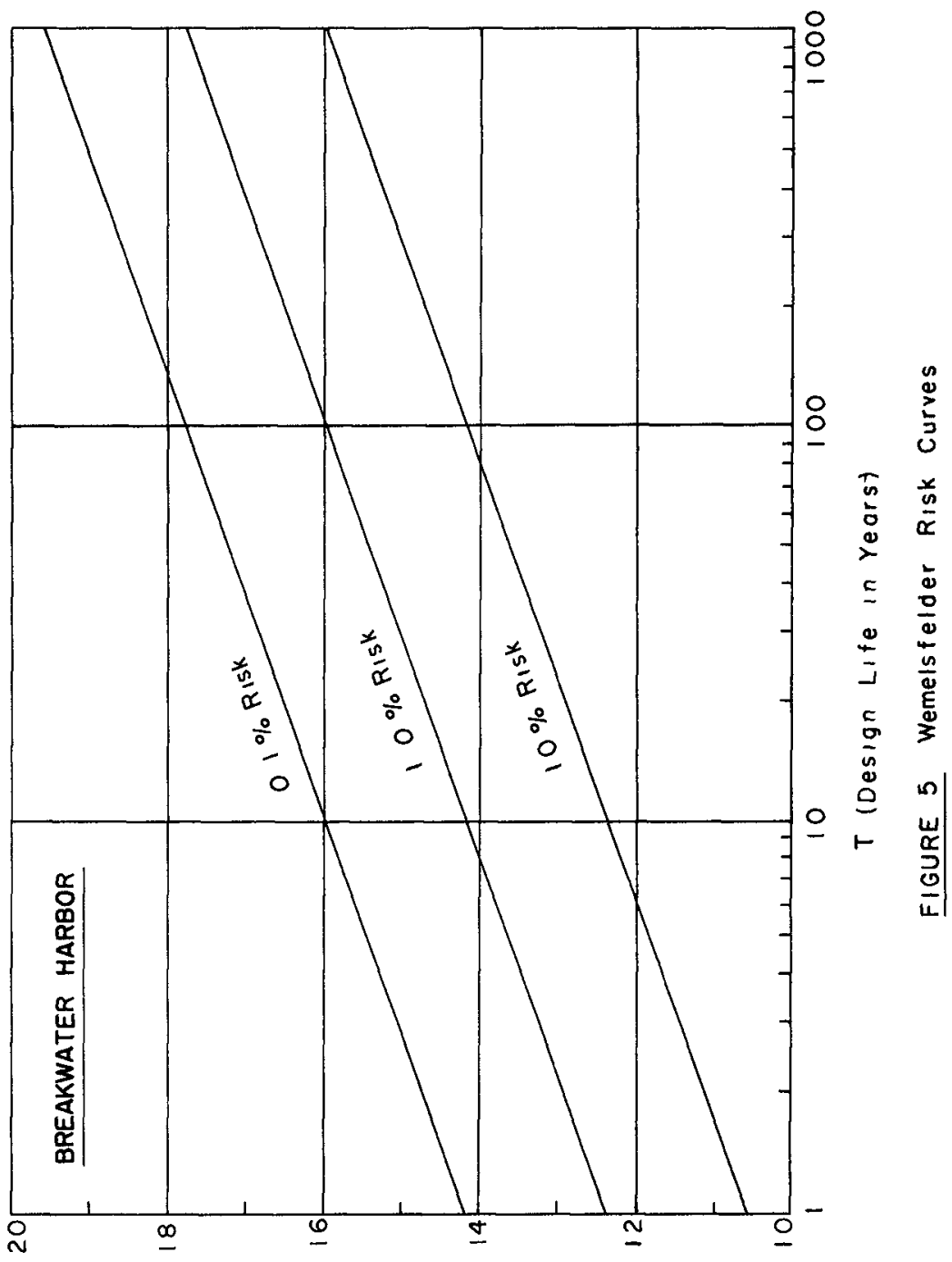

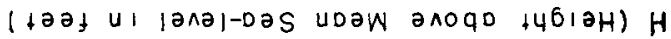




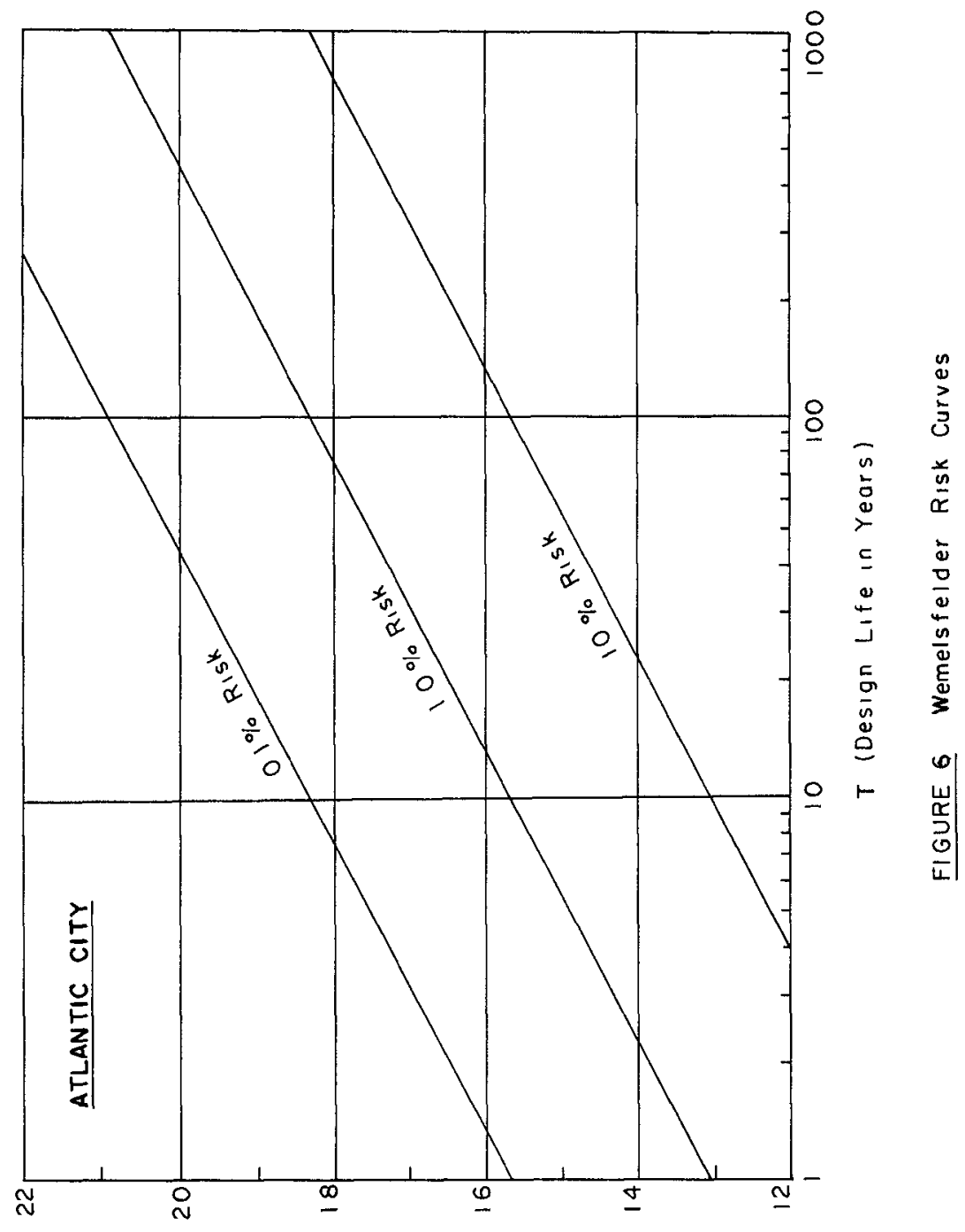

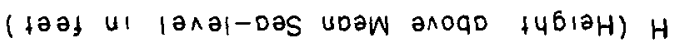




\section{BIBLIOGRAPHY}

1 Mather, J R et al "Storm Damage Hazard Along the East Coast of the United States," Journal Applied Meteorology, Vol 6, No 1 , February 1967 , p $20-30$

2 Harris, D I "The Hurricane Surge," Hurricane Symposium, Am So Oceanography, No 1 , October 1966 , Houston, Texas, pp 200228

3 Bretschnelder, C "Hurrlcane Surge Predictions for Delaware Bay and River," Misc Paper 4-59, November 1959, Beach Erosion Board, Corp of Engineers, Department Army

4 Laseur, $\mathbf{N}$ E "On the Description and Understanding of Hurricane Structure," Hurricane Symposium, Am So Oceanography, No 1 , October 1966, Houston, Texas, pp 71-81

5 Moore, P I "Forecasting and Warning Systems," Hurricane Symposium, Am So Oceanography, No 1, October 196⿸厂, Houston, Texas, pp 102-113

6 Wemelsfelder, P J "On the Use of Frequency Curves of Storm Floods," Proc Seventh Conference Coastal Englneers, Berkeley, California, The Englneering Foundation Council on Wave Research, 1961, pp 617-632

7 Plerson, $W \mathrm{~J}$ and Marks, $\mathrm{W}$ "The Power spectrum Analysis of Ocean Wave Records," Transactions, American Geophysical Union, $\mathrm{V} 33$, No 6 , December 1952, 835-844

8 Cartwright, D E and Longuet-Higgins, M S "The Statistical Distribution of Maxima of a Random Function," Proc Roy Soc. A237, 1956, 212-232

9 Freudenthal, A M "Probabilistic Evaluation of Design Criteria for Marine Structures," Bulletin of the Permanent International Association of Navigation Congresses, Vol III, 1968-1969, No $217-33$

10 Freudenthal, $A$ M and $W$ S Galther, "Probabilistic Approach to Economic Design of Marıtıme Structures," Sec 2, Subject 5, XXII and Permanent International Association of Navigation Congress, Parıs, France, 1969

11 Borgman, $\mathrm{L} E$ "The Statistical Distribution of Ocean Waves Forces on Vertical Plling," Institute of Englneering Res Tech Rep HEL-9-3, University of California, Berkeley, 1964 
12 Borgman, I E "The Spectral Density for Ocean Waves Forces," Coastal Englneerıng ASCE Special Conference, Santa, Barbara, 1965, Am Soc of Civil Englneers, 1966, 147-182

13 Welgel, R I Oceanographlcal Englneerıng, Prentıce Hall, 1964, pp 105-113

14 Gumbel, E J Statistics of Extremes, Columbla Press, 1958

15 Parzen, E Modern Probability Theory and Its Applications, John Wiley, 1960, p 251

16 Coxps of Englneers, U S Army, "Atlantıc Cıty, New Jersey," Letter from Chief of Englneers submitting Report of Interim Hurricane Survey," April 14, 1964 Item 334-A, D103, 22 AT6

17 Corps of Engıneers, U S Army, "Delaware River and Delaware Bay, Letter from Chief Engineers Submitting Report on Interim Hurricane Survey and Review of Reports, "August 17, 1964 Item $334-\mathrm{A}, \mathrm{D} 10322$ D3716

18 Parısı, A $M$ "Statistical Prediction of Design Still Water Level in Delaware Bay, $M S$ Thesıs, Civil Englneering Department, University of Delaware, July, 1970 


\title{
CHAPTER 123
}

\section{ANALYSIS OF HURRICANE TIDES AT PADRE ISLAND, TEXAS}

\author{
Frank D Maschl, Robert J Brandes ${ }^{2}$ \\ Floyd R Hill ${ }^{3}$ and William A White ${ }^{4}$
}

\begin{abstract}
To establish design data, a study was undertaken to determine expected hurricane tide elevations, durations and frequencies of occurrence for events of va rious magnitudes in the vicinity of a proposed development at Padre Island, Texas A set of synthetic hurricanes with selected sizes, translation speeds, wind fields, and pressure patterns were generated corresponding to various frequencies of occurrence of the CPI Two numerical computer models were developed to determine the offshore surge hydrograph and to route the surge through the bay waters for each synthetic hurricane moving directly over the site

Results included storm tide hydrographs at selected locations near the development Synthetic storms we re also routed across the coast at locations north and south of the site and storm hydrographs again computed
\end{abstract}

\section{INT RODUCTION}

This study was undertaken to investigate hurricane tides on the Laguna Madre and Corpus Christi Bay sides of Padre Island The location shown in Fig 1 represents an extensive property development on the northernmost end of Padre Island and is connected to the mainland near Corpus Christ1, Texas, by the John F Kennedy Causeway Of particular concern at the site were hurricane tide elevations, durations, and frequencies of occurrence of storm events of various magnitudes

The specific objectives of this study a re summarized as follows (1) Determine the offshore storm tide hydrographs near the site which result from hurricane events of various frequencies of occurrence, (2) Route these same hurricanes over the shallow waters of Corpus Christi Bay and the Laguna Madre and calculate the storm tide hydrographs generated on the bay side of the development site on both the Corpus Christi Bay and Laguna Madre sides

T Special Consultant on Fiscalın, Austın, Texas and Zurich, Switzerland, on leave from The University of Texas at Austin

2 Special Consultant on Fiscalin, Austin, Texas and Zurich, Switzerland

3 Engineer, Frank D Masch and Associates, Austin, Texas

4 Engineer, Texas Water Development Board, Austin, Texas 
of the causeway, and (3) Determine the effects of hurricanes moving across the coastline at distances north and south of the development site and calculate the storm tides generated on both sides of the causeway and at the development site

Because there are insufficient data to develop a reliable statistical representation of hurricane tides in Corpus Christi Bay and the Laguna Madre, two computer models based on the fundamental equations of motion were developed for computing offshore storm surge and for routing this surge through the shallow bay waters These models were first vexified for measured astronomical and hurricane tides They then were used to develop synthetic offshore hydrographs for storms of varjous frequencies of occurrence and to compute the storm tides at selected points in Corpus Christi Bay and the Laguna Madre resulting from these hurricanes

For calculating hurricane tides, many of the hydrographic and physiographic features characteristic of the region needed to be described These features include shallow water depths in Corpus Christi Bay and Laguna Madre, small islands, spoil dumps and limited exchange of waters between the Gulf of Mexico and the bay and lagoon In the immediate vicinity of the development, other conditions exist that could affect the magnitudes of hurricane tides A step type seawall, Ref [1], is now in existence over a portion of the Gulf side of the development site and is proposed to be extended to eliminate the possibility of Packery Channel cutting open to the Gulf of Mexico during hurricanes

The John $F$ Kennedy Causeway which connects Padre Island with the mainland is an earth-fill dike with openings for the Intracoastal Canal and the Humble Channel For hurricanes crossing the coast north of the development site, the causeway serves as a barrier and restricts the flow of water into the Laguna Madre until tides reach an elevation that produces overtopping of the causeway Similarly, the causeway restricts flow into Corpus Christi Bay for hurricanes moving up the coast from the south Islands formed by placement of dredge spoil from the construction of the Intracoastal Canal on the Laguna Madre side of the site also act as partial barriers to restrict somewhat the flow of water and consequently lower peak hurricane tide elevations slightly

\section{PROBLEM ANALYSIS}

To obtain answers to the stated objectives, the problem analysis was divided into the following three parts (1) Establishment of storm 
characteristics for synthetic hurricanes with various frequencies of occurrence, (2) Determination of synthetic hurricane surges offshore of the site, and (3) Determination of storm hydrographs at the development site utilizing the hydrographic and physiographic features of Corpus Christi Bay and Laguna Madre, the synthetic hurricane characteristics and the corresponding offshore surges

Frequency Analysis After an extensive literature review, it was decided that Within the scope and time frame avallable for this study, the most meaningful frequency analysis could be accomplished through the generation of a set of synthetic hurricanes The $U S$ Weather Bureau, Ref [2], has divided hurricanes affecting the Gulf Coast into three zones of approximately 80,000 square nautical miles each, one of which covers the Texas Coast and a small portion of the coasts of Loussiana and Mexico Hurricanes within each zone have been analyzed for frequency of occurrence of the Central Pressure Index (CPI) and for size, translation speed, wind magnitudes, and other characteristics From these analyses, the frequency of occurrence of the CPI is determined for selected locations along the Gulf Coast, including Corpus Christi The CPI is the minimum estimated pressure in a storm and is a good indicator of storm intensity

Once the CPI has been determined for a specified frequency, Ref [2] presents a series of steps by which synthetic hurricanes or "standard project storms" can be constructed Utilizing the CPI as basic information, it is possible to determine the radius to maximum winds for va rious radiu storms, the variations of this radius with longitude and latitude, the translation speed of the storm, probable azimuth, maximum cyclostrophic wind, maximum gradient wind and maximum wind speed 30 feet over water Table I is a summary of the principal characteristics of synthetic hurricanes developed at Corpus Christi with frequencies from 33 years to 100 years These characteristics were determined in part by the methods of Ref [2] and by adjustments based on judgment to more nearly reflect conditions near the Corpus Christi area

In reviewing the relation between CPI and the radius to maximum winds, it was noted that data for the Texas coastal zone and in particular that for the Corpus Christi area indicate that the local mean radius storm is smaller than the average mean radius storm for all events Similarly, the longitudinal correction to the radius to maximum winds shows a negative departure from the mean of about five nautical miles for a longitude near $96^{\circ} \mathrm{W}$ Hence, the values of radius to maximum wind were adjusted to reflect the se Corpus Christ characteristics Also in selecting the translation speed for the hurricane center, a modal value based on the cumulative percent of occurrences of all events was chosen From Ref [2], eleven knots is considered as a representative moderate speed and was taken as constant for all frequency hurricanes 
TABLE 1 SUMMARY OF SYNTHETIC HURRICANE CHARACTERISTICS

\begin{tabular}{|c|c|c|c|c|c|c|c|}
\hline $\begin{array}{l}\mathrm{S}_{\mathrm{o}} \\
(\mathrm{ft})\end{array}$ & $\begin{array}{c}T_{r} \\
(\mathrm{yrs})\end{array}$ & $\begin{array}{l}\text { CPl } \\
\text { (in ) }\end{array}$ & (n & $\begin{array}{l}\mathrm{R} \\
\mathrm{mi})\end{array}$ & $\begin{array}{l}V_{c x} \\
(m p h)\end{array}$ & $\begin{array}{c}\mathrm{V}_{\mathbf{g x}} \\
(\mathrm{mph})\end{array}$ & $\begin{array}{c}\mathrm{V}_{\mathbf{x}} \\
(\mathrm{mph})\end{array}$ \\
\hline 20 & 100 & 2744 & & 12 & 110 & 1082 & 999 \\
\hline 20 & 75 & 2750 & & 125 & 108 & 1062 & 983 \\
\hline 20 & 50 & $27 \quad 60$ & & 13 & 107 & 1051 & 973 \\
\hline 20 & 30 & 2773 & & 135 & 103 & 1011 & 938 \\
\hline 15 & 20 & 2788 & & 14 & 99 & 970 & 904 \\
\hline 15 & 15 & $27 \quad 94$ & & 145 & 97 & 950 & 88.3 \\
\hline 15 & 10 & $28 \quad 16$ & & 15 & 92 & 900 & 842 \\
\hline 10 & 5 & $28 \quad 56$ & & 155 & 82 & 799 & $\begin{array}{ll}75 & 3\end{array}$ \\
\hline 10 & 33 & $28 \quad 84$ & & 16 & 68 & 659 & 633 \\
\hline
\end{tabular}

\section{LIST OF SYMBOLS}

$S_{0}=$ lnitial hurricane surge

$T_{\mathbf{r}}=$ Return period of storm

CP1 = Central Pressure lndex or the estimated minumum pressure for a particular hurricane

$\mathrm{R}=$ Distance from center of storm to region of maximum winds

$\mathrm{V}_{c \mathrm{x}}=$ Maximum cyclostrophic wind

$\mathrm{V}_{\mathrm{gx}}=$ Maximum gradient wind

$\mathrm{V}_{\mathrm{x}}^{\mathrm{gx}}=$ Estimated maximum 30 foot over-water speed

Synthetic Storm Development Before the storm tide hydrographs could be determined at selected points in Corpus Christı Bay and Laguna Madre, it was necessary to know the hurricane surge hydrograph offshore in the vicinity of the development it is this storm surge that acts at the tidal inlets and, depending on magnitude, may overtop Padre lsland As such, offshore tide represents one of the boundary conditions necessary to calculate tides within the bay and lagoon

In hydrograph form the offshore surge gives a complete picture of the effects of the hurricane Information required for the solution of this tide includes definition of the bottom profile offshore from the site to the edge of the continental shelf and knowledge of the wind's magnitude and direction across the entire width of the hurricane U S C G S Hydrographic Charts provided the required data for the bottom profile Wind patterns for the design storms could be taken exther from actual historical hurricanes which have been documented or from synthetic storms such as illustrated in Ref [2] 
Total storm surge offshore associated with a given hurricane is normally broken into several components for calculation The total hurricane surge above mean sea level consists of an initial surge, an astronomical tide, a pressure tide and the components of wind tide due to winds blowing perpendicular and parallel to the coast The initial surge is that associated with Gulf hurricanes and usually varies from one to two feet To be conservative, the astronomical high tide above mean sea level was selected to coincide with the peak of the storm tide The pressure tide was taken as 114 times the pressure differential expressed in inches of mercury Wind tides were computed from wind magnitude, wind direction, duration and from a consideration of bottom and water surface shear stress coefficients

In determining hur ricane tide hydrographs there is the problem of appropriate values for the bottom friction and surface wind stress parameters used in the calculations The most direct way to evaluate such parameters is to compare actual measured hurricane tide hydrographs wath computed hydrographs, determined as above but with actual data on the wind patterns, wind magnitudes, storm directions, CPI, etc It then becomes a matter of adjusting the bottom friction and wind stress coefficients until the computed hydrograph can be made to agree reasonably well with that actually measured

The final hydrographs at the coast for a series of synthetic storms we re obtained by placing the hurricane wind patterns with their leading edge at the coast, calculating the wind tides shoreward from the edge of the continental shelf, and then moving the wind patterns shoreward a prescribed distance and again performing the wind tide calculations This process was repeated until the end of the hurricane passed over the coastline Synthetic surge hydrographs were computed for hurricanes with return periods of $33,5,10,20,30,50$ 75 and 100 years, and those for 10,30 and 100 year return periods are included in Fig 6 The isovel pattern used in the calculations is that of a mean radius moderate speed of translation standard project hurricane To reflect the lower winds in the more frequent hurricanes, the wind magnitudes at specified radil were scaled proportionally by the ratio of the maximum wind speeds of the lesser storms to that of the standard project storm.

Storm Hydrographs at the Development Site The calculation of the storm tide hydrographs in Corpus Christi Bay and the Laguna Madre involved a numerical solution to the two-dimensional vertically integrated equations of motion and continuity These equations were solved for the boundary configurations of the bay and lagoon and included such significant features as spoil banks, causeway, barrier islands, tidal inlets, flow controls, and the Intracoastal Canal The boundary conditions for the equations included the storm surge hydrographs off-coast at Port Aransas, other tidal inlets and locations where Padre 
Island was likely to be overtopped Wind was another external surface force applied to the water surface within Corpus Christı Bay and the Laguna Madre

The method utilized in the bay tide computations is based on work described in Ref [3] The first step was construction of an Eulerian type grid, one nautical mile square, which approximated the general physiographic and hydrographic features of the development area Figure 2 is a small scale map of Corpus Christi Bay, Laguna Madre and Baffin Bay with the computational grid superimposed Figure 3 illustrates the control features included within the computational model The dash lines correspond to reefs, spoil banks or other flow control situations The solid lines correspond to impermeable barriers such as 1slands and the causeway

In a manner similar to that used for the offshore hurricane surge hydrographs, it was also necessary to calibrate this computational model In a complicated configuration such as that represented by the combination of Corpus Christi Bay, Laguna Madre and Baffin Bay, this calibration was done in two steps The first involved calibrating the bay for normal astronomical tides without the influence of wind, or with wind effects minimized When the model was verified for astronomical tides, it was then operated using the offshore hurricane surge at tidal inlets and with varying wind on the water surface to compute the hurricane tide within the bay

Verification of the computational model for astronomical tide was obtained by comparing the computed tide with the actual tide measurements at the Naval Air Station in response to a known tide imposed at Aransas Pass The Naval Air Station 18 a location near the development site, Fig 2 , where normal astronomical tides were recorded by the Corps of Engineers Figure 4 illustrates an astronomical tide measured at Corpus Christi Naval Air Station compared with the computed tide from the numerical model The maximum departure of the computed curve from the measured tide is on the order of 01 feet For the purpose of this study, this agreement is considered adequate verification of the astronomical tides and bottom friction conditions in Corpus Christi Bay

Further hurricane tide verification was accomplished by introducing into the model, conditions associated with Hurricane Beulah The storm tide recorded at Port Aransas was introduced at the jetties, and utilizing the winds associated with Beulah, the hurricane storm tide was computed at the Corpus Christi Naval Air Station This hydrograph is illustrated in Fig 5 where it can be seen that the model reproduced the actual measured tide quite satisfactoril Tides were also computed at several othe $r$ locations but are not included in this paper The major difficulty in this calibration process was the inclusion 
of the high runoff resulting from the large rainfall which accompanied Beulah and the rainfall on the bay itself These conditions were approximated for this model from avalable data in Ref [4] Winds recorded at the Corpus Christi Airport during the passage of Hurricane Beulah we re applied in the time-dependent fashion measured at the alrport to duplicate the tides recorded at different stations within Corpus Christi Bay

To predict the tides on the Corpus Christi Bay and Laguna Madre side of the development and to simulate those conditions during which the Kennedy Causeway would be overtopped, the model had to be extended to include the Laguna Madre to a point south of Baffin Bay Another extensive data search was undertaken to acquire quantitative information on the hydrodynamic characteristics of the Laguna Madre-Baffin Bay area No tide data were avalable for this location and it was necessary to synthesize the behavor of tides south of the causeway Although it was not possible to verify the computed tides in the Laguna Madre or Baffin Bay area, it was possible to compare qualitatively the computed results with those obtained during Hurricanes Beulah and Carla, and to note that the same general trends and characteristics existed It was found that after allowing the Kennedy Causeway to be overtopped, very good verification of the Beulah hurricane tide at the Corpus Christi Naval Air Station was achieved For this reason, it is believed that the total model including Corpus Christı Bay, Laguna Madre and Baffin Bay was sufficiently verified for the analysis used in this study

\section{ASSUMPTIONS AND MODEL LIMITATIONS}

It is not difficult to envision a great number of combinations of hurricane events each of which may contribute to the storm hydrographs developed offshore and in the bays and lagoons Such factors as storm path, storm size, CPI, translation speed, winds, and storm azimuth are some of the variable hurricane characteristics Similarly, unpredictable factors such as the time during which the storm remains offshore and any unusual changes in storm course also control the formation of hurricane surge Once within a bay, such variables as the amount, intensity, distribution and duration of rainfall on the bay itself and the runoff from contiguous land areas are also factors which affect the height, shape, and duration of the storm tide hydrograph Obviously with so many variables very few of which are statistically predictable, caution must be used in attempts at generalizations of hurricane events at a given site

Although it was necessary to make assumptions on the distribution of rainfall during Hurricane Beulah for proper verification of storm tides at the Naval Air Station, the synthetic storms on which the results of this study are based do not include rainfall Since the storms themselves are synthetic and rainfall frequencies are not established relative to the hurricane frequencies, 
attempts at introducing rainfall and rainfall distributions within the time frame available for this study would not be meaningful it also was noted in the offshore storm hydrographs that the surges rise and fall rapidly, even though a decay factor was included in the model Part of these rapid changes is due to the synthetic nature of the storms, but part is also due to the absence of rainfall which normally accompanies such storm events

The computational model used in this study does not allow for inundation of adjacent land mass The 10 and 15 -foot contours lie very near the water line throughout most of the area in the vicinity of the development with the exception of the Laguna Madre side of Padre Island Actually the volume of water involved in land inundation in the area covered by the model is very small in relation to the volume of water in Corpus Christi Bay and the Laguna Madre, and omission of land inundation should not have a noticeable effect on the computed tides in this area Furthermore, this assumption is conservative, for if there is any effect at all, it would be to produce lower tides than those calculated

Although the results in the following section are based totally on synthetic storms, they provide an indication of the magnitude and duration of hurricane tides due to storms of specified frequencies The results are also conservative in that the lesser magnitude storms are based on the isovel patterns for a 100-year storm with a linear adjustment applied to the wind velocities for the less severe events Hence, the duration of the smaller events may be somewhat greater than would occur in an actual small storm On the other hand, these storms do not include rainfall and runoff and the computed tides could be of lower amplitude than those of a true storm with heavy precipitation

\section{DISCUSSION OF RESULTS}

The operation of the simulation model of Corpus Christi Bay, Laguna Madre, and Baffin Bay with synthetically generated offshore inputs at Port Aransas, Corpus Christi Pass, and Yarborough Pass has permitted the computation of tide hydrographs at selected points at the development site Synthetic hurricanes were routed across the shoreline with the eye crossing approximately 15 miles south of Port Aransas This produced maximum storm tides at the Port Aransas jetties, the major tidal inlet to the Corpus Christi Bay area Since the actual development site is protected on the Gulf side by a seawall and by proposed stabilization measures for Packery Channel and vicinity, it would appear that even though the maximum storm tides were allowed to exist immediately at the site, it would be unlikely that serious overtopping of Padre lsland would occur at that point However, in the computational model, allowances have been made for flow and tide at Corpus Christi Pass, which presumably will be opened and maintained in the future Port Aransas also was 
selected as the point for application of maximum storm tide since it was a location for which some surge data were available and was the input location for verification tides at the Naval Air Station during Hurricane Beulah Also, since frequencies are based on average conditions for the Texas Coast, the probabilities of an event crossing at a specific point are undoubtedly less Thus, the slight shift of the storm to permit tide computations at a point whe re some data were available is not considered significant

Development Site Tides Corresponding to the offshore hydrographs from the synthetic storms, tide hydrographs in the bay were computed near the development site at the points defined in Fig 1 The location of the two points considered most representative of the site were defined as Packery Channel and Padre Island Site West Three of the computed hydrographs for these two locations are shown in Fig 6 and are based on a causeway elevation of 45 feet which is near the present average elevation These hydrographs account for overtopping of the causeway when storm tides increase beyond an elevation of 45 feet For convenience, the input tides used in the calculations have also been included on these figures

The maximum storm tide elevations at Aransas Pass, Padre Island Site West, and at Packery Channel are summarized in Fig 10 where the maximum computed storm tide has been plotted against the probability of occurrence in any one year The input tide at Aransas Pass varies from 62 feet for a storm with 33 -year return period to 117 feet for the 100 -year storm The 30-year computed storm tide elevation is 112 feet although the curve indicates an elevation of about 108 feet Figure 10 also shows that the storm tides at Packery Channel, $1 \mathrm{e}$, on the Corpus Christi Bay side of the causeway, are always higher than tides on the Laguna Madre side of the development site The maximum storm tide at Packery Channel computed from the 100-year storm is 79 feet whereas at Padre Island $S_{1}$ te West it is 7 feet The 30-year storm for the same sites produces tides of 74 and 67 feet respectrvely

It is also significant to note that for the less frequent storms there is less change in the magnitude of storm tide at all locations including Aransas Pass For example, it can be seen from Fig 10 that for the existing cause.way elevation of 45 feet, the tides expected from a 30 -year storm differ from a 100-year storm by less than 06 feet This small change in maximum tide is due to the small difference in maximum average wind speed between the less frequent storms This can be noted in Table I

As also illustrated in Fig 6, the Padre Island Site West tide always lags the Packery Channel tide indicating the delaying influence of the causeway Actually the storm tides on the Laguna Madre side of the development do not begin to increase appreciably until the tides on the Corpus Christi Bay side are of sufficient magnitude to overtop the causeway at elevation of 45 
feet The Intracoastal Canal alone is not large enough to enable the exchange necessary for the tide to build up on the Laguna Madre side as rapidly as it does on the bay side This delay varies from about six to eight hours depending on storm magnitude It can also be noted that the Packery Channel tide reaches a peak shortly before the eye crosses the coastline and then falls rapidly as the eye crosses the coast and winds reverse direction This wind reversal also contributes to the rapid rise in the Padre lsland Site West tide immediately after the storm passes the coast and winds begin to blow up Laguna Madre When the Padre lsland Site West tide reaches its peak the eye has already crossed the coast and the Packery Channel tide has begun to fall Water from the Laguna Madre then flows back over the causeway into Corpus Christi Bay This, coupled with the fact that no sudden changes in wind direction occur after the eye has passed the site, produces a flatter peak on the Lag una Madre side The oscillation noted in the storm hydrog raphs after the passage of the peaks actually represents the astronomical component of the total tide

Further insight into the tide behavior on both sides of the causeway for different storm events can be obtained from Fig 7 These curves are computed for various frequency storms at points identified as Intracoastal Canal South, Intracoastal Canal North, Humble Channel South and Humble Channel North The locations of these points also are illustrated in Fig 1 These hydrographs give comparisons of the storm tide elevations for the three storm events of 10 , 30 and 100 years at two points on each side of the causeway it is not possible to construct a duration of overtopping-frequency relationship because by the nature of the synthetic hurricanes the more severe events tend to be somewhat tighter storms in order to produce the lower CPl's This leads to the apparently anomalous condition in which the less frequent events produce shorter periods of overtopping once overtopping is significant The study of the isovel patterns for less severe storms requires analysis considerably in excess of that available to this study

It can also be noted from Fig 7 that the tides on the south side of the causeway are characterized initially by very low tides followed by a rapid rise in the water surface In fact, the tide at Humble Channel South becomes less than mean sea level meaning that water is blown out of the computational cell at that point No verification data of this condition are avalable, however, qualitative data obtained during Carla, Ref [5], indicates very low tides in this area Also an astronomical tide study south of the causeway, Ref [6], shows that tides change very rapidly with relatively small changes in wind velocity or direction In the case of the synthetic hurricanes, the winds are northerly to northeasterly until the eye crosses the coast As already noted, overtopping of the causeway occurs at about this same time This overtopping together with the sudden reversal to winds from the south and southwest lead to this very rapid rise in wate $r$ surface elevation 
Storms at Other Locations In order to obtain some insight into the tides generated from hurricanes crossing the coast at locations other than at the development site, two conditions were considered These included the 30-year storm crossing the coast at points 50 miles south and 50 miles north of the site The storm hydrographs computed for the se conditions at Packery Channel and Padre Island Site West are shown in Fig 8 Also the corresponding tides on each side of the causeway are given in Fig 9 These computations have been carried out for a causeway elevation of 45 feet

Rather than re-rout the 30-year storm with its attendant adjustments into the coast over different offshore profiles, a linear adjustment based on observations made during Hurricane Carla, Ref [5] was applied to the input tide used in the previous computations It is well established that hurricane tides to the right of the eye when viewed in the direction of travel are larger than tides to the left of the eye This is because of the large component of tide resulting from onshore winds Thus, a hurricane crosising the Texas Coast south of the development site will produce a larger tide at the site than If the same storm moved inland north of the site Corresponding to the Carla results, the input tide at Aransas Pass was reduced to $90 \%$ for the storm south of the site and by $75 \%$ for the storm north of the site No adjustment was made to the storm duration

All the tides thus computed are lower than those produced by the $30-$ year storm at the site This can most easily be seen in Fig 11 which summarizes the maximum tides at Aransas Pass, Packery Channel and Padre Island Site West for the various storm locations One point of note is that for the storm south of the site, the Padre Island Site West tide is slightly higher than the Packery Channel tide Although the difference in actual magnitude may not be of numerical significane, the behavior of the tide in Laguna Madre relative to the Packery Channel tide is different than for a storm crossing at the site or to the north of the site It is possible that a slightly more severe storm or possibly one at some other location south of the site could produce a higher tide in the Laguna Madre than in Corpus Christi Bay This, of course, assumes that sufficient overtopping of Padre Island occurs so that a large enough volume of water is avalable for the tide buildup Although different storm paths were involved, a similar effect can be noted by comparing the computed tides with the spot tide elevations reported for Hurricane Carla and Beulah Carla which crossed the coast at Port O'Conner caused high tides at the Naval Air Station near the site and very low tides in the Laguna Madre Beulah, which affected the southern part of the coast, produced high tides in the Laguna Madre as well as Corpus Christi Bay 


\section{REFERENCES}

[1] Masch, F D et al, (1968), "Design Considerations for the Padre Island Seawall," Austın, Texas

[2] Graham, $\mathrm{E}$ E and Nunn, D E, (1959), National Hur ricane Project Report 33, "Meteorlogical Considerations Pertinent to Standard Project Hurricane, Atlantic and Gulf Coasts of the United States "

[3] Masch, F D et al, (1969), "A Numerical Model for the Simulation of Tidal Hydrodynamics in Shallow Irregular Estuaries, "Technical Report HYD 12-6901, The University of Texas at Austin

[4] U S Army Engineers District, Galveston, (1967), Report on Hurricane Beulah, U S Corps of Engineers, Galveston, Texas

[5] U S Army Engineers District, Galveston, (1962), Report on Hurricane Carla, U S Corps of Engineers, Galveston, Texas

[6] Copeland, $B \mathrm{~J}$ and Thompson, Jr, J H, (1968), Effects of Wind on Water Levels in the Texas Laguna Madre, The Texas Journal of Science, Vol VV, No 2, December, 1968 


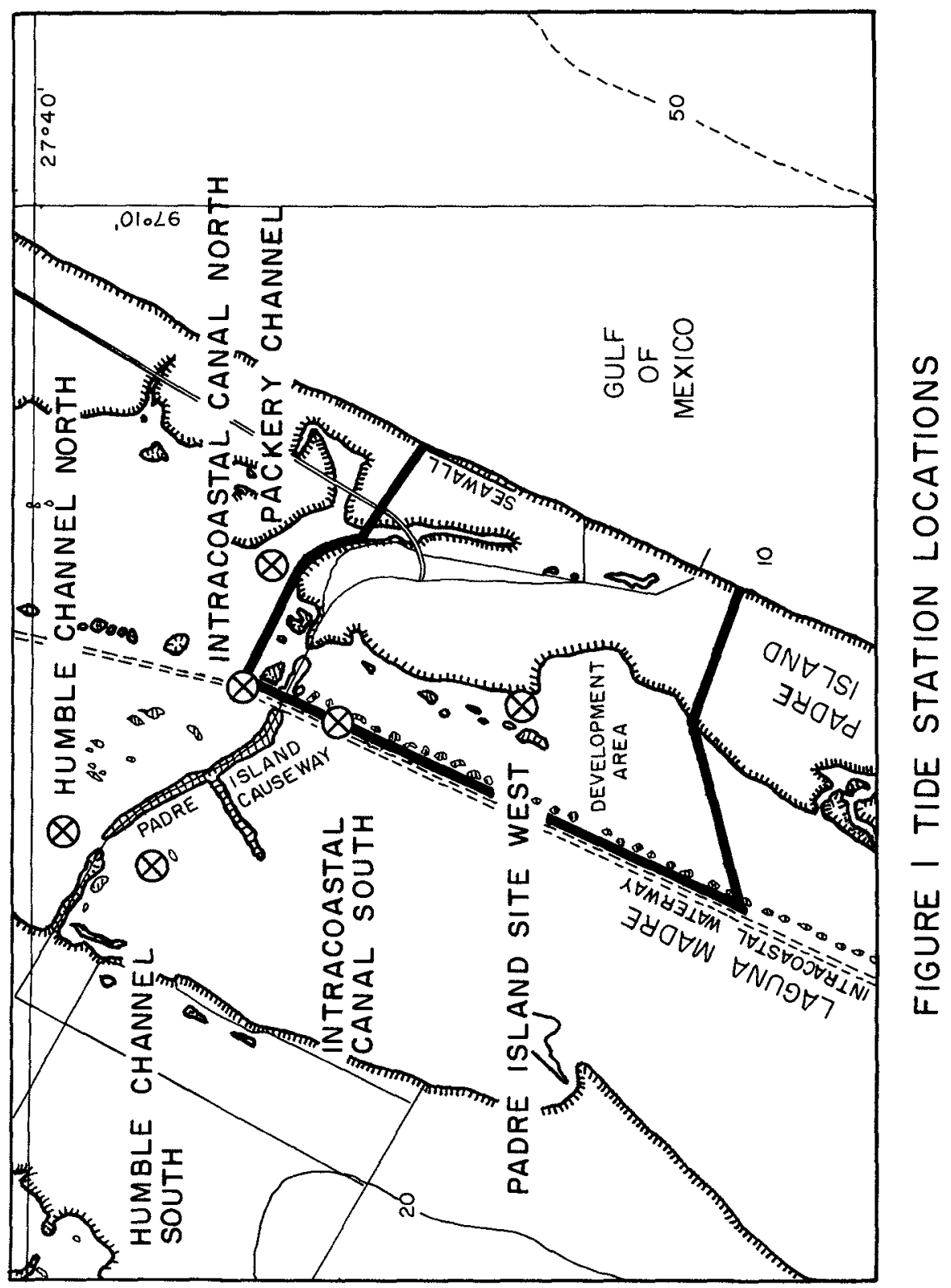




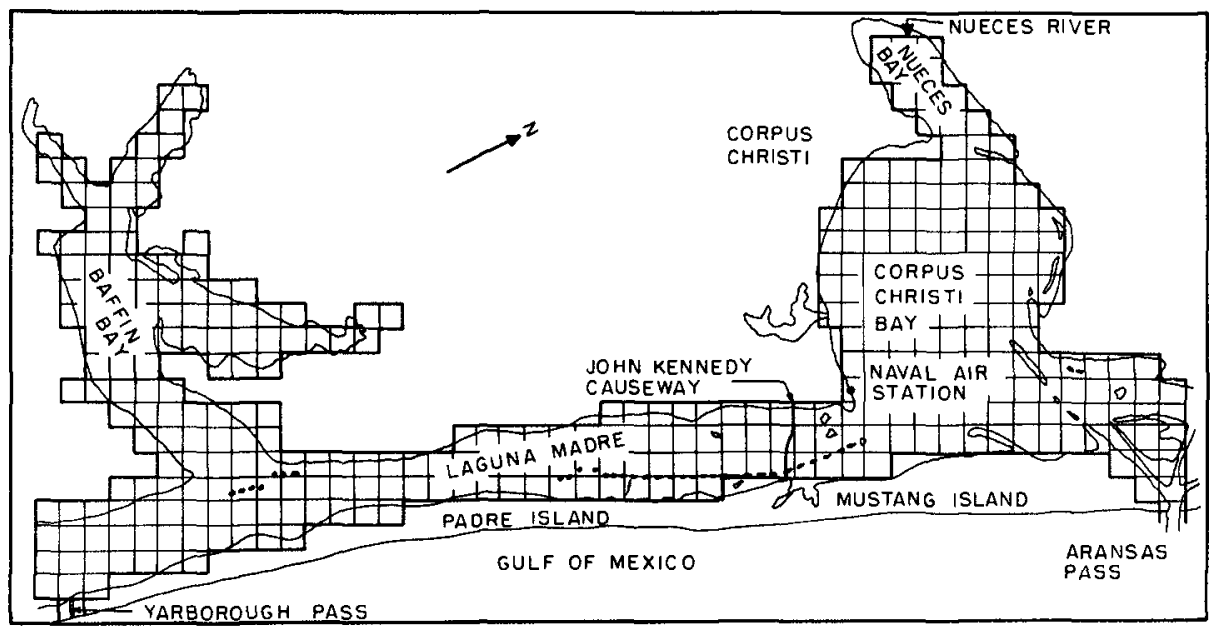

FIGURE 2 CORPUS CHRISTI AND BAFFIN BAY GRID LAYOUT

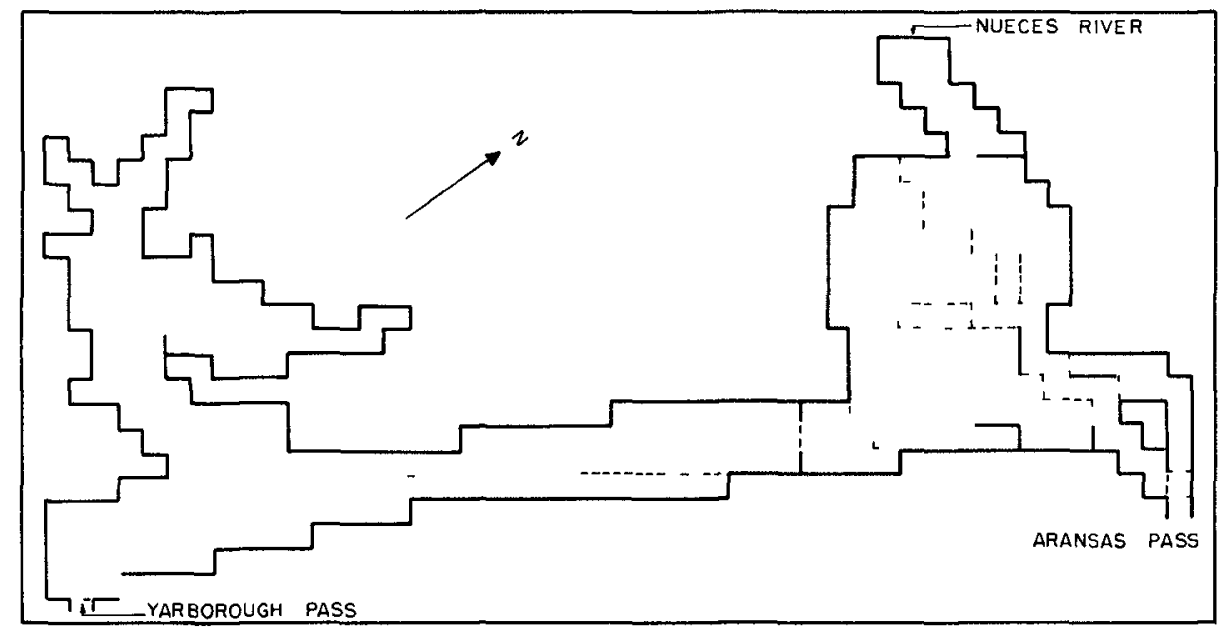

FIGURE 3 COMPUTATIONAL GRID SHOWING FLOW CONTROLS AND BARRIERS 


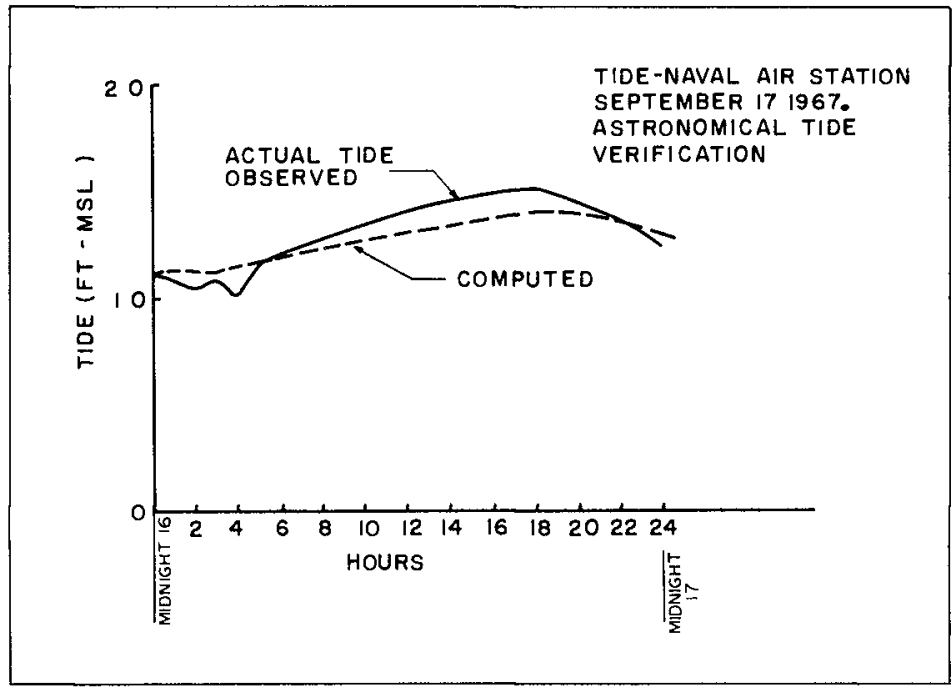

FIGURE 4 VERIFICATION OF ASTRONOMICAL TIDE NAVAL AIR STATION

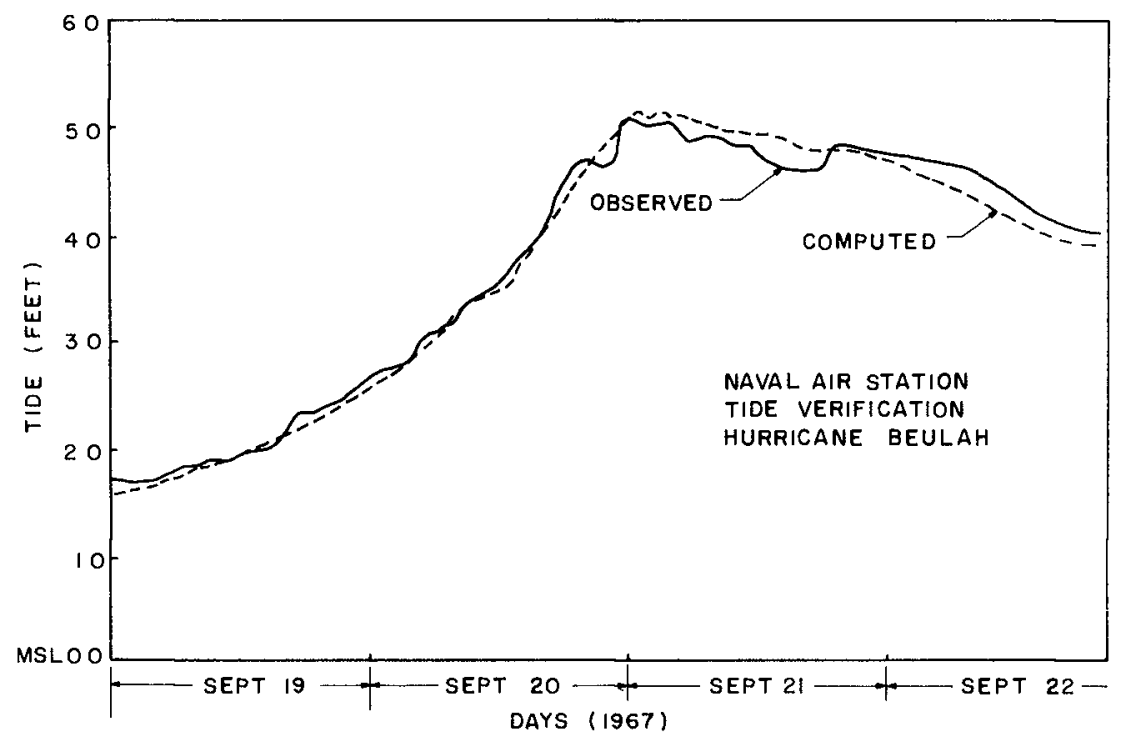

FIGURE 5 VERIFICATION OF HURRICANE TIDE-NAVAL AIR STATION 

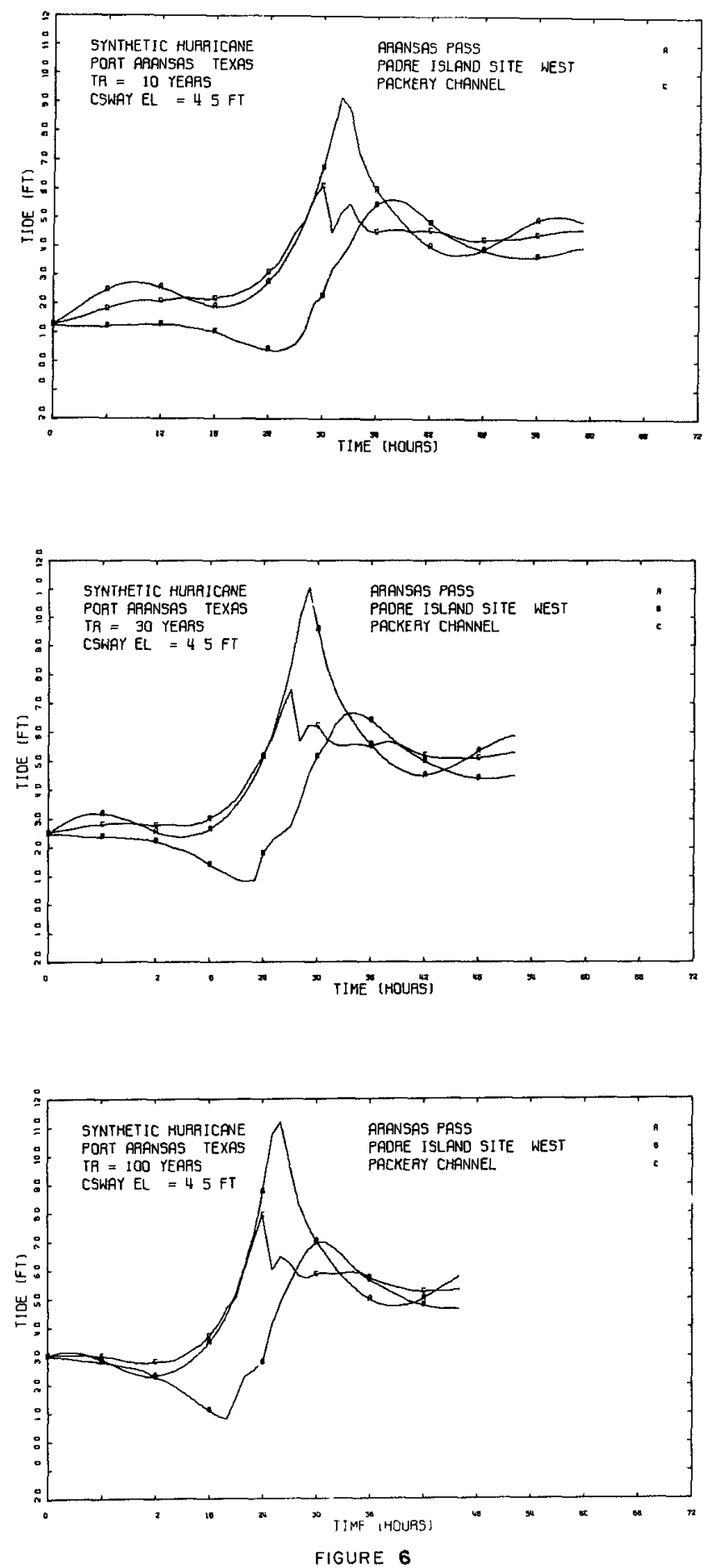

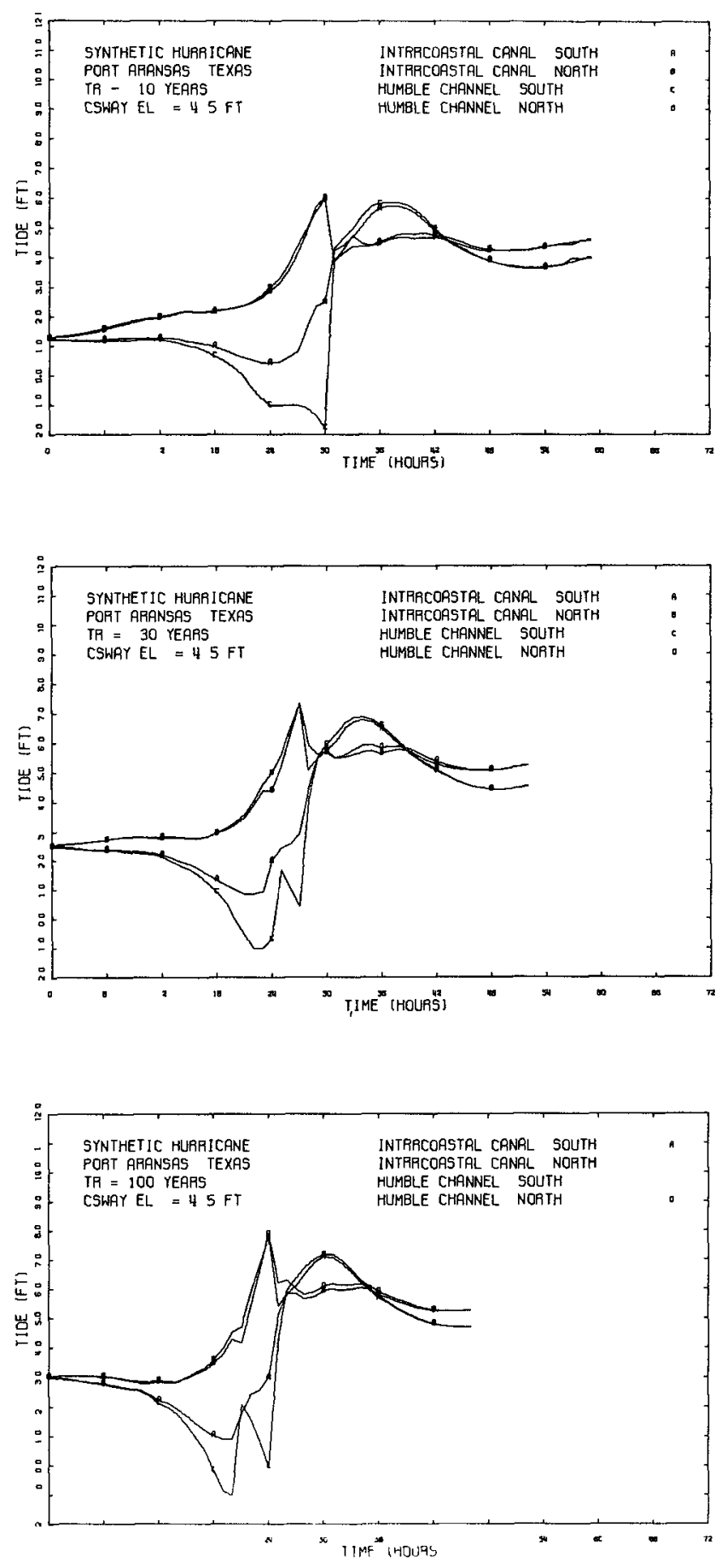

FIGURE 7 

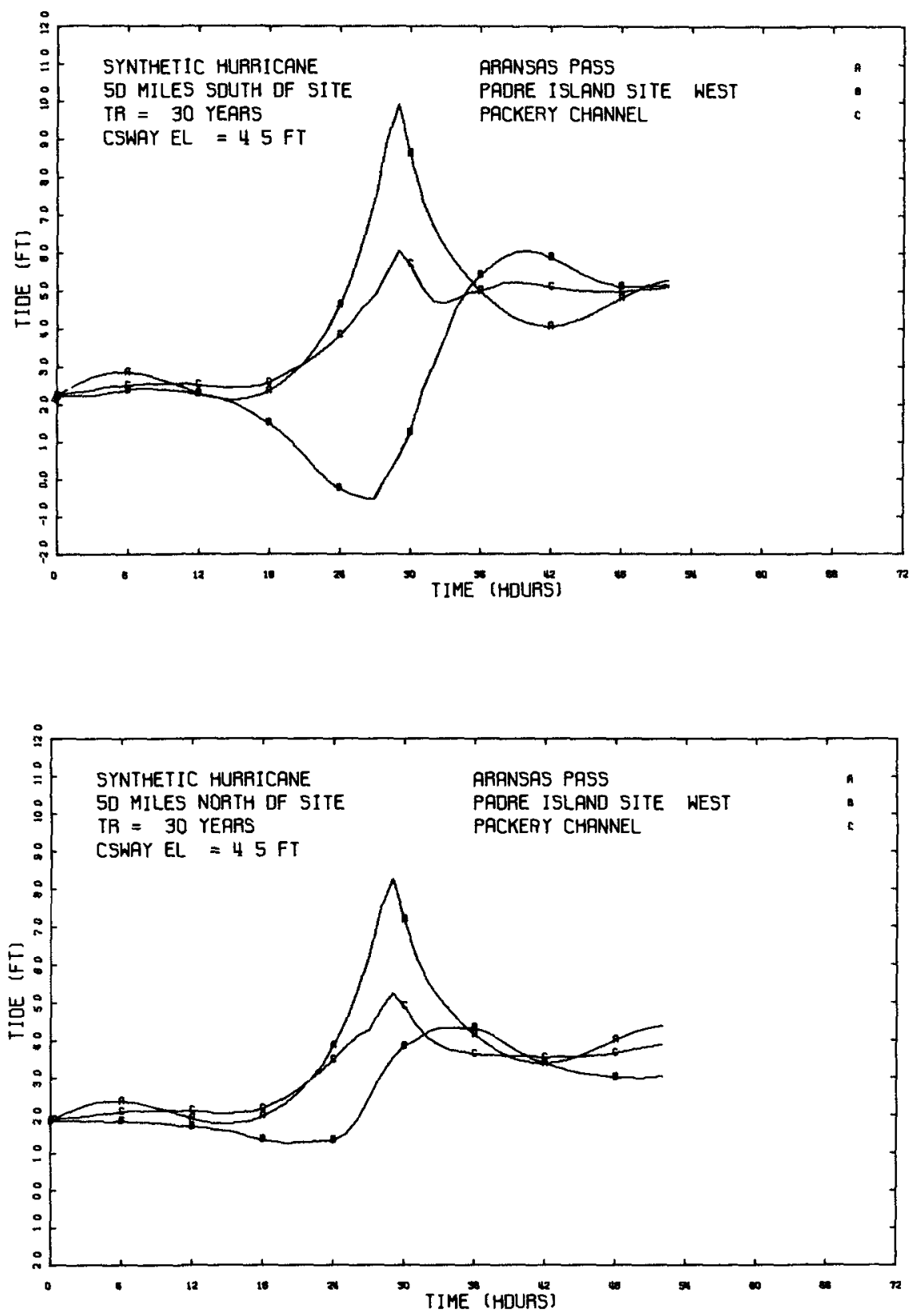

FIGURE 8 

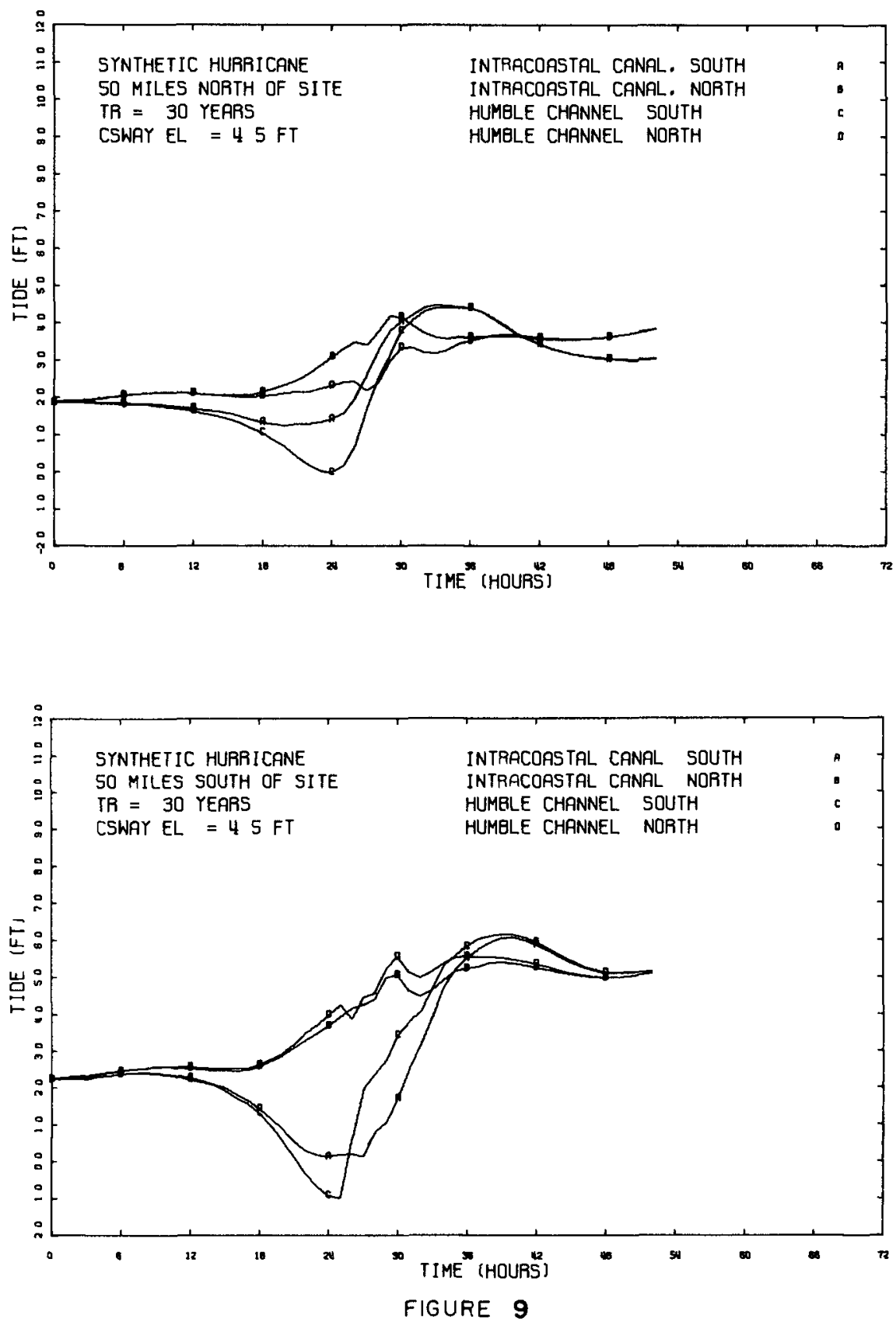


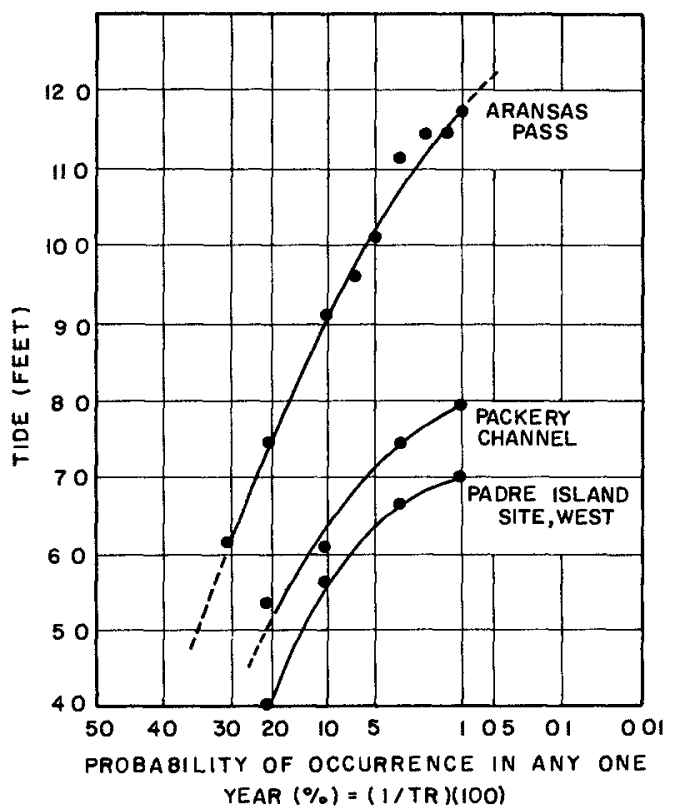

FIGUREIO OFFSHORE AND DEVELOPMENT SITE TIDES AS COMPUTED FROM VARIOUS FREQUENCY SYNTHETIC STORMS

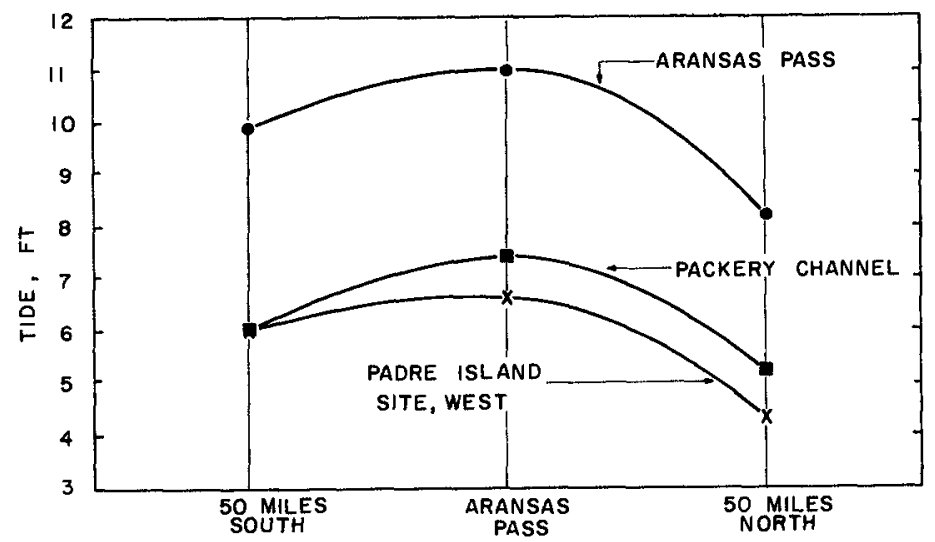

FIGURE 11 VARIATION OF MAXIMUM TIDE FOR STORMS CROSSING COASTLINE AT DIFFERENT LOCATIONS 


\title{
CHAPTER 124
}

\section{TURBULENCE IN HURRICANE-GENERATED COASTAL CURRENTS}

\author{
By Stephen P Murray \\ Coastal Studies Institute \\ Loulsiana State University \\ Baton Rouge, Loursiana 70803
}

\begin{abstract}
Wind and current meter records taken during the passage of a hurricane were subjected to time series analysis Filtering techniques 1solated the speed fluctuations in the 10-60 CPH frequency band These turbulent fluctuations proved to follow the Gaussian distribution for both wind and current W1th the passage of the storm front the turbulence intensity of the wind actually decreased, while, on the other hand, the turbulence intensity of the current rose to extremely large values, even exceeding 27 percent of the mean flow speed

Three phases of the storm were examined separately, and the energy density of the wind varied with the -1 power of the frequency in all phases With respect to the energy density of the current, a -1 power dependency on the frequency was approximated by the first two phases, whereas in the third phase, which was the most intense, the energy density varied approximately as the -05 power of the frequency The characteristics of the spectra indicate that there is little direct transfer of energy from the wind to the current in the frequency range studied Energy is passing into the 10-60 CPH band of the current from stı11 lower frequencies
\end{abstract}

\section{INTRODUCTION}

Hurrıcane-generated coastal currents can attaln enormous magnitude and are instrumental in inflicting material damage and producing changes in coastal topographies which result in great monetary losses The undermining of plers and jetties and the destruction of sea buoys and other navigation alds illustrate this point clearly However, owing to scarcity of data, the characteristics of currents produced by hurricane winds remaln essentially unknown

Hurricane Camille traversed the Gulf of Mexıco on a northwesterly track during the period August 15-17, 1969, and made 1ts landfall at Gulfport, Mississippi ( $F_{1 g}$ 1) During this time the Coastal Studies Institute was conducting a shallow-water oceanographic measuring program at its field facility on the Eglin Alr Force Base Santa Rosa Island Testing Grounds This locality, $160 \mathrm{~km}$ to the east of the hurricane landfall, was subjected to severe winds, high waves, and storm surge of several current meters installed on the nearshore bottom prior to the storm, one placed at the depth of 63 meters beyond the outer bar provided an excellent time series record of the current during the storm 


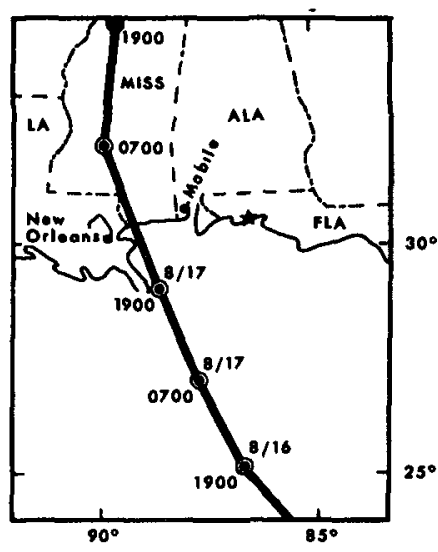

Fig 1 Track of the eye of Hurricane Camille, August 16 and 17,1969
An anemometer mounted 12 meters above the water level on the adjacent beach operated concurrently

The correlative aspects, especially in respect to the coastal c1rculation, of mean speeds and directions of both the current record and the wind record were discussed in Murray (1970) The purpose of the present paper is to discuss the properties of the turbulence associated with the hurricane winds and currents, with special reference to the turbulence, the energy density spectra, and cross spectra and coherence between these two signals

\section{INSTRUMENTATION}

The current sensor was a Marıne Advisers bottom-mounted Q-16 bidirectional integrating meter which utilizes a ducted impeller assembly of small mass with a threshold speed rated at 008 knots The duct is fitted w1th a vane that is long relative to the wave's orbltal diameter, thus the vane holds the meter with 1 ts axis aligned with the steady current A magnetic compass assemb1y within the instrument determines magnetic north, the orientation of the vane then determines the current direction

The current speed sensor contalns two reed switches that are activated by magnets on the blades of the lmpeller The reed switches are mounted so that they open and close at slightly different times, which allows the electronic circuitry to determine the direction of rotation of the Impeller If the Impeller is turning in the direction of the net current, a differential amplifier integrator integrates the pulses in the positive direction, if the impeller turns in the opposite direction, the amplifier integrates in the negative direction There is then produced at the output of the amplifier a DC voltage proportional to the net current through the speed sensor This voltage represents a time average over a time interval determined by the fixed time constant $\tau$ of the proper RC circuit Given a step function increase in the current speed up to a value $V_{O}$, the output speed $V$ lags the true speed $V_{0}$ according to

$$
V=V_{0}\left(1-e^{-t / \tau}\right)
$$

Thus one time constant is the time required for the output speed to reach $\left(1-e^{-1}\right) \sim 63$ percent of the true speed In the present case, the time constant was fixed at 62 seconds

Instruments with time constants function as filters which smooth, lag, and damp cycles in the output as a function of the frequency $f$ The frequency response $R(f)$ (the ratio of output amplitude to true amplitude) 
of a "time constant" filter is (Holloway, 1958)

$$
R(f)=\left(1+4 \pi^{2} \tau^{2} f^{2}\right)^{-1 / 2},
$$

and the phase lag is

$$
\phi=\tan ^{-1}(-2 \pi \tau f)
$$

The frequency response function with $T=62$ seconds, together with the phase lag, is shown in Figure 2 It is seen from the figure that there is at least 88 percent response for frequencies below $50 \mathrm{CPH}$ Since the frequency range of surface waves durıng the hurrıcane was between 450 and 720 CPH (5-8 second periods), the sensor response to waves was held to only 12-20 percent (frequency response at the output)

The Q-16 current meter assembly was situated on the bottom 360 meters seaward of the shoreline in 63 meters of water The speed sensor duct was 15 meters above the sandy bottom

The anemometer was a Sclence Assoclates No 162 General Purpose Wind Recording System The response characteristics of this particular instrument are not precisely known, but the sensitivity of this standard type three-cup anemometer has been well studied in the past Fergusson (1935) measured 230,060 , and 033 seconds for a standard three-cup anemometer to accelerate from zero speed up to 5,20 , and $35 \mathrm{~m} / \mathrm{sec}$ respectively It is conservative to say that our anemometer of this type has a full response for cycles with a period of 10 seconds or $360 \mathrm{CPH}$

\section{ANALYSIS TECHNIQUES}

Strıp chart recorders were used which had response tımes of 08 seconds for the current recorder and 05 seconds for the wand recorder The factors exerting ultimate control over the frequency response, however, were the recorder chart speeds--4 inches per hour for the current recorder and 3 inches per hour for the wind recorder The trace width allowed only frequencies less than $50 \mathrm{CPH}$ to be absolutely discriminated on both strip charts at these chart speeds

The strip chart records were digitized on a Calma Model 303 Digitızer, which transfers graphical analog data to digital magnetic tapes by visually tracing the graphic data Visual tracing in this case represents a smoothing filter which insures that no frequencles higher than about 60 $\mathrm{CPH}$ are transferred to the magnetic tape The digltizer was set to record incrementally the coordinates of 100 points per inch of strip chart data Owing to the two different chart speeds, this procedure produces a data density discrepancy which is eliminated by an interpolation option in the Calma software programs The selected interpolation procedure ylelded 100 data points per hour of record

Thirteen hours of data between 0400 and 1700 hours August 17 (2600 data points) were placed on the magnetic tape for analysis This interpolated version of the original observations was plotted by computer and 


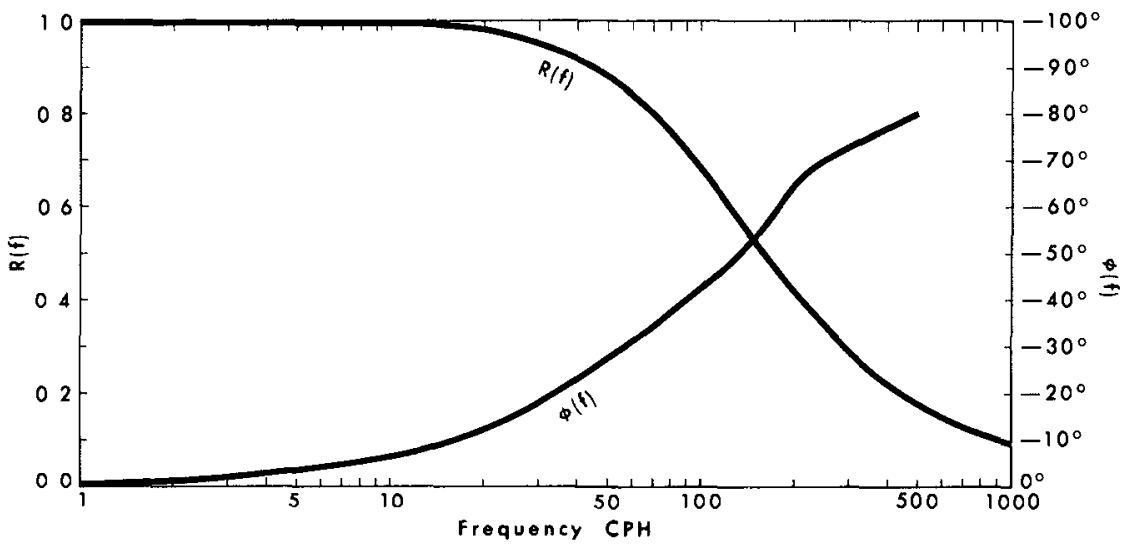

F1g 2 Frequency response and phase lag for a "tıme constant" filter w1 th $\tau=62$ seconds

Is shown as Figure 3 Inspection of the figure shows that (a) the Initial one third of this record (phase I) represents a pre-storm front phase, (b) the middle one third (II) represents an accelerative phase during which both the wind and current speeds increased rapidly after the storm front passage, and (c) the final third is a relatively steady phase (III) for both mean wind and mean current speeds

The data length and time increment selects the highest and lowest frequencies which are present in the data For $N$ observations taken at $\Delta \mathrm{T}$ seconds apart this spectral band $1 \mathrm{~s}$ approximately

$$
\frac{1}{2 \mathrm{~N} \Delta \mathrm{T}}<\mathrm{f}<\frac{1}{2 \Delta \mathrm{T}}
$$

In this study $\mathrm{N}=2600, \Delta \mathrm{T}=0005$ hours, and the observable frequency band $15004<\mathrm{f}<100 \mathrm{CPH}$ The frequency $1 / 2 \Delta \mathrm{T}=100 \mathrm{CPH} 1 \mathrm{~s}$ known as the Nyquist frequency $f_{C}$ In spectral analysis all power contalned in frequencles higher than $f_{C}$ is folded back into the spectra below $f_{C}$, resulting in allasing One method of avoiding this problem (Bendat and Plersol, 1966) is to select a Nyquist frequency sufficiently higher than the maximum frequency of interest to insure that there is little power in the frequencies $f>f_{c}$ From the discussion of the digitizing of the relatively slow chart speeds and the frequency response curve in Figure 2, it is clear that there is little power above $f_{c}=100 \mathrm{cPH}$ for aliasing

Before spectral analysis of time series data is performed, any trends or very low frequency oscillations must be eliminated from the data, or the resulting spectra may be considerably distorted (Bendat and Piersol, 1966) A smoothing function which is essentially a low 

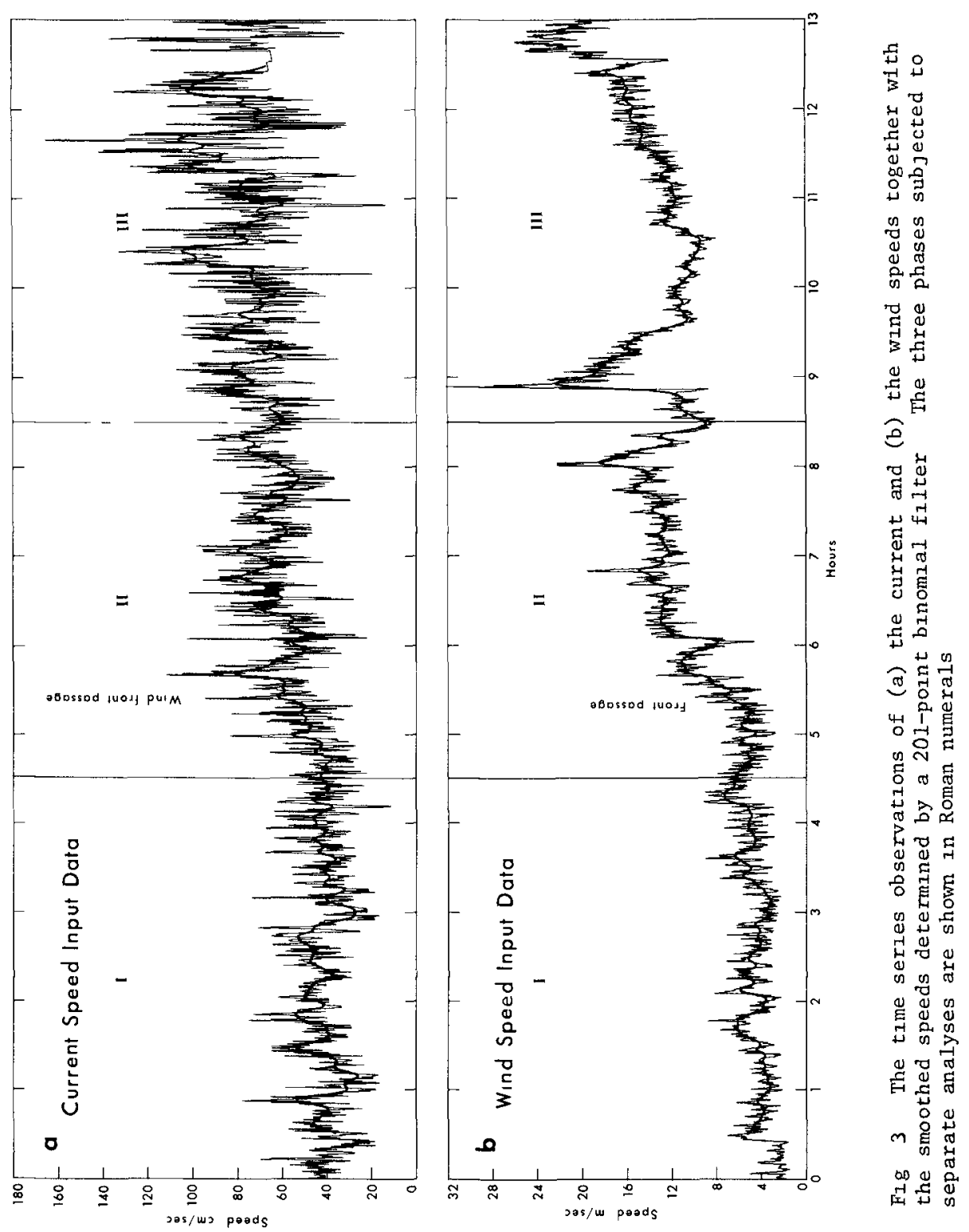
pass filter can be used to isolate the trends and low frequency components In Figure 3 the smoothed curve running through the observed data was derived from a 201-point binomial filter whose frequency response $R(f)_{B F 201}$ is plotted in Figure 4 using the relation $R(f)=$ $\cos ^{n}(\pi f \Delta T)$ where $n$ is the number of terms in the filter less one This smoothing function passes on ly 8 percent of the amplitude of frequencies above $10 \mathrm{CPH}$ and completely cuts out those amplitudes with $\mathrm{f} \geq 15 \mathrm{CPH}$

The next step is the subtraction of the smoothed time series from the original time series, which is a high pass filtering technique (Holloway, 1958, Panofsky and Brier, 1963) with a frequency response $R^{\prime}(f)$ in this case

$$
R^{\prime}(f)=1-R(f)_{B F 201}
$$

also plotted in Figure 4 All frequencies with $\mathrm{f} \geq 10 \mathrm{CPH}$ have at least 90 percent of their amplitudes transmitted through the filter, and, again considering the filtering performed in digitizing, the final form of the data 15 a band pass $10 \lesssim f \lesssim 60 \mathrm{CPH}$

Also plotted in Figure 4 for comparison is the response of a high pass filter produced by subtracting a 30-point equally weighted running mean from an original time series This type filter provides a sharper cut with minimal data loss but has distinct disadvantages in that 1 t amplifies and damps certain frequencies, the troughs in the response curve turn maxıma into minıma and may produce a falsely rippled power spectrum (Holloway, 1958) A preliminary analysis of the present data with a 30-point equally welghted running mean high pass filter did in fact produce pronounced peaks at 30 and $50 \mathrm{CPH}$ which could have been erroneously interpreted as surf beat effects

\section{TURBULENCE}

Let us denote the original time series observations of speed by $u_{o}$ and the smoothed record using the 201 binomial smoothing "function" by $U_{\mathrm{BF}}$ The turbulent speed u' may be defined following the conventiona1 theory by

$$
\mathbf{u}^{\prime} \equiv \mathrm{u}_{\mathrm{o}}-\mathbf{u}_{\mathrm{BF}}
$$

The random nature of $u^{\prime}$ is indicated by the histograms of $u^{\prime}$ (both wind and current), which show a good agreement with the corresponding best-fit Gaussian curves (Figure 5)--typical of most turbulence measurements

In Figure 6 the turbulence intensity $\left(\overline{u^{\prime 2}}\right)^{1 / 2}$ and the relative turbulence intensity $\left(\mathrm{u}^{\prime 2}\right)^{1 / 2} / \overline{\mathrm{U}}_{\mathrm{BF}}$ are plotted as a function of time The term $\left(u^{\prime 2}\right)^{1 / 2}$ was calculated each half hour as the root-mean-square value of the 100 observations in that time interval, whereas the term $\overline{\mathrm{U}}_{\mathrm{BF}}$ is the average of the corresponding 100 values of $U_{\mathrm{BF}}$ in that same time interval The turbulence intensity of the current increased fairly 


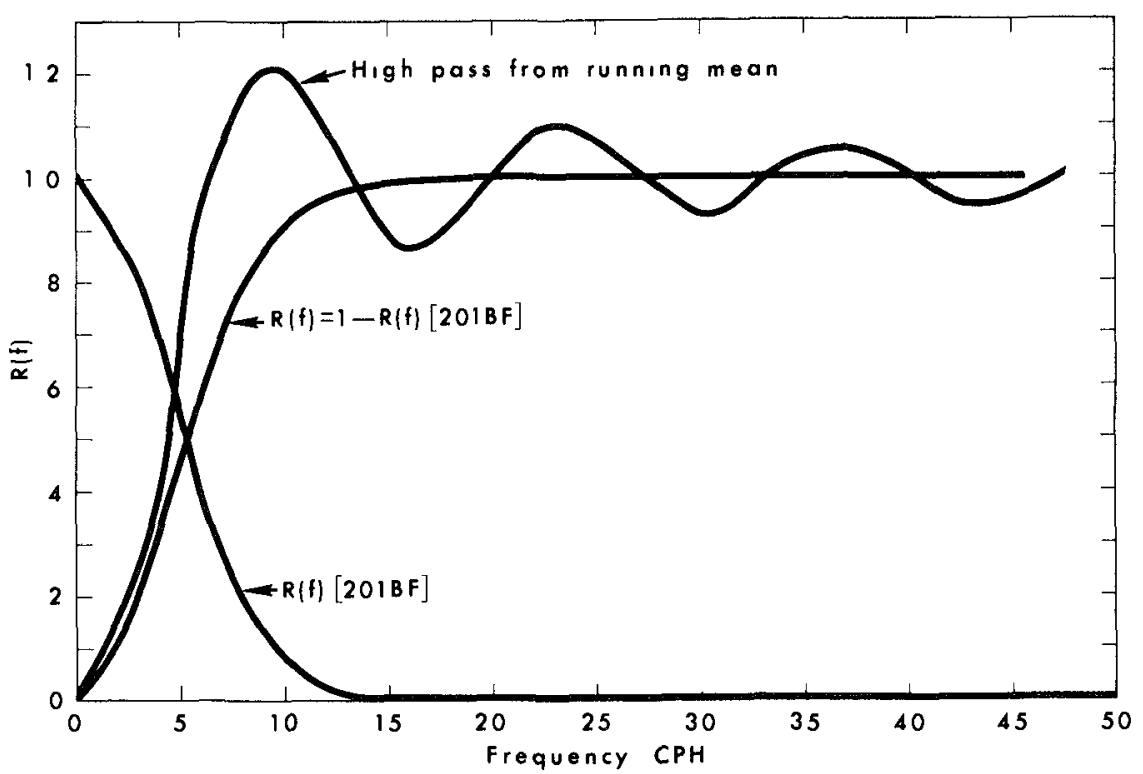

Fig 4 Frequency response curves for the high and low pass filters discussed in the text

regularly as the storm progressed from $8 \mathrm{~cm} / \mathrm{sec}$ up to $22 \mathrm{~cm} / \mathrm{sec}$, while relative to the mean speed it oscillated around a value of about 018 $\mathrm{cm} / \mathrm{sec}$, even reaching as high as $027 \mathrm{~cm} / \mathrm{sec}$ near the end of the record Many previous studies in both field and laboratory have shown that the turbulence intensity under more normal conditions varies between 005 and $015 \mathrm{~cm} / \mathrm{sec}$ of the mean flow speed The turbulence intensity of the wind increased from an initial value of $75 \mathrm{~cm} / \mathrm{sec}$ up to $150 \mathrm{~cm} / \mathrm{sec}$ in the seventh hour The relative value stayed at about $020 \mathrm{~cm} / \mathrm{sec}$ until it dropped precipitously with the arrival of the storm front and its high mean speeds at hour 53 (see Fig 3b)

It is notable that the turbulence intensity of the wind was decidedly damped after the front passed (excepting the effect of the large gust at 89 hours) The current turbulence intensity, on the other hand, inversed after the front passed It is significant that the current direction abruptly shifted (see Murray, 1970) after the wind front passed from westerly to southwesterly and south, reflecting, It 1 s believed, a seaward return flow caused by setup against the coast The increase in current turbulence intensity was probably caused by (a) the large vertical shear inherent within a reversing velocity profile and (b) the effects of large groups of wind-driven waves associated W1 th the suddenly increased mean wind 


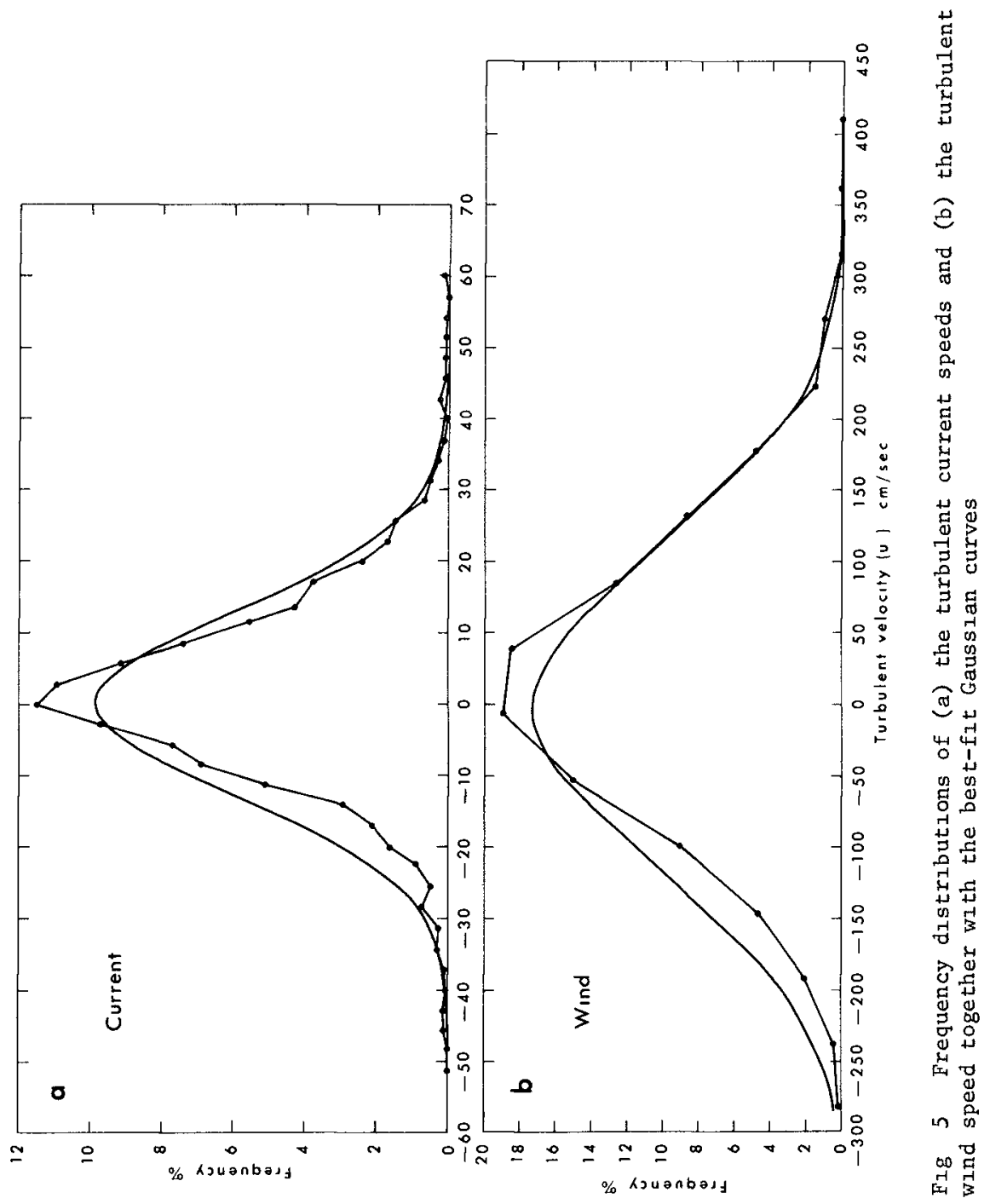




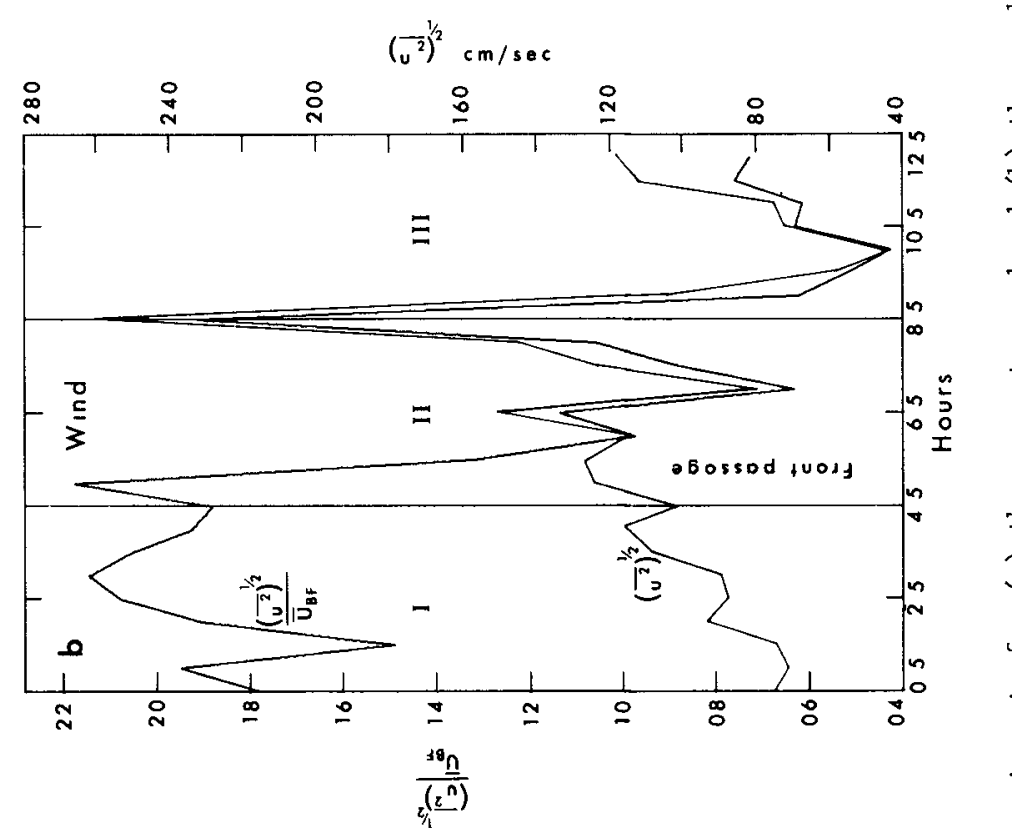

虸

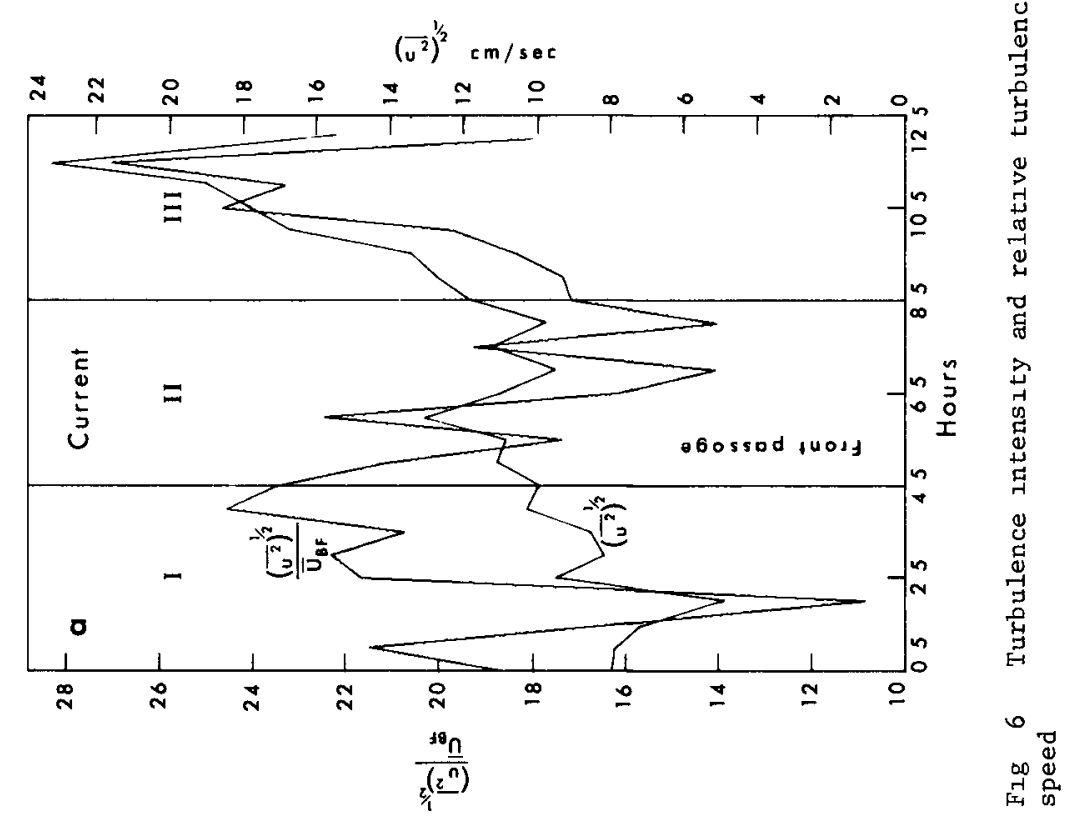




\section{TURBULENCE SPECTRA}

Energy density spectra $F(f)$ of the two $u^{\prime}$ time series were calculated from the procedure outlined in Bendat and Piersol (1966, p 291) and modified by the use of a Fast Fourier Transform The 2400 data points were first divided into three segments (I, II, and III), as discussed earlier, each containing 800 points, so that the change of the energy density with the progress of the storm could be investigated The resulting current speed spectra are shown in Figure 7 In the frequency range $15<f<60 \mathrm{CPH}$ there are no significant gaps or peaks apparent in any of the intervals, the energy density decreases smoothly with increasing frequency The apparent peak in spectrum II 1 s not significant at the 005 level at $f \simeq 50 \mathrm{CPH}$ The peaks at about $12 \mathrm{CPH}$, of course, are a result of the low frequency cutoff by the filter As shown in Table 1, the total relative energy (the area under the spectral curve) more than quadruples from intervals I to III There is considerably more energy at all frequencies in successively higher intervals, but the greatest increase is clearly at the lowest frequencies Also listed in Table 1 is the absolute energy $E$ associated with the turbulence in the mean flow direction, which is given by Taylor (1935) as

$$
E=\frac{\rho}{2} \overline{\left(u^{2}\right)}
$$

The spectra in Figure 7 have also been corrected for the instrument frequency response $R(f)$ plotted in Figure 2 The corrected spectral density $F^{\prime}(f)$, shown as a dashed line in Figure 7 and calculated from

$$
F^{\prime}(f)=\frac{F(f)}{R(f)^{2}},
$$

has 1ittle effect other than to increase the energy at the high frequencies

The wind speed spectra in Figure 8 display the same lack of peaks or gaps as the current spectra Most notable is the fact that the energy content (see Table 1) does not increase at all from intervals II to III, in sharp contrast to the twofold increase observed in the content of the current spectra between these intervals This suggests again a lack of direct energy transfer between wind and current in the frequency band $10<\mathrm{f}<60 \mathrm{CPH}$ under study here This inference is

\begin{tabular}{|c|c|c|c|c|}
\hline \multirow{2}{*}{ Interval } & \multicolumn{2}{|c|}{ RELATIVE $\int \mathrm{F}(\mathrm{f}) \mathrm{df}(\mathrm{cm} / \mathrm{sec})^{2}$} & \multicolumn{2}{|c|}{ ABSOLUTE E (ergs $/ \mathrm{cm}^{3}$ ) } \\
\hline & Current & Wind & Current & Wind \\
\hline I & 69 & 8281 & 34 & 41 \\
\hline II & 119 & 15006 & 59 & 75 \\
\hline III & 279 & 15126 & 139 & 76 \\
\hline
\end{tabular}

Table 1 Total Energy Associated with Energy Density Spectra 


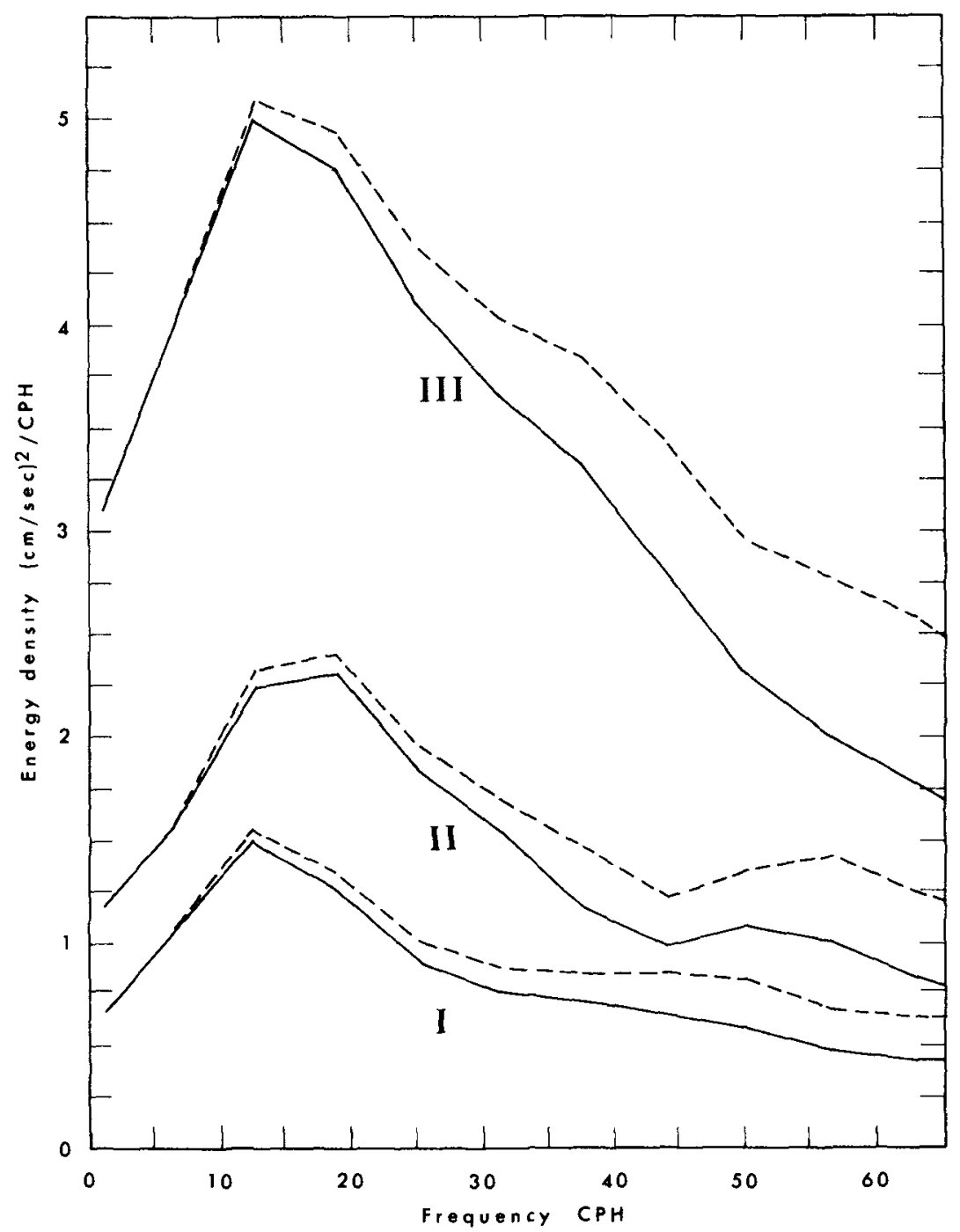

FIg 7 Energy density spectra of the current for the three phases of the storm The dashed IIne is a correction for the instrument frequency response 


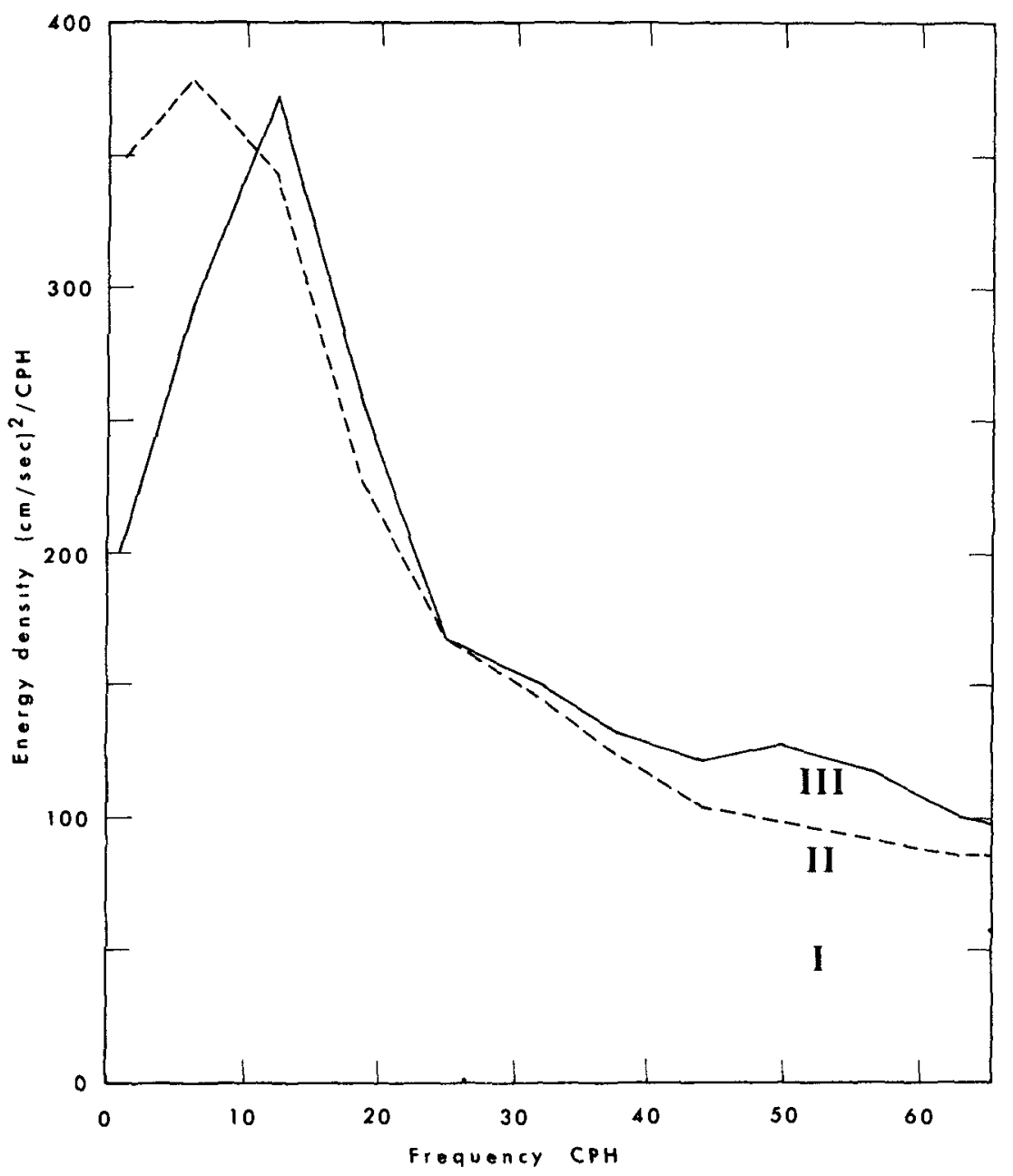

F1g 8 Energy density spectra of the wand speed for the three phases of the storm

supported by the data in Table 1, whuch shows that there are more ergs of energy per unit volume assoclated with the turbulence in the water than with that in the axr at these frequencies

The data seem to indicate that the energy is enterung the 10-60 CPH frequency band of the current from the lower frequency oscillations of the current itself, which are presumably generated by the low frequencies of the wind A good correlation does clearly exist between 
the DC levels (mean values) of the wind and the current (see Fig 3 and Murray, 1970)

In Figure 9 the $F(f)$ spectra of the wind are replotted on a log$10 \mathrm{~g}$ scale In studies of alr turbulence over water Pond et al (1966) found that in the low frequency ranges of their data the energy density dropped off with the -1 power of the frequency $F(f) \propto f-1$ Their -1 power range extended between $10^{-5}<k<3 \times 10^{-3}$ where $k$ is the wave number Using Taylor's hypothesis $f=U k / 2 \pi$ (Taylor, 1938) and the limits of the mean values for this study $500<\mathrm{U}_{\mathrm{BF}}<1300 \mathrm{~cm} / \mathrm{sec}$, the corresponding frequency band for their -1 power range is $3<\mathrm{f}<2300$ $\mathrm{CPH}$ The present data which fall in the low frequency end of the range are also represented very well by the -1 power slope Tchen (1953) has suggested that the -1 power law would hold below the inertial sub-range

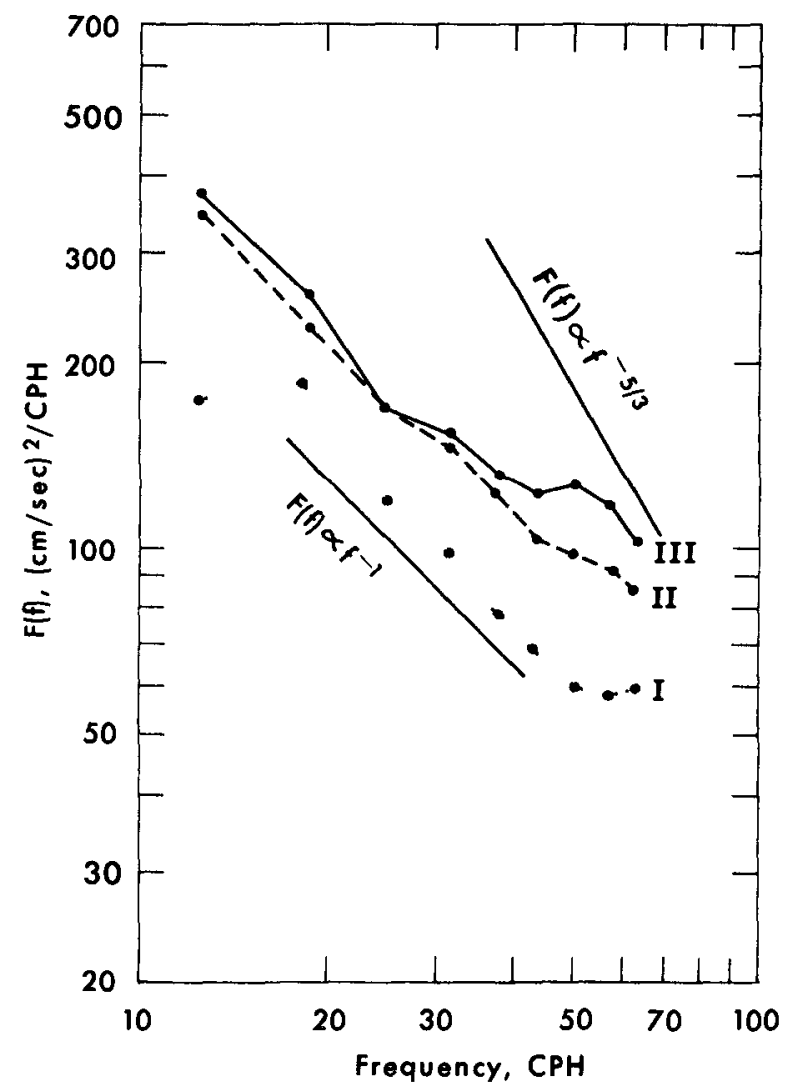

Fig 9 Energy density spectra of the wind on a log-log plot The stralght lines show $a^{-1}$ and a $-5 / 3$ power dependency on the frequency 
in the presence of strong vertical shear--a situation which certainly existed in the lower 10 meters of the atmosphere during this storm as a result of surface drag

So that the energy drop-off with frequency of the $F(f)$ spectra of the current speed may be similarly investigated, the spectra of Figure 7 are replotted on a log-log scale in Figure 10 Cannon (1969) has presented excellent and comprehensive data which clearly established the presence of a $-5 / 3$ law governing the drop-off of energy with frequency in an estuarine tidal flow in the frequency band $1<f<72 \mathrm{CPH}$ Cannon also reported, however, several experiments in which strong vertical shear was suspected and the log-log plots showed that $F(f) \propto f^{-1}$ During a large part of the 13-hour record used in this study the Q-16 meter was bounded by two shear zones (a) the bottom boundary layer and (b) the transition zone (probably near mid-depth) between upper-layer wind-driven

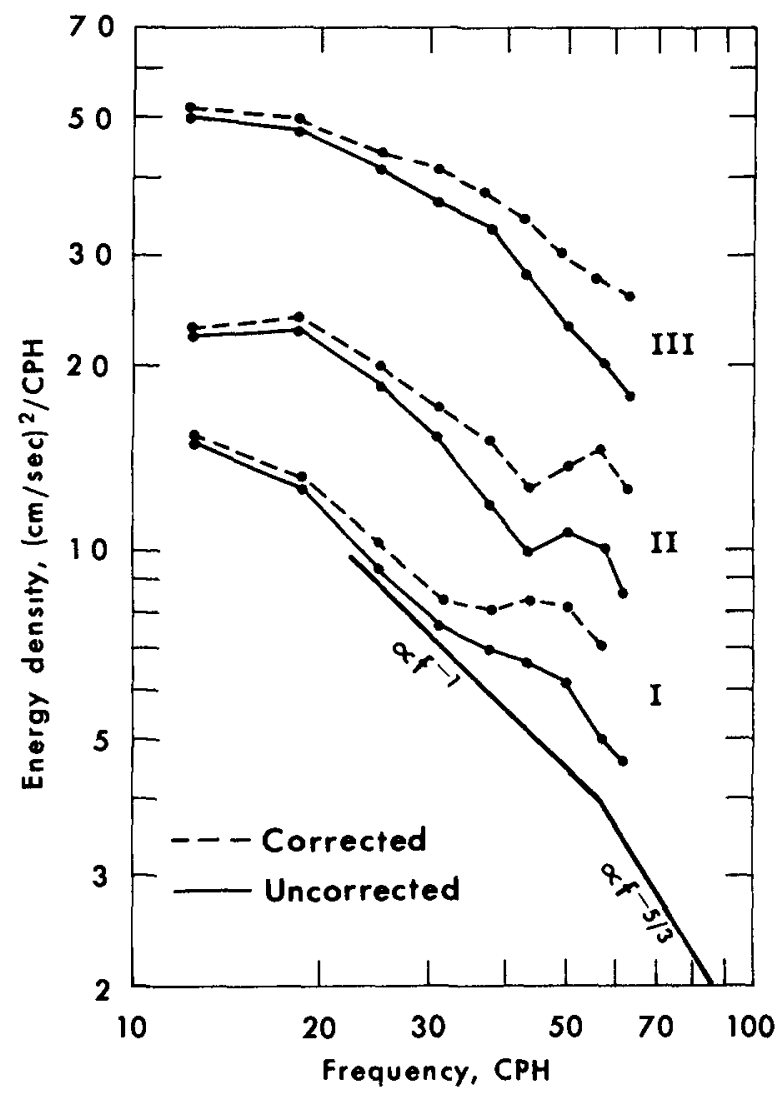

Fig 10 Energy density spectra of the current on a $\log -10 \mathrm{~g}$ plot The straight lines show a -1 and $a-5 / 3$ power dependency on the frequency 
currents and lower-layer return flows caused by the setup against the coast (Murray, 1970) Figure 10 may reflect that this phenomenon as the -1 power is a reasonable fit for the spectra (both corrected and uncorrected) in intervals $I$ and II However, in interval III of the current record, when the energy content was highest (Table 1), the slope of the spectra (on a log-log plot) was considerably flatter than the -1 value-having a value close to -05 These observations suggest that with increasing energy content (or perhaps increasing vertical shear) the $F(f)$ dependency on the frequency may shift from f-5/3 to f-1 to $f^{-0} 5$ in this intermediate frequency range

\section{CROSS CORRELATION}

A11 attempts to relate the turbulent energy distribution in the wind to that of the current yjelded negative results Figure 11 shows a typical wind-current coherence function (interval II), the coherence leve1 is insignificant at all frequencies Similarly, the phase lags (FIg 12) from the same data set oscillate with no apparent pattern Such results are not unexpected since Cannon (1969), in carefully controlled experiments, found no significant coherence between the records of adjacent current meters in frequencles above $10 \mathrm{CPH}$

\section{CONCLUDING REMARKS}

The principal conclusions from this study of wind and water turbulence in the frequency band $10<f<60 \mathrm{CPH}$ are as follows

1 As the storm progresses there is no systematic change in the energy spectra common to both wand and current

2 As the storm progresses the energy content of the current spectra increases markedly, the maxumum increase in energy is in the lowest frequencies

3 The coherence between wind and current is insignificant (< 1$)$ in this band

4 For reasons 1,2 , and 3 above it 1 s concluded that energy is fed into the 10-60 CPH frequency band of the current from the lower frequencies of the current itself

5 The energy densıty of the wind decreases proportionally to the -1 power of the frequency in all three storm intervals

6 The energy density of the current decreases proportionally to the -1 power of frequency in the first two intervals of the storm but proportionally to no 5 power of the frequency during the third storm interva1, which was the most intense

\section{ACKNOWLEDGMENTS}

Myron Young and Choule Sonu of Loulsiana State Unıversity developed the programs which permitted this time series analysis to be carried out The project was sponsored by the Geography Programs, Office of Naval Research, under Contract N00014-69-A-0211-0003, NR 388002 


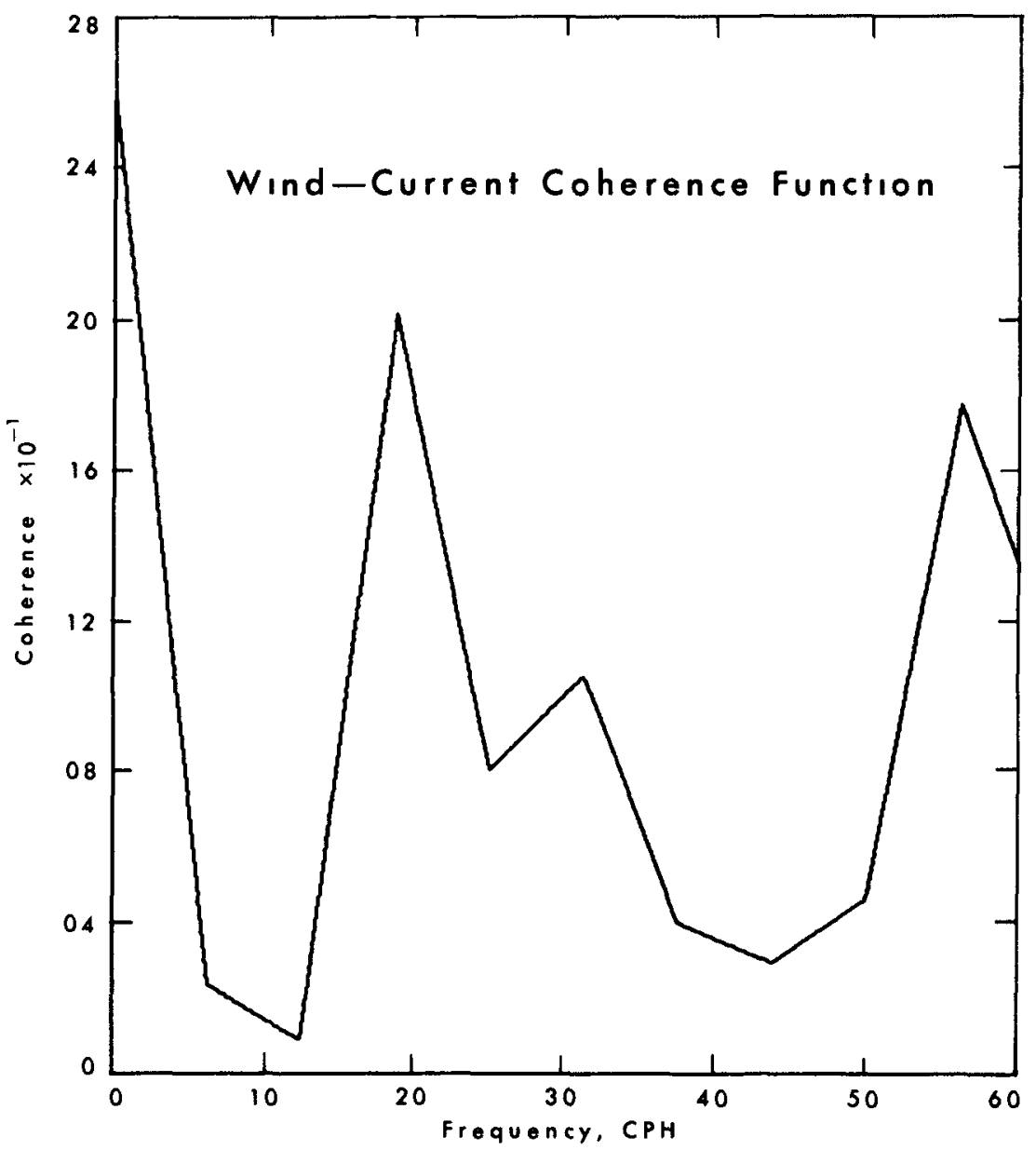

Fig 11 The coherence function between the wind and the current from interval II 


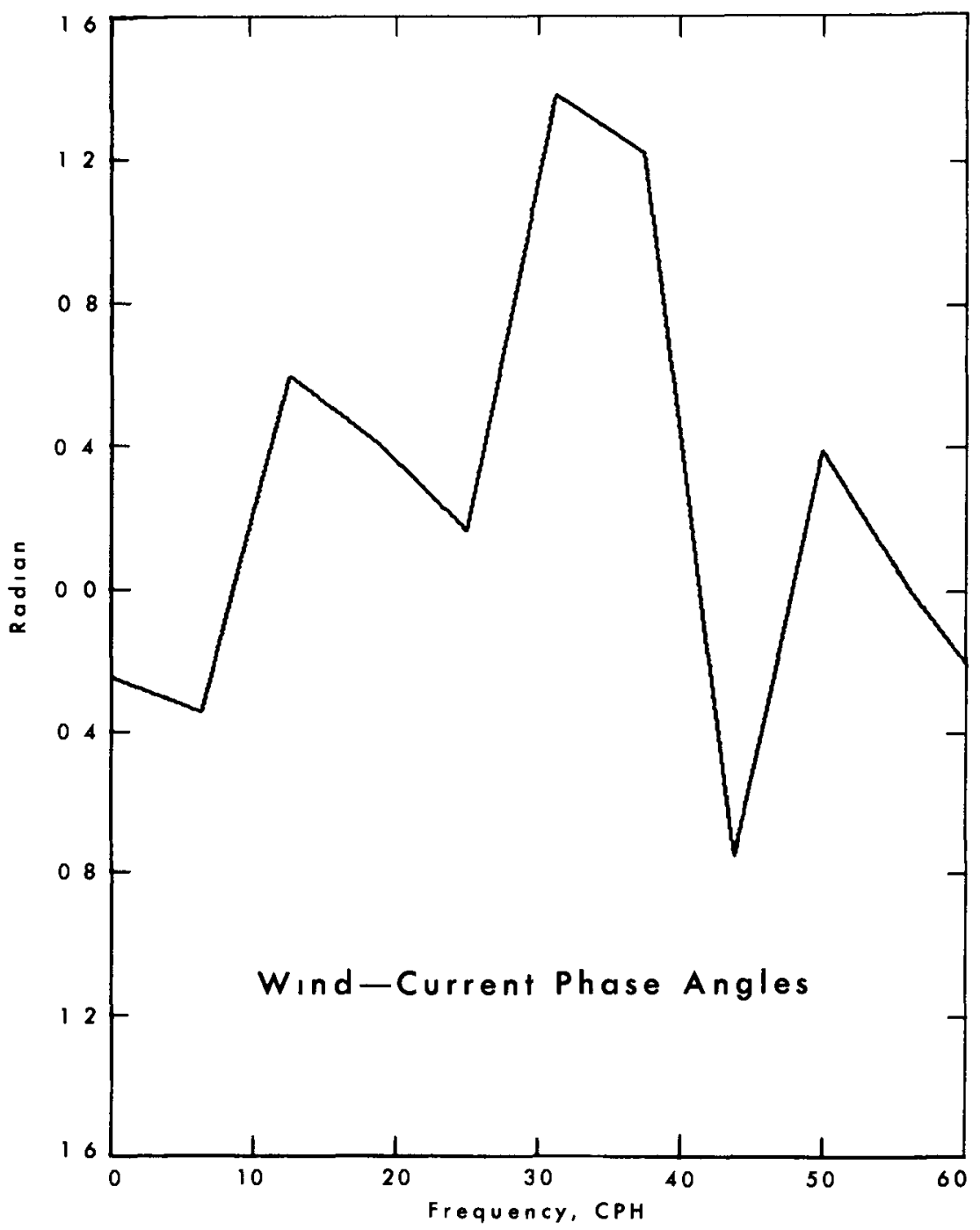

Fig 12 The phase lags of the wind and the current from interval II 
REFERENCES

Bendat, J S , and A G Plersol, 1966, Measurement and analysis of random no1se data New York (John Wiley), 390 pp

Cannon, G A, 1969, Observations of motion at intermediate and large scales in a coastal plain estuary Technical Report No 52, Ref 69-5, Chesapeake Bay Institute, The Johns Hopkins Univ, 113 pp

Fergusson, S P, 1935, The sensitiveness of anemometers Bull Am Meteorol Soc, 15 95-99

Holloway, J L, Jr, 1958, Smoothing and filterıng of time serles and space flelds In ( $H$ E Landsberg, ed ) Advances in geophysics, $4351-388$

Murray, S P, 1970, Bottom currents near the coast during Hurricane Camille J Geophys Res , 75(24) 4579-4582

Panofsky, H A , and G W Brıer, 1963, Some applıcatıons of statıstıcs to meteorology The Pennsylvania State Univ, Unıversıty Park, Pennsylvania, 224 pp

Pond, S, S D Sm1th, P F Hamblin, and R W Burling, 1966, Spectra of velocity and temperature fluctuations in the atmospheric boundary layer over the sea J Atmospherıc Sci, 23(4) 376-386

Taylor, G I, 1935, Statistical theory of turbulence Parts I-IV Proc Roy Soc London, A151, pp 421-478 , 1938, The spectrum of turbulence Proc Roy Soc London, Al64, pp 476-479

Tchen, C M, 1953, On the spectrum of energy in turbulent shear flow J Res NBS, $50(51)$ 


\title{
CHAPTER 125
}

\author{
Jamalca Bay Hurrıcane Barrier \\ by \\ Frank I Panuzio 1 ' \\ Fellow American Soclety of Civil Engineers
}

\section{ABSTRACT}

A $124 \mathrm{mlle}$ beach erosion control and hurricane flood protection project includes Jamaica Bay and the Rockaway Inlet in the southwest corner of Long Island, New York $n$ The project would provide $61 \mathrm{miles}$ of beach $\mathrm{fl}_{11}$ and floodwalls along the Atlantic Ocean shore and $63 \mathrm{mlles}$ of inland structures to tie back to high ground, including a $09 \mathrm{mlle}$ barrier across the $1 \mathrm{nlet}$ The barrier, with a 300 foot gated opening and a 300 foot ungated opening, would permit suppression of the design hurricane surge so as to eliminate the need of flood protection works within the bay Linear mathematical models were used to determine these openings Because of the 11mitation of these models to produce adequate data in the bay pertinent to environmental and ecological considerations, three hydraulic models were utilized General conclusions drawn from the hydraulic model test data are that the results of the mathematical models were upheld, a design storm with high peak is critical for determining the helght of protection, a design storm with high volume rather than high peak plus ralnfall runoff is critical in determining ungated openings and suppression of bay levels, and there is a combination of gated and ungated openings that would meet the flood protection, navigation, environmental and ecological ob jectives

\section{GENERAT}

Project The Jamaica Bay hurricane barrier is a part of a 124 mile, Federal beach erosion control and hurricane flood protection project, authorized by the Congress of the United States in June 1965 at more than 50 milion dollars 1,2

Location The project extends along the Atlantic Ocean side of the Rockaway peninsula from high ground in the vicinity of East Rockaway Inlet to high ground in the vicinity of Rockaway Inlet and includes Jamaica Bay (Figure 1) Jamaica Bay is a coastal body of water in the southwest corner of Long Island and connects to the lower bay of New York Harbor and the Atlantic Ocean through the Rockaway Inlet The bay is bounded by the boroughs of Brooklyn and Queens and Rockaway penınsula in New York City and by Nassau County, all in the southeast corner of New York State Long Island is a long, narrow island in the north Atlantic Ocean at a significant indentation of the northeastern coast line of the United States The Rockaway peninsula is a barrier beach which extends westward from the mainland of Long Island and separates Jamaica Bay from the Atlantic Ocean

objectives The project objective is to provide protection against storm water flooding due to hurricanes along the developed shore line of the Rockaway peninsula and of Jamaica Bay and to restore and to stabilize the beach along the ocean shore line of the Rockaway peninsula

The barrier objective is to provide a practicable solution of a tieback to high ground for the coastal works and to suppress the design hurricane surge to a nondamaging level in the bay so as to eliminate the need of protective works along the shores of the bay without any detrimental change in the existing bay environment and ecology during the no-storm period

Problem In the preauthorization studies, the size of the barrier openings during normal and storm periods was determined by the use of a simplified linear mathematical model While this model is adequate to predict water surface elevations and the discharges and average velocities in the openings, it did not produce adequate values of local hydraulic changes in the bay such as local

1/ Senior Engineer Consultant for Civil Works

U S Army, Corps of Engıneers, New York Distrıct 


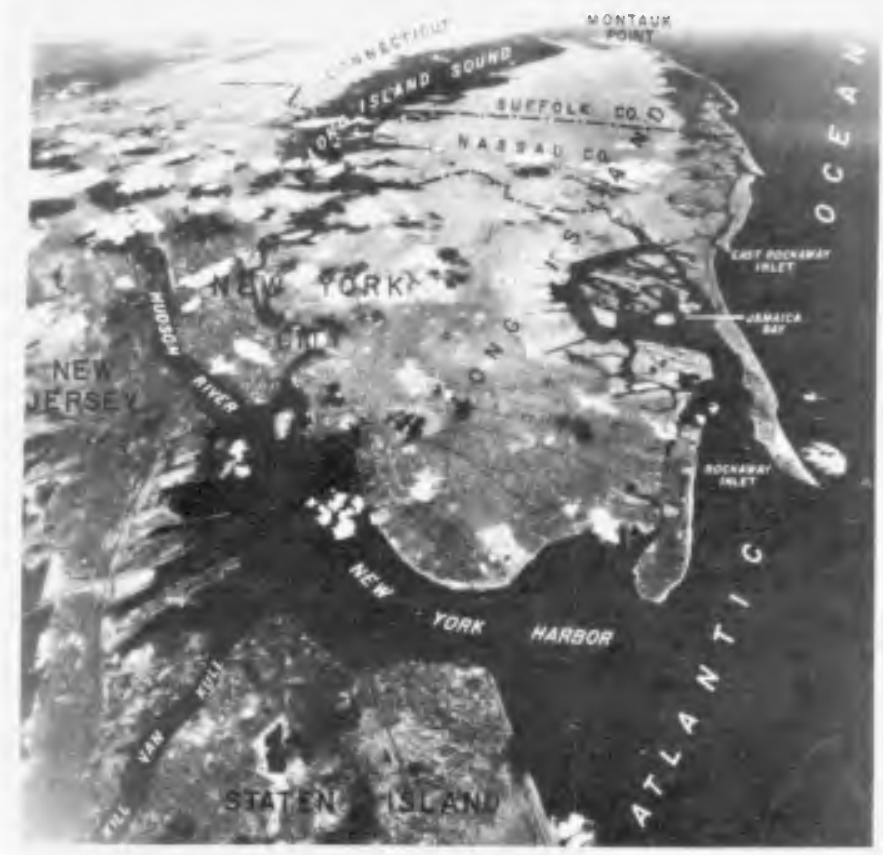

OBLIQUE LOOKING GENERALLY EASTWARD

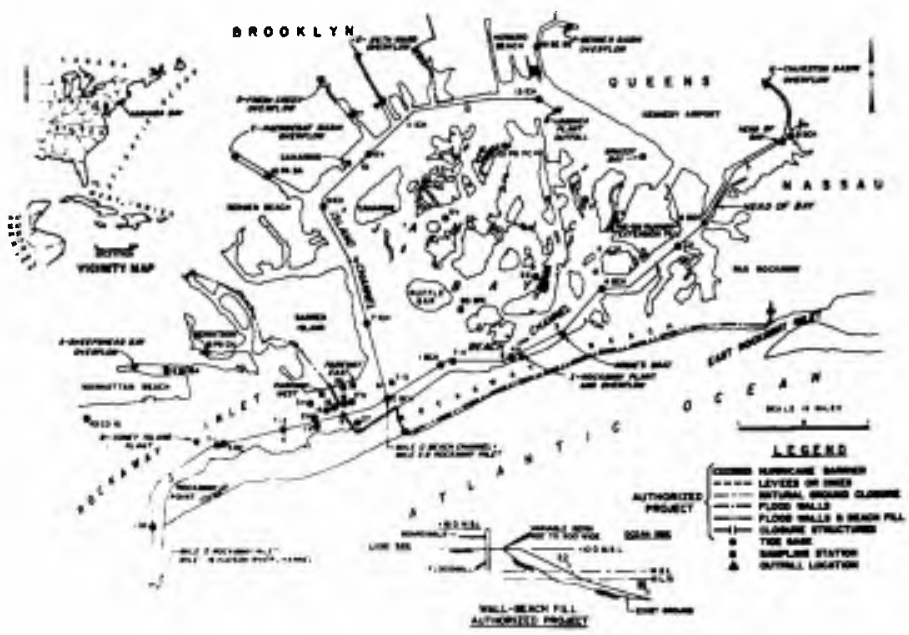

ROCKAWAY INLET TO EAST ROCKAWAY INLET

FIGURE！ JAMAICA BAY, LONG ISLAND, NEW YORK, U.S.A. 
velocitıes and currents, salinities, tıdal levels and circulation which are essential to pollution, fish and wildlife, and other environmental and ecological considerations $1,5,6$ Therefore, environmental and ecological interests, recognizing this deficiency, requested that additıonal field studies and hydraulic model investigations be conducted during the final design of the project to determine the specific effects of the hurricane barrier on water quality, fish and wildlife, and currents in Jamaica Bay The authorization by Congress was subject to this consideration

\section{AUTHORIZED PROJECI}

Description The authorized project from east to west, with all elevations referred to mean sea leve1, would consist of (Figure 1) a $03 \mathrm{mile}$ concrete closure wa 11 at East Rockaway Inlet w1th a top elevation from 18 to 15 feet at closure and with a 6 foot high and 40 foot wide stoplog structure, a beach fill of more than 4 milizon cubic yards with a 100 to 200 foot wide berm at an elevation of 10 feet and whth a 1 on 20 oceanward slope, backed up by a concrete-clad, stee 1 sheet pile floodwall with a top elevation of 18 feet along 61 miles of the ocean side of the Rockaway penınsula, a $09 \mathrm{mlle}$ flood dike wath a top elevation of 18 to 15 feet across the Rockaway peninsula to Rockaway Inlet, a $06 \mathrm{mlle}$ concrete floodwal1 and levee along the inlet wath a top elevation of 15 to 18 feet, a $09 \mathrm{mlle}$ gated barrier across the inlet with a top elevation of 18 feet, a $12 \mathrm{mile}$ levee and dike, and a $24 \mathrm{mlle}$ natural ground closure with a top elevation of 18 feet at the barrier to 15 feet at the closure

The Barrier The solid portion of the barrier would consist of (Figure 2) two sections with lengths of 1,860 and 1,670 feet, with a top width of 12 feet at an elevation of 18 feet, with side slopes of 1 on 15 and with fishing plat forms on both sides at an elevation of 80 feet The middle 1,000 feet of the barrier with a bottom elevation of minus 425 feet would consist of a 600 foot navigation openıng and two side rolling gates, each 150 feet wide with gate recesses, to permit closure of this opening to 300 feet during the storm periods

Sti11 Water Level The design still water leve 1 for the authorized project is the peak of the Standard Project Hurricane surge occurring at the mean astronomical tide (F1gure 3) For the prediction of surges at the mouth of the New York Harbor for a design storm, a research investigation was conducted at The $A$ and $M$ College of Texas 4 From this research, a correlation-prediction formula was developed empirically, with some degree of theoretical guidance, from observed tides and corresponding meteorological parameters of storms inducing them on this basis, using the meteorological parameters of the september 1944 hurricane transposed to a path critical to the New York Harbor area, the Standard Project Hurricane surge was determined to be 123 feet 3,4 The meteorological parameters were a maximum wind of 116 miles per hour, a central pressure range of 2755 to 2795 inches of mercury with a normal pressure of 3012 inches of mercury, a radius to maximum winds of 30 nautical miles and a foreward speed of 40 knots

Protection Height The design height of the protection works is the design still water level plus the wave runup Based on the solitary wave theory, the maximum breaking wave at the beach fill was found to be 205 feet with a 12 second period Using the composite slope method and experimental data, 7 the runup for this wave would be 57 feet Thus, the top of protection was placed at an elevation of 18 feet At the barrier, based on a generalızed relationshıp between winds and wave observations, 8 an effective fetch of $39 \mathrm{miles}$, a wind speed of $80 \mathrm{miles}$ per hour and an effective depth of 32 feet at the design still water level would result in a wave of 67 feet with a period of 5 seconds This wave, based on experimental data on riprap structures, 7,8 would produce a wind setup and runup of 57 feet Thus, the top of protection was also placed at elevation 18

Mathematical Model The propagation of tides inside a bay may be expressed by dynamic and continuity equations $1,5,6$ These equations, due to boundary conditions 

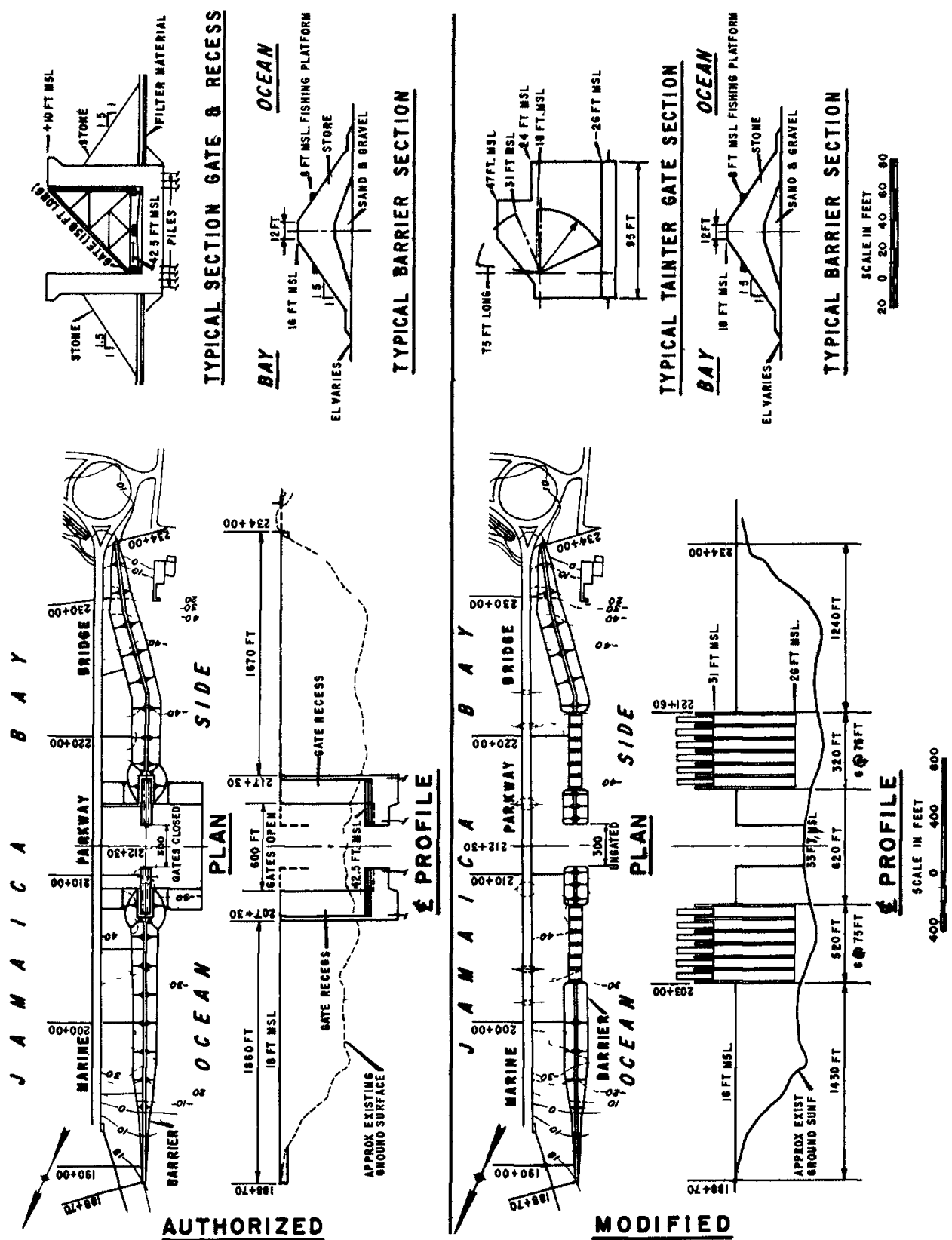

$$
\text { FIGURE } 2 \text { JAMAICA BAY BARRIER }
$$




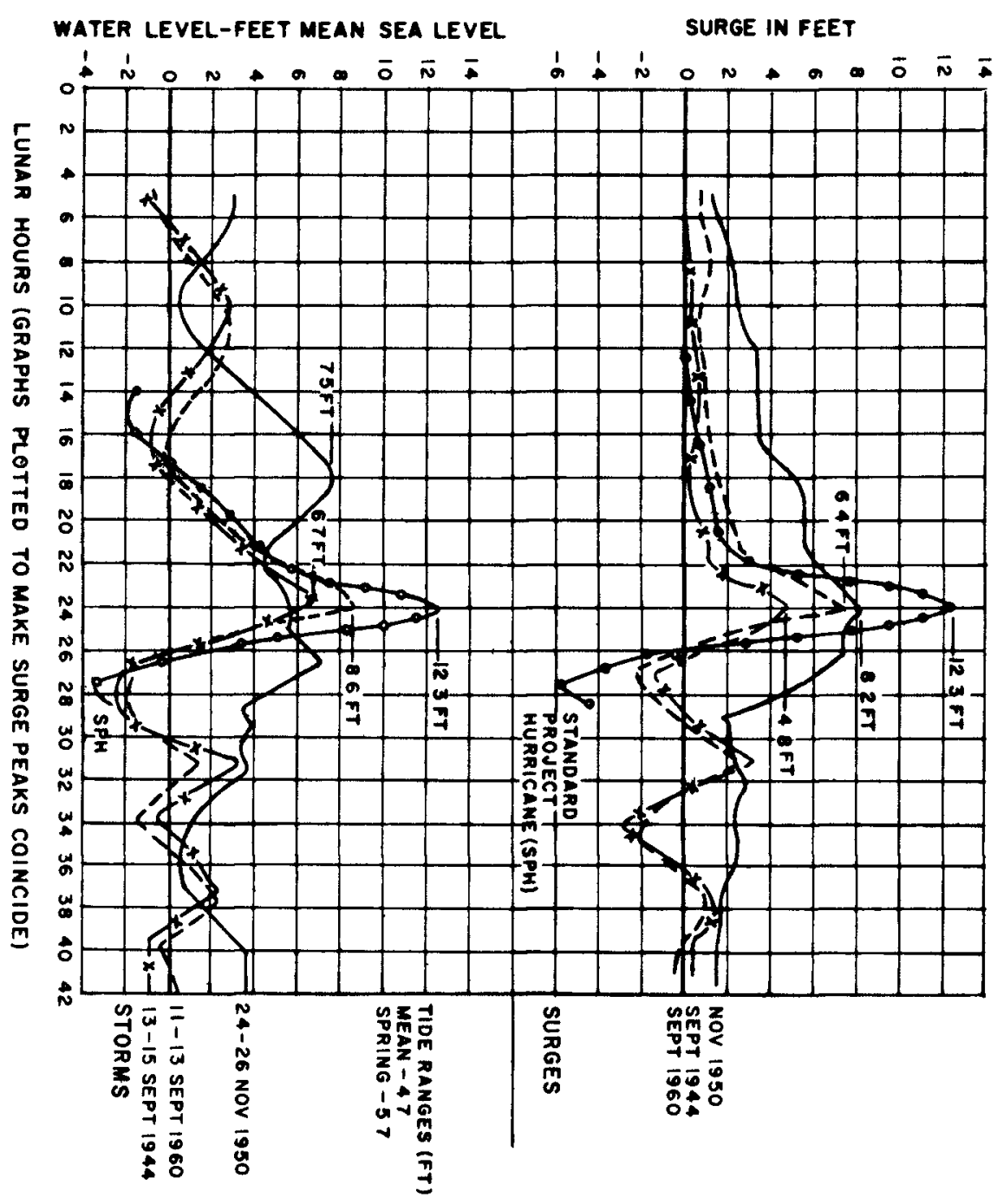

FIGURE 3 CRITICAL DESIGN HURRICANES-FORT HAMILTON,NY 
and many complex terms involved, could not be solved analytically within the scope and time frame of the study In order to obtain a practical solution, assumptions were made to simplify the equations by neglecting and simplifying the terms of the equations without substantial loss in accuracy The bay is, basically, a basin connected to the ocean by a relatively long and narrow channel of the Rockaway Inlet So, a simple, one-dimensional model for current natural conditions of the bay was developed on the assumption that the flow through the channel is governed only by the functional resistance in the channel with negligible inertia forces and that the level inside of the bay is variable only with time with an adjustment for wind setup and rainfall The adequacy and adjustment of this model were developed by routing the hurricane of September 1960 to produce recorded bay levels when the barrler is placed across the natural Rockaway Inlet, the mathematical model must be adjusted for the increased resistance to flow at the barrier Thus, the onedimensional model was based on two principal assumptions The resistance to flow through the barrier opening and the inlet channel is the only significant force acting on the dynamic system The water surface throughout the bay is assumed horizontal and related to the ocean surface elevation only by the law of continulty and the loss through the barrier and inlet channel With these basic assumptions, the dynamic relationships were expressed by the following equations

$$
Q= \pm C A_{b} \sqrt{2 g} \sqrt{Z_{a}-Z_{j}}
$$

In which $Q=$ discharge through barrier opening, $C=$ discharge coefficient, $A_{b}=$ area of openung, $z_{a}=$ water surface elevation on ocean side, and $Z_{j}=$ water surface elevation on bay side

$$
Q=\frac{s_{1} d_{Z_{1}}}{d t}-I_{r}
$$

In which $S_{j}=$ surface area of bay, and $\mathrm{dZ}_{\mathrm{J}}=$ change in bay water surface elevation for time interval of $\mathrm{dt}$ and $\mathrm{I}_{\mathrm{r}}=$ rainfall-runoff inflow rate

Barrier Opening The inltial barrier opening was sized to be large enough to minımize any change in the natural bay environment and ecology during no-storm periods, to satisfy the projected navigation vessel and traffic, and to suppress the design hurricane level to about zero damage stage in the bay To accomplish the first two objectives, 14 routings were made for the spring astronomical tidal range of 57 feet, utilizing the mathematical mode1, the area capacity curve, navigation depths of 15 to 426 feet mean sea level and openings from 100 to 1,000 feet wath results as shown on Figure 4 On the basis of these results, the minimum opening that would have minimal effect on the astronomical spring tidal range and would satisfy the navigation depths and velocities was the 600 foot opening with a depth of 425 feet at mean sea leve1, a $45 \mathrm{knot}$ maximum velocity in the opening, and a reduction in range of 010 feet The 1,000 foot opening showed no significant change in tidal range, and a 30 knot maximum velocity The average velocity in the Rockaway Inlet for the existing conditions is in the order of 27 knots However, average velocities up to 5 knots are considered tolerable for navigation

Based on a gross appreciation of the hydraulic system of Jamaica Bay and Rockaway Inlet, a quasi theoretical mathematical model of BOD vs Tidal Prism relationship for Jamalca Bay was developed 9 This relationship assumed that change in total waste load as measured by the BOD is directly related to the volume of the tidal prism This model was used with waste loads measured during the summer of 19591962 and anticlpated future waste loads obtalned from city sources to predict accumulation of waste in Jamaica Bay and Rockaway Inlet for the tidal prism change estimated from the effect of each barrier opening The results from the model for the 600 and 1,000 foot openings were that the change in waste load accumulation 

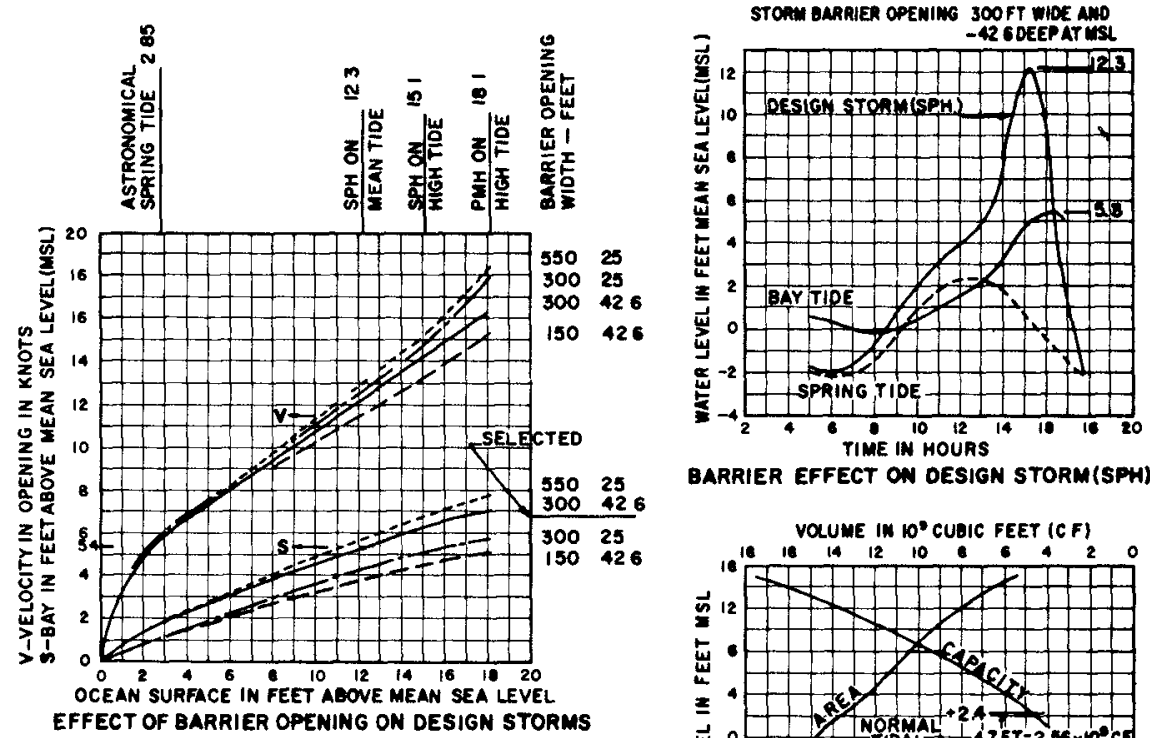

BARRIER EFFECT ON DESIGN STORM(SPH)
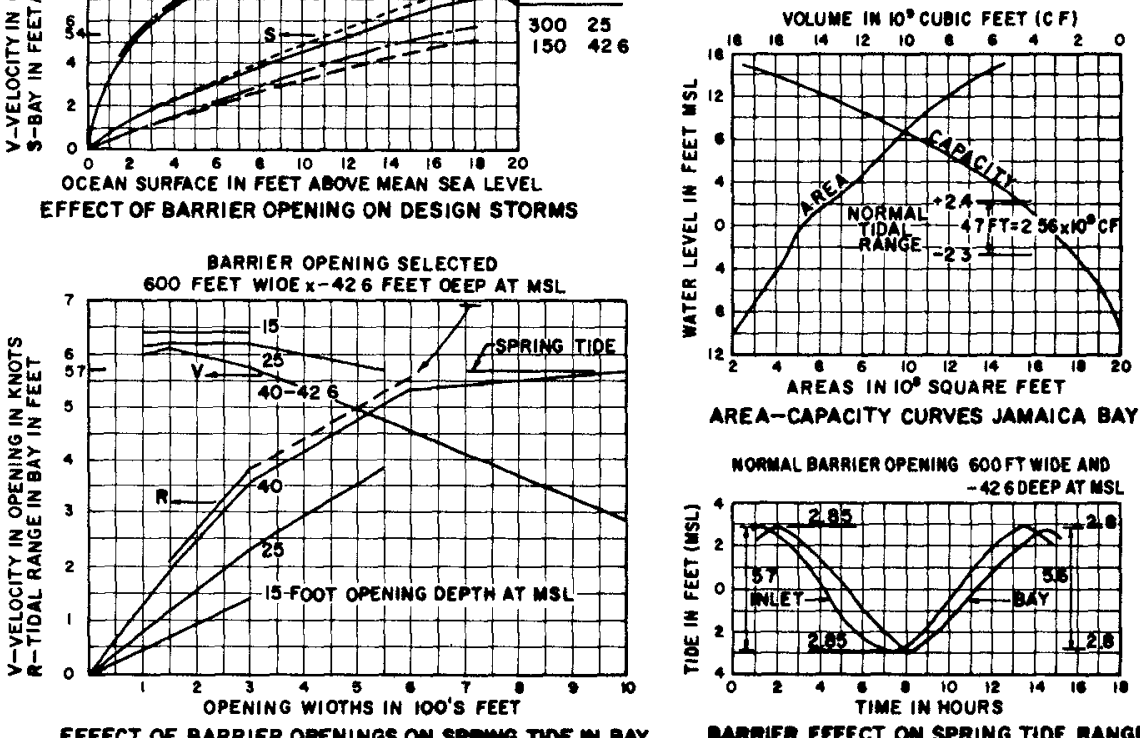

EFFECT OF BARRIER OPENINES ON SPAWNO THOE WI BAY

I- SECTION OF BARRIER OPENIMG

DAMRIER EFFECT ON SPRING TIOE RANCE

EFFECT OF

I- SELECTED BARRIER OPENING

FIGURE 4 BASIS OF AUTHORIZED BARRIER OPENING DESIGN 
would be virtually zero and that there would be no significant damage to water quality In the interest of minimum first cost, since the selected opening would have to be gated to obtain suppression of the design hurricane, the 600 foot opening was selected for the normal operating conditions

To obtain the third objective, to suppress the design hurricane level to about zero damage stage in the bay, 13 routings were performed utilizing the mathematica 1 model, the area capacity curve, navigation depths of 25 and 426 feet below mean sea leve 1 and openings of 150,300, and 550 feet with results as shown in Figure 4 On this basis, the storm opening of 300 feet with a depth of 426 feet at mean sea level was selected to produce a bay level for the standard Project surge on mean tide of 53 feet at mean sea leve1, about zero damage stage

I.mitations While these models are adequate to predict water surface elevations, discharges and average velocities in the opening, and gross evaluation of the water quality, they cannot produce adequate values of local hydraulic changes in various parts of the bay such as velocities and currents, salinities, tidal levels and circulation which are essential to pollution, fish and wildife, and other environmental and ecological considerations Therefore, recognizing this deficlency, the Congressional authorization was subject to the condition that additional field studies and hydraulic model investigations would be conducted in the final stage of design to evaluate and to minimize the specific effects of the hurricane barrier on water quality, fish and wild1ıfe, and currents in Jamalca Bay

\section{FIELD INVESTIGATIONS}

Measurements Field measurements were started in January 1967 with a view to developing data to construct and verify a hydraulic model of Jamaica Bay To develop the physical characteristics of the bay, a photographic and topographic survey of the area was made to a horizontal scale of 1 inch $=1,000$ feet and to a vertical relief of two foot contours up to elevation 20 feet mean sea level The underwater contours were developed by a sounding survey at sufficient sections to delineate the underwater topography For hydraulic verification, measurements were made on 12 and 13 June 1967 Current velocities were recorded at nıne stations in Jamaica Bay at locations shown on Figure 1 and identified by letter $V$ Current velocities were taken every one half hour over a complete tidal cycle at surface, mid-depth, and bottom Tidal current observations were timed generally to cover a period between successive low tides, during daylight hours, for a period in excess of 13 hours when the diurnal inequality was a minimum Simultaneous measurements were taken at stations $1 \mathrm{~V}, 2 \mathrm{~V}$, and $3 \mathrm{~V}$, at stations $2 \mathrm{~V}, 4 \mathrm{~V}, 5 \mathrm{~V}$, and $6 \mathrm{~V}$, and at stations $2 \mathrm{~V}, 7 \mathrm{~V}, 8 \mathrm{~V}$, and $9 \mathrm{~V}$ in order to obtain the distribution of flow in the channel system of the bay Each group of stations was tied into the other two groups through continuous reading at station $2 \mathrm{~V}$ Three ott meters with $\mathrm{F4}$ counter and two Gurley Price meters with 611 counter were used Each meter was connected to an electric revolution counter and watch Reduction of field data was by use of laboratory calibration curves for each meter At each station, current meter measurements were made for a total period of one minute at just below the surface, at 2 feet above the bottom and at mid-depth where depths exceed 6 feet at mean low water At each velocity station depth, water samples were taken with one or two 11ter Remmerer samplers after water was permitted to flow freely out of the sampler The temperature of each sample was taken immediately w1th armored thermometers with a range of $-1^{\circ}$ to $50^{\circ}$ Centigrade $1 n 01^{\circ}$ divisions The salinity of each sample was determined in the laboratory and recorded in parts per thousands of chlorides The hydrography at each of these stations was also obtained with depths referred to mean sea level Data were also developed as to sanitary and storm water inflow at four sewage treatment plant overflows and five storm water outfalls and on weather conditions as to rain, wind, and temperature 


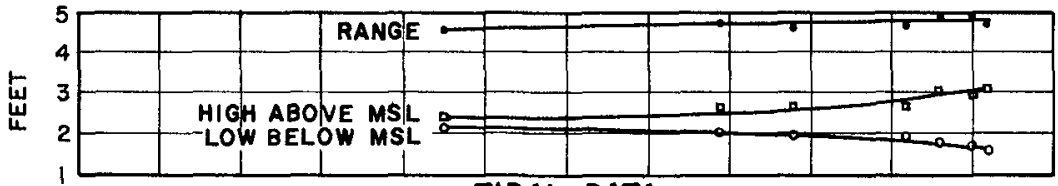

TIDAL DATA
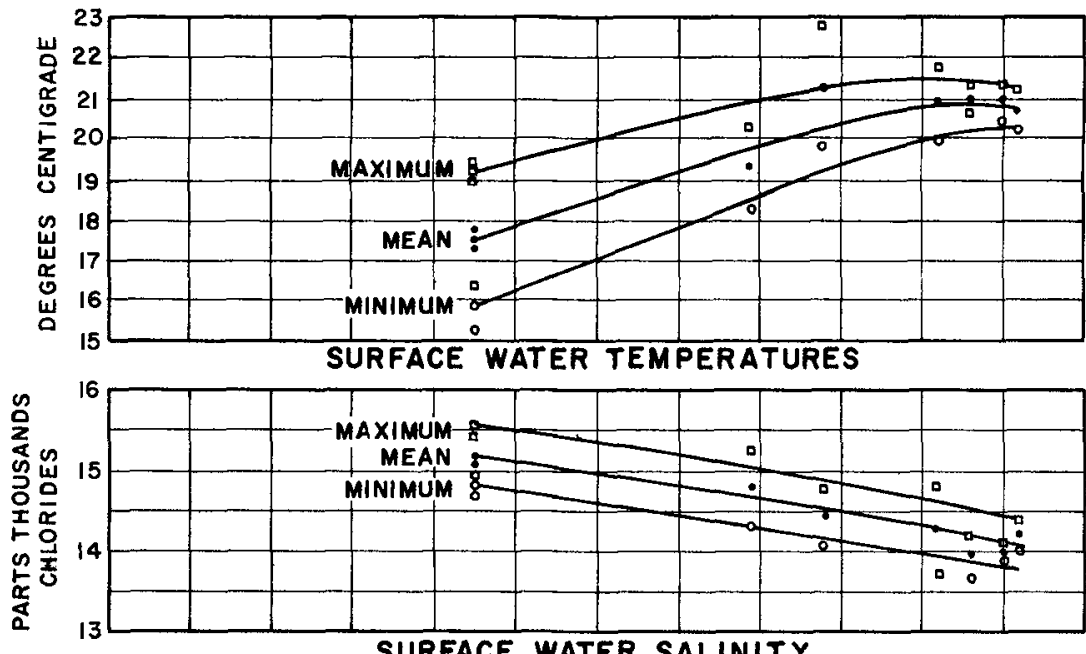

SURFACE WATER SALINITY

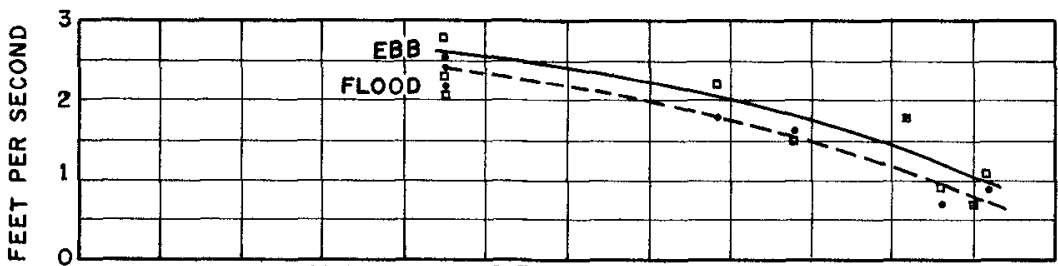

MAXIMUM SURFACE TIDAL CURRENT

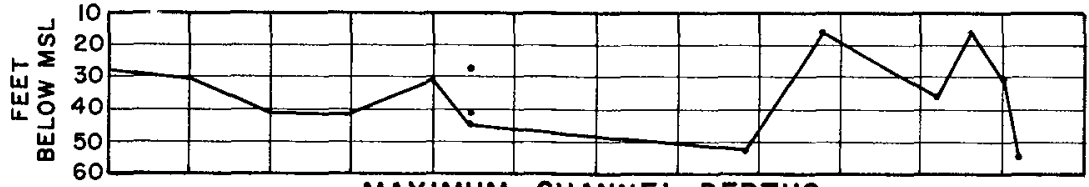

\section{MAXIMUM CHANNEL DEPTHS}

OBSERVATIONS ON 12 \& 13 JUNE 1967 AT NOTED STATIONS

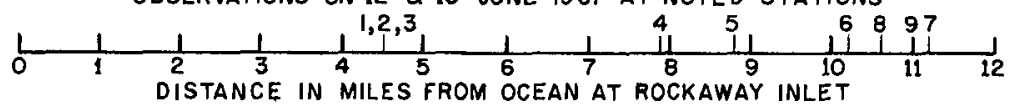

FIGURES 5 FIELD OBSERVATIONS-JAMAICA BAY CHARACTERISTICS 
Results The Jamaica Bay characteristics measured as to tidal currents, tidal range, temperatures, salinities and hydrography are shown in Figure 510 The water depth varies from 15 to 55 feet at mean sea level For the measured tidal cycle, the tidal range varies from 46 to 49 feet with the higher values in the interior of the bay and with time of high and low water almost identical at the five interior stations The surface tidal currents vary from 25 to 07 feet per second on flood and from 28 to 07 feet per second on ebb with the lower values at the interior The currents were found to be greatest at the surface and minimum at the bottom slack water was found to be colncident with or within one half hour of the times of high and low tides The surface water salinity varied between 152 and 140 parts per thousand of chloride with the higher values at the entrance The salinities increased with depth and were maximum near the end of flood and minimum near end of ebb The surface water temperature varied from $173^{\circ}$ to $210^{\circ}$ Centigrade with the higher temperatures in the interior The temperature decreased with depth and was greatest near end of ebb and least near end of flood

\section{HYDRAULIC MODEL INVESTIGATIONS}

Purpose The model studies of Jamalca Bay were to determine the effects of the hurricane surge protection barrier in the Rockaway Inlet on (a) water quality in the bay as to public health, recreation, and fish and wildiffe, (b) recreational and commercial navigation, and (c) suppression of the design storm surge to such a level as to provide protection to the area surrounding the bay from storm flooding

Facilities The hydraulic model studies were carrled out in three research facilities at the U S Army, Corps of Engineers, Waterways Experiment Station, Vicksburg, Mississipp1, Figure 6 Jamaica Bay is a small section of the basic comprehensive model of the New York Harbor complex This model, constructed to linear scale ratio (model to prototype) of 1100 verticaliy and 11,000 horizontaliy, produces velocities at the ratio of 110 , time at 1100 , discharge at $11,000,000$ and salinity at $1 \quad 1$ The Jamaica Bay area was reconstructed to reproduce prototype hydrographic and topographic conditions below +20 feet mean sea level as of June 1967 Tides and their associated flood and ebb tidal currents are controlled in the model by the interaction of a primary programmable tide generator located in the Atlantic Ocean at Sandy Hook and two secondary tide generators, one located in Long Island Sound and another located in the Hudson River at Hyde Park at the upstream limit of the model Weirs are used to control the upland freshwater inflow from tributaries A surge generator is used to produce the time-elevation history of the design storms This model is used to develop data on the tidal regime, currents, salinities, dispersion characteristics, surge suppression and the barrier composition

A second model reproduced a short reach of the Rockaway Inlet to an undistorted innear scale of 1100 The velocity or time ratio is 110 and the discharge ratio is 1100,000 Varlous designs of gated and ungated barrier openings were tested to determine their hydraulic efficiency under conditions of normal tides and storm surges and to determine in detall the flow patterns and velocities in and adjacent to the openings that might be significant to the design of the structure or to navigation through the structure

The third model, to the scale of the basic model, was used to develop the distorted scale structures that have the hydraulic efficiencies as developed in the undistorted model for use in the distorted comprehensive model This procedure ensured that when the distorted scale structures are placed in the comprehensive model they would pass the proper flows into and out of Jamalca Bay under any combination of head differentials imposed by the tides and storm surges

Bay Model Verification The Jamaica Bay portion of the comprehensive model was first corrected to reproduce the latest topographic and hydrographic conditions Then, the bay area was adjusted to reproduce prototype data for tides, tidal currents, and salinities, as observed on 12 and 13 June 1967 (Figure 5) 10 The high 

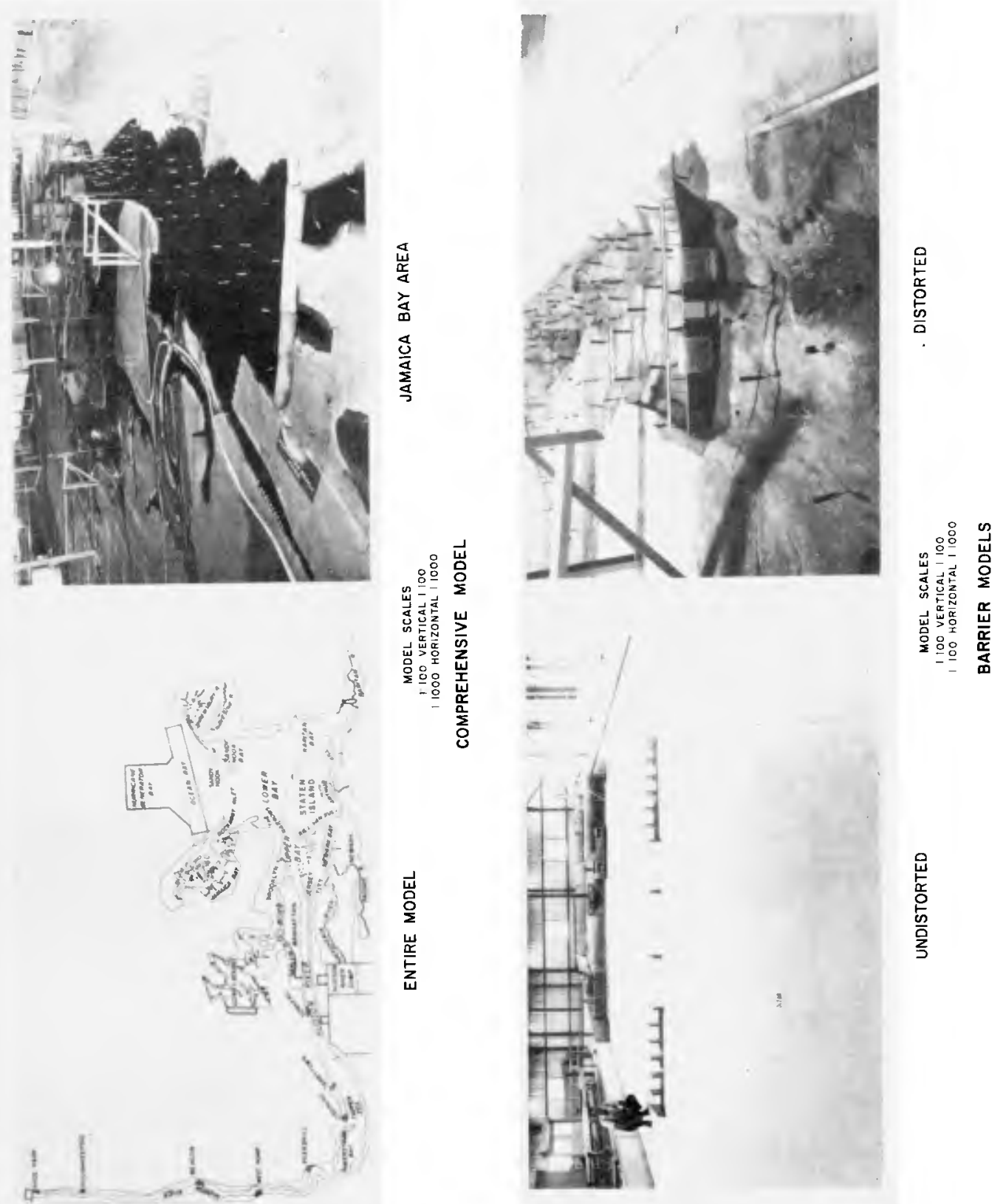

FIGURE 6 JAMAICA BAY MODELS 
degree of accuracy attained in the model verification as to tides, current velocities, salinities, and design storms is illustrated by the comparative model and prototype curves shown on Figure 7 il While there are some differences between model and prototype data, these differences were probably attributable to local winds and other disturbances that occurred when the prototype measurements were taken and that could neither be identified nor simulated to scale in the model verification tests

Barrier Model Verification The barrier gated and ungated openings were constructed to the undistorted scale of the second model (Figure 6) For steady flood flow conditions, and various combinations of the ungated opening with open gates, the ocean level was varied and the bay levels were determined The procedure was reversed for the ebb conditions, the bay levels were varied and the ocean levels were determuned The results of this procedure for a 150 foot opening are shown on Figure 8 A distorted model was designed and adjusted until its calibration by the same procedure would match the undistorted model calibration The results of this procedure for a 150 foot opening are shown on Figure 8 These tests were made to develop undistorted models for ungated openings of 300,150 , and 110 foot openings with combinations of 12 or 16 gate openings and sill levels in the ungated openings at natural ground, -23 feet and -26 feet at mean sea level The hydraulic efficlency of the openings expressed as a discharge coefficient for various degrees of submergence and differential head for various steady flow discharges were developed as shown for the 150 foot ungated opening on Figure 8 It is noticeable that with decrease of submergence and increase in differential head and discharge, the efficiency of the opening becomes constant as the discharge coefficient becomes constant, a value slightly greater than 090 The conditions of surface currents are developed by vertical photographs of three-second time lapse exposure of floating confetti in the undistorted model (Figure 8) The length of confetti streak is converted to surface velocity The barrler openings so developed in the distorted scales were inserted in the comprehensive model for water quality, navigation, and environmental tests

Base Tests Once the model verification was accepted as being sufficiently accurate, model base tests or tests of existing conditions under carefully controlled conditions of tides, freshwater inflow, pollution input and hurricane surges were conducted The results of these base tests (Figure 9), rather than the verification tests (Figure 7), were used to evaluate the effects of the barrier plans investigated in subsequent model tests Thus, the model tests with and without barriers were made under Identical and carefully controlled conditions In this manner, any differences noted in tides, tidal currents, salinities, dispersion of pollutants or surge elevations with various barrier plans installed are attributable to the barrier plan under study and are not affected in any way by minor differences between model and prototype phenomena noted during verification

The base tests were conducted with a mean repetitive tide, a constant source of salinity, freshwater inflows and pollution sources and rates All necessary phenomena were measured at predetermined sampling stations that would reflect conditions throughout the bay area The repetitive mean tide had a duration of 1245 hours and a range of 47 feet measured at Sandy Hook The sump salinity was manntained at 30 parts per thousand (ppt) The primary freshwater inflow was $12,000 \mathrm{c}$ f $\mathrm{s}$ from the Hudson River at Hyde Park and $1,770 \mathrm{c}$ f $\mathrm{s}$ from the Raritan River at head of tide Additional freshwater inflow sources were from treatment plant outfalls and storm water overflows in the amount of $13069 \mathrm{c} \mathrm{f} \mathrm{s}$ from two sources into the inlet downstream of any barrier and of $23693 \mathrm{c} \mathrm{f} \mathrm{s}$ from seven sources into the bay upstream of any barrier Under these conditions, the model was operated for 25 cycles, equivalent to 125 days in nature to assure that salinity stability had been obtained before data collection was begun Tides were measured with point gauges graduated to the nearest 001 foot ( 01 foot prototype) 

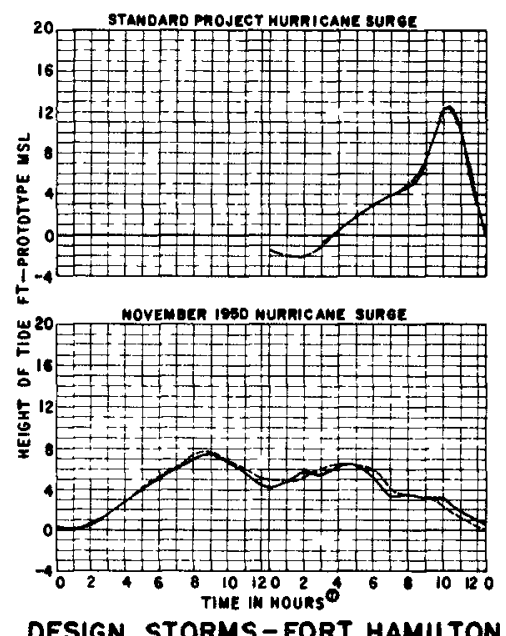

DESIGN STORMS-FORT HAMILTON
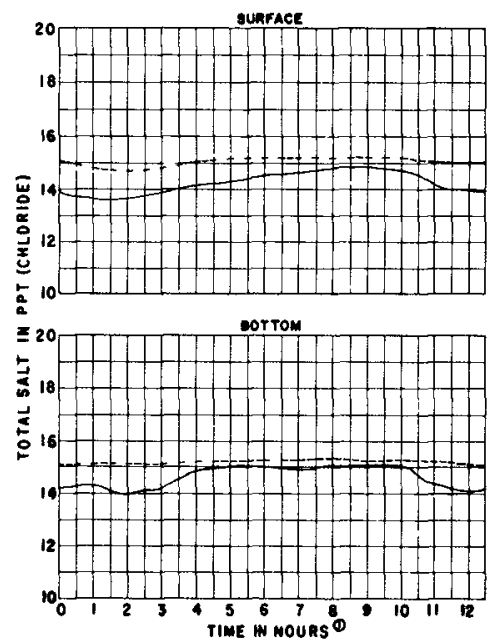

BAY SALINITIES-STATION 6V

- after moon s transit of 74Th Meriolan

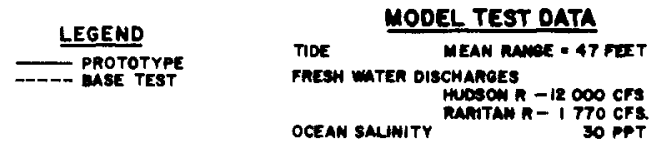

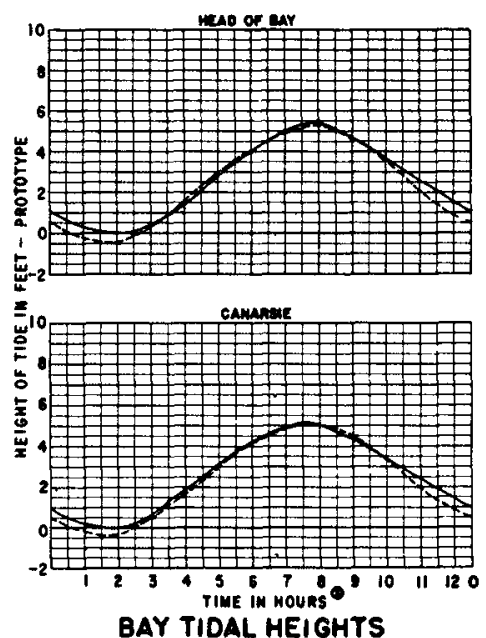

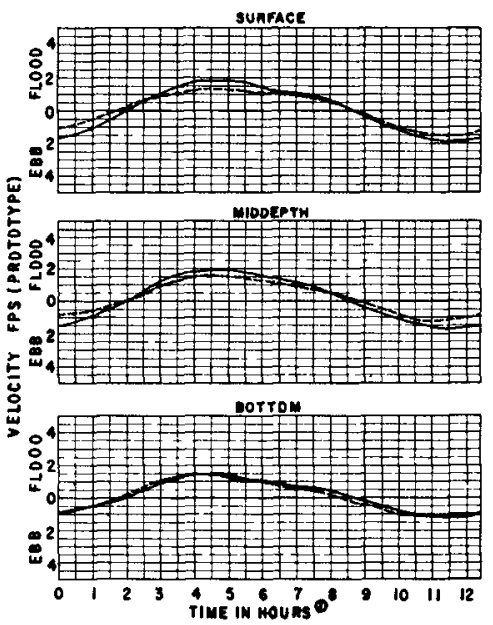

BAY CURRENT VELOCITIES-STATION GV

FIGURE 7 BASIC MODEL VERIFICATION 

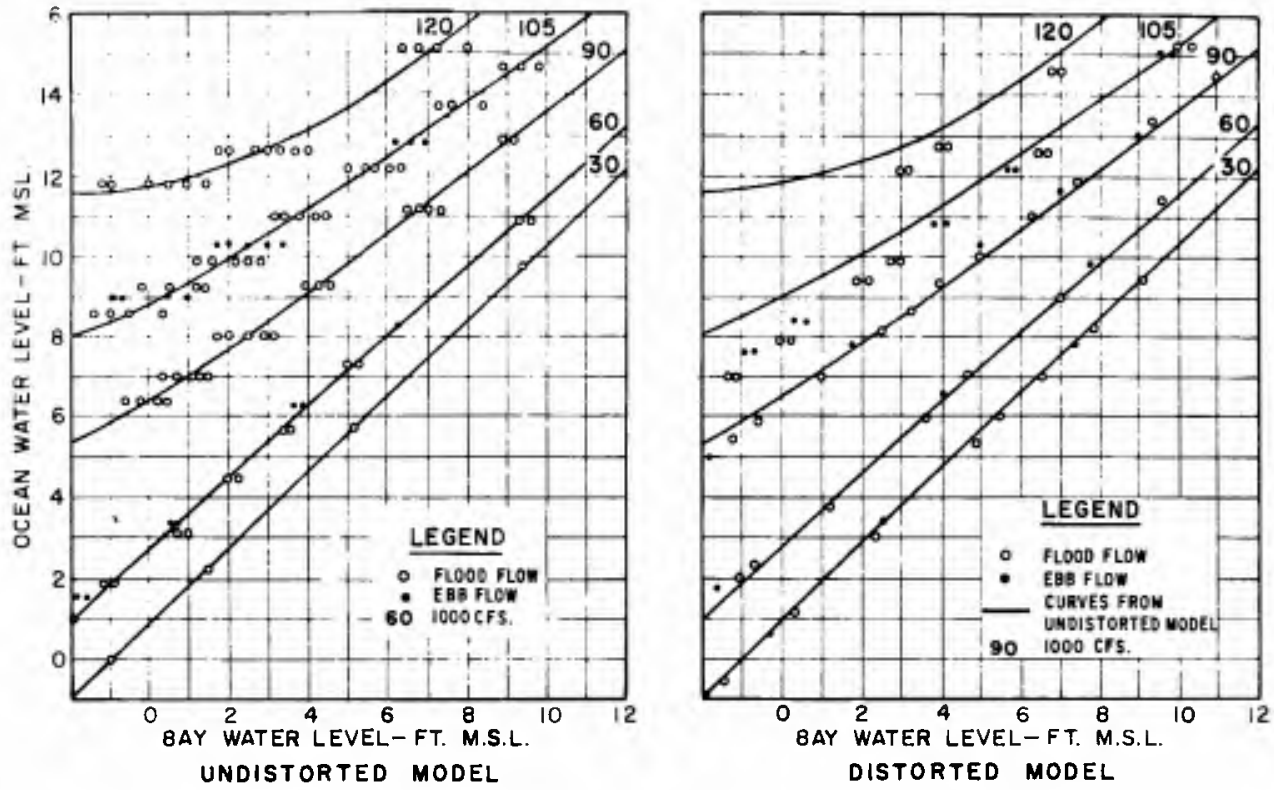

DEVELOPMENT AND CALIBRATION OF DISTORTED MODEL OPENING
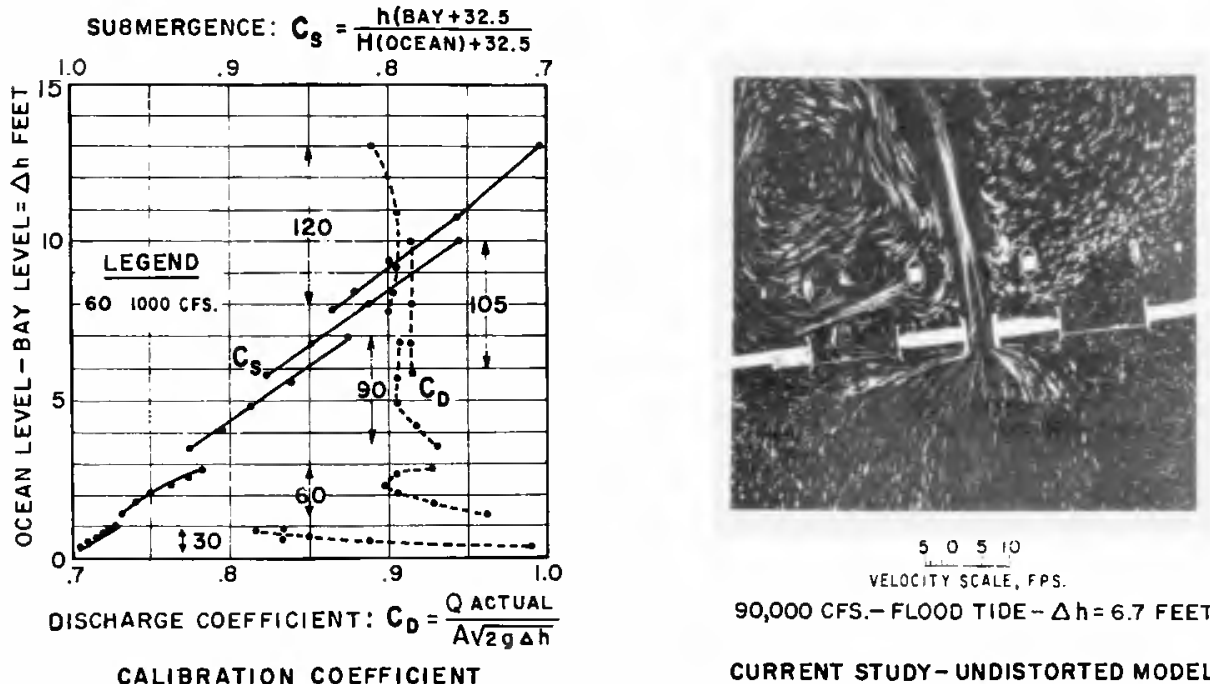

CURRENT STUDY - UNDISTORTED MODEL

FIGURE 8 CALIBRATION OF I5O FOOT UNGATED OPENING (ONLY) NO SILL 
Current velocities were measured for each lunar half hour at 23 locations throughout Rockaway Inlet and Jamalca Bay (Figure 1) At each location where depths were 15 feet or more, velocities were measured at surface and bottom depths Where depths were less than 15 feet, only mld-depth measurements were made Current velocity measurements were made in the model by using miniature Price-type current meters The meter cups are 0.02 foot in diameter, and the diameter of the cup wheels is about 008 foot Wheel revolutions per 10 seconds were visually counted and subsequently converted to current velocities in feet per second prototype by referring to calibration curves which were checked frequently The meters are capable of measuring actual velocities of 005 foct per second ( $05 \mathrm{fps}$ in prototype)

Salinities were measured for each lunar hour at surface and bottom depths at 18 stations located throughout Rockaway Inlet and Jamaica Bay At each station, 5 mililiter samples were taken, labeled as to depth and time, and stored under a constant temperature until salinities were determined Meters, operating on an electrical conductivity principle, were used to measure the sampled salinities The electrical output of the conductivity meter, attached to a dip cell, is calibrated in terms of total salts within the concentration range of 0 to 40 parts per thousand

Water samples for determination of dye concentration were taken at 34 stations at surface and bottom depths during high and low water slack periods The model was operated until salinity stability had been obtained Then, elther uranine or pontacyl, brilliant pink dye, was injected at the nine pollution sources (Figure 9) Freshwater with pontacyl dye adjusted to an initial concentration of 10,000 ppb was released continuously at the two sources in the Rockaway Inlet, located oceanward of Jamaica Bay Freshwater with uranine dye adjusted to an initial concentration of $10,000 \mathrm{ppb}$ was released continuously at the seven pollution sources inside Jamaica Bay. The introduction of the dyed inflows was continued for 100 tidal cycles, which is equivalent to about 50 lunar days During this time, water samples were taken and stored for later analysis Dye concentrations were measured by utilizing G K Turner Fluorometers capable of accurately measuring concentrations ranging from 0 to $10,000 \mathrm{ppb}$, (parts per billion)

The hurricane surge generator used to simulate hurricane surges in the model was a vertically displacing steel constructed box with variable speed automatic drive mechanism which could be programmed to cause the water surface elevation to rise and fall as required with respect to time to reproduce the desired hurricane surges Elevations were recorded throughout the problem area by means of automatic float-type recording gauges and by utilizing manual observations with the permanent point gauges described previously

Barrier Plan 3 The first plan subjected to model testing, Barrier Plan 3 , consisted of a 300 foot wide, ungated opening at natural bottom at about 325 feet below mean sea level, flanked by two gated sections (Figures 2 and 6) Each gate section consisted of six 75 foot wide tainter gates with sills at 26 feet below mean sea level (Figure 2) The tidal ranges with the gates fully open were not affected significantly (Figure 7) The time phasing of the tides in the interior of the bay was somewhat delayed, a matter of minutes The mean low water was raised slightly, a matter of tenths of a foot (Figure 7) The current velocities on the whole throughout the bay were not changed significantly (Figure 7)

The results of the dye dispersion test of plan 3 are sumnarized in Figure 9 For the purpose of this summary, the region seaward from the barrier was divided into three areas (the approach channel, Coney Island Beach, and the basins), and Jamaica Bay was divided Into four areas (Beach Channel, Island Channel, the tidal flats, and the basins) For the last 10 tidal cycles of the base and plan tests, the results of all sampling performed in the above seven areas were averaged, and the average concentrations are shown on Figure 9 for both dye sources seaward and 

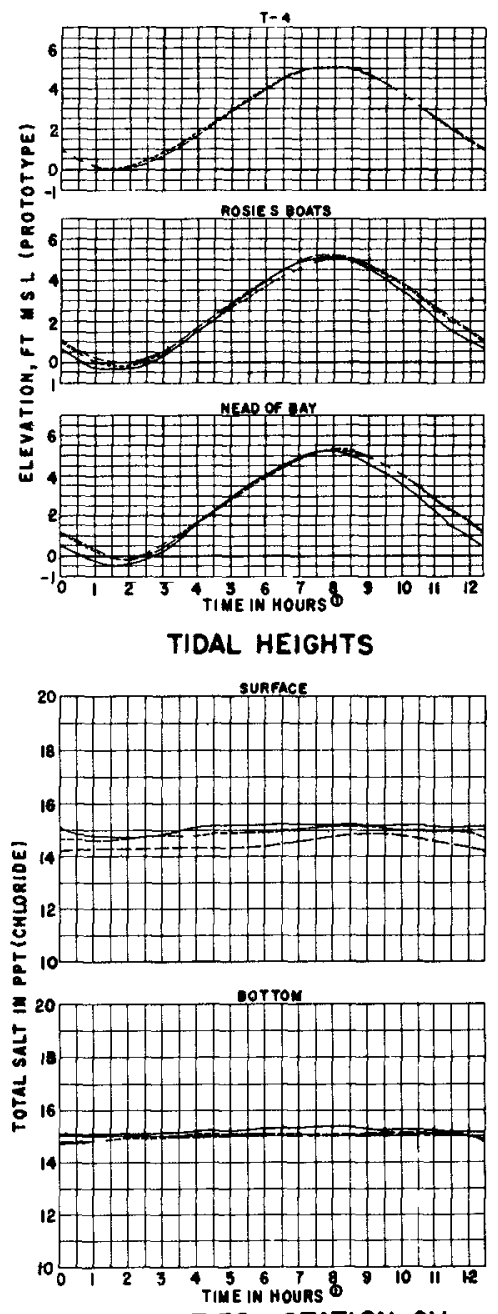

SALINITIES - STATION $6 V$

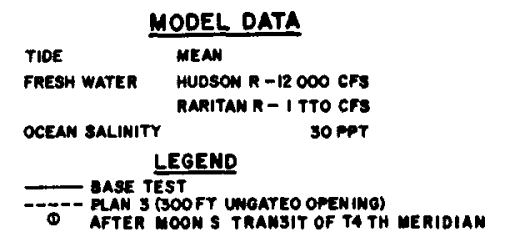

FIGURE 9 BASE TEST RESULTS

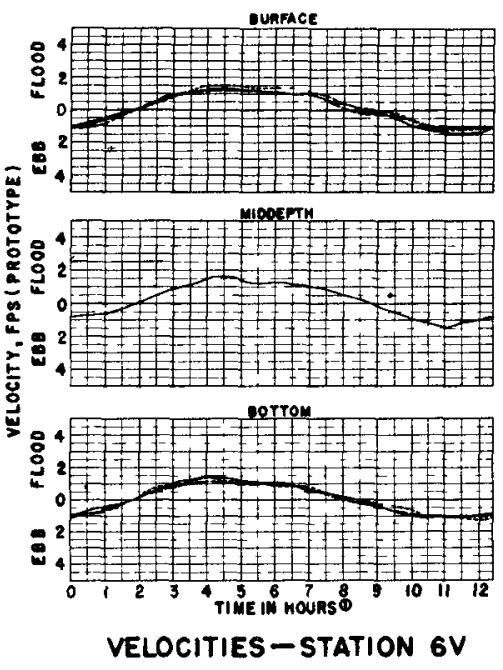

SIMULATED CONTMUOUS PNOTOTYPE IN NLW

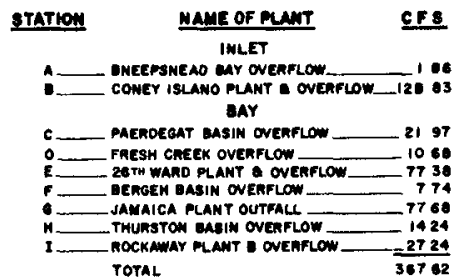

pon station Location gee figune:

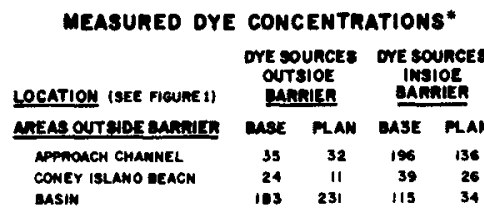

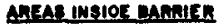

DEACN CMANHEL

ISLAMD GMANWE

TIOAL FLATS

easins

- part3 men altlion tera

inITIAL OVE CONCENTHATIONs AT RELEASE MOWT 100000 Pre

100 crcles so wman oare

\section{DYE DISPERSION TESTS}


landward from the barrier For dye sources seaward from the barrier, average dye concentrations for the plan test were reduced slightly in six of the seven areas from those observed in the base test For dye sources inside Jamalca Bay, the plan test showed slightly reduced concentrations in all seven areas as compared to the base test The results of these tests do not prove conclusively that pollutants w1 11 be flushed from Jamaica Bay as rapidly or more rapidly under plan 3 conditions than under existing conditions, since the sampling performed was not sufficiently comprehensive to account for all dye released in the model for the tests However, the fact that the average plan dye concentrations for plan 3 were less than those of the base test in essentially all areas used for this evaluation suggests strongly that the flushing characteristics of the bay will be as good or better under plan 3 conditions than for existing conditions

The two storm surges were used for the hurricane surge tests in the model as shown on Figures 2 and 7 One is an actual hurricane surge that occurred in November 1950 and the other is the Standard Project Hurricane (SPH) surge that would be produced by a hurricane of maximum recorded intensity moving over the problem area on a critical path It is important to note that the November 1950 surge with a peak of 82 feet is considerably less in amplitude but is of much longer duration than the SPH surge with a peak of 123 feet The duration of rise of the surge or the volume of the surge is critical in considering total flow through an ungated opening, and the resulting bay leve1 due to avallable bay storage

These surges were reproduced with the model water surface pooled at mean sea level For these tests, the gated openings were closed so that the passage of the surge into the bay was only through the ungated opening A comparison of levels with and wi thout Barrier P1an 3 is shawn on Tab1e 1 The maximum elevation recorded in Jamalca Bay for the Standard Project Hurricane base test was 113 feet above mean sea leve 1 for a natural bay suppression of about 1 foot Under the Barrier Plan 3, the bay elevation was reduced to 48 feet above mean sea level for an additional bay suppression of 65 feet For the November 1950 hurricane surge, the maximum elevation recorded in the bay was 84 feet above mean sea level for no natural bay suppression Under Barrier Plan 3, the bay elevation was reduced to 66 feet above mean sea level for a bay suppression of 18 feet These suppressions would have to be reduced because of the increase in bay level that would occur due to runoff from a coincidental rainfall No correction was made for wind setup

Bay Rise Due to Rainfa11 The drainage area that reaches Jamaica Bay through drainage systems or overland was determined from U S G S quadrangles as being 102 square miles of which 18 square miles would represent bay water area at mean sea leve1 A study of rainfall associated with hurricane storms and extra-tropical storms for the last 30 years showed that ralnfall accompanying the hurricane of September 1944 would yield the greatest rainfall excess The study also revealed that the bulk of the ralnfall would preceed the hurricane surge so as reasonably to assume that the rainfall excess would totally contribute to the bay rise The total rainfall for this storm would be 362 inches of which 115 inches would be rainfa11 excess on this basis, the runoff into the bay would result in a rise of 374 inches which, added to rainfall onto the bay of 362 inches, would equal 736 inches or about 06 foot rise in the bay surface

Discussion of Barrier P1an 3 The plan could meet the enviroment and navigation. objectives Further, the plan could meet the flood damage level objective of 3 feet mean sea level if the Standard Project Hurricane were the critical storm However, it is apparent from the surge tests that although the Standard Project Hurricane Surge with the higher peak is the basis for estab1ishment of the helght of the protective works, the November 1950 surge with the greater volume is critical for determining the size of the ungated opening that will obtain the no-damage leve 1 for the bay Therefore, the Barrier P1an 3 opening does not meet the zero flood damage bay leve 1 objective 
TABLE I - EFFECTS OF BARRIERS ON HURRICANE TIDES A - DIMENSIONS OF BARRIER OPENINGS FOR SURGE TESTS

\begin{tabular}{|c|c|c|c|c|c|c|c|}
\hline \multirow[b]{2}{*}{ Plan No } & \multicolumn{3}{|c|}{ Ungated Opemng a } & \multicolumn{3}{|c|}{ Gated Openıngs $a, b$} & Tota1 Opening \\
\hline & $\frac{\text { Width }}{\text { (FT) }}$ & $\begin{array}{l}\text { Depth } \\
\text { at MSL } \\
\text { (FT) }\end{array}$ & $\begin{array}{l}\text { Area Below } \\
\frac{\text { MSL }}{(\mathrm{SQ} \text { FT })}\end{array}$ & $\begin{array}{l}\text { No of } \\
\text { Gates }\end{array}$ & $\begin{array}{l}\text { Depth } \\
\text { at MSL } \\
\text { (FT) }\end{array}$ & $\begin{array}{l}\text { Area Below } \\
\frac{\text { MSL }}{(\text { SQ FT) }}\end{array}$ & $\begin{array}{c}\text { Area a of } \\
\text { Openungs Below MSL } \\
\text { (SQ FT) }\end{array}$ \\
\hline Base & 3,700 & -- & 117,750 & -- & -- & -- & 117,750 \\
\hline 6 & 110 & 33 & 3,630 & 16 & 26 & 31,200 & 34,830 \\
\hline 8 & 150 & 23 & 3,450 & 16 & 26 & 31,200 & 34,650 \\
\hline 7 & 150 & 26 & 3,900 & 16 & 26 & 31,200 & 35,100 \\
\hline 9 & 200 & 23 & 4,600 & 16 & 26 & 31,200 & 35,800 \\
\hline 3 & 300 & 33 & 9,900 & 12 & 26 & 23,400 & 33,300 \\
\hline
\end{tabular}

a - MSL = mean sea level b - a11 gates 75 feet wide

B - MAXIMUM BAY IEVELS FOR PLANS 3 AND 6 (FT MSL)

\begin{tabular}{|c|c|c|c|c|c|c|c|c|}
\hline \multirow[b]{2}{*}{ Location } & \multicolumn{4}{|c|}{1950 Surge } & \multicolumn{4}{|c|}{ Standard Project Hurricane (SPH) } \\
\hline & Without & Barrier & $\frac{\text { With }}{\text { pan } 3}$ & $\frac{\text { Barrier }}{3 \text { Plan } 6}$ & Without & Barrier & $\frac{\text { W1th }}{\text { plan } 3}$ & $\frac{\text { Barrier }}{\text { Plan } 6}$ \\
\hline OUTS IDE BARRIER & & & & & & & & \\
\hline Fort Haml1ton & 8 & 2 & 81 & 82 & & & 123 & 123 \\
\hline Parkway West & 8 & 2 & 80 & 83 & 11 & 7 & 116 & 116 \\
\hline INSIDE BARRIER & & & & & & & & \\
\hline Parkway East & 8 & 3 & 66 & 48 & 11 & 0 & 46 & 26 \\
\hline Canarsie & 8 & 3 & 67 & 50 & 11 & 3 & 48 & 29 \\
\hline Grassy Bay & 8 & 4 & 67 & 50 & 11 & 3 & 48 & 28 \\
\hline Rosie's Boats & 8 & 3 & 66 & 50 & 11 & 3 & 49 & 28 \\
\hline
\end{tabular}

C - SUMMARY OF MAXIMUM BAY LEVELS FOR ALL PLANS (FT MSL)

\begin{tabular}{|c|c|c|c|c|c|c|}
\hline Plan No & 1950 Surge & Rain Runoff* & Tota1 & Design Surge & Rain Runof f* & Tota \\
\hline Base & 84 & 06 & 90 & 113 & 05 & 118 \\
\hline 6 & 50 & 06 & 56 & 28 & 07 & 35 \\
\hline 8 & 53 & 06 & 59 & 29 & 07 & 36 \\
\hline 7 & 56 & 06 & 62 & 33 & 06 & 39 \\
\hline 9 & 60 & 06 & 66 & 37 & 06 & 43 \\
\hline 3 & 66 & 06 & 72 & 48 & 06 & 54 \\
\hline
\end{tabular}

* - computed based on 365 inch rainfall and 115 anch rainfall excess

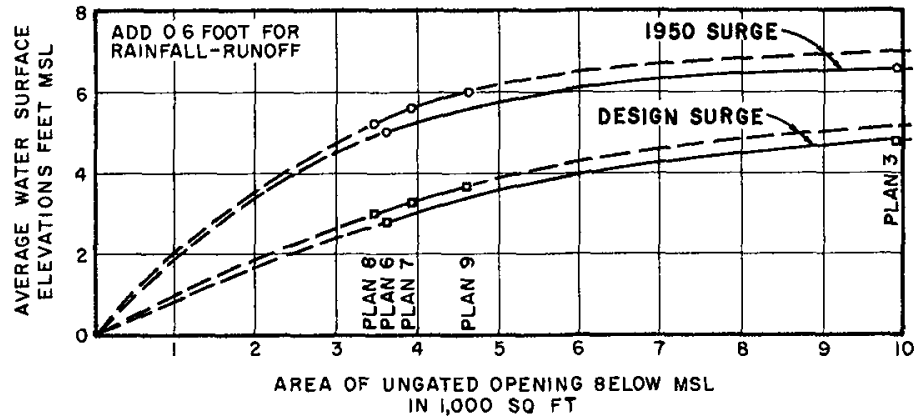


Other Barrier Plans Alternative plans were developed to meet the multiobjectives The overall open area through the barrier was generally maintalned to meet the environmental objective as to tides, currents, velocities, salinities, and diffusion and flushing of pollutants by compensating any loss in ungated area by an increase in the gated area (Table 1) The reduction in ungated area to meet the zero damage flood level was effected by reduction in width and depth of the ungated opening The principal elements of four alternative plans are shown in Table 1 The Barrier Plan 6 with 110 foot ungated opening and with all gates open had only minor effects on tides, current velocities, salinities, and dye dispersion, generally less than measured for Barrier Plan 3 The results of the suppression tests for both the November 1950 and Standard Project Hurricane surges, as shown in Table 1 , most closely meet the flood surge suppression ob jective However, the needs of commercial navigation require that the ungated opening be not less than 150 feet wide with a si11 at a depth not less than 285 feet below mean sea level From the test results for the plans tested, it is noted that elther a 110 foot wide opening at natural depth, or a 150 foot wide opening with a s111 at 23 feet below mean sea level, would hold the maximum water surface elevation in Jamaica Bay essentially at or below the critical zero damage level wider openings, or those with lower sill elevations, would have to be partially gated to insure necessary surge suppression during a recurrence of the November 1950 hurricane surge to about zero damage level in the bay

\section{CONCLUSIONS}

General Findıngs Hydraulıc model technıques, utılızıng distorted and undistorted scale models, can be used to resolve the multiobjectives with a moderate amount of field investigations to assure acceptable degrees of verification of the model required to obtain reliability and acceptability of results The results of the mathematical models were upheld The discharge coefficient of the ungated opening is fairly constant except for low discharges and submergence above 97 percent The suppression is more sensitive to width changes than depth changes The design storm for determination of height of protection must be based on a high-peak storm such as Standard Project Hurricane The design of ungated barrier openings to suppress bay levels must be based on the hurricane of critical volume, in this instance, the November 1950 hurricane Any future change in bay storage would effect the degree of suppression A bay barrier having the necessary combination of ungated and gated openings can be constructed that will simultaneously meet the requirements of hurricane surge suppression, recreational boating and commercial navigation, and enviromental and ecological objectives as water quality, recreation, and fish and wildilfe considerations

\section{ACKNOWLEDGEMENTS}

The material presented herein is the result of studies conducted by the US Army, Corps of Engineers, New York District, and model studies conducted at the Corps of Engineers Waterways Experiment Station at Vicksburg, Mississipp1 The permission of the Chief of Engineers to use this information is appreciated Corps of Engineers personne1 to whom acknowledgement is due for their assistance are colonel James $\mathrm{W}$ Barnett, District Engineer, Messrs Jesse Rosen, Herman Simensky, Joseph Tennen, Joseph Palminter1, Laszlo Maka1, and Mrs Arlene Posner of the New York District, Messrs Henry Simmons, Willıam $H$ Bobb, Thomas C Hil1, and Richard A Sager of Waterways Experiment Station Any conclusions drawn are those of the author and do not necessarily reflect the policy or views of the Corps of Engineers or the Chief of Engineers 


\section{REFERENCES}

1 New York District, Corps of Engineers - Cooperatıve Beach Erosion Control and Hurricane Study of the Atlantıc Coast of New York City from East Rockaway Inlet to Rockaway Inlet and Jamaica Bay (Interım Survey Report, 1964), printed as House Document No 215, 89th Congress, 1st Session, 1965

2 Panuzio, Frank L - The Atlantic Coast of Iong Island Proceedings of Eleventh Conference on Coastal Engıneerıng, Volume II, 1968, published by American Society of Civıl Engıneers

3 Gofseyeff, Samuel and Panuz10, Frank I - Hurricane Studies of New York. Harbor Journal of the Waterways and Harbors Division, Proceedings of American Society of Civil Engineers, February 1962

4 Wilson, Basil W - The Prediction of Hurricane Storm Tides in New York Bay Final Technical Report No 165-3, October 1959, Department of Oceanography and Meteorology, The $A$ and $M$ College of Texas

5 Tippetts-Abbett-McCarthy-Stratton, Engineers and Architects, New York Study of Hurrıcane Barriers Across Jamaica Bay for Corps of Englneers, U S Army Engineer District, New York (unpub11shed), February 1963

6 Balloffet, Armando and Kupferman, Arnold - Hydraulic Studies of Jamalca Bay Journal of the Hydraulics Division, Proceedings of American Society of Civil Englneers, November 1964

7 Coastal Engıneerıng Research Center, Corps of Engineers, U S Army, Technıca1 Report No 4 Shore Protection Planning and Design, 1966

8 Saville, J T, Mcclendon, E W, and Cochran, A L - Freeboard Allowances for Waves in Inland Reservoirs Journal of the Waterways and Harbors Division, Proceedings of American Society of Clvil Englneers, May 1962

9 Taft, Robert - Investigation of the Effects of Hurricane Surge contro1 Structures on the Water Quality of Jamalca Bay Division of Water Supply and Pollution Control, Public Health Service, U S Department of Health, Education, and Welfare, Appendix $\mathrm{N}$ of Reference No 1

10 Norman Porter Associates, Englneers - Current Velocity Measurements in Jamaica Bay on 12-13 June 1967, for U S Army, Corps of Engıneers, New York District, (unpublished)

11 U S Army, Corps of Engıneers, Waterways Experıment Station - Test Data, Mode1 Studies of Jamaica Bay Hurricane Barrier, 1970, (unpublished) 
CHAPTER 126

MODEL STUDY OF TRANSFORMATION OF TSUNAMIS IN URADO BAY

\author{
ShIgehisa NAKAMURA \\ Research Assistant, \\ Disaster Prevention Research Institute, \\ Yuichi IWAGAKI \\ Professor, \\ Department of Civil Enguneering \\ and \\ Yoshzto TSUCHIYA \\ Professor, \\ Disaster Prevention Research Institute, \\ Kyoto Unlversity, Kyoto, Japan
}

\begin{abstract}
For the city and harbour of Kochl, Including Urado Bay, facing the Pacific Ocean, an experimental study has been carried out on the problem of protection from tsunami disasters, and future harbour planning; that is, dredging, reclamation and construction of breakwaters against tsunamis. A hydraulic model of horizontal scale $1 / 250$ and vertical $1 / 100$ was used according to Froud's similitude. The transformation of the design tsunam in the bay was studied to find the effect of the tounami breakwaters, dredging and reclamations by use of the model which was able to reproduce the Chilean Tsunami.
\end{abstract}

\title{
INTRODUCTION
}

The clty and harbour of Kochl, Including Urado Bay, located in Shikoku Island facing the Pacific Ocean as shown in Fig.I, have in the past 30 years been severely damaged by several tsunamis, for instance, the one due to the Nankal Earthquake in 1946 and the Chllean Tsunami in 1960. Tounamis are malnly caused by Japan's position in a sesmic active zone so that a historioal review wall show plenty of damage by the tsunamis and their causat Ive earthquakes.

The characteristics of tsunamis have been studied theoretzcally, experimentally, and through fleld observations; but they should be studied too from the view point of coastal disaster prevention. A countermeasure has been the construction of seawalls. Recently the development of industries and of the harbour in Urado Bay have been planned, so it is necessary to study the effects of the construction of breakwaters and of the dredging andland reclamation in the bay on the behaviour of tsunamis. It 
was decided that the best approach would be conducting hydral zc model studies.

This paper deals to study the transformations of tsunamzs in the bay before and after completion of this project.

\section{TSUNAMI GENERATOR}

For this study, a tsunami generator of the plunger type was constructed, driven by an oll-hydraulic servo system. The plunger was made of steel, $7 \mathrm{~m} \times 3 \mathrm{~m} \times 0.5 \mathrm{~m}$, located as shown in Fig.2. Power Is supplied by an oll-hydraulic controller, shown in Fig.3. Transmission of an input signal is performed by a servo system, schematically given in the block diagram of Fig.l, to drive the plunger. The input signal is given by the cam of acrylite. In order to produce not only simisozdal regular waves but arbitrary waves, cams of various forms were used. According to the radius of the cam, the servo system controls the displacement and phase of the plunger through which the amplitude of the model tsunami wave is produced. By controlling the angular velocity of the cam, the perlod of the generated wave will be given. The stroke of the plunger is continuously variable and Its maximum $18 \pm 100 \mathrm{~mm}$, and the period of the cam for a cycle is variable contimuously in the range of 3 to $30 \mathrm{~min}$.

\section{SIMILITUNE AND THE MODEL}

First of all, the area of the model should be determined with consideration of tsunamis around the bay mouth.

The tsunamis which attacked this bay were from off Nankaldo, Hyuganada, the Aleutzan Islands, the Chilean coast, and so on. These tsunamis propagated and attacked the bay after refraction, deflection and rectifyzng the wave rays to the contours. The propagation maps of the tsunamis show that the fronts of the tsunamis were almost parallel to the contours in the coastal area of 15 to $20 \mathrm{~m}$ deep, whlch was taken as a criterion to limit the model area. The tsunamis which entered the bay should mun up the rivers so that the phenomena caused by the tsunamis should be found in the estuarys. The other criterion to lamit the model is to find the limit of the tidal and the tsunami influences refering to the field data.

Second, the distortion of the model should be checked in relation to the characteristics of the tsunamis. When the model experiment is carried out by use of a distorted model which has different vertical and horizontal scales, the slope of the bottom is distorted so that the reflection and refraction of the tsunamis might be generally different compared to those in the nondistorted and distorted models. And graphical analysis to give a refraction diagram of the tsunami in the bay shows a little 
difference up to the distortion of 2.5 ( $1 / 50$ of horizontal and $1 / 100$ of vertical ) with consideration of recording and processing errors of the data. It was reported that the Hilo harbour tsunam model gave a distortion 3.0 ( $1 / 600$ of horizontal and $1 / 200$ of vertical ) without effect of distortion to refraction and reflection of the tsunamis (Palmer et al., 1965).

In the estuary, saline water contacts fresh ruver water to mix vertically or to stratıfy into a stable double layer by the effect of the density difference of the waters. The river discharges flowng into the bay were so small compared to the tidal flushing that there should be prevalled vertical mixing which suggests that the consideration of the density difference might give little errors in the model experiment.

The similutude for the model was derived by the equation of motion and contimuly. Froud's similitude was applied to the model experiment; the scale ratios of the prototype to the model are 250 for horizontal length, 100 for vertioal length, 10 for current velocity, 25 for time, $2.5 \times 10^{5}$ for river discharge, and 100 for kinetic energy, respectively.

Generally, the flow regume og the prototype are turbulent so that the friction coefficient may be taken as a certain constant value. And for the model the cofficlent is a function of a Reynolds number, therefore it is difficult to hold tre similatude mentzoner riove for a rhol, peraol of experament.

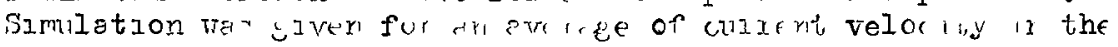
niouel rea.

Roughness of the bottom was simulated by use of Manning's formula for the current velocity as an average. The simulitude gives the scale ratıo 1.365 of Manning's roughness parameter. An artificial roughness was given in the model for simulation fulfilling the above condition. With the consideration of size distributions of sediments, the roughness parameter is 0.03 in the harbour part which is simulated as 0.022 in the model to give the roughness of 0.2 to $0.3 \mathrm{~cm}$ by brushing up the mortar surface. For the river part in the model, the roughness was given by fixing sands of 0.3 to $0.5 \mathrm{~cm}$ in size on the river bed mortar. Judging from the result of the experiment, the effect of the roughness was not distinctive compared to topographical influences.

The water level was recorder on the photographlc chart contimuously and simultaneously. For dynamical understanding, current velocity was obtalned from movies tracking floats of $1 \mathrm{~cm}$ in diameter in several areas in the model.

The model of Urado Bay is schematically shown in Fig.I for present topography and Is shown in Fig.4 as a burd's eye view. 


\section{REPRODUCTION OF CHILEAN TSUNAMI}

There are many records of damage caused by tsunam 1 inundation, there is a few records of tsunamis for Urado Bay. One of the representatiove tsunamis in Urado Bay was the Chilean tsunami of 1960 , which was recorded at three stations in the bay: Katsurahama(St.1), Urado(st.3), and Wakamatsu-cho(St.2l). The locations are shown in Fis.1. Trials were oarried out to reproduce this tsunami in the model basin. Tsunami records for the first four hours were reproduced us ing a cam as input under the dynamical consideration and repeating correction of the cam until the tsunami records of the prototype coincide with the wave records in the model experiments. One of the records is shown in Fig.5, in which the scales of elevation and time are in prototype according to the simlitude. From Fig.5 It is found that the wave forms in the model colncide well with each other in amplitude and phase as a whole. Especially, the wave form at St.l Is well reproduced. The detalls do not perfectly colnolde at $5 t .3$ and $S t .21$. At St.3, the experimental result shifts about $20 \mathrm{~cm}$ high compared to the prototype records. The distorted model might give the shift of the water level. At St.2I, the wave helght in the experiment is up to twlce as large as the tsunam records in the mareograms, which might be caused by the local topography around St.21. The river elevation increases from the end of the navigation cource to the Kagami river so that the water passing St.2l is affected by local topographical effects and shoaling effect. These detalled discrepancies between the prototype and the experiment should be studied for more accurate reproduction of the tsunami in the model experiment.

\section{DESIGN TSUNAMI AND ITS TRANSFORMATION}

Inundation from tsunamis in Kochi Harbour was studied by numerical computation ( Hamada et al., 1961). Scouring problems near the tsunami breakwaters at the entrance of KochI Harbour were studied experimentally by shibayama et al. ( 1964 ). The behaviour of tsunamis at the junctions of rivers were studied mumerically by Horıguch ( 1965). These computationg and experiments were carried out by use of a design ysunami determined by the records of the tsunami of the Nankaido Earthquake and of the ChIlean tsunam.. Hamada ( 1961 ) gave the design tsunaml at St. I such as the crest helght $1 \mathrm{~s} 2.4 \mathrm{~m}$ above and the trough $1.5 \mathrm{~m}$ below the mean high water level with the period of 30 min for the numerical computation.

Spectral analysis of the Chilean tsunami gave the significant period of 30 to $35 \mathrm{~min}$. For the Hyuganada Farthquake tsunami in 1968, the significant period was about $25 \mathrm{~min}$. 
These results suggest that the sultable period of the design tsunami is probably to be $30 \mathrm{mln}$ (Iwagakı et al., 1970). In this experiment the same conditions were given for the design tsunami. The inltial water level before the tsunami inundation was taken to be the mean high water level ( $1.1 .+1.89 \mathrm{~m})$.

The design tsunami that entered the bay propagates through the bay mouth; that is, the waves are transformed and their phases are delayed with the progress including the effects of the boundary and topography of the bay. There should be many factors affecting the transformation of the design tsunami, such as: phase velocity as a function of water depth, refraction caused by the bottom slope, deflection around a sharp edge, reflection at the coast and shoaling in the river mouth. The resultant effect of these factors gives the wave transformation. When the design tsunami at the entrance of the bay is given, the transformation of the wave in the model is obtalned through the experiment as shown in Fig. 6 in model scale. In Fig.6, wave height and time are shown in the model scale. The wave profiles are arranged ordinally for the stations, the locations of whlch are lllustrated by numbers in clrcles in this figure.

The wave profile at St.l is a simasoldal which is found the lowest part of Fig.6. The wave is transformed and decreases its helght at the narrow, the fact of which is found by comparison of the wave form at St.1, St.2 and St.5. There should be energy dissipation of the wave at the narrow caused by the confused configulation of the coast lines and to the curved water way. At the inlets, resonance occured by produce higher harmonics of the incidental tsunami. For example, the wave form at 10 suggests that the third harmonics of the tsunam is amplified in the small rectangular resonator in which the location of St.10 is included.

In the river part, the wave height increases with inundation of the tsunami as found the wave forms of St.22, 23 and 25 in Fig.6. In the Kuma river, the wave helght increases from St.23 to St.25. The wave form is transformed to be assymmetry; that 1s, the steep proflle before the crest and the gentle slope after the crest of the wave. These transformed wave profiles differ from the incldental sinusoldal wave at the entrance of the bay.

\section{CREST HEIGHT CHANGE AND TSUNAMI BREAKWATERS}

The crest height distributions of the design tounami were considered for the model of the present topography, the model without the tsunami breakwater for after dredging and reclamations, and the model with the tsunaml breakwaters for after dredging and reclamations. The distributions were obtalned as 
two dimentional or areal distrlbutzons whlch will give understanding about the complexity of the wave characteristics in the bay. In this paper, the distributions are shown only along the navigation course from the entrance to the head of the bay and along the Kagami River and Kokubu River or Kuma River, as shown in Fig.7. In Fig.7, the distances of the stations from the bay mouth are shown in reduced prototype scale in $\mathrm{Km}$ and the crest height of the tsunami in meter with reference to the elevation of the mean high water level. The locations of the stations in Fig.7 are shown by the numbers in circles. The dots, the circles and the semi-circles show respectively the case for the present topography, the case for after dredging and reclamations, and the case with the tsunami breakwaters for after dredgins and reclamations. The model for the conditions after dredging and reclamations is shown in Fig.8. Tsunami breokwaters are under planning for construction at the narrow point of the entrance of the bay, as shown in Fig. 8 .

The crest helght distributions are shown in Fig. 8 al ong the navigation course and the Kagami River. Comparing to the distribution for the present topography, the distribution for after dredging and reclamations suggests that the tsunami propagates with a little decrease of wave height because of water depth increase. But it is not clear why the crest helght at St, 2 beoame anomalously high after dredging. The effect of the reclamations is not so remarcable for the crest helght distribution. When the tsunami breakwaters are constructed for the harbour after dredging and reclamations, it is found a remarkable effect of the tsunaml-break-waters to diminish the wave helght in the bay and to protect the harbour from tsunami inundations around the bay mouth and in the bay. For the crest helght distributions, the effect of the tsunami breakwaters is not remarcable at the end of the navigation course. In the Kagaml River, there should be found a topographical effect for the tsunami inundations.

About the Kuma River or Kokubu River, the crest helght distributions are shown in the up-right part of $F_{18.7}$ to compare those to the main profiles of the crest helght in the same scale. There are found a little effect of the tsunami breakwater, dredging and reclamations in the profiles of the crest height distributions of the design tsunami.

\section{CONCLUSION}

From the results of the model experiment on tsunamis in Urado Bay, the authors obtalned the following conclusions.

1) By use of the tsunami generator with consideration of Froude's simllitude, the Chilean tsunami was reproduced in the model experimant. This result shows the possibllity of studying future problems in the bay. 
2) Transformation of the design tsunami and crest height distribution of the tsunami were studied to find the negative effect of dredging of the navigation course and to find the active effect of tsunami breakwaters for protection of the harbour from tsunami imundation. Reclamations gave a little influence on the crest height distribution and on the wave height of the tsunami.

Kochl was attacked directly by the typhoon 7010 on 21 , August, 1970 so that they suffered from heavy damages by the storm surge accompanied with the typhoon. Thls fact suggests that It should be promoted not only to study on tsunam but to investigate on storm surges in Urado Bay. Future plan of the harbour should be refered on the studies mentioned above to protect from coastal disasters.

\section{ACKNOWLPDGEMMENT}

The authors wish to express their thanks for the help given by the Manlstry of Transport and Kochl Prefecture.

\section{REF'ERENCES}

Hamada,T., Horıguchı,T., Kato,H. and Kaneko, 1". (1961). Calculation of tsunami inundation in a channel - - a case of Kochl Harbour ---, Proc. 8th Conf. Coastal Eng. In Japan, pp.30 - 35 ( In Japanese ).

Hor 1 guchı, T. and Ko, R。 (1965). Calculation of tsunamı Imundation in bay and river junctions, Proc. 12th Conf. Coastal Fing. In Japan, pp.14 - 18 ( In Japanese).

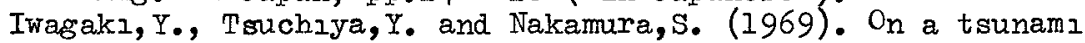
generator, Proc. 16th Conf. Coastal Eng. in Japan, pp.321 - 326 ( In Japanese).

Iwagakı, Y., Tsuchıya, Y. and Nakamura, S. (1970). Tsunamı model experiment of Kochl Harbour, Ann. Dis. Prev. Res. Inst. Kyoto University, No. 13 B, pp.47I - 488 (in Japanese).

Palmer,R.Q., Malvihlli,M.E. and Funasakı,G.T. (1965). H1lo Harbour tsunami model - - reflected waves superimposed, Castal Eng., Santa Barbara, Sp. Conf., ASCE, pp.21 - 31.

Shlbayama,H. Kimura,H. and Takemura,K. (1964). Model exper1ment on tsunami breakwater of Kochl Harbour, Rep. Port and Harbour Res. Inst., MInistry of Transport, Vol.3, No.2, pp.14 - I8 ( In Japanese). 


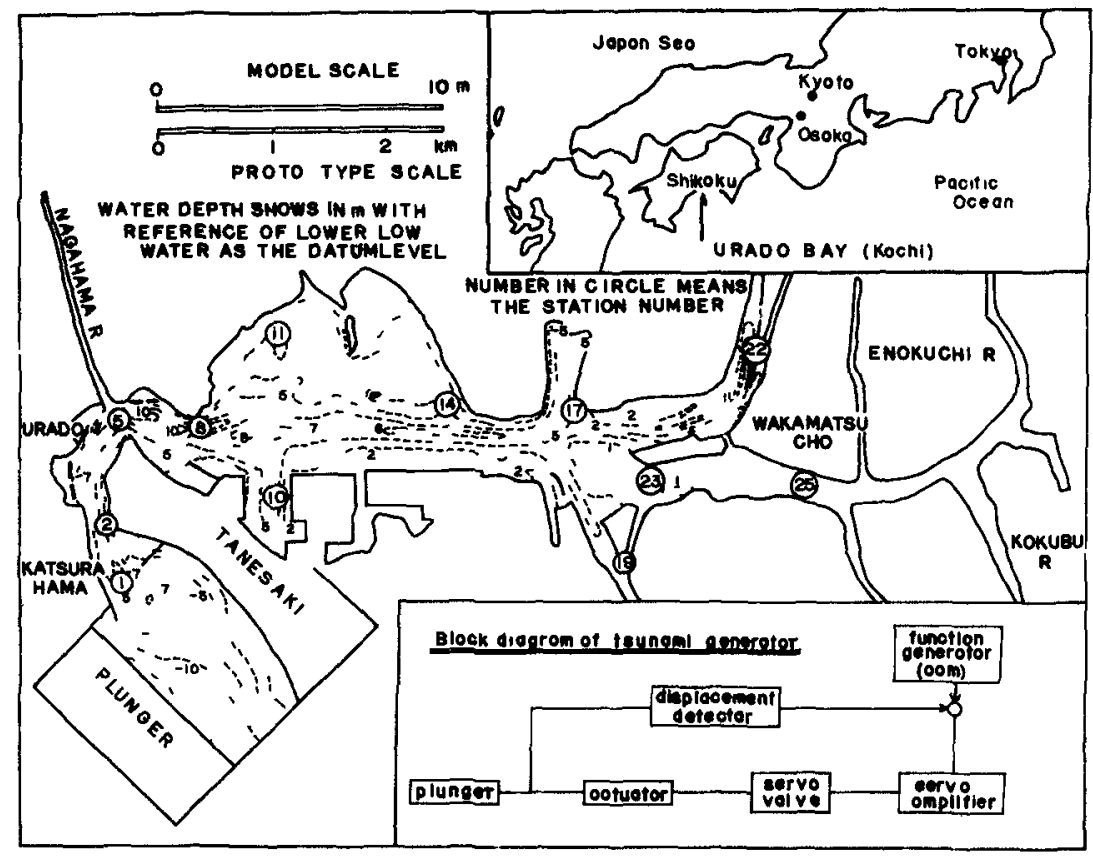

FIg.1 SCHEMATIC PLANE OF THE TSUNAMI MODEL OF URADO BAY ( Including locations of stations where water levels were recorded in the tsunaml model, and tide gauges are located at St.2, St.3, St.7, St.17 and St.21 in the prototype of the harbour) UP-RIGET: IOCATION OF URADO BAY DOWN-RIGET: BLOCK DIAGRAM OF THE SERVO SYSTEM 


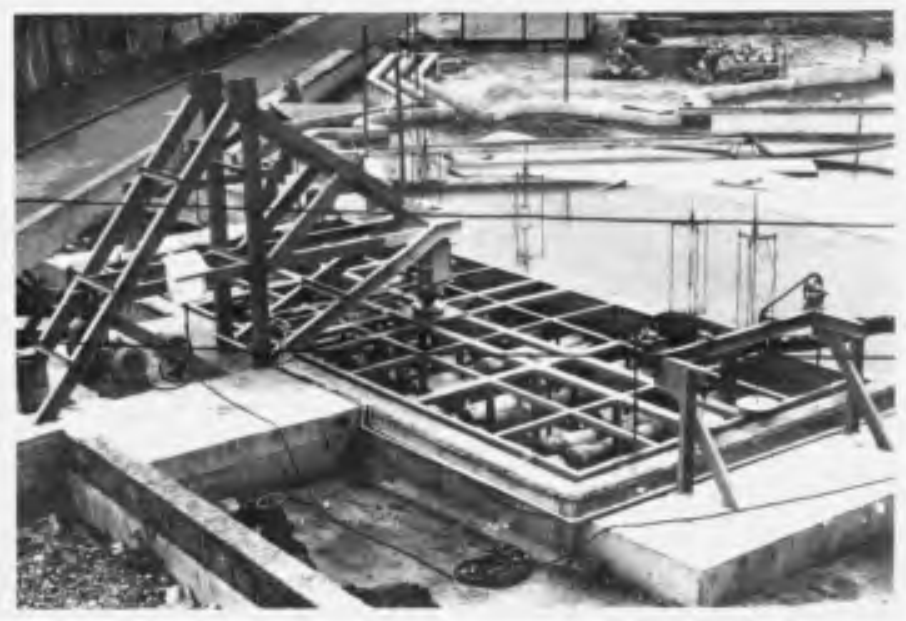

Pig. 2 PLUNGER OF THE TSUNAMI GENERATOR

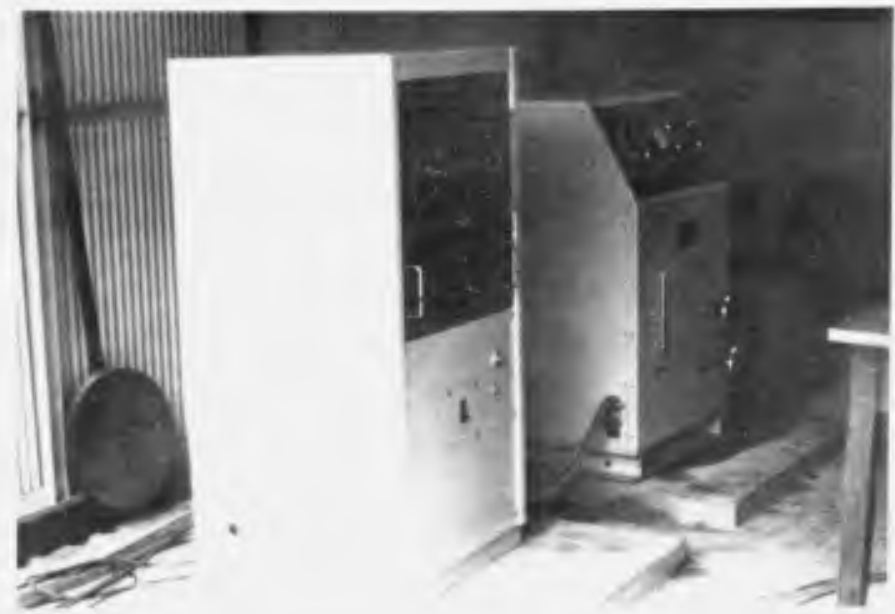

Fig. 3 OIL-HYDRAULIC CONTROLLER OF THE TSUNAMI GENERATOR 


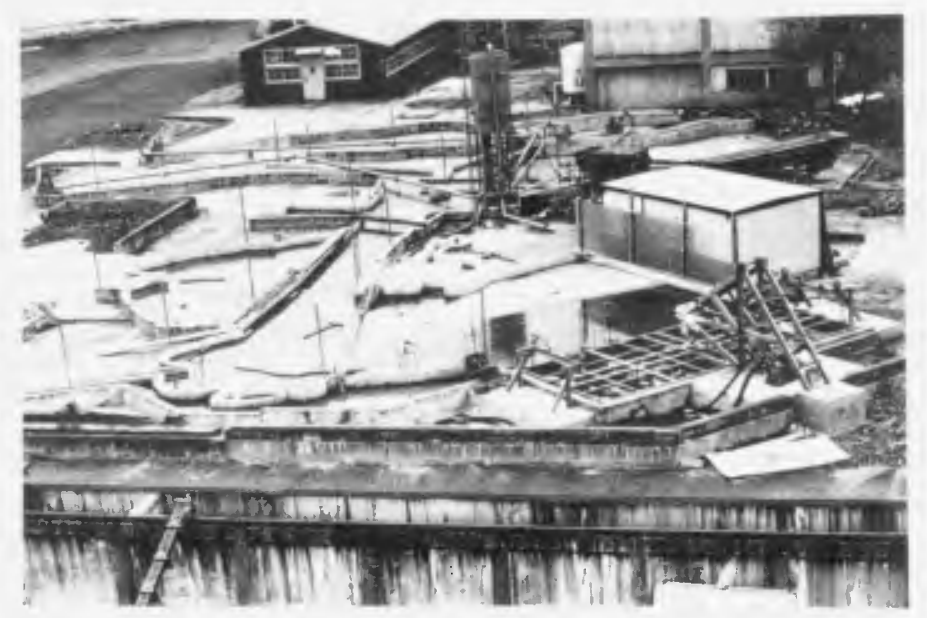

Fig.4 BIRD'S EYE VIEW OF THE TSUNAMI MODEL ( The plunger shown in Fig. 2 is at the end of the model. The controller is in the white cottage. The head tank at the center of this photograph supplys river discharges in the model.) 


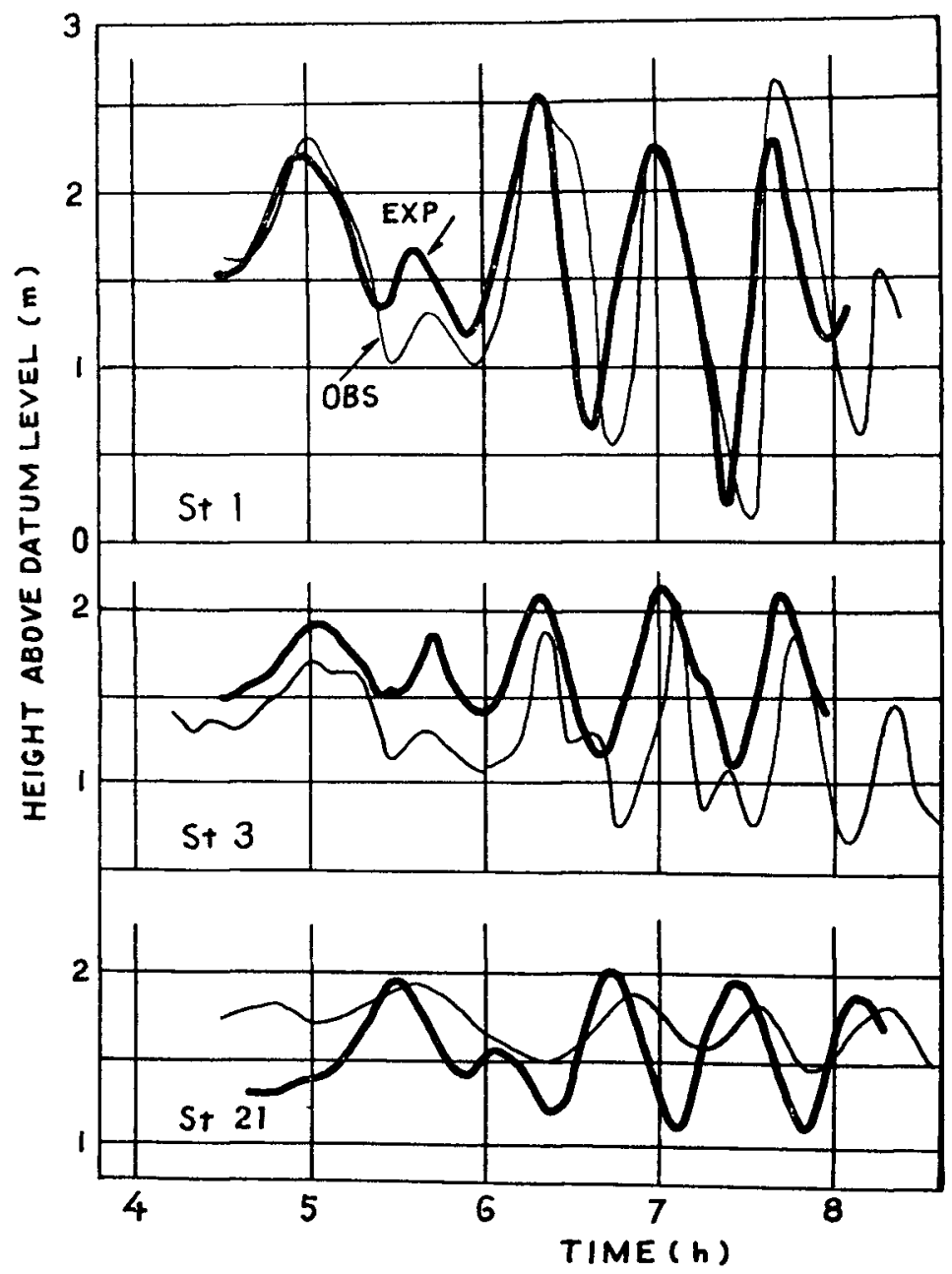

FIg. 5 RFPRODUCTION OF THE CHILEAN TSUNAMI IN URADO BAY 


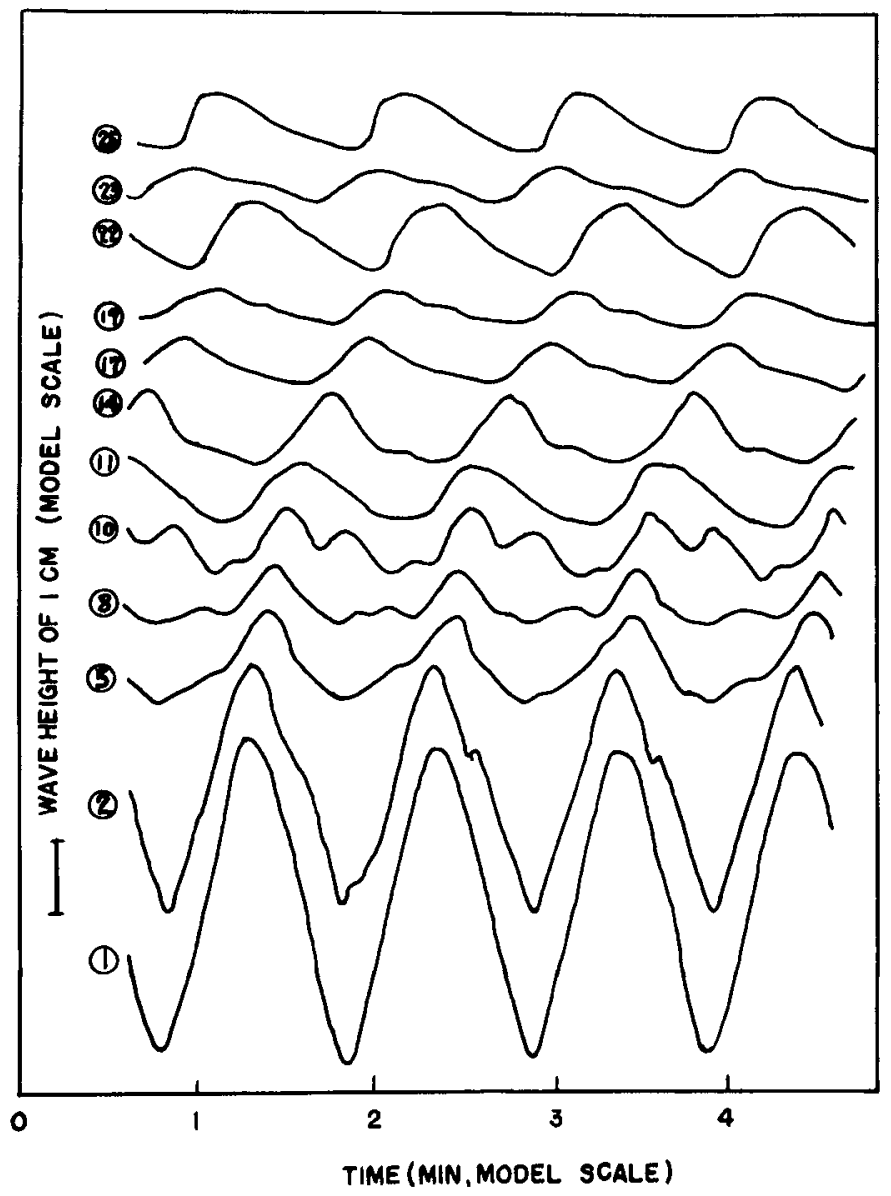

F1g. 6 TRANSFORMATION OF A DESIGN TSUNAMI 


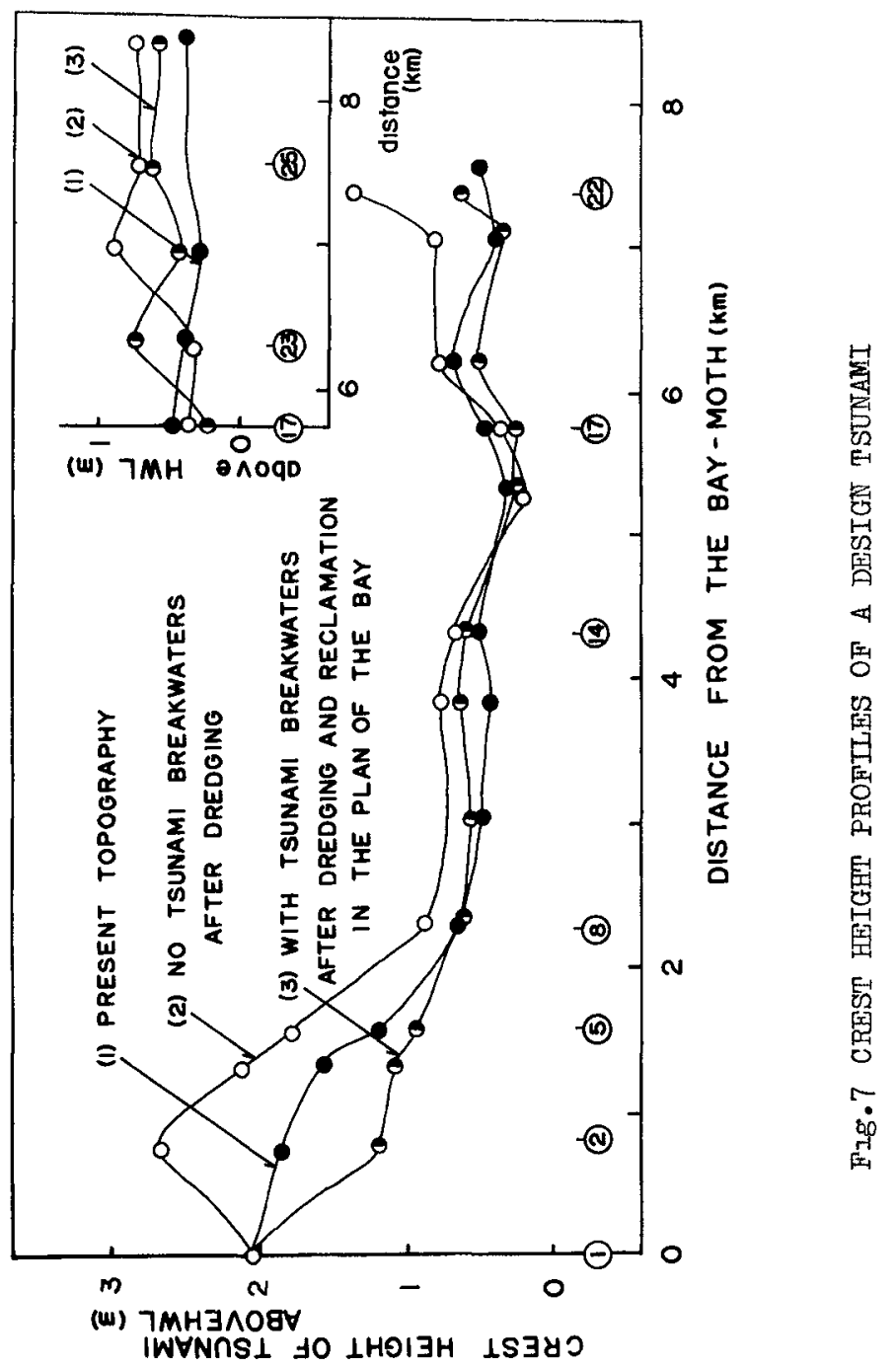




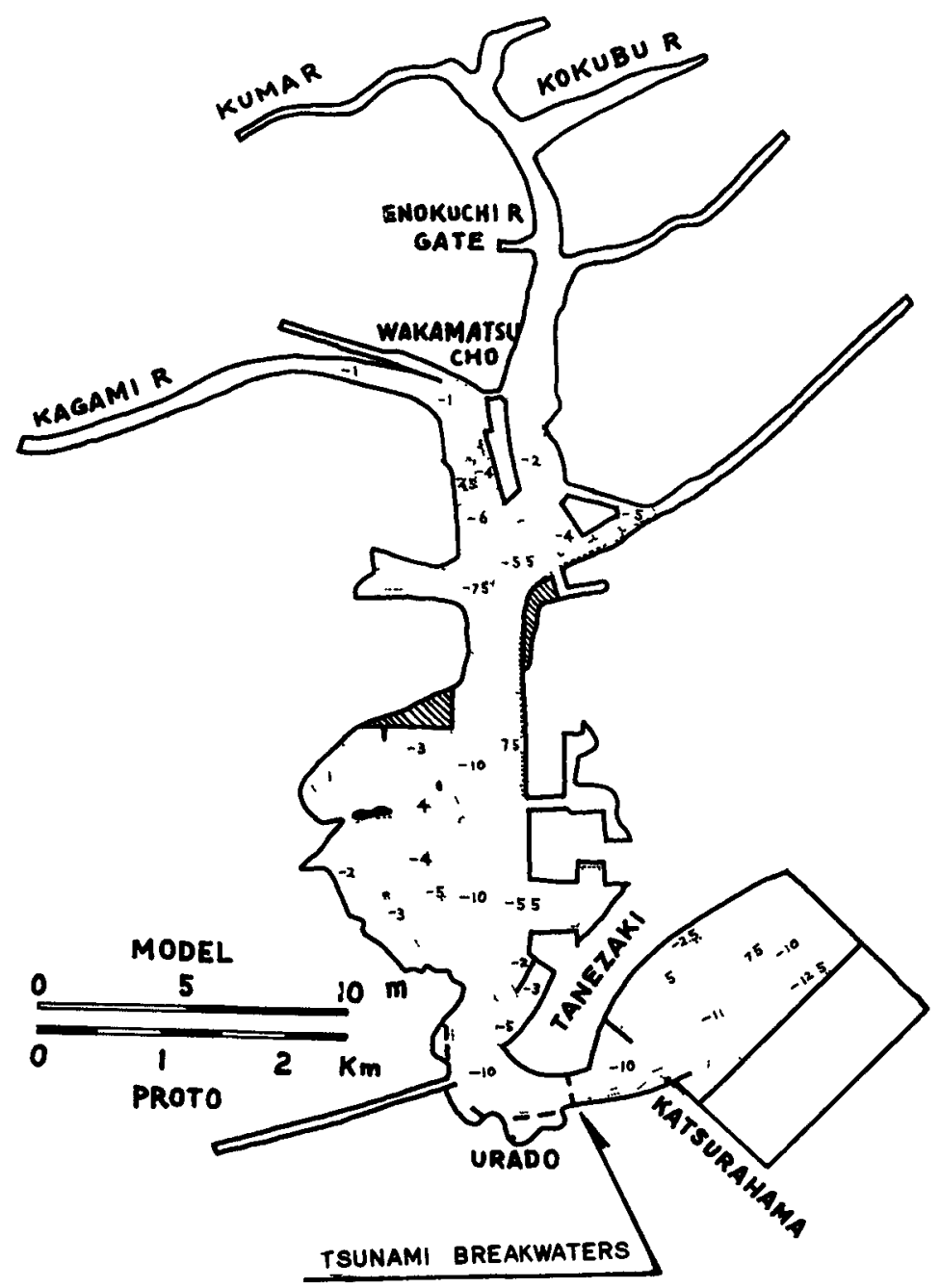

FIg.8 TSUNAMI MODEL AFTER DREDGING AND RECLAMATIONS ( The location of the tsunami breakwaters 18 indicated by an axrow.) 


\section{CHAPTER 127}

\section{TSUNAMIS SOME LABORATORY AND FIELD OBSER VATIONS}

by Fredric Rachlen Assoc Prof of Civil Engineering

$W M$ Keck Laboratory of Hydraulics and Water Resources, California Institute of Technology, Pasadena, California, U S A

\section{ABSTRACT}

A unique laboratory facility to generate waves by impulsive movements of the bottom of a wave tank is described and examples of the wave forms which result are shown An analytical model is presented for the case of rapid bottom movements which describes, in a qualitative way, certain features of the experiments

Marigrams from various field stations for the tsunamis from the Chilean earthquake of 1960 and the Alaskan earthquake of 1964 have been analyzed using spectral analysis techniques to determine the harmonic components of these records Comparisons are made between the spectra of the two tsunamis at each of several locations

\section{INTRODUCTION}

The problems associated whth earthquake generated sea waves, known as tsunamis are serious in many parts of the world particularly around the rim of the Pacific Ocean which 1 s such a seismically active region Perhaps one of the worse tsunamis of historical times was generated off the coast of Japan on June 15, 1896 The maximum wave which was generated ran up on the nearby 1 and to an elevation of $75 \mathrm{ft}$ to $100 \mathrm{ft}$ above the normal tide level resulting in the death of more than 27,000 persons and the destruction of over 10,000 homes A much more recent example of the widespread property damage that such a wave system can cause is the tsunami associated with the Alaskan earthquake of March 27, 1964 Th1s tsunami wh1ch or1ginated in the Gulf of Alaska caused loss of life and extensive damage as far away as Crescent City California. (\$11 million damage) In addition to this distant damage, an estimated $\$ 350$ million damage to a number of coastal towns in Alaska could be attributed to the combined effect of the tsunam1, soil failure and vibrations

To learn more about the generation, propagation, and coastal effects of the tsunamis a laboratory study has been initiated which deals with the generation of waves by impulsive bottom movements and the propagation of these waves in the near field (The near field region is of particular importance to Southern California which appears to be reasonably protected from distant tsunamis, but may be vulnerable to sea waves generated by earthquakes near the coastline) This investigation is directed toward understanding the phenomenon of bottom generation and the effect of linear and nonlinear dispersion on the resultant wave system Some preliminary results of the laboratory study are presented herein, thereby demonstrating some of the characteristics and capabilities of the wave generating system which has been developed

In addition to this laboratory program, field data have been analyzed to gain a better understanding of the interaction of tsunamis with the coastline 
Using spectral analysis techniques certain conclusions can be drawn regarding the effect of the tsunam which resulted from the Alaskan earthquake of 1964 and that resultang from the Chilean earthquake of 1960 on the harbors and embayments where tidal records are avalable Hence, this paper is divided into two parts the first dealing with the laboratory simulation of earthquake generated sea waves and the second dealing with the analysis of field data.

\section{LABORATORY OBSERVATIONS}

Experimental Equipment - The expermental program is being conducted in a wave tank which is $316 \mathrm{~m}$ long, $39 \mathrm{~cm}$ wide, and $61 \mathrm{~cm}$ deep with glass sidewalls throughout The impulsive wave generator is located at one end of the wave tank and consists, in the initial phases of this study, of a moveable section of the bottom of the wave tank $61 \mathrm{~cm}$ long and nearly the same with as the wave tank $(39 \mathrm{~cm})$ The section of the bottom can be moved in a predetermined fashion exther upwards or downwards, hence, this system can be thought of as modeling a tectonic movement in nature which is either a simple block upthrust or downthrow In the laboratory the maximum movement of the bottom in a vertical direction is $\pm 15 \mathrm{~cm}$ with one of the design criterion being that the bottom should be capable of moving $\pm 3 \mathrm{~cm}$ in $005 \mathrm{sec}$ With some modifications the surface area of the generator can be changed

A schematic drawing of the tsunami generator and the wave tank is presented in Fig 1 It should be noted that the wave system under investigation is two-dimensional and the rigid wall which exists at $x=0$ represents a plane of symmetry in the true problem Therefore, with reference to Fig 1 , the total length of the moveable section of the bottom, $l$, is one-half of the length of the block in nature which is being modeled

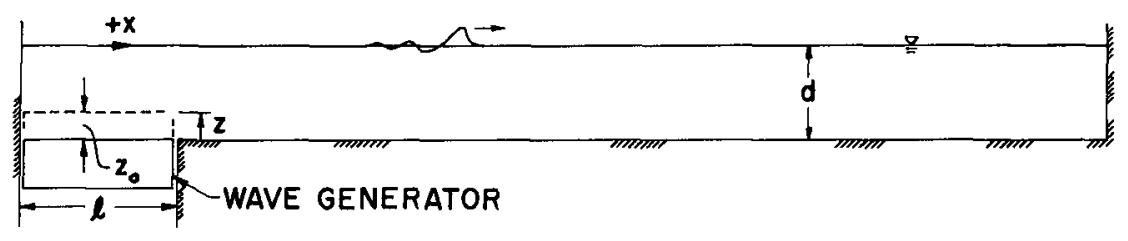

Fig 1 Schematic Drawing of Impulsıve Wave Generator

The wave generator is driven by a hydraulic-servo-system which accepts an input voltage which is proportional to the desired displacement of the bottom, and in this way a variety of bottom motions can be obtained Basically the syst $\in m$ can be divided into three parts the hydraulic supply unit, the servosystem, and the moveable bed unit assembly

The hydraulic supply system consisting of oll reservoir, pump, filter, and heat exchange unxt supplies the mann hydraulic cylinder which actuates the bed with hydraulic flud at a pressure of $3200 \mathrm{psi}$ An accumulator is installed in the system primarily to provide a sufficient supply of hydraulic fluid at high pressure to drive the bed for programs which initially demand large flow rates 
The servo-system consists of a servo-valve and its associated electronics used in conjunction with a linear variable differential transformer (LVDT) which senses the motion of the bed The LVDT with its core mounted to the moving bed and the coil fixed in position provides the electrical feed-back signal which is compared to the input signal supplied by the function generator, by comparing these two signals minor adjustments of the movement of the spool of the servovalve are made automatically to achieve the desired program of bed movement

A photograph is presented in Fig 2 showning in detall the moveable bed unit assembly consisting of the hydraulic cylinder, its supporting structure, two flexures, the load cell, the guide cylinder, the bearing support box, and the bed unit The hydraulic cylinder which drives the bed is a double-throw type mounted vertically and attached by means of flexures to the laboratory floor and to the bed unit The flexures provide a means of correcting for any small vertical misalignment of the hydraulic cylinder upon installation The upper flexure 15 connected to a load cell, for measuring the total applied force, which in turn is connected to the moveable bed unit The guide cylinder, seen in Fig 2 moves against two fixed bronze bearings and insures that the bed unit moves in a vertical direction The bed unit is firmly fixed to the upper surface of the guide cylinder

Fig 3 shows the tsunami gencrator attached to the upstream section of the wave tank wh the bed unit in its maximum upward position A problem in the design of the generator which had to be overcome for successful operation was the sealing of the bed unit around its four sides two of which are in proximity to glass walls and the other two near machined alumınum surfaces The seal which was designed and fabricated was a one-piece unit molded of a relatively flexible material and mounted directly to the moveable portion of the bed Detals of this seal can be seen in both Figs 2 and 3, this type of design (with water on only one side of the generator) minimizes the forces which arise in wave generation

Some Experimental Results - The time-displacement of the bed which 1s being used initially is an exponential motion described by the following expression

$$
\frac{z}{z_{0}}=1-e^{-\alpha t}
$$

where $z$ is the motion of the bed measured positive upwards from the fixed elevation of the bottom of the wave tank, $z_{0}$ is the maximum motion of the bed, varying time is represented by $t$, and $\alpha$ is ${ }^{\circ}$ a coefficient which 1 s equal to the bed velocity divided by the maximum bed dis placement, $z_{0}$ at $t=0$

Figs 4 and 5 are examples of the water surface motions which are generated by such exponential motions of the generator for a water depth of $10 \mathrm{~cm}$ and for positive and negative motions respectively The ratio of the maximum displacement to the depth, $z_{o} / d$, for all cases shown was 01 , and for positive and for negative motions examples are presented for two different non-dimensional times, $t_{R}(g / d){ }^{1 / 2}$, the time $t_{R}$ is defined as the time that it takes the bed to rise to one-half its maximum value The two values of the non-dimensional time for which results are shown in $\mathrm{F} 1 \mathrm{~g} \quad 4$ and 5 are $t_{R}(g / d)^{1 / 2}=04$ and 35 which, for these experiments, correspond to $\alpha=172 \mathrm{sec}^{-1}$ and $196 \mathrm{sec}^{-1}$ respectively The motion of the bottom is shown as the upper record in each of these figures and is labelled as $z$ 


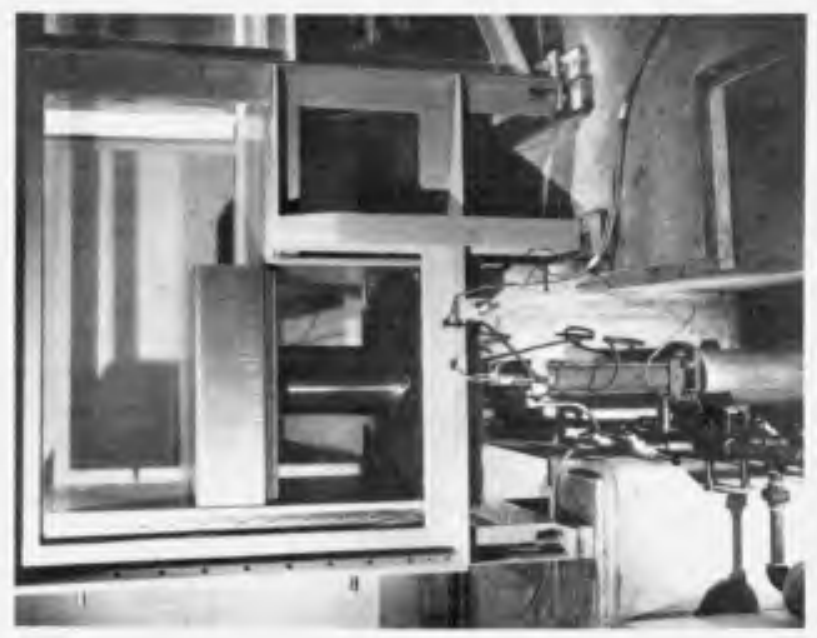

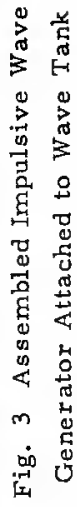
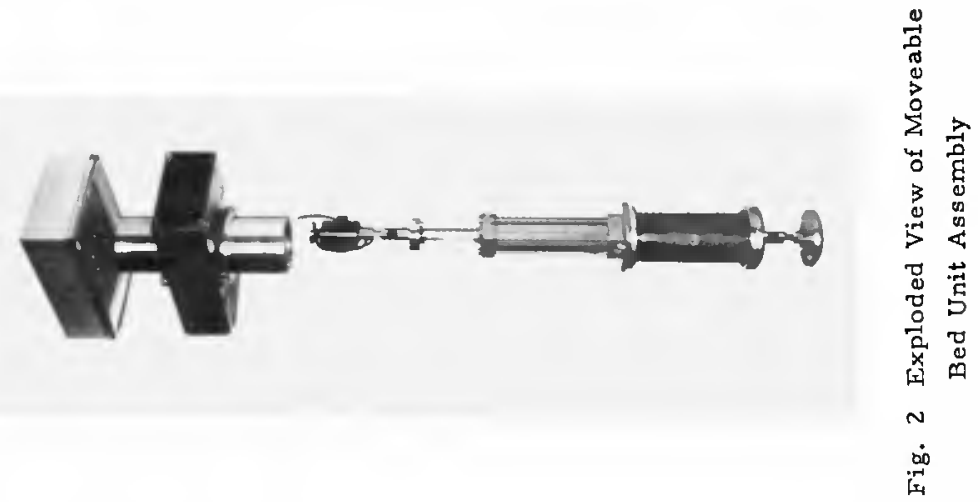


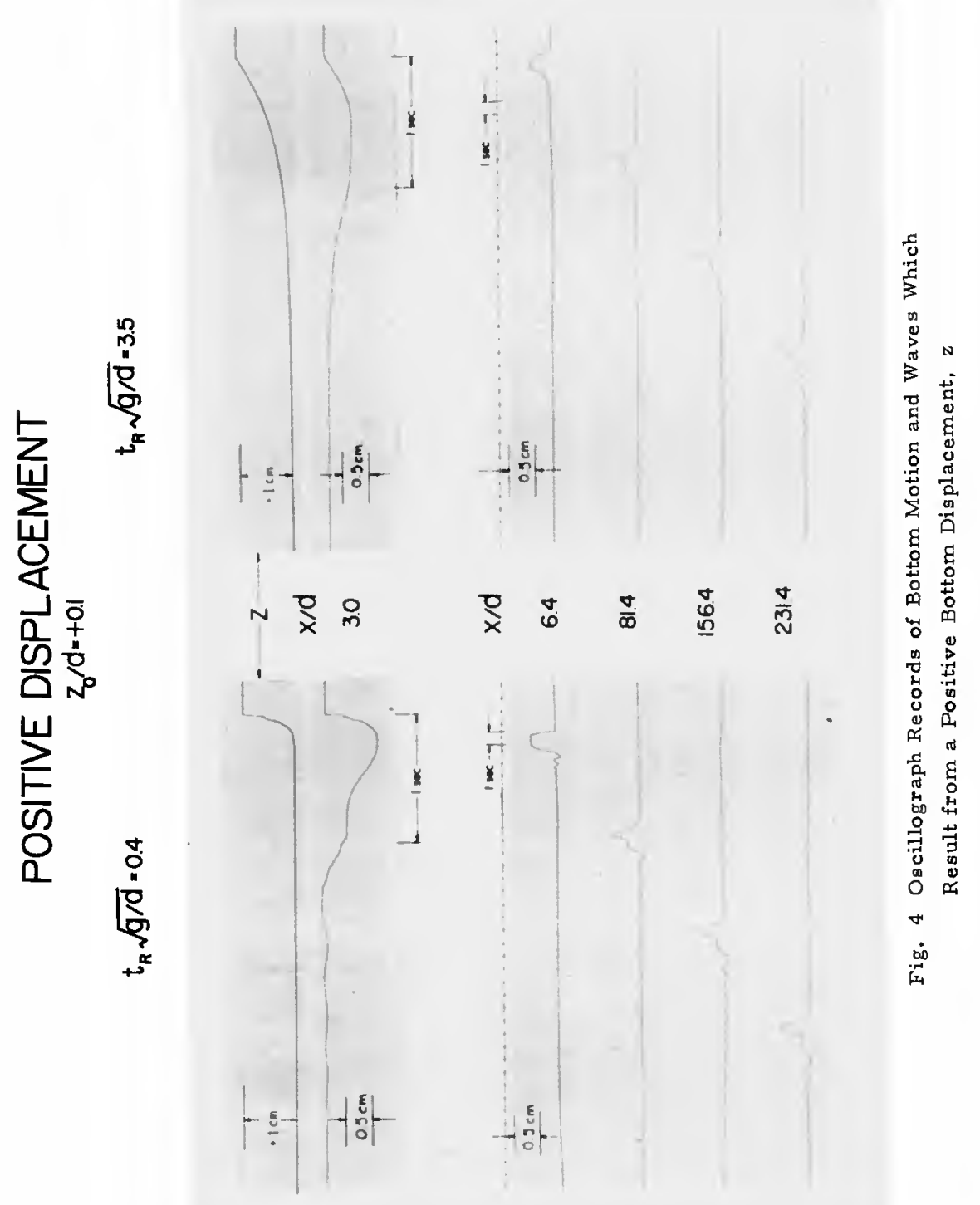




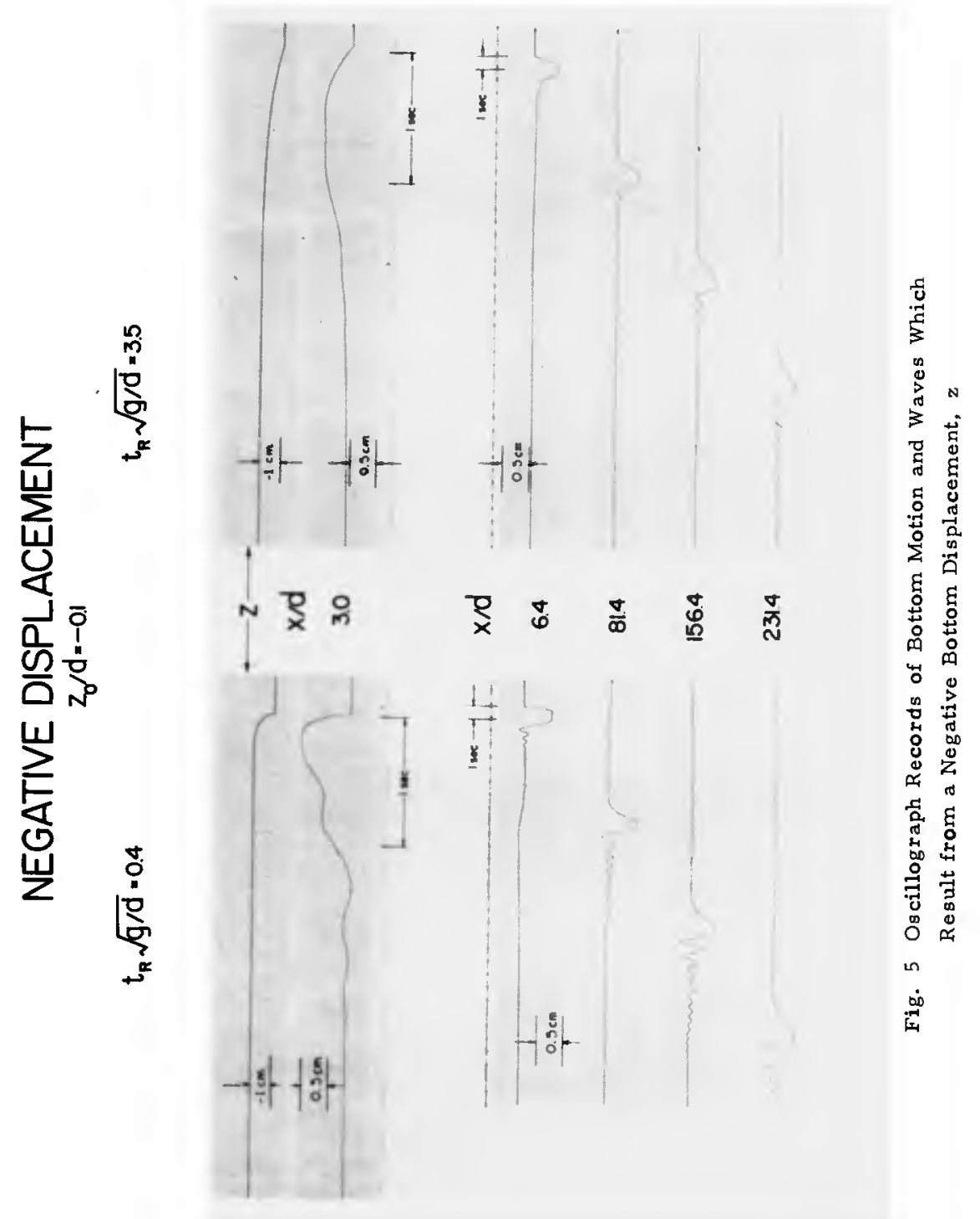


In Figs 4 and 5 the variation of the water surface with time measured using resistance wave gages is shown at five different locations along the wave tank The first location is $\mathrm{x} / \mathrm{d}=30$ which is approximately midway over the moveable bottom (Since the wall at $x / d=0$ also can be thought of as a plane of symmetry for the case of a moving block of length $2 l$ centered at $x / d=0$ for this depth the location $x / d=30$ represents the quarter-point of that moving block, 1 e $x / 2 \ell \approx 025$ ) It should be noted in the osc1llograph records which are shown, for the motion of the bottom ( $z$ ) and for the variation of the water surface at the first location $(x / d=30)$ positive movements and water surface displacements are downward at all other locations the positive water surface displacements which are shown on the oscillograph records are upwards In all of the records time increases to the left, the length of the record which corresponds to one second is indicated in the figure Four of the wave gages are equally spaced $(x / d=64,814,1564$, and 2314$)$ with the first gage located quite close to the "leading edge" of the moving bottom Since the electronic amplification of the various wave gages has been adjusted to be approximately the same and the gages are equally spaced, a characteristic diagram could be constructed from these four records

In Fig 4 for the two different non-dimensional times, the upper records show the exponential motion of the bottom At the location $x / d=3$ for the condition of rapid bottom movement the water surface rises in nearly the same way as does the bottom and begins to fall near the time of maximum bottom displacement For the experiment with a slower bottom motion the wate $r$ surface at the same location reaches a maximum before the bottom has fully risen and then begins to fall For the former case the ratio of the maximum amplitude to the maximum bottom displacement is approximately unity whereas for the latter this ratio is about one-half thus, one effect of the magnitude of the non-dimensional time, $t_{R}(g / d)^{1 / 2}$, can be seen

For a non-dimensional time of 04 , near the edge of the generator $(\mathrm{x} / \mathrm{d}=$ 6 4) the water surface rises quickly to a maximum and remains there for a short period of time before falling In falling, the water surface elevation first becomes less than the still water level, then rises above the still water level and then oscillates in a damped periodic manner about the quiescent level As the lead wave progresses downstream it tends to change radically as additional waves are generated from it In addition to this, groups of waves are evident in the lee of the main wave system and these waves appear to be left further behind in time as the distance from the generation area increases probably due to frequency dispersion

Similar effects can be seen for the example at a larger non-dimensional time (3 5) except that the dispersive effects are not nearly as pronounced as for the case of the faster bottom movement Comparing the amplitude of the lead wave for locations $x / d \geq 6 \quad 4$ to that at $x / d=30$ it 1 s seen that the waves are approximately 08 of the amplitude of the wave over the generator For the case of a more rapid bottom movement this same ratio was about 05 demonstrating another effect of the velocity of the generator on the waves generated

The comparable case for negative waves is presented in Fig 5 The conditions for the two cases shown are identical to those presented in Fig 4 except a negative motion of $z_{0} / d=-01$ is used In this case, for the 
displacement of the bottom and of the water surface at $x / d=30$ negative $d x$ placements are upwards on the oscillograph paper, at all other locations negative displacements are downwards Comparing the water surface displacements in Figs 4 and 5 at $x / d=3 \quad 0$ and 64 for both non-dimensional times it is seen that the negative displacements are nearly mirror images of the positive displacements However, for a normalized distance of 814 major differences are seen between the positive and negative cases Perhaps the most obvous is the significant growth of a train of waves in the lee of the negative system compared to the positive case which supports the arguments proposed by Keulegan and Patterson (1940) in their paper dealing with non-linear long waves The effect of dispersion on the lead wave can be seen readily in Fig 5 for the example whth the rapid bottom motion from the decrease of the slope of the leading edge of the main wave as the distance from the region of generation increases

Similar features are evident for the case of a slower bottom movement, 1 e , a larger non-dimensional time (3 5) Again the features in the lee of the main wave have grown much more rapidly than their positive counterpart If a comparison is made of the amplitude of the lead wave over the generator to those for $x / d \geq 64$, for the case of rapid motion it is found that away from the generator the amplitude is approximately 06 of that over the generator For the slower generator motion this ratio varies from about 085 to 090 The ratio of the amplitude of the lead wave to the maximum displacement of the generator for the two examples of negative displacements are comparable in magnitude to the corresponding positive cases Thus, with respect to wave amplitudes there is little difference between the lead wave amplitude resulting from the negative and positive bottom motions for comparable initial conditions

Some Analytical Considerations - To investigate, in a qualitative sense, some of the features of the wave systems observed in Figs 4 and 5 an analytical method developed by Peregrine (1966) for the treatment of non-linear long waves has been applied The equations of continuity and momentum can be written in nondimensional form as

$$
\begin{gathered}
\frac{\partial n}{\partial t}+\frac{\partial}{\partial x}[(1+\eta) u]=0 \\
\frac{\partial u}{\partial t}+u \frac{\partial u}{\partial x}+\frac{\partial \eta}{\partial x}=\frac{1}{3} \frac{\partial^{3} u}{\partial x^{2} \partial t}+0\left(\varepsilon^{2} \sigma^{3}\right)
\end{gathered}
$$

All length quantities in Eqs 2 and 3 have been normalized whth respect to the depth d, the velocities with respect to the shallow water wave velocity, (gd) $)^{1 / 2}$, and time by multiplying by $(\mathrm{g} / \mathrm{d})^{\mathrm{I} /} \mathrm{z}$ (In the following discussion the same notation as used in Eqs 2 and 3 will be used to denote dimensional and dimensionless quantities the meaming will be clear from the context of the discussion) In Eqs 2 and 3 the distance $\eta$ to the water surface is measured from the still water level, the velocity $u 1 s$ the horizontal velocity averaged over the depth, $\sigma$ is the ratio of water depth to wavelength, $\varepsilon$ is the ratio of wave amplitude to water depth, and the notation $0($ ) indicates terms of the order of the quantity in parentheses and smaller The solution of these two equations allows for waves traveling in the positive and the negative $x$-directions, Peregrine's finite difference $s$ cheme was used for the numerical solution

The problem of waves which are formed by an impulsive movement of the bottom was then treated as an intial value problem where the flud in a long tank is unitially at rest everywhere and a mass of fluxd is added to (or subtracted 
from) the still water surface over a portion of the tank at $t=0$ This theoretical model is crude in the sense that the movement of the bottom is not included instead an assumed form of the water surface $1 \mathrm{~s}$ chosen at a time near the end of the wave-maker stroke and before the wave has left the generation area (defined for this analysis as $t=0$ ) The assumption that the horizontal velocity is initially zero everywhere just when this surface disturbance is formed is probably reasonably close to the experimental conditions for rapid bottom movements In addition to these features of the analysis, similar to the laboratory conditions, the two ends of the tank in the theory are considered to be solid walls wath zero water particle velocity at these walls for all time This type of intial value problem is similar to that treated by Long (1964) using basically the same expressions as Eqs 2 and 3 but manipulated in such a way that solutions could be obtained by integrating along the characteristics in the $(x, t)$ plane

The intial amplitude distribution of the water surface which was used was

$$
\frac{\eta}{d}= \pm 0 \quad 05\left[1-\tanh \left(\frac{x}{d}-6\right)\right]
$$

From Eq 4 it is seen that for $x / d<3$ the amplitude of the water surface is essentially constant and approximately equal to 01 and for $\mathrm{x} / \mathrm{d}>9$ the amplitude is essentially zero Such an expression allows for a smooth transition from one water surface elevation to another for the conditions chosen the length of the moveable bed corresponds to $\mathrm{s} / \mathrm{d}=6 \quad$ With reference to Figs 4 and 5 it is evident that $\mathrm{Eq} 4$ compares more favorably with the example which corresponds to the rapid bottom movement Consider first the amplitude at $\mathrm{x} / \mathrm{d}=3$, Eq 4 gives this amplitude as essentially 01 times the depth In Figs 4 and 5 the amplitude at this location for the case of $r$ apid motions is approximately the same as the maximum bed motion which is, in relative terms, $\mathrm{z}_{\mathrm{o}} / \mathrm{d}= \pm 0 \quad 1$ From Eq 4 the relative amplitude at $\mathrm{x} / \mathrm{d}=6$ is \pm 0 o5 whch corresponds reasonably well to the observed values of t0 046 and -0057 at about this location in the experiments for $t_{R}(g / d)^{1 / z}=04$ Thus, although this representation of the water surface is perhaps crude, it does retain some of the important features of the observed distributions

The analytical results for the case of the positive disturbance are presented in Fig 6 where the variation in the water surface displacement, $\eta / d$ is plotted as a function of non-dimensional time $T=t(g / d)^{1 / a}$, for various values of normalized distance, 1 e $\mathrm{x} / \mathrm{d}=0 \quad 3,10203040$ A number of interesting features of the problem emerge from this analytical treatment and these will be dis cussed separately before comparing them to the experimental results At $x / d=0$ the wave amplitude decreases with time from the initial value of $\eta / d=0 \quad 1$ to a magnitude less than zero and then oscillates with time about the still water level with the amplitude of oscillation decreasing with time and approaching zero At $x / d=10$ the water surface increases gradually with time from zero reaches a maximum, decreases and then increases slightly before decreasing and approaching zero Hence, a second wave appears to be forming from the lead wave An additional interesting feature of Fig 6 is that another group of waves appears in the lee of the main wave for $\tau>30$ Both of these occurances is a strong indication of the dispersive nature of such a wave system For larger values of $\mathrm{x} / \mathrm{d}$ the main wave appears to begin to move out in front, leaving behind the secondary wave system If the computer storage had been sufficient to enable the computations to be made for a larger tank and thus to proceed to large values of $\mathrm{x} / \mathrm{d}$ and $\mathrm{T}$ it might have been possible to see the lead wave approach a solitary wave in form As it is, because of this limitation the reflection of the wave from the far tank wall can be seen at $x / d=40$ for $T>80$ 


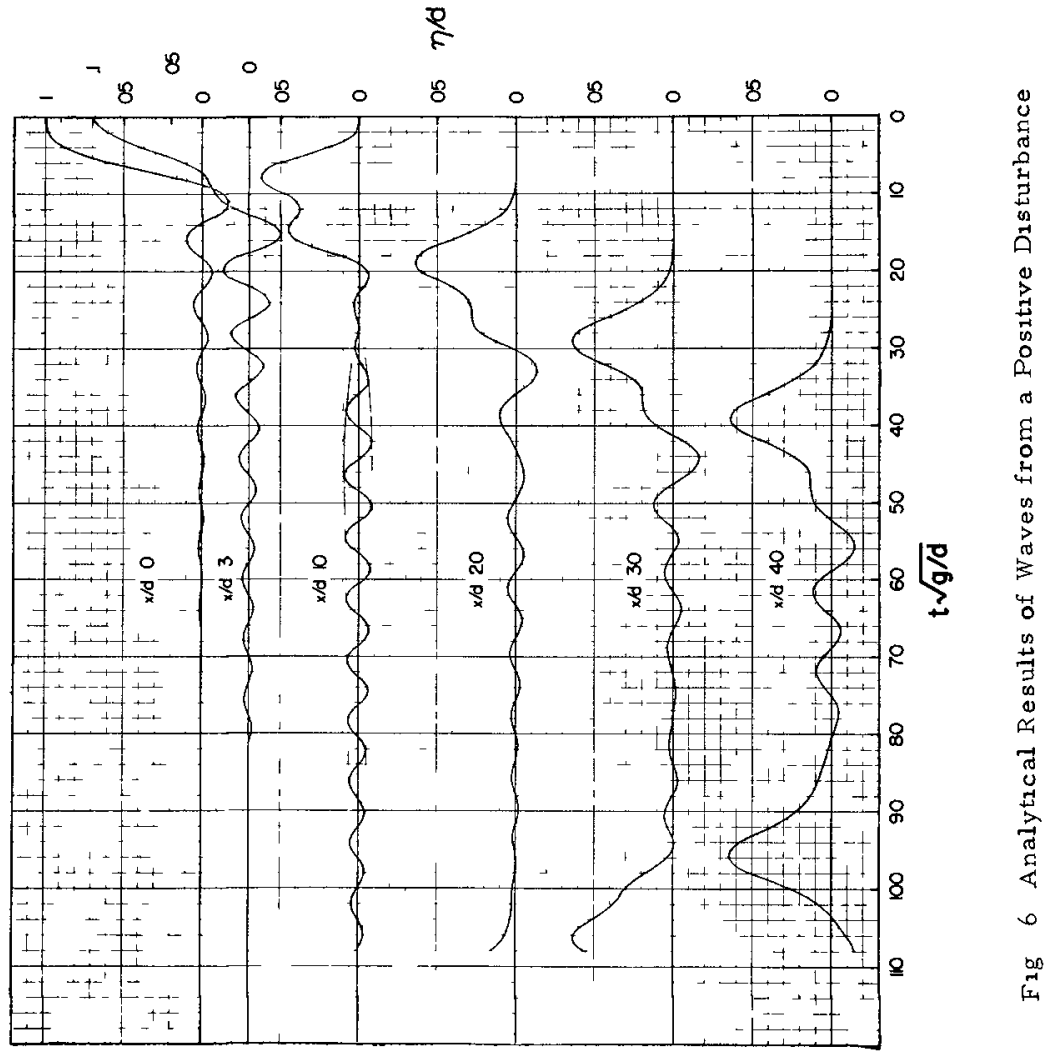


It is interesting to compare Figs 4 and 6 in a qualitative sense In Fig 4 , for elther $\mathrm{s} / \mathrm{d}=30$ or 64 the same tralling-edge wave system is observed as at $\mathrm{r} / \mathrm{d}=0$ in the analytical solution (F1g 6) The wave systems in both cases decrease to zero with increasing time Unfortunately, as mentioned previously the analytical solution could not be extended further than $x / d=40$ so that a station comparable to the next experimental station $x / d=814$ could not be reached However, in the analytical results similar changes in wave form are observed as in the experiments although perhaps the dispersion occurs more rapidly in the analytical solution than it does in the experiments

In Fig 7 analytical results are presented for the comparable case of a negative disturbance which propagates from the initial conditions given by Eq 4. At $x / d=0$ the shape of the disturbance is nearly the mirror image of the positive disturbance shown at the same location in Fig 6 , and again trailing waves are seen in the lee of the lead wave Fig 7 shows that the slope of the leading edge of the main wave decreases wath distance as the wave propagates downstream the same effect is observed when the results are plotted as a function of distance for constant times If Fig 6 and 7 are compared it is seen that the lead negative disturbance is well formed for a given location compared to the positive wave, and also the trailing waves appear to have grown more rapidly in the case of the negative disturbance compared to the positive one In addition it has been found that for the last station shown in Fig 7 the variation of the normalized velocity with time is nearly identical to the water surface variation even though at $x / d=0$ these distributions are different

If, as before, Fig 7 is compared to its experimental counterpart, Fig 5 , the same general trends can be seen the decreasing slope of the lead wave with distance, the rapid development of the form of the lead wave, and the rapid growth of the waves in the lee of the main disturbance

In summary, it is felt that this analytical method describes the changes in a wave system due to both linear and nonlinear dispersion Even though the $1 \mathrm{nit}$ al conditions assumed appear crude, the resultant wave forms agree qualitatively wath the experiments

\section{ANALYSIS OF FIELD OBSER VATIONS}

In addition to the experimental and analytical studies of which this paper presents only some preliminary results, tide gage records of various tsunamis obtained at certain locations around the Pacific Ocean have been studied to investigate the interaction of these waves with the coastine An obvious feature of such records is that, even though the particular earthquake has a relatively short duration (of the order of minutes or less), the tide gage records generally exhibit oscillations which last longer than $24 \mathrm{hrs}$ In addition, the major waves at many of these locations occur within the first few hours of the arrival of the lead wave An example of such records is presented in Fig 8 for three locations in Southern California for the tsunam which resulted from the Alaskan earthquake of March 27, 1964 these and subsequent records were taken from Spaeth \& Berkman (1965) and Berkman \& Symons (1964) Such water surface fluctuations at the coastline raise the question whether the wave train which is measured at the coast bears any resemblance to the wave which existed in the open-sea It is possible that the waves where are recorded are the result of a short impulsive wave train triggering the oscillation of the water masses in local embayments and coastal waters which then oscillate with small damping for long periods of time 


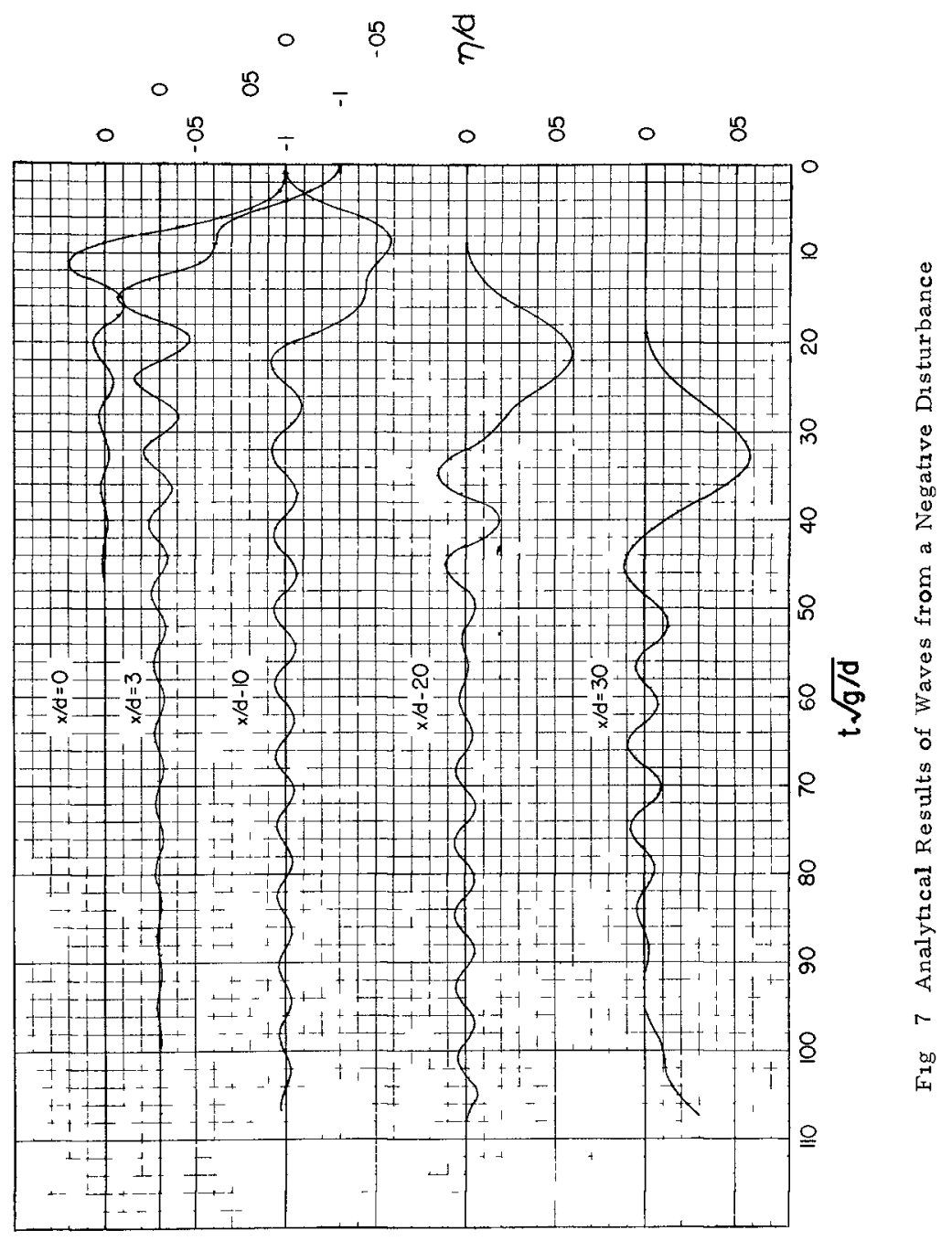



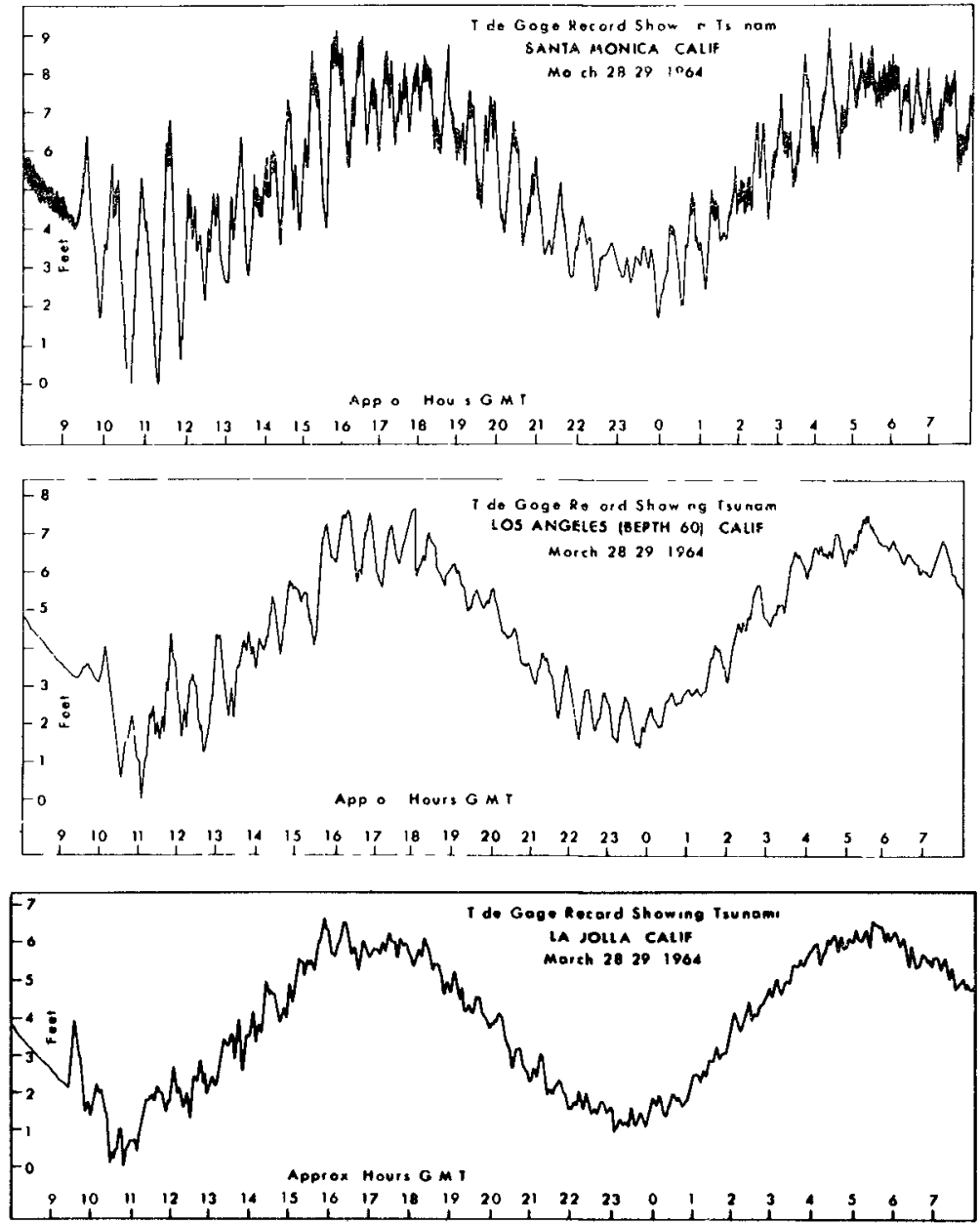

Fig 8 Tide Gage Records from the Tsunam of the Alaskan Earthquake of March 271964 (After Spaeth \& Berkman (1965)) 
To investigate this type of question by obtaining information regarding the distribution of wave energy in these records as a function of frequency (or wave period) the wave records were analyzed using spectral analysis procedures similar to those used for analyzing ocean wave records In essence these methods treat the record as if it were a stationary process This is not considered too serious a limitation since the primary objective is to compare spectra from various harbors for the same event and from the same harbor for various events, and if approximately the same length of record is treated in the same way for all cases a reasonable comparison can be made (Of course the same questions regarding stationarity also can be raised about other analytical methods such as harmonic analysis )

The spectra of the tsunami associated with the Alaskan earthquake of March 27, 1964 for three Southern California locations are presented in Fig 9 The ordinate in Fig 9 is the normalized energy density, normalized with respect to the mean square of the deviation of the water surface from the tide level in units of hours and the abscissa is the frequency in cycles per hour (cph) Therefore, the area under each spectrum is unity The spectra were obtained after subtracting from the marigram the effect of the tide so that this low frequency component would not completely distort the energy content of the spectrum This was accomplished by first subtracting the predicted or fitted tide from the record and then passing the resultant digitized record through a numerical high-pass filter such as that described by Kinsman (1965) This, as well as the spectral analysis computation was accomplished on a digital computer using the procedure for spectral analysis described by Blackman and Tukey (1958) and by Raichlen (1967) In all cases the confidence limits of the spectra were obtained by applying the method described by Kinsman (1965) For the spectra which are presented it is expected that, for a given frequency-band, if the process were stationary, there is a probability that $90 \%$ of all spectral estimates will exceed 069 times the ordinate and $10 \%$ of a all values will exceed 130 times the ordinate with $50 \%$ of all values exceeding 098 times the ordinate value The frequency resolution which was used was chosen based on the avallable record lengths, the confidence limits, the sampling interval, and the fact that spectra from different events were to be compared

Returning now to Fig 9, spectra are presented for Santa Monica, Los Angeles Harbor (Berth 60) and La Jolla, all in Southern California with the first and last of these locations approximately $125 \mathrm{ml}(200 \mathrm{~km})$ apart in a North-South direction Values of the root-mean-square of the water surface fluctuations about the tide ( $\mathrm{r} \mathrm{m}$ ) are shown in Fig 9 for these locations The $r \mathrm{~m} s$ tends to decrease in a Southerly direction with dislance from the tsunami source, in fact, the $r \mathrm{~m}$ decreases by a factor of nearly $25 \mathrm{com}$ paring the two furthest stations Considering the problems usually associated with spectral analysis, the spectra for these locations are remarkably similar indicating a major concentration of energy between frequencies of $16 \mathrm{cph}$ and $18 \mathrm{cph}$ (wave periods between $334 \mathrm{~min}$ and $375 \mathrm{~min}$ ) In addition, there is a secondary concentration of energy between frequencies of $04 \mathrm{cph}$ and $06 \mathrm{cph}$ (wave periods between $167 \mathrm{hrs}$ and $25 \mathrm{hrs}$ ) Before discussing indetall some conclusions which may be drawn from these spectra it is useful to view others that have been obtained

Spectra for four locations are presented in Figs 10 and 11 for both the tsunami which resulted from the earthquake in Alaska of March 27, 1964 and the earthquake in Chile of May 22, 1960 In all cases the spectra were obtained 


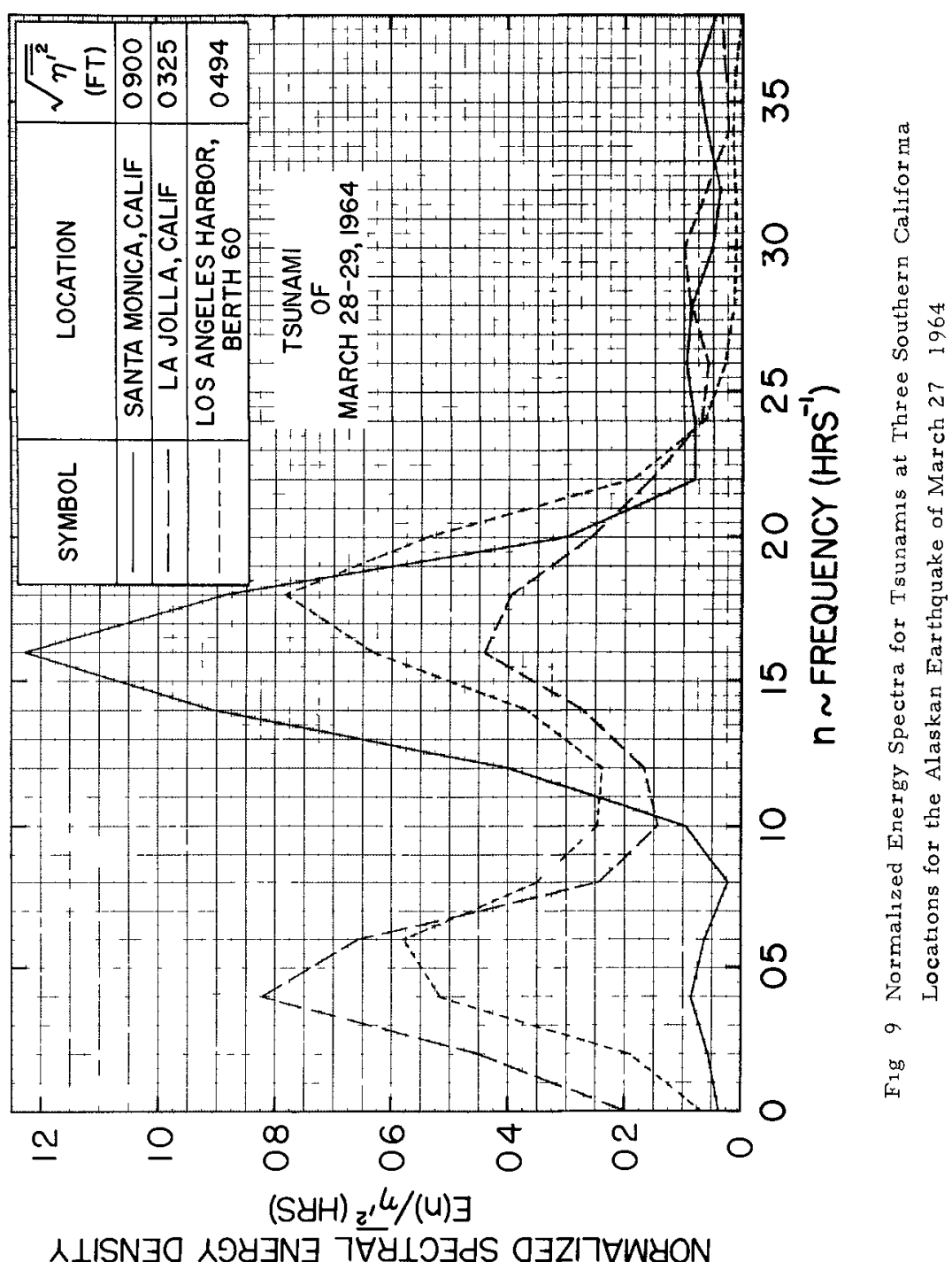




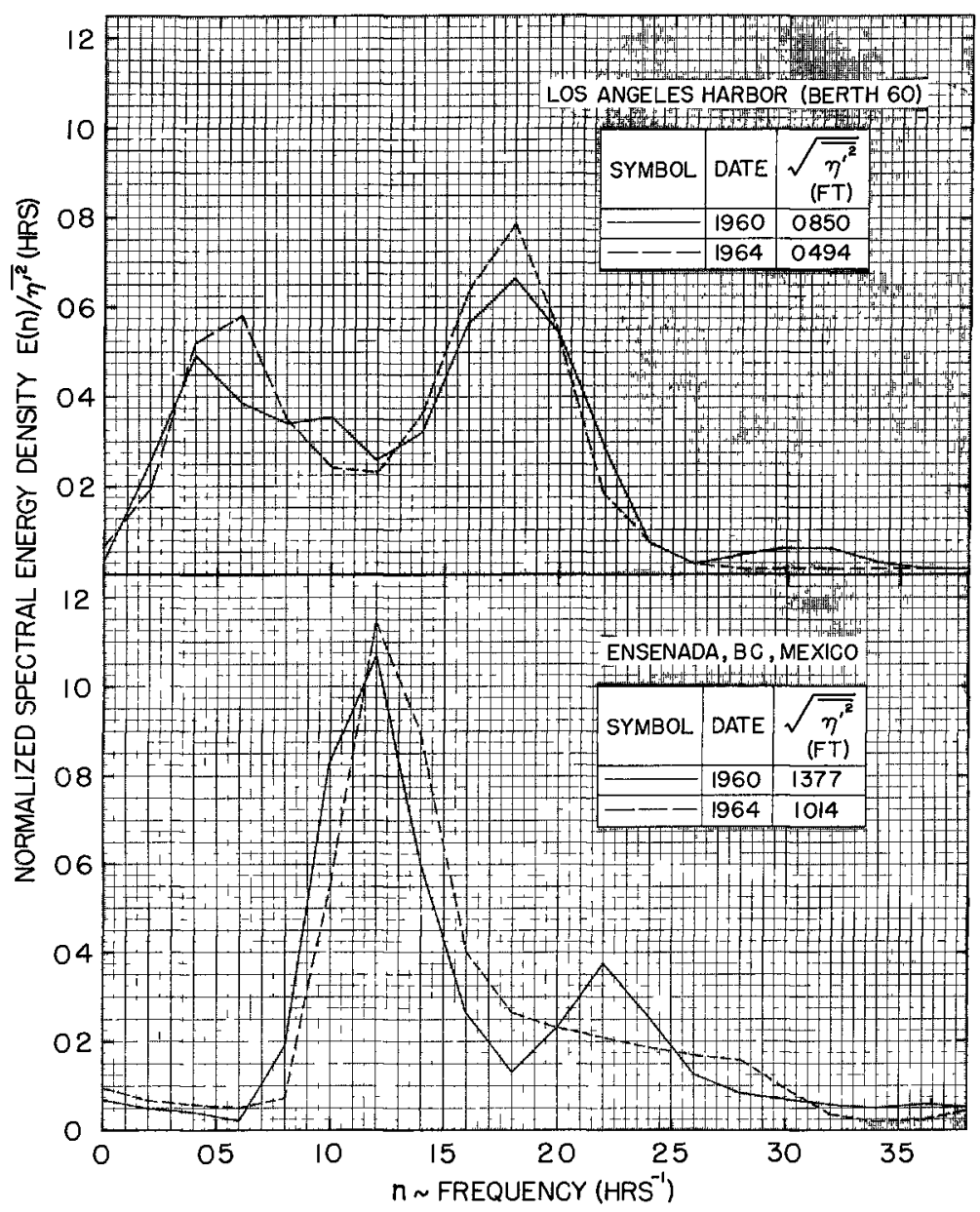

Fig 10 Normalized Energy Spectra for Tsunamis at

Los Angeles Harbor (Berth 60) and Ensenada B C, Mexico Caused by Chilean (1960) and Alaskan (1964) Earthquakes 


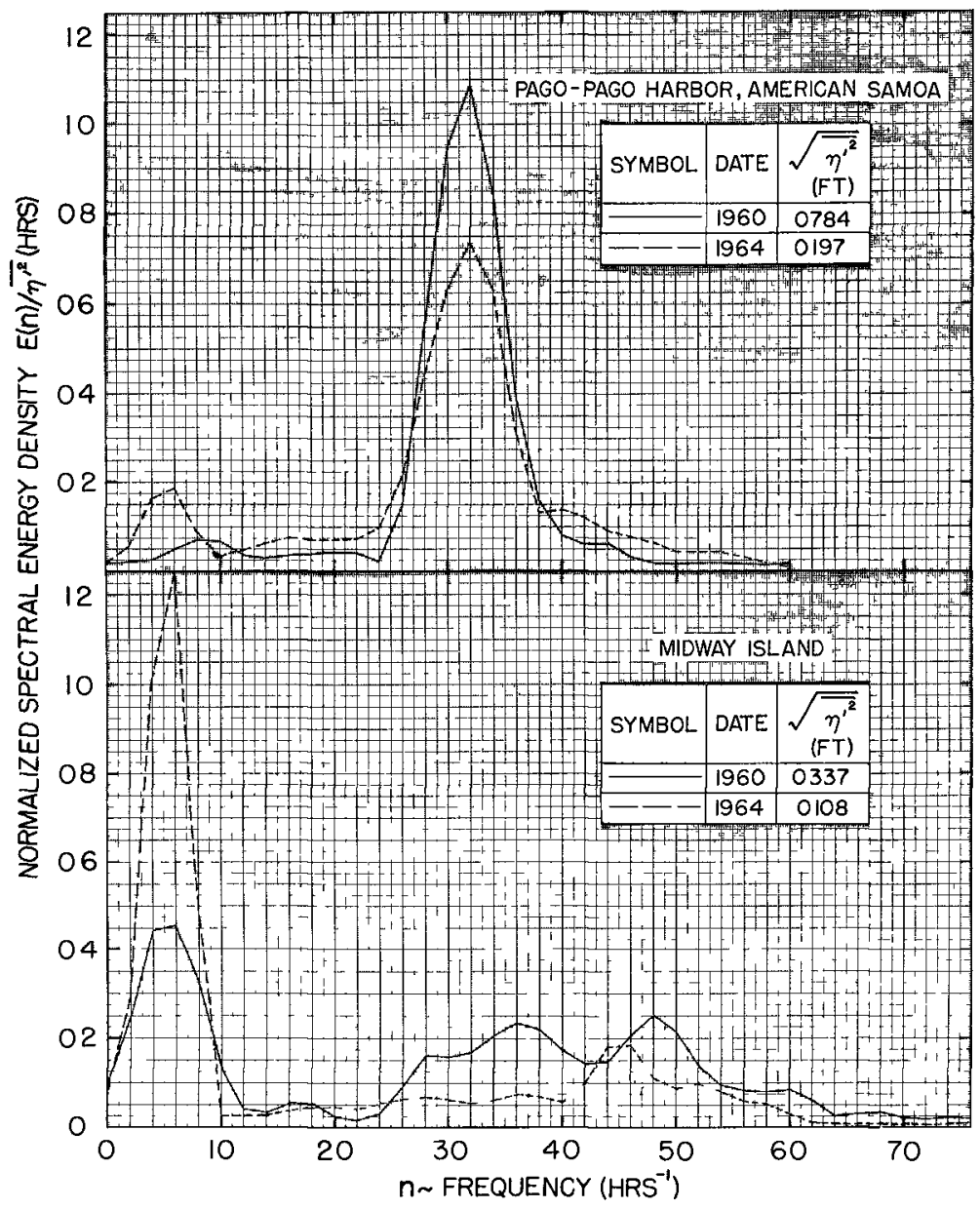

F1g 11 Normalized Energy Spectra for Tsunamis at Pago-Pago Harbor, American Samoa and Midway Island Caused by Chrlean (1960) and Alaskan (1964) Earthquakes 
and plotted in the same way as those shown in Fig 9 and the values of the $r \mathbf{m} s$ are indicated in the figures There are two obvious features of Figs 10 and 11 (1) the spectra at each location for the two different tsunamis are quite similar, and (2) in all cases the $r \mathrm{~m} s$ value for the Chilean tsunams is greater than that for the Alaskan tsunami (the ratio varying from nearly 14 to 4 )

Wilson (1969) in a general investigation of earthquake movements and tsunamis concludes that the tectonic movement associated with both the Chilean earthquake of 1960 and the Alaskan earthquake of 1964 were probably similar and that evidence indicates that a molar upthrust combined with a down-throw played an important role in the earth movement This similarity may be the reason for the relatively good agreement between spectra at each of the locations shown in Figs 10 and 11 It is also quite possible, as suggested earlier that each location is very strongly responsive to particular frequencies which are in the tsunami wave train Therefore, even if the tsunamis were quite different in the open-sea as long as a small amount of energy existed at the preferred frequencies for that particular location, due to the resonant nature of harbors and coastal waters, frequencies to which the harbor is not strongly responsive would be masked In this way it is possible that spectra at a particular location could be similar even though the spectra of the events in the open-sea were quite different For the records analyzed it is probable that both of these explanations are true in part, however, from these limited data a more definite conclusion cannot be made

Wilson (1969) and Munk et al (1959) also have discussed the possibility of the excitation of harbors by these transient wave systems Munk and Cepeda (1962) have attempted to analyse in some detall the marigram at Acapulco, Mexico due to the tsunami associated with the Mexican earthquake of 1957 to determine whether the response of Acapulco Harbor could be the cause of a sharp peak observed in the spectrum at a frequency of $198 \mathrm{cph}$ (a period of about $05 \mathrm{hrs}$ ) They treated the harbor as if it were a rectangular harbor of constant depth and applied the method of Miles and Munk (1961) to determine the frequencies of various modes of oscillation of this harbor Their conclusion was that the observed wave period of $05 \mathrm{hrs}$ was probably not due to the harbor alone but 1t was probably caused by the oscillation of a much larger body of water offshore Their method of determining the response was admittedly crude, and without determining the response by a more exact method such as that developed by Lee (1969) 1t is difficult to draw a definite conclusion as to the cause of the observed periodicity except to say that the peak which was observed in their spectrum was probably due to local excitations

Comparing Figs 9, 10, and 11 it is seen that for both tsunamis at three of the four locations investigated there is a peak in all spectra in the period range between 167 and $25 \mathrm{hrs}$ (For the Alaskan tsunams alone this periodicity is common to five of the six sites studied) Ensenada, Mexico is the only location which does not exhibit such a peak, and this may be due to the method of analysis If the tide is not completely subtracted in the procedure, and if small amounts of energy exist at frequencies near that of the tide, it is possible that the remaining tidal energy can completely mask pexiodicities with small energy content at low frequencies 
The pcaks whrch are observed in the spectra of Figs 10 and 11 at higher frequencies appear to be common for a particular location, however, these frequcncies are not common among locations Therefore, the energy concentrations which appear at wave periods between $20 \mathrm{~min}$ and $50 \mathrm{~min}$ are probably due to the bathymetric and coastal configuration of the particular location

$W_{1} 1$ son (1969) and Wilson and T $\phi r u m$ (1968) have concluded that the period of the primary tsunami wave for both the Alaskan tsunami of 1964 and the Chilean tsunami of 1960 was of the order of $17 \mathrm{hrs}$ Since the peaks at the lower frequencies in the spectra presented herein are approximately at the same frequency independent of location, the same conclusion may be made If one agrees with this conclusion then, considering the periodicity between 33 min and $38 \mathrm{~mm}$, Fig 9 indicates that the Alaskan tsunami of 1964 must also have excited a large mass of water off the coast of Southern Californa extending at least from Santa Monica, California to La Jolla California Therefore, in order to learn more about local tsunami effects such as run-up and forces on structures, which are important from engineering considerations, then more must be understood about the response of off-shore water masses to impulsive wave systems

\section{CONCLUSIONS}

The unique laboratory wave generating facility described herein is capable of producing an impulsively generated wave system which shows the same dispersive effects as predicted by a simple theory The analysis which is used considers the wave system to originate from an initial condition of a disturbed water surface in a still tank of water It shows, in addition to dispersive effects, the same distinct differences between positıve and negative initial disturbances as are observed in the laboratory

Spectra determined from the tide gage records at four locations around the Pacific Ocean for the tsunamis from the Alaskan earthquake of 1964 and the Chilean earthquake of 1960 had similar shapes at each location The results indicate that the $x$ e is a periodicity of approximate $\overline{l y} 2 \mathrm{hrs}$ which is common to nearly all of the locations investigated Other concentrations of energy at higher frequencies which for a given location are similar in period for both tsunamis are not similar for different locations This shows that local embayments and bathymetry tend to influence significantly the wave amplitudes due to tsunamis measured near the shore

\section{ACKNOWLEDGEMENTS}

This research is sponsored by the National Science Foundation under Grant No GK-2370 The work which deals with the analytical simulation of surface waves was conducted at the Technical University of Denmark while the author was on leave of absence there, the assistance of the Northern Europe University Computing Center in making those computations is appreciated 


\section{REFFRENCES}

Berkman S C and Symons, J M "The Tsunams of May 22, 1960 as Recorded at Tide Stations" U S Dept of Commerce, U S Coast and Geodetic Survey, Feb 1964

BJackman, $R$ B , and Tukey $J W$, The Measurement of Power Spectra Dover Publications New York $N \overline{\mathrm{Y}}, 1958$

Keulegan, G H, and Patterson, G W, "Mathematical Theory of Irrotational Translation Waves", Journal of Research of the National Bureau of Standards Vol 24, Jan 1940, pp 47-101

Kinsman, B Wind Waves Prentice-Hall, lnc, Inglewood Cliffs, New Jersey, 1965, pp 448-455

Lee, J - J "Wave Induced Oscillations in Harbors of Arbitrary Shape", Report KH-R-20, W M Keck Lab of Hydr \& Wal Res, California Institute of Technology Pasadena Calıf , Dec 1969

Long $R$ R "The Initial-Value Problem for Long Waves of Finte Amplitude" Journal of Flu1d Mechanics, Vol 20, Part 1, Sept 1964, pp 161-170

Miles J, and Munk W H , "The Harbor Paradox" Journal of Waterways and Harbors Division, ASCE, Vol 87, No WW3, 1961, pp 111-130

Munk, $W$ and Cepeda $H$, "Concerning a Remarkably Sharp Peak in the Sed Level Spectra at Acapulco", Contributions University of California, San Diego, Scripps Institution of Oceanography, Vol 32, 1962 pp 1031-1040

Munk $W H$ Snodgrass, F E and Tucker, $M J$, "Spectra of Low Frequency Ocean Waves", Bulln Scripps Instit of Oceanography Vol 7 (4), 1959 , pp 283-362

Peregrine, D H , "Calculations of the Development of an Undular Bore", Journal of Fluid Mechancs Vol 25 Part 2 June 1966, pp $321-330$

Ralchlen, F, "Some Turbulence Measurements in Water", Journal of the Engineering Mechanics Division, ASCE EM2, Paper 5195, April 1967, pp $78-80$

Spaeth, M B and Berkman, S C , "The Tsunam1 of March 28, 1964 as Recorded at Tide Stations", U S Dept of Commerce, U S Coast and Gi odetic Survey, April 1965

Wilson, B W "Earthquake Occurrence and Effects in Ocean Areas", Techn1cal Report CR 69 027, prepared for U S N C E L, Port Hueneme, Callf Feb 1969

Wilson, B W and Torum, A, "The Alaskan Tsunami of March 1964 Engineering Evaluation", Tech Memo No 25 Coastal Engr Res Cent , Corps Engrs , U S Army, Washington, D C , 1968 


\title{
CHAPTEK 128
}

HEAD LOSS AT TSUNAMI-BREAKWATER OPENING

by Yoshryukı ITO*

\begin{abstract}
The head loss at breakwater openung 18 inportant factor for evaluating the effect of breakwaters aganst tsunam. This paper examines the coefficient of the head loss term in the equation of motion by comparang the results of numerical calculations wath the actual record observed an the port of of unato at the time of 1968 tsunami. Numerical calculations are repeated changing the head loss coefficlent from zero to 3.0 whth the interval of 0.5 . The calculated water level variation is quite different from the record if no head loss is taken into consideratzon. Although the most suztable value of the coefficlent is not definitely determined, the value of 1.5, which has been adopted in our previous calculations, seems to be reasonable for practical purpose.
\end{abstract}

\section{Introduction}

The construction of tsunami-breakwaters in the Port of of unato and several other harbours was started after the dzsaster due to Chilean Earthquake Tsunami in 1960. Concerning this project the author studied the effect of breakwaters against tsunam mainly by numerical calculations The method of calculation and some results obtalned were reported to 10th Coastal Engineering Conference held in Tokyo in 1966 (Reference 1).

In May 1968, the tsunam-breakwater in the Port of ofunato experienced the first remarkable tsunam since its completion in 1967. The water level variation was recorded by two tide gauges located outside and inside the breakwater. The author immediately applied the method of calculation and obtained a good agreement between calculation and observation. The effect of the tsunami-breakwater was also confirmed by this onalysis. The detalls were already reported to 13th IAHR Congress in Kyoto, 1969 (Reference 2).

The term of head loss included in the equation of motion at the breakwater opening plays an important role in numerical calculation. If no head loss is taken into account, the effect of breakwaters against tsunam is not appropriately evaluated due to the appearance of so-called harbor-paradox. The author has so far adoted the head loss coefficlent of 1.5 in previous calculations. The influence of thrs coefficient on the calculated results is examned in this paper by comparing the calculations with the actual observation of 1968 tsunami in the Port of ofunato.

* Hydraulics Division, Port and Harbour Research Institute, Ministry of Transport 3-1-1, Nagase, Yokosuka, Japan 


\section{Basic Equations}

The principle of calculation $2 \mathrm{~s}$ to solve numerically the equations of motion and of continulty for two-dimensional long wave under certain initial and boundary conditions. Fundamental equations are as follows,

$$
\begin{aligned}
& \frac{\partial u}{\partial t}=-g \frac{\partial \zeta}{\partial x} \\
& \frac{\partial v}{\partial t}=-g \frac{\partial \zeta}{\partial y} \\
& \frac{\partial \zeta}{\partial t}=-\frac{\partial}{\partial x}(h u)-\frac{\partial}{\partial y}(h v)
\end{aligned}
$$

where, $u, v=v e l o c i t y$ component in $x$ - and $y$-direction, respectively $\zeta=w a t e r$ level elevation with respect to mean water level $h=$ water depth below mean water level

These differential equations are transformed into difference equations. Figure 1 shows the grid system around the breakwater in the Port of ofunato. The grid interval in this case is 280 metres and the time interval is 10 seconds.

The outer sea $2 \mathrm{~s}$ replaced by a channel of constant depth and wadth, where the computation is only almed at supplying the incoming tsunam whthout being affected by reflected waves from the breakwater and shorelines. As a boundary condztzon, velocity components normal to the breakwater and shorelines are gaven to be zero. The calculation 25 started from the time when the front of tsunami arrives at the bey-nouth. Inztial water level and velocity inside the bay are accordingly zero, whle those in the outer sea correspond to the incldent tsunami profile.
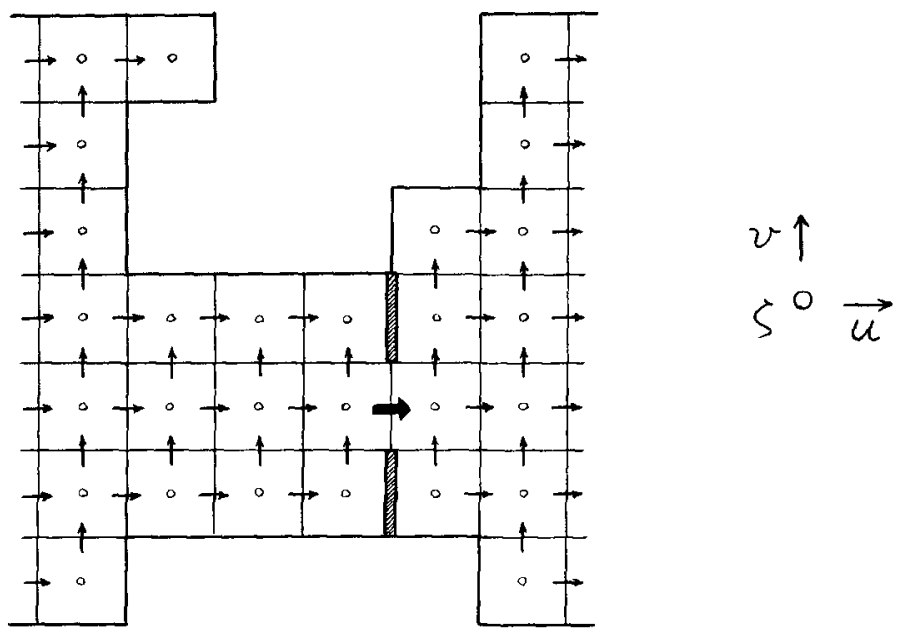

Figure 1 Grid System for Numerical Calculation 
Al though higher order terms for inertia or bottom friction are neglected in Equation (1), a term representing the head loss is added to the equation of motion at the breakwater opening as shown in the following difference equation,

$$
\frac{\Delta_{t} u}{\Delta t}=-g \frac{\Delta_{x} \zeta}{\Delta x}-\frac{f}{2 \Delta x}-u|u|
$$

The head loss coefficient $f$ in this equation has been assumed to be 1.5 in previous calculations. In this paper numerical calculations are carried out with $f$ of $0,05,1.0, \ldots, 3.0$.

1968 tsunams in the Port of of unato

Figure 2 shows the plan of Of unato Bay situated in the northeastern part of Japan along the Paclfic Coast. The tsunamibreakwater near the bay-mouth, where the maximum water depth reaches almost 40 metres, was constructed after ChIlean Earthquake Tsunami in 1960 and was completed in 1967.

on May 16, 1968, an earthquake of magnitude 7.8 occurred of $f$ this district A tsunami accompanied by this earthquake attacked the coast and caused certain damages to several ports. The tsunampbreakwater in the Port of Ofunato effectively protected the harbour and the city by reducing the water level elevation in the inner basin.

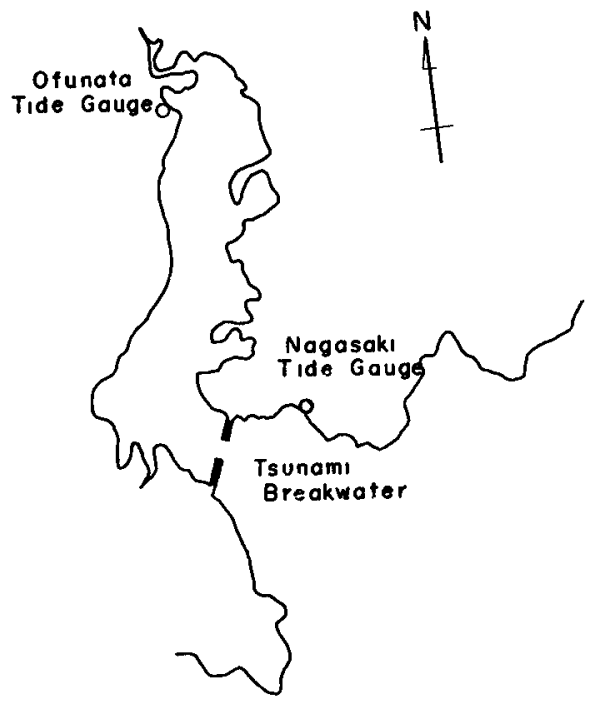

F1gure 2 Tsunam-breakwater and Tide Gauges in the port of of unato 
Actual records of water level variation were obtalned at two tide gauges, the one is located between the bay-mouth and the breakwater (Nagasakz) and the other is at the innermost part of the bay (Ofunato). The position of these tide gauges is indicated in Figure 2.

Figure 3 shows the water level variations during the first two hours, the astronomacal tide being subtracted.

In the analysis of this tsunamz by applying the author's method, the incident tsunami profile for calculation was determined so that the calculated water level variation at the outside tide gauge might be as close as possible to the observation. The outline of the procedure for this determination is as the following,

1) The water level variation at the outside tide gauge during the first 124 minutes, in which most of the major fluctuations are included, 28 analyzed into Fourier sine series.

2) Calculations are repeated for component regular sinusozdal waves in order to obtain the amplifyung factor at the outside tide gauge. The amplifyzng factor obtazned varies from 1.7 to 2.9.

3) The amplitude of each component obtalned in the Item 1) is divided by the corresponding amplifying factor in the item 2 ). The Fourier sine series consisting of thus determined amplitudes gives the approximate profile of the incident tsunam.

With this incident tsunam profile, a good agreement was confirmed between the calculation and the observation at the inside tide gauge. A calculation with the same incident tsunami was also carried out for the harbour before the construction of the tsunamibreakwater. The highest water level elevation in the harbour after the breakwater construction is 1.1 1.2 metres, whle without the breakwater It reaches more than 2 metres. The effect of the tsunamibreakwater 18 definitely evaluated by these numerical calculations

\section{Influence of the head loss coefficzent on calculated results}

The incident tsunami profile determined by the above-mentioned procedure will be affected to a certain extent by the head loss coefficient at the breakwater opening. However, the profile for $f=1.5$ is commonly used $2 n$ this paper to all the cases from $f=0$ to 3.0. The influence of the value of $f$ on the water level at the outside tide gauge is comparatively small, as shown in Figure 4.

Figure 5 is the comparison between calculation and observation at the inside tide gauge. This figure indicates that the curve for $f=0$ is quite different from the actual record, not only in the value at each peak or trough but also in the form of the curve itself.

Table 1 shows the computed and observed water level elevations at several peaks and troughs. The value of $f$ fitted to the observation differs at each position, as indicated by the mark (*) in the colum of calculation.

Figure 6 shows the goodness of fit of computed water level. The goodness is measured by the root mean square $(\sigma)$ of the residuals at every two minutes during two hours $(N=60)$.

$$
\sigma=\sqrt{\sum\left(\zeta_{\text {cal }}-\zeta_{\text {obs }}\right)^{2} / N}
$$




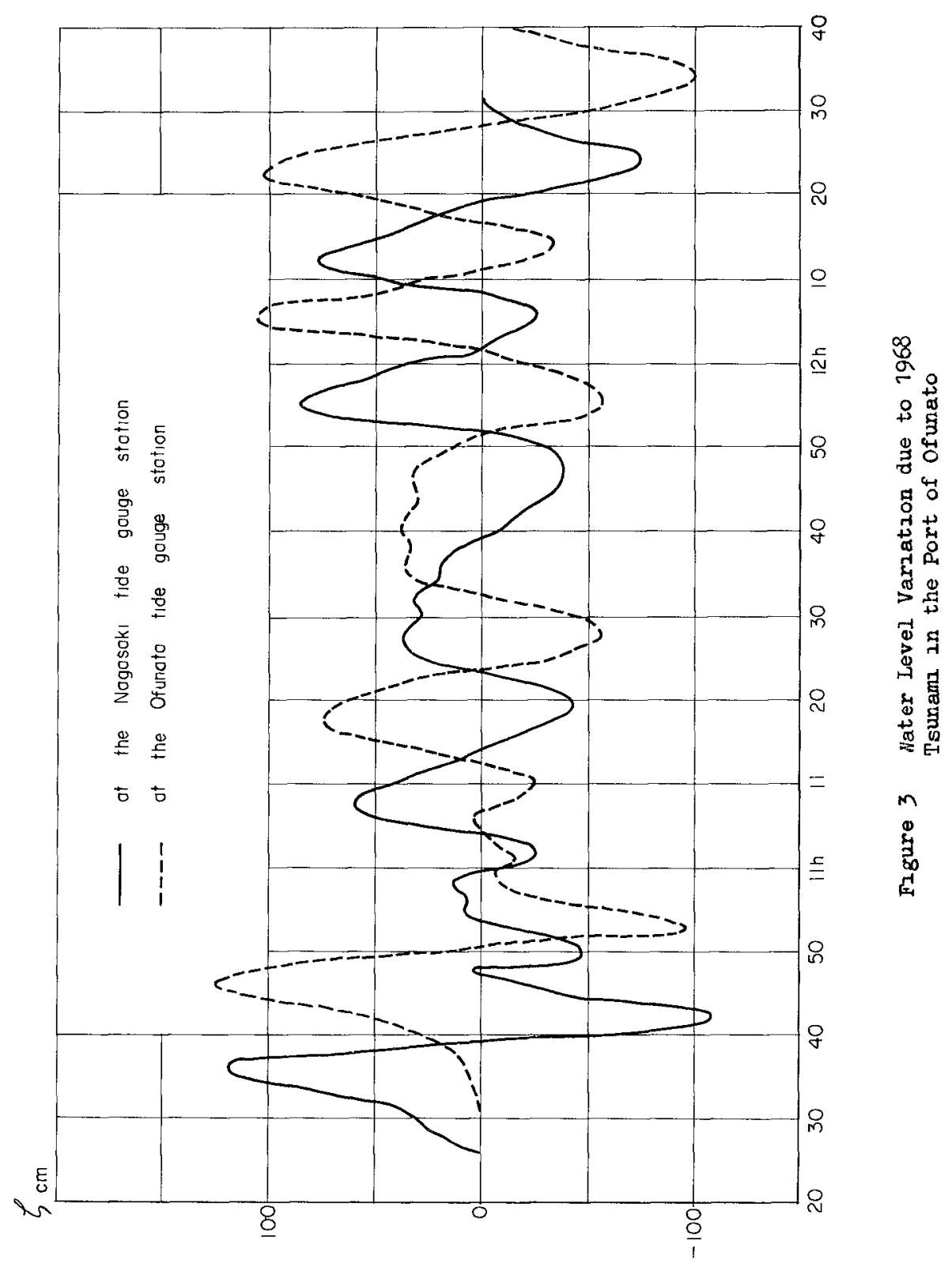




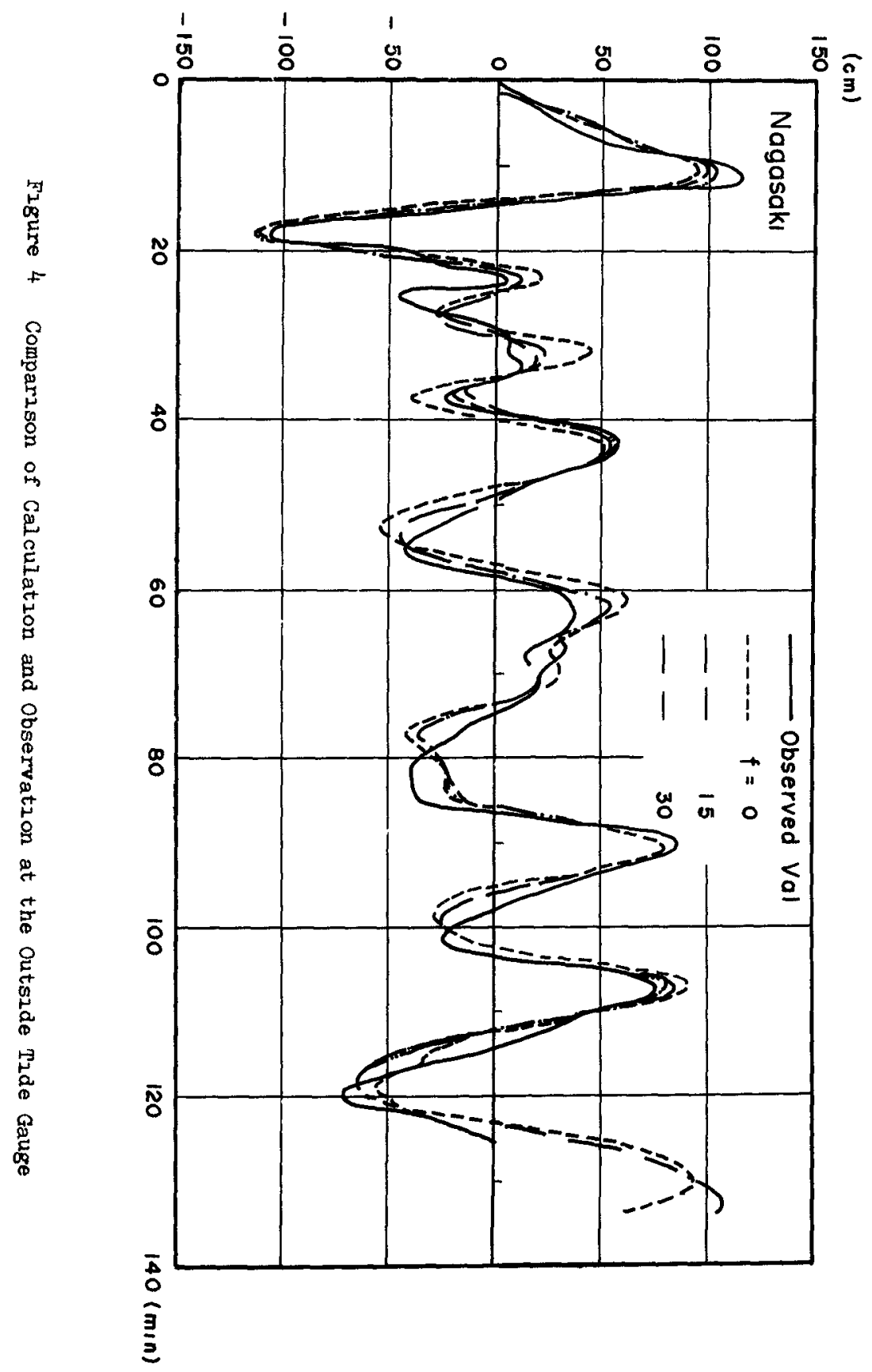




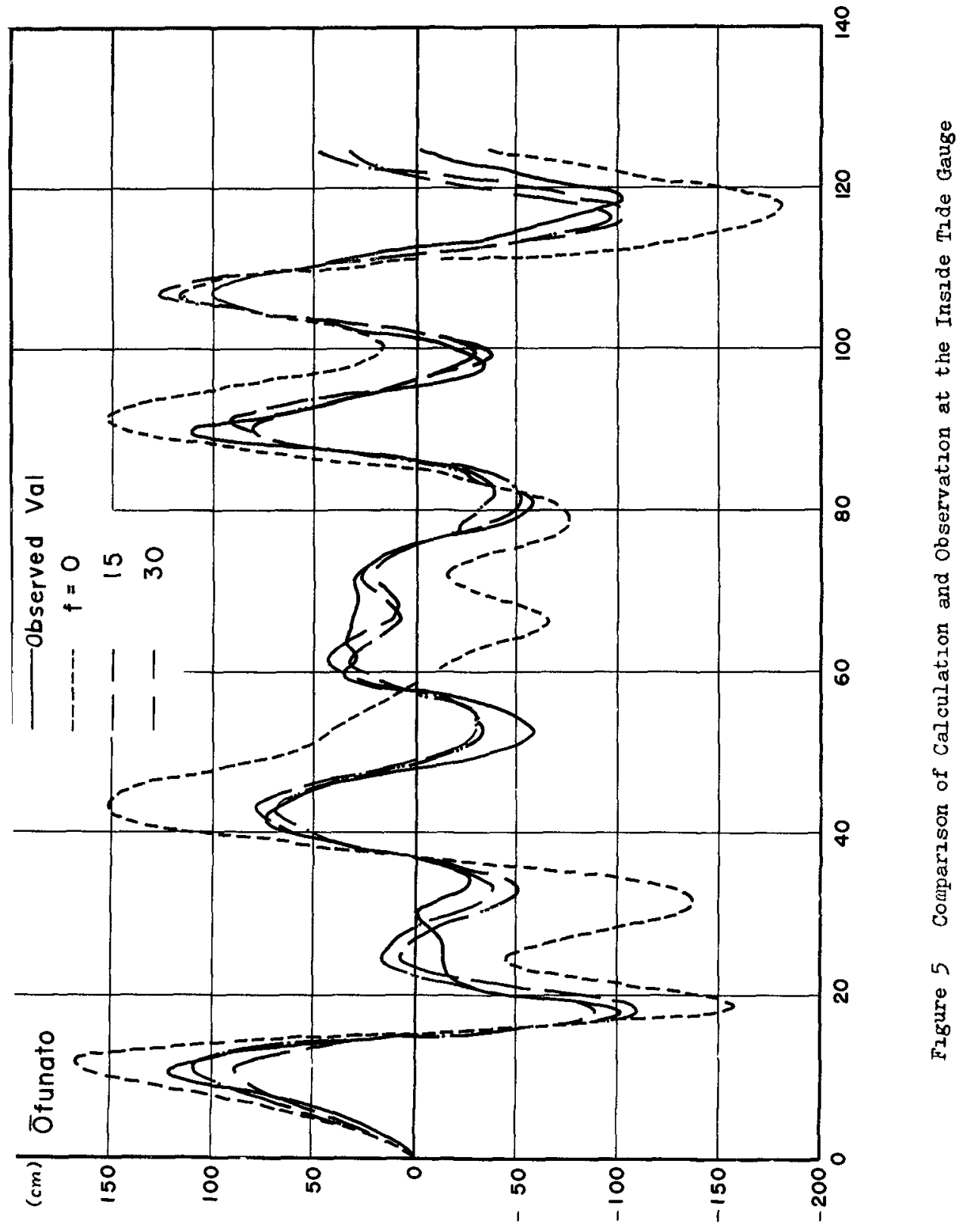


Table 1 Comparison of Calculation and Observation at Peaks and Troughs

(unzt $\mathrm{cm}$ )

\begin{tabular}{l|r|rrrrrrr}
\hline \multirow{2}{*}{ Position } & \multirow{2}{*}{ Observatzon } & \multicolumn{7}{|c}{ Calculation } \\
\cline { 3 - 8 } & & $f=0$ & 0.5 & 1.0 & 1.5 & 2.0 & 25 & 3.0 \\
\hline 1st peak & 124 & 169 & 140 & $123^{*}$ & 111 & 103 & 96 & 91 \\
1st trough & -102 & -159 & -137 & -120 & -108 & $-101 *$ & -95 & -90 \\
3rd peak & 74 & 153 & 104 & 88 & 80 & $75^{*}$ & 72 & 69 \\
4th peak & 112 & 153 & $108 *$ & 100 & 94 & 90 & 85 & 81 \\
6th peak & 102 & 119 & 138 & 134 & 129 & 123 & 119 & $115^{*}$ \\
6th trough & -101 & -180 & -121 & -111 & -105 & $-102^{*}$ & -99 & -97 \\
\hline
\end{tabular}

Al though the incident tsunam profile has been determined so that the computed water level at the outside tide gauge might colneide with the observed record, there still remain some differences between calculation and observation. The deviation of calculation for the anside tide gauge wil I depend on both the accuracy of the incident tsunam profile and the value of the head loss coefficient $f$.

At the inside tide gauge, the root mean square of the residuals is very bis at $f=0$. It decreases rapidly with the increase of $f$ and becomes almost constant for $f$ of bigger than 1.0 .

It is not easy to find out definitely the most suitable value of $f$ from these results. However, the analysis in this paper suggests the practical validity of the adoption of 1.5 or so as the head loss coefficlent at the tsunami-breakwater openzng, as far as the form of the equation of motion in our method of calculation is concerned.

References

1) H. Fukuuch2, Y. Ito on the Effect of Breakwaters against Tsunamı, Proc. 10th Conference on Coastal Englneering (Tokyo, 1966), pp.821 839

2) Y. Ito on the Ef fect of ofunato Tsunam-Breakwater against 1968 Tsunaml, Proc. 13th Congress of IAHR (Kyoto, 1969), Vol.3, pp.85 -93 


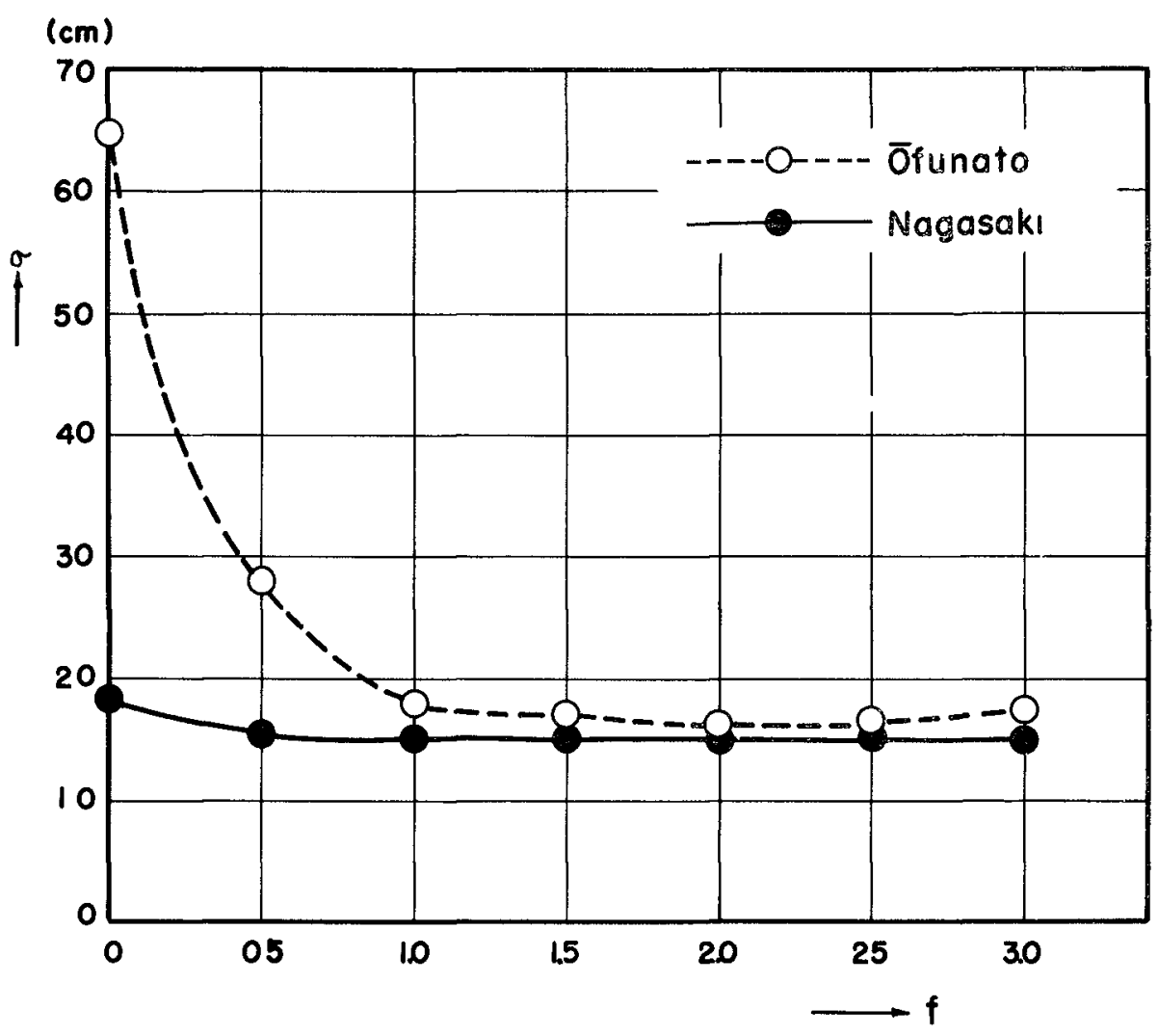

Figure 6 Goodness of Fit 

CHAPTER 129

TIDAL WAVES IN SCHEMATIC ESTUARIES

Hans Vollmers

Bundesanstalt fur Wasserbau Hamburg

and

Jurgen Sundermann

Institut fur Meereskunde der Universitat Hamburg

\begin{abstract}
An investigation of tidal waves in schematic estuaries has been carried out simultaneously for hydraulic and hydrodynamic-numerical (HN) models Thereby a far-reaching agreement of results is obtained for geometrically simple shapes. In these cases hydraulic investigations can be replaced equivalently by $\mathrm{HN}$-computations.
\end{abstract}

\title{
INTRODUCTION AND GENERAL VIEW
}

The knowledge of the tidal caused motion processes in the near shore area, especially in the estuaries, is of great importance for coastal englneering and navigation. The quantitative determination of these processes, e g. finding out of water levels and current velocities depending on space and time, can be done by direct measurements in the natural area In recent years for this purpose also hydraulic and hydrodynamic-numerical (HN) models are used which simulate the natural conditions The application of such models requires e $g$. much less effort and costs and gives, further on, the possibility to study the effect of prospected coastal engineering structures on these grounds model techniques succeeded in the last years on a large scale Especially, the HN-methods belong to the west effective tools of dynamical oceanography.

The following results on the propagation of tidal waves in estuaries are obtained by means of hydraulic and $\mathrm{HN}-$ models It was a main intention thereby to find out by a systematic investigation of geometrical simple estuarine shapes the degree of agreement and the possibilities and restrictions of the two principally different methods. It appears, that in this way a senseful mutual completion of both methods could be reached. Now, the more expensive hydraulic model experiments will be carried out only in those 
complicated cases, for which theoretical solutions are not yet available on the other hand, HN-Investigations can relieve the hydraulic model testing plants of time-consuming routine experiments and be very useful if the constructional equipment of the laboratory do not allow the study of certain questions e g. the influence of the Coriolis force The investigations which result from a co-operation of the Bundesanstalt fur Wasserbau Hamburg and the Institut fur Meereskunde der Universitat Hamburg, are sponsored by the Deutsche Forschungsgemeinschaft (Vo 153/1/2) The whole study will be reported later on We are very thankful to W Wulzinger for his assistance in the hydraulic model investigations

The hydraulic processes in a tidal estuary without a steady inflow are maniy influenced by two aspects

(a) Geometry of the estuary

(b) Shape of the incoming tidal wave

In the following only the effect (a) is discussed Fig. 1 shows the considered schematic estuaries The types $B$ and $E$ are developed from tidal rivers at the German coast By this time the comparison between the two models was realized for the types $A$ and $D$.

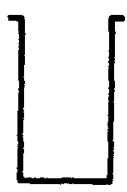

A

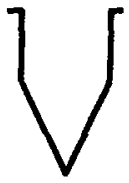

D

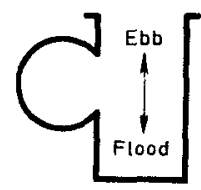

B

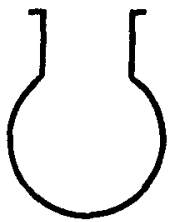

E

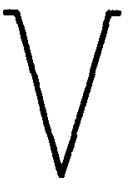

C

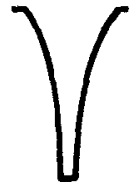

F

F1g 1 Types of Estuaries

In al1 cases a uniform $M_{2}$-tide with a mean amplitude of $1,5 \mathrm{~m}$ has been used

Actually, the HN-models produce vertically integrated horxzontal velocities It is intended, furtherly, to consider the vertical dimension too. 


\section{THE NUMERICAL MODEL}

The foundation of the mathematical model 1 s, that flow movements (especialiy instationary tidal waves which are of Interest here) take place according to the we11-known fundamental equations of hydrodynamics. Therefore, the mathematical treatment of these equations gives the possibilıty, in principal, to determine the motion processes in a certain area The mathematical model has to be adapted extensively to natural conditions

In general, due to the nonlinearity of the hydrodynamic differential equations, an analytic solution is not aval1able Therefore the application of numerical methods $1 \mathrm{~s}$ necessary, e $g$. the HN-difference method (HANSEN [1]) ThIs requires the application of electronic computers In the last years, the HN-method was proved for many natural sea areas (Technical Report [4], SUNDERMANN [2]) The following differential equations have been used

$$
\frac{\partial u}{\partial t}+\frac{r}{h+\xi} u \sqrt{u^{2}+v^{2}}-A_{H} \Delta u-f v+g \frac{\partial \xi}{\partial x}=0
$$

$$
\begin{aligned}
& \frac{\partial v}{\partial t}+\frac{r}{h+\xi} v \sqrt{u^{2}+v^{2}}-A_{H} \Delta v+f u+g \frac{\partial \xi}{\partial y}=0 \\
& \frac{\partial \xi}{\partial t}+\frac{\partial}{\partial x}((h+\xi) u)+\frac{\partial}{\partial y}((h+\xi) v)=0
\end{aligned}
$$

The first two equations are motion equations and 13 is the continulty equation The system is two-dimensional For this a cartesian system of coordinates has been used The $x-a x i s$ Is directed to East, the y-axis to North, the z-axis indicates the vertical direction (see Fig 2)

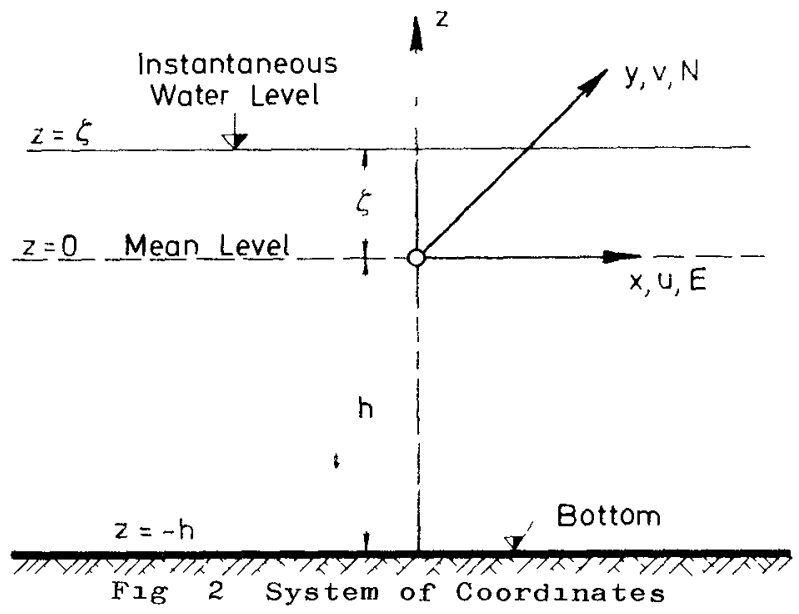




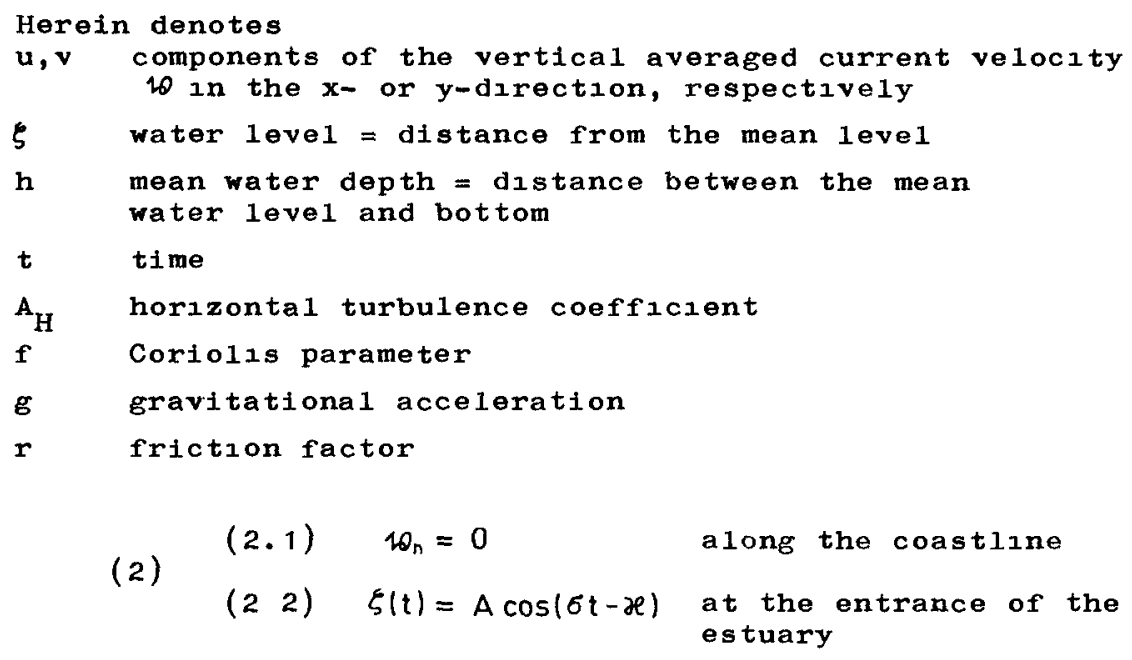

The boundary conditions are given in the equations (2) 2.1 signifies that no normal component of the velocity appears at the coast. In $2.2 \mathrm{~A}$ denotes the amplitude, $\sigma$ the frequency and $\mathcal{x}$ the phase of the incoming tidal wave Geometry, $A$ and $x$ must be given

The initial conditions are

$$
\text { (3) } \quad u=v=0, \quad \xi=0
$$

The water level $\xi$ is included in the total depth $\mathrm{H}=\mathrm{h}+\xi$ and the bottom friction is given by a quadratical law Therefore, the system (1) becomes nonlinear

As shown by computation with and without horizontal turbulence term, this effect can be neglected for the model types $A, C, D$ and $F\left(A_{H}=0\right)$ The Coriolis parameter has been taken as $f=0$, because the hydraulic model installations did not allow to realize this effect A numerical experiment has shown however the influence of the earth's rotation on the distribution of current velocities, while the water level nearly did not change

With these assumptions it is sufficient to use instead of (1) the one-dimensional equations for the types $A, C, D, F$

$$
\frac{\partial u}{\partial t}+\frac{r}{h+\xi} u|u|+g \frac{\partial \xi}{\partial x}=0
$$

$$
\text { (42) } \frac{\partial \xi}{\partial t}+\frac{\partial}{\partial x}((h+\xi) u)=0
$$


Because the used calculation method was utilized mainly for natiral areas, the hydraulic model was fitted with a corresponding scale.

For the difference method the use of a horizontal grid system is indispensable. Fig 3 illustrates the two-dimensional grid system of a rectangular estuary.

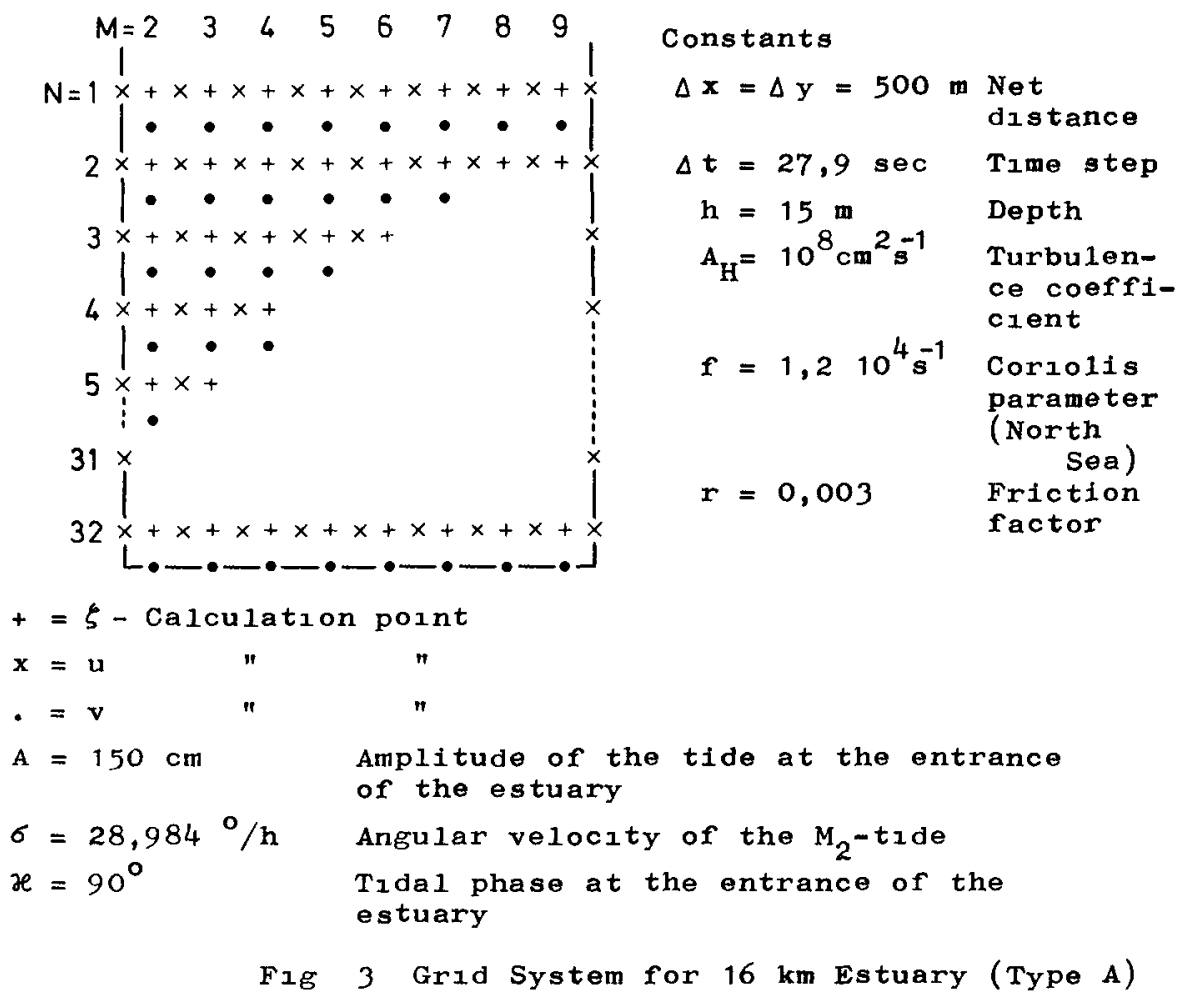

Steady flow conditions occur in the two-dimensional consideration after about 12 periods

The one-dimensional treatment $1 \mathrm{~s}$ based on a $55 \mathrm{~km}$ canal (Type A) The grid system and the constants are given in FIg 4 


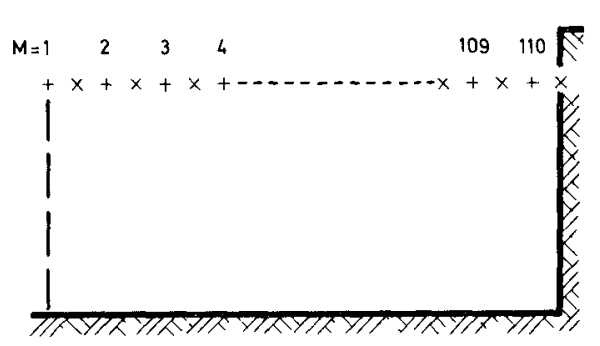

Constants

$$
\begin{aligned}
\Delta \mathrm{x} & =500 \mathrm{~m} \text { Net distance } \\
\Delta \mathrm{t} & =30 \mathrm{sec} \text { T1me step } \\
\mathrm{h} & =15 \mathrm{~m} \text { Depth } \\
\mathbf{r} & =0,003 \quad \begin{array}{l}
\text { Friction } \\
\text { factor }
\end{array}
\end{aligned}
$$

$+=\zeta$ - Calculation point

$\mathbf{x}=\mathbf{u}-" \|$

$A=162,5$ resp $164 \mathrm{~cm}$ Amplitude of the tide at the entrance of the estuary

$\sigma=28,984^{\circ} / \mathrm{h}$

$x=90^{\circ}$ Angular velocity of the $\mathrm{M}_{2}$-tide

Tidal phase at the entrance of the estuary

Fig 4 Grid System for $55 \mathrm{~km}$ Estuary (Type A)

In the HN-model, the amplitude of the incoming tidal wave was adapted to the value measured in the hydraulic model The strady state was reached after about 5 periods

A11 programs are written in FORTRAN IV

As an example, in Fig. 5 the tidal and the velocity curves are given for 3 selected points The tidal wave 1 ncreases to the end of the estuary The velocity curves show the typical steep gradient in the flood branch and the smoother distribution of the ebb

THE HYDRAULIC MODEL

The hydraulic model of type A $1 \mathrm{~s} 55 \mathrm{~m}$ long, $4 \mathrm{~m}$ wide, the mean water depth amounts $015 \mathrm{~m}$ Fig 6 shows the model 1nstallation. The entrance of the model has a fixed bed with artificial roughness. The estuary itself was filled by sand which was not moved by the current In this part of the model the roughness was simulated by artificial 1-cm-ripples This kind of roughness corresponded with the assumed friction factor $r=0003$ in the HN-model

According to natural conditions, the following scales were appointed the extensions of length and width 1 1000, depths 1 100, e.g the distortion is tenfold The whole hydraulic data were reduced by Froude. The tidal period is $745 \mathrm{minu}-$ tes in the model. The water levels were measured with mechanical and electrical water gages and the current velocities w1 th micro-current meters (VOLLMERS [3]) 


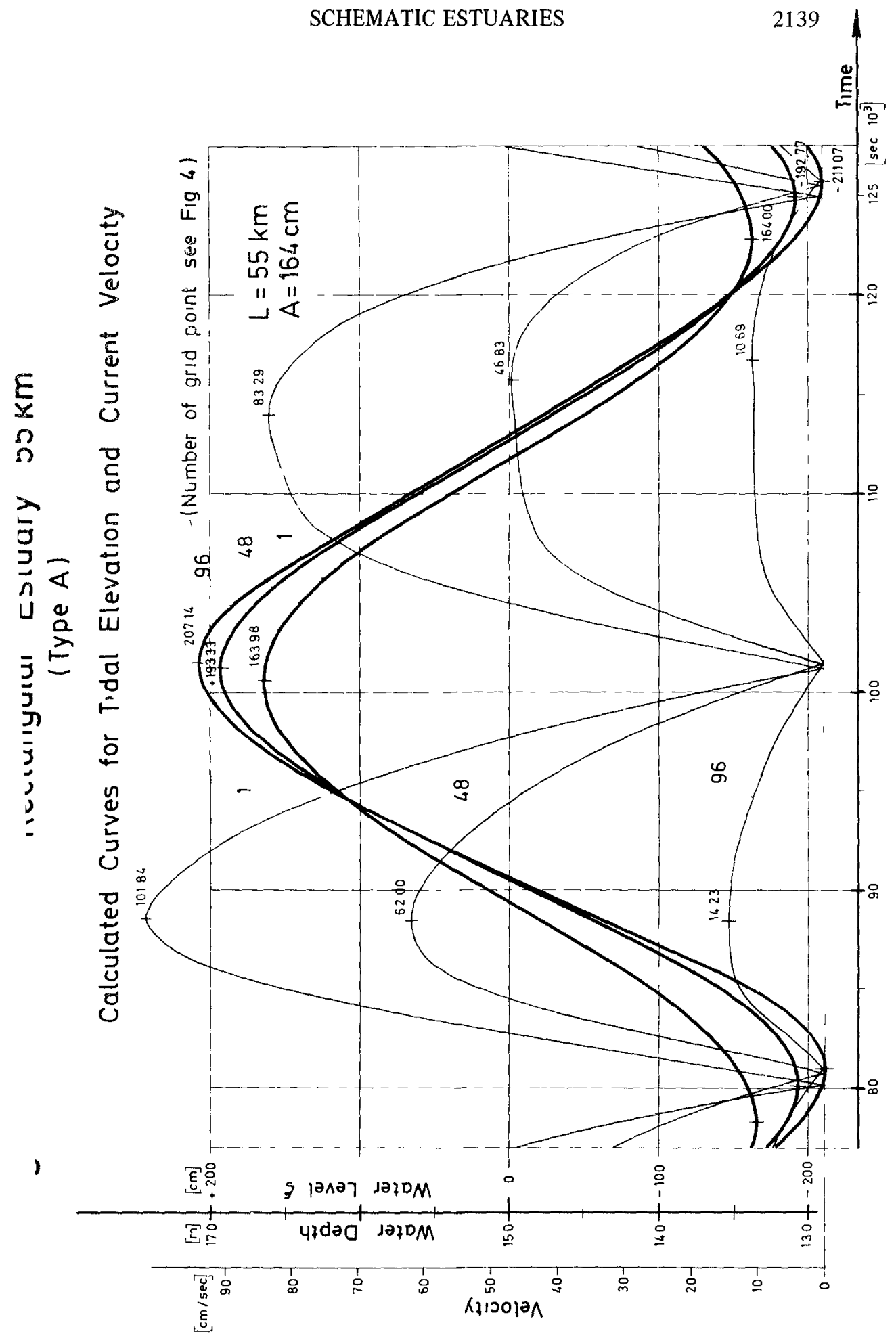




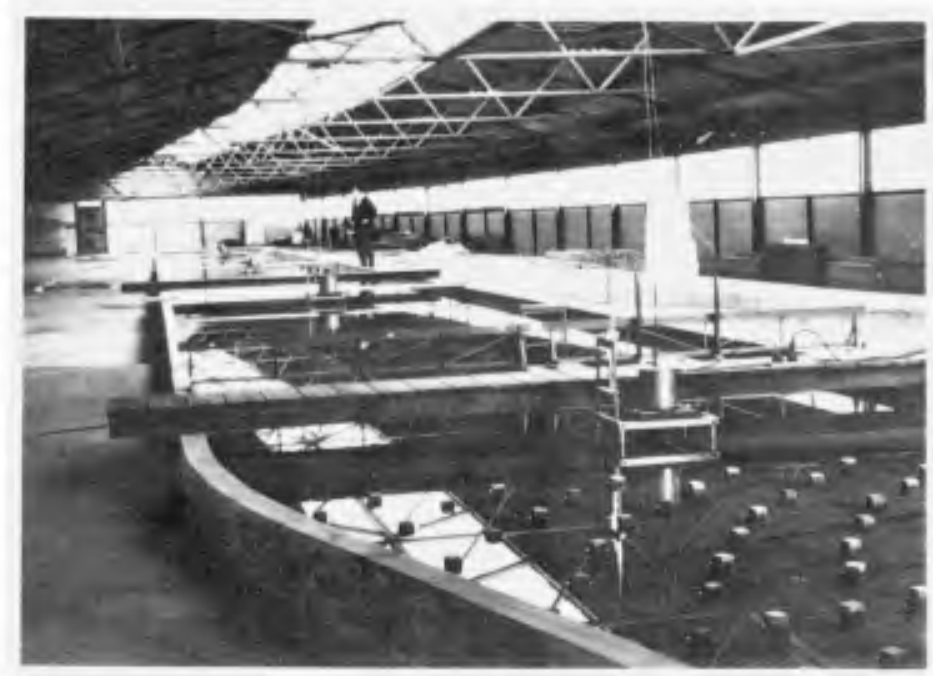

Fig. 6 Model Installation

The first tests were carried out in a $16 \mathrm{~m}$ long rectangular basin (e.g. $16 \mathrm{~km}$ in natur), but it was not possible to get clear results because the model was too short and therefore the differences for the speed are too small for measuring.

The measurement of the hydraulic data yields good results in the long canal ( $55 \mathrm{~km}$ in nature) of the Type A. Fig. 7 demonstrates side by side the measured and calculated water levels for different points during one tide. It was necessary to draw the curves side by side, because the good agreement shows nearly no differences between measurement and calculation. The tidal curve increases, as 1 t is well-known, at the end of the canal and the time ratio between flood and ebb will be displaced. The tidal range increases from 3.28 to $4.18 \mathrm{~m}$, the time ratio, tide low water to tide high water and tide high water to tide low water decreases from 1 at the entrance to about 0.80 at the end of the canal.

The current velocities were measured at different water depths.

Due to the restriction of the HN-model to vertically averaged velocities, the comparison between the two models was possible only for mean conditions. Fig. 8 shows this comparison for a mean calculated and measured curve. The agreement is good as well in shape as in order of magnitude. 


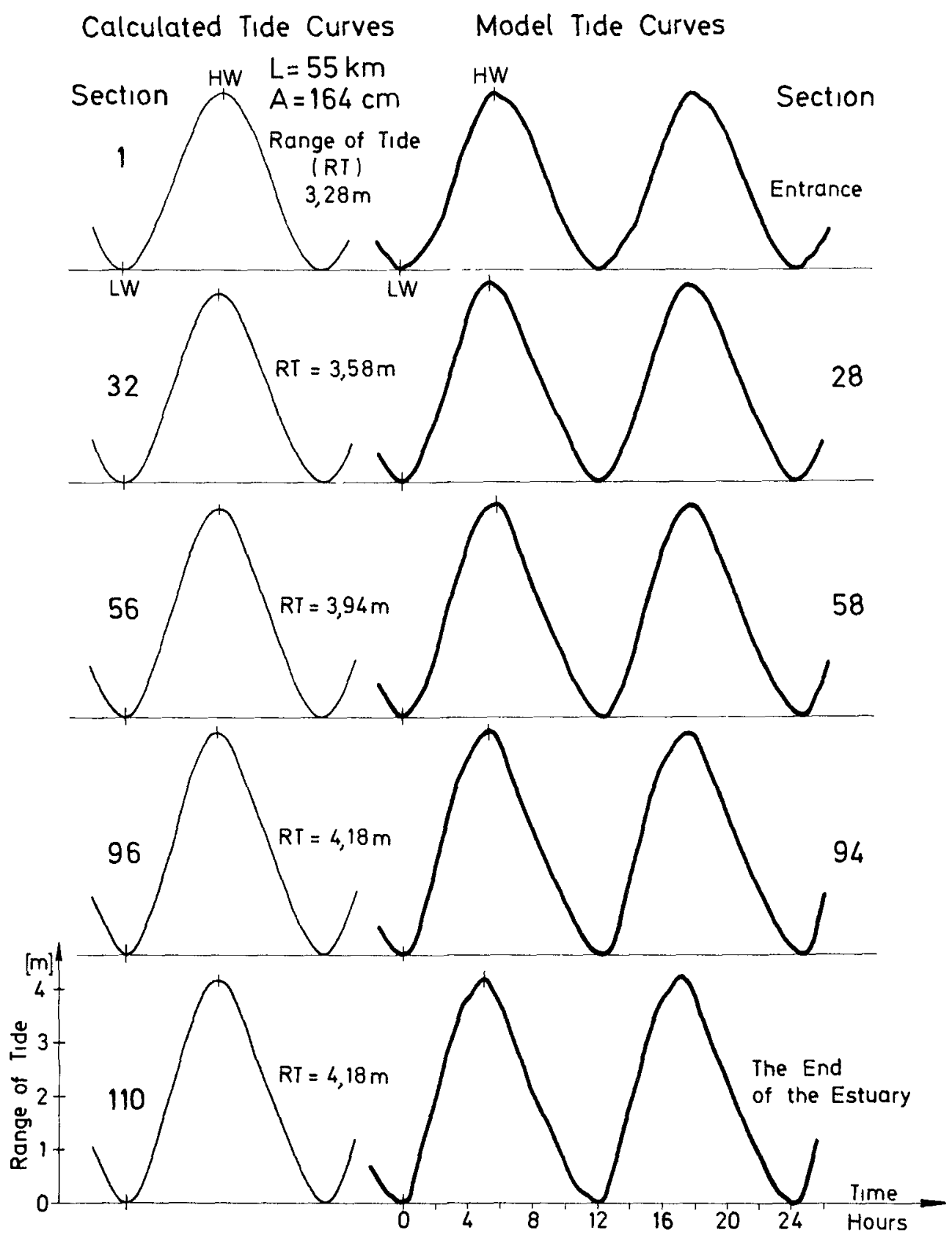

Fig 7 Rectangular Estuary $55 \mathrm{~km}$ 
The characteristic shows, that acceleration and deceleration are considerable and oscillations are small during the flood The distribution curve is more filled and the oscillations are greater during ebb stream

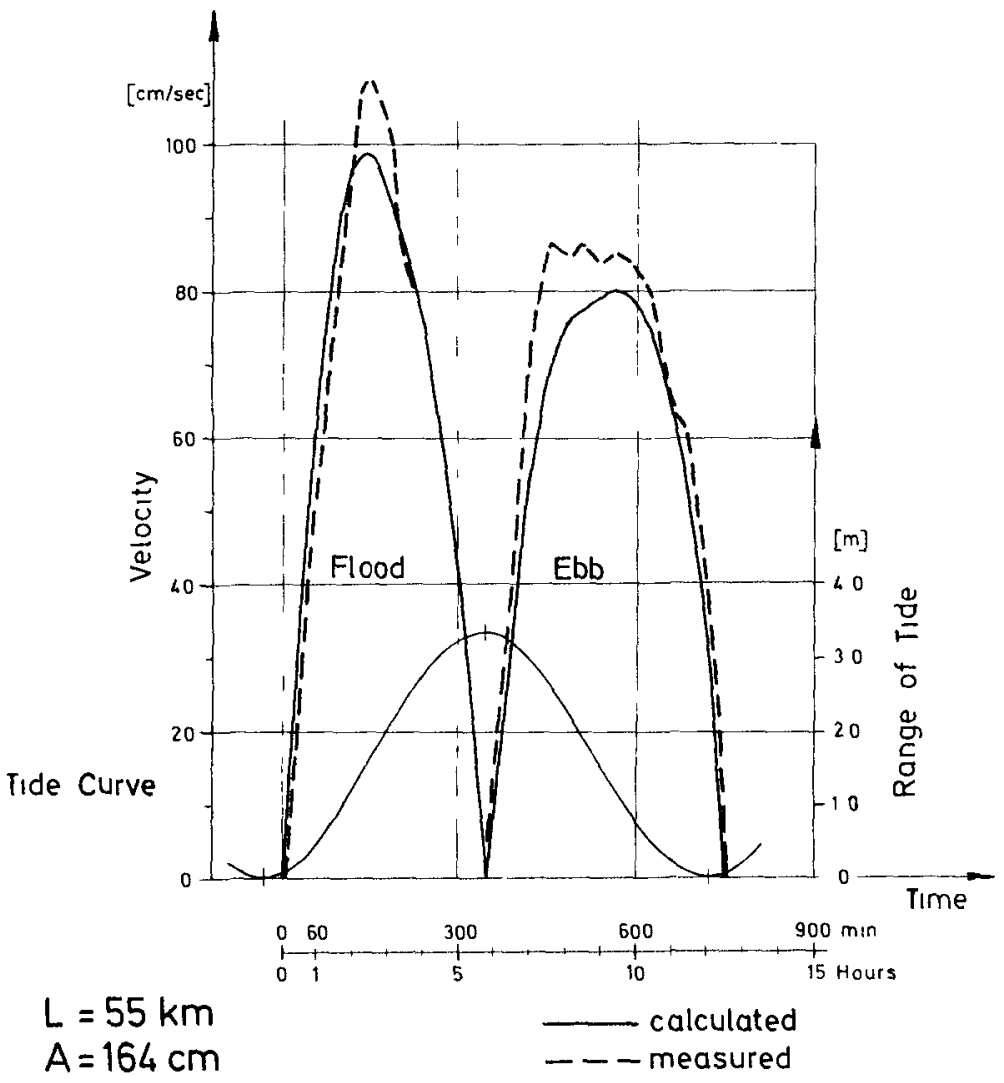

Fig 8 Typical velocity curve

The mean current velocities, averaged over cross section 5 (see Fig 4) for different water depths, are drawn in Fig. 9. The agreement with the corresponding calculated curve is satisfactory. 


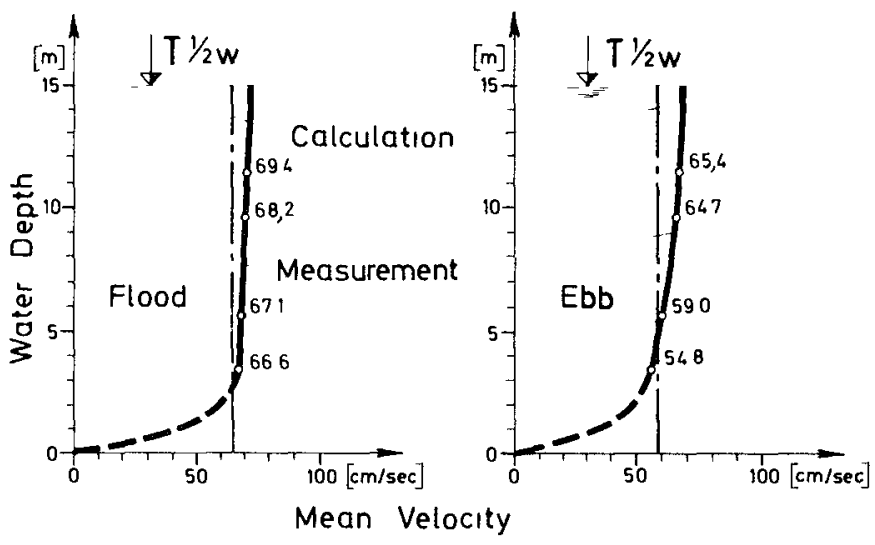

F1g. 9 Typical vertical distribution of velocity

Fig 10 shows the tidal ranges along the canal In order to make also a comparison between other canal sizes ( $16 \mathrm{~km}$ and 50 kilometer), the calculated curves are listed with another amplitude The differences between calculation and measurement for the $55-\mathrm{km}$ canal are small With regard to the model scale, only differences of 03 to $06 \mathrm{~mm}$ are present. 50 \% of these data are already inside of the accuracy of measurement

\section{SUMMARY}

It was shown by a systematic comparison between the hydrau$11 \mathrm{c}$ and the mathematical model, that the water levels and the mean current velocities for the investigated estuarine forms are in a fair agreement In these cases the one-dimensional method was sufficient

For the types $B$ and $E$, however, the cross extension place an important role. Therefore the corresponding HN-model must be considered two-dimensionally

Finally, further investigations will include the vertical dimension, in order to study the shear stress distribution at the bottom 


\section{REFERENCES}

\begin{tabular}{|c|c|c|}
\hline 1] & Hansen & $\begin{array}{l}\text { Hydrodynamical methods applied to } \\
\text { oceanographic problems } \\
\text { Mitt. Inst. f. Meereskunde, } \\
\text { Universitat Hamburg, Nr 1, } 1962\end{array}$ \\
\hline & Sundermann & $\begin{array}{l}\text { Ein Verglelch zwischen der analyti- } \\
\text { schen und der numerischen Rechnung } \\
\text { winderzeugter Stromungen und Wasser- } \\
\text { stande in einem Modelimeer mit An- } \\
\text { wendungen auf die Nordsee } \\
\text { Mitt Inst. f Meereskunde, } \\
\text { Universitat Hamburg, Nr 4, } 1966\end{array}$ \\
\hline & Volimers & $\begin{array}{l}\text { Grobflachige Tidemodelle } \\
\text { Das Gas-und Wasserfach, Heft } 42,1969\end{array}$ \\
\hline & $-\infty$ & $\begin{array}{l}\text { Die Reproduktion der Bewegungsvor- } \\
\text { gange im Meer mit Hilfe hydrodyna- } \\
\text { misch-numerischer Methoden } \\
\text { Mitt. Inst f. Meereskunde, } \\
\text { Un1versitat Hamburg, Nr. 5, } 1966\end{array}$ \\
\hline
\end{tabular}




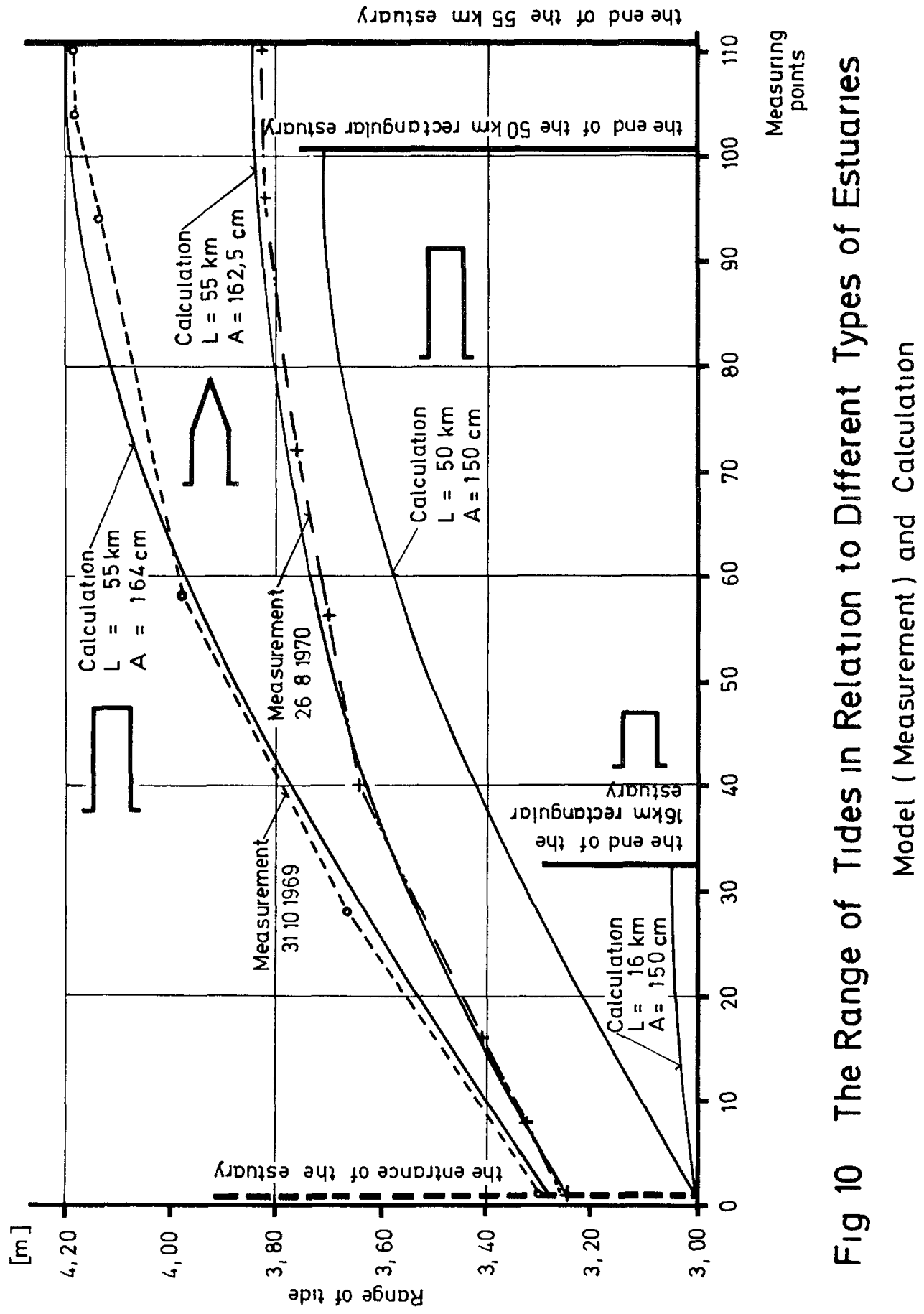



CHAPTER 130

DEFORMATION OF ROTATIONAL TIDAL

CURRENTS IN SHALLOW COASTAL WATER

\author{
Dr -Ing Harald Gohren \\ Strom - und Hafenbau Hamburg \\ Forschungsgruppe Neuwerk
}

Abstract

$=======$

Along gently sloping coasts of seas having rotary tidal currents, the symmetry of the rotary current is interrupted where the water is shallow As a result, residual counter currents are formed The dynamics of such a current system are described based on current measurements in the Elbe Estuary (southeastern North Sea) The residual currents of this area result in the movement of bottom sediment and are most likely responsible for a serıes of similar appearing morphologic features.

\title{
1 Rotary TIdal Waves
}

A typical effect of tidal motion in oceans as well as in bays is the formation of rotary tidal waves They are characterized by cotidal lines radiating out from a nodal or amphidromic point, where the tide vanishes to zero Amphidromic regions can be developed through the superposition of longitudinal and transverse oscillations Normally, however, they form as a result of the Corlolis 


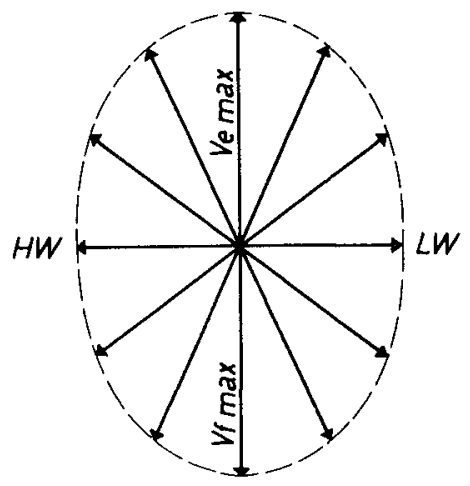

Polar diagram

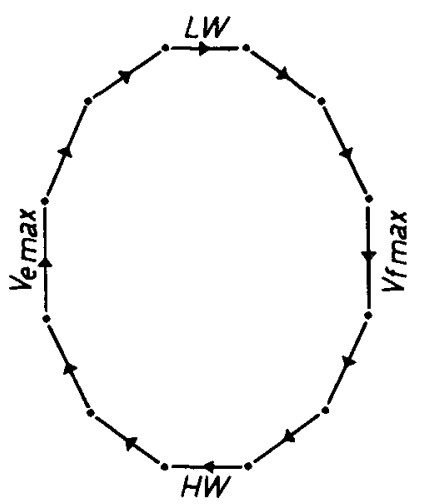

Vectorial track

Fig 1

Pattern of a rotational tidal current (shematic)
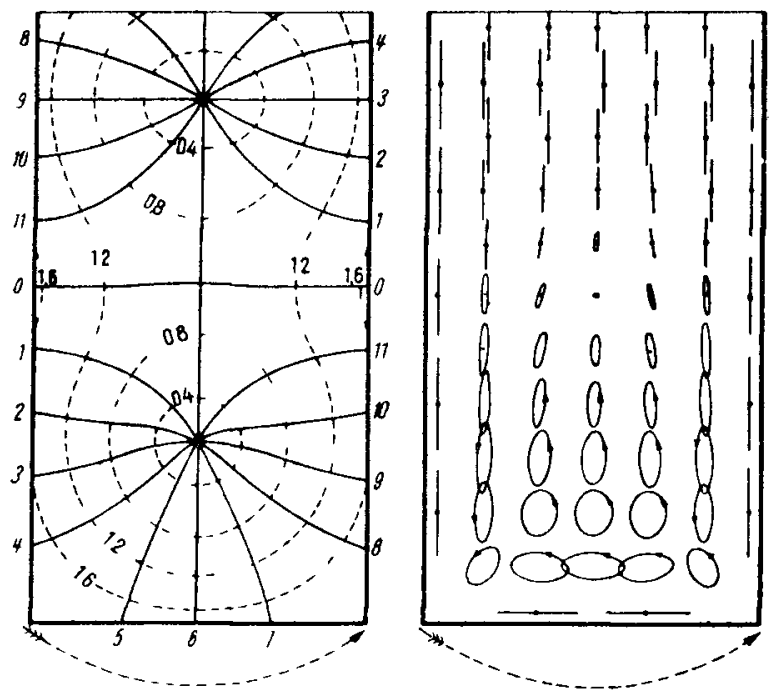

\section{Fig 2}

Reflection of a Kelvin Wave in a basin open at one end (from Dietrich, 1957)

Left cotidal Iines and tide range

Right currents 
force acting on progressive or on standing long-perlod waves This effect was described by $W$ Thomson (Lord Kelvin) in 1879 (Kelvin Waves)

Observations of tidal currents within an amphidromic system show characteristic circular or elliptical current patterns when the measurements from a complete tidal phase are plotted in vector form (Fig 1) Figure 2 shows Taylor's (1920) mathematical description of a Kelvin Wave in a basin open at one end The dimensions of his theoretical basin are comparable to the North Sea Two counterclockwiserotating amphidromıc systems are developed, the southernmost of which has rotating currents The general pattern of this example corresponds to the actual tidal motion found in the North Sea

\section{Deformation of Rotational Current in Shallow} Coastal Water

In the theoretical model of Fig 2, simplified marginal conditions result in the formation of reversing currents along the coastline on natural coasts the relationships are much more complex The deformation of the rotary current flow along the shallow coasts of the southern North Sea results in an interesting effect which can be of consequence for sand transport Large parts of this coast are composed of tidal flats ("Wattenmeere") (Fig 3), which are covered with water at high tide and fall dry at low tide Even further away from the coast the southern North Sea remains quite shallow, seldom reaching depths of over 30 or $40 \mathrm{~m}$

If symmetrical rotary currents with elliptical current patterns exist in deep water off such a shallow coast, then this symmetry will be altered in the shallower water zones This is illustrated in schematic form in Figure 4 The left drawing shows the symmetrical rotary current in deep water. The pattern is based on a counterclockwise current flow whose maximum velocity (reached during flood an ebb) is 


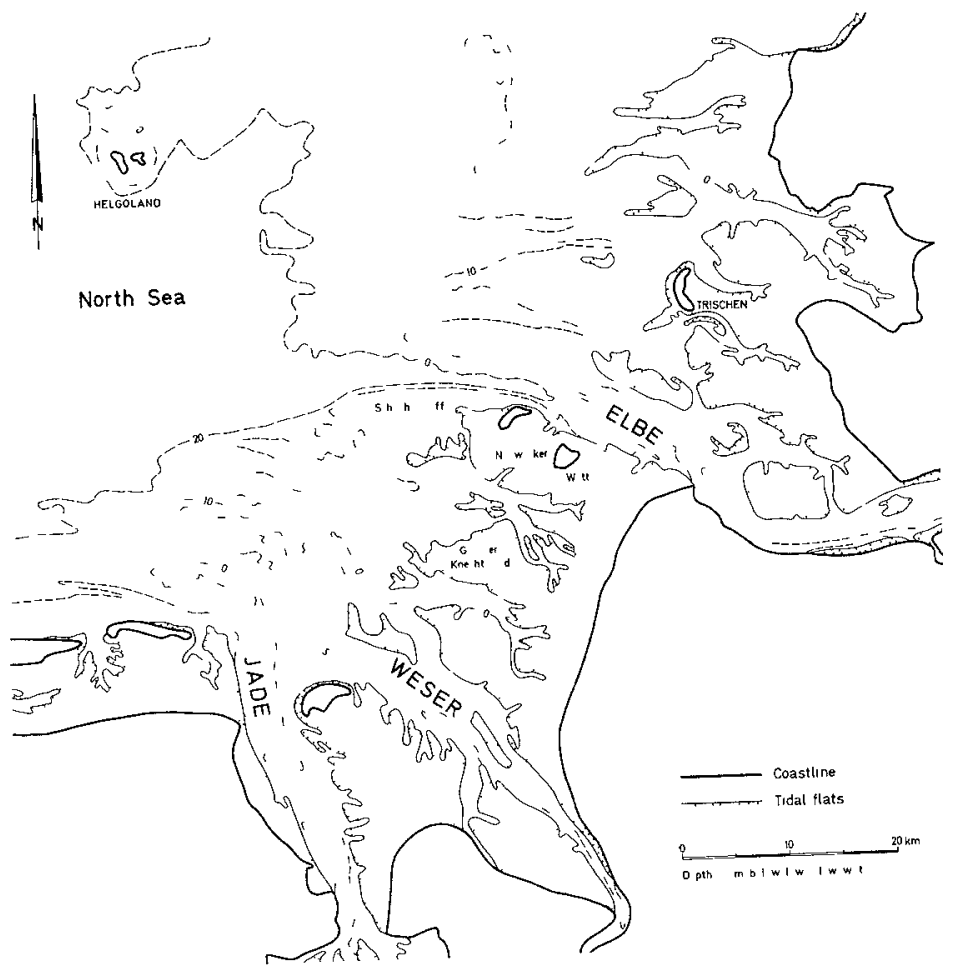

Fig 3

Map of the coastal area in the southeastern North Sea 
ROTATIONAL TIDAL CURRENTS
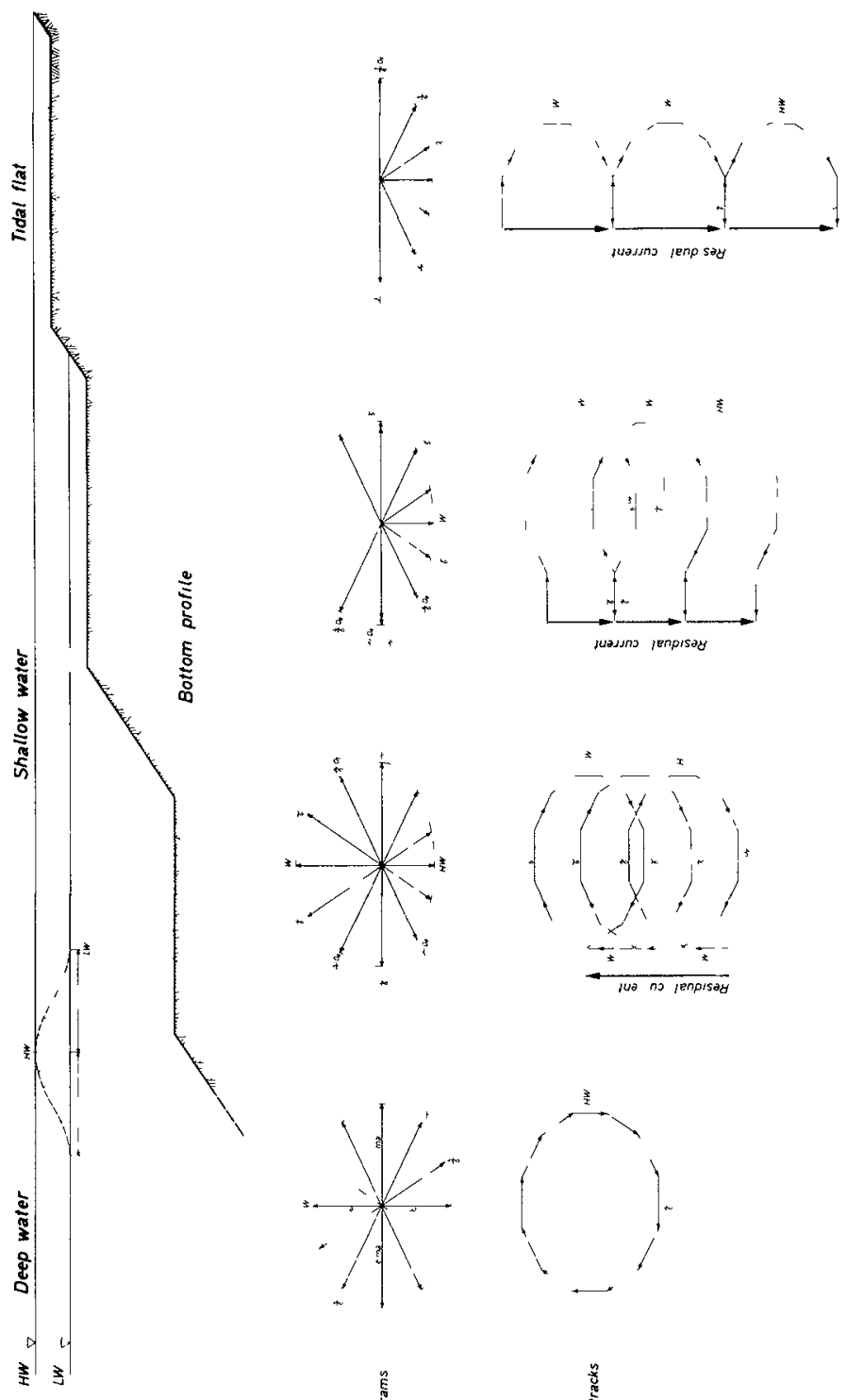

5
0
$+\infty$
3
3

7
0
0
0
0
0
0
3
0
-11
7
0
0
0

ज्ञ

$+40$

\& $\pi$
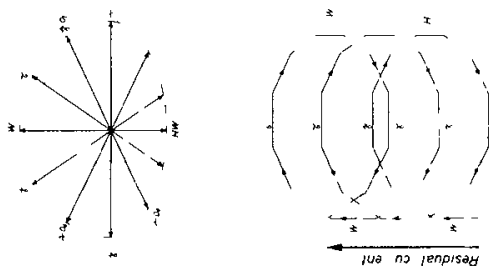

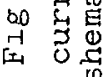

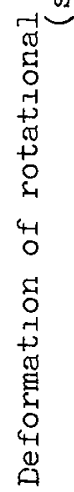
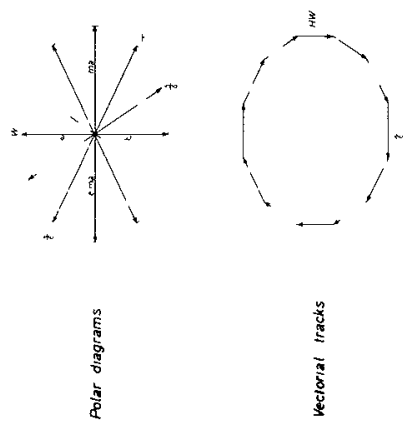
developed normal to the coast, and whose minimum velocity (at high and low water) runs parallel to the coast In general this compares to the relationships in the southern Elbe Estuary.

On the tidal flat (right in Fig 4) only the high water phase of the tidal current ellipse can be effective The current vectors yield a garland pattern with a strong residual current developed in the current direction which prevalls at hıgh tide

Two factors lead to the conclusion that opposing residual currents, like those on the tidal flat, must also develop in the shallow water zone between tidal flat and deep water (mıddle, Fig. 4) Even without a net transport of water, the current velocities reached during low water are greater because of the decreased water depth in comparison to the tidal range Consequently, the vectorial plot takes the form of a series of elongated loops showing a shift in the direction of flow at low water. This shift is intensifled through an actual transport of water in the same direction; the total movement counterbalances the residual flow in the tidal flat

The combination of these two factors changes the $t_{1-}$ dal ellıpse into a spiraling current pattern which has a residual flow corresponding to the current direction found at low tide In this way a system of opposing residual currents is developed which can influence both bottom morphology and sediment movement

\section{Data from the Elbe Estuary (southeastern North Sea)}

The Elbe Estuary is presently the site of a wideranging scientific program concerned with harbor planning (Laucht, 1968) Figure 5 shows the "Scharhornriff" portion of the investigation area (cf Fig. 3), a broad shallow- 


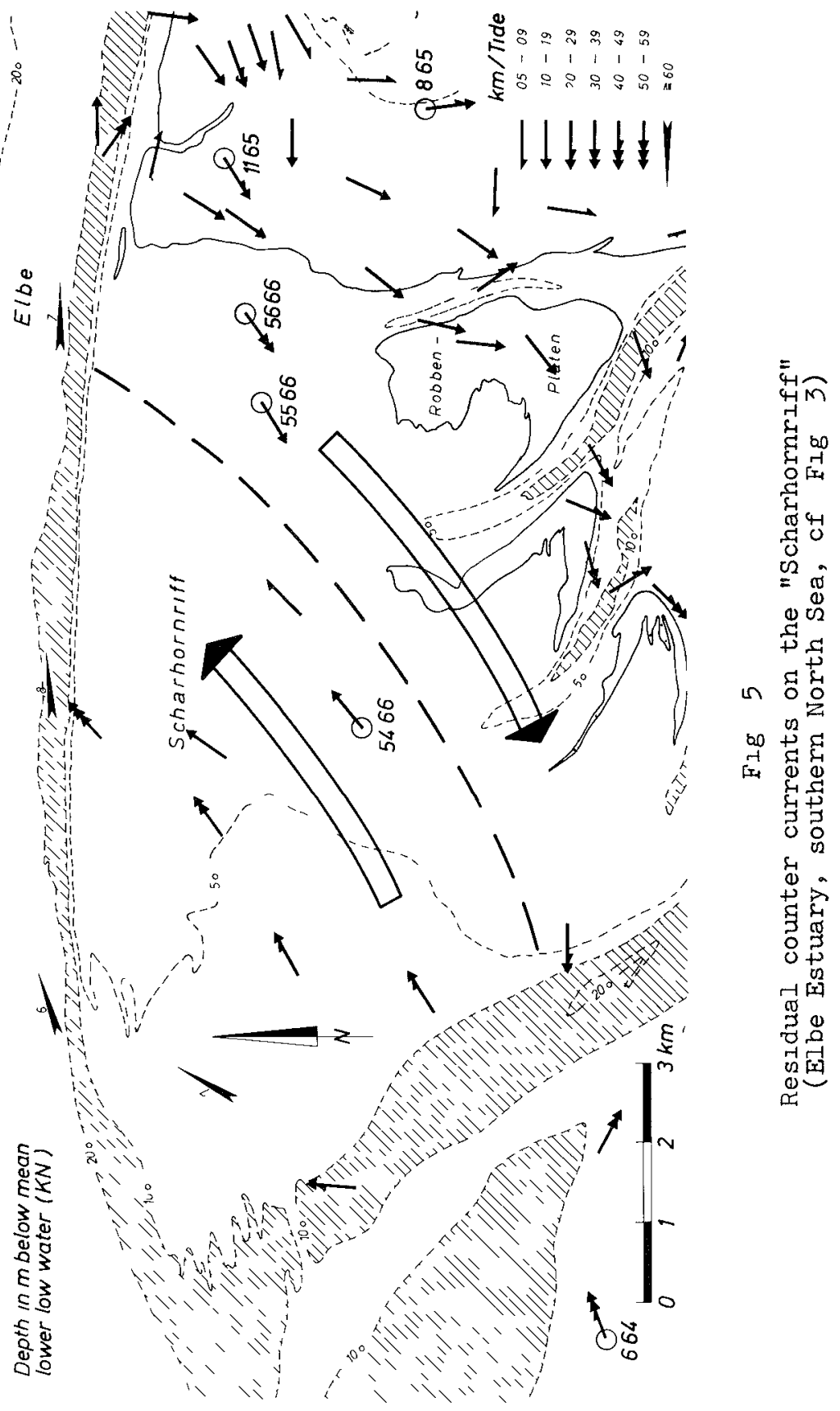


water zone bordering the Neuwerk tidal flat. Using the continuous recording current meters pictured in Figs. 10 and 11 , current measurements have been made at numerous points throughout the zone (Gohren, 1969) Each recording lasted approximately two weeks Calculations based on these records show a uniform residual current toward the northeast in the outer portion of the zone and an opposing southwest residual current in the inner portion and on the tidal flat. A sharp boundary line can be drawn between these two resldual current systems

Plots of current velocity and direction for several stations are given in Fig. 6 The rotary nature of the current can be clearly seen, especially at the outermost stations (6 64 and 5466 ).

At stations 5566 and 56.66 the decreased water depth is reflected in a disturbance of the rotary current pattern. On the tidal flat itself (stations 11.65 and 8.65 ) the motion is interrupted during the low tide period, however, during the time when the flat is covered with water, the rotary nature of the current is clearly present, especially at station 11.65

Figure 7 presents further substantiation that the residual counter currents lllustrated in Fig 5 are actually caused by the distortion of the rotary current in shallow water Here, the measurements from several stations are presented as vector tracks The closely spaced measurement intervals ( $5 \mathrm{~m} / \mathrm{n}$ ) result in nearly continuous curves. The loop-shaped current paths correspond exactly to the theoretical picture presented in Fig. 4. This seems to be confirmation that the residual current system in Fig 5 is not an isolated occurrence but rather a hydrodynamic process, which may be expected on any shallow coast where rotary tidal waves are developed Simllar measurements are not yet avallable for other coastal sections in the 

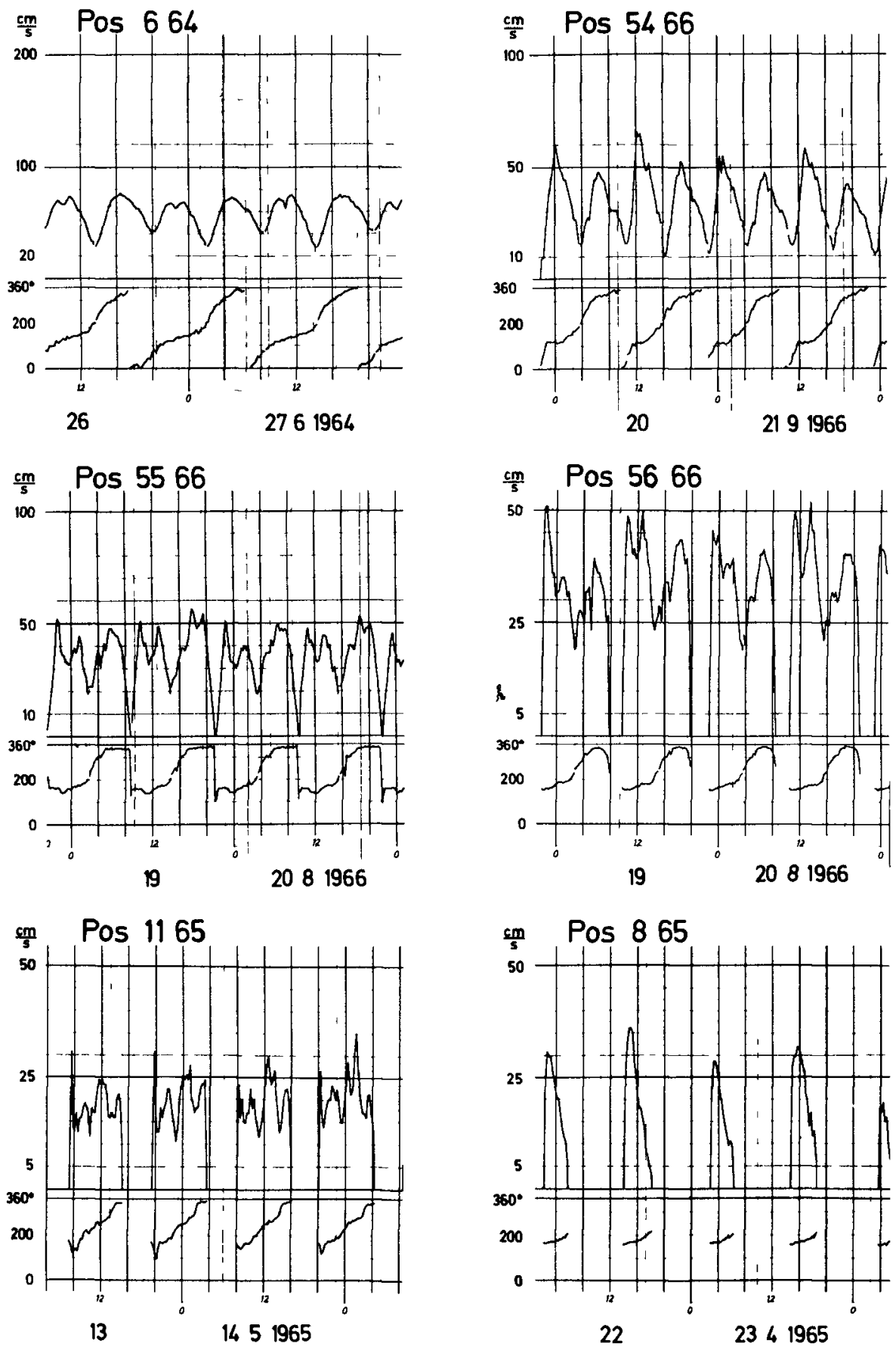

Fig 6

Results of current measurements from several stations on the "Scharhornriff" (cf Fig 5) 
2156

COASTAL ENGINEERING
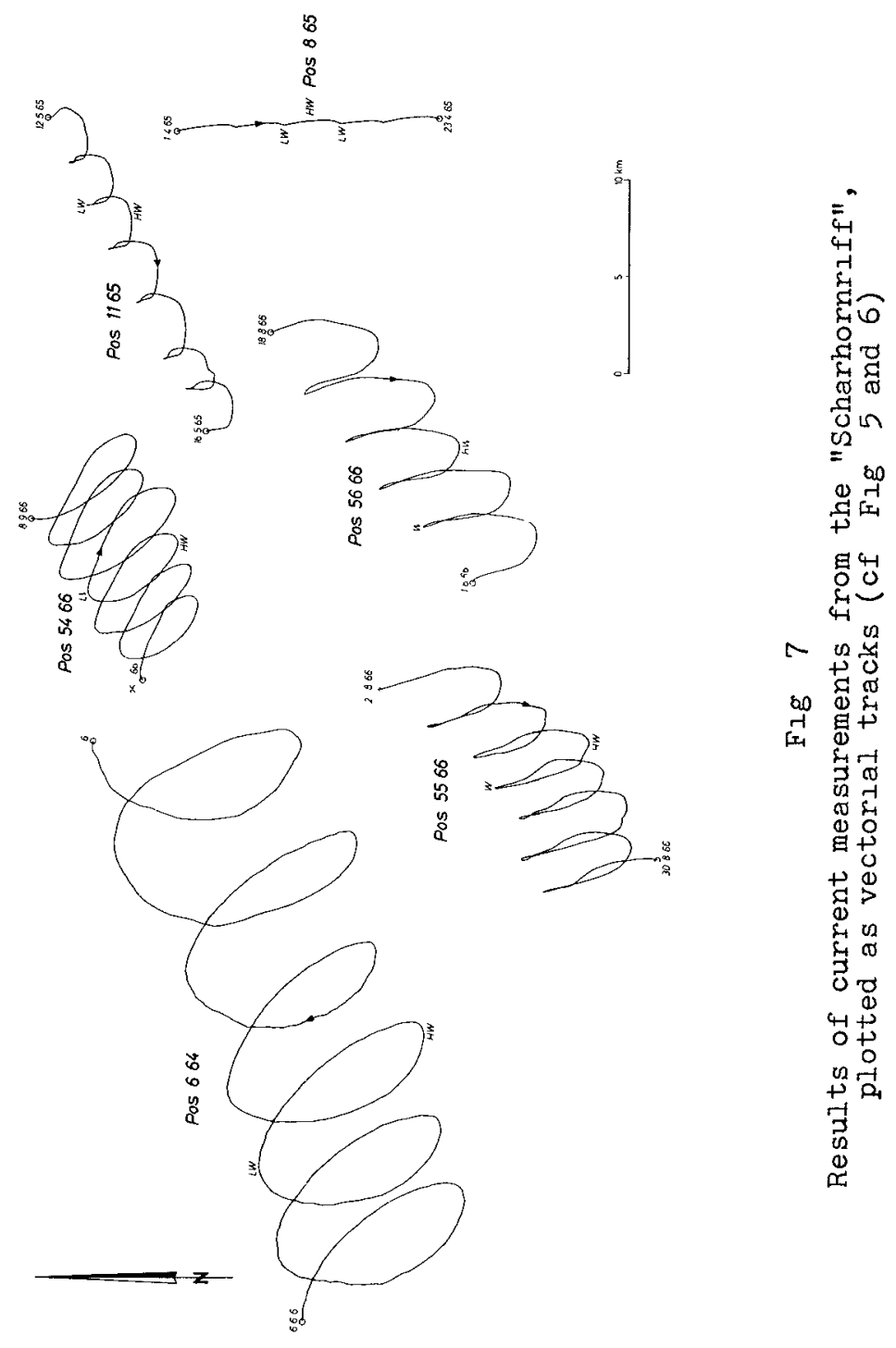
southeastern North Sea This is due to the fact that current measurements in exposed shallow water areas are extremely difficult to obtain and were possible in the Elbe Estuary only after the development of the equipment plctured in Figs 10 and 11

\section{Influence of Residual Counter Currents on Sand} Movement and Morphology

The direction of sand movement cannot be categorically equated with the residual current direction Figure 6 shows how difficult it is, when rotary tidal currents are present, to single out a dominant sand transport direction However, the maximum velocities developed in the "Scharhornriff" area are sufficlent to move sand grains, particularly when one conslders the added action of wind generated waves and surf

In order to establish the principal directions of sediment movement within this area of complex currents, a large number of tracer sand experıments were carrled out (Gohren, 1969) Figure 8 shows the dispersion of the tracer gralns at the conclusion of 5 of these experiments A comparison with Figure 5 shows good agreement between tracer movement and residual current direction the sediment movement on the outer portion of the "Scharhornriff" is toward the northeast or east, on the tidal flat near the Island of Scharhorn it is clearly toward the south A circulation of bed material is present which almost certainly exerts an influence on the bottom morphology

Southwest of the "Scharhornriff", the V-form sand banks of the "Robbenplaten" form consplcuous morphologlc features bordering the tidal flat Figure 9 shows this area together with the outlying parts of the Trischen and Knechtsand tidal flats, whlch lie north and south of the Elbe Estuary ( $f$ Fig 3) Isolated measurements 


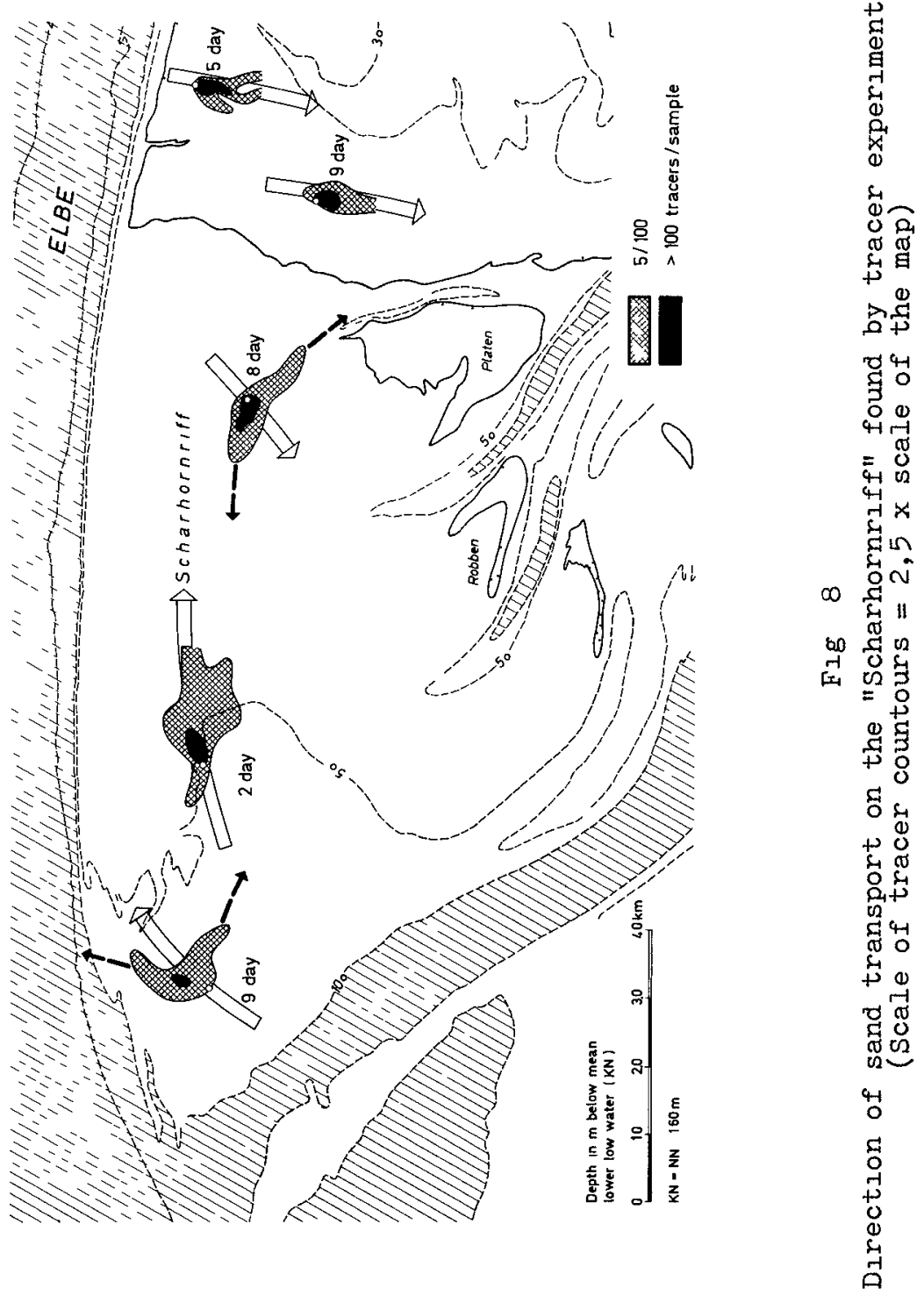


ROTATIONAL TIDAL CURRENTS

2159
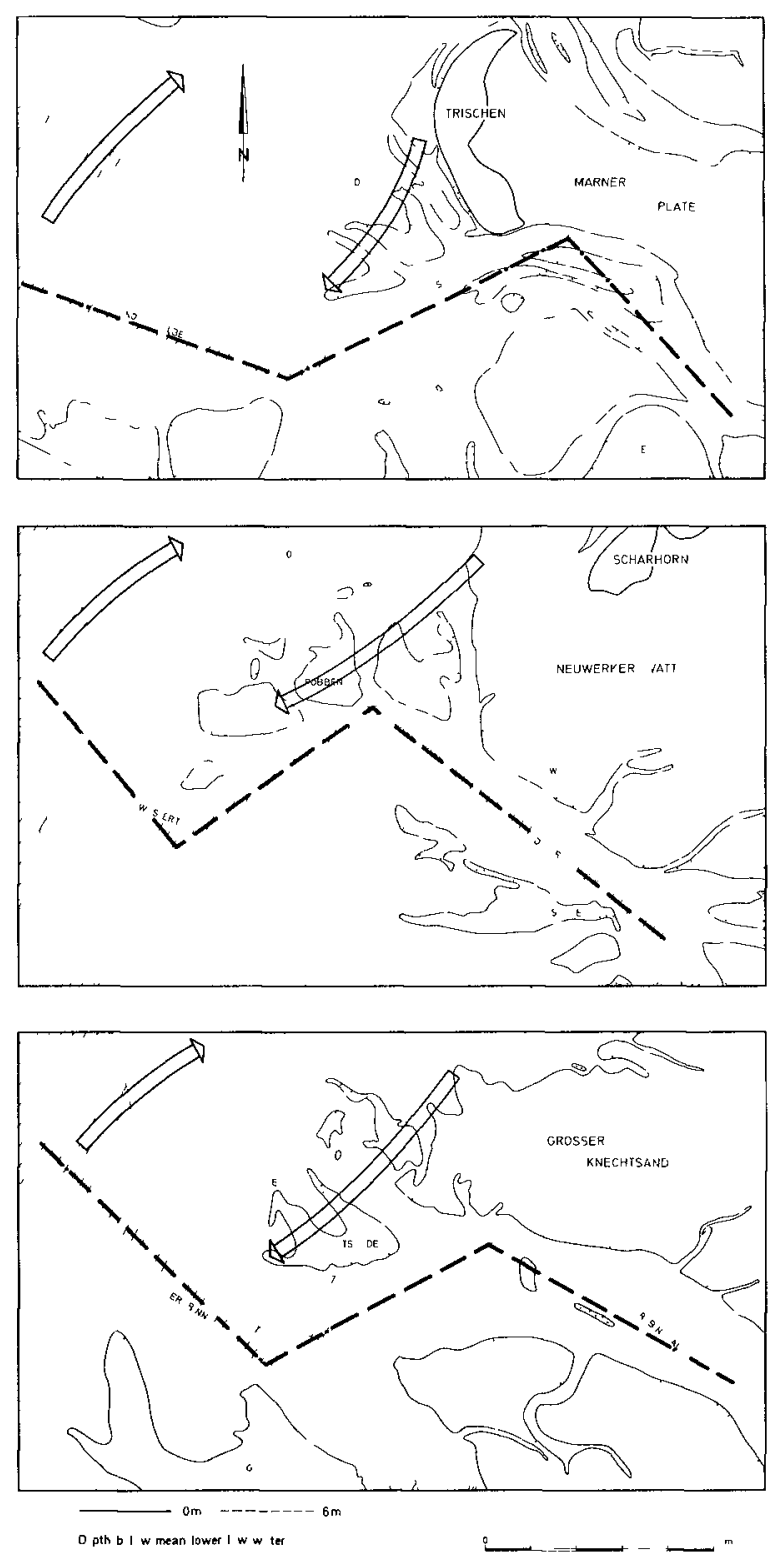

Fig 9

Influence of residual counter currents on the morphology of the outlying parts of the Tryschen, Neuwerk and knechtsand tidal flats (cf Fig 3) 


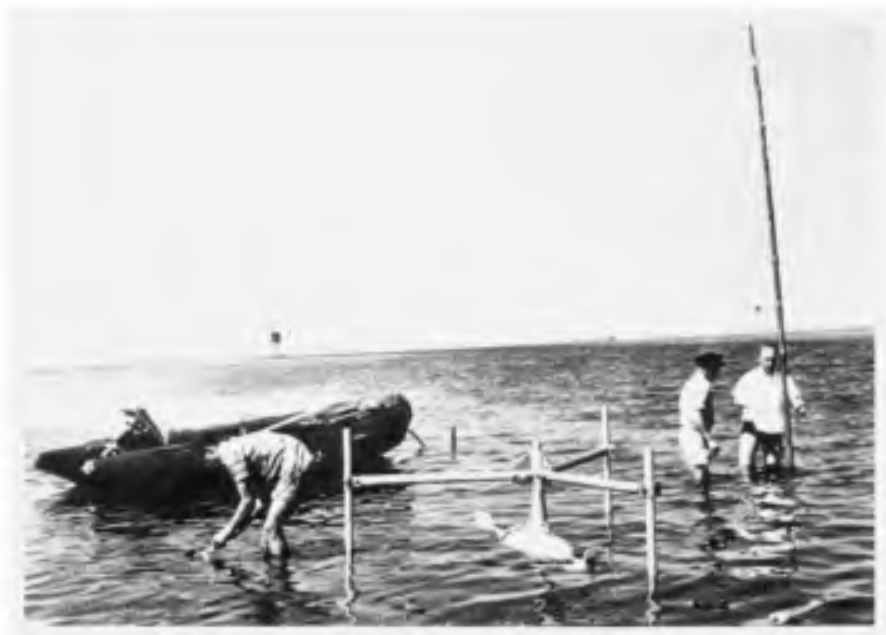

Fig. 10

Recording current meter for use in tidal flats

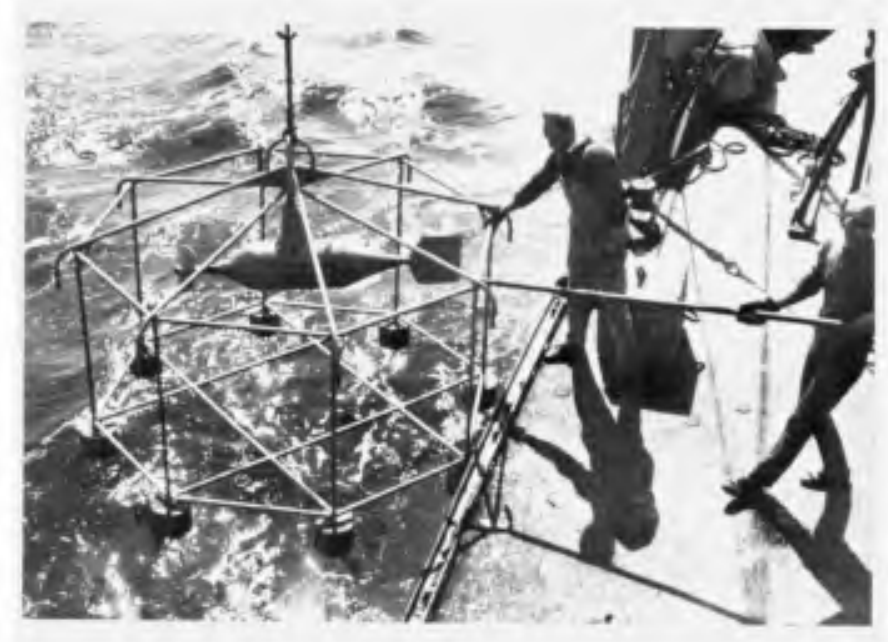

\author{
Fig. 11
}

Recording current meter for use in shallow coastal water 
have revealed opposing residual currents simllar to those of the "Scharhornriff" in these areas, and It is apparent that the morphologic development in all three areas exhlbits great simllarlty The sand banks seem to be areas of accumulation for the south or southwestward moving sediment This is also apparent from the configuration of the larger tidal channels south of the three flat areas (Neufahrwasser, TIll and Robinsbalje). All three exhlbit a decided S-form in their channels; the bends opposite the sandbanks reflect the southwestward directed forces. Further seaward the channels revert to a northwesterly course Here, as previously indicated, the sediment transport is directed toward the northeast and most likely exerts a force against the channels in this direction

The morphologic character and general similarity of the three areas mentioned above indicate an analogous mode of development which probably has its basis in comparable hydrodynamic conditions, le, the generation of residual counter currents caused by the interruption of the rotary tidal current in shallow water

REFERENCES

Dietrıch, G and Kalle, H. (1957) Allgemelne Meereskunde

Gohren, H (1969) Die Stromungsverhaltnisse im Elbmundungsgeblet Hamburger Kustenforschung, Heft 6

Gohren, H (1969) Untersuchungen mit fluoreszierenden Leitstoffen Im sudlichen Außenelbegeblet Hamburger Kustenforschung, Heft 10

Hansen, W (1952) Gezelten und Gezeltenstrome der halbtagıgen Hauptmondtıde $\mathrm{M}_{2}$ in der Nordsee Deutsche Hydrographlsche Zeltschrift, Erg Heft 1 


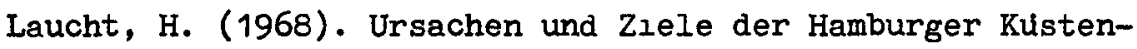
forschung. Hamburger Küstenforschung, Heft 1

Taylor, G.J (1920) Tidal oscillations in gulfs and rectangular basıns. Proc. London Math. Soc., 70

Thomson, W. (1879). On gravitational oscillations of rotating Water. Proc. Roy Soc Edinburgh 10 


\section{CHAPTER 131}

\section{RESONANCE 1N HARBORS OF ARBITRARY SHAPE}

by Jin-Jen Lee, Visiting Assistant Professor of Civil Engineering, University of Southern California Los Angeles, California, U S A and

Fredric Raichlen Associate Professor of Civil Engineering,

W M Keck Laboratory of Hydraulics \& Water Resources

Calıfornia lnstitute of Technology Pasadena Calıforna U S A

\section{ABSTRACT}

A theory is presented for analyzing the wave induced oscillations in an arbitrary shape harbor with constant depth which is connected to the open-sea The solution is formulated as an integral equation which is then approximated by a matrix equation The final solution is obtained by equating, at the harbor entrance the wave amplitude and its normal derivative obtained from the solutions for the regions outside and inside the harbor

The results of experiments conducted using a harbor model of the East and West Basins of Long Beach Harbor (Long Beach, Californa) are presented and compared to the theory Good agreement has been found between the theory and experiments

\section{INTRODUCTION}

Experience has shown that a natural or an artificial harbor can be excited by incident waves from the open-sea in such a way that large water surface osc1llations can result for certain wave periods This phenomena of resonance is similar to the dynamic response of mechanical or acoustical systems when exposed to time-varying forces, pressures, or displacements

Resonant oscillations in harbors (also termed seiche and harbor surging) can cause significant damage to moored ships and marine structures especially if the resonant period is close to that of the ship-mooring system In addition the currents induced by these oscillations can create navigation hazards Such resonant oscillations have occurred at many locations around the world and have damaged moored ships and dockside facilities, e g Table Bay Harbor Cape Town, South Africa In order to correct an existing resonance problem or design a harbor which is free of such problems one must be able to predict the resonant frequencies and the expected wave amplitude at various locations within the harbor as a function of the wave period

Many previous investigators have studied various aspect of the harbor resonance problem McNown (1952) studied seiches in circular harbors with small entrances by assuming an antinode occurred at the harbor entrance when the harbor was in resonance A similar method was applied to rectangular harbors by Kravtchenko and McNown (1955) Thus, due to this assumption the resonant periods were identical to those of the eigenvalues for the free oscillations of a completely closed circular (or rectangular) basin The problem of a rectangular harbor connected directly to the open-sea was investigated by Miles and Munk (1961) They included the effect of the wave radiation from the harbor * Formerly Research Fellow in Civil Engineerıng W M keck Labr of Hydr

\& Water Res, Californı Institute of Technology, Pasadena, Calif, U S A 
mouth to the open-sea thereby limiting the maximum wave amplitude within the harbor for the inviscid case to a finite value even at resonance Ippen and Goda (1963) also studied the problem of a rectangular harbor connected to the opensea by using the Fourier transformation method to evaluate the wave radiation from the entrance to the open-sea good agreement was found by them between the theory and experiments

In recent years studies on more complicated harbor geometries have been conducted Wilson Hendrickson and Kilmer (1965) studied the oscillations induccd by long waves in a basin with a complicated shape and variable depth incorporating an assumption of a nodal line (a line of zero amplitude) at the harbor entrance Leend $\epsilon$ tse (1967) developed a finite-diff $\epsilon$ rence numerical schenie for the propagation of long-period waves in an arbitrary shape basin of variable depth given the water surface elevations at the open boundary Recentiy, Hwang and Tuck (1970) in a study independent of the authors' study investigated the wave induced oscillations in an arbitrary shape basin with constant depth connected to the open-sea The1r approach was to superimpose scattered waves which were computed using a distribution of sources on the standing wave system In their analysis the calculation of the source stxengths along the entire reflecting boundary must be terminated at some distance from the entrance the location of such a termination is not obvious unless trial solutions are mad $\epsilon$

The present theoretical analysis is developed by applying Weber's solution of the Helmholtz equation in both the regions inside and outside the harbor with the final solution obtained by matching the wave amplitudes and their normal derivatives at the harbor entrance In this way some of the problems of application which other investigators have experienced are eliminated since only the wave characteristics at infinity in the open-sca need be specified to obtain a complete solution Experiments were performed in the laboratory to verify the theoretical solutions

\section{THEORETICAL ANALYSIS}

For the theoretical analysis the flow is assumed ir rotational and the fluid incompressible thus one can define a velocity potential $\Phi$ such that the fluid particle velocity can be expressed as a vector as $\vec{u}=\nabla \Phi$, where $\nabla$ is the gradient

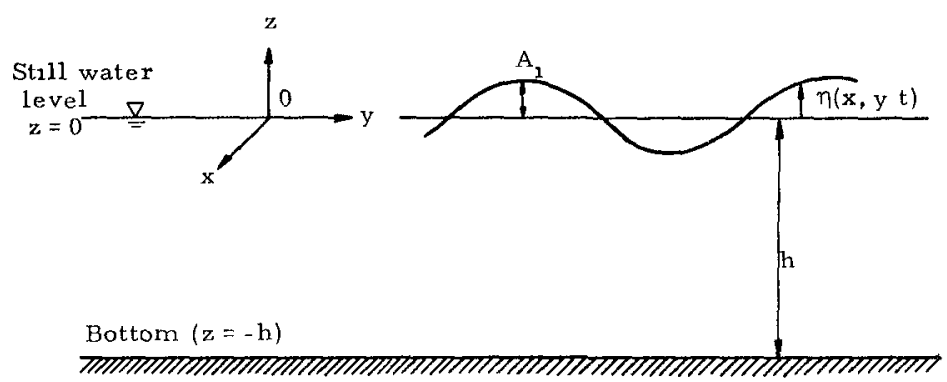

Fig 1 Definition sketch of the coordinate system 
operator (The coordinate system is defined in Fig I ) The velocity potential $\Phi$ must satisfy Laplace's cquation

$$
\frac{\partial^{2} \Phi}{\partial x^{2}}+\frac{\partial^{2} \Phi}{\partial y^{2}}+\frac{\partial^{2} \Phi}{\partial z^{2}}=0
$$

and number of prescribed boundary conditions one of these is that the flurd does not penetrate the solid boundaries which define the limits of the domain of interest $1 \mathrm{e} \quad \partial \Phi / \partial \mathrm{n}=0$ on solid boundaries (where $\mathrm{n}$ is normal to the boundary and directed outward)

If the water depth is considered constant the method of separation of variables can be used to obtain the function which represents the depthwise variation of the velocity potential Thus, within the limitation of small amplitude water wave theory the following form of the velocity potential $\Phi$ is found

$$
\Phi(x y z t)=\frac{1}{l \sigma} \frac{A_{1} g \cosh k(h+z)}{\cosh k h} f(x y) \epsilon^{-i \sigma t}
$$

where $\sigma$ is the angular frequency defined as $2 \pi / T$ (T is the wave period) $l=\sqrt{-1}$, $A_{1}$ is the wave amplitude at the crest of the incident wave $h$ is the water depth (assumed constant), $g$ is the acceleration of gravity $k$ is the wave number defined as $2 \pi / L$ ( $L$ is the wave length), and the function $f(x, y)$ termed the wave function describes the variation of $\Phi$ in the $x-y$ directions The function $f(x y)$ must satisfy the Helmholtz equation

$$
\frac{\partial^{2} f}{\partial x^{2}}+\frac{\partial^{2} f}{\partial y^{3}}+k^{2} f=0
$$

To complete the expression for the velocity potential $\Phi$ the remaining problem is to determine the function $f(x) y)$ which satisfies $\mathrm{Eq} \quad 3$ (known as the Helmholtz equation) and the following boundary conditions

(1) $\partial f / \partial n=0$ along all fuxed boundaries such as the coastline and the boundary of the harbor

(11) as $\sqrt{x^{2}+y^{2}} \rightarrow \infty$ there is no effect of the harbor on the wave system this is termed the radiation condition Mathematically the radiation condition is necessary to ensure a unique solution of the function $f(x y)$ in the unbounded domain

A muthod for solving the Helmholtz equation $\mathrm{Eq} 3$ for an arbitrary shape harbor is prcsented in the following The domain of interest is divided at the harbor entrance into two regions as shown in Fig 2 the infinite occan (Region 1) and the region bounded by the limits of the harbor (Region II) For convcnience the function $f$ of $\mathrm{Eq} 3$ in $R$ ingion II is denoted as $f_{2}$ and in Region I it is denoted as $f_{1}$

In Region II the function $f_{2}$ that satisfies the Helmholtz equation at any position $\vec{x}$ inside the harbor can be expressed as the following line integral

$$
f_{2}(\vec{x})=-\frac{\lambda}{4} \int_{s}\left\{f_{2}\left(\vec{x}_{0}\right) \frac{\partial}{\partial n}\left[H_{0}^{(l)}(k r)\right]-H_{0}^{(l)}(k r) \frac{\partial}{\partial n} f_{2}\left(\vec{x}_{0}\right)\right\} \text { d s }\left(\vec{x}_{o}\right)
$$

where $\vec{x}_{0}$ is the position vector of the boundary point $r$ is the dictance $\left|\vec{x}_{-}-\vec{x}_{0}\right|$ and $n$ is normal to the boundary and is directed outward The function 
$H_{0}^{(1)}(k r)=J_{0}(k r)+l Y_{0}(k r)$ and is termed the Hankel function of the $f_{1} r s t$ kind and zeroth order The integration in Eq 4 is to be performed along the boundary of the harbor traveling in a counter-clockwise direction

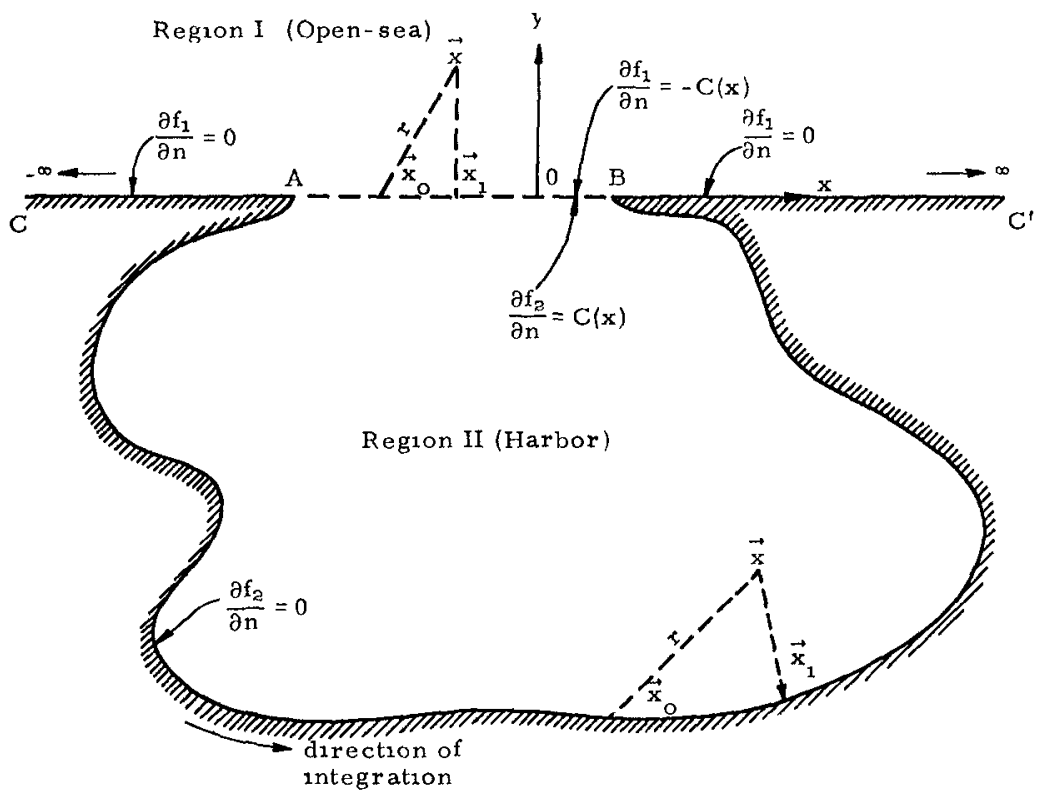

Fig 2 Defimition sketch of an arbitrary shape harbor

Eq 4 indicates that if one knows the value of $f_{2}$ and $\partial f_{2} / \partial n$ at the harbor boundary the function $f_{2}$ at any position $\vec{x}$ inside the harbor can be obtained readily However the value of $f_{z}$ at the boundary (including the harbor entrance) is not known at this stage of the development the value of $\partial f_{2} / \partial n$ at the harbor entrance is also not known although it is known that $\partial f_{2} / \partial n$ is zero on all solid boundaries within the harbor In order to evaluate $f_{2}\left(\vec{x}_{0}\right)$ at the boundary as a function of the value of $\partial f_{2} / \partial n$ at the harbor entrance the fleld point $\vec{x}$ is allowed to approach a boundary point $\vec{x}_{1}$ If the boundary is sectionally smooth an integral equation is obtained as follows

$$
f_{2}\left(\vec{x}_{1}\right)=-\frac{l}{2} \int_{s}\left[f_{2}\left(\vec{x}_{0}\right) \frac{\partial}{\partial n} H_{o}^{(1)}(k r)-H_{0}^{(1)}(k r)-\frac{\partial f_{2}}{\partial n}\left(\vec{x}_{0}\right)\right] d s\left(\vec{x}_{0}\right)
$$

where $r=\left|\vec{x}_{0}-\vec{x}_{1}\right|$ Although the exact solution of the integral q quation ( $\Gamma q$ 5) 
is difficult to obtain it is possible to obtain an approximate solution of Eq 5 by converting this integral equation into a matrix equation This is accomplished by dividing the boundary into a sufficiently large number of segments $(N)$ and the value of $f_{2}$ (or $\partial f_{2} / \partial n$ ) at each boundary segment is considered constant and equal to the value at the mid-point of each segment. Thus Eq 5 can be approximated by the following matrix equation

$$
(F)_{1}=-\frac{l}{2}\left[\sum_{j=1}^{N}\left(G_{n}\right)_{1 j}(F)_{j}-\sum_{j=1}^{N}(G)_{1 j}\left(F_{n}\right)_{j}\right] .
$$

$$
\text { for } 1=12
$$

using the notation

$$
\begin{aligned}
& (F)_{1}=f_{2}\left(\vec{x}_{1}\right) \text {, } \\
& 1=1,2 \\
& \text { N } \\
& \left(G_{n}\right)_{1 j}=-k H_{l}^{(1)}\left(k r_{1 j}\right) \frac{\partial r_{\mu J}}{\partial n} \Delta s_{j} \\
& =-k\left[J_{1}\left(k r_{1 j}\right)+l Y_{1}\left(k r_{1 j}\right)\right]\left[-\frac{x_{1}-x_{1}}{r_{1 j}}\left(\frac{\partial y}{\partial s}\right)+\frac{y_{1}-y_{j}}{r_{1 j}}\left(\frac{\partial x}{\partial s}\right)\right]_{j} \Delta s_{j}, \\
& \left(G_{n}\right)_{11}=2 \int_{0}^{\frac{1}{2} \Delta s} 1\left(-k_{1}^{(1)}(k r) \frac{\partial r}{\partial n}\right) d r \\
& x, j=1,2 \\
& N \quad i \neq j \\
& \approx \frac{i}{\pi}\left(\frac{\partial \mathrm{x}}{\partial \mathrm{s}} \frac{\partial^{2} \mathrm{y}}{\partial \mathrm{s}^{2}}-\frac{\partial^{2} \mathrm{x}}{\partial \mathrm{s}^{2}} \frac{\partial \mathrm{y}}{\partial \mathrm{s}}\right) \Delta_{1} \mathrm{~s}_{1}, \\
& 1=1,2 \\
& \text { N } \\
& (G)_{1 \jmath}=\mathrm{H}_{\mathrm{o}}^{(1)}\left(\mathrm{kr}_{1 \mathrm{j}}\right) \Delta \mathrm{s}_{\mathrm{j}} \text {, } \\
& 1 \mathrm{j}=1,2 \text {, } \\
& N \quad 1 \neq j \\
& \left(F_{n}\right)_{1}=\partial f_{2} / \partial n\left(\vec{x}_{1}\right) \text {, } \\
& 1=12 \text {, } \\
& N \\
& \text { (G) }=2 \int_{0}^{\frac{1}{2} \Delta s}{ }_{11} H_{0}^{(1)}(\mathrm{kr}) \mathrm{dr} \\
& \approx\left[1+l \frac{2}{\pi}\left[\log \frac{k \Delta s_{1}}{4}-042278\right]\right] \Delta s_{1} \quad 1=12 \quad N
\end{aligned}
$$

The vector $F_{n}$ in $E q \quad 6$ involves the unknown value of $\partial f_{2} / \partial n$ at the harbor entrance as well as the value of $\partial f_{z} / \partial n$ at the solid boundaries (these latter values are zero) Thus the vector $F_{n}$ can be represented as

$$
F_{n} \sum_{j=1}^{p} \delta_{1 j} C_{j}=U \underline{C}
$$

where $p$ is the total number of segments into which the harbor entrance is divided the vector $\underline{C}$ represents the $p$ unknown values of $\partial f_{\mathfrak{2}} / \partial \mathrm{n}$ at the midpoint of each entrance segment, and the matrix 
$\mathrm{j}=1,2$

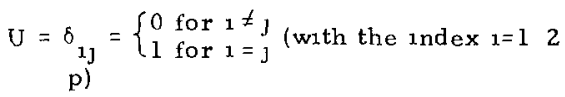

$\mathrm{N}$ and the index

Substituting Eq 8 into Eq 6 and rearranging one obtains

$$
f_{2}\left(\vec{x}_{1}\right)-\sum_{j=1}^{p} M_{1 j} C_{j}=M C \quad(\text { for } 1=12 \quad \text { N) . }
$$

in which the matrix $M=\left(\lambda / 2 G_{n}+I\right)^{-1}(\lambda / 2 G U)$ is a $N \times p$ matrix and can be computed directly (1 is an 1dentity matrix) Eq 9 shows that the function $f_{2}\left(\vec{x}_{1}\right)$ on the harbor boundary can be expressed as a function of the unk nown value of $\partial f_{p} / \partial \mathrm{n}$ at the entrance $\mathrm{Eq} 19$ can also be interpreted as the contribution to the value of $f_{2}$ on the harbor boundary from the superposition of the effect of $\mathrm{p}$ small harbor openings

In order to evaluate the unknown vector $\mathrm{C}$ defined in $\mathrm{Eq} 9,1$ e the value of $\partial f_{\partial} / \partial n$ at the entrance the function $f_{I}$ in Region $I$ at the harbor entrance must be expressed as a function of $\partial \mathrm{f}_{1} / \partial \mathrm{n}$ Thus using the "matching condition" at the entrance $f_{1}=f_{2}, \partial f_{1} / \partial n=-\partial f_{2} / \partial n$ one $1 \mathrm{~s}$ able to solve for the vector $\subseteq$ and the complete solution to the response problem can be obtained

For the region outside the harbor (Region 1 ) the function $f_{1}$ may be expressed as follows

$$
f_{1}(x, y)=f_{1}(x y)+f_{r}(x y)+f_{3}(x, y)
$$

where $f_{1}$ represents an incıdent wave function $f_{\mathbf{r}}$ represents a reflccted wave function considered to occur as if the harbor entrance were closed and $f_{3}$ (termed the radiated wave function) represents a correction to $f_{r}$ due to the presence of the harbor For the case of a periodic incident wave with the wave ray,perpendicular to the coastline the function $f_{1}(x y)$ can be represented by $\frac{1}{2} \mathrm{e}^{\text {tky }}$ (the factor $\frac{1}{2}$ is taken for convenience) Thus the reflected wave function $f_{r}$ can be represented by $f_{r}(x)=f_{1}(x, y)=\frac{1}{2} e-l k y$

The function $f_{3}(x)$ ) can be obtained by the same procedure as used in Eq 4 for determining the function $f_{2}$ the reader is referred to Lee (1969) for 1ts development as well as other details of the analysis

For the condition of a periodic incident wave propagating in a direction perpendicular to the coastline $\left(x\right.$-axis) the function $f_{1}$ at the harbor entrance can be expressed as

$$
f_{1}(x \quad 0)=1+\left(-\frac{l}{2}\right) \int_{\overline{A B}} H_{0}^{(1)}\left(k\left|x-x_{o}\right|\right)\left[\frac{\partial}{\partial n} f_{2}\left(x_{0} 0\right)\right] d x_{o}
$$

The first term on the $r$ ight-hand side of Eq 11 represents the incident wave plus the reflected wave if the harbor entrance is closed, the second term represents the contribution from the radiated wave function $f_{3}$ It is noted that in deriving $\mathrm{Eq} 11$ the relation $\partial \mathrm{f}_{1} / \partial \mathrm{n}=\partial \mathrm{f}_{3} / \partial \mathrm{n}=-\partial \mathrm{f}_{2} / \partial \mathrm{n}$ has been used at the harbor entrance 
Eq 11 can be approximated by a matrix equation as

$$
f_{1}\left(\vec{x}_{1}\right)=1+\left(-\frac{l}{2}\right) \sum_{j=1}^{p} H_{1 j} C_{j}, \quad \text { (for } 1=12 \quad \text { p) }
$$

where

$$
\begin{array}{ccc}
\mathrm{H}_{1 \mathrm{j}}=\mathrm{H}_{0}^{(1)}\left(\mathrm{kr}_{1 \mathrm{j}}\right) \Delta \mathrm{s}_{\mathrm{j}} & \text { for } 1 \mathrm{j}=1 \quad 2 \quad \mathrm{p} \quad 1 \neq \mathrm{J} \\
\mathrm{H}_{11}=\left[1+\lambda \frac{2}{\pi}\left(\log \left(\frac{\mathrm{k} \Delta \mathrm{s}_{1}}{4}\right)-0\right.\right. & 42278)] \Delta s_{1} \text { for } 1=1 \quad 2 \quad \mathrm{p},
\end{array}
$$

and the vector $\underline{C}$ is the derivative $\partial f_{2} / \partial n$ at the harbor entrance as defined in Eq 8

Eqs 9 and 12 can now be equated to solve for the unknown value of $\partial \mathrm{f}_{2} / \partial \mathrm{n}$ at the harbor entrance 1 e the vector $\underline{C}$ This is done by first taking the first p equations from $\mathrm{Eq} 9$

$$
f_{z}\left(x_{1}\right)=\sum_{j=1}^{p} M_{1 j} C_{j}=M_{p} \cdot \underline{C}
$$

In which the index $1=12, \quad \mathrm{p}$ and the matrix $M_{p}$ is a $p \times p$ matrix obtaned from the first $p$ rows of the matrix M Then by equating Eq 13 to $\mathrm{Eq} 12$ and solving for the unknown vector $\underline{\mathrm{C}}$ one obtains

$$
\underline{C}=\left(M_{\mathrm{p}}+\frac{l}{2} \mathrm{H}\right)^{-1} \frac{1}{1}
$$

where $\left(M_{\mathrm{p}}+\frac{l}{2} \mathrm{H}\right)^{-1}$ is the inverse of the matrix $\left(\mathrm{M}_{\mathrm{p}}+\frac{l}{2} \mathrm{H}\right)$, and 1 is the vector with each $p$ element equal to unity

With the value of $\partial f_{2} / \partial n$ at the entrance 1 e the vector $\subseteq$ determined from $\mathrm{Eq} 14$ the value of $\mathrm{f}_{2}\left(\overrightarrow{\mathrm{x}}_{1}\right)$ at the harbor boundary can be evaluated from Eq 9 and the value of $f_{2}(\vec{x})$ at any position inside the harbor can be obtained from the followng discrete form of Eq 4

$$
f_{z}(\vec{x})=-\frac{\lambda}{4}\left\{\sum_{j=1}^{N} f_{z}\left(\vec{x}_{j}\right)\left[-k H_{1}^{(1)}(k r) \frac{\partial r}{\partial r}\right] \Delta s_{j}-\sum_{j=1}^{p} H_{0}^{(1)}(k r) c_{j} \Delta s_{j}\right\}
$$

where $\vec{x}_{j}$ is at the midpoint of the $j^{\text {th }}$ boundary segment and $r=\left|\vec{x}-\vec{x}_{j}\right|$

The response of a harbor to incident waves is described by a parameter called the "amplification factor" which is defined as the ratio of the wave amplitude at any position ( $x$ y) inside the harbor to the sum of the incident and the reflected wave amplitude at the coastline (with the harbor entrance closed)

$$
R=\frac{\left|n_{2}(x y t)\right|}{\left|A_{2}\left(f_{1}+f_{r}\right) e^{-l \sigma t}\right|}=\frac{\left|A_{2} f_{2}(x, y) e^{-l \sigma t}\right|}{\left|A_{1} \cdot 1 \cdot e^{-l \sigma t}\right|}=\left|f_{2}(x, y)\right|
$$




\section{EXPERIMENTAL EQUIPMENT}

Experiments were conducted in the laboratory in a wave basin $1 \mathrm{ft} 9$ in deep $15 \mathrm{ft} 5 \mathrm{in}$ wide, and $31 \mathrm{ft} 5 \mathrm{in}$ long An overall view of the wave basin is presented in Fig 3 The wave generator was a pendulum type $11 \mathrm{ft} 8$ in long, $2 \mathrm{ft}$ high located at one end of the basin and 1 t was designed to operate either as a paddle- or piston-type wave generator with a maximun stroke of \pm 6 in (For detailed description and design considerations the reader is referred to Ralchlen (1965)) Wave perlods ranging from 034 see to 38 sec can be obtained

The wave period was determined by a pulse counting technique the pulses are generated by interrupting a light beam which was direetcd at a photo cell by a disc with 360 evenly spaced holes arranged in a cirele around its outer edge The voltage pulses which are produced in this manner are counted by an industrial counter over an interval of $10 \mathrm{sec}$ Thus the wave period measured was an average over $10 \mathrm{sec}$ and throughout an experiment this period varied at most by to $05 \%$

Wave amplitudes were measured electroncally using resistance wave gages and an oscillograph recorder The wave gage was calibrated before and after an experiment (approximately one hour apart) Even though these ealibrations showed very little difference a calibration eurve representing an average over the duration of an experiment was used in reducing the experimental data (see Lee (1969))

In order to simulate the open-sea in the laboratory wave basin two types of wave energy dissipators were employed a wave filter placed in front of the wave generator and wave absorbers located along the side-walls of the wave basin these can be seen in Fig 3 The wave filter was li ft 9 in long, $1 \mathrm{ft}$ $4 \mathrm{in} \mathrm{high} \mathrm{and} 5 \mathrm{ft}$ thick in the direction of wave propagation and was constructed of 70 sheets of galvanized iron wire sereen with each sheet spaced $08 \mathrm{in}$ apart The wire diameter of the screens was $0011 \mathrm{nn}$ with 18 wires per inch in one direction and 14 wres per inch in the other The wave absorbers placed along the sidewalls of the basin were each $1 \mathrm{ft} 6$ in high $1 \mathrm{ft} 10$ in thick normal to the sidewall, and $30 \mathrm{ft}$ long and consisted of 50 equally spaced layers of the same galvanized 1 ron screen as used in the wave filtcr The majority of waves used in the experiments were reduced in amplitude by at least $80 \%$ as the result of passing through the wave filter (or absorber) reflecting from the wave machine (or wall) and passing through the wave filter (or absorber) again Such wave encrgy dissipating materials were necessary in order to simulate the open-se a condition satisfactorily without this wave filter and these absorbers waves radiated from the harbor entrance would be reflected from the wave paddle and the sidewalls of the basin creating a wave system which is quite different from the open-sea This problem was described by Ralchlen and Ippen (1965) in which it was shown that, due to coupling between the harbor and the wave basin the response of a rectangular harbor in a highly reflective basin was radically different from that of a similar harbor connected to the open sea

In order to fully test the theory developed it was diended to use a model of Long Beach Harbor which in the past experienced problems from long period waves This model shown in Fig 4 was constructed from in thick lucite 


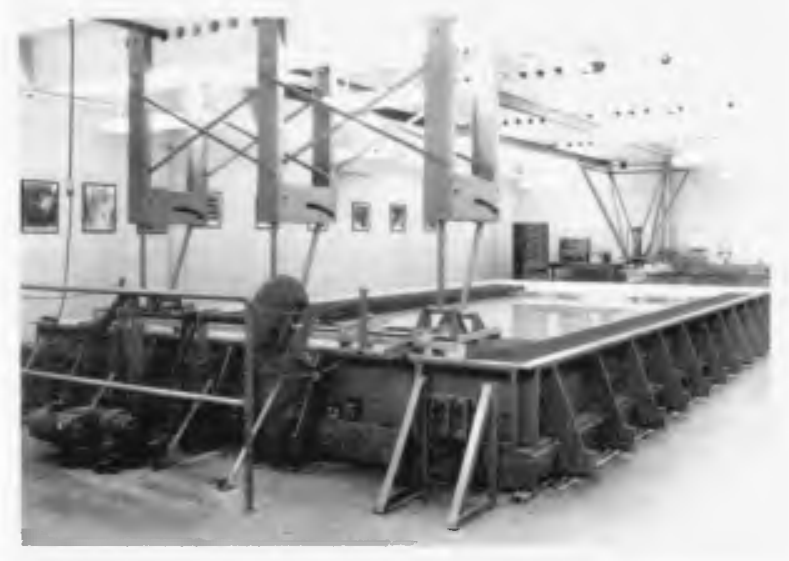

Fig. 3 Over-all view of the wave basin and wave generator with wave filter and absorbers in place

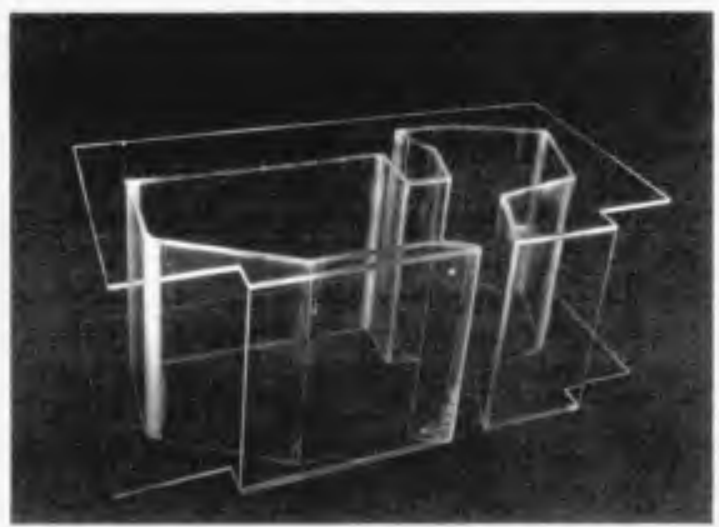

Fig. 4 Model of the East and West Basins of Long Beach Harbor (Long Beach, California, U.S.A.) 
plate with a planform which was simplified from the prototype East and West Basuns of Long Beach Harbor The harbor model bult to a horizontal scale equal to 14700 was designed so that it would fit into an opening at the center of a false wall simulating a perfectly reflecting coastline which was installed $27 \mathrm{ft} 6 \mathrm{in}$ from and parallel to the wave paddle

\section{PRESENTATION AND DISCUSSION OF RESULTS}

Prior to applying this theory to a complirated harbor the theory was applied to harbors of simpler shapes circular and rectangular The circular harbor represents an extreme where the harbor boundary is curved and the tangent to the boundary is continuously changing direction The rectangular shape harbor represents the other cxtreme since the harbor boundary is composed of stralght lines, along each line the direction of the tangent to the boundary remains the same It has been found (see Lee 1969 1970) that the results of this theory appljed to circular and rectangular harbors agree well with corresponding experiments

As mentioned previously in order to verify the theory for a harbor of complicated shape Long Beach Harbor was studied theoretically and experimentally in the laboratory A sketch of the harbor model which was used is presented in Fig 5 which shows the width of the entrance as $02 \mathrm{ft}$ and the characteristic dimension of the harbor a equal to $144 \mathrm{ft}$ The water depth was constant in both the harbor and the "open-sea" and equal to $1 \mathrm{ft}$

Re sponse curves at four different locations inside the harbor are presented in Figs 6 to 9 The four points are designated as $A$ B, C, D and their relative positions in the model are shown in Fig 5 as A $1030 \mathrm{ft}$, $-0525 \mathrm{ft}), \mathrm{B}(030 \mathrm{ft},-096 \mathrm{ft}) \mathrm{C}(132 \mathrm{ft},-096 \mathrm{ft})$, and $D(-045 \mathrm{ft}$, - $1245 \mathrm{ft}$ ), where the first number inside the parenthesis is the $x$-coordinate and the second number is the $y$-coordinate For all the response curves the abscissa is the wave number parameter $k a$ (where $k$ is the wave number, and "a" is shown in Fig 5) the ordinate is the amplification factor R, defined as the wave amplitude at point $A$ (or $B, C, D$ ) divided by the average standing wave amplitude at the harbor entrance when the entrance is closed (see $\mathrm{Eq} \mathrm{16)}$

The theoretical results ohtanned are shown as solid lines in the response curves whilf the experimental results are shown as circles In applying the theory the boundarv of the harbor is divided into 75 unequal straight-line segments including two segments for the harbor entrance The segments are numbered counter-clockwise starting from the right-hand limit of the harbor entrance and this numbering svstem is also shown in Fig 5 For accurate results the length of these boundary segments should be less than appros. mately one-tenth of the smallest wave length investigated (see Lee 1969)

From Figs 678 , and 9 it as seen that the theoretical results agree well with the experimental data at all four locations which werc unvestigated The complicated shape of the response curves are due to the ir regular shape of the harbor and the fact that thıs harbor $r e$ ally consists of two coupled basins One common feature of the four response curves is that while the theory has prediced the frequency of every resonant mode of oscillation correctly the theoretical amplification factor at resonance is slightly larger than the experimental data especially for the resonant modes at larger values of $\mathrm{ka}$ There are two possible reasons for this First in applying 


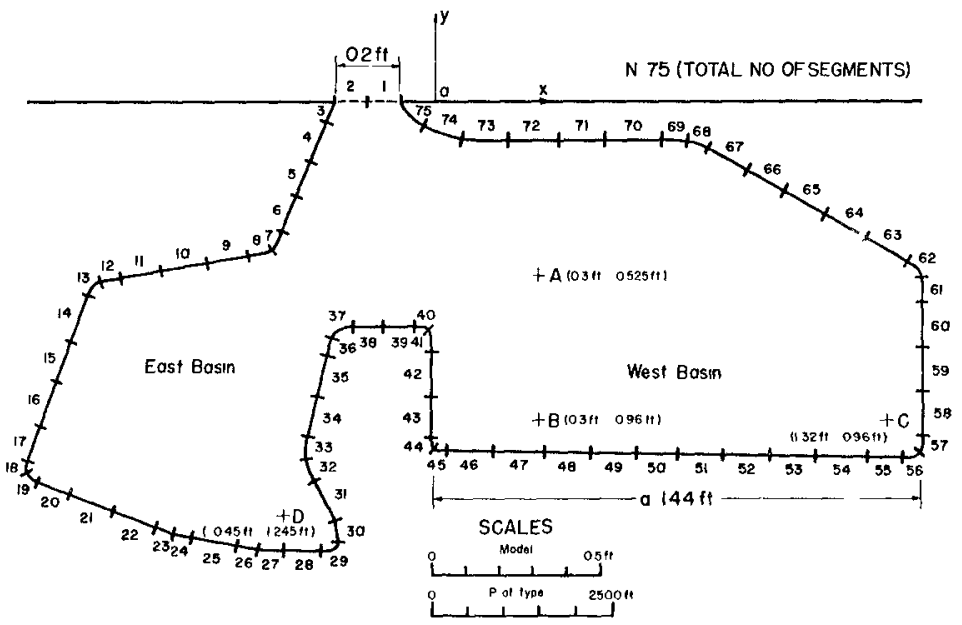

Fig 5 Dimensions sketch of the Long Beach Harbor model

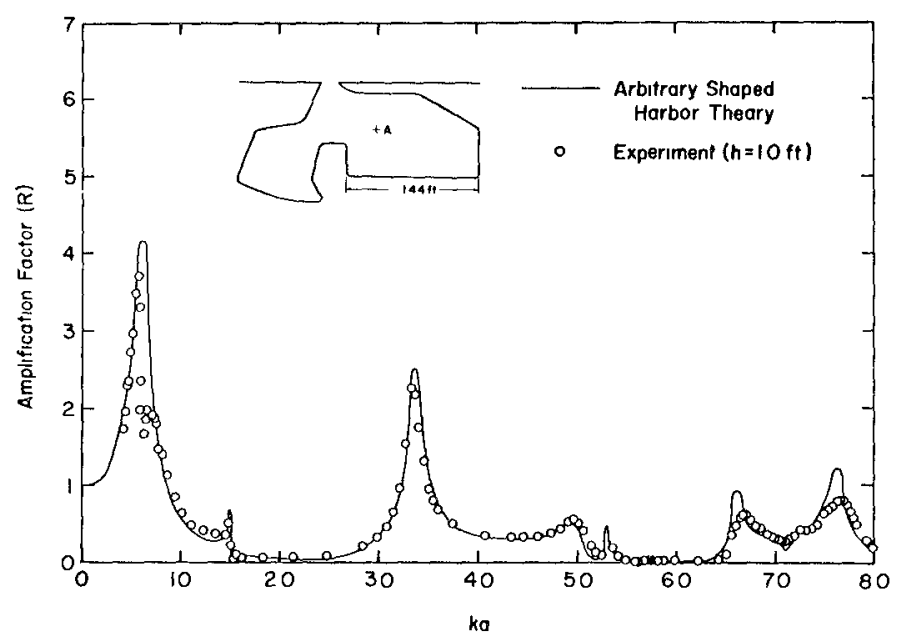

Fig 6 Response curve at point A of the Long Beach Farbor model 


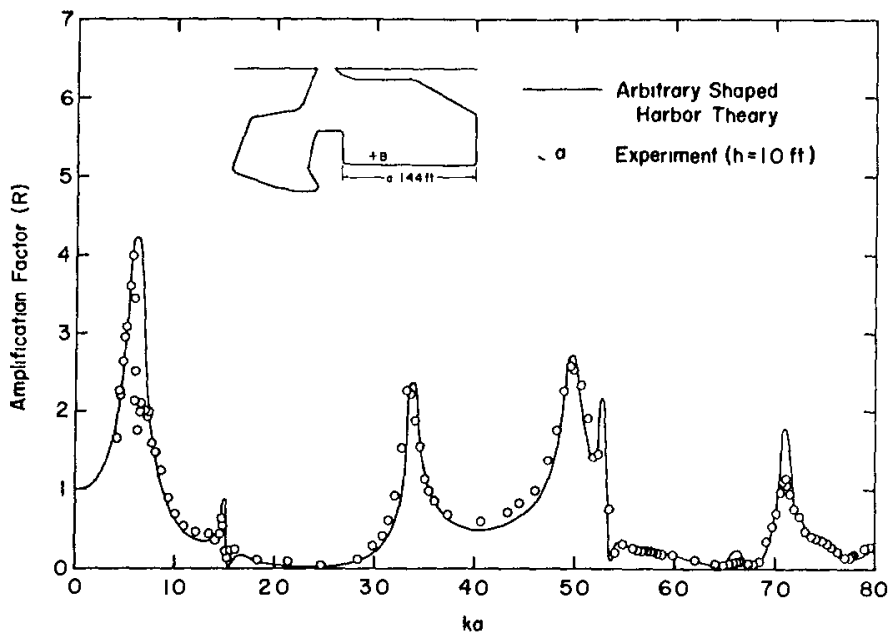

Fig 7 Response curve at point $B$ of the Long Beach Harbor model

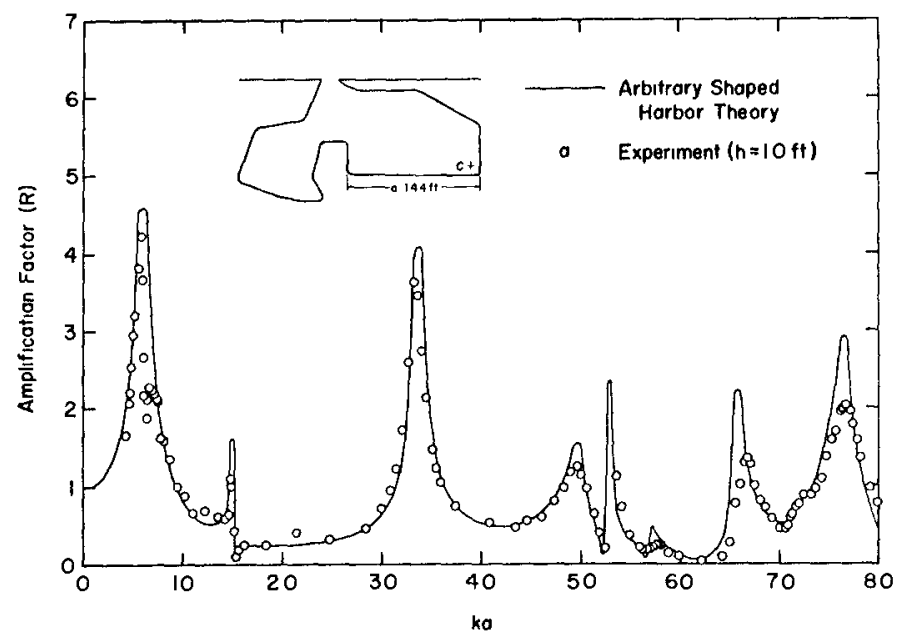

Fig 8 Response curve at point $C$ of the Long Beach Harbor model 


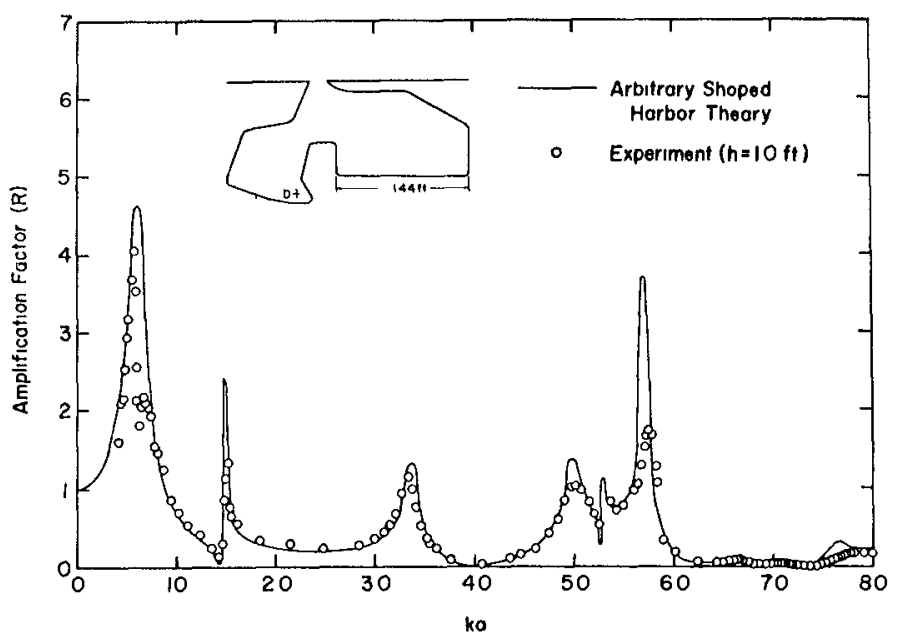

Fig 9 Response curve at point $D$ of the Long Beach Harbor model

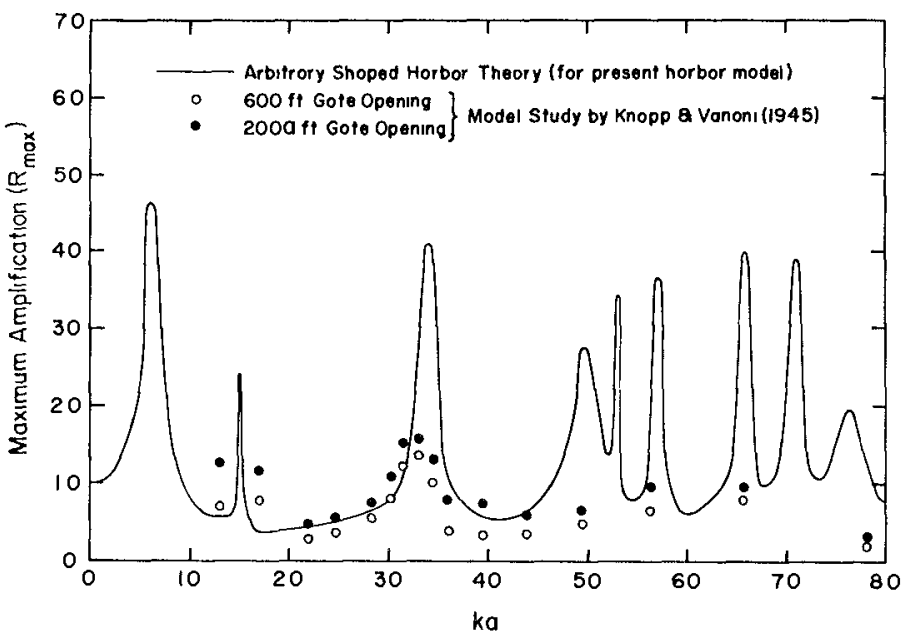

Fig 10 Response curve of the maximum amplification for the model of Long Beach Harbor compared with the data of the model study by Knapp and Vanoni (1945) 
the theory the harbor boundary was divided into the same number of segments for all wave numbers thus the ratio of the segment length $\Delta s$ to wave length $L$ is smaller for the smaller values of ka than for larger values of ka Therefore the theoretical results for small $\mathrm{ka}$ are more accurate than the results which correspond to large $\mathrm{ka}$ and thus better agreement between the theory and experiments is to be expected Second energy dissipation 15 larger near resonance for large values of $\mathrm{ka}$ which also tends to decrease the experimental amplification factors compared to those determined theoretically

It can also be seen from the response curves that the agrcement between the theoretical solution and the experimental data is unformly good at each of the locations This suggests that the theory has also accurately predicted the wave amplitude distribution inside the harbor for each mode of resonant oscillation

It is inter $\epsilon$ sting to note that for larger values of $\mathrm{ka}$, the shape of the mode of oscillation inside the harbor becomes more complex For example for the first resonant mode (ka 0 61) the amplification factors at the four positions (A $B$ C and D) are approximately the same In fact for this mode of oscillation the wave amplitude is fairly unform throughout the harbor and either positive or negative water surface displacements occur simultaneously throughout the harbor Thus the first resonant mode $(k a=061)$ is usually termed the "pumping mode" However for the mode corresponding to $\mathrm{ka}=762$ the amplification factors at the four locations differ considerably indicating that this is a much more complicated mode of oscillation than the "pumping mode"

The variation of the maximum amplification within the entire basin plotted as a function of $\mathrm{ka}$ is presented in the respone curve of $F / g \quad 10$ The ordinate is the ratio of the maximum wave amplitude within the harbor regardless of location to the standing wave amplitude with the entrance closed This curve shows every possible mode of resonant oscillation for the range of ka that has been investigated, as well as the maximum amplification for each mode Experimental data from a model study conducted by Knapp and Vanom (1945) are included in Fig 10 for comparison (The gate or entrance width used in the present model corresponds to a prototype wath of $940 \mathrm{ft}$ ) The original data of Knapp and Vanom were presented by them as the maximum amplification fdctor as a function of prototype wave period In order to convert their wave period to the wave number parameter (ka) used heren an average prototype wate $r$ depth of $40 \mathrm{ft}$ was used throughout the harbor along with a characteristic dimension of the harbor $a=6768 \mathrm{ft}$ These hydraulic model data and the present theoretical curve show qualitative agreement of the wave periods of resonant oscillations especially the mode of oscillation at $\mathrm{ka}=338$ However there is a considerable difference between the predicted maximum amplification and the measured There are two factors that may contribute to such differences First the maximum amplification factor used by Knapp and Vanoni was defined as the ratio of the maximum wave amplitude inside the harbor model to the maximum wave amplitude measured outside the harbor thus it differs from the definition used in the present theory Second, the water depth in the model used by Knapp and Vanon 1 was small increasing the importance of viscous effects in their model compared either to the inviscid theory or the experiments of this study 
Fig 11 shows the distribution of wave amplitude inside the harbor for the $\mathrm{r}$ sonant mode at $\mathrm{ka}=338$ determened from the present theory The wave amplitude has been normalszed with respect to the wave amplitude at point $\mathrm{C}$ (the coordinates of this position are shown in Fig 8) Two nodal lines (lines with zero amplutude) occur one in the East Basin and one in the West Basin with maxima at the ends of each basin and a minimum near the confluence of the two Data are presented in Fig 12 from Knapp and Vanon (1945) on the wave amplitude distribution for a prototype wave period of 6 minutes ( $\mathrm{ka}=330$ ) By comparing Figs 11 and 12 it 1 s seen that the gencral shape of wave amplitude are similar for the two models (e $\mathrm{g}$, the location of the two nodcs and the maximum) even though the boundary of the model used for present study is simplified compared to the hydraulic model

Fig 13 shows the average maximum total velocity at the harbor entrance (maximum in time averaged across the harbor entrance) as a function of the wave numbur parameter ka The ordinate has been nor malized with respect to the maximum horizontal water particle velocity for a small amplitude shallow water wave $\sqrt{\mathrm{gh}} \mathrm{A}_{1} / \mathrm{h}$ From this figure it is seen that there are nine maxima in the range of ka presented which correspond to the nine resonant modes shown in Fig 10 demonstrating that each miximum of the total entrance velocity is assiciated with a mode of resonant oscillation inside the harbor Fig 13 also shows that the velocity at the entrance for the pumpIng mode ( $\mathrm{ka}=0$ 61) is significantly larger than that of any other mode of oscillation Using the prototype dimensions mentioned previously the wave period of this mode is about 33 minutes and could possibly be excited by tsunamis If $A_{1}=05 \mathrm{ft}$ and $\mathrm{h}=40 \mathrm{ft}, F 1 \mathrm{~g} 12$ indicates that the maximum entrance velocity for the pumping mode would be $10 \mathrm{fps}$ and for other modes it would be of the order of $2 \mathrm{fps}$ such velocities may cause damage to structures located near the entrance as well as creating navigation problems

\section{CONCLUSIONS}

The following major conclusions may be drawn from this study

1 The present linear-inviscid-theory predicts the response to periodic incident waves of an arbitrary shape harbor with constant depth connectcd to the open-sea quite well even near resonance

2 The theoretical prediction of the resonant wave numbers for resonant frequencies) agree well with the experimental data The theoretical amplification factor at resonance is generally somewhat larger than the experimental data especially for the resonance modes at larger values of $\mathrm{ka}$

3 The average total velocity across the harbor entrance reaches a maximum when a resonant os cillation develops inside the harbor the magnitude of such entrance velocities may be much larger than the corresponding water particle velocity of the incident wave

4 The present theoretical results also agree qualitatively with the experimental data obtained from a model study conducted by Knapp and Vanon (1945) although the planform of the model investigated by them was more complicated and their study included depthwise variations 


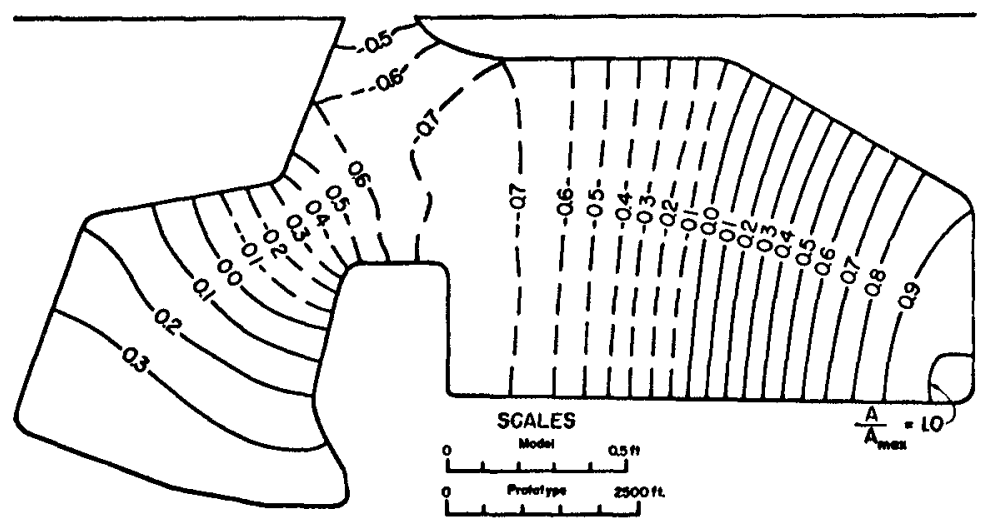

Fig 11 The theoretical wave amplitude distribution in the Long Beach Harbor model $(\mathrm{ka}=338)$

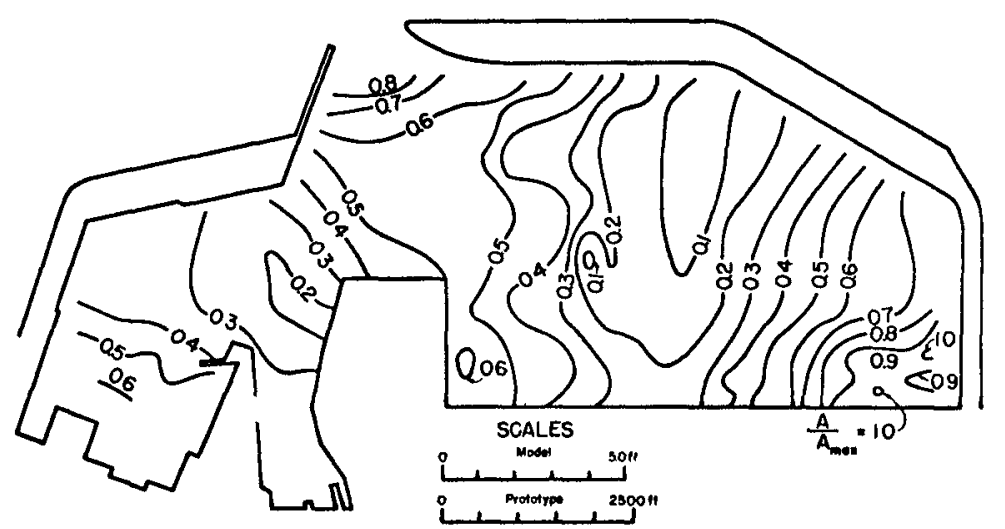

F2g 12 Wave amplitude distribution inside the harbor model of Knapp and Vanon (1945) for six minute waves ( $k a=3 \quad 30)$ (see Knapp and Vanom (1945) p 133) 


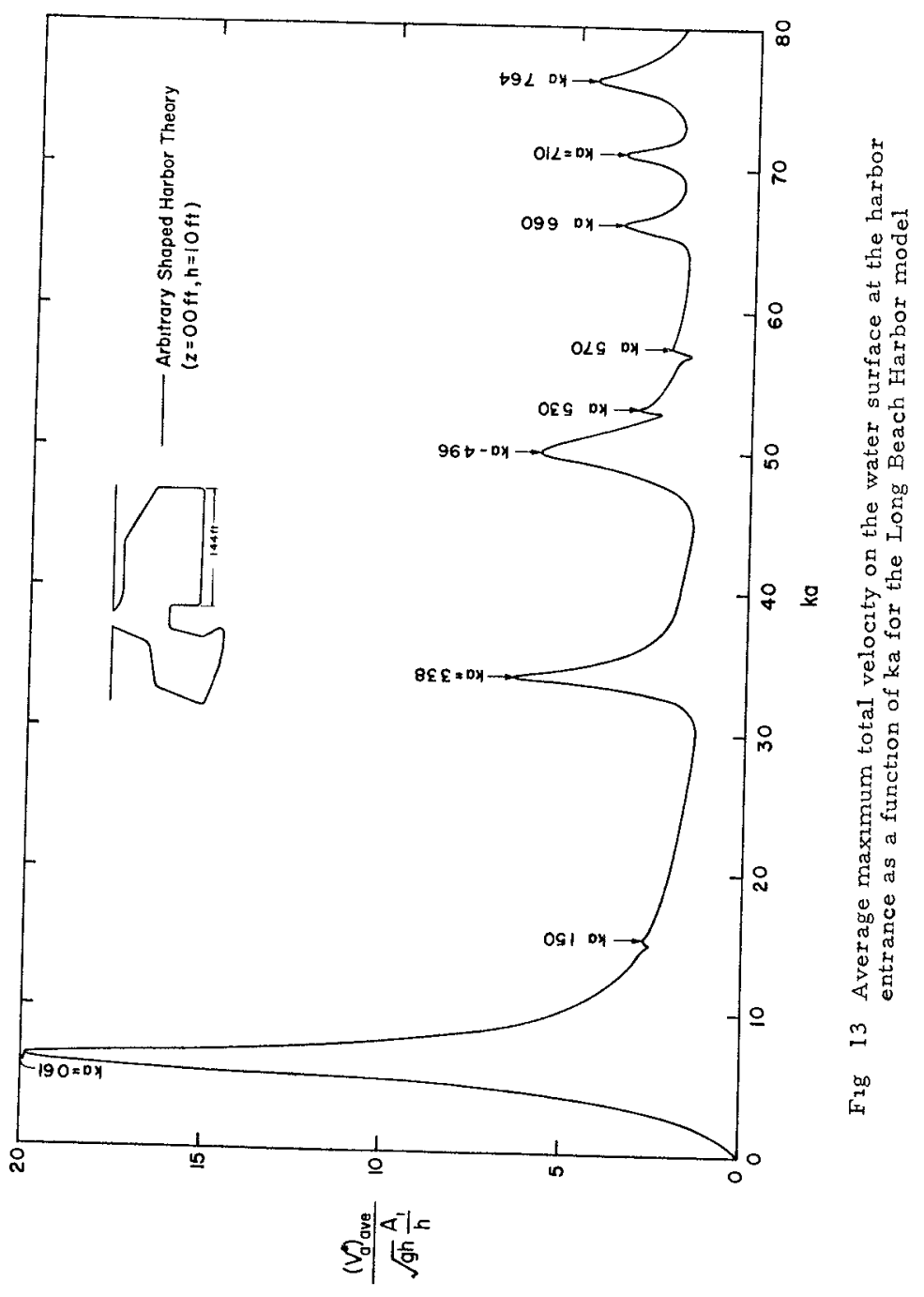




\section{ACKNOWLEDGEMENT}

This study was supported by the $U S$ Army Corps of Engineers under Contract No DA-22-079-CIVENG-64-11 and was conducted at the $W M$ Keck Laboratory of Hydraufics and Water Resources California Institute of Technology

\section{LIST OF REFERENCES}

Hwang $L S$ and Tuck, E O "On the Oscillations of Harbors of Arbitrary Shape", Journal of Flu1d Mechanics Vol 42, 1970, pp 447 464

Ippen, A T and Goda $\mathrm{Y}$, "Wave Induced Oscillations in Harbors The Solution for a Rectangular Harbor Connected to the Open-Sea", MIT Hydrodynamics Laboratory Report No 59, 1963

Knapp R T and Vanon1, V A "Wave and Surge Study for the Naval Operatıng Base Terminal Island Calıfornia", Hydraulic Structure Lab Calif Inst of Tech Pasadena, Calif, 1945

Kravtchenko, $J$ and McNown $J S$, "Seiche in Rectangular Ports", Quarterly of Applied Math, Vol 13 1955, pp 19-26

Lee, J $-J$, "Wave Induced Oscullations in Harbors of Arbitrary Shape", Report KH-R-20, W M Keck Lab of Hydraulics and Water Resources, Calıf Inst of Tech, Pasadena Calif 1969

Lee J - J , "Wave-Induced Oscillations in Harbours of Arbitrary Geometry", Journal of Fluid Mechanics, 1970 (1n press)

Leendertse, J J "Aspects of a Computational Model for Long-Perıod Water Wave Propagation", RM-5294-PR The Rand Corporation Santa Monica, Calıf 1967

McNown J S "Waves and Seiche in Idealized Ports" Gravity Waves Symposium N B S Circular 521, 1952

Miles, J, and Munk W, "Harbor Paradox" Journal of Waterways and Harbors Div, ASCE Vol 87 No WW3, 1961 pp 111-130

Raichlen $F \quad$ "Wave-Induced Oscillations of Small Moored Vessels", Report KH-R-10 W M Keck Lab of Hydraulics and Water Resources, Calif Inst of Tech Pasadena Calif, 1965

Ralchlen, $F$, and Ippen A $T$ "Wave Induced Oscillations in Harbors", Journal of Hydraulics Div, ASCE, Vol 91 , No HY2 1965, pp $1-26$

Wilson, B W, Hendrickson, J A, and Kilmer, R C "Feasibility Study for a Surge-Action Model of Monterey Harbor, Californa", Report 2-136, Science Enganeering Associates, San Marino, Calif , 1965 


\title{
CHAPTER 132
}

\author{
SPECTRAL RESPONSE OF HARBOR RESONATOR CONFIGURATIONS
}

\author{
William James*
}

\section{ABSTRACT}

An outline is given of methods devised recently by the author to predict the spectral response of rectangular resonators, and to improve the response of resonators generally A simple small scale acoustic model of the ocean-resonator-harbor configuration was developed and Is described The acoustic "ocean" Is effectively decoupled from the rest of the system by means of sound absorbent material placed along the "Infincte" boundarıes, and standard audio-frequency equipment is used The results demonstrate that the open end contraction for rectangular resonators may not differ significantly from the contraction for resonators of similar geometry placed in a semi-infinite (or effectively decoupled) wave channel, at least if the wavelengths are not smaller than the width of the harbor entrance channel

\section{INTRODUCTION}

It Is desirable to design harbor entrances to eliminate (as far as possible) those bandwldths in the incldent wave spectra that cause difficulties such as range action, high mooring forces, unreasonable wave Impact, wave overtopping and drift of littoral sediments into the harbor In many problems these difficulties are functionally related to approximately the second (or higher) power of the wave helght, and hence any reduction in the incident wave height will produce real benefits

Resonators placed at the harbor entrance can be tuned to radiate back into the ocean those frequency bandwldths considered to be harmful without hindering navigation ${ }^{1}$ Readers are cautioned against using the blanket quarter-wavelength recommendation ${ }^{2,3,4}$, the inltial design should accord with the fact that the tuning of individual resonators is considerably dependent on the width of the harbor entrance channel 5,6 Readers should also note that resonators are not effective

* Senzor Lecturer, Unversity of Natat, Durban, South Afmca, presently visiting at Queen's Unuersity. Canada. 
for wavelengths smaller than the wldth of the harbor entrance channe] Hence this paper relates to incldent wavelengths that exceed the horlzontal distance between the leading edges of symmetrically opposed rectangular resonators

The transmitted and reflected spectra can be predicted for given geometry and incldent spectra, assuming a linear system 7 , and this procedure is outlined below Certain innovations 8 that both broaden the tuning of the resonators and decrease the cost of construction are also briefly described in this paper An acoustic model has been devised 6 and this is used to check the ocean-resonator-harbor coupling The results of the latter tests constitute the major contribution of this paper

\section{SPECTRAI, RESPONSE}

The method devised for computing the spectral response of indlvidual rectangular resonators is based on experimental results It is usual to plot frequency response curves in the frequency doman 9 but in this study observed transmissivity and reflectivity were plotted against the tuning parameter $d / L$ ( 1 e, in the ka domain) For detalls of the wave measurement and wave analysis procedure, readers are referred to earlier publicationslo,1l The geometrical conditions for distinct resonance are wummarised in fig 1

Approximate rectilinear apexes were fitted into the ka domaın resonance curves, and the resulting maxıma and minlma are summarised In fig 2 The tuning parameter bandwidths at the half resonant values were measured and found to be reasonably constant for various values of $W / L$ The information is summarized in fig 3 Sufficlent data are avalable in these three figures to allow the computation of the spectral response of any rectangular resonator to given incident spectra, but only for the furst resonant mode

Briefly, the procedure is to transform the incident spectrum into an amplitude/wavelength relationship for the particular harbor entrance The dimensions of the resonator are chosen such that distinct resonance obtalns for the dominant wavelength, using fig I For discrete wavelengths the ratios $W / L, W / L$ and $d / L$ are calculated The resonant tuning parameter Is obtalned from fig 1 , and the resorance curves "reconstructed" graphically or digltally using fıgs 2 and 3 Values of transmissivity and reflectlvity are then interpolated for various tuning parameters

Finally, the incldent spectra are transformed using these values Two examples are presented elsewhere7, and it is shown that a standard quarter-wavelength resonator may reflect only one quarter of the peak energy, nearly all of which is reflected by a resonator designed dccordung to flg 1 


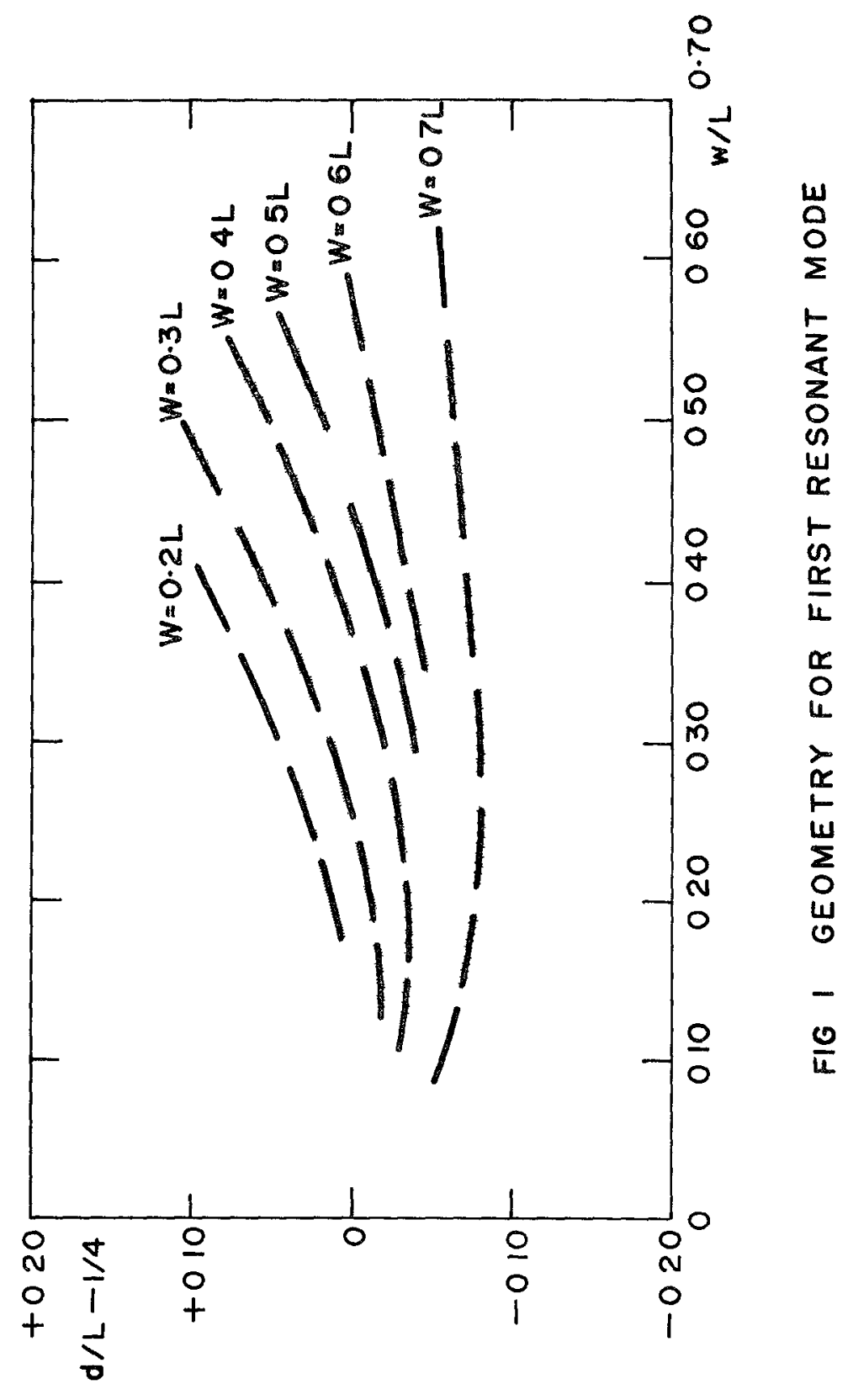




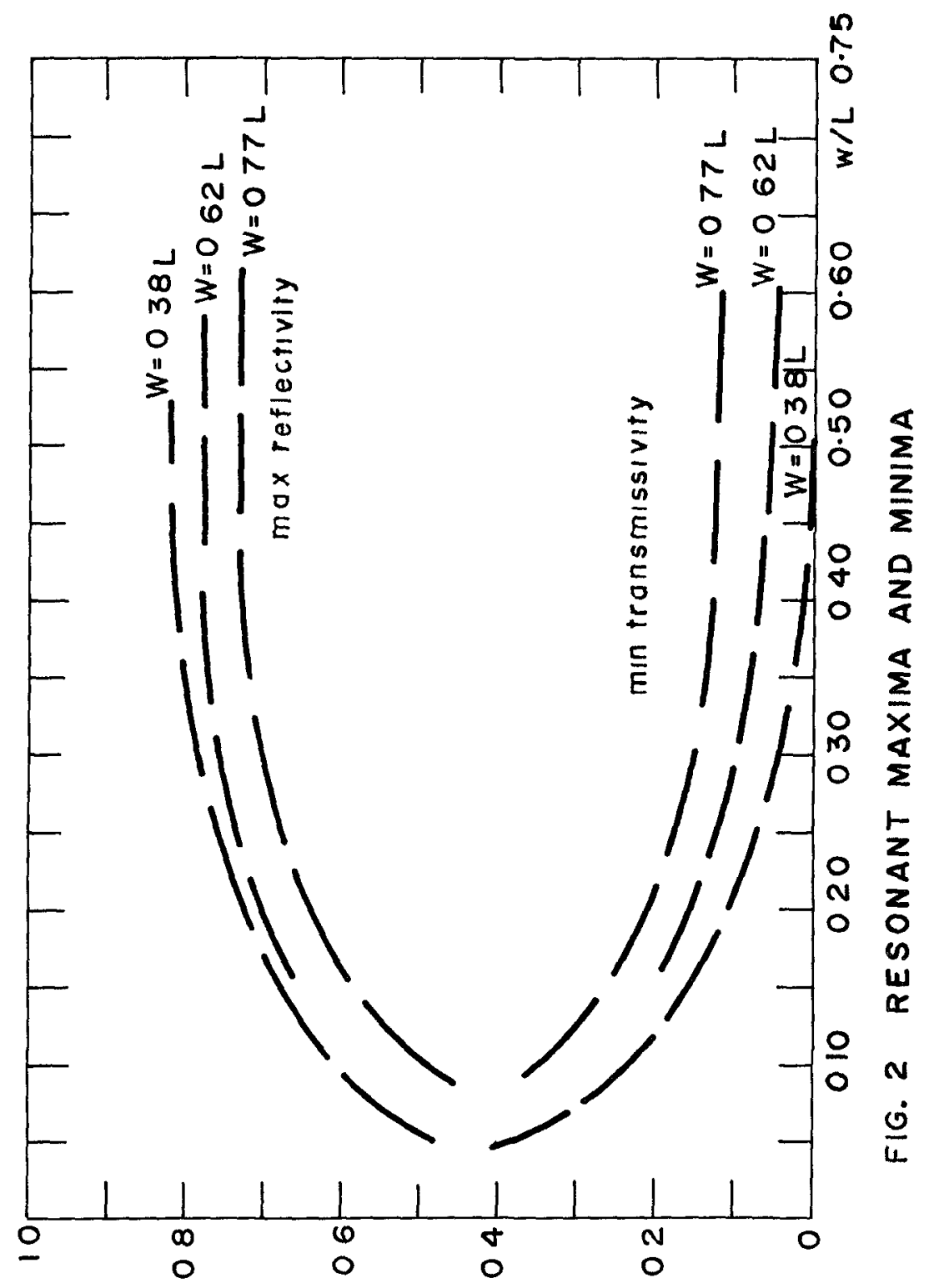




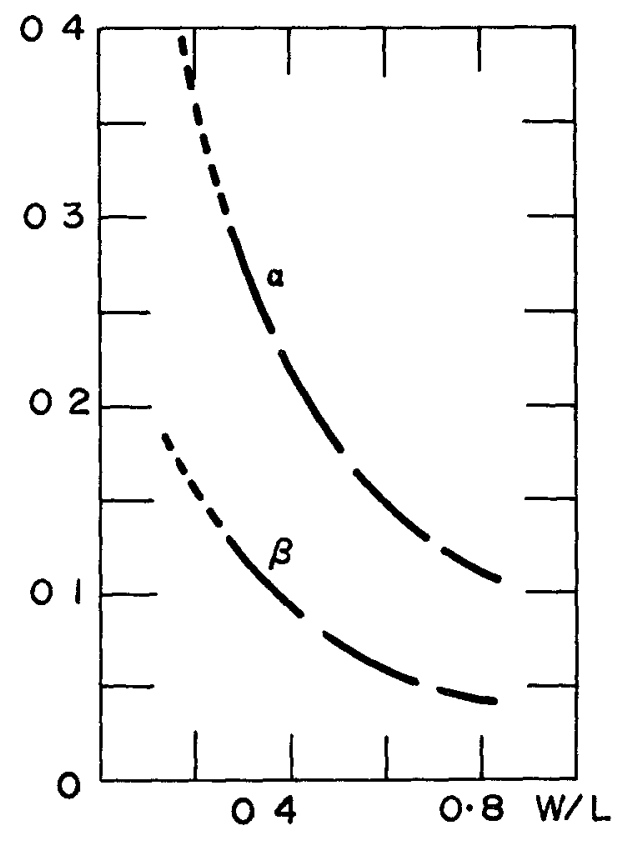

FIG. 3 TUNING PARAMETER BANDWIDTH AT HALF RESONANT VALUE 


\section{TWO INNOVATIONS}

Experiments were performed on

(a) variable distances between three resonators in a battery configuration, and

(b) non-uniform depths in a resonator/harbor entrance area

Trıple Resonator Batterıes

Raylezgh ${ }^{12}$ recommended that the ratıo of the fundamental perlods of the individual resonators in a battery configuration should be $1 \sqrt{2}$ In this study resonators were constructed from $3 / 4$ inch thlck "perspex", geometries are shown in fig 4

Reflectlvity and transmissivi.ty were measured at points approylmately one wavelength upstream and downstream of the battery respectively In these tests the water depth was held constant, and wavelengths were systematically varıed.

The results showed resonant peaks at those individual resonator frequencles obtained in earlier tests on single resonators, as is to be expected Hence fig $I$ can be used to predlct the response of battery conflgurations superimpose the equation (a stralght line) for each battery geometry (e g w/L = I 61 d/L) on $\mathrm{flg} 1$ and scan the line for the location such that the harbor entrance and resonator geometry satisfy the geometrical conditions for resonance This identifles the tuning paramete for resonance, and performance can be estimated using the same computational procedure for spectral response descrubed above

By increasing the distances between the resonators, the water mass in the entrance channel contiguous with the leading edges of two adjacent resonators is brought into the system response This effectively broadens the overall tuning of the battery configuration and hence improves the spectral performance of the battery Because of the end-contractions, the distance should be significant, e g commensurate with resonator dimensions, and chosen by careful ad hoe model tests (acoustic models preferably)

Non-Unı form Depths

In the experiments on non-uniform depths an artificlall invert was inserted into a single resonator, as depicted in fig 5 The stlll water depth and wavelength were held constant and the resonator planform was varıed systematically (by gradually retracting the rear will outwards) Reflectlvity and transmissivity were measured as in early tests ${ }^{1}$

The results indicated a reduced efficlency for resonators of comparatively small still water depth, but less upstream agltation 

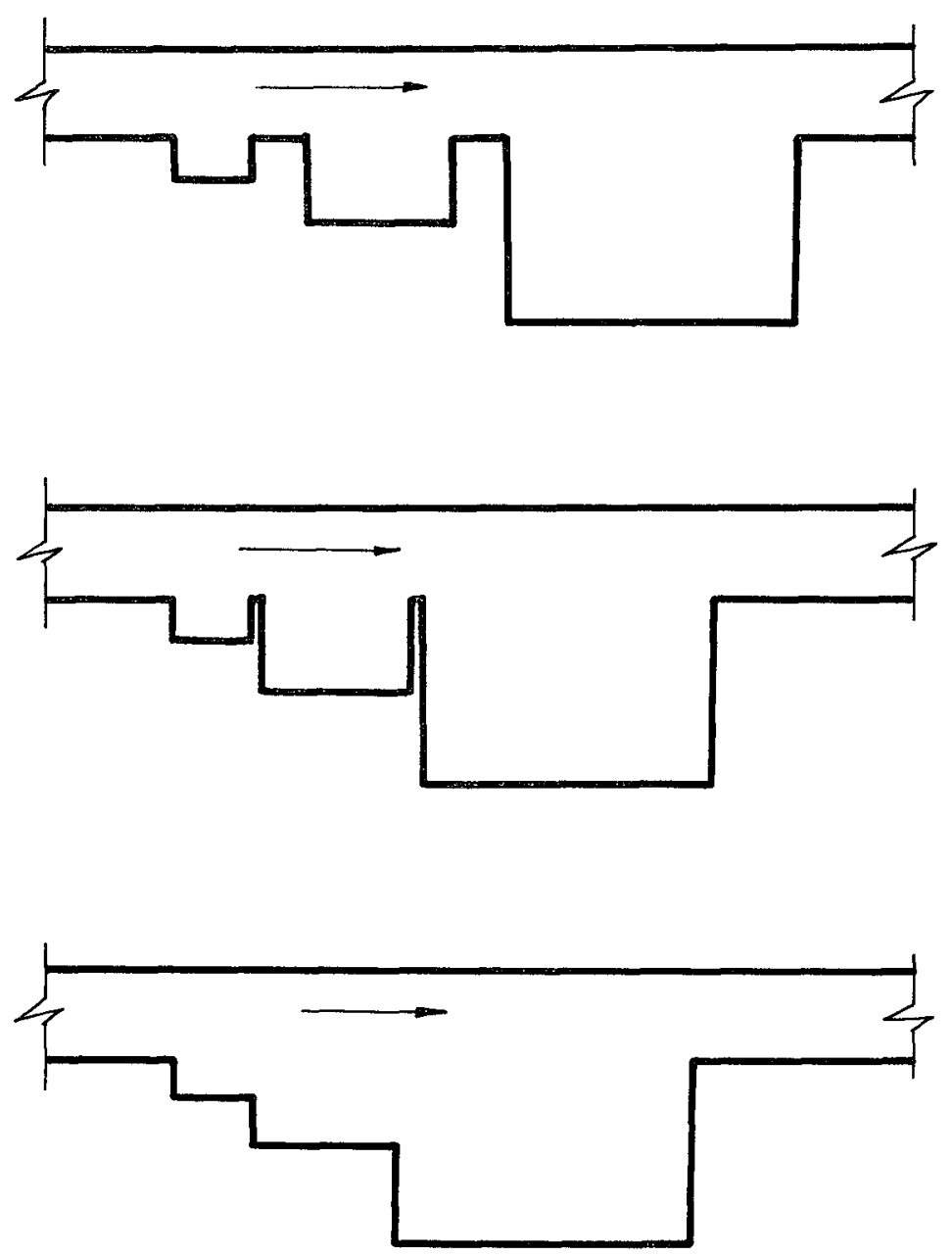

FIG 4 BATTERY GEOMETRIES EXAMINED 
2188

COASTAL ENGINEERING

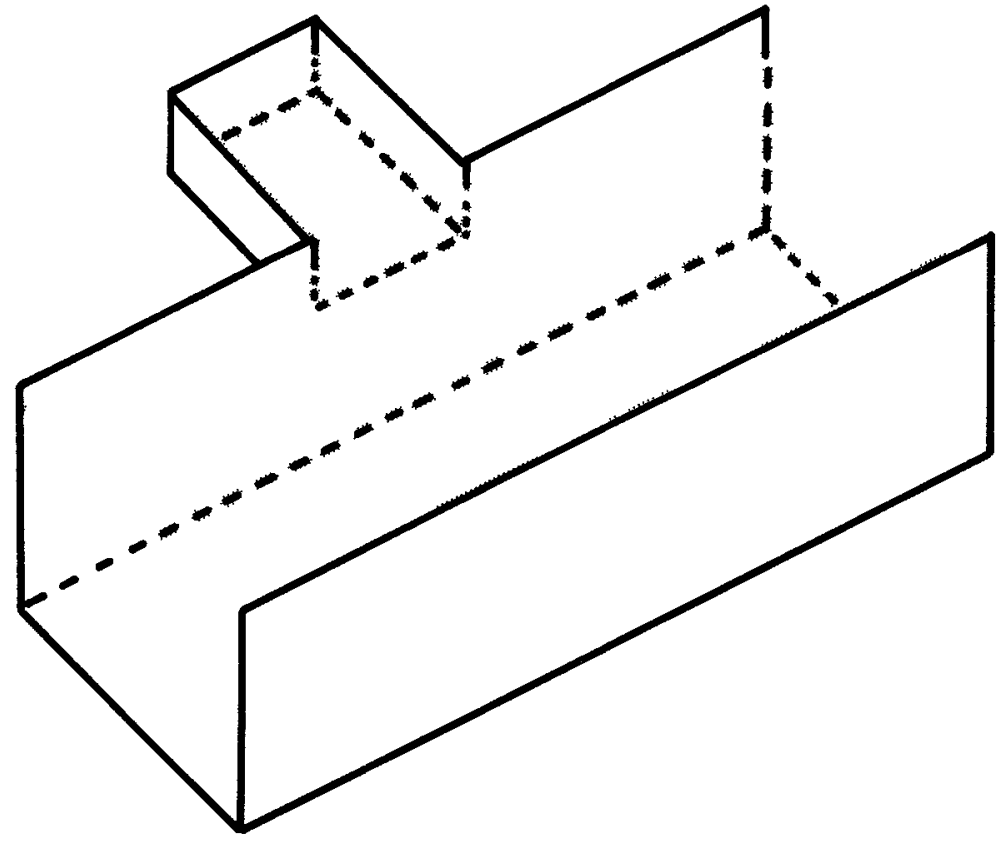

FIG. 5 NONUNIFORM DEPTHS 
(reflectlvity) was assoclated with glven transmissivity

It was found that the length of the resonator could easily be halved, if the still water depth in the resonator is reduced to a fraction of the harbor entrance channel depth, a point of considerable practical slgniflcance However, tidal ranges will detune the resonator, the effect being related to the proportional reduction in depth and to the actual tidal range

\section{THE ACOUSTIC MODEL}

An acoustic model evidently has certain advantages over a hydraulic model wave generation and absorption are easier, the fluid does not have to be isolated, wavelengths are generally shorter, and measuring equipment, speed and accuracy are generally better

To test the model, earlier experjments on resonators in a water wave channel were reproduced The varıable geometry was bullt up from 3 inch movable timber walls and placed on a glass plate on top of a desk A second glass plate was placed on top of the walls, and a loudspeaker was connected to an osclllator and placed agalnst sponge rubber at the entry to the mann duct A microphone was placed aganst the sponge rubber absorber at the harbor end of the main duct, and was connected through a small pre-amplifier to an oscllloscope By setting the wldths of the main entrance duct and of the resonator, and by holding the osclllator frequency constant, the length of the resonator was adjusted until resonance occurred This was monltored by a minimum signal on the oscllioscope

The effect of Imperfect sound absorption was checked and found to be unimportant Tests on scale effects were also negative, although these were not exhaustive In addition the effects of sound waves entering the duct along its length were also found to be insignifıcant The signal-to-nolse ratıo was easlly lmproved by means of the amplifier on the oscilioscope

The results showed slight differences from those of the water wave experiments Reasonably accurate predictions of harbor resonance modes for frequencies can be obtalned in this way, but further development of the method wll be necessary if absolute values of harbor amplification are required

\section{OCEAN-RESONATOR-HARBOR COUPLING}

Fig 6 shows the experimental apparatus used to examine the coupling problem In this case the model was set up on a reasonably clean office floor, agaln using 3 inch hlgh timber walls The cover to the ocean domaln was supported on 3 inch iron nalls Cotton batting (reflectivity about 5\%) was arranged along the "infinite" 


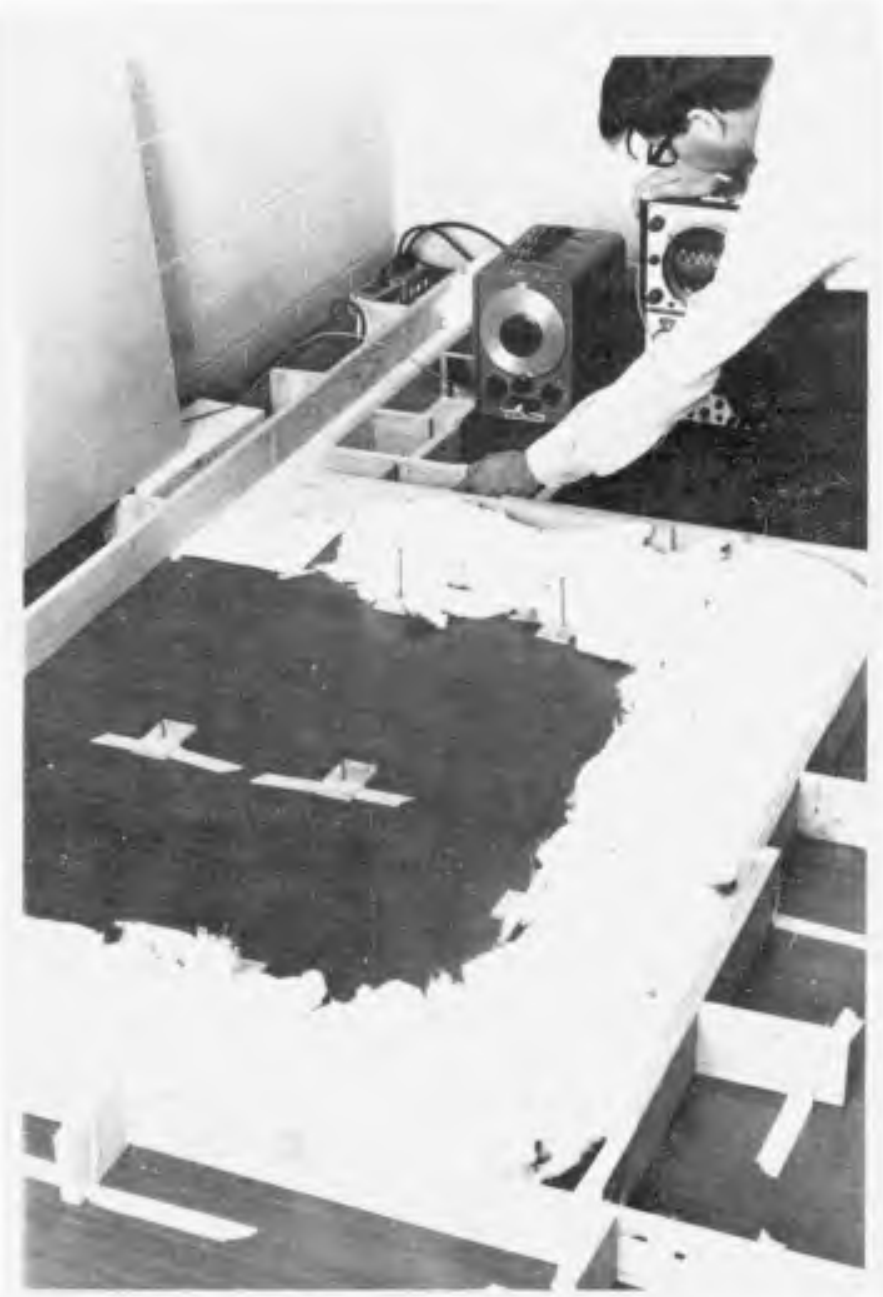

FIG. 6 ACOUSTIC MODEL: OCEAN-RESONATOR-HARBOR 
boundaries, and this effectively decoupled the ocean domain Total cost of the model excluding audio equipment was $\$ 850$

Acoustic waves were generated from a virtual point source, and so the ocean domain had to be long enough for the radiating incident waves to achieve an approximately parallel wave front at the harbor entrance It follows that re-reflections from the harbor off the loudspeaker were negliglble

The experimental procedure adopted was similar to that above resonance monltored by a minlmum acoustic pressure signal in the harbor, and achieved by systematic variation of resonator geometry This method was better than incldent wave frequency varlation, since system attenuatıon, transmissivity and reflectivity, parasitic vibrations, and also wave generation and recording were frequency dependent

Geometrical conditions obtalned for distinct resonance are presented in fig 7 Results for semi-infinite ocean coupling and seml-infinlte wave channel coupling almost colnclded, consequently both results could not be plotted on the figure However the ocean coupling curves were slightly flatter, as indlcated by the dashed curve

Evidently, then, harbor resonance studies may be carried out at the end of water wave channels (provided that the incident wavelength exceeds the width of the harbor entrance channel) wlthout materlal loss of accuracy

Further tests on the acoustic model conflrmed that resonators should be located at the ocean end of the entrance channel, and at an amplitude antinode in the wave envelope, if the harbor is reflective at the resonator tuning frequency Since partial re-reflections occur off the ocean end of the entrance channel, the downstream harbor and channel oscillations are generally not amplified by resonators For transparent frequencies there is no effect and for opaque frequencies there is no penetration

\section{CONCLUSIONS}

Three figures are presented for estimating the spectral response of individual rectangular resonators, but only for the first resonant mode No account is taken of the second resonant mode even though this is important when considering the response of a battery of resonators

Indıvidual resonators in a battery respond in an addltıve manner, and hence this response can also be predicted The mass of water contiguous with adjacent resonators in the harbor entrance channel can 


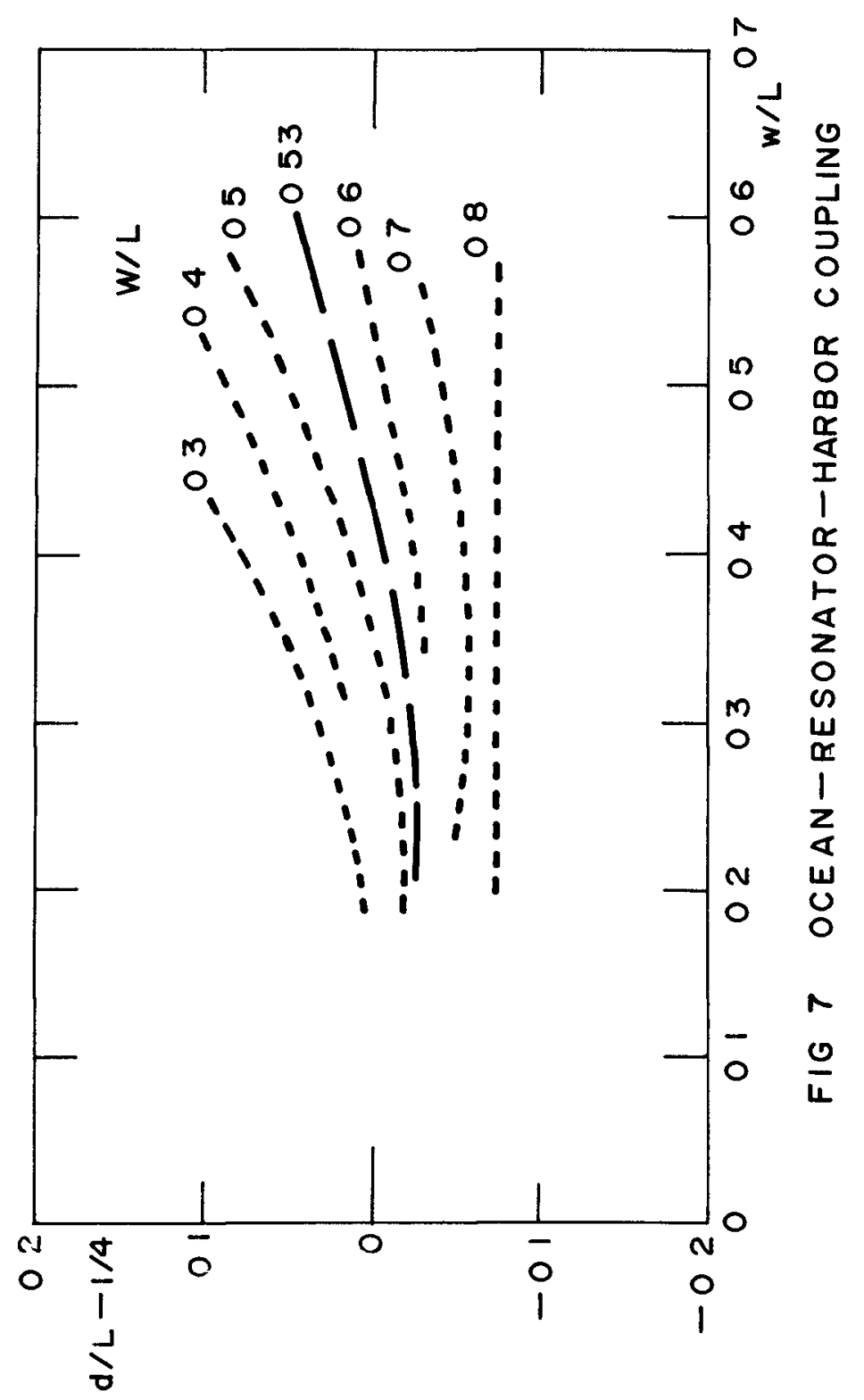


also be incorporated In the system response For this reason the resonators should not be contiguous, if at all possible

Comparatively shallow stıll water depths in individual resonators detunes the resonator downwards, I e towards smalier frequencles Hence, for a particular tuning, non-uniform depths, with smaller depths in the resonator, result in smaller geometry, and concommltant savings in construction and excavation costs Large tidal amplitudes would effectıvely detune such systems, however

The acoustuc analogy is an extremely fast and cheap method for evaluating ergen frequencues for any harbor planform The method could probably be elaborated for estimation of orbital velocities and even of mooring forces, for uniform water depths in the harbor and harbor entrance No scale effects were detected in the tests reported

An acoustic model was constructed to check the ocean-resonatorharbor coupling The results indrcated that the end-effect does not differ significantly from the contraction for resonators of simular geometry placed in a sem-infinute wave channel, at least for waveZengths greater than the harbor entrance whdth

This result validates the experimental results obtained in the wave channel, the method for predlcting the spectral response of resonators for a real situation ( 1 e on the edge of a seml-inflnite ocean) is evidently reliable to the first order

\section{REFERENCES}

I James, W, "Rectangular Resonators for Harbour Entrances", Chapter 98, Coastal Englneerıng, A S C E , August 1969, pp 1512 $-1530$

2 Valembols, $J$, "Etude de I'Action d'Ouvrages Resonants sur la Propagation de la Houle" Proc Mnnesota Int Hydraulics Conf, Minneapolis 1953, pp 193-200

3 Wiegel, R I, Oceanographlcal Englneerıng, Pub Prentıce Halı, $1964, \mathrm{p} 144$

4 Ippen, A T (Ed) Estuary and Coastlıne Hydrodynamıcs, pub McGraw HIII, 1966, p 397

5 James, W, "An Experimental Study on End-Effects for Rectangular Resonators", Journal of Fluid Mechanics, in press

6 James, W, "A Desk-top Model of Harbour Resonators", Journal of the Acoustrcal Socıety of Amerıca, in press 
7 James, W, "The Spectral Response of Harbour Resonators", The Institution of Civil Englneers (London), in press

8 James, W, "Two Innovatıons for Harbour Resonators", A S C E , In press

9 Mles, J, and Munk, W, "Harbor Paradox", Proc A S C E, Vol 87 No WW3, Aug 1961, pp 111-130

10 James, W, "Accurate Wave Measurements in the Presence of Reflections", Jour Instn Water Engrs (London), Vol 23, No 8, Nov 1969

11 James, W, "Resolution of Partial Clapotıs", Proc A S C E Vol 96, No WWl, Feb 1970

12 Strutt, J W (Lord Raylelgh), The Theory of Sound, Dover, 2nd Ed 1945, Chap XVI

NOTATION

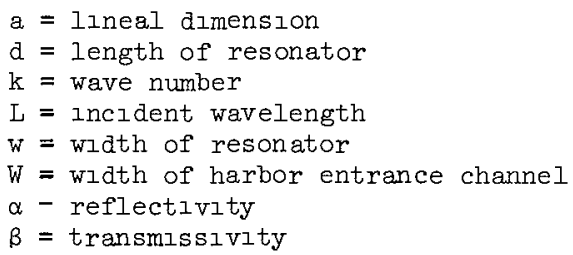

\section{ACKNOWLEDGEMENTS}

The work reported was developed and the paper wrltten whlle the author was visiting at Queen's University Thanks are due to that Institution for the opportunity to visıt Canada and to the University of Natal for leave of absence The work was based on experimental results obtalned mostly at Aberdeen Unlversıty, Scotland, where guldance was readlly glven by Professor Jack Allen and Dr G D Matthew Professor H Stewart, former Head of the Dept of Electrrcal Englneering at Queen's kindly loaned the audio equipment The ldea of the acoustic analogy crystallısed out of discussion in Honolulu and Washington wlth Dr Dick Shaw of Buffalo Unzversity 


\section{CHAPTER 133}

\section{HARBOR STUDY FOR SAN NICOLAS BAY, PERU}

By James $M$ KeIth ${ }^{1}$ and Emmett $J$ Murphy ${ }^{2}$

\section{ABSTRACT}

A pair of ducted impeller current meters, one mounted vertically and the other hor zontally, were used to measure wave action at San Nicolas Harbor, Peru The horizontal water velocity records are superior to conventional wave records because they measure directly the wave property which induces adverse horizontal ship motion, and provide directional wave data Spectral analysis methods proved well-suited to detalled interpretation of the particle velocity records, while considerable insight into the wave phenomena was gained by simple, rational inspections and interpretations of the records TIme-lapse movies of a moored shIp, when correlated with sımultaneous water particle velocity records, provided an exceptionally clear picture of ship response to wave action, and led to the rather surprisıng observation that long-period ship motion is not necessarily caused by long-period waves The foregoing ship response was duplicated in hydraulic model tests

\section{INTRODUCT ION}

The results of a study program were used to determine the source of difficulties in mooring ore carriers at Marcona Mining Company's iron ore loading facility at San Nicolas Harbor, Peru Observations of wave action were primarily obtained by time-lapse movies of a moored ship and by an orbital velocity meter consisting of two ducted impeller current meters, one mounted vertically and the other horizontally The horizontal water velocity records are superior to conventional wave height records in two Important respects (1) They provide direct data regarding the wave property most directly affecting horizontal ship movement, and (2) they provide directional wave data

Analyses were performed in two distınct stages A direct, rational application of the records as a time-history of the horizontal water velocity was used to derive the time-history of horizontal water displacement Using this approach, a mechanısm was establıshed whereby a relatively short-period swell of $16 \mathrm{sec}$ induced long-period (50 sec to $150 \mathrm{sec}$ ) ship motion transverse to the pier Spectral analyses by digital computer were then employed to derive a clear insight into the various wave trains existing at particular times, and to quantitatively evaluate the relative energy contents of selected wave frequencies simple hydraulic model tests were utılized to verify that swell generated periodic transverse ship motions having a period many times longer than the period of the swell This occurred when a quartering stern swell acted on a ship moored with slack elastic mooring lines to a pier with elastic fenders

\footnotetext{
1 Vice President, John A Blume \& Associates, Engıneers, San Francisco, Cal ifornia

2 Project Manager, Marcona Corporatıon, San Francısco, Calıfornıa
} 


\author{
BACKGROUNO
}

The port at San Nicolas Bay is utilized by Marcona for the annual shipment of approximately $9 \mathrm{mill}$ ion tons of Iron ore concentrates and pellets The bay is a typical hooked embayment not fully protected from the ocean At times, serious mooring problems have been experienced at the pier due to adverse water motion which has resulted in the breaking of many moorIng lines and damage to the pier by the ship's impact (see Fig i) Individual ships have broken more than twenty mooring 1 ines and some of the impacts against the pier have been estimated to exceed the pier's design load by a substantial margin

In general, rough water conditions in the bay occur during the winter months when storms in the lower southern latitudes frequently cause heavy swells along the coast ouring these periods, which vary from a few hours to three or four days, ships are forced to anchor well out from the pier awating calmer water, and many ships have been delayed in this manner

The pler deck (see Fig 2) is constructed of prestressed concrete sections supported on prestressed concrete piling The fender system consists of hollow, end-loaded rubber cylinder buffers 18 in $0 D, 9$ in 10 , by 21 in long for energy absorption Timber piles bear on the outer ends of rubber cylınders and support $12-1 n \times 12-1 n$ rubbing timbers

Fig 3 is a map of San Nicolas Bay showing the pier projecting into the bay from the south shore IIne The harbor is relatively deep, water depth at the pier when the latter was completed in 1962 was $45 \mathrm{ft}$ at the south end and $59 \mathrm{ft}$ at the north end

The mooring problem at San Nicolas Bay is further complicated by the large vessels currently using the pier facilities Marcona Corporation owns and operates a fleet of the largest dry bulk, ore, and combination ore011 carriers avallable These carriers are used to transport Peruvian I ron ore mined and concentrated by Marcona to Japan To date, ships of over 100,000 OWT have been loaded at San Nicolas pier, by early 1970, ships of 130,000 OWT will be in service Use of carriers exceeding 150,000 OWT is foreseen in the future, and the west side of the pier, where ships are moored for loading, has been dredged to $57 \mathrm{ft}$ to meet the draft requirements for vessels of this size Table 1 shows typical dimensions of bulk and ore carriers

The economic advantage of larger ships is easily demonstrated by comparison of crew requirements and tonnage capacities A crew of 45 was required to man a 10,000-ton Liberty ship, the highly automated 100,000ton ships of today require 35-man crews The 10-knot Liberty ship could make about four Peru-Japan voyages per year for a total annual ore lift of about 39,000 tons, or about 850 tons for each crew member, the 16knot 100,000 OWT ore carrier can average about $6-1 / 4$ voyages per year, lifting about 635,000 tons annually or over 18,000 tons per year for each crew member, 21 times the rate of the Liberty ship 


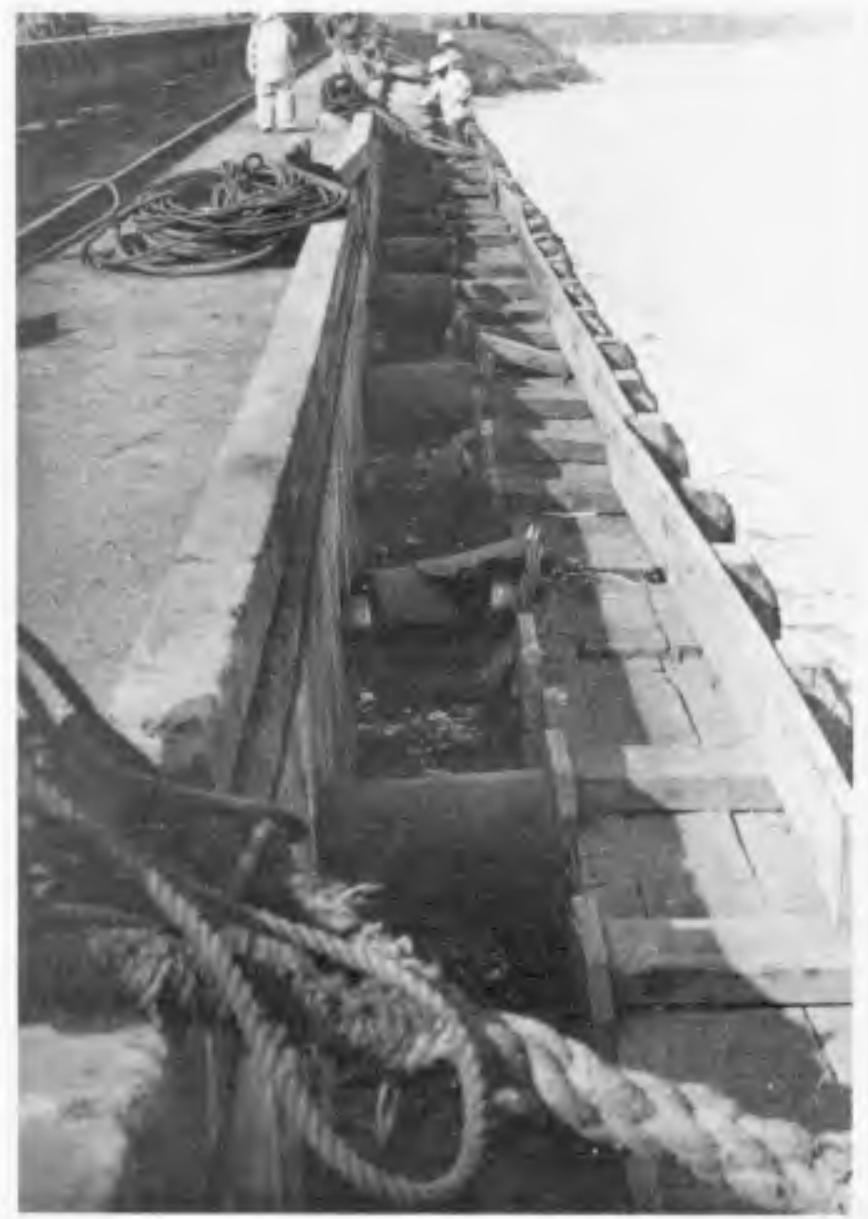

FIG. 1. - FENDER DAMAGED FROM IMPACT OF MOORED SHIP 


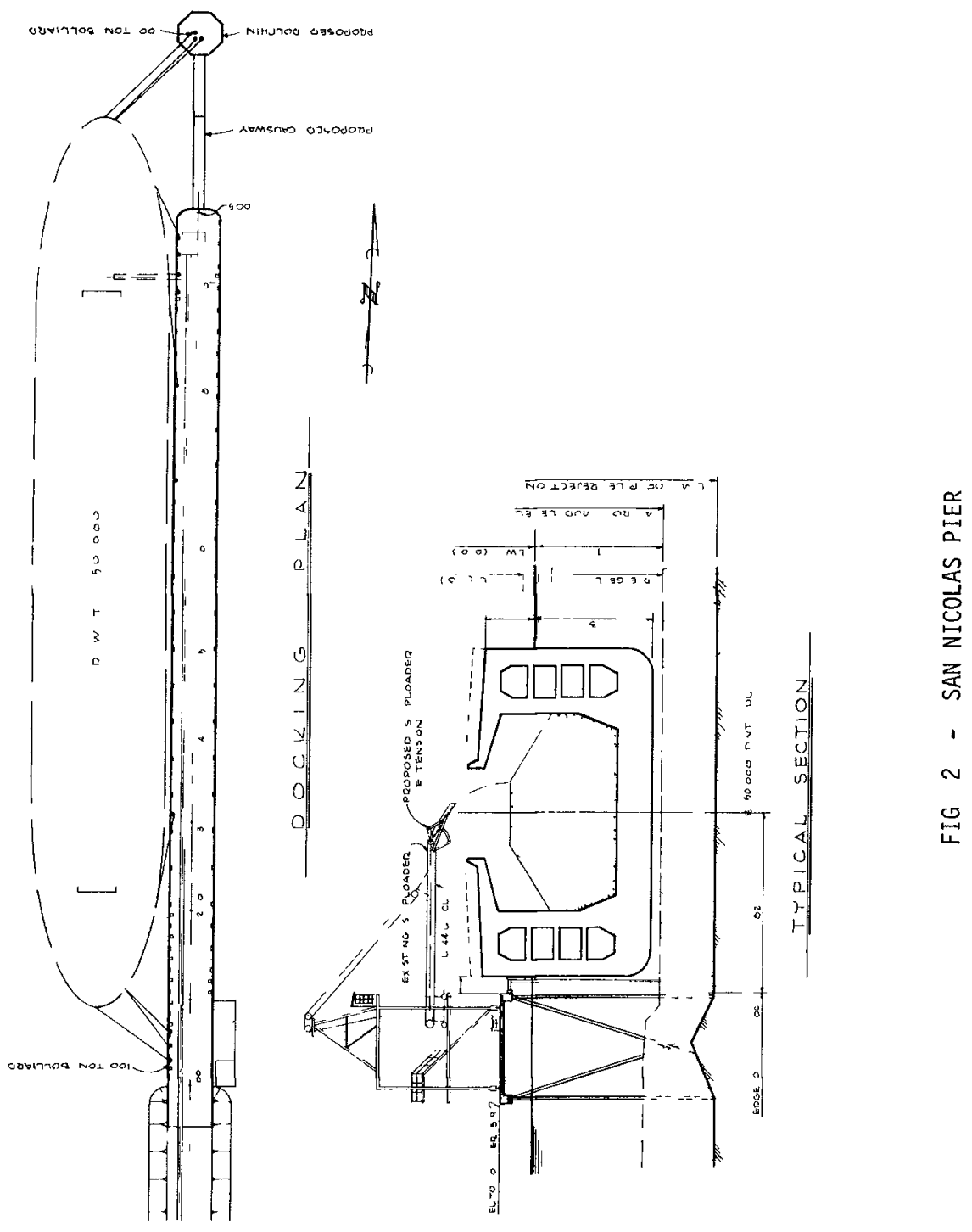




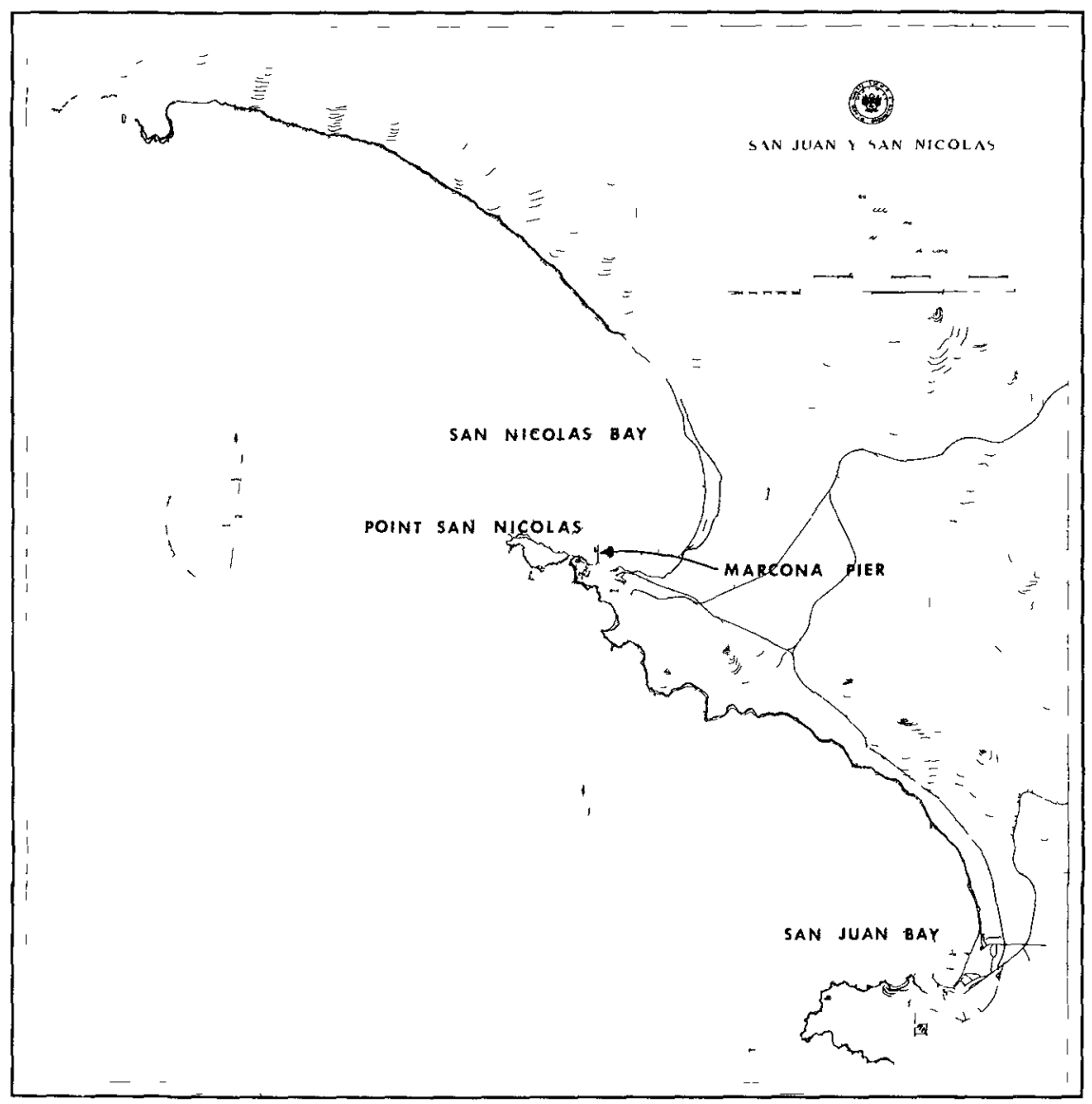

FIG 3 - SAN NICOLAS BAY, PERU 
TABLE 1 - TYPICAL DIMENSIONS, BULK AND ORE CARRIERS

\begin{tabular}{|c|c|c|c|c|c|c|}
\hline $\begin{array}{l}\text { Vessel } \\
\text { type } \\
\text { (1) }\end{array}$ & $\begin{array}{c}\text { Vessel } \\
\text { sıze, DWT } \\
\text { (2) }\end{array}$ & $\begin{array}{l}\text { Displace- } \\
\text { ment } \\
\text { (3) }\end{array}$ & $\begin{array}{c}\text { Length } \\
\text { overall, } \\
\text { In feet } \\
\text { and Inches } \\
\text { (4) }\end{array}$ & $\begin{array}{l}\text { Beam, in } \\
\text { feet and } \\
\text { Inches } \\
\text { (5) }\end{array}$ & $\begin{array}{c}\text { Depth, in } \\
\text { feet and } \\
\text { Inches } \\
(6)\end{array}$ & $\begin{array}{c}\text { Draft } \\
\text { summer, } \\
\text { in feet } \\
\text { and inches } \\
\text { (7) }\end{array}$ \\
\hline Liberty & 10,800 & 14,100 & $441-6$ & $56-11$ & $37-4$ & $27-8$ \\
\hline Oread & 31,662 & 41,641 & $655-0$ & $87-0$ & $46-6$ & $34-2$ \\
\hline $\begin{array}{l}\text { San Juan } \\
\text { Pathfinder }\end{array}$ & 71,205 & 89,122 & $835-0$ & $106-0$ & $65-0$ & $44-9$ \\
\hline $\begin{array}{l}\text { San Juan } \\
\text { Exporter }\end{array}$ & 106,000 & 123,852 & $865-3$ & $124-8$ & $68-11$ & $50-10$ \\
\hline- & $130,000^{\mathrm{a}}$ & 154,400 & $948-2$ & $138-10$ & $74-2$ & $51-9$ \\
\hline- & $150,000^{b}$ & 180,000 & $1,014-0$ & $150-0$ & $75-0$ & $53-0$ \\
\hline $\begin{array}{l}\text { a Under cor } \\
\text { b Possible }\end{array}$ & $\begin{array}{l}\text { uction } \\
\text { ign }\end{array}$ & & & & & \\
\hline
\end{tabular}


Consıderıng the trend toward larger ships and the problems encountered in mooring ships currently in service, Marcona Corporation retained John $A$ Blume \& Associates, Engıneers, to conduct a study to define wave action in the harbor This information was consıdered essential to an evaluation of various proposals for Improvement of the harbor

Initial observations suggested that the ship motion to and from the pier was caused by harbor surge, since the period of motion ranged from $1 \mathrm{~min}$ to $3 \mathrm{mIn}$, and since the pier tide gage ind Icated occurrence of long-perlod waves ranging up to $20 \mathrm{~min}$ Some ships at anchor appeared to swing on their anchors at perıods approximately $20 \mathrm{~min}$, an additional indıcation of surge Discussion of the problem with local pilots indicated that swell might be a dominant factor in disturbances to moored vessels The pilots relıed upon the height of breakers on the north side of Point San Nicolas and the height of the surf along the beach southeast of the pier to estimate mooring conditions it was therefore evident that if surge was the problem, it occurred only during periods of adverse weather as evidenced by the heavy swell

The offshore swell approach Ing San Nicolas Bay from the south and southwest is refracted around Point San Nicolas and directed toward the pier from a northwesterly direction It was noted that placement of a breakwater west of the pier would provide an excellent means of shielding the pier from the swell Additional information, however, was needed to determine the effects of the breakwater on ship motion caused by surge, since a breakwater might well have little effect on reducing a long-perlod surge An observation program was inıtıated to determıne whether swell, surge, or a combination of these forces was causing the adverse motion

\section{OBSERVATION PROGRAM}

Most conventional wave instruments record only wave height, and additional observations must be made to determıne wave direction Since wave direction at San Nicolas Bay is difficult to observe because of relatively low helghts of swell and surge, a method of measurement was required which would el Iminate the need to determine horizontal water motion from wave height data and observations of wave direction Hor izontal water motion from the swell or surge or both of these was the dominant factor causing ship motion, the most promısing approach to the problem, therefore, was to measure this motion directly at the pier location

Several marıne instrument manufacturers were contacted for proposals to supply instrumentation which would record horizontal water particle direction and velocity Two ducted impeller current meters arranged or thogonally on vertical and horizontal axes (see FIg 4) were purchased from Marıne Advisors, Inc These meters were suspended approximately $10 \mathrm{ft}$ below the low tide water surface and readıngs from each were simultaneously recorded on strip charts A direction-sensing vane was installed in addı$t$ ion to the meters, and data from the vane was also recorded on a strip chart Fig 5 shows the instruments being placed off the north end of the pier 


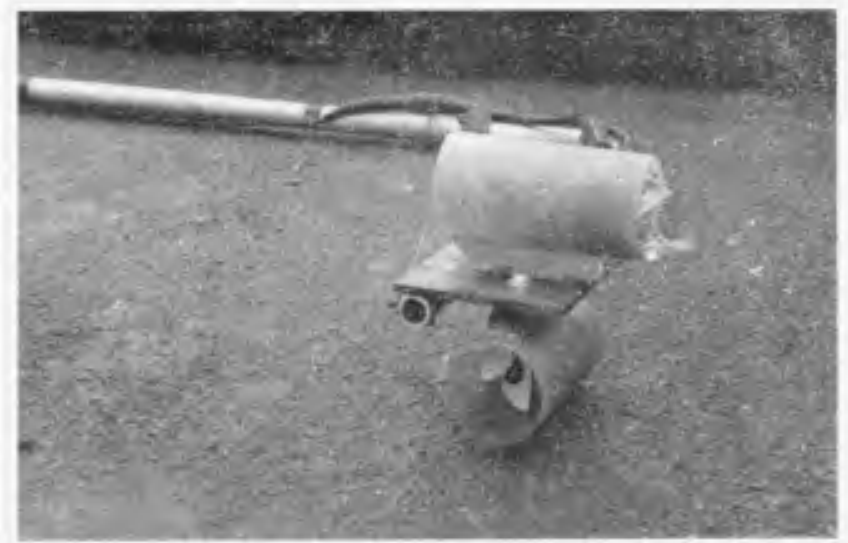

FIG. 4. - PROTOYPE: ORTHOGONALLY MOUNTED DUCTED IMPELLER CURRENT METERS

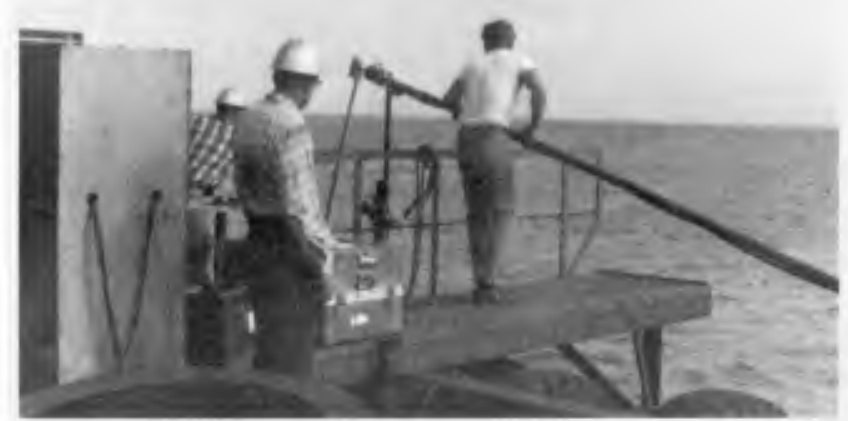

Fig. 5. - INSTALLING METER AT SAN NICOLAS PIER 
Marıne Advisors, Inc Indicated that threshold current velocity for operation of the meters would be less than $3 \mathrm{~cm}$ per sec $(006 \mathrm{knots})$, and that the meters would have a response time of $40 \mathrm{msec}$ to $70 \mathrm{msec}$ and a directional response approachıng a cosıne function of the angle between the meters' axes and the direction of water motion Development data for these meters, ovallable in another publication (3)

Since conditions causing mooring problems occurred relatively infrequently, readings were only uskan during the rough sea perıods Recordings were made at $30^{\circ}$ inc rements for six successive headings from $270^{\circ}$ to $60^{\circ}$, with a 4-hr recoroing period tor tach heading On April 8, April 15, and April 19, 1\% 58 , recordings of moderately rough conditions considered typical of problem-type $c$ torms were obtained on July 26-27, 1968, recordings of the most severe wave disturbances since construction of the pier were obtained These tisturbances were considerably worse than any conditions previously experienced at the pier The storm generating these waves caused damage to many marıne installatıons along the Chılean and Peruvian coasts In addition to the instrument records, a tıme-lapse motıon pıcLure study was filmed on April 19, 1968, of an ore carrier of 74,730 tons displacement inoored at the pier The camera mounted on the mole at the south end of the pier and focused on the bow of the ship recorded one frame every two seconds Data from the wave instruments and the camera recoras were used to analyze water motion conditions within the harbor

\section{INITIAL ANALYSIS}

The initial data analysis was a noncomputerized, rational comparison of the time-lapse movie and the horizontal water velocity record as shown in Fig 6 The movement of the ship's bow was obtained by plotting sequential positions of a point on the ship's bow as the film was projected one frame at a time The horizontal movement of a water particle was obtaıned by integratıng the hor izontal velocity record Integration was done by measuring the area between the velocity curve and the zero line with a planimeter The ship's bow movement consisted of a clockwise circular motion which showed excellent correlation with the swell period, but which was distorted by a long-period motion toward and away from the pier Although the horizontal water motion also has a longperiod component, the correlation between the long-period motions of the ship and the water was very poor

In seeking an explanation of the long-period ship movements, the following hypothesis was proposed the ship, fender system, and moor Ing 1 Ines comprised a vibratıng system where in the fender and the mooring lines were sor Ings having slack or free travel space and the ship represented a mass having circular motion excited by the swell Figs 7 and 8 depict the Idealized system A model test was run In a University of Calıfornia wave tank to confirm this hypothesis The model simulated the conditions depicted by Fig 8 at a linear scale of 1 to 159 Movies of the bow of the model ship indicated that a motion very similar to that shown in the lower portion of Fig 8 could be generated in the 


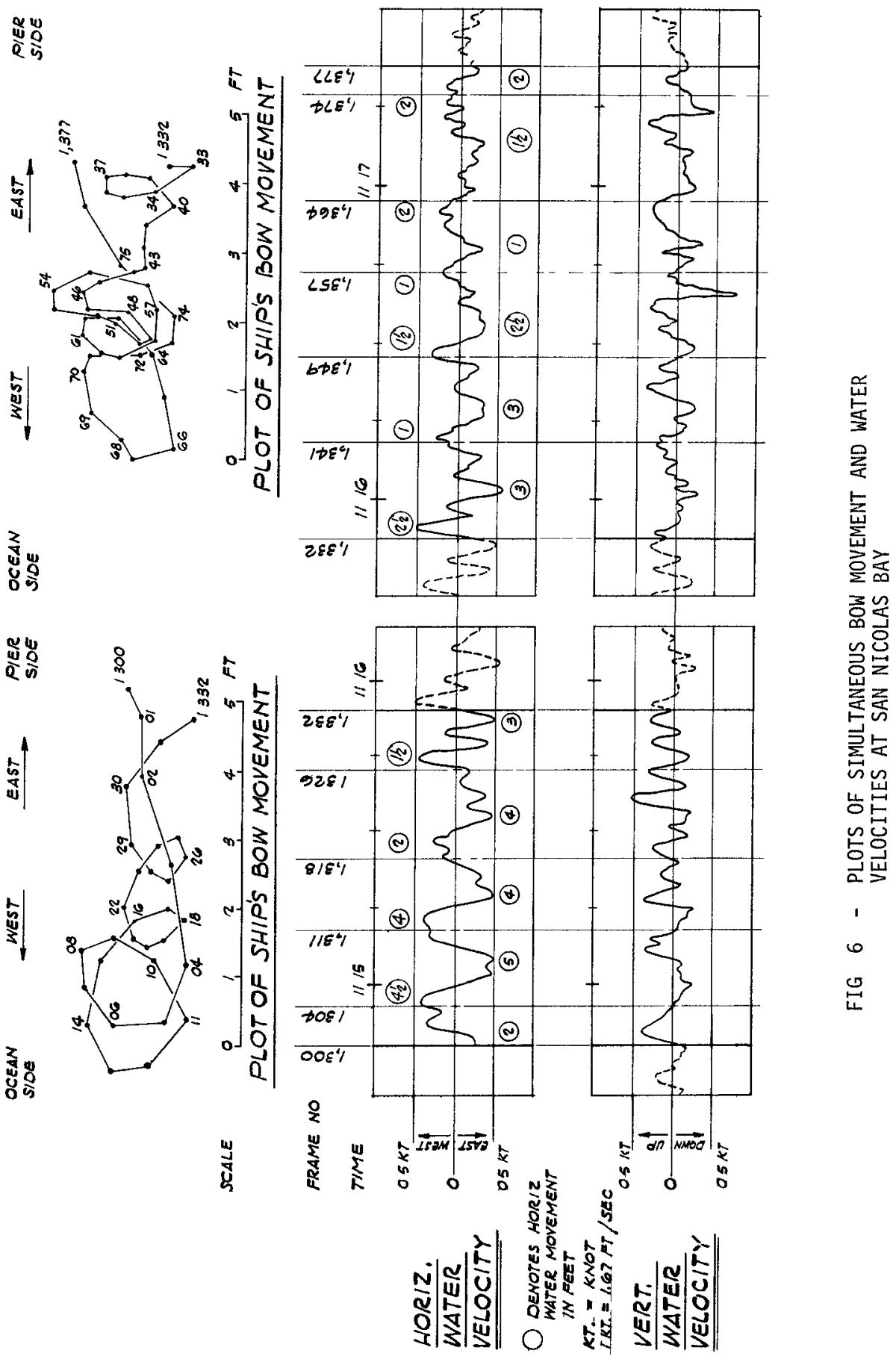



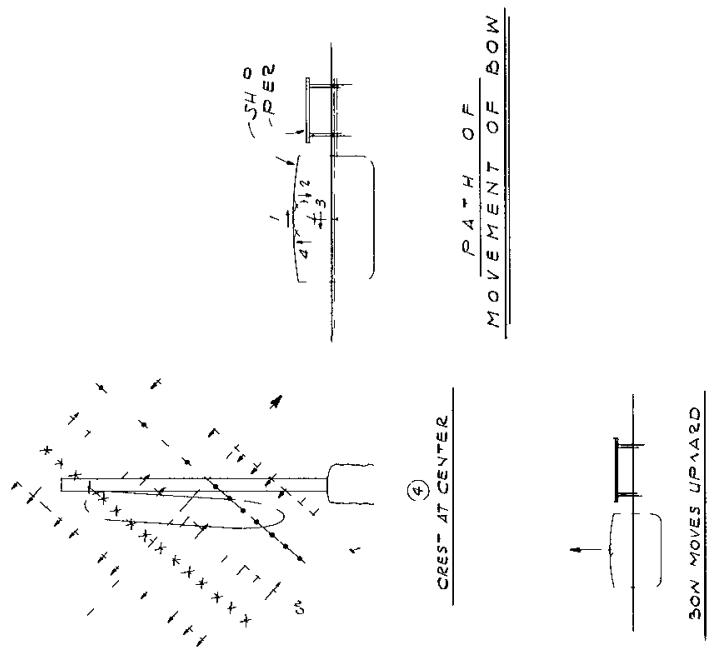

خั
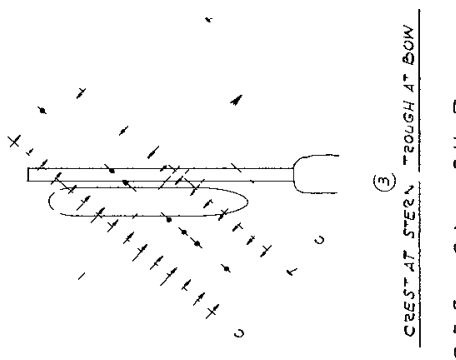

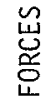
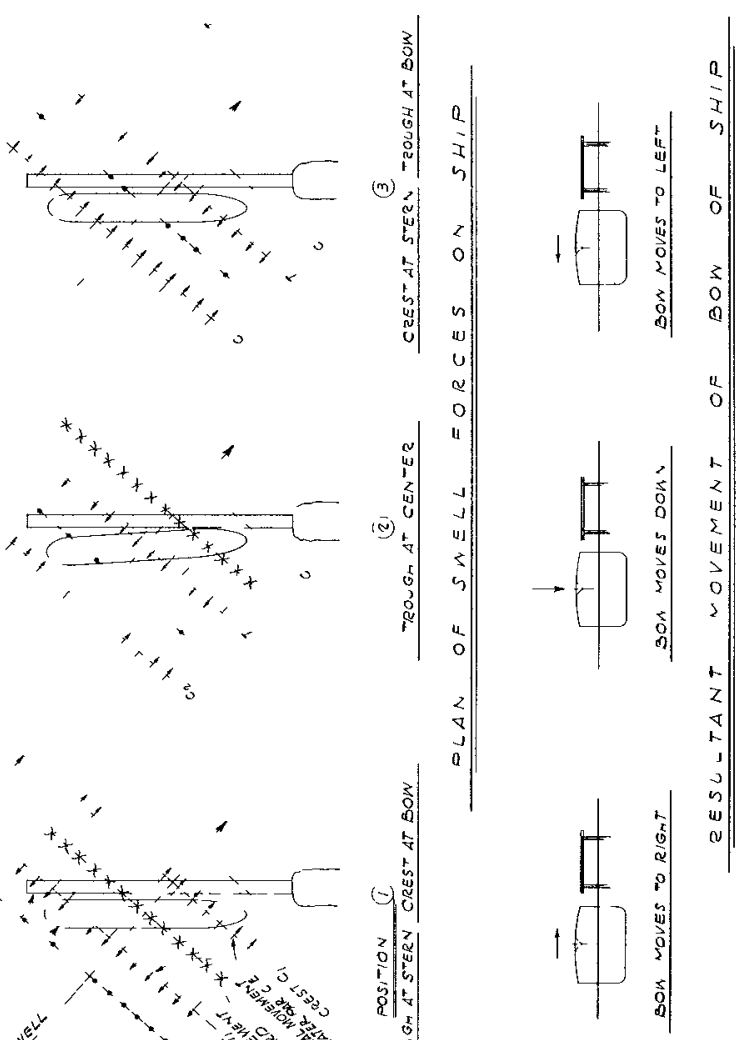

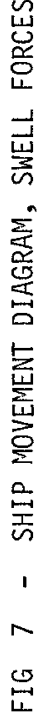

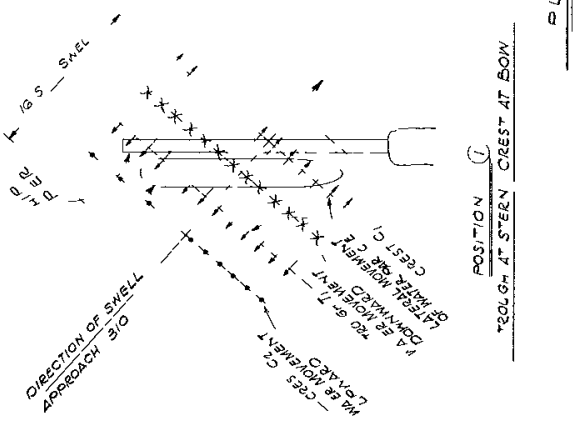




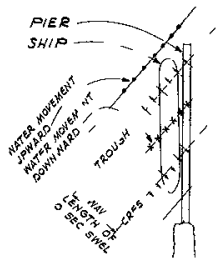

POSITION ON BOH (20)

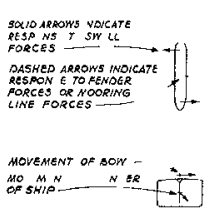

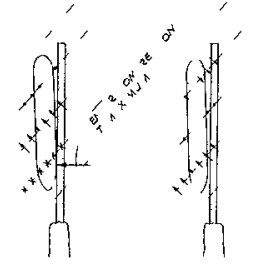

(1)

(c)
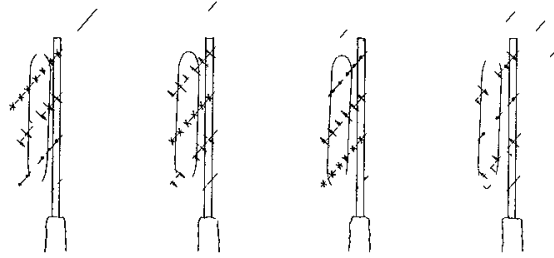

(3) 40

(4) $4(s)$

54

(6)

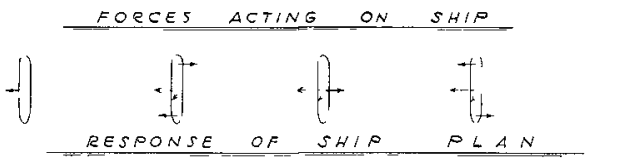

it]

[Y] [

[A]

[T]

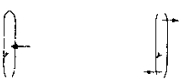

RESULTANT

MOVEMEN

$S H 10$

EEVA-ON
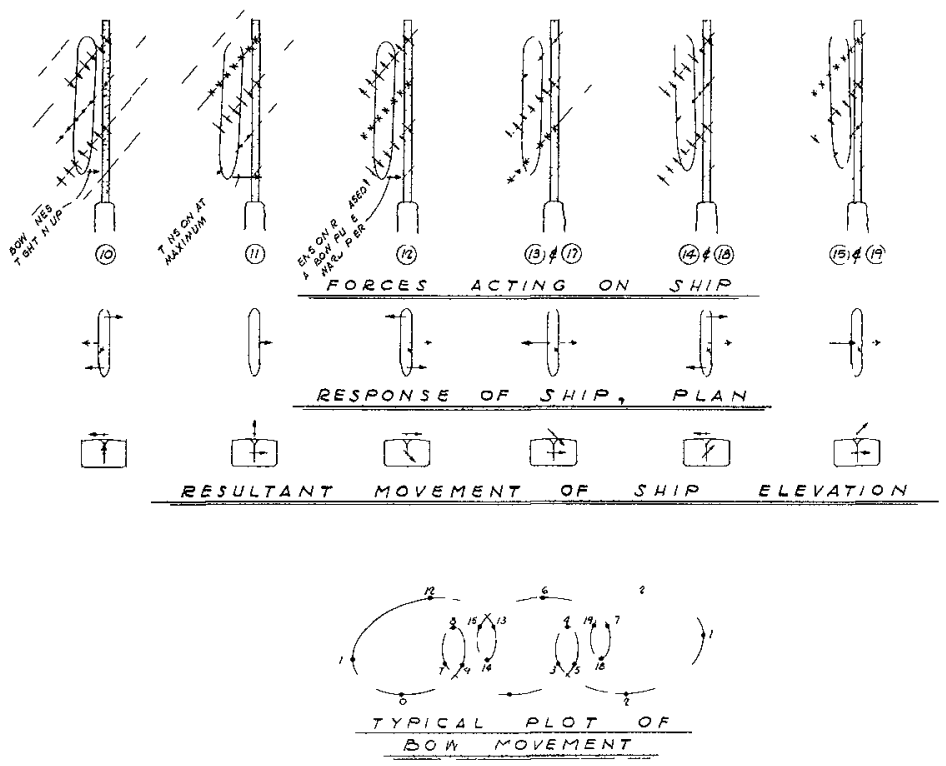

FIG 8 - SHIP MOVEMENT DIAGRAM, COMBINED SWELL AND MOORING FORCES 
model It was also noted that the amount of slack in the mooring lines directly controlled the period of the transverse excursions of the model ship Another interesting observation was that a nonelastic or energy dissipatıng fender system attenuated the long-period excursions irrespective of the amount of slack in the mooring lines

The directional response of the ducted impellers of the current meters was essentially flat for a $60^{\circ}$ sector centered parallel to the duct's axis, With the duct's axis at right angles to the water movement, however, a good null was obtained Direction was thus shown more clearly by a null position than by a centered position An inspection of the April, 1968, recordings confirmed the assumed swell approach azımuth of $\pm 300^{\circ}$

Upon completion of the water motion analysis and the model test observations, it was concluded that the major cause of the adverse ship motion was swell and not surge While the possibility of a surge problem could not be completely discounted, it was simply evident that swell was the greatest contributor to the ship motion problem The model tests confirmed also that the direction of swell approach to the pier caused the ship to oscillate with a yawing motion, and elastic fenders and mooring lines accentuated the problem

Additionally, it was establıshed by calculatıon and model testing that this swell could be effectively attenuated by the construction of a breakwater

\author{
SPECTRAL ANALYSIS
}

In comparing the April storms and the July storm, it was noted that the July storm contained significant long-period wave action of high energy content This surge was not evident during the April storms Because the July storm was of such exceptional intensity, a rather complete analysis of the water motion records was warranted

To precisely interpret this July record, a more sophisticated approach based on computer analysis was used The first objective was to obtain a power spectral density analysis for each direction of the horizontal velocity sensor by use of a digital computer Essentially, the computer program splits the total energy in the record into a number of frequency bands, and determines the uniform sinusoidal motion in every frequency band which would have the same power as the original record had in each band When performed on an analog computer, the analysis is more readily understood Here the input signal is an electrical current wherein voltage represents the quantity being measured, and the computer consists of a series of narrow band pass filters which ideally have square cut-offs The signal passing each filter is squared and averaged for the duration of the record to give a measure of the power in the original record which was contained in each frequency band Spectral analyses were computed by FORTRAN program ACAPS (Auto Correlation and Power Spectrum) ( 1 ) on a CDC 6600 computer 
For the program used, the equivalent uniform sinusoidal motion could be calculated from

$$
\begin{aligned}
& V_{\text {max }}=2 \sqrt{S_{e}}=\text { maximum velocity } \\
& D=2 \frac{V_{\text {max }}}{\omega}=\text { displacement } \\
& T=\frac{2 \pi}{\omega}=\text { period in seconds }
\end{aligned}
$$

and

In which $S=$ spectral dens Ity, $B_{e}=$ bandwidth in radians per second, and $\omega=$ mid-frequency of the band $1 n$ radians per second The equivalence can be applied to each bandwidth, Individual peaks, or a group of peaks

Record input for the spectral density calculation consists of discrete values of the velocity determined at a constant time interval, $\Delta t$, the sampling interval The number of "lags" used in the calculation, $m$, is predetermined The selection of total record length to be analyzed (which must be contınuous), the sampling interval, and the number of lags are influenced by the following factors

1 The maximum period for which the spectral density can be calculated with reasonable accuracy is approximately $1 / 10$ of the total record tıme

2 The minimum period for which the spectral density can be calculated is 2 times the sampling interval

3 The number of frequency bands is equal to the number of lags

4 The bandwidth is $\pi /(m \Delta t)$ or $1 / m$ tımes the frequency of the minımum calculated period

5 The maximum calculated period (irrespective of accuracy of computation) is $2 \mathrm{~m} \Delta t$

6 The sampling interval, $\Delta t$, should be not more than approximately $1 / 4$ of the minimum period in the record that contains a significant proportion of the total energy in the record

If criterion 6 is not observed, the energy in these shorter periods will show up at spurious frequencies of longer period for the instrument location at San Nicolas Harbor, the prevalling wind is offshore This lımıts the fetch for local wind waves and gives assurance that the energy content in waves of less than $10-$ sec period is nominal on this basis, a sampling interval of $3 \mathrm{sec}$ was considered ideal

For all wave records taken to date at San Nicolas Harbor, an attempt was made to obtain $4 \mathrm{hr}$ of contınuous record for each orientation of the horızontal velocity sensor This method was based on the assumption that periods up to $24 \mathrm{~m} / \mathrm{n}$ would be of interest Subsequent studies have shown 
that only tsunamıs such as from the Alaskan earthquake in 1964 have significant velocities in this period range, and that an upper period 1 imit of 6 min is reasonable for all wave action except tsunamis on this basis, one hour of record was considered ideal for each spectral analysis

Analyses were run with 720 lags and 360 lags for comparison The 720-lag solution gave better definition of the component peaks, showed essentially the same total energy content, and was used for most of the data analysis

Fig 9 is a typical spectral density plot for the horizontal water motion of the seven success Ive headings for the July 26-27 wave conditions

\section{CROSS SPECTRAL ANALYSIS}

A further development of spectral analysis is the cross-spectral analysis, in which two separate records are analyzed Output of this analysis includes the spectral densities of each record, the cross-spectra consistinq of two parts, real and Imaginary, the coherence, and the phase coherence

The cross-spectral analyses were computed for the study by FORTRAN program CROSPEC on a CDC 6600 computer CROSPEC computes cross-correlation and cross-power spectral density functions for two or more time-series, and was developed from subrout ines published by Robınson (2)

Cross-spectral analyses were run on the simultaneous vertical and hor zontal velocity records for the $270^{\circ}$ heading of July 26 , and for the simultaneous ship's horizontal bow velocity and the horizontal water velocity of April 19 For the latter analysis, the displacement plots from the time-lapse movie sequence were converted to horizontal velocity rates by determining the horizontal distance moved every $2 \mathrm{sec}$

The results of this analys Is are shown in FIgs 10,11 , and $12 \mathrm{FIg} 10$ shows the power spectrum of each motion plotted on the same graph The excellent correlation in the range of periods for swell is readily apparent, while in the long-period range, especially from $90 \mathrm{sec}$ to $110 \mathrm{sec}$, the ship motion has considerably more energy than the water motion These results are also shown in Fig 11 , which presents a tabulation of the equivalent uniform sinusoidal motions corresponding to the energy peaks of the two spectra

Fig 12 shows the coherence and phase coherence from this analysis for the coherence between the two records, a value of 10 shows excellent correlation and a value of 00 represents a completely random relationship Here again, good coherence is shown in the swell range, some coherence in the $90-\mathrm{sec}$ to $110-\mathrm{sec}$ range, and an essentially random relationship in all other frequencies

The phase coherence shows consistent values only in the swell range where the phase-angle values are clustered close to $0^{\circ}$ There is actually a $90^{\circ}$ phase lag in the ship's response to the swell, but this phase difference was compensated by a time shift in the input data for the ship's 


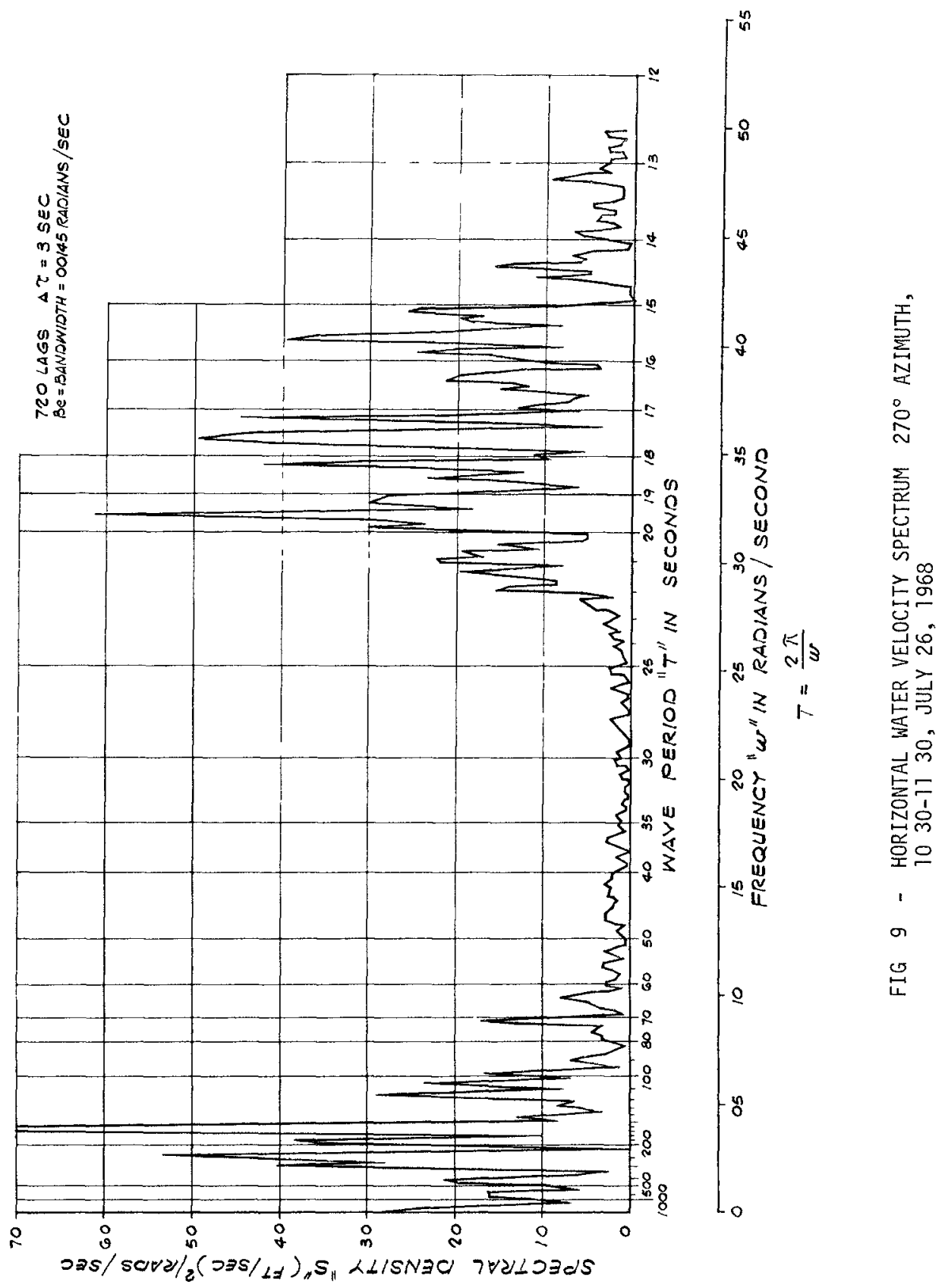




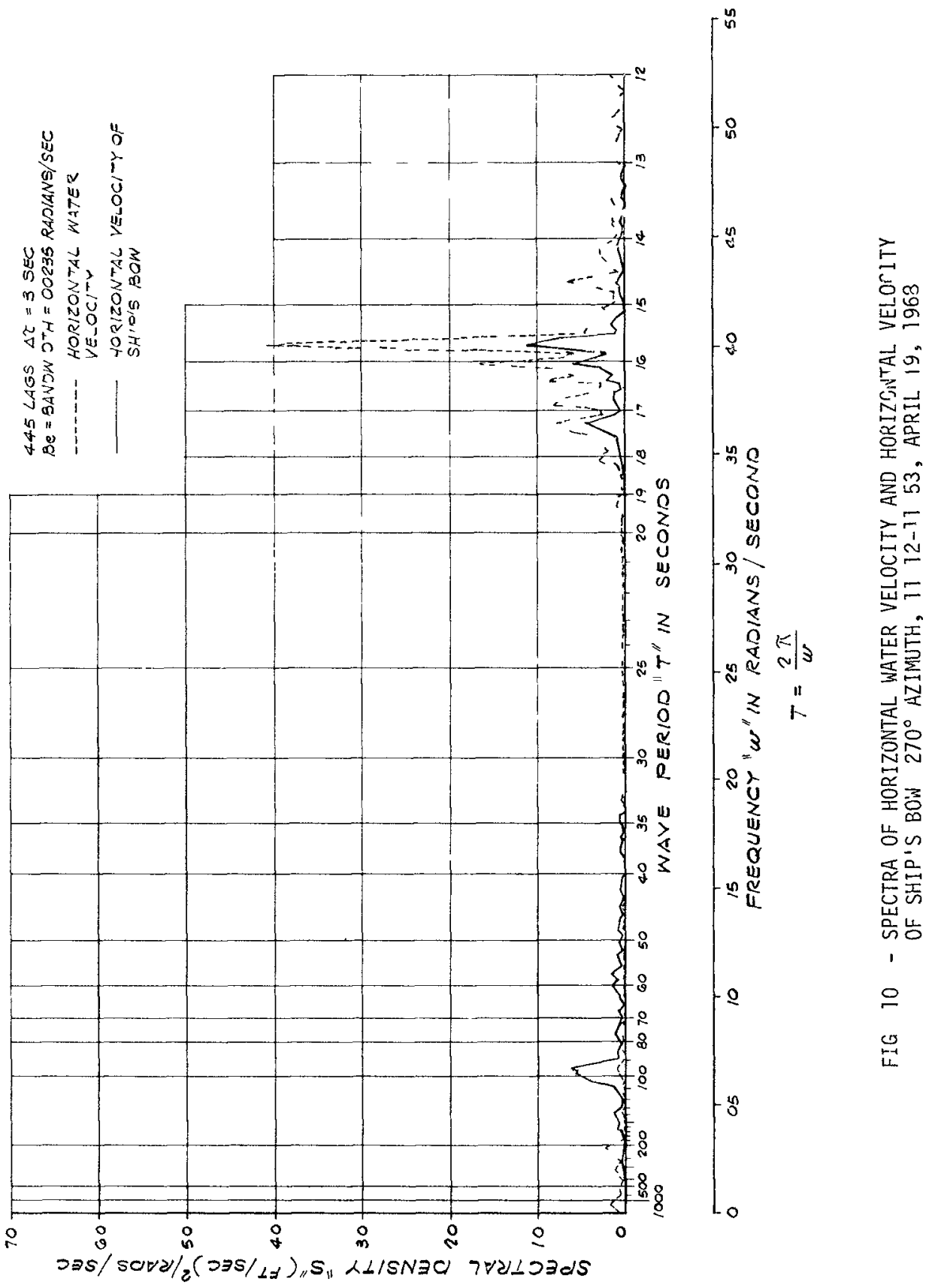




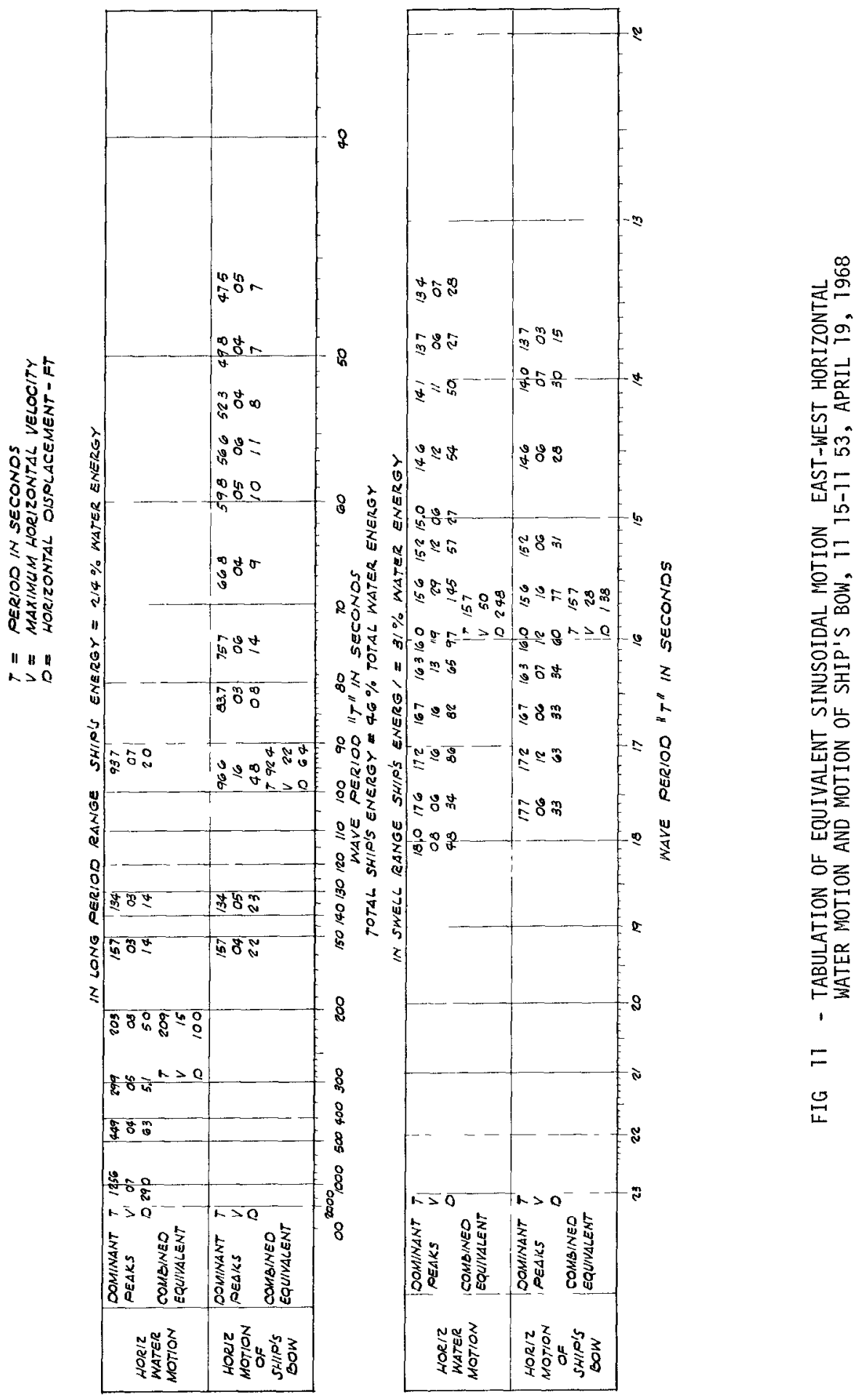




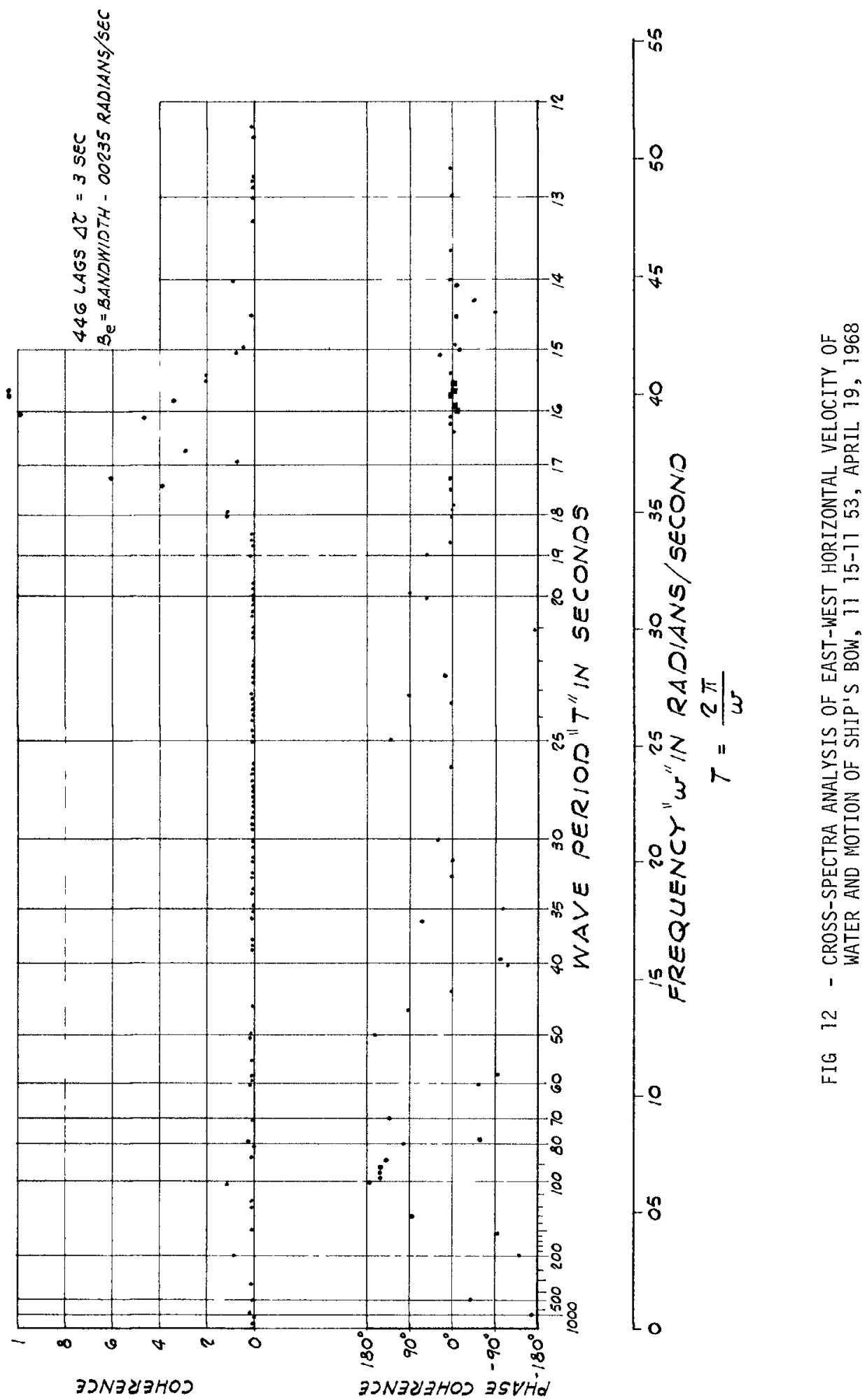


motion Elsewhere, the phase-angle relationship is essentially random except for a tendency of values to cluster around $180^{\circ}$ at $90 \mathrm{sec}$ to $110 \mathrm{sec}$ No firm explanation is offered for this last trend, but it might be that che time shift from $90^{\circ}$ to $0^{\circ}$ in the input data for the periods for swell may have shifted the long-period coherence in a direction opposite to the anticipated value of $90^{\circ}$ No clear physical significance of the real and Imaginary cross-spectral functions could be determıned, hence, plots of these values are not shown

The results for the vertical and horizontal water velocity cross-spectral analyses were not as consistent as analysis results for the ship motion and horizontal water motion Fig 13 shows a dual plot of the spectral densities for both the horizontal and the vertical water velocities, and Fig 14 shows a plot of the equivalent sinusoidal wave components corresponding to the peaks in each power spectrum In Fig 13, the spectral density scale for the vertical velocity is expanded to better illustrate the correlation between the two it can be noted that the period range for swe ll again has good correlation, but the long-period correlation is rather inconsistent Especially significant are a few frequencies, such as those near $36 \mathrm{sec}$, where the vertical spectral density function value 1- proportionally higher than elsewhere This relationship could be inuspreted in three possible ways

I The instrument was near an antı-node of a standıng wave For such a wave the water particle motion would be vertical only and a corresbonding horizontal peak would not exist

2 An incident wave of that perıod passed at right angles to the hor Izontal sensor in this null direction, the horizontal velocity component would not register

3 The relatilely high horizontal velocities, combined with floating seaweed caught on the instrumerit supporting pıpe, deflected the vertical duct out of plumb and thereby caused the vertical sensor to register some of the horizonta, velocity as a false downward component for a heavy swell, the false velocity component would not reverse since the false downward component would not be affected by the direction in which the instrument was out of plumb Such behavior would show up as an unduly high peak in the power spectrum of the vertical 'elocity, indicatıng a false downard displacement

This last interpretation was believed to be correct, since the displacemut plot derived from the vertical velocity record also showed a false tcwnward moverent of the water surface considerably greater than that norma'ly expected Fig 15 shows the displacement plot and the horizontal $v$-locity record to the same time scale Correlation between the rate of $\pi$ ) w iward displacemert and the magnitude of horizontal velocity is insufI sient for this condilion to be interpreted as the sole cause of the ano-

'o \& peaks in the zower spec'lum of the vertical velocity record The

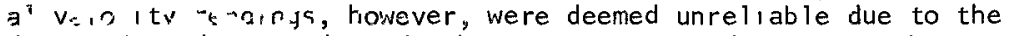
at dowl lald eading and no furtrer analysis was done using the vertical yulual-v records in further applications, this problem could probably be ¿ il iated by nodıficatıor of the instrument mountıng arrangement 


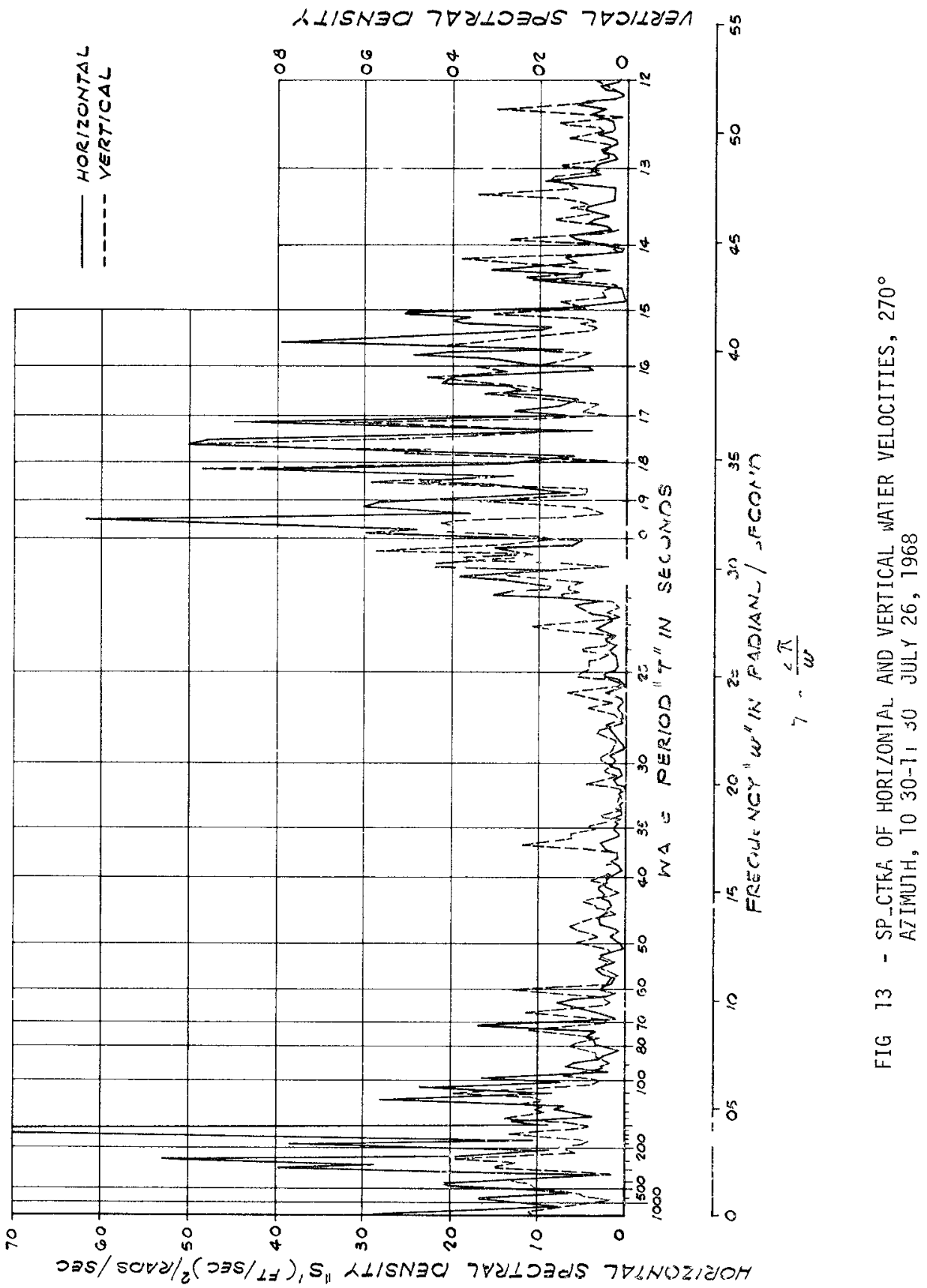




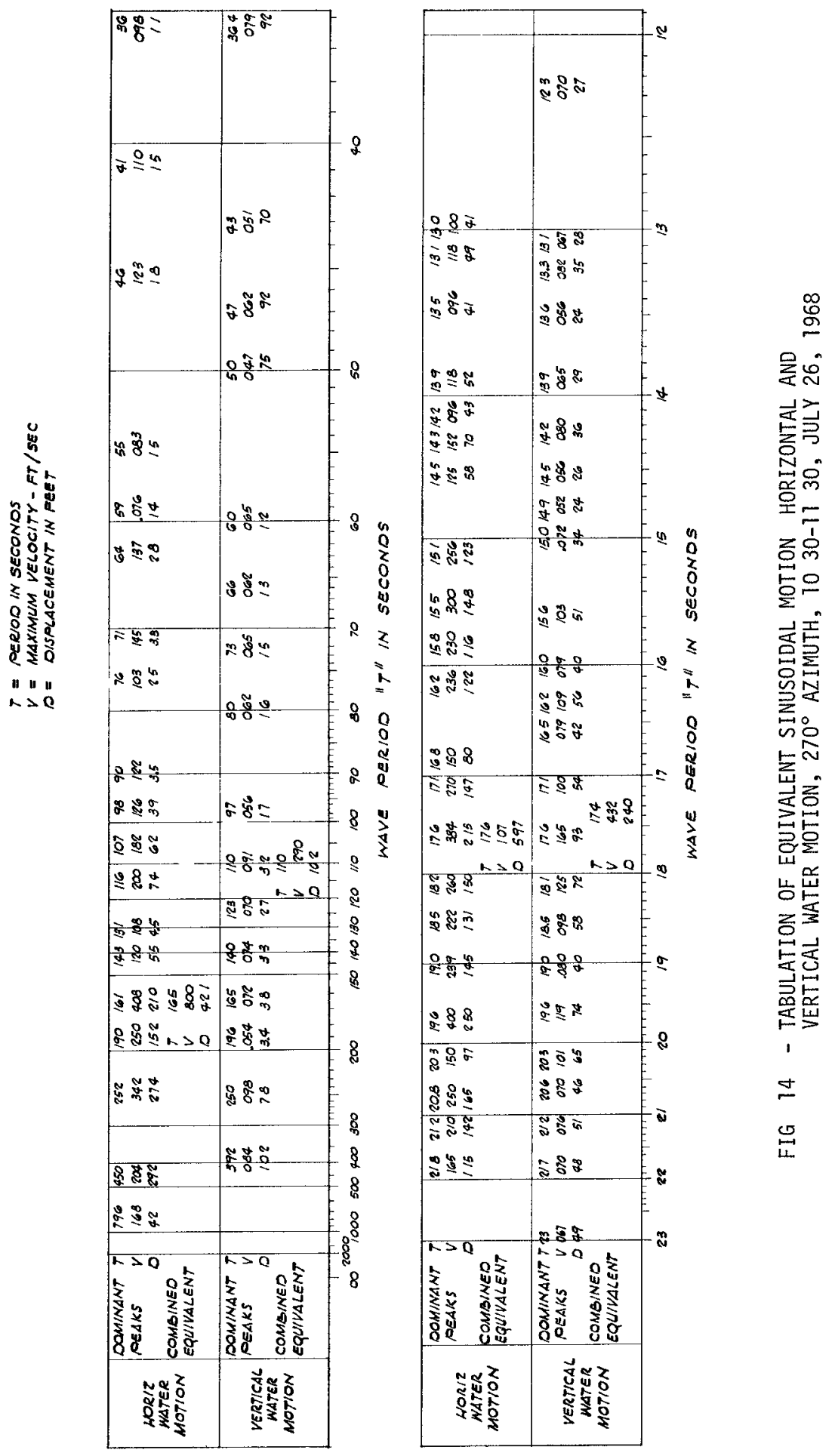




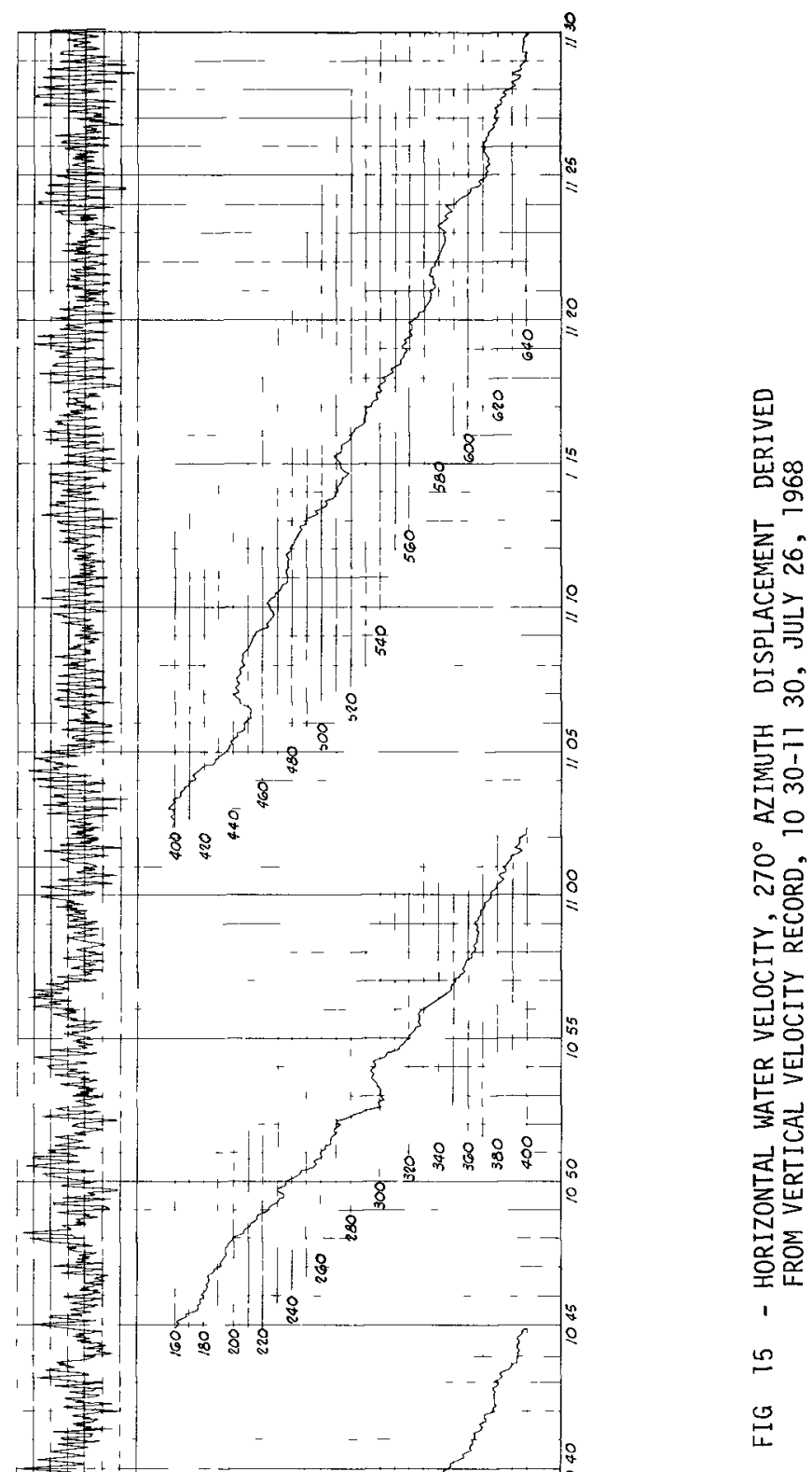


A primary purpose of the analysıs of the July 26-27 data was to determıne the magnitude and type of long-period waves The magnitude as shown by the power spectra of the horizontal velocity records left no doubt as to the Importance of the long-period waves Energy content was nearly as high as that of the swell, and the energy content recorded for both longperiod waves and swell depicted conditions much too rough for mooring a large ship alongside the pler The cross-spectral analysis of the vertical and horizontal velocity records was expected to provide a major contribution in determiring whether long-period waves were incident waves similar to the swell, resonant or standing waves, or a combination of incident and reflected waves since the questionable valıdity of the vertical velocity readings eliminated this possibility, the power spectra of the horizontal velocities were used instead

Fig 16 shows a tabulation of the equivalent unıform sınusoidal wave components corresponding to each energy peak of the horizontal spectra in the swell range, $F_{1 g} 17$ shows a similar tabulation for the energy peaks of the horizontal spectra in the long-period range For each heading a combined equivalent wave for each period range is also included

If the long-perıod waves were a resonance phenomenon, nearly identical peaks would be expected, especially for waves approaching from azımuths of $295^{\circ}$ and $300^{\circ}$, and long-period waves caused by a beat generated by the swell periods would be expected to change in period in accordance with shifts in the swell periods

A study of Fig 16 indicates that during the recorded interval

1 The periods of swell shifted towards the shorter ranqe

2 The total swell energy decreased in magnitude by a little more than $40 \%$

3 Most of the swell approached the north end of the pier from an azımuth of approximately $300^{\circ}$

4 Several of the peak periods were present throughout the entire recording period

A simılar study of Fig 17 indicates that during this interval

1 The energy content of the long-period waves decreased in magnitude by approximate ly $80 \%$

2 The null direction of the long-period waves was not as clearly defined as for the swell, the dominant direction, however, was simılar to the di rection of swell

3 Shifts in period were apparently rather random and did not correspond to the shift to shorter periods of the swell

4 Very few of the peaks were apparent throughout the recording period 


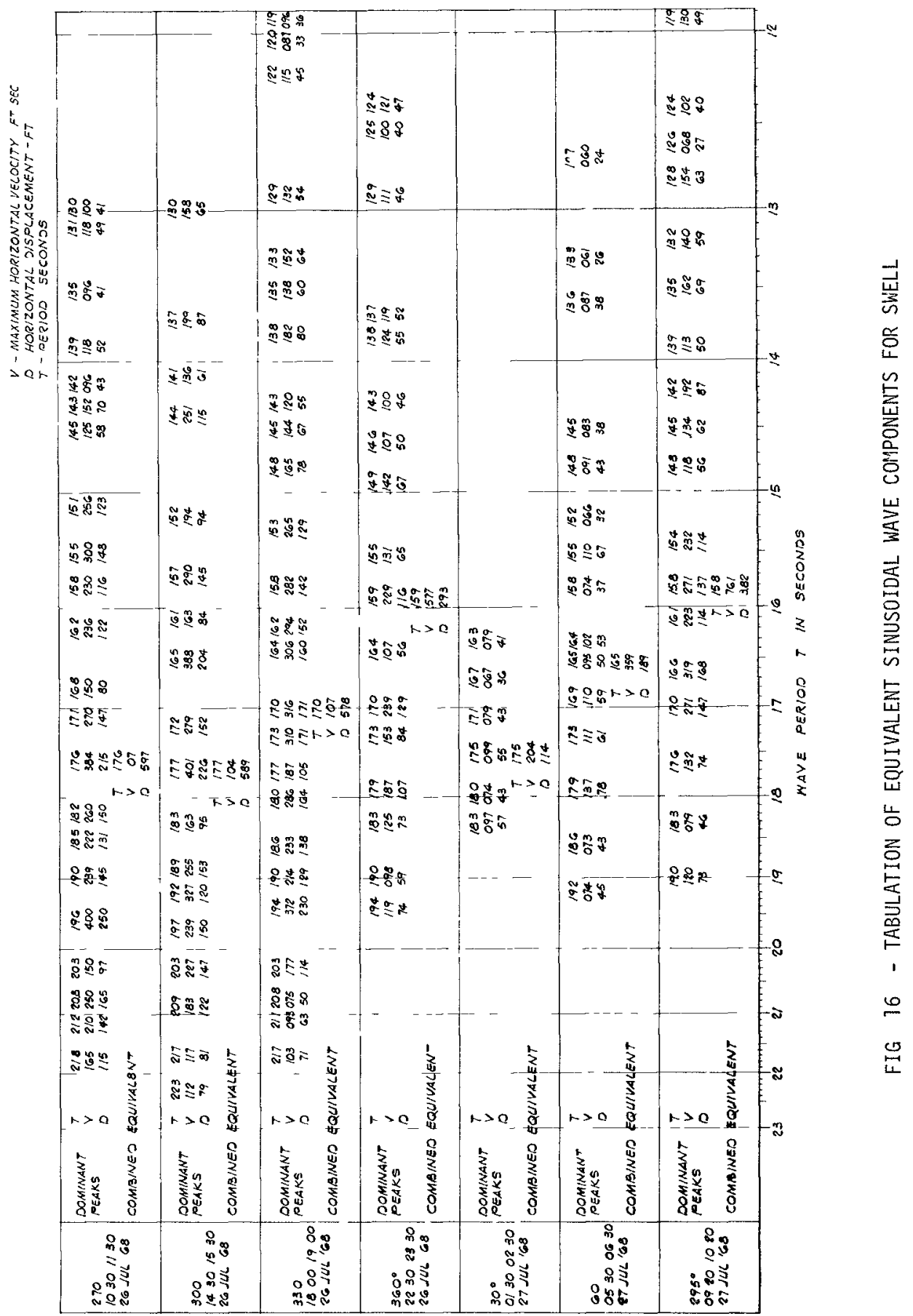




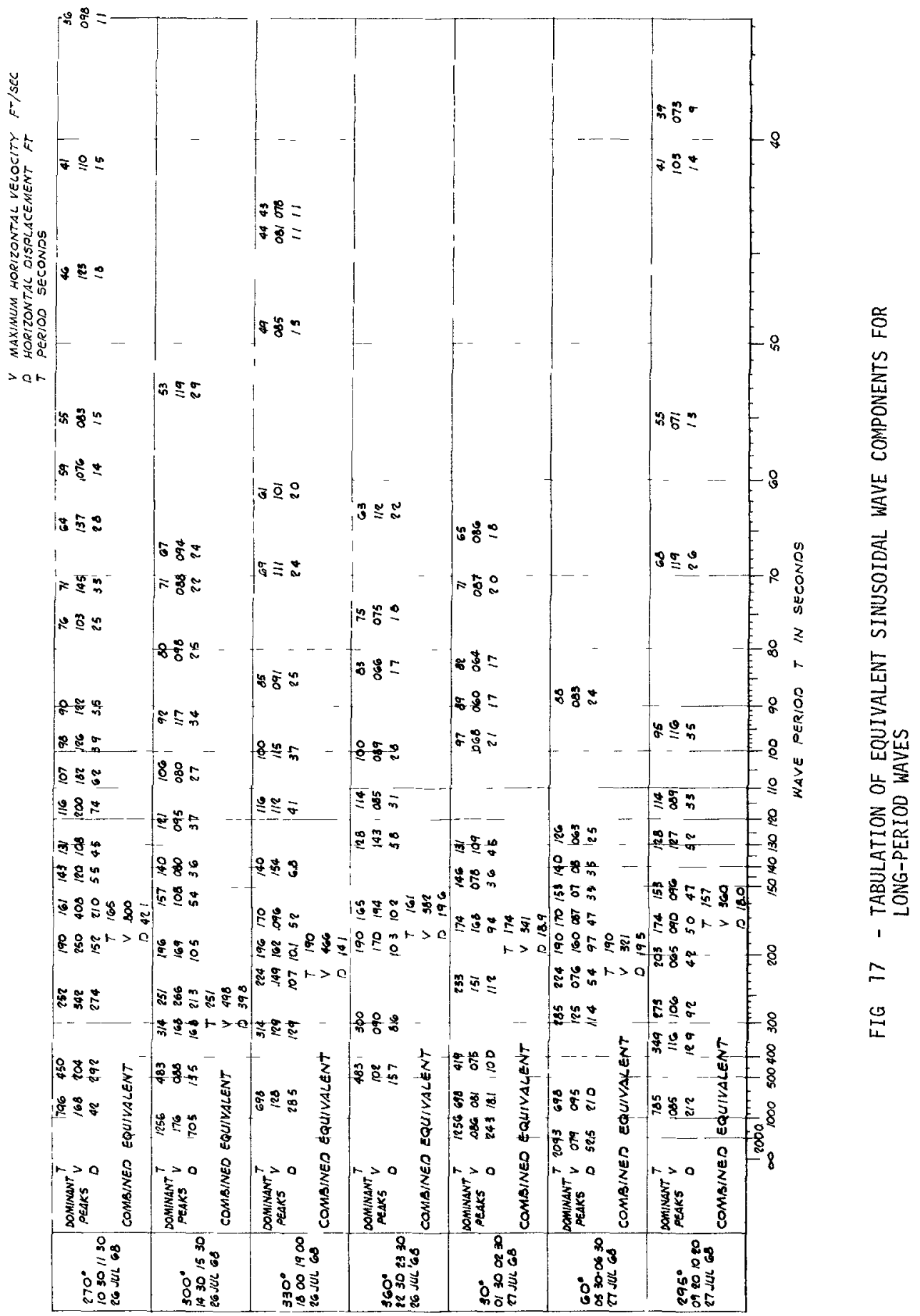


These observatıons Implied that the long-period waves are progressive waves originating from the same storm center as the swell, and that resonance of the bay is probably not an Important factor Unlike the energy of the swell, very little of the long-period wave energy is dissipated in breakers on the shore and most of the waves are reflected back to the ocean

Some of the long-period waves are probably reflected more than once before returning to the ocean, which would explain their lack of a clear null direction

\section{APPLICATION TO HARBOR PROGRAM}

Based on the preceding analysıs, it was concluded that ocean swells refracted around Point San Nicolas and approaching the pier from the $\pm 300^{\circ}$ azımuth were the primary source of adverse mooring conditions at the pier A breakwater similar to that shown in FIg 18 is currently under construction at San Nicolas The breakwater is expected to attenuate ocean swell so that wave action in the swell range will no longer cause interpretations to ore loading operations Attenuation of longuperiod waves of high energy content will be nominal, but for these unusual occurrences, the breakwater will have some beneficial effect by reorienting these longperiod waves so that their horizontal water motion will be primarily northsouth or parallel to moored ships rather than east-west as at present

\section{CONCLUSIONS}

The age-old problems of harbor design are becoming increasingly complex due to phenomenal increases in the sizes of modern ships Fortunately, our burgeoning technology is developing many new and powerful tools which can be applied in solving these problems The harbor study at San Nicolas adequately defined wave movement so that an economical solution could be obtained The full potential of the techniques used was not applied, but experience gained during the study indıcates the capabilıty for Improved results in similar applications

Ducted impeller meters provided an extremely valuable means for studying harbor wave action These instruments were especially useful in measuring long-period swells and surges which were difficult to identify by sight

Spectral analysis methods proved well-suited to detailed interpretation of the particle velocity records, consıderable insight into the wave phenomena was gaıned by simple, rational inspections and interpretations of the records

Time-lapse movies of a moored ship in motion, when correlated with sımultaneous water particle velocity records, provided an exceptionally clear picture of ship response to wave action, and led to the rather surprising observation that long-period ship motion is not necessarily caused by longperiod waves 


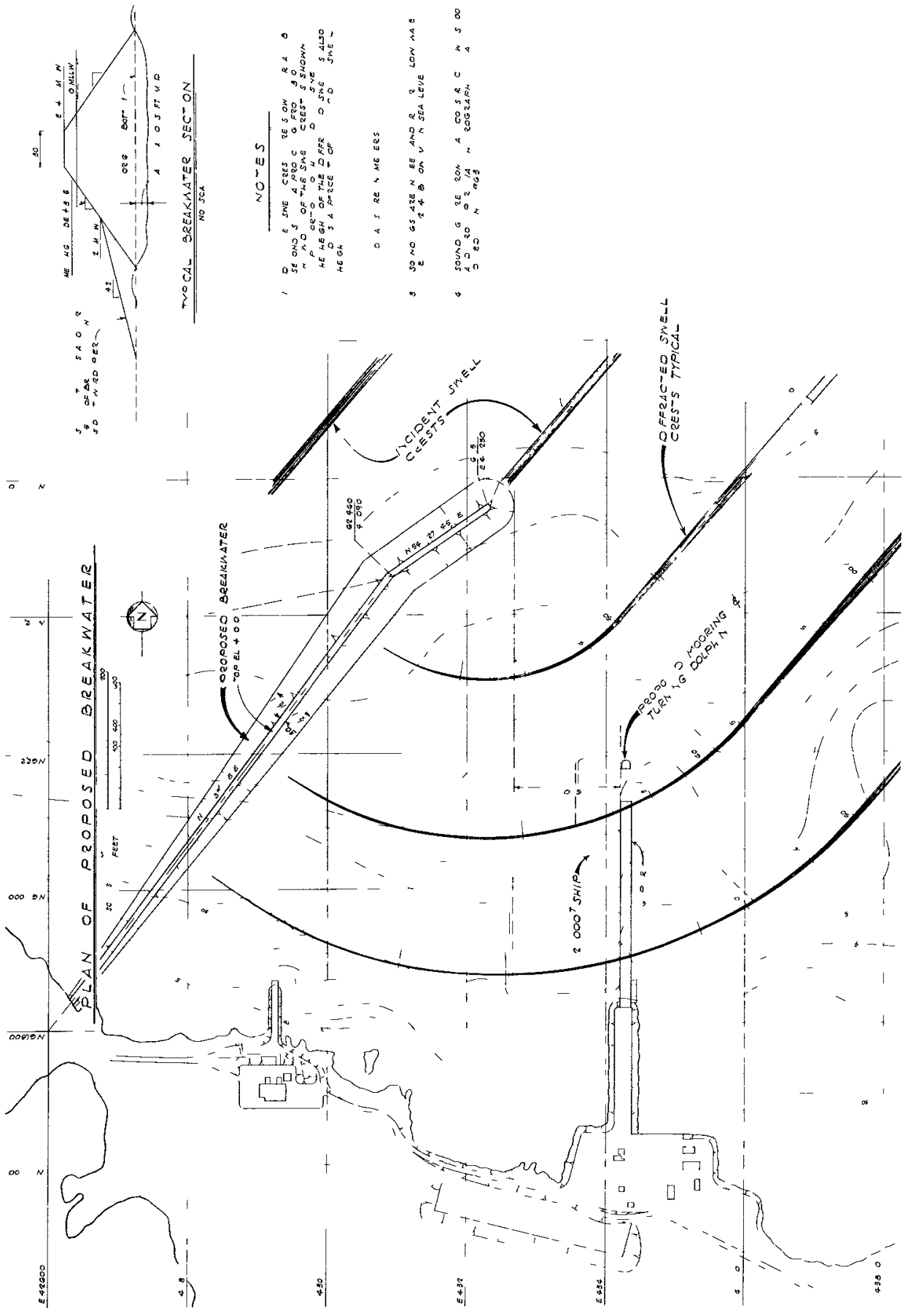

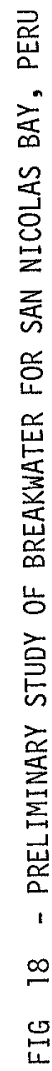


The usefulness of hydraulic model tests was again demonstrated, and it should be noted that very useful results can be obtalned from model tests at modest expense if the test goals are clearly defined and the test work performed simply and Judiciously

\section{ACKNOWLEDGMENTS}

The writers especially wish to express their appreciation to Marcona Corporation for permission to publish this paper The writers also wish to thank the many individuals within Marcona Corporation and John A Blume $\varepsilon$ Associates who participated in the harbor study Dilip Jhaverl, who selected and modified the computer programs used for the spectral analyses, and $\mathrm{J} W$ Johnson, who was an engineering consultant for the harbor study, deserve special mention for their contributions to the study program

\section{APPENDIX-REFERENCES}

1 LIU, S C, and Jhaverı, D P, "Spectral and Correlation Analysis of Ground-Motion Accelerograms," BulZetin, Selsmological Society of America, Vol 59, No 4, August, 1969, pp 1517-1534

2 Robinson, E A, Multuchannez Trme Semes Analysus wrth Drgital Computer Progroms Holden-Day, Inc, San Francisco, 1967

3 Shanting, D $H$, "Observations of Particle Motions in Ocean Waves," $A D$ 658876, Clearinghouse for Federal Scientıfic and Technical Information, Springfield, Virginia, July, 1967 



\section{CHAPTER 134}

TIDAL MOTION IN BAYS

by

O H Shemdin, Associate Professor

R M Forney, Research Assistant

Department of Coastal and Oceanographic Engineering

University of Florida, Gainesville, Florida

\section{ABSTRACT}

A method is proposed to investigate periodic tidal motion in single or multiple basins connected to the ocean by an inlet Non-sinusoidal tidal motion in the ocean and square friction law in the inlet are both considered The method is applied to Boca Raton inlet, Florida The calculated tidal elevation and velocity in the inlet are found to be in reasonable agreement $w_{1}$ th measured values The bottom shear friction coefficient is defined

$$
T_{\text {to }}=\rho r u|u|
$$

where $p$ is the water density, $T$ is the shear stress, and $u$ is the average velocity in the inlet The results of the study yleld $r=00039$, and predict net transport of sediment into the bay

\section{INTRODUCTION}

The dynamics of tidal motion in a bay connected to the ocean by an inlet wat investigated by Brown (1928) who considered only sinusoidal tidal motion in the ocean and linear bottom friction in the inlet Later, Keulegan (1951) treated the same problem but included tne square friction 1 aw in the inlet an predicted a non-sinusoidal oscillation in the bay elevation Van de Kreeke (1968) developed a scheme which predicted tidal oscillations in bays in the presence of freshwater inflow by rainfall or streams The above investigations were all 11mited to a sinusordal tidal oscillation in the ocean and all neglected flow acceleration in the inlet

An extensive treatise on tides and tidal propagation is given by Dronkers (1964) It is known that tidal motion is not simple but has many harmonic constituents The principal components are shown in Table I 
Table 1 Principal Tidal Components

\begin{tabular}{|c|c|c|}
\hline Symbol & Component & Perrods \\
\hline M2 & Principal lunar semidiurnal & 12421 \\
\hline \$2 & Principal solar semidiurnal & 12000 \\
\hline N2 & Lunar elliptic semidiurnal & 12658 \\
\hline K1 & Lunisolar declinational diurnal & 23936 \\
\hline 01 & Lunar declinational diurnal & 25819 \\
\hline
\end{tabular}

The relative importance of the different constituents depends on geographical location and depth of water The principal lunar semidiurnal component (M-2) has usually the highest amplitude

This study proposes a general method for analyzing tidal oscillation in hays connected to the ocean by inlets It includes acceleration of flow in the inlet, square friction law, and a non-sinusoidal tidal oscillation in the ocean The method utilizes the general equations of motion and approximates the periodic tidal oscillation by a series in circular functions Use is made of complex variables to simplify computations The method can be applied to multiple basins connected to each other and to the ocean The present study is restricted to relatively deep bays in which negligible spacial variation in water elevation exist The inlet area is assumed constant and equal to the mean area during one tidal cycle The method can be applied, however, to shallow bays The method has a resemblence to Dronker's (1964) harmonic method of tidal propagation al though he did not specifically apply the method to iniets A method proposed by Sidjabat (1970) for describing the nonlinear friction is employed in this study

\section{THEORETICAL APPROACH}

The theoretical development to follow describes the dynamics of flow in an inlet with a constant cross sectional area connected on one side to a basin with uniform water level and on the other side to an ocean which has harmonic but non-sinusoidal tidal oscillation The flow in the inlet is assumed onedimensional Resonance and bottom friction in the bay are neglected Quantitatively bays satisfying these conditions must be at least 20 feet deep when the longest dimension equals 5 miles

\section{A Single Bay Coupled to Ocean}

A definition sketch is shown in Figure 1 The equation of motion in the $x$-direction (direction of intet flow) is used to describe the inlet flow

$$
\frac{\partial u}{\partial t}+u \frac{\partial u}{\partial x}+w \frac{\partial u}{\partial z}=-\frac{1}{\rho} \frac{\partial p}{\partial x}+\frac{1}{\rho} \frac{\partial \mathrm{T}}{\partial z},
$$

where $u$ is the inlet velocity, $p$ is the pressure, $p$ is the water density, and $T_{v}$ is the viscous shear stress Denoting 


$$
\begin{aligned}
u(x, y, z, t) & =u_{m}(x, z, t)+u^{\prime}(x, y, z, t) \\
w(x, y, z, t) & =\quad w^{\prime}(x, y, z, t)
\end{aligned}
$$

where $U_{m}$ denotes the mean flow over a length of $t_{1} m e, T$, which 15 small compared to tidal period by long compared to time scale turbulence The overbar is taken to denote averaging over period $\mathrm{T}$

$$
\bar{u}=\frac{1}{T} \int_{0}^{T} u d t=U_{m}
$$

Equation (1) becomes

$$
\frac{\partial U_{m}}{\partial t}+\frac{\partial}{\partial x}\left(U_{m}^{2}+\overline{U^{T}}\right)=-\frac{1}{\rho} \frac{\partial p}{\partial x}+\frac{1}{\rho} \frac{\partial}{\partial z}\left(T_{v}+\tau_{t}\right),
$$

where $\tau_{v} 1 s$ the turbulent Reynolds stress defined by

$$
{ }^{T} t=-p \quad \overline{u^{\prime} w^{\top}}=-\rho \int_{0}^{T} u^{\prime} w^{\prime} d t
$$

Next, a vertical average over the depth $h$ is specified We define

$$
U(x, t)=\frac{1}{h} \int_{0}^{h} U_{m}(x, z, t) d t
$$

Integrating Equation (2) over depth and assuming hydrostatic pressure distribution

$$
\frac{\partial U}{\partial t}+\alpha \frac{\partial}{\partial x} U^{2}=-g \frac{\partial h}{\partial x}-\frac{1}{\rho h}\left(r_{v}+\tau_{t}\right)_{0},
$$

where

$$
\alpha U^{2}=\frac{1}{h} \int_{0}^{h}\left(U_{m}^{2}+\frac{\bar{u}}{u^{\prime z}}\right) d z \quad,
$$

and the subscript o denotes bottom values $(z=0)$ From open channel considerations o is the momentum factor and 15 of the order of 105 The value of 10 will be assumed sufficiently accurate In inlets, the flow is mostly turbulent exceot when $1 t$ approaches zero during a tidal reversal The bottom shear stress $w 111$ be assumed to be primarily due to turbulence rather than $v 1 s \cos 1$ ty $\left(1 \in{ }^{T} v \ll T_{t}\right)$ and to have the empirical square-law
form 


$$
r_{t_{0}}=\rho r U|U|
$$

where $r$ is the friction coefficient Equation (4) now becomes

$$
\frac{\partial U}{\partial t}+\frac{\partial}{\partial x} U^{2}=-g \frac{\partial h}{\partial x}-\frac{r}{h} U \mid U
$$

Equation (6) is applied to the inlet shown in Figure 1 Integration of Equation (6) along the $x$-direction $y_{1} e 1 d s$ an equation which relates the ocean level to that in the basin Assuming the inlet is finite in length and the velocity accelerates towards the inlet but achieves a constant velocity, $U_{1}$, in the inlet, Equation (6) is integrated to yield

$$
h_{s}=h_{b_{1}}+\frac{\partial U_{1}}{\partial t} \frac{L_{1}}{g}+\left(1+\frac{r}{h_{1}} L_{1}\right) \frac{U_{1}\left|U_{1}\right|}{g} \text {, }
$$

where $h_{s}, h_{b_{1}}, L_{1}$ and $h_{1}$ are defined in Figure 1 The equation of mass conservation consistant with the above assumptions becomes

$$
U_{1} A_{1}=A_{b_{1}} \frac{d h_{b_{1}}}{d t}
$$

where $A_{1}$ and $A_{b_{1}}$ are as defined in Figure 1 Equation (8) further assumes that the changes in $A_{b_{1}}$ and $A_{1}$ due to depth changes are small over a tidal cycle Eliminating $U_{1}$ in Equation (7) yields

$$
h_{s}=h_{b_{1}}+\left(\frac{A_{b_{1}}}{A_{1}}\right) \frac{L_{1}}{g} \frac{d^{2} h_{b_{1}}}{d t^{2}}+\left(1+\frac{r_{1}}{h_{1}} L_{1}\right) \frac{1}{g}\left(\frac{A_{b_{1}}}{A_{1}}\right)^{2} \frac{d h_{b_{1}}}{d t}\left|\frac{d h_{b_{1}}}{d t}\right|
$$

\section{The Linearized Solution for Simple Motion}

The tidal motion in the ocean is described by complex variables to simplify computations

$$
h_{S}(t)=\hat{h}_{S} e^{1 \sigma t} \quad,
$$

where $\hat{h}_{s}$ is a complex number and $\sigma$ is the $t_{1}$ dal frequency Let

$$
h_{b_{1}}=-1 \hat{h}_{b_{1}} e^{1 \sigma t}
$$


where $\hat{h}_{b}$ is a real number representing the bay tidal amplitude Linearization of the last term in Equation (9) implies

$$
\frac{d h_{b_{1}}}{d t}\left|\frac{d h_{b_{1}}}{d t}\right| \approx \frac{8}{3 \pi} \sigma^{2} h_{b_{1}} e^{1 \sigma t}+,
$$

which yields the solution

$$
\hat{h}_{s}=\hat{h}_{b_{1}}\left[\zeta \hat{h}_{b_{l}}-i n\right] \quad,
$$

where

$$
\zeta=\frac{8}{3 \pi}\left(1+\frac{r_{1}}{h_{1}} L_{1}\right) \frac{1}{g}\left(\frac{A_{b_{1}}}{A_{1}}\right)^{2} \sigma^{2} \quad,
$$

and

$$
n=\left[1-\left(\frac{A_{b_{1}}}{A_{1}}\right) \frac{L_{1}}{9} \sigma^{2}\right]
$$

The term $\left(\frac{A_{b_{1}}}{A_{1}}\right) \frac{L}{9} \sigma^{2}$ represents the flow acceleration and has been traditionally neglected $\bar{A}_{1}$ It heed not be always negligible compared to 10,1 t was found to be equal to 025 for Macquarie Harbor which was studied by Van de Kreeke (1968) The amplitude and phase shift of the ocean fluctuation are given respectively,

$$
\left|\hat{h}_{s}\right|=\hat{h}_{b_{1}} \sqrt{\left(\xi h_{b_{1}}\right)^{2}+n^{2}}
$$

and

$$
\tan \theta=-\frac{\eta}{\zeta \hat{h}_{b}}
$$

O'Brien (1969) proposed an empirical relationship between the tidal prism, $P$, and inlet area $A_{1}$

$$
A_{1}=C P
$$

where $C$ is a constant The present analysis yields an analytical representation for $C$ which can be used to test the empirical constant of $0^{\prime}$ Brien

$$
C=\frac{\left(\frac{8}{3 \pi}\right)\left(1+\frac{r_{1}}{h_{1}} L_{1}\right) \frac{\sigma^{2}}{g}\left(\frac{A_{b_{1}}}{A_{1}}\right)^{2} \tan \theta}{2\left[\left(\frac{A_{b_{1}}}{A_{1}}\right) \frac{L_{1}}{g} \sigma^{2}-1\right]}
$$




\section{Solution for Non-sinusoidal Motion}

The tidal motion in the ocean is not simple as indicated previously so that sinusoidal simplifications introduce errors Since the bay motion is affected by nonlinear friction, motion is not simple even when the ocean motion $1 \mathrm{~s}$ The latter case was investigated in detail by Keulegan (1951) When the tidal motion in the ocean is periodic but not simple, the bay motion is even more complex since each ocean harmonic constituent generates its own harmonic as well as multiple harmonics due to nonlinear friction Thus a linearized procedure becomes invalid, in fact it is unrealistic and suggests negative friction at the higher harmonics

In the following a method is proposed to describe the harmonic motion in both bay and ocean The complex representation is employed

$$
h_{s}=\sum_{n} \frac{1}{2}\left(r_{n}-1 s_{n}\right) e^{1 n \sigma t}
$$

and

$$
n_{b_{1}}=\sum_{n} \frac{1}{2}\left(c_{n}-1 d_{n}\right) e^{1 n \sigma t}
$$

where the summation ranges over both positive and negative values of the index $n$ which specifies the principal tidal frequencies, 1inear combinations thereof, and higher harmonics The terms corresponding to $n=0$ reflect mean levels in ocean and bay since $h_{s}$ and $h_{b}$ are real functions, the following relationships must be satisfied (Lee ${ }^{b_{1}}$ (1967))

$$
\begin{array}{lll}
r_{n}=r_{-n} & , & s_{n}=-s_{-n} \\
c_{n}=c_{-n} & , & d_{n}=-d_{-n}
\end{array}
$$

The average velocity is specified by

$$
u(t)=\sum_{n} \frac{1}{2}\left(a_{n}-1 b_{n}\right) e^{1 n_{\sigma} t}
$$

where

$$
a_{n}=a_{-n}, \quad b_{n}=-b_{-n}
$$

The representation of $\mathrm{T}$ in series form was considered by Sidjabat (1970) in conjunction with tidat propagation in wide shallow bays His description of the noninear term is conveniently adopted Equation (15) may be expressed

$$
T_{\text {to }}=\operatorname{pr}\left[\sum_{\ell} \sum_{m} \frac{1}{4}\left(a_{\ell}-1 b_{\ell}\right)\left(a_{m}-1 b_{m}\right) e^{7(\ell+m)} t^{\frac{1}{2}} \sum_{n}\left(a_{n}-1 b_{n}\right) e^{1 \sigma t}\right.
$$


which is equivalent to

$$
\begin{aligned}
& r_{\text {to }}=\operatorname{pr}\left\{\left[\sum_{\ell} \frac{1}{4}\left(a_{\ell}{ }^{2}+b_{\ell}{ }^{2}\right)+\sum_{\ell} \sum_{\ell \neq m} \sum_{m} \frac{1}{4}\left(a_{\ell}-1 b_{\ell}\right)\left(a_{m}-1 b_{m}\right) e^{l(\ell+m) t}\right]^{\frac{1}{2}}\right. \\
& \sum_{n}^{5}\left(a_{n}-1 b_{n}\right) e^{1 n o t}
\end{aligned}
$$

Denote

$$
\lambda^{2}=\sum_{\ell=-\infty}^{\infty} \frac{1}{4}\left(a_{\ell}{ }^{2}+b_{\ell}{ }^{2}\right)=\frac{1}{2} \sum_{\ell=0}^{\infty}\left(a_{\ell}{ }^{2}+b_{\ell}{ }^{2}\right)
$$

Then

$$
r_{\text {to }}=\rho r_{\lambda}(1+\varepsilon)^{\frac{1}{2}} \sum_{n}\left(a_{n}-1 b_{n}\right) e^{1 n \sigma t},
$$

where

$$
\varepsilon=\frac{1}{\lambda^{2}} \sum_{\ell} \sum_{\ell \neq m} \frac{1}{4}\left(a_{\ell}-1 b_{\ell}\right)\left(a_{m}-1 b_{m}\right) e^{1(\ell+m) \sigma t}
$$

It was found by sidjabat (1970) that in areas where M2 is the dominant tide $\lambda$ is determined primarily by the $M-2$ component and $\varepsilon$ does not exceed the value 025 Equation (22) is approximated by

$$
r_{\text {to }} \approx \operatorname{or\lambda } \sum_{n} \frac{1}{2}\left(a_{n}-1 b_{n}\right) e^{1 n \sigma t}
$$

The error in the friction term is less than $125 \%$ and corresponds to smaller errors in computing tidal elevation In studies where such an error is significant, it is possible to calculate $\varepsilon$ and to include its effect in Equation (22) The above method w17l be applied to Boca Raton inlet, the terms comprising $\varepsilon$ w 11 be neglected subject to comparison with measured tidal elevations

The non-linear term in Equation (9) becomes according to the above analysis (See Equation (17))

$$
\left|\frac{d h_{b_{1}}}{d t}\right| \frac{d h_{b_{1}}}{d t}=\lambda_{1} \sum_{n} \frac{1}{2}\left(1 n \sigma c_{n}+n \circ d_{n}\right) e^{1 n \sigma t},
$$

where 


$$
\lambda_{1}=\frac{1}{\sqrt{2}}\left[\sum_{\ell=0}^{\infty} \sigma^{2} \ell^{2}\left(c_{n}+d_{n}\right)\right]^{\frac{1}{2}}
$$

Substituting for $h_{s}, h_{b_{1}}$, and $\left|d h_{b_{1}} / d t\right|\left(d h_{b_{1}} / d t\right)$ and equating the real and imaginary coefficients, the following algebraic equations for the coefficients of different harmonic constituents are deduced

$$
\begin{aligned}
& r_{n}=\left[1-n^{2} \sigma^{2}\left(\frac{A_{b_{1}}}{A_{1}}\right) \frac{L_{1}}{g}\right] c_{n}+\frac{\lambda_{1}}{g}\left(1+\frac{r}{h_{1}} L_{1}\right)\left(\frac{A_{b_{1}}}{A_{1}}\right)^{2} n_{\sigma d_{n}} \\
& s_{n}=\left[1-n^{2} \sigma^{2}\left(\frac{A_{b_{1}}}{A_{1}}\right) \frac{L_{1}}{g}\right] d_{n}-\frac{\lambda_{1}}{g}\left(1+\frac{r}{h_{1}} L_{1}\right)\left(\frac{A_{b_{1}}}{A_{1}}\right)^{2} n_{\sigma c_{n}}
\end{aligned}
$$

Equations (26) relate the bay motion to the ocean tidal oscillation The non-1inear friction is specified by the term $\lambda_{1}$ which also couples the different harmonic constituents of the ocean elevation

The procedure for solution depends on the avallable information If the tidal elevations in both ocean and bay are measured, it is possible to evaluate the friction coefficient $r$ When only the motion in the bay is known, it is possible to predict the tidal motion in the ocean for any given $r$ However, when the tidal motion in the ocean is given the motion in the bay can only be computed by an iterative procedure for any given $r$

\section{B* Two Bays Coupled to Ocean}

A definition sketch for two bays coupled together and to the ocean 15 shown in Figure 2 The second bay is not connected to the ocean The tidal elevations in the ocean and first bay are specified by Equations (16) and (17), respectively The tidal motion in the second bay is specified by

$$
h_{b_{2}}=\sum_{n} \frac{1}{2}\left(p_{n}-r q_{n}\right) e^{1 n \sigma t}
$$

Subject to all assumptions stated previously, the equation which relates the motions in the two bays becomes

$$
h_{b_{1}}=h_{b_{2}}+\left(\frac{A_{b_{2}}}{A_{2}}\right) \frac{L_{2}}{g} \frac{d^{2} h_{b_{2}}}{d t^{2}}+\left(1+\frac{r}{h_{2}} L_{2}\right) \frac{l}{g}\left(\frac{A_{b_{2}}}{A_{2}}\right) \frac{d h_{b_{2}}}{d t}\left|\frac{d h_{b_{2}}}{d t}\right| \text {, }
$$

where the equation of continuity in the second inlet

$$
U_{2} A_{2}=A_{b_{2}} \frac{d h_{b_{2}}}{d t}
$$

was used The resulting algebraic coefficient equations relating the two bays become 


$$
\begin{aligned}
& c_{n}=\left[\begin{array}{ll}
1-n^{2} \sigma^{2} & \left(\frac{A_{b_{2}}}{A_{2}}\right) \frac{L_{2}}{g}
\end{array}\right] p_{n}+\frac{\lambda_{2}}{g}\left(1+\frac{r}{h_{2}} L_{2}\right)\left(\frac{A_{b_{2}}}{A_{2}}\right)^{2} n \sigma q_{n}, \\
& d_{n}=\left[\begin{array}{ll}
1-n^{2} \sigma^{2} & \left(\frac{A_{b_{2}}}{A_{2}}\right) \frac{L_{2}}{g}
\end{array}\right] a_{n}-\frac{\lambda_{2}}{g}\left(1+\frac{r}{h_{2}} L_{2}\right)\left(\frac{A_{b_{2}}}{A_{2}}\right)^{2} n \sigma p_{n},
\end{aligned}
$$

where

$$
\lambda_{2}=\frac{1}{\sqrt{2}}\left[\sum_{\ell=0}^{\infty} \sigma^{2} \ell^{2} \quad\left(p_{n}{ }^{2}+q_{n}{ }^{2}\right)\right]^{\frac{2}{2}}
$$

The motion of the first bay may now be related to the motions of both ocean and second bay Equation (7) remains valid for the first inlet, but the continuity equation now takes the form

$$
U_{1} A_{1}=A_{b_{1}} \frac{d h_{b}}{d t}+U_{2} A_{2}
$$

Using Equation (29)

$$
U_{1}=\frac{A_{b_{1}}}{A_{1}} \frac{d h_{b_{1}}}{d t}+\frac{A_{b_{2}}}{A_{1}} \frac{d h_{b_{2}}}{d t}
$$

and substituting for $U_{1}$ in Equation (7) the equation which relates the first bay to the ocean is obtained

$$
\begin{aligned}
h_{s}= & h_{b}+\frac{L_{1}}{g}\left(\frac{A_{b_{1}}}{A_{1}}\right) \frac{d^{2} h_{b_{1}}}{d t^{2}}+\frac{L_{1}}{g}\left(\frac{A_{b_{2}}}{A_{2}}\right) \frac{d^{2} h_{b_{2}}}{d t^{2}} \\
& +\left(1+\frac{r}{h_{1}} L_{1}\right) \frac{1}{g}\left(\frac{A_{b_{1}}}{A_{1}} \frac{d h_{b_{1}}}{d t}+\frac{A_{b_{2}}}{A_{1}} \frac{d h_{b_{2}}}{d t}\right)\left|\frac{A_{b_{1}}}{A_{1}} \frac{d h_{b_{1}}}{d t}+\frac{A_{b_{2}}}{A_{1}} \frac{d h_{b_{2}}}{d t}\right|
\end{aligned}
$$

Using the representation for $h_{s}, h_{b_{1}}$, and $h_{b_{2}}$, given by Equation (16), (17) and (27), respectively, the following algebraic coefficient equations are deduced

$$
\begin{aligned}
r_{n}= & c_{n}\left[1-n^{2} \sigma^{2} \frac{L_{1}}{g}\left(\frac{A_{b_{1}}}{A_{1}}\right)\right]-p_{n}\left[n^{2} \sigma^{2} \frac{L_{1}}{g}\left(\frac{A_{b_{2}}}{A_{1}}\right)\right] \\
+ & \frac{\lambda_{3}}{g}\left(1+\frac{r}{h_{1}} L_{1}\right)\left[\left(\frac{A_{1}}{A_{1}}\right) n \sigma d_{n}+\left(\frac{A_{b_{2}}}{A_{1}}\right) n \sigma q_{n}\right] \\
s_{n}= & d_{n}\left[1-n^{2} \sigma^{2} \frac{L_{1}}{g}\left(\frac{A_{b_{1}}}{A_{1}}\right)\right]-q_{n}\left[n^{2} \sigma^{2} \frac{L_{1}}{g}\left(\frac{A_{b_{2}}}{A_{1}}\right)\right] \\
& -\frac{\lambda_{3}}{g}\left(1+\frac{r}{h_{1}} L_{1}\right)\left[\left(\frac{b_{1}}{A_{1}}\right) n \sigma c_{n}+\left(\frac{A_{b_{2}}}{A_{1}}\right) n \sigma p_{n}\right]
\end{aligned}
$$

where 
$\lambda_{3}=\frac{1}{\sqrt{2}}\left[\sum_{\ell=0}^{\infty}\left\{\left(\frac{A_{b_{1}}}{A_{1}}\right) n \sigma d_{n}+\left(\frac{A_{b_{1}}}{A_{1}}\right) n \sigma q_{n}\right\}^{2}+\left\{\left(\frac{A_{b_{1}}}{A_{1}}\right) n \sigma c_{n}+\left(\frac{A_{b}}{A_{1}}\right) n \sigma p_{n}\right\}^{2}\right]^{\frac{1}{2}}$

The procedure for solution again depends on the avallable information The friction factor can be determined when the motion in the ocean and both bays are known The elevations in the first bay and ocean can be obtained deterministicaliy for any given $r$ when the motion in the second bay is known The motion in the bays can be determined by an interative scheme for any given $r$ when the motion in the ocean is known

\section{APPLICATION - BOCA RATON INLET}

The theoretical method outlined above was applied to Boca Raton Inlet, Florida The inlet connects the Atiantic Ocean to Boca Raton Lake which is also connected to Lake Wyman A plan view of these lakes is shown in Fiqure 3 The intercoastal waterway which connects Lake Wyman and Boca Raton Lake extends to South Lake Worth Inlet north and Hillsboro Inlet south Comparisons of tide records obtained at Boca Raton Lake and at a station on the Intercoastal Waterway south of Boca Raton indicated no possible flow to or from the Intercoastal Waterway south of Boca Raton Lake Boca Raton Inlet primarily influences Boca Raton Lake, Lake Wyman, and areas occupied by boating marinas north of Lake Wyman

Boca Raton Inlet was chosen for this study because tide records were avarlable at the Inlet North Jetty (denoted by Station 1 in Frqure 3), at Boca Raton Lake (denoted by Station 2), and at Lake Wyman (denoted by Station 3) Actually tide records at three stations around Boca Raton Lake were also avallable These records indicated no special variation of tide elevation in Boca Raton Lake and verifies the representation of conservation of mass given by Equation (8) The tide elevations recorded at Stations 1,2 and 3 are shown in Figure 4 They indicate the magnitude and phase shift of tidal motion in the two bays relative to the ocean other avarlable data included velocity measurements over a tidal cycle at Station 1 to be shown Tater in comparison with computed velocities

Since tidal records were available in both bays and at the inlet, the multiple bay analysis was used to determine the friction factor $r$ for the system The tidal record in Lake Wyman was used to predict the tidal elevation in Boca Raton Lake using Equation (30) The computed elevation was then compared with the measured one With the tidai elevation in Boca Raton Lake known, the ocean elevation in the ocean (Station 1) was computed using Equations (34) and the resuits compared with the measured values The variances between the computed and measured elevations were computed at Stations 1 and 2 for different friction coefficients The quantities which describe the bay system (see Figure 2) $A_{1}, L_{1}, A_{b_{1}}, h_{1}, A_{2}, L_{2}, h_{2}$ were al1 known The area $A_{b_{2}}$ was not known The computed motion which gave the best $f_{1} t$ to measured tidal elevations at Stations 1 and 2 corresponded to $r=00039$ and $A_{b_{2}}=23 \times 10^{6}$ $\mathrm{ft}^{2}$ The latter area is much larger than Lake Wyman's area and suggests that the tidal motion extends to areas occupied by marinas north of Lake Wyman Tabie 2 summarizes the physical properties of the above twc-bay system 
TABLE 2 Properties of the Two-Bay System

Inlet area $\left(\mathrm{ft}^{-}\right)$

Bay Area $\left(\mathrm{ft}^{2}\right)$

Inlet depth ( $\mathrm{ft}$ )

Inlet length ( $\mathrm{ft}$ )

$M-2$ measured ampi itude $(\mathrm{ft})$

\section{Boca Raton Lake Lake llyman}

$\begin{array}{ll}140 \times 10^{3} & 168 \times 10^{3} \\ 374 \times 10^{6} & 2300 \times 10^{6} \\ 70 & 80 \\ 267 \times 10^{3} & 733 \times 10^{3} \\ 157 & 140\end{array}$

The variances between computed and measured tidal elevations at Boca Raton Lake (station 2) and at the 0cean (station 1) were $19 \times 10^{-2}$ and $20 \times 10^{-2}$, respectively for $r=00039$ The difference in computed and measured phase shifts of the $1 \mathrm{H}-2$ component were 2 degrees for Boca Raton Lake and 4 degrees for the ocean

\section{SIGNIFICANCE OF NON-SINUSOIDAL TIDAL COMPUTATION}

A comparison between the measured tide record at station 1 and the $M-2$ constituent is shown in Figure 5 The agreement is reasonable only over part of the tide cycle A comparison between the measured tide elevation at station 1 and the computed elevation using six harmonics 15 shown in Figure 6 Better agreement is recognized over most of the tidal cycle The importance of considering the different harmonics in tidal computations appears more dramatically in velocity computations, however In Figure 7 , the measured velocity at station 1 is compared to the computed velocity using the M-2 constituent only Near the maximum three velocity peaks appear in the measured record but are absent in the sinusoldal computation These peaks appear in computations which include different harmonics as shown in Figure 8 since the contribution of each harmonic to velocity depends on frequency and amplitude of that harmonic the higher harmonic contributes significantly to velocity even when the amplitude 15 small

The velocity cycle has an important influence on sediment transport in the inlet The sand trapping capability of an inlet may play an important role in shoreline stability in the vicinity of the inlet The trapping capability of Boca Raton Inlet is investigated in what follows

The theoretical basis for describing movable beds is empirical lany relationships exist that relate volume of sediment transport to bottom shear stress While there 15 no one relationship which is far superior to others, the empirical result arrived at by Einstein (1942) is used

$$
q_{s}^{\prime}=40 \rho F \frac{U_{s}^{G}}{\left[g\left(s_{s}-1\right)\right]^{\% / 2}}
$$

where $q_{s}^{\prime}$ = the weight rate ( $1 n$ water) of sediment transport per unit width, $\rho=$ watér density, $U_{*}=$ shear velocity $\left(=t_{t o} / \rho\right), g$ gravitational acceleration, 
$s_{S}=$ specific gravity of sand, and $F$ is given by

$$
F=\left[\frac{2}{3}+\frac{36 v^{2}}{g d^{3}\left(s_{s}-1\right)^{1 / 2}}-\left[\frac{36 v^{2}}{g d^{3}\left(s_{s}-1\right)^{1 / 2}}\right]^{\frac{1}{2}},\right.
$$

Where $v=k i n e m a t i c$ viscosity of water Equation (35) was used successfully by Shemdin (1970) in modeling of sediment flow in the coastal zone Equation (5) relates the average velocity to the shear velocity

$$
U_{\star}=r^{\frac{1}{2}} u
$$

For a wide channel with width $B$ the weight rate of sediment transport, $q_{s}$, becomes

$$
q_{s}=q_{s}^{\prime} B=40 \rho F B \frac{r^{3} u^{6}}{\left[g\left(s_{s}-1\right) d\right]^{3 / 2}}
$$

The net sediment transport into Boca Raton Inlet was computed from results similar to those shown in Figure 8 Tide records were found to fluctuate in amplitude and typical records were used for two different days The net transport into the inlet for the two days was calculated to be 15 and 47 $\left(\mathrm{yd}^{3} / \mathrm{day}\right)$ which correspond to 5,400 and $17,000 \mathrm{yd}^{3} / \mathrm{yr}$, respectively $\mathrm{A}$ recent dredging operation in Boca Raton inlet have been removing 30,000 40,000 cubrc yards of sand per year from the inlet The computed transport in the inlet 15 of the same order of magnitude Fugure research on inlets may fruitfully include field measurements of sand transport

\section{CONCLUSIONS}

A non-linear coupled procedure is proposed to analyze tidal motion in $1 n$ lets and bays The importance of the different tidal constituents is shown to be more important in velocity computation compared to surface elevation The exchange of sediment between bays and the ocean 1 s dependent on the velocity variation over the tidal cycle and can only be computed accurately by considering different harmonic constituents in a tidal record The procedure 15 applied to Boca Raton Inlet and the result indicate that more sand transport occurs during the flood period compared to the ebb period The inlet consequently behaves like a sand trap

\section{REFERENCES}

1 Brown, E I (1928), Inlets on Sandy Coasts, Proc ASCE, Vol IIV

2 Dronkers, J J (1964), Tidal Computations in Rivers and Coastal Waters, North - Holland Publishing Col, Amsterdam

3 Einstern, H A (1942), Formulas for the Transportation of Bed Load, Trans ASCE, Vol 107, pp 561-577

4 Keulegan, GH (1951), Water-level Fluctuations of Basins in Communication with Seas, Report No 1146, National Bureau of Standards, Washington, D C 
5 Lee, $Y W$ (1967), Statistical Theory of Communication, John Wiley \& Sons, Inc, New York

6 O'Brien, M P (1969), Equilibrium Flow Areas of Inlets on Sandy Coasts, $\mathrm{J}$ Waterways and Harbors Div, Vol 95, No WWI, pp 43-52

7 Shemdin, O H (1970), River-Coast Interaction Laboratory Simulation, $\mathrm{J}$ Waterways and Harbors Div, Vol 96, WWl

8 Sidjabat, M M (1970), The Numerical Modeling of Tides in a Shallow Semi-enclosed Basin by a Modified Elliptic Method, Ph D Dissertation Submitted to the University of Miami, Coral Gables, Florida

9 Van de Kreeke, J (1968), Water Level Fluctuations and Flow in Tidal Inlets, J Waterways and Harbors Div, Vol 93, No WW4, pp $97-106$ 


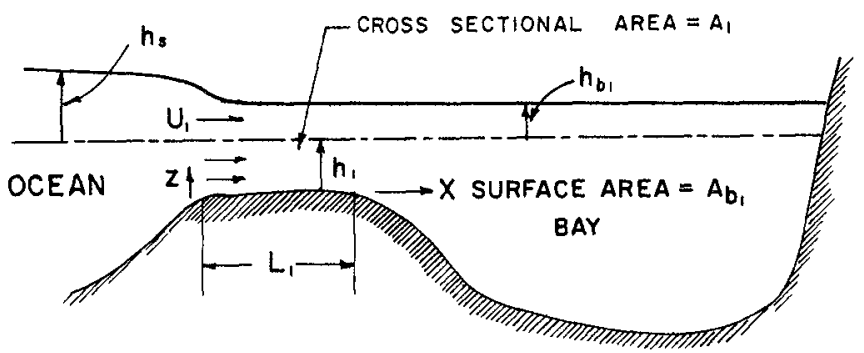

Figure 1 Definition sketch for a single bay coupled to ocean

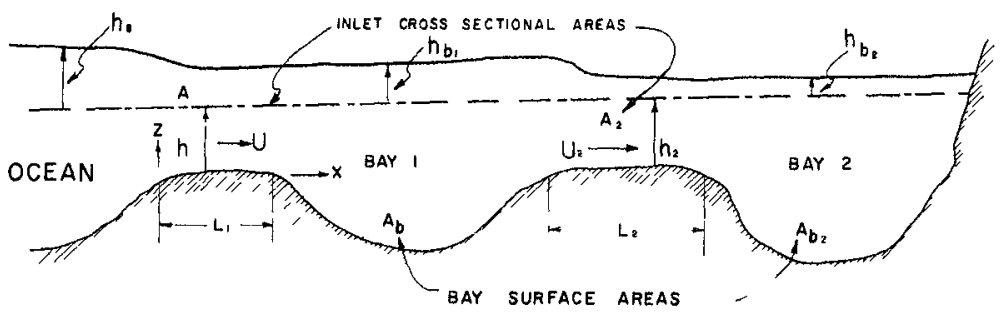

Figure 2 Definition sketch for two bays coupled to ocean 


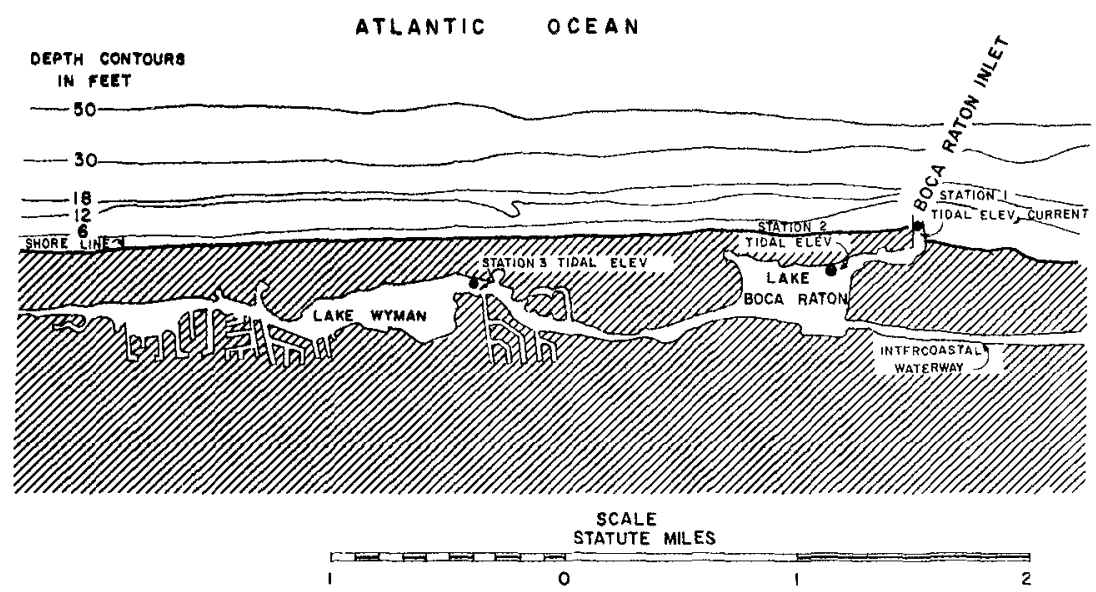

Figure 3 Plan view of Boca Raton Inlet and lake, and Lake Wyman

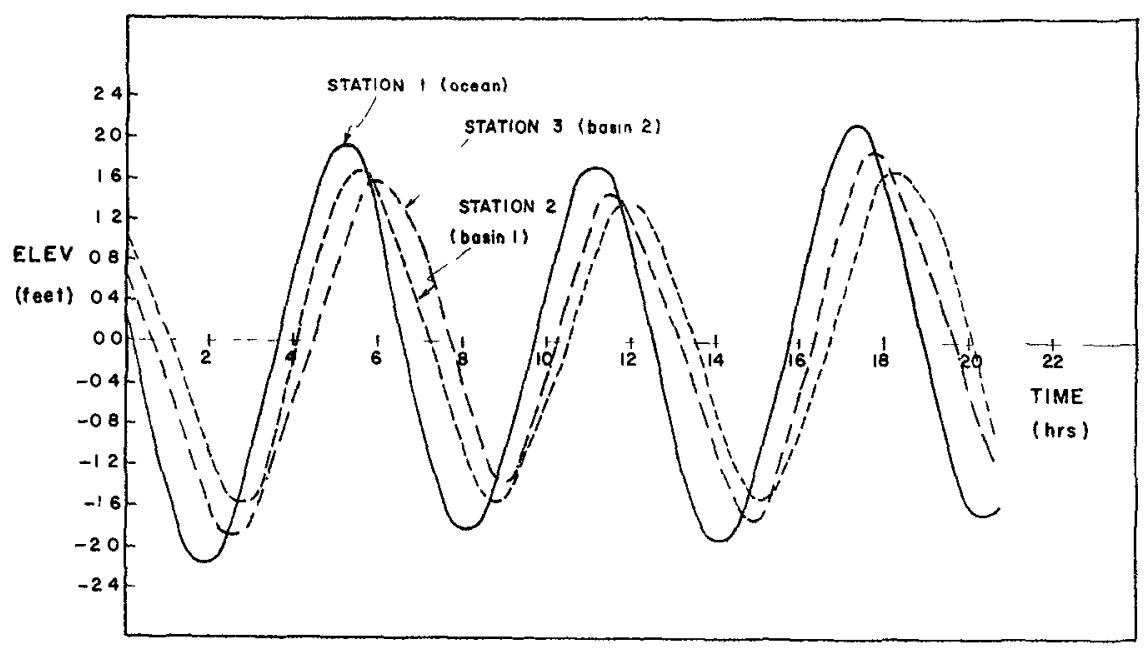

Figure 4 Tide elevations at Boca Raton Inlet, Boca Raton Lake, and Lake Wyman 


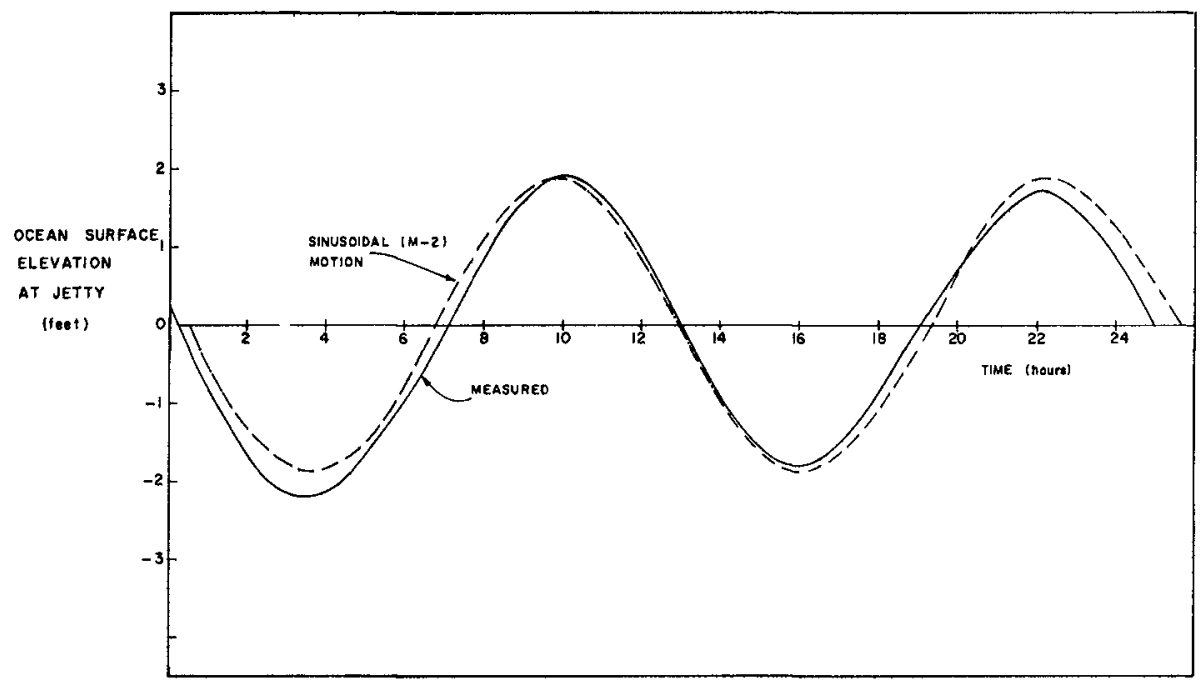

Figure 5 Comparison between measured and computed (M-2) component of tidal motion at Boca Raton Inlet

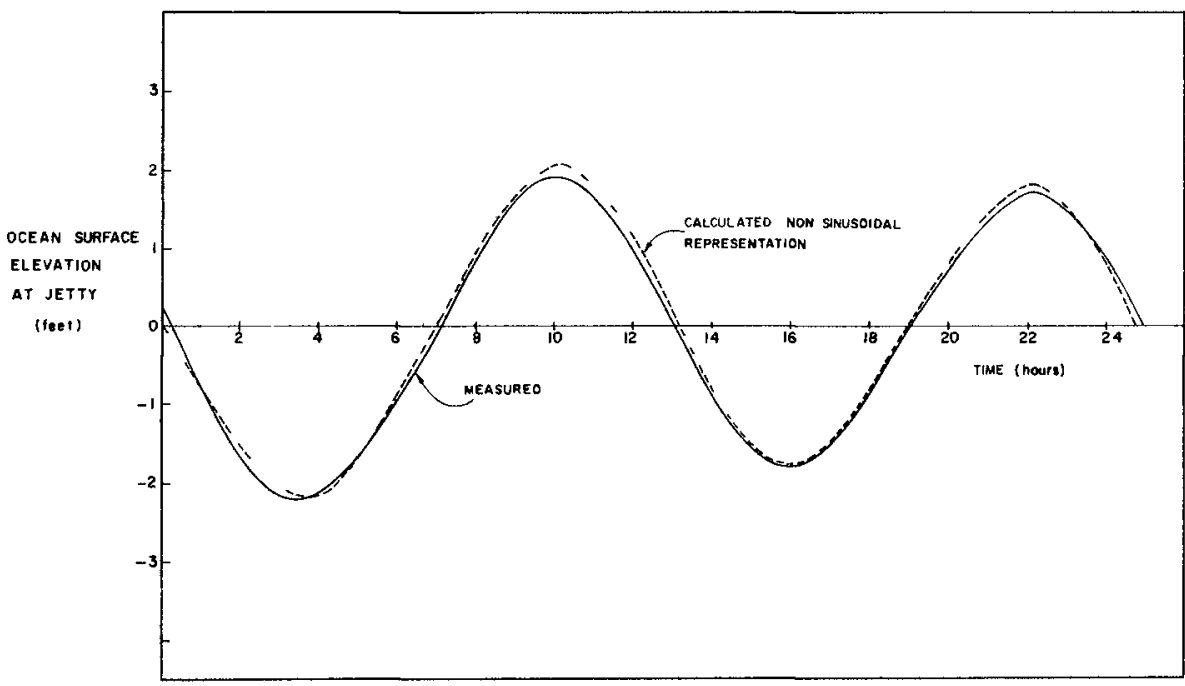

Figure 6 Comparison between measured and computed tidal motion using six harmonics at Boca Raton Inlet 


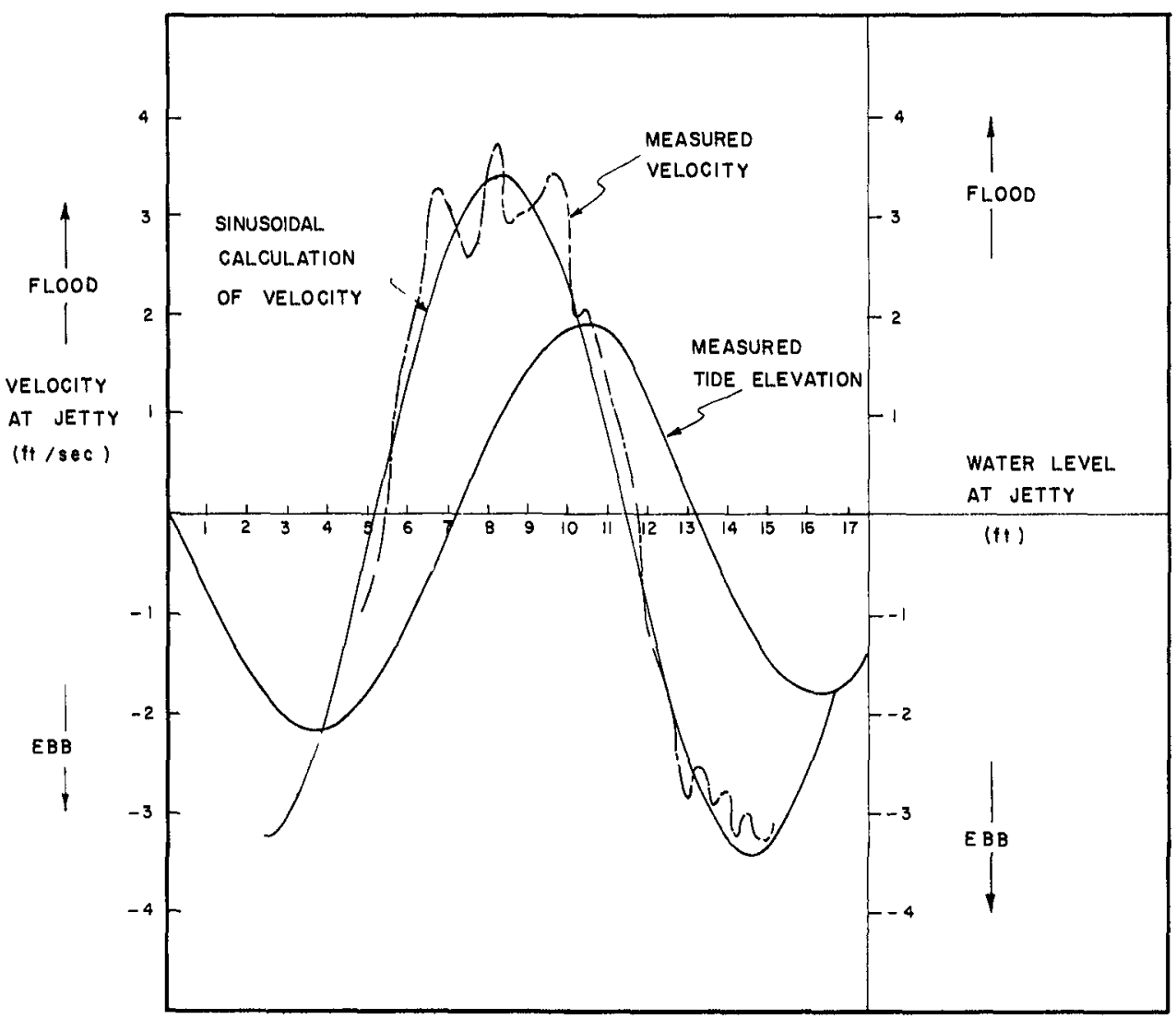

Figure 7 Comparison between measured and computed velocity using (M-2) component only at Boca Raton Inlet 


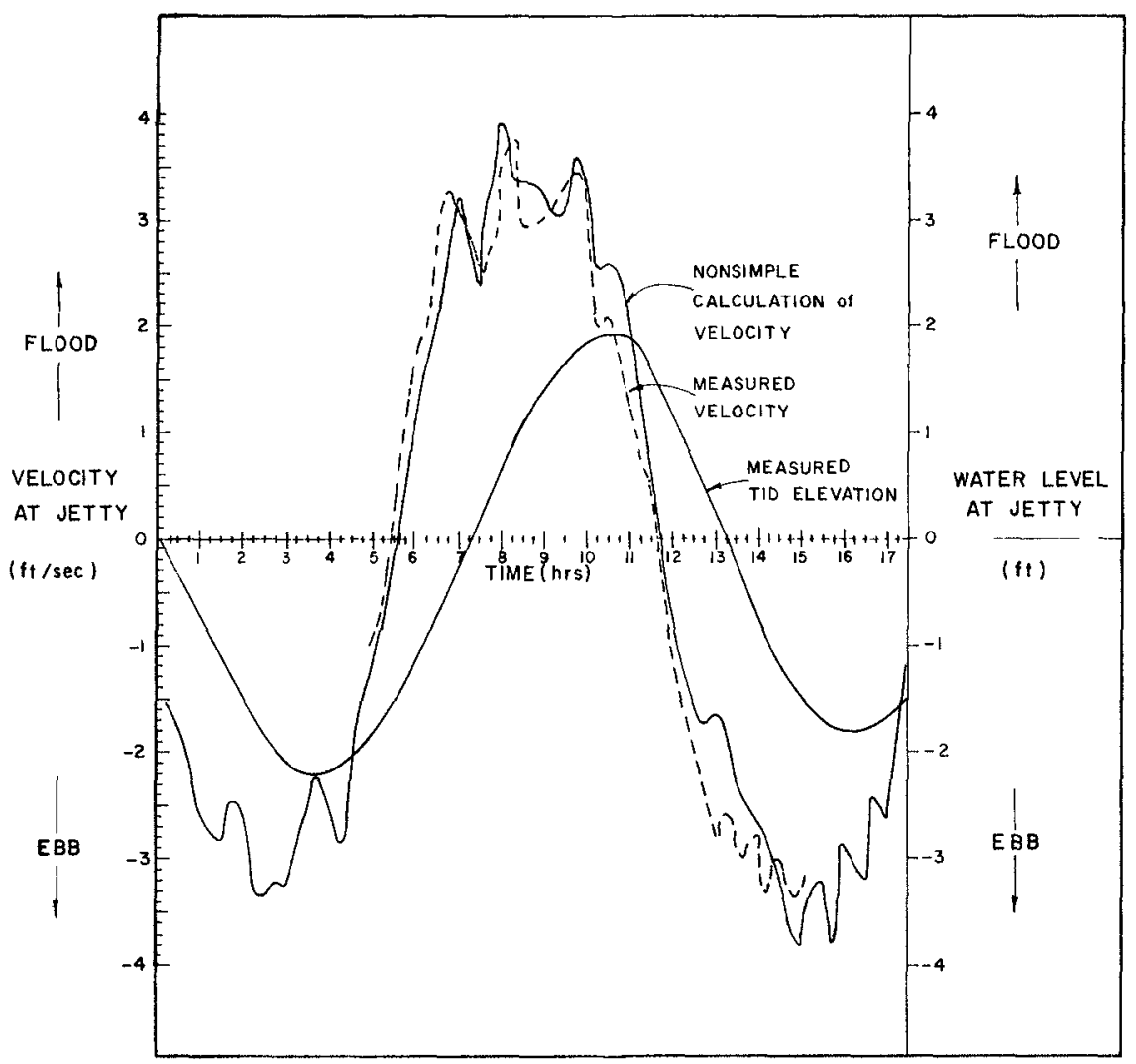

Figure 8 Comparison between measured and computed velocity using six harmonics at Boca Raton Inlet 


\title{
CHAPTER 135
}

SUNFACE USCILLATLONS IV A WATEK LANh CAUSED BY A OL BNE-GED JET

\author{
By \\ Juan B Font 1 \\ Orar Azpurua 2 \\ Flora Aranguren 3
}

ABS LKACT

In this paper are studied the surface oscillations that form in a water tank when a jet energes from the botton

The experimental syster consisted of a circular water tank with the water intake at the bottom close to its c.rcunference Surtace osc1 llat _ons were secorded for different situations

The experirental results are presented in both dirensional and dirensionless plottings where the oscillations anplitud is related with water depth and jet diameter and velocity

The dimensionless graphs show that the oscillation amplification is, with $\perp \mathrm{n}$ the variables ranges studied, rostly independent of tank diameter

1 VTL UDUCTIOA

When water flows into a shallow tank, in the form of a jet energing from the bottor, snall surface oscillations appear as a result of jet diffusion and supply pipe turbulence These oscillations are reflec ted in the tank walls increasing the water surface rovement which interferes with the jet flow, starting a horizontal oscillation of the latter A complex surface oscillation results, which under determined conditions is continuously anplified unt 1 wave breaking puts a limit on wave he,ght

The oscillition pattern, no nather the degree of amplification,

1 Asst Prof College of Engrg, Universidad Central - Venezuela

2 Civil Engineer - CONP OSA - Caracas - Venezuela

3

Civil Lngineer - inOS - Caracas - Venezuela 
is a conplex and unsteady one, with standing wave type tovenert being donindnt, with nodes and antinodes continuously changing their location

\author{
VAr LABLLD II VOJ VED ATUN IHEL LELATIVE IMPGRTAI UE
}

According to the ohysscal description of the phenonenon, the amplification is a function of the nagnitudes that describe the boundary georetry and surface novement, $x$ e tank and supply pipe georetry and froude vir ber in the amplification processes inertia and gravity forces are obviously ruch more important than viscous folces the wave breaking is dcterined by oscillation characteristics Thus the amplification and its upper limit will not be a function of Reynolds Nurber

The arplification nus result fron a resonant combination of jet movement, surface movenent and tank georetry However, and due to the surface oscillation variability, the tank geometry plays a secondary role as shown in Fig 1

To back tnis experimental conclusion we may consider several rea sons tirst, the boundary conditions imposed by the circular vertical walls of the tank, determinant of tne oscillation pattern in the steady case, are now almost irrelevant since a great number of intantaneous patterns $\left.w_{+} 1\right]$ exist, some of then being close to the maxit uin amplitic a tion situation for the existing set of depth and jet var $\llcorner$ ables This is specially true when tank diameter is several times the nean wave length As a natter of fact, $I L$ was observed in the experiments that the jet always followed a random secuence of quiet and oscillation periods becond spewally at resonance situations, when preaking takes place, waves are non-linear and, therefore, continuously changing wave helghts will cause that a range of frecuencies be allowed with the c15 cular boundary for every oscillation iode As it can be seen in the sample record shown in Fig 5, energy is mainly related Lo a wave frecuency, but nevertheless, the oscillation is not ronochromatic

\title{
Dimensionless paraneters
}

The variables thus involued are wave height $H$, jet diameter $d$, water depth $h$, jet velocity $V$ and gravity force per unit rass $g$ With these five secondary magnitudes, the theorer shows that, since onlylength and time are involved, there will exist a function $\emptyset$ such that

$$
\emptyset\left(\pi_{1}, \pi_{2}, \pi_{3}\right)=0
$$

where $\Pi_{1}, \Pi_{2}$, and $\Pi_{3}$ are independent dimensionless combinations of the above listed variables For ghaphical representation a convenient set of expresions is

$$
\Pi_{1}=\frac{\mathrm{H}}{\mathrm{d}}, \quad \Pi_{2}=\frac{\mathrm{v}}{\sqrt{\mathrm{gd}}}, \quad \Pi_{3}=\frac{\mathrm{v}}{\sqrt{\mathrm{gh}}}
$$




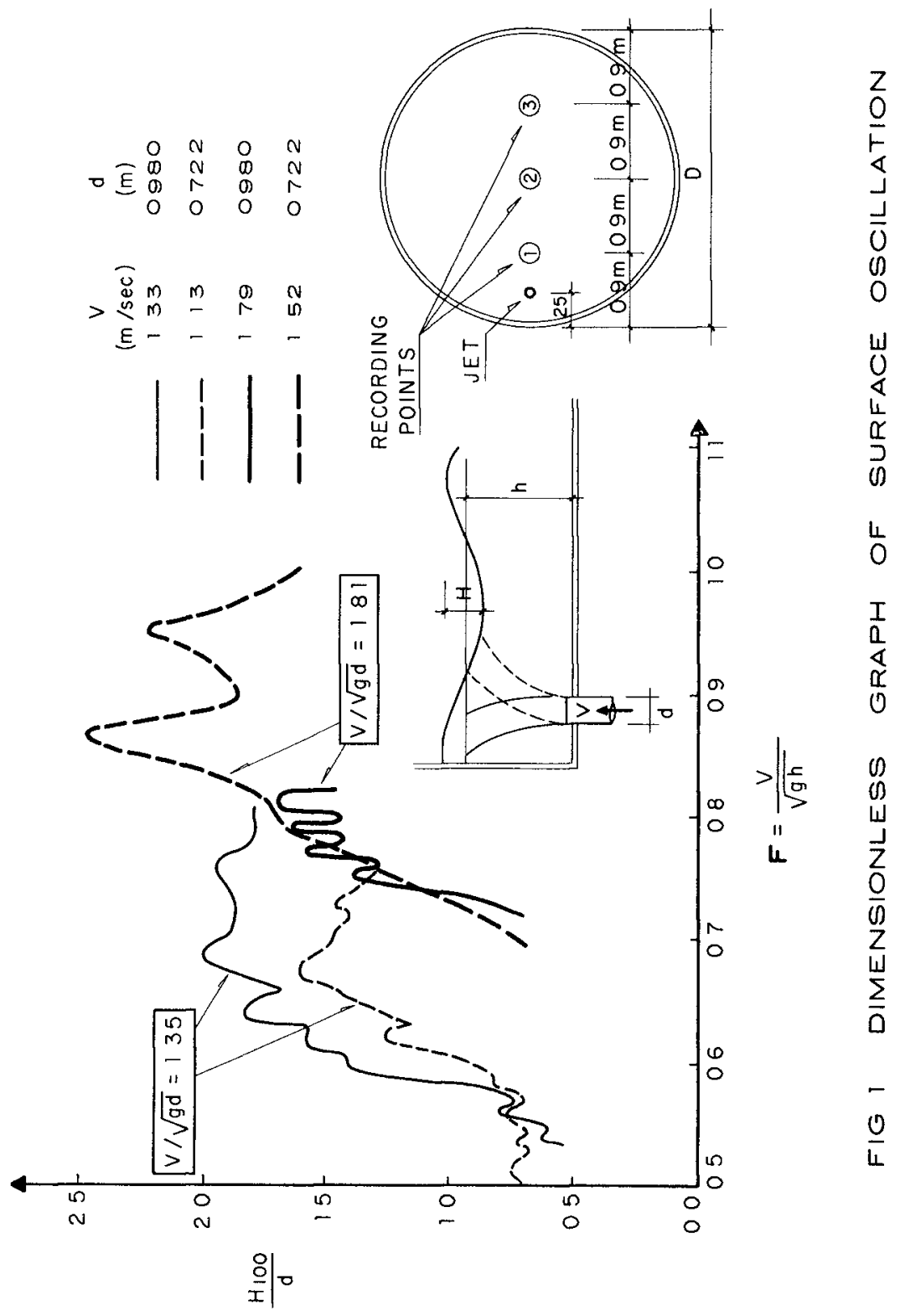




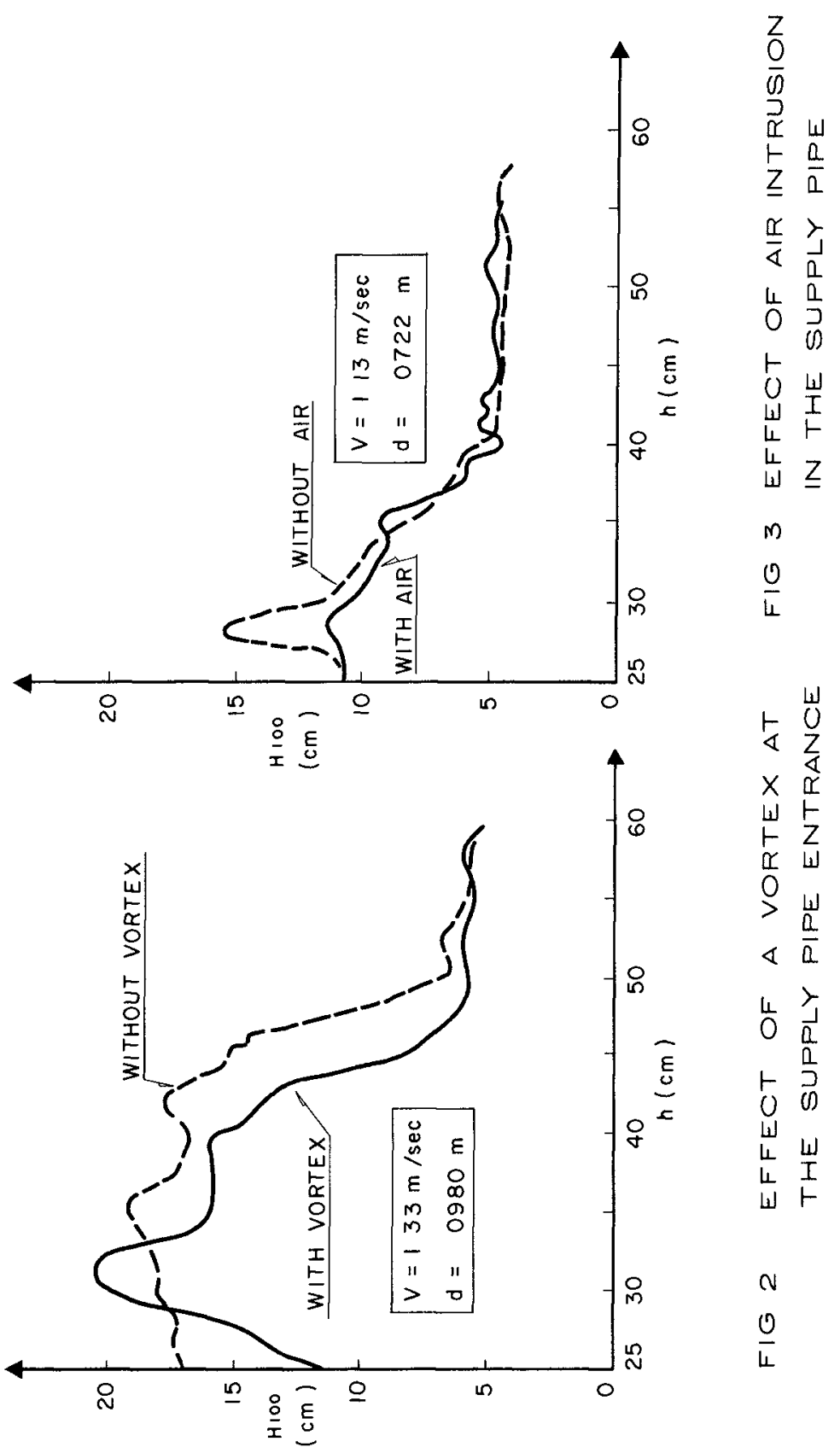




\section{EXPERIMENTAL CONDITIONS}

6

For a description of the experzmental set-up see figures 1, 5, and

Tests were run starting with the tank full of water, and allowing the outflow to be a little larger than the inflow such that water depth would slow and continuously decrease, until the possuble minimum was attained, the water discharge was held constant through the experiment by means of a weir controled elevated supply tank Two supply pipe dia neters were used with two different velocities por each one Velocity was measured indirectly in terms of discharge by weir and elbow neters, previously calibrated in the experimental system with $z$ weir neter

The water surface elevacion was recorded continuosly at the three points shown in Fig 1

As an indication of surface oscillation $\mathrm{H}_{100}$ has been defined as the average of the one percent largest wave of the two recordings taken at electrodes $N^{\circ} 2$ and $N^{3} 3$

Influence of supply pipe disturbances

To investigate the influence of water flow disturbances in the supply pipe, one test was run allowing the formation of a vortex in the supply tank In another test air was allowed to enter in the supply pipe Figures $A^{\circ} 2$ and 3 show the results with these conditions

Uscillation damping

In one of the tests, after maximum amplification was reached, wa ter outflow and inflow were stoped and the water surface was continuously recorded until oscillation nearly desapeared At the beggining, a rather fast damping took place as a result of wave breaking, thereaf ter having a very weak damping lalling $H_{100} t=U$ the one percent high est wave at an arbitrary tiue when wave breaking is over, the damping factor, in terms of the highest one perent height, has been plotted in fig 3 This graph shows that laminar type danping takes place As a matter of fact Reynolds numbers computed for both bothom and tank walls, in the manner shown in fig 3 , fall below the critical heynolds numbers given by several authors ( $L_{1}$, tef 2 , Jonsson, kef 1 ) for transition frorr laminar to turbulent regime on $a$ smooth boundary Comparison of the keynolds numbers for the bottom and the tank wall, and the respective areas of these boundaries, leads to the conclusion that wall fric tion is much nore important than botton friction in this specific case, and therefore that the tank diameter plays dn 1 mportant role in the free oscillation damping

\section{CONCLUSIONS}

1 The oscillation in the water surface that takes place in a water tank when a jet emerges from the bottom is a very complex one, with rrany frecuencias superimposed and nonlinearl- 

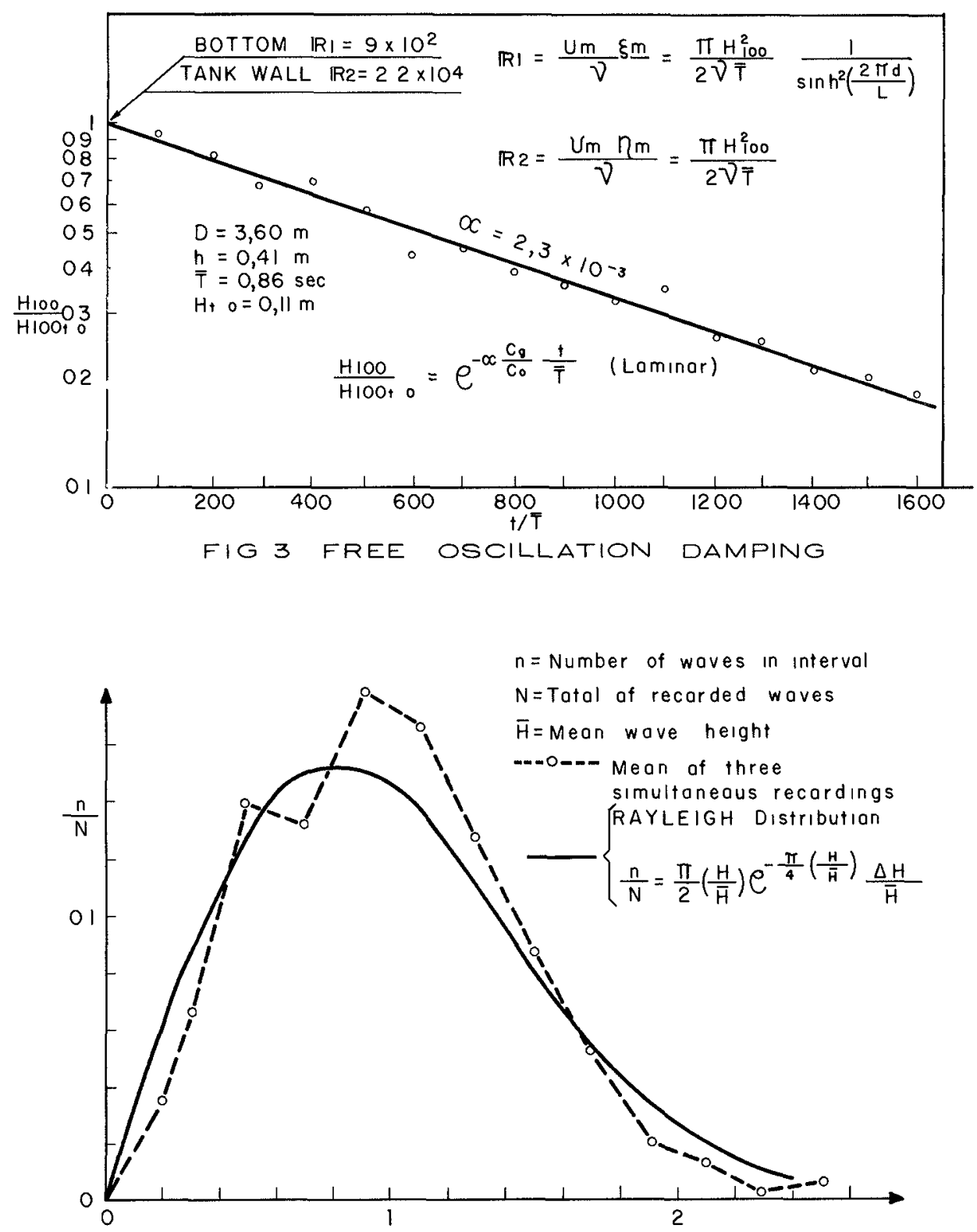

FIG 4 WAVE HEIGHTS HISTOGRAPH 


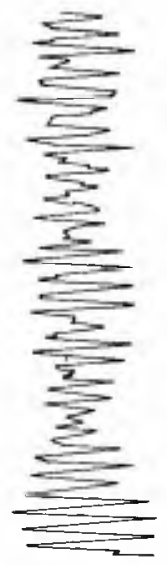

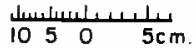
ELECTRODE ।
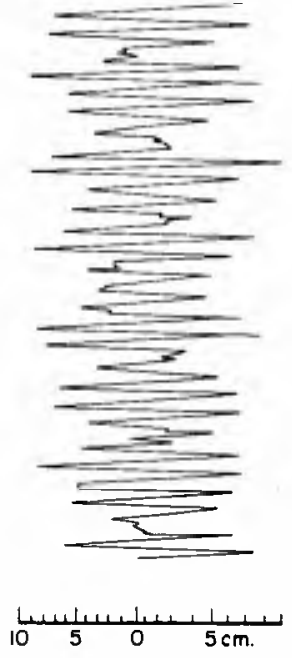

ELECTRODE 2
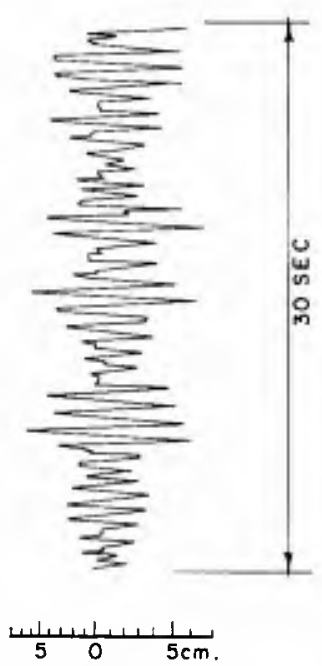

ELECTRODE 3

FIG.5 SAMPLE SURFACE RECORDS

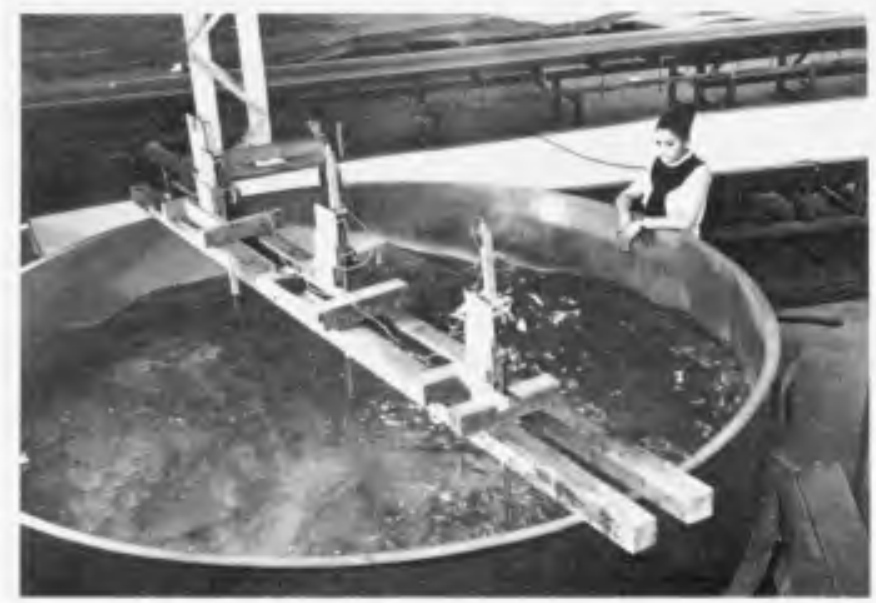

FIG. $G$ VIEW OF LABORATORY SET-UP 
ties characteristic of impulsive wave generation and breaking Wave breaking puts a limit to wave height in resonant situaLions

2 The maxinur amplification that reaches the oscillation is mainly due to a resonance anong the ererging jet and the free surface rovement

3 The experimental results surnarized in Fig 1 show that the resonance depends nostly on jet characteristics and water depth, rather than on tank diameter

4 Flow disturbances in the supply pipe, such as longitudinal vorticily and air intrusion, are of second order importance in the amplification phenomena

5 Once the jet flow has been stoped, the wave breaking disappear shortly and afler that, the oscillation damping is very slow

ACK OLEDGMENTS

It is appreciated the cooperation and assistance provided by the Laboratory "krnesto Leon D, LNOS" in the experiments reported in this paper

The authors are also grateful for the assistance given by the 11 I nistry of Public works, Ports and Airports Division, in the preparation of this report

\section{REFERENCES}

1 - Jonsson, lvar $G$ "Wave boundary layers and triction factors" Coasta1 Engineering 1966, Chapter 10, pp 127-48, Pub Arerican socjety of Civil Engineers, vew York, 1967

2 - L1, H "Stability of Oscillatory Laninar Flow Along a Wall" Beach Erosion Board Tech Meito $\mathrm{N}^{\circ} 47$, Washington, 1954 


\section{CHAPTER 136}

\section{EFFECT OF LONG PERIOD WAVES ON HYDROGRAPHIC SURVEYS}

by

Orville T Magoon

Coastal Engineering Branch, $U S$ Army Engineer Division, South Pacıfic, 630 Sansome Street, San Francısco, Calıfornı 94111

and

Willıam 0 Sarlin

Chief, Technical Service Branch, U S Army Engineer District, San Francisco, 100 McAllister Street, San Francisco, Calıfornıa 94102

\section{ABSTRACT}

In conjunction with routine hydrographic surveys at Santa Cruz Harbor, California, bottom elevation discrepancies were observed which were not associated with positional errors it was suspected that these errors were associated with long period wave activity, common at this particular location on the Pacific coast

The existing practıce for obtaining hydrographic soundings is by use of floating craft using either echo sounding techniques or a "lead line "Both of the above technıques utılıze the instantaneous water surface at the survey boat as a datum reference Normally the water surface elevation is determined by use of a water level recorder

Based on the analysis of 50 repetitions of a well monumented cross section in Santa Cruz Harbor, it was concluded that long period waves affect the results of hydrographic surveys by slowly varying the datum plane In the case of Santa Cruz Harbor, the maximum error due to this effect would be about \pm 15 feet

\section{INTRODUCT ION}

Santa Cruz Harbor is located at the northerly end of Monterey Bay, Calıfornia, about 65 nautical miles south of the entrance to San Francisco Bay, as shown in Figure 1 and Photo 1 The hydrography of the general coastal area and of Monterey Bay are shown on charts published by the United States Coast and Geodetic Survey, Nos 5402 and 5403 respectively In connection with studies of Monterey Harbor, located at the southerly end of Monterey Bay, and at Santa Cruz Harbor 
It has been shown that Monterey Bay and Santa Cruz Harbor are subject to pronounced selching Individual maximum trough to crest heights of over three feet have been observed Nominal periods associated with these seiches vary from 80 seconds to over 10 minutes with oscillations of about 3 minutes in period being most evident

During the analyses of surveys made in the vicinity of Santa Cruz Harbor, an unexplaıned ambıguıty in the sounding depths became apparent Although the cause of these ambiguities was unknown, it was hypothesized that they were caused by shifts in the sounding datum by long period waves Water level changes caused by these long period waves had previously been observed at Santa Cruz Harbor on numerous occasions by the authors These long wave oscillations of Monterey Bay and Santa Cruz Harbor are discussed by Wilson (1), Grauzinis(2), and Lynch(3) The existing practice for obtainıng hydrographic survey information is by use of floatıng craft and eıther echo soundıng techniques or by lead line The above techniques both utilıze the instantaneous water surface at the survey boat as a datum reference. Generally a recording tide gage mounted on a $4^{\prime \prime}$ pipe well with a suitable orifice, a float, and recorder, are used in determınıng the instantaneous water surface At tenuated long period fluctuations in the water surface may be observed in the resulting marigram trace Discussion of tide gage attenuation is discussed by Cross $(4)$ These long period undulations vary spacially both in period and height, however, it appears that their presence, but not their relatıve magnitude, may be determıned by conventıonal portable tide gages $0 n l y$ the relatively flat bottom areas of the channel were used for purposes of this study and, therefore, the side slope areas are not involved

\section{SURVEY TECHNIQUE}

In order to measure the effects of long period waves on hydrographic surveys, a test project was started at Santa Cruz Harbor by estab1ıshıng a "repetitive 1 Ine" and this line is indicated on Figure 2 Crosssection surveys were taken along this line using an aluminum hydrographic boat with a length of 14 feet, a beam of 62 inches, a draft of approximately 12 inches The boat was equipped with a conventional tagline reel with a cable marked at one foot intervals and a "Raytheon" fathometer, Model DE 1190 , operating at a frequency of 200 kılo Hertz Leadlıne soundıngs were then taken with an elght pound lead to verify fathometer soundings A typical method used for hydrographic surveys is shown in Figure 3

Fifty (50) measurements of the repetitive 1 Ines were taken on 21-22 March 1968 InItially, ten (10) cross-sections were taken of the repetitıve line on 20 March 1968, however, these lines were discarded from this

Numbers in parentheses indıcate listed references at end of text 
study because they were considered practice lines for the crew to become accustomed to the survey techniques The tide was recorded continuously during the survey period with an automatic tide recorder mounted on a 4inch pipe attached to a dock Tide corrections were applied to the fathometer soundings in order to correct the water depths The boat was controlled in the forward motion by a tag line calıbrated at one (1) foot intervals with the zero end attached to a baseline on shore The side motion of the boat was controlled by an instrumentman with a transit on shore, giving signals to the boat operator for keeping the boat precisely on line The marigrams shown on Figures 4 and 5 are taken from a previous project and are included for typical data Figure 4 shows a typical record with an open end well, Figure 5 shows a typical record with a damped well

Then the plotted lines of soundings were superimposed on a chart to compare the results A sampling of these lines is shown on Figures 6 , 7 and 8 These plottings indicate a data envelope of the echo sounding measurements of about one foot As the survey boat was traversing the reference line with closely controlled position, the sounding variations are then due to the datum changes as produced by the long period waves

These soundings are believed to be accurate since the location of the boat was controlled very carefully with an instrumentman on shore for the left and right directions or side motion of the boat The distances of the boat from the shore baselıne were read on the tag line which was calıbrated in feet Soundings were read to the closest tenth of a foot on the fathograms

\section{DISCUSSION}

The effect of long period waves on hydrographic surveys has been demonstrated at Santa Cruz Harbor, and the effect of these waves at other locations along the Northern Calıfornia Coast is believed to be of similar magnitude An effective monitor appears to be a recording tide gage located at an anti-nodal position in the survey area with a suitable orifice that would filter out short period waves A direct correction for the long wave effect could be obtained by use of a datum other than the water surface However, consideration of these techniques is beyond the scope of this study

In adjusting the soundings to account for the effect of tides, care must be taken to filter out the long wave excursıons on the applicable marıgrams In Santa Cruz Harbor, water elevations at locations away from the tide gage may be experiencing different water level elevations than at the tide gage In the extreme case, the water level at the tide gage could represent a maximum or peak on a wave and at the survey boat location in the harbor could represent a minimum or trough if the instantaneous water elevation is used at both the $t$ ide gage and at the survey boat, the sounding error will equal plus or minus the long period wave helght 
In the reduction of hydrographic sounding data, the water surface at the survey boat location is taken as a temporary datum This datum, is, of course, influenced by any water level fluctuations in the case presented in this paper, the actual fluctuation is considered to be due to long waves alone This is shown diagrammatically in Figure 9A, where the total long wave height is taken as " $H$ "' and it is further assumed that a recording gage and also the survey boat are located at anti-nodal positions in a standing wave situation Under these conditions, and with the recording tide gage operating correctly, a mean line may be drawn through the tide gage record (obviously the tide gage record may be damped mechanically or electrically) in this example, the maximum error due to the long wave effect will be equal to $+H / 2$ When the survey boat is at position $A$, the water level at the gage $T^{\prime} A^{\prime}$, but the average gage reading $w_{1} l l$ be $A^{\prime \prime}$ Simılarly, when the survey boat is at position $B$, the water level at the gage is $B^{\prime}$, and the average gage readıng will be $B^{\prime \prime}$ If the actual water surfaces at both the survey boat and the gage are used, then the situation shown in Figure 98 results In this case, when the survey boat is at position $C$, the water level and the tide gage datum are at position C' Similarly, when the survey boat is at position $D$, the water surface and tide gage datum are at position $D^{\prime}$ In this instance, the maximum error $w_{1} 11$ be $\pm H$ or twice the error as resulting In Case A, above

In a real harbor, the situation becomes much more complex due to the Inabılıty to describe the instantaneous water surface elevation at the gage and survey boat In instances where long waves are known to have occurred during surveys, it is suggested that a note be added to the survey information and resulting charts to indicate the situation

\section{CONCLUSIONS}

Based on the results of this study, it is concluded that long period waves affect the results of hydrographic surveys by slowly varying the datum plane in a manner that cannot be corrected by existing techniques Experience at Santa Cruz Harbor Indicates that long period waves reach heights slightly in excess of 3 feet and thus during times of occurrence of such waves, the maximum expected error in hydrographic surveys would be approximately equal to $1 / 2$ this value or about $\pm 1-1 / 2$ feet due to this effect If the tide gage record has been properly reduced Incorrect reduction of the tide gage record may result in the sounding error equal to plus or minus the long wave height, or in this example \pm 3 feet

\section{ACKNOWLEDGEMENT}

Acknowledgement is gratefully made to the Corps of Eng Ineers for access and permission to use this study material, avallable from the $U S$ Army Engıneer District, San Francisco, Calıfornia 


\section{REFERENCES}

1 Wilson, Basıl $W$, Hendrickson, James $A$, and Kilmer, Robert $E$, "Feasıbılity Study for a Surge Action Model of Monterey Harbor, Calıfornı," prepared for U S Army Engineer Waterways Experıment Station, Corps of Engineers, Vicksburg, MississıppI, under Contract No DA 22-079-CIVENG-65-10, 0ctober 1965 Science Engineering Associates, a Division of Kaman Aırcraft Corporation, San Marıno, Cal ifornia

2 Grauzınıs, V J, "An Analysıs of Seıche Condıtıons in Santa Cruz Harbor, Calıfornia, and Some Implications for the Proposed Harbor Extension," for $U$ S Army Engineer District, San Francisco, Corps of Englneers, Contract No DACW07-68-C-0034, March 1968

3 Lynch, Thomas John, "Long Wave Study of Monterey Bay," Thes Is at United States Naval Postgraduate School, September 1970

4 Cross, Ralph H, "Non-Linear Wave Effects on TIde Gages," Hydraulıc Engıneerıng Laboratory, College of Engıneerıng, Unıversıty of Calıfornıa, Berkeley, May 1967 


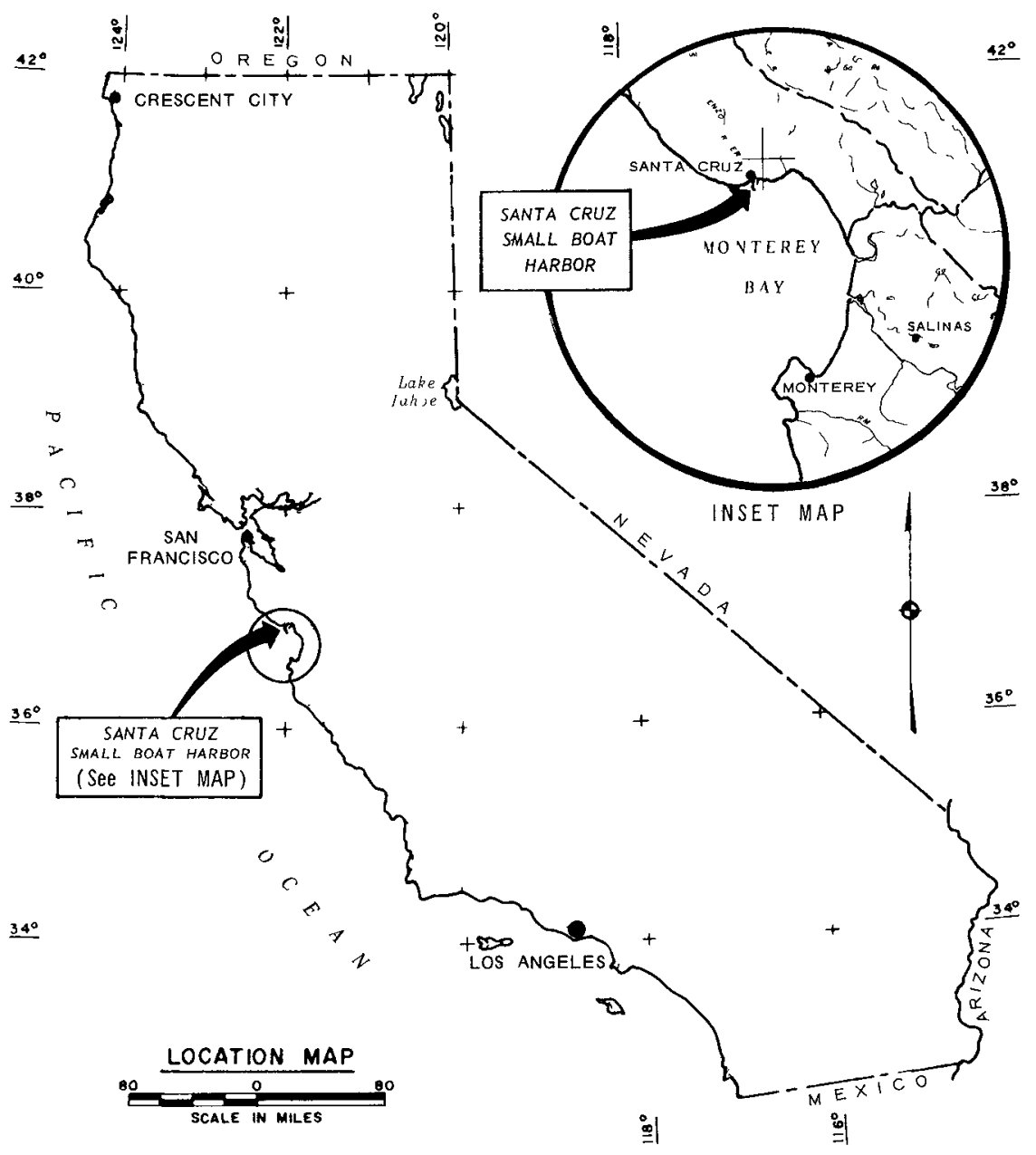

SANTA CRUZ HARBOR

FIGURE 1 


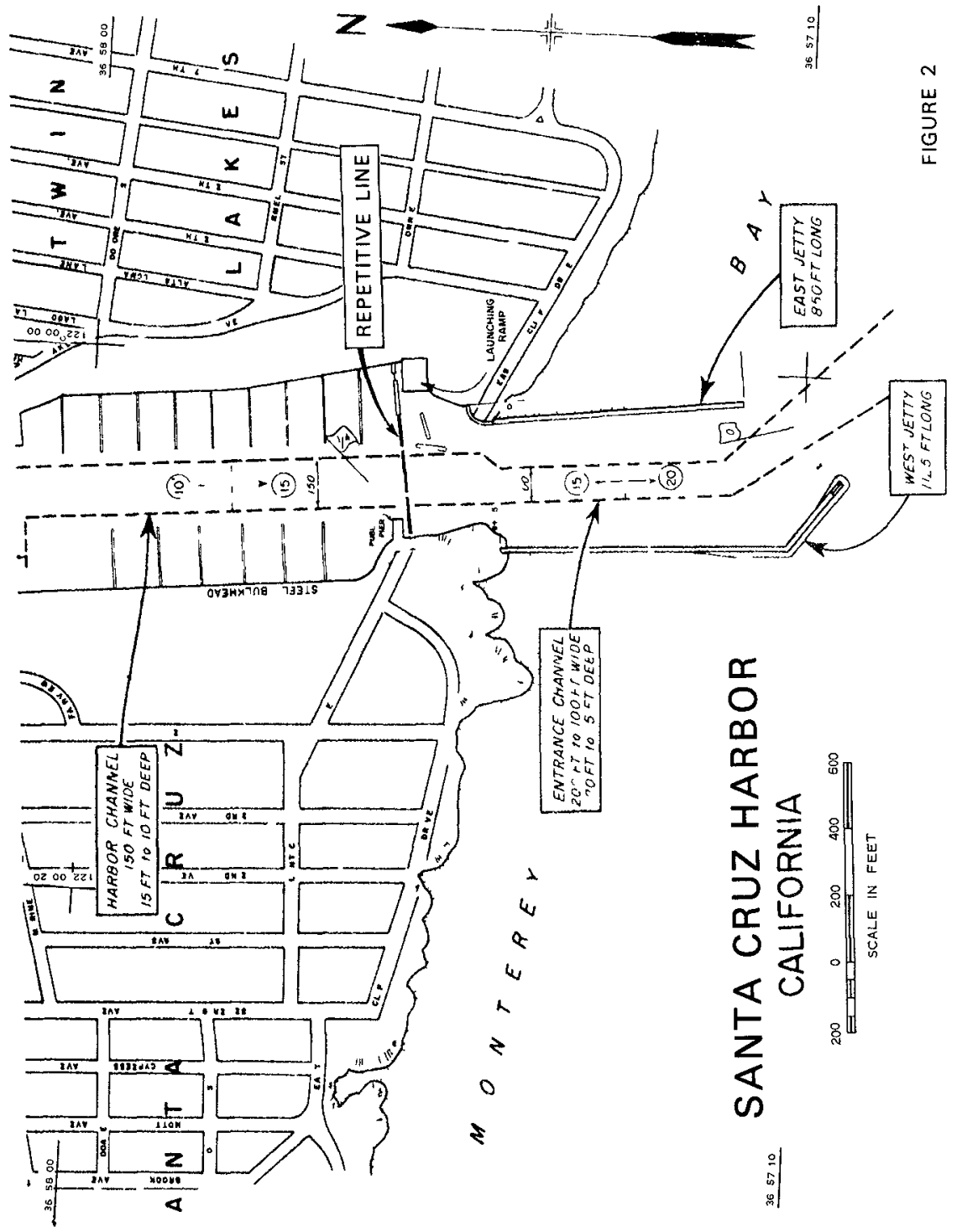




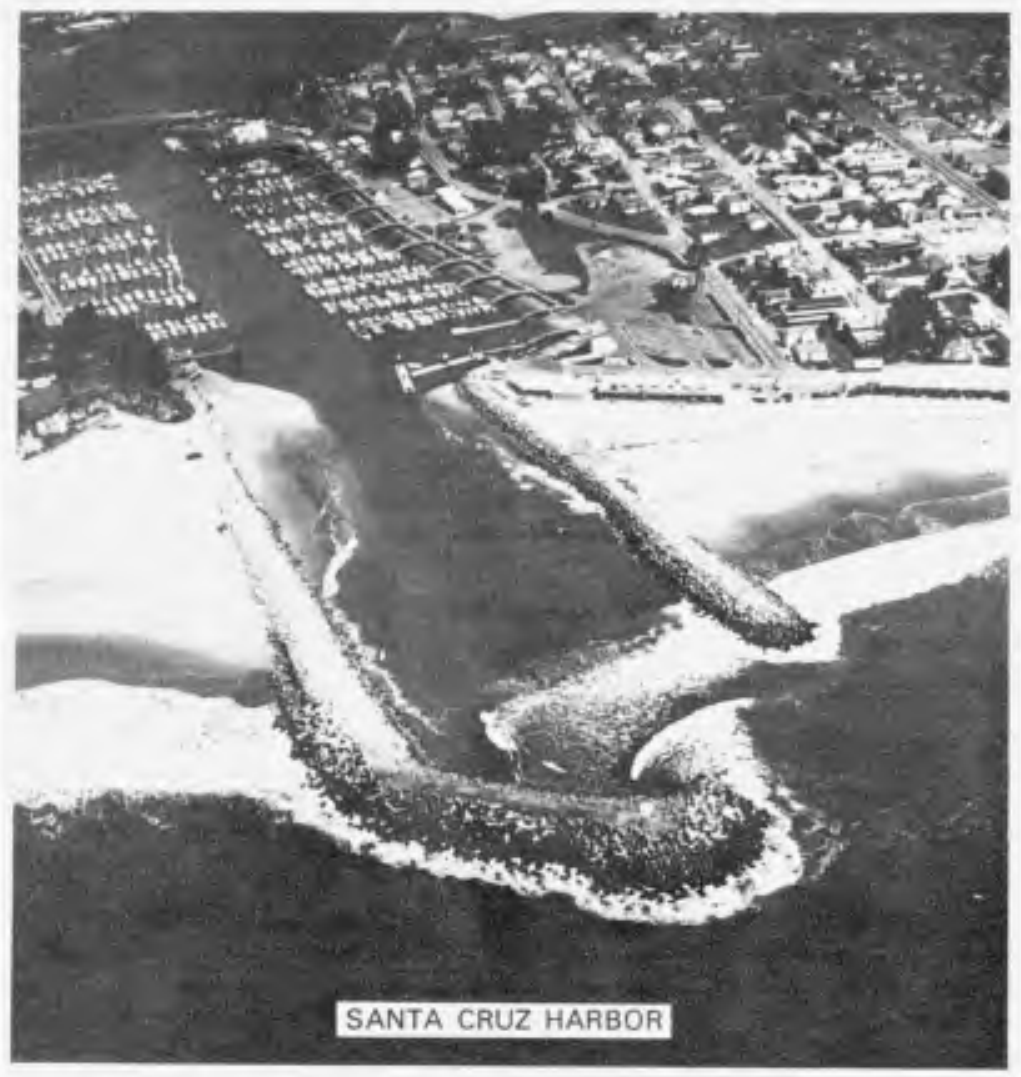

PHOTO । 


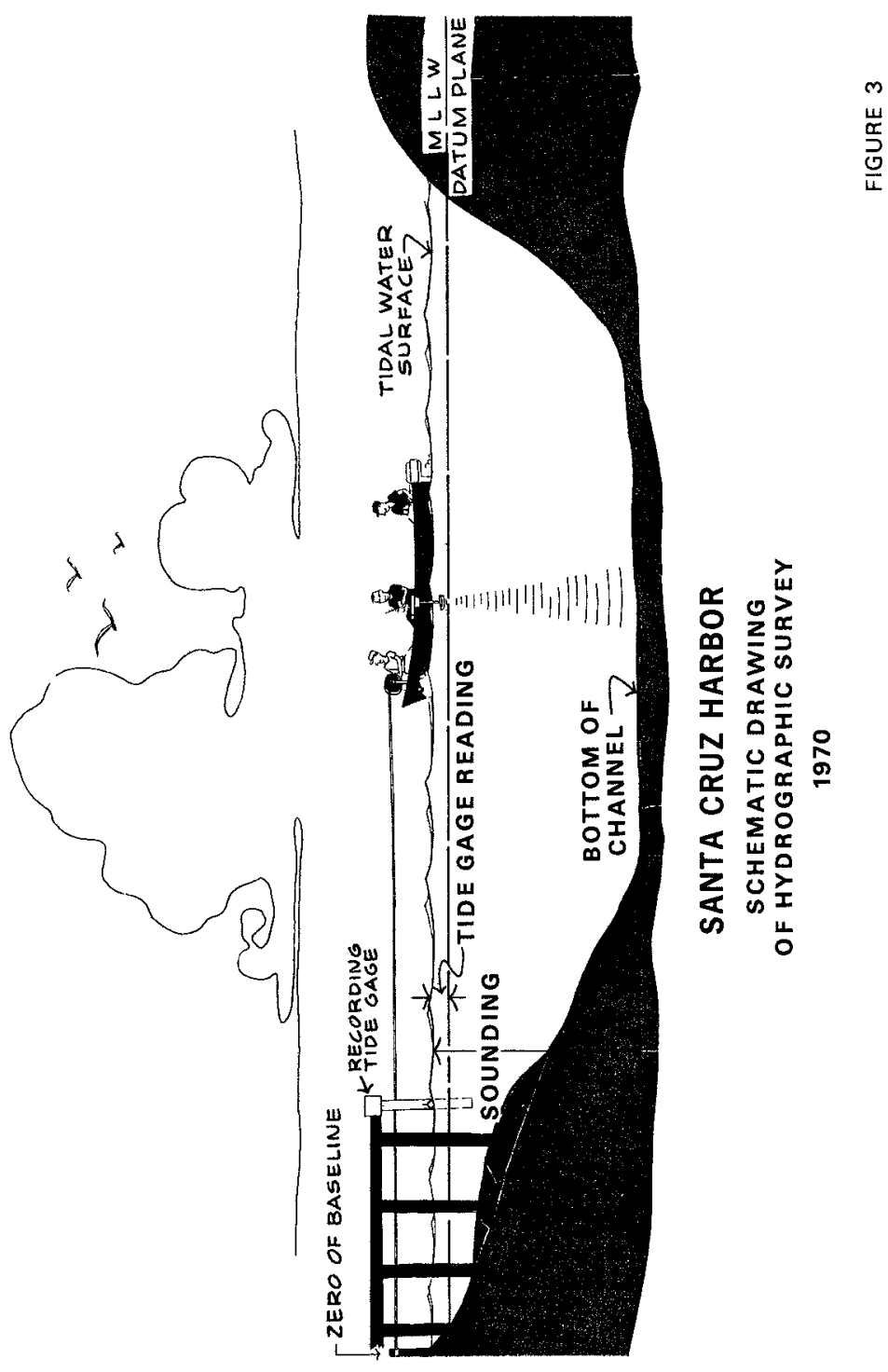


L $3 \exists \exists \quad N I$ JOII

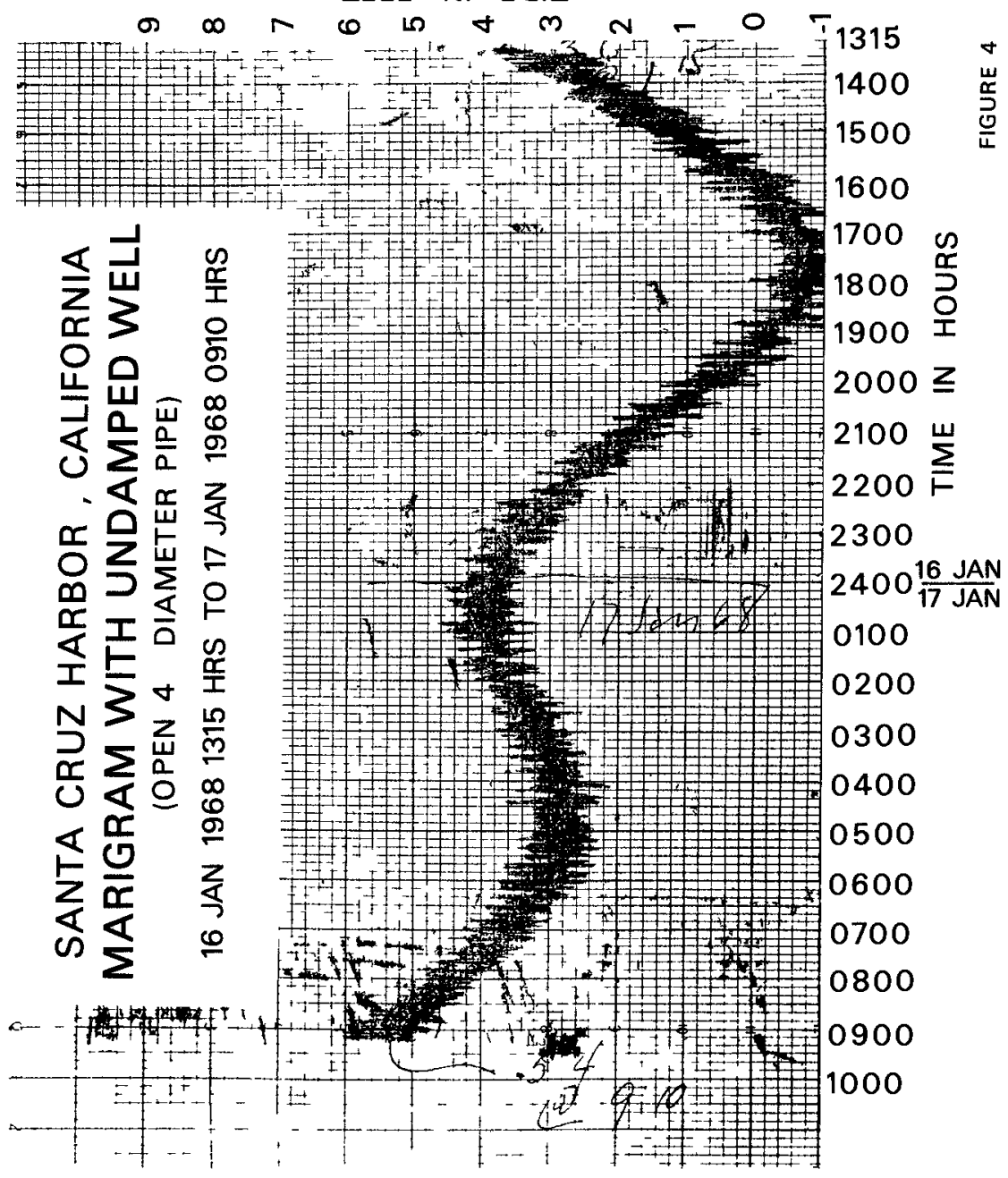


L $3 \exists$ J NI $\exists$ OII

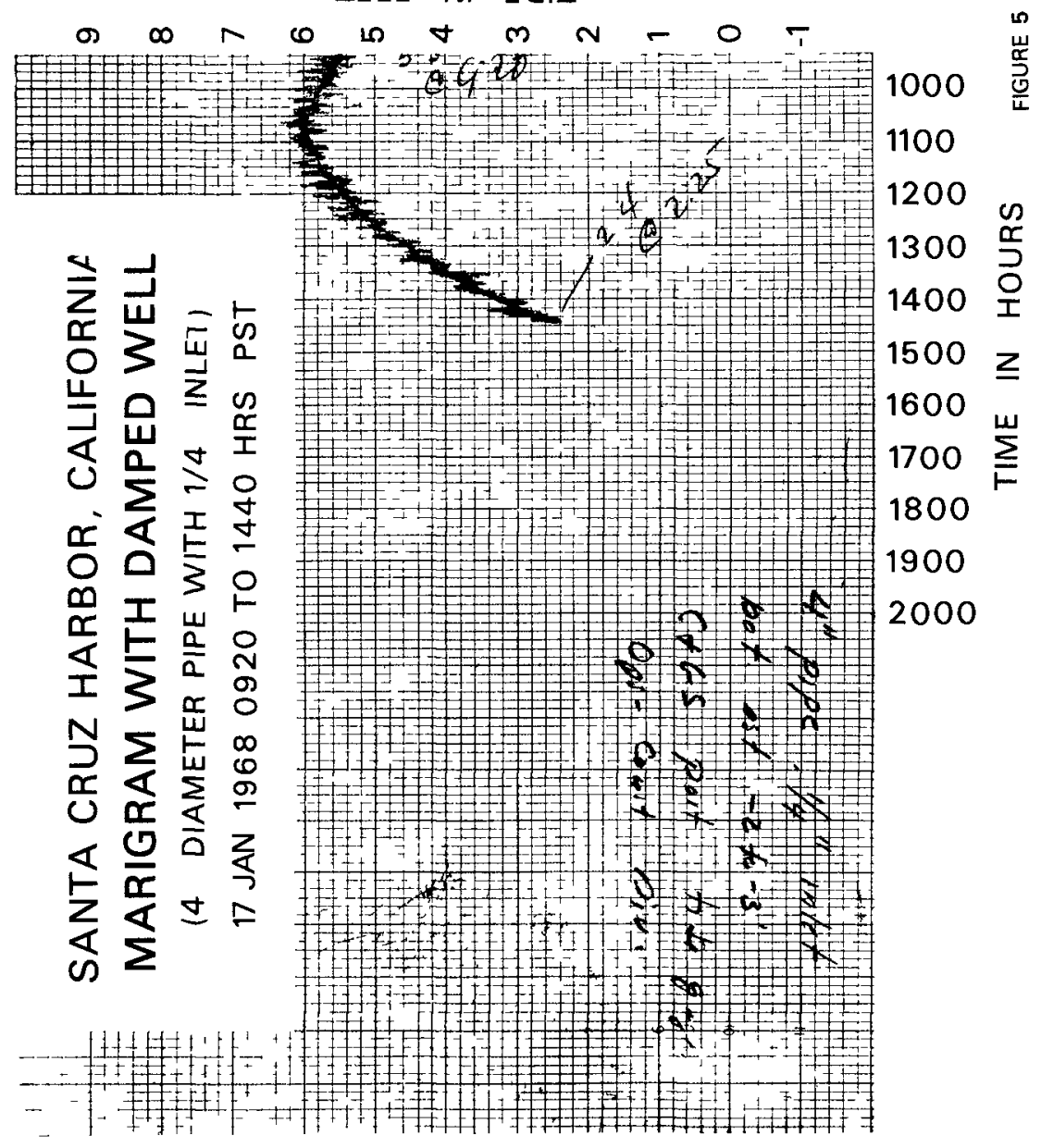




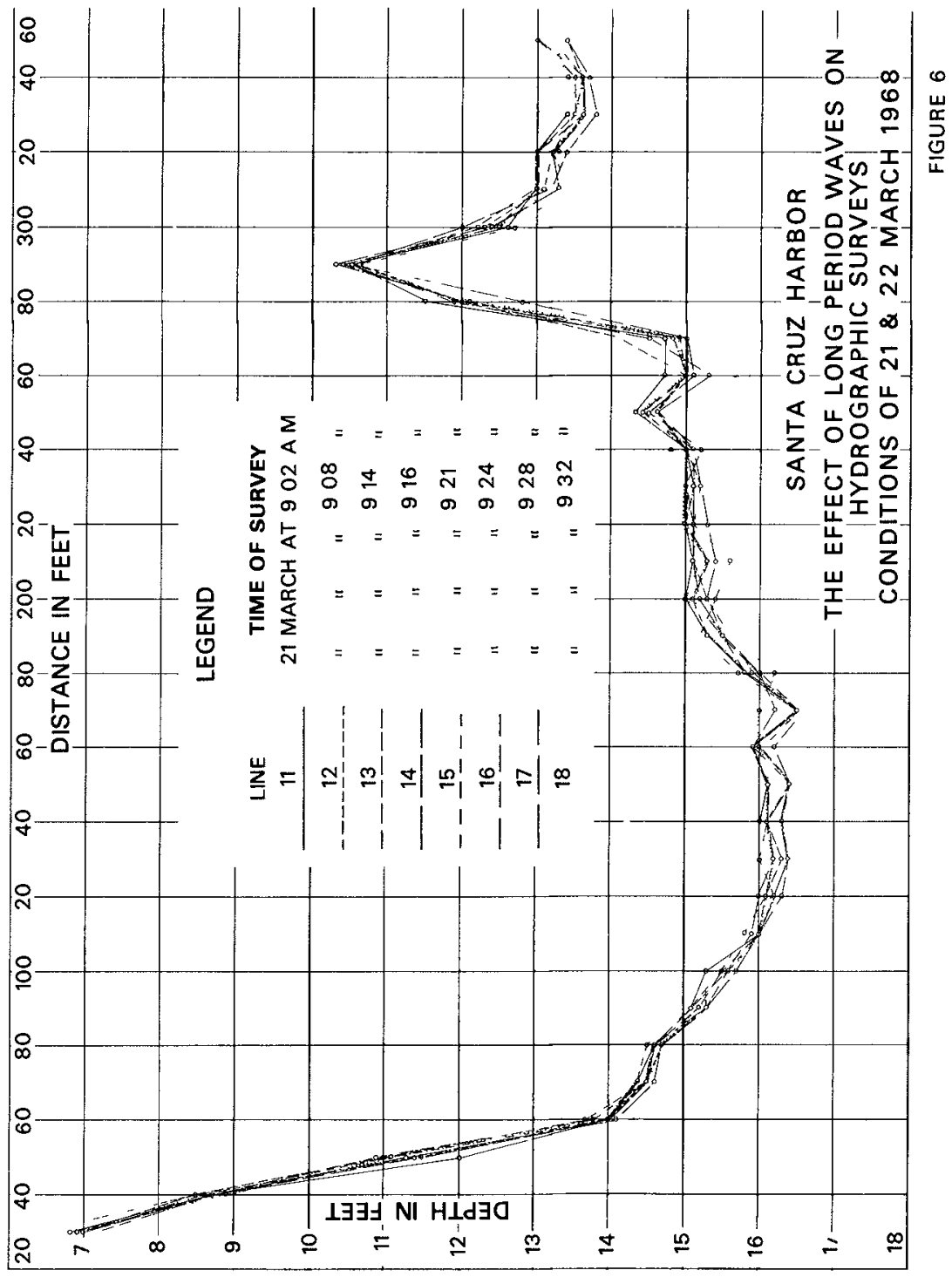




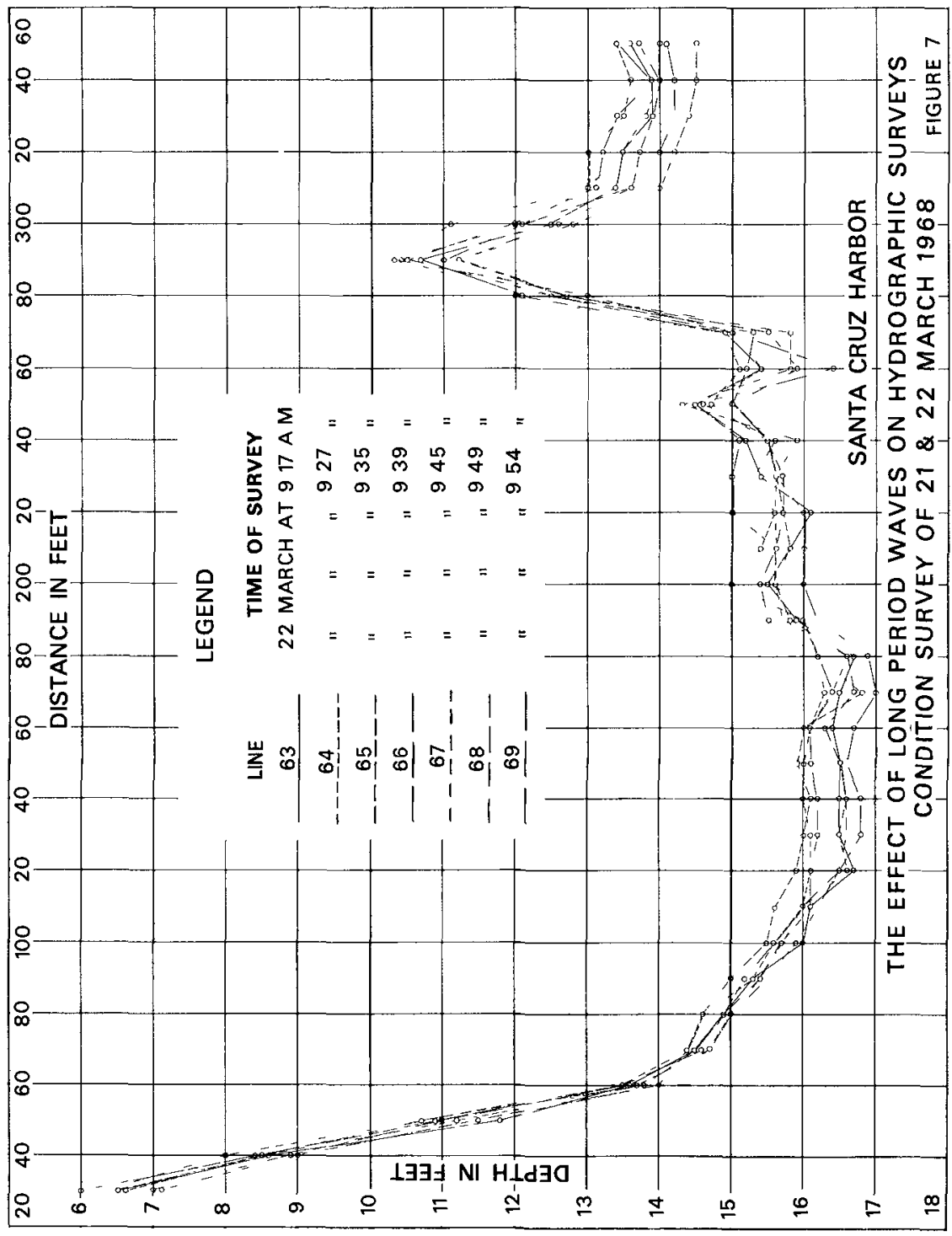




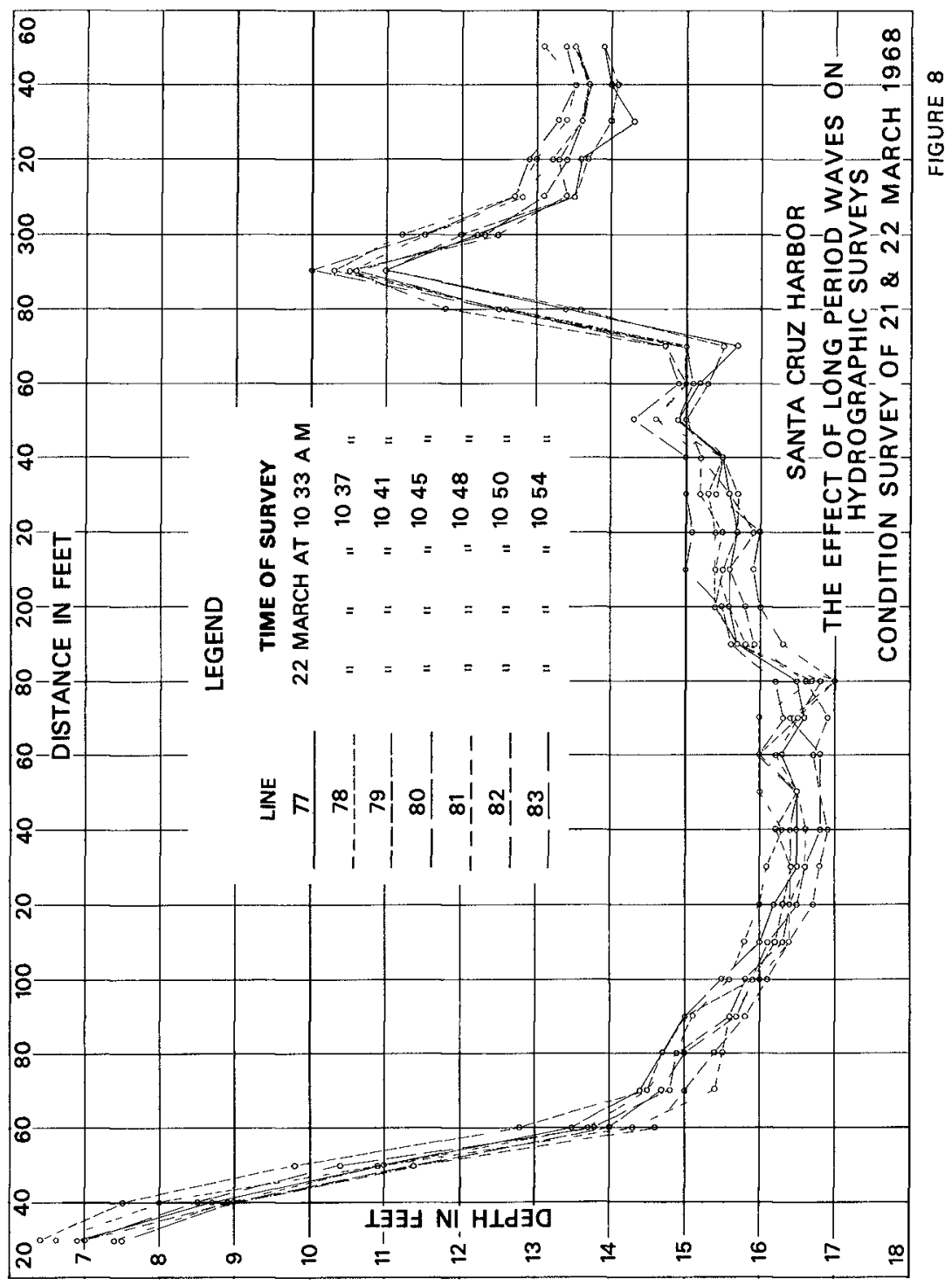




\section{SANTA CRUZ HARBOR}

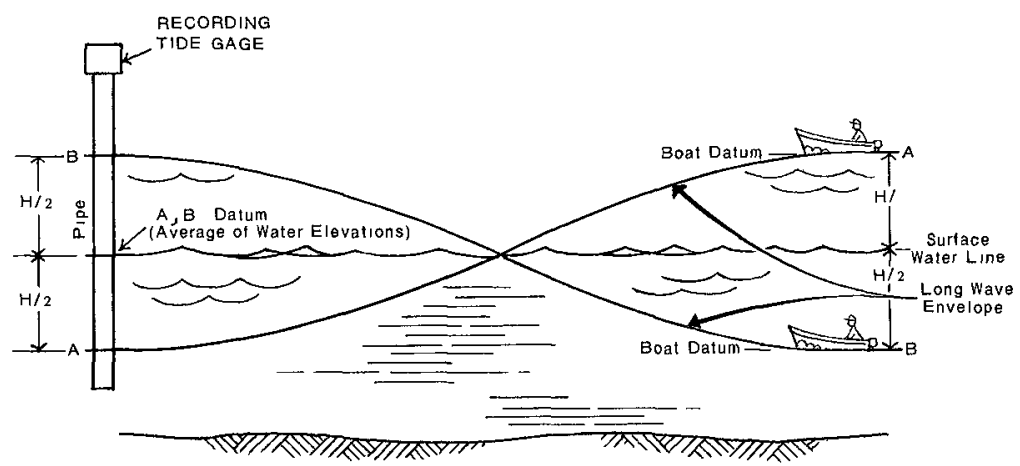

(A) NORMAL METHOD OF SOUNDING REDUCTION FOR TIDES

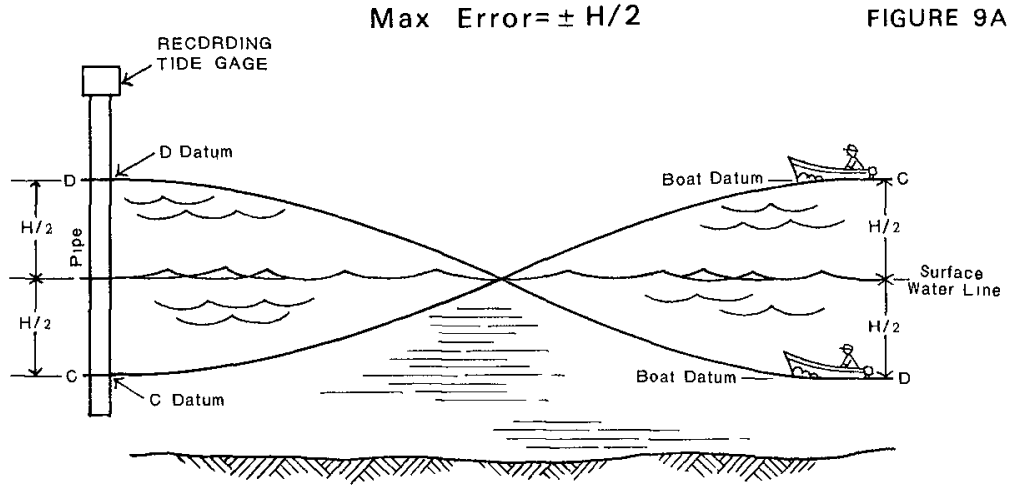

(B) INCORRECT METHOD OF SOUNDING REDUCTION FOR TIDES

Max Error $= \pm H$

$\mathrm{H}=$ Long Period Wave Height

FIGURE $9 B$ 



\title{
CHAPTER 137
}

\author{
SELECTION OF DISPOSAL AREAS FOR \\ SPOIL FROM BALTIMORE HARBOR
}

By John F Hoffman ${ }^{1}$, M ASCE

\section{ABSTRACT}

Selection of sites for the diked disposal of 100 million cubıc yards of spozl dredged from Baltimore Harbor presented a formidable problem Flfty percent of this spoll will be derived from harbor improvement in the next ten years The remalnder will result from maintenance dredging and some private dredging over a 20 to 25 year period

The number of sites avallable for diked disposal areas, the varlous methods used for dredgung and the fact that the dike material required traisportation to site gave rise to a number of varıables The decision maknng process was facilitated by an econometric model

Concentrations of metals such as chromium, cadium, zinc, lead, and copper have been found $\mathrm{In}$ the seduments of Chesapeake Bay An Investigation as to the mode of their occurrence in the material to be aredged was made to appracse any posslbility of toxic concentrations occurring in filter feeders such as oysters for shellfish constitute a sizeable Industry in the Chesapeake Bay

Stabilization of the deposited dredged spoll was investigated In order to determine whether the unstable loosely-deposited fine graln matemial extending over a three to five square mile area could be economically converted to a firm foundation material sultable for ındustrial parks, harbor terminals or water-orıented parks

\section{INTRODUCTION}

Deepening the 25 miles of channel extending from Baltumore Harbor to a depth of 50 feet from Its present depth of 42 feet and malntalning continuously that depth of channel will result in $100 \mathrm{mlll}$ ion cublc yards of spoll in about twenty to twenty-five years

\footnotetext{
I Assoclate Professor of Oceanography, $U S$ Naval Academy, Annapolis, Maryland and Senıor Assocıate, Trident Englneerıng Assoclates, Inc
} 
Senate Blll No 623 approved by the Congress of the state of Maryland on May 2, 1969 provides for the fundlng of " the design and construction of one or more diked disposal areas and other and appurtenant facilities to recelve dredging spoll from the harbor and the approach channels in the water and adjacent areas known generally as the Harbor of Baltimore $\mathrm{Cl}_{1} \mathrm{y}$

This paper deals with the method of the selection of candidate sites rather than the specific sites themselves Site selection Involved the following overall considerations

1 Economy as It is related to the transportation of spoli and the material for the construction of the dike

2

Economy of dike construction from a standpoint of partitioning the overall diked area as well as consolidation of the underlying sediments

3 Ecology of the Bay as it pertans to oyster beds, sports and commercual flshing, and flsh spawning areas

4 The varıou Federal and State regulations concerning the construction of diked areas

5 Possible utilization of the three to five square miles of land created

Disposal of dredged spoil Ln previous years was by open dumping in desıgnated submarine disposal points withın Chesapeake Bay, for the open ocean is more than 150 miles downbay However, the economic value of the fishing grounds and the lack of control of the deposition of the fine-grained sediments in the free fall through deep water has outmoded this method of disposal

Owlng to the essentıally free storage volume between the level of the Chesapeake Bay and Its bottom, dIked disposal areas within the Bay Itself were the ones evaluated primirily At first view this may appear to be agalnst the best juterests of the bay, however, valuable real estate is created by fllling the diked area Spoll dumped in a depth of water of 18 feet to an elevation of 9 feet above the bay level. would produce about $3 \frac{1}{2}$ square miles of land

\section{FITURE DREDG TNG REQUTREMENTS IN BALTIMORE HARBOR}

It is estimated that approximately ten years would be needed to dredge the channel from 42 feet to 50 feet without widening It Removal of some $50 \mathrm{mlll}$ ion cublc yards of spoll would be involved From 1975 to 1983 there should be a steady Increase In the total amount of spoll due to greater mantenance dredging and private dredging A slight decline should be realızed after 1983, and should level off at 
about 1985 to approximately 3 milion cublc yards annually As malntenance is a contınuıng operation, more than one diked disposal area wlll be required in the long range plcture

\section{CURRENT DREDGING PRACTICES AND COSTS}

Dredging in the vicinlty of Baltimore Harbor and its approach channels is accomplished by means of bucket and scow, hopper dredge, and pipeline dredge The first is used for new dredging work, the second for malntenance dredglng by the Corps of Englneers, and the third for special work

The cost projected. In every dredging contract will be pecullar to the specific job to be performed and wlll be based on the followIng factors

1 Dredging Site Distance from disposal area and depth of water in disposal area.

2 Type of material Sand, silt, and clay are dredged at different rates and have different effects on the equipment

3 Depth of water in the area to be aredged

4 Whether dredgıng is classıfıed "maıntenance" or "new work "

5 Volume of matenzal to be aredged

6 Financial posture of the bidding firm

7 Moblizzation and demoblizzation costs involved

8 Time of the year in whlch dredgung is to be performed

Contact was made with a number of dredging firms and various districts of the Corps of Englneers and approximate cost ranges were determined for the three types of dredging These were used in the Econometric Model discussed later in this paper The total costs involved in bucket and scow and hopper dredging included mobilizing and demobilizing equipment and cutting, loading, transporting, and. off-loading spoll Pipeline dredging costs, except for mobilization and demobilization, cannot easily be separated into component costs Accordingly, the figures used in the study represent the best estimates avalable from firms contacted 
SITE SELECTION

Federal and State Regulatıons Important to Spoll Disposal

Any dredglng project in navigable waters is affected by certaln Federal and State regulations as well as by local interests and concerns Followng is a resume of the baslc Federal and State statutes and authority Important to a spozl disposal project

1

Federal Jurisdiction

The Federal agency most critically involved in any dredging project lis the $U$ S Army Corps of Englneers Authorlty for this body's Jurisdiction goes back to, and Is still primarlly based on, the Rivers and Harbors Act of 1899 ChIef among the areas of jurisdiction axe the following

a Dams and dikes across navigable waterways

b Pıers, dredgıng, etc, In waterways Plans for wharves, plers, dolphins, booms, weirs, breakwaters, bulkheads, jettıes, or other structures, and excavation or flll in navigable waters must be recommended by the Chief of Enguneers and approved by the Secretary of the Army Section 10 of the RIver and Harbor Act of March 3, 1899 and Section $4(f)$ of the Outer Continental Shelf Lands Act of August 7, 1953, extend the authority of the Secretary of the Army to prevent obstructions to navigation by artIfIcIal Islands and structures on the Outer ContInental Shelf (Bridges and Causeways are now (1970) under Coast Guard Jurisdiction.)

c Use of government works

d Harbor Itines

e Improvement of any navigable river

f Pollution Section 13 of the River and Harbor Act of 1899 states that, "It shall not be lawful to throw, discharge or deposit any refuse matter of any kind or description whatever other than that flowing from streets and sewers Into any navigable water of the Unzted States, or Into any tributary of any navigable water "The River and Harbor Act of 1888 defines pollutants as "refuse, mud, sand, dredgings, sludge, acid or any other matter of any kind " Furthexmore, Executıve Order \#11288 specifles the responsıbllitıes of all Federal agencles to improve water qualıty through prevention, control, and abatement of water pollution 
from Federal governme nt actıvities in the Unıted States The provisions of this Order are applicable to the pollutional aspects of all dredging operations Including the drsposal of dredged materials

In any dredgrng project, the Maryland state agency most Intımately involved at present is the Board of Public Works Thls body Issues permits when state money Is Involved and also Is the coordinating volce of all state groups Involved While the Corps of Englneels' has the ultimate power in grantıng or refusing a permit, It wlll probably never override serious objections ralsed by the State's Board of Public Works In addition to the Board as a major volce of the State, the Submerged Lands Commıssion acts In an advasory capacıty partıcularly In very large or controversial projects

As a coordinating body, the Board of Publıc Works gathers opınlons from various state agencles in the process of considering an application for a permit Some of these agencies are

a Department of Water Resources

b Maryland Geological Survey

c Department of Chesapeake Bay Affaurs

d Departinent of Health

e Department of Game and Inland Fish

$f$ Department of For ests and Parls

g Department of Natural Resources

Selection of Potential Sites

In order to evaluate all possible spoll disposal sites avallable a comprehensive study was made of navigatıonal charts covering the Chesapeake Bay area from the mouth of the Susquehanna River south to Tangler Island

Potentıal sites that were consudered feasıble for spoll disposal purposes were categorızed as follows

- Island and shorelıne sites which could border a diked area and serve as a base for eventual fast land recovery 
- Marsh and swamp areas whlch could recelve dredged harbor spoll providıng eventual land recovery or possıble nutrıtıve enrichment of wetlands for wildlife use

Relatıvely shallow open water areas sultable for dıkıng for use as contalned spoll disposal areas

- Deep bay areas such as trenches or holes where spoll disposal would not be subject to the spreading of spoll

Seventy sıtes were selected on this basıs These were then critically examined on the basis of economic, ecological, and environmental factors These factors included site's proxumity to oyster beds and other valuable seafood areas, the size and distance from the area to be dredged, the impact of the contalnment on water flow and navigation, the potential utilization as a reclaimed land area including accessibility to populated areas, and the present value as wetlands or wildlufe refuge areas

Following this, a review of each site was made with personnel of varıous state, commercial, civic, and private interests to insure the fact that all groups were represented

Concurrently, the potentzal sjtes were examined from an economics standpolnt by means of a mathematical model, hereafter termed Econometric Model Detalls concerning this model are discussed below

The Econometric Model

Input data to the Econometric Model were based on current dredgIng practices and costs Assumed constant in the model were (1) the cost of excavating the dike material, (2) the cost of off-loading the spoll, and (3) the cost of purchasing the dike material at $\$ 10$ per cuble yard

Variables in the model included (1) transportation distance of the spoll to the disposal site, (2) cost of cutting channels to some sites, (3) transportation of dike material, (4) construction of the dike, (5) partitioning, and (6) compressublilty of the bottom sediments Costs were computed for purposes of the comparison of sites on the basis of unit cost of cuble yard of dredged spoll

For convenlence, the shlpplng channel to be dredged was duvided into five zones As the bottom contour of the bottom varies, the centrold of the volume to be cut for each zone was located after the channel had been divided Navigable distances to the sites were computed from these centrolds 
One of the most Important factors in choosing the site was the dike itself For comparisons and to enable certaln conclusions to be drawn, a structural model had to be formulated early in the study The dike used for the model had a I 4 slope on each face with elevation of +9 above mean low water (MLW) It was trapezodial in shape whth 4 yards across the top The water depth at each of the sltes was 15 feet or 18 feet MLW In order for the unlt cost of constructing the dike to be incorporated in the model, the following equation was used

$$
\text { Unzt cost } / \mathrm{yd}^{3}=\frac{\text { Volume of Dlke }}{\text { Vol of Spoll In SIte }}\left(\$ 277 / \mathrm{yd}^{3}\right)
$$

The flgure $\$ 277 / \mathrm{yd}^{3}$ was the unlt cost assumed for constructing the dike In keeping with the design of the model, the cost was divided by the amount of spoll in the site ThIs gives a unit cost per cublc yard of spozl

The four most common horizontal conflgurations for the flnished dike are the circle, square, rectangle, and equllateral triangle The followlng table gives the amount of diko material in cukls yards required to contain 200 milion cublc yards of spoll at 18 feet MLW Calculations 1or the volume of the dike material are based on dimenslons measured to the top irner edge of slope Furthermorc zt was assumed for the sake of umplicaty that the volume of dike material underlychg the face of the inner slope had a negliguble effect on the storage capability of this enclosure

\begin{tabular}{|c|c|c|c|}
\hline Curcle & Square & $\begin{array}{l}\text { Rectangle (3000 } x \\
3740 \text { yards) }\end{array}$ & Trzangle \\
\hline 4254 million $\mathrm{yd}^{3}$ & $480 \mathrm{mllllon} \mathrm{J}^{3}$ & $4826 \mathrm{ml} 11 \mathrm{ion} \mathrm{yd}^{3}$ & 5471 milizon $\mathrm{yd}^{3}$ \\
\hline
\end{tabular}

From the above table It is clear that the circle would be the best, since the dike required the least material This conclusion requires modiflcation when partıtıoning is consldered Detalls concerning this matter are discussed below

In the disposal of spoll in diked areas the question arises whether to build one continuous dike for the entire volume to be contalned, awalting a long perıod for flllıng and stabllization, or to partition the site into sections and thereby create stablizzed acreage at a much faster rate In partıtioning, however, extra dike material ıs required and the most economical dike shape may change Since the triangle was by far the most costly of the configurations, the effect of partitionlng was eyamined only on the clrcle, square, and rectangle A table of unit costs for the three shapes with varying numbers of partitions is glven below, assuming 100 milion cublc yards to be placed in the site and the bay bottom to be 18 feet below mean low water 


\begin{tabular}{|c|c|c|c|}
\hline Number of Partitions & Clrcle & Square & Rectangle \\
\hline 4 & $\$ 194 / \mathrm{yd}^{3}$ & $\$ 199 / \mathrm{yd}^{3}$ & $\$ 221 / \mathrm{yd}^{3}$ \\
\hline 5 & $\$ 210 / \mathrm{yd}^{3}$ & $\$ 210 / \mathrm{yd}^{3}$ & $\$ 253 / \mathrm{yd}^{3}$ \\
\hline 6 & $\$ .229 / \mathrm{yd}^{3}$ & $233 / \mathrm{yd}^{3}$ & $\$ 283 / \mathrm{yd}^{3}$ \\
\hline 7 & $\$ 248 / \mathrm{yd}^{3}$ & $\$ 243 / \mathrm{yd}^{3}$ & $\$ 314 / \mathrm{yd}^{3}$ \\
\hline 8 & $\$ 266 / y d^{3}$ & $\$ 255 / \mathrm{yd}^{3}$ & $\$ 344 / \mathrm{yd}^{3}$ \\
\hline 9 & $\$ 285 / \mathrm{yd}^{3}$ & $\$ 266 / \mathrm{yd}^{3}$ & $\$ 375 / \mathrm{yd}^{3}$ \\
\hline 10 & $\$ 303 / \mathrm{yd}^{3}$ & $\$ 277 / \mathrm{yd}^{3}$ & $\$ 406 / \mathrm{yd}^{3}$ \\
\hline
\end{tabular}

Although the circle does at times seem the best economically, the unlt cost for a square is never more than one-half cent higher than that of a circle per cubic yard. As the number of partitions increases, the square becomes the more desirable shape

Inltially, the compressibllity of the sub-bottom was assumed zero An analysis was pursued for the purpose of determining a multiplier to be applied to the unit cost for zero compressibllity to adjust for the estimated compressibllity of the bottom sediments When settlement takes place more dike materıal is required if a predetermined elevatıon of top of dike is to be mantained Thus, inutially the dike must be bullt to a higher elevation. Once the amount and rate of settling 15 estımated from tests on bottom core samples, compensation for settlIng can be achieved by considering a pad of trapezodial cross-section whth the same 14 side slope as existing beneath the dike The helght of the cross-section would be equal to the estimated settlement As a result both studies may proceed simultaneously

Economıc Projection

Selecting an area for a diked enclosure involves a decision whether to bulld for long range use at the present time or to bulld smaller enclosures fllling them in shorter intervals All are aware of the diminlshing value of the construction dollar with time, however, the more rapldly land is created the more rapldly its worth can be realized from both a sale and tax income standpoint

The Englneering News-Record Construction Cost Index, whych is an aggregate combination of common labor cost and material cost, was examined to determine the effects of inflation on construction The Construction Cost Index In December, 1949, for Baltimore was 424 In December 1969 , It was 1015 Thls 15 an 1 ncrease of about 240 percent. A construction project in 1949 costing $\$ 10$ million would cost $\$ 24$ million today 
Projections of the ENR Construction Cost Index were made to the year 2000 Three stralght line projections were made maximum, median, and minımum rates of growth based on past recorded growth rates Median rates have been extrapolated from the average trend line between 1950 and 1970 Maxumum rates are based upon the trend line between 1963 and 1970 and manlmum growth rates upon the trend between 1949 and 1963 Projection of the costs for any project to some future date were obtalned by multiplylng today's estrmated construction costs by a ratio of the estimated future cost index to the present cost index Space prevents further elaboration here of the studies made

\section{SUB-BOTTOM INVESTIGATION}

A program of sub-bottom investigation was undertaken for four purposes

1. To determine the types of sediment to be dredged

2 To determine the compresslbility of the material underlying the dıkes for dike design purposes

3 To obtain exact information as to the bathymetry in the candidate sponl areas

4 To obtain samples from the channel to be dredged for the chemical analysis of selected metal ions

The investigation was divided into two parts--a selsmac survey and a test boring program

The seismic survey technique chosen for sub-bottom profiling employed a $35 \mathrm{KH}$ output frequency sub-bottom profiling device provided and operated by personnel from the Oceans International Company, Mystıc, Connecticut

The profiling equipment used on this project emitted an acoustic signal strong enough to penetrate to approximately 30-50 feet of the sub-bottom About equivalent to the nolse of a small sledge-hammer blow on a hard surface, the signal energy which is transmltted through the water and then absorbed and/or reflected by the sub-bottom in no way disturbed the bottom of the bay

The position of the vessel at any time durlng the sezsmlc survey was automatically recorded by the Motorola Range Positioning System (RPS) RPS operates at line-of-sight ranges up to 50 nautzcal miles and the usual system range measurement accuracy is consaderably better 
than 50 feet The RPS was chosen for this project because of Its 50mile range, its all-weather capability, and Its unique coding for noninterference with shipping or other communzcations in the congested harbor area

To eliminate the necessity for shlfting the transponders to new line-of-site visible fixed reference stations, two high elevation sites were selected which could be "seen" electronically at all times from any planned position of the survey boat one of these, an outside observation-type terrace area of the Maryland National Bank BuzldIng In downtown Baltımore, whlch the Chesapeake and Potomac Telephone Company made avallable, is approximately 475 feet above sea level The other was atop one of the high towers on the suspension bridge of the Chesapeake Bay Bridge at an elevation of about 400 feet above sea level Each transponder and Its antennas weighed approximately four pounds

The selsmic data which were acquired in less than one week, when correlated whth test boring data acquired at a slightly later date, and over a perıod of six weeks, gave an extended plcture as to the composition and compressibility of the sub-stratum in the areas concerned

The test boring program conslsted of basıcally takıng contınuous samples with a four-foot long $3^{\prime \prime}$ diameter plston corer according to ASTM Specification DI587-63T Twenty borings in 411 cased holes spaced 6600 feet apart to a depth of 15 feet in the various channels to be deepened formed the backbone of thas phase of the program These were supplemented by 20 additional single four-foot piston cores (uncased) taken midway between the cased holes

Immediately upon removal from the drilling rig the sample tubes were placed in dry 1 ce and frozen in preparation for chemical analysis and to preserve intact any samples with a high water content at the laboratory the long cores were cut laterally into 6-inch long cylinders and then longitudinally into hemu-cylınders one of the hemicylınders was used for grazn size analysis of the sediments, the other was utilized for chemical analysis

The second part of the test borıng program consisted of making plston core borlngs at each of the candldate sutes to depths ranglng from 36 to 50 feet below the bay bottom Samples from this phase were used to obtann a quantitatıve estımate of dike settlement 


\section{FUTURE USES OF THE FILLED CONTAINMENT AREA}

Once fllled to capacity and stabllized, the filled contalnment area wll present the state with the plesant dilemma of deciding upon one or even several of the many possible uses of this newly created plece of real estate If land were a goal, the State could expect to spend upwards of $\$ 25,000$, O00 for sandfill alone Where the area is located offshore some means of access must be provided in order to accomplish any of the uses Accordingly, sites adjacent to existIng land where connections whth the existing transit facllities are possible are particularly attractıve

One often-proposed utilization for newly created waterfront land is the construction of an alrport The location of an alrport on or near water has a number of distinct advantages However, the problems and disadvantages of offshore alrports must also be considered The proymity to water presents a weather problem in that fog is always more prevalent near the water This fog or haze enhances the risk commerclal carriers must face in take-offs or landings

There wıll also be alr trafflc problems resultıng from the proxımity of Washıngton National, Dulles, Frıendshıp, and Andrews Alr Force Base to thls offshore alrport Even without a fifth major alrport, alr traffic controllers have clalmed that the alr space in this region Is too crowded

Industries of various types might be attracted to new land on a waterfront site The end result is more employment opportunities for Marylanders and a general increase in the tempo of economic activity in the Baltımore area A shlpping termınal would be another possibllıty The present Dundalk Marıne Termınal in Baltımore, old and new sections, totals about 540 acres

Prıces for Industrially-zoned land in metropolıtan areas start at about $\$ 6,500$ per acre whlle industrial sites with all utilitıes and rall frontage range upward from about $\$ 15,000$ per acre A conservative estimate of the worth of waterfront land in the large cities places Its value in a range between. $\$ 15,000$ and $\$ 25,000$ with the shoreline lots belng the most valuable Assuming the most conservative price $(\$ 15,000)$ as an average sale price per acre, the state could consider the stabilized filled disposal area as an asset worth in excess of $\$ 30,000,000$

Turnıng from industrial, development, lt can be advantageous to consider the new land in terms of a recreation area The State Department of Forests and Parks presently prefers at least 500 acres for a state park Each of the prime sites meets this requirement Along with a park, an 18-hole championshlp golf course (approximately 150 
acres) could be scenIcally positioned on the chesapeake Bay WIth golf and aquatic sports readlly avallable, It would be loglcal to also construct marınas, hotels, and motels Athletic stadiums and whldilfe preserves are other posslbillties

The Federal Government, through the Bureau of Outdoor Recreation of the Department of the Interior, provides matching grants to states to help defray the costs experienced in the construction of recreation areas Funds for thus type of outlay are authorızed under the Land and Water Conservation Fund Act of 1965 As a result, this recreation benefit could be realized for only half of the actual cost

\section{SPECIAL SITUATIONS STUDIED}

\section{Heavy Metal. Content}

In recent years brief investigations indicated that heavy metals exist In the bottom sediments of Chesapeake Bay Knowledge of the solublilty and concentration of these metals is necessary for the proper evaluation of the possible disposal sites concurrently, with the present investigation, a study is being made of the effect of these metals on shelifush This is being undertaken in a cooperative effort by the Chesapeake Blological Laboratory, the Chesapeake Bay Institute, the Department of Chesapeake Bay Affalrs, and the Department of Water Resources

Chemical analysis of the sediments by Atomac Absorption Method for the Chromıum, Copper, Cadmlum, Zinc, Lead, Nıckel, Cobalt, Mercury, Manganese, and Molybdenum was made Although other methods are avallable, Atomic Absorption Spectrometry was chosen as the method for makIng the desured analyses ThIs method Is generally accepted as the most desirable because It Is rapid and inexpensive and requires the least interpretation to obtain actual concentrations In addition, standards used can be prepared to take into account amounts of Interfering metals whych may be included in the samples

Inltıally, several digestion procedures for the sediments were utilized One was acetıc acid-sodium acetate buffer system Another was 10 Molar concentration of hydrochloric acId (HCL) at $70^{\circ} \mathrm{C}$ for 48 hours A third procedure used was digestion wath aqua regia.

There was little difference in the results obtalned using HCL and aqua regia digestions The latter method was therefore discontinued A conslderable dufference between the other two digestion procedures was observed, however, and, therefore, a dual set of analyses was made 
Digestion with the acetate buffer method would be eypected to extract all of the soluble portions or lightly-bound particles whlle the HCI method would extract the more tightly-bound particles These two methods, then, probably enable distinction between the readily avalable or soluble metal lons and the more tightly-bound metal lons

\section{Spozl Stabilization}

During the initial period of spoll disposal the spoll, which is largely silt, wll be covered by the water contalned in the diked area A large lake within the Bay wlll be delineated by the dike Subsequent spoll disposal wIIl cause the level of the spoll to rise above the water level wathin the dike slow movement of water entrapped in the interstitial spaces of the fine-grained material, however, will preclude the soll from bearing safely superimposed loads, such as bulldings, for a number of years In hopes of converting the created land into valuable real estate more rapıdly, a search was made of the technical literature pertalning to soll stabilızation by vibro-flotation, by electro-osmosis, and by chemicals Agencies concerned with this type of problem such as the Corps of Englneers in Buffalo and Detrolt and those concerned wath the dry land phase, such as the IIIghway Research Board, were contacted

The results of both parts of this investigation indicates that few Inroads, If any, have been made into the problem and no satisfactory method of rapidly stablizing the water-deposited dredged spolI is avallable

\section{ACKNOWLEDGMENTS}

This work was carried out for the State of Maryland under the general supervision of Mr George Lewls, Secretary of the Department of General Services Site selection and assoclated studies were performed by Trident Engıneering Assoclates, Annapolıs, Maryland The author was project manager of this phase Preliminary design of the duked disposal areas was performed by Green Associates, Inc, Baltimore, Maryland 



\title{
CHAPTER 138
}

\author{
WAVES GENERATED BY LARGE SHIPS
}

AND SMALL BOATS

$M \quad M$ Das and $J$ W Johnson

University of Calıfornia

Berkeley

The peak wave energy in a system of waves resulting from the passage of a ship is of importance in such problems as bank erosion, the motion of moored vessels, forces on fixed and floating docks, etc With respect to the bank erosion problem, the question of ten asked 18 whether the single passage of a large ship during a day, for example, is more damaging than numerous passages of small pleasure craft during the day With this in mind this study was conducted to determine the relative importance of the peak energy resulting from the passage of a Marıner class cargo ship and a pleasure cruıser The characteristics of these vessels are as follows

TABLE 1 MODEL CHARACTERISTICS

\begin{tabular}{|c|c|c|c|c|c|}
\hline & Scale & $\begin{array}{c}\text { Overall length } \\
\text { (Inches) }\end{array}$ & $\begin{array}{c}\text { Beam } \\
\text { (1nches) }\end{array}$ & $\begin{array}{c}\text { Draft } \\
\text { (1nches) }\end{array}$ & $\begin{array}{l}\text { Total Displace- } \\
\text { ment (1bs) }\end{array}$ \\
\hline $\begin{array}{l}\text { Mariner class } \\
\text { cargo ship }\end{array}$ & 196 & $70 \quad 75$ & 925 & 3 & $\begin{array}{ll}37 & 32\end{array}$ \\
\hline Crulser & 116 & $48 \quad 75$ & - & 2 & $24 \quad 91$ \\
\hline
\end{tabular}

The characteristics of the waves generated by these vessels moving at various speeds in deep and shallow water were determined from towing tests in a model basin (Ref 1)

The energy density or total energy per unit surface area is equivalent to the mean-square-helght of the waves The value of this term in a finite wave train varies with distance outward from the salling line From the point of view of the design of the banks of navigable channels and forces on docks or moored vessels, the peak wave energy associated with the maximum wave height in a wave train is of Importance For example, Figure 1 shows the peak energy density, $\mathrm{H}^{2}$, at various distances from the salling line resulting from the cruiser model moving through shallow water (prototype depth = $55 \mathrm{ft}$ ) at various speeds (Froude Numbers) It is obvious from Figure 1 how Important the ship speed is in generating high waves, 


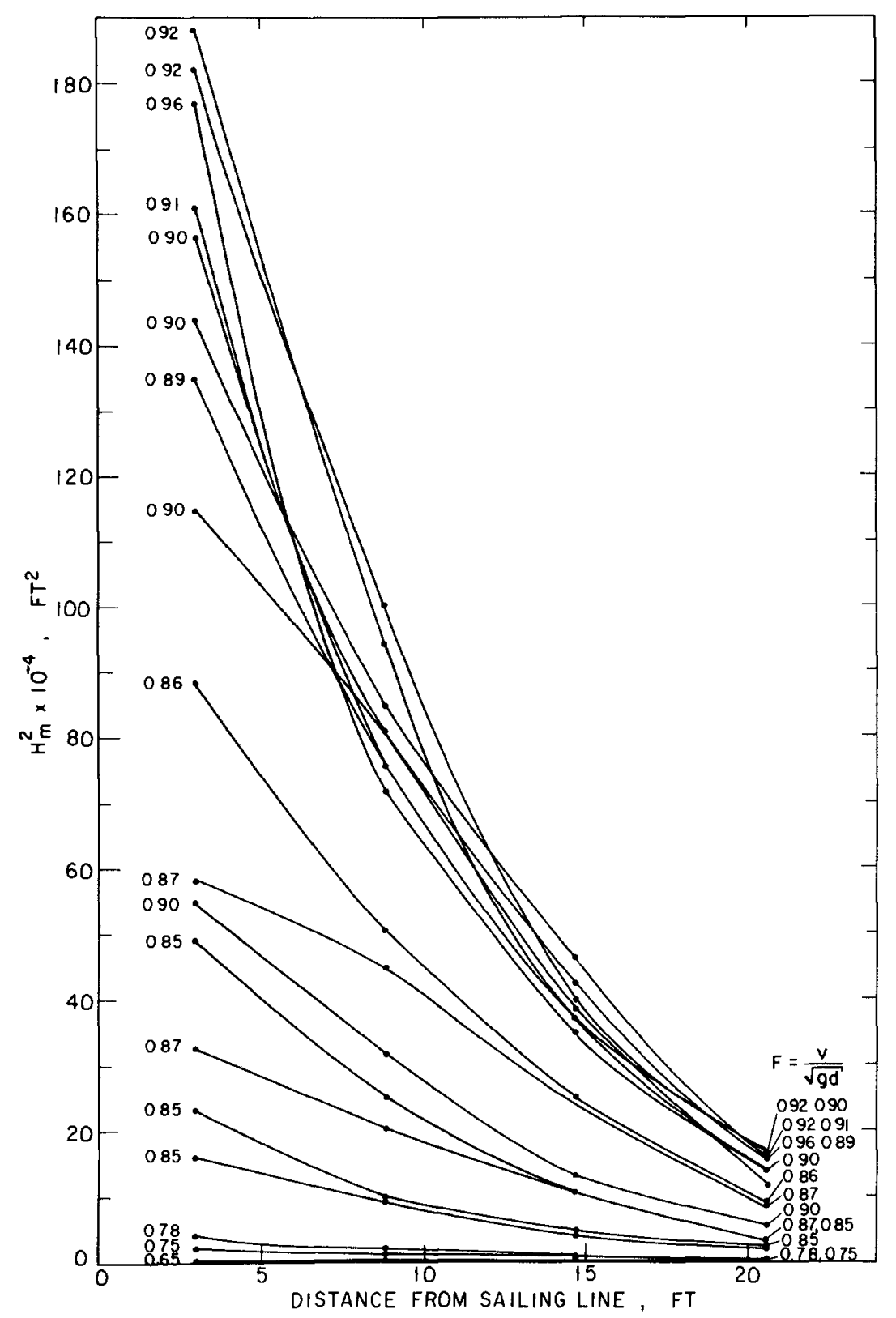

FIG I H HS A FUNCTION OF DISTANCE FROM SAILING LINE WITH FROUDE NUMBER ASA PARAMETER FOR CRUISER MOOEL IN SHALLOW WATER 


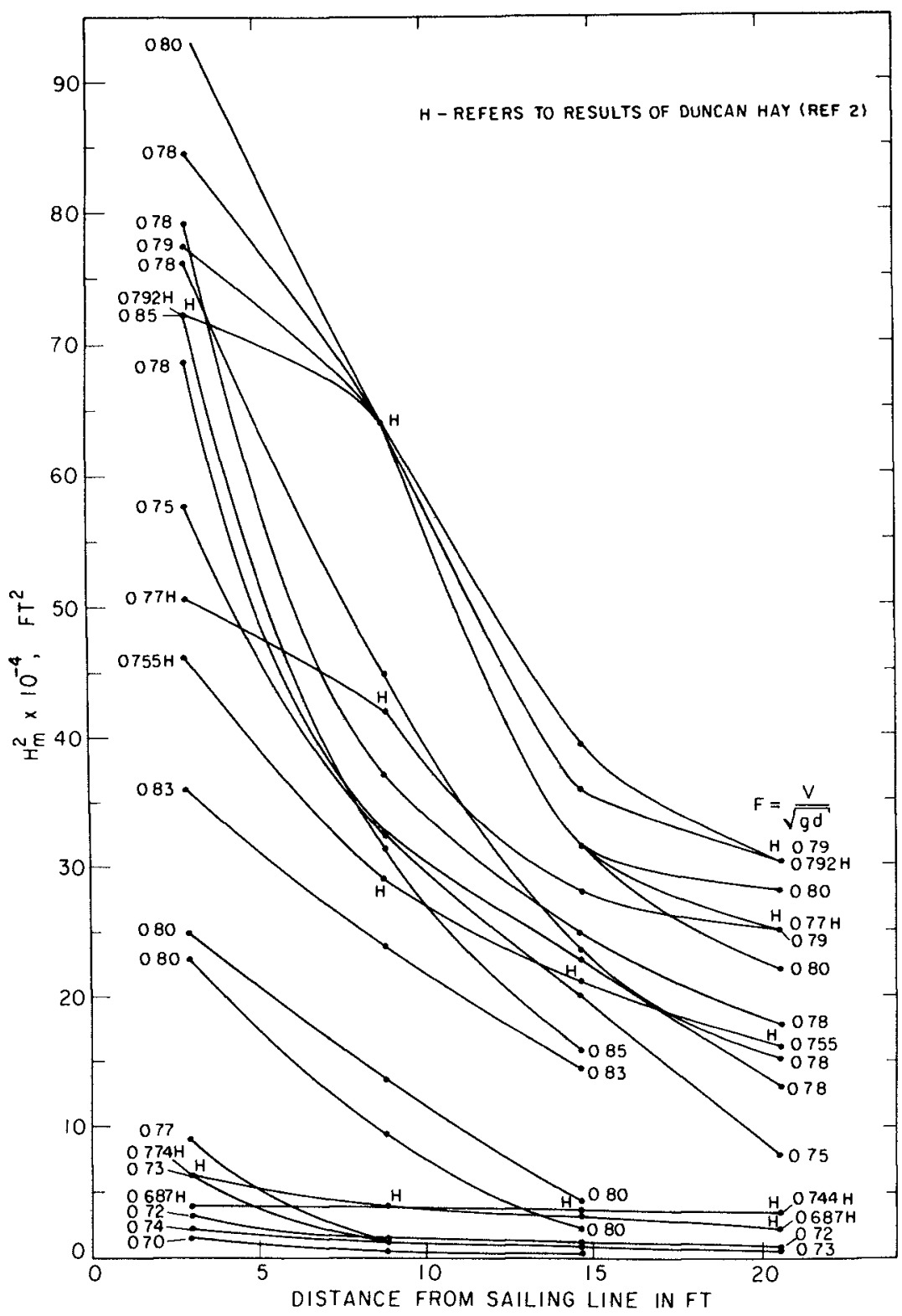

FIG 2 H ${ }_{m}^{2}$ AS A FUNCTION OF DISTANCE FROM SAILING LINE WITH FROUDE NUMBER AS A PARAMETER FOR MARINER MODEL IN SHALLOW WATER 


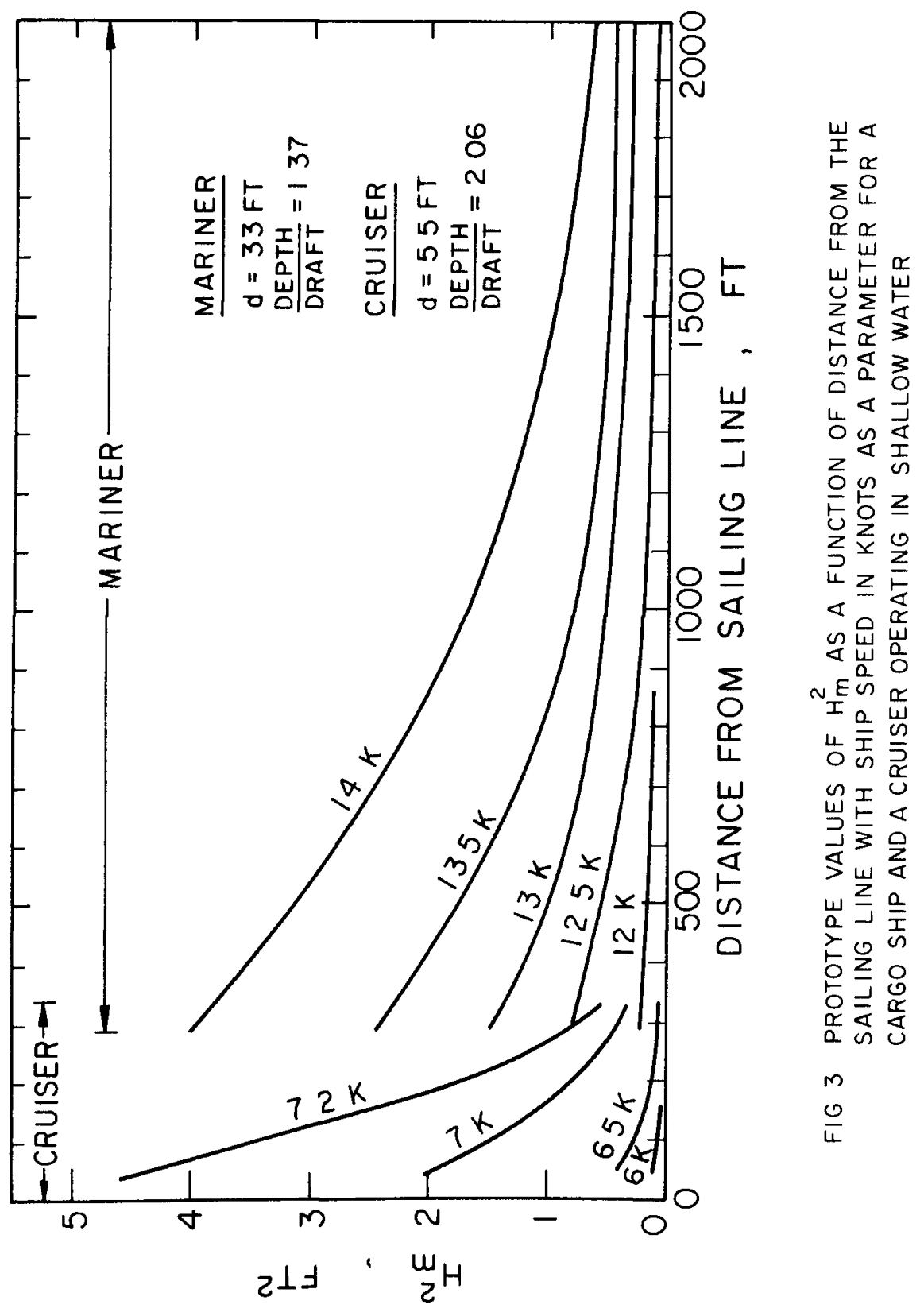


particularly near the salling line Figure 1 also shows that the peak energy density is reduced by about 90 percent at a distance of flve ship lengths from the sailing line for high ship speeds A plot similar to Figure 1, shown in Figure 2, is for the cargo ship model also moving in shallow water (prototype depth $=33 \mathrm{ft}$ ) It 1 s obvious from Figures 1 and 2 that the wave-making resistance of the cruiser is much greater than the cargo ship, especially at high speeds and near the salling line A cross-plot of data from the tests on the two models in prototype values is shown in Figure 3 where $\mathrm{H}^{2}$, a measure of peak energy density, is given as a function of distance from the salling line for varıous ship speeds in knots In both instances the models operated under shallow-water conditions, that 1s, a low value of the ratio of water depth to ship draft Comparısons of peak energy densıty for the two vessels can be made from Figure 3 For example, the cruiser travelling at 7 knots creates the same peak energy density at $150 \mathrm{ft}$ from the salling line as does the cargo ship travelling at 13 knots at $500 \mathrm{ft}$ from the salling line Another comparison is that at $300 \mathrm{ft}$ from the salling line the cruiser travelling at 7 knots creates the same peak density as the cargo ship does when travelling slightly more than 12 knots It therefore appears from these comparisons that for vessels of the sizes used in this study that small boats can induce more serıous wave conditıons than can a large shıp

\section{ACKNOWLEDGEMENTS}

The work reported both in this paper and in the references below was completed on a project supported by the $U$ S Army Coastal Englneerıng Research Center, Washington, D C 


\section{REFERENCES}

1 Das, M M (1969) Relatıve effect of waves generated by large ships and small boats in restrıcted waterways Unıv of Calif Hyd Eng1n Lab Rept No HEL-12-9, Nov 1969

2 Hay, D (1969) Ship waves in navigable waterways Proc Eleventh Conf of Coastal Engin, pp 1472-1487

3 Johnson, J W, (1969) Ship waves at recreatıona1 beaches Shore and Beach, Vol 37, No 1, pp 11-15

4 Johnson, J W, (1969) Ship waves in shoaling water Proc Eleventh Conf on Coasta1 Engln, pp 1488-1498

5 Sorensen, R M (1967) Investıgatıon of ship-generated waves Jour, Waterways and Harbors D1V, A S C E, Vol 93, Feb 1967, pp $\quad 85-99$

6 Sorensen, R M (1968) Stereo photogrammetric analysis of wave surfaces Jour, Hydraulics Div, A S C E, Vo1 94 Jan 1968 pp 181-194

7 Sorensen, R M (1969) Waves generated by model ship hull Jour, Waterways and Harbors Div, A S C E , Nov 1969, pp 513-538 